UNIVERSIDADE DE SÃO PAULO

INSTITUTO DE GEOCIÊNCIAS

\title{
STRATIGRAPHY AND ERUPTIVE MODEL OF THE DACITIC VOLCANISM IN THE REGION OF SÃO MARCOS (SOUTH PARANÁ MAGMATIC PROVINCE)
}

Letícia Freitas Guimarães

Orientador: Valdecir de Assis Janasi

TESE DE DOUTORAMENTO

Programa de Pós-Graduação em Mineralogia e Petrologia

São Paulo 
UNIVERSIDADE DE SÃO PAULO

INSTITUO DE GEOCIÊNCIAS

STRATIGRAPHY AND ERUPTIVE MODEL OF THE DACITIC VOLCANISM IN THE REGION OF SÃO MARCOS (SOUTH PARANÁ MAGMATIC PROVINCE)

\section{LETÍCIA FREITAS GUIMARÃES}

Tese apresentada ao Programa de PósGraduação em Mineralogia e Petrologia para obtenção do título de Doutor em Ciências

Área de concentração: Petrologia Ígnea e Metamórfica

Orientador: Prof. Dr. Valdecir de Assis Janasi

SÃO PAULO 
UNIVERSIDADE DE SÃO PAULO

INSTITUO DE GEOCIÊNCIAS

\section{STRATIGRAPHY AND ERUPTIVE MODEL OF THE DACITIC VOLCANISM IN THE REGION OF SÃO MARCOS (SOUTH PARANÁ MAGMATIC PROVINCE)}

\section{LETÍCIA FREITAS GUIMARÃES}

Orientador: Prof. Dr. Valdecir de Assis Janasi

Tese de Doutorado

No 599

COMISSÃO JULGADORA

Dr. Valdecir de Assis Janasi

Dra. Eleonora Maria Gouvêa Vasconcellos

Dra. Liza Angélica Polo

Dr. Carlos Augusto Sommer

Dr. Excelso Ruberti

SÃO PAULO 
Autorizo a reprodução e divulgação total ou parcial deste trabalho, por qualquer meio convencional ou eletrônico, para fins de estudo e pesquisa, desde que citada a fonte.

Serviço de Biblioteca e Documentação do IGc/USP

Ficha catalográfica gerada automaticamente com dados fornecidos pelo(a) autor(a) via programa desenvolvido pela Seção Técnica de Informática do ICMC/USP

Bibliotecários responsáveis pela estrutura de catalogação da publicação: Sonia Regina Yole Guerra - CRB-8/4208 | Anderson de Santana - CRB-8/6658

Guimarães, Letícia Freitas

Stratigraphy and eruptive model of the dacitic volcanism in the region of São Marcos (South Paraná Magmatic Province) / Letícia Freitas Guimarães; orientador Valdecir de Assis Janasi. -- São Paulo, 2019 .

$153 \mathrm{p}$.

Tese (Doutorado - Programa de Pós-Graduação em Mineralogia e Petrologia) -- Instituto de Geociências, Universidade de São Paulo, 2019.

1. Província Magmática Paraná. 2. Vulcanismo dacítico. 3. Mapeamento. 4. Análise Textural. 5. Geoquímica. I. Janasi, Valdecir de Assis, orient. II. Título. 
"Life need not be easy, provided only that it is not empty" Lise Meitner

(Austrian-Swedish physicist who worked on radioactivity and nuclear physics)

Para Lu, nosso exemplo de força e alto-astral mesmo nos momentos dificeis. 
"It is invaluable to have a friend who shares your interests and helps you stay motivated."Maryam Mirzakhani (Iranian math professor at Stanford, the first female scientist to win the prestigiuous Fields Medal in 2014 due to her work with the geometry of surfaces (that, for instance, when applied to how tectonic plates shift during an earthquake, allows predictions about their movement)

Esta tese foi financiada por agências de pesquisa nacionais; FAPESP (Fundação de Amparo à Pesquisa do Estado de São Paulo), através do projeto temático $\mathrm{n}^{\mathrm{o}}$ 2012/06082-6 “A Província Magmática Paraná-Etendeka no Brasil: relações temporais e petrológicas entre o magmatismo toleítico e alcalino e suas implicações geodinâmicas"; bolsa de doutorado pelo CNPq (Conselho Nacional de Desenvolvimento Científico e Tecnológico) processo ${ }^{0}$ Processo: 142084/2015-8 e de doutorado-sanduíche no exterior pela CAPES (Coordenação de Aperfeiçoamento de Pessoal de Nível Superior) processo no 88881.134384/2016-01.

A ciência brasileira (r)existe!

Impossível não começar este "muito obrigada" por quem sempre esteve ao meu lado, minha família. Ao Lucas, namorado, melhor amigo, companheiro de todas as horas e finalmente marido, obrigada por revisar meus textos, por cuidar de mim e da nossa casinha, por comemorar comigo minhas (nossas!) vitórias e não me deixar abater nas derrotas. Por todo seu apoio e agora também pelo Marx, nossa bola de pêlos que chegou pra alegrar ainda mais a casinha. Aos meus pais, Maria Helena e Gilberto, pelo apoio e amor incondicionais sempre, pelos incentivos, e por me ensinarem a ser uma pessoa que luta por aquilo que quer, sempre no caminho da honestidade e da justiça. Aos meus irmãos e à família que trouxeram junto, por serem sempre meus exemplos e orgulho, Matheus, Lu, Ju, Lô, Mone, Léo e Dieguinho. Obrigada por tudo. Amo muito vocês!

Ao meu orientador Valdecir, que desde o começo da minha vida acadêmica me deu todo suporte e incentivo, sendo sempre um exemplo de pesquisador correto e confiável. Obrigada por sempre responder minhas perguntas, mesmo que elas fossem repetitivas e parecessem tolas. Ao Evandro (Chinês), por sempre ter nos recebido tão 
bem, aceitado trabalhar conosco, pelos trabalhos de campo, pelas discussões sempre muito enriquecedoras e pelo alto astral. À Bis, pela ajuda de sempre e por não me deixar perder as esperanças quando achei que tudo estava perdido. À Cristina, por ter aberto as portas da LMU, pelos campos e pelas discussões produtivas.

Aos amigos de todas as horas, sem os quais a vida não valeria à pena. Às metralhas, mulherada forte que me enche de orgulho! Ao “oiem", Borba, Li, Kabong e Diroca, pelos almoços, cafés, mensagens, conselhos e apoio. À Orca e à Cami, que estiveram comigo desde o começo até o final desta tese, trabalhando comigo e sendo amigas tão incríveis. Às amigas do começo da vida geológica, Clô e Pig, pela amizade verdadeira e duradoura.

Vielen Dank to my German/LMU family! Thank you so much Don, for having me so well in your amazing department and for sharingall your knowledge. To the LMU professors, especially Werner and Kai, who taught me a lot in the labs.To Beth and Fabian for the best lectures, to Corrado and especially to Uli and Adrian, without whom I probably would not have a thesis. Thank you guys so much for helping me, for your patience and for everything you taught me. To the friends who made me feel at home: Matt, Pablo, Pancho, Micha, Joan, Damian, Ana, Vale, Cristian, Ale, Joali, Stephan,Lukas, Caron, Rike,Sid, Quentin and Jeremy. If I cried the whole flight back (14 hours!) it was because of you guys. Miss you all everyday! Balcony break?

Aos colegas, profesores e técnicos do IGc-USP, que estiveram comigo nesses 4 anos e um pouquinho, em especial ao Samuca, Marcos, Zé's, Audrey... enfim, todos aqueles que estiveram presentes nesse caminho e sem os quais a tese não existiria. Aos colegas do bloco B.Gracias a los profesores y amiginhos de Salta que, a pesar de los percances, también me enseñaron mucho.

À família de São Paulo, de sangue (Freitas Landi) ou de escolha (Villela Cassini), por sempre se fazerem presentes e pelo apoio de sempre. Às meninas do Coletivo Feminista Geóloga Dinalva e da Associação Brasileira de Mulheres nas Geociências, por compartilhar comigo a luta diária para sermos aquilo que queremos ser.

Aos sãomarquenses pela receptividade, pelas uvas, cafés e cucas, em especial: Seu Lauri, Dona Eva e família; Luciano; Dona Ivete; Manuela, Janete e família; Angélica e Vera; Pedro e Silvana Zanella; Constantino e Dalva DalPonte; Pedro, $\mathrm{M}^{\mathrm{a}}$ Inês Polidoro e seus filhos; Paulo; Janete; Nestor; Noredir; Flávio; e todas as pessoas que gentilmente nos permitiram acessar os afloramentos em suas propriedades. 
Meu muito obrigada a todos que estiveram comigo e meu pedido de desculpas sincero caso tenha me esquecido de mencionar alguém. No meu coração eu juro que não esqueço, mas agora na correria do fim da tese, a memória pode falhar. 


\begin{abstract}
Voluminous silicic volcanic rocks from the Paraná-Etendeka Magmatic Province are exposed in the southern Brazil, where a regional synformal structure (Torres Syncline) allowed the deposition and favored the preservation of a thick sequence of these units. In the São Marcos region (Rio Grande do Sul state) the volcanic sequence comprises basaltic rubbly pahoehoe flows (Vale do Sol Fm.) overlapped by dacitic deposits (Caxias do Sul sub-type), both intruded by basaltic dykes (Esmeralda Fm.). The genetic correlation between the Caxias do Sul dacites and the Vale do Sol basalts by an AFC process, already well described in the literature, is confirmed here; the fractionation of the basaltic magmas (with the extraction of plagioclase, pyroxene and Ti-magnetite) is clear through the patterns of major and trace elements, whereas the assimilation of crust is evidenced by variations in the traces elements ratios such as $\mathrm{Rb} / \mathrm{Ba}$ and $\mathrm{Th} / \mathrm{U}$. Part of the chemical variations observed in the Caxias do Sul dacites were interpreted as primary, revealing the existence of two distinct groups of samples, with subtle variations in trace elements contents $(\mathrm{Ce}, \mathrm{Sm}, \mathrm{Y}$ and $\mathrm{Th} / \mathrm{U})$, suggestive of different sources and/or variations in the assimilated crustal component.
\end{abstract}

Sub-parallel to the axis of the Torres Syncline, a NW-SE-trending zone constituted by the alignment of complex structures was interpreted as the feeding system of silicic volcanism, characterized as fissural. Detailed mapping allowed the description of distinct morphological domains, designated as "breccia domain", "fragmentation domain", "regular domain" and "filaments domain", the first domain being recognized as the host rock (together with underlying banded dacites) of the intrusive flow represented by the latter. The vertical intrusive flow exhibits two preferential directions: $\mathrm{NW}$, ranging from $\mathrm{N} 272^{\circ}$ to $\mathrm{N} 355^{\circ}$, and $\mathrm{NE}$, varying from $\mathrm{N} 20^{\circ}$ to $\mathrm{N} 85^{\circ}$. The stratigraphy of the dacitic sequence can be defined, from the base to the top, as: banded deposits, classified as rheoignimbrites, and volcanic breccias locally intruded by the dacitic feeder system referred to above, followed by hybrid deposits (characterized by synchronous volcanic breccias and lobated lavas) which gradually give place to lavas flows and finally to massive deposits. Macroscopic quantitative textural analysis (including size distribution and shape parameters analysis of fragments and vesicles) of the volcanic breccias indicate that these deposits were generated under low eruptive energy and comparative studies support the hypothesis that they correspond to block and ash deposits.

An eruptive model is proposed as follows: high temperature volatile-poor dacitic magma raised in a fissural conduit system with no significant crystallization. The low content (or even absence) of crystals and high temperatures inhibited the drastic increases in the viscosity of the shallow magmatic system. A magmatic rise fast enough to inhibit intense crystallization may have led to late-stage bubble growth and, together with high magma outflow rates, to boiling over eruptions responsible for the generation of PDCs (rheoignimbrites) related either to low-explosivity events or magma fountains. This first pyroclastic phase may have allowed efficient magma degassing and viscosity 
increase, and was succeeded by dome extrusions. The dome structure reduced the permeability and degassing of the system, leading to a new overpressure and causing the collapse of the dome structure and originating block and ash deposits. Additionally, the fissural architecture of the feeding system possibly permitted magma migration along an extended and variably open/closed fissure, leading to the occurrence of isolated magma batches evolving differently in terms of viscosity and degassing. This condition would be responsible for the occurrence of active and inactive vents, or vents with distinct eruptive dynamics spatially and/or temporally separated along the fissure system. Over time, the dome extrusion evolved towards new explosive events as relatively gas-rich magma ascent through the active shallow plumbing system or lava dome collapse caused rapid decompression and fragmentation. Finally, a gradual loss of eruptive energy and the widening of the active vent may have led to the explosiveeffusive transition responsible for the generation of the hybrid deposits and lava flows. 


\section{Resumo}

Grandes volumes de rochas vulcânicas ácidas relacionadas à Província Magmática Paraná Etendeka afloram na região sul do Brasil, onde uma estrutura sinformal regional, o Sinclinal de Torres, permitiu a colocação e favoreceu a preservação de espessas sequências vulcânicas. Na região de São Marcos (Rio Grande do Sul) a sequência vulcânica compreende derrames basálticos tipo rubbly pahoehoe (Fm. Vale do Sol) sobrepostos por depósitos dacíticos (sub-tipo Caxias do Sul), além de diques basálticos (Fm. Esmeralda) intrudindo todo o pacote. A correlação genética entre os dacitos Caxias do Sul e os basaltos Vale do Sol, através de processos de AFC, já bem definida na literatura, é aqui confirmada; o fracionamento dos basaltos (com extração de plagioclásio, piroxênio e ti-magnetita) fica claro através dos padrões de elementos maiores e traço, enquanto que a assimilação crustal é evidenciada pelas variações observadas nas razões de elementos traço como $\mathrm{Rb} / \mathrm{Ba}$ e $\mathrm{Th} / \mathrm{U}$. As variações químicas observadas nas amostras de dacito foram caracterizadas como primárias e revelam a existência de dois grupos distintos, com variações sutis no conteúdo de elementos traço como $\mathrm{Ce}, \mathrm{Sm}, \mathrm{Y}$ e nas razões $\mathrm{Th} / \mathrm{U}$, sugerindo variações de fonte e/ou do componente crustal assimilado.

Sub-paralela ao eixo do Sinclinal de Torres, com uma direção NW-SE, uma zona marcada pelo alinhamento de estruturas complexas foi interpretada como o sistema alimentador do vulcanismo ácido, caracterizado como fissural. $\mathrm{O}$ mapeamento de detalhe de tais estruturas permitiu o reconhecimento de domínios morfológicos distintos, denominados como "domínio de brecha", "domínio de fragmentação", "domínio regular" e "domínio de filamentos", sendo o primeiro reconhecido como a rocha encaixante (juntamente com os depósitos dacíticos bandados subjacentes) do fluxo intrusivo caracterizado pelos últimos domínios. O fluxo intrusivo vertical exibe

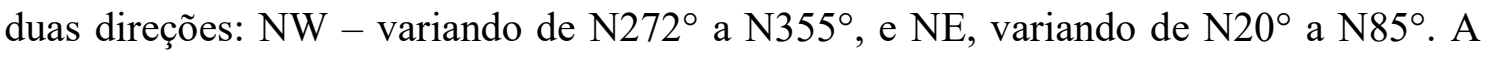
estratigrafia dos depósitos dacíticos pode ser definida, da base para o topo, em: depósitos bandados, interpretados como reoignimbritos, e brechas vulcânicas, ambos localmente intrudidos pelo sistema alimentador descrito anteriormente. O conjunto é sobreposto por depósitos híbridos (caracterizados pela ocorrência síncrona de brechas vulcânicas e lavas lobadas) que transicionam para derrames de lava e depósitos maciços. Análise textural quantitativa macroscópica das brechas vulcânicas (incluindo freqüência de distribuição de tamanho e parâmetros de forma tanto dos fragmentos quanto das vesículas) indica que tais depósitos foram gerados em eventos de baixa energia e um estudo comparativo dá suporte ao modelo de depósitos tipo block and ash.

O seguinte modelo eruptivo para o vulcanismo ácido da região é proposto: um magma dacítico de altas temperaturas e relativamente empobrecido em voláteis ascendeu por um conduto fissural sem cristalização significativa. O baixo conteúdo de cristais e as elevadas temperaturas inibiram o aumento da viscosidade do sistema raso. Uma ascensão suficientemente rápida para inibir intensa cristalização permitiu uma nucleação e crescimento de vesículas tardio que, juntamente com elevada taxa de 
extrusão, possivelmente acarretaram a ocorrência de erupções tipo boiling over, responsáveis pela geração dos $P D C s$ (reoignimbritos), relacionados a eventos de baixa explosividade ou a magma fountain. Esta fase inicial piroclástica permitiu uma desgaseificação eficiente e um consequente aumento na viscosidade do magma, resultando em uma fase de extrusão de corpos dômicos. A estrutura dômica gerada reduziu a permeabilidade e a degaseificação do sistema, resultando em uma nova sobrepressão, levando ao colapso da estrutura e gerando os depósitos block and ash. Ademais, a arquitetura fissural do sistema alimentador possivelmente permitiu a movimentação lateral do magma em ascensão ao longo de fissuras extensas e variavelmente abertas e fechadas, permitindo a ocorrência de corpos de magma isolados evoluindo distintamente em termos de viscosidade e desgaseificação. Esta condição favoreceu a ocorrência de vents ativos e inativos, ou mesmo com dinâmicas eruptivas distintas, espacial e temporalmente lado a lado ao longo do sistema de fissuras. Ao longo do tempo, a extrusão sob a forma de domos evoluiu para nova fase explosiva conforme novos fluxos magmáticos relativamente enriquecidos em voláteis ascenderam pelo conduto ativo ou o colapso do domo causou rápida descompressão e fragmentação do magma subjacente. Finalmente, a perda gradual da energia eruptiva, junto ao alargamento do vent ativo, levou à ocorrência dos depósitos híbridos e derrames de lavas. 


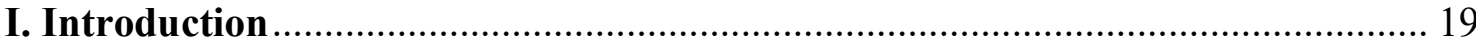

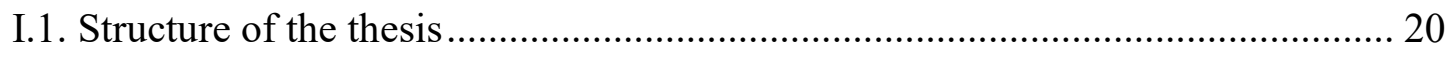

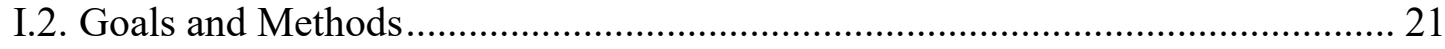

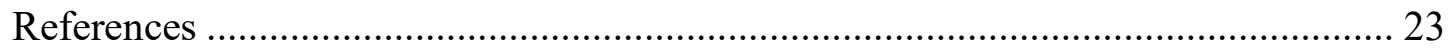

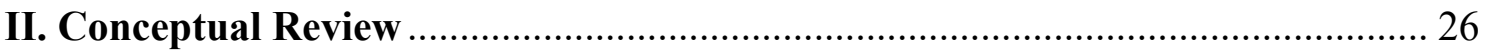

II.1. "To break or not to break": fragmentation, the dilemma of a rising magma ...... 26

II.1.1. Brittle fragmentation related to degassing and bubble overpressure ........... 28

II.1.2. Fragmentation by shear strain .................................................................. 31

II.2. Explosive-Effusive transitions on silicic volcanic systems ................................ 33

III. State of the art on the Paraná Etendeka Magmatic Province ……………….... 41

III.1. Origin and evolution of the PEMP magmatism ................................................ 50

III.2. The eruptive style of the PEMP silicic volcanism - explosive or effusive? ..... 52

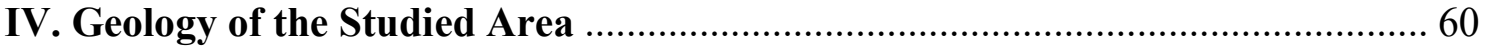

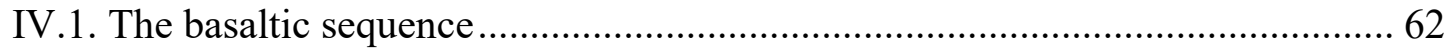

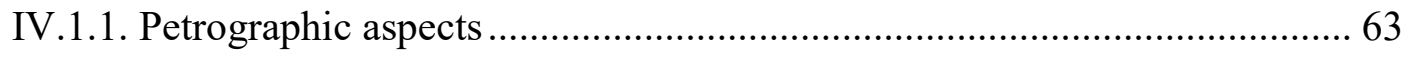

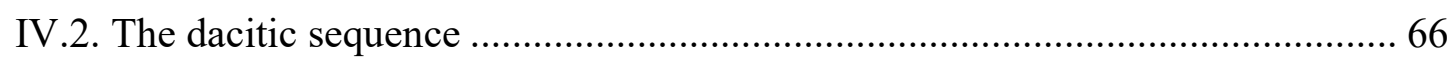

IV.2.1. Banded deposits (rheoignimbrites, high-grade ignimbrites or lava flows?)

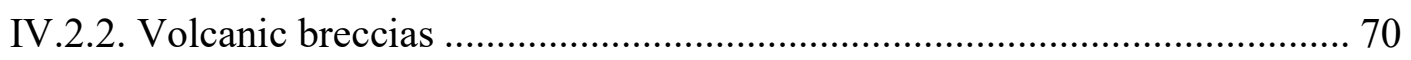

IV.2.3. Dacitic complex structures along linear trends ........................................... 71

IV.2.4. Hybrid deposits and lava flows ............................................................... 75

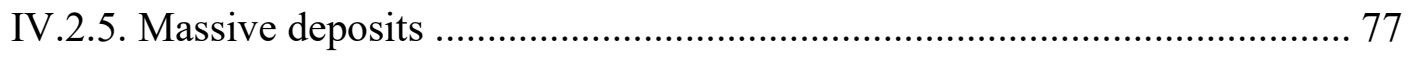

IV.2.6. Petrographic aspects and mineral chemistry .............................................. 78

V. The basal Caxias do Sul dacitic breccias in the Paraná Magmatic Province,

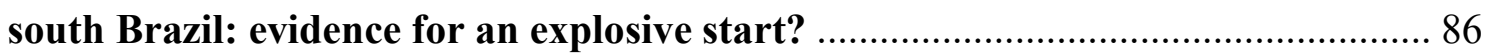




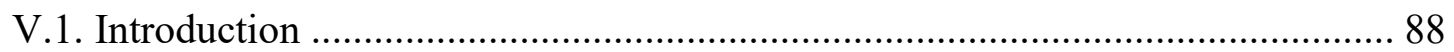

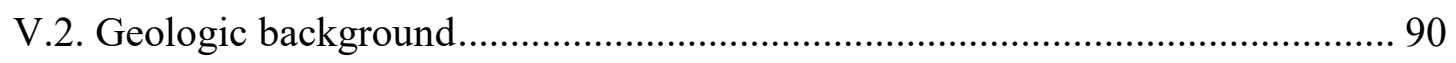

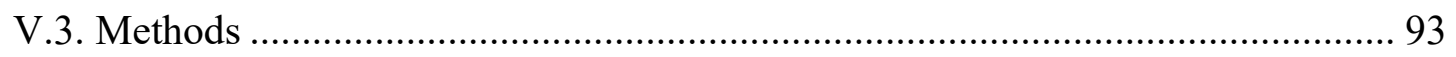

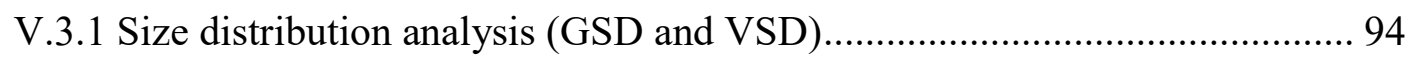

V.3.2 Shape Parameters and macro ............................................................... 95

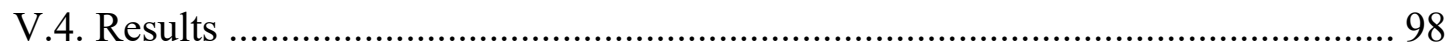

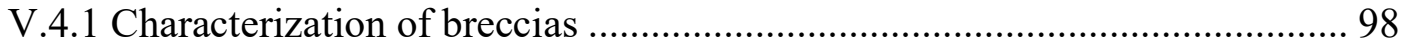

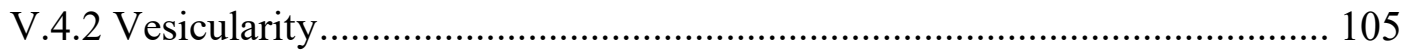

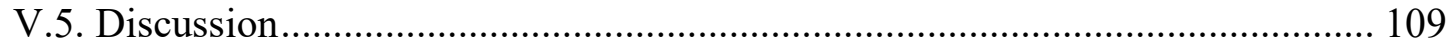

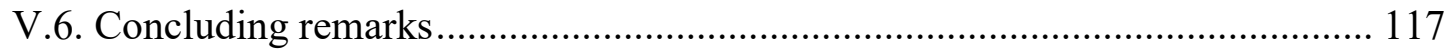

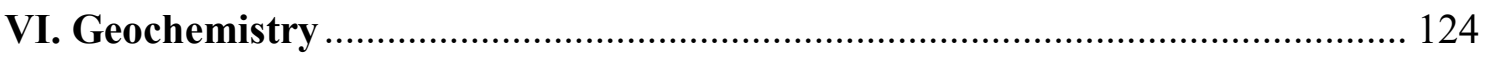

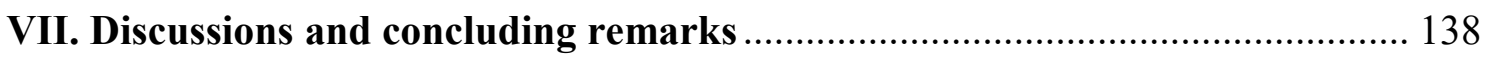

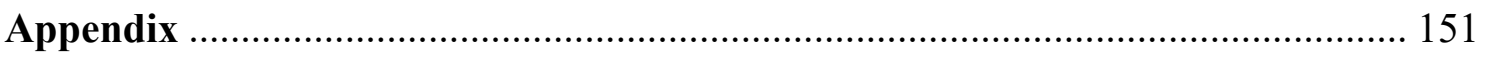




\section{List of Tables}

Table 1: Compilation of geochemical data for the high-Ti basalts; contents of some major, minor and trace elements and isotopic ratios for discrimination of magma types. (1) Peate et al. (1997); (2) Peate et al. (1992); (3) Garland et al. (1995).

Table 2: Compilation of geochemical data for the low-Ti basalts (Peate et al., 1997; Peate et al., 1992); contents of some major, minor elements and trace elements and isotopic ratios for the discrimination of magma types. 45

Table 3: Abbreviations used in particle parameter calculations, figures and results..... 96

Table 4: Description of macro outputs, parameter calculations and references

Table 5: Statistical parameters of the size and the fractal dimension for each sample.101

Table 6: Volcaniclastic deposit characteristics for lava flow auto breccias, block-andash flow, column collapse pyroclastic flows, lava fountain and lag breccias. Regarding the "lag breccias", we have taken into account exclusively those deposits classified by Walker (1985) as "lag breccias strictu sensu", which corresponds to coarse proximal deposits, lateral equivalents of ignimbrites, deposited at the deflation zone, due to gas loss (carrying fine particles) and consequent loss of flow energy (dense phase deposition). In this review work, the author also includes three other mechanisms of formation, which result in differences in the characteristics of the deposits formed (fine depletion, matrix or clast supported, etc), but not considered in this table. Highlighted cells correspond to those that best match the Caxias do Sul breccias studied. 112 


\section{List of Figures}

Figure 1: Schematic representation of primary fragmentation due to rapid magma ascent or rapid decompression. Fragmentation related to rapid magma ascent propagates upwards and the rising acceleration may be related to bubbles expansion, with fragmentation occurring when bubble overpressure exceeds the fragmentation threshold or when high strain rates cause the magma to cross the glass transition. Fragmentation by rapid decompression propagates downwards and may be a result of bubble-bearing magma (already under overpressure) is suddenly exposed to a lower pressure. Extracted from Cashman and Scheu (2015).

Figure 2: Schematic representation of degassing conditions. A) Closed system, with non-connected bubbles network. Both hetereogenous and homogeneous bubbles nucleation represented (a1), with possible restrict coalescence (a2). B) Open system outgassing, with development of permeable network due to bubbles coalescence (b3), collapse of coalesced bubbles (b4), fracturing (b5) and percolation through intergranular pore space (b6). C) Conceptual model for different degassing conditions and possibly related eruptive styles. Extracted from Colombier, 2018.

Figure 3: Schematic plot illustrating the hysteresis in permeability-porosity data. Initialy isolated bubbles expand up to the percolation threshold $(\mathrm{Pc})$ at around $60 \%$ vesicularity. Permeability rapidly increases to a permeability threshold (Pm) that limits further expansion. Deformation allows gas loss (porosity reduction) without loss of permeability, what happens later with bubbles collapse. Extracted from Cashman and Scheu (2015) 31

Figure 4: Deformation behaviors of silicate melts with various compositions, based on experimental studies by Webb and Dingwell (1990; fiber elongation experiments), Cordonnier et al. (2012b; uniaxial compression experiments), Kameda et al. (2008) and Witcher (2017; uniaxial compression experiments). The onset of elastic behavior is defined by $\mathrm{De}=0.01$, and the regime where brittle fracturing is the dominant response to stress occurred in this study when De $>0.04$. Adapted from Witcher (2017). 32

Figure 5: Paraná-Etendeka Magmatic Province, with the current geometry of the South American (Paraná Magmatic Province) and African (Etendeka Magmatic Province) portions. Compiled from Peate et al. (1992, 1999), Rossetti et al. (2014), and Ewart et al. (2004). 1: Basalts from PMP; 2: post-magmatic sedimentary rocks (Gr. Bauru) from PMP; 3: Intermediate and silicic volcanic rocks (andesites, dacites and rhyolites) from PMP; 4: PMP dikes swarms - Santos-Rio de Janeiro (trend N40-50E) at the north, Ponta Grossa arc (trend N40-50W) in the central region, and Florianopolis (trend N15-25E) at the south; 5: Basalts from PME; 6: Silicic rocks (latites and quartz latites) from PME; 7: Annular subvolcanic intrusions from the Damaraland and Messum Complexes. 42 
Figure 6: Geological map of the Paraná Magmatic Province, showing the geographic and stratigraphic distribution of the high-Ti and low-Ti basaltic rocks and related silicic rocks. The occurrence of dike swarms (Santos-Rio de Janeiro, Ponta Grossa and Florianópolis), the main tectonic lineaments (Rio Piquiri and Rio Uruguai) and the Torres Syncline axis are also indicated. The location of the study area is indicated by the red square. Modified from Janasi et al. (2011).

Figure 7: Discriminatory geochemical diagrams for the basaltic magma types from the PMP.

Figure 8: TAS (above) and Harker diagrams (at right) for the silicic rocks from PMP: Chapecó (green) and Palmas (red) Formation. Adapted from Nardy et al. (2008) 47

Figure 9: Discriminatory diagrams for the high-Ti (Chapecó type) and low-Ti (Palmas type) silicic rocks based on the $\mathrm{P} 2 \mathrm{O} 5$ and $\mathrm{TiO} 2$ contents and the $87 \mathrm{Sr} / 86 \mathrm{Sr}(\mathrm{i})$ isotopic ratio. Adapted from Nardy et al. (2008) and Garland et al. (1995). 48

Figure 10: Representative polygons of the minimum distribution areas of the different pyroclastic flows resulting from explosive volcanic events of great magnitude in the Etendeka region, according to Bryan et al. (2010). 53

Figure 11: Geological map of the studied area in the vicinity of São Marcos city, Rio Grande do Sul/Brazil. The Caxias do Sul dacites (Palmas Formation) and Vale do Sol and Esmeralda basalts belong to the Serra Geral Group. The rose diagrams present mainly vertical foliation from the filament domains (section IV.2.3). Their sizes are related to the amount of obtained data - ranging from 2 to 12 measurements in each outcrop, totaling 58 measurements from which ca. $90 \%$ comprises dips varying between $80^{\circ}-90^{\circ}$ and $10 \%$ comprises dips varying between $50^{\circ}-75^{\circ}$. Single measurements of vertical foliation are represented by single black lines. White arrows indicate the range of lava flow directions based on vesicles stretching. The geographic location of the stratigraphic sections (presented in figure 14) is highlighted by red circles on the map.

Figure 12: Stratigraphic section (left) for basalts from BR-116 profile - at least 8 flows were recognized based on their internal structure, comprising massive base and cores (A), with vesicles size and density increasing upwards (B1 to B3) transitioning to fragmented crust (C). Due to the lack of continuity, the whole structure was not observed at the higher elevations, hindering a precise delimitation of flow units. Basaltic dykes (Esmeralda Formation) of decimetric thickness, abrupt and irregular contact with the hosting basaltic flows (Vale do Sol Formation), were also observed (D).

Figure 13: Petrographic aspects of basaltic samples. A) Parallel polarizers at left and crossed at right. Hypocrystalline basalt from the basal portion of the flow, with intersertal, intergranular and subophitic texture. Plagioclase crystals (euhedral to subhedral, tabular), clinopyroxene (tabular euhedral to subhedral, granular) and opaque 
(subhedral) minerals are dispersed in a vitreous matrix. B) Parallel polarizers at left and crossed at right. Holocrystalline basalt from the inner portion of the flow; the groundmass is composed by microlites of plagioclase, clinopyroxene and magnetite and, locally by quartz+k-feldspar from recrystallization of previously glassy material. $\mathrm{C} 1$ and C2) Parallel and crossed polarizers respectively. Hypocrystalline porphyritic basalt; microphenocrysts of plagioclase, with oscillatory zoning, and clinopyroxene, also defining a subophitic texture. Yellow arrows indicate clinopyroxene $\rightarrow$ epidote alteration. D and E) Parallel polarizers at left and crossed at right. Hypocrystalline basalts with aligned euhedral tabular plagioclase crystals from the inner portion of a flow. Yellow arrows indicate irregular bubbles (diktytaxitic texture) filled by quartz. $\mathrm{F}$ and G) Samples from a thinner ( $\sim 30 \mathrm{~cm}$ thick) and a thicker ( $\sim 1 \mathrm{~m}$ thick) Esmeraldatype dykes respectively. Parallel polarizers at left and crossed at right. Radial distribution of acicular to euhedric tabular (with high aspect ratios) plagioclase crystals and interstitial granular clinopyroxene (intergranular texture). 65

Figure 14: Stratigraphic section for the dacitic volcanic sequence. Theirs geographic location is highlighted by red circles on the map at figure $11 . \mathrm{mD}$ : massive (gray) dacite; lfD: dacitic lava flows; bD: vesicle-rich dacite ((g) - grayish facies; (r) - reddish facies); bbD: vesicle-rich dacitic breccias; hdD: hybrid dacitic deposit; oD: dacitic obsidian; $\mathrm{bD}(\mathrm{r})$ : banded dacite (rheoignimbrite); vbD: dacitic volcanic breccias; fcD: dacitic feeder conduits; rB: rubbly basalts.

Figure 15: Field aspects of banded deposits: a, b, d) dark-colored bands, folded and rotated with spherulitic texture; c) lens-shaped discontinuous and horizontally folded banding; e) homogeneous lens-shaped banding; more (d, e) or less ( $a, c, b, f)$ continuous, flattened and commonly folded. The dark-colores bands suggest pseudotachylitic bands (, , b, c, d) or extremely stretched fiammes; g) reddish facies with horizontal banding and extremely stretched vesicles; h) millimetric to centimetric rounded lithic fragments deforming the banding.

Figure 16: Field aspects of volcanic breccias. A) Volcanic breccia overlaid by banded deposit at the vicinity of the complex structured zone. The yellow square indicates one of the selected portions for the quantitative textural analysis presented in Chapter $\mathrm{V}$ (sample SM-01). B and C) Detail of volcanic breccia in the complex structured zone. More details are given in Chapter V.

Figure 17: Field aspects of dacitic complex strutures. A) Exemples of breccia, regular and filaments domains. B) Interaction between different melts with local bubble-rich pumice (black arrow). At the bottom (yellow arrow) a level of giant-vesicles. C) Regular, breccia and previous filament domains are crosscut by a superimposed filament domain, indicating cyclicity and superposition of processes. D) General aspect of the filament domain with millimetric to centimetric chaotic filaments of aphyric material, which is folded, stretched and deformed by flow. E) Giant vesicle deforming the flow and promoting mingling between different melts. F) Isoclinal folds (viewed from above), with vertical hinge lines, from magmatic flow. 
Figure 18: Structural data from filaments domains. Exemples in a funil-like structure from quarry SM-20 (A) and from quarry SM-82 (B). The regular (Rd), filaments (Fd), fragmentation and regular $(\mathrm{Rd})$ domains are indicated. Stereograms indicates the range of flow directions and do not represent the magmatic flow dips. 73

Figure 19: Field aspects of dacite complex structures. A) Upwards transition between a filament and fragmentation domains. B) Breccia domain (reddish shades) intruded by filaments domains (grayish shades). C) Example of the breccia domain, with rounded bubble-rich centimetric fragments in a finer-grained (more angular, millimetric dense fragments), clay-rich matrix. More aspects of the breccias domain are shown in Chapter V.

Figure 20: Hybrid dacitic deposits with pyroclastic and effusive components; A) pyroclastic component at the base with flows inside or above; B) continuous deposit in the SW of the area (see geological map on figure 11) with the predominance of pyroclastics at the base (B1) grading into the predominance of compounded lava flows (B); B2) detail from the bottom of a lava flow, with stretched vesicles, along two flow directions: NNW-SSE and NE-SW.

Figure 21: Details from massive deposits. A) fine-graines, vesicle-rich (black arrows), banded dacite; B) coarse-grained, microporfiritic (red circles) gray dacite; C and D) aphanitic to fine-grained, locally microporphyritic dacite with "ghost-structures" (diffuse banding and crossed bedding).

Figure 22: Petrography of dacites. A1 and A2) Parallel and crossed polarizers respectively. Hypocrystalline glomeroporphiritic dacite. The two types of microcrystals are clearly recognized: euhedral (tabular) to subhedral rounded crystals with resorption features depicting a glomeroporphiritic texture and smaller euhedral crystals with fastcooling features; B) Parallel polarizers. Microlites from the complex structured zone are oriented along the magmatic flow. $\mathrm{C} 1$ and $\mathrm{C} 2$ ) Parallel and crossed polarizers respectively. Holocrystalline dacite. Tabular plagioclase crystals, with fast-cooling features. Subedral rounded-shaped pyroxene crystals and Ti-magnetite are dispersed on a completely devitrified groundmass. D) SEM image: microphenocrystals and microlites of plagioclase, pyroxene and Ti-magnetite lying on a micrographic groundmass; E) Parallel polarizers. Spherulitic texture in a partially devitrified groundmass, suggesting recrystallization along previous perlitic fractures; F) Parallel polarizers. Clusters (microxenoliths) of euhedral crystals of plagioclase, pyroxene, magnetite and quartz, with interfingered and diffuse edges, dispersed in the holohyaline dacite (obsidian); G) SEM image from the hipohyaline dacite (obsidian) with pigeonite and Ti-magnetite microlites and microxenolith constituted of plagioclase + clinopyroxene + magnetite; H) Parallel polarizers. Tabular, euhedral, microphenocrysts of plagioclase, with compositional zoning and resorption features; I) Parallel polarizers. Hipocrystalline dacite with subhedral microphenocrystals of plagioclase (crystals fragments - arrow), pyroxene and Ti-magnetite lying in a partialy devitrified 
groundmass (sperulithic texture). J) Parallel polarizers. Microlites (bead-crystals) of pyroxene and Ti-magnetite.

Figure 23: An-Ab-Or ternary diagrams for classification of feldspars from Caxias do Sul dacites. 81

Figure 24: Compositional map (for $\mathrm{Ca} \mathrm{Ka}-$ and $\mathrm{Mg} \mathrm{Ka}$-lines) of holocrystalline dacite with micrographic groundmass and glomeroporphiritic texture. Microprobe analysis with a Jeol JXA-FE-8530 from IGc/USP 82

Figure 25: Di-Hd-En-Fs diagrams for classification of pyroxenes from Caxias do Sul dacites.

Figure 26: Diagrams for \#mg variations as a function of crystals population of augite (left) and pigeonite (right) from Caxias do Sul dacites. Crystals from the microenclaves from the vitrophyric dacitic sample (SM-01D) are also presented, and examples from pyroxene compositions from basaltic samples (Vale do Sol Fm) from the literature are also shown for comparison purposes.

Figure 27:Simplified geological map and stratigraphic charts from the Paraná Magmatic Province - volcanic and sub volcanic (dykes swarms) occurrences - and younger sediments. Basalts are represented in green shades, while silicic rocks are represented in yellow, pink and red. The location of the study area is indicated by the red square. Open stars: sites of ${ }^{40} \mathrm{Ar} /{ }^{39} \mathrm{Ar}$ dating of lava flows and associated sills by stepped heating (Renne et al., 1992; Ernesto et al., 1999; Thiede and Vasconcelos, 2010); Close star: site of U-Pb baddeleyite/zircon dating (Chapecó dacite; Janasi et al., 2011). All dates in Ma. For interpretation of the colors references the reader is referred to the web version of this article. Modified after Peate et al., 1996; Nardy et al., 2008; Janasi et al., 2011 and Waichel et al., 2012. 91

Figure 28:Geologic map and resumed stratigraphic chart from the study area in the vicinity of São Marcos city. The black stars indicate the locations where the conduits system crop out, while the location of the analyzed breccias are represented by red stars. For interpretation of the colors references the reader is referred to the web version of this article. Simplified from Guimarães et al., (2018a).

Figure 29: Macro and microscopic aspects of the volcanic breccias. A) Quarry SM-82 dacitic volcanic breccias (reddish shades) are intruded by dacitic flows (gray shades). B, D, E) Detail of the volcanic breccias from quarry SM-82, with rounded to sub-angular, variable degrees of macroscopically visible flow-banding, dense to vesicle-bearing fragments in a fine-grained matrix, with more angular and smaller (millimetric) fragments. C, F) Detail of the volcanic breccias from quarry SM-20, with rounded to sub-angular, dense to vesicle-bearing fragments in a fine-grained matrix, with more angular and smaller (millimetric) fragments. The volcanic breccias (labeled as "b") in B and $\mathrm{C}$ are affected by the intrusive flow (labeled as "f") as suggested by rheomorphic features (labeled as "a"). G, H) Petrographic aspects of volcanic breccia from quarry 
SM-82. Sub-angular, microlite-rich, vesicle-bearing fragment in a devitrified matrix with sub-millimetric angular fragments.

Figure 30:Grain-size distribution histograms from the studied volcanic breccias. $\Phi$ is giving by $-\log _{2}$ (diameter), where diameter is giving in millimeters. SM-82_detail corresponds to the area delimited by the dashed yellow square on SM-82 sample. The fragments perimeters are marked by black lines on the breccias photos - these were used to calculate the parameters of interest (e.g. perimeter, area, GSDs, shape parameters) presented in Appendix IV. 102

Figure 31: $\log -\log$ plots of the cumulative frequency $(\log [\mathrm{M}(\mathrm{r}<\mathrm{R})])$ vs size $(\log (\mathrm{r}))$ for the same samples from figure 4. Linear fitting of GSDs, values of correlation coefficient $\left(\mathrm{r}^{2}\right)$ and the fractal dimension value (D), which corresponds to the slope of the linear fitting, are also presented. 103

Figure 32: Boxplots showing the variation of clasts' shape parameters (axial ratio, form factor, roundness and solidity) as a function of clasts' size $(\Phi=-\log 2$ [feret diameter]). The box limits represent the 1st and 3rd quartiles (lower and upper limits, respectively), the middle line represents the median, the " $x$ " is the average and the whiskers show the minimum and maximum values obtained. 104

Figure 33: Convexity vs Solidity plots for the SM-82 samples as a function of clasts' size $(\Phi=-\log 2$ [feret diameter]), divided by colors. The shaded fields corresponds to shards (red), vesicular particles (green), dense fragments (blue) and microcrystalline vesicular particles (orange) extracted from Liu et al. (2015). For interpretation of the colors references in the figure legend the reader is referred to the web version of this article. The convexity values were calculated using the convex hull values manually corrected by a factor of 0.968 .

Figure 34: Black and white thresholded images of isolated vesicles used to calculate the parameters of interest (e.g. area, VSDs, shape parameters) presented at the Suplementary Materials. Frequency distribution of Avesicle/Aclast histograms; simple area fraction vesicles-size distribution (VSD) histograms; VSD curves in terms of number density, after stereological conversion considering a sphere with equivalent diameter. 106

Figure 35: Representative boxplots for sample SM-82_Br1 showing the variation of vesicles' shape parameters (axial ratio, form factor, solidity and roundness) as a function of vesicles' size ( $\Phi=-\log 2$ [diameter], where diameter corresponds to the diameter of a circle with equivalent area). The highlighted black clasts correspond to those analyzed (with more than 50 vesicles with a minimum 10-pixel-area). The results presented here correspond to the vesicles from the numbered black clasts. At the diagrams, the box limits represent the 1 st and 3rd quartiles (lower and upper limits, respectively), the middle line represents the median, the " $x$ " is the average and the whiskers show the minimum and maximum values obtained. 107 
Figure 36: Representative boxplots for samples SM-82_Br2 (above) and SM-82_Br4 (below) showing the variation of vesicles' shape parameters (axial ratio, form factor, solidity and roundness) as a function of vesicles' size $(\Phi=-\log 2$ [diameter], where diameter corresponds to the diameter of a circle with equivalent area). The highlighted black clasts correspond to those analyzed (with more than 50 vesicles with a minimum 10-pixel-area). The results presented here correspond to the vesicles from the numbered black clasts. At the diagrams, the box limits represent the 1st and 3rd quartiles (lower and upper limits, respectively), the middle line represents the median, the " $\mathrm{x}$ " is the average and the whiskers show the minimum and maximum values obtained. 108

Figure 37: Vesicles orientation for the upper and lower portions of each sample. $0^{\circ}$ points upwards; " $n$ " indicates the number of vesicles measured and "max" represents the maximum the maximum proportion of vesicles obtained in the most measured orientation.

Figure 38: At left, MFW diagram from Ohta and Arai (2007) showing the weathering effects on both basaltic and dacitic samples - symbols for each unit and deposits' type are presented below the diagrams. Samples with red contour represent vitrophyric samples (SM-01D and SM-127Ba). M corresponds to mafic rocks, F to felsic rocks and W to weathered compositions: $\mathrm{M}=-0.395 \times \ln \left(\mathrm{SiO}_{2}\right)+0.206 \times \ln \left(\mathrm{TiO}_{2}\right)-0.316 \times$ $\ln \left(\mathrm{Al}_{2} \mathrm{O}_{3}\right)+0.160 \times \ln \left(\mathrm{Fe}_{2} \mathrm{O}_{3}\right)+0.246 \times \ln (\mathrm{MgO})+0.368 \times \ln \left(\mathrm{CaO}^{*}\right)+0.073 \times$ $\ln \left(\mathrm{Na}_{2} \mathrm{O}\right)-0.342 \times \ln \left(\mathrm{K}_{2} \mathrm{O}\right)+2.266 ; \mathrm{F}=0.191 \times \ln \left(\mathrm{SiO}_{2}\right)-0.397 \times \ln \left(\mathrm{TiO}_{2}\right)+0.020 \times$ $\ln \left(\mathrm{Al}_{2} \mathrm{O}_{3}\right)-0.375 \times \ln \left(\mathrm{Fe}_{2} \mathrm{O}_{3}\right)-0.243 \times \ln (\mathrm{MgO})+0.079 \times \ln (\mathrm{CaO} *)+0.392 \times$ $\ln \left(\mathrm{Na}_{2} \mathrm{O}\right)+0.333 \times \ln \left(\mathrm{K}_{2} \mathrm{O}\right)-0.892 ; \mathrm{W}=0.203 \times \ln \left(\mathrm{SiO}_{2}\right)+0.191 \times \ln \left(\mathrm{TiO}_{2}\right)+0.296 \times$ $\ln \left(\mathrm{Al}_{2} \mathrm{O}_{3}\right)+0.215 \times \ln \left(\mathrm{Fe}_{2} \mathrm{O}_{3}\right)-0.002 \times \ln (\mathrm{MgO})-0.448 \times \ln (\mathrm{CaO} *)-0.464 \times$ $\ln \left(\mathrm{Na}_{2} \mathrm{O}\right)+0.008 \times \ln \left(\mathrm{K}_{2} \mathrm{O}\right)-1.374$. Where $\mathrm{CaO}^{*}$ denotes $\mathrm{CaO}$ corrected for apatite and carbonates. At right, total alkali vs silica (TAS) diagram from Le Bas et al., (1986). The colored fields represent compositions of the units defined in the literature, according to Peate et al. (1992), Garland et al. (1995), Nardy et al. (2008) and Polo and Janasi (2014).

Figure 39: Binary diagrams evidencing compositional variations. Above, $\mathrm{K}_{2} \mathrm{O} / \mathrm{Na}_{2} \mathrm{O}$ vs. $\mathrm{SiO}_{2}$ (left) and $\mathrm{K}_{2} \mathrm{O} / \mathrm{Na}_{2} \mathrm{O}$ vs. $\mathrm{TiO}_{2}$ (right) showing chemical variations between vitrophyric and holocrystalline samples, possibly derived from devitrification processes, where the former presents lower $\mathrm{K}_{2} \mathrm{O} / \mathrm{Na}_{2} \mathrm{O}$ ratios in relation to the related holocrystalline sample (samples SM-01D and SM-01C highlighted by the yellow circle). The compositional range of less mobile elements such as $\mathrm{SiO}_{2}$ and $\mathrm{TiO}_{2}$ suggests also a primary component on the chemical variability. Below, principal component analysis (PCA) diagram showing the main elements responsible for compositional variability in basaltic (left) and dacitic (right) samples; symbols for each unit and deposits' type are presented below the diagrams. Samples with red contour represent vitrophyric rocks. $\mathrm{SiO}_{2}, \mathrm{FeO}_{\mathrm{t}}, \mathrm{MgO}$ and $\mathrm{Cao}$ are the main elements responsible for compositional variation in the basalts, while the dacitic rocks compositions vary mainly as a function of $\mathrm{SiO}_{2}, \mathrm{Al}_{2} \mathrm{O}_{3}, \mathrm{~K}_{2} \mathrm{O}, \mathrm{CaO}$ and $\mathrm{Na}_{2} \mathrm{O}$. Samples 
from the complex structures zone (conduits) are significantly enriched in $\mathrm{SiO}_{2}$, which may be related to post-magmatic hydrothermal (silicification) processes.

Figure 40: Classificatory diagrams for both basaltic and dacitic units. At left, AFM diagram defined by Irvine and Baragar, (1971), at the middle the $\mathrm{FeO}_{\mathrm{t}} / \mathrm{MgO}$ vs $\mathrm{SiO}_{2}$ plot by Miyashiro (1974), both defining the tholeiite and calc-alkaline series. At right, A/NK vs A/CNK plt by Shand (1943) for discrimation of metaluminous, peraluminous and peralkaline rocks. Symbols for each unit and deposits' type are the same from the previous figures.

Figure 41: Discriminatory diagrams or the different low-Ti formations from the PMP. In the upper diagrams (basalts at left and dacites at right) the colored fields refer to compositions published in the literature (Peate et al., 1992; Garland et al., 1995; Nardy et al., 2008; Polo and Janasi, 2014), while the gray symbols correspond to the samples from the present study. In the lower diagram, the colored symbols correspond to the compositions published in the literature and the gray symbols to the samples of the present study, as indicated in the legend next to the diagram. 128

Figure 42: Binary plots for major elements (in oxides, \%wt) vs \#mg. Symbols for each unit and deposits' type are presented below the diagrams; dacitic samples with red contour correspond to the vitrophyric samples.

Figure 43: Binary plots of \#mg and major elements (in oxides, \%wt) contents variations as a function of stratigraphy for the samples from the BR-116 profile. Black circles correspond to flow samples (Vale do Sol Fm.) while gray diamonds correspond to the basaltic dykes (Esmeralda Fm.). In the left profile (already shown in figure 12), solid lines represent contacts between mapped spills, while dotted lines represent inferred contacts. That way, the alternations between yellowish and white bands delimit different flows; at the uppermost portions the yellow dashed lines represent inferred (possible) limits between flows whose discontinuity of the outcrops prevented a better delimitation.

Figure 44: Binary plots for selected trace elements (in ppm) vs \#mg. All concentrations obtained by ICPMS, with the only exception of $\mathrm{Cr}$ and Ni, obtained by XRF. Symbols for each unit and deposits' type are presented below the diagrams; dacitic samples with red contour correspond to the vitrophyric samples.

Figure 45: Spidergrams for REE (above), normalized by the condrite (Boynton, 1984), and for trace elements (below), normalized by NMORB (Sun and McDonough, 1989). The basaltic samples are highlighted at the diagrams to the left, while the dacites are highlighted at the diagrams to the right. Symbols for each unit and deposits' type are presented below the diagrams; dacitic samples with red contour correspond to the vitrophyric samples.

Figure 46: Binary plots of selected trace elements (in ppm) contents variations as a function of stratigraphy for the samples from the BR-116 profile. Black circles 
correspond to flow samples (Vale do Sol Fm.) while gray lozenges correspond to the basaltic dykes (Esmeralda Fm.). All concentrations obtained by ICPMS, with the only exception of $\mathrm{Cr}$ and $\mathrm{Ni}$, obtained by XRF. In the left profile (already shown in figure 12), solid lines represent contacts between mapped spills, while dotted lines represent inferred contacts. That way, the alternations between yellowish and white bands delimit different flows; at the uppermost portions the yellow dashed lines represent inferred (possible) limits between flows whose discontinuity of the outcrops prevented a better delimitation.

Figure 47: Binary plots of selected trace elements contents (in ppm) and ratios variations as a function of $\mathrm{Zr}$ contentsfor the dacitic samples. Black dashed lines delimitate the two recognized groups of dacites. 136

Figure 48: AMS data (from Cañón-Tapia and Raposo, 2018) of the funil-like structure from quarry SM-20. The regular $(\mathrm{Rd})$, filaments $(\mathrm{Fd})$, fragmentation and regular $(\mathrm{Rd})$ domains are indicated. The data were divided and reinterpreted here according to the faciological domains defined in the item $I V .2 .3$ of this thesis and the data of each sampling site is separated by color as indicated in the figure. 141

Figure 49: AMS data (from Cañón-Tapia and Raposo, 2018) of quarry SM-82. The regular $(\mathrm{Rd})$, filaments $(\mathrm{Fd})$, fragmentation and regular $(\mathrm{Rd})$ domains are indicated. The data were divided and reinterpreted here according to the faciological domains defined in the item $I V .2 .3$ of this thesis and the data of each sampling site is separated by color as indicated in the figure.

Figure 50: Eruptive model for the dacitic volcanic sequence in the São Marcos region. 146 


\section{Introduction}

"Let us choose for ourselves our path in life, and let us try to strew that path with flowers"Émilie du Châtelet

(French natural philosopher, mathematician, physicist, and author)

Large Igneous Provinces (LIPs) can be briefly defined as magmatic provinces with volumes $>0.1 \mathrm{Mkm}^{3}$ and short lifespan (up to 50Myr), characterized by eruptive pulses of short duration ( 1-5 Myr) and developed in intraplate tectonic settings (Bryan and Ernst, 2008). These provinces are dominantly basaltic in composition, with subordinate amounts of more evolved magmas such as dacites and rhyolites. Many studies have attempted to define LIP formation cycles and trends throughout the geological history, and the work by Prokoph et al. (2004) has shown that LIP flux average is relatively constant, supporting the idea of continuous temporal emplacement of such magmatic provinces, although several semi-persistent weak cycles have been recognized.

The study of LIPs attracts much interest of the scientific community since the occurrence of such intense magmatic events has important tectonic and environmental implications, giving rise to several questions such as how they relate to rifting processes and to mass extinction events (Courtillot et al., 1999; Dalziel et al., 2000; Anderson, 2005; Saunders, 2005; Wignall, 2005; Bryan and Ferrari, 2013; Charbonnier et al., 2017). Indeed, a better understanding of the processes of generation and evolution of LIPs, the factors that control their compositional diversity and, mainly, their eruptive dynamics is of fundamental importance to understand current analogues, such as SnakeRiver Plain and its currently active portion, the Yellowstone Plateau, in the attempt to mitigate volcanic hazards.

In this context, the Paraná-Etendeka Magmatic Province (PEMP), with a preserved volume of volcanic rocks estimated around 800,000 $\mathrm{km}^{3}$ (Peate et al., 1990), easily accessible, stands out as an excellent opportunity of study. Preceding the Gondwana break-up (Juro-cretaceous period), the PEMP encompasses a large volume of tholeiitic basalts and basaltic andesites and related silicic rocks, mostly exposed at its South American portion. The silicic magmatism of the Province, even though 
subordinate to the associated mafic component (ca. 3\% vs. ca. 97\%), is still one of the best examples of silicic large igneous provinces (Bryan et al., 2010; Bryan and Ferrari, 2013) and comprise rocks with an expressive compositional diversity, with $\mathrm{SiO}_{2}$ contents varying from ca. $63 \%$ to $73 \%$. The mode of emplacement of these rocks is, however, a topic of intense debate. Opposing the most widely accepted models which propose an origin related to explosive eruptions of great magnitude (e.g. Milner and Ewart, 1989; Milner et al., 1992, 1995; Marsh et al., 2001; Ewart et al., 2002; Bryan et al., 2010), recent works carried out in the Brazilian portion of the province, based on geological mapping, petrography, geochemistry, geothermobarometry and anisotropy of magnetic susceptibility (ASM) (e.g. Janasi et al., 2007; Luchetti, 2010; Nardy et al., 2011; Lima et al., 2012; Guimarães, 2014; Guimarães et al., 2018; Polo et al., 2018b, 2018a) suggest an effusive occurrence for a substantial part of these deposits, with minor amounts of low-energy explosive phases (e.g. Luchetti et al., 2018a,b). The current efforts to characterize the feeder system of this magmatism (e.g. Lima et al., 2018; Simões et al., 2018), well exposed at São Marcos region (South Brazil), may help to shed some light on these issues. These conduits and their related deposits are the target of the present thesis, which provides a new stratigraphy for the dacitic deposits from the São Marcos region through the combination of detailed field descriptions, geochemistry, structural and textural analysis, and proposes an eruptive model for these deposits.

\section{I.1. Structure of the thesis}

Following this brief introduction where the motivation and main goal of this thesis are exposed, I present in Chapter II a brief conceptual review on the processes of magmatic fragmentation, with fundamental concepts for the structural and textural analysis of the studied deposits and the elaboration of the eruptive model of the dacitic rocks. Chapter III is dedicated to a review on the Paraná-Etendeka Magmatic Province, followed by Chapter IV where I present a detailed stratigraphy for the studied area, mainly focused on the dacitic volcanic rocks, as well as a structural characterization of the dacitic feeding structures and the petrography and mineral chemistry of the dacites. These data are part of the article titled "Flow and fragmentation patterns in the silicic feeder system and related deposits in the Paraná-Etendeka Magmatic Province, São 
Marcos, South Brazil", published at the Journal of Volcanology and Geothermal Research (doi.org/10.1016/j.jvolgeores.2018.03.021), presented in Appedinx I. In Chapter $V$ I present the results of the quantitative textural analysis carried out on the volcanic breccias that compose the base of the dacitic sequence in the form of an article submitted to the Bulletin of Volcanology. Chapter VI is dedicated to the whole-rock geochemistry of both basaltic and silicic rocks and Chapter VII presents an integrated discussion, making use of the whole set of data and summarizing the main conclusions of the thesis.

\section{I.2. Goals and Methods}

The present thesis aims at the definition of a detailed stratigraphy of the volcanic sequences at the São Marcos region, focusing on the dacitic rocks, as well as a structural, textural, and geochemical characterization of the dacites, concluding with the elaboration of an eruptive model for these rocks. In order to achieve this, detailed field work, petrography, quantitative textural analysis of volcanic breccias, whole rock and mineral geochemistry were carried out.

The field work was carried out in 5 missions (January 17-30, 2015, July 16-27, 2015, August 7-17, 2015, January 25-February 4, 2016, December 11-18 2017). The first missions aimed a regional mapping for recognition and understanding of the stratigraphic relationships between the volcanic deposits and were based on the 1:50000-scale metric topographic map "São Marcos - SH.22-V-B-VI-4". A total of 183 outcrops were described and their location is presented in Appendix II. Afterwards, the best expositions that allow greater detail were selected for structural, petrographic, textural and geochemical studies.

Petrographic analyzes were performed on 80 thin-sections $(30 \mu \mathrm{m})$ under transmitted light using the Olympus BX-50 and Zein AxioPlan apparatus from the Laboratory of Petrographic Microscopy (LMP) of the Geoanalitica-USP facility, University of São Paulo. These analyzes aimed to characterize the faciological varieties of the studied rocks, to recognize their main mineralogy, textures and microstructural relations. Based on these analyzes 5 dacitic samples from different types of deposits 
were selected for SEM (scanning electron microscopy) and electron microprobe analysis.

Quantitative chemical analyzes of the main mineral phases (plagioclase and clinopyroxene) were performed at the Laboratory of Electronic Microprobe (ME) from the Geoanalitica-USP facility at the University of São Paulo, using a JEOL JXA-FE8530 equipment, with 5 WDS and 1 EDS spectrometers and an electronic cannon supported by Field Emission (FE), which allows better spatial resolution. A CL (cathodoluminescence) detector allows obtaining compositional images in high resolution. Each spectrometer is equipped with crystals for standard analysis: 1. TAP ( $\mathrm{Si}, \mathrm{Al}), 2$. LIFL (Fe, Mn), 3.PETJ (Ca, K, Sr) and 5.TAPH (Na, Mg).The analyzes were performed under $15 \mathrm{kV}$, acceleration of $20 \mathrm{n} \AA$ and diameters from 8 to $10 \mu \mathrm{m}$ in the case of plagioclase and 3 to $5 \mu \mathrm{m}$ in the case of pyroxenes.

Textural analyses were carried out in 4 different spots from 2 outcrops of volcanic breccias aiming to obtain size distribution and shape parameters data of both the fragments and their vesicles. Due to the nature of these deposits, they are usually intensely weathered and locally well preserved due to post-magmatic silicification. This condition makes the most common analytical technique through sieving unfeasible, so the analyzes were carried out using HDR (high dynamic range) images. A complete and detailed description of the employed methodology is given in chapter $\mathrm{V}$.

Whole rock chemical analyses were performed in 23 basaltic samples ( 3 of which were collected in dykes) and 26 dacitic samples. The samples were prepared at the Preparation Lab of the Geosciences Institute, University of São Paulo, and basically followed four steps: i) discard of weathered parts; ii) sample fragmentation with a jawcrusher or hydraulic press; iii) quartering of the fragments; iv) pulverization to $<200$ mesh in a planetary type agate mill. All the geochemical analyses were performed at the X-Ray Fluorescence Laboratory and at the Chemistry and ICP Laboratory of the Geoanalitica-USP facility at the University of São Paulo. For the X-Ray Fluorescence method (XRF), trace-elements were analyzed by pressed-pellets (with $7.5 \mathrm{~g}$ of the sample for $20 \%$ wax) and major-elements through fused-beads (1g of sample for $9 \mathrm{~g}$ of flux agent - lithium metaborate), according to the description by Mori et al. 1999). The contents of additional trace-elements, including the rare-earth-elements, were determined in selected samples by ICP-MS (inductively coupled plasma mass 
spectrometer), as described at Navarro et al., (2008).The process involves acid dissolution in Parr-type pumps with concentrated $\mathrm{HNO}_{3}$ and $\mathrm{HF}$ under high temperatures $\left(200^{\circ} \mathrm{C}\right)$ for approximately 5 days in order to guarantee the complete dissolution of accessory minerals.

\section{References}

Anderson, D.L., 2005, Large Igneous Provinces, Delamination, and Fertile Mantle: Elements, v. 1, p. 271-275.

Bryan, S.E., and Ernst, R.E., 2008, Revised definition of Large Igneous Provinces (LIPs): $\quad$ Earth-Science Reviews, $\quad$ v. $\quad 86, \quad$ p. 175-202, doi:10.1016/j.earscirev.2007.08.008.

Bryan, S.E., and Ferrari, L., 2013, Large igneous provinces and silicic large igneous provinces: progress in our understanding over the last 25 years: Geological Society of America Bulletin, v. 125, p. 1053-1078.

Bryan, S.E., Peate, I.U., Peate, D.W., Self, S., Jerram, D.A., Mawby, M.R., Marsh, J.S.G., and Miller, J.A., 2010, The largest volcanic eruptions on Earth: EarthScience Reviews, v. 102, p. 207-229, doi:10.1016/j.earscirev.2010.07.001.

Charbonnier, G., Morales, C., Duchamp-alphonse, S., Westermann, S., Adatte, T., and Föllmi, K.B., 2017, Mercury enrichment indicates volcanic triggering of Valanginian environmental change: Nature, p. 1-6, doi:10.1038/srep40808.

Courtillot, V., Jaupart, C., Manighetti, I., Tapponnier, P., and Besse, J., 1999, On causal links between flood basalts and continental breakup: Earth and Planetary Science Letters, v. 166, p. 177-195.

Dalziel, I.W.D., Lawver, L.A., and Murphy, J.B., 2000, Plumes , orogenesis , and supercontinental fragmentation: Earth and Planetary Science Letters, v. 178, p. 111 .

Ewart, A., Milner, S.C., Duncan, A.R., and Bailey, M., 2002, The Cretaceous Messum igneous complex, S.W. Etendeka, Namibia: Reinterpretation in terms of a downsag-cauldron subsidence model: Journal of Volcanology and Geothermal Research, v. 114, p. 251-273, doi:10.1016/S0377-0273(01)00266-9.

Guimarães, L.F., 2014, Características físicas e químicas e modelo eruptivo para os riolitos tipo Santa Maria ( Província Magmática Paraná ) na região de Gramado Xavier, RS: Universidade de São Paulo.

Guimarães, L.F., Raposo, M.I.B., Janasi, V.A., Cañón-Tapia, E., and Polo, L.A., 2018, An AMS study of different silicic units from the southern Paraná-Etendeka 
Magmatic Province in Brazil: Implications for the identification of flow directions and local sources: Journal of Volcanology and Geothermal Research, v. 355, p. 304-318, doi:10.1016/j.jvolgeores.2017.11.014.

Janasi, V.A., Montanheiro, T.J., Freitas, V.A., Reis, P.M., Negri, F.A., and Dantas, F.A., 2007, Geology, petrography and geochemistry of the acid volcanism of the Parana magmatic province in the Piraju-Ourinhos region, SE Brazil: Revista Brasileira de Geociencias, v. 37, p. 745-759.

Lima, E.F., Philipp, R.P., Rizzon, G.C., Waichel, B.L., and Rossetti, L.M.M., 2012, Sucessões vulcânicas, modelo de alimentação e geração de domos de lava ácidos da Formação Serra Geral na região de São Marcos-Antônio Prado (RS): Geologia USP - Serie Cientifica, v. 12, p. 49-64, doi:10.5327/Z1519-874X2012000200004.

Lima, E.F., Waichel, B.L., Rossetti, L.M.M., Sommer, C.A., and Simões, M.S., 2018, Feeder systems of acidic lava flows from the Paraná-Etendeka Igneous Province in southern Brazil and their implications for eruption style: Journal of South American Earth Sciences, v. 81, p. 1-9, doi:10.1016/J.JSAMES.2017.11.004.

Luchetti, A.C.F., 2010, Aspectos vulcanológicos dos traquidacitos da Região de PirajuOurinhos (SP): Universidade de São Paulo, doi:10.1017/CBO9781107415324.004.

Luchetti, A.C.F., Gravley, D.M., Gualda, G.A.R., and Nardy, A.J.R., 2018a, Textural evidence for high-grade ignimbrites formed by low-explosivity eruptions, Paraná Magmatic Province, southern Brazil: Journal of Volcanology and Geothermal Research, v. 355, p. 87-97, doi:10.1016/J.JVOLGEORES.2017.04.012.

Luchetti, A.C.F., Nardy, A.J.R., and Madeira, J., 2018b, Silicic, high- to extremely high-grade ignimbrites and associated deposits from the Paraná Magmatic Province, southern Brazil: Journal of Volcanology and Geothermal Research, v. 355, p. 270-286, doi:10.1016/J.JVOLGEORES.2017.11.010.

Marsh, J.S., Ewart, A., Milner, S.C., Duncan, A.R., and Miller, R.M., 2001, The Etendeka Igneous Province: Magma types and their stratigraphic distribution with implications for the evolution of the Paraná-Etendeka flood basalt province: Bulletin of Volcanology, v. 62, p. 464-486, doi:10.1007/s004450000115.

Milner, S.C., and Ewart, A., 1989, The geology of the Goboboseb Mountain volcanics and their relationship to the Messum Complex , Namibia: Commun. Geol. Surv. Namib, v. 5, p. 33-42.

Milner, S.C., Duncan, A.R., and Ewart, A., 1992, Quartz latite rheoignimbrite flows of the Etendeka Formation, north-western Namibia: Bulletin of Volcanology, v. 54, p. 200-219, doi:10.1007/BF00278389.

Milner, S.C., Duncan, A.R., Whittingham, A.M., and Ewart, A., 1995a, Trans-Atlantic correlation of eruptive sequences and individual silicic volcanic units within the Paran??-Etendeka igneous province: Journal of Volcanology and Geothermal Research, v. 69, p. 137-157, doi:10.1016/0377-0273(95)00040-2.

Mori, P.E., Reeves, S., Correia, C.T., and Haukka, M., 1999, Development of a fused glass disc XRF facility and comparison with the pressed powder pellet technique at Instituto de Geociencias, Sao Paulo University: Revista Brasileira de Geociencias, 
v. 29, p. 441-446, doi:10.5327/rbg.v29i3.715.

Nardy, A.J.R., Rosa, M.C., Luchetti, A.C.F., Ferreira, M.L.C., Machado, F.B., and Oliveira, M.A.F., 2011, Parâmetros físicos pré-eruptivos do magmatismo ácido da província magmática do Paraná: Resultados preliminares: Geociencias, v. 30, p. $575-588$.

Navarro, M.S., Andrade, S., Ulbrich, H., Gomes, C.B., and Girardi, V.A.V., 2008, The direct determination of rare earth elements in basaltic and related rocks using ICPMS: Testing the efficiency of microwave oven sample decomposition procedures: Geostandards and Geoanalytical Research, v. 32, p. 167-180, doi:10.1111/j.1751908X.2008.00840.x.

Peate, D.W., Hawkesworth, C.J., Mantovani, M.S.M., and Shukowsky, W., 1990, Mantle plumes and flood-basalt stratigraphy in the Paraná, South America: Geology, v. 18, p. 1223-1226, doi:10.1130/00917613(1990)018<1223:MPAFBS>2.3.CO;2.

Polo, L.A., Giordano, D., Janasi, V.A., and Guimarães, L.F., 2018b, Effusive silicic volcanism in the Paraná Magmatic Province, South Brazil: Physico-chemical conditions of storage and eruption and considerations on the rheological behavior during emplacement: Journal of Volcanology and Geothermal Research, v. 355, doi:10.1016/j.jvolgeores.2017.05.027.

Polo, L.A., Giordano, D., Janasi, V.A., and Guimarães, L.F., 2018a, Effusive silicic volcanism in the Paraná Magmatic Province, South Brazil: Physico-chemical conditions of storage and eruption and considerations on the rheological behavior during emplacement: Journal of Volcanology and Geothermal Research, v. 355, p. 115-135, doi:10.1016/J.JVOLGEORES.2017.05.027.

Prokoph, A., Ernst, R.E., and Buchan, K.L., 2004, Time-Series Analysis of Large Igneous Provinces: 3500 Ma to Present: The Journal of Geology, v. 112, p. 1-22.

Saunders, A.D., 2005, Large Igneous Provinces: Origin and Environmental Consequences: Elements, v. 1, p. 259-263.

Simões, M.S., Lima, E.F., Sommer, C.A., and Rossetti, L.M.M., 2018, Structures and lithofacies of inferred silicic conduits in the Paraná-Etendeka LIP, southernmost Brazil: Journal of Volcanology and Geothermal Research, v. 355, p. 319-336, doi:10.1016/J.JVOLGEORES.2017.12.013.

Wignall, P.B., 2005, The link between large igneous province eruptions and mass extinctions: Elements, v. 1, p. 293-297, doi:10.2113/gselements.1.5.293. 


\section{Conceptual Review}

"Don't be afraid of hard work. Nothing worthwhile comes easily. Don't let others discourage you or tell you that you can't do it." - Gertrude Elion

(American biochemist and pharmacologist, 1988-Nobel Prize-winning, who developed a multiple of new drugs to treat leukemia, malaria, meningitis, herps, AIDS, and more... Also developed the first immunosuppressive drug used for organ transplants.)

Viscous fluid:behavior of ideal fluids (liquids and gases) that deform irreversibly (flowing) under stress. The applied stress is not recovered; it is dissipated through the fluid and converted into energy (heat).

Viscoelastic fluid: most natural liquids (including magmas) present a rheological behavior in which both viscous and elastic components are present. In the case of magmas, when the temperature decreases and / or the rate of deformation increases, the elastic behavior becomes predominant.

Brittle failure: violent rupture, brittle fragmentation due to the application of high strain rates.

Relaxation time: the time required by a material to adapt to the stress changes. If the real timescale of the transformation is lower (i.e. faster) than the relaxation time, the material deforms (or breaks).

\section{II.1. "To break or not to break":}

fragmentation, the dilemma of a rising magma

Magma fragmentation is a destructive, irreversible mechanical process that occurs when the stress applied to a material (in our case, magma) exceeds a critical value (Perugini et al., 2011). In other words, the magma which initially behaved as a viscous fluid* progress to a viscoelastic* behavior as the applied stress increases until it exceeds the magma's tensile strength, when the magma transitions from a ductile to a brittle* behavior and breaks (Marti et al., 1999). As a consequence, a cohesive amount of magma (consisting of silicate melt \pm crystals \pm volatiles) is broken into individual fragments called pyroclasts.

The fragmentation related to magmatic processes is known as primary fragmentation and may occur mainly related to (i) rapid magma ascent (acceleration) or (ii) rapid decompression (Cashman and Scheu, 2015). In the first case, the magma ascent can be triggered by, for example, new magma injection, volatile supersaturation due to crystallization or any other process that 


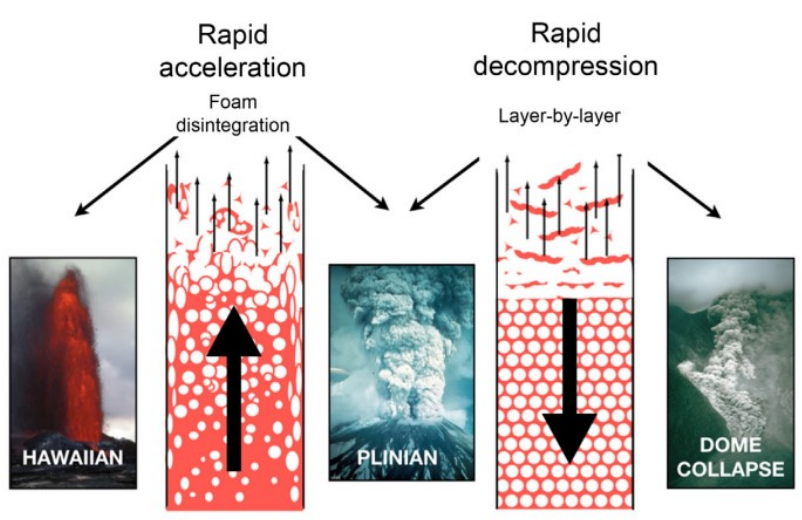

Figure 1: Schematic representation of primary fragmentation due to rapid magma ascent or rapid decompression. Fragmentation related to rapid magma ascent propagates upwards and the rising acceleration may be related to bubbles expansion, with fragmentation occurring when bubble overpressure exceeds the fragmentation threshold or when high strain rates cause the magma to cross the glass transition. Fragmentation by rapid decompression propagates downwards and may be a result of bubble-bearing magma (already under overpressure) is suddenly exposed to a lower pressure. Extracted from Cashman and Scheu (2015).

Scheu, 2015). In the later case, the rapid decompression is prompted by volcanic edifice or dome collapse and the stress applied to the magma, due to large overpressure at significantly fast timescale, results at brittle fragmentation. Here, the fragmentation front propagates layer-by-layer downwards (figure 1; Fowler et al., 2010; Cashman and Scheu, 2015).

All these processes can involve different mechanisms of fragmentation. In this review, I will follow the classification proposed by Gonnermann (2015), who have summarized the mechanisms of magma primary fragmentation in four types: (i) brittle fragmentation, (ii) fragmentation by shear, (iii) fluid-dynamic break-up and (iv) fragmentation by external water-magma interaction. Here, only the first two types will be addressed, and the conditions of each are explored in detail in the following sections.

Finally, processes acting at primarily formed pyroclasts during transport either in the conduit or on the surface (such as collision and abrasion) are known as secondary fragmentation and may be responsible for a reduction on fragments sizes. 


\section{II.1.1. Brittle fragmentation related to degassing and bubble overpressure}

The magma degassing corresponds to the exsolution of volatiles elements previously dissolved. The solubility of the volatile phase, which is mainly composed by $\mathrm{H}_{2} \mathrm{O}^{1}, \mathrm{CO}_{2}$ and $\mathrm{SO}_{2}$, is primary controlled by pressure and also by chemical properties; $\mathrm{SiO}_{2}$-rich magmas (intermediate to silicic) tend to present higher water-solubilities, at high pressures, than $\mathrm{SiO}_{2}$-poor magmas (Stolper, 1982a,1982b; Silver et al., 1990; Mysen and Richet, 2005). As a consequence, even though several processes may lead to degassing (such as heating, magma mixing and crystallization; Eichelberger, 1980; Lavallée et al., 2015) it mainly results from decompression, which occurs basically due to rapid magma ascent and/or collapse of volcanic edifices (e.g. Sparks, 1978; Mueller et al., 2008). The minimum pressure differential needed for fragmentation is known as fragmentation threshold and strongly depends on porosity (defined by the vesicle density of the magma), being inversely proportional to it (Spieler et al., 2004).

The vesiculation process is triggered by the bubble nucleation, which can be homogeneous (starting from the magma itself, without previous substrate) or heterogeneous (occurring on previously formed surfaces, such as crystals). Experimental works have shown that the energy and the supersaturation pressures $\left(\Delta P_{n}\right)$ needed are significantly higher for homogeneous nucleation (Shea, 2017and references therein). So that more viscous crystal-poor magmas with lower ascent rates delay bubble nucleation and, when it finally occurs, an intense and fast degassing will favor more explosive volcanism (Mangan and Sisson, 2000).

The next step after bubble nucleation is its expansion, due to both diffusion of the continuously exsolved volatiles and decrease in pressure. Since the volatile phase is more compressible than the hosting silicate melt, it also is more prone to a greater and faster expansion during magma ascent. However, the high viscosity of the magma and its high relaxation time (given by $\lambda=\mu / \mathrm{G}$; where $\mu$ is the magma viscosity and $\mathrm{G}$ is the shear modulus) offer a viscous resistance to volatile exsolution processes (diffusion, bubble nucleation, expansion and coalescence) leading to a gas overpressure in the

\footnotetext{
${ }^{1}$ Water, the most important volatile component, is also the one with higher solubility and, therefore, the last component to exsolve (commonly under low pressures).
} 
system (Mueller et al., (2008) and references therein). Additionally, if the decompression timescale (given by $\mathrm{T}_{\mathrm{p}} \sim \Delta \mathrm{p} / \dot{\mathrm{p}}$; where $\Delta \mathrm{p}$ is the difference in magma pressure during ascent and $\dot{\mathrm{p}}$ is the decompression rate) is lower (i.e. faster) than the timescale for bubble growth (given by $\mathrm{T}_{\mathrm{b}} \sim \mu_{\mathrm{m}} / \Delta \mathrm{p}$; where $\mu_{\mathrm{m}}$ is the effective melt viscosity) there would not be time enough for bubble expansion, contributing therefore to the gas overpressure (Gonnermann, 2015). Here it is worth mentioning that bubbles are a tricky component, since it can contribute both for an increase or a decrease in the effective viscosity of the system. (Llewellin and Manga, 2005) defined two different regimes where (i) shear viscosity increases with increasing bubbles volume-fraction and (ii) shear viscosity decreases with increasing bubbles volume-fraction. By defining the capillary number*, the authors correlate the viscous stress $\left(\mu / \gamma^{\prime}\right.$; here $\gamma^{\prime}$ is the strain rate), which tends to deform the bubbles, and interfacial tension $\left(\gamma_{s} / R\right.$; here $\gamma_{s}$ is the shear strain and $\mathrm{R}$ is the bubble's radius), which tends to restore bubble sphericity, and compares the bubble relaxation time and the shear strain rate (or, in unsteady flows, the timescale of shear strain rate changes). When the interfacial tension forces dominate and/or deformation timescale is higher than bubbles' relaxation time, the bubbles remain spherical, deforming the flow and consequently increasing effective viscosity. On the other hand, if the viscous forces dominate and/or deformation timescale is lower than bubbles' relaxation time, the bubbles will be deformed and the effective viscosity will decrease. This decrease in viscosity, although may delay the gas overpressure, is not able to prevent it. Thus, if there is no effective loss of the volatile component (which is not guaranteed by the exsolution process itself) and decreasing of the gas overpressure, magma fragmentation seems to be an unavoidable process. This way, among the processes that control fragmentation, gas overpressure is considered to be a crucial one and the permeability of the system (which in fact controls the volatile loss) is therefore a decisive parameter (Mueller et al., (2008) and references therein).

Permeability can be defined as the quantified amount of interconnected bubbles (through coalescence), fractures and void spaces in a magma body or rock, which allows the volatile loss due to a pressure gradient. In a rising magma, the permeability can vary between two extreme conditions: from a non-permeable system (closed system; figure 2), where an isolated impermeable network leads to a constantly increasing gas overpressure, to a permeable system (open system; figure 2), where a connected network allows an effective loss of gas, reducing considerably the 
overpressure. (Mueller et al., 2008) demonstrated that high permeability increases the overpressure needed to fragment the magma. The transitions between closed and open systems can be naturally observed through the common occurrence of switches between eruptive styles and even hybrid deposits (specially in silicic systems) reported by many authors (e.g. Castro et al., 2012; Schipper et al., 2013; Cabrera et al., 2015; Degruyter et al., 2017).

a) Closed-system degassing

b) Outgassing
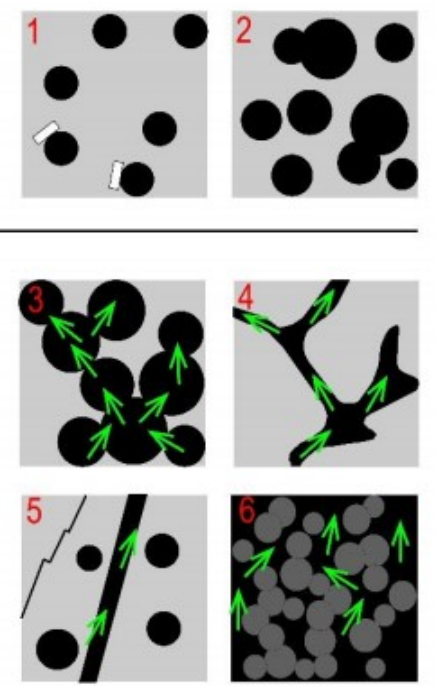

Figure 2: Schematic representation of degassing conditions. A) Closed system, with non-connected bubbles network. Both hetereogenous and homogeneous bubbles nucleation represented (a1), with possible restrict coalescence (a2). B) Open system outgassing, with development of permeable network due to bubbles coalescence (b3), collapse of coalesced bubbles (b4), fracturing (b5) and percolation through intergranular pore space (b6). C) Conceptual model for different degassing conditions and possibly related eruptive styles. Extracted from Colombier, 2018.

The critical porosity at which it this transition from an impermeable to a permeable system occurs is known as percolation threshold (Mueller et al., 2008; Colombier, 2018). Recent studies have shown that the magma percolation threshold $\left(\phi_{c}\right)$ is complex and depends on a set of parameters such as (i) bubbles size and spatial distributions, and deformation degree (higher diversity of bubbles' size distribution and higher packing results in higher $\phi_{c}$ and higher aspect ratios, i.e. deformation, lead to lower $\phi_{c}$ values; (e.g. Blower, 2001; Blower et al., 2001; Gaonac'h et al., 2003; Okumura et al., 2008; Burgisser et al., 2017); (ii) viscosity (higher viscosities hinders vesiculation processes and therefore results in higher $\phi_{c}$; (e.g. Lindoo et al., 2016); (iii) crystallinity (higher crystallinity enhances bubbles' nucleation - heterogeneously coalescence, migration and deformation, causing lower $\phi_{\mathrm{c}}$ values (e.g. Blower, 2001; Parmigiani et al., 2016; Lindoo et al., 2017; Colombier et al., 2017); and (iv) fracturing 
degree the presence of fractures increases permeability (even under low porosity conditions) and reduces the $\phi_{\mathrm{c}}$. Colombier(2018) showed that pore connectivity is a good metric for the percolation threshold of natural samples and, based on an extensive connectivity database revealed that effusive volcanic rocks present lower percolation thresholds than explosive volcanic rocks.

Last but not least, many authors (e.g. Rust and Cashman, 2004; Okumura et al., 2013; Cashman and Scheu, 2015; Colombier et al., 2017) have described an hysteretic time-dependence between connectivity and porosity (figure 3), which results from a cycle of bubble coalescence, increasing connectivity and permeability, gas escape, compaction and/or bubble collapse and finally reduction of connectivity and porosity. During the final stages, the connectivity and permeability can be maintained by cracks and fractures. Such cyclic behavior may collaborate for the occurrence of explosive-effusive transitions.

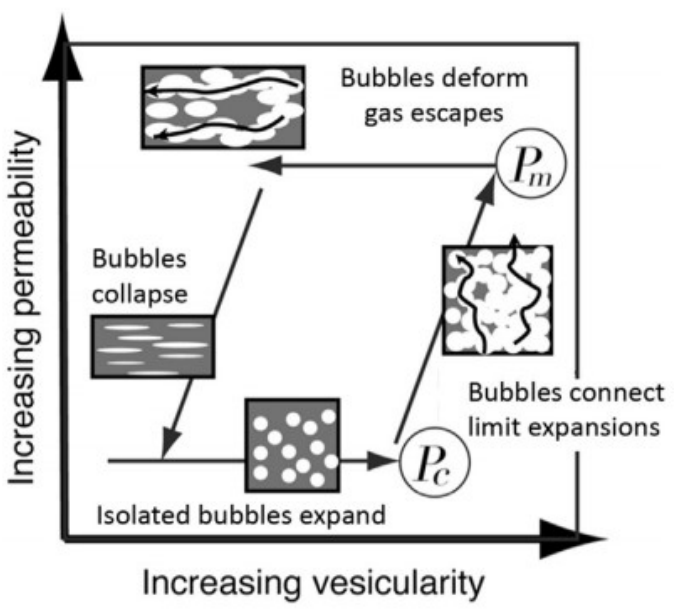

Figure 3: Schematic plot illustrating the hysteresis in permeability-porosity data. Initialy isolated bubbles expand up to the percolation threshold $(\mathrm{Pc})$ at around $60 \%$ vesicularity. Permeability rapidly increases to a permeability threshold (Pm) that limits further expansion. Deformation allows gas loss (porosity reduction) without loss of permeability, what happens later with bubbles collapse. Extracted from Cashman and Scheu (2015).

\section{II.1.2. Fragmentation by shear strain}

As already stated, magma is a natural viscoelastic fluid, meaning that under certain conditions such as high temperatures and low strain rates, it presents a Newtonian behavior (non-Arrhenian T-dependent), and flows viscously dissipating the applied stress (the viscosity, $\mu$, is proportional to the rate between the applied shear stress, $\tau$, and the shear strain rate, $\gamma^{\prime}$ ). In this condition, the magma is in a relaxed state or, in other words, the deformation timescale is large enough to permits relaxation. However, by increasing the shear stress applied to the magma, it turns into a viscoelastic non-Newtonian behavior (Webb and Dingwell, 1990). Under this condition, the silicate melt is in an unrelaxed state, not being able to successfully dissipate the stress, which is accumulated elastically and later released by fracturing (Wadsworth et 
al., 2017). When the strain rate is large enough to avoid stress dissipation, i.e. the relaxation timescale is higher than the deformation timescale, the melt experiences a brittle behavior and breaks. This relation between relaxation time $\left(\lambda_{r}\right)$ and deformation timescale $(\lambda)$ defines a dimensionless parameter termed Deborah number $\left(\mathrm{De}=\lambda_{r} / \lambda=\right.$ $\left.\left(\mu^{*} \gamma^{\prime}\right) / \mathrm{G}\right)$.

Several experimental works (e.g. Webb and Dingwell, 1990; Cordonnier et al., 2012a, 2012b; Witcher, 2017) covering a wide range of compositions and applied strain rates defined universal cutoff $D e$ values which mark the viscous-to-brittle transitions in magmas. At De $<0.01$, the silicate melt presents purely viscous relaxed behavior, which transitions to an unrelaxed regime $(0.01<$ De $<0.04$; where microfractures can propagate allowing the accumulated stress to release), until reaches the brittle regime (De $>0.04$ ), when the magma is "broken" (figure 4). In practical terms, this means that if the magma relaxation time is at least 100 times longer than the deformation timescale, it can be fragmented. These studies have shown that, regardless the composition, by knowing the correct parameters (i.e. viscosity and shear strain rate, which are easily accessed) it is possible to predict if a volcanic system is more prone to flow or fragment.

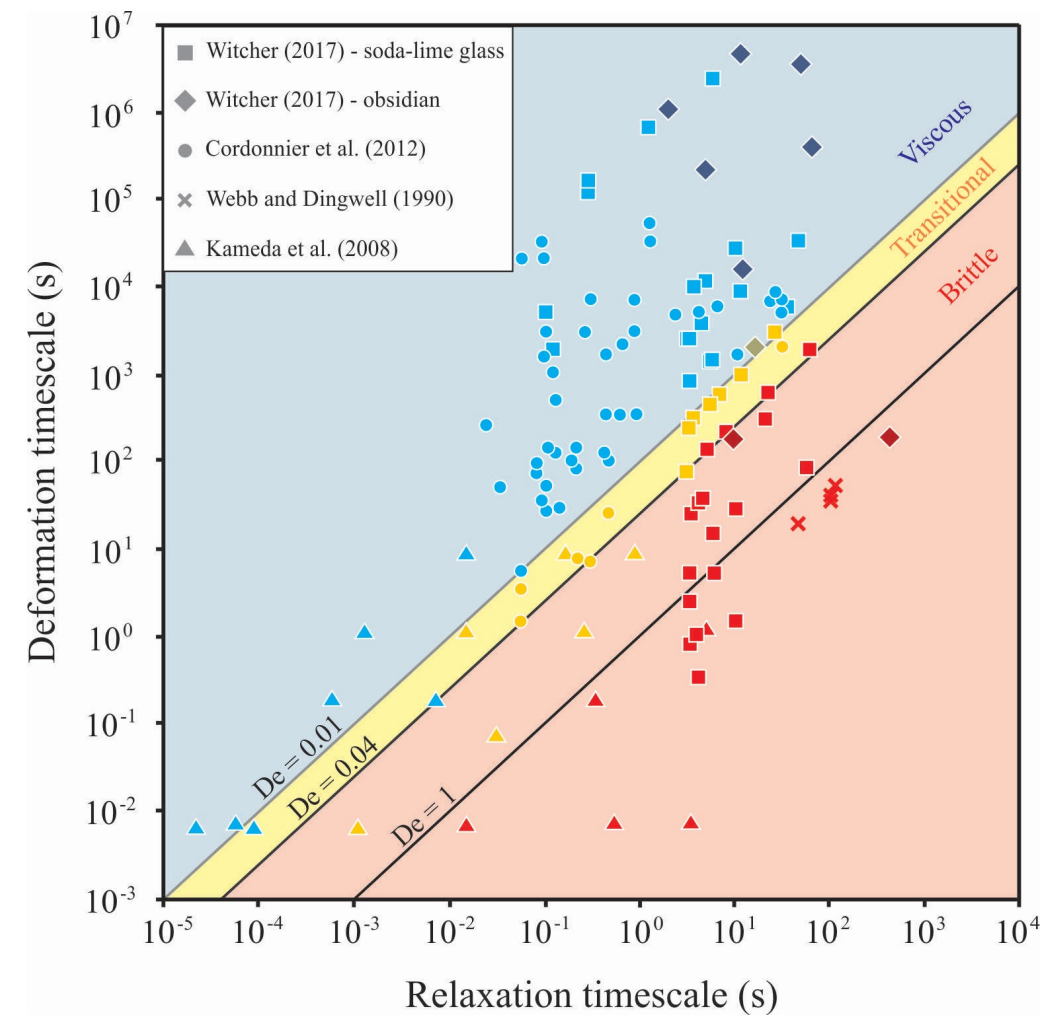

Figure 4: Deformation behaviors of silicate melts with various compositions, based on experimental studies by Webb and Dingwell (1990; fiber elongation experiments), Cordonnier et al. (2012b; uniaxial compression experiments), Kameda et al. (2008) and Witcher (2017; uniaxial compression experiments). The onset of elastic behavior is defined by $\mathrm{De}=0.01$, and the regime where brittle fracturing is the dominant response to stress occurred in this study when $\mathrm{De}>0.04$. Adapted from Witcher (2017). 
It is worth mentioning that the conditions defined above are valid for a singlephase magma (no crystals neither bubbles), which is not often the natural occurrence. It is evident that for two- or three-phase magmas, bulk viscosities and deformation rates should be considered, adding complexity to the system. Wadsworth et al., 2017 offers an overview on the topic based on recent studies on two-phase crystal-bearing (e.g. Cordonnier et al., 2012a) and bubble-bearing systems (e.g. Vasseur et al., 2013). In a very brief way, the second component of the system, either crystals or bubbles, would increase the bulk shear strain rate in the interstitial liquid, resulting in lower stress required to fragment the two-phase system in relation to a single-phase system. In the case of crystal-bearing magmas it would depend on the crystals shape and density, with decreasing stress required with increasing crystal contents. The same inverse relation is valid for bubble-bearing systems under high De regime, while more precise information regarding low De regime is still lacking (Vasseur et al., 2013; Wadsworth et al., 2017).

A practical example of the occurrence of such fragmentation mechanism would be the eruptions triggered by the collapse of lava domes or volcanic edifices. In this scenario, the volcanic structure acts as a cap for the upcoming magma and can lead to an increase in system overpressure. The collapse of the structure then leads to a fast decompression event and discharges a downwards elastic wave, subjecting the magma to a tensile stress which, if superior to the tensile strength of the material, results in its fragmentation (Alidibirov and Dingwell, 2000).

\section{II.2. Explosive-Effusive transitions on silicic volcanic systems}

Transitions between eruptive styles are quite common, especially in silicic systems. According to Cassidy et al., (2018), based on the Global Volcanism Program ${ }^{2}$ database, $61 \%$ of the volcanic events with $\mathrm{VEI}^{3}>3$ since 2000 were constituted by both

\footnotetext{
${ }^{2}$ The Global Volcanism Program is coordinated by the Smithsonian Institution from the National Museum of Natural History (Washington, USA) and offers an updated database with information about 9843 eruptions since the Pleistocene. https://volcano.si.edu/gvp_votw.cfm

${ }^{3}$ Volcano Explosivity Index: numeral scale for measuring the explosiveness of volcanic eruptions. It varies from 0 (non-explosive events, less than $10000 \mathrm{~m}^{3}$ of tephra ejected) to 8 (very large or megacolossal events, with more than $1000 \mathrm{~km}^{3}$ of tephra ejected and cloud column height of over $20 \mathrm{~km}$. The scale is logarithmic; each interval correspondstoa tenfold increase in observed ejecta (exceptions between VEI 0, VEI 1 and VEI 2).
} 
explosive and effusive phases. A complete understating about the behavior of the volcanic system and the advances in the ability to predict drastic shifts in eruptive style are essential for volcanic hazards assessment.

The shifts on eruptive style can occur along a single volcanic event, even forming hybrid deposits (e.g. Schipper et al., 2013; Cabrera et al., 2015; Suzuki et al., 2018), or over several eruptive events (e.g. Sulpizio et al., 2016; Degruyter et al., 2017). The first case is mainly controlled by shallow processes (within the conduit, during ascent, such as crystallization and bubble growth and overpressure, or even superficial processes as dome collapse), while the later is essentially controlled by deep processes occurring in the reservoir (such as magma mixing and variations on volatiles solubility) (Cassidy et al., 2018).

The transitions in eruptive styles within single volcanic events evolve more often from explosive to effusive and are more likely to be related to the conditions of shallow ascent than to the pre-eruptive volatile contents (Eichelberger et al., 1986). These conditions are controlled by (i) critical overpressures at the reservoirs, above which explosive eruptions occur (e.g. Woods and Koyaguchi, 1994) and (ii) the occurrence of a threshold decompression rate (e.g. Woods and Koyaguchi, 1994; Burgisser and Gardner, 2004; Andrews et al., 2018), that may be related to the overpressure. The decompression rate may control whether the system evolves under closed or open degassing conditions; rapid magma ascent prevents the processes which lead to a greater permeability of the system (e.g. bubbles coalescence) and the consequent loss of volatiles that, "locked" in the system, guarantee the maintenance of the high ascent rates related to the low density of the mixture (e.g. Woods and Koyaguchi, 1994; Burgisser and Gardner, 2004; Martel and Iacono-Marziano, 2015; Andrews et al., 2018). Slower decompression rates, on the other hand, where coalescence may occur, allow effective magma degassing resulting in effusive events. So, following this idea, an initially volatile-bearing magma would rise at higher rates (fast!) due to a combination of critical overpressure and low density, and would not be able to release the gas. The bubble overpressure, together with repetitive fracturing events (Cabrera et al., 2015) related to high-strain rates would cause magma fragmentation and, finally, its explosive extrusion. The explosion then allows a significant loss of volatiles and the magma will then lose speed. The explosive phase ceases when the eruption drive force reduces and the decompression rate drops below its threshold (Andrews et al., 2018). With lower ascent 
rates, bubbles coalescence may be achieved, creating permeable pathways which permit effective outgassing and effusive extrusions. At this stage, fracturing-healing events may still occur locally, contributing to an even more effective degassing (Cabrera et al., 2015).

Transitions in eruptive style over several volcanic events are, in turn, related to changes on the reservoir dynamics which are commonly associated to its replenishment. New batches of magma may however, cause both effusive and explosive eruptions. The very first effect of a new injection of magma into a reservoir is an increasing overpressure which may constitute the driven force for explosive eruptions (Degruyter et al., 2016). In this context, some authors (e.g. Degruyter et al., 2016, 2017) have shown that both the size of the reservoir and its pre-replenishment conditions (i.e. exsolved volatiles contents) should play a key role in the style of the eruption that will be triggered. On the model proposed by Degruyter et al. (2017), the overpressure to be built by the recharge would be damped in case of entering a bubbly system, and the increase in temperature would enhance outgassing, leading to effusive eruptions. Later, when new batches of hot magma entered an already degassed (and by consequence less compressible) chamber, the overpressure would be rapidly reached, even before allowing a reheating, resulting in an explosive event. Indeed, recharge events may result in shifts in eruptive dynamics due to variations in system temperature. In one sense, an increasing in temperature related to new hot magma injection may lead to vigorous convection, high vesiculation and consequently increasing in bubble overpressure (e.g. Sparks et al., 1977) or also promote higher assimilation of hosting rocks which can contribute for an increase on volatile contents (e.g. Costa et al., 2013), in all cases promoting explosive eruptions. Additionally, dome collapse may occur due to repetitive high magma + gas influx (e.g. Williamson et al., 2010). Alternatively, progressive heating of the system may lead to resorption and remobilization of crystal-rich magmas, resulting in viscosity decrease which, combined with the higher temperatures may accelerate volatile diffusion, bubbles nucleation and coalescence, building up permeability, leading to open degassing system (e.g. Ruprecht and Bachmann, 2010). Also, new high temperature volatile-poor magma may dilute the volatile contents of the system (e.g. Cassidy et al., 2016), all these last cases resuming in effusive events. 
The complexity of the parameters controlling the transitions between eruptive styles must be therefore analyzed in a holistic way, in order to obtain a better understanding and predictability of the evolution of a volcanic system.

\section{References}

Alidibirov, M., and Dingwell, D.B., 2000, Three fragmentation mechanisms for highly viscous magma under rapid decompression: Journal of Volcanology and Geothermal Research, v. 100, p. 413-421, doi:10.1016/S0377-0273(00)00149-9.

Andrews, B.J., Dufek, J., and Ponomareva, V., 2018, Eruption dynamics and explosiveeffusive transitions during the 1400 cal BP eruption of Opala volcano, Kamchatka, Russia: Journal of Volcanology and Geothermal Research, v. 356, p. 316-330, doi:10.1016/j.jvolgeores.2018.02.019.

Blower, J., 2001, Factors controlling permeability-porosity relationships in magma: Bulletin of Volcanology, v. 63, p. 497-504, doi:10.1007/s004450100172.

Blower, J.D., Keating, J.P., Mader, H.M., and Phillips, J.C., 2001, Inferring volcanic degassing processes from vesicle size distributions: Geophysical Research Letters, v. 28, p. 347-350, doi:10.1029/2000GL012188.

Burgisser, A., Chevalier, L., Gardner, J.E., and Castro, J.M., 2017, The percolation threshold and permeability evolution of ascending magmas: Earth and Planetary Science Letters, v. 470, p. 37-47, doi:10.1016/j.eps1.2017.04.023.

Burgisser, A., and Gardner, J.E., 2004, Experimental constraints on degassing and permeability in volcanic conduit flow: Bulletin of Volcanology, v. 67, p. 42-56, doi:10.1007/s00445-004-0359-5.

Cabrera, A., Weinberg, R.F., and Wright, H.M.N., 2015, Magma fracturing and degassing associated with obsidian formation: The explosive-effusive transition: Journal of Volcanology and Geothermal Research, v. 298, p. 71-84, doi:10.1016/j.jvolgeores.2014.12.014.

Cashman, K.V., and Scheu, B., 2015, Magmatic Fragmentation: Elsevier Inc., 459-471 p., doi:10.1016/B978-0-12-385938-9.00025-0.

Cassidy, M., Castro, J.M., Helo, C., Troll, V.R., Deegan, F.M., Muir, D., Neave, D.A., and Mueller, S.P., 2016, Volatile dilution during magma injections and implications for volcano explosivity: Geology, v. 44, p. 1027-1030, doi:10.1130/G38411.1.

Cassidy, M., Manga, M., Cashman, K., and Bachmann, O., 2018, Controls on explosive-effusive volcanic eruption styles: Nature Communications, v. 9, 
doi:10.1038/s41467-018-05293-3.

Castro, J.M., Cordonnier, B., Tuffen, H., Tobin, M.J., Puskar, L., Martin, M.C., and Bechtel, H.A., 2012, The role of melt-fracture degassing in defusing explosive rhyolite eruptions at volcán Chaitén: Earth and Planetary Science Letters, v. 333334, p. 63-69, doi:10.1016/j.eps1.2012.04.024.

Colombier, M., 2018, The role of percolation threshold and water-magma interaction on volcanic eruptive style: Ludwig-Maximilians-Universität München.

Colombier, M., Wadsworth, F.B., Gurioli, L., Scheu, B., Kueppers, U., Di Muro, A., and Dingwell, D.B., 2017, The evolution of pore connectivity in volcanic rocks: Earth and Planetary Science Letters, v. 462, p. 99-109, doi:10.1016/j.eps1.2017.01.011.

Cordonnier, B., Caricchi, L., Pistone, M., Castro, J., Hess, K.U., Gottschaller, S., Manga, M., Dingwell, D.B., and Burlini, L., 2012a, The viscous-brittle transition of crystal-bearing silicic melt: Direct observation of magma rupture and healing: Geology, v. 40, p. 611-614, doi:10.1130/G3914.1.

Cordonnier, B., Schmalholz, S.M., Hess, K.U., and Dingwell, D.B., 2012b, Viscous heating in silicate melts: An experimental and numerical comparison: Journal of Geophysical Research: Solid Earth, v. 117, p. 1-13, doi:10.1029/2010JB007982.

Costa, F., Andreastuti, S., Bouvet de Maisonneuve, C., and Pallister, J.S., 2013, Petrological insights into the storage conditions, and magmatic processes that yielded the centennial 2010 Merapi explosive eruption: Journal of Volcanology and Geothermal Research, v. 261, p. 209-235, doi:10.1016/j.jvolgeores.2012.12.025.

Degruyter, W., Huber, C., Bachmann, O., Cooper, K.M., and Kent, A.J.R., 2017, Influence of Exsolved Volatiles on Reheating Silicic Magmas by Recharge and Consequences for Eruptive Style at Volcán Quizapu (Chile): Geochemistry, Geophysics, Geosystems, v. 18, p. 4123-4135, doi:10.1002/2017GC007219.

Degruyter, W., Huber, C., Bachmann, O., Cooper, K.M., and Kent, A.J.R., 2016, Magma reservoir response to transient recharge events: The case of Santorini volcano (Greece): Geology, v. 44, p. 23-26, doi:10.1130/G37333.1.

Eichelberger, J.C., 1980, Vesiculation of mafic magma during replenishment of silicic magma reservoirs: Nature, v. 288, p. 446-450, doi:10.1038/288446a0.

Eichelberger, J.C., Carrigan, C.R., Westrich, H.R., and Price, R.H., 1986, Nonexplosive silicic volcanism: Nature, v. 323, p. 598-602, doi:10.1038/323598a0.

Fowler, A.C., Scheu, B., Lee, W.T., and McGuinness, M.J., 2010, A theoretical model of the explosive fragmentation of vesicular magma: Proceedings of the Royal Society A: Mathematical, Physical and Engineering Sciences, v. 466, p. 731-752, doi:10.1098/rspa.2009.0382.

Gaonac'h, H., Lovejoy, S., and Schertzer, D., 2003, Percolating magmas and explosive volcanism: Geophysical Research Letters, v. 30, p. 11-14, doi:10.1029/2002GL016022. 
Gonnermann, H.M., 2015, Magma Fragmentation: Annual Review of Earth and Planetary Sciences, v. 43, p. 431-458, doi:10.1146/annurev-earth-060614-105206.

Lavallée, Y. et al., 2015, Thermal vesiculation during volcanic eruptions: Nature, v. 528, p. 544-547, doi:10.1038/nature16153.

Lindoo, A., Larsen, J.F., Cashman, K.V., Dunn, A.L., and Neill, O.K., 2016, An experimental study of permeability development as a function of crystal-free melt viscosity: Earth and Planetary Science Letters, v. 435, p. 45-54, doi:10.1016/j.eps1.2015.11.035.

Llewellin, E.W., and Manga, M., 2005, Bubble suspension rheology and implications for conduit flow: Journal of Volcanology and Geothermal Research, v. 143, p. 205-217, doi:10.1016/j.jvolgeores.2004.09.018.

Mangan, M., and Sisson, T., 2000, Delayed, disequilibrium degassing in rhyolite magma: Decompression experiments and implications for explosive volcanism: Earth and Planetary Science Letters, v. 183, p. 441-455, doi:10.1016/S0012821X(00)00299-5.

Martel, C., and Iacono-Marziano, G., 2015, Timescales of bubble coalescence, outgassing, and foam collapse in decompressed rhyolitic melts: Earth and Planetary Science Letters, v. 412, p. 173-185, doi:10.1016/j.epsl.2014.12.010.

Marti, J., Soriano, C., and Dingwell, D.B., 1999, Tube pumices as strain markers of the ductile-brittle transition during magma fragmentation: Nature, v. 402, p. 650-653, doi:10.1038/45219.

McBirney, A.R., and Murase, T., 1970, Factors governing the formation of pyroclastic rocks: Bulletin Volcanologique, v. 34, p. 372-384, doi:10.1007/BF02596762.

Mueller, S., Scheu, B., Spieler, O., and Dingwell, D.B., 2008, Permeability control on magma fragmentation: Geology, v. 36, p. 399-402, doi:10.1130/G24605A.1.

Okumura, S., Nakamura, M., Tsuchiyama, A., Nakano, T., and Uesugi, K., 2008, Evolution of bubble microstructure in sheared rhyolite: Formation of a channellike bubble network: Journal of Geophysical Research: Solid Earth, v. 113, p. 118, doi:10.1029/2007JB005362.

Okumura, S., Nakamura, M., Uesugi, K., Nakano, T., and Fujioka, T., 2013, Coupled effect of magma degassing and rheology on silicic volcanism: Earth and Planetary Science Letters, v. 362, p. 163-170, doi:10.1016/j.epsl.2012.11.056.

Parmigiani, A., Faroughi, S., Huber, C., Bachmann, O., and Su, Y., 2016, Bubble accumulation and its role in the evolution of magma reservoirs in the upper crust: Nature, v. 532, p. 492-495, doi:10.1038/nature17401.

Perugini, D., Speziali, A., Caricchi, L., and Kueppers, U., 2011, Application of fractal fragmentation theory to natural pyroclastic deposits: Insights into volcanic explosivity of the Valentano scoria cone (Italy): Journal of Volcanology and Geothermal Research, v. 202, p. 200-210, doi:10.1016/j.jvolgeores.2011.02.008.

Ruprecht, P., and Bachmann, O., 2010, Pre-eruptive reheating during magma mixing at Quizapu volcano and the implications for the explosiveness of silicic arc 
volcanoes: Geology, v. 38, p. 919-922, doi:10.1130/G31110.1.

Rust, A.C., and Cashman, K.V., 2004, Permeability of vesicular silicic magma: Inertial and hysteresis effects: Earth and Planetary Science Letters, v. 228, p. 93-107, doi:10.1016/j.eps1.2004.09.025.

Sahagian, D., 1999, Magma fragmentation in eruptions: Nature, v. 402, p. 589-591, doi:10.1038/45099.

Schipper, C.I., Castro, J.M., Tuffen, H., James, M.R., and How, P., 2013, Shallow vent architecture during hybrid explosive-effusive activity at Cordón Caulle (Chile, 2011-12): Evidence from direct observations and pyroclast textures: Journal of Volcanology and Geothermal Research, v. 262, p. 25-37, doi:10.1016/j.jvolgeores.2013.06.005.

Shea, T., 2017, Bubble nucleation in magmas: A dominantly heterogeneous process? Journal of Volcanology and Geothermal Research, v. 343, p. 155-170, doi:10.1016/j.jvolgeores.2017.06.025.

Silver, L.A., Ihinger, P.D., and Stolper, E., 1990, The influence of bulk composition on the speciation of water in silicate glasses: Contributions to Mineralogy and Petrology, v. 104, p. 142-162, doi:10.1007/BF00306439.

Sparks, R.S.J., 1978, The dynamics of bubble formation and growth in magmas: A review and analysis: Journal of Volcanology and Geothermal Research, v. 3, p. 137, doi:10.1016/0377-0273(78)90002-1.

Sparks, S.R.J., Sigurdsson, H., and Wilson, L., 1977, Magma mixing: A mechanism for triggering acid explosive eruptions: Nature, v. 267, p. 315-318, doi: $10.1038 / 267315 \mathrm{a} 0$.

Spieler, O., Kennedy, B., Kueppers, U., Dingwell, D.B., Scheu, B., and Taddeucci, J., 2004, The fragmentation threshold of pyroclastic rocks: Earth and Planetary Science Letters, v. 226, p. 139-148, doi:10.1016/j.eps1.2004.07.016.

Stolper, E., 1982a, The speciation of water in silicate melts: Geochimica et Cosmochimica Acta, v. 46, p. 2609-2620, doi:10.1016/0016-7037(82)90381-7.

Stolper, E., 1982b, Water in silicate glasses: An infrared spectroscopic study: Contributions to Mineralogy and Petrology, v. 81, p. 1-17, doi:10.1007/BF00371154.

Sulpizio, R., Lucchi, F., Forni, F., Massaro, S., and Tranne, C., 2016, Unravelling the effusive-explosive transitions and the construction of a volcanic cone from geological data: The example of Monte dei Porri, Salina Island (Italy): Journal of Volcanology and Geothermal Research, v. 327, p. 1-22, doi:10.1016/j.jvolgeores.2016.06.024.

Suzuki, Y., Maeno, F., Nagai, M., Shibutani, H., Shimizu, S., and Nakada, S., 2018, Conduit processes during the climactic phase of the Shinmoe-dake 2011 eruption (Japan): Insights into intermittent explosive activity and transition in eruption style of andesitic magma: Elsevier B.V, v. 358, 87-104 p., doi:10.1016/j.jvolgeores.2018.02.008. 
Vasseur, J., Wadsworth, F.B., Lavallée, Y., Hess, K.U., and Dingwell, D.B., 2013, Volcanic sintering: Timescales of viscous densification and strength recovery: Geophysical Research Letters, v. 40, p. 5658-5664, doi:10.1002/2013GL058105.

Webb, S.L., and Dingwell, D.B., 1990, The onset of non-Newtonian rheology of silicate melts - A fiber elongation study: Physics and Chemistry of Minerals, v. 17, p. 125132, doi:10.1007/BF00199663.

Williamson, B.J., Di Muro, A., Horwell, C.J., Spieler, O., and Llewellin, E.W., 2010, Injection of vesicular magma into an andesitic dome at the effusive-explosive transition: Earth and Planetary Science Letters, v. 295, p. 83-90, doi:10.1016/j.eps1.2010.03.027.

Witcher, T.A., 2017, Relax or Fail: How silicate melts respond to stress: LudwigMaximilians Universität München.

Woods, A.W., and Koyaguchi, T., 1994, Transitions between explosive and effusive eruptions of silicic magmas: Nature, v. 370, p. 641-644, doi:10.1038/370641a0.

Zhang, Y., 1999, A criterion for the fragmentation of bubbly magma based on brittle failure theory: Nature, v. 402, p. 648-650, doi:10.1038/45210. 


\section{State of the art on the Paraná Etendeka}

\section{Magmatic Province}

"Life is not easy for any of us. But what of that? We must have perseverance and above all confidence in ourselves. We must believe that we are gifted for something and that this thing must be attained"-Marie Curie

(Polish physicist and chemist, pioneer in research on radioactivity. The first woman to win a Nobel Prize, the first (only woman) to win it twice, and the only to win it in two different sciences)

The Paraná Etendeka Magmatic Province (from now on referred to as PEMP) is the second largest continental igneous province on Earth in terms of areal extent, with a preserved volume estimated around $800,000 \mathrm{~km}^{3}$, from which at least $600,000 \mathrm{~km}^{3}$ occur at its South-American portion (figure5) (Peate et al., 1990; Frank et al., 2009).

The South-American portion of PEMP, named simply Paraná Magmatic Province (PMP), extends through the central-west, southeast and south of Brazil, southeast Paraguay, northern Argentina and eastern Uruguay (figure5) and piling up to $1700 \mathrm{~m}$ of volcanic rocks at its central axis. These rocks, grouped generically in the Serra Geral Group ${ }^{4}$, comprise tholeiitic basalts to andesites and subordinated amounts of chemically related dacites and rhyolites. According to Nardy (1995) the basaltic pile can reach thicknesses greater than $1000 \mathrm{~m}$, with individual flows as thick as $90 \mathrm{~m}$, while the silicic sequences do not exceed $400 \mathrm{~m}$, with individual flows up to $80 \mathrm{~m}$. Dyke swarms and sills are also related to this magmatism, extending through $650 \mathrm{~km}$ straight up from the southeast to south Brazil. The dykes are predominantly mafic (tholeiitic diabase) and present NE-SW, NW-SE and N-S trends, corresponding respectively to the Santos-Rio de Janeiro (northern), Ponta Grossa (central) and Florianópolis (southern) dyke swarms, which is interpreted by some authors as a triple junction related to the opening of the South Atlantic Ocean (Herz, 1977; Coutinho, 2008).

\footnotetext{
${ }^{4}$ The Serra Geral Group corresponds to the outcropping volcanic rocks in the Brazilian portion of the Province. At the other South American countries, these rocks are grouped in the Arapey and Cuaró Formations (Uruguay), Alto Paraná (Paraguay) and Serra Geral and Curuzú Cuatiá (Argentina). However, many authors use the term "Serra Geral" to designate the entire volcanic occurrence of the South American portion of PMPE.
} 
Covering a more restricted area $\left(\sim 0.08 \times 10^{6} \mathrm{~km}^{2}\right.$ versus $1.2 \times 10^{6} \mathrm{~km}^{2}$ at PMP, according to Peate et al., 1992), the African side of the Province, named Etendeka Magmatic Province (EMP), extends mainly through the northwest of Namibia ${ }^{5}$ (figure5). Basaltic lavas occur as sequences with up to $400 \mathrm{~m}$ in thickness, while the whole pack of silicic rocks, interpreted as rheoignimbrites, havean estimated thickness around 400-500 m. The EMP is divided into two domains: (i) the "coastal domain" where both basaltic and silicic rocks occur, dipping towards east and south, and strongly affected by normal faults; (ii) the "inland domain" where exclusively horizontal basaltic flows are preserved (Ewart, 2004). The province also comprises sills, dyke swarms and a range of annular subvolcanic intrusions grouped in the Damaraland (Martin et al., 1960; Milner et al., 1995b) and Messum Complexes (Ewart et al., 1998a, 2002).

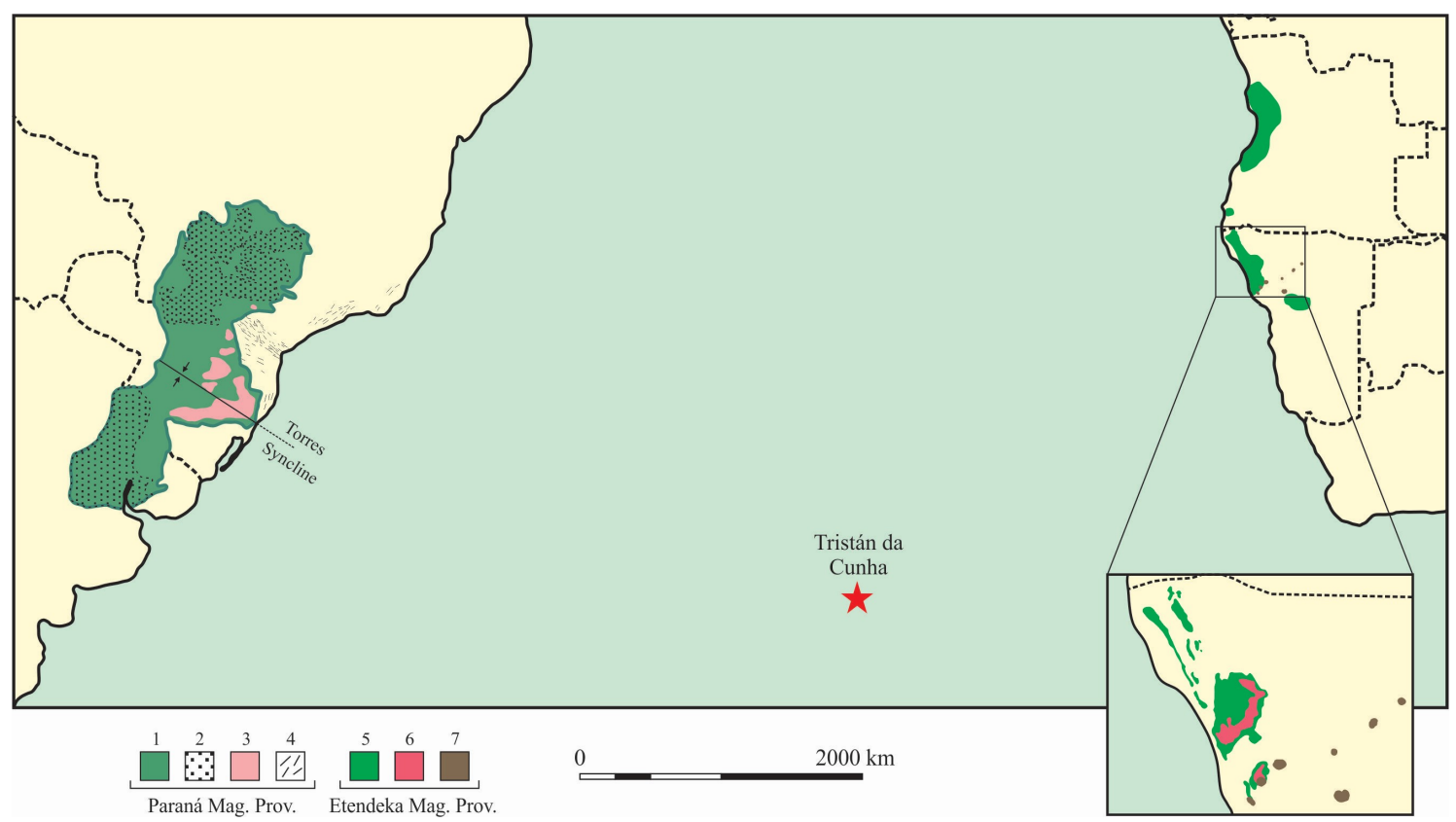

Figure 5: Paraná-Etendeka Magmatic Province, with the current geometry of the South American (Paraná Magmatic Province) and African (Etendeka Magmatic Province) portions. Compiled from Peate et al. (1992, 1999), Rossetti et al. (2014), and Ewart et al. (2004). 1: Basalts from PMP; 2: post-magmatic sedimentary rocks (Gr. Bauru) from PMP; 3: Intermediate and silicic volcanic rocks (andesites, dacites and rhyolites) from PMP; 4: PMP dikes swarms Santos-Rio de Janeiro (trend N40-50E) at the north, Ponta Grossa arc (trend N40-50W) in the central region,and Florianopolis (trend N15-25E) at the south; 5: Basalts from PME; 6: Silicic rocks (latites and quartz latites) from PME; 7: Annular subvolcanic intrusions from the Damaraland and Messum Complexes.

The volcanic rocks from PEMP were deposited over Juro-Cretaceous aeolian sandstones from Botucatu and Etjo Formations, which are part of the intracratonic

\footnotetext{
${ }^{5}$ Corresponds to the Etendeka Magmatic Province sensu stricto, since volcanic and subvolcanic rocks of the same age and, possibly related to the province, also occur in South Africa and Angola.
} 
Paraná (South America) and Huab (Africa) basins, respectively. The division and classification of the rocks from PEMP is based on chemical criteria proposed by Bellieni et al. (1984a) and Mantovani et al. (1985) for the basalts and later extended to the related silicic rocks. Two main groups of rocks are defined: high-Ti rocks present $\mathrm{TiO}_{2}$ contents higher than $2 \%$ and higher incompatible elements (e.g. Ba, $\mathrm{La}, \mathrm{Ce}, \mathrm{Ze}$ and $\mathrm{Sr}$ ) with lower $\mathrm{Rb}$, Th and $\mathrm{U}$ contents relative to the low-Ti rocks, which present $\mathrm{TiO}_{2}<$ $2 \%$ wt. Although intercalations between high and low-Ti rocks may occur locally in the Province, a general trend of geographical splitting between chemical types can be approximated, with high-Ti rocks predominating in the north, while the low-Ti sequences prevail in the central and southern regions (figure6).

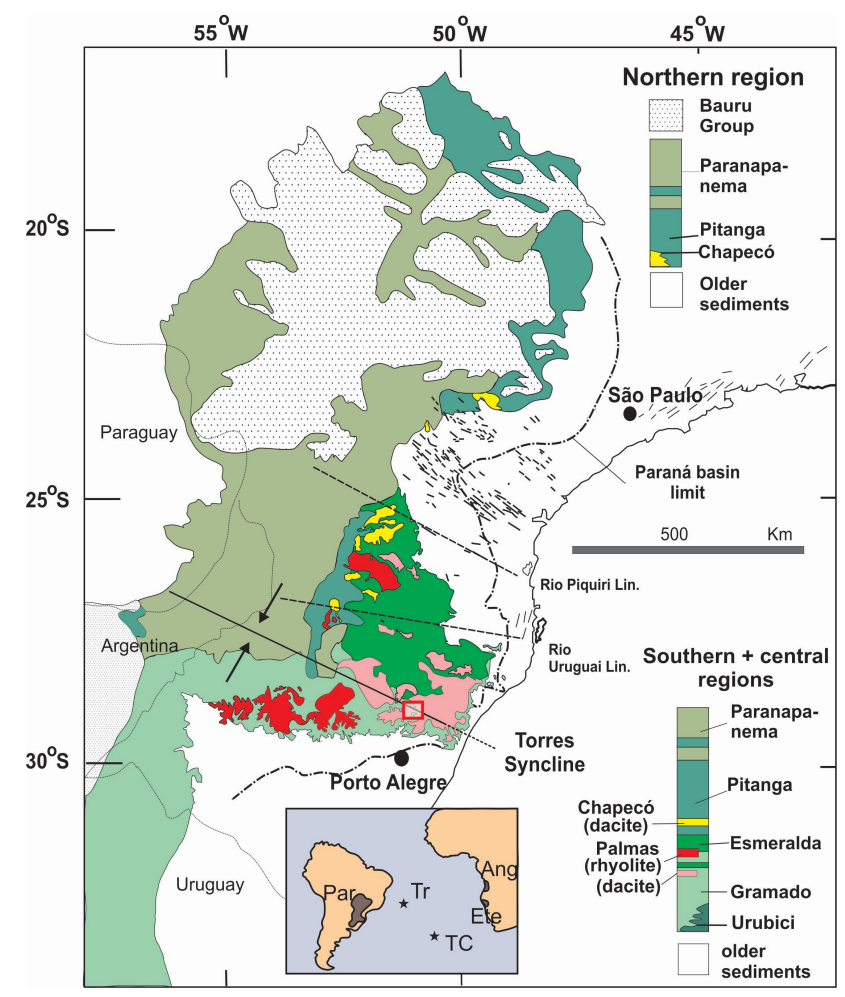

Figure 6: Geological map of the Paraná Magmatic Province, showing the geographic and stratigraphic distribution of the high-Ti and low-Ti basaltic rocks and related silicic rocks. The occurrence of dike swarms (Santos-Rio de Janeiro, Ponta Grossa and Florianópolis), the main tectonic lineaments (Rio Piquiri and Rio Uruguai) and the Torres Syncline axis are also indicated. The location of the study area is indicated by the red square. Modified from Janasi et al. (2011).

Regarding the PMP rocks, six main basaltic magma types have been recognized by (Peate et al., 1990): (i) Pitanga, Paranapanema and Ribeira (in order of decreasing Ti and P contents) correspond to high-Ti basalts from septentrional PMP; (ii) Gramado and Esmeralda, low-Ti basalts predominating in the south; and (iii) Urubici, high-Ti high-Sr basalts with restrict occurrence, interlayered to the Gramado basalts at the south PMP. 


\section{State of the Art on the Paraná Etendeka Magmatic Province}

The Pitanga basalts crop out at the $\mathrm{N}$ and $\mathrm{NE}$ of the Province, and have $\mathrm{SiO}_{2}$ contents ranging from $47 \%$ e $52 \%$ and $\mathrm{TiO}_{2}>2.8 \%$, while the Paranapanema basalts prevail at NW and central PMP, overlapping or intercalated with Pitanga basalts, with $\mathrm{SiO}_{2}$ contents from $48 \%$ to $53 \%$ and $\mathrm{TiO}_{2}$ from $1.7 \%$ to $3.2 \%$ (Peate et al., 1992; Garland et al., 1995). The Urubici basalts show $\mathrm{SiO}_{2}>49 \%$ and $\mathrm{TiO}_{2}>3.3 \%$ (Peate et al., 1992). The Ribeira basalts constitute a peculiar case; with restricted occurrence in the northern and central PMP and underlying the Pitanga basalts, these rocks were initially defined by Petrini et al. (1987) as low-Ti basalts but later reclassified by Peate (1997) as high-Ti basalts due to its affinity and continuum of composition with the Paranapanema-Pitanga association. The Ribeira basalts present $\mathrm{SiO}_{2}$ contents varying from $49 \%$ to $52 \%$ and $\mathrm{TiO}_{2}$ between $1.5 \%$ and $2.3 \%$ (Peate et al., 1992). With respect to the trace element contents, these basalts are similar to the Paranapanema basalts, being characterized by the high Ti/Y ratios typical of the Ti-high basalts. Due to the chemical similarities (even with respect to the ${ }^{87} \mathrm{Sr} /{ }^{86} \mathrm{Sr}$ and ${ }^{143} \mathrm{Nd} /{ }^{144} \mathrm{Nd}$ initial isotopic ratios), trace elements and $\mathrm{Fe}_{2} \mathrm{O}_{3}$ contents are the best discriminators for the high-Ti basalts (table 1; Bellieni et al., 1984b; Peate et al., 1992; Garland et al., 1995). The Urubici basalts have the higher contents of incompatible trace elements (specially $\mathrm{Sr}$ and $\mathrm{Ba}$ ) and $\mathrm{Ti} / \mathrm{Y}$ rations, while the lower contents are shown by the Parananema basalts. The higher $\mathrm{Fe}_{2} \mathrm{O}_{3}$ contents, on the other hand, are typical from Pitanga and Paranapanema basalts. Typical ${ }^{87} \mathrm{Sr}^{86} \mathrm{Sr}_{(\mathrm{i})}$ ratios are also presented in table 1.

Table 1: Compilation of geochemical data for the high-Ti basalts; contents of some major, minor and trace elements and isotopic ratios for discrimination of magma types.(1) Peate et al. (1997); (2) Peate et al. (1992); (3) Garland et al. (1995).

\begin{tabular}{|l|c|c|c|c|}
\hline & Ribeira & Pitanga & Paranapanema & Urubici \\
\hline $\mathbf{S i O}_{2}$ & $49-52 \%$ & $47-52 \%$ & $48-53 \%$ & $>49 \%$ \\
\hline $\mathbf{T i O}_{\mathbf{2}}$ & $1.5-2.3 \%^{(1)}$ & $>2.9 \%^{(1)}$ & $1.7-3.2 \%^{(1)}$ & $>3.3 \%{ }^{(1)}$ \\
\hline $\mathbf{F e}_{2} \mathbf{O}_{\mathbf{3}}$ & $12-16^{(1)}{ }^{(2)}$ & $12.5-18 \%^{(2)}$ & $12.5-17 \%^{(2)}$ & $<14.5 \%^{(2)}$ \\
\hline $\mathbf{S r}$ & $200-375^{(2)}$ & $210-625^{(3)}$ & $173-428^{(3)}$ & $550-1050^{(3)}$ \\
\hline $\mathbf{B a}$ & $200-600^{(2)}$ & $280-740^{(3)}$ & $116-489^{(3)}$ & $435-1135^{(3)}$ \\
\hline $\mathbf{T i} / \mathbf{Y}$ & $>310^{(1)}$ & $>350^{(1)}$ & $>330^{(1)}$ & $>500^{(1)}$ \\
\hline $\mathbf{T b} / \mathbf{Y b}$ & - & $0.35-0.44^{(3)}$ & $0.28-0.38^{(3)}$ & $0.47-0.56^{(3)}$ \\
\hline${ }^{87} \mathbf{S r} /{ }^{\mathbf{8 6}} \mathbf{S r}_{(\mathbf{i})}$ & $0.7055-0.7060$ & $0.7055-0.7062$ & $0.7055-0.7068$ & $0.7048-0.7066$ \\
& $(1)$ & $(3)$ & $(3)$ & $(3)$ \\
\hline
\end{tabular}




\section{Chapter III}

State of the Art on the Paraná Etendeka Magmatic Province

The Esmeralda basalts, restricted to the Brazilian PMP, cover the Gramado basalts. From a chemical point of view, comparing to the high-Ti, these low-Ti basalts present, in addition to the aforementioned lower $\mathrm{TiO}_{2}$ contents, lower $\mathrm{Ti} / \mathrm{Y}$ ratios (table 2, figure7; Peate, 1997). The Gramado basalts have $\mathrm{SiO}_{2}$ contents ranging between $49 \%$ and $60 \%, \mathrm{TiO}_{2}$ contents from $0.7 \%$ to $2 \%$ and are richer in incompatible elements (LILE) in relation to the HFSE and light-REE when compared to the Esmeralda basalts, which present higher $\mathrm{Ti} / \mathrm{Zr}$ ratios and $\mathrm{SiO}_{2}$ contents from $48 \%$ to $55 \%$ and $\mathrm{TiO}_{2}$ contents between $1.1 \%$ and $2.3 \%$. Isotopic data related to the low-Ti basalts are also presented in table 2 .

Table 2: Compilation of geochemical data for the low-Ti basalts (Peate et al., 1997; Peate et al., 1992); contents of some major, minor elements and trace elements and isotopic ratios for the discrimination of magma types.

\begin{tabular}{|l|c|c|}
\hline & Gramado & Esmeralda \\
\hline $\mathbf{S i O}_{\mathbf{2}}$ & $49-60 \%$ & $48-55 \%$ \\
\hline $\mathbf{T i O}_{\mathbf{2}}$ & $0.7-2.0 \%$ & $1.1-2.3 \%$ \\
\hline $\mathbf{F e}_{\mathbf{2}} \mathbf{O}_{\mathbf{3}}$ & $9-16 \%$ & $12-17 \%$ \\
\hline $\mathbf{S r}$ & $140-400$ & $<250$ \\
\hline $\mathbf{B a}$ & $100-700$ & $90-400$ \\
\hline $\mathbf{T i} / \mathbf{Y}$ & $<310$ & $<310$ \\
\hline${ }^{87} \mathbf{S r} /{ }^{86} \mathbf{S r}_{(i)}$ & $0.7075-0.7167$ & $0.7046-0.7086$ \\
\hline
\end{tabular}

For a better visualization of the chemical differences shown by the basaltic magma types from PMP a compilation of diagrams is presented in figure7.
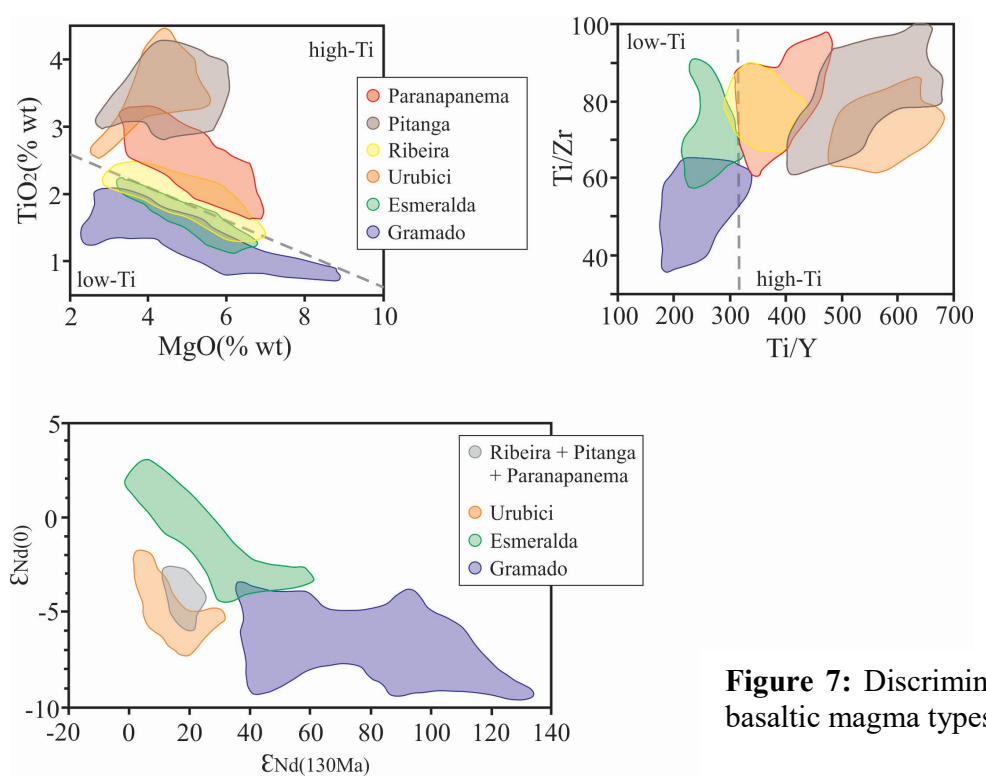

Figure 7: Discriminatory geochemical diagrams for the basaltic magma types from the PMP. 
Bellieni et al. (1984, 1986) and Piccirillo et al. (1987) recognized two main groups of silicic rocks in the PMP: (i) Chapecó type, with high-Ti dacites later divided into the Guarapuava, Ourinhos and Tamarana subtypes (Peate et al., 1992; Garland et al., 1995; Nardy et al., 2008); and (ii) Palmas type, comprising low-Ti dacites and rhyolites divided into the Santa Maria, Caxias do Sul, Anita Garibaldi, Clevelândia, Jacuí and Barros Cassal subtypes (Peate et al., 1992; Nardy et al., 2008; Polo and Janasi, 2014).

The Chapecó dacites are typically porphyritic, with phenocrysts of plagioclase, augite, pigeonite and magnetite making up to $25 \%$ of the volume of the rock. The matrix is composed by quartz, $\mathrm{k}$-feldspar, plagioclase, clinopyroxene and magnetite in a glassy groundmass. The Guarapuava subtype occurs at the base of the high-Ti sequence, presents the rocks with the highest phenocrysts contents, lower $\mathrm{SiO}_{2}(\sim 64-66 \%), \mathrm{Rb}, \mathrm{Pb}$ and Th contents and ${ }^{87} \mathrm{Sr} /{ }^{86} \mathrm{Sr}$ initial ratios (0.7055-0.7060; Peate et al., 1992; Garland et al., 1995). The Ourinhos rocks have lower contents of phenocrysts and the higher $\mathrm{SiO}_{2}$ $(\sim 65-68 \%)$ and ${ }^{87} \mathrm{Sr} /{ }^{86} \mathrm{Sr}$ initial ratios (0.7076-0.7080; Peate et al., 1992; Garland et al., 1995). Tamarana is geochemically intermediate between the Guarapuava and Ourinhos subtypes (Nardy et al., 2008).

The low-Ti silicic rocks (Palmas type) make up around $80 \%$ of the silicic volcanism volume, and crop out as an extensive E-W plateau at Southern Brazil or as sparse occurrences at the central region, where are overlapped by the high-Ti silicic rocks (Nardy, 1995). The Palmas rocks are hypohyaline, aphanitic to fine-grained, rarely porphyritic, when present low phenocrysts contents $(<10 \%$ vol. comprising plagioclase, clinopiroxene, magnetite and apatite), differing from the Chapecó crystals also for their smaller sizes. Chemically these rocks are characterized by a depletion in incompatible elements and enrichment in LILE when compared to the Chapecó rocks. Six subtypes have been defined based on $\mathrm{TiO}_{2}$ and $\mathrm{P}_{2} \mathrm{O}_{5}$ contents. The Santa Maria and Clevelândia subtypes comprise rhyolites with the highest $\mathrm{SiO}_{2}(69 \%-72 \%$ wt) and lowest $\mathrm{TiO}_{2}(\leq 0.87 \%)$ contents, with $\mathrm{P}_{2} \mathrm{O}_{5}$ lower than $0.2 \%$ or ranging between 0.21 and $0.23 \%$ respectively. These rocks also present the higher trace elements contents (with the exception of Sr, P and Ti). The Caxias do Sul, Jacuí and Anita Garibaldi subtypes comprise dacites with $\mathrm{SiO}_{2}$ contents ranging between $\sim 63 \%$ and $69 \%$ wt., $\mathrm{TiO}_{2}$ higher than $0,90 \%$ and $\mathrm{P}_{2} \mathrm{O}_{5}$ varying from $0.25 \%$ to $0.28 \%$, from $0.28 \%$ to $0.31 \%$ and from 
$0.32 \%$ to $0.36 \%$ respectively. These members also present higher $\mathrm{La} / \mathrm{Lu}$ rations compared to those with lower $\mathrm{TiO}_{2}$. The Palmas Formation is also characterized by high ${ }^{87} \mathrm{Sr} /{ }^{86} \mathrm{Sr}$ initial ratios (e.g. Santa Maria member $=0.7217$ to 0.7274; Caxias do Sul member $=0.7137$ to 0.7264 ; Anita Garibaldi member $=0.7137$ to 0.7147 ; Garland et al., 1995; Guimarães, 2014), which show a positive correlation with the $\mathrm{SiO}_{2}$ contents. A compilation of the geochemical (major elements) and isotopic data for discriminating the silicic volcanic rocks from the PMP is presented in figures 8 and 9.
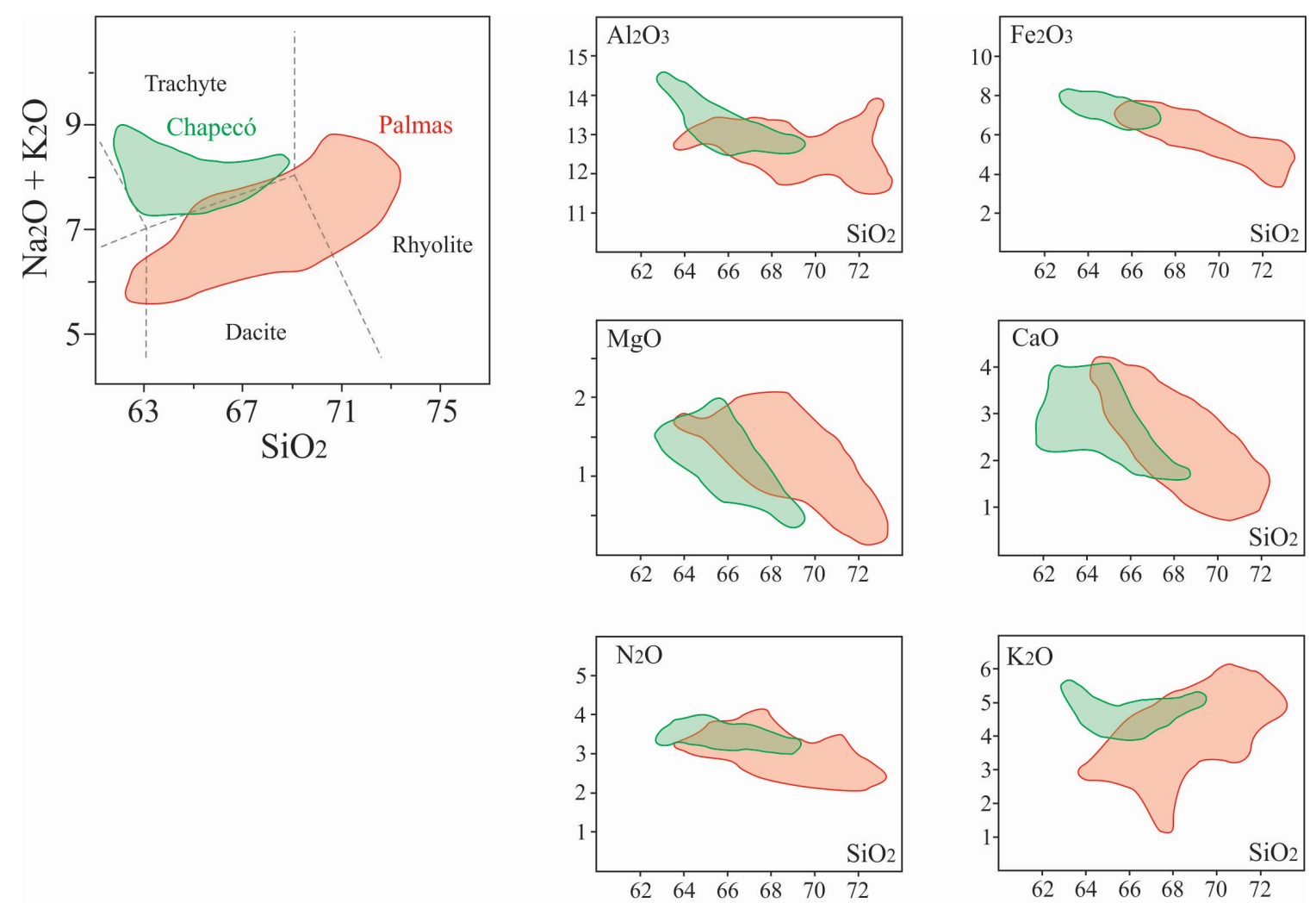

Figure 8: TAS (above) and Harker diagrams (at right) for the silicic rocks from PMP: Chapecó (green) and Palmas (red) Formation. Adapted from Nardy et al. (2008).
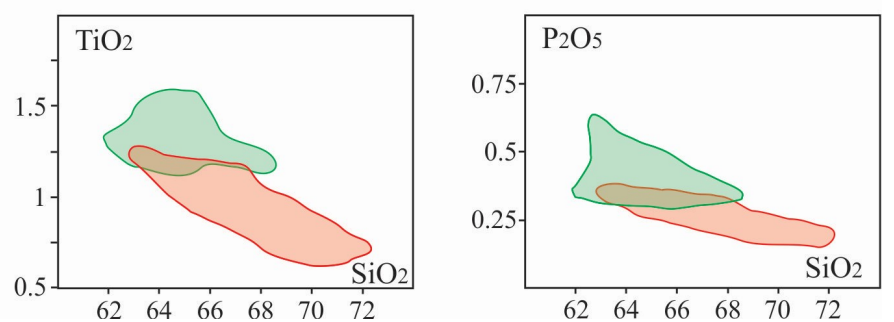

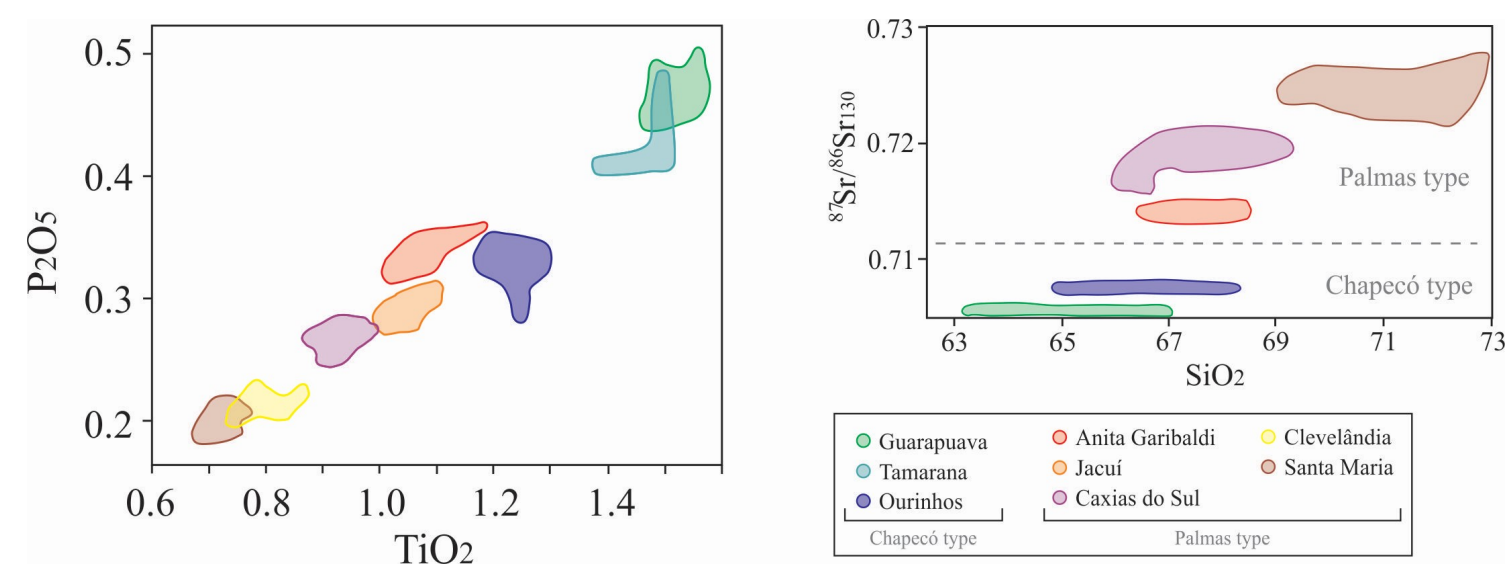

Figure 9: Discriminatory diagrams for the high-Ti (Chapecó type) and low-Ti (Palmas type) silicic rocks based on the $\mathrm{P} 2 \mathrm{O} 5$ and $\mathrm{TiO} 2$ contents and the $87 \mathrm{Sr} / 86 \mathrm{Sr}(\mathrm{i})$ isotopic ratio. Adapted from Nardy et al. (2008) and Garland et al. (1995).

Geochemical magma types of basalts and silicic rocks were also identified in the EMP; although occupying a small area, magma types correlated to most of those from PMP, in fact, a greater variety of magma types has been described, reflecting a more variable contents of elements such as $\mathrm{Mg}$ and $\mathrm{Si}$. High-Ti basalts from EMP are grouped into the Khumib type (geochemically correlated to the Urubici type from PMP; Marsh et al., 2001), which predominates at the Northern portion of this province, while the low-Ti basalts are divided into the types: Tafelberg (the most voluminous, correlated to the Gramado basalts from PMP; Erlank et al., 1984; Ewart et al., 1998 a, b), Albin, Horingbaai (diabase dykes with E-MORB signature), "regional diabases", Huab (Duncan et al., 1989; Erlank et al., 1984; Marsh et al., 2001), Kuidas (Duncan et al., 1988; Marsh et al., 2001), Esmeralda (correlated to the homonymous type from PMP; Peate et al., 1992; Marsh et al., 2001) and Tafelkop (Milner and leRoex, 1996; Ewart et al., 1998b; Peate, 1997).

The silicic volcanic rocks are described as quartz latites and latites $\left(>65 \% \mathrm{SiO}_{2}\right.$ and $<65 \% \mathrm{SiO}_{2}$, respectively; according to Erlank et al., 1984 and Marsh et al., 2001) whose compositional variety allowed the definition of 16 subtypes (Milner et al., 1995a; Marsh et al., 2001). The high-Ti domain comprises the subtypes Nadas, Sechomib, Hoarusib, Sarusas (correspondent to the Guarapuava subtype from PMP), Ventura, Khoraseb (equivalent to the Ourinhos subtype from PMP), Naudé, Elliot and Nil Desperandum - the first three comprising latites and the others consisting of quartz latites. The low-Ti domain encompasses the subtypes Hoas, Gobobosed, Springbok, 
Wereldsend, Grootberg, Beacon and Fria (equivalent to the Santa Maria subtype, from PMP), with the first consisting of latites and the others of quartz latites. The subtypes Nil Desperandum and Fria are "geographic exceptions": the former corresponds to highTi rocks cropping out in the Southern portion of the PME, while the latter is characterized by low-Ti rocks intercalated to the high-Ti rocks at the Northern portion of the PME.

The definition of a detailed stratigraphy and the regional stratigraphic correlation (e.g. Peate et al., 1990; 1992; Milner et al., 1995; and others) allowed not only to relate both sides of the Province, but also to infer a migration of the volcanism from the South to the North during the PEMP evolution.

Geochronological studies have been extensively employed aiming to infer the ages and lifespan of the PEMP volcanism. Dating based on K-Ar method suggested a long period of magmatic activity, from 138 to $115 \mathrm{Ma}$ (Rocha Campos et al., 1988). Later, the development of the ${ }^{40} \mathrm{Ar} /{ }^{39} \mathrm{Ar}$ technique during the 1990 's led to extensive and more precise dating of these rocks; Renne et al. (1992) and Hawkesworth et al. (1992) proposed a short lifespan (1 M.yr.), around $133 \pm 1$ Ma for the PEMP volcanic activity, while Turner et al. (1994) and Stewart et al. (1996), using the ${ }^{40} \mathrm{Ar} /{ }^{39} \mathrm{Ar}$ totalfusion technique suggested a 10 M.yr. long volcanism, from $\sim 138$ to $128 \mathrm{Ma}$. These results, however, were considered unreliable by Thiede and Vasconcelos (2010), which reanalyzed a group of 3 samples - that correspond to the whole $10 \mathrm{M}$.yr. range defined in the former works - through the ${ }^{40} \mathrm{Ar} /{ }^{39} \mathrm{Ar}$ stepped heating method and obtained ages around 134.2 to 134.8 Ma for all samples. Nevertheless, the small amount of data from the effusive occurrences at the Brazilian portion of the Province hinders a more precise definition of the time span for the entire extent of PMPE volcanism. Younger ages (ca. 133.6 - 131.5 Ma) were obtained for the high-Ti basalts by Ernesto et al. (1999) and are consistent with the hypothesis of volcanism migration from the South to the North based on the stratigraphic relations. Still talking about ${ }^{40} \mathrm{Ar} /{ }^{39} \mathrm{Ar}$ stepped heating method, ages between 131.4 e $129.2( \pm 0.4)$ Ma were obtained for the Ponta Grossa dykes swarm by Renne et al. (1996a). Data from TIMS U-Pb in zircon and baddeleyite were obtained by Janasi et al. (2011) for the Chapecó dacites (Ourinhos subtype), which corresponds to the base of the high-Ti sequence, and suggest a short period of high-Ti volcanism (around $3 \mathrm{Ma}$ ), starting at $134.3 \pm 0.8 \mathrm{Ma}$. Pinto et al. (2011) obtained ages around $135 \pm 2.3$ and $137.3 \pm 1.8 \mathrm{Ma}$ for the same dacites through $\mathrm{U}-\mathrm{Pb}$ in zircon by 
SHRIMP. Ongoing studies by Rocha et al. (submit) involving CA-ID-TIMS in zircon present the most consistent results for the low-Ti PMP silicic volcanism, with ages around 133.6 $\pm 0.1 \mathrm{Ma}$, suggesting a very short volcanism lifespan (about $100 \mathrm{ka}$ ). Regarding the EMP, data from volcanic samples from Namibia and Angola and from intrusions from Namibia were obtained by Renne et al. (1996 b) through the ${ }^{40} \mathrm{Ar} /{ }^{39} \mathrm{Ar}$ stepped heating method, and indicate ages between 132.3 and $131.7( \pm 0.7)$ Ma for the volcanic rocks and around 129.3 $\pm 0.7 \mathrm{Ma}$ for the syenites of the Messum Intrusive Complex. To summarize, a wide variation of ages based on different methods has been reported for the PEMP; the most consistent results suggest an initial phase of low-Ti volcanism, aged around 134 Ma with a short lifespan ( 1 M.yr.) and predominant in the southern portion of the Province, followed by slightly younger high-Ti volcanism, with a longer duration ( $\sim 3$ M.yr.), predominant in the north of the province, thus supporting the migration of the volcanism from the south to the north initially proposed based on stratigraphic relations.

\section{III.1. Origin and evolution of the PEMP magmatism}

The origin of large igneous provinces (LIPs) such as PEMP is still a topic of intense debate; some models propose an origin related to lithospheric stretching and consequent decompression (e.g. White and McKenzie, 1989; Lustrino, 2005), while others suggest a relation with mantle plumes (e.g. Morgan, 1971; Richards et al., 1989; Campbell and Griffiths, 1990; Campbell, 2005). Similarly, many different hypothesis have been proposed for the PEMP origin. Regarding the basaltic magmatism, some authors point the chemical similarities between EMP and Tristán da Cunha as an evidence for the mantle plume hypothesis (e.g. Gibson et al., 1995, 1999; Ewart et al., 1998b; Marsh et al., 2001; Ewart, 2004). Ewart (2004), however, recognize the existence of compositional variations and argues that they would possibly be related to the contribution of other materials such as asthenospheric mantle or residual subducted slabs preserved in the transition zone. Some authors, on the other hand, consider the role of the plume exclusively as a source of heating to melt lithospheric material, giving rise to a huge amount of magma (e.g. Peate, 1997; Peate et al., 1999; Marques et al., 1999). Ernesto et al. (2002) question the plume hypothesis for the generation of the PEMP magmatism based on paleomagnetic reconstructions that revealed a mismatch between the "real" position and the "ideal" position of the Tristán da Cunha plume. 
Geochemical evidence, especially $\mathrm{Sr}-\mathrm{Nd}-\mathrm{Pb}$ isotopes and trace elements contents, have been used to contest the mantle plume-originated models. The isotope signature of the PEMP rocks, characterized by high ${ }^{87} \mathrm{Sr} /{ }^{86} \mathrm{Sr}$ and negative ENd, suggest an enriched source such as the lithospheric mantle (e.g. Hawkesworth et al., 1988; Peate, 1997; Ernesto et al., 2002). The low Nb/La ratios of the PEMP basalts are distinct from the high ratios observed on the Tristán da Cunha plume and oceanic basalts, also favoring a lithospheric mantle-sourced magmatism (Peate et al., 1990; Peate, 1997; Hawkesworth et al., 1992; Turner and Hawkesworth, 1995). Indeed, the only restrict PEMP occurrences chemically compatible with the Tristán da Cunha plume are the Tafelkop basalts and some alkaline intrusions on the African portion of the Province (Milner and leRoex, 1996). The Esmeralda basalts from PMP, although volumetrically significant and with isotopic signature suggestive of asthenospheric mantle, are not compatible with the Tristán da Cunha magmatism (Peate, 1997). This author argues that the geochemical variations observed at the PEMP may be a consequence of different melting degrees of the source (e.g. the lithospheric mantle heterogeneities along with different degrees of melting at different depths would be responsible for the chemical variations of the high $\mathrm{Ti}$ rocks). On the other hand, also based on geochemical signature, the work of Gibson et al., (1995) suggests that the asthenospheric mantle is the predominant source of these basaltic magmas and may have mixed with volatileand K-rich mafic melts from the subcontinental lithospheric mantle and subsequently experienced some assimilation of upper crustal material. More recently, the work of Rocha-Júnior et al., (2012) is worth mentioning; based on $\mathrm{Os}, \mathrm{Sr}, \mathrm{Nd}$ and $\mathrm{Pb}$ isotope the authors suggest primary basaltic melts deriving from metasomatized asthenospheric mantle posteriorly mixed with enriched mantle components.

Like the basalts, the origin of the silicic magmas from the PEMP also carries some uncertainties. Based on isotopic data $\left({ }^{87} \mathrm{Sr} /{ }^{86} \mathrm{Sr}\right.$ and $\left.\delta^{18} \mathrm{O}\right)$ some authors related the silicic magmatism to crustal anatexis (e.g. Cordani et al., 1980; Hawkesworth et al., 1988; Harris et al., 1990; Bellieni et al., 1986). However, its clear stratigraphic and chemical relation to the basalts led some authors to propose a genetic link between them (Bellieni et al., 1984a; Piccirillo et al., 1987; Garland et al., 1995). With respect to the Chapecó type (high-Ti), the most accepted model proposes melting of basaltic underplates at the lower crust, without crustal contamination, as suggested by the low $\mathrm{Rb} / \mathrm{Sr}$ ratios (Bellieni et al., 1986; Garland et al., 1995). Freitas (2009), however, claims that these rocks underwent slight crustal contamination and could have been originated 
by AFC processes (assimilation and fractional crystallization). Regarding the Palmas type, the most accepted models indicate an origin related to the fractional crystallization of low-Ti basalts (Gramado member, mainly) together with crustal assimilation (Mantovani et al., 1985; Peate, 1997; Garlad et al., 1995; Guimarães, 2014). Guimarães (2014) suggests (based on geochemical modeling for the Santa Maria rhyolites) that the genesis of the low-Ti silicic magmatism involved assimilation of crustal material with similar compositions to the Neoproterozoic granites from the South PMP.

\section{III.2. The eruptive style of the PEMP silicic volcanism - explosive or effusive?}

Although subordinate to the related basaltic magmatism, the silicic volcanic rocks from the PMPE have the highest preserved volume among the continental flood basalt provinces $\left(\sim 15,300 \mathrm{~km}^{3}\right.$ in the Brazilian portion of the Province; Nardy et al., 2008). These rocks are chemically diverse $\left(63 \%<\mathrm{SiO}_{2}<72 \%\right)$ and occur in different stratigraphic positions marking important changes during the evolution of the province (e.g. Peate, 1997; Janasi et al., 2011). An aspect of particular interest is their eruptive style, a subject still quite controversial in the literature.

The current and most known model proposes an explosive volcanism, through pyroclastic flows (Bellieni et al., 1986; Milner and Ewart, 1989; Milner et al., 1992, 1995; Marsh et al., 2001; Ewart et al., 2002; Bryan et al., 2010) expelled from central conduits whose manifestation at the surface corresponds to the Messum complex (Bryan et al., 2010). Bryan et al. (2010) defined the coverage areas of these flows, considered by them as of great magnitude (figure 10). 

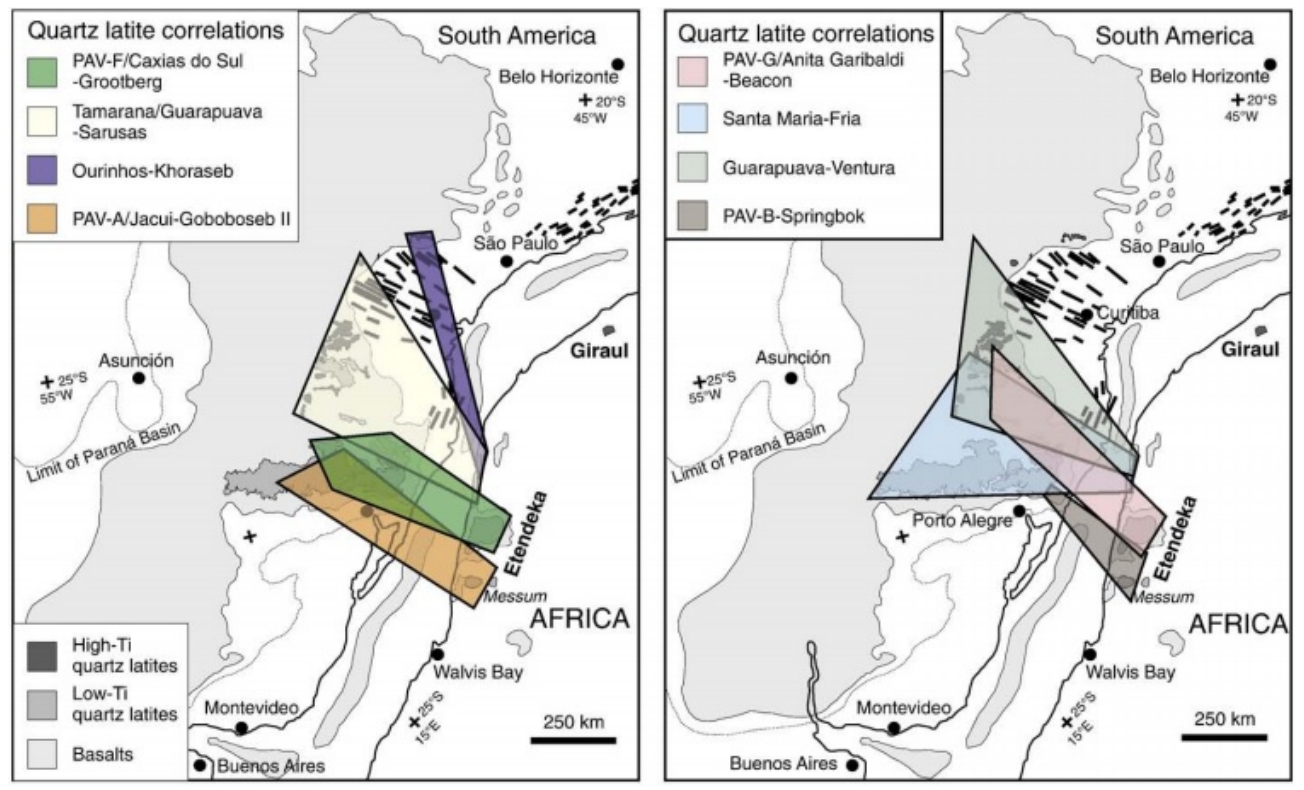

Figure 10: Representative polygons of the minimum distribution areas of the different pyroclastic flows resulting from explosive volcanic events of great magnitude in the Etendeka region, according to Bryan et al. (2010).

The lack of pyroclastic features, with the exception of those described by Milner et al. (1992) is attributed by these authors to the intensewelding of the deposits, which is a consequence to the high temperatures characteristic of these magmas. For these reasons, the above mentioned authors have classified all the silicic volcanic rocks as rheoignimbrites (lava-like deposits).

Although studies developed on the Brazilian portion of the PMPE propose based on geological mapping, petrography, geochemistry, geothermobarometry and anisotropy of magnetic susceptibility - the predominance of effusive silicic volcanism (e.g. Janasi et al., 2007; Luchetti, 2010; Nardy et al., 2011; Guimarães, 2014; Polo and Janasi, 2014; Guimarães et al., 2018b; Lima et al., 2018), some authors still endorse the occurrence of a significant volume of pyroclastic deposits (high-grade ignimbrites originated by low explosivity events; Luchetti et al., 2018 a, b; Guimarães et al., 2018 b). Evidence of effusive structures such as lava-domes and coulees is presented by Polo (2014), Guimarães (2014), Polo and Janasi (2014) and Guimarães et al. (2018a) and local feeding systems characterized by fissural conduits is suggested by Guimarães et al. (2018b), Lima et al. (2018) and Simões et al. (2018). 


\section{References}

Bellieni, G., Brotzu, P., Comin-chiaramonti, P., Ernesto, M., Melfi, A., Pacca, I.G., and Piccirillo, E.M., 1984a, Flood basalt to rhyolite suites in the Southern Paraná plateau (Brazil): Palaeomagnetism, petrogenesis and geodynamic implications: Journal of Petrology, v. 25, p. 579-618, doi:10.1093/petrology/25.3.579.

Bellieni, G., Comin-chiaramonti, P., Marques, L.S., Melfi, A.J., Nardy, A.J.R., Papatrechas, C., Piccirillo, E.M., Roisenberg, A., and Stolfa, D., 1986, Petrogenetic aspects of acid and basaltic lavas from the paraná plateau (Brazil): Geological, mineralogical and petrochemical relationships: Journal of Petrology, v. 27, p. 915-944, doi:10.1093/petrology/27.4.915.

Bellieni, G., Comin-Chiaramonti, P., Marques, L.S., Melfi, A.J., Piccirillo, E.M., Nardy, A.J.R., and Roisenberg, A., 1984b, High- and Low-TiO2 flood basalts from the Parana plateau (Brazil): petrology and geochemical aspects bearing on their mantle origin: Neus Jahrbuch Miner. Abh, v. 150, p. 273-306.

Bryan, S.E., Peate, I.U., Peate, D.W., Self, S., Jerram, D.A., Mawby, M.R., Marsh, J.S.G., and Miller, J.A., 2010, The largest volcanic eruptions on Earth: EarthScience Reviews, v. 102, p. 207-229, doi:10.1016/j.earscirev.2010.07.001.

Campbell, I.H., 2005, Large Igneous Provinces and the Mantle Plume Hypothesis: Elements, v. 1, p. 265-269, doi:10.2113/gselements.1.5.265.

Campbell, I.H., and Griffiths, R.W., 1990, Implications of mantle plume structure for the evolution of flood basalts: Earth and Planetary Science Letters, v. 99, p. 79-93, doi:10.1016/0012-821X(90)90072-6.

Ernesto, M., Marques, L.S., Piccirillo, E.M., Molina, E.C., Ussami, N., CominChiaramonti, P., and Bellieni, G., 2002, Paraná Magmatic Province-Tristan da Cunha plume system: Fixed versus mobile plume, petrogenetic considerations and alternative heat sources: Journal of Volcanology and Geothermal Research, v. 118, p. 15-36, doi:10.1016/S0377-0273(02)00248-2.

Ernesto, M., Raposo, M.I.B., Marques, L.S., Renne, P.R., Diogo, L.A., and De Min, A., 1999, Paleomagnetism, geochemistry and 40Ar/39Ar dating of the North-eastern Parana Magmatic Province: Tectonic implications: Journal of Geodynamics, v. 28, p. 321-340, doi:10.1016/S0264-3707(99)00013-7.

Ewart, A., 2004, Petrology and Geochemistry of Early Cretaceous Bimodal Continental Flood Volcanism of the NW Etendeka, Namibia. Part 1: Introduction, Mafic Lavas and Re-evaluation of Mantle Source Components: Journal of Petrology, v. 45, p. 59-105, doi:10.1093/petrology/egg083.

Ewart, A., Milner, S.C., Armstrong, R.A., and Duncan, A.R., 1998a, Etendeka Volcanism of the Goboboseb Mountains and Messum Igneous Complex, Namibia. Part I: Geochemical Evidence of Early Cretaceous Tristan Plume Melts and the Role of Crustal Contamination in the Paraná-Etendeka CFB: Journal of Petrology, v. 39, p. 227-253, doi:10.1093/petroj/39.2.191.

Ewart, A., Milner, S.C., Armstrong, R.A., and Duncan, A.R., 1998b, Etendeka Volcanism of the Goboboseb Mountains and Messum Igneous Complex, Namibia. 


\section{Chapter III \\ State of the Art on the Paraná Etendeka Magmatic Province}

Part II: Voluminous Quartz Latite Volcanism of the Awahab Magma System: Journal of Petrology, v. 39, p. 227-253, doi:10.1093/petroj/39.2.227.

Ewart, A., Milner, S.C., Duncan, A.R., and Bailey, M., 2002, The Cretaceous Messum igneous complex, S.W. Etendeka, Namibia: Reinterpretation in terms of a downsag-cauldron subsidence model: Journal of Volcanology and Geothermal Research, v. 114, p. 251-273, doi:10.1016/S0377-0273(01)00266-9.

Frank, H.T., Gomes, M.E.B., and Formoso, M.L.L., 2009, Review of the areal extent and the volume of the Serra Geral Formation, paraná Basin, South America: Pesquisas em Geociencias, v. 36, p. 49-57, http://www.scopus.com/inward/record.url?eid=2-s2.073949129716\&partnerID=40\&md5=e73384fle $685 \mathrm{~d} 0 \mathrm{f} 8 \mathrm{cc} 0082482 \mathrm{c} 4621 \mathrm{fa}$.

Freitas, V.A., 2009, A geração de magmas ácidos na Província Magmática Paraná, região de Piraju-Ourinhos (SP): uma contribuição da geoquímica isotópica e de elementos traço em rochas e minerais: Universidade de São Paulo.

Garland, F., Hawkesworth, C.J., and Mantovani, M.S.M., 1995, Description and Petrogenesis of the Paraná Rhyolites, Southern Brazil: Journal of Petrology, v. 36, p. 1193-1227.

Gibson, S.A., Thompson, R.N., Dickin, A.P., and Leonardos, O.H., 1995, High-Ti and low-Ti mafic potassic magmas: Key to plume-lithosphere interactions and continental flood-basalt genesis: Earth and Planetary Science Letters, v. 136, p. 149-165, doi:10.1016/0012-821X(95)00179-G.

Gibson, S.A., Thompson, R.N., Leonardos, O.H., Dickin, A.P., and Mitchell, J.G., 1999, The limited extent of plume-lithosphere interactions during continental flood-basalt genesis: Geochemical evidence from Cretaceous magmatism in southern Brazil: Contributions to Mineralogy and Petrology, v. 137, p. 147-169, doi: $10.1007 / \mathrm{s} 004100050588$.

Guimarães, L.F., 2014, Características físicas e químicas e modelo eruptivo para os riolitos tipo Santa Maria ( Província Magmática Paraná ) na região de Gramado Xavier, RS: Universidade de São Paulo.

Guimarães, L.F., De Campos, C.P., Janasi, V.A., Lima, E.F., and Dingwell, D.B., 2018a, Flow and fragmentation patterns in the silicic feeder system and related deposits in the Paraná-Etendeka Magmatic Province, São Marcos, South Brazil: Journal of Volcanology and Geothermal Research, doi:10.1016/J.JVOLGEORES.2018.03.021.

Guimarães, L.F., Raposo, M.I.B., Janasi, V.A., Cañón-Tapia, E., and Polo, L.A., 2018b, An AMS study of different silicic units from the southern Paraná-Etendeka Magmatic Province in Brazil: Implications for the identification of flow directions and local sources: Journal of Volcanology and Geothermal Research, v. 355, p. 304-318, doi:10.1016/j.jvolgeores.2017.11.014.

Hawkesworth, C.J., Gallagher, K., Kelley, S., Mantovani, M., Paete, D.W., Regelous, M., and Rogers, N.W., 1992, Paraná magmatism and the opening of the South Atlantic, in Storey, B.C., Alabaster, T., and Pankhurst, R.J. eds., Magmatism and the Causes of Continental Break-up, Geological Society Special Publication, p. 221-240. 
Hawkesworth, C.J., Mantovani, M.S.M., and Peate, D.W., 1988a, Lithosphere remobilization during Panamá CFB Magmatism: Journal of Petrology, p. 205-223, doi:10.1093/petrology/Special.

Hawkesworth, C., Mantovani, M., and Peate, D.W., 1988b, Lithosphere remobilization during parana cfb magmatism: Journal of Petrology, v. Special_Vo, p. 205-223, doi:10.1093/petrology/Special_Volume.1.205.

Herz, N., 1977, Timing of spreading in the South Atlantic: Information from Brazilian alkalic rocks: Bulletin of the Geological Society of America, v. 88, p. 101-112, doi:10.1130/0016-7606(1977)88<101:TOSITS >2.0.CO;2.

Janasi, V.A., Freitas, V.A., and Heaman, L.H., 2011, The onset of flood basalt volcanism, Northern Paraná Basin, Brazil: A precise U-Pb baddeleyite/zircon age for a Chapecó-type dacite: Earth and Planetary Science Letters, v. 302, p. 147-153, doi:10.1016/j.eps1.2010.12.005.

Janasi, V.A., Montanheiro, T.J., Freitas, V.A., Reis, P.M., Negri, F.A., and Dantas, F.A., 2007, Geology, petrography and geochemistry of the acid volcanism of the Parana magmatic province in the Piraju-Ourinhos region, SE Brazil: Revista Brasileira de Geociencias, v. 37, p. 745-759.

Lima, E.F., Waichel, B.L., Rossetti, L.M.M., Sommer, C.A., and Simões, M.S., 2018, Feeder systems of acidic lava flows from the Paraná-Etendeka Igneous Province in southern Brazil and their implications for eruption style: Journal of South American Earth Sciences, v. 81, p. 1-9, doi:10.1016/J.JSAMES.2017.11.004.

Luchetti, A.C.F., 2010, Aspectos vulcanológicos dos traquidacitos da Região de PirajuOurinhos (SP): Universidade de São Paulo, doi:10.1017/CBO9781107415324.004.

Luchetti, A.C.F., Gravley, D.M., Gualda, G.A.R., and Nardy, A.J.R., 2018a, Textural evidence for high-grade ignimbrites formed by low-explosivity eruptions, Paraná Magmatic Province, southern Brazil: Journal of Volcanology and Geothermal Research, v. 355, p. 87-97, doi:10.1016/J.JVOLGEORES.2017.04.012.

Luchetti, A.C.F., Nardy, A.J.R., and Madeira, J., 2018b, Silicic, high- to extremely high-grade ignimbrites and associated deposits from the Paraná Magmatic Province, southern Brazil: Journal of Volcanology and Geothermal Research, v. 355, p. 270-286, doi:10.1016/J.JVOLGEORES.2017.11.010.

Lustrino, M., 2005, How the delamination and detachment of lower crust can influence basaltic magmatism: Earth-Science Reviews, v. 72, p. 21-38, doi:10.1016/j.earscirev.2005.03.004.

Mantovani, M.S.M., Marques, L.S., De Sousa, M.A., Civetta, L., Atalla, L., and Innocenti, F., 1985, Trace Element and Strontium Isotope Constraints on the Origin and Evolution of Paraná Continental Flood Basalts of Santa Catarina State (Southern Brazil): Journal of Petrology, v. 26, p. 187-209, doi:10.1093/petrology/26.1.187.

Marques, L.S., Dupré, B., and Piccirillo, E.M., 1999, Mantle source compositions of the Parana Magmatic Province (southern Brazil): Evidence from trace element and Sr$\mathrm{Nd}-\mathrm{Pb}$ isotope geochemistry: Journal of Geodynamics, v. 28, p. 439-458, doi:10.1016/S0264-3707(99)00020-4. 


\section{Chapter III \\ State of the Art on the Paraná Etendeka Magmatic Province}

Marsh, J.S., Ewart, A., Milner, S.C., Duncan, A.R., and Miller, R.M., 2001, The Etendeka Igneous Province: Magma types and their stratigraphic distribution with implications for the evolution of the Paraná-Etendeka flood basalt province: Bulletin of Volcanology, v. 62, p. 464-486, doi:10.1007/s004450000115.

Martin, H., Mathias, M., Simpson, E.S.W., 1960, The Damaraland sub-volcanic ring complexes in South West Africa: Rep. Int. Geol. Congr. XXI Session, 13: 156-174.

Milner, S.C., Duncan, A.R., and Ewart, A., 1992, Quartz latite rheoignimbrite flows of the Etendeka Formation, north-western Namibia: Bulletin of Volcanology, v. 54, p. 200-219, doi:10.1007/BF00278389.

Milner, S.C., Duncan, A.R., Whittingham, A.M., and Ewart, A., 1995a, Trans-Atlantic correlation of eruptive sequences and individual silicic volcanic units within the Paran??-Etendeka igneous province: Journal of Volcanology and Geothermal Research, v. 69, p. 137-157, doi:10.1016/0377-0273(95)00040-2.

Milner, S.C., and Ewart, A., 1989, The geology of the Goboboseb Mountain volcanics and their relationship to the Messum Complex , Namibia: Commun. Geol. Surv. Namib, v. 5, p. 33-42.

Milner, S.C., and le Roex, A.P., 1996, Isotope characteristics of the Okenyenya igneous complex, northwestern Namibia: constraints on the composition of the early Tristan plume and the origin of the EM 1 mantle component: Earth and Planetary Science Letters, v. 141, p. 277-291, doi:http://dx.doi.org/10.1016/0012821X(96)00074-X.

Milner, S.C., Le Roex, A.P., and O’Connor, J.M., 1995b, Age of Mesozoic igneous rocks in northwestern Namibia, and their relationship to continental breakup: Journal - Geological Society (London), v. 152, p. 97-104, doi:10.1144/gsjgs.152.1.0097.

Morgan, W.J., 1971, Convection Plumes in the Lower Mantle: Nature, v. 230, p. 42-43.

Nardy, A.J.R., 1995, Geologia e petrologia do vulcanismo mesozóico da região central da Bacia do Paraná: Tese de doutoramento, Instituto de Geociências e Ciências Exatas - UNESP, Rio Claro (SP), 315 p.

Nardy, A.J.R., Machado, F.B., and Oliveira, M.A.F., 2008, As rochas vulcânicas mesozóicas ácidas da Bacia do Paraná: litoestratigrafia e considerações geoquímico-estratigráficas: Brazilian Journal of Geology, v. 38, p. 178-195, http://rbg.sbgeo.org.br/index.php/rbg/article/view/1353.

Nardy, A.J.R., Rosa, M.C., Luchetti, A.C.F., Ferreira, M.L.C., Machado, F.B., and Oliveira, M.A.F., 2011, Parâmetros físicos pré-eruptivos do magmatismo ácido da província magmática do Paraná: Resultados preliminares: Geociencias, v. 30, p. $575-588$.

Peate, D.W., Hawkesworth, C.J., and Mantovani, M.S.M., 1992, Chemical stratigraphy of the Paraná lavas (South America): classification of magma types and their spatial distribution: Bulletin of Volcanology, v. 55, p. 119-139.

Peate, D.W., Hawkesworth, C.J., Mantovani, M.S.M., Rogers, N.W., and Turner, S.P., 1999, Petrogenesis and Stratigraphy of the High-Ti/Y Urubici Magma Type in the 


\section{Chapter III \\ State of the Art on the Paraná Etendeka Magmatic Province}

Paraná Flood Basalt Province and Implications for the Nature of 'Dupal'-Type Mantle in the South Atlantic Region: Journal of Petrology, v. 40, p. 451-473, doi:10.1093/petroj/40.3.451.

Peate, D.W., Hawkesworth, C.J., Mantovani, M.S.M., and Shukowsky, W., 1990, Mantle plumes and flood-basalt stratigraphy in the Paraná, South America: Geology, v. 18, p. 1223-1226, doi:10.1130/00917613(1990)018<1223:MPAFBS>2.3.CO;2.

Petrini, R., Civetta, L., Piccirillo, E.M., Bellieni, G., Comin-chiaramonti, P., Marques, L.S., and Melfi, A.J., 1987, Mantle heterogeneity and crustal contamination in the genesis of low-ti continental flood basalts from the paranà plateau (brazil): Sr-nd isotope and geochemical evidence: Journal of Petrology, v. 28, p. 701-726, doi:10.1093/petrology/28.4.701.

Piccirillo, E.M., Raposo, M.I.B., Melfi, A.J., Comin-chiaramonti, P., Bellieni, G., Cordani, U.G., and Kawashita, K., 1987, Bimodal fissural volcanic suites from the Paraná Basin (Brazil): K-Ar age, Sr-isotopes and geochemistry: Geochimica Brasiliensis, v. 1, p. 53-69.

Pinto, V.M., Hartmann, L.A., Santos, J.O.S., McNaughton, N.J., and Wildner, W., 2011, Zircon U-Pb geochronology from the Paraná bimodal volcanic province support a brief eruptive cycle at $\sim 135 \mathrm{Ma}$ : Chemical Geology, v. 281, p. 93-102, doi:10.1016/j.chemgeo.2010.11.031.

Polo, L.A., 2014, O vulcanismo ácido da Província Magmática Paraná-Etendeka na região de Gramado Xavier, RS: estratigrafia, estruturas, petrogênese e modelo eruptivo: Universidade de São Paulo, 340 p., doi:10.11606/T.44.2014.tde26092014-101255.

Polo, L.A., and Janasi, V.A., 2014, Volcanic stratigraphy of intermediate to acidic rocks in the southern Paraná Magmatic Province, Brazil: Geologia USP - Serie Cientifica, v. 14, p. 83-100, doi:10.5327/Z1519-874X201400020005.

Renne, P.R., Deckart, K., Ernesto, M., Feraud, G., and Piccirillo, E.M., 1996a, Age of the Ponta Grossa dike swarm (Brazil), and implications to Parana flood volcanism: Earth and Planetary Science Letters, v. 144, p. 199-211, doi:10.1016/0012821X(96)00155-0.

Renne, P.R., Ernesto, M., Pacca, I.G., Coe, R.S., Glen, J.M., Prévot, M., and Perrin, M., 1992, The Age of Paraná Flood Volcanism, Rifting of Gondwanaland, and the Jurassic-Cretaceous Boundary: Science, v. 258, p. 975-979.

Renne, P.R., Glen, J.M., Milner, S.C., and Duncan, A.R., 1996b, Age of Etendeka flood volcanism and associated intrusions in southwestern Africa: Geology, v. 24, p. 659-662, doi:10.1130/0091-7613(1996)024<0659:AOEFVA>2.3.CO;2.

Richards, M.A., Duncan, R.A., and Courtillot, V.E., 1989, Flood Basalts and Hot Spot Tracks: Plumes Heads and Tails: Science, v. 246, p. 103-107.

Rocha-Júnior, E.R.V., Puchtel, I.S., Marques, L.S., Walker, R.J., Machado, F.B., Nardy, A.J.R., Babinski, M., and Figueiredo, A.M.G., 2012, Re-Os isotope and highly siderophile element systematics of the Paraná continental flood basalts (Brazil): Earth and Planetary Science Letters, v. 337-338, p. 164-173, 
doi:10.1016/J.EPSL.2012.04.050.

Simões, M.S., Lima, E.F., Sommer, C.A., and Rossetti, L.M.M., 2018, Structures and lithofacies of inferred silicic conduits in the Paraná-Etendeka LIP, southernmost Brazil: Journal of Volcanology and Geothermal Research, v. 355, p. 319-336, doi:10.1016/J.JVOLGEORES.2017.12.013.

Stewart, K., Turner, S., Kelley, S., Hawkesworth, C., Kirstein, L., and Mantovani, M., 1996, 3-D 40Ar-39Ar Geochronology in the Paraná Flood Basalt Province: Mineralogical Magazine, v. 143, p. 95-109, doi:10.1180/minmag.1994.58A.2.193.

Thiede, D.S., and Vasconcelos, P.M., 2010, Paraná flood basalts: Rapid extrusion hypothesis confirmed by new 40Ar/39Ar results: Geology, v. 38, p. 747-750, doi:10.1130/G30919.1.

Turner, S., and Hawkesworth, C.J., 1995, The nature of the sub-continental mantle: constraints from the major-element composition of continental flood basalts: Chemical Geology, v. 120, p. 295-314.

Turner, S., Regelous, M., Kelley, S., Hawkesworth, C., and Mantovani, M., 1994, Magmatism and continental break-up in the South Atlantic: high precision40Ar39Ar geochronology: Earth and Planetary Science Letters, v. 121, p. 333-348, doi:10.1016/0012-821X(94)90076-0.

Vianna Coutinho, J.M., 2008, Dyke swarms of the paraná triple junction, Southern Brazil: Geologia USP - Serie Cientifica, v. 8, p. 29-52.

White, R., and McKenzie, D., 1989, Magmatism at Rift Zones: The Generation of Volcanic Continental Margins and Flood Basalts: v. 94, p. 7685-7729. 


\section{Geology of the Studied Area}

"Don't let anyone rob you of your imagination, your creativity or your curiosity. It's your place in the world, it's your life. Go on and do all you can with it, and make it the life you want to live"-Mae Jemison

(North American engineer, physician and NASA astronaut - the first African American woman to travel in space)

The study region covers an area of about $225 \mathrm{~km}^{2}$ at the vicinities of São Marcos town, which is approximately $160 \mathrm{~km}$ to the north of the city of Porto Alegre (Rio Grande do Sul State) (figure 6). The existence of two large rivers (das Antas and São Marcos), two important highways (BR-116, RS-122) and access roads and many quarries favor the occurrence of good rock exposures in the region.

Considering the PMP context, the area belongs to the "low-Ti rocks" domain and is located in the central region of the Torres Syncline (TS), a regional concave structure with a NW-SE oriented major axis, which facilitated the accumulation and preservation of large volumes of silicic materials from the PMP. The complete stratigraphy preserved at the TS comprises, from the base to the top, (i) the Botucatu paleoerg, (ii) simple and compound pahoehoe basaltic flows,(iii) rubbly basaltic flows and (iv) silicic deposits (Waichel et al., 2012). Most of the works published in the region of the Torres syncline recognize the silicic deposits as lava domes (Lima et al., 2012) and lava flows (lobated and massive and extensive flows; Polo and Janasi, 2014; Polo et al., 2018; Simões et al., 2018; Cañón-Tapia and Raposo, 2018), although some recent studies suggest explosive deposits (high-grade ignimbrites) related to low energy explosive events (Luchetti et al., 2018a, 2018b).

In the study area, at the base of the outcropping sequence, along the valleys, aphanitic to finely phaneritic basalts crop out forming a profile with maximum thickness of ca. $400 \mathrm{~m}$ (figure 11). These basaltic rocks was recognized by Waichel et al., (2012) and Rossetti et al., (2014) as a sequence of pahoehoe and 'a 'a flows, and later reclassified by Rossetti et al., (2018) as rubbly flows. The dacites occurs at the top of the sequence, intruding and overlying the basaltic sequence (figure 11); the contact between the basaltic and the silicic sequences is located between elevations 620 and 680 meters a.s.l. The dacitic deposits were classified by Lima et al., (2012), Cañón-Tapia 
and Raposo, (2018) and Simões et al., (2018) as lava-domes and flows. Basaltic dykes occur intruding the whole sequence (figure 11).

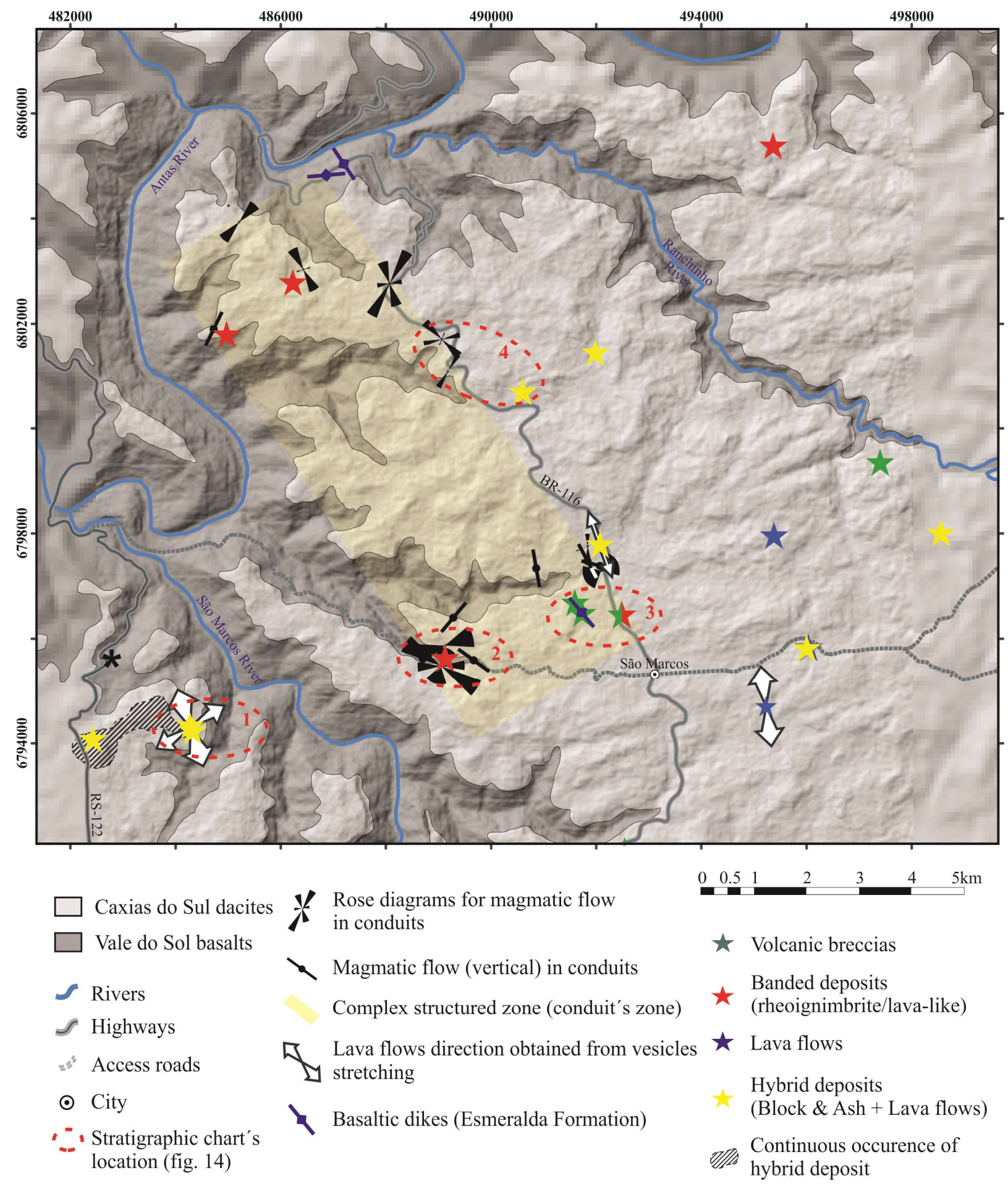

Figure 11: Geological map ofthe studied area in the vicinity ofSãoMarcos city, RioGrande do Sul/Brazil. The Caxias do Sul dacites (Palmas Formation) and Vale do Sol and Esmeraldabasalts belong to the SerraGeral Group. The rose diagramspresentmainly vertical foliation fromthefilament domains (section IV.2.3). Their sizes are related to the amount of obtained data - ranging from 2 to 12 measurements in each outcrop, totaling 58 measurements from which ca. $90 \%$ comprises dips varying between $80^{\circ}-90^{\circ}$ and $10 \%$ comprises dips varying between $50^{\circ}-75^{\circ}$. Single measurements of vertical foliation are represented by single black lines. White arrows indicate the range of lava flow 


\section{Chapter IV \\ Geology of the Studied Area}

directions based on vesicles stretching. The geographic location of the stratigraphic sections(presented in figure 14)is highlighted by red circles on the map.

\section{IV.1. The basaltic sequence}

The basaltic rocks belong to the Gramado type according to the definition by Peate et al. (1992), to the basic sequences II and III, according to Waichel et al.(2012), or to the Vale do Sol Formation according to Rossetti et al. (2018) (see Chapter VI for geochemical characterization). Here we will use the classification by Rossetti et al. (2018), since it is the most recent and proposes a standardization of the nomenclature used for PMP. These rocks build up a $\sim 400$ meters thick profile, with individual flows thickness ranging between 10 and 60 meters (avg. $\sim 35 \mathrm{~m}$ ). A type section was described along the BR-116 highway (figure 12) where at least 8 basaltic flows were recognized. These lavas present sheet-like tabular geometry and are constituted by (i) an aphanitic, massive to microvesiculated base followed by (ii) an aphanitic to finely phaneritic massive core (figure $12 \mathrm{~A}$ ), that transitions to to a (iii) vesiculated (figure $12 \mathrm{~B}$ ) and, finally, to a (iv) fragmented crust (figure $12 \mathrm{C}$ ) which characterizes rubbly flows. Horizontal joints are common at the base and core of the flows, and vertical columnar joints predominate at the cores. The vesiculation degree increases upwards together with vesicle size. The vesicles can reach up $5 \mathrm{~cm}$ and are mainly rounded to slightly stretched at the upper portions. The fragments in the rubbly crust are vesiculated, can reach up to $10 \mathrm{~cm}$ and are generally sub-rounded. The matrix between the clasts is constituted by secondary material (zeolites). Fragments were not observed in the first flow, as well as at the higher elevations of the section, where it was not possible to distinguish the limits between flows due to lack of continuity. Where the contact between flows can be observed the absence of sediments is noteworthy.

Basaltic dykes were also observed in the region and correspond to the Esmeralda Formation (figure $12 \mathrm{D}$; see Chapter VI for geochemical characterization). These dikes are oriented along NW-SE $\left(\mathrm{N}-320^{\circ}-326^{\circ}\right)$ and $\sim \mathrm{E}-\mathrm{W}\left(\mathrm{N} 85^{\circ}\right)$ trends (figure 11), with thicknesses varying from 0.3 to $1 \mathrm{~m}$. The basalts are typically aphanitic, with the exception of the finely phaneritic core of the $1 \mathrm{~m}$-thick dyke that intrudes the dacitic complex zone (see IV.2.3). 


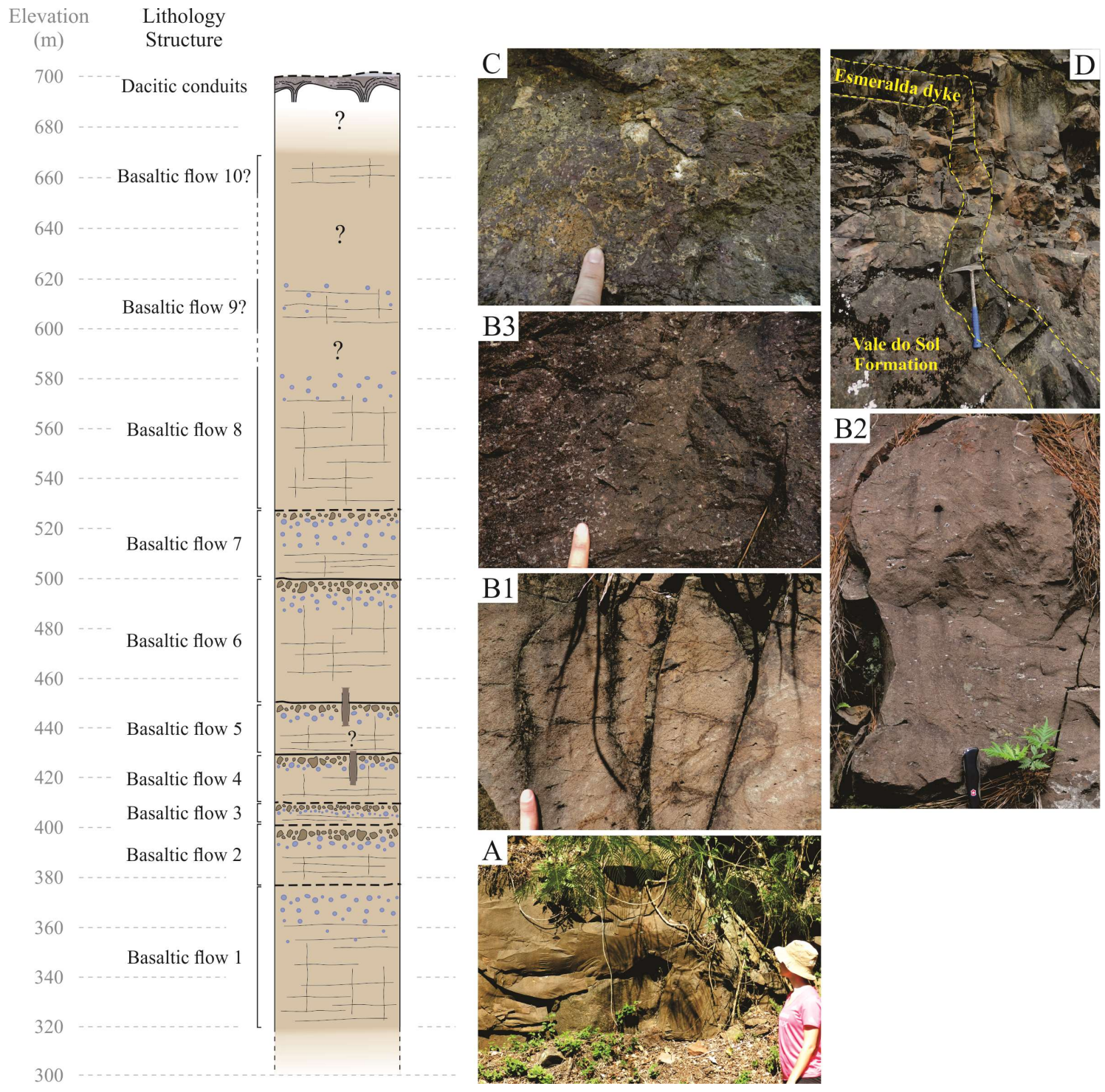

Figure 12:Stratigraphic section (left) for basalts from BR-116 profile - at least 8 flows were recognized based on their internal structure, comprising massive base and cores (A), with vesicles size and density increasing upwards (B1 to B3) transitioning to fragmented crust (C). Due to the lack of continuity, the whole structure was not observed at the higher elevations, hindering a precise delimitation of flow units. Basaltic dykes (Esmeralda Formation) of decimetric thickness, abrupt and irregular contact with the hosting basaltic flows (Vale do Sol Formation), were also observed (D).

\section{IV.1.1. Petrographic aspects}

Under the microscope the basalts range from hypo to holocrystalline, with equigranular to porphyritic texture. Hypocristalline rocks have a glassy matrix that can comprise up to $30 \%$ of its total volume, defining an intersertal texture (figure $13 \mathrm{~A}, \mathrm{C}$ ), and occurs mainly at the external portions (both base and top) of flows. This matrix is affected at variable degrees by recrystallization, when the groundmass turns into a quartz + feldspar intergrowth, defining a holocrystalline texture. Holocrystalline rocks 


\section{Chapter IV \\ Geology of the Studied Area}

are mainly found at the core of the flows (figure $13 \mathrm{~B}, \mathrm{D}, \mathrm{E}, \mathrm{F}$ and G). With high crystal contents (>70\% vol.) the basalts have plagioclase, clinopyroxene and magnetite as main constituents, while epidote/chlorite and quartz (+ feldspar) compose the accessory (post-magmatic) phases. Considering the internal structure of a single flow unit, it is evident that the outermost portions have finer grain-size and higher proportions of clinopyroxene in relation to plagioclase when compared to the core, where plagioclase with the lowest An contents (petrographically estimated) are observed. Plagioclase crystals alignment can be locally observed, especially at the inner portions of the flows (figure $13 \mathrm{D}$ and $\mathrm{E}$ ), and vesicles (filled mainly by quartz) become more common and bigger towards the upper portion of the flow, where they are usually stretched; vesicles with irregular shape define a diktytaxitic texture in some samples (figure $13 \mathrm{D}$ and E).

Plagioclase ranges between $30 \%$ and $55 \%$ vol. of the basalts, with euhedral to subhedral, commonly tabular crystals with medium size ranging between 0.1 and 0.2 mm (figure 13). Rapid cooling features such as swallowtail and hollow crystals can also occur, especially in the external portions of the flows. Microphenocrysts can show oscillatory zoning, reaching up to $1.5 \mathrm{~mm}$ (figure $13 \mathrm{C}$ ). Microlites are abundant in samples with higher volume of glassy matrix and have acicular morphologies. Labradorite $\left(\mathrm{An}_{50-65}\right)$ is the most common phase, although a few crystals of andesine $\left(\mathrm{An}_{40-50}\right)$ occur.

Clinopyroxene (augite) volumes range between $35 \%$ and $45 \%$ with subhedral to anhedral, granular crystals defining sub-ophitic and intergranular texture, and average sizes around $0.1 \mathrm{~mm}$ (figure 13). Microphenocrysts, although rare, can occur with up to $0.7 \mathrm{~mm}$ and are usually rimmed by magnetite microlites $(\sim 0.01 \mathrm{~mm})$ (figure $13 \mathrm{C}$ ). Clinopyronexe crystals are commonly altered to epidote/chlorite (figure $13 \mathrm{C}$ ).

Magnetite can reach up to $10 \%$ vol. and occurs as euhedral to subhedral crystals with up to $0.3 \mathrm{~mm}$, often included in clinopyroxenes (figure 13).

Regarding the basaltic dykes, the mineralogical content is basically the same, although plagioclases have lower anortite contents $\left(\mathrm{An}_{35-50}\right.$ - andesine). Additionally, the basaltic dykes presents a very characteristic texture with radial distribution of acicular to euhedric tabular (with high aspect ratios) plagioclase crystals and interstitial granular clinopyroxene (intergranular texture) (figure $13 \mathrm{~F}$ and $\mathrm{G}$ ). The thicker dyke has coarser crystals size and shows glomeroporphyritic texture with plagioclase $\left(\mathrm{An}_{>55}\right)$ and 
clinopyroxene phenocrysts occurs. Alignment of plagioclase crystals also could be locally recognized.
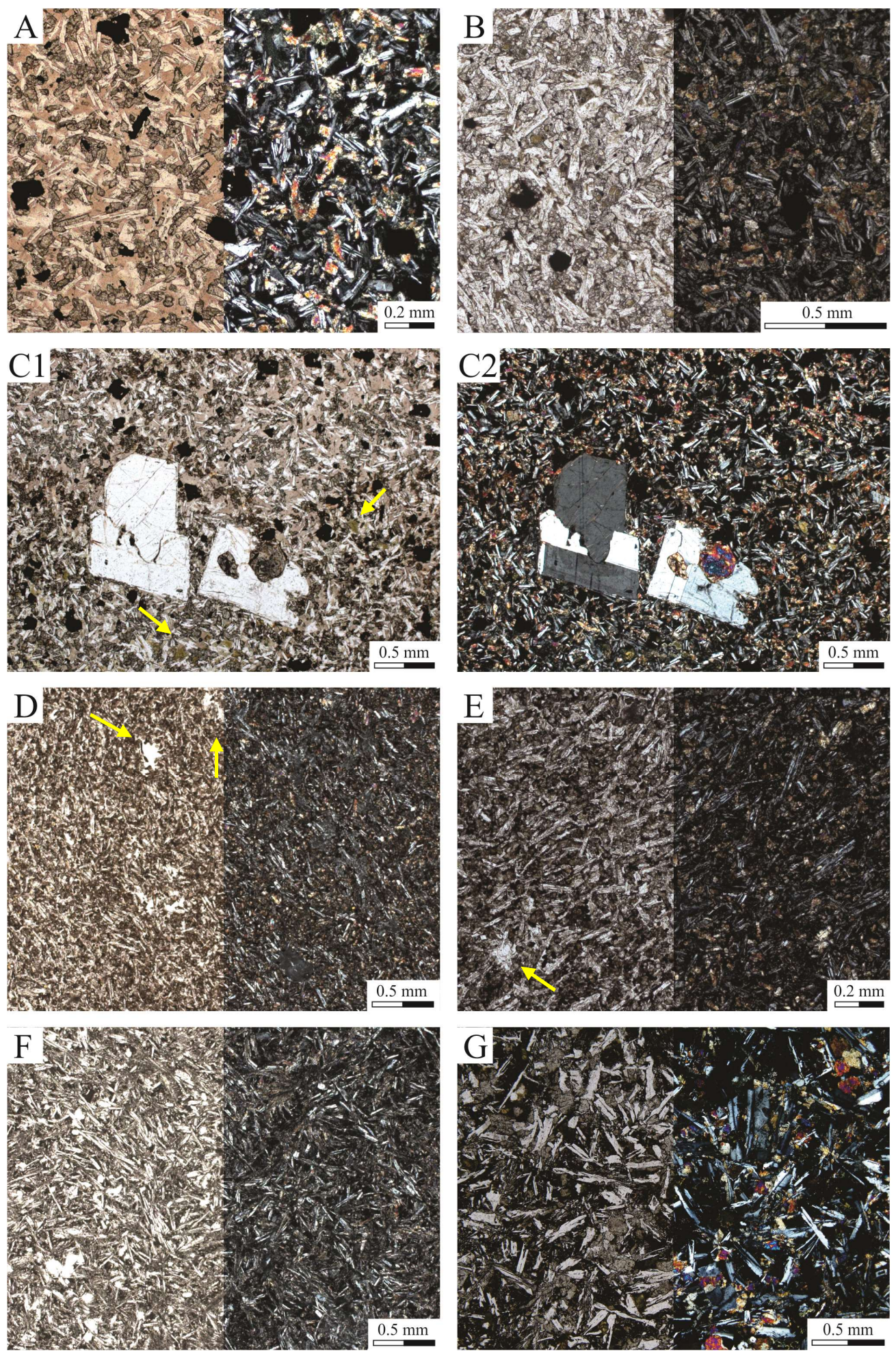

Figure 13: Petrographic aspects of basaltic samples. A) Parallel polarizers at left and crossed at right. Hypocrystalline basaltfrom the basal portion of the flow, with intersertal, intergranular and subophitic texture. Plagioclase crystals (euhedral to subhedral, tabular), clinopyroxene (tabular euhedral to subhedral, granular) and opaque (subhedral) minerals are dispersed in a vitreous matrix. B) Parallel polarizers at left and crossed at right. 


\section{Chapter IV \\ Geology of the Studied Area}

Holocrystalline basaltfrom the inner portion of the flow; the groundmass is composed by microlites of plagioclase, clinopyroxene and magnetite and, locally by quartz+k-feldspar from recrystallization of previously glassy material. $\mathrm{C} 1$ and C2) Parallel and crossed polarizers respectively. Hypocrystalline porphyritic basalt; microphenocrysts of plagioclase, with oscillatory zoning, and clinopyroxene, also defining a subophitic texture. Yellow arrows indicate clinopyroxene $\rightarrow$ epidote alteration. D and E) Parallel polarizers at left and crossed at right.Hypocrystalline basalts with aligned euhedral tabular plagioclase crystals from the inner portion of a flow. Yellow arrows indicate irregular bubbles (diktytaxitic texture) filled by quartz. F and G) Samples from a thinner ( $\sim 30 \mathrm{~cm}$ thick) and a thicker $(\sim 1 \mathrm{~m}$ thick) Esmeralda-type dykes respectively.Parallel polarizers at left and crossed at right.Radial distribution of acicular to euhedric tabular (with high aspect ratios) plagioclase crystals and interstitial granular clinopyroxene (intergranular texture).

\section{IV.2. The dacitic sequence}

The dacites belong to the Caxias do Sul subtype (see Chapter VI for geochemical characterization) and pile up a sequence of about 300 meters of total thickness, with the highest elevation at the region around $915 \mathrm{~m}$ a.s.l. However, individual deposits with thicknesses varying from a few meters to tens of meters have structural and textural variability and the detailed mapping allowed the recognition of different types of deposits (figure 14) that will be described in the following subsessions.

In a brief and generic description, the dacites have a wide range of crystallinity mainly related to the degree of devitrification. Hypohyaline portions may occur as irregular pockets preserved into the devitrified groundmass, as millimetric to decimetric bands either in complex structured zones or banded deposits (described below), or as layers with up to $10 \mathrm{~cm}$ in thickness. Holocrystalline and hypocrystalline textures occur throughout the area and the rocks vary from aphanitic to fine-grained and microporphyritic. Vesicles are common and their size, sphericity and stretching degree vary according to the type of deposit and position within the volcanic body. Flow banding and color variations may indicate changes in the oxidation degree. 


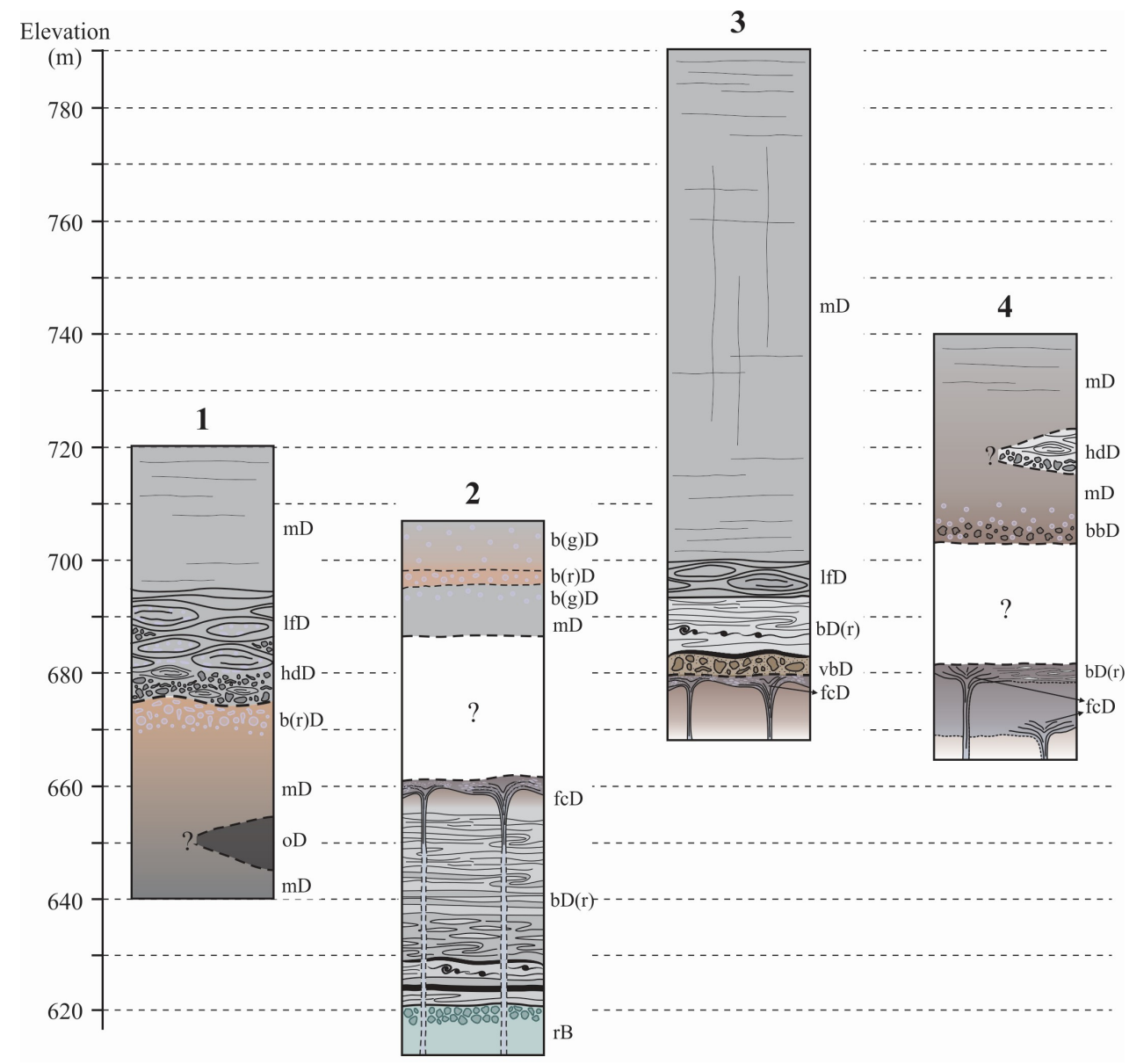

Figure 14: Stratigraphic section for the dacitic volcanic sequence. Theirs geographic location is highlighted by red circles on the map at figure 11. $\mathrm{mD}$ : massive (gray) dacite; lfD: dacitic lava flows; bD: vesicle-rich dacite $((\mathrm{g})-$ grayish facies; (r) - reddish facies); bbD: vesicle-rich dacitic breccias; hdD: hybrid dacitic deposit; oD: dacitic obsidian; $\mathrm{bD}(\mathrm{r})$ : banded dacite (rheoignimbrite); vbD: dacitic volcanic breccias; fcD: dacitic feeder conduits; rB: rubbly basalts.

\section{IV.2.1. Banded deposits (rheoignimbrites, high-grade ignimbrites or lava flows?)}

This unit may be more or less continuous with a horizontal morphology; however, its restricted occurrence and discontinuous outcropping (few dozen meters of extension and up to $10 \mathrm{~m}$ thick) hinder the delimitation of its extent and/or individual mapping of flow units.

The deposits occur at variable elevations and can be observed (i) at the base of the silicic sequence lying directly above the basaltic sequence, locally being intruded by the complex dacitic rocks described below, (ii) covering volcanic breccias or (iii) at 


\section{Chapter IV \\ Geology of the Studied Area}

higher elevations, where their stratigraphic relationship or lateral continuity with the massive deposits is unclear (figure 14). In the former case, the contact with the underlying basaltic sequence is characterized by a decimetric band (ca. $0.5 \mathrm{~m}$ ) of vitrophyric material with rootless fold hinge horizons and devitrified rounded structures(figure $15 \mathrm{~A}$ and $\mathrm{B}$ ). These rounded structures resemble sheath fold eye structures (Branney et al., 2004), from vitrophyric lithoclasts. A remarkable feature of these deposits is a discontinuous banding that originates a "zebra-like" texture (figure 15 A, B, C, D, E, F). The bands are lenticular, flattened to extremely flattened; the latter is common near the base of the deposit. In these horizons, centimetric to decimetric vitrophyric layers occur. Dark colored bands (millimetric to centimetric) are usually folded, refolded and rotated. Macroscopically, such features (figure 15 B, C and D) resemble extremely stretched fiammes or could be interpreted as deformed or remobilized pseudotachylitic bands (c.f. Kendrick et al., 2012, 2014); however, the complete recrystallization of these features (as described in the petrographic description session) makes its interpretation a tricky task. Massive to vesiculated lenses are typically dark red in color; vesicles range from millimetric to centimetric and are extremely stretched and elongated parallel to planar structures (figure $15 \mathrm{G}$ ). The gray facies is predominantly massive, rarely presenting (millimetric) spherical to stretched vesicles, and is characterized by spherulitic texture (figure $15 \mathrm{~A}$ and $\mathrm{B}$ ). Lithic fragments, although very rare, could be observed macroscopically (centimetric rounded clasts, deforming the characteristic banding of these rocks; figure $15 \mathrm{H}$ ) and microscopically. 

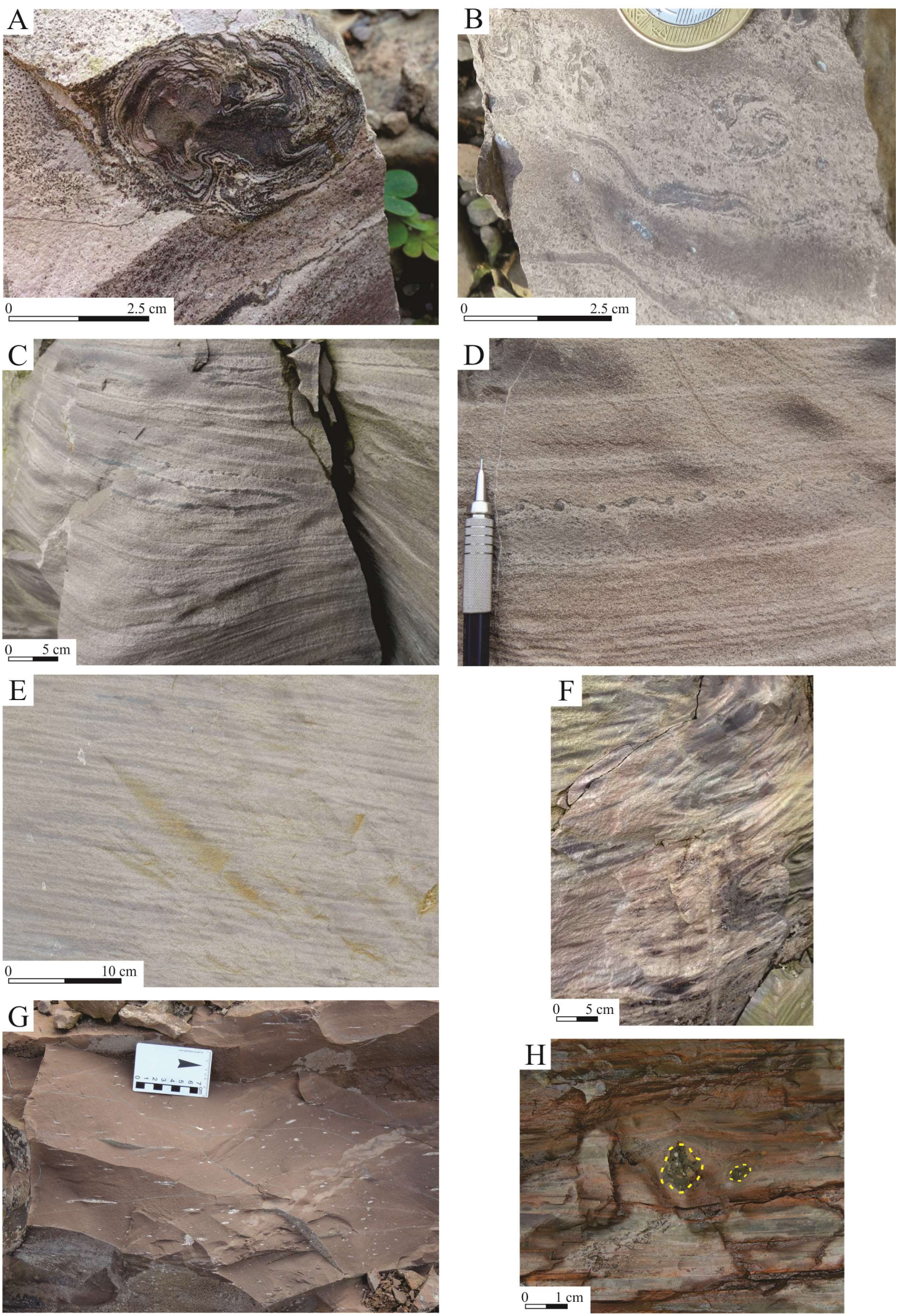

Figure 15: Field aspects of banded deposits: a, b, d) dark-colored bands, folded and rotated with spherulitic texture; c) lens-shaped discontinuous and horizontally folded banding; e) homogeneous lens-shaped banding; more (d, e) or less (a, c, b, f) continuous, flattened and commonly folded. The dark-colores bands suggest pseudotachylitic bands (a, $\mathrm{b}, \mathrm{c}, \mathrm{d}$ ) or extremely stretched fiammes; g) reddish facies with horizontal banding and extremely stretched vesicles; h) millimetric to centimetric rounded lithic fragments deforming the banding. 


\section{IV.2.2. Volcanic breccias}

The volcanic breccias occur at the base of the silicic sequence (i) at the vicinities of the "complex structured zone" (described in the following item), underneath the banded deposits or (ii) inside this zone, intercalated to the banded deposits, being intruded by the vertical flow structures. The deposits have restrict occurrence and the discontinuity of the outcrops makes it impossible to precisely delimit their volume or area.

The complete description of such deposits, including a quantitative textural analysis, is presented at Chapter V. Some general field aspects of the volcanic breccias are presented at figure 16; more details can be found in the next chapter.
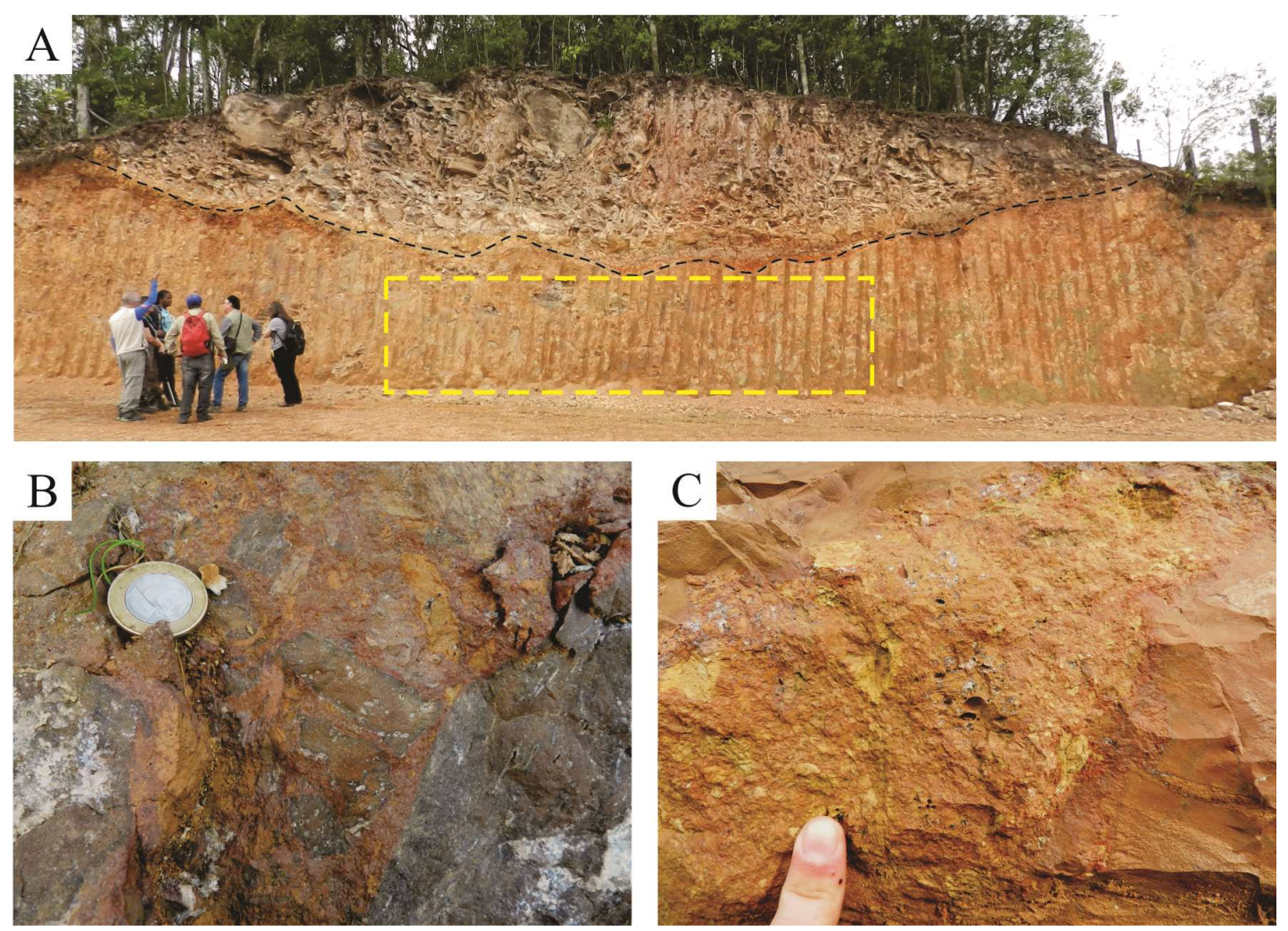

Figure 16:Field aspects of volcanic breccias. A) Volcanic breccia overlaid by banded deposit at the vicinity of the complex structured zone. The yellow square indicates one of the selected portions for the quantitative textural analysis presented in Chapter V (sample SM-01). B and C) Detail of volcanic breccia in the complex structured zone. More details are given in Chapter V. 


\section{IV.2.3. Dacitic complex structures along linear trends}

Dacitic complex structures crop out discontinuously along a NW-SE trend, defining a complex structured zone up to $4 \mathrm{~km}$ width (figure 11). These outcrops comprise aphanitic dacites, mainly hypocrystalline, with a notorious vertical magmatic banding. At least four different structural domains have been recognized: 1) regular; 2) filament; 3) fragmentation; and 4) breccia domains (figure $17 \mathrm{~A}$ ). Changes in color may reflect the co-existence of dacitic magmas with different oxidation conditions.

Large domains of predominantly gray rock, which can be massive and homogeneous to lens-shaped banded (“zebra-like pattern"), containing varying degrees of vesiculation, have been termed "regular domains" (figure 17 A, B, E and 18 B). In general, vesicles density increase upwards and pumice fragments are (locally) interlayered.

The "filament domain" (figures 17 A, B, C, D, E and 18) contains millimetric to centimetric filaments of aphyric/vitrophyric material, commonly folded and deformed by the flow. It can be strongly variable, grading from vertical to horizontal or funnellike structures marked by low to high angle divergent flow lines (figure $18 \mathrm{~A}$ ). Flow bands may either surround, be deformed by or deform pumice fragments. Vertical to sub vertical ductile shearing is common and millimetric to centimetric bands of holohyaline rock may result from remelting along shear flows. Giant vesicles (up to 50 $\mathrm{cm}$ in diameter) also deform the flow in the filament domains (figure $17 \mathrm{E}$ ). Structural data from magmatic flow (examples at figure 18) point towards two preferential directions of vertical flow: a NW trend, ranging from $\mathrm{N} 272^{\circ}$ to $\mathrm{N} 355^{\circ}$, and a $\mathrm{NE}$ trend varying between $\mathrm{N} 20^{\circ}$ and $\mathrm{N} 85^{\circ}$ (cf. rose diagrams in figure 11). 
Geology of the Studied Area
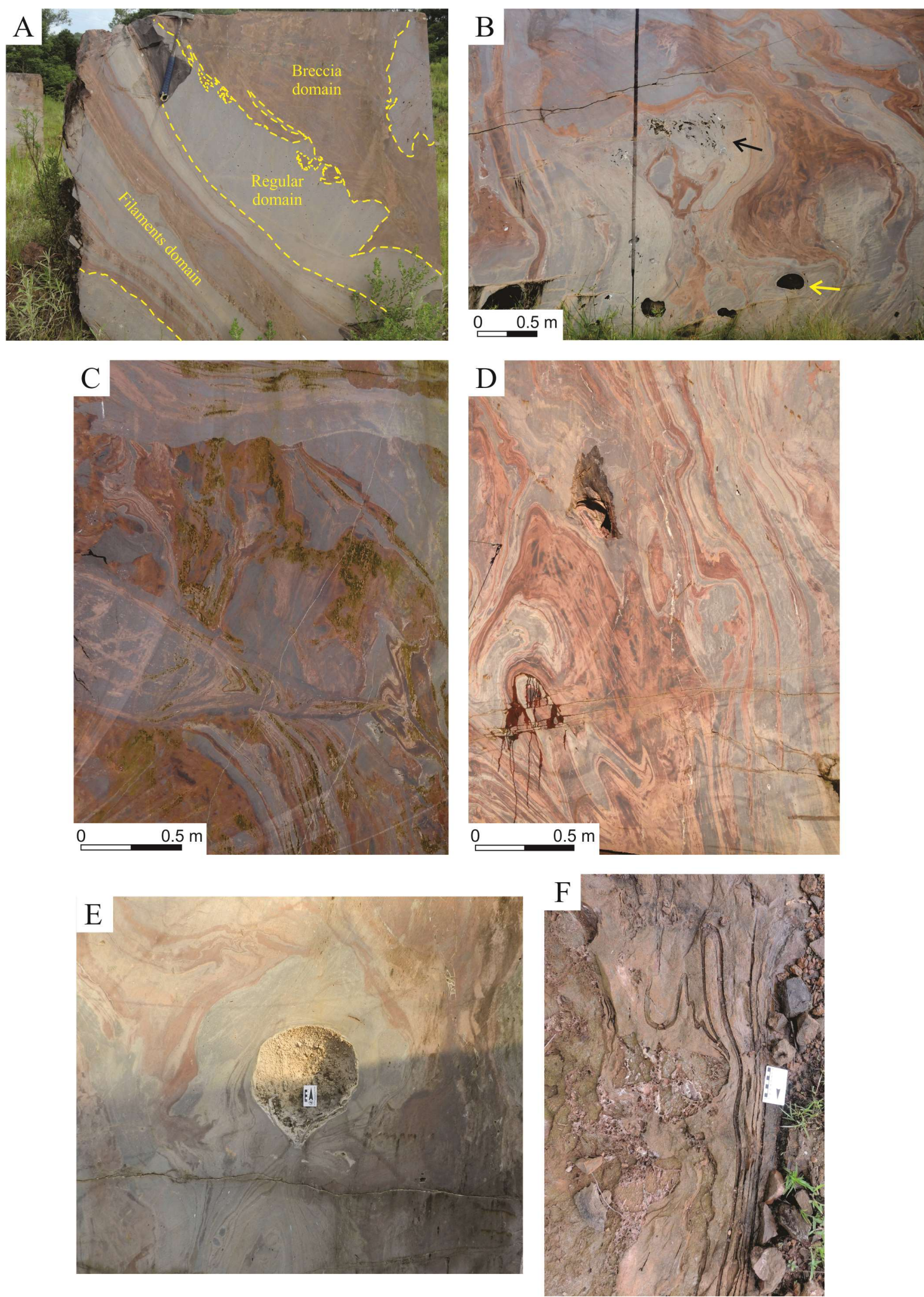

Figure 17: Field aspects of dacitic complex strutures. A) Exemples of breccia, regular and filaments domains. B) Interaction between different melts with local bubble-rich pumice (black arrow). At the bottom (yellow arrow) a level of giant-vesicles. C) Regular, breccia and previous filament domains are crosscut by a superimposed filament domain, indicating cyclicity and superposition of processes. D) General aspect of the filament domain with millimetric to centimetric chaotic filaments of aphyric material, which is folded, stretched and deformed by flow. E) Giant vesicle deforming the flow and promoting mingling between different melts. F) Isoclinal folds (viewed from above), with vertical hinge lines, from magmatic flow. 
A
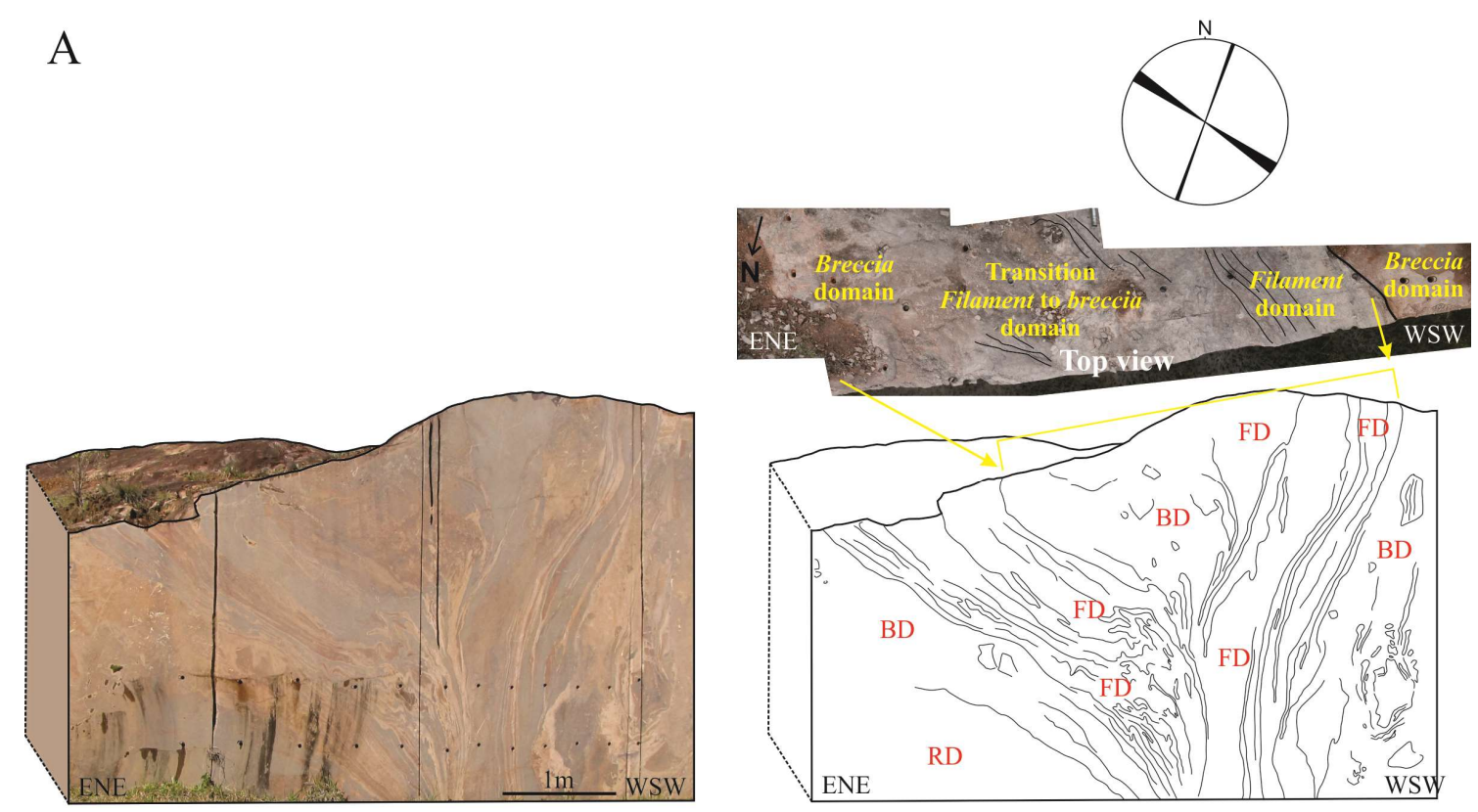

$\mathrm{B}$
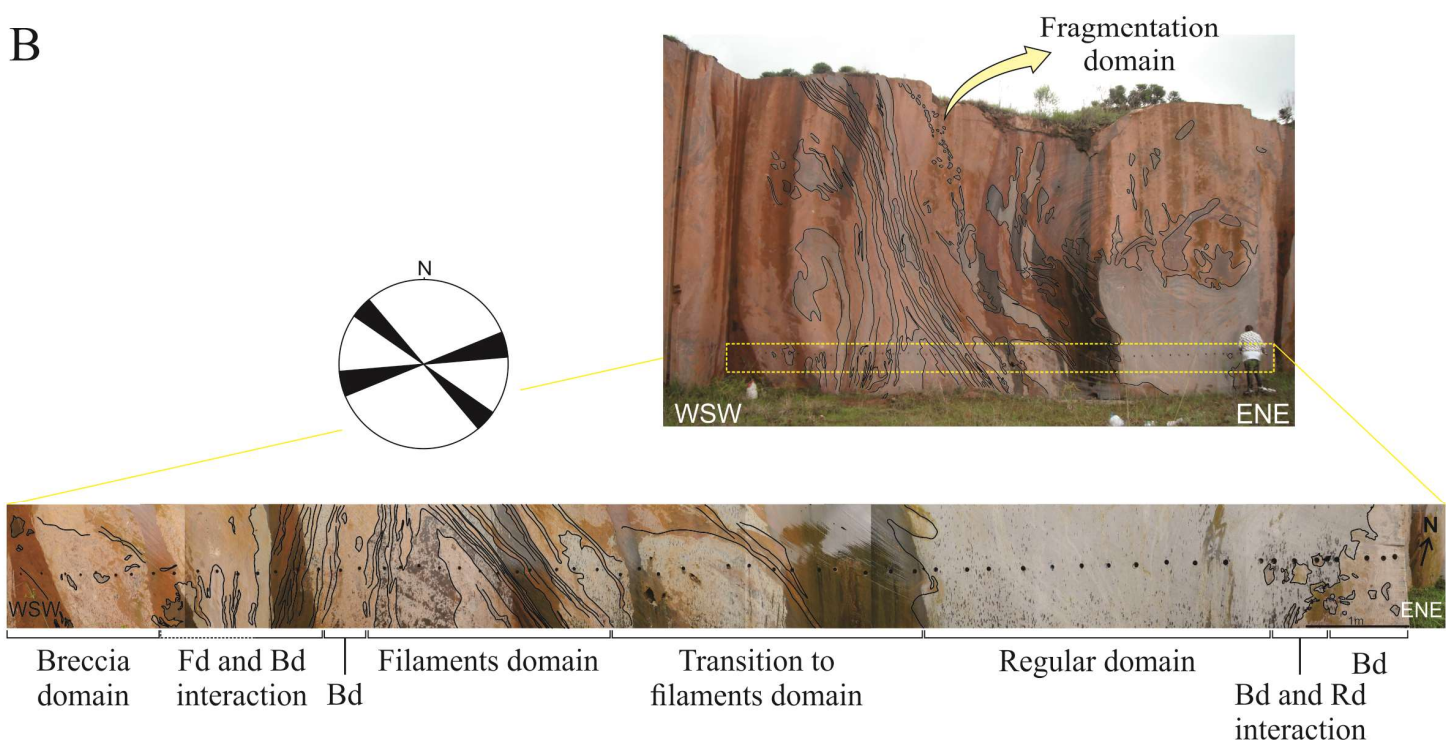

Figure 18: Structural data from filaments domains. Exemples in a funil-like structure from quarry SM-20 (A) and from quarry SM-82 (B). The regular (Rd), filaments (Fd), fragmentation and regular (Rd) domains are indicated. Stereograms indicates the range of flow directions and do not represent the magmatic flow dips.

In vertical and, more rarely, lateral/horizontal continuity with the filaments and regular domains, it is possible to recognize a "fragmentation domain" (figure $18 \mathrm{~B}$ and 19 A) responsible for generating angular and sub-angular centimetric fragments. The transition between these domains can be progressive, evolving from vertical and horizontal fracturing to the full fragmentation of the superimposed domain. Fragmentation increases towards the top together with the degree of vesiculation. 


\section{Chapter IV \\ Geology of the Studied Area}

The "breccia domains" (figure $19 \mathrm{~B}$ and C) have reddish to grayish colors, with purple shades. Rounded to sub-angular pumice fragments with variable vesicles sizes and shapes are suspended in a finer-grained matrix, with millimetric angular fragments.
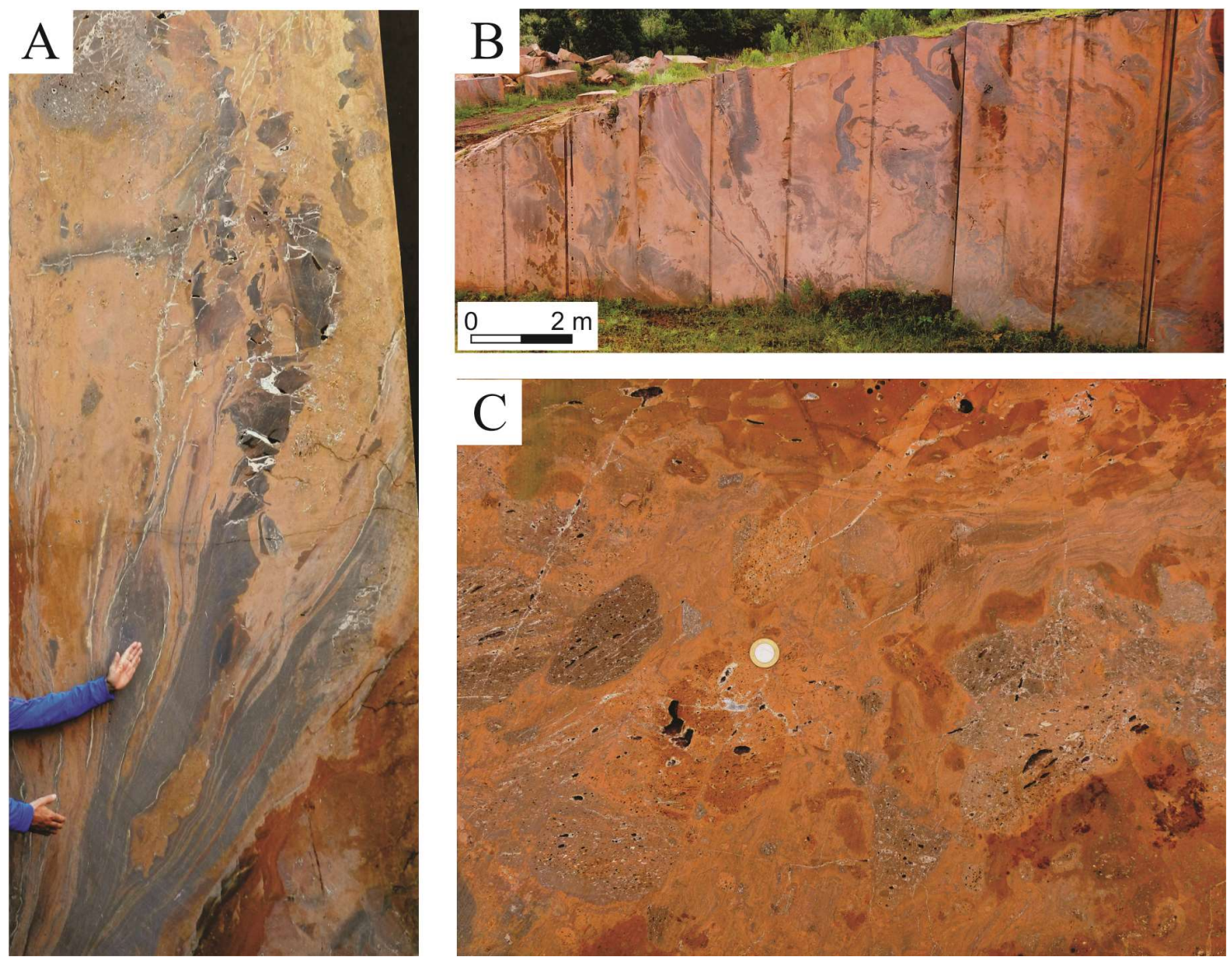

Figure 19: Field aspects of dacite complex structures. A) Upwards transition between a filament and fragmentation domains. B) Breccia domain (reddish shades) intruded by filaments domains (grayish shades). C) Example of the breccia domain, with rounded bubble-rich centimetric fragments in a finer-grained (more angular, millimetric dense fragments), clay-rich matrix. More aspects of the breccias domain are shown in Chapter V.

The above described domains present variable dimensions and are arranged in a repetitive way, occurring interspersed along the whole structure. Cross-cutting relations between them (figures $17 \mathrm{~A}, \mathrm{~B}, \mathrm{C}, 18 \mathrm{~B}$ and $19 \mathrm{~B}$ ) are quite common. Regional mapping, together with the stratigraphical relationships (figure 14), shows that breccia domains are the enclosing rocks. Additionally, a mostly flat-lying gray material consisting of blurred zebra-like lens-shaped domains in a massive, devitrified, sometimes spherulitic, matrix (similar to the banded deposits described above) was also recognized as enclosing rock. When visible, the limit between enclosing units and 


\section{Chapter IV \\ Geology of the Studied Area}

dacitic complex structures may be abrupt and well-marked by ductile shear zones. The contact between the complex structured zone and the basaltic sequence is, however, generally inferred, although an intrusive contact could be observed on the RS-122 highway, in the SW portion of the studied area. An upward magmatic flow in the filaments domain could be inferred from vesicle stretching and due to the vertical transition with the fragmentation domains. It is noteworthy that the vertical flow is restricted to this region, along the $4 \mathrm{~km}$ wide NW-SE trend. Low angle banding and related structures indicative of horizontal flow (including vesicle stretching) are uncommon and have been observed only locally described in this region. Horizontal banding, on the other hand, is typical for the enclosing units and overlapping deposits.

\section{IV.2.4. Hybrid deposits and lava flows}

Lava flows are lobate flows whose outcrops can reach up to $50 \mathrm{~m}$ of extension and 15-20 m height, but the lobes do not exceed $10 \mathrm{~m}$ wide vs $4 \mathrm{~m}$ thick. These deposits are characterized by aphanitic dacites, dark gray in color, with predominantly massive cores and vesicle-rich upper portions. In some cases, these deposits can occur covering the volcanic breccias (figure 14). Effusive flows can be observed locally inside these breccias, pointing towards a hybrid deposit (figure 20). When the hybrid deposits are continuous (e.g. SW portion of studied area, figure 11 and 20), it is possible to recognize the transition from the hybrid (with predominance of fragmented flows) to less significant contributions of pyroclastic events, and finally to an exclusive condition of effusive lobate lava flows (figure $20 \mathrm{~B}$ ).

In the case of coulées and the effusive portions of hybrid deposits, the dacites are aphanitic to finely phaneritic, gray when unweathered and brownish to pale pink when weathered. A remarkable feature is the occurrence of radial joints, following the edges of flow units (lobes). Similar structures were described by Polo et al. (2018a, 2018b) and Guimarães et al. (2018). These rocks range from massive to bubble-rich and spherulitic texture is common. Bubbles change from spherical to extremely flattened, up to $5 \mathrm{~cm}$ in diameter. Spherical vesicles occur preferentially in the nucleus and at the top of the flow, in some cases in larger sizes and higher density. On the other hand, the stretched bubbles tend to concentrate in the outer portions of the flow, especially at the bottom, and commonly deforming along the flow edges. This stretching allowed us to 
recognize and measure flow directions along two preferential trends: NE-SW and NW$\mathrm{SE}$, as shown in table 3.

The fragmented portion of the hybrid deposits (figure 20), in turn, is characterized by intensely weathered breccias, very similar to the volcanic breccias but with smaller fragments (mainly centimetric).

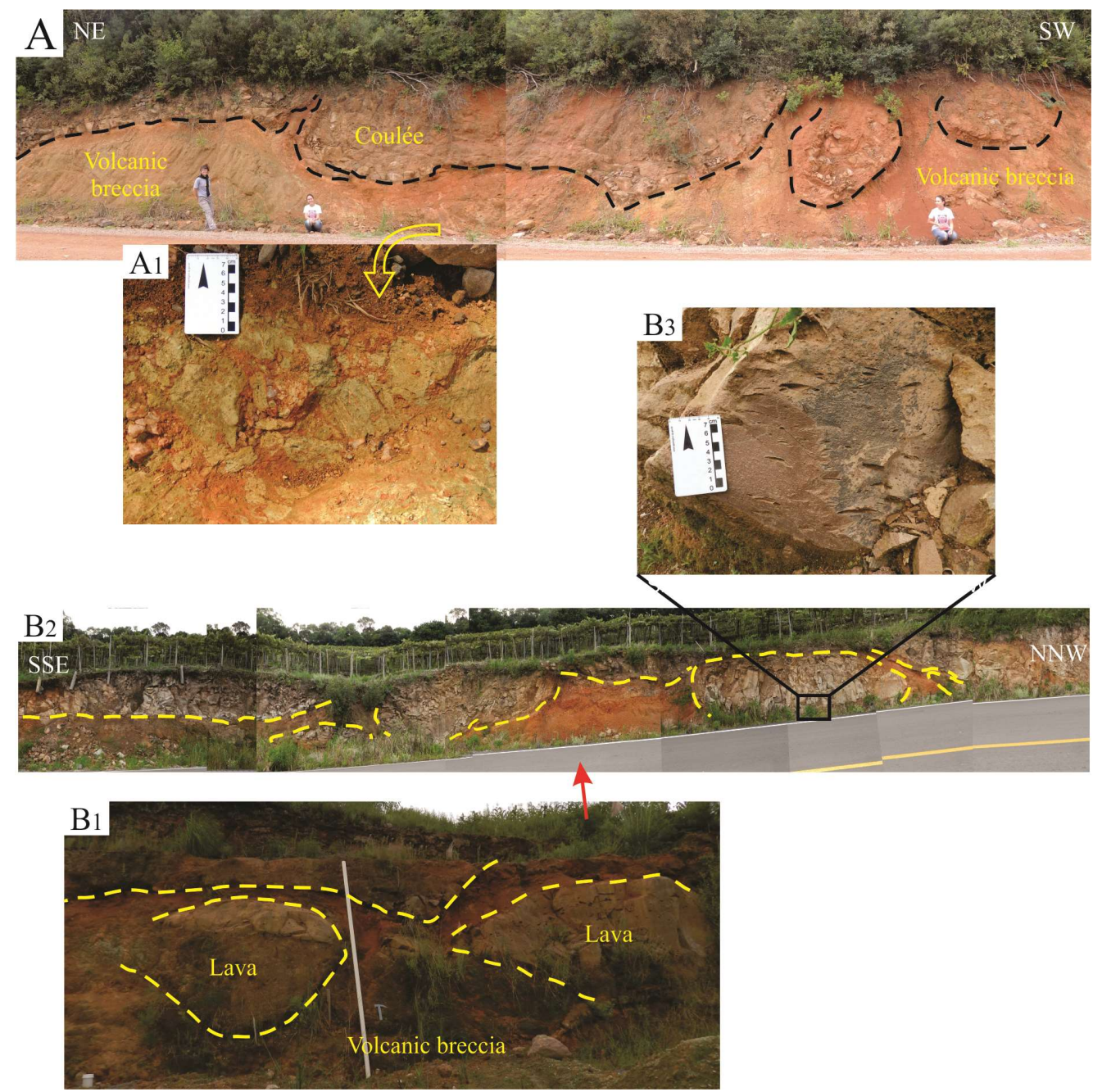

Figure 20: Hybrid dacitic deposits with pyroclastic and effusive components; A) pyroclastic component at the base with flows inside or above; B) continuous deposit in the SW of the area (see geological map on figure 11) with the predominance of pyroclastics at the base (B1) grading into the predominance of compounded lava flows (B); B2) detail from the bottom of a lava flow, with stretched vesicles, along two flow directions: NNW-SSE and NE-SW. 
Table 3: Measurements of flow directions from stretched bubbles in effusive portions from hybrid deposits. Two different flow units were measured at outcrop SM-65 and four at outcrop SM- 142. The directions plotted on the map (figure 11) correspond to the average of measurements.

Outcrop nr.

Location (UTM)

Flow direction

\begin{tabular}{|c|c|c|c|}
\hline \multirow{2}{*}{ SM-65 } & \multirow{2}{*}{495232} & \multirow{2}{*}{6794701} & N010 \\
\hline & & & $\mathrm{N} 335^{\circ}$ \\
\hline SM-126 & 492075 & 6797790 & $\mathrm{~N} 340^{\circ}$ \\
\hline \multirow{16}{*}{ SM-142 } & \multirow{16}{*}{484276} & \multirow{16}{*}{6794339} & N060 ${ }^{\circ}$ \\
\hline & & & N077 \\
\hline & & & $\mathrm{N} 306^{\circ}$ \\
\hline & & & $\mathrm{N} 310^{\circ}$ \\
\hline & & & $\mathrm{N} 354^{\circ}$ \\
\hline & & & $\mathrm{N} 327^{\circ}$ \\
\hline & & & $\mathrm{N} 320^{\circ}$ \\
\hline & & & N044 ${ }^{\circ}$ \\
\hline & & & $\mathrm{N}^{2} 26^{\circ}$ \\
\hline & & & N055 \\
\hline & & & N045 \\
\hline & & & N064 ${ }^{\circ}$ \\
\hline & & & N040 \\
\hline & & & $\mathrm{N} 310^{\circ}$ \\
\hline & & & N015 \\
\hline & & & $\mathrm{N} 040^{\circ}$ \\
\hline
\end{tabular}

\section{IV.2.5. Massive deposits}

Massive dacitic deposits are very homogeneous and extensive, and corresponds to the uppermost volcanic unit in São Marcos. The rocks are phaneritic, fine to coarse grained, microporphiritic gray dacites. They are massive, with spherulitic texture (spherulites with diameters up to $2 \mathrm{~mm}$ ) and locally contain plagioclase and pseudomorphs of pyroxene as acicular microphenocrysts (up to $1 \mathrm{~cm}$; figure $21 \mathrm{~A}, \mathrm{~B}$ ), mostly aligned along the flow. Horizontal joints are observed when the bottom of the deposit is exposed. This jointing may vary along the contact with the underlying deposit or with the edges of the volcanic body.

Vesicles, when present, are millimetric $(<0.5 \mathrm{~cm})$ and spherical, occurring preferentially in aphanitic to fine-grained deposits. Despite the significant predominance 


\section{Chapter IV \\ Geology of the Studied Area}

of massive structure, diffuse to blurred bands (smooth "ghost" structures) can be locally observed (figure $21 \mathrm{~A}, \mathrm{C}, \mathrm{D})$, with very subtle variations in color (light gray to gray).
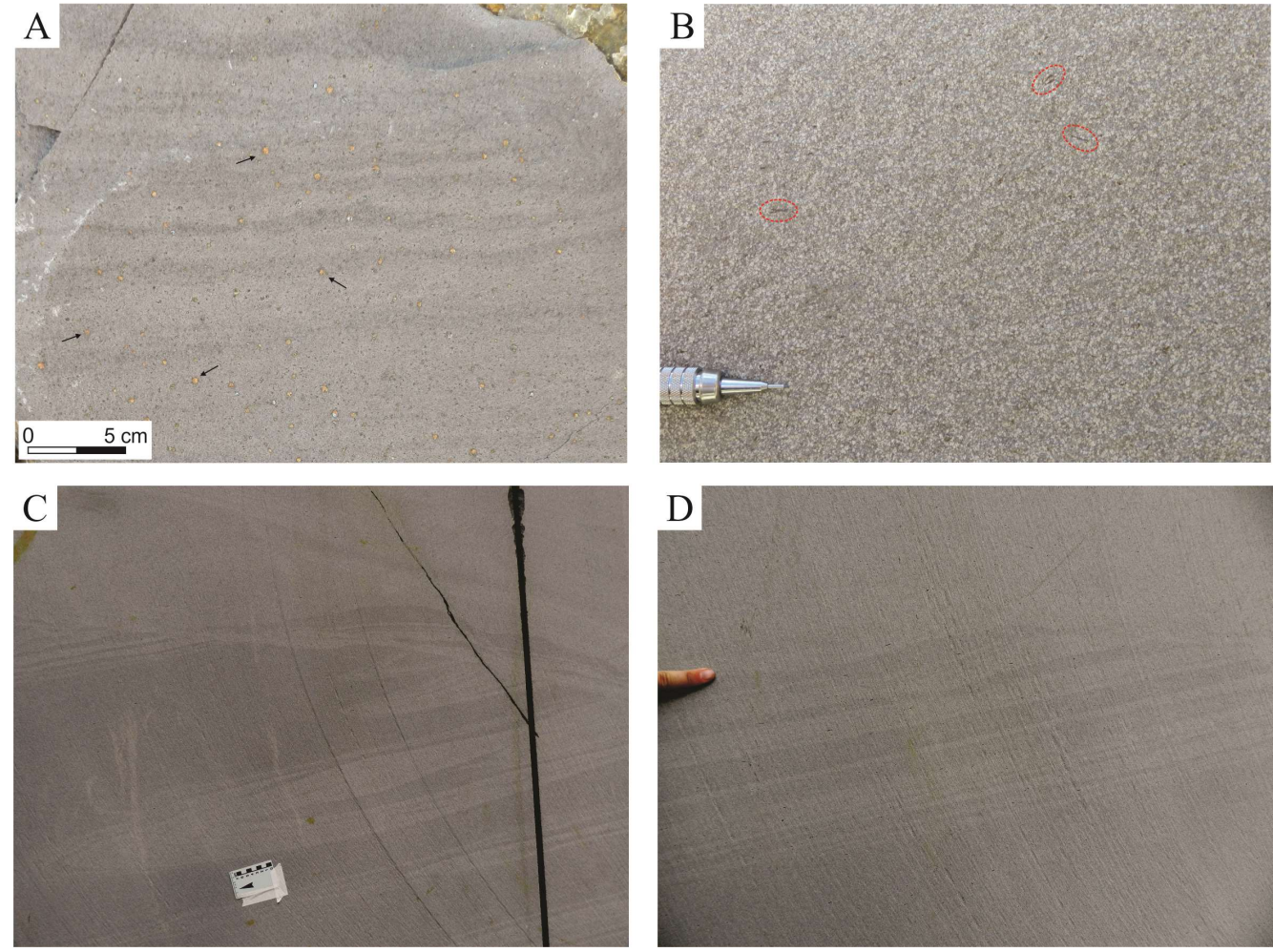

Figure 21: Details from massive deposits. A) fine-graines, vesicle-rich (black arrows), banded dacite; B) coarsegrained, microporfiritic (red circles) gray dacite; $\mathrm{C}$ and $\mathrm{D}$ ) aphanitic to fine-grained, locally microporphyritic dacite with "ghost-structures" (diffuse banding and crossed bedding).

\section{IV.2.6. Petrographic aspects and mineral chemistry}

Petrographic examination reveals that the dacites are characteristically hypohyaline, porphyritic, and locally glomeroporphyritic (figure 22 A). Extensive massive deposits (IV.2.5) show no visible crystal orientation in the groundmass. On the other hand, in the lava flows (IV.2.4), banded deposits (IV.2.1) and some specific filament-rich domains from the complex structured zone (IV.2.3), crystals are preferentially aligned along the flow direction (figure $22 \mathrm{~B}$ ). The crystals lie in an originally glassy groundmass, affected by varied degrees of devitrification and recrystallization which can make the interpretation of these rocks difficult. Hypocrystalline dacites, with spherulitic texture, and holocrystalline dacites, with a 


\section{Chapter IV \\ Geology of the Studied Area}

micrographic groundmass (figure $22 \mathrm{C}, \mathrm{D}$ ), may be a result of an intense, or even complete, recrystallization process. In samples with some degree of preservation of glassy groundmass, perlitic fractures can still be recognized (figure $22 \mathrm{E}$ ). Another important, but uncommon feature are lithoclasts consisting of euhedral plagioclase, pyroxene, magnetite and quartz. These lithoclasts were observed exclusively in the holohyaline dacite (obsidian) which is the bottom of the banded deposit closer to the feeder zone (SM-01). They present interfingered and diffuse edges with signs of dispersion of the crystals through the glassy host rock (figure $22 \mathrm{~F}, \mathrm{G}$ ). Other pyroclastic features are almost absent, being restricted to few occurrences of plagioclase fragments (figure 22 I).

The dacites exhibit low crystal contents, which vary according to the deposit type. Lava flows and massive and extensive deposits have the highest proportions of crystals, which can reach up to $15-30 \%$ of the volume of the rock. In contrast, crystal contents from complex structured zones and banded deposits in general, do not exceed 5-10\%. Three crystal populations, based on size and texture, could be recognized: a) large, or phenocrystals $(>0.3 \mathrm{~mm})$; b) small $(0.1 \mathrm{~mm}$ to $0.2 \mathrm{~mm})$; c) microlites $(<$ $0.1 \mathrm{~mm}$ ) (figure $22 \mathrm{~A}$ ). Large crystals are euhedral to subhedral, rarely reaching $1.5 \mathrm{~mm}$, with intense compositional zoning, resorption features, and depicting glomeroporphyritic texture (figure $22 \mathrm{~A}, \mathrm{H}$ ). The second group of small crystals are euhedral with fast-cooling features such as swallow-tail terminations (figure $22 \mathrm{I}$ ). Microlites $(<0.1 \mathrm{~mm})$, in turn, consist of tabular to acicular and bead-like crystals (figure $22 \mathrm{~J}$ ). They may follow the flow, in the case of the dacites from complex structured zones, or may be randomly arranged, in other deposits. The relative contents between different crystal populations vary according to the occurrence type; while microlites predominate in the complex structured zones, large and small crystals predominate in other deposits.

Despite local structural and textural variations, the dacites show a homogeneous mineralogy. Plagioclase, clinopyroxene and Ti-magnetite constitute the main primary mineral phases. 

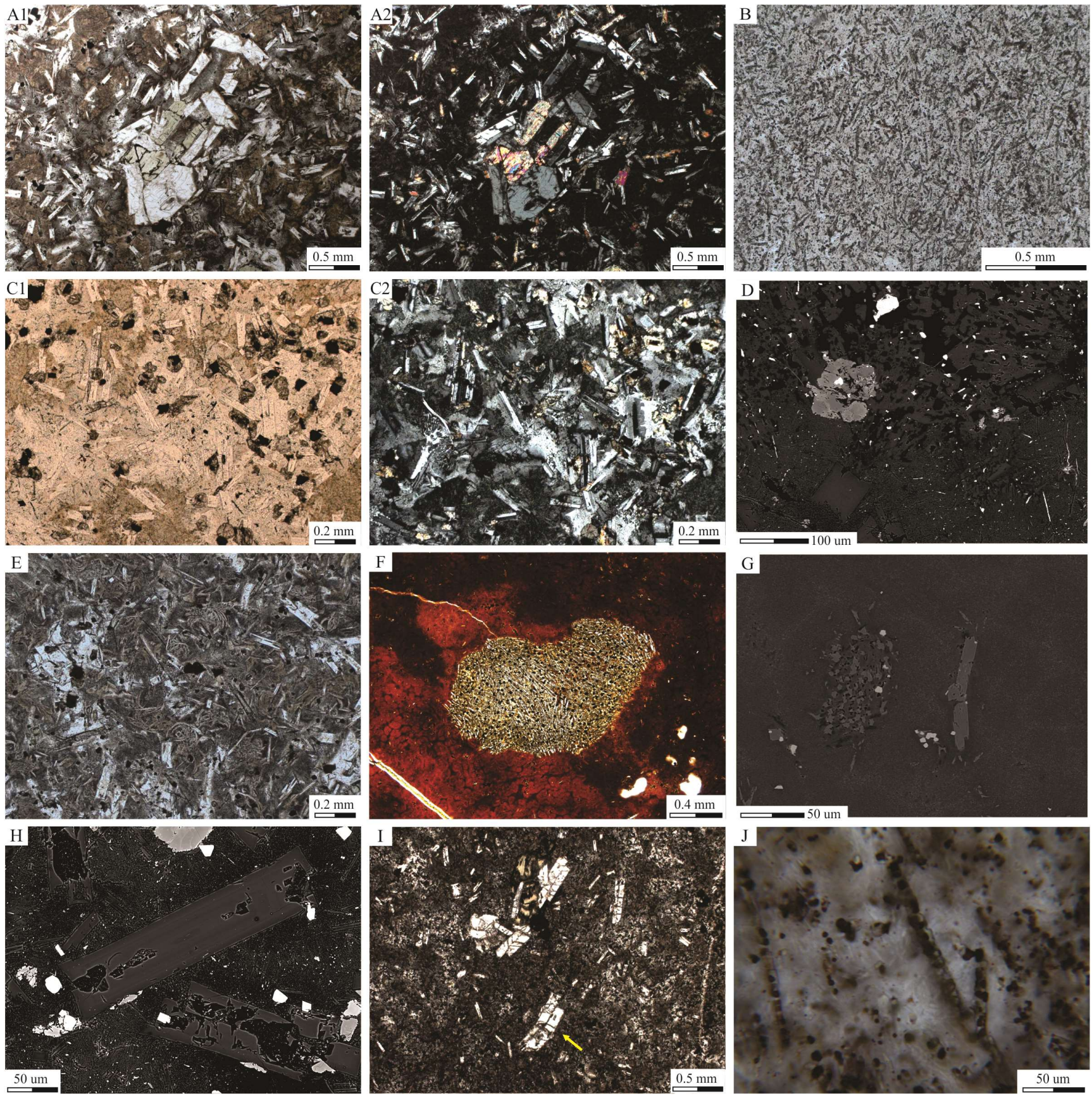

Figure 22: Petrography of dacites. A1 and A2) Parallel and crossed polarizers respectively. Hypocrystalline glomeroporphiritic dacite. The two types of microcrystals are clearly recognized: euhedral (tabular) to subhedral rounded crystals with resorption features depicting a glomeroporphiritic texture and smaller euhedral crystals with fast-cooling features; B) Parallel polarizers. Microlites from the complex structured zone are oriented along the magmatic flow. $\mathrm{C} 1$ and $\mathrm{C} 2$ ) Parallel and crossed polarizers respectively. Holocrystalline dacite.Tabular plagioclase crystals, with fast-cooling features. Subedral rounded-shaped pyroxene crystals and Ti-magnetite are dispersed on a completely devitrified groundmass. D) SEM image: microphenocrystals and microlites of plagioclase, pyroxene and Ti-magnetite lying on a micrographic groundmass; E) Parallel polarizers. Spherulitic texture in a partially devitrified groundmass, suggesting recrystallization along previous perlitic fractures; F) Parallel polarizers. Clusters (microxenoliths) of euhedral crystals of plagioclase, pyroxene, magnetite and quartz, with interfingered and diffuse edges, dispersed in the holohyaline dacite (obsidian); G) SEM image from the hipohyaline dacite (obsidian) with pigeonite and Ti-magnetite microlites and microxenolith constituted of plagioclase + clinopyroxene + magnetite; $\mathrm{H}$ ) Parallel polarizers. Tabular, euhedral, microphenocrysts of plagioclase, with compositional zoning and resorption features; I) Parallel polarizers.Hipocrystalline dacite with subhedral microphenocrystals of plagioclase (crystals 
fragments - arrow), pyroxene and Ti-magnetite lying in a partialy devitrified groundmass (sperulithic texture). J) Parallel polarizers. Microlites (bead-crystals) of pyroxene and Ti-magnetite.

Plagioclase is the main mineral phase and may represent up to $15 \%$ vol. of the dacites. Crystals are euhedral to subhedral, commonly tabular, showing oscillatory zoning (figure $22 \mathrm{H}$ ). Corroded cores rounded by very thin rims are typical in normal zoning, which predominates in these rocks. Indeed, the general trend of zoning and crystallization shows a normal evolution (figure 23$)$; labradorite $\left(\mathrm{An}_{50-60}\right)$ corresponds, in general, to the initial phases of crystallization, while the final phases are characterized by lower An-contents. Small embayment locally occurs and tabular crystals with up to $0.4 \mathrm{~mm}$ usually present textures of rapid cooling (e.g. swallow-tailed, skeletal and hollow crystals; figure 22 I). The microlites are abundant, show tabular to acicular morphologies, have oligoclase $\left(\mathrm{An}_{27-28}\right)$ and andesine $\left(\mathrm{An}_{30-49}\right)$ compositions (figure 23) and are in equilibrium with the borders of larger crystals (microphenocrysts). The feldspar occurring as groundmass, intergrown with quartz and probably resulting from post-magmatic processes (devitrification), corresponds to sanidine $\left(\mathrm{Or}_{54-67}\right)$ (figure 23). No significant difference in plagioclase chemistry and texture was observed between the deposits. The analytical results are fully presented in appendix III.

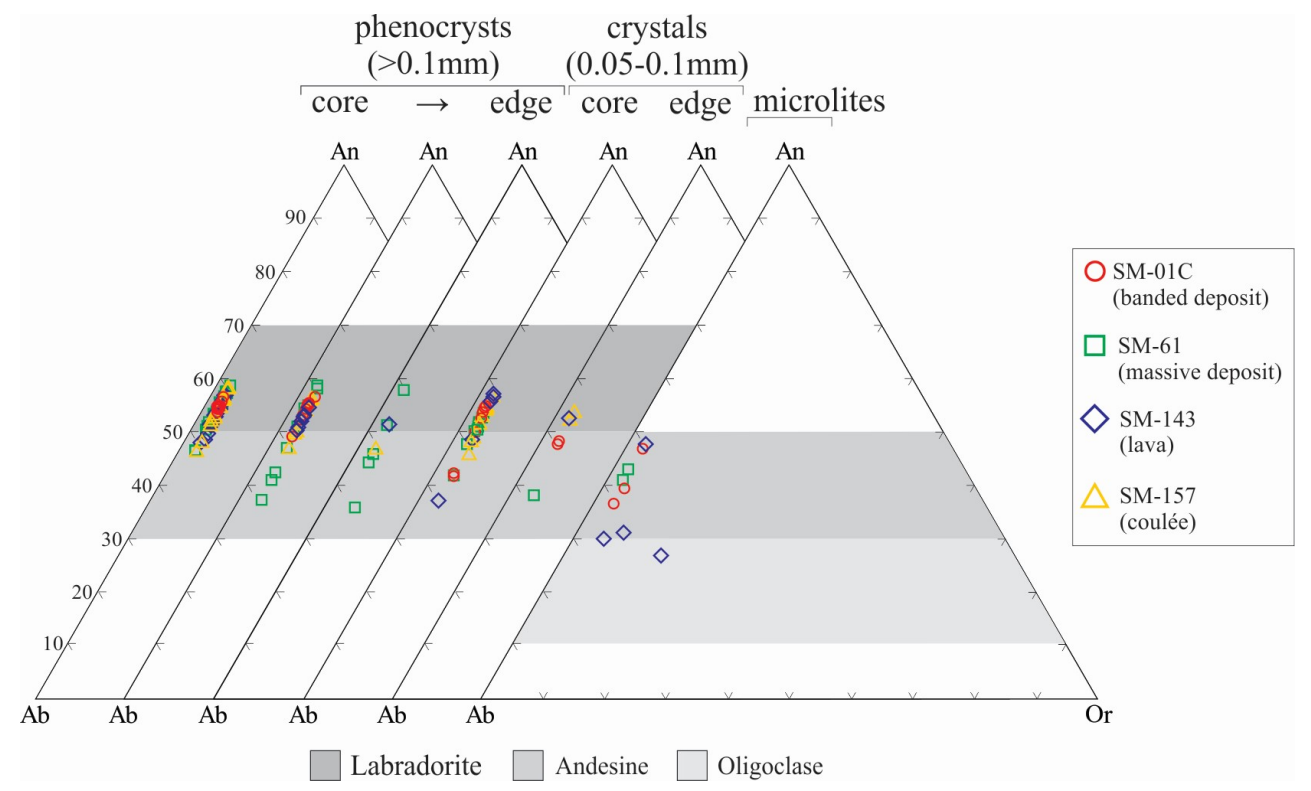

Figure 23: An-Ab-Or ternary diagrams for classification of feldspars from Caxias do Sul dacites. 


\section{Chapter IV \\ Geology of the Studied Area}

Clinopyroxene can make up to $8 \%$ vol. of dacites and, as plagioclase, can occur as (i) subhedral, tabular to acicular large crystals, with compositional zoning (figure 24), or as (ii) subhedral prismatic to granular, rounded crystals and granular (figure $22 \mathrm{C}, \mathrm{D}$ ), rounded microlites. Clusters of larger crystals and microlites are common. Complex clinopyroxene microphenocrysts with corroded cores of pigeonite $\left(\mathrm{Wo}_{6-11} \mathrm{En}_{48-62} \mathrm{Fs}_{32-44}\right.$; \#mg 53-67) mantled by augite $\left(\mathrm{Wo}_{34-37} \mathrm{En}_{38-42} \mathrm{Fs}_{22-26}\right.$; \#mg 60-68), in turn mantled by irregular rims of $\mathrm{Mg}$-poorer pigeonite $\left(\mathrm{Wo}_{9-11} \mathrm{En}_{32-28} \mathrm{Fs}_{52-59}\right.$; $\left.\# \mathrm{mg} 36-51\right)$ were observed exclusively in the samples from banded and massive deposits (figures 24 and 25). Augite microphenocrysts and smaller crystals occur with a small (clustered) range of compositions ( $\mathrm{Wo}_{33-38} \mathrm{En}_{36-43} \mathrm{Fs}_{21-29}$; \#mg56-68), not being possible to distinguish cores with augite compositions from different types of deposit. On the other hand, pigeonite crystals show a wide range of compositions $\left(\mathrm{Wo}_{7-8} \mathrm{En}_{42-61} \mathrm{Fs}_{33-49}\right.$; \#mg47-66), even for crystals cores, and microlites of both augite $\left(\mathrm{Wo}_{24-41} \mathrm{En}_{34-41} \mathrm{Fs}_{23-37} ; \# \mathrm{mg} 52-65\right)$ and pigeonite $\left(\mathrm{Wo}_{07-24} \mathrm{En}_{32-59} \mathrm{Fs}_{33-59}\right.$; \#mg39-65) are in equilibrium with the rims of coarser crystals (augite \#mg49-60 and pigeonite \#mg37-59) (figures 25). It is striking the continuum compositions of microlites from the banded deposits, where augites with progressively higher $\mathrm{Fe}$ contents and lower $\mathrm{Ca}$ contents converge to pigeonite compositions. Another feature that is important to highlight are the microlithoclasts observed in the obsidian base from a banded outcrop, which are composed exclusively by pigeonite ( $\mathrm{Wo}_{06-11} \mathrm{En}_{47-62} \mathrm{Fs}_{29-41}$; with high \#mg 54-69) (figures 25 and 26).
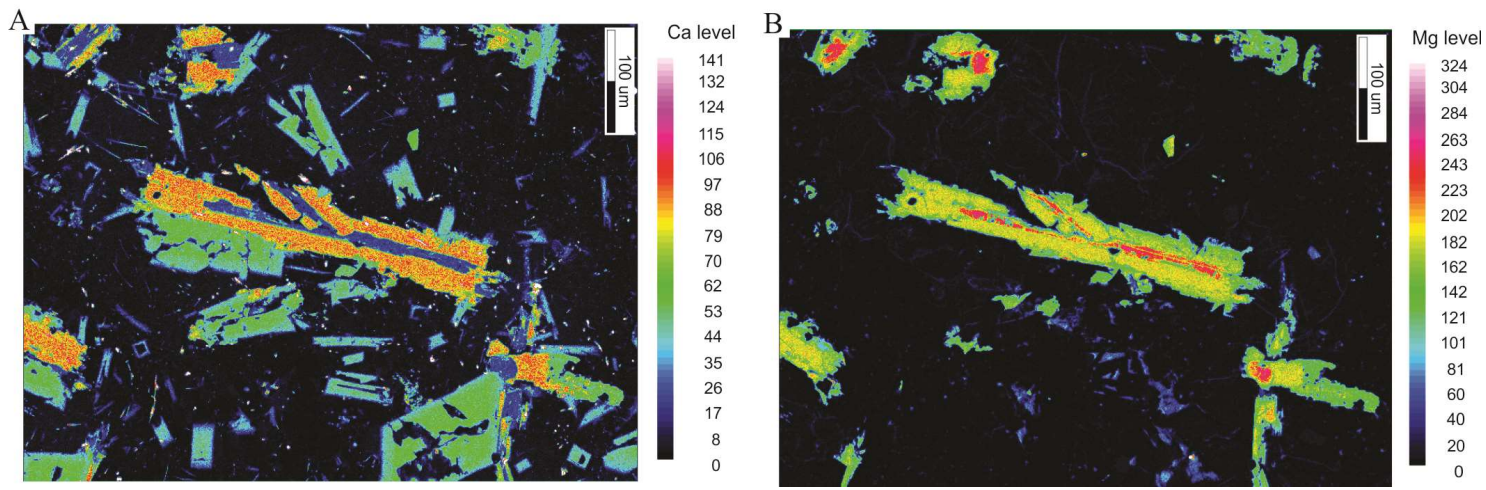

Figure 24: Compositional map (for $\mathrm{Ca} \mathrm{Ka}-$ and $\mathrm{Mg}$ Ka-lines) of holocrystalline dacite with micrographic groundmass and glomeroporphiritic texture. Microprobe analysis with a Jeol JXA-FE-8530 from IGc/USP.

The dacitic lavas present less varied clinopyroxene compositions. Microphenocrysts are mainly augite ( $\mathrm{Wo}_{34-37} \mathrm{En}_{34-42} \mathrm{Fs}_{21-31}$; \#mg 53-68); pigeonite is rare as a core $\left(\mathrm{Wo}_{7-8} \mathrm{En}_{61-60} \mathrm{Fs}_{33-41}\right.$; \#mg 56-66), and more common as rims with lower 


\section{Chapter IV}

\section{Geology of the Studied Area}

\#mg (Wo9-13 $\mathrm{En}_{33-53} \mathrm{Fs}_{38-53}$; \#mg 40-59). Both augite $\left(\mathrm{Wo}_{35-37} \mathrm{En}_{39-42} \mathrm{Fs}_{21-26}\right.$; \#mg 61-68) and pigeonite $\left(\mathrm{Wo}_{7-13} \mathrm{En}_{32-34} \mathrm{Fs}_{53-61} ; \# \mathrm{mg} 35-40\right)$ occurs as smaller crystals, and pigeonite predominates as microlites ( $\mathrm{Wo}_{9-11} \mathrm{En}_{29-38} \mathrm{Fs}_{53-58}$; \#mg 35-43) (figures 25 and 26). The analytical results are fully presented in appendix III.
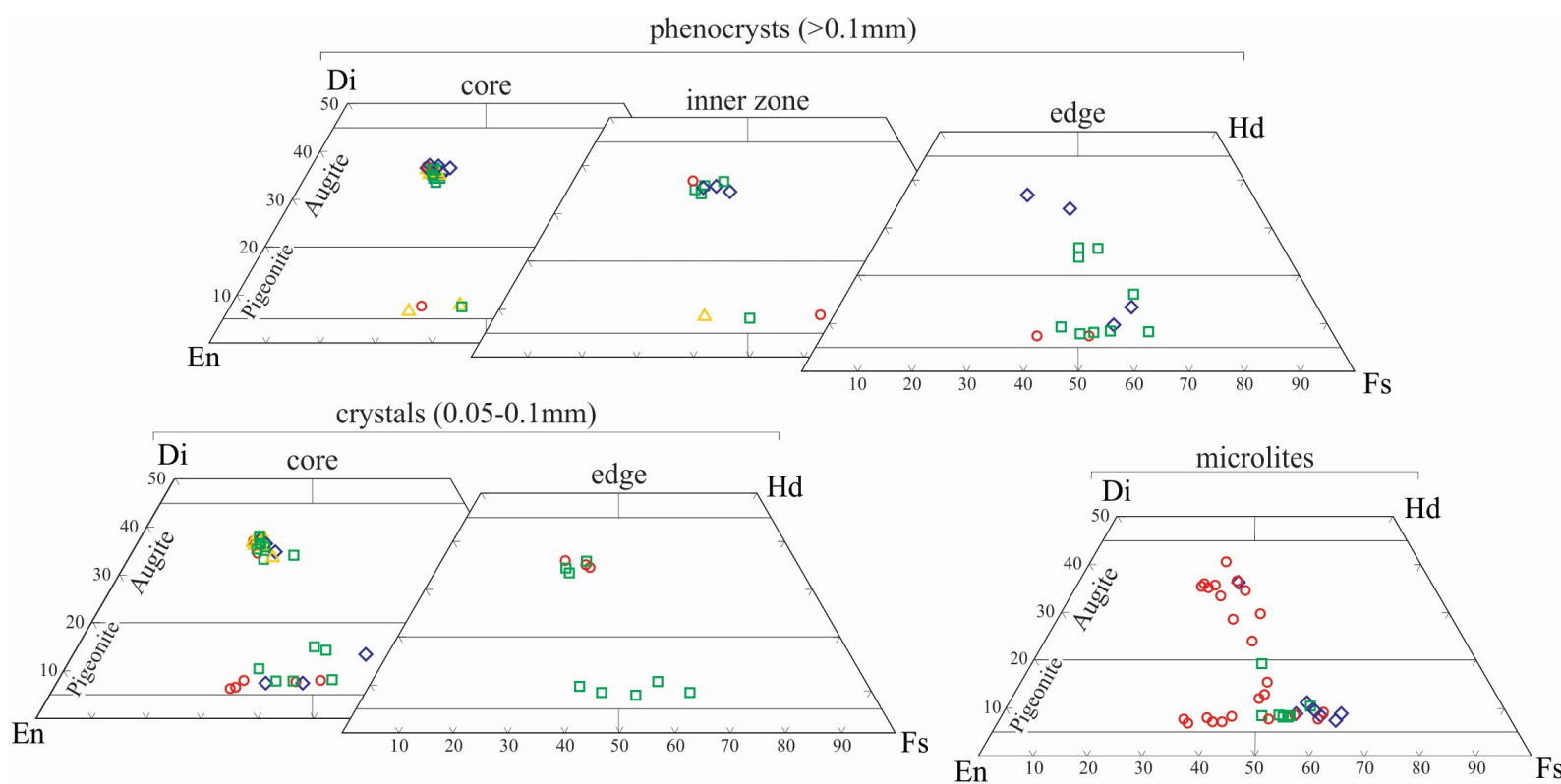

\begin{tabular}{|c|c|}
\hline $\begin{array}{l}\text { SM-01C } \\
\text { (banded deposit) }\end{array}$ & 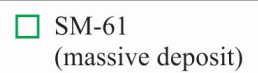 \\
\hline
\end{tabular}

Figure 25: Di-Hd-En-Fs diagrams for classification of pyroxenes from Caxias do Sul dacites.
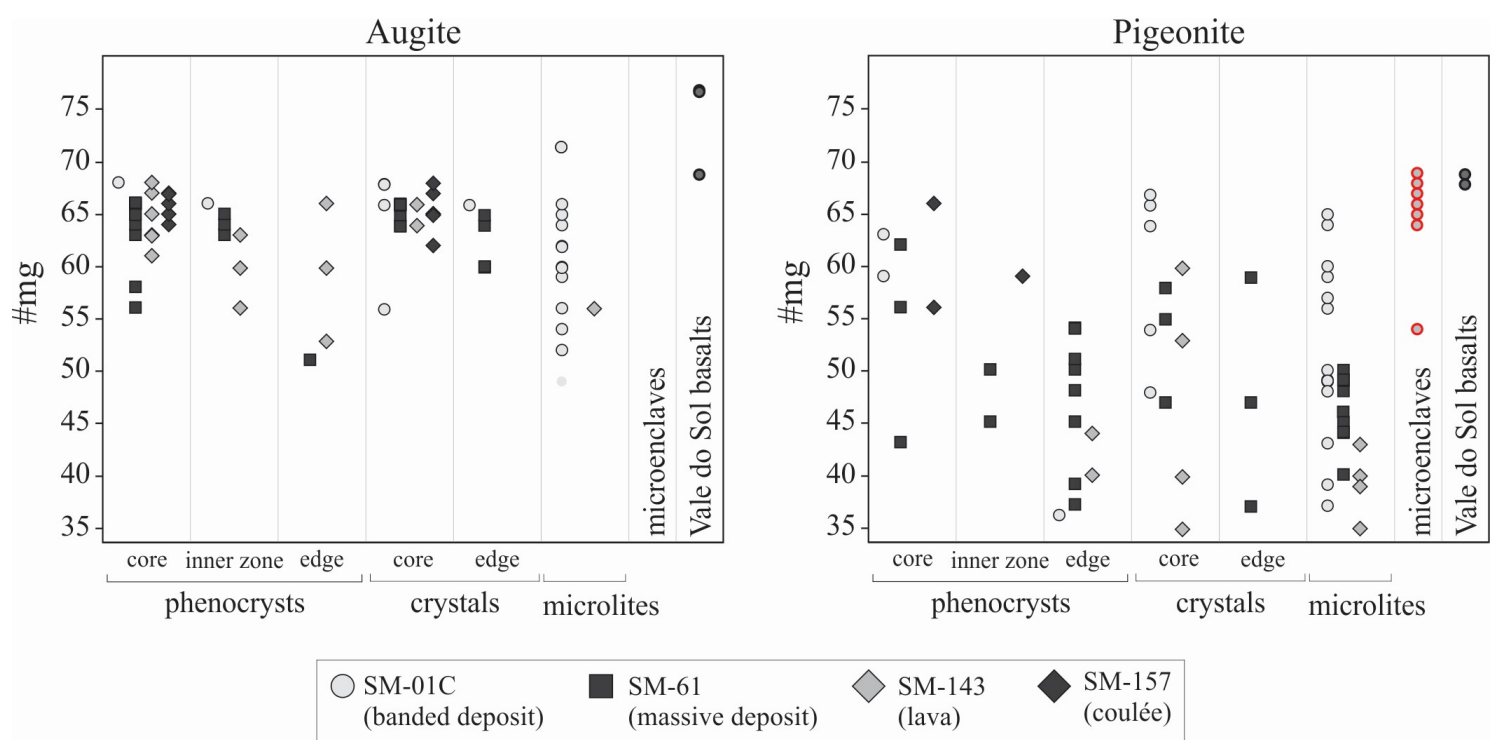

Figure 26: Diagrams for \#mg variations as a function of crystals population of augite (left) and pigeonite (right) from Caxias do Sul dacites. Crystals from the microenclaves from the vitrophyric dacitic sample (SM-01D) are also presented, and examples from pyroxene compositions from basaltic samples (Vale do Sol Fm) from the literature are also shown for comparison purposes. 
Ti-magnetite is usually associated to pyroxene and occurs in clusters of euheral to subhedral crystals (figure 22). Subhedral to anhedral, rounded-shaped, microlites are dispersed on the groundmass (figure $22 \mathrm{~J}$ ). Quantitative electron-microprobe analyses on Ti-magnetite were not feasible due to the crystal sizes and intense exsolution.

Apatite amounts to $<1 \%$ of the total volume and is a primary accessory phase. It occurs as acicular microlites randomly dispersed in the groundmass. Quartz and Kfeldspar (sanidine) are products of devitrification. Under optical and electronic microscopy (SEM), the bands macroscopically interpreted as possible extremely stretched fiammes or pseudotachylites are completely devitrified, forming a quartzfeldspar aggregate.

\section{References}

Branney, M.J., Barry, T.L., and Godchaux, M., 2004, Sheathfolds in rheomorphic ignimbrites: Bulletin of Volcanology, v. 66, p. 485-491, doi:10.1007/s00445-0030332-8.

Cañón-Tapia, E., and Raposo, M.I.B., 2018, Anisotropy of magnetic susceptibility of silicic rocks from quarries in the vicinity of São Marcos, Rio Grande do Sul, South Brazil: Implications for emplacement mechanisms: Journal of Volcanology and Geothermal Research, v. 355, p. 165-180, doi:10.1016/J.JVOLGEORES.2017.07.018.

Guimarães, L.F., Raposo, M.I.B., Janasi, V.A., Cañón-Tapia, E., and Polo, L.A., 2018, An AMS study of different silicic units from the southern Paraná-Etendeka Magmatic Province in Brazil: Implications for the identification of flow directions and local sources: Journal of Volcanology and Geothermal Research, v. 355, p. 304-318, doi:10.1016/j.jvolgeores.2017.11.014.

Kendrick, J.E., Lavallée, Y., Ferk, A., Perugini, D., Leonhardt, R., and Dingwell, D.B., 2012, Extreme frictional processes in the volcanic conduit of Mount St . Helens ( USA ) during the 2004 e 2008 eruption: Journal of Structural Geology, v. 38, p. 61-76, doi:10.1016/j.jsg.2011.10.003.

Kendrick, J.E., Lavallée, Y., Hess, K.-U., De Angelis, S., Ferk, A., Gaunt, H.E., Meredith, P.G., Dingwell, D.B., and Leonhardt, R., 2014, Seismogenic frictional melting in the magmatic column: Solid Earth, v. 5, p. 199-208, doi:10.5194/se-5199-2014.

Lima, E.F., Philipp, R.P., Rizzon, G.C., Waichel, B.L., and Rossetti, L.M.M., 2012, Sucessões vulcânicas, modelo de alimentação e geração de domos de lava ácidos da Formação Serra Geral na região de São Marcos-Antônio Prado (RS): Geologia 
USP - Serie Cientifica, v. 12, p. 49-64, doi:10.5327/Z1519-874X2012000200004.

Luchetti, A.C.F., Gravley, D.M., Gualda, G.A.R., and Nardy, A.J.R., 2018a, Textural evidence for high-grade ignimbrites formed by low-explosivity eruptions, Paraná Magmatic Province, southern Brazil: Journal of Volcanology and Geothermal Research, v. 355, p. 87-97, doi:10.1016/J.JVOLGEORES.2017.04.012.

Luchetti, A.C.F., Nardy, A.J.R., and Madeira, J., 2018b, Silicic, high- to extremely high-grade ignimbrites and associated deposits from the Paraná Magmatic Province, southern Brazil: Journal of Volcanology and Geothermal Research, v. 355, p. 270-286, doi:10.1016/J.JVOLGEORES.2017.11.010.

Peate, D.W., Hawkesworth, C.J., and Mantovani, M.S.M., 1992, Chemical stratigraphy of the Paraná lavas (South America): classification of magma types and their spatial distribution: Bulletin of Volcanology, v. 55, p. 119-139.

Polo, L.A., Giordano, D., Janasi, V.A., and Guimarães, L.F., 2018a, Effusive silicic volcanism in the Paraná Magmatic Province, South Brazil: Physico-chemical conditions of storage and eruption and considerations on the rheological behavior during emplacement: Journal of Volcanology and Geothermal Research, v. 355, p. 115-135, doi:10.1016/J.JVOLGEORES.2017.05.027.

Polo, L.A., and Janasi, V.A., 2014, Volcanic stratigraphy of intermediate to acidic rocks in the southern Paraná Magmatic Province, Brazil: Geologia USP - Serie Cientifica, v. 14, p. 83-100, doi:10.5327/Z1519-874X201400020005.

Polo, L.A., Janasi, V.A., Giordano, D., Lima, E.F., Cañón-Tapia, E., and Roverato, M., 2018b, Effusive silicic volcanism in the Paraná Magmatic Province, South Brazil: Evidence for locally-fed lava flows and domes from detailed field work: Journal of Volcanology and Geothermal Research, v. 355, p. 204-218, doi:10.1016/J.JVOLGEORES.2017.08.007.

Rossetti, L.M.M., Lima, E.F., Waichel, B.L., Hole, M.J., Simões, M.S., and Scherer, C.M.S., 2018, Lithostratigraphy and volcanology of the Serra Geral Group, Paraná-Etendeka Igneous Province in Southern Brazil: Towards a formal stratigraphical framework: Journal of Volcanology and Geothermal Research, v. 355, p. 98-114, doi:10.1016/j.jvolgeores.2017.05.008.

Rossetti, L.M.M., Lima, E.F., Waichel, B.L., Scherer, C.M., and Barreto, C.J.S., 2014, Stratigraphical framework of basaltic lavas in Torres Syncline main valley, southern Parana-Etendeka Volcanic Province: Journal of South American Earth Sciences, v. 56, p. 409-421, doi:10.1016/j.jsames.2014.09.025.

Simões, M.S., Lima, E.F., Sommer, C.A., and Rossetti, L.M.M., 2018, Structures and lithofacies of inferred silicic conduits in the Paraná-Etendeka LIP, southernmost Brazil: Journal of Volcanology and Geothermal Research, v. 355, p. 319-336, doi:10.1016/J.JVOLGEORES.2017.12.013.

Waichel, B.L., Lima, E.F., Viana, A.R., Scherer, C.M., Bueno, G.V., and Dutra, G., 2012, Stratigraphy and volcanic facies architecture of the Torres Syncline, Southern Brazil, and its role in understanding the Paraná-Etendeka Continental Flood Basalt Province: Journal of Volcanology and Geothermal Research, v. 215216, p. 74-82, doi:10.1016/j.jvolgeores.2011.12.004. 


\section{The basal Caxias do Sul dacitic breccias in the Paraná Magmatic Province, south Brazil: evidence for an explosive start?}

“Above all, don't fear difficult moments. The best comes from them."-Rita Levi-Montalcini (Nobel Prize-winning neurobiologist who co-discovered nerve growth factor)

Article to be submitted to the Bulletin of Volcanology.Here, the numbering of figures and tables follows the orderpresented in this thesis.

\section{The basal Caxias do Sul dacitic breccias in the Paraná Magmatic Province, south Brazil: evidence for an explosive start?}

Letícia Freitas Guimarães*,1

Adrian Hornby ${ }^{2}$

Ulrich Kueppers ${ }^{2}$

Adriana Alves ${ }^{1}$

Valdecir de Assis Janasi ${ }^{1}$

Evandro Fernandes de $\mathrm{Lima}^{3}$

Donald Bruce Dingwell ${ }^{2}$

\footnotetext{
*Corresponding author. E-mail address: leticia.guimaraes@usp.br

${ }^{1}$ Geosciences Institute, Universidade de São Paulo. São Paulo, Brazil.

${ }^{2}$ Earth and Environmental Sciences, Ludwig-Maximilians-Universität (LMU). Munich, Germany.

${ }^{3}$ Geoscience Institute, Universidade Federal do Rio Grande do Sul. Porto Alegre, Brasil.
} 


\begin{abstract}
In this work we perform textural analysis on volcanic breccias related to the silicic magmatism in the region of São Marcos (southern Brazil), Paraná Magmatic Province. The breccias are matrix-supported, coarse-grained, poorly-sorted with angular to rounded fragments in a fine-grained matrix. Juvenile fragments are dense to pumiceous, massive to banded dacites; lithic fragments were not recognized.

The analyses were performed on field photographs. Grain size distribution (GSD) analysis showed maximum fragment sizes between -8 and $-9.5 \phi$ and smallest recognized sizes between -3 and $1.5 \phi$; due to the resolution limit of the photographs and the weathered condition of the matrix, the fine fragments from the matrix were not analyzed. The GSD fractality could be quantified by a single power law, with low/moderate D values, suggesting low eruptive energies. Vesicle size distribution (VSD) and shape parameters of both fragments and vesicles were also measured. The fragments are characterized by low vesicularity, with median vesicle area fractions around 5\% for all samples, but pumiceous fragments with up to $43 \%$ area are found. VSDs exhibit single exponential curves, concave upwards, with vesicles becoming more deformed/complex with increasing size, suggesting a single continuous process of nucleation and growth under low degrees of unidirectional shear. Regarding the fragments shape parameters, all the samples have high values of solidity, and a positive correlation of roughness and angularity with fragment size, suggesting secondary fragmentation processes, although field evidence and GSD fractality characterizes proximal deposits. Based on a comparison of the characteristics described here with several types of volcanic breccias, we propose a block and ash flow source for these breccias, and develop a model where shallow magma migration and storage under a fissural conduit can lead to the formation and collapse of lava domes.
\end{abstract}




\section{V.1. Introduction}

Volcanic breccias result from a broad range of fragmentation processes that may occur under intrusive, effusive and explosive conditions. Discrimination of the mechanisms responsible for the generation and emplacement of volcanic breccias requires a non-trivial synthesis of careful textural and petrological description and field associations. However, volcanic breccias offer an opportunity to resolve processes and mechanisms where smaller-scaled textures or those in finer-grained deposits have been rendered intractable through weathering and alteration (e.g. Kirkpatrick and Rowe 2013). One of the enduring examples of this situation is the Parana-Etendeka magmatic province, the second-largest Large Igneous Province in the world. During the last decades, detailed mapping and stratigraphic descriptions (e.g. Peate et al. 1992; Milner et al. 1995; Marsh et al. 2001; Nardy et al. 2008; Polo and Janasi 2014; Rossetti et al. 2018), along with studies of the geochemistry, geochronology, and modelling of pre to syn-eruptive conditions (geothermobarometry and rheology) (e.g. Garland et al. 1995; Nardy et al. 2011; Rocha-Júnior et al. 2012, 2013; Guimarães 2014; Polo et al. 2018a) have ensured important advances in the understanding of the origin and evolution of the Province. Despite this, the eruption mode and processes responsible for emplacing the silicic (dacitic-rhyolitic) units remain controversial (cf. Bryan et al. 2010; Guimarães et al. 2018a,b; Lima et al. 2018; Luchetti et al. 2018a; Polo et al. 2018a). Quantitative textural analyses of the volcanic breccias derived from the initial phase of silicic volcanism in the studied region, related to the Paraná-Etendeka Magmatic Province are still lacking. In this context, the present work aims at a systematic quantitative analysis based on macroscopic textural characterization of the volcanic breccias through both frequency-size distribution and shape parameter analysis. We describe new methods to study and understand the magmatic processes and eruptive dynamics of this magmatism and present an initial effort to provide an eruptive model.

Textural analysis of pyroclastic deposits is most commonly conducted to interpret fragmentation and transport mechanisms, and eventually to make inferences on eruptive processes. In this regard, primary fragmentation corresponds to the initial fragmentation of coherent magma/lava. It typically occurs below the surface, either due to deformation rates exceeding the possibility for viscous response (i.e., the relaxation timescale of the magma; Wadsworth et al. 2018) or by gas overpressure from magmatic 
gas exsolution exceeding the magma's tensile strength. Above the surface, cooling lava breaks in a brittle fashion during flow. Secondary fragmentation concerns existing fragmental mixtures and affects the grain size distribution due to particle interaction during transport in the conduit, the eruption plume or above the ground (Kaminski and Jaupart 1998; Gonnermann 2015; Jones et al. 2016; Paredes-Mariño et al. 2017). It is well-established that examination of grain size distribution of volcanic fragments may be fruitful for the characterization of the fragmentation mechanisms (Turcotte 1986; Sheridan et al. 1987). Subsequent studies (e.g. Turcotte, 1992; Korvin, 1992; Kaminski and Jaupart 1998; Kueppers et al. 2006; Perugini and Kueppers 2012) have demonstrated that fragmentation is a scale-invariant process, i.e. fragmentation occurs to the same degree at different scales. Therefore, the frequency-size distribution of the resulting fragments follows fractal theory and the efficiency of the fragmentation process can be quantified; the higher the fractal dimension of the fragments size distribution, the higher the fragmentation efficiency (Kueppers et al. 2006).

In addition to grain size distribution studies, quantitative textural analysis of volcanic particles through comparison of shape parameters and particle components (such as vesicles and crystals) on 2-D stereoscopic projections can provide valuable information about eruption dynamics and fragmentation modes (Polacci et al. 2003; Maria and Carey 2007; Heilbronner and Barrett 2014; Liu et al. 2015, 2017 and references therein). Since the fragmentation of volcanic material produces fragments with complex and irregular shapes, their description requires the use of multiple parameters and dimensions, such as area, perimeter and diameter. Non-dimensional ratios of these measures define shape parameters such as axial ratio, form factor, roundness, convexity, solidity and others, which allow the quantification of particles' irregularities by contrasting with reference shapes such as convex hull (Heilbronner and Barrett 2014; Liu et al. 2015). Single-particle shape analysis also allows comparisons among volcanic particles and different samples to help infer and contrast the processes responsible for their generation (e.g. Nurfiani and Bouvet de Maisonneuve 2018; Jones et al., 2016; Hornby et al., 2019). Liu et al. (2015) assigned four main shape parameters to describe volcanic ash particles by categorizing them into 'morphological roughness', 'textural roughness' and 'form'. The latter describes the overall shape of particles based on differences between two dimensions, while roughness analysis describe both larger (morphological roughness) and smaller (textural roughness) concavities in a particles 2- 
D projection by measuring spatial variations based on area and perimeter, respectively. This set of analytical methods, together with field observations, provide a powerful tool for the physical characterization of volcanic deposits, thus allowing better inferences about their generating processes.

\section{V.2. Geologic background}

The opening of the South Atlantic during the Early Cretaceous ( 135-130 Ma) was preceded by intense magmatism, giving rise to the second largest LIP (Large Igneous Province) in the world, the Paraná-Etendeka Magmatic Province (PEMP). Most of this Province, around 75\% of its preserved volume (ca. 600,000 km 3 ), lies in South America (the Paraná Magmatic Province, or simply PMP) which, including subvolcanic and volcanic occurrences, covers a preserved area of $\sim 920,000 \mathrm{~km}^{2}$ (Frank et al. 2009) (figure27).

Tholeiitic basalts comprise the majority of these rocks (ca. 97 vol. \%), with subordinate, interbedded and chemically related dacites and rhyolites (ca. 3\% vol. \%) (figure 27). The classification of rocks in the PMP is based on geochemical criteria, which defined six main basaltic magma types (Pitanga, Paranapanema and Ribeira, characterized by high Ti contents; Gramado and Esmeralda, with low Ti contents; and Urubici, with high $\mathrm{Ti}$ and $\mathrm{Sr}$ contents) and two silicic magma types (Chapecó and Palmas, high and low Ti contents respectively) (Bellieni et al. 1984; Mantovani et al. 1985; Peate et al. 1990, 1992; Garland et al. 1995; Nardy et al. 2008). Regarding the silicic magmatism, a large number of geochemical and petrological studies have led to established models for its origin; remelting of basaltic underplates in the case of Chapecó rocks (Bellieni et al. 1986; Garland et al. 1995) and fractional crystallization of related basalts with crustal assimilation in the case of Palmas rocks (Mantovani et al. 1985; Garland et al. 1995; Peate, 1997; Guimarães 2014). However, recent studies have resumed the debate concerning the mode of emplacement for the silicic deposits, and contrasting theories support either an effusive or explosive origin. On the one hand, recent studies at the Brazilian portion of the province suggest multiple fissural feeder systems (e.g. Lima et al. 2018; Guimarães et al. 2018a; Simões et al. 2018), opposing the central cylindrical conduit model (e.g. Milner and Ewart 1989; Milner et al. 1992; 


\section{Chapter V}

The basal Caxias do Sul dacitic breccias in the Paraná Magmatic Province, south Brazil: evidence for an explosive start?

Bryan et al. 2010). On the other hand, there is ongoing debate regarding the occurrence or absence of pyroclastic phases (e.g. Guimarães et al. 2018a; Luchetti et al. 2018a,b; Polo et al. 2018a,b). Large-scale unimodal volcanic breccias have been found in the dacitic units at the PMP and have typically been interpreted as deposits from autobrecciation of lava flows.
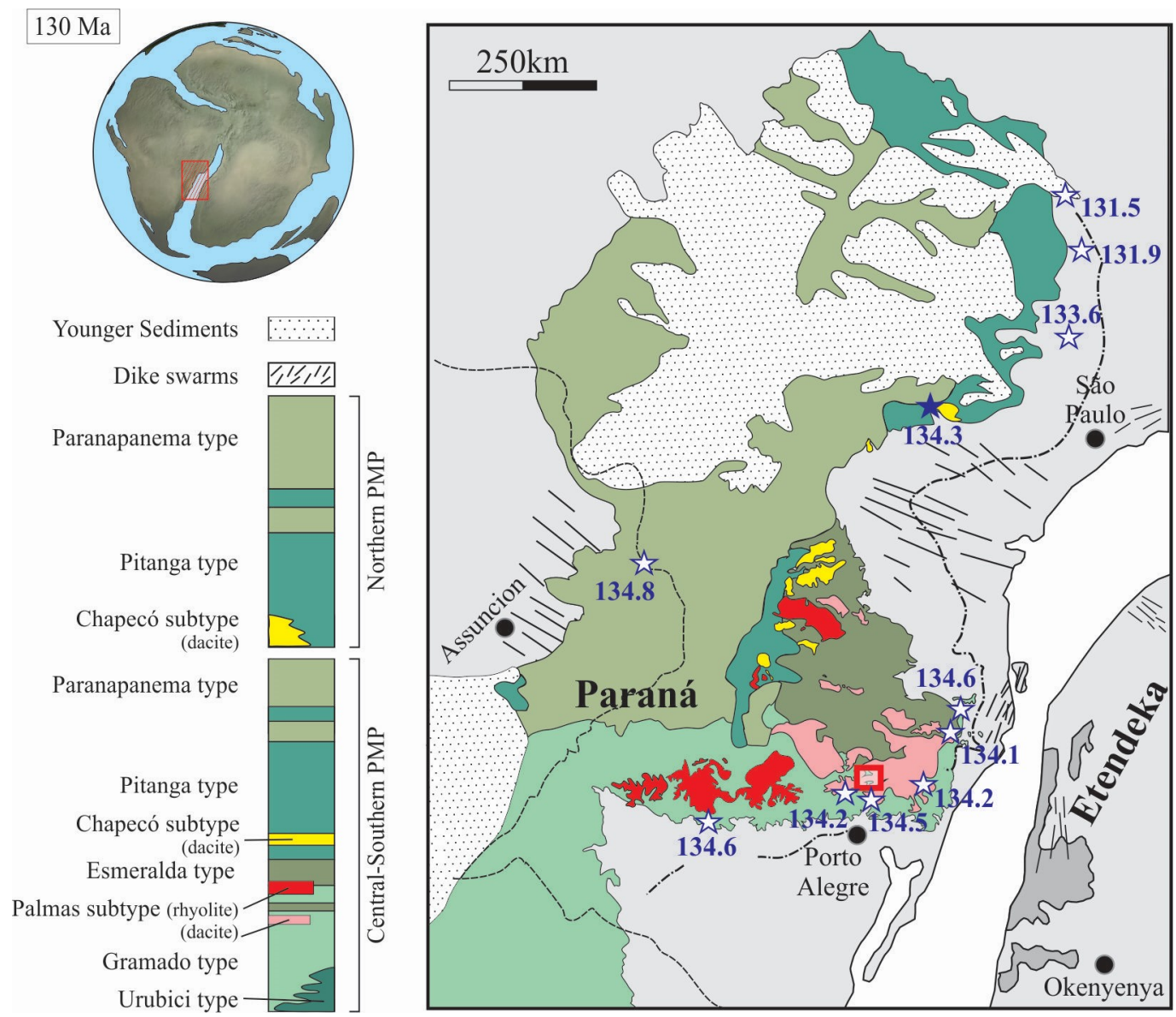

Figure 27:Simplified geological map and stratigraphic charts from the Paraná Magmatic Province - volcanic and sub volcanic (dykes swarms) occurrences - and younger sediments. Basalts are represented in green shades, while silicic rocks are represented in yellow, pink and red. The location of the study area is indicated by the red square. Open stars: sites of ${ }^{40} \mathrm{Ar} /{ }^{39} \mathrm{Ar}$ dating of lava flows and associated sills by stepped heating (Renne et al., 1992; Ernesto et al., 1999; Thiede and Vasconcelos, 2010); Close star: site of U-Pb baddeleyite/zircon dating (Chapecó dacite; Janasi et al., 2011). All dates in Ma. For interpretation of the colors references the reader is referred to the web version of this article. Modified after Peate et al., 1996; Nardy et al., 2008; Janasi et al., 2011 and Waichel et al., 2012.

The Palmas Formation, which makes up $80 \%$ of silicic rocks from PMP, outcropping an extensive E-W trending plateau in South Brazil (figure 27) and comprises dacites and rhyolites $\left(\mathrm{SiO}_{2}\right.$ contents ranging from $64 \%$ to $\left.73 \%\right)$ divided into six members: Santa Maria, Clevelândia, Caxias do Sul, Jacuí, Anita Garibaldi and Barros Cassal (in order of increasing $\mathrm{P}_{2} \mathrm{O}_{5}$ and $\mathrm{TiO}_{2}$ and decreasing $\mathrm{SiO}_{2}$ contents; Peate 
et al. 1992; Garland et al. 1995; Nardy et al. 2008; Polo and Janasi 2014). In the study region, at the vicinities of São Marcos city, South Brazil (figure 27), the Caxias do Sul dacites are chemically and mineralogically homogeneous, with $\mathrm{SiO} 2$ contents ranging from $65 \%$ to $69 \%$ and containing microphenocrysts of plagioclase (labradorite to andesine), clinopyroxene (augite and pigeonite) and Ti-magnetite lying in a partially to completely devitrified groundmass (Guimarães et al. 2018a). A variety of textures and structures have allowed the recognition of a fissural feeder system with a NW-SE trend, and proximal deposits have been interpreted as the products of an initially explosive volcanism that evolves to effusive events (figure 28; Guimarães et al. 2018a). The first silicic volcanic magmatism in the region corresponds to proximal rheoignimbrites and volcanic breccias, that have been interpreted as a result of an initial low-explosivity pyroclastic phase by Guimarães et al. (2018a). After this phase, a transition between explosive and effusive events is suggested based on the occurrence of hybrid deposits (characterized by synchronous volcanic breccias and coulées) which gradually gave place to lobate lavas (figure 28; Guimarães et al. 2018a).
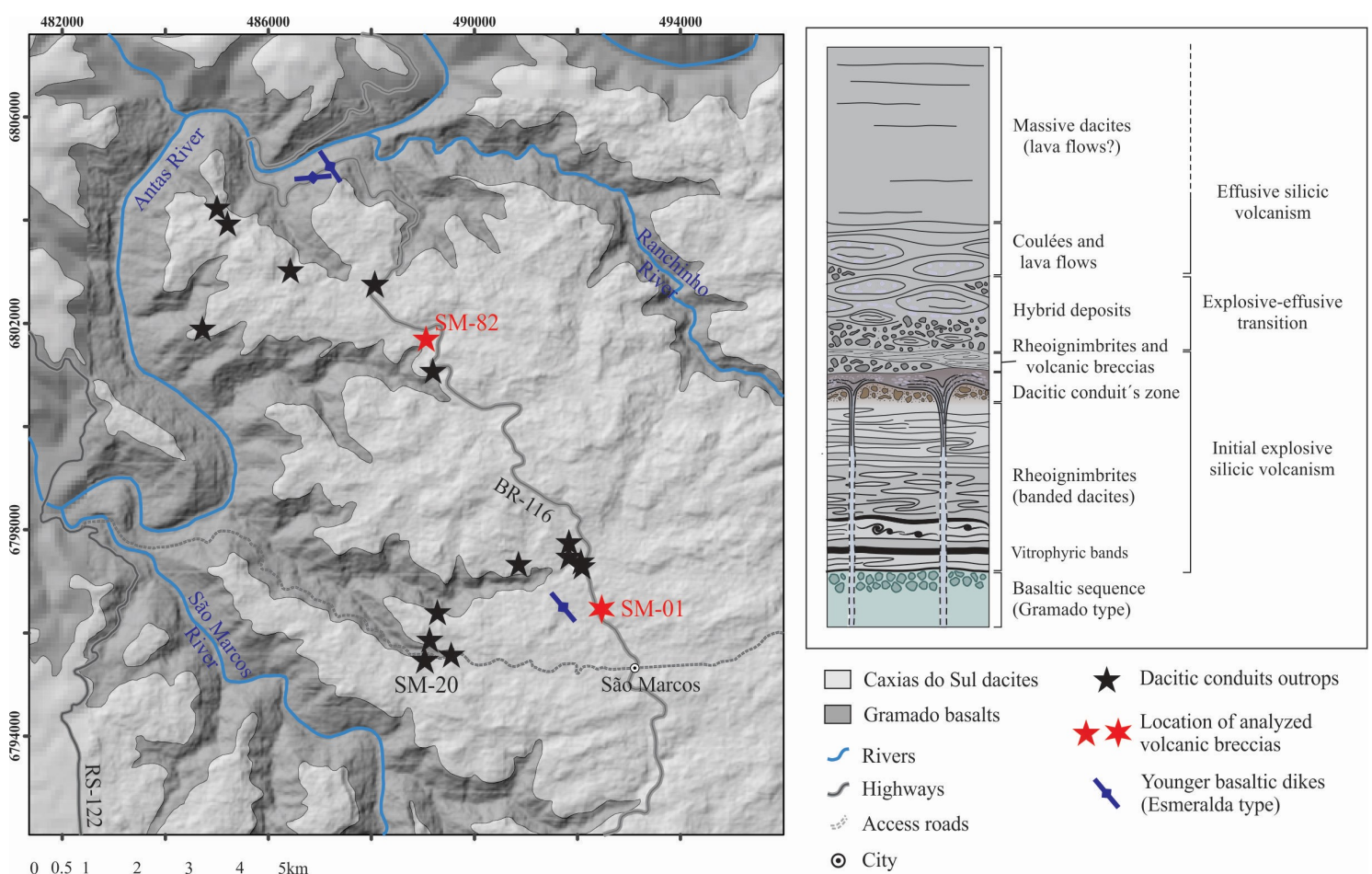

Figure 28:Geologic map and resumed stratigraphic chart from the study area in the vicinity of São Marcos city. The black stars indicate the locations where the conduits system crop out, while the location of the analyzed breccias are represented by red stars. For interpretation of the colors references the reader is referred to the web version of this article. Simplified from Guimarães et al., (2018a). 
The present work focuses precisely on dacitic breccias from the Caxias do Sul subtype. Dacitic volcanic breccias have been described throughout the Brazilian portion of the PMP, being generally classified as autobrecciation in lava flows (e.g. CañónTapia and Raposo 2018; Polo et al. 2018b; Simões et al. 2018) or as friction breccias at the margins of silicic conduit systems (Simões et al. 2018). More rarely an explosive origin has also been attributed to these deposits (Guimarães et al. 2018a). According to these authors, in the study area the volcanic breccias occur at the base of the silicic sequence, (i) in the vicinity of the locations recognized as a fissural conduit zone, underneath banded deposits (interpreted as rheoignimbrites by Guimarães et al. (2018a) and Luchetti et al. (2018b, a) or (ii) inside this zone, overlapping and/or intercalated to the banded deposits, which are intruded by the filamented vertical flow structures that characterize the silicic feeder system (Lima et al. 2018; Guimarães et al. 2018a). In the first case, the proximal deposits are visible in restricted exposures, covering areas of only a few dozen square meters with a maximum thickness up to 4 meters, although the discontinuity of the outcrops makes it impossible to precisely delimit its volume or area. At the latter case, these "breccia domains" (as named by Guimarães et al. 2018a), can reach up to 15 meters in length with a maximum outcrop height of 7 meters. In all scenarios, the contact with the related facies (either the banded deposits or the filamented vertical flow intrusions) is abrupt and irregular.

\section{V.3. Methods}

The analyses were carried out in four outcrops, one outcrop in the vicinity of the feeder zone (SM-01; Guimarães et al. 2018a), where the volcanic breccia underlies a rheoignimbrite, and three quarries where the breccias have been intruded from below, interpreted as a conduit zone (SM-05, SM-20, SM-82; Guimarães et al. 2018a). In the latter case, for the textural analysis we selected three different areas from quarry SM-82 (referred from here on as SM-82_Br1, SM-82_Br2 and SM-82_Br4), where the breccias appear to be unaffected by the intrusive flow and are most likely to preserve primary features. Due to the nature of these deposits and the tropical climate, they are usually intensely weathered and are well preserved in the SM82 quarry due to the silicification related to fluid percolation. The textural analyses of the breccias were carried out using HDR (high dynamic range) photographs. HDR images are taken from 3 shots of the 
same scene, one taken under normal exposure, one underexposed, and one overexposed. The combination of the photos results in an image with a range of illumination and contrast more similar to the human eye, allowing a better definition of the contours of the fragments to be drawn. After image acquisition, the fragment outlines were traced using CorelDraw, and saved as binary (black and white) images which were analyzed using the software ImageJ. Considering the average size of the fragments, the photos were taken covering a minimum area of $1 \mathrm{~m}^{2}$ and, due to the condition of the deposits and the resolution of the images, fine particles (i.e. $<16 \mathrm{~mm}$ in diameter for sample SM-01, $<0.4 \mathrm{~mm}$ for sample SM-82_Br1, $<1.1 \mathrm{~mm}$ for sample SM-82_Br2 and $<0.8$ $\mathrm{mm}$ for sample SM-82_Br4) and the matrix components were not included in the analysis. Vesicles were defined from the original images by grey scale thresholding. Two such images were combined to reproduce vesicle shapes that were partially in shadow.

\section{V.3.1 Size distribution analysis (GSD and VSD)}

Size measurements such as area, perimeter and the major and minor axis of the best fit ellipse were obtained from ImageJ for each fragment.

For the grain size distribution (GSD) analysis, the major axes of the best fit ellipses were used for calculation of $\phi$ parameter $[\phi=-\log 2$ (diameter in $\mathrm{mm}$ )], plotted on $2 \mathrm{D}$ size distribution diagrams. It is noteworthy that $3 \mathrm{D}$ histograms obtained after stereological conversion do not differ significantly from 2D diagrams. The fractal distribution of the size of the fragments was carried after 3D stereological conversion of the data using the software CSDCorrections (Higgins 2000). The shape parameters used for this conversion were tested by varying the ratio between the major, intermediate and minimum axes until the model data approximated those obtained for the sample; a bestfit ellipse with roundness 0.8 and axes ratio equal to 1.0:1.3:1.9 was used for all samples. To avoid "end-member effects" related to the lower statistical representativeness of the finest and coarsest fragments, these data were not taken into account for the elaboration of the log-log diagrams, ensuring minor errors in the analysis. 
For the vesicle size distribution (VSD) analysis we have considered the diameter of a circle with equivalent area, and the results were plotted on both 2D size frequency histograms and VSD curves after stereological conversion.

\section{V.3.2 Shape Parameters and macro}

Shape parameters were calculated for both fragments and vesicles using an intraparticle component (IPC) shape macro for ImageJ (Hornby 2018), which allows the calculation of the shape parameters of particles and multiple intra-particle components (e.g. crystals, vesicles) from sectorized (single-component) images of a sample, linking the components' results to their host particle. The macro uses shape definitions largely following Liu et al. (2015); abbreviations and descriptions measurement or calculation are listed in tables 3 and 4. The macro calls on the Shape Filter plugin (Wagner and Lipinski 2013) within ImageJ to make measurements rather than the native Analyze Particles function. The Shape Filter plugin uses connected contour labelling and 8connected contour tracing (Chang et al. 2004); 8-connected contour calculations allow pixels to be connected to any of the 8 surrounding pixels in a $3 \times 3$ grid, with 4 pixels connected orthogonally and 4 pixels connected diagonally. Perimeter measurements are made by conversion to 8-connected Chain Code (Freeman 1961) with correction factors applied to orthogonal and diagonal distances of 0.948 and 1.340 respectively. These correction factors, calculated by Proffitt and Rosen (1979), reduce the mean error for chain code measurements on lines of infinite length at any orientation. This is one of several approaches to minimizing error in measuring a linear distance between pixels in a raster (Fatemi et al. 2016). The Shape Filter perimeter measurements represent a significant improvement on perimeter measurements made using the Analyze Particles function, however it is unclear whether the same method is used within Shape Filter to calculate the convex hull perimeter. As convex hull measurements exceeded perimeter measurements in some cases, a manual correction factor of 0.968 was applied to all convex hull perimeter measurements for each set of results to reduce overestimation of convexity. The corrected values are labelled 'CH perimeter correction' and 'Convexity correction' respectively. 
Chapter V

The basal Caxias do Sul dacitic breccias in the Paraná Magmatic Province, south Brazil: evidence for an explosive start?

Table 3: Abbreviations used in particle parameter calculations, figures and results.

\begin{tabular}{|ll|}
\hline$A$ & Area \\
$A_{C H}$ & Convex hull area \\
$P$ & Perimeter \\
$\mathrm{P}_{\mathrm{CH}}$ & Convex hull perimeter \\
$\mathrm{D}_{\max }$ & Max. Feret diameter \\
$\mathrm{D}_{\min }$ & Min. Feret diameter \\
AR & Axial ratio (Feret) \\
$\mathrm{D}_{\mathrm{C}}$ & Diameter of equivalente-area circle \\
$\phi$ & $\mathrm{D}_{\mathrm{C}}$ in Phi $=-\log _{2} \frac{D_{C}}{1000}$ \\
$\mathrm{SLD}$ & Solidity \\
$\mathrm{CVX}$ & Convexity \\
RND & Roudness \\
CRC & Circularity \\
FMF & Form Factor \\
CCL & Chain code length \\
\hline
\end{tabular}

The macro first measures particle shapes in an image, including vesicles in the particles. The image is converted to binary, and a dialogue box prompts the user for pixel scale and the minimum particle area (particles under this size are ignored). The minimum particle size was set to 20 pixels, and pixel dimensions varied between 14.6 26.4 pixels $/ \mathrm{cm}$. The particle dimensions and shape parameters are measured and outlines are added the ROI manager. The macro then prompts the user to load singlecomponent images (these can be loaded as an image stack for multiple components, e.g. vesicles and crystals). All images in a containing folder are loaded at this stage. The components are measured sequentially within each ROI, so that components' results refer to the 'parent' particle (the ROI index in the results). A user prompt is generated for minimum phase area, which was set to 10 pixels in this study. The macro runs the Shape Filter plugin, before calculation of shape factors from the major dimensions of particle components and their respective reference shapes (e.g. convex hull), following the method in table 4. The results were plotted in box-plot diagrams as a function of $\phi$, here calculated based on the Feret diameter. For the vesicle analyzes we have considered the results from fragments with a minimum of 50 vesicles. 
Table 4: Description of macro outputs, parameter calculations and references

\begin{tabular}{|c|c|c|}
\hline Title & Calculation & References; notes \\
\hline Label & - & File name of image slice \\
\hline Area & Pixel count & Wagner and Lipinski (2013) \\
\hline Perimeter & $\begin{array}{l}0.948 \text { (orthogonal CCL) } \\
+1.34 \text { (diagonal CCL) }\end{array}$ & Freeman (1961); Proffitt and Rosen (1979) \\
\hline $\mathrm{CH}$ Area & $\mathrm{A}_{\mathrm{CH}}($ pixel count $)$ & Wagner and Lipinski (2013) \\
\hline $\mathrm{CH}$ Perimeter & $\mathrm{P}_{\mathrm{CH}}$ & Wagner and Lipinski (2013) \\
\hline Solidity (SLD) & $\frac{A}{A_{C H}}$ & Liu et al. (2015); 1 = fully convex \\
\hline Convexity (CVX) & $\frac{P_{C H}}{P}$ & Liu et al. (2015); 1 = fully convex \\
\hline Concavity Index & $\sqrt{\left(1-S L D^{2}\right)+\left(1-C V X^{2}\right)}$ & Liu et al. (2015); $0=$ fully convex \\
\hline $\begin{array}{l}\text { Form Factor (or Cox } \\
\text { Circularity) }\end{array}$ & $4 \pi \frac{A}{P^{2}}$ & $\begin{array}{l}\text { Heilbronner and Barrett (2014); Bagheri et } \\
\text { al. (2015); } 1 \text { = fully circular }\end{array}$ \\
\hline Circularity & $\frac{P}{2(\sqrt{\pi A})}$ & $\begin{array}{l}\text { Heilbronner and Barrett (2014); } 1=\text { fully } \\
\text { circular }\end{array}$ \\
\hline Roundness (RND) & $\frac{4 A}{\mu D \max ^{2}}$ & $\begin{array}{l}\text { Pons et al. (1999); Liu et al. (2015); } 1 \text { = fully } \\
\text { round }\end{array}$ \\
\hline AR Feret & $\frac{D_{\min }}{D_{\max }}$ & $1=$ equant; inverse of aspect ratio \\
\hline Feret d & $\begin{array}{l}D_{\max } \quad \text { (rotating calipers } \\
\text { algorithm*) }\end{array}$ & *https://github.com/bkiers/RotatingCalipers \\
\hline MinFeret d & $\begin{array}{l}D_{\min } \quad \text { (rotating calipers } \\
\text { algorithm*) }\end{array}$ & *https://github.com/bkiers/RotatingCalipers \\
\hline $\begin{array}{l}\text { Max. Insc. Circle } \\
\text { Diam. }\end{array}$ & $\begin{array}{l}\text { Diameter of largest circle fitting } \\
\text { inside the particle }\end{array}$ & Wagner and Lipinski (2013) \\
\hline Orientation & Major axis orientation & $0-180^{\circ}$ \\
\hline ROI Index & - & Particles labeled left to right, top to bottom \\
\hline
\end{tabular}




\section{V.4. Results}

\section{V.4.1 Characterization of breccias}

The dacitic volcanic breccias are massive (non-banded) and matrix-supported, with a brownish fine ash-dominated matrix. The fragments are rounded to sub-angular, with shapes ranging from low to high sphericity and sizes varying from $1 \mathrm{~mm}$ to $\sim 1 \mathrm{~m}$. They are mostly constituted of pumice, although dense fragments, massive or banded, also occur (figure 29; Guimarães et al. 2018a). Despite the textural variability of the fragments, they comprise exclusively juvenile material - lithic fragments related to previous units were not recognized. It is worth mentioning that in some outcrops within the conduit zone, the considerable volume of dacitic intrusive lava has visibly affected the "breccia domains". This thermal imprint may have remelted, remobilized and recrystallized the pyroclastic units and formed coherent, deformed and variably welded units. On the other hand, where these breccias outcrop with higher extension and lateral continuity, they are less affected (or even unaffected) by the intrusive flow (figure 29).

Using an optical microscope, it is possible to recognize submilimetric angular, cuneiform fragments. The fragments, which consist of a microlite-rich partially devitrified glass, are dispersed in a fine-grained altered matrix, partially recrystallised to quartz and feldspar (figure 29). Microlites can be locally aligned and comprise skeletal plagioclase and subhedral pyroxene and magnetite. 


\section{Chapter V}

The basal Caxias do Sul dacitic breccias in the Paraná Magmatic Province, south Brazil:

evidence for an explosive start?

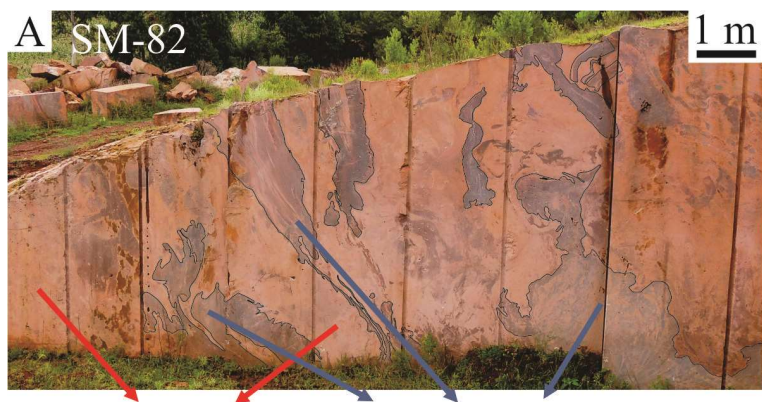

Volcanic breccias

Intrusive flow
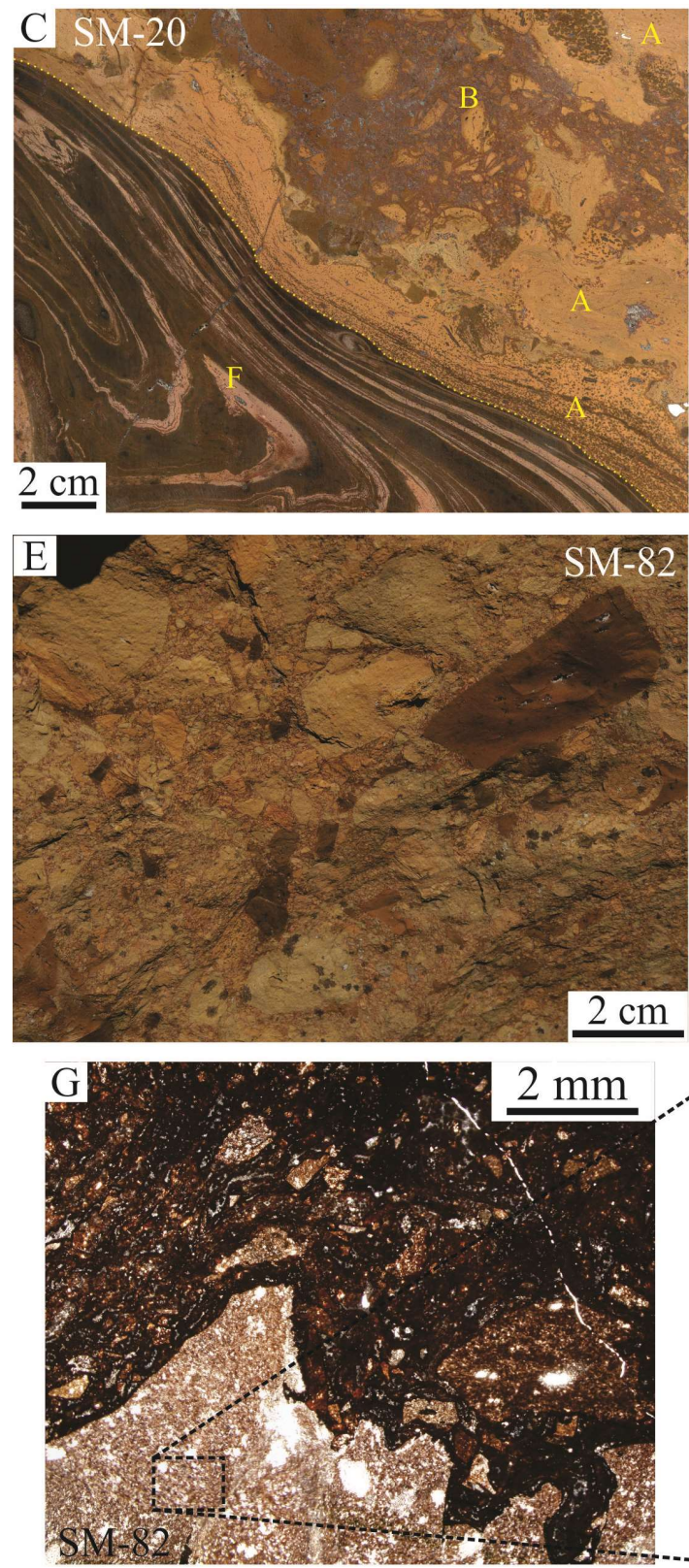
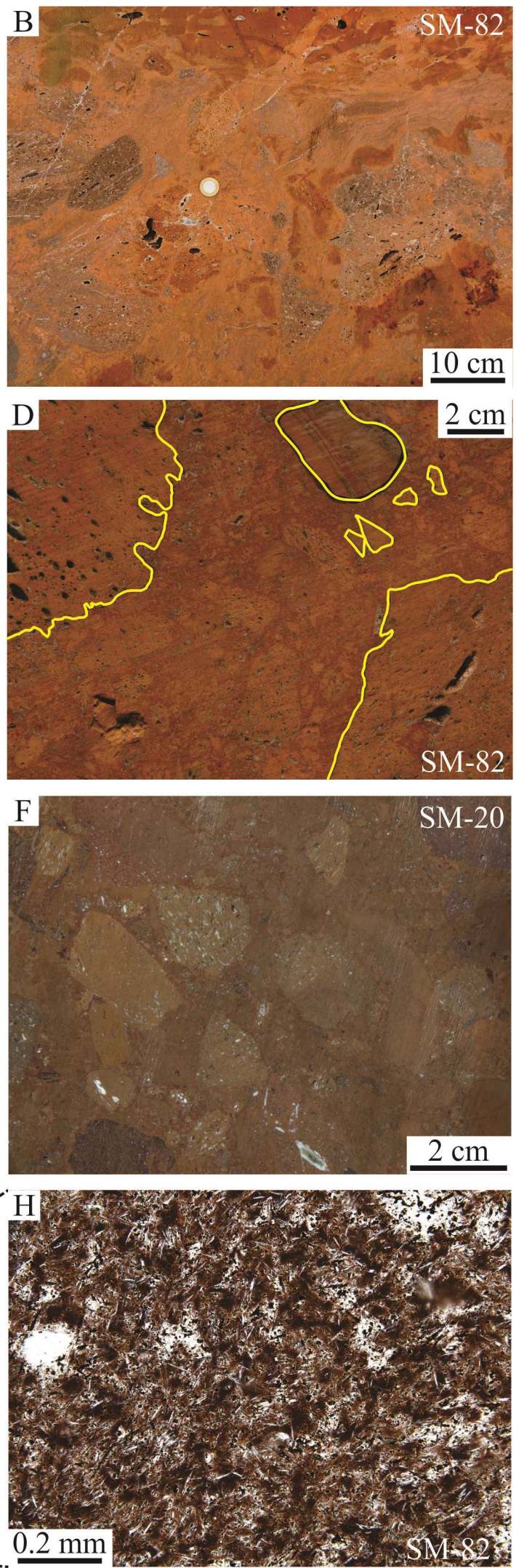

Figure 29: Macro and microscopic aspects of the volcanic breccias. A) Quarry SM- 82 - dacitic volcanic breccias (reddish shades) are intruded by dacitic flows (gray shades). B, D, E) Detail of the volcanic breccias from quarry SM- 


\section{Chapter V \\ The basal Caxias do Sul dacitic breccias in the Paraná Magmatic Province, south Brazil: evidence for an explosive start?}

82, with rounded to sub-angular,variable degrees of macroscopically visible flow-banding, dense to vesicle-bearing fragments in a fine-grained matrix, with more angular and smaller (millimetric) fragments. C, F) Detail of the volcanic breccias from quarry SM-20, with rounded to sub-angular, dense to vesicle-bearing fragments in a finegrained matrix, with more angular and smaller (millimetric) fragments. The volcanic breccias (labeled as "b") in B and $\mathrm{C}$ are affected by the intrusive flow (labeled as "f") as suggested by rheomorphic features (labeled as "a"). G, H) Petrographic aspects of volcanic breccia from quarry SM-82.Sub-angular, microlite-rich, vesicle-bearing fragmentin a devitrified matrix with sub-millimetric angular fragments.

\section{V.4.1.1 Grain Size Distribution and its Fractal Dimension}

The dacitic volcanic breccias are poorly sorted, with maximum measured fragments size ranging between -8 and $-9.5 \phi(256-725 \mathrm{~mm})$ and the smallest recognized sizes varying between -3 and $1.5 \phi(8-0.4 \mathrm{~mm})$. The median grain size (Md $\phi)$ obtained for sample SM-01, which corresponds to sample underlying the rheoignimbritic deposit, is $-6.4 \phi$ (around $84 \mathrm{~mm}$ ), while the samples from the quarry SM-82, that corresponds to the breccia which hosts the silicic feeder dykes, show Md $\phi$ ranging between -1.1 and $-3.6 \phi(2-12 \mathrm{~mm})$. Note that these values are dependent on the pixel resolution of the photographs and do not take into account fine particles in the deposits that are indistinguishable due to alteration. The results are presented in table 5, and the whole size ranges are presented in the 2D size distribution histograms showed in figure 30. The GSD histograms are unimodal and slightly negative skewed, i.e. a subtle majority of finer $(\leq 1 \mathrm{~cm})$ particles. Due to the applied method and the resolution limit of the photos, the analyzed fragments represent only $28.5 \%$ of the total analyzed area of sample SM-01, and between 31.2 and $48.4 \%$ of the areas evaluated in the SM-82 sample set. This means that a significant fraction of components, represented by the matrix and fragments finer than $16 \mathrm{~mm}$ in diameter in sample SM-01, $0.4 \mathrm{~mm}$ in sample SM-82_Br1, $1.1 \mathrm{~mm}$ in samples SM-82_Br2 and finer than $0.8 \mathrm{~mm}$ in sample SM82_Br4, was not taken into account and would certainly result in more asymmetric and positive skewed GSD curves and lower median diameters. 
Table 5: Statistical parameters of the size and the fractal dimension for each sample.

\begin{tabular}{|c|c|c|c|c|c|}
\hline \multirow{4}{*}{$\begin{array}{c}\text { Parameter } \\
\text { (cf. Inman, 1952) }\end{array}$} & \multicolumn{5}{|c|}{ Outcrop } \\
\hline & SM-01 & \multicolumn{4}{|c|}{ SM-82 } \\
\hline & \multicolumn{5}{|c|}{ Sample } \\
\hline & SM-01 & SM-82_Br1 & SM-82_Br2 & SM-82_Br2_detail & SM-82_Br4 \\
\hline $\begin{array}{l}\text { Phi mean diameter } \\
(\mathrm{M} \phi)\end{array}$ & -6.4 & -3.9 & -3.0 & -1.2 & -3.3 \\
\hline $\begin{array}{l}\text { Phi median diameter } \\
(\mathrm{Md} \phi)\end{array}$ & -6.4 & -3.6 & -2.9 & -1.1 & -3.2 \\
\hline $\begin{array}{l}\text { Phi skewness } \\
(\alpha \phi)\end{array}$ & -0.05 & -0.3 & -0.1 & -0.1 & -0.05 \\
\hline $\begin{array}{l}\text { Mean Size } \\
(\mathrm{mm})\end{array}$ & 84 & 12 & 7 & 2 & 9 \\
\hline $\mathbf{D}$ & 2.1 & 1.9 & 2.3 & 2.3 & 1.9 \\
\hline
\end{tabular}

The fractal character of the samples' grain size distribution was also calculated and the log-log plots (known as Mandelbrot-Richardson plot) are presented in figure 31. In order to verify any possible variation in the GSD parameters and the fractal dimension due to different scales, we have analyzed the same sample under different magnifications (named SM-82_Br2 and SM-82_Br2 detail). The results obtained for the samples SM-82_Br2 and the higher-resolution SM-82_Br2_detail were the same despite the different size ranges of fragments, demonstrating that the GSD of the sample follows a single power-law, regardless of the scale of analysis (Figure 31). It is clear from the plots that a single power-law relationship can be used to describe the GSDs regardless of scale or resolution. D-values are 2.1 for sample SM-01 and range from 1.9 to 2.3 for samples SM-82, with high correlation values $(\mathrm{r} 2>0.9)$ in all samples. 


\section{Chapter V}

The basal Caxias do Sul dacitic breccias in the Paraná Magmatic Province, south Brazil:

evidence for an explosive start?

\section{Outcrop SM-01:}
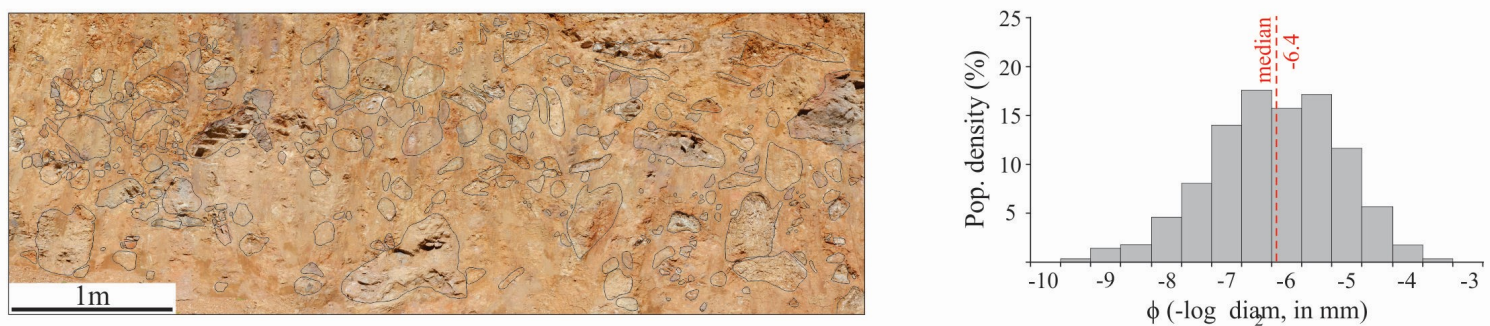

Outcrop SM-82:
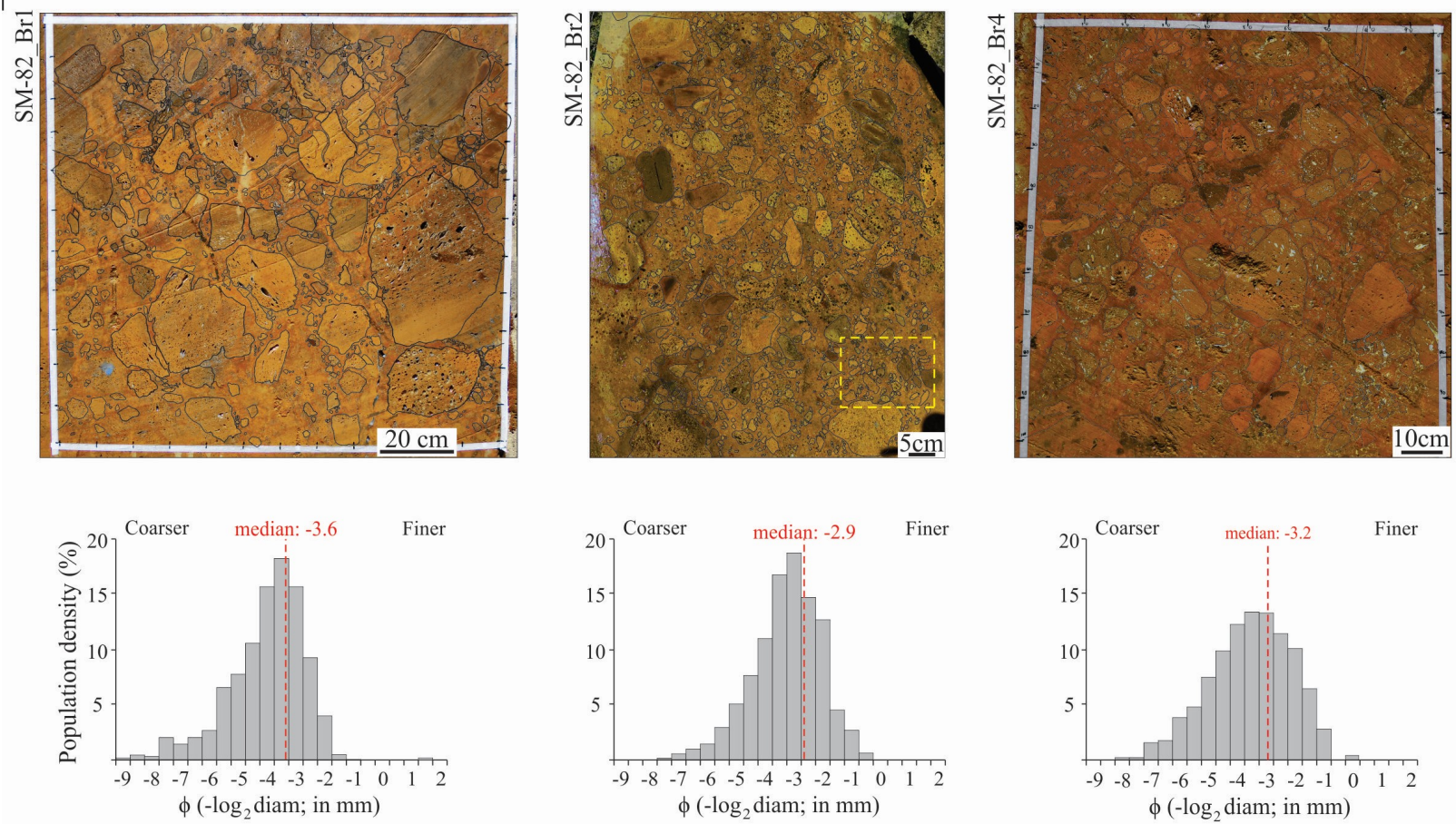

$\mathrm{SM}-82 \mathrm{Br} 2$ detail
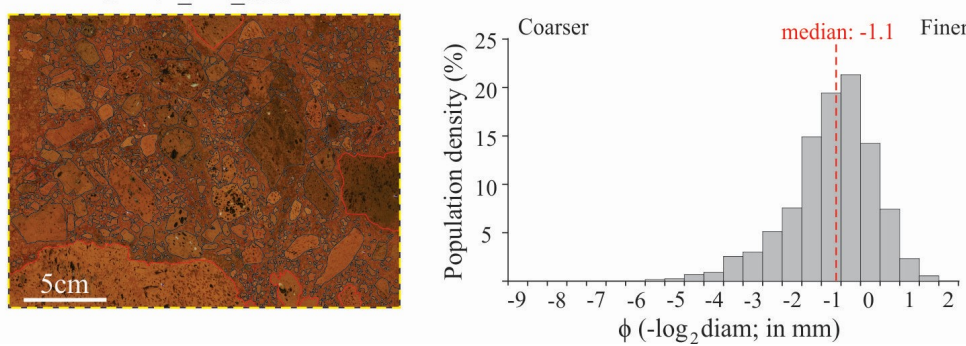

Figure 30:Grain-size distribution histograms from the studied volcanic breccias. $\Phi$ is giving by $-\log _{2}$ (diameter), where diameter is giving in millimeters. SM-82 detail corresponds to the area delimited by the dashed yellow square on SM-82 sample. The fragments perimeters are marked by black lines on the breccias photos - these were used to calculate the parameters of interest (e.g. perimeter, area, GSDs, shape parameters) presented inAppendix IV. 

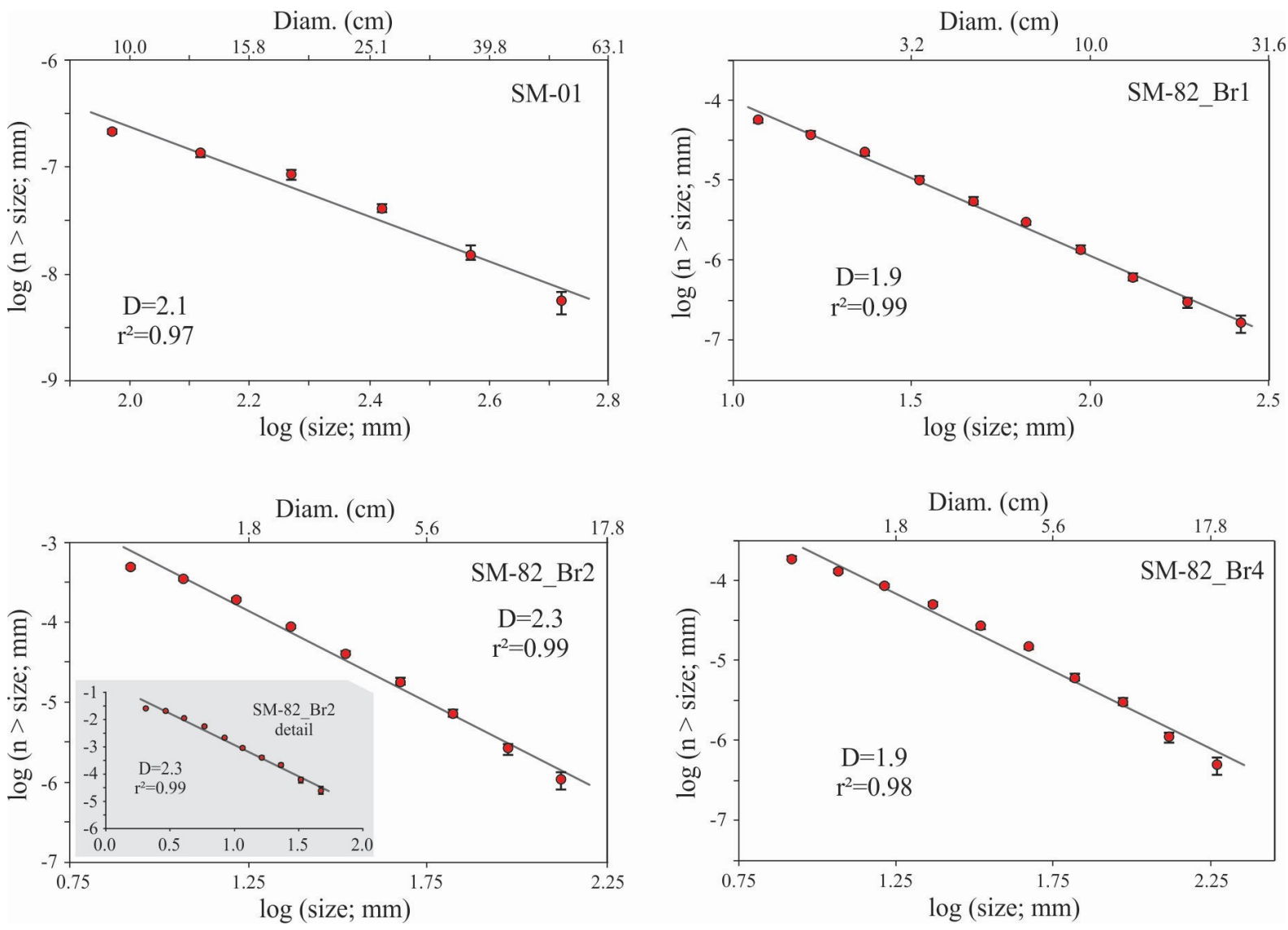

Figure 31: $\log -\log$ plots of the cumulative frequency $(\log [M(r<R)]) v s$ size $(\log (r))$ for the same samples from figure 4. Linear fitting of GSDs, values of correlation coefficient $\left(\mathrm{r}^{2}\right)$ and the fractal dimension value (D), which corresponds to the slope of the linear fitting, are also presented.

\section{V.4.1.2 Shape Parameters}

The samples were described in terms of axial ratio, form factor, roundness and solidity (see Table 4) as the main shape parameters needed for an effective description of morphological variance in volcanic particles (Liu et al. 2015). Particle shape is strongly influenced by intrinsic (petrographical textures, i.e. crystal number and size as well as vesicle shape, size and frequency) and extrinsic (e.g. fragmentation processes efficiency) factors. These parameters were plotted as a function of size classes $(\phi)$ in the boxplots presented in figure 32. Some trends could be defined; most evident is the decrease in form factor and roundness with increasing size of clasts (lower $\phi$ ). In other words, greater angularity with increasing size. Solidity values are high (SLD > 0.8) in all samples but an incipient declining trend towards larger sizes can be observed. Higher form factor, solidity values and aspect ratio could be observed for sample SM-82_Br04, 
Chapter V

The basal Caxias do Sul dacitic breccias in the Paraná Magmatic Province, south Brazil: evidence for an explosive start?

especially for its larger fragments indicating greater circularity and lesser angularity. No orientation trend could be distinguished. Convexity and solidity data are plotted against each other and compared to fields described in Liu et al. (2015) (figure 33), which also show sample SM-82_Br04 plotting in the dense fragments field. The complete tables with results are presented in the Appendix IV.
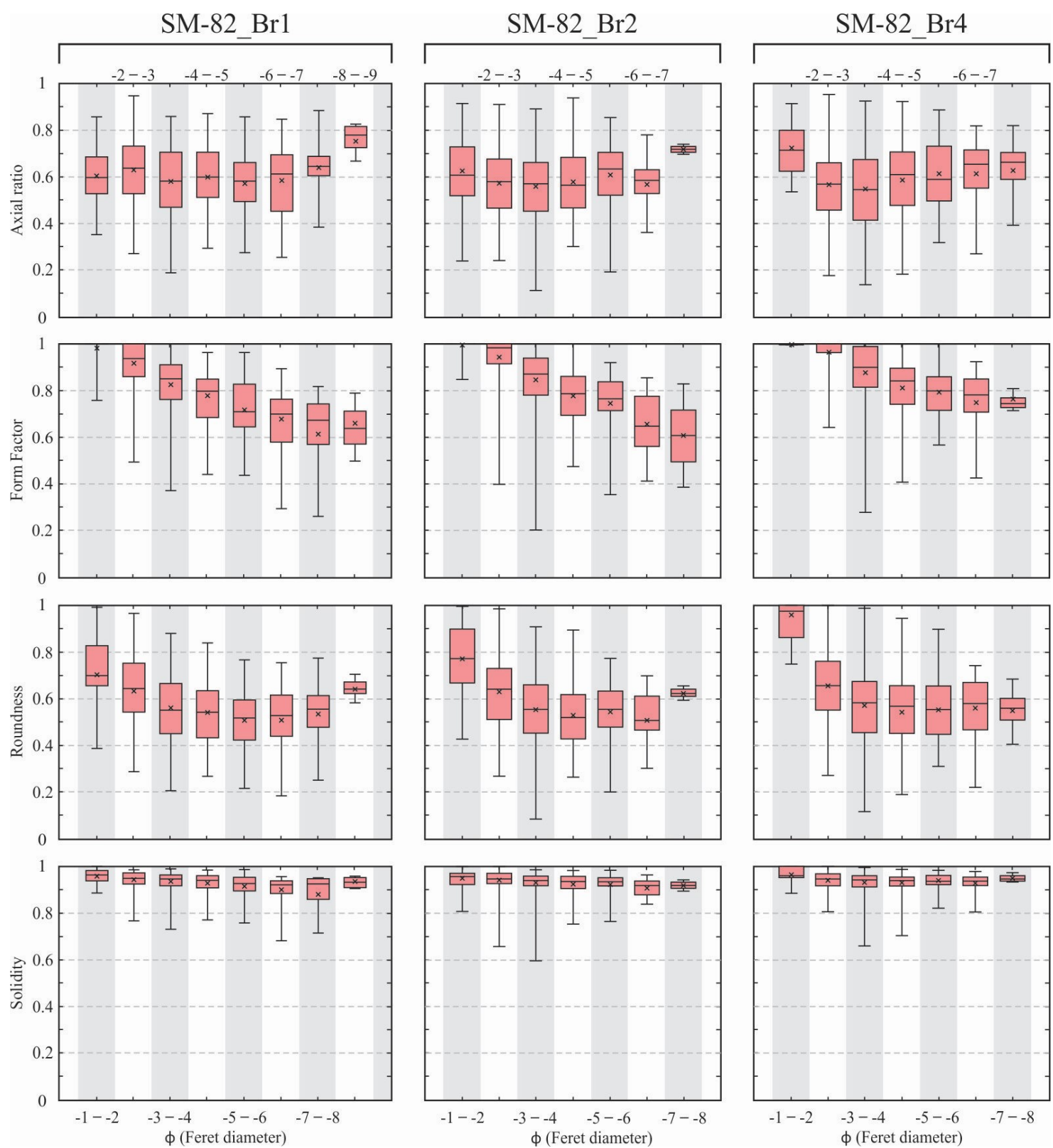

Figure 32: Boxplots showing the variation of clasts' shape parameters (axial ratio, form factor, roundness and solidity) as a function of clasts' size $(\Phi=-\log 2$ [feret diameter]). The box limits represent the 1 st and 3rd quartiles (lower and upper limits, respectively), the middle line represents the median, the " $\mathrm{x}$ " is the average and the whiskers show the minimum and maximum values obtained. 

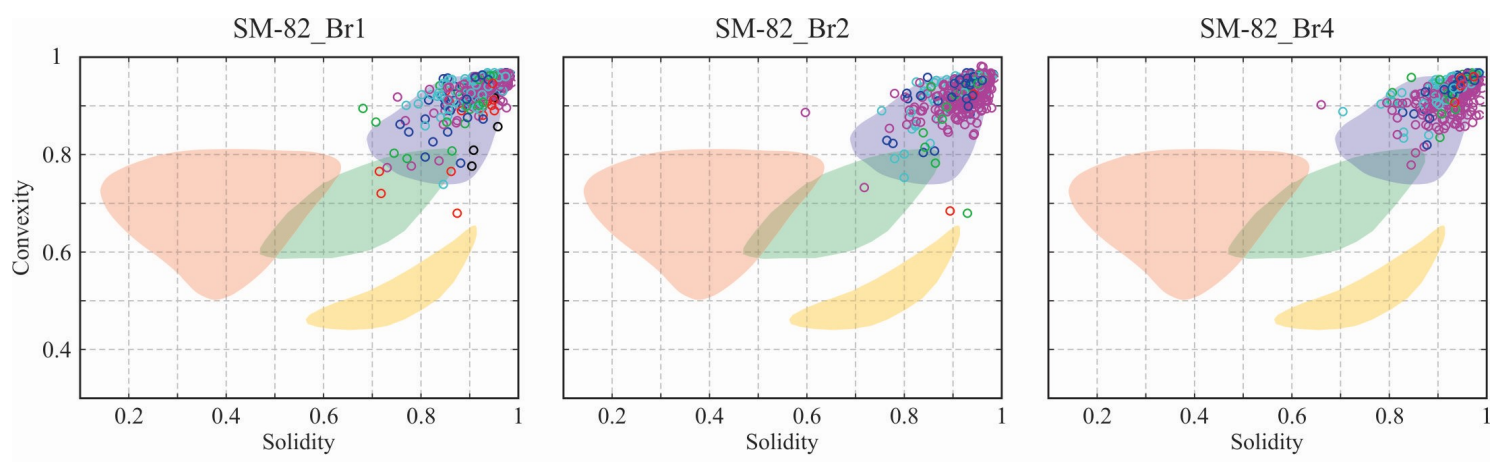

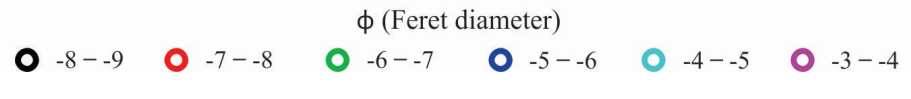

Figure 33: Convexity vs Solidity plots for the SM- 82 samples as a function of clasts' size $(\Phi=-\log 2$ [feret diameter]), divided by colors. The shaded fields corresponds to shards (red), vesicular particles (green), dense fragments (blue) and microcrystalline vesicular particles (orange) extracted from Liu et al. (2015). For interpretation of the colors references in the figure legend the reader is referred to the web version of this article. The convexity values were calculated using the convex hull values manually corrected by a factor of 0.968 .

\section{V.4.2 $\underline{\text { Vesicularity }}$}

\section{V.4.2.1 Vesicle Size Distribution and Shape Parameters}

The samples are characterized by low vesicularity, with area ratios (Avesicles/Aclast) up to 21\% for SM-82_Br01, 43\% for SM-82_Br02 and 23\% for SM82_Br04. 2D size distribution histograms are presented as a function of $\log (\operatorname{size})$, where the 'size' is defined as the diameter of a circle with equivalent area (figure 34), and show a single peak, better defined for samples SM-82_Br01 and SM-82_Br04. The working scale makes the analysis of submillimetric vesicles difficult, hindering the construction of a complete histogram. Vesicle size distribution curves (figure 34) built after stereological conversions present a single exponential curve, concave upwards for all samples. 

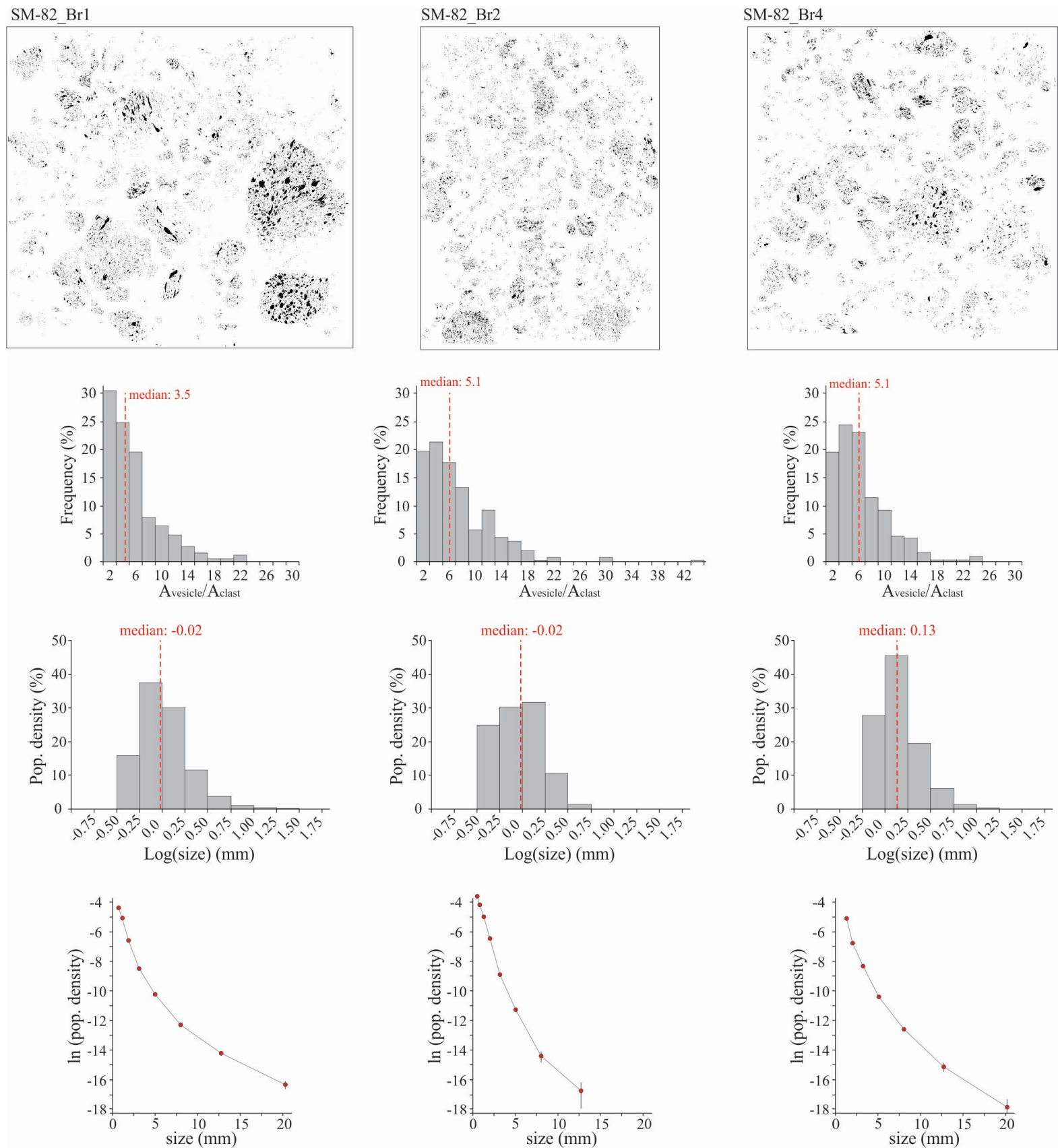

Figure 34: Black and white thresholded images of isolated vesicles used to calculate the parameters of interest (e.g. area, VSDs, shape parameters) presented at the Suplementary Materials. Frequency distribution of Avesicle/Aclast histograms; simple area fraction vesicles-size distribution (VSD) histograms; VSD curves in terms of number density, after stereological conversion considering a sphere with equivalent diameter.

The main shape parameters axial ratio, form factor, roundness and solidity were also analyzed for vesicles within a few of the largest clasts in each sample (figures 35 and 36). In general, vesicles become more deformed and/or more complex with increasing size. There appears to be no trend in preferred vesicle orientation for any of the samples (figure 37), supporting in-situ deposition and minimal welding or flow. 


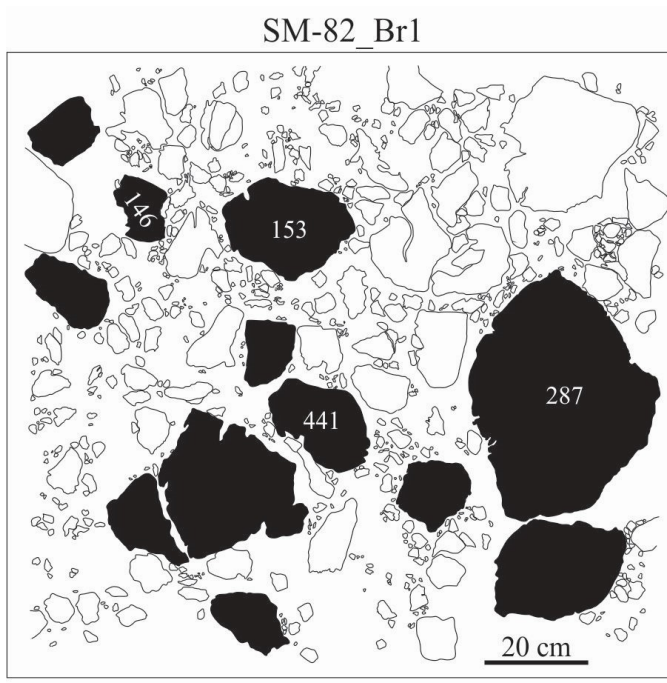

Figure 35: Representative boxplots for sample SM-82_Br1 showing the variation of vesicles shape parameters (axial ratio, form factor, solidity and roundness) as a function of vesicles' size ( $\Phi$ $-\log 2$ [diameter], where diameter corresponds to the diameter of a circle with equivalent area). The highlighted black clasts correspond to those analyzed (with more than 50 vesicles with minimum 10-pixel-area). The results presented here correspond to the vesicles from the numbered black clasts. At the diagrams, the box limits represent the 1st and 3rd quartiles (lower and upper limits, respectively), the middle line represents the median, the " $\mathrm{x}$ " is the average and the whiskers show the minimum and maximum values obtained.
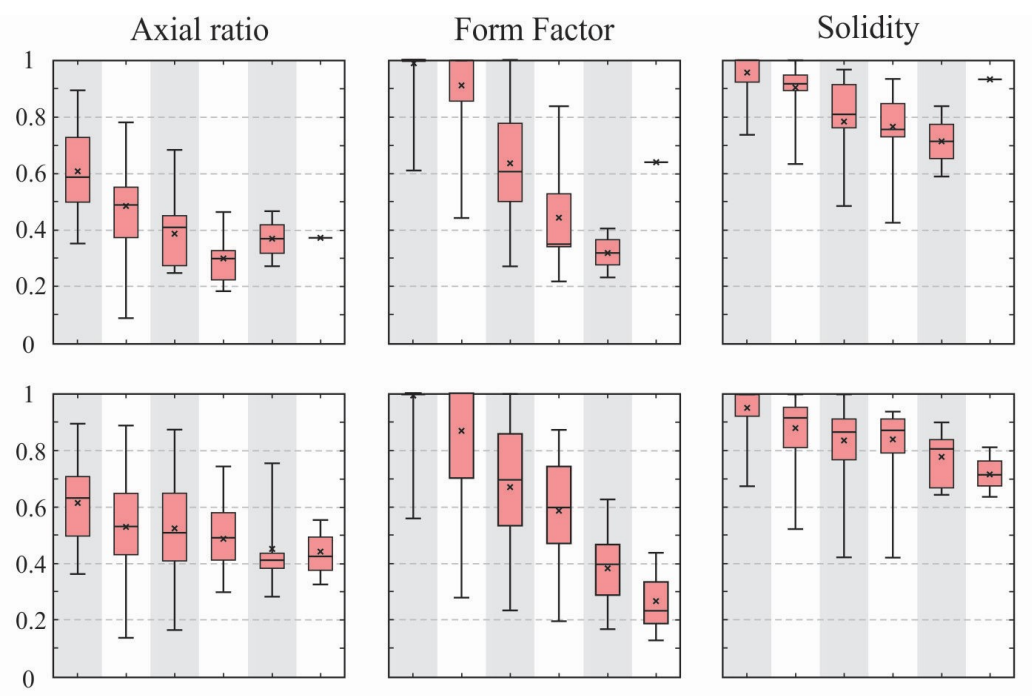

Roundness
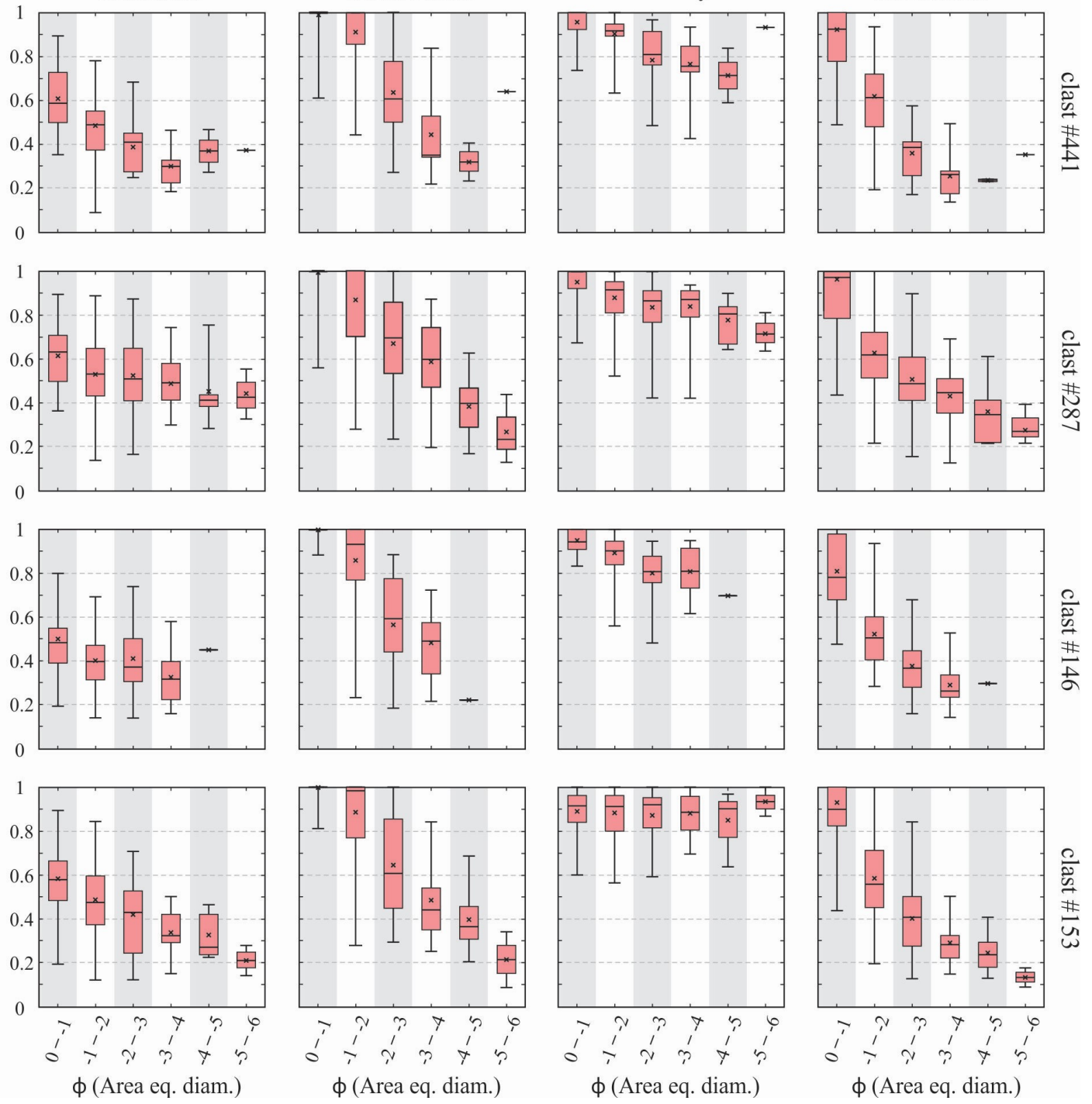

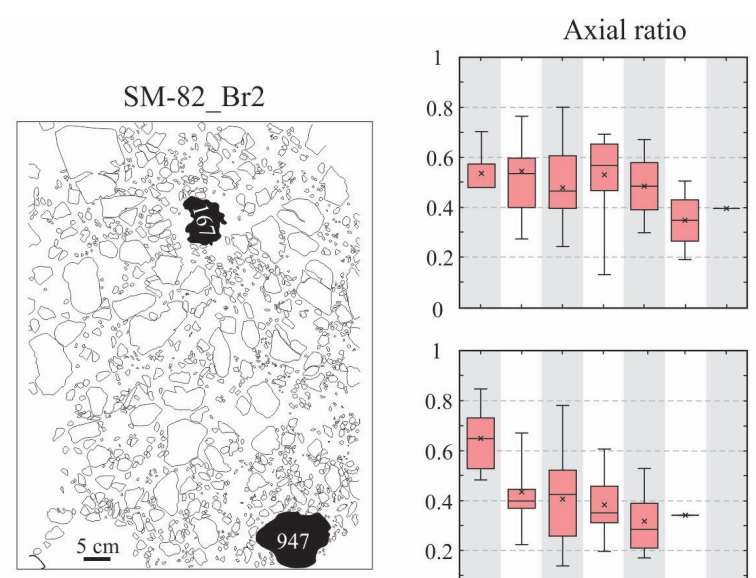

Form Factor
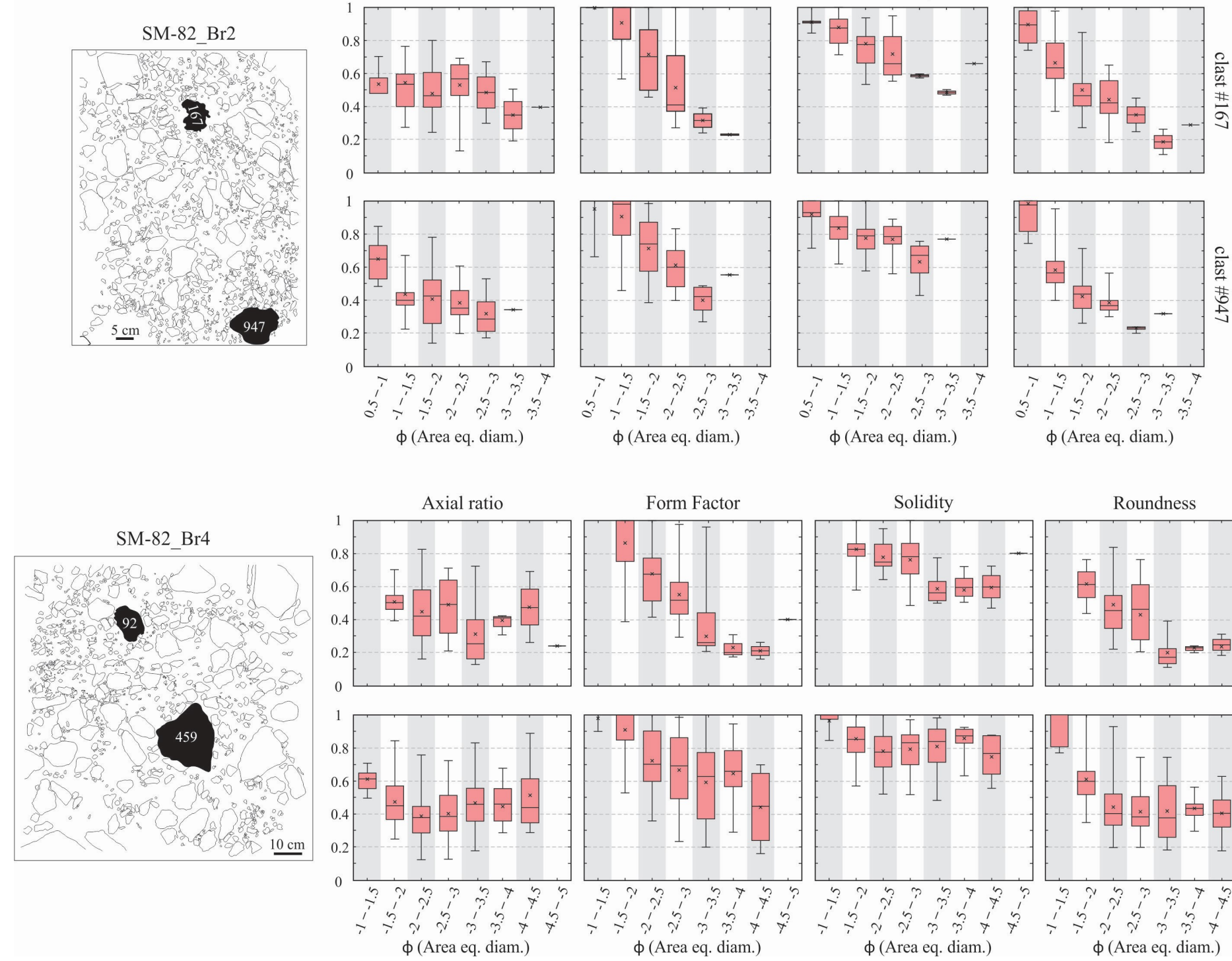
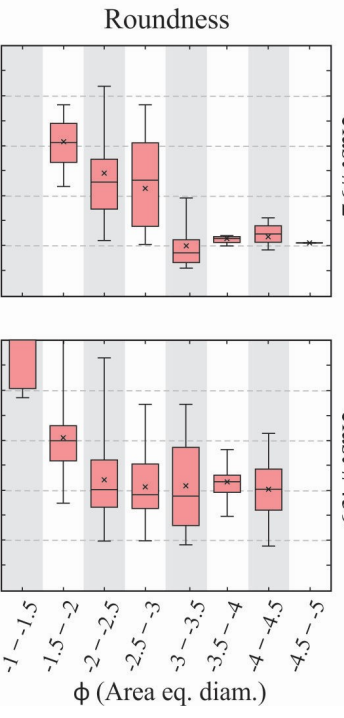

Roundness

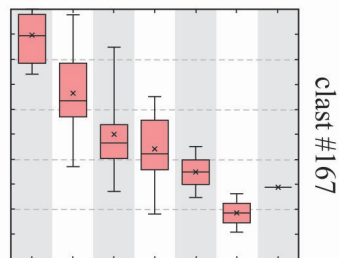

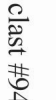

Figure 36: Representative boxplots for samples $\mathrm{SM}-82 \mathrm{Br} 2$ (above) and $\mathrm{SM}$ $82 \mathrm{Br} 4$ (below) showing the variation of vesicles' shape parameters (axial ratio, form factor, solidity and roundness) as a function of vesicles' size $(\Phi=-\log 2$ [diameter], where diameter corresponds to the diameter of a circle with equivalent area). The highlighted black clasts correspond to those analyzed (with more than 50 vesicles with a minimum 10-pixel-area). The results presented here correspond to o the vesicles from the numbered black * clasts. At the diagrams, the box limits represent the $1 \mathrm{st}$ and $3 \mathrm{rd}$ quartiles (lower and upper limits, respectively), the middle line represents the median, the " $x$ " is the average and the whiskers show the minimum and maximum values obtained. 
SM-82 Brl
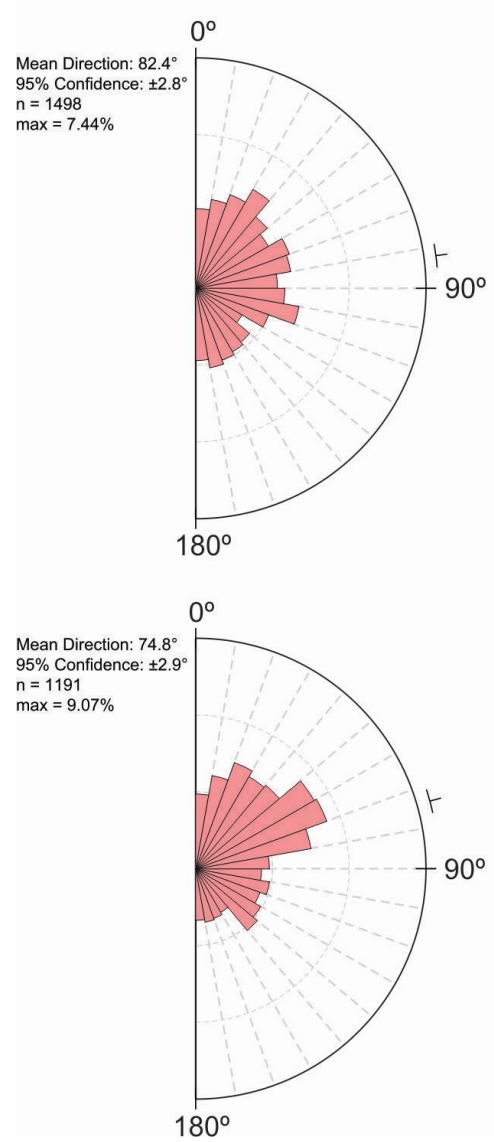

$\mathrm{SM}-82 \mathrm{Br} 2$
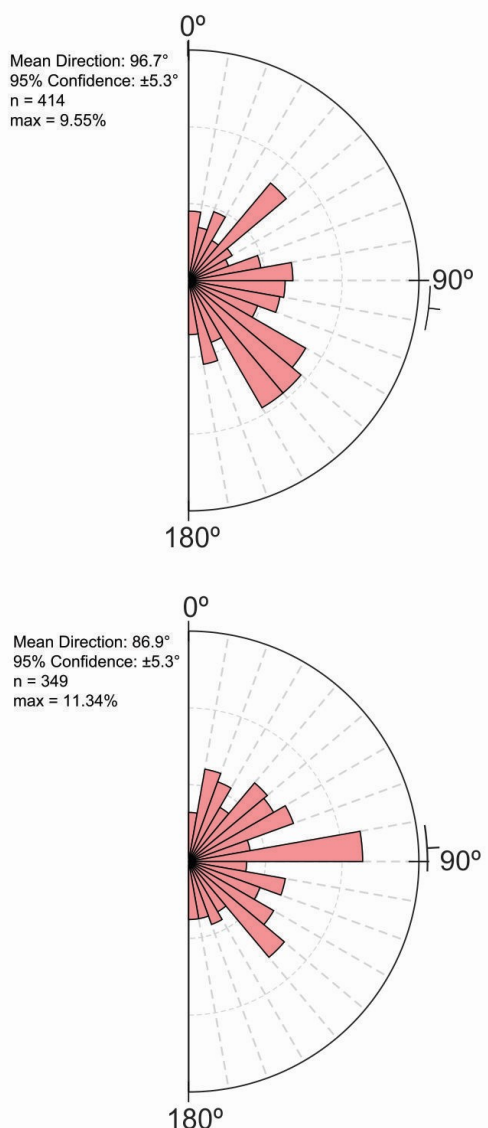

$\mathrm{SM}-82 \mathrm{Br} 4$
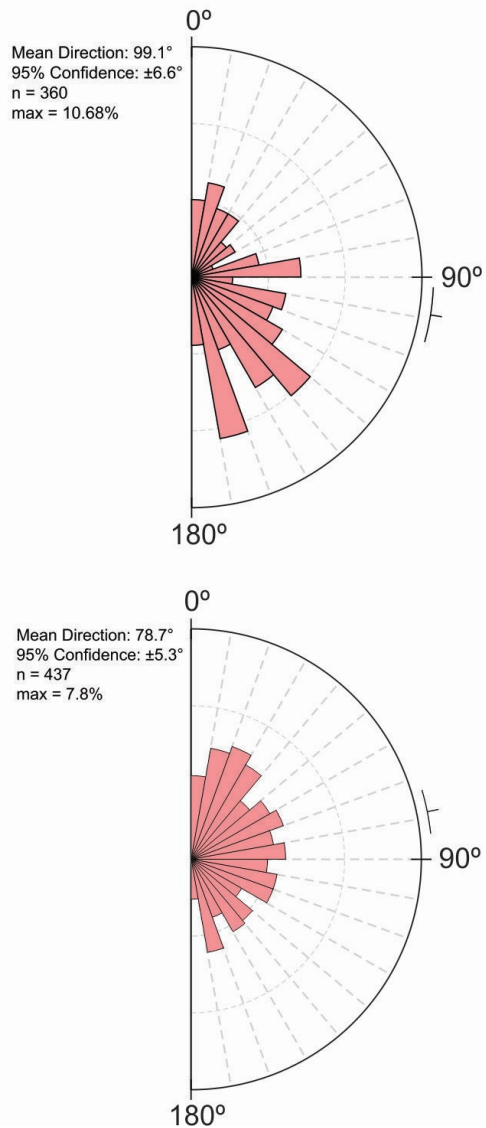

Figure 37: Vesicles orientation for the upper and lower portions of each sample. $0^{\circ}$ points upwards; " $n$ " indicates the number of vesicles measured and "max" represents the maximum the maximum proportion of vesicles obtained in the most measured orientation.

\section{V.5. Discussion}

Since fragments are products of scale-invariant processes, their size distribution is fractal and follows a power law behaviour, being quantifiable from the slope of the straight line defined in a Mandelbrot-Richardson plot (Turcotte, 1992; Kaminski and Jaupart 1998; Kueppers et al. 2006). Recent experimental studies (e.g. Kueppers et al. 2006; Perugini and Kueppers 2012), corroborated by investigations on natural occurrences (e.g. Pepe et al. 2008; Suzuki-Kamata et al. 2009; Perugini et al. 2011; Paredes-Mariño et al. 2017), demonstrated that the fractal dimension (D) is directly related to the open porosity of the samples and to the eruptive energy; in other words, the larger the value of $\mathrm{D}$, the more efficient the fragmentation process. Additionally, Kaminski and Jaupart (1998) suggested that high values of D (greater than 2.5) indicate 
deep fragmentation levels and consequently the significant effect of secondary fragmentation processes. Jones et al. (2017) showed that secondary fragmentation is inevitable during volcanic eruptions, and Paredes-Mariño et al. (2017) demonstrated that it can be recognized from the observation of kinks which define two fit straight lines in the M-R plots.

In our results, the grain size distributions could be quantified by a single power law for all samples, with low to moderate D values $(\leq 2.3)$ and, although Avesicle/Aclast ratios are broadly similar, it is still worth mentioning that the sample with the highest Avesicle/Aclast ratio also corresponds to the highest $\mathrm{D}$, in agreement with Kueppers et al. (2006) and Perugini and Kueppers (2012). Thus, if we consider our fragments as resulting from primary, magmatic fragmentation, these results are suggestive of shallow fragmentation processes characterized by low eruptive energy. Here it is important to note that the lack of information regarding fines from the matrix can mask the occurrence of "kinks" in the M-R plots, consequently hampering the recognition of secondary fragmentation. Similarly, the restricted number of individual outcrops, not far from each other, does not allow us to constrain transport-related sorting.

With respect to fragment shape parameters, we observe a positive correlation of roughness and angularity with fragment size. The small and large-scale recesses are typically due to vesicle nucleation or particle collision (Sarocchi et al. 2011); however particle interactions during secondary fragmentation processes (abrasion) tend to round larger particles by chipping away asperities and forming a secondary fine particle population (Caballero et al. 2012; Kueppers et al. 2012; Jones and Russell 2017). The commonly high values of solidity of our samples indicate smooth fragments; as a roughness textural indicator, the lower its value, the more recesses the surface of the particle will present (Liu et al. 2015). This condition points toward the operation of secondary fragmentation processes; however, field evidence, in accordance with the inferences from GSD and fractal analysis, characterizes proximal deposits. Indeed, these deposits have been described mainly at the vicinities of feeder system structures (e.g. Lima et al. 2018; Guimarães et al. 2018a; Simões et al. 2018) and, despite the characteristic absence of lithic fragments (typical of the deposits from the PMP), coarse volcanic breccias have been interpreted elsewhere as indicators of the location of the 
fissure type vents of the related (rheo)ignimbritic deposits (Aguirre-Díaz and LabartheHernández, 2003).

In summary, the coarse breccia deposits can be generated by (1) lava flow autobrecciation; (2) block-and-ash flows generated by collapse of proximal lava dome; (3) particle-rich pyroclastic density currents; or (4) lag breccias sensu stricto (Walker, 1985; Druitt and Bacon, 1986) (table 6). Although our analyses cannot definitively determine the fragmentation process, we present evidence from outcrops and clast characteristics that conform to different characterisation criteria for the abovementioned deposits, which are summarised in table 6.

Significant variability of the characteristics of the studied breccias has been identified. It is clear that similar volcanic breccia textures can originate from significantly different mechanisms, nonetheless we favour the block-and-ash flow deposit based on our comparative analysis. Indeed, a number of recent works advocate the occurrence of lava domes elsewhere in the PMP (e.g. Polo et al., 2018b; Guimarães et al., 2018a; Lima et al., 2012).

Finally, the pattern shown in the histograms and the VSD curves indicates a single continuous process of vesicle nucleation and growth. Vesicles appear to generally follow a pattern of increasing roundness with decreasing size, and do not have very low axial ratios, suggesting either (i) low degrees of shear experienced prior to quenching or primary fragmentation or (ii) unstable flow regime (heterogeneous/discontinuous shearing). It is worth mentioning that the low crystal content of the rising magma, attested by the exclusive occurrence of microlites in the analysed breccia samples, may lead to a late near-homogeneous vesicle nucleation. However, clast shape does not show a dependency on vesicle size, as corroborated by the high solidity values, due to the great clast size in relation to vesicle size. Indeed, the clasts can be classified as dense fragments derived from brittle fragmentation of poorly-vesicular magma (Liu et al. 2015).

According to the above discussion we attempt to elaborate an eruptive model for these rocks, as follows: 
Table 6: (part 1/4) Volcaniclastic deposit characteristics for lava flow auto breccias, block-and-ash flow, column collapse pyroclastic flows, lava fountain and lag breccias. Regarding the "lag breccias", we have taken into account exclusively those deposits classified by Walker (1985) as "lag breccias strictu sensu", which corresponds to coarse proximal deposits, lateral equivalents of ignimbrites, deposited at the deflation zone, due to gas loss (carrying fine particles) and consequent loss of flow energy (dense phase deposition). In this review work, the author also includes three other mechanisms of formation, which result in differences in the characteristics of the deposits formed (fine depletion, matrix or clast supported, etc), but not considered in this table. Highlighted cells correspond to those that best match the Caxias do Sul breccias studied.

\begin{tabular}{|c|c|c|c|c|c|c|c|}
\hline & $\begin{array}{c}\text { Autoclastic } \\
\text { breccia (effusive) }\end{array}$ & \multicolumn{4}{|c|}{ Pyroclastic breccias (explosive) } & \multirow{2}{*}{ Our data; notes } & \multirow[b]{2}{*}{ References } \\
\hline Parameter & $\begin{array}{c}\text { Lava flow } \\
\text { autobreccia }\end{array}$ & Block and ash & $\begin{array}{l}\text { Column collapse } \\
\text { pyroclastic flow }\end{array}$ & Lava fountain & $\begin{array}{l}\text { strictu }{ }^{1} \text { (Walker, } \\
\text { 1985; Druitt and } \\
\text { Bacon, 1986) }\end{array}$ & & \\
\hline GSD & $\begin{array}{l}\text { Broad; coarse- } \\
\text { skewed; unimodal }\end{array}$ & $\begin{array}{l}\text { Broad; bimodal; } \\
\text { finely skewed }\end{array}$ & $\begin{array}{l}\text { Broad; unimodal; } \\
\text { may vary from } \\
\text { coarse (fine deple- } \\
\text { ted) }\end{array}$ & $\begin{array}{l}\text { Unimodal; coarse- } \\
\text { skewed }\end{array}$ & $\begin{array}{l}\text { Unimodal; coarse- } \\
\text { skewed (strongly } \\
\text { fine-depleted) }\end{array}$ & $\begin{array}{l}\text { Unimodal; slightly } \\
\text { coarse skewed; } \\
\text { matrix/fines not } \\
\text { preserved/analyzed }\end{array}$ & Parsons (1969) \\
\hline $\begin{array}{c}\text { Fractal } \\
\text { Dimension }\end{array}$ & Low & Low - Intermediate & Low - Intermediate & Low - Intermediate & Intermediate -high & Intermediate & $\begin{array}{ll}\text { Kueppers } & \text { et } \text { al. } \\
(2006) ; & \text { Perugini } \\
\text { and } & \text { Kueppers } \\
(2012) & \end{array}$ \\
\hline Sorting & Poorly sorted & Very poorly sorted & $\begin{array}{l}\text { Poorly - very } \\
\text { poorly sorted }\end{array}$ & $\begin{array}{l}\text { Well - moderate } \\
\text { sorted }\end{array}$ & $\begin{array}{l}\text { Poorly to well } \\
\text { sorted }\end{array}$ & Poorly sorted* & $\begin{array}{lr}\text { Parsons } & \text { (1969); } \\
\text { Davies } & \text { et } \quad \text { al. } \\
(1978) ; & \text { Walker } \\
(1985) ; & \text { Pettijohn } \\
(1989) & \end{array}$ \\
\hline
\end{tabular}




\begin{tabular}{|c|c|c|c|c|c|c|c|}
\hline $\begin{array}{c}\text { Clast } \\
\text { orientation }\end{array}$ & Minor - none & Minor - none & $\begin{array}{l}\text { Imbrication may } \\
\text { occur }\end{array}$ & $\begin{array}{l}\text { Minor - none (un- } \\
\text { less viscous flow } \\
\text { occurs) }\end{array}$ & $\begin{array}{l}\text { Minor; local weak } \\
\text { imbrication can } \\
\text { occur }\end{array}$ & None & Pettijohn (1989) \\
\hline Shear & Varying in clasts & $\begin{array}{l}\text { None, unless wel- } \\
\text { ded; clasts may } \\
\text { vary }\end{array}$ & $\begin{array}{l}\text { Often stretched and } \\
\text { welded pumices; } \\
\text { clasts may vary }\end{array}$ & $\begin{array}{l}\text { May occur (viscous } \\
\text { clasts) }\end{array}$ & Varying in clasts & Varying in clasts & $\begin{array}{l}\text { Parsons } \quad(1969) ; \\
\text { Pettijohn (1989) }\end{array}$ \\
\hline Vesicularity & Low - high & Low - high & Moderate - high & $\begin{array}{l}\text { High (scoriaceous } \\
\text { clasts) }\end{array}$ & Low; dense clasts & Low (dense clasts) & $\begin{array}{l}\text { Parsons } \quad(1969) ; \\
\text { Pettijohn (1989) }\end{array}$ \\
\hline Vesicle shape & $\begin{array}{l}\text { As present in flow; } \\
\text { variable }\end{array}$ & $\begin{array}{l}\text { As present in } \\
\text { dome/lobe; variable }\end{array}$ & $\begin{array}{l}\text { Variable; from } \\
\text { rounded } \\
\text { extremely stretched }\end{array}$ & $\begin{array}{l}\text { Commonly } \\
\text { flattened and } \\
\text { stretched }\end{array}$ & Variable & $\begin{array}{l}\text { Variable, from } \\
\text { rounded } \\
\text { extremely stretched }\end{array}$ & \\
\hline Angularity & $\begin{array}{l}\text { Highly angular; can } \\
\text { be jagged with } \\
\text { spinose surface or } \\
\text { smooth poliedral. }\end{array}$ & $\begin{array}{l}\text { Variable (angular } \\
\text { to subrounded); } \\
\text { transport-related: } \\
\text { rounding increase } \\
\text { with distance. }\end{array}$ & $\begin{array}{l}\text { Angular, crushed to } \\
\text { rounded; transport- } \\
\text { related: collision } \\
\text { increase angularity } \\
\text { and spinosity while } \\
\text { abrasion decrease. }\end{array}$ & Subrounded & Variable & $\begin{array}{l}\text { Variable, mainly } \\
\text { subrounded. Fines } \\
\text { at matrix are more } \\
\text { angular, but were } \\
\text { not analyzed. }\end{array}$ & $\begin{array}{lr}\text { Fisher } & (1960) ; \\
\text { Parsons } & (1969) ; \\
\text { Pettijohn } & (1989) ; \\
\text { Freundt } & \text { and } \\
\text { Schmincke } & \text { (1992); } \\
\text { Sarocchi et al. } & \\
\text { (2011); Jones and } \\
\text { Russell (2017) }\end{array}$ \\
\hline
\end{tabular}




\begin{tabular}{|c|c|c|c|c|c|c|c|}
\hline Componetry & $\begin{array}{l}\text { Monomict; uni- } \\
\text { modal; lava flow } \\
\text { material. Fragments } \\
\text { may be glassy to } \\
\text { holocrystalline. } \\
\text { Clast supported. } \\
\text { Can (rarely) incur- } \\
\text { porate fragments of } \\
\text { previous deposits } \\
\text { /sediments. }\end{array}$ & $\begin{array}{l}\text { Monomict; uni- } \\
\text { modal; dome mate- } \\
\text { rial. Fragments can } \\
\text { be glassy to holo- } \\
\text { crystalline. } \\
\text { Groundmass: same } \\
\text { finer material. } \\
\text { Matrix to clast sup- } \\
\text { ported }\end{array}$ & $\begin{array}{l}\text { Polimict; juvenile }+ \\
\text { subordinated amo- } \\
\text { unts of acci-dental } \\
\text { and acessory mate- } \\
\text { rial. Clastscrystal- } \\
\text { linity may vary. } \\
\text { Groundmass: finer } \\
\text { juvenile material, } \\
\text { shards. Matrix to } \\
\text { clast supported } \\
\text { (diluted - concen- } \\
\text { trated flow) }\end{array}$ & $\begin{array}{l}\text { Monomict; juvenile } \\
\text { material, usually } \\
\text { glassy. Goundmass: } \\
\text { commonly lacking, } \\
\text { when present, com- } \\
\text { posed by coarse ash } \\
\text { of same material }\end{array}$ & $\begin{array}{l}\text { Polimict; lithic rich } \\
+ \text { juvenile (pumice, } \\
\text { scoria); clast } \\
\text { supported }\end{array}$ & $\begin{array}{l}\text { Monomict; juvenile } \\
\text { clasts }\end{array}$ & $\begin{array}{l}\text { Fisher } \quad(1960) \text {; } \\
\text { Parsons } \quad(1969) ; \\
\text { Pettijohn (1989) }\end{array}$ \\
\hline $\begin{array}{c}\text { Sedimentary } \\
\text { structures }\end{array}$ & $\begin{array}{l}\text { None (massive); } \\
\text { uniform } \\
\text { Possible massives } \\
\text { bedding } \\
\text { multiple flow units }\end{array}$ & $\begin{array}{l}\text { Possible, faint par- } \\
\text { allel bedding and } \\
\text { clast alignment; } \\
\text { non-uniform } \\
\text { thickness, thinning } \\
\text { away from source }\end{array}$ & $\begin{array}{l}\text { Possible, faint pa- } \\
\text { rallel bedding; non- } \\
\text { uniform thickness; } \\
\text { internally zoned }\end{array}$ & $\begin{array}{l}\text { Stratified; uniform } \\
\text { thickness }\end{array}$ & $\begin{array}{l}\text { Massive to crudely } \\
\text { stratified; bed late- } \\
\text { rally discontinuous; } \\
\text { thickening into } \\
\text { valleys }\end{array}$ & $\begin{array}{l}\text { None (massive); } \\
\text { non-uniform } \\
\text { thickness }\end{array}$ & $\begin{array}{l}\text { Parsons } \quad(1969) ; \\
\text { Pettijohn (1989) }\end{array}$ \\
\hline $\begin{array}{l}\text { Crystal } \\
\text { structure }\end{array}$ & $\begin{array}{l}\text { Intact, seldom bro- } \\
\text { ken; plastically de- } \\
\text { formed; may be mi- } \\
\text { nor alignment with } \\
\text { flow direction }\end{array}$ & $\begin{array}{l}\text { Broken; flow depo- } \\
\text { sits enriched in } \\
\text { dense minerals }\end{array}$ & Broken & $\begin{array}{l}\text { Intact, seldom bro- } \\
\text { ken; plastically de- } \\
\text { formed }\end{array}$ & Mainly broken & $\begin{array}{l}\text { Crystal-poor; clasts } \\
\text { and matrix con- } \\
\text { taining exclusively } \\
\text { microlites random- } \\
\text { ly dispersed }\end{array}$ & $\begin{array}{l}\text { Parsons } \quad(1969) ; \\
\text { Pettijohn } \quad(1989) ; \\
\text { Sparks and Walker } \\
(1977)\end{array}$ \\
\hline
\end{tabular}




\begin{tabular}{|c|c|c|c|c|c|c|c|}
\hline Grading & $\begin{array}{l}\text { None; possible len- } \\
\text { ses of finer material }\end{array}$ & $\begin{array}{l}\text { Lateral and vertical } \\
\text { zonation, } 15-50 \mathrm{~m} ; \\
\text { coarsest boulders } \\
\text { on top. }\end{array}$ & $\begin{array}{l}\text { Commonly reverse } \\
\text { graded }\end{array}$ & Non-graded & None & None apparent & $\begin{array}{l}\text { Parsons (1969); } \\
\text { Pettijohn (1989) }\end{array}$ \\
\hline $\begin{array}{c}\text { Associated } \\
\text { Deposits }\end{array}$ & $\begin{array}{l}\text { Lava flows cavities } \\
\text { and boundaries; } \\
\text { sometimes grading } \\
\text { into massive and } \\
\text { coherent cores. }\end{array}$ & $\begin{array}{l}\text { Fine-grained co- } \\
\text { PDC airfall depo- } \\
\text { sits capping, inter- } \\
\text { bedded and nearby; } \\
\text { valley fill; PDCs; } \\
\text { dome structures; } \\
\text { coarse fluvial depo- } \\
\text { sits and lahars. }\end{array}$ & $\begin{array}{l}\text { Ash fall, ignim- } \\
\text { brites, crater/cal- } \\
\text { dera structures, } \\
\text { debris flows and } \\
\text { lahars }\end{array}$ & $\begin{array}{l}\text { Thin-sheet lava } \\
\text { (under conditions } \\
\text { of heavy fountain), } \\
\text { scoria cone or } \\
\text { ramparts }\end{array}$ & $\begin{array}{l}\text { Commonly inter- } \\
\text { stratified with ig- } \\
\text { nimbrites; caldera } \\
\text { structures }\end{array}$ & $\begin{array}{l}\text { Banded dacites in- } \\
\text { terpreted as rheoig- } \\
\text { nimbrites (e.g. Gui- } \\
\text { marães et al., 2018; } \\
\text { Luchetti et al., } \\
2018 \text { ) or as lavas } \\
\text { (e.g. Simões et al., } \\
2018 \text { ); dacitic and } \\
\text { basaltic dykes; lava } \\
\text { flows }\end{array}$ & $\begin{array}{l}\text { Parsons (1969); } \\
\text { Sparks and Walker } \\
\text { (1977); Pettijohn } \\
(1989)\end{array}$ \\
\hline Score & 7 & 10 & 7 & 5 & 8 & & \\
\hline
\end{tabular}

Scoring system: 1 for matching criteria, -1 for non-matching criteria, -2 for well-constarined non-matching criteria

${ }^{1}$ Considering here exclusively those deposits classified by Walker (1985) as "lag breccias strictu sensu", which corresponds to coarse proximal deposits, lateral equivalents of ignimbrites, deposited at the deflation zone, due to gas loss (carring fine particles) and consequent loss of flow energy (dense phase deposition). In this review work, the author also includes 3 other mechanisms of formation, which result in differences in the characteristics of the deposits formed (fine depletion, matrix or clast supported, etc). 
A high-temperature, volatile-poor, dacitic magma $\left(\sim 1000^{\circ} \mathrm{C}\right.$ and $<1.3 \% \mathrm{H} 2 \mathrm{O}$, our unpublished data, which are in accordance with previous works on Caxias do Sul magma type Polo et al., 2018a) rises with no significant crystallization. The low content (or even absence) of crystals and high temperatures maintained the low viscosity of the shallow magmatic system.

The fissural architecture of the feeding system may have allowed lateral gas and magma migration along an extended and variably open fissure system (Leat 2008; Martí et al. 2017). This architecture would permit the emplacement of magma batches below non-erupting portions of the fissure, a particularly likely scenario in a rifted margin setting (Magee et al. 2016), which would evolve to distinct viscous and degassed states. Subsequently, intrusion could generate different eruptive scenario in space and time: i) mixing and remobilization by hot, volatile-rich magma may have lead to explosive eruptions, generating the underlying rheoignimbrites [interpreted by Luchetti et al (2018 a, b) and Guimarães (2019) as products of low energy explosions, possibly fountaining; cf. Bachmann et al. 2000], and ii) the ascent and emplacement of highly degassed and viscous magma promoting dome extrusion. Lava dome are inherently unstable, and prone to collapse through endogenous deformation, extrusive growth (Ui et al. 1999) and the development of cooling fractures (Smith 2018). Dome collapse and block-and-ash flows would be responsible for the generation of the proximal, coarse, monolithic volcanic breccias described here. Over time, the dome forming vents could evolve towards explosive behavior as greater volumes of relatively gas-rich magma started to migrate into the shallow plumbing system or rapidly switch to explosive activity as substantial lava dome collapse caused rapid decompression and downwards elastic waves subjected the magma to tensile stresses sufficient for magma fragmentation (Alidibirov and Dingwell 2000; Herd et al. 2005). This renewed explosive phase may be responsible for the related rheoignimbrites that locally overlie the volcanic breccias. Finally, opening/widening of the shallow fissural plumbing system, may have led to the explosive-effusive transition responsible for the generation of the hybrid deposits and lava flows described by Guimarães et al. (2018).

Active and inactive vents, or vents with distinct eruptive dynamics may have been spatially and/or temporally separated along the fissure system, and we may be describing a scenario that can be found elsewhere in the Province, or one that is unique to the local plumbing system. The results and models arising from this work needs to be tested for other breccia occurrences throughout the Province, considering the geographic limitations from a very large province and that the employment of the methodology under different 
The basal Caxias do Sul dacitic breccias in the Paraná Magmatic Province, south Brazil: evidence for an explosive start?

magnifications together with a more detailed analysis of porosity and vesicles properties may overcome the limitations of this first work.

\section{V.6. Concluding remarks}

Macroscopic textural analysis was carried out by means of grain size distribution and shape parameter analysis on the best available exposures of the volcanic breccias related to the initial phase of the silicic volcanism at the region of São Marcos (South Brazil). These breccias are coarse grained, matrix-supported and poorly sorted, with fragments dispersed in a finegrained matrix. The fragments are dense to pumiceous dacites, with variable degrees of macroscopically visible flow-banding, and do not appear to include any fragments derived from underlying units. Due to the limitations of the method used and the degree of alteration of the rocks, the matrix and the fine particle fraction were not included in our analysis.

The grain size distribution (GSD) of the studied breccias could be quantified by a single power law, with low to moderate D values (up to 2.3), suggesting low eruptive energy. The clasts are dense, with area ratios (Avesicles/Aclast) up to 21\% for sample SM-82_Br01, 43\% for SM-82_Br02 and 23\% for SM-82_Br04 and median values around 5\% for all samples. Shape analysis revealed a positive correlation of roughness and angularity with size; the typical high solidity values (i.e. smooth fragments) points towards the operation of secondary fragmentation processes, although field evidence together with GSD fractal distribution suggest proximal deposits. A comparison with several types of volcanic breccias originating both effusively and explosively (as summarized in table 6) shows the highest compatibility with block and ash deposits.

We present a preliminary model where fissural feeder systems and lateral magma migration may have allowed the occurrence of batches of magma evolving under different conditions of degassing and viscosity. Less degassed and viscous magmas may have extruded explosively, while highly degassed and viscous magma promoted dome extrusions. The collapse of these dome structures was responsible for the generation of the proximal, coarse, monolithic volcanic breccias. Subsequent explosive activity may have been caused by migration of relatively gas-rich magma into the shallow plumbing system, or due to rapid decompression and fragmentation related to the dome collapse itself. After a new explosive 
phase, loss of energy together with widening of the vent and/or fissural plumbing system allowed explosive-effusive transitions.

Despite drawbacks, namely the confined geographic area of the breccias and the size limit of the clast analysis, this first study provides a strong motivation for further textural studies on similar occurrences already described elsewhere in the Province to elucidate some still unsolved questions.

\section{References}

Aguirre-Díaz GJ, Labarthe-Hernández G (2003) Fissure ignimbrites: Fissure-source origin for voluminous ignimbrites of the Sierra Madre Occidental and its relationship with Basin and Range faulting. Geology 31:773-776. doi: 10.1130/G19665.1

Alidibirov M, Dingwell DB (2000) Three fragmentation mechanisms for highly viscous magma under rapid decompression. J Volcanol Geotherm Res 100:413-421. doi: 10.1016/S03770273(00)00149-9

Bachmann O, Dungan MA, Lipman PW (2000) Voluminous lava-like precursor to a major ashflow tuff: Low-column pyroclastic eruption of the Pagosa Peak Dacite, San Juan volcanic field, Colorado. J Volcanol Geotherm Res 98:153-171. doi: 10.1016/S03770273(99)00185-7

Bellieni G, Comin-chiaramonti P, Marques LS, et al (1986) Petrogenetic aspects of acid and basaltic lavas from the paraná plateau (Brazil): Geological, mineralogical and petrochemical relationships. J Petrol 27:915-944. doi: 10.1093/petrology/27.4.915

Bellieni G, Comin-Chiaramonti P, Marques LS, et al (1984) High- and Low-TiO2 flood basalts from the Parana plateau (Brazil): petrology and geochemical aspects bearing on their mantle origin. Neus Jahrb Miner Abh 150:273-306.

Bryan SE, Peate IU, Peate DW, et al (2010) The largest volcanic eruptions on Earth. EarthScience Rev 102:207-229. doi: 10.1016/j.earscirev.2010.07.001

Caballero L, Sarocchi D, Borselli L, Cárdenas AI (2012) Particle interaction inside debris flows: Evidence through experimental data and quantitative clast shape analysis. J Volcanol Geotherm Res 231-232:12-23. doi: 10.1016/j.jvolgeores.2012.04.007

Cañón-Tapia E, Raposo MIB (2018) Anisotropy of magnetic susceptibility of silicic rocks from quarries in the vicinity of São Marcos, Rio Grande do Sul, South Brazil: Implications for emplacement mechanisms. J Volcanol Geotherm Res 355:165-180. doi: 10.1016/J.JVOLGEORES.2017.07.018

Chang F, Chen C-J, Lu C-J (2004) A linear-time component-labeling algorithm using contour tracing technique. Comput Vis Image Underst 93:206-220. doi: 10.1016/J.CVIU.2003.09.002 
Fatemi M, Amini A, Baboulaz L, Vetterli M (2016) Shapes from Pixels. IEEE Trans Image Process 25:1193-1206. doi: 10.1109/TIP.2016.2514507

Frank HT, Gomes MEB, Formoso MLL (2009) Review of the areal extent and the volume of the Serra Geral Formation, paraná Basin, South America. Pesqui em Geociencias 36:4957.

Freeman H (1961) On the Encoding of Arbitrary Geometric Configurations. IRE Trans Electron Comput EC-10:260-268. doi: 10.1109/TEC.1961.5219197

Garland F, Hawkesworth CJ, Mantovani MSM (1995) Description and Petrogenesis of the Paraná Rhyolites, Southern Brazil. J Petrol 36:1193-1227.

Gonnermann HM (2015) Magma Fragmentation. Annu Rev Earth Planet Sci 43:431-458. doi: 10.1146/annurev-earth-060614-105206

Guimarães LF (2014) Características físicas e químicas e modelo eruptivo para os riolitos tipo Santa Maria ( Província Magmática Paraná ) na região de Gramado Xavier, RS. Universidade de São Paulo

Guimarães LF, De Campos CP, Janasi VA, et al (2018a) Flow and fragmentation patterns in the silicic feeder system and related deposits in the Paraná-Etendeka Magmatic Province, São Marcos, South Brazil. J Volcanol Geotherm Res. doi: 10.1016/J.JVOLGEORES.2018.03.021

Guimarães LF, Raposo MIB, Janasi VA, et al (2018b) An AMS study of different silicic units from the southern Paraná-Etendeka Magmatic Province in Brazil: Implications for the identification of flow directions and local sources. J Volcanol Geotherm Res 355:304318. doi: 10.1016/j.jvolgeores.2017.11.014

Heilbronner R, Barrett S (2014) Shape Descriptors. In: Image Analysis in Earth Sciences: Microstructures and Textures of Earth Materials. pp 323-347

Herd RA, Edmonds M, Bass VA (2005) Catastrophic lava dome failure at Soufrière Hills Volcano, Montserrat, 12-13 July 2003. J Volcanol Geotherm Res 148:234-252. doi: 10.1016/j.jvolgeores.2005.05.003

Higgins MD (2000) Measurement of crystal size distributions. Am Mineral 85:1105-1116. doi: 10.2138/am-2000-8-901

Hornby AJ (2018) IPC shape macro (Version 1.0.2). Zenodo. http://doi.org/10.5281/zenodo.1438432

Hornby AJ, Lavallée Y, Kendrick JE, et al (2019) Phase partitioning during fragmentation revealed by QEMSCAN Particle Mineralogical Analysis of volcanic ash. Sci Rep 9:1-12. doi: 10.1038/s41598-018-36857-4

Jones TJ, McNamara K, Eychenne J, et al (2016) Primary and secondary fragmentation of crystal-bearing intermediate magma. J Volcanol Geotherm Res 327:70-83. doi: 10.1016/j.jvolgeores.2016.06.022

Jones TJ, Russell JK (2017) Ash production by attrition in volcanic conduits and plumes. Sci Rep 7:5538. doi: 10.1038/s41598-017-05450-6 
Kaminski E, Jaupart C (1998) The size distribution of pyroclasts and the fragmentation sequence in explosive volcanic eruptions. J Geophys Res Solid Earth 103:29759-29779. doi: 10.1029/98JB02795

Kirkpatrick JD, Rowe CD (2013) Disappearing ink: How pseudotachylytes are lost from the rock record. J Struct Geol 52:183-198. doi: 10.1016/j.jsg.2013.03.003

Kueppers U, Perugini D, Dingwell DB (2006) "Explosive energy" during volcanic eruptions from fractal analysis of pyroclasts. Earth Planet Sci Lett 248:800-807. doi: 10.1016/j.epsl.2006.06.033

Kueppers U, Putz C, Spieler O, Dingwell DB (2012) Abrasion in pyroclastic density currents: Insights from tumbling experiments. Phys Chem Earth, Parts A/B/C 45-46:33-39. doi: 10.1016/J.PCE.2011.09.002

Leat PT (2008) On the long-distance transport of Ferrar magmas. In: Thomson K, Petford N (eds) Structure and Emplacement of High-Level Magmatic Systems, 302nd edn. Geological Society Special Publications, London, pp 45-61

Lima EF, Waichel BL, Rossetti LMM, et al (2018) Feeder systems of acidic lava flows from the Paraná-Etendeka Igneous Province in southern Brazil and their implications for eruption style. J South Am Earth Sci 81:1-9. doi: 10.1016/J.JSAMES.2017.11.004

Liu EJ, Cashman KV, Rust AC (2015) Optimising shape analysis to quantify volcanic ash morphology. GeoResJ 8:14-30. doi: 10.1016/j.grj.2015.09.001

Liu EJ, Cashman KV, Rust AC, Höskuldsson A (2017) Contrasting mechanisms of magma fragmentation during coeval magmatic and hydromagmatic activity: the Hverfjall Fires fissure eruption, Iceland. Bull Volcanol. doi: 10.1007/s00445-017-1150-8

Luchetti ACF, Gravley DM, Gualda GAR, Nardy AJR (2018a) Textural evidence for highgrade ignimbrites formed by low-explosivity eruptions, Paraná Magmatic Province, southern Brazil. J Volcanol Geotherm Res 355:87-97. doi: 10.1016/J.JVOLGEORES.2017.04.012

Luchetti ACF, Nardy AJR, Madeira J (2018b) Silicic, high- to extremely high-grade ignimbrites and associated deposits from the Paraná Magmatic Province, southern Brazil. J Volcanol Geotherm Res 355:270-286. doi: 10.1016/J.JVOLGEORES.2017.11.010

Magee C, Muirhead JD, Karvelas A, et al (2016) Lateral magma flow in mafic sill complexes. Geosphere 12:809-841. doi: 10.1130/GES01256.1

Mantovani MSM, Marques LS, De Sousa MA, et al (1985) Trace Element and Strontium Isotope Constraints on the Origin and Evolution of Paraná Continental Flood Basalts of Santa Catarina State (Southern Brazil). J Petrol 26:187-209. doi: 10.1093/petrology/26.1.187

Maria A, Carey S (2007) Quantitative discrimination of magma fragmentation and pyroclastic transport processes using the fractal spectrum technique. J Volcanol Geotherm Res 161:234-246. doi: 10.1016/j.jvolgeores.2006.12.006 
Marsh JS, Ewart A, Milner SC, et al (2001) The Etendeka Igneous Province: Magma types and their stratigraphic distribution with implications for the evolution of the Paraná-Etendeka flood basalt province. Bull Volcanol 62:464-486. doi: 10.1007/s004450000115

Martí J, Villaseñor A, Geyer A, et al (2017) Stress barriers controlling lateral migration of magma revealed by seismic tomography. Sci Rep 7:1-10. doi: 10.1038/srep40757

Milner SC, Duncan AR, Ewart A (1992) Quartz latite rheoignimbrite flows of the Etendeka Formation, north-western Namibia. Bull Volcanol 54:200-219. doi: 10.1007/BF00278389

Milner SC, Duncan AR, Whittingham AM, Ewart A (1995) Trans-Atlantic correlation of eruptive sequences and individual silicic volcanic units within the Paran??-Etendeka igneous province. J Volcanol Geotherm Res 69:137-157. doi: 10.1016/03770273(95)00040-2

Milner SC, Ewart A (1989) The geology of the Goboboseb Mountain volcanics and their relationship to the Messum Complex , Namibia. Commun Geol Surv Namib 5:33-42.

Nardy AJR, Machado FB, Oliveira MAF (2008) As rochas vulcânicas mesozóicas ácidas da Bacia do Paraná: litoestratigrafia e considerações geoquímico-estratigráficas. Brazilian J Geol 38:178-195.

Nardy AJR, Rosa MC, Luchetti ACF, et al (2011) Parâmetros físicos pré-eruptivos do magmatismo ácido da província magmática do Paraná: Resultados preliminares. Geociencias 30:575-588.

Nurfiani D, Bouvet de Maisonneuve C (2018) Furthering the investigation of eruption styles through quantitative shape analyses of volcanic ash particles. J Volcanol Geotherm Res 354:102-114. doi: 10.1016/j.jvolgeores.2017.12.001

Paredes-Mariño J, Morgavi D, Di Vito M, et al (2017) Syneruptive sequential fragmentation of pyroclasts from fractal modeling of grain size distributions of fall deposits: the Cretaio Tephra eruption (Ischia Island, Italy). J Volcanol Geotherm Res 345:161-171. doi: 10.1016/j.jvolgeores.2017.07.020

Peate DW, Hawkesworth CJ, Mantovani MSM (1992) Chemical stratigraphy of the Paraná lavas (South America): classification of magma types and their spatial distribution. Bull Volcanol 55:119-139.

Peate DW, Hawkesworth CJ, Mantovani MSM, Shukowsky W (1990) Mantle plumes and flood-basalt stratigraphy in the Paraná, South America. Geology 18:1223-1226. doi: 10.1130/0091-7613(1990)018<1223:MPAFBS >2.3.CO;2

Pepe S, Solaro G, Ricciardi GP, Tizzani P (2008) On the fractal dimension of the fallout deposits: A case study of the 79 A.D. Plinian eruption at Mt. Vesuvius. J Volcanol Geotherm Res 177:288-299. doi: 10.1016/j.jvolgeores.2008.01.023

Perugini D, Kueppers U (2012) Fractal analysis of experimentally generated pyroclasts: A tool for volcanic hazard assessment. Acta Geophys 60:682-698. doi: 10.2478/s11600-0120019-7 
Perugini D, Speziali A, Caricchi L, Kueppers U (2011) Application of fractal fragmentation theory to natural pyroclastic deposits: Insights into volcanic explosivity of the Valentano scoria cone (Italy). J Volcanol Geotherm Res 202:200-210. doi: 10.1016/j.jvolgeores.2011.02.008

Polacci M, Pioli L, Rosi M (2003) The Plinian phase of the Campanian Ignimbrite eruption (phlegrean fields, Italy): Evidence from density measurements and textural characterization of pumice. Bull Volcanol 65:418-432. doi: 10.1007/s00445-002-0268-4

Polo LA, Giordano D, Janasi VA, Guimarães LF (2018a) Effusive silicic volcanism in the Paraná Magmatic Province, South Brazil: Physico-chemical conditions of storage and eruption and considerations on the rheological behavior during emplacement. J Volcanol Geotherm Res 355:115-135. doi: 10.1016/J.JVOLGEORES.2017.05.027

Polo LA, Janasi VA (2014) Volcanic stratigraphy of intermediate to acidic rocks in the southern Paraná Magmatic Province, Brazil. Geol USP - Ser Cient 14:83-100. doi: 10.5327/Z1519-874X201400020005

Polo LA, Janasi VA, Giordano D, et al (2018b) Effusive silicic volcanism in the Paraná Magmatic Province, South Brazil: Evidence for locally-fed lava flows and domes from detailed field work. J Volcanol Geotherm Res 355:204-218. doi: 10.1016/J.JVOLGEORES.2017.08.007

Proffitt D, Rosen D (1979) Metrication errors and coding efficiency of chain-encoding schemes for the representation of lines and edges. Comput Graph Image Process 10:318-332. doi: 10.1016/S0146-664X(79)80041-6

Rocha-Júnior ERV, Marques LS, Babinski M, et al (2013) Sr-Nd-Pb isotopic constraints on the nature of the mantle sources involved in the genesis of the high-Ti tholeiites from northern Paraná Continental Flood Basalts (Brazil). J South Am Earth Sci 46:9-25. doi: 10.1016/J.JSAMES.2013.04.004

Rocha-Júnior ERV, Puchtel IS, Marques LS, et al (2012) Re-Os isotope and highly siderophile element systematics of the Paraná continental flood basalts (Brazil). Earth Planet Sci Lett 337-338:164-173. doi: 10.1016/J.EPSL.2012.04.050

Rossetti LMM, Lima EF, Waichel BL, et al (2018) Lithostratigraphy and volcanology of the Serra Geral Group, Paraná-Etendeka Igneous Province in Southern Brazil: Towards a formal stratigraphical framework. J Volcanol Geotherm Res 355:98-114. doi: 10.1016/j.jvolgeores.2017.05.008

Sarocchi D, Sulpizio R, Macías JL, Saucedo R (2011) The 17 July 1999 block-and-ash flow (BAF) at Colima Volcano: New insights on volcanic granular flows from textural analysis. J Volcanol Geotherm Res 204:40-56. doi: 10.1016/j.jvolgeores.2011.04.013

Sheridan MF, Wohletz KH, Dehn J (1987) Discrimination of grain-size subpopulations in pyroclastic deposits. Geology 15:367-370.

Simões MS, Lima EF, Sommer CA, Rossetti LMM (2018) Structures and lithofacies of inferred silicic conduits in the Paraná-Etendeka LIP, southernmost Brazil. J Volcanol Geotherm Res 355:319-336. doi: 10.1016/J.JVOLGEORES.2017.12.013 
Smith JV (2018) Susceptibility of lava domes to erosion and collapse by toppling on cooling joints. J Volcanol Geotherm Res 349:311-322. doi: 10.1016/J.JVOLGEORES.2017.11.015

Suzuki-Kamata K, Kusano T, Yamasaki K (2009) Fractal analysis of the fracture strength of lava dome material based on the grain size distribution of block-and-ash flow deposits at Unzen Volcano, Japan. Sediment Geol 220:162-168. doi: 10.1016/j.sedgeo.2009.04.026

Turcotte DL (1986) Fractals and Fragmentation. J Geophys Res 91:1921-1926.

Ui T, Matsuwo N, Sumita M, Fujinawa A (1999) Generation of block and ash flows during the 1990-1995 eruption of Unzen Volcano, Japan. J Volcanol Geotherm Res 89:123-137. doi: 10.1016/S0377-0273(98)00128-0

Wadsworth FB, Witcher T, Vossen CEJ, et al (2018) Combined effusive-explosive silicic volcanism straddles the multiphase viscous-to-brittle transition. Nat Commun 9:1-8. doi: $10.1038 / \mathrm{s} 41467-018-07187-w$

Wagner T, Lipinski H (2013) IJBlob : An ImageJ Library for Connected Component Analysis and Shape Analysis. J Open Res Softw 1:6-8. doi: http://dx.doi.org/10.5334/jors.ae 


\title{
VI. Geochemistry
}

\begin{abstract}
“All sorts of things can happen when you're open to new ideas and playing around with
things."-Stephanie Kwolek
\end{abstract}

(Chemist who invented Kevlar and winner of the Lavoisier Medal for technical achievements)

Whole rock chemical analysis were carried out on 23 basaltic samples, most of them along the BR-116 profile, and 26 dacitic samples, aiming at a wide geographical and stratigraphic coverage (see labelled outcrops at the map presented in Appendix II). As a first approach, in order to verify chemical variations due to weathering process all samples were plotted in the ternary diagram by Ohta and Arai (2007). Unlike the conventional models for quantifying weathering degree by either monitoring decomposition of unstable minerals or pursuing mass transfer of mobile elements, the model developed by Ohta and Arai (2007) is based on multivariate statistics from principal component analysis (PCA) which uses 8 major elements $\left(\mathrm{SiO}_{2}, \mathrm{TiO}_{2}, \mathrm{Al}_{2} \mathrm{O}_{3}\right.$, $\mathrm{Fe}_{2} \mathrm{O}_{3}, \mathrm{MgO}, \mathrm{CaO}, \mathrm{Na}_{2} \mathrm{O}$ and $\mathrm{K}_{2} \mathrm{O}$; wt $\%$ ) as variables. Besides the weathering index, the model allows correlating geochemical variations within a population of samples to the primary (magmatic) or secondary (post-magmatic and/or weathering) processes through a simple graphical analysis; the former will result in a dispersion of the samples parallel to the $M F$ edge of the ternary diagram, while the latter causes a dispersion towards $W$. The ternary diagram presented in figure 38 indicates that our samples are weakly weathered, and mainly spread along the MF edge, suggesting that the chemical variability is primary. It is worth mentioning that, considering the dacitic samples, the less modified compositions correspond to the vitrophyric samples (SM-01D, SM$127 \mathrm{Ba}$ ); this is in agreement with the proposal by Andrade et al. (2018) that glassy silicic samples from Palmas Fm. correspond (or at least are closer) to the original magma compositions, while the related hypocrystalline rocks correspond to their devitrified equivalents, with higher $\mathrm{K}_{2} \mathrm{O} / \mathrm{Na}_{2} \mathrm{O}$ ratios (figure 38). According to the authors, a decrease in $\mathrm{Na}$ contents, together with a relative increase in $\mathrm{K}$, and decrease in $\mathrm{Rb}$ and $\mathrm{Sr}$ occurs associated with the devitrification process. On the other hand, the 
samples with a dispersion towards the $\mathrm{W}$ vertex are those visibly weathered in the field descriptions (SM-23) and/or containing higher contents of microvesicles (SM-105).

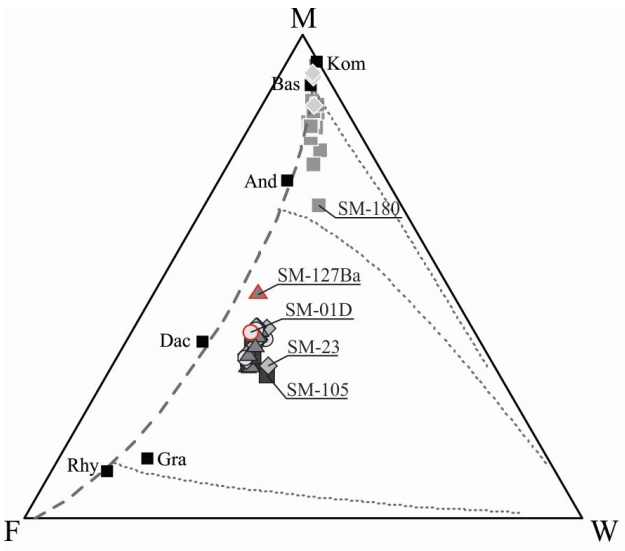

Basaltic Units

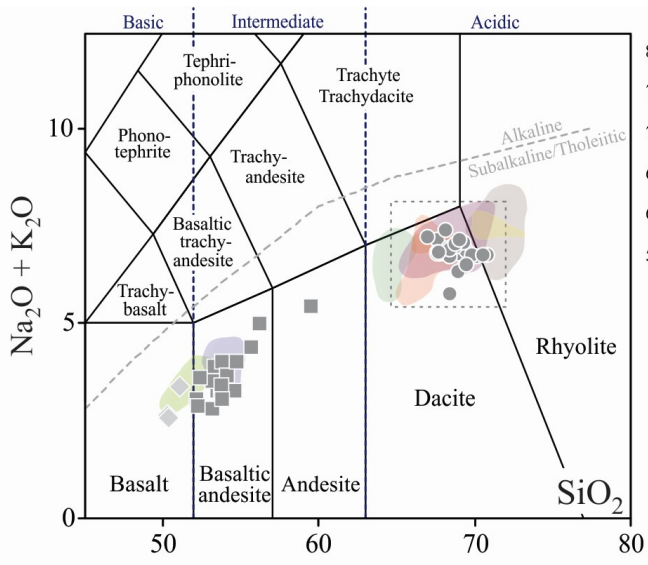

Dacites

Basaltic dykes (Esmeralda Fm.) $\quad \Delta$ Complex structures zone (conduits)

Basaltic flows (Vale do Sol Fm.)

○ Banded deposits (rheoignimbrite)
- Lava flows / coulées $\diamond$ Massive deposits

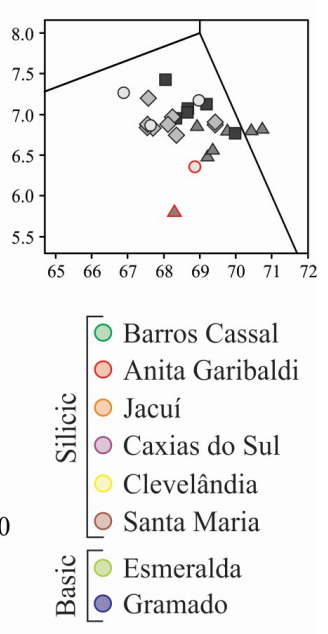

Figure 38: At left, MFW diagram from Ohta and Arai (2007) showing the weathering effects on both basaltic and dacitic samples - symbols for each unit and deposits' type are presented below the diagrams. Samples with red contour represent vitrophyric samples (SM-01D and SM-127Ba). M corresponds to mafic rocks, $\mathrm{F}$ to felsic rocks and W to weathered compositions: $\mathrm{M}=-0.395 \times \ln \left(\mathrm{SiO}_{2}\right)+0.206 \times \ln \left(\mathrm{TiO}_{2}\right)-0.316 \times \ln \left(\mathrm{Al}_{2} \mathrm{O}_{3}\right)+0.160 \times \ln \left(\mathrm{Fe}_{2} \mathrm{O}_{3}\right)+$ $0.246 \times \ln (\mathrm{MgO})+0.368 \times \ln \left(\mathrm{CaO}^{*}\right)+0.073 \times \ln \left(\mathrm{Na}_{2} \mathrm{O}\right)-0.342 \times \ln \left(\mathrm{K}_{2} \mathrm{O}\right)+2.266 ; \mathrm{F}=0.191 \times \ln \left(\mathrm{SiO}_{2}\right)-0.397 \times$ $\ln \left(\mathrm{TiO}_{2}\right)+0.020 \times \ln \left(\mathrm{Al}_{2} \mathrm{O}_{3}\right)-0.375 \times \ln \left(\mathrm{Fe}_{2} \mathrm{O}_{3}\right)-0.243 \times \ln (\mathrm{MgO})+0.079 \times \ln \left(\mathrm{CaO}^{*}\right)+0.392 \times \ln \left(\mathrm{Na}_{2} \mathrm{O}\right)+$ $0.333 \times \ln \left(\mathrm{K}_{2} \mathrm{O}\right)-0.892 ; \mathrm{W}=0.203 \times \ln \left(\mathrm{SiO}_{2}\right)+0.191 \times \ln \left(\mathrm{TiO}_{2}\right)+0.296 \times \ln \left(\mathrm{Al}_{2} \mathrm{O}_{3}\right)+0.215 \times \ln \left(\mathrm{Fe}_{2} \mathrm{O}_{3}\right)-0.002 \times$ $\ln (\mathrm{MgO})-0.448 \times \ln \left(\mathrm{CaO}^{*}\right)-0.464 \times \ln \left(\mathrm{Na}_{2} \mathrm{O}\right)+0.008 \times \ln \left(\mathrm{K}_{2} \mathrm{O}\right)-1.374$. Where $\mathrm{CaO} *$ denotes $\mathrm{CaO}$ corrected for apatite and carbonates. At right, total alkali vs silica (TAS) diagram from Le Bas et al., (1986). The colored fields represent compositions of the units defined in the literature, according to Peate et al. (1992), Garland et al. (1995), Nardy et al. (2008) and Polo and Janasi (2014).

The geochemical data show larger compositional variability among the basaltic samples, while the dacitic samples are more homogeneous; in the classificatory TAS diagram (figure 38) the first spreads through the compositional fields of basalts, basaltic andesites and andesites, with $\mathrm{SiO}_{2}$ contents ranging between $49.5 \%$ and $58.5 \%$ while the dacites have $\mathrm{SiO}_{2}$ contents from $65.2 \%$ to $69.6 \%$. It is noteworthy however that the highest $\mathrm{SiO}_{2}$ contents in both groups may be due to secondary processes (silicification), as indicated by the binary PCA diagrams (figure 39). 

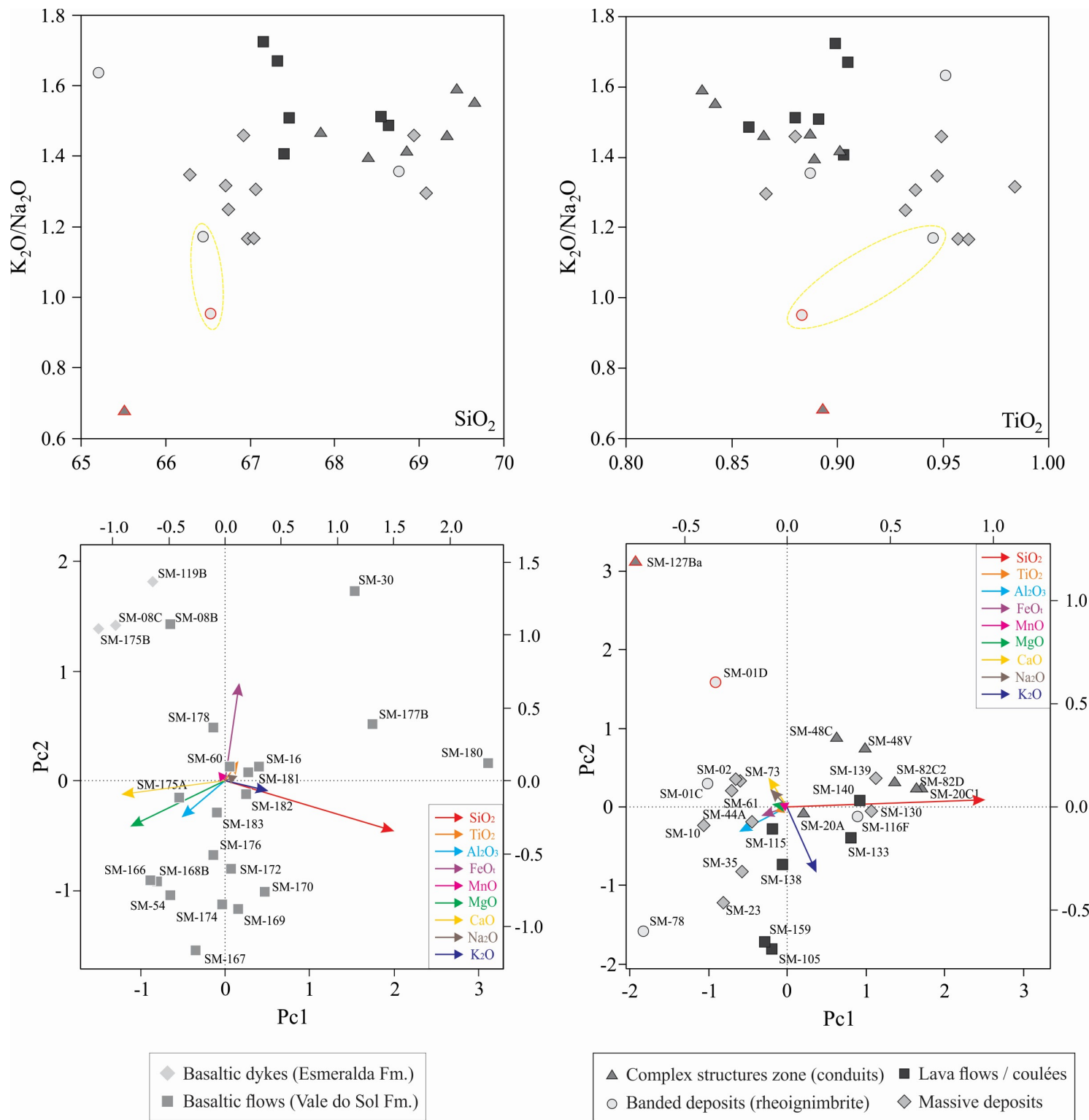

Figure 39: Binary diagrams evidencing compositional variations. Above, $\mathrm{K}_{2} \mathrm{O} / \mathrm{Na}_{2} \mathrm{O}$ vs. $\mathrm{SiO}_{2}$ (left) and $\mathrm{K}_{2} \mathrm{O} / \mathrm{Na}_{2} \mathrm{O}$ vs. $\mathrm{TiO}_{2}$ (right) showing chemical variations between vitrophyric and holocrystalline samples, possibly derived from devitrification processes, where the former presents lower $\mathrm{K}_{2} \mathrm{O} / \mathrm{Na}_{2} \mathrm{O}$ ratios in relation to the related holocrystalline sample (samples SM-01D and SM-01C highlighted by the yellow circle). The compositional range of less mobile elements such as $\mathrm{SiO}_{2}$ and $\mathrm{TiO}_{2}$ suggests also a primary component on the chemical variability. Below, principal component analysis (PCA) diagram showing the main elements responsible for compositional variability in basaltic (left) and dacitic (right) samples; symbols for each unit and deposits' type are presented below the diagrams. Samples with red contour represent vitrophyric rocks. $\mathrm{SiO}_{2}, \mathrm{FeO}_{\mathrm{t}}, \mathrm{MgO}$ and $\mathrm{Cao}$ are the main elements responsible for compositional variation in the basalts, while the dacitic rocks compositions vary mainly as a function of $\mathrm{SiO}_{2}, \mathrm{Al}_{2} \mathrm{O}_{3}$, $\mathrm{K}_{2} \mathrm{O}$, $\mathrm{CaO}$ and $\mathrm{Na}_{2} \mathrm{O}$. Samples from the complex structures zone (conduits) are significantly enriched in $\mathrm{SiO}_{2}$, which may be related to post-magmatic hydrothermal (silicification) processes.

The studied rocks belong to the tholeiitic series, with basalts and basaltic andesites poorer in alkalis, following a classical evolutionary trend where the intermediate samples show a slight enrichment in Fe and the most evolved samples 
(dacites) are depleted in $\mathrm{Fe}$ and $\mathrm{Mg}$ and enriched in alkalis. All the samples are characterized as metaluminous (figure 40).
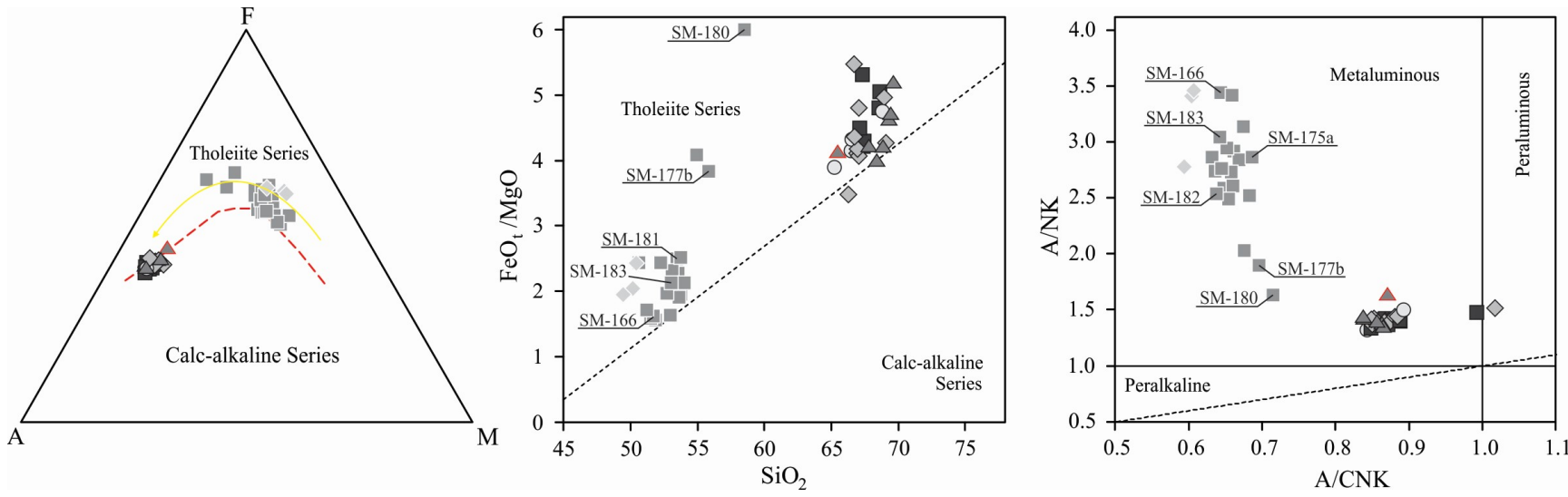

Figure 40: Classificatory diagrams for both basaltic and dacitic units. At left, AFM diagram defined by Irvine and Baragar, (1971), at the middle the $\mathrm{FeO}_{\mathrm{t}} / \mathrm{MgO}$ vs $\mathrm{SiO}_{2}$ plot by Miyashiro (1974), both defining the tholeiite and calcalkaline series. At right, A/NK vs A/CNK plt by Shand (1943) for discrimation of metaluminous, peraluminous and peralkaline rocks. Symbols for each unit and deposits' type are the same from the previous figures.

$\mathrm{TiO}_{2}$ contents vary from $0.99 \%$ to $1.9 \%$ for the basalts and basaltic andesites and from $0.84 \%$ to $0.98 \%$ for the dacites, $\mathrm{MgO}$ contents from $1.96 \%$ to $6.75 \%$ and $0.98 \%$ to $1.6 \%$ and $\mathrm{Fe}_{2} \mathrm{O}_{3}$ from $11.3 \%$ to $14.8 \%$ and $5.5 \%$ to $6.4 \%$ for the basaltic and dacitic samples respectively. The discriminatory diagrams commonly used for the PMP rocks (figure 41) correlate the studied samples with the Vale do Sol Fm. (basaltic flows), Esmeralda Fm. (basaltic dykes) and Caxias do Sul sub-type (dacites) (figure 41). The results of the chemical analysis and the values of the F, M and $\mathrm{W}$ parameters from Ohta and Arai (2007) are presented in appendix V. 

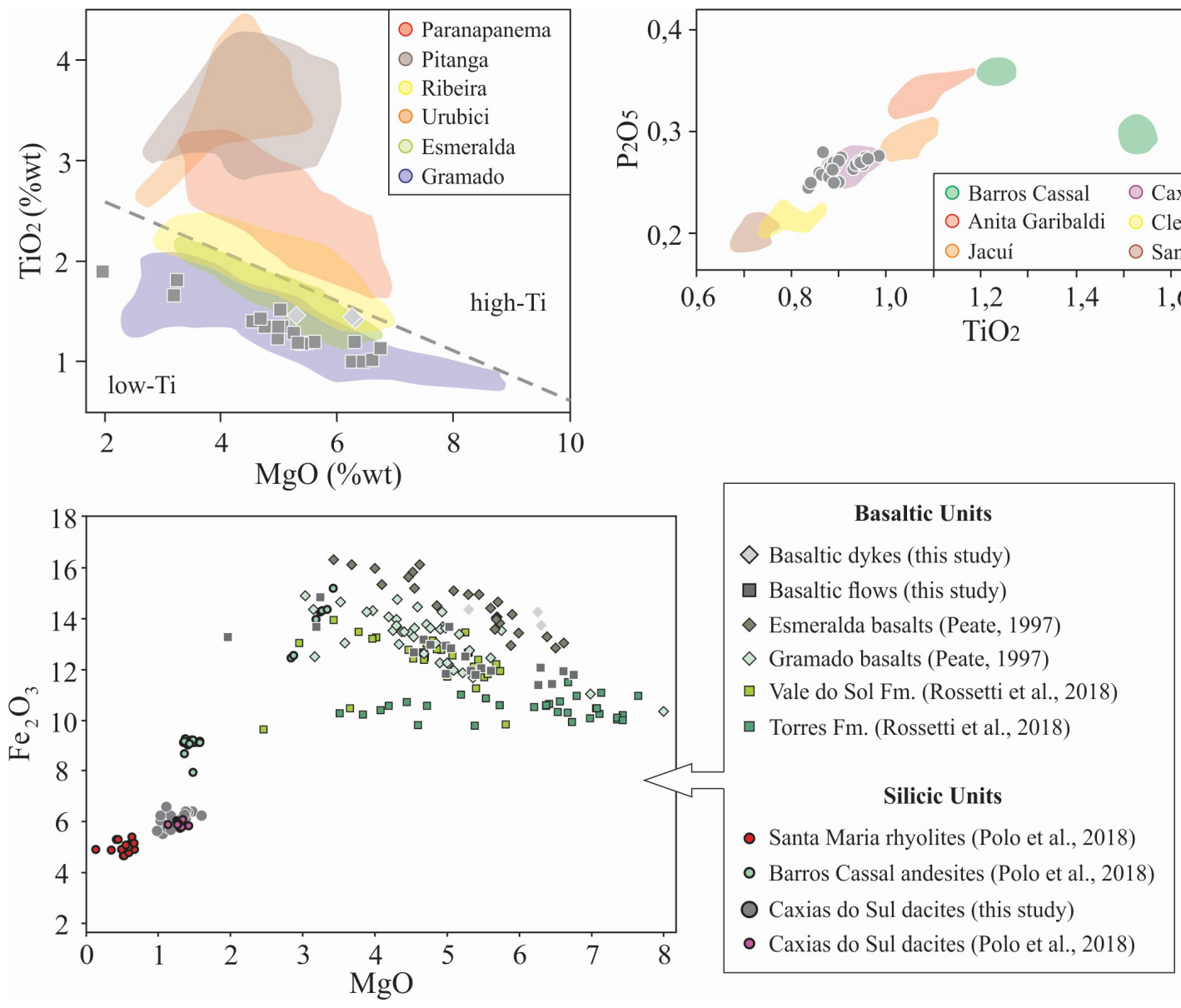

Figure 41: Discriminatory diagrams or the different low-Ti formations from the PMP. In the upper diagrams (basalts at left and dacites at right) the colored fields refer to compositions published in the literature (Peate et al., 1992; Garland et al., 1995; Nardy et al., 2008; Polo and Janasi, 2014), while the gray symbols correspond to the samples from the present study. In the lower diagram, the colored symbols correspond to the compositions published in the literature and the gray symbols to the samples of the present study, as indicated in the legend next to the diagram.

Considering \#mg as the differentiation index, linear trends are better observed in elements such as $\mathrm{MgO}$ and $\mathrm{P}_{2} \mathrm{O}_{5}$, which may indicate primary variations related to the magma differentiation. A gradual impoverishment of $\mathrm{MgO}$ and $\mathrm{CaO}$ of the basaltic rocks from Vale do Sol Fm. with an abrupt depletion of such elements on the Caxias do Sul dacites is clear (figure 42). A nearly opposite behaviour occurs for $\mathrm{K}_{2} \mathrm{O}$, while a continuous depletion in $\mathrm{Al}_{2} \mathrm{O}_{3}$ and enrichment in $\mathrm{Na}_{2} \mathrm{O}$ are also observed (figure 42). Regarding $\mathrm{TiO}_{2}$ and $\mathrm{FeO}_{\mathrm{t}}$, it is clear their gradual enrichment in the Vale do Sol rocks, with an abrupt depletion in the Caxias do Sul dacites (figure 42).Concerning the silicic samples the dispersion observed in the alkali contents $\left(\mathrm{Na}_{2} \mathrm{O}\right.$ and $\left.\mathrm{K}_{2} \mathrm{O}\right)$, which may blur the evolutionary trends, is probably due to secondary processes as devitrification as have been previously discussed. Also, the chemical variations and the deposit type do not appear to be related. 

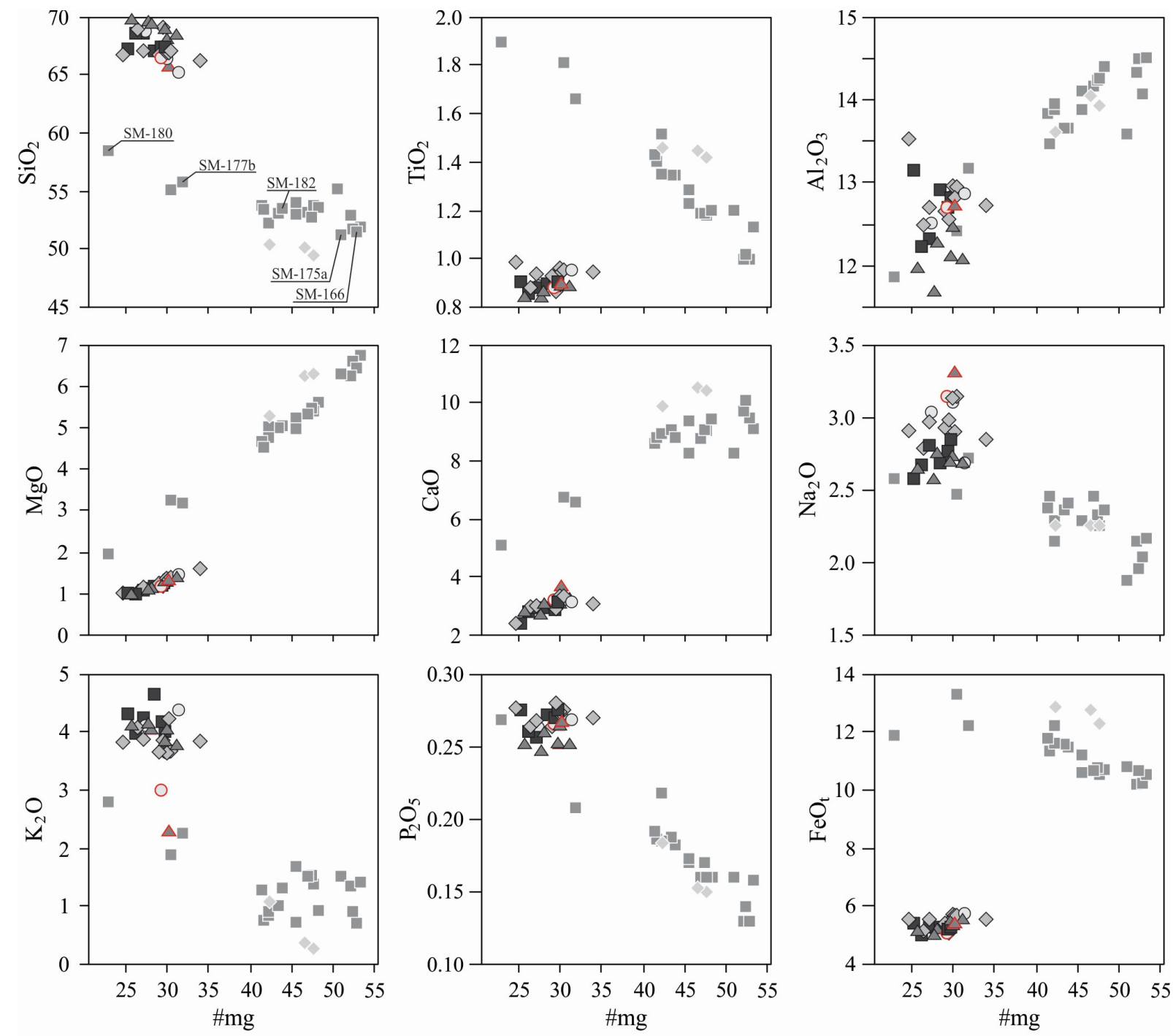

\begin{tabular}{|cll|}
\hline Basaltic Units & \multicolumn{2}{c|}{ Dacites } \\
Basaltic dykes (Esmeralda Fm.) & $\Delta$ Complex structures zone (conduits) & $\square$ Lava flows / coulées \\
Basaltic flows (Vale do Sol Fm.) & $\circ$ Banded deposits & $\diamond$ Massive deposits
\end{tabular}

Figure 42: Binary plots for major elements (in oxides, \%wt) vs \#mg. Symbols for each unit and deposits' type are presented below the diagrams; dacitic samples with red contour correspond to the vitrophyric samples.

Regarding the basaltic rocks, which present greater compositional variation, chemiostratigraphic analysis done through a continuous session along the BR-116 highway reveals that, despite the general evolution trend (i.e. higher differentiation upwards), the differentiation does not occur continuously, but through the intercalation between sequences slightly more and less differentiated (figure 43); thicker basaltic flows may, on their own, present internal geochemical variations, as is the case of flows numbered as 1, 4 and 7 in figure 12, with samples numbered as SM-166 to 168, SM174/175 and SM-177b/178 in figure 43. 


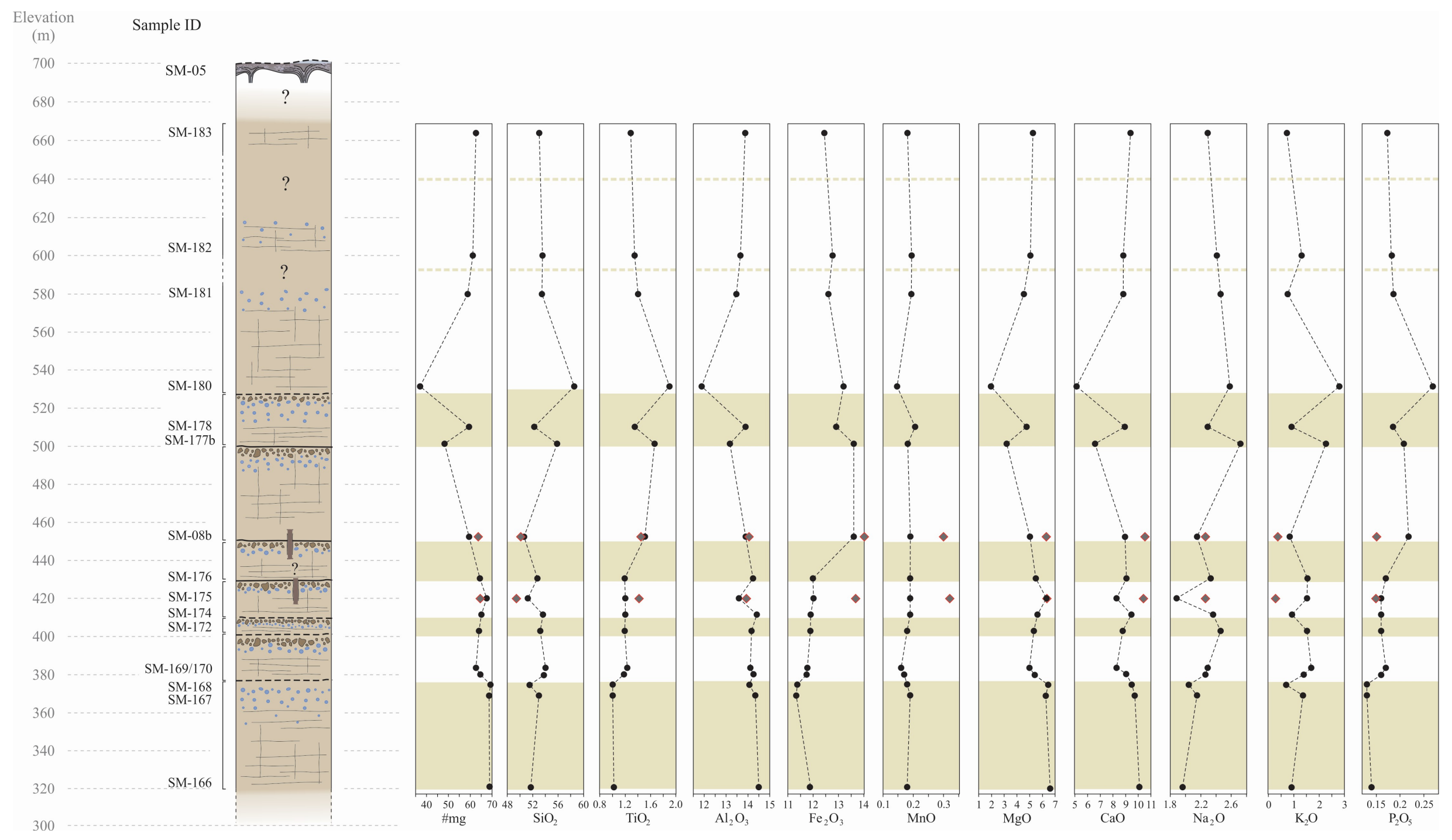

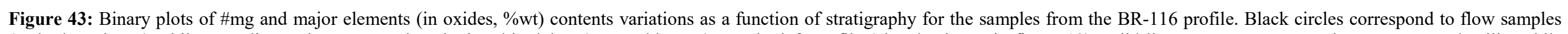

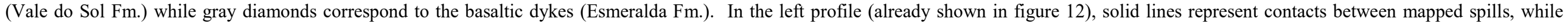

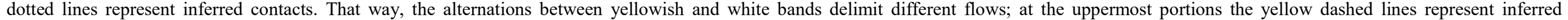
(possible) limits between flows whose discontinuity of the outcrops prevented a better delimitation. 
With respect to the trace elements, their contents variation as a function of \#mg, presented in figure 44, show a positive correlation for $\mathrm{Sr}$, $\mathrm{Ni}$ and $\mathrm{Cr}$, while other elements such as $\mathrm{Rb}, \mathrm{Y}, \mathrm{Zr}, \mathrm{Ba}, \mathrm{La}, \mathrm{Ce}, \mathrm{Sm}, \mathrm{Nd}$ and $\mathrm{Th}$ show negative correlations. Higher $\mathrm{Sr} / \mathrm{Y}$ ratios are observed for the Vale do Sol basalts (5.05 to 8.48), while the Esmeralda basaltic dykes show moderate ratios (5.17-6.27) and the Caxias do Sul dacites the lowest (3.49 to 4.09). In an opposite way, the Caxias do Sul dacites present higher $\mathrm{Rb} / \mathrm{Sr}$ ratios (1.05 to 1.49) than the basaltic rocks ( 0.1 to 0.72 in the Vale do Sol basalts and $0.08-0.25$ in the Esmeralda basaltic dykes).

In terms of REE, low to moderate fractionation patterns are observed for all samples (figure 45); the Caxias do Sul dacites have the higher LREE enrichment, with $\mathrm{La}_{\mathrm{N}} / \mathrm{Yb}_{\mathrm{N}}$ ratios ranging between 7.62 and 8.72, while the Vale do Sol basalts have $\mathrm{La}_{\mathrm{N}} / \mathrm{Yb}_{\mathrm{N}}$ ratios varying from 4.6 to 6.05 and the Esmeralda basaltic dykes show a nearly flat distribution with low $\mathrm{La}_{\mathrm{N}} / \mathrm{Yb}_{\mathrm{N}}$ ratios (1.69-3.87) typical of this formation (Peate, 1997). Subtle negative Eu anomalies are also observed for all samples, being more prominent in the dacites and absent to weak in the basaltic dykes; Caxias do Sul dacites show $\left(\mathrm{Eu} / \mathrm{Eu}^{*}\right)_{\mathrm{N}}$ ratios from 0.62 to 0.68 , Vale do Sol basalts between 0.76 and 0.95 and Esmeralda basaltic dykes from 0.87 to 1.04 . The spidergram for incompatible elements (figure 45) shows marked negative anomalies of $\mathrm{Nb}, \mathrm{SR}$ and $\mathrm{Ti}$ for all samples and significantly higher $\mathrm{Ba} / \mathrm{Rb}$ and $\mathrm{Th} / \mathrm{U}$ for the basalts when compared to the dacites (Vale do Sol basalts $-\mathrm{Ba} / \mathrm{Rb}=4.03$ to 15.80 and $\mathrm{Th} / \mathrm{U}=3.55$ to 6.85 ; Esmeralda basaltic dykes $-\mathrm{Ba} / \mathrm{Rb}=4.74-5.89$ and $\mathrm{Th} / \mathrm{U}=3.36-5.42$; Caxias do Sul dacites $-\mathrm{Ba} / \mathrm{Rb}=3.44$ to 5.93 and $\mathrm{Th} / \mathrm{U}=2.67$ to 3.93 ), highlighting the occurrence of two sets of Caxias do Sul dacites samples (one group with higher and other with lower $\mathrm{Th} / \mathrm{U}$ ). 

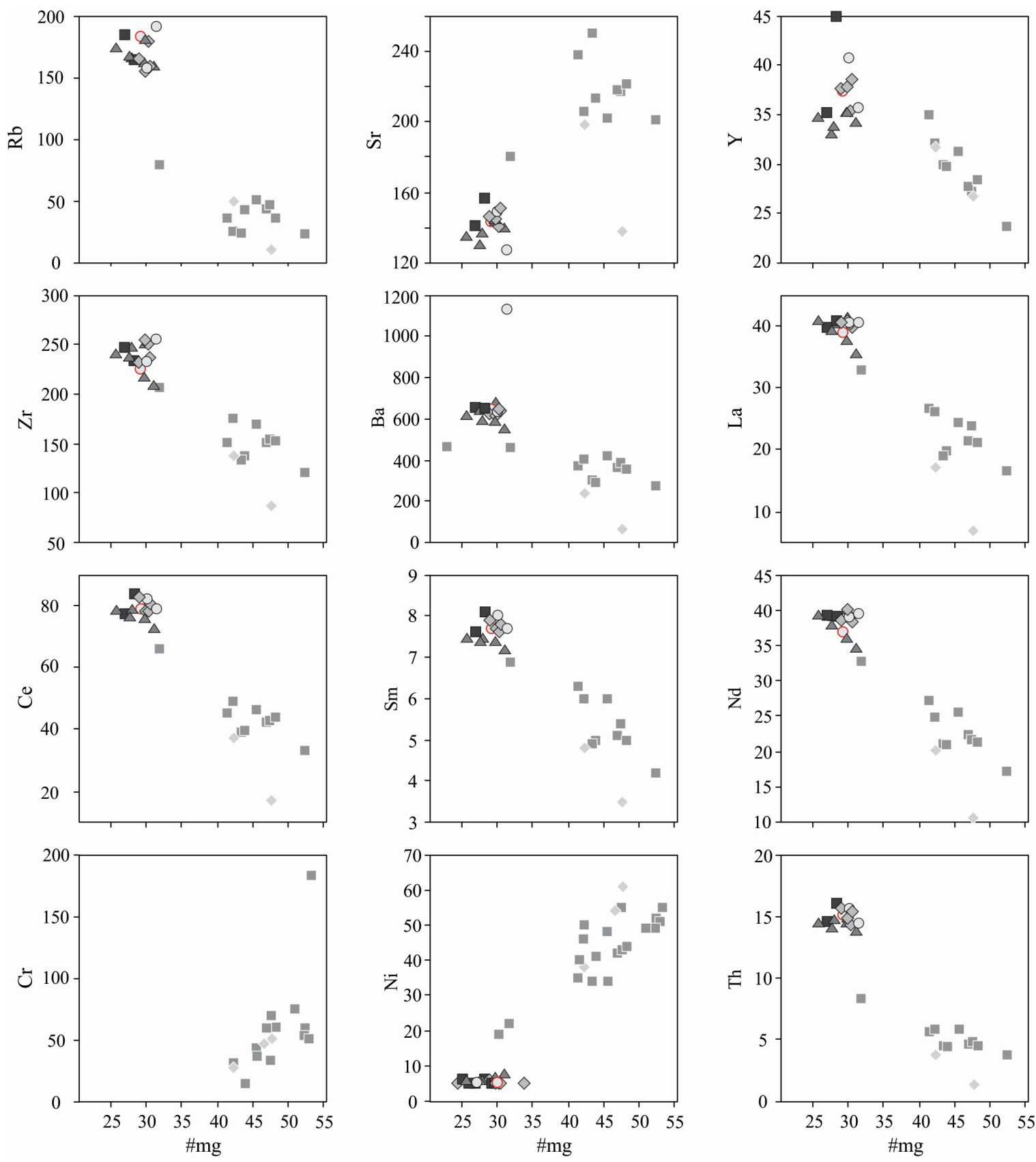

\begin{tabular}{|cll|}
\hline Basaltic Units & \multicolumn{1}{c|}{ Dacites } \\
Basaltic dykes (Esmeralda Fm.) & $\Delta$ Complex structures zone (conduits) & L Lava flows / coulées \\
Basaltic flows (Vale do Sol Fm.) & $\circ$ Banded deposits & $\diamond$ Massive deposits \\
\hline
\end{tabular}

Figure 44: Binary plots for selected trace elements (in ppm) vs \#mg. All concentrations obtained by ICPMS, with the only exception of $\mathrm{Cr}$ and $\mathrm{Ni}$, obtained by XRF. Symbols for each unit and deposits' type are presented below the diagrams; dacitic samples with red contour correspond to the vitrophyric samples. 
REE Spiderplot normalized by chondrite (Boyton, 1984)
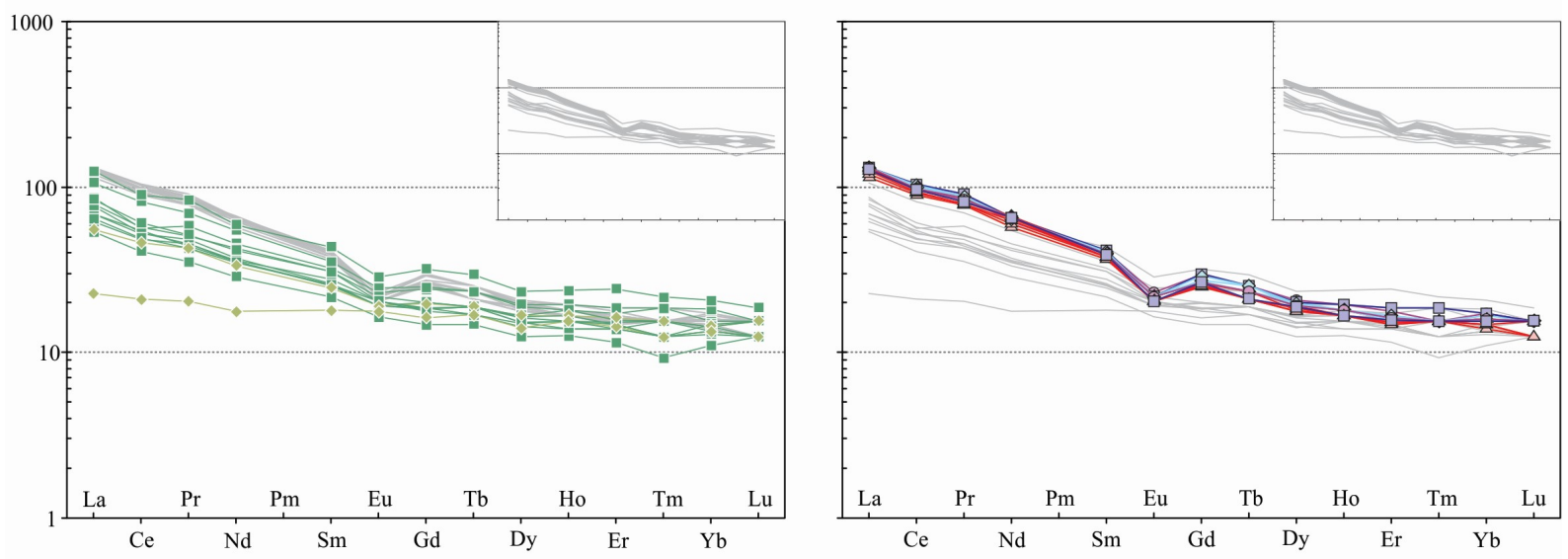

Spiderplot normalized by NMORB (Sun \& McDonough, 1989)
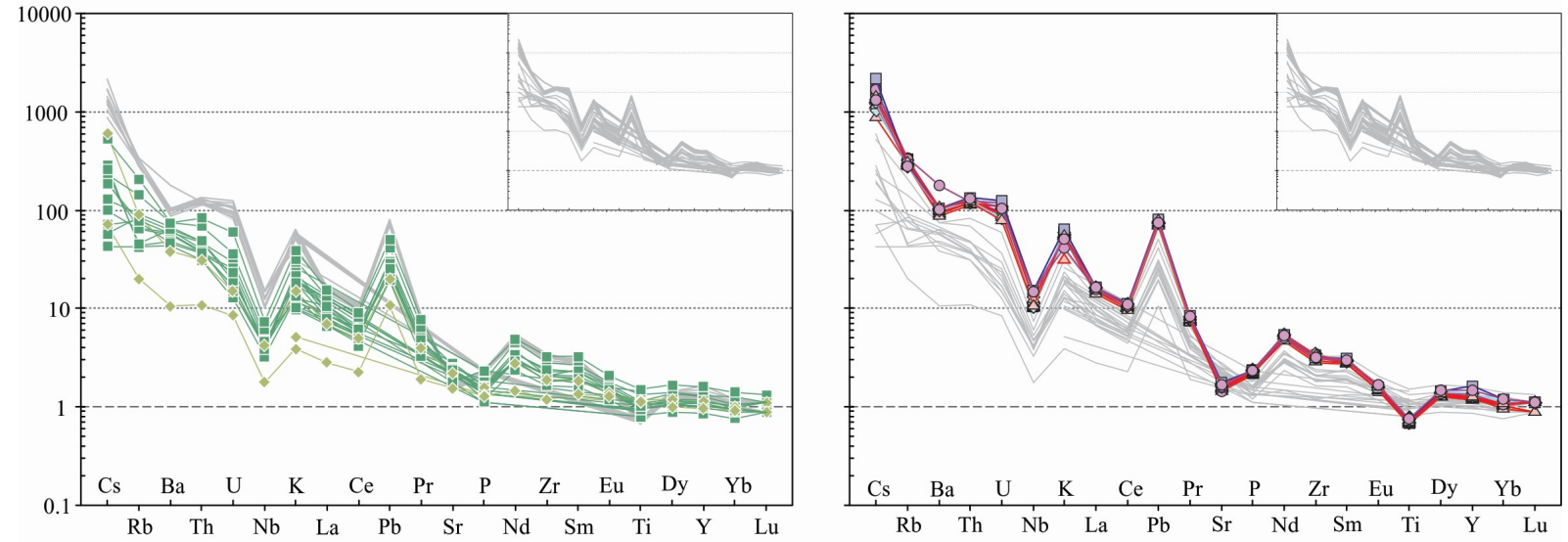

Basaltic dykes (Esmeralda Fm.)

- Basaltic flows (Vale do Sol Fm.)

$\triangle$ Complex structures zone (conduits) $\square$ Lava flows / coulées

○ Banded deposits (rheoignimbrite) $\diamond$ Massive deposits

Figure 45: Spidergrams for REE (above), normalized by the condrite (Boynton, 1984), and for trace elements (below), normalized by NMORB (Sun and McDonough, 1989). The basaltic samples are highlighted at the diagrams to the left, while the dacites are highlighted at the diagrams to the right. Symbols for each unit and deposits' type are presented below the diagrams; dacitic samples with red contour correspond to the vitrophyric samples.

The variation in the trace elements contents as a function of the stratigraphy (figure 46) is in agreement with the observed for the major elements in the case of the basaltic sequence; elements such as $\mathrm{Rb}, \mathrm{Sr}, \mathrm{Yr}, \mathrm{Zr}, \mathrm{Ba}, \mathrm{La}, \mathrm{Ce}, \mathrm{Nd}, \mathrm{Sm}$ and $\mathrm{Eu}$ exhibit an incompatible behaviour, with a general trend of subtle increase towards the top, whereas elements such as $\mathrm{Cr}$ and $\mathrm{Ni}$ present a slightly more complex behaviour. Small variations within flow units are observed and, in general, these elements are more abundant at the base of the flows, what is probably related to 
the higher relative contents of clinopyroxene and Ti-magnetite, petrographically verified at these portions; exceptions occur in flows 4 and 7 (the latter only for Ni), suggesting an alternative process, possibly replenishment during emplacement. Small oscillations between flows are also observed, however, a general trend of up-section decrease in the contents of $\mathrm{Cr}$ and $\mathrm{Ni}$, as expected from the more evolved compositions of the rocks, could be defined. The $\mathrm{La}_{\mathrm{N}} / \mathrm{Yb}_{\mathrm{N}}$ ratios show a subtle increase towards the top, also reflecting the progressively more differentiated compositions of the basaltic sequence.

With respect to the Caxias do Sul dacites, a more careful analysis of the contents of trace elements, especially those not (or less) affected by weathering and/or devitrification processes such as $\mathrm{Zr}, \mathrm{Ce}, \mathrm{Sm}, \mathrm{Y}, \mathrm{Th} / \mathrm{U}$ and $\mathrm{La}_{\mathrm{N}} / \mathrm{Yb}_{\mathrm{N}}$, reveals the existence of two groups of samples; one with higher $\mathrm{La}_{\mathrm{N}} / \mathrm{Yb}_{\mathrm{N}}, \mathrm{Ce}, \mathrm{Sm}$ and $\mathrm{Y}$ contents and lower Th/U and $\mathrm{Zr}$ contents, and another with the opposite pattern (figure 47). Considering that, unlike basaltic sequences, which comprise extensive sheet flows, silicic sequences consist of several deposits with varying geometry, thickness and extension, overlapping each other or occurring side by side depending on the relief and the location/distribution of the eruptive centers, an analysis based on elevation is not useful. However, in addition to differentiation by type of deposit, the samples were separated by color according to their location (NW, NE, SW or SE), showing that samples from the same chemical group occur geographically throughout the area. This observation is in agreement with the model presented in chapter IV of a fissural feeding system, composed by several synchronous eruptive centers. 


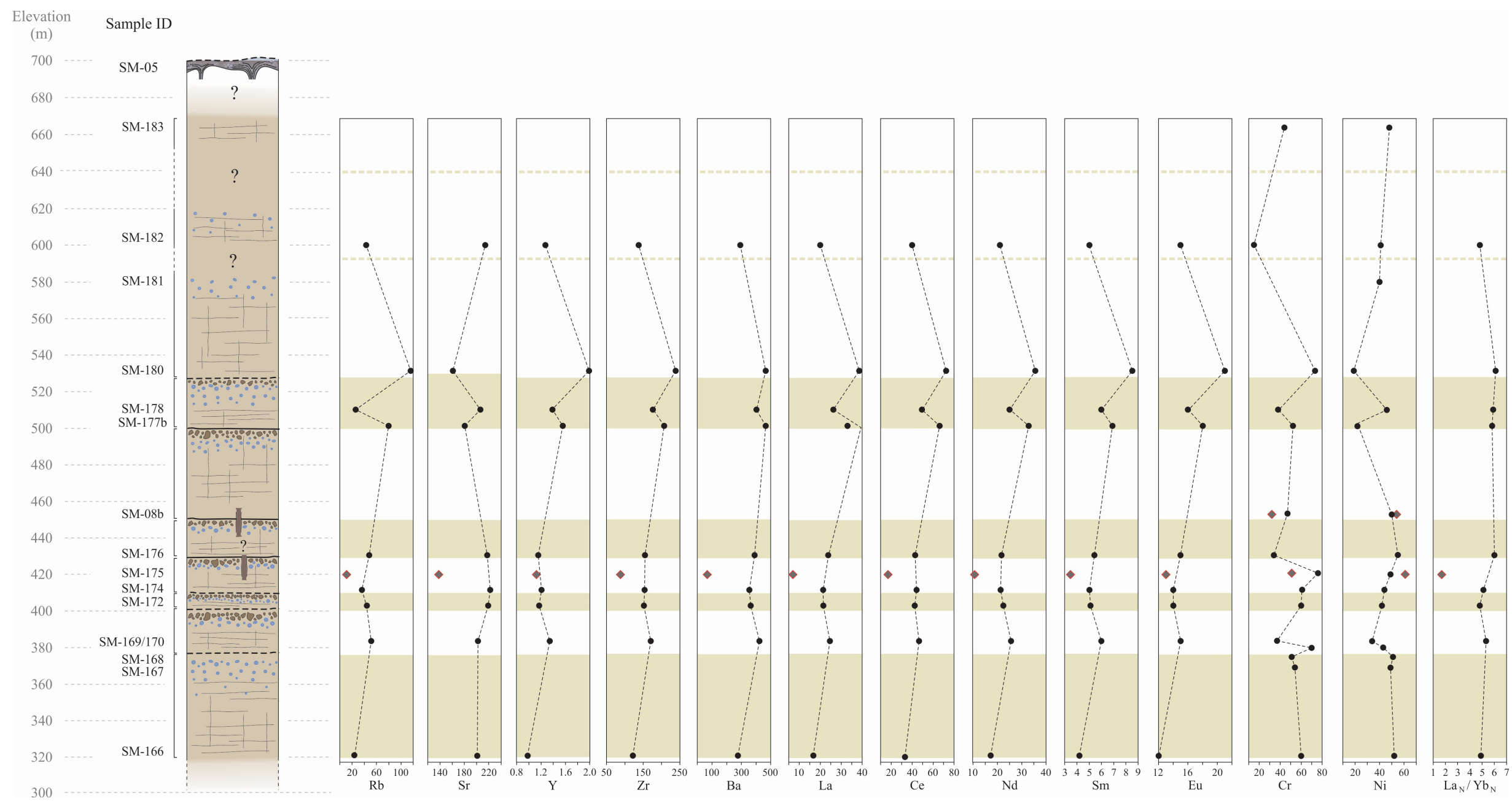

Figure 46: Binary plots of selected trace elements (in ppm) contents variations as a function of stratigraphy for the samples from the BR-116 profile. Black circles correspond to flow samples (Vale do Sol Fm.) while gray lozenges correspond to the basaltic dykes (Esmeralda Fm.). All concentrations obtained by ICPMS, with the only exception of Cr and Ni, obtained by XRF. In the left profile (already shown in figure 12), solid lines represent contacts between mapped spills, while dotted lines represent inferred contacts. That way, the alternations between yellowish and white bands delimit different flows; at the uppermost portions the yellow dashed lines represent inferred (possible) limits between flows whose discontinuity of the outcrops prevented a better delimitation. 

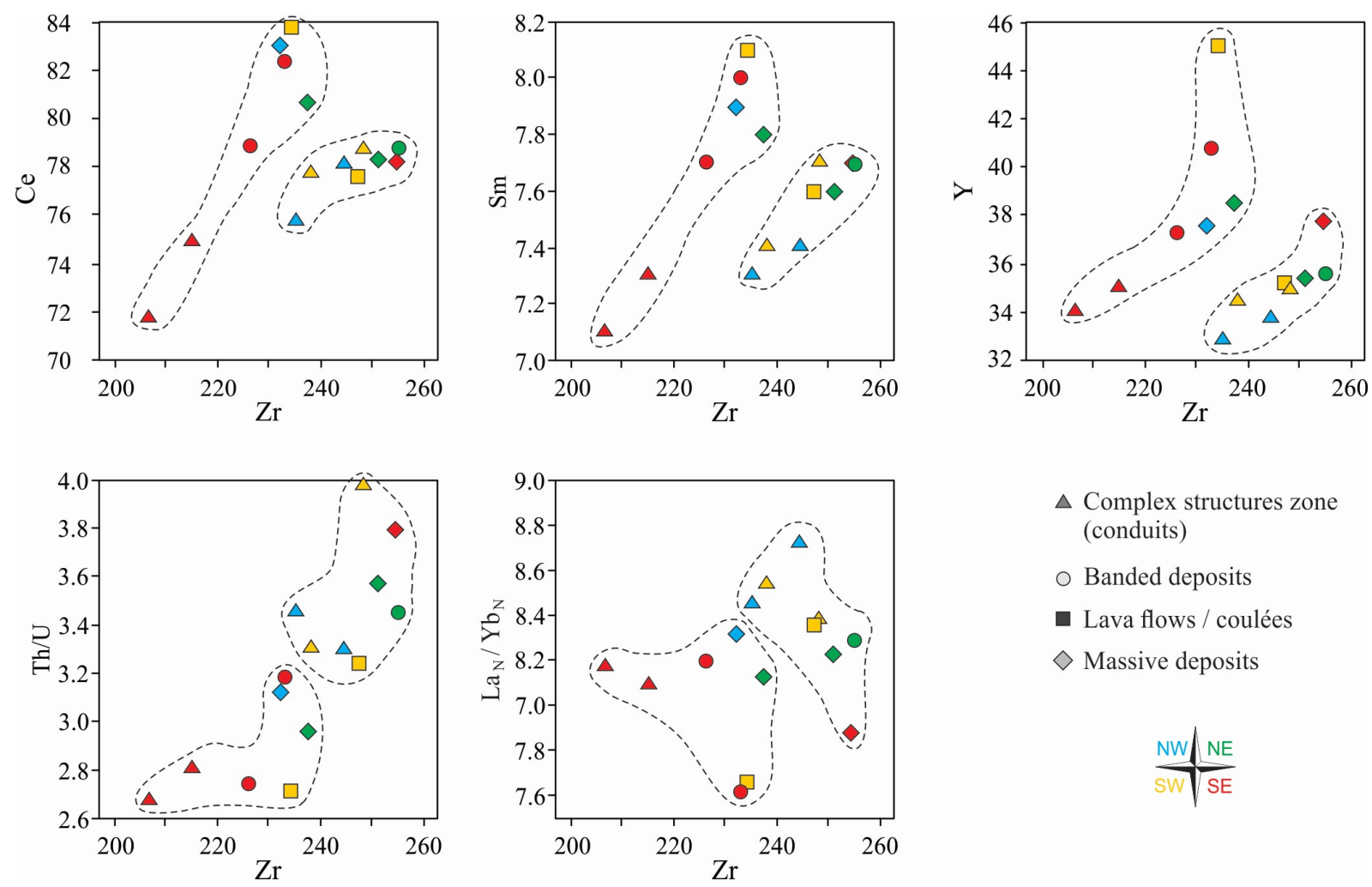

$\triangle$ Complex structures zone (conduits)

- Banded deposits

- Lava flows / coulées

$\diamond$ Massive deposits

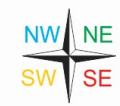

Figure 47: Binary plots of selected trace elements contents (in ppm) and ratios variations as a function of $\mathrm{Zr}$ contents for the dacitic samples. Black dashed lines delimitate the two recognized groups of dacites.

\section{References}

Andrade, F.R.D., Polo, L.A., Janasi, V.A., and Carvalho, F.M.S., 2018, Volcanic glass in Cretaceous dacites and rhyolites of the Paraná Magmatic Province, southern Brazil: Characterization and quantification by XRD-Rietveld: Journal of Volcanology and Geothermal Research, v. 355, p. 219-231, doi:10.1016/J.JVOLGEORES.2017.08.008.

Bas, M.J.L., Maitre, R.W.L., Streckeisen, A., and Zanettin, B., 1986, A chemical classification of volcanic rocks based on the total alkali-silica diagram: Journal of Petrology, v. 27, p. 745750, doi:10.1093/petrology/27.3.745.

Boynton, W. V., 1984, Cosmochemistry of the Rare Earth Elements: Meteorite Studies: Developments in Geochemistry, v. 2, p. 63-114, doi:10.1016/B978-0-444-42148-7.500083.

Garland, F., Hawkesworth, C.J., and Mantovani, M.S.M., 1995, Description and Petrogenesis of the Paraná Rhyolites, Southern Brazil: Journal of Petrology, v. 36, p. 1193-1227. 
Guimarães, L.F., 2014, Características físicas e químicas e modelo eruptivo para os riolitos tipo Santa Maria ( Província Magmática Paraná ) na região de Gramado Xavier , RS: Universidade de São Paulo.

Irvine, T.N., and Baragar, W.R.A., 1971, A Guide to the Chemical Classification of the Common Volcanic Rocks: Canadian Journal of Earth Sciences, v. 8, p. 523-548, doi:10.1139/e71055 .

Nardy, A.J.R., Machado, F.B., and Oliveira, M.A.F., 2008, As rochas vulcânicas mesozóicas ácidas da Bacia do Paraná: litoestratigrafia e considerações geoquímico-estratigráficas: Brazilian Journal of Geology, v. 38, p. 178-195, http://rbg.sbgeo.org.br/index.php/rbg/article/view/1353.

Ohta, T., and Arai, H., 2007, Statistical empirical index of chemical weathering in igneous rocks: A new tool for evaluating the degree of weathering: Chemical Geology, v. 240, p. 280-297, doi:10.1016/J.CHEMGEO.2007.02.017.

Peate, D.W., Hawkesworth, C.J., and Mantovani, M.S.M., 1992, Chemical stratigraphy of the Paraná lavas (South America): classification of magma types and their spatial distribution: Bulletin of Volcanology, v. 55, p. 119-139.

Peate, D.W., 1997, The Paraná-Etendeka Province. In: Mahoney, J.J. and Coffin (ed), Large Igneous Provinces: Continental, Oceanic and Planetary Flood Volcanism. Geophysical Monography Series, 100: 217-245.

Polo, L.A., and Janasi, V.A., 2014, Volcanic stratigraphy of intermediate to acidic rocks in the southern Paraná Magmatic Province, Brazil: Geologia USP - Serie Cientifica, v. 14, p. 83100, doi:10.5327/Z1519-874X201400020005.

Sun, S. -s., and McDonough, F., 1989, Chemical and isotopic systematics of oceanic basalts: implications for mantle composition and processes: Geological Society, London, Special Publications, v. 42, p. 313-345, doi:10.1144/gsl.sp.1989.042.01.19. 


\section{Discussions and concluding remarks}

"I'm in a charming state of confusion." - Ada Lovelace

(English mathematician and writer, mainly known for her work on the Analytical Engine, being the first to recognize the full potential of a "computing machine" and the first computer programmer)

Although some discussions have already been brought up in the previouschapters, an integration of the data presented in this thesis and some concluding remarks will be exposed in this final chapter.

The geochemical aspects of the PEMP have been extensively investigated and, even though discussions regarding the generation and evolution of magmas are not the focus of this thesis, some aspects showed here can be pointed out. The genetic connection between the Caxias do Sul dacites and the Vale do Sol basalts, already pointed out in previous works, is confirmed in the present study. Fractionation of the basaltic magmas, with the extraction of plagioclase, pyroxene and magnetite, is clear through the patterns of major and trace elements, whereas, despite the absence of isotopic data in this work, the presence of a crustal component (by means of assimilation, also already well established in the literature, e.g. Garland et al., 1995; Guimarães, 2014; Barreto et al., 2016) is suggested by variations in trace elements ratios such as $\mathrm{Rb} / \mathrm{Ba}$ and $\mathrm{Th} / \mathrm{U}$. Still regarding the basaltic sequence, besides the general trend of progressively more fractionated rocks towards the top of the sequence, subtle variations between flow units are evidence of small compositional regressions, suggesting changes in the source and/or replenishment events in crustal magma chambers where the magmas were stored. Chemical variations among the Caxias do Sul dacites (e.g. trace elements contents and ratios; e.g. Ce, Y and $\mathrm{Sm}$ and $\mathrm{Th} / \mathrm{U}$ ), characterized as primary, reveal the existence of at least two distinct sets of silicic rocks. Such variations can reflect different sources (or variations in the assimilated crustal component), and are not related to the type of deposit/eruptive style.

The mechanism of emplacement and eruptive style of the silicic volcanism is a topic still intensely debated in the PMP. The present work contradicts the model ofsingle central conduits located in the African portion of the province as responsible for the generation of huge pyroclastic deposits resulting from super-eruptions. In contrast, the occurrence of multiple 


\section{Chapter VII \\ Discussions and concluding remarks}

eruptive centers aligned along NW-SE trends, characterizing a fissural system is proposed. This feeder system is marked by zones with complex structures where vertical magmatic flow features (here called filament domain) are disposed side-by-side and in complex intrusive relations with other facies - here nominated breccia and regular domains. The breccia domain is interpreted to represent volcanic breccias that are, together with the underlying banded deposits, the hosting rocks of this feeder system. The regular domains, on the other hand, may be related to the filament domains, both composing the intrusive flow. These domains resemble those previously described by Poli and Perugini(2002), Perugini et al.(2003) and Morgavi et al.(2013a, 2013b) on natural outcrops and experimental samples and may represent regions within the convective flow with different energy/efficiency.

The model of fissural feeder system with multiple aligned eruptive centers is supported by some authors (e.g. Lima et al., 2012; Guimarães et al., 2018; Lima et al., 2018; Polo et al., 2018; Simões et al., 2018), although opposed by others (Cañón-Tapia and Raposo, 2018) who, based on AMS data and on macroscopic textural descriptions argue that the complex structures classified as feeder systems correspond simply to subaerial silicic blocky lava flows. According to these authors, the dominantly horizontal magnetic lineations $\left(\mathrm{K}_{\max }\right)$ measured in those outcrops where vertical magmatic flow features were classified as feeders, together with the low degree of anisotropy obtained, invalidate the hypothesis of feeder structures. Additionally they argue, based on field descriptions, that the portions characterized by the occurrence of fragments (nominated in this thesis as "breccia domain") correspond to the basal and upper auto brecciation of blocky flows, whereas those portions characterized by the vertical flow features (here called "filament domains") correspond to the interaction with later flows, generating "peperite-like" textures. In this sense, it is important to emphasize that (i) these features occur inspecific regions, at discontinuous and aligned outcrops, and that (ii) a more careful analysis of the AMS data presented by Cañón-Tapia and Raposo (2018) on the basis of the here-defined domains, reveals that the magnetic data presentparticular patterns for each domain described in this work. The AMS data is quite dispersed in the breccia domain, while less dispersed in regular and filament domains (examples in figures 48 and 49). Actually, higher dips of magnetic foliations and the higher degrees of anisotropy (in accordance with the values obtained for dyke swarms related to the PMP, e.g. Raposo and Ernesto, 1995; Raposo, 1997) are related to the filament domains (examples in figures 48 and 49), and the presence of horizontal lineations do not invalidate the fissural feeder systems if we consider that magmatic flows through dykes are not necessarily simple upward, continuous and homogeneous vertical flow. Indeed, lateral propagation in dykes 


\section{Chapter VII \\ Discussions and concluding remarks}

has been shown to be more common than previously thought and was already described on both natural outcrops based on either field descriptions and fabric and seismological analysis (e.g. Ernst and Baragar, 1992; Poland et al., 2008; Ágústsdóttir et al., 2016; Healy et al., 2018) and on numerical and experimental data (e.g. Lister and Kerr, 1991; Poland et al., 2008; Townsend et al., 2017). Similarly, convective movements and flow reversals (so-called drainback flows) have also been demonstrated to occur (e.g. Delaney and Pollard, 1981; Kavanagh et al., 2018), meaning that flow indicators such as vesicle and/or crystals orientation and anisotropy of magnetic susceptibility may be highly variable (Delaney and Pollard, 1981; Healy et al., 2018). Besides the possibility of generation of lateral and downwards flow related to the dyke ascent and propagation itself (defined as the "stage 2" of the 4 defined by Kavanagh et al., 2018), such patterns could be also generated by convective movements related to the occurrence of compositional and/or thermal gradients in the rising magma leading to chaotic dynamic flows. Indeed, Guimarães et al., (2018a) have suggested the occurrence of magmatic flow under chaotic dynamics, based on fractal dimension analysis of flow features that resemble patterns obtained experimentally by Morgavi et al.(2013), both presenting similar D values. Putting all the above mentioned factors together, it is clear that the processes of magma transportation and emplacement are highly complex, requiring a multidisciplinary investigation. In this sense, the well-exposed (yet discontinuous) and preserved outcrops of the fissural feeder system related to The PMP offer a valuable opportunity for its better understanding. It is also worth mentioning the cyclicity/reactivation of structures of the feeding system, corroborated by the structural compatibility between the dacitic conduits and the basaltic dyke swarms already well characterized in the province and by the occurrence of younger basaltic dikes (Esmeralda type)intruding the dacitic rocks from the conduits zone. 


\section{Chapter VII}

Discussions and concluding remarks
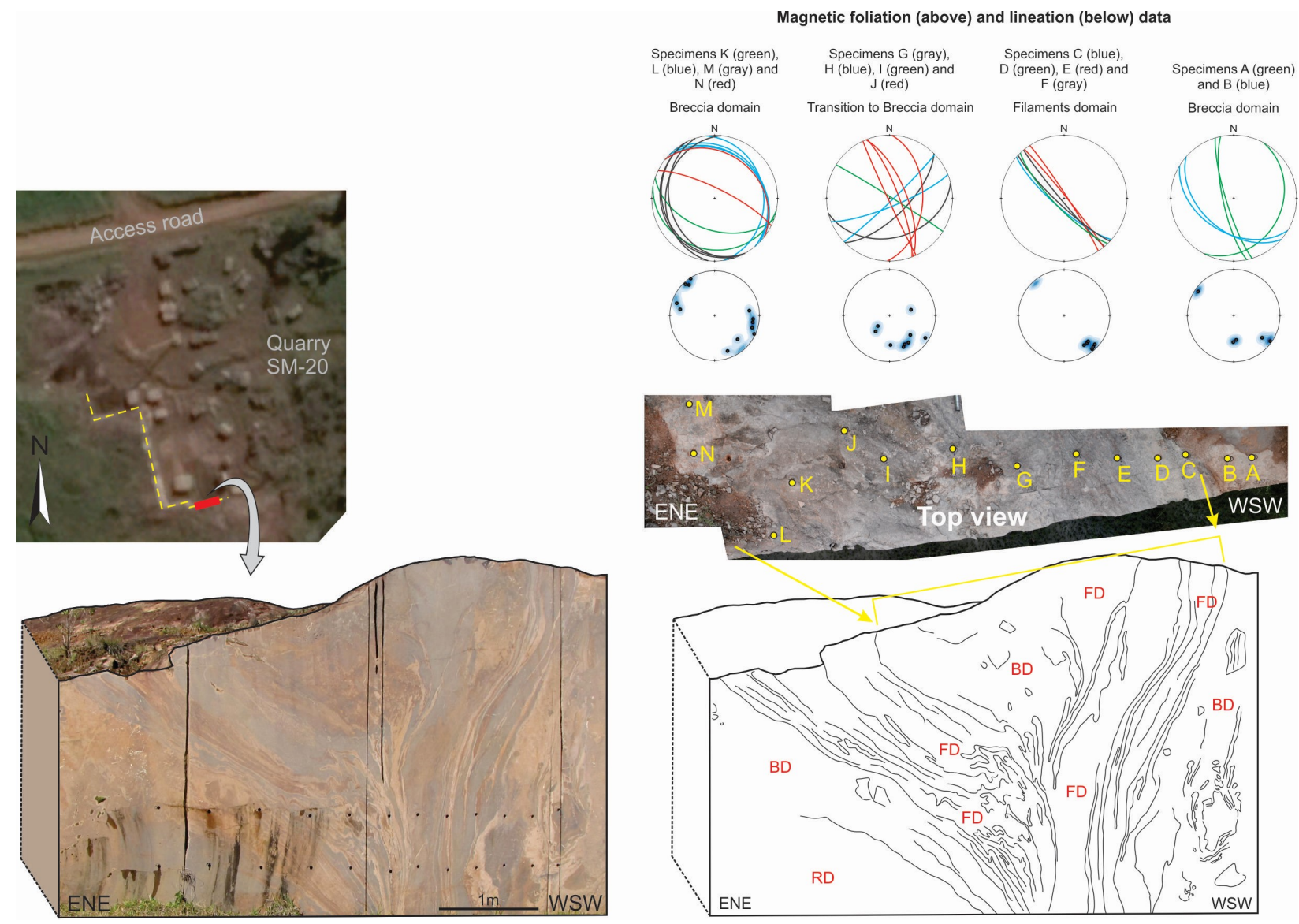

Figure 48: AMS data (from Cañón-Tapia and Raposo, 2018) of the funil-like structure from quarry SM-20. The regular (Rd), filaments $(\mathrm{Fd})$, fragmentation and regular $(\mathrm{Rd})$ domains are indicated. The data were divided and reinterpreted here according to the faciological domains defined in the item $I V .2 .3$ of this thesis and the data of each sampling site is separated by color as indicated in the figure. 

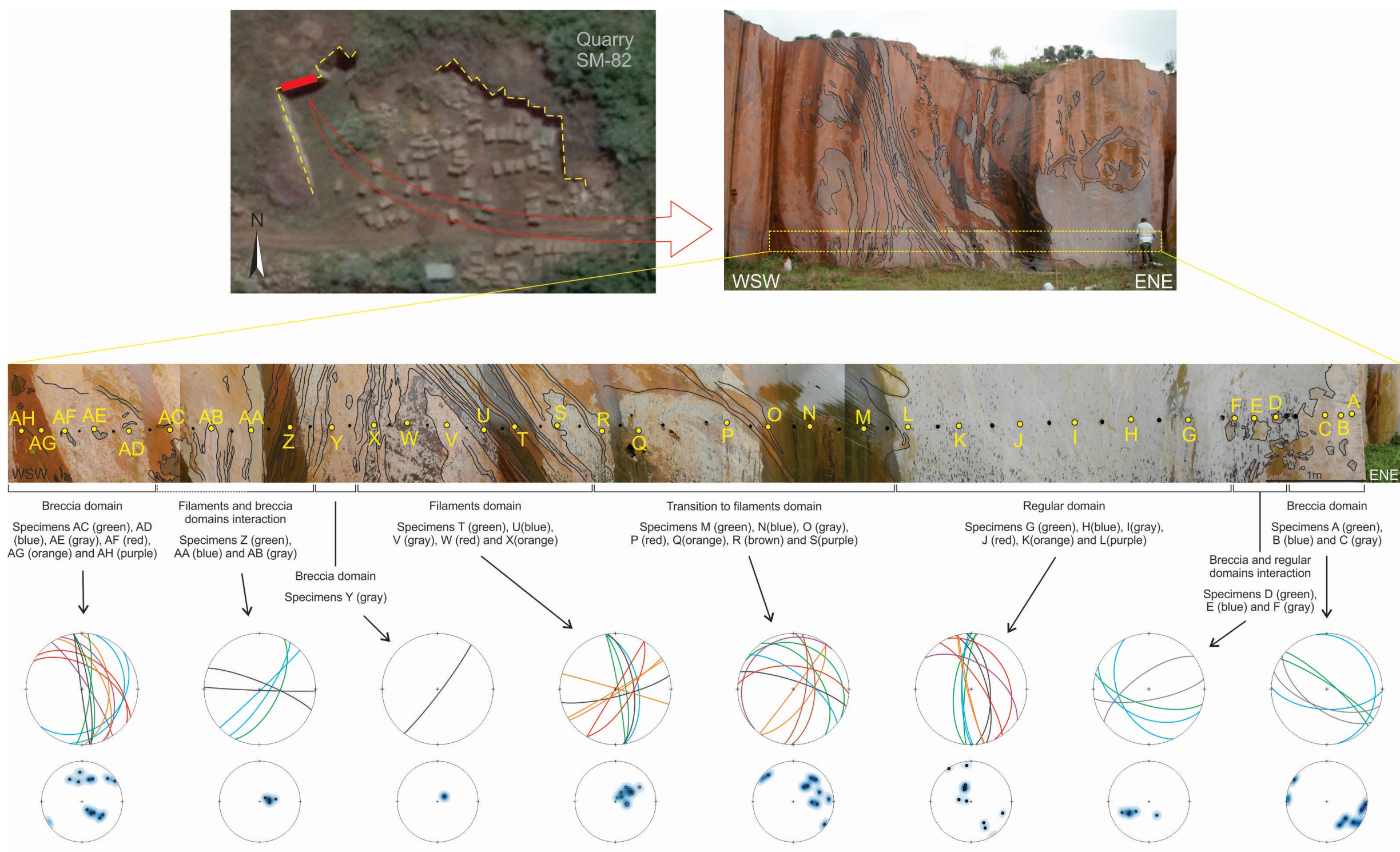

Magnetic foliation (above) and lineation (below) data

Figure 49: AMS data (from Cañón-Tapia and Raposo, 2018) of quarry SM-82. The regular (Rd), filaments (Fd), fragmentation and regular (Rd) domains are indicated. The data were divided and reinterpreted here according to the faciological domains defined in the item $I V .2 .3$ of this thesis and the data of each sampling site is separated by color as indicated in the figure. 
In addition to magma transportation function performed by the conduit, its architecture plays an important role on ascent and flow rates, on the permeability of the system and, as a consequence, on the degassing efficiency (Jaupart and Allegre, 1991; Sparks, 2003; de' Michieli Vitturi et al., 2008; Mueller et al., 2008). In this sense, the model of a fissure feeding system is quite satisfactory. This architecture may allow higher flow/discharge rates and a greater shearing that will allow a higher permeability of the system after volatile exsolution and vesicles formation (discussed later), favoring the explosive-effusive transitions.

Finally, the present thesis proposes a new detailed stratigraphy for the silicic volcanic rocks at the studied region. In summary, this silicic volcanic sequence presents at the base banded deposits followed by volcanic breccias, which are intruded by complex structures of dacitic composition. Hybrid deposits, lava flows and, finally, massive deposits occur overlapping the sequence.

Deposits similar to the banded deposits here described have been reported by Luchetti et al. (2018b, 2018a) throughout the Province and were interpreted as highgrade ignimbrites. In fact, the absence of clear pyroclastic features (e.g. shards and fiammes) turns their exact classification into a difficult task. However some other features such as lithic fragments, basal glassy layer and even the peculiar textural and compositional variation of pyroxenes (not observed in other deposits and possibly related to a heterogeneous remobilization of previously formed material) may support the hypothesis of an origin by pyroclastic flows. Regarding the eruptive dynamics responsible for the generation of such deposits, the pre-eruptive physico-chemical conditions of the magma must be considered. A high temperature volatile-poor dacitic magma $\left(\sim 1000^{\circ} \mathrm{C}\right.$ and $1-1.3 \% \mathrm{H}_{2} \mathrm{O}$, our unpublished data, which are in accordance with previous works on Caxias do Sul magma type - e.g. Polo et al., 2018a) rises in a fissural conduit system with no significant crystallization. The low content (or even absence) of crystals and high temperatures inhibited the drastic increases in the viscosity of the shallow magmatic system. A magmatic rise fast enough to inhibit intense crystallization can lead to late-stage bubble growth at shallow depth, and a fissural system allows high magma outflow rates, leading to boiling over eruptions responsible for the generation of pyroclastic density currents (PDC's) related either to low-explosivity events or magma fountains. Considering the first case, a combined effect of decreasing density (possibly increasing the velocity of magma ascent) and displacement of the glass transition 


\section{Chapter VII \\ Discussions and concluding remarks}

temperature due to the shallow volatile exsolution would cause the system to cross the ductile-brittle limit, leading to a fracture-dominated magma fragmentation at shallow depth resulting on low-explosivity eruptions (figure $50 \mathrm{~A} 1$ ). In the second case, slightly lower velocity of ascent and the exsolution of volatiles would not be enough to cross the ductile-brittle limit, favouring the occurrence of Etnean-style fountain (figure 50 A2). This type of eruption can be characterized by low-column pyroclastic fountaining with dense, poorly inflated pyroclastic flows that generate densely welded, rheomorphic proximal deposits (similar to layered lava flows), as suggested by Bachmann et al., (2000). Indeed, the banded deposits described in the present work resemble the extremely flattened blobs texture described by these authors. This model stands out also for guaranteeing the mobility/reomorphism needed for the generation of these structures even without a substrate with significant slope (which would be the case since the previous deposits corresponds to extensive sheet basaltic lava flows).

Moving upwards in the stratigraphy, the volcanic breccias also may have resulted from explosive volcanic events but, in this case, probably related to block and ash flows. As already discussed in Chapter V, the grain size distribution (GSD) fractality could be quantified by a single power law, with low to moderate D values, suggesting low eruptive energy. The fragments are characterized by low vesicularity and the vesicle size distribution (VSD) curves are represented by single exponential curves, concave upwards for all samples, with vesicles becoming more deformed/complex with increasing size, suggesting a single continuous process of vesicle nucleation and growth under low degrees of shear or unstable flow regime. The fragments are also characterized by high values of solidity, and a positive correlation of roughness and angularity with fragment size was recognized, suggesting the occurrence of secondary fragmentation processes, although field evidence, in accordance with the inferences from GSD and fractal analysis, characterizes proximal deposits. So for this scenario, I propose that the fountaining activity may allow an efficient degassing of the dacitic magma, inducing viscosity increase and shifting the eruptive dynamics to lavadome extrusions (figure $50 \mathrm{~B} 1$ ). Again, as already discussed in Chapter V, the lava dome may have worked as a cap for the rising magma, partially obstructing degassing and leading to a system overpressure increase that triggers the dome collapse generating the volcanic breccias (figure $50 \mathrm{~B} 2$ ). Additionally, it is important to highlight that thefissural architecture of the feeding system and the magma migration along an 
extended and variably erupting/non-erupting fissure, also with gas lateral migration, may have allowed the occurrence of isolated magma batches evolving to distinct viscous and degassed states. This condition may have permitted the existence of active and inactive vents, or vents with distinct eruptive dynamics spatially and/or temporally separated along the fissure system.

The rapid decompression provoked by the dome collapse triggers downwards elastic waves that subject the magma to a tensile stress which may have been responsible for a new magma fragmentation event (Alidibirov and Dingwell, 2000; Herd et al., 2005) and consequently generating the banded deposits (rheoignimbrites) that locally cover volcanic breccias. Another possibility for this new, brief and restrict pyroclastic phase would be the migration/ascent of new relatively gas-rich magma into the shallow plumbing system. Finally, a gradual loss of eruptive energy, together with opening/widening of the shallow plumbing system, may have led to the explosiveeffusive transition responsible for the generation of the hybrid deposits and lava flows (figure 50C). 
A1 Low-energy explosive eruptions generating rheoignimbrites
A2 Low-colunm lava fountain with dense, poorly inflated pyroclastic flows, generating welded, rheomorphic proximal deposits.
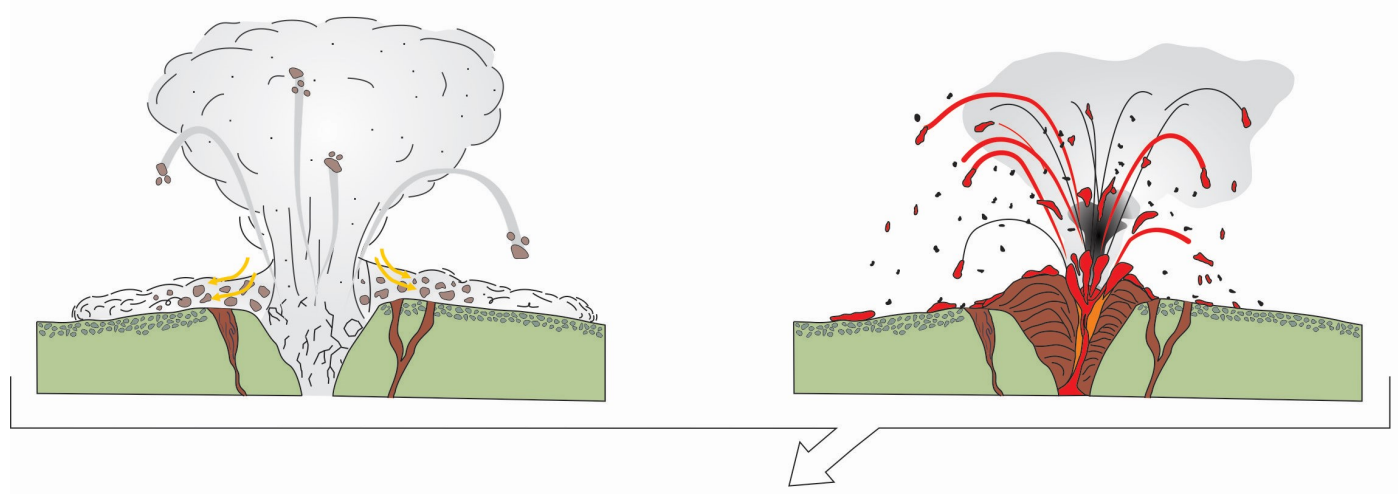

B1 Lava dome growth

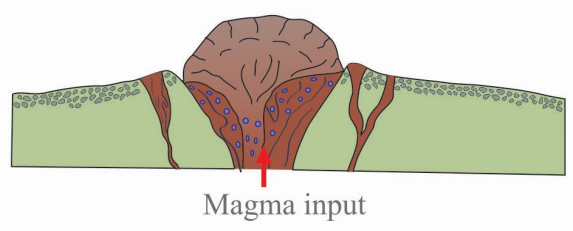

The lava dome partially seals the system, leading to an overpressure increase.
B2 Lava dome collapse

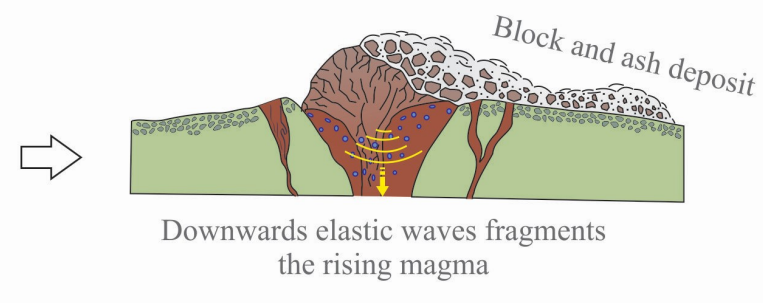

(C) Low-energy explosive eruptions may follow the dome collapse, locally generating rheoignimbrites. A loss of eruptive energy leads to explosive-effusive transitions, represented by the hybrid deposits followed by lava flows.

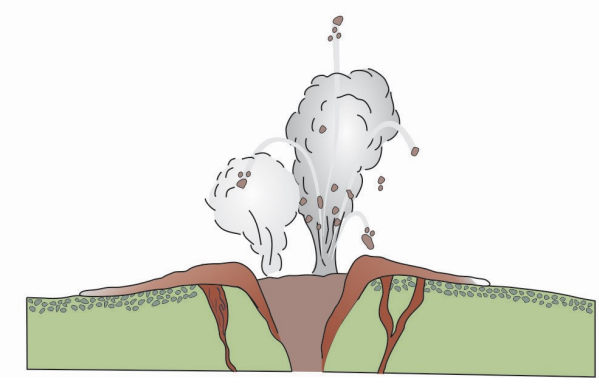

Figure 50: Eruptive model for the dacitic volcanic sequence in the São Marcos region. 


\section{SUMMARY OF CONCLUSIONS}

- Two slightly chemically distinct groups are recognized for the Caxias do Sul dacites, suggesting different sources and/or variations in the assimilated crustal component;

- A fissural feeder system with NW-SE trend and multiple aligned eruptive centers is proposed. This architecture favours lateral magma migration, allowing different magma batches to reach diverse viscous and degassed states, as well as the occurrence of active/non-active vents or vents with different eruptive dynamics spatially and/or temporally separated along the fissure. Additionally, the fissural architecture favours high flow/discharge rates and shearing, allowing the development of a highly permeable system and favouring explosive-effusive transitions.

- A new eruptive model and detailed stratigraphy of the silicic sequence are proposed and can be summarized as: (i) initial pyroclastic phase, related to either low-explosivity events or magma fountains, giving rise to proximal rheoignimbrites; (ii) transition to dome extrusions, as a result of the intense degasification promoted by the explosive phase or due to the occurrence of batches of magma under different viscous and degassed states, and posterior dome collapse giving rising to block and ash deposits; (iii) restricted occurrence of rheoignimbrites related to a new explosive phase, a consequence of fragmentation due to rapid decompression related to the dome collapse; (iv) explosive-effusive transition related to gradual loss of eruptive energy and widening of the active vent, resulting in hybrid deposits and afterwards to lava flows. 


\section{References}

Ágústsdóttir, T., Woods, T., Greenfield, T., Green, R.G., White, R.S., Winder, T., Brandsdóttir, B., Steinthórsson, S., and Soosalu, H., 2016, Strike-slip faulting during the 2014 Bárðarbunga- Holuhraun dike intrusion, central Iceland: Geophysical Research Letters, v. 43, p. 1495-1503, doi:10.1002/2014GL059364.Received.

Alidibirov, M., and Dingwell, D.B., 2000, Three fragmentation mechanisms for highly viscous magma under rapid decompression: Journal of Volcanology and Geothermal Research, v. 100, p. 413-421, doi:10.1016/S0377-0273(00)00149-9.

Bachmann, O., Dungan, M.A., and Lipman, P.W., 2000, Voluminous lava-like precursor to a major ash-flow tuff: Low-column pyroclastic eruption of the Pagosa Peak Dacite, San Juan volcanic field, Colorado: Journal of Volcanology and Geothermal Research, v. 98, p. 153-171, doi:10.1016/S0377-0273(99)00185-7.

Barreto, C.J.S., Lafon, J.M., Lima, E.F., and Sommer, C.A., 2016, Geochemical and Sr$\mathrm{Nd}-\mathrm{Pb}$ isotopic insight into the low-Ti basalts from southern Paraná Igneous Province, Brazil: The role of crustal contamination: International Geology Review, v. 58, p. 1324-1349, doi:10.1080/00206814.2016.1147988.

Cañón-Tapia, E., and Raposo, M.I.B., 2018, Anisotropy of magnetic susceptibility of silicic rocks from quarries in the vicinity of São Marcos, Rio Grande do Sul, South Brazil: Implications for emplacement mechanisms: Journal of Volcanology and Geothermal Research, v. 355, p. 165-180, doi:10.1016/J.JVOLGEORES.2017.07.018.

Delaney, P.T. and Pollard, D.D., 1981, Deformation ofhost rocks and flow of magma during growth of minettedikes and breccia-bearing intrusions near Ship Rock,New Mexico. Tech. rep. 102. USGPO.

de' Michieli Vitturi, M., Clarke, A.B., Neri, A., and Voight, B., 2008, Effects of conduit geometry on magma ascent dynamics in dome-forming eruptions: Earth and Planetary Science Letters, v. 272, p. 567-578, doi:10.1016/j.epsl.2008.05.025.

Ernst, R.E., and Baragar, W.R.A., 1992, Evidence from magnetic fabric for the flow patterns of magma in the Mackenzie giant radiating dyke swarm: Letters to Nature, v. 356.

Garland, F., Hawkesworth, C.J., and Mantovani, M.S.M., 1995, Description and Petrogenesis of the Paraná Rhyolites, Southern Brazil: Journal of Petrology, v. 36, p. 1193-1227.

Guimarães, L.F., 2014, Características físicas e químicas e modelo eruptivo para os riolitos tipo Santa Maria ( Província Magmática Paraná ) na região de Gramado Xavier, RS: Universidade de São Paulo.

Guimarães, L.F., De Campos, C.P., Janasi, V.A., Lima, E.F., and Dingwell, D.B., 2018a, Flow and fragmentation patterns in the silicic feeder system and related deposits in the Paraná-Etendeka Magmatic Province, São Marcos, South Brazil: Journal of Volcanology and Geothermal Research, v. 358, p. 149-164, doi:10.1016/J.JVOLGEORES.2018.03.021. 
Guimarães, L.F., Raposo, M.I.B., Janasi, V.A., Cañón-Tapia, E., and Polo, L.A., 2018b, An AMS study of different silicic units from the southern Paraná-Etendeka Magmatic Province in Brazil: Implications for the identification of flow directions and local sources: Journal of Volcanology and Geothermal Research, v. 355, p. 304-318, doi:10.1016/j.jvolgeores.2017.11.014.

Healy, D., Rizzo, R.E., Duffy, M., Farrell, N.J.C., Hole, M.J., and Muirhead, D., 2018, Field evidence for the lateral emplacement of igneous dykes: Implications for 3D mechanical models and the plumbing beneath fissure eruptions: Volcanica, v. 1, p. 85-105, doi:https://doi.org/10.30909/vol.01.02.85105.

Herd, R.A., Edmonds, M., and Bass, V.A., 2005, Catastrophic lava dome failure at Soufrière Hills Volcano, Montserrat, 12-13 July 2003: Journal of Volcanology and Geothermal Research, v. 148, p. 234-252, doi:10.1016/j.jvolgeores.2005.05.003.

Jaupart, C., and Allegre, C.J., 1991, Gas Content, Eruption Rate and Instabilities of Eruption Regime in Silicic Volcanos: Earth and Planetary Science Letters, v. 102, p. 413-429, doi:10.1016/0012-821x(91)90032-d.

Kavanagh, J.L., Burns, A.J., Hilmi Hazim, S., Wood, E.P., Martin, S.A., Hignett, S., and Dennis, D.J.C., 2018, Challenging dyke ascent models using novel laboratory experiments: Implications for reinterpreting evidence of magma ascent and volcanism: Journal of Volcanology and Geothermal Research, v. 354, p. 87-101, doi:10.1016/J.JVOLGEORES.2018.01.002.

Lima, E.F., Philipp, R.P., Rizzon, G.C., Waichel, B.L., and Rossetti, L.M.M., 2012, Sucessões vulcânicas, modelo de alimentação e geração de domos de lava ácidos da Formação Serra Geral na região de São Marcos-Antônio Prado (RS): Geologia USP - Serie Cientifica, v. 12, p. 49-64, doi:10.5327/Z1519-874X2012000200004.

Lima, E.F., Waichel, B.L., Rossetti, L.M.M., Sommer, C.A., and Simões, M.S., 2018, Feeder systems of acidic lava flows from the Paraná-Etendeka Igneous Province in southern Brazil and their implications for eruption style: Journal of South American Earth Sciences, v. 81, p. 1-9, doi:10.1016/J.JSAMES.2017.11.004.

Lister, J.R., and Kerr, R.C., 1991, Fluid-mechanical models of crack propagation and their application to magma transport in dykes: Journal of Geophysical Research, v. 96, p. 10049, doi:10.1029/91JB00600.

Luchetti, A.C.F., Gravley, D.M., Gualda, G.A.R., and Nardy, A.J.R., 2018a, Textural evidence for high-grade ignimbrites formed by low-explosivity eruptions, Paraná Magmatic Province, southern Brazil: Journal of Volcanology and Geothermal Research, v. 355, p. 87-97, doi:10.1016/J.JVOLGEORES.2017.04.012.

Luchetti, A.C.F., Nardy, A.J.R., and Madeira, J., 2018b, Silicic, high- to extremely high-grade ignimbrites and associated deposits from the Paraná Magmatic Province, southern Brazil: Journal of Volcanology and Geothermal Research, v. 355, p. 270-286, doi:10.1016/J.JVOLGEORES.2017.11.010.

Morgavi, D., Perugini, D., De Campos, C.P., Ertel-Ingrisch, W., and Dingwell, D.B., 2013a, Time evolution of chemical exchanges during mixing of rhyolitic and basaltic melts: Journal of Volcanology and Geothermal Research, v. 253, p. 87-96, doi:10.1007/s00410-013-0894-1. 


\section{Chapter VII \\ Discussions and concluding remarks}

Morgavi, D., Perugini, D., De Campos, C.P., Ertel-Ingrisch, W., Lavallée, Y., Morgan, L., and Dingwell, D.B., 2013b, Interactions between rhyolitic and basaltic melts unraveled by chaotic mixing experiments: Chemical Geology, v. 346, p. 119-212, doi:10.1016/j.chemgeo.2012.10.003.

Mueller, S., Scheu, B., Spieler, O., and Dingwell, D.B., 2008, Permeability control on magma fragmentation: Geology, v. 36, p. 399-402, doi:10.1130/G24605A.1.

Perugini, D., Poli, G., and Mazzuoli, R., 2003, Chaotic advection, fractals and diffusion during mixing of magmas: Evidence from lava flows: Journal of Volcanology and Geothermal Research, v. 124, p. 255-279, doi:10.1016/S0377-0273(03)00098-2.

Poland, M.P., Moats, W.P., and Fink, J.H., 2008, A model for radial dike emplacement in composite cones based on observations from Summer Coon volcano, Colorado, USA: Bulletin of Volcanology, v. 70, p. 861-875, doi:10.1007/s00445-007-01759.

Poli, G., and Perugini, D., 2002, Strange attractors in magmas: Evidence from lava flows: Lithos, v. 65, p. 287-297, doi:10.1016/S0024-4937(02)00196-2.

Polo, L.A., Giordano, D., Janasi, V.A., and Guimarães, L.F., 2018a, Effusive silicic volcanism in the Paraná Magmatic Province, South Brazil: Physico-chemical conditions of storage and eruption and considerations on the rheological behavior during emplacement: Journal of Volcanology and Geothermal Research, v. 355, p. 115-135, doi:10.1016/J.JVOLGEORES.2017.05.027.

Polo, L.A., Janasi, V.A., Giordano, D., Lima, E.F., Cañón-Tapia, E., and Roverato, M., 2018b, Effusive silicic volcanism in the Paraná Magmatic Province, South Brazil: Evidence for locally-fed lava flows and domes from detailed field work: Journal of Volcanology and Geothermal Research, v. 355, p. 204-218, doi:10.1016/J.JVOLGEORES.2017.08.007.

Raposo, M.I.B., 1997, Magnetic fabric and its significance in the Florianópolis dyke swarm, southern Brazil: Geophysical Journal International, v. 131, p. 159-170, doi:10.1111/j.1365-246X.1997.tb00602.x.

Raposo, M.I.B., and Ernesto, M., 1995, Anisotropy of magnetic susceptibility in the Ponta Grossa dyke swarm (Brazil) and its relationship with magma flow direction: Physics of the Earth and Planetary Interiors, v. 87, p. 183-196, doi:10.1016/00319201(94)02970-M.

Simões, M.S., Lima, E.F., Sommer, C.A., and Rossetti, L.M.M., 2018, Structures and lithofacies of inferred silicic conduits in the Paraná-Etendeka LIP, southernmost Brazil: Journal of Volcanology and Geothermal Research, v. 355, p. 319-336, doi:10.1016/J.JVOLGEORES.2017.12.013.

Sparks, R.S.J., 2003, Dynamics of magma degassing: Geological Society, London, Special Publications, v. 213, p. 5-22, doi:10.1144/GSL.SP.2003.213.01.02.

Townsend, M.R., Pollard, D.D., and Smith, R.P., 2017, Mechanical models for dikes: A third school of thought: Tectonophysics, v. 703-704, p. 98-118, doi:10.1016/J.TECTO.2017.03.008. 


\section{Appendix I}

Paper published at Journal of Volcanology and Geothermal Research 
https://doi.org/10.1016/j.jvolgeores.2018.03.021 
Appendix II

Outcrops location map 


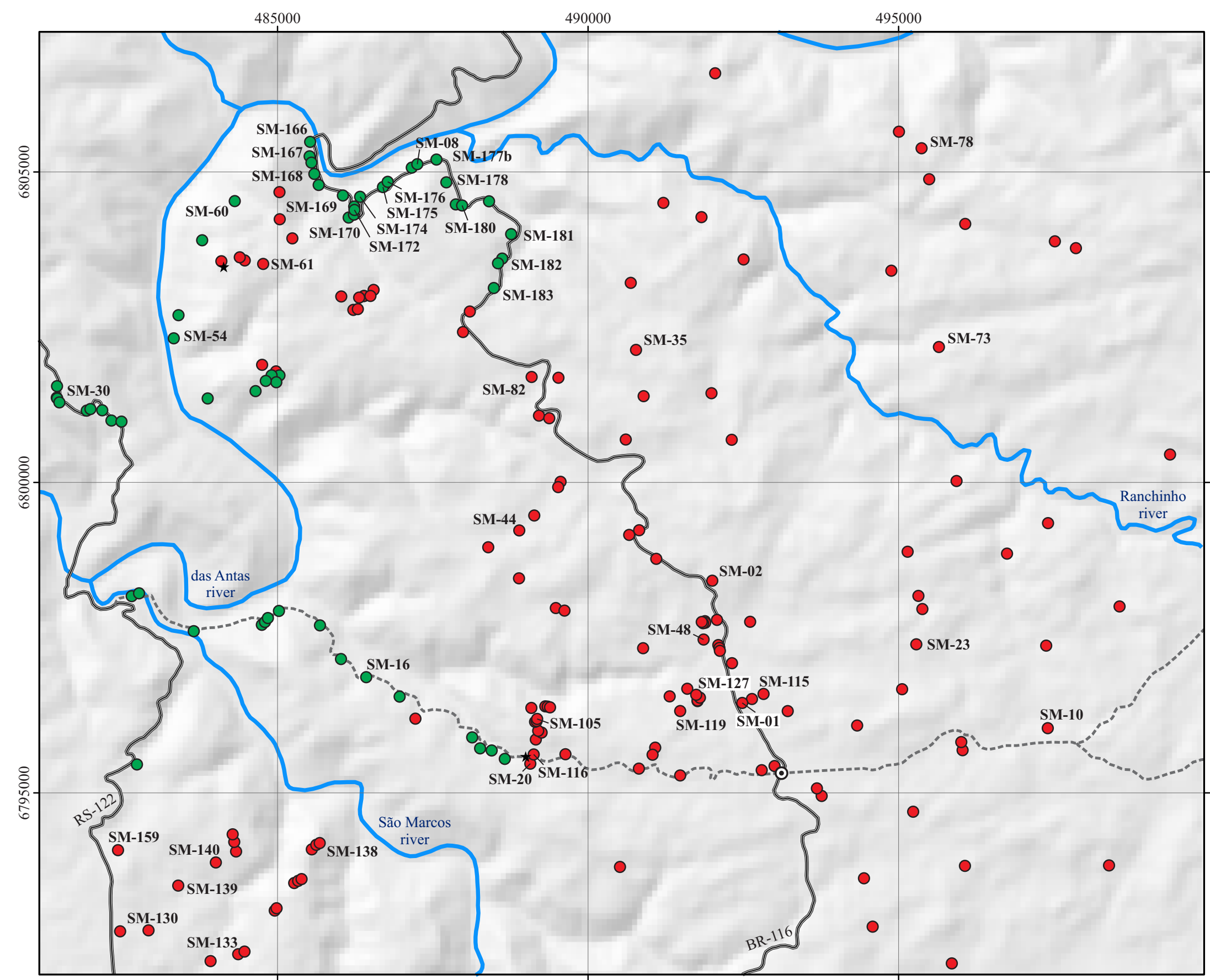

- Dacites

$\star$ Contact between basalts and dacites

- Basalts

$\int$ Rivers

Highways

- Access roads

$\odot$ City

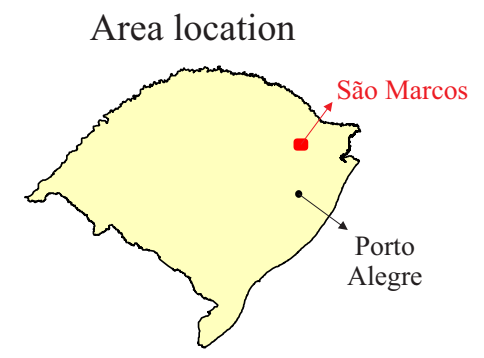

\begin{tabular}{|c|c|c|}
\hline \multicolumn{3}{|c|}{ Map sheets Index } \\
\hline $\begin{array}{l}\text { São Paulo } \\
\text { MI-2936/1 }\end{array}$ & $\begin{array}{c}\text { Guacho } \\
\text { MI-2936/2 }\end{array}$ & $\begin{array}{c}\text { Vacaria } \\
\text { MI-2937/1 }\end{array}$ \\
\hline $\begin{array}{c}\text { Antônio } \\
\text { Prado } \\
\text { MI-2936/3 }\end{array}$ & $\begin{array}{l}\text { São Marcos } \\
\text { MI-2936/4 }\end{array}$ & $\begin{array}{c}\text { Criúva } \\
\text { MI-2937/3 }\end{array}$ \\
\hline $\begin{array}{l}\text { Farroupilha } \\
\text { MI-2953/1 }\end{array}$ & $\begin{array}{c}\text { Caxias } \\
\text { do Sul } \\
\text { MI-2953/2 }\end{array}$ & $\begin{array}{l}\text { Oliva } \\
\text { MI-2954/1 }\end{array}$ \\
\hline
\end{tabular}


Appendix III

Mineral chemistry 
Chemical composition (\% wt) for plagioclase from Caxias do Sul dacites

\begin{tabular}{|c|c|c|c|c|c|c|c|c|c|c|c|c|c|}
\hline Sample & SM-01C & SM-01C & SM-01C & SM-01C & SM-01C & SM-01C & SM-01C & SM-01C & SM-01C & SM-01C & SM-01C & SM-01C & SM-01C \\
\hline Point ID & A.feno1.nucleo & A.fenol.borda & $\begin{array}{c}\text { A.microcristal } \\
1 \\
\end{array}$ & A.microlito & A.feno2.nucleo & A.micristal2 & B.fenol.nucleo & B.feno1.meio & B.microcristal & $\begin{array}{c}\text { B.microcristal. } \\
\text { borda }\end{array}$ & C.feno.nucleo & C.feno.meio & C.feno.borda \\
\hline Position & core & rim & core & core & core & core & core & intermediate & core & rim & core & intermediate & $\mathrm{rim}$ \\
\hline Size & \multicolumn{2}{|c|}{$0,21 \mathrm{~mm}$} & $0,056 \mathrm{~mm}$ & $0,033 \mathrm{~mm}$ & $0,13 \mathrm{~mm}$ & $0,066 \mathrm{~mm}$ & \multicolumn{2}{|c|}{$0,17 \mathrm{~mm}$} & \multicolumn{2}{|c|}{$0,053 \mathrm{~mm}$} & \multicolumn{3}{|c|}{$0,275 \mathrm{~mm}$} \\
\hline$\overline{\mathrm{SiO}_{2}}$ & 54,94 & 54,38 & 54,89 & 58,45 & 54,38 & 55,53 & 54,18 & 54,01 & 54,76 & 56,26 & 54,69 & 55,17 & 54,27 \\
\hline $\mathrm{TiO}_{2}$ & 0,07 & 0,01 & 0,06 & 0,12 & 0,01 & 0,09 & 0,04 & 0,04 & 0,11 & 0,09 & 0,02 & 0,02 & 0,04 \\
\hline $\mathrm{Al}_{2} \mathrm{O}_{3}$ & 28,61 & 28,75 & 28,89 & 26,37 & 28,88 & 28,00 & 28,55 & 28,77 & 29,01 & 27,69 & 28,74 & 28,53 & 28,75 \\
\hline $\mathrm{FeOt}$ & 1,11 & 1,13 & 1,22 & 0,92 & 1,13 & 1,08 & 1,33 & 1,23 & 1,27 & 1,03 & 1,21 & 0,99 & 1,26 \\
\hline $\mathrm{MnO}$ & 0,02 & 0,00 & 0,00 & 0,00 & 0,01 & 0,01 & 0,03 & 0,03 & 0,00 & 0,02 & 0,02 & 0,00 & 0,02 \\
\hline $\mathrm{MgO}$ & 0,04 & 0,07 & 0,05 & 0,04 & 0,07 & 0,01 & 0,06 & 0,04 & 0,04 & 0,00 & 0,09 & 0,07 & 0,06 \\
\hline $\mathrm{CaO}$ & 11,19 & 11,15 & 10,89 & 8,01 & 11,40 & 10,14 & 11,00 & 11,21 & 10,56 & 9,77 & 11,57 & 10,77 & 11,06 \\
\hline $\mathrm{Na}_{2} \mathrm{O}$ & 4,84 & 4,73 & 4,73 & 6,38 & 4,72 & 5,32 & 4,78 & 4,73 & 5,00 & 5,46 & 4,65 & 4,98 & 4,77 \\
\hline $\mathrm{K}_{2} \mathrm{O}$ & 0,39 & 0,38 & 0,32 & 0,57 & 0,36 & 0,44 & 0,37 & 0,38 & 0,41 & 0,44 & 0,36 & 0,39 & 0,34 \\
\hline $\mathrm{BaO}$ & 0,07 & 0,04 & 0,02 & 0,06 & 0,05 & 0,07 & 0,01 & 0,00 & 0,06 & 0,10 & 0,00 & 0,02 & 0,03 \\
\hline $\mathrm{SrO}$ & 0,09 & 0,04 & 0,13 & 0,08 & 0,08 & 0,08 & 0,10 & 0,06 & 0,10 & 0,07 & 0,09 & 0,02 & 0,07 \\
\hline Total & 101,38 & 100,68 & 101,20 & 101,00 & 101,10 & 100,78 & 100,45 & 100,49 & 101,33 & 100,93 & 101,44 & 100,95 & 100,68 \\
\hline \multicolumn{14}{|c|}{ Structural formula calculated for 8 oxygens } \\
\hline $\mathrm{Si}$ & 2,46 & 2,45 & 2,46 & 2,60 & 2,44 & 2,50 & 2,45 & 2,44 & 2,45 & 2,52 & 2,45 & 2,47 & 2,45 \\
\hline $\mathrm{Ti}$ & 0,00 & 0,00 & 0,00 & 0,00 & 0,00 & 0,00 & 0,00 & 0,00 & 0,00 & 0,00 & 0,00 & 0,00 & 0,00 \\
\hline $\mathrm{Al}$ & 1,51 & 1,53 & 1,53 & 1,38 & 1,53 & 1,48 & 1,52 & 1,53 & 1,53 & 1,46 & 1,52 & 1,51 & 1,53 \\
\hline $\mathrm{Fe}^{2+}$ & 0,04 & 0,04 & 0,05 & 0,03 & 0,04 & 0,04 & 0,05 & 0,05 & 0,05 & 0,04 & 0,05 & 0,04 & 0,05 \\
\hline $\mathrm{Mn}$ & 0,00 & 0,00 & 0,00 & 0,00 & 0,00 & 0,00 & 0,00 & 0,00 & 0,00 & 0,00 & 0,00 & 0,00 & 0,00 \\
\hline $\mathrm{Mg}$ & 0,00 & 0,00 & 0,00 & 0,00 & 0,00 & 0,00 & 0,00 & 0,00 & 0,00 & 0,00 & 0,01 & 0,00 & 0,00 \\
\hline $\mathrm{Ca}$ & 0,54 & 0,54 & 0,52 & 0,38 & 0,55 & 0,49 & 0,53 & 0,54 & 0,51 & 0,47 & 0,56 & 0,52 & 0,53 \\
\hline $\mathrm{Na}$ & 0,42 & 0,41 & 0,41 & 0,55 & 0,41 & 0,46 & 0,42 & 0,41 & 0,43 & 0,47 & 0,40 & 0,43 & 0,42 \\
\hline $\mathrm{K}$ & 0,02 & 0,02 & 0,02 & 0,03 & 0,02 & 0,03 & 0,02 & 0,02 & 0,02 & 0,03 & 0,02 & 0,02 & 0,02 \\
\hline $\mathrm{Ba}$ & 0,00 & 0,00 & 0,00 & 0,00 & 0,00 & 0,00 & 0,00 & 0,00 & 0,00 & 0,00 & 0,00 & 0,00 & 0,00 \\
\hline$\sum$ cations & 5,00 & 5,00 & 4,99 & 4,99 & 5,01 & 5,00 & 5,01 & 5,01 & 5,01 & 5,00 & 5,00 & 5,00 & 5,00 \\
\hline An & 54,82 & 55,31 & 54,92 & 39,59 & 55,96 & 49,98 & 54,74 & 55,43 & 52,54 & 48,42 & 56,70 & 53,19 & 55,03 \\
\hline $\mathbf{A b}$ & 42,91 & 42,46 & 43,17 & 57,06 & 41,92 & 47,45 & 43,04 & 42,32 & 45,02 & 48,96 & 41,23 & 44,51 & 42,95 \\
\hline Or & 2,27 & 2,23 & 1,91 & 3,35 & 2,12 & 2,56 & 2,22 & 2,24 & 2,44 & 2,62 & 2,07 & 2,30 & 2,02 \\
\hline
\end{tabular}


Chemical composition (\% wt) for plagioclase from Caxias do Sul dacites

\begin{tabular}{|c|c|c|c|c|c|c|c|c|c|c|c|c|c|}
\hline Sample & SM-01C & SM-01C & SM-01C & SM-01C & SM-01C & SM-01C & SM-01C & SM-01C & SM-01C & SM-01C & SM-01C & SM-01C & SM-01C \\
\hline Point ID & C.microlito & $\begin{array}{c}\text { EF.feno1.nucle } \\
\text { o }\end{array}$ & EF.micro1 & EF.feno2 & $\begin{array}{c}\text { EF.feno2.bord } \\
\mathrm{a}\end{array}$ & EF.micro2 & EF.matriz & GHI.feno1 & $\begin{array}{c}\text { GHI.feno1.bor } \\
\text { da }\end{array}$ & GHI.microlito1 & GHI.micro2 & $\begin{array}{c}\text { GHI.micro2.bo } \\
\text { rda }\end{array}$ & GHI.matriz \\
\hline Position & core & core & core & core & rim & core & core & core & rim & core & core & rim & core \\
\hline Size & $0,05 \mathrm{~mm}$ & $0,214 \mathrm{~mm}$ & $0,041 \mathrm{~mm}$ & \multicolumn{2}{|c|}{$0,242 \mathrm{~mm}$} & $0,085 \mathrm{~mm}$ & $0,016 \mathrm{~mm}$ & \multicolumn{2}{|c|}{$0,147 \mathrm{~mm}$} & $0,022 \mathrm{~mm}$ & \multicolumn{2}{|c|}{$0,046 \mathrm{~mm}$} & $0,011 \mathrm{~mm}$ \\
\hline $\mathrm{SiO}_{2}$ & 57,97 & 54,59 & 54,84 & 55,04 & 55,12 & 55,59 & 67,16 & 53,84 & 55,10 & 58,87 & 54,53 & 56,09 & 67,46 \\
\hline $\mathrm{TiO}_{2}$ & 0,09 & 0,05 & 0,07 & 0,04 & 0,04 & 0,09 & 0,06 & 0,04 & 0,07 & 0,04 & 0,09 & 0,05 & 0,10 \\
\hline $\mathrm{Al}_{2} \mathrm{O}_{3}$ & 26,56 & 28,50 & 28,42 & 28,32 & 28,81 & 28,16 & 19,45 & 29,35 & 28,34 & 25,71 & 28,73 & 27,68 & 18,65 \\
\hline $\mathrm{FeOt}$ & 1,02 & 1,11 & 1,12 & 1,15 & 1,19 & 1,12 & 0,32 & 1,18 & 1,24 & 0,74 & 1,16 & 1,06 & 0,41 \\
\hline $\mathrm{MnO}$ & 0,00 & 0,00 & 0,01 & 0,00 & 0,02 & 0,00 & 0,00 & 0,02 & 0,00 & 0,02 & 0,02 & 0,02 & 0,00 \\
\hline $\mathrm{MgO}$ & 0,00 & 0,09 & 0,07 & 0,07 & 0,02 & 0,02 & 0,01 & 0,04 & 0,06 & 0,03 & 0,04 & 0,01 & 0,02 \\
\hline $\mathrm{CaO}$ & 8,49 & 11,13 & 10,80 & 10,94 & 10,98 & 10,13 & 0,44 & 11,07 & 10,50 & 7,50 & 11,11 & 9,59 & 0,39 \\
\hline $\mathrm{Na}_{2} \mathrm{O}$ & 6,15 & 4,75 & 4,90 & 4,83 & 4,84 & 5,27 & 4,51 & 4,82 & 4,98 & 6,81 & 4,82 & 5,46 & 4,05 \\
\hline $\mathrm{K}_{2} \mathrm{O}$ & 0,54 & 0,41 & 0,35 & 0,39 & 0,38 & 0,42 & 9,82 & 0,32 & 0,40 & 0,54 & 0,37 & 0,47 & 9,92 \\
\hline $\mathrm{BaO}$ & 0,12 & 0,03 & 0,04 & 0,00 & 0,00 & 0,01 & 0,03 & 0,00 & 0,09 & 0,18 & 0,02 & 0,00 & 0,02 \\
\hline $\mathrm{SrO}$ & 0,12 & 0,09 & 0,09 & 0,06 & 0,07 & 0,10 & 0,01 & 0,04 & 0,11 & 0,05 & 0,07 & 0,06 & 0,04 \\
\hline Total & 101,06 & 100,74 & 100,72 & 100,84 & 101,48 & 100,91 & 101,81 & 100,73 & 100,89 & 100,50 & 100,96 & 100,49 & 101,05 \\
\hline \multicolumn{14}{|c|}{ Structural formula calculated for 8 oxygens } \\
\hline $\mathrm{Si}$ & 2,59 & 2,46 & 2,47 & 2,47 & 2,46 & 2,49 & 2,98 & 2,43 & 2,48 & 2,63 & 2,45 & 2,52 & 3,01 \\
\hline $\mathrm{Ti}$ & 0,00 & 0,00 & 0,00 & 0,00 & 0,00 & 0,00 & 0,00 & 0,00 & 0,00 & 0,00 & 0,00 & 0,00 & 0,00 \\
\hline $\mathrm{Al}$ & 1,40 & 1,51 & 1,51 & 1,50 & 1,52 & 1,49 & 1,02 & 1,56 & 1,50 & 1,35 & 1,52 & 1,47 & 0,98 \\
\hline $\mathrm{Fe}$ & 0,04 & 0,04 & 0,04 & 0,04 & 0,04 & 0,04 & 0,01 & 0,04 & 0,05 & 0,03 & 0,04 & 0,04 & 0,02 \\
\hline Mn & 0,00 & 0,00 & 0,00 & 0,00 & 0,00 & 0,00 & 0,00 & 0,00 & 0,00 & 0,00 & 0,00 & 0,00 & 0,00 \\
\hline $\mathrm{Mg}$ & 0,00 & 0,01 & 0,00 & 0,00 & 0,00 & 0,00 & 0,00 & 0,00 & 0,00 & 0,00 & 0,00 & 0,00 & 0,00 \\
\hline $\mathrm{Ca}$ & 0,41 & 0,54 & 0,52 & 0,53 & 0,53 & 0,49 & 0,02 & 0,53 & 0,51 & 0,36 & 0,54 & 0,46 & 0,02 \\
\hline $\mathrm{Na}$ & 0,53 & 0,42 & 0,43 & 0,42 & 0,42 & 0,46 & 0,39 & 0,42 & 0,43 & 0,59 & 0,42 & 0,48 & 0,35 \\
\hline K & 0,03 & 0,02 & 0,02 & 0,02 & 0,02 & 0,02 & 0,56 & 0,02 & 0,02 & 0,03 & 0,02 & 0,03 & 0,57 \\
\hline $\mathrm{Ba}$ & 0,00 & 0,00 & 0,00 & 0,00 & 0,00 & 0,00 & 0,00 & 0,00 & 0,00 & 0,00 & 0,00 & 0,00 & 0,00 \\
\hline$\sum$ cations & 4,99 & 5,00 & 5,00 & 5,00 & 5,00 & 5,00 & 4,98 & 5,01 & 5,00 & 5,00 & 5,00 & 5,00 & 4,95 \\
\hline An & 41,89 & 55,05 & 53,78 & 54,30 & 54,39 & 50,23 & 2,18 & 54,87 & 52,52 & 36,64 & 54,79 & 47,88 & 2,00 \\
\hline $\mathbf{A b}$ & 54,91 & 42,52 & 44,15 & 43,38 & 43,39 & 47,29 & 40,21 & 43,23 & 45,07 & 60,20 & 43,02 & 49,34 & 37,52 \\
\hline Or & 3,20 & 2,43 & 2,07 & 2,33 & 2,22 & 2,48 & 57,61 & 1,90 & 2,41 & 3,17 & 2,19 & 2,78 & 60,47 \\
\hline
\end{tabular}


Chemical composition (\% wt) for plagioclase from Caxias do Sul dacites

\begin{tabular}{|c|c|c|c|c|c|c|c|c|c|c|c|c|c|}
\hline Sample & SM-01C & SM-01C & SM-01C & SM-01C & SM-01C & SM-01C & SM-01C & SM-01C & SM-01C & SM-61 & SM-61 & SM-61 & SM-61 \\
\hline Point ID & J.fenol & J.feno1.borda & J.micro1 & J.microlito1 & J_matriz & K.micro & K.micro.meio & K.micro.borda & K.microlito & microcristalA1 & $\begin{array}{c}\text { microcristalA1 } \\
\text {.borda } \\
\end{array}$ & microcristalA2 & $\begin{array}{c}\text { microcristalA2 } \\
\text {.borda }\end{array}$ \\
\hline Position & core & rim & core & core & core & core & intermediate & rim & core & core & rim & core & rim \\
\hline Size & \multicolumn{2}{|c|}{$0,167 \mathrm{~mm}$} & $0,115 \mathrm{~mm}$ & $0,039 \mathrm{~mm}$ & $0,016 \mathrm{~mm}$ & & $0,138 \mathrm{~mm}$ & & $0,019 \mathrm{~mm}$ & \multicolumn{2}{|c|}{$0,243 \mathrm{~mm}$} & \multicolumn{2}{|c|}{$0,236 \mathrm{~mm}$} \\
\hline$\overline{\mathrm{SiO}_{2}}$ & 54,66 & 54,60 & 54,33 & 56,33 & 67,36 & 55,54 & 54,45 & 56,96 & 57,83 & 55,05 & 55,69 & 56,66 & 57,65 \\
\hline $\mathrm{TiO}_{2}$ & 0,11 & 0,06 & 0,08 & 0,08 & 0,02 & 0,06 & 0,05 & 0,06 & 0,15 & 0,13 & 0,06 & 0,06 & 0,03 \\
\hline $\mathrm{Al}_{2} \mathrm{O}_{3}$ & 28,35 & 28,73 & 28,79 & 27,35 & 18,59 & 28,27 & 28,64 & 27,40 & 26,63 & 28,18 & 28,12 & 27,43 & 26,95 \\
\hline $\mathrm{FeOt}$ & 1,16 & 1,15 & 1,15 & 1,11 & 0,27 & 1,10 & 1,10 & 0,99 & 1,14 & 1,22 & 1,06 & 0,98 & 0,92 \\
\hline $\mathrm{MnO}$ & 0,00 & 0,00 & 0,00 & 0,01 & 0,00 & 0,00 & 0,02 & 0,02 & 0,02 & 0,00 & 0,01 & 0,01 & 0,01 \\
\hline $\mathrm{MgO}$ & 0,06 & 0,05 & 0,05 & 0,01 & 0,03 & 0,10 & 0,08 & 0,02 & 0,05 & 0,02 & 0,02 & 0,02 & 0,00 \\
\hline $\mathrm{CaO}$ & 10,89 & 11,09 & 11,11 & 9,55 & 0,28 & 10,84 & 11,43 & 9,91 & 8,51 & 10,75 & 10,34 & 9,36 & 8,56 \\
\hline $\mathrm{Na}_{2} \mathrm{O}$ & 4,76 & 4,73 & 4,67 & 5,66 & 3,35 & 4,85 & 4,53 & 5,34 & 6,07 & 4,94 & 5,21 & 5,64 & 6,02 \\
\hline $\mathrm{K}_{2} \mathrm{O}$ & 0,42 & 0,32 & 0,38 & 0,49 & 11,06 & 0,44 & 0,43 & 0,43 & 0,52 & 0,38 & 0,42 & 0,43 & 0,53 \\
\hline $\mathrm{BaO}$ & 0,06 & 0,06 & 0,00 & 0,05 & 0,08 & 0,03 & 0,04 & 0,00 & 0,06 & 0,04 & 0,06 & 0,08 & 0,07 \\
\hline $\mathrm{SrO}$ & 0,07 & 0,06 & 0,12 & 0,12 & 0,02 & 0,03 & 0,08 & 0,08 & 0,11 & 0,09 & 0,07 & 0,08 & 0,09 \\
\hline Total & 100,55 & 100,86 & 100,68 & 100,75 & 101,04 & 101,25 & 100,85 & 101,21 & 101,09 & 100,81 & 101,05 & 100,74 & 100,82 \\
\hline \multicolumn{14}{|c|}{ Structural formula calculated for 8 oxygens } \\
\hline $\mathrm{Si}$ & 2,47 & 2,46 & 2,45 & 2,53 & 3,02 & 2,48 & 2,45 & 2,54 & 2,58 & 2,48 & 2,50 & 2,54 & 2,57 \\
\hline $\mathrm{Ti}$ & 0,00 & 0,00 & 0,00 & 0,00 & 0,00 & 0,00 & 0,00 & 0,00 & 0,01 & 0,00 & 0,00 & 0,00 & 0,00 \\
\hline $\mathrm{Al}$ & 1,51 & 1,52 & 1,53 & 1,45 & 0,98 & 1,49 & 1,52 & 1,44 & 1,40 & 1,50 & 1,49 & 1,45 & 1,42 \\
\hline $\mathrm{Fe}$ & 0,04 & 0,04 & 0,04 & 0,04 & 0,01 & 0,04 & 0,04 & 0,04 & 0,04 & 0,05 & 0,04 & 0,04 & 0,03 \\
\hline $\mathrm{Mn}$ & 0,00 & 0,00 & 0,00 & 0,00 & 0,00 & 0,00 & 0,00 & 0,00 & 0,00 & 0,00 & 0,00 & 0,00 & 0,00 \\
\hline $\mathrm{Mg}$ & 0,00 & 0,00 & 0,00 & 0,00 & 0,00 & 0,01 & 0,01 & 0,00 & 0,00 & 0,00 & 0,00 & 0,00 & 0,00 \\
\hline $\mathrm{Ca}$ & 0,53 & 0,53 & 0,54 & 0,46 & 0,01 & 0,52 & 0,55 & 0,47 & 0,41 & 0,52 & 0,50 & 0,45 & 0,41 \\
\hline $\mathrm{Na}$ & 0,42 & 0,41 & 0,41 & 0,49 & 0,29 & 0,42 & 0,40 & 0,46 & 0,52 & 0,43 & 0,45 & 0,49 & 0,52 \\
\hline K & 0,02 & 0,02 & 0,02 & 0,03 & 0,63 & 0,03 & 0,02 & 0,02 & 0,03 & 0,02 & 0,02 & 0,02 & 0,03 \\
\hline $\mathrm{Ba}$ & 0,00 & 0,00 & 0,00 & 0,00 & 0,00 & 0,00 & 0,00 & 0,00 & 0,00 & 0,00 & 0,00 & 0,00 & 0,00 \\
\hline$\sum$ cations & 5,00 & 5,00 & 5,00 & 5,00 & 4,95 & 4,99 & 5,00 & 4,98 & 4,99 & 5,00 & 5,00 & 4,99 & 4,99 \\
\hline An & 54,43 & 55,36 & 55,52 & 46,88 & 1,43 & 53,82 & 56,76 & 49,35 & 42,31 & 53,36 & 51,02 & 46,61 & 42,63 \\
\hline $\mathbf{A b}$ & 43,06 & 42,73 & 42,23 & 50,28 & 31,07 & 43,57 & 40,71 & 48,12 & 54,61 & 44,37 & 46,52 & 50,83 & 54,25 \\
\hline Or & 2,51 & 1,91 & 2,25 & 2,84 & 67,50 & 2,61 & 2,53 & 2,53 & 3,09 & 2,27 & 2,45 & 2,56 & 3,13 \\
\hline
\end{tabular}


Chemical composition (\% wt) for plagioclase from Caxias do Sul dacites

\begin{tabular}{|c|c|c|c|c|c|c|c|c|c|c|c|c|c|}
\hline Sample & SM-61 & SM-61 & SM-61 & SM-61 & SM-61 & SM-61 & SM-61 & SM-61 & SM-61 & SM-61 & SM-61 & SM-61 & SM-61 \\
\hline Point ID & microcristalA3 & $\begin{array}{c}\text { microcristalA3 } \\
.2 \\
\end{array}$ & microcristalA4 & $\begin{array}{c}\text { microcristalA5 } \\
\text { meio }\end{array}$ & $\begin{array}{c}\text { microcristalA5 } \\
\text { meio }\end{array}$ & $\begin{array}{c}\text { microcristalA5 } \\
\text {.borda }\end{array}$ & $\begin{array}{c}\text { microcristalA5 } \\
\text { a.nucleo }\end{array}$ & $\begin{array}{c}\text { microcristalA5 } \\
\text { a.nucleo2 }\end{array}$ & $\begin{array}{c}\text { microcristalA5 } \\
\text { a.meio }\end{array}$ & $\begin{array}{c}\text { microcristalA5 } \\
\text { a.borda }\end{array}$ & $\begin{array}{c}\text { microcristalA5 } \\
\text { nucleo }\end{array}$ & $\begin{array}{c}\text { microcristalA5 } \\
\text {.borda }\end{array}$ & A.microlito \\
\hline Position & core & & core & core & intermediate & rim & core & core & intermediate & rim & core & rim & core \\
\hline Size & $0,119 \mathrm{~mm}$ & & $0,240 \mathrm{~mm}$ & & $0,260 \mathrm{~mm}$ & & \multicolumn{4}{|c|}{$0,365 \mathrm{~mm}$} & \multicolumn{2}{|c|}{$0,128 \mathrm{~mm}$} & $0,026 \mathrm{~mm}$ \\
\hline$\overline{\mathrm{SiO}_{2}}$ & 54,99 & 55,03 & $\begin{array}{l}55,08 \\
\end{array}$ & 54,62 & 53,68 & $\begin{array}{l}55,37 \\
\end{array}$ & 54,34 & 54,54 & 53,69 & 53,86 & 54,77 & 58,15 & 57,65 \\
\hline $\mathrm{TiO}_{2}$ & 0,06 & 0,04 & 0,07 & 0,08 & 0,12 & 0,01 & 0,16 & 0,06 & 0,03 & 0,06 & 0,03 & 0,05 & 0,07 \\
\hline $\mathrm{Al}_{2} \mathrm{O}_{3}$ & 28,35 & 28,48 & 28,05 & 28,63 & 29,41 & 28,16 & 28,40 & 28,71 & 29,06 & 29,09 & 28,61 & 26,57 & 26,79 \\
\hline $\mathrm{FeOt}$ & 1,18 & 1,15 & 1,08 & 1,07 & 1,19 & 1,05 & 1,11 & 1,13 & 1,11 & 1,12 & 1,12 & 0,86 & 0,82 \\
\hline $\mathrm{MnO}$ & 0,00 & 0,00 & 0,00 & 0,01 & 0,01 & 0,00 & 0,00 & 0,00 & 0,00 & 0,00 & 0,04 & 0,01 & 0,00 \\
\hline $\mathrm{MgO}$ & 0,04 & 0,00 & 0,02 & 0,05 & 0,04 & 0,06 & 0,07 & 0,09 & 0,07 & 0,05 & 0,05 & 0,00 & 0,03 \\
\hline $\mathrm{CaO}$ & 10,93 & 10,87 & 10,70 & 11,24 & 12,19 & 10,34 & 11,25 & 11,44 & 11,76 & 11,71 & 11,15 & 8,39 & 8,71 \\
\hline $\mathrm{Na}_{2} \mathrm{O}$ & 4,91 & 4,98 & 5,21 & 4,72 & 4,28 & 5,13 & 4,68 & 4,56 & 4,42 & 4,50 & 4,77 & 6,25 & 6,11 \\
\hline $\mathrm{K}_{2} \mathrm{O}$ & 0,39 & 0,39 & 0,36 & 0,34 & 0,33 & 0,40 & 0,40 & 0,37 & 0,34 & 0,32 & 0,43 & 0,57 & 0,41 \\
\hline $\mathrm{BaO}$ & 0,05 & 0,04 & 0,07 & 0,00 & 0,02 & 0,08 & 0,04 & 0,05 & 0,03 & 0,00 & 0,00 & 0,08 & 0,09 \\
\hline $\mathrm{SrO}$ & 0,09 & 0,13 & 0,10 & 0,04 & 0,08 & 0,12 & 0,13 & 0,10 & 0,08 & 0,08 & 0,09 & 0,09 & 0,14 \\
\hline Total & 100,98 & 101,11 & 100,74 & 100,81 & 101,35 & 100,73 & 100,56 & 101,05 & 100,59 & 100,80 & 101,05 & 101,03 & 100,82 \\
\hline \multicolumn{14}{|c|}{ Structural formula calculated for 8 oxygens } \\
\hline $\mathrm{Si}$ & 2,47 & 2,47 & 2,48 & 2,46 & 2,41 & 2,49 & 2,46 & 2,45 & 2,43 & 2,43 & 2,46 & 2,59 & 2,58 \\
\hline $\mathrm{Ti}$ & 0,00 & 0,00 & 0,00 & 0,00 & 0,00 & 0,00 & 0,01 & 0,00 & 0,00 & 0,00 & 0,00 & 0,00 & 0,00 \\
\hline $\mathrm{Al}$ & 1,50 & 1,51 & 1,49 & 1,52 & 1,56 & 1,49 & 1,51 & 1,52 & 1,55 & 1,55 & 1,52 & 1,40 & 1,41 \\
\hline $\mathrm{Fe}$ & 0,04 & 0,04 & 0,04 & 0,04 & 0,04 & 0,04 & 0,04 & 0,04 & 0,04 & 0,04 & 0,04 & 0,03 & 0,03 \\
\hline $\mathrm{Mn}$ & 0,00 & 0,00 & 0,00 & 0,00 & 0,00 & 0,00 & 0,00 & 0,00 & 0,00 & 0,00 & 0,00 & 0,00 & 0,00 \\
\hline $\mathrm{Mg}$ & 0,00 & 0,00 & 0,00 & 0,00 & 0,00 & 0,00 & 0,00 & 0,01 & 0,00 & 0,00 & 0,00 & 0,00 & 0,00 \\
\hline $\mathrm{Ca}$ & 0,53 & 0,52 & 0,52 & 0,54 & 0,59 & 0,50 & 0,54 & 0,55 & 0,57 & 0,57 & 0,54 & 0,40 & 0,42 \\
\hline $\mathrm{Na}$ & 0,43 & 0,43 & 0,46 & 0,41 & 0,37 & 0,45 & 0,41 & 0,40 & 0,39 & 0,39 & 0,42 & 0,54 & 0,53 \\
\hline $\mathrm{K}$ & 0,02 & 0,02 & 0,02 & 0,02 & 0,02 & 0,02 & 0,02 & 0,02 & 0,02 & 0,02 & 0,02 & 0,03 & 0,02 \\
\hline $\mathrm{Ba}$ & 0,00 & 0,00 & 0,00 & 0,00 & 0,00 & 0,00 & 0,00 & 0,00 & 0,00 & 0,00 & 0,00 & 0,00 & 0,00 \\
\hline$\sum$ cations & 5,00 & 5,00 & 5,01 & 5,00 & 5,00 & 5,00 & 5,00 & 4,99 & 5,00 & 5,00 & 5,00 & 5,00 & 4,99 \\
\hline An & 53,89 & 53,43 & 52,05 & 55,68 & 59,95 & 51,44 & 55,72 & 56,81 & 58,32 & 57,86 & 54,95 & 41,16 & 42,99 \\
\hline $\mathbf{A b}$ & 43,81 & 44,30 & 45,86 & 42,31 & 38,09 & 46,18 & 41,95 & 40,98 & 39,67 & 40,24 & 42,54 & 55,49 & 54,57 \\
\hline Or & 2,31 & 2,27 & 2,09 & 2,02 & 1,95 & 2,39 & 2,33 & 2,21 & 2,01 & 1,90 & 2,50 & 3,35 & 2,43 \\
\hline
\end{tabular}


Chemical composition (\% wt) for plagioclase from Caxias do Sul dacites

\begin{tabular}{|c|c|c|c|c|c|c|c|c|c|c|c|c|c|c|}
\hline Sample & SM-61 & SM-61 & SM-61 & SM-61 & SM-61 & SM-61 & SM-61 & SM-61 & SM-61 & SM-61 & SM-61 & SM-61 & SM-61 & SM-61 \\
\hline Point ID & $\begin{array}{c}\begin{array}{c}\text { A.matriz.grano } \\
\text { firica }\end{array} \\
\end{array}$ & $\begin{array}{c}\text { B.fenocris.nucl } \\
\text { eo }\end{array}$ & B.microlitol & B.microlito2 & B.microlito3 & $\begin{array}{c}\text { C.microcris1.n } \\
\text { ucleo }\end{array}$ & $\begin{array}{c}\text { C.microcris1. } \\
\text { meioa }\end{array}$ & $\begin{array}{c}\text { C.microcris1. } \\
\text { meiob }\end{array}$ & $\begin{array}{c}\text { C.microcris1.b } \\
\text { orda }\end{array}$ & $\begin{array}{c}\text { C.microcris2.n } \\
\text { ucleo }\end{array}$ & $\begin{array}{c}\text { C.microcris } 2 . b \\
\text { orda }\end{array}$ & $\begin{array}{c}\text { C.microcris3.n } \\
\text { ucleo }\end{array}$ & $\begin{array}{c}\text { C.microcris } 3 . \\
\text { meio }\end{array}$ & $\begin{array}{c}\text { C.microcris3.b } \\
\text { orda }\end{array}$ \\
\hline Position & core & core & rim & core & core & core & intermediate & intermediate & rim & core & rim & core & intermediate & $\mathrm{rim}$ \\
\hline Size & $0,017 \mathrm{~mm}$ & $0,521 \mathrm{~mm}$ & $0,043 \mathrm{~mm}$ & $0,104 \mathrm{~mm}$ & $0,070 \mathrm{~mm}$ & \multicolumn{4}{|c|}{$0,195 \mathrm{~mm}$} & \multicolumn{2}{|c|}{$0,156 \mathrm{~mm}$} & \multicolumn{3}{|c|}{$0,208 \mathrm{~mm}$} \\
\hline$\overline{\mathrm{SiO}_{2}}$ & 67,01 & 54,33 & 58,44 & 54,81 & 56,22 & 54,39 & 53,70 & 54,49 & 68,01 & 54,95 & 59,62 & 56,70 & 55,07 & 57,31 \\
\hline $\mathrm{TiO}_{2}$ & 0,03 & 0,11 & 0,04 & 0,02 & 0,00 & 0,04 & 0,04 & 0,04 & 0,31 & 0,06 & 0,04 & 0,08 & 0,03 & 0,06 \\
\hline $\mathrm{Al}_{2} \mathrm{O}_{3}$ & 19,08 & 28,83 & 26,28 & 28,48 & 27,63 & 28,66 & 29,13 & 28,46 & 19,42 & 28,52 & 25,79 & 27,17 & 28,62 & 27,14 \\
\hline $\mathrm{FeOt}$ & 0,38 & 1,12 & 0,82 & 1,31 & 0,94 & 1,14 & 1,25 & 1,17 & 0,70 & 1,14 & 0,78 & 1,05 & 1,13 & 0,82 \\
\hline $\mathrm{MnO}$ & 0,01 & 0,00 & 0,02 & 0,01 & 0,03 & 0,00 & 0,03 & 0,00 & 0,00 & 0,00 & 0,00 & 0,00 & 0,01 & 0,01 \\
\hline $\mathrm{MgO}$ & 0,06 & 0,07 & 0,00 & 0,02 & 0,03 & 0,09 & 0,09 & 0,06 & 0,02 & 0,02 & 0,00 & 0,02 & 0,05 & 0,00 \\
\hline $\mathrm{CaO}$ & 0,37 & 11,40 & 8,25 & 10,86 & 9,54 & 11,38 & 11,95 & 11,24 & 4,59 & 10,86 & 7,69 & 9,35 & 11,15 & 9,08 \\
\hline $\mathrm{Na}_{2} \mathrm{O}$ & 4,25 & 4,42 & 6,25 & 4,93 & 5,44 & 4,56 & 4,40 & 4,79 & 5,14 & 4,98 & 6,74 & 5,63 & 4,93 & 5,96 \\
\hline $\mathrm{K}_{2} \mathrm{O}$ & 10,18 & 0,39 & 0,40 & 0,37 & 0,47 & 0,37 & 0,33 & 0,39 & 2,10 & 0,35 & 0,63 & 0,42 & 0,33 & 0,49 \\
\hline $\mathrm{BaO}$ & 0,00 & 0,08 & 0,05 & 0,00 & 0,05 & 0,00 & 0,08 & 0,03 & 0,00 & 0,00 & 0,00 & 0,00 & 0,09 & 0,09 \\
\hline $\mathrm{SrO}$ & 0,00 & 0,07 & 0,09 & 0,07 & 0,11 & 0,04 & 0,11 & 0,09 & 0,05 & 0,11 & 0,06 & 0,12 & 0,08 & 0,11 \\
\hline Total & 101,37 & 100,82 & 100,64 & 100,88 & 100,46 & 100,67 & 101,12 & 100,76 & 100,35 & 101,00 & 101,35 & 100,55 & 101,50 & 101,07 \\
\hline
\end{tabular}

\begin{tabular}{|c|c|c|c|c|c|c|c|c|c|c|c|c|c|c|}
\hline $\mathrm{Si}$ & 2,99 & 2,45 & 2,61 & 2,47 & 2,53 & 2,45 & 2,42 & 2,46 & 2,98 & 2,47 & 2,64 & 2,55 & 2,46 & 2,56 \\
\hline $\mathrm{Ti}$ & 0,00 & 0,00 & 0,00 & 0,00 & 0,00 & 0,00 & 0,00 & 0,00 & 0,01 & 0,00 & 0,00 & 0,00 & 0,00 & 0,00 \\
\hline $\mathrm{Al}$ & 1,00 & 1,53 & 1,38 & 1,51 & 1,46 & 1,52 & 1,55 & 1,51 & 1,00 & 1,51 & 1,35 & 1,44 & 1,51 & 1,43 \\
\hline $\mathrm{Fe}$ & 0,01 & 0,04 & 0,03 & 0,05 & 0,04 & 0,04 & 0,05 & 0,04 & 0,03 & 0,04 & 0,03 & 0,04 & 0,04 & 0,03 \\
\hline $\mathrm{Mn}$ & 0,00 & 0,00 & 0,00 & 0,00 & 0,00 & 0,00 & 0,00 & 0,00 & 0,00 & 0,00 & 0,00 & 0,00 & 0,00 & 0,00 \\
\hline $\mathrm{Mg}$ & 0,00 & 0,00 & 0,00 & 0,00 & 0,00 & 0,01 & 0,01 & 0,00 & 0,00 & 0,00 & 0,00 & 0,00 & 0,00 & 0,00 \\
\hline $\mathrm{Ca}$ & 0,02 & 0,55 & 0,39 & 0,52 & 0,46 & 0,55 & 0,58 & 0,54 & 0,22 & 0,52 & 0,36 & 0,45 & 0,53 & 0,43 \\
\hline $\mathrm{Na}$ & 0,37 & 0,39 & 0,54 & 0,43 & 0,47 & 0,40 & 0,38 & 0,42 & 0,44 & 0,43 & 0,58 & 0,49 & 0,43 & 0,52 \\
\hline $\mathrm{K}$ & 0,58 & 0,02 & 0,02 & 0,02 & 0,03 & 0,02 & 0,02 & 0,02 & 0,12 & 0,02 & 0,04 & 0,02 & 0,02 & 0,03 \\
\hline $\mathrm{Ba}$ & 0,00 & 0,00 & 0,00 & 0,00 & 0,00 & 0,00 & 0,00 & 0,00 & 0,00 & 0,00 & 0,00 & 0,00 & 0,00 & 0,00 \\
\hline$\sum$ cations & 4,98 & 4,99 & 4,98 & 5,00 & 4,99 & 4,99 & 5,01 & 5,00 & 4,79 & 5,00 & 4,99 & 4,99 & 5,00 & 5,00 \\
\hline An & 1,84 & 57,40 & 41,17 & 53,69 & 47,83 & 56,70 & 58,86 & 55,19 & 28,00 & 53,53 & 37,27 & 46,66 & 54,49 & 44,40 \\
\hline $\mathbf{A b}$ & 38,10 & 40,27 & 56,44 & 44,11 & 49,35 & 41,12 & 39,22 & 42,56 & 56,74 & 44,42 & 59,12 & 50,84 & 43,60 & 52,74 \\
\hline Or & 60,05 & 2,33 & 2,39 & 2,20 & 2,82 & 2,18 & 1,92 & 2,25 & 15,25 & 2,04 & 3,61 & 2,50 & 1,91 & 2,85 \\
\hline
\end{tabular}


Chemical composition (\% wt) for plagioclase from Caxias do Sul dacites

\begin{tabular}{|c|c|c|c|c|c|c|c|c|c|c|c|c|c|}
\hline Sample & SM-61 & SM-61 & SM-61 & SM-61 & SM-61 & SM-61 & SM-61 & SM-61 & SM-61 & SM-61 & SM-61 & SM-61 & SM-61 \\
\hline Point ID & D.microcris1.a & D.microcris $1 . b$ & D.microcris2.a & D.microcris $2 . b$ & D.microcris2.c & D.plagmatriz & D.microlito & E.fenol.nucleo & E.fenol.borda & E.microlito & F.fenocorroido & F.microcristall & F.microcristal2 \\
\hline Position & core & rim & core & intermeciate & rim & intermediate & intermediate & core & rim & core & core & core & rim \\
\hline Size & 0,09 & $1 \mathrm{~mm}$ & & $0,231 \mathrm{~mm}$ & & $0,016 \mathrm{~mm}$ & $0,088 \mathrm{~mm}$ & 0,356 & $5 \mathrm{~mm}$ & $0,089 \mathrm{~mm}$ & $0,260 \mathrm{~mm}$ & $0,074 \mathrm{~mm}$ & $0,290 \mathrm{~mm}$ \\
\hline $\mathrm{SiO}_{2}$ & 55,98 & 59,50 & 55,73 & 56,49 & 60,07 & 67,38 & 54,87 & 55,68 & 55,52 & 57,61 & 53,96 & 55,36 & 56,98 \\
\hline $\mathrm{TiO}_{2}$ & 0,02 & 0,09 & 0,06 & 0,06 & 0,06 & 0,04 & 0,03 & 0,05 & 0,07 & 0,08 & 0,03 & 0,12 & 0,00 \\
\hline $\mathrm{Al}_{2} \mathrm{O}_{3}$ & 27,42 & 25,45 & 27,80 & 27,31 & 25,36 & 19,43 & 27,92 & 27,68 & 28,29 & 26,37 & 28,88 & 27,81 & 27,11 \\
\hline $\mathrm{FeOt}$ & 0,96 & 0,87 & 1,15 & 1,06 & 0,79 & 0,27 & 1,10 & 1,14 & 1,06 & 0,82 & 1,18 & 1,14 & 1,00 \\
\hline $\mathrm{MnO}$ & 0,00 & 0,01 & 0,00 & 0,00 & 0,02 & 0,00 & 0,00 & 0,01 & 0,00 & 0,01 & 0,00 & 0,01 & 0,00 \\
\hline $\mathrm{MgO}$ & 0,05 & 0,05 & 0,05 & 0,06 & 0,00 & 0,00 & 0,01 & 0,03 & 0,03 & 0,00 & 0,07 & 0,02 & 0,03 \\
\hline $\mathrm{CaO}$ & 10,09 & 7,63 & 10,39 & 9,57 & 7,22 & 0,48 & 10,58 & 10,17 & 10,30 & 8,50 & 12,06 & 10,31 & 9,29 \\
\hline $\mathrm{Na}_{2} \mathrm{O}$ & 5,26 & 6,41 & 5,21 & 5,62 & 6,59 & 4,61 & 5,14 & 5,26 & 5,16 & 6,15 & 4,43 & 5,17 & 5,74 \\
\hline $\mathrm{K}_{2} \mathrm{O}$ & 0,43 & 0,61 & 0,39 & 0,47 & 0,83 & 9,41 & 0,38 & 0,40 & 0,40 & 0,55 & 0,35 & 0,44 & 0,47 \\
\hline $\mathrm{BaO}$ & 0,02 & 0,03 & 0,02 & 0,04 & 0,03 & 0,00 & 0,02 & 0,00 & 0,00 & 0,06 & 0,04 & 0,05 & 0,10 \\
\hline $\mathrm{SrO}$ & 0,08 & 0,10 & 0,12 & 0,11 & 0,09 & 0,00 & 0,10 & 0,10 & 0,11 & 0,07 & 0,05 & 0,10 & 0,09 \\
\hline Total & 100,31 & 100,75 & 100,91 & 100,78 & 101,05 & 101,62 & 100,15 & 100,52 & 100,95 & 100,22 & 101,06 & 100,53 & 100,82 \\
\hline \multicolumn{14}{|c|}{ Structural formula calculated for 8 oxygens } \\
\hline $\mathrm{Si}$ & 2,52 & 2,65 & 2,50 & 2,53 & 2,66 & 2,99 & 2,48 & 2,51 & 2,49 & 2,59 & 2,43 & 2,50 & 2,55 \\
\hline $\mathrm{Ti}$ & 0,00 & 0,00 & 0,00 & 0,00 & 0,00 & 0,00 & 0,00 & 0,00 & 0,00 & 0,00 & 0,00 & 0,00 & 0,00 \\
\hline $\mathrm{Al}$ & 1,46 & 1,34 & 1,47 & 1,44 & 1,33 & 1,02 & 1,49 & 1,47 & 1,50 & 1,40 & 1,53 & 1,48 & 1,43 \\
\hline $\mathrm{Fe}$ & 0,04 & 0,03 & 0,04 & 0,04 & 0,03 & 0,01 & 0,04 & 0,04 & 0,04 & 0,03 & 0,04 & 0,04 & 0,04 \\
\hline $\mathrm{Mn}$ & 0,00 & 0,00 & 0,00 & 0,00 & 0,00 & 0,00 & 0,00 & 0,00 & 0,00 & 0,00 & 0,00 & 0,00 & 0,00 \\
\hline $\mathrm{Mg}$ & 0,00 & 0,00 & 0,00 & 0,00 & 0,00 & 0,00 & 0,00 & 0,00 & 0,00 & 0,00 & 0,00 & 0,00 & 0,00 \\
\hline $\mathrm{Ca}$ & 0,49 & 0,36 & 0,50 & 0,46 & 0,34 & 0,02 & 0,51 & 0,49 & 0,49 & 0,41 & 0,58 & 0,50 & 0,45 \\
\hline $\mathrm{Na}$ & 0,46 & 0,55 & 0,45 & 0,49 & 0,57 & 0,40 & 0,45 & 0,46 & 0,45 & 0,54 & 0,39 & 0,45 & 0,50 \\
\hline K & 0,02 & 0,03 & 0,02 & 0,03 & 0,05 & 0,53 & 0,02 & 0,02 & 0,02 & 0,03 & 0,02 & 0,03 & 0,03 \\
\hline $\mathrm{Ba}$ & 0,00 & 0,00 & 0,00 & 0,00 & 0,00 & 0,00 & 0,00 & 0,00 & 0,00 & 0,00 & 0,00 & 0,00 & 0,00 \\
\hline$\sum$ cations & 4,99 & 4,97 & 5,00 & 5,00 & 4,98 & 4,97 & 5,01 & 5,00 & 5,00 & 5,00 & 5,00 & 5,00 & 5,00 \\
\hline An & 50,14 & 38,23 & 51,24 & 47,15 & 35,86 & 2,40 & 52,03 & 50,44 & 51,20 & 41,91 & 58,83 & 51,06 & 45,90 \\
\hline $\mathbf{A b}$ & 47,30 & 58,13 & 46,50 & 50,11 & 59,23 & 41,66 & 45,74 & 47,21 & 46,41 & 54,87 & 39,11 & 46,33 & 51,32 \\
\hline Or & 2,55 & 3,64 & 2,26 & 2,74 & 4,92 & 55,94 & 2,23 & 2,35 & 2,39 & 3,23 & 2,06 & 2,61 & 2,78 \\
\hline
\end{tabular}


Chemical composition (\% wt) for plagioclase from Caxias do Sul dacites

\begin{tabular}{|c|c|c|c|c|c|c|c|c|c|c|c|c|c|}
\hline Sample & SM-61 & SM-61 & SM-61 & SM-61 & SM-61 & SM-61 & SM-61 & SM-61 & SM-143 & SM-143 & SM-143 & SM-143 & SM-143 \\
\hline Point ID & F.microcristal3 & F.matriz & I.feno1 & I.feno2 & I.feno3 & I.microcristall & I.microcristal3 & I.matriz & plag1.1 & plag1.2 & plag1-2.3 & plag1-2.3 & plag1-2.3 \\
\hline Position & core & core & core & core & core & core & core & core & intermediate & intermediate & core & intermediate & rim \\
\hline Size & $0,064 \mathrm{~mm}$ & & $0,393 \mathrm{~mm}$ & $0,213 \mathrm{~mm}$ & $0,272 \mathrm{~mm}$ & $0,107 \mathrm{~mm}$ & 0,075 & $0,031 \mathrm{~mm}$ & $0,169 \mathrm{~mm}$ & $019 \mathrm{~mm}$ & & $0,145 \mathrm{~mm}$ & \\
\hline$\overline{\mathrm{SiO}_{2}}$ & $\begin{array}{l}55,74 \\
\end{array}$ & 67,18 & 54,29 & 54,21 & 54,46 & 54,98 & 55,78 & 67,21 & 54,69 & $\begin{array}{l}55,18 \\
\end{array}$ & 54,65 & 55,65 & 55,36 \\
\hline $\mathrm{TiO}_{2}$ & 0,03 & 0,07 & 0,02 & 0,03 & 0,00 & 0,07 & 0,01 & 0,08 & 0,02 & 0,04 & 0,03 & 0,01 & 0,03 \\
\hline $\mathrm{Al}_{2} \mathrm{O}_{3}$ & 28,11 & 19,32 & 28,69 & 29,02 & 28,98 & 28,04 & 27,83 & 19,42 & 28,29 & 27,88 & 27,74 & 27,53 & 27,70 \\
\hline $\mathrm{FeOt}$ & 1,10 & 0,39 & 1,13 & 1,09 & 1,13 & 1,14 & 1,14 & 0,31 & 1,13 & 1,16 & 1,08 & 1,14 & 1,08 \\
\hline $\mathrm{MnO}$ & 0,02 & 0,00 & 0,03 & 0,00 & 0,02 & 0,01 & 0,01 & 0,01 & 0,01 & 0,01 & 0,01 & 0,02 & 0,01 \\
\hline $\mathrm{MgO}$ & 0,06 & 0,03 & 0,07 & 0,05 & 0,10 & 0,08 & 0,03 & 0,00 & 0,03 & 0,03 & 0,06 & 0,03 & 0,01 \\
\hline $\mathrm{CaO}$ & 10,40 & 0,50 & 11,46 & 11,66 & 11,68 & 10,63 & 10,09 & 0,33 & 10,88 & 10,67 & 10,90 & 10,34 & 10,34 \\
\hline $\mathrm{Na}_{2} \mathrm{O}$ & 5,12 & 4,79 & 4,63 & 4,53 & 4,50 & 4,98 & 5,14 & 4,28 & 4,89 & 4,93 & 5,01 & 5,20 & 5,07 \\
\hline $\mathrm{K}_{2} \mathrm{O}$ & 0,42 & 9,14 & 0,37 & 0,33 & 0,34 & 0,39 & 0,46 & 10,15 & 0,42 & 0,47 & 0,40 & 0,47 & 0,46 \\
\hline $\mathrm{BaO}$ & 0,01 & 0,05 & 0,01 & 0,08 & 0,00 & 0,00 & 0,01 & 0,02 & 0,00 & 0,05 & 0,00 & 0,07 & 0,05 \\
\hline $\mathrm{SrO}$ & 0,08 & 0,00 & 0,11 & 0,08 & 0,03 & 0,11 & 0,09 & 0,03 & 0,01 & 0,02 & 0,00 & 0,03 & 0,02 \\
\hline Total & 101,09 & 101,47 & 100,81 & 101,07 & 101,24 & 100,43 & 100,60 & 101,85 & 100,37 & 100,44 & 99,88 & 100,50 & 100,12 \\
\hline \multicolumn{14}{|c|}{ Structural formula calculated for 8 oxygens } \\
\hline $\mathrm{Si}$ & 2,50 & 2,99 & 2,45 & 2,44 & 2,44 & 2,48 & 2,51 & 2,98 & 2,47 & 2,49 & 2,48 & 2,51 & 2,50 \\
\hline $\mathrm{Ti}$ & 0,00 & 0,00 & 0,00 & 0,00 & 0,00 & 0,00 & 0,00 & 0,00 & 0,00 & 0,00 & 0,00 & 0,00 & 0,00 \\
\hline $\mathrm{Al}$ & 1,48 & 1,01 & 1,52 & 1,54 & 1,53 & 1,49 & 1,48 & 1,02 & 1,51 & 1,48 & 1,48 & 1,46 & 1,48 \\
\hline $\mathrm{Fe}$ & 0,04 & 0,01 & 0,04 & 0,04 & 0,04 & 0,04 & 0,04 & 0,01 & 0,04 & 0,04 & 0,04 & 0,04 & 0,04 \\
\hline Mn & 0,00 & 0,00 & 0,00 & 0,00 & 0,00 & 0,00 & 0,00 & 0,00 & 0,00 & 0,00 & 0,00 & 0,00 & 0,00 \\
\hline $\mathrm{Mg}$ & 0,00 & 0,00 & 0,00 & 0,00 & 0,01 & 0,01 & 0,00 & 0,00 & 0,00 & 0,00 & 0,00 & 0,00 & 0,00 \\
\hline $\mathrm{Ca}$ & 0,50 & 0,02 & 0,55 & 0,56 & 0,56 & 0,51 & 0,49 & 0,02 & 0,53 & 0,52 & 0,53 & 0,50 & 0,50 \\
\hline $\mathrm{Na}$ & 0,44 & 0,41 & 0,40 & 0,40 & 0,39 & 0,44 & 0,45 & 0,37 & 0,43 & 0,43 & 0,44 & 0,45 & 0,44 \\
\hline K & 0,02 & 0,52 & 0,02 & 0,02 & 0,02 & 0,02 & 0,03 & 0,57 & 0,02 & 0,03 & 0,02 & 0,03 & 0,03 \\
\hline $\mathrm{Ba}$ & 0,00 & 0,00 & 0,00 & 0,00 & 0,00 & 0,00 & 0,00 & 0,00 & 0,00 & 0,00 & 0,00 & 0,00 & 0,00 \\
\hline$\sum$ cations & 5,00 & 4,97 & 5,00 & 5,00 & 5,00 & 5,00 & 4,99 & 4,98 & 5,00 & 5,00 & 5,01 & 5,00 & 4,99 \\
\hline An & 51,58 & 2,51 & 56,52 & 57,58 & 57,74 & 52,86 & 50,60 & 1,64 & 53,78 & 52,97 & 53,31 & 50,90 & 51,55 \\
\hline $\mathbf{A b}$ & 45,96 & 43,22 & 41,32 & 40,48 & 40,25 & 44,81 & 46,64 & 38,42 & 43,74 & 44,29 & 44,34 & 46,32 & 45,74 \\
\hline Or & 2,46 & 54,27 & 2,16 & 1,93 & 2,01 & 2,33 & 2,76 & 59,95 & 2,48 & 2,75 & 2,35 & 2,78 & 2,71 \\
\hline
\end{tabular}


Chemical composition (\% wt) for plagioclase from Caxias do Sul dacites

\begin{tabular}{|c|c|c|c|c|c|c|c|c|c|c|c|c|c|c|}
\hline Sample & SM-143 & SM-143 & SM-143 & SM-143 & SM-143 & SM-143 & SM-143 & SM-143 & SM-143 & SM-143 & SM-143 & SM-143 & SM-143 & SM-143 \\
\hline Point ID & plag3.1 & plag3.1 & plag 4.1 & plag 4.2 & plag5.1 & plag5.1 & plag5.2 & plag5.3 & plag5.3 & plag5.4 & plag7.1 & plag7.2 & plag7.3 & plag7.3 \\
\hline Position & core & intermediate & core & core & core & intermediate & core & core & int/rim & core & core & core & core & int/rim \\
\hline Size & \multicolumn{2}{|c|}{$0,26 \mathrm{~mm}$} & $0,087 \mathrm{~mm}$ & $0,12 \mathrm{~mm}$ & \multicolumn{2}{|c|}{$0,25 \mathrm{~mm}$} & $0,11 \mathrm{~mm}$ & \multicolumn{2}{|c|}{$0,16 \mathrm{~mm}$} & $0,46 \mathrm{~mm}$ & $0,165 \mathrm{~mm}$ & $0,163 \mathrm{~mm}$ & \multicolumn{2}{|c|}{$0,24 \mathrm{~mm}$} \\
\hline $\mathrm{SiO}_{2}$ & 53,97 & 55,04 & 54,24 & 54,79 & 54,22 & 54,80 & 55,18 & 53,94 & 55,32 & 55,61 & 55,15 & 56,15 & 55,37 & 54,98 \\
\hline $\mathrm{TiO}_{2}$ & 0,09 & 0,06 & 0,03 & 0,10 & 0,04 & 0,07 & 0,09 & 0,08 & 0,08 & 0,09 & 0,04 & 0,08 & 0,04 & 0,04 \\
\hline $\mathrm{Al}_{2} \mathrm{O}_{3}$ & 28,38 & 27,73 & 28,60 & 27,89 & 28,02 & 28,04 & 27,68 & 28,49 & 27,43 & 27,33 & 27,73 & 27,39 & 27,59 & 27,74 \\
\hline $\mathrm{FeOt}$ & 1,05 & 1,10 & 1,02 & 1,05 & 1,12 & 1,08 & 1,06 & 1,19 & 1,14 & 1,06 & 1,08 & 0,92 & 1,04 & 1,08 \\
\hline $\mathrm{MnO}$ & 0,01 & 0,00 & 0,00 & 0,01 & 0,00 & 0,00 & 0,01 & 0,00 & 0,02 & 0,00 & 0,01 & 0,00 & 0,01 & 0,00 \\
\hline $\mathrm{MgO}$ & 0,06 & 0,01 & 0,03 & 0,04 & 0,04 & 0,02 & 0,03 & 0,03 & 0,00 & 0,02 & 0,00 & 0,02 & 0,03 & 0,05 \\
\hline $\mathrm{CaO}$ & 11,32 & 10,67 & 11,47 & 10,77 & 11,10 & 10,72 & 10,41 & 11,61 & 10,18 & 9,89 & 10,32 & 9,91 & 10,47 & 10,71 \\
\hline $\mathrm{Na}_{2} \mathrm{O}$ & 4,70 & 5,13 & 4,58 & 4,85 & 4,74 & 4,98 & 5,16 & 4,63 & 5,23 & 5,42 & 5,10 & 5,63 & 5,16 & 4,93 \\
\hline $\mathrm{K}_{2} \mathrm{O}$ & 0,41 & 0,44 & 0,39 & 0,40 & 0,41 & 0,44 & 0,42 & 0,36 & 0,44 & 0,51 & 0,46 & 0,46 & 0,45 & 0,44 \\
\hline $\mathrm{BaO}$ & 0,02 & 0,10 & 0,02 & 0,03 & 0,03 & 0,07 & 0,02 & 0,00 & 0,10 & 0,01 & 0,03 & 0,09 & 0,04 & 0,00 \\
\hline $\mathrm{SrO}$ & 0,02 & 0,00 & 0,04 & 0,03 & 0,00 & 0,03 & 0,04 & 0,06 & 0,05 & 0,02 & 0,00 & 0,04 & 0,03 & 0,06 \\
\hline Total & 100,03 & 100,28 & 100,42 & 99,97 & 99,72 & 100,25 & 100,09 & 100,40 & 99,99 & 99,96 & 99,94 & 100,69 & 100,23 & 100,04 \\
\hline \multicolumn{15}{|c|}{ Structural formula calculated for 8 oxygens } \\
\hline $\mathrm{Si}$ & 2,45 & 2,49 & 2,45 & 2,48 & 2,47 & 2,48 & 2,50 & 2,44 & 2,51 & 2,52 & 2,50 & 2,52 & 2,50 & 2,49 \\
\hline $\mathrm{Ti}$ & 0,00 & 0,00 & 0,00 & 0,00 & 0,00 & 0,00 & 0,00 & 0,00 & 0,00 & 0,00 & 0,00 & 0,00 & 0,00 & 0,00 \\
\hline $\mathrm{Al}$ & 1,52 & 1,48 & 1,52 & 1,49 & 1,50 & 1,50 & 1,48 & 1,52 & 1,47 & 1,46 & 1,48 & 1,45 & 1,47 & 1,48 \\
\hline $\mathrm{Fe}$ & 0,04 & 0,04 & 0,04 & 0,04 & 0,04 & 0,04 & 0,04 & 0,05 & 0,04 & 0,04 & 0,04 & 0,03 & 0,04 & 0,04 \\
\hline $\mathrm{Mn}$ & 0,00 & 0,00 & 0,00 & 0,00 & 0,00 & 0,00 & 0,00 & 0,00 & 0,00 & 0,00 & 0,00 & 0,00 & 0,00 & 0,00 \\
\hline Mg & 0,00 & 0,00 & 0,00 & 0,00 & 0,00 & 0,00 & 0,00 & 0,00 & 0,00 & 0,00 & 0,00 & 0,00 & 0,00 & 0,00 \\
\hline $\mathrm{Ca}$ & 0,55 & 0,52 & 0,56 & 0,52 & 0,54 & 0,52 & 0,50 & 0,56 & 0,49 & 0,48 & 0,50 & 0,48 & 0,51 & 0,52 \\
\hline $\mathrm{Na}$ & 0,41 & 0,45 & 0,40 & 0,43 & 0,42 & 0,44 & 0,45 & 0,41 & 0,46 & 0,48 & 0,45 & 0,49 & 0,45 & 0,43 \\
\hline K & 0,02 & 0,03 & 0,02 & 0,02 & 0,02 & 0,03 & 0,02 & 0,02 & 0,03 & 0,03 & 0,03 & 0,03 & 0,03 & 0,03 \\
\hline $\mathrm{Ba}$ & 0,00 & 0,00 & 0,00 & 0,00 & 0,00 & 0,00 & 0,00 & 0,00 & 0,00 & 0,00 & 0,00 & 0,00 & 0,00 & 0,00 \\
\hline$\sum$ cations & 5,01 & 5,01 & 5,00 & 4,99 & 5,00 & 5,00 & 5,00 & 5,01 & 5,00 & 5,00 & 5,00 & 5,01 & 5,00 & 5,00 \\
\hline An & 55,74 & 52,11 & 56,71 & 53,78 & 55,06 & 52,92 & 51,42 & 56,86 & 50,47 & 48,71 & 51,36 & 48,01 & 51,47 & 53,13 \\
\hline $\mathbf{A b}$ & 41,88 & 45,34 & 40,98 & 43,83 & 42,55 & 44,49 & 46,12 & 41,04 & 46,92 & 48,31 & 45,93 & 49,36 & 45,90 & 44,26 \\
\hline Or & 2,37 & 2,55 & 2,31 & 2,39 & 2,39 & 2,59 & 2,46 & 2,10 & 2,60 & 2,98 & 2,72 & 2,63 & 2,63 & 2,60 \\
\hline
\end{tabular}


Chemical composition (\% wt) for plagioclase from Caxias do Sul dacites

\begin{tabular}{|c|c|c|c|c|c|c|c|c|c|c|c|c|c|}
\hline Sample & SM-143 & SM-143 & SM-143 & SM-143 & SM-143 & SM-143 & SM-143 & SM-143 & SM-143 & SM-143 & SM-143 & SM-143 & SM-143 \\
\hline Point ID & plag9.1 & plag9.2 & plag9.3 & plag9.4 & plag10.1 & plag10.2 & plag10.3 & plag10.4 & plag12.1 & $\begin{array}{l}\text { plag12.2_micr } \\
\text { olito }\end{array}$ & plag13.1 & plag13.2 & plag14.1 \\
\hline Position & core & core & core & core & core & core & core & core & core & core & core & core & core \\
\hline Size & $0,16 \mathrm{~mm}$ & $0,136 \mathrm{~mm}$ & $0,07 \mathrm{~mm}$ & $\begin{array}{l}0,024 \mathrm{~mm} \\
\text { (matrix) }\end{array}$ & $0,03 \mathrm{~mm}$ & $0,249 \mathrm{~mm}$ & $0,09 \mathrm{um}$ & $0,34 \mathrm{~mm}$ & $0,199 \mathrm{~mm}$ & $0,05 \mathrm{~mm}$ & $0,213 \mathrm{~mm}$ & $0,3 \mathrm{~mm}$ & $0,09 \mathrm{~mm}$ \\
\hline$\overline{\mathrm{SiO}_{2}}$ & $\begin{array}{l}55,49 \\
\end{array}$ & 54,90 & 64,99 & 70,86 & 56,08 & 54,65 & 53,73 & 54,03 & 54,16 & 64,83 & 54,10 & 53,87 & 55,89 \\
\hline $\mathrm{TiO}_{2}$ & 0,00 & 0,02 & 0,20 & 0,16 & 0,01 & 0,08 & 0,08 & 0,04 & 0,02 & 0,10 & 0,06 & 0,01 & 0,09 \\
\hline $\mathrm{Al}_{2} \mathrm{O}_{3}$ & 27,28 & 27,75 & 22,40 & 18,17 & 27,08 & 27,97 & 28,53 & 28,22 & 28,39 & 21,27 & 28,22 & 28,45 & 27,31 \\
\hline $\mathrm{FeOt}$ & 1,02 & 1,09 & 0,81 & 0,67 & 1,20 & 1,13 & 1,09 & 1,14 & 1,14 & 0,51 & 1,14 & 1,21 & 0,91 \\
\hline $\mathrm{MnO}$ & 0,00 & 0,02 & 0,00 & 0,02 & 0,01 & 0,00 & 0,03 & 0,00 & 0,00 & 0,00 & 0,00 & 0,02 & 0,00 \\
\hline $\mathrm{MgO}$ & 0,02 & 0,03 & 0,03 & 0,04 & 0,02 & 0,02 & 0,02 & 0,04 & 0,07 & 0,02 & 0,05 & 0,03 & 0,04 \\
\hline $\mathrm{CaO}$ & 10,14 & 10,49 & 6,02 & 4,77 & 9,62 & 10,76 & 11,48 & 11,26 & 11,14 & 5,01 & 11,22 & 11,40 & 9,72 \\
\hline $\mathrm{Na}_{2} \mathrm{O}$ & 5,29 & 5,10 & 5,31 & 5,71 & 5,48 & 4,87 & 4,53 & 4,60 & 4,55 & 5,87 & 4,77 & 4,50 & 5,34 \\
\hline $\mathrm{K}_{2} \mathrm{O}$ & 0,50 & 0,45 & 0,41 & 0,65 & 0,45 & 0,42 & 0,34 & 0,37 & 0,39 & 2,43 & 0,36 & 0,38 & 0,48 \\
\hline $\mathrm{BaO}$ & 0,03 & 0,04 & 0,12 & 0,08 & 0,00 & 0,07 & 0,03 & 0,10 & 0,03 & 0,15 & 0,06 & 0,00 & 0,08 \\
\hline $\mathrm{SrO}$ & 0,03 & 0,04 & 0,01 & 0,03 & 0,04 & 0,05 & 0,04 & 0,02 & 0,00 & 0,01 & 0,03 & 0,05 & 0,02 \\
\hline Total & 99,79 & 99,93 & 100,30 & 101,17 & 100,00 & 100,02 & 99,90 & 99,82 & 99,89 & 100,20 & 100,00 & 99,91 & 99,89 \\
\hline \multicolumn{14}{|c|}{ Structural formula calculated for 8 oxygens } \\
\hline $\mathrm{Si}$ & 2,52 & 2,49 & 2,85 & 3,05 & 2,53 & 2,48 & 2,44 & 2,46 & 2,46 & 2,87 & 2,46 & 2,45 & 2,53 \\
\hline $\mathrm{Ti}$ & 0,00 & 0,00 & 0,01 & 0,01 & 0,00 & 0,00 & 0,00 & 0,00 & 0,00 & 0,00 & 0,00 & 0,00 & 0,00 \\
\hline $\mathrm{Al}$ & 1,46 & 1,48 & 1,16 & 0,92 & 1,44 & 1,50 & 1,53 & 1,51 & 1,52 & 1,11 & 1,51 & 1,52 & 1,46 \\
\hline $\mathrm{Fe}$ & 0,04 & 0,04 & 0,03 & 0,02 & 0,05 & 0,04 & 0,04 & 0,04 & 0,04 & 0,02 & 0,04 & 0,05 & 0,03 \\
\hline $\mathrm{Mn}$ & 0,00 & 0,00 & 0,00 & 0,00 & 0,00 & 0,00 & 0,00 & 0,00 & 0,00 & 0,00 & 0,00 & 0,00 & 0,00 \\
\hline $\mathrm{Mg}$ & 0,00 & 0,00 & 0,00 & 0,00 & 0,00 & 0,00 & 0,00 & 0,00 & 0,00 & 0,00 & 0,00 & 0,00 & 0,00 \\
\hline $\mathrm{Ca}$ & 0,49 & 0,51 & 0,28 & 0,22 & 0,47 & 0,52 & 0,56 & 0,55 & 0,54 & 0,24 & 0,55 & 0,56 & 0,47 \\
\hline $\mathrm{Na}$ & 0,47 & 0,45 & 0,45 & 0,48 & 0,48 & 0,43 & 0,40 & 0,41 & 0,40 & 0,50 & 0,42 & 0,40 & 0,47 \\
\hline K & 0,03 & 0,03 & 0,02 & 0,04 & 0,03 & 0,02 & 0,02 & 0,02 & 0,02 & 0,14 & 0,02 & 0,02 & 0,03 \\
\hline $\mathrm{Ba}$ & 0,00 & 0,00 & 0,00 & 0,00 & 0,00 & 0,00 & 0,00 & 0,00 & 0,00 & 0,00 & 0,00 & 0,00 & 0,00 \\
\hline$\sum$ cations & 5,00 & 5,00 & 4,80 & 4,74 & 5,00 & 5,00 & 5,00 & 5,00 & 4,99 & 4,89 & 5,01 & 5,00 & 4,99 \\
\hline An & 49,93 & 51,78 & 37,34 & 30,05 & 47,93 & 53,60 & 57,16 & 56,22 & 56,15 & 27,04 & 55,31 & 57,01 & 48,71 \\
\hline $\mathbf{A b}$ & 47,14 & 45,55 & 59,60 & 65,09 & 49,41 & 43,90 & 40,82 & 41,56 & 41,50 & 57,34 & 42,55 & 40,72 & 48,43 \\
\hline Or & 2,93 & 2,67 & 3,06 & 4,87 & 2,66 & 2,49 & 2,02 & 2,21 & 2,35 & 15,62 & 2,13 & 2,27 & 2,86 \\
\hline
\end{tabular}


Chemical composition (\% wt) for plagioclase from Caxias do Sul dacites

\begin{tabular}{|c|c|c|c|c|c|c|c|c|c|c|c|c|c|}
\hline Sample & SM-143 & SM-143 & SM-143 & SM-143 & SM-143 & SM-157 & SM-157 & SM-157 & SM-157 & SM-157 & SM-157 & SM-157 & SM-157 \\
\hline Point ID & $\begin{array}{l}\text { plag14.2_micr } \\
\text { olito }\end{array}$ & plag14.3 & $\begin{array}{c}\text { plag14.4_nucle } \\
\text { o }\end{array}$ & $\begin{array}{c}\text { plag14.4_bord } \\
\text { a }\end{array}$ & plag14.5 & plag1.1 & plag1.2 & plag1.2 & plag1.3 & plag2.1 & plag2.2 & plag2.3 & plag2.4 \\
\hline Position & core & core & core & int/rim & core & core & core & int/rim & core & core & core & core & intermedia \\
\hline Size & $0,07 \mathrm{~mm}$ & $0,19 \mathrm{~mm}$ & \multicolumn{2}{|c|}{$0,1 \mathrm{~mm}$} & $0,22 \mathrm{~mm}$ & $0,17 \mathrm{~mm}$ & \multicolumn{2}{|c|}{$0,1 \mathrm{~mm}$} & $0,1 \mathrm{~mm}$ & $0,22 \mathrm{~mm}$ & $0,08 \mathrm{~mm}$ & $0,19 \mathrm{~mm}$ & $0,22 \mathrm{~mm}$ \\
\hline $\mathrm{SiO}_{2}$ & 64,45 & 54,34 & 53,96 & 54,67 & 54,80 & 54,26 & 55,03 & 55,37 & 54,79 & 53,98 & 55,98 & 56,15 & 55,28 \\
\hline $\mathrm{TiO}_{2}$ & 0,19 & 0,07 & 0,05 & 0,05 & 0,10 & 0,03 & 0,09 & 0,13 & 0,05 & 0,02 & 0,09 & 0,09 & 0,04 \\
\hline $\mathrm{Al}_{2} \mathrm{O}_{3}$ & 21,70 & 28,25 & 28,02 & 27,82 & 27,99 & 28,27 & 27,94 & 27,87 & 28,08 & 28,58 & 27,50 & 27,27 & 27,96 \\
\hline $\mathrm{FeOt}$ & 0,73 & 1,09 & 1,14 & 1,11 & 1,23 & 0,99 & 1,13 & 0,99 & 1,05 & 1,18 & 1,07 & 1,03 & 1,07 \\
\hline $\mathrm{MnO}$ & 0,01 & 0,00 & 0,00 & 0,00 & 0,01 & 0,00 & 0,01 & 0,00 & 0,00 & 0,00 & 0,00 & 0,00 & 0,03 \\
\hline $\mathrm{MgO}$ & 0,06 & 0,03 & 0,06 & 0,01 & 0,03 & 0,05 & 0,07 & 0,01 & 0,02 & 0,03 & 0,03 & 0,02 & 0,02 \\
\hline $\mathrm{CaO}$ & 5,62 & 10,99 & 11,11 & 10,63 & 10,98 & 11,52 & 10,58 & 10,65 & 10,82 & 11,39 & 9,97 & 9,75 & 10,57 \\
\hline $\mathrm{Na}_{2} \mathrm{O}$ & 6,10 & 4,79 & 4,59 & 5,04 & 4,76 & 4,68 & 4,95 & 5,12 & 4,78 & 4,61 & 5,42 & 5,50 & 4,96 \\
\hline $\mathrm{K}_{2} \mathrm{O}$ & 1,13 & 0,44 & 0,38 & 0,38 & 0,44 & 0,42 & 0,39 & 0,43 & 0,41 & 0,38 & 0,52 & 0,51 & 0,47 \\
\hline $\mathrm{BaO}$ & 0,12 & 0,06 & 0,00 & 0,04 & 0,00 & 0,03 & 0,04 & 0,08 & 0,08 & 0,04 & 0,00 & 0,00 & 0,00 \\
\hline $\mathrm{SrO}$ & 0,04 & 0,03 & 0,04 & 0,01 & 0,02 & 0,02 & 0,01 & 0,02 & 0,01 & 0,00 & 0,03 & 0,03 & 0,03 \\
\hline Total & 100,15 & 100,09 & 99,34 & 99,76 & 100,37 & 100,26 & 100,23 & 100,67 & 100,09 & 100,20 & 100,62 & 100,36 & 100,43 \\
\hline \multicolumn{14}{|c|}{ Structural formula calculated for 8 oxygens } \\
\hline $\mathrm{Si}$ & 2,85 & 2,46 & 2,46 & 2,48 & 2,48 & 2,46 & 2,49 & 2,49 & 2,48 & 2,45 & 2,52 & 2,53 & 2,49 \\
\hline $\mathrm{Ti}$ & 0,01 & 0,00 & 0,00 & 0,00 & 0,00 & 0,00 & 0,00 & 0,00 & 0,00 & 0,00 & 0,00 & 0,00 & 0,00 \\
\hline $\mathrm{Al}$ & 1,13 & 1,51 & 1,51 & 1,49 & 1,49 & 1,51 & 1,49 & 1,48 & 1,50 & 1,53 & 1,46 & 1,45 & 1,49 \\
\hline $\mathrm{Fe}$ & 0,03 & 0,04 & 0,04 & 0,04 & 0,05 & 0,04 & 0,04 & 0,04 & 0,04 & 0,04 & 0,04 & 0,04 & 0,04 \\
\hline $\mathrm{Mn}$ & 0,00 & 0,00 & 0,00 & 0,00 & 0,00 & 0,00 & 0,00 & 0,00 & 0,00 & 0,00 & 0,00 & 0,00 & 0,00 \\
\hline $\mathrm{Mg}$ & 0,00 & 0,00 & 0,00 & 0,00 & 0,00 & 0,00 & 0,00 & 0,00 & 0,00 & 0,00 & 0,00 & 0,00 & 0,00 \\
\hline $\mathrm{Ca}$ & 0,27 & 0,53 & 0,54 & 0,52 & 0,53 & 0,56 & 0,51 & 0,51 & 0,52 & 0,55 & 0,48 & 0,47 & 0,51 \\
\hline $\mathrm{Na}$ & 0,52 & 0,42 & 0,41 & 0,44 & 0,42 & 0,41 & 0,43 & 0,45 & 0,42 & 0,41 & 0,47 & 0,48 & 0,43 \\
\hline K & 0,06 & 0,03 & 0,02 & 0,02 & 0,03 & 0,02 & 0,02 & 0,02 & 0,02 & 0,02 & 0,03 & 0,03 & 0,03 \\
\hline $\mathrm{Ba}$ & 0,00 & 0,00 & 0,00 & 0,00 & 0,00 & 0,00 & 0,00 & 0,00 & 0,00 & 0,00 & 0,00 & 0,00 & 0,00 \\
\hline$\sum$ cations & 4,87 & 5,00 & 4,99 & 5,00 & 5,00 & 5,00 & 4,99 & 5,00 & 4,99 & 5,00 & 5,00 & 5,00 & 4,99 \\
\hline An & 31,22 & 54,45 & 55,92 & 52,61 & 54,59 & 56,24 & 52,90 & 52,12 & 54,21 & 56,44 & 48,87 & 47,99 & 52,58 \\
\hline $\mathbf{A b}$ & 61,32 & 42,95 & 41,81 & 45,14 & 42,82 & 41,34 & 44,79 & 45,35 & 43,34 & 41,34 & 48,08 & 48,99 & 44,65 \\
\hline Or & 7,46 & 2,60 & 2,27 & 2,25 & 2,59 & 2,42 & 2,31 & 2,53 & 2,45 & 2,22 & 3,06 & 3,01 & 2,77 \\
\hline
\end{tabular}


Chemical composition (\% wt) for plagioclase from Caxias do Sul dacites

\begin{tabular}{|c|c|c|c|c|c|c|c|c|c|c|c|c|}
\hline Sample & SM-157 & SM-157 & SM-157 & SM-157 & SM-157 & SM-157 & SM-157 & SM-157 & SM-157 & SM-157 & SM-157 & SM-157 \\
\hline Point ID & plag2.5 & pl1.1 & pl1.1 & pl1.2 & pl1.2 & pl1.2 & pl1.2 & pl1.3 & pl3.1 & $\mathrm{pl} 3.2$ & $\mathrm{pl} 3.3$ & $\mathrm{pl} 3.3$ \\
\hline Position & intermediate & core & int/rim & core & intermediate & intermediate & int/rim & core (matrix) & core & core & core & int/rim \\
\hline Size & $0,315 \mathrm{~mm}$ & \multicolumn{2}{|c|}{$0,173 \mathrm{~mm}$} & \multicolumn{4}{|c|}{$0,25 \mathrm{~mm}$} & $0,04 \mathrm{~mm}$ & $0,1 \mathrm{~mm}$ & $0,238 \mathrm{~mm}$ & \multicolumn{2}{|c|}{$0,229 \mathrm{~mm}$} \\
\hline$\overline{\mathrm{SiO}_{2}}$ & 56,60 & 53,80 & 56,05 & 55,30 & 54,65 & 55,72 & 56,36 & 67,05 & 54,55 & 54,58 & 54,24 & 54,24 \\
\hline $\mathrm{TiO}_{2}$ & 0,00 & 0,09 & 0,00 & 0,05 & 0,08 & 0,01 & 0,03 & 0,06 & 0,06 & 0,11 & 0,10 & 0,09 \\
\hline $\mathrm{Al}_{2} \mathrm{O}_{3}$ & 27,10 & 28,52 & 27,50 & 27,87 & 27,83 & 27,09 & 26,99 & 18,45 & 27,95 & 28,03 & 28,09 & 28,17 \\
\hline $\mathrm{FeOt}$ & 1,01 & 1,09 & 1,05 & 1,08 & 1,19 & 1,08 & 1,01 & 0,34 & 1,11 & 1,13 & 1,10 & 1,12 \\
\hline $\mathrm{MnO}$ & 0,02 & 0,02 & 0,02 & 0,01 & 0,01 & 0,01 & 0,01 & 0,00 & 0,00 & 0,01 & 0,01 & 0,00 \\
\hline $\mathrm{MgO}$ & 0,00 & 0,07 & 0,02 & 0,06 & 0,05 & 0,06 & 0,00 & 0,00 & 0,04 & 0,04 & 0,08 & 0,05 \\
\hline $\mathrm{CaO}$ & 9,40 & 11,54 & 9,88 & 10,78 & 10,90 & 10,10 & 9,38 & 0,27 & 11,06 & 10,97 & 11,14 & 11,16 \\
\hline $\mathrm{Na}_{2} \mathrm{O}$ & 5,70 & 4,35 & 5,25 & 5,00 & 4,90 & 5,27 & 5,54 & 3,87 & 4,82 & 4,75 & 4,60 & 4,56 \\
\hline $\mathrm{K}_{2} \mathrm{O}$ & 0,49 & 0,37 & 0,46 & 0,40 & 0,42 & 0,50 & 0,54 & 10,56 & 0,38 & 0,43 & 0,41 & 0,39 \\
\hline $\mathrm{BaO}$ & 0,05 & 0,05 & 0,02 & 0,05 & 0,02 & 0,03 & 0,07 & 0,00 & 0,07 & 0,04 & 0,00 & 0,01 \\
\hline $\mathrm{SrO}$ & 0,04 & 0,00 & 0,04 & 0,00 & 0,00 & 0,00 & 0,01 & 0,01 & 0,03 & 0,00 & 0,01 & 0,03 \\
\hline Total & 100,40 & 99,90 & 100,28 & 100,61 & 100,04 & 99,88 & 99,94 & 100,61 & 100,06 & 100,09 & 99,78 & 99,82 \\
\hline $\mathrm{Si}$ & 2,55 & 2,45 & 2,52 & 2,49 & 2,48 & 2,52 & 2,55 & 3,02 & 2,47 & 2,47 & 2,47 & 2,46 \\
\hline $\mathrm{Ti}$ & 0,00 & 0,00 & 0,00 & 0,00 & 0,00 & 0,00 & 0,00 & 0,00 & 0,00 & 0,00 & 0,00 & 0,00 \\
\hline $\mathrm{Al}$ & 1,44 & 1,53 & 1,46 & 1,48 & 1,49 & 1,45 & 1,44 & 0,98 & 1,49 & 1,50 & 1,51 & 1,51 \\
\hline $\mathrm{Fe}$ & 0,04 & 0,04 & 0,04 & 0,04 & 0,05 & 0,04 & 0,04 & 0,01 & 0,04 & 0,04 & 0,04 & 0,04 \\
\hline $\mathrm{Mn}$ & 0,00 & 0,00 & 0,00 & 0,00 & 0,00 & 0,00 & 0,00 & 0,00 & 0,00 & 0,00 & 0,00 & 0,00 \\
\hline $\mathrm{Mg}$ & 0,00 & 0,00 & 0,00 & 0,00 & 0,00 & 0,00 & 0,00 & 0,00 & 0,00 & 0,00 & 0,01 & 0,00 \\
\hline $\mathrm{Ca}$ & 0,45 & 0,56 & 0,48 & 0,52 & 0,53 & 0,49 & 0,45 & 0,01 & 0,54 & 0,53 & 0,54 & 0,54 \\
\hline $\mathrm{Na}$ & 0,50 & 0,38 & 0,46 & 0,44 & 0,43 & 0,46 & 0,49 & 0,34 & 0,42 & 0,42 & 0,41 & 0,40 \\
\hline K & 0,03 & 0,02 & 0,03 & 0,02 & 0,02 & 0,03 & 0,03 & 0,61 & 0,02 & 0,03 & 0,02 & 0,02 \\
\hline $\mathrm{Ba}$ & 0,00 & 0,00 & 0,00 & 0,00 & 0,00 & 0,00 & 0,00 & 0,00 & 0,00 & 0,00 & 0,00 & 0,00 \\
\hline$\sum$ cations & 5,00 & 4,99 & 4,99 & 5,00 & 5,00 & 5,00 & 4,99 & 4,97 & 5,00 & 5,00 & 4,99 & 4,99 \\
\hline An & 46,30 & 58,13 & 49,59 & 53,09 & 53,80 & 49,91 & 46,78 & 1,38 & 54,66 & 54,62 & 55,83 & 56,16 \\
\hline $\mathbf{A b}$ & 50,81 & 39,66 & 47,68 & 44,56 & 43,76 & 47,13 & 50,00 & 35,28 & 43,11 & 42,80 & 41,72 & 41,52 \\
\hline Or & 2,89 & 2,21 & 2,73 & 2,34 & 2,44 & 2,96 & 3,22 & 63,34 & 2,23 & 2,58 & 2,45 & 2,32 \\
\hline
\end{tabular}


Chemical composition (\% wt) for plagioclase from Caxias do Sul dacites

\begin{tabular}{|c|c|c|c|c|c|c|c|c|c|c|c|c|c|}
\hline Sample & SM-157 & SM-157 & SM-157 & SM-157 & SM-157 & SM-157 & SM-157 & SM-157 & SM-157 & SM-157 & SM-157 & SM-157 & SM-157 \\
\hline Point ID & $\mathrm{pl} 3-2.1$ & pl3-2.1 & $\mathrm{pl} 3-2.2$ & $\mathrm{pl} 3-2.2$ & $\mathrm{pl} 3-2.3$ & $\mathrm{p} 15.1$ & p15.1 & p15.2 & p15.3 & p15.4 & p15.5 & pl7.1 & p17.2 \\
\hline Position & core & rim & core & rim & core & core & int/rim & core & core & core & core & core & core \\
\hline Size & \multicolumn{2}{|c|}{$0,116 \mathrm{~mm}$} & \multicolumn{2}{|c|}{$0,1 \mathrm{~mm}$} & $0,07 \mathrm{~mm}$ & \multicolumn{2}{|c|}{$0,3 \mathrm{~mm}$} & $0,07 \mathrm{~mm}$ & $0,085 \mathrm{~mm}$ & $0,13 \mathrm{~mm}$ & $0,18 \mathrm{~mm}$ & $0,125 \mathrm{~mm}$ & $0,07 \mathrm{~mm}$ \\
\hline $\mathrm{SiO}_{2}$ & 53,77 & 56,72 & 54,06 & 55,24 & 54,85 & 54,04 & 54,29 & 56,25 & 55,30 & 54,25 & 55,44 & 55,44 & 56,84 \\
\hline $\mathrm{TiO}_{2}$ & 0,04 & 0,03 & 0,05 & 0,07 & 0,04 & 0,01 & 0,04 & 0,10 & 0,05 & 0,07 & 0,06 & 0,06 & 0,06 \\
\hline $\mathrm{Al}_{2} \mathrm{O}_{3}$ & 28,40 & 27,04 & 28,59 & 27,92 & 27,84 & 28,06 & 28,20 & 27,22 & 27,63 & 27,94 & 27,67 & 27,47 & 26,71 \\
\hline $\mathrm{FeOt}$ & 1,11 & 0,94 & 1,11 & 0,99 & 1,19 & 1,17 & 1,16 & 0,92 & 1,06 & 1,11 & 1,07 & 1,01 & 0,83 \\
\hline $\mathrm{MnO}$ & 0,02 & 0,03 & 0,00 & 0,01 & 0,01 & 0,01 & 0,02 & 0,01 & 0,02 & 0,02 & 0,01 & 0,00 & 0,00 \\
\hline $\mathrm{MgO}$ & 0,06 & 0,02 & 0,04 & 0,01 & 0,04 & 0,06 & 0,02 & 0,00 & 0,03 & 0,02 & 0,02 & 0,03 & 0,02 \\
\hline $\mathrm{CaO}$ & 11,73 & 9,38 & 11,41 & 10,79 & 10,93 & 11,23 & 11,35 & 9,69 & 10,32 & 11,40 & 10,49 & 10,34 & 9,26 \\
\hline $\mathrm{Na}_{2} \mathrm{O}$ & 4,48 & 5,59 & 4,59 & 4,86 & 4,88 & 4,64 & 4,70 & 5,45 & 5,09 & 4,72 & 5,08 & 5,17 & 5,66 \\
\hline $\mathrm{K}_{2} \mathrm{O}$ & 0,35 & 0,47 & 0,37 & 0,43 & 0,41 & 0,35 & 0,38 & 0,50 & 0,44 & 0,41 & 0,39 & 0,47 & 0,64 \\
\hline $\mathrm{BaO}$ & 0,02 & 0,14 & 0,00 & 0,07 & 0,07 & 0,00 & 0,04 & 0,08 & 0,00 & 0,03 & 0,04 & 0,03 & 0,07 \\
\hline $\mathrm{SrO}$ & 0,03 & 0,03 & 0,01 & 0,04 & 0,03 & 0,05 & 0,02 & 0,06 & 0,03 & 0,04 & 0,02 & 0,05 & 0,04 \\
\hline Total & 100,00 & 100,39 & 100,23 & 100,43 & 100,28 & 99,63 & 100,23 & 100,29 & 99,96 & 100,01 & 100,28 & 100,05 & 100,13 \\
\hline \multicolumn{14}{|c|}{ Structural formula calculated for 8 oxygens } \\
\hline $\mathrm{Si}$ & 2,44 & 2,55 & 2,45 & 2,49 & 2,48 & 2,46 & 2,46 & 2,53 & 2,50 & 2,47 & 2,50 & 2,51 & 2,56 \\
\hline $\mathrm{Ti}$ & 0,00 & 0,00 & 0,00 & 0,00 & 0,00 & 0,00 & 0,00 & 0,00 & 0,00 & 0,00 & 0,00 & 0,00 & 0,00 \\
\hline $\mathrm{Al}$ & 1,52 & 1,43 & 1,53 & 1,48 & 1,49 & 1,51 & 1,51 & 1,45 & 1,47 & 1,50 & 1,47 & 1,46 & 1,42 \\
\hline $\mathrm{Fe}$ & 0,04 & 0,04 & 0,04 & 0,04 & 0,04 & 0,04 & 0,04 & 0,03 & 0,04 & 0,04 & 0,04 & 0,04 & 0,03 \\
\hline Mn & 0,00 & 0,00 & 0,00 & 0,00 & 0,00 & 0,00 & 0,00 & 0,00 & 0,00 & 0,00 & 0,00 & 0,00 & 0,00 \\
\hline $\mathrm{Mg}$ & 0,00 & 0,00 & 0,00 & 0,00 & 0,00 & 0,00 & 0,00 & 0,00 & 0,00 & 0,00 & 0,00 & 0,00 & 0,00 \\
\hline $\mathrm{Ca}$ & 0,57 & 0,45 & 0,55 & 0,52 & 0,53 & 0,55 & 0,55 & 0,47 & 0,50 & 0,56 & 0,51 & 0,50 & 0,45 \\
\hline $\mathrm{Na}$ & 0,39 & 0,49 & 0,40 & 0,43 & 0,43 & 0,41 & 0,41 & 0,48 & 0,45 & 0,42 & 0,44 & 0,45 & 0,49 \\
\hline K & 0,02 & 0,03 & 0,02 & 0,02 & 0,02 & 0,02 & 0,02 & 0,03 & 0,03 & 0,02 & 0,02 & 0,03 & 0,04 \\
\hline $\mathrm{Ba}$ & 0,00 & 0,00 & 0,00 & 0,00 & 0,00 & 0,00 & 0,00 & 0,00 & 0,00 & 0,00 & 0,00 & 0,00 & 0,00 \\
\hline$\sum$ cations & 5,00 & 4,99 & 5,00 & 4,99 & 5,00 & 5,00 & 5,00 & 4,99 & 4,99 & 5,00 & 4,99 & 5,00 & 4,99 \\
\hline An & 57,93 & 46,76 & 56,60 & 53,68 & 53,97 & 56,03 & 55,88 & 48,08 & 51,46 & 55,80 & 52,05 & 51,05 & 45,71 \\
\hline $\mathbf{A b}$ & 40,04 & 50,42 & 41,21 & 43,75 & 43,60 & 41,89 & 41,87 & 48,94 & 45,93 & 41,81 & 45,62 & 46,19 & 50,55 \\
\hline Or & 2,03 & 2,82 & 2,19 & 2,57 & 2,43 & 2,08 & 2,25 & 2,98 & 2,60 & 2,39 & 2,33 & 2,75 & 3,74 \\
\hline
\end{tabular}


Chemical composition (\% wt) for pyroxene from Caxias do Sul dacites

\begin{tabular}{|c|c|c|c|c|c|c|c|c|c|c|c|c|c|}
\hline Sample & SM-01C & SM-01C & SM-01C & SM-01C & SM-01C & SM-01C & SM-01C & SM-01C & SM-01C & SM-01C & SM-01C & SM-01C & SM-01C \\
\hline Point ID* & A.fenola & A.fenolb & A.fenolc & A.feno2a & A.micro1 & A.micro2a & A.micro2b & A.microlito1 & A.micro3 & A.micro4 & A.feno3 & A.microlito2 & A.micro5 \\
\hline Position & intermediate & rim & core & core & core & core & core & core & core & core & core & intermediate & core \\
\hline Size & & $0,35 \mathrm{~mm}$ & & $0,075 \mathrm{~mm}$ & $0,02 \mathrm{~mm}$ & $0,05 \mathrm{~mm}$ & $0,01 \mathrm{~mm}$ & $0,01 \mathrm{~mm}$ & $0,03 \mathrm{~mm}$ & $0,03 \mathrm{~mm}$ & $0,15 \mathrm{~mm}$ & $\begin{array}{c}0,01 \mathrm{~mm} \\
\text { (acicular) }\end{array}$ & $0,025 \mathrm{~mm}$ \\
\hline$\overline{\mathrm{SiO}_{2}}$ & 50,99 & 49,50 & 52,09 & 51,52 & 51,04 & 51,61 & 51,42 & 49,45 & 51,20 & 52,60 & 51,71 & 50,52 & 52,69 \\
\hline $\mathrm{TiO}_{2}$ & 0,54 & 0,42 & 0,37 & 0,48 & 0,54 & 0,42 & 0,29 & 0,37 & 0,65 & 0,31 & 0,37 & 0,48 & 0,26 \\
\hline $\mathrm{Al}_{2} \mathrm{O}_{3}$ & 2,01 & 0,64 & 0,84 & 1,66 & 1,81 & 1,61 & 0,77 & 0,85 & 1,82 & 1,12 & 1,76 & 1,05 & 0,71 \\
\hline $\mathrm{FeO}$ & 13,49 & 33,28 & 23,78 & 12,66 & 14,29 & 12,59 & 25,19 & 30,93 & 13,62 & 20,56 & 12,69 & 26,63 & 21,40 \\
\hline $\mathrm{MnO}$ & 0,54 & 1,44 & 0,87 & 0,48 & 0,59 & 0,48 & 0,99 & 1,32 & 0,55 & 0,76 & 0,52 & 1,03 & 0,82 \\
\hline $\mathrm{MgO}$ & 14,61 & 10,73 & 19,11 & 14,91 & 14,22 & 14,79 & 17,64 & 13,09 & 14,31 & 21,16 & 14,82 & 14,30 & 21,13 \\
\hline $\mathrm{CaO}$ & 16,95 & 4,12 & 3,69 & 17,74 & 17,07 & 18,20 & 4,07 & 4,11 & 17,50 & 3,85 & 18,00 & 6,10 & 3,46 \\
\hline $\mathrm{Na}_{2} \mathrm{O}$ & 0,21 & 0,08 & 0,11 & 0,24 & 0,22 & 0,23 & 0,04 & 0,07 & 0,20 & 0,08 & 0,22 & 0,10 & 0,05 \\
\hline $\mathrm{K}_{2} \mathrm{O}$ & 0,01 & 0,08 & 0,01 & 0,02 & 0,06 & 0,01 & 0,03 & 0,08 & 0,04 & 0,00 & 0,04 & 0,05 & 0,02 \\
\hline $\mathrm{Cr}_{2} \mathrm{O}_{3}$ & 0,04 & 0,02 & 0,04 & 0,01 & 0,00 & 0,00 & 0,02 & 0,00 & 0,00 & 0,00 & 0,08 & 0,00 & 0,06 \\
\hline Total & 99,39 & 100,31 & 100,91 & 99,73 & 99,83 & 99,95 & 100,46 & 100,27 & 99,89 & 100,44 & 100,21 & 100,26 & 100,60 \\
\hline \multicolumn{14}{|c|}{ Structural formula calculated for 6 oxygens } \\
\hline $\mathrm{Si}$ & 1,93 & 1,97 & 1,96 & 1,94 & 1,93 & 1,94 & 1,96 & 1,95 & 1,94 & 1,96 & 1,94 & 1,96 & 1,96 \\
\hline $\mathrm{Ti}$ & 0,02 & 0,01 & 0,01 & 0,01 & 0,02 & 0,01 & 0,01 & 0,01 & 0,02 & 0,01 & 0,01 & 0,01 & 0,01 \\
\hline $\mathrm{Al}$ & 0,09 & 0,03 & 0,04 & 0,07 & 0,08 & 0,07 & 0,03 & 0,04 & 0,08 & 0,05 & 0,08 & 0,05 & 0,03 \\
\hline $\mathrm{Cr}$ & 0,00 & 0,00 & 0,00 & 0,00 & 0,00 & 0,00 & 0,00 & 0,00 & 0,00 & 0,00 & 0,00 & 0,00 & 0,00 \\
\hline $\mathrm{Fe}^{2+}$ & 0,43 & 1,11 & 0,75 & 0,40 & 0,45 & 0,40 & 0,80 & 1,02 & 0,43 & 0,64 & 0,40 & 0,86 & 0,67 \\
\hline $\mathrm{Mn}$ & 0,02 & 0,05 & 0,03 & 0,02 & 0,02 & 0,02 & 0,03 & 0,04 & 0,02 & 0,02 & 0,02 & 0,03 & 0,03 \\
\hline $\mathrm{Mg}$ & 0,83 & 0,64 & 1,07 & 0,84 & 0,80 & 0,83 & 1,00 & 0,77 & 0,81 & 1,17 & 0,83 & 0,83 & 1,17 \\
\hline $\mathrm{Ca}$ & 0,69 & 0,18 & 0,15 & 0,72 & 0,69 & 0,73 & 0,17 & 0,17 & 0,71 & 0,15 & 0,72 & 0,25 & 0,14 \\
\hline $\mathrm{Na}$ & 0,02 & 0,01 & 0,01 & 0,02 & 0,02 & 0,02 & 0,00 & 0,01 & 0,01 & 0,01 & 0,02 & 0,01 & 0,00 \\
\hline K & 0,00 & 0,00 & 0,00 & 0,00 & 0,00 & 0,00 & 0,00 & 0,00 & 0,00 & 0,00 & 0,00 & 0,00 & 0,00 \\
\hline$\sum$ cations & 4,01 & 4,00 & 4,01 & 4,02 & 4,02 & 4,02 & 4,01 & 4,02 & 4,01 & 4,01 & 4,02 & 4,01 & 4,01 \\
\hline En & 43,48 & 33,28 & 55,67 & 44,03 & 42,44 & 43,57 & 51,99 & 40,53 & 42,38 & 60,78 & 43,65 & 43,08 & 60,65 \\
\hline Fs & 20,27 & 57,54 & 36,61 & 18,31 & 20,95 & 17,90 & 39,39 & 50,32 & 20,38 & 31,27 & 18,25 & 43,71 & 32,22 \\
\hline Wo & 36,26 & 9,18 & 7,73 & 37,65 & 36,61 & 38,53 & 8,62 & 9,15 & 37,25 & 7,95 & 38,10 & 13,21 & 7,14 \\
\hline$\# \mathbf{m g}$ & 68,21 & 36,65 & 60,33 & 70,63 & 66,95 & 70,88 & 56,90 & 44,61 & 67,53 & 66,03 & 70,51 & 49,63 & 65,31 \\
\hline
\end{tabular}


Chemical composition (\% wt) for pyroxene from Caxias do Sul dacites

\begin{tabular}{|c|c|c|c|c|c|c|c|c|c|c|c|c|c|c|}
\hline Sample & SM-01C & SM-01C & SM-01C & SM-01C & SM-01C & SM-01C & SM-01C & SM-01C & SM-01C & SM-01C & SM-01C & SM-01C & SM-01C & SM-01C \\
\hline Point ID* & A.micro6a & A.micro6b & B.micro_a & B.micro_b & B.micro_c & D.microla & D.microlb & D.micro2 & D.micro3 & EF.fenola & EF.fenolb & EF.fenolc & EF.microlito1 & EF.microlitolb \\
\hline Position & core & core & core & core & core & intermediate & core & core & core & core & intermediate & rim & intermediate & intermediae \\
\hline Size & $0,03 \mathrm{~mm}$ & 0,01 & $0,05 \mathrm{~mm}$ & $0,05 \mathrm{~mm}$ & $0,05 \mathrm{~mm}$ & $0,05 \mathrm{~mm}$ & $0,05 \mathrm{~mm}$ & $0,04 \mathrm{~mm}$ & $0,07 \mathrm{~mm}$ & \multicolumn{2}{|c|}{$0,15 \mathrm{~mm}$} & $0,02 \mathrm{~mm}$ & \multicolumn{2}{|c|}{$0,015 \mathrm{~mm}$} \\
\hline$\overline{\mathrm{SiO}_{2}}$ & 50,43 & 47,89 & 49,25 & 50,83 & 50,55 & 51,65 & 51,24 & 51,34 & 51,30 & 52,40 & 51,01 & 50,62 & 50,34 & 49,91 \\
\hline $\mathrm{TiO}_{2}$ & 0,74 & 0,64 & 0,57 & 0,36 & 0,61 & 0,62 & 0,24 & 0,66 & 0,59 & 0,33 & 0,53 & 0,39 & 0,42 & 0,65 \\
\hline $\mathrm{Al}_{2} \mathrm{O}_{3}$ & 2,09 & 1,88 & 1,97 & 0,76 & 1,74 & 1,74 & 0,77 & 2,06 & 1,80 & 0,92 & 1,73 & 0,69 & 0,96 & 1,47 \\
\hline $\mathrm{FeO}$ & 14,51 & 14,65 & 15,74 & 28,35 & 16,39 & 13,46 & 25,87 & 13,59 & 13,71 & 21,20 & 13,23 & 28,58 & 32,53 & 21,06 \\
\hline $\mathrm{MnO}$ & 0,55 & 0,59 & 0,63 & 1,12 & 0,67 & 0,52 & 0,97 & 0,54 & 0,51 & 0,76 & 0,51 & 1,06 & 1,33 & 0,87 \\
\hline $\mathrm{MgO}$ & 13,34 & 11,99 & 13,31 & 15,54 & 13,09 & 14,57 & 17,05 & 14,71 & 14,84 & 20,68 & 14,69 & 15,32 & 10,85 & 11,57 \\
\hline $\mathrm{CaO}$ & 16,95 & 19,39 & 16,90 & 3,94 & 16,62 & 17,69 & 3,79 & 17,27 & 16,85 & 3,90 & 18,22 & 3,58 & 4,25 & 14,12 \\
\hline $\mathrm{Na}_{2} \mathrm{O}$ & 0,26 & 0,22 & 0,22 & 0,06 & 0,19 & 0,22 & 0,06 & 0,25 & 0,26 & 0,13 & 0,24 & 0,06 & 0,12 & 0,23 \\
\hline $\mathrm{K}_{2} \mathrm{O}$ & 0,19 & 0,06 & 0,05 & 0,05 & 0,02 & 0,04 & 0,00 & 0,02 & 0,00 & 0,00 & 0,03 & 0,01 & 0,12 & 0,04 \\
\hline $\mathrm{Cr}_{2} \mathrm{O}_{3}$ & 0,03 & 0,00 & 0,00 & 0,03 & 0,01 & 0,00 & 0,00 & 0,04 & 0,00 & 0,02 & 0,00 & 0,01 & 0,04 & 0,00 \\
\hline Total & 99,09 & 97,31 & 98,64 & 101,03 & 99,90 & 100,52 & 99,99 & 100,49 & 99,85 & 100,35 & 100,19 & 100,33 & 100,96 & 99,93 \\
\hline \multicolumn{15}{|c|}{ Structural formula calculated for 6 oxygens } \\
\hline $\mathrm{Si}$ & 1,93 & 1,89 & 1,91 & 1,96 & 1,93 & 1,94 & 1,97 & 1,93 & 1,94 & 1,96 & 1,92 & 1,96 & 1,98 & 1,94 \\
\hline $\mathrm{Ti}$ & 0,02 & 0,02 & 0,02 & 0,01 & 0,02 & 0,02 & 0,01 & 0,02 & 0,02 & 0,01 & 0,02 & 0,01 & 0,01 & 0,02 \\
\hline $\mathrm{Al}$ & 0,09 & 0,09 & 0,09 & 0,03 & 0,08 & 0,08 & 0,03 & 0,09 & 0,08 & 0,04 & 0,08 & 0,03 & 0,04 & 0,07 \\
\hline $\mathrm{Cr}$ & 0,00 & 0,00 & 0,00 & 0,00 & 0,00 & 0,00 & 0,00 & 0,00 & 0,00 & 0,00 & 0,00 & 0,00 & 0,00 & 0,00 \\
\hline $\mathrm{Fe}^{2+}$ & 0,46 & 0,48 & 0,51 & 0,91 & 0,52 & 0,42 & 0,83 & 0,43 & 0,43 & 0,66 & 0,42 & 0,93 & 1,07 & 0,68 \\
\hline $\mathrm{Mn}$ & 0,02 & 0,02 & 0,02 & 0,04 & 0,02 & 0,02 & 0,03 & 0,02 & 0,02 & 0,02 & 0,02 & 0,03 & 0,04 & 0,03 \\
\hline $\mathrm{Mg}$ & 0,76 & 0,71 & 0,77 & 0,89 & 0,75 & 0,81 & 0,98 & 0,82 & 0,83 & 1,15 & 0,83 & 0,89 & 0,64 & 0,67 \\
\hline $\mathrm{Ca}$ & 0,70 & 0,82 & 0,70 & 0,16 & 0,68 & 0,71 & 0,16 & 0,69 & 0,68 & 0,16 & 0,74 & 0,15 & 0,18 & 0,59 \\
\hline $\mathrm{Na}$ & 0,02 & 0,02 & 0,02 & 0,00 & 0,01 & 0,02 & 0,00 & 0,02 & 0,02 & 0,01 & 0,02 & 0,00 & 0,01 & 0,02 \\
\hline $\mathrm{K}$ & 0,01 & 0,00 & 0,00 & 0,00 & 0,00 & 0,00 & 0,00 & 0,00 & 0,00 & 0,00 & 0,00 & 0,00 & 0,01 & 0,00 \\
\hline$\sum$ cations & 4,01 & 4,05 & 4,04 & 4,02 & 4,02 & 4,02 & 4,01 & 4,02 & 4,02 & 4,02 & 4,03 & 4,01 & 3,99 & 4,02 \\
\hline En & 40,57 & 38,14 & 41,18 & 46,53 & 39,30 & 42,83 & 50,47 & 43,57 & 43,96 & 59,86 & 43,81 & 45,90 & 33,17 & 35,45 \\
\hline Fs & 22,38 & 17,53 & 21,23 & 44,99 & 24,83 & 19,79 & 41,47 & 19,67 & 20,17 & 32,02 & 17,13 & 46,40 & 57,49 & 33,46 \\
\hline Wo & 37,05 & 44,33 & 37,58 & 8,48 & 35,87 & 37,38 & 8,06 & 36,76 & 35,87 & 8,11 & 39,06 & 7,71 & 9,34 & 31,09 \\
\hline \#mg & 64,44 & 68,50 & 65,98 & 50,84 & 61,28 & 68,40 & 54,90 & 68,90 & 68,55 & 65,15 & 71,89 & 49,73 & 36,59 & 51,44 \\
\hline
\end{tabular}


Chemical composition (\% wt) for pyroxene from Caxias do Sul dacites

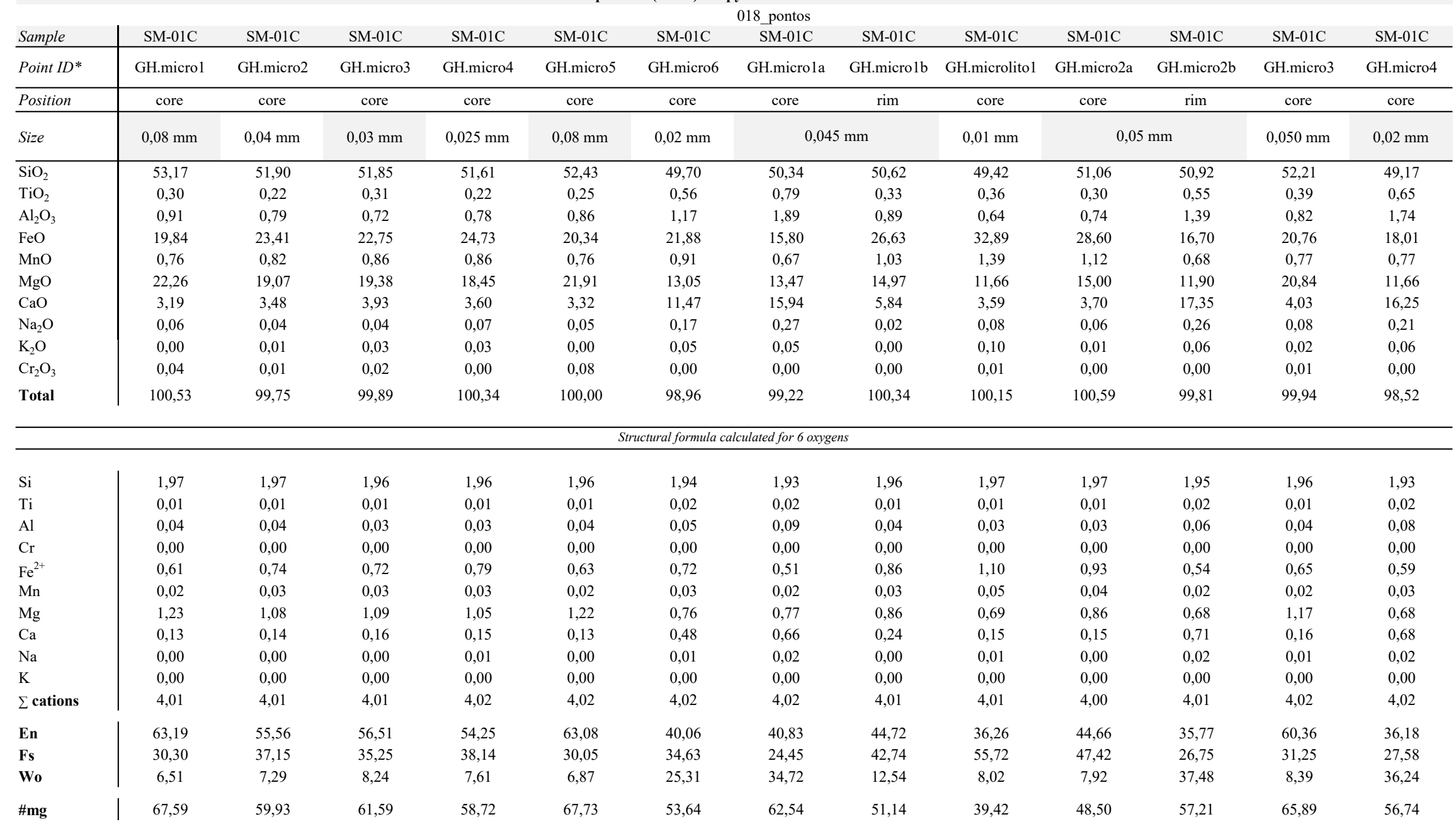


Chemical composition (\% wt) for pyroxene from Caxias do Sul dacites

\begin{tabular}{|c|c|c|c|c|c|c|c|c|c|c|c|c|c|c|}
\hline Sample & SM-01C & SM-01C & SM-01D & SM-01D & SM-01D & SM-01D & SM-01D & SM-01D & SM-01D & SM-01D & SM-01D & SM-01D & SM-01D & SM-01D \\
\hline Point ID* & GH.micro5 & GH.micro5b & microlitomatri & 3.enclavemicro & $\begin{array}{l}\text { B.enclavemicr } \\
02\end{array}$ & B.enclavemicro: & D.micro1 & D.micro2a & D.micro2b & E.microlito1 & E.microlito2 & E.microlito3 & E.microlito4 & E.microlito5 \\
\hline Position & core & rim & core & core & core & core & core & core & core & core & core & core & core & core \\
\hline Size & \multicolumn{2}{|c|}{$0,03 \mathrm{~mm}$} & $0,011 \mathrm{~mm}$ & $0,015 \mathrm{~mm}$ & $0,014 \mathrm{~mm}$ & $0,012 \mathrm{~mm}$ & $0,044 \mathrm{~mm}$ & \multicolumn{2}{|c|}{$0,036 \mathrm{~mm}$} & $0,040 \mathrm{~mm}$ & $0,025 \mathrm{~mm}$ & $0,0225 \mathrm{~mm}$ & $0,020 \mathrm{~mm}$ & $0,013 \mathrm{~mm}$ \\
\hline$\overline{\mathrm{SiO}_{2}}$ & 50,53 & 50,70 & 54,60 & 53,23 & 52,86 & 53,19 & 52,96 & 52,66 & 51,91 & 52,67 & 53,24 & $\begin{array}{l}53,09 \\
\end{array}$ & 53,36 & 53,03 \\
\hline $\mathrm{TiO}_{2}$ & 0,76 & 0,39 & 0,31 & 0,23 & 0,29 & 0,24 & 0,26 & 0,29 & 0,35 & 0,30 & 0,23 & 0,27 & 0,24 & 0,36 \\
\hline $\mathrm{Al}_{2} \mathrm{O}_{3}$ & 1,82 & 0,97 & 3,85 & 0,72 & 0,82 & 0,82 & 1,38 & 1,23 & 1,94 & 0,95 & 0,75 & 0,81 & 1,03 & 1,48 \\
\hline $\mathrm{FeO}$ & 18,64 & 25,97 & 16,37 & 19,42 & 19,57 & 18,88 & 19,61 & 20,18 & 20,13 & 19,09 & 18,87 & 19,38 & 17,86 & 19,61 \\
\hline $\mathrm{MnO}$ & 0,72 & 1,03 & 0,62 & 0,61 & 0,66 & 0,58 & 0,67 & 0,68 & 0,69 & 0,54 & 0,55 & 0,66 & 0,51 & 0,70 \\
\hline $\mathrm{MgO}$ & 13,53 & 13,70 & 18,35 & 22,01 & 22,46 & 22,11 & 20,78 & 21,58 & 20,10 & 22,48 & 22,03 & 22,12 & 21,83 & 20,94 \\
\hline $\mathrm{CaO}$ & 13,61 & 7,37 & 4,07 & 3,13 & 3,14 & 3,87 & 4,16 & 3,74 & 4,24 & 3,82 & 4,14 & 3,58 & 5,38 & 3,64 \\
\hline $\mathrm{Na}_{2} \mathrm{O}$ & 0,15 & 0,12 & 0,90 & 0,43 & 0,10 & 0,14 & 0,28 & 0,21 & 0,31 & 0,03 & 0,07 & 0,05 & 0,07 & 0,20 \\
\hline $\mathrm{K}_{2} \mathrm{O}$ & 0,04 & 0,08 & 0,45 & 0,05 & 0,06 & 0,06 & 0,18 & 0,15 & 0,24 & 0,02 & 0,07 & 0,05 & 0,04 & 0,05 \\
\hline $\mathrm{Cr}_{2} \mathrm{O}_{3}$ & 0,00 & 0,02 & 0,00 & 0,00 & 0,04 & 0,00 & 0,02 & 0,00 & 0,02 & 0,00 & 0,06 & 0,00 & 0,05 & 0,02 \\
\hline Total & 99,80 & 100,35 & 99,52 & 99,83 & 100,00 & 99,89 & 100,29 & 100,72 & 99,93 & 99,90 & 100,00 & 100,00 & 100,37 & 100,04 \\
\hline \multicolumn{15}{|c|}{ Structural formula calculated for 6 oxygens } \\
\hline $\mathrm{Si}$ & 1,94 & 1,97 & 2,00 & 1,98 & 1,96 & 1,97 & 1,97 & 1,95 & 1,94 & 1,96 & 1,97 & 1,97 & 1,97 & 1,97 \\
\hline $\mathrm{Ti}$ & 0,02 & 0,01 & 0,01 & 0,01 & 0,01 & 0,01 & 0,01 & 0,01 & 0,01 & 0,01 & 0,01 & 0,01 & 0,01 & 0,01 \\
\hline $\mathrm{Al}$ & 0,08 & 0,04 & 0,17 & 0,03 & 0,04 & 0,04 & 0,06 & 0,05 & 0,09 & 0,04 & 0,03 & 0,04 & 0,04 & 0,06 \\
\hline $\mathrm{Cr}$ & 0,00 & 0,00 & 0,00 & 0,00 & 0,00 & 0,00 & 0,00 & 0,00 & 0,00 & 0,00 & 0,00 & 0,00 & 0,00 & 0,00 \\
\hline $\mathrm{Fe}^{2+}$ & 0,60 & 0,84 & 0,50 & 0,60 & 0,61 & 0,59 & 0,61 & 0,63 & 0,63 & 0,59 & 0,59 & 0,60 & 0,55 & 0,61 \\
\hline $\mathrm{Mn}$ & 0,02 & 0,03 & 0,02 & 0,02 & 0,02 & 0,02 & 0,02 & 0,02 & 0,02 & 0,02 & 0,02 & 0,02 & 0,02 & 0,02 \\
\hline $\mathrm{Mg}$ & 0,77 & 0,79 & 1,00 & 1,22 & 1,24 & 1,22 & 1,15 & 1,19 & 1,12 & 1,24 & 1,22 & 1,22 & 1,20 & 1,16 \\
\hline $\mathrm{Ca}$ & 0,56 & 0,31 & 0,16 & 0,12 & 0,12 & 0,15 & 0,17 & 0,15 & 0,17 & 0,15 & 0,16 & 0,14 & 0,21 & 0,14 \\
\hline $\mathrm{Na}$ & 0,01 & 0,01 & 0,06 & 0,03 & 0,01 & 0,01 & 0,02 & 0,02 & 0,02 & 0,00 & 0,01 & 0,00 & 0,00 & 0,01 \\
\hline K & 0,00 & 0,00 & 0,02 & 0,00 & 0,00 & 0,00 & 0,01 & 0,01 & 0,01 & 0,00 & 0,00 & 0,00 & 0,00 & 0,00 \\
\hline$\sum$ cations & 4,01 & 4,01 & 3,95 & 4,02 & 4,02 & 4,01 & 4,01 & 4,02 & 4,02 & 4,02 & 4,01 & 4,01 & 4,01 & 4,00 \\
\hline En & 40,51 & 41,29 & 55,05 & 64,22 & 64,43 & 63,15 & 60,71 & 62,96 & 60,19 & 64,09 & 62,56 & 62,88 & 61,70 & 60,28 \\
\hline Fs & 30,21 & 42,74 & 36,17 & 29,21 & 29,10 & 28,90 & 30,56 & 29,20 & 30,68 & 28,08 & 28,99 & 29,80 & 27,37 & 32,18 \\
\hline Wo & 29,29 & 15,97 & 8,78 & 6,56 & 6,47 & 7,94 & 8,74 & 7,84 & 9,13 & 7,83 & 8,45 & 7,31 & 10,93 & 7,53 \\
\hline$\# \mathbf{m g}$ & 57,28 & 49,14 & 60,35 & 68,74 & 68,88 & 68,61 & 66,52 & 68,32 & 66,24 & 69,54 & 68,33 & 67,84 & 69,27 & 65,19 \\
\hline
\end{tabular}


Chemical composition (\% wt) for pyroxene from Caxias do Sul dacites

\begin{tabular}{|c|c|c|c|c|c|c|c|c|c|c|c|c|c|c|}
\hline Sample & SM-61 & SM-61 & SM-61 & SM-61 & SM-61 & SM-61 & SM-61 & SM-61 & SM-61 & SM-61 & SM-61 & SM-61 & SM-61 & SM-61 \\
\hline Point ID* & microborda & ,microbordamg & A.microlito2 & A.microlito3 & A.fenola & A.fenolb & A.microla & A.microlb & A.microlito1 & B.feno1.n2 & B.feno2.n1 & B.feno1.n2 & B.feno1.nclaro & B.microla \\
\hline Position & core & intermediate & core & core & core & rim & core & rim & core & intermediate & intermediate & intermediate & core & core \\
\hline Size & \multicolumn{2}{|c|}{$0,1 \mathrm{~mm}$} & $0,033 \mathrm{~mm}$ & $0,017 \mathrm{~mm}$ & $0,143 \mathrm{~mm}$ & $0,026 \mathrm{~mm}$ & \multicolumn{2}{|c|}{$0,082 \mathrm{~mm}$} & $0,013 \mathrm{~mm}$ & \multicolumn{2}{|c|}{$0,169 \mathrm{~mm}$} & \multicolumn{2}{|c|}{$0,305 \mathrm{~mm}$} & $0,1 \mathrm{~mm}$ \\
\hline$\overline{\mathrm{SiO}_{2}}$ & 51,36 & 51,64 & 50,61 & 50,56 & 51,42 & 50,26 & 51,64 & 51,03 & 50,50 & 50,95 & 51,22 & 51,21 & $\begin{array}{l}51,67 \\
\end{array}$ & 50,92 \\
\hline $\mathrm{TiO}_{2}$ & 0,55 & 0,46 & 0,41 & 0,37 & 0,67 & 0,25 & 0,34 & 0,65 & 0,37 & 0,74 & 0,51 & 0,49 & 0,32 & 0,72 \\
\hline $\mathrm{Al}_{2} \mathrm{O}_{3}$ & 1,77 & 0,83 & 0,89 & 0,77 & 1,86 & 0,70 & 0,90 & 1,70 & 0,62 & 2,09 & 1,92 & 1,65 & 0,79 & 1,85 \\
\hline $\mathrm{FeO}$ & 14,73 & 23,29 & 25,57 & 26,52 & 13,58 & 29,31 & 25,58 & 15,68 & 30,10 & 13,87 & 13,76 & 14,62 & 25,22 & 13,34 \\
\hline $\mathrm{MnO}$ & 0,53 & 0,79 & 0,93 & 1,00 & 0,47 & 1,06 & 0,92 & 0,59 & 1,08 & 0,42 & 0,46 & 0,54 & 0,85 & 0,51 \\
\hline $\mathrm{MgO}$ & 14,60 & 18,47 & 14,56 & 13,75 & 14,50 & 14,09 & 17,32 & 13,15 & 14,07 & 13,16 & 14,40 & 14,37 & 17,66 & 14,48 \\
\hline $\mathrm{CaO}$ & 16,16 & 4,83 & 7,20 & 6,82 & 17,87 & 4,11 & 3,87 & 17,43 & 3,88 & 16,43 & 16,40 & 16,61 & 3,72 & 17,50 \\
\hline $\mathrm{Na}_{2} \mathrm{O}$ & 0,59 & 0,16 & 0,13 & 0,13 & 0,24 & 0,04 & 0,06 & 0,22 & 0,04 & 1,22 & 0,50 & 0,21 & 0,13 & 0,25 \\
\hline $\mathrm{K}_{2} \mathrm{O}$ & 0,00 & 0,02 & 0,04 & 0,03 & 0,00 & 0,04 & 0,01 & 0,02 & 0,04 & 0,00 & 0,00 & 0,00 & 0,02 & 0,00 \\
\hline $\mathrm{Cr}_{2} \mathrm{O}_{3}$ & 0,00 & 0,00 & 0,05 & 0,00 & 0,00 & 0,00 & 0,00 & 0,00 & 0,03 & 0,00 & 0,03 & 0,05 & 0,02 & 0,00 \\
\hline Total & 100,29 & 100,49 & 100,39 & 99,95 & 100,60 & 99,86 & 100,62 & 100,48 & 100,73 & 98,88 & 99,20 & 99,74 & 100,41 & 99,56 \\
\hline \multicolumn{15}{|c|}{ Structural formula calculated for 6 oxygens } \\
\hline $\mathrm{Si}$ & 1,94 & 1,96 & 1,96 & 1,97 & 1,93 & 1,97 & 1,97 & 1,94 & 1,97 & 1,95 & 1,94 & 1,94 & 1,97 & 1,93 \\
\hline $\mathrm{Ti}$ & 0,02 & 0,01 & 0,01 & 0,01 & 0,02 & 0,01 & 0,01 & 0,02 & 0,01 & 0,02 & 0,01 & 0,01 & 0,01 & 0,02 \\
\hline $\mathrm{Al}$ & 0,08 & 0,04 & 0,04 & 0,04 & 0,08 & 0,03 & 0,04 & 0,08 & 0,03 & 0,09 & 0,09 & 0,07 & 0,04 & 0,08 \\
\hline $\mathrm{Cr}$ & 0,00 & 0,00 & 0,00 & 0,00 & 0,00 & 0,00 & 0,00 & 0,00 & 0,00 & 0,00 & 0,00 & 0,00 & 0,00 & 0,00 \\
\hline $\mathrm{Fe}^{2+}$ & 0,46 & 0,74 & 0,83 & 0,86 & 0,43 & 0,96 & 0,81 & 0,50 & 0,98 & 0,44 & 0,44 & 0,46 & 0,80 & 0,42 \\
\hline $\mathrm{Mn}$ & 0,02 & 0,03 & 0,03 & 0,03 & 0,01 & 0,04 & 0,03 & 0,02 & 0,04 & 0,01 & 0,01 & 0,02 & 0,03 & 0,02 \\
\hline $\mathrm{Mg}$ & 0,82 & 1,04 & 0,84 & 0,80 & 0,81 & 0,82 & 0,98 & 0,74 & 0,82 & 0,75 & 0,81 & 0,81 & 1,00 & 0,82 \\
\hline $\mathrm{Ca}$ & 0,65 & 0,20 & 0,30 & 0,28 & 0,72 & 0,17 & 0,16 & 0,71 & 0,16 & 0,67 & 0,67 & 0,67 & 0,15 & 0,71 \\
\hline $\mathrm{Na}$ & 0,04 & 0,01 & 0,01 & 0,01 & 0,02 & 0,00 & 0,00 & 0,02 & 0,00 & 0,09 & 0,04 & 0,02 & 0,01 & 0,02 \\
\hline K & 0,00 & 0,00 & 0,00 & 0,00 & 0,00 & 0,00 & 0,00 & 0,00 & 0,00 & 0,00 & 0,00 & 0,00 & 0,00 & 0,00 \\
\hline$\sum$ cations & 4,03 & 4,02 & 4,02 & 4,01 & 4,02 & 4,01 & 4,01 & 4,02 & 4,01 & 4,03 & 4,02 & 4,01 & 4,01 & 4,02 \\
\hline En & 44,40 & 54,36 & 43,83 & 41,49 & 42,71 & 42,60 & 50,75 & 39,12 & 42,28 & 42,26 & 43,57 & 42,58 & 52,00 & 43,11 \\
\hline Fs & 20,28 & 35,43 & 40,60 & 43,71 & 19,46 & 48,47 & 41,10 & 23,61 & 49,34 & 19,83 & 20,76 & 22,05 & 40,13 & 19,45 \\
\hline Wo & 35,32 & 10,22 & 15,58 & 14,79 & 37,83 & 8,93 & 8,15 & 37,27 & 8,38 & 37,92 & 35,67 & 35,37 & 7,87 & 37,44 \\
\hline$\# \mathbf{m g}$ & 68,65 & 60,54 & 51,91 & 48,70 & 68,69 & 46,77 & 55,25 & 62,36 & 46,15 & 68,06 & 67,73 & 65,88 & 56,44 & 68,91 \\
\hline
\end{tabular}


Chemical composition (\% wt) for pyroxene from Caxias do Sul dacites

\begin{tabular}{|c|c|c|c|c|c|c|c|c|c|c|c|c|c|}
\hline Sample & SM-61 & SM-61 & SM-61 & SM-61 & SM-61 & SM-61 & SM-61 & SM-61 & SM-61 & SM-61 & SM-61 & SM-61 & SM-61 \\
\hline Point ID* & B.microlb & B.fenola & B.fenolb & C.fenola & C.fenolb & C.feno2a & \multicolumn{3}{|c|}{ C.microcristal1\&C.microcristal1t C.microcristal2 } & C.microlito1 & \multicolumn{3}{|c|}{ D.microcristal1D.microcristal2 J.microcristal2b } \\
\hline Position & rim & core & rim & core & rim & core & core & rim & core & core & core & core & intermediate \\
\hline Size & $0,105 \mathrm{~mm}$ & \multicolumn{2}{|c|}{$0,305 \mathrm{~mm}$} & 0,107 & $0,039 \mathrm{~mm}$ & $0,124 \mathrm{~mm}$ & \multicolumn{2}{|c|}{$0,080 \mathrm{~mm}$} & $0,092 \mathrm{~mm}$ & $0,017 \mathrm{~mm}$ & $0,147 \mathrm{~mm}$ & \multicolumn{2}{|c|}{$0,108 \mathrm{~mm}$} \\
\hline$\overline{\mathrm{SiO}_{2}}$ & 50,59 & 50,93 & 50,91 & 51,56 & 50,48 & 51,08 & 51,34 & 250,81 & 51,22 & 50,34 & 50,88 & 50,41 & 50,29 \\
\hline $\mathrm{TiO}_{2}$ & 0,22 & 0,57 & 0,40 & 0,65 & 0,29 & 0,68 & 0,67 & 0,33 & 0,64 & 0,37 & 0,64 & 0,61 & 0,32 \\
\hline $\mathrm{Al}_{2} \mathrm{O}_{3}$ & 0,64 & 1,90 & 0,75 & 1,76 & 0,69 & 1,80 & 1,76 & 0,68 & 1,94 & 0,68 & 1,78 & 1,48 & 0,70 \\
\hline $\mathrm{FeO}$ & 28,78 & 13,75 & 27,39 & 13,52 & 28,20 & 13,45 & 14,09 & 29,28 & 13,52 & 30,40 & 13,27 & 15,95 & 30,17 \\
\hline $\mathrm{MnO}$ & 1,06 & 0,47 & 0,97 & 0,50 & 1,00 & 0,46 & 0,50 & 1,00 & 0,47 & 1,09 & 0,49 & 0,62 & 1,08 \\
\hline $\mathrm{MgO}$ & 14,74 & 14,67 & 15,75 & 14,44 & 15,49 & 14,74 & 14,09 & 14,86 & 14,35 & 13,60 & 14,22 & 12,24 & 13,64 \\
\hline $\mathrm{CaO}$ & 3,96 & 17,02 & 3,78 & 16,78 & 4,12 & 17,90 & 17,36 & 3,81 & 17,84 & 3,95 & 17,65 & 17,36 & 4,06 \\
\hline $\mathrm{Na}_{2} \mathrm{O}$ & 0,05 & 0,23 & 0,04 & 0,63 & 0,05 & 0,22 & 0,21 & 0,08 & 0,24 & 0,05 & 0,21 & 0,25 & 0,04 \\
\hline $\mathrm{K}_{2} \mathrm{O}$ & 0,01 & 0,00 & 0,01 & 0,02 & 0,03 & 0,02 & 0,02 & 0,02 & 0,00 & 0,00 & 0,02 & 0,01 & 0,03 \\
\hline $\mathrm{Cr}_{2} \mathrm{O}_{3}$ & 0,017 & 0,00 & 0,00 & 0,06 & 0,00 & 0,01 & 0,01 & 0,02 & 0,00 & 0,00 & 0,00 & 0,01 & 0,00 \\
\hline Total & 100,07 & 99,54 & 100,00 & 99,91 & 100,34 & 100,35 & 100,05 & 100,89 & 100,23 & 100,48 & 99,16 & 98,94 & 100,32 \\
\hline \multicolumn{14}{|c|}{ Structural formula calculated for 6 oxygens } \\
\hline $\mathrm{Si}$ & 1,97 & 1,93 & 1,97 & 1,94 & 1,96 & 1,92 & 1,94 & 1,97 & 1,93 & 1,97 & 1,94 & 1,95 & 1,97 \\
\hline $\mathrm{Cr}$ & 0,00 & 0,00 & 0,00 & 0,00 & 0,00 & 0,00 & 0,00 & 0,00 & 0,00 & 0,00 & 0,00 & 0,00 & 0,00 \\
\hline $\mathrm{Fe}^{2+}$ & 0,94 & 0,44 & 0,89 & 0,43 & 0,92 & 0,42 & 0,45 & 0,95 & 0,43 & 0,99 & 0,42 & 0,52 & 0,99 \\
\hline $\mathrm{Mn}$ & 0,04 & 0,02 & 0,03 & 0,02 & 0,03 & 0,01 & 0,02 & 0,03 & 0,01 & 0,04 & 0,02 & 0,02 & 0,04 \\
\hline $\mathrm{Mg}$ & 0,86 & 0,83 & 0,91 & 0,81 & 0,90 & 0,83 & 0,79 & 0,86 & 0,81 & 0,79 & 0,81 & 0,70 & 0,80 \\
\hline $\mathrm{Ca}$ & 0,17 & 0,69 & 0,16 & 0,68 & 0,17 & 0,72 & 0,70 & 0,16 & 0,72 & 0,17 & 0,72 & 0,72 & 0,17 \\
\hline $\mathrm{Na}$ & 0,00 & 0,02 & 0,00 & 0,05 & 0,00 & 0,02 & 0,02 & 0,01 & 0,02 & 0,00 & 0,02 & 0,02 & 0,00 \\
\hline K & 0,00 & 0,00 & 0,00 & 0,00 & 0,00 & 0,00 & 0,00 & 0,00 & 0,00 & 0,00 & 0,00 & 0,00 & 0,00 \\
\hline$\sum$ cations & 4,01 & 4,02 & 4,00 & 4,02 & 4,02 & 4,03 & 4,01 & 4,01 & 4,02 & 4,01 & 4,01 & 4,01 & 4,01 \\
\hline En & 44,27 & 43,68 & 46,75 & 43,82 & 46,58 & 43,67 & 41,56 & 44,42 & 42,47 & 40,97 & 42,32 & 36,96 & 41,17 \\
\hline Fs & 47,18 & 19,89 & 45,19 & 19,59 & 44,51 & 18,21 & 21,63 & 47,39 & 19,58 & 50,48 & 19,92 & 25,37 & 50,03 \\
\hline Wo & 8,55 & 36,43 & 8,06 & 36,59 & 8,90 & 38,12 & 36,80 & 8,19 & 37,95 & 8,55 & 37,76 & 37,67 & 8,81 \\
\hline$\# \mathbf{m g}$ & 48,41 & 68,71 & 50,85 & 69,10 & 51,13 & 70,58 & 65,77 & 48,38 & 68,44 & 44,80 & 68,00 & 59,30 & 45,14 \\
\hline
\end{tabular}


Chemical composition (\% wt) for pyroxene from Caxias do Sul dacites

\begin{tabular}{|c|c|c|c|c|c|c|c|c|c|c|c|c|c|}
\hline Sample & SM-61 & SM-61 & SM-61 & SM-61 & SM-61 & SM-61 & SM-61 & SM-61 & SM-61 & SM-61 & SM-61 & SM-61 & SM-61 \\
\hline Point ID* & E.fenola & E.fenolb & E.feno2a & E.feno $2 b$ & E.microl & F.fenola & F.fenolb & F.feno2a & F.feno2b & F.microla & F.feno3 & F.feno4a & F.feno4b \\
\hline Position & core & rim & core & rim & core & core & rim & core & core & core & core & core & intermediate \\
\hline Size & \multicolumn{2}{|c|}{$0,369 \mathrm{~mm}$} & \multicolumn{2}{|c|}{$0,245 \mathrm{~mm}$} & 0,09 & \multicolumn{2}{|c|}{$0,172 \mathrm{~mm}$} & \multicolumn{2}{|c|}{$0,139 \mathrm{~mm}$} & $0,057 \mathrm{~mm}$ & $0,097 \mathrm{~mm}$ & \multicolumn{2}{|c|}{$0,116 \mathrm{~mm}$} \\
\hline $\mathrm{SiO}_{2}$ & 51,49 & 49,40 & 51,01 & 51,27 & 51,27 & 50,70 & 50,83 & 50,95 & 47,02 & 50,31 & 50,83 & 51,77 & 48,56 \\
\hline $\mathrm{TiO}_{2}$ & 0,52 & 0,33 & 0,58 & 0,40 & 0,71 & 0,70 & 0,45 & 0,64 & 1,00 & 0,34 & 0,62 & 0,43 & 0,34 \\
\hline $\mathrm{Al}_{2} \mathrm{O}_{3}$ & 1,72 & 0,62 & 1,65 & 0,77 & 1,65 & 2,42 & 1,88 & 1,95 & 4,97 & 0,62 & 2,09 & 1,10 & 0,92 \\
\hline $\mathrm{FeO}$ & 13,59 & 33,51 & 13,58 & 25,20 & 12,85 & 14,46 & 20,48 & 13,94 & 19,76 & 29,38 & 13,42 & 21,27 & 29,60 \\
\hline $\mathrm{MnO}$ & 0,50 & 1,20 & 0,48 & 0,90 & 0,48 & 0,53 & 0,76 & 0,50 & 0,52 & 1,10 & 0,49 & 0,71 & 1,09 \\
\hline $\mathrm{MgO}$ & 14,63 & 10,99 & 14,10 & 16,86 & 14,13 & 14,03 & 11,94 & 14,33 & 11,12 & 14,59 & 14,40 & 19,14 & 12,54 \\
\hline $\mathrm{CaO}$ & 17,07 & 3,86 & 17,32 & 4,57 & 18,60 & 16,40 & 11,68 & 17,35 & 9,77 & 3,96 & 17,71 & 5,15 & 5,01 \\
\hline $\mathrm{Na}_{2} \mathrm{O}$ & 0,29 & 0,06 & 0,24 & 0,06 & 0,22 & 0,26 & 0,20 & 0,22 & 2,17 & 0,04 & 0,29 & 0,08 & 0,07 \\
\hline $\mathrm{K}_{2} \mathrm{O}$ & 0,01 & 0,03 & 0,01 & 0,02 & 0,02 & 0,03 & 0,04 & 0,00 & 1,01 & 0,00 & 0,00 & 0,02 & 0,04 \\
\hline $\mathrm{Cr}_{2} \mathrm{O}_{3}$ & 0,00 & 0,00 & 0,00 & 0,02 & 0,00 & 0,00 & 0,00 & 0,00 & 0,02 & 0,00 & 0,00 & 0,02 & 0,00 \\
\hline Total & 99,81 & 100,00 & 98,97 & 100,07 & 99,93 & 99,53 & 98,25 & 99,88 & 97,37 & 100,34 & 99,85 & 99,69 & 98,16 \\
\hline \multicolumn{14}{|c|}{ Structural formula calculated for 6 oxygens } \\
\hline $\mathrm{Si}$ & 1,94 & 1,98 & 1,94 & 1,97 & 1,94 & 1,92 & 1,98 & 1,93 & 1,87 & 1,96 & 1,92 & 1,96 & 1,95 \\
\hline $\mathrm{Cr}$ & 0,00 & 0,00 & 0,00 & 0,00 & 0,00 & 0,00 & 0,00 & 0,00 & 0,00 & 0,00 & 0,00 & 0,00 & 0,00 \\
\hline $\mathrm{Fe}^{2+}$ & 0,43 & 1,12 & 0,43 & 0,81 & 0,41 & 0,46 & 0,67 & 0,44 & 0,66 & 0,96 & 0,42 & 0,67 & 1,00 \\
\hline $\mathrm{Mn}$ & 0,02 & 0,04 & 0,02 & 0,03 & 0,02 & 0,02 & 0,02 & 0,02 & 0,02 & 0,04 & 0,02 & 0,02 & 0,04 \\
\hline $\mathrm{Mg}$ & 0,82 & 0,66 & 0,80 & 0,96 & 0,80 & 0,79 & 0,69 & 0,81 & 0,66 & 0,85 & 0,81 & 1,08 & 0,75 \\
\hline $\mathrm{Ca}$ & 0,69 & 0,17 & 0,71 & 0,19 & 0,75 & 0,67 & 0,49 & 0,70 & 0,42 & 0,17 & 0,72 & 0,21 & 0,22 \\
\hline $\mathrm{Na}$ & 0,02 & 0,00 & 0,02 & 0,00 & 0,02 & 0,02 & 0,01 & 0,02 & 0,17 & 0,00 & 0,02 & 0,01 & 0,01 \\
\hline K & 0,00 & 0,00 & 0,00 & 0,00 & 0,00 & 0,00 & 0,00 & 0,00 & 0,05 & 0,00 & 0,00 & 0,00 & 0,00 \\
\hline$\sum$ cations & 4,01 & 4,00 & 4,01 & 4,01 & 4,02 & 4,01 & 3,97 & 4,02 & 4,10 & 4,01 & 4,02 & 4,01 & 4,02 \\
\hline En & 43,35 & 33,89 & 41,98 & 49,73 & 41,71 & 42,08 & 35,83 & 42,59 & 45,63 & 43,97 & 43,13 & 55,81 & 39,32 \\
\hline Fs & 20,29 & 57,56 & 20,96 & 40,59 & 18,82 & 22,56 & 38,97 & 20,36 & 25,56 & 47,46 & 18,74 & 33,40 & 49,38 \\
\hline Wo & 36,36 & 8,55 & 37,06 & 9,69 & 39,47 & 35,36 & 25,19 & 37,06 & 28,81 & 8,58 & 38,13 & 10,79 & 11,29 \\
\hline$\# \mathbf{m g}$ & 68,12 & 37,06 & 66,70 & 55,06 & 68,91 & 65,10 & 47,90 & 67,66 & 64,09 & 48,09 & 69,72 & 62,56 & 44,33 \\
\hline
\end{tabular}


Chemical composition (\% wt) for pyroxene from Caxias do Sul dacites

\begin{tabular}{|c|c|c|c|c|c|c|c|c|c|c|c|c|c|c|c|}
\hline Sample & SM-61 & SM-61 & SM-61 & SM-61 & SM-61 & SM-61 & SM-61 & SM-61 & SM-61 & SM-61 & SM-61 & SM-61 & SM-61 & SM-61 & SM-61 \\
\hline Point ID* & F.micro2 & I.fenola & I.fenolb & I.fenold & I.microla & I.microlb & I.microlito1 & I.microlito2 & I.micro2a & I.microacicul & icroacicular.l & augita_n2 & pigeonita_n2 & augita_n3 & augita_n4 \\
\hline Position & core & core & intermediate & rim & core & rim & core & core & core & core & rim & intermediate & core & intermediate & rim \\
\hline Size & $0,037 \mathrm{~mm}$ & & $0,251 \mathrm{~mm}$ & & \multicolumn{2}{|c|}{$0,068 \mathrm{~mm}$} & $0,021 \mathrm{~mm}$ & $0,025 \mathrm{~mm}$ & $0,098 \mathrm{~mm}$ & \multicolumn{2}{|c|}{$0,148 \mathrm{~mm}$} & \multicolumn{4}{|c|}{$0,082 \mathrm{~mm}$} \\
\hline$\overline{\mathrm{SiO}_{2}}$ & 47,93 & $\begin{array}{l}51,22 \\
\end{array}$ & 51,19 & 47,26 & 50,93 & 49,82 & 52,45 & 51,25 & 51,56 & 50,92 & 51,37 & 51,07 & 51,76 & 51,00 & 51,09 \\
\hline $\mathrm{TiO}_{2}$ & 0,60 & 0,60 & 0,40 & 0,88 & 0,74 & 0,33 & 0,46 & 0,48 & 0,53 & 0,52 & 0,26 & 0,61 & 0,31 & 0,59 & 0,66 \\
\hline $\mathrm{Al}_{2} \mathrm{O}_{3}$ & 1,29 & 1,77 & 0,65 & 3,37 & 1,88 & 2,26 & 1,46 & 0,98 & 1,58 & 1,39 & 0,62 & 1,91 & 0,91 & 1,65 & 1,61 \\
\hline $\mathrm{FeO}$ & 24,91 & 14,29 & 27,58 & 27,81 & 12,90 & 30,19 & 28,53 & 30,40 & 13,31 & 17,64 & 25,57 & 14,07 & 24,06 & 14,58 & 16,04 \\
\hline $\mathrm{MnO}$ & 0,76 & 0,53 & 0,98 & 1,14 & 0,46 & 1,05 & 1,00 & 1,09 & 0,49 & 0,66 & 0,91 & 0,49 & 0,81 & 0,55 & 0,61 \\
\hline $\mathrm{MgO}$ & 13,37 & 14,57 & 15,75 & 9,80 & 14,12 & 10,01 & 12,78 & 11,33 & 14,75 & 12,58 & 16,97 & 14,85 & 18,54 & 14,68 & 13,48 \\
\hline $\mathrm{CaO}$ & 9,16 & 16,13 & 3,93 & 6,98 & 18,30 & 3,55 & 3,88 & 4,79 & 17,23 & 16,54 & 4,17 & 16,91 & 3,93 & 16,23 & 16,49 \\
\hline $\mathrm{Na}_{2} \mathrm{O}$ & 0,31 & 0,44 & 0,09 & 1,39 & 0,27 & 0,13 & 0,07 & 0,21 & 0,21 & 0,21 & 0,05 & 0,22 & 0,07 & 0,23 & 0,33 \\
\hline $\mathrm{K}_{2} \mathrm{O}$ & 0,28 & 0,02 & 0,02 & 0,57 & 0,01 & 0,06 & 0,05 & 0,08 & 0,01 & 0,03 & 0,01 & 0,00 & 0,01 & 0,01 & 0,03 \\
\hline $\mathrm{Cr}_{2} \mathrm{O}_{3}$ & 0,00 & 0,03 & 0,00 & 0,01 & 0,02 & 0,00 & 0,03 & 0,00 & 0,02 & 0,00 & 0,06 & 0,00 & 0,00 & 0,00 & 0,02 \\
\hline Total & 98,60 & 99,59 & 100,59 & 99,21 & 99,63 & 97,40 & 100,70 & 100,60 & 99,68 & 100,48 & 99,99 & 100,13 & 100,39 & 99,53 & 100,35 \\
\hline \multicolumn{16}{|c|}{ Structural formula calculated for 6 oxygens } \\
\hline $\mathrm{Si}$ & 1,91 & 1,94 & 1,97 & 1,89 & 1,93 & 2,00 & 2,01 & 2,00 & 1,95 & 1,95 & 1,97 & 1,93 & 1,96 & 1,94 & 1,94 \\
\hline $\mathrm{Ti}$ & 0,02 & 0,02 & 0,01 & 0,03 & 0,02 & 0,01 & 0,01 & 0,01 & 0,01 & 0,01 & 0,01 & 0,02 & 0,01 & 0,02 & 0,02 \\
\hline $\mathrm{Al}$ & 0,06 & 0,08 & 0,03 & 0,16 & 0,08 & 0,11 & 0,07 & 0,04 & 0,07 & 0,06 & 0,03 & 0,08 & 0,04 & 0,07 & 0,07 \\
\hline $\mathrm{Cr}$ & 0,00 & 0,00 & 0,00 & 0,00 & 0,00 & 0,00 & 0,00 & 0,00 & 0,00 & 0,00 & 0,00 & 0,00 & 0,00 & 0,00 & 0,00 \\
\hline $\mathrm{Fe}^{2+}$ & 0,83 & 0,45 & 0,89 & 0,93 & 0,41 & 1,02 & 0,92 & 0,99 & 0,42 & 0,56 & 0,82 & 0,44 & 0,76 & 0,46 & 0,51 \\
\hline $\mathrm{Mn}$ & 0,03 & 0,02 & 0,03 & 0,04 & 0,01 & 0,04 & 0,03 & 0,04 & 0,02 & 0,02 & 0,03 & 0,02 & 0,03 & 0,02 & 0,02 \\
\hline $\mathrm{Mg}$ & 0,79 & 0,82 & 0,90 & 0,59 & 0,80 & 0,60 & 0,73 & 0,66 & 0,83 & 0,72 & 0,97 & 0,83 & 1,05 & 0,83 & 0,76 \\
\hline $\mathrm{Ca}$ & 0,39 & 0,65 & 0,16 & 0,30 & 0,74 & 0,15 & 0,16 & 0,20 & 0,70 & 0,68 & 0,17 & 0,68 & 0,16 & 0,66 & 0,67 \\
\hline $\mathrm{Na}$ & 0,02 & 0,03 & 0,01 & 0,11 & 0,02 & 0,01 & 0,01 & 0,02 & 0,02 & 0,02 & 0,00 & 0,02 & 0,00 & 0,02 & 0,02 \\
\hline K & 0,01 & 0,00 & 0,00 & 0,03 & 0,00 & 0,00 & 0,00 & 0,00 & 0,00 & 0,00 & 0,00 & 0,00 & 0,00 & 0,00 & 0,00 \\
\hline$\sum$ cations & 4,06 & 4,02 & 4,01 & 4,07 & 4,02 & 3,94 & 3,94 & 3,97 & 4,01 & 4,02 & 4,01 & 4,02 & 4,01 & 4,02 & 4,02 \\
\hline En & 43,50 & 43,90 & 46,70 & 36,41 & 42,08 & 30,75 & 36,96 & 34,00 & 43,37 & 37,61 & 50,02 & 44,03 & 54,17 & 43,73 & 40,41 \\
\hline Fs & 35,08 & 21,16 & 44,92 & 44,95 & 18,72 & 61,42 & 54,97 & 55,67 & 20,22 & 26,85 & 41,14 & 19,94 & 37,58 & 21,52 & 24,06 \\
\hline Wo & 21,42 & 34,93 & 8,38 & 18,64 & 39,20 & 7,84 & 8,07 & 10,33 & 36,41 & 35,54 & 8,83 & 36,04 & 8,25 & 34,75 & 35,53 \\
\hline$\# \mathbf{m g}$ & 55,36 & 67,48 & 50,97 & 44,75 & 69,21 & 33,36 & 40,21 & 37,91 & 68,20 & 58,35 & 54,87 & 68,83 & 59,04 & 67,01 & 62,68 \\
\hline
\end{tabular}


Chemical composition (\% wt) for pyroxene from Caxias do Sul dacites

\begin{tabular}{|c|c|c|c|c|c|c|c|c|c|c|c|c|c|c|}
\hline Sample & SM-143 & SM-143 & SM-143 & SM-143 & SM-143 & SM-143 & SM-143 & SM-143 & SM-143 & SM-143 & SM-143 & SM-143 & SM-143 & SM-143 \\
\hline Point ID* & pirox 16.1a & pirox $16.1 \mathrm{~b}$ & pirox 16.2 & pirox 16.3 & pirox 16-2.1 & pirox 16-2.1 & pirox16-2.1 & pirox 16-3.1 & pirox 16-3.2 & pirox 17.1 & pirox 17.1 & pirox 17.1 & pirox 17.1 & pirox 17.2 \\
\hline Position & core & core & core & core & core & intermediate & rim & core & core & core & intermediate & rim (spot) & rim & core \\
\hline Size & $0.29 \mathrm{~mm}$ & $0.015 \mathrm{~mm}$ & $0.072 \mathrm{~mm}$ & $0.02 \mathrm{~mm}$ & & $0.29 \mathrm{~mm}$ & & $0.089 \mathrm{~mm}$ & $0.01 \mathrm{~mm}$ & \multicolumn{4}{|c|}{$0.183 \mathrm{~mm}$} & $0.011 \mathrm{~mm}$ \\
\hline$\overline{\mathrm{SiO}_{2}}$ & 50,67 & 49,65 & 50,70 & 49,78 & 50,95 & 50,93 & $\begin{array}{l}49,97 \\
\end{array}$ & 48,24 & 50,49 & 50,42 & 50,53 & 50,19 & 49,41 & 60,37 \\
\hline $\mathrm{TiO}_{2}$ & 0,63 & 0,53 & 0,74 & 0,35 & 0,64 & 0,57 & 0,86 & 0,46 & 0,57 & 0,74 & 0,70 & 0,76 & 0,45 & 0,41 \\
\hline $\mathrm{Al}_{2} \mathrm{O}_{3}$ & 2,08 & 0,76 & 2,03 & 0,72 & 2,02 & 1,96 & 2,06 & 0,84 & 0,75 & 1,94 & 1,85 & 2,01 & 0,97 & 4,58 \\
\hline $\mathrm{FeO}$ & 13,72 & 32,97 & 15,19 & 30,56 & 13,49 & 14,41 & 18,30 & 30,42 & 31,22 & 14,97 & 15,59 & 15,57 & 29,66 & 22,08 \\
\hline $\mathrm{MnO}$ & 0,47 & 1,26 & 0,58 & 1,29 & 0,52 & 0,53 & 0,68 & 1,20 & 1,23 & 0,56 & 0,56 & 0,57 & 1,10 & 0,82 \\
\hline $\mathrm{MgO}$ & 13,88 & 11,26 & 13,49 & 11,60 & 14,35 & 13,97 & 11,65 & 11,24 & 11,29 & 13,07 & 12,94 & 12,97 & 13,01 & 6,73 \\
\hline $\mathrm{CaO}$ & 17,90 & 3,95 & 16,61 & 5,24 & 17,18 & 17,14 & 15,99 & 6,25 & 4,34 & 17,55 & 17,10 & 17,09 & 4,60 & 2,80 \\
\hline $\mathrm{Na}_{2} \mathrm{O}$ & 0,23 & 0,05 & 0,22 & 0,08 & 0,24 & 0,24 & 0,25 & 0,08 & 0,07 & 0,25 & 0,26 & 0,22 & 0,05 & 0,79 \\
\hline $\mathrm{K}_{2} \mathrm{O}$ & 0,00 & 0,05 & 0,01 & 0,04 & 0,00 & 0,01 & 0,01 & 0,06 & 0,08 & 0,01 & 0,02 & 0,01 & 0,02 & 2,02 \\
\hline $\mathrm{Cr}_{2} \mathrm{O}_{3}$ & 0,02 & 0,03 & 0,02 & 0,01 & 0,01 & 0,01 & 0,00 & 0,00 & 0,00 & 0,00 & 0,01 & 0,00 & 0,00 & 0,00 \\
\hline Total & 99,59 & 100,50 & 99,59 & 99,68 & 99,39 & 99,77 & 99,77 & 98,79 & 100,04 & 99,50 & 99,57 & 99,39 & 99,27 & 100,60 \\
\hline \multicolumn{15}{|c|}{ Structural formula calculated for 6 oxygens } \\
\hline $\mathrm{Si}$ & 1,92 & 1,97 & 1,93 & 1,98 & 1,93 & 1,93 & 1,93 & 1,95 & 1,99 & 1,93 & 1,93 & 1,92 & 1,96 & 2,21 \\
\hline $\mathrm{Ti}$ & 0,02 & 0,02 & 0,02 & 0,01 & 0,02 & 0,02 & 0,03 & 0,01 & 0,02 & 0,02 & 0,02 & 0,02 & 0,01 & 0,01 \\
\hline $\mathrm{Al}$ & 0,09 & 0,04 & 0,09 & 0,03 & 0,09 & 0,09 & 0,09 & 0,04 & 0,03 & 0,09 & 0,08 & 0,09 & 0,05 & 0,20 \\
\hline $\mathrm{Cr}$ & 0,00 & 0,00 & 0,00 & 0,00 & 0,00 & 0,00 & 0,00 & 0,00 & 0,00 & 0,00 & 0,00 & 0,00 & 0,00 & 0,00 \\
\hline $\mathrm{Fe}^{2+}$ & 0,44 & 1,09 & 0,48 & 1,02 & 0,43 & 0,46 & 0,59 & 1,03 & 1,03 & 0,48 & 0,50 & 0,50 & 0,98 & 0,68 \\
\hline $\mathrm{Mn}$ & 0,02 & 0,04 & 0,02 & 0,04 & 0,02 & 0,02 & 0,02 & 0,04 & 0,04 & 0,02 & 0,02 & 0,02 & 0,04 & 0,03 \\
\hline $\mathrm{Mg}$ & 0,79 & 0,67 & 0,77 & 0,69 & 0,81 & 0,79 & 0,67 & 0,68 & 0,66 & 0,75 & 0,74 & 0,74 & 0,77 & 0,37 \\
\hline $\mathrm{Ca}$ & 0,73 & 0,17 & 0,68 & 0,22 & 0,70 & 0,70 & 0,66 & 0,27 & 0,18 & 0,72 & 0,70 & 0,70 & 0,20 & 0,11 \\
\hline $\mathrm{Na}$ & 0,02 & 0,00 & 0,02 & 0,01 & 0,02 & 0,02 & 0,02 & 0,01 & 0,01 & 0,02 & 0,02 & 0,02 & 0,00 & 0,06 \\
\hline $\mathrm{K}$ & 0,00 & 0,00 & 0,00 & 0,00 & 0,00 & 0,00 & 0,00 & 0,00 & 0,00 & 0,00 & 0,00 & 0,00 & 0,00 & 0,09 \\
\hline$\sum$ cations & 4,02 & 4,00 & 4,01 & 4,00 & 4,01 & 4,02 & 4,01 & 4,02 & 3,98 & 4,02 & 4,01 & 4,02 & 4,01 & 3,75 \\
\hline En & 41,49 & 34,49 & 40,30 & 35,63 & 42,72 & 41,68 & 35,40 & 35,57 & 34,06 & 39,35 & 38,95 & 39,12 & 39,92 & 19,38 \\
\hline Fs & 20,04 & 56,81 & 24,04 & 52,80 & 20,51 & 21,57 & 29,67 & 50,22 & 56,53 & 22,67 & 24,06 & 23,82 & 49,94 & 74,83 \\
\hline Wo & 38,46 & 8,70 & 35,66 & 11,57 & 36,76 & 36,75 & 34,92 & 14,21 & 9,41 & 37,98 & 36,99 & 37,05 & 10,14 & 5,79 \\
\hline \#mg & 67,43 & 37,78 & 62,63 & 40,29 & 67,56 & 65,90 & 54,40 & 41,46 & 37,60 & 63,45 & 61,81 & 62,15 & 44,43 & 20,57 \\
\hline
\end{tabular}


Chemical composition (\% wt) for pyroxene from Caxias do Sul dacites

\begin{tabular}{|c|c|c|c|c|c|c|c|c|c|c|c|c|c|c|}
\hline Sample & SM-143 & SM-143 & SM-143 & SM-143 & SM-143 & SM-143 & SM-143 & SM-143 & SM-143 & SM-143 & SM-143 & SM-143 & SM-143 & SM-143 \\
\hline Point ID* & pirox 18.1 & pirox 18.2 & pirox 18.3 & pirox24.1 & pirox 24.1 & pirox 24.2 & pirox 24.3 & pirox 24.4 & pirox 26.1 & pirox26.1 & pirox 26.1 & pirox 26.1 & pirox_nucl_pi & rox_nucl_pig' \\
\hline Position & core & core & core & core & rim & core & core & core & core & intermediate & rim & core & core & core \\
\hline Size & $0.112 \mathrm{~mm}$ & $0.08 \mathrm{~mm}$ & $0.120 \mathrm{~mm}$ & \multicolumn{2}{|c|}{$0.235 \mathrm{~mm}$} & $0.02 \mathrm{~mm}$ & $0.075 \mathrm{~mm}$ & $0.1 \mathrm{~mm}$ & & $0.273 \mathrm{~mm}$ & & $0.016 \mathrm{~mm}$ & $0.08 \mathrm{~mm}$ & $0.08 \mathrm{~mm}$ \\
\hline$\overline{\mathrm{SiO}_{2}}$ & 51,84 & 51,56 & 51,10 & 51,52 & 51,41 & 50,71 & 48,22 & 50,91 & 50,63 & 49,95 & 48,80 & 49,72 & 52,69 & 51,37 \\
\hline $\mathrm{TiO}_{2}$ & 0,57 & 0,57 & 0,64 & 0,63 & 0,65 & 0,77 & 0,97 & 0,62 & 0,62 & 0,79 & 0,51 & 0,53 & 0,30 & 0,27 \\
\hline $\mathrm{Al}_{2} \mathrm{O}_{3}$ & 1,64 & 1,67 & 1,75 & 1,91 & 1,80 & 1,28 & 0,70 & 1,94 & 1,70 & 1,93 & 2,61 & 0,81 & 0,84 & 0,76 \\
\hline $\mathrm{FeO}$ & 12,74 & 12,98 & 14,32 & 12,57 & 13,29 & 16,82 & 33,95 & 13,95 & 14,50 & 17,14 & 29,47 & 30,49 & 22,95 & 26,23 \\
\hline $\mathrm{MnO}$ & 0,46 & 0,47 & 0,56 & 0,46 & 0,52 & 0,62 & 1,28 & 0,51 & 0,57 & 0,66 & 1,10 & 1,14 & 0,81 & 1,02 \\
\hline $\mathrm{MgO}$ & 14,51 & 14,14 & 13,83 & 14,68 & 14,20 & 11,87 & 10,32 & 13,89 & 13,85 & 12,23 & 10,89 & 12,75 & 19,29 & 16,55 \\
\hline $\mathrm{CaO}$ & 18,17 & 18,07 & 17,11 & 17,64 & 17,90 & 17,22 & 3,39 & 17,77 & 17,09 & 16,40 & 6,08 & 4,16 & 3,74 & 3,57 \\
\hline $\mathrm{Na}_{2} \mathrm{O}$ & 0,21 & 0,21 & 0,25 & 0,25 & 0,24 & 0,22 & 0,06 & 0,23 & 0,28 & 0,25 & 0,13 & 0,09 & 0,05 & 0,03 \\
\hline $\mathrm{K}_{2} \mathrm{O}$ & 0,01 & 0,01 & 0,02 & 0,02 & 0,00 & 0,01 & 0,05 & 0,00 & 0,00 & 0,03 & 0,07 & 0,02 & 0,01 & 0,02 \\
\hline $\mathrm{Cr}_{2} \mathrm{O}_{3}$ & 0,00 & 0,00 & 0,00 & 0,00 & 0,00 & 0,07 & 0,02 & 0,00 & 0,01 & 0,03 & 0,00 & 0,02 & 0,00 & 0,00 \\
\hline Total & 100,15 & 99,69 & 99,58 & 99,67 & 100,02 & 99,59 & 98,94 & 99,82 & 99,24 & 99,41 & 99,66 & 99,72 & 100,67 & 99,82 \\
\hline \multicolumn{15}{|c|}{ Structural formula calculated for 6 oxygens } \\
\hline $\mathrm{Si}$ & 1,95 & 1,95 & 1,94 & 1,94 & 1,94 & 1,95 & 1,96 & 1,93 & 1,93 & 1,93 & 1,93 & 1,97 & 1,98 & 1,98 \\
\hline $\mathrm{Ti}$ & 0,02 & 0,02 & 0,02 & 0,02 & 0,02 & 0,02 & 0,03 & 0,02 & 0,02 & 0,02 & 0,02 & 0,02 & 0,01 & 0,01 \\
\hline $\mathrm{Al}$ & 0,07 & 0,07 & 0,08 & 0,08 & 0,08 & 0,06 & 0,03 & 0,09 & 0,08 & 0,09 & 0,12 & 0,04 & 0,04 & 0,03 \\
\hline $\mathrm{Cr}$ & 0,00 & 0,00 & 0,00 & 0,00 & 0,00 & 0,00 & 0,00 & 0,00 & 0,00 & 0,00 & 0,00 & 0,00 & 0,00 & 0,00 \\
\hline $\mathrm{Fe}^{2+}$ & 0,40 & 0,41 & 0,46 & 0,40 & 0,42 & 0,54 & 1,15 & 0,44 & 0,46 & 0,55 & 0,98 & 1,01 & 0,72 & 0,84 \\
\hline $\mathrm{Mn}$ & 0,01 & 0,02 & 0,02 & 0,01 & 0,02 & 0,02 & 0,04 & 0,02 & 0,02 & 0,02 & 0,04 & 0,04 & 0,03 & 0,03 \\
\hline $\mathrm{Mg}$ & 0,81 & 0,80 & 0,78 & 0,82 & 0,80 & 0,68 & 0,62 & 0,79 & 0,79 & 0,70 & 0,64 & 0,75 & 1,08 & 0,95 \\
\hline $\mathrm{Ca}$ & 0,73 & 0,73 & 0,70 & 0,71 & 0,72 & 0,71 & 0,15 & 0,72 & 0,70 & 0,68 & 0,26 & 0,18 & 0,15 & 0,15 \\
\hline $\mathrm{Na}$ & 0,02 & 0,02 & 0,02 & 0,02 & 0,02 & 0,02 & 0,00 & 0,02 & 0,02 & 0,02 & 0,01 & 0,01 & 0,00 & 0,00 \\
\hline $\mathrm{K}$ & 0,00 & 0,00 & 0,00 & 0,00 & 0,00 & 0,00 & 0,00 & 0,00 & 0,00 & 0,00 & 0,00 & 0,00 & 0,00 & 0,00 \\
\hline$\sum$ cations & 4,01 & 4,01 & 4,01 & 4,01 & 4,01 & 4,00 & 4,00 & 4,02 & 4,02 & 4,02 & 4,00 & 4,00 & 4,00 & 4,00 \\
\hline En & 42,38 & 41,54 & 41,17 & 43,25 & 41,89 & 35,46 & 32,37 & 41,38 & 41,68 & 37,24 & 34,13 & 38,98 & 55,29 & 48,82 \\
\hline Fs & 19,48 & 20,30 & 22,23 & 19,40 & 20,16 & 27,56 & 59,98 & 20,58 & 21,35 & 26,87 & 52,18 & 51,88 & 37,01 & 43,61 \\
\hline Wo & 38,14 & 38,16 & 36,61 & 37,35 & 37,95 & 36,98 & 7,64 & 38,04 & 36,97 & 35,89 & 13,70 & 9,14 & 7,70 & 7,57 \\
\hline \#mg & 68,51 & 67,17 & 64,94 & 69,04 & 67,51 & 56,27 & 35,05 & 66,78 & 66,13 & 58,09 & 39,55 & 42,90 & 59,91 & 52,82 \\
\hline
\end{tabular}


Chemical composition (\% wt) for pyroxene from Caxias do Sul dacites

\begin{tabular}{|c|c|c|c|c|c|c|c|c|c|c|c|c|c|c|}
\hline Sample & SM-157 & SM-157 & SM-157 & SM-157 & SM-157 & SM-157 & SM-157 & SM-157 & SM-157 & SM-157 & SM-157 & SM-157 & SM-157 & SM-157 \\
\hline Point ID* & pirox2-1 & pirox2-2 & pirox 2-3 & pirox 1.1 & pirox 1.2 & pirox 1.3 & pirox 1.4 & pirox6.1 & pirox6.2 & pirox6.3 & pirox6.4 & pirox 6.5 & pirox7-1.1 & pirox7-1.2 \\
\hline Position & core & core & core & core & core & core & core & core & core & core & core & core & intermediate & core \\
\hline Size & $0.068 \mathrm{~mm}$ & $0.073 \mathrm{~mm}$ & $0.055 \mathrm{~mm}$ & $0.19 \mathrm{~mm}$ & $0.29 \mathrm{~mm}$ & $0.25 \mathrm{~mm}$ & $0.13 \mathrm{~mm}$ & $0.139 \mathrm{~mm}$ & $0.062 \mathrm{~mm}$ & $0.256 \mathrm{~mm}$ & $0.075 \mathrm{~mm}$ & $0.082 \mathrm{~mm}$ & $0.247 \mathrm{~mm}$ & $0.118 \mathrm{~mm}$ \\
\hline$\overline{\mathrm{SiO}_{2}}$ & 51,48 & 51,35 & 51,57 & 51,42 & 52,93 & 50,72 & 51,61 & 51,58 & 51,84 & 51,39 & 51,10 & 51,64 & 52,04 & 52,75 \\
\hline $\mathrm{TiO}_{2}$ & 0,60 & 0,56 & 0,64 & 0,59 & 0,27 & 0,59 & 0,52 & 0,57 & 0,52 & 0,52 & 0,63 & 0,54 & 0,31 & 0,28 \\
\hline $\mathrm{Al}_{2} \mathrm{O}_{3}$ & 1,65 & 1,72 & 1,67 & 1,66 & 0,86 & 1,85 & 1,66 & 1,85 & 1,75 & 1,76 & 1,70 & 1,66 & 0,73 & 0,94 \\
\hline $\mathrm{FeO}$ & 15,12 & 13,16 & 13,48 & 14,22 & 23,99 & 13,75 & 12,78 & 13,32 & 12,46 & 13,16 & 13,40 & 12,70 & 22,88 & 20,16 \\
\hline $\mathrm{MnO}$ & 0,62 & 0,47 & 0,51 & 0,56 & 0,88 & 0,51 & 0,48 & 0,46 & 0,44 & 0,52 & 0,51 & 0,45 & 0,84 & 0,72 \\
\hline $\mathrm{MgO}$ & 13,80 & 13,95 & 14,14 & 14,15 & 17,25 & 14,38 & 14,87 & 14,74 & 14,74 & 14,78 & 13,86 & 14,63 & 18,84 & 21,66 \\
\hline $\mathrm{CaO}$ & 16,06 & 17,89 & 17,20 & 16,84 & 3,75 & 17,29 & 17,60 & 17,38 & 17,85 & 17,04 & 18,17 & 17,37 & 4,21 & 3,31 \\
\hline $\mathrm{Na}_{2} \mathrm{O}$ & 0,24 & 0,22 & 0,24 & 0,24 & 0,05 & 0,24 & 0,21 & 0,22 & 0,23 & 0,21 & 0,25 & 0,23 & 0,05 & 0,05 \\
\hline $\mathrm{K}_{2} \mathrm{O}$ & 0,01 & 0,01 & 0,08 & 0,01 & 0,09 & 0,03 & 0,02 & 0,01 & 0,01 & 0,02 & 0,00 & 0,12 & 0,03 & 0,01 \\
\hline $\mathrm{Cr}_{2} \mathrm{O}_{3}$ & 0,03 & 0,02 & 0,00 & 0,00 & 0,02 & 0,01 & 0,00 & 0,05 & 0,00 & 0,00 & 0,00 & 0,01 & 0,00 & 0,04 \\
\hline Total & 99,60 & 99,36 & 99,53 & 99,69 & 100,09 & 99,36 & 99,75 & 100,18 & 99,84 & 99,41 & 99,61 & 99,35 & 99,94 & 99,93 \\
\hline \multicolumn{15}{|c|}{ Structural formula calculated for 6 oxygens } \\
\hline $\mathrm{Si}$ & 1,96 & 1,95 & 1,95 & 1,95 & 2,00 & 1,93 & 1,94 & 1,94 & 1,95 & 1,94 & 1,94 & 1,95 & 1,97 & 1,97 \\
\hline $\mathrm{Ti}$ & 0,02 & 0,02 & 0,02 & 0,02 & 0,01 & 0,02 & 0,01 & 0,02 & 0,01 & 0,01 & 0,02 & 0,02 & 0,01 & 0,01 \\
\hline $\mathrm{Al}$ & 0,07 & 0,08 & 0,07 & 0,07 & 0,04 & 0,08 & 0,07 & 0,08 & 0,08 & 0,08 & 0,08 & 0,07 & 0,03 & 0,04 \\
\hline $\mathrm{Cr}$ & 0,00 & 0,00 & 0,00 & 0,00 & 0,00 & 0,00 & 0,00 & 0,00 & 0,00 & 0,00 & 0,00 & 0,00 & 0,00 & 0,00 \\
\hline $\mathrm{Fe}^{2+}$ & 0,48 & 0,42 & 0,43 & 0,45 & 0,76 & 0,44 & 0,40 & 0,42 & 0,39 & 0,42 & 0,43 & 0,40 & 0,72 & 0,63 \\
\hline $\mathrm{Mn}$ & 0,02 & 0,02 & 0,02 & 0,02 & 0,03 & 0,02 & 0,02 & 0,01 & 0,01 & 0,02 & 0,02 & 0,01 & 0,03 & 0,02 \\
\hline $\mathrm{Mg}$ & 0,78 & 0,79 & 0,80 & 0,80 & 0,97 & 0,82 & 0,84 & 0,83 & 0,83 & 0,83 & 0,78 & 0,82 & 1,06 & 1,20 \\
\hline $\mathrm{Ca}$ & 0,65 & 0,73 & 0,70 & 0,68 & 0,15 & 0,70 & 0,71 & 0,70 & 0,72 & 0,69 & 0,74 & 0,70 & 0,17 & 0,13 \\
\hline $\mathrm{Na}$ & 0,02 & 0,02 & 0,02 & 0,02 & 0,00 & 0,02 & 0,02 & 0,02 & 0,02 & 0,02 & 0,02 & 0,02 & 0,00 & 0,00 \\
\hline $\mathrm{K}$ & 0,00 & 0,00 & 0,00 & 0,00 & 0,00 & 0,00 & 0,00 & 0,00 & 0,00 & 0,00 & 0,00 & 0,01 & 0,00 & 0,00 \\
\hline$\sum$ cations & 4,00 & 4,01 & 4,00 & 4,01 & 3,97 & 4,02 & 4,01 & 4,01 & 4,01 & 4,01 & 4,01 & 4,01 & 4,01 & 4,01 \\
\hline En & 40,79 & 41,18 & 41,76 & 41,82 & 49,50 & 43,09 & 43,69 & 43,32 & 43,13 & 43,67 & 41,17 & 43,23 & 54,75 & 61,96 \\
\hline Fs & 25,10 & 20,86 & 21,73 & 22,42 & 42,77 & 19,67 & 19,14 & 19,96 & 19,33 & 20,15 & 20,05 & 19,89 & 36,45 & 31,24 \\
\hline Wo & 34,12 & 37,96 & 36,51 & 35,77 & 7,73 & 37,24 & 37,17 & 36,72 & 37,54 & 36,18 & 38,79 & 36,89 & 8,79 & 6,81 \\
\hline$\# \mathbf{m g}$ & 61,91 & 66,38 & 65,77 & 65,10 & 53,64 & 68,66 & 69,54 & 68,46 & 69,05 & 68,42 & 67,25 & 68,49 & 60,03 & 66,48 \\
\hline
\end{tabular}


Appendix IV

Size distribution and Shape parameters data for clasts and vesicle from the volcanic breccias 
lasts' data for sample SM-01 used for grain size distribution and fractal dimension analysis

$\phi=-\log _{2}($ diam $)$, considering the major axis of the best fit ellipses, in millimeters.

\section{Best fit ellipse}

\begin{tabular}{|c|c|c|c|c|c|c|}
\hline Label & Area $\left(\mathrm{cm}^{2}\right)$ & $\begin{array}{l}\text { Perimeter } \\
\text { (cm) }\end{array}$ & $\begin{array}{l}\text { Major axis } \\
\quad(\mathrm{cm})\end{array}$ & $\begin{array}{l}\text { Minor axis } \\
\quad \text { (cm) }\end{array}$ & $\begin{array}{c}\text { Diameter } \\
\text { (mm) }\end{array}$ & Phi \\
\hline 1 & 394,85 & 99,41 & 34,44 & 14,60 & 344,43 & $-8,4$ \\
\hline 2 & 260,21 & 75,21 & 31,30 & 10,58 & 313,02 & $-8,3$ \\
\hline 3 & 13,51 & 17,19 & 8,34 & 2,06 & 83,43 & $-6,4$ \\
\hline 4 & 27,90 & 22,88 & 9,58 & 3,71 & 95,77 & $-6,6$ \\
\hline 5 & 8,86 & 12,03 & 5,28 & 2,14 & 52,77 & $-5,7$ \\
\hline 6 & 785,94 & 216,12 & 60,38 & 16,57 & 603,82 & $-9,2$ \\
\hline 7 & 4,65 & 8,70 & 3,85 & 1,54 & 38,47 & $-5,3$ \\
\hline 8 & 34,33 & 22,88 & 7,58 & 5,77 & 75,77 & $-6,2$ \\
\hline 9 & 8,86 & 10,70 & 4,06 & 2,78 & 40,59 & $-5,3$ \\
\hline 10 & 18,16 & 16,57 & 6,48 & 3,57 & 64,82 & $-6,0$ \\
\hline 11 & 2,88 & 6,27 & 2,12 & 1,73 & 21,18 & $-4,4$ \\
\hline 12 & 1,33 & 3,60 & 1,59 & 1,06 & 15,93 & $-4,0$ \\
\hline 13 & 20,60 & 16,69 & 5,37 & 4,88 & 53,72 & $-5,7$ \\
\hline 14 & 14,84 & 15,24 & 6,27 & 3,01 & 62,69 & $-6,0$ \\
\hline 15 & 565,81 & 141,85 & 62,57 & 11,51 & 625,69 & $-9,3$ \\
\hline 16 & 8,42 & 10,81 & 3,36 & 3,19 & 33,63 & $-5,1$ \\
\hline 17 & 5,09 & 8,81 & 3,71 & 1,75 & 37,06 & $-5,2$ \\
\hline 18 & 134,42 & 48,06 & 16,25 & 10,53 & 162,51 & $-7,3$ \\
\hline 19 & 85,48 & 40,62 & 13,43 & 8,11 & 134,28 & $-7,1$ \\
\hline 20 & 6,64 & 10,14 & 3,67 & 2,31 & 36,66 & $-5,2$ \\
\hline 21 & 9,08 & 11,75 & 4,86 & 2,38 & 48,63 & $-5,6$ \\
\hline 22 & 368,50 & 73,31 & 25,01 & 18,76 & 250,09 & $-8,0$ \\
\hline 23 & 11,52 & 13,36 & 5,13 & 2,86 & 51,30 & $-5,7$ \\
\hline 24 & 73,52 & 35,41 & 13,54 & 6,91 & 135,45 & $-7,1$ \\
\hline 25 & 20,15 & 16,85 & 5,18 & 4,95 & 51,79 & $-5,7$ \\
\hline 26 & 13,95 & 13,75 & 5,01 & 3,55 & 50,10 & $-5,6$ \\
\hline 27 & 91,68 & 38,86 & 14,64 & 7,97 & 146,40 & $-7,2$ \\
\hline 28 & 480,33 & 88,32 & 26,85 & 22,78 & 268,47 & $-8,1$ \\
\hline 29 & 175,39 & 106,87 & 45,56 & 4,90 & 455,60 & $-8,8$ \\
\hline 30 & 187,13 & 64,01 & 18,58 & 12,82 & 185,83 & $-7,5$ \\
\hline 31 & 441,58 & 83,66 & 28,94 & 19,43 & 289,44 & $-8,2$ \\
\hline 32 & 50,49 & 28,05 & 9,06 & 7,10 & 90,60 & $-6,5$ \\
\hline 33 & 207,06 & 57,63 & 21,29 & 12,38 & 212,93 & $-7,7$ \\
\hline 34 & 19,49 & 18,84 & 7,30 & 3,40 & 73,00 & $-6,2$ \\
\hline 35 & 35,43 & 23,00 & 7,48 & 6,03 & 74,77 & $-6,2$ \\
\hline 36 & 8,42 & 10,42 & 3,67 & 2,92 & 36,71 & $-5,2$ \\
\hline 37 & 172,96 & 64,93 & 22,42 & 9,82 & 224,21 & $-7,8$ \\
\hline 38 & 34,99 & 22,84 & 8,29 & 5,37 & 82,93 & $-6,4$ \\
\hline 39 & 328,19 & 76,15 & 28,44 & 14,69 & 284,41 & $-8,2$ \\
\hline 40 & 65,11 & 50,20 & 23,49 & 3,53 & 234,91 & $-7,9$ \\
\hline 41 & 191,56 & 76,10 & 18,30 & 13,33 & 183,01 & $-7,5$ \\
\hline 42 & 36,32 & 22,45 & 7,88 & 5,87 & 78,76 & $-6,3$ \\
\hline 43 & 35,65 & 22,88 & 8,20 & 5,54 & 82,00 & $-6,4$ \\
\hline 44 & 114,27 & 42,94 & 12,78 & 11,38 & 127,83 & $-7,0$ \\
\hline 45 & 38,75 & 27,70 & 9,98 & 4,94 & 99,84 & $-6,6$ \\
\hline 46 & 4,21 & 6,93 & 2,49 & 2,15 & 24,95 & $-4,6$ \\
\hline 47 & 37,65 & 25,43 & 10,69 & 4,48 & 106,89 & $-6,7$ \\
\hline 48 & 53,81 & 41,47 & 18,71 & 3,66 & 187,10 & $-7,5$ \\
\hline 49 & 69,09 & 37,18 & 13,90 & 6,33 & 138,99 & $-7,1$ \\
\hline 50 & 27,90 & 19,78 & 6,94 & 5,12 & 69,44 & $-6,1$ \\
\hline 51 & 10,41 & 13,36 & 5,49 & 2,41 & 54,90 & $-5,8$ \\
\hline 52 & 224,33 & 56,85 & 17,58 & 16,25 & 175,79 & $-7,5$ \\
\hline 53 & 169,63 & 61,74 & 17,13 & 12,61 & 171,28 & $-7,4$ \\
\hline
\end{tabular}




\begin{tabular}{|c|c|c|c|c|c|c|}
\hline 54 & 229,20 & 73,05 & 27,68 & 10,54 & 276,83 & $-8,1$ \\
\hline 55 & 175,39 & 56,25 & 20,88 & 10,70 & 208,79 & $-7,7$ \\
\hline 56 & 248,69 & 77,79 & 33,08 & 9,57 & 330,76 & $-8,4$ \\
\hline 57 & 18,38 & 20,84 & 4,98 & 4,70 & 49,77 & $-5,6$ \\
\hline 58 & 27,46 & 26,05 & 11,69 & 2,99 & 116,86 & $-6,9$ \\
\hline 59 & 31,89 & 20,56 & 7,32 & 5,55 & 73,19 & $-6,2$ \\
\hline 60 & 27,24 & 18,96 & 6,16 & 5,63 & 61,58 & $-5,9$ \\
\hline 61 & 32,55 & 22,33 & 8,78 & 4,72 & 87,84 & $-6,5$ \\
\hline 62 & 37,43 & 28,60 & 12,62 & 3,78 & 126,23 & $-7,0$ \\
\hline 63 & 169,19 & 51,92 & 18,32 & 11,76 & 183,16 & $-7,5$ \\
\hline 64 & 92,57 & 44,60 & 19,74 & 5,97 & 197,42 & $-7,6$ \\
\hline 65 & 15,94 & 15,35 & 5,45 & 3,72 & 54,55 & $-5,8$ \\
\hline 66 & 15,06 & 15,91 & 6,31 & 3,04 & 63,07 & $-6,0$ \\
\hline 67 & 116,26 & 46,43 & 17,79 & 8,32 & 177,86 & $-7,5$ \\
\hline 68 & 81,49 & 38,40 & 15,65 & 6,63 & 156,49 & $-7,3$ \\
\hline 69 & 229,43 & 67,29 & 21,27 & 13,73 & 212,73 & $-7,7$ \\
\hline 70 & 5,54 & 8,54 & 2,88 & 2,45 & 28,76 & $-4,8$ \\
\hline 71 & 112,94 & 45,56 & 15,45 & 9,31 & 154,54 & $-7,3$ \\
\hline 72 & 168,30 & 52,15 & 17,97 & 11,92 & 179,74 & $-7,5$ \\
\hline 73 & 302,06 & 80,97 & 23,20 & 16,58 & 232,03 & $-7,9$ \\
\hline 74 & 84,82 & 38,12 & 15,12 & 7,14 & 151,16 & $-7,2$ \\
\hline 75 & 19,93 & 17,51 & 6,59 & 3,85 & 65,94 & $-6,0$ \\
\hline 76 & 39,86 & 25,66 & 10,28 & 4,94 & 102,84 & $-6,7$ \\
\hline 77 & 75,96 & 32,70 & 11,80 & 8,20 & 117,96 & $-6,9$ \\
\hline 78 & 83,93 & 44,94 & 13,96 & 7,66 & 139,56 & $-7,1$ \\
\hline 79 & 704,89 & 125,75 & 52,90 & 16,97 & 529,01 & $-9,0$ \\
\hline 80 & 33,44 & 27,27 & 10,26 & 4,15 & 102,58 & $-6,7$ \\
\hline 81 & 35,43 & 28,87 & 12,54 & 3,60 & 125,45 & $-7,0$ \\
\hline 82 & 9,30 & 12,46 & 5,65 & 2,10 & 56,46 & $-5,8$ \\
\hline 83 & 9,74 & 12,42 & 4,81 & 2,58 & 48,14 & $-5,6$ \\
\hline 84 & 35,88 & 24,72 & 9,11 & 5,01 & 91,14 & $-6,5$ \\
\hline 85 & 65,77 & 30,37 & 10,42 & 8,03 & 104,25 & $-6,7$ \\
\hline 86 & 16,83 & 15,35 & 5,44 & 3,94 & 54,40 & $-5,8$ \\
\hline 87 & 104,53 & 43,77 & 13,46 & 9,89 & 134,56 & $-7,1$ \\
\hline 88 & 37,87 & 26,21 & 9,44 & 5,11 & 94,42 & $-6,6$ \\
\hline 89 & 12,18 & 12,97 & 4,29 & 3,62 & 42,87 & $-5,4$ \\
\hline 90 & 11,96 & 15,24 & 6,03 & 2,52 & 60,33 & $-5,9$ \\
\hline 91 & 40,08 & 24,05 & 8,07 & 6,32 & 80,74 & $-6,3$ \\
\hline 92 & 38,75 & 27,33 & 11,69 & 4,22 & 116,93 & $-6,9$ \\
\hline 93 & 652,84 & 102,41 & 34,57 & 24,05 & 345,65 & $-8,4$ \\
\hline 94 & 21,26 & 17,35 & 6,00 & 4,51 & 60,00 & $-5,9$ \\
\hline 95 & 209,49 & 56,85 & 18,72 & 14,25 & 187,23 & $-7,5$ \\
\hline 96 & 27,68 & 19,78 & 6,71 & 5,26 & 67,07 & $-6,1$ \\
\hline 97 & 423,42 & 82,40 & 29,46 & 18,30 & 294,55 & $-8,2$ \\
\hline 98 & 23,25 & 17,24 & 6,04 & 4,91 & 60,35 & $-5,9$ \\
\hline 99 & 31,22 & 23,39 & 8,16 & 4,87 & 81,60 & $-6,4$ \\
\hline 100 & 13,95 & 13,75 & 4,75 & 3,74 & 47,47 & $-5,6$ \\
\hline 101 & 22,37 & 18,96 & 6,45 & 4,42 & 64,50 & $-6,0$ \\
\hline 102 & 125,12 & 48,22 & 17,95 & 8,88 & 179,47 & $-7,5$ \\
\hline 103 & 28,57 & 22,06 & 7,40 & 4,91 & 74,04 & $-6,2$ \\
\hline 104 & 8,64 & 10,81 & 3,82 & 2,88 & 38,17 & $-5,3$ \\
\hline 105 & 76,84 & 37,85 & 14,88 & 6,58 & 148,78 & $-7,2$ \\
\hline 106 & 8,42 & 10,92 & 3,85 & 2,78 & 38,54 & $-5,3$ \\
\hline 107 & 37,87 & 23,11 & 7,00 & 6,88 & 70,03 & $-6,1$ \\
\hline 108 & 7,53 & 9,48 & 3,33 & 2,88 & 33,29 & $-5,1$ \\
\hline 109 & 916,60 & 145,54 & 60,02 & 19,44 & 600,18 & $-9,2$ \\
\hline 110 & 52,48 & 31,58 & 12,90 & 5,18 & 129,01 & $-7,0$ \\
\hline 111 & 73,97 & 34,75 & 12,14 & 7,76 & 121,42 & $-6,9$ \\
\hline 112 & 110,28 & 41,06 & 13,43 & 10,46 & 134,30 & $-7,1$ \\
\hline 113 & 215,70 & 56,46 & 19,76 & 13,90 & 197,58 & $-7,6$ \\
\hline 114 & 22,37 & 18,13 & 6,72 & 4,24 & 67,20 & $-6,1$ \\
\hline 115 & 24,36 & 20,17 & 8,24 & 3,76 & 82,39 & $-6,4$ \\
\hline
\end{tabular}




\begin{tabular}{|c|c|c|c|c|c|c|}
\hline 116 & 5,54 & 8,26 & 3,41 & 2,07 & 34,12 & $-5,1$ \\
\hline 117 & 95,67 & 43,17 & 11,49 & 10,60 & 114,93 & $-6,8$ \\
\hline 118 & 6,42 & 10,42 & 4,12 & 1,98 & 41,23 & $-5,4$ \\
\hline 119 & 58,24 & 33,42 & 10,49 & 7,07 & 104,89 & $-6,7$ \\
\hline 120 & 117,81 & 48,93 & 19,18 & 7,82 & 191,75 & $-7,6$ \\
\hline 121 & 17,49 & 16,96 & 6,14 & 3,63 & 61,41 & $-5,9$ \\
\hline 122 & 24,80 & 19,51 & 7,74 & 4,08 & 77,43 & $-6,3$ \\
\hline 123 & 55,81 & 31,03 & 11,56 & 6,14 & 115,65 & $-6,9$ \\
\hline 124 & 49,83 & 37,52 & 14,73 & 4,31 & 147,31 & $-7,2$ \\
\hline 125 & 11,29 & 12,14 & 4,22 & 3,41 & 42,22 & $-5,4$ \\
\hline 126 & 17,05 & 20,84 & 9,40 & 2,31 & 93,97 & $-6,6$ \\
\hline 127 & 507,35 & 88,82 & 28,03 & 23,05 & 280,26 & $-8,1$ \\
\hline 128 & 35,21 & 23,43 & 9,18 & 4,89 & 91,76 & $-6,5$ \\
\hline 129 & 19,04 & 16,57 & 5,98 & 4,06 & 59,76 & $-5,9$ \\
\hline 130 & 14,62 & 14,46 & 4,61 & 4,03 & 46,13 & $-5,5$ \\
\hline 131 & 15,28 & 14,18 & 5,48 & 3,55 & 54,84 & $-5,8$ \\
\hline 132 & 27,02 & 19,90 & 7,08 & 4,86 & 70,79 & $-6,1$ \\
\hline 133 & 5,76 & 9,20 & 3,94 & 1,86 & 39,38 & $-5,3$ \\
\hline 134 & 31,45 & 22,72 & 9,15 & 4,38 & 91,47 & $-6,5$ \\
\hline 135 & 18,38 & 20,45 & 8,06 & 2,90 & 80,58 & $-6,3$ \\
\hline 136 & 38,09 & 24,44 & 9,20 & 5,27 & 92,02 & $-6,5$ \\
\hline 137 & 95,22 & 36,75 & 11,89 & 10,19 & 118,95 & $-6,9$ \\
\hline 138 & 7,75 & 10,14 & 3,37 & 2,92 & 33,75 & $-5,1$ \\
\hline 139 & 2,21 & 5,21 & 1,87 & 1,51 & 18,67 & $-4,2$ \\
\hline 140 & 49,83 & 26,65 & 9,38 & 6,76 & 93,79 & $-6,6$ \\
\hline 141 & 3,54 & 6,66 & 2,56 & 1,76 & 25,60 & $-4,7$ \\
\hline 142 & 31,22 & 21,90 & 8,00 & 4,97 & 79,96 & $-6,3$ \\
\hline 143 & 11,52 & 12,30 & 4,50 & 3,25 & 45,05 & $-5,5$ \\
\hline 144 & 8,64 & 12,03 & 4,47 & 2,46 & 44,66 & $-5,5$ \\
\hline 145 & 35,21 & 31,31 & 14,31 & 3,13 & 143,06 & $-7,2$ \\
\hline 146 & 81,49 & 39,57 & 16,37 & 6,34 & 163,75 & $-7,4$ \\
\hline 147 & 79,06 & 41,18 & 14,79 & 6,81 & 147,85 & $-7,2$ \\
\hline 148 & 154,80 & 65,44 & 28,03 & 7,03 & 280,32 & $-8,1$ \\
\hline 149 & 19,93 & 17,90 & 7,42 & 3,42 & 74,17 & $-6,2$ \\
\hline 150 & 19,93 & 16,85 & 6,02 & 4,22 & 60,20 & $-5,9$ \\
\hline 151 & 13,51 & 13,91 & 5,46 & 3,15 & 54,58 & $-5,8$ \\
\hline 152 & 104,08 & 38,19 & 12,55 & 10,56 & 125,47 & $-7,0$ \\
\hline 153 & 91,68 & 44,78 & 17,69 & 6,60 & 176,92 & $-7,5$ \\
\hline 154 & 191,34 & 54,65 & 18,55 & 13,13 & 185,55 & $-7,5$ \\
\hline 155 & 112,94 & 42,51 & 14,47 & 9,94 & 144,72 & $-7,2$ \\
\hline 156 & 20,37 & 16,57 & 5,86 & 4,43 & 58,60 & $-5,9$ \\
\hline 157 & 15,28 & 15,24 & 5,45 & 3,57 & 54,49 & $-5,8$ \\
\hline 158 & 25,69 & 26,33 & 10,60 & 3,09 & 106,02 & $-6,7$ \\
\hline 159 & 2,66 & 5,60 & 2,36 & 1,43 & 23,64 & $-4,6$ \\
\hline 160 & 14,62 & 14,02 & 4,90 & 3,79 & 49,04 & $-5,6$ \\
\hline 161 & 22,81 & 17,90 & 6,11 & 4,75 & 61,15 & $-5,9$ \\
\hline 162 & 9,97 & 11,87 & 4,27 & 2,97 & 42,70 & $-5,4$ \\
\hline 163 & 38,75 & 24,88 & 9,20 & 5,37 & 91,96 & $-6,5$ \\
\hline 164 & 70,87 & 38,90 & 16,24 & 5,56 & 162,37 & $-7,3$ \\
\hline 165 & 116,93 & 42,12 & 14,42 & 10,32 & 144,19 & $-7,2$ \\
\hline 166 & 74,85 & 40,97 & 17,48 & 5,45 & 174,75 & $-7,4$ \\
\hline 167 & 16,39 & 15,13 & 5,74 & 3,64 & 57,40 & $-5,8$ \\
\hline 168 & 26,13 & 19,28 & 6,94 & 4,79 & 69,41 & $-6,1$ \\
\hline 169 & 82,82 & 34,75 & 11,59 & 9,10 & 115,92 & $-6,9$ \\
\hline 170 & 38,31 & 24,88 & 9,61 & 5,08 & 96,05 & $-6,6$ \\
\hline 171 & 9,97 & 12,97 & 5,24 & 2,42 & 52,45 & $-5,7$ \\
\hline 172 & 66,44 & 31,26 & 11,15 & 7,59 & 111,46 & $-6,8$ \\
\hline 173 & 28,57 & 20,45 & 7,63 & 4,77 & 76,31 & $-6,3$ \\
\hline 174 & 166,98 & 64,27 & 26,03 & 8,17 & 260,26 & $-8,0$ \\
\hline 175 & 47,17 & 28,92 & 11,91 & 5,04 & 119,14 & $-6,9$ \\
\hline 176 & 31,45 & 25,11 & 9,35 & 4,28 & 93,52 & $-6,5$ \\
\hline 177 & 341,70 & 80,91 & 31,23 & 13,93 & 312,26 & $-8,3$ \\
\hline
\end{tabular}




\begin{tabular}{|c|c|c|c|c|c|c|}
\hline 178 & 77,51 & 33,97 & 12,30 & 8,03 & 122,95 & $-6,9$ \\
\hline 179 & 70,87 & 35,02 & 10,54 & 8,56 & 105,44 & $-6,7$ \\
\hline 180 & 397,51 & 79,02 & 23,06 & 21,94 & 230,65 & $-7,8$ \\
\hline 181 & 125,12 & 43,12 & 15,22 & 10,47 & 152,23 & $-7,3$ \\
\hline 182 & 80,17 & 33,81 & 10,86 & 9,40 & 108,63 & $-6,8$ \\
\hline 183 & 113,16 & 53,25 & 15,61 & 9,23 & 156,11 & $-7,3$ \\
\hline 184 & 3,54 & 7,32 & 2,81 & 1,60 & 28,12 & $-4,8$ \\
\hline 185 & 1009,61 & 141,15 & 47,80 & 26,89 & 478,05 & $-8,9$ \\
\hline 186 & 31,89 & 25,11 & 9,48 & 4,28 & 94,80 & $-6,6$ \\
\hline 187 & 7,31 & 9,75 & 3,43 & 2,72 & 34,26 & $-5,1$ \\
\hline 188 & 44,96 & 25,94 & 8,73 & 6,56 & 87,28 & $-6,4$ \\
\hline 189 & 53,15 & 27,93 & 9,89 & 6,84 & 98,89 & $-6,6$ \\
\hline 190 & 186,24 & 75,05 & 18,74 & 12,65 & 187,44 & $-7,6$ \\
\hline 191 & 1043,04 & 143,50 & 45,47 & 29,20 & 454,74 & $-8,8$ \\
\hline 192 & 5,76 & 8,65 & 3,31 & 2,22 & 33,09 & $-5,0$ \\
\hline 193 & 45,40 & 25,27 & 8,56 & 6,76 & 85,55 & $-6,4$ \\
\hline 194 & 1,99 & 5,32 & 2,20 & 1,15 & 22,04 & $-4,5$ \\
\hline 195 & 6,42 & 9,87 & 3,96 & 2,06 & 39,62 & $-5,3$ \\
\hline 196 & 3,99 & 6,82 & 2,52 & 2,02 & 25,17 & $-4,7$ \\
\hline 197 & 8,42 & 11,09 & 4,32 & 2,48 & 43,21 & $-5,4$ \\
\hline 198 & 5,09 & 7,87 & 2,73 & 2,38 & 27,29 & $-4,8$ \\
\hline 199 & 116,71 & 41,68 & 14,92 & 9,96 & 149,22 & $-7,2$ \\
\hline 200 & 9,08 & 12,97 & 5,20 & 2,22 & 52,03 & $-5,7$ \\
\hline 201 & 2,66 & 5,32 & 2,08 & 1,63 & 20,82 & $-4,4$ \\
\hline 202 & 7,75 & 11,09 & 4,34 & 2,27 & 43,42 & $-5,4$ \\
\hline 203 & 16,17 & 15,35 & 6,02 & 3,42 & 60,18 & $-5,9$ \\
\hline 204 & 1275,13 & 147,67 & 44,02 & 36,88 & 440,24 & $-8,8$ \\
\hline 205 & 3,99 & 6,82 & 2,49 & 2,04 & 24,86 & $-4,6$ \\
\hline 206 & 9,30 & 13,20 & 5,78 & 2,05 & 57,77 & $-5,9$ \\
\hline 207 & 131,32 & 48,82 & 18,16 & 9,21 & 181,57 & $-7,5$ \\
\hline 208 & 26,57 & 20,66 & 7,93 & 4,27 & 79,27 & $-6,3$ \\
\hline 209 & 12,40 & 13,08 & 4,46 & 3,54 & 44,56 & $-5,5$ \\
\hline 210 & 124,24 & 43,56 & 13,64 & 11,60 & 136,41 & $-7,1$ \\
\hline 211 & 2,66 & 5,49 & 2,12 & 1,59 & 21,24 & $-4,4$ \\
\hline 212 & 15,72 & 18,41 & 8,32 & 2,41 & 83,24 & $-6,4$ \\
\hline 213 & 6,64 & 9,20 & 3,27 & 2,59 & 32,68 & $-5,0$ \\
\hline 214 & 16,17 & 16,18 & 6,50 & 3,16 & 65,04 & $-6,0$ \\
\hline 215 & 3,10 & 6,82 & 2,64 & 1,49 & 26,42 & $-4,7$ \\
\hline 216 & 13,51 & 14,14 & 5,31 & 3,24 & 53,13 & $-5,7$ \\
\hline 217 & 207,94 & 61,28 & 21,30 & 12,43 & 213,00 & $-7,7$ \\
\hline 218 & 7,97 & 11,20 & 4,69 & 2,17 & 46,85 & $-5,6$ \\
\hline 219 & 12,84 & 14,41 & 5,67 & 2,88 & 56,71 & $-5,8$ \\
\hline 220 & 21,04 & 16,85 & 5,43 & 4,93 & 54,34 & $-5,8$ \\
\hline 221 & 25,69 & 19,00 & 6,98 & 4,69 & 69,81 & $-6,1$ \\
\hline 222 & 4,65 & 7,87 & 2,80 & 2,12 & 27,97 & $-4,8$ \\
\hline 223 & 23,70 & 20,17 & 7,95 & 3,79 & 79,52 & $-6,3$ \\
\hline 224 & 16,39 & 15,24 & 5,50 & 3,79 & 55,02 & $-5,8$ \\
\hline 225 & 2551,36 & 237,28 & 75,95 & 42,77 & 759,46 & $-9,6$ \\
\hline 226 & 3,99 & 6,82 & 2,63 & 1,93 & 26,29 & $-4,7$ \\
\hline 227 & 17,05 & 16,18 & 5,68 & 3,82 & 56,83 & $-5,8$ \\
\hline 228 & 25,47 & 20,45 & 8,18 & 3,96 & 81,81 & $-6,4$ \\
\hline 229 & 81,49 & 42,83 & 16,82 & 6,17 & 168,23 & $-7,4$ \\
\hline 230 & 10,41 & 12,14 & 4,42 & 3,00 & 44,22 & $-5,5$ \\
\hline 231 & 82,38 & 45,54 & 19,83 & 5,29 & 198,26 & $-7,6$ \\
\hline 232 & 93,90 & 42,23 & 13,94 & 8,58 & 139,38 & $-7,1$ \\
\hline 233 & 152,36 & 47,99 & 15,79 & 12,28 & 157,94 & $-7,3$ \\
\hline 234 & 26,57 & 21,28 & 7,68 & 4,41 & 76,78 & $-6,3$ \\
\hline 235 & 7,31 & 10,42 & 4,01 & 2,32 & 40,10 & $-5,3$ \\
\hline 236 & 44,07 & 26,88 & 9,85 & 5,70 & 98,47 & $-6,6$ \\
\hline 237 & 4,43 & 7,76 & 2,83 & 1,99 & 28,27 & $-4,8$ \\
\hline 238 & 9,08 & 11,48 & 4,17 & 2,77 & 41,74 & $-5,4$ \\
\hline 239 & 167,42 & 53,04 & 17,48 & 12,19 & 174,81 & $-7,4$ \\
\hline
\end{tabular}




\begin{tabular}{|c|c|c|c|c|c|c|}
\hline 240 & 20,15 & 20,89 & 8,97 & 2,86 & 89,70 & $-6,5$ \\
\hline 241 & 35,21 & 25,11 & 9,67 & 4,64 & 96,68 & $-6,6$ \\
\hline 242 & 9,97 & 16,41 & 4,16 & 3,05 & 41,59 & $-5,4$ \\
\hline 243 & 3,32 & 6,43 & 2,66 & 1,59 & 26,55 & $-4,7$ \\
\hline 244 & 9,74 & 12,25 & 4,85 & 2,56 & 48,45 & $-5,6$ \\
\hline 245 & 3,32 & 6,93 & 2,42 & 1,75 & 24,16 & $-4,6$ \\
\hline 246 & 25,91 & 20,22 & 8,11 & 4,07 & 81,11 & $-6,3$ \\
\hline 247 & 43,63 & 26,05 & 9,25 & 6,00 & 92,54 & $-6,5$ \\
\hline 248 & 31,00 & 21,83 & 7,77 & 5,08 & 77,73 & $-6,3$ \\
\hline 249 & 11,29 & 12,53 & 5,02 & 2,87 & 50,17 & $-5,6$ \\
\hline 250 & 3,32 & 7,60 & 2,95 & 1,43 & 29,52 & $-4,9$ \\
\hline 251 & 6,20 & 10,03 & 4,14 & 1,91 & 41,38 & $-5,4$ \\
\hline 252 & 12,40 & 13,63 & 4,70 & 3,36 & 46,95 & $-5,6$ \\
\hline 253 & 56,91 & 31,97 & 10,70 & 6,77 & 106,97 & $-6,7$ \\
\hline 254 & 99,21 & 44,34 & 17,69 & 7,14 & 176,86 & $-7,5$ \\
\hline 255 & 41,63 & 25,43 & 8,01 & 6,62 & 80,07 & $-6,3$ \\
\hline 256 & 7,31 & 9,87 & 3,63 & 2,56 & 36,34 & $-5,2$ \\
\hline 257 & 139,29 & 45,88 & 15,93 & 11,13 & 159,33 & $-7,3$ \\
\hline 258 & 44,29 & 26,99 & 10,44 & 5,40 & 104,39 & $-6,7$ \\
\hline 259 & 57,80 & 29,86 & 11,53 & 6,38 & 115,32 & $-6,8$ \\
\hline 260 & 58,02 & 30,76 & 11,95 & 6,18 & 119,45 & $-6,9$ \\
\hline 261 & 706,44 & 118,04 & 44,23 & 20,33 & 442,33 & $-8,8$ \\
\hline 262 & 72,64 & 33,14 & 12,36 & 7,48 & 123,61 & $-6,9$ \\
\hline 263 & 72,42 & 37,80 & 13,11 & 7,03 & 131,12 & $-7,0$ \\
\hline 264 & 17,49 & 20,56 & 8,98 & 2,48 & 89,78 & $-6,5$ \\
\hline 265 & 49,83 & 29,81 & 11,09 & 5,72 & 110,87 & $-6,8$ \\
\hline 266 & 96,11 & 40,51 & 12,19 & 10,04 & 121,92 & $-6,9$ \\
\hline 267 & 79,94 & 46,45 & 21,29 & 4,78 & 212,92 & $-7,7$ \\
\hline 268 & 8,19 & 10,53 & 3,86 & 2,71 & 38,56 & $-5,3$ \\
\hline 269 & 25,69 & 19,28 & 6,12 & 5,34 & 61,23 & $-5,9$ \\
\hline 270 & 19,49 & 18,57 & 7,31 & 3,40 & 73,09 & $-6,2$ \\
\hline 271 & 249,36 & 62,50 & 19,10 & 16,62 & 191,02 & $-7,6$ \\
\hline 272 & 93,45 & 39,29 & 12,69 & 9,37 & 126,93 & $-7,0$ \\
\hline 273 & 75,74 & 32,36 & 10,39 & 9,28 & 103,89 & $-6,7$ \\
\hline 274 & 22,81 & 18,02 & 6,72 & 4,32 & 67,15 & $-6,1$ \\
\hline 275 & 152,80 & 48,15 & 17,45 & 11,15 & 174,52 & $-7,4$ \\
\hline 276 & 5,54 & 8,54 & 3,41 & 2,07 & 34,12 & $-5,1$ \\
\hline 277 & 13,73 & 13,63 & 4,71 & 3,71 & 47,11 & $-5,6$ \\
\hline 278 & 9,74 & 12,42 & 5,10 & 2,43 & 51,01 & $-5,7$ \\
\hline 279 & 41,19 & 24,33 & 8,45 & 6,21 & 84,50 & $-6,4$ \\
\hline 280 & 18,16 & 15,91 & 5,31 & 4,36 & 53,07 & $-5,7$ \\
\hline 281 & 37,43 & 24,05 & 8,40 & 5,67 & 84,04 & $-6,4$ \\
\hline 282 & 93,67 & 38,67 & 14,66 & 8,14 & 146,60 & $-7,2$ \\
\hline 283 & 4,65 & 8,26 & 3,15 & 1,88 & 31,45 & $-5,0$ \\
\hline 284 & 22,59 & 18,57 & 5,79 & 4,97 & 57,92 & $-5,9$ \\
\hline 285 & 15,94 & 15,63 & 5,96 & 3,41 & 59,56 & $-5,9$ \\
\hline
\end{tabular}


Clasts data for sample SM-82_Br01 used for grain size distribution and fractal dimension analysis

$\phi=-\log _{2}(\mathrm{diam})$, considering the major axis of the best fit ellipses, in millimeters.

Best fit ellipse

\begin{tabular}{|c|c|c|c|c|c|c|}
\hline Label & Area $\left(\mathbf{c m}^{2}\right)$ & $\begin{array}{l}\text { Perimeter } \\
\quad(\mathrm{cm})\end{array}$ & $\begin{array}{l}\text { Major axis } \\
\quad \text { (cm) }\end{array}$ & $\begin{array}{l}\text { Minor axis } \\
\quad \text { (cm) }\end{array}$ & $\begin{array}{c}\text { Diameter } \\
(\mathbf{m m})\end{array}$ & Phi \\
\hline 1 & 33,44 & 25,03 & 8,50 & 5,01 & 85,02 & $-6,4$ \\
\hline 2 & 0,24 & 1,97 & 0,70 & 0,44 & 6,96 & $-2,8$ \\
\hline 3 & 0,39 & 2,55 & 0,83 & 0,60 & 8,33 & $-3,1$ \\
\hline 4 & 0,08 & 1,08 & 0,41 & 0,26 & 4,14 & $-2,0$ \\
\hline 5 & 8,46 & 11,58 & 3,88 & 2,77 & 38,84 & $-5,3$ \\
\hline 6 & 2,02 & 6,39 & 2,37 & 1,09 & 23,70 & $-4,6$ \\
\hline 7 & 7,54 & 11,17 & 4,01 & 2,39 & 40,13 & $-5,3$ \\
\hline 8 & 3,24 & 8,31 & 3,35 & 1,23 & 33,45 & $-5,1$ \\
\hline 9 & 0,49 & 2,79 & 1,06 & 0,59 & 10,62 & $-3,4$ \\
\hline 10 & 463,67 & 104,08 & 26,28 & 22,46 & 262,81 & $-8,0$ \\
\hline 11 & 0,46 & 2,63 & 0,99 & 0,59 & 9,86 & $-3,3$ \\
\hline 12 & 0,25 & 1,98 & 0,76 & 0,42 & 7,58 & $-2,9$ \\
\hline 13 & 0,31 & 2,36 & 0,91 & 0,44 & 9,09 & $-3,2$ \\
\hline 14 & 3,82 & 8,91 & 2,93 & 1,66 & 29,29 & $-4,9$ \\
\hline 15 & 0,60 & 2,93 & 1,07 & 0,71 & 10,69 & $-3,4$ \\
\hline 16 & 0,08 & 1,23 & 0,46 & 0,21 & 4,61 & $-2,2$ \\
\hline 17 & 0,55 & 2,97 & 1,09 & 0,64 & 10,91 & $-3,4$ \\
\hline 18 & 9,54 & 14,32 & 4,81 & 2,53 & 48,06 & $-5,6$ \\
\hline 19 & 0,21 & 1,80 & 0,57 & 0,47 & 5,71 & $-2,5$ \\
\hline 20 & 1,80 & 5,50 & 1,76 & 1,30 & 17,58 & $-4,1$ \\
\hline 21 & 78,71 & 49,57 & 10,54 & 9,51 & 105,40 & $-6,7$ \\
\hline 22 & 2,69 & 6,64 & 2,15 & 1,59 & 21,52 & $-4,4$ \\
\hline 23 & 7,86 & 12,11 & 3,75 & 2,67 & 37,46 & $-5,2$ \\
\hline 24 & 0,39 & 2,53 & 0,90 & 0,54 & 9,03 & $-3,2$ \\
\hline 25 & 0,34 & 2,39 & 0,94 & 0,47 & 9,37 & $-3,2$ \\
\hline 26 & 0,50 & 2,82 & 1,03 & 0,61 & 10,27 & $-3,4$ \\
\hline 27 & 0,89 & 4,57 & 1,61 & 0,71 & 16,05 & $-4,0$ \\
\hline 28 & 0,51 & 3,35 & 1,32 & 0,50 & 13,16 & $-3,7$ \\
\hline 29 & 0,32 & 2,20 & 0,75 & 0,54 & 7,54 & $-2,9$ \\
\hline 30 & 1,04 & 4,13 & 1,36 & 0,97 & 13,57 & $-3,8$ \\
\hline 31 & 1,04 & 5,00 & 2,15 & 0,62 & 21,50 & $-4,4$ \\
\hline 32 & 0,11 & 1,37 & 0,51 & 0,26 & 5,12 & $-2,4$ \\
\hline 33 & 108,92 & 43,02 & 13,41 & 10,34 & 134,11 & $-7,1$ \\
\hline 34 & 1,05 & 4,42 & 1,34 & 0,99 & 13,44 & $-3,7$ \\
\hline 35 & 5,01 & 9,44 & 2,74 & 2,33 & 27,40 & $-4,8$ \\
\hline 36 & 20,97 & 21,39 & 6,29 & 4,24 & 62,90 & $-6,0$ \\
\hline 37 & 0,29 & 2,24 & 0,72 & 0,51 & 7,19 & $-2,8$ \\
\hline 38 & 13,05 & 24,74 & 8,84 & 1,88 & 88,37 & $-6,5$ \\
\hline 39 & 3,71 & 10,82 & 2,56 & 1,84 & 25,64 & $-4,7$ \\
\hline 40 & 28,97 & 24,39 & 9,34 & 3,95 & 93,35 & $-6,5$ \\
\hline 41 & 0,66 & 3,47 & 1,19 & 0,70 & 11,89 & $-3,6$ \\
\hline 42 & 1,59 & 6,13 & 2,28 & 0,89 & 22,81 & $-4,5$ \\
\hline 43 & 5,32 & 10,85 & 3,68 & 1,84 & 36,75 & $-5,2$ \\
\hline 44 & 0,43 & 2,89 & 1,18 & 0,47 & 11,80 & $-3,6$ \\
\hline 45 & 0,89 & 4,07 & 1,27 & 0,90 & 12,70 & $-3,7$ \\
\hline 46 & 0,57 & 3,01 & 1,06 & 0,68 & 10,60 & $-3,4$ \\
\hline 47 & 0,08 & 1,22 & 0,42 & 0,24 & 4,23 & $-2,1$ \\
\hline 48 & 1,80 & 6,39 & 2,07 & 1,11 & 20,67 & $-4,4$ \\
\hline 49 & 1,44 & 4,93 & 1,45 & 1,26 & 14,52 & $-3,9$ \\
\hline 50 & 0,13 & 1,59 & 0,54 & 0,30 & 5,43 & $-2,4$ \\
\hline 51 & 1,34 & 4,80 & 1,64 & 1,04 & 16,39 & $-4,0$ \\
\hline 52 & 0,54 & 3,21 & 1,12 & 0,62 & 11,20 & $-3,5$ \\
\hline 53 & 0,61 & 3,05 & 1,09 & 0,71 & 10,85 & $-3,4$ \\
\hline
\end{tabular}




\begin{tabular}{|c|c|c|c|c|c|c|}
\hline 54 & 16,31 & 20,91 & 6,66 & 3,12 & 66,56 & $-6,1$ \\
\hline 55 & 22,44 & 19,81 & 7,17 & 3,98 & 71,72 & $-6,2$ \\
\hline 56 & 2,50 & 6,49 & 1,98 & 1,61 & 19,83 & $-4,3$ \\
\hline 57 & 0,30 & 2,56 & 1,08 & 0,35 & 10,83 & $-3,4$ \\
\hline 58 & 15,63 & 23,36 & 7,28 & 2,73 & 72,80 & $-6,2$ \\
\hline 59 & 1,88 & 6,89 & 1,78 & 1,35 & 17,76 & $-4,2$ \\
\hline 60 & 0,17 & 1,99 & 0,88 & 0,25 & 8,81 & $-3,1$ \\
\hline 61 & 0,45 & 2,90 & 1,14 & 0,51 & 11,35 & $-3,5$ \\
\hline 62 & 0,59 & 3,23 & 1,18 & 0,64 & 11,82 & $-3,6$ \\
\hline 63 & 0,14 & 1,40 & 0,48 & 0,38 & 4,76 & $-2,3$ \\
\hline 64 & 11,26 & 15,42 & 5,46 & 2,63 & 54,61 & $-5,8$ \\
\hline 65 & 1,78 & 5,67 & 1,58 & 1,44 & 15,75 & $-4,0$ \\
\hline 66 & 0,84 & 3,74 & 1,41 & 0,76 & 14,12 & $-3,8$ \\
\hline 67 & 0,37 & 2,62 & 0,97 & 0,48 & 9,68 & $-3,3$ \\
\hline 68 & 0,25 & 1,99 & 0,69 & 0,46 & 6,92 & $-2,8$ \\
\hline 69 & 0,34 & 2,41 & 0,92 & 0,48 & 9,22 & $-3,2$ \\
\hline 70 & 15,00 & 16,25 & 5,48 & 3,49 & 54,76 & $-5,8$ \\
\hline 71 & 4,22 & 8,21 & 2,85 & 1,89 & 28,46 & $-4,8$ \\
\hline 72 & 10,01 & 12,59 & 4,20 & 3,04 & 41,97 & $-5,4$ \\
\hline 73 & 1,30 & 4,38 & 1,47 & 1,12 & 14,72 & $-3,9$ \\
\hline 74 & 44,12 & 48,13 & 12,96 & 4,34 & 129,58 & $-7,0$ \\
\hline 75 & 0,19 & 1,60 & 0,53 & 0,47 & 5,25 & $-2,4$ \\
\hline 76 & 0,19 & 1,62 & 0,54 & 0,45 & 5,39 & $-2,4$ \\
\hline 77 & 1,28 & 4,69 & 1,43 & 1,14 & 14,33 & $-3,8$ \\
\hline 78 & 0,82 & 3,54 & 1,11 & 0,94 & 11,13 & $-3,5$ \\
\hline 79 & 1,75 & 5,94 & 1,93 & 1,15 & 19,29 & $-4,3$ \\
\hline 80 & 28,93 & 22,46 & 6,96 & 5,29 & 69,58 & $-6,1$ \\
\hline 81 & 0,74 & 3,66 & 1,13 & 0,84 & 11,29 & $-3,5$ \\
\hline 82 & 5,11 & 11,30 & 3,71 & 1,75 & 37,10 & $-5,2$ \\
\hline 83 & 0,99 & 4,12 & 1,43 & 0,88 & 14,31 & $-3,8$ \\
\hline 84 & 0,35 & 2,55 & 0,99 & 0,45 & 9,91 & $-3,3$ \\
\hline 85 & 11,58 & 16,81 & 6,73 & 2,19 & 67,27 & $-6,1$ \\
\hline 86 & 10,80 & 15,02 & 5,30 & 2,59 & 53,00 & $-5,7$ \\
\hline 87 & 0,05 & 0,82 & 0,32 & 0,18 & 3,18 & $-1,7$ \\
\hline 88 & 0,13 & 1,35 & 0,43 & 0,37 & 4,32 & $-2,1$ \\
\hline 89 & 0,10 & 1,43 & 0,40 & 0,31 & 4,00 & $-2,0$ \\
\hline 90 & 4,41 & 8,60 & 3,07 & 1,83 & 30,68 & $-4,9$ \\
\hline 91 & 0,15 & 1,49 & 0,52 & 0,36 & 5,15 & $-2,4$ \\
\hline 92 & 0,25 & 1,93 & 0,70 & 0,45 & 6,98 & $-2,8$ \\
\hline 93 & 0,78 & 3,61 & 1,44 & 0,69 & 14,37 & $-3,8$ \\
\hline 94 & 0,22 & 1,73 & 0,56 & 0,50 & 5,61 & $-2,5$ \\
\hline 95 & 1,17 & 4,29 & 1,28 & 1,16 & 12,77 & $-3,7$ \\
\hline 96 & 1,24 & 4,80 & 1,79 & 0,88 & 17,89 & $-4,2$ \\
\hline 97 & 0,62 & 3,48 & 1,15 & 0,68 & 11,52 & $-3,5$ \\
\hline 98 & 0,45 & 2,95 & 1,18 & 0,48 & 11,84 & $-3,6$ \\
\hline 99 & 0,12 & 1,31 & 0,42 & 0,37 & 4,20 & $-2,1$ \\
\hline 100 & 0,19 & 1,91 & 0,77 & 0,32 & 7,68 & $-2,9$ \\
\hline 101 & 0,49 & 2,69 & 0,92 & 0,68 & 9,20 & $-3,2$ \\
\hline 102 & 27,26 & 22,27 & 6,74 & 5,15 & 67,40 & $-6,1$ \\
\hline 103 & 0,11 & 1,29 & 0,42 & 0,33 & 4,23 & $-2,1$ \\
\hline 104 & 0,26 & 1,90 & 0,62 & 0,54 & 6,19 & $-2,6$ \\
\hline 105 & 6,31 & 12,51 & 4,07 & 1,97 & 40,69 & $-5,3$ \\
\hline 106 & 1,03 & 4,18 & 1,30 & 1,01 & 12,97 & $-3,7$ \\
\hline 107 & 2,38 & 6,24 & 2,10 & 1,44 & 20,99 & $-4,4$ \\
\hline 108 & 1,09 & 3,99 & 1,31 & 1,06 & 13,09 & $-3,7$ \\
\hline 109 & 0,90 & 4,24 & 1,65 & 0,70 & 16,46 & $-4,0$ \\
\hline 110 & 0,88 & 4,46 & 1,78 & 0,63 & 17,83 & $-4,2$ \\
\hline 111 & 0,27 & 2,01 & 0,71 & 0,48 & 7,10 & $-2,8$ \\
\hline 112 & 2,22 & 6,23 & 1,98 & 1,43 & 19,77 & $-4,3$ \\
\hline 113 & 0,08 & 1,02 & 0,35 & 0,30 & 3,50 & $-1,8$ \\
\hline 114 & 3,63 & 8,34 & 2,59 & 1,78 & 25,93 & $-4,7$ \\
\hline 115 & 1,50 & 5,40 & 2,09 & 0,91 & 20,93 & $-4,4$ \\
\hline
\end{tabular}




\begin{tabular}{|c|c|c|c|c|c|c|}
\hline 116 & 0,16 & 1,68 & 0,58 & 0,36 & 5,76 & $-2,5$ \\
\hline 117 & 1,16 & 4,49 & 1,75 & 0,84 & 17,45 & $-4,1$ \\
\hline 118 & 0,25 & 1,85 & 0,70 & 0,45 & 6,96 & $-2,8$ \\
\hline 119 & 0,43 & 2,90 & 1,00 & 0,55 & 9,95 & $-3,3$ \\
\hline 120 & 1,44 & 4,73 & 1,55 & 1,18 & 15,46 & $-4,0$ \\
\hline 121 & 0,93 & 3,83 & 1,36 & 0,87 & 13,62 & $-3,8$ \\
\hline 122 & 4,15 & 8,75 & 2,46 & 2,15 & 24,61 & $-4,6$ \\
\hline 123 & 0,53 & 2,90 & 0,98 & 0,68 & 9,84 & $-3,3$ \\
\hline 124 & 0,55 & 2,91 & 1,01 & 0,69 & 10,11 & $-3,3$ \\
\hline 125 & 0,46 & 2,81 & 1,07 & 0,54 & 10,73 & $-3,4$ \\
\hline 126 & 0,10 & 1,29 & 0,43 & 0,30 & 4,25 & $-2,1$ \\
\hline 127 & 57,03 & 35,43 & 11,52 & 6,31 & 115,17 & $-6,8$ \\
\hline 128 & 9,72 & 13,90 & 4,50 & 2,75 & 45,02 & $-5,5$ \\
\hline 129 & 0,61 & 3,18 & 1,08 & 0,72 & 10,77 & $-3,4$ \\
\hline 130 & 0,11 & 1,26 & 0,44 & 0,31 & 4,40 & $-2,1$ \\
\hline 131 & 0,19 & 1,80 & 0,71 & 0,34 & 7,14 & $-2,8$ \\
\hline 132 & 0,11 & 1,36 & 0,47 & 0,29 & 4,74 & $-2,2$ \\
\hline 133 & 0,06 & 0,89 & 0,32 & 0,22 & 3,23 & $-1,7$ \\
\hline 134 & 0,33 & 2,99 & 1,23 & 0,34 & 12,33 & $-3,6$ \\
\hline 135 & 59,82 & 34,42 & 10,21 & 7,46 & 102,08 & $-6,7$ \\
\hline 136 & 0,34 & 2,39 & 0,93 & 0,47 & 9,32 & $-3,2$ \\
\hline 137 & 3,10 & 7,27 & 2,18 & 1,81 & 21,76 & $-4,4$ \\
\hline 138 & 0,13 & 1,62 & 0,62 & 0,27 & 6,17 & $-2,6$ \\
\hline 139 & 0,21 & 2,12 & 0,89 & 0,30 & 8,85 & $-3,1$ \\
\hline 140 & 0,33 & 2,24 & 0,77 & 0,54 & 7,74 & $-3,0$ \\
\hline 141 & 3,01 & 7,99 & 2,68 & 1,43 & 26,82 & $-4,7$ \\
\hline 142 & 13,46 & 21,56 & 5,57 & 3,08 & 55,65 & $-5,8$ \\
\hline 143 & 0,17 & 1,57 & 0,55 & 0,40 & 5,45 & $-2,4$ \\
\hline 144 & 0,35 & 2,30 & 0,74 & 0,60 & 7,41 & $-2,9$ \\
\hline 145 & 0,07 & 0,98 & 0,36 & 0,26 & 3,57 & $-1,8$ \\
\hline 146 & 0,50 & 2,86 & 0,95 & 0,67 & 9,46 & $-3,2$ \\
\hline 147 & 87,69 & 43,06 & 13,35 & 8,36 & 133,54 & $-7,1$ \\
\hline 148 & 7,21 & 10,83 & 3,36 & 2,73 & 33,57 & $-5,1$ \\
\hline 149 & 0,09 & 1,11 & 0,38 & 0,29 & 3,77 & $-1,9$ \\
\hline 150 & 0,09 & 1,49 & 0,65 & 0,17 & 6,51 & $-2,7$ \\
\hline 151 & 1,12 & 4,40 & 1,55 & 0,92 & 15,47 & $-4,0$ \\
\hline 152 & 0,05 & 0,83 & 0,30 & 0,22 & 2,98 & $-1,6$ \\
\hline 153 & 0,75 & 3,27 & 1,19 & 0,80 & 11,90 & $-3,6$ \\
\hline 154 & 329,57 & 79,00 & 24,07 & 17,43 & 240,73 & $-7,9$ \\
\hline 155 & 0,77 & 3,56 & 1,10 & 0,89 & 11,00 & $-3,5$ \\
\hline 156 & 1,79 & 5,30 & 1,83 & 1,25 & 18,30 & $-4,2$ \\
\hline 157 & 1,18 & 4,71 & 1,71 & 0,88 & 17,14 & $-4,1$ \\
\hline 158 & 3,86 & 7,97 & 2,64 & 1,86 & 26,44 & $-4,7$ \\
\hline 159 & 0,14 & 1,42 & 0,47 & 0,38 & 4,70 & $-2,2$ \\
\hline 160 & 0,23 & 1,91 & 0,73 & 0,39 & 7,34 & $-2,9$ \\
\hline 161 & 0,27 & 2,08 & 0,74 & 0,46 & 7,37 & $-2,9$ \\
\hline 162 & 0,50 & 2,93 & 1,04 & 0,61 & 10,36 & $-3,4$ \\
\hline 163 & 0,05 & 0,90 & 0,38 & 0,16 & 3,78 & $-1,9$ \\
\hline 164 & 0,16 & 1,58 & 0,50 & 0,40 & 5,03 & $-2,3$ \\
\hline 165 & 0,09 & 1,13 & 0,40 & 0,27 & 4,03 & $-2,0$ \\
\hline 166 & 14,73 & 15,51 & 5,09 & 3,68 & 50,94 & $-5,7$ \\
\hline 167 & 0,88 & 4,36 & 1,52 & 0,73 & 15,23 & $-3,9$ \\
\hline 168 & 0,59 & 3,19 & 1,17 & 0,64 & 11,70 & $-3,5$ \\
\hline 169 & 3,62 & 7,85 & 2,27 & 2,03 & 22,74 & $-4,5$ \\
\hline 170 & 12,78 & 16,08 & 6,29 & 2,59 & 62,88 & $-6,0$ \\
\hline 171 & 0,95 & 4,63 & 1,74 & 0,69 & 17,44 & $-4,1$ \\
\hline 172 & 172,27 & 79,61 & 16,74 & 13,10 & 167,43 & $-7,4$ \\
\hline 173 & 0,49 & 2,70 & 0,85 & 0,74 & 8,45 & $-3,1$ \\
\hline 174 & 0,20 & 2,15 & 0,94 & 0,28 & 9,35 & $-3,2$ \\
\hline 175 & 6,08 & 10,70 & 3,75 & 2,07 & 37,45 & $-5,2$ \\
\hline 176 & 12,42 & 14,37 & 4,58 & 3,45 & 45,78 & $-5,5$ \\
\hline 177 & 0,83 & 4,85 & 1,96 & 0,54 & 19,55 & $-4,3$ \\
\hline
\end{tabular}




\begin{tabular}{|c|c|c|c|c|c|c|}
\hline 178 & 0,13 & 1,37 & 0,46 & 0,36 & 4,63 & $-2,2$ \\
\hline 179 & 4,02 & 10,41 & 4,07 & 1,26 & 40,74 & $-5,3$ \\
\hline 180 & 0,04 & 0,74 & 0,29 & 0,19 & 2,85 & $-1,5$ \\
\hline 181 & 0,23 & 1,95 & 0,68 & 0,43 & 6,81 & $-2,8$ \\
\hline 182 & 0,09 & 1,21 & 0,44 & 0,25 & 4,41 & $-2,1$ \\
\hline 183 & 14,61 & 16,91 & 4,74 & 3,92 & 47,41 & $-5,6$ \\
\hline 184 & 0,70 & 3,44 & 1,28 & 0,69 & 12,82 & $-3,7$ \\
\hline 185 & 0,58 & 3,11 & 0,93 & 0,80 & 9,27 & $-3,2$ \\
\hline 186 & 0,31 & 2,25 & 0,81 & 0,49 & 8,10 & $-3,0$ \\
\hline 187 & 1,67 & 5,70 & 2,34 & 0,91 & 23,36 & $-4,5$ \\
\hline 188 & 83,07 & 50,23 & 13,23 & 7,99 & 132,31 & $-7,0$ \\
\hline 189 & 1,90 & 5,54 & 1,95 & 1,24 & 19,45 & $-4,3$ \\
\hline 190 & 0,39 & 2,48 & 0,86 & 0,58 & 8,56 & $-3,1$ \\
\hline 191 & 3,62 & 7,90 & 2,90 & 1,59 & 28,99 & $-4,9$ \\
\hline 192 & 19,23 & 18,78 & 7,39 & 3,31 & 73,94 & $-6,2$ \\
\hline 193 & 3,55 & 8,36 & 2,75 & 1,64 & 27,53 & $-4,8$ \\
\hline 194 & 7,68 & 13,68 & 4,37 & 2,24 & 43,70 & $-5,4$ \\
\hline 195 & 0,54 & 2,79 & 0,98 & 0,70 & 9,78 & $-3,3$ \\
\hline 196 & 0,53 & 3,34 & 0,88 & 0,77 & 8,78 & $-3,1$ \\
\hline 197 & 2,83 & 8,09 & 3,16 & 1,14 & 31,59 & $-5,0$ \\
\hline 198 & 0,38 & 2,36 & 0,76 & 0,63 & 7,56 & $-2,9$ \\
\hline 199 & 1,90 & 6,91 & 2,39 & 1,01 & 23,94 & $-4,6$ \\
\hline 200 & 0,40 & 2,66 & 1,04 & 0,49 & 10,35 & $-3,4$ \\
\hline 201 & 4,82 & 8,69 & 2,89 & 2,13 & 28,90 & $-4,9$ \\
\hline 202 & 6,70 & 9,87 & 3,24 & 2,63 & 32,40 & $-5,0$ \\
\hline 203 & 0,55 & 3,48 & 1,08 & 0,65 & 10,81 & $-3,4$ \\
\hline 204 & 106,52 & 61,53 & 15,41 & 8,80 & 154,11 & $-7,3$ \\
\hline 205 & 0,00 & 0,11 & 0,04 & 0,04 & 0,43 & 1,2 \\
\hline 206 & 2,14 & 5,99 & 2,17 & 1,25 & 21,74 & $-4,4$ \\
\hline 207 & 0,35 & 2,34 & 0,81 & 0,54 & 8,14 & $-3,0$ \\
\hline 208 & 1,45 & 5,40 & 2,13 & 0,86 & 21,34 & $-4,4$ \\
\hline 209 & 1,09 & 4,14 & 1,34 & 1,04 & 13,39 & $-3,7$ \\
\hline 210 & 24,85 & 20,89 & 6,20 & 5,10 & 62,02 & $-6,0$ \\
\hline 211 & 1,66 & 5,00 & 1,74 & 1,22 & 17,36 & $-4,1$ \\
\hline 212 & 0,56 & 3,31 & 0,97 & 0,73 & 9,66 & $-3,3$ \\
\hline 213 & 0,80 & 4,05 & 1,50 & 0,68 & 15,01 & $-3,9$ \\
\hline 214 & 1,28 & 4,47 & 1,50 & 1,08 & 15,04 & $-3,9$ \\
\hline 215 & 0,42 & 2,74 & 1,07 & 0,49 & 10,73 & $-3,4$ \\
\hline 216 & 66,39 & 36,42 & 12,49 & 6,77 & 124,86 & $-7,0$ \\
\hline 217 & 0,58 & 2,80 & 0,93 & 0,79 & 9,34 & $-3,2$ \\
\hline 218 & 0,54 & 3,19 & 1,26 & 0,55 & 12,60 & $-3,7$ \\
\hline 219 & 9,67 & 12,51 & 4,21 & 2,92 & 42,13 & $-5,4$ \\
\hline 220 & 0,19 & 1,78 & 0,66 & 0,37 & 6,56 & $-2,7$ \\
\hline 221 & 11,47 & 14,02 & 4,65 & 3,14 & 46,48 & $-5,5$ \\
\hline 222 & 1,14 & 4,47 & 1,50 & 0,97 & 14,99 & $-3,9$ \\
\hline 223 & 6,45 & 10,72 & 3,69 & 2,23 & 36,86 & $-5,2$ \\
\hline 224 & 0,26 & 1,94 & 0,59 & 0,57 & 5,91 & $-2,6$ \\
\hline 225 & 0,27 & 2,76 & 0,77 & 0,44 & 7,68 & $-2,9$ \\
\hline 226 & 0,20 & 1,74 & 0,55 & 0,46 & 5,54 & $-2,5$ \\
\hline 227 & 4,27 & 9,22 & 3,55 & 1,53 & 35,54 & $-5,2$ \\
\hline 228 & 6,25 & 11,35 & 4,36 & 1,83 & 43,62 & $-5,4$ \\
\hline 229 & 6,61 & 11,51 & 3,73 & 2,26 & 37,27 & $-5,2$ \\
\hline 230 & 4,04 & 9,65 & 2,89 & 1,78 & 28,88 & $-4,9$ \\
\hline 231 & 0,81 & 3,43 & 1,12 & 0,92 & 11,18 & $-3,5$ \\
\hline 232 & 0,42 & 2,53 & 0,79 & 0,68 & 7,87 & $-3,0$ \\
\hline 233 & 0,20 & 1,71 & 0,60 & 0,43 & 5,96 & $-2,6$ \\
\hline 234 & 0,59 & 2,89 & 0,95 & 0,80 & 9,47 & $-3,2$ \\
\hline 235 & 0,28 & 2,87 & 1,33 & 0,27 & 13,29 & $-3,7$ \\
\hline 236 & 0,62 & 3,04 & 1,04 & 0,76 & 10,42 & $-3,4$ \\
\hline 237 & 0,13 & 1,36 & 0,44 & 0,37 & 4,42 & $-2,1$ \\
\hline 238 & 0,22 & 1,97 & 0,81 & 0,35 & 8,06 & $-3,0$ \\
\hline 239 & 0,06 & 0,94 & 0,35 & 0,21 & 3,51 & $-1,8$ \\
\hline
\end{tabular}




\begin{tabular}{|c|c|c|c|c|c|c|}
\hline 240 & 0,07 & 0,97 & 0,36 & 0,23 & 3,58 & $-1,8$ \\
\hline 241 & 0,15 & 1,61 & 0,58 & 0,33 & 5,75 & $-2,5$ \\
\hline 242 & 0,19 & 1,73 & 0,62 & 0,38 & 6,16 & $-2,6$ \\
\hline 243 & 0,72 & 3,40 & 1,24 & 0,74 & 12,41 & $-3,6$ \\
\hline 244 & 1,02 & 3,80 & 1,18 & 1,10 & 11,81 & $-3,6$ \\
\hline 245 & 0,71 & 3,18 & 1,07 & 0,85 & 10,69 & $-3,4$ \\
\hline 246 & 0,62 & 3,41 & 1,34 & 0,59 & 13,36 & $-3,7$ \\
\hline 247 & 1,31 & 5,13 & 1,93 & 0,86 & 19,34 & $-4,3$ \\
\hline 248 & 0,22 & 1,86 & 0,72 & 0,39 & 7,21 & $-2,8$ \\
\hline 249 & 0,17 & 1,57 & 0,54 & 0,39 & 5,39 & $-2,4$ \\
\hline 250 & 13,50 & 16,62 & 6,11 & 2,81 & 61,10 & $-5,9$ \\
\hline 251 & 0,74 & 3,57 & 1,33 & 0,70 & 13,31 & $-3,7$ \\
\hline 252 & 1,32 & 4,35 & 1,50 & 1,13 & 14,95 & $-3,9$ \\
\hline 253 & 0,91 & 3,70 & 1,34 & 0,86 & 13,43 & $-3,7$ \\
\hline 254 & 1,90 & 6,40 & 2,17 & 1,11 & 21,73 & $-4,4$ \\
\hline 255 & 0,14 & 1,59 & 0,58 & 0,31 & 5,81 & $-2,5$ \\
\hline 256 & 0,58 & 3,52 & 1,10 & 0,67 & 11,03 & $-3,5$ \\
\hline 257 & 0,07 & 1,35 & 0,47 & 0,18 & 4,71 & $-2,2$ \\
\hline 258 & 10,92 & 15,62 & 4,63 & 3,00 & 46,30 & $-5,5$ \\
\hline 259 & 138,55 & 50,09 & 17,75 & 9,94 & 177,47 & $-7,5$ \\
\hline 260 & 0,44 & 3,75 & 1,09 & 0,52 & 10,91 & $-3,4$ \\
\hline 261 & 1,86 & 6,50 & 2,13 & 1,11 & 21,33 & $-4,4$ \\
\hline 262 & 3,84 & 8,16 & 2,77 & 1,76 & 27,71 & $-4,8$ \\
\hline 263 & 0,41 & 2,78 & 1,18 & 0,44 & 11,83 & $-3,6$ \\
\hline 264 & 0,13 & 1,33 & 0,49 & 0,32 & 4,92 & $-2,3$ \\
\hline 265 & 0,28 & 2,11 & 0,71 & 0,51 & 7,05 & $-2,8$ \\
\hline 266 & 6,52 & 13,35 & 3,47 & 2,39 & 34,72 & $-5,1$ \\
\hline 267 & 0,26 & 1,94 & 0,69 & 0,48 & 6,91 & $-2,8$ \\
\hline 268 & 1,20 & 5,02 & 2,04 & 0,75 & 20,36 & $-4,3$ \\
\hline 269 & 0,45 & 3,27 & 0,94 & 0,61 & 9,36 & $-3,2$ \\
\hline 270 & 7,67 & 15,64 & 4,90 & 1,99 & 49,03 & $-5,6$ \\
\hline 271 & 0,22 & 1,92 & 0,73 & 0,37 & 7,34 & $-2,9$ \\
\hline 272 & 1,20 & 4,64 & 1,56 & 0,98 & 15,62 & $-4,0$ \\
\hline 273 & 36,24 & 24,81 & 7,60 & 6,07 & 76,01 & $-6,2$ \\
\hline 274 & 0,82 & 4,08 & 1,34 & 0,78 & 13,41 & $-3,7$ \\
\hline 275 & 0,26 & 2,23 & 0,77 & 0,44 & 7,69 & $-2,9$ \\
\hline 276 & 1,67 & 5,12 & 1,73 & 1,23 & 17,33 & $-4,1$ \\
\hline 277 & 2,52 & 7,52 & 3,13 & 1,03 & 31,28 & $-5,0$ \\
\hline 278 & 35,17 & 29,25 & 9,58 & 4,68 & 95,77 & $-6,6$ \\
\hline 279 & 0,11 & 1,23 & 0,42 & 0,32 & 4,20 & $-2,1$ \\
\hline 280 & 4,71 & 9,29 & 2,61 & 2,30 & 26,07 & $-4,7$ \\
\hline 281 & 0,27 & 2,40 & 0,99 & 0,35 & 9,91 & $-3,3$ \\
\hline 282 & 2,48 & 6,01 & 1,84 & 1,71 & 18,43 & $-4,2$ \\
\hline 283 & 0,46 & 3,11 & 1,24 & 0,47 & 12,35 & $-3,6$ \\
\hline 284 & 0,14 & 1,62 & 0,69 & 0,26 & 6,90 & $-2,8$ \\
\hline 285 & 0,15 & 1,59 & 0,52 & 0,37 & 5,19 & $-2,4$ \\
\hline 286 & 0,14 & 1,59 & 0,48 & 0,37 & 4,75 & $-2,2$ \\
\hline 287 & 0,10 & 1,24 & 0,48 & 0,26 & 4,79 & $-2,3$ \\
\hline 288 & 10,50 & 13,42 & 4,71 & 2,84 & 47,13 & $-5,6$ \\
\hline 289 & 1171,01 & 153,43 & 44,75 & 33,32 & 447,50 & $-8,8$ \\
\hline 290 & 7,20 & 11,05 & 3,76 & 2,44 & 37,63 & $-5,2$ \\
\hline 291 & 5,06 & 9,22 & 2,69 & 2,40 & 26,86 & $-4,7$ \\
\hline 292 & 1,29 & 4,51 & 1,55 & 1,06 & 15,45 & $-3,9$ \\
\hline 293 & 0,18 & 1,72 & 0,69 & 0,34 & 6,87 & $-2,8$ \\
\hline 294 & 0,63 & 4,30 & 1,41 & 0,57 & 14,10 & $-3,8$ \\
\hline 295 & 0,67 & 3,32 & 1,13 & 0,75 & 11,29 & $-3,5$ \\
\hline 296 & 0,19 & 1,63 & 0,57 & 0,42 & 5,71 & $-2,5$ \\
\hline 297 & 10,64 & 14,40 & 4,62 & 2,93 & 46,18 & $-5,5$ \\
\hline 298 & 0,12 & 1,33 & 0,48 & 0,32 & 4,79 & $-2,3$ \\
\hline 299 & 0,22 & 1,76 & 0,65 & 0,43 & 6,45 & $-2,7$ \\
\hline 300 & 0,25 & 2,03 & 0,75 & 0,43 & 7,47 & $-2,9$ \\
\hline 301 & 0,53 & 2,89 & 1,03 & 0,66 & 10,34 & $-3,4$ \\
\hline
\end{tabular}




\begin{tabular}{|c|c|c|c|c|c|c|}
\hline 302 & 0,90 & 3,65 & 1,22 & 0,94 & 12,21 & $-3,6$ \\
\hline 303 & 0,49 & 2,83 & 1,15 & 0,54 & 11,47 & $-3,5$ \\
\hline 304 & 0,11 & 1,32 & 0,52 & 0,27 & 5,15 & $-2,4$ \\
\hline 305 & 0,36 & 2,38 & 0,73 & 0,63 & 7,25 & $-2,9$ \\
\hline 306 & 0,12 & 1,27 & 0,45 & 0,35 & 4,46 & $-2,2$ \\
\hline 307 & 0,17 & 1,82 & 0,68 & 0,33 & 6,79 & $-2,8$ \\
\hline 308 & 0,22 & 1,77 & 0,63 & 0,46 & 6,26 & $-2,6$ \\
\hline 309 & 0,09 & 1,15 & 0,45 & 0,24 & 4,50 & $-2,2$ \\
\hline 310 & 1,77 & 5,69 & 1,71 & 1,32 & 17,13 & $-4,1$ \\
\hline 311 & 0,04 & 0,74 & 0,28 & 0,18 & 2,77 & $-1,5$ \\
\hline 312 & 4,86 & 9,04 & 2,73 & 2,27 & 27,30 & $-4,8$ \\
\hline 313 & 1,06 & 4,31 & 1,62 & 0,83 & 16,17 & $-4,0$ \\
\hline 314 & 0,10 & 1,29 & 0,49 & 0,26 & 4,89 & $-2,3$ \\
\hline 315 & 0,46 & 3,24 & 1,00 & 0,59 & 9,98 & $-3,3$ \\
\hline 316 & 0,75 & 3,71 & 1,39 & 0,69 & 13,89 & $-3,8$ \\
\hline 317 & 0,04 & 0,74 & 0,30 & 0,16 & 3,03 & $-1,6$ \\
\hline 318 & 0,25 & 1,99 & 0,77 & 0,40 & 7,74 & $-3,0$ \\
\hline 319 & 0,10 & 1,21 & 0,39 & 0,31 & 3,94 & $-2,0$ \\
\hline 320 & 0,13 & 1,37 & 0,46 & 0,35 & 4,64 & $-2,2$ \\
\hline 321 & 0,06 & 0,89 & 0,32 & 0,24 & 3,15 & $-1,7$ \\
\hline 322 & 2,90 & 6,99 & 2,30 & 1,61 & 23,00 & $-4,5$ \\
\hline 323 & 2,12 & 6,16 & 2,08 & 1,30 & 20,78 & $-4,4$ \\
\hline 324 & 2,65 & 7,89 & 2,99 & 1,13 & 29,87 & $-4,9$ \\
\hline 325 & 0,17 & 1,64 & 0,56 & 0,38 & 5,57 & $-2,5$ \\
\hline 326 & 3,69 & 8,88 & 3,42 & 1,37 & 34,16 & $-5,1$ \\
\hline 327 & 10,87 & 13,44 & 4,21 & 3,29 & 42,08 & $-5,4$ \\
\hline 328 & 0,75 & 3,61 & 1,31 & 0,73 & 13,05 & $-3,7$ \\
\hline 329 & 0,19 & 2,15 & 0,88 & 0,27 & 8,82 & $-3,1$ \\
\hline 330 & 1,29 & 4,43 & 1,36 & 1,21 & 13,57 & $-3,8$ \\
\hline 331 & 0,27 & 2,00 & 0,64 & 0,53 & 6,41 & $-2,7$ \\
\hline 332 & 0,27 & 1,97 & 0,62 & 0,55 & 6,19 & $-2,6$ \\
\hline 333 & 0,39 & 2,50 & 0,89 & 0,55 & 8,90 & $-3,2$ \\
\hline 334 & 0,30 & 2,25 & 0,77 & 0,49 & 7,72 & $-2,9$ \\
\hline 335 & 7,94 & 13,39 & 3,80 & 2,66 & 38,01 & $-5,2$ \\
\hline 336 & 0,54 & 3,09 & 1,20 & 0,58 & 11,97 & $-3,6$ \\
\hline 337 & 0,35 & 2,39 & 0,86 & 0,53 & 8,55 & $-3,1$ \\
\hline 338 & 0,18 & 1,81 & 0,66 & 0,36 & 6,59 & $-2,7$ \\
\hline 339 & 0,22 & 2,57 & 1,16 & 0,24 & 11,58 & $-3,5$ \\
\hline 340 & 11,45 & 13,94 & 4,95 & 2,95 & 49,49 & $-5,6$ \\
\hline 341 & 0,07 & 1,01 & 0,38 & 0,25 & 3,78 & $-1,9$ \\
\hline 342 & 10,91 & 16,68 & 5,78 & 2,40 & 57,79 & $-5,9$ \\
\hline 343 & 0,19 & 1,57 & 0,53 & 0,44 & 5,34 & $-2,4$ \\
\hline 344 & 0,39 & 2,50 & 0,96 & 0,52 & 9,57 & $-3,3$ \\
\hline 345 & 0,77 & 3,81 & 1,47 & 0,67 & 14,67 & $-3,9$ \\
\hline 346 & 0,71 & 3,26 & 1,03 & 0,88 & 10,33 & $-3,4$ \\
\hline 347 & 0,12 & 1,72 & 0,69 & 0,22 & 6,91 & $-2,8$ \\
\hline 348 & 5,34 & 12,50 & 5,18 & 1,31 & 51,75 & $-5,7$ \\
\hline 349 & 0,06 & 1,10 & 0,40 & 0,20 & 4,01 & $-2,0$ \\
\hline 350 & 1,58 & 5,39 & 1,90 & 1,06 & 18,95 & $-4,2$ \\
\hline 351 & 4,62 & 9,60 & 3,08 & 1,91 & 30,81 & $-4,9$ \\
\hline 352 & 0,87 & 3,89 & 1,22 & 0,91 & 12,20 & $-3,6$ \\
\hline 353 & 0,67 & 3,93 & 1,43 & 0,60 & 14,28 & $-3,8$ \\
\hline 354 & 0,63 & 3,26 & 1,27 & 0,63 & 12,71 & $-3,7$ \\
\hline 355 & 4,01 & 8,34 & 2,80 & 1,82 & 27,99 & $-4,8$ \\
\hline 356 & 0,21 & 1,78 & 0,59 & 0,45 & 5,92 & $-2,6$ \\
\hline 357 & 0,27 & 1,95 & 0,63 & 0,54 & 6,26 & $-2,6$ \\
\hline 358 & 2,68 & 7,98 & 2,72 & 1,25 & 27,20 & $-4,8$ \\
\hline 359 & 0,05 & 1,01 & 0,41 & 0,17 & 4,05 & $-2,0$ \\
\hline 360 & 10,22 & 12,70 & 4,19 & 3,11 & 41,90 & $-5,4$ \\
\hline 361 & 1,25 & 4,43 & 1,65 & 0,97 & 16,51 & $-4,0$ \\
\hline 362 & 0,44 & 2,78 & 1,14 & 0,50 & 11,41 & $-3,5$ \\
\hline 363 & 0,52 & 2,80 & 0,89 & 0,74 & 8,92 & $-3,2$ \\
\hline
\end{tabular}




\begin{tabular}{|c|c|c|c|c|c|c|}
\hline 364 & 72,75 & 40,91 & 13,62 & 6,80 & 136,17 & $-7,1$ \\
\hline 365 & 3,35 & 6,96 & 2,33 & 1,83 & 23,31 & $-4,5$ \\
\hline 366 & 0,22 & 1,83 & 0,62 & 0,45 & 6,18 & $-2,6$ \\
\hline 367 & 2,52 & 6,64 & 2,07 & 1,55 & 20,72 & $-4,4$ \\
\hline 368 & 0,19 & 1,76 & 0,61 & 0,39 & 6,12 & $-2,6$ \\
\hline 369 & 0,23 & 1,86 & 0,70 & 0,41 & 7,01 & $-2,8$ \\
\hline 370 & 0,16 & 1,72 & 0,58 & 0,34 & 5,82 & $-2,5$ \\
\hline 371 & 0,06 & 0,89 & 0,32 & 0,23 & 3,15 & $-1,7$ \\
\hline 372 & 4,60 & 8,75 & 2,69 & 2,18 & 26,89 & $-4,7$ \\
\hline 373 & 118,68 & 47,42 & 15,54 & 9,72 & 155,40 & $-7,3$ \\
\hline 374 & 2,72 & 6,59 & 2,25 & 1,54 & 22,46 & $-4,5$ \\
\hline 375 & 0,34 & 2,48 & 0,89 & 0,48 & 8,94 & $-3,2$ \\
\hline 376 & 11,06 & 13,54 & 4,74 & 2,97 & 47,38 & $-5,6$ \\
\hline 377 & 6,01 & 10,03 & 3,56 & 2,15 & 35,62 & $-5,2$ \\
\hline 378 & 0,38 & 2,41 & 0,85 & 0,57 & 8,54 & $-3,1$ \\
\hline 379 & 0,64 & 3,11 & 1,19 & 0,68 & 11,92 & $-3,6$ \\
\hline 380 & 0,03 & 0,69 & 0,26 & 0,17 & 2,55 & $-1,4$ \\
\hline 381 & 11,83 & 15,35 & 6,06 & 2,48 & 60,64 & $-5,9$ \\
\hline 382 & 0,08 & 1,09 & 0,43 & 0,24 & 4,26 & $-2,1$ \\
\hline 383 & 0,02 & 0,61 & 0,27 & 0,11 & 2,65 & $-1,4$ \\
\hline 384 & 0,34 & 2,41 & 0,86 & 0,50 & 8,64 & $-3,1$ \\
\hline 385 & 0,32 & 2,37 & 0,87 & 0,47 & 8,72 & $-3,1$ \\
\hline 386 & 0,78 & 4,19 & 1,78 & 0,56 & 17,78 & $-4,2$ \\
\hline 387 & 0,12 & 1,39 & 0,50 & 0,30 & 4,97 & $-2,3$ \\
\hline 388 & 1,11 & 4,31 & 1,36 & 1,04 & 13,57 & $-3,8$ \\
\hline 389 & 0,30 & 2,24 & 0,89 & 0,42 & 8,88 & $-3,2$ \\
\hline 390 & 0,81 & 3,97 & 1,30 & 0,79 & 12,98 & $-3,7$ \\
\hline 391 & 97,14 & 40,97 & 13,18 & 9,38 & 131,80 & $-7,0$ \\
\hline 392 & 0,32 & 2,29 & 0,78 & 0,52 & 7,75 & $-3,0$ \\
\hline 393 & 0,04 & 0,99 & 0,37 & 0,16 & 3,65 & $-1,9$ \\
\hline 394 & 0,18 & 1,72 & 0,58 & 0,39 & 5,81 & $-2,5$ \\
\hline 395 & 1,81 & 5,55 & 2,16 & 1,06 & 21,61 & $-4,4$ \\
\hline 396 & 16,21 & 16,57 & 5,83 & 3,54 & 58,31 & $-5,9$ \\
\hline 397 & 0,28 & 2,81 & 1,20 & 0,30 & 12,04 & $-3,6$ \\
\hline 398 & 0,22 & 1,90 & 0,82 & 0,34 & 8,15 & $-3,0$ \\
\hline 399 & 56,91 & 35,14 & 9,78 & 7,41 & 97,81 & $-6,6$ \\
\hline 400 & 10,74 & 15,13 & 5,03 & 2,72 & 50,32 & $-5,7$ \\
\hline 401 & 0,12 & 1,34 & 0,41 & 0,37 & 4,12 & $-2,0$ \\
\hline 402 & 3,08 & 7,65 & 3,02 & 1,30 & 30,23 & $-4,9$ \\
\hline 403 & 0,56 & 3,72 & 1,57 & 0,45 & 15,70 & $-4,0$ \\
\hline 404 & 0,52 & 3,35 & 1,00 & 0,67 & 9,99 & $-3,3$ \\
\hline 405 & 29,91 & 25,18 & 7,98 & 4,77 & 79,79 & $-6,3$ \\
\hline 406 & 0,28 & 2,06 & 0,76 & 0,47 & 7,61 & $-2,9$ \\
\hline 407 & 0,34 & 2,41 & 0,75 & 0,58 & 7,48 & $-2,9$ \\
\hline 408 & 1,33 & 4,38 & 1,50 & 1,13 & 15,04 & $-3,9$ \\
\hline 409 & 2,56 & 6,21 & 2,09 & 1,56 & 20,89 & $-4,4$ \\
\hline 410 & 0,21 & 1,70 & 0,63 & 0,42 & 6,26 & $-2,6$ \\
\hline 411 & 10,86 & 15,05 & 5,86 & 2,36 & 58,62 & $-5,9$ \\
\hline 412 & 3,89 & 8,18 & 2,93 & 1,69 & 29,31 & $-4,9$ \\
\hline 413 & 0,09 & 1,33 & 0,46 & 0,24 & 4,59 & $-2,2$ \\
\hline 414 & 0,81 & 4,25 & 1,36 & 0,75 & 13,62 & $-3,8$ \\
\hline 415 & 0,58 & 3,34 & 1,34 & 0,56 & 13,41 & $-3,7$ \\
\hline 416 & 1,04 & 4,35 & 1,72 & 0,77 & 17,16 & $-4,1$ \\
\hline 417 & 0,31 & 2,16 & 0,74 & 0,53 & 7,40 & $-2,9$ \\
\hline 418 & 0,51 & 3,20 & 1,06 & 0,61 & 10,60 & $-3,4$ \\
\hline 419 & 0,09 & 1,11 & 0,36 & 0,32 & 3,61 & $-1,9$ \\
\hline 420 & 2,77 & 7,63 & 3,03 & 1,17 & 30,32 & $-4,9$ \\
\hline 421 & 0,26 & 1,98 & 0,65 & 0,51 & 6,46 & $-2,7$ \\
\hline 422 & 2,45 & 6,13 & 2,13 & 1,47 & 21,28 & $-4,4$ \\
\hline 423 & 0,60 & 2,99 & 1,13 & 0,68 & 11,27 & $-3,5$ \\
\hline 424 & 1,03 & 4,06 & 1,37 & 0,96 & 13,68 & $-3,8$ \\
\hline 425 & 0,11 & 1,27 & 0,42 & 0,34 & 4,24 & $-2,1$ \\
\hline
\end{tabular}




\begin{tabular}{|c|c|c|c|c|c|c|}
\hline 426 & 1,66 & 5,10 & 1,59 & 1,34 & 15,85 & $-4,0$ \\
\hline 427 & 1,70 & 5,95 & 1,73 & 1,25 & 17,29 & $-4,1$ \\
\hline 428 & 0,14 & 1,61 & 0,66 & 0,27 & 6,58 & $-2,7$ \\
\hline 429 & 0,67 & 3,19 & 1,02 & 0,84 & 10,19 & $-3,3$ \\
\hline 430 & 0,93 & 4,10 & 1,49 & 0,80 & 14,91 & $-3,9$ \\
\hline 431 & 21,41 & 20,17 & 6,70 & 4,07 & 66,98 & $-6,1$ \\
\hline 432 & 70,18 & 34,62 & 11,73 & 7,62 & 117,34 & $-6,9$ \\
\hline 433 & 4,30 & 12,19 & 3,83 & 1,43 & 38,34 & $-5,3$ \\
\hline 434 & 1,19 & 4,54 & 1,67 & 0,91 & 16,71 & $-4,1$ \\
\hline 435 & 0,38 & 3,75 & 1,17 & 0,41 & 11,71 & $-3,5$ \\
\hline 436 & 23,18 & 21,23 & 6,87 & 4,29 & 68,74 & $-6,1$ \\
\hline 437 & 0,32 & 2,41 & 0,70 & 0,58 & 6,97 & $-2,8$ \\
\hline 438 & 0,33 & 2,38 & 0,89 & 0,47 & 8,90 & $-3,2$ \\
\hline 439 & 2,46 & 6,99 & 2,81 & 1,11 & 28,14 & $-4,8$ \\
\hline 440 & 0,38 & 2,84 & 1,04 & 0,46 & 10,36 & $-3,4$ \\
\hline 441 & 0,23 & 2,15 & 0,85 & 0,35 & 8,46 & $-3,1$ \\
\hline 442 & 3,06 & 7,02 & 2,18 & 1,79 & 21,80 & $-4,4$ \\
\hline 443 & 0,26 & 2,18 & 0,67 & 0,49 & 6,71 & $-2,7$ \\
\hline 444 & 224,05 & 67,41 & 21,58 & 13,22 & 215,83 & $-7,8$ \\
\hline 445 & 0,19 & 1,91 & 0,68 & 0,36 & 6,75 & $-2,8$ \\
\hline 446 & 1,19 & 4,24 & 1,53 & 0,99 & 15,30 & $-3,9$ \\
\hline 447 & 0,72 & 3,24 & 1,09 & 0,84 & 10,88 & $-3,4$ \\
\hline 448 & 20,81 & 22,36 & 9,24 & 2,87 & 92,41 & $-6,5$ \\
\hline 449 & 0,65 & 3,27 & 0,97 & 0,86 & 9,70 & $-3,3$ \\
\hline 450 & 0,19 & 1,71 & 0,63 & 0,39 & 6,32 & $-2,7$ \\
\hline 451 & 0,06 & 0,94 & 0,35 & 0,20 & 3,49 & $-1,8$ \\
\hline 452 & 1,24 & 4,81 & 1,72 & 0,92 & 17,18 & $-4,1$ \\
\hline 453 & 1,01 & 4,36 & 1,67 & 0,77 & 16,67 & $-4,1$ \\
\hline 454 & 0,08 & 1,06 & 0,39 & 0,25 & 3,91 & $-2,0$ \\
\hline 455 & 0,80 & 3,63 & 1,36 & 0,75 & 13,58 & $-3,8$ \\
\hline 456 & 0,20 & 1,83 & 0,63 & 0,41 & 6,29 & $-2,7$ \\
\hline 457 & 9,37 & 13,58 & 4,95 & 2,41 & 49,50 & $-5,6$ \\
\hline 458 & 0,87 & 4,26 & 1,42 & 0,78 & 14,16 & $-3,8$ \\
\hline 459 & 0,71 & 3,39 & 1,21 & 0,75 & 12,09 & $-3,6$ \\
\hline 460 & 0,25 & 2,15 & 0,90 & 0,35 & 8,98 & $-3,2$ \\
\hline 461 & 0,13 & 1,50 & 0,62 & 0,26 & 6,17 & $-2,6$ \\
\hline 462 & 0,51 & 3,05 & 0,97 & 0,67 & 9,72 & $-3,3$ \\
\hline 463 & 0,25 & 1,96 & 0,66 & 0,49 & 6,55 & $-2,7$ \\
\hline 464 & 2,08 & 8,14 & 2,94 & 0,90 & 29,43 & $-4,9$ \\
\hline 465 & 0,38 & 3,21 & 0,89 & 0,54 & 8,88 & $-3,2$ \\
\hline 466 & 11,43 & 17,85 & 4,92 & 2,96 & 49,21 & $-5,6$ \\
\hline 467 & 40,80 & 26,67 & 8,35 & 6,23 & 83,45 & $-6,4$ \\
\hline 468 & 9,08 & 14,00 & 4,23 & 2,73 & 42,31 & $-5,4$ \\
\hline 469 & 3,79 & 9,22 & 3,26 & 1,48 & 32,60 & $-5,0$ \\
\hline 470 & 1,12 & 4,00 & 1,32 & 1,08 & 13,20 & $-3,7$ \\
\hline 471 & 0,54 & 2,87 & 1,04 & 0,67 & 10,37 & $-3,4$ \\
\hline 472 & 510,36 & 119,00 & 27,27 & 23,83 & 272,69 & $-8,1$ \\
\hline 473 & 0,21 & 1,69 & 0,54 & 0,50 & 5,37 & $-2,4$ \\
\hline 474 & 0,83 & 3,57 & 1,20 & 0,88 & 12,02 & $-3,6$ \\
\hline 475 & 1,34 & 4,83 & 1,79 & 0,95 & 17,86 & $-4,2$ \\
\hline 476 & 1,61 & 5,58 & 2,11 & 0,97 & 21,12 & $-4,4$ \\
\hline 477 & 2,13 & 6,56 & 2,38 & 1,14 & 23,79 & $-4,6$ \\
\hline 478 & 0,45 & 2,65 & 0,98 & 0,58 & 9,82 & $-3,3$ \\
\hline 479 & 1,27 & 4,53 & 1,71 & 0,95 & 17,05 & $-4,1$ \\
\hline 480 & 0,26 & 2,18 & 0,77 & 0,44 & 7,65 & $-2,9$ \\
\hline 481 & 0,13 & 1,33 & 0,44 & 0,38 & 4,43 & $-2,1$ \\
\hline 482 & 0,37 & 2,44 & 0,78 & 0,60 & 7,79 & $-3,0$ \\
\hline 483 & 3,83 & 8,11 & 2,64 & 1,85 & 26,36 & $-4,7$ \\
\hline 484 & 1,94 & 5,91 & 1,98 & 1,25 & 19,80 & $-4,3$ \\
\hline 485 & 0,21 & 1,96 & 0,79 & 0,34 & 7,91 & $-3,0$ \\
\hline 486 & 0,94 & 3,78 & 1,38 & 0,86 & 13,82 & $-3,8$ \\
\hline 487 & 1,86 & 5,35 & 1,80 & 1,31 & 18,00 & $-4,2$ \\
\hline
\end{tabular}




\begin{tabular}{|c|c|c|c|c|c|c|}
\hline 488 & 0,34 & 2,73 & 0,94 & 0,46 & 9,41 & $-3,2$ \\
\hline 489 & 0,19 & 1,91 & 0,75 & 0,33 & 7,45 & $-2,9$ \\
\hline 490 & 9,50 & 12,64 & 4,46 & 2,71 & 44,57 & $-5,5$ \\
\hline 491 & 0,23 & 2,14 & 0,65 & 0,46 & 6,45 & $-2,7$ \\
\hline 492 & 9,35 & 12,27 & 4,47 & 2,67 & 44,66 & $-5,5$ \\
\hline 493 & 0,19 & 1,98 & 0,82 & 0,29 & 8,21 & $-3,0$ \\
\hline 494 & 0,30 & 2,45 & 0,98 & 0,39 & 9,84 & $-3,3$ \\
\hline 495 & 0,28 & 2,20 & 0,93 & 0,38 & 9,33 & $-3,2$ \\
\hline 496 & 2,96 & 7,10 & 2,50 & 1,51 & 24,96 & $-4,6$ \\
\hline 497 & 2,18 & 5,80 & 1,99 & 1,39 & 19,89 & $-4,3$ \\
\hline 498 & 4,95 & 9,43 & 3,38 & 1,87 & 33,77 & $-5,1$ \\
\hline 499 & 0,47 & 2,67 & 1,02 & 0,59 & 10,20 & $-3,4$ \\
\hline 500 & 0,06 & 0,86 & 0,32 & 0,22 & 3,16 & $-1,7$ \\
\hline 501 & 0,15 & 1,77 & 0,77 & 0,24 & 7,70 & $-2,9$ \\
\hline 502 & 1,52 & 4,99 & 1,76 & 1,10 & 17,58 & $-4,1$ \\
\hline 503 & 4,70 & 8,65 & 2,72 & 2,20 & 27,21 & $-4,8$ \\
\hline 504 & 1,19 & 4,16 & 1,39 & 1,09 & 13,91 & $-3,8$ \\
\hline 505 & 0,09 & 1,17 & 0,40 & 0,29 & 4,02 & $-2,0$ \\
\hline 506 & 0,35 & 2,53 & 1,04 & 0,43 & 10,36 & $-3,4$ \\
\hline 507 & 0,14 & 1,55 & 0,56 & 0,31 & 5,64 & $-2,5$ \\
\hline 508 & 1,14 & 4,27 & 1,66 & 0,88 & 16,60 & $-4,1$ \\
\hline 509 & 26,19 & 27,66 & 11,11 & 3,00 & 111,11 & $-6,8$ \\
\hline 510 & 0,25 & 1,91 & 0,68 & 0,46 & 6,79 & $-2,8$ \\
\hline 511 & 0,28 & 2,11 & 0,73 & 0,48 & 7,34 & $-2,9$ \\
\hline 512 & 0,23 & 1,78 & 0,62 & 0,47 & 6,20 & $-2,6$ \\
\hline 513 & 35,78 & 26,95 & 8,27 & 5,51 & 82,72 & $-6,4$ \\
\hline 514 & 2,19 & 5,92 & 2,03 & 1,37 & 20,25 & $-4,3$ \\
\hline 515 & 0,31 & 2,50 & 1,05 & 0,38 & 10,53 & $-3,4$ \\
\hline 516 & 0,17 & 1,74 & 0,73 & 0,30 & 7,33 & $-2,9$ \\
\hline 517 & 0,24 & 2,05 & 0,84 & 0,37 & 8,39 & $-3,1$ \\
\hline 518 & 0,31 & 2,48 & 1,04 & 0,38 & 10,38 & $-3,4$ \\
\hline 519 & 0,06 & 1,02 & 0,42 & 0,18 & 4,21 & $-2,1$ \\
\hline 520 & 0,45 & 2,57 & 0,79 & 0,73 & 7,87 & $-3,0$ \\
\hline 521 & 0,58 & 3,67 & 1,19 & 0,62 & 11,93 & $-3,6$ \\
\hline 522 & 2,81 & 7,83 & 3,31 & 1,08 & 33,09 & $-5,0$ \\
\hline 523 & 0,20 & 1,73 & 0,65 & 0,39 & 6,50 & $-2,7$ \\
\hline 524 & 0,23 & 1,99 & 0,74 & 0,40 & 7,39 & $-2,9$ \\
\hline 525 & 0,47 & 3,24 & 1,15 & 0,52 & 11,49 & $-3,5$ \\
\hline 526 & 0,35 & 2,26 & 0,78 & 0,57 & 7,80 & $-3,0$ \\
\hline 527 & 0,21 & 2,19 & 0,79 & 0,34 & 7,92 & $-3,0$ \\
\hline 528 & 0,63 & 3,60 & 1,49 & 0,54 & 14,85 & $-3,9$ \\
\hline 529 & 0,36 & 2,40 & 0,78 & 0,59 & 7,76 & $-3,0$ \\
\hline 530 & 0,41 & 2,69 & 0,81 & 0,65 & 8,08 & $-3,0$ \\
\hline 531 & 0,31 & 2,10 & 0,69 & 0,58 & 6,88 & $-2,8$ \\
\hline 532 & 0,39 & 2,38 & 0,82 & 0,59 & 8,24 & $-3,0$ \\
\hline 533 & 133,39 & 48,94 & 13,89 & 12,23 & 138,89 & $-7,1$ \\
\hline 534 & 2,44 & 7,29 & 2,76 & 1,12 & 27,64 & $-4,8$ \\
\hline 535 & 1,48 & 5,68 & 2,31 & 0,82 & 23,11 & $-4,5$ \\
\hline 536 & 0,36 & 2,27 & 0,82 & 0,56 & 8,22 & $-3,0$ \\
\hline 537 & 0,34 & 2,53 & 0,96 & 0,46 & 9,59 & $-3,3$ \\
\hline 538 & 3,37 & 7,69 & 2,88 & 1,49 & 28,82 & $-4,8$ \\
\hline 539 & 0,17 & 1,57 & 0,48 & 0,44 & 4,80 & $-2,3$ \\
\hline 540 & 1,13 & 4,61 & 1,37 & 1,05 & 13,71 & $-3,8$ \\
\hline 541 & 5,95 & 10,52 & 3,76 & 2,01 & 37,63 & $-5,2$ \\
\hline 542 & 137,63 & 55,92 & 16,59 & 10,56 & 165,88 & $-7,4$ \\
\hline 543 & 0,16 & 1,45 & 0,46 & 0,43 & 4,61 & $-2,2$ \\
\hline 544 & 5,64 & 9,88 & 3,56 & 2,02 & 35,62 & $-5,2$ \\
\hline 545 & 0,14 & 1,37 & 0,48 & 0,38 & 4,77 & $-2,3$ \\
\hline 546 & 0,23 & 2,15 & 0,92 & 0,32 & 9,16 & $-3,2$ \\
\hline 547 & 0,11 & 1,27 & 0,39 & 0,35 & 3,94 & $-2,0$ \\
\hline 548 & 0,13 & 1,60 & 0,61 & 0,28 & 6,11 & $-2,6$ \\
\hline 549 & 0,72 & 3,61 & 1,17 & 0,79 & 11,69 & $-3,5$ \\
\hline
\end{tabular}




\begin{tabular}{|c|c|c|c|c|c|c|}
\hline 550 & 0,18 & 1,66 & 0,59 & 0,39 & 5,88 & $-2,6$ \\
\hline 551 & 0,45 & 2,67 & 0,82 & 0,70 & 8,18 & $-3,0$ \\
\hline 552 & 0,60 & 3,30 & 1,32 & 0,58 & 13,16 & $-3,7$ \\
\hline 553 & 0,75 & 3,35 & 1,08 & 0,89 & 10,77 & $-3,4$ \\
\hline 554 & 0,25 & 1,88 & 0,61 & 0,51 & 6,14 & $-2,6$ \\
\hline 555 & 0,22 & 1,93 & 0,74 & 0,38 & 7,38 & $-2,9$ \\
\hline 556 & 0,98 & 3,99 & 1,56 & 0,80 & 15,63 & $-4,0$ \\
\hline 557 & 80,37 & 42,50 & 14,96 & 6,84 & 149,58 & $-7,2$ \\
\hline 558 & 0,29 & 2,17 & 0,81 & 0,45 & 8,12 & $-3,0$ \\
\hline 559 & 2,49 & 7,44 & 2,41 & 1,32 & 24,05 & $-4,6$ \\
\hline 560 & 1,35 & 4,99 & 1,73 & 0,99 & 17,25 & $-4,1$ \\
\hline 561 & 0,27 & 2,20 & 0,84 & 0,41 & 8,39 & $-3,1$ \\
\hline 562 & 4,24 & 10,62 & 3,49 & 1,55 & 34,86 & $-5,1$ \\
\hline 563 & 5,11 & 9,07 & 2,91 & 2,23 & 29,11 & $-4,9$ \\
\hline 564 & 0,25 & 2,25 & 0,73 & 0,45 & 7,26 & $-2,9$ \\
\hline 565 & 0,04 & 0,71 & 0,29 & 0,16 & 2,85 & $-1,5$ \\
\hline 566 & 0,96 & 4,01 & 1,26 & 0,97 & 12,59 & $-3,7$ \\
\hline 567 & 2,02 & 6,93 & 2,23 & 1,15 & 22,33 & $-4,5$ \\
\hline 568 & 0,11 & 1,36 & 0,48 & 0,30 & 4,84 & $-2,3$ \\
\hline 569 & 2,44 & 6,56 & 2,27 & 1,37 & 22,70 & $-4,5$ \\
\hline 570 & 0,67 & 3,69 & 1,40 & 0,61 & 13,98 & $-3,8$ \\
\hline 571 & 0,20 & 1,76 & 0,62 & 0,42 & 6,15 & $-2,6$ \\
\hline 572 & 0,38 & 2,46 & 0,82 & 0,60 & 8,16 & $-3,0$ \\
\hline 573 & 5,48 & 11,11 & 3,65 & 1,91 & 36,48 & $-5,2$ \\
\hline 574 & 1,91 & 5,63 & 2,03 & 1,20 & 20,31 & $-4,3$ \\
\hline 575 & 0,40 & 2,54 & 0,84 & 0,60 & 8,43 & $-3,1$ \\
\hline 576 & 0,09 & 1,11 & 0,39 & 0,30 & 3,94 & $-2,0$ \\
\hline 577 & 1,88 & 5,61 & 1,80 & 1,33 & 18,04 & $-4,2$ \\
\hline 578 & 2,92 & 7,36 & 2,44 & 1,52 & 24,42 & $-4,6$ \\
\hline 579 & 0,27 & 2,98 & 1,24 & 0,28 & 12,41 & $-3,6$ \\
\hline 580 & 0,10 & 1,19 & 0,39 & 0,33 & 3,87 & $-2,0$ \\
\hline 581 & 377,53 & 81,59 & 26,05 & 18,45 & 260,47 & $-8,0$ \\
\hline 582 & 0,66 & 3,39 & 1,32 & 0,64 & 13,16 & $-3,7$ \\
\hline 583 & 0,11 & 1,21 & 0,45 & 0,32 & 4,46 & $-2,2$ \\
\hline 584 & 0,86 & 3,83 & 1,38 & 0,79 & 13,81 & $-3,8$ \\
\hline 585 & 3,53 & 7,28 & 2,22 & 2,02 & 22,21 & $-4,5$ \\
\hline 586 & 0,44 & 2,85 & 1,10 & 0,51 & 10,95 & $-3,5$ \\
\hline 587 & 2,24 & 5,89 & 1,80 & 1,58 & 18,01 & $-4,2$ \\
\hline 588 & 0,30 & 2,65 & 1,13 & 0,34 & 11,34 & $-3,5$ \\
\hline 589 & 1,15 & 4,75 & 1,96 & 0,75 & 19,60 & $-4,3$ \\
\hline 590 & 1,54 & 5,41 & 1,95 & 1,00 & 19,49 & $-4,3$ \\
\hline 591 & 0,25 & 2,28 & 0,93 & 0,35 & 9,25 & $-3,2$ \\
\hline 592 & 2,77 & 8,65 & 2,69 & 1,31 & 26,85 & $-4,7$ \\
\hline 593 & 0,79 & 3,70 & 1,34 & 0,75 & 13,36 & $-3,7$ \\
\hline 594 & 0,95 & 3,72 & 1,26 & 0,96 & 12,58 & $-3,7$ \\
\hline 595 & 0,17 & 1,69 & 0,52 & 0,42 & 5,24 & $-2,4$ \\
\hline 596 & 17,45 & 18,72 & 5,66 & 3,92 & 56,62 & $-5,8$ \\
\hline 597 & 0,19 & 1,66 & 0,57 & 0,43 & 5,67 & $-2,5$ \\
\hline 598 & 0,24 & 2,00 & 0,78 & 0,39 & 7,81 & $-3,0$ \\
\hline 599 & 4,03 & 9,17 & 3,61 & 1,42 & 36,07 & $-5,2$ \\
\hline 600 & 0,64 & 3,04 & 0,93 & 0,87 & 9,30 & $-3,2$ \\
\hline 601 & 5,55 & 9,79 & 3,06 & 2,31 & 30,58 & $-4,9$ \\
\hline 602 & 0,53 & 2,89 & 1,03 & 0,66 & 10,34 & $-3,4$ \\
\hline 603 & 2,99 & 7,85 & 2,85 & 1,34 & 28,45 & $-4,8$ \\
\hline 604 & 0,27 & 2,06 & 0,62 & 0,56 & 6,18 & $-2,6$ \\
\hline 605 & 0,42 & 2,65 & 0,95 & 0,56 & 9,53 & $-3,3$ \\
\hline 606 & 0,31 & 2,09 & 0,70 & 0,56 & 7,01 & $-2,8$ \\
\hline 607 & 0,40 & 2,38 & 0,74 & 0,68 & 7,38 & $-2,9$ \\
\hline 608 & 0,60 & 3,23 & 1,01 & 0,76 & 10,08 & $-3,3$ \\
\hline 609 & 0,66 & 3,05 & 1,00 & 0,85 & 9,95 & $-3,3$ \\
\hline 610 & 38,29 & 26,49 & 8,59 & 5,68 & 85,85 & $-6,4$ \\
\hline 611 & 2,73 & 6,52 & 1,98 & 1,76 & 19,77 & $-4,3$ \\
\hline
\end{tabular}




\begin{tabular}{|c|c|c|c|c|c|c|}
\hline 612 & 0,84 & 3,71 & 1,39 & 0,77 & 13,88 & $-3,8$ \\
\hline 613 & 0,56 & 2,97 & 1,00 & 0,72 & 9,98 & $-3,3$ \\
\hline 614 & 39,28 & 26,73 & 8,10 & 6,18 & 80,95 & $-6,3$ \\
\hline 615 & 0,83 & 3,52 & 1,19 & 0,89 & 11,92 & $-3,6$ \\
\hline 616 & 32,55 & 22,61 & 6,96 & 5,96 & 69,58 & $-6,1$ \\
\hline 617 & 0,14 & 1,53 & 0,45 & 0,41 & 4,46 & $-2,2$ \\
\hline 618 & 0,13 & 1,33 & 0,47 & 0,34 & 4,67 & $-2,2$ \\
\hline 619 & 0,38 & 2,61 & 0,92 & 0,53 & 9,18 & $-3,2$ \\
\hline 620 & 1,40 & 4,76 & 1,47 & 1,21 & 14,69 & $-3,9$ \\
\hline 621 & 1,61 & 5,53 & 1,86 & 1,10 & 18,63 & $-4,2$ \\
\hline 622 & 2,11 & 6,29 & 2,22 & 1,21 & 22,17 & $-4,5$ \\
\hline 623 & 0,40 & 2,81 & 1,06 & 0,48 & 10,63 & $-3,4$ \\
\hline 624 & 1,47 & 4,93 & 1,65 & 1,14 & 16,49 & $-4,0$ \\
\hline 625 & 0,65 & 3,20 & 1,20 & 0,69 & 11,99 & $-3,6$ \\
\hline 626 & 0,79 & 3,58 & 1,29 & 0,78 & 12,90 & $-3,7$ \\
\hline 627 & 1,00 & 4,12 & 1,30 & 0,98 & 13,04 & $-3,7$ \\
\hline 628 & 0,77 & 3,56 & 1,31 & 0,74 & 13,13 & $-3,7$ \\
\hline 629 & 0,80 & 3,94 & 1,36 & 0,75 & 13,64 & $-3,8$ \\
\hline 630 & 0,75 & 3,47 & 1,03 & 0,93 & 10,33 & $-3,4$ \\
\hline 631 & 0,12 & 1,36 & 0,43 & 0,34 & 4,31 & $-2,1$ \\
\hline 632 & 7,92 & 11,62 & 3,65 & 2,77 & 36,45 & $-5,2$ \\
\hline 633 & 0,87 & 3,58 & 1,17 & 0,94 & 11,73 & $-3,6$ \\
\hline 634 & 0,05 & 0,89 & 0,36 & 0,18 & 3,55 & $-1,8$ \\
\hline 635 & 0,39 & 2,39 & 0,81 & 0,61 & 8,14 & $-3,0$ \\
\hline 636 & 0,65 & 3,18 & 1,18 & 0,70 & 11,82 & $-3,6$ \\
\hline 637 & 0,45 & 2,76 & 0,97 & 0,59 & 9,71 & $-3,3$ \\
\hline 638 & 0,94 & 4,56 & 1,64 & 0,73 & 16,41 & $-4,0$ \\
\hline 639 & 0,45 & 2,71 & 0,95 & 0,61 & 9,47 & $-3,2$ \\
\hline 640 & 0,59 & 3,32 & 1,23 & 0,61 & 12,32 & $-3,6$ \\
\hline 641 & 0,35 & 2,44 & 0,74 & 0,61 & 7,40 & $-2,9$ \\
\hline 642 & 0,21 & 2,01 & 0,84 & 0,31 & 8,39 & $-3,1$ \\
\hline 643 & 0,09 & 1,30 & 0,48 & 0,24 & 4,80 & $-2,3$ \\
\hline 644 & 0,23 & 2,06 & 0,81 & 0,37 & 8,05 & $-3,0$ \\
\hline 645 & 0,24 & 2,02 & 0,69 & 0,45 & 6,89 & $-2,8$ \\
\hline 646 & 0,21 & 1,80 & 0,68 & 0,39 & 6,84 & $-2,8$ \\
\hline 647 & 0,38 & 2,37 & 0,82 & 0,59 & 8,16 & $-3,0$ \\
\hline 648 & 2,78 & 8,51 & 3,22 & 1,10 & 32,22 & $-5,0$ \\
\hline 649 & 1,97 & 5,58 & 2,01 & 1,25 & 20,11 & $-4,3$ \\
\hline 650 & 0,18 & 1,70 & 0,62 & 0,38 & 6,16 & $-2,6$ \\
\hline 651 & 7,92 & 12,09 & 3,50 & 2,88 & 35,00 & $-5,1$ \\
\hline 652 & 0,19 & 1,85 & 0,56 & 0,43 & 5,63 & $-2,5$ \\
\hline 653 & 0,72 & 3,41 & 1,21 & 0,76 & 12,05 & $-3,6$ \\
\hline 654 & 11,38 & 15,61 & 4,63 & 3,13 & 46,31 & $-5,5$ \\
\hline 655 & 5,27 & 9,24 & 3,57 & 1,88 & 35,66 & $-5,2$ \\
\hline 656 & 5,36 & 10,18 & 3,96 & 1,72 & 39,60 & $-5,3$ \\
\hline 657 & 10,74 & 14,55 & 5,85 & 2,34 & 58,47 & $-5,9$ \\
\hline 658 & 0,06 & 1,02 & 0,37 & 0,22 & 3,71 & $-1,9$ \\
\hline 659 & 0,14 & 1,50 & 0,52 & 0,34 & 5,15 & $-2,4$ \\
\hline 660 & 0,20 & 2,11 & 0,89 & 0,28 & 8,87 & $-3,1$ \\
\hline 661 & 1,14 & 4,21 & 1,42 & 1,02 & 14,19 & $-3,8$ \\
\hline 662 & 19,79 & 18,71 & 6,11 & 4,12 & 61,11 & $-5,9$ \\
\hline 663 & 111,27 & 46,48 & 15,31 & 9,26 & 153,05 & $-7,3$ \\
\hline 664 & 22,03 & 18,88 & 5,90 & 4,76 & 58,96 & $-5,9$ \\
\hline 665 & 7,26 & 12,12 & 4,02 & 2,30 & 40,15 & $-5,3$ \\
\hline 666 & 0,24 & 1,99 & 0,78 & 0,39 & 7,80 & $-3,0$ \\
\hline 667 & 6,44 & 10,25 & 3,26 & 2,51 & 32,64 & $-5,0$ \\
\hline 668 & 1,12 & 4,23 & 1,48 & 0,96 & 14,77 & $-3,9$ \\
\hline 669 & 14,17 & 15,86 & 5,33 & 3,38 & 53,29 & $-5,7$ \\
\hline 670 & 0,34 & 2,25 & 0,76 & 0,58 & 7,57 & $-2,9$ \\
\hline 671 & 0,20 & 1,84 & 0,75 & 0,34 & 7,47 & $-2,9$ \\
\hline 672 & 0,45 & 2,53 & 0,95 & 0,60 & 9,51 & $-3,2$ \\
\hline 673 & 0,36 & 2,64 & 0,88 & 0,53 & 8,80 & $-3,1$ \\
\hline
\end{tabular}




$\begin{array}{lcccccr}674 & 0,48 & 2,74 & 0,93 & 0,66 & 9,29 & -3,2 \\ 675 & 1,84 & 5,45 & 1,92 & 1,22 & 19,22 & -4,3 \\ 676 & 4,73 & 8,70 & 2,95 & 2,05 & 29,45 & -4,9 \\ 677 & 0,17 & 1,91 & 0,73 & 0,30 & 7,26 & -2,9 \\ 678 & 0,18 & 1,62 & 0,60 & 0,39 & 6,02 & -2,6 \\ 679 & 0,41 & 2,67 & 0,93 & 0,56 & 9,33 & -3,2 \\ 680 & 0,19 & 1,75 & 0,56 & 0,42 & 5,64 & -2,5 \\ 681 & 0,45 & 2,87 & 1,12 & 0,51 & 11,23 & -3,5 \\ 682 & 55,03 & 29,99 & 9,08 & 7,72 & 90,76 & -6,5 \\ 683 & 2,45 & 6,52 & 2,36 & 1,32 & 23,57 & -4,6 \\ 684 & 0,33 & 2,87 & 1,23 & 0,34 & 12,33 & -3,6 \\ 685 & 0,18 & 2,04 & 0,80 & 0,29 & 8,00 & -3,0 \\ 686 & 1,18 & 4,38 & 1,38 & 1,09 & 13,75 & -3,8 \\ 687 & 3,75 & 7,66 & 2,42 & 1,97 & 24,23 & -4,6 \\ 688 & 0,46 & 2,97 & 1,15 & 0,51 & 11,45 & -3,5 \\ 689 & 0,19 & 1,75 & 0,67 & 0,36 & 6,69 & -2,7 \\ 690 & 0,57 & 3,02 & 0,98 & 0,74 & 9,77 & -3,3 \\ 691 & 0,24 & 2,14 & 0,84 & 0,37 & 8,37 & -3,1 \\ 692 & 3,86 & 7,73 & 2,63 & 1,87 & 26,32 & -4,7 \\ 693 & 0,20 & 1,64 & 0,56 & 0,46 & 5,61 & -2,5 \\ 694 & 0,31 & 2,47 & 0,74 & 0,53 & 7,39 & -2,9 \\ 695 & 3,58 & 7,90 & 2,83 & 1,61 & 28,28 & -4,8 \\ 696 & 0,24 & 1,89 & 0,75 & 0,40 & 7,51 & -2,9 \\ 697 & 1,88 & 5,40 & 2,15 & 1,11 & 21,53 & -4,4 \\ 698 & 2,25 & 5,82 & 1,73 & 1,66 & 17,33 & -4,1 \\ 699 & 0,66 & 3,41 & 1,36 & 0,62 & 13,63 & -3,8 \\ 700 & 0,25 & 1,87 & 0,70 & 0,46 & 6,97 & -2,8 \\ 701 & 5,94 & 9,64 & 3,30 & 2,29 & 33,01 & -5,0\end{array}$


Clasts' data for samples SM-82_Br02 and SM-82_Br02_detail, used for grain size distribution and fractal dimension analysis

$\phi=-\log _{2}($ diam $)$, considering the major axis of the best fit ellipses, in millimeters.

SM-82_Br02

Best fit ellipse

\begin{tabular}{|c|c|c|c|c|c|c|}
\hline Label & Area $\left(\mathrm{cm}^{2}\right)$ & $\begin{array}{l}\text { Perimeter } \\
\text { (cm) }\end{array}$ & $\begin{array}{l}\text { Major axis } \\
\quad(\mathrm{cm})\end{array}$ & $\begin{array}{l}\text { Minor axis } \\
\quad(\mathrm{cm})\end{array}$ & $\begin{array}{l}\text { Diameter } \\
\text { (mm) }\end{array}$ & Phi \\
\hline 1 & 5,19 & 9,12 & 2,86 & 2,31 & 28,63 & $-4,8$ \\
\hline 2 & 1,34 & 4,47 & 1,49 & 1,14 & 14,94 & $-3,9$ \\
\hline 3 & 2,47 & 8,37 & 2,75 & 1,14 & 27,53 & $-4,8$ \\
\hline 4 & 118,47 & 65,09 & 14,23 & 10,60 & 142,31 & $-7,2$ \\
\hline 5 & 11,24 & 13,65 & 4,44 & 3,22 & 44,42 & $-5,5$ \\
\hline 6 & 0,93 & 3,63 & 1,20 & 0,99 & 12,03 & $-3,6$ \\
\hline 7 & 0,36 & 2,25 & 0,78 & 0,58 & 7,82 & $-3,0$ \\
\hline 8 & 0,27 & 2,13 & 0,78 & 0,45 & 7,76 & $-3,0$ \\
\hline 9 & 2,02 & 5,85 & 1,79 & 1,43 & 17,90 & $-4,2$ \\
\hline 10 & 0,26 & 1,91 & 0,62 & 0,53 & 6,24 & $-2,6$ \\
\hline 11 & 0,08 & 1,39 & 0,52 & 0,21 & 5,17 & $-2,4$ \\
\hline 12 & 5,41 & 9,88 & 3,26 & 2,11 & 32,61 & $-5,0$ \\
\hline 13 & 4,26 & 8,88 & 3,58 & 1,52 & 35,78 & $-5,2$ \\
\hline 14 & 0,64 & 3,19 & 1,10 & 0,74 & 11,02 & $-3,5$ \\
\hline 15 & 2,67 & 7,75 & 2,51 & 1,35 & 25,13 & $-4,7$ \\
\hline 16 & 0,18 & 1,59 & 0,50 & 0,46 & 5,00 & $-2,3$ \\
\hline 17 & 0,18 & 1,73 & 0,70 & 0,32 & 7,04 & $-2,8$ \\
\hline 18 & 0,59 & 3,35 & 1,43 & 0,53 & 14,25 & $-3,8$ \\
\hline 19 & 0,02 & 0,49 & 0,22 & 0,11 & 2,20 & $-1,1$ \\
\hline 20 & 0,62 & 3,26 & 1,22 & 0,64 & 12,23 & $-3,6$ \\
\hline 21 & 0,03 & 0,63 & 0,25 & 0,18 & 2,49 & $-1,3$ \\
\hline 22 & 1,11 & 4,50 & 1,76 & 0,80 & 17,64 & $-4,1$ \\
\hline 23 & 1,44 & 4,79 & 1,86 & 0,99 & 18,63 & $-4,2$ \\
\hline 24 & 0,46 & 2,68 & 0,93 & 0,63 & 9,29 & $-3,2$ \\
\hline 25 & 0,15 & 1,55 & 0,59 & 0,33 & 5,89 & $-2,6$ \\
\hline 26 & 0,19 & 1,76 & 0,67 & 0,36 & 6,69 & $-2,7$ \\
\hline 27 & 0,15 & 1,53 & 0,50 & 0,39 & 4,99 & $-2,3$ \\
\hline 28 & 0,12 & 1,42 & 0,60 & 0,26 & 6,00 & $-2,6$ \\
\hline 29 & 1,28 & 4,67 & 1,49 & 1,09 & 14,91 & $-3,9$ \\
\hline 30 & 0,28 & 2,42 & 0,82 & 0,44 & 8,16 & $-3,0$ \\
\hline 31 & 0,28 & 2,33 & 0,95 & 0,38 & 9,45 & $-3,2$ \\
\hline 32 & 0,66 & 3,07 & 1,10 & 0,77 & 11,01 & $-3,5$ \\
\hline 33 & 2,14 & 6,66 & 2,54 & 1,08 & 25,36 & $-4,7$ \\
\hline 34 & 1,41 & 5,18 & 1,92 & 0,93 & 19,23 & $-4,3$ \\
\hline 35 & 0,16 & 1,49 & 0,48 & 0,44 & 4,77 & $-2,3$ \\
\hline 36 & 0,47 & 2,87 & 1,21 & 0,50 & 12,10 & $-3,6$ \\
\hline 37 & 4,11 & 9,78 & 3,56 & 1,47 & 35,64 & $-5,2$ \\
\hline 38 & 0,48 & 2,73 & 0,86 & 0,71 & 8,56 & $-3,1$ \\
\hline 39 & 0,13 & 1,45 & 0,59 & 0,29 & 5,88 & $-2,6$ \\
\hline 40 & 0,09 & 1,06 & 0,36 & 0,31 & 3,55 & $-1,8$ \\
\hline 41 & 0,17 & 1,57 & 0,63 & 0,34 & 6,34 & $-2,7$ \\
\hline 42 & 0,14 & 1,34 & 0,45 & 0,38 & 4,51 & $-2,2$ \\
\hline 43 & 0,14 & 1,41 & 0,49 & 0,35 & 4,94 & $-2,3$ \\
\hline 44 & 0,61 & 3,23 & 1,15 & 0,67 & 11,45 & $-3,5$ \\
\hline 45 & 0,04 & 0,75 & 0,32 & 0,16 & 3,24 & $-1,7$ \\
\hline 46 & 0,21 & 1,81 & 0,68 & 0,39 & 6,83 & $-2,8$ \\
\hline 47 & 0,35 & 2,48 & 1,02 & 0,44 & 10,22 & $-3,4$ \\
\hline 48 & 1,45 & 4,86 & 1,87 & 0,99 & 18,67 & $-4,2$ \\
\hline 49 & 0,15 & 1,45 & 0,51 & 0,38 & 5,09 & $-2,3$ \\
\hline 50 & 1,00 & 4,22 & 1,29 & 0,99 & 12,89 & $-3,7$ \\
\hline 51 & 1,78 & 6,88 & 2,83 & 0,80 & 28,25 & $-4,8$ \\
\hline
\end{tabular}




\begin{tabular}{|c|c|c|c|c|c|c|}
\hline 52 & 9,61 & 13,62 & 4,23 & 2,90 & 42,27 & $-5,4$ \\
\hline 53 & 0,21 & 1,88 & 0,75 & 0,36 & 7,47 & $-2,9$ \\
\hline 54 & 4,44 & 8,63 & 2,99 & 1,89 & 29,92 & $-4,9$ \\
\hline 55 & 2,47 & 6,30 & 2,06 & 1,53 & 20,55 & $-4,4$ \\
\hline 56 & 0,12 & 1,31 & 0,50 & 0,31 & 4,98 & $-2,3$ \\
\hline 57 & 0,93 & 3,87 & 1,37 & 0,87 & 13,66 & $-3,8$ \\
\hline 58 & 0,90 & 3,67 & 1,21 & 0,95 & 12,08 & $-3,6$ \\
\hline 59 & 0,15 & 1,64 & 0,60 & 0,31 & 5,99 & $-2,6$ \\
\hline 60 & 0,08 & 1,04 & 0,32 & 0,31 & 3,18 & $-1,7$ \\
\hline 61 & 0,02 & 0,56 & 0,25 & 0,11 & 2,51 & $-1,3$ \\
\hline 62 & 0,13 & 1,34 & 0,53 & 0,30 & 5,26 & $-2,4$ \\
\hline 63 & 0,26 & 1,87 & 0,61 & 0,55 & 6,10 & $-2,6$ \\
\hline 64 & 0,17 & 1,83 & 0,69 & 0,32 & 6,94 & $-2,8$ \\
\hline 65 & 0,15 & 1,49 & 0,51 & 0,38 & 5,08 & $-2,3$ \\
\hline 66 & 0,13 & 1,53 & 0,66 & 0,25 & 6,60 & $-2,7$ \\
\hline 67 & 1,05 & 4,01 & 1,50 & 0,90 & 14,99 & $-3,9$ \\
\hline 68 & 0,07 & 1,03 & 0,36 & 0,25 & 3,56 & $-1,8$ \\
\hline 69 & 2,96 & 6,77 & 2,47 & 1,53 & 24,66 & $-4,6$ \\
\hline 70 & 0,43 & 2,63 & 0,92 & 0,59 & 9,19 & $-3,2$ \\
\hline 71 & 0,17 & 1,64 & 0,57 & 0,37 & 5,70 & $-2,5$ \\
\hline 72 & 0,06 & 0,90 & 0,35 & 0,22 & 3,48 & $-1,8$ \\
\hline 73 & 4,73 & 9,63 & 3,09 & 1,95 & 30,92 & $-5,0$ \\
\hline 74 & 0,44 & 2,51 & 0,89 & 0,63 & 8,90 & $-3,2$ \\
\hline 75 & 0,07 & 1,08 & 0,45 & 0,19 & 4,54 & $-2,2$ \\
\hline 76 & 0,04 & 0,90 & 0,36 & 0,16 & 3,57 & $-1,8$ \\
\hline 77 & 0,47 & 2,91 & 1,02 & 0,59 & 10,18 & $-3,3$ \\
\hline 78 & 1,54 & 4,97 & 1,78 & 1,10 & 17,84 & $-4,2$ \\
\hline 79 & 0,11 & 1,46 & 0,64 & 0,21 & 6,43 & $-2,7$ \\
\hline 80 & 0,06 & 0,93 & 0,32 & 0,26 & 3,16 & $-1,7$ \\
\hline 81 & 0,56 & 3,14 & 1,05 & 0,68 & 10,46 & $-3,4$ \\
\hline 82 & 0,11 & 1,43 & 0,63 & 0,23 & 6,25 & $-2,6$ \\
\hline 83 & 0,05 & 0,90 & 0,28 & 0,23 & 2,80 & $-1,5$ \\
\hline 84 & 0,40 & 2,48 & 0,89 & 0,58 & 8,86 & $-3,1$ \\
\hline 85 & 0,06 & 0,97 & 0,35 & 0,21 & 3,50 & $-1,8$ \\
\hline 86 & 22,54 & 21,54 & 6,80 & 4,22 & 67,99 & $-6,1$ \\
\hline 87 & 0,04 & 0,73 & 0,31 & 0,15 & 3,06 & $-1,6$ \\
\hline 88 & 3,03 & 6,63 & 2,09 & 1,85 & 20,93 & $-4,4$ \\
\hline 89 & 0,33 & 2,15 & 0,73 & 0,57 & 7,32 & $-2,9$ \\
\hline 90 & 1,84 & 6,14 & 2,29 & 1,02 & 22,93 & $-4,5$ \\
\hline 91 & 0,36 & 2,22 & 0,80 & 0,57 & 7,98 & $-3,0$ \\
\hline 92 & 0,73 & 3,78 & 1,48 & 0,63 & 14,77 & $-3,9$ \\
\hline 93 & 0,86 & 3,75 & 1,40 & 0,78 & 14,03 & $-3,8$ \\
\hline 94 & 0,27 & 2,62 & 1,11 & 0,32 & 11,09 & $-3,5$ \\
\hline 95 & 0,33 & 2,55 & 0,98 & 0,43 & 9,80 & $-3,3$ \\
\hline 96 & 0,21 & 2,12 & 0,77 & 0,36 & 7,66 & $-2,9$ \\
\hline 97 & 0,15 & 1,74 & 0,78 & 0,25 & 7,77 & $-3,0$ \\
\hline 98 & 0,05 & 0,82 & 0,30 & 0,23 & 2,97 & $-1,6$ \\
\hline 99 & 7,44 & 14,80 & 4,47 & 2,12 & 44,68 & $-5,5$ \\
\hline 100 & 0,25 & 1,95 & 0,77 & 0,41 & 7,66 & $-2,9$ \\
\hline 101 & 2,18 & 6,13 & 2,32 & 1,20 & 23,17 & $-4,5$ \\
\hline 102 & 3,20 & 8,04 & 2,75 & 1,48 & 27,53 & $-4,8$ \\
\hline 103 & 2,19 & 7,29 & 2,39 & 1,17 & 23,89 & $-4,6$ \\
\hline 104 & 0,02 & 0,52 & 0,19 & 0,12 & 1,85 & $-0,9$ \\
\hline 105 & 0,67 & 3,38 & 1,28 & 0,67 & 12,78 & $-3,7$ \\
\hline 106 & 0,65 & 3,27 & 1,20 & 0,70 & 11,98 & $-3,6$ \\
\hline 107 & 0,15 & 1,56 & 0,55 & 0,34 & 5,45 & $-2,4$ \\
\hline 108 & 0,20 & 1,74 & 0,66 & 0,40 & 6,56 & $-2,7$ \\
\hline 109 & 0,45 & 2,65 & 0,92 & 0,62 & 9,24 & $-3,2$ \\
\hline 110 & 1,01 & 4,68 & 1,51 & 0,85 & 15,09 & $-3,9$ \\
\hline 111 & 0,12 & 1,34 & 0,47 & 0,31 & 4,72 & $-2,2$ \\
\hline 112 & 0,19 & 2,01 & 0,70 & 0,35 & 6,99 & $-2,8$ \\
\hline 113 & 0,25 & 2,66 & 1,08 & 0,29 & 10,81 & $-3,4$ \\
\hline
\end{tabular}




\begin{tabular}{|c|c|c|c|c|c|c|}
\hline 114 & 0,07 & 0,90 & 0,30 & 0,28 & 2,97 & $-1,6$ \\
\hline 115 & 0,12 & 1,27 & 0,43 & 0,36 & 4,31 & $-2,1$ \\
\hline 116 & 0,09 & 1,10 & 0,40 & 0,30 & 3,96 & $-2,0$ \\
\hline 117 & 0,35 & 2,23 & 0,88 & 0,51 & 8,75 & $-3,1$ \\
\hline 118 & 0,38 & 2,61 & 1,02 & 0,47 & 10,20 & $-3,4$ \\
\hline 119 & 0,24 & 1,84 & 0,64 & 0,48 & 6,38 & $-2,7$ \\
\hline 120 & 0,08 & 0,97 & 0,38 & 0,25 & 3,83 & $-1,9$ \\
\hline 121 & 0,11 & 1,29 & 0,47 & 0,28 & 4,74 & $-2,2$ \\
\hline 122 & 0,05 & 0,79 & 0,29 & 0,21 & 2,87 & $-1,5$ \\
\hline 123 & 0,18 & 1,69 & 0,57 & 0,40 & 5,65 & $-2,5$ \\
\hline 124 & 0,15 & 1,50 & 0,58 & 0,33 & 5,82 & $-2,5$ \\
\hline 125 & 0,33 & 2,40 & 0,75 & 0,55 & 7,49 & $-2,9$ \\
\hline 126 & 0,75 & 3,49 & 1,37 & 0,70 & 13,70 & $-3,8$ \\
\hline 127 & 0,36 & 2,24 & 0,83 & 0,55 & 8,26 & $-3,0$ \\
\hline 128 & 0,06 & 0,93 & 0,33 & 0,23 & 3,33 & $-1,7$ \\
\hline 129 & 0,15 & 1,55 & 0,57 & 0,33 & 5,68 & $-2,5$ \\
\hline 130 & 0,10 & 1,20 & 0,47 & 0,26 & 4,72 & $-2,2$ \\
\hline 131 & 0,11 & 1,34 & 0,52 & 0,28 & 5,16 & $-2,4$ \\
\hline 132 & 4,01 & 8,24 & 3,09 & 1,65 & 30,94 & $-5,0$ \\
\hline 133 & 0,15 & 1,55 & 0,59 & 0,33 & 5,92 & $-2,6$ \\
\hline 134 & 0,04 & 0,76 & 0,30 & 0,16 & 2,99 & $-1,6$ \\
\hline 135 & 0,07 & 0,97 & 0,33 & 0,28 & 3,25 & $-1,7$ \\
\hline 136 & 0,07 & 0,93 & 0,33 & 0,26 & 3,29 & $-1,7$ \\
\hline 137 & 0,17 & 1,77 & 0,74 & 0,30 & 7,40 & $-2,9$ \\
\hline 138 & 0,08 & 1,13 & 0,44 & 0,24 & 4,39 & $-2,1$ \\
\hline 139 & 0,97 & 3,73 & 1,20 & 1,03 & 11,95 & $-3,6$ \\
\hline 140 & 0,62 & 3,07 & 1,00 & 0,79 & 9,96 & $-3,3$ \\
\hline 141 & 0,09 & 1,20 & 0,37 & 0,30 & 3,74 & $-1,9$ \\
\hline 142 & 0,01 & 0,38 & 0,16 & 0,10 & 1,56 & $-0,6$ \\
\hline 143 & 0,21 & 1,69 & 0,59 & 0,45 & 5,86 & $-2,6$ \\
\hline 144 & 0,08 & 1,00 & 0,37 & 0,26 & 3,73 & $-1,9$ \\
\hline 145 & 0,05 & 0,76 & 0,29 & 0,21 & 2,88 & $-1,5$ \\
\hline 146 & 0,15 & 1,45 & 0,53 & 0,36 & 5,33 & $-2,4$ \\
\hline 147 & 0,11 & 1,31 & 0,49 & 0,29 & 4,93 & $-2,3$ \\
\hline 148 & 2,02 & 6,40 & 2,28 & 1,13 & 22,80 & $-4,5$ \\
\hline 149 & 2,16 & 9,21 & 3,43 & 0,80 & 34,25 & $-5,1$ \\
\hline 150 & 0,40 & 2,54 & 0,88 & 0,57 & 8,77 & $-3,1$ \\
\hline 151 & 0,03 & 0,72 & 0,26 & 0,16 & 2,62 & $-1,4$ \\
\hline 152 & 0,10 & 1,31 & 0,53 & 0,24 & 5,28 & $-2,4$ \\
\hline 153 & 0,16 & 2,13 & 0,80 & 0,25 & 8,04 & $-3,0$ \\
\hline 154 & 1,48 & 4,70 & 1,44 & 1,31 & 14,35 & $-3,8$ \\
\hline 155 & 0,08 & 1,04 & 0,41 & 0,26 & 4,09 & $-2,0$ \\
\hline 156 & 0,39 & 2,84 & 1,14 & 0,44 & 11,43 & $-3,5$ \\
\hline 157 & 0,30 & 2,09 & 0,76 & 0,51 & 7,62 & $-2,9$ \\
\hline 158 & 5,79 & 9,83 & 2,91 & 2,53 & 29,09 & $-4,9$ \\
\hline 159 & 0,17 & 1,52 & 0,57 & 0,39 & 5,66 & $-2,5$ \\
\hline 160 & 4,88 & 9,08 & 2,85 & 2,18 & 28,49 & $-4,8$ \\
\hline 161 & 0,31 & 2,18 & 0,73 & 0,54 & 7,30 & $-2,9$ \\
\hline 162 & 0,17 & 1,62 & 0,60 & 0,37 & 5,95 & $-2,6$ \\
\hline 163 & 0,31 & 2,21 & 0,80 & 0,49 & 8,04 & $-3,0$ \\
\hline 164 & 0,07 & 1,15 & 0,49 & 0,19 & 4,87 & $-2,3$ \\
\hline 165 & 0,94 & 4,58 & 1,88 & 0,64 & 18,78 & $-4,2$ \\
\hline 166 & 1,32 & 4,26 & 1,40 & 1,20 & 14,04 & $-3,8$ \\
\hline 167 & 0,15 & 1,43 & 0,49 & 0,38 & 4,86 & $-2,3$ \\
\hline 168 & 0,57 & 3,57 & 1,43 & 0,50 & 14,34 & $-3,8$ \\
\hline 169 & 0,26 & 2,32 & 0,94 & 0,35 & 9,44 & $-3,2$ \\
\hline 170 & 0,08 & 1,15 & 0,49 & 0,20 & 4,89 & $-2,3$ \\
\hline 171 & 0,18 & 1,66 & 0,64 & 0,35 & 6,35 & $-2,7$ \\
\hline 172 & 0,83 & 3,97 & 1,57 & 0,67 & 15,70 & $-4,0$ \\
\hline 173 & 1,66 & 5,08 & 1,95 & 1,08 & 19,49 & $-4,3$ \\
\hline 174 & 0,27 & 2,33 & 0,88 & 0,40 & 8,78 & $-3,1$ \\
\hline 175 & 0,10 & 1,14 & 0,38 & 0,32 & 3,76 & $-1,9$ \\
\hline
\end{tabular}




\begin{tabular}{|c|c|c|c|c|c|c|}
\hline 176 & 2,48 & 6,39 & 2,15 & 1,47 & 21,53 & $-4,4$ \\
\hline 177 & 0,42 & 2,83 & 1,03 & 0,52 & 10,26 & $-3,4$ \\
\hline 178 & 0,24 & 1,83 & 0,61 & 0,49 & 6,13 & $-2,6$ \\
\hline 179 & 20,55 & 21,87 & 7,86 & 3,33 & 78,58 & $-6,3$ \\
\hline 180 & 47,53 & 34,28 & 9,03 & 6,70 & 90,26 & $-6,5$ \\
\hline 181 & 0,15 & 1,50 & 0,45 & 0,43 & 4,46 & $-2,2$ \\
\hline 182 & 0,65 & 3,33 & 1,23 & 0,67 & 12,34 & $-3,6$ \\
\hline 183 & 0,05 & 0,79 & 0,30 & 0,21 & 2,99 & $-1,6$ \\
\hline 184 & 1,42 & 4,89 & 1,72 & 1,05 & 17,23 & $-4,1$ \\
\hline 185 & 0,26 & 1,91 & 0,65 & 0,52 & 6,46 & $-2,7$ \\
\hline 186 & 36,54 & 35,08 & 8,14 & 5,72 & 81,35 & $-6,3$ \\
\hline 187 & 0,04 & 0,80 & 0,27 & 0,17 & 2,69 & $-1,4$ \\
\hline 188 & 0,54 & 3,17 & 1,08 & 0,64 & 10,83 & $-3,4$ \\
\hline 189 & 6,16 & 10,40 & 3,22 & 2,43 & 32,21 & $-5,0$ \\
\hline 190 & 2,92 & 6,63 & 2,00 & 1,86 & 19,98 & $-4,3$ \\
\hline 191 & 0,11 & 1,17 & 0,44 & 0,31 & 4,42 & $-2,1$ \\
\hline 192 & 0,18 & 1,60 & 0,56 & 0,42 & 5,58 & $-2,5$ \\
\hline 193 & 0,07 & 1,00 & 0,38 & 0,24 & 3,84 & $-1,9$ \\
\hline 194 & 1,27 & 4,68 & 1,62 & 1,00 & 16,19 & $-4,0$ \\
\hline 195 & 0,06 & 0,83 & 0,31 & 0,24 & 3,12 & $-1,6$ \\
\hline 196 & 0,62 & 3,22 & 1,24 & 0,64 & 12,38 & $-3,6$ \\
\hline 197 & 0,95 & 3,81 & 1,27 & 0,95 & 12,67 & $-3,7$ \\
\hline 198 & 0,84 & 3,64 & 1,40 & 0,76 & 13,96 & $-3,8$ \\
\hline 199 & 0,15 & 1,52 & 0,54 & 0,35 & 5,43 & $-2,4$ \\
\hline 200 & 6,23 & 10,74 & 3,12 & 2,54 & 31,22 & $-5,0$ \\
\hline 201 & 0,03 & 0,59 & 0,25 & 0,14 & 2,52 & $-1,3$ \\
\hline 202 & 0,10 & 1,18 & 0,49 & 0,25 & 4,94 & $-2,3$ \\
\hline 203 & 0,05 & 0,80 & 0,31 & 0,21 & 3,06 & $-1,6$ \\
\hline 204 & 0,44 & 2,56 & 0,89 & 0,64 & 8,85 & $-3,1$ \\
\hline 205 & 0,36 & 2,27 & 0,79 & 0,58 & 7,92 & $-3,0$ \\
\hline 206 & 2,09 & 5,98 & 1,86 & 1,43 & 18,58 & $-4,2$ \\
\hline 207 & 0,07 & 1,06 & 0,44 & 0,20 & 4,43 & $-2,1$ \\
\hline 208 & 1,19 & 4,19 & 1,43 & 1,06 & 14,25 & $-3,8$ \\
\hline 209 & 0,64 & 3,21 & 1,10 & 0,74 & 10,96 & $-3,5$ \\
\hline 210 & 0,08 & 0,97 & 0,37 & 0,26 & 3,72 & $-1,9$ \\
\hline 211 & 0,32 & 2,49 & 1,01 & 0,41 & 10,10 & $-3,3$ \\
\hline 212 & 4,06 & 8,95 & 3,11 & 1,66 & 31,05 & $-5,0$ \\
\hline 213 & 0,20 & 1,94 & 0,81 & 0,31 & 8,06 & $-3,0$ \\
\hline 214 & 0,18 & 1,83 & 0,77 & 0,30 & 7,71 & $-2,9$ \\
\hline 215 & 0,27 & 2,15 & 0,81 & 0,43 & 8,10 & $-3,0$ \\
\hline 216 & 0,20 & 2,00 & 0,84 & 0,31 & 8,36 & $-3,1$ \\
\hline 217 & 30,26 & 29,27 & 8,14 & 4,73 & 81,43 & $-6,3$ \\
\hline 218 & 1,64 & 4,85 & 1,63 & 1,28 & 16,34 & $-4,0$ \\
\hline 219 & 1,97 & 6,43 & 1,77 & 1,42 & 17,66 & $-4,1$ \\
\hline 220 & 0,07 & 0,93 & 0,35 & 0,26 & 3,50 & $-1,8$ \\
\hline 221 & 0,11 & 1,50 & 0,66 & 0,22 & 6,63 & $-2,7$ \\
\hline 222 & 0,30 & 2,26 & 0,86 & 0,45 & 8,63 & $-3,1$ \\
\hline 223 & 6,74 & 11,49 & 4,56 & 1,88 & 45,59 & $-5,5$ \\
\hline 224 & 0,50 & 2,77 & 0,88 & 0,73 & 8,76 & $-3,1$ \\
\hline 225 & 0,10 & 1,35 & 0,51 & 0,24 & 5,06 & $-2,3$ \\
\hline 226 & 0,13 & 1,50 & 0,68 & 0,24 & 6,77 & $-2,8$ \\
\hline 227 & 0,04 & 0,69 & 0,23 & 0,20 & 2,33 & $-1,2$ \\
\hline 228 & 0,15 & 1,46 & 0,57 & 0,34 & 5,66 & $-2,5$ \\
\hline 229 & 0,18 & 1,70 & 0,62 & 0,37 & 6,19 & $-2,6$ \\
\hline 230 & 0,03 & 0,55 & 0,22 & 0,16 & 2,17 & $-1,1$ \\
\hline 231 & 0,18 & 1,73 & 0,62 & 0,38 & 6,22 & $-2,6$ \\
\hline 232 & 0,31 & 2,18 & 0,87 & 0,46 & 8,65 & $-3,1$ \\
\hline 233 & 0,53 & 2,64 & 0,88 & 0,77 & 8,79 & $-3,1$ \\
\hline 234 & 0,17 & 1,62 & 0,58 & 0,38 & 5,79 & $-2,5$ \\
\hline 235 & 0,09 & 1,11 & 0,44 & 0,25 & 4,37 & $-2,1$ \\
\hline 236 & 0,28 & 1,99 & 0,75 & 0,47 & 7,47 & $-2,9$ \\
\hline 237 & 1,74 & 4,96 & 1,74 & 1,27 & 17,37 & $-4,1$ \\
\hline
\end{tabular}




\begin{tabular}{|c|c|c|c|c|c|c|}
\hline 238 & 0,17 & 1,52 & 0,48 & 0,45 & 4,82 & $-2,3$ \\
\hline 239 & 2,13 & 6,02 & 2,27 & 1,19 & 22,72 & $-4,5$ \\
\hline 240 & 0,04 & 0,80 & 0,30 & 0,18 & 2,99 & $-1,6$ \\
\hline 241 & 0,05 & 0,86 & 0,29 & 0,22 & 2,90 & $-1,5$ \\
\hline 242 & 4,26 & 11,39 & 3,42 & 1,59 & 34,19 & $-5,1$ \\
\hline 243 & 0,20 & 1,64 & 0,52 & 0,48 & 5,24 & $-2,4$ \\
\hline 244 & 0,15 & 1,55 & 0,63 & 0,30 & 6,32 & $-2,7$ \\
\hline 245 & 0,32 & 2,19 & 0,82 & 0,50 & 8,22 & $-3,0$ \\
\hline 246 & 0,14 & 1,43 & 0,58 & 0,30 & 5,80 & $-2,5$ \\
\hline 247 & 2,31 & 6,85 & 2,23 & 1,32 & 22,33 & $-4,5$ \\
\hline 248 & 2,06 & 6,04 & 2,33 & 1,12 & 23,28 & $-4,5$ \\
\hline 249 & 0,30 & 2,16 & 0,86 & 0,45 & 8,60 & $-3,1$ \\
\hline 250 & 0,19 & 1,74 & 0,61 & 0,41 & 6,05 & $-2,6$ \\
\hline 251 & 0,18 & 1,76 & 0,66 & 0,34 & 6,57 & $-2,7$ \\
\hline 252 & 0,22 & 1,77 & 0,64 & 0,45 & 6,36 & $-2,7$ \\
\hline 253 & 15,98 & 16,27 & 5,68 & 3,58 & 56,82 & $-5,8$ \\
\hline 254 & 0,52 & 2,72 & 0,86 & 0,77 & 8,63 & $-3,1$ \\
\hline 255 & 1,02 & 3,97 & 1,42 & 0,91 & 14,18 & $-3,8$ \\
\hline 256 & 0,11 & 1,63 & 0,72 & 0,19 & 7,23 & $-2,9$ \\
\hline 257 & 1,38 & 5,05 & 1,98 & 0,88 & 19,80 & $-4,3$ \\
\hline 258 & 0,10 & 1,19 & 0,49 & 0,25 & 4,85 & $-2,3$ \\
\hline 259 & 0,02 & 0,49 & 0,20 & 0,13 & 2,00 & $-1,0$ \\
\hline 260 & 0,10 & 1,13 & 0,42 & 0,30 & 4,20 & $-2,1$ \\
\hline 261 & 0,05 & 0,80 & 0,28 & 0,21 & 2,82 & $-1,5$ \\
\hline 262 & 0,26 & 2,13 & 0,69 & 0,48 & 6,93 & $-2,8$ \\
\hline 263 & 0,42 & 2,59 & 0,96 & 0,56 & 9,58 & $-3,3$ \\
\hline 264 & 7,25 & 12,04 & 3,70 & 2,50 & 36,95 & $-5,2$ \\
\hline 265 & 0,36 & 2,60 & 1,02 & 0,45 & 10,24 & $-3,4$ \\
\hline 266 & 0,37 & 2,23 & 0,69 & 0,68 & 6,89 & $-2,8$ \\
\hline 267 & 0,07 & 1,00 & 0,37 & 0,24 & 3,71 & $-1,9$ \\
\hline 268 & 1,50 & 5,03 & 1,90 & 1,01 & 19,01 & $-4,2$ \\
\hline 269 & 0,06 & 0,87 & 0,34 & 0,22 & 3,36 & $-1,7$ \\
\hline 270 & 0,04 & 0,89 & 0,37 & 0,15 & 3,72 & $-1,9$ \\
\hline 271 & 0,76 & 3,73 & 1,46 & 0,66 & 14,60 & $-3,9$ \\
\hline 272 & 0,16 & 1,63 & 0,65 & 0,32 & 6,53 & $-2,7$ \\
\hline 273 & 0,90 & 3,81 & 1,33 & 0,87 & 13,27 & $-3,7$ \\
\hline 274 & 0,16 & 1,76 & 0,65 & 0,32 & 6,46 & $-2,7$ \\
\hline 275 & 1,31 & 5,03 & 1,44 & 1,16 & 14,37 & $-3,8$ \\
\hline 276 & 0,59 & 3,05 & 1,20 & 0,63 & 12,01 & $-3,6$ \\
\hline 277 & 8,02 & 11,10 & 3,69 & 2,77 & 36,88 & $-5,2$ \\
\hline 278 & 0,13 & 1,24 & 0,45 & 0,36 & 4,46 & $-2,2$ \\
\hline 279 & 19,28 & 17,71 & 5,85 & 4,20 & 58,52 & $-5,9$ \\
\hline 280 & 0,11 & 1,20 & 0,38 & 0,35 & 3,80 & $-1,9$ \\
\hline 281 & 0,45 & 2,54 & 0,85 & 0,67 & 8,46 & $-3,1$ \\
\hline 282 & 0,16 & 1,69 & 0,61 & 0,33 & 6,06 & $-2,6$ \\
\hline 283 & 1,12 & 4,18 & 1,38 & 1,03 & 13,75 & $-3,8$ \\
\hline 284 & 0,11 & 1,50 & 0,59 & 0,23 & 5,88 & $-2,6$ \\
\hline 285 & 0,17 & 1,57 & 0,61 & 0,36 & 6,12 & $-2,6$ \\
\hline 286 & 0,32 & 2,46 & 0,90 & 0,45 & 8,97 & $-3,2$ \\
\hline 287 & 0,28 & 2,01 & 0,73 & 0,49 & 7,31 & $-2,9$ \\
\hline 288 & 0,84 & 4,14 & 1,55 & 0,69 & 15,49 & $-4,0$ \\
\hline 289 & 0,16 & 1,60 & 0,55 & 0,38 & 5,54 & $-2,5$ \\
\hline 290 & 0,53 & 3,05 & 1,25 & 0,54 & 12,54 & $-3,6$ \\
\hline 291 & 0,08 & 1,20 & 0,46 & 0,22 & 4,55 & $-2,2$ \\
\hline 292 & 1,17 & 4,55 & 1,69 & 0,88 & 16,93 & $-4,1$ \\
\hline 293 & 0,03 & 0,73 & 0,31 & 0,14 & 3,10 & $-1,6$ \\
\hline 294 & 3,57 & 7,66 & 2,52 & 1,81 & 25,21 & $-4,7$ \\
\hline 295 & 1,46 & 5,30 & 2,12 & 0,88 & 21,17 & $-4,4$ \\
\hline 296 & 1,15 & 4,48 & 1,49 & 0,98 & 14,87 & $-3,9$ \\
\hline 297 & 0,15 & 1,45 & 0,54 & 0,35 & 5,40 & $-2,4$ \\
\hline 298 & 0,09 & 1,15 & 0,42 & 0,28 & 4,24 & $-2,1$ \\
\hline 299 & 1,16 & 4,95 & 1,52 & 0,97 & 15,19 & $-3,9$ \\
\hline
\end{tabular}




\begin{tabular}{|c|c|c|c|c|c|c|}
\hline 300 & 0,43 & 2,85 & 0,81 & 0,68 & 8,10 & $-3,0$ \\
\hline 301 & 0,67 & 3,88 & 1,24 & 0,68 & 12,43 & $-3,6$ \\
\hline 302 & 0,29 & 2,05 & 0,72 & 0,52 & 7,16 & $-2,8$ \\
\hline 303 & 0,21 & 1,74 & 0,66 & 0,40 & 6,57 & $-2,7$ \\
\hline 304 & 1,58 & 5,39 & 2,08 & 0,97 & 20,83 & $-4,4$ \\
\hline 305 & 0,23 & 1,80 & 0,58 & 0,50 & 5,84 & $-2,5$ \\
\hline 306 & 0,04 & 0,77 & 0,31 & 0,17 & 3,07 & $-1,6$ \\
\hline 307 & 0,05 & 1,00 & 0,37 & 0,19 & 3,70 & $-1,9$ \\
\hline 308 & 0,82 & 3,52 & 1,33 & 0,79 & 13,28 & $-3,7$ \\
\hline 309 & 0,21 & 1,90 & 0,80 & 0,34 & 8,01 & $-3,0$ \\
\hline 310 & 0,05 & 0,83 & 0,30 & 0,23 & 3,00 & $-1,6$ \\
\hline 311 & 0,11 & 1,31 & 0,47 & 0,29 & 4,66 & $-2,2$ \\
\hline 312 & 0,39 & 2,44 & 0,87 & 0,57 & 8,71 & $-3,1$ \\
\hline 313 & 0,55 & 2,98 & 1,12 & 0,63 & 11,17 & $-3,5$ \\
\hline 314 & 0,24 & 1,90 & 0,62 & 0,49 & 6,24 & $-2,6$ \\
\hline 315 & 0,03 & 0,66 & 0,25 & 0,17 & 2,53 & $-1,3$ \\
\hline 316 & 20,92 & 19,57 & 5,32 & 5,00 & 53,24 & $-5,7$ \\
\hline 317 & 0,10 & 1,21 & 0,47 & 0,28 & 4,69 & $-2,2$ \\
\hline 318 & 0,61 & 2,99 & 1,08 & 0,72 & 10,78 & $-3,4$ \\
\hline 319 & 0,04 & 0,76 & 0,28 & 0,20 & 2,81 & $-1,5$ \\
\hline 320 & 0,29 & 2,08 & 0,67 & 0,55 & 6,68 & $-2,7$ \\
\hline 321 & 0,18 & 1,79 & 0,75 & 0,31 & 7,47 & $-2,9$ \\
\hline 322 & 0,12 & 1,46 & 0,57 & 0,27 & 5,70 & $-2,5$ \\
\hline 323 & 52,99 & 30,34 & 10,59 & 6,37 & 105,90 & $-6,7$ \\
\hline 324 & 0,09 & 1,10 & 0,36 & 0,30 & 3,61 & $-1,9$ \\
\hline 325 & 1,39 & 5,02 & 1,67 & 1,06 & 16,68 & $-4,1$ \\
\hline 326 & 0,78 & 3,40 & 1,15 & 0,87 & 11,50 & $-3,5$ \\
\hline 327 & 0,21 & 1,97 & 0,83 & 0,33 & 8,27 & $-3,0$ \\
\hline 328 & 0,17 & 1,49 & 0,54 & 0,40 & 5,36 & $-2,4$ \\
\hline 329 & 0,16 & 1,74 & 0,72 & 0,28 & 7,22 & $-2,9$ \\
\hline 330 & 0,06 & 0,90 & 0,35 & 0,21 & 3,48 & $-1,8$ \\
\hline 331 & 0,08 & 0,96 & 0,36 & 0,27 & 3,64 & $-1,9$ \\
\hline 332 & 0,02 & 0,45 & 0,15 & 0,14 & 1,53 & $-0,6$ \\
\hline 333 & 0,25 & 1,85 & 0,67 & 0,47 & 6,74 & $-2,8$ \\
\hline 334 & 0,32 & 2,31 & 0,77 & 0,53 & 7,73 & $-3,0$ \\
\hline 335 & 0,30 & 2,20 & 0,85 & 0,44 & 8,51 & $-3,1$ \\
\hline 336 & 13,82 & 17,27 & 6,28 & 2,80 & 62,83 & $-6,0$ \\
\hline 337 & 0,04 & 0,69 & 0,24 & 0,20 & 2,36 & $-1,2$ \\
\hline 338 & 0,55 & 3,09 & 1,12 & 0,63 & 11,19 & $-3,5$ \\
\hline 339 & 0,02 & 0,48 & 0,21 & 0,11 & 2,06 & $-1,0$ \\
\hline 340 & 0,07 & 1,06 & 0,39 & 0,22 & 3,91 & $-2,0$ \\
\hline 341 & 0,19 & 2,04 & 0,86 & 0,29 & 8,62 & $-3,1$ \\
\hline 342 & 0,36 & 2,51 & 0,98 & 0,47 & 9,76 & $-3,3$ \\
\hline 343 & 1,02 & 4,40 & 1,40 & 0,93 & 14,00 & $-3,8$ \\
\hline 344 & 0,13 & 1,38 & 0,51 & 0,32 & 5,05 & $-2,3$ \\
\hline 345 & 0,39 & 2,54 & 1,08 & 0,46 & 10,78 & $-3,4$ \\
\hline 346 & 0,56 & 3,42 & 1,44 & 0,50 & 14,41 & $-3,8$ \\
\hline 347 & 0,16 & 1,57 & 0,54 & 0,37 & 5,42 & $-2,4$ \\
\hline 348 & 0,12 & 1,32 & 0,54 & 0,29 & 5,35 & $-2,4$ \\
\hline 349 & 8,34 & 11,70 & 3,56 & 2,99 & 35,56 & $-5,2$ \\
\hline 350 & 2,73 & 6,85 & 1,99 & 1,75 & 19,85 & $-4,3$ \\
\hline 351 & 15,88 & 16,98 & 5,12 & 3,95 & 51,22 & $-5,7$ \\
\hline 352 & 0,27 & 2,38 & 1,00 & 0,35 & 9,99 & $-3,3$ \\
\hline 353 & 0,07 & 1,14 & 0,41 & 0,22 & 4,12 & $-2,0$ \\
\hline 354 & 0,97 & 4,03 & 1,51 & 0,82 & 15,09 & $-3,9$ \\
\hline 355 & 0,17 & 1,62 & 0,64 & 0,33 & 6,43 & $-2,7$ \\
\hline 356 & 0,22 & 1,91 & 0,73 & 0,38 & 7,29 & $-2,9$ \\
\hline 357 & 21,98 & 23,39 & 9,14 & 3,06 & 91,42 & $-6,5$ \\
\hline 358 & 0,05 & 0,86 & 0,31 & 0,22 & 3,09 & $-1,6$ \\
\hline 359 & 0,18 & 1,70 & 0,63 & 0,37 & 6,25 & $-2,6$ \\
\hline 360 & 0,97 & 4,08 & 1,48 & 0,84 & 14,81 & $-3,9$ \\
\hline 361 & 0,10 & 1,39 & 0,56 & 0,23 & 5,60 & $-2,5$ \\
\hline
\end{tabular}




\begin{tabular}{|c|c|c|c|c|c|c|}
\hline 362 & 0,29 & 2,18 & 0,79 & 0,46 & 7,91 & $-3,0$ \\
\hline 363 & 0,22 & 1,79 & 0,63 & 0,44 & 6,30 & $-2,7$ \\
\hline 364 & 0,07 & 1,32 & 0,54 & 0,17 & 5,44 & $-2,4$ \\
\hline 365 & 1,87 & 6,00 & 1,80 & 1,32 & 17,97 & $-4,2$ \\
\hline 366 & 0,07 & 0,93 & 0,35 & 0,25 & 3,51 & $-1,8$ \\
\hline 367 & 1,10 & 4,32 & 1,63 & 0,86 & 16,30 & $-4,0$ \\
\hline 368 & 0,99 & 3,90 & 1,43 & 0,88 & 14,34 & $-3,8$ \\
\hline 369 & 0,52 & 3,05 & 1,24 & 0,54 & 12,39 & $-3,6$ \\
\hline 370 & 8,08 & 11,75 & 3,54 & 2,91 & 35,37 & $-5,1$ \\
\hline 371 & 0,23 & 1,78 & 0,57 & 0,51 & 5,70 & $-2,5$ \\
\hline 372 & 0,21 & 1,83 & 0,64 & 0,42 & 6,43 & $-2,7$ \\
\hline 373 & 0,02 & 0,61 & 0,34 & 0,06 & 3,35 & $-1,7$ \\
\hline 374 & 0,25 & 2,02 & 0,76 & 0,42 & 7,60 & $-2,9$ \\
\hline 375 & 0,01 & 0,38 & 0,16 & 0,10 & 1,56 & $-0,6$ \\
\hline 376 & 0,73 & 3,28 & 1,10 & 0,85 & 10,98 & $-3,5$ \\
\hline 377 & 0,55 & 3,36 & 1,28 & 0,55 & 12,82 & $-3,7$ \\
\hline 378 & 5,06 & 9,83 & 3,60 & 1,79 & 35,96 & $-5,2$ \\
\hline 379 & 1,52 & 5,78 & 2,32 & 0,84 & 23,17 & $-4,5$ \\
\hline 380 & 0,06 & 0,93 & 0,32 & 0,25 & 3,17 & $-1,7$ \\
\hline 381 & 0,76 & 3,59 & 1,36 & 0,71 & 13,60 & $-3,8$ \\
\hline 382 & 0,24 & 2,09 & 0,79 & 0,38 & 7,88 & $-3,0$ \\
\hline 383 & 0,29 & 2,06 & 0,71 & 0,52 & 7,11 & $-2,8$ \\
\hline 384 & 0,09 & 1,08 & 0,38 & 0,29 & 3,82 & $-1,9$ \\
\hline 385 & 26,60 & 21,79 & 7,60 & 4,46 & 76,03 & $-6,2$ \\
\hline 386 & 0,12 & 1,41 & 0,41 & 0,38 & 4,06 & $-2,0$ \\
\hline 387 & 0,29 & 2,33 & 0,97 & 0,38 & 9,69 & $-3,3$ \\
\hline 388 & 0,19 & 1,71 & 0,54 & 0,46 & 5,38 & $-2,4$ \\
\hline 389 & 0,51 & 2,71 & 0,97 & 0,67 & 9,67 & $-3,3$ \\
\hline 390 & 0,38 & 2,32 & 0,74 & 0,65 & 7,36 & $-2,9$ \\
\hline 391 & 0,33 & 2,23 & 0,71 & 0,59 & 7,10 & $-2,8$ \\
\hline 392 & 0,46 & 2,89 & 1,15 & 0,51 & 11,52 & $-3,5$ \\
\hline 393 & 0,31 & 2,32 & 0,87 & 0,45 & 8,65 & $-3,1$ \\
\hline 394 & 0,12 & 1,34 & 0,51 & 0,29 & 5,13 & $-2,4$ \\
\hline 395 & 0,09 & 1,15 & 0,51 & 0,23 & 5,12 & $-2,4$ \\
\hline 396 & 0,58 & 3,07 & 1,14 & 0,65 & 11,36 & $-3,5$ \\
\hline 397 & 0,24 & 1,98 & 0,70 & 0,44 & 7,03 & $-2,8$ \\
\hline 398 & 0,06 & 1,00 & 0,35 & 0,21 & 3,51 & $-1,8$ \\
\hline 399 & 0,01 & 0,38 & 0,14 & 0,09 & 1,35 & $-0,4$ \\
\hline 400 & 0,11 & 1,53 & 0,58 & 0,24 & 5,76 & $-2,5$ \\
\hline 401 & 0,01 & 0,28 & 0,12 & 0,08 & 1,17 & $-0,2$ \\
\hline 402 & 0,01 & 0,35 & 0,16 & 0,10 & 1,56 & $-0,6$ \\
\hline 403 & 0,90 & 3,81 & 1,42 & 0,81 & 14,16 & $-3,8$ \\
\hline 404 & 2,13 & 7,81 & 3,29 & 0,82 & 32,86 & $-5,0$ \\
\hline 405 & 0,41 & 3,01 & 1,09 & 0,48 & 10,94 & $-3,5$ \\
\hline 406 & 0,10 & 1,22 & 0,41 & 0,32 & 4,06 & $-2,0$ \\
\hline 407 & 0,13 & 1,39 & 0,57 & 0,28 & 5,66 & $-2,5$ \\
\hline 408 & 2,00 & 6,03 & 1,87 & 1,36 & 18,69 & $-4,2$ \\
\hline 409 & 0,27 & 2,12 & 0,85 & 0,40 & 8,53 & $-3,1$ \\
\hline 410 & 0,01 & 0,35 & 0,16 & 0,10 & 1,56 & $-0,6$ \\
\hline 411 & 0,06 & 0,96 & 0,34 & 0,21 & 3,43 & $-1,8$ \\
\hline 412 & 0,17 & 1,57 & 0,52 & 0,41 & 5,18 & $-2,4$ \\
\hline 413 & 0,26 & 2,04 & 0,68 & 0,48 & 6,84 & $-2,8$ \\
\hline 414 & 0,13 & 1,31 & 0,48 & 0,33 & 4,82 & $-2,3$ \\
\hline 415 & 0,17 & 2,18 & 0,92 & 0,23 & 9,23 & $-3,2$ \\
\hline 416 & 0,23 & 1,85 & 0,62 & 0,47 & 6,23 & $-2,6$ \\
\hline 417 & 0,50 & 2,96 & 1,08 & 0,59 & 10,76 & $-3,4$ \\
\hline 418 & 0,64 & 3,86 & 1,51 & 0,54 & 15,11 & $-3,9$ \\
\hline 419 & 0,09 & 1,17 & 0,48 & 0,23 & 4,83 & $-2,3$ \\
\hline 420 & 0,17 & 1,58 & 0,59 & 0,37 & 5,93 & $-2,6$ \\
\hline 421 & 0,19 & 1,73 & 0,53 & 0,46 & 5,32 & $-2,4$ \\
\hline 422 & 0,47 & 2,69 & 0,96 & 0,62 & 9,57 & $-3,3$ \\
\hline 423 & 0,33 & 2,19 & 0,81 & 0,52 & 8,11 & $-3,0$ \\
\hline
\end{tabular}




\begin{tabular}{|c|c|c|c|c|c|c|}
\hline 424 & 0,15 & 1,52 & 0,51 & 0,36 & 5,13 & $-2,4$ \\
\hline 425 & 0,05 & 0,93 & 0,37 & 0,18 & 3,67 & $-1,9$ \\
\hline 426 & 0,05 & 0,83 & 0,34 & 0,18 & 3,38 & $-1,8$ \\
\hline 427 & 0,10 & 1,22 & 0,47 & 0,28 & 4,65 & $-2,2$ \\
\hline 428 & 23,75 & 21,42 & 8,18 & 3,70 & 81,80 & $-6,4$ \\
\hline 429 & 0,03 & 0,62 & 0,21 & 0,18 & 2,14 & $-1,1$ \\
\hline 430 & 0,25 & 1,88 & 0,70 & 0,45 & 6,98 & $-2,8$ \\
\hline 431 & 0,35 & 2,23 & 0,79 & 0,56 & 7,85 & $-3,0$ \\
\hline 432 & 0,36 & 3,36 & 1,33 & 0,35 & 13,27 & $-3,7$ \\
\hline 433 & 0,02 & 0,42 & 0,15 & 0,14 & 1,53 & $-0,6$ \\
\hline 434 & 0,97 & 3,98 & 1,29 & 0,96 & 12,87 & $-3,7$ \\
\hline 435 & 0,95 & 3,94 & 1,36 & 0,89 & 13,61 & $-3,8$ \\
\hline 436 & 0,05 & 0,97 & 0,39 & 0,17 & 3,89 & $-2,0$ \\
\hline 437 & 0,63 & 3,40 & 1,27 & 0,63 & 12,72 & $-3,7$ \\
\hline 438 & 0,11 & 1,28 & 0,48 & 0,30 & 4,75 & $-2,2$ \\
\hline 439 & 1,71 & 5,71 & 1,67 & 1,30 & 16,67 & $-4,1$ \\
\hline 440 & 0,52 & 3,33 & 1,35 & 0,49 & 13,52 & $-3,8$ \\
\hline 441 & 3,35 & 7,74 & 2,22 & 1,92 & 22,18 & $-4,5$ \\
\hline 442 & 0,45 & 2,58 & 0,93 & 0,62 & 9,32 & $-3,2$ \\
\hline 443 & 0,10 & 1,29 & 0,47 & 0,28 & 4,65 & $-2,2$ \\
\hline 444 & 0,69 & 3,19 & 1,17 & 0,75 & 11,72 & $-3,6$ \\
\hline 445 & 55,87 & 38,05 & 11,81 & 6,02 & 118,12 & $-6,9$ \\
\hline 446 & 1,51 & 5,22 & 2,01 & 0,96 & 20,11 & $-4,3$ \\
\hline 447 & 0,19 & 1,83 & 0,75 & 0,32 & 7,52 & $-2,9$ \\
\hline 448 & 0,06 & 1,07 & 0,42 & 0,18 & 4,16 & $-2,1$ \\
\hline 449 & 0,16 & 1,94 & 0,84 & 0,24 & 8,43 & $-3,1$ \\
\hline 450 & 0,09 & 1,31 & 0,48 & 0,24 & 4,76 & $-2,3$ \\
\hline 451 & 3,44 & 8,29 & 3,07 & 1,43 & 30,73 & $-4,9$ \\
\hline 452 & 3,20 & 7,88 & 3,24 & 1,26 & 32,38 & $-5,0$ \\
\hline 453 & 6,56 & 10,78 & 3,45 & 2,43 & 34,45 & $-5,1$ \\
\hline 454 & 0,18 & 2,03 & 0,90 & 0,25 & 9,04 & $-3,2$ \\
\hline 455 & 0,30 & 2,75 & 1,11 & 0,34 & 11,11 & $-3,5$ \\
\hline 456 & 0,07 & 1,10 & 0,44 & 0,21 & 4,38 & $-2,1$ \\
\hline 457 & 4,90 & 9,45 & 3,16 & 1,97 & 31,63 & $-5,0$ \\
\hline 458 & 0,14 & 1,50 & 0,56 & 0,32 & 5,55 & $-2,5$ \\
\hline 459 & 0,43 & 3,38 & 1,45 & 0,38 & 14,45 & $-3,9$ \\
\hline 460 & 0,06 & 1,00 & 0,38 & 0,20 & 3,77 & $-1,9$ \\
\hline 461 & 0,35 & 2,63 & 1,07 & 0,42 & 10,68 & $-3,4$ \\
\hline 462 & 0,47 & 2,65 & 0,98 & 0,61 & 9,80 & $-3,3$ \\
\hline 463 & 5,35 & 9,98 & 3,43 & 1,99 & 34,28 & $-5,1$ \\
\hline 464 & 0,56 & 2,94 & 1,11 & 0,65 & 11,05 & $-3,5$ \\
\hline 465 & 0,08 & 1,10 & 0,34 & 0,30 & 3,41 & $-1,8$ \\
\hline 466 & 0,21 & 2,10 & 0,93 & 0,29 & 9,31 & $-3,2$ \\
\hline 467 & 0,89 & 3,70 & 1,43 & 0,79 & 14,31 & $-3,8$ \\
\hline 468 & 0,04 & 0,73 & 0,30 & 0,17 & 2,96 & $-1,6$ \\
\hline 469 & 0,03 & 0,72 & 0,26 & 0,17 & 2,62 & $-1,4$ \\
\hline 470 & 0,70 & 3,46 & 1,37 & 0,65 & 13,66 & $-3,8$ \\
\hline 471 & 0,98 & 3,98 & 1,39 & 0,89 & 13,93 & $-3,8$ \\
\hline 472 & 1,03 & 4,31 & 1,38 & 0,95 & 13,77 & $-3,8$ \\
\hline 473 & 1,46 & 4,68 & 1,59 & 1,17 & 15,85 & $-4,0$ \\
\hline 474 & 0,95 & 3,82 & 1,34 & 0,91 & 13,38 & $-3,7$ \\
\hline 475 & 0,04 & 0,80 & 0,32 & 0,17 & 3,22 & $-1,7$ \\
\hline 476 & 0,09 & 1,20 & 0,49 & 0,24 & 4,93 & $-2,3$ \\
\hline 477 & 7,21 & 10,97 & 3,15 & 2,92 & 31,45 & $-5,0$ \\
\hline 478 & 0,19 & 1,69 & 0,59 & 0,42 & 5,85 & $-2,5$ \\
\hline 479 & 4,11 & 8,20 & 2,77 & 1,89 & 27,70 & $-4,8$ \\
\hline 480 & 2,16 & 6,47 & 2,68 & 1,03 & 26,79 & $-4,7$ \\
\hline 481 & 0,20 & 1,71 & 0,61 & 0,41 & 6,10 & $-2,6$ \\
\hline 482 & 0,23 & 1,88 & 0,70 & 0,42 & 7,00 & $-2,8$ \\
\hline 483 & 2,94 & 6,85 & 2,40 & 1,56 & 24,04 & $-4,6$ \\
\hline 484 & 0,30 & 2,57 & 0,96 & 0,40 & 9,55 & $-3,3$ \\
\hline 485 & 0,21 & 1,85 & 0,61 & 0,44 & 6,09 & $-2,6$ \\
\hline
\end{tabular}




\begin{tabular}{|c|c|c|c|c|c|c|}
\hline 486 & 17,71 & 17,01 & 5,75 & 3,92 & 57,48 & $-5,8$ \\
\hline 487 & 12,78 & 14,34 & 5,22 & 3,11 & 52,24 & $-5,7$ \\
\hline 488 & 0,06 & 0,90 & 0,36 & 0,21 & 3,62 & $-1,9$ \\
\hline 489 & 1,42 & 5,14 & 1,66 & 1,09 & 16,56 & $-4,0$ \\
\hline 490 & 1,53 & 5,68 & 1,78 & 1,10 & 17,80 & $-4,2$ \\
\hline 491 & 0,05 & 0,80 & 0,31 & 0,19 & 3,12 & $-1,6$ \\
\hline 492 & 1,22 & 4,25 & 1,38 & 1,12 & 13,81 & $-3,8$ \\
\hline 493 & 0,15 & 1,62 & 0,55 & 0,35 & 5,47 & $-2,5$ \\
\hline 494 & 0,71 & 3,25 & 0,96 & 0,94 & 9,61 & $-3,3$ \\
\hline 495 & 0,22 & 1,88 & 0,72 & 0,39 & 7,20 & $-2,8$ \\
\hline 496 & 0,17 & 1,67 & 0,49 & 0,44 & 4,86 & $-2,3$ \\
\hline 497 & 0,12 & 1,34 & 0,51 & 0,29 & 5,05 & $-2,3$ \\
\hline 498 & 3,41 & 10,08 & 4,36 & 1,00 & 43,58 & $-5,4$ \\
\hline 499 & 0,75 & 3,36 & 1,17 & 0,81 & 11,65 & $-3,5$ \\
\hline 500 & 0,11 & 1,21 & 0,42 & 0,32 & 4,24 & $-2,1$ \\
\hline 501 & 0,29 & 2,19 & 0,76 & 0,48 & 7,63 & $-2,9$ \\
\hline 502 & 0,04 & 0,86 & 0,35 & 0,15 & 3,49 & $-1,8$ \\
\hline 503 & 0,26 & 1,98 & 0,63 & 0,51 & 6,34 & $-2,7$ \\
\hline 504 & 10,35 & 13,74 & 4,28 & 3,08 & 42,83 & $-5,4$ \\
\hline 505 & 0,12 & 1,42 & 0,59 & 0,25 & 5,93 & $-2,6$ \\
\hline 506 & 22,35 & 22,70 & 9,02 & 3,15 & 90,22 & $-6,5$ \\
\hline 507 & 0,13 & 1,34 & 0,44 & 0,37 & 4,44 & $-2,2$ \\
\hline 508 & 0,14 & 1,56 & 0,67 & 0,26 & 6,65 & $-2,7$ \\
\hline 509 & 1,30 & 4,61 & 1,47 & 1,12 & 14,72 & $-3,9$ \\
\hline 510 & 0,17 & 1,55 & 0,53 & 0,40 & 5,30 & $-2,4$ \\
\hline 511 & 0,19 & 1,62 & 0,61 & 0,41 & 6,05 & $-2,6$ \\
\hline 512 & 17,49 & 19,35 & 6,16 & 3,62 & 61,60 & $-5,9$ \\
\hline 513 & 0,84 & 3,54 & 1,28 & 0,84 & 12,81 & $-3,7$ \\
\hline 514 & 0,06 & 1,13 & 0,45 & 0,18 & 4,48 & $-2,2$ \\
\hline 515 & 0,90 & 3,57 & 1,17 & 0,98 & 11,67 & $-3,5$ \\
\hline 516 & 0,36 & 2,19 & 0,78 & 0,59 & 7,79 & $-3,0$ \\
\hline 517 & 0,02 & 0,45 & 0,18 & 0,14 & 1,75 & $-0,8$ \\
\hline 518 & 0,34 & 2,53 & 0,88 & 0,49 & 8,75 & $-3,1$ \\
\hline 519 & 0,13 & 1,34 & 0,49 & 0,33 & 4,89 & $-2,3$ \\
\hline 520 & 0,58 & 2,87 & 0,97 & 0,76 & 9,70 & $-3,3$ \\
\hline 521 & 0,12 & 1,29 & 0,45 & 0,33 & 4,54 & $-2,2$ \\
\hline 522 & 57,58 & 32,94 & 11,36 & 6,45 & 113,60 & $-6,8$ \\
\hline 523 & 0,09 & 1,43 & 0,62 & 0,19 & 6,24 & $-2,6$ \\
\hline 524 & 2,88 & 6,51 & 2,00 & 1,83 & 20,02 & $-4,3$ \\
\hline 525 & 0,05 & 0,86 & 0,31 & 0,21 & 3,14 & $-1,7$ \\
\hline 526 & 1,02 & 4,76 & 1,84 & 0,71 & 18,35 & $-4,2$ \\
\hline 527 & 0,03 & 0,72 & 0,28 & 0,16 & 2,78 & $-1,5$ \\
\hline 528 & 0,09 & 1,17 & 0,44 & 0,26 & 4,38 & $-2,1$ \\
\hline 529 & 0,08 & 1,13 & 0,43 & 0,25 & 4,31 & $-2,1$ \\
\hline 530 & 2,77 & 6,91 & 2,45 & 1,44 & 24,48 & $-4,6$ \\
\hline 531 & 0,55 & 3,15 & 1,24 & 0,56 & 12,42 & $-3,6$ \\
\hline 532 & 4,66 & 8,76 & 3,14 & 1,89 & 31,35 & $-5,0$ \\
\hline 533 & 1,10 & 4,81 & 1,76 & 0,80 & 17,62 & $-4,1$ \\
\hline 534 & 0,07 & 1,00 & 0,33 & 0,28 & 3,26 & $-1,7$ \\
\hline 535 & 0,01 & 0,38 & 0,16 & 0,10 & 1,56 & $-0,6$ \\
\hline 536 & 0,09 & 1,17 & 0,49 & 0,22 & 4,86 & $-2,3$ \\
\hline 537 & 0,18 & 1,86 & 0,75 & 0,31 & 7,48 & $-2,9$ \\
\hline 538 & 3,13 & 6,97 & 2,23 & 1,79 & 22,26 & $-4,5$ \\
\hline 539 & 0,34 & 2,33 & 0,86 & 0,50 & 8,62 & $-3,1$ \\
\hline 540 & 0,15 & 1,46 & 0,50 & 0,37 & 5,02 & $-2,3$ \\
\hline 541 & 0,23 & 1,73 & 0,57 & 0,50 & 5,72 & $-2,5$ \\
\hline 542 & 0,22 & 1,80 & 0,62 & 0,45 & 6,15 & $-2,6$ \\
\hline 543 & 0,54 & 3,56 & 1,59 & 0,43 & 15,88 & $-4,0$ \\
\hline 544 & 0,30 & 2,38 & 0,93 & 0,41 & 9,27 & $-3,2$ \\
\hline 545 & 2,77 & 7,14 & 2,16 & 1,63 & 21,57 & $-4,4$ \\
\hline 546 & 0,23 & 1,85 & 0,61 & 0,48 & 6,11 & $-2,6$ \\
\hline 547 & 0,04 & 0,90 & 0,37 & 0,13 & 3,74 & $-1,9$ \\
\hline
\end{tabular}




\begin{tabular}{|c|c|c|c|c|c|c|}
\hline 548 & 0,06 & 0,86 & 0,35 & 0,21 & 3,49 & $-1,8$ \\
\hline 549 & 0,15 & 1,53 & 0,56 & 0,34 & 5,58 & $-2,5$ \\
\hline 550 & 0,54 & 3,01 & 0,87 & 0,80 & 8,67 & $-3,1$ \\
\hline 551 & 0,11 & 1,28 & 0,44 & 0,33 & 4,41 & $-2,1$ \\
\hline 552 & 0,14 & 1,53 & 0,63 & 0,28 & 6,28 & $-2,7$ \\
\hline 553 & 1,73 & 5,26 & 1,79 & 1,23 & 17,92 & $-4,2$ \\
\hline 554 & 0,08 & 1,10 & 0,40 & 0,25 & 3,99 & $-2,0$ \\
\hline 555 & 0,05 & 0,76 & 0,30 & 0,20 & 3,03 & $-1,6$ \\
\hline 556 & 6,52 & 11,10 & 3,71 & 2,24 & 37,09 & $-5,2$ \\
\hline 557 & 0,25 & 2,06 & 0,76 & 0,41 & 7,64 & $-2,9$ \\
\hline 558 & 0,15 & 1,52 & 0,54 & 0,36 & 5,36 & $-2,4$ \\
\hline 559 & 0,24 & 2,95 & 0,65 & 0,47 & 6,46 & $-2,7$ \\
\hline 560 & 0,21 & 1,81 & 0,66 & 0,41 & 6,60 & $-2,7$ \\
\hline 561 & 0,17 & 1,63 & 0,55 & 0,40 & 5,54 & $-2,5$ \\
\hline 562 & 0,12 & 1,41 & 0,54 & 0,27 & 5,42 & $-2,4$ \\
\hline 563 & 0,69 & 3,24 & 1,18 & 0,74 & 11,76 & $-3,6$ \\
\hline 564 & 0,11 & 1,18 & 0,38 & 0,37 & 3,80 & $-1,9$ \\
\hline 565 & 0,22 & 1,91 & 0,70 & 0,40 & 7,02 & $-2,8$ \\
\hline 566 & 0,07 & 0,90 & 0,33 & 0,25 & 3,33 & $-1,7$ \\
\hline 567 & 0,15 & 1,48 & 0,58 & 0,32 & 5,82 & $-2,5$ \\
\hline 568 & 0,08 & 1,18 & 0,47 & 0,22 & 4,73 & $-2,2$ \\
\hline 569 & 0,18 & 1,98 & 0,90 & 0,25 & 9,01 & $-3,2$ \\
\hline 570 & 0,07 & 0,93 & 0,32 & 0,26 & 3,22 & $-1,7$ \\
\hline 571 & 0,65 & 3,56 & 1,37 & 0,60 & 13,70 & $-3,8$ \\
\hline 572 & 0,70 & 3,52 & 1,14 & 0,78 & 11,41 & $-3,5$ \\
\hline 573 & 1,67 & 5,50 & 2,10 & 1,01 & 20,96 & $-4,4$ \\
\hline 574 & 0,04 & 0,83 & 0,38 & 0,12 & 3,78 & $-1,9$ \\
\hline 575 & 0,27 & 2,28 & 0,74 & 0,47 & 7,39 & $-2,9$ \\
\hline 576 & 0,08 & 1,04 & 0,41 & 0,24 & 4,14 & $-2,0$ \\
\hline 577 & 0,12 & 1,34 & 0,45 & 0,34 & 4,52 & $-2,2$ \\
\hline 578 & 0,05 & 0,86 & 0,33 & 0,19 & 3,27 & $-1,7$ \\
\hline 579 & 0,05 & 0,83 & 0,28 & 0,24 & 2,84 & $-1,5$ \\
\hline 580 & 0,05 & 0,83 & 0,28 & 0,22 & 2,75 & $-1,5$ \\
\hline 581 & 0,07 & 1,13 & 0,46 & 0,20 & 4,59 & $-2,2$ \\
\hline 582 & 0,10 & 1,42 & 0,58 & 0,22 & 5,81 & $-2,5$ \\
\hline 583 & 0,19 & 1,92 & 0,76 & 0,32 & 7,61 & $-2,9$ \\
\hline 584 & 0,33 & 2,21 & 0,77 & 0,54 & 7,69 & $-2,9$ \\
\hline 585 & 0,70 & 3,35 & 1,10 & 0,81 & 11,04 & $-3,5$ \\
\hline 586 & 0,83 & 3,85 & 1,54 & 0,69 & 15,42 & $-3,9$ \\
\hline 587 & 0,05 & 0,96 & 0,40 & 0,15 & 4,02 & $-2,0$ \\
\hline 588 & 0,31 & 2,12 & 0,75 & 0,52 & 7,49 & $-2,9$ \\
\hline 589 & 0,83 & 3,67 & 1,12 & 0,94 & 11,22 & $-3,5$ \\
\hline 590 & 6,08 & 10,64 & 3,36 & 2,31 & 33,56 & $-5,1$ \\
\hline 591 & 0,15 & 1,42 & 0,50 & 0,37 & 5,02 & $-2,3$ \\
\hline 592 & 0,10 & 1,49 & 0,58 & 0,22 & 5,80 & $-2,5$ \\
\hline 593 & 0,23 & 1,87 & 0,66 & 0,44 & 6,60 & $-2,7$ \\
\hline 594 & 0,13 & 1,35 & 0,48 & 0,34 & 4,80 & $-2,3$ \\
\hline 595 & 1,66 & 5,47 & 2,06 & 1,02 & 20,63 & $-4,4$ \\
\hline 596 & 0,70 & 3,47 & 1,25 & 0,72 & 12,52 & $-3,6$ \\
\hline 597 & 0,05 & 0,89 & 0,33 & 0,20 & 3,27 & $-1,7$ \\
\hline 598 & 0,11 & 1,24 & 0,45 & 0,30 & 4,47 & $-2,2$ \\
\hline 599 & 0,04 & 0,73 & 0,26 & 0,18 & 2,57 & $-1,4$ \\
\hline 600 & 0,21 & 1,90 & 0,74 & 0,36 & 7,43 & $-2,9$ \\
\hline 601 & 0,08 & 1,14 & 0,46 & 0,21 & 4,63 & $-2,2$ \\
\hline 602 & 1,60 & 5,50 & 2,06 & 0,99 & 20,56 & $-4,4$ \\
\hline 603 & 0,05 & 0,83 & 0,29 & 0,20 & 2,92 & $-1,5$ \\
\hline 604 & 1,35 & 4,62 & 1,49 & 1,15 & 14,91 & $-3,9$ \\
\hline 605 & 0,17 & 1,46 & 0,51 & 0,42 & 5,05 & $-2,3$ \\
\hline 606 & 0,05 & 0,87 & 0,34 & 0,20 & 3,36 & $-1,7$ \\
\hline 607 & 0,39 & 2,50 & 0,99 & 0,50 & 9,92 & $-3,3$ \\
\hline 608 & 0,11 & 1,25 & 0,48 & 0,29 & 4,75 & $-2,2$ \\
\hline 609 & 0,62 & 3,05 & 1,02 & 0,77 & 10,23 & $-3,4$ \\
\hline
\end{tabular}




\begin{tabular}{|c|c|c|c|c|c|c|}
\hline 610 & 0,19 & 1,63 & 0,58 & 0,42 & 5,84 & $-2,5$ \\
\hline 611 & 0,09 & 1,14 & 0,40 & 0,28 & 3,99 & $-2,0$ \\
\hline 612 & 0,32 & 2,19 & 0,67 & 0,61 & 6,67 & $-2,7$ \\
\hline 613 & 0,03 & 0,80 & 0,34 & 0,13 & 3,41 & $-1,8$ \\
\hline 614 & 1,57 & 5,65 & 1,61 & 1,25 & 16,09 & $-4,0$ \\
\hline 615 & 0,09 & 1,27 & 0,53 & 0,23 & 5,26 & $-2,4$ \\
\hline 616 & 0,43 & 2,69 & 0,98 & 0,56 & 9,81 & $-3,3$ \\
\hline 617 & 0,12 & 1,46 & 0,56 & 0,28 & 5,57 & $-2,5$ \\
\hline 618 & 0,11 & 1,31 & 0,43 & 0,33 & 4,30 & $-2,1$ \\
\hline 619 & 0,04 & 0,76 & 0,30 & 0,19 & 2,96 & $-1,6$ \\
\hline 620 & 3,39 & 7,79 & 2,97 & 1,46 & 29,65 & $-4,9$ \\
\hline 621 & 0,05 & 0,87 & 0,30 & 0,23 & 2,99 & $-1,6$ \\
\hline 622 & 0,05 & 0,87 & 0,35 & 0,17 & 3,46 & $-1,8$ \\
\hline 623 & 0,54 & 3,36 & 0,94 & 0,73 & 9,40 & $-3,2$ \\
\hline 624 & 0,09 & 1,13 & 0,41 & 0,27 & 4,12 & $-2,0$ \\
\hline 625 & 0,06 & 0,86 & 0,30 & 0,24 & 3,00 & $-1,6$ \\
\hline 626 & 1,36 & 5,34 & 2,24 & 0,77 & 22,40 & $-4,5$ \\
\hline 627 & 2,11 & 6,40 & 2,08 & 1,29 & 20,84 & $-4,4$ \\
\hline 628 & 0,53 & 2,94 & 0,98 & 0,68 & 9,84 & $-3,3$ \\
\hline 629 & 0,01 & 0,38 & 0,16 & 0,10 & 1,56 & $-0,6$ \\
\hline 630 & 0,17 & 1,57 & 0,60 & 0,37 & 5,96 & $-2,6$ \\
\hline 631 & 2,83 & 7,10 & 2,71 & 1,33 & 27,07 & $-4,8$ \\
\hline 632 & 5,97 & 10,56 & 3,94 & 1,93 & 39,41 & $-5,3$ \\
\hline 633 & 0,40 & 2,59 & 0,88 & 0,58 & 8,77 & $-3,1$ \\
\hline 634 & 0,16 & 1,66 & 0,63 & 0,32 & 6,31 & $-2,7$ \\
\hline 635 & 0,08 & 1,18 & 0,49 & 0,21 & 4,91 & $-2,3$ \\
\hline 636 & 0,86 & 3,94 & 1,39 & 0,79 & 13,93 & $-3,8$ \\
\hline 637 & 6,21 & 11,74 & 3,29 & 2,40 & 32,87 & $-5,0$ \\
\hline 638 & 0,48 & 3,26 & 1,36 & 0,45 & 13,61 & $-3,8$ \\
\hline 639 & 0,03 & 0,52 & 0,20 & 0,16 & 1,96 & $-1,0$ \\
\hline 640 & 0,15 & 1,76 & 0,59 & 0,32 & 5,88 & $-2,6$ \\
\hline 641 & 1,93 & 6,12 & 2,36 & 1,04 & 23,61 & $-4,6$ \\
\hline 642 & 0,36 & 2,69 & 0,98 & 0,47 & 9,76 & $-3,3$ \\
\hline 643 & 1,57 & 4,79 & 1,69 & 1,19 & 16,91 & $-4,1$ \\
\hline 644 & 0,24 & 1,90 & 0,60 & 0,51 & 6,03 & $-2,6$ \\
\hline 645 & 0,04 & 0,93 & 0,39 & 0,12 & 3,90 & $-2,0$ \\
\hline 646 & 0,07 & 0,97 & 0,32 & 0,29 & 3,16 & $-1,7$ \\
\hline 647 & 0,14 & 1,52 & 0,59 & 0,29 & 5,88 & $-2,6$ \\
\hline 648 & 0,35 & 2,26 & 0,69 & 0,65 & 6,94 & $-2,8$ \\
\hline 649 & 0,11 & 1,34 & 0,54 & 0,26 & 5,43 & $-2,4$ \\
\hline 650 & 0,90 & 3,66 & 1,23 & 0,93 & 12,27 & $-3,6$ \\
\hline 651 & 0,51 & 3,03 & 1,06 & 0,61 & 10,55 & $-3,4$ \\
\hline 652 & 0,51 & 2,80 & 0,87 & 0,74 & 8,72 & $-3,1$ \\
\hline 653 & 1,77 & 7,07 & 2,33 & 0,97 & 23,25 & $-4,5$ \\
\hline 654 & 1,70 & 5,93 & 2,42 & 0,90 & 24,19 & $-4,6$ \\
\hline 655 & 0,12 & 1,22 & 0,42 & 0,36 & 4,18 & $-2,1$ \\
\hline 656 & 0,13 & 1,34 & 0,52 & 0,31 & 5,19 & $-2,4$ \\
\hline 657 & 0,15 & 1,71 & 0,60 & 0,32 & 6,00 & $-2,6$ \\
\hline 658 & 0,26 & 1,99 & 0,76 & 0,43 & 7,60 & $-2,9$ \\
\hline 659 & 24,71 & 20,13 & 6,67 & 4,72 & 66,72 & $-6,1$ \\
\hline 660 & 1,00 & 4,23 & 1,29 & 0,99 & 12,92 & $-3,7$ \\
\hline 661 & 0,13 & 1,29 & 0,48 & 0,33 & 4,79 & $-2,3$ \\
\hline 662 & 0,04 & 0,69 & 0,26 & 0,21 & 2,59 & $-1,4$ \\
\hline 663 & 0,20 & 1,84 & 0,70 & 0,37 & 6,95 & $-2,8$ \\
\hline 664 & 0,17 & 1,81 & 0,74 & 0,29 & 7,37 & $-2,9$ \\
\hline 665 & 1,45 & 5,46 & 2,03 & 0,91 & 20,30 & $-4,3$ \\
\hline 666 & 0,08 & 1,04 & 0,35 & 0,29 & 3,53 & $-1,8$ \\
\hline 667 & 0,62 & 3,08 & 1,03 & 0,77 & 10,29 & $-3,4$ \\
\hline 668 & 0,09 & 1,17 & 0,37 & 0,32 & 3,71 & $-1,9$ \\
\hline 669 & 0,36 & 2,25 & 0,80 & 0,58 & 8,03 & $-3,0$ \\
\hline 670 & 0,13 & 1,42 & 0,48 & 0,35 & 4,77 & $-2,3$ \\
\hline 671 & 0,09 & 1,21 & 0,45 & 0,26 & 4,53 & $-2,2$ \\
\hline
\end{tabular}




\begin{tabular}{|c|c|c|c|c|c|c|}
\hline 672 & 24,97 & 23,66 & 7,85 & 4,05 & 78,52 & $-6,3$ \\
\hline 673 & 0,25 & 1,90 & 0,70 & 0,45 & 7,01 & $-2,8$ \\
\hline 674 & 0,59 & 3,38 & 1,26 & 0,59 & 12,60 & $-3,7$ \\
\hline 675 & 2,83 & 9,67 & 4,35 & 0,83 & 43,51 & $-5,4$ \\
\hline 676 & 0,15 & 1,52 & 0,55 & 0,36 & 5,54 & $-2,5$ \\
\hline 677 & 13,95 & 15,32 & 5,31 & 3,34 & 53,10 & $-5,7$ \\
\hline 678 & 0,40 & 2,66 & 0,98 & 0,52 & 9,79 & $-3,3$ \\
\hline 679 & 0,09 & 1,35 & 0,59 & 0,20 & 5,89 & $-2,6$ \\
\hline 680 & 0,44 & 2,66 & 0,98 & 0,57 & 9,76 & $-3,3$ \\
\hline 681 & 2,21 & 6,04 & 2,16 & 1,30 & 21,56 & $-4,4$ \\
\hline 682 & 0,04 & 0,80 & 0,28 & 0,20 & 2,76 & $-1,5$ \\
\hline 683 & 0,90 & 3,87 & 1,39 & 0,83 & 13,90 & $-3,8$ \\
\hline 684 & 0,39 & 2,47 & 0,95 & 0,52 & 9,50 & $-3,2$ \\
\hline 685 & 0,10 & 1,20 & 0,43 & 0,30 & 4,26 & $-2,1$ \\
\hline 686 & 1,02 & 4,33 & 1,72 & 0,75 & 17,18 & $-4,1$ \\
\hline 687 & 0,03 & 0,66 & 0,23 & 0,18 & 2,30 & $-1,2$ \\
\hline 688 & 0,42 & 2,60 & 0,96 & 0,56 & 9,58 & $-3,3$ \\
\hline 689 & 0,07 & 1,00 & 0,42 & 0,20 & 4,22 & $-2,1$ \\
\hline 690 & 0,49 & 2,75 & 0,84 & 0,74 & 8,36 & $-3,1$ \\
\hline 691 & 0,60 & 3,23 & 1,26 & 0,60 & 12,60 & $-3,7$ \\
\hline 692 & 0,41 & 2,55 & 1,05 & 0,50 & 10,51 & $-3,4$ \\
\hline 693 & 0,08 & 1,07 & 0,42 & 0,25 & 4,20 & $-2,1$ \\
\hline 694 & 0,18 & 2,04 & 0,72 & 0,31 & 7,20 & $-2,8$ \\
\hline 695 & 0,64 & 3,17 & 1,12 & 0,72 & 11,21 & $-3,5$ \\
\hline 696 & 0,29 & 2,22 & 0,86 & 0,43 & 8,56 & $-3,1$ \\
\hline 697 & 5,94 & 9,62 & 3,27 & 2,32 & 32,65 & $-5,0$ \\
\hline 698 & 0,67 & 3,38 & 1,32 & 0,65 & 13,17 & $-3,7$ \\
\hline 699 & 0,08 & 1,06 & 0,39 & 0,26 & 3,92 & $-2,0$ \\
\hline 700 & 0,66 & 3,37 & 1,35 & 0,62 & 13,53 & $-3,8$ \\
\hline 701 & 0,12 & 1,32 & 0,50 & 0,31 & 4,98 & $-2,3$ \\
\hline 702 & 0,18 & 1,69 & 0,57 & 0,41 & 5,67 & $-2,5$ \\
\hline 703 & 1,97 & 6,02 & 2,16 & 1,16 & 21,59 & $-4,4$ \\
\hline 704 & 0,38 & 2,39 & 0,93 & 0,51 & 9,33 & $-3,2$ \\
\hline 705 & 0,19 & 1,92 & 0,79 & 0,30 & 7,89 & $-3,0$ \\
\hline 706 & 0,05 & 0,82 & 0,31 & 0,22 & 3,05 & $-1,6$ \\
\hline 707 & 0,44 & 2,95 & 1,12 & 0,50 & 11,24 & $-3,5$ \\
\hline 708 & 0,10 & 1,22 & 0,50 & 0,25 & 5,00 & $-2,3$ \\
\hline 709 & 17,16 & 16,33 & 5,89 & 3,71 & 58,92 & $-5,9$ \\
\hline 710 & 0,51 & 2,92 & 1,02 & 0,63 & 10,21 & $-3,4$ \\
\hline 711 & 0,95 & 3,77 & 1,12 & 1,08 & 11,24 & $-3,5$ \\
\hline 712 & 0,14 & 1,41 & 0,43 & 0,41 & 4,28 & $-2,1$ \\
\hline 713 & 0,41 & 2,48 & 0,90 & 0,58 & 9,00 & $-3,2$ \\
\hline 714 & 0,10 & 1,27 & 0,37 & 0,33 & 3,72 & $-1,9$ \\
\hline 715 & 4,99 & 10,45 & 3,66 & 1,74 & 36,60 & $-5,2$ \\
\hline 716 & 0,24 & 2,21 & 0,84 & 0,36 & 8,39 & $-3,1$ \\
\hline 717 & 0,03 & 0,84 & 0,39 & 0,11 & 3,89 & $-2,0$ \\
\hline 718 & 0,04 & 0,87 & 0,41 & 0,12 & 4,05 & $-2,0$ \\
\hline 719 & 0,06 & 0,90 & 0,34 & 0,23 & 3,43 & $-1,8$ \\
\hline 720 & 0,39 & 2,72 & 1,13 & 0,43 & 11,34 & $-3,5$ \\
\hline 721 & 1,38 & 5,04 & 1,78 & 0,99 & 17,80 & $-4,2$ \\
\hline 722 & 0,14 & 1,48 & 0,59 & 0,30 & 5,88 & $-2,6$ \\
\hline 723 & 0,07 & 0,96 & 0,36 & 0,23 & 3,62 & $-1,9$ \\
\hline 724 & 0,21 & 1,84 & 0,68 & 0,40 & 6,76 & $-2,8$ \\
\hline 725 & 0,04 & 0,73 & 0,24 & 0,19 & 2,43 & $-1,3$ \\
\hline 726 & 0,09 & 1,22 & 0,53 & 0,21 & 5,27 & $-2,4$ \\
\hline 727 & 0,04 & 0,73 & 0,25 & 0,21 & 2,49 & $-1,3$ \\
\hline 728 & 0,42 & 2,68 & 0,94 & 0,57 & 9,38 & $-3,2$ \\
\hline 729 & 0,08 & 1,03 & 0,32 & 0,30 & 3,18 & $-1,7$ \\
\hline 730 & 0,09 & 1,20 & 0,48 & 0,23 & 4,82 & $-2,3$ \\
\hline 731 & 0,18 & 1,63 & 0,59 & 0,39 & 5,94 & $-2,6$ \\
\hline 732 & 0,18 & 1,67 & 0,62 & 0,36 & 6,19 & $-2,6$ \\
\hline 733 & 0,15 & 1,64 & 0,70 & 0,27 & 6,95 & $-2,8$ \\
\hline
\end{tabular}




\begin{tabular}{|c|c|c|c|c|c|c|}
\hline 734 & 0,05 & 0,76 & 0,31 & 0,20 & 3,11 & $-1,6$ \\
\hline 735 & 0,13 & 1,43 & 0,51 & 0,33 & 5,09 & $-2,3$ \\
\hline 736 & 0,30 & 2,59 & 1,09 & 0,35 & 10,93 & $-3,5$ \\
\hline 737 & 0,82 & 4,42 & 1,82 & 0,57 & 18,23 & $-4,2$ \\
\hline 738 & 0,91 & 3,67 & 1,16 & 1,00 & 11,56 & $-3,5$ \\
\hline 739 & 0,06 & 1,06 & 0,36 & 0,22 & 3,63 & $-1,9$ \\
\hline 740 & 1,86 & 5,53 & 1,72 & 1,38 & 17,16 & $-4,1$ \\
\hline 741 & 47,93 & 29,37 & 9,24 & 6,60 & 92,44 & $-6,5$ \\
\hline 742 & 0,07 & 1,00 & 0,34 & 0,27 & 3,42 & $-1,8$ \\
\hline 743 & 0,56 & 2,85 & 1,07 & 0,67 & 10,66 & $-3,4$ \\
\hline 744 & 0,54 & 3,31 & 1,08 & 0,64 & 10,80 & $-3,4$ \\
\hline 745 & 0,05 & 0,89 & 0,38 & 0,16 & 3,79 & $-1,9$ \\
\hline 746 & 14,40 & 16,84 & 6,14 & 2,99 & 61,36 & $-5,9$ \\
\hline 747 & 3,54 & 8,67 & 3,10 & 1,45 & 31,02 & $-5,0$ \\
\hline 748 & 0,06 & 1,01 & 0,47 & 0,15 & 4,72 & $-2,2$ \\
\hline 749 & 0,37 & 2,53 & 0,83 & 0,56 & 8,33 & $-3,1$ \\
\hline 750 & 0,99 & 4,15 & 1,68 & 0,75 & 16,79 & $-4,1$ \\
\hline 751 & 0,22 & 1,71 & 0,62 & 0,46 & 6,23 & $-2,6$ \\
\hline 752 & 0,61 & 3,15 & 1,04 & 0,75 & 10,43 & $-3,4$ \\
\hline 753 & 0,48 & 2,77 & 0,96 & 0,64 & 9,62 & $-3,3$ \\
\hline 754 & 0,62 & 2,98 & 1,00 & 0,78 & 10,02 & $-3,3$ \\
\hline 755 & 0,06 & 0,90 & 0,32 & 0,25 & 3,21 & $-1,7$ \\
\hline 756 & 1,17 & 5,51 & 2,14 & 0,69 & 21,44 & $-4,4$ \\
\hline 757 & 0,19 & 1,69 & 0,59 & 0,41 & 5,92 & $-2,6$ \\
\hline 758 & 2,10 & 6,53 & 2,39 & 1,12 & 23,94 & $-4,6$ \\
\hline 759 & 0,33 & 2,43 & 0,80 & 0,53 & 7,96 & $-3,0$ \\
\hline 760 & 17,98 & 17,71 & 5,27 & 4,34 & 52,70 & $-5,7$ \\
\hline 761 & 0,29 & 2,02 & 0,65 & 0,56 & 6,48 & $-2,7$ \\
\hline 762 & 2,49 & 7,28 & 2,69 & 1,18 & 26,94 & $-4,8$ \\
\hline 763 & 0,05 & 0,93 & 0,28 & 0,25 & 2,76 & $-1,5$ \\
\hline 764 & 0,54 & 2,95 & 0,96 & 0,71 & 9,63 & $-3,3$ \\
\hline 765 & 0,15 & 1,48 & 0,54 & 0,36 & 5,38 & $-2,4$ \\
\hline 766 & 0,05 & 0,82 & 0,30 & 0,21 & 2,99 & $-1,6$ \\
\hline 767 & 0,20 & 2,10 & 0,88 & 0,29 & 8,80 & $-3,1$ \\
\hline 768 & 0,19 & 1,73 & 0,60 & 0,40 & 5,95 & $-2,6$ \\
\hline 769 & 0,95 & 3,78 & 1,37 & 0,88 & 13,69 & $-3,8$ \\
\hline 770 & 0,10 & 1,38 & 0,49 & 0,26 & 4,87 & $-2,3$ \\
\hline 771 & 0,03 & 0,59 & 0,26 & 0,12 & 2,63 & $-1,4$ \\
\hline 772 & 0,29 & 2,27 & 0,85 & 0,43 & 8,48 & $-3,1$ \\
\hline 773 & 0,22 & 1,83 & 0,62 & 0,45 & 6,17 & $-2,6$ \\
\hline 774 & 4,64 & 8,49 & 2,69 & 2,19 & 26,92 & $-4,8$ \\
\hline 775 & 0,13 & 1,36 & 0,48 & 0,35 & 4,78 & $-2,3$ \\
\hline 776 & 0,15 & 1,95 & 0,89 & 0,22 & 8,91 & $-3,2$ \\
\hline 777 & 0,04 & 0,76 & 0,28 & 0,17 & 2,80 & $-1,5$ \\
\hline 778 & 1,09 & 4,63 & 1,78 & 0,78 & 17,75 & $-4,1$ \\
\hline 779 & 0,07 & 1,03 & 0,36 & 0,25 & 3,59 & $-1,8$ \\
\hline 780 & 0,19 & 1,71 & 0,56 & 0,43 & 5,55 & $-2,5$ \\
\hline 781 & 0,06 & 0,90 & 0,32 & 0,24 & 3,18 & $-1,7$ \\
\hline 782 & 0,19 & 2,09 & 0,93 & 0,25 & 9,34 & $-3,2$ \\
\hline 783 & 0,45 & 2,86 & 0,83 & 0,70 & 8,26 & $-3,0$ \\
\hline 784 & 0,97 & 3,93 & 1,43 & 0,86 & 14,32 & $-3,8$ \\
\hline 785 & 0,37 & 2,63 & 1,05 & 0,45 & 10,48 & $-3,4$ \\
\hline 786 & 1,16 & 4,14 & 1,43 & 1,04 & 14,30 & $-3,8$ \\
\hline 787 & 1,29 & 4,89 & 1,80 & 0,92 & 17,97 & $-4,2$ \\
\hline 788 & 1,21 & 4,65 & 1,44 & 1,07 & 14,35 & $-3,8$ \\
\hline 789 & 0,16 & 1,59 & 0,64 & 0,31 & 6,41 & $-2,7$ \\
\hline 790 & 0,14 & 1,43 & 0,51 & 0,35 & 5,08 & $-2,3$ \\
\hline 791 & 0,57 & 3,17 & 1,11 & 0,65 & 11,10 & $-3,5$ \\
\hline 792 & 5,86 & 9,44 & 3,16 & 2,36 & 31,63 & $-5,0$ \\
\hline 793 & 0,15 & 1,49 & 0,55 & 0,36 & 5,45 & $-2,4$ \\
\hline 794 & 2,28 & 6,30 & 2,27 & 1,28 & 22,70 & $-4,5$ \\
\hline 795 & 0,30 & 2,26 & 0,81 & 0,48 & 8,05 & $-3,0$ \\
\hline
\end{tabular}




\begin{tabular}{|c|c|c|c|c|c|c|}
\hline 796 & 0,21 & 1,78 & 0,69 & 0,39 & 6,90 & $-2,8$ \\
\hline 797 & 0,30 & 2,06 & 0,66 & 0,59 & 6,58 & $-2,7$ \\
\hline 798 & 0,03 & 0,59 & 0,21 & 0,18 & 2,06 & $-1,0$ \\
\hline 799 & 2,81 & 7,23 & 2,54 & 1,41 & 25,41 & $-4,7$ \\
\hline 800 & 0,22 & 2,12 & 0,84 & 0,33 & 8,41 & $-3,1$ \\
\hline 801 & 0,05 & 0,89 & 0,36 & 0,18 & 3,59 & $-1,8$ \\
\hline 802 & 0,19 & 1,70 & 0,66 & 0,36 & 6,61 & $-2,7$ \\
\hline 803 & 12,13 & 14,07 & 4,38 & 3,53 & 43,83 & $-5,5$ \\
\hline 804 & 0,76 & 3,40 & 1,19 & 0,81 & 11,94 & $-3,6$ \\
\hline 805 & 0,16 & 1,64 & 0,59 & 0,35 & 5,94 & $-2,6$ \\
\hline 806 & 0,01 & 0,38 & 0,16 & 0,10 & 1,56 & $-0,6$ \\
\hline 807 & 12,13 & 15,85 & 6,01 & 2,57 & 60,07 & $-5,9$ \\
\hline 808 & 0,17 & 1,77 & 0,74 & 0,29 & 7,39 & $-2,9$ \\
\hline 809 & 0,91 & 3,74 & 1,24 & 0,94 & 12,41 & $-3,6$ \\
\hline 810 & 1,49 & 4,70 & 1,66 & 1,14 & 16,57 & $-4,1$ \\
\hline 811 & 0,08 & 1,07 & 0,37 & 0,27 & 3,70 & $-1,9$ \\
\hline 812 & 0,90 & 4,18 & 1,67 & 0,69 & 16,69 & $-4,1$ \\
\hline 813 & 0,44 & 2,60 & 0,84 & 0,67 & 8,36 & $-3,1$ \\
\hline 814 & 0,60 & 3,05 & 1,07 & 0,71 & 10,68 & $-3,4$ \\
\hline 815 & 0,27 & 2,78 & 1,08 & 0,32 & 10,78 & $-3,4$ \\
\hline 816 & 0,02 & 0,55 & 0,24 & 0,10 & 2,44 & $-1,3$ \\
\hline 817 & 0,74 & 3,61 & 1,12 & 0,85 & 11,19 & $-3,5$ \\
\hline 818 & 1,76 & 5,27 & 1,76 & 1,27 & 17,61 & $-4,1$ \\
\hline 819 & 0,01 & 0,28 & 0,12 & 0,08 & 1,17 & $-0,2$ \\
\hline 820 & 0,72 & 3,78 & 1,48 & 0,62 & 14,76 & $-3,9$ \\
\hline 821 & 0,45 & 2,81 & 1,11 & 0,52 & 11,05 & $-3,5$ \\
\hline 822 & 0,25 & 1,86 & 0,65 & 0,48 & 6,47 & $-2,7$ \\
\hline 823 & 0,15 & 1,57 & 0,64 & 0,30 & 6,42 & $-2,7$ \\
\hline 824 & 0,03 & 0,72 & 0,29 & 0,13 & 2,94 & $-1,6$ \\
\hline 825 & 0,22 & 1,77 & 0,65 & 0,43 & 6,45 & $-2,7$ \\
\hline 826 & 0,04 & 0,76 & 0,32 & 0,17 & 3,23 & $-1,7$ \\
\hline 827 & 0,43 & 2,61 & 0,92 & 0,60 & 9,17 & $-3,2$ \\
\hline 828 & 0,20 & 1,77 & 0,53 & 0,47 & 5,30 & $-2,4$ \\
\hline 829 & 0,45 & 2,69 & 0,99 & 0,58 & 9,89 & $-3,3$ \\
\hline 830 & 0,32 & 2,04 & 0,67 & 0,61 & 6,65 & $-2,7$ \\
\hline 831 & 0,02 & 0,52 & 0,20 & 0,13 & 2,00 & $-1,0$ \\
\hline 832 & 0,08 & 1,18 & 0,49 & 0,22 & 4,86 & $-2,3$ \\
\hline 833 & 1,56 & 4,97 & 1,87 & 1,06 & 18,67 & $-4,2$ \\
\hline 834 & 0,02 & 0,48 & 0,17 & 0,17 & 1,68 & $-0,7$ \\
\hline 835 & 0,53 & 2,85 & 0,93 & 0,72 & 9,32 & $-3,2$ \\
\hline 836 & 0,11 & 1,18 & 0,42 & 0,33 & 4,19 & $-2,1$ \\
\hline 837 & 0,03 & 0,62 & 0,24 & 0,15 & 2,35 & $-1,2$ \\
\hline 838 & 1,97 & 6,13 & 1,82 & 1,38 & 18,17 & $-4,2$ \\
\hline 839 & 0,04 & 0,76 & 0,25 & 0,19 & 2,51 & $-1,3$ \\
\hline 840 & 0,24 & 1,87 & 0,68 & 0,46 & 6,76 & $-2,8$ \\
\hline 841 & 0,14 & 1,48 & 0,54 & 0,33 & 5,35 & $-2,4$ \\
\hline 842 & 0,09 & 1,17 & 0,47 & 0,24 & 4,69 & $-2,2$ \\
\hline 843 & 0,17 & 1,97 & 0,92 & 0,23 & 9,20 & $-3,2$ \\
\hline 844 & 0,22 & 1,92 & 0,74 & 0,38 & 7,38 & $-2,9$ \\
\hline 845 & 0,03 & 0,55 & 0,22 & 0,16 & 2,17 & $-1,1$ \\
\hline 846 & 1,31 & 4,70 & 1,69 & 0,99 & 16,91 & $-4,1$ \\
\hline 847 & 4,82 & 8,47 & 2,72 & 2,26 & 27,18 & $-4,8$ \\
\hline 848 & 0,62 & 3,03 & 1,09 & 0,72 & 10,94 & $-3,5$ \\
\hline 849 & 2,11 & 6,48 & 2,31 & 1,17 & 23,05 & $-4,5$ \\
\hline 850 & 0,75 & 3,51 & 1,36 & 0,70 & 13,62 & $-3,8$ \\
\hline 851 & 0,02 & 0,45 & 0,17 & 0,13 & 1,74 & $-0,8$ \\
\hline 852 & 0,02 & 0,45 & 0,15 & 0,13 & 1,45 & $-0,5$ \\
\hline 853 & 0,36 & 2,42 & 0,89 & 0,52 & 8,92 & $-3,2$ \\
\hline 854 & 0,01 & 0,21 & 0,11 & 0,06 & 1,12 & $-0,2$ \\
\hline 855 & 0,23 & 1,96 & 0,80 & 0,37 & 8,04 & $-3,0$ \\
\hline 856 & 0,06 & 1,28 & 0,51 & 0,14 & 5,13 & $-2,4$ \\
\hline 857 & 0,58 & 3,00 & 0,93 & 0,80 & 9,27 & $-3,2$ \\
\hline
\end{tabular}




\begin{tabular}{|c|c|c|c|c|c|c|}
\hline 858 & 0,16 & 1,59 & 0,61 & 0,33 & 6,07 & $-2,6$ \\
\hline 859 & 0,65 & 3,13 & 1,05 & 0,78 & 10,47 & $-3,4$ \\
\hline 860 & 0,20 & 1,87 & 0,69 & 0,37 & 6,87 & $-2,8$ \\
\hline 861 & 0,06 & 1,03 & 0,39 & 0,20 & 3,85 & $-1,9$ \\
\hline 862 & 1,05 & 4,15 & 1,41 & 0,95 & 14,13 & $-3,8$ \\
\hline 863 & 2,87 & 7,03 & 2,53 & 1,45 & 25,27 & $-4,7$ \\
\hline 864 & 0,39 & 3,25 & 1,13 & 0,44 & 11,34 & $-3,5$ \\
\hline 865 & 0,55 & 3,28 & 1,27 & 0,56 & 12,71 & $-3,7$ \\
\hline 866 & 0,11 & 1,22 & 0,46 & 0,30 & 4,64 & $-2,2$ \\
\hline 867 & 0,17 & 1,52 & 0,57 & 0,39 & 5,66 & $-2,5$ \\
\hline 868 & 0,58 & 3,07 & 1,18 & 0,62 & 11,76 & $-3,6$ \\
\hline 869 & 0,01 & 0,34 & 0,17 & 0,06 & 1,68 & $-0,7$ \\
\hline 870 & 0,35 & 2,41 & 0,74 & 0,60 & 7,41 & $-2,9$ \\
\hline 871 & 0,56 & 3,04 & 1,03 & 0,69 & 10,29 & $-3,4$ \\
\hline 872 & 0,15 & 1,59 & 0,64 & 0,31 & 6,36 & $-2,7$ \\
\hline 873 & 2,19 & 7,88 & 2,30 & 1,21 & 23,02 & $-4,5$ \\
\hline 874 & 0,12 & 1,43 & 0,59 & 0,25 & 5,90 & $-2,6$ \\
\hline 875 & 0,02 & 0,45 & 0,20 & 0,10 & 1,97 & $-1,0$ \\
\hline 876 & 9,48 & 12,99 & 4,66 & 2,59 & 46,62 & $-5,5$ \\
\hline 877 & 0,81 & 3,84 & 1,37 & 0,75 & 13,68 & $-3,8$ \\
\hline 878 & 0,02 & 0,55 & 0,21 & 0,14 & 2,08 & $-1,1$ \\
\hline 879 & 0,08 & 1,14 & 0,43 & 0,25 & 4,28 & $-2,1$ \\
\hline 880 & 0,44 & 3,33 & 1,21 & 0,47 & 12,11 & $-3,6$ \\
\hline 881 & 2,04 & 5,70 & 1,69 & 1,54 & 16,88 & $-4,1$ \\
\hline 882 & 0,15 & 1,46 & 0,52 & 0,38 & 5,24 & $-2,4$ \\
\hline 883 & 0,74 & 3,64 & 1,19 & 0,79 & 11,92 & $-3,6$ \\
\hline 884 & 0,21 & 1,78 & 0,66 & 0,41 & 6,63 & $-2,7$ \\
\hline 885 & 0,88 & 3,85 & 1,49 & 0,75 & 14,85 & $-3,9$ \\
\hline 886 & 0,05 & 0,93 & 0,36 & 0,18 & 3,56 & $-1,8$ \\
\hline 887 & 0,81 & 3,94 & 1,46 & 0,71 & 14,57 & $-3,9$ \\
\hline 888 & 0,18 & 1,62 & 0,57 & 0,40 & 5,68 & $-2,5$ \\
\hline 889 & 0,11 & 1,45 & 0,64 & 0,21 & 6,35 & $-2,7$ \\
\hline 890 & 0,11 & 1,24 & 0,47 & 0,29 & 4,72 & $-2,2$ \\
\hline 891 & 0,18 & 1,69 & 0,59 & 0,38 & 5,86 & $-2,6$ \\
\hline 892 & 1,09 & 4,11 & 1,20 & 1,15 & 11,99 & $-3,6$ \\
\hline 893 & 0,07 & 1,00 & 0,38 & 0,24 & 3,78 & $-1,9$ \\
\hline 894 & 0,01 & 0,24 & 0,11 & 0,06 & 1,12 & $-0,2$ \\
\hline 895 & 0,01 & 0,24 & 0,11 & 0,06 & 1,12 & $-0,2$ \\
\hline 896 & 0,96 & 5,10 & 1,97 & 0,62 & 19,66 & $-4,3$ \\
\hline 897 & 0,19 & 1,95 & 0,83 & 0,29 & 8,26 & $-3,0$ \\
\hline 898 & 0,31 & 2,13 & 0,66 & 0,60 & 6,55 & $-2,7$ \\
\hline 899 & 0,44 & 2,69 & 0,93 & 0,61 & 9,29 & $-3,2$ \\
\hline 900 & 0,05 & 0,86 & 0,34 & 0,18 & 3,43 & $-1,8$ \\
\hline 901 & 0,05 & 0,80 & 0,36 & 0,17 & 3,59 & $-1,8$ \\
\hline 902 & 1,65 & 5,96 & 1,73 & 1,21 & 17,32 & $-4,1$ \\
\hline 903 & 0,07 & 0,93 & 0,31 & 0,28 & 3,12 & $-1,6$ \\
\hline 904 & 25,92 & 21,65 & 7,54 & 4,38 & 75,40 & $-6,2$ \\
\hline 905 & 0,82 & 3,73 & 1,29 & 0,81 & 12,89 & $-3,7$ \\
\hline 906 & 0,01 & 0,35 & 0,16 & 0,10 & 1,56 & $-0,6$ \\
\hline 907 & 0,54 & 3,00 & 1,17 & 0,59 & 11,67 & $-3,5$ \\
\hline 908 & 1,82 & 5,67 & 2,29 & 1,01 & 22,88 & $-4,5$ \\
\hline 909 & 0,05 & 0,86 & 0,35 & 0,20 & 3,45 & $-1,8$ \\
\hline 910 & 0,77 & 3,43 & 1,19 & 0,82 & 11,89 & $-3,6$ \\
\hline 911 & 7,82 & 12,12 & 3,40 & 2,93 & 34,00 & $-5,1$ \\
\hline 912 & 1,88 & 5,65 & 2,10 & 1,14 & 21,00 & $-4,4$ \\
\hline 913 & 0,08 & 1,10 & 0,43 & 0,24 & 4,30 & $-2,1$ \\
\hline 914 & 0,37 & 2,40 & 0,80 & 0,60 & 7,97 & $-3,0$ \\
\hline 915 & 0,37 & 2,29 & 0,81 & 0,57 & 8,13 & $-3,0$ \\
\hline 916 & 0,06 & 0,86 & 0,29 & 0,26 & 2,85 & $-1,5$ \\
\hline 917 & 0,06 & 1,06 & 0,45 & 0,18 & 4,52 & $-2,2$ \\
\hline 918 & 0,05 & 0,93 & 0,39 & 0,18 & 3,87 & $-2,0$ \\
\hline 919 & 5,53 & 9,67 & 3,04 & 2,32 & 30,37 & $-4,9$ \\
\hline
\end{tabular}




\begin{tabular}{|c|c|c|c|c|c|c|}
\hline 920 & 0,04 & 0,87 & 0,42 & 0,11 & 4,23 & $-2,1$ \\
\hline 921 & 0,48 & 3,28 & 1,30 & 0,47 & 12,98 & $-3,7$ \\
\hline 922 & 5,72 & 10,13 & 3,39 & 2,15 & 33,85 & $-5,1$ \\
\hline 923 & 0,33 & 2,28 & 0,74 & 0,56 & 7,43 & $-2,9$ \\
\hline 924 & 0,25 & 1,87 & 0,70 & 0,44 & 7,04 & $-2,8$ \\
\hline 925 & 1,88 & 6,09 & 2,50 & 0,96 & 25,02 & $-4,6$ \\
\hline 926 & 0,13 & 1,43 & 0,53 & 0,32 & 5,32 & $-2,4$ \\
\hline 927 & 0,07 & 0,97 & 0,39 & 0,22 & 3,91 & $-2,0$ \\
\hline 928 & 0,13 & 1,46 & 0,55 & 0,30 & 5,49 & $-2,5$ \\
\hline 929 & 1,85 & 5,17 & 1,61 & 1,46 & 16,10 & $-4,0$ \\
\hline 930 & 1,03 & 3,99 & 1,25 & 1,06 & 12,45 & $-3,6$ \\
\hline 931 & 0,21 & 1,88 & 0,74 & 0,36 & 7,44 & $-2,9$ \\
\hline 932 & 0,17 & 1,69 & 0,55 & 0,39 & 5,54 & $-2,5$ \\
\hline 933 & 2,79 & 6,99 & 2,37 & 1,50 & 23,68 & $-4,6$ \\
\hline 934 & 0,07 & 0,93 & 0,36 & 0,23 & 3,60 & $-1,8$ \\
\hline 935 & 0,31 & 2,48 & 0,92 & 0,43 & 9,15 & $-3,2$ \\
\hline 936 & 0,61 & 3,12 & 1,09 & 0,72 & 10,92 & $-3,4$ \\
\hline 937 & 0,18 & 1,64 & 0,61 & 0,38 & 6,09 & $-2,6$ \\
\hline 938 & 0,03 & 0,65 & 0,27 & 0,16 & 2,69 & $-1,4$ \\
\hline 939 & 0,19 & 1,88 & 0,68 & 0,36 & 6,76 & $-2,8$ \\
\hline 940 & 2,52 & 6,87 & 2,40 & 1,34 & 24,02 & $-4,6$ \\
\hline 941 & 0,21 & 1,81 & 0,69 & 0,39 & 6,89 & $-2,8$ \\
\hline 942 & 0,08 & 1,18 & 0,39 & 0,28 & 3,85 & $-1,9$ \\
\hline 943 & 5,56 & 10,66 & 4,34 & 1,63 & 43,37 & $-5,4$ \\
\hline 944 & 2,09 & 5,82 & 2,15 & 1,24 & 21,49 & $-4,4$ \\
\hline 945 & 0,29 & 2,22 & 0,87 & 0,43 & 8,67 & $-3,1$ \\
\hline 946 & 0,10 & 1,35 & 0,47 & 0,27 & 4,74 & $-2,2$ \\
\hline 947 & 0,63 & 3,55 & 1,37 & 0,59 & 13,68 & $-3,8$ \\
\hline 948 & 0,01 & 0,38 & 0,16 & 0,10 & 1,56 & $-0,6$ \\
\hline 949 & 0,48 & 2,63 & 0,95 & 0,64 & 9,53 & $-3,3$ \\
\hline 950 & 2,19 & 6,33 & 2,44 & 1,14 & 24,41 & $-4,6$ \\
\hline 951 & 0,27 & 2,69 & 1,00 & 0,34 & 10,02 & $-3,3$ \\
\hline 952 & 0,43 & 2,62 & 1,04 & 0,53 & 10,40 & $-3,4$ \\
\hline 953 & 8,74 & 12,98 & 4,08 & 2,73 & 40,80 & $-5,4$ \\
\hline 954 & 0,38 & 2,49 & 0,80 & 0,60 & 7,96 & $-3,0$ \\
\hline 955 & 0,02 & 0,38 & 0,17 & 0,11 & 1,68 & $-0,7$ \\
\hline 956 & 0,02 & 0,49 & 0,23 & 0,08 & 2,29 & $-1,2$ \\
\hline 957 & 0,39 & 2,40 & 0,88 & 0,56 & 8,83 & $-3,1$ \\
\hline 958 & 0,02 & 0,45 & 0,18 & 0,14 & 1,75 & $-0,8$ \\
\hline 959 & 0,15 & 1,48 & 0,56 & 0,33 & 5,63 & $-2,5$ \\
\hline 960 & 0,21 & 1,88 & 0,72 & 0,38 & 7,16 & $-2,8$ \\
\hline 961 & 0,53 & 2,93 & 1,02 & 0,66 & 10,19 & $-3,3$ \\
\hline 962 & 0,15 & 1,53 & 0,58 & 0,34 & 5,78 & $-2,5$ \\
\hline 963 & 0,98 & 5,22 & 1,57 & 0,79 & 15,68 & $-4,0$ \\
\hline 964 & 0,03 & 0,65 & 0,25 & 0,16 & 2,51 & $-1,3$ \\
\hline 965 & 0,03 & 0,55 & 0,22 & 0,14 & 2,21 & $-1,1$ \\
\hline 966 & 0,29 & 2,22 & 0,90 & 0,41 & 9,03 & $-3,2$ \\
\hline 967 & 0,15 & 1,70 & 0,72 & 0,28 & 7,16 & $-2,8$ \\
\hline 968 & 1,56 & 5,32 & 2,00 & 0,99 & 20,03 & $-4,3$ \\
\hline 969 & 0,07 & 1,00 & 0,42 & 0,20 & 4,22 & $-2,1$ \\
\hline 970 & 0,09 & 1,15 & 0,43 & 0,26 & 4,33 & $-2,1$ \\
\hline 971 & 2,48 & 6,27 & 1,87 & 1,69 & 18,66 & $-4,2$ \\
\hline 972 & 2,26 & 5,99 & 1,91 & 1,51 & 19,06 & $-4,3$ \\
\hline 973 & 0,02 & 0,69 & 0,28 & 0,09 & 2,76 & $-1,5$ \\
\hline 974 & 0,03 & 0,72 & 0,28 & 0,16 & 2,78 & $-1,5$ \\
\hline 975 & 0,74 & 3,36 & 1,17 & 0,81 & 11,65 & $-3,5$ \\
\hline 976 & 0,89 & 3,56 & 1,16 & 0,97 & 11,59 & $-3,5$ \\
\hline 977 & 0,30 & 2,12 & 0,72 & 0,54 & 7,19 & $-2,8$ \\
\hline 978 & 0,10 & 1,34 & 0,38 & 0,33 & 3,76 & $-1,9$ \\
\hline 979 & 0,16 & 1,64 & 0,60 & 0,34 & 5,98 & $-2,6$ \\
\hline 980 & 0,09 & 1,13 & 0,36 & 0,31 & 3,58 & $-1,8$ \\
\hline 981 & 0,68 & 3,55 & 1,26 & 0,69 & 12,57 & $-3,7$ \\
\hline
\end{tabular}




\begin{tabular}{|c|c|c|c|c|c|c|}
\hline 982 & 0,09 & 2,63 & 1,12 & 0,10 & 11,19 & $-3,5$ \\
\hline 983 & 0,15 & 1,57 & 0,56 & 0,34 & 5,63 & $-2,5$ \\
\hline 984 & 0,35 & 2,51 & 1,01 & 0,44 & 10,07 & $-3,3$ \\
\hline 985 & 0,34 & 2,26 & 0,82 & 0,52 & 8,23 & $-3,0$ \\
\hline 986 & 0,02 & 0,52 & 0,18 & 0,14 & 1,81 & $-0,9$ \\
\hline 987 & 0,24 & 2,08 & 0,79 & 0,38 & 7,94 & $-3,0$ \\
\hline 988 & 3,18 & 7,43 & 2,47 & 1,64 & 24,69 & $-4,6$ \\
\hline 989 & 0,16 & 1,63 & 0,69 & 0,30 & 6,94 & $-2,8$ \\
\hline 990 & 0,08 & 1,01 & 0,39 & 0,25 & 3,93 & $-2,0$ \\
\hline 991 & 0,01 & 0,38 & 0,16 & 0,10 & 1,56 & $-0,6$ \\
\hline 992 & 0,05 & 0,79 & 0,31 & 0,19 & 3,13 & $-1,6$ \\
\hline 993 & 0,32 & 2,25 & 0,83 & 0,50 & 8,33 & $-3,1$ \\
\hline 994 & 0,02 & 0,52 & 0,20 & 0,13 & 1,97 & $-1,0$ \\
\hline 995 & 0,28 & 2,01 & 0,68 & 0,52 & 6,79 & $-2,8$ \\
\hline 996 & 0,32 & 2,26 & 0,83 & 0,49 & 8,34 & $-3,1$ \\
\hline 997 & 0,29 & 2,49 & 0,91 & 0,41 & 9,11 & $-3,2$ \\
\hline 998 & 5,15 & 9,89 & 3,08 & 2,13 & 30,82 & $-4,9$ \\
\hline 999 & 0,03 & 0,55 & 0,22 & 0,16 & 2,17 & $-1,1$ \\
\hline 1000 & 0,13 & 1,35 & 0,50 & 0,33 & 5,02 & $-2,3$ \\
\hline 1001 & 0,05 & 0,96 & 0,41 & 0,17 & 4,14 & $-2,0$ \\
\hline 1002 & 0,10 & 1,22 & 0,43 & 0,31 & 4,29 & $-2,1$ \\
\hline 1003 & 0,78 & 3,71 & 1,34 & 0,75 & 13,41 & $-3,7$ \\
\hline 1004 & 0,07 & 1,15 & 0,46 & 0,18 & 4,59 & $-2,2$ \\
\hline 1005 & 0,46 & 2,69 & 1,07 & 0,55 & 10,65 & $-3,4$ \\
\hline 1006 & 0,10 & 1,13 & 0,38 & 0,33 & 3,78 & $-1,9$ \\
\hline 1007 & 1,76 & 5,52 & 1,88 & 1,19 & 18,84 & $-4,2$ \\
\hline 1008 & 1,97 & 5,32 & 1,69 & 1,48 & 16,92 & $-4,1$ \\
\hline 1009 & 0,09 & 1,18 & 0,48 & 0,24 & 4,76 & $-2,3$ \\
\hline 1010 & 0,58 & 2,93 & 1,07 & 0,69 & 10,73 & $-3,4$ \\
\hline 1011 & 3,38 & 8,18 & 2,44 & 1,76 & 24,42 & $-4,6$ \\
\hline 1012 & 0,91 & 3,90 & 1,46 & 0,79 & 14,61 & $-3,9$ \\
\hline 1013 & 2,32 & 7,57 & 3,05 & 0,97 & 30,51 & $-4,9$ \\
\hline 1014 & 0,02 & 0,52 & 0,22 & 0,11 & 2,20 & $-1,1$ \\
\hline 1015 & 2,45 & 7,55 & 3,04 & 1,03 & 30,38 & $-4,9$ \\
\hline 1016 & 0,18 & 1,77 & 0,75 & 0,31 & 7,45 & $-2,9$ \\
\hline 1017 & 0,03 & 0,62 & 0,22 & 0,20 & 2,22 & $-1,2$ \\
\hline 1018 & 0,02 & 0,42 & 0,15 & 0,13 & 1,45 & $-0,5$ \\
\hline 1019 & 0,18 & 2,49 & 1,01 & 0,22 & 10,14 & $-3,3$ \\
\hline 1020 & 0,49 & 3,05 & 1,00 & 0,62 & 10,00 & $-3,3$ \\
\hline 1021 & 0,44 & 2,79 & 0,98 & 0,58 & 9,80 & $-3,3$ \\
\hline 1022 & 0,31 & 2,59 & 1,16 & 0,34 & 11,59 & $-3,5$ \\
\hline 1023 & 0,25 & 2,11 & 0,75 & 0,43 & 7,54 & $-2,9$ \\
\hline 1024 & 0,32 & 2,54 & 0,78 & 0,51 & 7,83 & $-3,0$ \\
\hline 1025 & 0,13 & 1,50 & 0,60 & 0,28 & 5,95 & $-2,6$ \\
\hline 1026 & 0,34 & 2,54 & 0,81 & 0,54 & 8,11 & $-3,0$ \\
\hline 1027 & 1,12 & 4,58 & 1,83 & 0,78 & 18,28 & $-4,2$ \\
\hline 1028 & 0,19 & 1,85 & 0,66 & 0,37 & 6,61 & $-2,7$ \\
\hline 1029 & 0,03 & 0,66 & 0,26 & 0,15 & 2,64 & $-1,4$ \\
\hline 1030 & 0,15 & 1,66 & 0,66 & 0,30 & 6,64 & $-2,7$ \\
\hline 1031 & 1,77 & 5,30 & 1,64 & 1,37 & 16,44 & $-4,0$ \\
\hline 1032 & 0,28 & 2,35 & 0,94 & 0,38 & 9,36 & $-3,2$ \\
\hline 1033 & 1,67 & 5,01 & 1,72 & 1,24 & 17,22 & $-4,1$ \\
\hline 1034 & 0,02 & 0,42 & 0,18 & 0,10 & 1,84 & $-0,9$ \\
\hline 1035 & 0,28 & 2,03 & 0,64 & 0,57 & 6,35 & $-2,7$ \\
\hline 1036 & 0,17 & 1,56 & 0,54 & 0,40 & 5,37 & $-2,4$ \\
\hline 1037 & 0,23 & 1,84 & 0,64 & 0,45 & 6,42 & $-2,7$ \\
\hline 1038 & 0,45 & 2,49 & 0,81 & 0,71 & 8,10 & $-3,0$ \\
\hline 1039 & 0,05 & 0,79 & 0,33 & 0,18 & 3,26 & $-1,7$ \\
\hline 1040 & 0,23 & 2,15 & 0,97 & 0,30 & 9,65 & $-3,3$ \\
\hline 1041 & 9,14 & 14,91 & 4,69 & 2,48 & 46,86 & $-5,6$ \\
\hline 1042 & 0,43 & 2,63 & 0,95 & 0,58 & 9,48 & $-3,2$ \\
\hline 1043 & 0,39 & 2,54 & 0,92 & 0,54 & 9,23 & $-3,2$ \\
\hline
\end{tabular}




\begin{tabular}{|c|c|c|c|c|c|c|}
\hline 1044 & 0,13 & 1,52 & 0,63 & 0,27 & 6,32 & $-2,7$ \\
\hline 1045 & 4,45 & 8,19 & 2,65 & 2,14 & 26,51 & $-4,7$ \\
\hline 1046 & 1,63 & 5,11 & 1,76 & 1,18 & 17,59 & $-4,1$ \\
\hline 1047 & 0,58 & 3,01 & 1,15 & 0,64 & 11,53 & $-3,5$ \\
\hline 1048 & 0,22 & 2,65 & 1,06 & 0,27 & 10,59 & $-3,4$ \\
\hline 1049 & 0,34 & 2,14 & 0,70 & 0,62 & 7,00 & $-2,8$ \\
\hline 1050 & 0,07 & 1,08 & 0,44 & 0,22 & 4,35 & $-2,1$ \\
\hline 1051 & 0,06 & 0,90 & 0,31 & 0,24 & 3,12 & $-1,6$ \\
\hline 1052 & 0,19 & 1,71 & 0,62 & 0,38 & 6,17 & $-2,6$ \\
\hline 1053 & 0,33 & 3,50 & 0,86 & 0,48 & 8,59 & $-3,1$ \\
\hline 1054 & 0,30 & 2,01 & 0,75 & 0,50 & 7,53 & $-2,9$ \\
\hline 1055 & 0,21 & 1,80 & 0,63 & 0,43 & 6,27 & $-2,6$ \\
\hline 1056 & 0,07 & 0,97 & 0,36 & 0,23 & 3,62 & $-1,9$ \\
\hline 1057 & 101,03 & 41,16 & 12,80 & 10,05 & 127,96 & $-7,0$ \\
\hline 1058 & 0,03 & 0,69 & 0,29 & 0,15 & 2,87 & $-1,5$ \\
\hline 1059 & 0,04 & 0,76 & 0,27 & 0,17 & 2,69 & $-1,4$ \\
\hline 1060 & 3,09 & 7,22 & 2,62 & 1,50 & 26,23 & $-4,7$ \\
\hline 1061 & 0,29 & 2,14 & 0,78 & 0,48 & 7,81 & $-3,0$ \\
\hline 1062 & 4,57 & 9,14 & 3,59 & 1,62 & 35,85 & $-5,2$ \\
\hline 1063 & 0,03 & 0,76 & 0,29 & 0,15 & 2,85 & $-1,5$ \\
\hline 1064 & 0,01 & 0,24 & 0,11 & 0,06 & 1,12 & $-0,2$ \\
\hline 1065 & 0,19 & 1,80 & 0,69 & 0,36 & 6,89 & $-2,8$ \\
\hline 1066 & 1,73 & 7,15 & 1,64 & 1,35 & 16,41 & $-4,0$ \\
\hline 1067 & 0,13 & 1,31 & 0,44 & 0,38 & 4,44 & $-2,2$ \\
\hline 1068 & 0,10 & 1,29 & 0,49 & 0,26 & 4,88 & $-2,3$ \\
\hline 1069 & 0,39 & 2,50 & 0,94 & 0,53 & 9,37 & $-3,2$ \\
\hline 1070 & 0,63 & 3,73 & 1,54 & 0,52 & 15,36 & $-3,9$ \\
\hline 1071 & 1,26 & 5,26 & 1,88 & 0,85 & 18,77 & $-4,2$ \\
\hline 1072 & 1,78 & 6,66 & 2,07 & 1,10 & 20,70 & $-4,4$ \\
\hline 1073 & 0,07 & 1,00 & 0,37 & 0,23 & 3,72 & $-1,9$ \\
\hline 1074 & 0,34 & 2,56 & 1,01 & 0,43 & 10,06 & $-3,3$ \\
\hline 1075 & 0,06 & 0,90 & 0,36 & 0,22 & 3,56 & $-1,8$ \\
\hline 1076 & 0,06 & 0,96 & 0,39 & 0,20 & 3,93 & $-2,0$ \\
\hline 1077 & 1,42 & 4,74 & 1,58 & 1,14 & 15,80 & $-4,0$ \\
\hline 1078 & 0,40 & 2,70 & 0,88 & 0,59 & 8,76 & $-3,1$ \\
\hline 1079 & 19,16 & 18,72 & 5,88 & 4,15 & 58,82 & $-5,9$ \\
\hline 1080 & 0,25 & 2,44 & 0,86 & 0,37 & 8,58 & $-3,1$ \\
\hline 1081 & 0,13 & 1,38 & 0,48 & 0,34 & 4,82 & $-2,3$ \\
\hline 1082 & 0,06 & 0,97 & 0,37 & 0,22 & 3,67 & $-1,9$ \\
\hline 1083 & 0,27 & 1,87 & 0,62 & 0,55 & 6,22 & $-2,6$ \\
\hline 1084 & 0,08 & 1,17 & 0,46 & 0,23 & 4,61 & $-2,2$ \\
\hline 1085 & 1,44 & 5,29 & 1,98 & 0,93 & 19,76 & $-4,3$ \\
\hline 1086 & 0,69 & 3,37 & 1,41 & 0,63 & 14,06 & $-3,8$ \\
\hline 1087 & 0,02 & 0,52 & 0,20 & 0,13 & 1,97 & $-1,0$ \\
\hline 1088 & 0,15 & 1,41 & 0,50 & 0,39 & 5,01 & $-2,3$ \\
\hline 1089 & 0,39 & 2,60 & 1,03 & 0,48 & 10,25 & $-3,4$ \\
\hline 1090 & 0,06 & 0,94 & 0,31 & 0,26 & 3,08 & $-1,6$ \\
\hline 1091 & 1,70 & 5,33 & 1,85 & 1,17 & 18,49 & $-4,2$ \\
\hline 1092 & 1,75 & 5,21 & 1,83 & 1,22 & 18,34 & $-4,2$ \\
\hline 1093 & 0,29 & 2,16 & 0,86 & 0,43 & 8,59 & $-3,1$ \\
\hline 1094 & 0,05 & 0,86 & 0,33 & 0,19 & 3,27 & $-1,7$ \\
\hline 1095 & 5,06 & 9,44 & 3,31 & 1,95 & 33,09 & $-5,0$ \\
\hline 1096 & 1,80 & 5,41 & 1,94 & 1,18 & 19,38 & $-4,3$ \\
\hline 1097 & 0,02 & 0,56 & 0,26 & 0,08 & 2,64 & $-1,4$ \\
\hline 1098 & 0,34 & 2,16 & 0,70 & 0,61 & 7,03 & $-2,8$ \\
\hline 1099 & 0,27 & 2,59 & 1,01 & 0,34 & 10,12 & $-3,3$ \\
\hline 1100 & 0,05 & 0,83 & 0,33 & 0,19 & 3,33 & $-1,7$ \\
\hline 1101 & 0,26 & 1,90 & 0,65 & 0,50 & 6,47 & $-2,7$ \\
\hline 1102 & 0,19 & 1,73 & 0,59 & 0,41 & 5,93 & $-2,6$ \\
\hline 1103 & 0,02 & 0,52 & 0,21 & 0,14 & 2,08 & $-1,1$ \\
\hline 1104 & 0,38 & 2,42 & 0,73 & 0,66 & 7,32 & $-2,9$ \\
\hline 1105 & 0,47 & 2,70 & 1,01 & 0,60 & 10,07 & $-3,3$ \\
\hline
\end{tabular}




\begin{tabular}{|c|c|c|c|c|c|c|}
\hline 1106 & 0,05 & 0,89 & 0,35 & 0,19 & 3,48 & $-1,8$ \\
\hline 1107 & 2,32 & 6,45 & 2,23 & 1,33 & 22,25 & $-4,5$ \\
\hline 1108 & 0,02 & 0,52 & 0,20 & 0,13 & 1,97 & $-1,0$ \\
\hline 1109 & 1,22 & 5,03 & 1,83 & 0,84 & 18,34 & $-4,2$ \\
\hline 1110 & 0,06 & 1,06 & 0,42 & 0,19 & 4,18 & $-2,1$ \\
\hline 1111 & 0,06 & 0,93 & 0,36 & 0,20 & 3,61 & $-1,9$ \\
\hline 1112 & 0,52 & 2,91 & 0,94 & 0,71 & 9,36 & $-3,2$ \\
\hline 1113 & 0,04 & 0,76 & 0,32 & 0,15 & 3,19 & $-1,7$ \\
\hline 1114 & 0,13 & 1,63 & 0,66 & 0,24 & 6,56 & $-2,7$ \\
\hline 1115 & 0,27 & 2,16 & 0,66 & 0,53 & 6,60 & $-2,7$ \\
\hline 1116 & 0,07 & 1,00 & 0,38 & 0,24 & 3,78 & $-1,9$ \\
\hline 1117 & 0,49 & 3,03 & 1,24 & 0,50 & 12,35 & $-3,6$ \\
\hline 1118 & 0,65 & 3,25 & 1,29 & 0,64 & 12,85 & $-3,7$ \\
\hline 1119 & 0,04 & 0,73 & 0,26 & 0,18 & 2,58 & $-1,4$ \\
\hline 1120 & 0,11 & 1,60 & 0,69 & 0,20 & 6,88 & $-2,8$ \\
\hline 1121 & 0,01 & 0,52 & 0,22 & 0,07 & 2,22 & $-1,2$ \\
\hline 1122 & 0,09 & 1,10 & 0,38 & 0,30 & 3,79 & $-1,9$ \\
\hline 1123 & 0,39 & 2,50 & 0,88 & 0,56 & 8,80 & $-3,1$ \\
\hline 1124 & 0,12 & 1,29 & 0,46 & 0,32 & 4,58 & $-2,2$ \\
\hline 1125 & 0,34 & 2,14 & 0,70 & 0,62 & 7,00 & $-2,8$ \\
\hline 1126 & 0,07 & 1,07 & 0,40 & 0,24 & 3,95 & $-2,0$ \\
\hline 1127 & 0,10 & 1,27 & 0,43 & 0,30 & 4,31 & $-2,1$ \\
\hline 1128 & 0,05 & 0,86 & 0,36 & 0,19 & 3,55 & $-1,8$ \\
\hline 1129 & 0,05 & 0,76 & 0,28 & 0,22 & 2,83 & $-1,5$ \\
\hline 1130 & 0,29 & 2,25 & 0,83 & 0,45 & 8,29 & $-3,1$ \\
\hline 1131 & 0,11 & 1,42 & 0,61 & 0,23 & 6,12 & $-2,6$ \\
\hline 1132 & 0,02 & 0,52 & 0,19 & 0,15 & 1,94 & $-1,0$ \\
\hline 1133 & 0,60 & 3,21 & 1,10 & 0,69 & 10,96 & $-3,5$ \\
\hline 1134 & 2,13 & 5,56 & 1,72 & 1,57 & 17,22 & $-4,1$ \\
\hline 1135 & 0,60 & 2,95 & 0,99 & 0,77 & 9,88 & $-3,3$ \\
\hline 1136 & 0,76 & 3,48 & 1,18 & 0,82 & 11,80 & $-3,6$ \\
\hline 1137 & 6,90 & 10,67 & 3,84 & 2,29 & 38,36 & $-5,3$ \\
\hline 1138 & 0,12 & 1,32 & 0,50 & 0,30 & 5,03 & $-2,3$ \\
\hline 1139 & 0,75 & 3,79 & 1,58 & 0,61 & 15,84 & $-4,0$ \\
\hline 1140 & 0,40 & 2,65 & 0,94 & 0,54 & 9,43 & $-3,2$ \\
\hline 1141 & 0,37 & 2,36 & 0,74 & 0,64 & 7,35 & $-2,9$ \\
\hline 1142 & 0,03 & 0,66 & 0,25 & 0,17 & 2,53 & $-1,3$ \\
\hline 1143 & 0,12 & 1,67 & 0,59 & 0,26 & 5,93 & $-2,6$ \\
\hline 1144 & 0,05 & 0,86 & 0,34 & 0,19 & 3,38 & $-1,8$ \\
\hline 1145 & 0,42 & 2,38 & 0,81 & 0,66 & 8,08 & $-3,0$ \\
\hline 1146 & 0,31 & 2,07 & 0,75 & 0,53 & 7,48 & $-2,9$ \\
\hline 1147 & 2,60 & 7,33 & 2,67 & 1,24 & 26,70 & $-4,7$ \\
\hline 1148 & 0,70 & 3,44 & 1,29 & 0,70 & 12,85 & $-3,7$ \\
\hline 1149 & 1,16 & 4,04 & 1,41 & 1,05 & 14,12 & $-3,8$ \\
\hline 1150 & 0,06 & 0,86 & 0,31 & 0,24 & 3,12 & $-1,6$ \\
\hline 1151 & 4,87 & 8,88 & 2,90 & 2,13 & 29,04 & $-4,9$ \\
\hline 1152 & 0,09 & 1,32 & 0,53 & 0,22 & 5,31 & $-2,4$ \\
\hline 1153 & 0,88 & 3,91 & 1,26 & 0,89 & 12,63 & $-3,7$ \\
\hline 1154 & 0,64 & 3,04 & 1,09 & 0,75 & 10,90 & $-3,4$ \\
\hline 1155 & 0,04 & 0,73 & 0,25 & 0,21 & 2,49 & $-1,3$ \\
\hline 1156 & 0,07 & 1,03 & 0,35 & 0,27 & 3,51 & $-1,8$ \\
\hline 1157 & 0,50 & 2,88 & 1,06 & 0,60 & 10,61 & $-3,4$ \\
\hline 1158 & 0,06 & 0,89 & 0,32 & 0,24 & 3,15 & $-1,7$ \\
\hline 1159 & 0,06 & 1,04 & 0,45 & 0,17 & 4,47 & $-2,2$ \\
\hline 1160 & 0,10 & 1,17 & 0,44 & 0,29 & 4,39 & $-2,1$ \\
\hline 1161 & 0,38 & 2,68 & 1,05 & 0,46 & 10,47 & $-3,4$ \\
\hline 1162 & 0,34 & 2,26 & 0,84 & 0,52 & 8,35 & $-3,1$ \\
\hline 1163 & 0,04 & 0,72 & 0,25 & 0,20 & 2,48 & $-1,3$ \\
\hline 1164 & 0,06 & 0,90 & 0,36 & 0,22 & 3,59 & $-1,8$ \\
\hline 1165 & 0,10 & 1,52 & 0,68 & 0,19 & 6,76 & $-2,8$ \\
\hline 1166 & 0,18 & 1,80 & 0,67 & 0,34 & 6,74 & $-2,8$ \\
\hline 1167 & 0,25 & 2,05 & 0,74 & 0,42 & 7,43 & $-2,9$ \\
\hline
\end{tabular}




$\begin{array}{lllllll}1168 & 0,43 & 2,71 & 0,88 & 0,63 & 8,75 & -3,1 \\ 1169 & 0,27 & 2,08 & 0,82 & 0,41 & 8,23 & -3,0 \\ 1170 & 0,19 & 1,67 & 0,65 & 0,37 & 6,45 & -2,7 \\ 1171 & 0,20 & 1,76 & 0,62 & 0,40 & 6,19 & -2,6 \\ 1172 & 0,04 & 0,92 & 0,39 & 0,14 & 3,94 & -2,0 \\ 1173 & 0,06 & 0,90 & 0,37 & 0,20 & 3,71 & -1,9 \\ 1174 & 0,31 & 2,16 & 0,71 & 0,56 & 7,14 & -2,8 \\ 1175 & 0,16 & 1,62 & 0,52 & 0,38 & 5,24 & -2,4 \\ 1176 & 0,06 & 0,93 & 0,30 & 0,25 & 3,02 & -1,6 \\ 1177 & 0,47 & 2,64 & 0,91 & 0,65 & 9,07 & -3,2 \\ 1178 & 0,05 & 0,89 & 0,33 & 0,19 & 3,33 & -1,7 \\ 1179 & 0,20 & 1,84 & 0,70 & 0,37 & 6,99 & -2,8 \\ 1180 & 0,55 & 2,86 & 1,02 & 0,69 & 10,17 & -3,3 \\ 1181 & 0,43 & 2,84 & 1,10 & 0,49 & 11,02 & -3,5 \\ 1182 & 0,19 & 1,84 & 0,73 & 0,33 & 7,29 & -2,9 \\ 1183 & 0,05 & 0,82 & 0,27 & 0,25 & 2,73 & -1,4 \\ 1184 & 0,04 & 0,86 & 0,35 & 0,14 & 3,46 & -1,8 \\ 1185 & 0,09 & 1,11 & 0,39 & 0,29 & 3,91 & -2,0\end{array}$

SM-82_Br02_detail

Best fit ellipse

\begin{tabular}{|c|c|c|c|c|c|c|}
\hline Label & Area $\left(\mathbf{c m}^{2}\right)$ & $\begin{array}{l}\text { Perimeter } \\
\quad(\mathrm{cm})\end{array}$ & $\begin{array}{l}\text { Major axis } \\
\quad \text { (cm) }\end{array}$ & $\begin{array}{l}\text { Minor axis } \\
\quad(\mathbf{c m})\end{array}$ & $\begin{array}{c}\text { Diameter } \\
\text { (mm) }\end{array}$ & Phi \\
\hline 1 & 0,029 & 0,674 & 0,264 & 0,142 & 2,64 & $-1,4$ \\
\hline 2 & 0,040 & 0,852 & 0,330 & 0,155 & 3,30 & $-1,7$ \\
\hline 3 & 0,009 & 0,386 & 0,158 & 0,071 & 1,58 & $-0,7$ \\
\hline 4 & 0,007 & 0,300 & 0,111 & 0,080 & 1,11 & $-0,2$ \\
\hline 5 & 0,016 & 0,501 & 0,173 & 0,117 & 1,73 & $-0,8$ \\
\hline 6 & 0,007 & 0,287 & 0,113 & 0,079 & 1,13 & $-0,2$ \\
\hline 7 & 0,753 & 3,431 & 1,113 & 0,861 & 11,13 & $-3,5$ \\
\hline 8 & 0,014 & 0,478 & 0,177 & 0,104 & 1,77 & $-0,8$ \\
\hline 9 & 0,080 & 1,141 & 0,363 & 0,280 & 3,63 & $-1,9$ \\
\hline 10 & 0,006 & 0,244 & 0,084 & 0,084 & 0,84 & 0,2 \\
\hline 11 & 0,010 & 0,374 & 0,128 & 0,102 & 1,28 & $-0,4$ \\
\hline 12 & 0,008 & 0,348 & 0,141 & 0,071 & 1,41 & $-0,5$ \\
\hline 13 & 0,003 & 0,183 & 0,068 & 0,053 & 0,68 & 0,6 \\
\hline 14 & 0,005 & 0,226 & 0,096 & 0,062 & 0,96 & 0,1 \\
\hline 15 & 0,155 & 1,698 & 0,568 & 0,347 & 5,68 & $-2,5$ \\
\hline 16 & 0,007 & 0,282 & 0,101 & 0,082 & 1,01 & 0,0 \\
\hline 17 & 0,009 & 0,348 & 0,120 & 0,099 & 1,20 & $-0,3$ \\
\hline 18 & 0,079 & 1,091 & 0,366 & 0,274 & 3,66 & $-1,9$ \\
\hline 19 & 0,008 & 0,348 & 0,135 & 0,079 & 1,35 & $-0,4$ \\
\hline 20 & 0,031 & 0,668 & 0,252 & 0,155 & 2,52 & $-1,3$ \\
\hline 21 & 0,536 & 2,900 & 0,911 & 0,749 & 9,11 & $-3,2$ \\
\hline 22 & 0,066 & 1,108 & 0,452 & 0,185 & 4,52 & $-2,2$ \\
\hline 23 & 0,331 & 2,677 & 0,902 & 0,468 & 9,02 & $-3,2$ \\
\hline 24 & 0,032 & 0,709 & 0,269 & 0,152 & 2,69 & $-1,4$ \\
\hline 25 & 0,018 & 0,496 & 0,173 & 0,131 & 1,73 & $-0,8$ \\
\hline 26 & 0,019 & 0,539 & 0,187 & 0,127 & 1,87 & $-0,9$ \\
\hline 27 & 0,016 & 0,447 & 0,177 & 0,114 & 1,77 & $-0,8$ \\
\hline 28 & 0,009 & 0,361 & 0,122 & 0,093 & 1,22 & $-0,3$ \\
\hline 29 & 0,008 & 0,331 & 0,115 & 0,088 & 1,15 & $-0,2$ \\
\hline 30 & 0,015 & 0,435 & 0,164 & 0,116 & 1,64 & $-0,7$ \\
\hline 31 & 0,043 & 0,852 & 0,300 & 0,182 & 3,00 & $-1,6$ \\
\hline 32 & 0,042 & 0,882 & 0,270 & 0,198 & 2,70 & $-1,4$ \\
\hline 33 & 0,070 & 1,027 & 0,337 & 0,266 & 3,37 & $-1,8$ \\
\hline 34 & 0,021 & 0,539 & 0,212 & 0,123 & 2,12 & $-1,1$ \\
\hline 35 & 0,243 & 2,165 & 0,649 & 0,476 & 6,49 & $-2,7$ \\
\hline 36 & 0,013 & 0,404 & 0,136 & 0,122 & 1,36 & $-0,4$ \\
\hline 37 & 0,020 & 0,570 & 0,205 & 0,125 & 2,05 & $-1,0$ \\
\hline 38 & 0,013 & 0,435 & 0,157 & 0,102 & 1,57 & $-0,7$ \\
\hline
\end{tabular}




\begin{tabular}{|c|c|c|c|c|c|c|}
\hline 39 & 0,045 & 0,999 & 0,348 & 0,165 & 3,48 & $-1,8$ \\
\hline 40 & 0,029 & 0,648 & 0,204 & 0,183 & 2,04 & $-1,0$ \\
\hline 41 & 0,012 & 0,374 & 0,139 & 0,107 & 1,39 & $-0,5$ \\
\hline 42 & 0,017 & 0,496 & 0,188 & 0,117 & 1,88 & $-0,9$ \\
\hline 43 & 0,012 & 0,409 & 0,157 & 0,095 & 1,57 & $-0,6$ \\
\hline 44 & 0,016 & 0,539 & 0,169 & 0,120 & 1,69 & $-0,8$ \\
\hline 45 & 0,048 & 0,839 & 0,309 & 0,198 & 3,09 & $-1,6$ \\
\hline 46 & 0,033 & 0,722 & 0,283 & 0,147 & 2,83 & $-1,5$ \\
\hline 47 & 0,116 & 1,484 & 0,547 & 0,269 & 5,47 & $-2,5$ \\
\hline 48 & 0,011 & 0,409 & 0,139 & 0,102 & 1,39 & $-0,5$ \\
\hline 49 & 0,540 & 3,078 & 1,165 & 0,591 & 11,65 & $-3,5$ \\
\hline 50 & 0,015 & 0,478 & 0,164 & 0,120 & 1,64 & $-0,7$ \\
\hline 51 & 0,051 & 1,004 & 0,393 & 0,166 & 3,93 & $-2,0$ \\
\hline 52 & 0,014 & 0,440 & 0,160 & 0,115 & 1,60 & $-0,7$ \\
\hline 53 & 0,022 & 0,661 & 0,227 & 0,125 & 2,27 & $-1,2$ \\
\hline 54 & 0,048 & 0,821 & 0,316 & 0,195 & 3,16 & $-1,7$ \\
\hline 55 & 0,002 & 0,153 & 0,068 & 0,044 & 0,68 & 0,6 \\
\hline 56 & 0,087 & 1,306 & 0,500 & 0,222 & 5,00 & $-2,3$ \\
\hline 57 & 0,097 & 1,367 & 0,584 & 0,211 & 5,84 & $-2,5$ \\
\hline 58 & 0,084 & 1,108 & 0,387 & 0,276 & 3,87 & $-2,0$ \\
\hline 59 & 0,079 & 1,096 & 0,404 & 0,248 & 4,04 & $-2,0$ \\
\hline 60 & 0,050 & 0,900 & 0,343 & 0,185 & 3,43 & $-1,8$ \\
\hline 61 & 0,006 & 0,287 & 0,105 & 0,074 & 1,05 & $-0,1$ \\
\hline 62 & 0,005 & 0,226 & 0,089 & 0,067 & 0,89 & 0,2 \\
\hline 63 & 0,003 & 0,183 & 0,067 & 0,062 & 0,67 & 0,6 \\
\hline 64 & 0,164 & 1,693 & 0,677 & 0,310 & 6,77 & $-2,8$ \\
\hline 65 & 0,099 & 1,152 & 0,366 & 0,345 & 3,66 & $-1,9$ \\
\hline 66 & 0,017 & 0,508 & 0,190 & 0,116 & 1,90 & $-0,9$ \\
\hline 67 & 0,226 & 1,922 & 0,744 & 0,388 & 7,44 & $-2,9$ \\
\hline 68 & 0,003 & 0,226 & 0,083 & 0,050 & 0,83 & 0,3 \\
\hline 69 & 0,014 & 0,435 & 0,184 & 0,100 & 1,84 & $-0,9$ \\
\hline 70 & 0,004 & 0,257 & 0,104 & 0,052 & 1,04 & $-0,1$ \\
\hline 71 & 0,096 & 1,190 & 0,372 & 0,330 & 3,72 & $-1,9$ \\
\hline 72 & 0,013 & 0,392 & 0,143 & 0,112 & 1,43 & $-0,5$ \\
\hline 73 & 0,020 & 0,539 & 0,203 & 0,123 & 2,03 & $-1,0$ \\
\hline 74 & 0,007 & 0,287 & 0,103 & 0,087 & 1,03 & 0,0 \\
\hline 75 & 0,007 & 0,282 & 0,118 & 0,071 & 1,18 & $-0,2$ \\
\hline 76 & 0,063 & 0,956 & 0,352 & 0,229 & 3,52 & $-1,8$ \\
\hline 77 & 0,010 & 0,422 & 0,158 & 0,079 & 1,58 & $-0,7$ \\
\hline 78 & 0,026 & 0,656 & 0,205 & 0,162 & 2,05 & $-1,0$ \\
\hline 79 & 0,028 & 0,674 & 0,261 & 0,136 & 2,61 & $-1,4$ \\
\hline 80 & 0,008 & 0,348 & 0,114 & 0,094 & 1,14 & $-0,2$ \\
\hline 81 & 0,019 & 0,526 & 0,170 & 0,143 & 1,70 & $-0,8$ \\
\hline 82 & 0,042 & 0,877 & 0,334 & 0,160 & 3,34 & $-1,7$ \\
\hline 83 & 0,014 & 0,440 & 0,161 & 0,107 & 1,61 & $-0,7$ \\
\hline 84 & 0,010 & 0,379 & 0,155 & 0,085 & 1,55 & $-0,6$ \\
\hline 85 & 0,295 & 2,084 & 0,666 & 0,565 & 6,66 & $-2,7$ \\
\hline 86 & 0,016 & 0,483 & 0,183 & 0,110 & 1,83 & $-0,9$ \\
\hline 87 & 0,011 & 0,404 & 0,141 & 0,097 & 1,41 & $-0,5$ \\
\hline 88 & 0,021 & 0,526 & 0,181 & 0,144 & 1,81 & $-0,9$ \\
\hline 89 & 0,175 & 1,995 & 0,779 & 0,286 & 7,79 & $-3,0$ \\
\hline 90 & 0,011 & 0,409 & 0,126 & 0,113 & 1,26 & $-0,3$ \\
\hline 91 & 0,265 & 2,290 & 0,741 & 0,455 & 7,41 & $-2,9$ \\
\hline 92 & 0,002 & 0,165 & 0,068 & 0,044 & 0,68 & 0,6 \\
\hline 93 & 0,010 & 0,374 & 0,134 & 0,098 & 1,34 & $-0,4$ \\
\hline 94 & 0,133 & 1,484 & 0,518 & 0,326 & 5,18 & $-2,4$ \\
\hline 95 & 0,048 & 0,834 & 0,289 & 0,209 & 2,89 & $-1,5$ \\
\hline 96 & 0,019 & 0,539 & 0,183 & 0,130 & 1,83 & $-0,9$ \\
\hline 97 & 0,146 & 1,653 & 0,666 & 0,280 & 6,66 & $-2,7$ \\
\hline 98 & 0,016 & 0,465 & 0,193 & 0,104 & 1,93 & $-1,0$ \\
\hline 99 & 0,014 & 0,440 & 0,158 & 0,116 & 1,58 & $-0,7$ \\
\hline 100 & 0,003 & 0,183 & 0,063 & 0,056 & 0,63 & 0,7 \\
\hline
\end{tabular}




\begin{tabular}{|c|c|c|c|c|c|c|}
\hline 101 & 0,002 & 0,153 & 0,069 & 0,034 & 0,69 & 0,5 \\
\hline 102 & 0,011 & 0,379 & 0,150 & 0,091 & 1,50 & $-0,6$ \\
\hline 103 & 0,077 & 1,078 & 0,376 & 0,260 & 3,76 & $-1,9$ \\
\hline 104 & 2,889 & 7,774 & 3,060 & 1,202 & 30,60 & $-4,9$ \\
\hline 105 & 0,003 & 0,226 & 0,096 & 0,043 & 0,96 & 0,1 \\
\hline 106 & 0,012 & 0,453 & 0,165 & 0,090 & 1,65 & $-0,7$ \\
\hline 107 & 0,187 & 1,845 & 0,653 & 0,365 & 6,53 & $-2,7$ \\
\hline 108 & 0,015 & 0,503 & 0,194 & 0,101 & 1,94 & $-1,0$ \\
\hline 109 & 0,024 & 0,625 & 0,258 & 0,120 & 2,58 & $-1,4$ \\
\hline 110 & 0,068 & 0,951 & 0,312 & 0,278 & 3,12 & $-1,6$ \\
\hline 111 & 0,810 & 3,546 & 1,154 & 0,894 & 11,54 & $-3,5$ \\
\hline 112 & 0,003 & 0,226 & 0,092 & 0,045 & 0,92 & 0,1 \\
\hline 113 & 0,002 & 0,122 & 0,049 & 0,049 & 0,49 & 1,0 \\
\hline 114 & 0,024 & 0,631 & 0,200 & 0,151 & 2,00 & $-1,0$ \\
\hline 115 & 0,029 & 0,717 & 0,290 & 0,129 & 2,90 & $-1,5$ \\
\hline 116 & 0,003 & 0,208 & 0,080 & 0,044 & 0,80 & 0,3 \\
\hline 117 & 1,669 & 5,235 & 1,897 & 1,120 & 18,97 & $-4,2$ \\
\hline 118 & 0,031 & 0,674 & 0,259 & 0,151 & 2,59 & $-1,4$ \\
\hline 119 & 0,009 & 0,331 & 0,111 & 0,107 & 1,11 & $-0,2$ \\
\hline 120 & 0,030 & 0,648 & 0,214 & 0,177 & 2,14 & $-1,1$ \\
\hline 121 & 0,012 & 0,392 & 0,149 & 0,100 & 1,49 & $-0,6$ \\
\hline 122 & 0,058 & 0,907 & 0,308 & 0,241 & 3,08 & $-1,6$ \\
\hline 123 & 0,047 & 0,852 & 0,288 & 0,208 & 2,88 & $-1,5$ \\
\hline 124 & 0,431 & 2,957 & 1,167 & 0,471 & 11,67 & $-3,5$ \\
\hline 125 & 0,030 & 0,666 & 0,228 & 0,169 & 2,28 & $-1,2$ \\
\hline 126 & 0,019 & 0,526 & 0,191 & 0,128 & 1,91 & $-0,9$ \\
\hline 127 & 0,081 & 1,080 & 0,389 & 0,264 & 3,89 & $-2,0$ \\
\hline 128 & 0,094 & 1,286 & 0,418 & 0,286 & 4,18 & $-2,1$ \\
\hline 129 & 0,139 & 1,411 & 0,427 & 0,415 & 4,27 & $-2,1$ \\
\hline 130 & 0,021 & 0,514 & 0,211 & 0,124 & 2,11 & $-1,1$ \\
\hline 131 & 0,010 & 0,379 & 0,149 & 0,087 & 1,49 & $-0,6$ \\
\hline 132 & 0,055 & 0,943 & 0,322 & 0,218 & 3,22 & $-1,7$ \\
\hline 133 & 0,050 & 0,877 & 0,352 & 0,182 & 3,52 & $-1,8$ \\
\hline 134 & 0,004 & 0,226 & 0,091 & 0,059 & 0,91 & 0,1 \\
\hline 135 & 0,036 & 0,722 & 0,265 & 0,175 & 2,65 & $-1,4$ \\
\hline 136 & 0,219 & 1,810 & 0,563 & 0,496 & 5,63 & $-2,5$ \\
\hline 137 & 0,007 & 0,282 & 0,101 & 0,089 & 1,01 & 0,0 \\
\hline 138 & 0,101 & 1,238 & 0,376 & 0,341 & 3,76 & $-1,9$ \\
\hline 139 & 0,032 & 0,679 & 0,257 & 0,157 & 2,57 & $-1,4$ \\
\hline 140 & 0,579 & 3,296 & 1,088 & 0,678 & 10,88 & $-3,4$ \\
\hline 141 & 0,011 & 0,379 & 0,146 & 0,094 & 1,46 & $-0,5$ \\
\hline 142 & 0,002 & 0,165 & 0,068 & 0,044 & 0,68 & 0,6 \\
\hline 143 & 0,013 & 0,435 & 0,162 & 0,103 & 1,62 & $-0,7$ \\
\hline 144 & 0,010 & 0,386 & 0,123 & 0,106 & 1,23 & $-0,3$ \\
\hline 145 & 0,083 & 1,202 & 0,485 & 0,219 & 4,85 & $-2,3$ \\
\hline 146 & 0,013 & 0,508 & 0,227 & 0,071 & 2,27 & $-1,2$ \\
\hline 147 & 0,128 & 1,759 & 0,486 & 0,336 & 4,86 & $-2,3$ \\
\hline 148 & 0,055 & 0,882 & 0,290 & 0,241 & 2,90 & $-1,5$ \\
\hline 149 & 0,007 & 0,305 & 0,108 & 0,088 & 1,08 & $-0,1$ \\
\hline 150 & 0,011 & 0,422 & 0,177 & 0,077 & 1,77 & $-0,8$ \\
\hline 151 & 0,081 & 1,121 & 0,396 & 0,261 & 3,96 & $-2,0$ \\
\hline 152 & 0,024 & 0,582 & 0,209 & 0,148 & 2,09 & $-1,1$ \\
\hline 153 & 0,014 & 0,465 & 0,167 & 0,110 & 1,67 & $-0,7$ \\
\hline 154 & 0,047 & 0,915 & 0,370 & 0,162 & 3,70 & $-1,9$ \\
\hline 155 & 0,004 & 0,226 & 0,082 & 0,058 & 0,82 & 0,3 \\
\hline 156 & 0,004 & 0,226 & 0,085 & 0,063 & 0,85 & 0,2 \\
\hline 157 & 0,007 & 0,313 & 0,138 & 0,069 & 1,38 & $-0,5$ \\
\hline 158 & 0,005 & 0,226 & 0,089 & 0,067 & 0,89 & 0,2 \\
\hline 159 & 0,004 & 0,239 & 0,091 & 0,059 & 0,91 & 0,1 \\
\hline 160 & 0,312 & 2,476 & 0,905 & 0,439 & 9,05 & $-3,2$ \\
\hline 161 & 0,042 & 0,839 & 0,301 & 0,180 & 3,01 & $-1,6$ \\
\hline 162 & 0,007 & 0,300 & 0,106 & 0,084 & 1,06 & $-0,1$ \\
\hline
\end{tabular}




\begin{tabular}{|c|c|c|c|c|c|c|}
\hline 163 & 0,151 & 1,912 & 0,689 & 0,279 & 6,89 & $-2,8$ \\
\hline 164 & 0,025 & 0,631 & 0,230 & 0,139 & 2,30 & $-1,2$ \\
\hline 165 & 0,103 & 1,454 & 0,540 & 0,244 & 5,40 & $-2,4$ \\
\hline 166 & 0,005 & 0,244 & 0,086 & 0,069 & 0,86 & 0,2 \\
\hline 167 & 0,006 & 0,257 & 0,096 & 0,075 & 0,96 & 0,1 \\
\hline 168 & 0,006 & 0,287 & 0,100 & 0,071 & 1,00 & 0,0 \\
\hline 169 & 0,007 & 0,300 & 0,125 & 0,071 & 1,25 & $-0,3$ \\
\hline 170 & 0,021 & 0,618 & 0,237 & 0,115 & 2,37 & $-1,2$ \\
\hline 171 & 0,785 & 3,716 & 1,121 & 0,892 & 11,21 & $-3,5$ \\
\hline 172 & 0,158 & 1,563 & 0,612 & 0,330 & 6,12 & $-2,6$ \\
\hline 173 & 0,195 & 1,835 & 0,542 & 0,458 & 5,42 & $-2,4$ \\
\hline 174 & 0,059 & 0,984 & 0,406 & 0,185 & 4,06 & $-2,0$ \\
\hline 175 & 0,013 & 0,409 & 0,166 & 0,100 & 1,66 & $-0,7$ \\
\hline 176 & 0,002 & 0,183 & 0,083 & 0,036 & 0,83 & 0,3 \\
\hline 177 & 0,212 & 1,926 & 0,690 & 0,392 & 6,90 & $-2,8$ \\
\hline 178 & 0,006 & 0,257 & 0,096 & 0,075 & 0,96 & 0,1 \\
\hline 179 & 0,023 & 0,582 & 0,203 & 0,146 & 2,03 & $-1,0$ \\
\hline 180 & 0,007 & 0,270 & 0,092 & 0,090 & 0,92 & 0,1 \\
\hline 181 & 0,092 & 1,159 & 0,395 & 0,297 & 3,95 & $-2,0$ \\
\hline 182 & 0,177 & 1,815 & 0,576 & 0,392 & 5,76 & $-2,5$ \\
\hline 183 & 0,006 & 0,270 & 0,093 & 0,076 & 0,93 & 0,1 \\
\hline 184 & 0,036 & 0,872 & 0,349 & 0,131 & 3,49 & $-1,8$ \\
\hline 185 & 1,931 & 6,267 & 2,249 & 1,093 & 22,49 & $-4,5$ \\
\hline 186 & 0,021 & 0,539 & 0,191 & 0,139 & 1,91 & $-0,9$ \\
\hline 187 & 0,427 & 3,210 & 1,261 & 0,431 & 12,61 & $-3,7$ \\
\hline 188 & 0,011 & 0,447 & 0,178 & 0,080 & 1,78 & $-0,8$ \\
\hline 189 & 0,150 & 1,833 & 0,723 & 0,265 & 7,23 & $-2,9$ \\
\hline 190 & 0,162 & 1,556 & 0,507 & 0,407 & 5,07 & $-2,3$ \\
\hline 191 & 0,070 & 1,047 & 0,368 & 0,243 & 3,68 & $-1,9$ \\
\hline 192 & 0,010 & 0,392 & 0,138 & 0,094 & 1,38 & $-0,5$ \\
\hline 193 & 0,013 & 0,422 & 0,171 & 0,097 & 1,71 & $-0,8$ \\
\hline 194 & 0,013 & 0,435 & 0,162 & 0,099 & 1,62 & $-0,7$ \\
\hline 195 & 0,096 & 1,207 & 0,449 & 0,274 & 4,49 & $-2,2$ \\
\hline 196 & 0,400 & 3,119 & 1,162 & 0,439 & 11,62 & $-3,5$ \\
\hline 197 & 0,094 & 1,274 & 0,448 & 0,266 & 4,48 & $-2,2$ \\
\hline 198 & 0,013 & 0,404 & 0,137 & 0,117 & 1,37 & $-0,5$ \\
\hline 199 & 5,618 & 9,896 & 3,136 & 2,281 & 31,36 & $-5,0$ \\
\hline 200 & 0,005 & 0,244 & 0,086 & 0,069 & 0,86 & 0,2 \\
\hline 201 & 0,014 & 0,435 & 0,168 & 0,106 & 1,68 & $-0,7$ \\
\hline 202 & 0,012 & 0,440 & 0,192 & 0,077 & 1,92 & $-0,9$ \\
\hline 203 & 0,021 & 0,570 & 0,228 & 0,120 & 2,28 & $-1,2$ \\
\hline 204 & 0,095 & 1,312 & 0,402 & 0,302 & 4,02 & $-2,0$ \\
\hline 205 & 0,010 & 0,374 & 0,139 & 0,090 & 1,39 & $-0,5$ \\
\hline 206 & 0,037 & 0,765 & 0,272 & 0,174 & 2,72 & $-1,4$ \\
\hline 207 & 0,002 & 0,165 & 0,069 & 0,034 & 0,69 & 0,5 \\
\hline 208 & 2,743 & 6,575 & 2,055 & 1,699 & 20,55 & $-4,4$ \\
\hline 209 & 0,005 & 0,270 & 0,111 & 0,059 & 1,11 & $-0,2$ \\
\hline 210 & 0,110 & 1,391 & 0,439 & 0,321 & 4,39 & $-2,1$ \\
\hline 211 & 0,299 & 2,442 & 0,879 & 0,433 & 8,79 & $-3,1$ \\
\hline 212 & 0,007 & 0,287 & 0,125 & 0,066 & 1,25 & $-0,3$ \\
\hline 213 & 0,163 & 1,588 & 0,621 & 0,334 & 6,21 & $-2,6$ \\
\hline 214 & 0,027 & 0,625 & 0,202 & 0,173 & 2,02 & $-1,0$ \\
\hline 215 & 0,041 & 0,808 & 0,277 & 0,188 & 2,77 & $-1,5$ \\
\hline 216 & 0,080 & 1,116 & 0,393 & 0,258 & 3,93 & $-2,0$ \\
\hline 217 & 0,426 & 2,730 & 0,887 & 0,612 & 8,87 & $-3,1$ \\
\hline 218 & 0,008 & 0,331 & 0,125 & 0,081 & 1,25 & $-0,3$ \\
\hline 219 & 1,214 & 4,474 & 1,464 & 1,056 & 14,64 & $-3,9$ \\
\hline 220 & 0,010 & 0,361 & 0,125 & 0,100 & 1,25 & $-0,3$ \\
\hline 221 & 0,005 & 0,331 & 0,095 & 0,062 & 0,95 & 0,1 \\
\hline 222 & 0,012 & 0,392 & 0,140 & 0,110 & 1,40 & $-0,5$ \\
\hline 223 & 0,041 & 0,826 & 0,262 & 0,199 & 2,62 & $-1,4$ \\
\hline 224 & 0,028 & 0,737 & 0,321 & 0,111 & 3,21 & $-1,7$ \\
\hline
\end{tabular}




\begin{tabular}{|c|c|c|c|c|c|c|}
\hline 225 & 0,557 & 3,236 & 1,133 & 0,626 & 11,33 & $-3,5$ \\
\hline 226 & 0,044 & 0,948 & 0,410 & 0,136 & 4,10 & $-2,0$ \\
\hline 227 & 0,038 & 0,722 & 0,265 & 0,182 & 2,65 & $-1,4$ \\
\hline 228 & 0,199 & 1,751 & 0,576 & 0,440 & 5,76 & $-2,5$ \\
\hline 229 & 0,013 & 0,409 & 0,163 & 0,098 & 1,63 & $-0,7$ \\
\hline 230 & 0,075 & 1,067 & 0,326 & 0,291 & 3,26 & $-1,7$ \\
\hline 231 & 0,016 & 0,465 & 0,177 & 0,114 & 1,77 & $-0,8$ \\
\hline 232 & 0,007 & 0,287 & 0,102 & 0,082 & 1,02 & 0,0 \\
\hline 233 & 0,027 & 0,692 & 0,292 & 0,118 & 2,92 & $-1,5$ \\
\hline 234 & 0,002 & 0,165 & 0,059 & 0,040 & 0,59 & 0,8 \\
\hline 235 & 0,005 & 0,270 & 0,111 & 0,059 & 1,11 & $-0,2$ \\
\hline 236 & 0,028 & 0,631 & 0,202 & 0,179 & 2,02 & $-1,0$ \\
\hline 237 & 0,007 & 0,331 & 0,137 & 0,069 & 1,37 & $-0,5$ \\
\hline 238 & 0,040 & 0,778 & 0,261 & 0,194 & 2,61 & $-1,4$ \\
\hline 239 & 0,066 & 1,304 & 0,573 & 0,147 & 5,73 & $-2,5$ \\
\hline 240 & 0,008 & 0,503 & 0,199 & 0,054 & 1,99 & $-1,0$ \\
\hline 241 & 0,007 & 0,270 & 0,092 & 0,090 & 0,92 & 0,1 \\
\hline 242 & 0,054 & 0,887 & 0,331 & 0,206 & 3,31 & $-1,7$ \\
\hline 243 & 1,009 & 3,988 & 1,247 & 1,031 & 12,47 & $-3,6$ \\
\hline 244 & 0,016 & 0,478 & 0,164 & 0,123 & 1,64 & $-0,7$ \\
\hline 245 & 0,170 & 1,592 & 0,549 & 0,396 & 5,49 & $-2,5$ \\
\hline 246 & 0,028 & 0,651 & 0,224 & 0,159 & 2,24 & $-1,2$ \\
\hline 247 & 0,085 & 1,220 & 0,475 & 0,229 & 4,75 & $-2,2$ \\
\hline 248 & 0,019 & 0,508 & 0,182 & 0,130 & 1,82 & $-0,9$ \\
\hline 249 & 0,002 & 0,165 & 0,069 & 0,034 & 0,69 & 0,5 \\
\hline 250 & 0,299 & 2,145 & 0,815 & 0,467 & 8,15 & $-3,0$ \\
\hline 251 & 0,047 & 0,803 & 0,318 & 0,187 & 3,18 & $-1,7$ \\
\hline 252 & 0,025 & 0,570 & 0,218 & 0,144 & 2,18 & $-1,1$ \\
\hline 253 & 0,038 & 0,773 & 0,251 & 0,194 & 2,51 & $-1,3$ \\
\hline 254 & 0,003 & 0,183 & 0,068 & 0,053 & 0,68 & 0,6 \\
\hline 255 & 1,813 & 5,894 & 1,769 & 1,305 & 17,69 & $-4,1$ \\
\hline 256 & 0,014 & 0,453 & 0,191 & 0,093 & 1,91 & $-0,9$ \\
\hline 257 & 0,013 & 0,422 & 0,148 & 0,113 & 1,48 & $-0,6$ \\
\hline 258 & 0,408 & 3,243 & 0,961 & 0,540 & 9,61 & $-3,3$ \\
\hline 259 & 0,013 & 0,440 & 0,157 & 0,106 & 1,57 & $-0,6$ \\
\hline 260 & 0,025 & 0,661 & 0,272 & 0,118 & 2,72 & $-1,4$ \\
\hline 261 & 0,043 & 0,852 & 0,326 & 0,167 & 3,26 & $-1,7$ \\
\hline 262 & 0,001 & 0,122 & 0,051 & 0,035 & 0,51 & 1,0 \\
\hline 263 & 0,012 & 0,417 & 0,153 & 0,101 & 1,53 & $-0,6$ \\
\hline 264 & 0,069 & 1,030 & 0,379 & 0,232 & 3,79 & $-1,9$ \\
\hline 265 & 0,064 & 1,024 & 0,334 & 0,245 & 3,34 & $-1,7$ \\
\hline 266 & 0,010 & 0,348 & 0,144 & 0,091 & 1,44 & $-0,5$ \\
\hline 267 & 0,872 & 4,631 & 1,683 & 0,660 & 16,83 & $-4,1$ \\
\hline 268 & 0,010 & 0,361 & 0,128 & 0,098 & 1,28 & $-0,4$ \\
\hline 269 & 0,066 & 1,035 & 0,360 & 0,234 & 3,60 & $-1,8$ \\
\hline 270 & 0,118 & 1,479 & 0,518 & 0,290 & 5,18 & $-2,4$ \\
\hline 271 & 0,026 & 0,623 & 0,202 & 0,164 & 2,02 & $-1,0$ \\
\hline 272 & 0,005 & 0,226 & 0,091 & 0,066 & 0,91 & 0,1 \\
\hline 273 & 0,015 & 0,460 & 0,185 & 0,103 & 1,85 & $-0,9$ \\
\hline 274 & 0,036 & 0,765 & 0,311 & 0,149 & 3,11 & $-1,6$ \\
\hline 275 & 0,026 & 0,668 & 0,261 & 0,128 & 2,61 & $-1,4$ \\
\hline 276 & 0,545 & 3,315 & 0,966 & 0,718 & 9,66 & $-3,3$ \\
\hline 277 & 0,011 & 0,417 & 0,164 & 0,087 & 1,64 & $-0,7$ \\
\hline 278 & 0,007 & 0,331 & 0,128 & 0,065 & 1,28 & $-0,4$ \\
\hline 279 & 0,029 & 0,686 & 0,254 & 0,145 & 2,54 & $-1,3$ \\
\hline 280 & 0,211 & 1,810 & 0,612 & 0,440 & 6,12 & $-2,6$ \\
\hline 281 & 0,031 & 0,661 & 0,253 & 0,155 & 2,53 & $-1,3$ \\
\hline 282 & 0,361 & 2,478 & 0,952 & 0,483 & 9,52 & $-3,3$ \\
\hline 283 & 0,027 & 0,631 & 0,241 & 0,145 & 2,41 & $-1,3$ \\
\hline 284 & 0,083 & 1,215 & 0,473 & 0,223 & 4,73 & $-2,2$ \\
\hline 285 & 0,030 & 0,697 & 0,224 & 0,172 & 2,24 & $-1,2$ \\
\hline 286 & 0,031 & 0,704 & 0,279 & 0,141 & 2,79 & $-1,5$ \\
\hline
\end{tabular}




\begin{tabular}{|c|c|c|c|c|c|c|}
\hline 287 & 0,082 & 1,202 & 0,457 & 0,230 & 4,57 & $-2,2$ \\
\hline 288 & 0,015 & 0,465 & 0,166 & 0,114 & 1,66 & $-0,7$ \\
\hline 289 & 0,021 & 0,570 & 0,220 & 0,119 & 2,20 & $-1,1$ \\
\hline 290 & 0,009 & 0,348 & 0,112 & 0,101 & 1,12 & $-0,2$ \\
\hline 291 & 0,053 & 1,012 & 0,334 & 0,201 & 3,34 & $-1,7$ \\
\hline 292 & 0,102 & 1,360 & 0,453 & 0,287 & 4,53 & $-2,2$ \\
\hline 293 & 0,030 & 0,668 & 0,239 & 0,159 & 2,39 & $-1,3$ \\
\hline 294 & 0,027 & 0,631 & 0,214 & 0,158 & 2,14 & $-1,1$ \\
\hline 295 & 0,003 & 0,226 & 0,092 & 0,045 & 0,92 & 0,1 \\
\hline 296 & 0,017 & 0,471 & 0,155 & 0,138 & 1,55 & $-0,6$ \\
\hline 297 & 0,023 & 0,600 & 0,250 & 0,116 & 2,50 & $-1,3$ \\
\hline 298 & 0,016 & 0,600 & 0,261 & 0,079 & 2,61 & $-1,4$ \\
\hline 299 & 0,004 & 0,196 & 0,076 & 0,062 & 0,76 & 0,4 \\
\hline 300 & 0,173 & 1,762 & 0,574 & 0,385 & 5,74 & $-2,5$ \\
\hline 301 & 0,034 & 0,699 & 0,250 & 0,176 & 2,50 & $-1,3$ \\
\hline 302 & 0,047 & 0,808 & 0,302 & 0,197 & 3,02 & $-1,6$ \\
\hline 303 & 0,001 & 0,092 & 0,049 & 0,024 & 0,49 & 1,0 \\
\hline 304 & 0,006 & 0,270 & 0,103 & 0,075 & 1,03 & 0,0 \\
\hline 305 & 0,002 & 0,122 & 0,049 & 0,049 & 0,49 & 1,0 \\
\hline 306 & 0,022 & 0,570 & 0,208 & 0,134 & 2,08 & $-1,1$ \\
\hline 307 & 0,088 & 1,213 & 0,449 & 0,250 & 4,49 & $-2,2$ \\
\hline 308 & 0,034 & 0,692 & 0,246 & 0,176 & 2,46 & $-1,3$ \\
\hline 309 & 0,220 & 1,906 & 0,699 & 0,401 & 6,99 & $-2,8$ \\
\hline 310 & 0,003 & 0,196 & 0,067 & 0,062 & 0,67 & 0,6 \\
\hline 311 & 0,552 & 3,025 & 0,931 & 0,756 & 9,31 & $-3,2$ \\
\hline 312 & 0,004 & 0,244 & 0,111 & 0,048 & 1,11 & $-0,2$ \\
\hline 313 & 0,131 & 1,571 & 0,556 & 0,301 & 5,56 & $-2,5$ \\
\hline 314 & 0,006 & 0,270 & 0,103 & 0,075 & 1,03 & 0,0 \\
\hline 315 & 0,313 & 2,214 & 0,663 & 0,602 & 6,63 & $-2,7$ \\
\hline 316 & 0,007 & 0,300 & 0,106 & 0,084 & 1,06 & $-0,1$ \\
\hline 317 & 0,101 & 1,266 & 0,461 & 0,278 & 4,61 & $-2,2$ \\
\hline 318 & 0,031 & 0,717 & 0,295 & 0,135 & 2,95 & $-1,6$ \\
\hline 319 & 0,034 & 0,692 & 0,256 & 0,169 & 2,56 & $-1,4$ \\
\hline 320 & 1,390 & 4,519 & 1,517 & 1,166 & 15,17 & $-3,9$ \\
\hline 321 & 0,324 & 2,427 & 0,730 & 0,566 & 7,30 & $-2,9$ \\
\hline 322 & 0,008 & 0,348 & 0,112 & 0,096 & 1,12 & $-0,2$ \\
\hline 323 & 0,025 & 0,607 & 0,197 & 0,160 & 1,97 & $-1,0$ \\
\hline 324 & 12,669 & 15,659 & 4,819 & 3,347 & 48,19 & $-5,6$ \\
\hline 325 & 0,018 & 0,539 & 0,185 & 0,125 & 1,85 & $-0,9$ \\
\hline 326 & 0,385 & 2,707 & 1,009 & 0,486 & 10,09 & $-3,3$ \\
\hline 327 & 0,008 & 0,331 & 0,113 & 0,094 & 1,13 & $-0,2$ \\
\hline 328 & 0,012 & 0,427 & 0,146 & 0,101 & 1,46 & $-0,5$ \\
\hline 329 & 0,004 & 0,196 & 0,076 & 0,062 & 0,76 & 0,4 \\
\hline 330 & 0,042 & 0,943 & 0,359 & 0,151 & 3,59 & $-1,8$ \\
\hline 331 & 0,022 & 0,595 & 0,231 & 0,121 & 2,31 & $-1,2$ \\
\hline 332 & 0,043 & 0,760 & 0,265 & 0,206 & 2,65 & $-1,4$ \\
\hline 333 & 0,009 & 0,374 & 0,132 & 0,085 & 1,32 & $-0,4$ \\
\hline 334 & 0,004 & 0,196 & 0,076 & 0,062 & 0,76 & 0,4 \\
\hline 335 & 3,068 & 8,488 & 2,451 & 1,594 & 24,51 & $-4,6$ \\
\hline 336 & 0,051 & 0,882 & 0,323 & 0,202 & 3,23 & $-1,7$ \\
\hline 337 & 0,007 & 0,287 & 0,103 & 0,087 & 1,03 & 0,0 \\
\hline 338 & 0,002 & 0,165 & 0,068 & 0,044 & 0,68 & 0,6 \\
\hline 339 & 0,025 & 0,656 & 0,233 & 0,137 & 2,33 & $-1,2$ \\
\hline 340 & 0,003 & 0,183 & 0,068 & 0,053 & 0,68 & 0,6 \\
\hline 341 & 0,041 & 0,808 & 0,296 & 0,178 & 2,96 & $-1,6$ \\
\hline 342 & 1,502 & 5,080 & 1,894 & 1,010 & 18,94 & $-4,2$ \\
\hline 343 & 0,014 & 0,521 & 0,204 & 0,087 & 2,04 & $-1,0$ \\
\hline 344 & 0,105 & 1,732 & 0,438 & 0,305 & 4,38 & $-2,1$ \\
\hline 345 & 0,016 & 0,514 & 0,214 & 0,097 & 2,14 & $-1,1$ \\
\hline 346 & 0,055 & 0,963 & 0,374 & 0,189 & 3,74 & $-1,9$ \\
\hline 347 & 0,007 & 0,313 & 0,104 & 0,080 & 1,04 & $-0,1$ \\
\hline 348 & 0,282 & 2,115 & 0,728 & 0,494 & 7,28 & $-2,9$ \\
\hline
\end{tabular}




\begin{tabular}{|c|c|c|c|c|c|c|}
\hline 349 & 0,006 & 0,270 & 0,118 & 0,061 & 1,18 & $-0,2$ \\
\hline 350 & 0,021 & 0,582 & 0,222 & 0,123 & 2,22 & $-1,2$ \\
\hline 351 & 0,007 & 0,305 & 0,115 & 0,072 & 1,15 & $-0,2$ \\
\hline 352 & 0,098 & 1,360 & 0,561 & 0,222 & 5,61 & $-2,5$ \\
\hline 353 & 0,033 & 0,699 & 0,264 & 0,160 & 2,64 & $-1,4$ \\
\hline 354 & 0,003 & 0,183 & 0,081 & 0,051 & 0,81 & 0,3 \\
\hline 355 & 0,007 & 0,287 & 0,107 & 0,089 & 1,07 & $-0,1$ \\
\hline 356 & 0,008 & 0,348 & 0,129 & 0,079 & 1,29 & $-0,4$ \\
\hline 357 & 0,089 & 1,141 & 0,380 & 0,300 & 3,80 & $-1,9$ \\
\hline 358 & 0,042 & 0,796 & 0,281 & 0,192 & 2,81 & $-1,5$ \\
\hline 359 & 0,054 & 0,913 & 0,345 & 0,198 & 3,45 & $-1,8$ \\
\hline 360 & 0,030 & 0,638 & 0,241 & 0,158 & 2,41 & $-1,3$ \\
\hline 361 & 0,010 & 0,331 & 0,112 & 0,112 & 1,12 & $-0,2$ \\
\hline 362 & 0,301 & 2,478 & 0,839 & 0,458 & 8,39 & $-3,1$ \\
\hline 363 & 0,032 & 0,692 & 0,267 & 0,151 & 2,67 & $-1,4$ \\
\hline 364 & 0,071 & 1,017 & 0,342 & 0,264 & 3,42 & $-1,8$ \\
\hline 365 & 1,647 & 5,637 & 1,559 & 1,345 & 15,59 & $-4,0$ \\
\hline 366 & 0,041 & 0,877 & 0,321 & 0,162 & 3,21 & $-1,7$ \\
\hline 367 & 0,006 & 0,257 & 0,090 & 0,079 & 0,90 & 0,2 \\
\hline 368 & 0,022 & 0,600 & 0,227 & 0,123 & 2,27 & $-1,2$ \\
\hline 369 & 0,010 & 0,361 & 0,121 & 0,103 & 1,21 & $-0,3$ \\
\hline 370 & 0,009 & 0,331 & 0,111 & 0,102 & 1,11 & $-0,1$ \\
\hline 371 & 0,068 & 1,047 & 0,340 & 0,257 & 3,40 & $-1,8$ \\
\hline 372 & 0,011 & 0,460 & 0,190 & 0,075 & 1,90 & $-0,9$ \\
\hline 373 & 0,014 & 0,435 & 0,162 & 0,110 & 1,62 & $-0,7$ \\
\hline 374 & 0,024 & 0,600 & 0,243 & 0,124 & 2,43 & $-1,3$ \\
\hline 375 & 0,055 & 1,022 & 0,399 & 0,176 & 3,99 & $-2,0$ \\
\hline 376 & 0,009 & 0,331 & 0,117 & 0,097 & 1,17 & $-0,2$ \\
\hline 377 & 0,039 & 0,778 & 0,293 & 0,170 & 2,93 & $-1,6$ \\
\hline 378 & 0,011 & 0,361 & 0,128 & 0,106 & 1,28 & $-0,4$ \\
\hline 379 & 0,006 & 0,270 & 0,102 & 0,070 & 1,02 & 0,0 \\
\hline 380 & 0,063 & 1,225 & 0,486 & 0,166 & 4,86 & $-2,3$ \\
\hline 381 & 0,431 & 2,607 & 0,835 & 0,657 & 8,35 & $-3,1$ \\
\hline 382 & 0,067 & 0,961 & 0,317 & 0,270 & 3,17 & $-1,7$ \\
\hline 383 & 0,033 & 0,686 & 0,235 & 0,179 & 2,35 & $-1,2$ \\
\hline 384 & 0,136 & 1,624 & 0,424 & 0,408 & 4,24 & $-2,1$ \\
\hline 385 & 0,019 & 0,539 & 0,199 & 0,119 & 1,99 & $-1,0$ \\
\hline 386 & 0,029 & 0,661 & 0,253 & 0,145 & 2,53 & $-1,3$ \\
\hline 387 & 0,007 & 0,300 & 0,114 & 0,083 & 1,14 & $-0,2$ \\
\hline 388 & 0,036 & 0,692 & 0,235 & 0,197 & 2,35 & $-1,2$ \\
\hline 389 & 0,006 & 0,270 & 0,094 & 0,082 & 0,94 & 0,1 \\
\hline 390 & 1,263 & 5,250 & 2,080 & 0,773 & 20,80 & $-4,4$ \\
\hline 391 & 0,041 & 0,753 & 0,270 & 0,193 & 2,70 & $-1,4$ \\
\hline 392 & 0,019 & 0,526 & 0,210 & 0,113 & 2,10 & $-1,1$ \\
\hline 393 & 0,027 & 0,666 & 0,265 & 0,130 & 2,65 & $-1,4$ \\
\hline 394 & 0,043 & 0,791 & 0,284 & 0,192 & 2,84 & $-1,5$ \\
\hline 395 & 0,788 & 3,556 & 1,152 & 0,871 & 11,52 & $-3,5$ \\
\hline 396 & 0,058 & 0,968 & 0,384 & 0,191 & 3,84 & $-1,9$ \\
\hline 397 & 0,005 & 0,270 & 0,099 & 0,060 & 0,99 & 0,0 \\
\hline 398 & 0,009 & 0,374 & 0,163 & 0,069 & 1,63 & $-0,7$ \\
\hline 399 & 0,030 & 0,648 & 0,258 & 0,147 & 2,58 & $-1,4$ \\
\hline 400 & 0,040 & 0,785 & 0,256 & 0,199 & 2,56 & $-1,4$ \\
\hline 401 & 0,008 & 0,305 & 0,118 & 0,085 & 1,18 & $-0,2$ \\
\hline 402 & 0,037 & 0,771 & 0,326 & 0,144 & 3,26 & $-1,7$ \\
\hline 403 & 0,018 & 0,496 & 0,152 & 0,148 & 1,52 & $-0,6$ \\
\hline 404 & 0,804 & 3,564 & 1,130 & 0,905 & 11,30 & $-3,5$ \\
\hline 405 & 0,009 & 0,422 & 0,149 & 0,076 & 1,49 & $-0,6$ \\
\hline 406 & 0,243 & 1,993 & 0,590 & 0,525 & 5,90 & $-2,6$ \\
\hline 407 & 0,041 & 0,862 & 0,345 & 0,153 & 3,45 & $-1,8$ \\
\hline 408 & 0,017 & 0,465 & 0,181 & 0,122 & 1,81 & $-0,9$ \\
\hline 409 & 0,027 & 0,605 & 0,201 & 0,171 & 2,01 & $-1,0$ \\
\hline 410 & 0,015 & 0,514 & 0,185 & 0,106 & 1,85 & $-0,9$ \\
\hline
\end{tabular}




\begin{tabular}{|c|c|c|c|c|c|c|}
\hline 411 & 0,365 & 3,063 & 1,260 & 0,369 & 12,60 & $-3,7$ \\
\hline 412 & 0,702 & 4,465 & 1,124 & 0,795 & 11,24 & $-3,5$ \\
\hline 413 & 0,034 & 0,735 & 0,247 & 0,176 & 2,47 & $-1,3$ \\
\hline 414 & 0,022 & 0,557 & 0,215 & 0,133 & 2,15 & $-1,1$ \\
\hline 415 & 0,023 & 0,600 & 0,183 & 0,159 & 1,83 & $-0,9$ \\
\hline 416 & 0,012 & 0,392 & 0,145 & 0,102 & 1,45 & $-0,5$ \\
\hline 417 & 0,932 & 3,885 & 1,428 & 0,831 & 14,28 & $-3,8$ \\
\hline 418 & 0,095 & 1,202 & 0,417 & 0,290 & 4,17 & $-2,1$ \\
\hline 419 & 0,050 & 0,869 & 0,292 & 0,219 & 2,92 & $-1,5$ \\
\hline 420 & 0,010 & 0,392 & 0,133 & 0,094 & 1,33 & $-0,4$ \\
\hline 421 & 0,010 & 0,343 & 0,136 & 0,092 & 1,36 & $-0,4$ \\
\hline 422 & 0,010 & 0,422 & 0,174 & 0,072 & 1,74 & $-0,8$ \\
\hline 423 & 0,008 & 0,313 & 0,121 & 0,089 & 1,21 & $-0,3$ \\
\hline 424 & 0,023 & 0,587 & 0,225 & 0,129 & 2,25 & $-1,2$ \\
\hline 425 & 0,080 & 1,078 & 0,347 & 0,294 & 3,47 & $-1,8$ \\
\hline 426 & 0,852 & 5,750 & 1,418 & 0,765 & 14,18 & $-3,8$ \\
\hline 427 & 0,016 & 0,465 & 0,151 & 0,138 & 1,51 & $-0,6$ \\
\hline 428 & 0,036 & 0,826 & 0,339 & 0,137 & 3,39 & $-1,8$ \\
\hline 429 & 0,016 & 0,496 & 0,187 & 0,111 & 1,87 & $-0,9$ \\
\hline 430 & 0,014 & 0,453 & 0,163 & 0,113 & 1,63 & $-0,7$ \\
\hline 431 & 0,007 & 0,287 & 0,107 & 0,083 & 1,07 & $-0,1$ \\
\hline 432 & 0,010 & 0,356 & 0,137 & 0,091 & 1,37 & $-0,5$ \\
\hline 433 & 0,019 & 0,552 & 0,207 & 0,114 & 2,07 & $-1,1$ \\
\hline 434 & 0,052 & 0,877 & 0,273 & 0,243 & 2,73 & $-1,4$ \\
\hline 435 & 0,004 & 0,214 & 0,085 & 0,063 & 0,85 & 0,2 \\
\hline 436 & 0,006 & 0,270 & 0,093 & 0,076 & 0,93 & 0,1 \\
\hline 437 & 0,015 & 0,508 & 0,202 & 0,097 & 2,02 & $-1,0$ \\
\hline 438 & 0,010 & 0,361 & 0,131 & 0,100 & 1,31 & $-0,4$ \\
\hline 439 & 0,010 & 0,356 & 0,139 & 0,090 & 1,39 & $-0,5$ \\
\hline 440 & 0,010 & 0,379 & 0,148 & 0,088 & 1,48 & $-0,6$ \\
\hline 441 & 0,176 & 1,617 & 0,549 & 0,408 & 5,49 & $-2,5$ \\
\hline 442 & 0,166 & 1,881 & 0,736 & 0,287 & 7,36 & $-2,9$ \\
\hline 443 & 0,006 & 0,275 & 0,115 & 0,067 & 1,15 & $-0,2$ \\
\hline 444 & 0,008 & 0,374 & 0,128 & 0,083 & 1,28 & $-0,4$ \\
\hline 445 & 0,079 & 1,195 & 0,409 & 0,247 & 4,09 & $-2,0$ \\
\hline 446 & 0,010 & 0,374 & 0,139 & 0,094 & 1,39 & $-0,5$ \\
\hline 447 & 0,010 & 0,422 & 0,171 & 0,076 & 1,71 & $-0,8$ \\
\hline 448 & 0,019 & 0,557 & 0,185 & 0,131 & 1,85 & $-0,9$ \\
\hline 449 & 0,041 & 0,747 & 0,277 & 0,186 & 2,77 & $-1,5$ \\
\hline 450 & 0,013 & 0,409 & 0,172 & 0,097 & 1,72 & $-0,8$ \\
\hline 451 & 0,060 & 0,994 & 0,348 & 0,218 & 3,48 & $-1,8$ \\
\hline 452 & 0,029 & 0,643 & 0,259 & 0,145 & 2,59 & $-1,4$ \\
\hline 453 & 0,110 & 1,294 & 0,446 & 0,315 & 4,46 & $-2,2$ \\
\hline 454 & 0,015 & 0,508 & 0,202 & 0,097 & 2,02 & $-1,0$ \\
\hline 455 & 0,030 & 0,709 & 0,305 & 0,126 & 3,05 & $-1,6$ \\
\hline 456 & 0,004 & 0,214 & 0,075 & 0,063 & 0,75 & 0,4 \\
\hline 457 & 0,032 & 0,704 & 0,204 & 0,200 & 2,04 & $-1,0$ \\
\hline 458 & 0,097 & 1,324 & 0,426 & 0,290 & 4,26 & $-2,1$ \\
\hline 459 & 0,104 & 1,301 & 0,455 & 0,291 & 4,55 & $-2,2$ \\
\hline 460 & 0,040 & 0,839 & 0,297 & 0,170 & 2,97 & $-1,6$ \\
\hline 461 & 0,006 & 0,257 & 0,095 & 0,082 & 0,95 & 0,1 \\
\hline 462 & 0,044 & 0,760 & 0,254 & 0,220 & 2,54 & $-1,3$ \\
\hline 463 & 0,034 & 0,765 & 0,316 & 0,139 & 3,16 & $-1,7$ \\
\hline 464 & 0,017 & 0,514 & 0,187 & 0,114 & 1,87 & $-0,9$ \\
\hline 465 & 3,161 & 7,127 & 2,090 & 1,925 & 20,90 & $-4,4$ \\
\hline 466 & 0,045 & 0,796 & 0,290 & 0,198 & 2,90 & $-1,5$ \\
\hline 467 & 0,035 & 0,735 & 0,283 & 0,160 & 2,83 & $-1,5$ \\
\hline 468 & 3,218 & 8,781 & 3,328 & 1,231 & 33,28 & $-5,1$ \\
\hline 469 & 0,019 & 0,539 & 0,203 & 0,117 & 2,03 & $-1,0$ \\
\hline 470 & 0,181 & 1,716 & 0,569 & 0,406 & 5,69 & $-2,5$ \\
\hline 471 & 0,006 & 0,300 & 0,105 & 0,073 & 1,05 & $-0,1$ \\
\hline 472 & 0,018 & 0,478 & 0,167 & 0,135 & 1,67 & $-0,7$ \\
\hline
\end{tabular}




\begin{tabular}{|c|c|c|c|c|c|c|}
\hline 473 & 0,023 & 0,575 & 0,207 & 0,141 & 2,07 & $-1,0$ \\
\hline 474 & 0,011 & 0,392 & 0,152 & 0,090 & 1,52 & $-0,6$ \\
\hline 475 & 0,104 & 1,510 & 0,461 & 0,288 & 4,61 & $-2,2$ \\
\hline 476 & 0,020 & 0,534 & 0,195 & 0,128 & 1,95 & $-1,0$ \\
\hline 477 & 0,014 & 0,417 & 0,144 & 0,119 & 1,44 & $-0,5$ \\
\hline 478 & 0,005 & 0,239 & 0,096 & 0,062 & 0,96 & 0,1 \\
\hline 479 & 0,011 & 0,361 & 0,142 & 0,096 & 1,42 & $-0,5$ \\
\hline 480 & 0,024 & 0,613 & 0,216 & 0,143 & 2,16 & $-1,1$ \\
\hline 481 & 0,007 & 0,331 & 0,115 & 0,072 & 1,15 & $-0,2$ \\
\hline 482 & 0,005 & 0,257 & 0,089 & 0,073 & 0,89 & 0,2 \\
\hline 483 & 0,031 & 0,656 & 0,216 & 0,184 & 2,16 & $-1,1$ \\
\hline 484 & 0,002 & 0,165 & 0,069 & 0,034 & 0,69 & 0,5 \\
\hline 485 & 1,130 & 4,279 & 1,419 & 1,013 & 14,19 & $-3,8$ \\
\hline 486 & 0,013 & 0,435 & 0,166 & 0,100 & 1,66 & $-0,7$ \\
\hline 487 & 0,016 & 0,544 & 0,217 & 0,096 & 2,17 & $-1,1$ \\
\hline 488 & 0,006 & 0,287 & 0,100 & 0,077 & 1,00 & 0,0 \\
\hline 489 & 0,095 & 1,355 & 0,497 & 0,244 & 4,97 & $-2,3$ \\
\hline 490 & 0,029 & 0,692 & 0,274 & 0,134 & 2,74 & $-1,5$ \\
\hline 491 & 0,005 & 0,257 & 0,082 & 0,079 & 0,82 & 0,3 \\
\hline 492 & 0,005 & 0,275 & 0,109 & 0,060 & 1,09 & $-0,1$ \\
\hline 493 & 0,013 & 0,435 & 0,153 & 0,105 & 1,53 & $-0,6$ \\
\hline 494 & 0,171 & 2,296 & 0,524 & 0,415 & 5,24 & $-2,4$ \\
\hline 495 & 0,020 & 0,508 & 0,191 & 0,130 & 1,91 & $-0,9$ \\
\hline 496 & 0,115 & 1,385 & 0,536 & 0,274 & 5,36 & $-2,4$ \\
\hline 497 & 0,044 & 1,065 & 0,436 & 0,129 & 4,36 & $-2,1$ \\
\hline 498 & 0,003 & 0,196 & 0,075 & 0,047 & 0,75 & 0,4 \\
\hline 499 & 0,021 & 0,539 & 0,192 & 0,142 & 1,92 & $-0,9$ \\
\hline 500 & 0,009 & 0,361 & 0,142 & 0,079 & 1,42 & $-0,5$ \\
\hline 501 & 0,064 & 0,999 & 0,301 & 0,270 & 3,01 & $-1,6$ \\
\hline 502 & 0,006 & 0,257 & 0,108 & 0,066 & 1,08 & $-0,1$ \\
\hline 503 & 0,012 & 0,453 & 0,175 & 0,088 & 1,75 & $-0,8$ \\
\hline 504 & 0,002 & 0,191 & 0,097 & 0,024 & 0,97 & 0,0 \\
\hline 505 & 0,102 & 1,281 & 0,426 & 0,305 & 4,26 & $-2,1$ \\
\hline 506 & 0,008 & 0,348 & 0,139 & 0,073 & 1,39 & $-0,5$ \\
\hline 507 & 0,018 & 0,539 & 0,195 & 0,115 & 1,95 & $-1,0$ \\
\hline 508 & 0,388 & 2,936 & 0,963 & 0,513 & 9,63 & $-3,3$ \\
\hline 509 & 0,084 & 1,126 & 0,430 & 0,250 & 4,30 & $-2,1$ \\
\hline 510 & 0,060 & 0,936 & 0,329 & 0,231 & 3,29 & $-1,7$ \\
\hline 511 & 0,143 & 1,576 & 0,627 & 0,289 & 6,27 & $-2,6$ \\
\hline 512 & 0,018 & 0,570 & 0,199 & 0,116 & 1,99 & $-1,0$ \\
\hline 513 & 0,002 & 0,165 & 0,068 & 0,044 & 0,68 & 0,6 \\
\hline 514 & 0,007 & 0,318 & 0,112 & 0,074 & 1,12 & $-0,2$ \\
\hline 515 & 0,037 & 0,722 & 0,244 & 0,192 & 2,44 & $-1,3$ \\
\hline 516 & 0,013 & 0,435 & 0,175 & 0,091 & 1,75 & $-0,8$ \\
\hline 517 & 0,032 & 0,656 & 0,213 & 0,192 & 2,13 & $-1,1$ \\
\hline 518 & 0,056 & 1,157 & 0,392 & 0,183 & 3,92 & $-2,0$ \\
\hline 519 & 0,007 & 0,318 & 0,100 & 0,095 & 1,00 & 0,0 \\
\hline 520 & 0,171 & 1,795 & 0,673 & 0,324 & 6,73 & $-2,8$ \\
\hline 521 & 0,004 & 0,196 & 0,076 & 0,062 & 0,76 & 0,4 \\
\hline 522 & 0,009 & 0,348 & 0,139 & 0,081 & 1,39 & $-0,5$ \\
\hline 523 & 0,008 & 0,343 & 0,130 & 0,078 & 1,30 & $-0,4$ \\
\hline 524 & 0,035 & 0,686 & 0,234 & 0,190 & 2,34 & $-1,2$ \\
\hline 525 & 0,044 & 0,834 & 0,299 & 0,189 & 2,99 & $-1,6$ \\
\hline 526 & 0,012 & 0,404 & 0,136 & 0,109 & 1,36 & $-0,4$ \\
\hline 527 & 0,067 & 1,159 & 0,446 & 0,190 & 4,46 & $-2,2$ \\
\hline 528 & 0,093 & 1,202 & 0,426 & 0,279 & 4,26 & $-2,1$ \\
\hline 529 & 0,023 & 0,526 & 0,190 & 0,153 & 1,90 & $-0,9$ \\
\hline 530 & 0,030 & 0,709 & 0,284 & 0,134 & 2,84 & $-1,5$ \\
\hline 531 & 0,016 & 0,508 & 0,204 & 0,102 & 2,04 & $-1,0$ \\
\hline 532 & 0,165 & 1,599 & 0,479 & 0,440 & 4,79 & $-2,3$ \\
\hline 533 & 0,036 & 0,767 & 0,295 & 0,155 & 2,95 & $-1,6$ \\
\hline 534 & 0,042 & 0,816 & 0,257 & 0,210 & 2,57 & $-1,4$ \\
\hline
\end{tabular}




\begin{tabular}{|c|c|c|c|c|c|c|}
\hline 535 & 0,003 & 0,165 & 0,073 & 0,049 & 0,73 & 0,5 \\
\hline 536 & 0,004 & 0,214 & 0,085 & 0,063 & 0,85 & 0,2 \\
\hline 537 & 0,626 & 3,633 & 1,295 & 0,616 & 12,95 & $-3,7$ \\
\hline 538 & 0,014 & 0,422 & 0,138 & 0,125 & 1,38 & $-0,5$ \\
\hline 539 & 0,020 & 0,526 & 0,216 & 0,118 & 2,16 & $-1,1$ \\
\hline 540 & 0,008 & 0,318 & 0,108 & 0,099 & 1,08 & $-0,1$ \\
\hline 541 & 0,003 & 0,196 & 0,076 & 0,055 & 0,76 & 0,4 \\
\hline 542 & 0,027 & 0,684 & 0,279 & 0,126 & 2,79 & $-1,5$ \\
\hline 543 & 0,014 & 0,460 & 0,148 & 0,116 & 1,48 & $-0,6$ \\
\hline 544 & 0,037 & 0,803 & 0,319 & 0,147 & 3,19 & $-1,7$ \\
\hline 545 & 0,014 & 0,435 & 0,162 & 0,113 & 1,62 & $-0,7$ \\
\hline 546 & 0,532 & 3,231 & 1,185 & 0,572 & 11,85 & $-3,6$ \\
\hline 547 & 0,005 & 0,270 & 0,102 & 0,064 & 1,02 & 0,0 \\
\hline 548 & 0,032 & 0,656 & 0,215 & 0,190 & 2,15 & $-1,1$ \\
\hline 549 & 0,007 & 0,287 & 0,110 & 0,075 & 1,10 & $-0,1$ \\
\hline 550 & 1,254 & 5,154 & 1,770 & 0,903 & 17,70 & $-4,1$ \\
\hline 551 & 0,004 & 0,226 & 0,085 & 0,063 & 0,85 & 0,2 \\
\hline 552 & 0,027 & 0,668 & 0,231 & 0,149 & 2,31 & $-1,2$ \\
\hline 553 & 0,034 & 0,760 & 0,319 & 0,134 & 3,19 & $-1,7$ \\
\hline 554 & 0,008 & 0,336 & 0,119 & 0,085 & 1,19 & $-0,3$ \\
\hline 555 & 0,020 & 0,508 & 0,182 & 0,137 & 1,82 & $-0,9$ \\
\hline 556 & 0,007 & 0,287 & 0,102 & 0,088 & 1,02 & 0,0 \\
\hline 557 & 0,010 & 0,392 & 0,135 & 0,092 & 1,35 & $-0,4$ \\
\hline 558 & 0,022 & 0,618 & 0,266 & 0,107 & 2,66 & $-1,4$ \\
\hline 559 & 0,052 & 0,986 & 0,378 & 0,174 & 3,78 & $-1,9$ \\
\hline 560 & 0,057 & 1,058 & 0,419 & 0,173 & 4,19 & $-2,1$ \\
\hline 561 & 0,009 & 0,331 & 0,138 & 0,082 & 1,38 & $-0,5$ \\
\hline 562 & 0,007 & 0,318 & 0,127 & 0,065 & 1,27 & $-0,3$ \\
\hline 563 & 0,006 & 0,287 & 0,109 & 0,071 & 1,09 & $-0,1$ \\
\hline 564 & 0,021 & 0,577 & 0,239 & 0,112 & 2,39 & $-1,3$ \\
\hline 565 & 0,008 & 0,331 & 0,123 & 0,087 & 1,23 & $-0,3$ \\
\hline 566 & 0,014 & 0,447 & 0,151 & 0,118 & 1,51 & $-0,6$ \\
\hline 567 & 0,436 & 3,065 & 0,936 & 0,594 & 9,36 & $-3,2$ \\
\hline 568 & 0,028 & 0,753 & 0,336 & 0,106 & 3,36 & $-1,7$ \\
\hline 569 & 0,020 & 0,532 & 0,191 & 0,133 & 1,91 & $-0,9$ \\
\hline 570 & 0,021 & 0,633 & 0,272 & 0,098 & 2,72 & $-1,4$ \\
\hline 571 & 0,004 & 0,226 & 0,102 & 0,047 & 1,02 & 0,0 \\
\hline 572 & 0,017 & 0,478 & 0,164 & 0,130 & 1,64 & $-0,7$ \\
\hline 573 & 0,007 & 0,318 & 0,131 & 0,068 & 1,31 & $-0,4$ \\
\hline 574 & 0,002 & 0,165 & 0,068 & 0,044 & 0,68 & 0,6 \\
\hline 575 & 0,004 & 0,252 & 0,113 & 0,047 & 1,13 & $-0,2$ \\
\hline 576 & 0,052 & 0,979 & 0,392 & 0,169 & 3,92 & $-2,0$ \\
\hline 577 & 0,004 & 0,244 & 0,098 & 0,049 & 0,98 & 0,0 \\
\hline 578 & 0,027 & 0,753 & 0,282 & 0,124 & 2,82 & $-1,5$ \\
\hline 579 & 0,004 & 0,226 & 0,085 & 0,063 & 0,85 & 0,2 \\
\hline 580 & 0,008 & 0,300 & 0,113 & 0,089 & 1,13 & $-0,2$ \\
\hline 581 & 0,067 & 1,266 & 0,569 & 0,149 & 5,69 & $-2,5$ \\
\hline 582 & 0,003 & 0,183 & 0,068 & 0,053 & 0,68 & 0,6 \\
\hline 583 & 0,035 & 0,747 & 0,276 & 0,163 & 2,76 & $-1,5$ \\
\hline 584 & 0,017 & 0,483 & 0,188 & 0,117 & 1,88 & $-0,9$ \\
\hline 585 & 0,045 & 0,814 & 0,300 & 0,190 & 3,00 & $-1,6$ \\
\hline 586 & 0,012 & 0,404 & 0,143 & 0,104 & 1,43 & $-0,5$ \\
\hline 587 & 6,596 & 11,900 & 4,485 & 1,873 & 44,85 & $-5,5$ \\
\hline 588 & 0,017 & 0,496 & 0,183 & 0,117 & 1,83 & $-0,9$ \\
\hline 589 & 0,002 & 0,153 & 0,069 & 0,034 & 0,69 & 0,5 \\
\hline 590 & 0,042 & 0,778 & 0,282 & 0,189 & 2,82 & $-1,5$ \\
\hline 591 & 0,018 & 0,600 & 0,235 & 0,098 & 2,35 & $-1,2$ \\
\hline 592 & 0,027 & 0,783 & 0,281 & 0,120 & 2,81 & $-1,5$ \\
\hline 593 & 0,022 & 0,575 & 0,192 & 0,145 & 1,92 & $-0,9$ \\
\hline 594 & 0,007 & 0,287 & 0,103 & 0,086 & 1,03 & 0,0 \\
\hline 595 & 0,056 & 1,174 & 0,516 & 0,138 & 5,16 & $-2,4$ \\
\hline 596 & 0,128 & 1,495 & 0,538 & 0,303 & 5,38 & $-2,4$ \\
\hline
\end{tabular}




\begin{tabular}{|c|c|c|c|c|c|c|}
\hline 597 & 0,006 & 0,270 & 0,088 & 0,081 & 0,88 & 0,2 \\
\hline 598 & 0,130 & 1,515 & 0,495 & 0,336 & 4,95 & $-2,3$ \\
\hline 599 & 0,034 & 0,709 & 0,255 & 0,170 & 2,55 & $-1,4$ \\
\hline 600 & 0,163 & 1,664 & 0,588 & 0,353 & 5,88 & $-2,6$ \\
\hline 601 & 0,130 & 1,446 & 0,490 & 0,337 & 4,90 & $-2,3$ \\
\hline 602 & 0,041 & 0,765 & 0,277 & 0,189 & 2,77 & $-1,5$ \\
\hline 603 & 0,134 & 1,538 & 0,530 & 0,321 & 5,30 & $-2,4$ \\
\hline 604 & 0,019 & 0,521 & 0,182 & 0,130 & 1,82 & $-0,9$ \\
\hline 605 & 0,010 & 0,453 & 0,187 & 0,070 & 1,87 & $-0,9$ \\
\hline 606 & 0,060 & 0,948 & 0,309 & 0,246 & 3,09 & $-1,6$ \\
\hline 607 & 0,046 & 0,839 & 0,277 & 0,210 & 2,77 & $-1,5$ \\
\hline 608 & 0,057 & 0,968 & 0,316 & 0,231 & 3,16 & $-1,7$ \\
\hline 609 & 0,043 & 0,852 & 0,324 & 0,170 & 3,24 & $-1,7$ \\
\hline 610 & 0,751 & 3,609 & 1,130 & 0,847 & 11,30 & $-3,5$ \\
\hline 611 & 0,015 & 0,473 & 0,205 & 0,093 & 2,05 & $-1,0$ \\
\hline 612 & 0,023 & 0,600 & 0,214 & 0,139 & 2,14 & $-1,1$ \\
\hline 613 & 0,015 & 0,435 & 0,151 & 0,129 & 1,51 & $-0,6$ \\
\hline 614 & 0,053 & 0,875 & 0,314 & 0,214 & 3,14 & $-1,6$ \\
\hline 615 & 0,012 & 0,404 & 0,155 & 0,099 & 1,55 & $-0,6$ \\
\hline 616 & 0,051 & 0,857 & 0,283 & 0,231 & 2,83 & $-1,5$ \\
\hline 617 & 0,070 & 1,030 & 0,364 & 0,244 & 3,64 & $-1,9$ \\
\hline 618 & 0,959 & 4,230 & 1,308 & 0,933 & 13,08 & $-3,7$ \\
\hline 619 & 0,024 & 0,613 & 0,236 & 0,131 & 2,36 & $-1,2$ \\
\hline 620 & 0,003 & 0,196 & 0,067 & 0,062 & 0,67 & 0,6 \\
\hline 621 & 0,019 & 0,508 & 0,187 & 0,127 & 1,87 & $-0,9$ \\
\hline 622 & 0,063 & 0,986 & 0,359 & 0,225 & 3,59 & $-1,8$ \\
\hline 623 & 0,006 & 0,270 & 0,102 & 0,070 & 1,02 & 0,0 \\
\hline 624 & 0,019 & 0,539 & 0,194 & 0,125 & 1,94 & $-1,0$ \\
\hline 625 & 0,037 & 0,753 & 0,238 & 0,199 & 2,38 & $-1,3$ \\
\hline 626 & 0,025 & 0,613 & 0,217 & 0,148 & 2,17 & $-1,1$ \\
\hline 627 & 0,207 & 1,901 & 0,665 & 0,396 & 6,65 & $-2,7$ \\
\hline 628 & 0,005 & 0,239 & 0,095 & 0,069 & 0,95 & 0,1 \\
\hline 629 & 0,373 & 2,475 & 0,851 & 0,558 & 8,51 & $-3,1$ \\
\hline 630 & 0,027 & 0,613 & 0,214 & 0,158 & 2,14 & $-1,1$ \\
\hline 631 & 0,038 & 0,791 & 0,264 & 0,182 & 2,64 & $-1,4$ \\
\hline 632 & 0,023 & 0,613 & 0,241 & 0,123 & 2,41 & $-1,3$ \\
\hline 633 & 0,028 & 0,631 & 0,228 & 0,156 & 2,28 & $-1,2$ \\
\hline 634 & 0,022 & 0,557 & 0,195 & 0,146 & 1,95 & $-1,0$ \\
\hline 635 & 0,057 & 0,913 & 0,303 & 0,239 & 3,03 & $-1,6$ \\
\hline 636 & 0,011 & 0,417 & 0,183 & 0,078 & 1,83 & $-0,9$ \\
\hline 637 & 0,035 & 0,852 & 0,267 & 0,169 & 2,67 & $-1,4$ \\
\hline 638 & 0,278 & 2,211 & 0,837 & 0,423 & 8,37 & $-3,1$ \\
\hline 639 & 0,008 & 0,331 & 0,132 & 0,081 & 1,32 & $-0,4$ \\
\hline 640 & 0,097 & 1,457 & 0,563 & 0,219 & 5,63 & $-2,5$ \\
\hline 641 & 0,065 & 0,992 & 0,381 & 0,216 & 3,81 & $-1,9$ \\
\hline 642 & 0,008 & 0,331 & 0,115 & 0,093 & 1,15 & $-0,2$ \\
\hline 643 & 0,019 & 0,526 & 0,185 & 0,129 & 1,85 & $-0,9$ \\
\hline 644 & 0,080 & 1,091 & 0,395 & 0,258 & 3,95 & $-2,0$ \\
\hline 645 & 0,021 & 0,600 & 0,211 & 0,129 & 2,11 & $-1,1$ \\
\hline 646 & 0,766 & 4,282 & 1,895 & 0,515 & 18,95 & $-4,2$ \\
\hline 647 & 0,023 & 0,613 & 0,209 & 0,142 & 2,09 & $-1,1$ \\
\hline 648 & 0,024 & 0,613 & 0,233 & 0,130 & 2,33 & $-1,2$ \\
\hline 649 & 0,019 & 0,465 & 0,171 & 0,139 & 1,71 & $-0,8$ \\
\hline 650 & 0,010 & 0,404 & 0,154 & 0,081 & 1,54 & $-0,6$ \\
\hline 651 & 0,034 & 0,753 & 0,279 & 0,155 & 2,79 & $-1,5$ \\
\hline 652 & 0,051 & 0,951 & 0,377 & 0,173 & 3,77 & $-1,9$ \\
\hline 653 & 0,013 & 0,404 & 0,140 & 0,115 & 1,40 & $-0,5$ \\
\hline 654 & 0,083 & 1,073 & 0,359 & 0,294 & 3,59 & $-1,8$ \\
\hline 655 & 0,020 & 0,521 & 0,181 & 0,141 & 1,81 & $-0,9$ \\
\hline 656 & 0,051 & 0,907 & 0,364 & 0,178 & 3,64 & $-1,9$ \\
\hline 657 & 0,015 & 0,483 & 0,194 & 0,098 & 1,94 & $-1,0$ \\
\hline 658 & 0,008 & 0,361 & 0,153 & 0,066 & 1,53 & $-0,6$ \\
\hline
\end{tabular}




\begin{tabular}{|c|c|c|c|c|c|c|}
\hline 659 & 1,500 & 4,995 & 1,504 & 1,270 & 15,04 & $-3,9$ \\
\hline 660 & 0,015 & 0,491 & 0,182 & 0,104 & 1,82 & $-0,9$ \\
\hline 661 & 0,026 & 0,648 & 0,206 & 0,162 & 2,06 & $-1,0$ \\
\hline 662 & 0,019 & 0,557 & 0,178 & 0,136 & 1,78 & $-0,8$ \\
\hline 663 & 0,012 & 0,417 & 0,161 & 0,096 & 1,61 & $-0,7$ \\
\hline 664 & 0,009 & 0,361 & 0,132 & 0,090 & 1,32 & $-0,4$ \\
\hline 665 & 0,038 & 0,760 & 0,252 & 0,193 & 2,52 & $-1,3$ \\
\hline 666 & 0,020 & 0,570 & 0,230 & 0,108 & 2,30 & $-1,2$ \\
\hline 667 & 0,010 & 0,374 & 0,156 & 0,084 & 1,56 & $-0,6$ \\
\hline 668 & 0,536 & 2,804 & 0,918 & 0,744 & 9,18 & $-3,2$ \\
\hline 669 & 0,004 & 0,196 & 0,076 & 0,062 & 0,76 & 0,4 \\
\hline 670 & 0,005 & 0,226 & 0,096 & 0,062 & 0,96 & 0,1 \\
\hline 671 & 0,019 & 0,521 & 0,167 & 0,142 & 1,67 & $-0,7$ \\
\hline 672 & 0,006 & 0,270 & 0,108 & 0,066 & 1,08 & $-0,1$ \\
\hline 673 & 0,017 & 0,496 & 0,172 & 0,124 & 1,72 & $-0,8$ \\
\hline 674 & 0,036 & 0,704 & 0,256 & 0,178 & 2,56 & $-1,4$ \\
\hline 675 & 4,115 & 8,506 & 2,489 & 2,105 & 24,89 & $-4,6$ \\
\hline 676 & 0,012 & 0,404 & 0,139 & 0,107 & 1,39 & $-0,5$ \\
\hline 677 & 0,020 & 0,539 & 0,183 & 0,139 & 1,83 & $-0,9$ \\
\hline 678 & 0,075 & 1,256 & 0,417 & 0,228 & 4,17 & $-2,1$ \\
\hline 679 & 0,068 & 1,055 & 0,415 & 0,207 & 4,15 & $-2,1$ \\
\hline 680 & 0,012 & 0,379 & 0,129 & 0,115 & 1,29 & $-0,4$ \\
\hline 681 & 0,095 & 1,225 & 0,408 & 0,295 & 4,08 & $-2,0$ \\
\hline 682 & 0,015 & 0,447 & 0,175 & 0,109 & 1,75 & $-0,8$ \\
\hline 683 & 0,223 & 2,499 & 1,050 & 0,271 & 10,50 & $-3,4$ \\
\hline 684 & 0,006 & 0,287 & 0,100 & 0,077 & 1,00 & 0,0 \\
\hline 685 & 0,026 & 0,643 & 0,226 & 0,147 & 2,26 & $-1,2$ \\
\hline 686 & 0,019 & 0,557 & 0,219 & 0,111 & 2,19 & $-1,1$ \\
\hline 687 & 0,006 & 0,287 & 0,121 & 0,059 & 1,21 & $-0,3$ \\
\hline 688 & 0,049 & 0,877 & 0,335 & 0,186 & 3,35 & $-1,7$ \\
\hline 689 & 0,226 & 1,998 & 0,625 & 0,460 & 6,25 & $-2,6$ \\
\hline 690 & 0,028 & 0,661 & 0,251 & 0,142 & 2,51 & $-1,3$ \\
\hline 691 & 0,059 & 1,126 & 0,445 & 0,168 & 4,45 & $-2,2$ \\
\hline 692 & 0,014 & 0,430 & 0,157 & 0,110 & 1,57 & $-0,6$ \\
\hline 693 & 0,005 & 0,282 & 0,108 & 0,061 & 1,08 & $-0,1$ \\
\hline 694 & 0,060 & 0,986 & 0,382 & 0,200 & 3,82 & $-1,9$ \\
\hline 695 & 0,003 & 0,196 & 0,063 & 0,056 & 0,63 & 0,7 \\
\hline 696 & 3,351 & 7,718 & 2,892 & 1,475 & 28,92 & $-4,9$ \\
\hline 697 & 0,031 & 0,791 & 0,293 & 0,134 & 2,93 & $-1,5$ \\
\hline 698 & 0,003 & 0,208 & 0,090 & 0,046 & 0,90 & 0,2 \\
\hline 699 & 0,028 & 0,613 & 0,210 & 0,169 & 2,10 & $-1,1$ \\
\hline 700 & 0,054 & 0,981 & 0,345 & 0,198 & 3,45 & $-1,8$ \\
\hline 701 & 0,015 & 0,491 & 0,169 & 0,116 & 1,69 & $-0,8$ \\
\hline 702 & 0,007 & 0,336 & 0,110 & 0,086 & 1,10 & $-0,1$ \\
\hline 703 & 0,201 & 2,120 & 0,697 & 0,368 & 6,97 & $-2,8$ \\
\hline 704 & 0,060 & 1,093 & 0,460 & 0,167 & 4,60 & $-2,2$ \\
\hline 705 & 0,743 & 3,507 & 1,236 & 0,765 & 12,36 & $-3,6$ \\
\hline 706 & 0,040 & 0,773 & 0,281 & 0,181 & 2,81 & $-1,5$ \\
\hline 707 & 0,039 & 0,864 & 0,315 & 0,156 & 3,15 & $-1,7$ \\
\hline 708 & 0,079 & 1,195 & 0,490 & 0,205 & 4,90 & $-2,3$ \\
\hline 709 & 0,015 & 0,460 & 0,161 & 0,122 & 1,61 & $-0,7$ \\
\hline 710 & 0,111 & 1,276 & 0,401 & 0,352 & 4,01 & $-2,0$ \\
\hline 711 & 0,015 & 0,465 & 0,161 & 0,122 & 1,61 & $-0,7$ \\
\hline 712 & 0,204 & 1,822 & 0,530 & 0,491 & 5,30 & $-2,4$ \\
\hline 713 & 0,005 & 0,257 & 0,097 & 0,061 & 0,97 & 0,0 \\
\hline 714 & 0,289 & 2,694 & 1,037 & 0,355 & 10,37 & $-3,4$ \\
\hline 715 & 0,009 & 0,361 & 0,155 & 0,077 & 1,55 & $-0,6$ \\
\hline 716 & 0,661 & 3,454 & 1,311 & 0,642 & 13,11 & $-3,7$ \\
\hline 717 & 0,015 & 0,453 & 0,157 & 0,124 & 1,57 & $-0,7$ \\
\hline 718 & 0,034 & 0,674 & 0,218 & 0,196 & 2,18 & $-1,1$ \\
\hline 719 & 0,048 & 0,846 & 0,274 & 0,225 & 2,74 & $-1,5$ \\
\hline 720 & 0,035 & 0,755 & 0,250 & 0,180 & 2,50 & $-1,3$ \\
\hline
\end{tabular}




\begin{tabular}{|c|c|c|c|c|c|c|}
\hline 721 & 0,021 & 0,557 & 0,189 & 0,144 & 1,89 & $-0,9$ \\
\hline 722 & 0,027 & 0,643 & 0,232 & 0,151 & 2,32 & $-1,2$ \\
\hline 723 & 0,017 & 0,478 & 0,173 & 0,124 & 1,73 & $-0,8$ \\
\hline 724 & 0,013 & 0,447 & 0,175 & 0,091 & 1,75 & $-0,8$ \\
\hline 725 & 0,009 & 0,331 & 0,123 & 0,096 & 1,23 & $-0,3$ \\
\hline 726 & 0,209 & 2,183 & 0,772 & 0,345 & 7,72 & $-2,9$ \\
\hline 727 & 0,052 & 0,938 & 0,332 & 0,198 & 3,32 & $-1,7$ \\
\hline 728 & 0,014 & 0,447 & 0,171 & 0,104 & 1,71 & $-0,8$ \\
\hline 729 & 0,005 & 0,239 & 0,095 & 0,069 & 0,95 & 0,1 \\
\hline 730 & 0,128 & 1,670 & 0,699 & 0,233 & 6,99 & $-2,8$ \\
\hline 731 & 0,012 & 0,422 & 0,156 & 0,095 & 1,56 & $-0,6$ \\
\hline 732 & 0,012 & 0,417 & 0,136 & 0,113 & 1,36 & $-0,4$ \\
\hline 733 & 0,219 & 2,135 & 0,796 & 0,351 & 7,96 & $-3,0$ \\
\hline 734 & 0,020 & 0,539 & 0,180 & 0,139 & 1,80 & $-0,8$ \\
\hline 735 & 0,055 & 0,986 & 0,381 & 0,184 & 3,81 & $-1,9$ \\
\hline 736 & 0,023 & 0,539 & 0,198 & 0,147 & 1,98 & $-1,0$ \\
\hline 737 & 0,013 & 0,430 & 0,148 & 0,112 & 1,48 & $-0,6$ \\
\hline 738 & 0,024 & 0,575 & 0,199 & 0,155 & 1,99 & $-1,0$ \\
\hline 739 & 0,004 & 0,226 & 0,097 & 0,049 & 0,97 & 0,0 \\
\hline 740 & 0,014 & 0,447 & 0,179 & 0,100 & 1,79 & $-0,8$ \\
\hline 741 & 0,411 & 3,134 & 1,141 & 0,459 & 11,41 & $-3,5$ \\
\hline 742 & 0,023 & 0,539 & 0,194 & 0,150 & 1,94 & $-1,0$ \\
\hline 743 & 0,011 & 0,361 & 0,134 & 0,102 & 1,34 & $-0,4$ \\
\hline 744 & 0,328 & 2,498 & 0,919 & 0,454 & 9,19 & $-3,2$ \\
\hline 745 & 0,026 & 0,681 & 0,241 & 0,135 & 2,41 & $-1,3$ \\
\hline 746 & 0,273 & 2,715 & 0,986 & 0,352 & 9,86 & $-3,3$ \\
\hline 747 & 0,004 & 0,226 & 0,085 & 0,063 & 0,85 & 0,2 \\
\hline 748 & 0,018 & 0,552 & 0,225 & 0,103 & 2,25 & $-1,2$ \\
\hline 749 & 0,184 & 2,079 & 0,663 & 0,353 & 6,63 & $-2,7$ \\
\hline 750 & 0,006 & 0,257 & 0,095 & 0,082 & 0,95 & 0,1 \\
\hline 751 & 0,075 & 1,106 & 0,431 & 0,222 & 4,31 & $-2,1$ \\
\hline 752 & 0,014 & 0,435 & 0,157 & 0,117 & 1,57 & $-0,7$ \\
\hline 753 & 3,147 & 7,788 & 2,441 & 1,642 & 24,41 & $-4,6$ \\
\hline 754 & 0,029 & 0,668 & 0,247 & 0,151 & 2,47 & $-1,3$ \\
\hline 755 & 0,160 & 1,538 & 0,539 & 0,378 & 5,39 & $-2,4$ \\
\hline 756 & 0,007 & 0,287 & 0,106 & 0,079 & 1,06 & $-0,1$ \\
\hline 757 & 0,094 & 1,322 & 0,441 & 0,270 & 4,41 & $-2,1$ \\
\hline 758 & 0,024 & 0,692 & 0,285 & 0,106 & 2,85 & $-1,5$ \\
\hline 759 & 0,021 & 0,544 & 0,211 & 0,129 & 2,11 & $-1,1$ \\
\hline 760 & 0,014 & 0,422 & 0,160 & 0,108 & 1,60 & $-0,7$ \\
\hline 761 & 0,034 & 0,712 & 0,245 & 0,177 & 2,45 & $-1,3$ \\
\hline 762 & 0,065 & 1,022 & 0,355 & 0,234 & 3,55 & $-1,8$ \\
\hline 763 & 0,105 & 1,243 & 0,372 & 0,361 & 3,72 & $-1,9$ \\
\hline 764 & 0,041 & 0,785 & 0,288 & 0,181 & 2,88 & $-1,5$ \\
\hline 765 & 0,079 & 1,225 & 0,377 & 0,266 & 3,77 & $-1,9$ \\
\hline 766 & 0,013 & 0,447 & 0,157 & 0,106 & 1,57 & $-0,6$ \\
\hline 767 & 0,005 & 0,257 & 0,097 & 0,061 & 0,97 & 0,0 \\
\hline 768 & 0,025 & 0,570 & 0,196 & 0,164 & 1,96 & $-1,0$ \\
\hline 769 & 0,239 & 2,158 & 0,789 & 0,386 & 7,89 & $-3,0$ \\
\hline 770 & 0,136 & 1,617 & 0,503 & 0,346 & 5,03 & $-2,3$ \\
\hline 771 & 0,025 & 0,613 & 0,218 & 0,147 & 2,18 & $-1,1$ \\
\hline 772 & 0,007 & 0,331 & 0,120 & 0,074 & 1,20 & $-0,3$ \\
\hline 773 & 0,005 & 0,257 & 0,097 & 0,067 & 0,97 & 0,0 \\
\hline 774 & 0,004 & 0,208 & 0,097 & 0,049 & 0,97 & 0,0 \\
\hline 775 & 0,014 & 0,435 & 0,171 & 0,107 & 1,71 & $-0,8$ \\
\hline 776 & 0,027 & 0,661 & 0,253 & 0,134 & 2,53 & $-1,3$ \\
\hline 777 & 0,008 & 0,313 & 0,116 & 0,087 & 1,16 & $-0,2$ \\
\hline 778 & 0,036 & 0,808 & 0,319 & 0,145 & 3,19 & $-1,7$ \\
\hline 779 & 0,005 & 0,257 & 0,097 & 0,067 & 0,97 & 0,0 \\
\hline 780 & 0,044 & 0,832 & 0,337 & 0,166 & 3,37 & $-1,8$ \\
\hline 781 & 0,002 & 0,122 & 0,049 & 0,049 & 0,49 & 1,0 \\
\hline 782 & 0,002 & 0,165 & 0,059 & 0,040 & 0,59 & 0,8 \\
\hline
\end{tabular}




\begin{tabular}{|c|c|c|c|c|c|c|}
\hline 783 & 0,049 & 0,872 & 0,285 & 0,219 & 2,85 & $-1,5$ \\
\hline 784 & 0,113 & 1,315 & 0,390 & 0,368 & 3,90 & $-2,0$ \\
\hline 785 & 0,004 & 0,226 & 0,082 & 0,058 & 0,82 & 0,3 \\
\hline 786 & 0,031 & 0,712 & 0,282 & 0,139 & 2,82 & $-1,5$ \\
\hline 787 & 0,074 & 1,121 & 0,381 & 0,248 & 3,81 & $-1,9$ \\
\hline 788 & 0,014 & 0,496 & 0,181 & 0,102 & 1,81 & $-0,9$ \\
\hline 789 & 0,012 & 0,503 & 0,198 & 0,075 & 1,98 & $-1,0$ \\
\hline 790 & 0,025 & 0,595 & 0,225 & 0,140 & 2,25 & $-1,2$ \\
\hline 791 & 0,101 & 1,365 & 0,577 & 0,222 & 5,77 & $-2,5$ \\
\hline 792 & 0,006 & 0,300 & 0,109 & 0,065 & 1,09 & $-0,1$ \\
\hline 793 & 0,356 & 2,374 & 0,816 & 0,556 & 8,16 & $-3,0$ \\
\hline 794 & 0,123 & 1,637 & 0,516 & 0,305 & 5,16 & $-2,4$ \\
\hline 795 & 0,004 & 0,226 & 0,082 & 0,058 & 0,82 & 0,3 \\
\hline 796 & 0,016 & 0,539 & 0,215 & 0,094 & 2,15 & $-1,1$ \\
\hline 797 & 0,038 & 0,747 & 0,270 & 0,180 & 2,70 & $-1,4$ \\
\hline 798 & 0,004 & 0,244 & 0,086 & 0,062 & 0,86 & 0,2 \\
\hline 799 & 0,052 & 0,900 & 0,288 & 0,231 & 2,88 & $-1,5$ \\
\hline 800 & 0,013 & 0,404 & 0,137 & 0,122 & 1,37 & $-0,4$ \\
\hline 801 & 0,020 & 0,526 & 0,164 & 0,152 & 1,64 & $-0,7$ \\
\hline 802 & 0,020 & 0,508 & 0,183 & 0,140 & 1,83 & $-0,9$ \\
\hline 803 & 0,023 & 0,582 & 0,216 & 0,137 & 2,16 & $-1,1$ \\
\hline 804 & 0,060 & 0,981 & 0,312 & 0,245 & 3,12 & $-1,6$ \\
\hline 805 & 0,004 & 0,208 & 0,097 & 0,049 & 0,97 & 0,0 \\
\hline 806 & 0,013 & 0,465 & 0,192 & 0,087 & 1,92 & $-0,9$ \\
\hline 807 & 0,058 & 0,913 & 0,299 & 0,248 & 2,99 & $-1,6$ \\
\hline 808 & 0,009 & 0,356 & 0,145 & 0,082 & 1,45 & $-0,5$ \\
\hline 809 & 0,012 & 0,422 & 0,142 & 0,109 & 1,42 & $-0,5$ \\
\hline 810 & 0,017 & 0,508 & 0,194 & 0,110 & 1,94 & $-1,0$ \\
\hline 811 & 0,002 & 0,165 & 0,068 & 0,044 & 0,68 & 0,6 \\
\hline 812 & 0,018 & 0,491 & 0,178 & 0,130 & 1,78 & $-0,8$ \\
\hline 813 & 0,031 & 0,643 & 0,208 & 0,188 & 2,08 & $-1,1$ \\
\hline 814 & 0,092 & 1,495 & 0,573 & 0,204 & 5,73 & $-2,5$ \\
\hline 815 & 0,022 & 0,546 & 0,189 & 0,151 & 1,89 & $-0,9$ \\
\hline 816 & 0,631 & 3,429 & 1,144 & 0,702 & 11,44 & $-3,5$ \\
\hline 817 & 0,010 & 0,417 & 0,173 & 0,076 & 1,73 & $-0,8$ \\
\hline 818 & 0,016 & 0,471 & 0,182 & 0,111 & 1,82 & $-0,9$ \\
\hline 819 & 0,012 & 0,478 & 0,193 & 0,080 & 1,93 & $-0,9$ \\
\hline 820 & 0,011 & 0,417 & 0,173 & 0,079 & 1,73 & $-0,8$ \\
\hline 821 & 0,040 & 0,864 & 0,343 & 0,147 & 3,43 & $-1,8$ \\
\hline 822 & 0,031 & 0,668 & 0,241 & 0,163 & 2,41 & $-1,3$ \\
\hline 823 & 0,104 & 1,294 & 0,439 & 0,302 & 4,39 & $-2,1$ \\
\hline 824 & 0,014 & 0,404 & 0,135 & 0,127 & 1,35 & $-0,4$ \\
\hline 825 & 0,050 & 0,834 & 0,275 & 0,231 & 2,75 & $-1,5$ \\
\hline 826 & 0,003 & 0,208 & 0,090 & 0,046 & 0,90 & 0,2 \\
\hline 827 & 0,010 & 0,348 & 0,133 & 0,099 & 1,33 & $-0,4$ \\
\hline 828 & 0,046 & 0,931 & 0,348 & 0,169 & 3,48 & $-1,8$ \\
\hline 829 & 0,012 & 0,392 & 0,133 & 0,111 & 1,33 & $-0,4$ \\
\hline 830 & 0,009 & 0,348 & 0,123 & 0,096 & 1,23 & $-0,3$ \\
\hline 831 & 0,014 & 0,473 & 0,205 & 0,090 & 2,05 & $-1,0$ \\
\hline 832 & 0,012 & 0,435 & 0,159 & 0,097 & 1,59 & $-0,7$ \\
\hline 833 & 0,037 & 0,803 & 0,288 & 0,165 & 2,88 & $-1,5$ \\
\hline 834 & 0,004 & 0,226 & 0,091 & 0,059 & 0,91 & 0,1 \\
\hline 835 & 0,040 & 0,783 & 0,321 & 0,157 & 3,21 & $-1,7$ \\
\hline 836 & 0,073 & 1,162 & 0,363 & 0,256 & 3,63 & $-1,9$ \\
\hline 837 & 0,010 & 0,430 & 0,173 & 0,075 & 1,73 & $-0,8$ \\
\hline 838 & 0,040 & 0,890 & 0,331 & 0,153 & 3,31 & $-1,7$ \\
\hline 839 & 0,491 & 2,973 & 0,857 & 0,729 & 8,57 & $-3,1$ \\
\hline 840 & 0,014 & 0,453 & 0,140 & 0,127 & 1,40 & $-0,5$ \\
\hline 841 & 1,233 & 4,823 & 1,632 & 0,962 & 16,32 & $-4,0$ \\
\hline 842 & 0,023 & 0,656 & 0,255 & 0,116 & 2,55 & $-1,3$ \\
\hline 843 & 0,588 & 3,556 & 1,022 & 0,733 & 10,22 & $-3,4$ \\
\hline 844 & 0,064 & 1,055 & 0,315 & 0,258 & 3,15 & $-1,7$ \\
\hline
\end{tabular}




\begin{tabular}{|c|c|c|c|c|c|c|}
\hline 845 & 0,013 & 0,435 & 0,162 & 0,099 & 1,62 & $-0,7$ \\
\hline 846 & 4,174 & 10,265 & 4,082 & 1,302 & 40,82 & $-5,4$ \\
\hline 847 & 0,338 & 2,440 & 0,774 & 0,556 & 7,74 & $-3,0$ \\
\hline 848 & 0,016 & 0,460 & 0,150 & 0,138 & 1,50 & $-0,6$ \\
\hline 849 & 0,010 & 0,361 & 0,140 & 0,094 & 1,40 & $-0,5$ \\
\hline 850 & 0,017 & 0,473 & 0,159 & 0,138 & 1,59 & $-0,7$ \\
\hline 851 & 0,051 & 0,961 & 0,374 & 0,173 & 3,74 & $-1,9$ \\
\hline 852 & 0,009 & 0,331 & 0,123 & 0,092 & 1,23 & $-0,3$ \\
\hline 853 & 0,129 & 1,662 & 0,652 & 0,251 & 6,52 & $-2,7$ \\
\hline 854 & 0,021 & 0,526 & 0,182 & 0,147 & 1,82 & $-0,9$ \\
\hline 855 & 0,021 & 0,526 & 0,189 & 0,145 & 1,89 & $-0,9$ \\
\hline 856 & 0,200 & 2,000 & 0,666 & 0,383 & 6,66 & $-2,7$ \\
\hline 857 & 0,042 & 0,821 & 0,248 & 0,215 & 2,48 & $-1,3$ \\
\hline 858 & 0,050 & 0,893 & 0,288 & 0,220 & 2,88 & $-1,5$ \\
\hline 859 & 0,004 & 0,257 & 0,087 & 0,061 & 0,87 & 0,2 \\
\hline 860 & 0,006 & 0,270 & 0,093 & 0,076 & 0,93 & 0,1 \\
\hline 861 & 0,009 & 0,325 & 0,120 & 0,094 & 1,20 & $-0,3$ \\
\hline 862 & 0,006 & 0,300 & 0,103 & 0,075 & 1,03 & 0,0 \\
\hline 863 & 0,024 & 0,575 & 0,198 & 0,153 & 1,98 & $-1,0$ \\
\hline 864 & 0,028 & 0,686 & 0,269 & 0,134 & 2,69 & $-1,4$ \\
\hline 865 & 0,013 & 0,478 & 0,194 & 0,086 & 1,94 & $-1,0$ \\
\hline 866 & 0,023 & 0,552 & 0,184 & 0,161 & 1,84 & $-0,9$ \\
\hline 867 & 0,011 & 0,417 & 0,173 & 0,082 & 1,73 & $-0,8$ \\
\hline 868 & 0,289 & 2,262 & 0,817 & 0,451 & 8,17 & $-3,0$ \\
\hline 869 & 0,003 & 0,196 & 0,067 & 0,062 & 0,67 & 0,6 \\
\hline 870 & 0,594 & 3,199 & 0,892 & 0,848 & 8,92 & $-3,2$ \\
\hline 871 & 0,011 & 0,374 & 0,153 & 0,089 & 1,53 & $-0,6$ \\
\hline 872 & 0,006 & 0,356 & 0,152 & 0,051 & 1,52 & $-0,6$ \\
\hline 873 & 0,082 & 1,251 & 0,416 & 0,251 & 4,16 & $-2,1$ \\
\hline 874 & 0,024 & 0,587 & 0,206 & 0,147 & 2,06 & $-1,0$ \\
\hline 875 & 0,005 & 0,257 & 0,107 & 0,056 & 1,07 & $-0,1$ \\
\hline 876 & 0,068 & 1,053 & 0,394 & 0,221 & 3,94 & $-2,0$ \\
\hline 877 & 1,984 & 6,247 & 2,364 & 1,069 & 23,64 & $-4,6$ \\
\hline 878 & 0,002 & 0,122 & 0,049 & 0,049 & 0,49 & 1,0 \\
\hline 879 & 0,007 & 0,374 & 0,135 & 0,070 & 1,35 & $-0,4$ \\
\hline 880 & 0,054 & 0,931 & 0,346 & 0,197 & 3,46 & $-1,8$ \\
\hline 881 & 0,066 & 1,040 & 0,312 & 0,270 & 3,12 & $-1,6$ \\
\hline 882 & 0,013 & 0,435 & 0,135 & 0,118 & 1,35 & $-0,4$ \\
\hline 883 & 0,010 & 0,386 & 0,121 & 0,108 & 1,21 & $-0,3$ \\
\hline 884 & 0,012 & 0,435 & 0,167 & 0,089 & 1,67 & $-0,7$ \\
\hline 885 & 0,050 & 0,834 & 0,287 & 0,223 & 2,87 & $-1,5$ \\
\hline 886 & 0,013 & 0,422 & 0,142 & 0,117 & 1,42 & $-0,5$ \\
\hline 887 & 0,005 & 0,226 & 0,089 & 0,067 & 0,89 & 0,2 \\
\hline 888 & 0,142 & 1,629 & 0,579 & 0,312 & 5,79 & $-2,5$ \\
\hline 889 & 0,534 & 3,248 & 1,207 & 0,563 & 12,07 & $-3,6$ \\
\hline 890 & 0,927 & 4,876 & 1,412 & 0,836 & 14,12 & $-3,8$ \\
\hline 891 & 0,018 & 0,496 & 0,177 & 0,131 & 1,77 & $-0,8$ \\
\hline 892 & 0,018 & 0,514 & 0,156 & 0,148 & 1,56 & $-0,6$ \\
\hline 893 & 0,015 & 0,465 & 0,190 & 0,100 & 1,90 & $-0,9$ \\
\hline 894 & 0,011 & 0,422 & 0,159 & 0,086 & 1,59 & $-0,7$ \\
\hline 895 & 0,014 & 0,503 & 0,199 & 0,092 & 1,99 & $-1,0$ \\
\hline 896 & 0,011 & 0,422 & 0,157 & 0,090 & 1,57 & $-0,7$ \\
\hline 897 & 0,007 & 0,313 & 0,129 & 0,069 & 1,29 & $-0,4$ \\
\hline 898 & 0,027 & 0,643 & 0,222 & 0,152 & 2,22 & $-1,2$ \\
\hline 899 & 0,856 & 4,293 & 1,309 & 0,833 & 13,09 & $-3,7$ \\
\hline 900 & 0,013 & 0,404 & 0,144 & 0,111 & 1,44 & $-0,5$ \\
\hline 901 & 0,068 & 1,078 & 0,385 & 0,227 & 3,85 & $-1,9$ \\
\hline 902 & 0,074 & 1,035 & 0,379 & 0,249 & 3,79 & $-1,9$ \\
\hline 903 & 0,482 & 2,809 & 1,006 & 0,610 & 10,06 & $-3,3$ \\
\hline 904 & 0,163 & 1,687 & 0,671 & 0,309 & 6,71 & $-2,7$ \\
\hline 905 & 0,020 & 0,534 & 0,204 & 0,122 & 2,04 & $-1,0$ \\
\hline 906 & 0,014 & 0,503 & 0,207 & 0,086 & 2,07 & $-1,0$ \\
\hline
\end{tabular}




\begin{tabular}{|c|c|c|c|c|c|c|}
\hline 907 & 0,077 & 1,065 & 0,332 & 0,295 & 3,32 & $-1,7$ \\
\hline 908 & 0,023 & 0,600 & 0,248 & 0,117 & 2,48 & $-1,3$ \\
\hline 909 & 0,013 & 0,483 & 0,179 & 0,093 & 1,79 & $-0,8$ \\
\hline 910 & 0,071 & 1,139 & 0,448 & 0,201 & 4,48 & $-2,2$ \\
\hline 911 & 0,136 & 1,504 & 0,554 & 0,314 & 5,54 & $-2,5$ \\
\hline 912 & 0,032 & 0,643 & 0,215 & 0,188 & 2,15 & $-1,1$ \\
\hline 913 & 0,625 & 3,276 & 1,230 & 0,647 & 12,30 & $-3,6$ \\
\hline 914 & 0,011 & 0,361 & 0,132 & 0,108 & 1,32 & $-0,4$ \\
\hline 915 & 0,021 & 0,526 & 0,192 & 0,139 & 1,92 & $-0,9$ \\
\hline 916 & 0,031 & 0,686 & 0,230 & 0,173 & 2,30 & $-1,2$ \\
\hline 917 & 0,012 & 0,409 & 0,129 & 0,119 & 1,29 & $-0,4$ \\
\hline 918 & 0,791 & 3,626 & 1,275 & 0,790 & 12,75 & $-3,7$ \\
\hline 919 & 0,014 & 0,430 & 0,162 & 0,113 & 1,62 & $-0,7$ \\
\hline 920 & 0,027 & 0,613 & 0,211 & 0,166 & 2,11 & $-1,1$ \\
\hline 921 & 0,019 & 0,508 & 0,166 & 0,143 & 1,66 & $-0,7$ \\
\hline 922 & 0,332 & 2,584 & 0,983 & 0,430 & 9,83 & $-3,3$ \\
\hline 923 & 0,006 & 0,257 & 0,090 & 0,079 & 0,90 & 0,2 \\
\hline 924 & 0,012 & 0,392 & 0,149 & 0,103 & 1,49 & $-0,6$ \\
\hline 925 & 0,007 & 0,343 & 0,129 & 0,074 & 1,29 & $-0,4$ \\
\hline 926 & 0,050 & 0,948 & 0,373 & 0,170 & 3,73 & $-1,9$ \\
\hline 927 & 0,018 & 0,521 & 0,189 & 0,119 & 1,89 & $-0,9$ \\
\hline 928 & 0,027 & 0,631 & 0,254 & 0,133 & 2,54 & $-1,3$ \\
\hline 929 & 0,043 & 0,771 & 0,266 & 0,205 & 2,66 & $-1,4$ \\
\hline 930 & 0,008 & 0,331 & 0,112 & 0,090 & 1,12 & $-0,2$ \\
\hline 931 & 0,008 & 0,318 & 0,129 & 0,083 & 1,29 & $-0,4$ \\
\hline 932 & 0,019 & 0,539 & 0,205 & 0,116 & 2,05 & $-1,0$ \\
\hline 933 & 0,031 & 0,661 & 0,241 & 0,163 & 2,41 & $-1,3$ \\
\hline 934 & 0,003 & 0,183 & 0,081 & 0,051 & 0,81 & 0,3 \\
\hline 935 & 0,007 & 0,287 & 0,110 & 0,075 & 1,10 & $-0,1$ \\
\hline 936 & 0,012 & 0,465 & 0,175 & 0,085 & 1,75 & $-0,8$ \\
\hline 937 & 0,188 & 1,865 & 0,658 & 0,364 & 6,58 & $-2,7$ \\
\hline 938 & 0,153 & 1,696 & 0,704 & 0,277 & 7,04 & $-2,8$ \\
\hline 939 & 0,093 & 1,175 & 0,360 & 0,330 & 3,60 & $-1,8$ \\
\hline 940 & 0,131 & 1,513 & 0,430 & 0,388 & 4,30 & $-2,1$ \\
\hline 941 & 0,001 & 0,104 & 0,049 & 0,024 & 0,49 & 1,0 \\
\hline 942 & 0,112 & 1,403 & 0,541 & 0,264 & 5,41 & $-2,4$ \\
\hline 943 & 0,021 & 0,582 & 0,241 & 0,108 & 2,41 & $-1,3$ \\
\hline 944 & 0,079 & 1,218 & 0,419 & 0,240 & 4,19 & $-2,1$ \\
\hline 945 & 0,089 & 1,268 & 0,502 & 0,227 & 5,02 & $-2,3$ \\
\hline 946 & 0,028 & 0,638 & 0,246 & 0,145 & 2,46 & $-1,3$ \\
\hline 947 & 0,029 & 0,668 & 0,259 & 0,144 & 2,59 & $-1,4$ \\
\hline 948 & 0,097 & 1,146 & 0,380 & 0,325 & 3,80 & $-1,9$ \\
\hline 949 & 0,042 & 0,808 & 0,306 & 0,174 & 3,06 & $-1,6$ \\
\hline 950 & 0,005 & 0,270 & 0,097 & 0,067 & 0,97 & 0,0 \\
\hline 951 & 0,026 & 0,631 & 0,237 & 0,140 & 2,37 & $-1,2$ \\
\hline 952 & 0,023 & 0,557 & 0,194 & 0,153 & 1,94 & $-1,0$ \\
\hline 953 & 0,015 & 0,453 & 0,179 & 0,109 & 1,79 & $-0,8$ \\
\hline 954 & 0,041 & 0,839 & 0,291 & 0,177 & 2,91 & $-1,5$ \\
\hline 955 & 0,067 & 0,986 & 0,361 & 0,237 & 3,61 & $-1,9$ \\
\hline 956 & 0,011 & 0,379 & 0,127 & 0,108 & 1,27 & $-0,3$ \\
\hline 957 & 0,003 & 0,183 & 0,080 & 0,044 & 0,80 & 0,3 \\
\hline 958 & 0,019 & 0,521 & 0,211 & 0,112 & 2,11 & $-1,1$ \\
\hline 959 & 0,022 & 0,552 & 0,192 & 0,148 & 1,92 & $-0,9$ \\
\hline 960 & 0,015 & 0,546 & 0,228 & 0,086 & 2,28 & $-1,2$ \\
\hline 961 & 0,009 & 0,331 & 0,132 & 0,085 & 1,32 & $-0,4$ \\
\hline 962 & 0,021 & 0,577 & 0,237 & 0,113 & 2,37 & $-1,2$ \\
\hline 963 & 0,032 & 0,661 & 0,223 & 0,181 & 2,23 & $-1,2$ \\
\hline 964 & 0,055 & 0,864 & 0,333 & 0,210 & 3,33 & $-1,7$ \\
\hline 965 & 0,025 & 0,587 & 0,206 & 0,153 & 2,06 & $-1,0$ \\
\hline 966 & 0,009 & 0,361 & 0,129 & 0,092 & 1,29 & $-0,4$ \\
\hline 967 & 0,030 & 0,661 & 0,242 & 0,160 & 2,42 & $-1,3$ \\
\hline 968 & 0,085 & 1,162 & 0,391 & 0,276 & 3,91 & $-2,0$ \\
\hline
\end{tabular}




\begin{tabular}{|c|c|c|c|c|c|c|}
\hline 969 & 0,073 & 1,075 & 0,345 & 0,268 & 3,45 & $-1,8$ \\
\hline 970 & 0,194 & 1,886 & 0,665 & 0,372 & 6,65 & $-2,7$ \\
\hline 971 & 0,055 & 0,976 & 0,354 & 0,200 & 3,54 & $-1,8$ \\
\hline 972 & 0,021 & 0,526 & 0,214 & 0,127 & 2,14 & $-1,1$ \\
\hline 973 & 0,031 & 0,636 & 0,227 & 0,175 & 2,27 & $-1,2$ \\
\hline 974 & 0,031 & 0,722 & 0,215 & 0,182 & 2,15 & $-1,1$ \\
\hline 975 & 0,027 & 0,643 & 0,248 & 0,141 & 2,48 & $-1,3$ \\
\hline 976 & 0,042 & 0,839 & 0,297 & 0,182 & 2,97 & $-1,6$ \\
\hline 977 & 0,129 & 1,403 & 0,461 & 0,355 & 4,61 & $-2,2$ \\
\hline 978 & 0,029 & 0,656 & 0,209 & 0,176 & 2,09 & $-1,1$ \\
\hline 979 & 0,017 & 0,503 & 0,191 & 0,115 & 1,91 & $-0,9$ \\
\hline 980 & 0,009 & 0,331 & 0,125 & 0,090 & 1,25 & $-0,3$ \\
\hline 981 & 0,021 & 0,534 & 0,198 & 0,138 & 1,98 & $-1,0$ \\
\hline 982 & 0,109 & 1,281 & 0,450 & 0,308 & 4,50 & $-2,2$ \\
\hline 983 & 0,130 & 1,492 & 0,495 & 0,333 & 4,95 & $-2,3$ \\
\hline 984 & 0,015 & 0,465 & 0,196 & 0,097 & 1,96 & $-1,0$ \\
\hline 985 & 0,035 & 0,791 & 0,280 & 0,159 & 2,80 & $-1,5$ \\
\hline 986 & 0,079 & 1,060 & 0,385 & 0,262 & 3,85 & $-1,9$ \\
\hline 987 & 0,003 & 0,196 & 0,067 & 0,062 & 0,67 & 0,6 \\
\hline 988 & 0,025 & 0,679 & 0,273 & 0,117 & 2,73 & $-1,4$ \\
\hline 989 & 0,007 & 0,348 & 0,153 & 0,054 & 1,53 & $-0,6$ \\
\hline 990 & 0,001 & 0,122 & 0,051 & 0,035 & 0,51 & 1,0 \\
\hline 991 & 0,003 & 0,196 & 0,067 & 0,062 & 0,67 & 0,6 \\
\hline 992 & 0,025 & 0,668 & 0,269 & 0,117 & 2,69 & $-1,4$ \\
\hline 993 & 0,042 & 0,839 & 0,328 & 0,163 & 3,28 & $-1,7$ \\
\hline 994 & 0,148 & 1,586 & 0,588 & 0,320 & 5,88 & $-2,6$ \\
\hline 995 & 0,014 & 0,435 & 0,155 & 0,119 & 1,55 & $-0,6$ \\
\hline 996 & 0,013 & 0,409 & 0,142 & 0,113 & 1,42 & $-0,5$ \\
\hline 997 & 0,017 & 0,465 & 0,169 & 0,130 & 1,69 & $-0,8$ \\
\hline 998 & 0,014 & 0,435 & 0,143 & 0,129 & 1,43 & $-0,5$ \\
\hline 999 & 0,015 & 0,521 & 0,204 & 0,093 & 2,04 & $-1,0$ \\
\hline 1000 & 0,005 & 0,239 & 0,087 & 0,068 & 0,87 & 0,2 \\
\hline 1001 & 0,006 & 0,287 & 0,092 & 0,078 & 0,92 & 0,1 \\
\hline 1002 & 0,204 & 2,125 & 0,779 & 0,333 & 7,79 & $-3,0$ \\
\hline 1003 & 0,233 & 1,888 & 0,615 & 0,483 & 6,15 & $-2,6$ \\
\hline 1004 & 0,016 & 0,496 & 0,195 & 0,103 & 1,95 & $-1,0$ \\
\hline 1005 & 0,025 & 0,600 & 0,229 & 0,137 & 2,29 & $-1,2$ \\
\hline 1006 & 0,331 & 2,561 & 1,045 & 0,404 & 10,45 & $-3,4$ \\
\hline 1007 & 0,031 & 0,643 & 0,219 & 0,182 & 2,19 & $-1,1$ \\
\hline 1008 & 0,012 & 0,430 & 0,168 & 0,088 & 1,68 & $-0,7$ \\
\hline 1009 & 0,035 & 0,753 & 0,275 & 0,164 & 2,75 & $-1,5$ \\
\hline 1010 & 0,027 & 0,656 & 0,207 & 0,166 & 2,07 & $-1,1$ \\
\hline 1011 & 0,050 & 0,900 & 0,321 & 0,200 & 3,21 & $-1,7$ \\
\hline 1012 & 0,046 & 0,791 & 0,289 & 0,201 & 2,89 & $-1,5$ \\
\hline 1013 & 0,005 & 0,239 & 0,086 & 0,069 & 0,86 & 0,2 \\
\hline 1014 & 0,011 & 0,417 & 0,166 & 0,086 & 1,66 & $-0,7$ \\
\hline 1015 & 0,035 & 0,788 & 0,312 & 0,143 & 3,12 & $-1,6$ \\
\hline 1016 & 0,031 & 0,722 & 0,237 & 0,165 & 2,37 & $-1,2$ \\
\hline 1017 & 0,064 & 0,979 & 0,327 & 0,249 & 3,27 & $-1,7$ \\
\hline 1018 & 0,145 & 1,495 & 0,534 & 0,345 & 5,34 & $-2,4$ \\
\hline 1019 & 0,002 & 0,153 & 0,068 & 0,044 & 0,68 & 0,6 \\
\hline 1020 & 0,016 & 0,491 & 0,184 & 0,110 & 1,84 & $-0,9$ \\
\hline 1021 & 0,009 & 0,361 & 0,143 & 0,083 & 1,43 & $-0,5$ \\
\hline 1022 & 0,048 & 0,852 & 0,307 & 0,199 & 3,07 & $-1,6$ \\
\hline 1023 & 0,063 & 1,065 & 0,326 & 0,248 & 3,26 & $-1,7$ \\
\hline 1024 & 0,017 & 0,534 & 0,148 & 0,144 & 1,48 & $-0,6$ \\
\hline 1025 & 0,017 & 0,453 & 0,150 & 0,143 & 1,50 & $-0,6$ \\
\hline 1026 & 0,005 & 0,270 & 0,101 & 0,065 & 1,01 & 0,0 \\
\hline 1027 & 0,017 & 0,460 & 0,162 & 0,131 & 1,62 & $-0,7$ \\
\hline 1028 & 0,005 & 0,244 & 0,086 & 0,069 & 0,86 & 0,2 \\
\hline 1029 & 0,061 & 1,042 & 0,350 & 0,224 & 3,50 & $-1,8$ \\
\hline 1030 & 0,017 & 0,465 & 0,153 & 0,140 & 1,53 & $-0,6$ \\
\hline
\end{tabular}




\begin{tabular}{|c|c|c|c|c|c|c|}
\hline 1031 & 0,034 & 0,783 & 0,251 & 0,173 & 2,51 & $-1,3$ \\
\hline 1032 & 0,055 & 0,913 & 0,321 & 0,220 & 3,21 & $-1,7$ \\
\hline 1033 & 0,051 & 0,839 & 0,260 & 0,249 & 2,60 & $-1,4$ \\
\hline 1034 & 0,027 & 0,613 & 0,233 & 0,148 & 2,33 & $-1,2$ \\
\hline 1035 & 0,014 & 0,447 & 0,149 & 0,120 & 1,49 & $-0,6$ \\
\hline 1036 & 0,019 & 0,526 & 0,202 & 0,118 & 2,02 & $-1,0$ \\
\hline 1037 & 0,024 & 0,674 & 0,263 & 0,117 & 2,63 & $-1,4$ \\
\hline 1038 & 0,007 & 0,287 & 0,107 & 0,089 & 1,07 & $-0,1$ \\
\hline 1039 & 0,137 & 1,454 & 0,499 & 0,349 & 4,99 & $-2,3$ \\
\hline 1040 & 0,009 & 0,361 & 0,128 & 0,093 & 1,28 & $-0,4$ \\
\hline 1041 & 0,071 & 1,042 & 0,368 & 0,247 & 3,68 & $-1,9$ \\
\hline 1042 & 0,012 & 0,392 & 0,145 & 0,103 & 1,45 & $-0,5$ \\
\hline 1043 & 0,029 & 0,679 & 0,291 & 0,129 & 2,91 & $-1,5$ \\
\hline 1044 & 0,005 & 0,239 & 0,086 & 0,069 & 0,86 & 0,2 \\
\hline 1045 & 0,013 & 0,478 & 0,157 & 0,106 & 1,57 & $-0,7$ \\
\hline 1046 & 0,011 & 0,374 & 0,147 & 0,093 & 1,47 & $-0,6$ \\
\hline 1047 & 0,034 & 0,783 & 0,328 & 0,134 & 3,28 & $-1,7$ \\
\hline 1048 & 0,012 & 0,404 & 0,147 & 0,101 & 1,47 & $-0,6$ \\
\hline 1049 & 2,807 & 6,983 & 2,264 & 1,579 & 22,64 & $-4,5$ \\
\hline 1050 & 0,007 & 0,331 & 0,120 & 0,069 & 1,20 & $-0,3$ \\
\hline 1051 & 0,004 & 0,270 & 0,099 & 0,054 & 0,99 & 0,0 \\
\hline 1052 & 0,010 & 0,348 & 0,133 & 0,098 & 1,33 & $-0,4$ \\
\hline 1053 & 0,014 & 0,453 & 0,185 & 0,093 & 1,85 & $-0,9$ \\
\hline 1054 & 0,465 & 2,970 & 1,221 & 0,485 & 12,21 & $-3,6$ \\
\hline 1055 & 0,015 & 0,478 & 0,172 & 0,111 & 1,72 & $-0,8$ \\
\hline 1056 & 0,019 & 0,491 & 0,183 & 0,133 & 1,83 & $-0,9$ \\
\hline 1057 & 1,332 & 4,818 & 1,338 & 1,268 & 13,38 & $-3,7$ \\
\hline 1058 & 0,008 & 0,356 & 0,148 & 0,068 & 1,48 & $-0,6$ \\
\hline 1059 & 0,013 & 0,435 & 0,153 & 0,109 & 1,53 & $-0,6$ \\
\hline 1060 & 0,009 & 0,361 & 0,143 & 0,083 & 1,43 & $-0,5$ \\
\hline 1061 & 0,009 & 0,343 & 0,135 & 0,088 & 1,35 & $-0,4$ \\
\hline 1062 & 0,037 & 0,778 & 0,303 & 0,155 & 3,03 & $-1,6$ \\
\hline 1063 & 0,021 & 0,570 & 0,203 & 0,131 & 2,03 & $-1,0$ \\
\hline 1064 & 0,006 & 0,282 & 0,109 & 0,065 & 1,09 & $-0,1$ \\
\hline 1065 & 0,075 & 1,067 & 0,386 & 0,246 & 3,86 & $-1,9$ \\
\hline 1066 & 0,006 & 0,287 & 0,116 & 0,062 & 1,16 & $-0,2$ \\
\hline 1067 & 0,016 & 0,521 & 0,187 & 0,108 & 1,87 & $-0,9$ \\
\hline 1068 & 0,016 & 0,465 & 0,172 & 0,121 & 1,72 & $-0,8$ \\
\hline 1069 & 0,013 & 0,564 & 0,211 & 0,079 & 2,11 & $-1,1$ \\
\hline 1070 & 0,032 & 0,760 & 0,285 & 0,144 & 2,85 & $-1,5$ \\
\hline 1071 & 0,007 & 0,305 & 0,099 & 0,090 & 0,99 & 0,0 \\
\hline 1072 & 0,029 & 0,631 & 0,208 & 0,180 & 2,08 & $-1,1$ \\
\hline 1073 & 0,017 & 0,508 & 0,193 & 0,114 & 1,93 & $-0,9$ \\
\hline 1074 & 0,038 & 0,826 & 0,287 & 0,170 & 2,87 & $-1,5$ \\
\hline 1075 & 0,034 & 0,887 & 0,354 & 0,124 & 3,54 & $-1,8$ \\
\hline 1076 & 0,005 & 0,300 & 0,118 & 0,055 & 1,18 & $-0,2$ \\
\hline 1077 & 0,006 & 0,257 & 0,106 & 0,073 & 1,06 & $-0,1$ \\
\hline 1078 & 0,007 & 0,300 & 0,108 & 0,088 & 1,08 & $-0,1$ \\
\hline 1079 & 0,015 & 0,625 & 0,249 & 0,076 & 2,49 & $-1,3$ \\
\hline 1080 & 0,031 & 0,618 & 0,215 & 0,185 & 2,15 & $-1,1$ \\
\hline 1081 & 0,007 & 0,287 & 0,107 & 0,089 & 1,07 & $-0,1$ \\
\hline 1082 & 0,219 & 1,896 & 0,607 & 0,460 & 6,07 & $-2,6$ \\
\hline 1083 & 0,080 & 1,060 & 0,361 & 0,281 & 3,61 & $-1,9$ \\
\hline 1084 & 0,078 & 1,434 & 0,387 & 0,256 & 3,87 & $-2,0$ \\
\hline 1085 & 0,047 & 0,834 & 0,283 & 0,212 & 2,83 & $-1,5$ \\
\hline 1086 & 0,030 & 0,681 & 0,213 & 0,181 & 2,13 & $-1,1$ \\
\hline 1087 & 0,006 & 0,257 & 0,096 & 0,075 & 0,96 & 0,1 \\
\hline 1088 & 0,009 & 0,343 & 0,126 & 0,089 & 1,26 & $-0,3$ \\
\hline 1089 & 0,016 & 0,435 & 0,160 & 0,126 & 1,60 & $-0,7$ \\
\hline 1090 & 0,035 & 0,846 & 0,347 & 0,130 & 3,47 & $-1,8$ \\
\hline 1091 & 0,228 & 1,922 & 0,621 & 0,468 & 6,21 & $-2,6$ \\
\hline 1092 & 0,082 & 1,258 & 0,508 & 0,206 & 5,08 & $-2,3$ \\
\hline
\end{tabular}




\begin{tabular}{|c|c|c|c|c|c|c|}
\hline 1093 & 0,028 & 0,643 & 0,239 & 0,152 & 2,39 & $-1,3$ \\
\hline 1094 & 0,102 & 1,332 & 0,454 & 0,285 & 4,54 & $-2,2$ \\
\hline 1095 & 0,040 & 0,816 & 0,318 & 0,161 & 3,18 & $-1,7$ \\
\hline 1096 & 0,005 & 0,244 & 0,097 & 0,061 & 0,97 & 0,0 \\
\hline 1097 & 0,021 & 0,575 & 0,207 & 0,132 & 2,07 & $-1,0$ \\
\hline 1098 & 0,159 & 1,728 & 0,468 & 0,433 & 4,68 & $-2,2$ \\
\hline 1099 & 0,015 & 0,465 & 0,168 & 0,113 & 1,68 & $-0,7$ \\
\hline 1100 & 0,024 & 0,582 & 0,205 & 0,151 & 2,05 & $-1,0$ \\
\hline 1101 & 0,023 & 0,570 & 0,208 & 0,143 & 2,08 & $-1,1$ \\
\hline 1102 & 0,004 & 0,239 & 0,092 & 0,052 & 0,92 & 0,1 \\
\hline 1103 & 0,007 & 0,318 & 0,101 & 0,088 & 1,01 & 0,0 \\
\hline 1104 & 0,887 & 3,764 & 1,379 & 0,820 & 13,79 & $-3,8$ \\
\hline 1105 & 0,040 & 0,862 & 0,326 & 0,155 & 3,26 & $-1,7$ \\
\hline 1106 & 0,033 & 0,692 & 0,246 & 0,169 & 2,46 & $-1,3$ \\
\hline 1107 & 0,051 & 0,907 & 0,355 & 0,184 & 3,55 & $-1,8$ \\
\hline 1108 & 0,020 & 0,539 & 0,206 & 0,121 & 2,06 & $-1,0$ \\
\hline 1109 & 0,031 & 0,686 & 0,267 & 0,147 & 2,67 & $-1,4$ \\
\hline 1110 & 0,024 & 0,600 & 0,207 & 0,146 & 2,07 & $-1,0$ \\
\hline 1111 & 0,029 & 0,618 & 0,202 & 0,185 & 2,02 & $-1,0$ \\
\hline 1112 & 0,033 & 0,778 & 0,303 & 0,137 & 3,03 & $-1,6$ \\
\hline 1113 & 0,048 & 0,808 & 0,278 & 0,217 & 2,78 & $-1,5$ \\
\hline 1114 & 0,018 & 0,544 & 0,231 & 0,100 & 2,31 & $-1,2$ \\
\hline 1115 & 0,016 & 0,564 & 0,219 & 0,092 & 2,19 & $-1,1$ \\
\hline 1116 & 0,084 & 1,190 & 0,405 & 0,264 & 4,05 & $-2,0$ \\
\hline 1117 & 0,012 & 0,392 & 0,148 & 0,104 & 1,48 & $-0,6$ \\
\hline 1118 & 0,008 & 0,343 & 0,139 & 0,073 & 1,39 & $-0,5$ \\
\hline 1119 & 0,008 & 0,331 & 0,130 & 0,077 & 1,30 & $-0,4$ \\
\hline 1120 & 0,056 & 0,981 & 0,335 & 0,214 & 3,35 & $-1,7$ \\
\hline 1121 & 0,030 & 0,661 & 0,225 & 0,171 & 2,25 & $-1,2$ \\
\hline 1122 & 0,007 & 0,300 & 0,104 & 0,080 & 1,04 & $-0,1$ \\
\hline 1123 & 0,016 & 0,478 & 0,191 & 0,105 & 1,91 & $-0,9$ \\
\hline 1124 & 0,067 & 1,055 & 0,388 & 0,219 & 3,88 & $-2,0$ \\
\hline 1125 & 0,007 & 0,300 & 0,109 & 0,087 & 1,09 & $-0,1$ \\
\hline 1126 & 0,079 & 1,121 & 0,424 & 0,236 & 4,24 & $-2,1$ \\
\hline 1127 & 0,156 & 1,626 & 0,609 & 0,325 & 6,09 & $-2,6$ \\
\hline 1128 & 0,023 & 0,582 & 0,183 & 0,159 & 1,83 & $-0,9$ \\
\hline 1129 & 0,006 & 0,270 & 0,106 & 0,067 & 1,06 & $-0,1$ \\
\hline 1130 & 0,187 & 2,011 & 0,609 & 0,391 & 6,09 & $-2,6$ \\
\hline 1131 & 0,030 & 0,674 & 0,257 & 0,148 & 2,57 & $-1,4$ \\
\hline 1132 & 0,183 & 1,840 & 0,583 & 0,399 & 5,83 & $-2,5$ \\
\hline 1133 & 0,030 & 0,600 & 0,202 & 0,188 & 2,02 & $-1,0$ \\
\hline 1134 & 0,031 & 0,709 & 0,245 & 0,160 & 2,45 & $-1,3$ \\
\hline 1135 & 0,221 & 1,942 & 0,640 & 0,439 & 6,40 & $-2,7$ \\
\hline 1136 & 0,032 & 0,674 & 0,261 & 0,157 & 2,61 & $-1,4$ \\
\hline 1137 & 0,058 & 1,037 & 0,435 & 0,169 & 4,35 & $-2,1$ \\
\hline 1138 & 0,021 & 0,552 & 0,187 & 0,143 & 1,87 & $-0,9$ \\
\hline 1139 & 0,018 & 0,526 & 0,164 & 0,138 & 1,64 & $-0,7$ \\
\hline 1140 & 0,004 & 0,196 & 0,076 & 0,062 & 0,76 & 0,4 \\
\hline 1141 & 0,013 & 0,447 & 0,167 & 0,099 & 1,67 & $-0,7$ \\
\hline 1142 & 0,034 & 0,699 & 0,255 & 0,170 & 2,55 & $-1,3$ \\
\hline 1143 & 0,014 & 0,409 & 0,161 & 0,111 & 1,61 & $-0,7$ \\
\hline 1144 & 0,007 & 0,287 & 0,115 & 0,072 & 1,15 & $-0,2$ \\
\hline 1145 & 0,010 & 0,361 & 0,121 & 0,108 & 1,21 & $-0,3$ \\
\hline 1146 & 0,233 & 2,264 & 0,779 & 0,381 & 7,79 & $-3,0$ \\
\hline 1147 & 0,004 & 0,226 & 0,085 & 0,063 & 0,85 & 0,2 \\
\hline 1148 & 0,051 & 0,839 & 0,298 & 0,219 & 2,98 & $-1,6$ \\
\hline 1149 & 0,012 & 0,392 & 0,140 & 0,110 & 1,40 & $-0,5$ \\
\hline 1150 & 0,010 & 0,331 & 0,112 & 0,112 & 1,12 & $-0,2$ \\
\hline 1151 & 0,082 & 1,411 & 0,469 & 0,223 & 4,69 & $-2,2$ \\
\hline 1152 & 0,018 & 0,521 & 0,165 & 0,140 & 1,65 & $-0,7$ \\
\hline 1153 & 0,025 & 0,587 & 0,214 & 0,150 & 2,14 & $-1,1$ \\
\hline 1154 & 0,020 & 0,631 & 0,224 & 0,111 & 2,24 & $-1,2$ \\
\hline
\end{tabular}




\begin{tabular}{|c|c|c|c|c|c|c|}
\hline 1155 & 0,020 & 0,526 & 0,184 & 0,139 & 1,84 & $-0,9$ \\
\hline 1156 & 0,031 & 0,666 & 0,240 & 0,163 & 2,40 & $-1,3$ \\
\hline 1157 & 0,007 & 0,313 & 0,108 & 0,088 & 1,08 & $-0,1$ \\
\hline 1158 & 0,030 & 0,668 & 0,252 & 0,153 & 2,52 & $-1,3$ \\
\hline 1159 & 0,014 & 0,460 & 0,168 & 0,109 & 1,68 & $-0,8$ \\
\hline 1160 & 0,014 & 0,422 & 0,179 & 0,096 & 1,79 & $-0,8$ \\
\hline 1161 & 0,089 & 1,271 & 0,492 & 0,229 & 4,92 & $-2,3$ \\
\hline 1162 & 0,013 & 0,447 & 0,164 & 0,101 & 1,64 & $-0,7$ \\
\hline 1163 & 0,004 & 0,244 & 0,079 & 0,068 & 0,79 & 0,3 \\
\hline 1164 & 0,021 & 0,648 & 0,255 & 0,105 & 2,55 & $-1,3$ \\
\hline 1165 & 0,044 & 0,814 & 0,281 & 0,199 & 2,81 & $-1,5$ \\
\hline 1166 & 0,081 & 1,128 & 0,407 & 0,252 & 4,07 & $-2,0$ \\
\hline 1167 & 0,002 & 0,122 & 0,049 & 0,049 & 0,49 & 1,0 \\
\hline 1168 & 0,007 & 0,313 & 0,108 & 0,088 & 1,08 & $-0,1$ \\
\hline 1169 & 0,024 & 0,552 & 0,187 & 0,162 & 1,87 & $-0,9$ \\
\hline 1170 & 0,016 & 0,508 & 0,184 & 0,113 & 1,84 & $-0,9$ \\
\hline 1171 & 0,117 & 1,583 & 0,641 & 0,232 & 6,41 & $-2,7$ \\
\hline 1172 & 0,010 & 0,374 & 0,126 & 0,099 & 1,26 & $-0,3$ \\
\hline 1173 & 0,065 & 1,261 & 0,399 & 0,207 & 3,99 & $-2,0$ \\
\hline 1174 & 0,023 & 0,552 & 0,202 & 0,147 & 2,02 & $-1,0$ \\
\hline 1175 & 0,020 & 0,534 & 0,191 & 0,134 & 1,91 & $-0,9$ \\
\hline 1176 & 0,043 & 0,826 & 0,298 & 0,183 & 2,98 & $-1,6$ \\
\hline 1177 & 0,010 & 0,356 & 0,139 & 0,094 & 1,39 & $-0,5$ \\
\hline 1178 & 0,019 & 0,544 & 0,181 & 0,135 & 1,81 & $-0,9$ \\
\hline 1179 & 0,019 & 0,508 & 0,174 & 0,136 & 1,74 & $-0,8$ \\
\hline 1180 & 0,041 & 0,791 & 0,296 & 0,174 & 2,96 & $-1,6$ \\
\hline 1181 & 0,017 & 0,582 & 0,227 & 0,094 & 2,27 & $-1,2$ \\
\hline 1182 & 0,017 & 0,564 & 0,241 & 0,091 & 2,41 & $-1,3$ \\
\hline 1183 & 0,071 & 1,177 & 0,373 & 0,242 & 3,73 & $-1,9$ \\
\hline 1184 & 0,019 & 0,539 & 0,211 & 0,112 & 2,11 & $-1,1$ \\
\hline 1185 & 0,053 & 0,943 & 0,373 & 0,181 & 3,73 & $-1,9$ \\
\hline 1186 & 0,005 & 0,226 & 0,089 & 0,067 & 0,89 & 0,2 \\
\hline 1187 & 0,009 & 0,356 & 0,141 & 0,080 & 1,41 & $-0,5$ \\
\hline 1188 & 0,017 & 0,508 & 0,158 & 0,135 & 1,58 & $-0,7$ \\
\hline 1189 & 0,030 & 0,679 & 0,233 & 0,166 & 2,33 & $-1,2$ \\
\hline 1190 & 0,014 & 0,453 & 0,174 & 0,099 & 1,74 & $-0,8$ \\
\hline 1191 & 0,009 & 0,348 & 0,142 & 0,083 & 1,42 & $-0,5$ \\
\hline 1192 & 0,016 & 0,473 & 0,179 & 0,116 & 1,79 & $-0,8$ \\
\hline 1193 & 0,023 & 0,595 & 0,244 & 0,119 & 2,44 & $-1,3$ \\
\hline 1194 & 0,035 & 0,747 & 0,270 & 0,165 & 2,70 & $-1,4$ \\
\hline 1195 & 0,022 & 0,613 & 0,223 & 0,125 & 2,23 & $-1,2$ \\
\hline 1196 & 0,014 & 0,496 & 0,155 & 0,118 & 1,55 & $-0,6$ \\
\hline 1197 & 0,051 & 0,900 & 0,329 & 0,198 & 3,29 & $-1,7$ \\
\hline 1198 & 0,011 & 0,397 & 0,167 & 0,085 & 1,67 & $-0,7$ \\
\hline 1199 & 0,014 & 0,465 & 0,188 & 0,098 & 1,88 & $-0,9$ \\
\hline 1200 & 0,009 & 0,374 & 0,136 & 0,083 & 1,36 & $-0,4$ \\
\hline 1201 & 0,015 & 0,491 & 0,189 & 0,104 & 1,89 & $-0,9$ \\
\hline 1202 & 0,016 & 0,496 & 0,187 & 0,108 & 1,87 & $-0,9$ \\
\hline 1203 & 0,007 & 0,318 & 0,113 & 0,079 & 1,13 & $-0,2$ \\
\hline 1204 & 0,014 & 0,435 & 0,161 & 0,114 & 1,61 & $-0,7$ \\
\hline 1205 & 0,014 & 0,496 & 0,176 & 0,101 & 1,76 & $-0,8$ \\
\hline 1206 & 0,023 & 0,552 & 0,189 & 0,157 & 1,89 & $-0,9$ \\
\hline 1207 & 0,005 & 0,257 & 0,094 & 0,063 & 0,94 & 0,1 \\
\hline 1208 & 0,266 & 2,145 & 0,719 & 0,471 & 7,19 & $-2,8$ \\
\hline 1209 & 0,007 & 0,331 & 0,114 & 0,073 & 1,14 & $-0,2$ \\
\hline 1210 & 0,068 & 1,053 & 0,405 & 0,214 & 4,05 & $-2,0$ \\
\hline 1211 & 0,047 & 0,839 & 0,295 & 0,203 & 2,95 & $-1,6$ \\
\hline 1212 & 0,007 & 0,313 & 0,124 & 0,077 & 1,24 & $-0,3$ \\
\hline 1213 & 0,025 & 0,618 & 0,204 & 0,155 & 2,04 & $-1,0$ \\
\hline 1214 & 0,119 & 1,665 & 0,488 & 0,310 & 4,88 & $-2,3$ \\
\hline 1215 & 0,058 & 0,963 & 0,365 & 0,203 & 3,65 & $-1,9$ \\
\hline 1216 & 0,017 & 0,508 & 0,197 & 0,112 & 1,97 & $-1,0$ \\
\hline
\end{tabular}




$\begin{array}{lllllll}1217 & 0,045 & 0,869 & 0,362 & 0,159 & 3,62 & -1,9 \\ 1218 & 0,041 & 0,841 & 0,339 & 0,154 & 3,39 & -1,8 \\ 1219 & 0,022 & 0,539 & 0,194 & 0,147 & 1,94 & -1,0 \\ 1220 & 0,005 & 0,239 & 0,086 & 0,069 & 0,86 & 0,2 \\ 1221 & 0,170 & 1,736 & 0,651 & 0,333 & 6,51 & -2,7 \\ 1222 & 0,010 & 0,417 & 0,176 & 0,071 & 1,76 & -0,8 \\ 1223 & 0,024 & 0,600 & 0,239 & 0,127 & 2,39 & -1,3 \\ 1224 & 0,080 & 1,055 & 0,374 & 0,271 & 3,74 & -1,9 \\ 1225 & 0,021 & 0,539 & 0,199 & 0,131 & 1,99 & -1,0 \\ 1226 & 0,027 & 0,709 & 0,270 & 0,130 & 2,70 & -1,4 \\ 1227 & 0,019 & 0,514 & 0,194 & 0,123 & 1,94 & -1,0 \\ 1228 & 0,013 & 0,392 & 0,144 & 0,111 & 1,44 & -0,5 \\ 1229 & 0,020 & 0,508 & 0,193 & 0,129 & 1,93 & -0,9\end{array}$


Clasts' data for sample SM-82_Br04 used for grain size distribution and fractal dimension analysis

$\phi=-\log _{2}(\mathrm{diam})$, considering the major axis of the best fit ellipses, in millimeters.

Best fit ellipse

\begin{tabular}{|c|c|c|c|c|c|c|}
\hline Label & Area $\left(\mathbf{c m}^{2}\right)$ & $\begin{array}{l}\text { Perimeter } \\
\text { (cm) }\end{array}$ & $\begin{array}{l}\text { Major axis } \\
\quad(\mathrm{cm})\end{array}$ & $\begin{array}{l}\text { Minor axis } \\
\quad \text { (cm) }\end{array}$ & $\begin{array}{l}\text { Diameter } \\
\text { (mm) }\end{array}$ & Phi \\
\hline 1 & 16,44 & 15,49 & 4,83 & 4,34 & 48,30 & $-5,6$ \\
\hline 2 & 4,98 & 8,79 & 2,97 & 2,13 & 29,73 & $-4,9$ \\
\hline 3 & 8,56 & 12,16 & 4,21 & 2,59 & 42,07 & $-5,4$ \\
\hline 4 & 0,32 & 2,56 & 1,08 & 0,38 & 10,79 & $-3,4$ \\
\hline 5 & 1,79 & 5,82 & 2,23 & 1,02 & 22,32 & $-4,5$ \\
\hline 6 & 2,16 & 6,02 & 2,23 & 1,23 & 22,28 & $-4,5$ \\
\hline 7 & 13,20 & 16,18 & 5,49 & 3,06 & 54,86 & $-5,8$ \\
\hline 8 & 0,50 & 2,91 & 1,12 & 0,57 & 11,19 & $-3,5$ \\
\hline 9 & 0,08 & 0,95 & 0,39 & 0,25 & 3,87 & $-2,0$ \\
\hline 10 & 0,63 & 3,21 & 1,22 & 0,66 & 12,16 & $-3,6$ \\
\hline 11 & 20,38 & 18,26 & 6,57 & 3,95 & 65,66 & $-6,0$ \\
\hline 12 & 0,24 & 1,81 & 0,66 & 0,45 & 6,60 & $-2,7$ \\
\hline 13 & 2,44 & 6,93 & 2,66 & 1,17 & 26,63 & $-4,7$ \\
\hline 14 & 0,07 & 1,27 & 0,50 & 0,18 & 4,97 & $-2,3$ \\
\hline 15 & 1,39 & 5,45 & 1,57 & 1,13 & 15,69 & $-4,0$ \\
\hline 16 & 7,97 & 12,42 & 4,25 & 2,39 & 42,45 & $-5,4$ \\
\hline 17 & 0,30 & 2,28 & 0,72 & 0,52 & 7,18 & $-2,8$ \\
\hline 18 & 4,05 & 8,10 & 2,72 & 1,90 & 27,15 & $-4,8$ \\
\hline 19 & 0,29 & 2,54 & 1,12 & 0,33 & 11,19 & $-3,5$ \\
\hline 20 & 3,35 & 7,30 & 2,55 & 1,68 & 25,46 & $-4,7$ \\
\hline 21 & 0,84 & 4,34 & 1,71 & 0,63 & 17,06 & $-4,1$ \\
\hline 22 & 0,45 & 2,62 & 0,80 & 0,71 & 8,02 & $-3,0$ \\
\hline 23 & 0,30 & 2,50 & 1,10 & 0,34 & 11,03 & $-3,5$ \\
\hline 24 & 1,77 & 5,49 & 2,00 & 1,13 & 19,95 & $-4,3$ \\
\hline 25 & 0,04 & 0,72 & 0,25 & 0,19 & 2,50 & $-1,3$ \\
\hline 26 & 0,54 & 2,88 & 1,14 & 0,61 & 11,36 & $-3,5$ \\
\hline 27 & 0,25 & 2,10 & 0,73 & 0,45 & 7,25 & $-2,9$ \\
\hline 28 & 0,24 & 1,75 & 0,60 & 0,50 & 6,01 & $-2,6$ \\
\hline 29 & 0,23 & 1,85 & 0,60 & 0,49 & 6,00 & $-2,6$ \\
\hline 30 & 2,15 & 6,35 & 2,28 & 1,20 & 22,82 & $-4,5$ \\
\hline 31 & 0,26 & 1,96 & 0,66 & 0,50 & 6,64 & $-2,7$ \\
\hline 32 & 0,67 & 3,33 & 1,24 & 0,69 & 12,36 & $-3,6$ \\
\hline 33 & 0,90 & 3,67 & 1,21 & 0,94 & 12,14 & $-3,6$ \\
\hline 34 & 0,45 & 2,92 & 1,14 & 0,50 & 11,39 & $-3,5$ \\
\hline 35 & 0,12 & 1,42 & 0,54 & 0,28 & 5,38 & $-2,4$ \\
\hline 36 & 2,51 & 6,30 & 1,95 & 1,63 & 19,53 & $-4,3$ \\
\hline 37 & 5,65 & 8,91 & 2,87 & 2,50 & 28,73 & $-4,8$ \\
\hline 38 & 0,03 & 0,68 & 0,29 & 0,14 & 2,92 & $-1,5$ \\
\hline 39 & 6,14 & 10,10 & 3,32 & 2,35 & 33,24 & $-5,1$ \\
\hline 40 & 0,98 & 3,82 & 1,44 & 0,87 & 14,43 & $-3,9$ \\
\hline 41 & 0,16 & 1,56 & 0,47 & 0,44 & 4,71 & $-2,2$ \\
\hline 42 & 13,82 & 15,86 & 4,63 & 3,80 & 46,33 & $-5,5$ \\
\hline 43 & 0,82 & 3,56 & 1,37 & 0,76 & 13,73 & $-3,8$ \\
\hline 44 & 1,11 & 4,79 & 1,60 & 0,88 & 16,04 & $-4,0$ \\
\hline 45 & 0,61 & 3,42 & 1,06 & 0,73 & 10,59 & $-3,4$ \\
\hline 46 & 0,20 & 1,67 & 0,65 & 0,40 & 6,50 & $-2,7$ \\
\hline 47 & 1,84 & 5,80 & 2,27 & 1,03 & 22,70 & $-4,5$ \\
\hline 48 & 0,90 & 3,57 & 1,21 & 0,95 & 12,10 & $-3,6$ \\
\hline 49 & 0,67 & 3,11 & 1,23 & 0,69 & 12,31 & $-3,6$ \\
\hline 50 & 0,57 & 3,50 & 1,18 & 0,62 & 11,77 & $-3,6$ \\
\hline 51 & 0,34 & 2,41 & 0,91 & 0,48 & 9,12 & $-3,2$ \\
\hline 52 & 0,25 & 2,50 & 1,15 & 0,28 & 11,47 & $-3,5$ \\
\hline 53 & 0,24 & 1,81 & 0,61 & 0,49 & 6,14 & $-2,6$ \\
\hline
\end{tabular}




\begin{tabular}{|c|c|c|c|c|c|c|}
\hline 54 & 1,70 & 5,45 & 1,50 & 1,45 & 14,98 & $-3,9$ \\
\hline 55 & 0,58 & 3,19 & 1,22 & 0,61 & 12,18 & $-3,6$ \\
\hline 56 & 6,82 & 10,82 & 3,22 & 2,69 & 32,23 & $-5,0$ \\
\hline 57 & 0,45 & 3,17 & 1,41 & 0,40 & 14,10 & $-3,8$ \\
\hline 58 & 1,13 & 4,22 & 1,43 & 1,01 & 14,27 & $-3,8$ \\
\hline 59 & 0,67 & 3,71 & 1,53 & 0,56 & 15,31 & $-3,9$ \\
\hline 60 & 0,37 & 2,57 & 0,84 & 0,56 & 8,36 & $-3,1$ \\
\hline 61 & 4,53 & 8,06 & 2,80 & 2,07 & 27,95 & $-4,8$ \\
\hline 62 & 0,05 & 0,95 & 0,39 & 0,17 & 3,85 & $-1,9$ \\
\hline 63 & 0,77 & 3,75 & 1,52 & 0,65 & 15,19 & $-3,9$ \\
\hline 64 & 3,65 & 7,76 & 2,51 & 1,85 & 25,08 & $-4,6$ \\
\hline 65 & 0,69 & 3,29 & 1,10 & 0,81 & 10,96 & $-3,5$ \\
\hline 66 & 0,34 & 2,24 & 0,71 & 0,60 & 7,13 & $-2,8$ \\
\hline 67 & 1,30 & 4,43 & 1,41 & 1,18 & 14,09 & $-3,8$ \\
\hline 68 & 8,14 & 12,40 & 4,03 & 2,57 & 40,34 & $-5,3$ \\
\hline 69 & 0,24 & 1,86 & 0,78 & 0,39 & 7,75 & $-3,0$ \\
\hline 70 & 0,40 & 2,47 & 0,95 & 0,54 & 9,45 & $-3,2$ \\
\hline 71 & 0,84 & 3,54 & 1,31 & 0,81 & 13,11 & $-3,7$ \\
\hline 72 & 49,51 & 28,13 & 9,10 & 6,93 & 90,99 & $-6,5$ \\
\hline 73 & 0,04 & 0,72 & 0,29 & 0,19 & 2,88 & $-1,5$ \\
\hline 74 & 0,40 & 2,61 & 0,94 & 0,54 & 9,37 & $-3,2$ \\
\hline 75 & 3,10 & 7,12 & 2,28 & 1,73 & 22,83 & $-4,5$ \\
\hline 76 & 0,39 & 2,57 & 0,97 & 0,50 & 9,71 & $-3,3$ \\
\hline 77 & 16,98 & 23,63 & 8,67 & 2,49 & 86,73 & $-6,4$ \\
\hline 78 & 0,35 & 2,16 & 0,74 & 0,60 & 7,44 & $-2,9$ \\
\hline 79 & 3,23 & 6,84 & 2,40 & 1,72 & 23,96 & $-4,6$ \\
\hline 80 & 5,91 & 9,80 & 3,39 & 2,22 & 33,90 & $-5,1$ \\
\hline 81 & 0,82 & 5,28 & 2,34 & 0,45 & 23,35 & $-4,5$ \\
\hline 82 & 1,46 & 4,99 & 1,59 & 1,17 & 15,93 & $-4,0$ \\
\hline 83 & 0,64 & 3,68 & 1,54 & 0,53 & 15,37 & $-3,9$ \\
\hline 84 & 1,76 & 5,28 & 1,82 & 1,23 & 18,24 & $-4,2$ \\
\hline 85 & 0,03 & 0,62 & 0,27 & 0,13 & 2,73 & $-1,4$ \\
\hline 86 & 8,53 & 11,90 & 4,00 & 2,72 & 39,99 & $-5,3$ \\
\hline 87 & 0,09 & 1,09 & 0,36 & 0,33 & 3,58 & $-1,8$ \\
\hline 88 & 0,08 & 1,11 & 0,45 & 0,23 & 4,45 & $-2,2$ \\
\hline 89 & 0,53 & 2,74 & 0,97 & 0,70 & 9,70 & $-3,3$ \\
\hline 90 & 1,79 & 7,00 & 2,22 & 1,02 & 22,23 & $-4,5$ \\
\hline 91 & 0,52 & 3,09 & 1,16 & 0,57 & 11,61 & $-3,5$ \\
\hline 92 & 0,83 & 4,36 & 1,77 & 0,59 & 17,70 & $-4,1$ \\
\hline 93 & 1,39 & 4,47 & 1,62 & 1,09 & 16,24 & $-4,0$ \\
\hline 94 & 0,08 & 0,95 & 0,35 & 0,27 & 3,54 & $-1,8$ \\
\hline 95 & 0,06 & 1,05 & 0,45 & 0,16 & 4,49 & $-2,2$ \\
\hline 96 & 1,00 & 3,99 & 1,17 & 1,08 & 11,71 & $-3,5$ \\
\hline 97 & 0,05 & 0,86 & 0,35 & 0,19 & 3,53 & $-1,8$ \\
\hline 98 & 9,13 & 11,97 & 3,92 & 2,97 & 39,18 & $-5,3$ \\
\hline 99 & 0,01 & 0,47 & 0,23 & 0,08 & 2,32 & $-1,2$ \\
\hline 100 & 1,21 & 4,26 & 1,48 & 1,04 & 14,78 & $-3,9$ \\
\hline 101 & 0,62 & 3,21 & 1,26 & 0,63 & 12,55 & $-3,6$ \\
\hline 102 & 0,15 & 1,73 & 0,67 & 0,29 & 6,68 & $-2,7$ \\
\hline 103 & 2,41 & 6,23 & 1,93 & 1,59 & 19,28 & $-4,3$ \\
\hline 104 & 0,13 & 1,32 & 0,52 & 0,31 & 5,16 & $-2,4$ \\
\hline 105 & 0,07 & 0,90 & 0,37 & 0,22 & 3,73 & $-1,9$ \\
\hline 106 & 86,68 & 39,15 & 12,87 & 8,57 & 128,74 & $-7,0$ \\
\hline 107 & 0,12 & 1,38 & 0,40 & 0,39 & 4,03 & $-2,0$ \\
\hline 108 & 0,03 & 0,66 & 0,28 & 0,15 & 2,84 & $-1,5$ \\
\hline 109 & 1,91 & 5,70 & 1,72 & 1,41 & 17,23 & $-4,1$ \\
\hline 110 & 3,20 & 7,07 & 2,26 & 1,80 & 22,58 & $-4,5$ \\
\hline 111 & 0,20 & 2,25 & 0,99 & 0,26 & 9,94 & $-3,3$ \\
\hline 112 & 0,16 & 1,53 & 0,59 & 0,33 & 5,91 & $-2,6$ \\
\hline 113 & 0,40 & 3,07 & 1,18 & 0,43 & 11,84 & $-3,6$ \\
\hline 114 & 5,10 & 8,95 & 3,27 & 1,99 & 32,74 & $-5,0$ \\
\hline 115 & 13,48 & 16,87 & 5,71 & 3,00 & 57,12 & $-5,8$ \\
\hline
\end{tabular}




\begin{tabular}{|c|c|c|c|c|c|c|}
\hline 116 & 1,36 & 4,80 & 1,75 & 0,99 & 17,46 & $-4,1$ \\
\hline 117 & 1,59 & 5,35 & 2,06 & 0,98 & 20,61 & $-4,4$ \\
\hline 118 & 4,09 & 8,39 & 2,61 & 2,00 & 26,08 & $-4,7$ \\
\hline 119 & 0,36 & 2,80 & 1,11 & 0,42 & 11,09 & $-3,5$ \\
\hline 120 & 8,25 & 11,54 & 3,74 & 2,81 & 37,37 & $-5,2$ \\
\hline 121 & 2,38 & 6,53 & 2,61 & 1,16 & 26,05 & $-4,7$ \\
\hline 122 & 0,02 & 0,58 & 0,26 & 0,11 & 2,63 & $-1,4$ \\
\hline 123 & 0,35 & 2,53 & 1,12 & 0,40 & 11,16 & $-3,5$ \\
\hline 124 & 1,55 & 5,12 & 1,87 & 1,06 & 18,74 & $-4,2$ \\
\hline 125 & 0,34 & 2,24 & 0,90 & 0,48 & 8,95 & $-3,2$ \\
\hline 126 & 0,94 & 3,79 & 1,26 & 0,95 & 12,60 & $-3,7$ \\
\hline 127 & 1,47 & 5,52 & 2,17 & 0,87 & 21,65 & $-4,4$ \\
\hline 128 & 18,97 & 17,26 & 5,81 & 4,16 & 58,09 & $-5,9$ \\
\hline 129 & 1,21 & 4,84 & 1,70 & 0,90 & 17,04 & $-4,1$ \\
\hline 130 & 3,54 & 7,84 & 2,80 & 1,61 & 28,03 & $-4,8$ \\
\hline 131 & 1,80 & 5,20 & 1,58 & 1,45 & 15,77 & $-4,0$ \\
\hline 132 & 1,16 & 4,40 & 1,45 & 1,02 & 14,46 & $-3,9$ \\
\hline 133 & 0,27 & 2,22 & 0,92 & 0,38 & 9,24 & $-3,2$ \\
\hline 134 & 0,50 & 2,84 & 1,13 & 0,57 & 11,26 & $-3,5$ \\
\hline 135 & 0,11 & 1,24 & 0,39 & 0,35 & 3,94 & $-2,0$ \\
\hline 136 & 3,05 & 7,35 & 2,57 & 1,51 & 25,72 & $-4,7$ \\
\hline 137 & 0,10 & 1,20 & 0,51 & 0,26 & 5,07 & $-2,3$ \\
\hline 138 & 0,06 & 0,78 & 0,30 & 0,24 & 3,03 & $-1,6$ \\
\hline 139 & 0,80 & 3,34 & 1,05 & 0,97 & 10,48 & $-3,4$ \\
\hline 140 & 0,05 & 0,72 & 0,28 & 0,21 & 2,82 & $-1,5$ \\
\hline 141 & 0,29 & 2,14 & 0,81 & 0,46 & 8,13 & $-3,0$ \\
\hline 142 & 0,09 & 1,09 & 0,42 & 0,28 & 4,22 & $-2,1$ \\
\hline 143 & 0,25 & 1,96 & 0,69 & 0,46 & 6,85 & $-2,8$ \\
\hline 144 & 0,01 & 0,29 & 0,16 & 0,08 & 1,55 & $-0,6$ \\
\hline 145 & 0,55 & 3,58 & 1,44 & 0,49 & 14,36 & $-3,8$ \\
\hline 146 & 0,04 & 0,91 & 0,37 & 0,13 & 3,65 & $-1,9$ \\
\hline 147 & 0,12 & 1,24 & 0,41 & 0,38 & 4,11 & $-2,0$ \\
\hline 148 & 0,26 & 1,96 & 0,66 & 0,51 & 6,56 & $-2,7$ \\
\hline 149 & 1,68 & 5,83 & 2,27 & 0,95 & 22,66 & $-4,5$ \\
\hline 150 & 13,55 & 15,58 & 5,66 & 3,05 & 56,57 & $-5,8$ \\
\hline 151 & 0,29 & 2,06 & 0,87 & 0,42 & 8,71 & $-3,1$ \\
\hline 152 & 7,99 & 11,84 & 3,83 & 2,66 & 38,30 & $-5,3$ \\
\hline 153 & 1,59 & 5,41 & 2,07 & 0,98 & 20,74 & $-4,4$ \\
\hline 154 & 0,59 & 3,44 & 1,41 & 0,53 & 14,11 & $-3,8$ \\
\hline 155 & 84,05 & 39,90 & 12,53 & 8,54 & 125,27 & $-7,0$ \\
\hline 156 & 0,32 & 2,81 & 1,26 & 0,33 & 12,64 & $-3,7$ \\
\hline 157 & 0,16 & 1,46 & 0,56 & 0,35 & 5,58 & $-2,5$ \\
\hline 158 & 0,41 & 2,74 & 1,08 & 0,49 & 10,84 & $-3,4$ \\
\hline 159 & 3,48 & 7,67 & 2,62 & 1,69 & 26,24 & $-4,7$ \\
\hline 160 & 10,59 & 13,59 & 5,13 & 2,63 & 51,34 & $-5,7$ \\
\hline 161 & 4,14 & 8,41 & 2,97 & 1,77 & 29,69 & $-4,9$ \\
\hline 162 & 9,21 & 12,81 & 4,56 & 2,57 & 45,62 & $-5,5$ \\
\hline 163 & 0,17 & 1,69 & 0,76 & 0,29 & 7,57 & $-2,9$ \\
\hline 164 & 0,27 & 1,98 & 0,74 & 0,47 & 7,37 & $-2,9$ \\
\hline 165 & 0,06 & 0,99 & 0,36 & 0,21 & 3,63 & $-1,9$ \\
\hline 166 & 0,11 & 1,28 & 0,49 & 0,28 & 4,86 & $-2,3$ \\
\hline 167 & 0,21 & 1,67 & 0,53 & 0,51 & 5,25 & $-2,4$ \\
\hline 168 & 0,40 & 2,41 & 0,80 & 0,64 & 7,99 & $-3,0$ \\
\hline 169 & 0,32 & 2,22 & 0,83 & 0,49 & 8,27 & $-3,0$ \\
\hline 170 & 6,73 & 10,53 & 4,01 & 2,14 & 40,13 & $-5,3$ \\
\hline 171 & 0,06 & 0,95 & 0,42 & 0,18 & 4,21 & $-2,1$ \\
\hline 172 & 0,09 & 1,19 & 0,44 & 0,26 & 4,36 & $-2,1$ \\
\hline 173 & 0,96 & 3,78 & 1,37 & 0,89 & 13,74 & $-3,8$ \\
\hline 174 & 0,23 & 2,12 & 0,83 & 0,35 & 8,34 & $-3,1$ \\
\hline 175 & 0,05 & 0,76 & 0,30 & 0,22 & 3,00 & $-1,6$ \\
\hline 176 & 3,31 & 8,16 & 2,75 & 1,53 & 27,45 & $-4,8$ \\
\hline 177 & 0,59 & 2,86 & 0,97 & 0,78 & 9,65 & $-3,3$ \\
\hline
\end{tabular}




\begin{tabular}{|c|c|c|c|c|c|c|}
\hline 178 & 0,70 & 3,25 & 1,02 & 0,88 & 10,15 & $-3,3$ \\
\hline 179 & 0,17 & 1,57 & 0,58 & 0,37 & 5,82 & $-2,5$ \\
\hline 180 & 0,22 & 1,90 & 0,74 & 0,37 & 7,36 & $-2,9$ \\
\hline 181 & 0,03 & 0,58 & 0,22 & 0,17 & 2,15 & $-1,1$ \\
\hline 182 & 2,31 & 6,02 & 1,94 & 1,52 & 19,44 & $-4,3$ \\
\hline 183 & 0,15 & 1,40 & 0,55 & 0,34 & 5,47 & $-2,5$ \\
\hline 184 & 0,16 & 1,71 & 0,66 & 0,31 & 6,64 & $-2,7$ \\
\hline 185 & 1,95 & 6,32 & 2,68 & 0,93 & 26,79 & $-4,7$ \\
\hline 186 & 0,16 & 2,00 & 0,78 & 0,26 & 7,81 & $-3,0$ \\
\hline 187 & 1,29 & 4,43 & 1,61 & 1,02 & 16,06 & $-4,0$ \\
\hline 188 & 0,03 & 0,58 & 0,22 & 0,17 & 2,15 & $-1,1$ \\
\hline 189 & 0,16 & 1,65 & 0,66 & 0,30 & 6,62 & $-2,7$ \\
\hline 190 & 6,70 & 10,27 & 3,55 & 2,41 & 35,47 & $-5,1$ \\
\hline 191 & 0,07 & 1,05 & 0,37 & 0,25 & 3,66 & $-1,9$ \\
\hline 192 & 0,10 & 1,15 & 0,46 & 0,29 & 4,61 & $-2,2$ \\
\hline 193 & 0,64 & 2,99 & 1,01 & 0,81 & 10,07 & $-3,3$ \\
\hline 194 & 1,64 & 5,06 & 1,50 & 1,40 & 14,96 & $-3,9$ \\
\hline 195 & 1,46 & 5,31 & 1,91 & 0,98 & 19,10 & $-4,3$ \\
\hline 196 & 0,51 & 2,86 & 1,03 & 0,63 & 10,26 & $-3,4$ \\
\hline 197 & 0,20 & 1,71 & 0,68 & 0,38 & 6,81 & $-2,8$ \\
\hline 198 & 0,19 & 1,56 & 0,52 & 0,47 & 5,17 & $-2,4$ \\
\hline 199 & 4,57 & 9,20 & 3,45 & 1,69 & 34,52 & $-5,1$ \\
\hline 200 & 0,52 & 2,80 & 0,96 & 0,69 & 9,63 & $-3,3$ \\
\hline 201 & 11,18 & 13,01 & 4,37 & 3,26 & 43,65 & $-5,4$ \\
\hline 202 & 0,24 & 1,86 & 0,66 & 0,47 & 6,58 & $-2,7$ \\
\hline 203 & 27,77 & 21,41 & 7,67 & 4,61 & 76,74 & $-6,3$ \\
\hline 204 & 0,67 & 3,30 & 1,20 & 0,71 & 11,99 & $-3,6$ \\
\hline 205 & 0,04 & 0,72 & 0,26 & 0,18 & 2,62 & $-1,4$ \\
\hline 206 & 0,10 & 1,15 & 0,43 & 0,30 & 4,32 & $-2,1$ \\
\hline 207 & 4,15 & 8,07 & 2,75 & 1,92 & 27,54 & $-4,8$ \\
\hline 208 & 0,06 & 0,82 & 0,34 & 0,21 & 3,36 & $-1,7$ \\
\hline 209 & 0,63 & 3,57 & 1,37 & 0,59 & 13,66 & $-3,8$ \\
\hline 210 & 0,05 & 0,82 & 0,30 & 0,20 & 2,98 & $-1,6$ \\
\hline 211 & 2,35 & 6,37 & 2,00 & 1,49 & 20,02 & $-4,3$ \\
\hline 212 & 0,02 & 0,52 & 0,22 & 0,14 & 2,15 & $-1,1$ \\
\hline 213 & 0,12 & 1,20 & 0,41 & 0,37 & 4,08 & $-2,0$ \\
\hline 214 & 2,65 & 6,32 & 2,12 & 1,59 & 21,21 & $-4,4$ \\
\hline 215 & 0,84 & 3,68 & 1,28 & 0,83 & 12,84 & $-3,7$ \\
\hline 216 & 0,42 & 2,45 & 0,85 & 0,64 & 8,46 & $-3,1$ \\
\hline 217 & 0,02 & 0,58 & 0,24 & 0,13 & 2,37 & $-1,2$ \\
\hline 218 & 0,25 & 1,90 & 0,59 & 0,55 & 5,87 & $-2,6$ \\
\hline 219 & 0,06 & 0,91 & 0,37 & 0,21 & 3,65 & $-1,9$ \\
\hline 220 & 0,30 & 2,30 & 0,97 & 0,39 & 9,72 & $-3,3$ \\
\hline 221 & 0,34 & 2,57 & 1,11 & 0,39 & 11,06 & $-3,5$ \\
\hline 222 & 0,01 & 0,33 & 0,16 & 0,08 & 1,55 & $-0,6$ \\
\hline 223 & 0,09 & 1,11 & 0,39 & 0,29 & 3,86 & $-1,9$ \\
\hline 224 & 53,66 & 34,76 & 8,53 & 8,01 & 85,33 & $-6,4$ \\
\hline 225 & 0,31 & 2,18 & 0,74 & 0,53 & 7,38 & $-2,9$ \\
\hline 226 & 0,15 & 1,52 & 0,58 & 0,33 & 5,78 & $-2,5$ \\
\hline 227 & 0,83 & 4,15 & 1,62 & 0,65 & 16,22 & $-4,0$ \\
\hline 228 & 0,03 & 0,66 & 0,28 & 0,13 & 2,83 & $-1,5$ \\
\hline 229 & 0,14 & 1,79 & 0,75 & 0,24 & 7,52 & $-2,9$ \\
\hline 230 & 0,03 & 0,62 & 0,27 & 0,13 & 2,73 & $-1,4$ \\
\hline 231 & 1,09 & 4,25 & 1,46 & 0,95 & 14,56 & $-3,9$ \\
\hline 232 & 2,08 & 6,83 & 2,94 & 0,90 & 29,43 & $-4,9$ \\
\hline 233 & 0,08 & 1,19 & 0,45 & 0,24 & 4,45 & $-2,2$ \\
\hline 234 & 0,32 & 2,10 & 0,68 & 0,60 & 6,83 & $-2,8$ \\
\hline 235 & 13,46 & 16,22 & 6,29 & 2,73 & 62,90 & $-6,0$ \\
\hline 236 & 0,50 & 2,84 & 1,11 & 0,57 & 11,14 & $-3,5$ \\
\hline 237 & 1,30 & 4,55 & 1,56 & 1,06 & 15,58 & $-4,0$ \\
\hline 238 & 0,13 & 1,42 & 0,53 & 0,30 & 5,31 & $-2,4$ \\
\hline 239 & 7,53 & 11,48 & 3,55 & 2,70 & 35,52 & $-5,2$ \\
\hline
\end{tabular}




\begin{tabular}{|c|c|c|c|c|c|c|}
\hline 240 & 3,58 & 7,69 & 2,29 & 1,99 & 22,87 & $-4,5$ \\
\hline 241 & 0,03 & 0,86 & 0,34 & 0,13 & 3,36 & $-1,7$ \\
\hline 242 & 0,07 & 0,99 & 0,39 & 0,21 & 3,93 & $-2,0$ \\
\hline 243 & 2,48 & 6,55 & 2,31 & 1,37 & 23,12 & $-4,5$ \\
\hline 244 & 4,20 & 7,97 & 2,65 & 2,02 & 26,45 & $-4,7$ \\
\hline 245 & 1,23 & 4,01 & 1,27 & 1,24 & 12,65 & $-3,7$ \\
\hline 246 & 5,19 & 8,98 & 2,82 & 2,34 & 28,19 & $-4,8$ \\
\hline 247 & 0,19 & 1,57 & 0,52 & 0,46 & 5,22 & $-2,4$ \\
\hline 248 & 0,47 & 2,57 & 0,84 & 0,72 & 8,39 & $-3,1$ \\
\hline 249 & 0,62 & 3,05 & 0,99 & 0,80 & 9,90 & $-3,3$ \\
\hline 250 & 0,11 & 1,20 & 0,38 & 0,36 & 3,83 & $-1,9$ \\
\hline 251 & 0,09 & 1,01 & 0,36 & 0,31 & 3,63 & $-1,9$ \\
\hline 252 & 0,03 & 0,76 & 0,32 & 0,13 & 3,18 & $-1,7$ \\
\hline 253 & 0,18 & 1,56 & 0,58 & 0,41 & 5,75 & $-2,5$ \\
\hline 254 & 2,20 & 6,21 & 2,08 & 1,35 & 20,75 & $-4,4$ \\
\hline 255 & 0,45 & 3,15 & 1,28 & 0,44 & 12,77 & $-3,7$ \\
\hline 256 & 0,71 & 3,61 & 1,02 & 0,89 & 10,16 & $-3,3$ \\
\hline 257 & 0,07 & 0,97 & 0,41 & 0,21 & 4,08 & $-2,0$ \\
\hline 258 & 0,10 & 1,38 & 0,47 & 0,28 & 4,65 & $-2,2$ \\
\hline 259 & 2,39 & 6,29 & 2,03 & 1,50 & 20,26 & $-4,3$ \\
\hline 260 & 0,51 & 2,69 & 0,92 & 0,71 & 9,18 & $-3,2$ \\
\hline 261 & 0,15 & 1,69 & 0,61 & 0,31 & 6,08 & $-2,6$ \\
\hline 262 & 0,11 & 1,36 & 0,62 & 0,22 & 6,22 & $-2,6$ \\
\hline 263 & 0,03 & 0,58 & 0,22 & 0,17 & 2,15 & $-1,1$ \\
\hline 264 & 0,08 & 1,20 & 0,49 & 0,21 & 4,93 & $-2,3$ \\
\hline 265 & 0,12 & 1,24 & 0,48 & 0,31 & 4,76 & $-2,3$ \\
\hline 266 & 0,33 & 2,24 & 0,90 & 0,47 & 8,96 & $-3,2$ \\
\hline 267 & 0,04 & 0,62 & 0,24 & 0,20 & 2,42 & $-1,3$ \\
\hline 268 & 0,29 & 2,10 & 0,78 & 0,47 & 7,79 & $-3,0$ \\
\hline 269 & 1,11 & 4,84 & 1,93 & 0,73 & 19,28 & $-4,3$ \\
\hline 270 & 0,62 & 3,31 & 0,95 & 0,84 & 9,48 & $-3,2$ \\
\hline 271 & 1,93 & 6,23 & 2,44 & 1,01 & 24,38 & $-4,6$ \\
\hline 272 & 4,83 & 8,88 & 3,00 & 2,05 & 29,98 & $-4,9$ \\
\hline 273 & 0,10 & 1,19 & 0,48 & 0,26 & 4,80 & $-2,3$ \\
\hline 274 & 0,19 & 1,94 & 0,69 & 0,35 & 6,93 & $-2,8$ \\
\hline 275 & 0,09 & 1,23 & 0,54 & 0,21 & 5,38 & $-2,4$ \\
\hline 276 & 0,10 & 1,15 & 0,42 & 0,30 & 4,20 & $-2,1$ \\
\hline 277 & 0,01 & 0,39 & 0,16 & 0,11 & 1,62 & $-0,7$ \\
\hline 278 & 0,13 & 1,50 & 0,61 & 0,27 & 6,11 & $-2,6$ \\
\hline 279 & 0,10 & 1,20 & 0,45 & 0,28 & 4,46 & $-2,2$ \\
\hline 280 & 0,30 & 2,14 & 0,84 & 0,45 & 8,37 & $-3,1$ \\
\hline 281 & 0,28 & 1,98 & 0,73 & 0,49 & 7,31 & $-2,9$ \\
\hline 282 & 0,14 & 1,69 & 0,66 & 0,26 & 6,57 & $-2,7$ \\
\hline 283 & 0,08 & 0,99 & 0,38 & 0,28 & 3,83 & $-1,9$ \\
\hline 284 & 3,37 & 7,91 & 2,19 & 1,97 & 21,85 & $-4,4$ \\
\hline 285 & 1,91 & 5,79 & 2,11 & 1,15 & 21,06 & $-4,4$ \\
\hline 286 & 6,45 & 9,95 & 3,51 & 2,34 & 35,05 & $-5,1$ \\
\hline 287 & 0,24 & 1,82 & 0,61 & 0,51 & 6,08 & $-2,6$ \\
\hline 288 & 0,58 & 3,02 & 1,11 & 0,66 & 11,06 & $-3,5$ \\
\hline 289 & 3,03 & 8,19 & 2,48 & 1,55 & 24,80 & $-4,6$ \\
\hline 290 & 1,48 & 4,90 & 1,82 & 1,04 & 18,19 & $-4,2$ \\
\hline 291 & 0,82 & 3,77 & 1,53 & 0,68 & 15,27 & $-3,9$ \\
\hline 292 & 0,08 & 1,15 & 0,45 & 0,23 & 4,50 & $-2,2$ \\
\hline 293 & 0,04 & 0,86 & 0,36 & 0,13 & 3,63 & $-1,9$ \\
\hline 294 & 0,21 & 2,60 & 1,16 & 0,23 & 11,61 & $-3,5$ \\
\hline 295 & 0,44 & 2,69 & 0,98 & 0,57 & 9,79 & $-3,3$ \\
\hline 296 & 0,09 & 1,09 & 0,45 & 0,25 & 4,51 & $-2,2$ \\
\hline 297 & 0,03 & 0,72 & 0,30 & 0,14 & 3,04 & $-1,6$ \\
\hline 298 & 1,37 & 4,86 & 2,03 & 0,86 & 20,29 & $-4,3$ \\
\hline 299 & 0,04 & 0,86 & 0,36 & 0,13 & 3,63 & $-1,9$ \\
\hline 300 & 1,47 & 4,82 & 1,59 & 1,18 & 15,85 & $-4,0$ \\
\hline 301 & 0,08 & 1,09 & 0,34 & 0,32 & 3,35 & $-1,7$ \\
\hline
\end{tabular}




\begin{tabular}{|c|c|c|c|c|c|c|}
\hline 302 & 0,85 & 3,66 & 1,33 & 0,81 & 13,33 & $-3,7$ \\
\hline 303 & 3,95 & 8,02 & 2,77 & 1,81 & 27,72 & $-4,8$ \\
\hline 304 & 1,22 & 4,49 & 1,65 & 0,94 & 16,49 & $-4,0$ \\
\hline 305 & 0,03 & 0,62 & 0,27 & 0,13 & 2,73 & $-1,4$ \\
\hline 306 & 24,02 & 21,14 & 6,88 & 4,45 & 68,78 & $-6,1$ \\
\hline 307 & 0,67 & 3,36 & 1,21 & 0,71 & 12,07 & $-3,6$ \\
\hline 308 & 0,45 & 2,82 & 1,10 & 0,52 & 10,96 & $-3,5$ \\
\hline 309 & 34,27 & 24,97 & 8,05 & 5,42 & 80,52 & $-6,3$ \\
\hline 310 & 0,99 & 4,34 & 1,77 & 0,71 & 17,70 & $-4,1$ \\
\hline 311 & 0,15 & 1,32 & 0,44 & 0,42 & 4,37 & $-2,1$ \\
\hline 312 & 1,14 & 5,07 & 1,56 & 0,93 & 15,55 & $-4,0$ \\
\hline 313 & 0,88 & 4,61 & 1,69 & 0,66 & 16,92 & $-4,1$ \\
\hline 314 & 1,13 & 4,16 & 1,46 & 0,98 & 14,64 & $-3,9$ \\
\hline 315 & 1,11 & 3,96 & 1,27 & 1,11 & 12,68 & $-3,7$ \\
\hline 316 & 0,17 & 1,52 & 0,60 & 0,37 & 5,98 & $-2,6$ \\
\hline 317 & 0,51 & 3,09 & 1,13 & 0,57 & 11,31 & $-3,5$ \\
\hline 318 & 7,40 & 11,01 & 3,92 & 2,40 & 39,22 & $-5,3$ \\
\hline 319 & 1,50 & 5,51 & 1,83 & 1,04 & 18,32 & $-4,2$ \\
\hline 320 & 0,86 & 3,79 & 1,42 & 0,77 & 14,19 & $-3,8$ \\
\hline 321 & 0,89 & 4,08 & 1,55 & 0,73 & 15,50 & $-4,0$ \\
\hline 322 & 0,60 & 3,23 & 0,97 & 0,79 & 9,67 & $-3,3$ \\
\hline 323 & 0,04 & 0,72 & 0,29 & 0,16 & 2,91 & $-1,5$ \\
\hline 324 & 0,31 & 2,49 & 1,05 & 0,37 & 10,52 & $-3,4$ \\
\hline 325 & 1,90 & 5,36 & 1,81 & 1,34 & 18,10 & $-4,2$ \\
\hline 326 & 2,19 & 7,14 & 2,94 & 0,95 & 29,42 & $-4,9$ \\
\hline 327 & 0,13 & 1,44 & 0,48 & 0,35 & 4,79 & $-2,3$ \\
\hline 328 & 0,12 & 1,42 & 0,59 & 0,26 & 5,91 & $-2,6$ \\
\hline 329 & 1,04 & 3,81 & 1,30 & 1,02 & 13,01 & $-3,7$ \\
\hline 330 & 0,11 & 1,28 & 0,44 & 0,31 & 4,38 & $-2,1$ \\
\hline 331 & 0,37 & 2,37 & 0,86 & 0,54 & 8,59 & $-3,1$ \\
\hline 332 & 0,53 & 2,83 & 1,08 & 0,63 & 10,75 & $-3,4$ \\
\hline 333 & 0,10 & 1,23 & 0,49 & 0,26 & 4,91 & $-2,3$ \\
\hline 334 & 0,18 & 1,90 & 0,80 & 0,29 & 7,99 & $-3,0$ \\
\hline 335 & 0,48 & 3,06 & 1,27 & 0,48 & 12,66 & $-3,7$ \\
\hline 336 & 0,26 & 2,14 & 0,77 & 0,43 & 7,72 & $-2,9$ \\
\hline 337 & 10,76 & 15,08 & 5,99 & 2,29 & 59,90 & $-5,9$ \\
\hline 338 & 0,17 & 1,56 & 0,63 & 0,34 & 6,25 & $-2,6$ \\
\hline 339 & 13,99 & 15,17 & 4,58 & 3,89 & 45,79 & $-5,5$ \\
\hline 340 & 0,11 & 1,52 & 0,65 & 0,21 & 6,54 & $-2,7$ \\
\hline 341 & 0,17 & 1,63 & 0,63 & 0,35 & 6,30 & $-2,7$ \\
\hline 342 & 3,52 & 8,75 & 2,67 & 1,68 & 26,73 & $-4,7$ \\
\hline 343 & 0,12 & 1,59 & 0,73 & 0,21 & 7,32 & $-2,9$ \\
\hline 344 & 0,02 & 0,61 & 0,31 & 0,08 & 3,09 & $-1,6$ \\
\hline 345 & 6,80 & 12,04 & 4,76 & 1,82 & 47,58 & $-5,6$ \\
\hline 346 & 0,23 & 1,83 & 0,74 & 0,39 & 7,44 & $-2,9$ \\
\hline 347 & 0,13 & 1,38 & 0,48 & 0,35 & 4,80 & $-2,3$ \\
\hline 348 & 7,54 & 11,79 & 4,44 & 2,16 & 44,41 & $-5,5$ \\
\hline 349 & 0,33 & 2,61 & 0,86 & 0,50 & 8,57 & $-3,1$ \\
\hline 350 & 0,05 & 0,82 & 0,31 & 0,21 & 3,09 & $-1,6$ \\
\hline 351 & 0,06 & 1,15 & 0,46 & 0,17 & 4,61 & $-2,2$ \\
\hline 352 & 5,44 & 10,76 & 4,59 & 1,51 & 45,92 & $-5,5$ \\
\hline 353 & 0,28 & 2,28 & 0,82 & 0,43 & 8,20 & $-3,0$ \\
\hline 354 & 0,17 & 1,86 & 0,77 & 0,28 & 7,72 & $-2,9$ \\
\hline 355 & 0,04 & 0,76 & 0,29 & 0,19 & 2,88 & $-1,5$ \\
\hline 356 & 0,05 & 0,76 & 0,30 & 0,22 & 3,00 & $-1,6$ \\
\hline 357 & 0,25 & 2,28 & 0,97 & 0,33 & 9,67 & $-3,3$ \\
\hline 358 & 2,11 & 6,44 & 2,47 & 1,09 & 24,70 & $-4,6$ \\
\hline 359 & 0,16 & 1,67 & 0,57 & 0,37 & 5,68 & $-2,5$ \\
\hline 360 & 0,10 & 1,23 & 0,48 & 0,26 & 4,82 & $-2,3$ \\
\hline 361 & 0,16 & 1,44 & 0,52 & 0,41 & 5,16 & $-2,4$ \\
\hline 362 & 0,97 & 4,01 & 1,55 & 0,79 & 15,54 & $-4,0$ \\
\hline 363 & 0,05 & 0,82 & 0,26 & 0,25 & 2,61 & $-1,4$ \\
\hline
\end{tabular}




\begin{tabular}{|c|c|c|c|c|c|c|}
\hline 364 & 0,56 & 3,51 & 1,41 & 0,50 & 14,13 & $-3,8$ \\
\hline 365 & 0,33 & 2,35 & 0,99 & 0,42 & 9,85 & $-3,3$ \\
\hline 366 & 0,82 & 4,61 & 1,80 & 0,58 & 18,00 & $-4,2$ \\
\hline 367 & 0,29 & 2,22 & 0,86 & 0,42 & 8,64 & $-3,1$ \\
\hline 368 & 0,15 & 1,48 & 0,47 & 0,41 & 4,71 & $-2,2$ \\
\hline 369 & 0,07 & 0,91 & 0,36 & 0,25 & 3,59 & $-1,8$ \\
\hline 370 & 0,20 & 1,65 & 0,60 & 0,42 & 5,95 & $-2,6$ \\
\hline 371 & 9,29 & 11,46 & 3,55 & 3,34 & 35,45 & $-5,1$ \\
\hline 372 & 0,11 & 1,28 & 0,49 & 0,29 & 4,89 & $-2,3$ \\
\hline 373 & 6,03 & 9,38 & 3,05 & 2,52 & 30,53 & $-4,9$ \\
\hline 374 & 3,99 & 8,15 & 3,10 & 1,64 & 31,01 & $-5,0$ \\
\hline 375 & 0,16 & 1,53 & 0,55 & 0,38 & 5,50 & $-2,5$ \\
\hline 376 & 8,91 & 11,98 & 3,78 & 3,00 & 37,84 & $-5,2$ \\
\hline 377 & 0,19 & 1,68 & 0,62 & 0,40 & 6,20 & $-2,6$ \\
\hline 378 & 6,51 & 12,10 & 4,91 & 1,69 & 49,06 & $-5,6$ \\
\hline 379 & 0,02 & 0,52 & 0,22 & 0,14 & 2,15 & $-1,1$ \\
\hline 380 & 0,07 & 0,95 & 0,35 & 0,25 & 3,53 & $-1,8$ \\
\hline 381 & 0,04 & 0,72 & 0,31 & 0,17 & 3,13 & $-1,6$ \\
\hline 382 & 0,22 & 1,92 & 0,80 & 0,35 & 7,95 & $-3,0$ \\
\hline 383 & 0,06 & 0,90 & 0,32 & 0,25 & 3,17 & $-1,7$ \\
\hline 384 & 0,06 & 0,95 & 0,40 & 0,18 & 4,04 & $-2,0$ \\
\hline 385 & 0,04 & 0,72 & 0,30 & 0,16 & 3,04 & $-1,6$ \\
\hline 386 & 0,60 & 2,91 & 0,96 & 0,79 & 9,57 & $-3,3$ \\
\hline 387 & 0,09 & 1,11 & 0,35 & 0,34 & 3,48 & $-1,8$ \\
\hline 388 & 0,04 & 0,80 & 0,36 & 0,15 & 3,58 & $-1,8$ \\
\hline 389 & 0,10 & 1,24 & 0,48 & 0,27 & 4,83 & $-2,3$ \\
\hline 390 & 0,02 & 0,39 & 0,16 & 0,16 & 1,55 & $-0,6$ \\
\hline 391 & 0,31 & 2,29 & 0,94 & 0,41 & 9,40 & $-3,2$ \\
\hline 392 & 5,17 & 9,39 & 2,90 & 2,27 & 29,02 & $-4,9$ \\
\hline 393 & 1,87 & 5,43 & 1,76 & 1,35 & 17,60 & $-4,1$ \\
\hline 394 & 0,35 & 2,41 & 0,71 & 0,63 & 7,10 & $-2,8$ \\
\hline 395 & 1,27 & 4,65 & 1,76 & 0,92 & 17,63 & $-4,1$ \\
\hline 396 & 0,11 & 1,28 & 0,39 & 0,35 & 3,92 & $-2,0$ \\
\hline 397 & 0,32 & 2,20 & 0,91 & 0,45 & 9,07 & $-3,2$ \\
\hline 398 & 2,69 & 7,59 & 2,56 & 1,34 & 25,63 & $-4,7$ \\
\hline 399 & 0,12 & 1,19 & 0,44 & 0,34 & 4,39 & $-2,1$ \\
\hline 400 & 0,06 & 0,95 & 0,39 & 0,20 & 3,90 & $-2,0$ \\
\hline 401 & 0,14 & 1,42 & 0,56 & 0,32 & 5,60 & $-2,5$ \\
\hline 402 & 0,57 & 3,51 & 1,40 & 0,52 & 13,97 & $-3,8$ \\
\hline 403 & 12,90 & 16,32 & 6,12 & 2,68 & 61,18 & $-5,9$ \\
\hline 404 & 0,01 & 0,39 & 0,16 & 0,11 & 1,62 & $-0,7$ \\
\hline 405 & 0,03 & 0,58 & 0,26 & 0,14 & 2,55 & $-1,4$ \\
\hline 406 & 0,25 & 2,14 & 0,86 & 0,38 & 8,59 & $-3,1$ \\
\hline 407 & 0,26 & 1,92 & 0,74 & 0,46 & 7,35 & $-2,9$ \\
\hline 408 & 0,34 & 2,20 & 0,83 & 0,52 & 8,26 & $-3,0$ \\
\hline 409 & 0,80 & 3,52 & 1,32 & 0,77 & 13,15 & $-3,7$ \\
\hline 410 & 0,35 & 2,37 & 0,95 & 0,47 & 9,45 & $-3,2$ \\
\hline 411 & 0,32 & 2,06 & 0,77 & 0,53 & 7,70 & $-2,9$ \\
\hline 412 & 0,17 & 1,90 & 0,69 & 0,32 & 6,90 & $-2,8$ \\
\hline 413 & 1,47 & 4,61 & 1,57 & 1,19 & 15,71 & $-4,0$ \\
\hline 414 & 0,02 & 0,52 & 0,22 & 0,11 & 2,19 & $-1,1$ \\
\hline 415 & 0,13 & 1,46 & 0,45 & 0,37 & 4,52 & $-2,2$ \\
\hline 416 & 0,02 & 0,48 & 0,22 & 0,14 & 2,15 & $-1,1$ \\
\hline 417 & 3,12 & 7,69 & 2,84 & 1,40 & 28,43 & $-4,8$ \\
\hline 418 & 29,78 & 22,60 & 7,86 & 4,82 & 78,60 & $-6,3$ \\
\hline 419 & 0,21 & 1,82 & 0,64 & 0,42 & 6,35 & $-2,7$ \\
\hline 420 & 0,60 & 2,93 & 0,91 & 0,83 & 9,14 & $-3,2$ \\
\hline 421 & 0,30 & 2,11 & 0,87 & 0,44 & 8,68 & $-3,1$ \\
\hline 422 & 24,69 & 22,83 & 7,44 & 4,22 & 74,43 & $-6,2$ \\
\hline 423 & 0,12 & 1,34 & 0,54 & 0,28 & 5,43 & $-2,4$ \\
\hline 424 & 0,06 & 0,99 & 0,44 & 0,18 & 4,35 & $-2,1$ \\
\hline 425 & 0,16 & 1,86 & 0,84 & 0,25 & 8,42 & $-3,1$ \\
\hline
\end{tabular}




\begin{tabular}{|c|c|c|c|c|c|c|}
\hline 426 & 28,73 & 21,95 & 6,73 & 5,44 & 67,26 & $-6,1$ \\
\hline 427 & 0,05 & 0,87 & 0,41 & 0,15 & 4,07 & $-2,0$ \\
\hline 428 & 12,01 & 15,50 & 5,62 & 2,72 & 56,15 & $-5,8$ \\
\hline 429 & 41,43 & 27,51 & 8,76 & 6,02 & 87,63 & $-6,5$ \\
\hline 430 & 0,16 & 1,48 & 0,48 & 0,44 & 4,78 & $-2,3$ \\
\hline 431 & 0,22 & 1,75 & 0,69 & 0,40 & 6,91 & $-2,8$ \\
\hline 432 & 0,03 & 0,66 & 0,28 & 0,15 & 2,84 & $-1,5$ \\
\hline 433 & 1,40 & 6,54 & 2,99 & 0,60 & 29,90 & $-4,9$ \\
\hline 434 & 1,31 & 4,97 & 2,08 & 0,80 & 20,77 & $-4,4$ \\
\hline 435 & 0,01 & 0,39 & 0,16 & 0,11 & 1,62 & $-0,7$ \\
\hline 436 & 0,16 & 1,69 & 0,67 & 0,30 & 6,67 & $-2,7$ \\
\hline 437 & 0,23 & 1,79 & 0,69 & 0,43 & 6,85 & $-2,8$ \\
\hline 438 & 0,26 & 1,94 & 0,72 & 0,45 & 7,24 & $-2,9$ \\
\hline 439 & 0,02 & 0,52 & 0,22 & 0,14 & 2,15 & $-1,1$ \\
\hline 440 & 0,90 & 3,91 & 1,33 & 0,86 & 13,28 & $-3,7$ \\
\hline 441 & 0,04 & 0,68 & 0,30 & 0,16 & 3,04 & $-1,6$ \\
\hline 442 & 1,91 & 5,53 & 2,01 & 1,21 & 20,10 & $-4,3$ \\
\hline 443 & 2,88 & 7,33 & 2,13 & 1,72 & 21,28 & $-4,4$ \\
\hline 444 & 0,42 & 2,53 & 0,91 & 0,59 & 9,07 & $-3,2$ \\
\hline 445 & 5,86 & 9,67 & 3,32 & 2,25 & 33,24 & $-5,1$ \\
\hline 446 & 0,04 & 0,80 & 0,36 & 0,15 & 3,58 & $-1,8$ \\
\hline 447 & 0,02 & 0,57 & 0,31 & 0,08 & 3,09 & $-1,6$ \\
\hline 448 & 0,03 & 0,62 & 0,26 & 0,14 & 2,55 & $-1,4$ \\
\hline 449 & 0,03 & 0,58 & 0,26 & 0,16 & 2,58 & $-1,4$ \\
\hline 450 & 0,01 & 0,33 & 0,16 & 0,08 & 1,55 & $-0,6$ \\
\hline 451 & 0,01 & 0,39 & 0,16 & 0,11 & 1,62 & $-0,7$ \\
\hline 452 & 0,01 & 0,33 & 0,16 & 0,08 & 1,55 & $-0,6$ \\
\hline 453 & 0,03 & 0,72 & 0,32 & 0,13 & 3,17 & $-1,7$ \\
\hline 454 & 0,06 & 1,16 & 0,43 & 0,18 & 4,27 & $-2,1$ \\
\hline 455 & 0,01 & 0,29 & 0,16 & 0,08 & 1,55 & $-0,6$ \\
\hline 456 & 0,21 & 1,77 & 0,62 & 0,44 & 6,17 & $-2,6$ \\
\hline 457 & 0,01 & 0,19 & 0,08 & 0,08 & 0,77 & 0,4 \\
\hline 458 & 1,73 & 5,70 & 2,00 & 1,10 & 19,99 & $-4,3$ \\
\hline 459 & 0,07 & 0,91 & 0,35 & 0,24 & 3,50 & $-1,8$ \\
\hline 460 & 21,59 & 18,94 & 6,31 & 4,35 & 63,13 & $-6,0$ \\
\hline 461 & 0,26 & 1,83 & 0,60 & 0,55 & 6,03 & $-2,6$ \\
\hline 462 & 0,16 & 3,02 & 1,26 & 0,17 & 12,56 & $-3,7$ \\
\hline 463 & 0,14 & 1,46 & 0,63 & 0,28 & 6,33 & $-2,7$ \\
\hline 464 & 0,16 & 1,52 & 0,49 & 0,41 & 4,86 & $-2,3$ \\
\hline 465 & 1,33 & 4,59 & 1,54 & 1,10 & 15,43 & $-3,9$ \\
\hline 466 & 0,07 & 1,01 & 0,43 & 0,20 & 4,30 & $-2,1$ \\
\hline 467 & 0,90 & 3,59 & 1,19 & 0,96 & 11,90 & $-3,6$ \\
\hline 468 & 0,62 & 3,29 & 1,37 & 0,57 & 13,73 & $-3,8$ \\
\hline 469 & 0,04 & 0,62 & 0,24 & 0,20 & 2,42 & $-1,3$ \\
\hline 470 & 0,06 & 0,95 & 0,38 & 0,20 & 3,81 & $-1,9$ \\
\hline 471 & 0,05 & 0,72 & 0,30 & 0,20 & 3,04 & $-1,6$ \\
\hline 472 & 2,36 & 6,23 & 2,12 & 1,42 & 21,23 & $-4,4$ \\
\hline 473 & 0,56 & 3,15 & 1,20 & 0,60 & 11,99 & $-3,6$ \\
\hline 474 & 0,18 & 1,79 & 0,75 & 0,31 & 7,48 & $-2,9$ \\
\hline 475 & 3,59 & 7,49 & 2,31 & 1,98 & 23,11 & $-4,5$ \\
\hline 476 & 5,27 & 9,50 & 3,33 & 2,02 & 33,30 & $-5,1$ \\
\hline 477 & 0,03 & 0,87 & 0,40 & 0,09 & 3,96 & $-2,0$ \\
\hline 478 & 0,11 & 1,23 & 0,45 & 0,32 & 4,54 & $-2,2$ \\
\hline 479 & 12,66 & 15,14 & 4,97 & 3,25 & 49,67 & $-5,6$ \\
\hline 480 & 2,88 & 6,57 & 2,10 & 1,75 & 20,95 & $-4,4$ \\
\hline 481 & 0,10 & 1,28 & 0,49 & 0,25 & 4,94 & $-2,3$ \\
\hline 482 & 0,19 & 1,81 & 0,73 & 0,33 & 7,28 & $-2,9$ \\
\hline 483 & 0,03 & 0,80 & 0,34 & 0,12 & 3,38 & $-1,8$ \\
\hline 484 & 0,05 & 0,82 & 0,31 & 0,21 & 3,09 & $-1,6$ \\
\hline 485 & 0,17 & 1,53 & 0,61 & 0,35 & 6,13 & $-2,6$ \\
\hline 486 & 6,16 & 10,87 & 3,23 & 2,43 & 32,25 & $-5,0$ \\
\hline 487 & 7,29 & 11,16 & 4,12 & 2,25 & 41,22 & $-5,4$ \\
\hline
\end{tabular}




\begin{tabular}{|c|c|c|c|c|c|c|}
\hline 488 & 0,12 & 1,48 & 0,57 & 0,27 & 5,71 & $-2,5$ \\
\hline 489 & 0,35 & 3,16 & 1,48 & 0,30 & 14,83 & $-3,9$ \\
\hline 490 & 0,09 & 1,05 & 0,40 & 0,29 & 3,97 & $-2,0$ \\
\hline 491 & 0,01 & 0,19 & 0,08 & 0,08 & 0,77 & 0,4 \\
\hline 492 & 0,96 & 3,67 & 1,29 & 0,95 & 12,87 & $-3,7$ \\
\hline 493 & 0,40 & 2,33 & 0,87 & 0,59 & 8,67 & $-3,1$ \\
\hline 494 & 0,14 & 1,38 & 0,51 & 0,34 & 5,06 & $-2,3$ \\
\hline 495 & 0,10 & 1,24 & 0,47 & 0,28 & 4,66 & $-2,2$ \\
\hline 496 & 10,26 & 14,14 & 5,14 & 2,54 & 51,41 & $-5,7$ \\
\hline 497 & 0,14 & 1,52 & 0,58 & 0,30 & 5,79 & $-2,5$ \\
\hline 498 & 0,22 & 1,79 & 0,61 & 0,45 & 6,14 & $-2,6$ \\
\hline 499 & 0,03 & 0,72 & 0,31 & 0,13 & 3,13 & $-1,6$ \\
\hline 500 & 0,71 & 3,13 & 1,03 & 0,88 & 10,26 & $-3,4$ \\
\hline 501 & 0,03 & 0,58 & 0,26 & 0,16 & 2,58 & $-1,4$ \\
\hline 502 & 0,25 & 1,92 & 0,66 & 0,49 & 6,55 & $-2,7$ \\
\hline 503 & 9,91 & 13,97 & 5,23 & 2,41 & 52,33 & $-5,7$ \\
\hline 504 & 0,16 & 1,61 & 0,59 & 0,34 & 5,92 & $-2,6$ \\
\hline 505 & 0,07 & 1,01 & 0,42 & 0,20 & 4,23 & $-2,1$ \\
\hline 506 & 10,80 & 13,54 & 4,32 & 3,18 & 43,20 & $-5,4$ \\
\hline 507 & 0,01 & 0,33 & 0,16 & 0,08 & 1,55 & $-0,6$ \\
\hline 508 & 0,01 & 0,39 & 0,18 & 0,07 & 1,78 & $-0,8$ \\
\hline 509 & 0,03 & 0,68 & 0,25 & 0,15 & 2,45 & $-1,3$ \\
\hline 510 & 0,54 & 2,95 & 1,14 & 0,60 & 11,44 & $-3,5$ \\
\hline 511 & 0,38 & 2,49 & 0,92 & 0,52 & 9,16 & $-3,2$ \\
\hline 512 & 0,02 & 0,52 & 0,22 & 0,14 & 2,15 & $-1,1$ \\
\hline 513 & 4,16 & 7,81 & 2,46 & 2,15 & 24,57 & $-4,6$ \\
\hline 514 & 0,06 & 0,91 & 0,35 & 0,22 & 3,52 & $-1,8$ \\
\hline 515 & 0,01 & 0,39 & 0,16 & 0,11 & 1,62 & $-0,7$ \\
\hline 516 & 0,01 & 0,39 & 0,16 & 0,11 & 1,62 & $-0,7$ \\
\hline 517 & 0,07 & 0,91 & 0,34 & 0,26 & 3,41 & $-1,8$ \\
\hline 518 & 0,19 & 1,69 & 0,62 & 0,40 & 6,20 & $-2,6$ \\
\hline 519 & 0,07 & 0,95 & 0,39 & 0,23 & 3,85 & $-1,9$ \\
\hline 520 & 0,31 & 2,31 & 0,90 & 0,45 & 8,96 & $-3,2$ \\
\hline 521 & 15,90 & 15,70 & 4,96 & 4,08 & 49,61 & $-5,6$ \\
\hline 522 & 0,09 & 1,15 & 0,46 & 0,26 & 4,59 & $-2,2$ \\
\hline 523 & 0,16 & 1,81 & 0,82 & 0,25 & 8,19 & $-3,0$ \\
\hline 524 & 0,64 & 3,23 & 1,12 & 0,73 & 11,23 & $-3,5$ \\
\hline 525 & 0,02 & 0,72 & 0,27 & 0,11 & 2,74 & $-1,5$ \\
\hline 526 & 0,06 & 0,86 & 0,34 & 0,21 & 3,42 & $-1,8$ \\
\hline 527 & 0,03 & 0,62 & 0,27 & 0,13 & 2,73 & $-1,4$ \\
\hline 528 & 0,03 & 0,58 & 0,26 & 0,16 & 2,58 & $-1,4$ \\
\hline 529 & 0,04 & 0,72 & 0,27 & 0,20 & 2,68 & $-1,4$ \\
\hline 530 & 0,04 & 0,72 & 0,28 & 0,17 & 2,77 & $-1,5$ \\
\hline 531 & 0,06 & 0,95 & 0,41 & 0,19 & 4,08 & $-2,0$ \\
\hline 532 & 0,01 & 0,33 & 0,16 & 0,08 & 1,55 & $-0,6$ \\
\hline 533 & 0,01 & 0,33 & 0,16 & 0,08 & 1,55 & $-0,6$ \\
\hline 534 & 0,01 & 0,29 & 0,16 & 0,08 & 1,55 & $-0,6$ \\
\hline 535 & 0,01 & 0,39 & 0,16 & 0,11 & 1,62 & $-0,7$ \\
\hline 536 & 0,03 & 0,58 & 0,26 & 0,16 & 2,58 & $-1,4$ \\
\hline 537 & 0,03 & 0,72 & 0,24 & 0,17 & 2,41 & $-1,3$ \\
\hline 538 & 0,15 & 1,38 & 0,48 & 0,39 & 4,81 & $-2,3$ \\
\hline 539 & 0,05 & 0,91 & 0,35 & 0,17 & 3,54 & $-1,8$ \\
\hline 540 & 0,01 & 0,33 & 0,16 & 0,08 & 1,55 & $-0,6$ \\
\hline 541 & 9,08 & 12,26 & 4,62 & 2,50 & 46,15 & $-5,5$ \\
\hline 542 & 0,16 & 1,63 & 0,52 & 0,38 & 5,18 & $-2,4$ \\
\hline 543 & 1,57 & 4,94 & 1,78 & 1,12 & 17,82 & $-4,2$ \\
\hline 544 & 0,12 & 1,23 & 0,42 & 0,35 & 4,24 & $-2,1$ \\
\hline 545 & 0,02 & 0,66 & 0,27 & 0,09 & 2,72 & $-1,4$ \\
\hline 546 & 0,01 & 0,33 & 0,16 & 0,08 & 1,55 & $-0,6$ \\
\hline 547 & 0,02 & 0,66 & 0,28 & 0,11 & 2,79 & $-1,5$ \\
\hline 548 & 0,06 & 0,95 & 0,38 & 0,20 & 3,81 & $-1,9$ \\
\hline 549 & 0,04 & 0,76 & 0,34 & 0,14 & 3,38 & $-1,8$ \\
\hline
\end{tabular}




\begin{tabular}{|c|c|c|c|c|c|c|}
\hline 550 & 0,92 & 4,06 & 1,17 & 1,00 & 11,67 & $-3,5$ \\
\hline 551 & 0,02 & 0,48 & 0,22 & 0,11 & 2,19 & $-1,1$ \\
\hline 552 & 0,05 & 0,86 & 0,31 & 0,19 & 3,14 & $-1,7$ \\
\hline 553 & 2,30 & 6,23 & 2,11 & 1,39 & 21,05 & $-4,4$ \\
\hline 554 & 0,08 & 1,36 & 0,58 & 0,19 & 5,76 & $-2,5$ \\
\hline 555 & 1,96 & 6,06 & 2,21 & 1,13 & 22,07 & $-4,5$ \\
\hline 556 & 0,03 & 0,62 & 0,27 & 0,13 & 2,73 & $-1,4$ \\
\hline 557 & 0,09 & 1,09 & 0,38 & 0,32 & 3,77 & $-1,9$ \\
\hline 558 & 0,01 & 0,39 & 0,16 & 0,11 & 1,62 & $-0,7$ \\
\hline 559 & 0,06 & 0,86 & 0,30 & 0,24 & 2,95 & $-1,6$ \\
\hline 560 & 2,95 & 6,88 & 2,17 & 1,73 & 21,73 & $-4,4$ \\
\hline 561 & 3,26 & 8,85 & 2,87 & 1,44 & 28,74 & $-4,8$ \\
\hline 562 & 1,79 & 6,52 & 2,67 & 0,85 & 26,72 & $-4,7$ \\
\hline 563 & 35,76 & 32,28 & 12,47 & 3,65 & 124,74 & $-7,0$ \\
\hline 564 & 0,04 & 0,72 & 0,29 & 0,16 & 2,93 & $-1,6$ \\
\hline 565 & 0,23 & 2,25 & 0,98 & 0,30 & 9,76 & $-3,3$ \\
\hline 566 & 0,09 & 1,09 & 0,40 & 0,28 & 4,00 & $-2,0$ \\
\hline 567 & 2,94 & 6,49 & 2,19 & 1,71 & 21,90 & $-4,5$ \\
\hline 568 & 0,37 & 2,47 & 0,96 & 0,49 & 9,57 & $-3,3$ \\
\hline 569 & 0,02 & 0,48 & 0,22 & 0,11 & 2,20 & $-1,1$ \\
\hline 570 & 0,18 & 1,61 & 0,58 & 0,41 & 5,75 & $-2,5$ \\
\hline 571 & 0,04 & 0,72 & 0,25 & 0,21 & 2,52 & $-1,3$ \\
\hline 572 & 0,16 & 1,57 & 0,55 & 0,38 & 5,50 & $-2,5$ \\
\hline 573 & 0,74 & 3,43 & 1,28 & 0,74 & 12,84 & $-3,7$ \\
\hline 574 & 0,02 & 0,52 & 0,22 & 0,11 & 2,20 & $-1,1$ \\
\hline 575 & 0,06 & 0,95 & 0,33 & 0,24 & 3,27 & $-1,7$ \\
\hline 576 & 0,18 & 1,57 & 0,55 & 0,42 & 5,45 & $-2,4$ \\
\hline 577 & 0,04 & 0,72 & 0,27 & 0,20 & 2,68 & $-1,4$ \\
\hline 578 & 0,01 & 0,39 & 0,16 & 0,11 & 1,62 & $-0,7$ \\
\hline 579 & 0,01 & 0,47 & 0,23 & 0,08 & 2,32 & $-1,2$ \\
\hline 580 & 0,26 & 2,04 & 0,66 & 0,51 & 6,57 & $-2,7$ \\
\hline 581 & 0,17 & 1,71 & 0,65 & 0,33 & 6,50 & $-2,7$ \\
\hline 582 & 0,02 & 0,48 & 0,19 & 0,13 & 1,87 & $-0,9$ \\
\hline 583 & 0,06 & 0,99 & 0,38 & 0,21 & 3,77 & $-1,9$ \\
\hline 584 & 0,10 & 1,15 & 0,39 & 0,33 & 3,85 & $-1,9$ \\
\hline 585 & 0,14 & 1,59 & 0,71 & 0,25 & 7,05 & $-2,8$ \\
\hline 586 & 0,02 & 0,48 & 0,19 & 0,13 & 1,87 & $-0,9$ \\
\hline 587 & 0,12 & 1,28 & 0,53 & 0,30 & 5,26 & $-2,4$ \\
\hline 588 & 0,01 & 0,52 & 0,22 & 0,08 & 2,20 & $-1,1$ \\
\hline 589 & 1,54 & 5,09 & 1,90 & 1,03 & 19,04 & $-4,3$ \\
\hline 590 & 15,72 & 17,27 & 5,27 & 3,80 & 52,73 & $-5,7$ \\
\hline 591 & 0,06 & 0,87 & 0,30 & 0,24 & 3,03 & $-1,6$ \\
\hline 592 & 0,84 & 3,60 & 1,32 & 0,81 & 13,16 & $-3,7$ \\
\hline 593 & 0,07 & 1,16 & 0,52 & 0,16 & 5,15 & $-2,4$ \\
\hline 594 & 2,06 & 5,85 & 2,07 & 1,27 & 20,69 & $-4,4$ \\
\hline 595 & 0,08 & 1,13 & 0,49 & 0,20 & 4,85 & $-2,3$ \\
\hline 596 & 3,36 & 7,73 & 2,90 & 1,48 & 28,99 & $-4,9$ \\
\hline 597 & 0,08 & 1,56 & 0,67 & 0,14 & 6,67 & $-2,7$ \\
\hline 598 & 0,20 & 1,75 & 0,72 & 0,35 & 7,24 & $-2,9$ \\
\hline 599 & 0,03 & 0,68 & 0,29 & 0,12 & 2,93 & $-1,6$ \\
\hline 600 & 0,02 & 0,80 & 0,35 & 0,09 & 3,48 & $-1,8$ \\
\hline 601 & 0,01 & 0,19 & 0,08 & 0,08 & 0,77 & 0,4 \\
\hline 602 & 8,29 & 12,39 & 4,77 & 2,21 & 47,66 & $-5,6$ \\
\hline 603 & 0,05 & 0,76 & 0,31 & 0,20 & 3,05 & $-1,6$ \\
\hline 604 & 0,11 & 1,44 & 0,47 & 0,31 & 4,69 & $-2,2$ \\
\hline 605 & 2,85 & 6,67 & 2,26 & 1,61 & 22,61 & $-4,5$ \\
\hline 606 & 0,17 & 1,65 & 0,54 & 0,40 & 5,44 & $-2,4$ \\
\hline 607 & 0,10 & 1,19 & 0,47 & 0,28 & 4,69 & $-2,2$ \\
\hline 608 & 73,95 & 36,49 & 10,14 & 9,29 & 101,38 & $-6,7$ \\
\hline 609 & 11,84 & 14,20 & 4,77 & 3,16 & 47,67 & $-5,6$ \\
\hline 610 & 0,02 & 0,58 & 0,21 & 0,14 & 2,11 & $-1,1$ \\
\hline 611 & 0,04 & 0,68 & 0,26 & 0,18 & 2,60 & $-1,4$ \\
\hline
\end{tabular}




\begin{tabular}{|c|c|c|c|c|c|c|}
\hline 612 & 0,01 & 0,29 & 0,16 & 0,08 & 1,55 & $-0,6$ \\
\hline 613 & 0,02 & 0,48 & 0,22 & 0,11 & 2,20 & $-1,1$ \\
\hline 614 & 0,18 & 2,02 & 0,79 & 0,30 & 7,86 & $-3,0$ \\
\hline 615 & 0,06 & 0,95 & 0,38 & 0,20 & 3,84 & $-1,9$ \\
\hline 616 & 0,02 & 0,52 & 0,22 & 0,14 & 2,15 & $-1,1$ \\
\hline 617 & 0,59 & 2,95 & 1,08 & 0,69 & 10,76 & $-3,4$ \\
\hline 618 & 0,01 & 0,58 & 0,27 & 0,07 & 2,72 & $-1,4$ \\
\hline 619 & 0,01 & 0,39 & 0,16 & 0,11 & 1,62 & $-0,7$ \\
\hline 620 & 0,05 & 0,72 & 0,28 & 0,21 & 2,82 & $-1,5$ \\
\hline 621 & 51,17 & 27,87 & 9,74 & 6,69 & 97,39 & $-6,6$ \\
\hline 622 & 0,05 & 0,76 & 0,30 & 0,22 & 3,00 & $-1,6$ \\
\hline 623 & 0,05 & 0,72 & 0,30 & 0,20 & 3,04 & $-1,6$ \\
\hline 624 & 0,33 & 2,34 & 0,99 & 0,43 & 9,85 & $-3,3$ \\
\hline 625 & 0,01 & 0,19 & 0,08 & 0,08 & 0,77 & 0,4 \\
\hline 626 & 0,01 & 0,39 & 0,18 & 0,07 & 1,78 & $-0,8$ \\
\hline 627 & 1,08 & 5,27 & 2,24 & 0,61 & 22,37 & $-4,5$ \\
\hline 628 & 0,01 & 0,43 & 0,23 & 0,08 & 2,32 & $-1,2$ \\
\hline 629 & 0,18 & 1,61 & 0,57 & 0,40 & 5,69 & $-2,5$ \\
\hline 630 & 0,39 & 2,33 & 0,79 & 0,64 & 7,90 & $-3,0$ \\
\hline 631 & 0,33 & 2,20 & 0,83 & 0,50 & 8,29 & $-3,1$ \\
\hline 632 & 7,88 & 11,51 & 4,01 & 2,50 & 40,06 & $-5,3$ \\
\hline 633 & 0,23 & 1,85 & 0,72 & 0,40 & 7,22 & $-2,9$ \\
\hline 634 & 58,17 & 33,06 & 9,53 & 7,77 & 95,32 & $-6,6$ \\
\hline 635 & 0,03 & 0,66 & 0,26 & 0,14 & 2,55 & $-1,4$ \\
\hline 636 & 0,46 & 2,84 & 1,08 & 0,55 & 10,76 & $-3,4$ \\
\hline 637 & 322,58 & 74,63 & 20,95 & 19,61 & 209,50 & $-7,7$ \\
\hline 638 & 1,61 & 5,50 & 2,10 & 0,97 & 21,04 & $-4,4$ \\
\hline 639 & 1,19 & 4,12 & 1,54 & 0,99 & 15,39 & $-3,9$ \\
\hline 640 & 0,03 & 0,58 & 0,26 & 0,16 & 2,58 & $-1,4$ \\
\hline 641 & 0,12 & 1,53 & 0,65 & 0,24 & 6,53 & $-2,7$ \\
\hline 642 & 1,45 & 4,95 & 2,04 & 0,90 & 20,37 & $-4,3$ \\
\hline 643 & 0,10 & 1,28 & 0,52 & 0,25 & 5,22 & $-2,4$ \\
\hline 644 & 1,28 & 4,47 & 1,54 & 1,06 & 15,35 & $-3,9$ \\
\hline 645 & 0,01 & 0,47 & 0,23 & 0,08 & 2,32 & $-1,2$ \\
\hline 646 & 1,30 & 4,45 & 1,47 & 1,13 & 14,70 & $-3,9$ \\
\hline 647 & 2,15 & 6,21 & 2,21 & 1,24 & 22,13 & $-4,5$ \\
\hline 648 & 0,07 & 0,87 & 0,31 & 0,27 & 3,12 & $-1,6$ \\
\hline 649 & 0,10 & 1,15 & 0,44 & 0,29 & 4,41 & $-2,1$ \\
\hline 650 & 0,64 & 3,17 & 1,21 & 0,68 & 12,10 & $-3,6$ \\
\hline 651 & 0,36 & 2,49 & 1,06 & 0,43 & 10,61 & $-3,4$ \\
\hline 652 & 0,04 & 0,82 & 0,30 & 0,18 & 3,04 & $-1,6$ \\
\hline 653 & 1,06 & 4,03 & 1,45 & 0,93 & 14,45 & $-3,9$ \\
\hline 654 & 1,11 & 4,32 & 1,32 & 1,07 & 13,23 & $-3,7$ \\
\hline 655 & 0,22 & 2,49 & 1,12 & 0,25 & 11,16 & $-3,5$ \\
\hline 656 & 0,64 & 3,21 & 1,18 & 0,69 & 11,79 & $-3,6$ \\
\hline 657 & 0,31 & 2,24 & 0,82 & 0,49 & 8,21 & $-3,0$ \\
\hline 658 & 2,19 & 5,70 & 2,11 & 1,33 & 21,05 & $-4,4$ \\
\hline 659 & 7,52 & 11,49 & 4,35 & 2,20 & 43,49 & $-5,4$ \\
\hline 660 & 3,15 & 7,10 & 2,34 & 1,72 & 23,36 & $-4,5$ \\
\hline 661 & 0,94 & 4,11 & 1,67 & 0,72 & 16,66 & $-4,1$ \\
\hline 662 & 0,17 & 1,61 & 0,63 & 0,35 & 6,28 & $-2,7$ \\
\hline 663 & 1,91 & 5,35 & 1,84 & 1,32 & 18,39 & $-4,2$ \\
\hline 664 & 0,69 & 3,31 & 1,24 & 0,70 & 12,44 & $-3,6$ \\
\hline 665 & 0,21 & 1,86 & 0,75 & 0,35 & 7,52 & $-2,9$ \\
\hline 666 & 0,21 & 1,73 & 0,72 & 0,37 & 7,24 & $-2,9$ \\
\hline 667 & 0,08 & 0,99 & 0,37 & 0,28 & 3,68 & $-1,9$ \\
\hline 668 & 0,40 & 2,57 & 1,02 & 0,50 & 10,18 & $-3,3$ \\
\hline 669 & 1,27 & 4,40 & 1,60 & 1,01 & 15,98 & $-4,0$ \\
\hline 670 & 2,60 & 5,94 & 1,85 & 1,79 & 18,47 & $-4,2$ \\
\hline 671 & 0,29 & 2,14 & 0,65 & 0,57 & 6,49 & $-2,7$ \\
\hline 672 & 0,06 & 0,91 & 0,37 & 0,21 & 3,65 & $-1,9$ \\
\hline 673 & 2,34 & 6,50 & 2,45 & 1,21 & 24,51 & $-4,6$ \\
\hline
\end{tabular}




\begin{tabular}{|c|c|c|c|c|c|c|}
\hline 674 & 1,00 & 3,82 & 1,45 & 0,87 & 14,48 & $-3,9$ \\
\hline 675 & 4,74 & 9,04 & 2,96 & 2,04 & 29,60 & $-4,9$ \\
\hline 676 & 8,75 & 11,69 & 4,00 & 2,78 & 40,04 & $-5,3$ \\
\hline 677 & 2,45 & 6,78 & 2,47 & 1,26 & 24,71 & $-4,6$ \\
\hline 678 & 3,17 & 7,32 & 2,53 & 1,59 & 25,31 & $-4,7$ \\
\hline 679 & 47,05 & 27,82 & 10,38 & 5,77 & 103,79 & $-6,7$ \\
\hline 680 & 0,18 & 1,56 & 0,57 & 0,41 & 5,65 & $-2,5$ \\
\hline 681 & 0,22 & 2,04 & 0,76 & 0,37 & 7,62 & $-2,9$ \\
\hline 682 & 7,09 & 10,46 & 3,20 & 2,82 & 32,01 & $-5,0$ \\
\hline 683 & 0,72 & 4,43 & 1,89 & 0,48 & 18,94 & $-4,2$ \\
\hline 684 & 0,06 & 0,82 & 0,34 & 0,21 & 3,44 & $-1,8$ \\
\hline 685 & 0,03 & 0,72 & 0,30 & 0,14 & 3,04 & $-1,6$ \\
\hline 686 & 0,78 & 3,45 & 1,33 & 0,75 & 13,33 & $-3,7$ \\
\hline 687 & 0,26 & 1,98 & 0,72 & 0,45 & 7,23 & $-2,9$ \\
\hline 688 & 0,06 & 1,03 & 0,42 & 0,17 & 4,21 & $-2,1$ \\
\hline 689 & 0,08 & 1,32 & 0,56 & 0,19 & 5,61 & $-2,5$ \\
\hline 690 & 0,15 & 1,40 & 0,45 & 0,43 & 4,48 & $-2,2$ \\
\hline 691 & 1,33 & 4,36 & 1,33 & 1,27 & 13,33 & $-3,7$ \\
\hline 692 & 0,01 & 0,48 & 0,22 & 0,08 & 2,20 & $-1,1$ \\
\hline 693 & 0,08 & 1,11 & 0,48 & 0,22 & 4,83 & $-2,3$ \\
\hline 694 & 17,24 & 19,59 & 5,07 & 4,33 & 50,66 & $-5,7$ \\
\hline 695 & 0,14 & 1,34 & 0,51 & 0,35 & 5,08 & $-2,3$ \\
\hline 696 & 2,14 & 6,15 & 2,08 & 1,31 & 20,78 & $-4,4$ \\
\hline 697 & 0,15 & 1,57 & 0,65 & 0,30 & 6,46 & $-2,7$ \\
\hline 698 & 0,28 & 2,24 & 0,81 & 0,44 & 8,08 & $-3,0$ \\
\hline 699 & 0,34 & 2,18 & 0,86 & 0,50 & 8,58 & $-3,1$ \\
\hline 700 & 6,29 & 11,54 & 4,48 & 1,79 & 44,79 & $-5,5$ \\
\hline 701 & 0,08 & 1,07 & 0,47 & 0,21 & 4,66 & $-2,2$ \\
\hline 702 & 13,55 & 15,14 & 4,94 & 3,49 & 49,38 & $-5,6$ \\
\hline 703 & 0,04 & 0,62 & 0,24 & 0,20 & 2,42 & $-1,3$ \\
\hline 704 & 0,05 & 0,91 & 0,34 & 0,17 & 3,44 & $-1,8$ \\
\hline 705 & 23,80 & 19,70 & 6,56 & 4,62 & 65,57 & $-6,0$ \\
\hline 706 & 193,46 & 59,97 & 19,06 & 12,92 & 190,58 & $-7,6$ \\
\hline 707 & 0,17 & 1,94 & 0,77 & 0,29 & 7,66 & $-2,9$ \\
\hline 708 & 0,44 & 2,58 & 0,90 & 0,62 & 9,04 & $-3,2$ \\
\hline 709 & 0,54 & 2,94 & 1,13 & 0,61 & 11,31 & $-3,5$ \\
\hline 710 & 0,07 & 1,13 & 0,45 & 0,19 & 4,50 & $-2,2$ \\
\hline 711 & 2,84 & 6,92 & 2,39 & 1,51 & 23,88 & $-4,6$ \\
\hline 712 & 0,62 & 3,11 & 1,01 & 0,78 & 10,07 & $-3,3$ \\
\hline 713 & 0,51 & 2,74 & 0,88 & 0,74 & 8,84 & $-3,1$ \\
\hline 714 & 12,66 & 14,74 & 5,33 & 3,02 & 53,30 & $-5,7$ \\
\hline 715 & 0,11 & 1,23 & 0,49 & 0,28 & 4,90 & $-2,3$ \\
\hline 716 & 0,29 & 2,04 & 0,80 & 0,46 & 8,01 & $-3,0$ \\
\hline 717 & 56,53 & 33,26 & 13,50 & 5,33 & 134,96 & $-7,1$ \\
\hline 718 & 0,18 & 1,60 & 0,64 & 0,37 & 6,38 & $-2,7$ \\
\hline 719 & 0,55 & 2,82 & 1,12 & 0,62 & 11,21 & $-3,5$ \\
\hline 720 & 0,25 & 1,85 & 0,64 & 0,50 & 6,38 & $-2,7$ \\
\hline 721 & 1,21 & 4,30 & 1,49 & 1,04 & 14,89 & $-3,9$ \\
\hline 722 & 0,54 & 2,76 & 1,02 & 0,67 & 10,24 & $-3,4$ \\
\hline 723 & 1,15 & 4,14 & 1,47 & 0,99 & 14,67 & $-3,9$ \\
\hline 724 & 1,69 & 5,00 & 1,59 & 1,35 & 15,91 & $-4,0$ \\
\hline 725 & 0,24 & 2,20 & 0,94 & 0,32 & 9,40 & $-3,2$ \\
\hline 726 & 0,96 & 4,03 & 1,58 & 0,78 & 15,79 & $-4,0$ \\
\hline 727 & 0,56 & 3,57 & 1,58 & 0,45 & 15,79 & $-4,0$ \\
\hline 728 & 0,12 & 1,30 & 0,48 & 0,31 & 4,77 & $-2,3$ \\
\hline 729 & 0,11 & 1,24 & 0,45 & 0,30 & 4,53 & $-2,2$ \\
\hline 730 & 77,87 & 34,65 & 11,65 & 8,51 & 116,51 & $-6,9$ \\
\hline 731 & 2,32 & 6,31 & 2,16 & 1,37 & 21,64 & $-4,4$ \\
\hline 732 & 0,51 & 2,96 & 1,16 & 0,56 & 11,60 & $-3,5$ \\
\hline 733 & 1,82 & 5,38 & 1,95 & 1,18 & 19,53 & $-4,3$ \\
\hline 734 & 4,15 & 7,82 & 2,39 & 2,21 & 23,89 & $-4,6$ \\
\hline 735 & 1,13 & 4,37 & 1,33 & 1,08 & 13,28 & $-3,7$ \\
\hline
\end{tabular}




\begin{tabular}{|c|c|c|c|c|c|c|}
\hline 736 & 3,73 & 7,36 & 2,55 & 1,86 & 25,51 & $-4,7$ \\
\hline 737 & 1,95 & 5,57 & 1,93 & 1,29 & 19,30 & $-4,3$ \\
\hline 738 & 3,53 & 7,54 & 2,80 & 1,61 & 27,97 & $-4,8$ \\
\hline 739 & 1,32 & 4,47 & 1,64 & 1,03 & 16,39 & $-4,0$ \\
\hline 740 & 1,46 & 6,05 & 2,43 & 0,77 & 24,30 & $-4,6$ \\
\hline 741 & 0,24 & 2,24 & 0,91 & 0,34 & 9,05 & $-3,2$ \\
\hline 742 & 1,07 & 3,83 & 1,31 & 1,05 & 13,06 & $-3,7$ \\
\hline 743 & 0,07 & 0,91 & 0,36 & 0,25 & 3,59 & $-1,8$ \\
\hline 744 & 0,24 & 2,04 & 0,88 & 0,35 & 8,81 & $-3,1$ \\
\hline 745 & 0,25 & 2,10 & 0,90 & 0,35 & 9,03 & $-3,2$ \\
\hline 746 & 0,09 & 1,27 & 0,49 & 0,23 & 4,89 & $-2,3$ \\
\hline 747 & 0,06 & 0,82 & 0,34 & 0,21 & 3,44 & $-1,8$ \\
\hline 748 & 0,86 & 4,28 & 1,87 & 0,59 & 18,71 & $-4,2$ \\
\hline 749 & 0,17 & 1,69 & 0,63 & 0,34 & 6,25 & $-2,6$ \\
\hline 750 & 0,05 & 0,82 & 0,26 & 0,25 & 2,61 & $-1,4$ \\
\hline 751 & 0,05 & 0,76 & 0,31 & 0,20 & 3,05 & $-1,6$ \\
\hline 752 & 0,14 & 1,34 & 0,53 & 0,33 & 5,25 & $-2,4$ \\
\hline 753 & 1,86 & 5,74 & 2,01 & 1,18 & 20,06 & $-4,3$ \\
\hline 754 & 0,05 & 0,82 & 0,31 & 0,19 & 3,13 & $-1,6$ \\
\hline 755 & 0,04 & 0,68 & 0,27 & 0,20 & 2,68 & $-1,4$ \\
\hline 756 & 3,27 & 7,33 & 2,62 & 1,59 & 26,23 & $-4,7$ \\
\hline 757 & 0,38 & 2,58 & 0,93 & 0,52 & 9,27 & $-3,2$ \\
\hline 758 & 8,32 & 11,79 & 3,52 & 3,01 & 35,15 & $-5,1$ \\
\hline 759 & 0,08 & 1,34 & 0,57 & 0,19 & 5,73 & $-2,5$ \\
\hline 760 & 0,42 & 2,45 & 0,75 & 0,72 & 7,46 & $-2,9$ \\
\hline 761 & 0,12 & 1,52 & 0,61 & 0,26 & 6,07 & $-2,6$ \\
\hline 762 & 0,63 & 3,29 & 1,29 & 0,62 & 12,93 & $-3,7$ \\
\hline 763 & 1,70 & 4,94 & 1,76 & 1,23 & 17,63 & $-4,1$ \\
\hline 764 & 19,08 & 24,30 & 9,84 & 2,47 & 98,37 & $-6,6$ \\
\hline 765 & 2,25 & 7,10 & 2,42 & 1,19 & 24,18 & $-4,6$ \\
\hline 766 & 23,14 & 19,39 & 6,45 & 4,57 & 64,48 & $-6,0$ \\
\hline 767 & 1,02 & 3,85 & 1,32 & 0,99 & 13,16 & $-3,7$ \\
\hline 768 & 2,21 & 6,98 & 2,67 & 1,06 & 26,68 & $-4,7$ \\
\hline 769 & 9,23 & 11,97 & 3,51 & 3,35 & 35,06 & $-5,1$ \\
\hline 770 & 1,38 & 4,67 & 1,38 & 1,27 & 13,75 & $-3,8$ \\
\hline 771 & 51,25 & 31,47 & 12,12 & 5,39 & 121,18 & $-6,9$ \\
\hline 772 & 15,30 & 15,62 & 4,83 & 4,03 & 48,31 & $-5,6$ \\
\hline 773 & 0,07 & 1,05 & 0,43 & 0,19 & 4,34 & $-2,1$ \\
\hline 774 & 3,98 & 9,56 & 3,25 & 1,56 & 32,53 & $-5,0$ \\
\hline 775 & 0,54 & 2,80 & 1,01 & 0,69 & 10,09 & $-3,3$ \\
\hline 776 & 0,23 & 1,98 & 0,82 & 0,35 & 8,22 & $-3,0$ \\
\hline 777 & 1,41 & 6,97 & 2,75 & 0,65 & 27,50 & $-4,8$ \\
\hline 778 & 1,17 & 4,53 & 1,53 & 0,97 & 15,32 & $-3,9$ \\
\hline 779 & 1,91 & 6,03 & 2,03 & 1,20 & 20,31 & $-4,3$ \\
\hline 780 & 1,25 & 5,04 & 1,68 & 0,95 & 16,78 & $-4,1$ \\
\hline 781 & 1,53 & 5,02 & 1,86 & 1,05 & 18,63 & $-4,2$ \\
\hline 782 & 2,21 & 5,82 & 1,91 & 1,47 & 19,05 & $-4,3$ \\
\hline 783 & 0,60 & 3,31 & 1,18 & 0,64 & 11,80 & $-3,6$ \\
\hline 784 & 0,36 & 2,20 & 0,69 & 0,66 & 6,92 & $-2,8$ \\
\hline 785 & 0,16 & 1,56 & 0,50 & 0,41 & 4,96 & $-2,3$ \\
\hline 786 & 3,22 & 7,61 & 2,18 & 1,88 & 21,81 & $-4,4$ \\
\hline 787 & 4,80 & 9,98 & 3,80 & 1,61 & 37,96 & $-5,2$ \\
\hline 788 & 0,76 & 3,20 & 1,06 & 0,91 & 10,63 & $-3,4$ \\
\hline 789 & 0,65 & 3,40 & 1,42 & 0,58 & 14,22 & $-3,8$ \\
\hline 790 & 0,41 & 2,74 & 1,11 & 0,48 & 11,07 & $-3,5$ \\
\hline 791 & 1,64 & 5,78 & 2,26 & 0,92 & 22,64 & $-4,5$ \\
\hline 792 & 1,39 & 4,74 & 1,62 & 1,09 & 16,18 & $-4,0$ \\
\hline 793 & 0,44 & 2,47 & 0,84 & 0,67 & 8,42 & $-3,1$ \\
\hline 794 & 5,51 & 10,44 & 4,01 & 1,75 & 40,10 & $-5,3$ \\
\hline 795 & 0,82 & 3,49 & 1,33 & 0,78 & 13,33 & $-3,7$ \\
\hline 796 & 0,76 & 3,56 & 1,16 & 0,84 & 11,55 & $-3,5$ \\
\hline 797 & 0,77 & 4,14 & 1,71 & 0,58 & 17,08 & $-4,1$ \\
\hline
\end{tabular}




\begin{tabular}{|c|c|c|c|c|c|c|}
\hline 798 & 5,48 & 9,28 & 3,18 & 2,19 & 31,80 & $-5,0$ \\
\hline 799 & 3,80 & 7,95 & 2,72 & 1,78 & 27,23 & $-4,8$ \\
\hline 800 & 26,48 & 21,30 & 6,54 & 5,16 & 65,40 & $-6,0$ \\
\hline 801 & 0,26 & 2,08 & 0,70 & 0,48 & 6,96 & $-2,8$ \\
\hline 802 & 9,38 & 13,44 & 5,44 & 2,20 & 54,40 & $-5,8$ \\
\hline 803 & 0,47 & 2,62 & 0,88 & 0,68 & 8,77 & $-3,1$ \\
\hline 804 & 0,29 & 2,11 & 0,79 & 0,47 & 7,89 & $-3,0$ \\
\hline 805 & 0,27 & 2,55 & 1,04 & 0,33 & 10,37 & $-3,4$ \\
\hline 806 & 0,13 & 1,38 & 0,54 & 0,31 & 5,36 & $-2,4$ \\
\hline 807 & 0,12 & 1,24 & 0,47 & 0,33 & 4,65 & $-2,2$ \\
\hline 808 & 64,88 & 35,88 & 11,47 & 7,20 & 114,70 & $-6,8$ \\
\hline 809 & 0,55 & 2,78 & 0,87 & 0,80 & 8,70 & $-3,1$ \\
\hline 810 & 3,43 & 7,85 & 2,29 & 1,91 & 22,90 & $-4,5$ \\
\hline 811 & 0,21 & 2,26 & 0,92 & 0,29 & 9,21 & $-3,2$ \\
\hline 812 & 17,26 & 16,45 & 5,45 & 4,03 & 54,46 & $-5,8$ \\
\hline 813 & 0,49 & 2,90 & 1,19 & 0,53 & 11,85 & $-3,6$ \\
\hline 814 & 7,37 & 12,76 & 5,02 & 1,87 & 50,20 & $-5,6$ \\
\hline 815 & 0,07 & 1,11 & 0,45 & 0,19 & 4,52 & $-2,2$ \\
\hline 816 & 0,22 & 1,85 & 0,72 & 0,39 & 7,17 & $-2,8$ \\
\hline 817 & 29,47 & 24,49 & 9,64 & 3,89 & 96,35 & $-6,6$ \\
\hline 818 & 1,15 & 4,32 & 1,63 & 0,90 & 16,25 & $-4,0$ \\
\hline 819 & 1,40 & 5,52 & 2,41 & 0,74 & 24,08 & $-4,6$ \\
\hline 820 & 0,21 & 1,81 & 0,73 & 0,36 & 7,33 & $-2,9$ \\
\hline 821 & 55,41 & 31,08 & 9,65 & 7,31 & 96,52 & $-6,6$ \\
\hline 822 & 0,33 & 2,51 & 1,00 & 0,42 & 9,97 & $-3,3$ \\
\hline 823 & 0,85 & 3,56 & 1,28 & 0,85 & 12,82 & $-3,7$ \\
\hline 824 & 0,42 & 2,58 & 0,94 & 0,57 & 9,39 & $-3,2$ \\
\hline 825 & 5,70 & 10,54 & 3,42 & 2,13 & 34,15 & $-5,1$ \\
\hline 826 & 0,43 & 2,51 & 0,99 & 0,56 & 9,91 & $-3,3$ \\
\hline 827 & 1,30 & 4,92 & 1,61 & 1,02 & 16,10 & $-4,0$ \\
\hline 828 & 0,41 & 2,80 & 1,11 & 0,48 & 11,06 & $-3,5$ \\
\hline 829 & 2,20 & 5,94 & 2,06 & 1,36 & 20,56 & $-4,4$ \\
\hline 830 & 0,49 & 2,95 & 1,20 & 0,52 & 11,99 & $-3,6$ \\
\hline 831 & 0,24 & 1,85 & 0,67 & 0,46 & 6,74 & $-2,8$ \\
\hline 832 & 5,20 & 9,25 & 3,46 & 1,91 & 34,64 & $-5,1$ \\
\hline 833 & 3,71 & 7,69 & 2,56 & 1,84 & 25,64 & $-4,7$ \\
\hline 834 & 10,65 & 12,91 & 4,06 & 3,34 & 40,64 & $-5,3$ \\
\hline 835 & 12,93 & 17,28 & 4,64 & 3,55 & 46,40 & $-5,5$ \\
\hline 836 & 1,24 & 4,61 & 1,51 & 1,05 & 15,07 & $-3,9$ \\
\hline 837 & 0,52 & 2,76 & 0,95 & 0,69 & 9,53 & $-3,3$ \\
\hline 838 & 7,08 & 12,08 & 4,73 & 1,91 & 47,29 & $-5,6$ \\
\hline 839 & 0,31 & 2,00 & 0,67 & 0,58 & 6,72 & $-2,7$ \\
\hline 840 & 0,49 & 2,69 & 0,91 & 0,68 & 9,13 & $-3,2$ \\
\hline 841 & 0,58 & 3,35 & 1,33 & 0,55 & 13,30 & $-3,7$ \\
\hline 842 & 0,82 & 4,81 & 1,58 & 0,66 & 15,78 & $-4,0$ \\
\hline 843 & 5,16 & 10,10 & 3,80 & 1,73 & 37,96 & $-5,2$ \\
\hline 844 & 13,52 & 17,28 & 5,74 & 3,00 & 57,35 & $-5,8$ \\
\hline 845 & 0,26 & 2,08 & 0,78 & 0,43 & 7,79 & $-3,0$ \\
\hline 846 & 1,00 & 4,11 & 1,36 & 0,93 & 13,64 & $-3,8$ \\
\hline 847 & 2,09 & 5,35 & 1,69 & 1,57 & 16,93 & $-4,1$ \\
\hline 848 & 3,77 & 7,99 & 3,05 & 1,57 & 30,47 & $-4,9$ \\
\hline 849 & 0,10 & 1,56 & 0,61 & 0,22 & 6,11 & $-2,6$ \\
\hline 850 & 2,21 & 6,15 & 2,36 & 1,19 & 23,60 & $-4,6$ \\
\hline 851 & 0,19 & 1,71 & 0,63 & 0,39 & 6,32 & $-2,7$ \\
\hline 852 & 1,37 & 4,40 & 1,46 & 1,19 & 14,63 & $-3,9$ \\
\hline 853 & 1,45 & 5,41 & 2,08 & 0,89 & 20,84 & $-4,4$ \\
\hline 854 & 0,22 & 1,90 & 0,69 & 0,40 & 6,90 & $-2,8$ \\
\hline 855 & 2,43 & 7,23 & 2,63 & 1,18 & 26,30 & $-4,7$ \\
\hline 856 & 1,31 & 4,66 & 1,49 & 1,12 & 14,93 & $-3,9$ \\
\hline 857 & 0,17 & 1,63 & 0,68 & 0,32 & 6,78 & $-2,8$ \\
\hline 858 & 0,27 & 2,35 & 0,92 & 0,37 & 9,22 & $-3,2$ \\
\hline 859 & 0,13 & 1,81 & 0,79 & 0,20 & 7,89 & $-3,0$ \\
\hline
\end{tabular}




\begin{tabular}{|c|c|c|c|c|c|c|}
\hline 860 & 0,56 & 2,84 & 0,88 & 0,81 & 8,83 & $-3,1$ \\
\hline 861 & 1,63 & 5,25 & 1,86 & 1,12 & 18,59 & $-4,2$ \\
\hline 862 & 0,07 & 0,91 & 0,29 & 0,29 & 2,92 & $-1,5$ \\
\hline 863 & 0,54 & 2,86 & 0,94 & 0,74 & 9,42 & $-3,2$ \\
\hline 864 & 0,10 & 1,15 & 0,49 & 0,26 & 4,87 & $-2,3$ \\
\hline 865 & 5,10 & 10,10 & 4,17 & 1,56 & 41,65 & $-5,4$ \\
\hline 866 & 69,23 & 34,86 & 12,25 & 7,20 & 122,47 & $-6,9$ \\
\hline 867 & 0,06 & 1,05 & 0,44 & 0,16 & 4,37 & $-2,1$ \\
\hline 868 & 42,84 & 29,01 & 10,69 & 5,10 & 106,94 & $-6,7$ \\
\hline 869 & 0,10 & 1,11 & 0,43 & 0,29 & 4,29 & $-2,1$ \\
\hline 870 & 0,11 & 1,19 & 0,45 & 0,30 & 4,54 & $-2,2$ \\
\hline 871 & 0,17 & 1,53 & 0,57 & 0,39 & 5,72 & $-2,5$ \\
\hline 872 & 0,91 & 3,44 & 1,19 & 0,97 & 11,87 & $-3,6$ \\
\hline 873 & 36,61 & 24,49 & 7,66 & 6,09 & 76,56 & $-6,3$ \\
\hline 874 & 5,20 & 9,65 & 3,24 & 2,04 & 32,44 & $-5,0$ \\
\hline 875 & 0,08 & 1,07 & 0,41 & 0,25 & 4,14 & $-2,0$ \\
\hline 876 & 0,14 & 1,44 & 0,50 & 0,36 & 4,97 & $-2,3$ \\
\hline 877 & 3,40 & 7,57 & 2,81 & 1,54 & 28,11 & $-4,8$ \\
\hline 878 & 0,12 & 1,65 & 0,65 & 0,23 & 6,46 & $-2,7$ \\
\hline 879 & 0,15 & 1,81 & 0,74 & 0,25 & 7,37 & $-2,9$ \\
\hline 880 & 0,23 & 1,73 & 0,66 & 0,45 & 6,56 & $-2,7$ \\
\hline 881 & 1,38 & 4,82 & 1,85 & 0,96 & 18,45 & $-4,2$ \\
\hline 882 & 0,37 & 2,43 & 0,96 & 0,49 & 9,56 & $-3,3$ \\
\hline 883 & 0,29 & 2,12 & 0,83 & 0,44 & 8,34 & $-3,1$ \\
\hline 884 & 0,08 & 0,95 & 0,36 & 0,28 & 3,58 & $-1,8$ \\
\hline 885 & 0,08 & 1,15 & 0,42 & 0,25 & 4,24 & $-2,1$ \\
\hline 886 & 0,45 & 2,57 & 0,98 & 0,58 & 9,83 & $-3,3$ \\
\hline 887 & 0,17 & 2,01 & 0,93 & 0,23 & 9,25 & $-3,2$ \\
\hline 888 & 0,08 & 0,95 & 0,39 & 0,25 & 3,87 & $-2,0$ \\
\hline 889 & 6,23 & 9,83 & 3,03 & 2,62 & 30,31 & $-4,9$ \\
\hline 890 & 0,50 & 3,41 & 1,55 & 0,41 & 15,50 & $-4,0$ \\
\hline 891 & 18,11 & 18,15 & 6,59 & 3,50 & 65,85 & $-6,0$ \\
\hline 892 & 0,74 & 3,33 & 1,19 & 0,79 & 11,90 & $-3,6$ \\
\hline 893 & 0,41 & 2,67 & 1,04 & 0,50 & 10,35 & $-3,4$ \\
\hline 894 & 0,57 & 3,62 & 1,45 & 0,50 & 14,51 & $-3,9$ \\
\hline 895 & 5,82 & 10,16 & 3,30 & 2,25 & 32,95 & $-5,0$ \\
\hline 896 & 0,07 & 1,11 & 0,46 & 0,20 & 4,56 & $-2,2$ \\
\hline 897 & 0,58 & 2,87 & 0,97 & 0,76 & 9,74 & $-3,3$ \\
\hline 898 & 0,39 & 2,41 & 0,90 & 0,55 & 8,99 & $-3,2$ \\
\hline 899 & 0,38 & 2,95 & 1,15 & 0,42 & 11,52 & $-3,5$ \\
\hline 900 & 0,66 & 3,36 & 1,07 & 0,78 & 10,74 & $-3,4$ \\
\hline 901 & 4,06 & 8,27 & 2,86 & 1,81 & 28,61 & $-4,8$ \\
\hline 902 & 0,24 & 2,02 & 0,82 & 0,38 & 8,18 & $-3,0$ \\
\hline 903 & 0,08 & 1,15 & 0,39 & 0,26 & 3,90 & $-2,0$ \\
\hline 904 & 0,53 & 2,90 & 1,05 & 0,64 & 10,51 & $-3,4$ \\
\hline 905 & 13,61 & 17,16 & 6,79 & 2,55 & 67,87 & $-6,1$ \\
\hline 906 & 3,27 & 8,02 & 2,43 & 1,72 & 24,27 & $-4,6$ \\
\hline 907 & 1,65 & 5,09 & 1,69 & 1,24 & 16,94 & $-4,1$ \\
\hline 908 & 0,36 & 2,53 & 0,97 & 0,47 & 9,71 & $-3,3$ \\
\hline 909 & 1,15 & 4,12 & 1,34 & 1,09 & 13,44 & $-3,7$ \\
\hline 910 & 0,17 & 1,63 & 0,72 & 0,30 & 7,19 & $-2,8$ \\
\hline 911 & 0,63 & 2,90 & 0,97 & 0,82 & 9,71 & $-3,3$ \\
\hline 912 & 0,23 & 2,30 & 0,98 & 0,30 & 9,76 & $-3,3$ \\
\hline 913 & 2,51 & 6,43 & 1,92 & 1,66 & 19,24 & $-4,3$ \\
\hline 914 & 0,27 & 2,10 & 0,78 & 0,44 & 7,77 & $-3,0$ \\
\hline 915 & 0,68 & 3,40 & 1,37 & 0,63 & 13,68 & $-3,8$ \\
\hline 916 & 1,05 & 5,02 & 2,04 & 0,66 & 20,35 & $-4,3$ \\
\hline 917 & 0,23 & 1,77 & 0,66 & 0,45 & 6,56 & $-2,7$ \\
\hline 918 & 4,77 & 10,82 & 3,70 & 1,64 & 36,96 & $-5,2$ \\
\hline 919 & 1,78 & 5,35 & 1,95 & 1,17 & 19,47 & $-4,3$ \\
\hline 920 & 3,15 & 7,25 & 2,35 & 1,71 & 23,53 & $-4,6$ \\
\hline 921 & 0,16 & 1,48 & 0,49 & 0,42 & 4,87 & $-2,3$ \\
\hline
\end{tabular}




\begin{tabular}{|c|c|c|c|c|c|c|}
\hline 922 & 20,48 & 19,93 & 5,33 & 4,89 & 53,34 & $-5,7$ \\
\hline 923 & 0,41 & 2,74 & 1,14 & 0,46 & 11,39 & $-3,5$ \\
\hline 924 & 0,26 & 2,14 & 0,89 & 0,38 & 8,91 & $-3,2$ \\
\hline 925 & 0,13 & 1,32 & 0,52 & 0,31 & 5,18 & $-2,4$ \\
\hline 926 & 29,89 & 22,76 & 7,42 & 5,13 & 74,16 & $-6,2$ \\
\hline 927 & 0,23 & 1,71 & 0,62 & 0,47 & 6,18 & $-2,6$ \\
\hline 928 & 0,30 & 2,18 & 0,76 & 0,50 & 7,59 & $-2,9$ \\
\hline 929 & 0,17 & 1,67 & 0,66 & 0,33 & 6,64 & $-2,7$ \\
\hline 930 & 0,30 & 2,24 & 0,94 & 0,40 & 9,43 & $-3,2$ \\
\hline 931 & 0,84 & 3,71 & 1,30 & 0,83 & 13,02 & $-3,7$ \\
\hline 932 & 0,04 & 0,72 & 0,27 & 0,20 & 2,68 & $-1,4$ \\
\hline 933 & 0,10 & 1,09 & 0,42 & 0,30 & 4,19 & $-2,1$ \\
\hline 934 & 0,05 & 0,76 & 0,30 & 0,22 & 3,00 & $-1,6$ \\
\hline 935 & 0,48 & 2,82 & 0,99 & 0,62 & 9,94 & $-3,3$ \\
\hline 936 & 3,45 & 8,04 & 3,38 & 1,30 & 33,75 & $-5,1$ \\
\hline 937 & 0,04 & 0,72 & 0,25 & 0,19 & 2,50 & $-1,3$ \\
\hline 938 & 0,13 & 1,28 & 0,43 & 0,38 & 4,28 & $-2,1$ \\
\hline 939 & 0,08 & 1,05 & 0,44 & 0,25 & 4,36 & $-2,1$ \\
\hline 940 & 0,19 & 2,07 & 0,83 & 0,30 & 8,29 & $-3,1$ \\
\hline 941 & 0,07 & 0,91 & 0,33 & 0,25 & 3,32 & $-1,7$ \\
\hline 942 & 0,27 & 2,32 & 0,82 & 0,42 & 8,23 & $-3,0$ \\
\hline 943 & 0,08 & 0,95 & 0,39 & 0,25 & 3,87 & $-2,0$ \\
\hline 944 & 1,08 & 4,39 & 1,74 & 0,79 & 17,37 & $-4,1$ \\
\hline 945 & 0,17 & 1,71 & 0,59 & 0,38 & 5,90 & $-2,6$ \\
\hline 946 & 2,52 & 6,07 & 1,98 & 1,61 & 19,84 & $-4,3$ \\
\hline 947 & 0,06 & 1,07 & 0,47 & 0,17 & 4,67 & $-2,2$ \\
\hline 948 & 2,34 & 6,24 & 1,96 & 1,52 & 19,57 & $-4,3$ \\
\hline 949 & 0,04 & 0,62 & 0,24 & 0,20 & 2,42 & $-1,3$ \\
\hline 950 & 0,07 & 0,99 & 0,42 & 0,22 & 4,15 & $-2,1$ \\
\hline 951 & 3,25 & 6,90 & 2,26 & 1,83 & 22,58 & $-4,5$ \\
\hline 952 & 0,03 & 0,58 & 0,21 & 0,20 & 2,11 & $-1,1$ \\
\hline 953 & 0,15 & 1,52 & 0,56 & 0,34 & 5,61 & $-2,5$ \\
\hline 954 & 2,50 & 6,96 & 2,47 & 1,29 & 24,67 & $-4,6$ \\
\hline 955 & 0,31 & 1,96 & 0,71 & 0,55 & 7,13 & $-2,8$ \\
\hline 956 & 8,78 & 13,19 & 3,63 & 3,08 & 36,31 & $-5,2$ \\
\hline 957 & 16,00 & 16,68 & 5,96 & 3,42 & 59,56 & $-5,9$ \\
\hline 958 & 0,39 & 2,49 & 0,94 & 0,53 & 9,43 & $-3,2$ \\
\hline 959 & 3,04 & 7,30 & 2,72 & 1,43 & 27,15 & $-4,8$ \\
\hline 960 & 0,06 & 0,87 & 0,33 & 0,23 & 3,32 & $-1,7$ \\
\hline 961 & 0,22 & 1,89 & 0,66 & 0,43 & 6,56 & $-2,7$ \\
\hline
\end{tabular}


Clasts and bubbles' data for sample SM-82_Br01 used for shape parameters analysis

$\phi=-\log _{2}($ diam$)$, considering the Feret Diameter in millimeters

Clasts' data

\begin{tabular}{|c|c|c|c|c|c|c|c|c|c|c|c|c|c|c|c|c|c|c|c|}
\hline Label & Area $\left(\mathrm{cm}^{2}\right)$ & $\begin{array}{c}\text { Perimeter } \\
(\mathbf{c m})\end{array}$ & CH Area & CH Perim. & $\begin{array}{l}\text { CH Perim. } \\
\text { Correction }\end{array}$ & Solidity & Convexity & $\begin{array}{l}\text { Convexity } \\
\text { Correction }\end{array}$ & $\begin{array}{l}\text { Concavity } \\
\text { Index }\end{array}$ & Form Factor & Circularity & Roundness & $\begin{array}{c}\text { Axial Ratio } \\
\text { (Feret) }\end{array}$ & $\begin{array}{c}\text { Feret } \\
\text { diameter }(\mathbf{c m})\end{array}$ & $\begin{array}{l}\text { Min. Feret } \\
\text { diam. }\end{array}$ & $\begin{array}{l}\text { Max. } \\
\text { Insc. }\end{array}$ & Orientatior & $\begin{array}{c}\text { ROI } \\
\text { Index }\end{array}$ & Phi \\
\hline 0 & 33,4 & 23,7 & 36,1 & 23,4 & 22,7 & 0,927 & 0,988 & 0,956 & 0,074 & 0,747 & 1,157 & 0,508 & 0,566 & 9,154 & 5,182 & 4,576 & 90 & 0 & $-6,5$ \\
\hline 1 & 0,2 & 1,8 & 0,3 & 1,7 & 1,6 & 0,949 & 0,932 & 0,902 & 0,085 & 0,931 & 1,036 & 0,582 & 0,607 & 0,729 & 0,442 & 0,442 & 21 & 1 & $-2,9$ \\
\hline 2 & 0,4 & 2,4 & 0,4 & 2,3 & 2,2 & 0,919 & 0,970 & 0,939 & 0,086 & 0,872 & 1,071 & 0,646 & 0,714 & 0,879 & 0,627 & 0,577 & 98 & 2 & $-3,1$ \\
\hline 3 & 0,1 & 1,0 & 0,1 & 0,9 & 0,9 & 0,983 & 0,948 & 0,917 & 0,055 & 1,000 & 0,947 & 0,697 & 0,549 & 0,390 & 0,214 & 0,273 & 49 & 3 & $-2,0$ \\
\hline 4 & 8,5 & 11,0 & 8,8 & 10,8 & 10,4 & 0,956 & 0,985 & 0,953 & 0,046 & 0,885 & 1,063 & 0,645 & 0,683 & 4,085 & 2,792 & 2,630 & 78 & 4 & $-5,4$ \\
\hline 5 & 2,0 & 6,0 & 2,2 & 5,9 & 5,7 & 0,914 & 0,978 & 0,947 & 0,089 & 0,702 & 1,194 & 0,399 & 0,447 & 2,541 & 1,135 & 1,154 & 12 & 5 & $-4,7$ \\
\hline 6 & 7,5 & 10,6 & 7,9 & 10,5 & 10,1 & 0,954 & 0,994 & 0,962 & 0,046 & 0,852 & 1,084 & 0,575 & 0,595 & 4,088 & 2,431 & 2,334 & 112 & 6 & $-5,4$ \\
\hline 7 & 3,2 & 7,9 & 3,5 & 7,8 & 7,6 & 0,925 & 0,994 & 0,962 & 0,075 & 0,660 & 1,231 & 0,395 & 0,386 & 3,232 & 1,247 & 1,198 & 111 & 7 & $-5,0$ \\
\hline 8 & 0,5 & 2,6 & 0,5 & 2,5 & 2,4 & 0,950 & 0,969 & 0,938 & 0,059 & 0,907 & $\begin{array}{l}1,050 \\
0\end{array}$ & 0,536 & 0,562 & 1,078 & 0,606 & 0,611 & 100 & 8 & $-3,4$ \\
\hline 9 & 463,7 & 99,1 & 510,2 & 82,8 & 80,2 & 0,909 & 0,836 & 0,809 & 0,188 & 0,593 & 1,298 & 0,709 & 0,816 & 28,851 & 23,538 & 18,673 & 53 & 9 & $-8,2$ \\
\hline 10 & 0,5 & 2,5 & $\begin{array}{c}0,5 \\
0,5\end{array}$ & $\begin{array}{l}2,0,4 \\
2,4\end{array}$ & 2,3 & 0,961 & 0,979 & 0,948 & 0,045 & 0,951 & $\begin{array}{l}1,025 \\
1,25\end{array}$ & 0,631 & 0,600 & 0,958 & 0,575 & 0,546 & 101 & 10 & $\begin{array}{l}-3,2 \\
-3,3\end{array}$ \\
\hline 11 & 0,3 & 1,8 & 0,3 & 1,8 & 1,8 & 0,967 & 0,989 & 0,957 & 0,035 & 0,928 & 1,038 & 0,594 & 0,545 & 0,732 & 0,398 & 0,408 & 162 & 11 & $-2,9$ \\
\hline 12 & 0,3 & 2,2 & 0,3 & 2,1 & 2,1 & 0,940 & 0,967 & 0,936 & 0,069 & 0,817 & 1,107 & 0,545 & 0,476 & 0,855 & 0,407 & 0,408 & 155 & 12 & $-3,1$ \\
\hline 13 & 3,8 & 8,4 & 4,5 & 8,2 & 8,0 & 0,852 & 0,975 & 0,944 & 0,150 & 0,676 & 1,216 & 0,449 & 0,586 & 3,293 & 1,928 & 1,457 & 49 & 13 & $-5,0$ \\
\hline 14 & 0,6 & 2,7 & 0,6 & 2,7 & 2,6 & 0,979 & 0,974 & 0,943 & 0,034 & 1,000 & 1,000 & 0,702 & 0,619 & 1,040 & 0,644 & 0,682 & 7 & 14 & $-3,4$ \\
\hline 15 & 0,1 & 1,1 & 0,1 & 1,0 & 0,9 & 0,815 & 0,870 & 0,843 & 0,226 & 0,785 & 1,129 & 0,462 & 0,461 & 0,458 & 0,211 & 0,227 & 110 & 15 & $-2,2$ \\
\hline 16 & 0,5 & 2,8 & 0,6 & 2,7 & 2,6 & 0,960 & 0,971 & 0,940 & 0,050 & 0,904 & $\begin{array}{l}1,052 \\
\text { S }\end{array}$ & 0,553 & 0,570 & 1,122 & 0,639 & 0,643 & 80 & 16 & $-3,5$ \\
\hline 17 & 9,5 & 13,6 & 10,5 & 13,1 & 12,7 & 0,912 & 0,970 & 0,939 & 0,092 & 0,653 & 1,238 & 0,442 & 0,549 & 5,244 & 2,879 & 2,630 & 1 & 17 & $-5,7$ \\
\hline 18 & 0,2 & 1,6 & 0,2 & 1,6 & 1,5 & 0,967 & 0,944 & 0,913 & 0,065 & 0,987 & 1,007 & 0,754 & 0,791 & 0,599 & 0,474 & 0,429 & 120 & 18 & $\begin{array}{l}-2,6 \\
-2,6\end{array}$ \\
\hline 19 & 1,8 & 5,2 & 1,9 & 4,9 & 4,8 & 0,926 & 0,953 & 0,922 & 0,088 & 0,839 & $\begin{array}{l}1,092 \\
\text {. }\end{array}$ & 0,706 & 0,764 & 1,801 & 1,375 & 1,186 & 92 & 19 & $-4,2$ \\
\hline 20 & 78,7 & 47,1 & 101,9 & 38,5 & 37,3 & 0,772 & 0,817 & 0,791 & 0,292 & 0,445 & $\begin{array}{l}1,499 \\
\text { S }\end{array}$ & 0,611 & 0,854 & 12,804 & 10,934 & 1,948 & 128 & 20 & $\begin{array}{l}-7,0 \\
-1,0\end{array}$ \\
\hline 21 & 2,7 & 6,3 & 2,8 & 6,1 & 5,9 & 0,964 & 0,974 & 0,942 & 0,045 & 0,862 & $\begin{array}{l}1,077 \\
1\end{array}$ & 0,574 & 0,701 & 2,440 & 1,711 & 1,545 & 47 & 21 & $-4,6$ \\
\hline 22 & 7,9 & 11,5 & 8,6 & 11,3 & 11,0 & 0,912 & 0,990 & 0,958 & 0,089 & 0,751 & 1,154 & 0,585 & 0,718 & 4,136 & 2,971 & 2,260 & 163 & 22 & $-5,4$ \\
\hline 23 & 0,4 & 2,4 & 0,4 & 2,3 & 2,3 & 0,968 & 0,987 & 0,956 & 0,035 & 0,869 & 1,073 & 0,512 & 0,572 & 0,978 & 0,559 & 0,577 & 144 & 23 & $-3,3$ \\
\hline 24 & 0,3 & 2,2 & 0,4 & 2,2 & 2,1 & 0,960 & 0,987 & 0,955 & 0,042 & 0,869 & 1,073 & 0,506 & 0,487 & 0,929 & 0,452 & 0,455 & 89 & 24 & $-3,2$ \\
\hline 25 & 0,5 & 2,6 & 0,5 & 2,5 & 2,4 & 0,945 & 0,954 & 0,923 & 0,072 & 0,894 & 1,057 & 0,690 & 0,617 & 0,956 & 0,590 & 0,606 & 12 & 25 & $-3,3$ \\
\hline 26 & 0,9 & 4,3 & 1,1 & 4,1 & $\begin{array}{l}2,7 \\
3,9\end{array}$ & 0,842 & 0,950 & 0,920 & 0,166 & 0,609 & 1,281 & 0,410 & 0,513 & $\begin{array}{l}1,665 \\
\end{array}$ & 0,854 & 0,625 & 114 & 26 & $-4,1$ \\
\hline 27 & 0,5 & 3,1 & 0,5 & 3,0 & 2,9 & 0,940 & 0,974 & 0,943 & 0,066 & 0,665 & 1,226 & 0,347 & 0,396 & 1,372 & 0,543 & 0,530 & 99 & 27 & $-3,8$ \\
\hline 28 & 0,3 & 2,0 & 0,3 & 2,0 & 1,9 & 0,966 & 0,965 & 0,934 & 0,049 & 0,981 & 1,010 & 0,686 & 0,692 & 0,773 & 0,535 & 0,536 & 106 & 28 & $-3,0$ \\
\hline 29 & 1,0 & 3,9 & 1,1 & 3,7 & 3,6 & 0,941 & 0,966 & 0,935 & 0,068 & 0,878 & $\begin{array}{l}1,067 \\
\end{array}$ & 0,696 & 0,688 & 1,377 & 0,947 & 0,915 & 102 & 29 & $-3,8$ \\
\hline 30 & 1,0 & 4,7 & 1,1 & 4,6 & 4,4 & 0,912 & 0,965 & 0,935 & 0,095 & 0,581 & 1,312 & 0,280 & 0,296 & 2,174 & 0,643 & 0,455 & 139 & 30 & $-4,4$ \\
\hline 31 & 0,1 & 1,2 & 0,1 & 1,1 & 1,0 & 0,880 & 0,850 & 0,823 & 0,192 & 0,859 & 1,079 & 0,502 & 0,508 & 0,515 & 0,262 & 0,240 & 72 & 31 & $-2,4$ \\
\hline 32 & 108,9 & 40,9 & 114,5 & 39,9 & 38,6 & 0,951 & 0,976 & 0,945 & 0,054 & 0,818 & 1,105 & 0,724 & 0,706 & 13,836 & 9,769 & 9,645 & 41 & 32 & $-7,1$ \\
\hline 33 & 1,0 & 4,1 & 1,1 & 4,1 & 3,9 & 0,930 & 0,988 & 0,956 & 0,071 & 0,772 & 1,138 & 0,550 & 0,685 & 1,557 & 1,067 & 0,909 & 163 & 33 & $-4,0$ \\
\hline 34 & 5,0 & 8,9 & 5,6 & 8,6 & 8,3 & 0,902 & 0,964 & 0,933 & 0,105 & 0,790 & 1,125 & 0,637 & 0,750 & 3,165 & 2,373 & 2,186 & 93 & 34 & $-5,0$ \\
\hline 35 & 21,0 & 20,3 & 23,4 & 18,9 & 18,3 & 0,894 & 0,929 & 0,899 & 0,127 & 0,638 & 1,252 & 0,495 & 0,656 & 7,344 & 4,817 & 3,940 & 50 & 35 & $-6,2$ \\
\hline 36 & 0,3 & 2,1 & 0,3 & 2,0 & 1,9 & 0,918 & 0,961 & 0,930 & 0,091 & 0,859 & $\begin{array}{l}1,079 \\
\text { S }\end{array}$ & 0,573 & 0,652 & 0,803 & 0,523 & 0,485 & 147 & 36 & $\begin{array}{l}-3,0 \\
-3,0\end{array}$ \\
\hline 37 & 13,1 & 23,5 & 18,4 & 21,1 & 20,4 & 0,708 & 0,895 & 0,867 & 0,310 & 0,297 & 1,836 & 0,187 & 0,257 & 9,434 & 2,426 & 1,382 & 82 & 37 & $-6,6$ \\
\hline 38 & 3,7 & 10,3 & 4,4 & 7,8 & 7,6 & 0,846 & 0,762 & 0,738 & 0,283 & 0,443 & 1,503 & 0,560 & 0,665 & 2,905 & 1,932 & 0,915 & 7 & 38 & $-4,9$ \\
\hline $\begin{array}{l}50 \\
39\end{array}$ & $\begin{array}{r}29,0 \\
29,0\end{array}$ & 23,1 & $\begin{array}{l}4,4 \\
30,7\end{array}$ & $\begin{array}{l}23,0 \\
23,0\end{array}$ & 22,2 & $\begin{array}{l}0,0940 \\
0,943\end{array}$ & 0,994 & 0,962 & 0,058 & 0,681 & 1,212 & 0,434 & 0,446 & 9,218 & $\begin{array}{r}4,112 \\
4,12\end{array}$ & 3,966 & 73 & $\begin{array}{l}30 \\
39\end{array}$ & $-6,5$ \\
\hline 40 & 0,7 & 3,3 & 0,7 & 3,2 & 3,1 & 0,923 & 0,984 & 0,953 & 0,078 & 0,782 & 1,131 & 0,546 & 0,599 & 1,238 & 0,741 & 0,678 & 81 & 40 & $-3,6$ \\
\hline 41 & 1,6 & 5,8 & 1,9 & 5,5 & 5,3 & 0,834 & 0,949 & 0,918 & 0,174 & 0,598 & 1,293 & 0,359 & 0,479 & 2,373 & 1,137 & 0,546 & 23 & 41 & $-4,6$ \\
\hline 42 & $\begin{array}{l}5,0 \\
5,3\end{array}$ & $\begin{array}{r}10,0 \\
10,3\end{array}$ & 6,3 & 9,6 & 9,3 & 0,851 & 0,931 & 0,901 & 0,164 & 0,632 & $\begin{array}{l}1,258 \\
\end{array}$ & 0,487 & 0,562 & 3,728 & 2,094 & 1,677 & 75 & 42 & $-5,2$ \\
\hline 43 & 0,4 & 2,7 & 0,5 & 2,6 & 2,5 & 0,955 & 0,962 & 0,931 & 0,058 & 0,744 & 1,159 & 0,374 & 0,376 & 1,210 & 0,455 & 0,461 & 107 & 43 & $-3,6$ \\
\hline 44 & 0,9 & 3,8 & 1,0 & 3,6 & 3,5 & 0,866 & 0,937 & 0,907 & 0,148 & 0,769 & 1,140 & 0,712 & 0,821 & 1,264 & 1,038 & 0,816 & 28 & 44 & $-3,7$ \\
\hline 45 & 0,6 & 2,8 & 0,6 & 2,8 & 2,7 & 0,961 & 0,989 & 0,958 & 0,040 & 0,901 & $\begin{array}{l}1,054 \\
\end{array}$ & 0,583 & 0,619 & $\begin{array}{l}1,114 \\
1,10\end{array}$ & 0,690 & 0,678 & 107 & 45 & $-3,5$ \\
\hline 46 & 0,1 & 1,1 & 0,1 & 1,0 & 0,9 & 0,949 & 0,896 & 0,867 & 0,116 & 0,843 & 1,089 & 0,524 & 0,574 & 0,442 & 0,254 & 0,227 & 64 & 46 & $-2,1$ \\
\hline 47 & 1,8 & 6,0 & 2,3 & 5,6 & 5,5 & 0,795 & 0,934 & 0,904 & 0,215 & 0,620 & 1,270 & 0,455 & 0,599 & 2,246 & 1,345 & 0,964 & 170 & 47 & $-4,5$ \\
\hline 48 & 1,4 & 4,7 & 1,6 & 4,4 & 4,3 & 0,901 & 0,955 & 0,924 & 0,109 & 0,834 & 1,095 & 0,752 & 0,845 & 1,560 & 1,318 & 1,085 & 135 & 48 & $-4,0$ \\
\hline 49 & 0,1 & 1,5 & 0,1 & 1,2 & 1,2 & 0,900 & 0,857 & 0,830 & 0,174 & 0,772 & 1,138 & 0,458 & 0,453 & 0,599 & 0,271 & 0,312 & 23 & 49 & $-2,6$ \\
\hline
\end{tabular}




\begin{tabular}{|c|c|c|c|c|c|c|c|c|c|c|c|c|c|c|c|c|c|c|c|}
\hline 50 & 1,3 & 4,5 & 1,4 & 4,4 & 4,3 & 0,937 & 0,982 & 0,951 & 0,066 & 0,830 & 1,098 & 0,589 & 0,614 & 1,705 & 1,046 & 1,052 & 63 & 50 & $-4,1$ \\
\hline 51 & 0,5 & 3,0 & 0,6 & 2,9 & 2,8 & 0,924 & 0,966 & 0,935 & 0,083 & 0,765 & 1,143 & 0,461 & 0,526 & 1,224 & 0,644 & 0,606 & 81 & 51 & $-3,6$ \\
\hline 52 & 0,6 & 2,8 & 0,6 & 2,8 & 2,7 & 0,970 & 0,967 & 0,936 & 0,044 & 0,937 & 1,033 & 0,632 & 0,647 & 1,104 & 0,715 & 0,698 & 72 & 52 & $-3,5$ \\
\hline 53 & 16,3 & 19,8 & 18,9 & 16,5 & 16,0 & 0,865 & 0,833 & 0,806 & 0,215 & 0,521 & 1,386 & 0,446 & 0,540 & 6,823 & 3,684 & 2,304 & 120 & 53 & $-6,1$ \\
\hline 54 & 22,4 & 18,8 & 23,7 & 18,5 & 17,9 & 0,948 & 0,983 & 0,951 & 0,055 & 0,798 & 1,119 & 0,594 & 0,558 & 6,936 & 3,870 & 3,665 & 54 & 54 & $-6,1$ \\
\hline 55 & 2,5 & 6,1 & 2,6 & 5,9 & 5,8 & 0,953 & 0,970 & 0,939 & 0,056 & 0,836 & 1,094 & 0,634 & 0,688 & 2,240 & 1,542 & 1,534 & 97 & 55 & $-4,5$ \\
\hline 56 & 0,3 & 2,4 & 0,3 & 2,3 & 2,3 & 0,933 & 0,975 & 0,944 & 0,072 & 0,657 & 1,233 & 0,323 & 0,326 & 1,085 & 0,353 & 0,379 & 112 & 56 & $-3,4$ \\
\hline 57 & 15,6 & 22,1 & 22,9 & 20,4 & 19,8 & 0,682 & 0,924 & 0,894 & 0,327 & 0,401 & 1,578 & 0,275 & 0,408 & 8,510 & 3,473 & 0,321 & 61 & 57 & $-6,4$ \\
\hline 58 & 1,9 & 6,5 & 2,3 & 5,8 & 5,6 & 0,810 & 0,887 & 0,858 & 0,221 & 0,552 & 1,345 & 0,514 & 0,725 & 2,157 & 1,564 & 0,719 & 125 & 58 & $-4,4$ \\
\hline 59 & 0,2 & 1,8 & 0,2 & 1,8 & 1,7 & 0,922 & 0,984 & 0,953 & 0,079 & 0,643 & 1,247 & 0,303 & 0,280 & 0,847 & 0,237 & 0,240 & 116 & 59 & $-3,1$ \\
\hline 60 & 0,5 & 2,7 & 0,5 & 2,6 & 2,5 & 0,932 & 0,955 & 0,924 & 0,082 & 0,774 & 1,137 & 0,461 & 0,441 & 1,119 & 0,494 & 0,479 & 33 & 60 & $-3,5$ \\
\hline 61 & 0,6 & 3,0 & 0,6 & 3,0 & 2,9 & 0,952 & 0,990 & 0,959 & 0,050 & 0,827 & 1,100 & 0,475 & 0,498 & 1,259 & 0,628 & 0,643 & 17 & 61 & $-3,7$ \\
\hline 62 & 0,1 & 1,3 & 0,1 & 1,2 & 1,2 & 0,980 & 0,960 & 0,929 & 0,045 & 1,000 & 0,951 & 0,816 & 0,800 & 0,469 & 0,375 & 0,339 & 104 & 62 & $-2,2$ \\
\hline 63 & 11,3 & 14,6 & 12,0 & 14,5 & 14,0 & 0,939 & 0,994 & 0,962 & 0,062 & 0,664 & 1,227 & 0,434 & 0,525 & 5,750 & 3,021 & 2,677 & 60 & 63 & $-5,8$ \\
\hline 64 & 1,8 & 5,4 & 2,0 & 5,1 & 5,0 & 0,897 & 0,960 & 0,929 & 0,110 & 0,781 & 1,132 & 0,634 & 0,772 & 1,891 & 1,461 & 1,154 & 102 & 64 & $-4,2$ \\
\hline 65 & 0,8 & 3,5 & 0,9 & 3,4 & 3,3 & 0,964 & 0,980 & 0,948 & 0,042 & 0,861 & 1,078 & 0,534 & 0,551 & 1,415 & 0,779 & 0,773 & 101 & 65 & $-3,8$ \\
\hline 66 & 0,4 & 2,4 & 0,4 & 2,3 & 2,2 & 0,921 & 0,932 & 0,902 & 0,104 & 0,787 & 1,127 & 0,473 & 0,504 & 0,994 & 0,501 & 0,479 & 121 & 66 & $-3,3$ \\
\hline 67 & 0,3 & 1,9 & 0,3 & 1,6 & 1,5 & 0,911 & 0,861 & 0,833 & 0,165 & 0,916 & 1,045 & 0,655 & 0,636 & 0,696 & 0,443 & 0,408 & 62 & 67 & $-2,8$ \\
\hline 68 & 0,3 & 2,2 & 0,4 & 2,1 & 2,0 & 0,949 & 0,949 & 0,918 & 0,073 & 0,872 & 1,071 & 0,495 & 0,497 & 0,941 & 0,467 & 0,461 & 60 & 68 & $-3,2$ \\
\hline 69 & 15,0 & 15,4 & 15,9 & 14,8 & 14,3 & 0,941 & 0,958 & 0,927 & 0,073 & 0,795 & 1,122 & 0,586 & 0,674 & 5,710 & 3,849 & 3,592 & 149 & 69 & $-5,8$ \\
\hline 70 & 4,2 & 7,8 & 4,4 & 7,7 & 7,4 & 0,965 & 0,991 & 0,959 & 0,037 & 0,879 & 1,066 & 0,637 & 0,645 & 2,904 & 1,874 & 1,767 & 73 & 70 & $-4,9$ \\
\hline 71 & 10,0 & 11,9 & 10,4 & 11,8 & 11,4 & 0,962 & 0,987 & 0,955 & 0,040 & 0,883 & 1,064 & 0,655 & 0,677 & 4,409 & 2,983 & 2,844 & 63 & 71 & $-5,5$ \\
\hline 72 & 1,3 & 4,1 & 1,3 & 4,1 & 3,9 & 0,968 & 0,993 & 0,961 & 0,033 & 0,966 & 1,018 & 0,732 & 0,724 & 1,502 & 1,088 & 1,061 & 116 & 72 & $-3,9$ \\
\hline 73 & 44,1 & 45,9 & 61,4 & 34,1 & 33,0 & 0,719 & 0,744 & 0,720 & 0,380 & 0,264 & 1,947 & 0,253 & 0,386 & 14,896 & 5,746 & 2,868 & 129 & 73 & $-7,2$ \\
\hline 74 & 0,2 & 1,5 & 0,2 & 1,4 & 1,4 & 0,971 & 0,966 & 0,935 & 0,045 & 1,000 & 0,952 & 0,922 & 0,877 & 0,515 & 0,452 & 0,442 & 115 & 74 & $-2,4$ \\
\hline 75 & 0,2 & 1,5 & 0,2 & 1,5 & 1,4 & 0,978 & 0,966 & 0,935 & 0,040 & 1,000 & 0,973 & 0,832 & 0,796 & 0,538 & 0,429 & 0,408 & 64 & 75 & $-2,4$ \\
\hline 76 & 1,3 & 4,4 & 1,4 & 4,4 & 4,2 & 0,911 & 0,989 & 0,957 & 0,090 & 0,820 & 1,104 & 0,686 & 0,803 & 1,542 & 1,237 & 0,985 & 69 & 76 & $-3,9$ \\
\hline 77 & 0,8 & 3,3 & 0,9 & 3,1 & 3,0 & 0,961 & 0,931 & 0,901 & 0,079 & 0,938 & 1,033 & 0,718 & 0,754 & 1,206 & 0,909 & 0,857 & 70 & 77 & $-3,6$ \\
\hline 78 & 1,7 & 5,6 & 1,9 & 5,4 & 5,2 & 0,897 & 0,961 & 0,930 & 0,110 & 0,697 & 1,198 & 0,434 & 0,563 & 2,264 & 1,275 & 1,183 & 149 & 78 & $-4,5$ \\
\hline 79 & 28,9 & 21,3 & 30,8 & 20,5 & 19,8 & 0,938 & 0,960 & 0,929 & 0,073 & 0,799 & 1,119 & 0,610 & 0,696 & 7,772 & 5,409 & 5,037 & 65 & 79 & $-6,3$ \\
\hline 80 & 0,7 & 3,4 & 0,8 & 3,3 & 3,2 & 0,896 & 0,974 & 0,943 & 0,107 & 0,795 & 1,122 & 0,660 & 0,779 & 1,198 & 0,934 & 0,719 & 85 & 80 & $-3,6$ \\
\hline 81 & 5,1 & 10,7 & 5,9 & 9,8 & 9,5 & 0,861 & 0,918 & 0,889 & 0,161 & 0,559 & 1,338 & 0,373 & 0,505 & 4,173 & 2,107 & 1,728 & 156 & 81 & $-5,4$ \\
\hline 82 & 1,0 & 3,8 & 1,0 & 3,8 & 3,7 & 0,961 & 0,987 & 0,955 & 0,041 & 0,839 & 1,092 & 0,578 & 0,626 & 1,473 & 0,922 & 0,857 & 119 & 82 & $-3,9$ \\
\hline 83 & 0,3 & 2,4 & 0,4 & 2,3 & 2,2 & 0,946 & 0,957 & 0,926 & 0,069 & 0,788 & 1,127 & 0,410 & 0,437 & 1,040 & 0,455 & 0,455 & 97 & 83 & $-3,4$ \\
\hline 84 & 11,6 & 15,9 & 12,6 & 15,5 & 15,0 & 0,916 & 0,976 & 0,945 & 0,087 & 0,575 & 1,319 & 0,315 & 0,321 & 6,842 & 2,199 & 2,068 & 91 & 84 & $-6,1$ \\
\hline 85 & 10,8 & 14,3 & 11,9 & 13,5 & 13,0 & 0,905 & 0,939 & 0,909 & 0,113 & 0,660 & 1,231 & 0,456 & 0,481 & 5,489 & 2,641 & 2,252 & 134 & 85 & $-5,8$ \\
\hline 86 & 0,0 & 0,7 & 0,0 & 0,6 & 0,6 & 0,941 & 0,899 & 0,872 & 0,116 & 1,000 & 0,941 & 0,702 & 0,529 & 0,288 & 0,153 & 0,169 & 27 & 86 & $-1,5$ \\
\hline 87 & 0,1 & 1,2 & 0,1 & 1,2 & 1,1 & 0,946 & 0,958 & 0,928 & 0,068 & 1,000 & 0,970 & 0,818 & 0,725 & 0,443 & 0,321 & 0,339 & 39 & 87 & $-2,1$ \\
\hline 88 & 0,1 & 1,3 & 0,1 & 1,1 & 1,1 & 0,911 & 0,848 & 0,821 & 0,176 & 0,727 & 1,172 & 0,733 & 0,773 & 0,423 & 0,327 & 0,240 & 94 & 88 & $-2,1$ \\
\hline 89 & 4,4 & 8,1 & 4,5 & 8,1 & 7,8 & 0,974 & 0,993 & 0,961 & 0,027 & 0,843 & 1,089 & 0,544 & 0,576 & 3,215 & 1,851 & 1,832 & 149 & 89 & $-5,0$ \\
\hline 90 & 0,1 & 1,4 & 0,2 & 1,3 & 1,2 & 0,962 & 0,932 & 0,903 & 0,078 & 0,981 & 1,010 & 0,669 & 0,646 & 0,528 & 0,341 & 0,379 & 170 & 90 & $-2,4$ \\
\hline 91 & 0,2 & 1,8 & 0,3 & 1,7 & 1,7 & 0,972 & 0,971 & 0,940 & 0,040 & 0,982 & 1,009 & 0,680 & 0,593 & 0,678 & 0,402 & 0,408 & 41 & 91 & $-2,8$ \\
\hline 92 & 0,8 & 3,4 & 0,8 & 3,3 & 3,2 & 0,970 & 0,991 & 0,959 & 0,032 & 0,855 & 1,082 & 0,563 & 0,465 & 1,325 & 0,616 & 0,643 & 135 & 92 & $-3,7$ \\
\hline 93 & 0,2 & 1,6 & 0,2 & 1,6 & 1,5 & 0,981 & 0,968 & 0,937 & 0,037 & 1,000 & 0,965 & 0,881 & 0,840 & 0,563 & 0,473 & 0,479 & 67 & 93 & $-2,5$ \\
\hline 94 & 1,2 & 4,0 & 1,2 & 4,0 & 3,9 & 0,962 & 0,993 & 0,961 & 0,039 & 0,904 & 1,052 & 0,738 & 0,823 & 1,420 & 1,168 & 1,061 & 85 & 94 & $-3,8$ \\
\hline 95 & 1,2 & 4,5 & 1,3 & 4,4 & 4,3 & 0,963 & 0,982 & 0,951 & 0,041 & 0,764 & 1,144 & 0,483 & 0,504 & 1,807 & 0,911 & 0,864 & 47 & 95 & $-4,2$ \\
\hline 96 & 0,6 & 3,2 & 0,7 & 3,2 & 3,1 & 0,890 & 0,975 & 0,944 & 0,113 & 0,741 & 1,161 & 0,464 & 0,506 & 1,302 & 0,659 & 0,611 & 77 & 96 & $-3,7$ \\
\hline 97 & 0,4 & 2,8 & 0,5 & 2,7 & 2,6 & 0,931 & 0,966 & 0,935 & 0,077 & 0,743 & 1,160 & 0,446 & 0,418 & 1,130 & 0,472 & 0,485 & 39 & 97 & $-3,5$ \\
\hline 98 & 0,1 & 1,2 & 0,1 & 1,2 & 1,1 & 0,955 & 0,958 & 0,927 & 0,062 & 1,000 & 0,973 & 0,866 & 0,846 & 0,423 & 0,358 & 0,321 & 56 & 98 & $-2,1$ \\
\hline 99 & 0,2 & 1,8 & 0,2 & 1,6 & 1,6 & 0,944 & 0,919 & 0,890 & 0,099 & 0,766 & 1,142 & 0,401 & 0,412 & 0,781 & 0,321 & 0,339 & 49 & 99 & $-3,0$ \\
\hline 100 & 0,5 & 2,5 & 0,5 & 2,5 & 2,4 & 0,974 & 0,988 & 0,956 & 0,028 & 0,984 & 1,008 & 0,689 & 0,728 & 0,954 & 0,695 & 0,698 & 180 & 100 & $-3,3$ \\
\hline 101 & 27,3 & 21,2 & 29,6 & 19,7 & 19,1 & 0,920 & 0,931 & 0,901 & 0,106 & 0,764 & 1,144 & 0,708 & 0,734 & 7,001 & 5,141 & 4,697 & 84 & 101 & $-6,1$ \\
\hline 102 & 0,1 & 1,2 & 0,1 & 1,1 & 1,1 & 0,962 & 0,974 & 0,943 & 0,046 & 1,000 & 0,993 & 0,774 & 0,766 & 0,423 & 0,325 & 0,303 & 94 & 102 & $-2,1$ \\
\hline 103 & 0,3 & 1,8 & 0,3 & 1,7 & 1,7 & 0,968 & 0,983 & 0,952 & 0,036 & 1,000 & 0,973 & 0,874 & 0,852 & 0,617 & 0,525 & 0,530 & 90 & 103 & $-2,6$ \\
\hline 104 & 6,3 & 11,9 & 7,0 & 11,2 & 10,8 & 0,895 & 0,943 & 0,913 & 0,120 & 0,563 & 1,333 & 0,332 & 0,438 & 4,919 & 2,154 & 2,021 & 104 & 104 & $-5,6$ \\
\hline 105 & 1,0 & 3,9 & 1,1 & 3,8 & 3,7 & 0,915 & 0,960 & 0,930 & 0,094 & 0,835 & 1,094 & 0,658 & 0,729 & 1,414 & 1,031 & 0,925 & 148 & 105 & $-3,8$ \\
\hline 106 & 2,4 & 5,9 & 2,5 & 5,9 & 5,7 & 0,937 & 0,999 & 0,967 & 0,063 & 0,865 & 1,075 & 0,613 & 0,655 & 2,222 & 1,456 & 1,323 & 141 & 106 & $-4,5$ \\
\hline 107 & 1,1 & 3,7 & 1,1 & 3,7 & 3,6 & 0,977 & 0,992 & 0,960 & 0,025 & 0,988 & 1,006 & 0,777 & 0,736 & 1,334 & 0,982 & 0,970 & 118 & 107 & $-3,7$ \\
\hline
\end{tabular}




\begin{tabular}{|c|c|c|c|c|c|c|c|c|c|c|c|c|c|c|c|c|c|c|c|}
\hline 108 & 0,9 & 4,0 & 1,0 & 3,9 & 3,8 & 0,946 & 0,985 & 0,953 & 0,056 & 0,713 & 1,184 & 0,348 & 0,407 & 1,815 & 0,738 & 0,758 & 108 & 108 & $-4,2$ \\
\hline 109 & 0,9 & 4,2 & 1,0 & 4,1 & 4,0 & 0,860 & 0,986 & 0,954 & 0,140 & 0,628 & 1,262 & 0,345 & 0,379 & 1,797 & 0,682 & 0,606 & 109 & 109 & $-4,2$ \\
\hline 110 & 0,3 & 1,9 & 0,3 & 1,8 & 1,7 & 0,954 & 0,957 & 0,927 & 0,063 & 0,961 & 1,020 & 0,679 & 0,642 & 0,708 & 0,455 & 0,455 & 92 & 110 & $-2,8$ \\
\hline 111 & 2,2 & 5,9 & 2,4 & 5,8 & 5,6 & 0,944 & 0,988 & 0,956 & 0,057 & 0,811 & 1,111 & 0,592 & 0,654 & 2,186 & 1,429 & 1,349 & 85 & 111 & $-4,5$ \\
\hline 112 & 0,1 & 0,9 & 0,1 & 0,9 & 0,9 & 0,966 & 0,967 & 0,936 & 0,047 & 1,000 & 0,896 & 0,994 & 0,819 & 0,324 & 0,265 & 0,273 & 96 & 112 & $-1,7$ \\
\hline 113 & 3,6 & 7,9 & 4,0 & 7,4 & 7,2 & 0,899 & 0,942 & 0,912 & 0,116 & 0,732 & 1,169 & 0,569 & 0,628 & 2,848 & 1,788 & 1,728 & 83 & 113 & $-4,8$ \\
\hline 114 & 1,5 & 5,1 & 1,6 & 5,0 & 4,8 & 0,936 & 0,980 & 0,949 & 0,067 & 0,730 & 1,170 & 0,432 & 0,450 & 2,100 & 0,946 & 0,958 & 123 & 114 & $-4,4$ \\
\hline 115 & 0,2 & 1,5 & 0,2 & 1,5 & 1,4 & 0,898 & 0,953 & 0,922 & 0,113 & 0,878 & 1,067 & 0,634 & 0,652 & 0,573 & 0,374 & 0,321 & 23 & 115 & $-2,5$ \\
\hline 116 & 1,2 & 4,2 & 1,2 & 4,1 & 3,9 & 0,970 & 0,961 & 0,930 & 0,049 & 0,818 & 1,106 & 0,443 & 0,470 & 1,822 & 0,857 & 0,864 & 133 & 116 & $-4,2$ \\
\hline 117 & 0,2 & 1,7 & 0,3 & 1,6 & 1,6 & 0,983 & 0,953 & 0,923 & 0,050 & 1,000 & 0,972 & 0,648 & 0,614 & 0,698 & 0,429 & 0,479 & 127 & 117 & $-2,8$ \\
\hline 118 & 0,4 & 2,7 & 0,5 & 2,6 & 2,5 & 0,937 & 0,973 & 0,942 & 0,068 & 0,732 & 1,169 & 0,409 & 0,493 & 1,152 & 0,568 & 0,546 & 86 & 118 & $-3,5$ \\
\hline 119 & 1,4 & 4,4 & 1,5 & 4,4 & 4,2 & 0,974 & 0,989 & 0,957 & 0,029 & 0,921 & 1,042 & 0,675 & 0,685 & 1,646 & 1,128 & 1,119 & 137 & 119 & $-4,0$ \\
\hline 120 & 0,9 & 3,6 & 1,0 & 3,5 & 3,4 & 0,949 & 0,986 & 0,954 & 0,053 & 0,905 & 1,051 & 0,663 & 0,612 & 1,334 & 0,817 & 0,773 & 107 & 120 & $-3,7$ \\
\hline 121 & 4,2 & 8,3 & 4,5 & 7,7 & 7,4 & 0,914 & 0,926 & 0,897 & 0,113 & 0,759 & 1,148 & 0,677 & 0,753 & 2,794 & 2,104 & 1,970 & 93 & 121 & $-4,8$ \\
\hline 122 & 0,5 & 2,7 & 0,5 & 2,6 & 2,5 & 0,968 & 0,963 & 0,932 & 0,049 & 0,898 & 1,055 & 0,584 & 0,636 & 1,071 & 0,682 & 0,678 & 95 & 122 & $-3,4$ \\
\hline 123 & 0,5 & 2,7 & 0,6 & 2,6 & 2,6 & 0,960 & 0,981 & 0,950 & 0,044 & 0,945 & 1,029 & 0,641 & 0,616 & 1,042 & 0,642 & 0,678 & 87 & 123 & $-3,4$ \\
\hline 124 & 0,5 & 2,6 & 0,5 & 2,5 & 2,5 & 0,958 & 0,980 & 0,949 & 0,046 & 0,851 & 1,084 & 0,456 & 0,480 & 1,130 & 0,542 & 0,577 & 20 & 124 & $-3,5$ \\
\hline 125 & 0,1 & 1,2 & 0,1 & 1,1 & 1,0 & 0,946 & 0,913 & 0,883 & 0,103 & 0,934 & 1,035 & 0,602 & 0,658 & 0,461 & 0,303 & 0,273 & 77 & 125 & $-2,2$ \\
\hline 126 & 57,0 & 33,6 & 65,0 & 32,3 & 31,2 & 0,877 & 0,959 & 0,928 & 0,130 & 0,633 & 1,257 & 0,442 & 0,588 & 12,817 & 7,532 & 3,557 & 62 & 126 & $-7,0$ \\
\hline 127 & 9,7 & 13,2 & 10,5 & 12,6 & 12,2 & 0,930 & 0,959 & 0,928 & 0,081 & 0,703 & 1,193 & 0,513 & 0,584 & 4,910 & 2,868 & 2,572 & 141 & 127 & $-5,6$ \\
\hline 128 & 0,6 & 3,0 & 0,7 & 2,9 & 2,8 & 0,910 & 0,969 & 0,938 & 0,095 & 0,855 & 1,082 & 0,678 & 0,678 & 1,071 & 0,727 & 0,652 & 117 & 128 & $-3,4$ \\
\hline 129 & 0,1 & 1,2 & 0,1 & 1,1 & 1,1 & 0,961 & 0,945 & 0,915 & 0,067 & 1,000 & 0,999 & 0,688 & 0,674 & 0,443 & 0,299 & 0,303 & 108 & 129 & $-2,1$ \\
\hline 130 & 0,2 & 1,7 & 0,2 & 1,5 & 1,5 & 0,912 & 0,926 & 0,897 & 0,115 & 0,871 & 1,071 & 0,479 & 0,483 & 0,715 & 0,345 & 0,379 & 113 & 130 & $-2,8$ \\
\hline 131 & 0,1 & 1,2 & 0,1 & 1,1 & 1,1 & 0,916 & 0,892 & 0,863 & 0,137 & 0,881 & 1,065 & 0,605 & 0,593 & 0,479 & 0,284 & 0,303 & 17 & 131 & $-2,3$ \\
\hline 132 & 0,1 & 0,8 & 0,1 & 0,7 & 0,7 & 0,974 & 0,909 & 0,879 & 0,095 & 1,000 & 0,950 & 0,654 & 0,575 & 0,326 & 0,187 & 0,214 & 145 & 132 & $-1,7$ \\
\hline 133 & 0,3 & 2,8 & 0,4 & 2,7 & 2,6 & 0,903 & 0,963 & 0,932 & 0,104 & 0,552 & 1,346 & 0,255 & 0,259 & 1,288 & 0,334 & 0,379 & 32 & 133 & $-3,7$ \\
\hline 134 & 59,8 & 32,7 & 64,0 & 31,5 & 30,5 & 0,935 & 0,966 & 0,935 & 0,073 & 0,705 & 1,191 & 0,532 & 0,665 & 11,963 & 7,961 & 7,216 & 115 & 134 & $-6,9$ \\
\hline 135 & 0,3 & 2,2 & 0,4 & 2,2 & 2,1 & 0,952 & 0,977 & 0,946 & 0,053 & 0,872 & 1,071 & 0,549 & 0,483 & 0,892 & 0,431 & 0,442 & 111 & 135 & $-3,2$ \\
\hline 136 & 3,1 & 6,9 & 3,4 & 6,5 & 6,3 & 0,914 & 0,945 & 0,915 & 0,102 & 0,819 & 1,105 & 0,679 & 0,739 & 2,409 & 1,780 & 1,667 & 117 & 136 & $-4,6$ \\
\hline 137 & 0,1 & 1,5 & 0,1 & 1,4 & 1,3 & 0,919 & 0,945 & 0,914 & 0,098 & 0,781 & 1,132 & 0,395 & 0,409 & 0,648 & 0,265 & 0,303 & 91 & 137 & $-2,7$ \\
\hline 138 & 0,2 & 2,0 & 0,2 & 1,9 & 1,8 & 0,919 & 0,948 & 0,918 & 0,096 & 0,686 & 1,208 & 0,364 & 0,343 & 0,859 & 0,294 & 0,312 & 38 & 138 & $-3,1$ \\
\hline 139 & 0,3 & 2,1 & 0,3 & 2,0 & 1,9 & 0,942 & 0,965 & 0,934 & 0,068 & 0,962 & 1,020 & 0,663 & 0,664 & 0,791 & 0,525 & 0,530 & 78 & 139 & $-3,0$ \\
\hline 140 & 3,0 & 7,6 & 3,4 & 7,0 & 6,8 & 0,891 & 0,929 & 0,899 & 0,130 & 0,664 & 1,227 & 0,459 & 0,533 & 2,891 & 1,540 & 1,393 & 24 & 140 & $-4,9$ \\
\hline 141 & 13,5 & 20,6 & 18,1 & 17,1 & 16,5 & 0,745 & 0,828 & 0,802 & 0,307 & 0,398 & 1,584 & 0,343 & 0,526 & 7,063 & 3,718 & 2,737 & 50 & 141 & $-6,1$ \\
\hline 142 & 0,2 & 1,4 & 0,2 & 1,4 & 1,3 & 0,960 & 0,950 & 0,919 & 0,064 & 1,000 & 0,975 & 0,715 & 0,687 & 0,552 & 0,379 & 0,408 & 80 & 142 & $-2,5$ \\
\hline 143 & 0,3 & 2,1 & 0,4 & 2,0 & 2,0 & 0,942 & 0,943 & 0,913 & 0,082 & 0,954 & 1,024 & 0,802 & 0,783 & 0,744 & 0,583 & 0,552 & 71 & 143 & $-2,9$ \\
\hline 144 & 0,1 & 0,9 & 0,1 & 0,8 & 0,8 & 0,962 & 0,941 & 0,911 & 0,070 & 1,000 & 0,913 & 0,860 & 0,658 & 0,326 & 0,214 & 0,273 & 137 & 144 & $-1,7$ \\
\hline 145 & 0,5 & 2,7 & 0,5 & 2,6 & 2,5 & 0,933 & 0,959 & 0,928 & 0,079 & 0,876 & 1,068 & 0,607 & 0,646 & 1,023 & 0,661 & 0,647 & 155 & 145 & $-3,4$ \\
\hline 146 & 87,7 & 40,9 & 99,2 & 37,6 & 36,4 & 0,884 & 0,919 & 0,889 & 0,142 & 0,659 & 1,232 & 0,557 & 0,682 & 14,157 & 9,659 & 6,325 & 109 & 146 & $-7,1$ \\
\hline 147 & 7,2 & 10,3 & 7,6 & 10,0 & 9,7 & 0,954 & 0,972 & 0,941 & 0,054 & 0,859 & 1,079 & 0,744 & 0,804 & 3,514 & 2,825 & 2,541 & 128 & 147 & $-5,1$ \\
\hline 148 & 0,1 & 1,0 & 0,1 & 0,9 & 0,9 & 0,938 & 0,919 & 0,890 & 0,103 & 1,000 & 0,951 & 0,849 & 0,745 & 0,359 & 0,268 & 0,273 & 140 & 148 & $-1,8$ \\
\hline 149 & 0,1 & 1,3 & 0,1 & 1,2 & 1,2 & 0,816 & 0,925 & 0,895 & 0,199 & 0,615 & 1,275 & 0,316 & 0,273 & 0,599 & 0,163 & 0,169 & 14 & 149 & $-2,6$ \\
\hline 150 & 1,1 & 4,1 & 1,2 & 4,1 & 4,0 & 0,948 & 0,993 & 0,961 & 0,053 & 0,824 & 1,102 & 0,472 & 0,502 & 1,738 & 0,872 & 0,912 & 33 & 150 & $-4,1$ \\
\hline 151 & 0,1 & 0,7 & 0,1 & 0,7 & 0,7 & 0,973 & 0,959 & 0,928 & 0,049 & 1,000 & 0,897 & 0,865 & 0,680 & 0,276 & 0,187 & 0,214 & 110 & 151 & $-1,5$ \\
\hline 152 & 0,7 & 3,1 & 0,8 & 3,0 & 2,9 & 0,974 & 0,990 & 0,959 & 0,028 & 1,000 & 0,999 & 0,726 & 0,661 & 1,146 & 0,758 & 0,758 & 177 & 152 & $-3,5$ \\
\hline 153 & 329,6 & 75,1 & 346,2 & 69,0 & 66,8 & 0,952 & 0,918 & 0,889 & 0,095 & 0,734 & 1,167 & 0,639 & 0,714 & 25,634 & 18,297 & 16,608 & 174 & 153 & $-8,0$ \\
\hline 154 & 0,8 & 3,3 & 0,8 & 3,3 & 3,2 & 0,975 & 0,994 & 0,962 & 0,026 & 0,867 & 1,074 & 0,696 & 0,794 & 1,186 & 0,942 & 0,805 & 80 & 154 & $-3,6$ \\
\hline 155 & 1,8 & 5,0 & 1,9 & 4,9 & 4,8 & 0,963 & 0,984 & 0,952 & 0,040 & 0,898 & 1,055 & 0,586 & 0,653 & 1,973 & 1,288 & 1,176 & 2 & 155 & $-4,3$ \\
\hline 156 & 1,2 & 4,4 & 1,3 & 4,3 & 4,2 & 0,904 & 0,980 & 0,948 & 0,099 & 0,767 & 1,142 & 0,506 & 0,520 & 1,725 & 0,898 & 0,847 & 100 & 156 & $-4,1$ \\
\hline 157 & 3,9 & 7,5 & 4,0 & 7,4 & 7,2 & 0,958 & 0,988 & 0,956 & 0,043 & 0,854 & 1,082 & 0,618 & 0,651 & 2,819 & 1,835 & 1,762 & 163 & 157 & $-4,8$ \\
\hline 158 & 0,1 & 1,3 & 0,1 & 1,2 & 1,2 & 0,942 & 0,922 & 0,893 & 0,097 & 1,000 & 0,983 & 0,837 & 0,811 & 0,462 & 0,375 & 0,379 & 67 & 158 & $-2,2$ \\
\hline 159 & 0,2 & 1,8 & 0,2 & 1,7 & 1,7 & 0,929 & 0,983 & 0,952 & 0,073 & 0,904 & 1,052 & 0,551 & 0,537 & 0,724 & 0,389 & 0,379 & 14 & 159 & $-2,9$ \\
\hline 160 & 0,3 & 1,9 & 0,3 & 1,8 & 1,8 & 0,917 & 0,952 & 0,921 & 0,096 & 0,904 & 1,052 & 0,682 & 0,669 & 0,708 & 0,473 & 0,386 & 167 & 160 & $-2,8$ \\
\hline 161 & 0,5 & 2,7 & 0,5 & 2,6 & 2,5 & 0,941 & 0,955 & 0,925 & 0,074 & 0,836 & 1,093 & 0,527 & 0,540 & 1,098 & 0,593 & 0,577 & 150 & 161 & $-3,5$ \\
\hline 162 & 0,0 & 0,8 & 0,1 & 0,8 & 0,7 & 0,971 & 0,948 & 0,918 & 0,059 & 0,921 & 1,042 & 0,486 & 0,427 & 0,357 & 0,153 & 0,169 & 145 & 162 & $-1,8$ \\
\hline 163 & 0,2 & 1,5 & 0,2 & 1,4 & 1,3 & 0,910 & 0,951 & 0,921 & 0,103 & 0,941 & 1,031 & 0,764 & 0,825 & 0,515 & 0,425 & 0,379 & 89 & 163 & $-2,4$ \\
\hline 164 & 0,1 & 1,0 & 0,1 & 0,9 & 0,9 & 0,952 & 0,861 & 0,834 & 0,147 & 1,000 & 0,992 & 0,676 & 0,632 & 0,403 & 0,255 & 0,273 & 53 & 164 & $-2,0$ \\
\hline 165 & 14,7 & 14,7 & 15,4 & 14,5 & 14,0 & 0,956 & 0,988 & 0,956 & 0,046 & 0,861 & 1,078 & 0,604 & 0,645 & 5,574 & 3,597 & 3,328 & 106 & 165 & $-5,8$ \\
\hline
\end{tabular}




\begin{tabular}{|c|c|c|c|c|c|c|c|c|c|c|c|c|c|c|c|c|c|c|c|}
\hline 166 & 0,9 & 4,1 & 1,0 & 4,0 & 3,9 & 0,904 & 0,982 & 0,951 & 0,098 & 0,664 & 1,228 & 0,365 & 0,461 & 1,746 & 0,806 & 0,746 & 47 & 166 & $-4,1$ \\
\hline 167 & 0,6 & 3,0 & 0,6 & 2,9 & 2,8 & 0,943 & 0,963 & 0,932 & 0,068 & 0,832 & 1,096 & 0,511 & 0,545 & 1,210 & 0,659 & 0,647 & 106 & 167 & $-3,6$ \\
\hline 168 & 3,6 & 7,4 & 3,9 & 7,4 & 7,1 & 0,940 & 0,996 & 0,964 & 0,060 & 0,830 & 1,098 & 0,676 & 0,782 & 2,611 & 2,042 & 1,801 & 104 & 168 & $-4,7$ \\
\hline 169 & 12,8 & 15,2 & 13,7 & 15,0 & 14,5 & 0,931 & 0,984 & 0,953 & 0,071 & 0,693 & 1,201 & 0,383 & 0,436 & 6,523 & 2,841 & 2,576 & 173 & 169 & $-6,0$ \\
\hline 170 & 1,0 & 4,4 & 1,1 & 4,1 & 3,9 & 0,877 & 0,933 & 0,903 & 0,140 & 0,625 & 1,265 & 0,379 & 0,424 & 1,787 & 0,758 & 0,647 & 86 & 170 & $-4,2$ \\
\hline 171 & 172,3 & 75,6 & 196,9 & 52,9 & 51,2 & 0,875 & 0,700 & 0,677 & 0,325 & 0,379 & 1,625 & 0,556 & 0,730 & 19,868 & 14,496 & 5,807 & 74 & 171 & $-7,6$ \\
\hline 172 & 0,5 & 2,5 & 0,5 & 2,4 & 2,4 & 0,963 & 0,980 & 0,949 & 0,042 & 0,982 & 1,009 & 0,766 & 0,803 & 0,900 & 0,723 & 0,678 & 75 & 172 & $-3,2$ \\
\hline 173 & 0,2 & 2,0 & 0,2 & 1,9 & 1,8 & 0,910 & 0,959 & 0,929 & 0,099 & 0,649 & 1,241 & 0,330 & 0,291 & 0,883 & 0,257 & 0,273 & 59 & 173 & $-3,1$ \\
\hline 174 & 6,1 & 10,2 & 6,6 & 10,0 & 9,7 & 0,919 & 0,982 & 0,950 & 0,083 & 0,739 & 1,163 & 0,472 & 0,550 & 4,051 & 2,227 & 2,047 & 27 & 174 & $-5,3$ \\
\hline 175 & 12,4 & 13,6 & 13,2 & 13,4 & 13,0 & 0,943 & 0,985 & 0,953 & 0,059 & 0,841 & 1,090 & 0,687 & 0,774 & 4,797 & 3,711 & 3,258 & 79 & 175 & $-5,6$ \\
\hline 176 & 0,8 & 4,5 & 1,0 & 4,3 & 4,2 & 0,838 & 0,958 & 0,927 & 0,167 & 0,504 & 1,409 & 0,269 & 0,351 & 1,976 & 0,694 & 0,678 & 9 & 176 & $-4,3$ \\
\hline 177 & 0,1 & 1,2 & 0,1 & 1,1 & 1,0 & 0,938 & 0,867 & 0,839 & 0,147 & 1,000 & 0,972 & 0,836 & 0,683 & 0,443 & 0,303 & 0,339 & 6 & 177 & $-2,1$ \\
\hline 178 & 4,0 & 9,9 & 4,8 & 9,4 & 9,1 & 0,844 & 0,950 & 0,920 & 0,164 & 0,518 & 1,389 & 0,305 & 0,348 & 4,093 & 1,423 & 0,988 & 73 & 178 & $-5,4$ \\
\hline 179 & 0,0 & 0,7 & 0,0 & 0,6 & 0,6 & 1,000 & 0,892 & 0,863 & 0,108 & 1,000 & 0,918 & 0,738 & 0,600 & 0,268 & 0,161 & 0,169 & 143 & 179 & $-1,4$ \\
\hline 180 & 0,2 & 1,8 & 0,2 & 1,8 & 1,7 & 0,953 & 0,972 & 0,941 & 0,055 & 0,882 & 1,065 & 0,603 & 0,575 & 0,698 & 0,402 & 0,429 & 36 & 180 & $-2,8$ \\
\hline 181 & 0,1 & 1,1 & 0,1 & 1,0 & 0,9 & 0,884 & 0,906 & 0,878 & 0,149 & 0,939 & 1,032 & 0,597 & 0,562 & 0,432 & 0,243 & 0,240 & 54 & 181 & $-2,1$ \\
\hline 182 & 14,6 & 16,1 & 15,9 & 15,6 & 15,1 & 0,916 & 0,968 & 0,937 & 0,090 & 0,710 & 1,187 & 0,633 & 0,806 & 5,421 & 4,368 & 3,282 & 141 & 182 & $-5,8$ \\
\hline 183 & 0,7 & 3,2 & 0,7 & 3,2 & 3,1 & 0,968 & 0,988 & 0,956 & 0,034 & 0,846 & 1,087 & 0,531 & 0,537 & 1,295 & 0,695 & 0,698 & 120 & 183 & $-3,7$ \\
\hline 184 & 0,6 & 2,9 & 0,6 & 2,8 & 2,8 & 0,960 & 0,985 & 0,954 & 0,043 & 0,872 & 1,071 & 0,695 & 0,808 & 1,030 & 0,833 & 0,698 & 50 & 184 & $-3,4$ \\
\hline 185 & 0,3 & 2,1 & 0,3 & 2,1 & 2,0 & 0,961 & 0,986 & 0,954 & 0,042 & 0,905 & 1,051 & 0,580 & 0,580 & 0,831 & 0,482 & 0,485 & 128 & 185 & $-3,1$ \\
\hline 186 & 1,7 & 5,4 & 1,8 & 5,3 & 5,2 & 0,935 & 0,994 & 0,963 & 0,065 & 0,731 & 1,170 & 0,437 & 0,411 & 2,209 & 0,907 & 0,816 & 151 & 186 & $-4,5$ \\
\hline 187 & 83,1 & 47,8 & 96,4 & 37,7 & 36,5 & 0,862 & 0,789 & 0,764 & 0,252 & 0,458 & 1,478 & 0,528 & 0,649 & 14,156 & 9,192 & 6,612 & 60 & 187 & $-7,1$ \\
\hline 188 & 1,9 & 5,2 & 2,0 & 5,2 & 5,0 & 0,972 & 0,990 & 0,959 & 0,030 & 0,870 & 1,072 & 0,554 & 0,611 & 2,089 & 1,275 & 1,261 & 178 & 188 & $-4,4$ \\
\hline 189 & 0,4 & 2,3 & 0,4 & 2,2 & 2,2 & 0,941 & 0,969 & 0,938 & 0,066 & 0,924 & 1,040 & 0,638 & 0,643 & 0,884 & 0,568 & 0,577 & 103 & 189 & $-3,1$ \\
\hline 190 & 3,6 & 7,4 & 3,8 & 7,4 & 7,2 & 0,947 & 0,993 & 0,961 & 0,053 & 0,821 & 1,104 & 0,544 & 0,552 & 2,911 & 1,607 & 1,593 & 45 & 190 & $-4,9$ \\
\hline 191 & 19,2 & 17,8 & 20,2 & 17,4 & 16,8 & 0,953 & 0,976 & 0,945 & 0,052 & 0,762 & 1,145 & 0,468 & 0,454 & 7,232 & 3,283 & 3,172 & 96 & 191 & $-6,2$ \\
\hline 192 & 3,6 & 7,9 & 3,9 & 7,5 & 7,2 & 0,920 & 0,939 & 0,909 & 0,100 & 0,708 & 1,189 & 0,503 & 0,598 & 3,000 & 1,795 & 1,515 & 58 & 192 & $-4,9$ \\
\hline 193 & 7,7 & 13,0 & 9,5 & 11,7 & 11,3 & 0,810 & 0,901 & 0,872 & 0,215 & 0,572 & 1,323 & 0,484 & 0,581 & 4,495 & 2,612 & 1,762 & 33 & 193 & $-5,5$ \\
\hline 194 & 0,5 & 2,6 & 0,6 & 2,5 & 2,4 & 0,974 & 0,969 & 0,938 & 0,040 & 0,995 & 1,003 & 0,691 & 0,686 & 0,994 & 0,682 & 0,682 & 90 & 194 & $-3,3$ \\
\hline 195 & 0,5 & 3,2 & 0,6 & 2,8 & 2,7 & 0,846 & 0,891 & 0,863 & 0,189 & 0,669 & 1,223 & 0,695 & 0,826 & 0,983 & 0,812 & 0,592 & 168 & 195 & $-3,3$ \\
\hline 196 & 2,8 & 7,6 & 3,3 & 7,5 & 7,3 & 0,865 & 0,984 & 0,953 & 0,136 & 0,610 & 1,280 & 0,407 & 0,450 & 2,975 & 1,339 & 1,154 & 173 & 196 & $-4,9$ \\
\hline 197 & 0,4 & 2,2 & 0,4 & 2,1 & 2,1 & 0,939 & 0,986 & 0,955 & 0,062 & 1,000 & 1,000 & 0,847 & 0,819 & 0,752 & 0,616 & 0,592 & 131 & 197 & $-2,9$ \\
\hline 198 & 1,9 & 6,5 & 2,2 & 6,3 & 6,1 & 0,846 & 0,977 & 0,945 & 0,155 & 0,566 & 1,329 & 0,331 & 0,404 & 2,702 & 1,090 & 0,864 & 19 & 198 & $-4,8$ \\
\hline 199 & 0,4 & 2,5 & 0,4 & 2,4 & 2,4 & 0,930 & 0,980 & 0,948 & 0,073 & 0,811 & 1,110 & 0,448 & 0,463 & 1,063 & 0,492 & 0,508 & 5 & 199 & $-3,4$ \\
\hline 200 & 4,8 & 8,2 & 5,0 & 8,2 & 7,9 & 0,960 & 0,996 & 0,965 & 0,040 & 0,902 & 1,053 & 0,662 & 0,746 & 3,045 & 2,273 & 2,047 & 179 & 200 & $-4,9$ \\
\hline 201 & 6,7 & 9,3 & 6,8 & 9,3 & 9,0 & 0,986 & 0,997 & 0,965 & 0,014 & 0,970 & 1,015 & 0,763 & 0,804 & 3,345 & 2,689 & 2,669 & 21 & 201 & $-5,1$ \\
\hline 202 & 0,6 & 3,3 & 0,6 & 3,2 & 3,1 & 0,859 & 0,969 & 0,938 & 0,144 & 0,650 & 1,240 & 0,423 & 0,600 & 1,288 & 0,772 & 0,643 & 150 & 202 & $-3,7$ \\
\hline 203 & 106,5 & 58,5 & 149,0 & 46,2 & 44,7 & 0,715 & 0,789 & 0,764 & 0,355 & 0,391 & 1,599 & 0,415 & 0,630 & 18,080 & 11,396 & 3,788 & 47 & 203 & $-7,5$ \\
\hline 204 & 2,1 & 5,6 & 2,2 & 5,6 & 5,4 & 0,955 & 0,986 & 0,954 & 0,047 & 0,844 & 1,089 & 0,623 & 0,600 & 2,089 & 1,254 & 1,198 & 103 & 204 & $-4,4$ \\
\hline 205 & 0,3 & 2,2 & 0,4 & 2,1 & 2,1 & 0,957 & 0,986 & 0,955 & 0,046 & 0,935 & 1,034 & 0,593 & 0,651 & 0,864 & 0,562 & 0,536 & 161 & 205 & $-3,1$ \\
\hline 206 & 1,4 & 5,1 & 1,5 & 4,9 & 4,7 & 0,934 & 0,963 & 0,932 & 0,075 & 0,711 & 1,186 & 0,422 & 0,412 & 2,089 & 0,860 & 0,833 & 86 & 206 & $-4,4$ \\
\hline 207 & 1,1 & 3,9 & 1,1 & 3,8 & 3,7 & 0,968 & 0,987 & 0,955 & 0,034 & 0,910 & 1,049 & 0,664 & 0,712 & 1,447 & 1,030 & 0,985 & 64 & 207 & $-3,9$ \\
\hline 208 & 24,9 & 19,8 & 27,0 & 19,5 & 18,9 & 0,919 & 0,985 & 0,954 & 0,082 & 0,797 & 1,120 & 0,696 & 0,787 & 6,743 & 5,307 & 4,743 & 81 & 208 & $-6,1$ \\
\hline 209 & 1,7 & 4,7 & 1,7 & 4,6 & 4,5 & 0,972 & 0,985 & 0,953 & 0,032 & 0,948 & 1,027 & 0,701 & 0,703 & 1,738 & 1,222 & 1,212 & 81 & 209 & $-4,1$ \\
\hline 210 & 0,6 & 3,1 & 0,7 & 2,8 & 2,7 & 0,827 & 0,914 & 0,885 & 0,193 & 0,729 & 1,171 & 0,604 & 0,802 & 1,083 & 0,869 & 0,611 & 81 & 210 & $-3,4$ \\
\hline 211 & 0,8 & 3,8 & 0,9 & 3,7 & 3,5 & 0,910 & 0,963 & 0,932 & 0,097 & 0,694 & 1,200 & 0,416 & 0,485 & 1,560 & 0,758 & 0,715 & 2 & 211 & $-4,0$ \\
\hline 212 & 1,3 & 4,2 & 1,3 & 4,1 & 4,0 & 0,952 & 0,981 & 0,950 & 0,052 & 0,911 & 1,048 & 0,663 & 0,697 & 1,566 & 1,091 & 1,061 & 75 & 212 & $-4,0$ \\
\hline 213 & 0,4 & 2,5 & 0,4 & 2,4 & 2,3 & 0,939 & 0,952 & 0,921 & 0,078 & 0,810 & 1,111 & 0,470 & 0,461 & 1,061 & 0,489 & 0,485 & 127 & 213 & -3 \\
\hline 214 & 66,4 & 34,6 & 74,9 & 33,5 & 32,4 & 0,886 & 0,967 & 0,936 & 0,119 & 0,697 & 1,198 & 0,501 & 0,590 & 12,986 & 7,667 & 6,612 & 88 & 214 & $-7,0$ \\
\hline 215 & 0,6 & 2,6 & 0,6 & 2,6 & 2,5 & 0,976 & 0,997 & 0,965 & 0,024 & 1,000 & 0,967 & 0,881 & 0,819 & 0,916 & 0,750 & 0,805 & 135 & 215 & $-3,2$ \\
\hline 216 & 0,5 & 3,0 & 0,6 & 2,9 & 2,8 & 0,943 & 0,983 & 0,951 & 0,060 & 0,778 & 1,133 & 0,426 & 0,426 & 1,273 & 0,542 & 0,546 & 27 & 216 & -3 \\
\hline 217 & 9,7 & 11,8 & 9,9 & 11,8 & 11,4 & 0,976 & 0,999 & 0,967 & 0,024 & 0,870 & 1,072 & 0,584 & 0,655 & 4,593 & 3,011 & 2,875 & 38 & 217 & $-5,5$ \\
\hline 218 & 0,2 & 1,6 & 0,2 & 1,5 & 1,5 & 0,950 & 0,937 & 0,908 & 0,080 & 0,910 & 1,048 & 0,555 & 0,539 & 0,662 & 0,357 & 0,379 & 73 & 218 & 7 \\
\hline 219 & 11,5 & 13,3 & 12,1 & 13,2 & 12,8 & 0,949 & 0,994 & 0,962 & 0,051 & 0,819 & 1,105 & 0,575 & 0,632 & 5,040 & 3,187 & 3,165 & 49 & 219 & 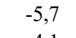 \\
\hline 220 & 1,1 & 4,2 & 1,2 & 4,1 & 4,0 & 0,931 & 0,988 & 0,956 & 0,070 & 0,812 & 1,110 & 0,518 & 0,597 & 1,674 & 1,000 & 0,964 & 95 & 220 & $-4,1$ \\
\hline 221 & 6,5 & 10,1 & 7,1 & 10,0 & 9,7 & 0,914 & 0,989 & 0,957 & 0,087 & 0,789 & 1,126 & 0,565 & 0,645 & 3,812 & 2,459 & 2,058 & 49 & 221 & \\
\hline 222 & 0,3 & 1,8 & 0,3 & 1,7 & 1,7 & 0,953 & 0,983 & 0,952 & 0,050 & 1,000 & 0,977 & 0,967 & 0,947 & 0,588 & 0,557 & 0,536 & 128 & 222 & $-2,6$ \\
\hline 223 & 0,3 & 2,6 & 0,3 & 1,9 & 1,8 & 0,873 & 0,733 & 0,710 & 0,295 & 0,496 & 1,420 & 0,557 & 0,592 & 0,781 & 0,463 & 0,214 & 60 & 223 & $-3,0$ \\
\hline
\end{tabular}




\begin{tabular}{|c|c|c|c|c|c|c|c|c|c|c|c|c|c|c|c|c|c|c|c|}
\hline 224 & 0,2 & 1,6 & 0,2 & 1,6 & 1,5 & 0,908 & 0,956 & 0,925 & 0,102 & 0,934 & 1,035 & 0,857 & 0,840 & 0,542 & 0,455 & 0,379 & 130 & 224 & $-2,4$ \\
\hline 225 & 4,3 & 8,7 & 4,6 & 8,6 & 8,3 & 0,926 & 0,985 & 0,953 & 0,075 & 0,706 & 1,190 & 0,396 & 0,452 & 3,707 & 1,675 & 1,591 & 101 & 225 & $-5,2$ \\
\hline 226 & 6,3 & 10,7 & 6,7 & 10,6 & 10,3 & 0,927 & 0,988 & 0,956 & 0,074 & 0,681 & 1,212 & 0,381 & 0,415 & 4,571 & 1,897 & 1,845 & 119 & 226 & $-5,5$ \\
\hline 227 & 6,6 & 10,9 & 7,7 & 10,8 & 10,4 & 0,855 & 0,989 & 0,957 & 0,146 & 0,703 & 1,193 & 0,512 & 0,689 & 4,055 & 2,792 & 2,249 & 0 & 227 & $-5,3$ \\
\hline 228 & 4,0 & 9,2 & 4,8 & 8,3 & 8,0 & 0,842 & 0,905 & 0,876 & 0,185 & 0,602 & 1,289 & 0,552 & 0,662 & 3,054 & 2,023 & 1,661 & 145 & 228 & $-4,9$ \\
\hline 229 & 0,8 & 3,2 & 0,8 & 3,0 & 2,9 & 0,969 & 0,938 & 0,908 & 0,069 & 0,988 & 1,006 & 0,690 & 0,744 & 1,222 & 0,909 & 0,909 & 14 & 229 & $-3,6$ \\
\hline 230 & 0,4 & 2,3 & 0,4 & 2,2 & 2,1 & 0,980 & 0,948 & 0,917 & 0,056 & 0,964 & 1,019 & 0,779 & 0,824 & 0,827 & 0,682 & 0,606 & 6 & 230 & $-3,0$ \\
\hline 231 & 0,2 & 1,6 & 0,2 & 1,5 & 1,4 & 0,972 & 0,941 & 0,911 & 0,065 & 0,998 & 1,001 & 0,678 & 0,619 & 0,612 & 0,379 & 0,442 & 4 & 231 & $-2,6$ \\
\hline 232 & 0,6 & 2,7 & 0,6 & 2,7 & 2,6 & 0,974 & 0,997 & 0,965 & 0,026 & 1,000 & 0,987 & 0,828 & 0,792 & 0,956 & 0,758 & 0,758 & 164 & 232 & $-3,3$ \\
\hline 233 & 0,3 & 2,7 & 0,3 & 2,6 & 2,5 & 0,912 & 0,973 & 0,942 & 0,092 & 0,497 & 1,418 & 0,232 & 0,213 & 1,249 & 0,266 & 0,303 & 168 & 233 & $-3,6$ \\
\hline 234 & 0,6 & 2,8 & 0,6 & 2,8 & 2,7 & 0,973 & 0,975 & 0,943 & 0,037 & 0,963 & 1,019 & 0,699 & 0,728 & 1,061 & 0,773 & 0,746 & 115 & 234 & $-3,4$ \\
\hline 235 & 0,1 & 1,2 & 0,1 & 1,2 & 1,1 & 0,928 & 0,926 & 0,896 & 0,104 & 1,000 & 0,979 & 0,843 & 0,787 & 0,442 & 0,347 & 0,339 & 154 & 235 & $-2,1$ \\
\hline 236 & 0,2 & 1,8 & 0,2 & 1,7 & 1,7 & 0,945 & 0,944 & 0,913 & 0,078 & 0,860 & 1,079 & 0,500 & 0,455 & 0,755 & 0,343 & 0,339 & 73 & 236 & $-2,9$ \\
\hline 237 & 0,1 & 0,8 & 0,1 & 0,7 & 0,7 & 0,952 & 0,885 & 0,856 & 0,125 & 1,000 & 0,951 & 0,637 & 0,559 & 0,339 & 0,189 & 0,214 & 23 & 237 & $-1,8$ \\
\hline 238 & 0,1 & 0,9 & 0,1 & 0,7 & 0,7 & 0,957 & 0,857 & 0,829 & 0,149 & 1,000 & 0,953 & 0,674 & 0,614 & 0,349 & 0,214 & 0,227 & 136 & 238 & $-1,8$ \\
\hline 239 & 0,1 & 1,5 & 0,2 & 1,4 & 1,4 & 0,904 & 0,965 & 0,934 & 0,103 & 0,863 & 1,077 & 0,525 & 0,557 & 0,599 & 0,334 & 0,339 & 115 & 239 & $-2,6$ \\
\hline 240 & 0,2 & 1,6 & 0,2 & 1,6 & 1,5 & 0,970 & 0,968 & 0,937 & 0,044 & 0,906 & 1,051 & 0,551 & 0,575 & 0,654 & 0,376 & 0,379 & 173 & 240 & $-2,7$ \\
\hline 241 & 0,7 & 3,2 & 0,7 & 3,2 & 3,1 & 0,967 & 0,991 & 0,959 & 0,034 & 0,890 & 1,060 & 0,543 & 0,584 & 1,297 & 0,758 & 0,773 & 179 & 241 & $-3,7$ \\
\hline 242 & 1,0 & 3,6 & 1,0 & 3,5 & 3,4 & 0,988 & 0,992 & 0,960 & 0,015 & 1,000 & 0,995 & 0,845 & 0,820 & 1,241 & 1,018 & 1,019 & 52 & 242 & $-3,6$ \\
\hline 243 & 0,7 & 3,0 & 0,7 & 2,9 & 2,8 & 0,974 & 0,990 & 0,958 & 0,027 & 1,000 & 0,995 & 0,836 & 0,782 & 1,040 & 0,813 & 0,816 & 73 & 243 & $-3,4$ \\
\hline 244 & 0,6 & 3,2 & 0,7 & 3,1 & 3,0 & 0,901 & 0,972 & 0,941 & 0,103 & 0,768 & 1,141 & 0,458 & 0,471 & 1,308 & 0,616 & 0,530 & 16 & 244 & $-3,7$ \\
\hline 245 & 1,3 & 4,9 & 1,4 & 4,7 & 4,6 & 0,906 & 0,966 & 0,935 & 0,100 & 0,691 & 1,203 & 0,465 & 0,523 & 1,894 & 0,991 & 0,791 & 129 & 245 & $-4,2$ \\
\hline 246 & 0,2 & 1,7 & 0,2 & 1,6 & 1,5 & 0,969 & 0,933 & 0,903 & 0,074 & 0,968 & 1,016 & 0,629 & 0,508 & 0,671 & 0,341 & 0,379 & 177 & 246 & $-2,7$ \\
\hline 247 & 0,2 & 1,4 & 0,2 & 1,3 & 1,3 & 0,958 & 0,914 & 0,885 & 0,095 & 1,000 & 0,992 & 0,759 & 0,676 & 0,526 & 0,356 & 0,379 & 151 & 247 & $-2,4$ \\
\hline 248 & 13,5 & 15,7 & 14,7 & 15,5 & 15,0 & 0,916 & 0,988 & 0,957 & 0,085 & 0,688 & 1,206 & 0,386 & 0,463 & 6,675 & 3,088 & 2,880 & 22 & 248 & $-6,1$ \\
\hline 249 & 0,7 & 3,3 & 0,8 & 3,3 & 3,2 & 0,957 & 0,991 & 0,959 & 0,044 & 0,825 & 1,101 & 0,485 & 0,526 & 1,390 & 0,731 & 0,715 & 18 & 249 & $-3,8$ \\
\hline 250 & 1,3 & 4,1 & 1,3 & 4,0 & 3,9 & 0,980 & 0,982 & 0,951 & 0,027 & 0,988 & 1,006 & 0,698 & 0,716 & 1,553 & 1,112 & 1,103 & 94 & 250 & $-4,0$ \\
\hline 251 & 0,9 & 3,5 & 0,9 & 3,4 & 3,3 & 0,963 & 0,985 & 0,954 & 0,039 & 0,938 & 1,032 & 0,689 & 0,659 & 1,296 & 0,854 & 0,816 & 40 & 251 & $-3,7$ \\
\hline 252 & 1,9 & 6,0 & 2,3 & 5,8 & 5,6 & 0,821 & 0,954 & 0,923 & 0,185 & 0,653 & 1,238 & 0,459 & 0,577 & 2,296 & 1,325 & 1,016 & 44 & 252 & $-4,5$ \\
\hline 253 & 0,1 & 1,5 & 0,2 & 1,4 & 1,4 & 0,935 & 0,971 & 0,940 & 0,071 & 0,833 & 1,095 & 0,488 & 0,496 & 0,612 & 0,303 & 0,312 & 161 & 253 & $-2,6$ \\
\hline 254 & 0,6 & 3,3 & 0,7 & 3,2 & 3,1 & 0,852 & 0,957 & 0,926 & 0,155 & 0,660 & 1,231 & 0,453 & 0,574 & 1,273 & 0,730 & 0,625 & 122 & 254 & $-3,7$ \\
\hline 255 & 0,1 & 1,2 & 0,1 & 1,0 & 1,0 & 0,767 & 0,837 & 0,811 & 0,284 & 0,580 & 1,313 & 0,293 & 0,354 & 0,536 & 0,189 & 0,227 & 9 & 255 & $-2,4$ \\
\hline 256 & 10,9 & 14,8 & 13,4 & 13,9 & 13,5 & 0,816 & 0,941 & 0,910 & 0,193 & 0,624 & 1,266 & 0,478 & 0,662 & 5,392 & 3,571 & 2,947 & 79 & 256 & $-5,8$ \\
\hline 257 & 138,6 & 47,6 & 146,0 & 46,2 & 44,7 & 0,949 & 0,971 & 0,940 & 0,059 & 0,770 & 1,140 & 0,589 & 0,623 & 17,306 & 10,782 & 9,709 & 144 & 257 & $-7,4$ \\
\hline 258 & 0,4 & 3,5 & 0,6 & 3,3 & 3,2 & 0,752 & 0,947 & 0,917 & 0,254 & 0,450 & 1,490 & 0,279 & 0,467 & 1,423 & 0,665 & 0,461 & 29 & 258 & $-3,8$ \\
\hline 259 & 1,9 & 6,1 & 2,3 & 5,8 & 5,7 & 0,802 & 0,953 & 0,923 & 0,204 & 0,622 & 1,268 & 0,435 & 0,596 & 2,330 & 1,388 & 0,996 & 168 & 259 & $-4,5$ \\
\hline 260 & 3,8 & 7,7 & 4,1 & 7,4 & 7,2 & 0,941 & 0,960 & 0,929 & 0,072 & 0,810 & 1,111 & 0,585 & 0,649 & 2,891 & 1,875 & 1,682 & 132 & 260 & $-4,9$ \\
\hline 261 & 0,4 & 2,6 & 0,4 & 2,5 & 2,4 & 0,953 & 0,961 & 0,930 & 0,062 & 0,764 & 1,144 & 0,393 & 0,376 & 1,145 & 0,431 & 0,442 & 52 & 261 & $-3,5$ \\
\hline 262 & 0,1 & 1,2 & 0,1 & 1,1 & 1,1 & 0,956 & 0,924 & 0,894 & 0,088 & 1,000 & 0,972 & 0,706 & 0,638 & 0,475 & 0,303 & 0,312 & 111 & 262 & $-2,2$ \\
\hline 263 & 0,3 & 1,9 & 0,3 & 1,8 & 1,8 & 0,947 & 0,948 & 0,918 & 0,074 & 0,944 & 1,029 & 0,665 & 0,666 & 0,735 & 0,490 & 0,479 & 86 & 263 & $-2,9$ \\
\hline 264 & 6,5 & 12,7 & 8,1 & 10,4 & 10,1 & 0,809 & 0,821 & 0,795 & 0,261 & 0,506 & 1,405 & 0,501 & 0,662 & 4,069 & 2,695 & 1,742 & 167 & 264 & $-5,3$ \\
\hline 265 & 0,3 & 1,8 & 0,3 & 1,7 & 1,7 & 0,973 & 0,971 & 0,940 & 0,039 & 1,000 & 0,982 & 0,713 & 0,649 & 0,683 & 0,443 & 0,442 & 63 & 265 & $-2,8$ \\
\hline 266 & 1,2 & 4,7 & 1,3 & 4,7 & 4,5 & 0,933 & 0,985 & 0,953 & 0,069 & 0,673 & 1,219 & 0,368 & 0,390 & 2,038 & 0,794 & 0,805 & 138 & 266 & $-4,3$ \\
\hline 267 & 0,5 & 3,1 & 0,6 & 2,7 & 2,7 & 0,768 & 0,898 & 0,869 & 0,254 & 0,606 & 1,284 & 0,514 & 0,721 & 1,057 & 0,762 & 0,485 & 58 & 267 & $-3,4$ \\
\hline 268 & 7,7 & 14,9 & 10,1 & 13,2 & 12,8 & 0,758 & 0,891 & 0,862 & 0,266 & 0,436 & 1,515 & 0,316 & 0,451 & 5,557 & 2,506 & 1,762 & 147 & 268 & $-5,8$ \\
\hline 269 & 0,2 & 1,8 & 0,2 & 1,8 & 1,7 & 0,909 & 0,983 & 0,952 & 0,092 & 0,850 & 1,085 & 0,536 & 0,524 & 0,715 & 0,375 & 0,339 & 127 & 269 & $-2,8$ \\
\hline 270 & 1,2 & 4,4 & 1,3 & 4,3 & 4,2 & 0,926 & 0,993 & 0,962 & 0,074 & 0,796 & 1,121 & 0,518 & 0,612 & 1,718 & 1,051 & 0,887 & 163 & 270 & $-4,1$ \\
\hline 271 & 36,2 & 23,6 & 38,4 & 23,0 & 22,2 & 0,944 & 0,975 & 0,944 & 0,061 & 0,820 & 1,104 & 0,667 & 0,754 & 8,316 & 6,269 & 5,869 & 148 & 271 & $-6,4$ \\
\hline 272 & 0,8 & 3,8 & 0,9 & 3,7 & 3,6 & 0,893 & 0,962 & 0,932 & 0,113 & 0,703 & 1,193 & 0,468 & 0,590 & 1,492 & 0,880 & 0,746 & 96 & 272 & $-3,9$ \\
\hline 273 & 0,3 & 2,1 & 0,3 & 1,9 & 1,9 & 0,924 & 0,950 & 0,920 & 0,091 & 0,785 & 1,129 & 0,502 & 0,552 & 0,816 & 0,451 & 0,442 & 120 & 273 & $-3,0$ \\
\hline 274 & 1,7 & 4,8 & 1,8 & 4,7 & 4,6 & 0,947 & 0,986 & 0,954 & 0,055 & 0,905 & 1,051 & 0,696 & 0,724 & 1,748 & 1,266 & 1,222 & 57 & 274 & $-4,1$ \\
\hline 275 & 2,5 & 7,1 & 2,8 & 6,9 & 6,7 & 0,909 & 0,983 & 0,952 & 0,092 & 0,634 & 1,256 & 0,359 & 0,363 & 2,989 & 1,084 & 0,985 & 93 & 275 & $-4,9$ \\
\hline 276 & 35,2 & 27,9 & 41,2 & 25,0 & 24,2 & 0,854 & 0,895 & 0,867 & 0,180 & 0,567 & 1,328 & 0,441 & 0,563 & 10,082 & 5,678 & 3,882 & 95 & 276 & $-6,7$ \\
\hline 277 & 0,1 & 1,1 & 0,1 & 1,0 & 1,0 & 0,987 & 0,917 & 0,888 & 0,084 & 1,000 & 0,973 & 0,754 & 0,716 & 0,423 & 0,303 & 0,303 & 15 & 277 & $-2,1$ \\
\hline 278 & 4,7 & 8,9 & 5,1 & 8,1 & 7,9 & 0,922 & 0,920 & 0,890 & 0,112 & 0,756 & 1,150 & 0,651 & 0,724 & 3,035 & 2,196 & 2,105 & 59 & 278 & $-4,9$ \\
\hline 279 & 0,3 & 2,2 & 0,3 & 2,2 & 2,1 & 0,917 & 0,968 & 0,937 & 0,089 & 0,682 & 1,211 & 0,351 & 0,350 & 0,991 & 0,347 & 0,339 & 14 & 279 & $-3,3$ \\
\hline 280 & 2,5 & 5,7 & 2,5 & 5,7 & 5,5 & 0,977 & 0,998 & 0,966 & 0,023 & 0,967 & 1,017 & 0,820 & 0,819 & 1,962 & 1,606 & 1,598 & 97 & 280 & $-4,3$ \\
\hline 281 & 0,5 & 2,9 & 0,5 & 2,8 & 2,7 & 0,947 & 0,965 & 0,934 & 0,064 & 0,690 & 1,204 & 0,345 & 0,363 & 1,302 & 0,473 & 0,479 & 11 & 281 & $-3,7$ \\
\hline
\end{tabular}




\begin{tabular}{|c|c|c|c|c|c|c|c|c|c|c|c|c|c|c|c|c|c|c|c|}
\hline 282 & 0,1 & 1,5 & 0,1 & 1,4 & 1,4 & 0,960 & 0,966 & 0,935 & 0,052 & 0,799 & 1,119 & 0,427 & 0,369 & 0,644 & 0,237 & 0,273 & 63 & 282 & $-2,7$ \\
\hline 283 & 0,2 & 1,4 & 0,2 & 1,3 & 1,3 & 0,921 & 0,944 & 0,913 & 0,097 & 0,927 & 1,038 & 0,605 & 0,673 & 0,563 & 0,379 & 0,379 & 82 & 283 & $-2,5$ \\
\hline 284 & 0,1 & 1,5 & 0,1 & 1,3 & 1,3 & 0,932 & 0,902 & 0,873 & 0,119 & 0,800 & 1,118 & 0,630 & 0,718 & 0,528 & 0,379 & 0,312 & 20 & 284 & $-2,4$ \\
\hline 285 & 0,1 & 1,1 & 0,1 & 1,1 & 1,0 & 0,944 & 0,955 & 0,924 & 0,072 & 0,941 & 1,031 & 0,588 & 0,524 & 0,456 & 0,239 & 0,240 & 9 & 285 & $-2,2$ \\
\hline 286 & 10,5 & 12,7 & 11,2 & 12,6 & 12,2 & 0,937 & 0,989 & 0,958 & 0,064 & 0,815 & 1,108 & 0,548 & 0,643 & 4,939 & 3,173 & 2,879 & 123 & 286 & $-5,6$ \\
\hline 287 & 1171,0 & 146,2 & 1222,6 & 129,3 & 125,1 & 0,958 & 0,884 & 0,856 & 0,123 & 0,689 & 1,205 & 0,632 & 0,741 & 48,582 & 35,993 & 29,622 & 91 & 287 & $-8,9$ \\
\hline 288 & 7,2 & 10,5 & 7,5 & 10,2 & 9,9 & 0,958 & 0,977 & 0,946 & 0,048 & 0,829 & 1,098 & 0,564 & 0,591 & 4,033 & 2,383 & 2,357 & 139 & 288 & $-5,3$ \\
\hline 289 & 5,1 & 8,8 & 5,4 & 8,4 & 8,2 & 0,932 & 0,962 & 0,931 & 0,078 & 0,825 & 1,101 & 0,633 & 0,749 & 3,190 & 2,390 & 2,185 & 83 & 289 & $-5,0$ \\
\hline 290 & 1,3 & 4,3 & 1,3 & 4,1 & 4,0 & 0,964 & 0,971 & 0,940 & 0,046 & 0,897 & 1,056 & 0,621 & 0,694 & 1,627 & 1,129 & 1,052 & 12 & 290 & $-4,0$ \\
\hline 291 & 0,2 & 1,6 & 0,2 & 1,5 & 1,5 & 0,962 & 0,955 & 0,925 & 0,059 & 0,909 & 1,049 & 0,519 & 0,493 & 0,671 & 0,331 & 0,339 & 75 & 291 & $-2,7$ \\
\hline 292 & 0,6 & 4,1 & 0,7 & 3,3 & 3,2 & 0,837 & 0,812 & 0,786 & 0,249 & 0,472 & 1,455 & 0,425 & 0,442 & 1,368 & 0,605 & 0,479 & 146 & 292 & $-3,8$ \\
\hline 293 & 0,7 & 3,1 & 0,7 & 3,1 & 3,0 & 0,955 & 0,990 & 0,959 & 0,046 & 0,864 & 1,076 & 0,586 & 0,646 & 1,203 & 0,777 & 0,750 & 57 & 293 & $-3,6$ \\
\hline 294 & 0,2 & 1,5 & 0,2 & 1,5 & 1,4 & 0,963 & 0,961 & 0,930 & 0,054 & 1,000 & 0,982 & 0,719 & 0,696 & 0,577 & 0,402 & 0,429 & 23 & 294 & $-2,5$ \\
\hline 295 & 10,6 & 13,6 & 11,7 & 13,0 & 12,6 & 0,913 & 0,953 & 0,922 & 0,099 & 0,720 & 1,179 & 0,598 & 0,646 & 4,760 & 3,075 & 2,209 & 113 & 295 & $-5,6$ \\
\hline 296 & 0,1 & 1,2 & 0,1 & 1,1 & 1,0 & 0,977 & 0,882 & 0,853 & 0,120 & 1,000 & 0,989 & 0,591 & 0,631 & 0,510 & 0,321 & 0,321 & 127 & 296 & $-2,4$ \\
\hline 297 & 0,2 & 1,6 & 0,2 & 1,6 & 1,6 & 0,975 & 0,995 & 0,963 & 0,026 & 1,000 & 0,982 & 0,639 & 0,630 & 0,662 & 0,417 & 0,442 & 163 & 297 & $-2,7$ \\
\hline 298 & 0,3 & 1,9 & 0,3 & 1,8 & 1,7 & 0,947 & 0,962 & 0,931 & 0,066 & 0,914 & 1,046 & 0,550 & 0,594 & 0,767 & 0,455 & 0,429 & 142 & 298 & $-2,9$ \\
\hline 299 & 0,5 & 2,7 & 0,6 & 2,6 & 2,5 & 0,961 & 0,973 & 0,942 & 0,047 & 0,922 & 1,041 & 0,571 & 0,590 & 1,091 & 0,644 & 0,678 & 172 & 299 & $-3,4$ \\
\hline 300 & 0,9 & 3,4 & 1,0 & 3,4 & 3,3 & 0,944 & 0,983 & 0,951 & 0,059 & 0,964 & 1,019 & 0,750 & 0,768 & 1,234 & 0,947 & 0,887 & 83 & 300 & $-3,6$ \\
\hline 301 & 0,5 & 2,6 & 0,5 & 2,6 & 2,5 & 0,974 & 0,989 & 0,957 & 0,028 & 0,886 & 1,062 & 0,539 & 0,450 & 1,072 & 0,482 & 0,536 & 46 & 301 & $-3,4$ \\
\hline 302 & 0,1 & 1,2 & 0,1 & 1,2 & 1,1 & 0,974 & 0,975 & 0,944 & 0,036 & 0,964 & 1,019 & 0,569 & 0,488 & 0,494 & 0,241 & 0,273 & 35 & 302 & $-2,3$ \\
\hline 303 & 0,4 & 2,2 & 0,4 & 2,1 & 2,1 & 0,954 & 0,967 & 0,936 & 0,056 & 0,940 & 1,031 & 0,848 & 0,868 & 0,735 & 0,638 & 0,577 & 102 & 303 & $-2,9$ \\
\hline 304 & 0,1 & 1,2 & 0,1 & 1,1 & 1,1 & 0,977 & 0,974 & 0,943 & 0,034 & 1,000 & 0,934 & 0,876 & 0,759 & 0,423 & 0,321 & 0,339 & 49 & 304 & $-2,1$ \\
\hline 305 & 0,2 & $\begin{array}{l}1,7 \\
\text {. }\end{array}$ & 0,2 & 1,5 & 1,4 & 0,945 & 0,896 & 0,867 & 0,118 & 0,787 & 1,127 & 0,397 & 0,456 & 0,746 & 0,340 & 0,339 & 109 & 305 & $-2,9$ \\
\hline 306 & 0,2 & 1,7 & 0,2 & 1,6 & 1,6 & 0,957 & 0,969 & 0,938 & 0,053 & 1,000 & 0,991 & 0,795 & 0,707 & 0,599 & 0,424 & 0,429 & 132 & 306 & $-2,6$ \\
\hline 307 & 0,1 & 1,0 & 0,1 & 1,0 & 0,9 & 0,938 & 0,930 & 0,901 & 0,094 & 1,000 & 0,992 & 0,558 & 0,513 & 0,443 & 0,227 & 0,240 & 159 & 307 & $-2,1$ \\
\hline 308 & 1,8 & 5,4 & 1,9 & 5,3 & 5,1 & 0,944 & 0,981 & 0,950 & 0,059 & 0,777 & 1,135 & 0,560 & 0,656 & 2,008 & 1,317 & 1,286 & 159 & 308 & $-4,3$ \\
\hline 309 & 0,0 & 0,6 & 0,0 & 0,6 & 0,5 & 0,931 & 0,875 & 0,846 & 0,143 & 1,000 & 0,921 & 0,764 & 0,621 & 0,254 & 0,158 & 0,169 & 124 & 309 & $-1,3$ \\
\hline 310 & 4,9 & 8,5 & 5,1 & 8,5 & 8,2 & 0,960 & 0,997 & 0,965 & 0,040 & 0,840 & 1,091 & 0,692 & 0,788 & 2,990 & 2,356 & 2,000 & 58 & 310 & $-4,9$ \\
\hline 311 & 1,1 & 4,0 & 1,1 & 4,0 & 3,9 & 0,958 & 0,993 & 0,961 & 0,042 & 0,811 & 1,110 & 0,586 & 0,504 & 1,514 & 0,762 & 0,773 & 65 & 311 & $-3,9$ \\
\hline 312 & 0,1 & 1,1 & 0,1 & 1,1 & 1,1 & 0,945 & 0,956 & 0,925 & 0,071 & 0,955 & 1,023 & 0,520 & 0,490 & 0,492 & 0,241 & 0,273 & 150 & 312 & $-2,3$ \\
\hline 313 & 0,5 & 3,0 & 0,5 & 2,8 & 2,7 & 0,854 & 0,933 & 0,903 & 0,161 & 0,641 & 1,249 & 0,425 & 0,545 & 1,176 & 0,640 & 0,577 & 7 & 313 & $-3,6$ \\
\hline 314 & 0,7 & 3,5 & 0,8 & 3,4 & 3,3 & 0,946 & 0,977 & 0,946 & 0,059 & 0,779 & 1,133 & 0,439 & 0,434 & 1,473 & 0,639 & 0,643 & 133 & 314 & $-3,9$ \\
\hline 315 & 0,0 & 0,6 & 0,0 & 0,6 & 0,5 & 0,964 & 0,875 & 0,846 & 0,130 & 1,000 & 0,921 & 0,661 & 0,490 & 0,273 & 0,134 & 0,169 & 56 & 315 & $-1,4$ \\
\hline 316 & 0,2 & 1,8 & 0,3 & 1,8 & 1,7 & 0,972 & 0,972 & 0,941 & 0,040 & 0,924 & 1,040 & 0,544 & 0,530 & 0,758 & 0,402 & 0,429 & 47 & 316 & $-2,9$ \\
\hline 317 & 0,1 & 1,1 & 0,1 & 1,0 & 1,0 & 0,985 & 0,935 & 0,905 & 0,067 & 0,978 & 1,011 & 0,718 & 0,740 & 0,410 & 0,303 & 0,273 & 59 & 317 & $-2,0$ \\
\hline 318 & 0,1 & 1,3 & 0,1 & 1,2 & 1,1 & 0,989 & 0,936 & 0,906 & 0,065 & 1,000 & 0,986 & 0,769 & 0,737 & 0,462 & 0,341 & 0,379 & 66 & 318 & $-2,2$ \\
\hline 319 & 0,1 & 0,8 & 0,1 & 0,7 & 0,7 & 1,000 & 0,935 & 0,905 & 0,065 & 1,000 & 0,903 & 0,823 & 0,702 & 0,305 & 0,214 & 0,227 & 59 & 319 & $-1,6$ \\
\hline 320 & 2,9 & 6,6 & 3,0 & 6,5 & 6,3 & 0,953 & 0,978 & 0,947 & 0,052 & 0,831 & 1,097 & 0,603 & 0,726 & 2,476 & 1,798 & 1,471 & 86 & 320 & $-4,6$ \\
\hline 321 & 2,1 & 5,8 & 2,3 & 5,7 & 5,5 & 0,908 & 0,974 & 0,943 & 0,096 & 0,787 & 1,127 & 0,544 & 0,646 & 2,228 & 1,439 & 1,270 & 100 & 321 & $-4,5$ \\
\hline 322 & 2,7 & 7,5 & 3,0 & 7,2 & 6,9 & 0,871 & 0,960 & 0,929 & 0,135 & 0,596 & 1,295 & 0,385 & 0,433 & 2,961 & 1,283 & 0,887 & 164 & 322 & $-4,9$ \\
\hline 323 & 0,2 & 1,5 & 0,2 & 1,3 & 1,3 & 0,927 & 0,904 & 0,875 & 0,121 & 0,931 & 1,036 & 0,605 & 0,633 & 0,589 & 0,373 & 0,379 & 62 & 323 & $-2,6$ \\
\hline 324 & 3,7 & 8,4 & 4,1 & 8,1 & 7,9 & 0,900 & 0,974 & 0,943 & 0,104 & 0,664 & 1,227 & 0,385 & 0,435 & 3,492 & 1,518 & 1,393 & 68 & 324 & $-5,1$ \\
\hline 325 & 10,9 & 12,7 & 11,6 & 12,5 & 12,1 & 0,936 & 0,980 & 0,949 & 0,067 & 0,845 & 1,088 & 0,726 & 0,737 & 4,365 & 3,218 & 2,988 & 35 & 325 & $-5,4$ \\
\hline 326 & 0,8 & 3,4 & 0,8 & 3,4 & 3,3 & 0,965 & 0,997 & 0,966 & 0,035 & 0,826 & 1,100 & 0,548 & 0,588 & 1,321 & 0,777 & 0,746 & 137 & 326 & $-3,7$ \\
\hline 327 & 0,2 & 2,0 & 0,2 & 1,8 & 1,7 & 0,921 & 0,922 & 0,892 & 0,111 & 0,610 & 1,280 & 0,305 & 0,302 & 0,879 & 0,265 & 0,303 & 91 & 327 & $-3,1$ \\
\hline 328 & 1,3 & 4,2 & 1,3 & 4,1 & 4,0 & 0,957 & 0,993 & 0,961 & 0,043 & 0,936 & 1,034 & 0,757 & 0,812 & 1,473 & 1,196 & 1,126 & 9 & 328 & $-3,9$ \\
\hline 329 & 0,3 & 1,8 & 0,3 & 1,8 & 1,7 & 0,969 & 0,972 & 0,941 & 0,042 & 0,991 & 1,005 & 0,772 & 0,755 & 0,662 & 0,500 & 0,508 & 61 & 329 & $-2,7$ \\
\hline 330 & 0,3 & 1,8 & 0,3 & 1,8 & 1,7 & 0,930 & 0,984 & 0,953 & 0,072 & 1,000 & 1,000 & 0,866 & 0,862 & 0,625 & 0,538 & 0,485 & 88 & 330 & $-2,6$ \\
\hline 331 & 0,4 & 2,3 & 0,4 & 2,2 & 2,2 & 0,954 & 0,974 & 0,943 & 0,053 & 0,914 & 1,046 & 0,581 & 0,575 & 0,922 & 0,530 & 0,536 & 87 & 331 & $-3,2$ \\
\hline 332 & 0,3 & 2,1 & 0,3 & 1,9 & 1,9 & 0,920 & 0,931 & 0,901 & 0,105 & 0,860 & 1,079 & 0,555 & 0,573 & 0,827 & 0,474 & 0,485 & 63 & 332 & $-3,0$ \\
\hline 333 & 7,9 & 12,7 & 9,3 & 11,1 & 10,8 & 0,856 & 0,874 & 0,846 & 0,191 & 0,617 & 1,273 & 0,628 & 0,685 & 4,013 & 2,750 & 2,308 & 169 & 333 & $-5,3$ \\
\hline 334 & 0,5 & 2,9 & 0,6 & 2,8 & 2,7 & 0,947 & 0,975 & 0,944 & 0,058 & 0,815 & 1,107 & 0,439 & 0,494 & 1,255 & 0,620 & 0,577 & 152 & 334 & $-3,6$ \\
\hline 335 & 0,4 & 2,2 & 0,4 & 2,2 & 2,1 & 0,915 & 0,977 & 0,946 & 0,088 & 0,901 & 1,054 & 0,638 & 0,616 & 0,841 & 0,518 & 0,485 & 51 & 335 & 3 \\
\hline 336 & 0,2 & 1,7 & 0,2 & 1,6 & 1,5 & 0,914 & 0,964 & 0,933 & 0,093 & 0,845 & 1,088 & 0,489 & 0,545 & 0,691 & 0,377 & 0,379 & 82 & 336 & $-2,8$ \\
\hline 337 & 0,2 & 2,4 & 0,2 & 2,2 & 2,2 & 0,888 & 0,940 & 0,909 & 0,127 & 0,483 & 1,439 & 0,213 & 0,191 & 1,137 & 0,217 & 0,227 & 177 & 337 & $-3,5$ \\
\hline 338 & 11,4 & 13,2 & 11,9 & 13,0 & 12,6 & 0,961 & 0,986 & 0,955 & 0,042 & 0,826 & 1,100 & 0,597 & 0,582 & 4,940 & 2,875 & 2,848 & 125 & 338 & \\
\hline 339 & 0,1 & 0,9 & 0,1 & 0,9 & 0,9 & 1,000 & 0,990 & 0,959 & 0,010 & 1,000 & 0,935 & 0,722 & 0,596 & 0,359 & 0,214 & 0,240 & 121 & 339 & 1,8 \\
\hline
\end{tabular}




\begin{tabular}{|c|c|c|c|c|c|c|c|c|c|c|c|c|c|c|c|c|c|c|c|}
\hline 340 & 10,9 & 15,8 & 12,2 & 14,3 & 13,9 & 0,897 & 0,905 & 0,876 & 0,140 & 0,547 & 1,352 & 0,354 & 0,448 & 6,263 & 2,803 & 2,350 & 4 & 340 & $-6,0$ \\
\hline 341 & 0,2 & 1,5 & 0,2 & 1,4 & 1,4 & 0,977 & 0,980 & 0,948 & 0,031 & 1,000 & 0,951 & 0,851 & 0,799 & 0,526 & 0,420 & 0,429 & 133 & 341 & $-2,4$ \\
\hline 342 & 0,4 & 2,3 & 0,4 & 2,3 & 2,2 & 0,951 & 0,987 & 0,956 & 0,050 & 0,904 & 1,052 & 0,583 & 0,548 & 0,925 & 0,507 & 0,536 & 130 & 342 & $-3,2$ \\
\hline 343 & 0,8 & 3,6 & 0,8 & 3,5 & 3,4 & 0,963 & 0,986 & 0,954 & 0,040 & 0,763 & 1,145 & 0,453 & 0,458 & 1,474 & 0,675 & 0,678 & 28 & 343 & $-3,9$ \\
\hline 344 & 0,7 & 3,1 & 0,7 & 3,0 & 2,9 & 0,967 & 0,983 & 0,952 & 0,037 & 0,960 & 1,021 & 0,743 & 0,798 & 1,104 & 0,881 & 0,837 & 150 & 344 & $-3,5$ \\
\hline 345 & 0,1 & 1,6 & 0,1 & 1,5 & 1,4 & 0,894 & 0,954 & 0,924 & 0,116 & 0,629 & 1,261 & 0,297 & 0,300 & 0,719 & 0,216 & 0,227 & 19 & 345 & $-2,8$ \\
\hline 346 & 5,3 & 11,8 & 6,3 & 11,2 & 10,8 & 0,849 & 0,945 & 0,914 & 0,161 & 0,480 & 1,444 & 0,259 & 0,277 & 5,128 & 1,421 & 1,415 & 0 & 346 & $-5,7$ \\
\hline 347 & 0,1 & 0,9 & 0,1 & 0,9 & 0,9 & 0,977 & 0,946 & 0,916 & 0,059 & 0,880 & 1,066 & 0,438 & 0,434 & 0,423 & 0,184 & 0,214 & 106 & 347 & $-2,1$ \\
\hline 348 & 1,6 & 5,0 & 1,7 & 5,0 & 4,9 & 0,947 & 0,994 & 0,962 & 0,053 & 0,777 & 1,134 & 0,467 & 0,548 & 2,072 & 1,136 & 1,103 & 99 & 348 & $-4,4$ \\
\hline 349 & 4,6 & 9,1 & 5,1 & 9,0 & 8,7 & 0,911 & 0,989 & 0,957 & 0,090 & 0,707 & 1,189 & 0,515 & 0,598 & 3,382 & 2,024 & 1,845 & 127 & 349 & $-5,1$ \\
\hline 350 & 0,9 & 3,6 & 0,9 & 3,6 & 3,5 & 0,927 & 0,980 & 0,949 & 0,076 & 0,826 & 1,101 & 0,649 & 0,721 & 1,308 & 0,944 & 0,833 & 11 & 350 & $-3,7$ \\
\hline 351 & 0,7 & 3,7 & 0,8 & 3,6 & 3,4 & 0,818 & 0,964 & 0,934 & 0,185 & 0,621 & 1,269 & 0,370 & 0,439 & 1,519 & 0,667 & 0,508 & 169 & 351 & $-3,9$ \\
\hline 352 & 0,6 & 3,0 & 0,7 & 3,0 & 2,9 & 0,961 & 0,983 & 0,952 & 0,043 & 0,854 & 1,082 & 0,507 & 0,481 & 1,259 & 0,606 & 0,678 & 2 & 352 & $-3,7$ \\
\hline 353 & 4,0 & 7,9 & 4,4 & 7,7 & 7,5 & 0,915 & 0,986 & 0,954 & 0,086 & 0,815 & 1,108 & 0,649 & 0,736 & 2,805 & 2,064 & 1,767 & 122 & 353 & $-4,8$ \\
\hline 354 & 0,2 & 1,6 & 0,2 & 1,6 & 1,5 & 0,961 & 0,969 & 0,938 & 0,050 & 0,999 & 1,000 & 0,678 & 0,721 & 0,627 & 0,452 & 0,442 & 152 & 354 & $-2,6$ \\
\hline 355 & 0,3 & 1,8 & 0,3 & 1,7 & 1,6 & 0,959 & 0,921 & 0,891 & 0,089 & 1,000 & 0,988 & 0,757 & 0,791 & 0,670 & 0,530 & 0,530 & 143 & 355 & $-2,7$ \\
\hline 356 & 2,7 & 7,5 & 3,1 & 7,0 & 6,7 & 0,858 & 0,922 & 0,893 & 0,162 & 0,591 & 1,300 & 0,420 & 0,523 & 2,849 & 1,491 & 1,212 & 37 & 356 & $-4,8$ \\
\hline 357 & 0,1 & 0,9 & 0,1 & 0,8 & 0,8 & 0,902 & 0,908 & 0,879 & 0,134 & 0,871 & 1,072 & 0,471 & 0,354 & 0,379 & 0,134 & 0,169 & 135 & 357 & $-1,9$ \\
\hline 358 & 10,2 & 12,0 & 10,5 & 11,8 & 11,5 & 0,969 & 0,988 & 0,956 & 0,033 & 0,895 & 1,057 & 0,650 & 0,704 & 4,475 & 3,148 & 3,047 & 152 & 358 & $-5,5$ \\
\hline 359 & 1,3 & 4,2 & 1,3 & 4,2 & 4,0 & 0,980 & 0,998 & 0,966 & 0,020 & 0,909 & 1,049 & 0,550 & 0,562 & 1,702 & 0,956 & 0,949 & 67 & 359 & $-4,1$ \\
\hline 360 & 0,4 & 2,6 & 0,5 & 2,5 & 2,4 & 0,966 & 0,972 & 0,941 & 0,044 & 0,824 & 1,102 & 0,437 & 0,406 & 1,136 & 0,461 & 0,479 & 37 & 360 & $-3,5$ \\
\hline 361 & 0,5 & 2,6 & 0,5 & 2,6 & 2,5 & 0,953 & 0,981 & 0,949 & 0,051 & 0,956 & 1,023 & 0,739 & 0,761 & 0,947 & 0,721 & 0,698 & 43 & 361 & $-3,2$ \\
\hline 362 & 72,8 & 38,8 & 84,7 & 36,5 & 35,3 & 0,859 & 0,940 & 0,910 & 0,153 & 0,608 & 1,282 & 0,442 & 0,598 & 14,472 & 8,656 & 6,213 & 55 & 362 & $-7,2$ \\
\hline 363 & 3,3 & 6,6 & 3,4 & 6,6 & 6,3 & 0,980 & 0,995 & 0,964 & 0,021 & 0,970 & 1,015 & 0,802 & 0,813 & 2,304 & 1,874 & 1,769 & 66 & 363 & $-4,5$ \\
\hline 364 & 0,2 & 1,7 & 0,2 & 1,7 & 1,6 & 0,968 & 0,982 & 0,951 & 0,036 & 0,954 & 1,024 & 0,712 & 0,728 & 0,625 & 0,455 & 0,408 & 12 & 364 & $-2,6$ \\
\hline 365 & 2,5 & 6,3 & 2,7 & 6,1 & 5,9 & 0,939 & 0,970 & 0,939 & 0,068 & 0,805 & 1,115 & 0,633 & 0,757 & 2,252 & 1,706 & 1,372 & 150 & 365 & $-4,5$ \\
\hline 366 & 0,2 & 1,6 & 0,2 & 1,7 & 1,6 & 0,949 & 1,013 & 0,980 & 0,053 & 0,873 & 1,070 & 0,562 & 0,602 & 0,647 & 0,390 & 0,379 & 150 & 366 & $-2,7$ \\
\hline 367 & 0,2 & 1,7 & 0,2 & 1,6 & 1,6 & 0,963 & 0,953 & 0,922 & 0,060 & 0,975 & 1,013 & 0,591 & 0,577 & 0,696 & 0,402 & 0,408 & 59 & 367 & $-2,8$ \\
\hline 368 & 0,2 & 1,6 & 0,2 & 1,4 & 1,4 & 0,844 & 0,909 & 0,880 & 0,181 & 0,788 & 1,127 & 0,564 & 0,573 & 0,592 & 0,339 & 0,303 & 30 & 368 & $-2,6$ \\
\hline 369 & 0,1 & 0,8 & 0,1 & 0,7 & 0,6 & 0,976 & 0,844 & 0,818 & 0,158 & 1,000 & 0,926 & 0,835 & 0,724 & 0,296 & 0,214 & 0,214 & 60 & 369 & $-1,6$ \\
\hline 370 & 4,6 & 8,3 & 4,9 & 8,1 & 7,8 & 0,941 & 0,977 & 0,946 & 0,063 & 0,841 & 1,091 & 0,665 & 0,737 & 2,969 & 2,189 & 2,090 & 20 & 370 & $-4,9$ \\
\hline 371 & 118,7 & 45,0 & 125,0 & 42,3 & 41,0 & 0,949 & 0,941 & 0,911 & 0,078 & 0,736 & 1,166 & 0,623 & 0,638 & 15,569 & 9,934 & 7,897 & 87 & 371 & $-7,3$ \\
\hline 372 & 2,7 & 6,2 & 2,8 & 6,1 & 5,9 & 0,961 & 0,985 & 0,954 & 0,042 & 0,879 & 1,067 & 0,648 & 0,653 & 2,311 & 1,510 & 1,492 & 67 & 372 & $-4,5$ \\
\hline 373 & 0,3 & 2,3 & 0,4 & 2,3 & 2,2 & 0,901 & 0,978 & 0,947 & 0,102 & 0,799 & 1,119 & 0,508 & 0,564 & 0,922 & 0,520 & 0,485 & 6 & 373 & $-3,2$ \\
\hline 374 & 11,1 & 12,8 & 11,6 & 12,6 & 12,2 & 0,953 & 0,986 & 0,954 & 0,049 & 0,846 & 1,087 & 0,600 & 0,620 & 4,843 & 3,004 & 2,745 & 53 & 374 & $-5,6$ \\
\hline 375 & 6,0 & 9,5 & 6,3 & 9,4 & 9,1 & 0,957 & 0,996 & 0,964 & 0,043 & 0,843 & 1,089 & 0,532 & 0,583 & 3,793 & 2,211 & 2,101 & 126 & 375 & $-5,2$ \\
\hline 376 & 0,4 & 2,2 & 0,4 & 2,2 & 2,1 & 0,981 & 0,972 & 0,941 & 0,034 & 0,945 & 1,029 & 0,649 & 0,614 & 0,864 & 0,530 & 0,552 & 90 & 376 & $-3,1$ \\
\hline 377 & 0,6 & 2,9 & 0,7 & 2,9 & 2,8 & 0,974 & 0,990 & 0,958 & 0,028 & 0,937 & 1,033 & 0,620 & 0,561 & 1,145 & 0,643 & 0,678 & 127 & 377 & $-3,5$ \\
\hline 378 & 0,0 & 0,6 & 0,0 & 0,5 & 0,5 & 0,958 & 0,879 & 0,850 & 0,128 & 1,000 & 0,919 & 0,791 & 0,589 & 0,230 & 0,136 & 0,152 & 21 & 378 & $-1,2$ \\
\hline 379 & 11,8 & 14,6 & 12,8 & 14,5 & 14,0 & 0,926 & 0,995 & 0,963 & 0,075 & 0,702 & 1,193 & 0,430 & 0,444 & 5,915 & 2,626 & 2,181 & 121 & 379 & $-5,9$ \\
\hline 380 & 0,1 & 1,0 & 0,1 & 0,9 & 0,9 & 0,982 & 0,885 & 0,857 & 0,116 & 1,000 & 0,988 & 0,686 & 0,490 & 0,386 & 0,189 & 0,227 & 90 & 380 & $-1,9$ \\
\hline 381 & 0,3 & 2,2 & 0,4 & 2,1 & 2,1 & 0,956 & 0,955 & 0,924 & 0,063 & 0,852 & 1,084 & 0,518 & 0,537 & 0,916 & 0,492 & 0,479 & 2 & 381 & $-3,2$ \\
\hline 382 & 0,3 & 2,2 & 0,3 & 2,1 & 2,0 & 0,937 & 0,958 & 0,927 & 0,076 & 0,822 & 1,103 & 0,487 & 0,498 & 0,912 & 0,455 & 0,485 & 2 & 382 & $-3,2$ \\
\hline 383 & 0,8 & 4,0 & 0,9 & 3,9 & 3,8 & 0,892 & 0,987 & 0,956 & 0,109 & 0,629 & 1,261 & 0,356 & 0,329 & 1,672 & 0,550 & 0,577 & 163 & 383 & $-4,1$ \\
\hline 384 & 0,1 & 1,3 & 0,1 & 1,2 & 1,2 & 0,953 & 0,960 & 0,929 & 0,062 & 0,938 & 1,033 & 0,614 & 0,582 & 0,494 & 0,288 & 0,303 & 9 & 384 & $-2,3$ \\
\hline 385 & 1,1 & 4,1 & 1,2 & 3,8 & 3,7 & 0,921 & 0,934 & 0,904 & 0,103 & 0,832 & 1,096 & 0,817 & 0,835 & 1,315 & 1,098 & 0,816 & 85 & 385 & $-3,7$ \\
\hline 386 & 0,3 & 2,1 & 0,3 & 2,0 & 2,0 & 0,945 & 0,976 & 0,944 & 0,060 & 0,855 & 1,081 & 0,488 & 0,474 & 0,879 & 0,417 & 0,455 & 177 & 386 & $-3,1$ \\
\hline 387 & 0,8 & 3,7 & 0,9 & 3,5 & 3,4 & 0,908 & 0,927 & 0,897 & 0,118 & 0,727 & 1,173 & 0,525 & 0,568 & 1,402 & 0,795 & 0,758 & 85 & 387 & $-3,8$ \\
\hline 388 & 97,1 & 38,8 & 102,5 & 38,0 & 36,7 & 0,948 & 0,977 & 0,946 & 0,057 & 0,809 & 1,112 & 0,612 & 0,669 & 14,220 & 9,518 & 8,707 & 74 & 388 & $-7,2$ \\
\hline 389 & 0,3 & 2,1 & 0,3 & 2,0 & 2,0 & 0,917 & 0,956 & 0,926 & 0,094 & 0,871 & 1,072 & 0,587 & 0,638 & 0,827 & 0,528 & 0,530 & 173 & 389 & $-3,0$ \\
\hline 390 & 0,0 & 0,9 & 0,1 & 0,7 & 0,7 & 0,886 & 0,823 & 0,797 & 0,211 & 0,759 & 1,148 & 0,391 & 0,362 & 0,381 & 0,138 & 0,152 & 100 & 390 & $-1,9$ \\
\hline 391 & 0,2 & 1,6 & 0,2 & 1,5 & 1,5 & 0,932 & 0,955 & 0,925 & 0,082 & 0,873 & 1,070 & 0,542 & 0,647 & 0,644 & 0,417 & 0,379 & 32 & 391 & $-2,7$ \\
\hline 392 & 1,8 & 5,2 & 1,8 & 5,2 & 5,0 & 0,977 & 0,990 & 0,959 & 0,025 & 0,832 & 1,097 & 0,477 & 0,479 & 2,196 & 1,052 & 1,077 & 150 & 392 & $-4,5$ \\
\hline 393 & 16,2 & 15,8 & 17,1 & 15,3 & 14,8 & 0,950 & 0,970 & 0,939 & 0,058 & 0,819 & 1,105 & 0,617 & 0,641 & 5,781 & 3,707 & 3,428 & 115 & 393 & \\
\hline 394 & 0,3 & 2,6 & 0,3 & 2,5 & 2,5 & 0,917 & 0,980 & 0,949 & 0,086 & 0,529 & 1,376 & 0,240 & 0,247 & 1,227 & 0,303 & 0,312 & 175 & 394 & 3,6 \\
\hline 395 & 0,2 & 1,8 & 0,2 & 1,5 & 1,4 & 0,987 & 0,842 & 0,815 & 0,159 & 0,871 & 1,071 & 0,507 & 0,361 & 0,735 & 0,265 & 0,303 & 90 & 395 & $-2,9$ \\
\hline 396 & 56,9 & 33,4 & 63,9 & 29,8 & 28,8 & 0,891 & 0,892 & 0,863 & 0,153 & 0,642 & 1,248 & 0,601 & 0,694 & 10,979 & 7,625 & 6,364 & 130 & 396 & , \\
\hline 397 & 10,7 & 14,3 & 12,4 & 13,5 & 13,0 & 0,868 & 0,940 & 0,910 & 0,145 & 0,658 & 1,233 & 0,463 & 0,536 & 5,437 & 2,916 & 2,348 & 72 & 397 & 5,8 \\
\hline
\end{tabular}




\begin{tabular}{|c|c|c|c|c|c|c|c|c|c|c|c|c|c|c|c|c|c|c|c|}
\hline 398 & 0,1 & 1,2 & 0,1 & 1,1 & 1,1 & 0,922 & 0,918 & 0,889 & 0,113 & 0,982 & 1,009 & 0,866 & 0,824 & 0,418 & 0,345 & 0,312 & 105 & 398 & $-2,1$ \\
\hline 399 & 3,1 & 7,2 & 3,1 & 7,2 & 6,9 & 0,979 & 0,996 & 0,964 & 0,022 & 0,748 & 1,156 & 0,363 & 0,432 & 3,285 & 1,419 & 1,429 & 112 & 399 & $-5,0$ \\
\hline 400 & 0,6 & 3,5 & 0,6 & 3,4 & 3,3 & 0,911 & 0,979 & 0,948 & 0,091 & 0,578 & 1,315 & 0,291 & 0,303 & 1,566 & 0,475 & 0,442 & 61 & 400 & $-4,0$ \\
\hline 401 & 0,5 & 3,2 & 0,6 & 2,8 & 2,7 & 0,875 & 0,898 & 0,870 & 0,161 & 0,662 & 1,229 & 0,501 & 0,588 & 1,154 & 0,678 & 0,592 & 145 & 401 & $-3,5$ \\
\hline 402 & 30,2 & 24,0 & 32,4 & 23,0 & 22,2 & 0,932 & 0,959 & 0,929 & 0,079 & 0,662 & 1,229 & 0,482 & 0,602 & 8,929 & 5,378 & 4,547 & 11 & 402 & $-6,5$ \\
\hline 403 & 0,3 & 1,9 & 0,3 & 1,9 & 1,8 & 0,965 & 0,984 & 0,953 & 0,038 & 0,985 & 1,008 & 0,643 & 0,611 & 0,744 & 0,455 & 0,461 & 85 & 403 & $-2,9$ \\
\hline 404 & 0,3 & 2,2 & 0,4 & 2,0 & 2,0 & 0,919 & 0,907 & 0,878 & 0,123 & 0,861 & 1,078 & 0,587 & 0,715 & 0,859 & 0,614 & 0,508 & 53 & 404 & $-3,1$ \\
\hline 405 & 1,3 & 4,1 & 1,4 & 4,1 & 4,0 & 0,971 & 0,998 & 0,966 & 0,029 & 0,991 & 1,005 & 0,772 & 0,739 & 1,482 & 1,095 & 1,119 & 29 & 405 & $-3,9$ \\
\hline 406 & 2,6 & 5,9 & 2,7 & 5,8 & 5,6 & 0,953 & 0,991 & 0,960 & 0,048 & 0,936 & 1,034 & 0,742 & 0,752 & 2,097 & 1,578 & 1,455 & 117 & 406 & $-4,4$ \\
\hline 407 & 0,2 & 1,6 & 0,2 & 1,5 & 1,5 & 0,979 & 0,968 & 0,937 & 0,038 & 1,000 & 0,979 & 0,698 & 0,676 & 0,612 & 0,413 & 0,429 & 67 & 407 & $-2,6$ \\
\hline 408 & 10,9 & 14,3 & 11,5 & 13,9 & 13,5 & 0,941 & 0,971 & 0,940 & 0,066 & 0,667 & 1,225 & 0,357 & 0,403 & 6,223 & 2,508 & 2,238 & 109 & 408 & $-6,0$ \\
\hline 409 & 3,9 & 7,7 & 4,0 & 7,7 & 7,4 & 0,974 & 0,996 & 0,964 & 0,027 & 0,821 & 1,104 & 0,501 & 0,551 & 3,144 & 1,734 & 1,694 & 124 & 409 & $-5,0$ \\
\hline 410 & 0,1 & 1,2 & 0,1 & 1,1 & 1,0 & 0,896 & 0,896 & 0,867 & 0,147 & 0,772 & 1,138 & 0,452 & 0,487 & 0,492 & 0,240 & 0,214 & 65 & 410 & $-2,3$ \\
\hline 411 & 0,8 & 4,0 & 0,9 & 3,7 & 3,6 & 0,858 & 0,941 & 0,910 & 0,154 & 0,637 & 1,253 & 0,403 & 0,495 & 1,595 & 0,790 & 0,719 & 88 & 411 & $-4,0$ \\
\hline 412 & 0,6 & 3,1 & 0,6 & 3,1 & 3,0 & 0,923 & 0,981 & 0,949 & 0,079 & 0,750 & 1,155 & 0,436 & 0,441 & 1,306 & 0,576 & 0,577 & 117 & 412 & $-3,7$ \\
\hline 413 & 1,0 & 4,1 & 1,1 & 4,0 & 3,9 & 0,940 & 0,993 & 0,961 & 0,060 & 0,790 & 1,125 & 0,495 & 0,461 & 1,632 & 0,752 & 0,746 & 29 & 413 & $-4,0$ \\
\hline 414 & 0,3 & 2,0 & 0,3 & 2,0 & 1,9 & 0,947 & 0,975 & 0,943 & 0,059 & 0,961 & 1,020 & 0,704 & 0,670 & 0,747 & 0,500 & 0,485 & 43 & 414 & $-2,9$ \\
\hline 415 & 0,5 & 3,0 & 0,6 & 2,7 & 2,7 & 0,851 & 0,918 & 0,888 & 0,170 & 0,713 & 1,185 & 0,557 & 0,561 & 1,077 & 0,604 & 0,536 & 75 & 415 & $-3,4$ \\
\hline 416 & 0,1 & 1,0 & 0,1 & 0,9 & 0,9 & 0,928 & 0,929 & 0,899 & 0,102 & 1,000 & 0,941 & 0,840 & 0,790 & 0,373 & 0,295 & 0,312 & 6 & 416 & $-1,9$ \\
\hline 417 & 2,8 & 7,2 & 3,1 & 7,1 & 6,9 & 0,908 & 0,992 & 0,960 & 0,092 & 0,677 & 1,216 & 0,446 & 0,404 & 2,815 & 1,138 & 1,085 & 103 & 417 & $-4,8$ \\
\hline 418 & 0,3 & 1,8 & 0,3 & 1,8 & 1,7 & 0,942 & 0,956 & 0,925 & 0,073 & 0,959 & 1,021 & 0,701 & 0,754 & 0,683 & 0,515 & 0,479 & 11 & 418 & $-2,8$ \\
\hline 419 & 2,5 & 5,8 & 2,5 & 5,7 & 5,5 & 0,972 & 0,993 & 0,961 & 0,029 & 0,926 & 1,039 & 0,621 & 0,681 & 2,243 & 1,527 & 1,515 & 127 & 419 & $-4,5$ \\
\hline 420 & 0,6 & 2,8 & 0,6 & 2,8 & 2,7 & 0,972 & 0,989 & 0,958 & 0,030 & 0,965 & 1,018 & 0,666 & 0,576 & 1,069 & 0,616 & 0,643 & 136 & 420 & $-3,4$ \\
\hline 421 & 1,0 & 3,8 & 1,1 & 3,8 & 3,6 & 0,963 & 0,987 & 0,955 & 0,040 & 0,893 & 1,058 & 0,692 & 0,680 & 1,379 & 0,938 & 0,909 & 88 & 421 & $-3,8$ \\
\hline 422 & 0,1 & 1,2 & 0,1 & 1,1 & 1,1 & 0,975 & 0,956 & 0,926 & 0,050 & 1,000 & 0,974 & 0,860 & 0,784 & 0,410 & 0,321 & 0,339 & 142 & 422 & $-2,0$ \\
\hline 423 & 1,7 & 4,8 & 1,7 & 4,7 & 4,6 & 0,961 & 0,983 & 0,952 & 0,042 & 0,910 & 1,048 & 0,758 & 0,770 & 1,672 & 1,288 & 1,249 & 173 & 423 & $-4,1$ \\
\hline 424 & 1,7 & 5,6 & 2,0 & 5,3 & 5,1 & 0,853 & 0,941 & 0,911 & 0,159 & 0,670 & 1,222 & 0,529 & 0,597 & 2,020 & 1,206 & 1,176 & 169 & 424 & $-4,3$ \\
\hline 425 & 0,1 & 1,5 & 0,2 & 1,3 & 1,3 & 0,891 & 0,887 & 0,859 & 0,157 & 0,826 & 1,100 & 0,478 & 0,435 & 0,612 & 0,266 & 0,273 & 102 & 425 & $-2,6$ \\
\hline 426 & 0,7 & 3,0 & 0,7 & 2,9 & 2,8 & 0,955 & 0,983 & 0,951 & 0,048 & 0,952 & 1,025 & 0,720 & 0,781 & 1,091 & 0,851 & 0,791 & 107 & 426 & $-3,4$ \\
\hline 427 & 0,9 & 3,8 & 1,0 & 3,8 & 3,7 & 0,938 & 0,987 & 0,955 & 0,063 & 0,795 & 1,122 & 0,475 & 0,546 & 1,583 & 0,865 & 0,805 & 59 & 427 & $-4,0$ \\
\hline 428 & 21,4 & 19,2 & 22,9 & 18,2 & 17,7 & 0,935 & 0,952 & 0,921 & 0,081 & 0,732 & 1,168 & 0,533 & 0,596 & 7,152 & 4,260 & 4,091 & 159 & 428 & $-6,2$ \\
\hline 429 & 70,2 & 32,8 & 73,9 & 32,6 & 31,6 & 0,949 & 0,993 & 0,961 & 0,051 & 0,818 & 1,106 & 0,619 & 0,645 & 12,010 & 7,747 & 7,245 & 152 & 429 & $-6,9$ \\
\hline 430 & 4,3 & 11,5 & 4,9 & 9,3 & 9,0 & 0,883 & 0,807 & 0,781 & 0,226 & 0,406 & 1,570 & 0,309 & 0,380 & 4,210 & 1,601 & 1,439 & 59 & 430 & $-5,4$ \\
\hline 431 & 1,2 & 4,3 & 1,2 & 4,2 & 4,1 & 0,970 & 0,988 & 0,957 & 0,033 & 0,822 & 1,103 & 0,513 & 0,551 & 1,718 & 0,947 & 0,912 & 6 & 431 & $-4,1$ \\
\hline 432 & 0,4 & 3,6 & 0,5 & 2,8 & 2,7 & 0,731 & 0,798 & 0,772 & 0,337 & 0,374 & 1,635 & 0,278 & 0,389 & 1,315 & 0,512 & 0,379 & 179 & 432 & $-3,7$ \\
\hline 433 & 23,2 & 20,1 & 25,0 & 19,4 & 18,8 & 0,928 & 0,964 & 0,933 & 0,081 & 0,718 & 1,180 & 0,529 & 0,624 & 7,470 & 4,660 & 3,964 & 14 & 433 & $-6,2$ \\
\hline 434 & 0,3 & 2,2 & 0,3 & 2,1 & 2,0 & 0,909 & 0,932 & 0,901 & 0,114 & 0,806 & 1,114 & 0,616 & 0,734 & 0,810 & 0,595 & 0,530 & 73 & 434 & $-3,0$ \\
\hline 435 & 0,3 & 2,2 & 0,3 & 2,2 & 2,1 & 0,954 & 0,981 & 0,949 & 0,050 & 0,858 & 1,080 & 0,512 & 0,506 & 0,904 & 0,457 & 0,479 & 59 & 435 & $-3,2$ \\
\hline 436 & 2,5 & 6,6 & 2,6 & 6,5 & 6,3 & 0,930 & 0,992 & 0,961 & 0,070 & 0,711 & 1,186 & 0,394 & 0,418 & 2,819 & 1,178 & 1,146 & 102 & 436 & $-4,8$ \\
\hline 437 & 0,4 & 2,6 & 0,4 & 2,5 & 2,5 & 0,910 & 0,966 & 0,935 & 0,096 & 0,683 & 1,210 & 0,365 & 0,419 & 1,145 & 0,479 & 0,479 & 131 & 437 & $-3,5$ \\
\hline 438 & 0,2 & 2,0 & 0,2 & 1,9 & 1,8 & 0,959 & 0,964 & 0,933 & 0,055 & 0,746 & 1,158 & 0,378 & 0,394 & 0,884 & 0,348 & 0,379 & 37 & 438 & $-3,1$ \\
\hline 439 & 3,1 & 6,6 & 3,2 & 6,6 & 6,4 & 0,953 & 0,992 & 0,961 & 0,048 & 0,879 & 1,067 & 0,662 & 0,700 & 2,425 & 1,697 & 1,674 & 114 & 439 & $-4,6$ \\
\hline 440 & 0,3 & 2,0 & 0,3 & 1,9 & 1,9 & 0,874 & 0,950 & 0,920 & 0,136 & 0,788 & 1,126 & 0,680 & 0,785 & 0,695 & 0,546 & 0,408 & 111 & 440 & $-2,8$ \\
\hline 441 & 224,0 & 64,1 & 241,1 & 58,2 & 56,4 & 0,929 & 0,909 & 0,880 & 0,115 & 0,686 & 1,207 & 0,586 & 0,652 & 22,063 & 14,395 & 11,811 & 143 & 441 & $-7,8$ \\
\hline 442 & 0,2 & 1,7 & 0,2 & 1,6 & 1,6 & 0,937 & 0,918 & 0,888 & 0,104 & 0,787 & 1,127 & 0,432 & 0,452 & 0,750 & 0,339 & 0,339 & 124 & 442 & $-2,9$ \\
\hline 443 & 1,2 & 4,0 & 1,2 & 4,0 & 3,8 & 0,973 & 0,998 & 0,966 & 0,027 & 0,948 & 1,027 & 0,665 & 0,657 & 1,512 & 0,993 & 0,985 & 60 & 443 & $-3,9$ \\
\hline 444 & 0,7 & 3,0 & 0,7 & 3,0 & 2,9 & 0,960 & 0,983 & 0,952 & 0,044 & 0,981 & 1,010 & 0,825 & 0,767 & 1,053 & 0,808 & 0,816 & 80 & 444 & $-3,4$ \\
\hline 445 & 20,8 & 21,2 & 22,9 & 20,8 & 20,2 & 0,910 & 0,982 & 0,951 & 0,092 & 0,581 & 1,312 & 0,311 & 0,336 & 9,224 & 3,102 & 2,652 & 7 & 445 & $-6,5$ \\
\hline 446 & 0,7 & 3,1 & 0,7 & 2,9 & 2,8 & 0,918 & 0,960 & 0,929 & 0,092 & 0,880 & 1,066 & 0,752 & 0,787 & 1,052 & 0,828 & 0,758 & 109 & 446 & $-3,4$ \\
\hline 447 & 0,2 & 1,6 & 0,2 & 1,5 & 1,4 & 0,957 & 0,941 & 0,910 & 0,073 & 0,995 & 1,002 & 0,649 & 0,553 & 0,617 & 0,341 & 0,379 & 96 & 447 & $-2,6$ \\
\hline 448 & 0,1 & 0,8 & 0,1 & 0,7 & 0,7 & 0,907 & 0,827 & 0,801 & 0,197 & 1,000 & 0,988 & 0,621 & 0,559 & 0,339 & 0,189 & 0,227 & 80 & 448 & $-1,8$ \\
\hline 449 & 1,2 & 4,5 & 1,3 & 4,4 & 4,3 & 0,924 & 0,982 & 0,951 & 0,078 & 0,764 & 1,144 & 0,447 & 0,556 & 1,881 & 1,047 & 0,915 & 22 & 449 & $-4,2$ \\
\hline 450 & 1,0 & 4,1 & 1,1 & 4,0 & 3,9 & 0,948 & 0,988 & 0,956 & 0,054 & 0,758 & 1,149 & 0,411 & 0,454 & 1,769 & 0,804 & 0,758 & 130 & 450 & $-4,1$ \\
\hline 451 & 0,1 & 1,0 & 0,1 & 0,9 & 0,9 & 0,930 & 0,926 & 0,896 & 0,102 & 1,000 & 0,990 & 0,696 & 0,574 & 0,373 & 0,214 & 0,240 & 138 & 451 & 10 \\
\hline 452 & 0,8 & 3,4 & 0,8 & 3,4 & 3,3 & 0,970 & 0,991 & 0,959 & 0,031 & 0,865 & 1,075 & 0,502 & 0,534 & 1,420 & 0,758 & 0,758 & 178 & 452 & $-3,8$ \\
\hline 453 & 0,2 & 1,7 & 0,2 & 1,6 & 1,5 & 0,916 & 0,939 & 0,909 & 0,104 & 0,907 & 1,050 & 0,619 & 0,664 & 0,645 & 0,429 & 0,379 & 133 & 453 & $-2,7$ \\
\hline 454 & 9,4 & 12,9 & 10,1 & 12,5 & 12,1 & 0,928 & 0,965 & 0,934 & 0,080 & 0,706 & 1,190 & 0,436 & 0,517 & 5,231 & 2,704 & 2,319 & 6 & 454 & 57 \\
\hline 455 & 0,9 & 4,0 & 1,0 & 3,6 & 3,5 & 0,873 & 0,894 & 0,865 & 0,165 & 0,675 & 1,217 & 0,532 & 0,591 & 1,442 & 0,853 & 0,746 & 2 & 455 & 3,8 \\
\hline
\end{tabular}




\begin{tabular}{|c|c|c|c|c|c|c|c|c|c|c|c|c|c|c|c|c|c|c|c|}
\hline 456 & 0,7 & 3,2 & 0,7 & 3,1 & 3,0 & 0,956 & 0,975 & 0,943 & 0,051 & 0,884 & 1,064 & 0,545 & 0,583 & 1,287 & 0,750 & 0,758 & 133 & 456 & $-3,7$ \\
\hline 457 & 0,3 & 2,0 & 0,3 & 1,9 & 1,9 & 0,951 & 0,954 & 0,924 & 0,067 & 0,766 & 1,143 & 0,412 & 0,387 & 0,879 & 0,340 & 0,379 & 9 & 457 & $-3,1$ \\
\hline 458 & 0,1 & 1,3 & 0,1 & 1,3 & 1,2 & 0,978 & 0,946 & 0,916 & 0,058 & 0,884 & 1,064 & 0,448 & 0,379 & 0,599 & 0,227 & 0,303 & 10 & 458 & $-2,6$ \\
\hline 459 & 0,5 & 2,8 & 0,6 & 2,8 & 2,7 & 0,911 & 0,975 & 0,944 & 0,093 & 0,793 & 1,123 & 0,536 & 0,625 & 1,103 & 0,689 & 0,643 & 85 & 459 & $-3,5$ \\
\hline 460 & 0,3 & 1,8 & 0,3 & 1,7 & 1,7 & 0,967 & 0,972 & 0,941 & 0,043 & 0,989 & 1,006 & 0,711 & 0,715 & 0,674 & 0,482 & 0,479 & 133 & 460 & $-2,8$ \\
\hline 461 & 2,1 & 7,7 & 2,5 & 7,6 & 7,3 & 0,846 & 0,987 & 0,955 & 0,155 & 0,445 & 1,499 & 0,219 & 0,287 & 3,469 & 0,996 & 0,958 & 8 & 461 & $-5,1$ \\
\hline 462 & 0,4 & 3,0 & 0,5 & 2,4 & 2,3 & 0,780 & 0,802 & 0,776 & 0,296 & 0,517 & 1,390 & 0,614 & 0,737 & 0,884 & 0,651 & 0,429 & 25 & 462 & $-3,1$ \\
\hline 463 & 11,4 & 17,0 & 13,9 & 14,5 & 14,0 & 0,825 & 0,852 & 0,825 & 0,229 & 0,497 & 1,419 & 0,457 & 0,578 & 5,642 & 3,259 & 2,727 & 93 & 463 & $-5,8$ \\
\hline 464 & 40,8 & 25,4 & 43,3 & 24,3 & 23,6 & 0,943 & 0,960 & 0,929 & 0,070 & 0,797 & 1,120 & 0,630 & 0,708 & 9,080 & 6,428 & 5,619 & 99 & 464 & $-6,5$ \\
\hline 465 & 9,1 & 13,3 & 10,2 & 12,3 & 11,9 & 0,886 & 0,926 & 0,896 & 0,136 & 0,643 & 1,247 & 0,515 & 0,639 & 4,739 & 3,029 & 2,279 & 32 & 465 & $-5,6$ \\
\hline 466 & 3,8 & 8,7 & 4,2 & 8,3 & 8,0 & 0,895 & 0,953 & 0,922 & 0,115 & 0,630 & 1,260 & 0,397 & 0,474 & 3,488 & 1,654 & 1,366 & 4 & 466 & $-5,1$ \\
\hline 467 & 1,1 & 3,7 & 1,1 & 3,7 & 3,6 & 0,975 & 0,992 & 0,960 & 0,026 & 0,999 & 1,000 & 0,772 & 0,753 & 1,358 & 1,023 & 1,061 & 69 & 467 & $-3,8$ \\
\hline 468 & 0,5 & 2,7 & 0,6 & 2,7 & 2,6 & 0,962 & 0,981 & 0,950 & 0,042 & 0,936 & 1,033 & 0,684 & 0,658 & 1,006 & 0,662 & 0,678 & 38 & 468 & $-3,3$ \\
\hline 469 & 510,3 & 113,2 & 564,2 & 90,7 & 87,8 & 0,905 & 0,801 & 0,775 & 0,221 & 0,500 & 1,414 & 0,660 & 0,826 & 31,386 & 25,936 & 6,413 & 147 & 469 & $-8,3$ \\
\hline 470 & 0,2 & 1,5 & 0,2 & 1,5 & 1,5 & 0,967 & 0,994 & 0,962 & 0,034 & 1,000 & 0,949 & 0,877 & 0,826 & 0,552 & 0,455 & 0,479 & 153 & 470 & $-2,5$ \\
\hline 471 & 0,8 & 3,3 & 0,9 & 3,3 & 3,2 & 0,958 & 0,985 & 0,953 & 0,044 & 0,941 & 1,031 & 0,627 & 0,700 & 1,296 & 0,908 & 0,909 & 51 & 471 & $-3,7$ \\
\hline 472 & 1,3 & 4,6 & 1,4 & 4,4 & 4,3 & 0,937 & 0,958 & 0,928 & 0,076 & 0,799 & 1,118 & 0,525 & 0,547 & 1,800 & 0,985 & 0,909 & 6 & 472 & $-4,2$ \\
\hline 473 & 1,6 & 5,2 & 1,7 & 5,2 & 5,0 & 0,959 & 0,994 & 0,962 & 0,041 & 0,742 & 1,161 & 0,420 & 0,450 & 2,213 & 0,995 & 0,985 & 172 & 473 & $-4,5$ \\
\hline 474 & 2,1 & 6,2 & 2,3 & 6,1 & 5,9 & 0,920 & 0,987 & 0,955 & 0,081 & 0,701 & 1,194 & 0,442 & 0,530 & 2,479 & 1,313 & 1,154 & 139 & 474 & $-4,6$ \\
\hline 475 & 0,4 & 2,4 & 0,5 & 2,4 & 2,3 & 0,954 & 0,988 & 0,956 & 0,047 & 0,942 & 1,030 & 0,638 & 0,607 & 0,947 & 0,575 & 0,577 & 115 & 475 & $-3,2$ \\
\hline 476 & 1,3 & 4,3 & 1,3 & 4,2 & 4,1 & 0,961 & 0,993 & 0,961 & 0,040 & 0,884 & 1,063 & 0,608 & 0,554 & 1,632 & 0,904 & 0,912 & 147 & 476 & $-4,0$ \\
\hline 477 & 0,3 & 2,0 & 0,3 & 2,0 & 1,9 & 0,906 & 0,965 & 0,934 & 0,100 & 0,806 & 1,114 & 0,522 & 0,528 & 0,803 & 0,424 & 0,408 & 115 & 477 & $-3,0$ \\
\hline 478 & 0,1 & 1,2 & 0,1 & 1,1 & 1,1 & 0,958 & 0,940 & 0,910 & 0,073 & 1,000 & 0,935 & 0,901 & 0,806 & 0,432 & 0,348 & 0,379 & 54 & 478 & $-2,1$ \\
\hline 479 & 0,4 & 2,3 & 0,4 & 2,2 & 2,1 & 0,928 & 0,959 & 0,928 & 0,083 & 0,910 & 1,049 & 0,751 & 0,704 & 0,791 & 0,557 & 0,536 & 22 & 479 & $-3,0$ \\
\hline 480 & 3,8 & 7,7 & 4,1 & 7,5 & 7,2 & 0,934 & 0,969 & 0,938 & 0,073 & 0,813 & 1,109 & 0,556 & 0,660 & 2,961 & 1,955 & 1,824 & 105 & 480 & $-4,9$ \\
\hline 481 & 1,9 & 5,6 & 2,0 & 5,5 & 5,3 & 0,951 & 0,980 & 0,949 & 0,052 & 0,781 & 1,131 & 0,591 & 0,677 & 2,043 & 1,383 & 1,146 & 105 & 481 & $-4,4$ \\
\hline 482 & 0,2 & 1,8 & 0,2 & 1,6 & 1,5 & 0,924 & 0,875 & 0,847 & 0,146 & 0,794 & 1,123 & 0,502 & 0,429 & 0,729 & 0,313 & 0,303 & 11 & 482 & $-2,9$ \\
\hline 483 & 0,9 & 3,5 & 1,0 & 3,5 & 3,4 & 0,970 & 0,992 & 0,960 & 0,031 & 0,937 & 1,033 & 0,627 & 0,649 & 1,379 & 0,894 & 0,857 & 54 & 483 & $-3,8$ \\
\hline 484 & 1,9 & 5,0 & 1,9 & 5,0 & 4,8 & 0,968 & 0,994 & 0,962 & 0,033 & 0,920 & 1,043 & 0,685 & 0,742 & 1,858 & 1,378 & 1,290 & 120 & 484 & $-4,2$ \\
\hline 485 & 0,3 & 2,5 & 0,4 & 2,4 & 2,4 & 0,929 & 0,963 & 0,932 & 0,080 & 0,671 & 1,221 & 0,383 & 0,458 & 1,063 & 0,487 & 0,461 & 180 & 485 & $-3,4$ \\
\hline 486 & 0,2 & 1,7 & 0,2 & 1,7 & 1,6 & 0,931 & 0,971 & 0,940 & 0,075 & 0,793 & 1,123 & 0,471 & 0,443 & 0,721 & 0,320 & 0,339 & 13 & 486 & $-2,8$ \\
\hline 487 & 9,5 & 12,0 & 10,0 & 11,9 & 11,5 & 0,951 & 0,991 & 0,959 & 0,050 & 0,828 & 1,099 & 0,562 & 0,619 & 4,641 & 2,872 & 2,612 & 148 & 487 & $-5,5$ \\
\hline 488 & 0,2 & 2,0 & 0,3 & 1,9 & 1,8 & 0,896 & 0,938 & 0,908 & 0,121 & 0,741 & 1,161 & 0,576 & 0,703 & 0,719 & 0,505 & 0,408 & 7 & 488 & $-2,8$ \\
\hline 489 & 9,4 & 11,6 & 9,6 & 11,6 & 11,2 & 0,976 & 0,999 & 0,967 & 0,024 & 0,875 & 1,069 & 0,542 & 0,582 & 4,685 & 2,726 & 2,652 & 98 & 489 & $-5,5$ \\
\hline 490 & 0,2 & 1,8 & 0,2 & 1,8 & 1,7 & 0,910 & 0,961 & 0,930 & 0,098 & 0,702 & 1,194 & 0,375 & 0,351 & 0,799 & 0,281 & 0,312 & 146 & 490 & $-3,0$ \\
\hline 491 & 0,3 & 2,3 & 0,4 & 2,2 & 2,1 & 0,861 & 0,956 & 0,926 & 0,146 & 0,717 & 1,181 & 0,440 & 0,435 & 0,936 & 0,407 & 0,379 & 152 & 491 & $-3,2$ \\
\hline 492 & 0,3 & 2,0 & 0,3 & 2,0 & 1,9 & 0,950 & 0,965 & 0,934 & 0,061 & 0,827 & 1,099 & 0,454 & 0,388 & 0,879 & 0,341 & 0,379 & 3 & 492 & $-3,1$ \\
\hline 493 & 3,0 & 6,7 & 3,2 & 6,6 & 6,4 & 0,923 & 0,989 & 0,958 & 0,077 & 0,836 & 1,094 & 0,606 & 0,625 & 2,493 & 1,558 & 1,391 & 106 & 493 & $-4,6$ \\
\hline 494 & 2,2 & 5,5 & 2,2 & 5,4 & 5,2 & 0,974 & 0,991 & 0,959 & 0,027 & 0,919 & 1,043 & 0,652 & 0,691 & 2,063 & 1,426 & 1,409 & 88 & 494 & $-4,4$ \\
\hline 495 & 5,0 & 8,9 & 5,2 & 8,7 & 8,4 & 0,947 & 0,977 & 0,946 & 0,058 & 0,782 & 1,131 & 0,537 & 0,572 & 3,429 & 1,963 & 1,871 & 145 & 495 & $-5,1$ \\
\hline 496 & 0,5 & 2,5 & 0,5 & 2,5 & 2,4 & 0,979 & 0,988 & 0,956 & 0,024 & 0,957 & 1,022 & 0,616 & 0,582 & 0,988 & 0,575 & 0,577 & 153 & 496 & $-3,3$ \\
\hline 497 & 0,1 & 0,8 & 0,1 & 0,6 & 0,6 & 0,950 & 0,838 & 0,811 & 0,170 & 1,000 & 0,914 & 0,834 & 0,657 & 0,288 & 0,189 & 0,214 & 92 & 497 & $-1,5$ \\
\hline 498 & 0,1 & 1,6 & 0,2 & 1,6 & 1,5 & 0,910 & 0,969 & 0,938 & 0,095 & 0,677 & 1,216 & 0,326 & 0,313 & 0,752 & 0,235 & 0,273 & 140 & 498 & $-2,9$ \\
\hline 499 & 1,5 & 4,7 & 1,6 & 4,7 & 4,5 & 0,981 & 0,994 & 0,962 & 0,020 & 0,869 & 1,073 & 0,556 & 0,617 & 1,865 & 1,150 & 1,085 & 160 & 499 & $-4,2$ \\
\hline 500 & 4,7 & 8,2 & 4,8 & 8,1 & 7,9 & 0,975 & 0,996 & 0,964 & 0,026 & 0,886 & 1,062 & 0,646 & 0,743 & 3,041 & 2,259 & 2,145 & 70 & 500 & $-4,9$ \\
\hline 501 & 1,2 & 3,9 & 1,2 & 3,9 & 3,8 & 0,982 & 0,998 & 0,966 & 0,018 & 0,982 & 1,009 & 0,715 & 0,754 & 1,457 & 1,098 & 1,077 & 180 & 501 & $-3,9$ \\
\hline 502 & 0,1 & 1,1 & 0,1 & 1,0 & 0,9 & 0,955 & 0,912 & 0,883 & 0,099 & 1,000 & 0,980 & 0,696 & 0,647 & 0,410 & 0,265 & 0,303 & 28 & 502 & $-2,0$ \\
\hline 503 & 0,4 & 2,4 & 0,4 & 2,3 & 2,2 & 0,932 & 0,970 & 0,938 & 0,075 & 0,794 & 1,122 & 0,459 & 0,446 & 0,988 & 0,440 & 0,408 & 115 & 503 & $-3,3$ \\
\hline 504 & 0,1 & 1,4 & 0,1 & 1,4 & 1,3 & 0,923 & 0,979 & 0,947 & 0,080 & 0,873 & 1,070 & 0,534 & 0,577 & 0,573 & 0,331 & 0,312 & 92 & 504 & $-2,5$ \\
\hline 505 & 1,1 & 4,0 & 1,2 & 4,0 & 3,9 & 0,984 & 0,993 & 0,961 & 0,018 & 0,894 & 1,058 & 0,538 & 0,521 & 1,645 & 0,857 & 0,883 & 43 & 505 & $-4,0$ \\
\hline 506 & 26,2 & 26,3 & 31,2 & 25,5 & 24,7 & 0,839 & 0,972 & 0,941 & 0,163 & 0,477 & 1,448 & 0,262 & 0,325 & 11,270 & 3,667 & 3,117 & 119 & 506 & $-6,8$ \\
\hline 507 & 0,2 & 1,7 & 0,3 & 1,7 & 1,6 & 0,945 & 0,971 & 0,940 & 0,062 & 1,000 & 0,991 & 0,697 & 0,677 & 0,671 & 0,455 & 0,461 & 20 & 507 & $-2,7$ \\
\hline 508 & 0,3 & 1,9 & 0,3 & 1,8 & 1,7 & 0,990 & 0,914 & 0,885 & 0,086 & 0,940 & 1,031 & 0,598 & 0,593 & 0,766 & 0,455 & 0,479 & 10 & 508 & $-2,9$ \\
\hline 509 & 0,2 & 1,6 & 0,2 & 1,6 & 1,5 & 0,963 & 0,969 & 0,938 & 0,048 & 1,000 & 0,974 & 0,774 & 0,734 & 0,611 & 0,448 & 0,442 & 48 & 509 & 2 \\
\hline 510 & 35,8 & 25,7 & 39,7 & 23,7 & 22,9 & 0,901 & 0,922 & 0,893 & 0,126 & 0,682 & 1,211 & 0,576 & 0,688 & 8,890 & 6,118 & 4,994 & 65 & 510 & $-6,5$ \\
\hline 511 & 2,2 & 5,6 & 2,3 & 5,5 & 5,3 & 0,957 & 0,991 & 0,959 & 0,044 & 0,885 & 1,063 & 0,609 & 0,673 & 2,138 & 1,439 & 1,323 & 129 & 511 & $-4,4$ \\
\hline 512 & 0,3 & 2,3 & 0,3 & 2,3 & 2,2 & 0,904 & 0,978 & 0,947 & 0,099 & 0,729 & 1,171 & 0,392 & 0,346 & 1,003 & 0,347 & 0,379 & 4 & 512 & 3 \\
\hline 513 & 0,2 & 1,6 & 0,2 & 1,6 & 1,5 & 0,960 & 0,981 & 0,950 & 0,044 & 0,849 & 1,085 & 0,438 & 0,380 & 0,705 & 0,268 & 0,321 & 42 & 513 & 2,8 \\
\hline
\end{tabular}




\begin{tabular}{|c|c|c|c|c|c|c|c|c|c|c|c|c|c|c|c|c|c|c|c|}
\hline 514 & 0,2 & 1,9 & 0,3 & 1,8 & 1,8 & 0,944 & 0,962 & 0,931 & 0,068 & 0,861 & 1,077 & 0,447 & 0,432 & 0,831 & 0,359 & 0,379 & 17 & 514 & $-3,1$ \\
\hline 515 & 0,3 & 2,3 & 0,3 & 2,2 & 2,2 & 0,973 & 0,978 & 0,946 & 0,035 & 0,742 & 1,161 & 0,395 & 0,375 & 1,000 & 0,375 & 0,379 & 132 & 515 & $-3,3$ \\
\hline 516 & 0,1 & 0,9 & 0,1 & 0,8 & 0,8 & 0,891 & 0,865 & 0,837 & 0,173 & 0,895 & 1,057 & 0,462 & 0,419 & 0,403 & 0,169 & 0,169 & 55 & 516 & $-2,0$ \\
\hline 517 & 0,4 & 2,4 & 0,5 & 2,3 & 2,3 & 0,963 & 0,970 & 0,939 & 0,048 & 0,979 & 1,011 & 0,859 & 0,882 & 0,816 & 0,720 & 0,643 & 67 & 517 & $-3,0$ \\
\hline 518 & 0,6 & 3,4 & 0,6 & 3,4 & 3,3 & 0,910 & 0,991 & 0,959 & 0,091 & 0,621 & 1,269 & 0,374 & 0,496 & 1,402 & 0,695 & 0,643 & 139 & 518 & $-3,8$ \\
\hline 519 & 2,8 & 7,4 & 3,1 & 7,3 & 7,0 & 0,909 & 0,982 & 0,950 & 0,093 & 0,646 & 1,245 & 0,349 & 0,358 & 3,201 & 1,146 & 1,016 & 134 & 519 & $-5,0$ \\
\hline 520 & 0,2 & 1,6 & 0,2 & 1,6 & 1,5 & 0,939 & 0,981 & 0,950 & 0,064 & 0,976 & 1,012 & 0,639 & 0,601 & 0,630 & 0,379 & 0,379 & 146 & 520 & $-2,7$ \\
\hline 521 & 0,2 & 1,9 & 0,2 & 1,7 & 1,7 & 0,976 & 0,938 & 0,908 & 0,066 & 0,847 & 1,086 & 0,527 & 0,558 & 0,747 & 0,417 & 0,408 & 75 & 521 & $-2,9$ \\
\hline 522 & 0,5 & 3,0 & 0,5 & 2,9 & 2,8 & 0,894 & 0,976 & 0,945 & 0,108 & 0,655 & 1,235 & 0,404 & 0,476 & 1,222 & 0,581 & 0,536 & 100 & 522 & $-3,6$ \\
\hline 523 & 0,4 & 2,1 & 0,4 & 2,1 & 2,0 & 0,957 & 0,976 & 0,945 & 0,049 & 0,993 & 1,004 & 0,674 & 0,699 & 0,813 & 0,568 & 0,577 & 33 & 523 & $-3,0$ \\
\hline 524 & 0,2 & 2,0 & 0,2 & 1,9 & 1,8 & 0,865 & 0,925 & 0,896 & 0,155 & 0,638 & 1,252 & 0,353 & 0,394 & 0,872 & 0,343 & 0,339 & 132 & 524 & $-3,1$ \\
\hline 525 & 0,6 & 3,4 & 0,7 & 3,3 & 3,2 & 0,952 & 0,985 & 0,953 & 0,050 & 0,703 & 1,193 & 0,390 & 0,393 & 1,432 & 0,562 & 0,577 & 55 & 525 & $-3,8$ \\
\hline 526 & 0,4 & 2,2 & 0,4 & 2,2 & 2,1 & 0,969 & 0,977 & 0,946 & 0,038 & 0,909 & 1,049 & 0,651 & 0,732 & 0,841 & 0,616 & 0,536 & 43 & 526 & $-3,1$ \\
\hline 527 & 0,4 & 2,5 & 0,5 & 2,4 & 2,3 & 0,891 & 0,959 & 0,928 & 0,116 & 0,835 & 1,095 & 0,630 & 0,728 & 0,912 & 0,664 & 0,577 & 65 & 527 & $-3,2$ \\
\hline 528 & 0,3 & 1,9 & 0,3 & 1,9 & 1,9 & 0,969 & 0,985 & 0,953 & 0,035 & 1,000 & 0,983 & 0,834 & 0,822 & 0,691 & 0,568 & 0,536 & 87 & 528 & $-2,8$ \\
\hline 529 & 0,4 & 2,2 & 0,4 & 2,2 & 2,1 & 0,971 & 0,977 & 0,946 & 0,037 & 0,985 & 1,008 & 0,692 & 0,676 & 0,841 & 0,568 & 0,606 & 1 & 529 & $-3,1$ \\
\hline 530 & 133,4 & 46,6 & 140,8 & 43,3 & 41,9 & 0,947 & 0,930 & 0,900 & 0,088 & 0,773 & 1,137 & 0,776 & 0,884 & 14,796 & 13,074 & 11,243 & 29 & 530 & $-7,2$ \\
\hline 531 & 2,4 & 6,9 & 2,7 & 6,8 & 6,6 & 0,919 & 0,991 & 0,960 & 0,082 & 0,649 & 1,242 & 0,395 & 0,453 & 2,802 & 1,270 & 1,186 & 156 & 531 & $-4,8$ \\
\hline 532 & 1,5 & 5,3 & 1,6 & 5,2 & 5,0 & 0,949 & 0,968 & 0,937 & 0,060 & 0,653 & 1,237 & 0,354 & 0,369 & 2,311 & 0,852 & 0,864 & 97 & 532 & $-4,5$ \\
\hline 533 & 0,4 & 2,1 & 0,4 & 2,0 & 2,0 & 0,988 & 0,966 & 0,935 & 0,036 & 1,000 & 0,995 & 0,697 & 0,655 & 0,810 & 0,530 & 0,536 & 11 & 533 & $-3,0$ \\
\hline 534 & 0,3 & 2,4 & 0,4 & 2,3 & 2,2 & 0,937 & 0,978 & 0,947 & 0,066 & 0,775 & 1,136 & 0,411 & 0,465 & 1,030 & 0,479 & 0,461 & 19 & 534 & $-3,4$ \\
\hline 535 & 3,4 & 7,3 & 3,5 & 7,2 & 6,9 & 0,951 & 0,985 & 0,953 & 0,051 & 0,800 & 1,118 & 0,545 & 0,532 & 2,807 & 1,492 & 1,439 & 17 & 535 & $-4,8$ \\
\hline 536 & 0,2 & 1,4 & 0,2 & 1,4 & 1,4 & 0,928 & 0,965 & 0,934 & 0,080 & 1,000 & 1,000 & 0,738 & 0,750 & 0,536 & 0,402 & 0,429 & 173 & 536 & $-2,4$ \\
\hline 537 & 1,1 & 4,3 & 1,2 & 4,3 & 4,2 & 0,915 & 0,991 & 0,960 & 0,085 & 0,759 & 1,148 & 0,654 & 0,773 & 1,485 & 1,147 & 0,925 & 95 & 537 & $-3,9$ \\
\hline 538 & 5,9 & 10,0 & 6,6 & 9,8 & 9,5 & 0,907 & 0,979 & 0,948 & 0,096 & 0,751 & 1,154 & 0,538 & 0,581 & 3,752 & 2,181 & 2,000 & 118 & 538 & $-5,2$ \\
\hline 539 & 137,6 & 53,1 & 161,6 & 51,1 & 49,4 & 0,852 & 0,961 & 0,930 & 0,153 & 0,612 & 1,278 & 0,489 & 0,608 & 18,926 & 11,503 & 2,837 & 122 & 539 & $-7,6$ \\
\hline 540 & 0,2 & 1,3 & 0,2 & 1,3 & 1,2 & 0,956 & 0,962 & 0,931 & 0,058 & 1,000 & 0,957 & 0,929 & 0,872 & 0,461 & 0,402 & 0,408 & 170 & 540 & $-2,2$ \\
\hline 541 & 5,6 & 9,4 & 6,0 & 9,1 & 8,8 & 0,934 & 0,970 & 0,939 & 0,072 & 0,808 & 1,112 & 0,598 & 0,623 & 3,465 & 2,159 & 1,912 & 79 & 541 & $-5,1$ \\
\hline 542 & 0,1 & 1,3 & 0,1 & 1,2 & 1,2 & 0,980 & 0,977 & 0,945 & 0,031 & 1,000 & 0,941 & 0,832 & 0,793 & 0,469 & 0,371 & 0,379 & 180 & 542 & $-2,2$ \\
\hline 543 & 0,2 & 2,0 & 0,2 & 1,9 & 1,8 & 0,931 & 0,949 & 0,919 & 0,086 & 0,739 & 1,163 & 0,394 & 0,361 & 0,864 & 0,312 & 0,303 & 106 & 543 & $-3,1$ \\
\hline 544 & 0,1 & 1,1 & 0,1 & 1,1 & 1,0 & 1,000 & 0,948 & 0,917 & 0,052 & 1,000 & 0,982 & 0,876 & 0,857 & 0,395 & 0,339 & 0,312 & 127 & 544 & $-2,0$ \\
\hline 545 & 0,1 & 1,5 & 0,1 & 1,4 & 1,4 & 0,930 & 0,966 & 0,935 & 0,078 & 0,766 & 1,143 & 0,406 & 0,419 & 0,647 & 0,271 & 0,273 & 155 & 545 & $-2,7$ \\
\hline 546 & 0,7 & 3,4 & 0,8 & 3,3 & 3,2 & 0,892 & 0,973 & 0,942 & 0,112 & 0,780 & 1,133 & 0,600 & 0,700 & 1,237 & 0,866 & 0,719 & 50 & 546 & $-3,6$ \\
\hline 547 & 0,2 & 1,5 & 0,2 & 1,5 & 1,4 & 0,940 & 0,961 & 0,930 & 0,071 & 0,986 & 1,007 & 0,736 & 0,640 & 0,559 & 0,358 & 0,339 & 54 & 547 & $-2,5$ \\
\hline 548 & 0,4 & 2,5 & 0,5 & 2,4 & 2,3 & 0,921 & 0,963 & 0,932 & 0,088 & 0,895 & 1,057 & 0,731 & 0,787 & 0,884 & 0,696 & 0,682 & 12 & 548 & $-3,1$ \\
\hline 549 & 0,6 & 3,1 & 0,6 & 3,0 & 2,9 & 0,955 & 0,981 & 0,949 & 0,049 & 0,806 & 1,114 & 0,502 & 0,445 & 1,237 & 0,551 & 0,577 & 53 & 549 & $-3,6$ \\
\hline 550 & 0,8 & 3,1 & 0,8 & 3,1 & 3,0 & 0,983 & 0,990 & 0,959 & 0,019 & 0,976 & 1,012 & 0,770 & 0,806 & 1,115 & 0,898 & 0,847 & 9 & 550 & $-3,5$ \\
\hline 551 & 0,2 & 1,7 & 0,3 & 1,7 & 1,7 & 0,977 & 0,995 & 0,963 & 0,023 & 1,000 & 0,989 & 0,735 & 0,751 & 0,654 & 0,491 & 0,485 & 5 & 551 & $-2,7$ \\
\hline 552 & 0,2 & 1,8 & 0,2 & 1,6 & 1,6 & 0,957 & 0,920 & 0,890 & 0,091 & 0,869 & 1,073 & 0,555 & 0,524 & 0,712 & 0,373 & 0,379 & 69 & 552 & $-2,8$ \\
\hline 553 & 1,0 & 3,7 & 1,0 & 3,7 & 3,6 & 0,977 & 0,998 & 0,966 & 0,023 & 0,878 & 1,067 & 0,550 & 0,495 & 1,502 & 0,743 & 0,750 & 139 & 553 & $-3,9$ \\
\hline 554 & 80,4 & 40,4 & 85,8 & 38,0 & 36,7 & 0,937 & 0,939 & 0,909 & 0,088 & 0,618 & 1,272 & 0,383 & 0,446 & 16,351 & 7,287 & 7,114 & 63 & 554 & $-7,4$ \\
\hline 555 & 0,3 & 2,0 & 0,3 & 2,0 & 1,9 & 0,939 & 0,985 & 0,953 & 0,063 & 0,897 & 1,056 & 0,564 & 0,587 & 0,803 & 0,471 & 0,479 & 21 & 555 & $-3,0$ \\
\hline 556 & 2,5 & 7,0 & 2,9 & 6,4 & 6,2 & 0,855 & 0,908 & 0,878 & 0,172 & 0,633 & 1,257 & 0,468 & 0,573 & 2,602 & 1,491 & 0,816 & 29 & 556 & $-4,7$ \\
\hline 557 & 1,3 & 4,7 & 1,5 & 4,5 & 4,3 & 0,904 & 0,957 & 0,927 & 0,105 & 0,776 & 1,135 & 0,606 & 0,657 & 1,682 & 1,105 & 0,912 & 19 & 557 & $-4,1$ \\
\hline 558 & 0,3 & 2,0 & 0,3 & 2,0 & 1,9 & 0,945 & 0,979 & 0,948 & 0,058 & 0,822 & 1,103 & 0,491 & 0,502 & 0,841 & 0,422 & 0,442 & 117 & 558 & $-3,1$ \\
\hline 559 & 4,2 & 10,1 & 5,5 & 8,8 & 8,5 & 0,775 & 0,873 & 0,845 & 0,259 & 0,521 & 1,386 & 0,425 & 0,509 & 3,562 & 1,814 & 0,915 & 134 & 559 & $-5,2$ \\
\hline 560 & 5,1 & 8,6 & 5,4 & 8,4 & 8,1 & 0,939 & 0,979 & 0,948 & 0,064 & 0,876 & 1,069 & 0,658 & 0,716 & 3,143 & 2,250 & 2,197 & 133 & 560 & $-5,0$ \\
\hline 561 & 0,3 & 2,1 & 0,3 & 2,0 & 1,9 & 0,847 & 0,961 & 0,931 & 0,158 & 0,744 & 1,159 & 0,510 & 0,606 & 0,796 & 0,483 & 0,408 & 151 & 561 & $-3,0$ \\
\hline 562 & 0,0 & 0,6 & 0,0 & 0,5 & 0,5 & 1,000 & 0,834 & 0,807 & 0,166 & 1,000 & 0,913 & 0,637 & 0,500 & 0,268 & 0,134 & 0,169 & 45 & 562 & $-1,4$ \\
\hline 563 & 1,0 & 3,8 & 1,1 & 3,6 & 3,4 & 0,913 & 0,945 & 0,914 & 0,103 & 0,853 & 1,083 & 0,745 & 0,792 & 1,282 & 1,015 & 0,805 & 167 & 563 & $-3,7$ \\
\hline 564 & 2,0 & 6,6 & 2,3 & 6,2 & 6,0 & 0,872 & 0,939 & 0,909 & 0,142 & 0,592 & 1,300 & 0,378 & 0,513 & 2,609 & 1,338 & 1,119 & 143 & 564 & $-4,7$ \\
\hline 565 & 0,1 & 1,3 & 0,1 & 1,1 & 1,1 & 0,952 & 0,887 & 0,858 & 0,123 & 0,886 & 1,062 & 0,559 & 0,596 & 0,508 & 0,303 & 0,303 & 25 & 565 & $-2,3$ \\
\hline 566 & 2,4 & 6,2 & 2,6 & 6,1 & 5,9 & 0,954 & 0,989 & 0,957 & 0,048 & 0,803 & 1,116 & 0,510 & 0,519 & 2,469 & 1,282 & 1,288 & 155 & 566 & $-4,6$ \\
\hline 567 & 0,7 & 3,5 & 0,8 & 3,3 & 3,2 & 0,856 & 0,967 & 0,936 & 0,148 & 0,701 & 1,194 & 0,466 & 0,488 & 1,349 & 0,659 & 0,552 & 134 & 567 & ? \\
\hline 568 & 0,2 & 1,6 & 0,2 & 1,6 & 1,5 & 0,959 & 0,981 & 0,950 & 0,045 & 0,999 & 1,001 & 0,655 & 0,623 & 0,625 & 0,389 & 0,408 & 111 & 568 & $-2,6$ \\
\hline 569 & 0,4 & 2,3 & 0,4 & 2,3 & 2,2 & 0,978 & 0,987 & 0,956 & 0,026 & 0,910 & 1,048 & 0,649 & 0,697 & 0,865 & 0,603 & 0,536 & 153 & 569 & $-3,1$ \\
\hline 570 & 5,5 & 10,5 & 6,4 & 9,9 & 9,6 & 0,855 & 0,941 & 0,911 & 0,156 & 0,623 & 1,267 & 0,430 & 0,513 & 4,025 & 2,066 & 1,876 & 52 & 570 & 53 \\
\hline 571 & 1,9 & 5,3 & 2,0 & 5,2 & 5,1 & 0,949 & 0,985 & 0,953 & 0,053 & 0,848 & 1,086 & 0,569 & 0,599 & 2,067 & 1,238 & 1,136 & 47 & 571 & 4,4 \\
\hline
\end{tabular}




\begin{tabular}{|c|c|c|c|c|c|c|c|c|c|c|c|c|c|c|c|c|c|c|c|}
\hline 572 & 0,4 & 2,4 & 0,4 & 2,2 & 2,2 & 0,942 & 0,948 & 0,918 & 0,077 & 0,894 & 1,057 & 0,626 & 0,689 & 0,900 & 0,620 & 0,577 & 93 & 572 & $-3,2$ \\
\hline 573 & 0,1 & 1,0 & 0,1 & 1,0 & 0,9 & 0,985 & 0,971 & 0,940 & 0,033 & 1,000 & 0,941 & 0,784 & 0,686 & 0,386 & 0,265 & 0,303 & 159 & 573 & $-1,9$ \\
\hline 574 & 1,9 & 5,3 & 2,0 & 5,2 & 5,1 & 0,956 & 0,994 & 0,962 & 0,044 & 0,849 & 1,085 & 0,667 & 0,714 & 1,894 & 1,353 & 1,295 & 149 & 574 & $-4,2$ \\
\hline 575 & 2,9 & 6,9 & 3,1 & 6,9 & 6,6 & 0,950 & 0,990 & 0,958 & 0,051 & 0,763 & 1,145 & 0,588 & 0,680 & 2,514 & 1,709 & 1,488 & 97 & 575 & $-4,7$ \\
\hline 576 & 0,3 & 2,8 & 0,3 & 2,7 & 2,6 & 0,878 & 0,963 & 0,932 & 0,127 & 0,443 & 1,503 & 0,212 & 0,208 & 1,270 & 0,264 & 0,303 & 106 & 576 & $-3,7$ \\
\hline 577 & 0,1 & 1,1 & 0,1 & 1,0 & 1,0 & 0,972 & 0,953 & 0,923 & 0,054 & 1,000 & 0,971 & 0,818 & 0,789 & 0,395 & 0,312 & 0,312 & 160 & 577 & $-2,0$ \\
\hline 578 & 377,5 & 77,5 & 396,5 & 73,3 & 71,0 & 0,952 & 0,946 & 0,916 & 0,072 & 0,790 & 1,125 & 0,584 & 0,668 & 28,684 & 19,160 & 18,152 & 17 & 578 & $-8,2$ \\
\hline 579 & 0,7 & 3,2 & 0,7 & 3,1 & 3,0 & 0,956 & 0,991 & 0,959 & 0,045 & 0,828 & 1,099 & 0,479 & 0,469 & 1,321 & 0,620 & 0,611 & 69 & 579 & $-3,7$ \\
\hline 580 & 0,1 & 1,1 & 0,1 & 1,1 & 1,0 & 0,975 & 0,954 & 0,923 & 0,052 & 1,000 & 0,930 & 0,795 & 0,707 & 0,423 & 0,300 & 0,312 & 158 & 580 & $-2,1$ \\
\hline 581 & 0,9 & 3,6 & 0,9 & 3,5 & 3,4 & 0,963 & 0,986 & 0,954 & 0,040 & 0,844 & 1,089 & 0,517 & 0,528 & 1,452 & 0,767 & 0,773 & 7 & 581 & $-3,9$ \\
\hline 582 & 3,5 & 6,9 & 3,7 & 6,8 & 6,6 & 0,960 & 0,990 & 0,958 & 0,041 & 0,929 & 1,038 & 0,823 & 0,871 & 2,336 & 2,034 & 1,871 & 92 & 582 & $-4,5$ \\
\hline 583 & 0,4 & 2,7 & 0,5 & 2,7 & 2,6 & 0,936 & 0,997 & 0,965 & 0,064 & 0,778 & 1,134 & 0,446 & 0,457 & 1,119 & 0,511 & 0,530 & 114 & 583 & $-3,5$ \\
\hline 584 & 2,2 & 5,5 & 2,3 & 5,5 & 5,3 & 0,962 & 0,991 & 0,959 & 0,039 & 0,918 & 1,044 & 0,678 & 0,751 & 2,051 & 1,541 & 1,500 & 89 & 584 & $-4,4$ \\
\hline 585 & 0,3 & 2,5 & 0,3 & 2,4 & 2,3 & 0,900 & 0,979 & 0,948 & 0,102 & 0,624 & 1,266 & 0,303 & 0,284 & 1,119 & 0,318 & 0,312 & 74 & 585 & $-3,5$ \\
\hline 586 & 1,2 & 4,5 & 1,3 & 4,4 & 4,2 & 0,922 & 0,975 & 0,944 & 0,082 & 0,720 & 1,179 & 0,392 & 0,388 & 1,933 & 0,749 & 0,678 & 4 & 586 & $-4,3$ \\
\hline 587 & 1,5 & 5,1 & 1,7 & 5,1 & 4,9 & 0,929 & 0,990 & 0,958 & 0,072 & 0,739 & 1,163 & 0,430 & 0,519 & 2,133 & 1,108 & 0,985 & 151 & 587 & $-4,4$ \\
\hline 588 & 0,3 & 2,1 & 0,3 & 2,0 & 1,9 & 0,880 & 0,952 & 0,921 & 0,129 & 0,716 & 1,182 & 0,417 & 0,391 & 0,879 & 0,344 & 0,379 & 99 & 588 & $-3,1$ \\
\hline 589 & 2,8 & 8,2 & 3,6 & 7,6 & 7,4 & 0,771 & 0,930 & 0,900 & 0,240 & 0,517 & 1,391 & 0,354 & 0,543 & 3,151 & 1,711 & 0,915 & 16 & 589 & $-5,0$ \\
\hline 590 & 0,8 & 3,5 & 0,8 & 3,4 & 3,3 & 0,946 & 0,968 & 0,937 & 0,062 & 0,819 & 1,105 & 0,448 & 0,520 & 1,494 & 0,777 & 0,780 & 119 & 590 & $-3,9$ \\
\hline 591 & 1,0 & 3,5 & 1,0 & 3,5 & 3,4 & 0,976 & 0,991 & 0,960 & 0,025 & 0,979 & 1,011 & 0,709 & 0,724 & 1,306 & 0,946 & 0,949 & 54 & 591 & $-3,7$ \\
\hline 592 & 0,2 & 1,5 & 0,2 & 1,4 & 1,4 & 0,953 & 0,947 & 0,916 & 0,071 & 0,947 & 1,028 & 0,673 & 0,709 & 0,573 & 0,407 & 0,408 & 88 & 592 & $-2,5$ \\
\hline 593 & 17,5 & 17,7 & 20,1 & 17,2 & 16,7 & 0,870 & 0,970 & 0,939 & 0,133 & 0,696 & 1,199 & 0,514 & 0,675 & 6,577 & 4,437 & 3,649 & 9 & 593 & $-6,0$ \\
\hline 594 & 0,2 & 1,5 & 0,2 & 1,5 & 1,4 & 0,937 & 0,967 & 0,936 & 0,072 & 1,000 & 0,988 & 0,730 & 0,723 & 0,577 & 0,417 & 0,429 & 120 & 594 & $-2,5$ \\
\hline 595 & 0,2 & 1,9 & 0,3 & 1,8 & 1,8 & 0,938 & 0,984 & 0,952 & 0,065 & 0,860 & 1,078 & 0,505 & 0,483 & 0,773 & 0,373 & 0,379 & 85 & 595 & $-3,0$ \\
\hline 596 & 4,0 & 8,7 & 4,3 & 8,5 & 8,3 & 0,939 & 0,985 & 0,953 & 0,063 & 0,673 & 1,219 & 0,369 & 0,373 & 3,728 & 1,391 & 1,393 & 137 & 596 & $-5,2$ \\
\hline 597 & 0,6 & 2,8 & 0,7 & 2,7 & 2,7 & 0,976 & 0,975 & 0,943 & 0,035 & 1,000 & 0,995 & 0,864 & 0,859 & 0,970 & 0,833 & 0,791 & 95 & 597 & $-3,3$ \\
\hline 598 & 5,6 & 9,3 & 5,9 & 8,9 & 8,6 & 0,937 & 0,954 & 0,924 & 0,078 & 0,810 & 1,111 & 0,697 & 0,763 & 3,186 & 2,430 & 1,931 & 131 & 598 & $-5,0$ \\
\hline 599 & 0,5 & 2,7 & 0,6 & 2,6 & 2,6 & 0,956 & 0,981 & 0,950 & 0,048 & 0,922 & 1,041 & 0,586 & 0,640 & 1,077 & 0,689 & 0,678 & 134 & 599 & $-3,4$ \\
\hline 600 & 3,0 & 7,4 & 3,3 & 7,3 & 7,0 & 0,893 & 0,980 & 0,949 & 0,109 & 0,684 & 1,209 & 0,445 & 0,529 & 2,922 & 1,545 & 1,323 & 88 & 600 & $-4,9$ \\
\hline 601 & 0,3 & 1,9 & 0,3 & 1,7 & 1,7 & 0,950 & 0,913 & 0,884 & 0,100 & 0,960 & 1,021 & 0,791 & 0,857 & 0,663 & 0,568 & 0,479 & 126 & 601 & $-2,7$ \\
\hline 602 & 0,4 & 2,5 & 0,4 & 2,3 & 2,2 & 0,964 & 0,941 & 0,912 & 0,069 & 0,873 & 1,070 & 0,609 & 0,606 & 0,936 & 0,567 & 0,577 & 93 & 602 & $-3,2$ \\
\hline 603 & 0,3 & 1,9 & 0,3 & 1,9 & 1,8 & 0,964 & 0,985 & 0,953 & 0,039 & 1,000 & 0,983 & 0,846 & 0,777 & 0,683 & 0,531 & 0,530 & 163 & 603 & $-2,8$ \\
\hline 604 & 0,4 & 2,2 & 0,4 & 2,2 & 2,1 & 0,962 & 0,987 & 0,955 & 0,041 & 1,000 & 0,993 & 0,827 & 0,868 & 0,781 & 0,678 & 0,611 & 142 & 604 & $-3,0$ \\
\hline 605 & 0,6 & 3,0 & 0,6 & 2,9 & 2,8 & 0,941 & 0,959 & 0,929 & 0,071 & 0,825 & 1,101 & 0,664 & 0,736 & 1,072 & 0,790 & 0,682 & 9 & 605 & $-3,4$ \\
\hline 606 & 0,7 & 2,9 & 0,7 & 2,8 & 2,7 & 0,975 & 0,990 & 0,958 & 0,027 & 1,000 & 0,988 & 0,841 & 0,802 & 1,003 & 0,805 & 0,805 & 113 & 606 & $-3,3$ \\
\hline 607 & 38,3 & 25,1 & 41,2 & 23,5 & 22,8 & 0,929 & 0,937 & 0,907 & 0,095 & 0,762 & 1,146 & 0,662 & 0,695 & 8,582 & 5,964 & 5,414 & 6 & 607 & $-6,4$ \\
\hline 608 & 2,7 & 6,1 & 2,9 & 6,1 & 5,9 & 0,952 & 0,999 & 0,967 & 0,048 & 0,910 & 1,048 & 0,747 & 0,792 & 2,157 & 1,709 & 1,591 & 6 & 608 & $-4,4$ \\
\hline 609 & 0,8 & 3,5 & 0,9 & 3,4 & 3,3 & 0,970 & 0,979 & 0,948 & 0,036 & 0,867 & 1,074 & 0,497 & 0,506 & 1,462 & 0,740 & 0,746 & 44 & 609 & $-3,9$ \\
\hline 610 & 0,6 & 2,8 & 0,6 & 2,6 & 2,6 & 0,951 & 0,953 & 0,922 & 0,068 & 0,916 & 1,045 & 0,721 & 0,686 & 0,996 & 0,684 & 0,682 & 75 & 610 & $-3,3$ \\
\hline 611 & 39,3 & 25,4 & 42,3 & 23,7 & 22,9 & 0,928 & 0,931 & 0,901 & 0,100 & 0,764 & 1,144 & 0,673 & 0,716 & 8,619 & 6,175 & 5,537 & 141 & 611 & $-6,4$ \\
\hline 612 & 0,8 & 3,3 & 0,8 & 3,2 & 3,1 & 0,980 & 0,976 & 0,944 & 0,032 & 0,959 & 1,021 & 0,722 & 0,697 & 1,209 & 0,842 & 0,847 & 140 & 612 & $-3,6$ \\
\hline 613 & 32,6 & 21,4 & 34,2 & 21,3 & 20,6 & 0,951 & 0,994 & 0,963 & 0,049 & 0,894 & 1,058 & 0,757 & 0,847 & 7,400 & 6,265 & 5,507 & 176 & 613 & $-6,2$ \\
\hline 614 & 0,1 & 1,4 & 0,2 & 1,3 & 1,3 & 0,952 & 0,928 & 0,898 & 0,086 & 0,910 & 1,048 & 0,776 & 0,811 & 0,485 & 0,393 & 0,379 & 49 & 614 & $-2,3$ \\
\hline 615 & 0,1 & 1,2 & 0,1 & 1,1 & 1,1 & 0,978 & 0,922 & 0,893 & 0,081 & 1,000 & 0,955 & 0,706 & 0,638 & 0,475 & 0,303 & 0,379 & 27 & 615 & $-2,2$ \\
\hline 616 & 0,4 & 2,4 & 0,4 & 2,3 & 2,2 & 0,902 & 0,942 & 0,911 & 0,114 & 0,838 & 1,092 & 0,566 & 0,571 & 0,929 & 0,530 & 0,479 & 89 & 616 & $-3,2$ \\
\hline 617 & 1,4 & 4,5 & 1,5 & 4,4 & 4,2 & 0,932 & 0,975 & 0,944 & 0,072 & 0,880 & 1,066 & 0,758 & 0,792 & 1,533 & 1,214 & 1,055 & 20 & 617 & $-3,9$ \\
\hline 618 & 1,6 & 5,2 & 1,8 & 5,1 & 4,9 & 0,914 & 0,974 & 0,943 & 0,090 & 0,741 & 1,162 & 0,519 & 0,578 & 1,987 & 1,148 & 1,016 & 159 & 618 & $-4,3$ \\
\hline 619 & 2,1 & 5,9 & 2,2 & 5,8 & 5,7 & 0,939 & 0,986 & 0,955 & 0,063 & 0,755 & 1,150 & 0,450 & 0,555 & 2,443 & 1,356 & 1,222 & 101 & 619 & -4 \\
\hline 620 & 0,4 & 2,6 & 0,5 & 2,5 & 2,4 & 0,883 & 0,972 & 0,941 & 0,121 & 0,741 & 1,161 & 0,438 & 0,496 & 1,077 & 0,534 & 0,536 & 145 & 620 & $-3,4$ \\
\hline 621 & 1,5 & 4,7 & 1,6 & 4,5 & 4,4 & 0,944 & 0,974 & 0,942 & 0,062 & 0,852 & 1,083 & 0,616 & 0,710 & 1,743 & 1,237 & 1,085 & 143 & 621 & $-4,1$ \\
\hline 622 & 0,7 & 3,0 & 0,7 & 2,9 & 2,8 & 0,962 & 0,983 & 0,952 & 0,042 & 0,914 & 1,046 & 0,566 & 0,560 & 1,210 & 0,678 & 0,682 & 173 & 622 & $-3,6$ \\
\hline 623 & 0,8 & 3,4 & 0,8 & 3,3 & 3,2 & 0,937 & 0,982 & 0,951 & 0,066 & 0,868 & 1,074 & 0,556 & 0,617 & 1,343 & 0,829 & 0,791 & 165 & 623 & 3 \\
\hline 624 & 1,0 & 3,9 & 1,1 & 3,8 & 3,7 & 0,912 & 0,976 & 0,945 & 0,091 & 0,822 & 1,103 & 0,611 & 0,728 & 1,442 & 1,050 & 0,887 & 175 & 624 & $-3,8$ \\
\hline 625 & 0,8 & 3,3 & 0,8 & 3,3 & 3,2 & 0,957 & 0,985 & 0,953 & 0,046 & 0,865 & 1,075 & 0,589 & 0,543 & 1,288 & 0,700 & 0,698 & 158 & 625 & 37 \\
\hline 626 & 0,8 & 3,7 & 0,9 & 3,6 & 3,5 & 0,881 & 0,969 & 0,939 & 0,123 & 0,726 & 1,174 & 0,475 & 0,487 & 1,464 & 0,712 & 0,698 & 161 & 626 & $-3,9$ \\
\hline 627 & 0,8 & 3,3 & 0,8 & 3,2 & 3,1 & 0,937 & 0,965 & 0,934 & 0,072 & 0,883 & 1,064 & 0,736 & 0,831 & 1,140 & 0,947 & 0,805 & 27 & 627 & $-3,5$ \\
\hline 628 & 0,1 & 1,2 & 0,1 & 1,2 & 1,1 & 0,889 & 0,941 & 0,911 & 0,126 & 0,960 & 1,021 & 0,749 & 0,791 & 0,442 & 0,349 & 0,303 & 88 & 628 & 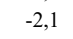 \\
\hline 629 & 7,9 & 11,0 & 8,3 & 10,9 & 10,5 & 0,952 & 0,988 & 0,956 & 0,049 & 0,824 & 1,102 & 0,599 & 0,680 & 4,105 & 2,791 & 2,745 & 126 & 629 & 5,4 \\
\hline
\end{tabular}




\begin{tabular}{|c|c|c|c|c|c|c|c|c|c|c|c|c|c|c|c|c|c|c|c|}
\hline 630 & 0,9 & 3,4 & 0,9 & 3,3 & 3,2 & 0,966 & 0,991 & 0,959 & 0,035 & 0,967 & 1,017 & 0,711 & 0,772 & 1,246 & 0,962 & 0,925 & 112 & 630 & $-3,6$ \\
\hline 631 & 0,0 & 0,8 & 0,1 & 0,7 & 0,7 & 0,944 & 0,911 & 0,882 & 0,105 & 0,941 & 1,031 & 0,528 & 0,442 & 0,343 & 0,152 & 0,214 & 166 & 631 & $-1,8$ \\
\hline 632 & 0,4 & 2,2 & 0,4 & 2,2 & 2,1 & 0,954 & 0,987 & 0,955 & 0,048 & 0,988 & 1,006 & 0,778 & 0,730 & 0,799 & 0,583 & 0,606 & 169 & 632 & $-3,0$ \\
\hline 633 & 0,6 & 3,0 & 0,7 & 2,9 & 2,8 & 0,968 & 0,959 & 0,928 & 0,052 & 0,921 & 1,042 & 0,587 & 0,543 & 1,186 & 0,644 & 0,678 & 100 & 633 & $-3,6$ \\
\hline 634 & 0,5 & 2,6 & 0,5 & 2,5 & 2,5 & 0,954 & 0,988 & 0,957 & 0,047 & 0,851 & 1,084 & 0,588 & 0,621 & 0,988 & 0,614 & 0,611 & 135 & 634 & $-3,3$ \\
\hline 635 & 0,9 & 4,3 & 1,0 & 4,0 & 3,9 & 0,900 & 0,935 & 0,905 & 0,120 & 0,644 & 1,246 & 0,375 & 0,424 & 1,787 & 0,758 & 0,773 & 176 & 635 & $-4,2$ \\
\hline 636 & 0,5 & 2,5 & 0,5 & 2,5 & 2,4 & 0,929 & 0,988 & 0,957 & 0,072 & 0,883 & 1,064 & 0,631 & 0,654 & 0,954 & 0,623 & 0,592 & 78 & 636 & $-3,3$ \\
\hline 637 & 0,6 & 3,1 & 0,6 & 3,0 & 2,9 & 0,954 & 0,951 & 0,920 & 0,067 & 0,770 & 1,140 & 0,482 & 0,482 & 1,249 & 0,602 & 0,606 & 169 & 637 & $-3,6$ \\
\hline 638 & 0,4 & 2,3 & 0,4 & 2,2 & 2,1 & 0,908 & 0,977 & 0,947 & 0,095 & 0,874 & 1,070 & 0,657 & 0,762 & 0,827 & 0,630 & 0,536 & 160 & 638 & $-3,0$ \\
\hline 639 & 0,2 & 1,8 & 0,2 & 1,8 & 1,8 & 0,941 & 0,984 & 0,953 & 0,061 & 0,759 & 1,148 & 0,409 & 0,395 & 0,799 & 0,315 & 0,312 & 125 & 639 & $-3,0$ \\
\hline 640 & 0,1 & 1,2 & 0,1 & 1,2 & 1,1 & 0,984 & 0,982 & 0,951 & 0,024 & 0,810 & 1,111 & 0,464 & 0,460 & 0,494 & 0,227 & 0,227 & 7 & 640 & $-2,3$ \\
\hline 641 & 0,2 & 1,9 & 0,2 & 1,8 & 1,7 & 0,976 & 0,925 & 0,896 & 0,079 & 0,800 & 1,118 & 0,423 & 0,407 & 0,837 & 0,341 & 0,379 & 180 & 641 & $-3,1$ \\
\hline 642 & 0,2 & 1,9 & 0,3 & 1,7 & 1,7 & 0,918 & 0,930 & 0,900 & 0,108 & 0,860 & 1,078 & 0,567 & 0,619 & 0,735 & 0,455 & 0,461 & 127 & 642 & $-2,9$ \\
\hline 643 & 0,2 & 1,7 & 0,2 & 1,6 & 1,6 & 0,967 & 0,975 & 0,944 & 0,041 & 0,956 & 1,023 & 0,576 & 0,579 & 0,683 & 0,395 & 0,408 & 151 & 643 & $-2,8$ \\
\hline 644 & 0,4 & 2,2 & 0,4 & 2,2 & 2,1 & 0,989 & 0,986 & 0,955 & 0,018 & 0,996 & 1,002 & 0,746 & 0,694 & 0,803 & 0,557 & 0,577 & 121 & 644 & $-3,0$ \\
\hline 645 & 2,8 & 8,1 & 3,1 & 7,8 & 7,6 & 0,899 & 0,971 & 0,940 & 0,105 & 0,540 & 1,361 & 0,276 & 0,338 & 3,585 & 1,212 & 1,214 & 4 & 645 & $-5,2$ \\
\hline 646 & 2,0 & 5,2 & 2,0 & 5,2 & 5,1 & 0,963 & 0,998 & 0,966 & 0,037 & 0,901 & 1,054 & 0,613 & 0,650 & 2,025 & 1,316 & 1,288 & 176 & 646 & $-4,3$ \\
\hline 647 & 0,2 & 1,6 & 0,2 & 1,5 & 1,5 & 0,955 & 0,967 & 0,937 & 0,055 & 0,959 & 1,021 & 0,668 & 0,601 & 0,592 & 0,356 & 0,339 & 29 & 647 & $-2,6$ \\
\hline 648 & 7,9 & 11,5 & 8,9 & 10,8 & 10,4 & 0,892 & 0,939 & 0,909 & 0,124 & 0,758 & 1,149 & 0,746 & 0,857 & 3,677 & 3,151 & 2,350 & 77 & 648 & $-5,2$ \\
\hline 649 & 0,2 & 1,7 & 0,2 & 1,6 & 1,5 & 0,898 & 0,927 & 0,897 & 0,126 & 0,849 & 1,085 & 0,613 & 0,726 & 0,627 & 0,455 & 0,379 & 137 & 649 & $-2,6$ \\
\hline 650 & 0,7 & 3,2 & 0,8 & 3,1 & 3,0 & 0,938 & 0,981 & 0,950 & 0,065 & 0,881 & 1,065 & 0,679 & 0,653 & 1,159 & 0,757 & 0,682 & 77 & 650 & $-3,5$ \\
\hline 651 & 11,4 & 14,8 & 12,2 & 13,3 & 12,9 & 0,929 & 0,902 & 0,873 & 0,121 & 0,653 & 1,237 & 0,539 & 0,640 & 5,187 & 3,321 & 2,652 & 80 & 651 & $-5,7$ \\
\hline 652 & 5,3 & 8,7 & 5,4 & 8,7 & 8,4 & 0,975 & 0,999 & 0,967 & 0,025 & 0,870 & 1,072 & 0,544 & 0,523 & 3,509 & 1,836 & 1,821 & 123 & 652 & $-5,1$ \\
\hline 653 & 5,4 & 9,6 & 5,6 & 9,6 & 9,3 & 0,965 & 0,995 & 0,963 & 0,036 & 0,727 & 1,173 & 0,375 & 0,432 & 4,265 & 1,842 & 1,832 & 168 & 653 & $-5,4$ \\
\hline 654 & 10,7 & 13,8 & 11,5 & 13,7 & 13,2 & 0,930 & 0,993 & 0,961 & 0,071 & 0,711 & 1,186 & 0,399 & 0,405 & 5,855 & 2,371 & 2,181 & 170 & 654 & $-5,9$ \\
\hline 655 & 0,1 & 0,9 & 0,1 & 0,8 & 0,8 & 0,898 & 0,888 & 0,859 & 0,151 & 0,961 & 1,020 & 0,659 & 0,605 & 0,349 & 0,211 & 0,227 & 90 & 655 & $-1,8$ \\
\hline 656 & 0,1 & 1,4 & 0,1 & 1,3 & 1,3 & 0,931 & 0,963 & 0,932 & 0,078 & 0,902 & 1,053 & 0,672 & 0,660 & 0,508 & 0,336 & 0,312 & 80 & 656 & $-2,3$ \\
\hline 657 & 0,2 & 1,9 & 0,2 & 1,8 & 1,7 & 0,926 & 0,925 & 0,896 & 0,105 & 0,673 & 1,219 & 0,330 & 0,303 & 0,874 & 0,265 & 0,303 & 1 & 657 & $-3,1$ \\
\hline 658 & 1,1 & 3,9 & 1,2 & 3,9 & 3,8 & 0,975 & 0,992 & 0,961 & 0,026 & 0,917 & 1,044 & 0,670 & 0,710 & 1,471 & 1,045 & 0,988 & 129 & 658 & $-3,9$ \\
\hline 659 & 19,8 & 17,7 & 21,5 & 17,2 & 16,6 & 0,921 & 0,970 & 0,939 & 0,084 & 0,793 & 1,123 & 0,649 & 0,719 & 6,229 & 4,479 & 3,939 & 93 & 659 & $-6,0$ \\
\hline 660 & 111,3 & 44,2 & 120,0 & 41,9 & 40,5 & 0,927 & 0,947 & 0,916 & 0,090 & 0,715 & 1,183 & 0,499 & 0,593 & 16,848 & 9,986 & 9,242 & 146 & 660 & $-7,4$ \\
\hline 661 & 22,0 & 17,9 & 22,9 & 17,3 & 16,8 & 0,961 & 0,965 & 0,934 & 0,053 & 0,860 & 1,078 & 0,766 & 0,795 & 6,049 & 4,807 & 4,736 & 138 & 661 & $-5,9$ \\
\hline 662 & 7,3 & 11,5 & 8,0 & 10,8 & 10,5 & 0,907 & 0,940 & 0,910 & 0,110 & 0,686 & 1,207 & 0,519 & 0,554 & 4,220 & 2,337 & 2,135 & 130 & 662 & $-5,4$ \\
\hline 663 & 0,2 & 1,9 & 0,2 & 1,8 & 1,7 & 0,960 & 0,950 & 0,920 & 0,064 & 0,857 & 1,080 & 0,508 & 0,493 & 0,773 & 0,381 & 0,408 & 14 & 663 & $-3,0$ \\
\hline 664 & 6,4 & 9,7 & 6,7 & 9,7 & 9,4 & 0,954 & 0,995 & 0,963 & 0,046 & 0,856 & 1,081 & 0,648 & 0,716 & 3,555 & 2,544 & 2,334 & 93 & 664 & $-5,2$ \\
\hline 665 & 1,1 & 4,0 & 1,2 & 3,9 & 3,8 & 0,937 & 0,993 & 0,961 & 0,063 & 0,887 & 1,062 & 0,603 & 0,617 & 1,535 & 0,947 & 0,985 & 78 & 665 & $-3,9$ \\
\hline 666 & 14,2 & 15,1 & 15,2 & 14,9 & 14,4 & 0,935 & 0,990 & 0,958 & 0,066 & 0,786 & 1,128 & 0,530 & 0,598 & 5,834 & 3,489 & 3,214 & 13 & 666 & $-5,9$ \\
\hline 667 & 0,3 & 2,1 & 0,4 & 2,1 & 2,0 & 0,972 & 0,986 & 0,955 & 0,032 & 0,984 & 1,008 & 0,767 & 0,743 & 0,755 & 0,561 & 0,552 & 154 & 667 & $-2,9$ \\
\hline 668 & 0,2 & 1,7 & 0,2 & 1,7 & 1,6 & 0,932 & 0,970 & 0,939 & 0,074 & 0,851 & 1,084 & 0,473 & 0,420 & 0,728 & 0,306 & 0,312 & 141 & 668 & $-2,9$ \\
\hline 669 & 0,4 & 2,4 & 0,5 & 2,3 & 2,2 & 0,987 & 0,979 & 0,948 & 0,025 & 1,000 & 0,999 & 0,628 & 0,558 & 0,950 & 0,530 & 0,606 & 12 & 669 & $-3,2$ \\
\hline 670 & 0,4 & 2,4 & 0,4 & 2,3 & 2,2 & 0,898 & 0,950 & 0,920 & 0,114 & 0,770 & 1,140 & 0,515 & 0,582 & 0,949 & 0,552 & 0,508 & 153 & 670 & $-3,2$ \\
\hline 671 & 0,5 & 2,6 & 0,5 & 2,5 & 2,4 & 0,923 & 0,964 & 0,933 & 0,085 & 0,911 & 1,048 & 0,638 & 0,706 & 0,983 & 0,694 & 0,647 & 31 & 671 & $-3,3$ \\
\hline 672 & 1,8 & 5,1 & 1,9 & 5,1 & 4,9 & 0,953 & 0,990 & 0,958 & 0,048 & 0,887 & 1,062 & 0,563 & 0,602 & 2,040 & 1,227 & 1,222 & 74 & 672 & $-4,4$ \\
\hline 673 & 4,7 & 8,2 & 5,0 & 8,2 & 7,9 & 0,952 & 0,995 & 0,964 & 0,048 & 0,882 & 1,064 & 0,644 & 0,706 & 3,060 & 2,160 & 1,898 & 15 & 673 & $-4,9$ \\
\hline 674 & 0,2 & 1,8 & 0,2 & 1,7 & 1,6 & 0,916 & 0,971 & 0,940 & 0,089 & 0,703 & 1,193 & 0,373 & 0,419 & 0,767 & 0,322 & 0,321 & 21 & 674 & $-2,9$ \\
\hline 675 & 0,2 & 1,5 & 0,2 & 1,4 & 1,4 & 0,977 & 0,966 & 0,935 & 0,041 & 1,000 & 0,982 & 0,696 & 0,588 & 0,579 & 0,341 & 0,379 & 177 & 675 & $-2,5$ \\
\hline 676 & 0,4 & 2,5 & 0,4 & 2,4 & 2,3 & 0,913 & 0,963 & 0,932 & 0,094 & 0,813 & 1,109 & 0,566 & 0,592 & 0,959 & 0,568 & 0,577 & 165 & 676 & $-3,3$ \\
\hline 677 & 0,2 & 1,6 & 0,2 & 1,5 & 1,5 & 0,949 & 0,955 & 0,924 & 0,068 & 0,913 & 1,046 & 0,662 & 0,717 & 0,599 & 0,429 & 0,379 & 72 & 677 & -2 \\
\hline 678 & 0,4 & 2,7 & 0,5 & 2,6 & 2,5 & 0,954 & 0,962 & 0,932 & 0,060 & 0,780 & 1,132 & 0,458 & 0,433 & 1,114 & 0,482 & 0,536 & 42 & 678 & $-3,5$ \\
\hline 679 & 55,0 & 28,4 & 57,5 & 27,7 & 26,8 & 0,956 & 0,975 & 0,944 & 0,050 & 0,858 & 1,079 & 0,698 & 0,744 & 10,020 & 7,450 & 7,093 & 114 & 679 & $-6,6$ \\
\hline 680 & 2,4 & 6,2 & 2,6 & 6,1 & 5,9 & 0,950 & 0,988 & 0,957 & 0,051 & 0,811 & 1,110 & 0,541 & 0,545 & 2,401 & 1,308 & 1,290 & 79 & 680 & -4 \\
\hline 681 & 0,3 & 2,7 & 0,4 & 2,6 & 2,5 & 0,935 & 0,981 & 0,950 & 0,068 & 0,587 & 1,305 & 0,276 & 0,270 & 1,233 & 0,333 & 0,379 & 98 & 681 & 2 \\
\hline 682 & 0,2 & 1,9 & 0,2 & 1,7 & 1,6 & 0,940 & 0,918 & 0,889 & 0,102 & 0,663 & 1,228 & 0,344 & 0,324 & 0,818 & 0,265 & 0,303 & 82 & 682 & 3,0 \\
\hline 683 & 1,2 & 4,1 & 1,2 & 4,1 & 4,0 & 0,965 & 0,993 & 0,961 & 0,036 & 0,875 & 1,069 & 0,626 & 0,742 & 1,547 & 1,149 & 1,016 & 107 & 683 & -4 \\
\hline 684 & 3,7 & 7,2 & 3,9 & 7,0 & 6,8 & 0,957 & 0,975 & 0,944 & 0,049 & 0,903 & 1,052 & 0,727 & 0,786 & 2,562 & 2,012 & 1,894 & 56 & 684 & $-4,7$ \\
\hline 685 & 0,5 & 2,8 & 0,5 & 2,6 & 2,5 & 0,955 & 0,952 & 0,922 & 0,065 & 0,762 & 1,146 & 0,407 & 0,425 & 1,198 & 0,509 & 0,536 & 52 & 685 & \\
\hline 686 & 0,2 & 1,6 & 0,2 & 1,5 & 1,5 & 0,963 & 0,937 & 0,907 & 0,073 & 0,913 & 1,046 & 0,589 & 0,529 & 0,635 & 0,336 & 0,339 & 67 & 686 & -27 \\
\hline 687 & 0,6 & 2,8 & 0,6 & 2,8 & 2,7 & 0,923 & 0,975 & 0,944 & 0,081 & 0,880 & 1,066 & 0,740 & 0,737 & 0,988 & 0,728 & 0,698 & 140 & 687 & 3,3 \\
\hline
\end{tabular}




\begin{tabular}{|c|c|c|c|c|c|c|c|c|c|c|c|c|c|c|c|c|c|c|c|}
\hline 688 & 0,2 & 1,9 & 0,3 & 1,9 & 1,9 & 0,949 & 0,985 & 0,953 & 0,053 & 0,798 & 1,120 & 0,392 & 0,420 & 0,884 & 0,371 & 0,379 & 16 & 688 & $-3,1$ \\
\hline 689 & 3,9 & 7,3 & 4,0 & 7,3 & 7,0 & 0,965 & 0,996 & 0,964 & 0,035 & 0,909 & 1,049 & 0,711 & 0,743 & 2,629 & $\begin{array}{l}1,953 \\
\end{array}$ & 1,821 & 132 & 689 & $-4,7$ \\
\hline 690 & 0,2 & 1,5 & 0,2 & 1,5 & 1,4 & 0,973 & 0,966 & 0,935 & 0,043 & 1,000 & 0,946 & 0,882 & 0,768 & 0,542 & 0,417 & 0,455 & 174 & 690 & $-2,4$ \\
\hline 691 & 0,3 & 2,3 & 0,3 & 2,1 & 2,1 & 0,895 & 0,933 & 0,903 & 0,124 & 0,743 & 1,160 & 0,523 & 0,611 & 0,865 & 0,528 & 0,508 & 71 & 691 & $-3,1$ \\
\hline 692 & 3,6 & 7,5 & 3,7 & 7,4 & 7,2 & 0,965 & 0,999 & 0,967 & 0,035 & 0,811 & 1,110 & 0,495 & 0,559 & 3,036 & $\begin{array}{l}1,698 \\
\end{array}$ & 1,593 & 145 & 692 & $-4,9$ \\
\hline 693 & 0,2 & 1,8 & 0,2 & 1,7 & 1,6 & 0,965 & 0,971 & 0,940 & 0,046 & 0,961 & 1,020 & 0,564 & 0,506 & 0,729 & 0,368 & 0,379 & 150 & 693 & $-2,9$ \\
\hline 694 & $\begin{array}{l}1,2 \\
1,9\end{array}$ & $\begin{array}{l}5,0 \\
5,1\end{array}$ & 1,9 & 5,1 & $\begin{array}{l}4,9 \\
4,9\end{array}$ & 0,984 & 0,998 & 0,966 & 0,016 & 0,914 & 1,046 & 0,564 & 0,497 & 2,058 & 1,023 & $\begin{array}{l}1,061 \\
\text { lo }\end{array}$ & 90 & 694 & $-4,4$ \\
\hline 695 & 2,3 & 5,5 & 2,3 & 5,4 & 5,3 & 0,979 & 0,995 & 0,963 & 0,022 & 0,944 & 1,029 & 0,843 & 0,827 & 1,845 & $\begin{array}{l}1,525 \\
\text { S }\end{array}$ & 1,525 & 27 & 695 & $-4,2$ \\
\hline 696 & 0,7 & 3,2 & 0,7 & 3,1 & 3,0 & 0,928 & 0,978 & 0,946 & 0,076 & 0,816 & 1,107 & 0,481 & 0,470 & 1,325 & 0,623 & 0,611 & 27 & 696 & $-3,7$ \\
\hline 697 & 0,3 & 1,7 & 0,3 & 1,7 & 1,6 & 0,967 & 0,971 & 0,940 & 0,044 & 1,000 & 0,976 & 0,728 & 0,647 & 0,663 & 0,429 & 0,479 & 55 & 697 & $-2,7$ \\
\hline 698 & 5,9 & 9,1 & 6,1 & 9,0 & 8,8 & 0,977 & 0,992 & 0,960 & 0,025 & 0,898 & 1,056 & 0,647 & 0,670 & 3,418 & 2,290 & 2,206 & 151 & 698 & $-5,1$ \\
\hline
\end{tabular}

Label Area

$\begin{array}{lll}0,027 & 0,592 & 0,029 \\ 0,014 & 0,347 & 0,06\end{array}$

$0,024 \quad 0,613$

0,693

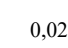

$0,029 \quad 0,520-0,029$
0,135

0,135

$0,039 \quad 0,757$

$\begin{array}{ll}0,030 & 0,562 \\ 0,113 & 1,226\end{array}$

0,113
0,022

0,016

$0,030 \quad 0,765$

$\begin{array}{ll}0,046 & 0,947 \\ 0,065 & 0,807\end{array}$

$0,052 \quad 0,744$

$\begin{array}{ll}0,023 & 0,511 \\ 0,019 & 0,440\end{array}$

$\begin{array}{ll}0,019 & 0,440 \\ 0,020 & 0,440\end{array}$

$0,413 \quad 2,553$

$\begin{array}{ll}0,024 & 0,461 \\ 0,093 & 1,040\end{array}$

$\begin{array}{ll}0,093 & 1,040 \\ 0,030 & 0,685\end{array}$

$0,017 \quad 0,622$

$\begin{array}{ll}0,016 & 0,397 \\ 0,108 & 1,458\end{array}$

$0,016 \quad 0,368$

$0,024 \quad 0,664$

$\begin{array}{ll}0,016 & 0,389 \\ 0,024 & 0,541\end{array}$
0,029
0,155
0,047
0,033
0,116
0,024
0,016
0,040
0,017
0,037
0,055
0,066
0,057
0,026
0,019
0,022
0,458
0,026
0,093
0,036
0,024
0,017
0,129
0,016
0,034
0,017
0,029
0,050

\section{0,805}

0,939
0,810
0,835

0,835
1,017
0,707
0,793

0,793
0,842

0,819
0,812
0,893

\begin{tabular}{|c|c|c|c|c|c|c|c|c|c|c|}
\hline $\begin{array}{l}\text { Concavity } \\
\text { Index }\end{array}$ & FormFactor $\mathrm{C}$ & Circularity & Roundness & AR Feret & Feret d & MinFeret d & $\begin{array}{l}\text { Max. Insc. } \\
\text { Circle Diam. }\end{array}$ & Orientation & $\begin{array}{c}\text { ROI } \\
\text { Index }\end{array}$ & Phi \\
\hline 0,248 & 0,978 & 1,011 & 0,484 & 0,300 & 0,268 & 0,080 & 0,107 & 131 & 0 & $-1,4$ \\
\hline 0,307 & 1,000 & 0,816 & 0,979 & 0,588 & 0,137 & 0,080 & 0,107 & 143 & 0 & $-0,5$ \\
\hline 0,141 & 1,000 & 0,901 & 0,749 & 0,524 & 0,221 & 0,116 & 0,152 & 149 & 0 & $-1,1$ \\
\hline 0,302 & 0,816 & 1,107 & 0,373 & 0,291 & 0,288 & 0,084 & 0,107 & 69 & 0 & $-1,5$ \\
\hline \multirow[t]{5}{*}{0,400} & 0,563 & 1,333 & 0,258 & 0,201 & 0,326 & 0,065 & 0,076 & 39 & 0 & $-1,7$ \\
\hline & nothing to measure & & & & & & & & 1 & - \\
\hline & nothing to measure & & & & & & & & 2 & - \\
\hline & nothing to measure & & & & & & & & 3 & - \\
\hline & nothing to measure & & & & & & & & 4 & - \\
\hline 0,195 & 1,000 & 0,866 & 0,878 & 0,657 & 0,204 & 0,134 & 0,152 & 120 & 5 & $-1,0$ \\
\hline 0,143 & 0,611 & 1,279 & 0,310 & 0,299 & 0,744 & 0,223 & 0,227 & 9 & 5 & $-2,9$ \\
\hline 0,263 & 0,851 & 1,084 & 0,593 & 0,619 & 0,288 & 0,179 & 0,152 & 180 & 6 & $-1,5$ \\
\hline 0,187 & 1,000 & 0,914 & 0,652 & 0,489 & 0,243 & 0,119 & 0,152 & 124 & 7 & $-1,3$ \\
\hline 0,030 & 0,948 & 1,027 & 0,491 & 0,441 & 0,542 & 0,239 & 0,273 & 109 & 7 & $-2,4$ \\
\hline 0,316 & 1,000 & 0,943 & 0,597 & 0,491 & 0,214 & 0,105 & 0,152 & 140 & 7 & $-1,1$ \\
\hline 0,207 & 1,000 & 0,778 & 1,077 & 0,588 & 0,137 & 0,080 & 0,107 & 135 & 7 & $-0,5$ \\
\hline \multirow[t]{2}{*}{0,158} & 1,000 & 0,904 & 0,686 & 0,490 & 0,273 & 0,134 & 0,169 & 123 & 7 & $-1,4$ \\
\hline & nothing to measure & & & & & & & & 8 & - \\
\hline 0,181 & 1,000 & 0,854 & 0,764 & 0,474 & 0,169 & 0,080 & 0,107 & 72 & 9 & $-0,8$ \\
\hline 0,269 & 0,647 & 1,243 & 0,315 & 0,298 & 0,349 & 0,104 & 0,152 & 37 & 9 & $-1,8$ \\
\hline 0,191 & 0,643 & 1,247 & 0,281 & 0,235 & 0,456 & 0,107 & 0,152 & 48 & 9 & $-2,2$ \\
\hline 0,153 & 1,000 & 0,896 & 0,988 & 0,788 & 0,288 & 0,227 & 0,273 & 101 & 9 & $-1,5$ \\
\hline 0,121 & 1,000 & 0,924 & 0,865 & 0,763 & 0,276 & 0,210 & 0,214 & 18 & 9 & $-1,5$ \\
\hline 0,357 & 1,000 & 0,952 & 0,784 & 0,588 & 0,193 & 0,114 & 0,152 & 165 & 9 & $-0,9$ \\
\hline 0,115 & 1,000 & 0,908 & 0,637 & 0,392 & 0,193 & 0,076 & 0,107 & 4 & 9 & $-0,9$ \\
\hline 0,334 & 1,000 & 0,875 & 0,990 & 0,633 & 0,161 & 0,102 & 0,152 & 66 & 9 & $-0,7$ \\
\hline 0,111 & 0,797 & 1,120 & 0,542 & 0,540 & 0,985 & 0,532 & 0,461 & 158 & 9 & $-3,3$ \\
\hline 0,166 & 1,000 & 0,832 & 1,082 & 0,671 & 0,169 & 0,114 & 0,152 & 162 & 9 & $-0,8$ \\
\hline 0,069 & 1,000 & 0,961 & 0,609 & 0,485 & 0,442 & 0,214 & 0,273 & 36 & 9 & $-2,1$ \\
\hline 0,218 & 0,808 & 1,113 & 0,393 & 0,338 & 0,312 & 0,106 & 0,152 & 72 & 9 & $-1,6$ \\
\hline 0,354 & 0,560 & 1,336 & 0,250 & 0,181 & 0,296 & 0,054 & 0,076 & 47 & 9 & $-1,6$ \\
\hline 0,269 & 1,000 & 0,892 & 0,778 & 0,500 & 0,161 & 0,080 & 0,107 & 90 & 9 & $-0,7$ \\
\hline 0,223 & 0,636 & 1,254 & 0,412 & 0,397 & 0,577 & 0,229 & 0,240 & 32 & 9 & $-2,5$ \\
\hline 0,333 & 1,000 & 0,826 & 1,077 & 0,745 & 0,137 & 0,102 & 0,107 & 135 & 9 & $-0,5$ \\
\hline 0,329 & 0,696 & 1,199 & 0,481 & 0,490 & 0,254 & 0,125 & 0,107 & 91 & 9 & $-1,3$ \\
\hline 0,305 & 1,000 & 0,873 & 0,700 & 0,400 & 0,169 & 0,068 & 0,076 & 21 & 9 & $-0,8$ \\
\hline 0,354 & 1,000 & 0,977 & 1,082 & 0,869 & 0,169 & 0,147 & 0,152 & 56 & 9 & $-0,8$ \\
\hline \multirow[t]{5}{*}{0,048} & & 0,977 & 0,528 & 0,442 & 0,343 & 0,152 & 0,214 & 11 & 9 & $-1,8$ \\
\hline & nothing to measure & & & & & & & & 10 & \\
\hline & nothing to measure & & & & & & & & 11 & - \\
\hline & nothing to measure & & & & & & & & 12 & - \\
\hline & nothing to measure & & & & & & & & 13 & \\
\hline
\end{tabular}




\begin{tabular}{|c|c|c|c|c|c|c|c|c|c|c|c|c|c|c|c|c|c|c|}
\hline 1 & $\begin{array}{l}14 \\
15 \\
16\end{array}$ & 0,014 & 0,368 & 0,016 & 0,245 & 0,909 & 0,667 & 0,346 & $\begin{array}{c}1,000 \\
\text { nothing to measure } \\
\text { nothing to measure }\end{array}$ & 0,866 & 0,749 & 0,485 & 0,156 & 0,076 & 0,107 & 90 & $\begin{array}{l}14 \\
15 \\
16\end{array}$ & $\begin{array}{c}-0,6 \\
- \\
-\end{array}$ \\
\hline 1 & 17 & 0,024 & 0,541 & 0,027 & 0,469 & 0,895 & 0,867 & 0,169 & 1,000 & 0,977 & 0,585 & 0,493 & 0,230 & 0,114 & 0,152 & 0 & 17 & $-1,2$ \\
\hline 1 & 18 & 0,017 & 0,643 & 0,024 & 0,499 & 0,706 & 0,776 & 0,369 & 0,524 & 1,381 & 0,250 & 0,261 & 0,296 & 0,077 & 0,076 & 90 & 18 & $-1,6$ \\
\hline 1 & 19 & 0,026 & 0,490 & 0,027 & 0,410 & 0,947 & 0,836 & 0,172 & 1,000 & 0,861 & 0,881 & 0,588 & 0,193 & 0,114 & 0,152 & 77 & 19 & $-0,9$ \\
\hline 1 & 20 & 0,090 & 1,336 & 0,112 & 1,061 & 0,808 & 0,794 & 0,282 & 0,636 & 1,253 & 0,542 & 0,568 & 0,461 & 0,262 & 0,214 & 4 & 20 & $-2,2$ \\
\hline 2 & 20 & 0,037 & 0,693 & 0,044 & 0,613 & 0,839 & 0,884 & 0,199 & 0,975 & 1,013 & 0,676 & 0,571 & 0,265 & 0,152 & 0,152 & 10 & 20 & $-1,4$ \\
\hline 3 & 20 & 0,036 & 0,837 & 0,050 & 0,676 & 0,714 & 0,808 & 0,344 & 0,643 & 1,247 & 0,388 & 0,437 & 0,343 & 0,150 & 0,152 & 1 & 20 & $-1,8$ \\
\hline 4 & 20 & 0,014 & 0,317 & 0,014 & 0,173 & 1,000 & 0,547 & 0,453 & 1,000 & 0,746 & 1,273 & 0,632 & 0,120 & 0,076 & 0,107 & 18 & 20 & $-0,3$ \\
\hline 5 & 20 & 0,014 & 0,418 & 0,017 & 0,215 & 0,833 & 0,515 & 0,513 & 1,000 & 0,986 & 0,637 & 0,447 & 0,169 & 0,076 & 0,076 & 152 & 20 & $-0,8$ \\
\hline 6 & 20 & 0,030 & 0,571 & 0,030 & 0,520 & 1,000 & 0,911 & 0,089 & 1,000 & 0,928 & 0,594 & 0,422 & 0,254 & 0,107 & 0,152 & 148 & 20 & $-1,3$ \\
\hline 7 & 20 & 0,217 & 2,101 & 0,263 & 1,746 & 0,825 & 0,831 & 0,243 & 0,617 & 1,273 & 0,540 & 0,675 & 0,715 & 0,482 & 0,379 & 148 & 20 & $-2,8$ \\
\hline 8 & 20 & 0,062 & 1,797 & 0,119 & 1,226 & 0,518 & 0,682 & 0,577 & 0,240 & 2,040 & 0,234 & 0,392 & 0,579 & 0,227 & 0,107 & 2 & 20 & $-2,5$ \\
\hline 9 & 20 & 0,070 & 1,492 & 0,100 & 1,327 & 0,700 & 0,890 & 0,320 & 0,397 & 1,587 & 0,195 & 0,225 & 0,678 & 0,152 & 0,152 & 157 & 20 & $-2,8$ \\
\hline 10 & 20 & 0,026 & 0,541 & 0,029 & 0,418 & 0,900 & 0,773 & 0,248 & 1,000 & 0,950 & 0,790 & 0,557 & 0,204 & 0,114 & 0,152 & 4 & 20 & $-1,0$ \\
\hline 11 & 20 & 0,395 & 2,879 & 0,472 & 2,317 & 0,836 & 0,805 & 0,255 & 0,598 & 1,293 & 0,700 & 0,770 & 0,847 & 0,652 & 0,530 & 116 & 20 & $-3,1$ \\
\hline 12 & 20 & 0,049 & 0,858 & 0,055 & 0,735 & 0,895 & 0,857 & 0,177 & 0,833 & 1,096 & 0,397 & 0,362 & 0,395 & 0,143 & 0,152 & 171 & 20 & $-2,0$ \\
\hline 13 & 20 & 0,204 & 2,612 & 0,261 & 1,725 & 0,780 & 0,660 & 0,405 & 0,375 & 1,633 & 0,535 & 0,692 & 0,696 & 0,482 & 0,273 & 99 & 20 & $-2,8$ \\
\hline 14 & 20 & 0,030 & 0,664 & 0,036 & 0,511 & 0,840 & 0,771 & 0,280 & 0,860 & 1,078 & 0,504 & 0,369 & 0,276 & 0,102 & 0,152 & 162 & 20 & $-1,5$ \\
\hline 15 & 20 & 0,063 & 1,315 & 0,095 & 1,061 & 0,667 & 0,807 & 0,385 & 0,459 & 1,476 & 0,409 & 0,604 & 0,443 & 0,268 & 0,107 & 142 & 20 & $-2,1$ \\
\hline 16 & 20 & 0,029 & 0,592 & 0,030 & 0,520 & 0,952 & 0,879 & 0,130 & 1,000 & 0,986 & 0,621 & 0,552 & 0,243 & 0,134 & 0,152 & 124 & 20 & $-1,3$ \\
\hline 17 & 20 & 0,026 & 0,643 & 0,029 & 0,469 & 0,900 & 0,730 & 0,288 & 0,786 & 1,128 & 0,395 & 0,294 & 0,288 & 0,085 & 0,107 & 115 & 20 & $-1,5$ \\
\hline 18 & 20 & 0,014 & 0,347 & 0,016 & 0,224 & 0,909 & 0,646 & 0,365 & 1,000 & 0,816 & 0,979 & 0,588 & 0,137 & 0,080 & 0,107 & 146 & 20 & $-0,5$ \\
\hline 19 & 20 & 0,016 & 0,461 & 0,022 & 0,245 & 0,733 & 0,532 & 0,538 & 0,935 & 1,034 & 0,700 & 0,600 & 0,169 & 0,102 & 0,076 & 0 & 20 & $-0,8$ \\
\hline 20 & 20 & 0,014 & 0,469 & 0,017 & 0,347 & 0,833 & 0,739 & 0,310 & 0,819 & 1,105 & 0,509 & 0,400 & 0,189 & 0,076 & 0,076 & 36 & 20 & $-0,9$ \\
\hline 21 & 20 & 0,014 & 0,317 & 0,014 & 0,173 & 1,000 & 0,547 & 0,453 & 1,000 & 0,746 & 1,273 & 0,632 & 0,120 & 0,076 & 0,107 & 90 & 20 & $-0,3$ \\
\hline 22 & 20 & 0,029 & 0,888 & 0,044 & 0,744 & 0,645 & 0,838 & 0,390 & 0,458 & 1,478 & 0,204 & 0,201 & 0,423 & 0,085 & 0,076 & 152 & 20 & $-2,1$ \\
\hline 23 & 20 & 0,014 & 0,347 & 0,014 & 0,224 & 1,000 & 0,646 & 0,354 & 1,000 & 0,816 & 0,707 & 0,333 & 0,161 & 0,054 & 0,107 & 135 & 20 & $-0,7$ \\
\hline 24 & 20 & 0,014 & 0,440 & 0,016 & 0,389 & 0,909 & 0,885 & 0,147 & 0,933 & 1,035 & 0,490 & 0,196 & 0,193 & 0,038 & 0,076 & 90 & 20 & $-0,9$ \\
\hline 25 & 20 & 0,162 & 2,266 & 0,212 & 1,662 & 0,764 & 0,733 & 0,356 & 0,397 & 1,587 & 0,351 & 0,423 & 0,767 & 0,325 & 0,152 & 142 & 20 & $-2,9$ \\
\hline 26 & 20 & 0,017 & 0,490 & 0,020 & 0,347 & 0,857 & 0,707 & 0,326 & 0,900 & 1,054 & 0,449 & 0,364 & 0,221 & 0,080 & 0,076 & 149 & 20 & $-1,1$ \\
\hline 27 & 20 & 0,014 & 0,410 & 0,016 & 0,397 & 0,909 & 0,970 & 0,096 & 1,000 & 0,965 & 0,490 & 0,196 & 0,193 & 0,038 & 0,076 & 0 & 20 & $-0,9$ \\
\hline 28 & 20 & 0,020 & 0,520 & 0,026 & 0,317 & 0,778 & 0,610 & 0,449 & 0,934 & 1,035 & 0,524 & 0,384 & 0,221 & 0,085 & 0,107 & 151 & 20 & $-1,1$ \\
\hline 29 & 20 & 0,019 & 0,440 & 0,022 & 0,541 & 0,867 & 1,231 & 0,267 & 1,000 & 0,908 & 0,828 & 0,671 & 0,169 & 0,114 & 0,152 & 90 & 20 & $-0,8$ \\
\hline 30 & 20 & 0,023 & 0,664 & 0,034 & 0,930 & 0,667 & 1,401 & 0,522 & 0,655 & 1,236 & 0,392 & 0,386 & 0,273 & 0,105 & 0,076 & 58 & 20 & $-1,4$ \\
\hline 31 & 20 & 0,070 & 1,530 & 0,103 & 0,317 & 0,681 & 0,207 & 0,855 & 0,377 & 1,628 & 0,624 & 0,797 & 0,379 & 0,302 & 0,152 & 24 & 20 & $-1,9$ \\
\hline 32 & 20 & 0,022 & 0,490 & 0,026 & 0,448 & 0,833 & 0,914 & 0,188 & 1,000 & 0,943 & 0,735 & 0,588 & 0,193 & 0,114 & 0,152 & 90 & 20 & $-0,9$ \\
\hline 33 & 20 & 0,026 & 0,490 & 0,027 & 0,735 & 0,947 & 1,500 & 0,503 & 1,000 & 0,861 & 0,917 & 0,707 & 0,189 & 0,134 & 0,152 & 122 & 20 & $-0,9$ \\
\hline 34 & 20 & 0,042 & 0,930 & 0,060 & 0,347 & 0,690 & 0,373 & 0,699 & 0,605 & 1,286 & 0,415 & 0,522 & 0,357 & 0,186 & 0,152 & 158 & 20 & $-1,8$ \\
\hline 35 & 20 & 0,016 & 0,469 & 0,020 & 0,541 & 0,786 & 1,153 & 0,263 & 0,901 & 1,054 & 0,412 & 0,276 & 0,221 & 0,061 & 0,076 & 50 & 20 & $-1,1$ \\
\hline 36 & 20 & 0,036 & 0,693 & 0,042 & 0,317 & 0,862 & 0,457 & 0,560 & 0,938 & 1,033 & 0,612 & 0,555 & 0,273 & 0,152 & 0,169 & 125 & 20 & $-1,4$ \\
\hline 37 & 20 & 0,023 & 0,440 & 0,024 & 0,245 & 0,941 & 0,558 & 0,446 & 1,000 & 0,818 & 1,019 & 0,671 & 0,169 & 0,114 & 0,152 & 110 & 20 & $-0,8$ \\
\hline 38 & 20 & 0,019 & 0,389 & 0,020 & 0,909 & 0,929 & 2,337 & 1,339 & 1,000 & 0,803 & 0,920 & 0,633 & 0,161 & 0,102 & 0,152 & 135 & 20 & $-0,7$ \\
\hline 39 & 20 & 0,072 & 1,010 & 0,079 & 0,296 & 0,909 & 0,293 & 0,713 & 0,883 & 1,064 & 0,490 & 0,496 & 0,432 & 0,214 & 0,240 & 138 & 20 & $-2,1$ \\
\hline 40 & 20 & 0,016 & 0,397 & 0,017 & 1,467 & 0,917 & 3,692 & 2,693 & 1,000 & 0,892 & 0,700 & 0,435 & 0,169 & 0,074 & 0,076 & 135 & 20 & $-0,8$ \\
\hline 41 & 20 & 0,146 & 1,818 & 0,202 & 0,499 & 0,723 & 0,275 & 0,776 & 0,557 & 1,340 & 0,519 & 0,621 & 0,599 & 0,372 & 0,273 & 128 & 20 & $-2,6$ \\
\hline 42 & 20 & 0,016 & 0,499 & 0,019 & 2,068 & 0,846 & 4,144 & 3,148 & 0,797 & 1,120 & 0,342 & 0,217 & 0,243 & 0,053 & 0,076 & 126 & 20 & $-1,3$ \\
\hline 43 & 20 & 0,128 & 2,782 & 0,214 & 0,194 & 0,597 & 0,070 & 1,014 & 0,207 & 2,196 & 0,162 & 0,295 & 1,001 & 0,295 & 0,152 & 146 & 20 & $-3,3$ \\
\hline 44 & 20 & 0,014 & 0,368 & 0,016 & 0,317 & 0,909 & 0,862 & 0,165 & 1,000 & 0,866 & 0,749 & 0,485 & 0,156 & 0,076 & 0,107 & 5 & 20 & $-0,6$ \\
\hline 45 & 20 & 0,024 & 0,482 & 0,024 & 0,347 & 1,000 & 0,720 & 0,280 & 1,000 & 0,870 & 0,746 & 0,371 & 0,204 & 0,076 & 0,152 & 175 & 20 & $-1,0$ \\
\hline 46 & 20 & 0,022 & 0,469 & 0,024 & 0,215 & 0,882 & 0,459 & 0,553 & 1,000 & 0,902 & 1,061 & 0,786 & 0,161 & 0,126 & 0,107 & 117 & 20 & $-0,7$ \\
\hline 47 & 20 & 0,017 & 0,368 & 0,017 & 1,124 & 1,000 & 3,057 & 2,057 & 1,000 & 0,791 & 0,899 & 0,485 & 0,156 & 0,076 & 0,152 & 0 & 20 & $-0,6$ \\
\hline 48 & 20 & 0,060 & 1,420 & 0,100 & 0,613 & 0,600 & 0,432 & 0,695 & 0,375 & 1,632 & 0,261 & 0,407 & 0,542 & 0,221 & 0,107 & 172 & 20 & $-2,4$ \\
\hline 49 & 20 & 0,030 & 0,693 & 0,033 & 1,737 & 0,913 & 2,505 & 1,508 & 0,788 & 1,127 & 0,366 & 0,262 & 0,324 & 0,085 & 0,107 & 156 & 20 & $-1,7$ \\
\hline 50 & 20 & 0,135 & 2,427 & 0,224 & 0,245 & 0,603 & 0,101 & 0,983 & 0,288 & 1,864 & 0,266 & 0,380 & 0,804 & 0,305 & 0,214 & 169 & 20 & $-3,0$ \\
\hline 51 & 20 & 0,016 & 0,368 & 0,017 & 0,245 & 0,917 & 0,667 & 0,344 & 1,000 & 0,826 & 0,824 & 0,485 & 0,156 & 0,076 & 0,107 & 18 & 20 & $-0,6$ \\
\hline 52 & 20 & 0,016 & 0,338 & 0,016 & 0,347 & 1,000 & 1,026 & 0,026 & 1,000 & 0,759 & 1,077 & 0,555 & 0,137 & 0,076 & 0,152 & 0 & 20 & $-0,5$ \\
\hline
\end{tabular}




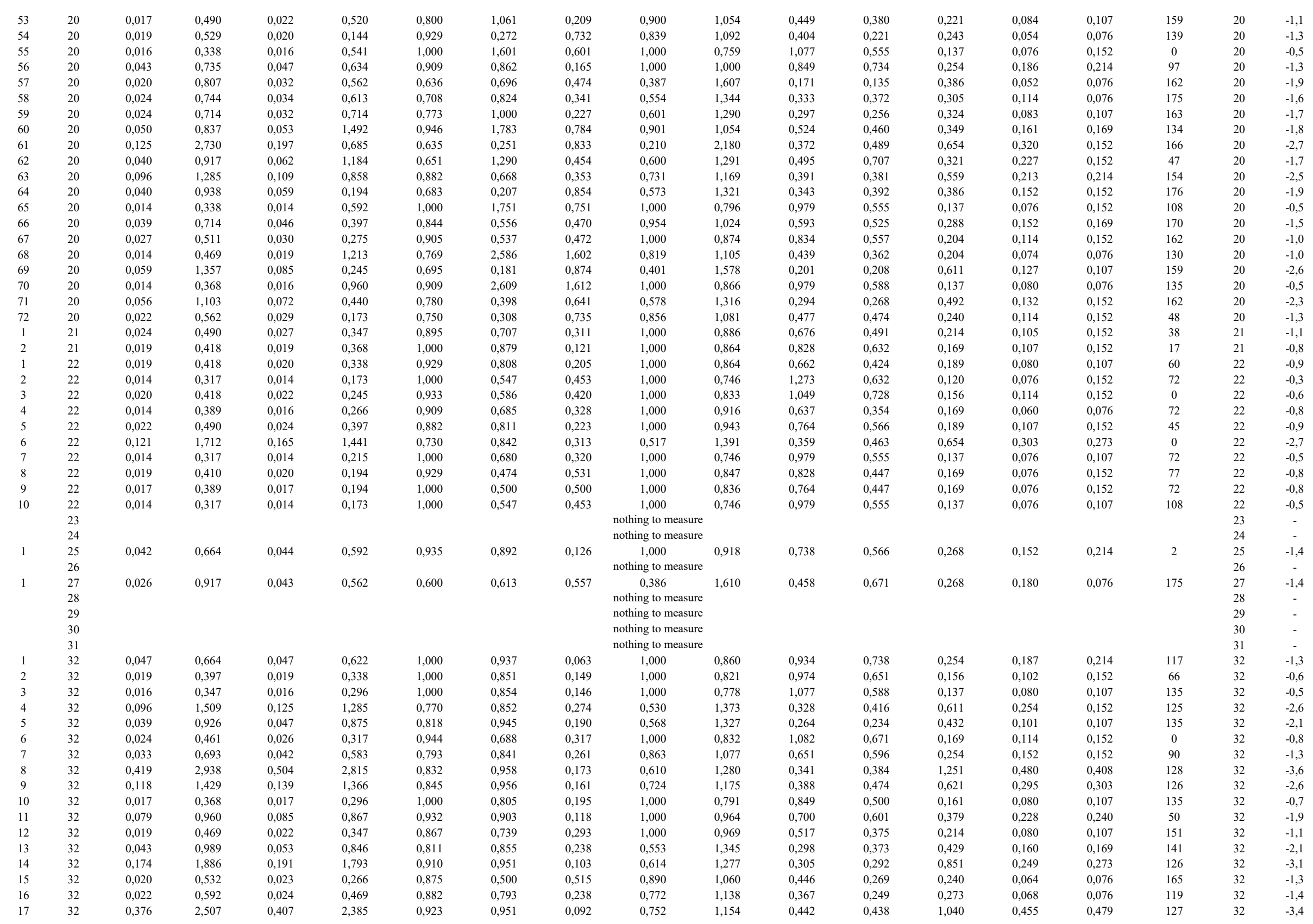




\begin{tabular}{|c|c|c|c|c|c|c|c|c|c|c|c|c|c|c|c|c|c|c|}
\hline 18 & 32 & 0,110 & 2,541 & 0,159 & 1,483 & 0,694 & 0,584 & 0,517 & 0,215 & 2,156 & 0,260 & 0,309 & 0,735 & 0,227 & 0,169 & 97 & 32 & $-2,9$ \\
\hline 19 & 32 & 0,132 & 1,480 & 0,152 & 1,247 & 0,868 & 0,843 & 0,205 & 0,758 & 1,149 & 0,689 & 0,690 & 0,494 & 0,341 & 0,273 & 116 & 32 & $-2,3$ \\
\hline 20 & 32 & 0,039 & 0,655 & 0,042 & 0,469 & 0,931 & 0,716 & 0,292 & 1,000 & 0,939 & 0,838 & 0,773 & 0,243 & 0,187 & 0,169 & 135 & 32 & $-1,3$ \\
\hline 21 & 32 & 0,019 & 0,397 & 0,019 & 0,376 & 1,000 & 0,947 & 0,053 & 1,000 & 0,821 & 0,974 & 0,686 & 0,156 & 0,107 & 0,152 & 163 & 32 & $-0,6$ \\
\hline 22 & 32 & 0,095 & 1,509 & 0,143 & 1,255 & 0,660 & 0,832 & 0,379 & 0,522 & 1,384 & 0,361 & 0,469 & 0,578 & 0,271 & 0,214 & 118 & 32 & $-2,5$ \\
\hline 23 & 32 & 0,014 & 0,520 & 0,019 & 0,397 & 0,769 & 0,764 & 0,330 & 0,667 & 1,225 & 0,311 & 0,221 & 0,243 & 0,054 & 0,076 & 147 & 32 & $-1,3$ \\
\hline 24 & 32 & 0,024 & 0,571 & 0,024 & 0,469 & 1,000 & 0,822 & 0,178 & 0,941 & 1,031 & 0,433 & 0,300 & 0,268 & 0,080 & 0,107 & 140 & 32 & $-1,4$ \\
\hline 25 & 32 & 0,077 & 1,683 & 0,106 & 1,133 & 0,730 & 0,673 & 0,424 & 0,344 & 1,705 & 0,331 & 0,463 & 0,546 & 0,253 & 0,152 & 107 & 32 & $-2,4$ \\
\hline 26 & 32 & 0,016 & 0,347 & 0,016 & 0,296 & 1,000 & 0,854 & 0,146 & 1,000 & 0,778 & 1,077 & 0,588 & 0,137 & 0,080 & 0,107 & 72 & 32 & $-0,5$ \\
\hline 27 & 32 & 0,017 & 0,418 & 0,020 & 0,317 & 0,857 & 0,757 & 0,282 & 1,000 & 0,900 & 0,611 & 0,400 & 0,189 & 0,076 & 0,152 & 90 & 32 & $-0,9$ \\
\hline 28 & 32 & 0,017 & 0,347 & 0,017 & 0,296 & 1,000 & 0,854 & 0,146 & 1,000 & 0,745 & 1,528 & 0,894 & 0,120 & 0,107 & 0,107 & 90 & 32 & $-0,3$ \\
\hline 29 & 32 & 0,076 & 1,163 & 0,095 & 1,091 & 0,803 & 0,938 & 0,206 & 0,707 & 1,189 & 0,493 & 0,498 & 0,443 & 0,221 & 0,214 & 136 & 32 & $-2,1$ \\
\hline 30 & 32 & 0,044 & 0,685 & 0,044 & 0,592 & 1,000 & 0,864 & 0,136 & 1,000 & 0,916 & 0,607 & 0,496 & 0,305 & 0,152 & 0,214 & 111 & 32 & $-1,6$ \\
\hline 31 & 32 & 0,017 & 0,550 & 0,020 & 0,520 & 0,857 & 0,946 & 0,153 & 0,716 & 1,182 & 0,306 & 0,196 & 0,268 & 0,053 & 0,076 & 144 & 32 & $-1,4$ \\
\hline 32 & 32 & 0,234 & 3,838 & 0,327 & 1,910 & 0,715 & 0,498 & 0,578 & 0,199 & 2,239 & 0,538 & 0,662 & 0,744 & 0,492 & 0,169 & 98 & 32 & $-2,9$ \\
\hline 33 & 32 & 0,036 & 0,592 & 0,037 & 0,490 & 0,962 & 0,828 & 0,176 & 1,000 & 0,881 & 0,776 & 0,552 & 0,243 & 0,134 & 0,169 & 130 & 32 & $-1,3$ \\
\hline 34 & 32 & 0,019 & 0,520 & 0,023 & 0,440 & 0,812 & 0,845 & 0,243 & 0,867 & 1,074 & 0,414 & 0,300 & 0,240 & 0,072 & 0,076 & 162 & 32 & $-1,3$ \\
\hline 35 & 32 & 0,014 & 0,338 & 0,014 & 0,194 & 1,000 & 0,575 & 0,425 & 1,000 & 0,796 & 0,979 & 0,555 & 0,137 & 0,076 & 0,152 & 90 & 32 & $-0,5$ \\
\hline 36 & 32 & 0,168 & 1,581 & 0,184 & 1,450 & 0,914 & 0,917 & 0,119 & 0,844 & 1,089 & 0,573 & 0,549 & 0,611 & 0,336 & 0,312 & 83 & 32 & $-2,6$ \\
\hline 37 & 32 & 0,022 & 0,440 & 0,022 & 0,338 & 1,000 & 0,769 & 0,231 & 1,000 & 0,845 & 0,735 & 0,392 & 0,193 & 0,076 & 0,152 & 90 & 32 & $-0,9$ \\
\hline 38 & 32 & 0,020 & 0,440 & 0,022 & 0,296 & 0,933 & 0,673 & 0,334 & 1,000 & 0,875 & 0,713 & 0,448 & 0,189 & 0,085 & 0,107 & 117 & 32 & $-0,9$ \\
\hline 39 & 32 & 0,022 & 0,562 & 0,023 & 0,511 & 0,938 & 0,910 & 0,110 & 0,856 & 1,081 & 0,390 & 0,286 & 0,265 & 0,076 & 0,107 & 92 & 32 & $-1,4$ \\
\hline 40 & 32 & 0,085 & 1,623 & 0,122 & 1,429 & 0,694 & 0,880 & 0,328 & 0,404 & 1,574 & 0,209 & 0,236 & 0,719 & 0,170 & 0,152 & 110 & 32 & $-2,8$ \\
\hline 41 & 32 & 0,019 & 0,613 & 0,026 & 0,482 & 0,722 & 0,786 & 0,351 & 0,624 & 1,266 & 0,312 & 0,275 & 0,276 & 0,076 & 0,107 & 72 & 32 & $-1,5$ \\
\hline 42 & 32 & 0,016 & 0,368 & 0,017 & 0,296 & 0,917 & 0,805 & 0,212 & 1,000 & 0,826 & 1,077 & 0,621 & 0,137 & 0,085 & 0,107 & 127 & 32 & $-0,5$ \\
\hline 43 & 32 & 0,016 & 0,368 & 0,016 & 0,224 & 1,000 & 0,609 & 0,391 & 1,000 & 0,826 & 0,778 & 0,500 & 0,161 & 0,080 & 0,107 & 135 & 32 & $-0,7$ \\
\hline 44 & 32 & 0,016 & 0,397 & 0,017 & 0,397 & 0,917 & 1,000 & 0,083 & 1,000 & 0,892 & 0,700 & 0,401 & 0,169 & 0,068 & 0,076 & 151 & 32 & $-0,8$ \\
\hline 45 & 32 & 0,023 & 0,490 & 0,024 & 0,418 & 0,941 & 0,854 & 0,158 & 1,000 & 0,913 & 0,702 & 0,529 & 0,204 & 0,108 & 0,152 & 79 & 32 & $-1,0$ \\
\hline 46 & 32 & 0,020 & 0,418 & 0,020 & 0,296 & 1,000 & 0,707 & 0,293 & 1,000 & 0,833 & 0,713 & 0,424 & 0,189 & 0,080 & 0,107 & 145 & 32 & $-0,9$ \\
\hline 47 & 32 & 0,019 & 0,418 & 0,019 & 0,296 & 1,000 & 0,707 & 0,293 & 1,000 & 0,864 & 0,662 & 0,424 & 0,189 & 0,080 & 0,107 & 147 & 32 & $-0,9$ \\
\hline 48 & 32 & 0,040 & 0,664 & 0,042 & 0,583 & 0,966 & 0,879 & 0,126 & 1,000 & 0,934 & 0,673 & 0,549 & 0,276 & 0,152 & 0,169 & 107 & 32 & $-1,5$ \\
\hline 49 & 32 & 0,016 & 0,368 & 0,017 & 0,194 & 0,917 & 0,529 & 0,479 & 1,000 & 0,826 & 0,778 & 0,500 & 0,161 & 0,080 & 0,107 & 135 & 32 & $-0,7$ \\
\hline 50 & 32 & 0,069 & 0,888 & 0,075 & 0,858 & 0,923 & 0,967 & 0,084 & 1,000 & 0,954 & 0,837 & 0,704 & 0,324 & 0,228 & 0,227 & 107 & 32 & $-1,7$ \\
\hline 51 & 32 & 0,019 & 0,529 & 0,020 & 0,448 & 0,929 & 0,848 & 0,168 & 0,839 & 1,092 & 0,404 & 0,221 & 0,243 & 0,054 & 0,107 & 135 & 32 & $-1,3$ \\
\hline 52 & 32 & 0,017 & 0,613 & 0,023 & 0,418 & 0,750 & 0,683 & 0,404 & 0,576 & 1,318 & 0,306 & 0,283 & 0,268 & 0,076 & 0,076 & 148 & 32 & $-1,4$ \\
\hline 53 & 32 & 0,016 & 0,397 & 0,017 & 0,245 & 0,917 & 0,617 & 0,392 & 1,000 & 0,892 & 1,077 & 0,784 & 0,137 & 0,107 & 0,107 & 108 & 32 & $-0,5$ \\
\hline 54 & 32 & 0,022 & 0,490 & 0,023 & 0,347 & 0,938 & 0,707 & 0,300 & 1,000 & 0,943 & 0,597 & 0,500 & 0,214 & 0,107 & 0,152 & 132 & 32 & $-1,1$ \\
\hline 55 & 32 & 0,049 & 0,723 & 0,053 & 0,643 & 0,919 & 0,889 & 0,138 & 1,000 & 0,923 & 0,866 & 0,700 & 0,268 & 0,187 & 0,214 & 76 & 32 & $-1,4$ \\
\hline 56 & 32 & 0,014 & 0,317 & 0,014 & 0,173 & 1,000 & 0,547 & 0,453 & 1,000 & 0,746 & 0,979 & 0,555 & 0,137 & 0,076 & 0,107 & 108 & 32 & $-0,5$ \\
\hline 57 & 32 & 0,019 & 0,418 & 0,019 & 0,275 & 1,000 & 0,657 & 0,343 & 1,000 & 0,864 & 0,662 & 0,424 & 0,189 & 0,080 & 0,107 & 132 & 32 & $-0,9$ \\
\hline 58 & 32 & 0,016 & 0,368 & 0,017 & 0,266 & 0,917 & 0,724 & 0,288 & 1,000 & 0,826 & 0,824 & 0,485 & 0,156 & 0,076 & 0,107 & 108 & 32 & $-0,6$ \\
\hline 59 & 32 & 0,023 & 0,571 & 0,029 & 0,418 & 0,800 & 0,733 & 0,333 & 0,886 & 1,063 & 0,784 & 0,762 & 0,193 & 0,147 & 0,152 & 52 & 32 & $-0,9$ \\
\hline 60 & 32 & 0,049 & 1,163 & 0,076 & 0,960 & 0,642 & 0,825 & 0,399 & 0,454 & 1,485 & 0,299 & 0,396 & 0,456 & 0,181 & 0,107 & 154 & 32 & $-2,2$ \\
\hline 61 & 32 & 0,016 & 0,418 & 0,019 & 0,317 & 0,846 & 0,757 & 0,287 & 1,000 & 0,940 & 0,560 & 0,358 & 0,189 & 0,068 & 0,076 & 125 & 32 & $-0,9$ \\
\hline 62 & 32 & 0,014 & 0,397 & 0,017 & 0,287 & 0,833 & 0,723 & 0,323 & 1,000 & 0,936 & 0,749 & 0,485 & 0,156 & 0,076 & 0,107 & 90 & 32 & $-0,6$ \\
\hline 63 & 32 & 0,042 & 0,685 & 0,047 & 0,634 & 0,879 & 0,926 & 0,142 & 1,000 & 0,947 & 0,710 & 0,621 & 0,273 & 0,170 & 0,169 & 63 & 32 & $-1,4$ \\
\hline 64 & 32 & 0,039 & 0,714 & 0,042 & 0,562 & 0,931 & 0,787 & 0,224 & 0,954 & 1,024 & 0,537 & 0,375 & 0,303 & 0,114 & 0,152 & 95 & 32 & $-1,6$ \\
\hline 65 & 32 & 0,014 & 0,347 & 0,014 & 0,347 & 1,000 & 1,000 & 0,000 & 1,000 & 0,816 & 0,749 & 0,485 & 0,156 & 0,076 & 0,107 & 162 & 32 & $-0,6$ \\
\hline 66 & 32 & 0,027 & 0,541 & 0,032 & 0,418 & 0,864 & 0,773 & 0,264 & 1,000 & 0,924 & 0,712 & 0,537 & 0,221 & 0,119 & 0,152 & 112 & 32 & $-1,1$ \\
\hline 67 & 32 & 0,030 & 0,870 & 0,050 & 0,613 & 0,600 & 0,704 & 0,498 & 0,500 & 1,415 & 0,411 & 0,610 & 0,305 & 0,186 & 0,076 & 82 & 32 & $-1,6$ \\
\hline 68 & 32 & 0,053 & 0,795 & 0,059 & 0,735 & 0,902 & 0,925 & 0,123 & 1,000 & 0,973 & 0,725 & 0,666 & 0,305 & 0,203 & 0,214 & 74 & 32 & $-1,6$ \\
\hline 69 & 32 & 0,017 & 0,368 & 0,017 & 0,287 & 1,000 & 0,781 & 0,219 & 1,000 & 0,791 & 1,175 & 0,745 & 0,137 & 0,102 & 0,152 & 45 & 32 & $-0,5$ \\
\hline 70 & 32 & 0,014 & 0,520 & 0,017 & 0,368 & 0,833 & 0,707 & 0,337 & 0,667 & 1,225 & 0,283 & 0,179 & 0,254 & 0,046 & 0,076 & 147 & 32 & $-1,3$ \\
\hline 71 & 32 & 0,016 & 0,440 & 0,020 & 0,245 & 0,786 & 0,558 & 0,491 & 1,000 & 0,987 & 0,539 & 0,373 & 0,193 & 0,072 & 0,076 & 90 & 32 & $-0,9$ \\
\hline 72 & 32 & 0,026 & 0,571 & 0,032 & 0,469 & 0,818 & 0,822 & 0,254 & 0,996 & 1,002 & 0,674 & 0,480 & 0,221 & 0,106 & 0,107 & 46 & 32 & $-1,1$ \\
\hline 73 & 32 & 0,014 & 0,317 & 0,014 & 0,173 & 1,000 & 0,547 & 0,453 & 1,000 & 0,746 & 0,979 & 0,555 & 0,137 & 0,076 & 0,107 & 108 & 32 & $-0,5$ \\
\hline 74 & 32 & 0,022 & 0,562 & 0,030 & 0,440 & 0,714 & 0,782 & 0,359 & 0,856 & 1,081 & 0,597 & 0,531 & 0,214 & 0,114 & 0,107 & 75 & 32 & $-1,1$ \\
\hline 75 & 32 & 0,024 & 0,571 & 0,026 & 0,499 & 0,944 & 0,874 & 0,138 & 0,941 & 1,031 & 0,433 & 0,300 & 0,268 & 0,080 & 0,107 & 132 & 32 & $-1,4$ \\
\hline
\end{tabular}




\begin{tabular}{|c|c|c|c|c|c|c|c|c|c|c|c|c|c|c|c|c|c|c|}
\hline 76 & 32 & 0,014 & 0,317 & 0,014 & 0,173 & 1,000 & 0,547 & 0,453 & 1,000 & 0,746 & 0,979 & 0,555 & 0,137 & 0,076 & 0,107 & 108 & 32 & $-0,5$ \\
\hline 77 & 32 & 0,059 & 0,837 & 0,066 & 0,765 & 0,891 & 0,914 & 0,138 & 1,000 & 0,973 & 0,900 & 0,802 & 0,288 & 0,231 & 0,240 & 112 & 32 & $-1,5$ \\
\hline 78 & 32 & 0,023 & 0,613 & 0,030 & 0,440 & 0,762 & 0,717 & 0,370 & 0,768 & 1,141 & 0,453 & 0,368 & 0,254 & 0,093 & 0,107 & 165 & 32 & $-1,3$ \\
\hline 79 & 32 & 0,014 & 0,338 & 0,014 & 0,194 & 1,000 & 0,575 & 0,425 & 1,000 & 0,796 & 0,979 & 0,555 & 0,137 & 0,076 & 0,152 & 18 & 32 & $-0,5$ \\
\hline 80 & 32 & 0,017 & 0,397 & 0,017 & 0,317 & 1,000 & 0,798 & 0,202 & 1,000 & 0,854 & 0,764 & 0,474 & 0,169 & 0,080 & 0,107 & 143 & 32 & $-0,8$ \\
\hline 81 & 32 & 0,108 & 1,255 & 0,119 & 1,154 & 0,904 & 0,919 & 0,126 & 0,858 & 1,080 & 0,552 & 0,476 & 0,498 & 0,237 & 0,240 & 106 & 32 & $-2,3$ \\
\hline 82 & 32 & 0,032 & 0,622 & 0,034 & 0,571 & 0,917 & 0,918 & 0,117 & 1,000 & 0,987 & 0,539 & 0,388 & 0,273 & 0,106 & 0,152 & 141 & 32 & $-1,4$ \\
\hline 83 & 32 & 0,016 & 0,338 & 0,016 & 0,245 & 1,000 & 0,725 & 0,275 & 1,000 & 0,759 & 1,077 & 0,555 & 0,137 & 0,076 & 0,152 & 0 & 32 & $-0,5$ \\
\hline 84 & 32 & 0,099 & 2,101 & 0,176 & 1,530 & 0,561 & 0,728 & 0,516 & 0,282 & 1,884 & 0,249 & 0,420 & 0,712 & 0,299 & 0,152 & 154 & 32 & $-2,8$ \\
\hline 85 & 32 & 0,057 & 0,828 & 0,060 & 0,685 & 0,952 & 0,827 & 0,180 & 1,000 & 0,975 & 0,637 & 0,495 & 0,339 & 0,168 & 0,214 & 110 & 32 & $-1,8$ \\
\hline 86 & 32 & 0,026 & 0,571 & 0,027 & 0,499 & 0,947 & 0,874 & 0,136 & 0,996 & 1,002 & 0,458 & 0,300 & 0,268 & 0,080 & 0,107 & 138 & 32 & $-1,4$ \\
\hline 87 & 32 & 0,019 & 0,622 & 0,022 & 0,448 & 0,867 & 0,721 & 0,309 & 0,607 & 1,284 & 0,271 & 0,181 & 0,296 & 0,054 & 0,076 & 138 & 32 & $-1,6$ \\
\hline 88 & 32 & 0,055 & 0,888 & 0,063 & 0,757 & 0,864 & 0,852 & 0,201 & 0,869 & 1,072 & 0,569 & 0,542 & 0,349 & 0,189 & 0,214 & 109 & 32 & $-1,8$ \\
\hline 89 & 32 & 0,033 & 0,541 & 0,033 & 0,461 & 1,000 & 0,851 & 0,149 & 1,000 & 0,840 & 0,861 & 0,606 & 0,221 & 0,134 & 0,169 & 62 & 32 & $-1,1$ \\
\hline 90 & 32 & 0,014 & 0,338 & 0,016 & 0,194 & 0,909 & 0,575 & 0,435 & 1,000 & 0,796 & 0,979 & 0,555 & 0,137 & 0,076 & 0,107 & 108 & 32 & $-0,5$ \\
\hline 91 & 32 & 0,036 & 0,888 & 0,047 & 0,727 & 0,758 & 0,819 & 0,303 & 0,572 & 1,322 & 0,354 & 0,316 & 0,359 & 0,114 & 0,107 & 95 & 32 & $-1,8$ \\
\hline 92 & 32 & 0,017 & 0,499 & 0,019 & 0,478 & 0,923 & 0,958 & 0,088 & 0,869 & 1,073 & 0,449 & 0,308 & 0,221 & 0,068 & 0,076 & 151 & 32 & $-1,1$ \\
\hline 93 & 32 & 0,016 & 0,651 & 0,027 & 0,562 & 0,579 & 0,863 & 0,443 & 0,468 & 1,462 & 0,264 & 0,324 & 0,276 & 0,089 & 0,076 & 135 & 32 & $-1,5$ \\
\hline 94 & 32 & 0,023 & 0,685 & 0,030 & 0,541 & 0,762 & 0,790 & 0,317 & 0,615 & 1,275 & 0,279 & 0,223 & 0,324 & 0,072 & 0,076 & 109 & 32 & $-1,7$ \\
\hline 95 & 32 & 0,024 & 0,744 & 0,033 & 0,562 & 0,739 & 0,755 & 0,358 & 0,554 & 1,344 & 0,271 & 0,248 & 0,339 & 0,084 & 0,076 & 166 & 32 & $-1,8$ \\
\hline 96 & 32 & 0,016 & 0,410 & 0,016 & 0,266 & 1,000 & 0,650 & 0,350 & 1,000 & 0,920 & 0,539 & 0,196 & 0,193 & 0,038 & 0,076 & 0 & 32 & $-0,9$ \\
\hline 97 & 32 & 0,044 & 0,960 & 0,059 & 0,846 & 0,756 & 0,881 & 0,271 & 0,607 & 1,283 & 0,372 & 0,408 & 0,390 & 0,159 & 0,169 & 132 & 32 & $-2,0$ \\
\hline 98 & 32 & 0,042 & 0,643 & 0,042 & 0,562 & 1,000 & 0,875 & 0,125 & 1,000 & 0,889 & 0,738 & 0,566 & 0,268 & 0,152 & 0,214 & 90 & 32 & $-1,4$ \\
\hline 99 & 32 & 0,016 & 0,469 & 0,017 & 0,418 & 0,917 & 0,892 & 0,137 & 0,901 & 1,054 & 0,412 & 0,231 & 0,221 & 0,051 & 0,076 & 117 & 32 & $-1,1$ \\
\hline 100 & 32 & 0,020 & 0,448 & 0,020 & 0,397 & 1,000 & 0,887 & 0,113 & 1,000 & 0,892 & 0,713 & 0,424 & 0,189 & 0,080 & 0,107 & 125 & 32 & $-0,9$ \\
\hline 101 & 32 & 0,075 & 1,458 & 0,095 & 1,142 & 0,788 & 0,783 & 0,304 & 0,441 & 1,506 & 0,343 & 0,407 & 0,526 & 0,214 & 0,169 & 120 & 32 & $-2,4$ \\
\hline 102 & 32 & 0,075 & 1,154 & 0,090 & 0,930 & 0,825 & 0,806 & 0,261 & 0,704 & 1,192 & 0,607 & 0,575 & 0,395 & 0,227 & 0,214 & 166 & 32 & $-2,0$ \\
\hline 103 & 32 & 0,020 & 0,410 & 0,020 & 0,194 & 1,000 & 0,474 & 0,526 & 1,000 & 0,816 & 0,891 & 0,447 & 0,169 & 0,076 & 0,152 & 90 & 32 & $-0,8$ \\
\hline 104 & 32 & 0,106 & 1,924 & 0,141 & 1,730 & 0,755 & 0,899 & 0,265 & 0,360 & 1,666 & 0,172 & 0,180 & 0,888 & 0,160 & 0,152 & 139 & 32 & $-3,2$ \\
\hline 105 & 32 & 0,029 & 0,664 & 0,033 & 0,520 & 0,870 & 0,784 & 0,253 & 0,819 & 1,105 & 0,417 & 0,441 & 0,296 & 0,130 & 0,152 & 128 & 32 & $-1,6$ \\
\hline 106 & 32 & 0,020 & 0,469 & 0,023 & 0,397 & 0,875 & 0,847 & 0,198 & 1,000 & 0,934 & 0,686 & 0,555 & 0,193 & 0,107 & 0,107 & 149 & 32 & $-0,9$ \\
\hline 107 & 32 & 0,020 & 0,469 & 0,022 & 0,347 & 0,933 & 0,739 & 0,270 & 1,000 & 0,934 & 0,713 & 0,424 & 0,189 & 0,080 & 0,107 & 147 & 32 & $-0,9$ \\
\hline 108 & 32 & 0,014 & 0,368 & 0,016 & 0,245 & 0,909 & 0,667 & 0,346 & 1,000 & 0,866 & 0,707 & 0,459 & 0,161 & 0,074 & 0,076 & 135 & 32 & $-0,7$ \\
\hline 109 & 32 & 0,027 & 0,571 & 0,032 & 0,469 & 0,864 & 0,822 & 0,224 & 1,000 & 0,975 & 0,712 & 0,606 & 0,221 & 0,134 & 0,152 & 119 & 32 & $-1,1$ \\
\hline 110 & 32 & 0,020 & 0,440 & 0,022 & 0,296 & 0,933 & 0,673 & 0,334 & 1,000 & 0,875 & 0,713 & 0,555 & 0,189 & 0,105 & 0,152 & 150 & 32 & $-0,9$ \\
\hline 111 & 32 & 0,019 & 0,389 & 0,019 & 0,245 & 1,000 & 0,631 & 0,369 & 1,000 & 0,803 & 0,828 & 0,447 & 0,169 & 0,076 & 0,152 & 98 & 32 & $-0,8$ \\
\hline 112 & 32 & 0,042 & 0,765 & 0,050 & 0,664 & 0,829 & 0,867 & 0,217 & 0,893 & 1,058 & 0,697 & 0,674 & 0,276 & 0,186 & 0,169 & 101 & 32 & $-1,5$ \\
\hline 113 & 32 & 0,014 & 0,317 & 0,014 & 0,173 & 1,000 & 0,547 & 0,453 & 1,000 & 0,746 & 0,979 & 0,555 & 0,137 & 0,076 & 0,107 & 108 & 32 & $-0,5$ \\
\hline 114 & 32 & 0,020 & 0,643 & 0,027 & 0,469 & 0,737 & 0,730 & 0,377 & 0,611 & 1,279 & 0,274 & 0,256 & 0,305 & 0,078 & 0,076 & 58 & 32 & $-1,6$ \\
\hline 115 & 32 & 0,014 & 0,368 & 0,016 & 0,194 & 0,909 & 0,529 & 0,480 & 1,000 & 0,866 & 0,749 & 0,461 & 0,156 & 0,072 & 0,076 & 90 & 32 & $-0,6$ \\
\hline 116 & 32 & 0,023 & 0,846 & 0,033 & 0,672 & 0,696 & 0,795 & 0,367 & 0,403 & 1,574 & 0,180 & 0,151 & 0,403 & 0,061 & 0,076 & 141 & 32 & $-2,0$ \\
\hline 117 & 32 & 0,026 & 0,490 & 0,027 & 0,389 & 0,947 & 0,793 & 0,214 & 1,000 & 0,861 & 0,790 & 0,557 & 0,204 & 0,114 & 0,152 & 153 & 32 & $-1,0$ \\
\hline 118 & 32 & 0,037 & 0,816 & 0,043 & 0,664 & 0,867 & 0,813 & 0,229 & 0,704 & 1,192 & 0,414 & 0,350 & 0,339 & 0,119 & 0,152 & 115 & 32 & $-1,8$ \\
\hline 119 & 32 & 0,020 & 0,592 & 0,029 & 0,418 & 0,700 & 0,707 & 0,419 & 0,721 & 1,178 & 0,482 & 0,493 & 0,230 & 0,114 & 0,076 & 83 & 32 & $-1,2$ \\
\hline 120 & 32 & 0,023 & 0,469 & 0,024 & 0,418 & 0,941 & 0,892 & 0,123 & 1,000 & 0,874 & 0,815 & 0,566 & 0,189 & 0,107 & 0,152 & 135 & 32 & $-0,9$ \\
\hline 121 & 32 & 0,019 & 0,440 & 0,020 & 0,275 & 0,929 & 0,625 & 0,381 & 1,000 & 0,908 & 0,662 & 0,424 & 0,189 & 0,080 & 0,107 & 128 & 32 & $-0,9$ \\
\hline 122 & 32 & 0,062 & 1,082 & 0,077 & 0,786 & 0,796 & 0,727 & 0,341 & 0,662 & 1,229 & 0,644 & 0,728 & 0,349 & 0,254 & 0,169 & 167 & 32 & $-1,8$ \\
\hline 123 & 32 & 0,033 & 0,562 & 0,034 & 0,541 & 0,958 & 0,963 & 0,056 & 1,000 & 0,873 & 0,861 & 0,686 & 0,221 & 0,152 & 0,169 & 142 & 32 & $-1,1$ \\
\hline 124 & 32 & 0,055 & 1,184 & 0,082 & 0,930 & 0,667 & 0,786 & 0,396 & 0,489 & 1,430 & 0,465 & 0,560 & 0,386 & 0,216 & 0,152 & 88 & 32 & $-1,9$ \\
\hline 125 & 32 & 0,019 & 0,368 & 0,019 & 0,245 & 1,000 & 0,667 & 0,333 & 1,000 & 0,760 & 1,273 & 0,784 & 0,137 & 0,107 & 0,152 & 90 & 32 & $-0,5$ \\
\hline 126 & 32 & 0,044 & 1,040 & 0,055 & 0,960 & 0,816 & 0,923 & 0,200 & 0,517 & 1,391 & 0,251 & 0,250 & 0,475 & 0,119 & 0,107 & 115 & 32 & $-2,2$ \\
\hline 127 & 32 & 0,016 & 0,461 & 0,017 & 0,296 & 0,917 & 0,642 & 0,367 & 0,935 & 1,034 & 0,483 & 0,371 & 0,204 & 0,076 & 0,107 & 135 & 32 & $-1,0$ \\
\hline 128 & 32 & 0,026 & 0,511 & 0,027 & 0,368 & 0,947 & 0,719 & 0,286 & 1,000 & 0,898 & 0,674 & 0,460 & 0,221 & 0,102 & 0,152 & 160 & 32 & $-1,1$ \\
\hline 129 & 32 & 0,020 & 0,440 & 0,022 & 0,296 & 0,933 & 0,673 & 0,334 & 1,000 & 0,875 & 0,713 & 0,500 & 0,189 & 0,095 & 0,107 & 125 & 32 & $-0,9$ \\
\hline 130 & 32 & 0,037 & 0,977 & 0,044 & 0,947 & 0,839 & 0,970 & 0,164 & 0,491 & 1,427 & 0,255 & 0,247 & 0,432 & 0,107 & 0,107 & 132 & 32 & $-2,1$ \\
\hline 131 & 32 & 0,019 & 0,389 & 0,019 & 0,347 & 1,000 & 0,892 & 0,108 & 1,000 & 0,803 & 0,974 & 0,485 & 0,156 & 0,076 & 0,152 & 172 & 32 & $-0,6$ \\
\hline 132 & 32 & 0,019 & 0,368 & 0,019 & 0,347 & 1,000 & 0,943 & 0,057 & 1,000 & 0,760 & 1,273 & 0,784 & 0,137 & 0,107 & 0,152 & 135 & 32 & $-0,5$ \\
\hline 133 & 32 & 0,046 & 0,858 & 0,055 & 0,706 & 0,842 & 0,823 & 0,238 & 0,784 & 1,130 & 0,479 & 0,434 & 0,349 & 0,152 & 0,169 & 98 & 32 & $-1,8$ \\
\hline
\end{tabular}




\begin{tabular}{|c|c|c|c|c|c|c|c|c|c|c|c|c|c|c|c|c|c|c|}
\hline 134 & 32 & 0,026 & 0,511 & 0,027 & 0,397 & 0,947 & 0,777 & 0,229 & 1,000 & 0,898 & 0,716 & 0,625 & 0,214 & 0,134 & 0,152 & 150 & 32 & $-1,1$ \\
\hline 135 & 32 & 0,020 & 0,469 & 0,022 & 0,347 & 0,933 & 0,739 & 0,270 & 1,000 & 0,934 & 0,686 & 0,527 & 0,193 & 0,102 & 0,107 & 158 & 32 & $-0,9$ \\
\hline 136 & 32 & 0,014 & 0,440 & 0,017 & 0,296 & 0,833 & 0,673 & 0,367 & 0,933 & 1,035 & 0,509 & 0,424 & 0,189 & 0,080 & 0,076 & 140 & 32 & $-0,9$ \\
\hline 137 & 32 & 0,046 & 0,960 & 0,056 & 0,825 & 0,821 & 0,859 & 0,228 & 0,627 & 1,263 & 0,300 & 0,263 & 0,442 & 0,116 & 0,152 & 122 & 32 & $-2,1$ \\
\hline 138 & 32 & 0,032 & 0,583 & 0,034 & 0,469 & 0,917 & 0,805 & 0,212 & 1,000 & 0,926 & 0,875 & 0,707 & 0,214 & 0,152 & 0,152 & 165 & 32 & $-1,1$ \\
\hline 139 & 32 & 0,014 & 0,368 & 0,014 & 0,296 & 1,000 & 0,805 & 0,195 & 1,000 & 0,866 & 0,637 & 0,400 & 0,169 & 0,068 & 0,076 & 159 & 32 & $-0,8$ \\
\hline 140 & 32 & 0,017 & 0,418 & 0,019 & 0,317 & 0,923 & 0,757 & 0,254 & $\begin{array}{l}1,000 \\
\text {. }\end{array}$ & 0,900 & 0,611 & 0,389 & 0,189 & 0,074 & 0,076 & 140 & 32 & $\begin{array}{l}-0,9 \\
-\end{array}$ \\
\hline 141 & 32 & 0,019 & 0,418 & 0,020 & 0,338 & 0,929 & 0,808 & 0,205 & 1,000 & 0,864 & 0,828 & 0,566 & 0,169 & 0,096 & 0,152 & 0 & 32 & $-0,8$ \\
\hline 142 & 32 & 0,019 & 0,482 & 0,019 & 0,266 & 1,000 & 0,553 & 0,447 & 1,000 & 0,995 & 0,447 & 0,164 & 0,230 & 0,038 & 0,076 & 90 & 32 & $-1,2$ \\
\hline 143 & 32 & 0,017 & 0,418 & 0,019 & 0,338 & 0,923 & 0,808 & 0,207 & 1,000 & 0,900 & 0,588 & 0,392 & 0,193 & 0,076 & 0,107 & 167 & 32 & $-0,9$ \\
\hline 144 & 32 & 0,024 & 0,600 & 0,024 & 0,520 & 1,000 & 0,866 & 0,134 & 0,850 & 1,085 & 0,355 & 0,181 & 0,296 & 0,054 & 0,107 & 135 & 32 & $-1,6$ \\
\hline 145 & 32 & 0,017 & 0,693 & 0,024 & 0,592 & 0,706 & 0,854 & 0,329 & 0,450 & 1,491 & 0,191 & 0,126 & 0,339 & 0,043 & 0,076 & 144 & 32 & $-1,8$ \\
\hline 1 & 33 & 0,056 & 0,998 & 0,065 & 0,947 & 0,867 & 0,949 & 0,143 & 0,706 & 1,190 & 0,342 & 0,294 & 0,456 & 0,134 & 0,169 & 45 & 33 & $-2,2$ \\
\hline 2 & 33 & 0,022 & 0,490 & 0,023 & 0,347 & 0,938 & 0,707 & 0,300 & 1,000 & 0,943 & 0,562 & 0,434 & 0,221 & 0,096 & 0,152 & 33 & 33 & $-1,1$ \\
\hline 3 & 33 & 0,020 & 0,440 & 0,020 & 0,347 & 1,000 & 0,789 & 0,211 & $\begin{array}{l}1,000 \\
\text {. }\end{array}$ & 0,875 & 0,686 & 0,392 & 0,193 & 0,076 & 0,152 & 0 & 33 & $\begin{array}{l}-0,9 \\
\end{array}$ \\
\hline 1 & 34 & 0,020 & 0,440 & 0,020 & 0,266 & 1,000 & 0,606 & 0,394 & 1,000 & 0,875 & 0,686 & 0,392 & 0,193 & 0,076 & 0,152 & 90 & 34 & $-0,9$ \\
\hline 2 & 34 & 0,123 & 1,268 & 0,129 & 1,217 & 0,956 & 0,960 & 0,060 & 0,965 & 1,018 & 0,615 & 0,525 & 0,505 & 0,265 & 0,303 & 1 & 34 & $-2,3$ \\
\hline 3 & 34 & 0,055 & 0,816 & 0,060 & 0,765 & 0,905 & 0,938 & 0,114 & 1,000 & 0,986 & 0,663 & 0,617 & 0,324 & 0,200 & 0,214 & 114 & 34 & $-1,7$ \\
\hline 4 & 34 & 0,017 & 0,397 & 0,017 & 0,296 & 1,000 & 0,745 & 0,255 & 1,000 & 0,854 & 0,764 & 0,474 & 0,169 & 0,080 & 0,107 & 72 & 34 & $-0,8$ \\
\hline 1 & 35 & 0,020 & 0,418 & 0,020 & 0,368 & 1,000 & 0,879 & 0,121 & 1,000 & 0,833 & 0,891 & 0,600 & 0,169 & 0,102 & 0,152 & 66 & 35 & $-0,8$ \\
\hline 2 & 35 & 0,027 & 0,562 & 0,030 & 0,389 & 0,905 & 0,692 & 0,323 & 1,000 & 0,960 & 0,712 & 0,514 & 0,221 & 0,114 & 0,152 & 10 & 35 & $-1,1$ \\
\hline 3 & 35 & 0,077 & 1,133 & 0,088 & 0,960 & 0,885 & 0,847 & 0,191 & 0,759 & 1,148 & 0,419 & 0,422 & 0,485 & 0,205 & 0,214 & 47 & 35 & $-2,3$ \\
\hline 4 & 35 & 0,445 & 2,511 & 0,461 & 2,409 & 0,966 & 0,960 & 0,053 & 0,887 & 1,062 & 0,544 & 0,578 & $\begin{array}{l}1,020 \\
\text { S }\end{array}$ & 0,589 & 0,592 & 26 & 35 & $-3,4$ \\
\hline 5 & 35 & 0,102 & 1,611 & 0,138 & 1,450 & 0,740 & 0,900 & 0,279 & 0,493 & 1,424 & 0,288 & 0,321 & 0,671 & 0,216 & 0,227 & 17 & 35 & $-2,7$ \\
\hline 6 & 35 & 0,024 & 0 & 0,024 & $\begin{array}{l}0,450 \\
0,389\end{array}$ & 1,000 & 0,793 & 0,207 & $\begin{array}{l}1,000 \\
1,07\end{array}$ & $\begin{array}{l}1,+247 \\
0,886\end{array}$ & $\begin{array}{l}0,200 \\
0,637\end{array}$ & $\begin{array}{l}0,321 \\
0,460\end{array}$ & 0,221 & 0,102 & 0,152 & 64 & 35 & $-1,1$ \\
\hline 7 & 35 & 0,409 & 2,672 & 0,452 & 2,439 & 0,905 & 0,913 & 0,129 & 0,720 & 1,179 & 0,468 & 0,530 & 1,055 & 0,559 & 0,536 & 22 & 35 & $-3,4$ \\
\hline 8 & 35 & 0,022 & 0,418 & 0,022 & 0,368 & 1,000 & 0,879 & 0,121 & 1,000 & 0,805 & 0,955 & 0,632 & 0,169 & 0,107 & 0,152 & 20 & 35 & $-0,8$ \\
\hline 9 & 35 & 0,017 & 0,461 & 0,017 & 0,389 & 1,000 & 0,844 & 0,156 & 1,000 & 0,990 & 0,413 & 0,164 & 0,230 & 0,038 & 0,076 & 0 & 35 & $-1,2$ \\
\hline 10 & 35 & 0,020 & 0,490 & 0,024 & 0,376 & 0,824 & 0,768 & 0,292 & 1,000 & 0,976 & 0,686 & 0,502 & 0,193 & 0,097 & 0,107 & 14 & 35 & $\begin{array}{l}-0,9 \\
\end{array}$ \\
\hline 11 & 35 & 0,030 & 0,693 & 0,037 & 0,664 & 0,808 & 0,957 & 0,197 & 0,788 & 1,127 & 0,438 & 0,453 & 0,296 & 0,134 & 0,152 & 40 & 35 & $-1,6$ \\
\hline 12 & 35 & 0,017 & 0,499 & 0,019 & 0,427 & 0,923 & 0,856 & 0,163 & 0,869 & 1,073 & 0,373 & 0,221 & 0,243 & 0,054 & 0,107 & 40 & 35 & $-1,3$ \\
\hline 13 & 35 & 0,308 & 3,175 & 0,392 & 2,434 & 0,788 & 0,767 & 0,315 & 0,385 & 1,612 & 0,363 & 0,379 & 1,040 & 0,394 & 0,169 & 94 & 35 & $-3,4$ \\
\hline 14 & 35 & 0,029 & 0,562 & 0,033 & 0,440 & 0,870 & 0,782 & 0,254 & 1,000 & 0,936 & 0,749 & 0,606 & 0,221 & 0,134 & 0,152 & 52 & 35 & $-1,1$ \\
\hline 15 & 35 & 0,033 & 0,541 & 0,033 & 0,482 & 1,000 & 0,890 & 0,110 & 1,000 & 0,840 & 0,861 & 0,606 & 0,221 & 0,134 & 0,169 & 32 & 35 & $-1,1$ \\
\hline 16 & 35 & 0,014 & 0,326 & 0,014 & 0,245 & 1,000 & 0,753 & 0,247 & 1,000 & 0,767 & 1,273 & 0,671 & 0,120 & 0,080 & 0,107 & 72 & 35 & $-0,3$ \\
\hline 17 & 35 & 0,043 & 0,634 & 0,043 & 0,511 & 1,000 & 0,807 & 0,193 & 1,000 & 0,862 & 0,932 & 0,769 & 0,243 & 0,186 & 0,214 & 49 & 35 & $-1,3$ \\
\hline 18 & 35 & 0,026 & 0,520 & 0,029 & 0,469 & 0,900 & 0,902 & 0,140 & 1,000 & 0,913 & 0,790 & 0,646 & 0,204 & 0,132 & 0,152 & 23 & 35 & $-1,0$ \\
\hline 19 & 35 & 0,057 & 0,858 & 0,069 & 0,714 & 0,833 & 0,833 & 0,236 & 0,980 & 1,010 & 0,784 & 0,722 & 0,305 & 0,220 & 0,214 & 134 & 35 & $-1,6$ \\
\hline 20 & 35 & 0,032 & 0,622 & 0,034 & 0,490 & 0,917 & 0,789 & 0,227 & 1,000 & 0,987 & 0,966 & 0,788 & 0,204 & 0,161 & 0,152 & 15 & 35 & $-1,0$ \\
\hline 21 & 35 & 0,056 & 0,778 & 0,057 & 0,685 & 0,975 & 0,881 & 0,122 & 1,000 & 0,927 & 0,730 & 0,485 & 0,312 & 0,152 & 0,227 & 4 & 35 & $-1,6$ \\
\hline & 36 & & & & & & & & nothing to measure & & & & & & & & 36 & - \\
\hline 1 & 37 & 0,042 & 1,163 & 0,059 & 0,744 & 0,707 & 0,640 & 0,464 & 0,387 & 1,608 & 0,410 & 0,425 & 0,359 & 0,153 & 0,152 & 64 & 37 & $-1,8$ \\
\hline 2 & 37 & 0,022 & 0,440 & 0,022 & 0,359 & 1,000 & 0,817 & 0,183 & 1,000 & 0,845 & 0,735 & 0,392 & 0,193 & 0,076 & 0,152 & 85 & 37 & $-0,9$ \\
\hline 3 & 37 & 0,019 & 0,440 & 0,020 & 0,317 & 0,929 & 0,721 & 0,288 & 1,000 & 0,908 & 0,828 & 0,600 & 0,169 & 0,102 & 0,152 & 52 & 37 & $-0,8$ \\
\hline 4 & 37 & 0,115 & 2,579 & 0,184 & 1,530 & 0,625 & 0,593 & 0,553 & 0,217 & 2,147 & 0,313 & 0,454 & 0,683 & 0,310 & 0,152 & 72 & 37 & $-2,8$ \\
\hline 5 & 37 & 0,079 & 0,981 & 0,080 & 0,879 & 0,982 & 0,896 & 0,105 & 1,000 & 0,985 & 0,778 & 0,738 & 0,359 & 0,265 & 0,273 & 76 & 37 & $-1,8$ \\
\hline 6 & 37 & 0,014 & 0,448 & 0,017 & 0,347 & 0,833 & 0,773 & 0,281 & 0,898 & 1,055 & 0,490 & 0,264 & 0,193 & 0,051 & 0,076 & 68 & 37 & $\begin{array}{l}-0,9 \\
\end{array}$ \\
\hline 7 & 37 & 0,020 & 0,520 & 0,023 & 0,418 & 0,875 & 0,805 & 0,232 & 0,934 & 1,035 & 0,524 & 0,384 & 0,221 & 0,085 & 0,076 & 148 & 37 & $-1,1$ \\
\hline 8 & 37 & 0,105 & 1,366 & 0,126 & 1,133 & 0,830 & 0,830 & 0,241 & 0,706 & 1,190 & 0,637 & 0,644 & 0,458 & 0,295 & 0,214 & 46 & 37 & $-2,2$ \\
\hline 9 & 37 & 0,185 & 3,602 & 0,310 & 2,227 & 0,597 & 0,618 & 0,555 & 0,179 & 2,362 & 0,261 & 0,424 & 0,950 & 0,402 & 0,152 & 83 & 37 & $-3,2$ \\
\hline 10 & 37 & 0,016 & 0,376 & 0,017 & 0,296 & 0,917 & 0,786 & 0,229 & 1,000 & 0,845 & 0,824 & 0,514 & 0,156 & 0,080 & 0,107 & 108 & 37 & $-0,6$ \\
\hline 11 & 37 & 0,024 & 0,440 & 0,024 & 0,317 & 1,000 & 0,721 & 0,279 & 1,000 & 0,794 & $\begin{array}{l}1,087 \\
1,082\end{array}$ & 0,671 & 0,169 & 0,114 & 0,152 & 90 & 37 & $-0,8$ \\
\hline 12 & 37 & 0,020 & 0,562 & 0,026 & 0,389 & 0,778 & 0,692 & 0,380 & 0,799 & 1,119 & 0,615 & 0,496 & 0,204 & 0,101 & 0,107 & 69 & 37 & $-1,0$ \\
\hline 13 & 37 & 0,016 & 0,368 & 0,016 & 0,368 & 1,000 & 1,000 & 0,000 & 1,000 & 0,826 & 0,824 & 0,485 & 0,156 & 0,076 & 0,107 & 0 & 37 & $-0,6$ \\
\hline 14 & 37 & 0,037 & 0,714 & 0,044 & 0,592 & 0,839 & 0,828 & 0,235 & 0,919 & 1,043 & 0,625 & 0,587 & 0,276 & 0,162 & 0,169 & 16 & 37 & $-1,5$ \\
\hline 15 & 37 & 0,019 & 0,562 & 0,024 & 0,317 & 0,765 & 0,564 & 0,496 & 0,742 & 1,161 & 0,828 & 0,671 & 0,169 & 0,114 & 0,107 & 162 & 37 & $-0,8$ \\
\hline 16 & 37 & 0,022 & 0,664 & 0,030 & 0,418 & 0,714 & 0,631 & 0,467 & 0,614 & 1,276 & 0,659 & 0,646 & 0,204 & 0,132 & 0,107 & 90 & 37 & $-1,0$ \\
\hline 17 & 37 & 0,024 & 0,499 & 0,026 & 0,469 & 0,944 & 0,940 & 0,081 & 1,000 & 0,901 & 0,833 & 0,693 & 0,193 & 0,134 & 0,169 & 30 & 37 & $\begin{array}{l}-0,9 \\
\end{array}$ \\
\hline
\end{tabular}




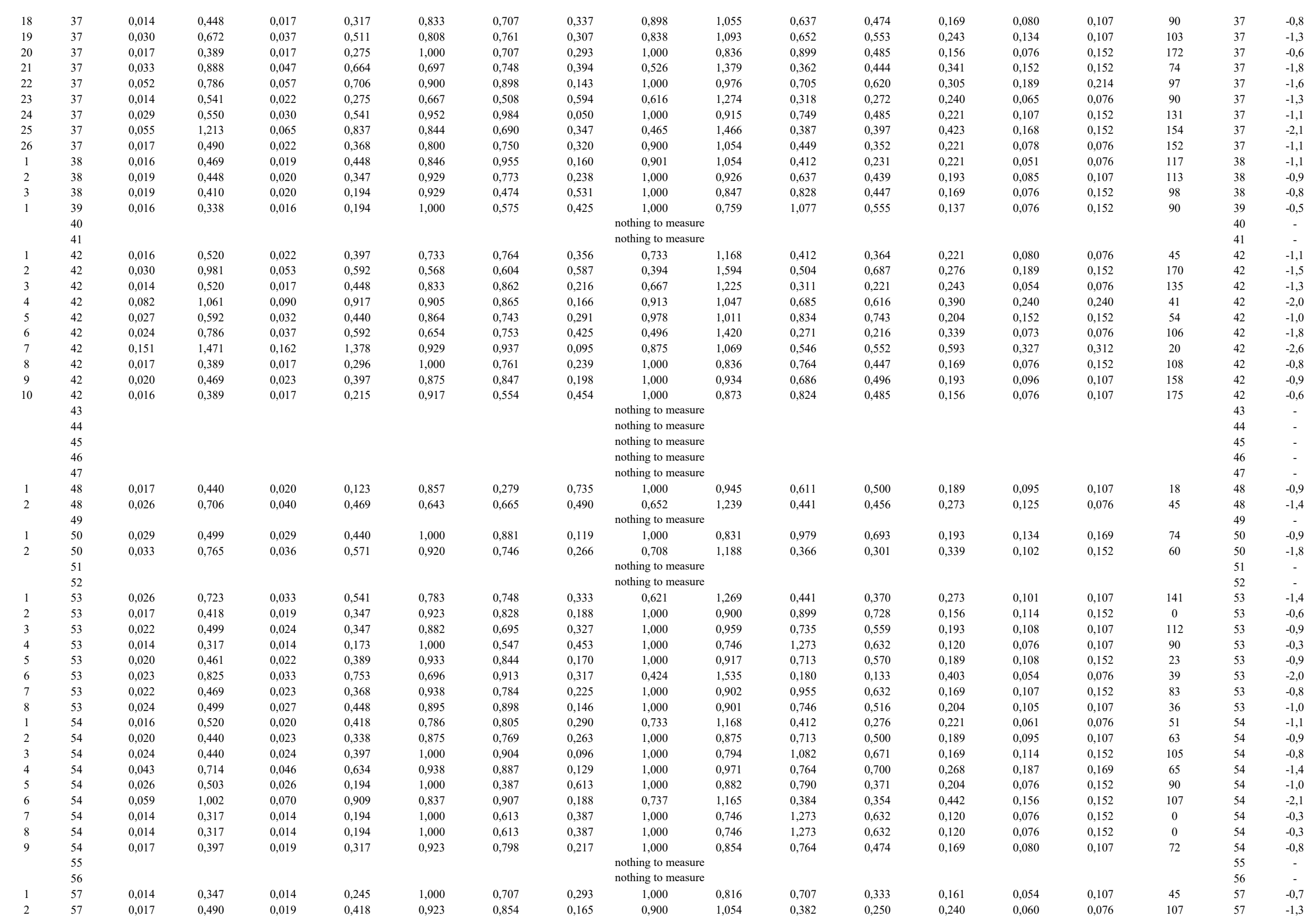




\begin{tabular}{|c|c|c|c|c|c|c|c|c|c|c|c|c|c|c|c|c|}
\hline 0,022 & 0,490 & 0,023 & 0,338 & 0,938 & 0,689 & 0,317 & 1,000 & 0,943 & 0,516 & 0,329 & 0,230 & 0,076 & 0,152 & 86 & 57 & $-1,2$ \\
\hline 0,042 & 0,727 & 0,044 & 0,490 & 0,935 & 0,675 & 0,332 & 0,990 & 1,005 & 0,543 & 0,364 & 0,312 & 0,114 & 0,152 & 94 & 57 & $-1,6$ \\
\hline 0,027 & 0,723 & 0,036 & 0,562 & 0,760 & 0,777 & 0,327 & 0,655 & 1,235 & 0,372 & 0,317 & 0,305 & 0,097 & 0,107 & 104 & 57 & $-1,6$ \\
\hline 0,016 & 0,376 & 0,016 & 0,317 & 1,000 & 0,842 & 0,158 & 1,000 & 0,845 & 0,824 & 0,514 & 0,156 & 0,080 & 0,107 & 108 & 57 & \\
\hline 0,026 & 0,714 & 0,033 & 0,440 & 0,783 & 0,615 & 0,442 & 0,636 & 1,254 & 0,509 & 0,527 & 0,254 & 0,134 & 0,107 & 31 & 57 & $-1,3$ \\
\hline 0,036 & 1,163 & 0,056 & 0,693 & 0,641 & 0,596 & 0,540 & 0,334 & 1,732 & 0,490 & 0,614 & 0,305 & 0,187 & 0,107 & 133 & 57 & $-1,6$ \\
\hline 0,179 & 1,712 & 0,197 & 1,581 & 0,912 & 0,923 & 0,116 & 0,769 & 1,141 & 0,490 & 0,431 & 0,683 & 0,295 & 0,312 & 60 & 57 & \\
\hline 0,024 & 0,685 & 0,033 & 0,520 & 0,739 & 0,759 & 0,355 & 0,654 & 1,237 & 0,373 & 0,342 & 0,288 & 0,099 & 0,107 & 109 & 57 & $-1,5$ \\
\hline 0,017 & 0,418 & 0,019 & 0,368 & 0,923 & 0,879 & 0,144 & 1,000 & 0,900 & 0,611 & 0,389 & 0,189 & 0,074 & 0,076 & 130 & 57 & $-0,9$ \\
\hline 0,083 & 1,061 & 0,088 & 0,989 & 0,951 & 0,932 & 0,084 & 0,929 & 1,038 & 0,509 & 0,461 & 0,456 & 0,210 & 0,214 & 45 & 57 & 2,2 \\
\hline 0,022 & 0,562 & 0,024 & 0,389 & 0,882 & 0,692 & 0,330 & 0,856 & 1,081 & 0,360 & 0,261 & 0,276 & 0,072 & 0,076 & 76 & 57 & -1 \\
\hline 0,030 & 0,685 & 0,040 & 0,562 & 0,750 & 0,821 & 0,307 & 0,808 & 1,113 & 0,594 & 0,639 & 0,254 & 0,162 & 0,152 & 122 & 57 & $-1,3$ \\
\hline 0,039 & 0,867 & 0,049 & 0,664 & 0,794 & 0,766 & 0,312 & 0,648 & 1,242 & 0,661 & 0,588 & 0,273 & 0,161 & 0,152 & 38 & 57 & \\
\hline 0,122 & 1,399 & 0,139 & 1,142 & 0,876 & 0,816 & 0,222 & 0,783 & 1,130 & 0,790 & 0,785 & 0,443 & 0,348 & 0,312 & 2 & 57 & 1 \\
\hline 0,040 & 0,664 & 0,043 & 0,541 & 0,933 & 0,815 & 0,196 & 1,000 & 0,934 & 0,686 & 0,588 & 0,273 & 0,161 & 0,169 & 127 & 57 & $-1,4$ \\
\hline 0,166 & 1,928 & 0,228 & 1,615 & 0,730 & 0,838 & 0,315 & 0,563 & 1,333 & 0,504 & 0,613 & 0,648 & 0,397 & 0,240 & 13 & 57 & \\
\hline 0,066 & 1,112 & 0,080 & 0,858 & 0,821 & 0,772 & 0,290 & 0,671 & 1,221 & 0,604 & 0,609 & 0,373 & 0,227 & 0,240 & 7 & 57 & -1 \\
\hline 0,075 & 1,124 & 0,088 & 0,981 & 0,852 & 0,872 & 0,195 & 0,742 & 1,161 & 0,414 & 0,400 & 0,479 & 0,192 & 0,227 & 16 & 57 & $-2,3$ \\
\hline 0,017 & 0,469 & 0,020 & 0,347 & 0,857 & 0,739 & 0,298 & 0,983 & 1,009 & 0,449 & 0,286 & 0,221 & 0,063 & 0,076 & 31 & 57 & $-1,1$ \\
\hline 0,194 & 2,786 & 0,306 & 2,182 & 0,634 & 0,783 & 0,426 & 0,314 & 1,786 & 0,231 & 0,335 & 1,034 & 0,346 & 0,303 & 29 & 57 & $-3,4$ \\
\hline \multirow[t]{2}{*}{0,029} & 0,592 & 0,030 & 0,541 & 0,952 & 0,914 & 0,098 & 1,000 & 0,986 & 0,509 & 0,424 & 0,268 & 0,114 & 0,152 & 169 & 57 & $-1,4$ \\
\hline & & & & & & & nothing to measure & & & & & & & & 58 & \\
\hline \multirow[t]{6}{*}{0,016} & 0,490 & 0,019 & 0,347 & 0,846 & 0,707 & 0,331 & 0,825 & 1,101 & 0,438 & 0,354 & 0,214 & 0,076 & 0,076 & 108 & 59 & $-1,1$ \\
\hline & & & & & & & nothing to measure & & & & & & & & 60 & \\
\hline & & & & & & & nothing to measure & & & & & & & & 61 & \\
\hline & & & & & & & nothing to measure & & & & & & & & 62 & \\
\hline & & & & & & & nothing to measure & & & & & & & & 63 & \\
\hline & & & & & & & nothing to measure & & & & & & & & 64 & \\
\hline 0,020 & 0,592 & 0,024 & 0,347 & 0,824 & 0,586 & 0,450 & 0,721 & 1,178 & 0,713 & 0,696 & 0,189 & 0,132 & 0,107 & 139 & 65 & $-0,9$ \\
\hline \multirow{4}{*}{0,020} & 0,490 & 0,023 & 0,397 & 0,875 & 0,811 & 0,227 & 1,000 & 0,976 & 0,524 & 0,384 & 0,221 & 0,085 & 0,107 & 33 & 65 & $-1,1$ \\
\hline & & & & & & & nothing to measure & & & & & & & & 66 & \\
\hline & & & & & & & nothing to measure & & & & & & & & 67 & \\
\hline & & & & & & & nothing to measure & & & & & & & & 68 & \\
\hline 0,067 & 0,930 & 0,077 & 0,837 & 0,870 & 0,900 & 0,164 & 0,980 & 1,010 & 0,880 & 0,825 & 0,312 & 0,258 & 0,227 & 144 & 69 & $-1,6$ \\
\hline 0,049 & 0,685 & 0,049 & 0,643 & 1,000 & 0,938 & 0,062 & 1,000 & 0,874 & 0,866 & 0,700 & 0,268 & 0,187 & 0,214 & 117 & 69 & -1 \\
\hline 0,060 & 1,704 & 0,100 & 1,031 & 0,600 & 0,605 & 0,562 & 0,261 & 1,958 & 0,326 & 0,521 & 0,485 & 0,253 & 0,107 & 125 & 69 & $-2,3$ \\
\hline 0,022 & 0,490 & 0,024 & 0,368 & 0,882 & 0,750 & 0,276 & 1,000 & 0,943 & 0,659 & 0,470 & 0,204 & 0,096 & 0,107 & 153 & 69 & $-1,0$ \\
\hline 0,017 & 0,389 & 0,017 & 0,245 & 1,000 & 0,631 & 0,369 & 1,000 & 0,836 & 0,899 & 0,485 & 0,156 & 0,076 & 0,107 & 0 & 69 & 0 \\
\hline 0,016 & 0,347 & 0,016 & 0,296 & 1,000 & 0,854 & 0,146 & 1,000 & 0,778 & 1,401 & 0,849 & 0,120 & 0,102 & 0,107 & 45 & 69 & $\begin{array}{l}-0,3 \\
\end{array}$ \\
\hline 0,016 & 0,368 & 0,016 & 0,347 & 1,000 & 0,943 & 0,057 & 1,000 & 0,826 & 0,824 & 0,485 & 0,156 & 0,076 & 0,107 & 162 & 69 & $-0,6$ \\
\hline 0,037 & 0,879 & 0,052 & 0,643 & 0,722 & 0,731 & 0,387 & 0,607 & 1,284 & 0,487 & 0,530 & 0,312 & 0,165 & 0,152 & 70 & 69 & 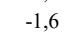 \\
\hline 0,032 & 0,520 & 0,032 & 0,469 & 1,000 & 0,902 & 0,098 & 1,000 & 0,826 & 0,966 & 0,657 & 0,204 & 0,134 & 0,169 & 148 & 69 & 1 \\
\hline 0,026 & 0,490 & 0,027 & 0,389 & 0,947 & 0,793 & 0,214 & 1,000 & 0,861 & 0,716 & 0,500 & 0,214 & 0,107 & 0,152 & 52 & 69 & -1 \\
\hline 0,037 & 0,592 & 0,039 & 0,541 & 0,963 & 0,914 & 0,093 & 1,000 & 0,864 & 0,895 & 0,658 & 0,230 & 0,152 & 0,214 & 168 & 69 & \\
\hline 0,020 & 0,389 & 0,020 & 0,296 & 1,000 & 0,761 & 0,239 & 1,000 & 0,774 & 1,371 & 0,832 & 0,137 & 0,114 & 0,152 & 135 & 69 & $\begin{array}{l}-0,5 \\
\end{array}$ \\
\hline 0,102 & 1,052 & 0,106 & 0,960 & 0,959 & 0,912 & 0,097 & 1,000 & 0,930 & 0,904 & 0,800 & 0,379 & 0,303 & 0,339 & 168 & 69 & 1 \\
\hline 0,023 & 0,440 & 0,023 & 0,317 & 1,000 & 0,721 & 0,279 & 1,000 & 0,818 & 1,019 & 0,671 & 0,169 & 0,114 & 0,152 & 70 & 69 & $-0,8$ \\
\hline 0,034 & 0,583 & 0,036 & 0,511 & 0,960 & 0,877 & 0,129 & 1,000 & 0,887 & 0,899 & 0,686 & 0,221 & 0,152 & 0,152 & 16 & 69 & -1 \\
\hline 0,027 & 0,511 & 0,029 & 0,389 & 0,950 & 0,760 & 0,245 & 1,000 & 0,874 & 0,968 & 0,759 & 0,189 & 0,144 & 0,152 & 143 & 69 & 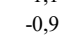 \\
\hline 0,156 & 1,522 & 0,172 & 1,369 & 0,908 & 0,900 & 0,136 & 0,849 & 1,085 & 0,655 & 0,614 & 0,552 & 0,338 & 0,379 & 167 & 69 & -2 \\
\hline 0,059 & 0,757 & 0,062 & 0,664 & 0,953 & 0,877 & 0,131 & 1,000 & 0,880 & 1,004 & 0,832 & 0,273 & 0,227 & 0,273 & 151 & 69 & -1 \\
\hline 0,037 & 0,664 & 0,042 & 0,520 & 0,897 & 0,784 & 0,240 & 1,000 & 0,969 & 0,637 & 0,559 & 0,273 & 0,153 & 0,169 & 138 & 69 & \\
\hline 0,055 & 0,938 & 0,069 & 0,735 & 0,792 & 0,784 & 0,300 & 0,778 & 1,134 & 0,663 & 0,628 & 0,324 & 0,203 & 0,169 & 67 & 69 & -1 \\
\hline 0,039 & 0,685 & 0,042 & 0,643 & 0,931 & 0,938 & 0,092 & 1,000 & 0,981 & 0,593 & 0,489 & 0,288 & 0,141 & 0,152 & 163 & 69 & 5 \\
\hline 0,044 & 0,634 & 0,044 & 0,511 & 1,000 & 0,807 & 0,193 & 1,000 & 0,848 & 1,161 & 0,857 & 0,221 & 0,189 & 0,214 & 45 & 69 & \\
\hline 0,022 & 0,714 & 0,030 & 0,469 & 0,714 & 0,657 & 0,447 & 0,530 & 1,374 & 0,659 & 0,657 & 0,204 & 0,134 & 0,076 & 13 & 69 & $-1,0$ \\
\hline 0,014 & 0,326 & 0,014 & 0,245 & 1,000 & 0,753 & 0,247 & $\begin{array}{l}1,000 \\
\text { S }\end{array}$ & 0,767 & 1,273 & 0,671 & 0,120 & 0,080 & 0,107 & 108 & 69 & \\
\hline 0,050 & 0,786 & 0,057 & 0,634 & 0,875 & 0,806 & 0,231 & 1,000 & 0,990 & 0,686 & 0,596 & 0,305 & 0,182 & 0,214 & 19 & 69 & \\
\hline
\end{tabular}




\begin{tabular}{|c|c|c|c|c|c|c|c|c|c|c|c|c|c|c|c|c|c|c|}
\hline 26 & 69 & 0,017 & 0,359 & 0,017 & 0,194 & 1,000 & 0,541 & 0,459 & 1,000 & 0,772 & 1,175 & 0,555 & 0,137 & 0,076 & 0,152 & 0 & 69 & $-0,5$ \\
\hline 27 & 69 & 0,092 & 1,031 & 0,096 & 1,031 & 0,955 & 1,000 & 0,045 & 1,000 & 0,960 & 0,627 & 0,558 & 0,432 & 0,241 & 0,273 & 127 & 69 & $-2,1$ \\
\hline 28 & 69 & 0,089 & 1,061 & 0,096 & 0,989 & 0,925 & 0,932 & 0,101 & 0,993 & 1,004 & 0,607 & 0,510 & 0,432 & 0,220 & 0,240 & 162 & 69 & $-2,1$ \\
\hline 29 & 69 & 0,251 & 1,797 & 0,268 & 1,707 & 0,936 & 0,950 & 0,081 & 0,978 & 1,011 & 0,793 & 0,760 & 0,635 & 0,482 & 0,479 & 99 & 69 & $-2,7$ \\
\hline 30 & 69 & 0,230 & 1,919 & 0,260 & 1,644 & 0,884 & 0,857 & 0,184 & 0,783 & 1,130 & 0,643 & 0,685 & 0,674 & 0,462 & 0,455 & 125 & 69 & $-2,8$ \\
\hline 31 & 69 & 0,026 & 0,706 & 0,032 & 0,541 & 0,818 & 0,767 & 0,296 & 0,652 & 1,239 & 0,353 & 0,276 & 0,305 & 0,084 & 0,107 & 156 & 69 & $-1,6$ \\
\hline 32 & 69 & 0,022 & 0,469 & 0,023 & 0,368 & 0,938 & 0,784 & 0,225 & 1,000 & 0,902 & 0,955 & 0,746 & 0,169 & 0,126 & 0,152 & 60 & 69 & $-0,8$ \\
\hline 33 & 69 & 0,029 & 0,930 & 0,046 & 0,664 & 0,625 & 0,714 & 0,472 & 0,417 & 1,548 & 0,300 & 0,424 & 0,349 & 0,148 & 0,076 & 66 & 69 & $-1,8$ \\
\hline 34 & 69 & 0,014 & 0,347 & 0,016 & 0,317 & 0,909 & 0,914 & 0,125 & 1,000 & 0,816 & 0,796 & 0,500 & 0,152 & 0,076 & 0,107 & 18 & 69 & $-0,6$ \\
\hline 35 & 69 & 0,022 & 0,418 & 0,023 & 0,347 & 0,938 & 0,828 & 0,183 & 1,000 & 0,805 & 1,123 & 0,728 & 0,156 & 0,114 & 0,152 & 12 & 69 & $-0,6$ \\
\hline 36 & 69 & 0,014 & 0,368 & 0,016 & 0,266 & 0,909 & 0,724 & 0,291 & 1,000 & 0,866 & 0,707 & 0,500 & 0,161 & 0,080 & 0,107 & 50 & 69 & $-0,7$ \\
\hline 37 & 69 & 0,052 & 0,723 & 0,053 & 0,714 & 0,973 & 0,988 & 0,030 & 1,000 & 0,897 & 0,917 & 0,759 & 0,268 & 0,203 & 0,214 & 171 & 69 & $-1,4$ \\
\hline 38 & 69 & 0,073 & 1,133 & 0,092 & 0,909 & 0,797 & 0,802 & 0,284 & 0,716 & 1,181 & 0,730 & 0,797 & 0,357 & 0,285 & 0,169 & 67 & 69 & $-1,8$ \\
\hline 39 & 69 & 0,014 & 0,317 & 0,014 & 0,173 & 1,000 & 0,547 & 0,453 & 1,000 & 0,746 & 1,273 & 0,632 & 0,120 & 0,076 & 0,107 & 18 & 69 & $-0,3$ \\
\hline 40 & 69 & 0,019 & 0,397 & 0,020 & 0,296 & 0,929 & 0,745 & 0,265 & 1,000 & 0,821 & 0,974 & 0,686 & 0,156 & 0,107 & 0,152 & 125 & 69 & $-0,6$ \\
\hline 41 & 69 & 0,032 & 0,511 & 0,032 & 0,469 & 1,000 & 0,918 & 0,082 & 1,000 & 0,812 & 1,120 & 0,800 & 0,189 & 0,152 & 0,214 & 135 & 69 & $-0,9$ \\
\hline 42 & 69 & 0,168 & 1,775 & 0,195 & 1,483 & 0,860 & 0,835 & 0,216 & 0,669 & 1,222 & 0,548 & 0,526 & 0,625 & 0,328 & 0,303 & 89 & 69 & $-2,6$ \\
\hline 43 & 69 & 0,030 & 0,511 & 0,032 & 0,389 & 0,955 & 0,760 & 0,244 & 1,000 & 0,831 & 1,070 & 0,800 & 0,189 & 0,152 & 0,169 & 149 & 69 & $-0,9$ \\
\hline 44 & 69 & 0,056 & 0,765 & 0,060 & 0,735 & 0,929 & 0,961 & 0,081 & 1,000 & 0,912 & 0,856 & 0,705 & 0,288 & 0,203 & 0,227 & 118 & 69 & $-1,5$ \\
\hline 45 & 69 & 0,016 & 0,338 & 0,016 & 0,245 & 1,000 & 0,725 & 0,275 & 1,000 & 0,759 & 1,077 & 0,555 & 0,137 & 0,076 & 0,152 & 90 & 69 & $-0,5$ \\
\hline 46 & 69 & 0,026 & 0,490 & 0,027 & 0,368 & 0,947 & 0,750 & 0,255 & 1,000 & 0,861 & 0,881 & 0,588 & 0,193 & 0,114 & 0,152 & 75 & 69 & $-0,9$ \\
\hline 47 & 69 & 0,119 & 1,124 & 0,122 & 1,052 & 0,976 & 0,936 & 0,068 & 1,000 & 0,919 & 0,903 & 0,819 & 0,410 & 0,335 & 0,379 & 130 & 69 & $-2,0$ \\
\hline 48 & 69 & 0,019 & 0,571 & 0,023 & 0,499 & 0,812 & 0,874 & 0,226 & 0,720 & 1,179 & 0,318 & 0,196 & 0,273 & 0,053 & 0,076 & 36 & 69 & $-1,4$ \\
\hline 49 & 69 & 0,042 & 0,765 & 0,044 & 0,643 & 0,935 & 0,840 & 0,173 & 0,893 & 1,058 & 0,499 & 0,411 & 0,326 & 0,134 & 0,152 & 40 & 69 & $-1,7$ \\
\hline 50 & 69 & 0,024 & 0,478 & 0,026 & 0,418 & 0,944 & 0,876 & 0,136 & 1,000 & 0,863 & 0,866 & 0,707 & 0,189 & 0,134 & 0,169 & 90 & 69 & $-0,9$ \\
\hline 51 & 69 & 0,089 & 1,010 & 0,093 & 0,981 & 0,954 & 0,971 & 0,055 & 1,000 & 0,956 & 0,782 & 0,697 & 0,381 & 0,265 & 0,303 & 88 & 69 & $-1,9$ \\
\hline 52 & 69 & 0,024 & 0,440 & 0,024 & 0,359 & 1,000 & 0,817 & 0,183 & 1,000 & 0,794 & 1,082 & 0,671 & 0,169 & 0,114 & 0,152 & 90 & 69 & $-0,8$ \\
\hline 53 & 69 & 0,029 & 0,613 & 0,034 & 0,469 & 0,833 & 0,766 & 0,288 & 0,960 & 1,021 & 0,490 & 0,413 & 0,273 & 0,113 & 0,152 & 23 & 69 & $-1,4$ \\
\hline 1 & 70 & 0,032 & 0,562 & 0,032 & 0,469 & 1,000 & 0,835 & 0,165 & 1,000 & 0,893 & 0,683 & 0,552 & 0,243 & 0,134 & 0,169 & 143 & 70 & $-1,3$ \\
\hline 2 & 70 & 0,014 & 0,359 & 0,014 & 0,440 & 1,000 & 1,224 & 0,224 & 1,000 & 0,846 & 0,749 & 0,243 & 0,156 & 0,038 & 0,076 & 0 & 70 & $-0,6$ \\
\hline 3 & 70 & 0,026 & 0,520 & 0,027 & 0,520 & 0,947 & 1,000 & 0,053 & 1,000 & 0,913 & 0,790 & 0,619 & 0,204 & 0,126 & 0,152 & 113 & 70 & $-1,0$ \\
\hline 4 & 70 & 0,032 & 0,541 & 0,032 & 0,592 & 1,000 & 1,094 & 0,094 & 1,000 & 0,859 & 0,824 & 0,606 & 0,221 & 0,134 & 0,152 & 119 & 70 & $-1,1$ \\
\hline 1 & 71 & 0,077 & 0,960 & 0,082 & 0,888 & 0,947 & 0,925 & 0,091 & 1,000 & 0,972 & 0,809 & 0,759 & 0,349 & 0,265 & 0,240 & 150 & 71 & $-1,8$ \\
\hline 2 & 71 & 0,026 & 0,469 & 0,027 & 0,418 & 0,947 & 0,892 & 0,120 & 1,000 & 0,824 & 1,146 & 0,791 & 0,169 & 0,134 & 0,169 & 131 & 71 & $-0,8$ \\
\hline 3 & 71 & 0,032 & 0,511 & 0,032 & 0,368 & 1,000 & 0,719 & 0,281 & 1,000 & 0,812 & 1,120 & 0,800 & 0,189 & 0,152 & 0,214 & 90 & 71 & $-0,9$ \\
\hline 4 & 71 & 0,113 & 2,042 & 0,176 & 1,399 & 0,642 & 0,685 & 0,476 & 0,342 & 1,711 & 0,474 & 0,614 & 0,552 & 0,338 & 0,169 & 12 & 71 & $-2,5$ \\
\hline 5 & 71 & 0,019 & 0,397 & 0,020 & 0,338 & 0,929 & 0,851 & 0,166 & 1,000 & 0,821 & 0,974 & 0,651 & 0,156 & 0,102 & 0,152 & 150 & 71 & $-0,6$ \\
\hline 6 & 71 & 0,034 & 0,816 & 0,040 & 0,744 & 0,857 & 0,912 & 0,168 & 0,650 & 1,240 & 0,306 & 0,258 & 0,379 & 0,098 & 0,107 & 147 & 71 & $-1,9$ \\
\hline 7 & 71 & 0,089 & 1,234 & 0,106 & 1,073 & 0,838 & 0,870 & 0,208 & 0,734 & 1,168 & 0,530 & 0,595 & 0,462 & 0,275 & 0,273 & 127 & 71 & $-2,2$ \\
\hline 8 & 71 & 0,077 & 0,909 & 0,080 & 0,858 & 0,964 & 0,944 & 0,066 & 1,000 & 0,921 & 0,859 & 0,783 & 0,339 & 0,265 & 0,303 & 42 & 71 & $-1,8$ \\
\hline 9 & 71 & 0,014 & 0,317 & 0,014 & 0,173 & 1,000 & 0,547 & 0,453 & 1,000 & 0,746 & 1,273 & 0,632 & 0,120 & 0,076 & 0,152 & 72 & 71 & $-0,3$ \\
\hline 10 & 71 & 0,020 & 0,440 & 0,022 & 0,338 & 1,000 & 0,769 & 0,231 & 1,000 & 0,875 & 0,686 & 0,392 & 0,193 & 0,076 & 0,152 & 0 & 71 & $-0,9$ \\
\hline 11 & 71 & 0,014 & 0,397 & 0,017 & 0,296 & 0,833 & 0,745 & 0,305 & 1,000 & 0,936 & 0,637 & 0,400 & 0,169 & 0,068 & 0,076 & 154 & 71 & $-0,8$ \\
\hline 12 & 71 & 0,030 & 0,693 & 0,036 & 0,541 & 0,840 & 0,780 & 0,272 & 0,788 & 1,127 & 0,594 & 0,527 & 0,254 & 0,134 & 0,169 & 42 & 71 & $-1,3$ \\
\hline 13 & 71 & 0,043 & 0,655 & 0,044 & 0,490 & 0,968 & 0,749 & 0,254 & 1,000 & 0,891 & 0,764 & 0,696 & 0,268 & 0,186 & 0,214 & 135 & 71 & $-1,4$ \\
\hline 14 & 71 & 0,126 & 1,205 & 0,133 & 1,154 & 0,946 & 0,958 & 0,068 & 1,000 & 0,956 & 0,896 & 0,803 & 0,423 & 0,340 & 0,312 & 76 & 71 & $-2,1$ \\
\hline 15 & 71 & 0,049 & 1,255 & 0,067 & 0,735 & 0,723 & 0,586 & 0,498 & 0,389 & 1,603 & 0,585 & 0,626 & 0,326 & 0,204 & 0,107 & 103 & 71 & $-1,7$ \\
\hline 16 & 71 & 0,020 & 0,440 & 0,022 & 0,317 & 0,933 & 0,721 & 0,287 & 1,000 & 0,875 & 0,713 & 0,537 & 0,189 & 0,102 & 0,152 & 150 & 71 & $-0,9$ \\
\hline 17 & 71 & 0,017 & 0,389 & 0,017 & 0,194 & 1,000 & 0,500 & 0,500 & 1,000 & 0,836 & 0,764 & 0,447 & 0,169 & 0,076 & 0,107 & 162 & 71 & $-0,8$ \\
\hline 18 & 71 & 0,207 & 2,625 & 0,261 & 1,611 & 0,791 & 0,614 & 0,439 & 0,377 & 1,629 & 0,674 & 0,780 & 0,625 & 0,487 & 0,152 & 59 & 71 & $-2,6$ \\
\hline 19 & 71 & 0,016 & 0,418 & 0,017 & 0,368 & 0,917 & 0,879 & 0,147 & 1,000 & 0,940 & 0,700 & 0,501 & 0,169 & 0,085 & 0,076 & 45 & 71 & $-0,8$ \\
\hline 20 & 71 & 0,086 & 1,408 & 0,113 & 1,163 & 0,759 & 0,826 & 0,297 & 0,546 & 1,353 & 0,424 & 0,439 & 0,508 & 0,223 & 0,227 & 53 & 71 & $-2,3$ \\
\hline 21 & 71 & 0,088 & 1,133 & 0,096 & 0,951 & 0,910 & 0,839 & 0,184 & 0,857 & 1,080 & 0,687 & 0,693 & 0,403 & 0,279 & 0,240 & 152 & 71 & $-2,0$ \\
\hline 22 & 71 & 0,022 & 0,613 & 0,029 & 0,520 & 0,750 & 0,848 & 0,292 & 0,720 & 1,178 & 0,367 & 0,333 & 0,273 & 0,091 & 0,076 & 127 & 71 & $-1,4$ \\
\hline 23 & 71 & 0,032 & 0,634 & 0,037 & 0,520 & 0,846 & 0,820 & 0,237 & 0,987 & 1,007 & 0,622 & 0,555 & 0,254 & 0,141 & 0,152 & 159 & 71 & $-1,3$ \\
\hline 1 & 72 & 0,023 & 0,461 & 0,023 & 0,266 & 1,000 & 0,578 & 0,422 & 1,000 & 0,858 & 0,784 & 0,392 & 0,193 & 0,076 & 0,152 & 5 & 72 & $-0,9$ \\
\hline 2 & 72 & 0,016 & 0,347 & 0,016 & 0,296 & 1,000 & 0,854 & 0,146 & 1,000 & 0,778 & 1,077 & 0,588 & 0,137 & 0,080 & 0,107 & 72 & 72 & $-0,5$ \\
\hline 1 & 73 & 0,108 & 1,234 & 0,123 & 1,103 & 0,872 & 0,894 & 0,166 & 0,887 & 1,062 & 0,654 & 0,686 & 0,458 & 0,314 & 0,273 & 46 & 73 & $-2,2$ \\
\hline
\end{tabular}




\begin{tabular}{|c|c|c|c|c|c|c|c|c|c|c|c|c|c|c|c|c|}
\hline 0,188 & 2,059 & 0,238 & 1,670 & 0,789 & 0,811 & 0,283 & 0,557 & 1,340 & 0,571 & 0,650 & 0,647 & 0,421 & 0,303 & 132 & 73 & $-2,7$ \\
\hline 0,067 & 0,837 & 0,070 & 0,786 & 0,959 & 0,939 & 0,073 & 1,000 & 0,909 & 0,880 & 0,728 & 0,312 & 0,227 & 0,240 & 1 & 73 & $-1,6$ \\
\hline 0,267 & 2,152 & 0,296 & 1,978 & 0,903 & 0,919 & 0,126 & 0,724 & 1,175 & 0,404 & 0,425 & 0,917 & 0,390 & 0,379 & 127 & 73 & $-3,2$ \\
\hline 0,034 & 0,672 & 0,037 & 0,592 & 0,923 & 0,880 & 0,142 & 0,957 & 1,022 & 0,501 & 0,362 & 0,296 & 0,107 & 0,152 & 139 & 73 & $-1,6$ \\
\hline 0,086 & 1,378 & 0,121 & 1,091 & 0,714 & 0,792 & 0,354 & 0,570 & 1,325 & 0,611 & 0,782 & 0,423 & 0,331 & 0,240 & 27 & 73 & $-2,1$ \\
\hline 0,049 & 0,744 & 0,053 & 0,714 & 0,919 & 0,960 & 0,090 & 1,000 & 0,950 & 0,666 & 0,526 & 0,305 & 0,161 & 0,214 & 143 & 73 & $-1,6$ \\
\hline 0,030 & 0,571 & 0,032 & 0,511 & 0,955 & 0,896 & 0,114 & 1,000 & 0,928 & 0,922 & 0,748 & 0,204 & 0,153 & 0,169 & 98 & 73 & $-1,0$ \\
\hline 0,023 & 0,469 & 0,023 & 0,397 & 1,000 & 0,847 & 0,153 & 1,000 & 0,874 & 0,637 & 0,375 & 0,214 & 0,080 & 0,107 & 135 & 73 & $-1,1$ \\
\hline 0,017 & 0,440 & 0,019 & 0,368 & 0,923 & 0,837 & 0,181 & 1,000 & 0,945 & 0,527 & 0,332 & 0,204 & 0,068 & 0,076 & 108 & 73 & $-1,0$ \\
\hline 0,020 & 0,440 & 0,022 & 0,317 & 0,933 & 0,721 & 0,287 & 1,000 & 0,875 & 0,713 & 0,537 & 0,189 & 0,102 & 0,152 & 60 & 73 & $-0,9$ \\
\hline 0,016 & 0,338 & 0,016 & 0,194 & 1,000 & 0,575 & 0,425 & 1,000 & 0,759 & 1,077 & 0,555 & 0,137 & 0,076 & 0,152 & 162 & 73 & $-0,5$ \\
\hline 0,022 & 0,461 & 0,023 & 0,338 & 0,938 & 0,734 & 0,273 & 1,000 & 0,886 & 0,764 & 0,506 & 0,189 & 0,096 & 0,152 & 117 & 73 & $-0,9$ \\
\hline 0,044 & 0,837 & 0,052 & 0,702 & 0,861 & 0,839 & 0,213 & 0,798 & 1,120 & 0,443 & 0,375 & 0,357 & 0,134 & 0,169 & 130 & 73 & $-1,8$ \\
\hline 0,019 & 0,418 & 0,020 & 0,317 & 0,929 & 0,757 & 0,253 & 1,000 & 0,864 & 0,828 & 0,566 & 0,169 & 0,096 & 0,152 & 123 & 73 & $-0,8$ \\
\hline 0,023 & 0,490 & 0,024 & 0,338 & 0,941 & 0,689 & 0,316 & 1,000 & 0,913 & 0,702 & 0,529 & 0,204 & 0,108 & 0,152 & 21 & 73 & $-1,0$ \\
\hline 0,033 & 0,664 & 0,034 & 0,550 & 0,958 & 0,828 & 0,177 & 0,942 & 1,031 & 0,451 & 0,333 & 0,305 & 0,102 & 0,152 & 117 & 73 & $-1,6$ \\
\hline 0,026 & 0,541 & 0,030 & 0,440 & 0,857 & 0,812 & 0,236 & 1,000 & 0,950 & 0,674 & 0,524 & 0,221 & 0,116 & 0,152 & 120 & 73 & $-1,1$ \\
\hline 0,020 & 0,448 & 0,022 & 0,368 & 0,933 & 0,820 & 0,191 & 1,000 & 0,892 & 1,049 & 0,686 & 0,156 & 0,107 & 0,107 & 97 & 73 & $-0,6$ \\
\hline 0,014 & 0,397 & 0,016 & 0,397 & 0,909 & 1,000 & 0,091 & 1,000 & 0,936 & 0,509 & 0,283 & 0,189 & 0,054 & 0,076 & 145 & 73 & $-0,9$ \\
\hline 0,016 & 0,520 & 0,022 & 0,368 & 0,733 & 0,707 & 0,396 & 0,733 & 1,168 & 0,483 & 0,371 & 0,204 & 0,076 & 0,107 & 96 & 73 & $-1,0$ \\
\hline 0,029 & 0,562 & 0,030 & 0,490 & 0,952 & 0,872 & 0,136 & 1,000 & 0,936 & 0,796 & 0,707 & 0,214 & 0,152 & 0,152 & 147 & 73 & $-1,1$ \\
\hline 0,027 & 0,693 & 0,033 & 0,592 & 0,826 & 0,854 & 0,227 & 0,713 & 1,185 & 0,331 & 0,261 & 0,324 & 0,085 & 0,107 & 146 & 73 & $-1,7$ \\
\hline 0,043 & 1,142 & 0,060 & 1,019 & 0,714 & 0,893 & 0,305 & 0,415 & 1,552 & 0,198 & 0,200 & 0,526 & 0,105 & 0,107 & 30 & 73 & $-2,4$ \\
\hline \multirow[t]{3}{*}{0,014} & 0,499 & 0,017 & 0,397 & 0,833 & 0,797 & 0,263 & 0,724 & 1,175 & 0,311 & 0,175 & 0,243 & 0,042 & 0,076 & 35 & 73 & $-1,3$ \\
\hline & & & & & & & nothing to measure & & & & & & & & 74 & - \\
\hline & & & & & & & nothing to measure & & & & & & & & 75 & - \\
\hline \multirow[t]{3}{*}{0,019} & 0,571 & 0,022 & 0,469 & 0,867 & 0,822 & 0,222 & 0,720 & 1,179 & 0,318 & 0,262 & 0,273 & 0,071 & 0,076 & 57 & 76 & $-1,4$ \\
\hline & & & & & & & nothing to measure & & & & & & & & 77 & - \\
\hline & & & & & & & nothing to measure & & & & & & & & 78 & - \\
\hline 0,027 & 0,490 & 0,029 & 0,368 & 0,950 & 0,750 & 0,255 & 1,000 & 0,838 & 1,210 & 0,894 & 0,169 & 0,152 & 0,169 & 0 & 79 & $-0,8$ \\
\hline 0,014 & 0,440 & 0,017 & 0,317 & 0,833 & 0,721 & 0,325 & 0,933 & 1,035 & 0,637 & 0,501 & 0,169 & 0,085 & 0,076 & 62 & 79 & $-0,8$ \\
\hline 0,014 & 0,368 & 0,014 & 0,266 & 1,000 & 0,724 & 0,276 & 1,000 & 0,866 & 0,637 & 0,400 & 0,169 & 0,068 & 0,076 & 69 & 79 & $-0,8$ \\
\hline 0,059 & 1,369 & 0,082 & 1,145 & 0,719 & 0,836 & 0,325 & 0,394 & 1,593 & 0,201 & 0,209 & 0,611 & 0,128 & 0,152 & 83 & 79 & $-2,6$ \\
\hline 0,017 & 0,418 & 0,017 & 0,296 & 1,000 & 0,707 & 0,293 & 1,000 & 0,900 & 0,611 & 0,424 & 0,189 & 0,080 & 0,107 & 50 & 79 & $-0,9$ \\
\hline 0,307 & 2,430 & 0,356 & 2,338 & 0,863 & 0,962 & 0,142 & 0,653 & 1,237 & 0,361 & 0,356 & 1,040 & 0,370 & 0,379 & 150 & 79 & $-3,4$ \\
\hline 0,027 & 0,490 & 0,027 & 0,418 & 1,000 & 0,854 & 0,146 & 1,000 & 0,838 & 0,834 & 0,557 & 0,204 & 0,114 & 0,152 & 17 & 79 & $-1,0$ \\
\hline 0,017 & 0,672 & 0,022 & 0,571 & 0,800 & 0,849 & 0,251 & 0,479 & 1,445 & 0,206 & 0,146 & 0,326 & 0,048 & 0,076 & 140 & 79 & $-1,7$ \\
\hline 0,020 & 0,490 & 0,023 & 0,368 & 0,875 & 0,750 & 0,280 & 1,000 & 0,976 & 0,615 & 0,411 & 0,204 & 0,084 & 0,107 & 105 & 79 & $-1,0$ \\
\hline 0,014 & 0,499 & 0,017 & 0,448 & 0,833 & 0,898 & 0,195 & 0,724 & 1,175 & 0,311 & 0,175 & 0,243 & 0,042 & 0,076 & 147 & 79 & $-1,3$ \\
\hline 0,020 & 0,418 & 0,020 & 0,376 & 1,000 & 0,899 & 0,101 & 1,000 & 0,833 & 0,891 & 0,600 & 0,169 & 0,102 & 0,152 & 153 & 79 & $-0,8$ \\
\hline 0,022 & 0,440 & 0,024 & 0,338 & 0,882 & 0,769 & 0,259 & $\begin{array}{l}1,000 \\
\text { S }\end{array}$ & 0,845 & 1,061 & 0,707 & 0,161 & 0,114 & 0,152 & 88 & 80 & $-0,7$ \\
\hline 0,020 & 0,592 & 0,026 & 0,418 & 0,778 & 0,707 & 0,368 & 0,721 & 1,178 & 0,396 & 0,328 & 0,254 & 0,083 & 0,076 & 32 & 81 & $-1,3$ \\
\hline 0,050 & 0,858 & 0,055 & 0,693 & 0,921 & 0,808 & 0,208 & 0,857 & 1,080 & 0,602 & 0,515 & 0,326 & 0,168 & 0,169 & 21 & 81 & $\begin{array}{l}-1,7 \\
\end{array}$ \\
\hline 0,024 & 0,490 & 0,027 & 0,397 & 0,895 & 0,811 & 0,217 & 1,000 & 0,886 & 0,746 & 0,557 & 0,204 & 0,114 & 0,152 & 21 & 81 & $-1,0$ \\
\hline 0,026 & 0,643 & 0,030 & 0,541 & 0,857 & 0,842 & 0,213 & 0,786 & 1,128 & 0,376 & 0,344 & 0,296 & 0,102 & 0,152 & 32 & 81 & $-1,6$ \\
\hline 0,022 & 0,634 & 0,024 & 0,511 & 0,882 & 0,807 & 0,226 & 0,673 & 1,219 & 0,329 & 0,311 & 0,288 & 0,090 & 0,076 & 23 & 81 & $-1,5$ \\
\hline \multirow[t]{3}{*}{0,016} & 0,376 & 0,016 & 0,347 & 1,000 & 0,921 & 0,079 & 1,000 & 0,845 & 0,824 & 0,514 & 0,156 & 0,080 & 0,107 & 37 & 81 & $-0,6$ \\
\hline & & & & & & & nothing to measure & & & & & & & & 82 & - \\
\hline & & & & & & & nothing to measure & & & & & & & & 83 & . \\
\hline 0,024 & 0,520 & 0,027 & 0,440 & 0,895 & 0,845 & 0,187 & 1,000 & 0,939 & 0,585 & 0,493 & 0,230 & 0,114 & 0,152 & 100 & 84 & $-1,2$ \\
\hline 0,014 & 0,389 & 0,014 & 0,317 & 1,000 & 0,815 & 0,185 & 1,000 & 0,916 & 0,490 & 0,196 & 0,193 & 0,038 & 0,076 & 90 & 84 & $-0,9$ \\
\hline 0,016 & 0,368 & 0,016 & 0,296 & 1,000 & 0,805 & 0,195 & 1,000 & 0,826 & 0,824 & 0,485 & 0,156 & 0,076 & 0,107 & 18 & 84 & $-0,6$ \\
\hline 0,014 & 0,368 & 0,014 & 0,296 & 1,000 & 0,805 & 0,195 & 1,000 & 0,866 & 0,637 & 0,400 & 0,169 & 0,068 & 0,076 & 108 & 85 & $-0,8$ \\
\hline 0,016 & 0,368 & 0,016 & 0,245 & 1,000 & 0,667 & 0,333 & $\begin{array}{l}1,000 \\
-100\end{array}$ & 0,826 & 1,077 & 0,745 & 0,137 & 0,102 & 0,107 & 117 & 85 & $-0,5$ \\
\hline 0,019 & 0,418 & 0,019 & 0,338 & 1,000 & 0,808 & 0,192 & 1,000 & 0,864 & 0,662 & 0,424 & 0,189 & 0,080 & 0,107 & 33 & 85 & $-0,9$ \\
\hline 0,198 & 1,674 & 0,211 & 1,644 & 0,939 & 0,982 & 0,064 & 0,888 & 1,061 & 0,475 & 0,463 & 0,729 & 0,338 & 0,379 & 9 & 85 & $-2,9$ \\
\hline 0,017 & 0,347 & 0,017 & 0,296 & 1,000 & 0,854 & 0,146 & $\begin{array}{l}1,000 \\
-1000\end{array}$ & 0,745 & 1,528 & 0,894 & 0,120 & 0,107 & 0,107 & 90 & 85 & $-0,3$ \\
\hline 0,020 & 0,520 & 0,023 & 0,418 & 0,875 & 0,805 & 0,232 & 0,934 & 1,035 & 0,435 & 0,331 & 0,243 & 0,080 & 0,107 & 33 & 85 & $-1,3$ \\
\hline
\end{tabular}


nothing to measure
nothing to measure

$\begin{array}{llll}0,016 & 0,338 & 0.016 & \text { nothing to measure }\end{array}$

$\begin{array}{lllllll}0,017 & 0,338 & 0,016 & 0,194 & 1,000 & 0,575 & 0,425 \\ 0,017 & 0,347 & 0,017 & 0,296 & 1,000 & 0,854 & 0,146\end{array}$

1,000

$\begin{array}{llllll}0,014 & 0,317 & 0,014 & 0,173 & 1,000 & 0,547\end{array}$

$\begin{array}{rrr}0,759 & 1,077 & 0,555 \\ 0,745 & 1,28 & 0,894\end{array}$

$\begin{array}{llllll}0,026 & 0,753 & 0,032 & 0,672 & 0,818 & 0,893 \\ 0,043 & 0,981 & 0,057 & 0,825 & 0,750 & 0,841 \\ 0,016 & 0,389 & 0,017 & 0,173 & 0,917 & 0,446\end{array}$

0,211

1,321

$1,528 \quad 0,8,273$

0,120

0,560

0,563

1,333

0,234

0,143

nothing to measure

mesure

nothing to measure

nothing to measure

nothing to measure

$\begin{array}{lllllllll}0,014 & 0,317 & 0,014 & 0,194 & 1,000 & 0,613 & 0,387 & 1,000 & 0,746\end{array}$

$\begin{array}{llllll}0,022 & 0,418 & 0,023 & 0,368 & 0,938 & 0,879\end{array}$

0,136

$\begin{array}{ll}1,000 & 0,746 \\ 1,000 & 0,805 \\ 1,000 & 0,746\end{array}$

$\begin{array}{ll}1,000 \\ 1,000\end{array} \quad 0,950$

$\begin{array}{ll}1,000 & 0,908 \\ 1,000 & 0,805\end{array}$

$\begin{array}{lll}0,019 & 0,440 & 0,020 \\ 0,022 & 0,418 & 0,022\end{array}$

$0,461 \quad 0,857$

$0,851 \quad 0,206$

$\begin{array}{ll}0,857 & 0,829 \\ 1,000 & 0,721 \\ 0,846 & 0,897\end{array}$

0,121

0,805
0,920
1,808

$\begin{array}{lll}0,063 & 1,611 & 0,126\end{array}$

$\begin{array}{lll}0,014 & 0,368 & 0,016\end{array}$

$\begin{array}{lll}0,047 & 1,040 & 0,067 \\ 0,030 & 0,562 & 0,033\end{array}$

$0,016 \quad 0,347$

$0,014 \quad 0,347$

$0,049 \quad 0,693$

$\begin{array}{lll}0,066 & 0,837 & 0,070\end{array}$

$\begin{array}{lll}0,030 & 0,685 & 0,034 \\ 0 & 0,034\end{array}$

$\begin{array}{ll}0,066 & 1,315\end{array}$

$\begin{array}{ll}0,185 & 3,386\end{array}$

$0,016 \quad 0,338$

$\begin{array}{ll}0,088 & 1,315 \\ 0,029 & 0,520\end{array}$

$\begin{array}{ll}0,032 & 0,989 \\ 0,017 & 0,347\end{array}$

$\begin{array}{lll}0,149 & 2,875 & 0,017\end{array}$

$\begin{array}{lll}0,032 & 0,583 & 0,034 \\ 0,024 & 0,469 & 0,024\end{array}$

$\begin{array}{lll}0,208 & 1,814 & 0,224\end{array}$

$\begin{array}{lll}0,039 & 0,938 & 0,053 \\ 0,126 & 2,642 & 0,198\end{array}$

0,368
1,159

0,500

0,719

0,573

0,306

$\begin{array}{rr}1,000 & 0,899 \\ 1,000 & 0,866\end{array}$

1,000
0,550

0,875

0,440

$\begin{array}{ll}0,909 & 0,529 \\ 0,702 & 0,842\end{array}$

0,337

0,913

$0,782 \quad 0,235$

1,000

$\begin{array}{ll}1,000 & 0,778\end{array}$

$\begin{array}{ll}1,000 & 0,888 \\ 1,000 & 0,816\end{array}$

$\begin{array}{ll}1,000 & 0,886 \\ 0,852 & 1,084\end{array}$

$\begin{array}{ll}1,000 & 0,874 \\ 1,000 & 0,796\end{array}$

0,577

$\quad 0,490$

$0,040 \quad 0,634$

$\begin{array}{ll}0,283 & 1,784 \\ 0,016 & 0,144\end{array}$

$\begin{array}{ll}0,108 & 1,103 \\ 032 & 0,469\end{array}$

0,469
0,469

0,727

2,017
0,448

0,368
1,551

0,723

1,543
2,355

$\begin{array}{lll}1,078 & 0,887 & 0,043 \\ 0,878 & 0,857\end{array}$

$0,909 \quad 0,425$

$\begin{array}{ll}0,982 & 0,968 \\ 0,697 & 0,743\end{array}$

$\begin{array}{ll}0,697 & 0,743 \\ 0,571 & 0,757\end{array}$

$\begin{array}{ll}0,655 & 0,527 \\ 1,000 & 0,425\end{array}$

$\begin{array}{ll}0,000 & 0,425 \\ 0,813 & 0,839\end{array}$

$\begin{array}{lll}1,000 & 0,425 & 0,276 \\ 0,909- & 0,902 & 0,246\end{array}$

$\begin{array}{lll}0,958 & 0,822 & 0,133 \\ 0,629 & 0,183\end{array}$

$\begin{array}{lll}0,629 & 0,735 & 0,456 \\ 1,000 & 0,854 & 0,146\end{array}$

$\begin{array}{lll}0,550 & 0,702 & 0,540 \\ 0,917 & 0,769 & 0,246\end{array}$

0,784

0,730

0,855
0,770

0,216
0,161

0,161
0,355

0,705

0,584

0,552
0,373

$1,000 \quad 1,16$

$\begin{array}{ll}0,412 & 1,558 \\ 0,203 & 2,220\end{array}$

$\begin{array}{ll}1,000 & 0,759\end{array}$

$\begin{array}{ll}1,636 & 1,254 \\ 1,000 & 0,866\end{array}$

$\begin{array}{ll}1,000 & 0,886 \\ 0,405 & 1,571\end{array}$

$\begin{array}{ll}1,000 & 0,745 \\ 0,227 & 2,099\end{array}$

$1,000 \quad 0,926$

$\begin{array}{ll}1,000 & 0,848 \\ 0,795 & 1,122\end{array}$

$0,553 \quad 1,345$

$\begin{array}{ll}0,227 & 2,098 \\ 0,360 & 1,667\end{array}$

$\begin{array}{ll}0,979 & 0,555 \\ 1,123 & 0,728\end{array}$

$\begin{array}{ll}0,979 & 0,755 \\ 0,979 & 0,555\end{array}$

$\begin{array}{ll}0,619 & 0,493 \\ 0,637 & 0,392\end{array}$

$\begin{array}{ll}1,123 & 0,728\end{array}$

$\begin{array}{ll}1,700 & 0,434 \\ 0,303 & 0,520\end{array}$

$\begin{array}{ll}0,857 & 0,686\end{array}$

$\begin{array}{ll}0,979 & 0,555 \\ 0,359 & 0,412\end{array}$

$\begin{array}{ll}0,922 & 0,743 \\ 1,077 & 0,588\end{array}$

$\begin{array}{ll}1,0789 & 0,566 \\ 0,749 & 0,485\end{array}$

$\begin{array}{ll}0,7482 & 0,894 \\ 0,695 & 0,677\end{array}$

$\begin{array}{ll}0,955 & 0,778 \\ 0,979 & 0,555\end{array}$

$1,010 \quad 0,860$

$\begin{array}{ll}0,294 & 0,311 \\ 0,723 & 0,658\end{array}$

$\begin{array}{ll}0,977 & 0,658\end{array}$

$\begin{array}{ll}0,428 & 0,499\end{array}$

$0,456 \quad 0,696$

$\begin{array}{ll}1,077 & 0,555 \\ 0,597 & 0,727\end{array}$

$\begin{array}{ll}0,979 & 0,784\end{array}$

$\begin{array}{ll}1,010 & 0,788 \\ 0,277 & 0,299\end{array}$

$\begin{array}{lll}1,010 & 0,788 & 0,384 \\ 1,528 & 0,894 & 0,120\end{array}$

$\begin{array}{lll}0,204 & 0,343 & 0,966 \\ 0,824 & 0,66 & 0,218\end{array}$

$\begin{array}{ll}0,824 & 0,666 \\ 0,866 & 0,566\end{array}$

$\begin{array}{ll}0,866 & 0,566 \\ 0,796 & 0,743\end{array}$

$\begin{array}{ll}0,404 & 0,405 \\ 0,423 & 0,632\end{array}$

0,318

0,632
0,392

0,189
0,577
0,349
0,617

$\begin{array}{ll}0,617 & 0,141 \\ 0,390\end{array}$ 


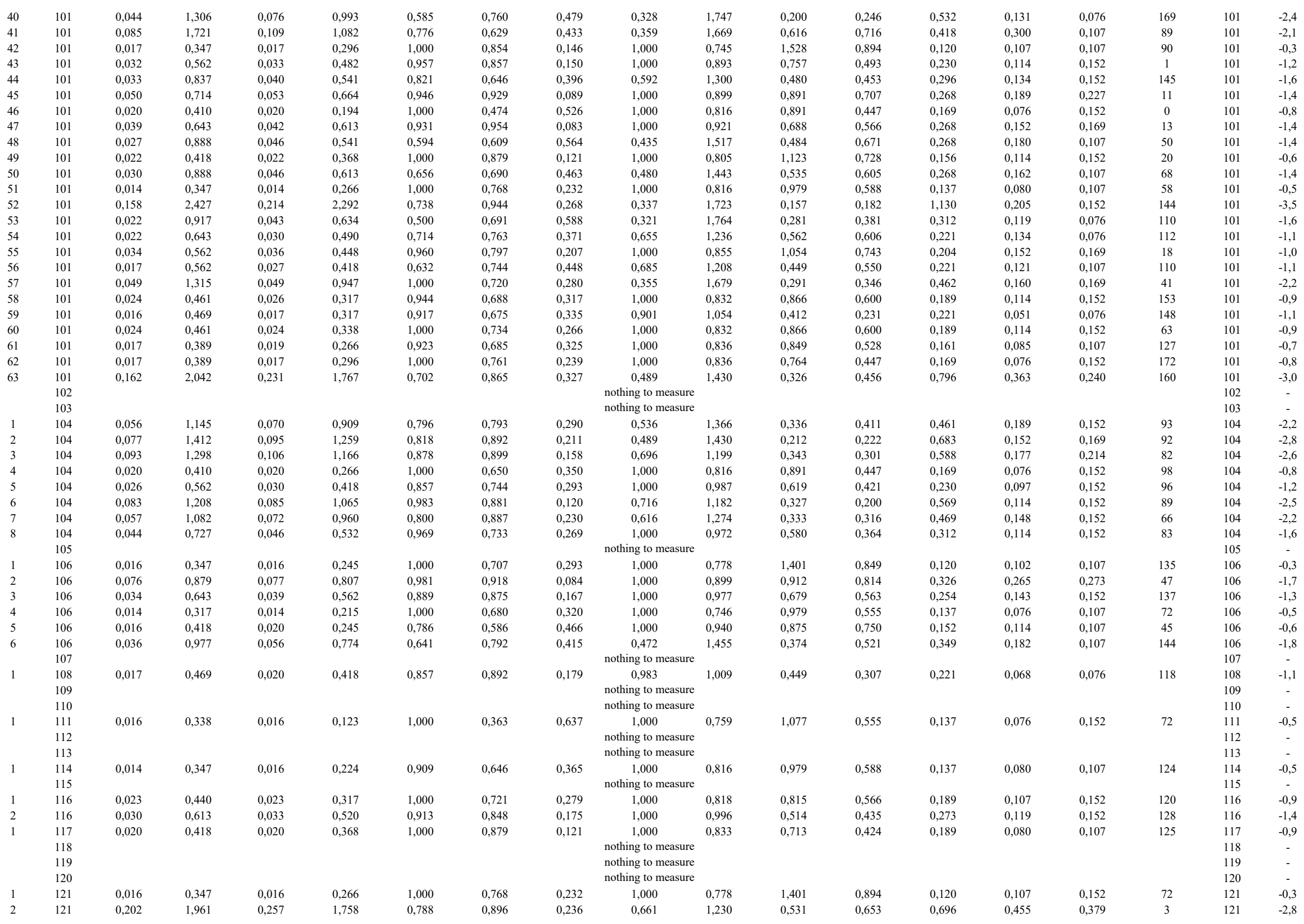


nothing to measure

nothing to measure

nothing to measure

$\begin{array}{lllll}0,034 & 0,723 & 0,040 & 0,592 & 0,857\end{array}$

$\begin{array}{ll}0,828 & 1,099 \\ 1,000 & 0,816\end{array}$

0,424

$0,321 \quad 0,106$

0,152 $\begin{array}{lll}0,936 & 0,509 & 0,280 \\ 1,075 & 0,520 & 0,028\end{array}$ $0,189-054$

$\begin{array}{llllll}0,169 & 1,569 & 0,176 & 1,518 & 0,959 & 0,868\end{array}$ $\begin{array}{lll}0,202 & 1,000 & 0,936 \\ 0,052 & 0,865 & 1,075\end{array}$ $0,869 \quad 1,073$

$\begin{array}{ll}1,000 & 0,791 \\ 1,000 & 0,746\end{array}$ $1,000 \quad 0,803$

$\begin{array}{llll}0,014 & 0,317 & 0,014 & 0,275\end{array}$

$\begin{array}{lll}0,039 & 0,389 & 0,019 \\ 0,049 & 0,867 & 0,046\end{array}$

$\begin{array}{lll}0,049 & 0,867 & 0,057\end{array}$

$0,056 \quad 1,112$

$\begin{array}{lll}0,026 & 0,643 & 0,356\end{array}$

$0,022-0,469-0,023$

$\begin{array}{ll}0,022 & 0,520 \\ 0,139 & 1,775\end{array}$

$\begin{array}{ll}0,133 & 1,264\end{array}$

$\begin{array}{lll}0,019 & 0,397 & 0,019\end{array}$

$\begin{array}{lll}0,040 & 0,757 & 0,047 \\ 0,027 & 0,490 & 0,029\end{array}$

$\begin{array}{lll}0,020 & 0,643 & 0,029\end{array}$

$\begin{array}{ll}0,228 & 2,879\end{array}$

$0,014 \quad 0,368$

0,016

$\begin{array}{ll}0,148 & 1,725 \\ 0,025 & 0,368\end{array}$

$0,017 \quad 0,368$

$0,027 \quad 0,744$

$\begin{array}{ll}0,016 & 0,520 \\ 0,017 & 0,368\end{array}$

$0,019 \quad 0,469$

$\begin{array}{ll}0,023 & 0,469 \\ 0,040 & 0,613\end{array}$

$\begin{array}{ll}0,017 & 0,550 \\ 0,014 & 0,368\end{array}$

0,019

$\begin{array}{ll}0,368 & 0,016 \\ 0,368 & 0,019\end{array}$

0,224
0,693
0,57

$0,923 \quad 0,667$

1,000

0,867

0,342

0,424
0,175

$\begin{array}{lll}0,844 & 0,921 & 0,175 \\ 0,850 & 0,873 & 0,197\end{array}$

$\begin{array}{ll}0,850 & 0,873 \\ 0,736 & 0,844\end{array}$

0,307

2,185

2,185
0,490

0,138

0,816

0,816
0,569

$\begin{array}{ll}0,569 & 1,326 \\ 0,710 & 1,187\end{array}$

$\begin{array}{ll}0,5610 & 1,187 \\ 0,786 & 1,128\end{array}$

$\begin{array}{ll}1,000 & 0,902\end{array}$

$\begin{array}{lll}0,938 & 0,829 & 0,182 \\ 0,882 & 0,845 & 0,194\end{array}$

$\begin{array}{ll}0,882 & 0,845 \\ 0,758 & 0,879\end{array}$

0,194
0,271
0,076
0,169

1,000
0,555

$\begin{array}{ll}1,000 & 0,976 \\ 0,927 & 1,039\end{array}$

$\begin{array}{ll}1,927 & 1,039 \\ 1,000 & 0,821\end{array}$

$\begin{array}{ll}0,882 & 1,065 \\ 1,000 & 0,838\end{array}$

$0,611 \quad 1,279$

$0,950 \quad 0,793$

$\begin{array}{ll}0,700 & 0,809\end{array}$

0,809
0,874
0,876
0,831

0,356

0,454
0,346

1,000

$\begin{array}{ll}1,000 & 0,952\end{array}$

$\begin{array}{ll}1,0624 & 1,266\end{array}$

$\begin{array}{ll}1,000 & 0,875\end{array}$

$\begin{array}{ll}1,619 & 1,271 \\ 1,000 & 0,852\end{array}$

$\begin{array}{ll}0,733 & 1,168 \\ 1,000 & 0,791\end{array}$

$\begin{array}{lll}0,020 & 0,389 & 0,786 \\ 0,017 & 0,317 & 1,006\end{array}$

0,022

0,023

0,042

0,019

0,368
0,418

0,418
0,511

$\begin{array}{ll}0,245 & 0,909 \\ 0,245 & 1,000\end{array}$

$\begin{array}{ll}1,000 & 0,969 \\ 1,000\end{array} \quad 0,874$

$\begin{array}{ll}0,716 & 1,182 \\ 1,000 & 0,866\end{array}$

1,000
thing to measure

0,760

$0,520 \quad 0,458$

$\begin{array}{ll}1,175 & 0,745 \\ 1,273 & 0,632\end{array}$

$\begin{array}{ll}1,273 & 0,632 \\ 1,273 & 0,832\end{array}$

$0,506 \quad 0,462$

$\begin{array}{ll}0,637 & 0,606 \\ 0,397 & 0,396 \\ 0,477 & 0,552\end{array}$

$\begin{array}{ll}0,477 & 0,552 \\ 0,441 & 0,392\end{array}$

$\begin{array}{ll}0,471 & 0,392 \\ 0,764 & 0,566\end{array}$

$\begin{array}{ll}0,562 & 0,485\end{array}$

$\begin{array}{ll}0,380 & 0,538 \\ 0,740 & 0,671\end{array}$

$\begin{array}{ll}0,509 & 0,448 \\ 0,828 & 0,474\end{array}$

$0,548 \quad 0,496$

$\begin{array}{ll}1,210 & 0,849 \\ 0,292 & 0,249\end{array}$

0,269

0,249
0,155

$\begin{array}{ll}0,239 & 0,305 \\ 0,244 & 0,327\end{array}$

$\begin{array}{ll}0,749 & 0,461\end{array}$

$\begin{array}{ll}0,367 & 0,294 \\ 0,509 & 0,316\end{array}$

$\begin{array}{ll}0,321 & 0,344 \\ 1,175 & 0,745\end{array}$

$\begin{array}{ll}0,175 & 0,745 \\ 0,527 & 0,362 \\ 0,713 & 0,506\end{array}$

$\begin{array}{ll}0,527 & 0,362 \\ 0,834 & 0,740 \\ 0,878 & 0,557\end{array}$

$\begin{array}{ll}0,834 & 0,557 \\ 0,379 & 0,261 \\ 1,175 & 0,784\end{array}$

1,175
0,662

$\begin{array}{ll}1,175 & 0,784 \\ 0,602 & 0,555 \\ 1,049 & 0,499 \\ 0,306 & 0,844\end{array}$

0,306
0,749

1,273

nothing to measure

nothing to measure
nothing to measure

$\begin{array}{lllllll}0,019 & 0,397 & 0,019 & 0,347 & 1,000 & 0,872 & 0,128 \\ 0,017 & 0,389 & 0,017 & 0,296 & 1,000 & 0,761 & 0,239 \\ 0,128 & 1,306 & 0,138 & 1,184 & 0,927 & 0,906 & 0,1 \\ 0,024 & 0,440 & 0,024 & 0,338 & 1,000 & 0,769 & 0,231 \\ 0,023 & 0,448 & 0,023 & 0,389 & 1,000 & 0,867 & 0,13 \\ 0,014 & 0,317 & 0,014 & 0,173 & 1,000 & 0,547 & 0,4\end{array}$

nothing to measure
1,000
1,000

$\begin{array}{ll}1,000 & 0,82 \\ 1,000 & 0,836 \\ 0,941 & 1,031 \\ 1,000 & 0,784 \\ 1,000 & 0,84 \\ 1,000 & 0,746\end{array}$

21

$\begin{array}{ll}0,221 & 0,061 \\ 0,137 & 0,02\end{array}$

$0,120 \quad 0,076$

$\begin{array}{ll}0,137 & 0,114 \\ 0,312 & 0,144\end{array}$

$0,312 \quad 0,189$

$0,916 \quad 0,506$

$\begin{array}{ll}0,916 & 0,107 \\ 0,189 & 0,107 \\ 0 & 2210\end{array}$

$0,221 \quad 0,107$

$\begin{array}{ll}0,479 & 0,367 \\ 0,254 & 0,321\end{array}$

$\begin{array}{ll}0,254 & 0,114 \\ 0,169 & 0,080\end{array}$

$0,305 \quad 0,152$

$\begin{array}{ll}0,152 & 0,152 \\ 0,144 & 0,152\end{array}$

$\begin{array}{lll}0,273 & 0,042 & 0,076 \\ 0,510 & 0,155 & 0,152\end{array}$

$\begin{array}{ll}1,091 & 0,357\end{array}$

$\begin{array}{lll}0,156 & 0,072 & 0,214 \\ 0,076\end{array}$

$\begin{array}{ll}0,273 & 0,080 \\ 0,240 & 0,076\end{array}$

$\begin{array}{lll}0,766 & 0,264 & 0,152 \\ 0,137 & 0,10273\end{array}$

$\begin{array}{lll}0,137 & 0,102 & 0,152 \\ 0,204 & 0,074 & 0,076\end{array}$

$\begin{array}{lll}0,189 & 0,096 & 0,152\end{array}$

$\begin{array}{lll}0,204 & 0,153 & 0,076 \\ 0,204 & 0,114 & 0,152\end{array}$

$\begin{array}{lll}0,230 & 0,060 & 0,076\end{array}$

$0,137 \quad 0,107$

$\begin{array}{lll}0,189 & 0,105 & 0,107 \\ 0,204 & 0,102 & 0,152 \\ 0,221 & 0,186 & 0,214\end{array}$

$\begin{array}{ll}0,268 & 0,054 \\ 0,156 & 0,072\end{array}$

$0,137 \quad 0,107$

$\begin{array}{ll}0,764 & 0,447 \\ 0,584 & 0,574 \\ 1,082 & 0,671 \\ 1,06 & 0,632\end{array}$

$\begin{array}{ll}1,019 & 0,671 \\ 1,273 & 0,632\end{array}$

0,074

0,528

0,528
0,169
0,169

0,169

$0,120 \quad 076$

0,076
0,303
0,114
0,107
0,076

0,152

0,152

$\begin{array}{ll}122 & - \\ 123 & - \\ 124 & - \\ 125 & - \\ 126 & -1,7 \\ 126 & -0,6 \\ 126 & -0,9 \\ 126 & -2,7 \\ 126 & -1,1 \\ 126 & -0,5 \\ 126 & -0,3 \\ 126 & -0,5 \\ 126 & -1,6 \\ 126 & -1,6 \\ 126 & -2,1 \\ 126 & -3,2 \\ 126 & -1,4 \\ 126 & -0,9 \\ 126 & -1,1 \\ 126 & -2,8 \\ 126 & -2,3 \\ 126 & -1,3 \\ 126 & -0,8 \\ 126 & -1,6 \\ 126 & -0,8 \\ 126 & -1,6 \\ 126 & -1,4 \\ 126 & -2,4 \\ 126 & -3,4 \\ 126 & -0,6 \\ 126 & -1,4 \\ 126 & -1,3 \\ 126 & -2,9 \\ 126 & -0,5 \\ 126 & -1,0 \\ 126 & -0,9 \\ 126 & -1,0 \\ 127 & -1,0 \\ 127 & -1,2 \\ 127 & -0,5 \\ 127 & -0,9 \\ 127 & -1,0 \\ 127 & -1,1 \\ 127 & -1,4 \\ 127 & -0,6 \\ 127 & -0,5 \\ 128 & - \\ 129 & - \\ 130 & - \\ 131 & - \\ 132 & - \\ 133 & - \\ 134 & -0,8 \\ 134 & -0,8 \\ 134 & -2,4 \\ 134 & -0,8 \\ 134 & -0,8 \\ 134 & -0,3 \\ & -\end{array}$




\begin{tabular}{|c|c|c|c|c|c|c|c|c|c|c|c|c|c|c|c|c|c|c|}
\hline 7 & 134 & 0,110 & 1,572 & 0,148 & 1,441 & 0,748 & 0,917 & 0,266 & 0,562 & 1,335 & 0,336 & 0,364 & 0,647 & 0,236 & 0,214 & 8 & 134 & $-2,7$ \\
\hline 8 & 134 & 0,310 & 2,101 & 0,339 & 1,978 & 0,915 & 0,942 & 0,103 & 0,882 & 1,065 & 0,672 & 0,721 & 0,766 & 0,553 & 0,485 & 5 & 134 & $-2,9$ \\
\hline 9 & 134 & 0,026 & 0,672 & 0,027 & 0,571 & 0,947 & 0,849 & 0,160 & 0,718 & 1,180 & 0,318 & 0,167 & 0,321 & 0,054 & 0,076 & 44 & 134 & $-1,7$ \\
\hline 10 & 134 & 0,037 & 0,634 & 0,040 & 0,490 & 0,929 & 0,773 & 0,238 & 1,000 & 0,926 & 0,828 & 0,632 & 0,240 & 0,152 & 0,169 & 178 & 134 & $-1,3$ \\
\hline 11 & 134 & 0,052 & 0,930 & 0,060 & 0,858 & 0,857 & 0,923 & 0,162 & 0,751 & 1,154 & 0,376 & 0,362 & 0,418 & 0,152 & 0,169 & 176 & 134 & $-2,1$ \\
\hline 12 & 134 & 0,016 & 0,347 & 0,016 & 0,266 & 1,000 & 0,768 & 0,232 & 1,000 & 0,778 & 1,077 & 0,588 & 0,137 & 0,080 & 0,107 & 45 & 134 & $-0,5$ \\
\hline 13 & 134 & 0,017 & 0,368 & 0,017 & 0,287 & 1,000 & 0,781 & 0,219 & 1,000 & 0,791 & 0,849 & 0,500 & 0,161 & 0,080 & 0,107 & 45 & 134 & $-0,7$ \\
\hline 14 & 134 & 0,184 & 1,835 & 0,215 & 1,632 & 0,853 & 0,889 & 0,184 & 0,686 & 1,208 & 0,497 & 0,559 & 0,686 & 0,383 & 0,312 & 93 & 134 & $-2,8$ \\
\hline 15 & 134 & 0,017 & 0,490 & 0,020 & 0,389 & 0,857 & 0,793 & 0,252 & 0,900 & 1,054 & 0,382 & 0,269 & 0,240 & 0,064 & 0,076 & 12 & 134 & $-1,3$ \\
\hline 16 & 134 & 0,016 & 0,338 & 0,016 & 0,194 & 1,000 & 0,575 & 0,425 & 1,000 & 0,759 & 1,077 & 0,555 & 0,137 & 0,076 & 0,152 & 162 & 134 & $-0,5$ \\
\hline 17 & 134 & 0,016 & 0,389 & 0,017 & 0,194 & 0,917 & 0,500 & 0,507 & 1,000 & 0,873 & 0,824 & 0,485 & 0,156 & 0,076 & 0,107 & 5 & 134 & $-0,6$ \\
\hline 18 & 134 & 0,066 & 1,052 & 0,077 & 0,799 & 0,852 & 0,759 & 0,283 & 0,749 & 1,156 & 0,658 & 0,636 & 0,357 & 0,227 & 0,214 & 54 & 134 & $-1,8$ \\
\hline 19 & 134 & 0,017 & 0,368 & 0,019 & 0,317 & 0,923 & 0,862 & 0,158 & 1,000 & 0,791 & 1,175 & 0,745 & 0,137 & 0,102 & 0,152 & 135 & 134 & $-0,5$ \\
\hline 20 & 134 & 0,014 & 0,317 & 0,014 & 0,275 & 1,000 & 0,867 & 0,133 & 1,000 & 0,746 & 1,273 & 0,632 & 0,120 & 0,076 & 0,107 & 162 & 134 & $-0,3$ \\
\hline 21 & 134 & 0,030 & 0,664 & 0,034 & 0,541 & 0,875 & 0,815 & 0,223 & 0,860 & 1,078 & 0,504 & 0,405 & 0,276 & 0,112 & 0,107 & 168 & 134 & $-1,5$ \\
\hline 22 & 134 & 0,016 & 0,338 & 0,016 & 0,245 & 1,000 & 0,725 & 0,275 & 1,000 & 0,759 & 1,077 & 0,555 & 0,137 & 0,076 & 0,152 & 0 & 134 & $-0,5$ \\
\hline 23 & 134 & 0,017 & 0,368 & 0,017 & 0,245 & 1,000 & 0,667 & 0,333 & 1,000 & 0,791 & 1,175 & 0,784 & 0,137 & 0,107 & 0,152 & 0 & 134 & $-0,5$ \\
\hline 24 & 134 & 0,023 & 0,592 & 0,030 & 0,440 & 0,762 & 0,743 & 0,351 & 0,824 & 1,102 & 0,551 & 0,493 & 0,230 & 0,114 & 0,107 & 157 & 134 & $-1,2$ \\
\hline \multirow[t]{6}{*}{25} & 134 & 0,016 & 0,347 & 0,016 & 0,338 & 1,000 & 0,975 & 0,025 & 1,000 & 0,778 & 0,875 & 0,500 & 0,152 & 0,076 & 0,152 & 0 & 134 & $-0,6$ \\
\hline & 135 & & & & & & & & nothing to measure & & & & & & & & 135 & - \\
\hline & 136 & & & & & & & & nothing to measure & & & & & & & & 136 & - \\
\hline & 137 & & & & & & & & nothing to measure & & & & & & & & 137 & - \\
\hline & 138 & & & & & & & & nothing to measure & & & & & & & & 138 & - \\
\hline & 139 & & & & & & & & nothing to measure & & & & & & & & 139 & - \\
\hline 1 & 140 & 0,020 & 0,478 & 0,020 & 0,418 & 1,000 & 0,876 & 0,124 & 1,000 & 0,951 & 0,686 & 0,527 & 0,193 & 0,102 & 0,107 & 39 & 140 & $-0,9$ \\
\hline 2 & 140 & 0,046 & 1,264 & 0,072 & 1,142 & 0,640 & 0,903 & 0,373 & 0,361 & 1,664 & 0,226 & 0,328 & 0,508 & 0,167 & 0,107 & 31 & 140 & $-2,3$ \\
\hline 3 & 140 & 0,033 & 0,816 & 0,043 & 0,693 & 0,767 & 0,850 & 0,278 & 0,623 & 1,267 & 0,293 & 0,231 & 0,379 & 0,088 & 0,107 & 51 & 140 & $-1,9$ \\
\hline 1 & 141 & 0,019 & 0,368 & 0,019 & 0,245 & 1,000 & 0,667 & 0,333 & 1,000 & 0,760 & 1,273 & 0,784 & 0,137 & 0,107 & 0,152 & 90 & 141 & $-0,5$ \\
\hline 2 & 141 & 0,326 & 3,157 & 0,376 & 3,005 & 0,866 & 0,952 & 0,142 & 0,411 & 1,561 & 0,182 & 0,202 & 1,509 & 0,305 & 0,303 & 75 & 141 & $-3,9$ \\
\hline 3 & 141 & 0,194 & 1,821 & 0,204 & 1,678 & 0,951 & 0,921 & 0,093 & 0,734 & 1,167 & 0,360 & 0,321 & 0,827 & 0,265 & 0,273 & 81 & 141 & $-3,0$ \\
\hline 4 & 141 & 0,042 & 0,837 & 0,052 & 0,693 & 0,806 & 0,828 & 0,259 & 0,746 & 1,157 & 0,462 & 0,528 & 0,339 & 0,179 & 0,152 & 59 & 141 & $-1,8$ \\
\hline 5 & 141 & 0,432 & 3,728 & 0,519 & 3,656 & 0,831 & 0,981 & 0,170 & 0,390 & 1,600 & 0,185 & 0,209 & 1,725 & 0,360 & 0,379 & 71 & 141 & $-4,1$ \\
\hline 6 & 141 & 0,052 & 0,807 & 0,055 & 0,685 & 0,947 & 0,848 & 0,161 & 0,996 & 1,002 & 0,509 & 0,422 & 0,359 & 0,152 & 0,169 & 77 & 141 & $-1,8$ \\
\hline 7 & 141 & 0,090 & 1,091 & 0,100 & 1,010 & 0,900 & 0,926 & 0,124 & 0,955 & 1,023 & 0,771 & 0,761 & 0,386 & 0,294 & 0,273 & 61 & 141 & $-1,9$ \\
\hline 8 & 141 & 0,030 & 0,520 & 0,030 & 0,490 & 1,000 & 0,943 & 0,057 & 1,000 & 0,845 & 0,922 & 0,657 & 0,204 & 0,134 & 0,152 & 31 & 141 & $-1,0$ \\
\hline 9 & 141 & 0,026 & 0,930 & 0,053 & 0,643 & 0,486 & 0,691 & 0,599 & 0,375 & 1,632 & 0,258 & 0,419 & 0,357 & 0,150 & 0,076 & 148 & 141 & $-1,8$ \\
\hline 10 & 141 & 0,016 & 0,389 & 0,017 & 0,266 & 0,917 & 0,685 & 0,326 & 1,000 & 0,873 & 0,778 & 0,597 & 0,161 & 0,096 & 0,152 & 72 & 141 & $-0,7$ \\
\hline 11 & 141 & 0,419 & 3,339 & 0,485 & 3,043 & 0,864 & 0,911 & 0,162 & 0,472 & 1,455 & 0,246 & 0,278 & 1,473 & 0,410 & 0,442 & 71 & 141 & $-3,9$ \\
\hline 12 & 141 & 0,253 & 2,063 & 0,291 & 1,860 & 0,867 & 0,902 & 0,165 & 0,746 & 1,158 & 0,527 & 0,626 & 0,781 & 0,489 & 0,429 & 71 & 141 & $-3,0$ \\
\hline 13 & 141 & 0,076 & 1,040 & 0,083 & 0,938 & 0,914 & 0,902 & 0,130 & 0,884 & 1,064 & 0,540 & 0,443 & 0,423 & 0,187 & 0,214 & 52 & 141 & $-2,1$ \\
\hline 14 & 141 & 0,039 & 0,921 & 0,046 & 0,778 & 0,844 & 0,844 & 0,221 & 0,574 & 1,320 & 0,264 & 0,219 & 0,432 & 0,095 & 0,107 & 80 & 141 & $-2,1$ \\
\hline \multirow[t]{5}{*}{15} & 141 & 0,067 & 0,909 & 0,076 & 0,786 & 0,887 & 0,865 & 0,176 & 1,000 & 0,987 & 0,672 & 0,589 & 0,357 & 0,210 & 0,214 & 66 & 141 & $-1,8$ \\
\hline & 142 & & & & & & & & nothing to measure & & & & & & & & 142 & - \\
\hline & 143 & & & & & & & & nothing to measure & & & & & & & & 143 & - \\
\hline & 144 & & & & & & & & nothing to measure & & & & & & & & 144 & - \\
\hline & 145 & & & & & & & & nothing to measure & & & & & & & & 145 & - \\
\hline 1 & 146 & 0,047 & 0,879 & 0,055 & 0,757 & 0,868 & 0,861 & 0,192 & 0,770 & 1,140 & 0,385 & 0,302 & 0,395 & 0,120 & 0,152 & 167 & 146 & $-2,0$ \\
\hline 2 & 146 & 0,016 & 0,418 & 0,017 & 0,317 & 0,917 & 0,757 & 0,256 & 1,000 & 0,940 & 0,483 & 0,332 & 0,204 & 0,068 & 0,076 & 159 & 146 & $-1,0$ \\
\hline 3 & 146 & 0,020 & 0,440 & 0,020 & 0,338 & 1,000 & 0,769 & 0,231 & 1,000 & 0,875 & 0,686 & 0,392 & 0,193 & 0,076 & 0,152 & 172 & 146 & $-0,9$ \\
\hline 4 & 146 & 0,030 & 0,541 & 0,032 & 0,490 & 0,955 & 0,906 & 0,104 & 1,000 & 0,879 & 0,723 & 0,493 & 0,230 & 0,114 & 0,152 & 0 & 146 & $-1,2$ \\
\hline 5 & 146 & 0,129 & 1,830 & 0,158 & 1,627 & 0,818 & 0,889 & 0,213 & 0,485 & 1,437 & 0,235 & 0,226 & 0,837 & 0,189 & 0,214 & 179 & 146 & $-3,1$ \\
\hline 6 & 146 & 0,020 & 0,503 & 0,020 & 0,215 & 1,000 & 0,429 & 0,571 & 0,999 & 1,001 & 0,482 & 0,164 & 0,230 & 0,038 & 0,076 & 0 & 146 & $-1,2$ \\
\hline 7 & 146 & 0,034 & 0,634 & 0,039 & 0,418 & 0,889 & 0,660 & 0,358 & 1,000 & 0,964 & 0,764 & 0,632 & 0,240 & 0,152 & 0,152 & 4 & 146 & $-1,3$ \\
\hline 8 & 146 & 0,030 & 0,562 & 0,033 & 0,469 & 0,913 & 0,835 & 0,187 & 1,000 & 0,914 & 0,723 & 0,493 & 0,230 & 0,114 & 0,152 & 174 & 146 & $-1,2$ \\
\hline 9 & 146 & 0,017 & 0,520 & 0,019 & 0,469 & 0,923 & 0,902 & 0,124 & 0,800 & 1,118 & 0,340 & 0,249 & 0,254 & 0,063 & 0,076 & 145 & 146 & $-1,3$ \\
\hline 10 & 146 & 0,392 & 2,925 & 0,428 & 2,874 & 0,916 & 0,983 & 0,086 & 0,576 & 1,318 & 0,265 & 0,262 & 1,372 & 0,359 & 0,379 & 172 & 146 & $-3,8$ \\
\hline 11 & 146 & 0,036 & 0,592 & 0,039 & 0,541 & 0,926 & 0,914 & 0,113 & 1,000 & 0,881 & 0,936 & 0,667 & 0,221 & 0,147 & 0,169 & 124 & 146 & $-1,1$ \\
\hline 12 & 146 & 0,023 & 0,490 & 0,026 & 0,347 & 0,889 & 0,707 & 0,313 & 1,000 & 0,913 & 0,784 & 0,588 & 0,193 & 0,114 & 0,152 & 0 & 146 & $-0,9$ \\
\hline
\end{tabular}




\begin{tabular}{|c|c|c|c|c|c|c|c|c|c|c|c|c|c|c|c|c|c|c|}
\hline 13 & 146 & 0,030 & 0,520 & 0,032 & 0,490 & 0,955 & 0,943 & 0,073 & 1,000 & 0,845 & 1,028 & 0,784 & 0,193 & 0,152 & 0,169 & 172 & 146 & $-0,9$ \\
\hline 14 & 146 & 0,095 & 2,000 & 0,154 & 1,910 & 0,617 & 0,955 & 0,386 & 0,298 & 1,833 & 0,145 & 0,162 & 0,911 & 0,147 & 0,152 & 161 & 146 & $-3,2$ \\
\hline 15 & 146 & 0,032 & 0,634 & 0,034 & 0,511 & 0,917 & 0,807 & 0,211 & 0,987 & 1,007 & 0,560 & 0,424 & 0,268 & 0,114 & 0,152 & 165 & 146 & $-1,4$ \\
\hline 16 & 146 & 0,022 & 0,511 & 0,022 & 0,440 & 1,000 & 0,860 & 0,140 & 1,000 & 0,983 & 0,477 & 0,316 & 0,240 & 0,076 & 0,152 & 171 & 146 & $-1,3$ \\
\hline 17 & 146 & 0,042 & 0,816 & 0,044 & 0,714 & 0,935 & 0,876 & 0,140 & 0,785 & 1,128 & 0,381 & 0,318 & 0,373 & 0,119 & 0,152 & 153 & 146 & $-1,9$ \\
\hline 18 & 146 & 0,014 & 0,317 & 0,014 & 0,173 & 1,000 & 0,547 & 0,453 & 1,000 & 0,746 & 0,979 & 0,555 & 0,137 & 0,076 & 0,107 & 108 & 146 & $-0,5$ \\
\hline 19 & 146 & 0,169 & 1,995 & 0,182 & 1,893 & 0,929 & 0,949 & 0,087 & 0,535 & 1,368 & 0,237 & 0,199 & 0,954 & 0,189 & 0,227 & 175 & 146 & $-3,3$ \\
\hline 20 & 146 & 0,060 & 0,972 & 0,063 & 0,849 & 0,955 & 0,874 & 0,134 & 0,802 & 1,117 & 0,369 & 0,249 & 0,456 & 0,114 & 0,152 & 1 & 146 & $-2,2$ \\
\hline 21 & 146 & 0,017 & 0,440 & 0,019 & 0,317 & 0,923 & 0,721 & 0,289 & 1,000 & 0,945 & 0,527 & 0,332 & 0,204 & 0,068 & 0,076 & 159 & 146 & $-1,0$ \\
\hline 22 & 146 & 0,072 & 1,285 & 0,089 & 0,981 & 0,806 & 0,763 & 0,306 & 0,546 & 1,354 & 0,465 & 0,503 & 0,443 & 0,223 & 0,214 & 1 & 146 & $-2,1$ \\
\hline 23 & 146 & 0,014 & 0,368 & 0,016 & 0,317 & 0,909 & 0,862 & 0,165 & 1,000 & 0,866 & 0,749 & 0,461 & 0,156 & 0,072 & 0,076 & 169 & 146 & $-0,6$ \\
\hline 24 & 146 & 0,142 & 1,758 & 0,168 & 1,636 & 0,846 & 0,930 & 0,169 & 0,577 & 1,316 & 0,312 & 0,305 & 0,761 & 0,233 & 0,227 & 172 & 146 & $-2,9$ \\
\hline 25 & 146 & 0,106 & 2,248 & 0,161 & 1,821 & 0,661 & 0,810 & 0,389 & 0,264 & 1,947 & 0,159 & 0,205 & 0,922 & 0,189 & 0,214 & 177 & 146 & $-3,2$ \\
\hline 26 & 146 & 0,132 & 1,615 & 0,156 & 1,462 & 0,844 & 0,906 & 0,182 & 0,636 & 1,254 & 0,360 & 0,363 & 0,683 & 0,248 & 0,227 & 170 & 146 & $-2,8$ \\
\hline 27 & 146 & 0,034 & 0,634 & 0,036 & 0,541 & 0,960 & 0,853 & 0,152 & 1,000 & 0,964 & 0,577 & 0,412 & 0,276 & 0,114 & 0,152 & 173 & 146 & $-1,5$ \\
\hline 28 & 146 & 0,014 & 0,317 & 0,014 & 0,245 & 1,000 & 0,773 & 0,227 & 1,000 & 0,746 & 0,979 & 0,555 & 0,137 & 0,076 & 0,107 & 162 & 146 & $-0,5$ \\
\hline 29 & 146 & 0,036 & 1,086 & 0,047 & 0,778 & 0,758 & 0,716 & 0,373 & 0,382 & 1,617 & 0,162 & 0,142 & 0,532 & 0,076 & 0,152 & 5 & 146 & $-2,4$ \\
\hline 30 & 146 & 0,069 & 0,921 & 0,070 & 0,727 & 0,980 & 0,789 & 0,212 & 1,000 & 0,990 & 0,561 & 0,383 & 0,395 & 0,152 & 0,227 & 174 & 146 & $-2,0$ \\
\hline 31 & 146 & 0,445 & 3,292 & 0,496 & 3,131 & 0,896 & 0,951 & 0,115 & 0,516 & 1,393 & 0,254 & 0,253 & 1,495 & 0,379 & 0,379 & 174 & 146 & $-3,9$ \\
\hline 32 & 146 & 0,100 & 1,255 & 0,113 & 1,154 & 0,886 & 0,919 & 0,140 & 0,801 & 1,118 & 0,420 & 0,409 & 0,552 & 0,225 & 0,240 & 162 & 146 & $-2,5$ \\
\hline 33 & 146 & 0,042 & 0,676 & 0,043 & 0,461 & 0,967 & 0,681 & 0,320 & 1,000 & 0,935 & 0,697 & 0,412 & 0,276 & 0,114 & 0,152 & 3 & 146 & $-1,5$ \\
\hline 34 & 146 & 0,014 & 0,368 & 0,016 & 0,317 & 0,909 & 0,862 & 0,165 & 1,000 & 0,866 & 0,749 & 0,434 & 0,156 & 0,068 & 0,076 & 159 & 146 & $-0,6$ \\
\hline 35 & 146 & 0,032 & 0,613 & 0,033 & 0,520 & 0,957 & 0,848 & 0,158 & 1,000 & 0,973 & 0,529 & 0,412 & 0,276 & 0,114 & 0,152 & 169 & 146 & $-1,5$ \\
\hline 36 & 146 & 0,020 & 0,418 & 0,020 & 0,376 & 1,000 & 0,899 & 0,101 & 1,000 & 0,833 & 0,891 & 0,600 & 0,169 & 0,102 & 0,152 & 153 & 146 & $-0,8$ \\
\hline 37 & 146 & 0,331 & 3,483 & 0,452 & 2,798 & 0,733 & 0,803 & 0,331 & 0,343 & 1,707 & 0,264 & 0,394 & 1,264 & 0,498 & 0,214 & 171 & 146 & $-3,7$ \\
\hline 38 & 146 & 0,052 & 0,909 & 0,057 & 0,837 & 0,900 & 0,921 & 0,127 & 0,786 & 1,128 & 0,376 & 0,351 & 0,418 & 0,147 & 0,152 & 174 & 146 & $-2,1$ \\
\hline 39 & 146 & 0,055 & 0,858 & 0,057 & 0,807 & 0,950 & 0,941 & 0,077 & 0,931 & 1,037 & 0,479 & 0,398 & 0,381 & 0,152 & 0,169 & 175 & 146 & $-1,9$ \\
\hline 40 & 146 & 0,251 & 2,532 & 0,340 & 2,126 & 0,738 & 0,840 & 0,307 & 0,492 & 1,425 & 0,348 & 0,422 & 0,958 & 0,404 & 0,303 & 177 & 146 & $-3,3$ \\
\hline 41 & 146 & 0,274 & 2,182 & 0,300 & 2,071 & 0,914 & 0,950 & 0,100 & 0,724 & 1,176 & 0,399 & 0,399 & 0,936 & 0,373 & 0,379 & 155 & 146 & $-3,2$ \\
\hline 42 & 146 & 0,037 & 0,706 & 0,042 & 0,562 & 0,897 & 0,796 & 0,228 & 0,941 & 1,031 & 0,487 & 0,364 & 0,312 & 0,114 & 0,152 & 173 & 146 & $-1,6$ \\
\hline 43 & 146 & 0,132 & 1,462 & 0,139 & 1,361 & 0,948 & 0,931 & 0,086 & 0,776 & 1,135 & 0,373 & 0,339 & 0,671 & 0,227 & 0,240 & 169 & 146 & $-2,7$ \\
\hline 44 & 146 & 0,014 & 0,317 & 0,014 & 0,245 & 1,000 & 0,773 & 0,227 & 1,000 & 0,746 & 0,979 & 0,555 & 0,137 & 0,076 & 0,107 & 162 & 146 & $-0,5$ \\
\hline 45 & 146 & 0,106 & 1,695 & 0,162 & 1,255 & 0,655 & 0,741 & 0,432 & 0,464 & 1,467 & 0,444 & 0,650 & 0,552 & 0,358 & 0,227 & 153 & 146 & $-2,5$ \\
\hline 46 & 146 & 0,062 & 1,073 & 0,070 & 0,951 & 0,878 & 0,886 & 0,167 & 0,673 & 1,219 & 0,308 & 0,271 & 0,505 & 0,137 & 0,152 & 168 & 146 & $-2,3$ \\
\hline 47 & 146 & 0,115 & 1,716 & 0,142 & 1,462 & 0,808 & 0,852 & 0,242 & 0,490 & 1,429 & 0,292 & 0,308 & 0,708 & 0,218 & 0,152 & 173 & 146 & $-2,8$ \\
\hline 48 & 146 & 0,024 & 0,622 & 0,032 & 0,541 & 0,773 & 0,871 & 0,262 & 0,794 & 1,123 & 0,408 & 0,307 & 0,276 & 0,085 & 0,107 & 153 & 146 & $-1,5$ \\
\hline 49 & 146 & 0,055 & 0,828 & 0,057 & 0,706 & 0,950 & 0,852 & 0,156 & 0,999 & 1,001 & 0,538 & 0,422 & 0,359 & 0,152 & 0,169 & 173 & 146 & $-1,8$ \\
\hline 50 & 146 & 0,019 & 0,511 & 0,019 & 0,368 & 1,000 & 0,719 & 0,281 & 0,896 & 1,056 & 0,571 & 0,496 & 0,204 & 0,101 & 0,152 & 162 & 146 & $-1,0$ \\
\hline 51 & 146 & 0,023 & 0,461 & 0,023 & 0,317 & 1,000 & 0,688 & 0,312 & 1,000 & 0,858 & 0,702 & 0,371 & 0,204 & 0,076 & 0,152 & 175 & 146 & $-1,0$ \\
\hline 52 & 146 & 0,172 & 1,564 & 0,184 & 1,399 & 0,938 & 0,895 & 0,122 & 0,885 & 1,063 & 0,501 & 0,458 & 0,662 & 0,303 & 0,312 & 176 & 146 & $-2,7$ \\
\hline 53 & 146 & 0,050 & 0,714 & 0,052 & 0,664 & 0,972 & 0,929 & 0,076 & 1,000 & 0,899 & 0,768 & 0,623 & 0,288 & 0,180 & 0,214 & 159 & 146 & $-1,5$ \\
\hline 54 & 146 & 0,027 & 0,562 & 0,029 & 0,469 & 0,950 & 0,835 & 0,173 & 1,000 & 0,960 & 0,605 & 0,474 & 0,240 & 0,114 & 0,152 & 162 & 146 & $-1,3$ \\
\hline 55 & 146 & 0,106 & 1,602 & 0,136 & 1,437 & 0,779 & 0,897 & 0,244 & 0,520 & 1,387 & 0,280 & 0,366 & 0,695 & 0,254 & 0,273 & 150 & 146 & $-2,8$ \\
\hline 56 & 146 & 0,141 & 1,695 & 0,162 & 1,441 & 0,867 & 0,850 & 0,200 & 0,615 & 1,275 & 0,486 & 0,465 & 0,607 & 0,282 & 0,214 & 168 & 146 & $-2,6$ \\
\hline 57 & 146 & 0,016 & 0,410 & 0,016 & 0,144 & 1,000 & 0,350 & 0,650 & 1,000 & 0,920 & 0,539 & 0,196 & 0,193 & 0,038 & 0,076 & 0 & 146 & $-0,9$ \\
\hline 58 & 146 & 0,016 & 0,338 & 0,016 & 0,194 & 1,000 & 0,575 & 0,425 & 1,000 & 0,759 & 1,077 & 0,555 & 0,137 & 0,076 & 0,152 & 162 & 146 & $-0,5$ \\
\hline 59 & 146 & 0,240 & 2,075 & 0,253 & 2,045 & 0,949 & 0,986 & 0,053 & 0,699 & 1,196 & 0,338 & 0,319 & 0,950 & 0,303 & 0,312 & 177 & 146 & $-3,2$ \\
\hline 60 & 146 & 0,073 & 1,082 & 0,085 & 0,917 & 0,864 & 0,848 & 0,204 & 0,785 & 1,128 & 0,500 & 0,549 & 0,432 & 0,237 & 0,214 & 132 & 146 & $-2,1$ \\
\hline 61 & 146 & 0,042 & 0,735 & 0,043 & 0,706 & 0,967 & 0,960 & 0,052 & 0,967 & 1,017 & 0,450 & 0,331 & 0,343 & 0,114 & 0,152 & 177 & 146 & $-1,8$ \\
\hline 62 & 146 & 0,182 & 3,242 & 0,240 & 1,839 & 0,760 & 0,567 & 0,495 & 0,218 & 2,142 & 0,318 & 0,370 & 0,855 & 0,316 & 0,152 & 165 & 146 & $-3,1$ \\
\hline 63 & 146 & 0,537 & 3,120 & 0,610 & 2,815 & 0,880 & 0,902 & 0,155 & 0,693 & 1,201 & 0,529 & 0,581 & 1,136 & 0,660 & 0,611 & 152 & 146 & $-3,5$ \\
\hline 64 & 146 & 0,016 & 0,368 & 0,016 & 0,317 & 1,000 & 0,862 & 0,138 & 1,000 & 0,826 & 0,824 & 0,485 & 0,156 & 0,076 & 0,107 & 0 & 146 & $-0,6$ \\
\hline 65 & 146 & 0,083 & 1,205 & 0,106 & 1,044 & 0,784 & 0,866 & 0,254 & 0,721 & 1,178 & 0,631 & 0,695 & 0,410 & 0,285 & 0,214 & 77 & 146 & $-2,0$ \\
\hline 66 & 146 & 0,072 & 1,196 & 0,093 & 0,951 & 0,769 & 0,795 & 0,309 & 0,630 & 1,260 & 0,490 & 0,523 & 0,432 & 0,226 & 0,227 & 1 & 146 & $-2,1$ \\
\hline 67 & 146 & 0,032 & 0,532 & 0,033 & 0,368 & 0,957 & 0,691 & 0,312 & 1,000 & 0,845 & 1,120 & 0,800 & 0,189 & 0,152 & 0,169 & 54 & 146 & $-0,9$ \\
\hline 68 & 146 & 0,016 & 0,368 & 0,017 & 0,317 & 0,917 & 0,862 & 0,161 & 1,000 & 0,826 & 0,824 & 0,485 & 0,156 & 0,076 & 0,107 & 167 & 146 & $-0,6$ \\
\hline 69 & 146 & 0,017 & 0,418 & 0,019 & 0,317 & 0,923 & 0,757 & 0,254 & 1,000 & 0,900 & 0,764 & 0,495 & 0,169 & 0,084 & 0,107 & 162 & 146 & $-0,8$ \\
\hline 70 & 146 & 0,034 & 0,714 & 0,043 & 0,613 & 0,800 & 0,858 & 0,245 & 0,848 & 1,086 & 0,470 & 0,451 & 0,305 & 0,138 & 0,152 & 4 & 146 & $-1,6$ \\
\hline
\end{tabular}




\begin{tabular}{|c|c|c|c|c|c|c|c|c|c|c|c|c|c|c|c|c|c|c|}
\hline 71 & 146 & 0,022 & 0,511 & 0,023 & 0,287 & 0,938 & 0,562 & 0,443 & 1,000 & 0,983 & 0,477 & 0,316 & 0,240 & 0,076 & 0,107 & 165 & 146 & $-1,3$ \\
\hline 72 & 146 & 0,034 & 0,693 & 0,042 & 0,592 & 0,828 & 0,854 & 0,226 & 0,900 & 1,054 & 0,577 & 0,492 & 0,276 & 0,136 & 0,152 & 142 & 146 & $-1,5$ \\
\hline 73 & 146 & 0,022 & 0,532 & 0,024 & 0,266 & 0,882 & 0,500 & 0,514 & 0,954 & 1,024 & 0,516 & 0,329 & 0,230 & 0,076 & 0,107 & 6 & 146 & $-1,2$ \\
\hline 74 & 146 & 0,024 & 0,490 & 0,026 & 0,418 & 0,944 & 0,854 & 0,157 & 1,000 & 0,886 & 0,746 & 0,529 & 0,204 & 0,108 & 0,152 & 163 & 146 & $-1,0$ \\
\hline 75 & 146 & 0,056 & 1,052 & 0,066 & 0,757 & 0,848 & 0,719 & 0,320 & 0,635 & 1,255 & 0,680 & 0,585 & 0,324 & 0,189 & 0,214 & 174 & 146 & $-1,7$ \\
\hline 76 & 146 & 0,043 & 0,685 & 0,043 & 0,634 & 1,000 & 0,926 & 0,074 & 1,000 & 0,931 & 0,588 & 0,372 & 0,305 & 0,114 & 0,152 & 177 & 146 & $-1,6$ \\
\hline 77 & 146 & 0,027 & 0,693 & 0,039 & 0,461 & 0,704 & 0,664 & 0,448 & 0,713 & 1,185 & 0,484 & 0,526 & 0,268 & 0,141 & 0,169 & 7 & 146 & $-1,4$ \\
\hline 78 & 146 & 0,742 & 6,447 & 1,063 & 4,218 & 0,698 & 0,654 & 0,459 & 0,224 & 2,112 & 0,299 & 0,452 & 1,779 & 0,804 & 0,169 & 20 & 146 & $-4,2$ \\
\hline 79 & 146 & 0,019 & 0,418 & 0,020 & 0,296 & 0,929 & 0,707 & 0,301 & 1,000 & 0,864 & 0,828 & 0,600 & 0,169 & 0,102 & 0,152 & 149 & 146 & $-0,8$ \\
\hline 80 & 146 & 0,158 & 1,860 & 0,201 & 1,602 & 0,786 & 0,861 & 0,255 & 0,573 & 1,321 & 0,393 & 0,503 & 0,715 & 0,359 & 0,303 & 152 & 146 & $-2,8$ \\
\hline 81 & 146 & 0,032 & 0,664 & 0,036 & 0,550 & 0,880 & 0,828 & 0,209 & 0,901 & 1,054 & 0,539 & 0,445 & 0,273 & 0,121 & 0,152 & 131 & 146 & $-1,4$ \\
\hline 82 & 146 & 0,063 & 0,879 & 0,065 & 0,807 & 0,978 & 0,918 & 0,085 & 1,000 & 0,987 & 0,514 & 0,383 & 0,395 & 0,152 & 0,227 & 7 & 146 & $-2,0$ \\
\hline 83 & 146 & 0,030 & 0,643 & 0,036 & 0,541 & 0,840 & 0,842 & 0,225 & 0,917 & 1,044 & 0,594 & 0,478 & 0,254 & 0,121 & 0,107 & 144 & 146 & $-1,3$ \\
\hline 84 & 146 & 0,014 & 0,389 & 0,014 & 0,266 & 1,000 & 0,685 & 0,315 & 1,000 & 0,916 & 0,490 & 0,196 & 0,193 & 0,038 & 0,076 & 0 & 146 & $-0,9$ \\
\hline 85 & 146 & 0,067 & 1,572 & 0,136 & 1,264 & 0,495 & 0,804 & 0,542 & 0,343 & 1,708 & 0,266 & 0,496 & 0,568 & 0,282 & 0,152 & 39 & 146 & $-2,5$ \\
\hline 86 & 146 & 0,029 & 0,541 & 0,029 & 0,418 & 1,000 & 0,773 & 0,227 & 1,000 & 0,901 & 0,621 & 0,442 & 0,243 & 0,107 & 0,152 & 146 & 146 & $-1,3$ \\
\hline 87 & 146 & 0,014 & 0,317 & 0,014 & 0,173 & 1,000 & 0,547 & 0,453 & 1,000 & 0,746 & 1,273 & 0,632 & 0,120 & 0,076 & 0,107 & 18 & 146 & $-0,3$ \\
\hline 88 & 146 & 0,029 & 0,583 & 0,032 & 0,448 & 0,909 & 0,769 & 0,249 & 1,000 & 0,971 & 0,637 & 0,474 & 0,240 & 0,114 & 0,152 & 21 & 146 & $-1,3$ \\
\hline 89 & 146 & 0,020 & 0,461 & 0,020 & 0,368 & 1,000 & 0,798 & 0,202 & 1,000 & 0,917 & 0,686 & 0,392 & 0,193 & 0,076 & 0,152 & 8 & 146 & $-0,9$ \\
\hline 90 & 146 & 0,033 & 0,807 & 0,039 & 0,655 & 0,852 & 0,811 & 0,240 & 0,636 & 1,254 & 0,293 & 0,200 & 0,379 & 0,076 & 0,152 & 178 & 146 & $-1,9$ \\
\hline 91 & 146 & 0,294 & 2,870 & 0,425 & 2,473 & 0,693 & 0,862 & 0,337 & 0,449 & 1,493 & 0,307 & 0,467 & 1,104 & 0,516 & 0,339 & 14 & 146 & $-3,5$ \\
\hline 92 & 146 & 0,072 & 1,572 & 0,096 & 1,205 & 0,746 & 0,766 & 0,345 & 0,365 & 1,656 & 0,255 & 0,319 & 0,599 & 0,191 & 0,152 & 162 & 146 & $-2,6$ \\
\hline 93 & 146 & 0,169 & 1,593 & 0,191 & 1,390 & 0,887 & 0,873 & 0,170 & 0,838 & 1,092 & 0,680 & 0,740 & 0,563 & 0,417 & 0,379 & 40 & 146 & $-2,5$ \\
\hline 94 & 146 & 0,039 & 0,879 & 0,047 & 0,757 & 0,818 & 0,861 & 0,229 & 0,630 & 1,260 & 0,282 & 0,272 & 0,418 & 0,114 & 0,152 & 0 & 146 & $-2,1$ \\
\hline 95 & 146 & 0,027 & 0,583 & 0,030 & 0,448 & 0,905 & 0,769 & 0,250 & 1,000 & 0,996 & 0,605 & 0,474 & 0,240 & 0,114 & 0,152 & 179 & 146 & $-1,3$ \\
\hline 96 & 146 & 0,024 & 0,714 & 0,036 & 0,448 & 0,680 & 0,627 & 0,491 & 0,601 & 1,290 & 0,637 & 0,653 & 0,221 & 0,144 & 0,107 & 62 & 146 & $-1,1$ \\
\hline 97 & 146 & 0,020 & 0,440 & 0,020 & 0,368 & 1,000 & 0,837 & 0,163 & 1,000 & 0,875 & 0,686 & 0,392 & 0,193 & 0,076 & 0,152 & 0 & 146 & $-0,9$ \\
\hline 98 & 146 & 0,019 & 0,511 & 0,020 & 0,317 & 0,929 & 0,620 & 0,387 & 0,896 & 1,056 & 0,414 & 0,307 & 0,240 & 0,074 & 0,076 & 173 & 146 & $-1,3$ \\
\hline 99 & 146 & 0,070 & 0,879 & 0,072 & 0,795 & 0,980 & 0,904 & 0,098 & 1,000 & 0,935 & 0,701 & 0,600 & 0,357 & 0,214 & 0,227 & 151 & 146 & $-1,8$ \\
\hline 100 & 146 & 0,014 & 0,368 & 0,016 & 0,296 & 0,909 & 0,805 & 0,215 & 1,000 & 0,866 & 0,637 & 0,400 & 0,169 & 0,068 & 0,076 & 159 & 146 & $-0,8$ \\
\hline 101 & 146 & 0,016 & 0,368 & 0,017 & 0,245 & 0,917 & 0,667 & 0,344 & 1,000 & 0,826 & 0,778 & 0,500 & 0,161 & 0,080 & 0,107 & 18 & 146 & $-0,7$ \\
\hline 102 & 146 & 0,014 & 0,397 & 0,017 & 0,347 & 0,833 & 0,872 & 0,210 & 1,000 & 0,936 & 0,637 & 0,373 & 0,169 & 0,063 & 0,107 & 145 & 146 & $-0,8$ \\
\hline 103 & 146 & 0,014 & 0,338 & 0,014 & 0,245 & 1,000 & 0,725 & 0,275 & 1,000 & 0,796 & 0,979 & 0,555 & 0,137 & 0,076 & 0,107 & 72 & 146 & $-0,5$ \\
\hline 104 & 146 & 0,030 & 0,778 & 0,043 & 0,541 & 0,700 & 0,696 & 0,427 & 0,626 & 1,264 & 0,361 & 0,448 & 0,326 & 0,146 & 0,152 & 108 & 146 & $-1,7$ \\
\hline 105 & 146 & 0,016 & 0,338 & 0,016 & 0,194 & 1,000 & 0,575 & 0,425 & 1,000 & 0,759 & 1,077 & 0,555 & 0,137 & 0,076 & 0,152 & 162 & 146 & $-0,5$ \\
\hline 106 & 146 & 0,014 & 0,368 & 0,016 & 0,173 & 0,909 & 0,471 & 0,536 & 1,000 & 0,866 & 0,749 & 0,485 & 0,156 & 0,076 & 0,107 & 90 & 146 & $-0,6$ \\
\hline 107 & 146 & 0,017 & 0,511 & 0,020 & 0,368 & 0,857 & 0,719 & 0,315 & 0,827 & 1,099 & 0,382 & 0,260 & 0,240 & 0,062 & 0,076 & 90 & 146 & $-1,3$ \\
\hline 108 & 146 & 0,023 & 0,490 & 0,024 & 0,410 & 0,941 & 0,836 & 0,174 & 1,000 & 0,913 & 0,599 & 0,434 & 0,221 & 0,096 & 0,152 & 24 & 146 & $-1,1$ \\
\hline 109 & 146 & 0,023 & 0,562 & 0,026 & 0,490 & 0,889 & 0,872 & 0,169 & 0,913 & 1,047 & 0,407 & 0,283 & 0,268 & 0,076 & 0,107 & 12 & 146 & $-1,4$ \\
\hline 110 & 146 & 0,044 & 0,837 & 0,049 & 0,765 & 0,912 & 0,914 & 0,123 & 0,798 & 1,120 & 0,407 & 0,358 & 0,373 & 0,134 & 0,152 & 26 & 146 & $-1,9$ \\
\hline 111 & 146 & 0,044 & 0,816 & 0,055 & 0,744 & 0,816 & 0,912 & 0,204 & 0,840 & 1,091 & 0,493 & 0,492 & 0,339 & 0,167 & 0,169 & 23 & 146 & $-1,8$ \\
\hline 112 & 146 & 0,019 & 0,520 & 0,023 & 0,397 & 0,812 & 0,764 & 0,301 & 0,867 & 1,074 & 0,404 & 0,304 & 0,243 & 0,074 & 0,076 & 40 & 146 & $-1,3$ \\
\hline 113 & 146 & 0,019 & 0,448 & 0,019 & 0,368 & 1,000 & 0,820 & 0,180 & 1,000 & 0,926 & 0,517 & 0,250 & 0,214 & 0,054 & 0,107 & 45 & 146 & $-1,1$ \\
\hline 114 & 146 & 0,014 & 0,368 & 0,017 & 0,245 & 0,833 & 0,667 & 0,373 & 1,000 & 0,866 & 0,979 & 0,621 & 0,137 & 0,085 & 0,107 & 0 & 146 & $-0,5$ \\
\hline 115 & 146 & 0,027 & 0,786 & 0,036 & 0,622 & 0,760 & 0,791 & 0,319 & 0,554 & 1,343 & 0,327 & 0,329 & 0,326 & 0,107 & 0,107 & 39 & 146 & $-1,7$ \\
\hline 116 & 146 & 0,016 & 0,389 & 0,017 & 0,144 & 0,917 & 0,369 & 0,636 & 1,000 & 0,873 & 0,824 & 0,485 & 0,156 & 0,076 & 0,107 & 11 & 146 & $-0,6$ \\
\hline 117 & 146 & 0,016 & 0,440 & 0,019 & 0,224 & 0,846 & 0,510 & 0,514 & 1,000 & 0,987 & 0,539 & 0,373 & 0,193 & 0,072 & 0,076 & 11 & 146 & $-0,9$ \\
\hline 118 & 146 & 0,033 & 1,327 & 0,059 & 0,685 & 0,561 & 0,516 & 0,654 & 0,235 & 2,061 & 0,401 & 0,650 & 0,324 & 0,210 & 0,076 & 35 & 146 & $-1,7$ \\
\hline 119 & 146 & 0,099 & 2,583 & 0,142 & 1,590 & 0,697 & 0,616 & 0,490 & 0,187 & 2,316 & 0,197 & 0,253 & 0,799 & 0,202 & 0,152 & 33 & 146 & $-3,0$ \\
\hline 120 & 146 & 0,030 & 0,613 & 0,032 & 0,520 & 0,955 & 0,848 & 0,158 & 1,000 & 0,996 & 0,504 & 0,369 & 0,276 & 0,102 & 0,152 & 20 & 146 & $-1,5$ \\
\hline 121 & 146 & 0,037 & 0,807 & 0,047 & 0,634 & 0,788 & 0,785 & 0,302 & 0,719 & 1,179 & 0,404 & 0,331 & 0,343 & 0,114 & 0,152 & 175 & 146 & $-1,8$ \\
\hline 122 & 146 & 0,016 & 0,532 & 0,019 & 0,338 & 0,846 & 0,635 & 0,396 & 0,700 & 1,196 & 0,286 & 0,143 & 0,265 & 0,038 & 0,076 & 0 & 146 & $-1,4$ \\
\hline 123 & 146 & 0,039 & 0,795 & 0,042 & 0,714 & 0,931 & 0,899 & 0,122 & 0,771 & 1,139 & 0,430 & 0,400 & 0,339 & 0,136 & 0,152 & 30 & 146 & $-1,8$ \\
\hline 124 & 146 & 0,027 & 0,562 & 0,027 & 0,469 & 1,000 & 0,835 & 0,165 & 1,000 & 0,960 & 0,538 & 0,400 & 0,254 & 0,102 & 0,152 & 24 & 146 & $-1,3$ \\
\hline 125 & 146 & 0,053 & 0,837 & 0,060 & 0,714 & 0,881 & 0,854 & 0,189 & 0,952 & 1,025 & 0,589 & 0,498 & 0,339 & 0,169 & 0,169 & 26 & 146 & $-1,8$ \\
\hline 126 & 146 & 0,020 & 0,490 & 0,022 & 0,389 & 0,933 & 0,793 & 0,218 & 1,000 & 0,976 & 0,446 & 0,316 & 0,240 & 0,076 & 0,107 & 15 & 146 & $-1,3$ \\
\hline 127 & 146 & 0,032 & 0,807 & 0,037 & 0,511 & 0,846 & 0,633 & 0,398 & 0,609 & 1,282 & 0,700 & 0,474 & 0,240 & 0,114 & 0,152 & 21 & 146 & $-1,3$ \\
\hline 128 & 146 & 0,029 & 0,744 & 0,034 & 0,672 & 0,833 & 0,903 & 0,193 & 0,651 & 1,239 & 0,318 & 0,251 & 0,339 & 0,085 & 0,107 & 31 & 146 & $-1,8$ \\
\hline
\end{tabular}




\begin{tabular}{|c|c|c|c|c|c|c|c|c|c|c|c|c|c|c|c|c|c|c|}
\hline 129 & 146 & 0,016 & 0,389 & 0,017 & 0,245 & 0,917 & 0,631 & 0,379 & 1,000 & 0,873 & 0,824 & 0,485 & 0,156 & 0,076 & 0,107 & 108 & 146 & $-0,6$ \\
\hline 130 & 146 & 0,022 & 0,418 & 0,022 & 0,347 & 1,000 & 0,828 & 0,172 & 1,000 & 0,805 & 1,123 & 0,728 & 0,156 & 0,114 & 0,152 & 0 & 146 & $-0,6$ \\
\hline 131 & 146 & 0,017 & 0,368 & 0,019 & 0,266 & 0,923 & 0,724 & 0,287 & 1,000 & 0,791 & 1,175 & 0,745 & 0,137 & 0,102 & 0,107 & 153 & 146 & $-0,5$ \\
\hline 132 & 146 & 0,033 & 0,786 & 0,044 & 0,664 & 0,742 & 0,844 & 0,302 & 0,671 & 1,221 & 0,366 & 0,294 & 0,339 & 0,100 & 0,107 & 24 & 146 & $-1,8$ \\
\hline 133 & 146 & 0,023 & 0,643 & 0,032 & 0,490 & 0,727 & 0,763 & 0,361 & 0,699 & 1,196 & 0,453 & 0,425 & 0,254 & 0,108 & 0,107 & 12 & 146 & $-1,3$ \\
\hline 134 & 146 & 0,095 & 2,050 & 0,159 & 1,446 & 0,595 & 0,705 & 0,501 & 0,283 & 1,880 & 0,259 & 0,374 & 0,683 & 0,256 & 0,107 & 31 & 146 & $-2,8$ \\
\hline 135 & 146 & 0,123 & 2,059 & 0,172 & 1,480 & 0,717 & 0,719 & 0,399 & 0,366 & 1,653 & 0,358 & 0,409 & 0,663 & 0,271 & 0,169 & 27 & 146 & $-2,7$ \\
\hline 136 & 146 & 0,029 & 0,562 & 0,032 & 0,410 & 0,909 & 0,729 & 0,286 & 1,000 & 0,936 & 0,796 & 0,633 & 0,214 & 0,136 & 0,169 & 32 & 146 & $-1,1$ \\
\hline 137 & 146 & 0,016 & 0,520 & 0,019 & 0,469 & 0,846 & 0,902 & 0,182 & 0,733 & 1,168 & 0,311 & 0,200 & 0,254 & 0,051 & 0,076 & 23 & 146 & $-1,3$ \\
\hline 138 & 146 & 0,044 & 0,837 & 0,049 & 0,765 & 0,912 & 0,914 & 0,123 & 0,798 & 1,120 & 0,407 & 0,353 & 0,373 & 0,132 & 0,152 & 27 & 146 & $-1,9$ \\
\hline 139 & 146 & 0,017 & 0,440 & 0,019 & 0,389 & 0,923 & 0,885 & 0,139 & 1,000 & 0,945 & 0,527 & 0,353 & 0,204 & 0,072 & 0,076 & 15 & 146 & $-1,0$ \\
\hline 1 & 147 & 0,020 & 0,389 & 0,020 & 0,287 & 1,000 & 0,739 & 0,261 & 1,000 & 0,774 & 0,990 & 0,667 & 0,161 & 0,107 & 0,152 & 45 & 147 & $-0,7$ \\
\hline 2 & 147 & 0,016 & 0,347 & 0,016 & 0,245 & 1,000 & 0,707 & 0,293 & 1,000 & 0,778 & 1,401 & 0,849 & 0,120 & 0,102 & 0,107 & 45 & 147 & $-0,3$ \\
\hline 3 & 147 & 0,131 & 1,420 & 0,154 & 1,247 & 0,850 & 0,878 & 0,193 & 0,814 & 1,109 & 0,597 & 0,574 & 0,528 & 0,303 & 0,303 & 10 & 147 & $-2,4$ \\
\hline 4 & 147 & 0,020 & 0,418 & 0,022 & 0,347 & 0,933 & 0,828 & 0,184 & 1,000 & 0,833 & 1,049 & 0,728 & 0,156 & 0,114 & 0,152 & 8 & 147 & $-0,6$ \\
\hline 5 & 147 & 0,014 & 0,317 & 0,014 & 0,173 & 1,000 & 0,547 & 0,453 & 1,000 & 0,746 & 1,273 & 0,632 & 0,120 & 0,076 & 0,107 & 18 & 147 & $-0,3$ \\
\hline 6 & 147 & 0,024 & 0,469 & 0,026 & 0,389 & 0,944 & 0,829 & 0,180 & 1,000 & 0,848 & 1,082 & 0,701 & 0,169 & 0,119 & 0,152 & 39 & 147 & $-0,8$ \\
\hline 7 & 147 & 0,016 & 0,347 & 0,016 & 0,296 & 1,000 & 0,854 & 0,146 & 1,000 & 0,778 & 1,401 & 0,849 & 0,120 & 0,102 & 0,107 & 45 & 147 & $-0,3$ \\
\hline 8 & 147 & 0,020 & 0,418 & 0,020 & 0,317 & 1,000 & 0,757 & 0,243 & 1,000 & 0,833 & 0,891 & 0,632 & 0,169 & 0,107 & 0,152 & 17 & 147 & $-0,8$ \\
\hline 9 & 147 & 0,047 & 0,685 & 0,050 & 0,583 & 0,943 & 0,852 & 0,159 & 1,000 & 0,888 & 0,934 & 0,723 & 0,254 & 0,184 & 0,227 & 10 & 147 & $-1,3$ \\
\hline 10 & 147 & 0,022 & 0,440 & 0,022 & 0,389 & 1,000 & 0,885 & 0,115 & 1,000 & 0,845 & 0,955 & 0,671 & 0,169 & 0,114 & 0,152 & 163 & 147 & $-0,8$ \\
\hline 11 & 147 & 0,050 & 0,714 & 0,052 & 0,613 & 0,972 & 0,858 & 0,145 & 1,000 & 0,899 & 0,990 & 0,828 & 0,254 & 0,210 & 0,214 & 43 & 147 & $-1,3$ \\
\hline 12 & 147 & 0,014 & 0,317 & 0,014 & 0,245 & 1,000 & 0,773 & 0,227 & 1,000 & 0,746 & 0,979 & 0,555 & 0,137 & 0,076 & 0,107 & 162 & 147 & $-0,5$ \\
\hline 13 & 147 & 0,016 & 0,418 & 0,017 & 0,368 & 0,917 & 0,879 & 0,147 & 1,000 & 0,940 & 0,560 & 0,389 & 0,189 & 0,074 & 0,076 & 162 & 147 & $-0,9$ \\
\hline 14 & 147 & 0,027 & 0,490 & 0,029 & 0,397 & 0,950 & 0,811 & 0,196 & 1,000 & 0,838 & 1,210 & 0,894 & 0,169 & 0,152 & 0,169 & 110 & 147 & $-0,8$ \\
\hline 15 & 147 & 0,023 & 0,418 & 0,023 & 0,368 & 1,000 & 0,879 & 0,121 & 1,000 & 0,779 & 1,198 & 0,728 & 0,156 & 0,114 & 0,152 & 0 & 147 & $-0,6$ \\
\hline 16 & 147 & 0,024 & 0,440 & 0,024 & 0,397 & 1,000 & 0,904 & 0,096 & 1,000 & 0,794 & 1,082 & 0,671 & 0,169 & 0,114 & 0,152 & 105 & 147 & $-0,8$ \\
\hline 17 & 147 & 0,019 & 0,389 & 0,019 & 0,317 & 1,000 & 0,815 & 0,185 & 1,000 & 0,803 & 0,974 & 0,485 & 0,156 & 0,076 & 0,152 & 98 & 147 & $-0,6$ \\
\hline & 148 & \multirow{2}{*}{\multicolumn{15}{|c|}{$\begin{array}{l}\text { nothing to measure } \\
\text { nothing to measure }\end{array}$}} & 148 & - \\
\hline & 149 & \multicolumn{9}{|c|}{ nothing to measure } & & & & & & & 149 & - \\
\hline & 150 & \multirow{2}{*}{\multicolumn{15}{|c|}{$\begin{array}{l}\text { nothing to measure } \\
\text { nothing to measure }\end{array}$}} & 150 & - \\
\hline & 151 & & & & & & & & & & & & & & & & 151 & - \\
\hline 1 & 152 & 0,033 & 0,888 & 0,049 & 0,571 & 0,676 & 0,643 & 0,482 & 0,526 & 1,379 & 0,431 & 0,489 & 0,312 & 0,153 & 0,107 & 22 & 152 & $-1,6$ \\
\hline 2 & 152 & 0,022 & 0,461 & 0,023 & 0,266 & 0,938 & 0,578 & 0,427 & 1,000 & 0,886 & 0,659 & 0,371 & 0,204 & 0,076 & 0,152 & 171 & 152 & $-1,0$ \\
\hline 1 & 153 & 0,029 & 0,511 & 0,029 & 0,397 & 1,000 & 0,777 & 0,223 & 1,000 & 0,852 & 1,019 & 0,800 & 0,189 & 0,152 & 0,169 & 135 & 153 & $-0,9$ \\
\hline 2 & 153 & 0,034 & 0,634 & 0,037 & 0,532 & 0,923 & 0,840 & 0,178 & 1,000 & 0,964 & 0,611 & 0,424 & 0,268 & 0,114 & 0,152 & 90 & 153 & $-1,4$ \\
\hline 3 & 153 & 0,024 & 0,825 & 0,030 & 0,723 & 0,810 & 0,877 & 0,227 & 0,451 & 1,489 & 0,192 & 0,132 & 0,403 & 0,053 & 0,076 & 141 & 153 & $-2,0$ \\
\hline 4 & 153 & 0,204 & 3,175 & 0,324 & 2,135 & 0,628 & 0,672 & 0,495 & 0,254 & 1,984 & 0,299 & 0,495 & 0,932 & 0,461 & 0,240 & 76 & 153 & $-3,2$ \\
\hline 5 & 153 & 0,037 & 1,091 & 0,063 & 0,795 & 0,591 & 0,729 & 0,491 & 0,394 & 1,593 & 0,283 & 0,379 & 0,410 & 0,155 & 0,107 & 122 & 153 & $-2,0$ \\
\hline 6 & 153 & 0,034 & 0,592 & 0,037 & 0,511 & 0,923 & 0,864 & 0,156 & 1,000 & 0,900 & 0,764 & 0,600 & 0,240 & 0,144 & 0,152 & 106 & 153 & $-1,3$ \\
\hline 7 & 153 & 0,030 & 0,786 & 0,036 & 0,714 & 0,840 & 0,909 & 0,184 & 0,613 & 1,278 & 0,276 & 0,225 & 0,373 & 0,084 & 0,107 & 111 & 153 & $-1,9$ \\
\hline 8 & 153 & 0,014 & 0,317 & 0,014 & 0,173 & 1,000 & 0,547 & 0,453 & 1,000 & 0,746 & 0,979 & 0,555 & 0,137 & 0,076 & 0,107 & 108 & 153 & $-0,5$ \\
\hline 9 & 153 & 0,043 & 0,664 & 0,044 & 0,583 & 0,968 & 0,879 & 0,125 & 1,000 & 0,902 & 0,721 & 0,549 & 0,276 & 0,152 & 0,169 & 75 & 153 & $-1,5$ \\
\hline 10 & 153 & 0,046 & 1,154 & 0,065 & 1,082 & 0,711 & 0,938 & 0,296 & 0,433 & 1,519 & 0,199 & 0,246 & 0,542 & 0,133 & 0,169 & 115 & 153 & $-2,4$ \\
\hline 11 & 153 & 0,032 & 0,634 & 0,036 & 0,397 & 0,880 & 0,627 & 0,392 & 0,987 & 1,007 & 0,539 & 0,476 & 0,273 & 0,130 & 0,152 & 114 & 153 & $-1,4$ \\
\hline 12 & 153 & 0,044 & 0,765 & 0,049 & 0,786 & 0,912 & 1,028 & 0,092 & 0,955 & 1,023 & 0,493 & 0,395 & 0,339 & 0,134 & 0,152 & 115 & 153 & $-1,8$ \\
\hline 13 & 153 & 0,046 & 1,433 & 0,069 & 0,714 & 0,667 & 0,499 & 0,602 & 0,281 & 1,886 & 0,458 & 0,525 & 0,357 & 0,188 & 0,076 & 97 & 153 & $-1,8$ \\
\hline 14 & 153 & 0,029 & 0,706 & 0,036 & 0,511 & 0,800 & 0,725 & 0,340 & 0,724 & 1,175 & 0,349 & 0,231 & 0,324 & 0,075 & 0,076 & 168 & 153 & $-1,7$ \\
\hline 15 & 153 & 0,278 & 2,080 & 0,300 & 2,000 & 0,928 & 0,961 & 0,082 & 0,808 & 1,112 & 0,504 & 0,503 & 0,838 & 0,422 & 0,442 & 110 & 153 & $-3,1$ \\
\hline 16 & 153 & 0,066 & 0,837 & 0,069 & 0,807 & 0,958 & 0,965 & 0,055 & 1,000 & 0,919 & 0,861 & 0,728 & 0,312 & 0,227 & 0,240 & 88 & 153 & $-1,6$ \\
\hline 17 & 153 & 0,020 & 0,440 & 0,023 & 0,338 & 0,875 & 0,769 & 0,263 & 1,000 & 0,875 & 0,713 & 0,500 & 0,189 & 0,095 & 0,107 & 63 & 153 & $-0,9$ \\
\hline 18 & 153 & 0,016 & 0,347 & 0,016 & 0,275 & 1,000 & 0,793 & 0,207 & 1,000 & 0,778 & 1,077 & 0,588 & 0,137 & 0,080 & 0,107 & 135 & 153 & $-0,5$ \\
\hline 19 & 153 & 0,116 & 1,399 & 0,145 & 1,247 & 0,802 & 0,891 & 0,226 & 0,746 & 1,158 & 0,503 & 0,575 & 0,542 & 0,312 & 0,227 & 111 & 153 & $-2,4$ \\
\hline 20 & 153 & 0,588 & 3,783 & 0,644 & 3,660 & 0,913 & 0,968 & 0,093 & 0,517 & 1,391 & 0,239 & 0,239 & 1,769 & 0,423 & 0,442 & 99 & 153 & $-4,1$ \\
\hline 21 & 153 & 0,020 & 0,418 & 0,020 & 0,317 & 1,000 & 0,757 & 0,243 & 1,000 & 0,833 & 1,049 & 0,728 & 0,156 & 0,114 & 0,107 & 83 & 153 & $-0,6$ \\
\hline 22 & 153 & 0,026 & 0,520 & 0,029 & 0,440 & 0,900 & 0,845 & 0,184 & 1,000 & 0,913 & 0,619 & 0,493 & 0,230 & 0,114 & 0,152 & 72 & 153 & $-1,2$ \\
\hline 23 & 153 & 0,036 & 0,613 & 0,039 & 0,490 & 0,926 & 0,800 & 0,213 & 1,000 & 0,913 & 0,796 & 0,614 & 0,240 & 0,147 & 0,152 & 106 & 153 & $-1,3$ \\
\hline 24 & 153 & 0,065 & 1,657 & 0,098 & 1,496 & 0,662 & 0,903 & 0,352 & 0,296 & 1,839 & 0,130 & 0,143 & 0,796 & 0,114 & 0,152 & 89 & 153 & $-3,0$ \\
\hline
\end{tabular}




\begin{tabular}{|c|c|c|c|c|c|c|c|c|c|c|c|c|c|c|c|c|c|c|}
\hline 25 & 153 & 0,017 & 0,368 & 0,017 & 0,287 & 1,000 & 0,781 & 0,219 & 1,000 & 0,791 & 0,899 & 0,485 & 0,156 & 0,076 & 0,152 & 82 & 153 & $-0,6$ \\
\hline 26 & 153 & 0,039 & 0,685 & 0,040 & 0,583 & 0,964 & 0,852 & 0,153 & 1,000 & 0,981 & 0,529 & 0,372 & 0,305 & 0,114 & 0,152 & 91 & 153 & $-1,6$ \\
\hline 27 & 153 & 0,017 & 0,389 & 0,020 & 0,245 & 0,857 & 0,631 & 0,396 & 1,000 & 0,836 & 0,849 & 0,633 & 0,161 & 0,102 & 0,152 & 143 & 153 & $-0,7$ \\
\hline 28 & 153 & 0,037 & 0,849 & 0,044 & 0,748 & 0,839 & 0,880 & 0,201 & 0,650 & 1,241 & 0,304 & 0,218 & 0,395 & 0,086 & 0,152 & 81 & 153 & $-2,0$ \\
\hline 29 & 153 & 0,016 & 0,410 & 0,016 & 0,144 & 1,000 & 0,350 & 0,650 & 1,000 & 0,920 & 0,539 & 0,196 & 0,193 & 0,038 & 0,076 & 90 & 153 & $-0,9$ \\
\hline 30 & 153 & 0,119 & 1,408 & 0,141 & 1,217 & 0,847 & 0,865 & 0,204 & 0,755 & 1,151 & 0,625 & 0,556 & 0,492 & 0,274 & 0,240 & 10 & 153 & $-2,3$ \\
\hline 31 & 153 & 0,032 & 0,968 & 0,049 & 0,664 & 0,647 & 0,685 & 0,473 & 0,423 & 1,537 & 0,529 & 0,680 & 0,276 & 0,187 & 0,076 & 144 & 153 & $-1,5$ \\
\hline 32 & 153 & 0,024 & 0,469 & 0,024 & 0,389 & 1,000 & 0,829 & 0,171 & 1,000 & 0,848 & 0,833 & 0,588 & 0,193 & 0,114 & 0,152 & 90 & 153 & $-0,9$ \\
\hline 33 & 153 & 0,040 & 0,613 & 0,040 & 0,562 & 1,000 & 0,917 & 0,083 & 1,000 & 0,863 & 1,049 & 0,844 & 0,221 & 0,186 & 0,214 & 63 & 153 & $-1,1$ \\
\hline 34 & 153 & 2,215 & 17,575 & 3,685 & 11,252 & 0,601 & 0,640 & 0,537 & 0,090 & 3,331 & 0,092 & 0,144 & 5,522 & 0,794 & 0,303 & 109 & 153 & $-5,8$ \\
\hline 35 & 153 & 0,017 & 0,418 & 0,019 & 0,347 & 0,923 & 0,828 & 0,188 & 1,000 & 0,900 & 0,764 & 0,501 & 0,169 & 0,085 & 0,107 & 123 & 153 & $-0,8$ \\
\hline 36 & 153 & 0,019 & 0,410 & 0,019 & 0,194 & 1,000 & 0,474 & 0,526 & 1,000 & 0,847 & 0,828 & 0,447 & 0,169 & 0,076 & 0,152 & 98 & 153 & $-0,8$ \\
\hline 37 & 153 & 0,214 & 2,430 & 0,275 & 1,991 & 0,776 & 0,819 & 0,288 & 0,455 & 1,483 & 0,342 & 0,449 & 0,892 & 0,400 & 0,273 & 104 & 153 & $-3,2$ \\
\hline 38 & 153 & 0,023 & 0,613 & 0,027 & 0,461 & 0,842 & 0,752 & 0,294 & 0,768 & 1,141 & 0,384 & 0,275 & 0,276 & 0,076 & 0,107 & 79 & 153 & $-1,5$ \\
\hline 39 & 153 & 0,019 & 0,744 & 0,029 & 0,490 & 0,650 & 0,659 & 0,489 & 0,423 & 1,537 & 0,487 & 0,537 & 0,221 & 0,119 & 0,076 & 117 & 153 & $-1,1$ \\
\hline 40 & 153 & 0,139 & 1,306 & 0,146 & 1,184 & 0,951 & 0,906 & 0,106 & 1,000 & 0,988 & 0,772 & 0,708 & 0,479 & 0,339 & 0,339 & 104 & 153 & $-2,3$ \\
\hline 41 & 153 & 0,023 & 0,448 & 0,024 & 0,418 & 0,941 & 0,934 & 0,089 & 1,000 & 0,834 & 1,198 & 0,857 & 0,156 & 0,134 & 0,169 & 18 & 153 & $-0,6$ \\
\hline 42 & 153 & 0,022 & 0,418 & 0,022 & 0,338 & 1,000 & 0,808 & 0,192 & 1,000 & 0,805 & 1,123 & 0,728 & 0,156 & 0,114 & 0,152 & 45 & 153 & $-0,6$ \\
\hline 43 & 153 & 0,017 & 0,448 & 0,020 & 0,296 & 0,857 & 0,660 & 0,369 & 1,000 & 0,964 & 0,899 & 0,651 & 0,156 & 0,102 & 0,107 & 153 & 153 & $-0,6$ \\
\hline 44 & 153 & 0,263 & 3,136 & 0,370 & 2,578 & 0,709 & 0,822 & 0,341 & 0,335 & 1,727 & 0,224 & 0,294 & 1,222 & 0,359 & 0,227 & 91 & 153 & $-3,6$ \\
\hline 45 & 153 & 0,052 & 0,714 & 0,053 & 0,643 & 0,973 & 0,899 & 0,104 & 1,000 & 0,887 & 0,881 & 0,686 & 0,273 & 0,187 & 0,214 & 134 & 153 & $-1,4$ \\
\hline 46 & 153 & 0,032 & 1,095 & 0,049 & 0,849 & 0,647 & 0,776 & 0,418 & 0,331 & 1,738 & 0,137 & 0,125 & 0,542 & 0,068 & 0,076 & 81 & 153 & $-2,4$ \\
\hline 47 & 153 & 0,017 & 0,490 & 0,022 & 0,296 & 0,800 & 0,604 & 0,444 & 0,900 & 1,054 & 0,764 & 0,637 & 0,169 & 0,108 & 0,107 & 108 & 153 & $-0,8$ \\
\hline 48 & 153 & 0,073 & 0,909 & 0,079 & 0,849 & 0,927 & 0,935 & 0,098 & 1,000 & 0,948 & 0,764 & 0,651 & 0,349 & 0,227 & 0,273 & 91 & 153 & $-1,8$ \\
\hline 49 & 153 & 0,030 & 0,520 & 0,032 & 0,418 & 0,955 & 0,805 & 0,200 & 1,000 & 0,845 & 1,028 & 0,784 & 0,193 & 0,152 & 0,169 & 99 & 153 & $-0,9$ \\
\hline 50 & 153 & 0,023 & 0,807 & 0,033 & 0,440 & 0,696 & 0,544 & 0,548 & 0,443 & 1,503 & 0,453 & 0,596 & 0,254 & 0,152 & 0,076 & 125 & 153 & $-1,3$ \\
\hline 51 & 153 & 0,307 & 2,637 & 0,360 & 2,476 & 0,853 & 0,939 & 0,160 & 0,555 & 1,343 & 0,300 & 0,317 & 1,142 & 0,362 & 0,303 & 95 & 153 & $-3,5$ \\
\hline 52 & 153 & 0,027 & 0,520 & 0,030 & 0,418 & 0,905 & 0,805 & 0,217 & 1,000 & 0,888 & 0,834 & 0,657 & 0,204 & 0,134 & 0,152 & 52 & 153 & $-1,0$ \\
\hline 53 & 153 & 0,129 & 1,369 & 0,135 & 1,319 & 0,957 & 0,963 & 0,056 & 0,865 & 1,075 & 0,418 & 0,410 & 0,627 & 0,257 & 0,303 & 108 & 153 & $-2,6$ \\
\hline 54 & 153 & 0,016 & 0,338 & 0,016 & 0,245 & 1,000 & 0,725 & 0,275 & 1,000 & 0,759 & 1,077 & 0,555 & 0,137 & 0,076 & 0,152 & 90 & 153 & $-0,5$ \\
\hline 55 & 153 & 0,036 & 0,613 & 0,040 & 0,490 & 0,893 & 0,800 & 0,227 & 1,000 & 0,913 & 0,796 & 0,600 & 0,240 & 0,144 & 0,152 & 67 & 153 & $-1,3$ \\
\hline 56 & 153 & 0,027 & 0,532 & 0,027 & 0,410 & 1,000 & 0,770 & 0,230 & 1,000 & 0,910 & 0,605 & 0,316 & 0,240 & 0,076 & 0,152 & 87 & 153 & $-1,3$ \\
\hline 57 & 153 & 0,016 & 0,562 & 0,024 & 0,418 & 0,647 & 0,744 & 0,436 & 0,628 & 1,262 & 0,350 & 0,316 & 0,240 & 0,076 & 0,076 & 90 & 153 & $-1,3$ \\
\hline 58 & 153 & 0,175 & 1,585 & 0,181 & 1,475 & 0,968 & 0,930 & 0,076 & 0,876 & 1,069 & 0,495 & 0,395 & 0,671 & 0,265 & 0,303 & 96 & 153 & $-2,7$ \\
\hline 59 & 153 & 0,017 & 0,604 & 0,020 & 0,461 & 0,857 & 0,762 & 0,277 & 0,593 & 1,299 & 0,235 & 0,124 & 0,305 & 0,038 & 0,076 & 90 & 153 & $-1,6$ \\
\hline 60 & 153 & 0,043 & 1,289 & 0,067 & 1,014 & 0,638 & 0,787 & 0,420 & 0,326 & 1,753 & 0,149 & 0,187 & 0,607 & 0,114 & 0,107 & 88 & 153 & $-2,6$ \\
\hline 61 & 153 & 0,915 & 7,461 & 1,544 & 5,553 & 0,593 & 0,744 & 0,481 & 0,207 & 2,200 & 0,182 & 0,273 & 2,529 & 0,691 & 0,530 & 100 & 153 & $-4,7$ \\
\hline 62 & 153 & 0,055 & 1,187 & 0,069 & 1,014 & 0,792 & 0,854 & 0,254 & 0,486 & 1,435 & 0,211 & 0,185 & 0,573 & 0,106 & 0,152 & 86 & 153 & $-2,5$ \\
\hline 63 & 153 & 0,030 & 0,613 & 0,032 & 0,541 & 0,955 & 0,883 & 0,126 & 1,000 & 0,996 & 0,668 & 0,632 & 0,240 & 0,152 & 0,152 & 99 & 153 & $-1,3$ \\
\hline 64 & 153 & 0,016 & 0,338 & 0,016 & 0,194 & 1,000 & 0,575 & 0,425 & 1,000 & 0,759 & 1,077 & 0,555 & 0,137 & 0,076 & 0,152 & 108 & 153 & $-0,5$ \\
\hline 65 & 153 & 0,102 & 1,196 & 0,115 & 1,095 & 0,887 & 0,915 & 0,141 & 0,895 & 1,057 & 0,523 & 0,456 & 0,498 & 0,227 & 0,240 & 90 & 153 & $-2,3$ \\
\hline 66 & 153 & 0,034 & 0,634 & 0,037 & 0,469 & 0,923 & 0,740 & 0,271 & 1,000 & 0,964 & 0,588 & 0,555 & 0,273 & 0,152 & 0,169 & 123 & 153 & $-1,4$ \\
\hline 67 & 153 & 0,029 & 0,622 & 0,034 & 0,541 & 0,833 & 0,871 & 0,211 & 0,934 & 1,035 & 0,566 & 0,497 & 0,254 & 0,126 & 0,152 & 52 & 153 & $-1,3$ \\
\hline 68 & 153 & 0,060 & 1,082 & 0,076 & 0,938 & 0,792 & 0,867 & 0,246 & 0,647 & 1,244 & 0,334 & 0,318 & 0,479 & 0,152 & 0,169 & 109 & 153 & $-2,3$ \\
\hline 69 & 153 & 0,024 & 0,583 & 0,026 & 0,410 & 0,944 & 0,703 & 0,302 & 0,901 & 1,053 & 0,408 & 0,275 & 0,276 & 0,076 & 0,107 & 78 & 153 & $-1,5$ \\
\hline 70 & 153 & 0,079 & 1,509 & 0,098 & 0,917 & 0,809 & 0,608 & 0,436 & 0,435 & 1,516 & 0,642 & 0,745 & 0,395 & 0,295 & 0,227 & 102 & 153 & $-2,0$ \\
\hline 71 & 153 & 0,014 & 0,469 & 0,020 & 0,368 & 0,714 & 0,784 & 0,358 & 0,819 & 1,105 & 0,439 & 0,311 & 0,204 & 0,063 & 0,076 & 20 & 153 & $-1,0$ \\
\hline 72 & 153 & 0,022 & 0,418 & 0,023 & 0,296 & 0,938 & 0,707 & 0,299 & 1,000 & 0,805 & 1,123 & 0,728 & 0,156 & 0,114 & 0,152 & 110 & 153 & $-0,6$ \\
\hline 73 & 153 & 0,037 & 0,613 & 0,039 & 0,511 & 0,963 & 0,834 & 0,170 & 1,000 & 0,895 & 0,828 & 0,632 & 0,240 & 0,152 & 0,169 & 107 & 153 & $-1,3$ \\
\hline 74 & 153 & 0,019 & 0,592 & 0,024 & 0,440 & 0,765 & 0,743 & 0,349 & 0,669 & 1,222 & 0,368 & 0,350 & 0,254 & 0,089 & 0,107 & 37 & 153 & $-1,3$ \\
\hline 75 & 153 & 0,066 & 0,849 & 0,067 & 0,676 & 0,979 & 0,796 & 0,205 & 1,000 & 0,933 & 0,791 & 0,581 & 0,326 & 0,189 & 0,227 & 81 & 153 & $-1,7$ \\
\hline 76 & 153 & 0,037 & 0,693 & 0,043 & 0,583 & 0,867 & 0,841 & 0,207 & 0,975 & 1,013 & 0,662 & 0,566 & 0,268 & 0,152 & 0,152 & 84 & 153 & $-1,4$ \\
\hline 77 & 153 & 0,024 & 0,461 & 0,024 & 0,338 & 1,000 & 0,734 & 0,266 & 1,000 & 0,832 & 0,866 & 0,600 & 0,189 & 0,114 & 0,152 & 63 & 153 & $-0,9$ \\
\hline 78 & 153 & 0,023 & 0,469 & 0,024 & 0,368 & 0,941 & 0,784 & 0,224 & 1,000 & 0,874 & 0,702 & 0,499 & 0,204 & 0,102 & 0,152 & 114 & 153 & $-1,0$ \\
\hline 79 & 153 & 0,043 & 0,735 & 0,047 & 0,634 & 0,909 & 0,862 & 0,165 & 1,000 & 1,000 & 0,588 & 0,496 & 0,305 & 0,152 & 0,169 & 91 & 153 & $-1,6$ \\
\hline 80 & 153 & 0,017 & 0,440 & 0,022 & 0,266 & 0,800 & 0,606 & 0,442 & 1,000 & 0,945 & 0,588 & 0,392 & 0,193 & 0,076 & 0,152 & 72 & 153 & $-0,9$ \\
\hline 81 & 153 & 0,040 & 0,706 & 0,044 & 0,592 & 0,903 & 0,839 & 0,188 & 1,000 & 0,993 & 0,548 & 0,439 & 0,305 & 0,134 & 0,152 & 106 & 153 & $-1,6$ \\
\hline 82 & 153 & 0,034 & 1,044 & 0,044 & 0,799 & 0,774 & 0,765 & 0,326 & 0,397 & 1,587 & 0,180 & 0,153 & 0,494 & 0,075 & 0,076 & 81 & 153 & $-2,3$ \\
\hline
\end{tabular}




\begin{tabular}{|c|c|c|c|c|c|c|c|c|c|c|c|c|c|c|c|c|c|c|}
\hline 83 & 153 & 0,046 & 0,765 & 0,050 & 0,685 & 0,914 & 0,895 & 0,136 & 0,986 & 1,007 & 0,558 & 0,487 & 0,324 & 0,158 & 0,169 & 111 & 153 & $-1,7$ \\
\hline 84 & 153 & 0,110 & 1,826 & 0,146 & 1,222 & 0,755 & 0,669 & 0,412 & 0,416 & 1,550 & 0,551 & 0,584 & 0,505 & 0,295 & 0,152 & 119 & 153 & $-2,3$ \\
\hline 85 & 153 & 0,057 & 1,327 & 0,096 & 0,938 & 0,597 & 0,707 & 0,498 & 0,409 & 1,563 & 0,374 & 0,643 & 0,442 & 0,284 & 0,169 & 52 & 153 & $-2,1$ \\
\hline 86 & 153 & 0,056 & 0,968 & 0,073 & 0,858 & 0,765 & 0,886 & 0,261 & 0,750 & 1,155 & 0,584 & 0,631 & 0,349 & 0,220 & 0,214 & 143 & 153 & $-1,8$ \\
\hline 87 & 153 & 0,014 & 0,410 & 0,014 & 0,245 & 1,000 & 0,598 & 0,402 & 1,000 & 0,965 & 0,490 & 0,196 & 0,193 & 0,038 & 0,076 & 90 & 153 & $-0,9$ \\
\hline 88 & 153 & 0,020 & 0,469 & 0,023 & 0,397 & 0,875 & 0,847 & 0,198 & 1,000 & 0,934 & 0,557 & 0,375 & 0,214 & 0,080 & 0,107 & 50 & 153 & $-1,1$ \\
\hline 89 & 153 & 0,179 & 1,754 & 0,197 & 1,551 & 0,912 & 0,884 & 0,145 & 0,732 & 1,169 & 0,478 & 0,485 & 0,691 & 0,336 & 0,312 & 103 & 153 & $-2,8$ \\
\hline 90 & 153 & 0,026 & 0,511 & 0,026 & 0,410 & 1,000 & 0,801 & 0,199 & 1,000 & 0,898 & 0,619 & 0,329 & 0,230 & 0,076 & 0,152 & 87 & 153 & $-1,2$ \\
\hline 91 & 153 & 0,017 & 0,347 & 0,017 & 0,296 & 1,000 & 0,854 & 0,146 & 1,000 & 0,745 & 1,528 & 0,894 & 0,120 & 0,107 & 0,107 & 90 & 153 & $-0,3$ \\
\hline 92 & 153 & 0,072 & 0,879 & 0,076 & 0,757 & 0,943 & 0,861 & 0,150 & 1,000 & 0,926 & 0,715 & 0,636 & 0,357 & 0,227 & 0,273 & 64 & 153 & $-1,8$ \\
\hline 93 & 153 & 0,037 & 0,613 & 0,040 & 0,511 & 0,929 & 0,834 & 0,180 & 1,000 & 0,895 & 0,828 & 0,614 & 0,240 & 0,147 & 0,152 & 110 & 153 & $-1,3$ \\
\hline 94 & 153 & 0,059 & 0,930 & 0,067 & 0,858 & 0,872 & 0,923 & 0,149 & 0,855 & 1,081 & 0,450 & 0,374 & 0,408 & 0,152 & 0,169 & 112 & 153 & $-2,0$ \\
\hline 95 & 153 & 0,083 & 1,112 & 0,093 & 1,002 & 0,892 & 0,901 & 0,146 & 0,846 & 1,087 & 0,499 & 0,468 & 0,461 & 0,216 & 0,227 & 77 & 153 & $-2,2$ \\
\hline 96 & 153 & 0,161 & 1,923 & 0,215 & 1,585 & 0,747 & 0,824 & 0,308 & 0,546 & 1,353 & 0,359 & 0,441 & 0,755 & 0,333 & 0,227 & 96 & 153 & $-2,9$ \\
\hline 97 & 153 & 0,359 & 2,473 & 0,383 & 2,371 & 0,936 & 0,959 & 0,076 & 0,737 & 1,165 & 0,378 & 0,379 & 1,099 & 0,417 & 0,442 & 86 & 153 & $-3,5$ \\
\hline 98 & 153 & 0,022 & 0,418 & 0,023 & 0,296 & 0,938 & 0,707 & 0,299 & 1,000 & 0,805 & 1,123 & 0,728 & 0,156 & 0,114 & 0,152 & 110 & 153 & $-0,6$ \\
\hline 99 & 153 & 0,080 & 1,775 & 0,135 & 1,420 & 0,596 & 0,800 & 0,451 & 0,320 & 1,767 & 0,202 & 0,235 & 0,712 & 0,168 & 0,152 & 103 & 153 & $-2,8$ \\
\hline 100 & 153 & 0,049 & 0,807 & 0,053 & 0,664 & 0,919 & 0,822 & 0,196 & 0,941 & 1,031 & 0,486 & 0,458 & 0,357 & 0,164 & 0,169 & 66 & 153 & $-1,8$ \\
\hline 101 & 153 & 0,033 & 0,634 & 0,034 & 0,511 & 0,958 & 0,807 & 0,198 & 1,000 & 0,984 & 0,553 & 0,412 & 0,276 & 0,114 & 0,152 & 79 & 153 & $-1,5$ \\
\hline 102 & 153 & 0,060 & 0,888 & 0,067 & 0,757 & 0,894 & 0,852 & 0,182 & 0,961 & 1,020 & 0,786 & 0,728 & 0,312 & 0,227 & 0,214 & 96 & 153 & $-1,6$ \\
\hline 103 & 153 & 0,046 & 0,643 & 0,046 & 0,613 & 1,000 & 0,954 & 0,046 & 1,000 & 0,846 & 1,019 & 0,783 & 0,240 & 0,187 & 0,214 & 70 & 153 & $-1,3$ \\
\hline 104 & 153 & 0,019 & 0,461 & 0,022 & 0,296 & 0,867 & 0,642 & 0,382 & 1,000 & 0,951 & 0,662 & 0,506 & 0,189 & 0,096 & 0,152 & 55 & 153 & $-0,9$ \\
\hline 105 & 153 & 0,029 & 0,541 & 0,030 & 0,461 & 0,952 & 0,851 & 0,156 & 1,000 & 0,901 & 0,688 & 0,493 & 0,230 & 0,114 & 0,152 & 85 & 153 & $-1,2$ \\
\hline 106 & 153 & 0,684 & 4,848 & 0,819 & 4,548 & 0,835 & 0,938 & 0,176 & 0,366 & 1,653 & 0,185 & 0,227 & 2,168 & 0,491 & 0,455 & 107 & 153 & $-4,4$ \\
\hline 107 & 153 & 0,059 & 0,778 & 0,060 & 0,685 & 0,976 & 0,881 & 0,122 & 1,000 & 0,904 & 0,900 & 0,657 & 0,288 & 0,189 & 0,214 & 103 & 153 & $-1,5$ \\
\hline 108 & 153 & 0,020 & 0,757 & 0,030 & 0,685 & 0,667 & 0,905 & 0,347 & 0,441 & 1,506 & 0,198 & 0,174 & 0,359 & 0,063 & 0,076 & 104 & 153 & $-1,8$ \\
\hline 109 & 153 & 0,089 & 1,399 & 0,113 & 1,145 & 0,785 & 0,819 & 0,281 & 0,571 & 1,323 & 0,427 & 0,484 & 0,515 & 0,249 & 0,214 & 101 & 153 & $-2,4$ \\
\hline 110 & 153 & 0,033 & 0,604 & 0,036 & 0,440 & 0,920 & 0,727 & 0,284 & 1,000 & 0,938 & 0,732 & 0,474 & 0,240 & 0,114 & 0,152 & 86 & 153 & $-1,3$ \\
\hline 111 & 153 & 0,123 & 1,150 & 0,123 & 1,120 & 1,000 & 0,974 & 0,026 & 1,000 & 0,924 & 0,842 & 0,682 & 0,432 & 0,295 & 0,321 & 41 & 153 & $-2,1$ \\
\hline 112 & 153 & 0,014 & 0,317 & 0,014 & 0,173 & 1,000 & 0,547 & 0,453 & 1,000 & 0,746 & 0,979 & 0,555 & 0,137 & 0,076 & 0,107 & 108 & 153 & $-0,5$ \\
\hline 113 & 153 & 0,090 & 1,002 & 0,095 & 0,888 & 0,955 & 0,886 & 0,122 & 1,000 & 0,940 & 0,757 & 0,676 & 0,390 & 0,264 & 0,273 & 68 & 153 & $-2,0$ \\
\hline 114 & 153 & 0,092 & 1,644 & 0,125 & 1,255 & 0,736 & 0,764 & 0,355 & 0,427 & 1,531 & 0,338 & 0,432 & 0,588 & 0,254 & 0,152 & 120 & 153 & $-2,6$ \\
\hline 115 & 153 & 0,095 & 1,399 & 0,112 & 1,196 & 0,846 & 0,855 & 0,211 & 0,608 & 1,283 & 0,359 & 0,362 & 0,579 & 0,210 & 0,152 & 79 & 153 & $-2,5$ \\
\hline 116 & 153 & 0,024 & 0,469 & 0,024 & 0,389 & 1,000 & 0,829 & 0,171 & 1,000 & 0,848 & 0,833 & 0,588 & 0,193 & 0,114 & 0,152 & 90 & 153 & $-0,9$ \\
\hline 117 & 153 & 0,468 & 2,642 & 0,492 & 2,655 & 0,950 & 1,005 & 0,050 & 0,842 & 1,090 & 0,488 & 0,495 & 1,104 & 0,547 & 0,577 & 117 & 153 & $-3,5$ \\
\hline 118 & 153 & 0,043 & 0,685 & 0,046 & 0,613 & 0,938 & 0,895 & 0,122 & 1,000 & 0,931 & 0,721 & 0,549 & 0,276 & 0,152 & 0,169 & 81 & 153 & $-1,5$ \\
\hline 119 & 153 & 0,024 & 0,714 & 0,036 & 0,520 & 0,680 & 0,728 & 0,420 & 0,601 & 1,290 & 0,416 & 0,527 & 0,273 & 0,144 & 0,152 & 110 & 153 & $-1,4$ \\
\hline 120 & 153 & 0,023 & 0,520 & 0,026 & 0,440 & 0,889 & 0,845 & 0,190 & 1,000 & 0,968 & 0,599 & 0,460 & 0,221 & 0,102 & 0,152 & 110 & 153 & $-1,1$ \\
\hline 121 & 153 & 0,067 & 0,846 & 0,072 & 0,816 & 0,940 & 0,965 & 0,070 & 1,000 & 0,919 & 0,880 & 0,686 & 0,312 & 0,214 & 0,240 & 120 & 153 & $-1,6$ \\
\hline 122 & 153 & 0,014 & 0,511 & 0,019 & 0,245 & 0,769 & 0,479 & 0,569 & 0,690 & 1,204 & 0,318 & 0,192 & 0,240 & 0,046 & 0,076 & 108 & 153 & $-1,3$ \\
\hline 123 & 153 & 0,029 & 0,490 & 0,029 & 0,440 & 1,000 & 0,896 & 0,104 & 1,000 & 0,816 & 1,019 & 0,707 & 0,189 & 0,134 & 0,169 & 45 & 153 & $-0,9$ \\
\hline 124 & 153 & 0,032 & 0,541 & 0,033 & 0,418 & 0,957 & 0,773 & 0,231 & 1,000 & 0,859 & 0,966 & 0,722 & 0,204 & 0,147 & 0,169 & 109 & 153 & $-1,0$ \\
\hline 125 & 153 & 0,017 & 0,440 & 0,020 & 0,317 & 0,857 & 0,721 & 0,313 & 1,000 & 0,945 & 0,588 & 0,392 & 0,193 & 0,076 & 0,107 & 90 & 153 & $-0,9$ \\
\hline 126 & 153 & 0,020 & 0,532 & 0,023 & 0,389 & 0,875 & 0,730 & 0,297 & 0,890 & 1,060 & 0,446 & 0,269 & 0,240 & 0,064 & 0,076 & 103 & 153 & $-1,3$ \\
\hline 127 & 153 & 0,019 & 0,389 & 0,019 & 0,245 & 1,000 & 0,631 & 0,369 & 1,000 & 0,803 & 0,828 & 0,447 & 0,169 & 0,076 & 0,152 & 98 & 153 & $-0,8$ \\
\hline 128 & 153 & 0,016 & 0,368 & 0,016 & 0,194 & 1,000 & 0,529 & 0,471 & 1,000 & 0,826 & 0,824 & 0,485 & 0,156 & 0,076 & 0,107 & 90 & 153 & $-0,6$ \\
\hline 129 & 153 & 0,052 & 0,786 & 0,052 & 0,685 & 1,000 & 0,871 & 0,129 & 1,000 & 0,976 & 0,559 & 0,442 & 0,343 & 0,152 & 0,214 & 88 & 153 & $-1,8$ \\
\hline 130 & 153 & 0,057 & 0,816 & 0,060 & 0,765 & 0,952 & 0,938 & 0,078 & 1,000 & 0,961 & 0,688 & 0,572 & 0,326 & 0,186 & 0,214 & 113 & 153 & $-1,7$ \\
\hline 131 & 153 & 0,106 & 1,175 & 0,115 & 1,031 & 0,925 & 0,878 & 0,143 & 0,966 & 1,017 & 0,645 & 0,642 & 0,458 & 0,294 & 0,303 & 119 & 153 & $-2,2$ \\
\hline 132 & 153 & 0,040 & 0,930 & 0,049 & 0,828 & 0,824 & 0,891 & 0,208 & 0,584 & 1,309 & 0,285 & 0,246 & 0,423 & 0,104 & 0,152 & 83 & 153 & $-2,1$ \\
\hline 133 & 153 & 0,215 & 2,376 & 0,264 & 1,995 & 0,815 & 0,840 & 0,245 & 0,479 & 1,445 & 0,326 & 0,331 & 0,916 & 0,303 & 0,303 & 94 & 153 & $-3,2$ \\
\hline 134 & 153 & 0,096 & 1,851 & 0,121 & 1,699 & 0,798 & 0,918 & 0,218 & 0,353 & 1,684 & 0,151 & 0,162 & 0,900 & 0,146 & 0,152 & 77 & 153 & $-3,2$ \\
\hline 135 & 153 & 0,034 & 0,592 & 0,034 & 0,490 & 1,000 & 0,828 & 0,172 & 1,000 & 0,900 & 0,826 & 0,658 & 0,230 & 0,152 & 0,169 & 85 & 153 & $-1,2$ \\
\hline 136 & 153 & 0,014 & 0,562 & 0,023 & 0,368 & 0,625 & 0,654 & 0,510 & 0,571 & 1,324 & 0,283 & 0,189 & 0,254 & 0,048 & 0,076 & 104 & 153 & $-1,3$ \\
\hline 137 & 153 & 0,106 & 1,957 & 0,152 & 1,530 & 0,698 & 0,782 & 0,372 & 0,348 & 1,695 & 0,262 & 0,341 & 0,719 & 0,245 & 0,227 & 65 & 153 & $-2,8$ \\
\hline 138 & 153 & 0,020 & 0,448 & 0,024 & 0,368 & 0,824 & 0,820 & 0,252 & 1,000 & 0,892 & 1,049 & 0,857 & 0,156 & 0,134 & 0,152 & 150 & 153 & $-0,6$ \\
\hline 139 & 153 & 0,056 & 0,757 & 0,060 & 0,685 & 0,929 & 0,905 & 0,119 & 1,000 & 0,902 & 0,955 & 0,832 & 0,273 & 0,227 & 0,240 & 137 & 153 & $-1,4$ \\
\hline 140 & 153 & 0,044 & 0,664 & 0,046 & 0,541 & 0,969 & 0,815 & 0,187 & 1,000 & 0,888 & 0,759 & 0,588 & 0,273 & 0,161 & 0,214 & 148 & 153 & $-1,4$ \\
\hline
\end{tabular}




\begin{tabular}{|c|c|c|c|c|c|c|c|c|c|c|c|c|c|c|c|c|c|c|}
\hline 141 & 153 & 0,034 & 0,765 & 0,044 & 0,613 & 0,774 & 0,801 & 0,301 & 0,739 & 1,163 & 0,501 & 0,549 & 0,296 & 0,162 & 0,169 & 115 & 153 & $-1,6$ \\
\hline 142 & 153 & 0,172 & 1,450 & 0,179 & 1,369 & 0,960 & 0,945 & 0,068 & 1,000 & 0,986 & 0,667 & 0,595 & 0,573 & 0,341 & 0,379 & 96 & 153 & $-2,5$ \\
\hline 143 & 153 & 0,156 & 1,754 & 0,181 & 1,513 & 0,865 & 0,862 & 0,193 & 0,639 & 1,251 & 0,411 & 0,416 & 0,696 & 0,290 & 0,273 & 114 & 153 & 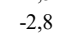 \\
\hline 144 & 153 & 0,014 & 0,359 & 0,014 & 1,889 & 1,000 & 5,262 & 4,262 & 1,000 & 0,846 & 0,749 & 0,243 & 0,156 & 0,038 & 0,076 & 90 & 153 & $-0,6$ \\
\hline 145 & 153 & 0,253 & 2,854 & 0,308 & 1,412 & 0,819 & 0,495 & 0,537 & 0,390 & 1,602 & 0,601 & 0,695 & 0,732 & 0,508 & 0,312 & 108 & 153 & $-2,9$ \\
\hline 146 & 153 & 0,128 & 1,644 & 0,154 & 0,613 & 0,832 & 0,373 & 0,649 & 0,594 & 1,298 & 0,372 & 0,401 & 0,662 & 0,265 & 0,107 & 100 & 153 & $-2,7$ \\
\hline 147 & 153 & 0,034 & 0,879 & 0,046 & 0,368 & 0,750 & 0,418 & 0,633 & 0,560 & 1,336 & 0,360 & 0,325 & 0,349 & 0,114 & 0,152 & 92 & 153 & $-1,8$ \\
\hline 148 & 153 & 0,024 & 0,469 & 0,024 & 0,583 & 1,000 & 1,243 & 0,243 & 1,000 & 0,848 & 0,746 & 0,525 & 0,204 & 0,107 & 0,152 & 69 & 153 & $-1,0$ \\
\hline 149 & 153 & 0,053 & 0,706 & 0,053 & 0,672 & 1,000 & 0,953 & 0,047 & 1,000 & 0,864 & 1,047 & 0,745 & 0,254 & 0,189 & 0,227 & 78 & 153 & $-1,3$ \\
\hline 150 & 153 & 0,042 & 0,765 & 0,044 & 0,930 & 0,935 & 1,215 & 0,225 & 0,893 & 1,058 & 0,415 & 0,375 & 0,357 & 0,134 & 0,152 & 126 & 153 & $-1,8$ \\
\hline 151 & 153 & 0,075 & 1,031 & 0,082 & 0,490 & 0,912 & 0,475 & 0,532 & 0,881 & 1,065 & 0,530 & 0,474 & 0,423 & 0,201 & 0,214 & 103 & 153 & $-2,1$ \\
\hline 152 & 153 & 0,030 & 0,685 & 0,037 & 0,744 & 0,808 & 1,087 & 0,211 & 0,808 & 1,113 & 0,668 & 0,651 & 0,240 & 0,156 & 0,152 & 93 & 153 & $-1,3$ \\
\hline 153 & 153 & 0,050 & 0,765 & 0,053 & 0,727 & 0,946 & 0,950 & 0,074 & 1,000 & 0,963 & 0,602 & 0,453 & 0,326 & 0,148 & 0,169 & 126 & 153 & $-1,7$ \\
\hline 154 & 153 & 0,026 & 0,849 & 0,034 & 5,080 & 0,750 & 5,981 & 4,987 & 0,450 & 1,491 & 0,188 & 0,158 & 0,418 & 0,066 & 0,076 & 96 & 153 & $-2,1$ \\
\hline 155 & 153 & 1,217 & 6,547 & 1,469 & 0,613 & 0,828 & 0,094 & 0,923 & 0,357 & 1,674 & 0,275 & 0,383 & 2,372 & 0,909 & 0,611 & 107 & 153 & $-4,6$ \\
\hline 156 & 153 & 0,036 & 0,706 & 0,039 & 1,623 & 0,926 & 2,300 & 1,302 & 0,905 & 1,051 & 0,436 & 0,322 & 0,324 & 0,104 & 0,152 & 102 & 153 & $-1,7$ \\
\hline 157 & 153 & 0,220 & 1,725 & 0,230 & 1,052 & 0,956 & 0,610 & 0,392 & 0,927 & 1,038 & 0,541 & 0,556 & 0,719 & 0,399 & 0,408 & 106 & 153 & $-2,8$ \\
\hline 158 & 153 & 0,108 & 1,103 & 0,112 & 0,287 & 0,962 & 0,260 & 0,741 & 1,000 & 0,949 & 0,735 & 0,614 & 0,432 & 0,265 & 0,303 & 92 & 153 & $-2,1$ \\
\hline 159 & 153 & 0,017 & 0,368 & 0,017 & 0,685 & 1,000 & 1,862 & 0,862 & 1,000 & 0,791 & 0,899 & 0,485 & 0,156 & 0,076 & 0,152 & 82 & 153 & $-0,6$ \\
\hline 160 & 153 & 0,029 & 0,786 & 0,034 & 0,583 & 0,833 & 0,742 & 0,307 & 0,583 & 1,309 & 0,263 & 0,203 & 0,373 & 0,076 & 0,076 & 69 & 153 & $-1,9$ \\
\hline 161 & 153 & 0,019 & 0,634 & 0,026 & 0,461 & 0,722 & 0,727 & 0,390 & 0,583 & 1,309 & 0,255 & 0,163 & 0,305 & 0,050 & 0,076 & 100 & 153 & $-1,6$ \\
\hline 162 & 153 & 0,026 & 0,520 & 0,030 & 0,173 & 0,857 & 0,333 & 0,682 & 1,000 & 0,913 & 0,790 & 0,582 & 0,204 & 0,119 & 0,152 & 75 & 153 & $-1,0$ \\
\hline 163 & 153 & 0,014 & 0,317 & 0,014 & 0,942 & 1,000 & 2,973 & 1,973 & 1,000 & 0,746 & 1,273 & 0,632 & 0,120 & 0,076 & 0,107 & 90 & 153 & $-0,3$ \\
\hline 164 & 153 & 0,063 & 1,166 & 0,072 & 1,163 & 0,880 & 0,997 & 0,120 & 0,583 & 1,310 & 0,280 & 0,212 & 0,536 & 0,114 & 0,152 & 90 & 153 & $-2,4$ \\
\hline 165 & 153 & 0,089 & 1,395 & 0,109 & 0,245 & 0,816 & 0,176 & 0,845 & 0,574 & 1,320 & 0,409 & 0,406 & 0,526 & 0,213 & 0,169 & 120 & 153 & $-2,4$ \\
\hline 166 & 153 & 0,016 & 0,368 & 0,017 & 0,245 & 0,917 & 0,667 & 0,344 & 1,000 & 0,826 & 0,824 & 0,485 & 0,156 & 0,076 & 0,107 & 108 & 153 & $-0,6$ \\
\hline 167 & 153 & 0,014 & 0,317 & 0,014 & 0,389 & 1,000 & 1,227 & 0,227 & 1,000 & 0,746 & 0,979 & 0,555 & 0,137 & 0,076 & 0,107 & 162 & 153 & $-0,5$ \\
\hline 168 & 153 & 0,020 & 0,511 & 0,029 & 0,541 & 0,700 & 1,058 & 0,306 & 0,965 & 1,018 & 0,524 & 0,514 & 0,221 & 0,114 & 0,076 & 107 & 153 & $-1,1$ \\
\hline 169 & 153 & 0,039 & 0,685 & 0,042 & 4,980 & 0,931 & 7,273 & 6,273 & 1,000 & 0,981 & 0,764 & 0,738 & 0,254 & 0,187 & 0,169 & 64 & 153 & $-1,3$ \\
\hline 170 & 153 & 0,607 & 5,437 & 1,075 & 0,389 & 0,565 & 0,072 & 1,025 & 0,258 & 1,969 & 0,132 & 0,252 & 2,421 & 0,611 & 0,227 & 64 & 153 & $-4,6$ \\
\hline 171 & 153 & 0,014 & 0,511 & 0,019 & 0,562 & 0,769 & 1,099 & 0,251 & 0,690 & 1,204 & 0,344 & 0,164 & 0,230 & 0,038 & 0,076 & 90 & 153 & $-1,2$ \\
\hline 172 & 153 & 0,037 & 0,664 & 0,043 & 0,643 & 0,867 & 0,968 & 0,137 & 1,000 & 0,969 & 0,662 & 0,566 & 0,268 & 0,152 & 0,169 & 65 & 153 & $-1,4$ \\
\hline 173 & 153 & 0,042 & 0,744 & 0,046 & 4,608 & 0,906 & 6,193 & 5,194 & 0,944 & 1,029 & 0,506 & 0,444 & 0,324 & 0,144 & 0,152 & 111 & 153 & $-1,7$ \\
\hline 174 & 153 & 1,240 & 4,760 & 1,326 & 0,440 & 0,935 & 0,092 & 0,910 & 0,687 & 1,206 & 0,409 & 0,459 & 1,965 & 0,902 & 0,912 & 125 & 153 & $-4,3$ \\
\hline 175 & 153 & 0,024 & 0,511 & 0,024 & 0,807 & 1,000 & 1,579 & 0,579 & 1,000 & 0,924 & 0,541 & 0,316 & 0,240 & 0,076 & 0,152 & 95 & 153 & $-1,3$ \\
\hline 176 & 153 & 0,037 & 0,981 & 0,049 & 0,828 & 0,765 & 0,845 & 0,282 & 0,488 & 1,432 & 0,242 & 0,189 & 0,443 & 0,084 & 0,107 & 108 & 153 & $-2,1$ \\
\hline 177 & 153 & 0,063 & 0,960 & 0,072 & 0,317 & 0,880 & 0,330 & 0,680 & 0,862 & 1,077 & 0,514 & 0,455 & 0,395 & 0,180 & 0,214 & 102 & 153 & $-2,0$ \\
\hline 178 & 153 & 0,019 & 0,418 & 0,020 & 0,511 & 0,929 & 1,222 & 0,233 & 1,000 & 0,864 & 0,828 & 0,632 & 0,169 & 0,107 & 0,152 & 53 & 153 & $-0,8$ \\
\hline 179 & 153 & 0,040 & 0,613 & 0,040 & 0,296 & 1,000 & 0,483 & 0,517 & 1,000 & 0,863 & 0,792 & 0,596 & 0,254 & 0,152 & 0,214 & 107 & 153 & $-1,3$ \\
\hline 180 & 153 & 0,020 & 0,418 & 0,022 & 0,469 & 0,933 & 1,121 & 0,138 & 1,000 & 0,833 & 0,891 & 0,600 & 0,169 & 0,102 & 0,152 & 114 & 153 & $-0,8$ \\
\hline 181 & 153 & 0,027 & 0,735 & 0,037 & 0,634 & 0,731 & 0,862 & 0,303 & 0,633 & 1,257 & 0,712 & 0,734 & 0,221 & 0,162 & 0,076 & 178 & 153 & $-1,1$ \\
\hline 182 & 153 & 0,047 & 0,900 & 0,059 & 2,363 & 0,805 & 2,626 & 1,637 & 0,734 & 1,167 & 0,576 & 0,585 & 0,324 & 0,189 & 0,152 & 96 & 153 & $-1,7$ \\
\hline 183 & 153 & 0,207 & 2,456 & 0,235 & 0,266 & 0,878 & 0,108 & 0,900 & 0,430 & 1,524 & 0,190 & 0,187 & 1,177 & 0,220 & 0,240 & 123 & 153 & $-3,6$ \\
\hline 184 & 153 & 0,020 & 0,410 & 0,020 & 0,469 & 1,000 & 1,145 & 0,145 & 1,000 & 0,816 & 0,891 & 0,447 & 0,169 & 0,076 & 0,152 & 98 & 153 & $-0,8$ \\
\hline 185 & 153 & 0,027 & 0,520 & 0,030 & 0,613 & 0,905 & 1,179 & 0,202 & 1,000 & 0,888 & 0,834 & 0,657 & 0,204 & 0,134 & 0,152 & 128 & 153 & $-1,0$ \\
\hline 186 & 153 & 0,049 & 0,757 & 0,050 & 1,593 & 0,971 & 2,106 & 1,107 & 1,000 & 0,966 & 0,585 & 0,468 & 0,326 & 0,153 & 0,169 & 118 & 153 & $-1,7$ \\
\hline 187 & 153 & 0,204 & 1,695 & 0,215 & 1,103 & 0,947 & 0,651 & 0,353 & 0,891 & 1,059 & 0,485 & 0,477 & 0,732 & 0,349 & 0,379 & 110 & 153 & $-2,9$ \\
\hline 188 & 153 & 0,113 & 1,285 & 0,126 & 0,469 & 0,898 & 0,365 & 0,643 & 0,862 & 1,077 & 0,641 & 0,665 & 0,475 & 0,316 & 0,273 & 119 & 153 & $-2,2$ \\
\hline 189 & 153 & 0,026 & 0,541 & 0,027 & 1,935 & 0,947 & 3,577 & 2,577 & 1,000 & 0,950 & 0,674 & 0,460 & 0,221 & 0,102 & 0,152 & 115 & 153 & $-1,1$ \\
\hline 190 & 153 & 0,126 & 2,177 & 0,161 & 0,440 & 0,786 & 0,202 & 0,826 & 0,335 & 1,728 & 0,165 & 0,153 & 0,988 & 0,152 & 0,214 & 94 & 153 & $-3,3$ \\
\hline 191 & 153 & 0,019 & 0,592 & 0,023 & 0,613 & 0,812 & 1,036 & 0,191 & 0,669 & 1,222 & 0,447 & 0,329 & 0,233 & 0,076 & 0,076 & 108 & 153 & $-1,2$ \\
\hline 192 & 153 & 0,043 & 0,714 & 0,044 & 5,153 & 0,968 & 7,213 & 6,213 & 1,000 & 0,971 & 0,588 & 0,526 & 0,305 & 0,161 & 0,169 & 123 & 153 & $-1,6$ \\
\hline 193 & 153 & 1,245 & 6,281 & 1,644 & 0,706 & 0,757 & 0,112 & 0,920 & 0,397 & 1,588 & 0,308 & 0,466 & 2,271 & 1,058 & 0,746 & 114 & 153 & $-4,5$ \\
\hline 194 & 153 & 0,046 & 0,807 & 0,055 & 0,338 & 0,842 & 0,419 & 0,602 & 0,885 & 1,063 & 0,558 & 0,468 & 0,324 & 0,152 & 0,169 & 91 & 153 & $-1,7$ \\
\hline 195 & 153 & 0,016 & 0,368 & 0,016 & 0,418 & 1,000 & 1,138 & 0,138 & 1,000 & 0,826 & 0,824 & 0,485 & 0,156 & 0,076 & 0,107 & 82 & 153 & $-0,6$ \\
\hline 196 & 153 & 0,026 & 0,583 & 0,029 & 0,338 & 0,900 & 0,580 & 0,432 & 0,954 & 1,024 & 0,559 & 0,501 & 0,243 & 0,121 & 0,107 & 136 & 153 & $-1,3$ \\
\hline 197 & 153 & 0,019 & 0,490 & 0,020 & 0,224 & 0,929 & 0,457 & 0,548 & 0,975 & 1,013 & 0,447 & 0,319 & 0,230 & 0,074 & 0,076 & 90 & 153 & $-1,2$ \\
\hline 198 & 153 & 0,014 & 0,317 & 0,014 & 0,296 & 1,000 & 0,934 & 0,066 & 1,000 & 0,746 & 1,273 & 0,632 & 0,120 & 0,076 & 0,152 & 108 & 153 & $\begin{array}{r}-0,3 \\
-2,\end{array}$ \\
\hline
\end{tabular}




\begin{tabular}{|c|c|c|c|c|c|c|c|c|c|c|c|c|c|c|c|c|c|c|}
\hline 199 & 153 & 0,014 & 0,368 & 0,016 & 0,828 & 0,909 & 2,253 & 1,256 & 1,000 & 0,866 & 0,637 & 0,400 & 0,169 & 0,068 & 0,076 & 111 & 153 & $-0,8$ \\
\hline 200 & 153 & 0,073 & 0,951 & 0,077 & 0,960 & 0,944 & 1,009 & 0,056 & 1,000 & 0,992 & 0,560 & 0,464 & 0,408 & 0,189 & 0,214 & 105 & 153 & $-2,0$ \\
\hline 201 & 153 & 0,055 & 1,052 & 0,069 & 0,676 & 0,792 & 0,642 & 0,414 & 0,619 & 1,271 & 0,308 & 0,299 & 0,475 & 0,142 & 0,152 & 107 & 153 & $-2,2$ \\
\hline 202 & 153 & 0,039 & 0,807 & 0,040 & 0,807 & 0,964 & 1,000 & 0,036 & 0,747 & 1,157 & 0,344 & 0,200 & 0,379 & 0,076 & 0,152 & 89 & 153 & $-1,9$ \\
\hline 203 & 153 & 0,060 & 0,900 & 0,063 & 0,664 & 0,955 & 0,737 & 0,267 & 0,935 & 1,034 & 0,491 & 0,383 & 0,395 & 0,152 & 0,214 & 100 & 153 & $-2,0$ \\
\hline 204 & 153 & 0,046 & 0,858 & 0,055 & 0,245 & 0,842 & 0,286 & 0,732 & 0,784 & 1,130 & 0,453 & 0,472 & 0,359 & 0,169 & 0,169 & 114 & 153 & $-1,8$ \\
\hline 205 & 153 & 0,014 & 0,338 & 0,014 & 3,423 & 1,000 & 10,128 & 9,128 & 1,000 & 0,796 & 0,979 & 0,555 & 0,137 & 0,076 & 0,107 & 72 & 153 & $-0,5$ \\
\hline 206 & 153 & 0,587 & 3,733 & 0,746 & 0,296 & 0,787 & 0,079 & 0,945 & 0,529 & 1,375 & 0,317 & 0,413 & 1,535 & 0,634 & 0,379 & 104 & 153 & $-3,9$ \\
\hline 207 & 153 & 0,017 & 0,397 & 0,019 & 3,087 & 0,923 & 7,766 & 6,767 & 1,000 & 0,854 & 0,899 & 0,651 & 0,156 & 0,102 & 0,107 & 98 & 153 & $-0,6$ \\
\hline 208 & 153 & 0,422 & 3,129 & 0,514 & 0,888 & 0,821 & 0,284 & 0,738 & 0,542 & 1,359 & 0,270 & 0,336 & 1,409 & 0,473 & 0,479 & 126 & 153 & $-3,8$ \\
\hline 209 & 153 & 0,033 & 1,010 & 0,052 & 0,613 & 0,639 & 0,607 & 0,534 & 0,406 & 1,569 & 0,201 & 0,186 & 0,458 & 0,085 & 0,107 & 62 & 153 & $-2,2$ \\
\hline 210 & 153 & 0,037 & 0,685 & 0,040 & 2,422 & 0,929 & 3,537 & 2,538 & 1,000 & 1,000 & 0,509 & 0,414 & 0,305 & 0,126 & 0,152 & 109 & 153 & $-1,6$ \\
\hline 211 & 153 & 0,235 & 2,693 & 0,339 & 1,399 & 0,695 & 0,520 & 0,569 & 0,408 & 1,566 & 0,252 & 0,294 & 1,091 & 0,320 & 0,227 & 71 & 153 & $-3,4$ \\
\hline 212 & 153 & 0,102 & 1,522 & 0,125 & 1,255 & 0,816 & 0,825 & 0,254 & 0,553 & 1,345 & 0,278 & 0,243 & 0,683 & 0,166 & 0,169 & 69 & 153 & $-2,8$ \\
\hline 213 & 153 & 0,099 & 1,348 & 0,113 & 0,194 & 0,873 & 0,144 & 0,865 & 0,684 & 1,209 & 0,337 & 0,300 & 0,612 & 0,184 & 0,214 & 111 & 153 & $-2,6$ \\
\hline 214 & 153 & 0,014 & 0,338 & 0,014 & 0,389 & 1,000 & 1,150 & 0,150 & 1,000 & 0,796 & 0,979 & 0,555 & 0,137 & 0,076 & 0,152 & 108 & 153 & $-0,5$ \\
\hline 215 & 153 & 0,023 & 0,469 & 0,023 & 0,795 & 1,000 & 1,694 & 0,694 & 1,000 & 0,874 & 0,784 & 0,588 & 0,193 & 0,114 & 0,152 & 77 & 153 & $-0,9$ \\
\hline 216 & 153 & 0,030 & 0,917 & 0,044 & 1,247 & 0,677 & 1,359 & 0,483 & 0,450 & 1,491 & 0,237 & 0,245 & 0,403 & 0,099 & 0,107 & 148 & 153 & $-2,0$ \\
\hline 217 & 153 & 0,092 & 1,450 & 0,115 & 0,347 & 0,800 & 0,239 & 0,787 & 0,549 & 1,350 & 0,312 & 0,311 & 0,612 & 0,190 & 0,169 & 105 & 153 & $-2,6$ \\
\hline 218 & 153 & 0,022 & 0,469 & 0,023 & 0,440 & 0,938 & 0,937 & 0,089 & 1,000 & 0,902 & 0,659 & 0,416 & 0,204 & 0,085 & 0,107 & 112 & 153 & $-1,0$ \\
\hline 219 & 153 & 0,024 & 0,490 & 0,024 & 0,317 & 1,000 & 0,646 & 0,354 & 1,000 & 0,886 & 0,833 & 0,588 & 0,193 & 0,114 & 0,152 & 90 & 153 & $\begin{array}{l}-0,9 \\
-6\end{array}$ \\
\hline 220 & 153 & 0,020 & 0,418 & 0,020 & 2,105 & 1,000 & 5,030 & 4,030 & 1,000 & 0,833 & 1,049 & 0,728 & 0,156 & 0,114 & 0,152 & 90 & 153 & $-0,6$ \\
\hline 221 & 153 & 0,195 & 2,574 & 0,275 & 1,243 & 0,708 & 0,483 & 0,594 & 0,370 & 1,644 & 0,253 & 0,320 & 0,991 & 0,317 & 0,273 & 87 & 153 & $-3,3$ \\
\hline 222 & 153 & 0,105 & 1,480 & 0,128 & 0,317 & 0,820 & 0,214 & 0,806 & 0,601 & 1,290 & 0,453 & 0,503 & 0,542 & 0,273 & 0,227 & 133 & 153 & $-2,4$ \\
\hline 223 & 153 & 0,017 & 0,397 & 0,019 & 0,317 & 0,923 & 0,798 & 0,217 & 1,000 & 0,854 & 0,899 & 0,651 & 0,156 & 0,102 & 0,107 & 45 & 153 & $-0,6$ \\
\hline 224 & 153 & 0,026 & 0,461 & 0,026 & 0,275 & 1,000 & 0,597 & 0,403 & 1,000 & 0,809 & 1,146 & 0,671 & 0,169 & 0,114 & 0,152 & 90 & 153 & $-0,8$ \\
\hline 225 & 153 & 0,019 & 0,440 & 0,022 & 0,266 & 0,867 & 0,606 & 0,416 & 1,000 & 0,908 & 0,662 & 0,506 & 0,189 & 0,096 & 0,152 & 90 & 153 & $-0,9$ \\
\hline 226 & 153 & 0,017 & 0,389 & 0,019 & 0,317 & 0,923 & 0,815 & 0,200 & 1,000 & 0,836 & 0,899 & 0,485 & 0,156 & 0,076 & 0,152 & 72 & 153 & $-0,6$ \\
\hline 227 & 153 & 0,023 & 0,440 & 0,023 & 0,368 & 1,000 & 0,837 & 0,163 & 1,000 & 0,818 & 1,132 & 0,707 & 0,161 & 0,114 & 0,152 & 0 & 153 & $-0,7$ \\
\hline 228 & 153 & 0,016 & 0,418 & 0,017 & 0,520 & 0,917 & 1,243 & 0,256 & 1,000 & 0,940 & 0,539 & 0,351 & 0,193 & 0,068 & 0,076 & 108 & 153 & $-0,9$ \\
\hline 229 & 153 & 0,032 & 0,613 & 0,033 & 0,296 & 0,957 & 0,483 & 0,519 & 1,000 & 0,973 & 0,539 & 0,471 & 0,273 & 0,129 & 0,152 & 113 & 153 & $-1,4$ \\
\hline 230 & 153 & 0,016 & 0,368 & 0,016 & 0,144 & 1,000 & 0,391 & 0,609 & 1,000 & 0,826 & 0,700 & 0,447 & 0,169 & 0,076 & 0,107 & 108 & 153 & $-0,8$ \\
\hline 231 & 153 & 0,016 & 0,338 & 0,016 & 0,448 & 1,000 & 1,326 & 0,326 & 1,000 & 0,759 & 1,077 & 0,555 & 0,137 & 0,076 & 0,152 & 0 & 153 & $-0,5$ \\
\hline 232 & 153 & 0,027 & 0,490 & 0,029 & 0,583 & 0,950 & 1,189 & 0,196 & 1,000 & 0,838 & 1,210 & 0,894 & 0,169 & 0,152 & 0,169 & 166 & 153 & $\begin{array}{l}-0,8 \\
\end{array}$ \\
\hline 233 & 153 & 0,032 & 0,795 & 0,039 & 1,247 & 0,815 & 1,569 & 0,598 & 0,628 & 1,262 & 0,384 & 0,351 & 0,324 & 0,114 & 0,152 & 99 & 153 & $-1,7$ \\
\hline 234 & 153 & 0,125 & 1,369 & 0,138 & 0,634 & 0,906 & 0,463 & 0,545 & 0,836 & 1,093 & 0,473 & 0,458 & 0,579 & 0,265 & 0,273 & 94 & 153 & $-2,5$ \\
\hline 235 & 153 & 0,049 & 0,735 & 0,052 & 0,368 & 0,944 & 0,500 & 0,503 & 1,000 & 0,939 & 0,817 & 0,687 & 0,276 & 0,189 & 0,214 & 95 & 153 & $-1,5$ \\
\hline 236 & 153 & 0,019 & 0,490 & 0,020 & 1,581 & 0,929 & 3,225 & 2,226 & 0,975 & 1,013 & 0,571 & 0,450 & 0,204 & 0,092 & 0,107 & 53 & 153 & $-1,0$ \\
\hline 237 & 153 & 0,184 & 1,814 & 0,208 & 0,583 & 0,883 & 0,322 & 0,689 & 0,702 & 1,194 & 0,482 & 0,490 & 0,696 & 0,341 & 0,312 & 113 & 153 & $-2,8$ \\
\hline 238 & 153 & 0,039 & 0,655 & 0,040 & 0,592 & 0,964 & 0,904 & 0,103 & 1,000 & 0,939 & 0,649 & 0,412 & 0,276 & 0,114 & 0,152 & 99 & 153 & $-1,5$ \\
\hline 239 & 153 & 0,033 & 0,714 & 0,040 & 0,317 & 0,821 & 0,444 & 0,584 & 0,813 & 1,109 & 0,431 & 0,407 & 0,312 & 0,127 & 0,107 & 62 & 153 & $-1,6$ \\
\hline 240 & 153 & 0,022 & 0,418 & 0,023 & 0,926 & 0,938 & 2,213 & 1,214 & 1,000 & 0,805 & 1,123 & 0,728 & 0,156 & 0,114 & 0,152 & 84 & 153 & $-0,6$ \\
\hline 241 & 153 & 0,047 & 1,049 & 0,055 & 8,592 & 0,868 & 8,194 & 7,195 & 0,541 & 1,359 & 0,256 & 0,221 & 0,485 & 0,107 & 0,169 & 131 & 153 & $-2,3$ \\
\hline 242 & 153 & 2,212 & 9,010 & 2,867 & 0,511 & 0,772 & 0,057 & 0,970 & 0,343 & 1,709 & 0,179 & 0,281 & 3,971 & 1,118 & 1,077 & 129 & 153 & $-5,3$ \\
\hline 243 & 153 & 0,032 & 0,664 & 0,039 & 1,061 & 0,815 & 1,599 & 0,627 & 0,901 & 1,054 & 0,757 & 0,658 & 0,230 & 0,152 & 0,169 & 1 & 153 & $-1,2$ \\
\hline 244 & 153 & 0,079 & 1,184 & 0,093 & 0,664 & 0,846 & 0,561 & 0,465 & 0,708 & 1,189 & 0,412 & 0,434 & 0,494 & 0,214 & 0,214 & 124 & 153 & $-2,3$ \\
\hline 245 & 153 & 0,046 & 0,744 & 0,050 & 0,714 & 0,914 & 0,960 & 0,095 & 1,000 & 0,980 & 0,702 & 0,547 & 0,288 & 0,158 & 0,169 & 123 & 153 & $-1,5$ \\
\hline 246 & 153 & 0,059 & 0,765 & 0,060 & 0,499 & 0,976 & 0,652 & 0,349 & 1,000 & 0,890 & 0,900 & 0,729 & 0,288 & 0,210 & 0,214 & 121 & 153 & $-1,5$ \\
\hline 247 & 153 & 0,032 & 0,643 & 0,034 & 0,765 & 0,917 & 1,191 & 0,208 & 0,961 & 1,020 & 0,459 & 0,355 & 0,296 & 0,105 & 0,152 & 128 & 153 & $-1,6$ \\
\hline 248 & 153 & 0,049 & 0,846 & 0,052 & 0,592 & 0,944 & 0,700 & 0,305 & 0,857 & 1,080 & 0,408 & 0,343 & 0,390 & 0,134 & 0,152 & 120 & 153 & $-2,0$ \\
\hline 249 & 153 & 0,030 & 0,693 & 0,037 & 0,317 & 0,808 & 0,457 & 0,576 & 0,788 & 1,127 & 0,411 & 0,379 & 0,305 & 0,116 & 0,152 & 27 & 153 & $-1,6$ \\
\hline 250 & 153 & 0,017 & 0,418 & 0,022 & 0,807 & 0,800 & 1,929 & 0,950 & 1,000 & 0,900 & 0,955 & 0,750 & 0,152 & 0,114 & 0,152 & 0 & 153 & $-0,6$ \\
\hline 251 & 153 & 0,057 & 0,930 & 0,065 & 0,194 & 0,889 & 0,209 & 0,799 & 0,834 & 1,095 & 0,439 & 0,360 & 0,408 & 0,147 & 0,152 & 102 & 153 & $-2,0$ \\
\hline 252 & 153 & 0,016 & 0,338 & 0,016 & 0,266 & 1,000 & 0,788 & 0,212 & 1,000 & 0,759 & 1,077 & 0,555 & 0,137 & 0,076 & 0,152 & 90 & 153 & $-0,5$ \\
\hline 253 & 153 & 0,016 & 0,347 & 0,016 & 0,499 & 1,000 & 1,439 & 0,439 & 1,000 & 0,778 & 1,401 & 0,894 & 0,120 & 0,107 & 0,152 & 72 & 153 & $-0,3$ \\
\hline 254 & 153 & 0,026 & 0,571 & 0,030 & 0,643 & 0,857 & 1,126 & 0,190 & 0,996 & 1,002 & 0,573 & 0,559 & 0,240 & 0,134 & 0,152 & 156 & 153 & $-1,3$ \\
\hline 255 & 153 & 0,040 & 0,807 & 0,044 & 0,448 & 0,903 & 0,555 & 0,455 & 0,775 & 1,136 & 0,488 & 0,468 & 0,324 & 0,152 & 0,152 & 111 & 153 & $-1,7$ \\
\hline 256 & 153 & 0,019 & 0,592 & 0,024 & 0,194 & 0,765 & 0,328 & 0,712 & 0,669 & 1,222 & 0,331 & 0,300 & 0,268 & 0,080 & 0,076 & 139 & 153 & $-1,4$ \\
\hline
\end{tabular}




\begin{tabular}{|c|c|c|c|c|c|c|c|c|c|c|c|c|c|c|c|c|c|c|}
\hline 257 & 153 & 0,016 & 0,368 & 0,016 & 0,592 & 1,000 & 1,609 & 0,609 & 1,000 & 0,826 & 0,824 & 0,485 & 0,156 & 0,076 & 0,107 & 90 & 153 & $-0,6$ \\
\hline 258 & 153 & 0,044 & 0,634 & 0,044 & 1,737 & 1,000 & 2,740 & 1,740 & 1,000 & 0,848 & 0,963 & 0,773 & 0,243 & 0,187 & 0,214 & 135 & 153 & $-1,3$ \\
\hline 259 & 153 & 0,250 & 1,860 & 0,265 & 2,465 & 0,941 & 1,325 & 0,331 & 0,907 & 1,050 & 0,526 & 0,555 & 0,777 & 0,432 & 0,442 & 135 & 153 & $-3,0$ \\
\hline 260 & 153 & 0,248 & 2,976 & 0,327 & 0,592 & 0,759 & 0,199 & 0,837 & 0,352 & 1,685 & 0,228 & 0,303 & 1,177 & 0,356 & 0,227 & 125 & 153 & $-3,6$ \\
\hline 261 & 153 & 0,039 & 0,786 & 0,049 & 0,490 & 0,794 & 0,624 & 0,429 & 0,788 & 1,127 & 0,529 & 0,526 & 0,305 & 0,161 & 0,152 & 140 & 153 & $-1,6$ \\
\hline 262 & 153 & 0,030 & 0,541 & 0,032 & 0,541 & 0,955 & 1,000 & 0,045 & 1,000 & 0,879 & 0,786 & 0,572 & 0,221 & 0,126 & 0,169 & 149 & 153 & $-1,1$ \\
\hline 263 & 153 & 0,039 & 0,613 & 0,039 & 0,469 & 1,000 & 0,766 & 0,234 & 1,000 & 0,878 & 0,688 & 0,500 & 0,268 & 0,134 & 0,169 & 135 & 153 & $-1,4$ \\
\hline 264 & 153 & 0,030 & 0,541 & 0,030 & 0,245 & 1,000 & 0,453 & 0,547 & 1,000 & 0,879 & 0,652 & 0,442 & 0,243 & 0,107 & 0,169 & 143 & 153 & $-1,3$ \\
\hline 265 & 153 & 0,019 & 0,389 & 0,019 & 0,846 & 1,000 & 2,175 & 1,175 & 1,000 & 0,803 & 0,828 & 0,447 & 0,169 & 0,076 & 0,152 & 98 & 153 & $-0,8$ \\
\hline 266 & 153 & 0,052 & 0,896 & 0,057 & 0,672 & 0,900 & 0,750 & 0,269 & 0,808 & 1,113 & 0,432 & 0,370 & 0,390 & 0,144 & 0,169 & 123 & 153 & $-2,0$ \\
\hline 267 & 153 & 0,040 & 0,714 & 0,043 & 1,865 & 0,933 & 2,610 & 1,611 & 0,989 & 1,005 & 0,548 & 0,432 & 0,305 & 0,132 & 0,152 & 116 & 153 & $-1,6$ \\
\hline 268 & 153 & 0,202 & 2,029 & 0,248 & 0,317 & 0,815 & 0,156 & 0,864 & 0,617 & 1,273 & 0,403 & 0,498 & 0,799 & 0,398 & 0,339 & 134 & 153 & $-3,0$ \\
\hline 269 & 153 & 0,019 & 0,490 & 0,022 & 0,173 & 0,867 & 0,354 & 0,660 & 0,975 & 1,013 & 0,828 & 0,671 & 0,169 & 0,114 & 0,107 & 90 & 153 & $-0,8$ \\
\hline 270 & 153 & 0,014 & 0,317 & 0,014 & 0,397 & 1,000 & 1,254 & 0,254 & 1,000 & 0,746 & 1,273 & 0,632 & 0,120 & 0,076 & 0,107 & 18 & 153 & $-0,3$ \\
\hline 271 & 153 & 0,019 & 0,469 & 0,020 & 0,541 & 0,929 & 1,153 & 0,169 & 1,000 & 0,969 & 0,517 & 0,375 & 0,214 & 0,080 & 0,107 & 136 & 153 & $-1,1$ \\
\hline 272 & $\begin{array}{l}153 \\
154\end{array}$ & 0,032 & 0,613 & 0,034 & 0,245 & 0,917 & 0,400 & 0,606 & $\begin{array}{c}1,000 \\
\text { nothing to measure }\end{array}$ & 0,973 & 0,622 & 0,506 & 0,254 & 0,129 & 0,152 & 153 & $\begin{array}{l}153 \\
154\end{array}$ & $-1,3$ \\
\hline 1 & 155 & 0,022 & 0,469 & 0,023 & 0,347 & 0,938 & 0,739 & 0,269 & 1,000 & 0,902 & 0,764 & 0,500 & 0,189 & 0,095 & 0,107 & 39 & 155 & $-0,9$ \\
\hline 2 & $\begin{array}{l}155 \\
156\end{array}$ & 0,014 & 0,397 & 0,016 & 0,368 & 0,909 & 0,925 & 0,118 & $\begin{array}{c}1,000 \\
\text { nothing to measure }\end{array}$ & 0,936 & 0,637 & 0,474 & 0,169 & 0,080 & 0,107 & 119 & $\begin{array}{l}155 \\
156\end{array}$ & $\begin{array}{l}-0,8 \\
-\end{array}$ \\
\hline 1 & $\begin{array}{l}160 \\
161\end{array}$ & 0,014 & 0,347 & 0,016 & 0,224 & 0,909 & 0,646 & 0,365 & $\begin{array}{l}1,000 \\
\text { nothing to measure }\end{array}$ & 0,816 & 0,979 & 0,588 & 0,137 & 0,080 & 0,107 & 124 & $\begin{array}{l}160 \\
161\end{array}$ & $\begin{array}{c}-0,5 \\
-\end{array}$ \\
\hline & 162 & & & & & & & & nothing to measure & & & & & & & & 162 & - \\
\hline 1 & 163 & 0,033 & 0,562 & 0,033 & 0,461 & 1,000 & 0,819 & 0,181 & 1,000 & 0,873 & 0,791 & 0,493 & 0,230 & 0,114 & 0,152 & 85 & 163 & $-1,2$ \\
\hline 1 & $\begin{array}{l}164 \\
165\end{array}$ & 0,014 & 0,440 & 0,017 & 0,245 & 0,833 & 0,558 & 0,473 & $\begin{array}{l}\text { nothing to measure } \\
0,933\end{array}$ & 1,035 & 0,509 & 0,424 & 0,189 & 0,080 & 0,076 & 62 & $\begin{array}{l}164 \\
165\end{array}$ & $\begin{array}{c}- \\
-0,9\end{array}$ \\
\hline & 166 & & & & & & & & nothing to measure & & & & & & & & 166 & \\
\hline 1 & 167 & 0,039 & 0,744 & 0,043 & 0,643 & 0,900 & 0,864 & 0,169 & 0,879 & 1,066 & 0,465 & 0,349 & 0,326 & 0,114 & 0,152 & 127 & 167 & $-1,7$ \\
\hline 1 & 168 & 0,020 & 0,550 & 0,020 & 0,520 & 1,000 & 0,946 & 0,054 & 0,835 & 1,094 & 0,357 & 0,200 & 0,268 & 0,054 & 0,107 & 42 & 168 & $-1,4$ \\
\hline 2 & 168 & 0,049 & 0,938 & 0,070 & 0,757 & 0,694 & 0,806 & 0,362 & 0,696 & 1,199 & 0,637 & 0,728 & 0,312 & 0,227 & 0,107 & 92 & 168 & $-1,6$ \\
\hline 3 & 168 & 0,017 & 0,397 & 0,019 & 0,317 & 0,923 & 0,798 & 0,217 & 1,000 & 0,854 & 0,899 & 0,614 & 0,156 & 0,096 & 0,107 & 18 & 168 & $-0,6$ \\
\hline 4 & 168 & 0,036 & 0,786 & 0,047 & 0,571 & 0,758 & 0,726 & 0,366 & 0,729 & 1,171 & 0,796 & 0,834 & 0,240 & 0,200 & 0,152 & 57 & 168 & $-1,3$ \\
\hline 5 & 168 & 0,016 & 0,338 & 0,016 & 0,144 & 1,000 & 0,425 & 0,575 & 1,000 & 0,759 & 1,077 & 0,555 & 0,137 & 0,076 & 0,152 & 0 & 168 & $-0,5$ \\
\hline 6 & 168 & 0,040 & 0,714 & 0,044 & 0,664 & 0,903 & 0,929 & 0,120 & 0,989 & 1,005 & 0,548 & 0,432 & 0,305 & 0,132 & 0,152 & 21 & 168 & $-1,6$ \\
\hline 7 & 168 & 0,032 & 0,643 & 0,034 & 0,592 & 0,917 & 0,921 & 0,115 & 0,961 & 1,020 & 0,529 & 0,369 & 0,276 & 0,102 & 0,152 & 24 & 168 & $-1,5$ \\
\hline 8 & 168 & 0,026 & 0,550 & 0,029 & 0,520 & 0,900 & 0,946 & 0,114 & 1,000 & 0,965 & 0,573 & 0,425 & 0,240 & 0,102 & 0,152 & 30 & 168 & $-1,3$ \\
\hline 1 & 169 & 0,065 & 1,086 & 0,067 & 0,942 & 0,957 & 0,868 & 0,139 & 0,688 & 1,206 & 0,337 & 0,230 & 0,494 & 0,114 & 0,152 & 88 & 169 & $-2,3$ \\
\hline 2 & 169 & 0,027 & 0,532 & 0,030 & 0,368 & 0,905 & 0,691 & 0,324 & 1,000 & 0,910 & 0,756 & 0,643 & 0,214 & 0,138 & 0,152 & 52 & 169 & $-1,1$ \\
\hline 3 & 169 & 0,014 & 0,368 & 0,016 & 0,245 & 0,909 & 0,667 & 0,346 & 1,000 & 0,866 & 0,749 & 0,461 & 0,156 & 0,072 & 0,076 & 101 & 169 & $-0,6$ \\
\hline 4 & 169 & 0,019 & 0,511 & 0,023 & 0,266 & 0,812 & 0,521 & 0,515 & 0,896 & 1,056 & 0,571 & 0,371 & 0,204 & 0,076 & 0,107 & 7 & 169 & $-1,0$ \\
\hline 1 & 170 & 0,017 & 0,571 & 0,023 & 0,469 & 0,750 & 0,822 & 0,307 & 0,664 & 1,227 & 0,294 & 0,187 & 0,273 & 0,051 & 0,076 & 27 & 170 & $-1,4$ \\
\hline 1 & 171 & 0,139 & 1,560 & 0,164 & 1,315 & 0,851 & 0,843 & 0,217 & 0,719 & 1,180 & 0,668 & 0,655 & 0,515 & 0,338 & 0,321 & 131 & 171 & $-2,4$ \\
\hline 2 & 171 & 0,027 & 0,541 & 0,030 & 0,448 & 0,905 & 0,828 & 0,196 & 1,000 & 0,924 & 0,590 & 0,442 & 0,243 & 0,107 & 0,152 & 128 & 171 & $-1,3$ \\
\hline 3 & 171 & 0,014 & 0,397 & 0,014 & 0,275 & 1,000 & 0,692 & 0,308 & 1,000 & 0,936 & 0,509 & 0,283 & 0,189 & 0,054 & 0,107 & 40 & 171 & $-0,9$ \\
\hline 4 & 171 & 0,046 & 0,685 & 0,049 & 0,562 & 0,941 & 0,821 & 0,188 & 1,000 & 0,901 & 0,784 & 0,658 & 0,273 & 0,180 & 0,214 & 162 & 171 & $-1,4$ \\
\hline 5 & 171 & 0,014 & 0,317 & 0,014 & 0,215 & 1,000 & 0,680 & 0,320 & 1,000 & 0,746 & 0,979 & 0,555 & 0,137 & 0,076 & 0,107 & 72 & 171 & $-0,5$ \\
\hline 6 & 171 & 0,055 & 0,930 & 0,065 & 0,765 & 0,844 & 0,823 & 0,236 & 0,792 & 1,123 & 0,494 & 0,440 & 0,375 & 0,165 & 0,169 & 126 & 171 & $-1,9$ \\
\hline 7 & 171 & 0,016 & 0,389 & 0,017 & 0,245 & 0,917 & 0,631 & 0,379 & 1,000 & 0,873 & 0,824 & 0,485 & 0,156 & 0,076 & 0,107 & 162 & 171 & $-0,6$ \\
\hline 8 & 171 & 0,016 & 0,418 & 0,019 & 0,317 & 0,846 & 0,757 & 0,287 & 1,000 & 0,940 & 0,700 & 0,566 & 0,169 & 0,096 & 0,152 & 72 & 171 & $-0,8$ \\
\hline 9 & 171 & 0,014 & 0,541 & 0,022 & 0,397 & 0,667 & 0,735 & 0,426 & 0,616 & 1,274 & 0,311 & 0,218 & 0,243 & 0,053 & 0,076 & 34 & 171 & $-1,3$ \\
\hline 10 & 171 & 0,749 & 3,432 & 0,803 & 3,246 & 0,932 & 0,946 & 0,087 & 0,799 & 1,119 & 0,532 & 0,556 & 1,339 & 0,744 & 0,698 & 72 & 171 & $-3,7$ \\
\hline 11 & 171 & 0,019 & 0,651 & 0,023 & 0,600 & 0,812 & 0,922 & 0,203 & 0,553 & 1,345 & 0,224 & 0,140 & 0,326 & 0,046 & 0,076 & 37 & 171 & $-1,7$ \\
\hline 12 & 171 & 0,019 & 0,693 & 0,019 & 0,448 & 1,000 & 0,646 & 0,354 & 0,488 & 1,432 & 0,331 & 0,300 & 0,268 & 0,080 & 0,076 & 45 & 171 & $-1,4$ \\
\hline 13 & 171 & 0,037 & 1,082 & 0,056 & 0,727 & 0,667 & 0,672 & 0,468 & 0,400 & 1,580 & 0,404 & 0,442 & 0,343 & 0,152 & 0,152 & 79 & 171 & $-1,8$ \\
\hline 14 & 171 & 0,027 & 0,511 & 0,029 & 0,368 & 0,950 & 0,719 & 0,285 & 1,000 & 0,874 & 0,834 & 0,557 & 0,204 & 0,114 & 0,152 & 105 & 171 & $-1,0$ \\
\hline
\end{tabular}




\begin{tabular}{|c|c|c|c|c|c|c|c|c|c|c|c|c|c|c|c|c|c|c|}
\hline 15 & 171 & 0,033 & 0,664 & 0,037 & 0,613 & 0,885 & 0,924 & 0,138 & 0,942 & 1,031 & 0,651 & 0,596 & 0,254 & 0,152 & 0,152 & 151 & 171 & $-1,3$ \\
\hline 16 & 171 & 0,020 & 0,714 & 0,036 & 0,541 & 0,560 & 0,757 & 0,502 & 0,495 & 1,422 & 0,336 & 0,369 & 0,276 & 0,102 & 0,107 & 0 & 171 & $-1,5$ \\
\hline 17 & 171 & 0,030 & 0,765 & 0,039 & 0,296 & 0,778 & 0,387 & 0,652 & 0,647 & 1,243 & 0,594 & 0,596 & 0,254 & 0,152 & 0,152 & 73 & 171 & $-1,3$ \\
\hline 18 & 171 & 0,014 & 0,440 & 0,017 & 0,194 & 0,833 & 0,442 & 0,582 & 0,933 & 1,035 & 0,439 & 0,271 & 0,204 & 0,055 & 0,076 & 20 & 171 & $-1,0$ \\
\hline 19 & 171 & 0,014 & 0,317 & 0,014 & 0,592 & 1,000 & 1,867 & 0,867 & 1,000 & 0,746 & 0,979 & 0,555 & 0,137 & 0,076 & 0,107 & 18 & 171 & $-0,5$ \\
\hline 20 & 171 & 0,027 & 0,693 & 0,032 & 0,347 & 0,864 & 0,500 & 0,518 & 0,713 & 1,185 & 0,417 & 0,401 & 0,288 & 0,116 & 0,152 & 35 & 171 & $-1,5$ \\
\hline 21 & 171 & 0,019 & 0,529 & 0,023 & 1,543 & 0,812 & 2,918 & 1,927 & 0,839 & 1,092 & 0,662 & 0,566 & 0,189 & 0,107 & 0,107 & 50 & 171 & $-0,9$ \\
\hline 22 & 171 & 0,136 & 2,765 & 0,224 & 0,532 & 0,609 & 0,193 & 0,897 & 0,224 & 2,112 & 0,484 & 0,627 & 0,599 & 0,376 & 0,169 & 168 & 171 & $-2,6$ \\
\hline 23 & 171 & 0,023 & 0,778 & 0,029 & 2,396 & 0,800 & 3,081 & 2,091 & 0,477 & 1,448 & 0,204 & 0,100 & 0,379 & 0,038 & 0,076 & 0 & 171 & $-1,9$ \\
\hline 24 & 171 & 0,184 & 2,637 & 0,265 & 0,490 & 0,692 & 0,186 & 0,870 & 0,332 & 1,736 & 0,169 & 0,222 & 1,177 & 0,261 & 0,214 & 3 & 171 & $-3,6$ \\
\hline 25 & 171 & 0,032 & 0,664 & 0,037 & 0,389 & 0,846 & 0,586 & 0,442 & 0,901 & 1,054 & 0,757 & 0,589 & 0,230 & 0,136 & 0,152 & 17 & 171 & $-1,2$ \\
\hline 26 & 171 & 0,020 & 0,469 & 0,022 & 0,900 & 0,933 & 1,918 & 0,921 & 1,000 & 0,934 & 0,713 & 0,555 & 0,189 & 0,105 & 0,152 & 42 & 171 & $-0,9$ \\
\hline 27 & 171 & 0,063 & 1,187 & 0,086 & 0,541 & 0,733 & 0,456 & 0,606 & 0,563 & 1,333 & 0,324 & 0,371 & 0,498 & 0,185 & 0,214 & 2 & 171 & $-2,3$ \\
\hline 28 & 171 & 0,026 & 0,622 & 0,032 & 0,778 & 0,818 & 1,251 & 0,310 & 0,840 & 1,091 & 0,458 & 0,395 & 0,268 & 0,106 & 0,152 & 19 & 171 & $-1,4$ \\
\hline 29 & 171 & 0,043 & 1,234 & 0,065 & 0,275 & 0,667 & 0,223 & 0,846 & 0,355 & 1,678 & 0,523 & 0,666 & 0,324 & 0,216 & 0,107 & 6 & 171 & $-1,7$ \\
\hline 30 & 171 & 0,016 & 0,347 & 0,016 & 0,765 & 1,000 & 2,207 & 1,207 & 1,000 & 0,778 & 1,077 & 0,588 & 0,137 & 0,080 & 0,107 & 135 & 171 & $-0,5$ \\
\hline 31 & 171 & 0,052 & 1,163 & 0,072 & 0,440 & 0,720 & 0,378 & 0,682 & 0,480 & 1,443 & 0,573 & 0,671 & 0,339 & 0,227 & 0,152 & 92 & 171 & $-1,8$ \\
\hline 32 & 171 & 0,016 & 0,550 & 0,027 & 0,448 & 0,579 & 0,815 & 0,460 & 0,656 & 1,234 & 0,412 & 0,485 & 0,221 & 0,107 & 0,076 & 66 & 171 & $-1,1$ \\
\hline 33 & 171 & 0,014 & 0,550 & 0,020 & 0,296 & 0,714 & 0,538 & 0,543 & 0,597 & 1,295 & 0,311 & 0,251 & 0,243 & 0,061 & 0,076 & 33 & 171 & $-1,3$ \\
\hline 34 & 171 & 0,014 & 0,440 & 0,017 & 1,217 & 0,833 & 2,769 & 1,777 & 0,933 & 1,035 & 0,439 & 0,271 & 0,204 & 0,055 & 0,076 & 20 & 171 & $-1,0$ \\
\hline 35 & 171 & 0,128 & 1,319 & 0,138 & 0,448 & 0,927 & 0,340 & 0,664 & 0,923 & 1,041 & 0,613 & 0,642 & 0,515 & 0,331 & 0,312 & 64 & 171 & $-2,4$ \\
\hline 36 & 171 & 0,017 & 0,592 & 0,026 & 0,418 & 0,667 & 0,707 & 0,444 & 0,618 & 1,272 & 0,382 & 0,401 & 0,240 & 0,096 & 0,076 & 79 & 171 & $-1,3$ \\
\hline 37 & 171 & 0,019 & 0,651 & 0,024 & 0,727 & 0,765 & 1,116 & 0,262 & 0,553 & 1,345 & 0,662 & 0,627 & 0,189 & 0,119 & 0,076 & 78 & 171 & $-0,9$ \\
\hline 38 & 171 & 0,043 & 0,909 & 0,055 & 0,461 & 0,789 & 0,507 & 0,536 & 0,655 & 1,236 & 0,466 & 0,442 & 0,343 & 0,152 & 0,152 & 88 & 171 & $-1,8$ \\
\hline 39 & 171 & 0,022 & 0,744 & 0,030 & 0,338 & 0,714 & 0,454 & 0,616 & 0,488 & 1,431 & 0,466 & 0,543 & 0,243 & 0,132 & 0,076 & 32 & 171 & $-1,3$ \\
\hline 40 & 171 & 0,022 & 0,562 & 0,027 & 0,410 & 0,789 & 0,729 & 0,343 & 0,856 & 1,081 & 0,659 & 0,511 & 0,204 & 0,104 & 0,107 & 165 & 171 & $-1,0$ \\
\hline 41 & 171 & 0,017 & 0,511 & 0,019 & 0,490 & 0,923 & 0,959 & 0,087 & 0,827 & 1,099 & 0,413 & 0,164 & 0,230 & 0,038 & 0,076 & 90 & 171 & $-1,2$ \\
\hline 42 & 171 & 0,024 & 0,685 & 0,033 & 2,012 & 0,739 & 2,938 & 1,956 & 0,654 & 1,237 & 0,408 & 0,367 & 0,276 & 0,101 & 0,107 & 16 & 171 & $-1,5$ \\
\hline 43 & 171 & 0,294 & 2,236 & 0,337 & 0,296 & 0,872 & 0,132 & 0,877 & 0,739 & 1,163 & 0,614 & 0,706 & 0,781 & 0,551 & 0,530 & 155 & 171 & $-3,0$ \\
\hline 44 & 171 & 0,016 & 0,368 & 0,016 & 1,399 & 1,000 & 3,805 & 2,805 & 1,000 & 0,826 & 0,700 & 0,447 & 0,169 & 0,076 & 0,107 & 18 & 171 & $-0,8$ \\
\hline 45 & 171 & 0,116 & 2,600 & 0,189 & 0,520 & 0,614 & 0,200 & 0,888 & 0,216 & 2,151 & 0,441 & 0,642 & 0,579 & 0,372 & 0,214 & 88 & 171 & $-2,5$ \\
\hline 46 & 171 & 0,022 & 0,744 & 0,033 & 0,867 & 0,652 & 1,165 & 0,385 & 0,488 & 1,431 & 0,382 & 0,443 & 0,268 & 0,119 & 0,107 & 49 & 171 & $-1,4$ \\
\hline 47 & 171 & 0,056 & 1,492 & 0,080 & 0,317 & 0,696 & 0,212 & 0,844 & 0,316 & 1,779 & 0,456 & 0,545 & 0,395 & 0,216 & 0,152 & 9 & 171 & $-2,0$ \\
\hline 48 & 171 & 0,016 & 0,368 & 0,017 & 0,296 & 0,917 & 0,805 & 0,212 & 1,000 & 0,826 & 1,077 & 0,745 & 0,137 & 0,102 & 0,152 & 135 & 171 & $-0,5$ \\
\hline 49 & 171 & 0,014 & 0,448 & 0,019 & 0,440 & 0,769 & 0,981 & 0,232 & 0,898 & 1,055 & 0,637 & 0,495 & 0,169 & 0,084 & 0,076 & 153 & 171 & $-0,8$ \\
\hline 50 & 171 & 0,029 & 0,541 & 0,030 & 0,757 & 0,952 & 1,398 & 0,401 & 1,000 & 0,901 & 0,749 & 0,537 & 0,221 & 0,119 & 0,152 & 155 & 171 & $-1,1$ \\
\hline 51 & 171 & 0,065 & 0,879 & 0,069 & 0,338 & 0,938 & 0,385 & 0,619 & 1,000 & 0,976 & 0,674 & 0,602 & 0,349 & 0,210 & 0,214 & 140 & 171 & $-1,8$ \\
\hline 52 & 171 & 0,022 & 0,440 & 0,023 & 1,767 & 0,938 & 4,020 & 3,020 & 1,000 & 0,845 & 0,955 & 0,671 & 0,169 & 0,114 & 0,152 & 98 & 171 & $-0,8$ \\
\hline 53 & 171 & 0,185 & 2,367 & 0,241 & 0,613 & 0,768 & 0,259 & 0,777 & 0,415 & 1,552 & 0,356 & 0,491 & 0,813 & 0,399 & 0,107 & 112 & 171 & $-3,0$ \\
\hline 54 & 171 & 0,023 & 0,735 & 0,034 & 2,055 & 0,667 & 2,794 & 1,825 & 0,533 & 1,369 & 0,255 & 0,213 & 0,339 & 0,072 & 0,076 & 163 & 171 & $-1,8$ \\
\hline 55 & 171 & 0,141 & 2,579 & 0,222 & 0,795 & 0,632 & 0,308 & 0,783 & 0,266 & 1,940 & 0,176 & 0,212 & 1,009 & 0,214 & 0,152 & 136 & 171 & $-3,3$ \\
\hline 56 & 171 & 0,043 & 0,989 & 0,055 & 0,960 & 0,789 & 0,970 & 0,213 & 0,553 & 1,345 & 0,326 & 0,370 & 0,410 & 0,152 & 0,152 & 41 & 171 & $-2,0$ \\
\hline 57 & 171 & 0,076 & 1,184 & 0,093 & 0,440 & 0,815 & 0,371 & 0,655 & 0,682 & 1,211 & 0,649 & 0,619 & 0,386 & 0,239 & 0,169 & 108 & 171 & $-1,9$ \\
\hline 58 & 171 & 0,020 & 0,622 & 0,027 & 0,368 & 0,737 & 0,592 & 0,486 & 0,653 & 1,237 & 0,357 & 0,377 & 0,268 & 0,101 & 0,107 & 73 & 171 & $-1,4$ \\
\hline 59 & 171 & 0,016 & 0,490 & 0,019 & 1,294 & 0,846 & 2,639 & 1,646 & 0,825 & 1,101 & 0,438 & 0,250 & 0,214 & 0,054 & 0,076 & 40 & 171 & $-1,1$ \\
\hline 60 & 171 & 0,065 & 1,653 & 0,108 & 5,334 & 0,600 & 3,227 & 2,263 & 0,297 & 1,835 & 0,220 & 0,265 & 0,611 & 0,162 & 0,169 & 142 & 171 & $-2,6$ \\
\hline 61 & 171 & 1,152 & 7,964 & 1,940 & 4,701 & 0,594 & 0,590 & 0,577 & 0,228 & 2,093 & 0,334 & 0,570 & 2,097 & 1,195 & 0,408 & 69 & 171 & $-4,4$ \\
\hline 62 & 171 & 1,456 & 5,191 & 1,616 & 0,317 & 0,901 & 0,061 & 0,944 & 0,679 & 1,214 & 0,538 & 0,618 & 1,857 & 1,148 & 0,925 & 71 & 171 & $-4,2$ \\
\hline 63 & 171 & 0,026 & 0,461 & 0,026 & 0,266 & 1,000 & 0,578 & 0,422 & 1,000 & 0,809 & 1,146 & 0,671 & 0,169 & 0,114 & 0,152 & 0 & 171 & $-0,8$ \\
\hline 64 & 171 & 0,014 & 0,490 & 0,019 & 1,839 & 0,769 & 3,750 & 2,760 & 0,750 & 1,155 & 0,749 & 0,485 & 0,156 & 0,076 & 0,076 & 85 & 171 & $-0,6$ \\
\hline 65 & 171 & 0,244 & 2,101 & 0,268 & 0,266 & 0,909 & 0,127 & 0,878 & 0,694 & 1,200 & 0,449 & 0,469 & 0,832 & 0,390 & 0,386 & 120 & 171 & $-3,1$ \\
\hline 66 & 171 & 0,016 & 0,389 & 0,017 & 0,368 & 0,917 & 0,946 & 0,099 & 1,000 & 0,873 & 0,700 & 0,425 & 0,169 & 0,072 & 0,076 & 101 & 171 & $-0,8$ \\
\hline 67 & 171 & 0,017 & 0,440 & 0,019 & 0,541 & 0,923 & 1,231 & 0,243 & 1,000 & 0,945 & 0,588 & 0,373 & 0,193 & 0,072 & 0,076 & 108 & 171 & $-0,9$ \\
\hline 68 & 171 & 0,040 & 0,685 & 0,043 & 0,296 & 0,933 & 0,432 & 0,572 & 1,000 & 0,964 & 0,713 & 0,535 & 0,268 & 0,143 & 0,152 & 11 & 171 & $-1,4$ \\
\hline 69 & 171 & 0,020 & 0,440 & 0,022 & 1,103 & 0,933 & 2,510 & 1,511 & 1,000 & 0,875 & 0,713 & 0,537 & 0,189 & 0,102 & 0,152 & 125 & 171 & $-0,9$ \\
\hline 70 & 171 & 0,099 & 1,488 & 0,118 & 0,490 & 0,841 & 0,329 & 0,689 & 0,562 & 1,334 & 0,574 & 0,566 & 0,469 & 0,265 & 0,273 & 169 & 171 & $-2,2$ \\
\hline 71 & 171 & 0,026 & 0,592 & 0,030 & 0,532 & 0,857 & 0,900 & 0,175 & 0,927 & 1,039 & 0,559 & 0,509 & 0,243 & 0,123 & 0,152 & 108 & 171 & $-1,3$ \\
\hline 72 & 171 & 0,030 & 1,010 & 0,043 & 1,065 & 0,700 & 1,054 & 0,305 & 0,371 & 1,642 & 0,652 & 0,769 & 0,243 & 0,186 & 0,107 & 126 & 171 & $-1,3$ \\
\hline
\end{tabular}




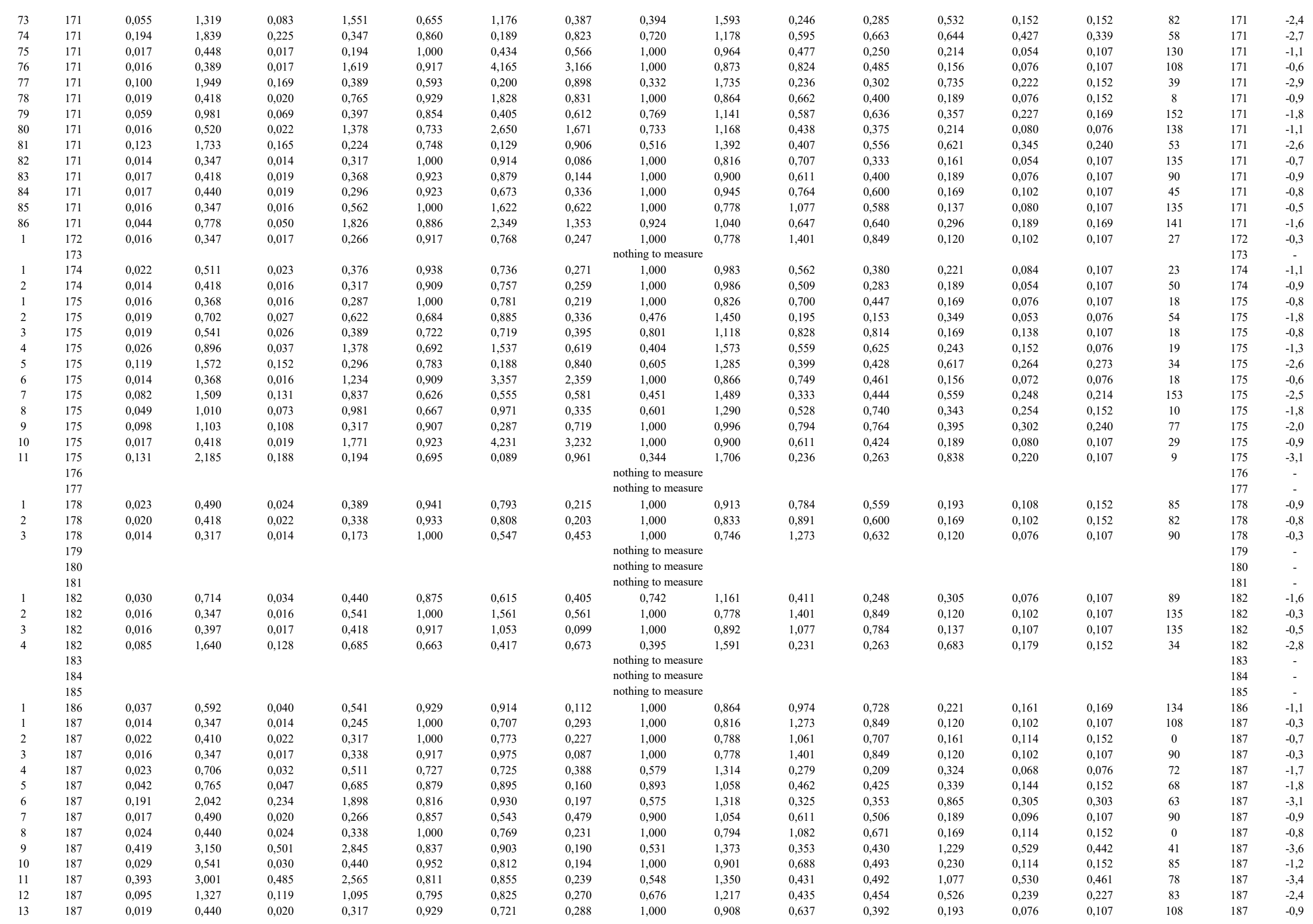




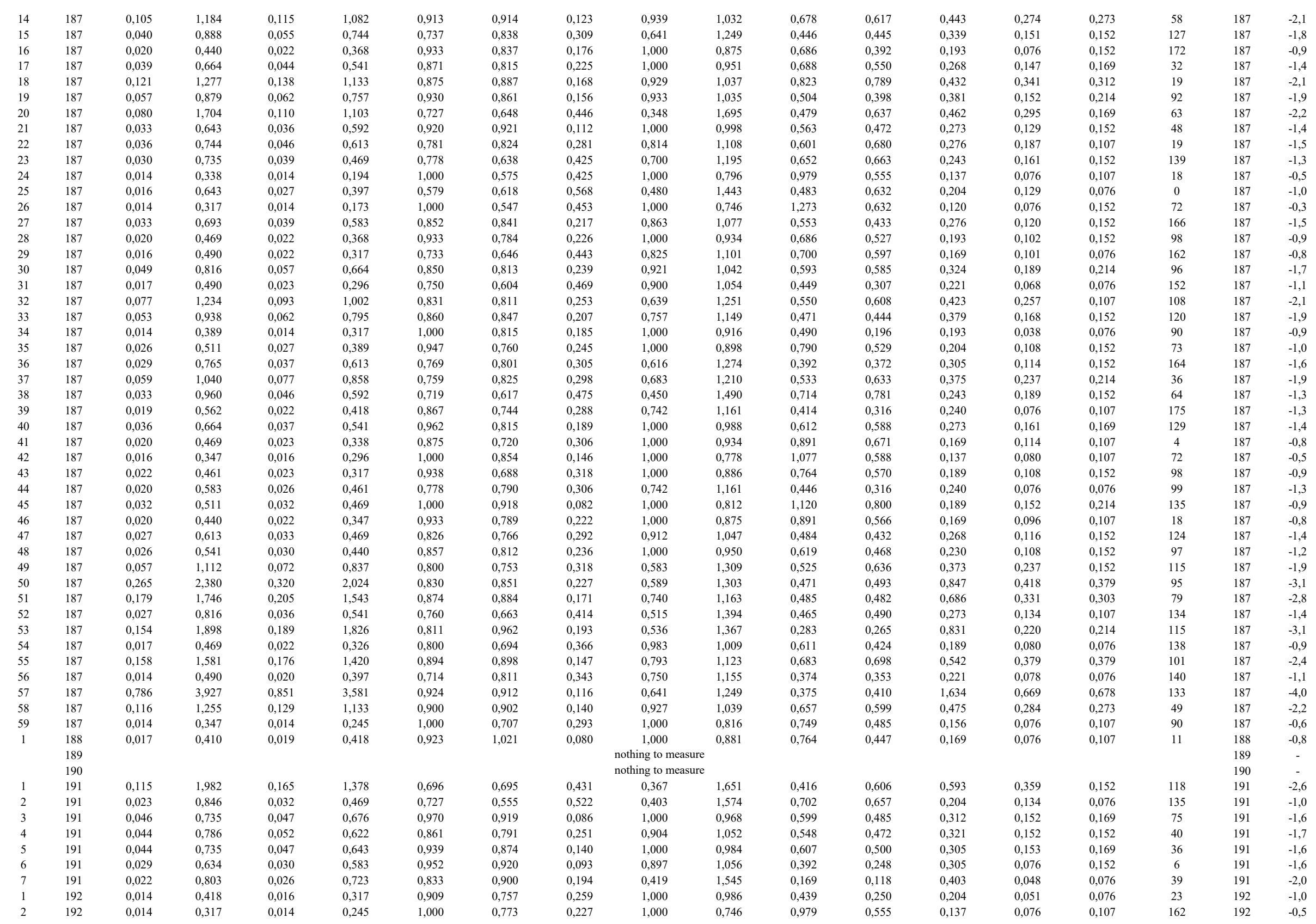




\begin{tabular}{|c|c|c|c|c|c|c|c|c|c|c|c|c|c|c|c|c|c|c|}
\hline 3 & 192 & 0,016 & 0,478 & 0,020 & 0,347 & 0,786 & 0,725 & 0,348 & 0,868 & 1,073 & 0,824 & 0,673 & 0,156 & 0,105 & 0,107 & 146 & 192 & $-0,6$ \\
\hline 4 & 192 & 0,043 & 0,960 & 0,052 & 0,714 & 0,833 & 0,745 & 0,305 & 0,587 & 1,305 & 0,477 & 0,466 & 0,339 & 0,158 & 0,169 & 136 & 192 & $-1,8$ \\
\hline 1 & 193 & 0,014 & 0,317 & 0,014 & 0,173 & 1,000 & 0,547 & 0,453 & 1,000 & 0,746 & 1,273 & 0,632 & 0,120 & 0,076 & 0,152 & 72 & 193 & $-0,3$ \\
\hline 2 & 193 & 0,022 & 0,469 & 0,022 & 0,368 & 1,000 & 0,784 & 0,216 & 1,000 & 0,902 & 0,597 & 0,375 & 0,214 & 0,080 & 0,107 & 40 & 193 & $-1,1$ \\
\hline 3 & 193 & 0,019 & 0,469 & 0,022 & 0,368 & 0,867 & 0,784 & 0,254 & 1,000 & 0,969 & 0,828 & 0,621 & 0,169 & 0,105 & 0,107 & 135 & 193 & $-0,8$ \\
\hline & 194 & & & & & & & & nothing to measure & & & & & & & & 194 & - \\
\hline & 195 & & & & & & & & nothing to measure & & & & & & & & 195 & - \\
\hline & 196 & & & & & & & & nothing to measure & & & & & & & & 196 & - \\
\hline & 197 & & & & & & & & nothing to measure & & & & & & & & 197 & - \\
\hline & 198 & & & & & & & & nothing to measure & & & & & & & & 198 & - \\
\hline 1 & 199 & 0,016 & 0,338 & 0,016 & 0,144 & 1,000 & 0,425 & 0,575 & 1,000 & 0,759 & 1,077 & 0,555 & 0,137 & 0,076 & 0,152 & 0 & 199 & $-0,5$ \\
\hline 1 & 200 & 0,019 & 0,368 & 0,019 & 0,245 & 1,000 & 0,667 & 0,333 & 1,000 & 0,760 & 1,273 & 0,784 & 0,137 & 0,107 & 0,152 & 90 & 200 & $-0,5$ \\
\hline 2 & 200 & 0,023 & 0,643 & 0,029 & 0,418 & 0,800 & 0,651 & 0,402 & 0,699 & 1,196 & 0,599 & 0,537 & 0,221 & 0,119 & 0,107 & 152 & 200 & $-1,1$ \\
\hline 3 & 200 & 0,067 & 0,828 & 0,069 & 0,757 & 0,979 & 0,913 & 0,089 & 1,000 & 0,900 & 0,981 & 0,768 & 0,296 & 0,227 & 0,303 & 135 & 200 & $-1,6$ \\
\hline 4 & 200 & 0,034 & 0,664 & 0,040 & 0,499 & 0,857 & 0,752 & 0,286 & 0,983 & 1,009 & 0,899 & 0,728 & 0,221 & 0,161 & 0,152 & 140 & 200 & $-1,1$ \\
\hline 5 & 200 & 0,265 & 1,809 & 0,277 & 1,737 & 0,959 & 0,960 & 0,057 & 1,000 & 0,990 & 0,675 & 0,589 & 0,708 & 0,417 & 0,455 & 1 & 200 & $-2,8$ \\
\hline 6 & 200 & 0,100 & 1,175 & 0,110 & 0,972 & 0,909 & 0,827 & 0,195 & 0,914 & 1,046 & 0,686 & 0,654 & 0,432 & 0,282 & 0,303 & 170 & 200 & $-2,1$ \\
\hline 7 & 200 & 0,016 & 0,643 & 0,026 & 0,368 & 0,611 & 0,572 & 0,578 & 0,480 & 1,443 & 0,539 & 0,588 & 0,193 & 0,114 & 0,076 & 45 & 200 & $-0,9$ \\
\hline 8 & 200 & 0,053 & 1,002 & 0,065 & 0,685 & 0,822 & 0,684 & 0,363 & 0,665 & 1,226 & 0,589 & 0,559 & 0,339 & 0,189 & 0,227 & 84 & 200 & $-1,8$ \\
\hline \multirow{4}{*}{10} & 200 & 0,017 & 0,397 & 0,020 & 0,347 & 0,857 & 0,872 & 0,192 & 1,000 & 0,854 & 0,955 & 0,671 & 0,152 & 0,102 & 0,152 & 0 & 200 & $-0,6$ \\
\hline & 200 & 0,033 & 0,562 & 0,034 & 0,469 & 0,958 & 0,835 & 0,170 & 1,000 & 0,873 & 0,791 & 0,493 & 0,230 & 0,114 & 0,152 & 176 & 200 & $-1,2$ \\
\hline & 201 & & & & & & & & nothing to measure & & & & & & & & 201 & - \\
\hline & 202 & & & & & & & & nothing to measure & & & & & & & & 202 & . \\
\hline 1 & 203 & 0,040 & 0,930 & 0,050 & 0,613 & 0,800 & 0,659 & 0,395 & 0,584 & 1,309 & 0,548 & 0,510 & 0,305 & 0,156 & 0,152 & 28 & 203 & $-1,6$ \\
\hline 2 & 203 & 0,016 & 0,389 & 0,016 & 0,194 & 1,000 & 0,500 & 0,500 & 1,000 & 0,873 & 0,700 & 0,447 & 0,169 & 0,076 & 0,107 & 167 & 203 & $-0,8$ \\
\hline 3 & 203 & 0,050 & 1,480 & 0,076 & 0,837 & 0,660 & 0,566 & 0,551 & 0,288 & 1,862 & 0,420 & 0,522 & 0,390 & 0,203 & 0,152 & 23 & 203 & $-2,0$ \\
\hline 4 & 203 & 0,056 & 1,399 & 0,100 & 1,217 & 0,557 & 0,870 & 0,462 & 0,359 & 1,668 & 0,191 & 0,272 & 0,611 & 0,166 & 0,107 & 175 & 203 & $-2,6$ \\
\hline 5 & 203 & 0,019 & 0,418 & 0,019 & 0,347 & 1,000 & 0,828 & 0,172 & 1,000 & 0,864 & 0,637 & 0,392 & 0,193 & 0,076 & 0,152 & 8 & 203 & $-0,9$ \\
\hline 6 & 203 & 0,029 & 0,735 & 0,037 & 0,532 & 0,769 & 0,724 & 0,360 & 0,667 & 1,225 & 0,509 & 0,424 & 0,268 & 0,114 & 0,152 & 5 & 203 & $-1,4$ \\
\hline 7 & 203 & 0,037 & 1,031 & 0,047 & 0,613 & 0,788 & 0,594 & 0,458 & 0,441 & 1,506 & 0,625 & 0,687 & 0,276 & 0,189 & 0,107 & 10 & 203 & $-1,5$ \\
\hline 8 & 203 & 0,032 & 0,735 & 0,037 & 0,520 & 0,846 & 0,707 & 0,331 & 0,733 & 1,168 & 0,757 & 0,658 & 0,230 & 0,152 & 0,152 & 37 & 203 & $-1,2$ \\
\hline 9 & 203 & 0,014 & 0,469 & 0,019 & 0,296 & 0,769 & 0,631 & 0,436 & 0,819 & 1,105 & 0,374 & 0,238 & 0,221 & 0,053 & 0,076 & 45 & 203 & $-1,1$ \\
\hline 10 & 203 & 0,095 & 1,429 & 0,119 & 1,273 & 0,795 & 0,891 & 0,232 & 0,583 & 1,310 & 0,307 & 0,332 & 0,627 & 0,208 & 0,214 & 32 & 203 & $-2,6$ \\
\hline 11 & 203 & 0,042 & 1,010 & 0,057 & 0,735 & 0,725 & 0,728 & 0,387 & 0,512 & 1,397 & 0,434 & 0,425 & 0,349 & 0,148 & 0,152 & 75 & 203 & $-1,8$ \\
\hline 12 & 203 & 0,014 & 0,418 & 0,016 & 0,368 & 0,909 & 0,879 & 0,152 & 1,000 & 0,986 & 0,439 & 0,294 & 0,204 & 0,060 & 0,076 & 18 & 203 & $-1,0$ \\
\hline 13 & 203 & 0,053 & 1,399 & 0,099 & 1,145 & 0,536 & 0,819 & 0,498 & 0,341 & 1,713 & 0,184 & 0,268 & 0,606 & 0,163 & 0,107 & 169 & 203 & $-2,6$ \\
\hline 14 & 203 & 0,039 & 0,888 & 0,047 & 0,613 & 0,818 & 0,690 & 0,359 & 0,618 & 1,272 & 0,859 & 0,778 & 0,240 & 0,186 & 0,152 & 130 & 203 & $-1,3$ \\
\hline 15 & 203 & 0,017 & 0,511 & 0,022 & 0,410 & 0,800 & 0,801 & 0,282 & 0,827 & 1,099 & 0,413 & 0,271 & 0,230 & 0,062 & 0,076 & 90 & 203 & $-1,2$ \\
\hline 16 & 203 & 0,014 & 0,317 & 0,014 & 0,245 & 1,000 & 0,773 & 0,227 & 1,000 & 0,746 & 0,979 & 0,555 & 0,137 & 0,076 & 0,107 & 162 & 203 & $-0,5$ \\
\hline 17 & 203 & 0,046 & 0,981 & 0,057 & 0,849 & 0,800 & 0,866 & 0,241 & 0,600 & 1,291 & 0,334 & 0,272 & 0,418 & 0,114 & 0,152 & 178 & 203 & $-2,1$ \\
\hline 18 & 203 & 0,067 & 1,437 & 0,096 & 1,052 & 0,701 & 0,732 & 0,401 & 0,410 & 1,561 & 0,413 & 0,623 & 0,456 & 0,284 & 0,152 & 52 & 203 & $-2,2$ \\
\hline 19 & 203 & 0,017 & 0,368 & 0,017 & 0,296 & 1,000 & 0,805 & 0,195 & 1,000 & 0,791 & 0,899 & 0,485 & 0,156 & 0,076 & 0,152 & 8 & 203 & $-0,6$ \\
\hline 20 & 203 & 0,017 & 0,664 & 0,027 & 0,347 & 0,632 & 0,522 & 0,603 & 0,491 & 1,427 & 0,382 & 0,435 & 0,240 & 0,104 & 0,076 & 10 & 203 & $-1,3$ \\
\hline 21 & 203 & 0,042 & 0,989 & 0,063 & 0,786 & 0,659 & 0,795 & 0,398 & 0,534 & 1,368 & 0,348 & 0,414 & 0,390 & 0,161 & 0,107 & 69 & 203 & $-2,0$ \\
\hline 22 & 203 & 0,017 & 0,440 & 0,020 & 0,296 & 0,857 & 0,673 & 0,357 & 1,000 & 0,945 & 0,611 & 0,424 & 0,189 & 0,080 & 0,107 & 35 & 203 & $-0,9$ \\
\hline 23 & 203 & 0,039 & 0,634 & 0,039 & 0,562 & 1,000 & 0,887 & 0,113 & 1,000 & 0,909 & 0,649 & 0,412 & 0,276 & 0,114 & 0,152 & 4 & 203 & $-1,5$ \\
\hline 24 & 203 & 0,171 & 2,371 & 0,221 & 1,940 & 0,773 & 0,818 & 0,291 & 0,382 & 1,619 & 0,242 & 0,260 & 0,947 & 0,246 & 0,240 & 14 & 203 & $-3,2$ \\
\hline 25 & 203 & 0,017 & 0,541 & 0,024 & 0,317 & 0,706 & 0,586 & 0,508 & 0,739 & 1,163 & 0,477 & 0,491 & 0,214 & 0,105 & 0,107 & 72 & 203 & $-1,1$ \\
\hline 26 & 203 & 0,022 & 0,461 & 0,023 & 0,368 & 0,938 & 0,798 & 0,211 & 1,000 & 0,886 & 0,735 & 0,392 & 0,193 & 0,076 & 0,152 & 171 & 203 & $-0,9$ \\
\hline 27 & 203 & 0,033 & 0,938 & 0,044 & 0,774 & 0,742 & 0,825 & 0,312 & 0,471 & 1,457 & 0,225 & 0,225 & 0,432 & 0,097 & 0,107 & 42 & 203 & $-2,1$ \\
\hline 28 & 203 & 0,023 & 0,714 & 0,029 & 0,511 & 0,800 & 0,716 & 0,348 & 0,565 & 1,330 & 0,551 & 0,493 & 0,230 & 0,114 & 0,076 & 23 & 203 & $-1,2$ \\
\hline 29 & 203 & 0,039 & 1,031 & 0,056 & 0,664 & 0,692 & 0,643 & 0,471 & 0,458 & 1,478 & 0,506 & 0,584 & 0,312 & 0,182 & 0,107 & 171 & 203 & $-1,6$ \\
\hline 30 & 203 & 0,017 & 0,511 & 0,020 & 0,245 & 0,857 & 0,479 & 0,540 & 0,827 & 1,099 & 0,764 & 0,447 & 0,169 & 0,076 & 0,076 & 84 & 203 & $-0,8$ \\
\hline 31 & 203 & 0,092 & 2,084 & 0,136 & 1,369 & 0,674 & 0,657 & 0,473 & 0,266 & 1,940 & 0,248 & 0,317 & 0,686 & 0,218 & 0,227 & 172 & 203 & $-2,8$ \\
\hline 32 & 203 & 0,032 & 0,706 & 0,039 & 0,562 & 0,815 & 0,796 & 0,275 & 0,796 & 1,121 & 0,412 & 0,346 & 0,312 & 0,108 & 0,152 & 16 & 203 & $-1,6$ \\
\hline 33 & 203 & 0,014 & 0,317 & 0,014 & 0,194 & 1,000 & 0,613 & 0,387 & 1,000 & 0,746 & 0,979 & 0,555 & 0,137 & 0,076 & 0,107 & 18 & 203 & $-0,5$ \\
\hline 34 & 203 & 0,014 & 0,604 & 0,019 & 0,461 & 0,769 & 0,762 & 0,331 & 0,494 & 1,423 & 0,196 & 0,123 & 0,305 & 0,038 & 0,076 & 0 & 203 & $-1,6$ \\
\hline 35 & 203 & 0,023 & 0,676 & 0,030 & 0,317 & 0,762 & 0,469 & 0,582 & 0,631 & 1,259 & 0,300 & 0,180 & 0,312 & 0,056 & 0,076 & 170 & 203 & $-1,6$ \\
\hline
\end{tabular}




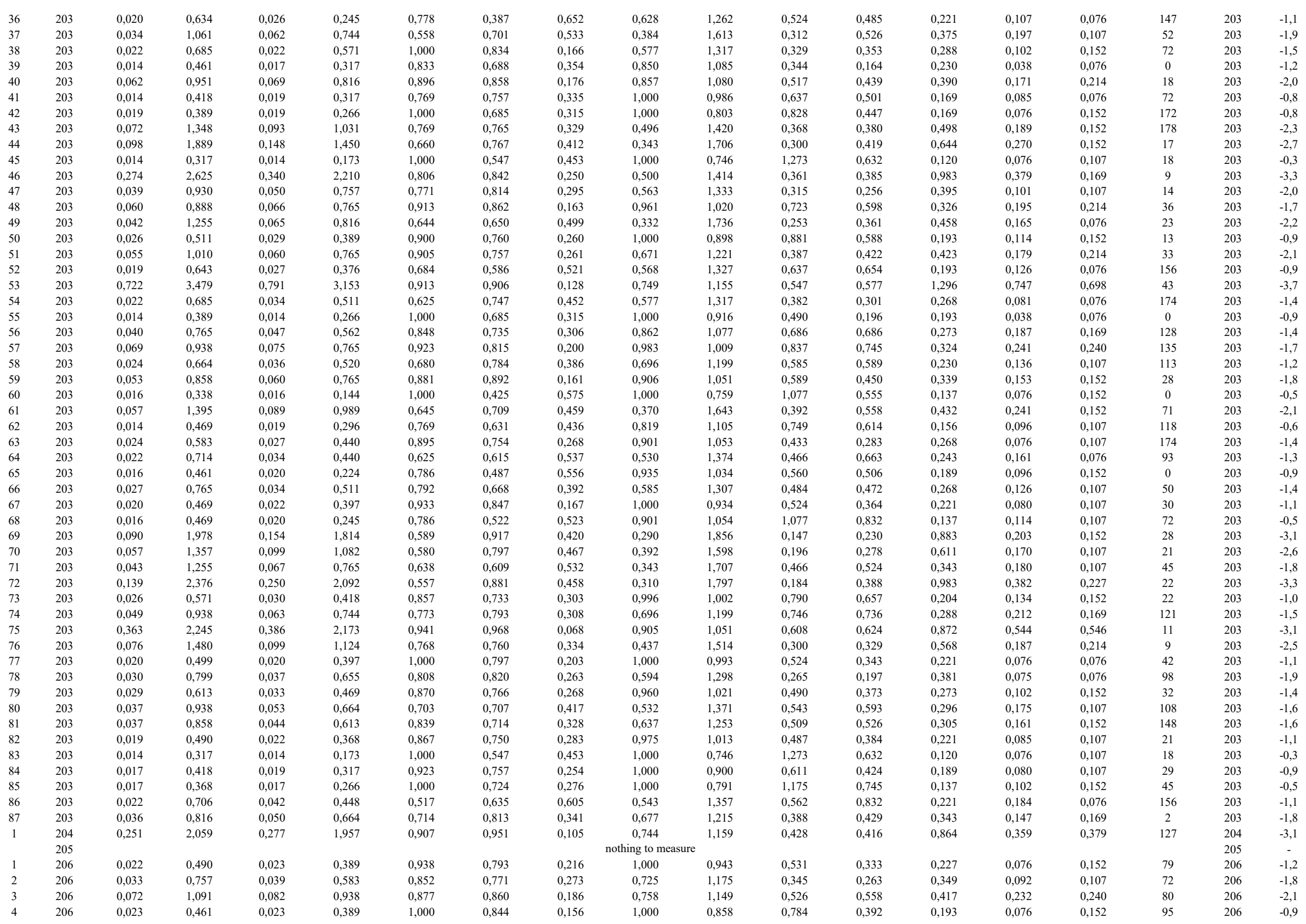


nothing to measure

nothing to measure
nothing to measure

nothing to measure
nothing to measure

$\begin{array}{lllllll}0,032 & 0,613 & 0,037 & 0,499 & 0,846 & 0,814 & 0,241\end{array}$

$1,000 \quad 0,973$
nothing to

nothing to measure

$\begin{array}{llllll}0,016 & 0,368 & 0,016 & 0,296 & 1,000 & 0,805 \\ 0,072 & 1,374 & 0,092 & 1,213 & 0,781 & 0,883\end{array}$

$\begin{array}{ll}1,000 & 0,826\end{array}$

0,826

0,757

0,559

0,23

0,129

0,152

$\begin{array}{lll}0,248 & 0,477 & 1,447 \\ 0,254 & 1,000 & 0,900\end{array}$

$0,824 \quad 0,485$

0,156

0,107

$\begin{array}{lll}0,156 & 0,108 & 0,167\end{array}$

$0,599 \quad 0,237$

$\begin{array}{llll}0,069 & 1,733 & 0,121 & 1,247 \\ 0,060 & 1,877 & 0,098 & 1,754\end{array}$

$\begin{array}{llll}0,060 & 1,877 & 0,098 & 1,754 \\ 0,023 & 0,562 & 0,026 & 0,490\end{array}$

$\begin{array}{llll}0,152 & 2,469 & 0,240 & 1,856 \\ 0,016 & 0,389 & 0,017 & 0,275\end{array}$

$0,016 \quad 0,389$

$0,040 \quad 0,837$

$\begin{array}{ll}0,020 & 0,951 \\ 0,080 & 1,150\end{array}$

$\begin{array}{ll}0,017 & 0,275 \\ 0,017 & 0,245\end{array}$

0,019

$\begin{array}{ll}0,088 & 1,091 \\ 0,123 & 1,082\end{array}$

$\begin{array}{ll}0,571 & 0,719 \\ 0,618 & 0,935\end{array}$

0,388

0,215

0,314

$\begin{array}{ll}0,635 & 0,752 \\ 0,917 & 0,707\end{array}$

0,442

0,305
0,333
0,243

0,243

1,000
1,000

1,786
0,873
0,791

$\begin{array}{ll}1,000 & 0,791 \\ 1,000 & 0,945\end{array}$

$\begin{array}{lll}0,174 & 0,721 & 1,178\end{array}$

0,097
0,138

$0,763-1,145$

nothing to measure

$\begin{array}{llllll}0,030 & 0,541 & 0,033 & 0,448 & 0,913 & 0,828\end{array}$

$0,023 \quad 0,562$

0,024

0,461

0,941

0,828

0,192

$\begin{array}{ll}1,000 & 0,879\end{array}$

$\begin{array}{lll}0,016 & 0,397 & 0,019\end{array}$

$\begin{array}{ll}0,026 & 0,634\end{array}$

$0,017 \quad 0,520$

0,017

0,023

0,019

0,520
0,461

0,846
0,857

0,74

0,534

0,913

1,000
1,000

0,808

0,275

0,275
0,225

0,800
1,000

0,901

$0,825 \quad 1,054$

$\begin{array}{lll}0,232 & 0,825 & 1,101 \\ 0,195 & 1,000 & 0,791\end{array}$

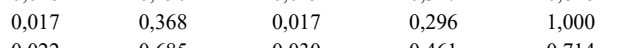

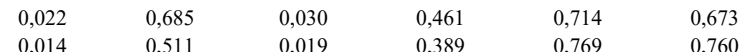

$\begin{array}{lll}0,019 & 0,469 & 0,020\end{array}$

0,397

0,929

0,847

$\begin{array}{lll}0,155 & 1,737-0,188\end{array}$

$\begin{array}{lll}0,039 & 0,613 \quad 0,042\end{array}$

$\begin{array}{ll}0,119 & 1,399 \\ 0,022 & 0,418\end{array}$

0,019

0,019

0,133
0,022
0,019
0,019

0,019
0,023

$\begin{array}{ll}0,929 & 0,906 \\ 0,739\end{array}$

$\begin{array}{ll}1,929 & 0,739 \\ 0,824 & 0,791\end{array}$

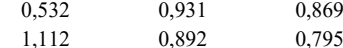

0,317

0,892
1,000

1,000

0,869
0,795
0,757
0,631
0,943

$\begin{array}{llllll}0,014 & 0,317 & 0,014 & 0,173 & 1,000 & 0,547\end{array}$

$\begin{array}{llllll}0,020 & 0,693 & 0,030 & 0,520 & 0,667 & 0,750\end{array}$

$\begin{array}{llllll}0,039 & 1,154 & 0,056 & 0,765 & 0,692 & 0,663 \\ 0,019 & 0,685 & 0,026 & 0,347 & 0,722 & 0,506\end{array}$

0,169
0,094
0,271
0,273
0,148
0,232
0,243
0,369
0,057
0,414
0,453
0,417
0,456
0,566

$1,000 \quad 0,969$

$\begin{array}{ll}1,645 & 1,24 \\ 1,000 & 0,878\end{array}$

$\begin{array}{ll}1,000 & 0,878 \\ 0,765 & 1,144\end{array}$

$\begin{array}{ll}1,000 & 0,805 \\ 1,000 & 0,803\end{array}$

$\begin{array}{rr}1,000 & 0,803 \\ 1,000 & 0,760\end{array}$

hing to measure

$\begin{array}{ll}1,000 & 0,746 \\ 0,525 & 1,380 \\ 0,366 & 1,654\end{array}$

$\begin{array}{ll}0,366 & 1,654 \\ 0,500 & 1,414\end{array}$

nothing to measure

nothing to measure

$\begin{array}{llllll}0,033 & 1,061 & 0,052 & 0,714 & 0,639 & 0,673 \\ 0,027 & 0,592 & 0,032 & 0,490 & 0,864 & 0,828\end{array}$

0,487

$\begin{array}{ll}0,368 & 1,648 \\ 0,978 & 1,01\end{array}$

$\begin{array}{lll}0,027 & 0,592 & 0,032\end{array}$

$\begin{array}{lll}0,042 & 0,837 & 0,049 \\ 0,016 & 0,490 & 0,020\end{array}$

$\begin{array}{lll}0,490 & 0,864 & 0,828 \\ 0,613 & 0,853 & 0,32\end{array}$

$\begin{array}{lll}0,613 & 0,853 & 0,732 \\ 0,389 & 0,786 & 0,793\end{array}$

$\begin{array}{lll}0,219 & 0,978 & 1,011 \\ 0,305 & 0,746 & 1,157\end{array}$

0,298

0,825

1,546

$0,244 \quad 0,396$

$\begin{array}{ll}0,092 & 0,111 \\ 0,416 & 0,286\end{array}$

$\begin{array}{ll}0,416 & 0,286 \\ 0,260 & 0,405\end{array}$

$\begin{array}{ll}0,700 & 0,400 \\ 1,175 & 0,745\end{array}$

$\begin{array}{ll}1,175 & 0,745 \\ 0,611 & 0,424\end{array}$

$0,336 \quad 0,333$

$\begin{array}{ll}0,394 & 0,305 \\ 0,725 & 0,683\end{array}$

$\begin{array}{ll}0,786 & 0,572 \\ 0,384 & 0,275\end{array}$

$\begin{array}{ll}0,384 & 0,275 \\ 0,597 & 0,554\end{array}$

$0,700 \quad 0,435$

$\begin{array}{ll}0,395 & 0,250 \\ 0,449 & 0,416\end{array}$

$0,527 \quad 0,332$

$\begin{array}{ll}0,529 & 0,492 \\ 0,350 & 0,212\end{array}$

$0,899 \quad 0,485$

$\begin{array}{ll}0,294 & 0,248 \\ 0,344 & 0,164\end{array}$

$\begin{array}{ll}0,487 & 0,334 \\ 1,010 & 0,743 \\ 0,662 & 0,537\end{array}$

$\begin{array}{ll}0,662 & 0,537 \\ 0,649 & 0,68\end{array}$

$\begin{array}{ll}0,011 & 0,680 \\ 0,514 & 0,814\end{array}$

$\begin{array}{ll}0,508 & 0,539 \\ 1,123 & 0,728\end{array}$

$\begin{array}{ll}1,123 & 0,728 \\ 0,974 & 0,485\end{array}$

$\begin{array}{ll}1,273 & 0,784 \\ 0,571 & 0,477\end{array}$

$\begin{array}{ll}1,273 & 0,784 \\ 0,273 & 0,632\end{array}$

$\begin{array}{ll}0,241 & 0,216 \\ 0,340 & 0,391\end{array}$

$\begin{array}{lll}0,265 & 0,076 & 0,107 \\ 0,152\end{array}$

$\begin{array}{lll}0,864 & 0,350 & 0,169 \\ 0,169 & 0,068 & 0,076\end{array}$

$\begin{array}{lll}0,169 & 0,068 & 0,076 \\ 0,137 & 0,102 & 0,107 \\ 0,189 & 0,080 & 0,076\end{array}$

$\begin{array}{lll}0,189 & 0,102 & 0,107 \\ 0,189 & 0,080 & 0,076\end{array}$

$\begin{array}{lll}0,455 & 0,038 & 0,076 \\ 0,510 & 0,155 & 0,169\end{array}$

0,44

0,221

0,076

$0,169 \quad 0,074$

$\begin{array}{ll}0,288 & 0,072 \\ 0,221 & 0,092\end{array}$

$0,204 \quad 0,068$

$\begin{array}{ll}0,276 & 0,136 \\ 0,240 & 0,051\end{array}$

$0,156 \quad 0,076$

$\begin{array}{ll}0,305 & 0,076 \\ 0,230 & 0,038\end{array}$

$\begin{array}{lll}0,204 & 0,152 & 0,076 \\ 0,189 & 0,169 & 0,169 \\ 0,552 & 0,375 & 0,107\end{array}$

$\begin{array}{lll}0,552 & 0,375 & 0,103\end{array}$

$\begin{array}{lll}0,221 & 0,180 & 0,214\end{array}$

$\begin{array}{lll}0,546 & 0,294 & 0,240 \\ 0,156 & 0,114 & 0,152\end{array}$

$0,156 \quad 0,076$

$0,137 \quad 0,107$

0,152

0,152

0,557

0,120

0,076

0,152

0,076

0,076

$\begin{array}{lllll}0,396 & 0,469 & 0,326 & 0,153 & 0,107\end{array}$

$\begin{array}{lllll}0,396 & 0,469 & 0,326 & 0,153 & 0,107 \\ 0,930 & 0,784 & 0,193 & 0,152 & 0,152 \\ 0,513 & 0,500 & 0,321 & 0,161 & 0,169\end{array}$

$0,513 \quad 0,500$

$\begin{array}{ll}0,412 & 0,231 \\ 0,216 & 0,293\end{array}$

0,221

0,051

0,169
0,076

0,303

55
61
51 


\begin{tabular}{|c|c|c|c|c|c|c|c|c|c|c|c|c|c|c|c|c|c|}
\hline 226 & 0,023 & 0,461 & 0,023 & 0,418 & 1,000 & 0,909 & 0,091 & 1,000 & 0,858 & 0,784 & 0,392 & 0,193 & 0,076 & 0,152 & 175 & 226 & $-0,9$ \\
\hline 226 & 0,020 & 0,440 & 0,022 & 0,338 & 0,933 & 0,769 & 0,240 & 1,000 & 0,875 & 0,713 & 0,537 & 0,189 & 0,102 & 0,152 & 45 & 226 & $-0,9$ \\
\hline 226 & 0,019 & 0,389 & 0,019 & 0,245 & 1,000 & 0,631 & 0,369 & 1,000 & 0,803 & 0,974 & 0,485 & 0,156 & 0,076 & 0,152 & 90 & 226 & $-0,6$ \\
\hline 226 & 0,019 & 0,490 & 0,022 & 0,368 & 0,867 & 0,750 & 0,283 & 0,975 & 1,013 & 0,571 & 0,412 & 0,204 & 0,084 & 0,107 & 120 & 226 & $-1,0$ \\
\hline 227 & 0,014 & 0,368 & 0,016 & 0,296 & 0,909 & 0,805 & 0,215 & 1,000 & 0,866 & 0,749 & 0,461 & 0,156 & 0,072 & 0,076 & 18 & 227 & $-0,6$ \\
\hline 227 & 0,016 & 0,368 & 0,017 & 0,266 & 0,917 & 0,724 & 0,288 & 1,000 & 0,826 & 0,778 & 0,500 & 0,161 & 0,080 & 0,107 & 37 & 227 & $-0,7$ \\
\hline 227 & 0,022 & 0,520 & 0,026 & 0,397 & 0,833 & 0,764 & 0,289 & 1,000 & 1,000 & 0,562 & 0,378 & 0,221 & 0,083 & 0,107 & 38 & 227 & $-1,1$ \\
\hline 227 & 0,062 & 1,133 & 0,079 & 0,879 & 0,782 & 0,776 & 0,313 & 0,604 & 1,287 & 0,403 & 0,449 & 0,442 & 0,199 & 0,169 & 18 & 227 & $-2,1$ \\
\hline 228 & & & & & & & & nothing to measure & & & & & & & & 228 & - \\
\hline 229 & & & & & & & & nothing to measure & & & & & & & & 229 & - \\
\hline 230 & & & & & & & & nothing to measure & & & & & & & & 230 & - \\
\hline 231 & & & & & & & & nothing to measure & & & & & & & & 231 & 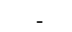 \\
\hline 232 & 0,024 & 0,490 & 0,024 & 0,368 & 1,000 & 0,750 & 0,250 & 1,000 & 0,886 & 0,746 & 0,557 & 0,204 & 0,114 & 0,152 & 107 & 232 & $-1,0$ \\
\hline 233 & & & & & & & & nothing to measure & & & & & & & & 233 & - \\
\hline 234 & & & & & & & & nothing to measure & & & & & & & & 234 & - \\
\hline 235 & & & & & & & & nothing to measure & & & & & & & & 235 & - \\
\hline 236 & & & & & & & & nothing to measure & & & & & & & & 236 & - \\
\hline 237 & & & & & & & & nothing to measure & & & & & & & & 237 & - \\
\hline 238 & & & & & & & & nothing to measure & & & & & & & & 238 & - \\
\hline 239 & & & & & & & & nothing to measure & & & & & & & & 239 & - \\
\hline 240 & & & & & & & & nothing to measure & & & & & & & & 240 & - \\
\hline 241 & & & & & & & & nothing to measure & & & & & & & & 241 & - \\
\hline 242 & & & & & & & & nothing to measure & & & & & & & & 242 & - \\
\hline 243 & & & & & & & & nothing to measure & & & & & & & & 243 & - \\
\hline 244 & & & & & & & & nothing to measure & & & & & & & & 244 & - \\
\hline 245 & & & & & & & & nothing to measure & & & & & & & & 245 & - \\
\hline 246 & & & & & & & & nothing to measure & & & & & & & & 246 & - \\
\hline 247 & & & & & & & & nothing to measure & & & & & & & & 247 & . \\
\hline 248 & 0,022 & 0,541 & 0,026 & 0,347 & 0,833 & 0,641 & 0,396 & 0,924 & 1,040 & 0,477 & 0,350 & 0,240 & 0,084 & 0,107 & 24 & 248 & $-1,3$ \\
\hline 248 & 0,026 & 0,989 & 0,043 & 0,520 & 0,600 & 0,526 & 0,620 & 0,332 & 1,736 & 0,432 & 0,583 & 0,276 & 0,161 & 0,076 & 117 & 248 & $-1,5$ \\
\hline 248 & 0,020 & 0,541 & 0,023 & 0,469 & 0,875 & 0,867 & 0,182 & 0,862 & 1,077 & 0,396 & 0,267 & 0,254 & 0,068 & 0,076 & 26 & 248 & $-1,3$ \\
\hline 248 & 0,195 & 2,355 & 0,242 & 1,889 & 0,805 & 0,802 & 0,278 & 0,442 & 1,504 & 0,343 & 0,394 & 0,851 & 0,335 & 0,321 & 22 & 248 & $-3,1$ \\
\hline 248 & 0,017 & 0,490 & 0,020 & 0,397 & 0,857 & 0,811 & 0,237 & 0,900 & 1,054 & 0,449 & 0,287 & 0,221 & 0,063 & 0,076 & 27 & 248 & $-1,1$ \\
\hline 248 & 0,121 & 1,809 & 0,168 & 1,387 & 0,718 & 0,767 & 0,366 & 0,463 & 1,470 & 0,418 & 0,558 & 0,606 & 0,338 & 0,169 & 172 & 248 & $-2,6$ \\
\hline 248 & 0,014 & 0,368 & 0,016 & 0,266 & 0,909 & 0,724 & 0,291 & 1,000 & 0,866 & 0,707 & 0,422 & 0,161 & 0,068 & 0,076 & 32 & 248 & $-0,7$ \\
\hline 248 & 0,055 & 0,989 & 0,067 & 0,867 & 0,809 & 0,876 & 0,228 & 0,700 & 1,195 & 0,387 & 0,391 & 0,423 & 0,165 & 0,214 & 15 & 248 & $-2,1$ \\
\hline 248 & 0,019 & 0,469 & 0,022 & 0,317 & 0,867 & 0,675 & 0,351 & 1,000 & 0,969 & 0,974 & 0,728 & 0,156 & 0,114 & 0,107 & 79 & 248 & $-0,6$ \\
\hline 248 & 0,077 & 2,308 & 0,138 & 1,369 & 0,562 & 0,593 & 0,597 & 0,183 & 2,339 & 0,237 & 0,352 & 0,645 & 0,227 & 0,152 & 172 & 248 & $-2,7$ \\
\hline 248 & 0,016 & 0,418 & 0,019 & 0,368 & 0,846 & 0,879 & 0,196 & 1,000 & 0,940 & 0,560 & 0,380 & 0,189 & 0,072 & 0,076 & 21 & 248 & $-0,9$ \\
\hline 248 & 0,016 & 0,440 & 0,017 & 0,368 & 0,917 & 0,837 & 0,183 & 1,000 & 0,987 & 0,483 & 0,315 & 0,204 & 0,064 & 0,076 & 12 & 248 & $-1,0$ \\
\hline 248 & 0,042 & 1,133 & 0,055 & 0,989 & 0,763 & 0,873 & 0,269 & 0,407 & 1,567 & 0,191 & 0,186 & 0,526 & 0,098 & 0,076 & 28 & 248 & $-2,4$ \\
\hline 249 & & & & & & & & nothing to measure & & & & & & & & 249 & - \\
\hline 250 & & & & & & & & nothing to measure & & & & & & & & 250 & \\
\hline 251 & 0,023 & 0,440 & 0,023 & 0,317 & 1,000 & 0,721 & 0,279 & 1,000 & 0,818 & 1,019 & 0,671 & 0,169 & 0,114 & 0,152 & 0 & 251 & $-0,8$ \\
\hline 252 & 0,016 & 0,347 & 0,016 & 0,317 & 1,000 & 0,914 & 0,086 & 1,000 & 0,778 & 1,401 & 0,849 & 0,120 & 0,102 & 0,107 & 90 & 252 & $-0,3$ \\
\hline 252 & 0,017 & 0,520 & 0,020 & 0,418 & 0,857 & 0,805 & 0,242 & 0,800 & 1,118 & 0,424 & 0,333 & 0,227 & 0,076 & 0,107 & 72 & 252 & $-1,2$ \\
\hline 252 & 0,014 & 0,317 & 0,014 & 0,215 & 1,000 & 0,680 & 0,320 & 1,000 & 0,746 & 0,979 & 0,555 & 0,137 & 0,076 & 0,107 & 72 & 252 & $-0,5$ \\
\hline 253 & 0,019 & 0,389 & 0,019 & 0,347 & 1,000 & 0,892 & 0,108 & 1,000 & 0,803 & 0,974 & 0,485 & 0,156 & 0,076 & 0,152 & 172 & 253 & $-0,6$ \\
\hline 254 & & & & & & & & nothing to measure & & & & & & & & 254 & - \\
\hline 255 & & & & & & & & nothing to measure & & & & & & & & 255 & \\
\hline 256 & 0,017 & 0,469 & 0,020 & 0,368 & 0,857 & 0,784 & 0,259 & 0,983 & 1,009 & 0,477 & 0,375 & 0,214 & 0,080 & 0,107 & 135 & 256 & -1 , \\
\hline 256 & 0,037 & 0,867 & 0,052 & 0,634 & 0,722 & 0,731 & 0,386 & 0,624 & 1,266 & 0,571 & 0,588 & 0,288 & 0,169 & 0,169 & 166 & 256 & -1 , \\
\hline 256 & 0,014 & 0,368 & 0,016 & 0,245 & 0,909 & 0,667 & 0,346 & 1,000 & 0,866 & 0,749 & 0,485 & 0,156 & 0,076 & 0,107 & 18 & 256 & $-0,6$ \\
\hline 256 & 0,046 & 0,757 & 0,050 & 0,562 & 0,914 & 0,743 & 0,271 & 1,000 & 0,996 & 0,702 & 0,525 & 0,288 & 0,152 & 0,169 & 88 & 256 & $-1,5$ \\
\hline 256 & 0,014 & 0,490 & 0,019 & 0,368 & 0,769 & 0,750 & 0,340 & 0,750 & 1,155 & 0,509 & 0,424 & 0,189 & 0,080 & 0,076 & 149 & 256 & 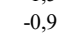 \\
\hline 256 & 0,049 & 1,327 & 0,069 & 0,837 & 0,708 & 0,631 & 0,471 & 0,348 & 1,695 & 0,416 & 0,490 & 0,386 & 0,189 & 0,107 & 174 & 256 & $-1,9$ \\
\hline 256 & 0,017 & 0,389 & 0,017 & 0,194 & 1,000 & 0,500 & 0,500 & 1,000 & 0,836 & 0,764 & 0,447 & 0,169 & 0,076 & 0,107 & 162 & 256 & $-0,8$ \\
\hline 256 & 0,019 & 0,592 & 0,023 & 0,389 & 0,812 & 0,657 & 0,391 & 0,669 & 1,222 & 0,828 & 0,671 & 0,169 & 0,114 & 0,107 & 18 & 256 & $-0,8$ \\
\hline
\end{tabular}




\begin{tabular}{|c|c|c|c|c|c|c|c|c|c|c|c|c|c|c|c|c|c|c|}
\hline 1 & 257 & 0,079 & 1,530 & 0,106 & 1,031 & 0,743 & 0,674 & 0,415 & 0,423 & 1,537 & 0,511 & 0,570 & 0,443 & 0,253 & 0,152 & 92 & 257 & $-2,1$ \\
\hline 2 & 257 & 0,019 & 0,389 & 0,019 & 0,224 & 1,000 & 0,576 & 0,424 & 1,000 & 0,803 & 0,920 & 0,633 & 0,161 & 0,102 & 0,152 & 135 & 257 & $-0,7$ \\
\hline 3 & 257 & 0,052 & 1,061 & 0,063 & 0,706 & 0,818 & 0,665 & 0,381 & 0,577 & 1,317 & 0,790 & 0,788 & 0,288 & 0,227 & 0,169 & 95 & 257 & $-1,5$ \\
\hline 4 & 257 & 0,020 & 0,765 & 0,030 & 0,389 & 0,667 & 0,508 & 0,594 & 0,431 & 1,523 & 0,686 & 0,784 & 0,193 & 0,152 & 0,076 & 41 & 257 & $-0,9$ \\
\hline 5 & 257 & 0,020 & 0,440 & 0,022 & 0,317 & 0,933 & 0,721 & 0,287 & 1,000 & 0,875 & 0,686 & 0,392 & 0,193 & 0,076 & 0,152 & 9 & 257 & $-0,9$ \\
\hline 6 & 257 & 0,222 & 3,778 & 0,354 & 3,380 & 0,628 & 0,895 & 0,387 & 0,196 & 2,260 & 0,086 & 0,113 & 1,819 & 0,205 & 0,214 & 91 & 257 & $-4,2$ \\
\hline 7 & 257 & 0,020 & 0,418 & 0,022 & 0,245 & 0,933 & 0,586 & 0,420 & 1,000 & 0,833 & 1,049 & 0,728 & 0,156 & 0,114 & 0,152 & 0 & 257 & $-0,6$ \\
\hline 8 & 257 & 0,019 & 0,520 & 0,022 & 0,469 & 0,867 & 0,902 & 0,165 & 0,867 & 1,074 & 0,404 & 0,294 & 0,243 & 0,071 & 0,107 & 135 & 257 & $-1,3$ \\
\hline 9 & 257 & 0,014 & 0,368 & 0,016 & 0,317 & 0,909 & 0,862 & 0,165 & 1,000 & 0,866 & 0,637 & 0,400 & 0,169 & 0,068 & 0,076 & 162 & 257 & $-0,8$ \\
\hline 10 & 257 & 0,020 & 0,397 & 0,020 & 0,338 & 1,000 & 0,851 & 0,149 & 1,000 & 0,791 & 1,049 & 0,686 & 0,156 & 0,107 & 0,152 & 73 & 257 & $-0,6$ \\
\hline 11 & 257 & 0,017 & 0,418 & 0,019 & 0,296 & 0,923 & 0,707 & 0,303 & 1,000 & 0,900 & 0,764 & 0,496 & 0,169 & 0,084 & 0,107 & 53 & 257 & $-0,8$ \\
\hline 12 & 257 & 0,036 & 0,858 & 0,047 & 0,643 & 0,758 & 0,749 & 0,349 & 0,612 & 1,278 & 0,650 & 0,783 & 0,265 & 0,208 & 0,152 & 73 & 257 & $-1,4$ \\
\hline 13 & 257 & 0,044 & 0,744 & 0,047 & 0,592 & 0,939 & 0,795 & 0,213 & 1,000 & 0,995 & 0,548 & 0,417 & 0,321 & 0,134 & 0,169 & 130 & 257 & $-1,7$ \\
\hline 14 & 257 & 0,020 & 0,520 & 0,024 & 0,397 & 0,824 & 0,764 & 0,294 & 0,934 & 1,035 & 0,524 & 0,429 & 0,221 & 0,095 & 0,107 & 140 & 257 & $-1,1$ \\
\hline 15 & 257 & 0,056 & 0,837 & 0,060 & 0,765 & 0,929 & 0,914 & 0,112 & 1,000 & 0,998 & 0,507 & 0,421 & 0,375 & 0,158 & 0,169 & 128 & 257 & $-1,9$ \\
\hline 16 & 257 & 0,019 & 0,389 & 0,020 & 0,245 & 0,929 & 0,631 & 0,376 & 1,000 & 0,803 & 0,920 & 0,633 & 0,161 & 0,102 & 0,152 & 135 & 257 & $-0,7$ \\
\hline 17 & 257 & 0,119 & 1,336 & 0,126 & 1,315 & 0,943 & 0,984 & 0,059 & 0,839 & 1,092 & 0,470 & 0,424 & 0,568 & 0,241 & 0,240 & 138 & 257 & $-2,5$ \\
\hline 18 & 257 & 0,033 & 0,735 & 0,040 & 0,592 & 0,821 & 0,805 & 0,265 & 0,767 & 1,142 & 0,401 & 0,314 & 0,324 & 0,102 & 0,107 & 160 & 257 & $-1,7$ \\
\hline 19 & 257 & 0,033 & 0,706 & 0,037 & 0,469 & 0,885 & 0,665 & 0,354 & 0,833 & 1,096 & 0,451 & 0,444 & 0,305 & 0,136 & 0,152 & 152 & 257 & $-1,6$ \\
\hline 20 & 257 & 0,047 & 0,875 & 0,055 & 0,803 & 0,868 & 0,918 & 0,155 & 0,777 & 1,135 & 0,420 & 0,321 & 0,379 & 0,121 & 0,152 & 126 & 257 & $-1,9$ \\
\hline 21 & 257 & 0,119 & 1,860 & 0,181 & 1,348 & 0,659 & 0,725 & 0,438 & 0,433 & 1,520 & 0,399 & 0,646 & 0,617 & 0,398 & 0,169 & 124 & 257 & $-2,6$ \\
\hline 22 & 257 & 0,016 & 0,440 & 0,019 & 0,317 & 0,846 & 0,721 & 0,318 & 1,000 & 0,987 & 0,539 & 0,311 & 0,193 & 0,060 & 0,076 & 158 & 257 & $-0,9$ \\
\hline 23 & 257 & 0,023 & 0,440 & 0,023 & 0,317 & 1,000 & 0,721 & 0,279 & 1,000 & 0,818 & 0,815 & 0,566 & 0,189 & 0,107 & 0,152 & 150 & 257 & $-0,9$ \\
\hline 24 & 257 & 0,133 & 2,089 & 0,195 & 1,530 & 0,684 & 0,733 & 0,414 & 0,384 & 1,613 & 0,408 & 0,605 & 0,645 & 0,390 & 0,169 & 15 & 257 & $-2,7$ \\
\hline 25 & 257 & 0,022 & 0,440 & 0,022 & 0,317 & 1,000 & 0,721 & 0,279 & 1,000 & 0,845 & 0,764 & 0,566 & 0,189 & 0,107 & 0,152 & 144 & 257 & $-0,9$ \\
\hline 26 & 257 & 0,019 & 0,520 & 0,022 & 0,418 & 0,867 & 0,805 & 0,236 & 0,867 & 1,074 & 0,447 & 0,416 & 0,230 & 0,096 & 0,107 & 149 & 257 & $-1,2$ \\
\hline 27 & 257 & 0,016 & 0,338 & 0,016 & 0,245 & 1,000 & 0,725 & 0,275 & 1,000 & 0,759 & 1,077 & 0,555 & 0,137 & 0,076 & 0,152 & 90 & 257 & $-0,5$ \\
\hline 28 & 257 & 0,024 & 0,490 & 0,027 & 0,368 & 0,895 & 0,750 & 0,271 & 1,000 & 0,886 & 0,746 & 0,557 & 0,204 & 0,114 & 0,152 & 110 & 257 & $-1,0$ \\
\hline 29 & 257 & 0,033 & 0,714 & 0,043 & 0,613 & 0,767 & 0,858 & 0,273 & 0,813 & 1,109 & 0,651 & 0,632 & 0,254 & 0,161 & 0,152 & 40 & 257 & $-1,3$ \\
\hline 30 & 257 & 0,040 & 0,837 & 0,046 & 0,786 & 0,875 & 0,939 & 0,139 & 0,721 & 1,178 & 0,327 & 0,256 & 0,395 & 0,101 & 0,152 & 17 & 257 & $-2,0$ \\
\hline 31 & 257 & 0,016 & 0,469 & 0,019 & 0,368 & 0,846 & 0,784 & 0,265 & 0,901 & 1,054 & 0,412 & 0,307 & 0,221 & 0,068 & 0,076 & 159 & 257 & $-1,1$ \\
\hline 32 & 257 & 0,030 & 0,930 & 0,040 & 0,541 & 0,750 & 0,582 & 0,487 & 0,438 & 1,511 & 0,668 & 0,632 & 0,240 & 0,152 & 0,076 & 58 & 257 & $-1,3$ \\
\hline 33 & 257 & 0,024 & 0,592 & 0,030 & 0,418 & 0,810 & 0,707 & 0,349 & 0,875 & 1,069 & 0,637 & 0,477 & 0,221 & 0,105 & 0,107 & 50 & 257 & $-1,1$ \\
\hline 34 & 257 & 0,016 & 0,389 & 0,017 & 0,194 & 0,917 & 0,500 & 0,507 & 1,000 & 0,873 & 0,700 & 0,400 & 0,169 & 0,068 & 0,076 & 159 & 257 & $-0,8$ \\
\hline 35 & 257 & 0,029 & 0,706 & 0,029 & 0,469 & 1,000 & 0,665 & 0,335 & 0,724 & 1,175 & 0,637 & 0,474 & 0,240 & 0,114 & 0,152 & 173 & 257 & $-1,3$ \\
\hline 36 & 257 & 0,034 & 0,672 & 0,037 & 0,613 & 0,923 & 0,912 & 0,117 & 0,957 & 1,022 & 0,527 & 0,411 & 0,288 & 0,119 & 0,152 & 152 & 257 & $-1,5$ \\
\hline 37 & 257 & 0,017 & 0,359 & 0,017 & 0,123 & 1,000 & 0,341 & 0,659 & 1,000 & 0,772 & 1,175 & 0,555 & 0,137 & 0,076 & 0,152 & 90 & 257 & $-0,5$ \\
\hline 38 & 257 & 0,027 & 0,550 & 0,029 & 0,469 & 0,950 & 0,854 & 0,155 & 1,000 & 0,939 & 0,605 & 0,447 & 0,240 & 0,107 & 0,152 & 119 & 257 & $-1,3$ \\
\hline 39 & 257 & 0,020 & 0,440 & 0,022 & 0,296 & 0,933 & 0,673 & 0,334 & 1,000 & 0,875 & 0,713 & 0,448 & 0,189 & 0,085 & 0,107 & 150 & 257 & $-0,9$ \\
\hline 40 & 257 & 0,019 & 0,389 & 0,019 & 0,266 & 1,000 & 0,685 & 0,315 & 1,000 & 0,803 & 0,974 & 0,485 & 0,156 & 0,076 & 0,152 & 8 & 257 & $-0,6$ \\
\hline 41 & 257 & 0,019 & 0,490 & 0,022 & 0,389 & 0,867 & 0,793 & 0,246 & 0,975 & 1,013 & 0,487 & 0,352 & 0,221 & 0,078 & 0,076 & 152 & 257 & $-1,1$ \\
\hline 42 & 257 & 0,016 & 0,389 & 0,019 & 0,266 & 0,846 & 0,685 & 0,351 & 1,000 & 0,873 & 0,700 & 0,447 & 0,169 & 0,076 & 0,107 & 159 & 257 & $-0,8$ \\
\hline 43 & 257 & 0,030 & 0,592 & 0,033 & 0,571 & 0,913 & 0,964 & 0,094 & 1,000 & 0,962 & 0,594 & 0,467 & 0,254 & 0,119 & 0,152 & 156 & 257 & $-1,3$ \\
\hline 44 & 257 & 0,016 & 0,461 & 0,019 & 0,245 & 0,846 & 0,532 & 0,492 & 0,935 & 1,034 & 0,483 & 0,271 & 0,204 & 0,055 & 0,076 & 163 & 257 & $-1,0$ \\
\hline 45 & 257 & 0,017 & 0,418 & 0,019 & 0,317 & 0,923 & 0,757 & 0,254 & 1,000 & 0,900 & 0,611 & 0,424 & 0,189 & 0,080 & 0,107 & 140 & 257 & $-0,9$ \\
\hline 46 & 257 & 0,022 & 0,499 & 0,023 & 0,418 & 0,938 & 0,839 & 0,173 & 1,000 & 0,959 & 0,659 & 0,499 & 0,204 & 0,102 & 0,107 & 23 & 257 & $-1,0$ \\
\hline 47 & 257 & 0,017 & 0,389 & 0,020 & 0,245 & 0,857 & 0,631 & 0,396 & 1,000 & 0,836 & 0,849 & 0,633 & 0,161 & 0,102 & 0,152 & 143 & 257 & $-0,7$ \\
\hline 48 & 257 & 0,027 & 0,757 & 0,036 & 0,592 & 0,760 & 0,782 & 0,324 & 0,599 & 1,293 & 0,302 & 0,248 & 0,339 & 0,084 & 0,107 & 158 & 257 & $-1,8$ \\
\hline 49 & 257 & 0,030 & 1,052 & 0,044 & 0,634 & 0,677 & 0,602 & 0,512 & 0,342 & 1,710 & 0,326 & 0,331 & 0,343 & 0,114 & 0,076 & 24 & 257 & $-1,8$ \\
\hline 50 & 257 & 0,023 & 0,744 & 0,034 & 0,490 & 0,667 & 0,659 & 0,477 & 0,521 & 1,385 & 0,702 & 0,743 & 0,204 & 0,152 & 0,107 & 36 & 257 & $-1,0$ \\
\hline 51 & 257 & 0,072 & 1,378 & 0,102 & 1,010 & 0,704 & 0,733 & 0,398 & 0,475 & 1,451 & 0,509 & 0,569 & 0,423 & 0,241 & 0,152 & 156 & 257 & $-2,1$ \\
\hline 52 & 257 & 0,017 & 0,389 & 0,017 & 0,296 & 1,000 & 0,761 & 0,239 & 1,000 & 0,836 & 0,899 & 0,485 & 0,156 & 0,076 & 0,152 & 8 & 257 & $-0,6$ \\
\hline 53 & 257 & 0,016 & 0,368 & 0,016 & 0,347 & 1,000 & 0,943 & 0,057 & 1,000 & 0,826 & 0,824 & 0,485 & 0,156 & 0,076 & 0,152 & 0 & 257 & $-0,6$ \\
\hline 54 & 257 & 0,218 & 3,335 & 0,296 & 1,826 & 0,738 & 0,548 & 0,523 & 0,246 & 2,015 & 0,484 & 0,597 & 0,758 & 0,452 & 0,152 & 135 & 257 & $-2,9$ \\
\hline 55 & 257 & 0,017 & 0,368 & 0,017 & 0,266 & 1,000 & 0,724 & 0,276 & 1,000 & 0,791 & 0,849 & 0,500 & 0,161 & 0,080 & 0,107 & 135 & 257 & $-0,7$ \\
\hline 56 & 257 & 0,024 & 0,723 & 0,032 & 0,490 & 0,773 & 0,678 & 0,394 & 0,586 & 1,306 & 0,585 & 0,581 & 0,230 & 0,134 & 0,076 & 29 & 257 & $-1,2$ \\
\hline 57 & 257 & 0,019 & 0,440 & 0,022 & 0,296 & 0,867 & 0,673 & 0,353 & 1,000 & 0,908 & 0,662 & 0,444 & 0,189 & 0,084 & 0,107 & 147 & 257 & $-0,9$ \\
\hline 58 & 257 & 0,026 & 0,511 & 0,027 & 0,389 & 0,947 & 0,760 & 0,245 & 1,000 & 0,898 & 0,881 & 0,588 & 0,193 & 0,114 & 0,152 & 165 & 257 & $-0,9$ \\
\hline
\end{tabular}




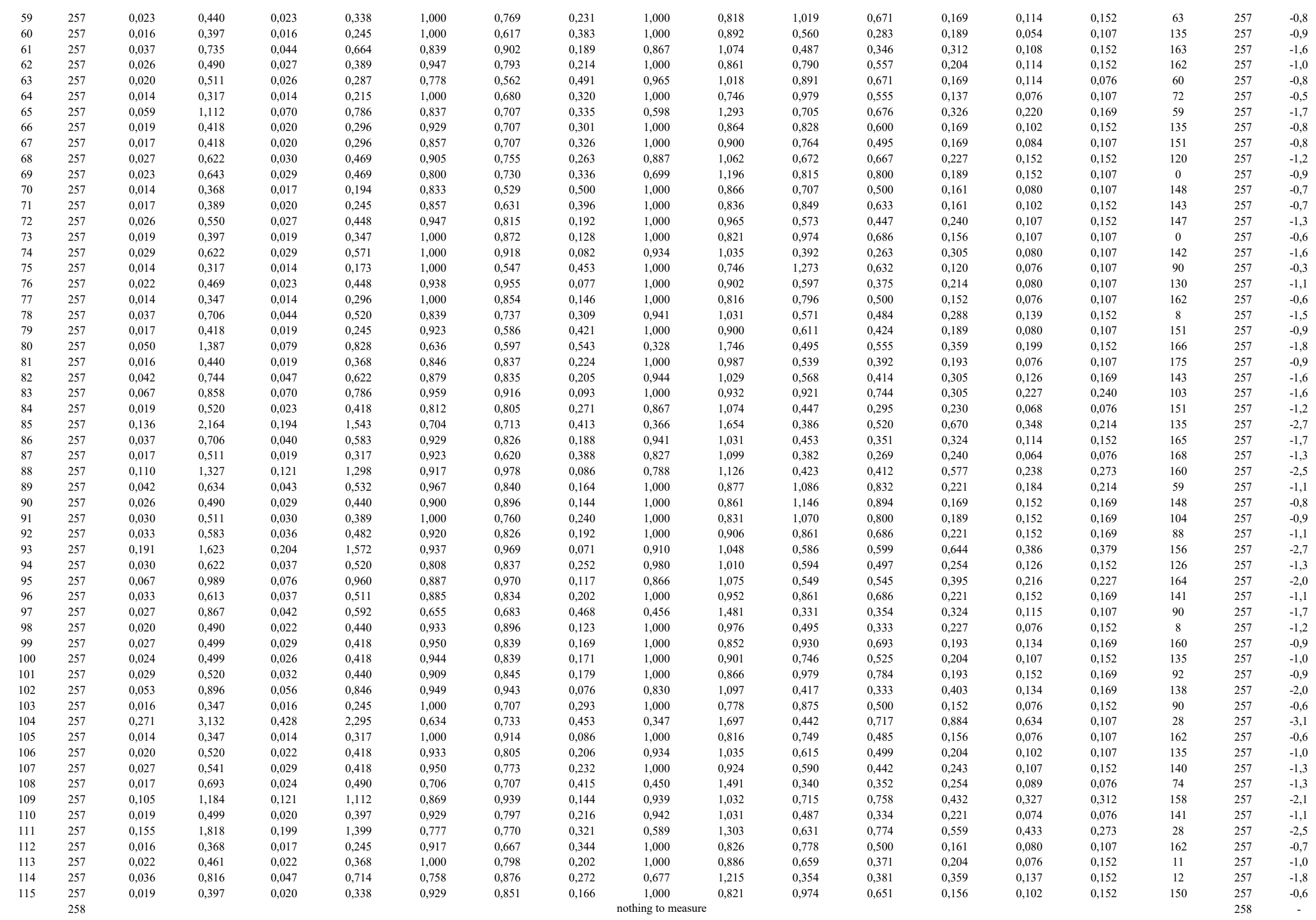


nothing to measure

nothing to measure

$\begin{array}{llllll}0,022 & 0,998 & 0,043 & 0,744 & 0,500 & 0,746 \\ 0,022 & 0,541 & 0,026 & 0,368 & 0,833 & 0,680\end{array}$

0,272

1,919
1,040

$0,163 \quad 0,308$

$\begin{array}{lllll}0,361 & 0,924 & 1,040 & 0,764 & 0,566 \\ 0,299 & 1,000 & 0,805 & 1,123 & 0,728\end{array}$

$\begin{array}{ll}0,938 & 0,707 \\ 0,769 & 0,707\end{array}$

$\begin{array}{lll}0,299 & 1,000 & 0,805 \\ 0,373 & 0,750 & 1,155\end{array}$

$\begin{array}{ll}0,750 & 1,155 \\ 0,848 & 1,086 \\ 0,559 & 1,338\end{array}$

$0,344 \quad 0,259$

$\begin{array}{llllll}0,034 & 0,714 & 0,046 & 0,613 & 0,750 & 0,858 \\ 0,020 & 0,672 & 0,029 & 0,520 & 0,700 & 0,773\end{array}$

0,030

$\begin{array}{lll}0,047 & 0,664 & 0,049\end{array}$

$\begin{array}{ll}0,027 & 0,410 \\ 0,030 & 0,592\end{array}$

$\begin{array}{ll}0,017 & 0,418 \\ 0,017 & 0,368\end{array}$

$\begin{array}{ll}0,032 & 0,643 \\ 0,019 & 0,397\end{array}$

$\begin{array}{ll}0,014 & 0,368 \\ 0,029 & 0,541\end{array}$

$\begin{array}{lll}0,511 & 0,840 & 0,807 \\ 0,613 & 0,971 & 0,924\end{array}$

0,376

0,559

1,030

0,307

0,332

$\begin{array}{ll}1,000 & 0,860 \\ 1,000 & 0,816\end{array}$

0,474
0,185

0,185
0,243

1,000
1,000

$\begin{array}{ll}1,000 & 0,962 \\ 1,000 & 0,900\end{array}$

$\begin{array}{ll}0,875 & 0,864 \\ 1,000 & 0,757\end{array}$

nothing to measure

nothing to meas

$\begin{array}{llll}0,020 & 0,723 & 0,027 & 0,418\end{array}$

0,737

0,497

0,483

$\begin{array}{ll}0,020 & 0,685 \\ 0,019 & 0,735\end{array}$

$\begin{array}{ll}0,016 & 0,397 \\ 0,016 & 0,397\end{array}$

$\begin{array}{ll}0,016 & 0,397 \\ 0,055 & 0,879\end{array}$

$\begin{array}{ll}0,055 & 0,879 \\ 0,024 & 0,744\end{array}$

$0,040 \quad 0,061$

0,017

0,030

$\begin{array}{ll}0,017 & 0,317\end{array}$

0,017
0,060

0,033

0,066

0,685

0,609
0,619

0,642

0,530

0,538

1,439

0,934

0,891

0,723
0,611
1,175

$1,175 \quad 0,78$

0,974

$\begin{array}{ll}0,749 & 0,485 \\ 0,796 & 0,625\end{array}$

0,421
0,219

0,269

$0,917 \quad 0,745$

$\begin{array}{ll}0,905 & 0,779 \\ 0,739 & 0,659\end{array}$

0,429

1,000

1,000
0,887

$\begin{array}{ll}0,464 & 1,467\end{array}$

hing to measu

$\begin{array}{llllll}0,014 & 0,347 & 0,014 & 0,296 & 1,000 & 0,854\end{array}$

0,026

0,410

1,000

0,873

0,127

$\begin{array}{lll}0,020 & 0,714 & 0,032 \\ 0,017 & 0,397 & 0,017\end{array}$

$\begin{array}{lll}0,448 & 0,636 & 0,627 \\ 0,296 & 1,000 & 0,745\end{array}$

$\begin{array}{ll}0,017 & 0,397 \\ 0,055 & 1,002\end{array}$

$0,176 \quad 2,688$

0,024

0,017
0,062
0,293

0,296

0,627
0,745

0,022

$\begin{array}{ll}0,030 & 1,230\end{array}$

0,293

2,045

$\begin{array}{ll}0,884 & 0,899 \\ 0,603 & 0,761\end{array}$

$\begin{array}{ll}0,803 & 0,761 \\ 0,944 & 0,760 \\ 0,789 & 0,764\end{array}$

$\begin{array}{ll}0,677 & 0,842 \\ 1,000 & 0,835\end{array}$

$\begin{array}{lll}0,044 & 1,035 & 0,677 \\ 0,026 & 0,469 & 1,000\end{array}$

0,842
0,835
0,764

$\begin{array}{ll}0,397 & 0,895 \\ 0,347 & 0,917\end{array}$

0,764
0,872
0,946

$\begin{array}{ll}1,000 & 0,946 \\ 0,694 & 0,607\end{array}$

$\begin{array}{ll}0,016 & 0,397 \\ 0,020 & 0,550\end{array}$

0,020

0,613

0,496

0,607
0,715
0,831

$\begin{array}{ll}0,574 & 0,831 \\ 0,870 & 0,879 \\ 0,952 & 0,76\end{array}$

$\begin{array}{ll}0,128 & 2,177 \\ 0,029 & 0,592\end{array}$

0,222

0,030

0,520

0,952

0,746
0,929

$\begin{array}{ll}0,929 & 0,097 \\ 0,660 & 0,476\end{array}$ nothing to measu 0,816

0,686

0,693

$0,202 \quad 0,192$

$\begin{array}{ll}0,700 & 0,474 \\ 0,700 & 0,401\end{array}$

$\begin{array}{ll}0,499 & 0,418 \\ 0,637 & 0,606\end{array}$

$0,435 \quad 0,331$

0,388
0,588

0,496

0,917

0,566

$\begin{array}{lll}0,521 & 0,495 & 1,422 \\ 0,255 & 1,000 & 0,8 \\ 0,154 & 0,683 & 1,2 \\ 0,463 & 0,307 & 1,805 \\ 0,246 & 1,000 & 0,924 \\ 0,316 & 1,000 & 1,000 \\ 0,359 & 0,250 & 1,998 \\ 0,165 & 1,000 & 0,987 \\ 0,258 & 1,000 & 0,939 \\ 0,152 & 1,000 & 0,82 \\ 0,054 & 0,835 & 1,094 \\ 0,498 & 0,442 & 1,505 \\ 0,579 & 0,235 & 2,063 \\ 0,458 & 0,339 & 1,78 \\ 0,178 & 1,000 & 0,986 \\ 0,258 & 1,000 & 0,887 \\ 0,097 & 0,989 & 1,005 \\ 0,476 & 0,389 & 1,603 \\ & & \end{array}$

1,422

$\begin{array}{ll}0,396 & 0,467\end{array}$

$\begin{array}{ll}0,955 & 0,671 \\ 0,331 & 0,236\end{array}$

$\begin{array}{ll}0,331 & 0,236 \\ 0,227 & 0,361\end{array}$

$\begin{array}{ll}0,833 & 0,588 \\ 0,659 & 0,646\end{array}$

$\begin{array}{ll}0,659 & 0,646 \\ 0,104 & 0,062\end{array}$

$\begin{array}{ll}0,509 & 0,400\end{array}$

0,400
0,525
0,474

$\begin{array}{ll}0,357 & 0,200 \\ 0,612 & 0,790\end{array}$

0,790

$\begin{array}{ll}0,2095 & 0,435 \\ 0,315 & 0,45\end{array}$

$\begin{array}{ll}0,621 & 0,445 \\ 0,749 & 0,514\end{array}$

$\begin{array}{llll}0,040 & 0,714 & 0,043 & 0,664 \\ 0,049 & 1,255 & 0,073 & 0,828\end{array}$

(1) 


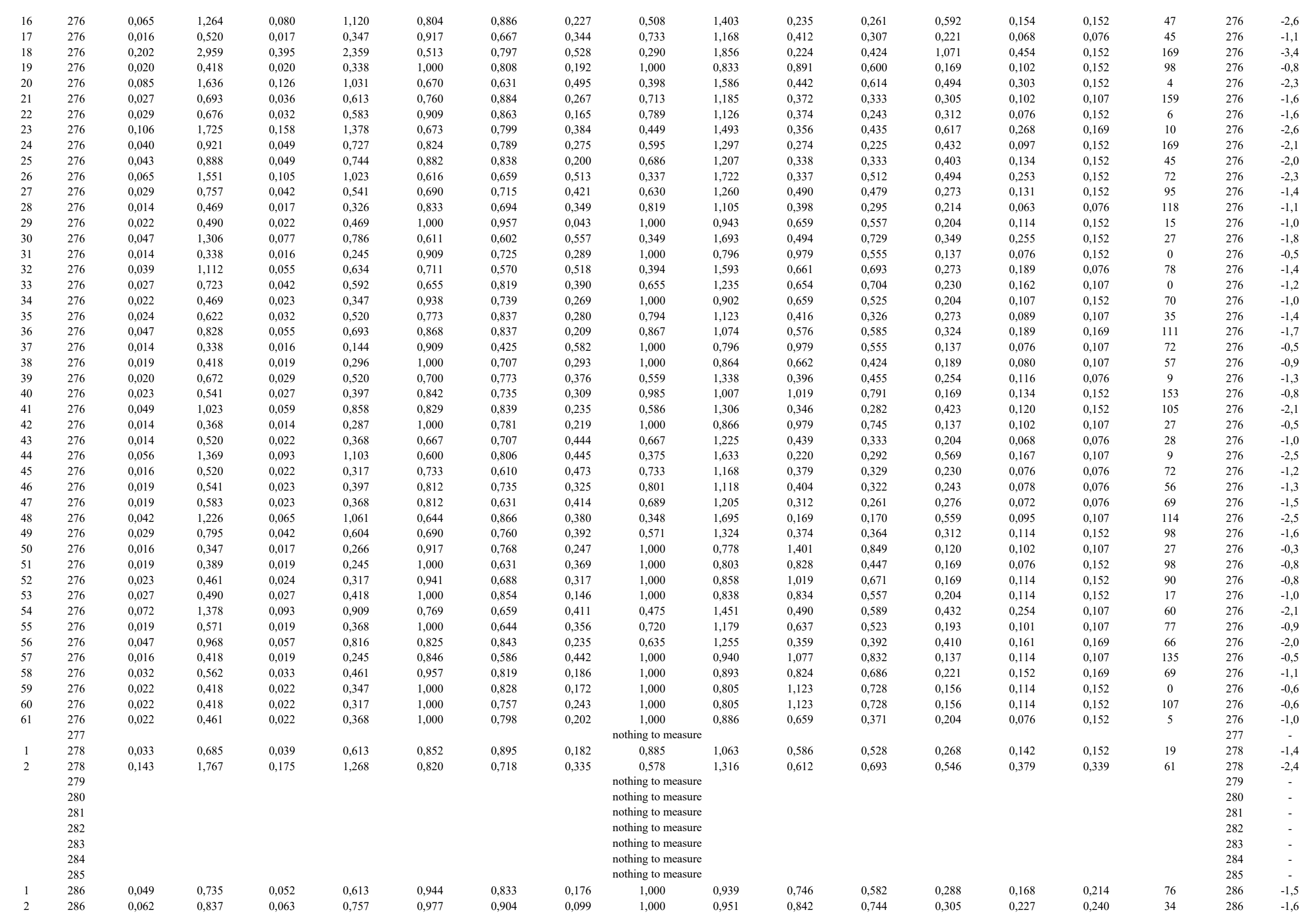




\begin{tabular}{|c|c|c|c|c|c|c|c|c|c|c|c|c|c|c|c|c|c|c|}
\hline 3 & 286 & 0,149 & 2,304 & 0,201 & 1,636 & 0,743 & 0,710 & 0,388 & 0,353 & 1,683 & 0,414 & 0,502 & 0,678 & 0,340 & 0,107 & 114 & 286 & $-2,8$ \\
\hline 4 & 286 & 0,055 & 1,775 & 0,106 & 1,002 & 0,514 & 0,564 & 0,653 & 0,217 & 2,145 & 0,308 & 0,531 & 0,475 & 0,252 & 0,107 & 4 & 286 & $-2,2$ \\
\hline 5 & 286 & 0,040 & 0,757 & 0,050 & 0,634 & 0,800 & 0,838 & 0,257 & 0,882 & 1,065 & 0,673 & 0,648 & 0,276 & 0,179 & 0,152 & 94 & 286 & $-1,5$ \\
\hline 6 & 286 & 0,066 & 0,837 & 0,069 & 0,786 & 0,958 & 0,939 & 0,074 & 1,000 & 0,919 & 0,791 & 0,658 & 0,326 & 0,214 & 0,240 & 130 & 286 & $-1,7$ \\
\hline 7 & 286 & 0,017 & 0,389 & 0,019 & 0,266 & 0,923 & 0,685 & 0,325 & 1,000 & 0,836 & 0,764 & 0,447 & 0,169 & 0,076 & 0,107 & 108 & 286 & $-0,8$ \\
\hline 8 & 286 & 0,014 & 0,347 & 0,014 & 0,224 & 1,000 & 0,646 & 0,354 & 1,000 & 0,816 & 0,979 & 0,588 & 0,137 & 0,080 & 0,107 & 122 & 286 & $-0,5$ \\
\hline 1 & 287 & 0,019 & 0,418 & 0,020 & 0,317 & 0,929 & 0,757 & 0,253 & 1,000 & 0,864 & 0,828 & 0,566 & 0,169 & 0,096 & 0,152 & 21 & 287 & $-0,8$ \\
\hline 2 & 287 & 0,046 & 1,073 & 0,060 & 0,634 & 0,762 & 0,591 & 0,474 & 0,501 & 1,413 & 0,627 & 0,560 & 0,305 & 0,171 & 0,169 & 73 & 287 & $-1,6$ \\
\hline 3 & 287 & 0,042 & 0,989 & 0,059 & 0,816 & 0,707 & 0,825 & 0,341 & 0,534 & 1,368 & 0,369 & 0,346 & 0,379 & 0,131 & 0,169 & 47 & 287 & $-1,9$ \\
\hline 4 & 287 & 2,093 & 8,658 & 2,689 & 7,166 & 0,779 & 0,828 & 0,281 & 0,351 & 1,688 & 0,260 & 0,313 & 3,204 & 1,003 & 0,937 & 65 & 287 & $-5,0$ \\
\hline 5 & 287 & 0,017 & 0,368 & 0,017 & 0,317 & 1,000 & 0,862 & 0,138 & 1,000 & 0,791 & 1,175 & 0,745 & 0,137 & 0,102 & 0,107 & 135 & 287 & $-0,5$ \\
\hline 6 & 287 & 0,024 & 0,499 & 0,027 & 0,469 & 0,895 & 0,940 & 0,121 & 1,000 & 0,901 & 0,833 & 0,555 & 0,193 & 0,107 & 0,152 & 27 & 287 & $-0,9$ \\
\hline 7 & 287 & 0,141 & 1,572 & 0,159 & 1,471 & 0,883 & 0,935 & 0,134 & 0,715 & 1,183 & 0,369 & 0,369 & 0,696 & 0,257 & 0,273 & 71 & 287 & $-2,8$ \\
\hline 8 & 287 & 0,047 & 0,786 & 0,052 & 0,706 & 0,917 & 0,898 & 0,132 & 0,963 & 1,019 & 0,467 & 0,422 & 0,359 & 0,152 & 0,169 & 72 & 287 & $-1,8$ \\
\hline 9 & 287 & 0,090 & 1,052 & 0,106 & 0,951 & 0,851 & 0,904 & 0,177 & 1,000 & 0,987 & 0,802 & 0,776 & 0,379 & 0,294 & 0,169 & 68 & 287 & $-1,9$ \\
\hline 10 & 287 & 0,016 & 0,389 & 0,017 & 0,245 & 0,917 & 0,631 & 0,379 & 1,000 & 0,873 & 0,700 & 0,425 & 0,169 & 0,072 & 0,076 & 69 & 287 & $-0,8$ \\
\hline 11 & 287 & 0,039 & 0,613 & 0,040 & 0,511 & 0,964 & 0,834 & 0,169 & 1,000 & 0,878 & 1,011 & 0,844 & 0,221 & 0,186 & 0,169 & 90 & 287 & $-1,1$ \\
\hline 12 & 287 & 0,036 & 0,592 & 0,039 & 0,541 & 0,926 & 0,914 & 0,113 & 1,000 & 0,881 & 0,936 & 0,728 & 0,221 & 0,161 & 0,169 & 38 & 287 & $-1,1$ \\
\hline 13 & 287 & 0,014 & 0,418 & 0,019 & 0,245 & 0,769 & 0,586 & 0,474 & 1,000 & 0,986 & 0,509 & 0,361 & 0,189 & 0,068 & 0,107 & 45 & 287 & $-0,9$ \\
\hline 14 & 287 & 0,026 & 0,795 & 0,036 & 0,693 & 0,720 & 0,872 & 0,308 & 0,514 & 1,395 & 0,270 & 0,230 & 0,349 & 0,080 & 0,076 & 46 & 287 & $-1,8$ \\
\hline 15 & 287 & 0,055 & 0,867 & 0,060 & 0,795 & 0,905 & 0,917 & 0,126 & 0,912 & 1,047 & 0,605 & 0,553 & 0,339 & 0,187 & 0,169 & 43 & 287 & $-1,8$ \\
\hline 16 & 287 & 0,037 & 0,583 & 0,037 & 0,440 & 1,000 & 0,754 & 0,246 & 1,000 & 0,852 & 0,974 & 0,686 & 0,221 & 0,152 & 0,214 & 97 & 287 & $-1,1$ \\
\hline 17 & 287 & 0,014 & 0,347 & 0,016 & 0,245 & 0,909 & 0,707 & 0,307 & 1,000 & 0,816 & 0,979 & 0,588 & 0,137 & 0,080 & 0,107 & 37 & 287 & $-0,5$ \\
\hline 18 & 287 & 0,099 & 1,437 & 0,121 & 1,196 & 0,821 & 0,832 & 0,245 & 0,602 & 1,289 & 0,453 & 0,488 & 0,528 & 0,257 & 0,273 & 79 & 287 & $-2,4$ \\
\hline 19 & 287 & 0,088 & 1,298 & 0,108 & 1,061 & 0,813 & 0,818 & 0,261 & 0,653 & 1,237 & 0,621 & 0,624 & 0,423 & 0,264 & 0,227 & 101 & 287 & $-2,1$ \\
\hline 20 & 287 & 0,158 & 1,560 & 0,176 & 1,467 & 0,894 & 0,940 & 0,121 & 0,815 & 1,108 & 0,521 & 0,468 & 0,621 & 0,291 & 0,273 & 47 & 287 & $-2,6$ \\
\hline 21 & 287 & 0,014 & 0,448 & 0,017 & 0,296 & 0,833 & 0,660 & 0,378 & 0,898 & 1,055 & 0,439 & 0,332 & 0,204 & 0,068 & 0,076 & 18 & 287 & $-1,0$ \\
\hline 22 & 287 & 0,020 & 0,440 & 0,022 & 0,296 & 0,933 & 0,673 & 0,334 & 1,000 & 0,875 & 0,891 & 0,637 & 0,169 & 0,108 & 0,152 & 0 & 287 & $-0,8$ \\
\hline 23 & 287 & 0,032 & 0,541 & 0,033 & 0,461 & 0,957 & 0,851 & 0,155 & 1,000 & 0,859 & 0,966 & 0,665 & 0,204 & 0,136 & 0,152 & 67 & 287 & $-1,0$ \\
\hline 24 & 287 & 0,039 & 0,634 & 0,042 & 0,511 & 0,931 & 0,807 & 0,205 & 1,000 & 0,909 & 0,764 & 0,596 & 0,254 & 0,152 & 0,169 & 82 & 287 & $-1,3$ \\
\hline 25 & 287 & 0,046 & 1,234 & 0,072 & 0,825 & 0,640 & 0,668 & 0,490 & 0,379 & 1,625 & 0,479 & 0,614 & 0,349 & 0,214 & 0,169 & 109 & 287 & $-1,8$ \\
\hline 26 & 287 & 0,034 & 0,583 & 0,034 & 0,440 & 1,000 & 0,754 & 0,246 & 1,000 & 0,887 & 0,764 & 0,474 & 0,240 & 0,114 & 0,152 & 94 & 287 & $-1,3$ \\
\hline 27 & 287 & 0,053 & 0,837 & 0,057 & 0,765 & 0,925 & 0,914 & 0,114 & 0,952 & 1,025 & 0,529 & 0,475 & 0,357 & 0,170 & 0,152 & 60 & 287 & $-1,8$ \\
\hline 28 & 287 & 0,042 & 0,693 & 0,044 & 0,643 & 0,935 & 0,927 & 0,098 & 1,000 & 0,959 & 0,605 & 0,453 & 0,296 & 0,134 & 0,169 & 47 & 287 & $-1,6$ \\
\hline 29 & 287 & 0,044 & 1,040 & 0,057 & 0,706 & 0,775 & 0,679 & 0,392 & 0,517 & 1,391 & 0,681 & 0,657 & 0,288 & 0,189 & 0,107 & 103 & 287 & $-1,5$ \\
\hline 30 & 287 & 0,024 & 0,541 & 0,030 & 0,418 & 0,810 & 0,773 & 0,296 & 1,000 & 0,977 & 0,585 & 0,468 & 0,230 & 0,108 & 0,152 & 66 & 287 & $-1,2$ \\
\hline 31 & 287 & 0,022 & 0,410 & 0,022 & 0,317 & 1,000 & 0,773 & 0,227 & 1,000 & 0,788 & 1,061 & 0,707 & 0,161 & 0,114 & 0,152 & 0 & 287 & $-0,7$ \\
\hline 32 & 287 & 0,017 & 0,368 & 0,017 & 0,245 & 1,000 & 0,667 & 0,333 & 1,000 & 0,791 & 1,175 & 0,784 & 0,137 & 0,107 & 0,152 & 135 & 287 & $-0,5$ \\
\hline 33 & 287 & 0,367 & 3,648 & 0,415 & 2,320 & 0,886 & 0,636 & 0,381 & 0,347 & 1,698 & 0,432 & 0,437 & 1,040 & 0,455 & 0,152 & 79 & 287 & $-3,4$ \\
\hline 34 & 287 & 0,024 & 0,469 & 0,024 & 0,389 & 1,000 & 0,829 & 0,171 & 1,000 & 0,848 & 1,082 & 0,791 & 0,169 & 0,134 & 0,169 & 70 & 287 & $-0,8$ \\
\hline 35 & 287 & 0,291 & 2,143 & 0,326 & 1,970 & 0,894 & 0,919 & 0,133 & 0,797 & 1,120 & 0,537 & 0,632 & 0,831 & 0,525 & 0,455 & 70 & 287 & $-3,1$ \\
\hline 36 & 287 & 0,023 & 0,440 & 0,023 & 0,317 & 1,000 & 0,721 & 0,279 & 1,000 & 0,818 & 1,019 & 0,671 & 0,169 & 0,114 & 0,152 & 63 & 287 & $-0,8$ \\
\hline 37 & 287 & 0,103 & 1,581 & 0,131 & 1,163 & 0,791 & 0,735 & 0,337 & 0,519 & 1,388 & 0,542 & 0,607 & 0,492 & 0,299 & 0,273 & 58 & 287 & $-2,3$ \\
\hline 38 & 287 & 0,073 & 0,879 & 0,073 & 0,807 & 1,000 & 0,918 & 0,082 & 1,000 & 0,917 & 0,812 & 0,671 & 0,339 & 0,227 & 0,273 & 15 & 287 & $-1,8$ \\
\hline 39 & 287 & 0,023 & 0,490 & 0,023 & 0,448 & 1,000 & 0,914 & 0,086 & 1,000 & 0,913 & 0,784 & 0,588 & 0,193 & 0,114 & 0,152 & 6 & 287 & $-0,9$ \\
\hline 40 & 287 & 0,029 & 0,917 & 0,044 & 0,532 & 0,645 & 0,580 & 0,550 & 0,428 & 1,528 & 0,621 & 0,674 & 0,243 & 0,164 & 0,152 & 0 & 287 & $-1,3$ \\
\hline 41 & 287 & 0,481 & 2,828 & 0,522 & 2,655 & 0,920 & 0,939 & 0,101 & 0,755 & 1,151 & 0,449 & 0,512 & 1,167 & 0,598 & 0,606 & 104 & 287 & $-3,5$ \\
\hline 42 & 287 & 0,020 & 0,448 & 0,022 & 0,368 & 0,933 & 0,820 & 0,191 & 1,000 & 0,892 & 0,891 & 0,632 & 0,169 & 0,107 & 0,107 & 107 & 287 & $-0,8$ \\
\hline 43 & 287 & 0,343 & 2,156 & 0,364 & 2,003 & 0,941 & 0,929 & 0,092 & 0,927 & 1,038 & 0,900 & 0,869 & 0,696 & 0,605 & 0,552 & 103 & 287 & $-2,8$ \\
\hline 44 & 287 & 0,056 & 0,807 & 0,059 & 0,714 & 0,951 & 0,885 & 0,125 & 1,000 & 0,963 & 0,680 & 0,585 & 0,324 & 0,189 & 0,227 & 11 & 287 & $-1,7$ \\
\hline 45 & 287 & 0,047 & 1,091 & 0,063 & 0,765 & 0,750 & 0,701 & 0,389 & 0,500 & 1,414 & 0,646 & 0,714 & 0,305 & 0,218 & 0,152 & 119 & 287 & $-1,6$ \\
\hline 46 & 287 & 0,024 & 0,672 & 0,033 & 0,562 & 0,739 & 0,836 & 0,308 & 0,678 & 1,214 & 0,433 & 0,403 & 0,268 & 0,108 & 0,107 & 163 & 287 & $-1,4$ \\
\hline 47 & 287 & 0,050 & 1,501 & 0,076 & 0,735 & 0,660 & 0,490 & 0,613 & 0,280 & 1,889 & 0,602 & 0,728 & 0,326 & 0,237 & 0,107 & 120 & 287 & $-1,7$ \\
\hline 48 & 287 & 0,032 & 0,613 & 0,034 & 0,520 & 0,917 & 0,848 & 0,173 & 1,000 & 0,973 & 0,560 & 0,424 & 0,268 & 0,114 & 0,152 & 1 & 287 & $-1,4$ \\
\hline 49 & 287 & 0,109 & 1,480 & 0,145 & 1,217 & 0,752 & 0,823 & 0,305 & 0,626 & 1,264 & 0,523 & 0,563 & 0,515 & 0,290 & 0,214 & 14 & 287 & $-2,4$ \\
\hline 50 & 287 & 0,059 & 1,560 & 0,085 & 0,900 & 0,695 & 0,577 & 0,522 & 0,304 & 1,814 & 0,418 & 0,537 & 0,423 & 0,227 & 0,107 & 96 & 287 & $-2,1$ \\
\hline 51 & 287 & 0,019 & 0,389 & 0,019 & 0,266 & 1,000 & 0,685 & 0,315 & 1,000 & 0,803 & 0,974 & 0,485 & 0,156 & 0,076 & 0,152 & 90 & 287 & $-0,6$ \\
\hline 52 & 287 & 0,033 & 0,664 & 0,034 & 0,562 & 0,958 & 0,847 & 0,159 & 0,942 & 1,031 & 0,505 & 0,421 & 0,288 & 0,121 & 0,152 & 58 & 287 & $-1,5$ \\
\hline
\end{tabular}




\begin{tabular}{|c|c|c|c|c|c|c|c|c|c|c|c|c|c|c|c|c|c|c|}
\hline 53 & 287 & 0,116 & 2,338 & 0,201 & 1,602 & 0,579 & 0,685 & 0,526 & 0,267 & 1,934 & 0,306 & 0,500 & 0,695 & 0,347 & 0,107 & 149 & 287 & $-2,8$ \\
\hline 54 & 287 & 0,112 & 2,021 & 0,155 & 1,255 & 0,722 & 0,621 & 0,470 & 0,344 & 1,704 & 0,667 & 0,788 & 0,462 & 0,364 & 0,240 & 178 & 287 & $-2,2$ \\
\hline 55 & 287 & 0,386 & 2,861 & 0,426 & 2,760 & 0,906 & 0,965 & 0,101 & 0,592 & 1,299 & 0,294 & 0,322 & 1,293 & 0,417 & 0,442 & 84 & 287 & $-3,7$ \\
\hline 56 & 287 & 0,063 & 1,184 & 0,086 & 1,010 & 0,733 & 0,854 & 0,304 & 0,566 & 1,329 & 0,366 & 0,411 & 0,469 & 0,192 & 0,169 & 20 & 287 & $-2,2$ \\
\hline 57 & 287 & 0,026 & 0,837 & 0,039 & 0,622 & 0,667 & 0,743 & 0,421 & 0,463 & 1,469 & 0,353 & 0,351 & 0,305 & 0,107 & 0,076 & 51 & 287 & $-1,6$ \\
\hline 58 & 287 & 0,016 & 0,338 & 0,016 & 0,245 & 1,000 & 0,725 & 0,275 & 1,000 & 0,759 & 1,077 & 0,555 & 0,137 & 0,076 & 0,152 & 0 & 287 & $-0,5$ \\
\hline 59 & 287 & 0,079 & 0,938 & 0,083 & 0,888 & 0,948 & 0,946 & 0,075 & 1,000 & 0,942 & 0,824 & 0,728 & 0,349 & 0,254 & 0,273 & 162 & 287 & $-1,8$ \\
\hline 60 & 287 & 0,020 & 0,418 & 0,020 & 0,296 & 1,000 & 0,707 & 0,293 & 1,000 & 0,833 & 1,049 & 0,728 & 0,156 & 0,114 & 0,152 & 0 & 287 & $-0,6$ \\
\hline 61 & 287 & 0,023 & 0,469 & 0,023 & 0,418 & 1,000 & 0,892 & 0,108 & 1,000 & 0,874 & 0,702 & 0,525 & 0,204 & 0,107 & 0,152 & 150 & 287 & $-1,0$ \\
\hline 62 & 287 & 0,016 & 0,347 & 0,016 & 0,266 & 1,000 & 0,768 & 0,232 & 1,000 & 0,778 & 1,401 & 0,894 & 0,120 & 0,107 & 0,152 & 72 & 287 & $-0,3$ \\
\hline 63 & 287 & 0,060 & 1,010 & 0,069 & 0,981 & 0,875 & 0,971 & 0,128 & 0,742 & 1,161 & 0,390 & 0,399 & 0,443 & 0,177 & 0,214 & 162 & 287 & $-2,1$ \\
\hline 64 & 287 & 0,191 & 1,543 & 0,201 & 1,420 & 0,950 & 0,921 & 0,094 & 1,000 & 0,996 & 0,753 & 0,716 & 0,568 & 0,407 & 0,386 & 71 & 287 & $-2,5$ \\
\hline 65 & 287 & 0,435 & 2,714 & 0,476 & 2,511 & 0,913 & 0,925 & 0,115 & 0,742 & 1,161 & 0,500 & 0,580 & 1,052 & 0,610 & 0,577 & 127 & 287 & $-3,4$ \\
\hline 66 & 287 & 0,016 & 0,448 & 0,016 & 0,418 & 1,000 & 0,934 & 0,066 & 0,987 & 1,006 & 0,412 & 0,243 & 0,221 & 0,054 & 0,076 & 35 & 287 & $-1,1$ \\
\hline 67 & 287 & 0,034 & 0,613 & 0,036 & 0,490 & 0,960 & 0,800 & 0,204 & 1,000 & 0,932 & 0,679 & 0,566 & 0,254 & 0,144 & 0,152 & 18 & 287 & $-1,3$ \\
\hline 68 & 287 & 0,083 & 1,082 & 0,089 & 0,981 & 0,935 & 0,906 & 0,114 & 0,893 & 1,058 & 0,434 & 0,399 & 0,494 & 0,197 & 0,214 & 60 & 287 & $-2,3$ \\
\hline 69 & 287 & 0,040 & 0,714 & 0,043 & 0,613 & 0,933 & 0,858 & 0,157 & 0,989 & 1,005 & 0,548 & 0,432 & 0,305 & 0,132 & 0,152 & 63 & 287 & $-1,6$ \\
\hline 70 & 287 & 0,017 & 0,389 & 0,017 & 0,194 & 1,000 & 0,500 & 0,500 & 1,000 & 0,836 & 0,899 & 0,485 & 0,156 & 0,076 & 0,107 & 0 & 287 & $-0,6$ \\
\hline 71 & 287 & 0,057 & 0,837 & 0,063 & 0,757 & 0,909 & 0,904 & 0,132 & 1,000 & 0,986 & 0,784 & 0,682 & 0,305 & 0,208 & 0,227 & 106 & 287 & $-1,6$ \\
\hline 72 & 287 & 0,067 & 1,437 & 0,085 & 1,336 & 0,797 & 0,929 & 0,215 & 0,410 & 1,561 & 0,178 & 0,167 & 0,695 & 0,116 & 0,152 & 155 & 287 & $-2,8$ \\
\hline 73 & 287 & 0,118 & 1,306 & 0,131 & 1,133 & 0,901 & 0,867 & 0,166 & 0,867 & 1,074 & 0,644 & 0,611 & 0,482 & 0,295 & 0,303 & 57 & 287 & $-2,3$ \\
\hline 74 & 287 & 0,037 & 0,571 & 0,037 & 0,541 & 1,000 & 0,948 & 0,052 & 1,000 & 0,834 & 1,142 & 0,788 & 0,204 & 0,161 & 0,214 & 45 & 287 & $-1,0$ \\
\hline 75 & 287 & 0,095 & 1,306 & 0,112 & 1,082 & 0,846 & 0,828 & 0,230 & 0,697 & 1,197 & 0,568 & 0,575 & 0,461 & 0,265 & 0,273 & 178 & 287 & $-2,2$ \\
\hline 76 & 287 & 0,032 & 0,643 & 0,034 & 0,541 & 0,917 & 0,842 & 0,179 & 0,961 & 1,020 & 0,459 & 0,355 & 0,296 & 0,105 & 0,152 & 37 & 287 & $-1,6$ \\
\hline 77 & 287 & 0,096 & 1,399 & 0,128 & 1,268 & 0,753 & 0,906 & 0,264 & 0,617 & 1,273 & 0,416 & 0,433 & 0,542 & 0,235 & 0,227 & 105 & 287 & $-2,4$ \\
\hline 78 & 287 & 0,052 & 0,714 & 0,053 & 0,643 & 0,973 & 0,899 & 0,104 & 1,000 & 0,887 & 1,019 & 0,843 & 0,254 & 0,214 & 0,227 & 37 & 287 & $-1,3$ \\
\hline 79 & 287 & 0,069 & 1,040 & 0,082 & 0,917 & 0,842 & 0,882 & 0,197 & 0,800 & 1,118 & 0,605 & 0,697 & 0,381 & 0,265 & 0,214 & 99 & 287 & $-1,9$ \\
\hline 80 & 287 & 0,043 & 0,930 & 0,057 & 0,664 & 0,750 & 0,714 & 0,380 & 0,626 & 1,264 & 0,516 & 0,605 & 0,326 & 0,197 & 0,152 & 117 & 287 & $-1,7$ \\
\hline 81 & 287 & 0,017 & 0,368 & 0,017 & 0,287 & 1,000 & 0,781 & 0,219 & 1,000 & 0,791 & 1,175 & 0,745 & 0,137 & 0,102 & 0,152 & 45 & 287 & $-0,5$ \\
\hline 82 & 287 & 0,019 & 0,368 & 0,019 & 0,245 & 1,000 & 0,667 & 0,333 & 1,000 & 0,760 & 1,273 & 0,784 & 0,137 & 0,107 & 0,152 & 90 & 287 & $-0,5$ \\
\hline 83 & 287 & 0,019 & 0,389 & 0,019 & 0,245 & 1,000 & 0,631 & 0,369 & 1,000 & 0,803 & 0,974 & 0,485 & 0,156 & 0,076 & 0,152 & 90 & 287 & $-0,6$ \\
\hline 84 & 287 & 0,072 & 1,196 & 0,098 & 1,023 & 0,735 & 0,855 & 0,302 & 0,630 & 1,260 & 0,439 & 0,498 & 0,456 & 0,227 & 0,169 & 85 & 287 & $-2,2$ \\
\hline 85 & 287 & 0,287 & 2,317 & 0,329 & 2,042 & 0,873 & 0,881 & 0,174 & 0,672 & 1,220 & 0,453 & 0,512 & 0,898 & 0,459 & 0,386 & 62 & 287 & $-3,2$ \\
\hline 86 & 287 & 0,042 & 0,643 & 0,042 & 0,571 & 1,000 & 0,888 & 0,112 & 1,000 & 0,889 & 0,710 & 0,490 & 0,273 & 0,134 & 0,169 & 39 & 287 & $-1,4$ \\
\hline 87 & 287 & 0,020 & 0,389 & 0,020 & 0,287 & 1,000 & 0,739 & 0,261 & 1,000 & 0,774 & 0,990 & 0,667 & 0,161 & 0,107 & 0,152 & 45 & 287 & $-0,7$ \\
\hline 88 & 287 & 0,020 & 0,410 & 0,020 & 0,317 & 1,000 & 0,773 & 0,227 & 1,000 & 0,816 & 0,990 & 0,707 & 0,161 & 0,114 & 0,152 & 45 & 287 & $-0,7$ \\
\hline 89 & 287 & 0,023 & 0,440 & 0,023 & 0,347 & 1,000 & 0,789 & 0,211 & 1,000 & 0,818 & 1,019 & 0,671 & 0,169 & 0,114 & 0,152 & 15 & 287 & $-0,8$ \\
\hline 90 & 287 & 0,947 & 6,417 & 1,468 & 5,149 & 0,645 & 0,802 & 0,406 & 0,289 & 1,860 & 0,222 & 0,381 & 2,328 & 0,887 & 0,682 & 63 & 287 & $-4,5$ \\
\hline 91 & 287 & 0,020 & 0,469 & 0,023 & 0,389 & 0,875 & 0,829 & 0,212 & 1,000 & 0,934 & 0,686 & 0,527 & 0,193 & 0,102 & 0,107 & 174 & 287 & $-0,9$ \\
\hline 92 & 287 & 0,096 & 1,184 & 0,108 & 1,091 & 0,893 & 0,922 & 0,132 & 0,862 & 1,077 & 0,520 & 0,524 & 0,485 & 0,254 & 0,227 & 38 & 287 & $-2,3$ \\
\hline 93 & 287 & 0,019 & 0,634 & 0,023 & 0,490 & 0,812 & 0,773 & 0,294 & 0,583 & 1,309 & 0,255 & 0,181 & 0,305 & 0,055 & 0,076 & 15 & 287 & $-1,6$ \\
\hline 94 & 287 & 0,766 & 4,721 & 0,934 & 3,758 & 0,820 & 0,796 & 0,272 & 0,432 & 1,521 & 0,349 & 0,427 & 1,672 & 0,713 & 0,606 & 98 & 287 & $-4,1$ \\
\hline 95 & 287 & 0,019 & 0,418 & 0,020 & 0,347 & 0,929 & 0,828 & 0,186 & 1,000 & 0,864 & 0,828 & 0,600 & 0,169 & 0,102 & 0,152 & 161 & 287 & $-0,8$ \\
\hline 96 & 287 & 0,109 & 2,283 & 0,164 & 1,327 & 0,667 & 0,581 & 0,535 & 0,263 & 1,950 & 0,523 & 0,676 & 0,515 & 0,348 & 0,273 & 62 & 287 & $-2,4$ \\
\hline 97 & 287 & 0,023 & 0,461 & 0,026 & 0,338 & 0,889 & 0,734 & 0,288 & 1,000 & 0,858 & 1,019 & 0,671 & 0,169 & 0,114 & 0,152 & 88 & 287 & $-0,8$ \\
\hline 98 & 287 & 0,067 & 0,960 & 0,076 & 0,816 & 0,887 & 0,850 & 0,188 & 0,920 & 1,042 & 0,665 & 0,612 & 0,359 & 0,220 & 0,214 & 116 & 287 & $-1,8$ \\
\hline 99 & 287 & 0,060 & 1,243 & 0,075 & 0,867 & 0,808 & 0,697 & 0,359 & 0,490 & 1,428 & 0,594 & 0,596 & 0,359 & 0,214 & 0,214 & 147 & 287 & $-1,8$ \\
\hline 100 & 287 & 0,047 & 0,786 & 0,055 & 0,664 & 0,868 & 0,844 & 0,204 & 0,963 & 1,019 & 0,568 & 0,572 & 0,326 & 0,186 & 0,169 & 36 & 287 & $-1,7$ \\
\hline 101 & 287 & 0,014 & 0,397 & 0,014 & 0,317 & 1,000 & 0,798 & 0,202 & 1,000 & 0,936 & 0,509 & 0,283 & 0,189 & 0,054 & 0,076 & 40 & 287 & $-0,9$ \\
\hline 102 & 287 & 0,014 & 0,490 & 0,020 & 0,194 & 0,714 & 0,396 & 0,668 & 0,750 & 1,155 & 0,979 & 0,832 & 0,137 & 0,114 & 0,076 & 135 & 287 & $-0,5$ \\
\hline 103 & 287 & 0,179 & 1,492 & 0,189 & 1,378 & 0,947 & 0,924 & 0,093 & 1,000 & 0,994 & 0,658 & 0,661 & 0,589 & 0,390 & 0,408 & 31 & 287 & $-2,6$ \\
\hline 104 & 287 & 0,022 & 0,562 & 0,026 & 0,389 & 0,833 & 0,692 & 0,351 & 0,856 & 1,081 & 0,562 & 0,479 & 0,221 & 0,106 & 0,076 & 162 & 287 & $-1,1$ \\
\hline 105 & 287 & 0,231 & 1,746 & 0,242 & 1,644 & 0,953 & 0,942 & 0,075 & 0,952 & 1,025 & 0,700 & 0,643 & 0,648 & 0,417 & 0,455 & 86 & 287 & $-2,7$ \\
\hline 106 & 287 & 0,022 & 0,440 & 0,024 & 0,317 & 0,882 & 0,721 & 0,303 & 1,000 & 0,845 & 0,955 & 0,671 & 0,169 & 0,114 & 0,152 & 125 & 287 & $-0,8$ \\
\hline 107 & 287 & 0,030 & 0,520 & 0,032 & 0,490 & 0,955 & 0,943 & 0,073 & 1,000 & 0,845 & 0,922 & 0,657 & 0,204 & 0,134 & 0,152 & 67 & 287 & $-1,0$ \\
\hline 108 & 287 & 0,037 & 0,643 & 0,037 & 0,541 & 1,000 & 0,842 & 0,158 & 1,000 & 0,939 & 0,662 & 0,566 & 0,268 & 0,152 & 0,169 & 76 & 287 & $-1,4$ \\
\hline 109 & 287 & 0,016 & 0,368 & 0,016 & 0,275 & 1,000 & 0,747 & 0,253 & 1,000 & 0,826 & 1,077 & 0,784 & 0,137 & 0,107 & 0,107 & 90 & 287 & $-0,5$ \\
\hline 110 & 287 & 0,022 & 0,461 & 0,022 & 0,317 & 1,000 & 0,688 & 0,312 & 1,000 & 0,886 & 0,659 & 0,371 & 0,204 & 0,076 & 0,152 & 11 & 287 & $-1,0$ \\
\hline
\end{tabular}




\begin{tabular}{|c|c|c|c|c|c|c|c|c|c|c|c|c|c|c|c|c|c|c|}
\hline 111 & 287 & 0,034 & 0,613 & 0,036 & 0,490 & 0,960 & 0,800 & 0,204 & 1,000 & 0,932 & 0,745 & 0,552 & 0,243 & 0,134 & 0,152 & 44 & 287 & $-1,3$ \\
\hline 112 & 287 & 0,065 & 0,960 & 0,072 & 0,930 & 0,900 & 0,969 & 0,105 & 0,881 & 1,065 & 0,494 & 0,463 & 0,408 & 0,189 & 0,227 & 75 & 287 & $-2,0$ \\
\hline 113 & 287 & 0,063 & 0,786 & 0,066 & 0,735 & 0,957 & 0,935 & 0,078 & 1,000 & 0,883 & 1,057 & 0,824 & 0,276 & 0,227 & 0,273 & 176 & 287 & $-1,5$ \\
\hline 114 & 287 & 0,033 & 0,583 & 0,034 & 0,418 & 0,958 & 0,718 & 0,285 & 1,000 & 0,906 & 0,714 & 0,559 & 0,243 & 0,136 & 0,169 & 58 & 287 & $-1,3$ \\
\hline 115 & 287 & 0,027 & 0,541 & 0,030 & 0,490 & 0,905 & 0,906 & 0,134 & 1,000 & 0,924 & 0,654 & 0,493 & 0,230 & 0,114 & 0,152 & 15 & 287 & $-1,2$ \\
\hline 116 & 287 & 0,166 & 1,674 & 0,191 & 1,501 & 0,872 & 0,896 & 0,164 & 0,746 & 1,157 & 0,603 & 0,586 & 0,593 & 0,347 & 0,321 & 27 & 287 & $-2,6$ \\
\hline 117 & 287 & 0,014 & 0,347 & 0,016 & 0,224 & 0,909 & 0,646 & 0,365 & 1,000 & 0,816 & 0,979 & 0,588 & 0,137 & 0,080 & 0,107 & 124 & 287 & $-0,5$ \\
\hline 118 & 287 & 0,022 & 0,418 & 0,023 & 0,317 & 0,938 & 0,757 & 0,251 & 1,000 & 0,805 & 1,123 & 0,728 & 0,156 & 0,114 & 0,152 & 84 & 287 & $-0,6$ \\
\hline 119 & 287 & 0,029 & 0,592 & 0,033 & 0,469 & 0,870 & 0,793 & 0,245 & 1,000 & 0,986 & 0,621 & 0,501 & 0,243 & 0,121 & 0,152 & 151 & 287 & $-1,3$ \\
\hline 120 & 287 & 0,175 & 1,509 & 0,189 & 1,441 & 0,924 & 0,955 & 0,088 & 0,966 & 1,018 & 0,840 & 0,809 & 0,515 & 0,417 & 0,408 & 85 & 287 & $-2,4$ \\
\hline 121 & 287 & 0,020 & 0,418 & 0,022 & 0,317 & 0,933 & 0,757 & 0,252 & 1,000 & 0,833 & 0,990 & 0,667 & 0,161 & 0,107 & 0,152 & 17 & 287 & $-0,7$ \\
\hline 122 & 287 & 0,016 & 0,541 & 0,022 & 0,317 & 0,733 & 0,586 & 0,493 & 0,677 & 1,215 & 0,778 & 0,667 & 0,161 & 0,107 & 0,076 & 135 & 287 & $-0,7$ \\
\hline 123 & 287 & 0,024 & 0,571 & 0,027 & 0,490 & 0,895 & 0,859 & 0,176 & 0,941 & 1,031 & 0,528 & 0,331 & 0,243 & 0,080 & 0,107 & 40 & 287 & $-1,3$ \\
\hline 124 & 287 & 0,027 & 0,511 & 0,027 & 0,389 & 1,000 & 0,760 & 0,240 & 1,000 & 0,874 & 0,712 & 0,514 & 0,221 & 0,114 & 0,152 & 66 & 287 & $-1,1$ \\
\hline 125 & 287 & 0,115 & 1,112 & 0,118 & 1,082 & 0,976 & 0,973 & 0,036 & 1,000 & 0,926 & 0,878 & 0,780 & 0,408 & 0,318 & 0,321 & 144 & 287 & $-2,0$ \\
\hline 126 & 287 & 0,019 & 0,389 & 0,020 & 0,245 & 0,929 & 0,631 & 0,376 & 1,000 & 0,803 & 1,273 & 0,832 & 0,137 & 0,114 & 0,152 & 27 & 287 & $-0,5$ \\
\hline 127 & 287 & 0,019 & 0,397 & 0,019 & 0,317 & 1,000 & 0,798 & 0,202 & 1,000 & 0,821 & 0,974 & 0,651 & 0,156 & 0,102 & 0,107 & 59 & 287 & $-0,6$ \\
\hline 128 & 287 & 0,023 & 0,440 & 0,023 & 0,317 & 1,000 & 0,721 & 0,279 & 1,000 & 0,818 & 0,815 & 0,566 & 0,189 & 0,107 & 0,152 & 60 & 287 & $-0,9$ \\
\hline 129 & 287 & 0,019 & 0,368 & 0,019 & 0,245 & 1,000 & 0,667 & 0,333 & 1,000 & 0,760 & 1,273 & 0,784 & 0,137 & 0,107 & 0,152 & 90 & 287 & $-0,5$ \\
\hline 130 & 287 & 0,062 & 1,040 & 0,070 & 0,858 & 0,878 & 0,825 & 0,214 & 0,717 & 1,181 & 0,472 & 0,464 & 0,408 & 0,189 & 0,169 & 81 & 287 & $-2,0$ \\
\hline 131 & 287 & 0,024 & 0,461 & 0,026 & 0,296 & 0,944 & 0,642 & 0,362 & 1,000 & 0,832 & 1,082 & 0,671 & 0,169 & 0,114 & 0,152 & 27 & 287 & $-0,8$ \\
\hline 132 & 287 & 0,027 & 0,511 & 0,029 & 0,368 & 0,950 & 0,719 & 0,285 & 1,000 & 0,874 & 0,968 & 0,759 & 0,189 & 0,144 & 0,152 & 75 & 287 & $-0,9$ \\
\hline 133 & 287 & 0,019 & 0,389 & 0,020 & 0,287 & 0,929 & 0,739 & 0,271 & 1,000 & 0,803 & 0,920 & 0,633 & 0,161 & 0,102 & 0,152 & 60 & 287 & $-0,7$ \\
\hline 134 & 287 & 0,020 & 0,520 & 0,024 & 0,347 & 0,824 & 0,667 & 0,377 & 0,934 & 1,035 & 0,891 & 0,671 & 0,169 & 0,114 & 0,107 & 35 & 287 & $-0,8$ \\
\hline 135 & 287 & 0,034 & 0,786 & 0,042 & 0,643 & 0,828 & 0,817 & 0,251 & 0,700 & 1,195 & 0,360 & 0,316 & 0,349 & 0,111 & 0,152 & 36 & 287 & $-1,8$ \\
\hline 136 & 287 & 0,016 & 0,714 & 0,030 & 0,583 & 0,524 & 0,816 & 0,510 & 0,389 & 1,604 & 0,219 & 0,250 & 0,303 & 0,076 & 0,076 & 169 & 287 & $-1,6$ \\
\hline 137 & 287 & 0,026 & 0,714 & 0,033 & 0,469 & 0,783 & 0,657 & 0,406 & 0,636 & 1,254 & 0,441 & 0,373 & 0,273 & 0,102 & 0,107 & 30 & 287 & $-1,4$ \\
\hline 138 & 287 & 0,057 & 0,968 & 0,062 & 0,846 & 0,930 & 0,873 & 0,145 & 0,769 & 1,140 & 0,351 & 0,282 & 0,456 & 0,129 & 0,169 & 136 & 287 & $-2,2$ \\
\hline 139 & 287 & 0,077 & 0,981 & 0,083 & 0,879 & 0,931 & 0,896 & 0,124 & 1,000 & 0,994 & 0,688 & 0,626 & 0,379 & 0,237 & 0,240 & 24 & 287 & $-1,9$ \\
\hline 140 & 287 & 0,141 & 1,970 & 0,171 & 1,319 & 0,824 & 0,669 & 0,375 & 0,455 & 1,482 & 0,674 & 0,767 & 0,515 & 0,395 & 0,214 & 111 & 287 & $-2,4$ \\
\hline 141 & 287 & 0,027 & 0,541 & 0,029 & 0,440 & 0,950 & 0,812 & 0,194 & 1,000 & 0,924 & 0,654 & 0,493 & 0,230 & 0,114 & 0,152 & 95 & 287 & $-1,2$ \\
\hline 142 & 287 & 0,020 & 0,389 & 0,020 & 0,296 & 1,000 & 0,761 & 0,239 & 1,000 & 0,774 & 1,371 & 0,832 & 0,137 & 0,114 & 0,152 & 135 & 287 & $-0,5$ \\
\hline 143 & 287 & 0,016 & 0,347 & 0,016 & 0,266 & 1,000 & 0,768 & 0,232 & 1,000 & 0,778 & 1,401 & 0,894 & 0,120 & 0,107 & 0,152 & 108 & 287 & $-0,3$ \\
\hline 144 & 287 & 0,017 & 0,482 & 0,019 & 0,215 & 0,923 & 0,447 & 0,558 & 0,933 & 1,036 & 0,413 & 0,164 & 0,230 & 0,038 & 0,076 & 0 & 287 & $-1,2$ \\
\hline 145 & 287 & 0,029 & 0,807 & 0,043 & 0,583 & 0,667 & 0,722 & 0,434 & 0,553 & 1,344 & 0,300 & 0,325 & 0,349 & 0,114 & 0,152 & 6 & 287 & $-1,8$ \\
\hline 146 & 287 & 0,039 & 1,306 & 0,066 & 0,786 & 0,587 & 0,602 & 0,574 & 0,285 & 1,872 & 0,382 & 0,588 & 0,359 & 0,211 & 0,152 & 110 & 287 & $-1,8$ \\
\hline 147 & 287 & 0,126 & 1,255 & 0,135 & 1,184 & 0,936 & 0,943 & 0,086 & 1,000 & 0,997 & 0,683 & 0,650 & 0,485 & 0,315 & 0,312 & 39 & 287 & $-2,3$ \\
\hline 148 & 287 & 0,037 & 0,837 & 0,053 & 0,664 & 0,703 & 0,793 & 0,362 & 0,669 & 1,222 & 0,625 & 0,589 & 0,276 & 0,162 & 0,152 & 154 & 287 & $-1,5$ \\
\hline 149 & 287 & 0,024 & 0,440 & 0,024 & 0,338 & 1,000 & 0,769 & 0,231 & 1,000 & 0,794 & 1,082 & 0,671 & 0,169 & 0,114 & 0,152 & 75 & 287 & $-0,8$ \\
\hline 150 & 287 & 0,042 & 0,672 & 0,044 & 0,562 & 0,935 & 0,836 & 0,176 & 1,000 & 0,930 & 0,738 & 0,600 & 0,268 & 0,161 & 0,214 & 26 & 287 & $-1,4$ \\
\hline 151 & 287 & 0,014 & 0,376 & 0,017 & 0,296 & 0,833 & 0,786 & 0,271 & 1,000 & 0,886 & 0,749 & 0,514 & 0,156 & 0,080 & 0,107 & 34 & 287 & $-0,6$ \\
\hline 152 & 287 & 0,014 & 0,418 & 0,017 & 0,275 & 0,833 & 0,657 & 0,382 & 1,000 & 0,986 & 0,509 & 0,388 & 0,189 & 0,074 & 0,076 & 162 & 287 & $-0,9$ \\
\hline 153 & 287 & 0,014 & 0,317 & 0,014 & 0,173 & 1,000 & 0,547 & 0,453 & 1,000 & 0,746 & 1,273 & 0,632 & 0,120 & 0,076 & 0,107 & 18 & 287 & $-0,3$ \\
\hline 154 & 287 & 0,158 & 1,686 & 0,184 & 1,534 & 0,859 & 0,910 & 0,167 & 0,697 & 1,197 & 0,431 & 0,398 & 0,683 & 0,272 & 0,303 & 77 & 287 & $-2,8$ \\
\hline $\begin{array}{l}154 \\
155\end{array}$ & 287 & 0,024 & $\begin{array}{l}1,000 \\
0,520\end{array}$ & 0,026 & 0,469 & 0,944 & 0,902 & 0,112 & 1,000 & 0,939 & 0,601 & 0,500 & 0,227 & 0,114 & 0,152 & 21 & 287 & $-1,2$ \\
\hline 156 & 287 & 0,032 & 0,553 & 0,033 & 0,389 & 0,957 & 0,702 & 0,301 & 1,000 & 0,879 & 0,824 & 0,514 & 0,221 & 0,114 & 0,152 & 169 & 287 & $-1,1$ \\
\hline 157 & 287 & 0,020 & 0,418 & 0,020 & 0,397 & 1,000 & 0,950 & 0,050 & 1,000 & 0,833 & 0,891 & 0,632 & 0,169 & 0,107 & 0,152 & 163 & 287 & $-0,8$ \\
\hline 158 & 287 & 0,017 & 0,368 & 0,017 & 0,317 & 1,000 & 0,862 & 0,138 & 1,000 & 0,791 & 0,899 & 0,485 & 0,156 & 0,076 & 0,152 & 172 & 287 & $-0,6$ \\
\hline 159 & 287 & 0,023 & 0,482 & 0,024 & 0,266 & 0,941 & 0,553 & 0,451 & 1,000 & 0,897 & 0,702 & 0,371 & 0,204 & 0,076 & 0,152 & 171 & 287 & $-1,0$ \\
\hline 160 & 287 & 0,027 & 0,520 & 0,029 & 0,490 & 0,950 & 0,943 & 0,076 & 1,000 & 0,888 & 0,654 & 0,493 & 0,230 & 0,114 & 0,152 & 9 & 287 & $-1,2$ \\
\hline 161 & 287 & 0,014 & 0,347 & 0,017 & 0,266 & 0,833 & 0,768 & 0,286 & 1,000 & 0,816 & 1,273 & 0,849 & 0,120 & 0,102 & 0,107 & 108 & 287 & $-0,3$ \\
\hline 162 & 287 & 0,123 & 1,412 & 0,131 & 1,205 & 0,945 & 0,853 & 0,156 & 0,778 & 1,134 & 0,400 & 0,385 & 0,627 & 0,241 & 0,273 & 74 & 287 & $-2,6$ \\
\hline 163 & 287 & 0,017 & 0,368 & 0,017 & 0,266 & 1,000 & 0,724 & 0,276 & 1,000 & 0,791 & 1,175 & 0,784 & 0,137 & 0,107 & 0,152 & 90 & 287 & $-0,5$ \\
\hline 164 & 287 & 0,231 & 2,329 & 0,291 & 2,063 & 0,793 & 0,886 & 0,236 & 0,535 & 1,367 & 0,328 & 0,405 & 0,947 & 0,383 & 0,312 & 76 & 287 & $-3,2$ \\
\hline 165 & 287 & 0,036 & 0,634 & 0,037 & 0,511 & 0,962 & 0,807 & 0,197 & 1,000 & 0,944 & 0,637 & 0,424 & 0,268 & 0,114 & 0,152 & 81 & 287 & $-1,4$ \\
\hline 166 & 287 & 0,066 & 0,816 & 0,067 & 0,786 & 0,979 & 0,964 & 0,042 & 1,000 & 0,896 & 0,861 & 0,728 & 0,312 & 0,227 & 0,240 & 167 & 287 & $-1,6$ \\
\hline 167 & 287 & 0,016 & 0,368 & 0,016 & 0,317 & 1,000 & 0,862 & 0,138 & 1,000 & 0,826 & 0,700 & 0,447 & 0,169 & 0,076 & 0,107 & 162 & 287 & $-0,8$ \\
\hline 168 & 287 & 0,049 & 0,960 & 0,062 & 0,888 & 0,791 & 0,925 & 0,222 & 0,666 & 1,226 & 0,333 & 0,338 & 0,432 & 0,146 & 0,152 & 145 & 287 & $-2,1$ \\
\hline
\end{tabular}




\begin{tabular}{|c|c|c|c|c|c|c|c|c|c|c|c|c|c|c|c|c|c|c|}
\hline 169 & 287 & 0,024 & 0,469 & 0,024 & 0,389 & 1,000 & 0,829 & 0,171 & 1,000 & 0,848 & 1,082 & 0,791 & 0,169 & 0,134 & 0,169 & 41 & 287 & $-0,8$ \\
\hline 170 & 287 & 0,017 & 0,368 & 0,017 & 0,266 & 1,000 & 0,724 & 0,276 & 1,000 & 0,791 & 1,175 & 0,745 & 0,137 & 0,102 & 0,107 & 45 & 287 & $-0,5$ \\
\hline 171 & 287 & 0,014 & 0,317 & 0,014 & 0,194 & 1,000 & 0,613 & 0,387 & 1,000 & 0,746 & 1,273 & 0,632 & 0,120 & 0,076 & 0,152 & 0 & 287 & $-0,3$ \\
\hline 172 & 287 & 0,042 & 0,765 & 0,049 & 0,643 & 0,853 & 0,840 & 0,217 & 0,893 & 1,058 & 0,499 & 0,466 & 0,326 & 0,152 & 0,152 & 49 & 287 & $-1,7$ \\
\hline 173 & 287 & 0,020 & 0,418 & 0,020 & 0,317 & 1,000 & 0,757 & 0,243 & 1,000 & 0,833 & 0,891 & 0,632 & 0,169 & 0,107 & 0,152 & 59 & 287 & $-0,8$ \\
\hline 174 & 287 & 0,029 & 0,520 & 0,030 & 0,511 & 0,952 & 0,983 & 0,050 & 1,000 & 0,866 & 0,979 & 0,784 & 0,193 & 0,152 & 0,152 & 14 & 287 & $-0,9$ \\
\hline 175 & 287 & 0,073 & 1,133 & 0,080 & 1,031 & 0,911 & 0,910 & 0,126 & 0,716 & 1,181 & 0,336 & 0,305 & 0,526 & 0,161 & 0,169 & 61 & 287 & $-2,4$ \\
\hline 176 & 287 & 0,023 & 0,461 & 0,023 & 0,317 & 1,000 & 0,688 & 0,312 & 1,000 & 0,858 & 0,784 & 0,392 & 0,193 & 0,076 & 0,152 & 90 & 287 & $-0,9$ \\
\hline 177 & 287 & 0,026 & 0,511 & 0,026 & 0,368 & 1,000 & 0,719 & 0,281 & 1,000 & 0,898 & 0,619 & 0,329 & 0,230 & 0,076 & 0,152 & 3 & 287 & $-1,2$ \\
\hline 178 & 287 & 0,057 & 1,070 & 0,073 & 0,816 & 0,784 & 0,763 & 0,321 & 0,630 & 1,260 & 0,525 & 0,621 & 0,373 & 0,232 & 0,152 & 23 & 287 & $-1,9$ \\
\hline 179 & 287 & 0,017 & 0,368 & 0,017 & 0,296 & 1,000 & 0,805 & 0,195 & 1,000 & 0,791 & 0,899 & 0,485 & 0,156 & 0,076 & 0,152 & 8 & 287 & $-0,6$ \\
\hline 180 & 287 & 0,043 & 0,723 & 0,046 & 0,693 & 0,938 & 0,959 & 0,075 & 1,000 & 0,983 & 0,659 & 0,529 & 0,288 & 0,153 & 0,169 & 36 & 287 & $-1,5$ \\
\hline 181 & 287 & 0,067 & 0,930 & 0,073 & 0,879 & 0,922 & 0,945 & 0,096 & 0,980 & 1,010 & 0,549 & 0,455 & 0,395 & 0,180 & 0,214 & 169 & 287 & $-2,0$ \\
\hline 182 & 287 & 0,066 & 1,031 & 0,075 & 0,909 & 0,885 & 0,881 & 0,166 & 0,780 & 1,132 & 0,480 & 0,450 & 0,418 & 0,188 & 0,227 & 9 & 287 & $-2,1$ \\
\hline 183 & 287 & 0,030 & 0,511 & 0,032 & 0,389 & 0,955 & 0,760 & 0,244 & 1,000 & 0,831 & 1,070 & 0,800 & 0,189 & 0,152 & 0,169 & 149 & 287 & $-0,9$ \\
\hline 184 & 287 & 0,022 & 0,490 & 0,023 & 0,440 & 0,938 & 0,896 & 0,121 & 1,000 & 0,943 & 0,516 & 0,329 & 0,230 & 0,076 & 0,152 & 169 & 287 & $-1,2$ \\
\hline 185 & 287 & 0,062 & 0,947 & 0,072 & 0,858 & 0,860 & 0,906 & 0,169 & 0,864 & 1,076 & 0,676 & 0,661 & 0,341 & 0,225 & 0,227 & 22 & 287 & $-1,8$ \\
\hline 186 & 287 & 0,861 & 7,212 & 1,334 & 4,975 & 0,645 & 0,690 & 0,471 & 0,208 & 2,193 & 0,218 & 0,390 & 2,244 & 0,874 & 0,152 & 164 & 287 & $-4,5$ \\
\hline 187 & 287 & 0,016 & 0,550 & 0,023 & 0,469 & 0,688 & 0,854 & 0,345 & 0,656 & 1,234 & 0,379 & 0,306 & 0,230 & 0,070 & 0,076 & 152 & 287 & $-1,2$ \\
\hline 188 & 287 & 0,020 & 0,389 & 0,020 & 0,296 & 1,000 & 0,761 & 0,239 & 1,000 & 0,774 & 1,371 & 0,832 & 0,137 & 0,114 & 0,152 & 135 & 287 & $-0,5$ \\
\hline 189 & 287 & 0,037 & 0,693 & 0,043 & 0,541 & 0,867 & 0,780 & 0,257 & 0,975 & 1,013 & 0,807 & 0,674 & 0,243 & 0,164 & 0,152 & 63 & 287 & $-1,3$ \\
\hline 190 & 287 & 0,976 & 4,426 & 1,083 & 4,104 & 0,901 & 0,927 & 0,123 & 0,626 & 1,264 & 0,415 & 0,429 & 1,731 & 0,743 & 0,715 & 70 & 287 & $-4,1$ \\
\hline 191 & 287 & 0,014 & 0,397 & 0,016 & 0,296 & 0,909 & 0,745 & 0,271 & 1,000 & 0,936 & 0,637 & 0,447 & 0,169 & 0,076 & 0,107 & 108 & 287 & $-0,8$ \\
\hline 192 & 287 & 0,020 & 0,389 & 0,020 & 0,287 & 1,000 & 0,739 & 0,261 & 1,000 & 0,774 & 0,990 & 0,667 & 0,161 & 0,107 & 0,152 & 45 & 287 & $-0,7$ \\
\hline 193 & 287 & 0,367 & 3,111 & 0,439 & 2,786 & 0,837 & 0,895 & 0,194 & 0,477 & 1,448 & 0,267 & 0,304 & 1,323 & 0,402 & 0,303 & 66 & 287 & $-3,7$ \\
\hline 194 & 287 & 0,032 & 0,622 & 0,034 & 0,490 & 0,917 & 0,789 & 0,227 & 1,000 & 0,987 & 0,824 & 0,728 & 0,221 & 0,161 & 0,152 & 124 & 287 & $-1,1$ \\
\hline 195 & 287 & 0,017 & 0,368 & 0,019 & 0,245 & 0,923 & 0,667 & 0,342 & 1,000 & 0,791 & 1,175 & 0,745 & 0,137 & 0,102 & 0,107 & 135 & 287 & $-0,5$ \\
\hline 196 & 287 & 0,085 & 1,205 & 0,102 & 0,989 & 0,831 & 0,821 & 0,246 & 0,733 & 1,168 & 0,515 & 0,583 & 0,458 & 0,267 & 0,273 & 118 & 287 & $-2,2$ \\
\hline 197 & 287 & 0,062 & 0,960 & 0,070 & 0,807 & 0,878 & 0,841 & 0,200 & 0,842 & 1,090 & 0,472 & 0,457 & 0,408 & 0,186 & 0,214 & 71 & 287 & $-2,0$ \\
\hline 198 & 287 & 0,082 & 1,725 & 0,108 & 1,095 & 0,760 & 0,635 & 0,437 & 0,346 & 1,701 & 0,354 & 0,349 & 0,542 & 0,189 & 0,169 & 94 & 287 & $-2,4$ \\
\hline 199 & 287 & 0,142 & 1,733 & 0,181 & 1,458 & 0,786 & 0,841 & 0,267 & 0,594 & 1,297 & 0,485 & 0,510 & 0,611 & 0,312 & 0,273 & 24 & 287 & $-2,6$ \\
\hline 200 & 287 & 0,019 & 0,490 & 0,023 & 0,347 & 0,812 & 0,707 & 0,348 & 0,975 & 1,013 & 0,662 & 0,570 & 0,189 & 0,108 & 0,152 & 26 & 287 & $-0,9$ \\
\hline 201 & 287 & 0,063 & 0,807 & 0,063 & 0,757 & 1,000 & 0,937 & 0,063 & 1,000 & 0,906 & 0,767 & 0,585 & 0,324 & 0,189 & 0,227 & 9 & 287 & $-1,7$ \\
\hline 202 & 287 & 0,156 & 1,742 & 0,184 & 1,471 & 0,852 & 0,844 & 0,215 & 0,648 & 1,243 & 0,540 & 0,521 & 0,607 & 0,316 & 0,312 & 108 & 287 & $-2,6$ \\
\hline 203 & 287 & 0,166 & 2,033 & 0,217 & 1,615 & 0,768 & 0,794 & 0,310 & 0,506 & 1,406 & 0,381 & 0,434 & 0,746 & 0,324 & 0,240 & 11 & 287 & $-2,9$ \\
\hline 204 & 287 & 0,032 & 0,583 & 0,034 & 0,368 & 0,917 & 0,631 & 0,379 & 1,000 & 0,926 & 0,700 & 0,474 & 0,240 & 0,114 & 0,152 & 100 & 287 & $-1,3$ \\
\hline 205 & 287 & 0,040 & 0,714 & 0,043 & 0,643 & 0,933 & 0,899 & 0,121 & 0,989 & 1,005 & 0,548 & 0,500 & 0,305 & 0,153 & 0,152 & 35 & 287 & $-1,6$ \\
\hline 206 & 287 & 0,356 & 2,329 & 0,402 & 2,147 & 0,886 & 0,922 & 0,138 & 0,824 & 1,101 & 0,710 & 0,715 & 0,799 & 0,572 & 0,530 & 175 & 287 & $-3,0$ \\
\hline 207 & 287 & 0,062 & 1,593 & 0,098 & 0,900 & 0,632 & 0,565 & 0,570 & 0,305 & 1,810 & 0,468 & 0,647 & 0,410 & 0,265 & 0,107 & 96 & 287 & $-2,0$ \\
\hline 208 & 287 & 0,022 & 0,431 & 0,022 & 0,194 & 1,000 & 0,451 & 0,549 & 1,000 & 0,829 & 0,955 & 0,447 & 0,169 & 0,076 & 0,152 & 90 & 287 & $-0,8$ \\
\hline 209 & 287 & 0,653 & 3,948 & 0,739 & 3,145 & 0,883 & 0,797 & 0,234 & 0,526 & 1,379 & 0,513 & 0,526 & 1,273 & 0,669 & 0,152 & 26 & 287 & $-3,7$ \\
\hline 210 & 287 & 0,016 & 0,368 & 0,016 & 0,296 & 1,000 & 0,805 & 0,195 & 1,000 & 0,826 & 0,824 & 0,485 & 0,156 & 0,076 & 0,107 & 18 & 287 & $-0,6$ \\
\hline 211 & 287 & 0,098 & 1,361 & 0,108 & 1,238 & 0,907 & 0,910 & 0,130 & 0,662 & 1,229 & 0,299 & 0,235 & 0,645 & 0,152 & 0,227 & 178 & 287 & $-2,7$ \\
\hline 212 & 287 & 0,016 & 0,469 & 0,019 & 0,338 & 0,846 & 0,720 & 0,319 & 0,901 & 1,054 & 0,483 & 0,371 & 0,204 & 0,076 & 0,107 & 69 & 287 & $-1,0$ \\
\hline 213 & 287 & 0,019 & 0,397 & 0,020 & 0,389 & 0,929 & 0,978 & 0,075 & 1,000 & 0,821 & 1,035 & 0,707 & 0,152 & 0,107 & 0,152 & 135 & 287 & $-0,6$ \\
\hline 214 & 287 & 0,022 & 0,469 & 0,022 & 0,418 & 1,000 & 0,892 & 0,108 & 1,000 & 0,902 & 0,562 & 0,364 & 0,221 & 0,080 & 0,107 & 32 & 287 & $-1,1$ \\
\hline 215 & 287 & 0,067 & 0,828 & 0,069 & 0,706 & 0,979 & 0,852 & 0,149 & 1,000 & 0,900 & 0,921 & 0,744 & 0,305 & 0,227 & 0,273 & 11 & 287 & $-1,6$ \\
\hline 216 & 287 & 0,016 & 0,368 & 0,017 & 0,245 & 0,917 & 0,667 & 0,344 & 1,000 & 0,826 & 0,778 & 0,500 & 0,161 & 0,080 & 0,107 & 127 & 287 & $-0,7$ \\
\hline 217 & 287 & 0,083 & 1,543 & 0,102 & 0,981 & 0,817 & 0,636 & 0,408 & 0,439 & 1,509 & 0,738 & 0,761 & 0,379 & 0,288 & 0,214 & 153 & 287 & $-1,9$ \\
\hline 218 & 287 & 0,014 & 0,440 & 0,017 & 0,368 & 0,833 & 0,837 & 0,233 & 0,933 & 1,035 & 0,439 & 0,271 & 0,204 & 0,055 & 0,076 & 105 & 287 & $-1,0$ \\
\hline 219 & 287 & 0,047 & 0,714 & 0,047 & 0,643 & 1,000 & 0,899 & 0,101 & 1,000 & 0,926 & 0,646 & 0,496 & 0,305 & 0,152 & 0,214 & 4 & 287 & $-1,6$ \\
\hline 220 & 287 & 0,161 & 3,157 & 0,380 & 2,658 & 0,423 & 0,842 & 0,599 & 0,203 & 2,222 & 0,129 & 0,301 & 1,259 & 0,379 & 0,169 & 105 & 287 & $-3,7$ \\
\hline 221 & 287 & 0,037 & 0,846 & 0,052 & 0,643 & 0,722 & 0,760 & 0,367 & 0,656 & 1,235 & 0,447 & 0,479 & 0,326 & 0,156 & 0,152 & 36 & 287 & $-1,7$ \\
\hline 222 & 287 & 0,016 & 0,368 & 0,017 & 0,296 & 0,917 & 0,805 & 0,212 & 1,000 & 0,826 & 0,824 & 0,485 & 0,156 & 0,076 & 0,107 & 85 & 287 & $-0,6$ \\
\hline 223 & 287 & 0,072 & 0,960 & 0,079 & 0,837 & 0,909 & 0,872 & 0,157 & 0,979 & 1,011 & 0,563 & 0,496 & 0,403 & 0,200 & 0,214 & 37 & 287 & $-2,0$ \\
\hline 224 & 287 & 0,073 & 1,082 & 0,083 & 1,040 & 0,879 & 0,961 & 0,127 & 0,785 & 1,128 & 0,414 & 0,393 & 0,475 & 0,186 & 0,214 & 151 & 287 & $-2,2$ \\
\hline 225 & 287 & 0,032 & 0,583 & 0,033 & 0,418 & 0,957 & 0,718 & 0,286 & 1,000 & 0,926 & 0,622 & 0,447 & 0,254 & 0,114 & 0,152 & 17 & 287 & $-1,3$ \\
\hline 226 & 287 & 0,030 & 0,643 & 0,033 & 0,520 & 0,913 & 0,809 & 0,210 & 0,917 & 1,044 & 0,594 & 0,534 & 0,254 & 0,136 & 0,152 & 60 & 287 & $-1,3$ \\
\hline
\end{tabular}




\begin{tabular}{|c|c|c|c|c|c|c|c|c|c|c|c|c|c|c|c|c|c|c|}
\hline 227 & 287 & 0,069 & 0,909 & 0,077 & 0,786 & 0,889 & 0,865 & 0,175 & 1,000 & 0,977 & 0,849 & 0,738 & 0,321 & 0,237 & 0,240 & 46 & 287 & $-1,7$ \\
\hline 228 & 287 & 0,014 & 0,317 & 0,014 & 0,194 & 1,000 & 0,613 & 0,387 & 1,000 & 0,746 & 0,979 & 0,555 & 0,137 & 0,076 & 0,107 & 18 & 287 & $-0,5$ \\
\hline 229 & 287 & 0,032 & 0,592 & 0,034 & 0,532 & 0,917 & 0,900 & 0,130 & 1,000 & 0,940 & 0,700 & 0,600 & 0,240 & 0,144 & 0,169 & 79 & 287 & $-1,3$ \\
\hline 230 & 287 & 0,154 & 2,355 & 0,221 & 1,653 & 0,695 & 0,702 & 0,427 & 0,348 & 1,695 & 0,404 & 0,561 & 0,695 & 0,390 & 0,152 & 65 & 287 & $-2,8$ \\
\hline 231 & 287 & 0,040 & 0,643 & 0,042 & 0,520 & 0,966 & 0,809 & 0,194 & 1,000 & 0,904 & 0,686 & 0,490 & 0,273 & 0,134 & 0,169 & 51 & 287 & $-1,4$ \\
\hline 232 & 287 & 0,043 & 0,685 & 0,046 & 0,583 & 0,938 & 0,852 & 0,161 & 1,000 & 0,931 & 0,849 & 0,745 & 0,254 & 0,189 & 0,214 & 175 & 287 & $-1,3$ \\
\hline 233 & 287 & 0,076 & 1,082 & 0,090 & 0,960 & 0,841 & 0,887 & 0,195 & 0,816 & 1,107 & 0,519 & 0,521 & 0,432 & 0,225 & 0,227 & 176 & 287 & $-2,1$ \\
\hline 234 & 287 & 0,017 & 0,389 & 0,017 & 0,296 & 1,000 & 0,761 & 0,239 & 1,000 & 0,836 & 0,764 & 0,447 & 0,169 & 0,076 & 0,107 & 18 & 287 & $-0,8$ \\
\hline 235 & 287 & 0,112 & 1,501 & 0,139 & 1,348 & 0,804 & 0,899 & 0,221 & 0,625 & 1,265 & 0,375 & 0,430 & 0,617 & 0,265 & 0,214 & 4 & 287 & $-2,6$ \\
\hline 236 & 287 & 0,026 & 0,490 & 0,026 & 0,397 & 1,000 & 0,811 & 0,189 & 1,000 & 0,861 & 0,917 & 0,667 & 0,189 & 0,126 & 0,152 & 41 & 287 & $-0,9$ \\
\hline 237 & 287 & 0,022 & 0,541 & 0,027 & 0,461 & 0,789 & 0,851 & 0,258 & 0,924 & 1,040 & 0,516 & 0,441 & 0,230 & 0,102 & 0,107 & 59 & 287 & $-1,2$ \\
\hline 238 & 287 & 0,142 & 1,429 & 0,154 & 1,277 & 0,925 & 0,893 & 0,130 & 0,874 & 1,069 & 0,485 & 0,472 & 0,611 & 0,288 & 0,303 & 66 & 287 & $-2,6$ \\
\hline 239 & 287 & 0,016 & 0,440 & 0,019 & 0,317 & 0,846 & 0,721 & 0,318 & 1,000 & 0,987 & 0,700 & 0,597 & 0,169 & 0,101 & 0,107 & 18 & 287 & $-0,8$ \\
\hline 240 & 287 & 0,022 & 0,440 & 0,023 & 0,389 & 0,938 & 0,885 & 0,131 & 1,000 & 0,845 & 0,955 & 0,637 & 0,169 & 0,108 & 0,152 & 117 & 287 & $-0,8$ \\
\hline 241 & 287 & 0,014 & 0,347 & 0,014 & 0,266 & 1,000 & 0,768 & 0,232 & 1,000 & 0,816 & 0,749 & 0,485 & 0,156 & 0,076 & 0,107 & 72 & 287 & $-0,6$ \\
\hline 242 & 287 & 0,055 & 1,306 & 0,092 & 0,917 & 0,594 & 0,702 & 0,504 & 0,402 & 1,578 & 0,372 & 0,591 & 0,432 & 0,255 & 0,107 & 16 & 287 & $-2,1$ \\
\hline 243 & 287 & 0,024 & 0,469 & 0,027 & 0,440 & 0,895 & 0,937 & 0,123 & 1,000 & 0,848 & 1,082 & 0,791 & 0,169 & 0,134 & 0,169 & 138 & 287 & $-0,8$ \\
\hline 244 & 287 & 0,022 & 0,541 & 0,024 & 0,389 & 0,882 & 0,719 & 0,305 & 0,924 & 1,040 & 0,764 & 0,667 & 0,189 & 0,126 & 0,152 & 0 & 287 & $-0,9$ \\
\hline 245 & 287 & 0,017 & 0,368 & 0,017 & 0,287 & 1,000 & 0,781 & 0,219 & 1,000 & 0,791 & 0,849 & 0,500 & 0,161 & 0,080 & 0,107 & 45 & 287 & $-0,7$ \\
\hline 246 & 287 & 0,141 & 1,429 & 0,154 & 1,378 & 0,916 & 0,964 & 0,091 & 0,866 & 1,075 & 0,480 & 0,468 & 0,611 & 0,286 & 0,273 & 151 & 287 & $-2,6$ \\
\hline 247 & 287 & 0,016 & 0,368 & 0,016 & 0,368 & 1,000 & 1,000 & 0,000 & 1,000 & 0,826 & 0,824 & 0,485 & 0,156 & 0,076 & 0,107 & 0 & 287 & $-0,6$ \\
\hline 248 & 287 & 0,065 & 0,858 & 0,067 & 0,765 & 0,957 & 0,892 & 0,116 & 1,000 & 0,953 & 0,716 & 0,637 & 0,339 & 0,216 & 0,227 & 19 & 287 & $-1,8$ \\
\hline 249 & 287 & 0,096 & 1,665 & 0,126 & 1,298 & 0,761 & 0,779 & 0,325 & 0,436 & 1,515 & 0,327 & 0,344 & 0,612 & 0,210 & 0,152 & 111 & 287 & $-2,6$ \\
\hline 250 & 287 & 0,088 & 1,695 & 0,123 & 1,052 & 0,709 & 0,621 & 0,478 & 0,383 & 1,616 & 0,521 & 0,682 & 0,462 & 0,315 & 0,214 & 134 & 287 & $-2,2$ \\
\hline 251 & 287 & 0,019 & 0,482 & 0,022 & 0,347 & 0,867 & 0,720 & 0,310 & 1,000 & 0,995 & 0,571 & 0,365 & 0,204 & 0,074 & 0,076 & 7 & 287 & $-1,0$ \\
\hline 252 & 287 & 0,027 & 0,490 & 0,027 & 0,410 & 1,000 & 0,836 & 0,164 & 1,000 & 0,838 & 0,968 & 0,707 & 0,189 & 0,134 & 0,169 & 45 & 287 & $-0,9$ \\
\hline 253 & 287 & 0,024 & 0,550 & 0,029 & 0,490 & 0,850 & 0,892 & 0,185 & 1,000 & 0,993 & 0,541 & 0,396 & 0,240 & 0,095 & 0,107 & 63 & 287 & $-1,3$ \\
\hline 254 & 287 & 0,274 & 2,021 & 0,296 & 1,940 & 0,927 & 0,960 & 0,083 & 0,843 & 1,089 & 0,510 & 0,545 & 0,827 & 0,451 & 0,442 & 110 & 287 & $-3,0$ \\
\hline 255 & 287 & 0,020 & 0,469 & 0,023 & 0,368 & 0,875 & 0,784 & 0,250 & 1,000 & 0,934 & 0,713 & 0,566 & 0,189 & 0,107 & 0,152 & 18 & 287 & $-0,9$ \\
\hline 256 & 287 & 0,016 & 0,347 & 0,016 & 0,296 & 1,000 & 0,854 & 0,146 & 1,000 & 0,778 & 1,077 & 0,588 & 0,137 & 0,080 & 0,107 & 72 & 287 & $-0,5$ \\
\hline 257 & 287 & 0,029 & 0,786 & 0,039 & 0,583 & 0,741 & 0,742 & 0,366 & 0,583 & 1,309 & 0,392 & 0,372 & 0,305 & 0,114 & 0,152 & 178 & 287 & $-1,6$ \\
\hline 258 & 287 & 0,026 & 0,744 & 0,036 & 0,550 & 0,720 & 0,739 & 0,383 & 0,586 & 1,306 & 0,573 & 0,664 & 0,240 & 0,159 & 0,152 & 142 & 287 & $-1,3$ \\
\hline 259 & 287 & 0,088 & 1,019 & 0,093 & 0,989 & 0,938 & 0,971 & 0,068 & 1,000 & 0,972 & 0,670 & 0,591 & 0,408 & 0,241 & 0,273 & 29 & 287 & $-2,0$ \\
\hline 260 & 287 & 0,023 & 0,469 & 0,024 & 0,389 & 0,941 & 0,829 & 0,181 & 1,000 & 0,874 & 1,019 & 0,701 & 0,169 & 0,119 & 0,152 & 49 & 287 & $-0,8$ \\
\hline 261 & 287 & 0,468 & 3,115 & 0,552 & 2,756 & 0,847 & 0,885 & 0,192 & 0,606 & 1,285 & 0,392 & 0,455 & 1,233 & 0,561 & 0,152 & 5 & 287 & $-3,6$ \\
\hline 262 & 287 & 0,029 & 0,511 & 0,030 & 0,347 & 0,952 & 0,678 & 0,326 & 1,000 & 0,852 & 1,019 & 0,800 & 0,189 & 0,152 & 0,152 & 75 & 287 & $-0,9$ \\
\hline 263 & 287 & 0,143 & 1,429 & 0,156 & 1,298 & 0,917 & 0,908 & 0,123 & 0,883 & 1,064 & 0,621 & 0,559 & 0,542 & 0,303 & 0,303 & 83 & 287 & $-2,4$ \\
\hline 264 & 287 & 0,024 & 0,499 & 0,024 & 0,448 & 1,000 & 0,898 & 0,102 & 1,000 & 0,901 & 0,637 & 0,364 & 0,221 & 0,080 & 0,107 & 40 & 287 & $-1,1$ \\
\hline 265 & 287 & 0,077 & 0,879 & 0,077 & 0,757 & 1,000 & 0,861 & 0,139 & 1,000 & 0,891 & 0,809 & 0,690 & 0,349 & 0,241 & 0,273 & 46 & 287 & $-1,8$ \\
\hline 266 & 287 & 0,069 & 1,243 & 0,092 & 0,938 & 0,750 & 0,755 & 0,350 & 0,560 & 1,336 & 0,421 & 0,499 & 0,456 & 0,228 & 0,169 & 59 & 287 & $-2,2$ \\
\hline 267 & 287 & 0,020 & 0,490 & 0,023 & 0,389 & 0,875 & 0,793 & 0,242 & 1,000 & 0,976 & 0,686 & 0,392 & 0,193 & 0,076 & 0,107 & 165 & 287 & $-0,9$ \\
\hline 268 & 287 & 0,067 & 1,019 & 0,076 & 0,947 & 0,887 & 0,930 & 0,133 & 0,816 & 1,107 & 0,460 & 0,412 & 0,432 & 0,178 & 0,169 & 49 & 287 & $-2,1$ \\
\hline 269 & 287 & 0,023 & 0,440 & 0,024 & 0,317 & 0,941 & 0,721 & 0,285 & 1,000 & 0,818 & 1,132 & 0,707 & 0,161 & 0,114 & 0,152 & 0 & 287 & $-0,7$ \\
\hline 270 & 287 & 0,020 & 0,440 & 0,022 & 0,338 & 0,933 & 0,769 & 0,240 & 1,000 & 0,875 & 0,686 & 0,392 & 0,193 & 0,076 & 0,152 & 81 & 287 & $-0,9$ \\
\hline 271 & 287 & 0,042 & 0,909 & 0,052 & 0,706 & 0,806 & 0,777 & 0,296 & 0,633 & 1,257 & 0,434 & 0,325 & 0,349 & 0,114 & 0,107 & 91 & 287 & $-1,8$ \\
\hline 272 & 287 & 0,565 & 2,963 & 0,613 & 2,688 & 0,923 & 0,907 & 0,121 & 0,809 & 1,112 & 0,632 & 0,641 & 1,067 & 0,685 & 0,682 & 89 & 287 & $-3,4$ \\
\hline 273 & 287 & 0,019 & 0,368 & 0,019 & 0,245 & 1,000 & 0,667 & 0,333 & 1,000 & 0,760 & 1,273 & 0,784 & 0,137 & 0,107 & 0,152 & 90 & 287 & $-0,5$ \\
\hline 274 & 287 & 0,024 & 0,511 & 0,027 & 0,368 & 0,895 & 0,719 & 0,300 & 1,000 & 0,924 & 0,833 & 0,588 & 0,193 & 0,114 & 0,152 & 5 & 287 & $-0,9$ \\
\hline 275 & 287 & 0,100 & 1,285 & 0,121 & 1,052 & 0,833 & 0,819 & 0,246 & 0,764 & 1,144 & 0,686 & 0,702 & 0,432 & 0,303 & 0,273 & 71 & 287 & $-2,1$ \\
\hline 276 & 287 & 0,419 & 2,591 & 0,455 & 2,388 & 0,921 & 0,922 & 0,111 & 0,784 & 1,129 & 0,533 & 0,562 & 1,000 & 0,562 & 0,546 & 134 & 287 & $-3,3$ \\
\hline 277 & 287 & 0,027 & 0,511 & 0,029 & 0,410 & 0,950 & 0,801 & 0,205 & 1,000 & 0,874 & 0,968 & 0,759 & 0,189 & 0,144 & 0,152 & 80 & 287 & $-0,9$ \\
\hline 278 & 287 & 0,075 & 0,947 & 0,077 & 0,909 & 0,963 & 0,960 & 0,055 & 1,000 & 0,978 & 0,662 & 0,633 & 0,379 & 0,240 & 0,240 & 92 & 287 & $-1,9$ \\
\hline 279 & 287 & 0,083 & 2,110 & 0,128 & 1,142 & 0,652 & 0,541 & 0,576 & 0,235 & 2,063 & 0,568 & 0,818 & 0,432 & 0,353 & 0,107 & 78 & 287 & $-2,1$ \\
\hline 280 & 287 & 0,022 & 0,440 & 0,024 & 0,317 & 0,882 & 0,721 & 0,303 & 1,000 & 0,845 & 1,061 & 0,707 & 0,161 & 0,114 & 0,152 & 90 & 287 & $-0,7$ \\
\hline 281 & 287 & 0,360 & 3,449 & 0,459 & 2,591 & 0,784 & 0,751 & 0,329 & 0,380 & 1,621 & 0,378 & 0,459 & 1,101 & 0,505 & 0,152 & 51 & 287 & $-3,5$ \\
\hline 282 & 287 & 0,023 & 0,520 & 0,024 & 0,418 & 0,941 & 0,805 & 0,204 & 1,000 & 0,968 & 0,497 & 0,331 & 0,243 & 0,080 & 0,107 & 52 & 287 & $-1,3$ \\
\hline 283 & 287 & 0,033 & 0,778 & 0,039 & 0,655 & 0,852 & 0,842 & 0,216 & 0,686 & 1,207 & 0,293 & 0,200 & 0,379 & 0,076 & 0,152 & 173 & 287 & $-1,9$ \\
\hline 284 & 287 & 0,024 & 0,520 & 0,027 & 0,490 & 0,895 & 0,943 & 0,120 & 1,000 & 0,939 & 0,585 & 0,441 & 0,230 & 0,102 & 0,152 & 23 & 287 & $-1,2$ \\
\hline
\end{tabular}




\begin{tabular}{|c|c|c|c|c|c|c|c|c|c|c|c|c|c|c|c|c|c|c|}
\hline 285 & 287 & 0,053 & 0,807 & 0,059 & 0,672 & 0,902 & 0,833 & 0,194 & 1,000 & 0,988 & 0,654 & 0,583 & 0,321 & 0,187 & 0,214 & 46 & 287 & $-1,7$ \\
\hline 286 & 287 & 0,108 & 1,234 & 0,116 & 1,163 & 0,926 & 0,942 & 0,094 & 0,887 & 1,062 & 0,492 & 0,469 & 0,528 & 0,248 & 0,273 & 148 & 287 & $-2,4$ \\
\hline 287 & 287 & 0,036 & 0,613 & 0,039 & 0,541 & 0,926 & 0,883 & 0,139 & 1,000 & 0,913 & 0,776 & 0,663 & 0,243 & 0,161 & 0,214 & 47 & 287 & $-1,3$ \\
\hline 288 & 287 & 0,052 & 0,714 & 0,053 & 0,643 & 0,973 & 0,899 & 0,104 & 1,000 & 0,887 & 1,019 & 0,800 & 0,254 & 0,203 & 0,214 & 34 & 287 & $-1,3$ \\
\hline 289 & 287 & 0,036 & 0,571 & 0,036 & 0,520 & 1,000 & 0,911 & 0,089 & 1,000 & 0,850 & 0,860 & 0,658 & 0,230 & 0,152 & 0,214 & 12 & 287 & $-1,2$ \\
\hline 290 & 287 & 0,987 & 4,332 & 1,055 & 3,795 & 0,936 & 0,876 & 0,140 & 0,661 & 1,230 & 0,515 & 0,481 & 1,562 & 0,751 & 0,758 & 94 & 287 & $-4,0$ \\
\hline 291 & 287 & 0,040 & 0,917 & 0,052 & 0,634 & 0,778 & 0,691 & 0,381 & 0,600 & 1,291 & 0,524 & 0,485 & 0,312 & 0,152 & 0,169 & 93 & 287 & $-1,6$ \\
\hline 292 & 287 & 0,125 & 1,462 & 0,143 & 1,369 & 0,870 & 0,936 & 0,145 & 0,734 & 1,168 & 0,418 & 0,430 & 0,617 & 0,265 & 0,273 & 5 & 287 & $-2,6$ \\
\hline 293 & 287 & 0,027 & 0,490 & 0,027 & 0,368 & 1,000 & 0,750 & 0,250 & 1,000 & 0,838 & 0,968 & 0,707 & 0,189 & 0,134 & 0,152 & 32 & 287 & $-0,9$ \\
\hline 294 & 287 & 0,024 & 0,461 & 0,026 & 0,317 & 0,944 & 0,688 & 0,317 & 1,000 & 0,832 & 1,082 & 0,671 & 0,169 & 0,114 & 0,152 & 84 & 287 & $-0,8$ \\
\hline 295 & 287 & 0,055 & 0,968 & 0,072 & 0,816 & 0,760 & 0,843 & 0,287 & 0,731 & 1,170 & 0,479 & 0,555 & 0,381 & 0,211 & 0,169 & 106 & 287 & $-1,9$ \\
\hline 296 & 287 & 0,024 & 0,592 & 0,029 & 0,469 & 0,850 & 0,793 & 0,256 & 0,875 & 1,069 & 0,433 & 0,300 & 0,268 & 0,080 & 0,107 & 45 & 287 & $-1,4$ \\
\hline 297 & 287 & 0,014 & 0,317 & 0,014 & 0,194 & 1,000 & 0,613 & 0,387 & 1,000 & 0,746 & 0,979 & 0,555 & 0,137 & 0,076 & 0,107 & 18 & 287 & $-0,5$ \\
\hline 298 & 287 & 0,056 & 0,786 & 0,057 & 0,714 & 0,975 & 0,909 & 0,095 & 1,000 & 0,938 & 0,621 & 0,531 & 0,339 & 0,180 & 0,214 & 22 & 287 & $-1,8$ \\
\hline 299 & 287 & 0,014 & 0,317 & 0,014 & 0,194 & 1,000 & 0,613 & 0,387 & 1,000 & 0,746 & 1,273 & 0,632 & 0,120 & 0,076 & 0,152 & 0 & 287 & $-0,3$ \\
\hline 300 & 287 & 0,020 & 0,397 & 0,020 & 0,338 & 1,000 & 0,851 & 0,149 & 1,000 & 0,791 & 1,049 & 0,686 & 0,156 & 0,107 & 0,152 & 73 & 287 & $-0,6$ \\
\hline 301 & 287 & 0,069 & 0,896 & 0,073 & 0,837 & 0,941 & 0,934 & 0,089 & 1,000 & 0,964 & 0,687 & 0,525 & 0,357 & 0,187 & 0,214 & 45 & 287 & $-1,8$ \\
\hline 302 & 287 & 0,069 & 1,031 & 0,086 & 0,909 & 0,800 & 0,881 & 0,233 & 0,814 & 1,109 & 0,611 & 0,696 & 0,379 & 0,264 & 0,227 & 39 & 287 & $-1,9$ \\
\hline 303 & 287 & 0,095 & 1,133 & 0,106 & 1,019 & 0,892 & 0,899 & 0,148 & 0,927 & 1,039 & 0,771 & 0,745 & 0,395 & 0,295 & 0,273 & 149 & 287 & $-2,0$ \\
\hline 304 & 287 & 0,037 & 0,613 & 0,040 & 0,532 & 0,929 & 0,869 & 0,149 & 1,000 & 0,895 & 0,736 & 0,580 & 0,254 & 0,147 & 0,169 & 66 & 287 & $-1,3$ \\
\hline 305 & 287 & 0,022 & 0,410 & 0,022 & 0,266 & 1,000 & 0,650 & 0,350 & 1,000 & 0,788 & 1,061 & 0,707 & 0,161 & 0,114 & 0,152 & 135 & 287 & $-0,7$ \\
\hline 306 & 287 & 0,024 & 0,469 & 0,024 & 0,440 & 1,000 & 0,937 & 0,063 & 1,000 & 0,848 & 0,866 & 0,566 & 0,189 & 0,107 & 0,152 & 45 & 287 & $-0,9$ \\
\hline 307 & 287 & 0,023 & 0,735 & 0,032 & 0,592 & 0,727 & 0,805 & 0,335 & 0,533 & 1,369 & 0,255 & 0,248 & 0,339 & 0,084 & 0,107 & 28 & 287 & $-1,8$ \\
\hline 308 & 287 & 0,628 & 4,346 & 0,821 & 3,323 & 0,766 & 0,765 & 0,332 & 0,418 & 1,546 & 0,452 & 0,616 & 1,331 & 0,819 & 0,152 & 2 & 287 & $-3,7$ \\
\hline 309 & 287 & 0,032 & 0,562 & 0,034 & 0,469 & 0,917 & 0,835 & 0,185 & 1,000 & 0,893 & 0,875 & 0,633 & 0,214 & 0,136 & 0,152 & 23 & 287 & $-1,1$ \\
\hline 310 & 287 & 0,022 & 0,469 & 0,023 & 0,368 & 0,938 & 0,784 & 0,225 & 1,000 & 0,902 & 0,955 & 0,791 & 0,169 & 0,134 & 0,152 & 30 & 287 & $-0,8$ \\
\hline 311 & 287 & 0,019 & 0,418 & 0,020 & 0,338 & 0,929 & 0,808 & 0,205 & 1,000 & 0,864 & 0,828 & 0,501 & 0,169 & 0,085 & 0,107 & 23 & 287 & $-0,8$ \\
\hline 312 & 287 & 0,016 & 0,389 & 0,017 & 0,245 & 0,917 & 0,631 & 0,379 & 1,000 & 0,873 & 0,824 & 0,485 & 0,156 & 0,076 & 0,107 & 5 & 287 & $-0,6$ \\
\hline 313 & 287 & 0,077 & 1,234 & 0,098 & 1,052 & 0,794 & 0,853 & 0,253 & 0,639 & 1,251 & 0,397 & 0,409 & 0,498 & 0,204 & 0,214 & 103 & 287 & $-2,3$ \\
\hline 314 & 287 & 0,044 & 0,693 & 0,046 & 0,592 & 0,969 & 0,854 & 0,150 & 1,000 & 0,927 & 0,759 & 0,588 & 0,273 & 0,161 & 0,214 & 35 & 287 & $-1,4$ \\
\hline 315 & 287 & 0,027 & 0,664 & 0,033 & 0,571 & 0,826 & 0,860 & 0,223 & 0,778 & 1,134 & 0,417 & 0,374 & 0,288 & 0,108 & 0,152 & 21 & 287 & $-1,5$ \\
\hline 316 & 287 & 0,125 & 1,602 & 0,171 & 1,378 & 0,731 & 0,860 & 0,303 & 0,611 & 1,279 & 0,426 & 0,502 & 0,611 & 0,306 & 0,214 & 25 & 287 & $-2,6$ \\
\hline 317 & 287 & 0,049 & 0,960 & 0,062 & 0,774 & 0,791 & 0,806 & 0,285 & 0,666 & 1,226 & 0,541 & 0,553 & 0,339 & 0,187 & 0,169 & 43 & 287 & $-1,8$ \\
\hline 318 & 287 & 0,155 & 1,754 & 0,178 & 1,306 & 0,871 & 0,745 & 0,286 & 0,633 & 1,257 & 0,611 & 0,660 & 0,568 & 0,375 & 0,312 & 67 & 287 & $-2,5$ \\
\hline 319 & 287 & 0,024 & 0,541 & 0,027 & 0,418 & 0,895 & 0,773 & 0,250 & 1,000 & 0,977 & 0,637 & 0,550 & 0,221 & 0,121 & 0,152 & 135 & 287 & $-1,1$ \\
\hline 320 & 287 & 0,044 & 0,795 & 0,046 & 0,723 & 0,969 & 0,910 & 0,096 & 0,885 & 1,063 & 0,395 & 0,283 & 0,379 & 0,107 & 0,169 & 40 & 287 & $-1,9$ \\
\hline 321 & 287 & 0,017 & 0,368 & 0,017 & 0,215 & 1,000 & 0,586 & 0,414 & 1,000 & 0,791 & 0,899 & 0,485 & 0,156 & 0,076 & 0,152 & 0 & 287 & $-0,6$ \\
\hline 322 & 287 & 0,082 & 1,408 & 0,098 & 1,154 & 0,838 & 0,820 & 0,242 & 0,519 & 1,389 & 0,333 & 0,371 & 0,559 & 0,207 & 0,227 & 31 & 287 & $-2,5$ \\
\hline 323 & 287 & 0,050 & 0,714 & 0,053 & 0,613 & 0,946 & 0,858 & 0,152 & 1,000 & 0,899 & 0,990 & 0,800 & 0,254 & 0,203 & 0,227 & 135 & 287 & $-1,3$ \\
\hline 324 & 287 & 0,106 & 1,133 & 0,106 & 1,082 & 1,000 & 0,955 & 0,045 & 1,000 & 0,981 & 0,558 & 0,462 & 0,492 & 0,227 & 0,303 & 3 & 287 & $-2,3$ \\
\hline 325 & 287 & 0,072 & 1,285 & 0,093 & 1,073 & 0,769 & 0,835 & 0,284 & 0,546 & 1,354 & 0,430 & 0,399 & 0,461 & 0,184 & 0,214 & 77 & 287 & $-2,2$ \\
\hline 326 & 287 & 0,023 & 0,541 & 0,027 & 0,376 & 0,842 & 0,696 & 0,343 & 0,985 & 1,007 & 1,019 & 0,869 & 0,169 & 0,147 & 0,152 & 130 & 287 & $-0,8$ \\
\hline 327 & 287 & 0,119 & 1,226 & 0,133 & 1,103 & 0,892 & 0,900 & 0,147 & 0,996 & 1,002 & 0,771 & 0,769 & 0,443 & 0,341 & 0,339 & 60 & 287 & $-2,1$ \\
\hline 328 & 287 & 0,027 & 0,520 & 0,029 & 0,440 & 0,950 & 0,845 & 0,163 & 1,000 & 0,888 & 0,712 & 0,485 & 0,221 & 0,107 & 0,152 & 47 & 287 & $-1,1$ \\
\hline 329 & 287 & 0,014 & 0,347 & 0,014 & 0,296 & 1,000 & 0,854 & 0,146 & 1,000 & 0,816 & 0,749 & 0,485 & 0,156 & 0,076 & 0,107 & 18 & 287 & $-0,6$ \\
\hline 330 & 287 & 0,270 & 2,346 & 0,324 & 2,139 & 0,832 & 0,912 & 0,190 & 0,616 & 1,274 & 0,437 & 0,471 & 0,887 & 0,418 & 0,386 & 38 & 287 & $-3,1$ \\
\hline 331 & 287 & 0,057 & 1,378 & 0,077 & 0,816 & 0,741 & 0,592 & 0,483 & 0,380 & 1,623 & 0,520 & 0,588 & 0,375 & 0,220 & 0,107 & 33 & 287 & $-1,9$ \\
\hline 332 & 287 & 0,014 & 0,317 & 0,014 & 0,173 & 1,000 & 0,547 & 0,453 & 1,000 & 0,746 & 1,273 & 0,632 & 0,120 & 0,076 & 0,107 & 18 & 287 & $-0,3$ \\
\hline 333 & 287 & 0,098 & 1,763 & 0,142 & 1,234 & 0,687 & 0,700 & 0,434 & 0,394 & 1,592 & 0,500 & 0,686 & 0,498 & 0,342 & 0,169 & 164 & 287 & $-2,3$ \\
\hline 334 & 287 & 0,076 & 2,017 & 0,179 & 1,543 & 0,424 & 0,765 & 0,622 & 0,235 & 2,063 & 0,208 & 0,445 & 0,682 & 0,303 & 0,107 & 85 & 287 & $-2,8$ \\
\hline 335 & 287 & 0,020 & 0,448 & 0,023 & 0,418 & 0,875 & 0,934 & 0,141 & 1,000 & 0,892 & 1,049 & 0,809 & 0,156 & 0,126 & 0,107 & 117 & 287 & $-0,6$ \\
\hline 336 & 287 & 0,032 & 0,693 & 0,037 & 0,592 & 0,846 & 0,854 & 0,212 & 0,825 & 1,101 & 0,459 & 0,453 & 0,296 & 0,134 & 0,152 & 50 & 287 & $-1,6$ \\
\hline 337 & 287 & 0,022 & 0,440 & 0,023 & 0,317 & 0,938 & 0,721 & 0,286 & 1,000 & 0,845 & 0,955 & 0,671 & 0,169 & 0,114 & 0,152 & 45 & 287 & $-0,8$ \\
\hline 338 & 287 & 0,036 & 0,960 & 0,036 & 0,592 & 1,000 & 0,617 & 0,383 & 0,490 & 1,429 & 0,707 & 0,723 & 0,254 & 0,184 & 0,169 & 39 & 287 & $-1,3$ \\
\hline 339 & 287 & 0,116 & 1,268 & 0,131 & 1,175 & 0,890 & 0,927 & 0,132 & 0,909 & 1,049 & 0,674 & 0,647 & 0,469 & 0,303 & 0,303 & 13 & 287 & $-2,2$ \\
\hline 340 & 287 & 0,036 & 0,685 & 0,039 & 0,613 & 0,926 & 0,895 & 0,128 & 0,961 & 1,020 & 0,490 & 0,388 & 0,305 & 0,119 & 0,152 & 21 & 287 & $-1,6$ \\
\hline 341 & 287 & 0,050 & 0,825 & 0,055 & 0,795 & 0,921 & 0,964 & 0,087 & 0,928 & 1,038 & 0,501 & 0,375 & 0,357 & 0,134 & 0,169 & 45 & 287 & $-1,8$ \\
\hline 342 & 287 & 0,019 & 0,511 & 0,022 & 0,368 & 0,867 & 0,719 & 0,311 & 0,896 & 1,056 & 0,414 & 0,251 & 0,240 & 0,060 & 0,076 & 17 & 287 & $-1,3$ \\
\hline
\end{tabular}




\begin{tabular}{|c|c|c|c|c|c|c|c|c|c|c|c|c|c|c|c|c|c|c|}
\hline 343 & 287 & 0,049 & 0,714 & 0,050 & 0,613 & 0,971 & 0,858 & 0,145 & 1,000 & 0,912 & 0,833 & 0,686 & 0,273 & 0,187 & 0,214 & 131 & 287 & $-1,4$ \\
\hline 344 & 287 & 0,032 & 0,592 & 0,033 & 0,490 & 0,957 & 0,828 & 0,177 & 1,000 & 0,940 & 0,757 & 0,658 & 0,230 & 0,152 & 0,169 & 99 & 287 & $-1,2$ \\
\hline 345 & 287 & 0,020 & 0,397 & 0,020 & 0,368 & 1,000 & 0,925 & 0,075 & 1,000 & 0,791 & 1,049 & 0,686 & 0,156 & 0,107 & 0,152 & 45 & 287 & $-0,6$ \\
\hline 346 & 287 & 0,017 & 0,490 & 0,023 & 0,368 & 0,750 & 0,750 & 0,354 & 0,900 & 1,054 & 0,477 & 0,354 & 0,214 & 0,076 & 0,076 & 58 & 287 & $-1,1$ \\
\hline 347 & 287 & 0,083 & 1,399 & 0,103 & 0,981 & 0,806 & 0,701 & 0,357 & 0,534 & 1,368 & 0,543 & 0,575 & 0,442 & 0,254 & 0,240 & 76 & 287 & $-2,1$ \\
\hline 348 & 287 & 0,036 & 0,807 & 0,049 & 0,613 & 0,735 & 0,759 & 0,358 & 0,692 & 1,202 & 0,436 & 0,451 & 0,324 & 0,146 & 0,152 & 12 & 287 & $-1,7$ \\
\hline 349 & 287 & 0,027 & 0,541 & 0,027 & 0,469 & 1,000 & 0,867 & 0,133 & 1,000 & 0,924 & 0,590 & 0,442 & 0,243 & 0,107 & 0,152 & 38 & 287 & $-1,3$ \\
\hline 350 & 287 & 0,050 & 0,765 & 0,053 & 0,693 & 0,946 & 0,906 & 0,108 & 1,000 & 0,963 & 0,602 & 0,493 & 0,326 & 0,161 & 0,214 & 30 & 287 & $-1,7$ \\
\hline 351 & 287 & 0,202 & 1,851 & 0,224 & 1,627 & 0,904 & 0,879 & 0,155 & 0,742 & 1,161 & 0,572 & 0,508 & 0,671 & 0,341 & 0,379 & 87 & 287 & $-2,7$ \\
\hline 352 & 287 & 0,020 & 0,461 & 0,020 & 0,368 & 1,000 & 0,798 & 0,202 & 1,000 & 0,917 & 0,615 & 0,371 & 0,204 & 0,076 & 0,152 & 9 & 287 & $-1,0$ \\
\hline 353 & 287 & 0,027 & 0,562 & 0,029 & 0,490 & 0,950 & 0,872 & 0,137 & 1,000 & 0,960 & 0,605 & 0,450 & 0,240 & 0,108 & 0,152 & 163 & 287 & $-1,3$ \\
\hline 354 & 287 & 0,066 & 0,837 & 0,069 & 0,786 & 0,958 & 0,939 & 0,074 & 1,000 & 0,919 & 1,010 & 0,892 & 0,288 & 0,257 & 0,273 & 65 & 287 & $-1,5$ \\
\hline 355 & 287 & 0,032 & 0,592 & 0,034 & 0,541 & 0,917 & 0,914 & 0,120 & 1,000 & 0,940 & 0,622 & 0,527 & 0,254 & 0,134 & 0,152 & 23 & 287 & $-1,3$ \\
\hline 356 & 287 & 0,016 & 0,347 & 0,016 & 0,338 & 1,000 & 0,975 & 0,025 & 1,000 & 0,778 & 0,875 & 0,500 & 0,152 & 0,076 & 0,152 & 0 & 287 & $-0,6$ \\
\hline 357 & 287 & 0,044 & 0,837 & 0,053 & 0,685 & 0,838 & 0,818 & 0,244 & 0,798 & 1,120 & 0,607 & 0,496 & 0,305 & 0,152 & 0,152 & 85 & 287 & $-1,6$ \\
\hline 358 & 287 & 0,017 & 0,397 & 0,019 & 0,347 & 0,923 & 0,872 & 0,149 & 1,000 & 0,854 & 0,899 & 0,543 & 0,156 & 0,085 & 0,107 & 23 & 287 & $-0,6$ \\
\hline 359 & 287 & 0,026 & 0,562 & 0,027 & 0,469 & 0,947 & 0,835 & 0,173 & 1,000 & 0,987 & 0,509 & 0,377 & 0,254 & 0,096 & 0,152 & 20 & 287 & $-1,3$ \\
\hline 360 & 287 & 0,138 & 1,644 & 0,159 & 1,420 & 0,865 & 0,864 & 0,192 & 0,640 & 1,250 & 0,421 & 0,411 & 0,645 & 0,265 & 0,214 & 91 & 287 & $-2,7$ \\
\hline 361 & 287 & 0,023 & 0,490 & 0,026 & 0,368 & 0,889 & 0,750 & 0,274 & 1,000 & 0,913 & 0,702 & 0,496 & 0,204 & 0,101 & 0,152 & 11 & 287 & $-1,0$ \\
\hline 362 & 287 & 0,023 & 0,490 & 0,026 & 0,440 & 0,889 & 0,896 & 0,152 & 1,000 & 0,913 & 0,702 & 0,496 & 0,204 & 0,101 & 0,152 & 77 & 287 & $-1,0$ \\
\hline 363 & 287 & 0,016 & 0,397 & 0,019 & 0,338 & 0,846 & 0,851 & 0,214 & 1,000 & 0,892 & 0,875 & 0,633 & 0,152 & 0,096 & 0,107 & 34 & 287 & $-0,6$ \\
\hline 364 & 287 & 0,032 & 0,664 & 0,034 & 0,541 & 0,917 & 0,815 & 0,203 & 0,901 & 1,054 & 0,539 & 0,423 & 0,273 & 0,116 & 0,152 & 34 & 287 & $-1,4$ \\
\hline 365 & 287 & 0,042 & 0,981 & 0,060 & 0,634 & 0,690 & 0,646 & 0,470 & 0,544 & 1,356 & 0,434 & 0,577 & 0,349 & 0,201 & 0,152 & 46 & 287 & $-1,8$ \\
\hline 366 & 287 & 0,024 & 0,511 & 0,024 & 0,418 & 1,000 & 0,818 & 0,182 & 1,000 & 0,924 & 0,585 & 0,329 & 0,230 & 0,076 & 0,152 & 5 & 287 & $-1,2$ \\
\hline 367 & 287 & 0,027 & 0,532 & 0,027 & 0,418 & 1,000 & 0,786 & 0,214 & 1,000 & 0,910 & 0,605 & 0,316 & 0,240 & 0,076 & 0,152 & 3 & 287 & $-1,3$ \\
\hline 368 & 287 & 0,017 & 0,389 & 0,019 & 0,275 & 0,923 & 0,707 & 0,303 & 1,000 & 0,836 & 0,764 & 0,447 & 0,169 & 0,076 & 0,107 & 13 & 287 & $-0,8$ \\
\hline 369 & 287 & 0,019 & 0,418 & 0,020 & 0,317 & 0,929 & 0,757 & 0,253 & 1,000 & 0,864 & 0,974 & 0,686 & 0,156 & 0,107 & 0,107 & 135 & 287 & $-0,6$ \\
\hline 370 & 287 & 0,017 & 0,397 & 0,017 & 0,317 & 1,000 & 0,798 & 0,202 & 1,000 & 0,854 & 0,764 & 0,474 & 0,169 & 0,080 & 0,107 & 45 & 287 & $-0,8$ \\
\hline 371 & 287 & 0,014 & 0,317 & 0,014 & 0,194 & 1,000 & 0,613 & 0,387 & 1,000 & 0,746 & 1,273 & 0,632 & 0,120 & 0,076 & 0,152 & 0 & 287 & $-0,3$ \\
\hline 372 & 287 & 0,080 & 1,082 & 0,086 & 1,010 & 0,933 & 0,934 & 0,094 & 0,862 & 1,077 & 0,422 & 0,411 & 0,492 & 0,202 & 0,214 & 22 & 287 & $-2,3$ \\
\hline 373 & 287 & 0,019 & 0,490 & 0,024 & 0,397 & 0,765 & 0,811 & 0,302 & 0,975 & 1,013 & 0,571 & 0,499 & 0,204 & 0,102 & 0,107 & 0 & 287 & $-1,0$ \\
\hline 374 & 287 & 0,108 & 1,915 & 0,133 & 1,306 & 0,806 & 0,682 & 0,372 & 0,369 & 1,647 & 0,351 & 0,424 & 0,625 & 0,265 & 0,214 & 15 & 287 & $-2,6$ \\
\hline 375 & 287 & 0,304 & 2,418 & 0,350 & 2,367 & 0,869 & 0,979 & 0,133 & 0,654 & 1,237 & 0,362 & 0,352 & 1,035 & 0,364 & 0,379 & 22 & 287 & $-3,4$ \\
\hline 376 & 287 & 0,029 & 0,520 & 0,029 & 0,469 & 1,000 & 0,902 & 0,098 & 1,000 & 0,866 & 0,878 & 0,657 & 0,204 & 0,134 & 0,169 & 27 & 287 & $-1,0$ \\
\hline 377 & 287 & 0,040 & 0,622 & 0,040 & 0,571 & 1,000 & 0,918 & 0,082 & 1,000 & 0,875 & 0,964 & 0,814 & 0,230 & 0,187 & 0,214 & 166 & 287 & $-1,2$ \\
\hline 378 & 287 & 0,049 & 0,917 & 0,065 & 0,786 & 0,756 & 0,857 & 0,283 & 0,728 & 1,172 & 0,666 & 0,689 & 0,305 & 0,210 & 0,152 & 61 & 287 & $-1,6$ \\
\hline 379 & 287 & 0,017 & 0,418 & 0,017 & 0,296 & 1,000 & 0,707 & 0,293 & 1,000 & 0,900 & 0,611 & 0,389 & 0,189 & 0,074 & 0,076 & 50 & 287 & $-0,9$ \\
\hline 380 & 287 & 0,016 & 0,418 & 0,017 & 0,317 & 0,917 & 0,757 & 0,256 & 1,000 & 0,940 & 0,560 & 0,358 & 0,189 & 0,068 & 0,076 & 32 & 287 & $-0,9$ \\
\hline 381 & 287 & 0,017 & 0,359 & 0,017 & 0,194 & 1,000 & 0,541 & 0,459 & 1,000 & 0,772 & 1,175 & 0,555 & 0,137 & 0,076 & 0,152 & 0 & 287 & $-0,5$ \\
\hline 382 & 287 & 0,043 & 0,706 & 0,046 & 0,562 & 0,938 & 0,796 & 0,213 & 1,000 & 0,960 & 0,626 & 0,543 & 0,296 & 0,161 & 0,169 & 50 & 287 & $-1,6$ \\
\hline 383 & 287 & 0,040 & 0,816 & 0,050 & 0,613 & 0,800 & 0,751 & 0,319 & 0,758 & 1,148 & 0,548 & 0,572 & 0,305 & 0,175 & 0,152 & 66 & 287 & $-1,6$ \\
\hline 384 & 287 & 0,030 & 0,888 & 0,039 & 0,571 & 0,778 & 0,643 & 0,421 & 0,480 & 1,443 & 0,594 & 0,632 & 0,254 & 0,161 & 0,152 & 128 & 287 & $-1,3$ \\
\hline 385 & 287 & 0,022 & 0,440 & 0,023 & 0,317 & 0,938 & 0,721 & 0,286 & 1,000 & 0,845 & 0,955 & 0,600 & 0,169 & 0,102 & 0,152 & 27 & 287 & $-0,8$ \\
\hline 386 & 287 & 0,066 & 1,154 & 0,077 & 0,786 & 0,852 & 0,681 & 0,351 & 0,623 & 1,267 & 0,689 & 0,673 & 0,349 & 0,235 & 0,214 & 34 & 287 & $-1,8$ \\
\hline 387 & 287 & 0,052 & 0,786 & 0,055 & 0,685 & 0,947 & 0,871 & 0,139 & 1,000 & 0,976 & 0,619 & 0,575 & 0,326 & 0,187 & 0,214 & 49 & 287 & $-1,7$ \\
\hline 388 & 287 & 0,273 & 1,847 & 0,281 & 1,767 & 0,969 & 0,956 & 0,053 & 1,000 & 0,998 & 0,712 & 0,690 & 0,698 & 0,482 & 0,461 & 53 & 287 & $-2,8$ \\
\hline 389 & 287 & 0,014 & 0,347 & 0,014 & 0,296 & 1,000 & 0,854 & 0,146 & 1,000 & 0,816 & 0,979 & 0,588 & 0,137 & 0,080 & 0,107 & 127 & 287 & $-0,5$ \\
\hline 390 & 287 & 0,080 & 1,615 & 0,099 & 0,900 & 0,812 & 0,558 & 0,481 & 0,387 & 1,607 & 0,792 & 0,738 & 0,359 & 0,265 & 0,214 & 170 & 287 & $-1,8$ \\
\hline 391 & 287 & 0,014 & 0,317 & 0,014 & 0,275 & 1,000 & 0,867 & 0,133 & 1,000 & 0,746 & 1,273 & 0,632 & 0,120 & 0,076 & 0,107 & 162 & 287 & $-0,3$ \\
\hline 392 & 287 & 0,055 & 0,807 & 0,060 & 0,664 & 0,905 & 0,822 & 0,202 & 1,000 & 0,975 & 0,605 & 0,559 & 0,339 & 0,189 & 0,227 & 22 & 287 & $-1,8$ \\
\hline 393 & 287 & 3,448 & 9,630 & 4,109 & 7,267 & 0,839 & 0,755 & 0,294 & 0,467 & 1,463 & 0,602 & 0,705 & 2,700 & 1,902 & 0,303 & 68 & 287 & $-4,8$ \\
\hline 394 & 287 & 0,026 & 0,490 & 0,026 & 0,389 & 1,000 & 0,793 & 0,207 & 1,000 & 0,861 & 0,917 & 0,707 & 0,189 & 0,134 & 0,152 & 45 & 287 & $-0,9$ \\
\hline 395 & 287 & 1,804 & 7,914 & 2,287 & 5,596 & 0,789 & 0,707 & 0,361 & 0,362 & 1,662 & 0,521 & 0,666 & 2,100 & 1,399 & 0,912 & 51 & 287 & $-4,4$ \\
\hline 396 & 287 & 0,014 & 0,511 & 0,020 & 0,368 & 0,714 & 0,719 & 0,401 & 0,690 & 1,204 & 0,318 & 0,230 & 0,240 & 0,055 & 0,076 & 0 & 287 & $-1,3$ \\
\hline 397 & 287 & 0,016 & 0,368 & 0,016 & 0,296 & 1,000 & 0,805 & 0,195 & 1,000 & 0,826 & 0,824 & 0,485 & 0,156 & 0,076 & 0,107 & 18 & 287 & $-0,6$ \\
\hline 398 & 287 & 0,019 & 0,389 & 0,019 & 0,347 & 1,000 & 0,892 & 0,108 & 1,000 & 0,803 & 0,974 & 0,485 & 0,156 & 0,076 & 0,152 & 172 & 287 & $-0,6$ \\
\hline 399 & 287 & 0,034 & 0,837 & 0,047 & 0,664 & 0,727 & 0,793 & 0,342 & 0,618 & 1,272 & 0,588 & 0,559 & 0,273 & 0,153 & 0,152 & 69 & 287 & $-1,4$ \\
\hline 400 & 287 & 0,050 & 1,112 & 0,067 & 0,837 & 0,745 & 0,753 & 0,355 & 0,511 & 1,400 & 0,441 & 0,498 & 0,381 & 0,189 & 0,169 & 154 & 287 & $-1,9$ \\
\hline
\end{tabular}




\begin{tabular}{|c|c|c|c|c|c|c|c|c|c|c|c|c|c|c|c|c|c|c|}
\hline 401 & 287 & 0,019 & 0,418 & 0,020 & 1,369 & 0,929 & 3,272 & 2,273 & 1,000 & 0,864 & 0,828 & 0,566 & 0,169 & 0,096 & 0,152 & 21 & 287 & $-0,8$ \\
\hline 402 & 287 & 0,046 & 1,073 & 0,060 & 0,562 & 0,762 & 0,524 & 0,533 & 0,501 & 1,413 & 0,627 & 0,560 & 0,305 & 0,171 & 0,169 & 73 & 287 & $-1,6$ \\
\hline 403 & 287 & 0,042 & 0,989 & 0,059 & 0,490 & 0,707 & 0,496 & 0,583 & 0,534 & 1,368 & 0,369 & 0,346 & 0,379 & 0,131 & 0,169 & 47 & 287 & $-1,9$ \\
\hline 404 & 287 & 2,093 & 8,658 & 2,689 & 0,909 & 0,779 & 0,105 & 0,922 & 0,351 & 1,688 & 0,260 & 0,313 & 3,204 & 1,003 & 0,937 & 65 & 287 & $-5,0$ \\
\hline 405 & 287 & 0,017 & 0,368 & 0,017 & 0,418 & 1,000 & 1,138 & 0,138 & 1,000 & 0,791 & 1,175 & 0,745 & 0,137 & 0,102 & 0,107 & 135 & 287 & $-0,5$ \\
\hline 406 & 287 & 0,024 & 0,499 & 0,027 & 0,816 & 0,895 & 1,635 & 0,644 & 1,000 & 0,901 & 0,833 & 0,555 & 0,193 & 0,107 & 0,152 & 27 & 287 & $-0,9$ \\
\hline 407 & 287 & 0,141 & 1,572 & 0,159 & 0,706 & 0,883 & 0,449 & 0,563 & 0,715 & 1,183 & 0,369 & 0,369 & 0,696 & 0,257 & 0,273 & 71 & 287 & $-2,8$ \\
\hline 408 & 287 & 0,047 & 0,786 & 0,052 & 0,541 & 0,917 & 0,688 & 0,323 & 0,963 & 1,019 & 0,467 & 0,422 & 0,359 & 0,152 & 0,169 & 72 & 287 & $-1,8$ \\
\hline 409 & 287 & 0,090 & 1,052 & 0,106 & 0,296 & 0,851 & 0,281 & 0,734 & 1,000 & 0,987 & 0,802 & 0,776 & 0,379 & 0,294 & 0,169 & 68 & 287 & $-1,9$ \\
\hline 410 & 287 & 0,016 & 0,389 & 0,017 & 0,765 & 0,917 & 1,968 & 0,972 & 1,000 & 0,873 & 0,700 & 0,425 & 0,169 & 0,072 & 0,076 & 69 & 287 & $-0,8$ \\
\hline 411 & 287 & 0,039 & 0,613 & 0,040 & 2,604 & 0,964 & 4,248 & 3,249 & 1,000 & 0,878 & 1,011 & 0,844 & 0,221 & 0,186 & 0,169 & 90 & 287 & $-1,1$ \\
\hline 412 & 287 & 0,036 & 0,592 & 0,039 & 0,245 & 0,926 & 0,414 & 0,590 & 1,000 & 0,881 & 0,936 & 0,728 & 0,221 & 0,161 & 0,169 & 38 & 287 & $-1,1$ \\
\hline 413 & 287 & 0,014 & 0,418 & 0,019 & 0,541 & 0,769 & 1,293 & 0,373 & 1,000 & 0,986 & 0,509 & 0,361 & 0,189 & 0,068 & 0,107 & 45 & 287 & $-0,9$ \\
\hline 414 & 287 & 0,026 & 0,795 & 0,036 & 2,050 & 0,720 & 2,579 & 1,604 & 0,514 & 1,395 & 0,270 & 0,230 & 0,349 & 0,080 & 0,076 & 46 & 287 & $-1,8$ \\
\hline 415 & 287 & 0,055 & 0,867 & 0,060 & 0,245 & 0,905 & 0,283 & 0,723 & 0,912 & 1,047 & 0,605 & 0,553 & 0,339 & 0,187 & 0,169 & 43 & 287 & $-1,8$ \\
\hline 416 & 287 & 0,037 & 0,583 & 0,037 & 0,571 & 1,000 & 0,979 & 0,021 & 1,000 & 0,852 & 0,974 & 0,686 & 0,221 & 0,152 & 0,214 & 97 & 287 & $-1,1$ \\
\hline 417 & 287 & 0,014 & 0,347 & 0,016 & 0,643 & 0,909 & 1,854 & 0,858 & 1,000 & 0,816 & 0,979 & 0,588 & 0,137 & 0,080 & 0,107 & 37 & 287 & $-0,5$ \\
\hline 418 & 287 & 0,099 & 1,437 & 0,121 & 0,693 & 0,821 & 0,482 & 0,548 & 0,602 & 1,289 & 0,453 & 0,488 & 0,528 & 0,257 & 0,273 & 79 & 287 & $-2,4$ \\
\hline 419 & 287 & 0,088 & 1,298 & 0,108 & 2,933 & 0,813 & 2,261 & 1,274 & 0,653 & 1,237 & 0,621 & 0,624 & 0,423 & 0,264 & 0,227 & 101 & 287 & $-2,1$ \\
\hline 420 & 287 & 0,158 & 1,560 & 0,176 & 0,245 & 0,894 & 0,157 & 0,849 & 0,815 & 1,108 & 0,521 & 0,468 & 0,621 & 0,291 & 0,273 & 47 & 287 & $-2,6$ \\
\hline 421 & 287 & 0,014 & 0,448 & 0,017 & 0,807 & 0,833 & 1,801 & 0,818 & 0,898 & 1,055 & 0,439 & 0,332 & 0,204 & 0,068 & 0,076 & 18 & 287 & $-1,0$ \\
\hline 422 & 287 & 0,020 & 0,440 & 0,022 & 1,061 & 0,933 & 2,414 & 1,416 & 1,000 & 0,875 & 0,891 & 0,637 & 0,169 & 0,108 & 0,152 & 0 & 287 & $-0,8$ \\
\hline 423 & 287 & 0,032 & 0,541 & 0,033 & 0,592 & 0,957 & 1,094 & 0,103 & 1,000 & 0,859 & 0,966 & 0,665 & 0,204 & 0,136 & 0,152 & 67 & 287 & $-1,0$ \\
\hline 424 & 287 & 0,039 & 0,634 & 0,042 & 1,234 & 0,931 & 1,947 & 0,950 & 1,000 & 0,909 & 0,764 & 0,596 & 0,254 & 0,152 & 0,169 & 82 & 287 & $-1,3$ \\
\hline 425 & 287 & 0,046 & 1,234 & 0,072 & 0,317 & 0,640 & 0,257 & 0,826 & 0,379 & 1,625 & 0,479 & 0,614 & 0,349 & 0,214 & 0,169 & 109 & 287 & $-1,8$ \\
\hline 426 & 287 & 0,034 & 0,583 & 0,034 & 0,347 & 1,000 & 0,594 & 0,406 & 1,000 & 0,887 & 0,764 & 0,474 & 0,240 & 0,114 & 0,152 & 94 & 287 & $-1,3$ \\
\hline 427 & 287 & 0,053 & 0,837 & 0,057 & 1,467 & 0,925 & 1,753 & 0,757 & 0,952 & 1,025 & 0,529 & 0,475 & 0,357 & 0,170 & 0,152 & 60 & 287 & $-1,8$ \\
\hline 428 & 287 & 0,042 & 0,693 & 0,044 & 0,490 & 0,935 & 0,707 & 0,300 & 1,000 & 0,959 & 0,605 & 0,453 & 0,296 & 0,134 & 0,169 & 47 & 287 & $-1,6$ \\
\hline 429 & 287 & 0,044 & 1,040 & 0,057 & 0,765 & 0,775 & 0,736 & 0,347 & 0,517 & 1,391 & 0,681 & 0,657 & 0,288 & 0,189 & 0,107 & 103 & 287 & $-1,5$ \\
\hline 430 & 287 & 0,024 & 0,541 & 0,030 & 1,572 & 0,810 & 2,906 & 1,916 & 1,000 & 0,977 & 0,585 & 0,468 & 0,230 & 0,108 & 0,152 & 66 & 287 & $-1,2$ \\
\hline 431 & 287 & 0,022 & 0,410 & 0,022 & 0,490 & 1,000 & 1,196 & 0,196 & 1,000 & 0,788 & 1,061 & 0,707 & 0,161 & 0,114 & 0,152 & 0 & 287 & $-0,7$ \\
\hline 432 & 287 & 0,017 & 0,368 & 0,017 & 0,541 & 1,000 & 1,471 & 0,471 & 1,000 & 0,791 & 1,175 & 0,784 & 0,137 & 0,107 & 0,152 & 135 & 287 & $-0,5$ \\
\hline 433 & 287 & 0,367 & 3,648 & 0,415 & 1,366 & 0,886 & 0,374 & 0,636 & 0,347 & 1,698 & 0,432 & 0,437 & 1,040 & 0,455 & 0,152 & 79 & 287 & $-3,4$ \\
\hline 434 & 287 & 0,024 & 0,469 & 0,024 & 0,275 & 1,000 & 0,586 & 0,414 & 1,000 & 0,848 & 1,082 & 0,791 & 0,169 & 0,134 & 0,169 & 70 & 287 & $-0,8$ \\
\hline 435 & 287 & 0,291 & 2,143 & 0,326 & 3,246 & 0,894 & 1,515 & 0,525 & 0,797 & 1,120 & 0,537 & 0,632 & 0,831 & 0,525 & 0,455 & 70 & 287 & $-3,1$ \\
\hline 436 & 287 & 0,023 & 0,440 & 0,023 & 0,469 & 1,000 & 1,068 & 0,068 & 1,000 & 0,818 & 1,019 & 0,671 & 0,169 & 0,114 & 0,152 & 63 & 287 & $-0,8$ \\
\hline 437 & 287 & 0,103 & 1,581 & 0,131 & 0,296 & 0,791 & 0,187 & 0,839 & 0,519 & 1,388 & 0,542 & 0,607 & 0,492 & 0,299 & 0,273 & 58 & 287 & $-2,3$ \\
\hline 438 & 287 & 0,073 & 0,879 & 0,073 & 0,786 & 1,000 & 0,894 & 0,106 & 1,000 & 0,917 & 0,812 & 0,671 & 0,339 & 0,227 & 0,273 & 15 & 287 & $-1,8$ \\
\hline 439 & 287 & 0,023 & 0,490 & 0,023 & 0,440 & 1,000 & 0,896 & 0,104 & 1,000 & 0,913 & 0,784 & 0,588 & 0,193 & 0,114 & 0,152 & 6 & 287 & $-0,9$ \\
\hline 440 & 287 & 0,029 & 0,917 & 0,044 & 0,583 & 0,645 & 0,636 & 0,509 & 0,428 & 1,528 & 0,621 & 0,674 & 0,243 & 0,164 & 0,152 & 0 & 287 & $-1,3$ \\
\hline 441 & 287 & 0,481 & 2,828 & 0,522 & 0,266 & 0,920 & 0,094 & 0,909 & 0,755 & 1,151 & 0,449 & 0,512 & 1,167 & 0,598 & 0,606 & 104 & 287 & $-3,5$ \\
\hline 442 & 287 & 0,020 & 0,448 & 0,022 & 0,672 & 0,933 & 1,500 & 0,504 & 1,000 & 0,892 & 0,891 & 0,632 & 0,169 & 0,107 & 0,107 & 107 & 287 & $-0,8$ \\
\hline 443 & 287 & 0,343 & 2,156 & 0,364 & 8,307 & 0,941 & 3,853 & 2,854 & 0,927 & 1,038 & 0,900 & 0,869 & 0,696 & 0,605 & 0,552 & 103 & 287 & $-2,8$ \\
\hline 444 & 287 & 0,056 & 0,807 & 0,059 & 0,930 & 0,951 & 1,152 & 0,159 & 1,000 & 0,963 & 0,680 & 0,585 & 0,324 & 0,189 & 0,227 & 11 & 287 & $-1,7$ \\
\hline 445 & 287 & 0,047 & 1,091 & 0,063 & 0,418 & 0,750 & 0,384 & 0,665 & 0,500 & 1,414 & 0,646 & 0,714 & 0,305 & 0,218 & 0,152 & 119 & 287 & $-1,6$ \\
\hline 446 & 287 & 0,024 & 0,672 & 0,033 & 0,317 & 0,739 & 0,471 & 0,589 & 0,678 & 1,214 & 0,433 & 0,403 & 0,268 & 0,108 & 0,107 & 163 & 287 & $-1,4$ \\
\hline 447 & 287 & 0,050 & 1,501 & 0,076 & 0,440 & 0,660 & 0,293 & 0,784 & 0,280 & 1,889 & 0,602 & 0,728 & 0,326 & 0,237 & 0,107 & 120 & 287 & $-1,7$ \\
\hline 448 & 287 & 0,032 & 0,613 & 0,034 & 0,368 & 0,917 & 0,600 & 0,409 & 1,000 & 0,973 & 0,560 & 0,424 & 0,268 & 0,114 & 0,152 & 1 & 287 & $-1,4$ \\
\hline 449 & 287 & 0,109 & 1,480 & 0,145 & 0,613 & 0,752 & 0,414 & 0,636 & 0,626 & 1,264 & 0,523 & 0,563 & 0,515 & 0,290 & 0,214 & 14 & 287 & $-2,4$ \\
\hline 450 & 287 & 0,059 & 1,560 & 0,085 & 0,960 & 0,695 & 0,615 & 0,491 & 0,304 & 1,814 & 0,418 & 0,537 & 0,423 & 0,227 & 0,107 & 96 & 287 & $-2,1$ \\
\hline 451 & 287 & 0,019 & 0,389 & 0,019 & 0,643 & 1,000 & 1,653 & 0,653 & 1,000 & 0,803 & 0,974 & 0,485 & 0,156 & 0,076 & 0,152 & 90 & 287 & $-0,6$ \\
\hline 452 & 287 & 0,033 & 0,664 & 0,034 & 0,592 & 0,958 & 0,892 & 0,116 & 0,942 & 1,031 & 0,505 & 0,421 & 0,288 & 0,121 & 0,152 & 58 & 287 & $-1,5$ \\
\hline 453 & 287 & 0,116 & 2,338 & 0,201 & 2,609 & 0,579 & 1,116 & 0,437 & 0,267 & 1,934 & 0,306 & 0,500 & 0,695 & 0,347 & 0,107 & 149 & 287 & $-2,8$ \\
\hline 454 & 287 & 0,112 & 2,021 & 0,155 & 3,141 & 0,722 & 1,555 & 0,620 & 0,344 & 1,704 & 0,667 & 0,788 & 0,462 & 0,364 & 0,240 & 178 & 287 & $-2,2$ \\
\hline 455 & 287 & 0,386 & 2,861 & 0,426 & 2,012 & 0,906 & 0,703 & 0,311 & 0,592 & 1,299 & 0,294 & 0,322 & 1,293 & 0,417 & 0,442 & 84 & 287 & $-3,7$ \\
\hline 456 & 287 & 0,063 & 1,184 & 0,086 & 7,758 & 0,733 & 6,555 & 5,561 & 0,566 & 1,329 & 0,366 & 0,411 & 0,469 & 0,192 & 0,169 & 20 & 287 & $-2,2$ \\
\hline 457 & 287 & 0,026 & 0,837 & 0,039 & 1,175 & 0,667 & 1,404 & 0,524 & 0,463 & 1,469 & 0,353 & 0,351 & 0,305 & 0,107 & 0,076 & 51 & 287 & $-1,6$ \\
\hline 458 & 287 & 0,016 & 0,338 & 0,016 & 0,317 & 1,000 & 0,938 & 0,062 & 1,000 & 0,759 & 1,077 & 0,555 & 0,137 & 0,076 & 0,152 & 0 & 287 & $-0,5$ \\
\hline
\end{tabular}




\begin{tabular}{|c|c|c|c|c|c|c|c|c|c|c|c|c|c|c|c|c|c|c|}
\hline 459 & 287 & 0,079 & 0,938 & 0,083 & 0,520 & 0,948 & 0,554 & 0,449 & 1,000 & 0,942 & 0,824 & 0,728 & 0,349 & 0,254 & 0,273 & 162 & 287 & $-1,8$ \\
\hline 460 & 287 & 0,020 & 0,418 & 0,020 & 0,613 & 1,000 & 1,465 & 0,465 & 1,000 & 0,833 & 1,049 & 0,728 & 0,156 & 0,114 & 0,152 & 0 & 287 & $-0,6$ \\
\hline 461 & 287 & 0,023 & 0,469 & 0,023 & 0,317 & 1,000 & 0,675 & 0,325 & 1,000 & 0,874 & 0,702 & 0,525 & 0,204 & 0,107 & 0,152 & 150 & 287 & $-1,0$ \\
\hline 462 & 287 & 0,016 & 0,347 & 0,016 & 0,541 & 1,000 & 1,561 & 0,561 & 1,000 & 0,778 & 1,401 & 0,894 & 0,120 & 0,107 & 0,152 & 72 & 287 & $-0,3$ \\
\hline 463 & 287 & 0,060 & 1,010 & 0,069 & 0,562 & 0,875 & 0,556 & 0,461 & 0,742 & 1,161 & 0,390 & 0,399 & 0,443 & 0,177 & 0,214 & 162 & 287 & $-2,1$ \\
\hline 464 & 287 & 0,191 & 1,543 & 0,201 & 0,397 & 0,950 & 0,258 & 0,744 & 1,000 & 0,996 & 0,753 & 0,716 & 0,568 & 0,407 & 0,386 & 71 & 287 & $-2,5$ \\
\hline 465 & 287 & 0,435 & 2,714 & 0,476 & 1,357 & 0,913 & 0,500 & 0,508 & 0,742 & 1,161 & 0,500 & 0,580 & 1,052 & 0,610 & 0,577 & 127 & 287 & $-3,4$ \\
\hline 466 & 287 & 0,016 & 0,448 & 0,016 & 0,296 & 1,000 & 0,660 & 0,340 & 0,987 & 1,006 & 0,412 & 0,243 & 0,221 & 0,054 & 0,076 & 35 & 287 & $-1,1$ \\
\hline 467 & 287 & 0,034 & 0,613 & 0,036 & 0,938 & 0,960 & 1,531 & 0,533 & 1,000 & 0,932 & 0,679 & 0,566 & 0,254 & 0,144 & 0,152 & 18 & 287 & $-1,3$ \\
\hline 468 & 287 & 0,083 & 1,082 & 0,089 & 0,714 & 0,935 & 0,660 & 0,346 & 0,893 & 1,058 & 0,434 & 0,399 & 0,494 & 0,197 & 0,214 & 60 & 287 & $-2,3$ \\
\hline 469 & 287 & 0,040 & 0,714 & 0,043 & 0,368 & 0,933 & 0,515 & 0,490 & 0,989 & 1,005 & 0,548 & 0,432 & 0,305 & 0,132 & 0,152 & 63 & 287 & $-1,6$ \\
\hline 470 & 287 & 0,017 & 0,389 & 0,017 & 0,541 & 1,000 & 1,392 & 0,392 & 1,000 & 0,836 & 0,899 & 0,485 & 0,156 & 0,076 & 0,107 & 0 & 287 & $-0,6$ \\
\hline 471 & 287 & 0,057 & 0,837 & 0,063 & 0,875 & 0,909 & 1,046 & 0,102 & 1,000 & 0,986 & 0,784 & 0,682 & 0,305 & 0,208 & 0,227 & 106 & 287 & $-1,6$ \\
\hline 472 & 287 & 0,067 & 1,437 & 0,085 & 0,410 & 0,797 & 0,285 & 0,743 & 0,410 & 1,561 & 0,178 & 0,167 & 0,695 & 0,116 & 0,152 & 155 & 287 & $-2,8$ \\
\hline 473 & 287 & 0,118 & 1,306 & 0,131 & 0,469 & 0,901 & 0,359 & 0,648 & 0,867 & 1,074 & 0,644 & 0,611 & 0,482 & 0,295 & 0,303 & 57 & 287 & $-2,3$ \\
\hline 474 & 287 & 0,037 & 0,571 & 0,037 & 0,347 & 1,000 & 0,607 & 0,393 & 1,000 & 0,834 & 1,142 & 0,788 & 0,204 & 0,161 & 0,214 & 45 & 287 & $-1,0$ \\
\hline 475 & 287 & 0,095 & 1,306 & 0,112 & 0,613 & 0,846 & 0,469 & 0,553 & 0,697 & 1,197 & 0,568 & 0,575 & 0,461 & 0,265 & 0,273 & 178 & 287 & $-2,2$ \\
\hline 476 & 287 & 0,032 & 0,643 & 0,034 & 0,562 & 0,917 & 0,875 & 0,150 & 0,961 & 1,020 & 0,459 & 0,355 & 0,296 & 0,105 & 0,152 & 37 & 287 & $-1,6$ \\
\hline 477 & 287 & 0,096 & 1,399 & 0,128 & 0,592 & 0,753 & 0,423 & 0,628 & 0,617 & 1,273 & 0,416 & 0,433 & 0,542 & 0,235 & 0,227 & 105 & 287 & $-2,4$ \\
\hline 478 & 287 & 0,052 & 0,714 & 0,053 & 0,723 & 0,973 & 1,012 & 0,030 & 1,000 & 0,887 & 1,019 & 0,843 & 0,254 & 0,214 & 0,227 & 37 & 287 & $-1,3$ \\
\hline 479 & 287 & 0,069 & 1,040 & 0,082 & 0,245 & 0,842 & 0,236 & 0,780 & 0,800 & 1,118 & 0,605 & 0,697 & 0,381 & 0,265 & 0,214 & 99 & 287 & $-1,9$ \\
\hline 480 & 287 & 0,043 & 0,930 & 0,057 & 0,418 & 0,750 & 0,450 & 0,604 & 0,626 & 1,264 & 0,516 & 0,605 & 0,326 & 0,197 & 0,152 & 117 & 287 & $-1,7$ \\
\hline 481 & 287 & 0,017 & 0,368 & 0,017 & 0,461 & 1,000 & 1,253 & 0,253 & 1,000 & 0,791 & 1,175 & 0,745 & 0,137 & 0,102 & 0,152 & 45 & 287 & $-0,5$ \\
\hline 482 & 287 & 0,019 & 0,368 & 0,019 & 0,389 & 1,000 & 1,057 & 0,057 & 1,000 & 0,760 & 1,273 & 0,784 & 0,137 & 0,107 & 0,152 & 90 & 287 & $-0,5$ \\
\hline 483 & 287 & 0,019 & 0,389 & 0,019 & 1,632 & 1,000 & 4,197 & 3,197 & 1,000 & 0,803 & 0,974 & 0,485 & 0,156 & 0,076 & 0,152 & 90 & 287 & $-0,6$ \\
\hline 484 & 287 & 0,072 & 1,196 & 0,098 & 0,194 & 0,735 & 0,163 & 0,878 & 0,630 & 1,260 & 0,439 & 0,498 & 0,456 & 0,227 & 0,169 & 85 & 287 & $-2,2$ \\
\hline 485 & 287 & 0,287 & 2,317 & 0,329 & 0,757 & 0,873 & 0,327 & 0,685 & 0,672 & 1,220 & 0,453 & 0,512 & 0,898 & 0,459 & 0,386 & 62 & 287 & $-3,2$ \\
\hline 486 & 287 & 0,042 & 0,643 & 0,042 & 0,643 & 1,000 & 1,000 & 0,000 & 1,000 & 0,889 & 0,710 & 0,490 & 0,273 & 0,134 & 0,169 & 39 & 287 & $-1,4$ \\
\hline 487 & 287 & 0,020 & 0,389 & 0,020 & 0,266 & 1,000 & 0,685 & 0,315 & 1,000 & 0,774 & 0,990 & 0,667 & 0,161 & 0,107 & 0,152 & 45 & 287 & $-0,7$ \\
\hline 488 & 287 & 0,020 & 0,410 & 0,020 & 9,511 & 1,000 & 23,207 & 22,207 & 1,000 & 0,816 & 0,990 & 0,707 & 0,161 & 0,114 & 0,152 & 45 & 287 & $-0,7$ \\
\hline 489 & 287 & 0,023 & 0,440 & 0,023 & 0,511 & 1,000 & 1,163 & 0,163 & 1,000 & 0,818 & 1,019 & 0,671 & 0,169 & 0,114 & 0,152 & 15 & 287 & $-0,8$ \\
\hline 490 & 287 & 0,947 & 6,417 & 1,468 & 0,909 & 0,645 & 0,142 & 0,929 & 0,289 & 1,860 & 0,222 & 0,381 & 2,328 & 0,887 & 0,682 & 63 & 287 & $-4,5$ \\
\hline 491 & 287 & 0,020 & 0,469 & 0,023 & 0,490 & 0,875 & 1,045 & 0,133 & 1,000 & 0,934 & 0,686 & 0,527 & 0,193 & 0,102 & 0,107 & 174 & 287 & $-0,9$ \\
\hline 492 & 287 & 0,096 & 1,184 & 0,108 & 1,889 & 0,893 & 1,596 & 0,606 & 0,862 & 1,077 & 0,520 & 0,524 & 0,485 & 0,254 & 0,227 & 38 & 287 & $-2,3$ \\
\hline 493 & 287 & 0,019 & 0,634 & 0,023 & 0,541 & 0,812 & 0,853 & 0,238 & 0,583 & 1,309 & 0,255 & 0,181 & 0,305 & 0,055 & 0,076 & 15 & 287 & $-1,6$ \\
\hline 494 & 287 & 0,766 & 4,721 & 0,934 & 0,634 & 0,820 & 0,134 & 0,884 & 0,432 & 1,521 & 0,349 & 0,427 & 1,672 & 0,713 & 0,606 & 98 & 287 & $-4,1$ \\
\hline 495 & 287 & 0,019 & 0,418 & 0,020 & 1,040 & 0,929 & 2,485 & 1,487 & 1,000 & 0,864 & 0,828 & 0,600 & 0,169 & 0,102 & 0,152 & 161 & 287 & $-0,8$ \\
\hline 496 & 287 & 0,109 & 2,283 & 0,164 & 0,194 & 0,667 & 0,085 & 0,974 & 0,263 & 1,950 & 0,523 & 0,676 & 0,515 & 0,348 & 0,273 & 62 & 287 & $-2,4$ \\
\hline 497 & 287 & 0,023 & 0,461 & 0,026 & 0,685 & 0,889 & 1,487 & 0,499 & 1,000 & 0,858 & 1,019 & 0,671 & 0,169 & 0,114 & 0,152 & 88 & 287 & $-0,8$ \\
\hline 498 & 287 & 0,067 & 0,960 & 0,076 & 0,338 & 0,887 & 0,352 & 0,658 & 0,920 & 1,042 & 0,665 & 0,612 & 0,359 & 0,220 & 0,214 & 116 & 287 & $-1,8$ \\
\hline 499 & 287 & 0,060 & 1,243 & 0,075 & 0,245 & 0,808 & 0,197 & 0,825 & 0,490 & 1,428 & 0,594 & 0,596 & 0,359 & 0,214 & 0,214 & 147 & 287 & $-1,8$ \\
\hline 500 & 287 & 0,047 & 0,786 & 0,055 & 0,245 & 0,868 & 0,312 & 0,701 & 0,963 & 1,019 & 0,568 & 0,572 & 0,326 & 0,186 & 0,169 & 36 & 287 & $-1,7$ \\
\hline 501 & 287 & 0,014 & 0,397 & 0,014 & 2,000 & 1,000 & 5,031 & 4,031 & 1,000 & 0,936 & 0,509 & 0,283 & 0,189 & 0,054 & 0,076 & 40 & 287 & $-0,9$ \\
\hline 502 & 287 & 0,014 & 0,490 & 0,020 & 2,236 & 0,714 & 4,561 & 3,572 & 0,750 & 1,155 & 0,979 & 0,832 & 0,137 & 0,114 & 0,076 & 135 & 287 & $-0,5$ \\
\hline 503 & 287 & 0,179 & 1,492 & 0,189 & 0,448 & 0,947 & 0,300 & 0,702 & 1,000 & 0,994 & 0,658 & 0,661 & 0,589 & 0,390 & 0,408 & 31 & 287 & $-2,6$ \\
\hline 504 & 287 & 0,022 & 0,562 & 0,026 & 0,266 & 0,833 & 0,474 & 0,552 & 0,856 & 1,081 & 0,562 & 0,479 & 0,221 & 0,106 & 0,076 & 162 & 287 & $-1,1$ \\
\hline 505 & 287 & 0,231 & 1,746 & 0,242 & 0,296 & 0,953 & 0,170 & 0,832 & 0,952 & 1,025 & 0,700 & 0,643 & 0,648 & 0,417 & 0,455 & 86 & 287 & $-2,7$ \\
\hline 506 & 287 & 0,022 & 0,440 & 0,024 & 0,194 & 0,882 & 0,442 & 0,570 & 1,000 & 0,845 & 0,955 & 0,671 & 0,169 & 0,114 & 0,152 & 125 & 287 & $-0,8$ \\
\hline 507 & 287 & 0,030 & 0,520 & 0,032 & 0,266 & 0,955 & 0,512 & 0,490 & 1,000 & 0,845 & 0,922 & 0,657 & 0,204 & 0,134 & 0,152 & 67 & 287 & $-1,0$ \\
\hline 508 & 287 & 0,037 & 0,643 & 0,037 & 1,995 & 1,000 & 3,104 & 2,104 & 1,000 & 0,939 & 0,662 & 0,566 & 0,268 & 0,152 & 0,169 & 76 & 287 & $-1,4$ \\
\hline 509 & 287 & 0,016 & 0,368 & 0,016 & 0,368 & 1,000 & 1,000 & 0,000 & 1,000 & 0,826 & 1,077 & 0,784 & 0,137 & 0,107 & 0,107 & 90 & 287 & $-0,5$ \\
\hline 510 & 287 & 0,022 & 0,461 & 0,022 & 0,296 & 1,000 & 0,642 & 0,358 & 1,000 & 0,886 & 0,659 & 0,371 & 0,204 & 0,076 & 0,152 & 11 & 287 & $-1,0$ \\
\hline 511 & 287 & 0,034 & 0,613 & 0,036 & 0,989 & 0,960 & 1,614 & 0,615 & 1,000 & 0,932 & 0,745 & 0,552 & 0,243 & 0,134 & 0,152 & 44 & 287 & $-1,3$ \\
\hline 512 & 287 & 0,065 & 0,960 & 0,072 & 0,440 & 0,900 & 0,458 & 0,551 & 0,881 & 1,065 & 0,494 & 0,463 & 0,408 & 0,189 & 0,227 & 75 & 287 & $-2,0$ \\
\hline 513 & 287 & 0,063 & 0,786 & 0,066 & 1,124 & 0,957 & 1,430 & 0,432 & 1,000 & 0,883 & 1,057 & 0,824 & 0,276 & 0,227 & 0,273 & 176 & 287 & $-1,5$ \\
\hline 514 & 287 & 0,033 & 0,583 & 0,034 & 0,440 & 0,958 & 0,754 & 0,250 & 1,000 & 0,906 & 0,714 & 0,559 & 0,243 & 0,136 & 0,169 & 58 & 287 & $-1,3$ \\
\hline 515 & 287 & 0,027 & 0,541 & 0,030 & 0,418 & 0,905 & 0,773 & 0,246 & 1,000 & 0,924 & 0,654 & 0,493 & 0,230 & 0,114 & 0,152 & 15 & 287 & $-1,2$ \\
\hline 516 & 287 & 0,166 & 1,674 & 0,191 & 0,592 & 0,872 & 0,354 & 0,659 & 0,746 & 1,157 & 0,603 & 0,586 & 0,593 & 0,347 & 0,321 & 27 & 287 & $-2,6$ \\
\hline
\end{tabular}




\begin{tabular}{|c|c|c|c|c|c|c|c|c|c|c|c|c|c|c|c|c|c|c|}
\hline 517 & 287 & 0,014 & 0,347 & 0,016 & 1,480 & 0,909 & 4,268 & 3,269 & 1,000 & 0,816 & 0,979 & 0,588 & 0,137 & 0,080 & 0,107 & 124 & 287 & $-0,5$ \\
\hline 518 & 287 & 0,022 & 0,418 & 0,023 & 2,443 & 0,938 & 5,837 & 4,838 & 1,000 & 0,805 & 1,123 & 0,728 & 0,156 & 0,114 & 0,152 & 84 & 287 & $-0,6$ \\
\hline 519 & 287 & 0,029 & 0,592 & 0,033 & 0,490 & 0,870 & 0,828 & 0,215 & 1,000 & 0,986 & 0,621 & 0,501 & 0,243 & 0,121 & 0,152 & 151 & 287 & $-1,3$ \\
\hline 520 & 287 & 0,175 & 1,509 & 0,189 & 0,592 & 0,924 & 0,392 & 0,613 & 0,966 & 1,018 & 0,840 & 0,809 & 0,515 & 0,417 & 0,408 & 85 & 287 & $-2,4$ \\
\hline 521 & 287 & 0,020 & 0,418 & 0,022 & 0,448 & 0,933 & 1,071 & 0,097 & 1,000 & 0,833 & 0,990 & 0,667 & 0,161 & 0,107 & 0,152 & 17 & 287 & $-0,7$ \\
\hline 522 & 287 & 0,016 & 0,541 & 0,022 & 0,368 & 0,733 & 0,680 & 0,417 & 0,677 & 1,215 & 0,778 & 0,667 & 0,161 & 0,107 & 0,076 & 135 & 287 & $-0,7$ \\
\hline 523 & 287 & 0,024 & 0,571 & 0,027 & 1,091 & 0,895 & 1,911 & 0,917 & 0,941 & 1,031 & 0,528 & 0,331 & 0,243 & 0,080 & 0,107 & 40 & 287 & $-1,3$ \\
\hline 524 & 287 & 0,027 & 0,511 & 0,027 & 0,266 & 1,000 & 0,521 & 0,479 & 1,000 & 0,874 & 0,712 & 0,514 & 0,221 & 0,114 & 0,152 & 66 & 287 & $-1,1$ \\
\hline 525 & 287 & 0,115 & 1,112 & 0,118 & 0,123 & 0,976 & 0,110 & 0,890 & 1,000 & 0,926 & 0,878 & 0,780 & 0,408 & 0,318 & 0,321 & 144 & 287 & $-2,0$ \\
\hline 526 & 287 & 0,019 & 0,389 & 0,020 & 5,572 & 0,929 & 14,330 & 13,331 & 1,000 & 0,803 & 1,273 & 0,832 & 0,137 & 0,114 & 0,152 & 27 & 287 & $-0,5$ \\
\hline 527 & 287 & 0,019 & 0,397 & 0,019 & 0,532 & 1,000 & 1,340 & 0,340 & 1,000 & 0,821 & 0,974 & 0,651 & 0,156 & 0,102 & 0,107 & 59 & 287 & $-0,6$ \\
\hline 528 & 287 & 0,023 & 0,440 & 0,023 & 0,266 & 1,000 & 0,606 & 0,394 & 1,000 & 0,818 & 0,815 & 0,566 & 0,189 & 0,107 & 0,152 & 60 & 287 & $-0,9$ \\
\hline 529 & 287 & 0,019 & 0,368 & 0,019 & 0,338 & 1,000 & 0,919 & 0,081 & 1,000 & 0,760 & 1,273 & 0,784 & 0,137 & 0,107 & 0,152 & 90 & 287 & $-0,5$ \\
\hline 530 & 287 & 0,062 & 1,040 & 0,070 & 0,643 & 0,878 & 0,618 & 0,401 & 0,717 & 1,181 & 0,472 & 0,464 & 0,408 & 0,189 & 0,169 & 81 & 287 & $-2,0$ \\
\hline 531 & 287 & 0,024 & 0,461 & 0,026 & 1,991 & 0,944 & 4,322 & 3,323 & 1,000 & 0,832 & 1,082 & 0,671 & 0,169 & 0,114 & 0,152 & 27 & 287 & $-0,8$ \\
\hline 532 & 287 & 0,027 & 0,511 & 0,029 & 0,968 & 0,950 & 1,893 & 0,895 & 1,000 & 0,874 & 0,968 & 0,759 & 0,189 & 0,144 & 0,152 & 75 & 287 & $-0,9$ \\
\hline 533 & 287 & 0,019 & 0,389 & 0,020 & 0,511 & 0,929 & 1,315 & 0,323 & 1,000 & 0,803 & 0,920 & 0,633 & 0,161 & 0,102 & 0,152 & 60 & 287 & $-0,7$ \\
\hline 534 & 287 & 0,020 & 0,520 & 0,024 & 0,600 & 0,824 & 1,155 & 0,235 & 0,934 & 1,035 & 0,891 & 0,671 & 0,169 & 0,114 & 0,107 & 35 & 287 & $-0,8$ \\
\hline 535 & 287 & 0,034 & 0,786 & 0,042 & 0,266 & 0,828 & 0,339 & 0,684 & 0,700 & 1,195 & 0,360 & 0,316 & 0,349 & 0,111 & 0,152 & 36 & 287 & $-1,8$ \\
\hline 536 & 287 & 0,016 & 0,714 & 0,030 & 0,266 & 0,524 & 0,373 & 0,788 & 0,389 & 1,604 & 0,219 & 0,250 & 0,303 & 0,076 & 0,076 & 169 & 287 & $-1,6$ \\
\hline 537 & 287 & 0,026 & 0,714 & 0,033 & 0,469 & 0,783 & 0,657 & 0,406 & 0,636 & 1,254 & 0,441 & 0,373 & 0,273 & 0,102 & 0,107 & 30 & 287 & $-1,4$ \\
\hline 538 & 287 & 0,057 & 0,968 & 0,062 & 0,266 & 0,930 & 0,275 & 0,728 & 0,769 & 1,140 & 0,351 & 0,282 & 0,456 & 0,129 & 0,169 & 136 & 287 & $-2,2$ \\
\hline 539 & 287 & 0,077 & 0,981 & 0,083 & 0,287 & 0,931 & 0,293 & 0,710 & 1,000 & 0,994 & 0,688 & 0,626 & 0,379 & 0,237 & 0,240 & 24 & 287 & $-1,9$ \\
\hline 540 & 287 & 0,141 & 1,970 & 0,171 & 0,347 & 0,824 & 0,176 & 0,843 & 0,455 & 1,482 & 0,674 & 0,767 & 0,515 & 0,395 & 0,214 & 111 & 287 & $-2,4$ \\
\hline 541 & 287 & 0,027 & 0,541 & 0,029 & 1,061 & 0,950 & 1,961 & 0,962 & 1,000 & 0,924 & 0,654 & 0,493 & 0,230 & 0,114 & 0,152 & 95 & 287 & $-1,2$ \\
\hline 542 & 287 & 0,020 & 0,389 & 0,020 & 0,440 & 1,000 & 1,131 & 0,131 & 1,000 & 0,774 & 1,371 & 0,832 & 0,137 & 0,114 & 0,152 & 135 & 287 & $-0,5$ \\
\hline 543 & 287 & 0,016 & 0,347 & 0,016 & 0,909 & 1,000 & 2,622 & 1,622 & 1,000 & 0,778 & 1,401 & 0,894 & 0,120 & 0,107 & 0,152 & 108 & 287 & $-0,3$ \\
\hline 544 & 287 & 0,017 & 0,482 & 0,019 & 0,224 & 0,923 & 0,465 & 0,540 & 0,933 & 1,036 & 0,413 & 0,164 & 0,230 & 0,038 & 0,076 & 0 & 287 & $-1,2$ \\
\hline 545 & 287 & 0,029 & 0,807 & 0,043 & 0,795 & 0,667 & 0,985 & 0,334 & 0,553 & 1,344 & 0,300 & 0,325 & 0,349 & 0,114 & 0,152 & 6 & 287 & $-1,8$ \\
\hline 546 & 287 & 0,039 & 1,306 & 0,066 & 0,917 & 0,587 & 0,702 & 0,509 & 0,285 & 1,872 & 0,382 & 0,588 & 0,359 & 0,211 & 0,152 & 110 & 287 & $-1,8$ \\
\hline 547 & 287 & 0,126 & 1,255 & 0,135 & 0,245 & 0,936 & 0,195 & 0,807 & 1,000 & 0,997 & 0,683 & 0,650 & 0,485 & 0,315 & 0,312 & 39 & 287 & $-2,3$ \\
\hline 548 & 287 & 0,037 & 0,837 & 0,053 & 0,562 & 0,703 & 0,672 & 0,443 & 0,669 & 1,222 & 0,625 & 0,589 & 0,276 & 0,162 & 0,152 & 154 & 287 & $-1,5$ \\
\hline 549 & 287 & 0,024 & 0,440 & 0,024 & 1,467 & 1,000 & 3,338 & 2,338 & 1,000 & 0,794 & 1,082 & 0,671 & 0,169 & 0,114 & 0,152 & 75 & 287 & $-0,8$ \\
\hline 550 & 287 & 0,042 & 0,672 & 0,044 & 0,490 & 0,935 & 0,729 & 0,278 & 1,000 & 0,930 & 0,738 & 0,600 & 0,268 & 0,161 & 0,214 & 26 & 287 & $-1,4$ \\
\hline 551 & 287 & 0,014 & 0,376 & 0,017 & 0,389 & 0,833 & 1,033 & 0,170 & 1,000 & 0,886 & 0,749 & 0,514 & 0,156 & 0,080 & 0,107 & 34 & 287 & $-0,6$ \\
\hline 552 & 287 & 0,014 & 0,418 & 0,017 & 0,511 & 0,833 & 1,222 & 0,278 & 1,000 & 0,986 & 0,509 & 0,388 & 0,189 & 0,074 & 0,076 & 162 & 287 & $-0,9$ \\
\hline 553 & 287 & 0,014 & 0,317 & 0,014 & 1,399 & 1,000 & 4,414 & 3,414 & 1,000 & 0,746 & 1,273 & 0,632 & 0,120 & 0,076 & 0,107 & 18 & 287 & $-0,3$ \\
\hline 554 & 287 & 0,158 & 1,686 & 0,184 & 0,888 & 0,859 & 0,526 & 0,494 & 0,697 & 1,197 & 0,431 & 0,398 & 0,683 & 0,272 & 0,303 & 77 & 287 & $-2,8$ \\
\hline 555 & 287 & 0,024 & 0,520 & 0,026 & 0,490 & 0,944 & 0,943 & 0,080 & 1,000 & 0,939 & 0,601 & 0,500 & 0,227 & 0,114 & 0,152 & 21 & 287 & $-1,2$ \\
\hline 556 & 287 & 0,032 & 0,553 & 0,033 & 0,296 & 0,957 & 0,535 & 0,467 & 1,000 & 0,879 & 0,824 & 0,514 & 0,221 & 0,114 & 0,152 & 169 & 287 & $-1,1$ \\
\hline 557 & 287 & 0,020 & 0,418 & 0,020 & 3,115 & 1,000 & 7,444 & 6,444 & 1,000 & 0,833 & 0,891 & 0,632 & 0,169 & 0,107 & 0,152 & 163 & 287 & $-0,8$ \\
\hline 558 & 287 & 0,017 & 0,368 & 0,017 & 0,440 & 1,000 & 1,195 & 0,195 & 1,000 & 0,791 & 0,899 & 0,485 & 0,156 & 0,076 & 0,152 & 172 & 287 & $-0,6$ \\
\hline 559 & 287 & 0,023 & 0,482 & 0,024 & 1,907 & 0,941 & 3,958 & 2,959 & 1,000 & 0,897 & 0,702 & 0,371 & 0,204 & 0,076 & 0,152 & 171 & 287 & $-1,0$ \\
\hline 560 & 287 & 0,027 & 0,520 & 0,029 & 1,784 & 0,950 & 3,431 & 2,431 & 1,000 & 0,888 & 0,654 & 0,493 & 0,230 & 0,114 & 0,152 & 9 & 287 & $-1,2$ \\
\hline 561 & 287 & 0,014 & 0,347 & 0,017 & 1,298 & 0,833 & 3,743 & 2,748 & 1,000 & 0,816 & 1,273 & 0,849 & 0,120 & 0,102 & 0,107 & 108 & 287 & $-0,3$ \\
\hline 562 & 287 & 0,123 & 1,412 & 0,131 & 0,765 & 0,945 & 0,542 & 0,461 & 0,778 & 1,134 & 0,400 & 0,385 & 0,627 & 0,241 & 0,273 & 74 & 287 & $-2,6$ \\
\hline 563 & 287 & 0,017 & 0,368 & 0,017 & 1,420 & 1,000 & 3,862 & 2,862 & 1,000 & 0,791 & 1,175 & 0,784 & 0,137 & 0,107 & 0,152 & 90 & 287 & $-0,5$ \\
\hline 564 & 287 & 0,231 & 2,329 & 0,291 & 1,070 & 0,793 & 0,459 & 0,579 & 0,535 & 1,367 & 0,328 & 0,405 & 0,947 & 0,383 & 0,312 & 76 & 287 & $-3,2$ \\
\hline 565 & 287 & 0,036 & 0,634 & 0,037 & 0,562 & 0,962 & 0,887 & 0,120 & 1,000 & 0,944 & 0,637 & 0,424 & 0,268 & 0,114 & 0,152 & 81 & 287 & $-1,4$ \\
\hline 566 & 287 & 0,066 & 0,816 & 0,067 & 0,490 & 0,979 & 0,601 & 0,400 & 1,000 & 0,896 & 0,861 & 0,728 & 0,312 & 0,227 & 0,240 & 167 & 287 & $-1,6$ \\
\hline 567 & 287 & 0,016 & 0,368 & 0,016 & 0,664 & 1,000 & 1,805 & 0,805 & 1,000 & 0,826 & 0,700 & 0,447 & 0,169 & 0,076 & 0,107 & 162 & 287 & $-0,8$ \\
\hline 568 & 287 & 0,049 & 0,960 & 0,062 & 2,870 & 0,791 & 2,991 & 2,002 & 0,666 & 1,226 & 0,333 & 0,338 & 0,432 & 0,146 & 0,152 & 145 & 287 & $-2,1$ \\
\hline 569 & 287 & 0,024 & 0,469 & 0,024 & 0,144 & 1,000 & 0,306 & 0,694 & 1,000 & 0,848 & 1,082 & 0,791 & 0,169 & 0,134 & 0,169 & 41 & 287 & $-0,8$ \\
\hline 570 & 287 & 0,017 & 0,368 & 0,017 & 0,613 & 1,000 & 1,667 & 0,667 & 1,000 & 0,791 & 1,175 & 0,745 & 0,137 & 0,102 & 0,107 & 45 & 287 & $-0,5$ \\
\hline 571 & 287 & 0,014 & 0,317 & 0,014 & 0,837 & 1,000 & 2,641 & 1,641 & 1,000 & 0,746 & 1,273 & 0,632 & 0,120 & 0,076 & 0,152 & 0 & 287 & $-0,3$ \\
\hline 572 & 287 & 0,042 & 0,765 & 0,049 & 0,317 & 0,853 & 0,414 & 0,604 & 0,893 & 1,058 & 0,499 & 0,466 & 0,326 & 0,152 & 0,152 & 49 & 287 & $-1,7$ \\
\hline 573 & 287 & 0,020 & 0,418 & 0,020 & 0,837 & 1,000 & 2,000 & 1,000 & 1,000 & 0,833 & 0,891 & 0,632 & 0,169 & 0,107 & 0,152 & 59 & 287 & $-0,8$ \\
\hline 574 & 287 & 0,029 & 0,520 & 0,030 & 0,347 & 0,952 & 0,667 & 0,337 & 1,000 & 0,866 & 0,979 & 0,784 & 0,193 & 0,152 & 0,152 & 14 & 287 & $-0,9$ \\
\hline
\end{tabular}




\begin{tabular}{|c|c|c|c|c|c|c|c|c|c|c|c|c|c|c|c|c|c|c|}
\hline 575 & 287 & 0,073 & 1,133 & 0,080 & 0,245 & 0,911 & 0,216 & 0,789 & 0,716 & 1,181 & 0,336 & 0,305 & 0,526 & 0,161 & 0,169 & 61 & 287 & $-2,4$ \\
\hline 576 & 287 & 0,023 & 0,461 & 0,023 & 2,320 & 1,000 & 5,037 & 4,037 & 1,000 & 0,858 & 0,784 & 0,392 & 0,193 & 0,076 & 0,152 & 90 & 287 & $-0,9$ \\
\hline 577 & 287 & 0,026 & 0,511 & 0,026 & 0,643 & 1,000 & 1,257 & 0,257 & 1,000 & 0,898 & 0,619 & 0,329 & 0,230 & 0,076 & 0,152 & 3 & 287 & $-1,2$ \\
\hline 578 & 287 & 0,057 & 1,070 & 0,073 & 0,541 & 0,784 & 0,506 & 0,539 & 0,630 & 1,260 & 0,525 & 0,621 & 0,373 & 0,232 & 0,152 & 23 & 287 & $-1,9$ \\
\hline 579 & 287 & 0,017 & 0,368 & 0,017 & 0,266 & 1,000 & 0,724 & 0,276 & 1,000 & 0,791 & 0,899 & 0,485 & 0,156 & 0,076 & 0,152 & 8 & 287 & $-0,6$ \\
\hline 580 & 287 & 0,043 & 0,723 & 0,046 & 0,245 & 0,938 & 0,339 & 0,664 & 1,000 & 0,983 & 0,659 & 0,529 & 0,288 & 0,153 & 0,169 & 36 & 287 & $-1,5$ \\
\hline 581 & 287 & 0,067 & 0,930 & 0,073 & 0,317 & 0,922 & 0,341 & 0,664 & 0,980 & 1,010 & 0,549 & 0,455 & 0,395 & 0,180 & 0,214 & 169 & 287 & $-2,0$ \\
\hline 582 & 287 & 0,066 & 1,031 & 0,075 & 0,735 & 0,885 & 0,713 & 0,309 & 0,780 & 1,132 & 0,480 & 0,450 & 0,418 & 0,188 & 0,227 & 9 & 287 & $-2,1$ \\
\hline 583 & 287 & 0,030 & 0,511 & 0,032 & 0,368 & 0,955 & 0,719 & 0,285 & 1,000 & 0,831 & 1,070 & 0,800 & 0,189 & 0,152 & 0,169 & 149 & 287 & $-0,9$ \\
\hline 584 & 287 & 0,022 & 0,490 & 0,023 & 1,061 & 0,938 & 2,164 & 1,166 & 1,000 & 0,943 & 0,516 & 0,329 & 0,230 & 0,076 & 0,152 & 169 & 287 & $-1,2$ \\
\hline 585 & 287 & 0,062 & 0,947 & 0,072 & 1,910 & 0,860 & 2,017 & 1,027 & 0,864 & 1,076 & 0,676 & 0,661 & 0,341 & 0,225 & 0,227 & 22 & 287 & $-1,8$ \\
\hline 586 & 287 & 0,861 & 7,212 & 1,334 & 0,858 & 0,645 & 0,119 & 0,950 & 0,208 & 2,193 & 0,218 & 0,390 & 2,244 & 0,874 & 0,152 & 164 & 287 & $-4,5$ \\
\hline 587 & 287 & 0,016 & 0,550 & 0,023 & 0,296 & 0,688 & 0,538 & 0,558 & 0,656 & 1,234 & 0,379 & 0,306 & 0,230 & 0,070 & 0,076 & 152 & 287 & $-1,2$ \\
\hline 588 & 287 & 0,020 & 0,389 & 0,020 & 3,420 & 1,000 & 8,796 & 7,796 & 1,000 & 0,774 & 1,371 & 0,832 & 0,137 & 0,114 & 0,152 & 135 & 287 & $-0,5$ \\
\hline 589 & 287 & 0,037 & 0,693 & 0,043 & 0,287 & 0,867 & 0,414 & 0,601 & 0,975 & 1,013 & 0,807 & 0,674 & 0,243 & 0,164 & 0,152 & 63 & 287 & $-1,3$ \\
\hline 590 & 287 & 0,976 & 4,426 & 1,083 & 4,472 & 0,901 & 1,010 & 0,100 & 0,626 & 1,264 & 0,415 & 0,429 & 1,731 & 0,743 & 0,715 & 70 & 287 & $-4,1$ \\
\hline 591 & 287 & 0,014 & 0,397 & 0,016 & 1,213 & 0,909 & 3,053 & 2,055 & 1,000 & 0,936 & 0,637 & 0,447 & 0,169 & 0,076 & 0,107 & 108 & 287 & $-0,8$ \\
\hline 592 & 287 & 0,020 & 0,389 & 0,020 & 3,352 & 1,000 & 8,621 & 7,621 & 1,000 & 0,774 & 0,990 & 0,667 & 0,161 & 0,107 & 0,152 & 45 & 287 & $-0,7$ \\
\hline 593 & 287 & 0,367 & 3,111 & 0,439 & 0,499 & 0,837 & 0,160 & 0,855 & 0,477 & 1,448 & 0,267 & 0,304 & 1,323 & 0,402 & 0,303 & 66 & 287 & $-3,7$ \\
\hline 594 & 287 & 0,032 & 0,622 & 0,034 & 0,368 & 0,917 & 0,592 & 0,417 & 1,000 & 0,987 & 0,824 & 0,728 & 0,221 & 0,161 & 0,152 & 124 & 287 & $-1,1$ \\
\hline 595 & 287 & 0,017 & 0,368 & 0,019 & 0,317 & 0,923 & 0,862 & 0,158 & 1,000 & 0,791 & 1,175 & 0,745 & 0,137 & 0,102 & 0,107 & 135 & 287 & $-0,5$ \\
\hline 596 & 287 & 0,085 & 1,205 & 0,102 & 0,532 & 0,831 & 0,442 & 0,583 & 0,733 & 1,168 & 0,515 & 0,583 & 0,458 & 0,267 & 0,273 & 118 & 287 & $-2,2$ \\
\hline 597 & 287 & 0,062 & 0,960 & 0,070 & 0,592 & 0,878 & 0,617 & 0,402 & 0,842 & 1,090 & 0,472 & 0,457 & 0,408 & 0,186 & 0,214 & 71 & 287 & $-2,0$ \\
\hline 598 & 287 & 0,082 & 1,725 & 0,108 & 1,070 & 0,760 & 0,620 & 0,449 & 0,346 & 1,701 & 0,354 & 0,349 & 0,542 & 0,189 & 0,169 & 94 & 287 & $-2,4$ \\
\hline 599 & 287 & 0,142 & 1,733 & 0,181 & 0,245 & 0,786 & 0,141 & 0,885 & 0,594 & 1,297 & 0,485 & 0,510 & 0,611 & 0,312 & 0,273 & 24 & 287 & $-2,6$ \\
\hline 600 & 287 & 0,019 & 0,490 & 0,023 & 0,397 & 0,812 & 0,811 & 0,267 & 0,975 & 1,013 & 0,662 & 0,570 & 0,189 & 0,108 & 0,152 & 26 & 287 & $-0,9$ \\
\hline 601 & 287 & 0,063 & 0,807 & 0,063 & 0,296 & 1,000 & 0,367 & 0,633 & 1,000 & 0,906 & 0,767 & 0,585 & 0,324 & 0,189 & 0,227 & 9 & 287 & $-1,7$ \\
\hline 602 & 287 & 0,156 & 1,742 & 0,184 & 0,592 & 0,852 & 0,340 & 0,677 & 0,648 & 1,243 & 0,540 & 0,521 & 0,607 & 0,316 & 0,312 & 108 & 287 & $-2,6$ \\
\hline 603 & 287 & 0,166 & 2,033 & 0,217 & 0,562 & 0,768 & 0,276 & 0,760 & 0,506 & 1,406 & 0,381 & 0,434 & 0,746 & 0,324 & 0,240 & 11 & 287 & $-2,9$ \\
\hline 604 & 287 & 0,032 & 0,583 & 0,034 & 0,706 & 0,917 & 1,210 & 0,226 & 1,000 & 0,926 & 0,700 & 0,474 & 0,240 & 0,114 & 0,152 & 100 & 287 & $-1,3$ \\
\hline 605 & 287 & 0,040 & 0,714 & 0,043 & 1,247 & 0,933 & 1,745 & 0,748 & 0,989 & 1,005 & 0,548 & 0,500 & 0,305 & 0,153 & 0,152 & 35 & 287 & $-1,6$ \\
\hline 606 & 287 & 0,356 & 2,329 & 0,402 & 0,757 & 0,886 & 0,325 & 0,685 & 0,824 & 1,101 & 0,710 & 0,715 & 0,799 & 0,572 & 0,530 & 175 & 287 & $-3,0$ \\
\hline 607 & 287 & 0,062 & 1,593 & 0,098 & 0,368 & 0,632 & 0,231 & 0,853 & 0,305 & 1,810 & 0,468 & 0,647 & 0,410 & 0,265 & 0,107 & 96 & 287 & $-2,0$ \\
\hline 608 & 287 & 0,022 & 0,431 & 0,022 & 0,275 & 1,000 & 0,638 & 0,362 & 1,000 & 0,829 & 0,955 & 0,447 & 0,169 & 0,076 & 0,152 & 90 & 287 & $-0,8$ \\
\hline 609 & 287 & 0,653 & 3,948 & 0,739 & 0,613 & 0,883 & 0,155 & 0,853 & 0,526 & 1,379 & 0,513 & 0,526 & 1,273 & 0,669 & 0,152 & 26 & 287 & $-3,7$ \\
\hline 610 & 287 & 0,016 & 0,368 & 0,016 & 0,440 & 1,000 & 1,195 & 0,195 & 1,000 & 0,826 & 0,824 & 0,485 & 0,156 & 0,076 & 0,107 & 18 & 287 & $-0,6$ \\
\hline 611 & 287 & 0,098 & 1,361 & 0,108 & 3,026 & 0,907 & 2,224 & 1,227 & 0,662 & 1,229 & 0,299 & 0,235 & 0,645 & 0,152 & 0,227 & 178 & 287 & $-2,7$ \\
\hline 612 & 287 & 0,016 & 0,469 & 0,019 & 0,592 & 0,846 & 1,261 & 0,303 & 0,901 & 1,054 & 0,483 & 0,371 & 0,204 & 0,076 & 0,107 & 69 & 287 & $-1,0$ \\
\hline 613 & 287 & 0,019 & 0,397 & 0,020 & 0,144 & 0,929 & 0,361 & 0,643 & 1,000 & 0,821 & 1,035 & 0,707 & 0,152 & 0,107 & 0,152 & 135 & 287 & $-0,6$ \\
\hline 614 & 287 & 0,022 & 0,469 & 0,022 & 0,245 & 1,000 & 0,522 & 0,478 & 1,000 & 0,902 & 0,562 & 0,364 & 0,221 & 0,080 & 0,107 & 32 & 287 & $-1,1$ \\
\hline 615 & 287 & 0,067 & 0,828 & 0,069 & 0,440 & 0,979 & 0,531 & 0,470 & 1,000 & 0,900 & 0,921 & 0,744 & 0,305 & 0,227 & 0,273 & 11 & 287 & $-1,6$ \\
\hline 616 & 287 & 0,016 & 0,368 & 0,017 & 0,296 & 0,917 & 0,805 & 0,212 & 1,000 & 0,826 & 0,778 & 0,500 & 0,161 & 0,080 & 0,107 & 127 & 287 & $-0,7$ \\
\hline 617 & 287 & 0,083 & 1,543 & 0,102 & 0,194 & 0,817 & 0,126 & 0,893 & 0,439 & 1,509 & 0,738 & 0,761 & 0,379 & 0,288 & 0,214 & 153 & 287 & $-1,9$ \\
\hline 618 & 287 & 0,014 & 0,440 & 0,017 & 0,317 & 0,833 & 0,721 & 0,325 & 0,933 & 1,035 & 0,439 & 0,271 & 0,204 & 0,055 & 0,076 & 105 & 287 & $-1,0$ \\
\hline 619 & 287 & 0,047 & 0,714 & 0,047 & 0,347 & 1,000 & 0,485 & 0,515 & 1,000 & 0,926 & 0,646 & 0,496 & 0,305 & 0,152 & 0,214 & 4 & 287 & $-1,6$ \\
\hline 620 & 287 & 0,161 & 3,157 & 0,380 & 0,613 & 0,423 & 0,194 & 0,991 & 0,203 & 2,222 & 0,129 & 0,301 & 1,259 & 0,379 & 0,169 & 105 & 287 & $-3,7$ \\
\hline 621 & 287 & 0,037 & 0,846 & 0,052 & 0,368 & 0,722 & 0,435 & 0,630 & 0,656 & 1,235 & 0,447 & 0,479 & 0,326 & 0,156 & 0,152 & 36 & 287 & $-1,7$ \\
\hline 622 & 287 & 0,016 & 0,368 & 0,017 & 1,390 & 0,917 & 3,781 & 2,782 & 1,000 & 0,826 & 0,824 & 0,485 & 0,156 & 0,076 & 0,107 & 85 & 287 & $-0,6$ \\
\hline 623 & 287 & 0,072 & 0,960 & 0,079 & 1,898 & 0,909 & 1,978 & 0,982 & 0,979 & 1,011 & 0,563 & 0,496 & 0,403 & 0,200 & 0,214 & 37 & 287 & $-2,0$ \\
\hline 624 & 287 & 0,073 & 1,082 & 0,083 & 3,711 & 0,879 & 3,429 & 2,432 & 0,785 & 1,128 & 0,414 & 0,393 & 0,475 & 0,186 & 0,214 & 151 & 287 & $\begin{array}{l}-2,2 \\
-2\end{array}$ \\
\hline 625 & 287 & 0,032 & 0,583 & 0,033 & 1,031 & 0,957 & 1,769 & 0,770 & 1,000 & 0,926 & 0,622 & 0,447 & 0,254 & 0,114 & 0,152 & 17 & 287 & $-1,3$ \\
\hline 626 & 287 & 0,030 & 0,643 & 0,033 & 0,418 & 0,913 & 0,651 & 0,359 & 0,917 & 1,044 & 0,594 & 0,534 & 0,254 & 0,136 & 0,152 & 60 & 287 & $-1,3$ \\
\hline 627 & 287 & 0,069 & 0,909 & 0,077 & 2,042 & 0,889 & 2,247 & 1,252 & 1,000 & 0,977 & 0,849 & 0,738 & 0,321 & 0,237 & 0,240 & 46 & 287 & $-1,7$ \\
\hline 628 & 287 & 0,014 & 0,317 & 0,014 & 1,002 & 1,000 & 3,160 & 2,160 & 1,000 & 0,746 & 0,979 & 0,555 & 0,137 & 0,076 & 0,107 & 18 & 287 & $-0,5$ \\
\hline 629 & 287 & 0,032 & 0,592 & 0,034 & 0,664 & 0,917 & 1,121 & 0,147 & 1,000 & 0,940 & 0,700 & 0,600 & 0,240 & 0,144 & 0,169 & 79 & 287 & $-1,3$ \\
\hline 630 & 287 & 0,154 & 2,355 & 0,221 & 0,347 & 0,695 & 0,147 & 0,906 & 0,348 & 1,695 & 0,404 & 0,561 & 0,695 & 0,390 & 0,152 & 65 & 287 & $-2,8$ \\
\hline 631 & 287 & 0,040 & 0,643 & 0,042 & 0,532 & 0,966 & 0,829 & 0,175 & 1,000 & 0,904 & 0,686 & 0,490 & 0,273 & 0,134 & 0,169 & 51 & 287 & $-1,4$ \\
\hline 632 & 287 & 0,043 & 0,685 & 0,046 & 1,103 & 0,938 & 1,611 & 0,614 & 1,000 & 0,931 & 0,849 & 0,745 & 0,254 & 0,189 & 0,214 & 175 & 287 & $-1,3$ \\
\hline
\end{tabular}




\begin{tabular}{|c|c|c|c|c|c|c|c|c|c|c|c|c|c|c|c|c|c|c|}
\hline 633 & 287 & 0,076 & 1,082 & 0,090 & 0,347 & 0,841 & 0,320 & 0,698 & 0,816 & 1,107 & 0,519 & 0,521 & 0,432 & 0,225 & 0,227 & 176 & 287 & $-2,1$ \\
\hline 634 & 287 & 0,017 & 0,389 & 0,017 & 0,541 & 1,000 & 1,392 & 0,392 & 1,000 & 0,836 & 0,764 & 0,447 & 0,169 & 0,076 & 0,107 & 18 & 287 & $-0,8$ \\
\hline 635 & 287 & 0,112 & 1,501 & 0,139 & 0,448 & 0,804 & 0,299 & 0,728 & 0,625 & 1,265 & 0,375 & 0,430 & 0,617 & 0,265 & 0,214 & 4 & 287 & $-2,6$ \\
\hline 636 & 287 & 0,026 & 0,490 & 0,026 & 0,203 & 1,000 & 0,414 & 0,586 & 1,000 & 0,861 & 0,917 & 0,667 & 0,189 & 0,126 & 0,152 & 41 & 287 & $-0,9$ \\
\hline 637 & 287 & 0,022 & 0,541 & 0,027 & 0,194 & 0,789 & 0,359 & 0,674 & 0,924 & 1,040 & 0,516 & 0,441 & 0,230 & 0,102 & 0,107 & 59 & 287 & $-1,2$ \\
\hline 638 & 287 & 0,142 & 1,429 & 0,154 & 0,317 & 0,925 & 0,222 & 0,782 & 0,874 & 1,069 & 0,485 & 0,472 & 0,611 & 0,288 & 0,303 & 66 & 287 & $-2,6$ \\
\hline 639 & 287 & 0,016 & 0,440 & 0,019 & 0,368 & 0,846 & 0,837 & 0,224 & 1,000 & 0,987 & 0,700 & 0,597 & 0,169 & 0,101 & 0,107 & 18 & 287 & $-0,8$ \\
\hline 640 & 287 & 0,022 & 0,440 & 0,023 & 0,757 & 0,938 & 1,721 & 0,724 & 1,000 & 0,845 & 0,955 & 0,637 & 0,169 & 0,108 & 0,152 & 117 & 287 & $-0,8$ \\
\hline 641 & 287 & 0,014 & 0,347 & 0,014 & 0,469 & 1,000 & 1,354 & 0,354 & 1,000 & 0,816 & 0,749 & 0,485 & 0,156 & 0,076 & 0,107 & 72 & 287 & $-0,6$ \\
\hline 642 & 287 & 0,055 & 1,306 & 0,092 & 0,245 & 0,594 & 0,188 & 0,908 & 0,402 & 1,578 & 0,372 & 0,591 & 0,432 & 0,255 & 0,107 & 16 & 287 & $-2,1$ \\
\hline 643 & 287 & 0,024 & 0,469 & 0,027 & 0,440 & 0,895 & 0,937 & 0,123 & 1,000 & 0,848 & 1,082 & 0,791 & 0,169 & 0,134 & 0,169 & 138 & 287 & $-0,8$ \\
\hline 644 & 287 & 0,022 & 0,541 & 0,024 & 0,245 & 0,882 & 0,453 & 0,559 & 0,924 & 1,040 & 0,764 & 0,667 & 0,189 & 0,126 & 0,152 & 0 & 287 & $-0,9$ \\
\hline 645 & 287 & 0,017 & 0,368 & 0,017 & 0,368 & 1,000 & 1,000 & 0,000 & 1,000 & 0,791 & 0,849 & 0,500 & 0,161 & 0,080 & 0,107 & 45 & 287 & $-0,7$ \\
\hline 646 & 287 & 0,141 & 1,429 & 0,154 & 3,534 & 0,916 & 2,473 & 1,476 & 0,866 & 1,075 & 0,480 & 0,468 & 0,611 & 0,286 & 0,273 & 151 & 287 & $-2,6$ \\
\hline 647 & 287 & 0,016 & 0,368 & 0,016 & 0,837 & 1,000 & 2,276 & 1,276 & 1,000 & 0,826 & 0,824 & 0,485 & 0,156 & 0,076 & 0,107 & 0 & 287 & $-0,6$ \\
\hline 648 & 287 & 0,065 & 0,858 & 0,067 & 1,564 & 0,957 & 1,823 & 0,824 & 1,000 & 0,953 & 0,716 & 0,637 & 0,339 & 0,216 & 0,227 & 19 & 287 & $-1,8$ \\
\hline 649 & 287 & 0,096 & 1,665 & 0,126 & 0,490 & 0,761 & 0,294 & 0,745 & 0,436 & 1,515 & 0,327 & 0,344 & 0,612 & 0,210 & 0,152 & 111 & 287 & $-2,6$ \\
\hline 650 & 287 & 0,088 & 1,695 & 0,123 & 1,040 & 0,709 & 0,614 & 0,484 & 0,383 & 1,616 & 0,521 & 0,682 & 0,462 & 0,315 & 0,214 & 134 & 287 & $-2,2$ \\
\hline 651 & 287 & 0,019 & 0,482 & 0,022 & 0,266 & 0,867 & 0,553 & 0,467 & 1,000 & 0,995 & 0,571 & 0,365 & 0,204 & 0,074 & 0,076 & 7 & 287 & $-1,0$ \\
\hline 652 & 287 & 0,027 & 0,490 & 0,027 & 0,693 & 1,000 & 1,414 & 0,414 & 1,000 & 0,838 & 0,968 & 0,707 & 0,189 & 0,134 & 0,169 & 45 & 287 & $-0,9$ \\
\hline 653 & 287 & 0,024 & 0,550 & 0,029 & 0,858 & 0,850 & 1,561 & 0,581 & 1,000 & 0,993 & 0,541 & 0,396 & 0,240 & 0,095 & 0,107 & 63 & 287 & $-1,3$ \\
\hline 654 & 287 & 0,274 & 2,021 & 0,296 & 3,817 & 0,927 & 1,889 & 0,892 & 0,843 & 1,089 & 0,510 & 0,545 & 0,827 & 0,451 & 0,442 & 110 & 287 & $-3,0$ \\
\hline 655 & 287 & 0,020 & 0,469 & 0,023 & 4,515 & 0,875 & 9,623 & 8,623 & 1,000 & 0,934 & 0,713 & 0,566 & 0,189 & 0,107 & 0,152 & 18 & 287 & $-0,9$ \\
\hline 656 & 287 & 0,016 & 0,347 & 0,016 & 0,858 & 1,000 & 2,475 & 1,475 & 1,000 & 0,778 & 1,077 & 0,588 & 0,137 & 0,080 & 0,107 & 72 & 287 & $-0,5$ \\
\hline 657 & 287 & 0,029 & 0,786 & 0,039 & 0,664 & 0,741 & 0,844 & 0,303 & 0,583 & 1,309 & 0,392 & 0,372 & 0,305 & 0,114 & 0,152 & 178 & 287 & $-1,6$ \\
\hline 658 & 287 & 0,026 & 0,744 & 0,036 & 0,469 & 0,720 & 0,631 & 0,464 & 0,586 & 1,306 & 0,573 & 0,664 & 0,240 & 0,159 & 0,152 & 142 & 287 & $-1,3$ \\
\hline 659 & 287 & 0,088 & 1,019 & 0,093 & 0,947 & 0,938 & 0,930 & 0,094 & 1,000 & 0,972 & 0,670 & 0,591 & 0,408 & 0,241 & 0,273 & 29 & 287 & $-2,0$ \\
\hline 660 & 287 & 0,023 & 0,469 & 0,024 & 0,275 & 0,941 & 0,586 & 0,418 & 1,000 & 0,874 & 1,019 & 0,701 & 0,169 & 0,119 & 0,152 & 49 & 287 & $-0,8$ \\
\hline 661 & 287 & 0,468 & 3,115 & 0,552 & 0,541 & 0,847 & 0,174 & 0,840 & 0,606 & 1,285 & 0,392 & 0,455 & 1,233 & 0,561 & 0,152 & 5 & 287 & $-3,6$ \\
\hline 662 & 287 & 0,029 & 0,511 & 0,030 & 1,716 & 0,952 & 3,356 & 2,356 & 1,000 & 0,852 & 1,019 & 0,800 & 0,189 & 0,152 & 0,152 & 75 & 287 & $-0,9$ \\
\hline 663 & 287 & 0,143 & 1,429 & 0,156 & 0,917 & 0,917 & 0,642 & 0,367 & 0,883 & 1,064 & 0,621 & 0,559 & 0,542 & 0,303 & 0,303 & 83 & 287 & $-2,4$ \\
\hline 664 & 287 & 0,024 & 0,499 & 0,024 & 0,511 & 1,000 & 1,025 & 0,025 & 1,000 & 0,901 & 0,637 & 0,364 & 0,221 & 0,080 & 0,107 & 40 & 287 & $-1,1$ \\
\hline 665 & 287 & 0,077 & 0,879 & 0,077 & 1,336 & 1,000 & 1,520 & 0,520 & 1,000 & 0,891 & 0,809 & 0,690 & 0,349 & 0,241 & 0,273 & 46 & 287 & $-1,8$ \\
\hline 666 & 287 & 0,069 & 1,243 & 0,092 & 0,706 & 0,750 & 0,568 & 0,499 & 0,560 & 1,336 & 0,421 & 0,499 & 0,456 & 0,228 & 0,169 & 59 & 287 & $-2,2$ \\
\hline 667 & 287 & 0,020 & 0,490 & 0,023 & 0,245 & 0,875 & 0,500 & 0,515 & 1,000 & 0,976 & 0,686 & 0,392 & 0,193 & 0,076 & 0,107 & 165 & 287 & $-0,9$ \\
\hline 668 & 287 & 0,067 & 1,019 & 0,076 & 0,317 & 0,887 & 0,311 & 0,698 & 0,816 & 1,107 & 0,460 & 0,412 & 0,432 & 0,178 & 0,169 & 49 & 287 & $-2,1$ \\
\hline 669 & 287 & 0,023 & 0,440 & 0,024 & 0,461 & 0,941 & 1,048 & 0,076 & 1,000 & 0,818 & 1,132 & 0,707 & 0,161 & 0,114 & 0,152 & 0 & 287 & $-0,7$ \\
\hline 670 & 287 & 0,020 & 0,440 & 0,022 & 0,571 & 0,933 & 1,299 & 0,306 & 1,000 & 0,875 & 0,686 & 0,392 & 0,193 & 0,076 & 0,152 & 81 & 287 & $-0,9$ \\
\hline 671 & 287 & 0,042 & 0,909 & 0,052 & 0,592 & 0,806 & 0,651 & 0,399 & 0,633 & 1,257 & 0,434 & 0,325 & 0,349 & 0,114 & 0,107 & 91 & 287 & $-1,8$ \\
\hline 672 & 287 & 0,565 & 2,963 & 0,613 & 0,490 & 0,923 & 0,165 & 0,838 & 0,809 & 1,112 & 0,632 & 0,641 & 1,067 & 0,685 & 0,682 & 89 & 287 & $-3,4$ \\
\hline 673 & 287 & 0,019 & 0,368 & 0,019 & 2,071 & 1,000 & 5,633 & 4,633 & 1,000 & 0,760 & 1,273 & 0,784 & 0,137 & 0,107 & 0,152 & 90 & 287 & $-0,5$ \\
\hline 674 & 287 & 0,024 & 0,511 & 0,027 & 1,480 & 0,895 & 2,893 & 1,896 & 1,000 & 0,924 & 0,833 & 0,588 & 0,193 & 0,114 & 0,152 & 5 & 287 & $-0,9$ \\
\hline 675 & 287 & 0,100 & 1,285 & 0,121 & 0,765 & 0,833 & 0,595 & 0,438 & 0,764 & 1,144 & 0,686 & 0,702 & 0,432 & 0,303 & 0,273 & 71 & 287 & $-2,1$ \\
\hline 676 & 287 & 0,419 & 2,591 & 0,455 & 0,368 & 0,921 & 0,142 & 0,862 & 0,784 & 1,129 & 0,533 & 0,562 & 1,000 & 0,562 & 0,546 & 134 & 287 & $-3,3$ \\
\hline 677 & 287 & 0,027 & 0,511 & 0,029 & 0,706 & 0,950 & 1,380 & 0,383 & 1,000 & 0,874 & 0,968 & 0,759 & 0,189 & 0,144 & 0,152 & 80 & 287 & $-0,9$ \\
\hline 678 & 287 & 0,075 & 0,947 & 0,077 & 0,287 & 0,963 & 0,303 & 0,698 & 1,000 & 0,978 & 0,662 & 0,633 & 0,379 & 0,240 & 0,240 & 92 & 287 & $-1,9$ \\
\hline 679 & 287 & 0,083 & 2,110 & 0,128 & 0,245 & 0,652 & 0,116 & 0,950 & 0,235 & 2,063 & 0,568 & 0,818 & 0,432 & 0,353 & 0,107 & 78 & 287 & $-2,1$ \\
\hline 680 & 287 & 0,022 & 0,440 & 0,024 & 8,477 & 0,882 & 19,286 & 18,286 & 1,000 & 0,845 & 1,061 & 0,707 & 0,161 & 0,114 & 0,152 & 90 & 287 & $-0,7$ \\
\hline 681 & 287 & 0,360 & 3,449 & 0,459 & 0,571 & 0,784 & 0,165 & 0,862 & 0,380 & 1,621 & 0,378 & 0,459 & 1,101 & 0,505 & 0,152 & 51 & 287 & $-3,5$ \\
\hline 682 & 287 & 0,023 & 0,520 & 0,024 & 0,795 & 0,941 & 1,529 & 0,532 & 1,000 & 0,968 & 0,497 & 0,331 & 0,243 & 0,080 & 0,107 & 52 & 287 & $-1,3$ \\
\hline 683 & 287 & 0,033 & 0,778 & 0,039 & 0,482 & 0,852 & 0,619 & 0,408 & 0,686 & 1,207 & 0,293 & 0,200 & 0,379 & 0,076 & 0,152 & 173 & 287 & $-1,9$ \\
\hline 684 & 287 & 0,024 & 0,520 & 0,027 & 0,541 & 0,895 & 1,041 & 0,113 & 1,000 & 0,939 & 0,585 & 0,441 & 0,230 & 0,102 & 0,152 & 23 & 287 & $-1,2$ \\
\hline 685 & 287 & 0,053 & 0,807 & 0,059 & 0,368 & 0,902 & 0,456 & 0,553 & 1,000 & 0,988 & 0,654 & 0,583 & 0,321 & 0,187 & 0,214 & 46 & 287 & $-1,7$ \\
\hline 686 & 287 & 0,108 & 1,234 & 0,116 & 0,562 & 0,926 & 0,455 & 0,550 & 0,887 & 1,062 & 0,492 & 0,469 & 0,528 & 0,248 & 0,273 & 148 & 287 & $-2,4$ \\
\hline 687 & 287 & 0,036 & 0,613 & 0,039 & 0,490 & 0,926 & 0,800 & 0,213 & 1,000 & 0,913 & 0,776 & 0,663 & 0,243 & 0,161 & 0,214 & 47 & 287 & $-1,3$ \\
\hline 688 & 287 & 0,052 & 0,714 & 0,053 & 0,765 & 0,973 & 1,071 & 0,076 & 1,000 & 0,887 & 1,019 & 0,800 & 0,254 & 0,203 & 0,214 & 34 & 287 & $-1,3$ \\
\hline 689 & 287 & 0,036 & 0,571 & 0,036 & 1,154 & 1,000 & 2,022 & 1,022 & 1,000 & 0,850 & 0,860 & 0,658 & 0,230 & 0,152 & 0,214 & 12 & 287 & $-1,2$ \\
\hline 690 & 287 & 0,987 & 4,332 & 1,055 & 2,418 & 0,936 & 0,558 & 0,446 & 0,661 & 1,230 & 0,515 & 0,481 & 1,562 & 0,751 & 0,758 & 94 & 287 & $\begin{array}{l}-4,0 \\
\end{array}$ \\
\hline
\end{tabular}




\begin{tabular}{|c|c|c|c|c|c|c|c|c|c|c|c|c|c|c|c|c|c|c|}
\hline 691 & 287 & 0,040 & 0,917 & 0,052 & 0,613 & 0,778 & 0,668 & 0,399 & 0,600 & 1,291 & 0,524 & 0,485 & 0,312 & 0,152 & 0,169 & 93 & 287 & $-1,6$ \\
\hline 692 & 287 & 0,125 & 1,462 & 0,143 & 0,735 & 0,870 & 0,503 & 0,514 & 0,734 & 1,168 & 0,418 & 0,430 & 0,617 & 0,265 & 0,273 & 5 & 287 & $-2,6$ \\
\hline 693 & 287 & 0,027 & 0,490 & 0,027 & 1,095 & 1,000 & 2,232 & 1,232 & 1,000 & 0,838 & 0,968 & 0,707 & 0,189 & 0,134 & 0,152 & 32 & 287 & $-0,9$ \\
\hline 694 & 287 & 0,024 & 0,461 & 0,026 & 0,562 & 0,944 & 1,220 & 0,227 & 1,000 & 0,832 & 1,082 & 0,671 & 0,169 & 0,114 & 0,152 & 84 & 287 & $-0,8$ \\
\hline 695 & 287 & 0,055 & 0,968 & 0,072 & 1,184 & 0,760 & 1,223 & 0,327 & 0,731 & 1,170 & 0,479 & 0,555 & 0,381 & 0,211 & 0,169 & 106 & 287 & $-1,9$ \\
\hline 696 & 287 & 0,024 & 0,592 & 0,029 & 1,163 & 0,850 & 1,964 & 0,976 & 0,875 & 1,069 & 0,433 & 0,300 & 0,268 & 0,080 & 0,107 & 45 & 287 & $-1,4$ \\
\hline 697 & 287 & 0,014 & 0,317 & 0,014 & 0,786 & 1,000 & 2,480 & 1,480 & 1,000 & 0,746 & 0,979 & 0,555 & 0,137 & 0,076 & 0,107 & 18 & 287 & $-0,5$ \\
\hline 698 & 287 & 0,056 & 0,786 & 0,057 & 0,266 & 0,975 & 0,339 & 0,662 & 1,000 & 0,938 & 0,621 & 0,531 & 0,339 & 0,180 & 0,214 & 22 & 287 & $-1,8$ \\
\hline 699 & 287 & 0,014 & 0,317 & 0,014 & 2,194 & 1,000 & 6,922 & 5,922 & 1,000 & 0,746 & 1,273 & 0,632 & 0,120 & 0,076 & 0,152 & 0 & 287 & $-0,3$ \\
\hline 700 & 287 & 0,020 & 0,397 & 0,020 & 0,981 & 1,000 & 2,467 & 1,467 & 1,000 & 0,791 & 1,049 & 0,686 & 0,156 & 0,107 & 0,152 & 73 & 287 & $-0,6$ \\
\hline 701 & 287 & 0,069 & 0,896 & 0,073 & 0,511 & 0,941 & 0,570 & 0,434 & 1,000 & 0,964 & 0,687 & 0,525 & 0,357 & 0,187 & 0,214 & 45 & 287 & $-1,8$ \\
\hline 702 & 287 & 0,069 & 1,031 & 0,086 & 1,725 & 0,800 & 1,672 & 0,701 & 0,814 & 1,109 & 0,611 & 0,696 & 0,379 & 0,264 & 0,227 & 39 & 287 & $-1,9$ \\
\hline 703 & 287 & 0,095 & 1,133 & 0,106 & 0,144 & 0,892 & 0,127 & 0,880 & 0,927 & 1,039 & 0,771 & 0,745 & 0,395 & 0,295 & 0,273 & 149 & 287 & $-2,0$ \\
\hline 704 & 287 & 0,037 & 0,613 & 0,040 & 0,714 & 0,929 & 1,166 & 0,180 & 1,000 & 0,895 & 0,736 & 0,580 & 0,254 & 0,147 & 0,169 & 66 & 287 & $-1,3$ \\
\hline 705 & 287 & 0,022 & 0,410 & 0,022 & 0,287 & 1,000 & 0,701 & 0,299 & 1,000 & 0,788 & 1,061 & 0,707 & 0,161 & 0,114 & 0,152 & 135 & 287 & $-0,7$ \\
\hline 706 & 287 & 0,024 & 0,469 & 0,024 & 0,786 & 1,000 & 1,675 & 0,675 & 1,000 & 0,848 & 0,866 & 0,566 & 0,189 & 0,107 & 0,152 & 45 & 287 & $-0,9$ \\
\hline 707 & 287 & 0,023 & 0,735 & 0,032 & 0,368 & 0,727 & 0,500 & 0,570 & 0,533 & 1,369 & 0,255 & 0,248 & 0,339 & 0,084 & 0,107 & 28 & 287 & $-1,8$ \\
\hline 708 & 287 & 0,628 & 4,346 & 0,821 & 1,644 & 0,766 & 0,378 & 0,664 & 0,418 & 1,546 & 0,452 & 0,616 & 1,331 & 0,819 & 0,152 & 2 & 287 & $-3,7$ \\
\hline 709 & 287 & 0,032 & 0,562 & 0,034 & 0,368 & 0,917 & 0,654 & 0,356 & 1,000 & 0,893 & 0,875 & 0,633 & 0,214 & 0,136 & 0,152 & 23 & 287 & $-1,1$ \\
\hline 710 & 287 & 0,022 & 0,469 & 0,023 & 0,266 & 0,938 & 0,567 & 0,437 & 1,000 & 0,902 & 0,955 & 0,791 & 0,169 & 0,134 & 0,152 & 30 & 287 & $-0,8$ \\
\hline 711 & 287 & 0,019 & 0,418 & 0,020 & 0,418 & 0,929 & 1,000 & 0,071 & 1,000 & 0,864 & 0,828 & 0,501 & 0,169 & 0,085 & 0,107 & 23 & 287 & $-0,8$ \\
\hline 712 & 287 & 0,016 & 0,389 & 0,017 & 0,723 & 0,917 & 1,860 & 0,864 & 1,000 & 0,873 & 0,824 & 0,485 & 0,156 & 0,076 & 0,107 & 5 & 287 & $-0,6$ \\
\hline 713 & 287 & 0,077 & 1,234 & 0,098 & 2,033 & 0,794 & 1,647 & 0,679 & 0,639 & 1,251 & 0,397 & 0,409 & 0,498 & 0,204 & 0,214 & 103 & 287 & $-2,3$ \\
\hline 714 & 287 & 0,044 & 0,693 & 0,046 & 0,655 & 0,969 & 0,945 & 0,064 & 1,000 & 0,927 & 0,759 & 0,588 & 0,273 & 0,161 & 0,214 & 35 & 287 & $-1,4$ \\
\hline 715 & 287 & 0,027 & 0,664 & 0,033 & 0,714 & 0,826 & 1,076 & 0,190 & 0,778 & 1,134 & 0,417 & 0,374 & 0,288 & 0,108 & 0,152 & 21 & 287 & $-1,5$ \\
\hline 716 & 287 & 0,125 & 1,602 & 0,171 & 0,440 & 0,731 & 0,274 & 0,774 & 0,611 & 1,279 & 0,426 & 0,502 & 0,611 & 0,306 & 0,214 & 25 & 287 & $-2,6$ \\
\hline 717 & 287 & 0,049 & 0,960 & 0,062 & 4,143 & 0,791 & 4,317 & 3,324 & 0,666 & 1,226 & 0,541 & 0,553 & 0,339 & 0,187 & 0,169 & 43 & 287 & $-1,8$ \\
\hline 718 & 287 & 0,155 & 1,754 & 0,178 & 4,886 & 0,871 & 2,785 & 1,790 & 0,633 & 1,257 & 0,611 & 0,660 & 0,568 & 0,375 & 0,312 & 67 & 287 & $-2,5$ \\
\hline 719 & 287 & 0,024 & 0,541 & 0,027 & 0,440 & 0,895 & 0,812 & 0,215 & 1,000 & 0,977 & 0,637 & 0,550 & 0,221 & 0,121 & 0,152 & 135 & 287 & $-1,1$ \\
\hline 720 & 287 & 0,044 & 0,795 & 0,046 & 1,010 & 0,969 & 1,271 & 0,273 & 0,885 & 1,063 & 0,395 & 0,283 & 0,379 & 0,107 & 0,169 & 40 & 287 & $-1,9$ \\
\hline 721 & 287 & 0,017 & 0,368 & 0,017 & 1,564 & 1,000 & 4,253 & 3,253 & 1,000 & 0,791 & 0,899 & 0,485 & 0,156 & 0,076 & 0,152 & 0 & 287 & $-0,6$ \\
\hline 722 & 287 & 0,082 & 1,408 & 0,098 & 4,828 & 0,838 & 3,430 & 2,435 & 0,519 & 1,389 & 0,333 & 0,371 & 0,559 & 0,207 & 0,227 & 31 & 287 & $-2,5$ \\
\hline 723 & 287 & 0,050 & 0,714 & 0,053 & 1,399 & 0,946 & 1,958 & 0,960 & 1,000 & 0,899 & 0,990 & 0,800 & 0,254 & 0,203 & 0,227 & 135 & 287 & $-1,3$ \\
\hline 724 & 287 & 0,106 & 1,133 & 0,106 & 0,968 & 1,000 & 0,855 & 0,145 & 1,000 & 0,981 & 0,558 & 0,462 & 0,492 & 0,227 & 0,303 & 3 & 287 & $-2,3$ \\
\hline 725 & 287 & 0,072 & 1,285 & 0,093 & 0,562 & 0,769 & 0,437 & 0,608 & 0,546 & 1,354 & 0,430 & 0,399 & 0,461 & 0,184 & 0,214 & 77 & 287 & $-2,2$ \\
\hline 726 & 287 & 0,023 & 0,541 & 0,027 & 0,215 & 0,842 & 0,398 & 0,622 & 0,985 & 1,007 & 1,019 & 0,869 & 0,169 & 0,147 & 0,152 & 130 & 287 & $-0,8$ \\
\hline 727 & 287 & 0,119 & 1,226 & 0,133 & 1,991 & 0,892 & 1,624 & 0,633 & 0,996 & 1,002 & 0,771 & 0,769 & 0,443 & 0,341 & 0,339 & 60 & 287 & $-2,1$ \\
\hline 728 & 287 & 0,027 & 0,520 & 0,029 & 0,490 & 0,950 & 0,943 & 0,076 & 1,000 & 0,888 & 0,712 & 0,485 & 0,221 & 0,107 & 0,152 & 47 & 287 & $-1,1$ \\
\hline 729 & 287 & 0,014 & 0,347 & 0,014 & 0,448 & 1,000 & 1,293 & 0,293 & 1,000 & 0,816 & 0,749 & 0,485 & 0,156 & 0,076 & 0,107 & 18 & 287 & $-0,6$ \\
\hline 730 & 287 & 0,270 & 2,346 & 0,324 & 0,765 & 0,832 & 0,326 & 0,695 & 0,616 & 1,274 & 0,437 & 0,471 & 0,887 & 0,418 & 0,386 & 38 & 287 & $-3,1$ \\
\hline 731 & 287 & 0,057 & 1,378 & 0,077 & 0,807 & 0,741 & 0,586 & 0,489 & 0,380 & 1,623 & 0,520 & 0,588 & 0,375 & 0,220 & 0,107 & 33 & 287 & $-1,9$ \\
\hline 732 & 287 & 0,014 & 0,317 & 0,014 & 1,758 & 1,000 & 5,547 & 4,547 & 1,000 & 0,746 & 1,273 & 0,632 & 0,120 & 0,076 & 0,107 & 18 & 287 & $-0,3$ \\
\hline 733 & 287 & 0,098 & 1,763 & 0,142 & 0,837 & 0,687 & 0,475 & 0,612 & 0,394 & 1,592 & 0,500 & 0,686 & 0,498 & 0,342 & 0,169 & 164 & 287 & $-2,3$ \\
\hline 734 & 287 & 0,076 & 2,017 & 0,179 & 1,488 & 0,424 & 0,738 & 0,633 & 0,235 & 2,063 & 0,208 & 0,445 & 0,682 & 0,303 & 0,107 & 85 & 287 & $-2,8$ \\
\hline 735 & 287 & 0,020 & 0,448 & 0,023 & 0,440 & 0,875 & 0,981 & 0,126 & 1,000 & 0,892 & 1,049 & 0,809 & 0,156 & 0,126 & 0,107 & 117 & 287 & $-0,6$ \\
\hline 736 & 287 & 0,032 & 0,693 & 0,037 & 0,194 & 0,846 & 0,280 & 0,736 & 0,825 & 1,101 & 0,459 & 0,453 & 0,296 & 0,134 & 0,152 & 50 & 287 & $-1,6$ \\
\hline 737 & 287 & 0,022 & 0,440 & 0,023 & 0,173 & 0,938 & 0,394 & 0,609 & 1,000 & 0,845 & 0,955 & 0,671 & 0,169 & 0,114 & 0,152 & 45 & 287 & $-0,8$ \\
\hline 738 & 287 & 0,036 & 0,960 & 0,036 & 0,643 & 1,000 & 0,670 & 0,330 & 0,490 & 1,429 & 0,707 & 0,723 & 0,254 & 0,184 & 0,169 & 39 & 287 & $-1,3$ \\
\hline 739 & 287 & 0,116 & 1,268 & 0,131 & 0,245 & 0,890 & 0,193 & 0,814 & 0,909 & 1,049 & 0,674 & 0,647 & 0,469 & 0,303 & 0,303 & 13 & 287 & $-2,2$ \\
\hline 740 & 287 & 0,036 & 0,685 & 0,039 & 0,583 & 0,926 & 0,852 & 0,166 & 0,961 & 1,020 & 0,490 & 0,388 & 0,305 & 0,119 & 0,152 & 21 & 287 & $-1,6$ \\
\hline 741 & 287 & 0,050 & 0,825 & 0,055 & 1,758 & 0,921 & 2,132 & 1,135 & 0,928 & 1,038 & 0,501 & 0,375 & 0,357 & 0,134 & 0,169 & 45 & 287 & $-1,8$ \\
\hline 742 & 287 & 0,019 & 0,511 & 0,022 & 0,397 & 0,867 & 0,777 & 0,260 & 0,896 & 1,056 & 0,414 & 0,251 & 0,240 & 0,060 & 0,076 & 17 & 287 & $-1,3$ \\
\hline 743 & 287 & 0,049 & 0,714 & 0,050 & 0,643 & 0,971 & 0,899 & 0,105 & 1,000 & 0,912 & 0,833 & 0,686 & 0,273 & 0,187 & 0,214 & 131 & 287 & $-1,4$ \\
\hline 744 & 287 & 0,032 & 0,592 & 0,033 & 1,133 & 0,957 & 1,914 & 0,915 & 1,000 & 0,940 & 0,757 & 0,658 & 0,230 & 0,152 & 0,169 & 99 & 287 & $-1,2$ \\
\hline 745 & 287 & 0,020 & 0,397 & 0,020 & 0,173 & 1,000 & 0,436 & 0,564 & 1,000 & 0,791 & 1,049 & 0,686 & 0,156 & 0,107 & 0,152 & 45 & 287 & $-0,6$ \\
\hline 746 & 287 & 0,017 & 0,490 & 0,023 & 0,368 & 0,750 & 0,750 & 0,354 & 0,900 & 1,054 & 0,477 & 0,354 & 0,214 & 0,076 & 0,076 & 58 & 287 & $-1,1$ \\
\hline 747 & 287 & 0,083 & 1,399 & 0,103 & 0,440 & 0,806 & 0,314 & 0,713 & 0,534 & 1,368 & 0,543 & 0,575 & 0,442 & 0,254 & 0,240 & 76 & 287 & $-2,1$ \\
\hline 748 & 287 & 0,036 & 0,807 & 0,049 & 0,735 & 0,735 & 0,911 & 0,279 & 0,692 & 1,202 & 0,436 & 0,451 & 0,324 & 0,146 & 0,152 & 12 & 287 & $-1,7$ \\
\hline
\end{tabular}




\begin{tabular}{|c|c|c|c|c|c|c|c|c|c|c|c|c|c|c|c|c|c|c|}
\hline 749 & 287 & 0,027 & 0,541 & 0,027 & 0,685 & 1,000 & 1,265 & 0,265 & 1,000 & 0,924 & 0,590 & 0,442 & 0,243 & 0,107 & 0,152 & 38 & 287 & $-1,3$ \\
\hline 750 & 287 & 0,050 & 0,765 & 0,053 & 0,317 & 0,946 & 0,414 & 0,588 & 1,000 & 0,963 & 0,602 & 0,493 & 0,326 & 0,161 & 0,214 & 30 & 287 & $-1,7$ \\
\hline 751 & 287 & 0,202 & 1,851 & 0,224 & 1,830 & 0,904 & 0,989 & 0,097 & 0,742 & 1,161 & 0,572 & 0,508 & 0,671 & 0,341 & 0,379 & 87 & 287 & $-2,7$ \\
\hline 752 & 287 & 0,020 & 0,461 & 0,020 & 0,397 & 1,000 & 0,863 & 0,137 & 1,000 & 0,917 & 0,615 & 0,371 & 0,204 & 0,076 & 0,152 & 9 & 287 & $-1,0$ \\
\hline 753 & 287 & 0,027 & 0,562 & 0,029 & 0,490 & 0,950 & 0,872 & 0,137 & 1,000 & 0,960 & 0,605 & 0,450 & 0,240 & 0,108 & 0,152 & 163 & 287 & $-1,3$ \\
\hline 754 & 287 & 0,066 & 0,837 & 0,069 & 0,571 & 0,958 & 0,682 & 0,321 & 1,000 & 0,919 & 1,010 & 0,892 & 0,288 & 0,257 & 0,273 & 65 & 287 & $-1,5$ \\
\hline 755 & 287 & 0,032 & 0,592 & 0,034 & 0,144 & 0,917 & 0,243 & 0,762 & 1,000 & 0,940 & 0,622 & 0,527 & 0,254 & 0,134 & 0,152 & 23 & 287 & $-1,3$ \\
\hline 756 & 287 & 0,016 & 0,347 & 0,016 & 1,611 & 1,000 & 4,646 & 3,646 & 1,000 & 0,778 & 0,875 & 0,500 & 0,152 & 0,076 & 0,152 & 0 & 287 & $-0,6$ \\
\hline 757 & 287 & 0,044 & 0,837 & 0,053 & 1,031 & 0,838 & 1,232 & 0,283 & 0,798 & 1,120 & 0,607 & 0,496 & 0,305 & 0,152 & 0,152 & 85 & 287 & $-1,6$ \\
\hline 758 & 287 & 0,017 & 0,397 & 0,019 & 0,765 & 0,923 & 1,925 & 0,928 & 1,000 & 0,854 & 0,899 & 0,543 & 0,156 & 0,085 & 0,107 & 23 & 287 & $-0,6$ \\
\hline 759 & 287 & 0,026 & 0,562 & 0,027 & 0,643 & 0,947 & 1,143 & 0,152 & 1,000 & 0,987 & 0,509 & 0,377 & 0,254 & 0,096 & 0,152 & 20 & 287 & $-1,3$ \\
\hline 760 & 287 & 0,138 & 1,644 & 0,159 & 0,266 & 0,865 & 0,162 & 0,849 & 0,640 & 1,250 & 0,421 & 0,411 & 0,645 & 0,265 & 0,214 & 91 & 287 & $-2,7$ \\
\hline 761 & 287 & 0,023 & 0,490 & 0,026 & 0,583 & 0,889 & 1,189 & 0,220 & 1,000 & 0,913 & 0,702 & 0,496 & 0,204 & 0,101 & 0,152 & 11 & 287 & $-1,0$ \\
\hline 762 & 287 & 0,023 & 0,490 & 0,026 & 1,133 & 0,889 & 2,311 & 1,315 & 1,000 & 0,913 & 0,702 & 0,496 & 0,204 & 0,101 & 0,152 & 77 & 287 & $-1,0$ \\
\hline 763 & 287 & 0,016 & 0,397 & 0,019 & 0,347 & 0,846 & 0,872 & 0,200 & 1,000 & 0,892 & 0,875 & 0,633 & 0,152 & 0,096 & 0,107 & 34 & 287 & $-0,6$ \\
\hline 764 & 287 & 0,032 & 0,664 & 0,034 & 0,144 & 0,917 & 0,216 & 0,788 & 0,901 & 1,054 & 0,539 & 0,423 & 0,273 & 0,116 & 0,152 & 34 & 287 & $-1,4$ \\
\hline 765 & 287 & 0,019 & 0,418 & 0,020 & 0,144 & 0,929 & 0,343 & 0,661 & 1,000 & 0,864 & 0,828 & 0,566 & 0,169 & 0,096 & 0,152 & 21 & 287 & $-0,8$ \\
\hline 766 & 287 & 0,046 & 1,073 & 0,060 & 0,562 & 0,762 & 0,524 & 0,533 & 0,501 & 1,413 & 0,627 & 0,560 & 0,305 & 0,171 & 0,169 & 73 & 287 & $-1,6$ \\
\hline 767 & 287 & 0,042 & 0,989 & 0,059 & 0,347 & 0,707 & 0,350 & 0,712 & 0,534 & 1,368 & 0,369 & 0,346 & 0,379 & 0,131 & 0,169 & 47 & 287 & $-1,9$ \\
\hline 768 & 287 & 2,093 & 8,658 & 2,689 & 1,175 & 0,779 & 0,136 & 0,892 & 0,351 & 1,688 & 0,260 & 0,313 & 3,204 & 1,003 & 0,937 & 65 & 287 & $-5,0$ \\
\hline 769 & 287 & 0,017 & 0,368 & 0,017 & 0,418 & 1,000 & 1,138 & 0,138 & 1,000 & 0,791 & 1,175 & 0,745 & 0,137 & 0,102 & 0,107 & 135 & 287 & $-0,5$ \\
\hline 770 & 287 & 0,024 & 0,499 & 0,027 & 0,440 & 0,895 & 0,881 & 0,159 & 1,000 & 0,901 & 0,833 & 0,555 & 0,193 & 0,107 & 0,152 & 27 & 287 & $-0,9$ \\
\hline 771 & 287 & 0,141 & 1,572 & 0,159 & 0,347 & 0,883 & 0,220 & 0,788 & 0,715 & 1,183 & 0,369 & 0,369 & 0,696 & 0,257 & 0,273 & 71 & 287 & $-2,8$ \\
\hline 772 & 287 & 0,047 & 0,786 & 0,052 & 0,440 & 0,917 & 0,559 & 0,449 & 0,963 & 1,019 & 0,467 & 0,422 & 0,359 & 0,152 & 0,169 & 72 & 287 & $-1,8$ \\
\hline 773 & 287 & 0,090 & 1,052 & 0,106 & 0,592 & 0,851 & 0,562 & 0,462 & 1,000 & 0,987 & 0,802 & 0,776 & 0,379 & 0,294 & 0,169 & 68 & 287 & $-1,9$ \\
\hline 774 & 287 & 0,016 & 0,389 & 0,017 & 0,317 & 0,917 & 0,815 & 0,203 & 1,000 & 0,873 & 0,700 & 0,425 & 0,169 & 0,072 & 0,076 & 69 & 287 & $-0,8$ \\
\hline 775 & 287 & 0,039 & 0,613 & 0,040 & 2,820 & 0,964 & 4,602 & 3,602 & 1,000 & 0,878 & 1,011 & 0,844 & 0,221 & 0,186 & 0,169 & 90 & 287 & $-1,1$ \\
\hline 776 & 287 & 0,036 & 0,592 & 0,039 & 0,224 & 0,926 & 0,379 & 0,626 & 1,000 & 0,881 & 0,936 & 0,728 & 0,221 & 0,161 & 0,169 & 38 & 287 & $-1,1$ \\
\hline 777 & 287 & 0,014 & 0,418 & 0,019 & 0,368 & 0,769 & 0,879 & 0,261 & 1,000 & 0,986 & 0,509 & 0,361 & 0,189 & 0,068 & 0,107 & 45 & 287 & $-0,9$ \\
\hline 778 & 287 & 0,026 & 0,795 & 0,036 & 0,643 & 0,720 & 0,808 & 0,339 & 0,514 & 1,395 & 0,270 & 0,230 & 0,349 & 0,080 & 0,076 & 46 & 287 & $-1,8$ \\
\hline 779 & 287 & 0,055 & 0,867 & 0,060 & 0,765 & 0,905 & 0,883 & 0,151 & 0,912 & 1,047 & 0,605 & 0,553 & 0,339 & 0,187 & 0,169 & 43 & 287 & $-1,8$ \\
\hline 780 & 287 & 0,037 & 0,583 & 0,037 & 0,571 & 1,000 & 0,979 & 0,021 & 1,000 & 0,852 & 0,974 & 0,686 & 0,221 & 0,152 & 0,214 & 97 & 287 & $-1,1$ \\
\hline 781 & 287 & 0,014 & 0,347 & 0,016 & 0,490 & 0,909 & 1,414 & 0,424 & 1,000 & 0,816 & 0,979 & 0,588 & 0,137 & 0,080 & 0,107 & 37 & 287 & $-0,5$ \\
\hline 782 & 287 & 0,099 & 1,437 & 0,121 & 0,837 & 0,821 & 0,582 & 0,454 & 0,602 & 1,289 & 0,453 & 0,488 & 0,528 & 0,257 & 0,273 & 79 & 287 & $-2,4$ \\
\hline 783 & 287 & 0,088 & 1,298 & 0,108 & 0,224 & 0,813 & 0,173 & 0,848 & 0,653 & 1,237 & 0,621 & 0,624 & 0,423 & 0,264 & 0,227 & 101 & 287 & $-2,1$ \\
\hline 784 & 287 & 0,158 & 1,560 & 0,176 & 0,757 & 0,894 & 0,485 & 0,526 & 0,815 & 1,108 & 0,521 & 0,468 & 0,621 & 0,291 & 0,273 & 47 & 287 & $-2,6$ \\
\hline 785 & 287 & 0,014 & 0,448 & 0,017 & 0,592 & 0,833 & 1,320 & 0,361 & 0,898 & 1,055 & 0,439 & 0,332 & 0,204 & 0,068 & 0,076 & 18 & 287 & $-1,0$ \\
\hline 786 & 287 & 0,020 & 0,440 & 0,022 & 1,040 & 0,933 & 2,366 & 1,368 & 1,000 & 0,875 & 0,891 & 0,637 & 0,169 & 0,108 & 0,152 & 0 & 287 & $-0,8$ \\
\hline 787 & 287 & 0,032 & 0,541 & 0,033 & 0,571 & 0,957 & 1,055 & 0,070 & 1,000 & 0,859 & 0,966 & 0,665 & 0,204 & 0,136 & 0,152 & 67 & 287 & $-1,0$ \\
\hline 788 & 287 & 0,039 & 0,634 & 0,042 & 0,613 & 0,931 & 0,967 & 0,077 & 1,000 & 0,909 & 0,764 & 0,596 & 0,254 & 0,152 & 0,169 & 82 & 287 & $-1,3$ \\
\hline 789 & 287 & 0,046 & 1,234 & 0,072 & 0,440 & 0,640 & 0,356 & 0,738 & 0,379 & 1,625 & 0,479 & 0,614 & 0,349 & 0,214 & 0,169 & 109 & 287 & $-1,8$ \\
\hline 790 & 287 & 0,034 & 0,583 & 0,034 & 0,347 & 1,000 & 0,594 & 0,406 & 1,000 & 0,887 & 0,764 & 0,474 & 0,240 & 0,114 & 0,152 & 94 & 287 & $-1,3$ \\
\hline 791 & 287 & 0,053 & 0,837 & 0,057 & 0,520 & 0,925 & 0,621 & 0,386 & 0,952 & 1,025 & 0,529 & 0,475 & 0,357 & 0,170 & 0,152 & 60 & 287 & $-1,8$ \\
\hline 792 & 287 & 0,042 & 0,693 & 0,044 & 0,858 & 0,935 & 1,238 & 0,246 & 1,000 & 0,959 & 0,605 & 0,453 & 0,296 & 0,134 & 0,169 & 47 & 287 & $-1,6$ \\
\hline 793 & 287 & 0,044 & 1,040 & 0,057 & 0,275 & 0,775 & 0,264 & 0,769 & 0,517 & 1,391 & 0,681 & 0,657 & 0,288 & 0,189 & 0,107 & 103 & 287 & $-1,5$ \\
\hline 794 & 287 & 0,024 & 0,541 & 0,030 & 0,347 & 0,810 & 0,641 & 0,407 & 1,000 & 0,977 & 0,585 & 0,468 & 0,230 & 0,108 & 0,152 & 66 & 287 & $-1,2$ \\
\hline 795 & 287 & 0,022 & 0,410 & 0,022 & 0,317 & 1,000 & 0,773 & 0,227 & 1,000 & 0,788 & 1,061 & 0,707 & 0,161 & 0,114 & 0,152 & 0 & 287 & $-0,7$ \\
\hline 796 & 287 & 0,017 & 0,368 & 0,017 & 0,266 & 1,000 & 0,724 & 0,276 & 1,000 & 0,791 & 1,175 & 0,784 & 0,137 & 0,107 & 0,152 & 135 & 287 & $-0,5$ \\
\hline 797 & 287 & 0,367 & 3,648 & 0,415 & 0,347 & 0,886 & 0,095 & 0,912 & 0,347 & 1,698 & 0,432 & 0,437 & 1,040 & 0,455 & 0,152 & 79 & 287 & $-3,4$ \\
\hline 798 & 287 & 0,024 & 0,469 & 0,024 & 0,245 & 1,000 & 0,522 & 0,478 & 1,000 & 0,848 & 1,082 & 0,791 & 0,169 & 0,134 & 0,169 & 70 & 287 & $-0,8$ \\
\hline 799 & 287 & 0,291 & 2,143 & 0,326 & 1,082 & 0,894 & 0,505 & 0,506 & 0,797 & 1,120 & 0,537 & 0,632 & 0,831 & 0,525 & 0,455 & 70 & 287 & $-3,1$ \\
\hline 800 & 287 & 0,023 & 0,440 & 0,023 & 0,461 & 1,000 & 1,048 & 0,048 & 1,000 & 0,818 & 1,019 & 0,671 & 0,169 & 0,114 & 0,152 & 63 & 287 & $-0,8$ \\
\hline 801 & 287 & 0,103 & 1,581 & 0,131 & 0,389 & 0,791 & 0,246 & 0,782 & 0,519 & 1,388 & 0,542 & 0,607 & 0,492 & 0,299 & 0,273 & 58 & 287 & $-2,3$ \\
\hline 802 & 287 & 0,073 & 0,879 & 0,073 & 0,490 & 1,000 & 0,558 & 0,442 & 1,000 & 0,917 & 0,812 & 0,671 & 0,339 & 0,227 & 0,273 & 15 & 287 & $-1,8$ \\
\hline 803 & 287 & 0,023 & 0,490 & 0,023 & 0,604 & 1,000 & 1,232 & 0,232 & 1,000 & 0,913 & 0,784 & 0,588 & 0,193 & 0,114 & 0,152 & 6 & 287 & $-0,9$ \\
\hline 804 & 287 & 0,029 & 0,917 & 0,044 & 2,959 & 0,645 & 3,225 & 2,254 & 0,428 & 1,528 & 0,621 & 0,674 & 0,243 & 0,164 & 0,152 & 0 & 287 & $-1,3$ \\
\hline 805 & 287 & 0,481 & 2,828 & 0,522 & 0,613 & 0,920 & 0,217 & 0,787 & 0,755 & 1,151 & 0,449 & 0,512 & 1,167 & 0,598 & 0,606 & 104 & 287 & $-3,5$ \\
\hline 806 & 287 & 0,020 & 0,448 & 0,022 & 1,860 & 0,933 & 4,149 & 3,150 & 1,000 & 0,892 & 0,891 & 0,632 & 0,169 & 0,107 & 0,107 & 107 & 287 & $-0,8$ \\
\hline
\end{tabular}




\begin{tabular}{|c|c|c|c|c|c|c|c|c|c|c|c|c|c|c|c|c|c|c|}
\hline 807 & 287 & 0,343 & 2,156 & 0,364 & 0,317 & 0,941 & 0,147 & 0,855 & 0,927 & 1,038 & 0,900 & 0,869 & 0,696 & 0,605 & 0,552 & 103 & 287 & $-2,8$ \\
\hline 808 & 287 & 0,056 & 0,807 & 0,059 & 0,643 & 0,951 & 0,796 & 0,210 & 1,000 & 0,963 & 0,680 & 0,585 & 0,324 & 0,189 & 0,227 & 11 & 287 & $-1,7$ \\
\hline 809 & 287 & 0,047 & 1,091 & 0,063 & 0,389 & 0,750 & 0,356 & 0,690 & 0,500 & 1,414 & 0,646 & 0,714 & 0,305 & 0,218 & 0,152 & 119 & 287 & $-1,6$ \\
\hline 810 & 287 & 0,024 & 0,672 & 0,033 & 1,336 & 0,739 & 1,987 & 1,021 & 0,678 & 1,214 & 0,433 & 0,403 & 0,268 & 0,108 & 0,107 & 163 & 287 & $-1,4$ \\
\hline 811 & 287 & 0,050 & 1,501 & 0,076 & 0,571 & 0,660 & 0,380 & 0,707 & 0,280 & 1,889 & 0,602 & 0,728 & 0,326 & 0,237 & 0,107 & 120 & 287 & $-1,7$ \\
\hline 812 & 287 & 0,032 & 0,613 & 0,034 & 0,368 & 0,917 & 0,600 & 0,409 & 1,000 & 0,973 & 0,560 & 0,424 & 0,268 & 0,114 & 0,152 & 1 & 287 & $-1,4$ \\
\hline 813 & 287 & 0,109 & 1,480 & 0,145 & 1,429 & 0,752 & 0,966 & 0,250 & 0,626 & 1,264 & 0,523 & 0,563 & 0,515 & 0,290 & 0,214 & 14 & 287 & $-2,4$ \\
\hline 814 & 287 & 0,059 & 1,560 & 0,085 & 0,397 & 0,695 & 0,255 & 0,805 & 0,304 & 1,814 & 0,418 & 0,537 & 0,423 & 0,227 & 0,107 & 96 & 287 & $-2,1$ \\
\hline 815 & 287 & 0,019 & 0,389 & 0,019 & 1,390 & 1,000 & 3,576 & 2,576 & 1,000 & 0,803 & 0,974 & 0,485 & 0,156 & 0,076 & 0,152 & 90 & 287 & $-0,6$ \\
\hline 816 & 287 & 0,033 & 0,664 & 0,034 & 0,714 & 0,958 & 1,076 & 0,087 & 0,942 & 1,031 & 0,505 & 0,421 & 0,288 & 0,121 & 0,152 & 58 & 287 & $-1,5$ \\
\hline 817 & 287 & 0,116 & 2,338 & 0,201 & 0,440 & 0,579 & 0,188 & 0,915 & 0,267 & 1,934 & 0,306 & 0,500 & 0,695 & 0,347 & 0,107 & 149 & 287 & $-2,8$ \\
\hline 818 & 287 & 0,112 & 2,021 & 0,155 & 0,347 & 0,722 & 0,172 & 0,874 & 0,344 & 1,704 & 0,667 & 0,788 & 0,462 & 0,364 & 0,240 & 178 & 287 & $-2,2$ \\
\hline 819 & 287 & 0,386 & 2,861 & 0,426 & 0,930 & 0,906 & 0,325 & 0,682 & 0,592 & 1,299 & 0,294 & 0,322 & 1,293 & 0,417 & 0,442 & 84 & 287 & $-3,7$ \\
\hline 820 & 287 & 0,063 & 1,184 & 0,086 & 0,317 & 0,733 & 0,268 & 0,779 & 0,566 & 1,329 & 0,366 & 0,411 & 0,469 & 0,192 & 0,169 & 20 & 287 & $-2,2$ \\
\hline 821 & 287 & 0,026 & 0,837 & 0,039 & 0,245 & 0,667 & 0,293 & 0,782 & 0,463 & 1,469 & 0,353 & 0,351 & 0,305 & 0,107 & 0,076 & 51 & 287 & $-1,6$ \\
\hline 822 & 287 & 0,016 & 0,338 & 0,016 & 0,469 & 1,000 & 1,388 & 0,388 & 1,000 & 0,759 & 1,077 & 0,555 & 0,137 & 0,076 & 0,152 & 0 & 287 & $-0,5$ \\
\hline 823 & 287 & 0,079 & 0,938 & 0,083 & 0,469 & 0,948 & 0,500 & 0,503 & 1,000 & 0,942 & 0,824 & 0,728 & 0,349 & 0,254 & 0,273 & 162 & 287 & $-1,8$ \\
\hline 824 & 287 & 0,020 & 0,418 & 0,020 & 0,562 & 1,000 & 1,343 & 0,343 & 1,000 & 0,833 & 1,049 & 0,728 & 0,156 & 0,114 & 0,152 & 0 & 287 & $-0,6$ \\
\hline 825 & 287 & 0,023 & 0,469 & 0,023 & 1,551 & 1,000 & 3,306 & 2,306 & 1,000 & 0,874 & 0,702 & 0,525 & 0,204 & 0,107 & 0,152 & 150 & 287 & $-1,0$ \\
\hline 826 & 287 & 0,016 & 0,347 & 0,016 & 0,951 & 1,000 & 2,743 & 1,743 & 1,000 & 0,778 & 1,401 & 0,894 & 0,120 & 0,107 & 0,152 & 72 & 287 & $-0,3$ \\
\hline 827 & 287 & 0,060 & 1,010 & 0,069 & 0,440 & 0,875 & 0,435 & 0,579 & 0,742 & 1,161 & 0,390 & 0,399 & 0,443 & 0,177 & 0,214 & 162 & 287 & $-2,1$ \\
\hline 828 & 287 & 0,191 & 1,543 & 0,201 & 5,933 & 0,950 & 3,846 & 2,846 & 1,000 & 0,996 & 0,753 & 0,716 & 0,568 & 0,407 & 0,386 & 71 & 287 & $-2,5$ \\
\hline 829 & 287 & 0,435 & 2,714 & 0,476 & 0,266 & 0,913 & 0,098 & 0,906 & 0,742 & 1,161 & 0,500 & 0,580 & 1,052 & 0,610 & 0,577 & 127 & 287 & $-3,4$ \\
\hline 830 & 287 & 0,016 & 0,448 & 0,016 & 0,266 & 1,000 & 0,594 & 0,406 & 0,987 & 1,006 & 0,412 & 0,243 & 0,221 & 0,054 & 0,076 & 35 & 287 & $-1,1$ \\
\hline 831 & 287 & 0,034 & 0,613 & 0,036 & 0,888 & 0,960 & 1,448 & 0,450 & 1,000 & 0,932 & 0,679 & 0,566 & 0,254 & 0,144 & 0,152 & 18 & 287 & $-1,3$ \\
\hline 832 & 287 & 0,083 & 1,082 & 0,089 & 0,613 & 0,935 & 0,566 & 0,438 & 0,893 & 1,058 & 0,434 & 0,399 & 0,494 & 0,197 & 0,214 & 60 & 287 & $-2,3$ \\
\hline 833 & 287 & 0,040 & 0,714 & 0,043 & 0,347 & 0,933 & 0,485 & 0,519 & 0,989 & 1,005 & 0,548 & 0,432 & 0,305 & 0,132 & 0,152 & 63 & 287 & $-1,6$ \\
\hline 834 & 287 & 0,017 & 0,389 & 0,017 & 0,317 & 1,000 & 0,815 & 0,185 & 1,000 & 0,836 & 0,899 & 0,485 & 0,156 & 0,076 & 0,107 & 0 & 287 & $-0,6$ \\
\hline 835 & 287 & 0,057 & 0,837 & 0,063 & 0,397 & 0,909 & 0,475 & 0,533 & 1,000 & 0,986 & 0,784 & 0,682 & 0,305 & 0,208 & 0,227 & 106 & 287 & $-1,6$ \\
\hline 836 & 287 & 0,067 & 1,437 & 0,085 & 0,541 & 0,797 & 0,376 & 0,656 & 0,410 & 1,561 & 0,178 & 0,167 & 0,695 & 0,116 & 0,152 & 155 & 287 & $-2,8$ \\
\hline 837 & 287 & 0,118 & 1,306 & 0,131 & 0,706 & 0,901 & 0,540 & 0,470 & 0,867 & 1,074 & 0,644 & 0,611 & 0,482 & 0,295 & 0,303 & 57 & 287 & $-2,3$ \\
\hline 838 & 287 & 0,037 & 0,571 & 0,037 & 1,217 & 1,000 & 2,132 & 1,132 & 1,000 & 0,834 & 1,142 & 0,788 & 0,204 & 0,161 & 0,214 & 45 & 287 & $-1,0$ \\
\hline 839 & 287 & 0,095 & 1,306 & 0,112 & 0,245 & 0,846 & 0,188 & 0,827 & 0,697 & 1,197 & 0,568 & 0,575 & 0,461 & 0,265 & 0,273 & 178 & 287 & $-2,2$ \\
\hline 840 & 287 & 0,032 & 0,643 & 0,034 & 0,317 & 0,917 & 0,493 & 0,514 & 0,961 & 1,020 & 0,459 & 0,355 & 0,296 & 0,105 & 0,152 & 37 & 287 & $-1,6$ \\
\hline 841 & 287 & 0,096 & 1,399 & 0,128 & 0,296 & 0,753 & 0,212 & 0,826 & 0,617 & 1,273 & 0,416 & 0,433 & 0,542 & 0,235 & 0,227 & 105 & 287 & $-2,4$ \\
\hline 842 & 287 & 0,052 & 0,714 & 0,053 & 0,123 & 0,973 & 0,172 & 0,829 & 1,000 & 0,887 & 1,019 & 0,843 & 0,254 & 0,214 & 0,227 & 37 & 287 & $-1,3$ \\
\hline 843 & 287 & 0,069 & 1,040 & 0,082 & 0,397 & 0,842 & 0,382 & 0,638 & 0,800 & 1,118 & 0,605 & 0,697 & 0,381 & 0,265 & 0,214 & 99 & 287 & $-1,9$ \\
\hline 844 & 287 & 0,043 & 0,930 & 0,057 & 0,317 & 0,750 & 0,341 & 0,705 & 0,626 & 1,264 & 0,516 & 0,605 & 0,326 & 0,197 & 0,152 & 117 & 287 & $-1,7$ \\
\hline 845 & 287 & 0,017 & 0,368 & 0,017 & 0,469 & 1,000 & 1,276 & 0,276 & 1,000 & 0,791 & 1,175 & 0,745 & 0,137 & 0,102 & 0,152 & 45 & 287 & $-0,5$ \\
\hline 846 & 287 & 0,019 & 0,368 & 0,019 & 0,511 & 1,000 & 1,391 & 0,391 & 1,000 & 0,760 & 1,273 & 0,784 & 0,137 & 0,107 & 0,152 & 90 & 287 & $-0,5$ \\
\hline 847 & 287 & 0,019 & 0,389 & 0,019 & 0,347 & 1,000 & 0,892 & 0,108 & 1,000 & 0,803 & 0,974 & 0,485 & 0,156 & 0,076 & 0,152 & 90 & 287 & $-0,6$ \\
\hline 848 & 287 & 0,072 & 1,196 & 0,098 & 0,795 & 0,735 & 0,665 & 0,427 & 0,630 & 1,260 & 0,439 & 0,498 & 0,456 & 0,227 & 0,169 & 85 & 287 & $-2,2$ \\
\hline 849 & 287 & 0,287 & 2,317 & 0,329 & 0,583 & 0,873 & 0,252 & 0,759 & 0,672 & 1,220 & 0,453 & 0,512 & 0,898 & 0,459 & 0,386 & 62 & 287 & $-3,2$ \\
\hline 850 & 287 & 0,042 & 0,643 & 0,042 & 0,347 & 1,000 & 0,539 & 0,461 & 1,000 & 0,889 & 0,710 & 0,490 & 0,273 & 0,134 & 0,169 & 39 & 287 & $-1,4$ \\
\hline 851 & 287 & 0,020 & 0,389 & 0,020 & 0,693 & 1,000 & 1,783 & 0,783 & 1,000 & 0,774 & 0,990 & 0,667 & 0,161 & 0,107 & 0,152 & 45 & 287 & $-0,7$ \\
\hline 852 & 287 & 0,020 & 0,410 & 0,020 & 0,338 & 1,000 & 0,825 & 0,175 & 1,000 & 0,816 & 0,990 & 0,707 & 0,161 & 0,114 & 0,152 & 45 & 287 & $-0,7$ \\
\hline 853 & 287 & 0,023 & 0,440 & 0,023 & 0,317 & 1,000 & 0,721 & 0,279 & 1,000 & 0,818 & 1,019 & 0,671 & 0,169 & 0,114 & 0,152 & 15 & 287 & $-0,8$ \\
\hline 854 & 287 & 0,947 & 6,417 & 1,468 & 0,757 & 0,645 & 0,118 & 0,951 & 0,289 & 1,860 & 0,222 & 0,381 & 2,328 & 0,887 & 0,682 & 63 & 287 & $-4,5$ \\
\hline 855 & 287 & 0,020 & 0,469 & 0,023 & 0,613 & 0,875 & 1,306 & 0,331 & 1,000 & 0,934 & 0,686 & 0,527 & 0,193 & 0,102 & 0,107 & 174 & 287 & $-0,9$ \\
\hline 856 & 287 & 0,096 & 1,184 & 0,108 & 0,440 & 0,893 & 0,371 & 0,638 & 0,862 & 1,077 & 0,520 & 0,524 & 0,485 & 0,254 & 0,227 & 38 & 287 & $-2,3$ \\
\hline 857 & 287 & 0,019 & 0,634 & 0,023 & 0,930 & 0,812 & 1,467 & 0,503 & 0,583 & 1,309 & 0,255 & 0,181 & 0,305 & 0,055 & 0,076 & 15 & 287 & $-1,6$ \\
\hline 858 & 287 & 0,766 & 4,721 & 0,934 & 0,613 & 0,820 & 0,130 & 0,889 & 0,432 & 1,521 & 0,349 & 0,427 & 1,672 & 0,713 & 0,606 & 98 & 287 & $-4,1$ \\
\hline 859 & 287 & 0,019 & 0,418 & 0,020 & 0,469 & 0,929 & 1,121 & 0,141 & 1,000 & 0,864 & 0,828 & 0,600 & 0,169 & 0,102 & 0,152 & 161 & 287 & $-0,8$ \\
\hline 860 & 287 & 0,109 & 2,283 & 0,164 & 0,664 & 0,667 & 0,291 & 0,784 & 0,263 & 1,950 & 0,523 & 0,676 & 0,515 & 0,348 & 0,273 & 62 & 287 & $-2,4$ \\
\hline 861 & 287 & 0,023 & 0,461 & 0,026 & 0,410 & 0,889 & 0,890 & 0,156 & 1,000 & 0,858 & 1,019 & 0,671 & 0,169 & 0,114 & 0,152 & 88 & 287 & $-0,8$ \\
\hline 862 & 287 & 0,067 & 0,960 & 0,076 & 0,296 & 0,887 & 0,308 & 0,701 & 0,920 & 1,042 & 0,665 & 0,612 & 0,359 & 0,220 & 0,214 & 116 & 287 & $-1,8$ \\
\hline 863 & 287 & 0,060 & 1,243 & 0,075 & 0,693 & 0,808 & 0,558 & 0,482 & 0,490 & 1,428 & 0,594 & 0,596 & 0,359 & 0,214 & 0,214 & 147 & 287 & $-1,8$ \\
\hline 864 & 287 & 0,047 & 0,786 & 0,055 & 0,592 & 0,868 & 0,753 & 0,280 & 0,963 & 1,019 & 0,568 & 0,572 & 0,326 & 0,186 & 0,169 & 36 & 287 & $-1,7$ \\
\hline
\end{tabular}




\begin{tabular}{|c|c|c|c|c|c|c|c|c|c|c|c|c|c|c|c|c|c|c|}
\hline 865 & 287 & 0,014 & 0,397 & 0,014 & 1,319 & 1,000 & 3,318 & 2,318 & 1,000 & 0,936 & 0,509 & 0,283 & 0,189 & 0,054 & 0,076 & 40 & 287 & $-0,9$ \\
\hline 866 & 287 & 0,014 & 0,490 & 0,020 & 0,664 & 0,714 & 1,354 & 0,455 & 0,750 & 1,155 & 0,979 & 0,832 & 0,137 & 0,114 & 0,076 & 135 & 287 & $-0,5$ \\
\hline 867 & 287 & 0,179 & 1,492 & 0,189 & 0,532 & 0,947 & 0,357 & 0,645 & 1,000 & 0,994 & 0,658 & 0,661 & 0,589 & 0,390 & 0,408 & 31 & 287 & $-2,6$ \\
\hline 868 & 287 & 0,022 & 0,562 & 0,026 & 0,317 & 0,833 & 0,564 & 0,467 & 0,856 & 1,081 & 0,562 & 0,479 & 0,221 & 0,106 & 0,076 & 162 & 287 & $-1,1$ \\
\hline 869 & 287 & 0,231 & 1,746 & 0,242 & 0,245 & 0,953 & 0,140 & 0,861 & 0,952 & 1,025 & 0,700 & 0,643 & 0,648 & 0,417 & 0,455 & 86 & 287 & $-2,7$ \\
\hline 870 & 287 & 0,022 & 0,440 & 0,024 & 0,461 & 0,882 & 1,048 & 0,127 & 1,000 & 0,845 & 0,955 & 0,671 & 0,169 & 0,114 & 0,152 & 125 & 287 & $-0,8$ \\
\hline 871 & 287 & 0,030 & 0,520 & 0,032 & 3,166 & 0,955 & 6,088 & 5,088 & 1,000 & 0,845 & 0,922 & 0,657 & 0,204 & 0,134 & 0,152 & 67 & 287 & $-1,0$ \\
\hline 872 & 287 & 0,037 & 0,643 & 0,037 & 1,387 & 1,000 & 2,158 & 1,158 & 1,000 & 0,939 & 0,662 & 0,566 & 0,268 & 0,152 & 0,169 & 76 & 287 & $-1,4$ \\
\hline 873 & 287 & 0,016 & 0,368 & 0,016 & 0,338 & 1,000 & 0,919 & 0,081 & 1,000 & 0,826 & 1,077 & 0,784 & 0,137 & 0,107 & 0,107 & 90 & 287 & $-0,5$ \\
\hline 874 & 287 & 0,022 & 0,461 & 0,022 & 1,704 & 1,000 & 3,699 & 2,699 & 1,000 & 0,886 & 0,659 & 0,371 & 0,204 & 0,076 & 0,152 & 11 & 287 & $-1,0$ \\
\hline 875 & 287 & 0,034 & 0,613 & 0,036 & 1,336 & 0,960 & 2,180 & 1,180 & 1,000 & 0,932 & 0,745 & 0,552 & 0,243 & 0,134 & 0,152 & 44 & 287 & $-1,3$ \\
\hline 876 & 287 & 0,065 & 0,960 & 0,072 & 0,389 & 0,900 & 0,405 & 0,603 & 0,881 & 1,065 & 0,494 & 0,463 & 0,408 & 0,189 & 0,227 & 75 & 287 & $-2,0$ \\
\hline 877 & 287 & 0,063 & 0,786 & 0,066 & 1,429 & 0,957 & 1,817 & 0,818 & 1,000 & 0,883 & 1,057 & 0,824 & 0,276 & 0,227 & 0,273 & 176 & 287 & $-1,5$ \\
\hline 878 & 287 & 0,033 & 0,583 & 0,034 & 1,665 & 0,958 & 2,856 & 1,856 & 1,000 & 0,906 & 0,714 & 0,559 & 0,243 & 0,136 & 0,169 & 58 & 287 & $-1,3$ \\
\hline 879 & 287 & 0,027 & 0,541 & 0,030 & 0,440 & 0,905 & 0,812 & 0,210 & 1,000 & 0,924 & 0,654 & 0,493 & 0,230 & 0,114 & 0,152 & 15 & 287 & $-1,2$ \\
\hline 880 & 287 & 0,166 & 1,674 & 0,191 & 0,245 & 0,872 & 0,146 & 0,863 & 0,746 & 1,157 & 0,603 & 0,586 & 0,593 & 0,347 & 0,321 & 27 & 287 & $-2,6$ \\
\hline 881 & 287 & 0,014 & 0,347 & 0,016 & 0,634 & 0,909 & 1,829 & 0,834 & 1,000 & 0,816 & 0,979 & 0,588 & 0,137 & 0,080 & 0,107 & 124 & 287 & $-0,5$ \\
\hline 882 & 287 & 0,022 & 0,418 & 0,023 & 1,052 & 0,938 & 2,515 & 1,516 & 1,000 & 0,805 & 1,123 & 0,728 & 0,156 & 0,114 & 0,152 & 84 & 287 & $-0,6$ \\
\hline 883 & 287 & 0,029 & 0,592 & 0,033 & 0,347 & 0,870 & 0,586 & 0,434 & 1,000 & 0,986 & 0,621 & 0,501 & 0,243 & 0,121 & 0,152 & 151 & 287 & $-1,3$ \\
\hline 884 & 287 & 0,175 & 1,509 & 0,189 & 0,490 & 0,924 & 0,325 & 0,679 & 0,966 & 1,018 & 0,840 & 0,809 & 0,515 & 0,417 & 0,408 & 85 & 287 & $-2,4$ \\
\hline 885 & 287 & 0,020 & 0,418 & 0,022 & 0,194 & 0,933 & 0,465 & 0,540 & 1,000 & 0,833 & 0,990 & 0,667 & 0,161 & 0,107 & 0,152 & 17 & 287 & $-0,7$ \\
\hline 886 & 287 & 0,016 & 0,541 & 0,022 & 0,490 & 0,733 & 0,906 & 0,283 & 0,677 & 1,215 & 0,778 & 0,667 & 0,161 & 0,107 & 0,076 & 135 & 287 & $-0,7$ \\
\hline 887 & 287 & 0,024 & 0,571 & 0,027 & 1,923 & 0,895 & 3,369 & 2,371 & 0,941 & 1,031 & 0,528 & 0,331 & 0,243 & 0,080 & 0,107 & 40 & 287 & $-1,3$ \\
\hline 888 & 287 & 0,027 & 0,511 & 0,027 & 0,469 & 1,000 & 0,918 & 0,082 & 1,000 & 0,874 & 0,712 & 0,514 & 0,221 & 0,114 & 0,152 & 66 & 287 & $-1,1$ \\
\hline 889 & 287 & 0,115 & 1,112 & 0,118 & 0,296 & 0,976 & 0,266 & 0,734 & 1,000 & 0,926 & 0,878 & 0,780 & 0,408 & 0,318 & 0,321 & 144 & 287 & $-2,0$ \\
\hline 890 & 287 & 0,019 & 0,389 & 0,020 & 0,469 & 0,929 & 1,207 & 0,219 & 1,000 & 0,803 & 1,273 & 0,832 & 0,137 & 0,114 & 0,152 & 27 & 287 & $-0,5$ \\
\hline 891 & 287 & 0,019 & 0,397 & 0,019 & 0,837 & 1,000 & 2,106 & 1,106 & 1,000 & 0,821 & 0,974 & 0,651 & 0,156 & 0,102 & 0,107 & 59 & 287 & $-0,6$ \\
\hline 892 & 287 & 0,023 & 0,440 & 0,023 & 0,765 & 1,000 & 1,741 & 0,741 & 1,000 & 0,818 & 0,815 & 0,566 & 0,189 & 0,107 & 0,152 & 60 & 287 & $-0,9$ \\
\hline 893 & 287 & 0,019 & 0,368 & 0,019 & 0,296 & 1,000 & 0,805 & 0,195 & 1,000 & 0,760 & 1,273 & 0,784 & 0,137 & 0,107 & 0,152 & 90 & 287 & $-0,5$ \\
\hline 894 & 287 & 0,062 & 1,040 & 0,070 & 1,653 & 0,878 & 1,589 & 0,602 & 0,717 & 1,181 & 0,472 & 0,464 & 0,408 & 0,189 & 0,169 & 81 & 287 & $-2,0$ \\
\hline 895 & 287 & 0,024 & 0,461 & 0,026 & 0,317 & 0,944 & 0,688 & 0,317 & 1,000 & 0,832 & 1,082 & 0,671 & 0,169 & 0,114 & 0,152 & 27 & 287 & $-0,8$ \\
\hline 896 & 287 & 0,027 & 0,511 & 0,029 & 1,315 & 0,950 & 2,571 & 1,572 & 1,000 & 0,874 & 0,968 & 0,759 & 0,189 & 0,144 & 0,152 & 75 & 287 & $-0,9$ \\
\hline 897 & 287 & 0,019 & 0,389 & 0,020 & 0,879 & 0,929 & 2,261 & 1,263 & 1,000 & 0,803 & 0,920 & 0,633 & 0,161 & 0,102 & 0,152 & 60 & 287 & $-0,7$ \\
\hline 898 & 287 & 0,020 & 0,520 & 0,024 & 0,448 & 0,824 & 0,862 & 0,224 & 0,934 & 1,035 & 0,891 & 0,671 & 0,169 & 0,114 & 0,107 & 35 & 287 & $-0,8$ \\
\hline 899 & 287 & 0,034 & 0,786 & 0,042 & 0,520 & 0,828 & 0,661 & 0,380 & 0,700 & 1,195 & 0,360 & 0,316 & 0,349 & 0,111 & 0,152 & 36 & 287 & $-1,8$ \\
\hline 900 & 287 & 0,016 & 0,714 & 0,030 & 0,266 & 0,524 & 0,373 & 0,788 & 0,389 & 1,604 & 0,219 & 0,250 & 0,303 & 0,076 & 0,076 & 169 & 287 & $-1,6$ \\
\hline 901 & 287 & 0,026 & 0,714 & 0,033 & 0,858 & 0,783 & 1,201 & 0,296 & 0,636 & 1,254 & 0,441 & 0,373 & 0,273 & 0,102 & 0,107 & 30 & 287 & $-1,4$ \\
\hline 902 & 287 & 0,057 & 0,968 & 0,062 & 0,440 & 0,930 & 0,454 & 0,550 & 0,769 & 1,140 & 0,351 & 0,282 & 0,456 & 0,129 & 0,169 & 136 & 287 & $-2,2$ \\
\hline 903 & 287 & 0,077 & 0,981 & 0,083 & 0,541 & 0,931 & 0,552 & 0,454 & 1,000 & 0,994 & 0,688 & 0,626 & 0,379 & 0,237 & 0,240 & 24 & 287 & $-1,9$ \\
\hline 904 & 287 & 0,141 & 1,970 & 0,171 & 3,238 & 0,824 & 1,644 & 0,667 & 0,455 & 1,482 & 0,674 & 0,767 & 0,515 & 0,395 & 0,214 & 111 & 287 & $-2,4$ \\
\hline 905 & 287 & 0,027 & 0,541 & 0,029 & 0,144 & 0,950 & 0,265 & 0,736 & 1,000 & 0,924 & 0,654 & 0,493 & 0,230 & 0,114 & 0,152 & 95 & 287 & $-1,2$ \\
\hline 906 & 287 & 0,020 & 0,389 & 0,020 & 0,287 & 1,000 & 0,739 & 0,261 & 1,000 & 0,774 & 1,371 & 0,832 & 0,137 & 0,114 & 0,152 & 135 & 287 & $-0,5$ \\
\hline 907 & 287 & 0,016 & 0,347 & 0,016 & 0,735 & 1,000 & 2,122 & 1,122 & 1,000 & 0,778 & 1,401 & 0,894 & 0,120 & 0,107 & 0,152 & 108 & 287 & $-0,3$ \\
\hline 908 & 287 & 0,017 & 0,482 & 0,019 & 0,389 & 0,923 & 0,807 & 0,208 & 0,933 & 1,036 & 0,413 & 0,164 & 0,230 & 0,038 & 0,076 & 0 & 287 & $-1,2$ \\
\hline 909 & 287 & 0,029 & 0,807 & 0,043 & 0,317 & 0,667 & 0,393 & 0,693 & 0,553 & 1,344 & 0,300 & 0,325 & 0,349 & 0,114 & 0,152 & 6 & 287 & $-1,8$ \\
\hline 910 & 287 & 0,039 & 1,306 & 0,066 & 0,194 & 0,587 & 0,149 & 0,946 & 0,285 & 1,872 & 0,382 & 0,588 & 0,359 & 0,211 & 0,152 & 110 & 287 & $-1,8$ \\
\hline 911 & 287 & 0,126 & 1,255 & 0,135 & 0,418 & 0,936 & 0,333 & 0,670 & 1,000 & 0,997 & 0,683 & 0,650 & 0,485 & 0,315 & 0,312 & 39 & 287 & $-2,3$ \\
\hline 912 & 287 & 0,037 & 0,837 & 0,053 & 0,418 & 0,703 & 0,500 & 0,582 & 0,669 & 1,222 & 0,625 & 0,589 & 0,276 & 0,162 & 0,152 & 154 & 287 & $-1,5$ \\
\hline 913 & 287 & 0,024 & 0,440 & 0,024 & 0,368 & 1,000 & 0,837 & 0,163 & 1,000 & 0,794 & 1,082 & 0,671 & 0,169 & 0,114 & 0,152 & 75 & 287 & $-0,8$ \\
\hline 914 & 287 & 0,042 & 0,672 & 0,044 & 0,562 & 0,935 & 0,836 & 0,176 & 1,000 & 0,930 & 0,738 & 0,600 & 0,268 & 0,161 & 0,214 & 26 & 287 & $-1,4$ \\
\hline 915 & 287 & 0,014 & 0,376 & 0,017 & 1,234 & 0,833 & 3,280 & 2,286 & 1,000 & 0,886 & 0,749 & 0,514 & 0,156 & 0,080 & 0,107 & 34 & 287 & $-0,6$ \\
\hline 916 & 287 & 0,014 & 0,418 & 0,017 & 0,562 & 0,833 & 1,343 & 0,382 & 1,000 & 0,986 & 0,509 & 0,388 & 0,189 & 0,074 & 0,076 & 162 & 287 & $-0,9$ \\
\hline 917 & 287 & 0,014 & 0,317 & 0,014 & 0,368 & 1,000 & 1,160 & 0,160 & 1,000 & 0,746 & 1,273 & 0,632 & 0,120 & 0,076 & 0,107 & 18 & 287 & $-0,3$ \\
\hline 918 & 287 & 0,158 & 1,686 & 0,184 & 0,194 & 0,859 & 0,115 & 0,896 & 0,697 & 1,197 & 0,431 & 0,398 & 0,683 & 0,272 & 0,303 & 77 & 287 & $-2,8$ \\
\hline 919 & 287 & 0,024 & 0,520 & 0,026 & 0,562 & 0,944 & 1,081 & 0,098 & 1,000 & 0,939 & 0,601 & 0,500 & 0,227 & 0,114 & 0,152 & 21 & 287 & $-1,2$ \\
\hline 920 & 287 & 0,032 & 0,553 & 0,033 & 0,410 & 0,957 & 0,740 & 0,263 & 1,000 & 0,879 & 0,824 & 0,514 & 0,221 & 0,114 & 0,152 & 169 & 287 & $-1,1$ \\
\hline 921 & 287 & 0,020 & 0,418 & 0,020 & 0,490 & 1,000 & 1,172 & 0,172 & 1,000 & 0,833 & 0,891 & 0,632 & 0,169 & 0,107 & 0,152 & 163 & 287 & $-0,8$ \\
\hline 922 & 287 & 0,017 & 0,368 & 0,017 & 1,602 & 1,000 & 4,357 & 3,357 & 1,000 & 0,791 & 0,899 & 0,485 & 0,156 & 0,076 & 0,152 & 172 & 287 & $-0,6$ \\
\hline
\end{tabular}




\begin{tabular}{|c|c|c|c|c|c|c|c|c|c|c|c|c|c|c|c|c|c|c|}
\hline 923 & 287 & 0,023 & 0,482 & 0,024 & 0,571 & 0,941 & 1,185 & 0,194 & 1,000 & 0,897 & 0,702 & 0,371 & 0,204 & 0,076 & 0,152 & 171 & 287 & $-1,0$ \\
\hline 924 & 287 & 0,027 & 0,520 & 0,029 & 1,809 & 0,950 & 3,479 & 2,479 & 1,000 & 0,888 & 0,654 & 0,493 & 0,230 & 0,114 & 0,152 & 9 & 287 & $-1,2$ \\
\hline 925 & 287 & 0,014 & 0,347 & 0,017 & 0,368 & 0,833 & 1,061 & 0,177 & 1,000 & 0,816 & 1,273 & 0,849 & 0,120 & 0,102 & 0,107 & 108 & 287 & $-0,3$ \\
\hline 926 & 287 & 0,123 & 1,412 & 0,131 & 0,765 & 0,945 & 0,542 & 0,461 & 0,778 & 1,134 & 0,400 & 0,385 & 0,627 & 0,241 & 0,273 & 74 & 287 & $-2,6$ \\
\hline 927 & 287 & 0,017 & 0,368 & 0,017 & 0,194 & 1,000 & 0,529 & 0,471 & 1,000 & 0,791 & 1,175 & 0,784 & 0,137 & 0,107 & 0,152 & 90 & 287 & $-0,5$ \\
\hline 928 & 287 & 0,231 & 2,329 & 0,291 & 0,347 & 0,793 & 0,149 & 0,876 & 0,535 & 1,367 & 0,328 & 0,405 & 0,947 & 0,383 & 0,312 & 76 & 287 & $-3,2$ \\
\hline 929 & 287 & 0,036 & 0,634 & 0,037 & 0,693 & 0,962 & 1,094 & 0,101 & 1,000 & 0,944 & 0,637 & 0,424 & 0,268 & 0,114 & 0,152 & 81 & 287 & $-1,4$ \\
\hline 930 & 287 & 0,066 & 0,816 & 0,067 & 1,856 & 0,979 & 2,275 & 1,275 & 1,000 & 0,896 & 0,861 & 0,728 & 0,312 & 0,227 & 0,240 & 167 & 287 & $-1,6$ \\
\hline 931 & 287 & 0,016 & 0,368 & 0,016 & 1,082 & 1,000 & 2,943 & 1,943 & 1,000 & 0,826 & 0,700 & 0,447 & 0,169 & 0,076 & 0,107 & 162 & 287 & $-0,8$ \\
\hline 932 & 287 & 0,049 & 0,960 & 0,062 & 1,166 & 0,791 & 1,216 & 0,300 & 0,666 & 1,226 & 0,333 & 0,338 & 0,432 & 0,146 & 0,152 & 145 & 287 & $-2,1$ \\
\hline 933 & 287 & 0,024 & 0,469 & 0,024 & 0,511 & 1,000 & 1,090 & 0,090 & 1,000 & 0,848 & 1,082 & 0,791 & 0,169 & 0,134 & 0,169 & 41 & 287 & $-0,8$ \\
\hline 934 & 287 & 0,017 & 0,368 & 0,017 & 0,317 & 1,000 & 0,862 & 0,138 & 1,000 & 0,791 & 1,175 & 0,745 & 0,137 & 0,102 & 0,107 & 45 & 287 & $-0,5$ \\
\hline 935 & 287 & 0,014 & 0,317 & 0,014 & 0,418 & 1,000 & 1,320 & 0,320 & 1,000 & 0,746 & 1,273 & 0,632 & 0,120 & 0,076 & 0,152 & 0 & 287 & $-0,3$ \\
\hline 936 & 287 & 0,042 & 0,765 & 0,049 & 0,389 & 0,853 & 0,508 & 0,513 & 0,893 & 1,058 & 0,499 & 0,466 & 0,326 & 0,152 & 0,152 & 49 & 287 & $-1,7$ \\
\hline 937 & 287 & 0,020 & 0,418 & 0,020 & 0,317 & 1,000 & 0,757 & 0,243 & 1,000 & 0,833 & 0,891 & 0,632 & 0,169 & 0,107 & 0,152 & 59 & 287 & $-0,8$ \\
\hline 938 & 287 & 0,029 & 0,520 & 0,030 & 1,184 & 0,952 & 2,276 & 1,277 & 1,000 & 0,866 & 0,979 & 0,784 & 0,193 & 0,152 & 0,152 & 14 & 287 & $-0,9$ \\
\hline 939 & 287 & 0,073 & 1,133 & 0,080 & 0,960 & 0,911 & 0,847 & 0,177 & 0,716 & 1,181 & 0,336 & 0,305 & 0,526 & 0,161 & 0,169 & 61 & 287 & $-2,4$ \\
\hline 940 & 287 & 0,023 & 0,461 & 0,023 & 1,450 & 1,000 & 3,148 & 2,148 & 1,000 & 0,858 & 0,784 & 0,392 & 0,193 & 0,076 & 0,152 & 90 & 287 & $-0,9$ \\
\hline 941 & 287 & 0,026 & 0,511 & 0,026 & 1,243 & 1,000 & 2,431 & 1,431 & 1,000 & 0,898 & 0,619 & 0,329 & 0,230 & 0,076 & 0,152 & 3 & 287 & $-1,2$ \\
\hline 942 & 287 & 0,057 & 1,070 & 0,073 & 0,296 & 0,784 & 0,277 & 0,755 & 0,630 & 1,260 & 0,525 & 0,621 & 0,373 & 0,232 & 0,152 & 23 & 287 & $-1,9$ \\
\hline 943 & 287 & 0,017 & 0,368 & 0,017 & 0,765 & 1,000 & 2,081 & 1,081 & 1,000 & 0,791 & 0,899 & 0,485 & 0,156 & 0,076 & 0,152 & 8 & 287 & $-0,6$ \\
\hline 944 & 287 & 0,043 & 0,723 & 0,046 & 0,541 & 0,938 & 0,748 & 0,259 & 1,000 & 0,983 & 0,659 & 0,529 & 0,288 & 0,153 & 0,169 & 36 & 287 & $-1,5$ \\
\hline 945 & 287 & 0,067 & 0,930 & 0,073 & 0,490 & 0,922 & 0,527 & 0,479 & 0,980 & 1,010 & 0,549 & 0,455 & 0,395 & 0,180 & 0,214 & 169 & 287 & $-2,0$ \\
\hline 946 & 287 & 0,066 & 1,031 & 0,075 & 1,458 & 0,885 & 1,414 & 0,430 & 0,780 & 1,132 & 0,480 & 0,450 & 0,418 & 0,188 & 0,227 & 9 & 287 & $-2,1$ \\
\hline 947 & 287 & 0,030 & 0,511 & 0,032 & 0,592 & 0,955 & 1,157 & 0,164 & 1,000 & 0,831 & 1,070 & 0,800 & 0,189 & 0,152 & 0,169 & 149 & 287 & $-0,9$ \\
\hline 948 & 287 & 0,022 & 0,490 & 0,023 & 0,296 & 0,938 & 0,604 & 0,401 & 1,000 & 0,943 & 0,516 & 0,329 & 0,230 & 0,076 & 0,152 & 169 & 287 & $-1,2$ \\
\hline 949 & 287 & 0,062 & 0,947 & 0,072 & 0,266 & 0,860 & 0,281 & 0,732 & 0,864 & 1,076 & 0,676 & 0,661 & 0,341 & 0,225 & 0,227 & 22 & 287 & $-1,8$ \\
\hline 950 & 287 & 0,861 & 7,212 & 1,334 & 1,010 & 0,645 & 0,140 & 0,930 & 0,208 & 2,193 & 0,218 & 0,390 & 2,244 & 0,874 & 0,152 & 164 & 287 & $-4,5$ \\
\hline 951 & 287 & 0,016 & 0,550 & 0,023 & 0,735 & 0,688 & 1,338 & 0,460 & 0,656 & 1,234 & 0,379 & 0,306 & 0,230 & 0,070 & 0,076 & 152 & 287 & $-1,2$ \\
\hline 952 & 287 & 0,020 & 0,389 & 0,020 & 0,490 & 1,000 & 1,261 & 0,261 & 1,000 & 0,774 & 1,371 & 0,832 & 0,137 & 0,114 & 0,152 & 135 & 287 & $-0,5$ \\
\hline 953 & 287 & 0,037 & 0,693 & 0,043 & 0,317 & 0,867 & 0,457 & 0,559 & 0,975 & 1,013 & 0,807 & 0,674 & 0,243 & 0,164 & 0,152 & 63 & 287 & $-1,3$ \\
\hline 954 & 287 & 0,976 & 4,426 & 1,083 & 0,917 & 0,901 & 0,207 & 0,799 & 0,626 & 1,264 & 0,415 & 0,429 & 1,731 & 0,743 & 0,715 & 70 & 287 & $-4,1$ \\
\hline 955 & 287 & 0,014 & 0,397 & 0,016 & 0,397 & 0,909 & 1,000 & 0,091 & 1,000 & 0,936 & 0,637 & 0,447 & 0,169 & 0,076 & 0,107 & 108 & 287 & $-0,8$ \\
\hline 956 & 287 & 0,020 & 0,389 & 0,020 & 0,368 & 1,000 & 0,946 & 0,054 & 1,000 & 0,774 & 0,990 & 0,667 & 0,161 & 0,107 & 0,152 & 45 & 287 & $-0,7$ \\
\hline 957 & 287 & 0,367 & 3,111 & 0,439 & 0,541 & 0,837 & 0,174 & 0,842 & 0,477 & 1,448 & 0,267 & 0,304 & 1,323 & 0,402 & 0,303 & 66 & 287 & $-3,7$ \\
\hline 958 & 287 & 0,032 & 0,622 & 0,034 & 0,664 & 0,917 & 1,068 & 0,107 & 1,000 & 0,987 & 0,824 & 0,728 & 0,221 & 0,161 & 0,152 & 124 & 287 & $-1,1$ \\
\hline 959 & 287 & 0,017 & 0,368 & 0,019 & 3,213 & 0,923 & 8,737 & 7,738 & 1,000 & 0,791 & 1,175 & 0,745 & 0,137 & 0,102 & 0,107 & 135 & 287 & $-0,5$ \\
\hline 960 & 287 & 0,085 & 1,205 & 0,102 & 0,194 & 0,831 & 0,161 & 0,855 & 0,733 & 1,168 & 0,515 & 0,583 & 0,458 & 0,267 & 0,273 & 118 & 287 & $-2,2$ \\
\hline 961 & 287 & 0,062 & 0,960 & 0,070 & 0,440 & 0,878 & 0,458 & 0,556 & 0,842 & 1,090 & 0,472 & 0,457 & 0,408 & 0,186 & 0,214 & 71 & 287 & $-2,0$ \\
\hline 962 & 287 & 0,082 & 1,725 & 0,108 & 0,765 & 0,760 & 0,444 & 0,606 & 0,346 & 1,701 & 0,354 & 0,349 & 0,542 & 0,189 & 0,169 & 94 & 287 & $-2,4$ \\
\hline 963 & 287 & 0,142 & 1,733 & 0,181 & 0,744 & 0,786 & 0,429 & 0,610 & 0,594 & 1,297 & 0,485 & 0,510 & 0,611 & 0,312 & 0,273 & 24 & 287 & $-2,6$ \\
\hline 964 & 287 & 0,019 & 0,490 & 0,023 & 1,602 & 0,812 & 3,268 & 2,275 & 0,975 & 1,013 & 0,662 & 0,570 & 0,189 & 0,108 & 0,152 & 26 & 287 & $-0,9$ \\
\hline 965 & 287 & 0,063 & 0,807 & 0,063 & 1,670 & 1,000 & 2,069 & 1,069 & 1,000 & 0,906 & 0,767 & 0,585 & 0,324 & 0,189 & 0,227 & 9 & 287 & $-1,7$ \\
\hline 966 & 287 & 0,156 & 1,742 & 0,184 & 0,448 & 0,852 & 0,257 & 0,757 & 0,648 & 1,243 & 0,540 & 0,521 & 0,607 & 0,316 & 0,312 & 108 & 287 & $-2,6$ \\
\hline 967 & 287 & 0,166 & 2,033 & 0,217 & 0,397 & 0,768 & 0,195 & 0,837 & 0,506 & 1,406 & 0,381 & 0,434 & 0,746 & 0,324 & 0,240 & 11 & 287 & $-2,9$ \\
\hline 968 & 287 & 0,032 & 0,583 & 0,034 & 1,040 & 0,917 & 1,783 & 0,788 & 1,000 & 0,926 & 0,700 & 0,474 & 0,240 & 0,114 & 0,152 & 100 & 287 & $-1,3$ \\
\hline 969 & 287 & 0,040 & 0,714 & 0,043 & 1,366 & 0,933 & 1,912 & 0,914 & 0,989 & 1,005 & 0,548 & 0,500 & 0,305 & 0,153 & 0,152 & 35 & 287 & $-1,6$ \\
\hline 970 & 287 & 0,356 & 2,329 & 0,402 & 0,511 & 0,886 & 0,220 & 0,789 & 0,824 & 1,101 & 0,710 & 0,715 & 0,799 & 0,572 & 0,530 & 175 & 287 & $-3,0$ \\
\hline 971 & 287 & 0,062 & 1,593 & 0,098 & 1,070 & 0,632 & 0,671 & 0,493 & 0,305 & 1,810 & 0,468 & 0,647 & 0,410 & 0,265 & 0,107 & 96 & 287 & $-2,0$ \\
\hline 972 & 287 & 0,022 & 0,431 & 0,022 & 0,499 & 1,000 & 1,158 & 0,158 & 1,000 & 0,829 & 0,955 & 0,447 & 0,169 & 0,076 & 0,152 & 90 & 287 & $-0,8$ \\
\hline 973 & 287 & 0,653 & 3,948 & 0,739 & 1,564 & 0,883 & 0,396 & 0,615 & 0,526 & 1,379 & 0,513 & 0,526 & 1,273 & 0,669 & 0,152 & 26 & 287 & $-3,7$ \\
\hline 974 & 287 & 0,016 & 0,368 & 0,016 & 7,644 & 1,000 & 20,787 & 19,787 & 1,000 & 0,826 & 0,824 & 0,485 & 0,156 & 0,076 & 0,107 & 18 & 287 & $-0,6$ \\
\hline 975 & 287 & 0,098 & 1,361 & 0,108 & 0,368 & 0,907 & 0,270 & 0,736 & 0,662 & 1,229 & 0,299 & 0,235 & 0,645 & 0,152 & 0,227 & 178 & 287 & $-2,7$ \\
\hline 976 & 287 & 0,016 & 0,469 & 0,019 & 0,643 & 0,846 & 1,369 & 0,400 & 0,901 & 1,054 & 0,483 & 0,371 & 0,204 & 0,076 & 0,107 & 69 & 287 & $-1,0$ \\
\hline 977 & 287 & 0,019 & 0,397 & 0,020 & 0,714 & 0,929 & 1,798 & 0,801 & 1,000 & 0,821 & 1,035 & 0,707 & 0,152 & 0,107 & 0,152 & 135 & 287 & $-0,6$ \\
\hline 978 & 287 & 0,022 & 0,469 & 0,022 & 0,681 & 1,000 & 1,451 & 0,451 & 1,000 & 0,902 & 0,562 & 0,364 & 0,221 & 0,080 & 0,107 & 32 & 287 & $-1,1$ \\
\hline 979 & 287 & 0,067 & 0,828 & 0,069 & 0,368 & 0,979 & 0,444 & 0,556 & 1,000 & 0,900 & 0,921 & 0,744 & 0,305 & 0,227 & 0,273 & 11 & 287 & $-1,6$ \\
\hline 980 & 287 & 0,016 & 0,368 & 0,017 & 0,765 & 0,917 & 2,081 & 1,084 & 1,000 & 0,826 & 0,778 & 0,500 & 0,161 & 0,080 & 0,107 & 127 & 287 & $-0,7$ \\
\hline
\end{tabular}




\begin{tabular}{|c|c|c|c|c|c|c|c|c|c|c|c|c|c|c|c|c|c|c|}
\hline 981 & 287 & 0,083 & 1,543 & 0,102 & 0,338 & 0,817 & 0,219 & 0,802 & 0,439 & 1,509 & 0,738 & 0,761 & 0,379 & 0,288 & 0,214 & 153 & 287 & $-1,9$ \\
\hline 982 & 287 & 0,014 & 0,440 & 0,017 & 0,245 & 0,833 & 0,558 & 0,473 & 0,933 & 1,035 & 0,439 & 0,271 & 0,204 & 0,055 & 0,076 & 105 & 287 & $-1,0$ \\
\hline 983 & 287 & 0,047 & 0,714 & 0,047 & 0,541 & 1,000 & 0,757 & 0,243 & 1,000 & 0,926 & 0,646 & 0,496 & 0,305 & 0,152 & 0,214 & 4 & 287 & $-1,6$ \\
\hline 984 & 287 & 0,161 & 3,157 & 0,380 & 0,685 & 0,423 & 0,217 & 0,973 & 0,203 & 2,222 & 0,129 & 0,301 & 1,259 & 0,379 & 0,169 & 105 & 287 & $-3,7$ \\
\hline 985 & 287 & 0,037 & 0,846 & 0,052 & 0,757 & 0,722 & 0,895 & 0,297 & 0,656 & 1,235 & 0,447 & 0,479 & 0,326 & 0,156 & 0,152 & 36 & 287 & $-1,7$ \\
\hline 986 & 287 & 0,016 & 0,368 & 0,017 & 0,816 & 0,917 & 2,219 & 1,222 & 1,000 & 0,826 & 0,824 & 0,485 & 0,156 & 0,076 & 0,107 & 85 & 287 & $-0,6$ \\
\hline 987 & 287 & 0,072 & 0,960 & 0,079 & 0,685 & 0,909 & 0,714 & 0,301 & 0,979 & 1,011 & 0,563 & 0,496 & 0,403 & 0,200 & 0,214 & 37 & 287 & $-2,0$ \\
\hline 988 & 287 & 0,073 & 1,082 & 0,083 & 0,194 & 0,879 & 0,180 & 0,829 & 0,785 & 1,128 & 0,414 & 0,393 & 0,475 & 0,186 & 0,214 & 151 & 287 & $-2,2$ \\
\hline 989 & 287 & 0,032 & 0,583 & 0,033 & 0,296 & 0,957 & 0,507 & 0,495 & 1,000 & 0,926 & 0,622 & 0,447 & 0,254 & 0,114 & 0,152 & 17 & 287 & $-1,3$ \\
\hline 990 & 287 & 0,030 & 0,643 & 0,033 & 1,467 & 0,913 & 2,283 & 1,286 & 0,917 & 1,044 & 0,594 & 0,534 & 0,254 & 0,136 & 0,152 & 60 & 287 & $-1,3$ \\
\hline 991 & 287 & 0,069 & 0,909 & 0,077 & 0,930 & 0,889 & 1,023 & 0,114 & 1,000 & 0,977 & 0,849 & 0,738 & 0,321 & 0,237 & 0,240 & 46 & 287 & $-1,7$ \\
\hline 992 & 287 & 0,014 & 0,317 & 0,014 & 1,226 & 1,000 & 3,867 & 2,867 & 1,000 & 0,746 & 0,979 & 0,555 & 0,137 & 0,076 & 0,107 & 18 & 287 & $-0,5$ \\
\hline 993 & 287 & 0,032 & 0,592 & 0,034 & 0,173 & 0,917 & 0,293 & 0,712 & 1,000 & 0,940 & 0,700 & 0,600 & 0,240 & 0,144 & 0,169 & 79 & 287 & $-1,3$ \\
\hline 994 & 287 & 0,154 & 2,355 & 0,221 & 0,778 & 0,695 & 0,330 & 0,736 & 0,348 & 1,695 & 0,404 & 0,561 & 0,695 & 0,390 & 0,152 & 65 & 287 & $-2,8$ \\
\hline 995 & 287 & 0,040 & 0,643 & 0,042 & 1,112 & 0,966 & 1,730 & 0,731 & 1,000 & 0,904 & 0,686 & 0,490 & 0,273 & 0,134 & 0,169 & 51 & 287 & $-1,4$ \\
\hline 996 & 287 & 0,043 & 0,685 & 0,046 & 0,440 & 0,938 & 0,642 & 0,363 & 1,000 & 0,931 & 0,849 & 0,745 & 0,254 & 0,189 & 0,214 & 175 & 287 & $-1,3$ \\
\hline 997 & 287 & 0,076 & 1,082 & 0,090 & 0,693 & 0,841 & 0,641 & 0,393 & 0,816 & 1,107 & 0,519 & 0,521 & 0,432 & 0,225 & 0,227 & 176 & 287 & $-2,1$ \\
\hline 998 & 287 & 0,017 & 0,389 & 0,017 & 0,194 & 1,000 & 0,500 & 0,500 & 1,000 & 0,836 & 0,764 & 0,447 & 0,169 & 0,076 & 0,107 & 18 & 287 & $-0,8$ \\
\hline 999 & 287 & 0,112 & 1,501 & 0,139 & 1,623 & 0,804 & 1,082 & 0,212 & 0,625 & 1,265 & 0,375 & 0,430 & 0,617 & 0,265 & 0,214 & 4 & 287 & $-2,6$ \\
\hline 1000 & 287 & 0,026 & 0,490 & 0,026 & 1,408 & 1,000 & 2,871 & 1,871 & 1,000 & 0,861 & 0,917 & 0,667 & 0,189 & 0,126 & 0,152 & 41 & 287 & $-0,9$ \\
\hline 1001 & 287 & 0,022 & 0,541 & 0,027 & 0,469 & 0,789 & 0,867 & 0,249 & 0,924 & 1,040 & 0,516 & 0,441 & 0,230 & 0,102 & 0,107 & 59 & 287 & $-1,2$ \\
\hline 1002 & 287 & 0,142 & 1,429 & 0,154 & 0,245 & 0,925 & 0,172 & 0,832 & 0,874 & 1,069 & 0,485 & 0,472 & 0,611 & 0,288 & 0,303 & 66 & 287 & $-2,6$ \\
\hline 1003 & 287 & 0,016 & 0,440 & 0,019 & 0,858 & 0,846 & 1,952 & 0,964 & 1,000 & 0,987 & 0,700 & 0,597 & 0,169 & 0,101 & 0,107 & 18 & 287 & $-0,8$ \\
\hline 1004 & 287 & 0,022 & 0,440 & 0,023 & 0,440 & 0,938 & 1,000 & 0,062 & 1,000 & 0,845 & 0,955 & 0,637 & 0,169 & 0,108 & 0,152 & 117 & 287 & $-0,8$ \\
\hline 1005 & 287 & 0,014 & 0,347 & 0,014 & 0,317 & 1,000 & 0,914 & 0,086 & 1,000 & 0,816 & 0,749 & 0,485 & 0,156 & 0,076 & 0,107 & 72 & 287 & $-0,6$ \\
\hline 1006 & 287 & 0,055 & 1,306 & 0,092 & 1,010 & 0,594 & 0,773 & 0,465 & 0,402 & 1,578 & 0,372 & 0,591 & 0,432 & 0,255 & 0,107 & 16 & 287 & $-2,1$ \\
\hline 1007 & 287 & 0,024 & 0,469 & 0,027 & 0,418 & 0,895 & 0,892 & 0,151 & 1,000 & 0,848 & 1,082 & 0,791 & 0,169 & 0,134 & 0,169 & 138 & 287 & $-0,8$ \\
\hline 1008 & 287 & 0,022 & 0,541 & 0,024 & 1,285 & 0,882 & 2,375 & 1,380 & 0,924 & 1,040 & 0,764 & 0,667 & 0,189 & 0,126 & 0,152 & 0 & 287 & $-0,9$ \\
\hline 1009 & 287 & 0,017 & 0,368 & 0,017 & 0,338 & 1,000 & 0,919 & 0,081 & 1,000 & 0,791 & 0,849 & 0,500 & 0,161 & 0,080 & 0,107 & 45 & 287 & $-0,7$ \\
\hline 1010 & 287 & 0,141 & 1,429 & 0,154 & 0,685 & 0,916 & 0,479 & 0,528 & 0,866 & 1,075 & 0,480 & 0,468 & 0,611 & 0,286 & 0,273 & 151 & 287 & $-2,6$ \\
\hline 1011 & 287 & 0,016 & 0,368 & 0,016 & 1,399 & 1,000 & 3,805 & 2,805 & 1,000 & 0,826 & 0,824 & 0,485 & 0,156 & 0,076 & 0,107 & 0 & 287 & $-0,6$ \\
\hline 1012 & 287 & 0,065 & 0,858 & 0,067 & 0,837 & 0,957 & 0,975 & 0,049 & 1,000 & 0,953 & 0,716 & 0,637 & 0,339 & 0,216 & 0,227 & 19 & 287 & $-1,8$ \\
\hline 1013 & 287 & 0,096 & 1,665 & 0,126 & 0,520 & 0,761 & 0,312 & 0,728 & 0,436 & 1,515 & 0,327 & 0,344 & 0,612 & 0,210 & 0,152 & 111 & 287 & $-2,6$ \\
\hline 1014 & 287 & 0,088 & 1,695 & 0,123 & 0,245 & 0,709 & 0,145 & 0,903 & 0,383 & 1,616 & 0,521 & 0,682 & 0,462 & 0,315 & 0,214 & 134 & 287 & $-2,2$ \\
\hline 1015 & 287 & 0,019 & 0,482 & 0,022 & 0,541 & 0,867 & 1,123 & 0,182 & 1,000 & 0,995 & 0,571 & 0,365 & 0,204 & 0,074 & 0,076 & 7 & 287 & $-1,0$ \\
\hline 1016 & 287 & 0,027 & 0,490 & 0,027 & 0,592 & 1,000 & 1,207 & 0,207 & 1,000 & 0,838 & 0,968 & 0,707 & 0,189 & 0,134 & 0,169 & 45 & 287 & $-0,9$ \\
\hline 1017 & 287 & 0,024 & 0,550 & 0,029 & 0,317 & 0,850 & 0,577 & 0,449 & 1,000 & 0,993 & 0,541 & 0,396 & 0,240 & 0,095 & 0,107 & 63 & 287 & $-1,3$ \\
\hline 1018 & 287 & 0,274 & 2,021 & 0,296 & 0,795 & 0,927 & 0,393 & 0,611 & 0,843 & 1,089 & 0,510 & 0,545 & 0,827 & 0,451 & 0,442 & 110 & 287 & $-3,0$ \\
\hline 1019 & 287 & 0,020 & 0,469 & 0,023 & 0,397 & 0,875 & 0,847 & 0,198 & 1,000 & 0,934 & 0,713 & 0,566 & 0,189 & 0,107 & 0,152 & 18 & 287 & $-0,9$ \\
\hline 1020 & 287 & 0,016 & 0,347 & 0,016 & 0,448 & 1,000 & 1,293 & 0,293 & 1,000 & 0,778 & 1,077 & 0,588 & 0,137 & 0,080 & 0,107 & 72 & 287 & $-0,5$ \\
\hline 1021 & 287 & 0,029 & 0,786 & 0,039 & 0,347 & 0,741 & 0,441 & 0,616 & 0,583 & 1,309 & 0,392 & 0,372 & 0,305 & 0,114 & 0,152 & 178 & 287 & $-1,6$ \\
\hline 1022 & 287 & 0,026 & 0,744 & 0,036 & 0,511 & 0,720 & 0,687 & 0,420 & 0,586 & 1,306 & 0,573 & 0,664 & 0,240 & 0,159 & 0,152 & 142 & 287 & $-1,3$ \\
\hline 1023 & 287 & 0,088 & 1,019 & 0,093 & 1,226 & 0,938 & 1,203 & 0,212 & 1,000 & 0,972 & 0,670 & 0,591 & 0,408 & 0,241 & 0,273 & 29 & 287 & $-2,0$ \\
\hline 1024 & 287 & 0,023 & 0,469 & 0,024 & 0,634 & 0,941 & 1,351 & 0,356 & 1,000 & 0,874 & 1,019 & 0,701 & 0,169 & 0,119 & 0,152 & 49 & 287 & $-0,8$ \\
\hline 1025 & 287 & 0,468 & 3,115 & 0,552 & 1,112 & 0,847 & 0,357 & 0,661 & 0,606 & 1,285 & 0,392 & 0,455 & 1,233 & 0,561 & 0,152 & 5 & 287 & $-3,6$ \\
\hline 1026 & 287 & 0,029 & 0,511 & 0,030 & 0,287 & 0,952 & 0,562 & 0,441 & 1,000 & 0,852 & 1,019 & 0,800 & 0,189 & 0,152 & 0,152 & 75 & 287 & $-0,9$ \\
\hline 1027 & 287 & 0,143 & 1,429 & 0,156 & 1,234 & 0,917 & 0,864 & 0,159 & 0,883 & 1,064 & 0,621 & 0,559 & 0,542 & 0,303 & 0,303 & 83 & 287 & $-2,4$ \\
\hline 1028 & 287 & 0,024 & 0,499 & 0,024 & 0,418 & 1,000 & 0,839 & 0,161 & 1,000 & 0,901 & 0,637 & 0,364 & 0,221 & 0,080 & 0,107 & 40 & 287 & $-1,1$ \\
\hline 1029 & 287 & 0,077 & 0,879 & 0,077 & 0,727 & 1,000 & 0,827 & 0,173 & 1,000 & 0,891 & 0,809 & 0,690 & 0,349 & 0,241 & 0,273 & 46 & 287 & $-1,8$ \\
\hline 1030 & 287 & 0,069 & 1,243 & 0,092 & 0,194 & 0,750 & 0,156 & 0,880 & 0,560 & 1,336 & 0,421 & 0,499 & 0,456 & 0,228 & 0,169 & 59 & 287 & $-2,2$ \\
\hline 1031 & 287 & 0,020 & 0,490 & 0,023 & 2,084 & 0,875 & 4,250 & 3,252 & 1,000 & 0,976 & 0,686 & 0,392 & 0,193 & 0,076 & 0,107 & 165 & 287 & $-0,9$ \\
\hline 1032 & 287 & 0,067 & 1,019 & 0,076 & 0,676 & 0,887 & 0,663 & 0,355 & 0,816 & 1,107 & 0,460 & 0,412 & 0,432 & 0,178 & 0,169 & 49 & 287 & $-2,1$ \\
\hline 1033 & 287 & 0,023 & 0,440 & 0,024 & 0,583 & 0,941 & 1,327 & 0,332 & 1,000 & 0,818 & 1,132 & 0,707 & 0,161 & 0,114 & 0,152 & 0 & 287 & $-0,7$ \\
\hline 1034 & 287 & 0,020 & 0,440 & 0,022 & 0,592 & 0,933 & 1,346 & 0,353 & 1,000 & 0,875 & 0,686 & 0,392 & 0,193 & 0,076 & 0,152 & 81 & 287 & $-0,9$ \\
\hline 1035 & 287 & 0,042 & 0,909 & 0,052 & 0,816 & 0,806 & 0,898 & 0,220 & 0,633 & 1,257 & 0,434 & 0,325 & 0,349 & 0,114 & 0,107 & 91 & 287 & $-1,8$ \\
\hline 1036 & 287 & 0,565 & 2,963 & 0,613 & 0,541 & 0,923 & 0,183 & 0,821 & 0,809 & 1,112 & 0,632 & 0,641 & 1,067 & 0,685 & 0,682 & 89 & 287 & $-3,4$ \\
\hline 1037 & 287 & 0,019 & 0,368 & 0,019 & 0,296 & 1,000 & 0,805 & 0,195 & 1,000 & 0,760 & 1,273 & 0,784 & 0,137 & 0,107 & 0,152 & 90 & 287 & $-0,5$ \\
\hline 1038 & 287 & 0,024 & 0,511 & 0,027 & 0,541 & 0,895 & 1,058 & 0,120 & 1,000 & 0,924 & 0,833 & 0,588 & 0,193 & 0,114 & 0,152 & 5 & 287 & $-0,9$ \\
\hline
\end{tabular}




\begin{tabular}{|c|c|c|c|c|c|c|c|c|c|c|c|c|c|c|c|c|c|c|}
\hline 1039 & 287 & 0,100 & 1,285 & 0,121 & 0,461 & 0,833 & 0,358 & 0,663 & 0,764 & 1,144 & 0,686 & 0,702 & 0,432 & 0,303 & 0,273 & 71 & 287 & $-2,1$ \\
\hline 1040 & 287 & 0,419 & 2,591 & 0,455 & 1,746 & 0,921 & 0,674 & 0,336 & 0,784 & 1,129 & 0,533 & 0,562 & 1,000 & 0,562 & 0,546 & 134 & 287 & $-3,3$ \\
\hline 1041 & 287 & 0,027 & 0,511 & 0,029 & 0,326 & 0,950 & 0,637 & 0,367 & 1,000 & 0,874 & 0,968 & 0,759 & 0,189 & 0,144 & 0,152 & 80 & 287 & $-0,9$ \\
\hline 1042 & 287 & 0,075 & 0,947 & 0,077 & 0,490 & 0,963 & 0,518 & 0,484 & 1,000 & 0,978 & 0,662 & 0,633 & 0,379 & 0,240 & 0,240 & 92 & 287 & $-1,9$ \\
\hline 1043 & 287 & 0,083 & 2,110 & 0,128 & 0,909 & 0,652 & 0,431 & 0,667 & 0,235 & 2,063 & 0,568 & 0,818 & 0,432 & 0,353 & 0,107 & 78 & 287 & $-2,1$ \\
\hline 1044 & 287 & 0,022 & 0,440 & 0,024 & 0,461 & 0,882 & 1,048 & 0,127 & 1,000 & 0,845 & 1,061 & 0,707 & 0,161 & 0,114 & 0,152 & 90 & 287 & $-0,7$ \\
\hline 1045 & 287 & 0,360 & 3,449 & 0,459 & 1,133 & 0,784 & 0,328 & 0,705 & 0,380 & 1,621 & 0,378 & 0,459 & 1,101 & 0,505 & 0,152 & 51 & 287 & $-3,5$ \\
\hline 1046 & 287 & 0,023 & 0,520 & 0,024 & 0,461 & 0,941 & 0,886 & 0,128 & 1,000 & 0,968 & 0,497 & 0,331 & 0,243 & 0,080 & 0,107 & 52 & 287 & $-1,3$ \\
\hline 1047 & 287 & 0,033 & 0,778 & 0,039 & 1,712 & 0,852 & 2,202 & 1,211 & 0,686 & 1,207 & 0,293 & 0,200 & 0,379 & 0,076 & 0,152 & 173 & 287 & $-1,9$ \\
\hline 1048 & 287 & 0,024 & 0,520 & 0,027 & 0,368 & 0,895 & 0,707 & 0,311 & 1,000 & 0,939 & 0,585 & 0,441 & 0,230 & 0,102 & 0,152 & 23 & 287 & $-1,2$ \\
\hline 1049 & 287 & 0,053 & 0,807 & 0,059 & 0,744 & 0,902 & 0,922 & 0,125 & 1,000 & 0,988 & 0,654 & 0,583 & 0,321 & 0,187 & 0,214 & 46 & 287 & $-1,7$ \\
\hline 1050 & 287 & 0,108 & 1,234 & 0,116 & 1,408 & 0,926 & 1,140 & 0,159 & 0,887 & 1,062 & 0,492 & 0,469 & 0,528 & 0,248 & 0,273 & 148 & 287 & $-2,4$ \\
\hline 1051 & 287 & 0,036 & 0,613 & 0,039 & 0,469 & 0,926 & 0,766 & 0,246 & 1,000 & 0,913 & 0,776 & 0,663 & 0,243 & 0,161 & 0,214 & 47 & 287 & $-1,3$ \\
\hline 1052 & 287 & 0,052 & 0,714 & 0,053 & 0,592 & 0,973 & 0,828 & 0,174 & 1,000 & 0,887 & 1,019 & 0,800 & 0,254 & 0,203 & 0,214 & 34 & 287 & $-1,3$ \\
\hline 1053 & 287 & 0,036 & 0,571 & 0,036 & 0,786 & 1,000 & 1,377 & 0,377 & 1,000 & 0,850 & 0,860 & 0,658 & 0,230 & 0,152 & 0,214 & 12 & 287 & $-1,2$ \\
\hline 1054 & 287 & 0,987 & 4,332 & 1,055 & 0,440 & 0,936 & 0,101 & 0,901 & 0,661 & 1,230 & 0,515 & 0,481 & 1,562 & 0,751 & 0,758 & 94 & 287 & $-4,0$ \\
\hline 1055 & 287 & 0,040 & 0,917 & 0,052 & 0,714 & 0,778 & 0,779 & 0,314 & 0,600 & 1,291 & 0,524 & 0,485 & 0,312 & 0,152 & 0,169 & 93 & 287 & $-1,6$ \\
\hline 1056 & 287 & 0,125 & 1,462 & 0,143 & 1,919 & 0,870 & 1,312 & 0,338 & 0,734 & 1,168 & 0,418 & 0,430 & 0,617 & 0,265 & 0,273 & 5 & 287 & $-2,6$ \\
\hline 1057 & 287 & 0,027 & 0,490 & 0,027 & 0,571 & 1,000 & 1,164 & 0,164 & 1,000 & 0,838 & 0,968 & 0,707 & 0,189 & 0,134 & 0,152 & 32 & 287 & $-0,9$ \\
\hline 1058 & 287 & 0,024 & 0,461 & 0,026 & 0,592 & 0,944 & 1,285 & 0,290 & 1,000 & 0,832 & 1,082 & 0,671 & 0,169 & 0,114 & 0,152 & 84 & 287 & $-0,8$ \\
\hline 1059 & 287 & 0,055 & 0,968 & 0,072 & 0,664 & 0,760 & 0,685 & 0,396 & 0,731 & 1,170 & 0,479 & 0,555 & 0,381 & 0,211 & 0,169 & 106 & 287 & $-1,9$ \\
\hline 1060 & 287 & 0,024 & 0,592 & 0,029 & 0,613 & 0,850 & 1,036 & 0,154 & 0,875 & 1,069 & 0,433 & 0,300 & 0,268 & 0,080 & 0,107 & 45 & 287 & $-1,4$ \\
\hline 1061 & 287 & 0,014 & 0,317 & 0,014 & 1,095 & 1,000 & 3,453 & 2,453 & 1,000 & 0,746 & 0,979 & 0,555 & 0,137 & 0,076 & 0,107 & 18 & 287 & $-0,5$ \\
\hline 1062 & 287 & 0,056 & 0,786 & 0,057 & 0,418 & 0,975 & 0,532 & 0,468 & 1,000 & 0,938 & 0,621 & 0,531 & 0,339 & 0,180 & 0,214 & 22 & 287 & $-1,8$ \\
\hline 1063 & 287 & 0,014 & 0,317 & 0,014 & 0,397 & 1,000 & 1,254 & 0,254 & 1,000 & 0,746 & 1,273 & 0,632 & 0,120 & 0,076 & 0,152 & 0 & 287 & $-0,3$ \\
\hline 1064 & 287 & 0,020 & 0,397 & 0,020 & 0,338 & 1,000 & 0,851 & 0,149 & 1,000 & 0,791 & 1,049 & 0,686 & 0,156 & 0,107 & 0,152 & 73 & 287 & $-0,6$ \\
\hline 1065 & 287 & 0,069 & 0,896 & 0,073 & 0,245 & 0,941 & 0,273 & 0,729 & 1,000 & 0,964 & 0,687 & 0,525 & 0,357 & 0,187 & 0,214 & 45 & 287 & $-1,8$ \\
\hline 1066 & 287 & 0,069 & 1,031 & 0,086 & 0,338 & 0,800 & 0,328 & 0,701 & 0,814 & 1,109 & 0,611 & 0,696 & 0,379 & 0,264 & 0,227 & 39 & 287 & $-1,9$ \\
\hline 1067 & 287 & 0,095 & 1,133 & 0,106 & 0,744 & 0,892 & 0,657 & 0,360 & 0,927 & 1,039 & 0,771 & 0,745 & 0,395 & 0,295 & 0,273 & 149 & 287 & $-2,0$ \\
\hline 1068 & 287 & 0,037 & 0,613 & 0,040 & 0,655 & 0,929 & 1,069 & 0,099 & 1,000 & 0,895 & 0,736 & 0,580 & 0,254 & 0,147 & 0,169 & 66 & 287 & $-1,3$ \\
\hline 1069 & 287 & 0,022 & 0,410 & 0,022 & 0,194 & 1,000 & 0,474 & 0,526 & 1,000 & 0,788 & 1,061 & 0,707 & 0,161 & 0,114 & 0,152 & 135 & 287 & $-0,7$ \\
\hline 1070 & 287 & 0,024 & 0,469 & 0,024 & 0,643 & 1,000 & 1,369 & 0,369 & 1,000 & 0,848 & 0,866 & 0,566 & 0,189 & 0,107 & 0,152 & 45 & 287 & $-0,9$ \\
\hline 1071 & 287 & 0,023 & 0,735 & 0,032 & 0,520 & 0,727 & 0,707 & 0,400 & 0,533 & 1,369 & 0,255 & 0,248 & 0,339 & 0,084 & 0,107 & 28 & 287 & $-1,8$ \\
\hline 1072 & 287 & 0,628 & 4,346 & 0,821 & 0,490 & 0,766 & 0,113 & 0,918 & 0,418 & 1,546 & 0,452 & 0,616 & 1,331 & 0,819 & 0,152 & 2 & 287 & $-3,7$ \\
\hline 1073 & 287 & 0,032 & 0,562 & 0,034 & 1,213 & 0,917 & 2,158 & 1,161 & 1,000 & 0,893 & 0,875 & 0,633 & 0,214 & 0,136 & 0,152 & 23 & 287 & $-1,1$ \\
\hline 1074 & 287 & 0,022 & 0,469 & 0,023 & 0,532 & 0,938 & 1,135 & 0,148 & 1,000 & 0,902 & 0,955 & 0,791 & 0,169 & 0,134 & 0,152 & 30 & 287 & $-0,8$ \\
\hline 1075 & 287 & 0,019 & 0,418 & 0,020 & 0,592 & 0,929 & 1,414 & 0,420 & 1,000 & 0,864 & 0,828 & 0,501 & 0,169 & 0,085 & 0,107 & 23 & 287 & $-0,8$ \\
\hline 1076 & 287 & 0,016 & 0,389 & 0,017 & 1,695 & 0,917 & 4,360 & 3,361 & 1,000 & 0,873 & 0,824 & 0,485 & 0,156 & 0,076 & 0,107 & 5 & 287 & $-0,6$ \\
\hline 1077 & 287 & 0,077 & 1,234 & 0,098 & 0,296 & 0,794 & 0,240 & 0,788 & 0,639 & 1,251 & 0,397 & 0,409 & 0,498 & 0,204 & 0,214 & 103 & 287 & $-2,3$ \\
\hline 1078 & 287 & 0,044 & 0,693 & 0,046 & 0,499 & 0,969 & 0,720 & 0,282 & 1,000 & 0,927 & 0,759 & 0,588 & 0,273 & 0,161 & 0,214 & 35 & 287 & $-1,4$ \\
\hline 1079 & 287 & 0,027 & 0,664 & 0,033 & 1,112 & 0,826 & 1,675 & 0,697 & 0,778 & 1,134 & 0,417 & 0,374 & 0,288 & 0,108 & 0,152 & 21 & 287 & $-1,5$ \\
\hline 1080 & 287 & 0,125 & 1,602 & 0,171 & 0,917 & 0,731 & 0,573 & 0,505 & 0,611 & 1,279 & 0,426 & 0,502 & 0,611 & 0,306 & 0,214 & 25 & 287 & $-2,6$ \\
\hline 1081 & 287 & 0,049 & 0,960 & 0,062 & 1,319 & 0,791 & 1,374 & 0,429 & 0,666 & 1,226 & 0,541 & 0,553 & 0,339 & 0,187 & 0,169 & 43 & 287 & $-1,8$ \\
\hline 1082 & 287 & 0,155 & 1,754 & 0,178 & 0,469 & 0,871 & 0,267 & 0,744 & 0,633 & 1,257 & 0,611 & 0,660 & 0,568 & 0,375 & 0,312 & 67 & 287 & $-2,5$ \\
\hline 1083 & 287 & 0,024 & 0,541 & 0,027 & 0,275 & 0,895 & 0,508 & 0,503 & 1,000 & 0,977 & 0,637 & 0,550 & 0,221 & 0,121 & 0,152 & 135 & 287 & $-1,1$ \\
\hline 1084 & 287 & 0,044 & 0,795 & 0,046 & 0,723 & 0,969 & 0,910 & 0,096 & 0,885 & 1,063 & 0,395 & 0,283 & 0,379 & 0,107 & 0,169 & 40 & 287 & $-1,9$ \\
\hline 1085 & 287 & 0,017 & 0,368 & 0,017 & 1,560 & 1,000 & 4,242 & 3,242 & 1,000 & 0,791 & 0,899 & 0,485 & 0,156 & 0,076 & 0,152 & 0 & 287 & $-0,6$ \\
\hline 1086 & 287 & 0,082 & 1,408 & 0,098 & 4,265 & 0,838 & 3,030 & 2,036 & 0,519 & 1,389 & 0,333 & 0,371 & 0,559 & 0,207 & 0,227 & 31 & 287 & $-2,5$ \\
\hline 1087 & 287 & 0,050 & 0,714 & 0,053 & 0,440 & 0,946 & 0,615 & 0,389 & 1,000 & 0,899 & 0,990 & 0,800 & 0,254 & 0,203 & 0,227 & 135 & 287 & $-1,3$ \\
\hline 1088 & 287 & 0,106 & 1,133 & 0,106 & 1,264 & 1,000 & 1,116 & 0,116 & 1,000 & 0,981 & 0,558 & 0,462 & 0,492 & 0,227 & 0,303 & 3 & 287 & $-2,3$ \\
\hline 1089 & 287 & 0,072 & 1,285 & 0,093 & 0,440 & 0,769 & 0,342 & 0,697 & 0,546 & 1,354 & 0,430 & 0,399 & 0,461 & 0,184 & 0,214 & 77 & 287 & $-2,2$ \\
\hline 1090 & 287 & 0,023 & 0,541 & 0,027 & 0,368 & 0,842 & 0,680 & 0,357 & 0,985 & 1,007 & 1,019 & 0,869 & 0,169 & 0,147 & 0,152 & 130 & 287 & $-0,8$ \\
\hline 1091 & 287 & 0,119 & 1,226 & 0,133 & 1,010 & 0,892 & 0,824 & 0,206 & 0,996 & 1,002 & 0,771 & 0,769 & 0,443 & 0,341 & 0,339 & 60 & 287 & $-2,1$ \\
\hline 1092 & 287 & 0,027 & 0,520 & 0,029 & 1,103 & 0,950 & 2,122 & 1,123 & 1,000 & 0,888 & 0,712 & 0,485 & 0,221 & 0,107 & 0,152 & 47 & 287 & $-1,1$ \\
\hline 1093 & 287 & 0,014 & 0,347 & 0,014 & 1,277 & 1,000 & 3,682 & 2,682 & 1,000 & 0,816 & 0,749 & 0,485 & 0,156 & 0,076 & 0,107 & 18 & 287 & $-0,6$ \\
\hline 1094 & 287 & 0,270 & 2,346 & 0,324 & 0,347 & 0,832 & 0,148 & 0,869 & 0,616 & 1,274 & 0,437 & 0,471 & 0,887 & 0,418 & 0,386 & 38 & 287 & $-3,1$ \\
\hline 1095 & 287 & 0,057 & 1,378 & 0,077 & 0,296 & 0,741 & 0,215 & 0,827 & 0,380 & 1,623 & 0,520 & 0,588 & 0,375 & 0,220 & 0,107 & 33 & 287 & $-1,9$ \\
\hline 1096 & 287 & 0,014 & 0,317 & 0,014 & 0,960 & 1,000 & 3,027 & 2,027 & 1,000 & 0,746 & 1,273 & 0,632 & 0,120 & 0,076 & 0,107 & 18 & 287 & $-0,3$ \\
\hline
\end{tabular}




\begin{tabular}{|c|c|c|c|c|c|c|c|c|c|c|c|c|c|c|c|c|c|c|}
\hline 1097 & 287 & 0,098 & 1,763 & 0,142 & 0,613 & 0,687 & 0,348 & 0,724 & 0,394 & 1,592 & 0,500 & 0,686 & 0,498 & 0,342 & 0,169 & 164 & 287 & $-2,3$ \\
\hline 1098 & 287 & 0,076 & 2,017 & 0,179 & 0,418 & 0,424 & 0,207 & 0,980 & 0,235 & 2,063 & 0,208 & 0,445 & 0,682 & 0,303 & 0,107 & 85 & 287 & $-2,8$ \\
\hline 1099 & 287 & 0,020 & 0,448 & 0,023 & 0,397 & 0,875 & 0,887 & 0,169 & 1,000 & 0,892 & 1,049 & 0,809 & 0,156 & 0,126 & 0,107 & 117 & 287 & $-0,6$ \\
\hline 1100 & 287 & 0,032 & 0,693 & 0,037 & 0,194 & 0,846 & 0,280 & 0,736 & 0,825 & 1,101 & 0,459 & 0,453 & 0,296 & 0,134 & 0,152 & 50 & 287 & $-1,6$ \\
\hline 1101 & 287 & 0,022 & 0,440 & 0,023 & 0,989 & $\begin{array}{l}0,090 \\
0,938\end{array}$ & 2,251 & 1,252 & 1,000 & 0,845 & $\begin{array}{l}0,4595 \\
0,95\end{array}$ & $\begin{array}{l}0,+153 \\
0,671\end{array}$ & $\begin{array}{l}0,270 \\
0,169\end{array}$ & 0,114 & 0,152 & 45 & 287 & $\begin{array}{l}-1,0 \\
-0,8\end{array}$ \\
\hline 1102 & 287 & 0,036 & 0,960 & 0,036 & 0,541 & 1,000 & 0,564 & 0,436 & 0,490 & 1,429 & 0,707 & 0,723 & 0,254 & 0,184 & 0,169 & 39 & 287 & $-1,3$ \\
\hline 1103 & 287 & 0,116 & 1,268 & 0,131 & 0,440 & 0,890 & 0,347 & 0,662 & 0,909 & 1,049 & 0,674 & 0,647 & 0,469 & 0,303 & 0,303 & 13 & 287 & $-2,2$ \\
\hline 1104 & 287 & 0,036 & 0,685 & 0,039 & 0,541 & 0,926 & 0,790 & 0,222 & 0,961 & 1,020 & 0,490 & 0,388 & 0,305 & 0,119 & 0,152 & 21 & 287 & $-1,6$ \\
\hline 1105 & 287 & 0,050 & 0,825 & 0,055 & 0,541 & 0,921 & 0,656 & 0,353 & 0,928 & 1,038 & 0,501 & 0,375 & 0,357 & 0,134 & 0,169 & 45 & 287 & $-1,8$ \\
\hline 1106 & 287 & 0,019 & 0,511 & 0,022 & 0,541 & 0,867 & 1,058 & 0,145 & 0,896 & 1,056 & 0,414 & 0,251 & 0,240 & 0,060 & 0,076 & 17 & 287 & $-1,3$ \\
\hline 1107 & 287 & 0,049 & 0,714 & 0,050 & 0,469 & 0,971 & 0,657 & 0,344 & 1,000 & 0,912 & 0,833 & 0,686 & 0,273 & 0,187 & 0,214 & 131 & 287 & $-1,4$ \\
\hline 1108 & 287 & 0,032 & 0,592 & 0,033 & 3,191 & 0,957 & 5,391 & 4,392 & 1,000 & 0,940 & 0,757 & 0,658 & 0,230 & 0,152 & 0,169 & 99 & 287 & $-1,2$ \\
\hline 1109 & 287 & 0,020 & 0,397 & 0,020 & 0,296 & 1,000 & 0,745 & 0,255 & 1,000 & 0,791 & 1,049 & 0,686 & 0,156 & 0,107 & 0,152 & 45 & 287 & $-0,6$ \\
\hline 1110 & 287 & 0,017 & 0,490 & 0,023 & 0,440 & 0,750 & 0,896 & 0,271 & 0,900 & 1,054 & 0,477 & 0,354 & 0,214 & 0,076 & 0,076 & 58 & 287 & $-1,1$ \\
\hline 1111 & 287 & 0,083 & 1,399 & 0,103 & 0,714 & 0,806 & 0,511 & 0,527 & 0,534 & 1,368 & 0,543 & 0,575 & 0,442 & 0,254 & 0,240 & 76 & 287 & $-2,1$ \\
\hline 1112 & 287 & 0,036 & 0,807 & 0,049 & 1,171 & 0,735 & 1,451 & 0,523 & 0,692 & 1,202 & 0,436 & 0,451 & 0,324 & 0,146 & 0,152 & 12 & 287 & $-1,7$ \\
\hline 1113 & 287 & 0,027 & 0,541 & 0,027 & 0,490 & 1,000 & 0,906 & 0,094 & 1,000 & 0,924 & 0,590 & 0,442 & 0,243 & 0,107 & 0,152 & 38 & 287 & $-1,3$ \\
\hline 1114 & 287 & 0,050 & 0,765 & 0,053 & 2,388 & 0,946 & 3,121 & 2,122 & 1,000 & 0,963 & 0,602 & 0,493 & 0,326 & 0,161 & 0,214 & 30 & 287 & $-1,7$ \\
\hline 1115 & 287 & 0,202 & 1,851 & 0,224 & 0,368 & 0,904 & 0,199 & 0,807 & 0,742 & 1,161 & 0,572 & 0,508 & 0,671 & 0,341 & 0,379 & 87 & 287 & $-2,7$ \\
\hline 1116 & 287 & 0,020 & 0,461 & 0,020 & 0,418 & 1,000 & 0,909 & 0,091 & 1,000 & 0,917 & 0,615 & 0,371 & 0,204 & 0,076 & 0,152 & 9 & 287 & $-1,0$ \\
\hline $\begin{array}{l}1117 \\
1117\end{array}$ & 287 & 0,027 & $\begin{array}{l}0,401 \\
0,562\end{array}$ & 0,029 & $\begin{array}{l}0,410 \\
0,816\end{array}$ & 0,950 & 1,451 & 0,454 & 1,000 & 0,960 & 0,605 & 0,450 & $\begin{array}{l}0,204 \\
0,240\end{array}$ & 0,108 & 0,152 & 163 & 287 & $\begin{array}{l}-1,0 \\
-1,3\end{array}$ \\
\hline 1118 & 287 & 0,066 & 0,837 & 0,069 & 0,347 & 0,958 & 0,414 & 0,587 & 1,000 & 0,919 & 1,010 & 0,892 & 0,288 & 0,257 & 0,273 & 65 & 287 & $-1,5$ \\
\hline 1119 & 287 & 0,032 & 0,592 & 0,034 & 5,309 & 0,917 & 8,971 & 7,971 & 1,000 & 0,940 & 0,622 & 0,527 & 0,254 & 0,134 & 0,152 & 23 & 287 & $-1,3$ \\
\hline 1120 & 287 & 0,016 & 0,347 & 0,016 & 0,440 & 1,000 & 1,268 & 0,268 & 1,000 & 0,778 & 0,875 & 0,500 & 0,152 & 0,076 & 0,152 & 0 & 287 & $-0,6$ \\
\hline 1121 & 287 & 0,044 & 0,837 & 0,053 & 3,859 & 0,838 & 4,611 & 3,615 & 0,798 & 1,120 & 0,607 & 0,496 & 0,305 & 0,152 & 0,152 & 85 & 287 & $-1,6$ \\
\hline 1122 & 287 & 0,017 & 0,397 & 0,019 & 0,418 & 0,923 & 1,053 & 0,093 & 1,000 & 0,854 & 0,899 & 0,543 & 0,156 & 0,085 & 0,107 & 23 & 287 & $-0,6$ \\
\hline 1123 & 287 & 0,026 & 0,562 & 0,027 & 0,368 & 0,947 & 0,654 & 0,350 & 1,000 & 0,987 & 0,509 & 0,377 & 0,254 & 0,096 & 0,152 & 20 & 287 & $-1,3$ \\
\hline 1124 & 287 & 0,138 & 1,644 & 0,159 & 0,723 & 0,865 & 0,440 & 0,576 & 0,640 & 1,250 & 0,421 & 0,411 & 0,645 & 0,265 & 0,214 & 91 & 287 & $-2,7$ \\
\hline 1125 & 287 & 0,023 & 0,490 & 0,026 & 1,818 & 0,889 & 3,707 & 2,709 & 1,000 & 0,913 & 0,702 & 0,496 & 0,204 & 0,101 & 0,152 & 11 & 287 & $-1,0$ \\
\hline 1126 & 287 & 0,023 & 0,490 & 0,026 & 0,714 & 0,889 & 1,457 & 0,470 & 1,000 & 0,913 & 0,702 & 0,496 & 0,204 & 0,101 & 0,152 & 77 & 287 & $-1,0$ \\
\hline 1127 & 287 & 0,016 & 0,397 & 0,019 & 1,910 & 0,846 & 4,807 & 3,810 & 1,000 & 0,892 & 0,875 & 0,633 & 0,152 & 0,096 & 0,107 & 34 & 287 & $-0,6$ \\
\hline 1128 & 287 & 0,032 & 0,664 & 0,034 & 1,357 & 0,917 & 2,045 & 1,048 & 0,901 & 1,054 & 0,539 & 0,423 & 0,273 & 0,116 & 0,152 & 34 & 287 & $-1,4$ \\
\hline 1129 & 287 & 0,042 & 0,981 & 0,060 & 0,144 & 0,690 & 0,146 & 0,908 & 0,544 & 1,356 & 0,434 & 0,577 & 0,349 & 0,201 & 0,152 & 46 & 287 & $-1,8$ \\
\hline 1130 & 287 & 0,024 & 0,511 & 0,024 & 0,296 & 1,000 & 0,579 & 0,421 & 1,000 & 0,924 & 0,585 & 0,329 & 0,230 & 0,076 & 0,152 & 5 & 287 & $-1,2$ \\
\hline 1131 & 287 & 0,027 & 0,532 & 0,027 & 0,173 & 1,000 & 0,326 & 0,674 & 1,000 & 0,910 & 0,605 & 0,316 & 0,240 & 0,076 & 0,152 & 3 & 287 & $\begin{array}{l}-1,3 \\
\end{array}$ \\
\hline 1132 & 287 & 0,017 & 0,389 & 0,019 & 0,685 & 0,923 & 1,761 & 0,765 & 1,000 & 0,836 & 0,764 & 0,447 & 0,169 & 0,076 & 0,107 & 13 & 287 & $-0,8$ \\
\hline 1133 & 287 & 0,019 & 0,418 & 0,020 & 0,347 & 0,929 & 0,828 & 0,186 & 1,000 & 0,864 & 0,974 & 0,686 & 0,156 & 0,107 & 0,107 & 135 & 287 & $-0,6$ \\
\hline 1134 & 287 & 0,017 & 0,397 & 0,017 & 0,896 & 1,000 & 2,255 & 1,255 & 1,000 & 0,854 & 0,764 & 0,474 & 0,169 & 0,080 & 0,107 & 45 & 287 & $-0,8$ \\
\hline 1135 & 287 & 0,014 & 0,317 & 0,014 & 0,520 & 1,000 & 1,641 & 0,641 & 1,000 & 0,746 & 1,273 & 0,632 & 0,120 & 0,076 & 0,152 & 0 & 287 & $-0,3$ \\
\hline 1136 & 287 & 0,080 & 1,082 & 0,086 & 0,664 & 0,933 & 0,613 & 0,392 & 0,862 & 1,077 & 0,422 & 0,411 & 0,492 & 0,202 & 0,214 & 22 & 287 & $-2,3$ \\
\hline 1137 & 287 & 0,019 & 0,490 & 0,024 & 0,296 & 0,765 & 0,604 & 0,461 & 0,975 & 1,013 & 0,571 & 0,499 & 0,204 & 0,102 & 0,107 & 0 & 287 & $-1,0$ \\
\hline 1138 & 287 & 0,108 & 1,915 & 0,133 & 0,600 & 0,806 & 0,314 & 0,713 & 0,369 & 1,647 & 0,351 & 0,424 & 0,625 & 0,265 & 0,214 & 15 & 287 & $-2,6$ \\
\hline 1139 & 287 & 0,304 & 2,418 & 0,350 & 0,511 & 0,869 & 0,211 & 0,799 & 0,654 & 1,237 & 0,362 & 0,352 & 1,035 & 0,364 & 0,379 & 22 & 287 & $-3,4$ \\
\hline 1140 & 287 & 0,029 & 0,520 & 0,029 & 0,938 & 1,000 & 1,805 & 0,805 & 1,000 & 0,866 & 0,878 & 0,657 & 0,204 & 0,134 & 0,169 & 27 & 287 & $-1,0$ \\
\hline 1141 & 287 & 0,040 & 0,622 & 0,040 & 0,499 & 1,000 & 0,803 & 0,197 & 1,000 & 0,875 & 0,964 & 0,814 & 0,230 & 0,187 & 0,214 & 166 & 287 & $-1,2$ \\
\hline 1142 & 287 & 0,049 & 0,917 & 0,065 & 1,070 & 0,756 & 1,166 & 0,295 & 0,728 & 1,172 & 0,666 & 0,689 & 0,305 & 0,210 & 0,152 & 61 & 287 & $-1,6$ \\
\hline 1143 & 287 & 0,017 & 0,418 & 0,017 & 0,266 & 1,000 & 0,636 & 0,364 & 1,000 & 0,900 & 0,611 & 0,389 & 0,189 & 0,074 & 0,076 & 50 & 287 & $-0,9$ \\
\hline 1144 & 287 & 0,016 & 0,418 & 0,017 & 0,296 & 0,917 & 0,707 & 0,305 & 1,000 & 0,940 & 0,560 & 0,358 & 0,189 & 0,068 & 0,076 & 32 & 287 & $-0,9$ \\
\hline 1145 & 287 & 0,017 & 0,359 & 0,017 & 0,490 & 1,000 & 1,365 & 0,365 & 1,000 & 0,772 & 1,175 & 0,555 & 0,137 & 0,076 & 0,152 & 0 & 287 & $-0,5$ \\
\hline 1146 & 287 & 0,043 & 0,706 & 0,046 & 0,685 & 0,938 & 0,970 & 0,069 & 1,000 & 0,960 & 0,626 & 0,543 & 0,296 & 0,161 & 0,169 & 50 & 287 & $-1,6$ \\
\hline 1147 & 287 & 0,040 & 0,816 & 0,050 & 1,154 & 0,800 & 1,414 & 0,460 & 0,758 & 1,148 & 0,548 & 0,572 & 0,305 & 0,175 & 0,152 & 66 & 287 & $-1,6$ \\
\hline 1148 & 287 & 0,030 & 0,888 & 0,039 & 0,440 & 0,778 & 0,495 & 0,552 & 0,480 & 1,443 & 0,594 & 0,632 & 0,254 & 0,161 & 0,152 & 128 & 287 & $-1,3$ \\
\hline 1149 & 287 & 0,022 & 0,440 & 0,023 & 0,275 & 0,938 & 0,625 & 0,380 & 1,000 & 0,845 & 0,955 & 0,600 & 0,169 & 0,102 & 0,152 & 27 & 287 & $-0,8$ \\
\hline 1150 & 287 & 0,066 & 1,154 & 0,077 & 1,091 & 0,852 & 0,945 & 0,158 & 0,623 & 1,267 & 0,689 & 0,673 & 0,349 & 0,235 & 0,214 & 34 & 287 & $-1,8$ \\
\hline 1151 & 287 & 0,052 & 0,786 & 0,055 & 0,571 & 0,947 & 0,726 & 0,279 & 1,000 & 0,976 & 0,619 & 0,575 & 0,326 & 0,187 & 0,214 & 49 & 287 & $-1,7$ \\
\hline 1152 & 287 & 0,273 & 1,847 & 0,281 & 0,592 & 0,969 & 0,320 & 0,680 & 1,000 & 0,998 & 0,712 & 0,690 & 0,698 & 0,482 & 0,461 & 53 & 287 & $-2,8$ \\
\hline 1153 & 287 & 0,014 & 0,347 & 0,014 & 0,144 & 1,000 & 0,414 & 0,586 & 1,000 & 0,816 & 0,979 & 0,588 & 0,137 & 0,080 & 0,107 & 127 & 287 & $-0,5$ \\
\hline 1154 & 287 & 0,080 & 1,615 & 0,099 & 1,061 & 0,812 & 0,657 & 0,391 & 0,387 & 1,607 & 0,792 & 0,738 & 0,359 & 0,265 & 0,214 & 170 & 287 & -1 \\
\hline
\end{tabular}




\begin{tabular}{|c|c|c|c|c|c|c|c|c|c|c|c|c|c|c|c|c|c|c|}
\hline 1155 & 287 & 0,014 & 0,317 & 0,014 & 0,735 & 1,000 & 2,320 & 1,320 & 1,000 & 0,746 & 1,273 & 0,632 & 0,120 & 0,076 & 0,107 & 162 & 287 & $-0,3$ \\
\hline 1156 & 287 & 0,055 & 0,807 & 0,060 & 0,245 & 0,905 & 0,304 & 0,703 & 1,000 & 0,975 & 0,605 & 0,559 & 0,339 & 0,189 & 0,227 & 22 & 287 & $-1,8$ \\
\hline 1157 & 287 & 3,448 & 9,630 & 4,109 & 0,693 & 0,839 & 0,072 & 0,942 & 0,467 & 1,463 & 0,602 & 0,705 & 2,700 & 1,902 & 0,303 & 68 & 287 & $-4,8$ \\
\hline 1158 & 287 & 0,026 & 0,490 & 0,026 & 0,338 & 1,000 & 0,689 & 0,311 & 1,000 & 0,861 & 0,917 & 0,707 & 0,189 & 0,134 & 0,152 & 45 & 287 & $-0,9$ \\
\hline 1159 & 287 & 1,804 & 7,914 & 2,287 & 1,763 & 0,789 & 0,223 & 0,805 & 0,362 & 1,662 & 0,521 & 0,666 & 2,100 & 1,399 & 0,912 & 51 & 287 & $-4,4$ \\
\hline 1160 & 287 & 0,014 & 0,511 & 0,020 & 0,461 & 0,714 & 0,901 & 0,302 & 0,690 & 1,204 & 0,318 & 0,230 & 0,240 & 0,055 & 0,076 & 0 & 287 & $-1,3$ \\
\hline 1161 & 287 & 0,016 & 0,368 & 0,016 & 0,338 & 1,000 & 0,919 & 0,081 & 1,000 & 0,826 & 0,824 & 0,485 & 0,156 & 0,076 & 0,107 & 18 & 287 & $-0,6$ \\
\hline 1162 & 287 & 0,019 & 0,389 & 0,019 & 0,418 & 1,000 & 1,076 & 0,076 & 1,000 & 0,803 & 0,974 & 0,485 & 0,156 & 0,076 & 0,152 & 172 & 287 & $-0,6$ \\
\hline 1163 & 287 & 0,034 & 0,837 & 0,047 & 0,440 & 0,727 & 0,525 & 0,548 & 0,618 & 1,272 & 0,588 & 0,559 & 0,273 & 0,153 & 0,152 & 69 & 287 & $-1,4$ \\
\hline 1164 & 287 & 0,050 & 1,112 & 0,067 & 2,334 & 0,745 & 2,099 & 1,128 & 0,511 & 1,400 & 0,441 & 0,498 & 0,381 & 0,189 & 0,169 & 154 & 287 & $-1,9$ \\
\hline 1165 & 287 & 0,133 & 1,868 & 0,185 & 1,455 & 0,721 & 0,779 & 0,356 & 0,480 & 1,443 & 0,569 & 0,723 & 0,546 & 0,395 & 0,240 & 124 & 287 & $-2,4$ \\
\hline 1166 & 287 & 0,034 & 0,655 & 0,037 & 0,389 & 0,923 & 0,594 & 0,414 & 1,000 & 0,996 & 0,577 & 0,412 & 0,276 & 0,114 & 0,152 & 177 & 287 & $-1,5$ \\
\hline 1167 & 287 & 0,033 & 0,541 & 0,033 & 0,418 & 1,000 & 0,773 & 0,227 & 1,000 & 0,840 & 1,126 & 0,784 & 0,193 & 0,152 & 0,169 & 15 & 287 & $-0,9$ \\
\hline 1168 & 287 & 0,027 & 1,010 & 0,027 & 0,714 & 1,000 & 0,707 & 0,293 & 0,336 & 1,726 & 0,158 & 0,171 & 0,469 & 0,080 & 0,076 & 12 & 287 & $-2,2$ \\
\hline 1169 & 287 & 0,026 & 0,490 & 0,026 & 2,448 & 1,000 & 4,992 & 3,992 & 1,000 & 0,861 & 0,881 & 0,588 & 0,193 & 0,114 & 0,152 & 0 & 287 & $-0,9$ \\
\hline 1170 & 287 & 0,059 & 0,968 & 0,069 & 0,469 & 0,854 & 0,485 & 0,536 & 0,789 & 1,126 & 0,587 & 0,584 & 0,357 & 0,209 & 0,214 & 51 & 287 & $-1,8$ \\
\hline 1171 & 287 & 0,070 & 0,849 & 0,072 & 0,275 & 0,980 & 0,324 & 0,677 & 1,000 & 0,904 & 0,734 & 0,651 & 0,349 & 0,227 & 0,273 & 63 & 287 & $-1,8$ \\
\hline 1172 & 287 & 0,029 & 0,765 & 0,036 & 1,103 & 0,800 & 1,442 & 0,485 & 0,616 & 1,274 & 0,749 & 0,728 & 0,221 & 0,161 & 0,152 & 50 & 287 & $-1,1$ \\
\hline 1173 & 287 & 0,016 & 0,397 & 0,019 & 1,205 & 0,846 & 3,031 & 2,037 & 1,000 & 0,892 & 0,700 & 0,435 & 0,169 & 0,074 & 0,076 & 32 & 287 & $-0,8$ \\
\hline 1174 & 287 & 0,069 & 0,858 & 0,072 & 1,488 & 0,960 & 1,734 & 0,736 & 1,000 & 0,922 & 0,940 & 0,842 & 0,305 & 0,257 & 0,227 & 74 & 287 & $-1,6$ \\
\hline 1175 & 287 & 0,412 & 2,950 & 0,479 & 0,469 & 0,859 & 0,159 & 0,853 & 0,594 & 1,297 & 0,417 & 0,507 & 1,122 & 0,568 & 0,546 & 9 & 287 & $-3,5$ \\
\hline 1176 & 287 & 0,019 & 0,368 & 0,019 & 1,501 & 1,000 & 4,081 & 3,081 & 1,000 & 0,760 & 1,273 & 0,784 & 0,137 & 0,107 & 0,152 & 90 & 287 & $-0,5$ \\
\hline 1177 & 287 & 0,037 & 0,643 & 0,042 & 0,562 & 0,897 & 0,875 & 0,162 & 1,000 & 0,939 & 0,637 & 0,472 & 0,273 & 0,129 & 0,169 & 56 & 287 & $-1,4$ \\
\hline 1178 & 287 & 0,337 & 2,490 & 0,380 & 3,868 & 0,887 & 1,553 & 0,565 & 0,683 & 1,210 & 0,840 & 0,848 & 0,715 & 0,606 & 0,536 & 37 & 287 & $-2,8$ \\
\hline 1179 & 287 & 0,019 & 0,368 & 0,019 & 0,541 & 1,000 & 1,471 & 0,471 & 1,000 & 0,760 & 1,273 & 0,784 & 0,137 & 0,107 & 0,152 & 90 & 287 & $-0,5$ \\
\hline 1180 & 287 & 0,033 & 0,643 & 0,039 & 0,224 & 0,852 & 0,349 & 0,668 & 1,000 & 0,998 & 0,553 & 0,478 & 0,276 & 0,132 & 0,152 & 24 & 287 & $-1,5$ \\
\hline 1181 & 287 & 0,040 & 0,693 & 0,046 & 0,389 & 0,875 & 0,561 & 0,457 & 1,000 & 0,976 & 0,673 & 0,553 & 0,276 & 0,152 & 0,169 & 17 & 287 & $-1,5$ \\
\hline 1182 & 287 & 0,047 & 0,765 & 0,050 & 0,144 & 0,943 & 0,188 & 0,814 & 1,000 & 0,992 & 0,494 & 0,383 & 0,349 & 0,134 & 0,169 & 41 & 287 & $-1,8$ \\
\hline 1183 & 287 & 0,428 & 3,381 & 0,538 & 0,685 & 0,795 & 0,202 & 0,824 & 0,470 & 1,459 & 0,302 & 0,393 & 1,342 & 0,528 & 0,152 & 18 & 287 & $-3,7$ \\
\hline 1184 & 287 & 0,017 & 0,368 & 0,017 & 0,418 & 1,000 & 1,138 & 0,138 & 1,000 & 0,791 & 1,175 & 0,784 & 0,137 & 0,107 & 0,152 & 0 & 287 & $-0,5$ \\
\hline 1185 & 287 & 0,059 & 1,133 & 0,079 & 0,266 & 0,745 & 0,235 & 0,806 & 0,576 & 1,318 & 0,614 & 0,753 & 0,349 & 0,263 & 0,169 & 29 & 287 & $-1,8$ \\
\hline 1186 & 287 & 0,093 & 1,154 & 0,102 & 0,634 & 0,915 & 0,549 & 0,458 & 0,880 & 1,066 & 0,460 & 0,434 & 0,508 & 0,221 & 0,227 & 21 & 287 & $-2,3$ \\
\hline 1187 & 287 & 0,040 & 0,693 & 0,040 & 4,794 & 1,000 & 6,914 & 5,914 & 1,000 & 0,976 & 0,584 & 0,453 & 0,296 & 0,134 & 0,169 & 43 & 287 & $-1,6$ \\
\hline 1188 & 287 & 0,139 & 1,357 & 0,149 & 1,327 & 0,933 & 0,978 & 0,071 & 0,950 & 1,026 & 0,567 & 0,575 & 0,559 & 0,321 & 0,339 & 29 & 287 & $-2,5$ \\
\hline 1189 & 287 & 0,017 & 0,397 & 0,017 & 3,243 & 1,000 & 8,159 & 7,159 & 1,000 & 0,854 & 0,899 & 0,651 & 0,156 & 0,102 & 0,107 & 31 & 287 & $-0,6$ \\
\hline 1190 & 287 & 0,016 & 0,592 & 0,020 & 0,765 & 0,786 & 1,293 & 0,363 & 0,566 & 1,329 & 0,778 & 0,707 & 0,161 & 0,114 & 0,076 & 130 & 287 & $-0,7$ \\
\hline 1191 & 287 & 0,156 & 1,560 & 0,172 & 0,440 & 0,908 & 0,282 & 0,724 & 0,808 & 1,113 & 0,427 & 0,397 & 0,683 & 0,271 & 0,303 & 37 & 287 & $-2,8$ \\
\hline 1192 & 287 & 0,030 & 0,562 & 0,033 & 0,592 & 0,913 & 1,053 & 0,102 & 1,000 & 0,914 & 0,723 & 0,493 & 0,230 & 0,114 & 0,152 & 168 & 287 & $-1,2$ \\
\hline 1193 & 287 & 0,062 & 0,867 & 0,066 & 0,397 & 0,935 & 0,459 & 0,545 & 1,000 & 0,984 & 0,644 & 0,500 & 0,349 & 0,174 & 0,214 & 52 & 287 & $-1,8$ \\
\hline 1194 & 287 & 0,218 & 1,847 & 0,232 & 0,245 & 0,938 & 0,133 & 0,869 & 0,803 & 1,116 & 0,876 & 0,874 & 0,563 & 0,492 & 0,455 & 3 & 287 & $-2,5$ \\
\hline 1195 & 287 & 0,033 & 0,562 & 0,033 & 0,520 & 1,000 & 0,925 & 0,075 & 1,000 & 0,873 & 0,732 & 0,474 & 0,240 & 0,114 & 0,152 & 11 & 287 & $-1,3$ \\
\hline 1196 & 287 & 0,034 & 0,735 & 0,042 & 0,685 & 0,828 & 0,931 & 0,186 & 0,800 & 1,118 & 0,611 & 0,566 & 0,268 & 0,152 & 0,152 & 10 & 287 & $-1,4$ \\
\hline 1197 & 287 & 0,135 & 1,501 & 0,154 & 0,397 & 0,879 & 0,265 & 0,745 & 0,753 & 1,153 & 0,479 & 0,447 & 0,599 & 0,268 & 0,227 & 47 & 287 & $-2,6$ \\
\hline 1198 & 287 & 0,014 & 0,317 & 0,014 & 0,448 & 1,000 & 1,414 & 0,414 & 1,000 & 0,746 & 1,273 & 0,632 & 0,120 & 0,076 & 0,107 & 162 & 287 & $-0,3$ \\
\hline 1199 & 287 & 0,558 & 4,803 & 0,727 & 0,194 & 0,767 & 0,040 & 0,987 & 0,304 & 1,813 & 0,343 & 0,479 & 1,440 & 0,690 & 0,214 & 37 & 287 & $-3,8$ \\
\hline 1200 & 287 & 0,033 & 0,571 & 0,033 & 0,672 & 1,000 & 1,178 & 0,178 & 1,000 & 0,886 & 0,714 & 0,442 & 0,243 & 0,107 & 0,169 & 47 & 287 & $-1,3$ \\
\hline 1201 & 287 & 0,016 & 0,368 & 0,016 & 0,266 & 1,000 & 0,724 & 0,276 & 1,000 & 0,826 & 0,700 & 0,447 & 0,169 & 0,076 & 0,107 & 18 & 287 & $-0,8$ \\
\hline 1202 & 287 & 0,063 & 0,858 & 0,069 & 0,735 & 0,917 & 0,857 & 0,165 & 1,000 & 0,963 & 0,659 & 0,542 & 0,349 & 0,189 & 0,214 & 13 & 287 & $-1,8$ \\
\hline 1203 & 287 & 0,027 & 0,520 & 0,027 & 0,714 & 1,000 & 1,374 & 0,374 & 1,000 & 0,888 & 0,756 & 0,500 & 0,214 & 0,107 & 0,169 & 53 & 287 & $-1,1$ \\
\hline 1204 & 287 & 0,039 & 0,643 & 0,042 & 0,917 & 0,931 & 1,428 & 0,433 & 1,000 & 0,921 & 0,688 & 0,566 & 0,268 & 0,152 & 0,169 & 83 & 287 & $-1,4$ \\
\hline 1205 & 287 & 0,014 & 0,347 & 0,016 & 2,460 & 0,909 & 7,097 & 6,097 & 1,000 & 0,816 & 0,979 & 0,588 & 0,137 & 0,080 & 0,107 & 18 & 287 & $-0,5$ \\
\hline 1206 & 287 & 0,036 & 0,837 & 0,046 & 0,613 & 0,781 & 0,732 & 0,346 & 0,643 & 1,247 & 0,318 & 0,283 & 0,379 & 0,107 & 0,152 & 39 & 287 & $-1,9$ \\
\hline 1207 & 287 & 3,774 & 10,404 & 4,619 & 0,317 & 0,817 & 0,030 & 0,987 & 0,438 & 1,511 & 0,396 & 0,556 & 3,482 & 1,937 & 0,152 & 31 & 287 & $-5,1$ \\
\hline 1208 & 287 & 0,083 & 0,960 & 0,088 & 1,061 & 0,951 & 1,106 & 0,117 & 1,000 & 0,938 & 0,761 & 0,707 & 0,373 & 0,264 & 0,273 & 29 & 287 & $-1,9$ \\
\hline 1209 & 287 & 0,030 & 0,490 & 0,030 & 0,194 & 1,000 & 0,396 & 0,604 & 1,000 & 0,797 & 1,337 & 0,894 & 0,169 & 0,152 & 0,214 & 90 & 287 & $-0,8$ \\
\hline 1210 & 287 & 0,022 & 0,418 & 0,022 & 0,389 & 1,000 & 0,929 & 0,071 & 1,000 & 0,805 & 1,123 & 0,728 & 0,156 & 0,114 & 0,152 & 73 & 287 & $-0,6$ \\
\hline 1211 & 287 & 0,029 & 0,520 & 0,029 & 1,010 & 1,000 & 1,943 & 0,943 & 1,000 & 0,866 & 0,979 & 0,762 & 0,193 & 0,147 & 0,169 & 45 & 287 & $-0,9$ \\
\hline 1212 & 287 & 0,020 & 0,571 & 0,024 & 0,613 & 0,824 & 1,074 & 0,191 & 0,775 & 1,136 & 0,524 & 0,461 & 0,221 & 0,102 & 0,107 & 11 & 287 & $-1,1$ \\
\hline
\end{tabular}




\begin{tabular}{|c|c|c|c|c|c|c|c|c|c|c|c|c|c|c|c|c|c|c|}
\hline 1213 & 287 & 0,053 & 0,706 & 0,053 & 0,448 & 1,000 & 0,635 & 0,365 & 1,000 & 0,864 & 0,906 & 0,693 & 0,273 & 0,189 & 0,227 & 21 & 287 & $-1,4$ \\
\hline 1214 & 287 & 0,050 & 1,205 & 0,086 & 0,368 & 0,583 & 0,305 & 0,810 & 0,435 & 1,517 & 0,343 & 0,574 & 0,432 & 0,248 & 0,152 & 91 & 287 & $-2,1$ \\
\hline 1215 & 287 & 0,027 & 0,765 & 0,037 & 0,347 & 0,731 & 0,453 & 0,610 & 0,585 & 1,307 & 0,285 & 0,239 & 0,349 & 0,083 & 0,076 & 34 & 287 & $-1,8$ \\
\hline 1216 & 287 & 0,036 & 0,622 & 0,037 & 0,296 & 0,962 & 0,476 & 0,525 & 1,000 & 0,926 & 0,707 & 0,527 & 0,254 & 0,134 & 0,169 & 57 & 287 & $-1,3$ \\
\hline 1217 & 287 & 0,327 & 4,570 & 0,492 & 0,989 & 0,665 & 0,216 & 0,852 & 0,197 & 2,254 & 0,330 & 0,456 & 1,124 & 0,513 & 0,169 & 59 & 287 & $-3,5$ \\
\hline 1218 & 287 & 0,565 & 3,577 & 0,644 & 0,938 & 0,878 & 0,262 & 0,748 & 0,555 & 1,342 & 0,356 & 0,406 & 1,422 & 0,578 & 0,546 & 39 & 287 & $-3,8$ \\
\hline 1219 & 287 & 0,227 & 2,050 & 0,258 & 0,448 & 0,878 & 0,219 & 0,791 & 0,678 & 1,215 & 0,348 & 0,412 & 0,911 & 0,375 & 0,379 & 13 & 287 & $-3,2$ \\
\hline 1220 & 287 & 3,939 & 10,107 & 4,653 & 0,562 & 0,846 & 0,056 & 0,957 & 0,485 & 1,437 & 0,614 & 0,753 & 2,857 & 2,152 & 0,240 & 82 & 287 & $-4,8$ \\
\hline 1221 & 287 & 0,133 & 1,247 & 0,141 & 0,440 & 0,949 & 0,353 & 0,649 & 1,000 & 0,963 & 0,754 & 0,718 & 0,475 & 0,341 & 0,339 & 103 & 287 & $-2,2$ \\
\hline 1222 & 287 & 0,014 & 0,347 & 0,014 & 0,368 & 1,000 & 1,061 & 0,061 & 1,000 & 0,816 & 0,749 & 0,485 & 0,156 & 0,076 & 0,107 & 18 & 287 & $-0,6$ \\
\hline 1223 & 287 & 0,026 & 0,592 & 0,033 & 0,757 & 0,783 & 1,278 & 0,353 & 0,927 & 1,039 & 0,573 & 0,441 & 0,240 & 0,106 & 0,107 & 163 & 287 & $-1,3$ \\
\hline 1224 & 287 & 0,040 & 0,672 & 0,040 & 0,144 & 1,000 & 0,214 & 0,786 & 1,000 & 0,946 & 0,584 & 0,362 & 0,296 & 0,107 & 0,169 & 46 & 287 & $-1,6$ \\
\hline 1225 & 287 & 0,019 & 0,511 & 0,022 & 0,368 & 0,867 & 0,719 & 0,311 & 0,896 & 1,056 & 0,414 & 0,283 & 0,240 & 0,068 & 0,076 & 21 & 287 & $-1,3$ \\
\hline 1226 & 287 & 0,024 & 0,664 & 0,034 & 0,245 & 0,708 & 0,369 & 0,695 & 0,696 & 1,199 & 0,528 & 0,514 & 0,243 & 0,125 & 0,107 & 13 & 287 & $-1,3$ \\
\hline 1227 & 287 & 0,040 & 0,613 & 0,042 & 0,296 & 0,966 & 0,483 & 0,518 & 1,000 & 0,863 & 0,891 & 0,632 & 0,240 & 0,152 & 0,214 & 12 & 287 & $-1,3$ \\
\hline 1228 & 287 & 0,026 & 0,490 & 0,027 & 1,560 & 0,947 & 3,182 & 2,182 & 1,000 & 0,861 & 0,790 & 0,557 & 0,204 & 0,114 & 0,152 & 110 & 287 & $-1,0$ \\
\hline 1229 & 287 & 0,155 & 1,530 & 0,172 & 0,469 & 0,900 & 0,307 & 0,701 & 0,832 & 1,097 & 0,622 & 0,673 & 0,563 & 0,379 & 0,379 & 27 & 287 & $-2,5$ \\
\hline 1230 & 287 & 0,016 & 0,389 & 0,017 & 0,511 & 0,917 & 1,315 & 0,326 & 1,000 & 0,873 & 0,824 & 0,485 & 0,156 & 0,076 & 0,152 & 108 & 287 & $-0,6$ \\
\hline 1231 & 287 & 0,063 & 1,264 & 0,083 & 0,368 & 0,759 & 0,291 & 0,749 & 0,496 & 1,419 & 0,438 & 0,474 & 0,429 & 0,203 & 0,169 & 49 & 287 & $-2,1$ \\
\hline 1232 & 287 & 0,039 & 0,795 & 0,047 & 1,395 & 0,818 & 1,755 & 0,777 & 0,771 & 1,139 & 0,593 & 0,650 & 0,288 & 0,187 & 0,152 & 153 & 287 & $-1,5$ \\
\hline 1233 & 287 & 0,029 & 0,511 & 0,029 & 2,211 & 1,000 & 4,324 & 3,324 & 1,000 & 0,852 & 0,796 & 0,625 & 0,214 & 0,134 & 0,152 & 124 & 287 & $-1,1$ \\
\hline 1234 & 287 & 0,033 & 0,643 & 0,036 & 0,960 & 0,920 & 1,493 & 0,500 & 1,000 & 0,998 & 0,651 & 0,568 & 0,254 & 0,144 & 0,169 & 41 & 287 & $-1,3$ \\
\hline 1235 & 287 & 0,070 & 0,968 & 0,072 & 0,541 & 0,980 & 0,559 & 0,442 & 0,943 & 1,030 & 0,480 & 0,372 & 0,432 & 0,161 & 0,214 & 39 & 287 & $-2,1$ \\
\hline 1236 & 287 & 0,022 & 0,583 & 0,032 & 1,285 & 0,682 & 2,204 & 1,245 & 0,795 & 1,121 & 0,659 & 0,705 & 0,204 & 0,144 & 0,107 & 26 & 287 & $-1,0$ \\
\hline 1237 & 287 & 0,029 & 0,520 & 0,030 & 0,266 & 0,952 & 0,512 & 0,490 & 1,000 & 0,866 & 0,878 & 0,657 & 0,204 & 0,134 & 0,169 & 34 & 287 & $-1,0$ \\
\hline 1238 & 287 & 0,022 & 0,418 & 0,023 & 1,543 & 0,938 & 3,686 & 2,687 & 1,000 & 0,805 & 1,123 & 0,728 & 0,156 & 0,114 & 0,152 & 12 & 287 & $-0,6$ \\
\hline 1239 & 287 & 0,033 & 0,837 & 0,052 & 3,822 & 0,639 & 4,566 & 3,585 & 0,592 & 1,300 & 0,553 & 0,687 & 0,276 & 0,189 & 0,152 & 80 & 287 & $-1,5$ \\
\hline 1240 & 287 & 0,043 & 0,735 & 0,049 & 0,287 & 0,882 & 0,391 & 0,621 & 1,000 & 1,000 & 0,659 & 0,588 & 0,288 & 0,169 & 0,169 & 166 & 287 & $-1,5$ \\
\hline 1241 & 287 & 0,040 & 0,655 & 0,040 & 0,287 & 1,000 & 0,439 & 0,561 & 1,000 & 0,922 & 0,673 & 0,412 & 0,276 & 0,114 & 0,152 & 0 & 287 & $-1,5$ \\
\hline 1242 & 287 & 0,043 & 1,112 & 0,065 & 0,275 & 0,667 & 0,247 & 0,823 & 0,438 & 1,512 & 0,659 & 0,802 & 0,288 & 0,231 & 0,152 & 22 & 287 & $-1,5$ \\
\hline 1243 & 287 & 0,016 & 0,347 & 0,016 & 3,365 & 1,000 & 9,707 & 8,707 & 1,000 & 0,778 & 1,401 & 0,849 & 0,120 & 0,102 & 0,107 & 45 & 287 & $-0,3$ \\
\hline 1244 & 287 & 0,023 & 0,469 & 0,023 & 1,949 & 1,000 & 4,153 & 3,153 & 1,000 & 0,874 & 1,019 & 0,791 & 0,169 & 0,134 & 0,152 & 35 & 287 & $-0,8$ \\
\hline 1245 & 287 & 0,030 & 0,592 & 0,034 & 0,490 & 0,875 & 0,828 & 0,212 & 1,000 & 0,962 & 0,723 & 0,493 & 0,230 & 0,114 & 0,152 & 91 & 287 & $-1,2$ \\
\hline 1246 & 287 & 0,014 & 0,469 & 0,019 & 0,194 & 0,769 & 0,414 & 0,630 & 0,819 & 1,105 & 0,637 & 0,489 & 0,169 & 0,083 & 0,076 & 79 & 287 & $-0,8$ \\
\hline 1247 & 287 & 0,195 & 1,949 & 0,222 & 0,389 & 0,877 & 0,200 & 0,810 & 0,646 & 1,244 & 0,459 & 0,463 & 0,735 & 0,341 & 0,321 & 42 & 287 & $-2,9$ \\
\hline 1248 & 287 & 0,014 & 0,338 & 0,014 & 0,685 & 1,000 & 2,026 & 1,026 & 1,000 & 0,796 & 0,979 & 0,555 & 0,137 & 0,076 & 0,152 & 90 & 287 & $-0,5$ \\
\hline 1249 & 287 & 0,053 & 1,091 & 0,067 & 1,623 & 0,787 & 1,488 & 0,532 & 0,561 & 1,335 & 0,772 & 0,768 & 0,296 & 0,227 & 0,214 & 48 & 287 & $-1,6$ \\
\hline 1250 & 287 & 0,042 & 0,960 & 0,057 & 2,021 & 0,725 & 2,106 & 1,139 & 0,568 & 1,327 & 0,697 & 0,782 & 0,276 & 0,216 & 0,152 & 13 & 287 & $-1,5$ \\
\hline 1251 & 287 & 0,016 & 0,347 & 0,016 & 1,019 & 1,000 & 2,939 & 1,939 & 1,000 & 0,778 & 1,077 & 0,588 & 0,137 & 0,080 & 0,107 & 45 & 287 & $-0,5$ \\
\hline 1252 & 287 & 3,865 & 14,366 & 5,391 & 1,467 & 0,717 & 0,102 & 0,941 & 0,235 & 2,061 & 0,274 & 0,424 & 4,235 & 1,795 & 0,442 & 41 & 287 & $-5,4$ \\
\hline 1253 & 287 & 0,032 & 0,613 & 0,036 & 0,418 & 0,880 & 0,683 & 0,339 & 1,000 & 0,973 & 0,700 & 0,575 & 0,240 & 0,138 & 0,152 & 155 & 287 & $-1,3$ \\
\hline 1254 & 287 & 0,067 & 1,031 & 0,085 & 1,437 & 0,797 & 1,394 & 0,443 & 0,797 & 1,120 & 0,575 & 0,633 & 0,386 & 0,245 & 0,227 & 24 & 287 & $-1,9$ \\
\hline 1255 & 287 & 0,042 & 0,634 & 0,042 & 2,033 & 1,000 & 3,207 & 2,207 & 1,000 & 0,877 & 0,738 & 0,600 & 0,268 & 0,161 & 0,214 & 48 & 287 & $-1,4$ \\
\hline 1256 & 287 & 0,237 & 1,978 & 0,280 & 0,469 & 0,846 & 0,237 & 0,778 & 0,760 & 1,147 & 0,514 & 0,537 & 0,766 & 0,411 & 0,408 & 177 & 287 & $-2,9$ \\
\hline 1257 & 287 & 0,034 & 0,592 & 0,036 & 0,347 & 0,960 & 0,586 & 0,416 & 1,000 & 0,900 & 0,764 & 0,632 & 0,240 & 0,152 & 0,169 & 152 & 287 & $-1,3$ \\
\hline 1258 & 287 & 0,033 & 1,002 & 0,047 & 0,418 & 0,697 & 0,418 & 0,656 & 0,413 & 1,555 & 0,325 & 0,422 & 0,359 & 0,152 & 0,107 & 19 & 287 & $-1,8$ \\
\hline 1259 & 287 & 0,102 & 1,133 & 0,109 & 0,643 & 0,934 & 0,567 & 0,438 & 0,997 & 1,001 & 0,619 & 0,556 & 0,458 & 0,254 & 0,273 & 28 & 287 & $-2,2$ \\
\hline 1260 & 287 & 0,014 & 0,317 & 0,014 & 0,397 & 1,000 & 1,254 & 0,254 & 1,000 & 0,746 & 0,979 & 0,555 & 0,137 & 0,076 & 0,107 & 18 & 287 & $-0,5$ \\
\hline 1261 & 287 & 0,052 & 0,735 & 0,053 & 0,622 & 0,973 & 0,845 & 0,157 & 1,000 & 0,913 & 0,790 & 0,646 & 0,288 & 0,186 & 0,214 & 68 & 287 & $-1,5$ \\
\hline 1262 & 287 & 0,023 & 0,440 & 0,023 & 0,275 & 1,000 & 0,625 & 0,375 & 1,000 & 0,818 & 1,019 & 0,671 & 0,169 & 0,114 & 0,152 & 70 & 287 & $-0,8$ \\
\hline 1263 & 287 & 0,016 & 0,410 & 0,019 & 0,215 & 0,846 & 0,526 & 0,499 & 1,000 & 0,920 & 0,700 & 0,434 & 0,169 & 0,074 & 0,076 & 101 & 287 & $-0,8$ \\
\hline 1264 & 287 & 0,016 & 0,338 & 0,016 & 0,448 & 1,000 & 1,326 & 0,326 & 1,000 & 0,759 & 1,077 & 0,555 & 0,137 & 0,076 & 0,152 & 0 & 287 & $-0,5$ \\
\hline 1265 & 287 & 0,275 & 2,122 & 0,311 & 0,541 & 0,885 & 0,255 & 0,754 & 0,769 & 1,141 & 0,549 & 0,594 & 0,799 & 0,475 & 0,455 & 69 & 287 & $-3,0$ \\
\hline 1266 & 287 & 0,283 & 2,744 & 0,362 & 0,266 & 0,782 & 0,097 & 0,929 & 0,472 & 1,456 & 0,423 & 0,606 & 0,922 & 0,559 & 0,107 & 122 & 287 & $-3,2$ \\
\hline 1267 & 287 & 0,022 & 0,562 & 0,024 & 0,867 & 0,882 & 1,542 & 0,554 & 0,856 & 1,081 & 0,424 & 0,352 & 0,254 & 0,089 & 0,152 & 17 & 287 & $-1,3$ \\
\hline 1268 & 287 & 0,016 & 0,347 & 0,017 & 2,579 & 0,917 & 7,439 & 6,440 & 1,000 & 0,778 & 1,401 & 0,849 & 0,120 & 0,102 & 0,107 & 135 & 287 & $-0,3$ \\
\hline 1269 & 287 & 0,016 & 0,347 & 0,016 & 0,448 & 1,000 & 1,293 & 0,293 & 1,000 & 0,778 & 1,401 & 0,894 & 0,120 & 0,107 & 0,152 & 0 & 287 & $-0,3$ \\
\hline 1270 & 287 & 0,016 & 0,338 & 0,016 & 0,930 & 1,000 & 2,751 & 1,751 & 1,000 & 0,759 & 1,077 & 0,555 & 0,137 & 0,076 & 0,152 & 162 & 287 & $-0,5$ \\
\hline
\end{tabular}




\begin{tabular}{|c|c|c|c|c|c|c|c|c|c|c|c|c|c|c|c|c|c|c|}
\hline 1271 & 287 & 0,016 & 0,347 & 0,016 & 0,266 & 1,000 & 0,768 & 0,232 & 1,000 & 0,778 & 1,077 & 0,588 & 0,137 & 0,080 & 0,107 & 45 & 287 & $-0,5$ \\
\hline 1272 & 287 & 0,273 & 2,562 & 0,341 & 2,392 & 0,798 & 0,934 & 0,212 & 0,522 & 1,384 & 0,503 & 0,661 & 0,831 & 0,549 & 0,442 & 37 & 287 & $-3,1$ \\
\hline 1273 & 287 & 0,022 & 0,499 & 0,023 & 0,266 & 0,938 & 0,534 & 0,471 & 1,000 & 0,959 & 0,764 & 0,555 & 0,189 & 0,105 & 0,107 & 45 & 287 & $-0,9$ \\
\hline 1274 & 287 & 0,016 & 0,368 & 0,016 & 0,317 & 1,000 & 0,862 & 0,138 & 1,000 & 0,826 & 0,824 & 0,485 & 0,156 & 0,076 & 0,107 & 18 & 287 & $-0,6$ \\
\hline 1275 & 287 & 0,067 & 1,226 & 0,092 & 0,469 & 0,734 & 0,383 & 0,672 & 0,564 & 1,332 & 0,381 & 0,458 & 0,475 & 0,218 & 0,107 & 30 & 287 & $-2,2$ \\
\hline 1276 & 287 & 0,019 & 0,541 & 0,026 & 0,858 & 0,722 & 1,586 & 0,648 & 0,801 & 1,118 & 0,571 & 0,451 & 0,204 & 0,092 & 0,076 & 72 & 287 & $-1,0$ \\
\hline 1277 & 287 & 0,095 & 1,298 & 0,103 & 0,368 & 0,917 & 0,283 & 0,721 & 0,707 & 1,189 & 0,410 & 0,376 & 0,542 & 0,204 & 0,214 & 71 & 287 & $-2,4$ \\
\hline 1278 & 287 & 0,024 & 0,532 & 0,026 & 0,347 & 0,944 & 0,651 & 0,353 & 1,000 & 0,962 & 0,541 & 0,316 & 0,240 & 0,076 & 0,152 & 10 & 287 & $-1,3$ \\
\hline 1279 & 287 & 0,027 & 0,490 & 0,029 & 1,142 & 0,950 & 2,328 & 1,329 & 1,000 & 0,838 & 0,968 & 0,707 & 0,189 & 0,134 & 0,169 & 135 & 287 & $-0,9$ \\
\hline 1280 & 287 & 0,040 & 0,765 & 0,046 & 0,368 & 0,875 & 0,481 & 0,534 & 0,862 & 1,077 & 0,792 & 0,632 & 0,254 & 0,161 & 0,169 & 60 & 287 & $-1,3$ \\
\hline 1281 & 287 & 0,197 & 1,560 & 0,205 & 2,541 & 0,958 & 1,629 & 0,630 & 1,000 & 0,993 & 0,698 & 0,626 & 0,599 & 0,375 & 0,408 & 51 & 287 & $-2,6$ \\
\hline 1282 & 287 & 0,333 & 3,673 & 0,492 & 1,154 & 0,676 & 0,314 & 0,758 & 0,310 & 1,796 & 0,461 & 0,625 & 0,959 & 0,600 & 0,339 & 98 & 287 & $-3,3$ \\
\hline 1283 & 287 & 0,040 & 0,634 & 0,042 & 0,448 & 0,966 & 0,707 & 0,295 & 1,000 & 0,892 & 0,870 & 0,699 & 0,243 & 0,169 & 0,214 & 67 & 287 & $-1,3$ \\
\hline 1284 & 287 & 0,042 & 0,693 & 0,046 & 0,144 & 0,906 & 0,207 & 0,798 & 1,000 & 0,959 & 0,923 & 0,783 & 0,240 & 0,187 & 0,214 & 66 & 287 & $-1,3$ \\
\hline 1285 & 287 & 0,024 & 0,520 & 0,027 & 0,541 & 0,895 & 1,041 & 0,113 & 1,000 & 0,939 & 0,637 & 0,447 & 0,221 & 0,099 & 0,107 & 130 & 287 & $-1,1$ \\
\hline 1286 & 287 & 0,022 & 0,418 & 0,022 & 0,714 & 1,000 & 1,707 & 0,707 & 1,000 & 0,805 & 0,955 & 0,632 & 0,169 & 0,107 & 0,152 & 27 & 287 & $-0,8$ \\
\hline 1287 & 287 & 0,123 & 1,205 & 0,128 & 0,347 & 0,966 & 0,288 & 0,713 & 1,000 & 0,967 & 0,697 & 0,677 & 0,475 & 0,321 & 0,339 & 63 & 287 & $-2,2$ \\
\hline 1288 & 287 & 0,017 & 0,368 & 0,019 & 3,073 & 0,923 & 8,357 & 7,357 & 1,000 & 0,791 & 1,175 & 0,745 & 0,137 & 0,102 & 0,107 & 27 & 287 & $-0,5$ \\
\hline 1289 & 287 & 0,014 & 0,338 & 0,016 & 0,723 & 0,909 & 2,139 & 1,143 & 1,000 & 0,796 & 0,979 & 0,555 & 0,137 & 0,076 & 0,107 & 18 & 287 & $-0,5$ \\
\hline 1290 & 287 & 1,139 & 9,192 & 1,689 & 0,960 & 0,675 & 0,104 & 0,953 & 0,169 & 2,429 & 0,222 & 0,350 & 2,554 & 0,893 & 0,240 & 31 & 287 & $-4,7$ \\
\hline 1291 & 287 & 0,043 & 0,778 & 0,050 & 0,490 & 0,857 & 0,631 & 0,396 & 0,895 & 1,057 & 0,659 & 0,497 & 0,288 & 0,143 & 0,152 & 176 & 287 & $-1,5$ \\
\hline 1292 & 287 & 0,017 & 0,368 & 0,017 & 0,266 & 1,000 & 0,724 & 0,276 & 1,000 & 0,791 & 0,899 & 0,485 & 0,156 & 0,076 & 0,152 & 0 & 287 & $-0,6$ \\
\hline 1293 & 287 & 0,019 & 0,469 & 0,023 & 0,296 & 0,812 & 0,631 & 0,414 & 1,000 & 0,969 & 0,828 & 0,671 & 0,169 & 0,114 & 0,107 & 72 & 287 & $-0,8$ \\
\hline 1294 & 287 & 0,043 & 0,816 & 0,050 & 0,541 & 0,857 & 0,663 & 0,366 & 0,813 & 1,109 & 0,659 & 0,620 & 0,288 & 0,179 & 0,169 & 40 & 287 & $-1,5$ \\
\hline 1295 & 287 & 0,281 & 2,257 & 0,313 & 0,317 & 0,899 & 0,140 & 0,865 & 0,694 & 1,201 & 0,468 & 0,493 & 0,874 & 0,431 & 0,429 & 69 & 287 & $-3,1$ \\
\hline 1296 & 287 & 0,062 & 0,989 & 0,066 & 0,448 & 0,935 & 0,453 & 0,551 & 0,792 & 1,123 & 0,403 & 0,308 & 0,442 & 0,136 & 0,152 & 30 & 287 & $-2,1$ \\
\hline 1297 & 287 & 0,030 & 0,634 & 0,034 & 0,613 & 0,875 & 0,967 & 0,129 & 0,942 & 1,030 & 0,514 & 0,352 & 0,273 & 0,096 & 0,152 & 22 & 287 & $-1,4$ \\
\hline 1298 & 287 & 0,017 & 0,672 & 0,027 & 0,296 & 0,632 & 0,440 & 0,670 & 0,479 & 1,445 & 0,235 & 0,208 & 0,305 & 0,064 & 0,076 & 39 & 287 & $-1,6$ \\
\hline 1299 & 287 & 0,014 & 0,376 & 0,016 & 0,786 & 0,909 & 2,089 & 1,093 & 1,000 & 0,886 & 0,979 & 0,588 & 0,137 & 0,080 & 0,107 & 41 & 287 & $-0,5$ \\
\hline 1300 & 287 & 0,017 & 0,418 & 0,019 & 0,194 & 0,923 & 0,465 & 0,541 & 1,000 & 0,900 & 0,764 & 0,447 & 0,169 & 0,076 & 0,107 & 82 & 287 & $-0,8$ \\
\hline 1301 & 287 & 0,032 & 0,604 & 0,033 & 0,397 & 0,957 & 0,658 & 0,345 & 1,000 & 0,959 & 0,622 & 0,447 & 0,254 & 0,114 & 0,152 & 103 & 287 & $-1,3$ \\
\hline 1302 & 287 & 0,022 & 0,410 & 0,022 & 0,368 & 1,000 & 0,897 & 0,103 & 1,000 & 0,788 & 1,061 & 0,707 & 0,161 & 0,114 & 0,152 & 135 & 287 & $-0,7$ \\
\hline 1303 & 287 & 0,019 & 0,389 & 0,020 & 0,440 & 0,929 & 1,131 & 0,149 & 1,000 & 0,803 & 0,920 & 0,633 & 0,161 & 0,102 & 0,152 & 60 & 287 & $-0,7$ \\
\hline 1304 & 287 & 0,016 & 0,440 & 0,017 & 0,317 & 0,917 & 0,721 & 0,291 & 1,000 & 0,987 & 0,483 & 0,294 & 0,204 & 0,060 & 0,076 & 17 & 287 & $-1,0$ \\
\hline 1305 & 287 & 0,095 & 1,226 & 0,109 & 0,368 & 0,868 & 0,300 & 0,712 & 0,792 & 1,124 & 0,512 & 0,552 & 0,485 & 0,268 & 0,152 & 43 & 287 & $-2,3$ \\
\hline 1306 & 287 & 0,023 & 0,478 & 0,023 & 0,317 & 1,000 & 0,663 & 0,337 & 1,000 & 0,890 & 0,815 & 0,707 & 0,189 & 0,134 & 0,169 & 0 & 287 & $-0,9$ \\
\hline 1307 & 287 & 0,086 & 0,960 & 0,089 & 2,523 & 0,968 & 2,630 & 1,630 & 1,000 & 0,923 & 0,788 & 0,707 & 0,373 & 0,264 & 0,303 & 21 & 287 & $-1,9$ \\
\hline 1308 & 287 & 0,014 & 0,368 & 0,014 & 0,968 & 1,000 & 2,633 & 1,633 & 1,000 & 0,866 & 0,707 & 0,500 & 0,161 & 0,080 & 0,107 & 45 & 287 & $-0,7$ \\
\hline 1309 & 287 & 0,036 & 1,023 & 0,056 & 3,369 & 0,641 & 3,294 & 2,322 & 0,431 & 1,523 & 0,300 & 0,391 & 0,390 & 0,152 & 0,152 & 159 & 287 & $-2,0$ \\
\hline 1310 & 287 & 0,049 & 1,387 & 0,069 & 0,389 & 0,708 & 0,280 & 0,776 & 0,319 & 1,771 & 0,397 & 0,505 & 0,395 & 0,200 & 0,152 & 20 & 287 & $-2,0$ \\
\hline 1311 & 287 & 0,019 & 0,440 & 0,020 & 0,144 & 0,929 & 0,327 & 0,677 & 1,000 & 0,908 & 0,662 & 0,537 & 0,189 & 0,102 & 0,152 & 150 & 287 & $-0,9$ \\
\hline 1312 & 287 & 0,040 & 0,622 & 0,040 & 0,685 & 1,000 & 1,102 & 0,102 & 1,000 & 0,875 & 0,891 & 0,671 & 0,240 & 0,161 & 0,214 & 45 & 287 & $-1,3$ \\
\hline 1313 & 287 & 0,100 & 1,662 & 0,128 & 0,714 & 0,787 & 0,430 & 0,609 & 0,457 & 1,479 & 0,226 & 0,231 & 0,752 & 0,173 & 0,169 & 40 & 287 & $-2,9$ \\
\hline 1314 & 287 & 0,024 & 0,643 & 0,029 & 3,423 & 0,850 & 5,328 & 4,330 & 0,742 & 1,161 & 0,373 & 0,291 & 0,288 & 0,084 & 0,107 & 19 & 287 & $-1,5$ \\
\hline 1315 & 287 & 0,026 & 0,490 & 0,027 & 0,368 & 0,947 & 0,750 & 0,255 & 1,000 & 0,861 & 0,917 & 0,707 & 0,189 & 0,134 & 0,152 & 105 & 287 & $-0,9$ \\
\hline 1316 & 287 & 0,030 & 0,613 & 0,030 & 0,368 & 1,000 & 0,600 & 0,400 & 1,000 & 0,996 & 0,594 & 0,467 & 0,254 & 0,119 & 0,152 & 64 & 287 & $-1,3$ \\
\hline 1317 & 287 & 0,135 & 1,754 & 0,184 & 0,317 & 0,734 & 0,181 & 0,861 & 0,551 & 1,348 & 0,523 & 0,661 & 0,573 & 0,379 & 0,240 & 91 & 287 & $-2,5$ \\
\hline 1318 & 287 & 0,082 & 0,981 & 0,088 & 0,368 & 0,934 & 0,375 & 0,628 & 1,000 & 0,967 & 0,748 & 0,661 & 0,373 & 0,247 & 0,240 & 68 & 287 & $-1,9$ \\
\hline 1319 & 287 & 0,032 & 0,613 & 0,037 & 0,693 & 0,846 & 1,131 & 0,202 & 1,000 & 0,973 & 0,700 & 0,632 & 0,240 & 0,152 & 0,152 & 38 & 287 & $-1,3$ \\
\hline 1320 & 287 & 0,014 & 0,347 & 0,014 & 0,917 & 1,000 & 2,646 & 1,646 & 1,000 & 0,816 & 0,796 & 0,500 & 0,152 & 0,076 & 0,107 & 162 & 287 & $-0,6$ \\
\hline 1321 & 287 & 0,548 & 3,449 & 0,633 & 0,714 & 0,866 & 0,207 & 0,804 & 0,579 & 1,314 & 0,353 & 0,414 & 1,406 & 0,582 & 0,479 & 66 & 287 & $-3,8$ \\
\hline 1322 & 287 & 0,033 & 0,562 & 0,034 & 0,613 & 0,958 & 1,090 & 0,099 & 1,000 & 0,873 & 0,861 & 0,686 & 0,221 & 0,152 & 0,169 & 80 & 287 & $-1,1$ \\
\hline 1323 & 287 & 0,257 & 2,113 & 0,306 & 1,040 & 0,840 & 0,492 & 0,532 & 0,723 & 1,176 & 0,487 & 0,518 & 0,819 & 0,425 & 0,386 & 116 & 287 & $-3,0$ \\
\hline 1324 & 287 & 0,260 & 1,835 & 0,280 & 1,429 & 0,928 & 0,779 & 0,233 & 0,969 & 1,016 & 0,787 & 0,749 & 0,648 & 0,486 & 0,479 & 114 & 287 & $-2,7$ \\
\hline 1325 & 287 & 0,151 & 1,450 & 0,169 & 0,224 & 0,890 & 0,155 & 0,853 & 0,901 & 1,054 & 0,786 & 0,767 & 0,494 & 0,379 & 0,339 & 4 & 287 & $-2,3$ \\
\hline 1326 & 287 & 0,047 & 0,867 & 0,053 & 0,245 & 0,892 & 0,283 & 0,725 & 0,792 & 1,124 & 0,494 & 0,483 & 0,349 & 0,169 & 0,169 & 62 & 287 & $-1,8$ \\
\hline 1327 & 287 & 0,119 & 1,763 & 0,164 & 0,469 & 0,728 & 0,266 & 0,783 & 0,481 & 1,441 & 0,355 & 0,422 & 0,654 & 0,276 & 0,152 & 77 & 287 & $-2,7$ \\
\hline 1328 & 287 & 0,098 & 1,120 & 0,103 & 1,264 & 0,944 & 1,128 & 0,140 & 0,977 & 1,012 & 0,666 & 0,620 & 0,432 & 0,268 & 0,303 & 35 & 287 & $-2,1$ \\
\hline
\end{tabular}




\begin{tabular}{|c|c|c|c|c|c|c|c|c|c|c|c|c|c|c|c|c|c|c|}
\hline 1329 & 287 & 0,037 & 0,613 & 0,039 & 0,418 & 0,963 & 0,683 & 0,319 & 1,000 & 0,895 & 0,736 & 0,566 & 0,254 & 0,144 & 0,152 & 71 & 287 & $-1,3$ \\
\hline 1330 & 287 & 0,032 & 0,592 & 0,034 & 2,422 & 0,917 & 4,092 & 3,093 & 1,000 & 0,940 & 0,622 & 0,467 & 0,254 & 0,119 & 0,152 & 66 & 287 & $-1,3$ \\
\hline 1331 & 287 & 0,055 & 0,816 & 0,059 & 1,298 & 0,927 & 1,590 & 0,595 & 1,000 & 0,986 & 0,913 & 0,824 & 0,276 & 0,227 & 0,214 & 55 & 287 & $-1,5$ \\
\hline 1332 & 287 & 0,613 & 3,369 & 0,696 & 0,685 & 0,880 & 0,203 & 0,806 & 0,678 & 1,214 & 0,565 & 0,608 & 1,175 & 0,715 & 0,107 & 79 & 287 & $-3,6$ \\
\hline 1333 & 287 & 0,014 & 0,338 & 0,016 & 1,099 & 0,909 & 3,252 & 2,254 & 1,000 & 0,796 & 0,979 & 0,555 & 0,137 & 0,076 & 0,152 & 90 & 287 & $-0,5$ \\
\hline 1334 & 287 & 0,042 & 0,643 & 0,043 & 0,938 & 0,967 & 1,461 & 0,462 & 1,000 & 0,889 & 0,821 & 0,632 & 0,254 & 0,161 & 0,214 & 59 & 287 & $-1,3$ \\
\hline 1335 & 287 & 0,070 & 0,989 & 0,080 & 1,471 & 0,875 & 1,487 & 0,503 & 0,903 & 1,052 & 0,701 & 0,675 & 0,357 & 0,241 & 0,240 & 112 & 287 & $-1,8$ \\
\hline 1336 & 287 & 0,014 & 0,347 & 0,014 & 0,296 & 1,000 & 0,854 & 0,146 & 1,000 & 0,816 & 0,749 & 0,485 & 0,156 & 0,076 & 0,107 & 18 & 287 & $-0,6$ \\
\hline 1337 & 287 & 0,073 & 1,061 & 0,083 & 0,651 & 0,879 & 0,614 & 0,405 & 0,817 & 1,107 & 0,649 & 0,700 & 0,379 & 0,265 & 0,214 & 52 & 287 & $-1,9$ \\
\hline 1338 & 287 & 0,017 & 0,440 & 0,019 & 0,440 & 0,923 & 1,000 & 0,077 & 1,000 & 0,945 & 0,527 & 0,332 & 0,204 & 0,068 & 0,076 & 18 & 287 & $-1,0$ \\
\hline 1339 & 287 & 0,016 & 0,338 & 0,016 & 0,275 & 1,000 & 0,813 & 0,187 & 1,000 & 0,759 & 1,077 & 0,555 & 0,137 & 0,076 & 0,152 & 0 & 287 & $-0,5$ \\
\hline 1340 & 287 & 0,339 & 3,150 & 0,430 & 0,368 & 0,787 & 0,117 & 0,909 & 0,429 & 1,527 & 0,481 & 0,576 & 0,947 & 0,546 & 0,107 & 84 & 287 & $-3,2$ \\
\hline 1341 & 287 & 0,039 & 0,968 & 0,050 & 0,592 & 0,771 & 0,611 & 0,451 & 0,519 & 1,388 & 0,649 & 0,687 & 0,276 & 0,189 & 0,107 & 95 & 287 & $-1,5$ \\
\hline 1342 & 287 & 0,030 & 0,735 & 0,039 & 1,632 & 0,778 & 2,219 & 1,239 & 0,700 & 1,195 & 0,411 & 0,372 & 0,305 & 0,114 & 0,152 & 163 & 287 & $-1,6$ \\
\hline 1343 & 287 & 0,022 & 0,482 & 0,023 & 0,296 & 0,938 & 0,614 & 0,391 & 1,000 & 0,926 & 0,659 & 0,371 & 0,204 & 0,076 & 0,152 & 170 & 287 & $-1,0$ \\
\hline 1344 & 287 & 0,014 & 0,347 & 0,016 & 0,368 & 0,909 & 1,061 & 0,109 & 1,000 & 0,816 & 1,273 & 0,708 & 0,120 & 0,085 & 0,107 & 34 & 287 & $-0,3$ \\
\hline 1345 & 287 & 0,020 & 0,418 & 0,022 & 0,744 & 0,933 & 1,778 & 0,781 & 1,000 & 0,833 & 0,990 & 0,667 & 0,161 & 0,107 & 0,152 & 17 & 287 & $-0,7$ \\
\hline 1346 & 287 & 0,060 & 0,765 & 0,060 & 1,061 & 1,000 & 1,387 & 0,387 & 1,000 & 0,879 & 1,009 & 0,824 & 0,276 & 0,227 & 0,240 & 103 & 287 & $-1,5$ \\
\hline 1347 & 287 & 0,019 & 0,469 & 0,023 & 0,266 & 0,812 & 0,567 & 0,472 & 1,000 & 0,969 & 0,637 & 0,588 & 0,193 & 0,114 & 0,107 & 72 & 287 & $-0,9$ \\
\hline 1348 & 287 & 0,106 & 1,112 & 0,113 & 0,397 & 0,937 & 0,357 & 0,646 & 1,000 & 0,963 & 0,754 & 0,716 & 0,423 & 0,303 & 0,321 & 78 & 287 & $-2,1$ \\
\hline 1349 & 287 & 0,300 & 2,113 & 0,330 & 0,562 & 0,909 & 0,266 & 0,740 & 0,844 & 1,089 & 0,783 & 0,729 & 0,698 & 0,509 & 0,442 & 83 & 287 & $-2,8$ \\
\hline 1350 & 287 & 0,053 & 1,255 & 0,075 & 0,418 & 0,712 & 0,333 & 0,726 & 0,423 & 1,537 & 0,432 & 0,512 & 0,395 & 0,202 & 0,107 & 75 & 287 & $-2,0$ \\
\hline 1351 & 287 & 0,016 & 0,397 & 0,017 & 0,317 & 0,917 & 0,798 & 0,219 & 1,000 & 0,892 & 0,824 & 0,614 & 0,156 & 0,096 & 0,107 & 34 & 287 & $-0,6$ \\
\hline 1352 & 287 & 0,791 & 3,458 & 0,842 & 2,054 & 0,939 & 0,594 & 0,411 & 0,831 & 1,097 & 0,633 & 0,744 & 1,261 & 0,937 & 0,816 & 141 & 287 & $-3,7$ \\
\hline 1353 & 287 & 0,019 & 0,583 & 0,022 & 0,499 & 0,867 & 0,856 & 0,197 & 0,689 & 1,205 & 0,331 & 0,141 & 0,268 & 0,038 & 0,076 & 90 & 287 & $-1,4$ \\
\hline 1354 & 287 & 1,112 & 5,144 & 1,286 & 0,735 & 0,865 & 0,143 & 0,868 & 0,528 & 1,376 & 0,337 & 0,446 & 2,049 & 0,915 & 0,773 & 60 & 287 & $-4,4$ \\
\hline 1355 & 287 & 0,125 & 1,285 & 0,129 & 1,040 & 0,967 & 0,809 & 0,194 & 0,950 & 1,026 & 0,492 & 0,424 & 0,568 & 0,241 & 0,273 & 53 & 287 & $-2,5$ \\
\hline 1356 & 287 & 0,763 & 3,551 & 0,847 & 0,397 & 0,902 & 0,112 & 0,894 & 0,761 & 1,147 & 0,553 & 0,658 & 1,326 & 0,872 & 0,791 & 31 & 287 & $-3,7$ \\
\hline 1357 & 287 & 0,024 & 0,664 & 0,032 & 0,338 & 0,773 & 0,509 & 0,541 & 0,696 & 1,199 & 0,355 & 0,272 & 0,296 & 0,080 & 0,107 & 44 & 287 & $-1,6$ \\
\hline 1358 & 287 & 0,022 & 0,511 & 0,023 & 0,786 & 0,938 & 1,537 & 0,541 & 1,000 & 0,983 & 0,562 & 0,416 & 0,221 & 0,092 & 0,107 & 67 & 287 & $-1,1$ \\
\hline 1359 & 287 & 0,020 & 0,440 & 0,022 & 0,173 & 0,933 & 0,394 & 0,609 & 1,000 & 0,875 & 0,990 & 0,633 & 0,161 & 0,102 & 0,152 & 45 & 287 & $-0,7$ \\
\hline 1360 & 287 & 0,039 & 0,613 & 0,042 & 0,440 & 0,931 & 0,717 & 0,291 & 1,000 & 0,878 & 0,859 & 0,632 & 0,240 & 0,152 & 0,214 & 90 & 287 & $-1,3$ \\
\hline 1361 & 287 & 0,042 & 0,672 & 0,044 & 0,215 & 0,935 & 0,320 & 0,683 & 1,000 & 0,930 & 0,923 & 0,671 & 0,240 & 0,161 & 0,169 & 35 & 287 & $-1,3$ \\
\hline 1362 & 287 & 0,080 & 1,306 & 0,112 & 0,368 & 0,718 & 0,282 & 0,772 & 0,592 & 1,300 & 0,524 & 0,568 & 0,442 & 0,251 & 0,169 & 52 & 287 & $-2,1$ \\
\hline 1363 & 287 & 0,019 & 0,389 & 0,020 & 0,592 & 0,929 & 1,522 & 0,527 & 1,000 & 0,803 & 1,273 & 0,832 & 0,137 & 0,114 & 0,152 & 27 & 287 & $-0,5$ \\
\hline 1364 & 287 & 0,022 & 0,520 & 0,024 & 0,664 & 0,882 & 1,276 & 0,300 & 1,000 & 1,000 & 0,735 & 0,588 & 0,193 & 0,114 & 0,152 & 8 & 287 & $-0,9$ \\
\hline 1365 & 287 & 0,016 & 0,347 & 0,016 & 0,520 & 1,000 & 1,500 & 0,500 & 1,000 & 0,778 & 1,077 & 0,588 & 0,137 & 0,080 & 0,107 & 72 & 287 & $-0,5$ \\
\hline 1366 & 287 & 0,037 & 0,664 & 0,042 & 0,338 & 0,897 & 0,509 & 0,501 & 1,000 & 0,969 & 0,625 & 0,461 & 0,276 & 0,127 & 0,152 & 159 & 287 & $-1,5$ \\
\hline 1367 & 287 & 0,046 & 0,685 & 0,049 & 1,171 & 0,941 & 1,711 & 0,713 & 1,000 & 0,901 & 0,784 & 0,621 & 0,273 & 0,169 & 0,214 & 63 & 287 & $-1,4$ \\
\hline 1368 & 287 & 0,044 & 0,938 & 0,059 & 0,613 & 0,756 & 0,653 & 0,424 & 0,635 & 1,255 & 0,493 & 0,495 & 0,339 & 0,168 & 0,169 & 99 & 287 & $-1,8$ \\
\hline 1369 & 287 & 0,102 & 1,910 & 0,143 & 0,490 & 0,710 & 0,257 & 0,798 & 0,351 & 1,689 & 0,426 & 0,549 & 0,552 & 0,303 & 0,152 & 114 & 287 & $-2,5$ \\
\hline 1370 & 287 & 0,055 & 1,154 & 0,066 & 2,541 & 0,826 & 2,202 & 1,214 & 0,515 & 1,394 & 0,569 & 0,542 & 0,349 & 0,189 & 0,152 & 90 & 287 & $-1,8$ \\
\hline 1371 & 287 & 0,022 & 0,418 & 0,022 & 0,245 & 1,000 & 0,586 & 0,414 & 1,000 & 0,805 & 1,123 & 0,728 & 0,156 & 0,114 & 0,152 & 20 & 287 & $-0,6$ \\
\hline 1372 & 287 & 0,019 & 0,389 & 0,019 & 0,123 & 1,000 & 0,315 & 0,685 & 1,000 & 0,803 & 1,273 & 0,832 & 0,137 & 0,114 & 0,152 & 45 & 287 & $-0,5$ \\
\hline 1373 & 287 & 0,050 & 0,706 & 0,053 & 0,643 & 0,946 & 0,910 & 0,105 & 1,000 & 0,888 & 1,087 & 0,781 & 0,243 & 0,189 & 0,227 & 76 & 287 & $-1,3$ \\
\hline 1374 & 287 & 0,020 & 0,672 & 0,027 & 0,643 & 0,737 & 0,956 & 0,267 & 0,559 & 1,338 & 0,713 & 0,707 & 0,189 & 0,134 & 0,076 & 56 & 287 & $-0,9$ \\
\hline 1375 & 287 & 0,682 & 3,352 & 0,758 & 0,469 & 0,900 & 0,140 & 0,866 & 0,762 & 1,145 & 0,694 & 0,711 & 1,119 & 0,795 & 0,761 & 98 & 287 & $-3,5$ \\
\hline 1376 & 287 & 0,033 & 0,825 & 0,042 & 0,296 & 0,793 & 0,359 & 0,674 & 0,610 & 1,280 & 0,732 & 0,671 & 0,240 & 0,161 & 0,169 & 117 & 287 & $-1,3$ \\
\hline 1377 & 287 & 0,014 & 0,338 & 0,016 & 0,410 & 0,909 & 1,212 & 0,231 & 1,000 & 0,796 & 0,979 & 0,555 & 0,137 & 0,076 & 0,107 & 162 & 287 & $-0,5$ \\
\hline 1378 & 287 & 0,014 & 0,418 & 0,016 & 0,664 & 0,909 & 1,586 & 0,593 & 1,000 & 0,986 & 0,490 & 0,310 & 0,193 & 0,060 & 0,076 & 18 & 287 & $-0,9$ \\
\hline 1379 & 287 & 0,024 & 0,520 & 0,027 & 0,368 & 0,895 & 0,707 & 0,311 & 1,000 & 0,939 & 0,637 & 0,429 & 0,221 & 0,095 & 0,107 & 36 & 287 & $-1,1$ \\
\hline 1380 & 287 & 0,014 & 0,359 & 0,014 & 0,266 & 1,000 & 0,741 & 0,259 & 1,000 & 0,846 & 0,749 & 0,243 & 0,156 & 0,038 & 0,076 & 0 & 287 & $-0,6$ \\
\hline 1381 & 287 & 0,019 & 0,389 & 0,019 & 1,527 & 1,000 & 3,926 & 2,926 & 1,000 & 0,803 & 0,974 & 0,485 & 0,156 & 0,076 & 0,152 & 0 & 287 & $-0,6$ \\
\hline 1382 & 287 & 0,014 & 0,317 & 0,014 & 0,194 & 1,000 & 0,613 & 0,387 & 1,000 & 0,746 & 0,979 & 0,555 & 0,137 & 0,076 & 0,107 & 18 & 287 & $-0,5$ \\
\hline 1383 & 287 & 0,023 & 0,440 & 0,023 & 0,482 & 1,000 & 1,096 & 0,096 & 1,000 & 0,818 & 1,019 & 0,671 & 0,169 & 0,114 & 0,152 & 30 & 287 & $-0,8$ \\
\hline 1384 & 287 & 0,026 & 0,490 & 0,027 & 0,338 & 0,947 & 0,689 & 0,315 & 1,000 & 0,861 & 0,917 & 0,667 & 0,189 & 0,126 & 0,152 & 139 & 287 & $-0,9$ \\
\hline 1385 & 287 & 0,052 & 0,735 & 0,056 & 1,593 & 0,923 & 2,167 & 1,169 & 1,000 & 0,913 & 0,881 & 0,770 & 0,273 & 0,210 & 0,152 & 117 & 287 & $-1,4$ \\
\hline 1386 & 287 & 0,023 & 0,418 & 0,023 & 0,296 & 1,000 & 0,707 & 0,293 & 1,000 & 0,779 & 1,198 & 0,728 & 0,156 & 0,114 & 0,152 & 0 & 287 & $-0,6$ \\
\hline
\end{tabular}




\begin{tabular}{|c|c|c|c|c|c|c|c|c|c|c|c|c|c|c|c|c|c|c|}
\hline 1387 & 287 & 0,116 & 2,194 & 0,182 & 0,846 & 0,638 & 0,385 & 0,713 & 0,303 & 1,815 & 0,557 & 0,703 & 0,515 & 0,362 & 0,152 & 7 & 287 & $-2,4$ \\
\hline 1388 & 287 & 0,265 & 2,418 & 0,304 & 1,408 & 0,873 & 0,582 & 0,437 & 0,570 & 1,324 & 0,593 & 0,628 & 0,755 & 0,474 & 0,379 & 105 & 287 & $-2,9$ \\
\hline 1389 & 287 & 0,611 & 4,138 & 0,717 & 2,021 & 0,852 & 0,488 & 0,533 & 0,449 & 1,493 & 0,244 & 0,265 & 1,785 & 0,473 & 0,479 & 76 & 287 & $-4,2$ \\
\hline 1390 & 287 & 0,089 & 1,611 & 0,115 & 0,613 & 0,775 & 0,380 & 0,659 & 0,431 & 1,523 & 0,745 & 0,874 & 0,390 & 0,341 & 0,169 & 111 & 287 & $-2,0$ \\
\hline 1391 & 287 & 0,032 & 0,541 & 0,032 & 0,296 & 1,000 & 0,547 & 0,453 & 1,000 & 0,859 & 1,077 & 0,784 & 0,193 & 0,152 & 0,169 & 75 & 287 & $-0,9$ \\
\hline 1392 & 287 & 0,316 & 2,135 & 0,341 & 0,490 & 0,924 & 0,230 & 0,774 & 0,871 & 1,072 & 0,574 & 0,588 & 0,837 & 0,492 & 0,485 & 171 & 287 & $-3,1$ \\
\hline 1393 & 287 & 0,093 & 1,175 & 0,103 & 0,347 & 0,903 & 0,295 & 0,712 & 0,849 & 1,085 & 0,490 & 0,462 & 0,492 & 0,227 & 0,240 & 76 & 287 & $-2,3$ \\
\hline 1394 & 287 & 0,026 & 0,714 & 0,036 & 0,194 & 0,720 & 0,272 & 0,780 & 0,636 & 1,254 & 0,337 & 0,335 & 0,312 & 0,105 & 0,107 & 20 & 287 & $-1,6$ \\
\hline 1395 & 287 & 0,024 & 0,511 & 0,027 & 0,532 & 0,895 & 1,041 & 0,113 & 1,000 & 0,924 & 0,746 & 0,557 & 0,204 & 0,114 & 0,152 & 11 & 287 & $-1,0$ \\
\hline 1396 & 287 & 0,029 & 0,938 & 0,043 & 1,348 & 0,667 & 1,437 & 0,549 & 0,409 & 1,563 & 0,566 & 0,596 & 0,254 & 0,152 & 0,107 & 24 & 287 & $-1,3$ \\
\hline 1397 & 287 & 0,082 & 1,408 & 0,100 & 0,389 & 0,814 & 0,276 & 0,747 & 0,519 & 1,389 & 0,427 & 0,436 & 0,494 & 0,215 & 0,214 & 57 & 287 & $-2,3$ \\
\hline 1398 & 287 & 0,017 & 0,550 & 0,023 & 0,765 & 0,750 & 1,392 & 0,465 & 0,716 & 1,182 & 0,611 & 0,534 & 0,189 & 0,101 & 0,107 & 50 & 287 & $-0,9$ \\
\hline 1399 & 287 & 0,027 & 0,774 & 0,040 & 0,317 & 0,679 & 0,410 & 0,672 & 0,572 & 1,322 & 0,538 & 0,512 & 0,254 & 0,130 & 0,152 & 68 & 287 & $-1,3$ \\
\hline 1400 & 287 & 0,014 & 0,571 & 0,020 & 0,714 & 0,714 & 1,252 & 0,381 & 0,553 & 1,344 & 0,245 & 0,194 & 0,273 & 0,053 & 0,076 & 135 & 287 & $-1,4$ \\
\hline 1401 & 287 & 0,016 & 0,389 & 0,017 & 0,562 & 0,917 & 1,446 & 0,454 & 1,000 & 0,873 & 1,077 & 0,784 & 0,137 & 0,107 & 0,107 & 45 & 287 & $-0,5$ \\
\hline 1402 & 287 & 0,014 & 0,317 & 0,014 & 0,317 & 1,000 & 1,000 & 0,000 & 1,000 & 0,746 & 1,273 & 0,632 & 0,120 & 0,076 & 0,152 & 0 & 287 & $-0,3$ \\
\hline 1403 & 287 & 0,017 & 0,490 & 0,022 & 0,664 & 0,800 & 1,354 & 0,406 & 0,900 & 1,054 & 0,449 & 0,307 & 0,221 & 0,068 & 0,076 & 32 & 287 & $-1,1$ \\
\hline 1404 & 287 & 0,026 & 0,807 & 0,034 & 1,416 & 0,750 & 1,755 & 0,795 & 0,498 & 1,417 & 0,674 & 0,686 & 0,221 & 0,152 & 0,076 & 173 & 287 & $-1,1$ \\
\hline 1405 & 287 & 0,052 & 1,010 & 0,070 & 0,571 & 0,735 & 0,565 & 0,510 & 0,636 & 1,254 & 0,573 & 0,662 & 0,339 & 0,224 & 0,152 & 2 & 287 & $-1,8$ \\
\hline 1406 & 287 & 0,032 & 0,541 & 0,033 & 0,461 & 0,957 & 0,851 & 0,155 & 1,000 & 0,859 & 0,966 & 0,743 & 0,204 & 0,152 & 0,169 & 171 & 287 & $-1,0$ \\
\hline 1407 & 287 & 0,016 & 0,338 & 0,016 & 0,499 & 1,000 & 1,476 & 0,476 & 1,000 & 0,759 & 1,077 & 0,555 & 0,137 & 0,076 & 0,152 & 0 & 287 & $-0,5$ \\
\hline 1408 & 287 & 0,017 & 0,541 & 0,023 & 1,010 & 0,750 & 1,867 & 0,903 & 0,739 & 1,163 & 0,413 & 0,323 & 0,230 & 0,074 & 0,076 & 169 & 287 & $-1,2$ \\
\hline 1409 & 287 & 0,014 & 0,347 & 0,016 & 0,266 & 0,909 & 0,768 & 0,249 & 1,000 & 0,816 & 0,979 & 0,588 & 0,137 & 0,080 & 0,107 & 37 & 287 & $-0,5$ \\
\hline 1410 & 287 & 0,024 & 0,490 & 0,026 & 0,144 & 0,944 & 0,293 & 0,709 & 1,000 & 0,886 & 0,676 & 0,475 & 0,214 & 0,102 & 0,152 & 55 & 287 & $-1,1$ \\
\hline 1411 & 287 & 0,661 & 5,314 & 0,913 & 1,928 & 0,725 & 0,363 & 0,694 & 0,294 & 1,843 & 0,382 & 0,510 & 1,486 & 0,758 & 0,169 & 3 & 287 & $-3,9$ \\
\hline 1412 & 287 & 0,057 & 0,846 & 0,059 & 0,643 & 0,976 & 0,760 & 0,241 & 1,000 & 0,996 & 0,572 & 0,450 & 0,357 & 0,161 & 0,214 & 38 & 287 & $-1,8$ \\
\hline 1413 & 287 & 0,179 & 1,970 & 0,218 & 0,571 & 0,822 & 0,290 & 0,732 & 0,581 & 1,312 & 0,422 & 0,512 & 0,735 & 0,377 & 0,321 & 64 & 287 & $-2,9$ \\
\hline 1414 & 287 & 0,027 & 0,541 & 0,030 & 0,317 & 0,905 & 0,586 & 0,425 & 1,000 & 0,924 & 0,654 & 0,493 & 0,230 & 0,114 & 0,152 & 2 & 287 & $-1,2$ \\
\hline 1415 & 287 & 0,106 & 1,133 & 0,110 & 0,541 & 0,961 & 0,478 & 0,524 & 1,000 & 0,981 & 0,632 & 0,547 & 0,462 & 0,253 & 0,273 & 41 & 287 & $-2,2$ \\
\hline 1416 & 287 & 0,024 & 0,461 & 0,024 & 0,541 & 1,000 & 1,175 & 0,175 & 1,000 & 0,832 & 1,082 & 0,671 & 0,169 & 0,114 & 0,152 & 0 & 287 & $-0,8$ \\
\hline 1417 & 287 & 0,040 & 0,774 & 0,050 & 2,325 & 0,800 & 3,005 & 2,015 & 0,843 & 1,089 & 0,584 & 0,525 & 0,296 & 0,155 & 0,169 & 49 & 287 & $-1,6$ \\
\hline 1418 & 287 & 0,076 & 0,960 & 0,082 & 1,040 & 0,930 & 1,084 & 0,109 & 1,000 & 0,982 & 0,637 & 0,549 & 0,390 & 0,214 & 0,240 & 36 & 287 & $-2,0$ \\
\hline 1419 & 287 & 0,984 & 5,123 & 1,179 & 0,440 & 0,835 & 0,086 & 0,929 & 0,471 & 1,457 & 0,596 & 0,712 & 1,450 & 1,032 & 0,152 & 82 & 287 & $-3,9$ \\
\hline 1420 & 287 & 1,093 & 5,538 & 1,309 & 0,296 & 0,836 & 0,053 & 0,961 & 0,448 & 1,494 & 0,345 & 0,389 & 2,008 & 0,782 & 0,625 & 51 & 287 & $-4,3$ \\
\hline 1421 & 287 & 0,080 & 0,909 & 0,082 & 0,490 & 0,982 & 0,540 & 0,461 & 1,000 & 0,904 & 0,792 & 0,632 & 0,359 & 0,227 & 0,273 & 168 & 287 & $-1,8$ \\
\hline 1422 & 287 & 0,042 & 0,846 & 0,052 & 0,490 & 0,806 & 0,580 & 0,463 & 0,731 & 1,169 & 0,543 & 0,499 & 0,312 & 0,156 & 0,169 & 170 & 287 & $-1,6$ \\
\hline 1423 & 287 & 0,022 & 0,520 & 0,024 & 0,296 & 0,882 & 0,569 & 0,447 & 1,000 & 1,000 & 0,531 & 0,485 & 0,227 & 0,110 & 0,107 & 172 & 287 & $-1,2$ \\
\hline 1424 & 287 & 0,086 & 1,040 & 0,088 & 0,245 & 0,984 & 0,236 & 0,764 & 1,000 & 1,000 & 0,513 & 0,405 & 0,462 & 0,187 & 0,214 & 37 & 287 & $-2,2$ \\
\hline 1425 & 287 & 0,019 & 0,389 & 0,019 & 0,368 & 1,000 & 0,946 & 0,054 & 1,000 & 0,803 & 1,273 & 0,832 & 0,137 & 0,114 & 0,152 & 45 & 287 & $-0,5$ \\
\hline 1426 & 287 & 0,026 & 0,744 & 0,032 & 0,440 & 0,818 & 0,591 & 0,448 & 0,586 & 1,306 & 0,509 & 0,401 & 0,254 & 0,102 & 0,107 & 26 & 287 & $-1,3$ \\
\hline 1427 & 287 & 0,142 & 2,071 & 0,205 & 0,194 & 0,692 & 0,094 & 0,957 & 0,416 & 1,550 & 0,270 & 0,391 & 0,818 & 0,320 & 0,214 & 96 & 287 & $-3,0$ \\
\hline 1428 & 287 & 0,065 & 1,091 & 0,077 & 0,397 & 0,833 & 0,364 & 0,657 & 0,682 & 1,211 & 0,541 & 0,532 & 0,390 & 0,208 & 0,214 & 47 & 287 & $-2,0$ \\
\hline 1429 & 287 & 0,027 & 0,622 & 0,032 & 0,706 & 0,864 & 1,136 & 0,192 & 0,887 & 1,062 & 0,494 & 0,429 & 0,265 & 0,114 & 0,107 & 3 & 287 & $-1,4$ \\
\hline 1430 & 287 & 0,093 & 1,522 & 0,119 & 0,194 & 0,783 & 0,128 & 0,899 & 0,506 & 1,406 & 0,270 & 0,323 & 0,663 & 0,214 & 0,214 & 144 & 287 & $-2,7$ \\
\hline 1431 & 287 & 0,046 & 1,103 & 0,062 & 0,266 & 0,744 & 0,241 & 0,801 & 0,474 & 1,452 & 0,509 & 0,559 & 0,339 & 0,189 & 0,152 & 23 & 287 & $-1,8$ \\
\hline 1432 & 287 & 0,016 & 0,368 & 0,017 & 0,317 & 0,917 & 0,862 & 0,161 & 1,000 & 0,826 & 0,824 & 0,485 & 0,156 & 0,076 & 0,107 & 18 & 287 & $-0,6$ \\
\hline 1433 & 287 & 0,023 & 0,440 & 0,024 & 0,296 & 0,941 & 0,673 & 0,332 & 1,000 & 0,818 & 1,019 & 0,671 & 0,169 & 0,114 & 0,152 & 90 & 287 & $-0,8$ \\
\hline 1434 & 287 & 0,026 & 0,541 & 0,026 & 0,317 & 1,000 & 0,586 & 0,414 & 1,000 & 0,950 & 0,573 & 0,425 & 0,240 & 0,102 & 0,152 & 66 & 287 & $-1,3$ \\
\hline 1435 & 287 & 0,037 & 0,664 & 0,037 & 0,317 & 1,000 & 0,478 & 0,522 & 1,000 & 0,969 & 0,571 & 0,470 & 0,288 & 0,136 & 0,152 & 158 & 287 & $-1,5$ \\
\hline 1436 & 287 & 0,040 & 0,643 & 0,042 & 0,469 & 0,966 & 0,730 & 0,272 & 1,000 & 0,904 & 0,891 & 0,778 & 0,240 & 0,186 & 0,169 & 55 & 287 & $-1,3$ \\
\hline 1437 & 287 & 0,034 & 0,592 & 0,034 & 0,440 & 1,000 & 0,743 & 0,257 & 1,000 & 0,900 & 0,826 & 0,658 & 0,230 & 0,152 & 0,169 & 90 & 287 & $-1,2$ \\
\hline 1438 & 287 & 0,230 & 2,367 & 0,293 & 0,296 & 0,784 & 0,125 & 0,901 & 0,515 & 1,394 & 0,292 & 0,373 & 1,000 & 0,373 & 0,312 & 64 & 287 & $-3,3$ \\
\hline 1439 & 287 & 0,197 & 1,695 & 0,215 & 0,592 & 0,913 & 0,349 & 0,657 & 0,860 & 1,078 & 0,698 & 0,694 & 0,599 & 0,415 & 0,408 & 20 & 287 & $-2,6$ \\
\hline 1440 & 287 & 0,059 & 1,408 & 0,079 & 0,571 & 0,745 & 0,405 & 0,647 & 0,373 & 1,637 & 0,538 & 0,620 & 0,373 & 0,231 & 0,152 & 42 & 287 & $-1,9$ \\
\hline 1441 & 287 & 0,022 & 0,440 & 0,023 & 0,194 & 0,938 & 0,442 & 0,561 & 1,000 & 0,845 & 0,764 & 0,566 & 0,189 & 0,107 & 0,152 & 45 & 287 & $-0,9$ \\
\hline 1442 & 287 & 0,063 & 0,786 & 0,063 & 0,266 & 1,000 & 0,339 & 0,661 & 1,000 & 0,883 & 1,057 & 0,824 & 0,276 & 0,227 & 0,273 & 0 & 287 & $-1,5$ \\
\hline 1443 & 287 & 0,017 & 0,389 & 0,019 & 0,469 & 0,923 & 1,207 & 0,221 & 1,000 & 0,836 & 0,764 & 0,447 & 0,169 & 0,076 & 0,107 & 167 & 287 & -08 \\
\hline 1444 & 287 & 0,019 & 0,389 & 0,020 & 0,368 & 0,929 & 0,946 & 0,090 & 1,000 & 0,803 & 1,273 & 0,832 & 0,137 & 0,114 & 0,152 & 27 & 287 & $-0,5$ \\
\hline
\end{tabular}




\begin{tabular}{|c|c|c|c|c|c|c|c|c|c|c|c|c|c|c|c|c|c|c|}
\hline 1445 & 287 & 2,498 & 15,367 & 3,924 & 0,215 & 0,637 & 0,014 & 1,051 & 0,133 & 2,743 & 0,219 & 0,326 & 3,813 & 1,244 & 0,169 & 41 & 287 & $-5,3$ \\
\hline 1446 & 287 & 0,026 & 0,714 & 0,037 & 0,440 & 0,692 & 0,615 & 0,493 & 0,636 & 1,254 & 0,395 & 0,366 & 0,288 & 0,105 & 0,107 & 145 & 287 & $-1,5$ \\
\hline 1447 & 287 & 0,047 & 0,917 & 0,066 & 0,224 & 0,717 & 0,244 & 0,807 & 0,707 & 1,189 & 0,646 & 0,745 & 0,305 & 0,228 & 0,152 & 110 & 287 & $-1,6$ \\
\hline 1448 & 287 & 0,032 & 0,541 & 0,032 & 0,440 & 1,000 & 0,812 & 0,188 & 1,000 & 0,859 & 0,824 & 0,606 & 0,221 & 0,134 & 0,152 & 31 & 287 & $-1,1$ \\
\hline 1449 & 287 & 0,029 & 0,600 & 0,032 & 0,461 & 0,909 & 0,767 & 0,250 & 1,000 & 1,000 & 0,566 & 0,414 & 0,254 & 0,105 & 0,152 & 53 & 287 & $-1,3$ \\
\hline 1450 & 287 & 0,017 & 0,469 & 0,023 & 0,296 & 0,750 & 0,631 & 0,446 & 0,983 & 1,009 & 0,764 & 0,701 & 0,169 & 0,119 & 0,107 & 63 & 287 & $-0,8$ \\
\hline 2 & 288 & 0,022 & 0,418 & 0,022 & 0,317 & 1,000 & 0,757 & 0,243 & 1,000 & 0,805 & 0,955 & 0,632 & 0,169 & 0,107 & 0,152 & 107 & 288 & $-0,8$ \\
\hline 3 & 288 & 0,014 & 0,418 & 0,017 & 0,296 & 0,833 & 0,707 & 0,337 & 1,000 & 0,986 & 0,509 & 0,280 & 0,189 & 0,053 & 0,076 & 144 & 288 & $-0,9$ \\
\hline 1 & 289 & 0,042 & 0,735 & 0,050 & 0,613 & 0,829 & 0,833 & 0,239 & 0,967 & 1,017 & 0,697 & 0,567 & 0,276 & 0,156 & 0,169 & 174 & 289 & $-1,5$ \\
\hline 2 & 289 & 0,014 & 0,317 & 0,014 & 0,173 & 1,000 & 0,547 & 0,453 & 1,000 & 0,746 & 0,979 & 0,555 & 0,137 & 0,076 & 0,107 & 108 & 289 & $-0,5$ \\
\hline 3 & 289 & 0,042 & 1,091 & 0,063 & 0,723 & 0,659 & 0,663 & 0,479 & 0,439 & 1,508 & 0,499 & 0,572 & 0,326 & 0,186 & 0,107 & 134 & 289 & $-1,7$ \\
\hline 4 & 289 & 0,076 & 1,378 & 0,092 & 1,061 & 0,828 & 0,770 & 0,287 & 0,503 & 1,410 & 0,365 & 0,388 & 0,515 & 0,200 & 0,169 & 149 & 289 & $-2,4$ \\
\hline 5 & 289 & 0,036 & 1,327 & 0,072 & 1,163 & 0,500 & 0,876 & 0,515 & 0,256 & 1,977 & 0,122 & 0,182 & 0,611 & 0,111 & 0,076 & 20 & 289 & $-2,6$ \\
\hline 6 & 289 & 0,019 & 0,469 & 0,020 & 0,317 & 0,929 & 0,675 & 0,332 & 1,000 & 0,969 & 0,637 & 0,392 & 0,193 & 0,076 & 0,107 & 175 & 289 & $-0,9$ \\
\hline 7 & 289 & 0,016 & 0,368 & 0,017 & 0,245 & 0,917 & 0,667 & 0,344 & $\begin{array}{l}1,000 \\
\text { not }\end{array}$ & 0,826 & 0,778 & 0,500 & 0,161 & 0,080 & 0,107 & 162 & 289 & $-0,7$ \\
\hline 8 & 289 & 0,016 & 0,397 & 0,017 & 0,296 & 0,917 & 0,745 & 0,269 & 1,000 & 0,892 & 0,700 & 0,435 & 0,169 & 0,074 & 0,076 & 135 & 289 & $-0,8$ \\
\hline 9 & 289 & 0,017 & 0,541 & 0,022 & 0,397 & 0,800 & 0,735 & 0,332 & 0,739 & 1,163 & 0,373 & 0,331 & 0,243 & 0,080 & 0,107 & 135 & 289 & $-1,3$ \\
\hline 10 & 289 & 0,037 & 0,613 & 0,037 & 0,520 & 1,000 & 0,848 & 0,152 & 1,000 & 0,895 & 0,807 & 0,663 & 0,243 & 0,161 & 0,169 & 134 & 289 & $-1,3$ \\
\hline 11 & 289 & 0,024 & 0,440 & 0,024 & 0,326 & 1,000 & 0,741 & 0,259 & 1,000 & 0,794 & 1,082 & 0,671 & 0,169 & 0,114 & 0,152 & 0 & 289 & $-0,8$ \\
\hline 12 & 289 & 0,039 & 0,786 & 0,049 & 0,664 & 0,794 & 0,844 & 0,258 & 0,788 & 1,127 & 0,465 & 0,417 & 0,326 & 0,136 & 0,152 & 160 & 289 & $-1,7$ \\
\hline 13 & 289 & 0,020 & 0,592 & 0,027 & 0,389 & 0,737 & 0,657 & 0,432 & 0,721 & 1,178 & 0,524 & 0,460 & 0,221 & 0,102 & 0,107 & 18 & 289 & $-1,1$ \\
\hline 14 & 289 & 0,030 & 0,541 & 0,032 & 0,418 & 0,955 & 0,773 & 0,231 & 1,000 & 0,879 & 0,922 & 0,646 & 0,204 & 0,132 & 0,152 & 158 & 289 & $-1,0$ \\
\hline 15 & 289 & 0,037 & 1,061 & 0,060 & 0,757 & 0,619 & 0,713 & 0,477 & 0,416 & $\begin{array}{l}1,550 \\
\end{array}$ & 0,318 & 0,366 & 0,386 & 0,142 & 0,107 & 171 & 289 & $-1,9$ \\
\hline 16 & 289 & 0,017 & 0,389 & 0,017 & 0,296 & 1,000 & 0,761 & 0,239 & 1,000 & 0,836 & 0,764 & 0,447 & 0,169 & 0,076 & 0,152 & 8 & 289 & $-0,8$ \\
\hline & 290 & & & & & & & & nothing to measure & & & & & & & & 290 & - \\
\hline & 291 & & & & & & & & nothing to measure & & & & & & & & 291 & - \\
\hline & 292 & & & & & & & & nothing to measure & & & & & & & & 292 & - \\
\hline 1 & 293 & 0,016 & 0,562 & 0,019 & 0,389 & 0,846 & 0,692 & 0,345 & 0,628 & 1,262 & 0,264 & 0,174 & 0,276 & 0,048 & 0,076 & 70 & 293 & $-1,5$ \\
\hline & 294 & & & & & & & & nothing to measure & & & & & & & & 294 & \\
\hline 1 & 295 & 0,096 & 1,420 & 0,123 & 1,327 & 0,779 & 0,935 & 0,230 & 0,599 & 1,292 & 0,327 & 0,386 & 0,612 & 0,236 & 0,227 & 159 & 295 & $-2,6$ \\
\hline 2 & 295 & 0,033 & 0,634 & 0,036 & 0,511 & 0,920 & 0,807 & 0,209 & 1,000 & 0,984 & 0,563 & 0,463 & 0,273 & 0,126 & 0,152 & 153 & 295 & $-1,4$ \\
\hline 3 & 295 & 0,043 & 0,664 & 0,044 & 0,562 & 0,968 & 0,847 & 0,156 & 1,000 & 0,902 & 0,735 & 0,588 & 0,273 & 0,161 & 0,214 & 69 & 295 & $-1,4$ \\
\hline 4 & 295 & 0,040 & 0,634 & 0,042 & 0,511 & 0,966 & 0,807 & 0,196 & 1,000 & 0,892 & 0,792 & 0,596 & 0,254 & 0,152 & 0,169 & 96 & 295 & $-1,3$ \\
\hline 5 & 295 & 0,020 & 0,448 & 0,022 & 0,368 & 0,933 & 0,820 & 0,191 & 1,000 & 0,892 & 0,713 & 0,537 & 0,189 & 0,102 & 0,152 & 82 & 295 & $-0,9$ \\
\hline 6 & 295 & 0,030 & 0,511 & 0,030 & 0,440 & 1,000 & 0,860 & 0,140 & 1,000 & 0,831 & 0,922 & 0,557 & 0,204 & 0,114 & 0,152 & 97 & 295 & $-1,0$ \\
\hline 7 & 295 & 0,044 & 0,744 & 0,047 & 0,664 & 0,939 & 0,892 & 0,124 & 1,000 & 0,995 & 0,533 & 0,411 & 0,326 & 0,134 & 0,169 & 51 & 295 & $-1,7$ \\
\hline 8 & 295 & 0,057 & 0,765 & 0,059 & 0,714 & 0,976 & 0,934 & 0,071 & 1,000 & 0,901 & 0,878 & 0,743 & 0,288 & 0,214 & 0,227 & 71 & 295 & $-1,5$ \\
\hline 9 & 295 & 0,093 & 1,674 & 0,146 & 1,462 & 0,637 & 0,874 & 0,384 & 0,418 & 1,546 & 0,285 & 0,407 & 0,645 & 0,262 & 0,169 & 95 & 295 & $-2,7$ \\
\hline 10 & 295 & 0,017 & 0,359 & 0,017 & 0,194 & 1,000 & 0,541 & 0,459 & 1,000 & 0,772 & 1,175 & 0,555 & 0,137 & 0,076 & 0,152 & 0 & 295 & $-0,5$ \\
\hline 11 & 295 & 0,040 & 0,613 & 0,040 & 0,562 & 1,000 & 0,917 & 0,083 & 1,000 & 0,863 & 0,891 & 0,632 & 0,240 & 0,152 & 0,214 & 78 & 295 & $-1,3$ \\
\hline 12 & 295 & 0,022 & 0,418 & 0,023 & 0,338 & 0,938 & 0,808 & 0,202 & 1,000 & 0,805 & 1,123 & 0,728 & 0,156 & 0,114 & 0,152 & 82 & 295 & $-0,6$ \\
\hline 13 & 295 & 0,059 & 0,888 & 0,063 & 0,837 & 0,932 & 0,943 & 0,089 & 0,938 & 1,032 & 0,517 & 0,465 & 0,381 & 0,177 & 0,214 & 6 & 295 & $-1,9$ \\
\hline 14 & 295 & 0,024 & 0,440 & 0,024 & 0,338 & 1,000 & 0,769 & 0,231 & 1,000 & 0,794 & 1,082 & 0,671 & 0,169 & 0,114 & 0,152 & 0 & 295 & $-0,8$ \\
\hline 15 & 295 & 0,047 & 0,714 & $\begin{array}{l}0,027 \\
0,040\end{array}$ & 0,664 & 0,971 & 0,929 & 0,077 & $\begin{array}{l}1,000 \\
\end{array}$ & 0,926 & 0,840 & 0,696 & 0,268 & 0,186 & 0,214 & 72 & 295 & $-1,4$ \\
\hline 16 & 295 & 0,014 & 0,368 & 0,014 & 0,245 & 1,000 & 0,667 & 0,333 & $\begin{array}{l}1,000 \\
\text {. }\end{array}$ & 0,866 & 0,637 & 0,400 & 0,169 & 0,068 & 0,076 & 58 & 295 & $-0,8$ \\
\hline 17 & 295 & 0,019 & 0,368 & 0,019 & 0,287 & 1,000 & 0,781 & 0,219 & 1,000 & 0,760 & 1,273 & 0,784 & 0,137 & 0,107 & 0,152 & 45 & 295 & $-0,5$ \\
\hline 18 & 295 & 0,026 & 0,499 & 0,027 & 0,418 & 0,947 & 0,839 & 0,170 & $\begin{array}{l}1,000 \\
\text { not }\end{array}$ & 0,876 & 0,881 & 0,693 & 0,193 & 0,134 & 0,169 & 81 & 295 & $-0,9$ \\
\hline 19 & 295 & 0,280 & 1,991 & 0,310 & 1,940 & 0,903 & 0,975 & 0,101 & 0,887 & 1,062 & 0,625 & 0,604 & 0,755 & 0,456 & 0,386 & 109 & 295 & $-2,9$ \\
\hline 20 & 295 & 0,184 & 1,543 & 0,199 & 1,471 & 0,921 & 0,953 & 0,092 & 0,970 & 1,016 & 0,699 & 0,724 & 0,578 & 0,418 & 0,379 & 127 & 295 & $-2,5$ \\
\hline 21 & 295 & 0,082 & 0,938 & 0,088 & 0,888 & 0,934 & 0,946 & 0,085 & 1,000 & 0,926 & 0,981 & 0,822 & 0,326 & 0,268 & 0,312 & 140 & 295 & $-1,7$ \\
\hline 22 & 295 & 0,033 & 0,562 & 0,033 & 0,440 & 1,000 & 0,782 & 0,218 & 1,000 & 0,873 & 0,732 & 0,474 & 0,240 & 0,114 & 0,152 & 173 & 295 & $-1,3$ \\
\hline 23 & 295 & 0,019 & 0,418 & 0,019 & 0,296 & 1,000 & 0,707 & 0,293 & 1,000 & 0,864 & 0,974 & 0,728 & 0,156 & 0,114 & 0,152 & 82 & 295 & $-0,6$ \\
\hline 24 & 295 & 0,022 & 0,541 & 0,027 & 0,368 & 0,789 & 0,680 & 0,383 & 0,924 & 1,040 & 0,735 & 0,588 & 0,193 & 0,114 & 0,152 & 0 & 295 & $-0,9$ \\
\hline 25 & 295 & 0,039 & 0,592 & $\begin{array}{l}0,0279 \\
0,030\end{array}$ & 0,520 & 1,000 & 0,879 & $\begin{array}{l}0,303 \\
0,121\end{array}$ & $\begin{array}{l}1,000 \\
1\end{array}$ & $\begin{array}{l}0,840 \\
0,848\end{array}$ & 1,011 & $\begin{array}{l}0,700 \\
0,728\end{array}$ & 0,221 & 0,161 & 0,214 & 137 & 295 & $\begin{array}{l}-1,1 \\
-1,1\end{array}$ \\
\hline 26 & 295 & 0,039 & 0,613 & 0,040 & 0,532 & 0,964 & 0,869 & 0,136 & 1,000 & 0,878 & 0,859 & 0,632 & 0,240 & 0,152 & 0,169 & 20 & 295 & $-1,3$ \\
\hline 27 & 295 & 0,030 & 0,490 & 0,030 & 0,418 & 1,000 & 0,854 & 0,146 & 1,000 & 0,797 & 1,337 & 0,894 & 0,169 & 0,152 & 0,214 & 90 & 295 & $-0,8$ \\
\hline \multirow[t]{2}{*}{28} & 295 & 0,103 & 1,319 & 0,118 & 1,247 & 0,878 & 0,946 & 0,134 & 0,747 & 1,157 & 0,380 & 0,341 & 0,588 & 0,201 & 0,214 & 15 & 295 & $-2,6$ \\
\hline & 296 & & & & & & & & othing to $\mathrm{m}$ & & & & & & & & 296 & \\
\hline
\end{tabular}




\begin{tabular}{|c|c|c|c|c|c|c|c|c|c|c|c|c|c|c|c|c|c|}
\hline 297 & & & & & & & & nothing to measure & & & & & & & & 297 & - \\
\hline 298 & & & & & & & & nothing to measure & & & & & & & & 298 & - \\
\hline 299 & & & & & & & & nothing to measure & & & & & & & & 299 & - \\
\hline 300 & 0,024 & 0,837 & 0,039 & 0,511 & 0,630 & 0,611 & 0,537 & 0,438 & 1,512 & 0,481 & 0,734 & 0,254 & 0,186 & 0,076 & 144 & 300 & $-1,3$ \\
\hline 303 & 0,014 & 0,440 & 0,019 & 0,326 & 0,769 & 0,741 & 0,347 & 0,933 & 1,035 & 0,509 & 0,424 & 0,189 & 0,080 & 0,107 & 0 & 303 & $-0,9$ \\
\hline 304 & & & & & & & & nothing to measure & & & & & & & & 304 & - \\
\hline 305 & & & & & & & & nothing to measure & & & & & & & & 305 & - \\
\hline 306 & & & & & & & & nothing to measure & & & & & & & & 306 & - \\
\hline 307 & & & & & & & & nothing to measure & & & & & & & & 307 & - \\
\hline 308 & 0,024 & 0,816 & 0,036 & 0,571 & 0,680 & 0,700 & 0,439 & 0,460 & 1,474 & 0,333 & 0,389 & 0,305 & 0,119 & 0,076 & 27 & 308 & $-1,6$ \\
\hline 309 & & & & & & & & nothing to measure & & & & & & & & 309 & \\
\hline 310 & 0,024 & 0,765 & 0,039 & 0,520 & 0,630 & 0,680 & 0,490 & 0,524 & 1,382 & 0,585 & 0,697 & 0,230 & 0,161 & 0,076 & 106 & 310 & $-1,2$ \\
\hline 310 & 0,016 & 0,389 & 0,017 & 0,275 & 0,917 & 0,707 & 0,305 & 1,000 & 0,873 & 0,700 & 0,400 & 0,169 & 0,068 & 0,076 & 21 & 310 & $-0,8$ \\
\hline 310 & 0,060 & 1,154 & 0,082 & 0,816 & 0,737 & 0,707 & 0,394 & 0,569 & 1,326 & 0,723 & 0,863 & 0,326 & 0,281 & 0,214 & 8 & 310 & $-1,7$ \\
\hline 310 & 0,017 & 0,723 & 0,026 & 0,469 & 0,667 & 0,649 & 0,484 & 0,414 & 1,554 & 0,588 & 0,693 & 0,193 & 0,134 & 0,076 & 135 & 310 & $-0,9$ \\
\hline 311 & & & & & & & & nothing to measure & & & & & & & & 311 & - \\
\hline 312 & & & & & & & & nothing to measure & & & & & & & & 312 & - \\
\hline 313 & & & & & & & & nothing to measure & & & & & & & & 313 & - \\
\hline 314 & 0,034 & 0,592 & 0,037 & 0,490 & 0,923 & 0,828 & 0,188 & 1,000 & 0,900 & 1,054 & 0,877 & 0,204 & 0,179 & 0,169 & 21 & 314 & $-1,0$ \\
\hline 315 & & & & & & & & nothing to measure & & & & & & & & 315 & - \\
\hline 316 & & & & & & & & nothing to measure & & & & & & & & 316 & - \\
\hline 317 & & & & & & & & nothing to measure & & & & & & & & 317 & - \\
\hline 318 & & & & & & & & nothing to measure & & & & & & & & 318 & - \\
\hline 319 & & & & & & & & nothing to measure & & & & & & & & 319 & - \\
\hline 320 & 0,131 & 2,295 & 0,174 & 1,348 & 0,752 & 0,587 & 0,481 & 0,311 & 1,792 & 0,565 & 0,768 & 0,542 & 0,417 & 0,214 & 148 & 320 & $-2,4$ \\
\hline 320 & 0,039 & 0,786 & 0,049 & 0,643 & 0,794 & 0,817 & 0,275 & 0,788 & 1,127 & 0,529 & 0,496 & 0,305 & 0,152 & 0,152 & 169 & 320 & $-1,6$ \\
\hline 321 & 0,034 & 0,613 & 0,040 & 0,490 & 0,857 & 0,800 & 0,246 & 1,000 & 0,932 & 0,899 & 0,759 & 0,221 & 0,168 & 0,107 & 109 & 321 & $-1,1$ \\
\hline 322 & 0,029 & 0,951 & 0,037 & 0,757 & 0,769 & 0,796 & 0,308 & 0,399 & 1,584 & 0,166 & 0,118 & 0,469 & 0,055 & 0,076 & 166 & 322 & $-2,2$ \\
\hline 323 & & & & & & & & nothing to measure & & & & & & & & 323 & - \\
\hline 324 & 0,022 & 0,541 & 0,024 & 0,418 & 0,882 & 0,773 & 0,255 & 0.924 & 1,040 & 0,424 & 0,330 & 0,254 & 0,084 & 0,107 & 67 & 324 & $-1,3$ \\
\hline 324 & 0,022 & 0,520 & 0,024 & 0,440 & 0,882 & 0,845 & 0,194 & 1,000 & 1,000 & 0,466 & 0,331 & 0,243 & 0,080 & 0,107 & 60 & 324 & $-1,3$ \\
\hline 325 & 0,033 & 0,989 & 0,050 & 0,867 & 0,657 & 0,876 & 0,365 & 0,424 & 1,536 & 0,215 & 0,250 & 0,442 & 0,111 & 0,152 & 33 & 325 & $-2,1$ \\
\hline 325 & 0,075 & 1,010 & 0,080 & 0,960 & 0,929 & 0,950 & 0,087 & 0,919 & 1,043 & 0,509 & 0,462 & 0,432 & 0,200 & 0,214 & 39 & 325 & $-2,1$ \\
\hline 325 & 0,014 & 0,389 & 0,016 & 0,266 & 0,909 & 0,685 & 0,328 & 1,000 & 0,916 & 0,637 & 0,354 & 0,169 & 0,060 & 0,076 & 72 & 325 & $-0,8$ \\
\hline 325 & 0,085 & 1,133 & 0,095 & 1,019 & 0,894 & 0,899 & 0,146 & 0,829 & 1,098 & 0,458 & 0,442 & 0,485 & 0,214 & 0,214 & 40 & 325 & $-2,3$ \\
\hline 325 & 0,030 & 0,562 & 0,033 & 0,490 & 0,913 & 0,872 & 0,155 & 1,000 & 0,914 & 0,723 & 0,493 & 0,230 & 0,114 & 0,152 & 11 & 325 & $-1,2$ \\
\hline 325 & 0,039 & 0,592 & 0,039 & 0,490 & 1,000 & 0,828 & 0,172 & 1,000 & 0,848 & 1,185 & 0,914 & 0,204 & 0,186 & 0,214 & 121 & 325 & $-1,0$ \\
\hline 325 & 0,059 & 0,896 & 0,063 & 0,867 & 0,932 & 0,967 & 0,076 & 0,920 & 1,043 & 0,522 & 0,424 & 0,379 & 0,161 & 0,214 & 40 & 325 & $-1,9$ \\
\hline 325 & 0,016 & 0,410 & 0,016 & 0,317 & 1,000 & 0,773 & 0,227 & 1,000 & 0,920 & 0,539 & 0,196 & 0,193 & 0,038 & 0,076 & 90 & 325 & $-0,9$ \\
\hline 325 & 0,019 & 0,571 & 0,023 & 0,499 & 0,812 & 0,874 & 0,226 & 0,720 & 1,179 & 0,487 & 0,461 & 0,221 & 0,102 & 0,107 & 145 & 325 & $-1,1$ \\
\hline 325 & 0,065 & 1,070 & 0,079 & 0,968 & 0,818 & 0,905 & 0,205 & 0,709 & 1,188 & 0,365 & 0,368 & 0,475 & 0,174 & 0,214 & 28 & 325 & $-2,2$ \\
\hline 325 & 0,014 & 0,317 & 0,014 & 0,194 & 1,000 & 0,613 & 0,387 & 1,000 & 0,746 & 0,979 & 0,555 & 0,137 & 0,076 & 0,107 & 18 & 325 & $-0,5$ \\
\hline 325 & 0,024 & 0,550 & 0,024 & 0,499 & 1,000 & 0,908 & 0,092 & 1,000 & 0,993 & 0,481 & 0,316 & 0,254 & 0,080 & 0,107 & 38 & 325 & $-1,3$ \\
\hline 325 & 0,014 & 0,347 & 0,014 & 0,245 & 1,000 & 0,707 & 0,293 & 1,000 & 0,816 & 0,707 & 0,333 & 0,161 & 0,054 & 0,107 & 45 & 325 & $-0,7$ \\
\hline 325 & 0,046 & 0,714 & 0,050 & 0,634 & 0,914 & 0,887 & 0,142 & 1,000 & 0,941 & 0,994 & 0,781 & 0,243 & 0,189 & 0,214 & 102 & 325 & $-1,3$ \\
\hline 325 & 0,027 & 0,672 & 0,032 & 0,622 & 0,864 & 0,924 & 0,156 & 0,758 & 1,149 & 0,372 & 0,333 & 0,305 & 0,102 & 0,152 & 28 & 325 & $-1,6$ \\
\hline 325 & 0,042 & 0,837 & 0,052 & 0,672 & 0,806 & 0,803 & 0,277 & 0,746 & 1,157 & 0,568 & 0,523 & 0,305 & 0,160 & 0,152 & 36 & 325 & $-1,6$ \\
\hline 325 & 0,088 & 1,539 & 0,123 & 1,315 & 0,709 & 0,854 & 0,325 & 0,464 & 1,467 & 0,311 & 0,415 & 0,599 & 0,249 & 0,152 & 42 & 325 & $-2,6$ \\
\hline 325 & 0,023 & 0,541 & 0,023 & 0,469 & 1,000 & 0,867 & 0,133 & 0,985 & 1,007 & 0,453 & 0,334 & 0,254 & 0,085 & 0,107 & 30 & 325 & $-1,3$ \\
\hline 325 & 0,050 & 1,040 & 0,070 & 0,799 & 0,714 & 0,768 & 0,368 & 0,583 & 1,309 & 0,610 & 0,585 & 0,324 & 0,189 & 0,152 & 92 & 325 & $-1,7$ \\
\hline 325 & 0,047 & 0,714 & 0,047 & 0,664 & 1,000 & 0,929 & 0,071 & 1,000 & 0,926 & 0,646 & 0,526 & 0,305 & 0,161 & 0,214 & 29 & 325 & $-1,6$ \\
\hline 325 & 0,026 & 0,765 & 0,036 & 0,541 & 0,720 & 0,707 & 0,405 & 0,554 & 1,343 & 0,559 & 0,610 & 0,243 & 0,148 & 0,107 & 136 & 325 & $-1,3$ \\
\hline 325 & 0,017 & 0,368 & 0,017 & 0,245 & 1,000 & 0,667 & 0,333 & 1,000 & 0,791 & 1,175 & 0,784 & 0,137 & 0,107 & 0,152 & 45 & 325 & $-0,5$ \\
\hline 325 & 0,040 & 0,685 & 0,043 & 0,613 & 0,933 & 0,895 & 0,124 & 1,000 & 0,964 & 0,615 & 0,478 & 0,288 & 0,138 & 0,152 & 16 & 325 & $-1,5$ \\
\hline 325 & 0,024 & 0,571 & 0,026 & 0,499 & 0,944 & 0,874 & 0,138 & 0,941 & 1,031 & 0,416 & 0,294 & 0,273 & 0,080 & 0,107 & 34 & 325 & $-1,4$ \\
\hline 325 & 0,046 & 0,867 & 0,059 & 0,693 & 0,780 & 0,800 & 0,297 & 0,768 & 1,141 & 0,668 & 0,674 & 0,296 & 0,199 & 0,169 & 15 & 325 & 6 \\
\hline
\end{tabular}




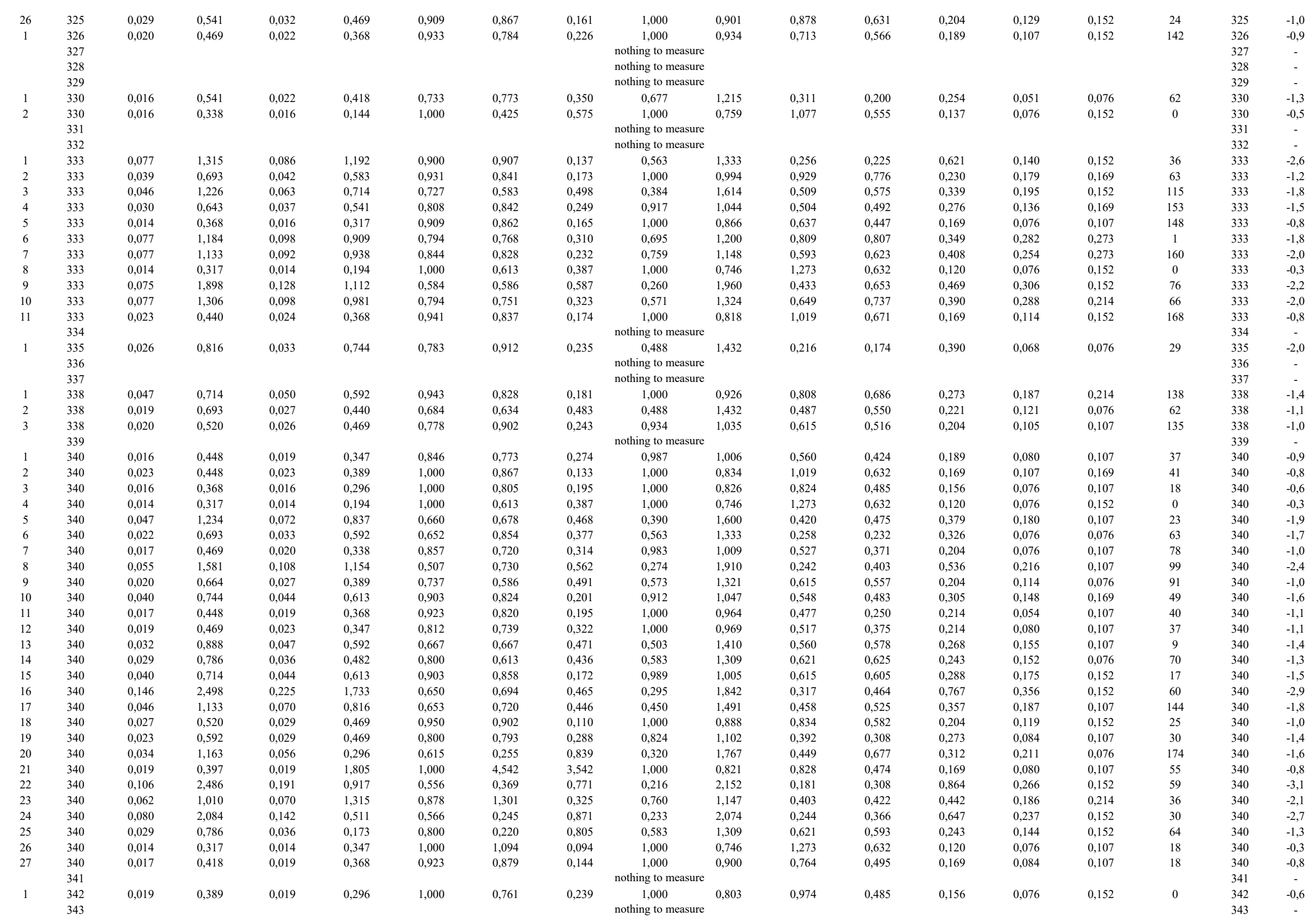


nothing to measure

$\begin{array}{ll}1,000 & 0,875 \\ 1,000 & 0,0,75 \\ 1,00 & 0,91\end{array}$

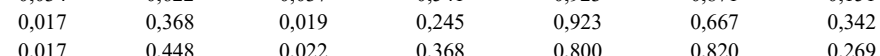

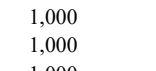

nothing to measure

0,791

$0,686 \quad 0,392$

0,175
0,764

0,745

0,193

$0,230 \quad 0,076 \quad 0,152$

$\begin{array}{lll}0,137 & 0,152\end{array}$

othing to measure

$\begin{array}{lllllll}0,014 & 0,317 & 0,014 & 0,215 & 1,000 & 0,680 & 0,320\end{array}$

$\begin{array}{lllllll}0,014 & 0,317 & 0,014 & 0,215 & 1,000 & 0,680 & 0,320 \\ 0,082 & 1,357 & 0,096 & 1,010 & 0,051 & 0,7458 & 0,296\end{array}$

0,746

$0.979 \quad 0.555$ 


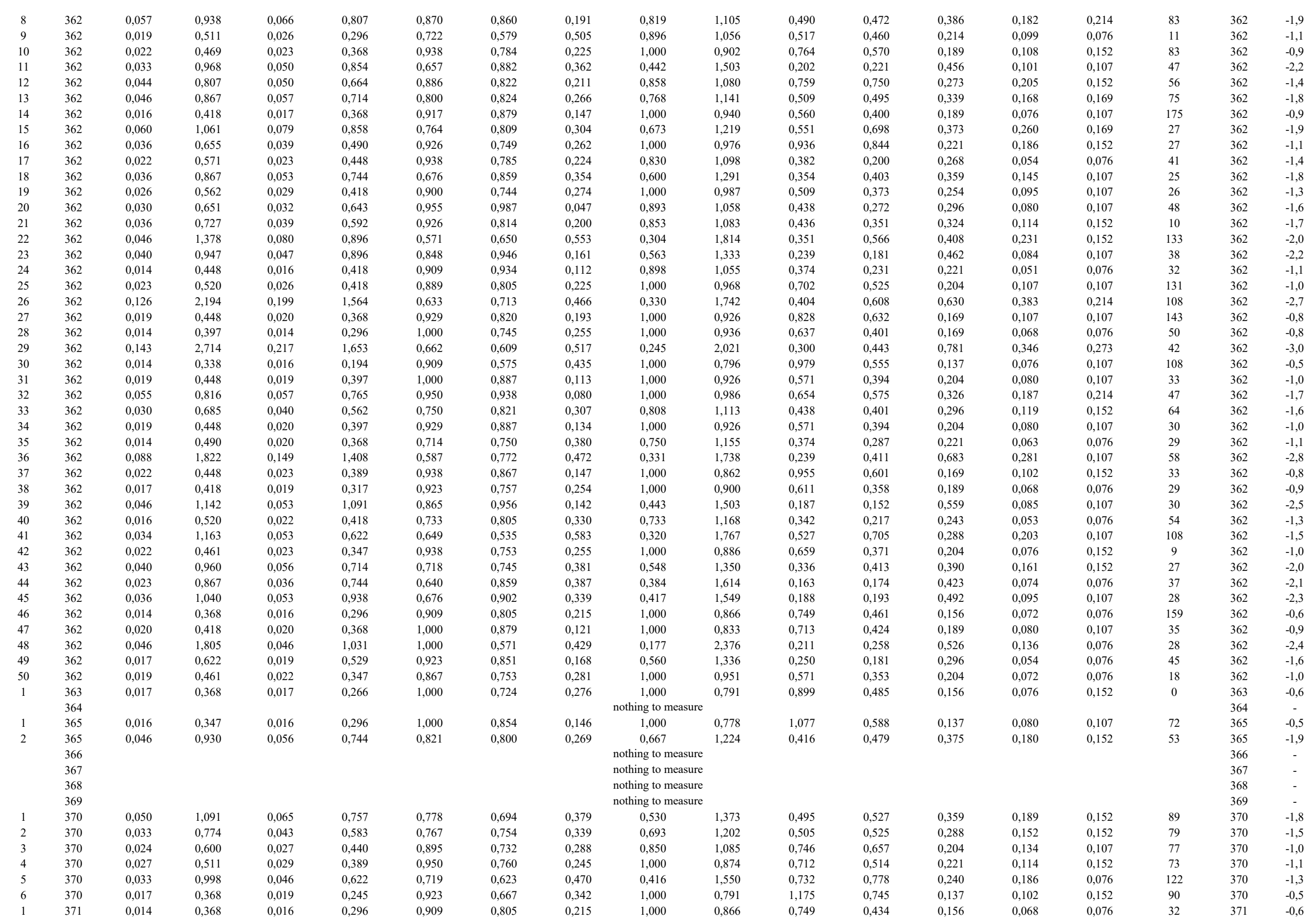




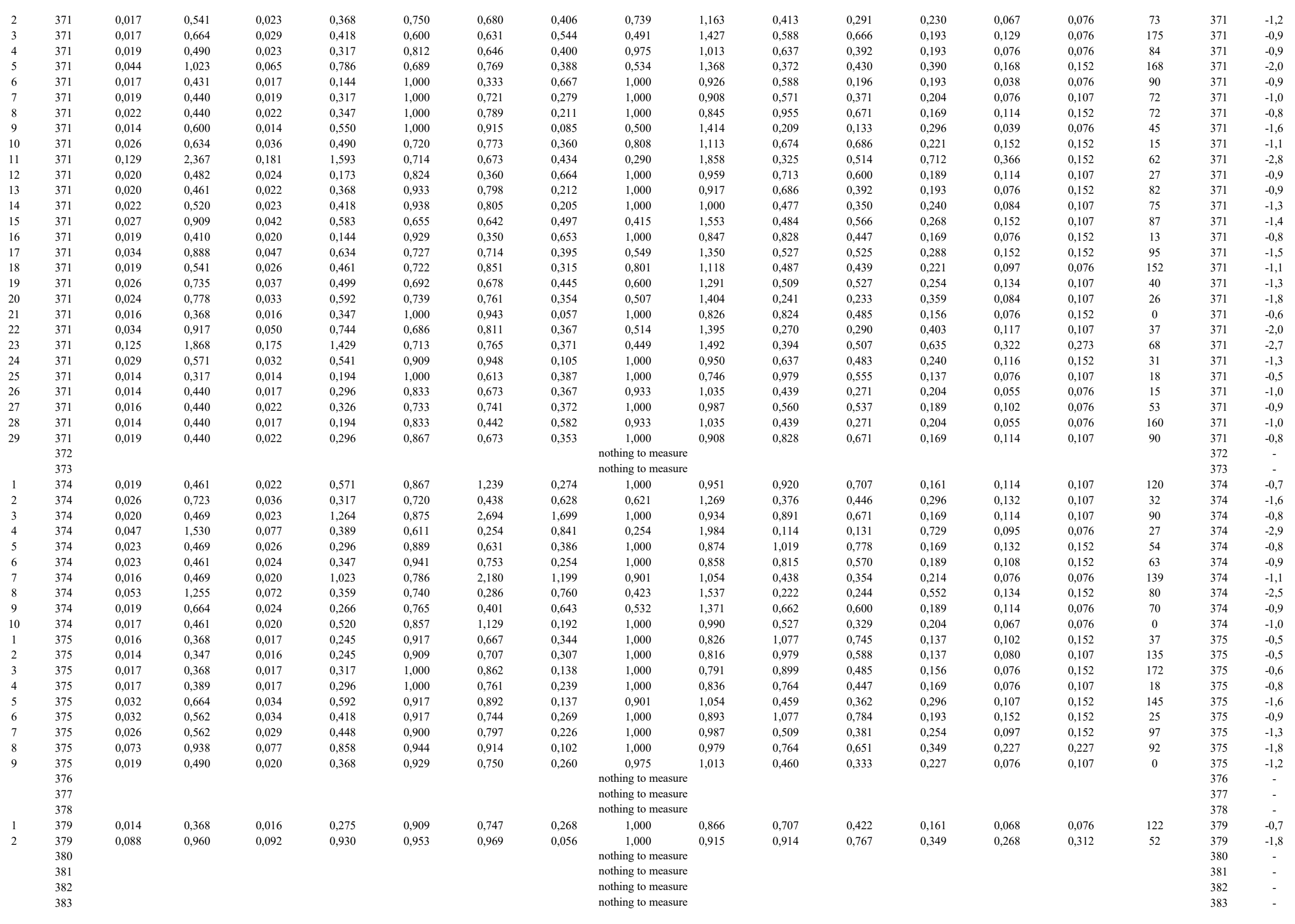




\begin{tabular}{|c|c|c|c|c|c|c|c|c|c|c|c|c|c|c|c|c|c|c|}
\hline \multirow[b]{2}{*}{1} & 384 & \multicolumn{15}{|c|}{ nothing to measure } & 384 & - \\
\hline & $\begin{array}{l}385 \\
386\end{array}$ & 0,017 & 0,448 & 0,019 & 0,418 & 0,923 & 0,934 & 0,102 & $\begin{array}{c}1,000 \\
\text { nothing to measure }\end{array}$ & 0,964 & 0,588 & 0,439 & 0,193 & 0,085 & 0,076 & 32 & $\begin{array}{l}385 \\
386\end{array}$ & $-0,9$ \\
\hline 1 & 387 & 0,014 & 0,368 & 0,017 & 0,245 & 0,833 & 0,667 & 0,373 & 1,000 & 0,866 & 0,979 & 0,702 & 0,137 & 0,096 & 0,107 & 45 & 387 & $-0,5$ \\
\hline 1 & 388 & 0,029 & 0,706 & 0,034 & 0,389 & 0,833 & 0,551 & 0,479 & 0,724 & 1,175 & 0,480 & 0,275 & 0,276 & 0,076 & 0,152 & 89 & 388 & $-1,5$ \\
\hline 2 & 388 & 0,030 & 0,613 & 0,032 & 0,490 & 0,955 & 0,800 & 0,205 & 1,000 & 0,996 & 0,504 & 0,412 & 0,276 & 0,114 & 0,152 & 14 & 388 & $-1,5$ \\
\hline 3 & 388 & 0,030 & 0,921 & 0,039 & 0,706 & 0,778 & 0,766 & 0,323 & 0,446 & 1,497 & 0,181 & 0,149 & 0,461 & 0,069 & 0,076 & 8 & 388 & $-2,2$ \\
\hline 4 & 388 & 0,023 & 0,938 & 0,043 & 0,520 & 0,533 & 0,554 & 0,645 & 0,328 & 1,747 & 0,407 & 0,600 & 0,268 & 0,161 & 0,076 & 105 & 388 & $-1,4$ \\
\hline 5 & 388 & 0,043 & 1,298 & 0,062 & 0,613 & 0,698 & 0,472 & 0,608 & 0,321 & 1,764 & 0,562 & 0,606 & 0,312 & 0,189 & 0,152 & 164 & 388 & $-1,6$ \\
\hline 6 & 388 & 0,016 & 0,490 & 0,020 & 0,296 & 0,786 & 0,604 & 0,451 & 0,825 & 1,101 & 0,412 & 0,287 & 0,221 & 0,063 & 0,076 & 18 & 388 & $-1,1$ \\
\hline 7 & 388 & 0,014 & 0,562 & 0,020 & 0,461 & 0,714 & 0,819 & 0,338 & 0,571 & 1,324 & 0,260 & 0,143 & 0,265 & 0,038 & 0,076 & 0 & 388 & $-1,4$ \\
\hline 8 & 388 & 0,024 & 0,757 & 0,032 & 0,583 & 0,773 & 0,771 & 0,323 & 0,536 & 1,366 & 0,255 & 0,202 & 0,349 & 0,071 & 0,076 & 5 & 388 & $-1,8$ \\
\hline 9 & 388 & 0,036 & 0,900 & 0,044 & 0,583 & 0,806 & 0,648 & 0,402 & 0,556 & 1,341 & 0,255 & 0,179 & 0,423 & 0,076 & 0,107 & 2 & 388 & $-2,1$ \\
\hline 10 & 388 & 0,063 & 1,492 & 0,102 & 1,340 & 0,620 & 0,898 & 0,394 & 0,356 & 1,675 & 0,172 & 0,209 & 0,683 & 0,143 & 0,107 & 9 & 388 & $-2,8$ \\
\hline 11 & 388 & 0,016 & 0,541 & 0,019 & 0,287 & 0,846 & 0,531 & 0,494 & 0,677 & 1,215 & 0,379 & 0,164 & 0,230 & 0,038 & 0,076 & 0 & 388 & $-1,2$ \\
\hline 12 & 388 & 0,116 & 1,420 & 0,141 & 1,255 & 0,827 & 0,884 & 0,209 & 0,724 & 1,175 & 0,486 & 0,533 & 0,552 & 0,294 & 0,240 & 13 & 388 & $-2,5$ \\
\hline 13 & 388 & 0,039 & 1,154 & 0,056 & 1,010 & 0,692 & 0,876 & 0,332 & 0,366 & 1,654 & 0,168 & 0,141 & 0,542 & 0,076 & 0,076 & 23 & 388 & $-2,4$ \\
\hline 14 & 388 & 0,023 & 0,807 & 0,034 & 0,655 & 0,667 & 0,811 & 0,383 & 0,443 & 1,503 & 0,204 & 0,200 & 0,379 & 0,076 & 0,076 & 178 & 388 & $-1,9$ \\
\hline 15 & 388 & 0,069 & 1,953 & 0,119 & 1,636 & 0,578 & 0,838 & 0,452 & 0,227 & 2,099 & 0,108 & 0,152 & 0,900 & 0,137 & 0,152 & 13 & 388 & $-3,2$ \\
\hline 16 & 388 & 0,027 & 0,583 & 0,029 & 0,338 & 0,950 & 0,580 & 0,423 & 1,000 & 0,996 & 0,605 & 0,316 & 0,240 & 0,076 & 0,152 & 179 & 388 & $-1,3$ \\
\hline 17 & 388 & 0,014 & 0,317 & 0,014 & 0,215 & 1,000 & 0,680 & 0,320 & 1,000 & 0,746 & 0,979 & 0,555 & 0,137 & 0,076 & 0,107 & 72 & 388 & $-0,5$ \\
\hline 18 & 388 & 0,093 & 2,637 & 0,178 & 1,357 & 0,524 & 0,515 & 0,680 & 0,169 & 2,436 & 0,374 & 0,597 & 0,563 & 0,336 & 0,152 & 152 & 388 & $-2,5$ \\
\hline 19 & 388 & 0,019 & 0,482 & 0,019 & 0,317 & 1,000 & 0,658 & 0,342 & 1,000 & 0,995 & 0,447 & 0,164 & 0,230 & 0,038 & 0,076 & 0 & 388 & $-1,2$ \\
\hline 20 & 388 & 0,027 & 0,828 & 0,039 & 0,583 & 0,704 & 0,704 & 0,419 & 0,499 & 1,415 & 0,240 & 0,284 & 0,381 & 0,108 & 0,107 & 18 & 388 & $-1,9$ \\
\hline 21 & 388 & 0,014 & 0,368 & 0,014 & 0,245 & 1,000 & 0,667 & 0,333 & 1,000 & 0,866 & 0,637 & 0,447 & 0,169 & 0,076 & 0,107 & 162 & 388 & $-0,8$ \\
\hline 22 & 388 & 0,046 & 0,879 & 0,052 & 0,765 & 0,889 & 0,870 & 0,171 & 0,747 & 1,157 & 0,392 & 0,392 & 0,386 & 0,152 & 0,169 & 0 & 388 & $-1,9$ \\
\hline 23 & 388 & 0,079 & 1,982 & 0,129 & 1,504 & 0,611 & 0,759 & 0,458 & 0,252 & 1,991 & 0,158 & 0,206 & 0,796 & 0,164 & 0,152 & 4 & 388 & $-3,0$ \\
\hline 24 & 388 & 0,016 & 0,389 & 0,017 & 0,296 & 0,917 & 0,761 & 0,253 & 1,000 & 0,873 & 0,700 & 0,425 & 0,169 & 0,072 & 0,076 & 21 & 388 & $-0,8$ \\
\hline 25 & 388 & 0,027 & 0,613 & 0,032 & 0,541 & 0,864 & 0,883 & 0,180 & 0,912 & 1,047 & 0,456 & 0,412 & 0,276 & 0,114 & 0,152 & 10 & 388 & $-1,5$ \\
\hline 26 & 388 & 0,022 & 0,541 & 0,026 & 0,418 & 0,833 & 0,773 & 0,281 & 0,924 & 1,040 & 0,516 & 0,421 & 0,230 & 0,097 & 0,107 & 162 & 388 & $-1,2$ \\
\hline 27 & 388 & 0,022 & 0,490 & 0,026 & 0,389 & 0,833 & 0,793 & 0,266 & 1,000 & 0,943 & 0,955 & 0,746 & 0,169 & 0,126 & 0,107 & 125 & 388 & $-0,8$ \\
\hline 28 & 388 & 0,030 & 0,706 & 0,039 & 0,490 & 0,778 & 0,695 & 0,378 & 0,760 & 1,147 & 0,504 & 0,522 & 0,276 & 0,144 & 0,107 & 9 & 388 & $-1,5$ \\
\hline 29 & 388 & 0,024 & 0,469 & 0,024 & 0,389 & 1,000 & 0,829 & 0,171 & 1,000 & 0,848 & 1,082 & 0,791 & 0,169 & 0,134 & 0,152 & 40 & 388 & $-0,8$ \\
\hline 30 & 388 & 0,014 & 0,368 & 0,016 & 0,296 & 0,909 & 0,805 & 0,215 & 1,000 & 0,866 & 0,637 & 0,400 & 0,169 & 0,068 & 0,076 & 18 & 388 & $-0,8$ \\
\hline 31 & 388 & 0,016 & 0,541 & 0,019 & 0,347 & 0,846 & 0,641 & 0,391 & 0,677 & 1,215 & 0,700 & 0,632 & 0,169 & 0,107 & 0,076 & 162 & 388 & $-0,8$ \\
\hline 32 & 388 & 0,017 & 0,541 & 0,022 & 0,368 & 0,800 & 0,680 & 0,378 & 0,739 & 1,163 & 0,382 & 0,265 & 0,240 & 0,063 & 0,076 & 148 & 388 & $-1,3$ \\
\hline 33 & 388 & 0,016 & 0,634 & 0,022 & 0,532 & 0,733 & 0,840 & 0,311 & 0,494 & 1,423 & 0,219 & 0,125 & 0,303 & 0,038 & 0,076 & 0 & 388 & $-1,6$ \\
\hline 34 & 388 & 0,059 & 1,154 & 0,076 & 1,103 & 0,774 & 0,956 & 0,231 & 0,555 & 1,342 & 0,261 & 0,240 & 0,536 & 0,129 & 0,152 & 15 & 388 & $-2,4$ \\
\hline 35 & 388 & 0,023 & 0,643 & 0,030 & 0,490 & 0,762 & 0,763 & 0,336 & 0,699 & 1,196 & 0,384 & 0,348 & 0,276 & 0,096 & 0,076 & 18 & 388 & $-1,5$ \\
\hline 36 & 388 & 0,016 & 0,482 & 0,017 & 0,194 & 0,917 & 0,404 & 0,602 & 0,855 & 1,082 & 0,379 & 0,164 & 0,230 & 0,038 & 0,076 & 0 & 388 & $-1,2$ \\
\hline 37 & 388 & 0,149 & 2,515 & 0,248 & 1,881 & 0,601 & 0,748 & 0,472 & 0,297 & 1,836 & 0,228 & 0,321 & 0,912 & 0,293 & 0,169 & 2 & 388 & $-3,2$ \\
\hline 38 & 388 & 0,034 & 0,664 & 0,042 & 0,541 & 0,828 & 0,815 & 0,253 & 0,983 & 1,009 & 0,679 & 0,526 & 0,254 & 0,134 & 0,152 & 26 & 388 & $-1,3$ \\
\hline 39 & 388 & 0,022 & 0,461 & 0,024 & 0,296 & 0,882 & 0,642 & 0,376 & 1,000 & 0,886 & 0,764 & 0,506 & 0,189 & 0,096 & 0,152 & 27 & 388 & $-0,9$ \\
\hline 40 & 388 & 0,099 & 1,366 & 0,122 & 1,192 & 0,812 & 0,873 & 0,227 & 0,667 & 1,224 & 0,475 & 0,513 & 0,515 & 0,264 & 0,214 & 134 & 388 & $-2,4$ \\
\hline 41 & 388 & 0,108 & 1,369 & 0,116 & 1,187 & 0,926 & 0,867 & 0,152 & 0,721 & 1,178 & 0,466 & 0,349 & 0,542 & 0,189 & 0,227 & 172 & 388 & $-2,4$ \\
\hline 42 & 388 & 0,113 & 1,910 & 0,155 & 1,412 & 0,731 & 0,739 & 0,375 & 0,390 & 1,601 & 0,220 & 0,234 & 0,810 & 0,189 & 0,227 & 2 & 388 & $-3,0$ \\
\hline 43 & 388 & 0,080 & 1,184 & 0,096 & 0,989 & 0,836 & 0,836 & 0,232 & 0,721 & 1,178 & 0,368 & 0,386 & 0,528 & 0,203 & 0,214 & 154 & 388 & $-2,4$ \\
\hline 44 & 388 & 0,033 & 0,685 & 0,037 & 0,562 & 0,885 & 0,821 & 0,213 & 0,885 & 1,063 & 0,586 & 0,607 & 0,268 & 0,162 & 0,152 & 56 & 388 & $-1,4$ \\
\hline 45 & 388 & 0,017 & 0,368 & 0,017 & 0,245 & 1,000 & 0,667 & 0,333 & 1,000 & 0,791 & 1,175 & 0,784 & 0,137 & 0,107 & 0,152 & 45 & 388 & $-0,5$ \\
\hline 46 & 388 & 0,014 & 0,532 & 0,019 & 0,389 & 0,769 & 0,730 & 0,355 & 0,636 & 1,254 & 0,260 & 0,143 & 0,265 & 0,038 & 0,076 & 0 & 388 & $-1,4$ \\
\hline 47 & 388 & 0,040 & 0,951 & 0,055 & 0,706 & 0,737 & 0,742 & 0,368 & 0,558 & 1,338 & 0,548 & 0,620 & 0,305 & 0,189 & 0,152 & 170 & 388 & $-1,6$ \\
\hline 48 & 388 & 0,047 & 0,735 & 0,053 & 0,664 & 0,892 & 0,902 & 0,146 & 1,000 & 0,953 & 0,689 & 0,634 & 0,296 & 0,187 & 0,214 & 46 & 388 & $-1,6$ \\
\hline 49 & 388 & 0,100 & 1,399 & 0,113 & 1,298 & 0,886 & 0,927 & 0,135 & 0,645 & 1,245 & 0,317 & 0,314 & 0,635 & 0,199 & 0,214 & 163 & 388 & $-2,7$ \\
\hline 50 & 388 & 0,026 & 0,511 & 0,026 & 0,368 & 1,000 & 0,719 & 0,281 & 1,000 & 0,898 & 0,674 & 0,514 & 0,221 & 0,114 & 0,152 & 150 & 388 & $-1,1$ \\
\hline 51 & 388 & 0,059 & 1,327 & 0,077 & 1,184 & 0,759 & 0,892 & 0,264 & 0,420 & 1,544 & 0,186 & 0,189 & 0,635 & 0,120 & 0,152 & 157 & 388 & $-2,7$ \\
\hline 52 & 388 & 0,019 & 0,368 & 0,019 & 0,245 & 1,000 & 0,667 & 0,333 & 1,000 & 0,760 & 1,273 & 0,784 & 0,137 & 0,107 & 0,152 & 90 & 388 & $-0,5$ \\
\hline 53 & 388 & 0,143 & 2,430 & 0,191 & 1,501 & 0,752 & 0,617 & 0,456 & 0,305 & 1,810 & 0,374 & 0,434 & 0,698 & 0,303 & 0,227 & 9 & 388 & $-2,8$ \\
\hline 54 & 388 & 0,080 & 0,930 & 0,082 & 0,837 & 0,982 & 0,900 & 0,101 & 1,000 & 0,925 & 0,792 & 0,632 & 0,359 & 0,227 & 0,273 & 168 & 388 & $-1,8$ \\
\hline
\end{tabular}




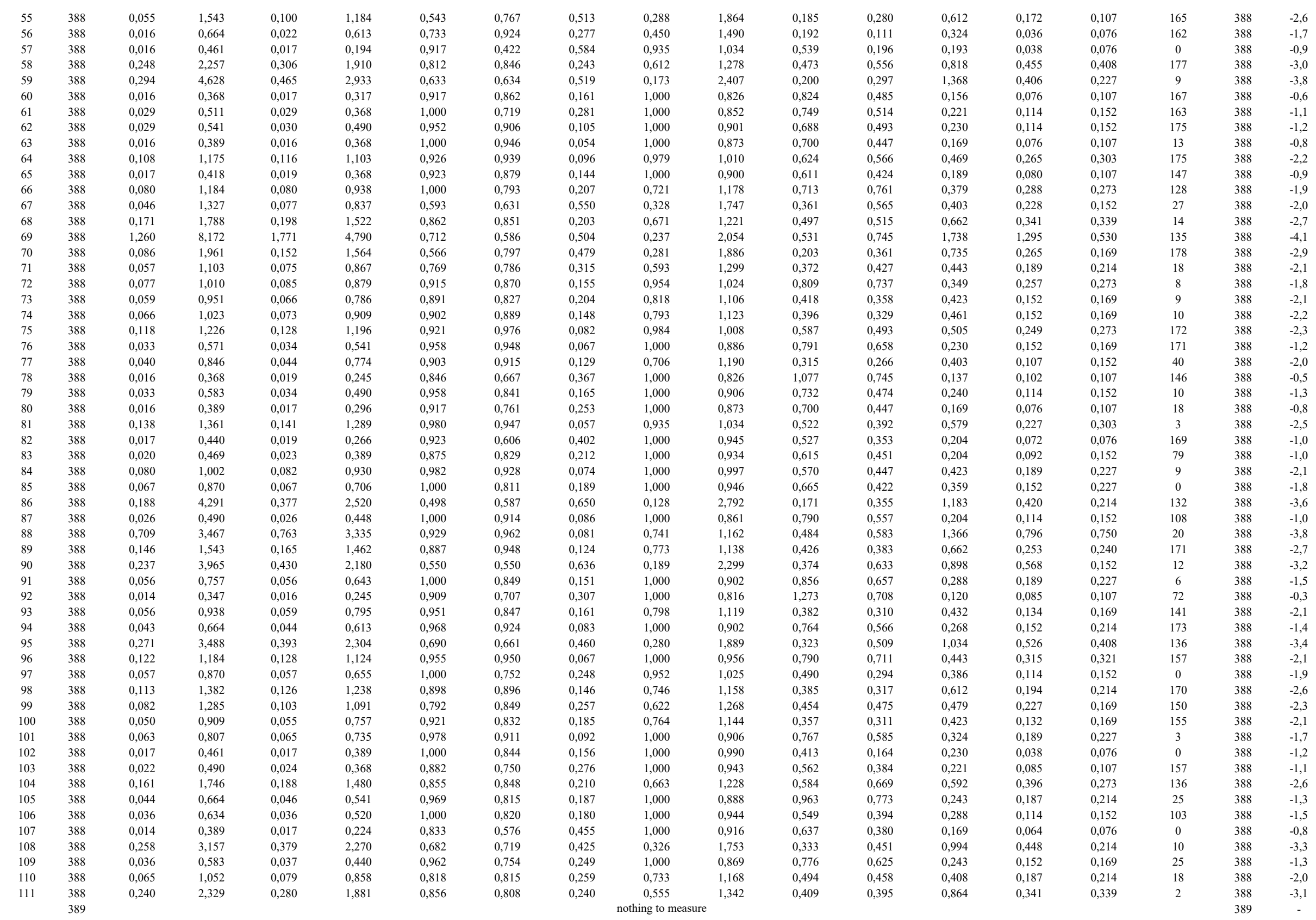


nothing to measure

nothing to measure

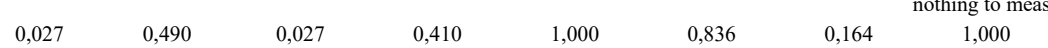

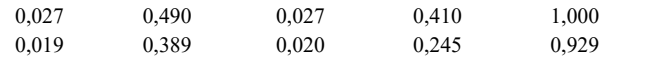

$\begin{array}{llllll}0,023 & 0,511 & 0,024 & 0,418 & 0,941 & 0,818\end{array}$

$\begin{array}{ll}1,000 & 0,838 \\ 1,000 & 0,803\end{array}$

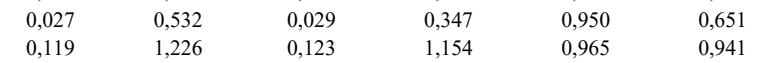

0,352

$\begin{array}{llll}1,000 & 0,838 & & \\ 1,068 & & 0,707 \\ 1,000 & 0,952 & 0,273 & 0,832 \\ & 0,566 & 0,333\end{array}$

0,910

0,566
0,712

$\begin{array}{ll}0,712 & 0,514 \\ 0,673 & 0,638\end{array}$

$\begin{array}{lll}0,014 & 0,389 & 0,016\end{array}$

$\begin{array}{lll}0,194 & 0,909 & 0,500\end{array}$

0,508

1,000

0,916

$0,637 \quad 0,425$

0,425
0,353

$\begin{array}{lll}0,055 & 1,348 & 0,092 \\ 0,022 & 0,816 & 0,033\end{array}$

$0,014 \quad 0,389$

$0,014 \quad 0,389$

0,039

$0,069 \quad 0,917$

$\begin{array}{ll}0,047 & 0,685 \\ 0,046 & 0,714\end{array}$

$\begin{array}{lll}0,165 & 2,287 & 0,220 \\ 0,052 & 0,807 & 0,055\end{array}$

$\begin{array}{ll}0,059 & 0,858 \\ 0,016 & 0,347\end{array}$

$0,397 \quad 4,282$

$\begin{array}{ll}0,040 & 0,735 \\ 0,057 & 0,807\end{array}$

$\begin{array}{ll}0,030 & 0,532 \\ 0,04 & 0,778\end{array}$

$0,044 \quad 0,778$

$0,062 \quad 1,133$

$\begin{array}{ll}0,122 & 1,725 \\ 0,112 & 1,315\end{array}$

$0,062 \quad 1,226$

$\begin{array}{ll}0,026 & 0,562 \\ 0,030 & 0,511\end{array}$

$\begin{array}{ll}0,065 & 0,849\end{array}$

$\begin{array}{ll}0,146 & 1,868\end{array}$

$0,032 \quad 0,634$

$\begin{array}{ll}0,023 & 0,490 \\ 0,083 & 1,103\end{array}$

$\begin{array}{ll}0,017 & 0,397\end{array}$

0,016

1,166
0,714

0,594

0,865

0,428

0,675
0,377

1,217
1,629
1,569

0,395

$\begin{array}{ll}0,186 & 0,248 \\ 0,180 & 0,13\end{array}$

0,406
1,000

0,455
0,446

0,215

$\begin{array}{ll}1,000 & 0.554\end{array}$

0,446

1,000
0,975

$\begin{array}{ll}1,075 & 0,916 \\ 0,928 & 1,013\end{array}$

$\begin{array}{ll}0,628 & 1,262 \\ 0,742 & 1,161\end{array}$

$\begin{array}{lll}0,807 & 0,894 & 0,799\end{array}$

0,837

0,931

0,912

0,227

1,000
1,000

1,000
1,000

0,909
0,986
0,888

0,613

0,914
1,000

0,719

0,166

1,000
1,000
1,000

0,888
0,941
0,852

1,602

0,752
0,947
0,957

0,701

0,312
0,389

1,000

0,062

0,016
0,548

0,046
0,060

0,807
0,714

0,957

0,941

0,136
0,073

0,396

0,996
1,000

1,000

0,707

0,293

3,242
0,613
0,757
0,347

0,875
0,952

0,833

0,367

1,000
1,000

0,272

$\begin{array}{ll}0,933 & 1,035 \\ 1,000 & 0,951\end{array}$

$\begin{array}{ll}1,000 & 0,865 \\ 0,924 & 1,040\end{array}$

$\begin{array}{ll}1,9024 & 1,040 \\ 1,000 & 0,963\end{array}$

$\begin{array}{llll}0,063 & 0,664 & 0,886 & 0,853 \\ 0,714 & 0,886 & 0,885\end{array}$

$0,076 \quad 0,765$

0,126

0,029

0,030

0,067
0,179

0,037
0,143

0,024

0,052

0,043
0,151

$\begin{array}{ll}0,040 & 0,765\end{array}$

$\begin{array}{ll}0,046 & 0,664 \\ 0,014 & 0,317\end{array}$

$\begin{array}{ll}0,886 & 0,885 \\ 0,811- & 0,162\end{array}$

$\begin{array}{lll}0,811 & 0,675 & 0,375 \\ 0,850 & 0,875 & 0,95\end{array}$

0,604

0,813

0,179
0,393

0,516

$\begin{array}{lll}20 & 29 \\ 39 & 39\end{array}$ 


\begin{tabular}{|c|c|c|c|c|c|c|c|c|c|c|c|c|c|c|c|c|c|}
\hline $\begin{array}{l}398 \\
399\end{array}$ & & & & & & & & $\begin{array}{l}\text { nothing to measure } \\
\text { nothing to measure }\end{array}$ & & & & & & & & $\begin{array}{l}398 \\
399\end{array}$ & - \\
\hline $\begin{array}{l}400 \\
401\end{array}$ & 0,020 & 0,520 & 0,026 & 0,347 & 0,778 & 0,667 & 0,401 & $\begin{array}{c}0,934 \\
\text { nothing to measure }\end{array}$ & 1,035 & 0,990 & 0,895 & 0,161 & 0,144 & 0,107 & 71 & $\begin{array}{l}400 \\
401\end{array}$ & $-0,7$ \\
\hline 402 & 0,019 & 0,469 & 0,022 & 0,347 & 0,867 & 0,739 & 0,293 & 1,000 & 0,969 & 0,637 & 0,476 & 0,193 & 0,092 & 0,107 & 75 & 402 & $-0,9$ \\
\hline 402 & 0,044 & 1,052 & 0,055 & 0,592 & 0,816 & 0,562 & 0,475 & 0,505 & 1,408 & 0,759 & 0,683 & 0,273 & 0,186 & 0,107 & 59 & 402 & $-1,4$ \\
\hline 402 & 0,017 & 0,440 & 0,022 & 0,245 & 0,800 & 0,558 & 0,485 & 1,000 & 0,945 & 0,611 & 0,506 & 0,189 & 0,096 & 0,152 & 45 & 402 & $-0,9$ \\
\hline 402 & 0,016 & 0,368 & 0,016 & 0,368 & 1,000 & 1,000 & 0,000 & 1,000 & 0,826 & 0,824 & 0,485 & 0,156 & 0,076 & 0,107 & 0 & 402 & $-0,6$ \\
\hline 402 & 0,042 & 0,727 & 0,049 & 0,541 & 0,853 & 0,744 & 0,295 & 0,990 & 1,005 & 0,821 & 0,707 & 0,254 & 0,180 & 0,152 & 20 & 402 & $-1,3$ \\
\hline 402 & 0,020 & 0,461 & 0,022 & 0,266 & 0,933 & 0,578 & 0,427 & 1,000 & 0,917 & 0,615 & 0,371 & 0,204 & 0,076 & 0,107 & 165 & 402 & $-1,0$ \\
\hline 402 & 0,039 & 1,031 & 0,056 & 0,706 & 0,692 & 0,684 & 0,441 & 0,458 & 1,478 & 0,354 & 0,406 & 0,373 & 0,152 & 0,107 & 15 & 402 & $-1,9$ \\
\hline 402 & 0,032 & 0,520 & 0,032 & 0,490 & 1,000 & 0,943 & 0,057 & 1,000 & 0,826 & 0,966 & 0,657 & 0,204 & 0,134 & 0,169 & 62 & 402 & $-1,0$ \\
\hline 402 & 0,014 & 0,389 & 0,016 & 0,245 & 0,909 & 0,631 & 0,380 & $\begin{array}{l}1,000 \\
\end{array}$ & 0,916 & 0,637 & 0,447 & 0,169 & 0,076 & 0,107 & 32 & 402 & $-0,8$ \\
\hline 402 & 0,016 & 0,368 & 0,016 & 0,275 & 1,000 & 0,747 & 0,253 & 1,000 & 0,826 & 1,077 & 0,745 & 0,137 & 0,102 & 0,107 & 117 & 402 & $-0,5$ \\
\hline 402 & 0,017 & 0,427 & 0,019 & 0,347 & 0,923 & 0,812 & 0,203 & 1,000 & 0,918 & 0,764 & 0,474 & 0,169 & 0,080 & 0,107 & 130 & 402 & $-0,8$ \\
\hline 402 & 0,017 & 0,368 & 0,017 & 0,266 & 1,000 & 0,724 & 0,276 & 1,000 & 0,791 & 1,175 & 0,784 & 0,137 & 0,107 & 0,152 & 90 & 402 & $-0,5$ \\
\hline 402 & 0,017 & 0,440 & 0,019 & 0,347 & 0,923 & 0,789 & 0,225 & 1,000 & 0,945 & 0,588 & 0,392 & 0,193 & 0,076 & 0,107 & 77 & 402 & $-0,9$ \\
\hline 402 & 0,014 & 0,389 & 0,016 & 0,194 & 0,909 & 0,500 & 0,508 & 1,000 & 0,916 & 0,637 & 0,380 & 0,169 & 0,064 & 0,076 & 90 & 402 & $-0,8$ \\
\hline 402 & 0,039 & 0,672 & 0,043 & 0,562 & 0,900 & 0,836 & 0,192 & 1,000 & 0,964 & 0,929 & 0,809 & 0,230 & 0,186 & 0,214 & 117 & 402 & $-1,2$ \\
\hline 402 & 0,019 & 0,469 & 0,022 & 0,347 & 0,867 & 0,739 & 0,293 & 1,000 & 0,969 & 0,920 & 0,667 & 0,161 & 0,107 & 0,107 & 90 & 402 & $-0,7$ \\
\hline 402 & 0,029 & 0,744 & 0,039 & 0,490 & 0,741 & 0,659 & 0,428 & 0,651 & 1,239 & 0,509 & 0,480 & 0,268 & 0,129 & 0,152 & 108 & 402 & $-1,4$ \\
\hline 402 & 0,023 & 0,440 & 0,023 & 0,338 & 1,000 & 0,769 & 0,231 & 1,000 & 0,818 & 1,132 & 0,707 & 0,161 & 0,114 & 0,152 & 90 & 402 & $-0,7$ \\
\hline 402 & 0,042 & 0,846 & 0,050 & 0,795 & 0,829 & 0,940 & 0,182 & 0,731 & $\begin{array}{l}1,169 \\
\end{array}$ & 0,381 & 0,364 & 0,373 & 0,136 & 0,169 & 35 & 402 & $-1,9$ \\
\hline 402 & 0,016 & 0,368 & 0,016 & 0,245 & 1,000 & 0,667 & 0,333 & 1,000 & 0,826 & 0,824 & 0,485 & 0,156 & 0,076 & 0,107 & 95 & 402 & $-0,6$ \\
\hline 402 & 0,016 & 0,469 & 0,020 & 0,347 & 0,786 & 0,739 & 0,338 & 0,901 & 1,054 & 0,560 & 0,424 & 0,189 & 0,080 & 0,076 & 126 & 402 & $-0,9$ \\
\hline 403 & & & & & & & & nothing to measure & & & & & & & & & - \\
\hline 404 & & & & & & & & nothing to measure & & & & & & & & 404 & - \\
\hline 405 & & & & & & & & nothing to measure & & & & & & & & 405 & - \\
\hline 406 & & & & & & & & nothing to measure & & & & & & & & 406 & - \\
\hline 407 & & & & & & & & nothing to measure & & & & & & & & 407 & - \\
\hline 408 & & & & & & & & nothing to measure & & & & & & & & 408 & - \\
\hline 409 & & & & & & & & nothing to measure & & & & & & & & 409 & - \\
\hline 410 & & & & & & & & nothing to measure & & & & & & & & 410 & - \\
\hline 411 & & & & & & & & nothing to measure & & & & & & & & 411 & - \\
\hline 412 & & & & & & & & nothing to measure & & & & & & & & 412 & - \\
\hline 413 & 0,024 & 0,592 & 0,027 & 0,440 & 0,895 & 0,743 & 0,278 & 0,875 & 1,069 & 0,408 & 0,333 & 0,276 & 0,092 & 0,107 & 17 & 413 & $-1,5$ \\
\hline 413 & 0,017 & 0,490 & 0,020 & 0,368 & 0,857 & 0,750 & 0,288 & 0,900 & 1,054 & 0,382 & 0,283 & 0,240 & 0,068 & 0,076 & 21 & 413 & $-1,3$ \\
\hline 414 & & & & & & & & nothing to measure & & & & & & & & 414 & - \\
\hline 415 & & & & & & & & nothing to measure & & & & & & & & 415 & - \\
\hline 416 & & & & & & & & nothing to measure & & & & & & & & 416 & - \\
\hline 417 & 0,016 & 0,520 & 0,020 & 0,338 & 0,786 & 0,650 & 0,410 & 0,733 & 1,168 & 0,539 & 0,392 & 0,193 & 0,076 & 0,107 & 45 & 417 & $-0,9$ \\
\hline 417 & 0,022 & 0,490 & 0,024 & 0,347 & 0,882 & 0,707 & 0,316 & 1,000 & 0,943 & 0,597 & 0,475 & 0,214 & 0,102 & 0,152 & 125 & 417 & $-1,1$ \\
\hline 418 & & & & & & & & nothing to measure & & & & & & & & 418 & t \\
\hline 419 & 0,024 & 0,490 & 0,024 & 0,440 & 1,000 & 0,896 & 0,104 & 1,000 & 0,886 & 0,676 & 0,500 & 0,214 & 0,107 & 0,152 & 52 & 419 & $-1,1$ \\
\hline 419 & 0,019 & 0,397 & 0,019 & 0,347 & 1,000 & 0,872 & 0,128 & 1,000 & 0,821 & 0,828 & 0,474 & 0,169 & 0,080 & 0,107 & 125 & 419 & $-0,8$ \\
\hline 419 & 0,067 & 1,023 & 0,075 & 0,921 & 0,904 & 0,901 & 0,138 & 0,810 & 1,111 & 0,404 & 0,311 & 0,461 & 0,143 & 0,152 & 99 & 419 & $-2,2$ \\
\hline 419 & 0,014 & 0,317 & 0,014 & 0,215 & 1,000 & 0,680 & 0,320 & 1,000 & 0,746 & 1,273 & 0,632 & 0,120 & 0,076 & 0,152 & 90 & 419 & $-0,3$ \\
\hline 419 & 0,019 & 0,389 & 0,019 & 0,245 & 1,000 & 0,631 & 0,369 & 1,000 & 0,803 & 0,974 & 0,485 & 0,156 & 0,076 & 0,152 & 82 & 419 & $-0,6$ \\
\hline 419 & 0,017 & 0,368 & 0,017 & 0,266 & 1,000 & 0,724 & 0,276 & 1,000 & 0,791 & 0,899 & 0,485 & 0,156 & 0,076 & 0,152 & 90 & 419 & $-0,6$ \\
\hline 420 & 0,019 & 0,389 & 0,019 & 0,287 & 1,000 & 0,739 & 0,261 & $\begin{array}{l}1,000 \\
-10\end{array}$ & 0,803 & 0,828 & 0,447 & 0,169 & 0,076 & 0,152 & 82 & 420 & $-0,8$ \\
\hline 423 & 0,017 & 0,389 & 0,017 & 0,296 & 1,000 & 0,761 & 0,239 & 1,000 & 0,836 & 0,764 & 0,447 & 0,169 & 0,076 & 0,152 & 172 & 423 & $-0,8$ \\
\hline 424 & & & & & & & & nothing to measure & & & & & & & & 424 & - \\
\hline
\end{tabular}


nothing to measure

$\begin{array}{ll}425 & - \\ 426 & - \\ 427 & -2,6 \\ 427 & -1,3 \\ 428 & -1,2 \\ 428 & -0,7 \\ 428 & -1,7 \\ 428 & -0,9 \\ 428 & -1,7 \\ 428 & -0,5 \\ 428 & -0,3 \\ 428 & -1,3 \\ 428 & -0,5 \\ 428 & -1,3 \\ 429 & -2,0 \\ 429 & -1,6 \\ 429 & -1,8 \\ 429 & -1,3 \\ 429 & -3,6 \\ 429 & -1,8 \\ 429 & -2,2 \\ 429 & -1,1 \\ 429 & -1,5 \\ 429 & -0,9 \\ 429 & -1,7 \\ 429 & -0,8 \\ 429 & -2,0 \\ 429 & -0,7 \\ 429 & -1,8 \\ 429 & -1,0 \\ 429 & -0,9 \\ 429 & -0,8 \\ 429 & -1,1 \\ 429 & -1,0 \\ 429 & -0,6 \\ 429 & -0,8 \\ 429 & -0,6 \\ 429 & -0,9 \\ 429 & -0,9 \\ 429 & -1,5 \\ 429 & -1,0 \\ 429 & -1,3 \\ 429 & -0,8 \\ 429 & -1,8 \\ 429 & -0,5 \\ 429 & -2,1 \\ 430 & -2,4 \\ 431 & - \\ 432 & - \\ 433 & -0,6 \\ 433 & -2,1 \\ 433 & -0,8 \\ 433 & -1,3 \\ 433 & -1,3 \\ 433 & -0,6 \\ 433 & -2,6 \\ 433 & -1,1 \\ 433 & -0,5 \\ & -\end{array}$


$0,909 \quad 0,862$

0,165

1,000

0,866
0,746
1,208

0,749

0,434

0,156

0,068 $\begin{array}{ll}0,750 & 0,654 \\ 0,667 & 0,559\end{array}$ $\begin{array}{lll}0,427 & 0,685 & 1,208 \\ 0,553 & 0,467 & 1,464\end{array}$

1,000 0,816

nothing to measure

$\begin{array}{lll}0,017 & 0,376 & 0,017\end{array}$

0,296

1,000

0,786

0,214 1,000
0,346

0,809 0,476
0,239 


\begin{tabular}{|c|c|c|c|c|c|c|c|c|c|c|c|c|c|c|c|c|c|c|}
\hline 47 & 441 & 0,022 & 0,418 & 0,022 & 0,338 & 1,000 & 0,808 & 0,192 & 1,000 & 0,805 & 1,123 & 0,728 & 0,156 & 0,114 & 0,152 & 70 & 441 & $-0,6$ \\
\hline 48 & 441 & 0,020 & 0,418 & 0,022 & 0,376 & 0,933 & 0,899 & 0,121 & 1,000 & 0,833 & 0,891 & 0,600 & 0,169 & 0,102 & 0,152 & 117 & 441 & $-0,8$ \\
\hline 49 & 441 & 0,030 & 0,520 & 0,030 & 0,490 & 1,000 & 0,943 & 0,057 & 1,000 & 0,845 & 0,922 & 0,657 & 0,204 & 0,134 & 0,152 & 149 & 441 & $-1,0$ \\
\hline 50 & 441 & 0,055 & 0,795 & 0,059 & 0,735 & 0,927 & 0,925 & 0,105 & 1,000 & 0,960 & 0,744 & 0,614 & 0,305 & 0,187 & 0,214 & 128 & 441 & $-1,6$ \\
\hline 51 & 441 & 0,019 & 0,727 & 0,027 & 0,511 & 0,684 & 0,704 & 0,433 & 0,444 & 1,501 & 0,195 & 0,136 & 0,349 & 0,047 & 0,076 & 0 & 441 & $-1,8$ \\
\hline 52 & 441 & 0,016 & 0,347 & 0,016 & 0,338 & 1,000 & 0,975 & 0,025 & 1,000 & 0,778 & 0,875 & 0,500 & 0,152 & 0,076 & 0,152 & 0 & 441 & $-0,6$ \\
\hline 53 & 441 & 0,022 & 0,490 & 0,023 & 0,440 & 0,938 & 0,896 & 0,121 & 1,000 & 0,943 & 0,562 & 0,384 & 0,221 & 0,085 & 0,107 & 115 & 441 & $-1,1$ \\
\hline 54 & 441 & 0,032 & 0,693 & 0,034 & 0,613 & 0,917 & 0,884 & 0,143 & 0,825 & 1,101 & 0,431 & 0,389 & 0,305 & 0,119 & 0,152 & 113 & 441 & $-1,6$ \\
\hline 55 & 441 & 0,017 & 0,541 & 0,022 & 0,347 & 0,800 & 0,641 & 0,411 & 0,739 & 1,163 & 0,477 & 0,375 & 0,214 & 0,080 & 0,107 & 140 & 441 & $-1,1$ \\
\hline 56 & 441 & 0,046 & 0,735 & 0,049 & 0,613 & 0,941 & 0,833 & 0,177 & 1,000 & 0,968 & 0,627 & 0,517 & 0,305 & 0,158 & 0,169 & 121 & 441 & $-1,6$ \\
\hline 57 & 441 & 0,019 & 0,368 & 0,019 & 0,347 & 1,000 & 0,943 & 0,057 & 1,000 & 0,760 & 1,273 & 0,784 & 0,137 & 0,107 & 0,152 & 135 & 441 & $-0,5$ \\
\hline 58 & 441 & 0,043 & 0,735 & 0,049 & 0,592 & 0,882 & 0,805 & 0,228 & 1,000 & 1,000 & 0,626 & 0,608 & 0,296 & 0,180 & 0,214 & 125 & 441 & $-1,6$ \\
\hline 59 & 441 & 0,019 & 0,418 & 0,020 & 0,397 & 0,929 & 0,950 & 0,087 & 1,000 & 0,864 & 0,662 & 0,424 & 0,189 & 0,080 & 0,107 & 138 & 441 & $-0,9$ \\
\hline 60 & 441 & 0,263 & 3,864 & 0,616 & 3,322 & 0,427 & 0,860 & 0,590 & 0,221 & 2,127 & 0,139 & 0,305 & 1,553 & 0,475 & 0,214 & 103 & 441 & $-4,0$ \\
\hline 61 & 441 & 0,066 & 0,896 & 0,070 & 0,867 & 0,939 & 0,967 & 0,070 & 1,000 & 0,984 & 0,604 & 0,503 & 0,373 & 0,187 & 0,214 & 139 & 441 & $-1,9$ \\
\hline 62 & 441 & 0,443 & 4,033 & 0,607 & 3,022 & 0,730 & 0,749 & 0,368 & 0,343 & 1,708 & 0,315 & 0,404 & 1,339 & 0,541 & 0,312 & 105 & 441 & $-3,7$ \\
\hline 63 & 441 & 0,029 & 0,490 & 0,029 & 0,418 & 1,000 & 0,854 & 0,146 & 1,000 & 0,816 & 0,979 & 0,588 & 0,193 & 0,114 & 0,152 & 0 & 441 & $-0,9$ \\
\hline 64 & 441 & 0,037 & 0,643 & 0,040 & 0,541 & 0,929 & 0,842 & 0,173 & 1,000 & 0,939 & 0,637 & 0,490 & 0,273 & 0,134 & 0,169 & 133 & 441 & $-1,4$ \\
\hline 65 & 441 & 0,016 & 0,490 & 0,020 & 0,347 & 0,786 & 0,707 & 0,363 & 0,825 & 1,101 & 0,539 & 0,392 & 0,193 & 0,076 & 0,107 & 81 & 441 & $-0,9$ \\
\hline 66 & 441 & 0,014 & 0,317 & 0,014 & 0,173 & 1,000 & 0,547 & 0,453 & 1,000 & 0,746 & 0,979 & 0,555 & 0,137 & 0,076 & 0,107 & 108 & 441 & $-0,5$ \\
\hline 67 & 441 & 0,039 & 0,816 & 0,046 & 0,774 & 0,844 & 0,948 & 0,165 & 0,731 & 1,169 & 0,386 & 0,285 & 0,357 & 0,102 & 0,152 & 121 & 441 & $-1,8$ \\
\hline 68 & 441 & 0,020 & 0,643 & 0,027 & 0,410 & 0,737 & 0,638 & 0,448 & 0,611 & 1,279 & 0,686 & 0,588 & 0,193 & 0,114 & 0,076 & 12 & 441 & $-0,9$ \\
\hline 69 & 441 & 0,052 & 0,858 & 0,057 & 0,765 & 0,900 & 0,892 & 0,147 & 0,882 & 1,065 & 0,432 & 0,391 & 0,390 & 0,152 & 0,169 & 115 & 441 & $-2,0$ \\
\hline 70 & 441 & 0,020 & 0,744 & 0,027 & 0,622 & 0,737 & 0,835 & 0,310 & 0,456 & 1,481 & 0,200 & 0,128 & 0,357 & 0,046 & 0,076 & 120 & 441 & $-1,8$ \\
\hline 71 & 441 & 0,115 & 1,590 & 0,131 & 1,467 & 0,879 & 0,923 & 0,143 & 0,571 & 1,324 & 0,260 & 0,250 & 0,750 & 0,187 & 0,214 & 137 & 441 & $-2,9$ \\
\hline 72 & 441 & 0,076 & 1,010 & 0,083 & 0,989 & 0,914 & 0,979 & 0,089 & 0,936 & 1,034 & 0,519 & 0,452 & 0,432 & 0,195 & 0,214 & 129 & 441 & $-2,1$ \\
\hline 73 & 441 & 0,032 & 0,541 & 0,033 & 0,511 & 0,957 & 0,945 & 0,070 & 1,000 & 0,859 & 0,966 & 0,743 & 0,204 & 0,152 & 0,169 & 122 & 441 & $-1,0$ \\
\hline 74 & 441 & 0,043 & 0,765 & 0,047 & 0,672 & 0,909 & 0,879 & 0,152 & 0,924 & 1,040 & 0,449 & 0,348 & 0,349 & 0,121 & 0,169 & 129 & 441 & $-1,8$ \\
\hline 75 & 441 & 0,063 & 0,900 & 0,066 & 0,706 & 0,957 & 0,784 & 0,220 & 0,979 & 1,011 & 0,622 & 0,422 & 0,359 & 0,152 & 0,214 & 83 & 441 & $-1,8$ \\
\hline 76 & 441 & 0,405 & 3,036 & 0,452 & 2,862 & 0,895 & 0,943 & 0,119 & 0,552 & 1,346 & 0,265 & 0,272 & 1,394 & 0,379 & 0,339 & 138 & 441 & $-3,8$ \\
\hline 77 & 441 & 0,065 & 0,837 & 0,067 & 0,735 & 0,957 & 0,879 & 0,129 & 1,000 & 0,929 & 0,674 & 0,537 & 0,349 & 0,187 & 0,214 & 131 & 441 & $-1,8$ \\
\hline 78 & 441 & 0,085 & 1,336 & 0,103 & 1,234 & 0,819 & 0,924 & 0,196 & 0,596 & 1,295 & 0,289 & 0,302 & 0,611 & 0,185 & 0,214 & 121 & 441 & $-2,6$ \\
\hline 79 & 441 & 0,139 & 1,501 & 0,152 & 1,399 & 0,915 & 0,932 & 0,109 & 0,777 & 1,135 & 0,405 & 0,452 & 0,662 & 0,299 & 0,303 & 110 & 441 & $-2,7$ \\
\hline 80 & 441 & 0,017 & 0,347 & 0,017 & 0,296 & 1,000 & 0,854 & 0,146 & 1,000 & 0,745 & 1,528 & 0,894 & 0,120 & 0,107 & 0,107 & 90 & 441 & $-0,3$ \\
\hline 81 & 441 & 0,063 & 0,930 & 0,069 & 0,828 & 0,917 & 0,891 & 0,137 & 0,918 & 1,044 & 0,514 & 0,394 & 0,395 & 0,156 & 0,169 & 105 & 441 & $-2,0$ \\
\hline 82 & 441 & 0,050 & 0,858 & 0,053 & 0,735 & 0,946 & 0,857 & 0,153 & 0,857 & 1,080 & 0,441 & 0,398 & 0,381 & 0,152 & 0,214 & 173 & 441 & $-1,9$ \\
\hline 83 & 441 & 0,029 & 0,541 & 0,029 & 0,418 & 1,000 & 0,773 & 0,227 & 1,000 & 0,901 & 0,621 & 0,442 & 0,243 & 0,107 & 0,152 & 143 & 441 & $-1,3$ \\
\hline 84 & 441 & 0,032 & 1,019 & 0,056 & 0,909 & 0,564 & 0,892 & 0,449 & 0,382 & 1,618 & 0,192 & 0,263 & 0,458 & 0,120 & 0,107 & 108 & 441 & $-2,2$ \\
\hline 85 & 441 & 0,185 & 1,957 & 0,241 & 1,793 & 0,768 & 0,916 & 0,247 & 0,607 & 1,283 & 0,411 & 0,411 & 0,758 & 0,311 & 0,273 & 127 & 441 & $-2,9$ \\
\hline 86 & 441 & 0,027 & 0,541 & 0,030 & 0,368 & 0,905 & 0,680 & 0,334 & 1,000 & 0,924 & 0,712 & 0,550 & 0,221 & 0,121 & 0,152 & 148 & 441 & $-1,1$ \\
\hline 87 & 441 & 0,020 & 0,469 & 0,023 & 0,368 & 0,875 & 0,784 & 0,250 & 1,000 & 0,934 & 0,891 & 0,778 & 0,169 & 0,132 & 0,152 & 117 & 441 & $-0,8$ \\
\hline 88 & 441 & 3,997 & 8,810 & 4,270 & 8,540 & 0,936 & 0,969 & 0,071 & 0,647 & 1,243 & 0,356 & 0,376 & 3,779 & 1,420 & 1,447 & 138 & 441 & $-5,2$ \\
\hline 89 & 441 & 0,042 & 0,634 & 0,042 & 0,490 & 1,000 & 0,773 & 0,227 & 1,000 & 0,877 & 0,710 & 0,555 & 0,273 & 0,152 & 0,214 & 159 & 441 & $-1,4$ \\
\hline 90 & 441 & 0,033 & 0,909 & 0,049 & 0,702 & 0,676 & 0,772 & 0,396 & 0,502 & 1,411 & 0,259 & 0,265 & 0,403 & 0,107 & 0,152 & 138 & 441 & $-2,0$ \\
\hline 91 & 441 & 0,017 & 0,579 & 0,017 & 0,550 & 1,000 & 0,949 & 0,051 & 0,645 & 1,246 & 0,250 & 0,091 & 0,296 & 0,027 & 0,076 & 135 & 441 & $-1,6$ \\
\hline 92 & 441 & 0,067 & 0,981 & 0,075 & 0,858 & 0,904 & 0,875 & 0,158 & 0,881 & 1,065 & 0,617 & 0,685 & 0,373 & 0,255 & 0,227 & 102 & 441 & $-1,9$ \\
\hline 93 & 441 & 0,027 & 0,490 & 0,027 & 0,418 & 1,000 & 0,854 & 0,146 & 1,000 & 0,838 & 0,756 & 0,500 & 0,214 & 0,107 & 0,169 & 135 & 441 & $-1,1$ \\
\hline 94 & 441 & 0,017 & 0,693 & 0,024 & 0,600 & 0,706 & 0,866 & 0,323 & 0,450 & 1,491 & 0,206 & 0,161 & 0,326 & 0,053 & 0,076 & 147 & 441 & $-1,7$ \\
\hline 95 & 441 & 0,057 & 0,888 & 0,062 & 0,744 & 0,930 & 0,838 & 0,176 & 0,915 & 1,045 & 0,451 & 0,395 & 0,403 & 0,159 & 0,169 & 136 & 441 & $-2,0$ \\
\hline 96 & 441 & 0,273 & 2,570 & 0,329 & 2,295 & 0,830 & 0,893 & 0,201 & 0,519 & 1,389 & 0,280 & 0,329 & 1,114 & 0,367 & 0,321 & 147 & 441 & $-3,5$ \\
\hline 97 & 441 & 0,079 & 1,226 & 0,098 & 1,031 & 0,809 & 0,841 & 0,248 & 0,660 & 1,231 & 0,387 & 0,420 & 0,510 & 0,214 & 0,214 & 134 & 441 & $-2,4$ \\
\hline 98 & 441 & 0,231 & 2,101 & 0,296 & 1,898 & 0,782 & 0,903 & 0,239 & 0,658 & 1,233 & 0,576 & 0,684 & 0,715 & 0,489 & 0,455 & 167 & 441 & $-2,8$ \\
\hline 99 & 441 & 0,017 & 0,347 & 0,017 & 0,296 & 1,000 & 0,854 & 0,146 & 1,000 & 0,745 & 1,528 & 0,894 & 0,120 & 0,107 & 0,107 & 90 & 441 & $-0,3$ \\
\hline 100 & 441 & 0,032 & 0,541 & 0,033 & 0,440 & 0,957 & 0,812 & 0,193 & 1,000 & 0,859 & 0,824 & 0,606 & 0,221 & 0,134 & 0,169 & 126 & 441 & $-1,1$ \\
\hline 101 & 441 & 0,026 & 0,469 & 0,027 & 0,418 & 0,947 & 0,892 & 0,120 & 1,000 & 0,824 & 1,146 & 0,791 & 0,169 & 0,134 & 0,169 & 131 & 441 & $-0,8$ \\
\hline 102 & 441 & 0,019 & 0,469 & 0,024 & 0,347 & 0,765 & 0,739 & 0,352 & 1,000 & 0,969 & 0,662 & 0,537 & 0,189 & 0,102 & 0,107 & 157 & 441 & $-0,9$ \\
\hline 103 & 441 & 0,039 & 0,634 & 0,043 & 0,490 & 0,900 & 0,773 & 0,248 & 1,000 & 0,909 & 0,838 & 0,769 & 0,243 & 0,186 & 0,169 & 154 & 441 & $-1,3$ \\
\hline 104 & 441 & 0,202 & 2,702 & 0,273 & 2,321 & 0,742 & 0,859 & 0,294 & 0,348 & 1,694 & 0,208 & 0,226 & 1,114 & 0,252 & 0,227 & 140 & 441 & $-3,5$ \\
\hline
\end{tabular}




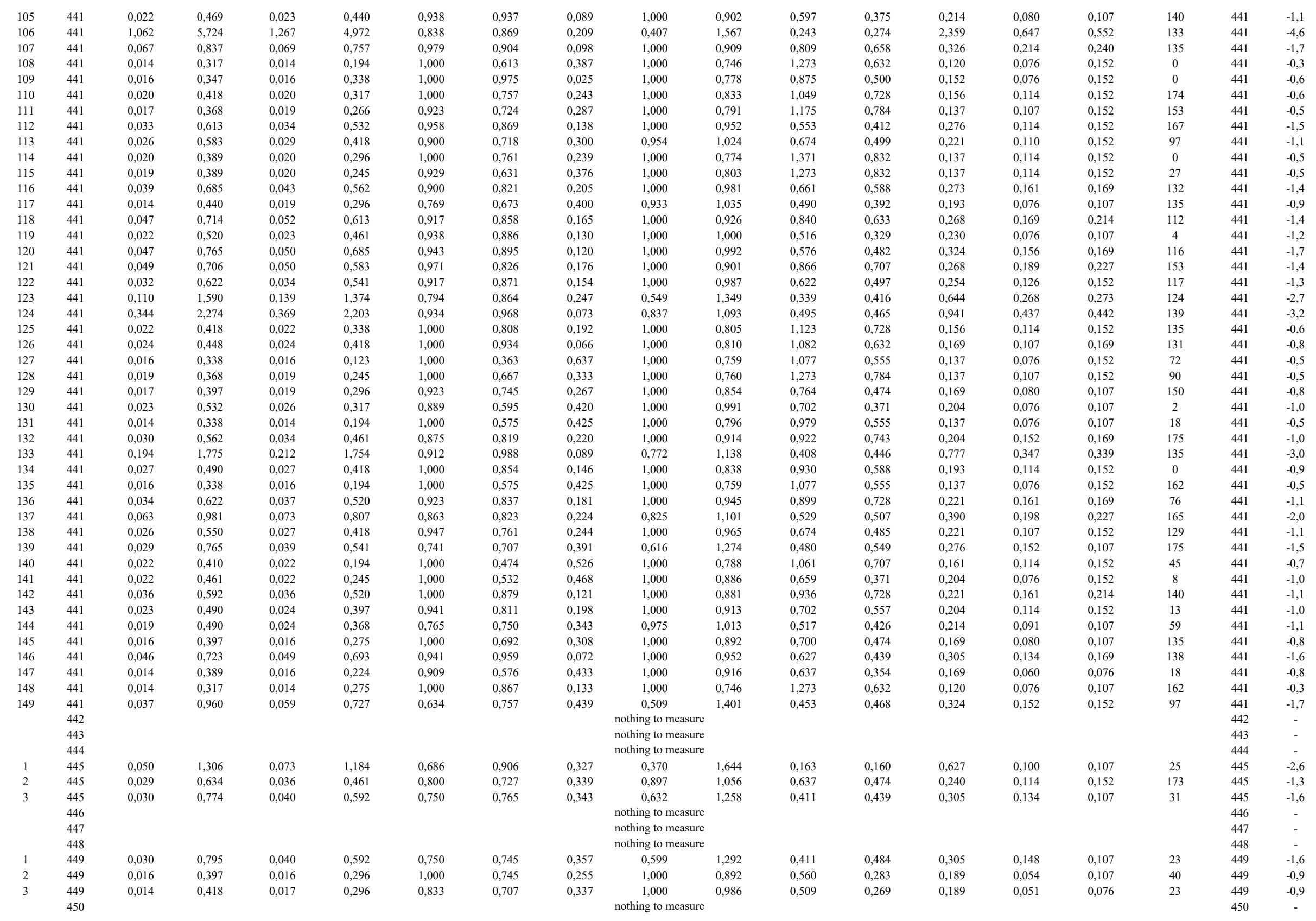


nothing to measure

nothing to measure

$\begin{array}{lllllll}0,026 & 0,532 & 0,026 & 0,287 & 1,000 & 0,540 & 0,460 \\ 0,014 & 0,440 & 0,017 & 0,317 & 0,833 & 0,721 & 0,325\end{array}$ 0,935 0,619 0,933
nothing to measure

nothing to measure

nothing to measure

nothing to measure

\begin{tabular}{|c|c|c|c|c|c|c|}
\hline 0,024 & 0,622 & 0,033 & 0,562 & 0,739 & 0,904 & 0,278 \\
\hline 0,062 & 0,837 & 0,065 & 0,757 & 0,956 & 0,904 & 0,106 \\
\hline 0,060 & 0,909 & 0,066 & 0,786 & 0,913 & 0,865 & 0,160 \\
\hline 0,037 & 0,714 & 0,044 & 0,592 & 0,839 & 0,828 & 0,235 \\
\hline 0,141 & 1,665 & 0,188 & 1,513 & 0,748 & 0,909 & 0,268 \\
\hline 0,037 & 0,643 & 0,040 & 0,592 & 0,929 & 0,921 & 0,106 \\
\hline 0,040 & 0,786 & 0,050 & 0,592 & 0,800 & 0,753 & 0,318 \\
\hline 0,187 & 3,120 & 0,303 & 2,185 & 0,616 & 0,700 & 0,487 \\
\hline 0,016 & 0,338 & 0,016 & 0,194 & 1,000 & 0,575 & 0,425 \\
\hline 0,019 & 0,448 & 0,022 & 0,347 & 0,867 & 0,773 & 0,263 \\
\hline 0,471 & 3,830 & 0,638 & 3,001 & 0,737 & 0,784 & 0,340 \\
\hline 0,019 & 0,418 & 0,019 & 0,245 & 1,000 & 0,586 & 0,414 \\
\hline 0,020 & 0,410 & 0,020 & 0,266 & 1,000 & 0,650 & 0,350 \\
\hline 0,063 & 0,837 & 0,067 & 0,765 & 0,936 & 0,914 & 0,107 \\
\hline 0,016 & 0,397 & 0,019 & 0,266 & 0,846 & 0,670 & 0,364 \\
\hline 0,017 & 0,347 & 0,017 & 0,296 & 1,000 & 0,854 & 0,146 \\
\hline 0,014 & 0,338 & 0,016 & 0,144 & 0,909 & 0,425 & 0,582 \\
\hline 0,027 & 0,511 & 0,029 & 0,368 & 0,950 & 0,719 & 0,285 \\
\hline 0,080 & 1,277 & 0,108 & 1,061 & 0,747 & 0,831 & 0,304 \\
\hline 0,055 & 0,786 & 0,056 & 0,706 & 0,974 & 0,898 & 0,105 \\
\hline 0,022 & 0,461 & 0,024 & 0,194 & 0,882 & 0,422 & 0,590 \\
\hline 0,019 & 0,482 & 0,022 & 0,296 & 0,867 & 0,614 & 0,408 \\
\hline 0,017 & 0,368 & 0,019 & 0,266 & 0,923 & 0,724 & 0,287 \\
\hline 0,053 & 0,858 & 0,059 & 0,778 & 0,902 & 0,906 & 0,135 \\
\hline 0,082 & 0,981 & 0,086 & 0,858 & 0,950 & 0,875 & 0,135 \\
\hline 0,053 & 1,234 & 0,082 & 0,938 & 0,649 & 0,760 & 0,425 \\
\hline 0,019 & 0,368 & 0,019 & 0,245 & 1,000 & 0,667 & 0,333 \\
\hline 0,040 & 0,693 & 0,040 & 0,643 & 1,000 & 0,927 & 0,073 \\
\hline 0,037 & 1,019 & 0,053 & 0,816 & 0,703 & 0,801 & 0,358 \\
\hline 0,131 & 1,847 & 0,179 & 1,653 & 0,728 & 0,895 & 0,292 \\
\hline 0,020 & 0,469 & 0,022 & 0,368 & 0,933 & 0,784 & 0,226 \\
\hline 0,080 & 1,285 & 0,098 & 1,124 & 0,824 & 0,875 & 0,216 \\
\hline 0,046 & 0,706 & 0,050 & 0,562 & 0,914 & 0,796 & 0,221 \\
\hline 0,023 & 0,520 & 0,026 & 0,418 & 0,889 & 0,805 & 0,225 \\
\hline 0,032 & 0,676 & 0,033 & 0,511 & 0,957 & 0,756 & 0,247 \\
\hline 0,020 & 0,541 & 0,026 & 0,418 & 0,778 & 0,773 & 0,317 \\
\hline 0,023 & 0,575 & 0,023 & 0,144 & 1,000 & 0,250 & 0,750 \\
\hline 0,016 & 0,376 & 0,017 & 0,296 & 0,917 & 0,786 & 0,229 \\
\hline 0,022 & 0,511 & 0,023 & 0,418 & 0,938 & 0,818 & 0,192 \\
\hline 0,027 & 0,655 & 0,030 & 0,461 & 0,905 & 0,703 & 0,312 \\
\hline 0,024 & 0,469 & 0,024 & 0,410 & 1,000 & 0,873 & 0,127 \\
\hline 0,066 & 1,154 & 0,088 & 0,867 & 0,754 & 0,751 & 0,350 \\
\hline 0,155 & 2,075 & 0,211 & 1,585 & 0,735 & 0,764 & 0,355 \\
\hline 0,024 & 0,511 & 0,026 & 0,440 & 0,944 & 0,860 & 0,151 \\
\hline
\end{tabular}

$\begin{array}{ll}0,794 & 1,123 \\ 1,000 & 0,951 \\ 0,917 & 1,044 \\ 0,919 & 1,043 \\ 0,637 & 1,253 \\ 1,000 & 0,939 \\ 0,817 & 1,107 \\ 0,241 & 2,038 \\ 1,000 & 0,759 \\ 1,000 & 0,926 \\ 0,403 & 1,575 \\ 1,000 & 0,864 \\ 1,000 & 0,816 \\ 1,000 & 0,940 \\ 1,000 & 0,892 \\ 1,000 & 0,745 \\ 1,000 & 0,796 \\ 1,000 & 0,874 \\ 0,620 & 1,270 \\ 1,000 & 0,950 \\ 1,000 & 0,886 \\ 1,000 & 0,995 \\ 1,000 & 0,791 \\ 0,906 & 1,051 \\ 1,000 & 0,967 \\ 0,438 & 1,511 \\ 1,000 & 0,760 \\ 1,000 & 0,976 \\ 0,452 & 1,488 \\ 0,481 & 1,442 \\ 1,000 & 0,934 \\ 0,611 & 1,279 \\ 1,000 & 0,929 \\ 1,000 & 0,968 \\ 0,868 & 1,073 \\ 0,862 & 1,077 \\ 0,874 & 1,070 \\ 1,000 & 0,845 \\ 1,000 & 0,983 \\ 0,798 & 1,119 \\ 1,000 & 0,848 \\ 0,623 & 1,267 \\ 0,452 & 1,487 \\ 1,000 & 0,924 \\ & \\ & \end{array}$

0,408
0,805
0,551
0,543
0,409
0,807
0,482
0,230
1,077
0,637
0,360
0,637
0,891
0,757
0,700
1,528
0,979
0,834
0,369
0,569
0,764
0,571
1,175
0,453
0,748
0,362
1,273
0,673
0,304
0,317
0,615
0,348
0,769
0,551
0,412
0,482
0,407
0,824
0,562
0,372
0,833
0,537
0,382
0,585

0,354

0,606
0,500
0,501

0,501
0,476
0,625

0,625

0,441
0,379
0,555
0,435

0,435
0,445
0,392

0,447

0,658
0,474

$\begin{array}{ll}0,474 & 0,169\end{array}$

0,894
0,555

0,557

0,434

0,434
0,600
0,365
0,784

0,784
0,375

0,375
0,609

0,435
0,784
0,615

0,784
0,615
0,386

0,386
0,396

0,396
0,470

0,419
0,549

0,549
0,365

0,243

0,141
0,514

0,460

0,248
0,588

0,635

0,474
0,329

0,276
0,312
0,373
0,296
0,662
0,243
0,326
1,016
0,137
0,19
1,29
0,193
0,169
0,326
0,169
0,12
0,137
0,204
0,526
0,349
0,189
0,204
0,137
0,386
0,373
0,432
0,137
0,276
0,395
0,724
0,204
0,542
0,276
0,23
0,312
0,23
0,268
0,156
0,221
0,305
0,193
0,395
0,719
0,230

0,276
0,312
0,373
0,296
0,622
0,243
0,326
1,016
0,137
0,193
1,290
0,193
0,169
0,326
0,169
0,120
0,137
0,204
0,526
0,349
0,189
0,204
0,137
0,386
0,373
0,432
0,137
0,276
0,395
0,724
0,204
0,542
0,276
0,230
0,312
0,230
0,268
0,156
0,221
0,305
0,193
0,395
0,119
0,230

0,098
0,189
0,186
0,148
0,315
0,152
0,144
0,385
0,076
0,084
0,574
0,076
0,076
0,214
0,080
0,107
0,076
0,114
0,231
0,152
0,114
0,074
0,107
0,145
0,227
0,188
0,107
0,169
0,153
0,287
0,096
0,227
0,152
0,084
0,076
0,083
0,038
0,080
0,102
0,076
0,114
0,251
0,341
0,076

0,214
0,152

0,152
0,214

0,169
0,152

0,152
0,169

0,152
0,107
0,152

0,152

0,152
0,152

0,152
0,227

0,107

0,107

0,107
0,107
0,152
0,214

0,214

0,152

0,076

0,152

0,273
0,152
0,152

0,152
0,152

0,169
0,107

0,107
0,214
0,107

0,107
0,214

0,214
0,214

0,107
0,152

0,152
0,107

0,107
0,076
0,107

0,107
0,152

0,107

0,152
0,227

0,227
0,240

0,152 


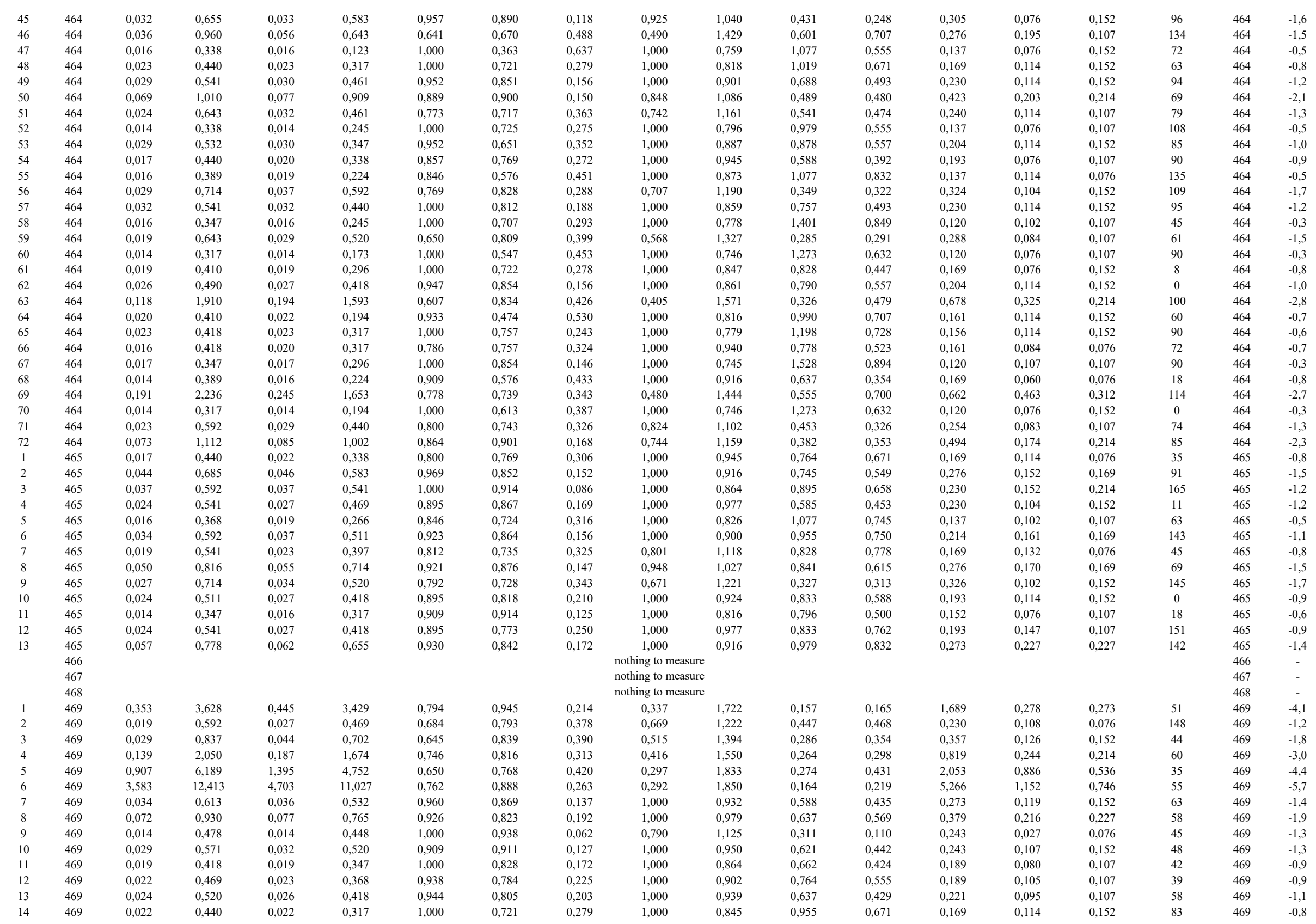




\begin{tabular}{|c|c|c|c|c|c|c|c|c|c|c|c|c|c|c|c|c|c|c|}
\hline 15 & 469 & 0,047 & 0,938 & 0,053 & 0,837 & 0,892 & 0,892 & 0,153 & 0,676 & 1,217 & 0,288 & 0,265 & 0,458 & 0,121 & 0,169 & 59 & 469 & $-2,2$ \\
\hline 16 & 469 & 0,149 & 2,367 & 0,194 & 2,338 & 0,770 & 0,987 & 0,230 & 0,335 & 1,729 & 0,152 & 0,158 & 1,119 & 0,177 & 0,214 & 69 & 469 & $-3,5$ \\
\hline 17 & 469 & 0,034 & 0,672 & 0,036 & 0,571 & 0,960 & 0,849 & 0,156 & 0,957 & 1,022 & 0,470 & 0,351 & 0,305 & 0,107 & 0,152 & 60 & 469 & $-1,6$ \\
\hline 18 & 469 & 0,014 & 0,368 & 0,014 & 0,245 & 1,000 & 0,667 & 0,333 & 1,000 & 0,866 & 0,637 & 0,400 & 0,169 & 0,068 & 0,076 & 58 & 469 & $-0,8$ \\
\hline 19 & 469 & 0,046 & 1,052 & 0,062 & 0,951 & 0,744 & 0,904 & 0,273 & 0,521 & 1,386 & 0,255 & 0,205 & 0,479 & 0,098 & 0,107 & 73 & 469 & $-2,3$ \\
\hline 20 & 469 & 0,014 & 0,368 & 0,016 & 0,266 & 0,909 & 0,724 & 0,291 & 1,000 & 0,866 & 0,707 & 0,422 & 0,161 & 0,068 & 0,076 & 58 & 469 & $-0,7$ \\
\hline 21 & 469 & 0,033 & 0,664 & 0,040 & 0,562 & 0,821 & 0,847 & 0,235 & 0,942 & 1,031 & 0,791 & 0,658 & 0,230 & 0,152 & 0,152 & 162 & 469 & $-1,2$ \\
\hline 22 & 469 & 0,063 & 1,040 & 0,072 & 0,938 & 0,880 & 0,902 & 0,155 & 0,733 & 1,168 & 0,357 & 0,310 & 0,475 & 0,147 & 0,169 & 63 & 469 & $-2,2$ \\
\hline 23 & 469 & 0,019 & 0,410 & 0,020 & 0,215 & 0,929 & 0,526 & 0,480 & 1,000 & 0,847 & 0,828 & 0,447 & 0,169 & 0,076 & 0,152 & 0 & 469 & $-0,8$ \\
\hline 24 & 469 & 0,014 & 0,338 & 0,016 & 0,123 & 0,909 & 0,363 & 0,644 & 1,000 & 0,796 & 0,979 & 0,555 & 0,137 & 0,076 & 0,107 & 18 & 469 & $-0,5$ \\
\hline 25 & 469 & 0,030 & 0,643 & 0,034 & 0,571 & 0,875 & 0,888 & 0,168 & 0,917 & 1,044 & 0,504 & 0,358 & 0,276 & 0,099 & 0,152 & 21 & 469 & $-1,5$ \\
\hline 26 & 469 & 0,019 & 0,418 & 0,019 & 0,347 & 1,000 & 0,828 & 0,172 & 1,000 & 0,864 & 0,662 & 0,424 & 0,189 & 0,080 & 0,107 & 42 & 469 & $-0,9$ \\
\hline 27 & 469 & 0,092 & 1,763 & 0,116 & 1,704 & 0,790 & 0,966 & 0,213 & 0,371 & 1,641 & 0,177 & 0,173 & 0,813 & 0,140 & 0,169 & 60 & 469 & $-3,0$ \\
\hline 28 & 469 & 0,017 & 0,448 & 0,019 & 0,418 & 0,923 & 0,934 & 0,102 & 1,000 & 0,964 & 0,588 & 0,439 & 0,193 & 0,085 & 0,076 & 32 & 469 & $-0,9$ \\
\hline 29 & 469 & 0,037 & 0,989 & 0,047 & 0,867 & 0,788 & 0,876 & 0,246 & 0,479 & 1,445 & 0,243 & 0,224 & 0,442 & 0,099 & 0,076 & 54 & 469 & $-2,1$ \\
\hline 30 & 469 & 0,017 & 0,418 & 0,020 & 0,296 & 0,857 & 0,707 & 0,326 & 1,000 & 0,900 & 0,899 & 0,614 & 0,156 & 0,096 & 0,107 & 15 & 469 & $-0,6$ \\
\hline 31 & 469 & 0,027 & 0,693 & 0,032 & 0,571 & 0,864 & 0,823 & 0,223 & 0,713 & 1,185 & 0,372 & 0,310 & 0,305 & 0,095 & 0,107 & 39 & 469 & $-1,6$ \\
\hline 32 & 469 & 0,019 & 0,389 & 0,019 & 0,245 & 1,000 & 0,631 & 0,369 & 1,000 & 0,803 & 0,920 & 0,633 & 0,161 & 0,102 & 0,152 & 45 & 469 & $-0,7$ \\
\hline 33 & 469 & 0,014 & 0,317 & 0,014 & 0,215 & 1,000 & 0,680 & 0,320 & 1,000 & 0,746 & 0,979 & 0,555 & 0,137 & 0,076 & 0,107 & 72 & 469 & $-0,5$ \\
\hline 34 & 469 & 0,023 & 0,490 & 0,024 & 0,410 & 0,941 & 0,836 & 0,174 & 1,000 & 0,913 & 0,599 & 0,434 & 0,221 & 0,096 & 0,152 & 24 & 469 & $-1,1$ \\
\hline 35 & 469 & 0,017 & 0,368 & 0,017 & 0,266 & 1,000 & 0,724 & 0,276 & 1,000 & 0,791 & 1,175 & 0,784 & 0,137 & 0,107 & 0,152 & 72 & 469 & $-0,5$ \\
\hline 36 & 469 & 0,171 & 2,304 & 0,205 & 2,152 & 0,832 & 0,934 & 0,180 & 0,404 & 1,573 & 0,201 & 0,185 & 1,040 & 0,192 & 0,169 & 58 & 469 & $-3,4$ \\
\hline 37 & 469 & 0,062 & 1,002 & 0,070 & 0,900 & 0,878 & 0,899 & 0,159 & 0,773 & 1,138 & 0,400 & 0,378 & 0,443 & 0,168 & 0,227 & 76 & 469 & $-2,1$ \\
\hline 38 & 469 & 0,027 & 0,786 & 0,037 & 0,592 & 0,731 & 0,753 & 0,366 & 0,554 & 1,343 & 0,327 & 0,355 & 0,326 & 0,116 & 0,107 & 31 & 469 & $-1,7$ \\
\hline 39 & 469 & 0,036 & 0,643 & 0,037 & 0,520 & 0,962 & 0,809 & 0,195 & 1,000 & 0,957 & 0,522 & 0,362 & 0,296 & 0,107 & 0,169 & 47 & 469 & $-1,6$ \\
\hline 40 & 469 & 0,017 & 0,520 & 0,022 & 0,418 & 0,800 & 0,805 & 0,279 & 0,800 & 1,118 & 0,373 & 0,251 & 0,243 & 0,061 & 0,076 & 55 & 469 & $-1,3$ \\
\hline 41 & 469 & 0,016 & 0,368 & 0,016 & 0,266 & 1,000 & 0,724 & 0,276 & 1,000 & 0,826 & 0,700 & 0,447 & 0,169 & 0,076 & 0,107 & 72 & 469 & $-0,8$ \\
\hline 42 & 469 & 0,014 & 0,317 & 0,014 & 0,215 & 1,000 & 0,680 & 0,320 & 1,000 & 0,746 & 0,979 & 0,555 & 0,137 & 0,076 & 0,107 & 72 & 469 & $-0,5$ \\
\hline 43 & 469 & 0,020 & 0,469 & 0,022 & 0,389 & 0,933 & 0,829 & 0,184 & 1,000 & 0,934 & 0,524 & 0,364 & 0,221 & 0,080 & 0,107 & 60 & 469 & $-1,1$ \\
\hline 44 & 469 & 0,027 & 0,664 & 0,032 & 0,520 & 0,864 & 0,784 & 0,256 & 0,778 & 1,134 & 0,372 & 0,333 & 0,305 & 0,102 & 0,076 & 32 & 469 & $-1,6$ \\
\hline 45 & 469 & 0,020 & 0,469 & 0,023 & 0,389 & 0,875 & 0,829 & 0,212 & 1,000 & 0,934 & 0,615 & 0,499 & 0,204 & 0,102 & 0,107 & 66 & 469 & $-1,0$ \\
\hline 46 & 469 & 0,016 & 0,418 & 0,019 & 0,368 & 0,846 & 0,879 & 0,196 & 1,000 & 0,940 & 0,700 & 0,489 & 0,169 & 0,083 & 0,107 & 77 & 469 & $-0,8$ \\
\hline 47 & 469 & 0,027 & 0,520 & 0,029 & 0,418 & 0,950 & 0,805 & 0,202 & 1,000 & 0,888 & 0,712 & 0,485 & 0,221 & 0,107 & 0,152 & 33 & 469 & $-1,1$ \\
\hline 48 & 469 & 0,022 & 0,418 & 0,022 & 0,317 & 1,000 & 0,757 & 0,243 & 1,000 & 0,805 & 0,955 & 0,632 & 0,169 & 0,107 & 0,152 & 73 & 469 & $-0,8$ \\
\hline 49 & 469 & 0,017 & 0,397 & 0,019 & 0,296 & 0,923 & 0,745 & 0,267 & 1,000 & 0,854 & 0,764 & 0,474 & 0,169 & 0,080 & 0,107 & 60 & 469 & $-0,8$ \\
\hline 50 & 469 & 0,049 & 0,714 & 0,049 & 0,655 & 1,000 & 0,917 & 0,083 & 1,000 & 0,912 & 0,637 & 0,485 & 0,312 & 0,152 & 0,214 & 77 & 469 & $-1,6$ \\
\hline 51 & 469 & 0,039 & 0,643 & 0,039 & 0,541 & 1,000 & 0,842 & 0,158 & 1,000 & 0,921 & 0,649 & 0,549 & 0,276 & 0,152 & 0,169 & 71 & 469 & $-1,5$ \\
\hline 52 & 469 & 0,062 & 1,061 & 0,072 & 0,968 & 0,860 & 0,912 & 0,165 & 0,689 & 1,205 & 0,334 & 0,350 & 0,485 & 0,170 & 0,169 & 42 & 469 & $-2,3$ \\
\hline 53 & 469 & 0,029 & 0,622 & 0,032 & 0,562 & 0,909 & 0,904 & 0,132 & 0,934 & 1,035 & 0,490 & 0,347 & 0,273 & 0,095 & 0,107 & 57 & 469 & $-1,4$ \\
\hline 54 & 469 & 0,016 & 0,448 & 0,020 & 0,347 & 0,786 & 0,773 & 0,312 & 0,987 & 1,006 & 0,700 & 0,600 & 0,169 & 0,102 & 0,107 & 135 & 469 & $-0,8$ \\
\hline 55 & 469 & 0,043 & 0,917 & 0,052 & 0,807 & 0,833 & 0,880 & 0,205 & 0,643 & 1,247 & 0,326 & 0,290 & 0,410 & 0,119 & 0,152 & 65 & 469 & $-2,0$ \\
\hline 56 & 469 & 0,070 & 1,163 & 0,077 & 1,082 & 0,907 & 0,931 & 0,116 & 0,654 & 1,237 & 0,323 & 0,278 & 0,526 & 0,146 & 0,152 & 61 & 469 & $-2,4$ \\
\hline 57 & 469 & 0,059 & 0,867 & 0,066 & 0,786 & 0,891 & 0,907 & 0,143 & 0,984 & 1,008 & 0,637 & 0,629 & 0,343 & 0,216 & 0,214 & 93 & 469 & $-1,8$ \\
\hline 58 & 469 & 0,046 & 0,846 & 0,052 & 0,765 & 0,889 & 0,905 & 0,146 & 0,807 & 1,113 & 0,384 & 0,317 & 0,390 & 0,123 & 0,152 & 59 & 469 & $-2,0$ \\
\hline 59 & 469 & 0,036 & 0,664 & 0,039 & 0,541 & 0,926 & 0,815 & 0,199 & 1,000 & 0,988 & 0,490 & 0,389 & 0,305 & 0,119 & 0,152 & 65 & 469 & $-1,6$ \\
\hline 60 & 469 & 0,027 & 0,592 & 0,030 & 0,490 & 0,905 & 0,828 & 0,196 & 0,978 & 1,011 & 0,538 & 0,400 & 0,254 & 0,102 & 0,107 & 64 & 469 & $-1,3$ \\
\hline 61 & 469 & 0,019 & 0,418 & 0,020 & 0,317 & 0,929 & 0,757 & 0,253 & 1,000 & 0,864 & 0,828 & 0,501 & 0,169 & 0,085 & 0,107 & 63 & 469 & $-0,8$ \\
\hline 62 & 469 & 0,024 & 0,541 & 0,027 & 0,448 & 0,895 & 0,828 & 0,201 & 1,000 & 0,977 & 0,541 & 0,400 & 0,240 & 0,096 & 0,107 & 75 & 469 & $-1,3$ \\
\hline 63 & 469 & 0,027 & 0,511 & 0,029 & 0,368 & 0,950 & 0,719 & 0,285 & 1,000 & 0,874 & 0,712 & 0,514 & 0,221 & 0,114 & 0,152 & 75 & 469 & $-1,1$ \\
\hline 64 & 469 & 0,079 & 1,163 & 0,089 & 1,040 & 0,887 & 0,895 & 0,154 & 0,734 & 1,167 & 0,379 & 0,348 & 0,515 & 0,179 & 0,214 & 57 & 469 & $-2,4$ \\
\hline 65 & 469 & 0,014 & 0,490 & 0,019 & 0,368 & 0,769 & 0,750 & 0,340 & 0,750 & 1,155 & 0,344 & 0,259 & 0,230 & 0,060 & 0,076 & 20 & 469 & $-1,2$ \\
\hline 66 & 469 & 0,014 & 0,347 & 0,014 & 0,266 & 1,000 & 0,768 & 0,232 & 1,000 & 0,816 & 0,979 & 0,588 & 0,137 & 0,080 & 0,107 & 58 & 469 & $-0,5$ \\
\hline 67 & 469 & 0,047 & 1,070 & 0,065 & 0,938 & 0,733 & 0,877 & 0,294 & 0,520 & 1,387 & 0,288 & 0,357 & 0,458 & 0,163 & 0,107 & 33 & 469 & $-2,2$ \\
\hline 68 & 469 & 0,017 & 0,376 & 0,017 & 0,368 & 1,000 & 0,977 & 0,023 & 1,000 & 0,809 & 0,899 & 0,514 & 0,156 & 0,080 & 0,107 & 135 & 469 & $-0,6$ \\
\hline 69 & 469 & 0,020 & 0,490 & 0,022 & 0,317 & 0,933 & 0,646 & 0,360 & 1,000 & 0,976 & 0,524 & 0,413 & 0,221 & 0,091 & 0,107 & 55 & 469 & $-1,1$ \\
\hline 70 & 469 & 0,022 & 0,520 & 0,023 & 0,389 & 0,938 & 0,748 & 0,260 & 1,000 & 1,000 & 0,516 & 0,329 & 0,230 & 0,076 & 0,107 & 90 & 469 & $-1,2$ \\
\hline 71 & 469 & 0,023 & 0,499 & 0,024 & 0,418 & 0,941 & 0,839 & 0,172 & 1,000 & 0,929 & 0,702 & 0,499 & 0,204 & 0,102 & 0,152 & 58 & 469 & $-1,0$ \\
\hline 72 & 469 & 0,016 & 0,368 & 0,016 & 0,245 & 1,000 & 0,667 & 0,333 & 1,000 & 0,826 & 0,778 & 0,500 & 0,161 & 0,080 & 0,107 & 45 & 469 & $-0,7$ \\
\hline
\end{tabular}




\begin{tabular}{|c|c|c|c|c|c|c|c|c|c|c|c|c|c|c|c|c|c|c|}
\hline 73 & 469 & 0,016 & 0,368 & 0,017 & 0,296 & 0,917 & 0,805 & 0,212 & 1,000 & 0,826 & 0,778 & 0,500 & 0,161 & 0,080 & 0,107 & 72 & 469 & $-0,7$ \\
\hline 74 & 469 & 0,023 & 0,643 & 0,024 & 0,541 & 0,941 & 0,842 & 0,169 & 0,699 & 1,196 & 0,313 & 0,222 & 0,305 & 0,068 & 0,076 & 64 & 469 & $-1,6$ \\
\hline 75 & 469 & 0,263 & 4,262 & 0,476 & 3,311 & 0,551 & 0,777 & 0,501 & 0,182 & 2,346 & 0,131 & 0,272 & 1,598 & 0,435 & 0,152 & 57 & 469 & $-4,0$ \\
\hline 76 & 469 & 0,062 & 0,837 & 0,066 & 0,735 & 0,935 & 0,879 & 0,138 & 1,000 & 0,951 & 0,842 & 0,702 & 0,305 & 0,214 & 0,214 & 44 & 469 & $-1,6$ \\
\hline 77 & 469 & 0,024 & 0,520 & 0,024 & 0,448 & 1,000 & 0,862 & 0,138 & 1,000 & 0,939 & 0,637 & 0,447 & 0,221 & 0,099 & 0,107 & 40 & 469 & $-1,1$ \\
\hline 78 & 469 & 0,039 & 1,040 & 0,055 & 0,693 & 0,711 & 0,667 & 0,441 & 0,450 & 1,491 & 0,506 & 0,599 & 0,312 & 0,187 & 0,107 & 170 & 469 & $-1,6$ \\
\hline 79 & 469 & 0,032 & 0,714 & 0,036 & 0,643 & 0,880 & 0,899 & 0,157 & 0,777 & 1,134 & 0,384 & 0,314 & 0,324 & 0,102 & 0,107 & 22 & 469 & $-1,7$ \\
\hline 80 & 469 & 0,020 & 0,440 & 0,023 & 0,347 & 0,875 & 0,789 & 0,246 & 1,000 & 0,875 & 0,713 & 0,537 & 0,189 & 0,102 & 0,152 & 30 & 469 & $-0,9$ \\
\hline 81 & 469 & 0,024 & 0,622 & 0,029 & 0,541 & 0,850 & 0,871 & 0,198 & 0,794 & 1,123 & 0,416 & 0,286 & 0,273 & 0,078 & 0,076 & 62 & 469 & $-1,4$ \\
\hline 82 & 469 & 0,019 & 0,440 & 0,020 & 0,224 & 0,929 & 0,510 & 0,495 & 1,000 & 0,908 & 0,662 & 0,537 & 0,189 & 0,102 & 0,152 & 18 & 469 & $-0,9$ \\
\hline 83 & 469 & 0,026 & 0,592 & 0,029 & 0,418 & 0,900 & 0,707 & 0,309 & 0,927 & 1,039 & 0,716 & 0,554 & 0,214 & 0,119 & 0,152 & 72 & 469 & $-1,1$ \\
\hline 84 & 469 & 0,016 & 0,440 & 0,019 & 0,296 & 0,846 & 0,673 & 0,361 & 1,000 & 0,987 & 0,700 & 0,559 & 0,169 & 0,095 & 0,076 & 45 & 469 & $-0,8$ \\
\hline 85 & 469 & 0,059 & 1,255 & 0,080 & 1,124 & 0,732 & 0,895 & 0,288 & 0,469 & 1,460 & 0,225 & 0,223 & 0,577 & 0,129 & 0,152 & 71 & 469 & $-2,5$ \\
\hline 86 & 469 & 0,022 & 0,448 & 0,022 & 0,397 & 1,000 & 0,887 & 0,113 & 1,000 & 0,862 & 0,955 & 0,632 & 0,169 & 0,107 & 0,152 & 66 & 469 & $-0,8$ \\
\hline 87 & 469 & 0,043 & 0,735 & 0,047 & 0,592 & 0,909 & 0,805 & 0,215 & 1,000 & 1,000 & 0,516 & 0,416 & 0,326 & 0,136 & 0,152 & 61 & 469 & $-1,7$ \\
\hline 88 & 469 & 0,086 & 1,112 & 0,095 & 1,031 & 0,909 & 0,928 & 0,116 & 0,875 & 1,069 & 0,452 & 0,413 & 0,492 & 0,203 & 0,214 & 66 & 469 & $-2,3$ \\
\hline 89 & 469 & 0,030 & 0,592 & 0,032 & 0,490 & 0,955 & 0,828 & 0,177 & 1,000 & 0,962 & 0,514 & 0,392 & 0,273 & 0,107 & 0,152 & 56 & 469 & $-1,4$ \\
\hline 90 & 469 & 0,014 & 0,326 & 0,014 & 0,296 & 1,000 & 0,909 & 0,091 & 1,000 & 0,767 & 1,273 & 0,671 & 0,120 & 0,080 & 0,107 & 45 & 469 & $-0,3$ \\
\hline 91 & 469 & 0,055 & 0,917 & 0,059 & 0,837 & 0,927 & 0,912 & 0,114 & 0,814 & 1,108 & 0,387 & 0,321 & 0,423 & 0,136 & 0,169 & 60 & 469 & $-2,1$ \\
\hline 92 & 469 & 0,052 & 1,082 & 0,062 & 0,981 & 0,837 & 0,906 & 0,188 & 0,554 & 1,343 & 0,255 & 0,233 & 0,508 & 0,119 & 0,152 & 66 & 469 & $-2,3$ \\
\hline 93 & 469 & 0,027 & 0,562 & 0,029 & 0,440 & 0,950 & 0,782 & 0,224 & 1,000 & 0,960 & 0,654 & 0,493 & 0,230 & 0,114 & 0,152 & 73 & 469 & $-1,2$ \\
\hline 94 & 469 & 0,027 & 0,693 & 0,036 & 0,541 & 0,760 & 0,780 & 0,325 & 0,713 & 1,185 & 0,417 & 0,416 & 0,288 & 0,120 & 0,076 & 28 & 469 & $-1,5$ \\
\hline 95 & 469 & 0,016 & 0,440 & 0,019 & 0,317 & 0,846 & 0,721 & 0,318 & 1,000 & 0,987 & 0,560 & 0,424 & 0,189 & 0,080 & 0,107 & 58 & 469 & $-0,9$ \\
\hline 96 & 469 & 0,027 & 0,520 & 0,030 & 0,461 & 0,905 & 0,886 & 0,149 & 1,000 & 0,888 & 0,834 & 0,657 & 0,204 & 0,134 & 0,169 & 52 & 469 & $-1,0$ \\
\hline 97 & 469 & 0,017 & 0,368 & 0,019 & 0,296 & 0,923 & 0,805 & 0,210 & 1,000 & 0,791 & 1,175 & 0,745 & 0,137 & 0,102 & 0,107 & 60 & 469 & $-0,5$ \\
\hline 98 & 469 & 0,020 & 0,541 & 0,024 & 0,418 & 0,824 & 0,773 & 0,287 & 0,862 & 1,077 & 0,446 & 0,345 & 0,240 & 0,083 & 0,107 & 73 & 469 & $-1,3$ \\
\hline 99 & 469 & 0,024 & 0,643 & 0,029 & 0,550 & 0,850 & 0,855 & 0,208 & 0,742 & 1,161 & 0,333 & 0,234 & 0,305 & 0,071 & 0,076 & 60 & 469 & $-1,6$ \\
\hline 100 & 469 & 0,024 & 0,511 & 0,026 & 0,410 & 0,944 & 0,801 & 0,206 & 1,000 & 0,924 & 0,637 & 0,489 & 0,221 & 0,108 & 0,152 & 71 & 469 & $-1,1$ \\
\hline 101 & 469 & 0,019 & 0,418 & 0,019 & 0,296 & 1,000 & 0,707 & 0,293 & 1,000 & 0,864 & 0,662 & 0,424 & 0,189 & 0,080 & 0,107 & 57 & 469 & $-0,9$ \\
\hline 102 & 469 & 0,017 & 0,368 & 0,017 & 0,287 & 1,000 & 0,781 & 0,219 & 1,000 & 0,791 & 0,849 & 0,500 & 0,161 & 0,080 & 0,107 & 45 & 469 & $-0,7$ \\
\hline 103 & 469 & 0,017 & 0,397 & 0,017 & 0,296 & 1,000 & 0,745 & 0,255 & 1,000 & 0,854 & 0,764 & 0,474 & 0,169 & 0,080 & 0,107 & 57 & 469 & $-0,8$ \\
\hline 104 & 469 & 0,032 & 0,693 & 0,037 & 0,592 & 0,846 & 0,854 & 0,212 & 0,825 & 1,101 & 0,389 & 0,333 & 0,321 & 0,107 & 0,152 & 46 & 469 & $-1,7$ \\
\hline 105 & 469 & 0,040 & 0,867 & 0,047 & 0,765 & 0,848 & 0,883 & 0,192 & 0,672 & 1,220 & 0,419 & 0,460 & 0,349 & 0,161 & 0,169 & 122 & 469 & $-1,8$ \\
\hline 106 & 469 & 0,024 & 0,469 & 0,026 & 0,368 & 0,944 & 0,784 & 0,223 & 1,000 & 0,848 & 0,833 & 0,588 & 0,193 & 0,114 & 0,152 & 92 & 469 & $-0,9$ \\
\hline 107 & 469 & 0,059 & 0,909 & 0,066 & 0,778 & 0,891 & 0,856 & 0,181 & 0,895 & 1,057 & 0,637 & 0,552 & 0,343 & 0,189 & 0,227 & 109 & 469 & $-1,8$ \\
\hline 108 & 469 & 0,149 & 2,824 & 0,275 & 2,430 & 0,542 & 0,861 & 0,479 & 0,235 & 2,062 & 0,150 & 0,312 & 1,124 & 0,351 & 0,152 & 65 & 469 & $-3,5$ \\
\hline 109 & 469 & 0,014 & 0,347 & 0,014 & 0,317 & 1,000 & 0,914 & 0,086 & 1,000 & 0,816 & 0,749 & 0,485 & 0,156 & 0,076 & 0,107 & 18 & 469 & $-0,6$ \\
\hline 110 & 469 & 0,016 & 0,397 & 0,019 & 0,296 & 0,846 & 0,745 & 0,298 & 1,000 & 0,892 & 0,824 & 0,607 & 0,156 & 0,095 & 0,107 & 69 & 469 & $-0,6$ \\
\hline 111 & 469 & 0,014 & 0,368 & 0,016 & 0,317 & 0,909 & 0,862 & 0,165 & 1,000 & 0,866 & 0,637 & 0,447 & 0,169 & 0,076 & 0,107 & 18 & 469 & $-0,8$ \\
\hline 112 & 469 & 0,037 & 0,858 & 0,044 & 0,613 & 0,839 & 0,714 & 0,328 & 0,637 & 1,253 & 0,625 & 0,549 & 0,276 & 0,152 & 0,152 & 84 & 469 & $-1,5$ \\
\hline 113 & 469 & 0,030 & 0,685 & 0,034 & 0,583 & 0,875 & 0,852 & 0,194 & 0,808 & 1,113 & 0,411 & 0,275 & 0,305 & 0,084 & 0,107 & 72 & 469 & $-1,6$ \\
\hline 114 & 469 & 0,014 & 0,317 & 0,014 & 0,194 & 1,000 & 0,613 & 0,387 & 1,000 & 0,746 & 0,979 & 0,555 & 0,137 & 0,076 & 0,107 & 18 & 469 & $-0,5$ \\
\hline 115 & 469 & 0,060 & 1,061 & 0,069 & 0,938 & 0,875 & 0,884 & 0,170 & 0,673 & 1,219 & 0,330 & 0,327 & 0,482 & 0,158 & 0,169 & 46 & 469 & $-2,3$ \\
\hline 116 & 469 & 0,040 & 0,714 & 0,044 & 0,643 & 0,903 & 0,899 & 0,140 & 0,989 & 1,005 & 0,495 & 0,393 & 0,321 & 0,126 & 0,169 & 52 & 469 & $-1,7$ \\
\hline 117 & 469 & 0,014 & 0,347 & 0,016 & 0,266 & 0,909 & 0,768 & 0,249 & 1,000 & 0,816 & 1,273 & 0,849 & 0,120 & 0,102 & 0,107 & 0 & 469 & $-0,3$ \\
\hline 118 & 469 & 0,016 & 0,418 & 0,019 & 0,317 & 0,846 & 0,757 & 0,287 & 1,000 & 0,940 & 0,560 & 0,389 & 0,189 & 0,074 & 0,076 & 18 & 469 & $-0,9$ \\
\hline 119 & 469 & 0,024 & 0,613 & 0,032 & 0,440 & 0,773 & 0,717 & 0,363 & 0,816 & 1,107 & 0,746 & 0,743 & 0,204 & 0,152 & 0,152 & 178 & 469 & $-1,0$ \\
\hline 120 & 469 & 0,016 & 0,418 & 0,019 & 0,338 & 0,846 & 0,808 & 0,246 & 1,000 & 0,940 & 0,824 & 0,651 & 0,156 & 0,102 & 0,107 & 45 & 469 & $-0,6$ \\
\hline 121 & 469 & 0,439 & 4,139 & 0,580 & 3,319 & 0,757 & 0,802 & 0,313 & 0,322 & 1,762 & 0,244 & 0,256 & 1,514 & 0,388 & 0,169 & 55 & 469 & $-3,9$ \\
\hline 122 & 469 & 0,016 & 0,397 & 0,016 & 0,296 & 1,000 & 0,745 & 0,255 & 1,000 & 0,892 & 0,560 & 0,283 & 0,189 & 0,054 & 0,107 & 50 & 469 & $-0,9$ \\
\hline 123 & 469 & 0,017 & 0,368 & 0,017 & 0,287 & 1,000 & 0,781 & 0,219 & 1,000 & 0,791 & 0,849 & 0,500 & 0,161 & 0,080 & 0,107 & 45 & 469 & $-0,7$ \\
\hline 124 & 469 & 0,049 & 0,867 & 0,053 & 0,807 & 0,919 & 0,931 & 0,106 & 0,816 & 1,107 & 0,397 & 0,333 & 0,395 & 0,132 & 0,152 & 69 & 469 & $-2,0$ \\
\hline 125 & 469 & 0,029 & 0,592 & 0,033 & 0,511 & 0,870 & 0,864 & 0,188 & 1,000 & 0,986 & 0,566 & 0,425 & 0,254 & 0,108 & 0,152 & 18 & 469 & $-1,3$ \\
\hline 126 & 469 & 0,023 & 0,499 & 0,024 & 0,461 & 0,941 & 0,923 & 0,097 & 1,000 & 0,929 & 0,702 & 0,465 & 0,204 & 0,095 & 0,107 & 58 & 469 & $-1,0$ \\
\hline 127 & 469 & 0,030 & 0,541 & 0,032 & 0,461 & 0,955 & 0,851 & 0,155 & 1,000 & 0,879 & 0,723 & 0,493 & 0,230 & 0,114 & 0,152 & 92 & 469 & $-1,2$ \\
\hline 128 & 469 & 0,253 & 3,652 & 0,376 & 2,439 & 0,672 & 0,668 & 0,467 & 0,238 & 2,050 & 0,224 & 0,334 & 1,198 & 0,399 & 0,312 & 52 & 469 & $-3,6$ \\
\hline 129 & 469 & 0,090 & 1,826 & 0,125 & 1,674 & 0,724 & 0,917 & 0,288 & 0,341 & 1,713 & 0,154 & 0,204 & 0,865 & 0,176 & 0,214 & 70 & 469 & $-3,1$ \\
\hline 130 & 469 & 0,085 & 1,404 & 0,095 & 1,395 & 0,894 & 0,994 & 0,106 & 0,540 & 1,361 & 0,237 & 0,199 & 0,674 & 0,134 & 0,169 & 50 & 469 & $-2,8$ \\
\hline
\end{tabular}




\begin{tabular}{|c|c|c|c|c|c|c|c|c|c|c|c|c|c|c|c|c|c|c|}
\hline 131 & 469 & 0,016 & 0,338 & 0,016 & 0,245 & 1,000 & 0,725 & 0,275 & 1,000 & 0,759 & 1,077 & 0,555 & 0,137 & 0,076 & 0,152 & 90 & 469 & $-0,5$ \\
\hline 132 & 469 & 0,043 & 0,867 & 0,057 & 0,706 & 0,750 & 0,814 & 0,311 & 0,720 & 1,178 & 0,588 & 0,653 & 0,305 & 0,199 & 0,152 & 93 & 469 & $-1,6$ \\
\hline 133 & 469 & 0,016 & 0,368 & 0,016 & 0,338 & 1,000 & 0,919 & 0,081 & 1,000 & 0,826 & 0,824 & 0,485 & 0,156 & 0,076 & 0,107 & 82 & 469 & $-0,6$ \\
\hline 134 & 469 & 0,039 & 0,867 & 0,043 & 0,744 & 0,900 & 0,859 & 0,173 & 0,648 & 1,242 & 0,304 & 0,263 & 0,403 & 0,106 & 0,152 & 52 & 469 & $-2,0$ \\
\hline 135 & 469 & 0,016 & 0,347 & 0,016 & 0,266 & 1,000 & 0,768 & 0,232 & 1,000 & 0,778 & 1,077 & 0,588 & 0,137 & 0,080 & 0,107 & 45 & 469 & $-0,5$ \\
\hline 136 & 469 & 0,032 & 0,685 & 0,040 & 0,541 & 0,786 & 0,790 & 0,300 & 0,846 & 1,087 & 0,539 & 0,476 & 0,273 & 0,130 & 0,107 & 56 & 469 & $-1,4$ \\
\hline 137 & 469 & 0,022 & 0,440 & 0,023 & 0,338 & 0,938 & 0,769 & 0,239 & 1,000 & 0,845 & 0,764 & 0,537 & 0,189 & 0,102 & 0,152 & 67 & 469 & $-0,9$ \\
\hline 138 & 469 & 0,020 & 0,440 & 0,020 & 0,338 & 1,000 & 0,769 & 0,231 & 1,000 & 0,875 & 0,713 & 0,500 & 0,189 & 0,095 & 0,107 & 55 & 469 & $-0,9$ \\
\hline 139 & 469 & 0,022 & 0,418 & 0,022 & 0,338 & 1,000 & 0,808 & 0,192 & 1,000 & 0,805 & 0,955 & 0,632 & 0,169 & 0,107 & 0,152 & 63 & 469 & $-0,8$ \\
\hline 140 & 469 & 0,019 & 0,397 & 0,019 & 0,368 & 1,000 & 0,925 & 0,075 & 1,000 & 0,821 & 0,828 & 0,474 & 0,169 & 0,080 & 0,107 & 35 & 469 & $-0,8$ \\
\hline 141 & 469 & 0,019 & 0,418 & 0,020 & 0,418 & 0,929 & 1,000 & 0,071 & 1,000 & 0,864 & 0,828 & 0,566 & 0,169 & 0,096 & 0,152 & 72 & 469 & $-0,8$ \\
\hline 142 & 469 & 0,016 & 0,440 & 0,019 & 0,317 & 0,846 & 0,721 & 0,318 & 1,000 & 0,987 & 0,539 & 0,392 & 0,193 & 0,076 & 0,107 & 90 & 469 & $-0,9$ \\
\hline 143 & 469 & 0,042 & 0,867 & 0,046 & 0,744 & 0,906 & 0,859 & 0,170 & 0,696 & 1,199 & 0,316 & 0,261 & 0,410 & 0,107 & 0,152 & 50 & 469 & $-2,0$ \\
\hline 144 & 469 & 0,019 & 0,397 & 0,019 & 0,347 & 1,000 & 0,872 & 0,128 & 1,000 & 0,821 & 0,828 & 0,474 & 0,169 & 0,080 & 0,107 & 45 & 469 & $-0,8$ \\
\hline 145 & 469 & 0,020 & 0,613 & 0,032 & 0,440 & 0,636 & 0,717 & 0,461 & 0,672 & 1,220 & 0,524 & 0,575 & 0,221 & 0,127 & 0,076 & 98 & 469 & $-1,1$ \\
\hline 146 & 469 & 0,019 & 0,389 & 0,020 & 0,287 & 0,929 & 0,739 & 0,271 & 1,000 & 0,803 & 0,920 & 0,633 & 0,161 & 0,102 & 0,152 & 60 & 469 & $-0,7$ \\
\hline 147 & 469 & 0,056 & 1,070 & 0,069 & 0,888 & 0,812 & 0,830 & 0,253 & 0,615 & 1,276 & 0,342 & 0,352 & 0,456 & 0,161 & 0,169 & 32 & 469 & $-2,2$ \\
\hline 148 & 469 & 0,026 & 0,571 & 0,029 & 0,511 & 0,900 & 0,896 & 0,144 & 0,996 & 1,002 & 0,573 & 0,447 & 0,240 & 0,107 & 0,152 & 51 & 469 & $-1,3$ \\
\hline 149 & 469 & 0,019 & 0,418 & 0,020 & 0,338 & 0,929 & 0,808 & 0,205 & 1,000 & 0,864 & 0,662 & 0,424 & 0,189 & 0,080 & 0,107 & 60 & 469 & $-0,9$ \\
\hline 150 & 469 & 0,027 & 0,541 & 0,030 & 0,469 & 0,905 & 0,867 & 0,163 & 1,000 & 0,924 & 0,712 & 0,537 & 0,221 & 0,119 & 0,152 & 38 & 469 & $-1,1$ \\
\hline 151 & 469 & 0,040 & 0,867 & 0,044 & 0,786 & 0,903 & 0,907 & 0,134 & 0,672 & 1,220 & 0,285 & 0,241 & 0,423 & 0,102 & 0,152 & 62 & 469 & $-2,1$ \\
\hline 152 & 469 & 0,026 & 0,541 & 0,029 & 0,418 & 0,900 & 0,773 & 0,248 & 1,000 & 0,950 & 0,559 & 0,434 & 0,243 & 0,105 & 0,152 & 135 & 469 & $-1,3$ \\
\hline 153 & 469 & 0,029 & 0,541 & 0,032 & 0,469 & 0,909 & 0,867 & 0,161 & 1,000 & 0,901 & 0,749 & 0,606 & 0,221 & 0,134 & 0,152 & 56 & 469 & $-1,1$ \\
\hline 154 & 469 & 0,023 & 0,490 & 0,024 & 0,389 & 0,941 & 0,793 & 0,215 & 1,000 & 0,913 & 0,599 & 0,384 & 0,221 & 0,085 & 0,107 & 65 & 469 & $-1,1$ \\
\hline 155 & 469 & 0,017 & 0,418 & 0,019 & 0,317 & 0,923 & 0,757 & 0,254 & 1,000 & 0,900 & 0,611 & 0,424 & 0,189 & 0,080 & 0,107 & 57 & 469 & $-0,9$ \\
\hline 156 & 469 & 0,022 & 0,520 & 0,026 & 0,448 & 0,833 & 0,862 & 0,216 & 1,000 & 1,000 & 0,562 & 0,447 & 0,221 & 0,099 & 0,107 & 50 & 469 & $-1,1$ \\
\hline 157 & 469 & 0,079 & 1,378 & 0,103 & 1,145 & 0,764 & 0,831 & 0,290 & 0,522 & 1,384 & 0,330 & 0,412 & 0,552 & 0,227 & 0,152 & 76 & 469 & $-2,5$ \\
\hline 158 & 469 & 0,077 & 1,530 & 0,125 & 1,345 & 0,621 & 0,879 & 0,398 & 0,416 & 1,551 & 0,248 & 0,344 & 0,630 & 0,217 & 0,169 & 55 & 469 & $-2,7$ \\
\hline 159 & 469 & 0,023 & 0,490 & 0,024 & 0,368 & 0,941 & 0,750 & 0,257 & 1,000 & 0,913 & 1,019 & 0,849 & 0,169 & 0,144 & 0,152 & 55 & 469 & $-0,8$ \\
\hline 160 & 469 & 0,016 & 0,368 & 0,016 & 0,296 & 1,000 & 0,805 & 0,195 & 1,000 & 0,826 & 0,778 & 0,500 & 0,161 & 0,080 & 0,107 & 53 & 469 & $-0,7$ \\
\hline 161 & 469 & 0,343 & 2,917 & 0,412 & 2,684 & 0,833 & 0,920 & 0,185 & 0,506 & 1,405 & 0,285 & 0,285 & 1,237 & 0,352 & 0,379 & 65 & 469 & $-3,6$ \\
\hline 162 & 469 & 0,016 & 0,347 & 0,016 & 0,296 & 1,000 & 0,854 & 0,146 & 1,000 & 0,778 & 1,401 & 0,849 & 0,120 & 0,102 & 0,107 & 45 & 469 & $-0,3$ \\
\hline 163 & 469 & 0,034 & 0,613 & 0,037 & 0,499 & 0,923 & 0,814 & 0,201 & 1,000 & 0,932 & 0,745 & 0,552 & 0,243 & 0,134 & 0,152 & 142 & 469 & $-1,3$ \\
\hline 164 & 469 & 0,034 & 0,693 & 0,040 & 0,592 & 0,857 & 0,854 & 0,205 & 0,900 & 1,054 & 0,527 & 0,383 & 0,288 & 0,111 & 0,152 & 62 & 469 & $-1,5$ \\
\hline 165 & 469 & 0,069 & 1,142 & 0,083 & 0,917 & 0,828 & 0,804 & 0,261 & 0,664 & 1,227 & 0,489 & 0,544 & 0,423 & 0,230 & 0,214 & 51 & 469 & $-2,1$ \\
\hline 166 & 469 & 0,030 & 0,757 & 0,037 & 0,664 & 0,808 & 0,877 & 0,228 & 0,662 & 1,229 & 0,297 & 0,249 & 0,359 & 0,089 & 0,152 & 15 & 469 & $-1,8$ \\
\hline 167 & 469 & 0,019 & 0,490 & 0,022 & 0,389 & 0,867 & 0,793 & 0,246 & 0,975 & 1,013 & 0,487 & 0,307 & 0,221 & 0,068 & 0,076 & 62 & 469 & $-1,1$ \\
\hline 168 & 469 & 0,060 & 0,968 & 0,066 & 0,909 & 0,913 & 0,939 & 0,106 & 0,808 & 1,113 & 0,393 & 0,343 & 0,442 & 0,152 & 0,169 & 59 & 469 & $-2,1$ \\
\hline 169 & 469 & 0,022 & 0,440 & 0,023 & 0,317 & 0,938 & 0,721 & 0,286 & 1,000 & 0,845 & 0,764 & 0,537 & 0,189 & 0,102 & 0,152 & 63 & 469 & $-0,9$ \\
\hline 170 & 469 & 0,119 & 1,327 & 0,132 & 1,226 & 0,902 & 0,924 & 0,124 & 0,849 & 1,085 & 0,485 & 0,471 & 0,559 & 0,264 & 0,303 & 64 & 469 & $-2,5$ \\
\hline 171 & 469 & 0,016 & 0,397 & 0,017 & 0,347 & 0,917 & 0,872 & 0,152 & 1,000 & 0,892 & 0,700 & 0,474 & 0,169 & 0,080 & 0,107 & 56 & 469 & $-0,8$ \\
\hline 172 & 469 & 0,029 & 0,723 & 0,036 & 0,693 & 0,800 & 0,959 & 0,204 & 0,690 & 1,204 & 0,344 & 0,291 & 0,326 & 0,095 & 0,107 & 55 & 469 & $-1,7$ \\
\hline 173 & 469 & 0,057 & 0,837 & 0,066 & 0,786 & 0,870 & 0,939 & 0,144 & 1,000 & 0,986 & 0,688 & 0,598 & 0,326 & 0,195 & 0,214 & 51 & 469 & $-1,7$ \\
\hline 174 & 469 & 0,020 & 0,410 & 0,020 & 0,266 & 1,000 & 0,650 & 0,350 & 1,000 & 0,816 & 0,891 & 0,447 & 0,169 & 0,076 & 0,152 & 98 & 469 & $-0,8$ \\
\hline 175 & 469 & 0,033 & 0,592 & 0,033 & 0,490 & 1,000 & 0,828 & 0,172 & 1,000 & 0,919 & 0,586 & 0,400 & 0,268 & 0,107 & 0,169 & 42 & 469 & $-1,4$ \\
\hline 176 & 469 & 0,039 & 0,723 & 0,043 & 0,693 & 0,900 & 0,959 & 0,108 & 0,931 & 1,036 & 0,529 & 0,439 & 0,305 & 0,134 & 0,152 & 61 & 469 & $-1,6$ \\
\hline 177 & 469 & 0,023 & 0,583 & 0,026 & 0,461 & 0,889 & 0,790 & 0,238 & 0,848 & 1,086 & 0,384 & 0,275 & 0,276 & 0,076 & 0,107 & 7 & 469 & $-1,5$ \\
\hline 178 & 469 & 0,017 & 0,389 & 0,017 & 0,347 & 1,000 & 0,892 & 0,108 & 1,000 & 0,836 & 0,899 & 0,485 & 0,156 & 0,076 & 0,152 & 0 & 469 & $-0,6$ \\
\hline 179 & 469 & 0,020 & 0,397 & 0,020 & 0,368 & 1,000 & 0,925 & 0,075 & 1,000 & 0,791 & 1,049 & 0,686 & 0,156 & 0,107 & 0,152 & 20 & 469 & $-0,6$ \\
\hline 180 & 469 & 0,027 & 0,643 & 0,029 & 0,520 & 0,950 & 0,809 & 0,197 & 0,830 & 1,098 & 0,372 & 0,275 & 0,305 & 0,084 & 0,107 & 60 & 469 & $-1,6$ \\
\hline 181 & 469 & 0,036 & 0,989 & 0,055 & 0,837 & 0,658 & 0,846 & 0,375 & 0,461 & 1,473 & 0,255 & 0,241 & 0,423 & 0,102 & 0,107 & 24 & 469 & $-2,1$ \\
\hline 182 & 469 & 0,014 & 0,418 & 0,019 & 0,296 & 0,769 & 0,707 & 0,373 & 1,000 & 0,986 & 0,509 & 0,358 & 0,189 & 0,068 & 0,076 & 162 & 469 & $-0,9$ \\
\hline 183 & 469 & 0,040 & 0,765 & 0,046 & 0,664 & 0,875 & 0,867 & 0,182 & 0,862 & 1,077 & 0,446 & 0,395 & 0,339 & 0,134 & 0,152 & 57 & 469 & $-1,8$ \\
\hline 184 & 469 & 0,034 & 0,643 & 0,036 & 0,592 & 0,960 & 0,921 & 0,089 & 1,000 & 0,977 & 0,501 & 0,362 & 0,296 & 0,107 & 0,152 & 36 & 469 & $-1,6$ \\
\hline 185 & 469 & 0,046 & 0,930 & 0,059 & 0,786 & 0,780 & 0,846 & 0,268 & 0,667 & 1,224 & 0,326 & 0,326 & 0,423 & 0,138 & 0,152 & 71 & 469 & $-2,1$ \\
\hline 186 & 469 & 0,033 & 0,744 & 0,040 & 0,613 & 0,821 & 0,824 & 0,251 & 0,749 & 1,155 & 0,401 & 0,419 & 0,324 & 0,136 & 0,169 & 65 & 469 & $-1,7$ \\
\hline 187 & 469 & 0,032 & 0,571 & 0,032 & 0,490 & 1,000 & 0,859 & 0,141 & 1,000 & 0,906 & 0,622 & 0,422 & 0,254 & 0,107 & 0,169 & 56 & 469 & $-1,3$ \\
\hline 188 & 469 & 0,022 & 0,571 & 0,026 & 0,440 & 0,833 & 0,770 & 0,284 & 0,830 & 1,098 & 0,562 & 0,485 & 0,221 & 0,107 & 0,107 & 55 & 469 & $-1,1$ \\
\hline
\end{tabular}




\begin{tabular}{|c|c|c|c|c|c|c|c|c|c|c|c|c|c|c|c|c|c|c|}
\hline 189 & 469 & 0,092 & 1,294 & 0,102 & 1,213 & 0,901 & 0,938 & 0,117 & 0,689 & 1,204 & 0,326 & 0,312 & 0,599 & 0,187 & 0,214 & 57 & 469 & $-2,6$ \\
\hline 190 & 469 & 0,032 & 0,541 & 0,033 & 0,511 & 0,957 & 0,945 & 0,070 & 1,000 & 0,859 & 0,966 & 0,743 & 0,204 & 0,152 & 0,152 & 27 & 469 & $-1,0$ \\
\hline 191 & 469 & 0,017 & 0,368 & 0,017 & 0,266 & 1,000 & 0,724 & 0,276 & 1,000 & 0,791 & 1,175 & 0,745 & 0,137 & 0,102 & 0,152 & 45 & 469 & $-0,5$ \\
\hline 192 & 469 & 0,032 & 0,592 & 0,036 & 0,499 & 0,880 & 0,843 & 0,198 & 1,000 & 0,940 & 0,824 & 0,690 & 0,221 & 0,152 & 0,169 & 78 & 469 & $-1,1$ \\
\hline 193 & 469 & 0,014 & 0,317 & 0,014 & 0,194 & 1,000 & 0,613 & 0,387 & 1,000 & 0,746 & 0,979 & 0,555 & 0,137 & 0,076 & 0,107 & 18 & 469 & $-0,5$ \\
\hline 194 & 469 & 0,023 & 0,448 & 0,024 & 0,418 & 0,941 & 0,934 & 0,089 & 1,000 & 0,834 & 1,198 & 0,857 & 0,156 & 0,134 & 0,169 & 18 & 469 & $-0,6$ \\
\hline 195 & 469 & 0,014 & 0,317 & 0,014 & 0,194 & 1,000 & 0,613 & 0,387 & 1,000 & 0,746 & 0,979 & 0,555 & 0,137 & 0,076 & 0,107 & 18 & 469 & $-0,5$ \\
\hline 196 & 469 & 0,014 & 0,338 & 0,014 & 0,194 & 1,000 & 0,575 & 0,425 & 1,000 & 0,796 & 0,979 & 0,555 & 0,137 & 0,076 & 0,107 & 18 & 469 & $-0,5$ \\
\hline 197 & 469 & 0,026 & 0,490 & 0,026 & 0,410 & 1,000 & 0,836 & 0,164 & 1,000 & 0,861 & 0,674 & 0,485 & 0,221 & 0,107 & 0,152 & 66 & 469 & $-1,1$ \\
\hline 198 & 469 & 0,026 & 0,744 & 0,036 & 0,469 & 0,720 & 0,631 & 0,464 & 0,586 & 1,306 & 0,573 & 0,566 & 0,240 & 0,136 & 0,107 & 113 & 469 & $-1,3$ \\
\hline 199 & 469 & 0,034 & 0,643 & 0,034 & 0,562 & 1,000 & 0,875 & 0,125 & 1,000 & 0,977 & 0,501 & 0,362 & 0,296 & 0,107 & 0,169 & 41 & 469 & $-1,6$ \\
\hline 200 & 469 & 0,027 & 0,592 & 0,030 & 0,461 & 0,905 & 0,778 & 0,241 & 0,978 & 1,011 & 0,712 & 0,514 & 0,221 & 0,114 & 0,152 & 77 & 469 & $-1,1$ \\
\hline 201 & 469 & 0,026 & 0,643 & 0,033 & 0,541 & 0,783 & 0,842 & 0,269 & 0,786 & 1,128 & 0,441 & 0,373 & 0,273 & 0,102 & 0,152 & 59 & 469 & $-1,4$ \\
\hline 202 & 469 & 0,023 & 0,511 & 0,024 & 0,418 & 0,941 & 0,818 & 0,191 & 1,000 & 0,952 & 0,551 & 0,329 & 0,230 & 0,076 & 0,152 & 85 & 469 & $-1,2$ \\
\hline 203 & 469 & 0,032 & 0,613 & 0,033 & 0,562 & 0,957 & 0,917 & 0,094 & 1,000 & 0,973 & 0,529 & 0,412 & 0,276 & 0,114 & 0,152 & 11 & 469 & $-1,5$ \\
\hline 204 & 469 & 0,027 & 0,541 & 0,030 & 0,389 & 0,905 & 0,719 & 0,297 & 1,000 & 0,924 & 0,834 & 0,557 & 0,204 & 0,114 & 0,152 & 92 & 469 & $-1,0$ \\
\hline 205 & 469 & 0,059 & 1,052 & 0,076 & 0,858 & 0,774 & 0,815 & 0,292 & 0,667 & 1,224 & 0,418 & 0,366 & 0,423 & 0,155 & 0,152 & 71 & 469 & $-2,1$ \\
\hline 206 & 469 & 0,047 & 0,858 & 0,056 & 0,714 & 0,846 & 0,833 & 0,227 & 0,808 & 1,112 & 0,420 & 0,389 & 0,379 & 0,147 & 0,169 & 53 & 469 & $-1,9$ \\
\hline 207 & 469 & 0,039 & 0,795 & 0,047 & 0,685 & 0,818 & 0,861 & 0,229 & 0,771 & 1,139 & 0,430 & 0,425 & 0,339 & 0,144 & 0,152 & 69 & 469 & $-1,8$ \\
\hline 208 & 469 & 0,017 & 0,622 & 0,026 & 0,499 & 0,667 & 0,803 & 0,387 & 0,560 & 1,336 & 0,340 & 0,316 & 0,254 & 0,080 & 0,107 & 90 & 469 & $-1,3$ \\
\hline 209 & 469 & 0,017 & 0,490 & 0,019 & 0,368 & 0,923 & 0,750 & 0,262 & 0,900 & 1,054 & 0,424 & 0,333 & 0,227 & 0,076 & 0,107 & 86 & 469 & $-1,2$ \\
\hline 210 & 469 & 0,036 & 0,714 & 0,040 & 0,571 & 0,893 & 0,799 & 0,228 & 0,883 & 1,064 & 0,442 & 0,333 & 0,321 & 0,107 & 0,152 & 47 & 469 & $-1,7$ \\
\hline 211 & 469 & 0,014 & 0,347 & 0,014 & 0,245 & 1,000 & 0,707 & 0,293 & 1,000 & 0,816 & 0,979 & 0,588 & 0,137 & 0,080 & 0,107 & 72 & 469 & $-0,5$ \\
\hline 212 & 469 & 0,033 & 0,592 & 0,037 & 0,511 & 0,885 & 0,864 & 0,178 & 1,000 & 0,919 & 0,714 & 0,552 & 0,243 & 0,134 & 0,169 & 55 & 469 & $-1,3$ \\
\hline 213 & 469 & 0,121 & 1,336 & 0,123 & 1,213 & 0,977 & 0,908 & 0,095 & 0,849 & 1,086 & 0,404 & 0,347 & 0,617 & 0,214 & 0,273 & 47 & 469 & $-2,6$ \\
\hline 214 & 469 & 0,019 & 0,440 & 0,020 & 0,338 & 0,929 & 0,769 & 0,242 & 1,000 & 0,908 & 0,662 & 0,443 & 0,189 & 0,084 & 0,107 & 68 & 469 & $-0,9$ \\
\hline 215 & 469 & 0,027 & 0,511 & 0,029 & 0,389 & 0,950 & 0,760 & 0,245 & 1,000 & 0,874 & 0,834 & 0,557 & 0,204 & 0,114 & 0,152 & 97 & 469 & $-1,0$ \\
\hline 216 & 469 & 0,022 & 0,469 & 0,023 & 0,368 & 0,938 & 0,784 & 0,225 & 1,000 & 0,902 & 0,659 & 0,416 & 0,204 & 0,085 & 0,107 & 65 & 469 & $-1,0$ \\
\hline 217 & 469 & 0,033 & 0,714 & 0,037 & 0,643 & 0,885 & 0,899 & 0,153 & 0,813 & 1,109 & 0,396 & 0,339 & 0,326 & 0,111 & 0,152 & 62 & 469 & $-1,7$ \\
\hline 218 & 469 & 0,016 & 0,368 & 0,016 & 0,245 & 1,000 & 0,667 & 0,333 & 1,000 & 0,826 & 0,700 & 0,447 & 0,169 & 0,076 & 0,107 & 72 & 469 & $-0,8$ \\
\hline 219 & 469 & 0,024 & 0,592 & 0,030 & 0,511 & 0,810 & 0,864 & 0,234 & 0,875 & 1,069 & 0,433 & 0,388 & 0,268 & 0,104 & 0,152 & 63 & 469 & $-1,4$ \\
\hline 220 & 469 & 0,017 & 0,389 & 0,019 & 0,266 & 0,923 & 0,685 & 0,325 & 1,000 & 0,836 & 0,849 & 0,528 & 0,161 & 0,085 & 0,107 & 37 & 469 & $-0,7$ \\
\hline 221 & 469 & 0,067 & 1,357 & 0,085 & 1,226 & 0,797 & 0,903 & 0,225 & 0,460 & 1,474 & 0,218 & 0,229 & 0,627 & 0,144 & 0,152 & 70 & 469 & $-2,6$ \\
\hline 222 & 469 & 0,017 & 0,397 & 0,019 & 0,296 & 0,923 & 0,745 & 0,267 & 1,000 & 0,854 & 0,764 & 0,474 & 0,169 & 0,080 & 0,107 & 60 & 469 & $-0,8$ \\
\hline 223 & 469 & 0,102 & 1,175 & 0,115 & 0,981 & 0,887 & 0,835 & 0,200 & 0,927 & 1,039 & 0,773 & 0,798 & 0,410 & 0,327 & 0,303 & 139 & 469 & $-2,0$ \\
\hline 224 & 469 & 0,014 & 0,317 & 0,014 & 0,275 & 1,000 & 0,867 & 0,133 & 1,000 & 0,746 & 1,273 & 0,632 & 0,120 & 0,076 & 0,107 & 90 & 469 & $-0,3$ \\
\hline 225 & 469 & 0,022 & 0,520 & 0,023 & 0,418 & 0,938 & 0,805 & 0,205 & 1,000 & 1,000 & 0,466 & 0,304 & 0,243 & 0,074 & 0,076 & 55 & 469 & $-1,3$ \\
\hline 226 & 469 & 0,099 & 1,247 & 0,118 & 1,061 & 0,841 & 0,851 & 0,218 & 0,800 & 1,118 & 0,646 & 0,773 & 0,442 & 0,342 & 0,273 & 23 & 469 & $-2,1$ \\
\hline 227 & 469 & 0,020 & 0,440 & 0,022 & 0,347 & 0,933 & 0,789 & 0,222 & 1,000 & 0,875 & 0,891 & 0,671 & 0,169 & 0,114 & 0,152 & 135 & 469 & $-0,8$ \\
\hline 228 & 469 & 0,082 & 1,184 & 0,099 & 1,010 & 0,826 & 0,854 & 0,227 & 0,734 & 1,168 & 0,558 & 0,685 & 0,432 & 0,296 & 0,214 & 77 & 469 & $-2,1$ \\
\hline 229 & 469 & 0,022 & 0,571 & 0,024 & 0,541 & 0,882 & 0,948 & 0,129 & 0,830 & 1,098 & 0,382 & 0,275 & 0,268 & 0,074 & 0,076 & 56 & 469 & $-1,4$ \\
\hline 230 & 469 & 0,019 & 0,397 & 0,020 & 0,368 & 0,929 & 0,925 & 0,103 & 1,000 & 0,821 & 0,974 & 0,651 & 0,156 & 0,102 & 0,107 & 71 & 469 & $-0,6$ \\
\hline 231 & 469 & 0,014 & 0,368 & 0,014 & 0,245 & 1,000 & 0,667 & 0,333 & 1,000 & 0,866 & 0,637 & 0,400 & 0,169 & 0,068 & 0,076 & 58 & 469 & $-0,8$ \\
\hline 232 & 469 & 0,030 & 0,685 & 0,036 & 0,511 & 0,840 & 0,747 & 0,299 & 0,808 & 1,113 & 0,411 & 0,333 & 0,305 & 0,102 & 0,107 & 62 & 469 & $-1,6$ \\
\hline 233 & 469 & 0,027 & 0,499 & 0,027 & 0,469 & 1,000 & 0,940 & 0,060 & 1,000 & 0,852 & 0,834 & 0,525 & 0,204 & 0,107 & 0,169 & 37 & 469 & $-1,0$ \\
\hline 234 & 469 & 0,016 & 0,469 & 0,020 & 0,347 & 0,786 & 0,739 & 0,338 & 0,901 & 1,054 & 0,483 & 0,411 & 0,204 & 0,084 & 0,107 & 72 & 469 & $-1,0$ \\
\hline 235 & 469 & 0,017 & 0,389 & 0,017 & 0,266 & 1,000 & 0,685 & 0,315 & 1,000 & 0,836 & 0,764 & 0,447 & 0,169 & 0,076 & 0,107 & 72 & 469 & $-0,8$ \\
\hline 236 & 469 & 0,016 & 0,397 & 0,019 & 0,296 & 0,846 & 0,745 & 0,298 & 1,000 & 0,892 & 0,700 & 0,435 & 0,169 & 0,074 & 0,076 & 58 & 469 & $-0,8$ \\
\hline 237 & 469 & 0,016 & 0,397 & 0,016 & 0,275 & 1,000 & 0,692 & 0,308 & 1,000 & 0,892 & 0,560 & 0,283 & 0,189 & 0,054 & 0,107 & 45 & 469 & $-0,9$ \\
\hline 238 & 469 & 0,017 & 0,397 & 0,019 & 0,296 & 0,923 & 0,745 & 0,267 & 1,000 & 0,854 & 0,764 & 0,474 & 0,169 & 0,080 & 0,107 & 135 & 469 & $-0,8$ \\
\hline 239 & 469 & 0,016 & 0,368 & 0,016 & 0,287 & 1,000 & 0,781 & 0,219 & 1,000 & 0,826 & 0,700 & 0,447 & 0,169 & 0,076 & 0,107 & 18 & 469 & $-0,8$ \\
\hline 240 & 469 & 0,049 & 1,061 & 0,070 & 0,867 & 0,694 & 0,817 & 0,357 & 0,545 & 1,355 & 0,370 & 0,481 & 0,410 & 0,197 & 0,169 & 40 & 469 & $-2,0$ \\
\hline 241 & 469 & 0,026 & 0,613 & 0,029 & 0,490 & 0,900 & 0,800 & 0,224 & 0,864 & 1,076 & 0,509 & 0,447 & 0,254 & 0,114 & 0,152 & 80 & 469 & $-1,3$ \\
\hline 242 & 469 & 0,037 & 0,765 & 0,046 & 0,592 & 0,812 & 0,773 & 0,294 & 0,801 & 1,118 & 0,637 & 0,627 & 0,273 & 0,171 & 0,169 & 41 & 469 & $-1,4$ \\
\hline 243 & 469 & 0,023 & 0,440 & 0,023 & 0,389 & 1,000 & 0,885 & 0,115 & 1,000 & 0,818 & 0,815 & 0,566 & 0,189 & 0,107 & 0,152 & 27 & 469 & $-0,9$ \\
\hline 244 & 469 & 0,088 & 1,357 & 0,112 & 1,112 & 0,782 & 0,819 & 0,283 & 0,597 & 1,294 & 0,351 & 0,427 & 0,563 & 0,241 & 0,214 & 59 & 469 & $-2,5$ \\
\hline 245 & 469 & 0,119 & 1,285 & 0,131 & 1,226 & 0,912 & 0,954 & 0,099 & 0,906 & 1,051 & 0,536 & 0,499 & 0,532 & 0,265 & 0,303 & 90 & 469 & $-2,4$ \\
\hline 246 & 469 & 0,024 & 0,461 & 0,026 & 0,317 & 0,944 & 0,688 & 0,317 & 1,000 & 0,832 & 1,082 & 0,671 & 0,169 & 0,114 & 0,152 & 84 & 469 & $-0,8$ \\
\hline
\end{tabular}




\begin{tabular}{|c|c|c|c|c|c|c|c|c|c|c|c|c|c|c|c|c|c|c|}
\hline 247 & 469 & 0,026 & 0,643 & 0,027 & 0,592 & 0,947 & 0,921 & 0,095 & 0,786 & 1,128 & 0,353 & 0,275 & 0,305 & 0,084 & 0,107 & 62 & 469 & $-1,6$ \\
\hline 248 & 469 & 0,016 & 0,368 & 0,016 & 0,245 & 1,000 & 0,667 & 0,333 & 1,000 & 0,826 & 0,778 & 0,500 & 0,161 & 0,080 & 0,107 & 45 & 469 & $-0,7$ \\
\hline 249 & 469 & 0,016 & 0,418 & 0,017 & 0,317 & 0,917 & 0,757 & 0,256 & 1,000 & 0,940 & 0,560 & 0,358 & 0,189 & 0,068 & 0,076 & 61 & 469 & $-0,9$ \\
\hline 250 & 469 & 0,023 & 0,461 & 0,024 & 0,338 & 0,941 & 0,734 & 0,273 & 1,000 & 0,858 & 1,019 & 0,671 & 0,169 & 0,114 & 0,152 & 90 & 469 & $-0,8$ \\
\hline 251 & 469 & 0,023 & 0,469 & 0,024 & 0,397 & 0,941 & 0,847 & 0,164 & 1,000 & 0,874 & 0,702 & 0,499 & 0,204 & 0,102 & 0,152 & 23 & 469 & $-1,0$ \\
\hline 252 & 469 & 0,090 & 1,285 & 0,108 & 1,196 & 0,840 & 0,931 & 0,174 & 0,688 & 1,206 & 0,378 & 0,412 & 0,552 & 0,227 & 0,227 & 79 & 469 & $-2,5$ \\
\hline 253 & 469 & 0,027 & 0,622 & 0,029 & 0,520 & 0,950 & 0,837 & 0,171 & 0,887 & 1,062 & 0,397 & 0,272 & 0,296 & 0,080 & 0,107 & 51 & 469 & $-1,6$ \\
\hline 254 & 469 & 0,062 & 1,480 & 0,093 & 1,243 & 0,662 & 0,840 & 0,374 & 0,354 & 1,680 & 0,179 & 0,178 & 0,663 & 0,118 & 0,152 & 53 & 469 & $-2,7$ \\
\hline 255 & 469 & 0,022 & 0,469 & 0,023 & 0,368 & 0,938 & 0,784 & 0,225 & 1,000 & 0,902 & 0,597 & 0,375 & 0,214 & 0,080 & 0,107 & 35 & 469 & $-1,1$ \\
\hline 256 & 469 & 0,056 & 0,837 & 0,062 & 0,714 & 0,907 & 0,854 & 0,173 & 1,000 & 0,998 & 0,621 & 0,550 & 0,339 & 0,186 & 0,214 & 31 & 469 & $-1,8$ \\
\hline 257 & 469 & 0,016 & 0,338 & 0,016 & 0,245 & 1,000 & 0,725 & 0,275 & 1,000 & 0,759 & 1,077 & 0,555 & 0,137 & 0,076 & 0,152 & 90 & 469 & $-0,5$ \\
\hline 258 & 469 & 0,044 & 0,837 & 0,055 & 0,735 & 0,816 & 0,879 & 0,221 & 0,798 & 1,120 & 0,443 & 0,381 & 0,357 & 0,136 & 0,152 & 56 & 469 & $-1,8$ \\
\hline 259 & 469 & 0,044 & 0,723 & 0,046 & 0,643 & 0,969 & 0,889 & 0,116 & 1,000 & 0,967 & 0,745 & 0,583 & 0,276 & 0,161 & 0,214 & 37 & 469 & $-1,5$ \\
\hline 260 & 469 & 0,014 & 0,317 & 0,014 & 0,194 & 1,000 & 0,613 & 0,387 & 1,000 & 0,746 & 0,979 & 0,555 & 0,137 & 0,076 & 0,107 & 18 & 469 & $-0,5$ \\
\hline 261 & 469 & 0,016 & 0,338 & 0,016 & 0,144 & 1,000 & 0,425 & 0,575 & 1,000 & 0,759 & 1,077 & 0,555 & 0,137 & 0,076 & 0,152 & 18 & 469 & $-0,5$ \\
\hline 262 & 469 & 0,039 & 0,693 & 0,042 & 0,613 & 0,931 & 0,884 & 0,135 & 1,000 & 0,994 & 0,564 & 0,453 & 0,296 & 0,134 & 0,152 & 44 & 469 & $-1,6$ \\
\hline 263 & 469 & 0,033 & 0,562 & 0,033 & 0,469 & 1,000 & 0,835 & 0,165 & 1,000 & 0,873 & 0,732 & 0,474 & 0,240 & 0,114 & 0,152 & 7 & 469 & $-1,3$ \\
\hline 264 & 469 & 0,023 & 0,469 & 0,026 & 0,368 & 0,889 & 0,784 & 0,243 & 1,000 & 0,874 & 0,815 & 0,555 & 0,189 & 0,105 & 0,152 & 38 & 469 & $-0,9$ \\
\hline 265 & 469 & 0,056 & 0,917 & 0,062 & 0,867 & 0,907 & 0,945 & 0,108 & 0,835 & 1,094 & 0,428 & 0,367 & 0,408 & 0,150 & 0,152 & 28 & 469 & $-2,0$ \\
\hline 266 & 469 & 0,023 & 0,440 & 0,023 & 0,317 & 1,000 & 0,721 & 0,279 & 1,000 & 0,818 & 1,019 & 0,671 & 0,169 & 0,114 & 0,152 & 63 & 469 & $-0,8$ \\
\hline 267 & 469 & 0,020 & 0,440 & 0,022 & 0,368 & 0,933 & 0,837 & 0,176 & 1,000 & 0,875 & 0,713 & 0,566 & 0,189 & 0,107 & 0,152 & 45 & 469 & $-0,9$ \\
\hline 268 & 469 & 0,019 & 0,469 & 0,022 & 0,368 & 0,867 & 0,784 & 0,254 & 1,000 & 0,969 & 0,517 & 0,375 & 0,214 & 0,080 & 0,107 & 46 & 469 & $-1,1$ \\
\hline 269 & 469 & 0,016 & 0,368 & 0,016 & 0,266 & 1,000 & 0,724 & 0,276 & 1,000 & 0,826 & 1,077 & 0,745 & 0,137 & 0,102 & 0,107 & 45 & 469 & $-0,5$ \\
\hline 270 & 469 & 0,143 & 2,409 & 0,187 & 2,299 & 0,769 & 0,954 & 0,235 & 0,311 & 1,794 & 0,144 & 0,139 & 1,126 & 0,156 & 0,152 & 71 & 469 & $-3,5$ \\
\hline 271 & 469 & 0,022 & 0,440 & 0,023 & 0,317 & 0,938 & 0,721 & 0,286 & 1,000 & 0,845 & 0,955 & 0,671 & 0,169 & 0,114 & 0,152 & 8 & 469 & $-0,8$ \\
\hline 272 & 469 & 0,036 & 0,685 & 0,042 & 0,511 & 0,862 & 0,747 & 0,288 & 0,961 & 1,020 & 0,707 & 0,539 & 0,254 & 0,137 & 0,152 & 12 & 469 & $-1,3$ \\
\hline 273 & 469 & 0,016 & 0,347 & 0,016 & 0,266 & 1,000 & 0,768 & 0,232 & 1,000 & 0,778 & 1,077 & 0,588 & 0,137 & 0,080 & 0,107 & 45 & 469 & $-0,5$ \\
\hline 274 & 469 & 0,037 & 0,714 & 0,039 & 0,643 & 0,963 & 0,899 & 0,107 & 0,919 & 1,043 & 0,447 & 0,329 & 0,326 & 0,107 & 0,152 & 38 & 469 & $-1,7$ \\
\hline 275 & 469 & 0,026 & 0,520 & 0,026 & 0,440 & 1,000 & 0,845 & 0,155 & 1,000 & 0,913 & 0,674 & 0,476 & 0,221 & 0,105 & 0,152 & 55 & 469 & $-1,1$ \\
\hline 276 & 469 & 0,033 & 0,672 & 0,036 & 0,664 & 0,920 & 0,987 & 0,081 & 0,918 & 1,044 & 0,480 & 0,362 & 0,296 & 0,107 & 0,169 & 48 & 469 & $-1,6$ \\
\hline 277 & 469 & 0,014 & 0,317 & 0,014 & 0,215 & 1,000 & 0,680 & 0,320 & 1,000 & 0,746 & 0,979 & 0,555 & 0,137 & 0,076 & 0,107 & 72 & 469 & $-0,5$ \\
\hline 278 & 469 & 0,050 & 1,243 & 0,082 & 0,951 & 0,614 & 0,765 & 0,452 & 0,408 & 1,565 & 0,305 & 0,407 & 0,458 & 0,186 & 0,152 & 17 & 469 & $-2,2$ \\
\hline 279 & 469 & 0,040 & 0,714 & 0,043 & 0,643 & 0,933 & 0,899 & 0,121 & 0,989 & 1,005 & 0,548 & 0,499 & 0,305 & 0,152 & 0,169 & 64 & 469 & $-1,6$ \\
\hline 280 & 469 & 0,017 & 0,368 & 0,017 & 0,287 & 1,000 & 0,781 & 0,219 & 1,000 & 0,791 & 0,899 & 0,485 & 0,156 & 0,076 & 0,152 & 82 & 469 & $-0,6$ \\
\hline 281 & 469 & 0,014 & 0,347 & 0,014 & 0,245 & 1,000 & 0,707 & 0,293 & 1,000 & 0,816 & 0,707 & 0,333 & 0,161 & 0,054 & 0,107 & 45 & 469 & $-0,7$ \\
\hline 282 & 469 & 0,016 & 0,389 & 0,017 & 0,266 & 0,917 & 0,685 & 0,326 & 1,000 & 0,873 & 0,700 & 0,400 & 0,169 & 0,068 & 0,076 & 69 & 469 & $-0,8$ \\
\hline 283 & 469 & 0,020 & 0,440 & 0,020 & 0,389 & 1,000 & 0,885 & 0,115 & 1,000 & 0,875 & 0,713 & 0,566 & 0,189 & 0,107 & 0,152 & 30 & 469 & $-0,9$ \\
\hline 284 & 469 & 0,019 & 0,440 & 0,020 & 0,338 & 0,929 & 0,769 & 0,242 & 1,000 & 0,908 & 0,974 & 0,728 & 0,156 & 0,114 & 0,152 & 0 & 469 & $-0,6$ \\
\hline 285 & 469 & 0,017 & 0,389 & 0,017 & 0,266 & 1,000 & 0,685 & 0,315 & 1,000 & 0,836 & 0,764 & 0,447 & 0,169 & 0,076 & 0,107 & 72 & 469 & $-0,8$ \\
\hline 286 & 469 & 0,090 & 1,366 & 0,121 & 1,255 & 0,750 & 0,919 & 0,263 & 0,609 & 1,281 & 0,391 & 0,438 & 0,542 & 0,238 & 0,214 & 57 & 469 & $-2,4$ \\
\hline 287 & 469 & 0,033 & 0,685 & 0,036 & 0,583 & 0,920 & 0,852 & 0,168 & 0,885 & 1,063 & 0,451 & 0,333 & 0,305 & 0,102 & 0,152 & 65 & 469 & $-1,6$ \\
\hline 288 & 469 & 0,017 & 0,347 & 0,017 & 0,296 & 1,000 & 0,854 & 0,146 & 1,000 & 0,745 & 1,528 & 0,894 & 0,120 & 0,107 & 0,107 & 90 & 469 & $-0,3$ \\
\hline 289 & 469 & 0,047 & 0,968 & 0,055 & 0,888 & 0,868 & 0,917 & 0,156 & 0,635 & 1,255 & 0,309 & 0,250 & 0,442 & 0,111 & 0,152 & 58 & 469 & $-2,1$ \\
\hline 290 & 469 & 0,016 & 0,338 & 0,016 & 0,245 & 1,000 & 0,725 & 0,275 & 1,000 & 0,759 & 1,077 & 0,555 & 0,137 & 0,076 & 0,152 & 90 & 469 & $-0,5$ \\
\hline 291 & 469 & 0,027 & 0,511 & 0,027 & 0,389 & 1,000 & 0,760 & 0,240 & 1,000 & 0,874 & 0,834 & 0,557 & 0,204 & 0,114 & 0,152 & 88 & 469 & $-1,0$ \\
\hline 292 & 469 & 0,014 & 0,317 & 0,014 & 0,275 & 1,000 & 0,867 & 0,133 & 1,000 & 0,746 & 1,273 & 0,632 & 0,120 & 0,076 & 0,107 & 162 & 469 & $-0,3$ \\
\hline 293 & 469 & 0,016 & 0,368 & 0,016 & 0,296 & 1,000 & 0,805 & 0,195 & 1,000 & 0,826 & 0,778 & 0,500 & 0,161 & 0,080 & 0,107 & 53 & 469 & $-0,7$ \\
\hline 294 & 469 & 0,042 & 0,765 & 0,044 & 0,664 & 0,935 & 0,867 & 0,148 & 0,893 & 1,058 & 0,415 & 0,375 & 0,357 & 0,134 & 0,152 & 59 & 469 & $-1,8$ \\
\hline 295 & 469 & 0,016 & 0,368 & 0,016 & 0,266 & 1,000 & 0,724 & 0,276 & 1,000 & 0,826 & 0,778 & 0,500 & 0,161 & 0,080 & 0,107 & 45 & 469 & $-0,7$ \\
\hline 296 & 469 & 0,151 & 1,910 & 0,192 & 1,686 & 0,784 & 0,883 & 0,246 & 0,519 & 1,388 & 0,287 & 0,292 & 0,818 & 0,239 & 0,169 & 79 & 469 & $-3,0$ \\
\hline 297 & 469 & 0,014 & 0,317 & 0,014 & 0,194 & 1,000 & 0,613 & 0,387 & 1,000 & 0,746 & 0,979 & 0,555 & 0,137 & 0,076 & 0,107 & 18 & 469 & $-0,5$ \\
\hline 298 & 469 & 0,022 & 0,418 & 0,022 & 0,368 & 1,000 & 0,879 & 0,121 & 1,000 & 0,805 & 0,955 & 0,632 & 0,169 & 0,107 & 0,152 & 30 & 469 & $-0,8$ \\
\hline 299 & 469 & 0,072 & 1,399 & 0,096 & 1,175 & 0,746 & 0,840 & 0,300 & 0,461 & 1,474 & 0,264 & 0,265 & 0,588 & 0,156 & 0,169 & 72 & 469 & $-2,6$ \\
\hline 300 & 469 & 0,020 & 0,389 & 0,020 & 0,296 & 1,000 & 0,761 & 0,239 & 1,000 & 0,774 & 1,371 & 0,832 & 0,137 & 0,114 & 0,152 & 0 & 469 & $-0,5$ \\
\hline 301 & 469 & 0,019 & 0,418 & 0,019 & 0,266 & 1,000 & 0,636 & 0,364 & 1,000 & 0,864 & 0,828 & 0,600 & 0,169 & 0,102 & 0,107 & 31 & 469 & $-0,8$ \\
\hline 302 & 469 & 0,040 & 1,061 & 0,055 & 0,917 & 0,737 & 0,865 & 0,296 & 0,448 & 1,493 & 0,210 & 0,187 & 0,494 & 0,092 & 0,076 & 60 & 469 & $-2,3$ \\
\hline 303 & 469 & 0,014 & 0,368 & 0,016 & 0,266 & 0,909 & 0,724 & 0,291 & 1,000 & 0,866 & 0,637 & 0,400 & 0,169 & 0,068 & 0,076 & 21 & 469 & $-0,8$ \\
\hline 304 & 469 & 0,049 & 0,765 & 0,055 & 0,634 & 0,895 & 0,829 & 0,201 & 1,000 & 0,977 & 0,817 & 0,676 & 0,276 & 0,186 & 0,214 & 165 & 469 & $-1,5$ \\
\hline
\end{tabular}




\begin{tabular}{|c|c|c|c|c|c|c|c|c|c|c|c|c|c|c|c|c|c|c|}
\hline 305 & 469 & 0,024 & 0,490 & 0,026 & 0,368 & 0,944 & 0,750 & 0,256 & 1,000 & 0,886 & 0,866 & 0,627 & 0,189 & 0,119 & 0,152 & 30 & 469 & $-0,9$ \\
\hline 306 & 469 & 0,016 & 0,347 & 0,016 & 0,266 & 1,000 & 0,768 & 0,232 & 1,000 & 0,778 & 1,077 & 0,588 & 0,137 & 0,080 & 0,107 & 45 & 469 & $-0,5$ \\
\hline 307 & 469 & 0,017 & 0,389 & 0,017 & 0,296 & 1,000 & 0,761 & 0,239 & 1,000 & 0,836 & 0,764 & 0,447 & 0,169 & 0,076 & 0,107 & 18 & 469 & $-0,8$ \\
\hline 308 & 469 & 0,032 & 0,816 & 0,044 & 0,744 & 0,710 & 0,912 & 0,303 & 0,596 & 1,295 & 0,330 & 0,333 & 0,349 & 0,116 & 0,152 & 39 & 469 & $-1,8$ \\
\hline 309 & 469 & 0,168 & 2,084 & 0,224 & 1,716 & 0,750 & 0,824 & 0,306 & 0,486 & 1,435 & 0,285 & 0,337 & 0,865 & 0,292 & 0,214 & 19 & 469 & $-3,1$ \\
\hline 310 & 469 & 0,023 & 0,490 & 0,026 & 0,418 & 0,889 & 0,854 & 0,184 & 1,000 & 0,913 & 0,702 & 0,529 & 0,204 & 0,108 & 0,152 & 27 & 469 & $-1,0$ \\
\hline 311 & 469 & 0,032 & 0,613 & 0,034 & 0,461 & 0,917 & 0,752 & 0,262 & 1,000 & 0,973 & 0,622 & 0,447 & 0,254 & 0,114 & 0,152 & 77 & 469 & $-1,3$ \\
\hline 312 & 469 & 0,024 & 0,490 & 0,026 & 0,368 & 0,944 & 0,750 & 0,256 & 1,000 & 0,886 & 0,676 & 0,500 & 0,214 & 0,107 & 0,152 & 43 & 469 & $-1,1$ \\
\hline 313 & 469 & 0,016 & 0,368 & 0,017 & 0,266 & 0,917 & 0,724 & 0,288 & 1,000 & 0,826 & 0,778 & 0,500 & 0,161 & 0,080 & 0,107 & 53 & 469 & $-0,7$ \\
\hline 314 & 469 & 0,027 & 0,532 & 0,029 & 0,296 & 0,950 & 0,556 & 0,447 & 1,000 & 0,910 & 0,834 & 0,557 & 0,204 & 0,114 & 0,152 & 5 & 469 & $-1,0$ \\
\hline 315 & 469 & 0,026 & 0,520 & 0,027 & 0,461 & 0,947 & 0,886 & 0,126 & 1,000 & 0,913 & 0,790 & 0,567 & 0,204 & 0,116 & 0,152 & 66 & 469 & $-1,0$ \\
\hline 316 & 469 & 0,017 & 0,520 & 0,022 & 0,410 & 0,800 & 0,788 & 0,291 & 0,800 & 1,118 & 0,849 & 0,707 & 0,161 & 0,114 & 0,107 & 45 & 469 & $-0,7$ \\
\hline 317 & 469 & 0,019 & 0,389 & 0,019 & 0,287 & 1,000 & 0,739 & 0,261 & 1,000 & 0,803 & 0,828 & 0,447 & 0,169 & 0,076 & 0,152 & 82 & 469 & $-0,8$ \\
\hline 318 & 469 & 0,014 & 0,347 & 0,016 & 0,245 & 0,909 & 0,707 & 0,307 & 1,000 & 0,816 & 0,979 & 0,588 & 0,137 & 0,080 & 0,107 & 37 & 469 & $-0,5$ \\
\hline 319 & 469 & 0,019 & 0,427 & 0,019 & 0,368 & 1,000 & 0,861 & 0,139 & 1,000 & 0,882 & 0,828 & 0,474 & 0,169 & 0,080 & 0,107 & 48 & 469 & $-0,8$ \\
\hline 320 & 469 & 0,017 & 0,368 & 0,017 & 0,317 & 1,000 & 0,862 & 0,138 & 1,000 & 0,791 & 1,175 & 0,745 & 0,137 & 0,102 & 0,107 & 135 & 469 & $-0,5$ \\
\hline 321 & 469 & 0,026 & 0,520 & 0,027 & 0,440 & 0,947 & 0,845 & 0,163 & 1,000 & 0,913 & 0,674 & 0,461 & 0,221 & 0,102 & 0,152 & 32 & 469 & $-1,1$ \\
\hline 322 & 469 & 0,020 & 0,440 & 0,022 & 0,338 & 0,933 & 0,769 & 0,240 & 1,000 & 0,875 & 0,713 & 0,448 & 0,189 & 0,085 & 0,107 & 63 & 469 & $-0,9$ \\
\hline 323 & 469 & 0,162 & 2,714 & 0,245 & 2,490 & 0,661 & 0,917 & 0,349 & 0,277 & 1,901 & 0,138 & 0,179 & 1,224 & 0,219 & 0,152 & 64 & 469 & $-3,6$ \\
\hline 324 & 469 & 0,032 & 0,583 & 0,033 & 0,482 & 0,957 & 0,826 & 0,179 & 1,000 & 0,926 & 0,622 & 0,447 & 0,254 & 0,114 & 0,152 & 77 & 469 & $-1,3$ \\
\hline 325 & 469 & 0,040 & 0,723 & 0,046 & 0,643 & 0,875 & 0,889 & 0,167 & 0,966 & 1,018 & 0,584 & 0,453 & 0,296 & 0,134 & 0,169 & 50 & 469 & $-1,6$ \\
\hline 326 & 469 & 0,056 & 0,858 & 0,060 & 0,735 & 0,929 & 0,857 & 0,160 & 0,955 & 1,023 & 0,558 & 0,427 & 0,357 & 0,153 & 0,169 & 61 & 469 & $-1,8$ \\
\hline 327 & 469 & 0,022 & 0,490 & 0,023 & 0,389 & 0,938 & 0,793 & 0,216 & 1,000 & 0,943 & 0,562 & 0,384 & 0,221 & 0,085 & 0,107 & 65 & 469 & $-1,1$ \\
\hline 328 & 469 & 0,014 & 0,317 & 0,014 & 0,194 & 1,000 & 0,613 & 0,387 & 1,000 & 0,746 & 0,979 & 0,555 & 0,137 & 0,076 & 0,107 & 18 & 469 & $-0,5$ \\
\hline 329 & 469 & 0,049 & 0,807 & 0,053 & 0,685 & 0,919 & 0,848 & 0,172 & 0,941 & 1,031 & 0,481 & 0,422 & 0,359 & 0,152 & 0,169 & 72 & 469 & $-1,8$ \\
\hline 330 & 469 & 0,014 & 0,368 & 0,016 & 0,266 & 0,909 & 0,724 & 0,291 & 1,000 & 0,866 & 0,637 & 0,400 & 0,169 & 0,068 & 0,076 & 69 & 469 & $-0,8$ \\
\hline 331 & 469 & 0,027 & 0,520 & 0,029 & 0,461 & 0,950 & 0,886 & 0,125 & 1,000 & 0,888 & 0,834 & 0,582 & 0,204 & 0,119 & 0,152 & 66 & 469 & $-1,0$ \\
\hline 332 & 469 & 0,050 & 0,837 & 0,057 & 0,714 & 0,875 & 0,854 & 0,193 & 0,901 & 1,054 & 0,501 & 0,427 & 0,357 & 0,153 & 0,152 & 29 & 469 & $-1,8$ \\
\hline 333 & 469 & 0,029 & 0,592 & 0,034 & 0,469 & 0,833 & 0,793 & 0,266 & 1,000 & 0,986 & 0,566 & 0,448 & 0,254 & 0,114 & 0,152 & 63 & 469 & $-1,3$ \\
\hline 334 & 469 & 0,095 & 1,581 & 0,119 & 1,551 & 0,795 & 0,981 & 0,206 & 0,476 & 1,449 & 0,225 & 0,229 & 0,732 & 0,168 & 0,214 & 68 & 469 & $-2,9$ \\
\hline 335 & 469 & 0,026 & 0,571 & 0,029 & 0,418 & 0,900 & 0,733 & 0,285 & 0,996 & 1,002 & 0,619 & 0,493 & 0,230 & 0,114 & 0,107 & 90 & 469 & $-1,2$ \\
\hline 336 & 469 & 0,022 & 0,490 & 0,023 & 0,368 & 0,938 & 0,750 & 0,258 & 1,000 & 0,943 & 0,531 & 0,333 & 0,227 & 0,076 & 0,152 & 11 & 469 & $-1,2$ \\
\hline 337 & 469 & 0,016 & 0,448 & 0,017 & 0,368 & 0,917 & 0,820 & 0,198 & 0,987 & 1,006 & 0,483 & 0,332 & 0,204 & 0,068 & 0,076 & 65 & 469 & $-1,0$ \\
\hline 338 & 469 & 0,063 & 1,061 & 0,080 & 0,828 & 0,786 & 0,781 & 0,307 & 0,705 & 1,191 & 0,578 & 0,573 & 0,373 & 0,214 & 0,214 & 7 & 469 & $-1,9$ \\
\hline 339 & 469 & 0,014 & 0,376 & 0,014 & 0,326 & 1,000 & 0,865 & 0,135 & 1,000 & 0,886 & 0,637 & 0,316 & 0,169 & 0,054 & 0,107 & 45 & 469 & $-0,8$ \\
\hline 340 & 469 & 0,098 & 2,008 & 0,139 & 1,712 & 0,701 & 0,853 & 0,333 & 0,304 & 1,814 & 0,163 & 0,191 & 0,872 & 0,166 & 0,169 & 52 & 469 & $-3,1$ \\
\hline 341 & 469 & 0,017 & 0,418 & 0,019 & 0,296 & 0,923 & 0,707 & 0,303 & 1,000 & 0,900 & 0,611 & 0,358 & 0,189 & 0,068 & 0,076 & 61 & 469 & $-0,9$ \\
\hline 342 & 469 & 0,016 & 0,440 & 0,019 & 0,317 & 0,846 & 0,721 & 0,318 & 1,000 & 0,987 & 0,483 & 0,315 & 0,204 & 0,064 & 0,076 & 78 & 469 & $-1,0$ \\
\hline 343 & 469 & 0,036 & 0,664 & 0,040 & 0,541 & 0,893 & 0,815 & 0,214 & 1,000 & 0,988 & 0,612 & 0,490 & 0,273 & 0,134 & 0,152 & 53 & 469 & $-1,4$ \\
\hline 344 & 469 & 0,100 & 1,886 & 0,136 & 1,784 & 0,737 & 0,946 & 0,269 & 0,355 & 1,678 & 0,164 & 0,174 & 0,883 & 0,154 & 0,152 & 60 & 469 & $-3,1$ \\
\hline 345 & 469 & 0,098 & 1,112 & 0,102 & 1,061 & 0,958 & 0,954 & 0,062 & 0,992 & 1,004 & 0,541 & 0,525 & 0,479 & 0,252 & 0,273 & 20 & 469 & $-2,3$ \\
\hline 346 & 469 & 0,014 & 0,347 & 0,014 & 0,266 & 1,000 & 0,768 & 0,232 & 1,000 & 0,816 & 0,979 & 0,588 & 0,137 & 0,080 & 0,107 & 58 & 469 & $-0,5$ \\
\hline 347 & 469 & 0,022 & 0,469 & 0,023 & 0,397 & 0,938 & 0,847 & 0,165 & 1,000 & 0,902 & 0,597 & 0,375 & 0,214 & 0,080 & 0,107 & 50 & 469 & $-1,1$ \\
\hline 348 & 469 & 0,014 & 0,347 & 0,016 & 0,287 & 0,909 & 0,829 & 0,194 & 1,000 & 0,816 & 0,979 & 0,588 & 0,137 & 0,080 & 0,107 & 72 & 469 & $-0,5$ \\
\hline $\begin{array}{l}340 \\
349\end{array}$ & 469 & 0,050 & 0,795 & 0,055 & 0,735 & 0,921 & 0,925 & 0,109 & 0,999 & 1,001 & 0,610 & 0,518 & 0,324 & 0,168 & 0,214 & 20 & 469 & $-1,7$ \\
\hline 350 & 469 & 0,020 & 0,418 & 0,022 & 0,338 & 0,933 & 0,808 & 0,203 & 1,000 & 0,833 & 0,891 & 0,600 & 0,169 & 0,102 & 0,107 & 63 & 469 & $-0,8$ \\
\hline 351 & 469 & 0,065 & 1,031 & 0,075 & 0,960 & 0,865 & 0,930 & 0,152 & 0,763 & 1,145 & 0,418 & 0,351 & 0,443 & 0,156 & 0,152 & 16 & 469 & $-2,1$ \\
\hline 352 & 469 & 0,026 & 0,592 & 0,027 & 0,490 & 0,947 & 0,828 & 0,179 & 0,927 & 1,039 & 0,509 & 0,401 & 0,254 & 0,102 & 0,107 & 31 & 469 & $-1,3$ \\
\hline 353 & 469 & 0,128 & 1,970 & 0,159 & 1,818 & 0,802 & 0,923 & 0,213 & 0,414 & 1,555 & 0,202 & 0,194 & 0,898 & 0,174 & 0,214 & 68 & 469 & $-3,2$ \\
\hline 354 & 469 & 0,037 & 0,786 & 0,044 & 0,706 & 0,839 & 0,898 & 0,191 & 0,758 & 1,148 & 0,404 & 0,304 & 0,343 & 0,104 & 0,152 & 12 & 469 & $-1,8$ \\
\hline 355 & 469 & 0,055 & 0,917 & 0,062 & 0,846 & 0,884 & 0,922 & 0,140 & 0,814 & 1,108 & 0,372 & 0,302 & 0,432 & 0,130 & 0,169 & 37 & 469 & $-2,1$ \\
\hline 356 & 469 & 0,014 & 0,368 & 0,016 & 0,266 & 0,909 & 0,724 & 0,291 & 1,000 & 0,866 & 0,749 & 0,485 & 0,156 & 0,076 & 0,107 & 175 & 469 & $-0,6$ \\
\hline 357 & 469 & 0,019 & 0,490 & 0,022 & 0,389 & 0,867 & 0,793 & 0,246 & 0,975 & 1,013 & 0,571 & 0,371 & 0,204 & 0,076 & 0,076 & 75 & 469 & $-1,0$ \\
\hline 358 & 469 & 0,020 & 0,418 & 0,022 & 0,338 & 0,933 & 0,808 & 0,203 & 1,000 & 0,833 & 1,049 & 0,728 & 0,156 & 0,114 & 0,152 & 7 & 469 & $-0,6$ \\
\hline 359 & 469 & 0,023 & 0,469 & 0,024 & 0,418 & 0,941 & 0,892 & 0,123 & 1,000 & 0,874 & 0,815 & 0,566 & 0,189 & 0,107 & 0,152 & 49 & 469 & $-0,9$ \\
\hline 360 & 469 & 0,024 & 0,541 & 0,029 & 0,397 & 0,850 & 0,735 & 0,305 & 1,000 & 0,977 & 0,637 & 0,485 & 0,221 & 0,107 & 0,152 & 38 & 469 & $-1,1$ \\
\hline 361 & 469 & 0,016 & 0,368 & 0,016 & 0,275 & 1,000 & 0,747 & 0,253 & 1,000 & 0,826 & 0,824 & 0,485 & 0,156 & 0,076 & 0,107 & 0 & 469 & $-0,6$ \\
\hline 362 & 469 & 0,072 & 1,091 & 0,082 & 0,989 & 0,877 & 0,907 & 0,154 & 0,758 & 1,149 & 0,388 & 0,367 & 0,485 & 0,178 & 0,169 & 44 & 469 & $-2,3$ \\
\hline
\end{tabular}




\begin{tabular}{|c|c|c|c|c|c|c|c|c|c|c|c|c|c|c|c|c|c|c|}
\hline 363 & 469 & 0,020 & 0,448 & 0,022 & 0,368 & 0,933 & 0,820 & 0,191 & 1,000 & 0,892 & 0,713 & 0,424 & 0,189 & 0,080 & 0,107 & 40 & 469 & $-0,9$ \\
\hline 364 & 469 & 0,123 & 1,978 & 0,174 & 1,450 & 0,711 & 0,733 & 0,394 & 0,396 & 1,589 & 0,403 & 0,564 & 0,625 & 0,352 & 0,169 & 14 & 469 & $-2,6$ \\
\hline 365 & 469 & 0,024 & 0,562 & 0,029 & 0,461 & 0,850 & 0,819 & 0,235 & 0,970 & 1,015 & 0,481 & 0,334 & 0,254 & 0,085 & 0,107 & 68 & 469 & $-1,3$ \\
\hline 366 & 469 & 0,040 & 0,917 & 0,055 & 0,774 & 0,737 & 0,843 & 0,306 & 0,600 & 1,291 & 0,357 & 0,348 & 0,379 & 0,132 & 0,107 & 52 & 469 & $-1,9$ \\
\hline 367 & 469 & 0,016 & 0,418 & 0,019 & 0,296 & 0,846 & 0,707 & 0,331 & 1,000 & 0,940 & 0,560 & 0,358 & 0,189 & 0,068 & 0,076 & 58 & 469 & $-0,9$ \\
\hline 368 & 469 & 0,019 & 0,397 & 0,019 & 0,296 & 1,000 & 0,745 & 0,255 & 1,000 & 0,821 & 0,828 & 0,474 & 0,169 & 0,080 & 0,107 & 55 & 469 & $-0,8$ \\
\hline 369 & 469 & 0,016 & 0,338 & 0,016 & 0,194 & 1,000 & 0,575 & 0,425 & 1,000 & 0,759 & 1,077 & 0,555 & 0,137 & 0,076 & 0,152 & 162 & 469 & $-0,5$ \\
\hline 370 & 469 & 0,022 & 0,469 & 0,022 & 0,418 & 1,000 & 0,892 & 0,108 & 1,000 & 0,902 & 0,764 & 0,555 & 0,189 & 0,105 & 0,152 & 49 & 469 & $-0,9$ \\
\hline 371 & 469 & 0,386 & 3,027 & 0,469 & 2,642 & 0,823 & 0,873 & 0,218 & 0,529 & 1,375 & 0,317 & 0,395 & 1,246 & 0,492 & 0,508 & 67 & 469 & $-3,6$ \\
\hline 372 & 469 & 0,019 & 0,389 & 0,019 & 0,287 & 1,000 & 0,739 & 0,261 & 1,000 & 0,803 & 0,920 & 0,633 & 0,161 & 0,102 & 0,152 & 45 & 469 & $-0,7$ \\
\hline 373 & 469 & 0,019 & 0,389 & 0,019 & 0,287 & 1,000 & 0,739 & 0,261 & 1,000 & 0,803 & 0,828 & 0,447 & 0,169 & 0,076 & 0,152 & 82 & 469 & $-0,8$ \\
\hline 374 & 469 & 0,050 & 0,989 & 0,060 & 0,909 & 0,833 & 0,919 & 0,185 & 0,645 & 1,245 & 0,299 & 0,250 & 0,462 & 0,116 & 0,152 & 59 & 469 & $-2,2$ \\
\hline 375 & 469 & 0,044 & 0,706 & 0,047 & 0,604 & 0,939 & 0,856 & 0,156 & 1,000 & 0,944 & 0,681 & 0,510 & 0,288 & 0,147 & 0,152 & 76 & 469 & $-1,5$ \\
\hline 376 & 469 & 0,017 & 0,520 & 0,020 & 0,418 & 0,857 & 0,805 & 0,242 & 0,800 & 1,118 & 0,373 & 0,221 & 0,243 & 0,054 & 0,076 & 45 & 469 & $-1,3$ \\
\hline 377 & 469 & 0,016 & 0,347 & 0,016 & 0,338 & 1,000 & 0,975 & 0,025 & 1,000 & 0,778 & 0,875 & 0,500 & 0,152 & 0,076 & 0,152 & 0 & 469 & $-0,6$ \\
\hline 378 & 469 & 0,046 & 0,917 & 0,059 & 0,786 & 0,780 & 0,857 & 0,262 & 0,686 & 1,208 & 0,392 & 0,431 & 0,386 & 0,166 & 0,152 & 5 & 469 & $-1,9$ \\
\hline 379 & 469 & 0,020 & 0,490 & 0,022 & 0,397 & 0,933 & 0,811 & 0,201 & 1,000 & 0,976 & 0,524 & 0,307 & 0,221 & 0,068 & 0,076 & 28 & 469 & $-1,1$ \\
\hline 380 & 469 & 0,083 & 1,082 & 0,092 & 0,951 & 0,906 & 0,879 & 0,153 & 0,893 & 1,058 & 0,591 & 0,530 & 0,423 & 0,224 & 0,214 & 99 & 469 & $-2,1$ \\
\hline 381 & 469 & 0,020 & 0,440 & 0,022 & 0,317 & 0,933 & 0,721 & 0,287 & 1,000 & 0,875 & 0,713 & 0,537 & 0,189 & 0,102 & 0,152 & 60 & 469 & $-0,9$ \\
\hline 382 & 469 & 0,027 & 0,532 & 0,030 & 0,368 & 0,905 & 0,691 & 0,324 & 1,000 & 0,910 & 0,712 & 0,499 & 0,221 & 0,110 & 0,152 & 172 & 469 & $-1,1$ \\
\hline 383 & 469 & 0,014 & 0,347 & 0,014 & 0,245 & 1,000 & 0,707 & 0,293 & 1,000 & 0,816 & 0,707 & 0,333 & 0,161 & 0,054 & 0,107 & 45 & 469 & $\begin{array}{l}-0,7 \\
\end{array}$ \\
\hline 384 & 469 & 0,027 & 0,511 & 0,027 & 0,347 & 1,000 & 0,678 & 0,322 & 1,000 & 0,874 & 0,834 & 0,557 & 0,204 & 0,114 & 0,152 & 175 & 469 & $-1,0$ \\
\hline 385 & 469 & 0,017 & 0,440 & 0,019 & 0,317 & 0,923 & 0,721 & 0,289 & 1,000 & 0,945 & 0,527 & 0,353 & 0,204 & 0,072 & 0,076 & 69 & 469 & $-1,0$ \\
\hline 386 & 469 & 0,022 & 0,541 & 0,026 & 0,397 & 0,833 & 0,735 & 0,313 & 0,924 & 1,040 & 0,477 & 0,354 & 0,240 & 0,085 & 0,107 & 75 & 469 & $-1,3$ \\
\hline 387 & 469 & 0,033 & 0,592 & 0,034 & 0,511 & 0,958 & 0,864 & 0,142 & 1,000 & 0,919 & 0,732 & 0,550 & 0,240 & 0,132 & 0,152 & 24 & 469 & $-1,3$ \\
\hline 388 & 469 & 0,027 & 0,664 & 0,037 & 0,511 & 0,731 & 0,771 & 0,354 & 0,778 & 1,134 & 0,605 & 0,632 & 0,240 & 0,152 & 0,107 & 119 & 469 & $-1,3$ \\
\hline 389 & 469 & 0,036 & 0,664 & 0,039 & 0,541 & 0,926 & 0,815 & 0,199 & 1,000 & 0,988 & 0,490 & 0,439 & 0,305 & 0,134 & 0,152 & 60 & 469 & $-1,6$ \\
\hline 390 & 469 & 0,014 & 0,317 & 0,014 & 0,194 & 1,000 & 0,613 & 0,387 & 1,000 & 0,746 & 0,979 & 0,555 & 0,137 & 0,076 & 0,107 & 18 & 469 & $-0,5$ \\
\hline 391 & 469 & 0,023 & 0,520 & 0,024 & 0,418 & 0,941 & 0,805 & 0,204 & 1,000 & 0,968 & 0,551 & 0,416 & 0,230 & 0,096 & 0,152 & 71 & 469 & $-1,2$ \\
\hline 392 & 469 & 0,063 & 0,930 & 0,069 & 0,786 & 0,917 & 0,846 & 0,176 & 0,918 & 1,044 & 0,514 & 0,479 & 0,395 & 0,189 & 0,227 & 79 & 469 & $-2,0$ \\
\hline 393 & 469 & 0,029 & 0,643 & 0,034 & 0,520 & 0,833 & 0,809 & 0,253 & 0,873 & 1,070 & 0,439 & 0,374 & 0,288 & 0,108 & 0,152 & 108 & 469 & $-1,5$ \\
\hline 394 & 469 & 0,016 & 0,389 & 0,017 & 0,317 & 0,917 & 0,815 & 0,203 & 1,000 & 0,873 & 0,700 & 0,425 & 0,169 & 0,072 & 0,076 & 79 & 469 & $-0,8$ \\
\hline 395 & 469 & 0,125 & 1,742 & 0,141 & 1,683 & 0,888 & 0,966 & 0,117 & 0,517 & 1,391 & 0,240 & 0,222 & 0,813 & 0,180 & 0,214 & 59 & 469 & $-3,0$ \\
\hline 396 & 469 & 0,014 & 0,347 & 0,016 & 0,245 & 0,909 & 0,707 & 0,307 & 1,000 & 0,816 & 1,273 & 0,849 & 0,120 & 0,102 & 0,107 & 34 & 469 & $-0,3$ \\
\hline 397 & 469 & 0,056 & 1,061 & 0,067 & 0,960 & 0,830 & 0,904 & 0,195 & 0,625 & 1,265 & 0,316 & 0,281 & 0,475 & 0,133 & 0,152 & 66 & 469 & $\begin{array}{l}-2,2 \\
\text {. }\end{array}$ \\
\hline 398 & 469 & 0,016 & 0,418 & 0,019 & 0,338 & 0,846 & 0,808 & 0,246 & 1,000 & 0,940 & 0,560 & 0,358 & 0,189 & 0,068 & 0,076 & 69 & 469 & $-0,9$ \\
\hline 399 & 469 & 0,098 & 1,476 & 0,116 & 1,416 & 0,840 & 0,960 & 0,165 & 0,563 & 1,333 & 0,293 & 0,288 & 0,652 & 0,187 & 0,214 & 55 & 469 & $-2,7$ \\
\hline 400 & 469 & 0,017 & 0,397 & 0,019 & 0,317 & 0,923 & 0,798 & 0,217 & 1,000 & 0,854 & 0,764 & 0,474 & 0,169 & 0,080 & 0,107 & 45 & 469 & $-0,8$ \\
\hline 401 & 469 & 0,034 & 0,888 & 0,047 & 0,723 & 0,727 & 0,814 & 0,330 & 0,549 & 1,350 & 0,270 & 0,251 & 0,403 & 0,101 & 0,107 & 52 & 469 & $-2,0$ \\
\hline 402 & 469 & 0,079 & 1,184 & 0,090 & 0,989 & 0,873 & 0,836 & 0,208 & 0,708 & 1,189 & 0,427 & 0,442 & 0,485 & 0,214 & 0,214 & 42 & 469 & $-2,3$ \\
\hline 403 & 469 & 0,016 & 0,478 & 0,020 & 0,317 & 0,786 & 0,663 & 0,399 & 0,868 & 1,073 & 0,483 & 0,394 & 0,204 & 0,080 & 0,107 & 72 & 469 & $-1,0$ \\
\hline 404 & 469 & 0,050 & 0,828 & 0,056 & 0,727 & 0,897 & 0,877 & 0,160 & 0,920 & 1,043 & 0,495 & 0,403 & 0,359 & 0,145 & 0,152 & 85 & 469 & $-1,8$ \\
\hline 405 & 469 & 0,017 & 0,368 & 0,017 & 0,245 & 1,000 & 0,667 & 0,333 & 1,000 & 0,791 & 1,175 & 0,784 & 0,137 & 0,107 & 0,152 & 135 & 469 & $-0,5$ \\
\hline 406 & 469 & 0,020 & 0,461 & 0,022 & 0,389 & 0,933 & 0,844 & 0,170 & 1,000 & 0,917 & 0,686 & 0,392 & 0,193 & 0,076 & 0,107 & 170 & 469 & $-0,9$ \\
\hline 407 & 469 & 0,027 & 0,672 & 0,034 & 0,622 & 0,792 & 0,924 & 0,222 & 0,758 & 1,149 & 0,465 & 0,390 & 0,273 & 0,107 & 0,107 & 48 & 469 & $-1,4$ \\
\hline 408 & 469 & 0,053 & 0,909 & 0,063 & 0,807 & 0,841 & 0,888 & 0,194 & 0,808 & 1,113 & 0,453 & 0,392 & 0,386 & 0,152 & 0,152 & 10 & 469 & $-1,9$ \\
\hline 409 & 469 & 0,022 & 0,541 & 0,027 & 0,418 & 0,789 & 0,773 & 0,309 & 0,924 & 1,040 & 0,562 & 0,461 & 0,221 & 0,102 & 0,152 & 45 & 469 & $-1,1$ \\
\hline 410 & 469 & 0,023 & 0,520 & 0,023 & 0,469 & 1,000 & 0,902 & 0,098 & 1,000 & 0,968 & 0,453 & 0,316 & 0,254 & 0,080 & 0,107 & 32 & 469 & $-1,3$ \\
\hline 411 & 469 & 0,019 & 0,368 & 0,019 & 0,287 & 1,000 & 0,781 & 0,219 & 1,000 & 0,760 & 1,273 & 0,784 & 0,137 & 0,107 & 0,152 & 45 & 469 & $-0,5$ \\
\hline 412 & 469 & 0,024 & 0,520 & 0,024 & 0,440 & 1,000 & 0,845 & 0,155 & 1,000 & 0,939 & 0,528 & 0,331 & 0,243 & 0,080 & 0,107 & 52 & 469 & $-1,3$ \\
\hline 413 & 469 & 0,040 & 1,061 & 0,055 & 0,968 & 0,737 & 0,912 & 0,277 & 0,448 & 1,493 & 0,210 & 0,206 & 0,494 & 0,102 & 0,152 & 27 & 469 & $-2,3$ \\
\hline 414 & 469 & 0,033 & 0,685 & 0,034 & 0,562 & 0,958 & 0,821 & 0,184 & 0,885 & 1,063 & 0,451 & 0,248 & 0,305 & 0,076 & 0,152 & 2 & 469 & $-1,6$ \\
\hline 415 & 469 & 0,085 & 1,357 & 0,108 & 1,213 & 0,787 & 0,894 & 0,238 & 0,578 & 1,316 & 0,307 & 0,369 & 0,593 & 0,219 & 0,214 & 60 & 469 & $-2,6$ \\
\hline 416 & 469 & 0,079 & 1,154 & 0,093 & 1,010 & 0,846 & 0,876 & 0,198 & 0,745 & 1,159 & 0,515 & 0,494 & 0,442 & 0,218 & 0,152 & 55 & 469 & $-2,1$ \\
\hline 417 & 469 & 0,033 & 0,765 & 0,042 & 0,693 & 0,793 & 0,906 & 0,227 & 0,708 & 1,188 & 0,451 & 0,439 & 0,305 & 0,134 & 0,152 & 43 & 469 & $-1,6$ \\
\hline 418 & 469 & 0,017 & 0,469 & 0,020 & 0,397 & 0,857 & 0,847 & 0,209 & 0,983 & 1,009 & 0,449 & 0,286 & 0,221 & 0,063 & 0,076 & 30 & 469 & $-1,1$ \\
\hline 419 & 469 & 0,017 & 0,389 & 0,017 & 0,194 & 1,000 & 0,500 & 0,500 & 1,000 & 0,836 & 0,899 & 0,485 & 0,156 & 0,076 & 0,107 & 0 & 469 & $-0,6$ \\
\hline 420 & 469 & 0,042 & 0,909 & 0,049 & 0,837 & 0,853 & 0,921 & 0,167 & 0,633 & 1,257 & 0,295 & 0,267 & 0,423 & 0,113 & 0,152 & 66 & 469 & $-2,1$ \\
\hline
\end{tabular}




\begin{tabular}{|c|c|c|c|c|c|c|c|c|c|c|c|c|c|c|c|c|c|c|}
\hline 421 & 469 & 0,019 & 0,389 & 0,019 & 0,296 & 1,000 & 0,761 & 0,239 & 1,000 & 0,803 & 0,828 & 0,447 & 0,169 & 0,076 & 0,152 & 8 & 469 & $-0,8$ \\
\hline 422 & 469 & 0,023 & 0,469 & 0,024 & 0,368 & 0,941 & 0,784 & 0,224 & 1,000 & 0,874 & 0,702 & 0,499 & 0,204 & 0,102 & 0,152 & 67 & 469 & $-1,0$ \\
\hline 423 & 469 & 0,098 & 1,522 & 0,129 & 1,205 & 0,756 & 0,792 & 0,321 & 0,530 & 1,374 & 0,433 & 0,449 & 0,536 & 0,241 & 0,214 & 13 & 469 & $-2,4$ \\
\hline 424 & 469 & 0,019 & 0,418 & 0,019 & 0,317 & 1,000 & 0,757 & 0,243 & 1,000 & 0,864 & 0,662 & 0,424 & 0,189 & 0,080 & 0,107 & 55 & 469 & $-0,9$ \\
\hline 425 & 469 & 0,066 & 0,909 & 0,070 & 0,858 & 0,939 & 0,944 & 0,083 & 1,000 & 0,998 & 0,604 & 0,529 & 0,373 & 0,197 & 0,214 & 24 & 469 & $-1,9$ \\
\hline 426 & 469 & 0,014 & 0,440 & 0,016 & 0,389 & 0,909 & 0,885 & 0,147 & 0,933 & 1,035 & 0,490 & 0,196 & 0,193 & 0,038 & 0,076 & 0 & 469 & $-0,9$ \\
\hline 427 & 469 & 0,036 & 0,583 & 0,036 & 0,469 & 1,000 & 0,805 & 0,195 & 1,000 & 0,869 & 0,796 & 0,474 & 0,240 & 0,114 & 0,152 & 0 & 469 & $-1,3$ \\
\hline 428 & 469 & 0,413 & 2,693 & 0,469 & 2,570 & 0,881 & 0,954 & 0,128 & 0,716 & 1,182 & 0,421 & 0,518 & 1,119 & 0,579 & 0,530 & 34 & 469 & $-3,5$ \\
\hline 429 & 469 & 0,062 & 1,175 & 0,073 & 1,073 & 0,843 & 0,914 & 0,179 & 0,562 & 1,334 & 0,258 & 0,217 & 0,552 & 0,120 & 0,152 & 75 & 469 & $-2,5$ \\
\hline 430 & 469 & 0,022 & 0,469 & 0,023 & 0,368 & 0,938 & 0,784 & 0,225 & 1,000 & 0,902 & 0,764 & 0,555 & 0,189 & 0,105 & 0,107 & 50 & 469 & $-0,9$ \\
\hline 431 & 469 & 0,017 & 0,541 & 0,024 & 0,368 & 0,706 & 0,680 & 0,435 & 0,739 & 1,163 & 0,527 & 0,475 & 0,204 & 0,097 & 0,076 & 21 & 469 & $-1,0$ \\
\hline 432 & 469 & 0,057 & 0,828 & 0,062 & 0,685 & 0,930 & 0,827 & 0,187 & 1,000 & 0,975 & 0,599 & 0,534 & 0,349 & 0,186 & 0,214 & 63 & 469 & $-1,8$ \\
\hline 433 & 469 & 0,037 & 0,837 & 0,042 & 0,735 & 0,897 & 0,879 & 0,159 & 0,669 & 1,222 & 0,312 & 0,218 & 0,390 & 0,085 & 0,107 & 63 & 469 & $-2,0$ \\
\hline 434 & 469 & 0,017 & 0,418 & 0,019 & 0,338 & 0,923 & 0,808 & 0,207 & 1,000 & 0,900 & 0,611 & 0,424 & 0,189 & 0,080 & 0,107 & 72 & 469 & $-0,9$ \\
\hline 435 & 469 & 0,019 & 0,418 & 0,020 & 0,368 & 0,929 & 0,879 & 0,141 & 1,000 & 0,864 & 0,828 & 0,600 & 0,169 & 0,102 & 0,107 & 45 & 469 & $-0,8$ \\
\hline 436 & 469 & 0,036 & 0,816 & 0,043 & 0,744 & 0,833 & 0,912 & 0,188 & 0,677 & 1,215 & 0,325 & 0,286 & 0,375 & 0,107 & 0,107 & 43 & 469 & $-1,9$ \\
\hline 437 & 469 & 0,079 & 1,695 & 0,109 & 1,543 & 0,724 & 0,910 & 0,291 & 0,345 & 1,702 & 0,156 & 0,156 & 0,803 & 0,125 & 0,107 & 77 & 469 & $-3,0$ \\
\hline 438 & 469 & 0,023 & 0,490 & 0,023 & 0,389 & 1,000 & 0,793 & 0,207 & 1,000 & 0,913 & 0,637 & 0,491 & 0,214 & 0,105 & 0,152 & 38 & 469 & $-1,1$ \\
\hline 439 & 469 & 0,023 & 0,490 & 0,023 & 0,389 & 1,000 & 0,793 & 0,207 & 1,000 & 0,913 & 0,566 & 0,333 & 0,227 & 0,076 & 0,152 & 5 & 469 & $-1,2$ \\
\hline 440 & 469 & 0,014 & 0,338 & 0,016 & 0,194 & 0,909 & 0,575 & 0,435 & 1,000 & 0,796 & 0,979 & 0,555 & 0,137 & 0,076 & 0,152 & 162 & 469 & $-0,5$ \\
\hline 441 & 469 & 0,016 & 0,368 & 0,016 & 0,266 & 1,000 & 0,724 & 0,276 & 1,000 & 0,826 & 0,700 & 0,447 & 0,169 & 0,076 & 0,107 & 72 & 469 & $-0,8$ \\
\hline 442 & 469 & 0,014 & 0,418 & 0,017 & 0,275 & 0,833 & 0,657 & 0,382 & 1,000 & 0,986 & 0,490 & 0,351 & 0,193 & 0,068 & 0,076 & 18 & 469 & $-0,9$ \\
\hline 443 & 469 & 0,043 & 1,124 & 0,063 & 1,023 & 0,682 & 0,910 & 0,331 & 0,428 & 1,529 & 0,206 & 0,208 & 0,515 & 0,107 & 0,107 & 76 & 469 & $-2,4$ \\
\hline 444 & 469 & 0,014 & 0,338 & 0,014 & 0,245 & 1,000 & 0,725 & 0,275 & 1,000 & 0,796 & 0,979 & 0,555 & 0,137 & 0,076 & 0,107 & 72 & 469 & $-0,5$ \\
\hline 445 & 469 & 0,027 & 0,600 & 0,032 & 0,541 & 0,864 & 0,901 & 0,168 & 0,950 & 1,026 & 0,538 & 0,414 & 0,254 & 0,105 & 0,152 & 51 & 469 & $-1,3$ \\
\hline 446 & 469 & 0,016 & 0,427 & 0,019 & 0,347 & 0,846 & 0,812 & 0,243 & 1,000 & 0,959 & 0,824 & 0,673 & 0,156 & 0,105 & 0,076 & 59 & 469 & $-0,6$ \\
\hline 447 & 469 & 0,029 & 0,499 & 0,029 & 0,418 & 1,000 & 0,839 & 0,161 & 1,000 & 0,831 & 0,979 & 0,693 & 0,193 & 0,134 & 0,169 & 106 & 469 & $-0,9$ \\
\hline 448 & 469 & 0,022 & 0,418 & 0,022 & 0,317 & 1,000 & 0,757 & 0,243 & 1,000 & 0,805 & 0,955 & 0,632 & 0,169 & 0,107 & 0,152 & 70 & 469 & $-0,8$ \\
\hline 449 & 469 & 0,065 & 0,938 & 0,072 & 0,816 & 0,900 & 0,869 & 0,164 & 0,921 & 1,042 & 0,674 & 0,631 & 0,349 & 0,220 & 0,214 & 18 & 469 & $-1,8$ \\
\hline 450 & 469 & 0,020 & 0,490 & 0,024 & 0,440 & 0,824 & 0,896 & 0,205 & 1,000 & 0,976 & 0,557 & 0,396 & 0,214 & 0,085 & 0,107 & 68 & 469 & $-1,1$ \\
\hline 451 & 469 & 0,077 & 1,010 & 0,079 & 0,917 & 0,982 & 0,908 & 0,094 & 0,954 & 1,024 & 0,471 & 0,460 & 0,458 & 0,210 & 0,214 & 67 & 469 & $-2,2$ \\
\hline 452 & 469 & 0,072 & 1,480 & 0,095 & 1,429 & 0,758 & 0,966 & 0,245 & 0,412 & 1,558 & 0,188 & 0,193 & 0,696 & 0,134 & 0,152 & 68 & 469 & $-2,8$ \\
\hline 453 & 469 & 0,042 & 0,858 & 0,049 & 0,786 & 0,853 & 0,916 & 0,169 & 0,710 & 1,187 & 0,348 & 0,289 & 0,390 & 0,113 & 0,152 & 71 & 469 & $-2,0$ \\
\hline 454 & 469 & 0,024 & 0,490 & 0,024 & 0,440 & 1,000 & 0,896 & 0,104 & 1,000 & 0,886 & 0,637 & 0,485 & 0,221 & 0,107 & 0,152 & 24 & 469 & $-1,1$ \\
\hline 455 & 469 & 0,042 & 0,786 & 0,047 & 0,706 & 0,879 & 0,898 & 0,159 & 0,846 & 1,087 & 0,410 & 0,347 & 0,359 & 0,125 & 0,152 & 75 & 469 & $-1,8$ \\
\hline 456 & 469 & 0,083 & 0,960 & 0,086 & 0,930 & 0,967 & 0,969 & 0,045 & 1,000 & 0,938 & 0,761 & 0,711 & 0,373 & 0,265 & 0,303 & 20 & 469 & $-1,9$ \\
\hline 457 & 469 & 0,024 & 0,613 & 0,032 & 0,440 & 0,773 & 0,717 & 0,363 & 0,816 & 1,107 & 0,637 & 0,607 & 0,221 & 0,134 & 0,152 & 2 & 469 & $-1,1$ \\
\hline 458 & 469 & 0,085 & 1,255 & 0,102 & 1,124 & 0,831 & 0,895 & 0,199 & 0,675 & 1,217 & 0,366 & 0,373 & 0,542 & 0,202 & 0,214 & 81 & 469 & $-2,4$ \\
\hline 459 & 469 & 0,024 & 0,541 & 0,027 & 0,440 & 0,895 & 0,812 & 0,215 & 1,000 & 0,977 & 0,833 & 0,761 & 0,193 & 0,147 & 0,152 & 26 & 469 & $-0,9$ \\
\hline 460 & 469 & 0,017 & 0,418 & 0,020 & 0,296 & 0,857 & 0,707 & 0,326 & 1,000 & 0,900 & 0,899 & 0,728 & 0,156 & 0,114 & 0,107 & 90 & 469 & $-0,6$ \\
\hline 461 & 469 & 0,195 & 1,704 & 0,208 & 1,632 & 0,938 & 0,958 & 0,075 & 0,845 & 1,088 & 0,500 & 0,470 & 0,705 & 0,331 & 0,339 & 40 & 469 & $-2,8$ \\
\hline 462 & 469 & 0,022 & 0,461 & 0,022 & 0,338 & 1,000 & 0,734 & 0,266 & 1,000 & 0,886 & 0,659 & 0,371 & 0,204 & 0,076 & 0,152 & 79 & 469 & $-1,0$ \\
\hline 463 & 469 & 0,016 & 0,368 & 0,016 & 0,296 & 1,000 & 0,805 & 0,195 & 1,000 & 0,826 & 0,700 & 0,447 & 0,169 & 0,076 & 0,107 & 18 & 469 & $-0,8$ \\
\hline 464 & 469 & 0,014 & 0,368 & 0,016 & 0,245 & 0,909 & 0,667 & 0,346 & 1,000 & 0,866 & 0,979 & 0,745 & 0,137 & 0,102 & 0,076 & 58 & 469 & $-0,5$ \\
\hline 465 & 469 & 0,017 & 0,418 & 0,019 & 0,347 & 0,923 & 0,828 & 0,188 & 1,000 & 0,900 & 0,611 & 0,424 & 0,189 & 0,080 & 0,107 & 42 & 469 & $-0,9$ \\
\hline 466 & 469 & 0,030 & 0,613 & 0,034 & 0,482 & 0,875 & 0,786 & 0,248 & 1,000 & 0,996 & 0,668 & 0,474 & 0,240 & 0,114 & 0,152 & 80 & 469 & $-1,3$ \\
\hline 467 & 469 & 0,024 & 0,541 & 0,027 & 0,440 & 0,895 & 0,812 & 0,215 & 1,000 & 0,977 & 0,528 & 0,349 & 0,243 & 0,085 & 0,107 & 56 & 469 & $-1,3$ \\
\hline 468 & 469 & 0,017 & 0,397 & 0,019 & 0,368 & 0,923 & 0,925 & 0,107 & 1,000 & 0,854 & 0,899 & 0,651 & 0,156 & 0,102 & 0,152 & 30 & 469 & $-0,6$ \\
\hline 469 & 469 & 0,044 & 1,010 & 0,059 & 0,837 & 0,756 & 0,828 & 0,298 & 0,548 & 1,351 & 0,288 & 0,306 & 0,443 & 0,136 & 0,152 & 26 & 469 & $-2,1$ \\
\hline 470 & 469 & 0,016 & 0,338 & 0,016 & 0,123 & 1,000 & 0,363 & 0,637 & 1,000 & 0,759 & 1,077 & 0,555 & 0,137 & 0,076 & 0,152 & 72 & 469 & $-0,5$ \\
\hline 471 & 469 & 0,029 & 0,562 & 0,030 & 0,440 & 0,952 & 0,782 & 0,223 & 1,000 & 0,936 & 0,637 & 0,474 & 0,240 & 0,114 & 0,152 & 77 & 469 & $-1,3$ \\
\hline 472 & 469 & 0,024 & 0,499 & 0,026 & 0,389 & 0,944 & 0,779 & 0,228 & 1,000 & 0,901 & 0,866 & 0,566 & 0,189 & 0,107 & 0,169 & 41 & 469 & $-0,9$ \\
\hline 473 & 469 & 0,023 & 0,469 & 0,024 & 0,410 & 0,941 & 0,873 & 0,140 & 1,000 & 0,874 & 0,815 & 0,537 & 0,189 & 0,102 & 0,152 & 63 & 469 & $-0,9$ \\
\hline 474 & 469 & 0,019 & 0,448 & 0,020 & 0,368 & 0,929 & 0,820 & 0,193 & 1,000 & 0,926 & 0,571 & 0,394 & 0,204 & 0,080 & 0,107 & 60 & 469 & $-1,0$ \\
\hline 475 & 469 & 0,022 & 0,795 & 0,022 & 0,664 & 1,000 & 0,835 & 0,165 & 0,428 & 1,528 & 0,212 & 0,216 & 0,359 & 0,078 & 0,076 & 35 & 469 & $-1,8$ \\
\hline 476 & 469 & 0,040 & 0,825 & 0,047 & 0,714 & 0,848 & 0,866 & 0,202 & 0,743 & 1,160 & 0,401 & 0,361 & 0,357 & 0,129 & 0,169 & 33 & 469 & $-1,8$ \\
\hline 477 & 469 & 0,014 & 0,347 & 0,014 & 0,296 & 1,000 & 0,854 & 0,146 & 1,000 & 0,816 & 0,749 & 0,485 & 0,156 & 0,076 & 0,107 & 18 & 469 & $-0,6$ \\
\hline 478 & 469 & 0,036 & 0,634 & 0,037 & 0,482 & 0,962 & 0,760 & 0,243 & 1,000 & 0,944 & 0,601 & 0,412 & 0,276 & 0,114 & 0,152 & 12 & 469 & $-1,5$ \\
\hline
\end{tabular}




\begin{tabular}{|c|c|c|c|c|c|c|c|c|c|c|c|c|c|c|c|c|c|c|}
\hline 479 & 469 & 0,040 & 0,664 & 0,042 & 0,592 & 0,966 & 0,892 & 0,114 & 1,000 & 0,934 & 0,615 & 0,499 & 0,288 & 0,144 & 0,152 & 23 & 469 & $-1,5$ \\
\hline 480 & 469 & 0,171 & 1,784 & 0,197 & 1,480 & 0,869 & 0,829 & 0,215 & 0,674 & 1,218 & 0,618 & 0,716 & 0,593 & 0,424 & 0,379 & 44 & 469 & $-2,6$ \\
\hline 481 & 469 & 0,088 & 1,429 & 0,112 & 1,357 & 0,782 & 0,950 & 0,224 & 0,539 & 1,362 & 0,269 & 0,316 & 0,644 & 0,204 & 0,214 & 64 & 469 & $\begin{array}{l}-2,7 \\
\end{array}$ \\
\hline 482 & 469 & 0,024 & 0,490 & 0,029 & 0,389 & 0,850 & 0,793 & 0,256 & 1,000 & 0,886 & 1,082 & 0,849 & 0,169 & 0,144 & 0,152 & 144 & 469 & $-0,8$ \\
\hline 483 & 469 & 0,019 & 0,389 & 0,019 & 0,296 & 1,000 & 0,761 & 0,239 & 1,000 & 0,803 & 0,828 & 0,447 & 0,169 & 0,076 & 0,152 & 8 & 469 & $-0,8$ \\
\hline 484 & 469 & 0,016 & 0,368 & 0,016 & 0,296 & 1,000 & 0,805 & 0,195 & 1,000 & 0,826 & 0,824 & 0,485 & 0,156 & 0,076 & 0,107 & 18 & 469 & $-0,6$ \\
\hline 485 & 469 & 0,033 & 0,613 & 0,037 & 0,418 & 0,885 & 0,683 & 0,338 & 1,000 & 0,952 & 0,651 & 0,534 & 0,254 & 0,136 & 0,169 & 18 & 469 & $-1,3$ \\
\hline 486 & 469 & 0,251 & 2,287 & 0,303 & 1,991 & 0,829 & 0,871 & 0,214 & 0,603 & 1,287 & 0,406 & 0,512 & 0,888 & 0,454 & 0,379 & 54 & 469 & $-3,2$ \\
\hline 487 & 469 & 0,016 & 0,338 & 0,016 & 0,123 & 1,000 & 0,363 & 0,637 & 1,000 & 0,759 & 1,077 & 0,555 & 0,137 & 0,076 & 0,152 & 72 & 469 & $-0,5$ \\
\hline 488 & 469 & 0,057 & 0,757 & 0,059 & 0,643 & 0,976 & 0,849 & 0,153 & 1,000 & 0,891 & 0,835 & 0,688 & 0,296 & 0,203 & 0,240 & 39 & 469 & $-1,6$ \\
\hline 489 & 469 & 0,016 & 0,389 & 0,017 & 0,296 & 0,917 & 0,761 & 0,253 & 1,000 & 0,873 & 0,700 & 0,425 & 0,169 & 0,072 & 0,076 & 21 & 469 & $-0,8$ \\
\hline 490 & 469 & 0,016 & 0,347 & 0,016 & 0,245 & 1,000 & 0,707 & 0,293 & 1,000 & 0,778 & 0,875 & 0,500 & 0,152 & 0,076 & 0,152 & 90 & 469 & $-0,6$ \\
\hline 491 & 469 & 0,014 & 0,376 & 0,016 & 0,317 & 0,909 & 0,842 & 0,182 & 1,000 & 0,886 & 0,749 & 0,435 & 0,156 & 0,068 & 0,076 & 32 & 469 & $-0,6$ \\
\hline 492 & 469 & 0,022 & 0,511 & 0,024 & 0,359 & 0,882 & 0,702 & 0,320 & 1,000 & 0,983 & 0,516 & 0,329 & 0,230 & 0,076 & 0,152 & 79 & 469 & $-1,2$ \\
\hline 493 & 469 & 0,026 & 0,511 & 0,027 & 0,389 & 0,947 & 0,760 & 0,245 & 1,000 & 0,898 & 0,790 & 0,557 & 0,204 & 0,114 & 0,152 & 81 & 469 & $-1,0$ \\
\hline 494 & 469 & 0,022 & 0,541 & 0,024 & 0,440 & 0,882 & 0,812 & 0,221 & 0,924 & 1,040 & 0,516 & 0,329 & 0,230 & 0,076 & 0,076 & 6 & 469 & $-1,2$ \\
\hline 495 & 469 & 0,019 & 0,389 & 0,019 & 0,287 & 1,000 & 0,739 & 0,261 & 1,000 & 0,803 & 0,828 & 0,447 & 0,169 & 0,076 & 0,152 & 82 & 469 & $-0,8$ \\
\hline 496 & 469 & 0,017 & 0,440 & 0,019 & 0,317 & 0,923 & 0,721 & 0,289 & 1,000 & 0,945 & 0,527 & 0,353 & 0,204 & 0,072 & 0,076 & 75 & 469 & $-1,0$ \\
\hline 497 & 469 & 0,022 & 0,469 & 0,022 & 0,418 & 1,000 & 0,892 & 0,108 & 1,000 & 0,902 & 0,659 & 0,465 & 0,204 & 0,095 & 0,107 & 32 & 469 & $-1,0$ \\
\hline 498 & 469 & 0,023 & 0,469 & 0,024 & 0,368 & 0,941 & 0,784 & 0,224 & 1,000 & 0,874 & 0,815 & 0,566 & 0,189 & 0,107 & 0,152 & 135 & 469 & $-0,9$ \\
\hline 499 & 469 & 0,020 & 0,490 & 0,024 & 0,347 & 0,824 & 0,707 & 0,342 & 1,000 & 0,976 & 0,615 & 0,411 & 0,204 & 0,084 & 0,107 & 24 & 469 & $-1,0$ \\
\hline 500 & 469 & 2,230 & 10,914 & 2,931 & 6,720 & 0,761 & 0,616 & 0,453 & 0,235 & 2,062 & 0,422 & 0,475 & 2,594 & 1,232 & 0,303 & 78 & 469 & $-4,7$ \\
\hline 501 & 469 & 0,022 & 0,511 & 0,026 & 0,368 & 0,833 & 0,719 & 0,327 & 1,000 & 0,983 & 0,597 & 0,500 & 0,214 & 0,107 & 0,107 & 48 & 469 & $-1,1$ \\
\hline 502 & 469 & 0,062 & 1,412 & 0,095 & 1,023 & 0,652 & 0,725 & 0,444 & 0,389 & 1,603 & 0,342 & 0,474 & 0,479 & 0,227 & 0,152 & 101 & 469 & $-2,3$ \\
\hline 503 & 469 & 0,042 & 0,643 & 0,044 & 0,592 & 0,935 & 0,921 & 0,102 & 1,000 & 0,889 & 0,998 & 0,814 & 0,230 & 0,187 & 0,214 & 179 & 469 & $-1,2$ \\
\hline 504 & 469 & 0,022 & 0,440 & 0,022 & 0,338 & 1,000 & 0,769 & 0,231 & 1,000 & 0,845 & 0,764 & 0,566 & 0,189 & 0,107 & 0,152 & 35 & 469 & $\begin{array}{l}-0,9 \\
-0,9\end{array}$ \\
\hline 505 & 469 & 0,036 & 0,634 & 0,039 & 0,541 & 0,926 & 0,853 & 0,164 & 1,000 & 0,944 & 0,707 & 0,519 & 0,254 & 0,132 & 0,152 & 21 & 469 & $-1,3$ \\
\hline 506 & 469 & 0,044 & 0,735 & 0,049 & 0,613 & 0,912 & 0,833 & 0,189 & 1,000 & 0,984 & 0,607 & 0,496 & 0,305 & 0,152 & 0,169 & 93 & 469 & $\begin{array}{l}-1,6 \\
-1,\end{array}$ \\
\hline 507 & 469 & 0,014 & 0,347 & 0,016 & 0,266 & 0,909 & 0,768 & 0,249 & 1,000 & 0,816 & 0,979 & 0,588 & 0,137 & 0,080 & 0,107 & 53 & 469 & $-0,5$ \\
\hline 508 & 469 & 0,024 & 0,685 & 0,030 & 0,511 & 0,810 & 0,747 & 0,317 & 0,654 & 1,237 & 0,333 & 0,248 & 0,305 & 0,076 & 0,076 & 178 & 469 & $-1,6$ \\
\hline 509 & 469 & 0,016 & 0,397 & 0,016 & 0,296 & 1,000 & 0,745 & 0,255 & 1,000 & 0,892 & 0,560 & 0,283 & 0,189 & 0,054 & 0,107 & 50 & 469 & $\begin{array}{l}-0,9 \\
-0,\end{array}$ \\
\hline 510 & 469 & 0,023 & 0,469 & 0,023 & 0,440 & 1,000 & 0,937 & 0,063 & 1,000 & 0,874 & 0,702 & 0,525 & 0,204 & 0,107 & 0,152 & 66 & 469 & $-1,0$ \\
\hline 511 & 469 & 0,016 & 0,368 & 0,016 & 0,266 & 1,000 & 0,724 & 0,276 & 1,000 & 0,826 & 0,824 & 0,485 & 0,156 & 0,076 & 0,107 & 72 & 469 & $-0,6$ \\
\hline 512 & 469 & 0,024 & 0,469 & 0,024 & 0,368 & 1,000 & 0,784 & 0,216 & 1,000 & 0,848 & 1,082 & 0,791 & 0,169 & 0,134 & 0,169 & 108 & 469 & $-0,8$ \\
\hline 513 & 469 & 0,027 & 0,532 & 0,027 & 0,338 & 1,000 & 0,635 & 0,365 & 1,000 & 0,910 & 0,605 & 0,316 & 0,240 & 0,076 & 0,152 & 177 & 469 & $-1,3$ \\
\hline 514 & 469 & 0,014 & 0,317 & 0,014 & 0,173 & 1,000 & 0,547 & 0,453 & 1,000 & 0,746 & 1,273 & 0,632 & 0,120 & 0,076 & 0,152 & 72 & 469 & $-0,3$ \\
\hline 515 & 469 & 0,022 & 0,448 & 0,023 & 0,389 & 0,938 & 0,867 & 0,147 & 1,000 & 0,862 & 0,955 & 0,632 & 0,169 & 0,107 & 0,152 & 45 & 469 & $-0,8$ \\
\hline 516 & 469 & 0,017 & 0,368 & 0,017 & 0,245 & 1,000 & 0,667 & 0,333 & 1,000 & 0,791 & 1,175 & 0,784 & 0,137 & 0,107 & 0,152 & 0 & 469 & $-0,5$ \\
\hline 517 & 469 & 0,019 & 0,482 & 0,022 & 0,194 & 0,867 & 0,404 & 0,611 & 1,000 & 0,995 & 0,571 & 0,365 & 0,204 & 0,074 & 0,076 & 173 & 469 & $-1,0$ \\
\hline 518 & 469 & 0,102 & 1,399 & 0,119 & 1,298 & 0,855 & 0,927 & 0,162 & 0,654 & 1,237 & 0,332 & 0,345 & 0,625 & 0,216 & 0,227 & 74 & 469 & $-2,6$ \\
\hline 519 & 469 & 0,022 & 0,685 & 0,032 & 0,397 & 0,682 & 0,580 & 0,527 & 0,577 & 1,317 & 0,562 & 0,606 & 0,221 & 0,134 & 0,076 & 115 & 469 & $-1,1$ \\
\hline 520 & 469 & 0,014 & 0,347 & 0,017 & 0,287 & 0,833 & 0,829 & 0,239 & 1,000 & 0,816 & 1,273 & 0,849 & 0,120 & 0,102 & 0,107 & 108 & 469 & $\begin{array}{l}-0,3 \\
-0,3\end{array}$ \\
\hline 521 & 469 & 0,076 & 1,429 & 0,118 & 1,120 & 0,646 & 0,784 & 0,414 & 0,468 & 1,462 & 0,441 & 0,742 & 0,469 & 0,347 & 0,169 & 58 & 469 & $-2,2$ \\
\hline 522 & 469 & 0,022 & 0,571 & 0,027 & 0,541 & 0,789 & 0,948 & 0,217 & 0,830 & 1,098 & 0,424 & 0,331 & 0,254 & 0,084 & 0,107 & 27 & 469 & $\begin{array}{l}-2,2 \\
-1,3\end{array}$ \\
\hline 523 & 469 & 0,146 & 2,452 & 0,221 & 2,206 & 0,662 & 0,900 & 0,352 & 0,306 & 1,808 & 0,153 & 0,210 & 1,104 & 0,232 & 0,152 & 71 & 469 & $-3,5$ \\
\hline 524 & 469 & 0,019 & 0,389 & 0,019 & 0,287 & 1,000 & 0,739 & 0,261 & 1,000 & 0,803 & 0,828 & 0,447 & 0,169 & 0,076 & 0,152 & 82 & 469 & $-0,8$ \\
\hline 525 & 469 & 0,014 & 0,347 & 0,014 & 0,266 & 1,000 & 0,768 & 0,232 & 1,000 & 0,816 & 0,979 & 0,588 & 0,137 & 0,080 & 0,107 & 56 & 469 & $\begin{array}{l}-0,5 \\
-0,5\end{array}$ \\
\hline 526 & 469 & 0,024 & 0,541 & 0,029 & 0,490 & 0,850 & 0,906 & 0,177 & 1,000 & 0,977 & 0,637 & 0,434 & 0,221 & 0,096 & 0,152 & 71 & 469 & $-1,1$ \\
\hline 527 & 469 & 0,039 & 0,807 & 0,044 & 0,685 & 0,871 & 0,848 & 0,199 & 0,747 & 1,157 & 0,386 & 0,332 & 0,357 & 0,119 & 0,152 & 65 & 469 & $-1,8$ \\
\hline 528 & 469 & 0,036 & 0,562 & 0,036 & 0,520 & 1,000 & 0,925 & 0,075 & 1,000 & 0,837 & 1,098 & 0,743 & 0,204 & 0,152 & 0,214 & 90 & 469 & $\begin{array}{l}-1,0 \\
-1,0\end{array}$ \\
\hline 529 & 469 & 0,014 & 0,317 & 0,014 & 0,173 & 1,000 & 0,547 & 0,453 & 1,000 & 0,746 & 1,273 & 0,632 & 0,120 & 0,076 & 0,107 & 90 & 469 & $\begin{array}{l}-0,3 \\
\end{array}$ \\
\hline 530 & 469 & 0,026 & 0,490 & 0,029 & 0,389 & 0,900 & 0,793 & 0,230 & 1,000 & 0,861 & 0,917 & 0,707 & 0,189 & 0,134 & 0,169 & 53 & 469 & $-0,9$ \\
\hline 531 & 469 & 0,014 & 0,368 & 0,016 & 0,245 & 0,909 & 0,667 & 0,346 & 1,000 & 0,866 & 0,749 & 0,461 & 0,156 & 0,072 & 0,076 & 58 & 469 & $-0,6$ \\
\hline 532 & 469 & 0,032 & 0,786 & 0,039 & 0,634 & 0,815 & 0,806 & 0,268 & 0,642 & 1,248 & 0,311 & 0,230 & 0,359 & 0,083 & 0,107 & 78 & 469 & $-1,8$ \\
\hline 533 & 469 & 0,027 & 0,571 & 0,029 & 0,469 & 0,950 & 0,822 & 0,185 & 1,000 & 0,975 & 0,605 & 0,495 & 0,240 & 0,119 & 0,152 & 66 & 469 & $-1,3$ \\
\hline 534 & 469 & 0,020 & 0,511 & 0,022 & 0,397 & 0,933 & 0,777 & 0,233 & 0,965 & 1,018 & 0,524 & 0,384 & 0,221 & 0,085 & 0,107 & 31 & 469 & $-1,1$ \\
\hline 535 & 469 & 0,017 & 0,418 & 0,017 & 0,317 & 1,000 & 0,757 & 0,243 & 1,000 & 0,900 & 0,611 & 0,424 & 0,189 & 0,080 & 0,107 & 60 & 469 & $\begin{array}{l}-0,9 \\
\end{array}$ \\
\hline 536 & 469 & 0,023 & 0,448 & 0,023 & 0,389 & 1,000 & 0,867 & 0,133 & 1,000 & 0,834 & 1,019 & 0,632 & 0,169 & 0,107 & 0,169 & 70 & 469 & $-0,8$ \\
\hline
\end{tabular}




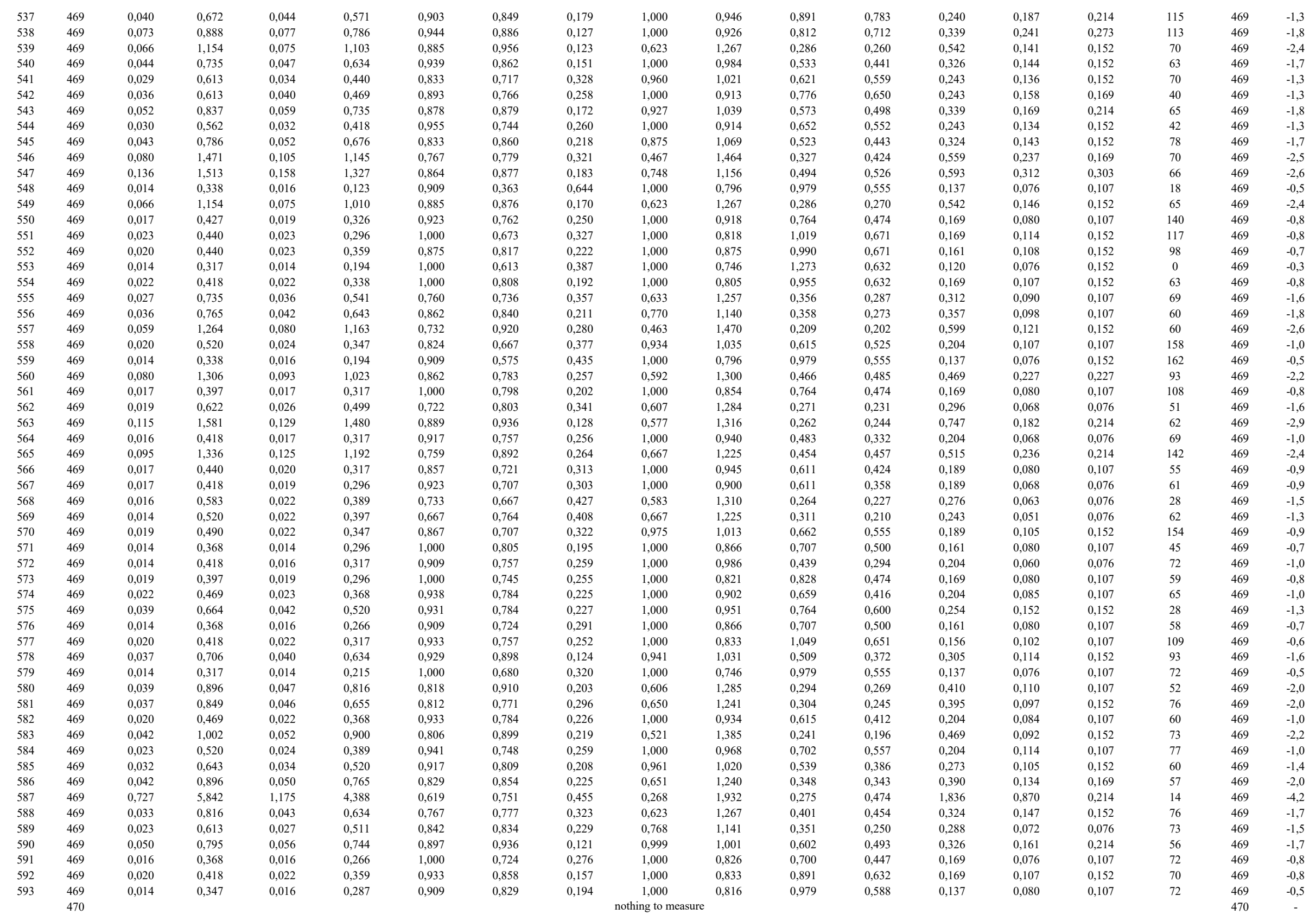




\begin{tabular}{|c|c|c|c|c|c|c|c|c|c|c|c|c|c|c|c|c|c|}
\hline 471 & & & & & & & & nothing to measure & & & & & & & & 471 & - \\
\hline 472 & 0,047 & 1,142 & 0,065 & 0,938 & 0,733 & 0,822 & 0,321 & 0,457 & 1,480 & 0,268 & 0,250 & 0,475 & 0,119 & 0,152 & 25 & 472 & $-2,2$ \\
\hline 472 & 0,014 & 0,389 & 0,017 & 0,224 & 0,833 & 0,576 & 0,455 & 1,000 & 0,916 & 0,637 & 0,354 & 0,169 & 0,060 & 0,076 & 23 & 472 & $-0,8$ \\
\hline 473 & 0,029 & 0,816 & 0,037 & 0,600 & 0,769 & 0,736 & 0,351 & 0,542 & 1,359 & 0,439 & 0,411 & 0,288 & 0,119 & 0,107 & 41 & 473 & $-1,5$ \\
\hline 474 & & & & & & & & nothing to measure & & & & & & & & 474 & - \\
\hline 475 & & & & & & & & nothing to measure & & & & & & & & 475 & - \\
\hline 476 & & & & & & & & nothing to measure & & & & & & & & 476 & - \\
\hline 477 & & & & & & & & nothing to measure & & & & & & & & 477 & - \\
\hline 478 & & & & & & & & nothing to measure & & & & & & & & 478 & - \\
\hline 479 & & & & & & & & nothing to measure & & & & & & & & 479 & - \\
\hline 480 & 0,023 & 0,469 & 0,024 & 0,448 & 0,941 & 0,955 & 0,074 & 1,000 & 0,874 & 0,815 & 0,537 & 0,189 & 0,102 & 0,152 & 125 & 480 & $-0,9$ \\
\hline 480 & 0,014 & 0,347 & 0,016 & 0,245 & 0,909 & 0,707 & 0,307 & 1,000 & 0,816 & 1,273 & 0,849 & 0,120 & 0,102 & 0,107 & 108 & 480 & $-0,3$ \\
\hline 480 & 0,014 & 0,317 & 0,014 & 0,173 & 1,000 & 0,547 & 0,453 & 1,000 & 0,746 & 0,979 & 0,555 & 0,137 & 0,076 & 0,107 & 108 & 480 & $-0,5$ \\
\hline 480 & 0,039 & 0,837 & 0,047 & 0,643 & 0,818 & 0,768 & 0,295 & 0,695 & 1,200 & 0,593 & 0,547 & 0,288 & 0,158 & 0,152 & 146 & 480 & $-1,5$ \\
\hline 481 & 0,016 & 0,389 & 0,017 & 0,266 & 0,917 & 0,685 & 0,326 & 1,000 & 0,873 & 0,824 & 0,485 & 0,156 & 0,076 & 0,107 & 77 & 481 & $-0,6$ \\
\hline 482 & & & & & & & & nothing to measure & & & & & & & & 482 & \\
\hline 483 & 0,020 & 0,418 & 0,022 & 0,317 & 0,933 & 0,757 & 0,252 & 1,000 & 0,833 & 0,891 & 0,600 & 0,169 & 0,102 & 0,152 & 71 & 483 & $-0,8$ \\
\hline 483 & 0,076 & 0,960 & 0,088 & 0,888 & 0,869 & 0,925 & 0,151 & 1,000 & 0,982 & 0,844 & 0,800 & 0,339 & 0,271 & 0,273 & 55 & 483 & $-1,8$ \\
\hline 484 & 0,049 & 0,951 & 0,060 & 0,613 & 0,810 & 0,645 & 0,403 & 0,678 & 1,215 & 0,585 & 0,618 & 0,326 & 0,201 & 0,152 & 144 & 484 & $-1,7$ \\
\hline 484 & 0,044 & 1,205 & 0,063 & 0,837 & 0,705 & 0,695 & 0,425 & 0,385 & 1,611 & 0,443 & 0,596 & 0,357 & 0,213 & 0,152 & 38 & 484 & $-1,8$ \\
\hline 484 & 0,039 & 0,622 & 0,040 & 0,571 & 0,964 & 0,918 & 0,089 & 1,000 & 0,891 & 0,859 & 0,671 & 0,240 & 0,161 & 0,214 & 130 & 484 & $-1,3$ \\
\hline 485 & & & & & & & & nothing to measure & & & & & & & & 485 & - \\
\hline 486 & & & & & & & & nothing to measure & & & & & & & & 486 & - \\
\hline 487 & & & & & & & & nothing to measure & & & & & & & & 487 & - \\
\hline 488 & & & & & & & & nothing to measure & & & & & & & & 488 & - \\
\hline 489 & 0,026 & 0,511 & 0,029 & 0,368 & 0,900 & 0,719 & 0,298 & 1,000 & 0,898 & 0,790 & 0,541 & 0,204 & 0,110 & 0,152 & 172 & 489 & $-1,0$ \\
\hline 489 & 0,017 & 0,655 & 0,026 & 0,224 & 0,667 & 0,342 & 0,738 & 0,504 & 1,408 & 0,340 & 0,447 & 0,254 & 0,114 & 0,076 & 90 & 489 & $-1,3$ \\
\hline 489 & 0,034 & 0,735 & 0,043 & 0,634 & 0,800 & 0,862 & 0,243 & 0,800 & 1,118 & 0,577 & 0,533 & 0,276 & 0,147 & 0,152 & 82 & 489 & $-1,5$ \\
\hline 489 & 0,033 & 0,571 & 0,033 & 0,511 & 1,000 & 0,896 & 0,104 & 1,000 & 0,886 & 0,791 & 0,658 & 0,230 & 0,152 & 0,169 & 170 & 489 & $-1,2$ \\
\hline 489 & 0,044 & 0,960 & 0,056 & 0,757 & 0,795 & 0,788 & 0,295 & 0,607 & 1,283 & 0,326 & 0,350 & 0,417 & 0,146 & 0,152 & 92 & 489 & $-2,1$ \\
\hline 489 & 0,016 & 0,418 & 0,019 & 0,317 & 0,846 & 0,757 & 0,287 & 1,000 & 0,940 & 0,560 & 0,334 & 0,189 & 0,063 & 0,076 & 31 & 489 & $-0,9$ \\
\hline 490 & & & & & & & & nothing to measure & & & & & & & & 490 & - \\
\hline 491 & & & & & & & & nothing to measure & & & & & & & & 491 & - \\
\hline 492 & & & & & & & & nothing to measure & & & & & & & & 492 & - \\
\hline 493 & 0,082 & 1,277 & 0,112 & 1,023 & 0,731 & 0,801 & 0,335 & 0,631 & 1,259 & 0,685 & 0,794 & 0,390 & 0,310 & 0,152 & 158 & 493 & $-2,0$ \\
\hline 494 & & & & & & & & nothing to measure & & & & & & & & 494 & \\
\hline 495 & 0,055 & 0,888 & 0,059 & 0,837 & 0,927 & 0,943 & 0,093 & 0,869 & 1,072 & 0,428 & 0,379 & 0,403 & 0,153 & 0,169 & 119 & 495 & $-2,0$ \\
\hline 495 & 0,033 & 0,541 & 0,033 & 0,520 & 1,000 & 0,961 & 0,039 & 1,000 & 0,840 & 0,861 & 0,606 & 0,221 & 0,134 & 0,169 & 122 & 495 & $-1,1$ \\
\hline 495 & 0,040 & 0,693 & 0,046 & 0,643 & 0,875 & 0,927 & 0,145 & 1,000 & 0,976 & 0,686 & 0,588 & 0,273 & 0,161 & 0,169 & 132 & 495 & $-1,4$ \\
\hline 496 & & & & & & & & nothing to measure & & & & & & & & 496 & - \\
\hline 497 & & & & & & & & nothing to measure & & & & & & & & 497 & - \\
\hline 498 & & & & & & & & nothing to measure & & & & & & & & 498 & - \\
\hline 499 & & & & & & & & nothing to measure & & & & & & & & 499 & - \\
\hline 500 & 0,032 & 0,951 & 0,043 & 0,511 & 0,733 & 0,538 & 0,534 & 0,439 & 1,510 & 0,683 & 0,625 & 0,243 & 0,152 & 0,107 & 94 & 500 & $-1,3$ \\
\hline 500 & 0,227 & 2,338 & 0,288 & 1,800 & 0,786 & 0,770 & 0,314 & 0,521 & 1,385 & 0,473 & 0,534 & 0,781 & 0,417 & 0,303 & 83 & 500 & $-3,0$ \\
\hline 501 & 0,016 & 0,613 & 0,022 & 0,347 & 0,733 & 0,566 & 0,510 & 0,528 & 1,376 & 0,700 & 0,671 & 0,169 & 0,114 & 0,076 & 124 & 501 & $-0,8$ \\
\hline 501 & 0,019 & 0,418 & 0,019 & 0,326 & 1,000 & 0,778 & 0,222 & 1,000 & 0,864 & 0,828 & 0,600 & 0,169 & 0,102 & 0,107 & 156 & 501 & $-0,8$ \\
\hline 501 & 0,019 & 0,389 & 0,019 & 0,224 & 1,000 & 0,576 & 0,424 & 1,000 & 0,803 & 0,920 & 0,633 & 0,161 & 0,102 & 0,152 & 135 & 501 & $-0,7$ \\
\hline 502 & & & & & & & & nothing to measure & & & & & & & & 502 & - \\
\hline 506 & 0,016 & 0,418 & 0,017 & 0,296 & 0,917 & 0,707 & 0,305 & 1,000 & 0,940 & 0,560 & 0,424 & 0,189 & 0,080 & 0,107 & 58 & 506 & $-0,9$ \\
\hline 506 & 0,014 & 0,326 & 0,014 & 0,224 & 1,000 & 0,688 & 0,312 & 1,000 & 0,767 & 1,273 & 0,671 & 0,120 & 0,080 & 0,107 & 135 & 506 & $-0,3$ \\
\hline 506 & 0,026 & 0,664 & 0,033 & 0,592 & 0,783 & 0,892 & 0,243 & 0,737 & 1,165 & 0,376 & 0,286 & 0,296 & 0,085 & 0,107 & 128 & 506 & $-1,6$ \\
\hline
\end{tabular}




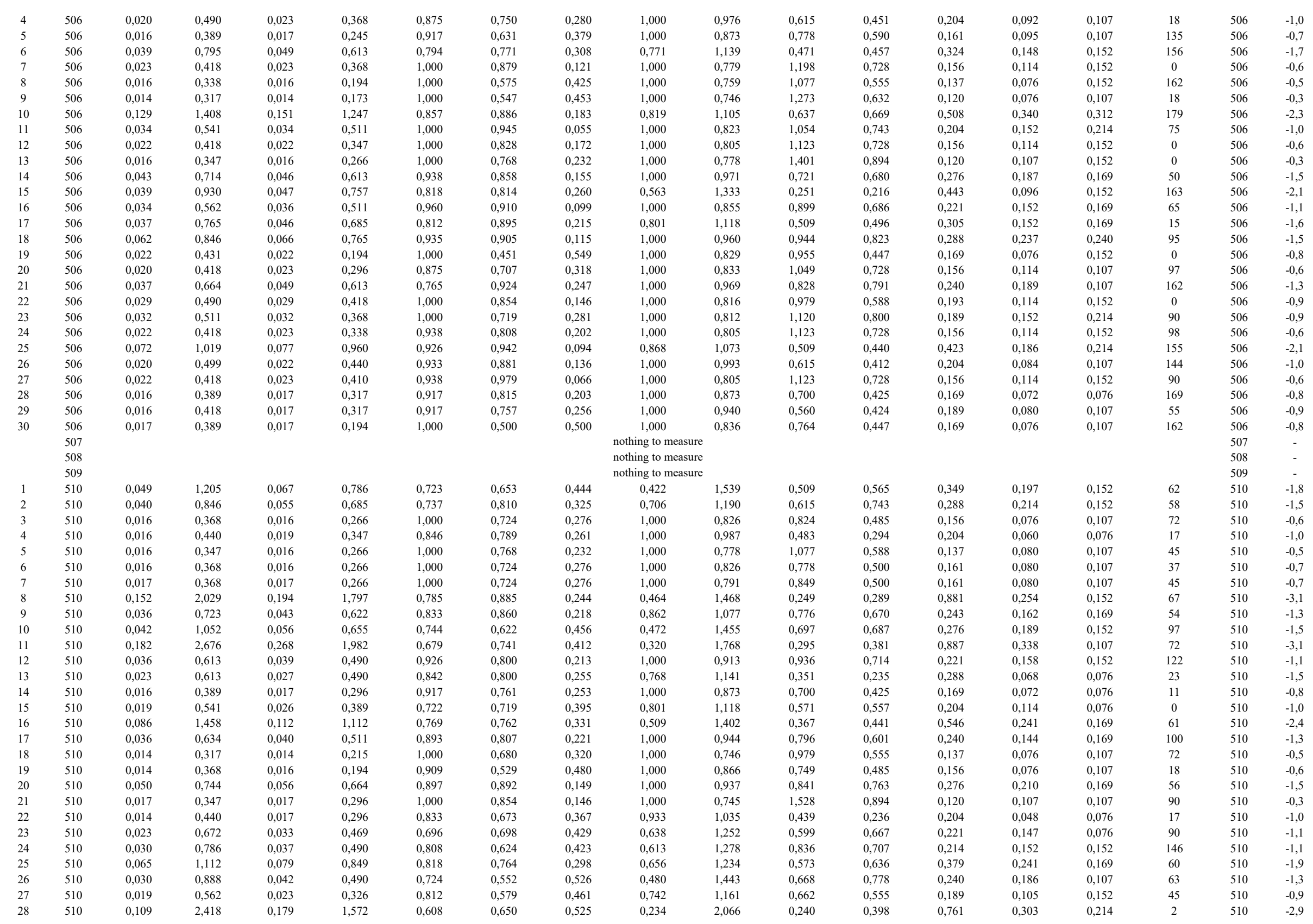




\begin{tabular}{|c|c|c|c|c|c|c|c|c|c|c|c|c|c|c|c|c|c|c|}
\hline 29 & 510 & 0,135 & 2,135 & 0,161 & 1,306 & 0,839 & 0,612 & 0,420 & 0,372 & 1,640 & 0,549 & 0,576 & 0,559 & 0,322 & 0,240 & 25 & 510 & $-2,5$ \\
\hline 30 & 510 & 0,080 & 1,142 & 0,096 & 0,960 & 0,836 & 0,841 & 0,229 & 0,775 & 1,136 & 0,570 & 0,620 & 0,423 & 0,263 & 0,273 & 61 & 510 & $-2,1$ \\
\hline 31 & 510 & 0,022 & 0,461 & 0,023 & 0,317 & 0,938 & 0,688 & 0,318 & 1,000 & 0,886 & 0,735 & 0,392 & 0,193 & 0,076 & 0,152 & 0 & 510 & $-0,9$ \\
\hline 32 & 510 & 0,014 & 0,389 & 0,017 & 0,266 & 0,833 & 0,685 & 0,357 & 1,000 & 0,916 & 0,637 & 0,380 & 0,169 & 0,064 & 0,076 & 0 & 510 & $-0,8$ \\
\hline 33 & 510 & 0,063 & 1,091 & 0,076 & 0,909 & 0,830 & 0,833 & 0,238 & 0,667 & 1,225 & 0,431 & 0,496 & 0,432 & 0,214 & 0,214 & 56 & 510 & $-2,1$ \\
\hline 34 & 510 & 0,023 & 0,511 & 0,024 & 0,368 & 0,941 & 0,719 & 0,287 & 1,000 & 0,952 & 0,551 & 0,329 & 0,230 & 0,076 & 0,152 & 10 & 510 & $-1,2$ \\
\hline 35 & 510 & 0,036 & 0,714 & 0,040 & 0,643 & 0,893 & 0,899 & 0,147 & 0,883 & 1,064 & 0,436 & 0,349 & 0,324 & 0,113 & 0,152 & 23 & 510 & $-1,7$ \\
\hline \multirow[t]{4}{*}{36} & 510 & 0,100 & 1,830 & 0,154 & 1,471 & 0,654 & 0,804 & 0,398 & 0,377 & 1,629 & 0,176 & 0,240 & 0,851 & 0,204 & 0,152 & 16 & 510 & $-3,1$ \\
\hline & 511 & & & & & & & & nothing to measure & & & & & & & & 511 & - \\
\hline & 512 & & & & & & & & nothing to measure & & & & & & & & 512 & - \\
\hline & 513 & & & & & & & & nothing to measure & & & & & & & & 513 & - \\
\hline \multirow[t]{16}{*}{1} & 514 & 0,027 & 0,714 & 0,034 & 0,600 & 0,792 & 0,841 & 0,262 & 0,671 & 1,221 & 0,327 & 0,291 & 0,326 & 0,095 & 0,107 & 36 & 514 & $-1,7$ \\
\hline & 515 & & & & & & & & nothing to measure & & & & & & & & 515 & - \\
\hline & 516 & & & & & & & & nothing to measure & & & & & & & & 516 & - \\
\hline & 517 & & & & & & & & nothing to measure & & & & & & & & 517 & - \\
\hline & 518 & & & & & & & & nothing to measure & & & & & & & & 518 & - \\
\hline & 519 & & & & & & & & nothing to measure & & & & & & & & 519 & - \\
\hline & 520 & & & & & & & & nothing to measure & & & & & & & & 520 & - \\
\hline & 521 & & & & & & & & nothing to measure & & & & & & & & 521 & - \\
\hline & 522 & & & & & & & & nothing to measure & & & & & & & & 522 & - \\
\hline & 523 & & & & & & & & nothing to measure & & & & & & & & 523 & - \\
\hline & 524 & & & & & & & & nothing to measure & & & & & & & & 524 & - \\
\hline & 525 & & & & & & & & nothing to measure & & & & & & & & 525 & - \\
\hline & 526 & & & & & & & & nothing to measure & & & & & & & & 526 & - \\
\hline & 527 & & & & & & & & nothing to measure & & & & & & & & 527 & - \\
\hline & 528 & & & & & & & & nothing to measure & & & & & & & & 528 & - \\
\hline & 529 & & & & & & & & nothing to measure & & & & & & & & 529 & - \\
\hline 1 & 530 & 0,095 & 1,551 & 0,128 & 1,213 & 0,742 & 0,782 & 0,338 & 0,494 & 1,422 & 0,373 & 0,419 & 0,568 & 0,238 & 0,169 & 37 & 530 & $-2,5$ \\
\hline 2 & 530 & 0,070 & 1,560 & 0,108 & 1,171 & 0,653 & 0,751 & 0,427 & 0,363 & 1,660 & 0,304 & 0,474 & 0,542 & 0,257 & 0,152 & 150 & 530 & $-2,4$ \\
\hline 3 & 530 & 0,036 & 0,592 & 0,036 & 0,541 & 1,000 & 0,914 & 0,086 & 1,000 & 0,881 & 0,776 & 0,552 & 0,243 & 0,134 & 0,169 & 49 & 530 & $-1,3$ \\
\hline 4 & 530 & 0,128 & 1,437 & 0,145 & 1,336 & 0,881 & 0,929 & 0,138 & 0,777 & 1,135 & 0,488 & 0,436 & 0,577 & 0,252 & 0,240 & 53 & 530 & $-2,5$ \\
\hline 5 & 530 & 0,023 & 0,511 & 0,026 & 0,368 & 0,889 & 0,719 & 0,302 & 1,000 & 0,952 & 0,702 & 0,557 & 0,204 & 0,114 & 0,152 & 125 & 530 & $-1,0$ \\
\hline 6 & 530 & 0,029 & 0,511 & 0,029 & 0,368 & 1,000 & 0,719 & 0,281 & 1,000 & 0,852 & 0,749 & 0,514 & 0,221 & 0,114 & 0,152 & 165 & 530 & $-1,1$ \\
\hline 7 & 530 & 0,047 & 0,735 & 0,049 & 0,655 & 0,971 & 0,891 & 0,113 & 1,000 & 0,953 & 0,618 & 0,485 & 0,312 & 0,152 & 0,169 & 76 & 530 & $-1,6$ \\
\hline 8 & 530 & 0,019 & 0,397 & 0,019 & 0,368 & 1,000 & 0,925 & 0,075 & 1,000 & 0,821 & 0,828 & 0,474 & 0,169 & 0,080 & 0,107 & 35 & 530 & $-0,8$ \\
\hline 9 & 530 & 0,057 & 0,981 & 0,065 & 0,867 & 0,889 & 0,884 & 0,161 & 0,750 & 1,155 & 0,374 & 0,334 & 0,442 & 0,148 & 0,169 & 124 & 530 & $-2,1$ \\
\hline 10 & 530 & 0,017 & 0,440 & 0,020 & 0,296 & 0,857 & 0,673 & 0,357 & 1,000 & 0,945 & 0,611 & 0,506 & 0,189 & 0,096 & 0,152 & 145 & 530 & $-0,9$ \\
\hline 11 & 530 & 0,017 & 0,368 & 0,017 & 0,287 & 1,000 & 0,781 & 0,219 & 1,000 & 0,791 & 0,849 & 0,500 & 0,161 & 0,080 & 0,107 & 45 & 530 & $-0,7$ \\
\hline 12 & 530 & 0,112 & 1,602 & 0,141 & 1,205 & 0,796 & 0,752 & 0,321 & 0,548 & 1,351 & 0,537 & 0,662 & 0,515 & 0,341 & 0,273 & 80 & 530 & $-2,4$ \\
\hline 13 & 530 & 0,192 & 2,346 & 0,268 & 1,704 & 0,717 & 0,726 & 0,394 & 0,439 & 1,509 & 0,525 & 0,675 & 0,683 & 0,461 & 0,152 & 99 & 530 & $-2,8$ \\
\hline 14 & 530 & 0,082 & 1,957 & 0,141 & 1,366 & 0,582 & 0,698 & 0,516 & 0,268 & 1,931 & 0,270 & 0,411 & 0,621 & 0,255 & 0,169 & 126 & 530 & $-2,6$ \\
\hline 15 & 530 & 0,030 & 0,592 & 0,034 & 0,490 & 0,875 & 0,828 & 0,212 & 1,000 & 0,962 & 0,668 & 0,559 & 0,240 & 0,134 & 0,152 & 89 & 530 & $-1,3$ \\
\hline 16 & 530 & 0,022 & 0,440 & 0,023 & 0,317 & 0,938 & 0,721 & 0,286 & 1,000 & 0,845 & 0,764 & 0,537 & 0,189 & 0,102 & 0,152 & 125 & 530 & $-0,9$ \\
\hline 17 & 530 & 0,043 & 0,706 & 0,043 & 0,604 & 1,000 & 0,856 & 0,144 & 1,000 & 0,960 & 0,562 & 0,364 & 0,312 & 0,114 & 0,152 & 84 & 530 & $-1,6$ \\
\hline 18 & 530 & 0,063 & 1,175 & 0,089 & 0,989 & 0,710 & 0,842 & 0,331 & 0,575 & 1,319 & 0,350 & 0,398 & 0,479 & 0,190 & 0,152 & 113 & 530 & $-2,3$ \\
\hline 19 & 530 & 0,019 & 0,368 & 0,019 & 0,287 & 1,000 & 0,781 & 0,219 & 1,000 & 0,760 & 1,273 & 0,784 & 0,137 & 0,107 & 0,152 & 45 & 530 & $-0,5$ \\
\hline 20 & 530 & 0,042 & 0,807 & 0,046 & 0,655 & 0,906 & 0,811 & 0,211 & 0,802 & 1,116 & 0,434 & 0,325 & 0,349 & 0,114 & 0,152 & 86 & 530 & $-1,8$ \\
\hline 21 & 530 & 0,725 & 3,234 & 0,785 & 2,993 & 0,923 & 0,925 & 0,107 & 0,871 & 1,072 & 0,853 & 0,840 & 1,040 & 0,874 & 0,833 & 11 & 530 & $-3,4$ \\
\hline 22 & 530 & 0,129 & 3,234 & 0,225 & 1,847 & 0,573 & 0,571 & 0,605 & 0,155 & 2,539 & 0,220 & 0,326 & 0,864 & 0,282 & 0,169 & 73 & 530 & $-3,1$ \\
\hline 23 & 530 & 0,014 & 0,368 & 0,016 & 0,296 & 0,909 & 0,805 & 0,215 & 1,000 & 0,866 & 0,637 & 0,400 & 0,169 & 0,068 & 0,076 & 111 & 530 & $-0,8$ \\
\hline 24 & 530 & 0,017 & 0,397 & 0,017 & 0,317 & 1,000 & 0,798 & 0,202 & 1,000 & 0,854 & 0,764 & 0,474 & 0,169 & 0,080 & 0,107 & 59 & 530 & $-0,8$ \\
\hline 25 & 530 & 0,020 & 0,410 & 0,020 & 0,266 & 1,000 & 0,650 & 0,350 & 1,000 & 0,816 & 0,891 & 0,447 & 0,169 & 0,076 & 0,152 & 98 & 530 & $-0,8$ \\
\hline 26 & 530 & 0,029 & 0,706 & 0,039 & 0,440 & 0,741 & 0,623 & 0,458 & 0,724 & 1,175 & 0,439 & 0,367 & 0,288 & 0,106 & 0,107 & 9 & 530 & $-1,5$ \\
\hline 27 & 530 & 0,016 & 0,368 & 0,017 & 0,194 & 0,917 & 0,529 & 0,479 & 1,000 & 0,826 & 0,778 & 0,500 & 0,161 & 0,080 & 0,107 & 135 & 530 & $-0,7$ \\
\hline 28 & 530 & 0,014 & 0,368 & 0,017 & 0,275 & 0,833 & 0,747 & 0,303 & 1,000 & 0,866 & 0,749 & 0,485 & 0,156 & 0,076 & 0,107 & 0 & 530 & $-0,6$ \\
\hline 29 & 530 & 0,026 & 0,613 & 0,029 & 0,410 & 0,900 & 0,669 & 0,346 & 0,864 & 1,076 & 0,458 & 0,283 & 0,268 & 0,076 & 0,152 & 85 & 530 & $-1,4$ \\
\hline 30 & 530 & 0,062 & 1,031 & 0,086 & 0,938 & 0,717 & 0,910 & 0,297 & 0,729 & 1,171 & 0,438 & 0,537 & 0,423 & 0,227 & 0,152 & 168 & 530 & $-2,1$ \\
\hline 31 & 530 & 0,037 & 0,968 & 0,057 & 0,735 & 0,650 & 0,760 & 0,425 & 0,500 & $\begin{array}{l}1,414 \\
\text { S }\end{array}$ & 0,487 & 0,687 & 0,312 & 0,215 & 0,152 & 148 & 530 & $-1,6$ \\
\hline
\end{tabular}




\begin{tabular}{|c|c|c|c|c|c|c|c|c|c|c|c|c|c|c|c|c|c|c|}
\hline 32 & 530 & 0,760 & 4,925 & 0,887 & 4,219 & 0,858 & 0,857 & 0,202 & 0,394 & 1,593 & 0,248 & 0,264 & 1,977 & 0,523 & 0,530 & 53 & 530 & $-4,3$ \\
\hline 33 & 530 & 0,026 & 0,592 & 0,030 & 0,499 & 0,857 & 0,843 & 0,212 & 0,927 & 1,039 & 0,458 & 0,316 & 0,268 & 0,085 & 0,107 & 56 & 530 & $-1,4$ \\
\hline 34 & 530 & 0,083 & 1,340 & 0,116 & 1,070 & 0,716 & 0,798 & 0,348 & 0,583 & 1,310 & 0,434 & 0,511 & 0,494 & 0,252 & 0,227 & 127 & 530 & $-2,3$ \\
\hline 35 & 530 & 0,123 & 2,418 & 0,201 & 1,662 & 0,614 & 0,687 & 0,497 & 0,265 & 1,942 & 0,314 & 0,360 & 0,708 & 0,255 & 0,169 & 115 & 530 & $-2,8$ \\
\hline 36 & 530 & 0,131 & 1,399 & 0,148 & 1,298 & 0,883 & 0,927 & 0,137 & 0,838 & 1,092 & 0,644 & 0,738 & 0,508 & 0,375 & 0,312 & 47 & 530 & $-2,3$ \\
\hline 37 & 530 & 0,014 & 0,562 & 0,022 & 0,347 & 0,667 & 0,617 & 0,508 & 0,571 & 1,324 & 0,637 & 0,621 & 0,169 & 0,105 & 0,076 & 0 & 530 & $-0,8$ \\
\hline 38 & 530 & 0,046 & 1,061 & 0,070 & 0,774 & 0,653 & 0,729 & 0,440 & 0,512 & 1,397 & 0,361 & 0,519 & 0,403 & 0,209 & 0,152 & 129 & 530 & $-2,0$ \\
\hline 39 & 530 & 0,014 & 0,389 & 0,017 & 0,224 & 0,833 & 0,576 & 0,455 & 1,000 & 0,916 & 0,637 & 0,354 & 0,169 & 0,060 & 0,076 & 23 & 530 & $-0,8$ \\
\hline 40 & 530 & 0,017 & 0,368 & 0,017 & 0,296 & 1,000 & 0,805 & 0,195 & 1,000 & 0,791 & 0,899 & 0,485 & 0,156 & 0,076 & 0,152 & 98 & 530 & $-0,6$ \\
\hline 41 & 530 & 0,032 & 0,664 & 0,037 & 0,541 & 0,846 & 0,815 & 0,240 & 0,901 & 1,054 & 0,431 & 0,388 & 0,305 & 0,119 & 0,152 & 19 & 530 & $-1,6$ \\
\hline 42 & 530 & 0,023 & 0,693 & 0,034 & 0,490 & 0,667 & 0,707 & 0,444 & 0,600 & 1,291 & 0,509 & 0,546 & 0,240 & 0,131 & 0,076 & 98 & 530 & $-1,3$ \\
\hline 43 & 530 & 0,019 & 0,520 & 0,022 & 0,418 & 0,867 & 0,805 & 0,236 & 0,867 & 1,074 & 0,414 & 0,283 & 0,240 & 0,068 & 0,076 & 30 & 530 & $-1,3$ \\
\hline 44 & 530 & 0,047 & 0,879 & 0,056 & 0,664 & 0,846 & 0,755 & 0,289 & 0,770 & 1,140 & 0,467 & 0,384 & 0,359 & 0,138 & 0,152 & 73 & 530 & $-1,8$ \\
\hline 45 & 530 & 0,016 & 0,397 & 0,017 & 0,317 & 0,917 & 0,798 & 0,219 & 1,000 & 0,892 & 0,778 & 0,500 & 0,161 & 0,080 & 0,107 & 135 & 530 & $-0,7$ \\
\hline 46 & 530 & 0,027 & 0,571 & 0,032 & 0,490 & 0,864 & 0,859 & 0,196 & 1,000 & 0,975 & 0,712 & 0,606 & 0,221 & 0,134 & 0,152 & 46 & 530 & $-1,1$ \\
\hline 47 & 530 & 0,037 & 1,061 & 0,059 & 0,714 & 0,634 & 0,673 & 0,491 & 0,416 & 1,550 & 0,625 & 0,755 & 0,276 & 0,208 & 0,107 & 103 & 530 & $-1,5$ \\
\hline 48 & 530 & 0,125 & 1,234 & 0,131 & 1,205 & 0,956 & 0,976 & 0,050 & 1,000 & 0,986 & 0,599 & 0,581 & 0,515 & 0,300 & 0,303 & 23 & 530 & $-2,4$ \\
\hline 49 & 530 & 0,138 & 1,919 & 0,191 & 1,593 & 0,722 & 0,830 & 0,326 & 0,470 & 1,459 & 0,324 & 0,419 & 0,735 & 0,308 & 0,214 & 107 & 530 & $-2,9$ \\
\hline 50 & 530 & 0,014 & 0,359 & 0,014 & 0,448 & 1,000 & 1,248 & 0,248 & 1,000 & 0,846 & 0,749 & 0,243 & 0,156 & 0,038 & 0,076 & 90 & 530 & $-0,6$ \\
\hline 51 & 530 & 0,027 & 0,520 & 0,027 & 0,693 & 1,000 & 1,333 & 0,333 & 1,000 & 0,888 & 0,712 & 0,485 & 0,221 & 0,107 & 0,152 & 123 & 530 & $-1,1$ \\
\hline 52 & 530 & 0,042 & 0,909 & 0,055 & 1,103 & 0,763 & 1,214 & 0,319 & 0,633 & 1,257 & 0,410 & 0,460 & 0,359 & 0,165 & 0,169 & 79 & 530 & $-1,8$ \\
\hline 53 & 530 & 0,100 & 1,285 & 0,113 & 0,469 & 0,886 & 0,365 & 0,645 & 0,764 & 1,144 & 0,524 & 0,460 & 0,494 & 0,227 & 0,214 & 94 & 530 & $-2,3$ \\
\hline 54 & 530 & 0,022 & 0,613 & 0,029 & 0,368 & 0,750 & 0,600 & 0,472 & 0,720 & 1,178 & 0,382 & 0,300 & 0,268 & 0,080 & 0,076 & 42 & 530 & $-1,4$ \\
\hline 55 & 530 & 0,020 & 0,592 & 0,026 & 0,511 & 0,778 & 0,864 & 0,261 & 0,721 & 1,178 & 0,713 & 0,611 & 0,189 & 0,116 & 0,107 & 148 & 530 & $-0,9$ \\
\hline 56 & 530 & 0,034 & 0,930 & 0,043 & 4,664 & 0,800 & 5,016 & 4,021 & 0,500 & 1,414 & 0,611 & 0,700 & 0,268 & 0,187 & 0,107 & 38 & 530 & $-1,4$ \\
\hline 57 & 530 & 0,911 & 7,018 & 1,547 & 0,938 & 0,589 & 0,134 & 0,959 & 0,232 & 2,074 & 0,336 & 0,602 & 1,858 & 1,118 & 0,273 & 71 & 530 & $-4,2$ \\
\hline 58 & 530 & 0,076 & 1,124 & 0,092 & 0,418 & 0,828 & 0,372 & 0,651 & 0,756 & 1,150 & 0,619 & 0,637 & 0,395 & 0,252 & 0,227 & 70 & 530 & $-2,0$ \\
\hline 59 & 530 & 0,022 & 0,735 & 0,034 & 0,592 & 0,625 & 0,805 & 0,423 & 0,500 & 1,414 & 0,562 & 0,686 & 0,221 & 0,152 & 0,107 & 118 & 530 & $-1,1$ \\
\hline 60 & 530 & 0,037 & 0,714 & 0,040 & 0,490 & 0,929 & 0,686 & 0,322 & 0,919 & 1,043 & 0,543 & 0,453 & 0,296 & 0,134 & 0,152 & 34 & 530 & $-1,6$ \\
\hline 61 & 530 & 0,023 & 0,765 & 0,033 & 1,602 & 0,696 & 2,094 & 1,135 & 0,493 & 1,425 & 0,702 & 0,743 & 0,204 & 0,152 & 0,076 & 126 & 530 & $-1,0$ \\
\hline 62 & 530 & 0,221 & 1,674 & 0,237 & 0,520 & 0,933 & 0,311 & 0,693 & 0,991 & 1,005 & 0,838 & 0,781 & 0,579 & 0,453 & 0,461 & 11 & 530 & $-2,5$ \\
\hline 63 & 530 & 0,032 & 0,693 & 0,039 & 1,268 & 0,815 & 1,829 & 0,849 & 0,825 & 1,101 & 0,683 & 0,629 & 0,243 & 0,152 & 0,107 & 52 & 530 & $-1,3$ \\
\hline 64 & 530 & 0,083 & 1,644 & 0,123 & 0,317 & 0,674 & 0,193 & 0,870 & 0,387 & 1,608 & 0,284 & 0,372 & 0,611 & 0,227 & 0,214 & 86 & 530 & $-2,6$ \\
\hline 65 & 530 & 0,014 & 0,389 & 0,014 & 0,520 & 1,000 & 1,337 & 0,337 & 1,000 & 0,916 & 0,490 & 0,196 & 0,193 & 0,038 & 0,076 & 90 & 530 & $-0,9$ \\
\hline 66 & 530 & 0,016 & 0,714 & 0,029 & 0,553 & 0,550 & 0,775 & 0,503 & 0,389 & 1,604 & 0,264 & 0,369 & 0,276 & 0,102 & 0,076 & 152 & 530 & $-1,5$ \\
\hline 67 & 530 & 0,037 & 0,799 & 0,040 & 1,420 & 0,929 & 1,778 & 0,781 & 0,735 & 1,166 & 0,389 & 0,217 & 0,349 & 0,076 & 0,152 & 86 & 530 & $-1,8$ \\
\hline 68 & 530 & 0,142 & 1,877 & 0,171 & 0,469 & 0,832 & 0,250 & 0,769 & 0,507 & 1,405 & 0,460 & 0,513 & 0,627 & 0,322 & 0,169 & 117 & 530 & $-2,6$ \\
\hline 69 & 530 & 0,029 & 0,592 & 0,030 & 0,368 & 0,952 & 0,621 & 0,382 & 1,000 & 0,986 & 0,509 & 0,400 & 0,268 & 0,107 & 0,152 & 52 & 530 & $-1,4$ \\
\hline 70 & 530 & 0,017 & 0,469 & 0,019 & 0,583 & 0,923 & 1,243 & 0,255 & 0,983 & 1,009 & 0,477 & 0,250 & 0,214 & 0,054 & 0,076 & 46 & 530 & $-1,1$ \\
\hline 71 & 530 & 0,037 & 0,879 & 0,047 & 0,981 & 0,788 & 1,115 & 0,242 & 0,607 & 1,284 & 0,637 & 0,673 & 0,273 & 0,184 & 0,152 & 118 & 530 & $-1,4$ \\
\hline 72 & 530 & 0,052 & 1,480 & 0,083 & 0,896 & 0,621 & 0,606 & 0,547 & 0,297 & 1,836 & 0,316 & 0,422 & 0,456 & 0,192 & 0,152 & 70 & 530 & $-2,2$ \\
\hline 73 & 530 & 0,039 & 1,070 & 0,053 & 0,981 & 0,730 & 0,917 & 0,283 & 0,425 & 1,533 & 0,264 & 0,264 & 0,432 & 0,114 & 0,152 & 36 & 530 & $-2,1$ \\
\hline 74 & 530 & 0,080 & 1,674 & 0,099 & 0,693 & 0,812 & 0,414 & 0,615 & 0,360 & 1,666 & 0,385 & 0,468 & 0,515 & 0,241 & 0,107 & 126 & 530 & $-2,4$ \\
\hline 75 & 530 & 0,057 & 0,786 & 0,060 & 0,347 & 0,952 & 0,441 & 0,561 & 1,000 & 0,926 & 0,961 & 0,824 & 0,276 & 0,227 & 0,227 & 36 & 530 & $-1,5$ \\
\hline 76 & 530 & 0,026 & 0,490 & 0,027 & 0,469 & 0,947 & 0,957 & 0,068 & 1,000 & 0,861 & 1,146 & 0,894 & 0,169 & 0,152 & 0,152 & 63 & 530 & $-0,8$ \\
\hline 77 & 530 & 0,027 & 0,520 & 0,029 & 0,757 & 0,950 & 1,455 & 0,458 & 1,000 & 0,888 & 0,834 & 0,646 & 0,204 & 0,132 & 0,152 & 25 & 530 & $-1,0$ \\
\hline 78 & 530 & 0,052 & 0,858 & 0,062 & 1,255 & 0,837 & 1,463 & 0,491 & 0,882 & 1,065 & 0,539 & 0,490 & 0,349 & 0,171 & 0,169 & 78 & 530 & $-1,8$ \\
\hline 79 & 530 & 0,105 & 2,050 & 0,154 & 0,541 & 0,682 & 0,264 & 0,802 & 0,313 & 1,787 & 0,453 & 0,640 & 0,542 & 0,347 & 0,214 & 18 & 530 & $-2,4$ \\
\hline 80 & 530 & 0,037 & 0,786 & 0,044 & 0,562 & 0,839 & 0,715 & 0,327 & 0,758 & 1,148 & 0,736 & 0,738 & 0,254 & 0,187 & 0,152 & 39 & 530 & $-1,3$ \\
\hline 81 & 530 & 0,039 & 1,031 & 0,053 & 0,685 & 0,730 & 0,664 & 0,431 & 0,458 & 1,478 & 0,564 & 0,621 & 0,296 & 0,184 & 0,152 & 36 & 530 & $-1,6$ \\
\hline 82 & 530 & 0,029 & 1,091 & 0,052 & 0,744 & 0,556 & 0,682 & 0,546 & 0,303 & 1,816 & 0,392 & 0,571 & 0,305 & 0,174 & 0,076 & 118 & 530 & $-1,6$ \\
\hline 83 & 530 & 0,044 & 1,306 & 0,063 & 0,368 & 0,705 & 0,282 & 0,777 & 0,328 & 1,747 & 0,403 & 0,485 & 0,375 & 0,182 & 0,107 & 52 & 530 & $-1,9$ \\
\hline 84 & 530 & 0,027 & 0,511 & 0,029 & 0,490 & 0,950 & 0,959 & 0,065 & 1,000 & 0,874 & 0,968 & 0,800 & 0,189 & 0,152 & 0,152 & 31 & 530 & $-0,9$ \\
\hline 85 & 530 & 0,027 & 0,571 & 0,030 & 1,049 & 0,905 & 1,837 & 0,843 & 1,000 & 0,975 & 0,538 & 0,422 & 0,254 & 0,107 & 0,152 & 24 & 530 & $-1,3$ \\
\hline 86 & 530 & 0,062 & 1,234 & 0,083 & 0,317 & 0,741 & 0,257 & 0,787 & 0,509 & 1,402 & 0,271 & 0,249 & 0,538 & 0,134 & 0,152 & 41 & 530 & $-2,4$ \\
\hline 87 & 530 & 0,016 & 0,397 & 0,017 & 0,613 & 0,917 & 1,542 & 0,548 & 1,000 & 0,892 & 0,824 & 0,543 & 0,156 & 0,085 & 0,107 & 15 & 530 & $-0,6$ \\
\hline 88 & 530 & 0,026 & 0,828 & 0,042 & 2,113 & 0,621 & 2,551 & 1,597 & 0,473 & 1,454 & 0,286 & 0,324 & 0,339 & 0,110 & 0,107 & 64 & 530 & $-1,8$ \\
\hline 89 & 530 & 0,205 & 3,327 & 0,387 & 0,418 & 0,530 & 0,126 & 0,993 & 0,233 & 2,072 & 0,398 & 0,737 & 0,810 & 0,597 & 0,152 & 93 & 530 & $-3,0$ \\
\hline
\end{tabular}




\begin{tabular}{|c|c|c|c|c|c|c|c|c|c|c|c|c|c|c|c|c|c|c|}
\hline 90 & 530 & 0,019 & 0,520 & 0,022 & 0,389 & 0,867 & 0,748 & 0,285 & 0,867 & 1,074 & 0,404 & 0,260 & 0,243 & 0,063 & 0,076 & 30 & 530 & $-1,3$ \\
\hline 91 & 530 & 0,023 & 0,490 & 0,026 & 0,583 & 0,889 & 1,189 & 0,220 & 1,000 & 0,913 & 0,784 & 0,588 & 0,193 & 0,114 & 0,152 & 150 & 530 & $-0,9$ \\
\hline 92 & 530 & 0,047 & 0,820 & 0,053 & 0,317 & 0,892 & 0,387 & 0,623 & 0,886 & 1,063 & 0,618 & 0,485 & 0,312 & 0,152 & 0,169 & 170 & 530 & $-1,6$ \\
\hline 93 & 530 & 0,017 & 0,418 & 0,020 & 0,389 & 0,857 & 0,929 & 0,160 & 1,000 & 0,900 & 0,764 & 0,501 & 0,169 & 0,085 & 0,107 & 37 & 530 & $-0,8$ \\
\hline 94 & 530 & 0,020 & 0,490 & 0,022 & 0,440 & 0,933 & 0,896 & 0,123 & 1,000 & 0,976 & 0,524 & 0,380 & 0,221 & 0,084 & 0,107 & 26 & 530 & $-1,1$ \\
\hline 95 & 530 & 0,020 & 0,541 & 0,026 & 0,541 & 0,778 & 1,000 & 0,222 & 0,862 & 1,077 & 0,482 & 0,479 & 0,230 & 0,110 & 0,107 & 23 & 530 & $-1,2$ \\
\hline 96 & 530 & 0,030 & 0,643 & 0,037 & 0,807 & 0,808 & 1,256 & 0,320 & 0,917 & 1,044 & 0,594 & 0,580 & 0,254 & 0,147 & 0,169 & 125 & 530 & $-1,3$ \\
\hline 97 & 530 & 0,056 & 1,154 & 0,069 & 0,338 & 0,812 & 0,293 & 0,732 & 0,528 & 1,376 & 0,680 & 0,585 & 0,324 & 0,189 & 0,214 & 83 & 530 & $-1,7$ \\
\hline 98 & 530 & 0,020 & 0,440 & 0,022 & 0,440 & 0,933 & 1,000 & 0,067 & 1,000 & 0,875 & 0,686 & 0,392 & 0,193 & 0,076 & 0,152 & 81 & 530 & $-0,9$ \\
\hline 99 & 530 & 0,026 & 0,541 & 0,032 & 0,643 & 0,818 & 1,188 & 0,261 & 1,000 & 0,950 & 0,674 & 0,583 & 0,221 & 0,129 & 0,152 & 107 & 530 & $-1,1$ \\
\hline 100 & 530 & 0,034 & 1,010 & 0,050 & 0,389 & 0,686 & 0,385 & 0,691 & 0,424 & 1,536 & 0,501 & 0,569 & 0,296 & 0,168 & 0,076 & 3 & 530 & $-1,6$ \\
\hline \multirow[t]{8}{*}{101} & 530 & 0,022 & 0,490 & 0,022 & 0,368 & 1,000 & 0,750 & 0,250 & 1,000 & 0,943 & 0,597 & 0,460 & 0,214 & 0,099 & 0,107 & 45 & 530 & $-1,1$ \\
\hline & 531 & & & & & & & & nothing to measure & & & & & & & & 531 & - \\
\hline & 532 & & & & & & & & nothing to measure & & & & & & & & 532 & - \\
\hline & 533 & & & & & & & & nothing to measure & & & & & & & & 533 & - \\
\hline & 534 & & & & & & & & nothing to measure & & & & & & & & 534 & - \\
\hline & 535 & & & & & & & & nothing to measure & & & & & & & & 535 & - \\
\hline & 536 & & & & & & & & nothing to measure & & & & & & & & 536 & - \\
\hline & 537 & & & & & & & & nothing to measure & & & & & & & & 537 & - \\
\hline 1 & 538 & 0,039 & 0,879 & 0,047 & 0,807 & 0,818 & 0,918 & 0,199 & 0,630 & 1,260 & 0,315 & 0,233 & 0,395 & 0,092 & 0,152 & 165 & 538 & $-2,0$ \\
\hline 2 & 538 & 0,076 & 1,133 & 0,098 & 1,061 & 0,779 & 0,937 & 0,230 & 0,745 & 1,159 & 0,540 & 0,641 & 0,423 & 0,271 & 0,227 & 63 & 538 & $-2,1$ \\
\hline 3 & 538 & 0,017 & 0,440 & 0,020 & 0,368 & 0,857 & 0,837 & 0,217 & 1,000 & 0,945 & 0,588 & 0,373 & 0,193 & 0,072 & 0,076 & 105 & 538 & $-0,9$ \\
\hline 4 & 538 & 0,014 & 0,440 & 0,017 & 0,296 & 0,833 & 0,673 & 0,367 & 0,933 & 1,035 & 0,439 & 0,236 & 0,204 & 0,048 & 0,076 & 17 & 538 & $-1,0$ \\
\hline 5 & 538 & 0,019 & 0,410 & 0,020 & 0,778 & 0,929 & 1,897 & 0,900 & 1,000 & 0,847 & 0,828 & 0,447 & 0,169 & 0,076 & 0,152 & 90 & 538 & $-0,8$ \\
\hline 6 & 538 & 0,043 & 0,900 & 0,044 & 1,944 & 0,968 & 2,160 & 1,160 & 0,668 & 1,224 & 0,313 & 0,181 & 0,418 & 0,076 & 0,152 & 92 & 538 & $-2,1$ \\
\hline 7 & 538 & 0,132 & 2,189 & 0,182 & 0,410 & 0,724 & 0,187 & 0,858 & 0,346 & 1,700 & 0,172 & 0,228 & 0,988 & 0,225 & 0,169 & 89 & 538 & $-3,3$ \\
\hline 1 & 539 & 0,016 & 0,511 & 0,024 & 0,245 & 0,647 & 0,479 & 0,629 & 0,758 & 1,148 & 0,560 & 0,641 & 0,189 & 0,121 & 0,107 & 90 & 539 & $-0,9$ \\
\hline 2 & 539 & 0,030 & 0,592 & 0,032 & 0,550 & 0,955 & 0,929 & 0,084 & 1,000 & 0,962 & 0,652 & 0,442 & 0,243 & 0,107 & 0,107 & 139 & 539 & $-1,3$ \\
\hline 3 & 539 & 0,030 & 0,786 & 0,043 & 0,672 & 0,700 & 0,855 & 0,333 & 0,613 & 1,278 & 0,334 & 0,334 & 0,339 & 0,113 & 0,076 & 117 & 539 & $-1,8$ \\
\hline 4 & 539 & 0,023 & 0,490 & 0,024 & 0,397 & 0,941 & 0,811 & 0,198 & 1,000 & 0,913 & 0,702 & 0,496 & 0,204 & 0,101 & 0,152 & 103 & 539 & $-1,0$ \\
\hline 5 & 539 & 0,020 & 0,693 & 0,029 & 0,418 & 0,700 & 0,604 & 0,497 & 0,525 & 1,380 & 0,524 & 0,606 & 0,221 & 0,134 & 0,076 & 119 & 539 & $-1,1$ \\
\hline 6 & 539 & 0,016 & 0,592 & 0,023 & 0,418 & 0,688 & 0,707 & 0,428 & 0,566 & 1,329 & 0,342 & 0,344 & 0,243 & 0,083 & 0,076 & 127 & 539 & $-1,3$ \\
\hline 7 & 539 & 0,024 & 0,553 & 0,027 & 0,317 & 0,895 & 0,573 & 0,440 & 1,000 & 1,000 & 0,541 & 0,316 & 0,240 & 0,076 & 0,152 & 83 & 539 & $-1,3$ \\
\hline 8 & 539 & 0,036 & 0,664 & 0,040 & 0,541 & 0,893 & 0,815 & 0,214 & 1,000 & 0,988 & 0,601 & 0,467 & 0,276 & 0,129 & 0,152 & 108 & 539 & $-1,5$ \\
\hline 9 & 539 & 0,019 & 0,418 & 0,020 & 0,275 & 0,929 & 0,657 & 0,351 & 1,000 & 0,864 & 0,828 & 0,501 & 0,169 & 0,085 & 0,107 & 108 & 539 & $-0,8$ \\
\hline 10 & 539 & 0,039 & 0,858 & 0,050 & 0,765 & 0,771 & 0,892 & 0,253 & 0,661 & 1,230 & 0,354 & 0,383 & 0,373 & 0,143 & 0,152 & 147 & 539 & $-1,9$ \\
\hline 11 & 539 & 0,019 & 0,418 & 0,019 & 0,296 & 1,000 & 0,707 & 0,293 & 1,000 & 0,864 & 0,662 & 0,424 & 0,189 & 0,080 & 0,107 & 135 & 539 & $-0,9$ \\
\hline 12 & 539 & 0,022 & 0,469 & 0,024 & 0,347 & 0,882 & 0,739 & 0,286 & 1,000 & 0,902 & 0,955 & 0,683 & 0,169 & 0,116 & 0,152 & 49 & 539 & $-0,8$ \\
\hline 13 & 539 & 0,024 & 0,816 & 0,043 & 0,511 & 0,567 & 0,627 & 0,572 & 0,460 & 1,474 & 0,355 & 0,573 & 0,296 & 0,169 & 0,107 & 159 & 539 & $-1,6$ \\
\hline 14 & 539 & 0,032 & 1,049 & 0,047 & 0,968 & 0,667 & 0,923 & 0,342 & 0,361 & 1,665 & 0,192 & 0,223 & 0,458 & 0,102 & 0,076 & 121 & 539 & $-2,2$ \\
\hline 15 & 539 & 0,034 & 0,592 & 0,036 & 0,571 & 0,960 & 0,964 & 0,054 & 1,000 & 0,900 & 0,745 & 0,552 & 0,243 & 0,134 & 0,169 & 135 & 539 & $-1,3$ \\
\hline 16 & 539 & 0,141 & 1,539 & 0,152 & 1,387 & 0,925 & 0,901 & 0,124 & 0,746 & 1,158 & 0,430 & 0,374 & 0,645 & 0,241 & 0,273 & 133 & 539 & $-2,7$ \\
\hline 17 & 539 & 0,014 & 0,397 & 0,017 & 0,837 & 0,833 & 2,106 & 1,118 & 1,000 & 0,936 & 1,273 & 0,849 & 0,120 & 0,102 & 0,076 & 124 & 539 & $-0,3$ \\
\hline 18 & 539 & 0,046 & 0,960 & 0,059 & 0,541 & 0,780 & 0,564 & 0,488 & 0,627 & 1,263 & 0,326 & 0,304 & 0,423 & 0,129 & 0,152 & 109 & 539 & $-2,1$ \\
\hline 19 & 539 & 0,020 & 0,693 & 0,029 & 0,266 & 0,700 & 0,384 & 0,685 & 0,525 & 1,380 & 0,396 & 0,534 & 0,254 & 0,136 & 0,076 & 108 & 539 & $-1,3$ \\
\hline 20 & 539 & 0,017 & 0,368 & 0,017 & 1,252 & 1,000 & 3,404 & 2,404 & 1,000 & 0,791 & 0,849 & 0,500 & 0,161 & 0,080 & 0,107 & 135 & 539 & $-0,7$ \\
\hline 21 & 539 & 0,066 & 1,425 & 0,098 & 0,296 & 0,676 & 0,208 & 0,856 & 0,408 & 1,565 & 0,218 & 0,257 & 0,621 & 0,160 & 0,107 & 134 & 539 & $-2,6$ \\
\hline 22 & 539 & 0,014 & 0,368 & 0,016 & 0,613 & 0,909 & 1,667 & 0,673 & 1,000 & 0,866 & 0,637 & 0,400 & 0,169 & 0,068 & 0,076 & 159 & 539 & $-0,8$ \\
\hline 23 & 539 & 0,047 & 0,706 & 0,052 & 0,397 & 0,917 & 0,563 & 0,445 & 1,000 & 0,915 & 0,934 & 0,745 & 0,254 & 0,189 & 0,214 & 88 & 539 & $-1,3$ \\
\hline 24 & 539 & 0,017 & 0,469 & 0,019 & 0,520 & 0,923 & 1,108 & 0,133 & 0,983 & 1,009 & 0,449 & 0,310 & 0,221 & 0,068 & 0,107 & 135 & 539 & $-1,1$ \\
\hline 25 & 539 & 0,022 & 0,571 & 0,024 & 0,368 & 0,882 & 0,644 & 0,375 & 0,830 & 1,098 & 0,367 & 0,250 & 0,273 & 0,068 & 0,076 & 145 & 539 & $-1,4$ \\
\hline 26 & 539 & 0,014 & 0,490 & 0,019 & 0,347 & 0,769 & 0,707 & 0,373 & 0,750 & 1,155 & 0,344 & 0,227 & 0,230 & 0,052 & 0,076 & 15 & 539 & $-1,2$ \\
\hline 27 & 539 & 0,019 & 0,469 & 0,023 & 0,275 & 0,812 & 0,586 & 0,455 & 1,000 & 0,969 & 0,571 & 0,412 & 0,204 & 0,084 & 0,107 & 149 & 539 & $-1,0$ \\
\hline 28 & 539 & 0,016 & 0,389 & 0,017 & 0,592 & 0,917 & 1,522 & 0,529 & 1,000 & 0,873 & 0,824 & 0,485 & 0,156 & 0,076 & 0,107 & 90 & 539 & $-0,6$ \\
\hline 29 & 539 & 0,039 & 0,795 & 0,046 & 0,397 & 0,844 & 0,500 & 0,524 & 0,771 & 1,139 & 0,529 & 0,444 & 0,305 & 0,136 & 0,169 & 119 & 539 & $-1,6$ \\
\hline 30 & 539 & 0,022 & 0,490 & 0,024 & 0,326 & 0,882 & 0,664 & 0,356 & 1,000 & 0,943 & 0,597 & 0,491 & 0,214 & 0,105 & 0,152 & 135 & 539 & $-1,1$ \\
\hline 31 & 539 & 0,020 & 0,397 & 0,020 & 0,735 & 1,000 & 1,851 & 0,851 & 1,000 & 0,791 & 1,049 & 0,686 & 0,156 & 0,107 & 0,152 & 160 & 539 & $-0,6$ \\
\hline 32 & 539 & 0,042 & 1,061 & 0,057 & 0,418 & 0,725 & 0,394 & 0,665 & 0,464 & 1,467 & 0,506 & 0,702 & 0,324 & 0,227 & 0,152 & 110 & 539 & $-1,7$ \\
\hline
\end{tabular}




\begin{tabular}{|c|c|c|c|c|c|c|c|c|c|c|c|c|c|c|c|c|c|c|}
\hline 33 & 539 & 0,016 & 0,448 & 0,017 & 0,368 & 0,917 & 0,820 & 0,198 & 0,987 & 1,006 & 0,412 & 0,243 & 0,221 & 0,054 & 0,076 & 125 & 539 & $-1,1$ \\
\hline 34 & 539 & 0,019 & 0,448 & 0,020 & 0,317 & 0,929 & 0,707 & 0,301 & 1,000 & 0,926 & 0,662 & 0,424 & 0,189 & 0,080 & 0,107 & 143 & 539 & $-0,9$ \\
\hline 35 & 539 & 0,059 & 1,061 & 0,075 & 0,757 & 0,788 & 0,713 & 0,357 & 0,657 & 1,234 & 0,492 & 0,549 & 0,390 & 0,214 & 0,169 & 144 & 539 & $-2,0$ \\
\hline 36 & 539 & 0,019 & 0,389 & 0,019 & 0,368 & 1,000 & 0,946 & 0,054 & 1,000 & 0,803 & 0,974 & 0,485 & 0,156 & 0,076 & 0,152 & 98 & 539 & $-0,6$ \\
\hline 37 & 539 & 0,049 & 0,795 & 0,053 & 1,031 & 0,919 & 1,298 & 0,308 & 0,970 & 1,015 & 0,509 & 0,446 & 0,349 & 0,156 & 0,169 & 107 & 539 & $-1,8$ \\
\hline 38 & 539 & 0,020 & 0,490 & 0,026 & 0,389 & 0,778 & 0,793 & 0,304 & 1,000 & 0,976 & 0,686 & 0,588 & 0,193 & 0,114 & 0,107 & 11 & 539 & $-0,9$ \\
\hline 39 & 539 & 0,082 & 1,264 & 0,082 & 3,467 & 1,000 & 2,742 & 1,742 & 0,643 & 1,247 & 0,558 & 0,554 & 0,432 & 0,239 & 0,227 & 119 & 539 & $-2,1$ \\
\hline 40 & 539 & 0,023 & 0,490 & 0,026 & 0,583 & 0,889 & 1,189 & 0,220 & 1,000 & 0,913 & 0,702 & 0,470 & 0,204 & 0,096 & 0,152 & 101 & 539 & $-1,0$ \\
\hline 41 & 539 & 0,583 & 4,477 & 0,733 & 0,685 & 0,795 & 0,153 & 0,872 & 0,365 & 1,655 & 0,318 & 0,381 & 1,527 & 0,582 & 0,107 & 128 & 539 & $-3,9$ \\
\hline 42 & 539 & 0,017 & 0,693 & 0,027 & 0,418 & 0,632 & 0,604 & 0,541 & 0,450 & 1,491 & 0,235 & 0,228 & 0,305 & 0,070 & 0,076 & 11 & 539 & $-1,6$ \\
\hline 43 & 539 & 0,046 & 0,879 & 0,053 & 0,820 & 0,865 & 0,932 & 0,151 & 0,747 & 1,157 & 0,374 & 0,349 & 0,395 & 0,138 & 0,152 & 109 & 539 & $-2,0$ \\
\hline 44 & 539 & 0,019 & 0,541 & 0,024 & 0,938 & 0,765 & 1,735 & 0,771 & 0,801 & 1,118 & 0,414 & 0,324 & 0,240 & 0,078 & 0,076 & 152 & 539 & $-1,3$ \\
\hline 45 & 539 & 0,050 & 1,010 & 0,063 & 0,440 & 0,795 & 0,435 & 0,601 & 0,618 & 1,272 & 0,409 & 0,383 & 0,395 & 0,152 & 0,152 & 85 & 539 & $-2,0$ \\
\hline 46 & 539 & 0,057 & 1,387 & 0,095 & 0,317 & 0,606 & 0,229 & 0,866 & 0,375 & 1,633 & 0,525 & 0,778 & 0,373 & 0,290 & 0,152 & 69 & 539 & $-1,9$ \\
\hline 47 & 539 & 0,024 & 0,490 & 0,026 & 0,368 & 0,944 & 0,750 & 0,256 & 1,000 & 0,886 & 0,637 & 0,485 & 0,221 & 0,107 & 0,152 & 117 & 539 & $-1,1$ \\
\hline 48 & 539 & 0,019 & 0,397 & 0,019 & 0,613 & 1,000 & 1,542 & 0,542 & 1,000 & 0,821 & 0,974 & 0,686 & 0,156 & 0,107 & 0,152 & 90 & 539 & $-0,6$ \\
\hline 49 & 539 & 0,019 & 0,469 & 0,022 & 0,397 & 0,867 & 0,847 & 0,203 & 1,000 & 0,969 & 0,571 & 0,411 & 0,204 & 0,084 & 0,107 & 162 & 539 & $-1,0$ \\
\hline 50 & 539 & 0,050 & 0,685 & 0,050 & 0,888 & 1,000 & 1,297 & 0,297 & 1,000 & 0,862 & 0,990 & 0,745 & 0,254 & 0,189 & 0,214 & 107 & 539 & $-1,3$ \\
\hline 51 & 539 & 0,023 & 0,571 & 0,026 & 0,469 & 0,889 & 0,822 & 0,210 & 0,886 & 1,063 & 0,497 & 0,331 & 0,243 & 0,080 & 0,107 & 144 & 539 & $-1,3$ \\
\hline 52 & 539 & 0,049 & 1,082 & 0,065 & 2,185 & 0,756 & 2,019 & 1,048 & 0,524 & 1,382 & 0,276 & 0,282 & 0,475 & 0,134 & 0,152 & 118 & 539 & $-2,2$ \\
\hline 53 & 539 & 0,026 & 0,613 & 0,030 & 0,245 & 0,857 & 0,400 & 0,617 & 0,864 & 1,076 & 0,458 & 0,424 & 0,268 & 0,114 & 0,152 & 152 & 539 & $-1,4$ \\
\hline 54 & 539 & 0,283 & 2,541 & 0,360 & 0,296 & 0,785 & 0,116 & 0,909 & 0,550 & 1,348 & 0,482 & 0,589 & 0,864 & 0,508 & 0,379 & 111 & 539 & $-3,1$ \\
\hline 55 & 539 & 0,016 & 0,338 & 0,016 & 0,592 & 1,000 & 1,751 & 0,751 & 1,000 & 0,759 & 1,077 & 0,555 & 0,137 & 0,076 & 0,152 & 90 & 539 & $-0,5$ \\
\hline 56 & 539 & 0,014 & 0,368 & 0,016 & 0,550 & 0,909 & 1,495 & 0,503 & 1,000 & 0,866 & 0,749 & 0,461 & 0,156 & 0,072 & 0,076 & 18 & 539 & $-0,6$ \\
\hline 57 & 539 & 0,029 & 0,735 & 0,040 & 0,224 & 0,714 & 0,305 & 0,752 & 0,667 & 1,225 & 0,480 & 0,435 & 0,276 & 0,120 & 0,107 & 109 & 539 & $-1,5$ \\
\hline 58 & 539 & 0,027 & 0,651 & 0,030 & 0,693 & 0,905 & 1,065 & 0,115 & 0,808 & 1,113 & 0,372 & 0,263 & 0,305 & 0,080 & 0,107 & 125 & 539 & $-1,6$ \\
\hline 59 & 539 & 0,014 & 0,347 & 0,014 & 0,744 & 1,000 & 2,146 & 1,146 & 1,000 & 0,816 & 0,707 & 0,333 & 0,161 & 0,054 & 0,107 & 135 & 539 & $-0,7$ \\
\hline 60 & 539 & 0,037 & 0,938 & 0,053 & 1,873 & 0,703 & 1,996 & 1,039 & 0,532 & 1,371 & 0,447 & 0,493 & 0,326 & 0,161 & 0,107 & 149 & 539 & $-1,7$ \\
\hline 61 & 539 & 0,059 & 0,960 & 0,070 & 2,029 & 0,837 & 2,115 & 1,127 & 0,803 & 1,116 & 0,614 & 0,573 & 0,349 & 0,200 & 0,107 & 132 & 539 & $-1,8$ \\
\hline 62 & 539 & 0,136 & 2,038 & 0,164 & 0,672 & 0,833 & 0,330 & 0,691 & 0,412 & 1,557 & 0,190 & 0,198 & 0,956 & 0,189 & 0,214 & 127 & 539 & $-3,3$ \\
\hline 63 & 539 & 0,178 & 2,702 & 0,317 & 0,592 & 0,561 & 0,219 & 0,896 & 0,306 & 1,807 & 0,316 & 0,621 & 0,847 & 0,526 & 0,240 & 83 & 539 & $-3,1$ \\
\hline 64 & 539 & 0,039 & 0,774 & 0,043 & 0,418 & 0,900 & 0,541 & 0,470 & 0,813 & 1,109 & 0,430 & 0,316 & 0,339 & 0,107 & 0,152 & 126 & 539 & $-1,8$ \\
\hline 65 & 539 & 0,020 & 0,744 & 0,034 & 0,685 & 0,583 & 0,920 & 0,424 & 0,456 & 1,481 & 0,336 & 0,416 & 0,276 & 0,115 & 0,076 & 140 & 539 & $-1,5$ \\
\hline 66 & 539 & 0,022 & 0,448 & 0,023 & 2,520 & 0,938 & 5,622 & 4,622 & 1,000 & 0,862 & 0,955 & 0,632 & 0,169 & 0,107 & 0,152 & 110 & 539 & $-0,8$ \\
\hline 67 & 539 & 0,043 & 0,807 & 0,052 & 1,023 & 0,833 & 1,267 & 0,315 & 0,830 & 1,098 & 0,429 & 0,386 & 0,357 & 0,138 & 0,152 & 111 & 539 & $-1,8$ \\
\hline 68 & 539 & 0,430 & 2,714 & 0,475 & 0,173 & 0,906 & 0,064 & 0,941 & 0,734 & 1,167 & 0,516 & 0,543 & 1,030 & 0,559 & 0,508 & 122 & 539 & $-3,4$ \\
\hline 69 & 539 & 0,075 & 1,429 & 0,103 & 0,368 & 0,722 & 0,257 & 0,793 & 0,459 & 1,476 & 0,389 & 0,445 & 0,494 & 0,220 & 0,152 & 98 & 539 & $-2,3$ \\
\hline 70 & 539 & 0,014 & 0,317 & 0,014 & 0,275 & 1,000 & 0,867 & 0,133 & 1,000 & 0,746 & 0,979 & 0,555 & 0,137 & 0,076 & 0,107 & 108 & 539 & $-0,5$ \\
\hline 71 & 539 & 0,022 & 0,418 & 0,023 & 0,275 & 0,938 & 0,657 & 0,349 & 1,000 & 0,805 & 1,123 & 0,728 & 0,156 & 0,114 & 0,152 & 168 & 539 & $-0,6$ \\
\hline 72 & 539 & 0,014 & 0,317 & 0,014 & 0,347 & 1,000 & 1,094 & 0,094 & 1,000 & 0,746 & 1,273 & 0,632 & 0,120 & 0,076 & 0,107 & 162 & 539 & $-0,3$ \\
\hline 73 & 539 & 0,016 & 0,469 & 0,019 & 0,541 & 0,846 & 1,153 & 0,217 & 0,901 & 1,054 & 0,539 & 0,496 & 0,193 & 0,096 & 0,076 & 118 & 539 & $-0,9$ \\
\hline 74 & 539 & 0,014 & 0,418 & 0,014 & 0,816 & 1,000 & 1,950 & 0,950 & 1,000 & 0,986 & 0,509 & 0,358 & 0,189 & 0,068 & 0,076 & 108 & 539 & $-0,9$ \\
\hline 75 & 539 & 0,032 & 0,816 & 0,040 & 0,960 & 0,786 & 1,176 & 0,277 & 0,596 & 1,295 & 0,384 & 0,351 & 0,324 & 0,114 & 0,107 & 14 & 539 & $-1,7$ \\
\hline 76 & 539 & 0,046 & 1,070 & 0,066 & 0,389 & 0,696 & 0,363 & 0,706 & 0,504 & 1,408 & 0,326 & 0,460 & 0,423 & 0,195 & 0,152 & 103 & 539 & $-2,1$ \\
\hline 77 & 539 & 0,085 & 1,031 & 0,093 & 0,571 & 0,908 & 0,553 & 0,456 & 1,000 & 1,000 & 0,642 & 0,621 & 0,410 & 0,254 & 0,273 & 127 & 539 & $-2,0$ \\
\hline 78 & 539 & 0,019 & 0,469 & 0,022 & 0,896 & 0,867 & 1,910 & 0,920 & 1,000 & 0,969 & 0,571 & 0,362 & 0,204 & 0,074 & 0,076 & 58 & 539 & $-1,0$ \\
\hline 79 & 539 & 0,037 & 0,714 & 0,042 & 0,672 & 0,897 & 0,941 & 0,119 & 0,919 & 1,043 & 0,543 & 0,453 & 0,296 & 0,134 & 0,152 & 135 & 539 & $-1,6$ \\
\hline 80 & 539 & 0,066 & 1,091 & 0,080 & 0,520 & 0,821 & 0,477 & 0,553 & 0,697 & 1,198 & 0,658 & 0,675 & 0,357 & 0,241 & 0,169 & 125 & 539 & $-1,8$ \\
\hline 81 & 539 & 0,032 & 0,858 & 0,042 & 0,541 & 0,759 & 0,631 & 0,441 & 0,539 & 1,362 & 0,330 & 0,307 & 0,349 & 0,107 & 0,152 & 146 & 539 & $-1,8$ \\
\hline 82 & 539 & 0,024 & 0,651 & 0,030 & 0,317 & 0,810 & 0,487 & 0,547 & 0,723 & 1,176 & 0,481 & 0,397 & 0,254 & 0,101 & 0,107 & 132 & 539 & $-1,3$ \\
\hline 83 & 539 & 0,023 & 0,592 & 0,027 & 0,397 & 0,842 & 0,672 & 0,364 & 0,824 & 1,102 & 0,407 & 0,300 & 0,268 & 0,080 & 0,107 & 125 & 539 & $-1,4$ \\
\hline 84 & 539 & 0,016 & 0,520 & 0,023 & 0,867 & 0,688 & 1,667 & 0,736 & 0,733 & 1,168 & 0,483 & 0,470 & 0,204 & 0,096 & 0,076 & 148 & 539 & $-1,0$ \\
\hline 85 & 539 & 0,023 & 0,562 & 0,026 & 0,397 & 0,889 & 0,707 & 0,313 & 0,913 & 1,047 & 0,497 & 0,350 & 0,243 & 0,085 & 0,107 & 125 & 539 & $-1,3$ \\
\hline 86 & 539 & 0,050 & 1,378 & 0,083 & 4,853 & 0,603 & 3,522 & 2,553 & 0,332 & 1,735 & 0,384 & 0,605 & 0,408 & 0,247 & 0,107 & 53 & 539 & $-2,0$ \\
\hline 87 & 539 & 0,023 & 0,440 & 0,023 & 0,490 & 1,000 & 1,115 & 0,115 & 1,000 & 0,818 & 1,019 & 0,671 & 0,169 & 0,114 & 0,152 & 0 & 539 & $-0,8$ \\
\hline 88 & 539 & 1,121 & 5,120 & 1,274 & 0,613 & 0,880 & 0,120 & 0,888 & 0,537 & 1,364 & 0,294 & 0,318 & 2,202 & 0,700 & 0,678 & 125 & 539 & $-4,5$ \\
\hline 89 & 539 & 0,017 & 0,664 & 0,032 & 0,266 & 0,545 & 0,401 & 0,752 & 0,491 & 1,427 & 0,288 & 0,370 & 0,276 & 0,102 & 0,076 & 28 & 539 & $-1,5$ \\
\hline 90 & 539 & 0,033 & 0,685 & 0,037 & 0,550 & 0,885 & 0,803 & 0,228 & 0,885 & 1,063 & 0,451 & 0,372 & 0,305 & 0,114 & 0,152 & 8 & 539 & $-1,6$ \\
\hline
\end{tabular}




\begin{tabular}{|c|c|c|c|c|c|c|c|c|c|c|c|c|c|c|c|c|c|c|}
\hline 91 & 539 & 0,017 & 0,368 & 0,017 & 0,807 & 1,000 & 2,195 & 1,195 & 1,000 & 0,791 & 1,175 & 0,745 & 0,137 & 0,102 & 0,152 & 90 & 539 & $-0,5$ \\
\hline 92 & 539 & 0,017 & 0,571 & 0,022 & 0,194 & 0,800 & 0,341 & 0,689 & 0,664 & 1,227 & 0,306 & 0,200 & 0,268 & 0,054 & 0,107 & 130 & 539 & $-1,4$ \\
\hline 93 & 539 & 0,042 & 1,163 & 0,063 & 1,061 & 0,659 & 0,913 & 0,352 & 0,387 & 1,608 & 0,327 & 0,399 & 0,403 & 0,161 & 0,107 & 141 & 539 & $-2,0$ \\
\hline 94 & 539 & 0,020 & 0,532 & 0,022 & 1,264 & 0,933 & 2,374 & 1,376 & 0,890 & 1,060 & 0,446 & 0,316 & 0,240 & 0,076 & 0,152 & 172 & 539 & $-1,3$ \\
\hline 95 & 539 & 0,093 & 1,163 & 0,102 & 0,490 & 0,915 & 0,422 & 0,584 & 0,867 & 1,074 & 0,505 & 0,442 & 0,485 & 0,214 & 0,240 & 134 & 539 & $-2,3$ \\
\hline 96 & 539 & 0,076 & 2,139 & 0,149 & 0,347 & 0,510 & 0,162 & 0,971 & 0,209 & 2,189 & 0,343 & 0,677 & 0,532 & 0,360 & 0,152 & 115 & 539 & $-2,4$ \\
\hline 97 & 539 & 0,023 & 0,592 & 0,033 & 1,175 & 0,696 & 1,985 & 1,031 & 0,824 & 1,102 & 0,702 & 0,694 & 0,204 & 0,142 & 0,107 & 103 & 539 & $-1,0$ \\
\hline 98 & 539 & 0,014 & 0,397 & 0,014 & 2,959 & 1,000 & 7,446 & 6,446 & 1,000 & 0,936 & 0,509 & 0,283 & 0,189 & 0,054 & 0,076 & 125 & 539 & $-0,9$ \\
\hline 99 & 539 & 0,126 & 1,234 & 0,132 & 0,888 & 0,957 & 0,719 & 0,284 & 1,000 & 0,980 & 0,714 & 0,620 & 0,475 & 0,294 & 0,312 & 51 & 539 & $-2,2$ \\
\hline 100 & 539 & 0,570 & 3,082 & 0,621 & 0,550 & 0,917 & 0,178 & 0,826 & 0,754 & 1,152 & 0,507 & 0,549 & 1,196 & 0,656 & 0,577 & 112 & 539 & $-3,6$ \\
\hline 101 & 539 & 0,056 & 1,010 & 0,067 & 0,317 & 0,830 & 0,314 & 0,707 & 0,689 & 1,205 & 0,362 & 0,344 & 0,443 & 0,152 & 0,169 & 109 & 539 & $-2,1$ \\
\hline 102 & 539 & 0,023 & 0,723 & 0,032 & 0,317 & 0,727 & 0,438 & 0,624 & 0,552 & 1,346 & 0,313 & 0,253 & 0,305 & 0,077 & 0,076 & 40 & 539 & $-1,6$ \\
\hline 103 & 539 & 0,016 & 0,368 & 0,016 & 0,643 & 1,000 & 1,747 & 0,747 & 1,000 & 0,826 & 0,700 & 0,447 & 0,169 & 0,076 & 0,107 & 108 & 539 & $-0,8$ \\
\hline 104 & 539 & 0,019 & 0,418 & 0,022 & 0,735 & 0,867 & 1,757 & 0,769 & 1,000 & 0,864 & 0,828 & 0,600 & 0,169 & 0,102 & 0,107 & 135 & 539 & $-0,8$ \\
\hline 105 & 539 & 0,049 & 0,744 & 0,052 & 0,368 & 0,944 & 0,494 & 0,509 & 1,000 & 0,950 & 0,746 & 0,650 & 0,288 & 0,187 & 0,214 & 57 & 539 & $-1,5$ \\
\hline 106 & 539 & 0,055 & 0,917 & 0,070 & 1,763 & 0,776 & 1,922 & 0,949 & 0,814 & 1,108 & 0,605 & 0,600 & 0,339 & 0,203 & 0,214 & 115 & 539 & $-1,8$ \\
\hline 107 & 539 & 0,026 & 0,532 & 0,029 & 1,501 & 0,900 & 2,818 & 1,821 & 1,000 & 0,935 & 0,674 & 0,514 & 0,221 & 0,114 & 0,152 & 107 & 539 & $-1,1$ \\
\hline 108 & 539 & 0,155 & 1,814 & 0,184 & 0,469 & 0,844 & 0,259 & 0,758 & 0,592 & 1,300 & 0,285 & 0,325 & 0,832 & 0,270 & 0,273 & 144 & 539 & $-3,1$ \\
\hline 109 & 539 & 0,136 & 2,017 & 0,179 & 1,175 & 0,760 & 0,583 & 0,481 & 0,421 & 1,541 & 0,385 & 0,508 & 0,671 & 0,341 & 0,273 & 4 & 539 & $-2,7$ \\
\hline 110 & 539 & 0,026 & 0,693 & 0,034 & 0,440 & 0,750 & 0,634 & 0,443 & 0,675 & $\begin{array}{l}1,217 \\
\end{array}$ & 0,674 & 0,728 & 0,221 & 0,161 & 0,107 & 43 & 539 & $-1,1$ \\
\hline 111 & 539 & 0,115 & 1,560 & 0,146 & 0,960 & 0,784 & 0,615 & 0,441 & 0,593 & $\begin{array}{l}1,299 \\
\text {. }\end{array}$ & 0,684 & 0,737 & 0,462 & 0,341 & 0,227 & 90 & 539 & $-2,2$ \\
\hline 112 & 539 & 0,022 & 0,511 & 0,024 & 1,052 & 0,882 & 2,058 & 1,065 & 1,000 & 0,983 & 0,659 & 0,371 & 0,204 & 0,076 & 0,152 & 171 & 539 & $-1,0$ \\
\hline 113 & 539 & 0,075 & 1,213 & 0,093 & 1,103 & 0,800 & 0,909 & 0,220 & 0,637 & 1,253 & 0,625 & 0,755 & 0,390 & 0,295 & 0,214 & 105 & 539 & $\begin{array}{l}-2,0 \\
\end{array}$ \\
\hline 114 & 539 & 0,060 & 1,154 & 0,093 & 1,061 & 0,646 & 0,920 & 0,363 & 0,569 & $\begin{array}{l}1,326 \\
\text { n }\end{array}$ & 0,366 & 0,521 & 0,458 & 0,238 & 0,214 & 166 & 539 & $-2,2$ \\
\hline 115 & 539 & 0,121 & 1,205 & 0,126 & 0,998 & 0,955 & 0,828 & 0,178 & 1,000 & 0,979 & 0,733 & 0,662 & 0,458 & 0,303 & 0,312 & 111 & 539 & $-2,2$ \\
\hline 116 & 539 & 0,063 & 1,450 & 0,103 & 0,499 & 0,611 & 0,344 & 0,762 & 0,377 & 1,628 & 0,310 & 0,501 & 0,510 & 0,255 & 0,152 & 129 & 539 & $-2,4$ \\
\hline 117 & 539 & 0,066 & 1,163 & 0,082 & 0,634 & 0,807 & 0,545 & 0,494 & 0,614 & 1,277 & 0,317 & 0,312 & 0,515 & 0,161 & 0,152 & 133 & 539 & $-2,4$ \\
\hline 118 & 539 & 0,023 & 0,693 & 0,030 & 0,651 & 0,762 & 0,939 & 0,246 & 0,600 & $\begin{array}{l}1,291 \\
\end{array}$ & 0,283 & 0,244 & 0,321 & 0,077 & 0,076 & 130 & 539 & $-1,7$ \\
\hline 119 & 539 & 0,039 & 0,723 & 0,046 & 0,347 & 0,844 & 0,479 & 0,543 & 0,931 & $\begin{array}{l}1,036 \\
\text { S }\end{array}$ & 0,764 & 0,734 & 0,254 & 0,186 & 0,169 & 103 & 539 & $-1,3$ \\
\hline 120 & 539 & 0,026 & 0,867 & 0,039 & 0,440 & 0,667 & 0,507 & 0,595 & 0,432 & 1,521 & 0,229 & 0,250 & 0,379 & 0,095 & 0,076 & 142 & 539 & $-1,9$ \\
\hline 121 & 539 & 0,014 & 0,520 & 0,020 & 0,296 & 0,714 & 0,569 & 0,517 & 0,667 & 1,225 & 0,311 & 0,221 & 0,243 & 0,054 & 0,076 & 152 & 539 & $-1,3$ \\
\hline 122 & 539 & 0,029 & 0,541 & 0,030 & 0,317 & 0,952 & 0,586 & 0,417 & 1,000 & 0,901 & 0,878 & 0,657 & 0,204 & 0,134 & 0,152 & 122 & 539 & $-1,0$ \\
\hline 123 & 539 & 0,017 & 0,461 & 0,020 & 1,277 & 0,857 & 2,771 & 1,777 & 1,000 & 0,990 & 0,527 & 0,315 & 0,204 & 0,064 & 0,076 & 17 & 539 & $-1,0$ \\
\hline 124 & 539 & 0,016 & 0,368 & 0,016 & 0,245 & 1,000 & 0,667 & 0,333 & 1,000 & 0,826 & 1,077 & 0,745 & 0,137 & 0,102 & 0,152 & 108 & 539 & $-0,5$ \\
\hline 125 & 539 & 0,115 & 1,602 & 0,149 & 0,744 & 0,769 & 0,464 & 0,583 & 0,562 & 1,334 & 0,504 & 0,595 & 0,538 & 0,320 & 0,240 & 140 & 539 & $-2,4$ \\
\hline 126 & 539 & 0,014 & 0,389 & 0,017 & 0,541 & 0,833 & 1,392 & 0,426 & 1,000 & 0,916 & 0,749 & 0,471 & 0,156 & 0,074 & 0,076 & 108 & 539 & $-0,6$ \\
\hline 127 & 539 & 0,032 & 0,888 & 0,042 & 0,461 & 0,759 & 0,519 & 0,538 & 0,503 & 1,410 & 0,239 & 0,219 & 0,410 & 0,090 & 0,107 & 123 & 539 & $-2,0$ \\
\hline 128 & 539 & 0,023 & 0,706 & 0,033 & 0,194 & 0,696 & 0,275 & 0,786 & 0,579 & 1,314 & 0,392 & 0,496 & 0,273 & 0,136 & 0,076 & 104 & 539 & $-1,4$ \\
\hline & 540 & & & & & & & & nothing to measure & & & & & & & & 540 & - \\
\hline 1 & 541 & 0,014 & 0,368 & 0,014 & 0,296 & 1,000 & 0,805 & 0,195 & 1,000 & 0,866 & 0,637 & 0,400 & 0,169 & 0,068 & 0,076 & 32 & 541 & $-0,8$ \\
\hline & 542 & & & & & & & & nothing to measure & & & & & & & & 542 & - \\
\hline & 543 & & & & & & & & nothing to measure & & & & & & & & 543 & - \\
\hline & 544 & & & & & & & & nothing to measure & & & & & & & & 544 & - \\
\hline & 545 & & & & & & & & nothing to measure & & & & & & & & 545 & - \\
\hline & 546 & & & & & & & & nothing to measure & & & & & & & & 546 & - \\
\hline & 547 & & & & & & & & nothing to measure & & & & & & & & 547 & - \\
\hline & 548 & & & & & & & & nothing to measure & & & & & & & & 548 & - \\
\hline & 549 & & & & & & & & nothing to measure & & & & & & & & 549 & - \\
\hline & 550 & & & & & & & & nothing to measure & & & & & & & & 550 & - \\
\hline & 551 & & & & & & & & nothing to measure & & & & & & & & 551 & - \\
\hline & 552 & & & & & & & & nothing to measure & & & & & & & & 552 & - \\
\hline & 553 & & & & & & & & nothing to measure & & & & & & & & 553 & 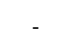 \\
\hline 1 & 554 & 0,020 & 0,735 & 0,026 & 0,418 & 0,778 & 0,569 & 0,485 & 0,467 & 1,464 & 0,615 & 0,557 & 0,204 & 0,114 & 0,076 & 131 & 554 & $-1,0$ \\
\hline 2 & 554 & 0,022 & 0,714 & 0,030 & 0,440 & 0,714 & 0,615 & 0,479 & 0,530 & 1,374 & 0,329 & 0,294 & 0,288 & 0,085 & 0,107 & 72 & 554 & $-1,5$ \\
\hline 3 & 554 & 0,023 & 0,490 & 0,026 & 0,418 & 0,889 & 0,854 & 0,184 & 1,000 & 0,913 & 0,702 & 0,470 & 0,204 & 0,096 & 0,107 & 109 & 554 & $-1,0$ \\
\hline 4 & 554 & 0,052 & 0,858 & 0,057 & 0,643 & 0,900 & 0,749 & 0,270 & 0,882 & 1,065 & 0,637 & 0,647 & 0,321 & 0,208 & 0,214 & 63 & 554 & $-1,7$ \\
\hline 5 & 554 & 0,172 & 2,152 & 0,212 & 1,940 & 0,811 & 0,902 & 0,213 & 0,467 & 1,463 & 0,243 & 0,286 & 0,949 & 0,271 & 0,273 & 21 & 554 & $-3,2$ \\
\hline 6 & 554 & 3,588 & 9,952 & 4,246 & 9,250 & 0,845 & 0,929 & 0,170 & 0,455 & 1,482 & 0,265 & 0,338 & 4,152 & 1,405 & 0,152 & 40 & 554 & $-5,4$ \\
\hline
\end{tabular}




\begin{tabular}{|c|c|c|c|c|c|c|c|c|c|c|c|c|c|c|c|c|c|c|}
\hline 7 & 554 & 0,043 & 1,133 & 0,059 & 0,989 & 0,732 & 0,873 & 0,297 & 0,421 & 1,540 & 0,186 & 0,188 & 0,542 & 0,102 & 0,076 & 61 & 554 & $-2,4$ \\
\hline 8 & 554 & 0,052 & 1,416 & 0,076 & 1,171 & 0,679 & 0,827 & 0,364 & 0,324 & 1,758 & 0,170 & 0,187 & 0,621 & 0,116 & 0,107 & 35 & 554 & $-2,6$ \\
\hline 9 & 554 & 0,024 & 0,490 & 0,026 & 0,368 & 0,944 & 0,750 & 0,256 & 1,000 & 0,886 & 0,866 & 0,667 & 0,189 & 0,126 & 0,152 & 38 & 554 & $-0,9$ \\
\hline 10 & 554 & 0,684 & 4,950 & 0,858 & 4,539 & 0,798 & 0,917 & 0,219 & 0,351 & 1,688 & 0,191 & 0,231 & 2,133 & 0,493 & 0,455 & 78 & 554 & $-4,4$ \\
\hline 11 & 554 & 0,026 & 0,714 & 0,034 & 0,541 & 0,750 & 0,757 & 0,348 & 0,636 & 1,254 & 0,458 & 0,566 & 0,268 & 0,152 & 0,107 & 57 & 554 & $-1,4$ \\
\hline 12 & 554 & 0,017 & 0,520 & 0,020 & 0,418 & 0,857 & 0,805 & 0,242 & 0,800 & 1,118 & 0,382 & 0,316 & 0,240 & 0,076 & 0,107 & 15 & 554 & $-1,3$ \\
\hline 13 & 554 & 0,141 & 1,814 & 0,168 & 1,721 & 0,838 & 0,949 & 0,170 & 0,537 & 1,364 & 0,259 & 0,252 & 0,831 & 0,209 & 0,214 & 51 & 554 & $-3,1$ \\
\hline 14 & 554 & 0,108 & 1,458 & 0,129 & 1,264 & 0,833 & 0,867 & 0,213 & 0,636 & 1,254 & 0,424 & 0,418 & 0,568 & 0,238 & 0,214 & 62 & 554 & $-2,5$ \\
\hline 15 & 554 & 0,014 & 0,448 & 0,016 & 0,296 & 0,909 & 0,660 & 0,352 & 0,898 & 1,055 & 0,439 & 0,250 & 0,204 & 0,051 & 0,076 & 62 & 554 & $-1,0$ \\
\hline 16 & 554 & 0,030 & 0,655 & 0,036 & 0,541 & 0,840 & 0,826 & 0,236 & 0,883 & 1,064 & 0,594 & 0,567 & 0,254 & 0,144 & 0,152 & 91 & 554 & $-1,3$ \\
\hline 17 & 554 & 0,017 & 0,368 & 0,017 & 0,245 & 1,000 & 0,667 & 0,333 & 1,000 & 0,791 & 1,175 & 0,784 & 0,137 & 0,107 & 0,152 & 0 & 554 & $-0,5$ \\
\hline 18 & 554 & 0,030 & 0,693 & 0,034 & 0,469 & 0,875 & 0,677 & 0,347 & 0,788 & 1,127 & 0,411 & 0,351 & 0,305 & 0,107 & 0,152 & 60 & 554 & $-1,6$ \\
\hline 19 & 554 & 0,020 & 0,541 & 0,023 & 0,440 & 0,875 & 0,812 & 0,225 & 0,862 & 1,077 & 0,396 & 0,267 & 0,254 & 0,068 & 0,076 & 60 & 554 & $-1,3$ \\
\hline 20 & 554 & 0,016 & 0,368 & 0,016 & 0,275 & 1,000 & 0,747 & 0,253 & 1,000 & 0,826 & 1,077 & 0,745 & 0,137 & 0,102 & 0,107 & 117 & 554 & $-0,5$ \\
\hline 21 & 554 & 0,050 & 1,704 & 0,092 & 1,196 & 0,547 & 0,702 & 0,542 & 0,217 & 2,145 & 0,185 & 0,268 & 0,588 & 0,158 & 0,107 & 77 & 554 & $-2,6$ \\
\hline 22 & 554 & 0,296 & 3,458 & 0,399 & 2,168 & 0,741 & 0,627 & 0,454 & 0,311 & 1,794 & 0,525 & 0,620 & 0,847 & 0,525 & 0,169 & 87 & 554 & $-3,1$ \\
\hline 23 & 554 & 0,123 & 1,746 & 0,151 & 1,268 & 0,819 & 0,726 & 0,328 & 0,509 & 1,402 & 0,547 & 0,566 & 0,536 & 0,303 & 0,240 & 85 & 554 & $-2,4$ \\
\hline 24 & 554 & 1,392 & 8,677 & 1,926 & 7,349 & 0,723 & 0,847 & 0,317 & 0,232 & 2,075 & 0,145 & 0,197 & 3,490 & 0,687 & 0,339 & 85 & 554 & $-5,1$ \\
\hline 25 & 554 & 0,019 & 0,571 & 0,020 & 0,397 & 0,929 & 0,696 & 0,312 & 0,720 & 1,179 & 0,318 & 0,249 & 0,273 & 0,068 & 0,076 & 55 & 554 & $-1,4$ \\
\hline 26 & 554 & 0,033 & 0,744 & 0,040 & 0,643 & 0,821 & 0,864 & 0,225 & 0,749 & 1,155 & 0,401 & 0,355 & 0,324 & 0,115 & 0,107 & 57 & 554 & $-1,7$ \\
\hline 27 & 554 & 0,023 & 0,622 & 0,029 & 0,499 & 0,800 & 0,803 & 0,281 & 0,747 & 1,157 & 0,334 & 0,261 & 0,296 & 0,077 & 0,076 & 47 & 554 & $-1,6$ \\
\hline 28 & 554 & 0,030 & 0,960 & 0,043 & 0,909 & 0,700 & 0,947 & 0,305 & 0,411 & 1,559 & 0,183 & 0,154 & 0,458 & 0,070 & 0,076 & 69 & 554 & $-2,2$ \\
\hline 29 & 554 & 0,023 & 0,520 & 0,027 & 0,389 & 0,842 & 0,748 & 0,298 & 1,000 & 0,968 & 0,815 & 0,667 & 0,189 & 0,126 & 0,152 & 120 & 554 & $-0,9$ \\
\hline 30 & 554 & 0,036 & 0,664 & 0,040 & 0,562 & 0,893 & 0,847 & 0,187 & 1,000 & 0,988 & 0,707 & 0,632 & 0,254 & 0,161 & 0,152 & 41 & 554 & $-1,3$ \\
\hline 31 & 554 & 0,014 & 0,418 & 0,017 & 0,266 & 0,833 & 0,636 & 0,400 & 1,000 & 0,986 & 0,979 & 0,621 & 0,137 & 0,085 & 0,076 & 143 & 554 & $-0,5$ \\
\hline 32 & 554 & 0,063 & 1,306 & 0,089 & 0,960 & 0,710 & 0,735 & 0,393 & 0,465 & 1,467 & 0,324 & 0,456 & 0,498 & 0,227 & 0,214 & 85 & 554 & $-2,3$ \\
\hline 33 & 554 & 0,014 & 0,490 & 0,020 & 0,317 & 0,714 & 0,646 & 0,455 & 0,750 & 1,155 & 0,354 & 0,327 & 0,227 & 0,074 & 0,076 & 90 & 554 & $-1,2$ \\
\hline 34 & 554 & 0,016 & 0,511 & 0,020 & 0,338 & 0,786 & 0,661 & 0,401 & 0,758 & 1,148 & 0,483 & 0,353 & 0,204 & 0,072 & 0,076 & 45 & 554 & $-1,0$ \\
\hline 35 & 554 & 0,057 & 1,103 & 0,075 & 0,778 & 0,769 & 0,705 & 0,375 & 0,593 & 1,299 & 0,688 & 0,809 & 0,326 & 0,264 & 0,152 & 9 & 554 & $-1,7$ \\
\hline 36 & 554 & 0,016 & 0,541 & 0,022 & 0,440 & 0,733 & 0,812 & 0,326 & 0,677 & 1,215 & 0,311 & 0,230 & 0,254 & 0,059 & 0,076 & 45 & 554 & $-1,3$ \\
\hline 37 & 554 & 0,989 & 7,122 & 1,225 & 6,420 & 0,807 & 0,901 & 0,217 & 0,245 & 2,021 & 0,122 & 0,129 & 3,216 & 0,415 & 0,379 & 77 & 554 & $-5,0$ \\
\hline 38 & 554 & 0,116 & 1,348 & 0,129 & 1,247 & 0,900 & 0,925 & 0,125 & 0,803 & 1,116 & 0,441 & 0,417 & 0,579 & 0,242 & 0,240 & 81 & 554 & $-2,5$ \\
\hline 39 & 554 & 0,016 & 0,389 & 0,017 & 0,266 & 0,917 & 0,685 & 0,326 & 1,000 & 0,873 & 0,700 & 0,400 & 0,169 & 0,068 & 0,076 & 69 & 554 & $-0,8$ \\
\hline 40 & 554 & 0,024 & 0,562 & 0,026 & 0,418 & 0,944 & 0,744 & 0,261 & 0,970 & 1,015 & 0,481 & 0,400 & 0,254 & 0,102 & 0,107 & 109 & 554 & $-1,3$ \\
\hline 41 & 554 & 0,316 & 3,040 & 0,379 & 2,591 & 0,833 & 0,853 & 0,223 & 0,429 & 1,526 & 0,270 & 0,341 & 1,220 & 0,415 & 0,386 & 62 & 554 & $-3,6$ \\
\hline 42 & 554 & 0,069 & 1,205 & 0,085 & 0,951 & 0,814 & 0,789 & 0,281 & 0,596 & 1,295 & 0,399 & 0,404 & 0,469 & 0,189 & 0,214 & 100 & 554 & $-2,2$ \\
\hline 43 & 554 & 0,093 & 1,623 & 0,128 & 1,226 & 0,730 & 0,755 & 0,364 & 0,445 & 1,499 & 0,361 & 0,460 & 0,573 & 0,264 & 0,169 & 91 & 554 & $-2,5$ \\
\hline 44 & 554 & 0,022 & 0,634 & 0,026 & 0,562 & 0,833 & 0,887 & 0,202 & 0,673 & 1,219 & 0,281 & 0,215 & 0,312 & 0,067 & 0,076 & 98 & 554 & $-1,6$ \\
\hline 45 & 554 & 0,149 & 2,075 & 0,197 & 1,830 & 0,759 & 0,882 & 0,268 & 0,435 & 1,515 & 0,243 & 0,286 & 0,884 & 0,253 & 0,152 & 77 & 554 & $-3,1$ \\
\hline 46 & 554 & 0,027 & 0,757 & 0,034 & 0,562 & 0,792 & 0,743 & 0,331 & 0,599 & 1,293 & 0,417 & 0,374 & 0,288 & 0,108 & 0,107 & 104 & 554 & $-1,5$ \\
\hline 47 & 554 & 0,106 & 1,226 & 0,119 & 1,061 & 0,892 & 0,866 & 0,173 & 0,888 & 1,061 & 0,589 & 0,528 & 0,479 & 0,253 & 0,273 & 6 & 554 & $-2,3$ \\
\hline 48 & 554 & 0,126 & 1,826 & 0,161 & 1,390 & 0,786 & 0,761 & 0,321 & 0,476 & 1,450 & 0,436 & 0,499 & 0,607 & 0,303 & 0,227 & 93 & 554 & $-2,6$ \\
\hline 49 & 554 & 0,043 & 1,205 & 0,065 & 0,685 & 0,667 & 0,568 & 0,545 & 0,373 & 1,638 & 0,721 & 0,824 & 0,276 & 0,227 & 0,107 & 153 & 554 & $-1,5$ \\
\hline 50 & 554 & 0,014 & 0,389 & 0,014 & 0,266 & 1,000 & 0,685 & 0,315 & 1,000 & 0,916 & 0,509 & 0,200 & 0,189 & 0,038 & 0,076 & 90 & 554 & $-0,9$ \\
\hline 51 & 554 & 0,023 & 0,562 & 0,027 & 0,389 & 0,842 & 0,692 & 0,346 & 0,913 & 1,047 & 0,416 & 0,286 & 0,265 & 0,076 & 0,152 & 95 & 554 & $-1,4$ \\
\hline 52 & 554 & 0,014 & 0,368 & 0,016 & 0,266 & 0,909 & 0,724 & 0,291 & 1,000 & 0,866 & 0,979 & 0,702 & 0,137 & 0,096 & 0,107 & 146 & 554 & $-0,5$ \\
\hline 53 & 554 & 0,014 & 0,397 & 0,017 & 0,338 & 0,833 & 0,851 & 0,224 & 1,000 & 0,936 & 0,637 & 0,401 & 0,169 & 0,068 & 0,076 & 69 & 554 & $-0,8$ \\
\hline 54 & 554 & 0,076 & 1,348 & 0,099 & 1,103 & 0,768 & 0,818 & 0,295 & 0,526 & 1,379 & 0,329 & 0,393 & 0,542 & 0,213 & 0,214 & 73 & 554 & $-2,4$ \\
\hline 55 & 554 & 0,138 & 1,750 & 0,184 & 1,399 & 0,750 & 0,800 & 0,320 & 0,565 & 1,330 & 0,382 & 0,455 & 0,678 & 0,308 & 0,240 & 99 & 554 & $-2,8$ \\
\hline 56 & 554 & 0,089 & 1,606 & 0,126 & 1,031 & 0,705 & 0,642 & 0,464 & 0,433 & 1,519 & 0,409 & 0,504 & 0,526 & 0,265 & 0,227 & 70 & 554 & $-2,4$ \\
\hline 57 & 554 & 0,135 & 1,881 & 0,204 & 1,480 & 0,662 & 0,787 & 0,400 & 0,479 & 1,445 & 0,440 & 0,667 & 0,625 & 0,417 & 0,214 & 112 & 554 & $-2,6$ \\
\hline 58 & 554 & 0,020 & 0,461 & 0,022 & 0,359 & 0,933 & 0,780 & 0,230 & 1,000 & 0,917 & 0,615 & 0,371 & 0,204 & 0,076 & 0,107 & 80 & 554 & $-1,0$ \\
\hline 59 & 554 & 0,060 & 1,002 & 0,067 & 0,778 & 0,894 & 0,776 & 0,248 & 0,755 & 1,151 & 0,461 & 0,371 & 0,408 & 0,152 & 0,169 & 98 & 554 & $-2,0$ \\
\hline 60 & 554 & 0,088 & 1,091 & 0,096 & 0,938 & 0,910 & 0,860 & 0,166 & 0,924 & 1,040 & 0,733 & 0,672 & 0,390 & 0,262 & 0,273 & 128 & 554 & $-2,0$ \\
\hline & 555 & & & & & & & & othing to measure & & & & & & & & 555 & \\
\hline \multirow[t]{3}{*}{1} & 556 & 0,019 & 0,389 & 0,019 & 0,173 & 1,000 & 0,446 & 0,554 & & 0,803 & 0,920 & 0,667 & 0,161 & 0,107 & 0,152 & 45 & 556 & $-0,7$ \\
\hline & 557 & & & & & & & & nothing to measure & & & & & & & & 557 & 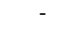 \\
\hline & 558 & & & & & & & & & & & & & & & & 558 & - \\
\hline
\end{tabular}




\begin{tabular}{|c|c|c|c|c|c|c|c|c|c|c|c|c|c|c|c|c|c|c|}
\hline 1 & 559 & 0,014 & 0,347 & 0,016 & 0,266 & 0,909 & 0,768 & 0,249 & 1,000 & 0,816 & 0,979 & 0,588 & 0,137 & 0,080 & 0,107 & 72 & 559 & $-0,5$ \\
\hline 2 & 559 & 0,026 & 0,685 & 0,034 & 0,418 & 0,750 & 0,611 & 0,462 & 0,692 & 1,202 & 0,509 & 0,534 & 0,254 & 0,136 & 0,152 & 32 & 559 & $-1,3$ \\
\hline 3 & 559 & 0,032 & 0,960 & 0,052 & 0,714 & 0,611 & 0,745 & 0,465 & 0,431 & 1,524 & 0,342 & 0,524 & 0,343 & 0,180 & 0,107 & 135 & 559 & $-1,8$ \\
\hline 4 & 559 & 0,016 & 0,368 & 0,017 & 0,266 & 0,917 & 0,724 & 0,288 & 1,000 & 0,826 & 0,778 & 0,500 & 0,161 & 0,080 & 0,107 & 37 & 559 & $-0,7$ \\
\hline \multirow[t]{7}{*}{5} & 559 & 0,016 & 0,490 & 0,019 & 0,296 & 0,846 & 0,604 & 0,425 & 0,825 & 1,101 & 0,483 & 0,447 & 0,204 & 0,091 & 0,076 & 118 & 559 & $-1,0$ \\
\hline & 560 & & & & & & & & nothing to measure & & & & & & & & 560 & - \\
\hline & 561 & & & & & & & & nothing to measure & & & & & & & & 561 & - \\
\hline & 562 & & & & & & & & nothing to measure & & & & & & & & 562 & - \\
\hline & 563 & & & & & & & & nothing to measure & & & & & & & & 563 & - \\
\hline & 564 & & & & & & & & nothing to measure & & & & & & & & 564 & - \\
\hline & 565 & & & & & & & & nothing to measure & & & & & & & & 565 & - \\
\hline 1 & 566 & 0,019 & 0,440 & 0,019 & 0,296 & 1,000 & 0,673 & 0,327 & 1,000 & 0,908 & 0,637 & 0,392 & 0,193 & 0,076 & 0,107 & 176 & 566 & $-0,9$ \\
\hline & 567 & & & & & & & & nothing to measure & & & & & & & & 567 & - \\
\hline & 568 & & & & & & & & nothing to measure & & & & & & & & 568 & - \\
\hline & 569 & & & & & & & & nothing to measure & & & & & & & & 569 & - \\
\hline 1 & 570 & 0,220 & 1,797 & 0,245 & 1,585 & 0,895 & 0,882 & 0,158 & 0,855 & 1,082 & 0,658 & 0,697 & 0,652 & 0,455 & 0,455 & 73 & 570 & $-2,7$ \\
\hline 1 & 571 & 0,017 & 0,418 & 0,019 & 0,317 & 0,923 & 0,757 & 0,254 & 1,000 & 0,900 & 0,611 & 0,389 & 0,189 & 0,074 & 0,076 & 140 & 571 & $-0,9$ \\
\hline 2 & 571 & 0,014 & 0,347 & 0,016 & 0,266 & 0,909 & 0,768 & 0,249 & 1,000 & 0,816 & 0,979 & 0,588 & 0,137 & 0,080 & 0,107 & 53 & 571 & $-0,5$ \\
\hline \multirow[t]{4}{*}{3} & 571 & 0,023 & 0,816 & 0,034 & 0,634 & 0,667 & 0,777 & 0,401 & 0,433 & 1,519 & 0,226 & 0,230 & 0,359 & 0,083 & 0,076 & 12 & 571 & $-1,8$ \\
\hline & 572 & & & & & & & & nothing to measure & & & & & & & & 572 & - \\
\hline & 573 & & & & & & & & nothing to measure & & & & & & & & 573 & - \\
\hline & 574 & & & & & & & & nothing to measure & & & & & & & & 574 & - \\
\hline 1 & 575 & 0,040 & 0,896 & 0,046 & 0,867 & 0,875 & 0,967 & 0,129 & 0,628 & 1,262 & 0,279 & 0,222 & 0,429 & 0,095 & 0,107 & 51 & 575 & $-2,1$ \\
\hline 2 & 575 & 0,017 & 0,469 & 0,020 & 0,317 & 0,857 & 0,675 & 0,355 & 0,983 & 1,009 & 0,527 & 0,371 & 0,204 & 0,076 & 0,107 & 85 & 575 & $-1,0$ \\
\hline 3 & 575 & 0,050 & 1,378 & 0,077 & 0,858 & 0,648 & 0,623 & 0,516 & 0,332 & 1,735 & 0,409 & 0,479 & 0,395 & 0,189 & 0,107 & 73 & 575 & $-2,0$ \\
\hline 4 & 575 & 0,037 & 0,693 & 0,042 & 0,571 & 0,897 & 0,823 & 0,205 & 0,975 & 1,013 & 0,637 & 0,555 & 0,273 & 0,152 & 0,169 & 45 & 575 & $-1,4$ \\
\hline 5 & 575 & 0,040 & 0,909 & 0,047 & 0,622 & 0,848 & 0,684 & 0,351 & 0,611 & 1,279 & 0,482 & 0,443 & 0,326 & 0,144 & 0,152 & 41 & 575 & $-1,7$ \\
\hline 6 & 575 & 0,017 & 0,389 & 0,017 & 0,275 & 1,000 & 0,707 & 0,293 & 1,000 & 0,836 & 0,899 & 0,485 & 0,156 & 0,076 & 0,152 & 172 & 575 & $-0,6$ \\
\hline \multirow[t]{3}{*}{7} & 575 & 0,022 & 0,562 & 0,026 & 0,389 & 0,833 & 0,692 & 0,351 & 0,856 & 1,081 & 0,477 & 0,474 & 0,240 & 0,114 & 0,152 & 55 & 575 & $-1,3$ \\
\hline & 576 & & & & & & & & nothing to measure & & & & & & & & 576 & - \\
\hline & 577 & & & & & & & & nothing to measure & & & & & & & & 577 & - \\
\hline 1 & 578 & 0,016 & 0,368 & 0,017 & 0,317 & 0,917 & 0,862 & 0,161 & 1,000 & 0,826 & 0,824 & 0,485 & 0,156 & 0,076 & 0,107 & 167 & 578 & $-0,6$ \\
\hline 2 & 578 & 0,106 & 1,175 & 0,116 & 1,031 & 0,914 & 0,878 & 0,150 & 0,966 & 1,017 & 0,688 & 0,620 & 0,443 & 0,275 & 0,273 & 113 & 578 & $-2,1$ \\
\hline 3 & 578 & 0,145 & 1,298 & 0,152 & 1,226 & 0,953 & 0,945 & 0,073 & 1,000 & 0,962 & 0,743 & 0,608 & 0,498 & 0,303 & 0,379 & 5 & 578 & $-2,3$ \\
\hline 4 & 578 & 0,174 & 1,581 & 0,188 & 1,509 & 0,924 & 0,955 & 0,089 & 0,873 & 1,070 & 0,556 & 0,510 & 0,630 & 0,321 & 0,312 & 39 & 578 & $-2,7$ \\
\hline 5 & 578 & 0,049 & 0,816 & 0,053 & 0,693 & 0,919 & 0,850 & 0,171 & 0,921 & 1,042 & 0,486 & 0,427 & 0,357 & 0,152 & 0,169 & 26 & 578 & $-1,8$ \\
\hline 6 & 578 & 0,024 & 0,592 & 0,029 & 0,469 & 0,850 & 0,793 & 0,256 & 0,875 & 1,069 & 0,637 & 0,485 & 0,221 & 0,107 & 0,107 & 65 & 578 & $-1,1$ \\
\hline 7 & 578 & 0,484 & 2,917 & 0,557 & 2,565 & 0,869 & 0,879 & 0,178 & 0,714 & 1,183 & 0,657 & 0,744 & 0,968 & 0,720 & 0,152 & 70 & 578 & $-3,3$ \\
\hline 8 & 578 & 0,014 & 0,317 & 0,014 & 0,194 & 1,000 & 0,613 & 0,387 & 1,000 & 0,746 & 1,273 & 0,632 & 0,120 & 0,076 & 0,152 & 0 & 578 & $-0,3$ \\
\hline 9 & 578 & 0,022 & 0,511 & 0,024 & 0,389 & 0,882 & 0,760 & 0,267 & 1,000 & 0,983 & 0,659 & 0,371 & 0,204 & 0,076 & 0,107 & 178 & 578 & $-1,0$ \\
\hline 10 & 578 & 0,416 & 2,291 & 0,435 & 2,168 & 0,957 & 0,946 & 0,069 & 0,997 & 1,002 & 0,749 & 0,711 & 0,841 & 0,598 & 0,606 & 163 & 578 & $-3,1$ \\
\hline 11 & 578 & 0,109 & 1,480 & 0,145 & 1,264 & 0,752 & 0,854 & 0,287 & 0,626 & 1,264 & 0,491 & 0,669 & 0,532 & 0,356 & 0,273 & 8 & 578 & $-2,4$ \\
\hline 12 & 578 & 0,014 & 0,338 & 0,016 & 0,194 & 0,909 & 0,575 & 0,435 & 1,000 & 0,796 & 0,979 & 0,555 & 0,137 & 0,076 & 0,107 & 108 & 578 & $-0,5$ \\
\hline 13 & 578 & 0,036 & 0,592 & 0,037 & 0,562 & 0,962 & 0,950 & 0,063 & 1,000 & 0,881 & 0,796 & 0,632 & 0,240 & 0,152 & 0,169 & 25 & 578 & $-1,3$ \\
\hline 14 & 578 & 0,106 & 1,082 & 0,112 & 1,031 & 0,949 & 0,953 & 0,069 & 1,000 & 0,937 & 0,864 & 0,766 & 0,395 & 0,303 & 0,312 & 174 & 578 & $-2,0$ \\
\hline 15 & 578 & 0,055 & 1,222 & 0,082 & 1,031 & 0,667 & 0,844 & 0,368 & 0,459 & 1,476 & 0,316 & 0,313 & 0,469 & 0,147 & 0,169 & 22 & 578 & $-2,2$ \\
\hline 16 & 578 & 0,271 & 2,633 & 0,359 & 2,084 & 0,756 & 0,791 & 0,321 & 0,491 & 1,427 & 0,566 & 0,725 & 0,781 & 0,566 & 0,408 & 37 & 578 & $-3,0$ \\
\hline 17 & 578 & 0,069 & 1,112 & 0,077 & 0,947 & 0,889 & 0,852 & 0,185 & 0,700 & 1,195 & 0,360 & 0,358 & 0,494 & 0,177 & 0,169 & 36 & 578 & $-2,3$ \\
\hline 18 & 578 & 0,017 & 0,418 & 0,019 & 0,317 & 0,923 & 0,757 & 0,254 & 1,000 & 0,900 & 0,764 & 0,474 & 0,169 & 0,080 & 0,107 & 57 & 578 & $-0,8$ \\
\hline 19 & 578 & 1,692 & 7,478 & 2,093 & 5,318 & 0,808 & 0,711 & 0,347 & 0,380 & 1,622 & 0,609 & 0,838 & 1,881 & 1,576 & 0,321 & 137 & 578 & $-4,2$ \\
\hline 20 & 578 & 0,086 & 1,196 & 0,103 & 0,989 & 0,833 & 0,827 & 0,240 & 0,756 & 1,150 & 0,513 & 0,576 & 0,462 & 0,266 & 0,240 & 55 & 578 & $-2,2$ \\
\hline 21 & 578 & 0,014 & 0,592 & 0,023 & 0,397 & 0,625 & 0,672 & 0,499 & 0,515 & 1,394 & 0,245 & 0,217 & 0,273 & 0,059 & 0,076 & 135 & 578 & $-1,4$ \\
\hline 22 & 578 & 0,070 & 0,837 & 0,072 & 0,807 & 0,980 & 0,965 & 0,041 & 1,000 & 0,890 & 0,917 & 0,728 & 0,312 & 0,227 & 0,273 & 1 & 578 & $-1,6$ \\
\hline 23 & 578 & 0,022 & 0,490 & 0,023 & 0,397 & 0,938 & 0,811 & 0,199 & 1,000 & 0,943 & 0,735 & 0,588 & 0,193 & 0,114 & 0,107 & 24 & 578 & $-0,9$ \\
\hline 24 & 578 & 0,154 & 1,450 & 0,165 & 1,336 & 0,930 & 0,921 & 0,105 & 0,918 & 1,044 & 0,625 & 0,515 & 0,559 & 0,288 & 0,303 & 25 & 578 & $-2,5$ \\
\hline 25 & 578 & 0,149 & 1,348 & 0,159 & 1,277 & 0,937 & 0,947 & 0,083 & 1,000 & 0,985 & 0,784 & 0,729 & 0,492 & 0,359 & 0,379 & 10 & 578 & $-2,3$ \\
\hline 26 & 578 & 0,049 & 1,205 & 0,070 & 0,786 & 0,694 & 0,653 & 0,463 & 0,422 & 1,539 & 0,585 & 0,692 & 0,326 & 0,225 & 0,152 & 46 & 578 & $-1,7$ \\
\hline 27 & 578 & 0,022 & 0,469 & 0,024 & 0,440 & 0,882 & 0,937 & 0,134 & 1,000 & 0,902 & 0,735 & 0,559 & 0,193 & 0,108 & 0,152 & 13 & 578 & $\begin{array}{r}-0,9 \\
\end{array}$ \\
\hline
\end{tabular}




\begin{tabular}{|c|c|c|c|c|c|c|c|c|c|c|c|c|c|c|c|c|c|c|}
\hline 28 & 578 & 0,129 & 1,551 & 0,146 & 1,387 & 0,882 & 0,894 & 0,158 & 0,674 & 1,218 & 0,387 & 0,379 & 0,652 & 0,247 & 0,273 & 36 & 578 & $-2,7$ \\
\hline 29 & 578 & 0,024 & 0,490 & 0,024 & 0,389 & 1,000 & 0,793 & 0,207 & 1,000 & 0,886 & 0,676 & 0,500 & 0,214 & 0,107 & 0,152 & 45 & 578 & $-1,1$ \\
\hline 30 & 578 & 0,016 & 0,347 & 0,016 & 0,296 & 1,000 & 0,854 & 0,146 & 1,000 & 0,778 & 1,401 & 0,894 & 0,120 & 0,107 & 0,152 & 0 & 578 & $-0,3$ \\
\hline 31 & 578 & 0,026 & 0,511 & 0,026 & 0,461 & 1,000 & 0,901 & 0,099 & 1,000 & 0,898 & 0,619 & 0,329 & 0,230 & 0,076 & 0,152 & 177 & 578 & $-1,2$ \\
\hline 32 & 578 & 0,027 & 0,664 & 0,033 & 0,490 & 0,826 & 0,739 & 0,314 & 0,778 & 1,134 & 0,605 & 0,474 & 0,240 & 0,114 & 0,152 & 162 & 578 & $-1,3$ \\
\hline 33 & 578 & 0,047 & 0,765 & 0,053 & 0,655 & 0,892 & 0,856 & 0,180 & 1,000 & 0,992 & 0,808 & 0,735 & 0,273 & 0,201 & 0,227 & 110 & 578 & $-1,4$ \\
\hline 34 & 578 & 0,416 & 3,073 & 0,505 & 2,452 & 0,824 & 0,798 & 0,268 & 0,554 & 1,344 & 0,576 & 0,632 & 0,959 & 0,606 & 0,530 & 180 & 578 & $-3,3$ \\
\hline 35 & 578 & 0,022 & 0,418 & 0,023 & 0,347 & 0,938 & 0,828 & 0,183 & 1,000 & 0,805 & 1,123 & 0,728 & 0,156 & 0,114 & 0,152 & 12 & 578 & $-0,6$ \\
\hline 36 & 578 & 0,169 & 1,420 & 0,181 & 1,327 & 0,937 & 0,935 & 0,091 & 1,000 & 0,974 & 0,774 & 0,690 & 0,528 & 0,364 & 0,379 & 14 & 578 & $-2,4$ \\
\hline 37 & 578 & 0,039 & 0,888 & 0,055 & 0,634 & 0,711 & 0,714 & 0,407 & 0,618 & 1,272 & 0,529 & 0,702 & 0,305 & 0,214 & 0,152 & 73 & 578 & $-1,6$ \\
\hline 38 & 578 & 0,019 & 0,389 & 0,019 & 0,287 & 1,000 & 0,739 & 0,261 & 1,000 & 0,803 & 0,974 & 0,485 & 0,156 & 0,076 & 0,152 & 8 & 578 & $-0,6$ \\
\hline 39 & 578 & 0,023 & 0,714 & 0,034 & 0,440 & 0,667 & 0,615 & 0,509 & 0,565 & 1,330 & 0,599 & 0,686 & 0,221 & 0,152 & 0,107 & 68 & 578 & $-1,1$ \\
\hline 40 & 578 & 0,037 & 0,613 & 0,039 & 0,541 & 0,963 & 0,883 & 0,123 & 1,000 & 0,895 & 0,736 & 0,596 & 0,254 & 0,152 & 0,169 & 26 & 578 & $-1,3$ \\
\hline 41 & 578 & 0,036 & 0,693 & 0,042 & 0,592 & 0,862 & 0,854 & 0,201 & 0,938 & 1,033 & 0,497 & 0,500 & 0,303 & 0,152 & 0,169 & 8 & 578 & $-1,6$ \\
\hline 42 & 578 & 0,023 & 0,418 & 0,023 & 0,317 & 1,000 & 0,757 & 0,243 & 1,000 & 0,779 & 1,198 & 0,728 & 0,156 & 0,114 & 0,152 & 90 & 578 & $-0,6$ \\
\hline 43 & 578 & 0,456 & 3,040 & 0,545 & 2,646 & 0,837 & 0,870 & 0,208 & 0,621 & 1,269 & 0,578 & 0,659 & 1,003 & 0,661 & 0,479 & 0 & 578 & $-3,3$ \\
\hline 44 & 578 & 0,017 & 0,410 & 0,019 & 0,173 & 0,923 & 0,423 & 0,582 & 1,000 & 0,881 & 0,849 & 0,667 & 0,161 & 0,107 & 0,107 & 45 & 578 & $-0,7$ \\
\hline 45 & 578 & 0,030 & 0,571 & 0,032 & 0,541 & 0,955 & 0,948 & 0,069 & 1,000 & 0,928 & 0,668 & 0,483 & 0,240 & 0,116 & 0,152 & 27 & 578 & $-1,3$ \\
\hline 46 & 578 & 0,057 & 1,243 & 0,083 & 0,846 & 0,690 & 0,680 & 0,446 & 0,467 & 1,464 & 0,566 & 0,744 & 0,359 & 0,267 & 0,169 & 74 & 578 & $-1,8$ \\
\hline 47 & 578 & 0,019 & 0,397 & 0,019 & 0,368 & 1,000 & 0,925 & 0,075 & 1,000 & 0,821 & 0,974 & 0,651 & 0,156 & 0,102 & 0,152 & 19 & 578 & $-0,6$ \\
\hline 48 & 578 & 0,023 & 0,499 & 0,024 & 0,418 & 0,941 & 0,839 & 0,172 & 1,000 & 0,929 & 0,815 & 0,707 & 0,189 & 0,134 & 0,152 & 45 & 578 & $-0,9$ \\
\hline 49 & 578 & 0,027 & 0,490 & 0,029 & 0,410 & 0,950 & 0,836 & 0,172 & 1,000 & 0,838 & 1,210 & 0,849 & 0,169 & 0,144 & 0,152 & 53 & 578 & $-0,8$ \\
\hline 50 & 578 & 0,023 & 0,490 & 0,024 & 0,368 & 0,941 & 0,750 & 0,257 & 1,000 & 0,913 & 0,702 & 0,529 & 0,204 & 0,108 & 0,107 & 156 & 578 & $-1,0$ \\
\hline 51 & 578 & 0,179 & 2,092 & 0,222 & 1,522 & 0,806 & 0,727 & 0,334 & 0,515 & 1,394 & 0,660 & 0,772 & 0,588 & 0,454 & 0,214 & 97 & 578 & $-2,6$ \\
\hline 52 & 578 & 0,462 & 5,419 & 0,816 & 3,259 & 0,566 & 0,601 & 0,589 & 0,198 & 2,249 & 0,376 & 0,729 & 1,251 & 0,912 & 0,240 & 169 & 578 & $-3,6$ \\
\hline 53 & 578 & 0,105 & 1,184 & 0,116 & 1,052 & 0,901 & 0,889 & 0,148 & 0,939 & 1,032 & 0,929 & 0,895 & 0,379 & 0,339 & 0,312 & 84 & 578 & $-1,9$ \\
\hline 54 & 578 & 0,017 & 0,418 & 0,019 & 0,317 & 0,923 & 0,757 & 0,254 & 1,000 & 0,900 & 0,588 & 0,392 & 0,193 & 0,076 & 0,107 & 18 & 578 & $-0,9$ \\
\hline 55 & 578 & 0,204 & 1,919 & 0,241 & 1,662 & 0,845 & 0,866 & 0,205 & 0,695 & 1,199 & 0,611 & 0,616 & 0,652 & 0,402 & 0,312 & 136 & 578 & $-2,7$ \\
\hline 56 & 578 & 0,069 & 1,234 & 0,103 & 0,981 & 0,667 & 0,794 & 0,392 & 0,568 & 1,327 & 0,527 & 0,645 & 0,408 & 0,263 & 0,152 & 174 & 578 & $-2,0$ \\
\hline 57 & 578 & 0,326 & 2,071 & 0,341 & 1,957 & 0,954 & 0,945 & 0,072 & 0,954 & 1,024 & 0,714 & 0,686 & 0,762 & 0,523 & 0,536 & 57 & 578 & $-2,9$ \\
\hline 58 & 578 & 0,643 & 3,022 & 0,679 & 2,929 & 0,947 & 0,969 & 0,061 & 0,884 & 1,063 & 0,570 & 0,566 & 1,198 & 0,678 & 0,647 & 66 & 578 & $-3,6$ \\
\hline 59 & 578 & 0,016 & 0,368 & 0,016 & 0,368 & 1,000 & 1,000 & 0,000 & 1,000 & 0,826 & 0,824 & 0,485 & 0,156 & 0,076 & 0,107 & 0 & 578 & $-0,6$ \\
\hline 60 & 578 & 0,339 & 2,215 & 0,369 & 2,084 & 0,918 & 0,941 & 0,101 & 0,867 & 1,074 & 0,707 & 0,744 & 0,781 & 0,581 & 0,606 & 173 & 578 & $-3,0$ \\
\hline 61 & 578 & 0,026 & 0,490 & 0,027 & 0,347 & 0,947 & 0,707 & 0,298 & 1,000 & 0,861 & 1,146 & 0,800 & 0,169 & 0,136 & 0,169 & 150 & 578 & $-0,8$ \\
\hline 62 & 578 & 0,646 & 2,968 & 0,671 & 2,815 & 0,962 & 0,949 & 0,064 & 0,921 & 1,042 & 0,678 & 0,632 & 1,101 & 0,696 & 0,746 & 42 & 578 & $-3,5$ \\
\hline 63 & 578 & 0,055 & 0,960 & 0,069 & 0,786 & 0,792 & 0,819 & 0,276 & 0,744 & 1,159 & 0,499 & 0,418 & 0,373 & 0,156 & 0,169 & 112 & 578 & $-1,9$ \\
\hline 64 & 578 & 0,187 & 1,674 & 0,204 & 1,450 & 0,915 & 0,866 & 0,158 & 0,836 & 1,093 & 0,634 & 0,672 & 0,612 & 0,411 & 0,379 & 14 & 578 & $-2,6$ \\
\hline 65 & 578 & 0,020 & 0,418 & 0,020 & 0,347 & 1,000 & 0,828 & 0,172 & 1,000 & 0,833 & 1,049 & 0,728 & 0,156 & 0,114 & 0,152 & 163 & 578 & $-0,6$ \\
\hline 66 & 578 & 0,046 & 0,672 & 0,046 & 0,643 & 1,000 & 0,956 & 0,044 & 1,000 & 0,885 & 0,815 & 0,700 & 0,268 & 0,187 & 0,214 & 13 & 578 & $-1,4$ \\
\hline 67 & 578 & 0,022 & 0,469 & 0,023 & 0,397 & 0,938 & 0,847 & 0,165 & 1,000 & 0,902 & 0,735 & 0,588 & 0,193 & 0,114 & 0,152 & 150 & 578 & $-0,9$ \\
\hline 68 & 578 & 0,022 & 0,440 & 0,023 & 0,317 & 0,938 & 0,721 & 0,286 & 1,000 & 0,845 & 0,955 & 0,600 & 0,169 & 0,102 & 0,152 & 27 & 578 & $-0,8$ \\
\hline 69 & 578 & 0,027 & 0,520 & 0,029 & 0,418 & 0,950 & 0,805 & 0,202 & 1,000 & 0,888 & 0,968 & 0,800 & 0,189 & 0,152 & 0,169 & 93 & 578 & $-0,9$ \\
\hline 70 & 578 & 0,580 & 2,866 & 0,613 & 2,786 & 0,946 & 0,972 & 0,061 & 0,887 & 1,062 & 0,595 & 0,596 & 1,114 & 0,664 & 0,647 & 19 & 578 & $-3,5$ \\
\hline 71 & 578 & 0,065 & 0,867 & 0,069 & 0,816 & 0,938 & 0,941 & 0,086 & 1,000 & 0,962 & 0,674 & 0,618 & 0,349 & 0,216 & 0,227 & 19 & 578 & $-1,8$ \\
\hline 72 & 578 & 0,019 & 0,368 & 0,019 & 0,287 & 1,000 & 0,781 & 0,219 & 1,000 & 0,760 & 1,273 & 0,784 & 0,137 & 0,107 & 0,152 & 45 & 578 & $-0,5$ \\
\hline 73 & 578 & 0,341 & 2,287 & 0,373 & 2,143 & 0,915 & 0,937 & 0,105 & 0,821 & 1,104 & 0,491 & 0,500 & 0,941 & 0,470 & 0,442 & 41 & 578 & $-3,2$ \\
\hline 74 & 578 & 0,032 & 0,592 & 0,034 & 0,469 & 0,917 & 0,793 & 0,223 & 1,000 & 0,940 & 0,700 & 0,550 & 0,240 & 0,132 & 0,152 & 105 & 578 & $-1,3$ \\
\hline 75 & 578 & 0,037 & 0,583 & 0,039 & 0,461 & 0,963 & 0,790 & 0,213 & 1,000 & 0,852 & 0,974 & 0,686 & 0,221 & 0,152 & 0,214 & 9 & 578 & $-1,1$ \\
\hline 76 & 578 & 0,037 & 0,693 & 0,043 & 0,613 & 0,867 & 0,884 & 0,177 & 0,975 & 1,013 & 0,662 & 0,627 & 0,268 & 0,168 & 0,169 & 15 & 578 & $-1,4$ \\
\hline 77 & 578 & 0,032 & 0,562 & 0,033 & 0,440 & 0,957 & 0,782 & 0,222 & 1,000 & 0,893 & 0,966 & 0,743 & 0,204 & 0,152 & 0,169 & 65 & 578 & $-1,0$ \\
\hline 78 & 578 & 0,125 & 1,327 & 0,136 & 1,226 & 0,916 & 0,924 & 0,114 & 0,890 & 1,060 & 0,540 & 0,512 & 0,542 & 0,278 & 0,303 & 26 & 578 & $-2,4$ \\
\hline 79 & 578 & 0,056 & 0,757 & 0,057 & 0,634 & 0,975 & 0,838 & 0,164 & 1,000 & 0,902 & 0,937 & 0,687 & 0,276 & 0,189 & 0,227 & 21 & 578 & $-1,5$ \\
\hline 80 & 578 & 0,047 & 0,807 & 0,055 & 0,634 & 0,868 & 0,785 & 0,252 & 0,913 & 1,047 & 0,618 & 0,485 & 0,312 & 0,152 & 0,152 & 178 & 578 & $-1,6$ \\
\hline 81 & 578 & 0,020 & 0,418 & 0,020 & 0,296 & 1,000 & 0,707 & 0,293 & 1,000 & 0,833 & 1,049 & 0,728 & 0,156 & 0,114 & 0,107 & 0 & 578 & $-0,6$ \\
\hline 82 & 578 & 0,062 & 0,778 & 0,065 & 0,664 & 0,956 & 0,853 & 0,153 & 1,000 & 0,883 & 1,053 & 0,832 & 0,273 & 0,227 & 0,273 & 0 & 578 & $-1,4$ \\
\hline 83 & 578 & 0,053 & 0,765 & 0,057 & 0,693 & 0,925 & 0,906 & 0,120 & 1,000 & 0,937 & 0,889 & 0,824 & 0,276 & 0,227 & 0,227 & 159 & 578 & $-1,5$ \\
\hline 84 & 578 & 0,047 & 0,714 & 0,050 & 0,613 & 0,943 & 0,858 & 0,153 & 1,000 & 0,926 & 0,840 & 0,707 & 0,268 & 0,189 & 0,169 & 6 & 578 & $-1,4$ \\
\hline 85 & 578 & 0,083 & 0,909 & 0,085 & 0,858 & 0,983 & 0,944 & 0,058 & 1,000 & 0,889 & 0,923 & 0,783 & 0,339 & 0,265 & 0,303 & 107 & 578 & $-1,8$ \\
\hline
\end{tabular}




\begin{tabular}{|c|c|c|c|c|c|c|c|c|c|c|c|c|c|c|c|c|c|c|}
\hline 86 & 578 & 0,020 & 0,461 & 0,023 & 0,317 & 0,875 & 0,688 & 0,336 & 1,000 & 0,917 & 0,990 & 0,707 & 0,161 & 0,114 & 0,107 & 114 & 578 & $-0,7$ \\
\hline 87 & 578 & 0,052 & 1,234 & 0,075 & 0,828 & 0,692 & 0,671 & 0,450 & 0,426 & 1,532 & 0,458 & 0,600 & 0,379 & 0,227 & 0,107 & 65 & 578 & $-1,9$ \\
\hline 88 & 578 & 0,039 & 0,613 & 0,040 & 0,520 & 0,964 & 0,848 & 0,156 & 1,000 & 0,878 & 1,011 & 0,814 & 0,221 & 0,180 & 0,214 & 90 & 578 & $-1,1$ \\
\hline 89 & 578 & 0,014 & 0,368 & 0,016 & 0,296 & 0,909 & 0,805 & 0,215 & 1,000 & 0,866 & 0,749 & 0,485 & 0,156 & 0,076 & 0,107 & 0 & 578 & $-0,6$ \\
\hline 90 & 578 & 0,030 & 0,583 & 0,033 & 0,469 & 0,913 & 0,805 & 0,214 & 1,000 & 0,948 & 0,594 & 0,447 & 0,254 & 0,114 & 0,152 & 20 & 578 & $-1,3$ \\
\hline 91 & 578 & 0,976 & 3,530 & 1,004 & 3,500 & 0,971 & 0,992 & 0,030 & 0,984 & 1,008 & 0,728 & 0,701 & 1,306 & 0,915 & 0,912 & 21 & 578 & $-3,7$ \\
\hline 92 & 578 & 0,050 & 1,378 & 0,069 & 0,837 & 0,729 & 0,607 & 0,477 & 0,332 & 1,735 & 0,384 & 0,464 & 0,408 & 0,189 & 0,152 & 157 & 578 & $-2,0$ \\
\hline 93 & 578 & 0,014 & 0,389 & 0,014 & 0,215 & 1,000 & 0,554 & 0,446 & 1,000 & 0,916 & 0,509 & 0,200 & 0,189 & 0,038 & 0,076 & 0 & 578 & $-0,9$ \\
\hline 94 & 578 & 0,991 & 5,276 & 1,116 & 3,724 & 0,888 & 0,706 & 0,315 & 0,448 & 1,495 & 0,626 & 0,720 & 1,420 & 1,023 & 0,107 & 91 & 578 & $-3,8$ \\
\hline 95 & 578 & 0,083 & 1,031 & 0,090 & 0,981 & 0,921 & 0,951 & 0,093 & 0,983 & 1,009 & 0,591 & 0,519 & 0,423 & 0,220 & 0,227 & 9 & 578 & $-2,1$ \\
\hline 96 & 578 & 0,029 & 0,735 & 0,042 & 0,541 & 0,690 & 0,736 & 0,408 & 0,667 & 1,225 & 0,480 & 0,542 & 0,276 & 0,150 & 0,107 & 73 & 578 & $-1,5$ \\
\hline 97 & 578 & 0,017 & 0,359 & 0,017 & 0,194 & 1,000 & 0,541 & 0,459 & 1,000 & 0,772 & 1,175 & 0,555 & 0,137 & 0,076 & 0,152 & 0 & 578 & $-0,5$ \\
\hline 98 & 578 & 0,116 & 1,234 & 0,123 & 1,163 & 0,942 & 0,942 & 0,082 & 0,958 & 1,021 & 0,657 & 0,577 & 0,475 & 0,274 & 0,273 & 38 & 578 & $-2,2$ \\
\hline 99 & 578 & 0,644 & 2,879 & 0,666 & 2,819 & 0,968 & 0,979 & 0,038 & 0,977 & 1,012 & 0,740 & 0,763 & 1,053 & 0,804 & 0,816 & 57 & 578 & $-3,4$ \\
\hline 100 & 578 & 0,022 & 0,418 & 0,022 & 0,317 & 1,000 & 0,757 & 0,243 & 1,000 & 0,805 & 1,123 & 0,728 & 0,156 & 0,114 & 0,152 & 20 & 578 & $-0,6$ \\
\hline 101 & 578 & 0,037 & 0,744 & 0,042 & 0,643 & 0,897 & 0,864 & 0,171 & 0,847 & 1,087 & 0,447 & 0,323 & 0,326 & 0,105 & 0,107 & 37 & 578 & $-1,7$ \\
\hline 102 & 578 & 0,065 & 1,163 & 0,080 & 0,858 & 0,804 & 0,738 & 0,327 & 0,600 & 1,291 & 0,458 & 0,499 & 0,423 & 0,211 & 0,152 & 69 & 578 & $-2,1$ \\
\hline 103 & 578 & 0,387 & 3,204 & 0,469 & 2,553 & 0,826 & 0,797 & 0,268 & 0,474 & 1,452 & 0,431 & 0,501 & 1,069 & 0,535 & 0,461 & 112 & 578 & $-3,4$ \\
\hline 104 & 578 & 0,036 & 0,786 & 0,047 & 0,592 & 0,758 & 0,753 & 0,346 & 0,729 & 1,171 & 0,707 & 0,738 & 0,254 & 0,187 & 0,152 & 144 & 578 & $-1,3$ \\
\hline 105 & 578 & 0,211 & 1,843 & 0,237 & 1,649 & 0,891 & 0,895 & 0,152 & 0,780 & 1,132 & 0,666 & 0,633 & 0,635 & 0,402 & 0,408 & 132 & 578 & $-2,7$ \\
\hline 106 & 578 & 0,154 & 1,277 & 0,155 & 1,247 & 0,991 & 0,977 & 0,025 & 1,000 & 0,919 & 0,933 & 0,828 & 0,458 & 0,379 & 0,408 & 19 & 578 & $-2,2$ \\
\hline 107 & 578 & 0,037 & 0,613 & 0,043 & 0,520 & 0,867 & 0,848 & 0,202 & 1,000 & 0,895 & 1,035 & 0,870 & 0,214 & 0,186 & 0,214 & 23 & 578 & $-1,1$ \\
\hline 108 & 578 & 0,026 & 0,490 & 0,027 & 0,410 & 0,947 & 0,836 & 0,172 & 1,000 & 0,861 & 0,881 & 0,588 & 0,193 & 0,114 & 0,152 & 15 & 578 & $-0,9$ \\
\hline 109 & 578 & 0,016 & 0,368 & 0,017 & 0,266 & 0,917 & 0,724 & 0,288 & 1,000 & 0,826 & 0,778 & 0,500 & 0,161 & 0,080 & 0,107 & 37 & 578 & $-0,7$ \\
\hline 110 & 578 & 0,060 & 1,091 & 0,075 & 0,888 & 0,808 & 0,814 & 0,268 & 0,636 & 1,253 & 0,428 & 0,453 & 0,423 & 0,192 & 0,169 & 31 & 578 & $-2,1$ \\
\hline 111 & 578 & 0,019 & 0,389 & 0,019 & 0,296 & 1,000 & 0,761 & 0,239 & 1,000 & 0,803 & 0,974 & 0,485 & 0,156 & 0,076 & 0,152 & 0 & 578 & $-0,6$ \\
\hline 112 & 578 & 0,017 & 0,418 & 0,019 & 0,317 & 0,923 & 0,757 & 0,254 & 1,000 & 0,900 & 0,611 & 0,358 & 0,189 & 0,068 & 0,076 & 29 & 578 & $-0,9$ \\
\hline 113 & 578 & 0,056 & 0,888 & 0,065 & 0,765 & 0,867 & 0,862 & 0,192 & 0,892 & 1,059 & 0,558 & 0,503 & 0,357 & 0,180 & 0,169 & 26 & 578 & $-1,8$ \\
\hline 114 & 578 & 0,029 & 0,634 & 0,033 & 0,440 & 0,870 & 0,693 & 0,333 & 0,897 & 1,056 & 0,566 & 0,527 & 0,254 & 0,134 & 0,152 & 41 & 578 & $-1,3$ \\
\hline 115 & 578 & 0,022 & 0,418 & 0,022 & 0,368 & 1,000 & 0,879 & 0,121 & 1,000 & 0,805 & 1,123 & 0,728 & 0,156 & 0,114 & 0,152 & 20 & 578 & $-0,6$ \\
\hline 116 & 578 & 0,049 & 0,714 & 0,050 & 0,685 & 0,971 & 0,958 & 0,050 & 1,000 & 0,912 & 0,666 & 0,496 & 0,305 & 0,152 & 0,227 & 173 & 578 & $-1,6$ \\
\hline 117 & 578 & 0,046 & 0,735 & 0,047 & 0,664 & 0,970 & 0,902 & 0,102 & 1,000 & 0,968 & 0,627 & 0,531 & 0,305 & 0,162 & 0,169 & 23 & 578 & $-1,6$ \\
\hline 118 & 578 & 0,032 & 0,562 & 0,033 & 0,389 & 0,957 & 0,692 & 0,311 & 1,000 & 0,893 & 0,966 & 0,743 & 0,204 & 0,152 & 0,169 & 90 & 578 & $-1,0$ \\
\hline 119 & 578 & 0,925 & 3,516 & 0,970 & 3,406 & 0,954 & 0,969 & 0,056 & 0,941 & 1,031 & 0,753 & 0,788 & 1,251 & 0,985 & 0,985 & 40 & 578 & $-3,6$ \\
\hline 120 & 578 & 0,020 & 0,541 & 0,024 & 0,397 & 0,824 & 0,735 & 0,319 & 0,862 & 1,077 & 0,446 & 0,324 & 0,240 & 0,078 & 0,076 & 26 & 578 & $-1,3$ \\
\hline 121 & 578 & 0,242 & 1,839 & 0,265 & 1,674 & 0,914 & 0,910 & 0,125 & 0,901 & 1,053 & 0,633 & 0,651 & 0,698 & 0,455 & 0,508 & 12 & 578 & $-2,8$ \\
\hline 122 & 578 & 0,030 & 0,655 & 0,039 & 0,511 & 0,778 & 0,781 & 0,312 & 0,883 & 1,064 & 0,535 & 0,424 & 0,268 & 0,114 & 0,152 & 4 & 578 & $-1,4$ \\
\hline 123 & 578 & 0,046 & 0,714 & 0,049 & 0,643 & 0,941 & 0,899 & 0,116 & 1,000 & 0,941 & 0,668 & 0,543 & 0,296 & 0,161 & 0,214 & 27 & 578 & $-1,6$ \\
\hline 124 & 578 & 0,046 & 0,706 & 0,050 & 0,562 & 0,914 & 0,796 & 0,221 & 1,000 & 0,929 & 0,905 & 0,703 & 0,254 & 0,179 & 0,214 & 11 & 578 & $-1,3$ \\
\hline 125 & 578 & 0,042 & 0,685 & 0,047 & 0,562 & 0,879 & 0,821 & 0,216 & 1,000 & 0,947 & 0,821 & 0,745 & 0,254 & 0,189 & 0,214 & 27 & 578 & $-1,3$ \\
\hline 126 & 578 & 0,024 & 0,532 & 0,026 & 0,338 & 0,944 & 0,635 & 0,369 & 1,000 & 0,962 & 0,585 & 0,329 & 0,230 & 0,076 & 0,152 & 177 & 578 & $-1,2$ \\
\hline 127 & 578 & 0,105 & 1,095 & 0,112 & 0,930 & 0,936 & 0,850 & 0,164 & 1,000 & 0,954 & 0,929 & 0,800 & 0,379 & 0,303 & 0,312 & 10 & 578 & $-1,9$ \\
\hline 128 & 578 & 0,300 & 1,898 & 0,304 & 1,826 & 0,986 & 0,962 & 0,040 & 1,000 & 0,978 & 0,747 & 0,696 & 0,715 & 0,497 & 0,530 & 33 & 578 & $-2,8$ \\
\hline 129 & 578 & 0,032 & 0,613 & 0,036 & 0,541 & 0,880 & 0,883 & 0,168 & 1,000 & 0,973 & 0,700 & 0,588 & 0,240 & 0,141 & 0,152 & 22 & 578 & $-1,3$ \\
\hline 130 & 578 & 0,017 & 0,376 & 0,017 & 0,368 & 1,000 & 0,977 & 0,023 & 1,000 & 0,809 & 0,955 & 0,707 & 0,152 & 0,107 & 0,152 & 0 & 578 & $-0,6$ \\
\hline 131 & 578 & 0,155 & 1,522 & 0,174 & 1,399 & 0,893 & 0,919 & 0,134 & 0,841 & 1,090 & 0,631 & 0,704 & 0,559 & 0,394 & 0,379 & 49 & 578 & $-2,5$ \\
\hline 132 & 578 & 0,403 & 2,532 & 0,436 & 2,270 & 0,924 & 0,896 & 0,128 & 0,790 & 1,125 & 0,629 & 0,689 & 0,904 & 0,623 & 0,647 & 58 & 578 & $-3,2$ \\
\hline 133 & 578 & 0,403 & 2,418 & 0,435 & 2,224 & 0,927 & 0,920 & 0,108 & 0,867 & 1,074 & 0,661 & 0,679 & 0,881 & 0,599 & 0,592 & 36 & 578 & $-3,1$ \\
\hline 134 & 578 & 0,175 & 1,420 & 0,187 & 1,348 & 0,938 & 0,949 & 0,080 & 1,000 & 0,958 & 0,947 & 0,859 & 0,485 & 0,417 & 0,442 & 176 & 578 & $-2,3$ \\
\hline 135 & 578 & 0,033 & 0,655 & 0,033 & 0,583 & 1,000 & 0,890 & 0,110 & 0,967 & 1,017 & 0,451 & 0,248 & 0,305 & 0,076 & 0,152 & 0 & 578 & $-1,6$ \\
\hline 136 & 578 & 0,014 & 0,418 & 0,017 & 0,296 & 0,833 & 0,707 & 0,337 & 1,000 & 0,986 & 0,637 & 0,543 & 0,169 & 0,092 & 0,107 & 0 & 578 & $-0,8$ \\
\hline 137 & 578 & 0,033 & 0,622 & 0,037 & 0,520 & 0,885 & 0,837 & 0,200 & 1,000 & 0,965 & 0,732 & 0,566 & 0,240 & 0,136 & 0,152 & 29 & 578 & $-1,3$ \\
\hline 138 & 578 & 0,313 & 2,012 & 0,336 & 1,889 & 0,932 & 0,939 & 0,092 & 0,971 & 1,015 & 0,887 & 0,898 & 0,670 & 0,602 & 0,577 & 52 & 578 & $-2,7$ \\
\hline 139 & 578 & 0,040 & 0,858 & 0,052 & 0,676 & 0,778 & 0,788 & 0,307 & 0,686 & 1,208 & 0,488 & 0,462 & 0,324 & 0,150 & 0,169 & 90 & 578 & $-1,7$ \\
\hline 140 & 578 & 0,019 & 0,389 & 0,019 & 0,275 & 1,000 & 0,707 & 0,293 & 1,000 & 0,803 & 1,273 & 0,832 & 0,137 & 0,114 & 0,152 & 45 & 578 & $-0,5$ \\
\hline 141 & 578 & 0,019 & 0,461 & 0,023 & 0,245 & 0,812 & 0,532 & 0,504 & 1,000 & 0,951 & 0,662 & 0,500 & 0,189 & 0,095 & 0,076 & 27 & 578 & $-0,9$ \\
\hline 142 & 578 & 0,077 & 0,909 & 0,080 & 0,786 & 0,964 & 0,865 & 0,140 & 1,000 & 0,921 & 0,942 & 0,819 & 0,324 & 0,265 & 0,273 & 26 & 578 & $-1,7$ \\
\hline 143 & 578 & 0,030 & 0,562 & 0,032 & 0,469 & 0,955 & 0,835 & 0,171 & 1,000 & 0,914 & 0,723 & 0,493 & 0,230 & 0,114 & 0,152 & 5 & 578 & $-1,2$ \\
\hline
\end{tabular}




\begin{tabular}{|c|c|c|c|c|c|c|c|c|c|c|c|c|c|c|c|c|c|c|}
\hline 144 & 578 & 0,020 & 0,389 & 0,020 & 0,296 & 1,000 & 0,761 & 0,239 & 1,000 & 0,774 & 1,371 & 0,832 & 0,137 & 0,114 & 0,152 & 135 & 578 & $-0,5$ \\
\hline 145 & 578 & 0,257 & 1,767 & 0,265 & 1,716 & 0,968 & 0,971 & 0,043 & 1,000 & 0,983 & 0,701 & 0,666 & 0,683 & 0,455 & 0,479 & 15 & 578 & $-2,8$ \\
\hline 146 & 578 & 0,027 & 0,562 & 0,030 & 0,418 & 0,905 & 0,744 & 0,273 & 1,000 & 0,960 & 0,834 & 0,694 & 0,204 & 0,142 & 0,152 & 83 & 578 & $-1,0$ \\
\hline 147 & 578 & 0,135 & 1,226 & 0,141 & 1,175 & 0,959 & 0,959 & 0,058 & 1,000 & 0,942 & 0,880 & 0,788 & 0,442 & 0,348 & 0,379 & 48 & 578 & $-2,1$ \\
\hline 148 & 578 & 0,024 & 0,541 & 0,032 & 0,440 & 0,773 & 0,812 & 0,295 & 1,000 & 0,977 & 0,746 & 0,676 & 0,204 & 0,138 & 0,152 & 13 & 578 & $-1,0$ \\
\hline 149 & 578 & 0,020 & 0,410 & 0,020 & 0,224 & 1,000 & 0,547 & 0,453 & 1,000 & 0,816 & 0,990 & 0,707 & 0,161 & 0,114 & 0,152 & 120 & 578 & $-0,7$ \\
\hline 150 & 578 & 0,020 & 0,410 & 0,020 & 0,224 & 1,000 & 0,547 & 0,453 & 1,000 & 0,816 & 0,990 & 0,707 & 0,161 & 0,114 & 0,152 & 120 & 578 & $-0,7$ \\
\hline 151 & 578 & 0,014 & 0,347 & 0,014 & 0,245 & 1,000 & 0,707 & 0,293 & 1,000 & 0,816 & 1,273 & 0,849 & 0,120 & 0,102 & 0,107 & 117 & 578 & $-0,3$ \\
\hline 152 & 578 & 0,014 & 0,359 & 0,014 & 0,194 & 1,000 & 0,541 & 0,459 & 1,000 & 0,846 & 0,749 & 0,243 & 0,156 & 0,038 & 0,076 & 0 & 578 & $-0,6$ \\
\hline 153 & 578 & 0,017 & 0,359 & 0,017 & 1,793 & 1,000 & 4,992 & 3,992 & 1,000 & 0,772 & 1,175 & 0,555 & 0,137 & 0,076 & 0,152 & 0 & 578 & $-0,5$ \\
\hline 154 & 578 & 0,191 & 2,266 & 0,253 & 0,418 & 0,756 & 0,185 & 0,851 & 0,467 & 1,463 & 0,413 & 0,448 & 0,767 & 0,343 & 0,273 & 51 & 578 & $-2,9$ \\
\hline 155 & 578 & 0,024 & 0,469 & 0,024 & 0,520 & 1,000 & 1,108 & 0,108 & 1,000 & 0,848 & 0,833 & 0,588 & 0,193 & 0,114 & 0,152 & 27 & 578 & $-0,9$ \\
\hline 156 & 578 & 0,037 & 0,685 & 0,046 & 0,368 & 0,812 & 0,537 & 0,499 & 1,000 & 1,000 & 0,807 & 0,750 & 0,243 & 0,182 & 0,169 & 16 & 578 & $-1,3$ \\
\hline 157 & 578 & 0,014 & 0,499 & 0,019 & 0,786 & 0,769 & 1,576 & 0,620 & 0,724 & 1,175 & 0,490 & 0,434 & 0,193 & 0,084 & 0,076 & 28 & 578 & $-0,9$ \\
\hline 158 & 578 & 0,062 & 0,816 & 0,065 & 0,389 & 0,956 & 0,477 & 0,525 & 1,000 & 0,927 & 1,033 & 0,861 & 0,276 & 0,237 & 0,273 & 119 & 578 & $-1,5$ \\
\hline 159 & 578 & 0,020 & 0,499 & 0,023 & 1,471 & 0,875 & 2,948 & 1,952 & 1,000 & 0,993 & 0,686 & 0,527 & 0,193 & 0,102 & 0,107 & 68 & 578 & $-0,9$ \\
\hline 160 & 578 & 0,175 & 1,522 & 0,191 & 0,317 & 0,917 & 0,208 & 0,796 & 0,950 & 1,026 & 0,805 & 0,838 & 0,526 & 0,441 & 0,408 & 37 & 578 & $-2,4$ \\
\hline 161 & 578 & 0,019 & 0,418 & 0,020 & 0,245 & 0,929 & 0,586 & 0,420 & 1,000 & 0,864 & 0,974 & 0,691 & 0,156 & 0,108 & 0,107 & 169 & 578 & $-0,6$ \\
\hline 162 & 578 & 0,020 & 0,389 & 0,020 & 1,835 & 1,000 & 4,719 & 3,719 & 1,000 & 0,774 & 1,371 & 0,832 & 0,137 & 0,114 & 0,152 & 90 & 578 & $-0,5$ \\
\hline 163 & 578 & 0,277 & 1,978 & 0,304 & 0,245 & 0,910 & 0,124 & 0,881 & 0,889 & 1,061 & 0,825 & 0,794 & 0,654 & 0,519 & 0,530 & 48 & 578 & $-2,7$ \\
\hline 164 & 578 & 0,016 & 0,368 & 0,017 & 0,245 & 0,917 & 0,667 & 0,344 & 1,000 & 0,826 & 1,077 & 0,745 & 0,137 & 0,102 & 0,152 & 72 & 578 & $-0,5$ \\
\hline 165 & 578 & 0,016 & 0,338 & 0,016 & 0,215 & 1,000 & 0,637 & 0,363 & 1,000 & 0,759 & 1,077 & 0,555 & 0,137 & 0,076 & 0,152 & 0 & 578 & $-0,5$ \\
\hline 166 & 578 & 0,017 & 0,389 & 0,019 & 0,368 & 0,923 & 0,946 & 0,094 & 1,000 & 0,836 & 0,899 & 0,485 & 0,156 & 0,076 & 0,107 & 13 & 578 & $-0,6$ \\
\hline 167 & 578 & 0,027 & 0,490 & 0,027 & 0,583 & 1,000 & 1,189 & 0,189 & 1,000 & 0,838 & 0,968 & 0,707 & 0,189 & 0,134 & 0,169 & 45 & 578 & $-0,9$ \\
\hline 168 & 578 & 0,039 & 0,735 & 0,044 & 0,816 & 0,871 & 1,109 & 0,169 & 0,900 & 1,054 & 0,593 & 0,465 & 0,288 & 0,134 & 0,152 & 16 & 578 & $-1,5$ \\
\hline 169 & 578 & 0,063 & 0,981 & 0,076 & 0,368 & 0,830 & 0,375 & 0,648 & 0,825 & 1,101 & 0,700 & 0,651 & 0,339 & 0,220 & 0,152 & 33 & 578 & $-1,8$ \\
\hline 170 & 578 & 0,016 & 0,469 & 0,020 & 0,368 & 0,786 & 0,784 & 0,305 & 0,901 & 1,054 & 0,539 & 0,373 & 0,193 & 0,072 & 0,076 & 164 & 578 & $-0,9$ \\
\hline 171 & 578 & 0,019 & 0,440 & 0,020 & 0,389 & 0,929 & 0,885 & 0,136 & 1,000 & 0,908 & 0,662 & 0,444 & 0,189 & 0,084 & 0,107 & 123 & 578 & $-0,9$ \\
\hline 172 & 578 & 0,030 & 0,562 & 0,033 & 0,981 & 0,913 & 1,744 & 0,750 & 1,000 & 0,914 & 1,070 & 0,800 & 0,189 & 0,152 & 0,152 & 75 & 578 & $-0,9$ \\
\hline 173 & 578 & 0,096 & 1,010 & 0,098 & 0,317 & 0,985 & 0,314 & 0,686 & 1,000 & 0,919 & 0,783 & 0,670 & 0,395 & 0,265 & 0,303 & 14 & 578 & $-2,0$ \\
\hline 174 & 578 & 0,020 & 0,418 & 0,022 & 0,287 & 0,933 & 0,686 & 0,321 & 1,000 & 0,833 & 0,891 & 0,600 & 0,169 & 0,102 & 0,152 & 125 & 578 & $-0,8$ \\
\hline 175 & 578 & 0,014 & 0,389 & 0,014 & 0,571 & 1,000 & 1,468 & 0,468 & 1,000 & 0,916 & 0,509 & 0,200 & 0,189 & 0,038 & 0,076 & 0 & 578 & $-0,9$ \\
\hline 176 & 578 & 0,039 & 0,600 & 0,039 & 0,583 & 1,000 & 0,971 & 0,029 & 1,000 & 0,861 & 0,929 & 0,697 & 0,230 & 0,161 & 0,214 & 26 & 578 & $-1,2$ \\
\hline 177 & 578 & 0,039 & 0,664 & 0,043 & 0,173 & 0,900 & 0,261 & 0,746 & 1,000 & 0,951 & 0,661 & 0,527 & 0,273 & 0,144 & 0,152 & 30 & 578 & $-1,4$ \\
\hline 178 & 578 & 0,014 & 0,317 & 0,014 & 0,266 & 1,000 & 0,840 & 0,160 & 1,000 & 0,746 & 1,273 & 0,632 & 0,120 & 0,076 & 0,107 & 90 & 578 & $-0,3$ \\
\hline 179 & 578 & 0,022 & 0,440 & 0,023 & 0,144 & 0,938 & 0,327 & 0,676 & 1,000 & 0,845 & 0,955 & 0,671 & 0,169 & 0,114 & 0,152 & 6 & 578 & $-0,8$ \\
\hline 180 & 578 & 0,014 & 0,338 & 0,016 & 0,735 & 0,909 & 2,176 & 1,179 & 1,000 & 0,796 & 0,979 & 0,555 & 0,137 & 0,076 & 0,107 & 162 & 578 & $-0,5$ \\
\hline 181 & 578 & 0,059 & 0,816 & 0,059 & 0,714 & 1,000 & 0,876 & 0,124 & 1,000 & 0,949 & 0,803 & 0,670 & 0,305 & 0,205 & 0,214 & 40 & 578 & $-1,6$ \\
\hline 182 & 578 & 0,053 & 0,765 & 0,062 & 0,338 & 0,860 & 0,442 & 0,575 & 1,000 & 0,937 & 0,889 & 0,800 & 0,276 & 0,221 & 0,169 & 177 & 578 & $-1,5$ \\
\hline 183 & 578 & 0,016 & 0,347 & 0,016 & 0,215 & 1,000 & 0,622 & 0,378 & 1,000 & 0,778 & 0,875 & 0,500 & 0,152 & 0,076 & 0,152 & 0 & 578 & $-0,6$ \\
\hline 184 & 578 & 0,014 & 0,317 & 0,014 & 0,469 & 1,000 & 1,480 & 0,480 & 1,000 & 0,746 & 1,273 & 0,632 & 0,120 & 0,076 & 0,152 & 90 & 578 & $-0,3$ \\
\hline 185 & 578 & 0,030 & 0,520 & 0,030 & 5,618 & 1,000 & 10,805 & 9,805 & 1,000 & 0,845 & 1,028 & 0,784 & 0,193 & 0,152 & 0,169 & 171 & 578 & $-0,9$ \\
\hline 186 & 578 & 1,953 & 7,673 & 2,426 & 0,317 & 0,805 & 0,041 & 0,978 & 0,417 & 1,549 & 0,503 & 0,637 & 2,222 & 1,416 & 0,746 & 39 & 578 & $-4,5$ \\
\hline 187 & 578 & 0,019 & 0,418 & 0,020 & 0,643 & 0,929 & 1,535 & 0,540 & 1,000 & 0,864 & 0,974 & 0,651 & 0,156 & 0,102 & 0,107 & 11 & 578 & $-0,6$ \\
\hline 188 & 578 & 0,046 & 0,714 & 0,049 & 0,266 & 0,941 & 0,373 & 0,630 & 1,000 & 0,941 & 0,769 & 0,666 & 0,276 & 0,184 & 0,169 & 4 & 578 & $-1,5$ \\
\hline 189 & 578 & 0,014 & 0,368 & 0,017 & 0,440 & 0,833 & 1,195 & 0,257 & 1,000 & 0,866 & 0,979 & 0,702 & 0,137 & 0,096 & 0,107 & 45 & 578 & $-0,5$ \\
\hline 190 & 578 & 0,024 & 0,469 & 0,026 & 1,366 & 0,944 & 2,910 & 1,911 & 1,000 & 0,848 & 0,833 & 0,588 & 0,193 & 0,114 & 0,152 & 15 & 578 & $-0,9$ \\
\hline 191 & 578 & 0,168 & 1,674 & 0,182 & 0,828 & 0,921 & 0,495 & 0,511 & 0,753 & 1,153 & 0,683 & 0,651 & 0,559 & 0,364 & 0,339 & 32 & 578 & $-2,5$ \\
\hline 192 & 578 & 0,060 & 0,989 & 0,073 & 0,448 & 0,824 & 0,453 & 0,575 & 0,774 & 1,137 & 0,668 & 0,572 & 0,339 & 0,194 & 0,169 & 103 & 578 & $-1,8$ \\
\hline 193 & 578 & 0,022 & 0,541 & 0,026 & 0,613 & 0,833 & 1,133 & 0,213 & 0,924 & 1,040 & 0,466 & 0,419 & 0,243 & 0,102 & 0,152 & 145 & 578 & $-1,3$ \\
\hline 194 & 578 & 0,039 & 0,643 & 0,040 & 0,397 & 0,964 & 0,618 & 0,383 & 1,000 & 0,921 & 0,764 & 0,601 & 0,254 & 0,153 & 0,152 & 28 & 578 & $-1,3$ \\
\hline 195 & 578 & 0,022 & 0,427 & 0,022 & 0,541 & 1,000 & 1,267 & 0,267 & 1,000 & 0,821 & 1,123 & 0,686 & 0,156 & 0,107 & 0,169 & 45 & 578 & $-0,6$ \\
\hline 196 & 578 & 0,037 & 0,592 & 0,039 & 3,064 & 0,963 & 5,178 & 4,178 & 1,000 & 0,864 & 1,142 & 0,831 & 0,204 & 0,169 & 0,169 & 153 & 578 & $-1,0$ \\
\hline 197 & 578 & 0,700 & 3,217 & 0,755 & 0,757 & 0,928 & 0,235 & 0,768 & 0,850 & 1,084 & 0,728 & 0,699 & 1,106 & 0,773 & 0,746 & 72 & 578 & $-3,5$ \\
\hline 198 & 578 & 0,067 & 0,837 & 0,069 & 0,685 & 0,979 & 0,818 & 0,183 & 1,000 & 0,909 & 0,880 & 0,728 & 0,312 & 0,227 & 0,240 & 4 & 578 & $-1,6$ \\
\hline 199 & 578 & 0,052 & 0,786 & 0,056 & 0,600 & 0,923 & 0,764 & 0,248 & 1,000 & 0,976 & 0,790 & 0,644 & 0,288 & 0,186 & 0,169 & 176 & 578 & $-1,5$ \\
\hline 200 & 578 & 0,036 & 0,651 & 0,036 & 0,326 & 1,000 & 0,500 & 0,500 & 1,000 & 0,970 & 0,601 & 0,486 & 0,276 & 0,134 & 0,169 & 33 & 578 & $-1,5$ \\
\hline 201 & 578 & 0,022 & 0,440 & 0,023 & 2,287 & 0,938 & 5,203 & 4,203 & 1,000 & 0,845 & 0,955 & 0,671 & 0,169 & 0,114 & 0,152 & 30 & 578 & $-0,8$ \\
\hline
\end{tabular}




\begin{tabular}{|c|c|c|c|c|c|c|c|c|c|c|c|c|c|c|c|c|c|c|}
\hline 202 & 578 & 0,415 & 2,418 & 0,445 & 1,031 & 0,932 & 0,427 & 0,577 & 0,891 & 1,059 & 0,690 & 0,693 & 0,874 & 0,606 & 0,592 & 1 & 578 & $-3,1$ \\
\hline 203 & 578 & 0,086 & 1,306 & 0,110 & 0,786 & 0,779 & 0,602 & 0,455 & 0,634 & 1,256 & 0,558 & 0,541 & 0,443 & 0,240 & 0,214 & 70 & 578 & $-2,1$ \\
\hline 204 & 578 & 0,055 & 0,938 & 0,066 & 3,952 & 0,826 & 4,211 & 3,216 & 0,778 & 1,134 & 0,712 & 0,647 & 0,312 & 0,202 & 0,169 & 71 & 578 & $-1,6$ \\
\hline 205 & 578 & 1,039 & 6,290 & 1,303 & 0,368 & 0,797 & 0,058 & 0,963 & 0,330 & 1,741 & 0,737 & 0,905 & 1,339 & 1,212 & 0,312 & 178 & 578 & $-3,7$ \\
\hline 206 & 578 & 0,019 & 0,490 & 0,022 & 1,298 & 0,867 & 2,646 & 1,652 & 0,975 & 1,013 & 0,571 & 0,465 & 0,204 & 0,095 & 0,107 & 130 & 578 & $-1,0$ \\
\hline 207 & 578 & 0,164 & 1,327 & 0,171 & 2,135 & 0,958 & 1,608 & 0,610 & 1,000 & 0,926 & 0,994 & 0,910 & 0,458 & 0,417 & 0,429 & 2 & 578 & $-2,2$ \\
\hline 208 & 578 & 0,253 & 2,574 & 0,300 & 1,163 & 0,842 & 0,452 & 0,571 & 0,479 & 1,445 & 0,339 & 0,387 & 0,974 & 0,377 & 0,227 & 163 & 578 & $-3,3$ \\
\hline 209 & 578 & 0,100 & 1,327 & 0,123 & 0,440 & 0,814 & 0,331 & 0,694 & 0,716 & 1,181 & 0,482 & 0,499 & 0,515 & 0,257 & 0,214 & 42 & 578 & $-2,4$ \\
\hline 210 & 578 & 0,030 & 0,511 & 0,030 & 0,520 & 1,000 & 1,017 & 0,017 & 1,000 & 0,831 & 0,922 & 0,557 & 0,204 & 0,114 & 0,152 & 97 & 578 & $-1,0$ \\
\hline 211 & 578 & 0,024 & 0,571 & 0,027 & 0,520 & 0,895 & 0,911 & 0,138 & 0,941 & 1,031 & 0,481 & 0,388 & 0,254 & 0,099 & 0,152 & 112 & 578 & $-1,3$ \\
\hline 212 & 578 & 0,030 & 0,592 & 0,032 & 0,418 & 0,955 & 0,707 & 0,296 & 1,000 & 0,962 & 0,594 & 0,519 & 0,254 & 0,132 & 0,152 & 117 & 578 & $-1,3$ \\
\hline 213 & 578 & 0,027 & 0,541 & 0,029 & 0,418 & 0,950 & 0,773 & 0,232 & 1,000 & 0,924 & 0,712 & 0,537 & 0,221 & 0,119 & 0,152 & 147 & 578 & $-1,1$ \\
\hline 214 & 578 & 0,030 & 0,511 & 0,030 & 1,234 & 1,000 & 2,414 & 1,414 & 1,000 & 0,831 & 0,922 & 0,557 & 0,204 & 0,114 & 0,152 & 7 & 578 & $-1,0$ \\
\hline 215 & 578 & 0,096 & 1,306 & 0,116 & 1,142 & 0,827 & 0,874 & 0,214 & 0,708 & 1,188 & 0,386 & 0,386 & 0,563 & 0,217 & 0,214 & 26 & 578 & $-2,5$ \\
\hline 216 & 578 & 0,099 & 1,522 & 0,139 & 0,469 & 0,711 & 0,308 & 0,749 & 0,537 & 1,364 & 0,488 & 0,567 & 0,508 & 0,288 & 0,152 & 44 & 578 & $-2,3$ \\
\hline 217 & 578 & 0,019 & 0,550 & 0,024 & 0,389 & 0,765 & 0,707 & 0,376 & 0,776 & 1,135 & 0,404 & 0,294 & 0,243 & 0,071 & 0,107 & 45 & 578 & $-1,3$ \\
\hline 218 & 578 & 0,024 & 0,469 & 0,024 & 1,124 & 1,000 & 2,396 & 1,396 & 1,000 & 0,848 & 0,746 & 0,525 & 0,204 & 0,107 & 0,152 & 153 & 578 & $-1,0$ \\
\hline 219 & 578 & 0,125 & 1,154 & 0,128 & 1,665 & 0,978 & 1,443 & 0,444 & 1,000 & 0,921 & 1,016 & 0,862 & 0,395 & 0,341 & 0,379 & 75 & 578 & $-2,0$ \\
\hline 220 & 578 & 0,227 & 1,746 & 0,241 & 0,520 & 0,940 & 0,298 & 0,705 & 0,935 & 1,034 & 0,604 & 0,571 & 0,691 & 0,395 & 0,408 & 71 & 578 & $-2,8$ \\
\hline 221 & 578 & 0,033 & 0,613 & 0,034 & 2,891 & 0,958 & 4,717 & 3,717 & 1,000 & 0,952 & 0,553 & 0,412 & 0,276 & 0,114 & 0,152 & 15 & 578 & $-1,5$ \\
\hline 222 & 578 & 0,683 & 2,921 & 0,702 & 0,879 & 0,973 & 0,301 & 0,700 & 1,000 & 0,997 & 0,821 & 0,736 & $\begin{array}{l}1,029 \\
\text { S }\end{array}$ & 0,758 & 0,791 & 91 & 578 & $-3,4$ \\
\hline 223 & 578 & 0,072 & 0,989 & 0,079 & 1,133 & 0,909 & 1,145 & 0,171 & 0,921 & 1,042 & 0,549 & 0,510 & 0,408 & 0,208 & 0,227 & 111 & 578 & $-2,0$ \\
\hline 224 & 578 & 0,106 & 1,348 & 0,125 & 1,154 & 0,851 & 0,856 & 0,208 & 0,734 & 1,167 & 0,486 & 0,546 & 0,528 & 0,288 & 0,312 & 26 & 578 & $-2,4$ \\
\hline 225 & 578 & 0,108 & 1,450 & 0,131 & 1,623 & 0,824 & 1,120 & 0,213 & 0,643 & 1,247 & 0,459 & 0,477 & 0,546 & 0,260 & 0,227 & 71 & 578 & $-2,4$ \\
\hline 226 & 578 & 0,230 & 1,683 & 0,241 & 0,960 & 0,952 & 0,570 & 0,432 & 1,000 & 0,991 & 0,781 & 0,744 & 0,612 & 0,455 & 0,461 & 34 & 578 & $-2,6$ \\
\hline 227 & 578 & 0,086 & 0,968 & 0,089 & 1,052 & 0,968 & 1,087 & 0,093 & 1,000 & 0,931 & 0,849 & 0,745 & 0,359 & 0,268 & 0,273 & 106 & 578 & $-1,8$ \\
\hline 228 & 578 & 0,090 & 1,247 & 0,115 & 0,418 & 0,788 & 0,336 & 0,698 & 0,731 & 1,170 & 0,553 & 0,604 & 0,456 & 0,276 & 0,273 & 69 & 578 & $-2,2$ \\
\hline 229 & 578 & 0,027 & 0,511 & 0,029 & 1,112 & 0,950 & 2,174 & 1,175 & 1,000 & 0,874 & 0,834 & 0,557 & 0,204 & 0,114 & 0,152 & 13 & 578 & $-1,0$ \\
\hline 230 & 578 & 0,121 & 1,234 & 0,125 & 1,357 & 0,966 & 1,099 & 0,105 & 0,994 & 1,003 & 0,681 & 0,613 & 0,475 & 0,291 & 0,312 & 39 & 578 & $-2,2$ \\
\hline 231 & 578 & 0,155 & 1,683 & 0,176 & 1,797 & 0,878 & 1,068 & 0,139 & 0,688 & 1,206 & 0,709 & 0,800 & 0,528 & 0,422 & 0,339 & 105 & 578 & $-2,4$ \\
\hline 232 & 578 & 0,268 & 1,826 & 0,281 & 0,418 & 0,954 & 0,229 & 0,772 & 1,000 & 0,995 & 0,661 & 0,671 & 0,719 & 0,482 & 0,442 & 69 & 578 & $-2,8$ \\
\hline 233 & 578 & 0,036 & 0,562 & 0,036 & 0,410 & 1,000 & 0,729 & 0,271 & 1,000 & 0,837 & 1,098 & 0,743 & 0,204 & 0,152 & 0,214 & 24 & 578 & $-1,0$ \\
\hline 234 & 578 & 0,022 & 0,418 & 0,023 & 6,037 & 0,938 & 14,426 & 13,426 & 1,000 & 0,805 & 1,123 & 0,728 & 0,156 & 0,114 & 0,152 & 90 & 578 & $-0,6$ \\
\hline 235 & 578 & 2,501 & 7,373 & 2,852 & 3,567 & 0,877 & 0,484 & 0,531 & 0,578 & 1,315 & 0,613 & 0,758 & 2,279 & 1,727 & 0,611 & 109 & 578 & $-4,5$ \\
\hline 236 & 578 & 0,760 & 3,719 & 0,864 & 0,888 & 0,880 & 0,239 & 0,771 & 0,691 & 1,203 & 0,397 & 0,437 & 1,562 & 0,682 & 0,682 & 79 & 578 & $-4,0$ \\
\hline 237 & 578 & 0,065 & 1,010 & 0,073 & 0,215 & 0,882 & 0,213 & 0,795 & 0,795 & 1,122 & 0,507 & 0,472 & 0,403 & 0,190 & 0,214 & 42 & 578 & $-2,0$ \\
\hline 238 & 578 & 0,014 & 0,317 & 0,014 & 1,366 & 1,000 & 4,308 & 3,308 & 1,000 & 0,746 & 1,273 & 0,632 & 0,120 & 0,076 & 0,152 & 90 & 578 & $-0,3$ \\
\hline 239 & 578 & 0,148 & 1,437 & 0,161 & 0,418 & 0,920 & 0,291 & 0,713 & 0,899 & 1,055 & 0,563 & 0,502 & 0,578 & 0,290 & 0,312 & 43 & 578 & $-2,5$ \\
\hline 240 & 578 & 0,023 & 0,469 & 0,023 & 0,634 & 1,000 & 1,351 & 0,351 & 1,000 & 0,874 & 0,702 & 0,499 & 0,204 & 0,102 & 0,152 & 27 & 578 & $-1,0$ \\
\hline 241 & 578 & 0,034 & 0,849 & 0,040 & 0,418 & 0,857 & 0,493 & 0,527 & 0,600 & 1,291 & 0,303 & 0,199 & 0,381 & 0,076 & 0,152 & 4 & 578 & $-1,9$ \\
\hline 242 & 578 & 0,024 & 0,469 & 0,024 & 5,558 & 1,000 & 11,845 & 10,845 & 1,000 & 0,848 & 0,833 & 0,588 & 0,193 & 0,114 & 0,152 & 0 & 578 & $-0,9$ \\
\hline 243 & 578 & 1,831 & 5,875 & 2,020 & 0,693 & 0,906 & 0,118 & 0,887 & 0,667 & 1,225 & 0,456 & 0,502 & 2,261 & 1,134 & 1,063 & 83 & 578 & $-4,5$ \\
\hline 244 & 578 & 0,052 & 0,816 & 0,057 & 0,714 & 0,900 & 0,876 & 0,160 & 0,975 & 1,013 & 0,559 & 0,552 & 0,343 & 0,189 & 0,214 & 9 & 578 & $-1,8$ \\
\hline 245 & 578 & 0,046 & 0,786 & 0,053 & 0,296 & 0,865 & 0,376 & 0,638 & 0,933 & 1,035 & 0,551 & 0,549 & 0,326 & 0,179 & 0,214 & 46 & 578 & $-1,7$ \\
\hline 246 & 578 & 0,017 & 0,389 & 0,019 & 1,091 & 0,923 & 2,806 & 1,807 & 1,000 & 0,836 & 0,764 & 0,447 & 0,169 & 0,076 & 0,152 & 0 & 578 & $-0,8$ \\
\hline 247 & 578 & 0,062 & 1,408 & 0,099 & 0,245 & 0,623 & 0,174 & 0,908 & 0,391 & 1,599 & 0,296 & 0,364 & 0,515 & 0,187 & 0,214 & 139 & 578 & $-2,4$ \\
\hline 248 & 578 & 0,017 & 0,368 & 0,017 & 1,163 & 1,000 & 3,162 & 2,162 & 1,000 & 0,791 & 1,175 & 0,784 & 0,137 & 0,107 & 0,152 & 0 & 578 & $-0,5$ \\
\hline 249 & 578 & 0,109 & 1,306 & 0,119 & 0,655 & 0,916 & 0,501 & 0,506 & 0,803 & 1,116 & 0,523 & 0,502 & 0,515 & 0,259 & 0,273 & 50 & 578 & $-2,4$ \\
\hline 250 & 578 & 0,055 & 0,735 & 0,056 & 1,205 & 0,974 & 1,638 & 0,639 & 1,000 & 0,889 & 0,913 & 0,687 & 0,276 & 0,189 & 0,227 & 74 & 578 & $-1,5$ \\
\hline 251 & 578 & 0,066 & 1,306 & 0,083 & 1,441 & 0,793 & 1,103 & 0,231 & 0,486 & 1,434 & 0,234 & 0,222 & 0,599 & 0,133 & 0,152 & 68 & 578 & $-2,6$ \\
\hline 252 & 578 & 0,141 & 2,050 & 0,187 & 0,520 & 0,754 & 0,254 & 0,786 & 0,420 & 1,542 & 0,536 & 0,749 & 0,578 & 0,433 & 0,214 & 68 & 578 & $-2,5$ \\
\hline 253 & 578 & 0,039 & 0,613 & 0,039 & 2,080 & 1,000 & 3,394 & 2,394 & 1,000 & 0,878 & 0,764 & 0,596 & 0,254 & 0,152 & 0,214 & 67 & 578 & $-1,3$ \\
\hline 254 & 578 & 0,281 & 2,388 & 0,319 & 0,194 & 0,883 & 0,081 & 0,926 & 0,620 & 1,270 & 0,439 & 0,413 & 0,904 & 0,373 & 0,379 & 32 & 578 & $-3,2$ \\
\hline 255 & 578 & 0,014 & 0,317 & 0,014 & 0,397 & 1,000 & 1,254 & 0,254 & 1,000 & 0,746 & 1,273 & 0,632 & 0,120 & 0,076 & 0,152 & 0 & 578 & $-0,3$ \\
\hline 256 & 578 & 0,027 & 0,490 & 0,027 & 0,440 & 1,000 & 0,896 & 0,104 & 1,000 & 0,838 & 0,834 & 0,557 & 0,204 & 0,114 & 0,152 & 15 & 578 & $-1,0$ \\
\hline 257 & 578 & 0,022 & 0,478 & 0,023 & 0,672 & 0,938 & 1,407 & 0,412 & 1,000 & 0,919 & 0,735 & 0,555 & 0,193 & 0,107 & 0,152 & 69 & 578 & $-0,9$ \\
\hline 258 & 578 & 0,049 & 0,765 & 0,059 & 1,868 & 0,829 & 2,442 & 1,452 & 1,000 & 0,977 & 0,817 & 0,824 & 0,276 & 0,227 & 0,152 & 172 & 578 & $-1,5$ \\
\hline 259 & 578 & 0,275 & 2,236 & 0,317 & 1,252 & 0,869 & 0,560 & 0,459 & 0,692 & 1,202 & 0,670 & 0,736 & 0,724 & 0,533 & 0,455 & 135 & 578 & $\begin{array}{l}-2,9 \\
\end{array}$ \\
\hline
\end{tabular}




\begin{tabular}{|c|c|c|c|c|c|c|c|c|c|c|c|c|c|c|c|c|c|c|}
\hline 260 & 578 & 0,108 & 2,182 & 0,155 & 0,562 & 0,694 & 0,258 & 0,803 & 0,284 & 1,876 & 0,552 & 0,733 & 0,498 & 0,365 & 0,169 & 67 & 578 & $-2,3$ \\
\hline 261 & 578 & 0,047 & 0,685 & 0,049 & 1,623 & 0,971 & 2,371 & 1,371 & 1,000 & 0,888 & 0,934 & 0,745 & 0,254 & 0,189 & 0,227 & 9 & 578 & $-1,3$ \\
\hline 262 & 578 & 0,217 & 1,653 & 0,222 & 1,940 & 0,974 & 1,174 & 0,176 & 0,997 & 1,002 & 0,601 & 0,593 & 0,678 & 0,402 & 0,429 & 55 & 578 & $-2,8$ \\
\hline 263 & 578 & 0,277 & 2,194 & 0,323 & 0,714 & 0,858 & 0,326 & 0,689 & 0,723 & 1,176 & 0,601 & 0,681 & 0,766 & 0,522 & 0,386 & 29 & 578 & $-2,9$ \\
\hline 264 & 578 & 0,060 & 0,858 & 0,069 & 1,716 & 0,875 & 2,000 & 1,008 & 1,000 & 0,986 & 0,668 & 0,637 & 0,339 & 0,216 & 0,214 & 31 & 578 & $-1,8$ \\
\hline 265 & 578 & 0,222 & 1,788 & 0,235 & 1,818 & 0,945 & 1,017 & 0,057 & 0,874 & 1,069 & 0,481 & 0,464 & 0,767 & 0,356 & 0,379 & 73 & 578 & $-2,9$ \\
\hline 266 & 578 & 0,253 & 1,919 & 0,270 & 0,144 & 0,936 & 0,075 & 0,927 & 0,862 & 1,077 & 0,486 & 0,458 & 0,813 & 0,373 & 0,379 & 67 & 578 & $-3,0$ \\
\hline 267 & 578 & 0,014 & 0,338 & 0,016 & 0,317 & 0,909 & 0,938 & 0,110 & 1,000 & 0,796 & 0,979 & 0,555 & 0,137 & 0,076 & 0,107 & 162 & 578 & $-0,5$ \\
\hline 268 & 578 & 0,016 & 0,397 & 0,017 & 0,799 & 0,917 & 2,010 & 1,013 & 1,000 & 0,892 & 0,700 & 0,474 & 0,169 & 0,080 & 0,107 & 29 & 578 & $-0,8$ \\
\hline 269 & 578 & 0,070 & 0,879 & 0,072 & 0,194 & 0,980 & 0,221 & 0,779 & 1,000 & 0,935 & 0,843 & 0,697 & 0,326 & 0,227 & 0,273 & 75 & 578 & $-1,7$ \\
\hline 270 & 578 & 0,022 & 0,431 & 0,022 & 0,245 & 1,000 & 0,569 & 0,431 & 1,000 & 0,829 & 0,955 & 0,447 & 0,169 & 0,076 & 0,152 & 0 & 578 & $-0,8$ \\
\hline 271 & 578 & 0,014 & 0,461 & 0,019 & 0,440 & 0,769 & 0,954 & 0,235 & 0,850 & 1,085 & 0,509 & 0,389 & 0,189 & 0,074 & 0,076 & 143 & 578 & $-0,9$ \\
\hline 272 & 578 & 0,024 & 0,469 & 0,024 & 0,550 & 1,000 & 1,171 & 0,171 & 1,000 & 0,848 & 0,866 & 0,566 & 0,189 & 0,107 & 0,152 & 45 & 578 & $-0,9$ \\
\hline 273 & 578 & 0,032 & 0,592 & 0,034 & 0,338 & 0,917 & 0,571 & 0,437 & 1,000 & 0,940 & 0,622 & 0,467 & 0,254 & 0,119 & 0,152 & 115 & 578 & $-1,3$ \\
\hline 274 & 578 & 0,019 & 0,418 & 0,019 & 0,338 & 1,000 & 0,808 & 0,192 & 1,000 & 0,864 & 0,662 & 0,424 & 0,189 & 0,080 & 0,107 & 33 & 578 & $-0,9$ \\
\hline 275 & 578 & 0,020 & 0,461 & 0,022 & 0,685 & 0,933 & 1,487 & 0,491 & 1,000 & 0,917 & 0,615 & 0,371 & 0,204 & 0,076 & 0,107 & 75 & 578 & $-1,0$ \\
\hline 276 & 578 & 0,055 & 0,786 & 0,059 & 0,511 & 0,927 & 0,650 & 0,357 & 1,000 & 0,950 & 0,793 & 0,634 & 0,296 & 0,187 & 0,214 & 41 & 578 & $-1,6$ \\
\hline 277 & 578 & 0,034 & 0,541 & 0,034 & 0,825 & 1,000 & 1,524 & 0,524 & 1,000 & 0,823 & 1,054 & 0,743 & 0,204 & 0,152 & 0,214 & 15 & 578 & $-1,0$ \\
\hline 278 & 578 & 0,063 & 0,989 & 0,073 & 0,469 & 0,863 & 0,474 & 0,543 & 0,811 & 1,111 & 0,572 & 0,501 & 0,375 & 0,188 & 0,169 & 32 & 578 & $-1,9$ \\
\hline 279 & 578 & 0,032 & 0,541 & 0,033 & 0,634 & 0,957 & 1,172 & 0,177 & 1,000 & 0,859 & 0,966 & 0,743 & 0,204 & 0,152 & 0,169 & 9 & 578 & $-1,0$ \\
\hline 280 & 578 & 0,053 & 0,757 & 0,056 & 1,163 & 0,949 & 1,537 & 0,539 & 1,000 & 0,926 & 0,772 & 0,675 & 0,296 & 0,200 & 0,214 & 41 & 578 & $-1,6$ \\
\hline 281 & 578 & 0,106 & 1,243 & 0,115 & 0,520 & 0,925 & 0,418 & 0,586 & 0,864 & 1,076 & 0,488 & 0,440 & 0,526 & 0,232 & 0,273 & 53 & 578 & $-2,4$ \\
\hline 282 & 578 & 0,036 & 0,571 & 0,036 & 1,644 & 1,000 & 2,881 & 1,881 & 1,000 & 0,850 & 1,098 & 0,788 & 0,204 & 0,161 & 0,214 & 45 & 578 & $-1,0$ \\
\hline 283 & 578 & 0,235 & 1,767 & 0,251 & 0,461 & 0,937 & 0,261 & 0,742 & 0,947 & 1,027 & 0,682 & 0,682 & 0,663 & 0,452 & 0,408 & 26 & 578 & $-2,7$ \\
\hline 284 & 578 & 0,033 & 0,541 & 0,033 & 0,224 & 1,000 & 0,414 & 0,586 & 1,000 & 0,840 & 0,861 & 0,606 & 0,221 & 0,134 & 0,169 & 62 & 578 & $-1,1$ \\
\hline 285 & 578 & 0,014 & 0,347 & 0,014 & 0,440 & 1,000 & 1,268 & 0,268 & 1,000 & 0,816 & 1,273 & 0,849 & 0,120 & 0,102 & 0,107 & 0 & 578 & $-0,3$ \\
\hline 286 & 578 & 0,029 & 0,520 & 0,029 & 1,091 & 1,000 & 2,098 & 1,098 & 1,000 & 0,866 & 0,878 & 0,657 & 0,204 & 0,134 & 0,152 & 53 & 578 & $-1,0$ \\
\hline 287 & 578 & 0,100 & 1,255 & 0,112 & 0,338 & 0,897 & 0,269 & 0,738 & 0,801 & 1,118 & 0,462 & 0,442 & 0,526 & 0,232 & 0,240 & 48 & 578 & $-2,4$ \\
\hline 288 & 578 & 0,022 & 0,418 & 0,022 & 0,410 & 1,000 & 0,979 & 0,021 & 1,000 & 0,805 & 0,955 & 0,632 & 0,169 & 0,107 & 0,152 & 45 & 578 & $-0,8$ \\
\hline 289 & 578 & 0,020 & 0,685 & 0,026 & 1,306 & 0,778 & 1,908 & 0,935 & 0,538 & 1,363 & 0,482 & 0,329 & 0,230 & 0,076 & 0,107 & 90 & 578 & $-1,2$ \\
\hline 290 & 578 & 0,146 & 1,826 & 0,168 & 0,947 & 0,872 & 0,519 & 0,498 & 0,551 & 1,347 & 0,812 & 0,814 & 0,479 & 0,390 & 0,303 & 89 & 578 & $-2,3$ \\
\hline 291 & 578 & 0,083 & 1,175 & 0,095 & 2,227 & 0,879 & 1,896 & 0,904 & 0,757 & 1,149 & 0,509 & 0,482 & 0,456 & 0,220 & 0,214 & 39 & 578 & $-2,2$ \\
\hline 292 & 578 & 0,340 & 3,327 & 0,448 & 1,653 & 0,760 & 0,497 & 0,558 & 0,386 & 1,609 & 0,517 & 0,621 & 0,915 & 0,568 & 0,152 & 11 & 578 & $-3,2$ \\
\hline 293 & 578 & 0,179 & 2,000 & 0,232 & 0,837 & 0,772 & 0,419 & 0,625 & 0,564 & 1,332 & 0,502 & 0,596 & 0,674 & 0,402 & 0,107 & 66 & 578 & $-2,8$ \\
\hline 294 & 578 & 0,055 & 1,103 & 0,072 & 0,469 & 0,760 & 0,425 & 0,623 & 0,563 & 1,333 & 0,499 & 0,517 & 0,373 & 0,193 & 0,152 & 164 & 578 & $-1,9$ \\
\hline 295 & 578 & 0,030 & 0,613 & 0,036 & 0,338 & 0,840 & 0,552 & 0,476 & 1,000 & 0,996 & 0,668 & 0,559 & 0,240 & 0,134 & 0,152 & 17 & 578 & $-1,3$ \\
\hline 296 & 578 & 0,024 & 0,532 & 0,026 & 0,541 & 0,944 & 1,016 & 0,058 & 1,000 & 0,962 & 0,541 & 0,316 & 0,240 & 0,076 & 0,152 & 172 & 578 & $-1,3$ \\
\hline 297 & 578 & 0,042 & 0,613 & 0,042 & 1,205 & 1,000 & 1,966 & 0,966 & 1,000 & 0,848 & 1,086 & 0,844 & 0,221 & 0,186 & 0,214 & 36 & 578 & $-1,1$ \\
\hline 298 & 578 & 0,139 & 1,277 & 0,145 & 1,581 & 0,960 & 1,239 & 0,242 & 1,000 & 0,965 & 0,668 & 0,592 & 0,515 & 0,305 & 0,312 & 64 & 578 & $-2,4$ \\
\hline 299 & 578 & 0,205 & 1,632 & 0,214 & 0,266 & 0,960 & 0,163 & 0,838 & 0,968 & 1,016 & 0,630 & 0,570 & 0,644 & 0,367 & 0,339 & 35 & 578 & $-2,7$ \\
\hline 300 & 578 & 0,017 & 0,368 & 0,017 & 0,989 & 1,000 & 2,690 & 1,690 & 1,000 & 0,791 & 1,175 & 0,745 & 0,137 & 0,102 & 0,107 & 45 & 578 & $-0,5$ \\
\hline 301 & 578 & 0,075 & 1,805 & 0,100 & 2,320 & 0,743 & 1,285 & 0,384 & 0,288 & 1,864 & 0,530 & 0,571 & 0,423 & 0,242 & 0,152 & 42 & 578 & $-2,1$ \\
\hline 302 & 578 & 0,435 & 2,604 & 0,473 & 0,520 & 0,918 & 0,200 & 0,804 & 0,806 & 1,114 & 0,647 & 0,671 & 0,925 & 0,621 & 0,577 & 68 & 578 & $-3,2$ \\
\hline 303 & 578 & 0,029 & 0,600 & 0,032 & 0,397 & 0,909 & 0,662 & 0,350 & 1,000 & 1,000 & 0,637 & 0,559 & 0,240 & 0,134 & 0,169 & 20 & 578 & $-1,3$ \\
\hline 304 & 578 & 0,027 & 0,490 & 0,029 & 0,846 & 0,950 & 1,725 & 0,726 & 1,000 & 0,838 & 1,210 & 0,894 & 0,169 & 0,152 & 0,169 & 110 & 578 & $-0,8$ \\
\hline 305 & 578 & 0,049 & 0,896 & 0,056 & 0,664 & 0,872 & 0,740 & 0,290 & 0,763 & 1,145 & 0,370 & 0,327 & 0,410 & 0,134 & 0,169 & 37 & 578 & $-2,0$ \\
\hline 306 & 578 & 0,053 & 0,786 & 0,055 & 0,571 & 0,974 & 0,726 & 0,275 & 1,000 & 0,963 & 0,693 & 0,485 & 0,312 & 0,152 & 0,227 & 1 & 578 & $-1,6$ \\
\hline 307 & 578 & 0,043 & 0,685 & 0,046 & 0,917 & 0,938 & 1,340 & 0,346 & 1,000 & 0,931 & 0,932 & 0,781 & 0,243 & 0,189 & 0,169 & 1 & 578 & $-1,3$ \\
\hline 308 & 578 & 0,095 & 1,061 & 0,099 & 0,664 & 0,957 & 0,625 & 0,377 & 1,000 & 0,973 & 0,618 & 0,546 & 0,442 & 0,241 & 0,273 & 57 & 578 & $-2,1$ \\
\hline 309 & 578 & 0,039 & 0,765 & 0,047 & 2,092 & 0,818 & 2,735 & 1,744 & 0,832 & 1,097 & 0,506 & 0,463 & 0,312 & 0,145 & 0,152 & 153 & 578 & $-1,6$ \\
\hline 310 & 578 & 0,248 & 3,162 & 0,369 & 0,592 & 0,673 & 0,187 & 0,876 & 0,312 & 1,790 & 0,436 & 0,655 & 0,851 & 0,557 & 0,227 & 43 & 578 & $-3,1$ \\
\hline 311 & 578 & 0,034 & 0,757 & 0,046 & 0,368 & 0,750 & 0,486 & 0,572 & 0,756 & 1,150 & 0,577 & 0,639 & 0,276 & 0,176 & 0,152 & 36 & 578 & $-1,5$ \\
\hline 312 & 578 & 0,023 & 0,448 & 0,024 & 0,655 & 0,941 & 1,461 & 0,465 & 1,000 & 0,834 & 1,019 & 0,632 & 0,169 & 0,107 & 0,169 & 138 & 578 & $-0,8$ \\
\hline 313 & 578 & 0,044 & 0,757 & 0,047 & 1,192 & 0,939 & 1,576 & 0,579 & 0,977 & 1,012 & 0,580 & 0,364 & 0,312 & 0,114 & 0,152 & 174 & 578 & $-1,6$ \\
\hline 314 & 578 & 0,089 & 1,294 & 0,106 & 0,144 & 0,838 & 0,111 & 0,904 & 0,668 & 1,224 & 0,439 & 0,474 & 0,508 & 0,241 & 0,107 & 37 & 578 & $-2,3$ \\
\hline 315 & 578 & 0,014 & 0,338 & 0,016 & 1,116 & 0,909 & 3,300 & 2,302 & 1,000 & 0,796 & 0,979 & 0,555 & 0,137 & 0,076 & 0,107 & 162 & 578 & $-0,5$ \\
\hline 316 & 578 & 0,119 & 1,277 & 0,131 & 0,816 & 0,912 & 0,639 & 0,371 & 0,918 & 1,043 & 0,652 & 0,668 & 0,482 & 0,322 & 0,321 & 131 & 578 & $-2,3$ \\
\hline 317 & 578 & 0,036 & 0,909 & 0,050 & 0,389 & 0,714 & 0,428 & 0,640 & 0,546 & 1,354 & 0,292 & 0,258 & 0,395 & 0,102 & 0,107 & 25 & 578 & $-2,0$ \\
\hline
\end{tabular}




\begin{tabular}{|c|c|c|c|c|c|c|c|c|c|c|c|c|c|c|c|c|c|c|}
\hline 318 & 578 & 0,024 & 0,469 & 0,024 & 0,765 & 1,000 & 1,631 & 0,631 & 1,000 & 0,848 & 1,082 & 0,791 & 0,169 & 0,134 & 0,152 & 90 & 578 & $-0,8$ \\
\hline 319 & 578 & 0,060 & 0,875 & 0,065 & 0,765 & 0,933 & 0,874 & 0,142 & 0,988 & 1,006 & 0,723 & 0,575 & 0,326 & 0,187 & 0,214 & 49 & 578 & $-1,7$ \\
\hline 320 & 578 & 0,059 & 0,816 & 0,063 & 5,393 & 0,932 & 6,610 & 5,611 & 1,000 & 0,949 & 0,715 & 0,620 & 0,324 & 0,201 & 0,214 & 17 & 578 & $-1,7$ \\
\hline 321 & 578 & 1,911 & 6,188 & 2,141 & 0,440 & 0,893 & 0,071 & 0,935 & 0,627 & 1,263 & 0,615 & 0,736 & 1,989 & 1,463 & 0,429 & 98 & 578 & $-4,3$ \\
\hline 322 & 578 & 0,029 & 0,541 & 0,030 & 0,571 & 0,952 & 1,055 & 0,073 & 1,000 & 0,901 & 0,621 & 0,442 & 0,243 & 0,107 & 0,152 & 34 & 578 & $-1,3$ \\
\hline 323 & 578 & 0,037 & 0,643 & 0,039 & 0,469 & 0,963 & 0,730 & 0,272 & 1,000 & 0,939 & 0,736 & 0,601 & 0,254 & 0,153 & 0,169 & 30 & 578 & $-1,3$ \\
\hline 324 & 578 & 0,032 & 0,541 & 0,033 & 0,338 & 0,957 & 0,625 & 0,378 & 1,000 & 0,859 & 0,966 & 0,743 & 0,204 & 0,152 & 0,169 & 14 & 578 & $-1,0$ \\
\hline 325 & 578 & 0,016 & 0,397 & 0,017 & 0,490 & 0,917 & 1,234 & 0,248 & 1,000 & 0,892 & 0,824 & 0,485 & 0,156 & 0,076 & 0,107 & 0 & 578 & $-0,6$ \\
\hline 326 & 578 & 0,034 & 0,592 & 0,037 & 5,123 & 0,923 & 8,657 & 7,657 & 1,000 & 0,900 & 0,899 & 0,667 & 0,221 & 0,147 & 0,169 & 46 & 578 & $-1,1$ \\
\hline 327 & 578 & 1,369 & 8,028 & 1,885 & 0,765 & 0,726 & 0,095 & 0,945 & 0,267 & 1,936 & 0,403 & 0,550 & 2,080 & 1,143 & 0,227 & 58 & 578 & $-4,4$ \\
\hline 328 & 578 & 0,073 & 0,879 & 0,075 & 0,469 & 0,981 & 0,534 & 0,467 & 1,000 & 0,917 & 0,764 & 0,690 & 0,349 & 0,241 & 0,273 & 42 & 578 & $-1,8$ \\
\hline 329 & 578 & 0,039 & 0,583 & 0,039 & 0,338 & 1,000 & 0,580 & 0,420 & 1,000 & 0,836 & 1,011 & 0,686 & 0,221 & 0,152 & 0,214 & 0 & 578 & $-1,1$ \\
\hline 330 & 578 & 0,016 & 0,490 & 0,017 & 0,807 & 0,917 & 1,646 & 0,652 & 0,825 & 1,101 & 0,389 & 0,167 & 0,227 & 0,038 & 0,076 & 0 & 578 & $-1,2$ \\
\hline 331 & 578 & 0,063 & 0,909 & 0,066 & 1,569 & 0,957 & 1,726 & 0,727 & 0,961 & 1,020 & 0,529 & 0,542 & 0,390 & 0,211 & 0,227 & 61 & 578 & $-2,0$ \\
\hline 332 & 578 & 0,168 & 1,856 & 0,205 & 1,826 & 0,818 & 0,984 & 0,183 & 0,612 & 1,278 & 0,378 & 0,392 & 0,752 & 0,295 & 0,227 & 135 & 578 & $-2,9$ \\
\hline 333 & 578 & 0,283 & 1,877 & 0,294 & 1,767 & 0,961 & 0,941 & 0,070 & 1,000 & 0,996 & 0,705 & 0,610 & 0,715 & 0,436 & 0,442 & 47 & 578 & $-2,8$ \\
\hline 334 & 578 & 0,261 & 1,818 & 0,274 & 0,786 & 0,953 & 0,433 & 0,569 & 0,993 & 1,003 & 0,688 & 0,673 & 0,695 & 0,468 & 0,455 & 32 & 578 & $-2,8$ \\
\hline 335 & 578 & 0,062 & 0,837 & 0,063 & 0,418 & 0,977 & 0,500 & 0,501 & 1,000 & 0,951 & 0,740 & 0,646 & 0,326 & 0,210 & 0,227 & 35 & 578 & $-1,7$ \\
\hline 336 & 578 & 0,029 & 0,490 & 0,029 & 0,317 & 1,000 & 0,646 & 0,354 & 1,000 & 0,816 & 1,273 & 0,894 & 0,169 & 0,152 & 0,169 & 0 & 578 & $-0,8$ \\
\hline 337 & 578 & 0,019 & 0,397 & 0,019 & 0,714 & 1,000 & 1,798 & 0,798 & 1,000 & 0,821 & 0,974 & 0,686 & 0,156 & 0,107 & 0,107 & 83 & 578 & $-0,6$ \\
\hline 338 & 578 & 0,057 & 0,765 & 0,059 & 0,520 & 0,976 & 0,680 & 0,321 & 1,000 & 0,901 & 0,961 & 0,799 & 0,276 & 0,220 & 0,240 & 26 & 578 & $-1,5$ \\
\hline 339 & 578 & 0,040 & 0,613 & 0,042 & 1,805 & 0,966 & 2,945 & 1,946 & 1,000 & 0,863 & 0,891 & 0,632 & 0,240 & 0,152 & 0,214 & 178 & 578 & $-1,3$ \\
\hline 340 & 578 & 0,238 & 1,978 & 0,263 & 5,208 & 0,907 & 2,632 & 1,635 & 0,765 & 1,144 & 0,488 & 0,480 & 0,788 & 0,378 & 0,379 & 57 & 578 & $-3,0$ \\
\hline 341 & 578 & 1,948 & 5,783 & 2,108 & 0,245 & 0,924 & 0,042 & 0,961 & 0,732 & 1,169 & 0,665 & 0,645 & 1,931 & 1,246 & 1,249 & 71 & 578 & $-4,3$ \\
\hline 342 & 578 & 0,014 & 0,317 & 0,014 & 0,960 & 1,000 & 3,027 & 2,027 & 1,000 & 0,746 & 0,979 & 0,555 & 0,137 & 0,076 & 0,107 & 162 & 578 & $-0,5$ \\
\hline 343 & 578 & 0,082 & 1,205 & 0,100 & 1,408 & 0,814 & 1,169 & 0,251 & 0,708 & 1,188 & 0,581 & 0,562 & 0,423 & 0,238 & 0,152 & 71 & 578 & $-2,1$ \\
\hline 344 & 578 & 0,166 & 1,581 & 0,188 & 0,562 & 0,885 & 0,356 & 0,655 & 0,837 & 1,093 & 0,656 & 0,660 & 0,568 & 0,375 & 0,379 & 40 & 578 & $-2,5$ \\
\hline 345 & 578 & 0,032 & 0,600 & 0,036 & 0,266 & 0,880 & 0,443 & 0,569 & 1,000 & 0,953 & 0,700 & 0,559 & 0,240 & 0,134 & 0,152 & 32 & 578 & $-1,3$ \\
\hline 346 & 578 & 0,014 & 0,440 & 0,017 & 0,397 & 0,833 & 0,904 & 0,192 & 0,933 & 1,035 & 0,439 & 0,294 & 0,204 & 0,060 & 0,076 & 72 & 578 & $-1,0$ \\
\hline 347 & 578 & 0,030 & 0,511 & 0,030 & 1,298 & 1,000 & 2,537 & 1,537 & 1,000 & 0,831 & 0,922 & 0,557 & 0,204 & 0,114 & 0,152 & 0 & 578 & $-1,0$ \\
\hline 348 & 578 & 0,155 & 1,378 & 0,165 & 0,888 & 0,939 & 0,644 & 0,361 & 1,000 & 0,988 & 0,838 & 0,781 & 0,485 & 0,379 & 0,379 & 71 & 578 & $-2,3$ \\
\hline 349 & 578 & 0,072 & 1,010 & 0,076 & 0,858 & 0,943 & 0,849 & 0,161 & 0,883 & 1,064 & 0,563 & 0,466 & 0,403 & 0,187 & 0,214 & 39 & 578 & $-2,0$ \\
\hline 350 & 578 & 0,072 & 0,981 & 0,080 & 0,981 & 0,893 & 1,000 & 0,107 & 0,938 & 1,033 & 0,715 & 0,655 & 0,357 & 0,234 & 0,227 & 27 & 578 & $-1,8$ \\
\hline 351 & 578 & 0,099 & 1,133 & 0,105 & 0,296 & 0,945 & 0,261 & 0,741 & 0,969 & 1,016 & 0,757 & 0,646 & 0,408 & 0,264 & 0,240 & 14 & 578 & $-2,0$ \\
\hline 352 & 578 & 0,016 & 0,418 & 0,017 & 0,368 & 0,917 & 0,879 & 0,147 & 1,000 & 0,940 & 0,539 & 0,392 & 0,193 & 0,076 & 0,107 & 18 & 578 & $-0,9$ \\
\hline 353 & 578 & 0,027 & 0,490 & 0,029 & 0,981 & 0,950 & 2,000 & 1,001 & 1,000 & 0,838 & 1,210 & 0,894 & 0,169 & 0,152 & 0,169 & 55 & 578 & $-0,8$ \\
\hline 354 & 578 & 0,100 & 1,052 & 0,103 & 0,245 & 0,972 & 0,233 & 0,768 & 1,000 & 0,937 & 0,789 & 0,732 & 0,403 & 0,295 & 0,303 & 34 & 578 & $-2,0$ \\
\hline 355 & 578 & 0,016 & 0,389 & 0,017 & 2,523 & 0,917 & 6,490 & 5,491 & 1,000 & 0,873 & 0,824 & 0,485 & 0,156 & 0,076 & 0,107 & 167 & 578 & $-0,6$ \\
\hline 356 & 578 & 0,519 & 2,655 & 0,550 & 0,245 & 0,945 & 0,092 & 0,909 & 0,926 & 1,039 & 0,733 & 0,718 & 0,950 & 0,682 & 0,678 & 76 & 578 & $-3,2$ \\
\hline 357 & 578 & 0,014 & 0,326 & 0,014 & 0,368 & 1,000 & 1,129 & 0,129 & 1,000 & 0,767 & 1,273 & 0,671 & 0,120 & 0,080 & 0,107 & 108 & 578 & $-0,3$ \\
\hline 358 & 578 & 0,024 & 0,469 & 0,024 & 4,938 & 1,000 & 10,522 & 9,522 & 1,000 & 0,848 & 0,833 & 0,588 & 0,193 & 0,114 & 0,152 & 103 & 578 & $-0,9$ \\
\hline 359 & 578 & 1,917 & 5,090 & 1,984 & 1,175 & 0,966 & 0,231 & 0,770 & 0,930 & 1,037 & 0,735 & 0,738 & 1,822 & 1,345 & 1,349 & 57 & 578 & $-4,2$ \\
\hline 360 & 578 & 0,113 & 1,387 & 0,113 & 0,541 & 1,000 & 0,390 & 0,610 & 0,741 & 1,162 & 0,734 & 0,769 & 0,443 & 0,341 & 0,339 & 48 & 578 & $-2,1$ \\
\hline 361 & 578 & 0,034 & 0,592 & 0,037 & 3,238 & 0,923 & 5,471 & 4,471 & 1,000 & 0,900 & 0,899 & 0,620 & 0,221 & 0,137 & 0,169 & 41 & 578 & $-1,1$ \\
\hline 362 & 578 & 0,855 & 3,310 & 0,891 & 2,777 & 0,960 & 0,839 & 0,166 & 0,981 & 1,010 & 0,787 & 0,757 & 1,176 & 0,891 & 0,864 & 99 & 578 & $-3,6$ \\
\hline 363 & 578 & 0,597 & 3,234 & 0,667 & 0,397 & 0,895 & 0,123 & 0,883 & 0,717 & 1,181 & 0,744 & 0,787 & 1,011 & 0,795 & 0,530 & 108 & 578 & $-3,3$ \\
\hline 364 & 578 & 0,022 & 0,499 & 0,026 & 0,245 & 0,833 & 0,491 & 0,535 & 1,000 & 0,959 & 0,735 & 0,546 & 0,193 & 0,105 & 0,107 & 29 & 578 & $-0,9$ \\
\hline 365 & 578 & 0,017 & 0,368 & 0,017 & 0,245 & 1,000 & 0,667 & 0,333 & 1,000 & 0,791 & 1,175 & 0,784 & 0,137 & 0,107 & 0,152 & 45 & 578 & $-0,5$ \\
\hline 366 & 578 & 0,014 & 0,317 & 0,014 & 0,440 & 1,000 & 1,387 & 0,387 & 1,000 & 0,746 & 0,979 & 0,555 & 0,137 & 0,076 & 0,107 & 162 & 578 & $-0,5$ \\
\hline 367 & 578 & 0,033 & 0,583 & 0,036 & 0,592 & 0,920 & 1,015 & 0,081 & 1,000 & 0,906 & 0,861 & 0,641 & 0,221 & 0,142 & 0,152 & 7 & 578 & $-1,1$ \\
\hline 368 & 578 & 0,036 & 0,664 & 0,040 & 0,287 & 0,893 & 0,433 & 0,577 & 1,000 & 0,988 & 0,601 & 0,497 & 0,276 & 0,137 & 0,152 & 9 & 578 & $-1,5$ \\
\hline 369 & 578 & 0,014 & 0,368 & 0,016 & 2,528 & 0,909 & 6,875 & 5,876 & 1,000 & 0,866 & 0,979 & 0,588 & 0,137 & 0,080 & 0,076 & 45 & 578 & $-0,5$ \\
\hline 370 & 578 & 0,509 & 2,642 & 0,532 & 0,266 & 0,957 & 0,101 & 0,900 & 0,917 & 1,044 & 0,665 & 0,617 & 0,988 & 0,610 & 0,611 & 53 & 578 & $-3,3$ \\
\hline 371 & 578 & 0,016 & 0,347 & 0,016 & 0,347 & 1,000 & 1,000 & 0,000 & 1,000 & 0,778 & 1,401 & 0,894 & 0,120 & 0,107 & 0,152 & 72 & 578 & $-0,3$ \\
\hline 372 & 578 & 0,016 & 0,376 & 0,017 & 0,511 & 0,917 & 1,359 & 0,368 & 1,000 & 0,845 & 0,875 & 0,671 & 0,152 & 0,102 & 0,107 & 45 & 578 & $-0,6$ \\
\hline 373 & 578 & 0,034 & 0,541 & 0,034 & 0,786 & 1,000 & 1,453 & 0,453 & 1,000 & 0,823 & 1,054 & 0,743 & 0,204 & 0,152 & 0,214 & 105 & 578 & $-1,0$ \\
\hline 374 & 578 & 0,060 & 0,938 & 0,069 & 0,469 & 0,875 & 0,500 & 0,515 & 0,860 & 1,078 & 0,535 & 0,500 & 0,379 & 0,190 & 0,214 & 33 & 578 & $-1,9$ \\
\hline 375 & 578 & 0,036 & 0,562 & 0,036 & 0,296 & 1,000 & 0,526 & 0,474 & 1,000 & 0,837 & 0,936 & 0,686 & 0,221 & 0,152 & 0,214 & 26 & 578 & $-1,1$ \\
\hline
\end{tabular}




\begin{tabular}{|c|c|c|c|c|c|c|c|c|c|c|c|c|c|c|c|c|c|c|}
\hline 376 & 578 & 0,014 & 0,347 & 0,014 & 2,494 & 1,000 & 7,193 & 6,193 & 1,000 & 0,816 & 0,796 & 0,500 & 0,152 & 0,076 & 0,107 & 18 & 578 & $-0,6$ \\
\hline 377 & 578 & 0,475 & 2,676 & 0,525 & 0,266 & 0,904 & 0,099 & 0,906 & 0,834 & 1,095 & 0,612 & 0,698 & 0,994 & 0,694 & 0,606 & 40 & 578 & $-3,3$ \\
\hline 378 & 578 & 0,019 & 0,389 & 0,019 & 0,930 & 1,000 & 2,392 & 1,392 & 1,000 & 0,803 & 1,273 & 0,832 & 0,137 & 0,114 & 0,152 & 0 & 578 & $-0,5$ \\
\hline 379 & 578 & 0,086 & 1,184 & 0,096 & 1,082 & 0,896 & 0,914 & 0,135 & 0,772 & 1,138 & 0,849 & 0,738 & 0,359 & 0,265 & 0,214 & 90 & 578 & $-1,8$ \\
\hline 380 & 578 & 0,105 & 1,205 & 0,113 & 1,860 & 0,924 & 1,544 & 0,549 & 0,907 & 1,050 & 0,628 & 0,575 & 0,461 & 0,265 & 0,303 & 171 & 578 & $-2,2$ \\
\hline 381 & 578 & 0,280 & 1,889 & 0,296 & 0,562 & 0,947 & 0,298 & 0,705 & 0,985 & 1,008 & 0,643 & 0,581 & 0,744 & 0,433 & 0,455 & 80 & 578 & $-2,9$ \\
\hline 382 & 578 & 0,033 & 0,664 & 0,039 & 0,144 & 0,852 & 0,216 & 0,797 & 0,942 & 1,031 & 0,791 & 0,757 & 0,230 & 0,174 & 0,152 & 8 & 578 & $-1,2$ \\
\hline 383 & 578 & 0,016 & 0,338 & 0,016 & 0,347 & 1,000 & 1,026 & 0,026 & 1,000 & 0,759 & 1,077 & 0,555 & 0,137 & 0,076 & 0,152 & 0 & 578 & $-0,5$ \\
\hline 384 & 578 & 0,023 & 0,469 & 0,023 & 2,021 & 1,000 & 4,306 & 3,306 & 1,000 & 0,874 & 1,019 & 0,746 & 0,169 & 0,126 & 0,152 & 45 & 578 & $-0,8$ \\
\hline 385 & 578 & 0,347 & 2,122 & 0,367 & 1,040 & 0,945 & 0,490 & 0,513 & 0,969 & 1,016 & 0,753 & 0,738 & 0,766 & 0,565 & 0,577 & 91 & 578 & $-2,9$ \\
\hline 386 & 578 & 0,102 & 1,154 & 0,112 & 0,296 & 0,910 & 0,256 & 0,749 & 0,961 & 1,020 & 0,695 & 0,620 & 0,432 & 0,268 & 0,273 & 47 & 578 & $-2,1$ \\
\hline 387 & 578 & 0,016 & 0,347 & 0,016 & 2,786 & 1,000 & 8,036 & 7,036 & 1,000 & 0,778 & 1,401 & 0,849 & 0,120 & 0,102 & 0,107 & 45 & 578 & $-0,3$ \\
\hline 388 & 578 & 0,630 & 2,879 & 0,659 & 0,389 & 0,956 & 0,135 & 0,866 & 0,955 & 1,023 & 0,755 & 0,714 & 1,030 & 0,736 & 0,746 & 36 & 578 & $-3,4$ \\
\hline 389 & 578 & 0,020 & 0,490 & 0,022 & 0,347 & 0,933 & 0,707 & 0,300 & 1,000 & 0,976 & 0,524 & 0,364 & 0,221 & 0,080 & 0,107 & 42 & 578 & $-1,1$ \\
\hline 390 & 578 & 0,014 & 0,347 & 0,014 & 0,693 & 1,000 & 2,000 & 1,000 & 1,000 & 0,816 & 0,749 & 0,485 & 0,156 & 0,076 & 0,107 & 162 & 578 & $-0,6$ \\
\hline 391 & 578 & 0,055 & 0,816 & 0,060 & 0,727 & 0,905 & 0,891 & 0,145 & 1,000 & 0,986 & 0,654 & 0,535 & 0,326 & 0,174 & 0,214 & 36 & 578 & $-1,7$ \\
\hline 392 & 578 & 0,046 & 0,837 & 0,056 & 1,716 & 0,821 & 2,050 & 1,066 & 0,824 & 1,102 & 0,668 & 0,640 & 0,296 & 0,189 & 0,214 & 147 & 578 & $-1,6$ \\
\hline 393 & 578 & 0,148 & 2,164 & 0,238 & 1,234 & 0,620 & 0,570 & 0,573 & 0,396 & 1,588 & 0,386 & 0,606 & 0,698 & 0,424 & 0,169 & 74 & 578 & $-2,8$ \\
\hline 394 & 578 & 0,146 & 1,285 & 0,151 & 0,562 & 0,971 & 0,437 & 0,563 & 1,000 & 0,948 & 0,872 & 0,753 & 0,462 & 0,348 & 0,379 & 49 & 578 & $-2,2$ \\
\hline 395 & 578 & 0,049 & 0,778 & 0,053 & 0,672 & 0,919 & 0,865 & 0,158 & 1,000 & 0,993 & 0,541 & 0,447 & 0,339 & 0,152 & 0,169 & 15 & 578 & $-1,8$ \\
\hline 396 & 578 & 0,037 & 0,837 & 0,046 & 0,317 & 0,812 & 0,379 & 0,649 & 0,669 & 1,222 & 0,414 & 0,373 & 0,339 & 0,126 & 0,152 & 115 & 578 & $-1,8$ \\
\hline 397 & 578 & 0,023 & 0,440 & 0,023 & 1,839 & 1,000 & 4,183 & 3,183 & 1,000 & 0,818 & 1,019 & 0,671 & 0,169 & 0,114 & 0,152 & 45 & 578 & $-0,8$ \\
\hline 398 & 578 & 0,253 & 1,868 & 0,263 & 0,194 & 0,962 & 0,104 & 0,897 & 0,909 & 1,049 & 0,539 & 0,539 & 0,773 & 0,417 & 0,429 & 81 & 578 & $-3,0$ \\
\hline 399 & 578 & 0,016 & 0,368 & 0,016 & 1,572 & 1,000 & 4,276 & 3,276 & 1,000 & 0,826 & 0,824 & 0,485 & 0,156 & 0,076 & 0,107 & 90 & 578 & $-0,6$ \\
\hline 400 & 578 & 0,187 & 1,788 & 0,220 & 1,907 & 0,850 & 1,066 & 0,164 & 0,733 & 1,168 & 0,453 & 0,503 & 0,724 & 0,364 & 0,312 & 65 & 578 & $-2,9$ \\
\hline 401 & 578 & 0,310 & 1,957 & 0,330 & 0,858 & 0,939 & 0,438 & 0,565 & 1,000 & 0,992 & 0,902 & 0,889 & 0,662 & 0,588 & 0,546 & 81 & 578 & $-2,7$ \\
\hline 402 & 578 & 0,080 & 0,888 & 0,082 & 1,010 & 0,982 & 1,138 & 0,139 & 1,000 & 0,883 & 0,977 & 0,819 & 0,324 & 0,265 & 0,273 & 100 & 578 & $-1,7$ \\
\hline 403 & 578 & 0,083 & 1,205 & 0,109 & 1,175 & 0,763 & 0,975 & 0,238 & 0,721 & 1,178 & 0,543 & 0,617 & 0,442 & 0,273 & 0,227 & 41 & 578 & $-2,1$ \\
\hline 404 & 578 & 0,141 & 1,226 & 0,143 & 0,317 & 0,980 & 0,259 & 0,742 & 1,000 & 0,922 & 0,911 & 0,769 & 0,443 & 0,341 & 0,379 & 76 & 578 & $-2,1$ \\
\hline 405 & 578 & 0,020 & 0,562 & 0,024 & 0,622 & 0,824 & 1,106 & 0,206 & 0,799 & 1,119 & 0,615 & 0,557 & 0,204 & 0,114 & 0,107 & 36 & 578 & $-1,0$ \\
\hline 406 & 578 & 0,033 & 0,714 & 0,037 & 0,469 & 0,885 & 0,657 & 0,362 & 0,813 & 1,109 & 0,396 & 0,329 & 0,326 & 0,107 & 0,152 & 39 & 578 & $-1,7$ \\
\hline 407 & 578 & 0,029 & 0,562 & 0,033 & 0,816 & 0,870 & 1,451 & 0,470 & 1,000 & 0,936 & 0,688 & 0,493 & 0,230 & 0,114 & 0,152 & 5 & 578 & $-1,2$ \\
\hline 408 & 578 & 0,069 & 0,888 & 0,076 & 0,287 & 0,906 & 0,324 & 0,683 & 1,000 & 0,954 & 0,899 & 0,794 & 0,312 & 0,248 & 0,240 & 27 & 578 & $-1,6$ \\
\hline 409 & 578 & 0,020 & 0,389 & 0,020 & 0,968 & 1,000 & 2,490 & 1,490 & 1,000 & 0,774 & 0,990 & 0,667 & 0,161 & 0,107 & 0,152 & 45 & 578 & $-0,7$ \\
\hline 410 & 578 & 0,093 & 1,019 & 0,098 & 0,583 & 0,956 & 0,572 & 0,430 & 1,000 & 0,941 & 0,974 & 0,844 & 0,349 & 0,295 & 0,312 & 117 & 578 & $-1,8$ \\
\hline 411 & 578 & 0,040 & 0,685 & 0,044 & 3,077 & 0,903 & 4,494 & 3,495 & 1,000 & 0,964 & 0,792 & 0,596 & 0,254 & 0,152 & 0,214 & 161 & 578 & $-1,3$ \\
\hline 412 & 578 & 0,505 & 3,259 & 0,584 & 0,469 & 0,865 & 0,144 & 0,867 & 0,598 & 1,294 & 0,316 & 0,336 & 1,426 & 0,479 & 0,479 & 83 & 578 & $-3,8$ \\
\hline 413 & 578 & 0,030 & 0,592 & 0,033 & 0,490 & 0,913 & 0,828 & 0,192 & 1,000 & 0,962 & 0,652 & 0,442 & 0,243 & 0,107 & 0,152 & 42 & 578 & $-1,3$ \\
\hline 414 & 578 & 0,033 & 0,571 & 0,036 & 1,154 & 0,920 & 2,022 & 1,025 & 1,000 & 0,886 & 1,010 & 0,788 & 0,204 & 0,161 & 0,169 & 104 & 578 & $-1,0$ \\
\hline 415 & 578 & 0,123 & 1,255 & 0,136 & 2,730 & 0,905 & 2,175 & 1,178 & 0,984 & 1,008 & 0,684 & 0,641 & 0,479 & 0,307 & 0,303 & 113 & 578 & $-2,3$ \\
\hline 416 & 578 & 0,323 & 3,572 & 0,517 & 1,467 & 0,625 & 0,411 & 0,698 & 0,318 & 1,773 & 0,276 & 0,421 & 1,220 & 0,513 & 0,152 & 13 & 578 & $-3,6$ \\
\hline 417 & 578 & 0,168 & 1,898 & 0,207 & 0,774 & 0,812 & 0,408 & 0,621 & 0,586 & 1,307 & 0,727 & 0,840 & 0,542 & 0,455 & 0,379 & 77 & 578 & $-2,4$ \\
\hline 418 & 578 & 0,047 & 0,938 & 0,050 & 0,532 & 0,943 & 0,567 & 0,436 & 0,676 & 1,217 & 0,328 & 0,250 & 0,429 & 0,107 & 0,152 & 47 & 578 & $-2,1$ \\
\hline 419 & 578 & 0,030 & 0,757 & 0,040 & 1,420 & 0,750 & 1,877 & 0,912 & 0,662 & 1,229 & 0,393 & 0,364 & 0,312 & 0,114 & 0,152 & 1 & 578 & $-1,6$ \\
\hline 420 & 578 & 0,123 & 1,898 & 0,169 & 3,285 & 0,729 & 1,731 & 0,779 & 0,430 & 1,524 & 0,375 & 0,427 & 0,647 & 0,276 & 0,107 & 69 & 578 & $-2,7$ \\
\hline 421 & 578 & 0,844 & 3,335 & 0,874 & 0,613 & 0,966 & 0,184 & 0,817 & 0,953 & 1,024 & 0,628 & 0,642 & 1,308 & 0,840 & 0,837 & 79 & 578 & $-3,7$ \\
\hline 422 & 578 & 0,055 & 0,757 & 0,057 & 1,868 & 0,950 & 2,470 & 1,471 & 1,000 & 0,914 & 1,075 & 0,867 & 0,254 & 0,220 & 0,227 & 114 & 578 & $-1,3$ \\
\hline 423 & 578 & 0,303 & 1,919 & 0,314 & 0,816 & 0,963 & 0,425 & 0,576 & 1,000 & 0,984 & 0,746 & 0,738 & 0,719 & 0,530 & 0,530 & 103 & 578 & $-2,8$ \\
\hline 424 & 578 & 0,056 & 0,888 & 0,065 & 1,010 & 0,867 & 1,138 & 0,192 & 0,892 & 1,059 & 0,552 & 0,551 & 0,359 & 0,198 & 0,214 & 26 & 578 & $-1,8$ \\
\hline 425 & 578 & 0,062 & 1,142 & 0,085 & 2,498 & 0,729 & 2,189 & 1,219 & 0,595 & 1,296 & 0,342 & 0,431 & 0,479 & 0,207 & 0,214 & 177 & 578 & $-2,3$ \\
\hline 426 & 578 & 0,294 & 3,745 & 0,463 & 0,643 & 0,635 & 0,172 & 0,905 & 0,264 & 1,948 & 0,446 & 0,744 & 0,916 & 0,681 & 0,107 & 77 & 578 & $-3,2$ \\
\hline 427 & 578 & 0,044 & 0,744 & 0,050 & 0,613 & 0,886 & 0,824 & 0,210 & 1,000 & 0,995 & 0,647 & 0,540 & 0,296 & 0,160 & 0,169 & 130 & 578 & $-1,6$ \\
\hline 428 & 578 & 0,044 & 0,643 & 0,044 & 1,133 & 1,000 & 1,763 & 0,763 & 1,000 & 0,859 & 0,987 & 0,783 & 0,240 & 0,187 & 0,214 & 61 & 578 & $-1,3$ \\
\hline 429 & 578 & 0,131 & 1,306 & 0,142 & 0,981 & 0,919 & 0,751 & 0,262 & 0,962 & 1,020 & 0,715 & 0,722 & 0,482 & 0,348 & 0,273 & 51 & 578 & $-2,3$ \\
\hline 430 & 578 & 0,103 & 1,052 & 0,106 & 0,338 & 0,973 & 0,321 & 0,679 & 1,000 & 0,924 & 0,945 & 0,812 & 0,373 & 0,303 & 0,312 & 5 & 578 & $-1,9$ \\
\hline 431 & 578 & 0,016 & 0,347 & 0,017 & 0,490 & 0,917 & 1,414 & 0,423 & 1,000 & 0,778 & 1,401 & 0,849 & 0,120 & 0,102 & 0,107 & 90 & 578 & $-0,3$ \\
\hline 432 & 578 & 0,030 & 0,541 & 0,030 & 1,133 & 1,000 & 2,094 & 1,094 & 1,000 & 0,879 & 0,723 & 0,493 & 0,230 & 0,114 & 0,152 & 0 & 578 & $-1,2$ \\
\hline 433 & 578 & 0,108 & 1,378 & 0,128 & 1,902 & 0,843 & 1,380 & 0,411 & 0,712 & 1,185 & 0,516 & 0,584 & 0,515 & 0,301 & 0,273 & 105 & 578 & $-2,4$ \\
\hline
\end{tabular}




\begin{tabular}{|c|c|c|c|c|c|c|c|c|c|c|c|c|c|c|c|c|c|c|}
\hline 434 & 578 & 0,199 & 2,224 & 0,273 & 0,685 & 0,732 & 0,308 & 0,742 & 0,507 & 1,405 & 0,387 & 0,468 & 0,810 & 0,379 & 0,273 & 176 & 578 & $-3,0$ \\
\hline 435 & 578 & 0,034 & 0,858 & 0,046 & 1,441 & 0,750 & 1,680 & 0,724 & 0,588 & 1,304 & 0,360 & 0,325 & 0,349 & 0,114 & 0,152 & 5 & 578 & $-1,8$ \\
\hline 436 & 578 & 0,194 & 1,572 & 0,205 & 0,368 & 0,944 & 0,234 & 0,768 & 0,984 & 1,008 & 0,891 & 0,792 & 0,526 & 0,417 & 0,408 & 99 & 578 & $-2,4$ \\
\hline 437 & 578 & 0,020 & 0,448 & 0,022 & 0,643 & 0,933 & 1,434 & 0,439 & 1,000 & 0,892 & 0,713 & 0,424 & 0,189 & 0,080 & 0,107 & 123 & 578 & $-0,9$ \\
\hline 438 & 578 & 0,059 & 0,786 & 0,060 & 4,138 & 0,976 & 5,263 & 4,263 & 1,000 & 0,914 & 0,900 & 0,764 & 0,288 & 0,220 & 0,240 & 76 & 578 & $-1,5$ \\
\hline 439 & 578 & 1,148 & 4,358 & 1,235 & 2,439 & 0,929 & 0,560 & 0,446 & 0,759 & 1,148 & 0,548 & 0,574 & 1,633 & 0,937 & 0,922 & 89 & 578 & $-4,0$ \\
\hline 440 & 578 & 0,445 & 2,705 & 0,506 & 0,245 & 0,878 & 0,091 & 0,918 & 0,764 & 1,144 & 0,597 & 0,652 & 0,974 & 0,635 & 0,592 & 109 & 578 & $-3,3$ \\
\hline 441 & 578 & 0,014 & 0,326 & 0,014 & 0,317 & 1,000 & 0,973 & 0,027 & 1,000 & 0,767 & 1,273 & 0,671 & 0,120 & 0,080 & 0,107 & 108 & 578 & $-0,3$ \\
\hline 442 & 578 & 0,019 & 0,418 & 0,020 & 0,469 & 0,929 & 1,121 & 0,141 & 1,000 & 0,864 & 1,273 & 0,832 & 0,137 & 0,114 & 0,107 & 90 & 578 & $-0,5$ \\
\hline 443 & 578 & 0,029 & 0,592 & 0,032 & 0,418 & 0,909 & 0,707 & 0,307 & 1,000 & 0,986 & 0,749 & 0,670 & 0,221 & 0,148 & 0,152 & 30 & 578 & $-1,1$ \\
\hline 444 & 578 & 0,029 & 0,511 & 0,030 & 2,122 & 0,952 & 4,150 & 3,150 & 1,000 & 0,852 & 1,019 & 0,716 & 0,189 & 0,136 & 0,169 & 39 & 578 & $-0,9$ \\
\hline 445 & 578 & 0,313 & 2,520 & 0,359 & 0,317 & 0,872 & 0,126 & 0,884 & 0,619 & 1,271 & 0,491 & 0,521 & 0,900 & 0,469 & 0,152 & 110 & 578 & $-3,2$ \\
\hline 446 & 578 & 0,023 & 0,440 & 0,023 & 0,634 & 1,000 & 1,442 & 0,442 & 1,000 & 0,818 & 1,019 & 0,671 & 0,169 & 0,114 & 0,152 & 90 & 578 & $-0,8$ \\
\hline 447 & 578 & 0,055 & 0,735 & 0,057 & 0,173 & 0,950 & 0,236 & 0,766 & 1,000 & 0,889 & 1,075 & 0,867 & 0,254 & 0,220 & 0,240 & 55 & 578 & $-1,3$ \\
\hline 448 & 578 & 0,014 & 0,338 & 0,014 & 3,153 & 1,000 & 9,329 & 8,329 & 1,000 & 0,796 & 0,979 & 0,555 & 0,137 & 0,076 & 0,107 & 162 & 578 & $-0,5$ \\
\hline 449 & 578 & 0,660 & 4,164 & 0,792 & 0,583 & 0,833 & 0,140 & 0,876 & 0,478 & 1,446 & 0,548 & 0,684 & 1,238 & 0,847 & 0,652 & 115 & 578 & $-3,6$ \\
\hline 450 & 578 & 0,046 & 0,664 & 0,049 & 1,797 & 0,941 & 2,707 & 1,708 & 1,000 & 0,874 & 0,994 & 0,773 & 0,243 & 0,187 & 0,214 & 123 & 578 & $-1,3$ \\
\hline 451 & 578 & 0,238 & 2,021 & 0,280 & 0,215 & 0,851 & 0,107 & 0,906 & 0,733 & 1,168 & 0,650 & 0,664 & 0,683 & 0,454 & 0,312 & 153 & 578 & $-2,8$ \\
\hline 452 & 578 & 0,016 & 0,368 & 0,017 & 0,879 & 0,917 & 2,391 & 1,393 & 1,000 & 0,826 & 0,824 & 0,485 & 0,156 & 0,076 & 0,107 & 162 & 578 & $-0,6$ \\
\hline 453 & 578 & 0,090 & 0,981 & 0,093 & 0,757 & 0,969 & 0,771 & 0,231 & 1,000 & 0,920 & 0,827 & 0,711 & 0,373 & 0,265 & 0,273 & 79 & 578 & $-1,9$ \\
\hline 454 & 578 & 0,067 & 0,837 & 0,069 & 0,440 & 0,979 & 0,525 & 0,475 & 1,000 & 0,909 & 0,820 & 0,702 & 0,324 & 0,227 & 0,240 & 98 & 578 & $-1,7$ \\
\hline 455 & 578 & 0,034 & 0,583 & 0,034 & 1,674 & 1,000 & 2,870 & 1,870 & 1,000 & 0,887 & 0,826 & 0,493 & 0,230 & 0,114 & 0,152 & 0 & 578 & $-1,2$ \\
\hline 456 & 578 & 0,254 & 1,746 & 0,268 & 1,399 & 0,947 & 0,801 & 0,206 & 1,000 & 0,977 & 0,920 & 0,889 & 0,593 & 0,527 & 0,530 & 39 & 578 & $-2,6$ \\
\hline 457 & 578 & 0,171 & 1,450 & 0,187 & 1,052 & 0,915 & 0,726 & 0,287 & 1,000 & 0,990 & 0,891 & 0,868 & 0,494 & 0,429 & 0,321 & 134 & 578 & $-2,3$ \\
\hline 458 & 578 & 0,103 & 1,103 & 0,112 & 0,643 & 0,923 & 0,582 & 0,425 & 1,000 & 0,968 & 0,881 & 0,784 & 0,386 & 0,303 & 0,303 & 79 & 578 & $-1,9$ \\
\hline 459 & 578 & 0,046 & 0,723 & 0,046 & 0,338 & 1,000 & 0,468 & 0,532 & 1,000 & 0,952 & 0,769 & 0,583 & 0,276 & 0,161 & 0,214 & 49 & 578 & $-1,5$ \\
\hline 460 & 578 & 0,020 & 0,418 & 0,020 & 1,636 & 1,000 & 3,908 & 2,908 & 1,000 & 0,833 & 1,114 & 0,750 & 0,152 & 0,114 & 0,152 & 90 & 578 & $-0,6$ \\
\hline 461 & 578 & 0,231 & 1,665 & 0,240 & 0,960 & 0,964 & 0,576 & 0,425 & 1,000 & 0,977 & 0,730 & 0,647 & 0,635 & 0,411 & 0,442 & 103 & 578 & $-2,7$ \\
\hline 462 & 578 & 0,086 & 1,010 & 0,092 & 0,317 & 0,938 & 0,314 & 0,689 & 1,000 & 0,971 & 0,611 & 0,560 & 0,423 & 0,237 & 0,273 & 157 & 578 & $-2,1$ \\
\hline 463 & 578 & 0,024 & 0,553 & 0,026 & 2,003 & 0,944 & 3,619 & 2,620 & 1,000 & 1,000 & 0,541 & 0,316 & 0,240 & 0,076 & 0,152 & 172 & 578 & $-1,3$ \\
\hline 464 & 578 & 0,317 & 2,054 & 0,333 & 0,448 & 0,953 & 0,218 & 0,783 & 0,944 & 1,029 & 0,625 & 0,558 & 0,804 & 0,448 & 0,455 & 91 & 578 & $-3,0$ \\
\hline 465 & 578 & 0,029 & 0,562 & 0,029 & 1,522 & 1,000 & 2,707 & 1,707 & 1,000 & 0,936 & 0,566 & 0,447 & 0,254 & 0,114 & 0,152 & 109 & 578 & $-1,3$ \\
\hline 466 & 578 & 0,201 & 1,623 & 0,214 & 1,522 & 0,940 & 0,937 & 0,087 & 0,958 & 1,022 & 0,728 & 0,686 & 0,593 & 0,407 & 0,408 & 115 & 578 & $-2,6$ \\
\hline 467 & 578 & 0,220 & 1,572 & 0,224 & 0,418 & 0,981 & 0,266 & 0,734 & 1,000 & 0,947 & 0,866 & 0,801 & 0,568 & 0,455 & 0,485 & 135 & 578 & $-2,5$ \\
\hline 468 & 578 & 0,037 & 0,604 & 0,039 & 4,008 & 0,963 & 6,633 & 5,633 & 1,000 & 0,883 & 0,807 & 0,625 & 0,243 & 0,152 & 0,214 & 157 & 578 & $-1,3$ \\
\hline 469 & 578 & 1,083 & 4,274 & 1,235 & 0,520 & 0,877 & 0,122 & 0,887 & 0,745 & 1,158 & 0,583 & 0,664 & 1,538 & 1,021 & 0,883 & 91 & 578 & $-3,9$ \\
\hline 470 & 578 & 0,027 & 0,571 & 0,030 & 0,266 & 0,905 & 0,466 & 0,542 & 1,000 & 0,975 & 0,605 & 0,528 & 0,240 & 0,126 & 0,152 & 20 & 578 & $-1,3$ \\
\hline 471 & 578 & 0,014 & 0,347 & 0,016 & 0,490 & 0,909 & 1,414 & 0,424 & 1,000 & 0,816 & 0,979 & 0,588 & 0,137 & 0,080 & 0,107 & 53 & 578 & $-0,5$ \\
\hline 472 & 578 & 0,027 & 0,592 & 0,033 & 3,103 & 0,826 & 5,243 & 4,246 & 0,978 & 1,011 & 0,654 & 0,582 & 0,230 & 0,134 & 0,152 & 164 & 578 & $-1,2$ \\
\hline 473 & 578 & 0,729 & 3,407 & 0,778 & 0,245 & 0,937 & 0,072 & 0,930 & 0,789 & 1,126 & 0,585 & 0,638 & 1,259 & 0,804 & 0,780 & 113 & 578 & $-3,7$ \\
\hline 474 & 578 & 0,019 & 0,368 & 0,019 & 0,583 & 1,000 & 1,586 & 0,586 & 1,000 & 0,760 & 1,273 & 0,784 & 0,137 & 0,107 & 0,152 & 90 & 578 & $-0,5$ \\
\hline 475 & 578 & 0,046 & 0,685 & 0,049 & 1,369 & 0,941 & 2,000 & 1,002 & 1,000 & 0,901 & 0,994 & 0,781 & 0,243 & 0,189 & 0,214 & 45 & 578 & $-1,3$ \\
\hline 476 & 578 & 0,125 & 2,008 & 0,178 & 0,664 & 0,702 & 0,330 & 0,733 & 0,389 & 1,603 & 0,508 & 0,686 & 0,559 & 0,384 & 0,312 & 103 & 578 & $-2,5$ \\
\hline 477 & 578 & 0,044 & 0,714 & 0,046 & 0,245 & 0,969 & 0,343 & 0,658 & 1,000 & 0,956 & 0,580 & 0,485 & 0,312 & 0,152 & 0,169 & 167 & 578 & $-1,6$ \\
\hline 478 & 578 & 0,014 & 0,338 & 0,016 & 0,998 & 0,909 & 2,952 & 1,954 & 1,000 & 0,796 & 0,979 & 0,555 & 0,137 & 0,076 & 0,152 & 0 & 578 & $-0,5$ \\
\hline 479 & 578 & 0,053 & 1,120 & 0,063 & 0,275 & 0,841 & 0,245 & 0,771 & 0,531 & 1,372 & 0,233 & 0,205 & 0,538 & 0,111 & 0,152 & 123 & 578 & $-2,4$ \\
\hline 480 & 578 & 0,014 & 0,317 & 0,014 & 0,583 & 1,000 & 1,840 & 0,840 & 1,000 & 0,746 & 1,273 & 0,632 & 0,120 & 0,076 & 0,107 & 162 & 578 & $-0,3$ \\
\hline 481 & 578 & 0,046 & 0,685 & 0,049 & 0,368 & 0,941 & 0,537 & 0,467 & 1,000 & 0,901 & 0,905 & 0,723 & 0,254 & 0,184 & 0,214 & 173 & 578 & $-1,3$ \\
\hline 482 & 578 & 0,020 & 0,397 & 0,020 & 0,389 & 1,000 & 0,978 & 0,022 & 1,000 & 0,791 & 1,049 & 0,686 & 0,156 & 0,107 & 0,152 & 45 & 578 & $-0,6$ \\
\hline 483 & 578 & 0,027 & 0,490 & 0,029 & 1,052 & 0,950 & 2,146 & 1,148 & 1,000 & 0,838 & 1,210 & 0,894 & 0,169 & 0,152 & 0,169 & 37 & 578 & $-0,8$ \\
\hline 484 & 578 & 0,090 & 1,205 & 0,105 & 0,613 & 0,863 & 0,509 & 0,510 & 0,783 & 1,130 & 0,524 & 0,532 & 0,469 & 0,249 & 0,273 & 106 & 578 & $-2,2$ \\
\hline 485 & 578 & 0,046 & 0,643 & 0,046 & 0,461 & 1,000 & 0,717 & 0,283 & 1,000 & 0,846 & 1,019 & 0,783 & 0,240 & 0,187 & 0,214 & 160 & 578 & $-1,3$ \\
\hline 486 & 578 & 0,032 & 0,562 & 0,034 & 2,523 & 0,917 & 4,489 & 3,490 & 1,000 & 0,893 & 0,966 & 0,743 & 0,204 & 0,152 & 0,169 & 78 & 578 & $-1,0$ \\
\hline 487 & 578 & 0,525 & 2,604 & 0,550 & 0,469 & 0,956 & 0,180 & 0,821 & 0,973 & 1,014 & 0,764 & 0,746 & 0,936 & 0,698 & 0,678 & 105 & 578 & $-3,2$ \\
\hline 488 & 578 & 0,033 & 0,562 & 0,034 & 0,245 & 0,958 & 0,436 & 0,565 & 1,000 & 0,873 & 1,010 & 0,743 & 0,204 & 0,152 & 0,169 & 165 & 578 & $-1,0$ \\
\hline 489 & 578 & 0,016 & 0,338 & 0,016 & 0,532 & 1,000 & 1,575 & 0,575 & 1,000 & 0,759 & 1,077 & 0,555 & 0,137 & 0,076 & 0,152 & 0 & 578 & $-0,5$ \\
\hline 490 & 578 & 0,036 & 0,571 & 0,036 & 1,593 & 1,000 & 2,792 & 1,792 & 1,000 & 0,850 & 1,098 & 0,788 & 0,204 & 0,161 & 0,214 & 25 & 578 & $-1,0$ \\
\hline 491 & 578 & 0,217 & 1,674 & 0,228 & 0,389 & 0,950 & 0,232 & 0,769 & 0,972 & 1,014 & 0,715 & 0,654 & 0,621 & 0,407 & 0,429 & 121 & 578 & $-2,6$ \\
\hline
\end{tabular}




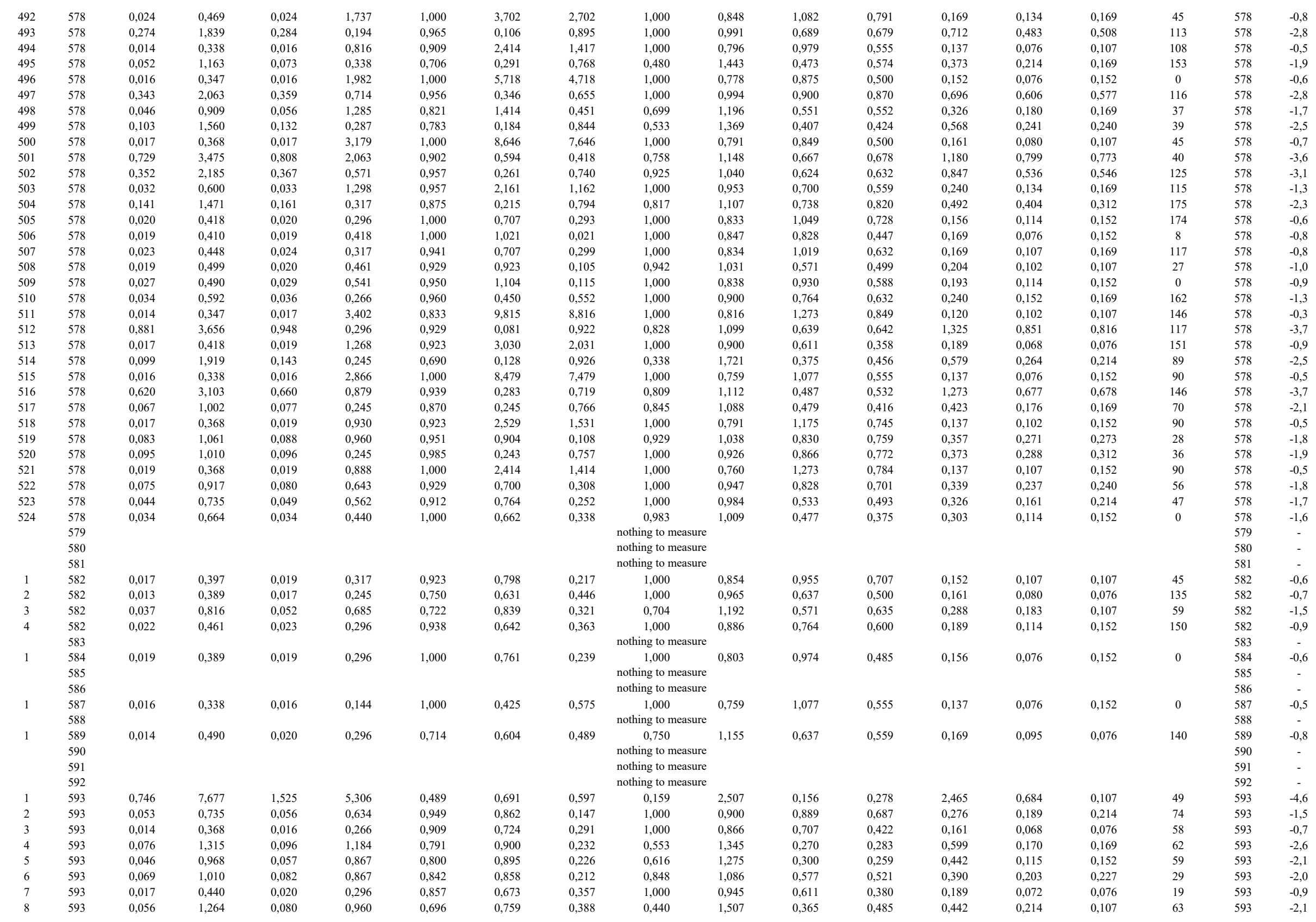




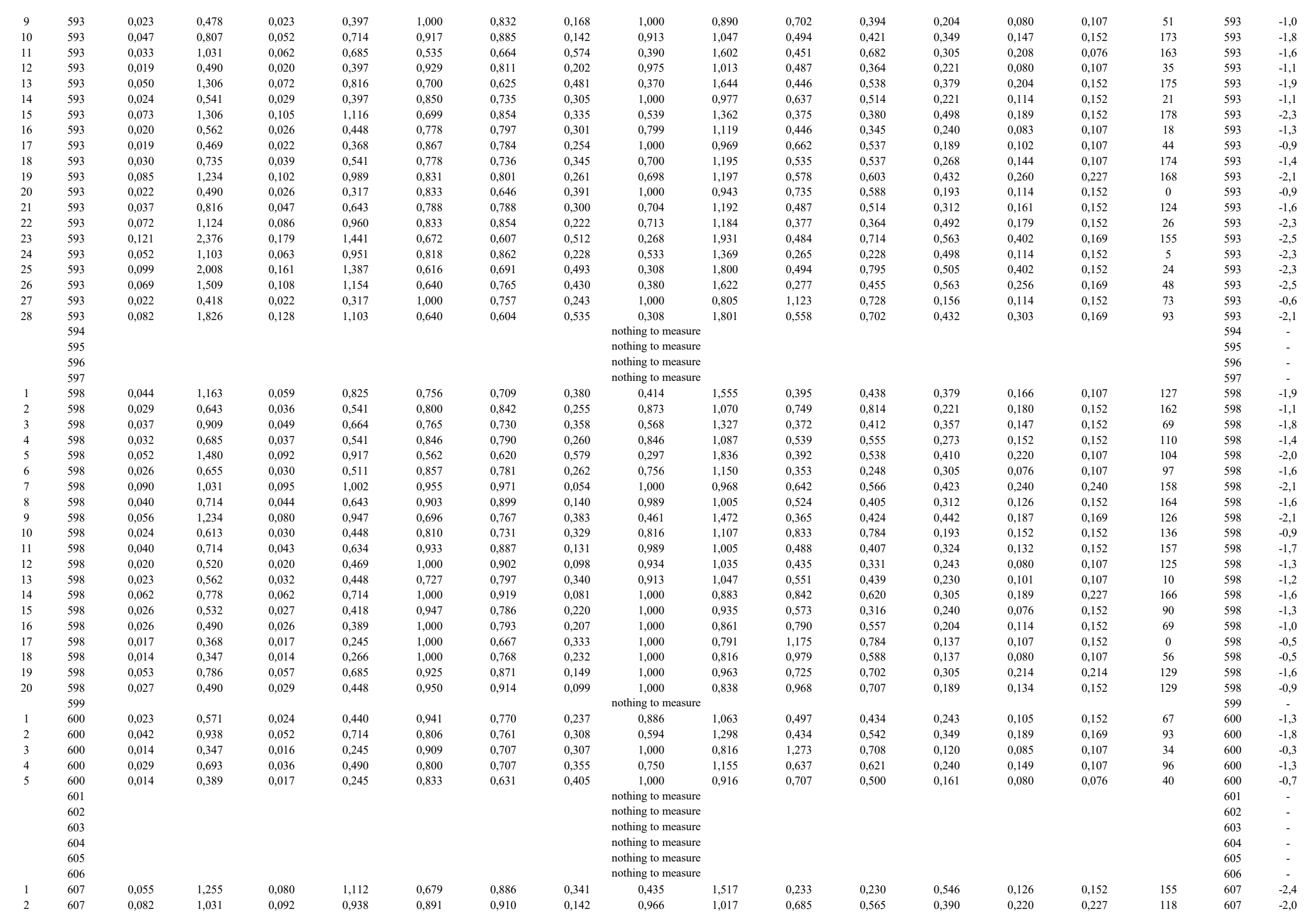




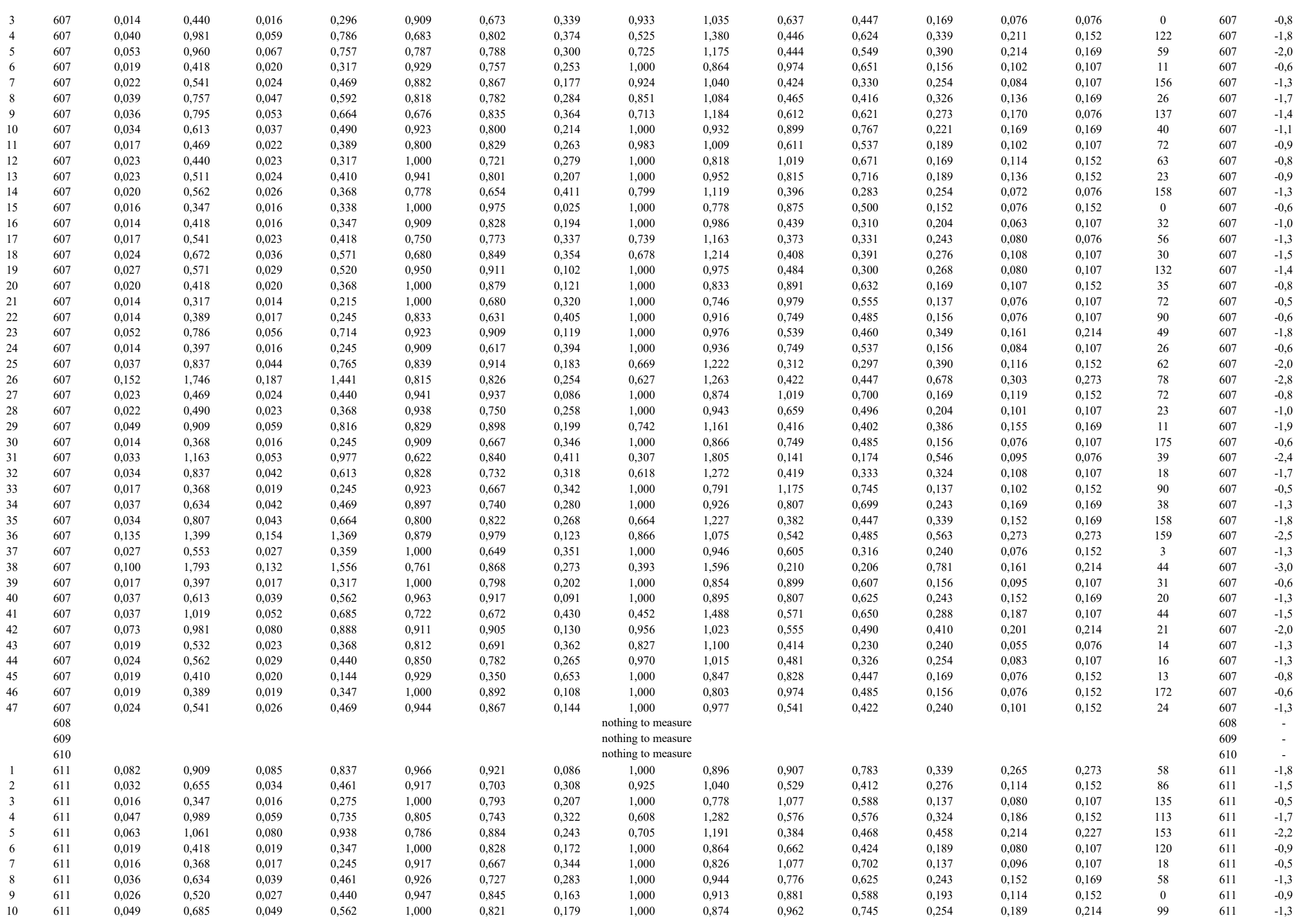




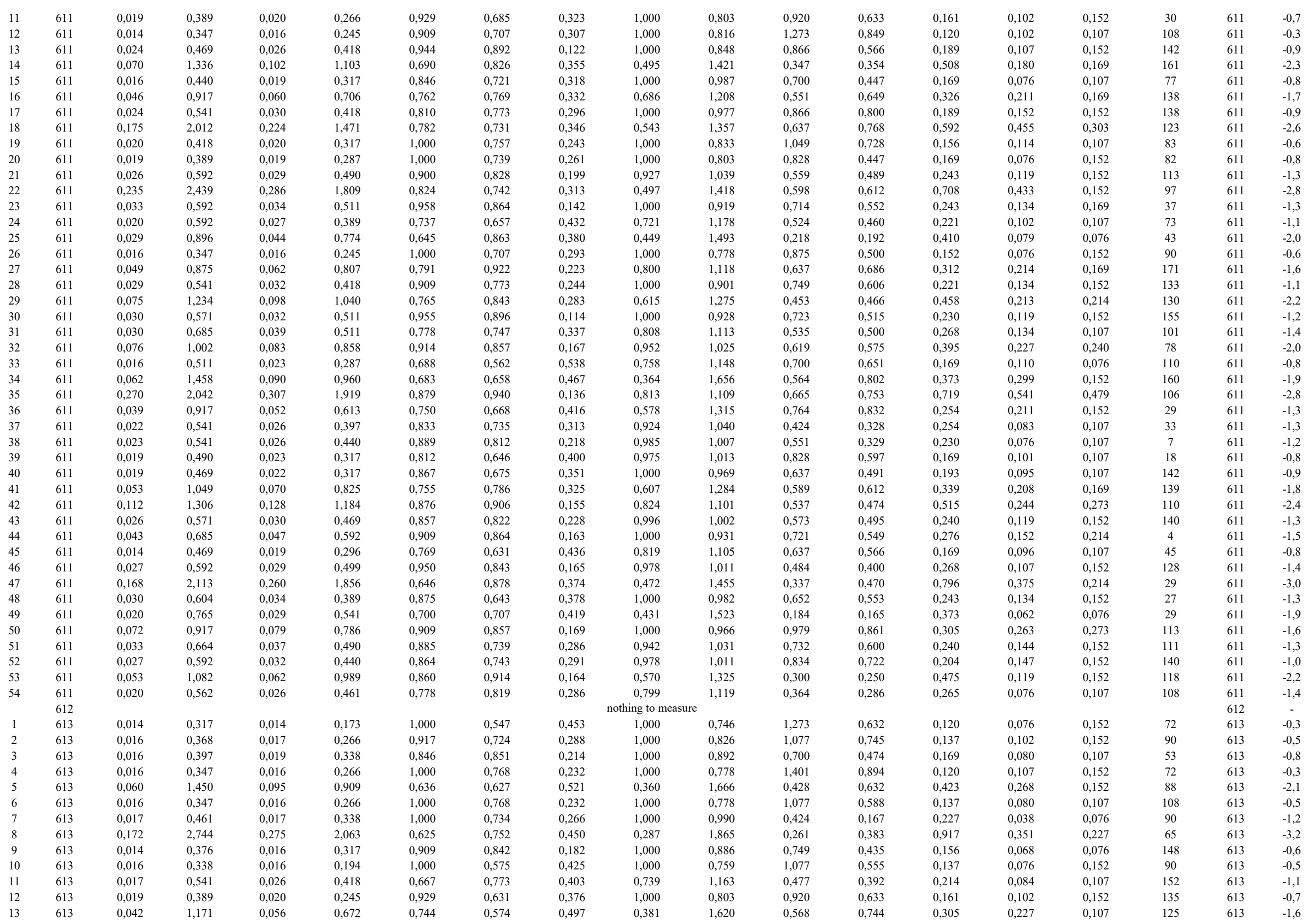




\begin{tabular}{|c|c|c|c|c|c|c|c|c|c|c|c|c|c|c|c|c|c|c|}
\hline 14 & 613 & 0,033 & 1,040 & 0,052 & 0,735 & 0,639 & 0,707 & 0,465 & 0,383 & 1,615 & 0,293 & 0,403 & 0,379 & 0,153 & 0,107 & 46 & 613 & $-1,9$ \\
\hline 15 & 613 & 0,014 & 0,368 & 0,017 & 0,245 & 0,833 & 0,667 & 0,373 & 1,000 & 0,866 & 0,979 & 0,702 & 0,137 & 0,096 & 0,107 & 45 & 613 & $-0,5$ \\
\hline 16 & 613 & 0,044 & 0,714 & 0,046 & 0,613 & 0,969 & 0,858 & 0,145 & 1,000 & 0,956 & 0,607 & 0,517 & 0,305 & 0,158 & 0,169 & 65 & 613 & $-1,6$ \\
\hline 17 & 613 & 0,132 & 1,978 & 0,194 & 1,471 & 0,681 & 0,743 & 0,409 & 0,424 & 1,536 & 0,451 & 0,551 & 0,611 & 0,336 & 0,240 & 93 & 613 & $-2,6$ \\
\hline 18 & 613 & 0,024 & 0,511 & 0,026 & 0,368 & 0,944 & 0,719 & 0,286 & 1,000 & 0,924 & 0,746 & 0,557 & 0,204 & 0,114 & 0,152 & 144 & 613 & $-1,0$ \\
\hline 19 & 613 & 0,036 & 1,163 & 0,052 & 0,706 & 0,694 & 0,607 & 0,498 & 0,334 & 1,732 & 0,468 & 0,537 & 0,312 & 0,168 & 0,076 & 160 & 613 & $-1,6$ \\
\hline 20 & 613 & 0,037 & 1,226 & 0,063 & 0,909 & 0,591 & 0,741 & 0,484 & 0,312 & 1,790 & 0,207 & 0,307 & 0,479 & 0,147 & 0,107 & 74 & 613 & $-2,3$ \\
\hline 21 & 613 & 0,044 & 0,989 & 0,056 & 0,727 & 0,795 & 0,735 & 0,335 & 0,571 & 1,323 & 0,464 & 0,405 & 0,349 & 0,142 & 0,152 & 97 & 613 & $-1,8$ \\
\hline 22 & 613 & 0,020 & 0,765 & 0,030 & 0,461 & 0,667 & 0,602 & 0,519 & 0,431 & 1,523 & 0,615 & 0,743 & 0,204 & 0,152 & 0,076 & 62 & 613 & $-1,0$ \\
\hline 23 & 613 & 0,026 & 0,867 & 0,040 & 0,592 & 0,643 & 0,683 & 0,478 & 0,432 & 1,521 & 0,318 & 0,401 & 0,321 & 0,129 & 0,107 & 125 & 613 & $-1,7$ \\
\hline 24 & 613 & 0,022 & 0,520 & 0,023 & 0,448 & 0,938 & 0,862 & 0,152 & 1,000 & 1,000 & 0,466 & 0,331 & 0,243 & 0,080 & 0,107 & 123 & 613 & $-1,3$ \\
\hline 25 & 613 & 0,016 & 0,368 & 0,017 & 0,296 & 0,917 & 0,805 & 0,212 & 1,000 & 0,826 & 1,077 & 0,702 & 0,137 & 0,096 & 0,107 & 90 & 613 & $-0,5$ \\
\hline 26 & 613 & 0,159 & 2,050 & 0,198 & 1,704 & 0,804 & 0,831 & 0,259 & 0,476 & 1,449 & 0,375 & 0,422 & 0,735 & 0,310 & 0,107 & 72 & 613 & $-2,9$ \\
\hline 27 & 613 & 0,014 & 0,389 & 0,014 & 0,144 & 1,000 & 0,369 & 0,631 & 1,000 & 0,916 & 0,509 & 0,200 & 0,189 & 0,038 & 0,076 & 90 & 613 & $-0,9$ \\
\hline 28 & 613 & 0,024 & 0,520 & 0,026 & 0,490 & 0,944 & 0,943 & 0,080 & 1,000 & 0,939 & 0,746 & 0,567 & 0,204 & 0,116 & 0,152 & 62 & 613 & $-1,0$ \\
\hline 29 & 613 & 0,029 & 0,938 & 0,046 & 0,592 & 0,625 & 0,631 & 0,526 & 0,409 & 1,563 & 0,480 & 0,549 & 0,276 & 0,152 & 0,107 & 19 & 613 & $-1,5$ \\
\hline \multirow[t]{5}{*}{30} & 613 & 0,022 & 0,490 & 0,023 & 0,440 & 0,938 & 0,896 & 0,121 & 1,000 & 0,943 & 0,531 & 0,333 & 0,227 & 0,076 & 0,152 & 171 & 613 & $-1,2$ \\
\hline & 614 & & & & & & & & nothing to measure & & & & & & & & 614 & - \\
\hline & 615 & & & & & & & & nothing to measure & & & & & & & & 615 & - \\
\hline & 616 & & & & & & & & nothing to measure & & & & & & & & 616 & - \\
\hline & 617 & & & & & & & & nothing to measure & & & & & & & & 617 & - \\
\hline 1 & 618 & 0.017 & 0.359 & 0.017 & 0,194 & 1,000 & 0.541 & 0.459 & 1.000 & 0,772 & 1.175 & 0.555 & 0.137 & 0,076 & 0.152 & 0 & 618 & -0.5 \\
\hline \multirow[t]{3}{*}{2} & 618 & 0,095 & 1,073 & 0,103 & 0,951 & 0,917 & 0,886 & 0,141 & 1,000 & 0,984 & 0,646 & 0,571 & 0,432 & 0,247 & 0,240 & 151 & 618 & $-2,1$ \\
\hline & 619 & & & & & & & & nothing to measure & & & & & & & & 619 & - \\
\hline & 620 & & & & & & & & nothing to measure & & & & & & & & 620 & - \\
\hline \multirow[t]{8}{*}{1} & 621 & 0,014 & 0,347 & 0,016 & 0,194 & 0,909 & 0,561 & 0,449 & 1,000 & 0,816 & 1,273 & 0,849 & 0,120 & 0,102 & 0,107 & 0 & 621 & $-0,3$ \\
\hline & 622 & & & & & & & & nothing to measure & & & & & & & & 622 & - \\
\hline & 623 & & & & & & & & nothing to measure & & & & & & & & 623 & - \\
\hline & 624 & & & & & & & & nothing to measure & & & & & & & & 624 & - \\
\hline & 625 & & & & & & & & nothing to measure & & & & & & & & 625 & - \\
\hline & 626 & & & & & & & & nothing to measure & & & & & & & & 626 & - \\
\hline & 627 & & & & & & & & nothing to measure & & & & & & & & 627 & - \\
\hline & 628 & & & & & & & & nothing to measure & & & & & & & & 628 & - \\
\hline \multirow[t]{6}{*}{1} & 629 & 0,056 & 1,429 & 0,098 & 1,061 & 0,574 & 0,743 & 0,498 & 0,344 & 1,704 & 0,292 & 0,419 & 0,494 & 0,207 & 0,152 & 57 & 629 & $-2,3$ \\
\hline & 630 & & & & & & & & nothing to measure & & & & & & & & 630 & - \\
\hline & 631 & & & & & & & & nothing to measure & & & & & & & & 631 & - \\
\hline & 632 & & & & & & & & nothing to measure & & & & & & & & 632 & - \\
\hline & 633 & & & & & & & & nothing to measure & & & & & & & & 633 & - \\
\hline & 634 & & & & & & & & nothing to measure & & & & & & & & 634 & - \\
\hline 1 & 635 & 0,033 & 0,553 & 0,033 & 0,275 & 1,000 & 0,497 & 0,503 & 1,000 & 0,859 & 0,861 & 0,514 & 0,221 & 0,114 & 0,152 & 7 & 635 & $-1,1$ \\
\hline \multirow[t]{10}{*}{2} & 635 & 0,027 & 0,562 & 0,030 & 0,418 & 0,905 & 0,744 & 0,273 & 1,000 & 0,960 & 0,605 & 0,450 & 0,240 & 0,108 & 0,152 & 169 & 635 & $-1,3$ \\
\hline & 636 & & & & & & & & nothing to measure & & & & & & & & 636 & - \\
\hline & 637 & & & & & & & & nothing to measure & & & & & & & & 637 & - \\
\hline & 638 & & & & & & & & nothing to measure & & & & & & & & 638 & - \\
\hline & 639 & & & & & & & & nothing to measure & & & & & & & & 639 & - \\
\hline & 640 & & & & & & & & nothing to measure & & & & & & & & 640 & - \\
\hline & 641 & & & & & & & & nothing to measure & & & & & & & & 641 & - \\
\hline & 642 & & & & & & & & nothing to measure & & & & & & & & 642 & - \\
\hline & 643 & & & & & & & & nothing to measure & & & & & & & & 643 & - \\
\hline & 644 & & & & & & & & nothing to measure & & & & & & & & 644 & - \\
\hline 1 & 645 & 0,096 & 1,814 & 0,159 & 1,357 & 0,604 & 0,748 & 0,470 & 0,367 & 1,650 & 0,322 & 0,553 & 0,617 & 0,341 & 0,107 & 176 & 645 & $-2,6$ \\
\hline 2 & 645 & 0,049 & 0,981 & 0,057 & 0,858 & 0,850 & 0,875 & 0,195 & 0,638 & 1,252 & 0,318 & 0,345 & 0,442 & 0,153 & 0,152 & 23 & 645 & $-2,1$ \\
\hline 1 & 646 & 0,036 & 0,765 & 0,042 & 0,511 & 0,862 & 0,668 & 0,359 & 0,770 & 1,140 & 0,707 & 0,596 & 0,254 & 0,152 & 0,169 & 102 & 646 & $-1,3$ \\
\hline \multirow[t]{2}{*}{2} & 646 & 0,022 & 0,685 & 0,029 & 0,368 & 0,750 & 0,537 & 0,526 & 0,577 & 1,317 & 0,955 & 0,778 & 0,169 & 0,132 & 0,076 & 67 & 646 & $-0,8$ \\
\hline & 647 & & & & & & & & nothing to measure & & & & & & & & 647 & \\
\hline 1 & 648 & 0,023 & 0,511 & 0,026 & 0,368 & 0,889 & 0,719 & 0,302 & 1,000 & 0,952 & 0,599 & 0,380 & 0,221 & 0,084 & 0,107 & 162 & 648 & $-1,1$ \\
\hline 2 & 648 & 0,036 & 0,592 & 0,036 & 0,571 & 1,000 & 0,964 & 0,036 & 1,000 & 0,881 & 0,776 & 0,552 & 0,243 & 0,134 & 0,169 & 129 & 648 & $-1,3$ \\
\hline 3 & 648 & 0,043 & 0,714 & 0,047 & 0,634 & 0,909 & 0,887 & 0,145 & 1,000 & 0,971 & 0,588 & 0,472 & 0,305 & 0,144 & 0,169 & 150 & 648 & $-1,6$ \\
\hline
\end{tabular}




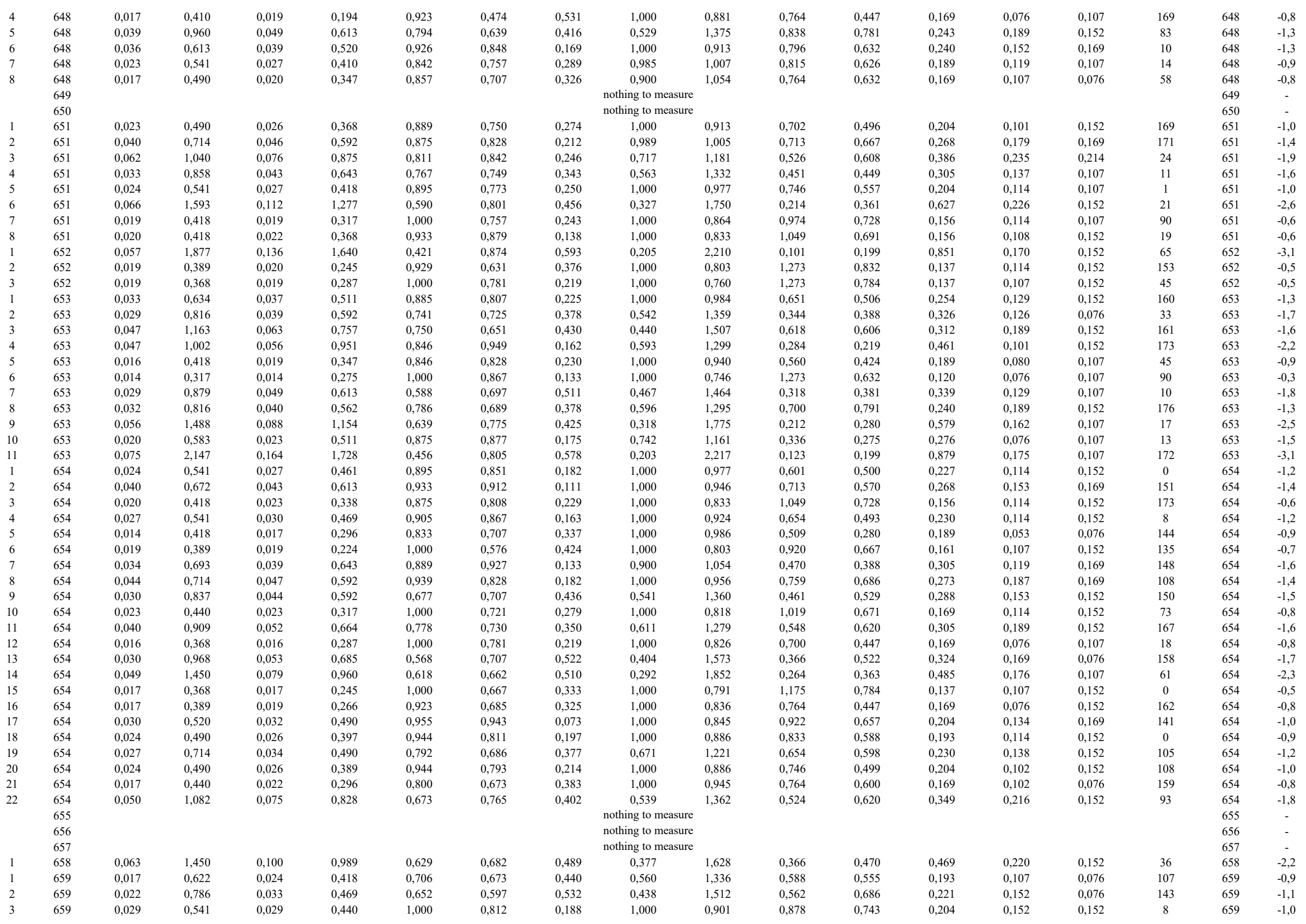




\begin{tabular}{|c|c|c|c|c|c|c|c|c|c|c|c|c|c|c|c|c|c|c|}
\hline 4 & 659 & 0,020 & 0,461 & 0,020 & 0,368 & 1,000 & 0,798 & 0,202 & 1,000 & 0,917 & 0,686 & 0,392 & 0,193 & 0,076 & 0,152 & 8 & 659 & $-0,9$ \\
\hline 1 & 660 & 0,017 & 0,389 & 0,017 & 0,275 & 1,000 & 0,707 & 0,293 & 1,000 & 0,836 & 1,175 & 0,832 & 0,137 & 0,114 & 0,107 & 117 & 660 & $-0,5$ \\
\hline 2 & 660 & 0,019 & 0,583 & 0,024 & 0,389 & 0,765 & 0,667 & 0,408 & 0,689 & 1,205 & 0,414 & 0,269 & 0,240 & 0,064 & 0,076 & 168 & 660 & $-1,3$ \\
\hline 3 & 660 & 0,047 & 0,803 & 0,052 & 0,714 & 0,917 & 0,889 & 0,139 & 0,922 & 1,042 & 0,646 & 0,523 & 0,305 & 0,160 & 0,169 & 129 & 660 & $-1,6$ \\
\hline 4 & 660 & 0,052 & 0,825 & 0,056 & 0,714 & 0,923 & 0,866 & 0,154 & 0,955 & 1,023 & 0,865 & 0,777 & 0,276 & 0,214 & 0,214 & 128 & 660 & $-1,5$ \\
\hline 5 & 660 & 0,044 & 0,930 & 0,062 & 0,685 & 0,721 & 0,736 & 0,384 & 0,646 & 1,244 & 0,533 & 0,625 & 0,326 & 0,204 & 0,152 & 101 & 660 & $-1,7$ \\
\hline 6 & 660 & 0,092 & 1,826 & 0,129 & 1,112 & 0,711 & 0,609 & 0,486 & 0,346 & 1,700 & 0,519 & 0,642 & 0,475 & 0,305 & 0,107 & 54 & 660 & $-2,2$ \\
\hline 7 & 660 & 0,043 & 0,960 & 0,059 & 0,664 & 0,732 & 0,692 & 0,409 & 0,587 & 1,305 & 0,659 & 0,743 & 0,288 & 0,214 & 0,152 & 23 & 660 & $-1,5$ \\
\hline 8 & 660 & 0,026 & 0,622 & 0,030 & 0,469 & 0,857 & 0,755 & 0,284 & 0,840 & 1,091 & 0,619 & 0,639 & 0,230 & 0,147 & 0,107 & 76 & 660 & $-1,2$ \\
\hline 9 & 660 & 0,026 & 0,664 & 0,033 & 0,461 & 0,783 & 0,694 & 0,375 & 0,737 & 1,165 & 0,395 & 0,284 & 0,288 & 0,082 & 0,107 & 167 & 660 & $-1,5$ \\
\hline 10 & 660 & 0,034 & 0,693 & 0,037 & 0,592 & 0,923 & 0,854 & 0,165 & 0,900 & 1,054 & 0,679 & 0,527 & 0,254 & 0,134 & 0,152 & 40 & 660 & $-1,3$ \\
\hline 11 & 660 & 0,053 & 1,163 & 0,070 & 0,807 & 0,755 & 0,694 & 0,392 & 0,494 & 1,423 & 0,554 & 0,605 & 0,349 & 0,211 & 0,169 & 4 & 660 & $-1,8$ \\
\hline 12 & 660 & 0,014 & 0,368 & 0,017 & 0,245 & 0,833 & 0,667 & 0,373 & 1,000 & 0,866 & 0,979 & 0,745 & 0,137 & 0,102 & 0,076 & 18 & 660 & $-0,5$ \\
\hline 13 & 660 & 0,014 & 0,317 & 0,014 & 0,194 & 1,000 & 0,613 & 0,387 & 1,000 & 0,746 & 1,273 & 0,632 & 0,120 & 0,076 & 0,152 & 0 & 660 & $-0,3$ \\
\hline 14 & 660 & 0,118 & 2,253 & 0,154 & 1,306 & 0,766 & 0,580 & 0,481 & 0,291 & 1,853 & 0,492 & 0,618 & 0,552 & 0,341 & 0,169 & 166 & 660 & $-2,5$ \\
\hline 15 & 660 & 0,019 & 0,592 & 0,024 & 0,541 & 0,765 & 0,914 & 0,250 & 0,669 & 1,222 & 0,285 & 0,196 & 0,288 & 0,057 & 0,076 & 21 & 660 & $-1,5$ \\
\hline 16 & 660 & 0,046 & 0,828 & 0,053 & 0,655 & 0,865 & 0,791 & 0,249 & 0,841 & 1,091 & 0,815 & 0,707 & 0,268 & 0,189 & 0,169 & 160 & 660 & $-1,4$ \\
\hline 17 & 660 & 0,017 & 0,410 & 0,019 & 1,294 & 0,923 & 3,157 & 2,158 & 1,000 & 0,881 & 0,764 & 0,447 & 0,169 & 0,076 & 0,107 & 11 & 660 & $-0,8$ \\
\hline 18 & 660 & 0,136 & 1,345 & 0,143 & 1,378 & 0,950 & 1,025 & 0,056 & 0,947 & 1,027 & 0,599 & 0,498 & 0,538 & 0,268 & 0,321 & 42 & 660 & $-2,4$ \\
\hline 19 & 660 & 0,171 & 1,501 & 0,185 & 0,397 & 0,922 & 0,265 & 0,739 & 0,953 & 1,024 & 0,715 & 0,678 & 0,552 & 0,374 & 0,379 & 162 & 660 & $-2,5$ \\
\hline 20 & 660 & 0,024 & 0,440 & 0,024 & 0,735 & 1,000 & 1,673 & 0,673 & 1,000 & 0,794 & 1,082 & 0,671 & 0,169 & 0,114 & 0,152 & 105 & 660 & $-0,8$ \\
\hline 21 & 660 & 0,030 & 0,998 & 0,047 & 0,520 & 0,636 & 0,521 & 0,601 & 0,380 & 1,622 & 0,297 & 0,434 & 0,359 & 0,156 & 0,107 & 71 & 660 & $-1,8$ \\
\hline 22 & 660 & 0,030 & 0,685 & 0,039 & 1,133 & 0,778 & 1,655 & 0,691 & 0,808 & 1,113 & 0,514 & 0,417 & 0,273 & 0,114 & 0,107 & 146 & 660 & $-1,4$ \\
\hline 23 & 660 & 0,085 & 1,255 & 0,115 & 0,655 & 0,738 & 0,522 & 0,546 & 0,675 & 1,217 & 0,548 & 0,676 & 0,443 & 0,300 & 0,214 & 11 & 660 & $-2,1$ \\
\hline 24 & 660 & 0,039 & 0,968 & 0,049 & 0,397 & 0,794 & 0,410 & 0,624 & 0,519 & 1,388 & 0,649 & 0,549 & 0,276 & 0,152 & 0,107 & 93 & 660 & $-1,5$ \\
\hline 25 & 660 & 0,019 & 0,469 & 0,020 & 0,326 & 0,929 & 0,694 & 0,314 & 1,000 & 0,969 & 0,571 & 0,411 & 0,204 & 0,084 & 0,107 & 21 & 660 & $-1,0$ \\
\hline 26 & 660 & 0,014 & 0,490 & 0,019 & 0,266 & 0,769 & 0,543 & 0,512 & 0,750 & 1,155 & 0,509 & 0,424 & 0,189 & 0,080 & 0,076 & 135 & 660 & $-0,9$ \\
\hline 27 & 660 & 0,014 & 0,347 & 0,014 & 0,571 & 1,000 & 1,646 & 0,646 & 1,000 & 0,816 & 1,273 & 0,849 & 0,120 & 0,102 & 0,107 & 72 & 660 & $-0,3$ \\
\hline 28 & 660 & 0,026 & 0,735 & 0,037 & 0,520 & 0,692 & 0,707 & 0,425 & 0,600 & 1,291 & 0,432 & 0,369 & 0,276 & 0,102 & 0,152 & 17 & 660 & $-1,5$ \\
\hline 29 & 660 & 0,020 & 0,693 & 0,030 & 0,613 & 0,667 & 0,884 & 0,353 & 0,525 & 1,380 & 0,336 & 0,358 & 0,276 & 0,099 & 0,076 & 139 & 660 & $-1,5$ \\
\hline 30 & 660 & 0,032 & 0,714 & 0,044 & 0,490 & 0,710 & 0,686 & 0,427 & 0,777 & 1,134 & 0,483 & 0,450 & 0,288 & 0,130 & 0,107 & 18 & 660 & $-1,5$ \\
\hline 31 & 660 & 0,023 & 0,795 & 0,036 & 0,723 & 0,640 & 0,910 & 0,371 & 0,457 & 1,480 & 0,509 & 0,632 & 0,240 & 0,152 & 0,076 & 83 & 660 & $-1,3$ \\
\hline 32 & 660 & 0,037 & 0,960 & 0,055 & 0,917 & 0,684 & 0,956 & 0,319 & 0,509 & 1,401 & 0,293 & 0,333 & 0,403 & 0,134 & 0,152 & 48 & 660 & $-2,0$ \\
\hline 33 & 660 & 0,055 & 1,091 & 0,069 & 0,275 & 0,792 & 0,252 & 0,776 & 0,576 & 1,318 & 0,295 & 0,276 & 0,485 & 0,134 & 0,152 & 42 & 660 & $-2,3$ \\
\hline 34 & 660 & 0,016 & 0,440 & 0,019 & 0,368 & 0,846 & 0,837 & 0,224 & 1,000 & 0,987 & 0,539 & 0,392 & 0,193 & 0,076 & 0,076 & 169 & 660 & $-0,9$ \\
\hline 35 & 660 & 0,017 & 0,448 & 0,019 & 0,368 & 0,923 & 0,820 & 0,195 & 1,000 & 0,964 & 0,527 & 0,394 & 0,204 & 0,080 & 0,107 & 151 & 660 & $-1,0$ \\
\hline 36 & 660 & 0,022 & 0,448 & 0,022 & 0,562 & 1,000 & 1,254 & 0,254 & 1,000 & 0,862 & 0,955 & 0,632 & 0,169 & 0,107 & 0,152 & 55 & 660 & $-0,8$ \\
\hline 37 & 660 & 0,020 & 0,714 & 0,034 & 0,672 & 0,583 & 0,941 & 0,421 & 0,495 & 1,422 & 0,279 & 0,375 & 0,303 & 0,114 & 0,076 & 4 & 660 & $-1,6$ \\
\hline 38 & 660 & 0,027 & 0,938 & 0,047 & 0,461 & 0,576 & 0,491 & 0,663 & 0,389 & 1,603 & 0,285 & 0,458 & 0,349 & 0,160 & 0,152 & 30 & 660 & $-1,8$ \\
\hline 39 & 660 & 0,029 & 0,562 & 0,033 & 0,317 & 0,870 & 0,564 & 0,455 & 1,000 & 0,936 & 0,749 & 0,624 & 0,221 & 0,138 & 0,152 & 83 & 660 & $-1,1$ \\
\hline 40 & 660 & 0,017 & 0,469 & 0,022 & 0,266 & 0,800 & 0,567 & 0,477 & 0,983 & 1,009 & 0,764 & 0,600 & 0,169 & 0,102 & 0,107 & 18 & 660 & $-0,8$ \\
\hline 41 & 660 & 0,016 & 0,368 & 0,017 & 0,368 & 0,917 & 1,000 & 0,083 & 1,000 & 0,826 & 0,778 & 0,500 & 0,161 & 0,080 & 0,107 & 37 & 660 & $-0,7$ \\
\hline 42 & 660 & 0,017 & 0,440 & 0,019 & 0,347 & 0,923 & 0,789 & 0,225 & 1,000 & 0,945 & 0,527 & 0,353 & 0,204 & 0,072 & 0,076 & 11 & 660 & $-1,0$ \\
\hline 43 & 660 & 0,022 & 0,469 & 0,024 & 0,418 & 0,882 & 0,892 & 0,160 & 1,000 & 0,902 & 1,061 & 0,833 & 0,161 & 0,134 & 0,152 & 117 & 660 & $-0,7$ \\
\hline 44 & 660 & 0,024 & 0,520 & 0,027 & 0,296 & 0,895 & 0,569 & 0,444 & 1,000 & 0,939 & 1,082 & 0,894 & 0,169 & 0,152 & 0,152 & 72 & 660 & $-0,8$ \\
\hline 45 & 660 & 0,019 & 0,418 & 0,022 & 0,224 & 0,867 & 0,535 & 0,483 & 1,000 & 0,864 & 0,828 & 0,600 & 0,169 & 0,102 & 0,152 & 90 & 660 & $-0,8$ \\
\hline 46 & 660 & 0,014 & 0,397 & 0,016 & 0,338 & 0,909 & 0,851 & 0,175 & 1,000 & 0,936 & 0,707 & 0,500 & 0,161 & 0,080 & 0,107 & 146 & 660 & $-0,7$ \\
\hline 47 & 660 & 0,019 & 0,440 & 0,020 & 2,346 & 0,929 & 5,338 & 4,338 & 1,000 & 0,908 & 0,637 & 0,392 & 0,193 & 0,076 & 0,107 & 85 & 660 & $-0,9$ \\
\hline 48 & 660 & 0,389 & 2,439 & 0,415 & 0,613 & 0,938 & 0,251 & 0,751 & 0,821 & 1,103 & 0,510 & 0,534 & 0,985 & 0,526 & 0,530 & 29 & 660 & $-3,3$ \\
\hline 49 & 660 & 0,036 & 0,714 & 0,043 & 0,562 & 0,833 & 0,787 & 0,271 & 0,883 & 1,064 & 0,637 & 0,639 & 0,268 & 0,171 & 0,152 & 3 & 660 & $-1,4$ \\
\hline 50 & 660 & 0,032 & 0,786 & 0,044 & 0,643 & 0,710 & 0,817 & 0,343 & 0,642 & 1,248 & 0,459 & 0,634 & 0,296 & 0,187 & 0,152 & 47 & 660 & $-1,6$ \\
\hline 51 & 660 & 0,030 & 0,765 & 0,039 & 0,592 & 0,778 & 0,773 & 0,317 & 0,647 & 1,243 & 0,334 & 0,291 & 0,339 & 0,099 & 0,107 & 23 & 660 & $-1,8$ \\
\hline 52 & 660 & 0,036 & 0,888 & 0,046 & 0,499 & 0,781 & 0,562 & 0,490 & 0,572 & 1,322 & 0,860 & 0,814 & 0,230 & 0,187 & 0,107 & 97 & 660 & $-1,2$ \\
\hline 53 & 660 & 0,033 & 0,643 & 0,039 & 0,317 & 0,852 & 0,493 & 0,528 & 1,000 & 0,998 & 0,563 & 0,452 & 0,273 & 0,123 & 0,152 & 32 & 660 & $-1,4$ \\
\hline 54 & 660 & 0,024 & 0,461 & 0,026 & 1,615 & 0,944 & 3,505 & 2,506 & 1,000 & 0,832 & 0,866 & 0,600 & 0,189 & 0,114 & 0,152 & 117 & 660 & $-0,9$ \\
\hline 55 & 660 & 0,098 & 2,460 & 0,198 & 1,192 & 0,493 & 0,485 & 0,723 & 0,203 & 2,222 & 0,223 & 0,443 & 0,746 & 0,331 & 0,107 & 159 & 660 & $-2,9$ \\
\hline 56 & 660 & 0,100 & 1,716 & 0,142 & 0,296 & 0,707 & 0,172 & 0,878 & 0,429 & 1,528 & 0,610 & 0,713 & 0,458 & 0,327 & 0,169 & 131 & 660 & $-2,2$ \\
\hline 57 & 660 & 0,020 & 0,613 & 0,024 & 0,266 & 0,824 & 0,434 & 0,593 & 0,672 & 1,220 & 0,713 & 0,600 & 0,189 & 0,114 & 0,107 & 117 & 660 & $-0,9$ \\
\hline
\end{tabular}




\begin{tabular}{|c|c|c|c|c|c|c|c|c|c|c|c|c|c|c|c|c|c|c|}
\hline 58 & 660 & 0,016 & 0,389 & 0,017 & 0,296 & 0,917 & 0,761 & 0,253 & 1,000 & 0,873 & 0,700 & 0,447 & 0,169 & 0,076 & 0,107 & 72 & 660 & $-0,8$ \\
\hline 59 & 660 & 0,019 & 0,440 & 0,020 & 0,368 & 0,929 & 0,837 & 0,178 & 1,000 & 0,908 & 0,662 & 0,424 & 0,189 & 0,080 & 0,107 & 40 & 660 & $-0,9$ \\
\hline 60 & 660 & 0,022 & 0,418 & 0,022 & 0,296 & 1,000 & 0,707 & 0,293 & 1,000 & 0,805 & 1,123 & 0,728 & 0,156 & 0,114 & 0,152 & 20 & 660 & $-0,6$ \\
\hline 61 & 660 & 0,016 & 0,368 & 0,016 & 0,490 & 1,000 & 1,333 & 0,333 & 1,000 & 0,826 & 0,824 & 0,485 & 0,156 & 0,076 & 0,107 & 18 & 660 & $-0,6$ \\
\hline 62 & 660 & 0,032 & 0,541 & 0,033 & 0,144 & 0,957 & 0,265 & 0,736 & 1,000 & 0,859 & 0,966 & 0,665 & 0,204 & 0,136 & 0,152 & 22 & 660 & $-1,0$ \\
\hline 63 & 660 & 0,014 & 0,338 & 0,016 & 0,275 & 0,909 & 0,813 & 0,208 & 1,000 & 0,796 & 0,979 & 0,555 & 0,137 & 0,076 & 0,107 & 72 & 660 & $-0,5$ \\
\hline 64 & 660 & 0,014 & 0,317 & 0,014 & 0,296 & 1,000 & 0,934 & 0,066 & 1,000 & 0,746 & 1,273 & 0,632 & 0,120 & 0,076 & 0,107 & 90 & 660 & $-0,3$ \\
\hline 65 & 660 & 0,014 & 0,520 & 0,020 & 0,389 & 0,714 & 0,748 & 0,381 & 0,667 & 1,225 & 0,749 & 0,728 & 0,156 & 0,114 & 0,076 & 95 & 660 & $-0,6$ \\
\hline 66 & 660 & 0,017 & 0,520 & 0,022 & 0,296 & 0,800 & 0,569 & 0,475 & 0,800 & 1,118 & 0,413 & 0,329 & 0,230 & 0,076 & 0,107 & 79 & 660 & $-1,2$ \\
\hline 67 & 660 & 0,019 & 0,418 & 0,019 & 1,133 & 1,000 & 2,707 & 1,707 & 1,000 & 0,864 & 0,662 & 0,424 & 0,189 & 0,080 & 0,107 & 57 & 660 & $-0,9$ \\
\hline 68 & 660 & 0,059 & 1,437 & 0,115 & 0,245 & 0,513 & 0,171 & 0,962 & 0,358 & 1,672 & 0,350 & 0,743 & 0,462 & 0,343 & 0,169 & 66 & 660 & $-2,2$ \\
\hline 69 & 660 & 0,014 & 0,389 & 0,017 & 0,469 & 0,833 & 1,207 & 0,266 & 1,000 & 0,916 & 0,707 & 0,633 & 0,161 & 0,102 & 0,107 & 127 & 660 & $-0,7$ \\
\hline 70 & 660 & 0,027 & 0,520 & 0,029 & 0,397 & 0,950 & 0,764 & 0,241 & 1,000 & 0,888 & 0,712 & 0,485 & 0,221 & 0,107 & 0,152 & 45 & 660 & $-1,1$ \\
\hline 71 & 660 & 0,014 & 0,541 & 0,020 & 0,786 & 0,714 & 1,453 & 0,536 & 0,616 & 1,274 & 0,439 & 0,483 & 0,204 & 0,099 & 0,076 & 34 & 660 & $-1,0$ \\
\hline 72 & 660 & 0,043 & 0,989 & 0,057 & 0,541 & 0,750 & 0,547 & 0,517 & 0,553 & 1,345 & 0,394 & 0,468 & 0,373 & 0,175 & 0,152 & 21 & 660 & $-1,9$ \\
\hline 73 & 660 & 0,040 & 0,664 & 0,043 & 0,900 & 0,933 & 1,356 & 0,363 & 1,000 & 0,934 & 0,792 & 0,707 & 0,254 & 0,180 & 0,214 & 18 & 660 & $-1,3$ \\
\hline 74 & 660 & 0,057 & 1,572 & 0,096 & 2,122 & 0,597 & 1,350 & 0,533 & 0,292 & 1,852 & 0,525 & 0,701 & 0,373 & 0,262 & 0,152 & 163 & 660 & $-1,9$ \\
\hline 75 & 660 & 0,326 & 2,355 & 0,367 & 0,917 & 0,887 & 0,390 & 0,621 & 0,738 & 1,164 & 0,649 & 0,715 & 0,799 & 0,571 & 0,485 & 59 & 660 & $-3,0$ \\
\hline 76 & 660 & 0,060 & 1,133 & 0,076 & 0,972 & 0,792 & 0,858 & 0,251 & 0,590 & 1,302 & 0,461 & 0,573 & 0,408 & 0,234 & 0,214 & 19 & 660 & $-2,0$ \\
\hline 77 & 660 & 0,057 & 1,255 & 0,082 & 4,903 & 0,702 & 3,905 & 2,921 & 0,458 & 1,478 & 0,344 & 0,358 & 0,461 & 0,165 & 0,107 & 89 & 660 & $-2,2$ \\
\hline 78 & 660 & 1,769 & 5,085 & 1,905 & 0,296 & 0,928 & 0,058 & 0,945 & 0,860 & 1,078 & 0,737 & 0,802 & 1,748 & 1,402 & 1,212 & 9 & 660 & $-4,1$ \\
\hline 79 & 660 & 0,017 & 0,520 & 0,022 & 0,828 & 0,800 & 1,593 & 0,626 & 0,800 & 1,118 & 0,764 & 0,651 & 0,169 & 0,110 & 0,076 & 21 & 660 & $-0,8$ \\
\hline 80 & 660 & 0,067 & 1,040 & 0,082 & 0,389 & 0,825 & 0,374 & 0,650 & 0,783 & 1,130 & 0,809 & 0,814 & 0,326 & 0,265 & 0,214 & 91 & 660 & $-1,7$ \\
\hline 81 & 660 & 0,017 & 0,490 & 0,020 & 0,461 & 0,857 & 0,939 & 0,155 & 0,900 & 1,054 & 0,449 & 0,307 & 0,221 & 0,068 & 0,076 & 62 & 660 & $-1,1$ \\
\hline 82 & 660 & 0,026 & 0,786 & 0,034 & 0,490 & 0,750 & 0,624 & 0,452 & 0,525 & 1,380 & 0,573 & 0,474 & 0,240 & 0,114 & 0,152 & 83 & 660 & $-1,3$ \\
\hline 83 & 660 & 0,026 & 0,592 & 0,029 & 0,347 & 0,900 & 0,586 & 0,426 & 0,927 & 1,039 & 0,559 & 0,442 & 0,243 & 0,107 & 0,152 & 59 & 660 & $-1,3$ \\
\hline 84 & 660 & 0,017 & 0,490 & 0,020 & 0,440 & 0,857 & 0,896 & 0,176 & 0,900 & 1,054 & 0,764 & 0,600 & 0,169 & 0,102 & 0,076 & 121 & 660 & $-0,8$ \\
\hline 85 & 660 & 0,022 & 0,520 & 0,027 & 0,664 & 0,789 & 1,276 & 0,347 & 1,000 & 1,000 & 0,659 & 0,557 & 0,204 & 0,114 & 0,107 & 18 & 660 & $-1,0$ \\
\hline 86 & 660 & 0,053 & 0,828 & 0,062 & 0,930 & 0,860 & 1,123 & 0,186 & 0,972 & 1,014 & 0,725 & 0,721 & 0,305 & 0,220 & 0,214 & 32 & 660 & $-1,6$ \\
\hline 87 & 660 & 0,050 & 1,458 & 0,088 & 1,031 & 0,574 & 0,707 & 0,517 & 0,297 & 1,836 & 0,357 & 0,626 & 0,423 & 0,265 & 0,152 & 20 & 660 & $-2,1$ \\
\hline 88 & 660 & 0,102 & 1,133 & 0,109 & 0,194 & 0,934 & 0,172 & 0,831 & 0,997 & 1,001 & 0,706 & 0,688 & 0,429 & 0,295 & 0,312 & 47 & 660 & $-2,1$ \\
\hline 89 & 660 & 0,014 & 0,347 & 0,016 & 1,154 & 0,909 & 3,329 & 2,330 & 1,000 & 0,816 & 1,273 & 0,849 & 0,120 & 0,102 & 0,107 & 0 & 660 & $-0,3$ \\
\hline 90 & 660 & 0,095 & 1,830 & 0,121 & 0,448 & 0,786 & 0,245 & 0,785 & 0,355 & 1,678 & 0,433 & 0,452 & 0,528 & 0,239 & 0,152 & 75 & 660 & $-2,4$ \\
\hline 91 & 660 & 0,022 & 0,613 & 0,026 & 1,488 & 0,833 & 2,428 & 1,438 & 0,720 & 1,178 & 0,382 & 0,393 & 0,268 & 0,105 & 0,152 & 33 & 660 & $-1,4$ \\
\hline 92 & 660 & 0,179 & 1,826 & 0,217 & 1,725 & 0,828 & 0,944 & 0,181 & 0,676 & 1,216 & 0,704 & 0,847 & 0,569 & 0,482 & 0,386 & 4 & 660 & $-2,5$ \\
\hline 93 & 660 & 0,152 & 2,101 & 0,231 & 0,245 & 0,658 & 0,117 & 0,947 & 0,433 & 1,520 & 0,453 & 0,759 & 0,654 & 0,496 & 0,240 & 107 & 660 & $-2,7$ \\
\hline 94 & 660 & 0,016 & 0,368 & 0,017 & 0,930 & 0,917 & 2,529 & 1,531 & 1,000 & 0,826 & 1,077 & 0,745 & 0,137 & 0,102 & 0,152 & 37 & 660 & $-0,5$ \\
\hline 95 & 660 & 0,060 & 1,052 & 0,073 & 1,247 & 0,824 & 1,185 & 0,255 & 0,684 & 1,209 & 0,334 & 0,300 & 0,479 & 0,144 & 0,152 & 74 & 660 & $-2,3$ \\
\hline 96 & 660 & 0,139 & 1,348 & 0,154 & 0,296 & 0,907 & 0,219 & 0,786 & 0,962 & 1,020 & 0,846 & 0,785 & 0,458 & 0,359 & 0,379 & 73 & 660 & $-2,2$ \\
\hline 97 & 660 & 0,014 & 0,347 & 0,014 & 0,714 & 1,000 & 2,061 & 1,061 & 1,000 & 0,816 & 0,749 & 0,485 & 0,156 & 0,076 & 0,107 & 108 & 660 & $-0,6$ \\
\hline 98 & 660 & 0,037 & 0,786 & 0,046 & 0,317 & 0,812 & 0,403 & 0,626 & 0,758 & 1,148 & 0,662 & 0,814 & 0,268 & 0,218 & 0,169 & 64 & 660 & $-1,4$ \\
\hline 99 & 660 & 0,014 & 0,440 & 0,019 & 0,541 & 0,769 & 1,231 & 0,326 & 0,933 & 1,035 & 0,490 & 0,392 & 0,193 & 0,076 & 0,107 & 135 & 660 & $-0,9$ \\
\hline 100 & 660 & 0,042 & 0,643 & 0,043 & 0,266 & 0,967 & 0,414 & 0,587 & 1,000 & 0,889 & 0,998 & 0,814 & 0,230 & 0,187 & 0,214 & 93 & 660 & $-1,2$ \\
\hline 101 & 660 & 0,019 & 0,389 & 0,020 & 0,338 & 0,929 & 0,869 & 0,149 & 1,000 & 0,803 & 0,920 & 0,633 & 0,161 & 0,102 & 0,152 & 30 & 660 & $-0,7$ \\
\hline 102 & 660 & 0,022 & 0,440 & 0,023 & 0,490 & 0,938 & 1,115 & 0,131 & 1,000 & 0,845 & 0,955 & 0,671 & 0,169 & 0,114 & 0,152 & 90 & 660 & $-0,8$ \\
\hline 103 & 660 & 0,026 & 0,714 & 0,034 & 0,828 & 0,750 & 1,159 & 0,297 & 0,636 & 1,254 & 0,559 & 0,607 & 0,243 & 0,147 & 0,107 & 130 & 660 & $-1,3$ \\
\hline 104 & 660 & 0,047 & 1,095 & 0,070 & 0,266 & 0,673 & 0,243 & 0,824 & 0,497 & 1,419 & 0,290 & 0,306 & 0,456 & 0,139 & 0,152 & 171 & 660 & $-2,2$ \\
\hline 105 & 660 & 0,016 & 0,347 & 0,016 & 1,408 & 1,000 & 4,061 & 3,061 & 1,000 & 0,778 & 1,401 & 0,894 & 0,120 & 0,107 & 0,152 & 72 & 660 & $-0,3$ \\
\hline 106 & 660 & 0,171 & 1,551 & 0,187 & 0,571 & 0,915 & 0,368 & 0,638 & 0,892 & 1,059 & 0,653 & 0,696 & 0,577 & 0,402 & 0,339 & 76 & 660 & $-2,5$ \\
\hline 107 & 660 & 0,036 & 0,622 & 0,039 & 0,368 & 0,926 & 0,592 & 0,415 & 1,000 & 0,926 & 0,776 & 0,552 & 0,243 & 0,134 & 0,152 & 57 & 660 & $-1,3$ \\
\hline 108 & 660 & 0,017 & 0,511 & 0,020 & 0,541 & 0,857 & 1,058 & 0,154 & 0,827 & 1,099 & 0,477 & 0,375 & 0,214 & 0,080 & 0,076 & 50 & 660 & $-1,1$ \\
\hline 109 & 660 & 0,029 & 0,592 & 0,036 & 0,194 & 0,800 & 0,328 & 0,701 & 1,000 & 0,986 & 0,566 & 0,497 & 0,254 & 0,126 & 0,152 & 27 & 660 & $-1,3$ \\
\hline 110 & 660 & 0,022 & 0,410 & 0,022 & 0,938 & 1,000 & 2,290 & 1,290 & 1,000 & 0,788 & 1,061 & 0,707 & 0,161 & 0,114 & 0,152 & 45 & 660 & $\begin{array}{l}-0,7 \\
\end{array}$ \\
\hline 111 & 660 & 0,069 & 1,509 & 0,090 & 0,735 & 0,762 & 0,487 & 0,565 & 0,380 & 1,622 & 0,719 & 0,844 & 0,349 & 0,295 & 0,152 & 130 & 660 & $-1,8$ \\
\hline 112 & 660 & 0,049 & 0,858 & 0,052 & 0,317 & 0,944 & 0,369 & 0,633 & 0,833 & 1,096 & 0,397 & 0,333 & 0,395 & 0,132 & 0,152 & 106 & 660 & $-2,0$ \\
\hline 113 & 660 & 0,016 & 0,418 & 0,020 & 0,368 & 0,786 & 0,879 & 0,246 & 1,000 & 0,940 & 0,824 & 0,651 & 0,156 & 0,102 & 0,076 & 159 & 660 & $-0,6$ \\
\hline 114 & 660 & 0,023 & 0,562 & 0,029 & 0,338 & 0,800 & 0,601 & 0,446 & 0,913 & 1,047 & 0,637 & 0,590 & 0,214 & 0,126 & 0,152 & 45 & 660 & $-1,1$ \\
\hline 115 & 660 & 0,019 & 0,418 & 0,020 & 0,194 & 0,929 & 0,465 & 0,540 & 1,000 & 0,864 & 0,828 & 0,632 & 0,169 & 0,107 & 0,152 & 153 & 660 & $\begin{array}{l}-0,8 \\
\end{array}$ \\
\hline
\end{tabular}




\begin{tabular}{|c|c|c|c|c|c|c|c|c|c|c|c|c|c|c|c|c|c|c|}
\hline 116 & 660 & 0,020 & 0,410 & 0,020 & 0,266 & 1,000 & 0,650 & 0,350 & 1,000 & 0,816 & 0,891 & 0,447 & 0,169 & 0,076 & 0,152 & 82 & 660 & $-0,8$ \\
\hline 117 & 660 & 0,014 & 0,389 & 0,016 & 0,266 & 0,909 & 0,685 & 0,328 & 1,000 & 0,916 & 0,637 & 0,380 & 0,169 & 0,064 & 0,076 & 18 & 660 & $-0,8$ \\
\hline 118 & 660 & 0,019 & 0,389 & 0,020 & 0,368 & 0,929 & 0,946 & 0,090 & 1,000 & 0,803 & 0,920 & 0,633 & 0,161 & 0,102 & 0,152 & 30 & 660 & $-0,7$ \\
\hline 119 & 660 & 0,022 & 0,418 & 0,022 & 0,296 & 1,000 & 0,707 & 0,293 & 1,000 & 0,805 & 0,955 & 0,632 & 0,169 & 0,107 & 0,152 & 27 & 660 & $-0,8$ \\
\hline 120 & 660 & 0,014 & 0,397 & 0,016 & 0,998 & 0,909 & 2,511 & 1,514 & 1,000 & 0,936 & 0,637 & 0,474 & 0,169 & 0,080 & 0,107 & 55 & 660 & $-0,8$ \\
\hline 121 & 660 & 0,049 & 1,192 & 0,066 & 0,828 & 0,739 & 0,695 & 0,402 & 0,431 & 1,523 & 0,239 & 0,263 & 0,510 & 0,134 & 0,152 & 40 & 660 & $-2,4$ \\
\hline 122 & 660 & 0,057 & 1,348 & 0,077 & 2,773 & 0,741 & 2,057 & 1,088 & 0,397 & 1,588 & 0,688 & 0,784 & 0,326 & 0,255 & 0,169 & 63 & 660 & $-1,7$ \\
\hline 123 & 660 & 0,499 & 3,264 & 0,598 & 0,541 & 0,835 & 0,166 & 0,850 & 0,589 & 1,303 & 0,485 & 0,602 & 1,145 & 0,690 & 0,577 & 134 & 660 & $-3,5$ \\
\hline 124 & 660 & 0,033 & 0,960 & 0,046 & 0,215 & 0,719 & 0,225 & 0,825 & 0,450 & 1,490 & 0,651 & 0,832 & 0,254 & 0,211 & 0,107 & 106 & 660 & $-1,3$ \\
\hline 125 & 660 & 0,016 & 0,389 & 0,017 & 0,418 & 0,917 & 1,076 & 0,113 & 1,000 & 0,873 & 0,824 & 0,485 & 0,156 & 0,076 & 0,107 & 90 & 660 & $-0,6$ \\
\hline 126 & 660 & 0,022 & 0,520 & 0,027 & 0,245 & 0,789 & 0,471 & 0,569 & 1,000 & 1,000 & 0,659 & 0,557 & 0,204 & 0,114 & 0,107 & 85 & 660 & $-1,0$ \\
\hline 127 & 660 & 0,016 & 0,368 & 0,017 & 0,245 & 0,917 & 0,667 & 0,344 & 1,000 & 0,826 & 0,824 & 0,485 & 0,156 & 0,076 & 0,107 & 18 & 660 & $-0,6$ \\
\hline 128 & 660 & 0,017 & 0,490 & 0,022 & 0,368 & 0,800 & 0,750 & 0,320 & 0,900 & 1,054 & 0,764 & 0,651 & 0,169 & 0,110 & 0,076 & 21 & 660 & $-0,8$ \\
\hline 129 & 660 & 0,017 & 0,440 & 0,019 & 0,397 & 0,923 & 0,904 & 0,123 & 1,000 & 0,945 & 0,588 & 0,392 & 0,193 & 0,076 & 0,107 & 5 & 660 & $-0,9$ \\
\hline 130 & 660 & 0,020 & 0,541 & 0,026 & 0,389 & 0,778 & 0,719 & 0,359 & 0,862 & 1,077 & 0,713 & 0,537 & 0,189 & 0,102 & 0,152 & 117 & 660 & $-0,9$ \\
\hline 131 & 660 & 0,022 & 0,541 & 0,026 & 0,837 & 0,833 & 1,547 & 0,572 & 0,924 & 1,040 & 0,516 & 0,468 & 0,230 & 0,108 & 0,107 & 0 & 660 & $-1,2$ \\
\hline 132 & 660 & 0,057 & 0,909 & 0,066 & 0,144 & 0,870 & 0,158 & 0,852 & 0,873 & 1,070 & 0,566 & 0,500 & 0,359 & 0,180 & 0,169 & 25 & 660 & $-1,8$ \\
\hline 133 & 660 & 0,016 & 0,338 & 0,016 & 0,888 & 1,000 & 2,626 & 1,626 & 1,000 & 0,759 & 1,077 & 0,555 & 0,137 & 0,076 & 0,152 & 0 & 660 & $-0,5$ \\
\hline 134 & 660 & 0,055 & 1,277 & 0,083 & 0,461 & 0,655 & 0,361 & 0,726 & 0,420 & 1,542 & 0,465 & 0,666 & 0,386 & 0,257 & 0,152 & 160 & 660 & $-1,9$ \\
\hline 135 & 660 & 0,024 & 0,727 & 0,040 & 0,215 & 0,607 & 0,296 & 0,806 & 0,580 & 1,313 & 0,373 & 0,470 & 0,288 & 0,136 & 0,076 & 63 & 660 & $-1,5$ \\
\hline 136 & 660 & 0,020 & 0,410 & 0,020 & 0,368 & 1,000 & 0,897 & 0,103 & 1,000 & 0,816 & 0,891 & 0,447 & 0,169 & 0,076 & 0,152 & 0 & 660 & $-0,8$ \\
\hline 137 & 660 & 0,016 & 0,469 & 0,019 & 0,266 & 0,846 & 0,567 & 0,459 & 0,901 & 1,054 & 0,483 & 0,371 & 0,204 & 0,076 & 0,107 & 0 & 660 & $-1,0$ \\
\hline 138 & 660 & 0,016 & 0,368 & 0,016 & 1,019 & 1,000 & 2,771 & 1,771 & 1,000 & 0,826 & 1,077 & 0,745 & 0,137 & 0,102 & 0,107 & 72 & 660 & $-0,5$ \\
\hline 139 & 660 & 0,080 & 1,285 & 0,102 & 0,368 & 0,789 & 0,286 & 0,744 & 0,611 & 1,279 & 0,466 & 0,566 & 0,469 & 0,265 & 0,214 & 178 & 660 & $-2,2$ \\
\hline 140 & 660 & 0,014 & 0,490 & 0,017 & 0,245 & 0,833 & 0,500 & 0,527 & 0,750 & 1,155 & 0,439 & 0,294 & 0,204 & 0,060 & 0,076 & 90 & 660 & $-1,0$ \\
\hline 141 & 660 & 0,014 & 0,389 & 0,017 & 0,693 & 0,833 & 1,783 & 0,801 & 1,000 & 0,916 & 0,707 & 0,500 & 0,161 & 0,080 & 0,076 & 135 & 660 & $-0,7$ \\
\hline 142 & 660 & 0,053 & 0,837 & 0,059 & 0,879 & 0,902 & 1,050 & 0,110 & 0,952 & 1,025 & 0,812 & 0,743 & 0,288 & 0,214 & 0,169 & 142 & 660 & $-1,5$ \\
\hline 143 & 660 & 0,062 & 1,277 & 0,080 & 0,583 & 0,768 & 0,457 & 0,591 & 0,476 & 1,450 & 0,421 & 0,439 & 0,432 & 0,189 & 0,169 & 178 & 660 & $-2,1$ \\
\hline 144 & 660 & 0,036 & 0,664 & 0,039 & 0,469 & 0,926 & 0,707 & 0,302 & 1,000 & 0,988 & 0,601 & 0,492 & 0,276 & 0,136 & 0,152 & 22 & 660 & $-1,5$ \\
\hline 145 & 660 & 0,019 & 0,643 & 0,024 & 0,338 & 0,765 & 0,526 & 0,529 & 0,568 & 1,327 & 0,331 & 0,300 & 0,268 & 0,080 & 0,107 & 54 & 660 & $-1,4$ \\
\hline 146 & 660 & 0,016 & 0,397 & 0,017 & 2,113 & 0,917 & 5,318 & 4,319 & 1,000 & 0,892 & 0,824 & 0,485 & 0,156 & 0,076 & 0,076 & 0 & 660 & $-0,6$ \\
\hline 147 & 660 & 0,333 & 2,236 & 0,372 & 0,600 & 0,896 & 0,269 & 0,739 & 0,837 & 1,093 & 0,613 & 0,729 & 0,832 & 0,606 & 0,508 & 61 & 660 & $-3,1$ \\
\hline 148 & 660 & 0,023 & 0,723 & 0,030 & 2,012 & 0,762 & 2,783 & 1,799 & 0,552 & 1,346 & 0,240 & 0,205 & 0,349 & 0,071 & 0,076 & 137 & 660 & $-1,8$ \\
\hline 149 & 660 & 0,224 & 2,164 & 0,290 & 0,693 & 0,772 & 0,320 & 0,717 & 0,601 & 1,290 & 0,353 & 0,427 & 0,898 & 0,383 & 0,303 & 19 & 660 & $-3,2$ \\
\hline 150 & 660 & 0,042 & 0,947 & 0,059 & 0,541 & 0,707 & 0,571 & 0,519 & 0,583 & 1,310 & 0,506 & 0,585 & 0,324 & 0,189 & 0,107 & 73 & 660 & $-1,7$ \\
\hline 151 & 660 & 0,027 & 0,744 & 0,039 & 0,194 & 0,704 & 0,261 & 0,796 & 0,619 & 1,271 & 0,465 & 0,435 & 0,273 & 0,119 & 0,076 & 57 & 660 & $-1,4$ \\
\hline 152 & 660 & 0,014 & 0,317 & 0,014 & 0,397 & 1,000 & 1,254 & 0,254 & 1,000 & 0,746 & 1,273 & 0,632 & 0,120 & 0,076 & 0,152 & 0 & 660 & $-0,3$ \\
\hline 153 & 660 & 0,016 & 0,418 & 0,017 & 1,052 & 0,917 & 2,515 & 1,517 & 1,000 & 0,940 & 0,560 & 0,424 & 0,189 & 0,080 & 0,107 & 130 & 660 & $-0,9$ \\
\hline 154 & 660 & 0,088 & 1,581 & 0,112 & 0,296 & 0,782 & 0,187 & 0,842 & 0,440 & 1,508 & 0,769 & 0,869 & 0,381 & 0,331 & 0,214 & 140 & 660 & $-1,9$ \\
\hline 155 & 660 & 0,014 & 0,397 & 0,016 & 0,541 & 0,909 & 1,361 & 0,373 & 1,000 & 0,936 & 0,509 & 0,283 & 0,189 & 0,054 & 0,107 & 45 & 660 & $-0,9$ \\
\hline 156 & 660 & 0,036 & 0,765 & 0,046 & 0,389 & 0,781 & 0,508 & 0,538 & 0,770 & 1,140 & 0,707 & 0,660 & 0,254 & 0,168 & 0,152 & 55 & 660 & $-1,3$ \\
\hline 157 & 660 & 0,019 & 0,418 & 0,020 & 0,440 & 0,929 & 1,050 & 0,087 & 1,000 & 0,864 & 0,662 & 0,400 & 0,189 & 0,076 & 0,152 & 172 & 660 & $-0,9$ \\
\hline 158 & 660 & 0,026 & 0,592 & 0,030 & 1,142 & 0,857 & 1,929 & 0,940 & 0,927 & 1,039 & 0,790 & 0,743 & 0,204 & 0,152 & 0,152 & 111 & 660 & $-1,0$ \\
\hline 159 & 660 & 0,119 & 1,243 & 0,128 & 0,735 & 0,933 & 0,592 & 0,414 & 0,969 & 1,016 & 0,845 & 0,822 & 0,423 & 0,348 & 0,321 & 39 & 660 & $-2,1$ \\
\hline 160 & 660 & 0,065 & 0,879 & 0,066 & 0,461 & 0,978 & 0,524 & 0,477 & 1,000 & 0,976 & 0,674 & 0,542 & 0,349 & 0,189 & 0,214 & 179 & 660 & $-1,8$ \\
\hline 161 & 660 & 0,023 & 0,693 & 0,023 & 1,002 & 1,000 & 1,445 & 0,445 & 0,600 & 1,291 & 0,702 & 0,665 & 0,204 & 0,136 & 0,152 & 11 & 660 & $-1,0$ \\
\hline 162 & 660 & 0,092 & 1,073 & 0,095 & 0,735 & 0,970 & 0,685 & 0,316 & 1,000 & 0,999 & 0,551 & 0,411 & 0,461 & 0,189 & 0,227 & 0 & 660 & $-2,2$ \\
\hline 1 & 661 & 0,020 & 0,490 & 0,022 & 0,347 & 0,933 & 0,707 & 0,300 & 1,000 & 0,976 & 0,524 & 0,416 & 0,221 & 0,092 & 0,152 & 29 & 661 & $-1,1$ \\
\hline 2 & 661 & 0,016 & 0,490 & 0,020 & 0,347 & 0,786 & 0,707 & 0,363 & 0,825 & 1,101 & 0,389 & 0,327 & 0,227 & 0,074 & 0,076 & 18 & 661 & $-1,2$ \\
\hline 3 & 661 & 0,017 & 0,440 & 0,019 & 0,389 & 0,923 & 0,885 & 0,139 & 1,000 & 0,945 & 0,527 & 0,353 & 0,204 & 0,072 & 0,076 & 15 & 661 & $-1,0$ \\
\hline 4 & 661 & 0,017 & 0,550 & 0,024 & 0,448 & 0,706 & 0,815 & 0,347 & 0,716 & 1,182 & 0,373 & 0,331 & 0,243 & 0,080 & 0,076 & 44 & 661 & $-1,3$ \\
\hline 5 & 661 & 0,014 & 0,410 & 0,016 & 0,266 & 0,909 & 0,650 & 0,362 & 1,000 & 0,965 & 0,490 & 0,196 & 0,193 & 0,038 & 0,076 & 0 & 661 & $-0,9$ \\
\hline 6 & 661 & 0,023 & 0,461 & 0,023 & 0,266 & 1,000 & 0,578 & 0,422 & 1,000 & 0,858 & 0,784 & 0,392 & 0,193 & 0,076 & 0,152 & 5 & 661 & $-0,9$ \\
\hline 7 & 661 & 0,019 & 0,440 & 0,020 & 0,368 & 0,929 & 0,837 & 0,178 & 1,000 & 0,908 & 0,637 & 0,392 & 0,193 & 0,076 & 0,107 & 15 & 661 & $-0,9$ \\
\hline 8 & 661 & 0,027 & 0,655 & 0,030 & 0,418 & 0,905 & 0,639 & 0,373 & 0,798 & 1,119 & 0,372 & 0,248 & 0,305 & 0,076 & 0,107 & 4 & 661 & $-1,6$ \\
\hline 9 & 661 & 0,016 & 0,461 & 0,017 & 0,338 & 0,917 & 0,734 & 0,279 & 0,935 & 1,034 & 0,379 & 0,164 & 0,230 & 0,038 & 0,076 & 0 & 661 & $-1,2$ \\
\hline 10 & 661 & 0,017 & 0,368 & 0,019 & 0,266 & 0,923 & 0,724 & 0,287 & 1,000 & 0,791 & 1,175 & 0,745 & 0,137 & 0,102 & 0,107 & 27 & 661 & $-0,5$ \\
\hline 11 & 661 & 0,092 & 1,809 & 0,138 & 1,298 & 0,667 & 0,717 & 0,437 & 0,353 & 1,684 & 0,397 & 0,543 & 0,542 & 0,295 & 0,107 & 179 & 661 & $-2,4$ \\
\hline
\end{tabular}




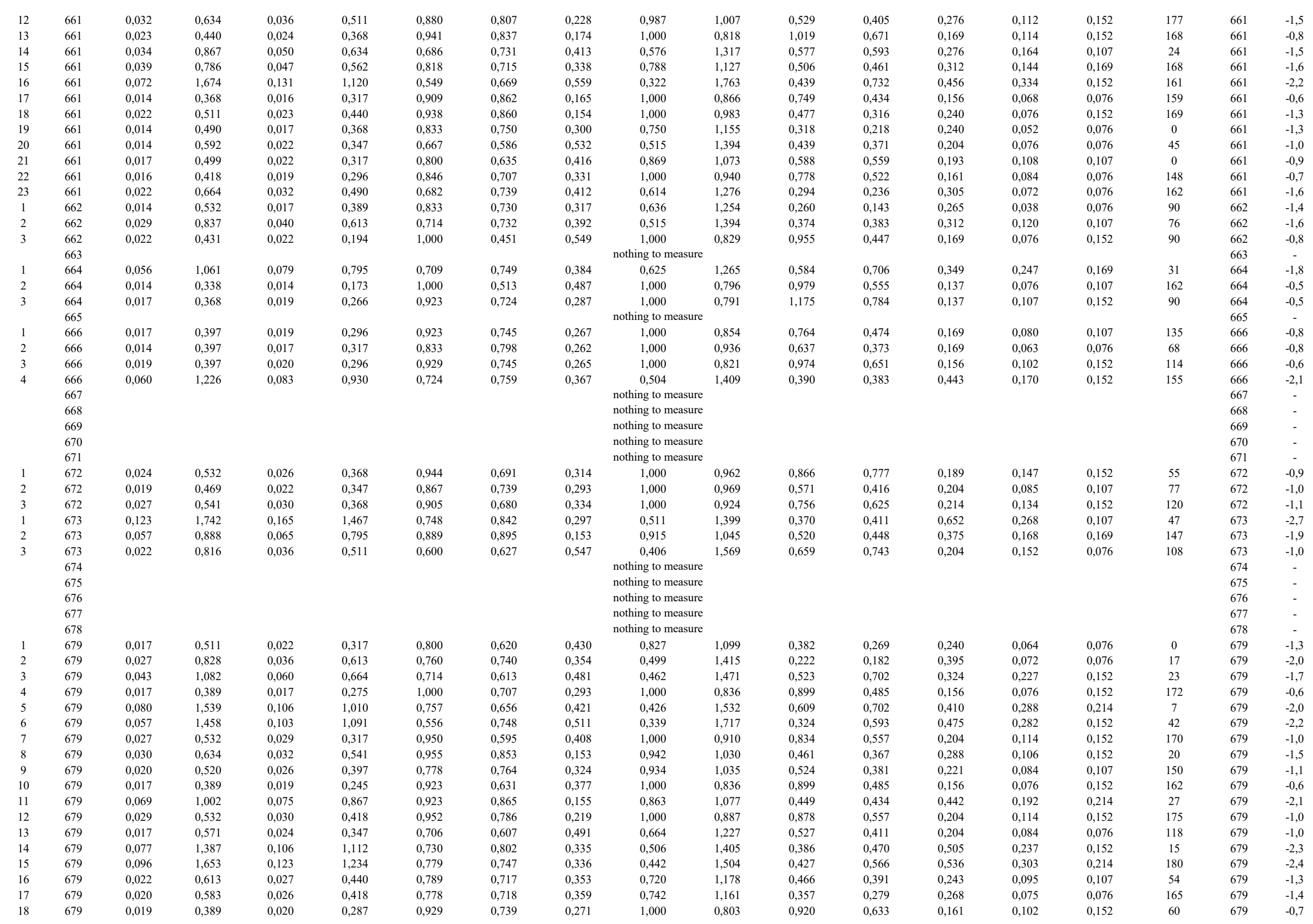




\begin{tabular}{|c|c|c|c|c|c|c|c|c|c|c|c|c|c|c|c|c|c|c|}
\hline 19 & 679 & 0,066 & 1,839 & 0,102 & 0,972 & 0,648 & 0,529 & 0,588 & 0,245 & 2,019 & 0,451 & 0,651 & 0,432 & 0,281 & 0,169 & 49 & 679 & $-2,1$ \\
\hline 20 & 679 & 0,047 & 1,082 & 0,069 & 0,786 & 0,688 & 0,727 & 0,415 & 0,508 & 1,403 & 0,396 & 0,400 & 0,390 & 0,156 & 0,152 & 20 & 679 & $\begin{array}{l}-2,1 \\
-2,0\end{array}$ \\
\hline 21 & 679 & 0,056 & 1,247 & 0,083 & 0,909 & 0,672 & 0,729 & 0,425 & 0,452 & 1,487 & 0,382 & 0,501 & 0,432 & 0,216 & 0,152 & 151 & 679 & $-2,1$ \\
\hline 22 & 679 & 0,030 & 0,685 & 0,037 & 0,562 & 0,808 & 0,821 & 0,263 & 0,808 & 1,113 & 0,504 & 0,412 & 0,276 & 0,114 & 0,152 & 7 & 679 & $-1,5$ \\
\hline 23 & 679 & 0,135 & 1,970 & 0,187 & $\begin{array}{l}1,581 \\
\end{array}$ & 0,723 & 0,803 & 0,340 & 0,437 & 1,513 & 0,354 & 0,387 & 0,696 & 0,269 & 0,227 & 17 & 679 & $-2,8$ \\
\hline 24 & 679 & 0,027 & 0,622 & 0,034 & 0,490 & 0,792 & 0,789 & 0,297 & 0,887 & 1,062 & 0,930 & 0,817 & 0,193 & 0,158 & 0,107 & 4 & 679 & $-0,9$ \\
\hline 25 & 679 & 0,030 & 0,592 & 0,032 & 0,541 & 0,955 & 0,914 & 0,097 & 1,000 & 0,962 & 0,594 & 0,401 & 0,254 & 0,102 & 0,152 & 32 & 679 & $-1,3$ \\
\hline 26 & 679 & 0,191 & 3,525 & 0,314 & 2,245 & 0,607 & 0,637 & 0,535 & 0,193 & 2,276 & 0,235 & 0,380 & 1,016 & 0,386 & 0,152 & 31 & 679 & $-3,3$ \\
\hline 27 & 679 & 0,416 & 2,401 & 0,443 & 2,320 & 0,939 & 0,966 & 0,070 & 0,907 & 1,050 & 0,674 & 0,598 & 0,887 & 0,530 & 0,552 & 88 & 679 & $-3,1$ \\
\hline 28 & 679 & 0,036 & 0,613 & 0,037 & 0,490 & 0,962 & 0,800 & 0,204 & 1,000 & 0,913 & 0,936 & 0,728 & 0,221 & 0,161 & 0,152 & 128 & 679 & $-1,1$ \\
\hline \multirow[t]{2}{*}{1} & 680 & 0,014 & 0,347 & 0,016 & 0,266 & 0,909 & 0,768 & 0,249 & 1,000 & 0,816 & 0,979 & 0,588 & 0,137 & 0,080 & 0,107 & 53 & 680 & $-0,5$ \\
\hline & 681 & & & & & & & & nothing to measure & & & & & & & & 681 & \\
\hline \multirow[t]{2}{*}{1} & 682 & 0,027 & 0,592 & 0,029 & 0,520 & 0,950 & 0,879 & 0,131 & $\begin{array}{l}0,978 \\
\text { nothing to measure }\end{array}$ & 1,011 & 0,465 & 0,372 & 0,273 & 0,102 & 0,152 & 62 & 682 & $-1,4$ \\
\hline & $\begin{array}{l}683 \\
684\end{array}$ & 0039 & 0968 & 0050 & 0,634 & 0.771 & 0,655 & 0,414 & $\begin{array}{l}\text { nothing to measure } \\
0.519\end{array}$ & 1388 & 0.859 & 0849 & 0240 & 0203 & 0152 & 97 & $\begin{array}{l}683 \\
684\end{array}$ & -13 \\
\hline \multirow[t]{5}{*}{2} & 684 & 0,014 & 0,389 & 0,016 & 0,123 & 0,909 & 0,315 & 0,691 & 1,000 & 0,916 & 0,637 & 0,380 & 0,169 & 0,064 & 0,076 & 162 & 684 & $-0,8$ \\
\hline & 685 & & & & & & & & nothing to measure & & & & & & & & 685 & - \\
\hline & 686 & & & & & & & & nothing to measure & & & & & & & & 686 & - \\
\hline & 687 & & & & & & & & nothing to measure & & & & & & & & 687 & - \\
\hline & 688 & & & & & & & & nothing to measure & & & & & & & & 688 & - \\
\hline 1 & 689 & 0,014 & 0,347 & 0,014 & 0,245 & 1,000 & 0,707 & 0,293 & 1,000 & 0,816 & 0,979 & 0,588 & 0,137 & 0,080 & 0,107 & 135 & 689 & $-0,5$ \\
\hline 2 & 689 & 0,016 & 0,338 & 0,016 & 0,194 & 1,000 & 0,575 & 0,425 & 1,000 & 0,759 & 1,077 & 0,555 & 0,137 & 0,076 & 0,152 & 162 & 689 & $-0,5$ \\
\hline 3 & 689 & 0,029 & 0,562 & 0,032 & 0,418 & 0,909 & 0,744 & 0,271 & 1,000 & 0,936 & 0,688 & 0,493 & 0,230 & 0,114 & 0,152 & 0 & 689 & $-1,2$ \\
\hline 4 & 689 & 0,023 & 0,469 & 0,024 & 0,368 & 0,941 & 0,784 & 0,224 & 1,000 & 0,874 & 1,019 & 0,778 & 0,169 & 0,132 & 0,152 & 11 & 689 & $-0,8$ \\
\hline 5 & 689 & 0,026 & 0,735 & 0,034 & 0,592 & 0,750 & 0,805 & 0,317 & 0,600 & 1,291 & 0,286 & 0,236 & 0,339 & 0,080 & 0,076 & 157 & 689 & $-1,8$ \\
\hline 6 & 689 & 0,020 & 0,469 & 0,022 & 0,317 & 0,933 & 0,675 & 0,331 & 1,000 & 0,934 & 0,990 & 0,707 & 0,161 & 0,114 & 0,107 & 162 & 689 & $-0,7$ \\
\hline 7 & 689 & 0,040 & 0,664 & 0,044 & 0,562 & 0,903 & 0,847 & 0,181 & 1,000 & 0,934 & 0,686 & 0,578 & 0,273 & 0,158 & 0,169 & 115 & 689 & $-1,4$ \\
\hline 8 & 689 & 0,022 & 0,418 & 0,022 & 0,296 & 1,000 & 0,707 & 0,293 & 1,000 & 0,805 & 1,123 & 0,728 & 0,156 & 0,114 & 0,152 & 110 & 689 & $-0,6$ \\
\hline 9 & 689 & 0,023 & 0,469 & 0,024 & 0,418 & 0,941 & 0,892 & 0,123 & 1,000 & 0,874 & 1,019 & 0,791 & 0,169 & 0,134 & 0,152 & 56 & 689 & $-0,8$ \\
\hline \multirow[t]{6}{*}{10} & 689 & 0,024 & 0,664 & 0,030 & 0,541 & 0,810 & 0,815 & 0,265 & 0,696 & 1,199 & 0,297 & 0,262 & 0,324 & 0,085 & 0,076 & 25 & 689 & $-1,7$ \\
\hline & 690 & & & & & & & & nothing to measure & & & & & & & & 690 & - \\
\hline & 691 & & & & & & & & nothing to measure & & & & & & & & 691 & - \\
\hline & 692 & & & & & & & & nothing to measure & & & & & & & & 692 & - \\
\hline & 693 & & & & & & & & nothing to measure & & & & & & & & 693 & - \\
\hline & 694 & & & & & & & & nothing to measure & & & & & & & & 694 & $\ldots$ \\
\hline 1 & 695 & 0,040 & 0,634 & 0,043 & 0,511 & 0,933 & 0,807 & 0,205 & 1,000 & 0,892 & 0,964 & 0,658 & 0,230 & 0,152 & 0,214 & 6 & 695 & $-1,2$ \\
\hline \multirow[t]{3}{*}{2} & 695 & 0,030 & 0,541 & 0,030 & 0,440 & 1,000 & 0,812 & 0,188 & 1,000 & 0,879 & 0,922 & 0,743 & 0,204 & 0,152 & 0,169 & 111 & 695 & $-1,0$ \\
\hline & 696 & & & & & & & & nothing to measure & & & & & & & & 696 & - \\
\hline & 697 & & & & & & & & nothing to measure & & & & & & & & 697 & . \\
\hline 1 & 698 & 0,105 & 1,818 & 0,142 & 1,501 & 0,737 & 0,826 & 0,315 & 0,398 & 1,584 & 0,258 & 0,285 & 0,719 & 0,205 & 0,152 & 158 & 698 & $-2,8$ \\
\hline 2 & 698 & 0,043 & 1,255 & 0,062 & 0,714 & 0,698 & 0,569 & 0,526 & 0,343 & 1,707 & 0,516 & 0,616 & 0,326 & 0,201 & 0,152 & 137 & 698 & $-1,7$ \\
\hline
\end{tabular}


Clasts' data

\begin{tabular}{|c|c|c|c|c|c|c|c|c|c|c|c|c|c|c|c|c|c|c|}
\hline Label & Area & Perim. & CH Area & CH Perim. & $\begin{array}{l}\text { CH Perim. } \\
\text { Correction }\end{array}$ & Solidity & Convexity & $\begin{array}{l}\text { Convexity } \\
\text { Correction }\end{array}$ & $\begin{array}{l}\text { Concavity } \\
\text { Index }\end{array}$ & FormFactor & Circularity & Roundness & AR Feret & Feret d & MinFeret d & $\begin{array}{l}\text { Max. } \\
\text { Insc. } \\
\text { Circle } \\
\text { Diam. }\end{array}$ & Orientatior & $\begin{array}{l}\text { ROI } \\
\text { Index }\end{array}$ \\
\hline 0 & 5,2 & 8,6 & 5,4 & 8,35 & 8,08 & 0,961 & 0,975 & 0,944 & 0,047 & 0,890 & 1,060 & 0,638 & 0,695 & 3,218 & 2,236 & 2,214 & 135,932 & 0 \\
\hline 1 & 1,3 & 4,2 & 1,4 & 4,17 & 4,04 & 0,977 & 0,997 & 0,965 & 0,023 & 0,963 & 1,019 & 0,706 & 0,728 & 1,554 & 1,132 & 1,107 & 108,440 & 1 \\
\hline 2 & 2,5 & 7,9 & 3,0 & 7,04 & 6,81 & 0,824 & 0,887 & 0,859 & 0,209 & 0,493 & 1,424 & 0,359 & 0,444 & 2,959 & 1,314 & 1,034 & 152,231 & 2 \\
\hline 3 & 118,5 & 61,9 & 132,4 & 43,81 & 42,41 & 0,895 & 0,707 & 0,685 & 0,311 & 0,388 & 1,605 & 0,599 & 0,697 & 15,869 & 11,060 & 4,450 & 112,724 & 3 \\
\hline 4 & 11,2 & 12,9 & 11,7 & 12,70 & 12,30 & 0,961 & 0,983 & 0,952 & 0,042 & 0,846 & 1,087 & 0,593 & 0,680 & 4,910 & 3,337 & 3,257 & 177,843 & 4 \\
\hline 5 & 0,9 & 3,4 & 1,0 & 3,30 & 3,19 & 0,965 & 0,972 & 0,941 & 0,045 & 1,000 & 0,990 & 0,828 & 0,785 & 1,198 & 0,941 & 0,975 & 79,149 & 5 \\
\hline 6 & 0,4 & 2,1 & 0,4 & 1,98 & 1,91 & 0,948 & 0,950 & 0,919 & 0,072 & 1,000 & 0,982 & 0,759 & 0,714 & 0,775 & 0,554 & 0,577 & 164,765 & 6 \\
\hline 7 & 0,3 & 1,9 & 0,3 & 1,84 & 1,78 & 0,949 & 0,946 & 0,916 & 0,074 & 0,912 & 1,047 & 0,570 & 0,598 & 0,783 & 0,468 & 0,495 & 111,211 & 7 \\
\hline 8 & 2,0 & 5,5 & 2,1 & 5,36 & 5,19 & 0,937 & 0,971 & 0,940 & 0,069 & 0,831 & 1,097 & 0,644 & 0,732 & 1,996 & 1,460 & 1,291 & 39,227 & 8 \\
\hline 9 & 0,3 & 1,7 & 0,3 & 1,67 & 1,61 & 0,955 & 0,962 & 0,931 & 0,059 & 1,000 & 0,965 & 0,791 & 0,765 & 0,644 & 0,492 & 0,505 & 76,807 & 9 \\
\hline 10 & 0,1 & 1,2 & 0,1 & 0,97 & 0,94 & 0,872 & 0,800 & 0,774 & 0,237 & 0,709 & 1,188 & 0,370 & 0,392 & 0,535 & 0,210 & 0,198 & 114,122 & 10 \\
\hline 11 & 5,4 & 9,3 & 5,8 & 9,16 & 8,86 & 0,935 & 0,980 & 0,949 & 0,068 & 0,779 & 1,133 & 0,498 & 0,570 & 3,720 & 2,120 & 2,082 & 35,305 & 11 \\
\hline 12 & 4,3 & 8,4 & 4,4 & 8,33 & 8,06 & 0,965 & 0,989 & 0,957 & 0,037 & 0,755 & $\begin{array}{l}1,151 \\
\text { n }\end{array}$ & 0,416 & 0,422 & 3,611 & 1,525 & 1,508 & 122,631 & 12 \\
\hline 13 & 0,6 & 3,0 & 0,7 & 2,82 & 2,73 & 0,953 & 0,946 & 0,916 & 0,072 & 0,910 & 1,048 & 0,617 & 0,626 & 1,151 & 0,720 & 0,721 & 165,393 & 13 \\
\hline 14 & 2,7 & 7,3 & 2,9 & 7,03 & 6,81 & 0,913 & 0,967 & 0,936 & 0,093 & 0,635 & 1,255 & 0,379 & 0,494 & 2,997 & 1,479 & 1,328 & 88,475 & 14 \\
\hline 15 & 0,2 & 1,5 & 0,2 & 1,36 & 1,32 & 0,974 & 0,935 & 0,905 & 0,070 & 1,000 & 0,963 & 0,906 & 0,832 & 0,505 & 0,420 & 0,420 & 10,037 & 15 \\
\hline 16 & 0,2 & 1,6 & 0,2 & 1,50 & 1,45 & 0,936 & 0,941 & 0,911 & 0,087 & 0,888 & 1,061 & 0,502 & 0,441 & 0,673 & 0,297 & 0,357 & 174,396 & 16 \\
\hline 17 & 0,6 & 3,1 & 0,6 & 3,01 & 2,91 & 0,957 & 0,970 & 0,939 & 0,053 & 0,775 & 1,136 & 0,422 & 0,364 & 1,338 & 0,487 & 0,495 & 131,321 & 17 \\
\hline 18 & 0,6 & 3,0 & 0,6 & 2,95 & 2,85 & 0,951 & 0,978 & 0,947 & 0,054 & 0,852 & 1,083 & 0,539 & 0,501 & 1,206 & 0,604 & 0,626 & 119,559 & 18 \\
\hline 19 & 1,1 & 4,2 & 1,1 & 4,06 & 3,93 & 0,964 & 0,968 & 0,937 & 0,048 & 0,792 & 1,124 & 0,415 & 0,444 & 1,842 & 0,819 & 0,840 & 120,605 & 19 \\
\hline 20 & 1,4 & 4,5 & 1,5 & 4,48 & 4,33 & 0,969 & 0,997 & 0,966 & 0,031 & 0,899 & 1,054 & 0,576 & 0,505 & 1,785 & 0,901 & 0,913 & 137,286 & 20 \\
\hline 21 & 0,5 & 2,5 & 0,5 & 2,27 & 2,20 & 0,969 & 0,924 & 0,894 & 0,082 & 0,953 & 1,024 & 0,560 & 0,617 & 1,021 & 0,630 & 0,664 & 141,903 & 21 \\
\hline 22 & 0,2 & 1,4 & 0,2 & 1,28 & 1,24 & 0,940 & 0,924 & 0,895 & 0,097 & 1,000 & 0,996 & 0,586 & 0,567 & 0,579 & 0,329 & 0,357 & 70,246 & 22 \\
\hline 23 & 0,2 & 1,6 & 0,2 & 1,55 & 1,50 & 0,907 & 0,959 & 0,928 & 0,102 & 0,923 & 1,041 & 0,584 & 0,554 & 0,645 & 0,357 & 0,357 & 10,620 & 23 \\
\hline 24 & 0,2 & 1,4 & 0,2 & 1,28 & 1,24 & 0,939 & 0,913 & 0,885 & 0,106 & 0,970 & 1,015 & 0,724 & 0,670 & 0,517 & 0,347 & 0,357 & 168,743 & 24 \\
\hline 25 & 0,1 & 1,3 & 0,1 & 1,16 & 1,12 & 0,925 & 0,897 & 0,868 & 0,127 & 0,903 & 1,052 & 0,480 & 0,414 & 0,564 & 0,234 & 0,280 & 140,420 & 25 \\
\hline 26 & 1,3 & 4,4 & 1,4 & 4,28 & 4,14 & 0,894 & 0,972 & 0,941 & 0,110 & 0,830 & 1,098 & 0,717 & 0,721 & 1,506 & 1,086 & 0,975 & 33,419 & 26 \\
\hline 27 & 0,3 & 2,2 & 0,3 & 1,98 & 1,92 & 0,826 & 0,896 & 0,867 & 0,203 & 0,715 & 1,182 & 0,429 & 0,538 & 0,910 & 0,490 & 0,396 & 145,062 & 27 \\
\hline 28 & 0,3 & 2,1 & 0,3 & 1,86 & 1,80 & 0,891 & 0,873 & 0,845 & 0,167 & 0,783 & 1,130 & 0,433 & 0,411 & 0,910 & 0,374 & 0,396 & 80,992 & 28 \\
\hline 29 & 0,7 & 2,9 & 0,7 & 2,85 & 2,75 & 0,971 & 0,996 & 0,964 & 0,029 & 1,000 & 0,990 & 0,681 & 0,667 & 1,112 & 0,743 & 0,754 & 161,152 & 29 \\
\hline 30 & 2,1 & 6,3 & 2,3 & 6,23 & 6,03 & 0,934 & 0,989 & 0,958 & 0,067 & 0,680 & 1,213 & 0,386 & 0,416 & 2,659 & 1,107 & 1,066 & 152,176 & 30 \\
\hline 31 & 1,4 & 4,9 & 1,5 & 4,63 & 4,48 & 0,917 & 0,946 & 0,916 & 0,099 & 0,741 & 1,162 & 0,529 & 0,530 & 1,842 & 0,975 & 0,840 & 126,852 & 31 \\
\hline 32 & 0,2 & 1,4 & 0,2 & 1,19 & 1,15 & 0,985 & 0,871 & 0,843 & 0,130 & 1,000 & 0,950 & 0,870 & 0,808 & 0,490 & 0,396 & 0,396 & 53,044 & 32 \\
\hline 33 & 0,5 & 2,7 & 0,5 & 2,51 & 2,43 & 0,965 & 0,940 & 0,910 & 0,069 & 0,827 & 1,099 & 0,433 & 0,379 & 1,177 & 0,446 & 0,495 & 96,536 & 33 \\
\hline 34 & 4,1 & 9,3 & 5,1 & 8,77 & 8,49 & 0,808 & 0,945 & 0,915 & 0,200 & 0,599 & 1,292 & 0,411 & 0,510 & 3,565 & 1,819 & 1,328 & 137,215 & 34 \\
\hline 35 & 0,5 & 2,5 & 0,5 & 2,29 & 2,22 & 0,933 & 0,914 & 0,885 & 0,109 & 0,950 & 1,026 & 0,714 & 0,746 & 0,921 & 0,687 & 0,664 & 129,716 & 35 \\
\hline 36 & 0,1 & 1,3 & 0,1 & 1,19 & 1,15 & 0,931 & 0,900 & 0,871 & 0,122 & 0,954 & 1,024 & 0,506 & 0,460 & 0,577 & 0,266 & 0,280 & 153,999 & 36 \\
\hline 37 & 0,1 & 0,9 & 0,1 & 0,68 & 0,66 & 0,972 & 0,737 & 0,714 & 0,265 & 1,000 & 0,888 & 0,990 & 0,745 & 0,332 & 0,248 & 0,297 & 170,042 & 37 \\
\hline 38 & 0,2 & 1,4 & 0,2 & 1,32 & 1,28 & 0,986 & 0,916 & 0,886 & 0,085 & 1,000 & 0,982 & 0,543 & 0,497 & 0,634 & 0,315 & 0,357 & 145,578 & 38 \\
\hline 39 & 0,1 & 1,2 & 0,1 & 1,09 & 1,06 & 0,982 & 0,921 & 0,891 & 0,081 & 1,000 & 0,913 & 0,946 & 0,814 & 0,426 & 0,347 & 0,396 & 106,084 & 39 \\
\hline 40 & 0,1 & 1,3 & 0,1 & 1,19 & 1,15 & 0,966 & 0,947 & 0,917 & 0,063 & 1,000 & 0,955 & 0,713 & 0,636 & 0,495 & 0,315 & 0,357 & 143,868 & 40 \\
\hline 41 & 0,6 & 3,0 & 0,7 & 2,89 & 2,80 & 0,929 & 0,960 & 0,929 & 0,082 & 0,839 & 1,092 & 0,544 & 0,540 & 1,190 & 0,642 & 0,664 & 152,964 & 41 \\
\hline 42 & 0,2 & 1,7 & 0,2 & 1,64 & 1,59 & 0,966 & 0,984 & 0,952 & 0,038 & 0,941 & 1,031 & 0,585 & 0,588 & 0,673 & 0,396 & 0,396 & 170,753 & 42 \\
\hline 43 & 0,4 & 2,4 & 0,4 & 2,17 & 2,10 & 0,911 & 0,920 & 0,891 & 0,119 & 0,797 & 1,120 & 0,465 & 0,427 & 0,983 & 0,420 & 0,420 & 136,986 & 43 \\
\hline 44 & 1,4 & 4,5 & 1,5 & 4,46 & 4,32 & 0,975 & 0,985 & 0,954 & 0,029 & 0,887 & 1,062 & 0,569 & 0,510 & 1,800 & 0,918 & 0,934 & 121,460 & 44 \\
\hline 45 & 0,2 & 1,3 & 0,2 & 1,23 & 1,19 & 0,969 & 0,929 & 0,900 & 0,078 & 1,000 & 0,956 & 0,789 & 0,707 & 0,495 & 0,350 & 0,357 & 137,674 & 45 \\
\hline 46 & 1,0 & 4,0 & 1,1 & 3,69 & 3,57 & 0,908 & 0,923 & 0,893 & 0,120 & 0,783 & 1,130 & 0,610 & 0,655 & 1,443 & 0,945 & 0,934 & 41,215 & 46 \\
\hline 47 & 1,8 & 6,5 & 2,1 & 6,27 & 6,07 & 0,848 & 0,961 & 0,930 & 0,156 & 0,526 & 1,378 & 0,277 & 0,303 & 2,861 & 0,866 & 0,913 & 145,241 & 47 \\
\hline & 96 & 129 & & & & 0869 & & & & & & & & & & & & \\
\hline
\end{tabular}




\begin{tabular}{|c|c|c|c|c|c|c|c|c|c|c|c|c|c|c|c|c|c|c|}
\hline 49 & 0,2 & 1,7 & 0,2 & 1,65 & 1,60 & 0,977 & 0,955 & 0,924 & 0,050 & 0,875 & 1,069 & 0,520 & 0,441 & 0,714 & 0,315 & 0,357 & 128,140 & 49 \\
\hline 50 & 4,4 & 8,1 & 4,7 & 8,06 & 7,80 & 0,944 & 0,992 & 0,960 & 0,057 & 0,846 & 1,088 & 0,651 & 0,662 & 2,948 & 1,952 & 1,782 & 104,257 & 50 \\
\hline 51 & 2,5 & 5,9 & 2,6 & 5,85 & 5,66 & 0,961 & 0,989 & 0,957 & 0,041 & 0,886 & 1,063 & 0,680 & 0,742 & 2,149 & 1,594 & 1,485 & 77,051 & 51 \\
\hline 52 & 0,1 & 1,2 & 0,1 & 1,00 & 0,97 & 0,961 & 0,862 & 0,834 & 0,144 & 1,000 & 0,944 & 0,701 & 0,600 & 0,467 & 0,280 & 0,313 & 138,375 & 52 \\
\hline 53 & 0,9 & 3,6 & 1,0 & 3,55 & 3,43 & 0,948 & 0,982 & 0,950 & 0,056 & 0,894 & 1,058 & 0,640 & 0,586 & 1,359 & 0,797 & 0,798 & 157,418 & 53 \\
\hline 54 & 0,9 & 3,4 & 1,0 & 3,35 & 3,24 & 0,938 & 0,977 & 0,946 & 0,066 & 0,961 & 1,020 & 0,819 & 0,806 & 1,181 & 0,952 & 0,891 & 152,977 & 54 \\
\hline 55 & 0,1 & 1,5 & 0,2 & 1,32 & 1,28 & 0,896 & 0,892 & 0,863 & 0,150 & 0,843 & 1,089 & 0,466 & 0,484 & 0,634 & 0,307 & 0,313 & 131,561 & 55 \\
\hline 56 & 0,1 & 0,9 & 0,1 & 0,84 & 0,81 & 0,970 & 0,927 & 0,897 & 0,079 & 1,000 & 0,913 & 1,019 & 0,894 & 0,313 & 0,280 & 0,280 & 110,918 & 56 \\
\hline 57 & 0,1 & 1,2 & 0,1 & 1,15 & 1,11 & 0,944 & 0,945 & 0,915 & 0,078 & 1,000 & 0,970 & 0,669 & 0,567 & 0,488 & 0,276 & 0,297 & 165,747 & 57 \\
\hline 58 & 0,3 & 1,7 & 0,3 & 1,68 & 1,63 & 0,955 & 0,977 & 0,947 & 0,050 & 1,000 & 0,947 & 0,921 & 0,814 & 0,602 & 0,490 & 0,533 & 60,355 & 58 \\
\hline 59 & 0,2 & 1,6 & 0,2 & 1,47 & 1,42 & 0,910 & 0,902 & 0,872 & 0,133 & 0,823 & 1,102 & 0,461 & 0,429 & 0,693 & 0,297 & 0,313 & 101,814 & 59 \\
\hline 60 & 0,2 & 1,3 & 0,2 & 1,22 & 1,18 & 0,954 & 0,909 & 0,880 & 0,102 & 1,000 & 0,967 & 0,699 & 0,673 & 0,526 & 0,354 & 0,396 & 50,919 & 60 \\
\hline 61 & 0,1 & 1,4 & 0,1 & 1,29 & 1,25 & 0,946 & 0,943 & 0,914 & 0,078 & 0,869 & 1,073 & 0,453 & 0,348 & 0,604 & 0,210 & 0,280 & 130,674 & 61 \\
\hline 62 & 1,1 & 3,7 & 1,1 & 3,65 & 3,53 & 0,968 & 0,979 & 0,948 & 0,038 & 0,955 & 1,023 & 0,632 & 0,578 & 1,457 & 0,842 & 0,891 & 90,001 & 62 \\
\hline 63 & 0,1 & 0,9 & 0,1 & 0,76 & 0,74 & 0,935 & 0,879 & 0,851 & 0,137 & 1,000 & 0,918 & 0,738 & 0,566 & 0,350 & 0,198 & 0,280 & 16,686 & 63 \\
\hline 64 & 3,0 & 6,4 & 3,1 & 6,32 & 6,12 & 0,959 & 0,990 & 0,958 & 0,043 & 0,912 & 1,047 & 0,674 & 0,628 & 2,366 & 1,485 & 1,472 & 89,267 & 64 \\
\hline 65 & 0,4 & 2,4 & 0,5 & 2,20 & 2,13 & 0,946 & 0,911 & 0,882 & 0,104 & 0,921 & 1,042 & 0,557 & 0,601 & 0,990 & 0,595 & 0,594 & 62,557 & 65 \\
\hline 66 & 0,2 & 1,5 & 0,2 & 1,31 & 1,27 & 0,971 & 0,868 & 0,840 & 0,135 & 0,921 & 1,042 & 0,693 & 0,626 & 0,553 & 0,347 & 0,357 & 83,116 & 66 \\
\hline 67 & 0,1 & 0,8 & 0,1 & 0,64 & 0,62 & 1,000 & 0,800 & 0,775 & 0,200 & 1,000 & 0,913 & 0,776 & 0,629 & 0,317 & 0,199 & 0,221 & 120,964 & 67 \\
\hline 68 & 4,7 & 9,1 & 5,6 & 9,01 & 8,72 & 0,849 & 0,990 & 0,958 & 0,152 & 0,717 & 1,181 & 0,527 & 0,642 & 3,382 & 2,172 & 1,729 & 152,644 & 68 \\
\hline 69 & 0,4 & 2,3 & 0,5 & 2,23 & 2,16 & 0,962 & 0,955 & 0,924 & 0,059 & 1,000 & 0,993 & 0,745 & 0,682 & 0,866 & 0,591 & 0,626 & 35,798 & 69 \\
\hline 70 & 0,1 & 0,9 & 0,1 & 0,84 & 0,81 & 1,000 & 0,915 & 0,887 & 0,085 & 1,000 & 0,988 & 0,548 & 0,351 & 0,399 & 0,140 & 0,221 & 46,506 & 70 \\
\hline 71 & 0,5 & 2,7 & 0,5 & 2,52 & 2,44 & 0,923 & 0,921 & 0,892 & 0,110 & 0,787 & 1,128 & 0,550 & 0,594 & 1,041 & 0,619 & 0,533 & 27,752 & 71 \\
\hline 72 & 1,5 & 4,6 & 1,6 & 4,59 & 4,44 & 0,952 & 0,992 & 0,960 & 0,049 & 0,904 & 1,052 & 0,595 & 0,601 & 1,816 & 1,091 & 1,089 & 83,811 & 72 \\
\hline 73 & 0,1 & 1,3 & 0,1 & 1,14 & 1,11 & 0,936 & 0,877 & 0,849 & 0,138 & 0,797 & 1,120 & 0,386 & 0,294 & 0,596 & 0,175 & 0,221 & 128,030 & 73 \\
\hline 74 & 0,1 & 0,8 & 0,1 & 0,67 & 0,65 & 0,929 & 0,834 & 0,807 & 0,180 & 1,000 & 0,895 & 0,974 & 0,767 & 0,289 & 0,222 & 0,221 & 72,704 & 74 \\
\hline 75 & 0,6 & 2,9 & 0,6 & 2,79 & 2,70 & 0,923 & 0,964 & 0,933 & 0,085 & 0,834 & 1,095 & 0,487 & 0,547 & 1,206 & 0,659 & 0,693 & 77,931 & 75 \\
\hline 76 & 0,1 & 1,3 & 0,1 & 1,15 & 1,11 & 0,920 & 0,896 & 0,868 & 0,131 & 0,863 & 1,077 & 0,428 & 0,369 & 0,579 & 0,214 & 0,280 & 73,128 & 76 \\
\hline 77 & 0,1 & 0,8 & 0,1 & 0,64 & 0,62 & 0,808 & 0,828 & 0,803 & 0,258 & 1,000 & 0,962 & 1,028 & 0,904 & 0,252 & 0,228 & 0,198 & 165,510 & 77 \\
\hline 78 & 0,4 & 2,3 & 0,4 & 2,23 & 2,16 & 0,937 & 0,971 & 0,940 & 0,069 & 0,957 & 1,022 & 0,614 & 0,604 & 0,913 & 0,551 & 0,577 & 119,132 & 78 \\
\hline 79 & 0,1 & 0,9 & 0,1 & 0,67 & 0,65 & 0,889 & 0,771 & 0,746 & 0,255 & 0,983 & 1,009 & 0,611 & 0,566 & 0,350 & 0,198 & 0,198 & 126,853 & 79 \\
\hline 80 & 22,5 & 20,4 & 25,4 & 18,96 & 18,35 & 0,888 & 0,927 & 0,898 & 0,133 & 0,678 & 1,215 & 0,508 & 0,613 & 7,516 & 4,610 & 4,021 & 108,290 & 80 \\
\hline 81 & 3,0 & 6,3 & 3,1 & 6,17 & 5,97 & 0,969 & 0,985 & 0,954 & 0,035 & 0,973 & 1,014 & 0,772 & 0,815 & 2,237 & 1,822 & 1,826 & 177,597 & 81 \\
\hline 82 & 0,3 & 2,0 & 0,3 & 1,95 & 1,89 & 0,978 & 0,980 & 0,949 & 0,029 & 1,000 & 0,979 & 0,732 & 0,741 & 0,756 & 0,560 & 0,560 & 135,647 & 82 \\
\hline 83 & 1,8 & 5,8 & 2,0 & 5,52 & 5,35 & 0,924 & 0,958 & 0,927 & 0,087 & 0,694 & 1,201 & 0,342 & 0,418 & 2,614 & 1,092 & 1,089 & 69,067 & 83 \\
\hline 84 & 0,4 & 2,1 & 0,4 & 1,98 & 1,91 & 0,961 & 0,962 & 0,931 & 0,055 & 1,000 & 0,969 & 0,759 & 0,703 & 0,775 & 0,545 & 0,560 & 109,687 & 84 \\
\hline 85 & 0,7 & 3,5 & 0,8 & 3,47 & 3,36 & 0,940 & 0,981 & 0,950 & 0,063 & 0,734 & 1,167 & 0,388 & 0,436 & 1,547 & 0,674 & 0,664 & 174,317 & 85 \\
\hline 86 & 0,9 & 3,5 & 0,9 & 3,43 & 3,32 & 0,946 & 0,978 & 0,946 & 0,058 & 0,881 & 1,065 & 0,618 & 0,559 & 1,333 & 0,745 & 0,792 & 109,471 & 86 \\
\hline 87 & 0,3 & 2,4 & 0,3 & 2,27 & 2,20 & 0,848 & 0,956 & 0,925 & 0,158 & 0,611 & 1,279 & 0,327 & 0,314 & 1,034 & 0,325 & 0,357 & 17,373 & 87 \\
\hline 88 & 0,3 & 2,4 & 0,3 & 2,29 & 2,22 & 0,950 & 0,956 & 0,926 & 0,066 & 0,718 & 1,180 & 0,386 & 0,426 & 1,041 & 0,443 & 0,495 & 29,336 & 88 \\
\hline 89 & 0,2 & 1,9 & 0,2 & 1,80 & 1,74 & 0,888 & 0,931 & 0,901 & 0,132 & 0,717 & 1,181 & 0,404 & 0,449 & 0,819 & 0,368 & 0,313 & 23,658 & 89 \\
\hline 90 & 0,2 & 1,6 & 0,2 & 1,53 & 1,48 & 0,955 & 0,975 & 0,944 & 0,052 & 0,788 & 1,127 & 0,386 & 0,294 & 0,714 & 0,210 & 0,280 & 133,736 & 90 \\
\hline 91 & 0,1 & 0,7 & 0,1 & 0,48 & 0,47 & 0,957 & 0,691 & 0,669 & 0,312 & 1,000 & 0,845 & 0,875 & 0,707 & 0,280 & 0,198 & 0,221 & 144,485 & 91 \\
\hline 92 & 7,4 & 14,1 & 9,6 & 11,96 & 11,58 & 0,778 & 0,849 & 0,822 & 0,268 & 0,471 & 1,457 & 0,377 & 0,481 & 5,011 & 2,409 & 2,297 & 138,825 & 92 \\
\hline 93 & 0,2 & 1,8 & 0,3 & 1,76 & 1,71 & 0,943 & 0,978 & 0,947 & 0,061 & 0,949 & 1,026 & 0,556 & 0,529 & 0,749 & 0,396 & 0,396 & 8,637 & 93 \\
\hline 94 & 2,2 & 5,8 & 2,3 & 5,59 & 5,41 & 0,932 & 0,960 & 0,930 & 0,079 & 0,808 & 1,112 & 0,515 & 0,542 & 2,320 & 1,257 & 1,107 & 42,573 & 94 \\
\hline 95 & 3,2 & 7,6 & 3,8 & 7,39 & 7,15 & 0,841 & 0,970 & 0,939 & 0,161 & 0,693 & 1,201 & 0,456 & 0,548 & 2,990 & 1,639 & 1,332 & 144,745 & 95 \\
\hline 96 & 2,2 & 6,9 & 2,5 & 6,71 & 6,50 & 0,862 & 0,978 & 0,947 & 0,139 & 0,586 & 1,307 & 0,376 & 0,552 & 2,727 & 1,506 & 1,205 & 159,969 & 96 \\
\hline 97 & 0,7 & 3,1 & 0,7 & 3,15 & 3,05 & 0,955 & 1,000 & 0,968 & 0,045 & 0,851 & 1,084 & 0,500 & 0,491 & 1,308 & 0,642 & 0,664 & 153,967 & 97 \\
\hline 98 & 0,7 & 3,1 & 0,7 & 3,01 & 2,91 & 0,947 & 0,987 & 0,956 & 0,055 & 0,884 & 1,064 & 0,531 & 0,587 & 1,252 & 0,735 & 0,700 & 113,453 & 98 \\
\hline 99 & 0,1 & 1,4 & 0,2 & 1,29 & 1,25 & 0,937 & 0,907 & 0,878 & 0,112 & 0,909 & 1,049 & 0,653 & 0,634 & 0,535 & 0,340 & 0,313 & 52,268 & 99 \\
\hline 100 & 0,2 & 1,6 & 0,2 & 1,44 & 1,40 & 0,943 & 0,916 & 0,887 & 0,102 & 1,000 & 0,985 & 0,644 & 0,578 & 0,634 & 0,367 & 0,408 & 44,878 & 100 \\
\hline 101 & 0,4 & 2,5 & 0,5 & 2,34 & 2,26 & 0,929 & 0,940 & 0,910 & 0,093 & 0,907 & 1,050 & 0,635 & 0,665 & 0,946 & 0,628 & 0,577 & 58,262 & 101 \\
\hline 102 & 1,0 & 4,4 & 1,2 & 4,20 & 4,06 & 0,841 & 0,952 & 0,922 & 0,166 & 0,653 & 1,238 & 0,443 & 0,519 & 1,703 & 0,885 & 0,846 & 140,849 & 102 \\
\hline 103 & 0,1 & 1,2 & 0,1 & 1,06 & 1,02 & 0,922 & 0,888 & 0,860 & 0,136 & 1,000 & 0,987 & 0,704 & 0,639 & 0,456 & 0,292 & 0,297 & 89,359 & 103 \\
\hline 104 & 0,2 & 1,8 & 0,2 & 1,67 & 1,61 & 0,867 & 0,926 & 0,897 & 0,152 & 0,741 & 1,162 & 0,424 & 0,473 & 0,757 & 0,358 & 0,357 & 71,072 & 104 \\
\hline 105 & 0,2 & 2,4 & 0,3 & 2,34 & 2,26 & 0,781 & 0,957 & 0,926 & 0,223 & 0,517 & 1,391 & 0,255 & 0,278 & 1,107 & 0,308 & 0,297 & 168,193 & 105 \\
\hline 106 & 0,1 & 0,8 & 0,1 & 0,71 & 0,68 & 0,964 & 0,914 & 0,885 & 0,093 & 1,000 & 0,848 & 1,185 & 0,914 & 0,267 & 0,244 & 0,280 & 116,565 & 106 \\
\hline
\end{tabular}




\begin{tabular}{|c|c|c|c|c|c|c|c|c|c|c|c|c|c|c|c|c|c|c|c|}
\hline 107 & 0,1 & 1,1 & 0,1 & 1,09 & 1,06 & 1,000 & 0,952 & 0,922 & 0,048 & 1,000 & 0,935 & 0,917 & 0,728 & 0,408 & 0,297 & 0,313 & 80,070 & 107 & $-2,0$ \\
\hline 108 & 0,1 & 1,0 & 0,1 & 0,87 & 0,84 & 0,950 & 0,902 & 0,873 & 0,110 & 1,000 & 0,889 & 0,913 & 0,687 & 0,360 & 0,248 & 0,280 & 0,000 & 108 & $-1,8$ \\
\hline 109 & 0,3 & 2,1 & 0,4 & 2,07 & 2,00 & 0,979 & 1,000 & 0,968 & 0,021 & 1,000 & 0,988 & 0,611 & 0,534 & 0,852 & 0,455 & 0,495 & 134,754 & 109 & $-3,1$ \\
\hline 110 & 0,4 & 2,4 & 0,4 & 2,24 & 2,17 & 0,939 & 0,923 & 0,893 & 0,098 & 0,811 & 1,110 & 0,437 & 0,432 & 1,052 & 0,455 & 0,495 & 45,240 & 110 & $-3,4$ \\
\hline 111 & 0,2 & 1,7 & 0,3 & 1,60 & 1,55 & 0,951 & 0,960 & 0,930 & 0,063 & 1,000 & 0,960 & 0,816 & 0,728 & 0,612 & 0,446 & 0,495 & 1,853 & 111 & $-2,6$ \\
\hline 112 & 0,1 & 0,9 & 0,1 & 0,81 & 0,79 & 1,000 & 0,937 & 0,907 & 0,063 & 1,000 & 0,888 & 0,759 & 0,588 & 0,357 & 0,210 & 0,280 & 128,641 & 112 & $-1,8$ \\
\hline 113 & 0,1 & 1,1 & 0,1 & 0,96 & 0,93 & 0,935 & 0,857 & 0,830 & 0,157 & 1,000 & 0,974 & 0,608 & 0,527 & 0,470 & 0,248 & 0,297 & 160,900 & 113 & $-2,2$ \\
\hline 114 & 0,2 & 1,5 & 0,2 & 1,39 & 1,34 & 0,960 & 0,930 & 0,900 & 0,081 & 0,996 & 1,002 & 0,733 & 0,750 & 0,553 & 0,415 & 0,396 & 71,565 & 114 & $-2,5$ \\
\hline 115 & 0,1 & 1,4 & 0,2 & 1,34 & 1,29 & 0,968 & 0,972 & 0,941 & 0,042 & 0,993 & 1,004 & 0,597 & 0,526 & 0,564 & 0,297 & 0,313 & 166,041 & 115 & $-2,5$ \\
\hline 116 & 0,3 & 2,2 & 0,4 & 2,19 & 2,12 & 0,905 & 0,983 & 0,951 & 0,097 & 0,826 & 1,100 & 0,584 & 0,619 & 0,843 & 0,522 & 0,495 & 152,381 & 116 & $-3,1$ \\
\hline 117 & 0,7 & 3,2 & 0,8 & 3,14 & 3,04 & 0,965 & 0,971 & 0,940 & 0,045 & 0,900 & 1,054 & 0,536 & 0,520 & 1,333 & 0,693 & 0,754 & 106,437 & 117 & $-3,7$ \\
\hline 118 & 0,4 & 2,1 & 0,4 & 1,95 & 1,89 & 0,967 & 0,941 & 0,911 & 0,067 & 1,000 & 0,977 & 0,712 & 0,619 & 0,800 & 0,495 & 0,533 & 7,244 & 118 & $-3,0$ \\
\hline 119 & 0,1 & 0,8 & 0,1 & 0,67 & 0,65 & 1,000 & 0,864 & 0,837 & 0,136 & 1,000 & 0,881 & 0,796 & 0,632 & 0,313 & 0,198 & 0,221 & 77,235 & 119 & $-1,6$ \\
\hline 120 & 0,1 & 1,4 & 0,2 & 1,27 & 1,22 & 0,937 & 0,912 & 0,883 & 0,108 & 0,961 & 1,020 & 0,558 & 0,573 & 0,579 & 0,332 & 0,357 & 161,531 & 120 & $-2,5$ \\
\hline 121 & 0,1 & 1,1 & 0,1 & 0,87 & 0,84 & 1,000 & 0,822 & 0,796 & 0,178 & 1,000 & 0,951 & 0,572 & 0,525 & 0,467 & 0,245 & 0,280 & 62,140 & 121 & $-2,2$ \\
\hline 122 & 0,1 & 1,2 & 0,1 & 1,12 & 1,09 & 0,958 & 0,944 & 0,913 & 0,070 & 1,000 & 0,998 & 0,604 & 0,514 & 0,488 & 0,251 & 0,280 & 67,247 & 122 & $-2,3$ \\
\hline 123 & 4,0 & 7,8 & 4,2 & 7,62 & 7,38 & 0,946 & 0,979 & 0,948 & 0,058 & 0,832 & 1,097 & 0,542 & 0,564 & 3,069 & 1,732 & 1,683 & 1,611 & 123 & $-4,9$ \\
\hline 124 & 0,2 & 1,4 & 0,2 & 1,32 & 1,28 & 0,940 & 0,952 & 0,921 & 0,077 & 1,000 & 0,996 & 0,590 & 0,546 & 0,577 & 0,315 & 0,357 & 141,911 & 124 & $-2,5$ \\
\hline 125 & 0,1 & 0,8 & 0,1 & 0,77 & 0,75 & 0,967 & 0,921 & 0,891 & 0,086 & 1,000 & 0,889 & 0,923 & 0,778 & 0,313 & 0,244 & 0,280 & 177,703 & 125 & $-1,6$ \\
\hline 126 & 0,1 & 0,8 & 0,1 & 0,67 & 0,65 & 1,000 & 0,864 & 0,837 & 0,136 & 1,000 & 0,848 & 0,929 & 0,658 & 0,301 & 0,198 & 0,280 & 87,350 & 126 & $-1,6$ \\
\hline 127 & 0,2 & 1,6 & 0,2 & 1,47 & 1,42 & 0,933 & 0,917 & 0,888 & 0,106 & 0,840 & 1,091 & 0,403 & 0,376 & 0,736 & 0,277 & 0,297 & 103,353 & 127 & $-2,9$ \\
\hline 128 & 0,1 & 1,0 & 0,1 & 0,90 & 0,87 & 0,971 & 0,905 & 0,876 & 0,099 & 1,000 & 0,966 & 0,637 & 0,485 & 0,408 & 0,198 & 0,280 & 10,600 & 128 & $-2,0$ \\
\hline 129 & 1,0 & 3,5 & 1,0 & 3,42 & 3,31 & 0,970 & 0,989 & 0,957 & 0,032 & 1,000 & 0,993 & 0,772 & 0,749 & 1,262 & 0,945 & 0,980 & 108,448 & 129 & $-3,7$ \\
\hline 130 & 0,6 & 2,9 & 0,6 & 2,74 & 2,65 & 0,969 & 0,957 & 0,927 & 0,052 & 0,951 & 1,025 & 0,736 & 0,766 & 1,034 & 0,792 & 0,721 & 179,519 & 130 & $-3,4$ \\
\hline 131 & 0,1 & 1,0 & 0,1 & 0,84 & 0,81 & 0,878 & 0,817 & 0,791 & 0,220 & 1,000 & 0,976 & 0,865 & 0,799 & 0,360 & 0,288 & 0,297 & 76,660 & 131 & $-1,8$ \\
\hline 132 & 0,2 & 1,5 & 0,2 & 1,48 & 1,43 & 0,977 & 0,974 & 0,944 & 0,035 & 1,000 & 0,945 & 0,856 & 0,759 & 0,553 & 0,420 & 0,420 & 44,832 & 132 & $-2,5$ \\
\hline 133 & 0,1 & 0,9 & 0,1 & 0,76 & 0,74 & 0,939 & 0,852 & 0,824 & 0,160 & 1,000 & 0,916 & 0,789 & 0,633 & 0,350 & 0,222 & 0,280 & 142,018 & 133 & $-1,8$ \\
\hline 134 & 0,1 & 1,3 & 0,2 & 1,28 & 1,24 & 0,984 & 0,971 & 0,940 & 0,034 & 1,000 & 0,963 & 0,777 & 0,627 & 0,495 & 0,310 & 0,313 & 150,662 & 134 & $-2,3$ \\
\hline 135 & 0,1 & 1,2 & 0,1 & 1,13 & 1,10 & 0,957 & 0,976 & 0,945 & 0,049 & 1,000 & 0,986 & 0,591 & 0,545 & 0,488 & 0,266 & 0,280 & 159,000 & 135 & $-2,3$ \\
\hline 136 & 2,0 & 6,0 & 2,2 & 5,98 & 5,79 & 0,917 & 0,989 & 0,957 & 0,084 & 0,694 & 1,200 & 0,448 & 0,524 & 2,395 & 1,254 & 1,155 & 131,694 & 136 & $-4,6$ \\
\hline 137 & 2,2 & 8,7 & 2,6 & 7,25 & 7,02 & 0,839 & 0,830 & 0,803 & 0,234 & 0,356 & 1,676 & 0,247 & 0,252 & 3,340 & 0,843 & 0,721 & 92,423 & 137 & $-5,1$ \\
\hline 138 & 0,4 & 2,4 & 0,4 & 2,28 & 2,21 & 0,936 & 0,967 & 0,936 & 0,072 & 0,891 & 1,059 & 0,625 & 0,647 & 0,897 & 0,580 & 0,533 & 88,273 & 138 & $-3,2$ \\
\hline 139 & 0,1 & 1,1 & 0,1 & 1,07 & 1,03 & 0,953 & 0,941 & 0,911 & 0,075 & 0,984 & 1,008 & 0,492 & 0,412 & 0,510 & 0,210 & 0,280 & 33,363 & 139 & $-2,4$ \\
\hline 140 & 0,2 & 1,9 & 0,2 & 1,69 & 1,63 & 0,771 & 0,881 & 0,854 & 0,258 & 0,540 & 1,361 & 0,267 & 0,327 & 0,865 & 0,283 & 0,280 & 88,198 & 140 & $-3,1$ \\
\hline 141 & 1,5 & 4,4 & 1,5 & 4,34 & 4,20 & 0,965 & 0,985 & 0,953 & 0,038 & 0,958 & 1,022 & 0,779 & 0,796 & 1,554 & 1,238 & 1,188 & 172,750 & 141 & $-4,0$ \\
\hline 142 & 0,1 & 0,9 & 0,1 & 0,87 & 0,84 & 1,000 & 0,957 & 0,926 & 0,043 & 1,000 & 0,886 & 0,833 & 0,588 & 0,357 & 0,210 & 0,280 & 42,723 & 142 & $-1,8$ \\
\hline 143 & 0,4 & 2,6 & 0,4 & 2,57 & 2,49 & 0,947 & 0,975 & 0,943 & 0,059 & 0,707 & 1,190 & 0,352 & 0,367 & 1,190 & 0,437 & 0,495 & 161,361 & 143 & $-3,6$ \\
\hline 144 & 0,3 & 1,9 & 0,3 & 1,87 & 1,81 & 0,954 & 0,966 & 0,934 & 0,058 & 1,000 & 0,990 & 0,655 & 0,644 & 0,769 & 0,495 & 0,533 & 172,157 & 144 & $-2,9$ \\
\hline 145 & 5,8 & 9,2 & 6,2 & 9,23 & 8,94 & 0,938 & 0,999 & 0,967 & 0,062 & 0,851 & 1,084 & 0,650 & 0,738 & 3,367 & 2,485 & 2,380 & 72,904 & 145 & $-5,1$ \\
\hline 146 & 0,2 & 1,4 & 0,2 & 1,32 & 1,28 & 0,986 & 0,971 & 0,940 & 0,032 & 1,000 & 0,926 & 0,768 & 0,657 & 0,533 & 0,350 & 0,408 & 30,772 & 146 & $-2,4$ \\
\hline 147 & 4,9 & 8,6 & 5,2 & 8,31 & 8,05 & 0,940 & 0,971 & 0,940 & 0,067 & 0,837 & 1,093 & 0,612 & 0,659 & 3,186 & 2,099 & 1,922 & 91,381 & 147 & $-5,0$ \\
\hline 148 & 0,3 & 2,0 & 0,3 & 1,90 & 1,83 & 0,962 & 0,953 & 0,922 & 0,061 & 0,989 & 1,006 & 0,706 & 0,661 & 0,749 & 0,495 & 0,495 & 12,667 & 148 & $-2,9$ \\
\hline 149 & 0,2 & 1,4 & 0,2 & 1,39 & 1,34 & 0,972 & 0,973 & 0,942 & 0,039 & 1,000 & 0,971 & 0,651 & 0,593 & 0,579 & 0,344 & 0,357 & 115,872 & 149 & $-2,5$ \\
\hline 150 & 0,3 & 2,0 & 0,3 & 1,91 & 1,85 & 0,940 & 0,935 & 0,905 & 0,088 & 0,922 & 1,042 & 0,551 & 0,574 & 0,842 & 0,483 & 0,495 & 62,740 & 150 & $-3,1$ \\
\hline 151 & 0,1 & 1,0 & 0,1 & 0,95 & 0,92 & 0,968 & 0,961 & 0,930 & 0,051 & 0,945 & 1,029 & 0,449 & 0,325 & 0,456 & 0,149 & 0,198 & 98,521 & 151 & $-2,2$ \\
\hline 152 & 0,9 & 4,3 & 1,0 & 4,08 & 3,95 & 0,946 & 0,950 & 0,920 & 0,074 & 0,643 & 1,247 & 0,315 & 0,345 & 1,953 & 0,673 & 0,700 & 59,290 & 152 & $-4,3$ \\
\hline 153 & 1,3 & 4,0 & 1,4 & 3,95 & 3,82 & 0,975 & 0,990 & 0,959 & 0,027 & 1,000 & 0,978 & 0,858 & 0,834 & $\begin{array}{l}1,401 \\
\text { S }\end{array}$ & 1,168 & 1,188 & 144,253 & 153 & $-3,8$ \\
\hline 154 & 0,1 & 1,3 & 0,1 & 1,16 & 1,12 & 0,983 & 0,925 & 0,895 & 0,077 & 1,000 & 0,930 & 0,835 & 0,738 & 0,470 & 0,347 & 0,396 & 117,086 & 154 & $-2,2$ \\
\hline 155 & 0,6 & 3,3 & 0,6 & 3,21 & 3,11 & 0,909 & 0,964 & 0,933 & 0,098 & 0,641 & 1,249 & 0,318 & 0,362 & 1,506 & 0,546 & 0,533 & 147,845 & 155 & $-3,9$ \\
\hline 156 & 0,3 & 2,1 & 0,3 & 2,01 & 1,95 & 0,891 & 0,963 & 0,932 & 0,115 & 0,748 & 1,156 & 0,415 & 0,379 & 0,892 & 0,338 & 0,357 & 57,107 & 156 & $-3,2$ \\
\hline 157 & 0,1 & 1,0 & 0,1 & 0,80 & 0,78 & 0,939 & 0,788 & 0,763 & 0,220 & 0,924 & 1,040 & 0,481 & 0,331 & 0,448 & 0,149 & 0,198 & 176,517 & 157 & $-2,2$ \\
\hline 158 & 0,2 & 1,5 & 0,2 & 1,43 & 1,38 & 0,935 & 0,956 & 0,925 & 0,079 & 0,996 & 1,002 & 0,628 & 0,552 & 0,598 & 0,330 & 0,357 & 58,830 & 158 & $-2,6$ \\
\hline 159 & 0,8 & 3,7 & 0,9 & 3,60 & 3,48 & 0,934 & 0,969 & 0,938 & 0,073 & 0,755 & 1,151 & 0,436 & 0,433 & 1,554 & 0,673 & 0,664 & 84,124 & 159 & $-4,0$ \\
\hline 160 & 1,7 & 4,8 & 1,7 & 4,73 & 4,58 & 0,964 & 0,992 & 0,960 & 0,037 & 0,916 & 1,045 & 0,586 & 0,550 & $\begin{array}{l}1,897 \\
\end{array}$ & 1,044 & 1,089 & 170,138 & 160 & $-4,2$ \\
\hline 161 & 0,3 & 2,1 & 0,3 & 1,91 & 1,85 & 0,911 & 0,899 & 0,870 & 0,135 & 0,763 & 1,145 & 0,454 & 0,451 & 0,877 & 0,396 & 0,408 & 94,312 & 161 & $-3,1$ \\
\hline 162 & 0,1 & 1,0 & 0,1 & 0,91 & 0,88 & 0,975 & 0,906 & 0,877 & 0,097 & 1,000 & 0,912 & 0,955 & 0,784 & 0,357 & 0,280 & 0,280 & $\begin{array}{l}108,999 \\
\end{array}$ & 162 & $-1,8$ \\
\hline 163 & 2,5 & 6,0 & 2,6 & 5,82 & 5,64 & 0,955 & 0,970 & 0,939 & 0,055 & 0,864 & 1,076 & 0,579 & 0,626 & 2,335 & 1,461 & 1,472 & 105,323 & 163 & $-4,5$ \\
\hline $\begin{array}{l}163 \\
164\end{array}$ & 0,4 & 2,6 & 0,5 & $\begin{array}{l}2,02 \\
2,50\end{array}$ & $\begin{array}{l}2,47 \\
2,42\end{array}$ & 0,891 & 0,950 & 0,919 & 0,120 & 0,758 & 1,149 & 0,456 & 0,490 & 1,081 & $\begin{array}{l}0,530 \\
0,431\end{array}$ & 0,443 & 122,843 & 164 & $-3,4$ \\
\hline
\end{tabular}




\begin{tabular}{|c|c|c|c|c|c|c|c|c|c|c|c|c|c|c|c|c|c|c|}
\hline 165 & 0,2 & 1,7 & 0,2 & 1,59 & 1,54 & 0,950 & 0,960 & 0,929 & 0,064 & 1,000 & 0,961 & 0,799 & 0,743 & 0,612 & 0,455 & 0,495 & 28,252 & 165 \\
\hline 166 & 20,6 & 20,7 & 22,4 & 20,16 & 19,52 & 0,919 & 0,973 & 0,941 & 0,086 & 0,601 & 1,290 & 0,348 & 0,433 & 8,671 & 3,757 & 3,564 & 15,927 & 166 \\
\hline 167 & 47,5 & 32,7 & 56,4 & 27,48 & 26,60 & 0,843 & 0,841 & 0,814 & 0,224 & 0,559 & 1,338 & 0,630 & 0,780 & 9,802 & 7,642 & 5,307 & 101,425 & 167 \\
\hline 168 & 0,2 & 1,4 & 0,2 & 1,19 & 1,15 & 0,925 & 0,864 & 0,836 & 0,156 & 1,000 & 0,995 & 0,877 & 0,843 & 0,470 & 0,396 & 0,396 & 37,118 & 168 \\
\hline 169 & 0,7 & 3,1 & 0,7 & 2,90 & 2,80 & 0,957 & 0,939 & 0,909 & 0,075 & 0,862 & 1,077 & 0,454 & 0,513 & 1,352 & 0,693 & 0,700 & 112,978 & 169 \\
\hline 170 & 0,0 & 0,7 & 0,1 & 0,48 & 0,47 & 0,952 & 0,719 & 0,697 & 0,285 & 1,000 & 0,852 & 0,878 & 0,557 & 0,267 & 0,149 & 0,198 & 170,583 & 170 \\
\hline 171 & 1,4 & 4,6 & 1,5 & 4,49 & 4,35 & 0,922 & 0,985 & 0,954 & 0,079 & 0,861 & 1,078 & 0,615 & 0,637 & 1,716 & 1,094 & 0,990 & 131,313 & 171 \\
\hline 172 & 0,3 & 1,7 & 0,3 & 1,70 & 1,64 & 0,955 & 0,978 & 0,946 & 0,050 & 1,000 & 0,956 & 0,890 & 0,777 & 0,612 & 0,476 & 0,495 & 15,507 & 172 \\
\hline 173 & 36,5 & 33,3 & 39,3 & 23,36 & 22,61 & 0,930 & 0,701 & 0,679 & 0,307 & 0,414 & 1,554 & 0,612 & 0,657 & 8,720 & 5,726 & 3,414 & 157,266 & 173 \\
\hline 174 & 0,5 & 2,9 & 0,6 & 2,75 & 2,66 & 0,902 & 0,940 & 0,910 & 0,115 & 0,794 & 1,122 & 0,590 & 0,595 & 1,081 & 0,644 & 0,626 & 96,897 & 174 \\
\hline 175 & 6,2 & 9,8 & 6,6 & 9,77 & 9,46 & 0,938 & 0,996 & 0,964 & 0,062 & 0,805 & 1,115 & 0,690 & 0,768 & 3,371 & 2,589 & 2,303 & 37,445 & 175 \\
\hline 176 & 2,9 & 6,2 & 3,0 & 6,12 & 5,92 & 0,968 & 0,981 & 0,949 & 0,038 & 0,943 & 1,030 & 0,756 & 0,801 & 2,217 & 1,775 & 1,785 & 0,797 & 176 \\
\hline 177 & 0,1 & 1,1 & 0,1 & 0,90 & 0,87 & 0,978 & 0,848 & 0,821 & 0,153 & 1,000 & 0,906 & 0,757 & 0,658 & 0,426 & 0,280 & 0,313 & 129,837 & 177 \\
\hline 178 & 0,2 & 1,4 & 0,2 & 1,38 & 1,33 & 0,949 & 0,954 & 0,923 & 0,068 & 1,000 & 0,949 & 0,876 & 0,732 & 0,517 & 0,378 & 0,396 & 102,125 & 178 \\
\hline 179 & 0,1 & 0,9 & 0,1 & 0,80 & 0,78 & 0,968 & 0,924 & 0,894 & 0,083 & 1,000 & 0,902 & 0,764 & 0,566 & 0,350 & 0,198 & 0,280 & 5,982 & 179 \\
\hline 180 & 1,3 & 4,4 & 1,4 & 4,32 & 4,18 & 0,935 & 0,979 & 0,947 & 0,068 & 0,824 & 1,102 & 0,539 & 0,584 & 1,735 & 1,014 & 0,975 & 9,295 & 180 \\
\hline 181 & 0,1 & 0,7 & 0,1 & 0,61 & 0,59 & 0,960 & 0,835 & 0,807 & 0,170 & 1,000 & 0,855 & 1,054 & 0,743 & 0,267 & 0,198 & 0,221 & 159,615 & 181 \\
\hline 182 & 0,6 & 3,0 & 0,6 & 2,94 & 2,84 & 0,951 & 0,978 & 0,947 & 0,054 & 0,859 & 1,079 & 0,553 & 0,514 & 1,192 & 0,612 & 0,626 & 82,169 & 182 \\
\hline 183 & 0,9 & 3,6 & 1,0 & 3,52 & 3,41 & 0,955 & 0,989 & 0,957 & 0,046 & 0,939 & 1,032 & 0,767 & 0,771 & 1,253 & 0,966 & 0,886 & 155,565 & 183 \\
\hline 184 & 0,8 & 3,4 & 0,9 & 3,33 & 3,23 & 0,961 & 0,980 & 0,949 & 0,044 & 0,910 & 1,049 & 0,562 & 0,534 & 1,376 & 0,735 & 0,773 & 43,898 & 184 \\
\hline 185 & 0,1 & 1,4 & 0,2 & 1,18 & 1,14 & 0,952 & 0,862 & 0,835 & 0,146 & 0,993 & 1,004 & 0,588 & 0,577 & 0,564 & 0,326 & 0,357 & 63,988 & 185 \\
\hline 186 & 6,2 & 10,1 & 6,5 & 10,01 & 9,69 & 0,959 & 0,991 & 0,959 & 0,042 & 0,767 & 1,142 & 0,602 & 0,785 & 3,631 & 2,850 & 2,258 & 37,769 & 186 \\
\hline 187 & 0,1 & 1,0 & 0,1 & 1,00 & 0,97 & 0,975 & 0,963 & 0,932 & 0,045 & 1,000 & 0,948 & 0,621 & 0,474 & 0,443 & 0,210 & 0,280 & 122,361 & 187 \\
\hline 188 & 0,1 & 0,7 & 0,1 & 0,59 & 0,57 & 1,000 & 0,828 & 0,802 & 0,172 & 1,000 & 0,879 & 0,786 & 0,606 & 0,289 & 0,175 & 0,221 & 128,802 & 188 \\
\hline 189 & 0,4 & 2,4 & 0,5 & 2,35 & 2,28 & 0,968 & 0,988 & 0,957 & 0,034 & 0,984 & 1,008 & 0,756 & 0,672 & 0,865 & 0,581 & 0,594 & 176,778 & 189 \\
\hline 190 & 0,4 & 2,1 & 0,4 & 1,97 & 1,91 & 0,942 & 0,937 & 0,907 & 0,086 & 1,000 & 0,993 & 0,771 & 0,684 & 0,769 & 0,526 & 0,560 & 58,134 & 190 \\
\hline 191 & 2,1 & 5,6 & 2,2 & 5,57 & 5,40 & 0,937 & 0,993 & 0,961 & 0,063 & 0,834 & 1,095 & 0,622 & 0,725 & 2,068 & 1,500 & 1,287 & 173,536 & 191 \\
\hline 192 & 0,1 & 0,9 & 0,1 & 0,83 & 0,80 & 1,000 & 0,926 & 0,897 & 0,074 & 1,000 & 0,964 & 0,524 & 0,364 & 0,408 & 0,149 & 0,198 & 8,850 & 192 \\
\hline 193 & 1,2 & 3,9 & 1,2 & 3,88 & 3,76 & 0,958 & 0,990 & 0,959 & 0,043 & 0,971 & 1,015 & 0,682 & 0,731 & 1,489 & 1,089 & 1,034 & 107,287 & 193 \\
\hline 194 & 0,6 & 3,0 & 0,7 & 2,86 & 2,76 & 0,970 & 0,956 & 0,925 & 0,054 & 0,893 & 1,058 & 0,602 & 0,664 & 1,159 & 0,769 & 0,714 & 113,393 & 194 \\
\hline 195 & 0,1 & 0,8 & 0,1 & 0,77 & 0,75 & 1,000 & 0,921 & 0,891 & 0,079 & 1,000 & 0,859 & 0,877 & 0,632 & 0,332 & 0,210 & 0,280 & 123,306 & 195 \\
\hline 196 & 0,3 & 2,3 & 0,3 & 2,24 & 2,17 & 0,957 & 0,983 & 0,951 & 0,047 & 0,781 & 1,132 & 0,385 & 0,394 & 1,034 & 0,407 & 0,408 & 16,548 & 196 \\
\hline 197 & 4,1 & 8,5 & 4,4 & 8,04 & 7,78 & 0,923 & 0,950 & 0,919 & 0,092 & 0,712 & 1,185 & 0,518 & 0,533 & 3,158 & 1,682 & 1,596 & 13,731 & 197 \\
\hline 198 & 0,2 & 1,8 & 0,2 & 1,63 & 1,58 & 0,880 & 0,925 & 0,895 & 0,141 & 0,803 & 1,116 & 0,486 & 0,392 & 0,721 & 0,282 & 0,280 & 73,021 & 198 \\
\hline 199 & 0,2 & 1,7 & 0,2 & 1,50 & 1,45 & 0,949 & 0,903 & 0,875 & 0,109 & 0,841 & 1,090 & 0,450 & 0,383 & 0,721 & 0,276 & 0,297 & 78,047 & 199 \\
\hline 200 & 0,3 & 1,9 & 0,3 & 1,80 & 1,74 & 0,949 & 0,931 & 0,901 & 0,086 & 0,914 & 1,046 & 0,541 & 0,526 & 0,800 & 0,421 & 0,443 & 62,108 & 200 \\
\hline 201 & 0,2 & 1,8 & 0,2 & 1,70 & 1,64 & 0,865 & 0,933 & 0,904 & 0,151 & 0,774 & 1,137 & 0,461 & 0,396 & 0,749 & 0,297 & 0,297 & 94,098 & 201 \\
\hline 202 & 30,3 & 28,0 & 35,0 & 22,62 & 21,89 & 0,865 & 0,808 & 0,782 & 0,235 & 0,485 & 1,435 & 0,507 & 0,586 & 8,714 & 5,104 & 4,275 & 111,056 & 202 \\
\hline 203 & 1,6 & 4,5 & 1,7 & 4,51 & 4,36 & 0,974 & 0,991 & 0,960 & 0,028 & 0,998 & 1,001 & 0,772 & 0,758 & 1,646 & 1,248 & 1,241 & 65,758 & 203 \\
\hline 204 & 2,0 & 6,1 & 2,2 & 5,60 & 5,42 & 0,878 & 0,914 & 0,885 & 0,149 & 0,660 & 1,231 & 0,555 & 0,722 & 2,126 & 1,535 & 1,089 & 1,368 & 204 \\
\hline 205 & 0,1 & 0,8 & 0,1 & 0,64 & 0,62 & 1,000 & 0,800 & 0,775 & 0,200 & 1,000 & 0,848 & 0,901 & 0,663 & 0,317 & 0,210 & 0,280 & 47,080 & 205 \\
\hline 206 & 0,1 & 1,3 & 0,1 & 1,23 & 1,19 & 0,939 & 0,910 & 0,881 & 0,109 & 0,780 & 1,132 & 0,373 & 0,321 & 0,620 & 0,199 & 0,221 & 112,726 & 206 \\
\hline 207 & 0,3 & 2,1 & 0,3 & 1,95 & 1,88 & 0,904 & 0,929 & 0,899 & 0,119 & 0,864 & 1,076 & 0,529 & 0,565 & 0,852 & 0,481 & 0,443 & 141,796 & 207 \\
\hline 208 & 6,7 & 10,9 & 7,2 & 10,72 & 10,38 & 0,932 & 0,986 & 0,955 & 0,069 & 0,717 & 1,181 & 0,438 & 0,445 & 4,424 & 1,971 & 1,950 & 28,743 & 208 \\
\hline 209 & 0,5 & 2,6 & 0,5 & 2,47 & 2,39 & 0,962 & 0,959 & 0,929 & 0,055 & 0,953 & 1,025 & 0,733 & 0,743 & 0,934 & 0,694 & 0,700 & 43,826 & 209 \\
\hline 210 & 0,1 & 1,2 & 0,1 & 1,12 & 1,09 & 0,886 & 0,935 & 0,905 & 0,131 & 0,835 & 1,094 & 0,497 & 0,417 & 0,495 & 0,206 & 0,221 & 143,025 & 210 \\
\hline 211 & 0,1 & 1,4 & 0,1 & 1,33 & 1,29 & 0,962 & 0,972 & 0,941 & 0,047 & 0,836 & 1,094 & 0,436 & 0,348 & 0,604 & 0,210 & 0,280 & 129,876 & 211 \\
\hline 212 & 0,1 & 1,3 & 0,2 & 1,18 & 1,14 & 0,968 & 0,899 & 0,870 & 0,106 & 1,000 & 0,955 & 0,670 & 0,557 & 0,533 & 0,297 & 0,357 & 105,471 & 212 \\
\hline 213 & 0,2 & 1,6 & 0,2 & 1,44 & 1,40 & 0,949 & 0,922 & 0,893 & 0,093 & 0,933 & 1,035 & 0,616 & 0,566 & 0,612 & 0,347 & 0,396 & 177,285 & 213 \\
\hline 214 & 0,2 & 1,6 & 0,2 & 1,52 & 1,47 & 0,882 & 0,958 & 0,927 & 0,125 & 0,919 & 1,043 & 0,659 & 0,589 & 0,596 & 0,351 & 0,357 & 156,601 & 214 \\
\hline 215 & 0,3 & 2,0 & 0,3 & 1,90 & 1,83 & 0,934 & 0,940 & 0,910 & 0,089 & 0,962 & 1,019 & 0,594 & 0,524 & 0,816 & 0,427 & 0,443 & 9,687 & 215 \\
\hline 216 & 0,5 & 2,5 & 0,5 & 2,41 & 2,34 & 0,978 & 0,984 & 0,953 & 0,027 & 1,000 & 0,947 & 0,910 & 0,850 & 0,865 & 0,735 & 0,754 & 16,218 & 216 \\
\hline 217 & 0,2 & 1,5 & 0,2 & 1,41 & 1,37 & 0,909 & 0,955 & 0,924 & 0,101 & 0,983 & 1,008 & 0,686 & 0,678 & 0,564 & 0,383 & 0,396 & 95,445 & 217 \\
\hline 218 & 0,1 & 1,0 & 0,1 & 0,87 & 0,84 & 0,946 & 0,867 & 0,839 & 0,143 & 1,000 & 0,963 & 0,619 & 0,500 & 0,420 & 0,210 & 0,280 & 122,668 & 218 \\
\hline 219 & 0,3 & 1,8 & 0,3 & 1,71 & 1,65 & 0,983 & 0,942 & 0,912 & 0,061 & 1,000 & 0,971 & 0,692 & 0,588 & 0,714 & 0,420 & 0,495 & 131,830 & 219 \\
\hline 220 & 1,7 & 4,6 & 1,8 & 4,61 & 4,46 & 0,981 & 0,992 & 0,960 & 0,021 & 1,000 & 0,995 & 0,739 & 0,687 & 1,729 & 1,188 & 1,287 & 95,615 & 220 \\
\hline 221 & 0,2 & 1,4 & 0,2 & 1,25 & 1,21 & 0,986 & 0,923 & 0,893 & 0,079 & 1,000 & 0,926 & 0,990 & 0,820 & 0,470 & 0,385 & 0,420 & 143,277 & 221 \\
\hline 222 & 2,1 & 5,6 & 2,2 & 5,57 & 5,39 & 0,949 & 0,988 & 0,957 & 0,053 & 0,842 & 1,090 & 0,581 & 0,512 & 2,158 & 1,105 & 1,089 & 126,932 & 222 \\
\hline
\end{tabular}




\begin{tabular}{|c|c|c|c|c|c|c|c|c|c|c|c|c|c|c|c|c|c|c|c|}
\hline 223 & 0,0 & 0,7 & 0,1 & 0,57 & 0,56 & 0,909 & 0,812 & 0,786 & 0,208 & 1,000 & 0,901 & 0,878 & 0,665 & 0,267 & 0,177 & 0,198 & 143,130 & 223 & $-1,4$ \\
\hline 224 & 4,3 & 10,8 & 5,6 & 9,25 & 8,95 & 0,765 & 0,856 & 0,829 & 0,275 & 0,459 & 1,476 & 0,375 & 0,512 & 3,802 & 1,946 & 1,462 & 6,232 & 224 & $-5,2$ \\
\hline 225 & 0,2 & 1,5 & 0,2 & 1,41 & 1,37 & 0,931 & 0,938 & 0,908 & 0,093 & 1,000 & 0,955 & 0,946 & 0,862 & 0,517 & 0,446 & 0,495 & 133,025 & 225 & $-2,4$ \\
\hline 226 & 0,1 & 1,4 & 0,2 & 1,28 & 1,24 & 0,968 & 0,924 & 0,895 & 0,082 & 0,977 & 1,012 & 0,567 & 0,459 & 0,579 & 0,266 & 0,280 & 62,680 & 226 & $-2,5$ \\
\hline 227 & 0,3 & 2,0 & 0,3 & 1,91 & 1,85 & 0,957 & 0,940 & 0,910 & 0,074 & 0,989 & 1,006 & 0,607 & 0,589 & 0,824 & 0,485 & 0,495 & 142,976 & 227 & $-3,0$ \\
\hline 228 & 0,1 & 1,3 & 0,1 & 1,16 & 1,12 & 0,982 & 0,905 & 0,876 & 0,096 & 1,000 & 0,976 & 0,548 & 0,496 & 0,564 & 0,280 & 0,313 & 122,999 & 228 & $-2,5$ \\
\hline 229 & 2,3 & 6,5 & 2,7 & 5,85 & 5,66 & 0,870 & 0,900 & 0,872 & 0,164 & 0,690 & 1,204 & 0,544 & 0,641 & 2,327 & 1,493 & 0,840 & 71,902 & 229 & $-4,5$ \\
\hline 230 & 2,1 & 5,7 & 2,3 & 5,55 & 5,37 & 0,912 & 0,974 & 0,943 & 0,092 & 0,795 & 1,122 & 0,502 & 0,533 & 2,284 & 1,218 & 1,188 & 26,719 & 230 & $-4,5$ \\
\hline 231 & 0,3 & 2,0 & 0,3 & 1,93 & 1,87 & 0,961 & 0,967 & 0,936 & 0,051 & 0,947 & 1,028 & 0,572 & 0,513 & 0,819 & 0,421 & 0,443 & 152,996 & 231 & $-3,0$ \\
\hline 232 & 0,2 & 1,6 & 0,2 & 1,53 & 1,48 & 0,963 & 0,975 & 0,944 & 0,044 & 0,988 & 1,006 & 0,657 & 0,611 & 0,612 & 0,374 & 0,357 & 32,694 & 232 & $-2,6$ \\
\hline 233 & 0,2 & 1,6 & 0,2 & 1,47 & 1,42 & 0,867 & 0,924 & 0,894 & 0,153 & 0,876 & 1,068 & 0,573 & 0,543 & 0,626 & 0,340 & 0,297 & 176,050 & 233 & $-2,6$ \\
\hline 234 & 0,2 & 1,6 & 0,2 & 1,57 & 1,52 & 0,978 & 0,966 & 0,935 & 0,040 & 1,000 & 0,973 & 0,706 & 0,664 & 0,634 & 0,421 & 0,443 & 121,640 & 234 & $-2,7$ \\
\hline 235 & 16,0 & 15,4 & 16,6 & 15,35 & 14,86 & 0,964 & 0,997 & 0,966 & 0,036 & 0,847 & 1,086 & 0,592 & 0,597 & 5,859 & 3,498 & 3,366 & 157,305 & 235 & $-5,9$ \\
\hline 236 & 0,5 & 2,5 & 0,5 & 2,40 & 2,33 & 0,959 & 0,962 & 0,932 & 0,055 & 1,000 & 0,977 & 0,882 & 0,857 & 0,866 & 0,743 & 0,700 & 135,145 & 236 & $-3,1$ \\
\hline 237 & 1,0 & 3,7 & 1,0 & 3,70 & 3,58 & 0,972 & 0,997 & 0,965 & 0,028 & 0,928 & 1,038 & 0,618 & 0,617 & 1,446 & 0,891 & 0,913 & 129,817 & 237 & $-3,9$ \\
\hline 238 & 0,1 & 1,5 & 0,1 & 1,43 & 1,38 & 0,880 & 0,973 & 0,943 & 0,123 & 0,632 & 1,258 & 0,303 & 0,265 & 0,673 & 0,178 & 0,198 & 124,193 & 238 & $-2,8$ \\
\hline 239 & 1,4 & 4,7 & 1,5 & 4,57 & 4,42 & 0,895 & 0,964 & 0,933 & 0,111 & 0,769 & 1,140 & 0,504 & 0,489 & 1,864 & 0,911 & 0,816 & 171,556 & 239 & $-4,2$ \\
\hline 240 & 0,1 & 1,1 & 0,1 & 1,04 & 1,01 & 0,951 & 0,940 & 0,910 & 0,077 & 0,983 & 1,008 & 0,621 & 0,474 & 0,443 & 0,210 & 0,280 & 143,022 & 240 & $-2,1$ \\
\hline 241 & 0,1 & 1,0 & 0,1 & 0,81 & 0,79 & 1,000 & 0,799 & 0,774 & 0,201 & 1,000 & 0,916 & 0,784 & 0,620 & 0,399 & 0,248 & 0,297 & 164,993 & 241 & $-2,0$ \\
\hline 242 & 0,3 & 1,9 & 0,3 & 1,79 & 1,73 & 0,922 & 0,918 & 0,888 & 0,114 & 0,863 & 1,076 & 0,582 & 0,647 & 0,754 & 0,488 & 0,443 & 134,640 & 242 & $-2,9$ \\
\hline 243 & 0,4 & 2,4 & 0,5 & 2,31 & 2,24 & 0,935 & 0,972 & 0,941 & 0,071 & 0,939 & 1,032 & 0,608 & 0,600 & 0,939 & 0,563 & 0,560 & 103,291 & 243 & $-3,2$ \\
\hline 244 & 7,2 & 11,4 & 7,9 & 10,41 & 10,07 & 0,919 & 0,909 & 0,880 & 0,122 & 0,695 & 1,199 & 0,551 & 0,662 & 4,092 & 2,709 & 2,380 & 128,919 & 244 & $-5,4$ \\
\hline 245 & 0,4 & 2,4 & 0,4 & 2,29 & 2,22 & 0,869 & 0,950 & 0,919 & 0,140 & 0,771 & 1,139 & 0,437 & 0,448 & 1,021 & 0,457 & 0,420 & 120,519 & 245 & $-3,4$ \\
\hline 246 & 0,4 & 2,0 & 0,4 & 1,85 & 1,79 & 0,980 & 0,908 & 0,879 & 0,094 & 1,000 & 0,949 & 0,912 & 0,832 & 0,714 & 0,594 & 0,602 & 72,576 & 246 & $-2,8$ \\
\hline 247 & 0,1 & 0,9 & 0,1 & 0,80 & 0,78 & 0,933 & 0,924 & 0,894 & 0,101 & 1,000 & 0,934 & 0,686 & 0,588 & 0,357 & 0,210 & 0,280 & 29,064 & 247 & $-1,8$ \\
\hline 248 & 1,5 & 4,7 & 1,6 & 4,65 & 4,50 & 0,955 & 0,986 & 0,954 & 0,047 & 0,850 & 1,084 & 0,531 & 0,541 & 1,897 & 1,027 & 1,034 & 175,590 & 248 & $-4,2$ \\
\hline 249 & 0,1 & 0,7 & 0,1 & 0,67 & 0,65 & 0,960 & 0,896 & 0,867 & 0,111 & 1,000 & 0,868 & 0,899 & 0,606 & 0,289 & 0,175 & 0,221 & 41,758 & 249 & $-1,5$ \\
\hline 250 & 0,8 & 3,5 & 0,8 & 3,42 & 3,31 & 0,939 & 0,981 & 0,949 & 0,064 & 0,783 & 1,130 & 0,475 & 0,433 & 1,425 & 0,617 & 0,626 & 107,645 & 250 & $-3,8$ \\
\hline 251 & 0,2 & 1,5 & 0,2 & 1,31 & 1,27 & 0,944 & 0,891 & 0,863 & 0,123 & 0,956 & 1,023 & 0,533 & 0,474 & 0,626 & 0,297 & 0,313 & 100,945 & 251 & $-2,6$ \\
\hline 252 & 0,9 & 3,5 & 0,9 & 3,47 & 3,36 & 0,956 & 0,981 & 0,950 & 0,048 & 0,907 & 1,050 & 0,612 & 0,631 & 1,369 & 0,863 & 0,886 & 129,907 & 252 & $-3,8$ \\
\hline 253 & 0,2 & 1,6 & 0,2 & 1,38 & 1,33 & 0,870 & 0,865 & 0,837 & 0,188 & 0,815 & 1,107 & 0,461 & 0,491 & 0,673 & 0,331 & 0,357 & 62,846 & 253 & $-2,8$ \\
\hline 254 & 1,3 & 4,8 & 1,5 & 4,19 & 4,06 & 0,889 & 0,879 & 0,851 & 0,165 & 0,722 & 1,177 & 0,682 & 0,793 & 1,563 & 1,240 & 0,980 & 5,426 & 254 & $-4,0$ \\
\hline 255 & 0,6 & 2,8 & 0,6 & 2,75 & 2,66 & 0,964 & 0,967 & 0,936 & 0,049 & 0,921 & 1,042 & 0,578 & 0,515 & 1,143 & 0,588 & 0,594 & 78,559 & 255 & $-3,5$ \\
\hline 256 & 8,0 & 10,5 & 8,3 & 10,40 & 10,06 & 0,968 & 0,993 & 0,961 & 0,033 & 0,919 & 1,043 & 0,667 & 0,721 & 3,912 & 2,822 & 2,788 & 143,660 & 256 & $-5,3$ \\
\hline 257 & 0,1 & 1,1 & 0,1 & 1,08 & 1,05 & 1,000 & 0,965 & 0,935 & 0,035 & 1,000 & 0,895 & 0,890 & 0,702 & 0,423 & 0,297 & 0,357 & 8,507 & 257 & $-2,1$ \\
\hline 258 & 19,3 & 16,8 & 20,0 & 16,51 & 15,98 & 0,965 & 0,982 & 0,951 & 0,039 & 0,857 & 1,080 & 0,669 & 0,710 & 6,056 & 4,298 & 4,156 & 161,466 & 258 & $-5,9$ \\
\hline 259 & 0,1 & 1,1 & 0,1 & 0,87 & 0,84 & 0,935 & 0,822 & 0,796 & 0,190 & 1,000 & 0,917 & 1,033 & 0,824 & 0,360 & 0,297 & 0,357 & 113,576 & 259 & $-1,8$ \\
\hline 260 & 0,4 & 2,4 & 0,5 & 2,32 & 2,25 & 0,933 & 0,984 & 0,952 & 0,069 & 1,000 & 0,996 & 0,825 & 0,774 & 0,830 & 0,642 & 0,626 & 146,147 & 260 & $-3,1$ \\
\hline 261 & 0,2 & 1,5 & 0,2 & 1,39 & 1,34 & 0,929 & 0,930 & 0,900 & 0,100 & 0,899 & 1,054 & 0,559 & 0,535 & 0,602 & 0,322 & 0,313 & 110,041 & 261 & $-2,6$ \\
\hline 262 & 1,1 & 3,9 & 1,2 & 3,82 & 3,69 & 0,934 & 0,976 & 0,945 & 0,070 & 0,916 & 1,045 & 0,749 & 0,728 & 1,376 & 1,002 & 0,975 & 14,496 & 262 & $-3,8$ \\
\hline 263 & 0,1 & 1,3 & 0,1 & 1,22 & 1,18 & 0,936 & 0,902 & 0,872 & 0,117 & 0,746 & 1,158 & 0,366 & 0,362 & 0,612 & 0,222 & 0,280 & 161,496 & 263 & $-2,6$ \\
\hline 264 & 0,2 & 1,4 & 0,2 & 1,38 & 1,33 & 0,947 & 0,954 & 0,923 & 0,070 & 1,000 & 0,975 & 0,695 & 0,596 & 0,564 & 0,336 & 0,396 & 173,919 & 264 & $-2,5$ \\
\hline 265 & 0,3 & 2,3 & 0,4 & 2,12 & 2,05 & 0,896 & 0,941 & 0,911 & 0,120 & 0,782 & 1,131 & 0,450 & 0,507 & 0,946 & 0,480 & 0,443 & 143,510 & 265 & $-3,2$ \\
\hline 266 & 0,3 & 1,9 & 0,3 & 1,79 & 1,73 & 0,974 & 0,964 & 0,933 & 0,044 & 1,000 & 0,988 & 0,708 & 0,647 & 0,709 & 0,459 & 0,495 & 151,931 & 266 & $-2,8$ \\
\hline 267 & 0,8 & 3,9 & 0,9 & 3,70 & 3,58 & 0,890 & 0,956 & 0,925 & 0,118 & 0,700 & 1,195 & 0,384 & 0,452 & 1,664 & 0,753 & 0,626 & 160,170 & 267 & $-4,1$ \\
\hline 268 & 0,2 & 1,4 & 0,2 & 1,39 & 1,34 & 0,971 & 0,962 & 0,931 & 0,048 & 0,993 & 1,004 & 0,656 & 0,614 & 0,564 & 0,347 & 0,396 & 0,426 & 268 & $-2,5$ \\
\hline 269 & 0,5 & 2,8 & 0,6 & 2,81 & 2,72 & 0,943 & 0,986 & 0,955 & 0,058 & 0,826 & 1,100 & 0,467 & 0,432 & 1,205 & 0,520 & 0,577 & 4,354 & 269 & $-3,6$ \\
\hline 270 & 0,1 & 1,1 & 0,1 & 0,84 & 0,81 & 0,914 & 0,796 & 0,771 & 0,221 & 0,885 & 1,063 & 0,453 & 0,422 & 0,470 & 0,198 & 0,280 & 10,105 & 270 & $-2,2$ \\
\hline 271 & 1,2 & 4,3 & 1,3 & 4,14 & 4,01 & 0,925 & 0,971 & 0,940 & 0,081 & 0,813 & 1,109 & 0,475 & 0,519 & 1,775 & 0,921 & 0,891 & 153,836 & 271 & $-4,1$ \\
\hline 272 & 3,6 & 7,2 & 3,7 & 7,17 & 6,94 & 0,954 & 0,991 & 0,959 & 0,047 & 0,857 & 1,080 & 0,593 & 0,651 & 2,770 & 1,803 & 1,732 & 36,304 & 272 & $-4,8$ \\
\hline 273 & 1,5 & 5,0 & 1,6 & 4,88 & 4,72 & 0,918 & 0,981 & 0,950 & 0,084 & 0,740 & 1,163 & 0,420 & 0,422 & 2,101 & 0,886 & 0,846 & 145,355 & 273 & $-4,4$ \\
\hline 274 & 1,1 & 4,2 & 1,3 & 3,93 & 3,80 & 0,916 & 0,937 & 0,907 & 0,105 & 0,822 & 1,103 & 0,564 & 0,680 & 1,610 & 1,096 & 0,990 & 131,015 & 274 & $-4,0$ \\
\hline 275 & 0,1 & 1,3 & 0,2 & 1,25 & 1,21 & 0,984 & 0,970 & 0,939 & 0,034 & 1,000 & 0,943 & 0,733 & 0,618 & 0,510 & 0,315 & 0,357 & 144,827 & 275 & $-2,4$ \\
\hline 276 & 0,1 & 1,0 & 0,1 & 0,80 & 0,78 & 0,927 & 0,788 & 0,763 & 0,224 & 1,000 & 0,939 & 0,834 & 0,657 & 0,377 & 0,248 & 0,297 & 77,925 & 276 & $-1,9$ \\
\hline 277 & 1,2 & 4,6 & 1,3 & 4,55 & 4,40 & 0,887 & 0,986 & 0,954 & 0,113 & 0,684 & 1,209 & 0,436 & 0,586 & 1,840 & 1,079 & 0,913 & 148,308 & 277 & $-4,2$ \\
\hline 278 & 0,4 & 2,7 & 0,5 & 2,52 & 2,44 & 0,889 & 0,950 & 0,919 & 0,121 & 0,775 & 1,136 & 0,663 & 0,800 & 0,913 & 0,730 & 0,533 & 57,661 & 278 & $-3,2$ \\
\hline 279 & 0,7 & 3,6 & 0,8 & 3,44 & 3,33 & 0,880 & 0,955 & 0,925 & 0,128 & 0,647 & 1,243 & 0,446 & 0,574 & 1,379 & 0,791 & 0,664 & 143,589 & 279 & $-3,8$ \\
\hline 280 & 0,3 & 1,9 & 0,3 & 1,77 & 1,72 & 0,960 & 0,950 & 0,919 & 0,064 & 1,000 & 0,971 & 0,721 & 0,687 & 0,721 & 0,495 & 0,533 & 174,924 & 280 & $-2,8$ \\
\hline
\end{tabular}




\begin{tabular}{|c|c|c|c|c|c|c|c|c|c|c|c|c|c|c|c|c|c|c|c|}
\hline 281 & 0,2 & 1,6 & 0,2 & 1,56 & 1,51 & 0,955 & 0,976 & 0,944 & 0,051 & 1,000 & 0,987 & 0,707 & 0,580 & 0,612 & 0,355 & 0,357 & 142,026 & 281 & $-2,6$ \\
\hline 282 & 1,6 & 5,1 & 1,6 & 5,00 & 4,84 & 0,963 & 0,987 & 0,955 & 0,040 & 0,775 & 1,136 & 0,454 & 0,466 & 2,105 & 0,980 & 1,019 & 47,193 & 282 & $-4,4$ \\
\hline 283 & 0,2 & 1,7 & 0,2 & 1,57 & 1,52 & 0,969 & 0,953 & 0,922 & 0,056 & 1,000 & 0,971 & 0,809 & 0,809 & 0,602 & 0,487 & 0,495 & 109,432 & 283 & $-2,6$ \\
\hline 284 & 0,1 & 0,9 & 0,1 & 0,77 & 0,75 & 0,957 & 0,892 & 0,863 & 0,117 & 0,901 & 1,054 & 0,459 & 0,453 & 0,387 & 0,175 & 0,198 & 146,280 & 284 & $-2,0$ \\
\hline 285 & 0,8 & 3,3 & 0,9 & 3,25 & 3,15 & 0,965 & 0,980 & 0,949 & 0,040 & 0,939 & 1,032 & 0,633 & 0,572 & 1,285 & 0,735 & 0,773 & 141,564 & 285 & $-3,7$ \\
\hline 286 & 0,2 & 1,7 & 0,2 & 1,72 & 1,66 & 0,978 & 0,984 & 0,952 & 0,027 & 0,879 & 1,067 & 0,426 & 0,395 & 0,798 & 0,315 & 0,357 & 148,494 & 286 & $-3,0$ \\
\hline 287 & 0,1 & 0,7 & 0,1 & 0,55 & 0,53 & 0,957 & 0,773 & 0,749 & 0,231 & 1,000 & 0,859 & 0,966 & 0,743 & 0,267 & 0,198 & 0,221 & 7,573 & 287 & $-1,4$ \\
\hline 288 & 0,1 & 1,2 & 0,1 & 1,12 & 1,09 & 0,898 & 0,944 & 0,913 & 0,116 & 0,961 & 1,020 & 0,659 & 0,600 & 0,456 & 0,274 & 0,297 & 85,431 & 288 & $-2,2$ \\
\hline 289 & 0,4 & 2,3 & 0,4 & 2,13 & 2,06 & 0,970 & 0,941 & 0,912 & 0,066 & 0,954 & 1,024 & 0,623 & 0,616 & 0,892 & 0,550 & 0,533 & 118,876 & 289 & $-3,2$ \\
\hline 290 & 0,6 & 2,8 & 0,6 & 2,71 & 2,62 & 0,942 & 0,976 & 0,945 & 0,063 & 0,905 & 1,051 & 0,584 & 0,579 & 1,099 & 0,637 & 0,626 & 161,252 & 290 & $-3,5$ \\
\hline 291 & 0,2 & 1,8 & 0,3 & 1,59 & 1,54 & 0,951 & 0,909 & 0,880 & 0,104 & 0,974 & 1,013 & 0,682 & 0,703 & 0,666 & 0,468 & 0,495 & 141,850 & 291 & $-2,7$ \\
\hline 292 & 20,9 & 18,6 & 22,5 & 17,58 & 17,02 & 0,929 & 0,945 & 0,915 & 0,090 & 0,760 & 1,147 & 0,754 & 0,854 & 5,941 & 5,075 & 4,509 & 80,621 & 292 & $-5,9$ \\
\hline 293 & 0,1 & 1,1 & 0,1 & 1,06 & 1,02 & 0,977 & 0,989 & 0,958 & 0,026 & 1,000 & 0,938 & 0,652 & 0,552 & 0,448 & 0,248 & 0,280 & 168,494 & 293 & $-2,2$ \\
\hline 294 & 0,6 & 2,8 & 0,6 & 2,75 & 2,66 & 0,976 & 0,986 & 0,954 & 0,027 & 0,989 & 1,006 & 0,701 & 0,632 & 1,052 & 0,665 & 0,700 & $\begin{array}{l}137,732 \\
\end{array}$ & 294 & $-3,4$ \\
\hline 295 & 0,3 & 1,9 & 0,3 & 1,82 & 1,76 & 0,967 & 0,945 & 0,915 & 0,064 & 0,983 & 1,008 & 0,774 & 0,771 & 0,690 & 0,532 & 0,495 & 85,879 & 295 & $-2,8$ \\
\hline 296 & 0,2 & 1,6 & 0,2 & 1,47 & 1,42 & 0,961 & 0,908 & 0,879 & 0,100 & 0,859 & $\begin{array}{l}1,079 \\
1\end{array}$ & 0,453 & 0,349 & 0,709 & 0,248 & 0,297 & 1,491 & 296 & $\begin{array}{l}-2,8 \\
-2\end{array}$ \\
\hline 297 & 0,1 & 1,3 & 0,1 & 1,12 & 1,09 & 0,891 & 0,857 & 0,829 & 0,180 & 0,881 & 1,066 & 0,538 & 0,470 & 0,533 & 0,251 & 0,297 & 14,109 & 297 & $-2,4$ \\
\hline 298 & 53,0 & 28,8 & 56,5 & 28,25 & 27,35 & 0,938 & 0,982 & 0,951 & 0,064 & 0,805 & 1,115 & 0,620 & 0,631 & 10,434 & 6,585 & 5,477 & 100,375 & 298 & $-6,7$ \\
\hline 299 & 0,1 & 1,0 & 0,1 & 0,83 & 0,80 & 0,921 & 0,862 & 0,835 & 0,159 & 1,000 & 0,926 & 0,891 & 0,759 & 0,350 & 0,266 & 0,313 & 39,578 & 299 & $-1,8$ \\
\hline 300 & $\begin{array}{l}, 1 \\
1,4\end{array}$ & 4,7 & 1,5 & $\begin{array}{l}\text {, } \\
4,64\end{array}$ & $\begin{array}{l}4,60 \\
4,49\end{array}$ & 0,904 & $\begin{array}{l}0,002 \\
0,986\end{array}$ & $\begin{array}{l}0,0954 \\
0,954\end{array}$ & 0,097 & 0,787 & $\begin{array}{l}1,127 \\
\end{array}$ & 0,522 & 0,600 & 1,840 & $\begin{array}{l}1,104 \\
\end{array}$ & 1,052 & 150,555 & 300 & $\begin{array}{l}-1,0 \\
-4,2\end{array}$ \\
\hline 301 & 0,8 & 3,2 & 0,8 & 3,11 & 3,01 & 0,949 & 0,979 & 0,948 & 0,055 & 0,974 & 1,013 & 0,733 & 0,720 & 1,165 & 0,838 & 0,886 & 177,337 & 301 & $-3,5$ \\
\hline 302 & 0,2 & 1,8 & 0,2 & 1,66 & 1,60 & 0,956 & 0,926 & 0,896 & 0,086 & 0,836 & 1,093 & 0,394 & 0,358 & 0,830 & 0,297 & 0,396 & 77,660 & 302 & $-3,1$ \\
\hline 303 & 0,2 & 1,3 & 0,2 & 1,12 & 1,09 & 0,986 & 0,839 & 0,812 & 0,162 & 1,000 & 0,924 & 0,817 & 0,680 & 0,510 & 0,347 & 0,396 & 97,478 & 303 & $-2,4$ \\
\hline 304 & 0,2 & 1,6 & 0,2 & 1,43 & 1,38 & 0,901 & 0,905 & 0,877 & 0,137 & 0,795 & 1,121 & 0,422 & 0,387 & 0,688 & 0,266 & 0,280 & 64,184 & 304 & $-2,8$ \\
\hline 305 & $\begin{array}{l}0,2 \\
0,1\end{array}$ & 0,7 & 0,1 & 0,71 & 0,68 & 0,958 & 0,948 & 0,917 & 0,067 & 1,000 & 0,886 & $\begin{array}{l}0,732 \\
0\end{array}$ & 0,559 & 0,313 & 0,175 & 0,198 & $\begin{array}{l}150,965 \\
\end{array}$ & 305 & $-1,6$ \\
\hline 306 & 0,1 & 0,8 & 0,1 & 0,70 & 0,67 & 1,000 & 0,840 & 0,813 & 0,160 & 1,000 & 0,848 & 0,987 & 0,632 & 0,313 & 0,198 & 0,297 & 90,000 & 306 & $-1,6$ \\
\hline 307 & $\begin{array}{l}0,1 \\
0,3\end{array}$ & 1,7 & 0,3 & 1,67 & 1,61 & 0,962 & 0,977 & 0,946 & 0,044 & 1,000 & $\begin{array}{l}0,960 \\
0,963\end{array}$ & 0,768 & 0,701 & 0,644 & 0,451 & 0,443 & 159,258 & 307 & $\begin{array}{l}-2,0 \\
-2,7\end{array}$ \\
\hline 308 & 0,3 & 2,1 & 0,3 & 1,95 & 1,89 & 0,949 & 0,924 & 0,895 & 0,091 & 0,906 & 1,051 & 0,609 & 0,604 & 0,819 & 0,495 & 0,495 & 79,535 & 308 & $-3,0$ \\
\hline 309 & 0,3 & 2,0 & 0,3 & 1,82 & 1,76 & 0,931 & 0,894 & 0,866 & 0,127 & 0,902 & 1,053 & 0,562 & 0,535 & 0,819 & 0,438 & 0,495 & 164,857 & 309 & $-3,0$ \\
\hline 310 & 13,8 & 16,4 & 15,4 & 15,52 & 15,02 & 0,899 & 0,947 & 0,916 & 0,115 & 0,646 & 1,244 & 0,420 & 0,453 & 6,472 & 2,933 & 2,475 & 135,719 & 310 & $-6,0$ \\
\hline 311 & 0,5 & 2,9 & 0,6 & 2,81 & 2,72 & 0,949 & 0,977 & 0,945 & 0,056 & 0,833 & 1,096 & 0,538 & 0,584 & 1,140 & 0,665 & 0,626 & 131,425 & 311 & $-3,5$ \\
\hline 312 & 0,1 & 0,9 & 0,1 & 0,80 & 0,78 & 0,900 & 0,895 & 0,866 & 0,145 & 1,000 & 0,981 & 0,593 & 0,510 & 0,377 & 0,192 & 0,198 & $\begin{array}{l}109,802 \\
\end{array}$ & 312 & $-1,9$ \\
\hline 313 & 0,2 & 1,9 & 0,2 & 1,75 & 1,69 & 0,888 & 0,929 & 0,899 & 0,133 & 0,689 & 1,204 & 0,380 & 0,333 & 0,806 & 0,268 & 0,280 & 143,500 & 313 & $-3,0$ \\
\hline 314 & $\begin{array}{l}0,2 \\
0,4\end{array}$ & 2,3 & 0,4 & 2,07 & 2,00 & $\begin{array}{l}0,060 \\
0,925\end{array}$ & 0,891 & 0,862 & 0,133 & 0,843 & $\begin{array}{l}1,089 \\
1,09\end{array}$ & 0,465 & 0,447 & 0,996 & 0,446 & 0,495 & $\begin{array}{l}166,105 \\
\end{array}$ & 314 & $-3,3$ \\
\hline 315 & 1,0 & 4,1 & 1,1 & 3,95 & 3,82 & 0,939 & 0,958 & 0,928 & 0,074 & 0,754 & 1,151 & 0,492 & 0,615 & 1,622 & 0,997 & 0,886 & 26,326 & 315 & $-4,0$ \\
\hline 316 & $\begin{array}{l}1,0 \\
0,1\end{array}$ & 1,3 & 0,1 & 1,15 & $\begin{array}{l}1,02 \\
1,11\end{array}$ & 0,944 & 0,916 & 0,887 & 0,101 & 0,999 & $\begin{array}{l}1,001 \\
1,1\end{array}$ & 0,643 & 0,597 & 0,498 & 0,297 & 0,313 & 0,838 & 316 & $-2,3$ \\
\hline 317 & 0,4 & 2,3 & 0,4 & 2,27 & 2,19 & 0,970 & 0,972 & 0,940 & 0,042 & 0,906 & $\begin{array}{l}1,050 \\
\text { n }\end{array}$ & 0,484 & 0,412 & 1,016 & 0,418 & 0,420 & 130,894 & 317 & $-3,3$ \\
\hline 318 & $\begin{array}{l}0,4 \\
0,6\end{array}$ & 3,2 & 0,6 & 3,06 & $\begin{array}{l}2,196 \\
2,96\end{array}$ & 0,916 & 0,967 & 0,936 & $\begin{array}{l}0,090 \\
0,09\end{array}$ & 0,706 & $\begin{array}{l}1,190 \\
1,190\end{array}$ & $\begin{array}{l}0,404 \\
0,379\end{array}$ & $\begin{array}{l}0,412 \\
0,349\end{array}$ & 1,373 & $\begin{array}{l}0,480 \\
0,480\end{array}$ & $\begin{array}{l}0,+205 \\
0,495\end{array}$ & 114,845 & 318 & $\begin{array}{l}-3,3 \\
-3,8\end{array}$ \\
\hline 319 & 0,2 & 1,4 & 0,2 & 1,23 & 1,19 & 0,928 & 0,867 & 0,840 & 0,151 & 0,985 & 1,007 & 0,702 & 0,697 & 0,533 & 0,372 & 0,357 & 27,016 & 319 & $-2,4$ \\
\hline 320 & 0,1 & 1,2 & 0,1 & 0,99 & 0,96 & 0,980 & 0,840 & 0,814 & 0,161 & 1,000 & 0,948 & 0,656 & 0,508 & 0,488 & 0,248 & 0,280 & 77,262 & 320 & $-2,3$ \\
\hline 321 & 8,3 & $\begin{array}{l}1,2 \\
11,0\end{array}$ & 8,7 & 10,88 & 10,54 & 0,958 & 0,986 & 0,954 & 0,045 & 0,860 & 1,078 & 0,745 & 0,802 & 3,776 & 3,029 & 2,675 & 28,578 & 321 & $-5,2$ \\
\hline 322 & 2,7 & 6,5 & 3,0 & 6,29 & 6,09 & 0,906 & 0,969 & 0,938 & 0,099 & 0,813 & 1,109 & 0,696 & 0,824 & 2,234 & 1,841 & 1,596 & 103,709 & 322 & $-4,5$ \\
\hline 323 & 15,9 & 16,1 & 17,3 & 15,62 & 15,12 & 0,917 & 0,968 & 0,937 & 0,089 & 0,767 & 1,142 & 0,642 & 0,715 & 5,614 & 4,015 & 3,577 & 35,112 & 323 & $-5,8$ \\
\hline 324 & 0,3 & 2,2 & 0,3 & 2,11 & 2,04 & 0,910 & 0,957 & 0,927 & 0,100 & 0,704 & 1,192 & 0,345 & 0,317 & 1,002 & 0,317 & 0,357 & 165,459 & 324 & $-3,3$ \\
\hline 325 & 0,1 & 1,0 & 0,1 & 0,84 & 0,81 & 0,829 & 0,840 & 0,813 & 0,235 & 0,893 & $\begin{array}{l}1,058 \\
1,10\end{array}$ & 0,568 & 0,574 & 0,399 & 0,229 & 0,198 & 138,442 & 325 & $-2,0$ \\
\hline 326 & 1,0 & 3,8 & 1,0 & 3,58 & 3,46 & 0,940 & 0,950 & 0,920 & 0,078 & 0,855 & $\begin{array}{l}1,081 \\
\text { S }\end{array}$ & 0,536 & 0,514 & 1,515 & 0,779 & 0,792 & 85,363 & 326 & $-3,9$ \\
\hline $\begin{array}{l}520 \\
327\end{array}$ & $\begin{array}{l}1,0 \\
0,2\end{array}$ & $\begin{array}{l}3,6 \\
1,5\end{array}$ & 0,2 & 年, & $\begin{array}{l}1,+0 \\
1,41\end{array}$ & 0,944 & 1,000 & 0,968 & 0,056 & $\begin{array}{l}0,035 \\
0,992\end{array}$ & $\begin{array}{l}1,001 \\
1,004\end{array}$ & 0,581 & 0,500 & 0,604 & 0,302 & 0,357 & $\begin{array}{l}124,240 \\
124,\end{array}$ & 327 & $-2,6$ \\
\hline 328 & 0,2 & 1,7 & 0,2 & 1,63 & 1,58 & 0,927 & 0,939 & 0,909 & 0,095 & 0,911 & 1,048 & 0,520 & 0,514 & 0,731 & 0,376 & 0,396 & 119,154 & 328 & $-2,9$ \\
\hline 329 & 22,0 & 22,2 & 24,3 & 21,16 & 20,48 & 0,905 & 0,954 & 0,924 & 0,105 & 0,562 & 1,334 & 0,308 & 0,363 & 9,527 & 3,462 & 3,147 & 97,201 & 329 & $-6,6$ \\
\hline 330 & 0,1 & 0,7 & 0,1 & 0,57 & 0,56 & 0,880 & 0,782 & 0,756 & 0,249 & 1,000 & 0,893 & 0,875 & 0,687 & 0,280 & 0,192 & 0,221 & 18,937 & 330 & $-1,5$ \\
\hline 331 & 0,2 & 1,5 & 0,2 & 1,49 & 1,44 & 0,962 & 0,975 & 0,943 & 0,046 & 0,986 & 1,007 & 0,645 & 0,571 & 0,602 & 0,344 & 0,357 & 147,793 & 331 & $-2,6$ \\
\hline 332 & $\begin{array}{l}, 2 \\
1,0\end{array}$ & 3,8 & 1,0 & 3,72 & 3,60 & 0,966 & 0,982 & 0,951 & 0,038 & 0,854 & $\begin{array}{l}1,082 \\
\end{array}$ & 0,546 & 0,530 & 1,506 & 0,797 & 0,798 & $\begin{array}{l}106,734 \\
\end{array}$ & 332 & $\begin{array}{l}-2,0 \\
-3,9\end{array}$ \\
\hline 333 & 0,1 & 1,2 & 0,1 & 1,12 & 1,09 & 0,932 & 0,923 & 0,893 & 0,103 & 0,855 & 1,081 & 0,450 & 0,380 & 0,533 & 0,202 & 0,221 & 108,080 & 333 & $-2,4$ \\
\hline 334 & $\begin{array}{l}0,1 \\
0,3\end{array}$ & $\begin{array}{l}1,2 \\
2,0\end{array}$ & 0,3 & 1,87 & 1,81 & 0,959 & 0,952 & 0,922 & 0,063 & 0,937 & $\begin{array}{l}1,033 \\
1,0\end{array}$ & 0,580 & 0,561 & 0,794 & 0,446 & 0,495 & $\begin{array}{l}10,735 \\
\end{array}$ & 334 & $\begin{array}{l}-2,+ \\
-3,0\end{array}$ \\
\hline 335 & 0,2 & 1,6 & 0,2 & 1,41 & 1,37 & 0,946 & 0,874 & 0,846 & 0,137 & 1,000 & 0,983 & 0,683 & 0,619 & 0,634 & 0,392 & 0,396 & 15,947 & 335 & $-2,7$ \\
\hline 336 & 0,1 & 1,1 & 0,1 & 1,08 & 1,04 & 0,967 & 0,942 & 0,912 & 0,067 & 0,682 & 1,210 & 0,327 & 0,266 & 0,526 & 0,140 & 0,198 & 137,125 & 336 & $-2,4$ \\
\hline 337 & 1,9 & 5,6 & 2,1 & 5,60 & 5,42 & 0,906 & 0,993 & 0,961 & 0,094 & 0,739 & 1,163 & 0,524 & 0,720 & 2,129 & 1,533 & 1,120 & 78,707 & 337 & $-4,4$ \\
\hline 338 & 0,1 & 0,8 & 0,1 & 0,77 & 0,75 & 0,966 & 0,966 & 0,934 & 0,049 & 1,000 & 0,863 & 0,870 & 0,663 & 0,317 & 0,210 & 0,280 & 140,330 & 338 & $-1,7$ \\
\hline
\end{tabular}




\begin{tabular}{|c|c|c|c|c|c|c|c|c|c|c|c|c|c|c|c|c|c|c|c|}
\hline 339 & 1,1 & 4,0 & 1,2 & 3,93 & 3,80 & 0,938 & 0,971 & 0,940 & 0,069 & 0,848 & 1,086 & 0,601 & 0,573 & 1,529 & 0,875 & 0,840 & 75,007 & 339 & $-3,9$ \\
\hline 340 & 1,0 & 3,7 & 1,0 & 3,61 & 3,49 & 0,969 & 0,982 & 0,950 & 0,036 & 0,924 & 1,040 & 0,583 & 0,547 & 1,472 & 0,805 & 0,840 & 34,558 & 340 & $-3,9$ \\
\hline 341 & 0,5 & 2,8 & 0,6 & 2,77 & 2,68 & 0,934 & 0,977 & 0,945 & 0,070 & 0,817 & 1,106 & 0,457 & 0,452 & 1,209 & 0,547 & 0,533 & 120,038 & 341 & $-3,6$ \\
\hline 342 & 8,1 & 11,1 & 8,6 & 10,85 & 10,50 & 0,935 & 0,973 & 0,942 & 0,070 & 0,818 & 1,106 & 0,683 & 0,746 & 3,881 & 2,896 & 2,666 & 99,690 & 342 & $-5,3$ \\
\hline 343 & 0,2 & 1,6 & 0,2 & 1,55 & 1,50 & 0,949 & 0,943 & 0,913 & 0,076 & 1,000 & 0,964 & 0,921 & 0,824 & 0,564 & 0,465 & 0,495 & 148,904 & 343 & $-2,5$ \\
\hline 344 & 0,2 & 1,7 & 0,2 & 1,55 & 1,50 & 0,916 & 0,936 & 0,906 & 0,106 & 0,982 & 1,009 & 0,743 & 0,646 & 0,604 & 0,391 & 0,408 & 48,417 & 344 & $-2,6$ \\
\hline 345 & 0,3 & 1,9 & 0,3 & 1,68 & 1,63 & 0,936 & 0,899 & 0,871 & 0,119 & 0,901 & 1,054 & 0,500 & 0,505 & 0,798 & 0,403 & 0,408 & 140,861 & 345 & $-3,0$ \\
\hline 346 & 0,7 & 3,1 & 0,8 & 3,01 & 2,91 & 0,965 & 0,984 & 0,952 & 0,039 & 0,987 & 1,007 & 0,700 & 0,728 & 1,155 & 0,840 & 0,846 & 121,462 & 346 & $-3,5$ \\
\hline 347 & 0,6 & 3,1 & 0,6 & 3,07 & 2,97 & 0,934 & 0,979 & 0,947 & 0,069 & 0,709 & 1,187 & 0,352 & 0,396 & 1,416 & 0,560 & 0,626 & 140,578 & 347 & $-3,8$ \\
\hline 348 & 5,1 & 9,3 & 5,4 & 8,97 & 8,68 & 0,937 & 0,968 & 0,937 & 0,071 & 0,740 & 1,162 & 0,460 & 0,523 & 3,742 & 1,957 & 1,818 & 108,043 & 348 & $-5,2$ \\
\hline 349 & 1,5 & 5,4 & 1,7 & 5,31 & 5,14 & 0,877 & 0,978 & 0,946 & 0,125 & 0,650 & 1,241 & 0,358 & 0,373 & 2,329 & 0,869 & 0,721 & 12,541 & 349 & $-4,5$ \\
\hline 350 & 0,1 & 0,8 & 0,1 & 0,67 & 0,65 & 0,926 & 0,834 & 0,807 & 0,181 & 1,000 & 0,913 & 0,776 & 0,663 & 0,317 & 0,210 & 0,280 & 104,872 & 350 & $-1,7$ \\
\hline 351 & 0,8 & 3,3 & 0,8 & 3,34 & 3,23 & 0,954 & 0,997 & 0,965 & 0,046 & 0,852 & 1,084 & 0,509 & 0,500 & 1,379 & 0,689 & 0,700 & 156,303 & 351 & $-3,8$ \\
\hline 352 & 0,2 & 1,9 & 0,3 & 1,81 & 1,75 & 0,923 & 0,951 & 0,920 & 0,091 & 0,814 & 1,109 & 0,454 & 0,458 & 0,812 & 0,372 & 0,357 & 38,467 & 352 & $-3,0$ \\
\hline 353 & 0,3 & 1,9 & 0,3 & 1,84 & 1,78 & 0,960 & 0,965 & 0,934 & 0,053 & 1,000 & 0,996 & 0,739 & 0,688 & 0,709 & 0,487 & 0,495 & 171,343 & 353 & $-2,8$ \\
\hline 354 & 0,1 & 1,0 & 0,1 & 0,74 & 0,71 & 0,946 & 0,773 & 0,749 & 0,233 & 1,000 & 0,915 & 0,731 & 0,640 & 0,387 & 0,248 & 0,280 & 145,054 & 354 & $-2,0$ \\
\hline 355 & 26,6 & 20,7 & 27,6 & 20,42 & 19,77 & 0,964 & 0,988 & 0,957 & 0,037 & 0,783 & 1,130 & 0,522 & 0,531 & 8,056 & 4,279 & 4,206 & 139,340 & 355 & $-6,3$ \\
\hline 356 & 0,1 & 1,2 & 0,1 & 1,07 & 1,03 & 0,891 & 0,869 & 0,841 & 0,170 & 1,000 & 0,998 & 0,843 & 0,822 & 0,426 & 0,350 & 0,313 & 86,930 & 356 & $-2,1$ \\
\hline 357 & 0,3 & 2,1 & 0,3 & 1,94 & 1,88 & 0,929 & 0,912 & 0,883 & 0,114 & 0,797 & 1,120 & 0,427 & 0,364 & 0,925 & 0,337 & 0,396 & 96,741 & 357 & $-3,2$ \\
\hline 358 & 0,2 & 1,6 & 0,2 & 1,44 & 1,40 & 0,952 & 0,900 & 0,871 & 0,111 & 0,948 & 1,027 & 0,734 & 0,769 & 0,579 & 0,446 & 0,408 & 113,527 & 358 & $-2,5$ \\
\hline 359 & 0,5 & 2,5 & 0,5 & 2,46 & 2,39 & 0,976 & 0,980 & 0,949 & 0,031 & 1,000 & 0,996 & 0,775 & 0,652 & 0,913 & 0,595 & 0,634 & 142,469 & 359 & $-3,2$ \\
\hline 360 & 0,4 & 2,1 & 0,4 & 2,02 & 1,95 & 0,945 & 0,938 & 0,908 & 0,083 & 1,000 & 0,987 & 0,845 & 0,793 & 0,754 & 0,598 & 0,602 & 78,096 & 360 & $-2,9$ \\
\hline 361 & 0,3 & 2,0 & 0,3 & 1,79 & 1,73 & 0,950 & 0,881 & 0,853 & 0,129 & 0,999 & 1,001 & 0,682 & 0,696 & 0,783 & 0,545 & 0,594 & 64,468 & 361 & $-3,0$ \\
\hline 362 & 0,5 & 2,7 & 0,5 & 2,65 & 2,57 & 0,969 & 0,986 & 0,954 & 0,034 & 0,804 & 1,115 & 0,423 & 0,415 & 1,181 & 0,490 & 0,533 & 141,962 & 362 & $-3,6$ \\
\hline 363 & 0,3 & 2,1 & 0,3 & 2,09 & 2,03 & 0,933 & 0,974 & 0,943 & 0,072 & 0,834 & 1,095 & 0,522 & 0,496 & 0,865 & 0,429 & 0,443 & 166,558 & 363 & $-3,1$ \\
\hline 364 & 0,1 & 1,2 & 0,1 & 1,09 & 1,06 & 0,980 & 0,900 & 0,872 & 0,102 & 1,000 & 1,000 & 0,561 & 0,479 & 0,517 & 0,248 & 0,297 & 105,446 & 364 & $-2,4$ \\
\hline 365 & 0,1 & 1,0 & 0,1 & 1,07 & 1,03 & 1,000 & 1,026 & 0,993 & 0,026 & 1,000 & 0,973 & 0,554 & 0,383 & 0,456 & 0,175 & 0,221 & 136,534 & 365 & $-2,2$ \\
\hline 366 & 0,6 & 2,9 & 0,6 & 2,76 & 2,67 & 0,956 & 0,967 & 0,936 & 0,055 & 0,899 & 1,055 & 0,590 & 0,533 & 1,122 & 0,598 & 0,626 & 35,290 & 366 & $-3,5$ \\
\hline 367 & 0,2 & 1,8 & 0,3 & 1,71 & 1,65 & 0,951 & 0,948 & 0,917 & 0,071 & 0,930 & 1,037 & 0,589 & 0,567 & 0,721 & 0,408 & 0,408 & 17,784 & 367 & $-2,8$ \\
\hline 368 & 0,1 & 0,9 & 0,1 & 0,64 & 0,62 & 0,889 & 0,739 & 0,716 & 0,284 & 0,983 & 1,009 & 0,588 & 0,497 & 0,357 & 0,177 & 0,221 & 156,697 & 368 & $-1,8$ \\
\hline 369 & 0,1 & 1,3 & 0,1 & 1,15 & 1,11 & 0,880 & 0,870 & 0,843 & 0,177 & 0,777 & 1,134 & 0,389 & 0,369 & 0,594 & 0,219 & 0,221 & 103,070 & 369 & $-2,6$ \\
\hline 370 & 0,9 & 3,6 & 0,9 & 3,52 & 3,41 & 0,956 & 0,989 & 0,957 & 0,045 & 0,895 & 1,057 & 0,599 & 0,564 & 1,387 & 0,782 & 0,754 & 62,690 & 370 & $-3,8$ \\
\hline 371 & 2,1 & 7,4 & 2,6 & 7,24 & 7,01 & 0,826 & 0,980 & 0,949 & 0,175 & 0,491 & 1,428 & 0,266 & 0,312 & 3,193 & 0,996 & 0,934 & 151,618 & 371 & $-5,0$ \\
\hline 372 & 0,4 & 2,8 & 0,5 & 2,47 & 2,39 & 0,805 & 0,891 & 0,862 & 0,224 & 0,674 & 1,218 & 0,484 & 0,481 & 1,044 & 0,502 & 0,420 & 100,485 & 372 & $-3,4$ \\
\hline 373 & 0,1 & 1,1 & 0,1 & 0,93 & 0,90 & 0,955 & 0,862 & 0,835 & 0,145 & 1,000 & 0,952 & 0,823 & 0,738 & 0,399 & 0,295 & 0,297 & 76,511 & 373 & $-2,0$ \\
\hline 374 & 0,1 & 1,2 & 0,1 & 1,12 & 1,09 & 0,962 & 0,902 & 0,873 & 0,105 & 1,000 & 0,992 & 0,560 & 0,464 & 0,533 & 0,248 & 0,280 & 13,545 & 374 & $-2,4$ \\
\hline 375 & 2,0 & 5,7 & 2,2 & 5,43 & 5,26 & 0,897 & 0,953 & 0,923 & 0,113 & 0,775 & 1,136 & 0,611 & 0,712 & 2,041 & 1,453 & 1,252 & 150,225 & 375 & $-4,4$ \\
\hline 376 & 0,3 & 1,9 & 0,3 & 1,83 & 1,77 & 0,965 & 0,946 & 0,915 & 0,065 & 0,898 & 1,055 & 0,494 & 0,454 & 0,830 & 0,377 & 0,408 & 70,495 & 376 & $-3,1$ \\
\hline 377 & 0,1 & 0,8 & 0,1 & 0,64 & 0,62 & 1,000 & 0,800 & 0,775 & 0,200 & 1,000 & 0,952 & 0,651 & 0,596 & 0,332 & 0,198 & 0,198 & 107,137 & 377 & $-1,7$ \\
\hline 378 & 0,2 & 1,4 & 0,2 & 1,32 & 1,28 & 0,932 & 0,952 & 0,921 & 0,084 & 1,000 & 0,958 & 0,794 & 0,766 & 0,517 & 0,396 & 0,396 & 26,008 & 378 & $-2,4$ \\
\hline 379 & 0,3 & 1,9 & 0,3 & 1,80 & 1,74 & 0,946 & 0,956 & 0,925 & 0,069 & 0,920 & 1,042 & 0,658 & 0,685 & 0,709 & 0,486 & 0,443 & 165,541 & 379 & $-2,8$ \\
\hline 380 & 0,1 & 1,2 & 0,1 & 0,80 & 0,78 & 0,981 & 0,687 & 0,666 & 0,313 & 1,000 & 0,930 & 0,812 & 0,559 & 0,443 & 0,248 & 0,297 & 2,179 & 380 & $-2,1$ \\
\hline 381 & 0,2 & 2,0 & 0,2 & 1,80 & 1,74 & 0,812 & 0,906 & 0,877 & 0,211 & 0,537 & 1,364 & 0,280 & 0,244 & 0,877 & 0,214 & 0,221 & 110,265 & 381 & $-3,1$ \\
\hline 382 & 0,2 & 1,7 & 0,2 & 1,55 & 1,50 & 0,939 & 0,921 & 0,891 & 0,100 & 1,000 & 0,992 & 0,817 & 0,753 & 0,596 & 0,449 & 0,443 & 134,966 & 382 & $-2,6$ \\
\hline 383 & 0,5 & 2,7 & 0,5 & 2,66 & 2,57 & 0,940 & 0,976 & 0,945 & 0,065 & 0,843 & 1,089 & 0,508 & 0,532 & 1,117 & 0,594 & 0,594 & 89,581 & 383 & $-3,5$ \\
\hline 384 & 0,6 & 3,6 & 0,7 & 3,51 & 3,40 & 0,912 & 0,981 & 0,950 & 0,090 & 0,623 & 1,267 & 0,307 & 0,349 & 1,622 & 0,566 & 0,594 & 85,642 & 384 & $-4,0$ \\
\hline 385 & 0,1 & 1,0 & 0,1 & 0,93 & 0,90 & 0,972 & 0,909 & 0,879 & 0,095 & 1,000 & 0,990 & 0,557 & 0,450 & 0,443 & 0,199 & 0,221 & 25,664 & 385 & $-2,1$ \\
\hline 386 & 0,2 & 1,4 & 0,2 & 1,18 & 1,14 & 0,986 & 0,829 & 0,802 & 0,172 & 1,000 & 0,960 & 0,723 & 0,537 & 0,553 & 0,297 & 0,396 & 5,597 & 386 & $-2,5$ \\
\hline 387 & 0,2 & 1,6 & 0,2 & 1,49 & 1,44 & 0,940 & 0,957 & 0,927 & 0,073 & 1,000 & 0,999 & 0,774 & 0,779 & 0,564 & 0,440 & 0,408 & 68,929 & 387 & $-2,5$ \\
\hline 388 & 0,5 & 2,5 & 0,5 & 2,35 & 2,27 & 0,950 & 0,951 & 0,921 & 0,070 & 0,965 & 1,018 & 0,741 & 0,642 & 0,897 & 0,576 & 0,577 & 18,553 & 388 & $-3,2$ \\
\hline 389 & 0,3 & 2,0 & 0,3 & 1,99 & 1,93 & 0,971 & 0,981 & 0,949 & 0,034 & 1,000 & 0,991 & 0,637 & 0,606 & 0,816 & 0,495 & 0,560 & 74,306 & 389 & $-3,0$ \\
\hline 390 & 0,1 & 1,4 & 0,2 & 1,28 & 1,24 & 0,952 & 0,924 & 0,895 & 0,090 & 0,945 & 1,029 & 0,689 & 0,714 & 0,517 & 0,369 & 0,357 & 93,113 & 390 & $-2,4$ \\
\hline 391 & 0,1 & 0,8 & 0,1 & 0,74 & 0,71 & 0,955 & 0,917 & 0,888 & 0,094 & 1,000 & 0,996 & 0,535 & 0,424 & 0,350 & 0,149 & 0,198 & 168,576 & 391 & $-1,8$ \\
\hline 392 & 0,1 & 1,1 & 0,1 & 0,87 & 0,84 & 0,932 & 0,801 & 0,775 & 0,210 & 1,000 & 0,963 & 0,653 & 0,559 & 0,443 & 0,248 & 0,297 & 14,564 & 392 & $-2,1$ \\
\hline 393 & 23,8 & 20,3 & 24,9 & 20,19 & 19,54 & 0,954 & 0,995 & 0,964 & 0,046 & 0,726 & 1,174 & 0,468 & 0,486 & 8,039 & 3,910 & 3,588 & 18,733 & 393 & $-6,3$ \\
\hline 394 & 0,2 & 1,8 & 0,3 & 1,67 & 1,61 & 0,971 & 0,947 & 0,916 & 0,061 & 1,000 & 0,999 & 0,653 & 0,570 & 0,695 & 0,396 & 0,495 & 0,509 & 394 & $-2,8$ \\
\hline 395 & 0,3 & 2,0 & 0,4 & 2,03 & 1,96 & 0,973 & 0,994 & 0,963 & 0,028 & 1,000 & 0,975 & 0,723 & 0,671 & 0,783 & 0,525 & 0,560 & 147,419 & 395 & $-3,0$ \\
\hline 396 & 0,4 & 3,1 & 0,4 & 2,86 & 2,76 & 0,841 & 0,927 & 0,897 & 0,175 & 0,480 & 1,444 & 0,230 & 0,297 & 1,418 & 0,420 & 0,396 & 105,474 & 396 & $-3,8$ \\
\hline
\end{tabular}




\begin{tabular}{|c|c|c|c|c|c|c|c|c|c|c|c|c|c|c|c|c|c|c|}
\hline 397 & 1,0 & 3,7 & 1,0 & 3,59 & 3,47 & 0,934 & 0,972 & 0,940 & 0,072 & 0,893 & 1,058 & 0,621 & 0,644 & 1,409 & 0,907 & 0,886 & 116,508 & 397 \\
\hline 398 & 1,0 & 3,7 & 1,0 & 3,65 & 3,53 & 0,949 & 0,982 & 0,951 & 0,054 & 0,867 & 1,074 & 0,653 & 0,668 & 1,362 & 0,910 & 0,816 & 128,594 & 398 \\
\hline 399 & 0,1 & 0,8 & 0,1 & 0,68 & 0,66 & 0,955 & 0,837 & 0,811 & 0,170 & 0,980 & 1,010 & 0,514 & 0,385 & 0,357 & 0,137 & 0,198 & 58,225 & 399 \\
\hline 400 & 0,6 & 3,2 & 0,7 & 2,96 & 2,87 & 0,895 & 0,932 & 0,902 & 0,125 & 0,781 & 1,131 & 0,473 & 0,519 & 1,299 & 0,675 & 0,634 & 128,671 & 400 \\
\hline 401 & 0,1 & 1,1 & 0,1 & 1,07 & 1,03 & 0,958 & 0,941 & 0,911 & 0,072 & 1,000 & 0,952 & 0,689 & 0,582 & 0,456 & 0,266 & 0,280 & 19,172 & 401 \\
\hline 402 & 1,7 & 5,3 & 1,9 & 5,07 & 4,91 & 0,898 & 0,950 & 0,920 & 0,113 & 0,753 & 1,153 & 0,586 & 0,775 & 1,926 & 1,493 & 1,120 & 38,957 & 402 \\
\hline 403 & 0,5 & 3,1 & 0,5 & 3,01 & 2,91 & 0,955 & 0,978 & 0,947 & 0,050 & 0,696 & 1,199 & 0,332 & 0,349 & 1,418 & 0,496 & 0,533 & 57,700 & 403 \\
\hline 404 & 3,3 & 7,3 & 3,5 & 7,18 & 6,95 & 0,943 & 0,989 & 0,958 & 0,058 & 0,798 & 1,120 & 0,695 & 0,835 & 2,475 & 2,067 & 1,624 & 20,381 & 404 \\
\hline 405 & 0,5 & 2,4 & 0,5 & 2,30 & 2,23 & 0,968 & 0,972 & 0,941 & 0,042 & 1,000 & 0,996 & 0,721 & 0,628 & 0,892 & 0,560 & 0,626 & 38,785 & 405 \\
\hline 406 & 0,1 & 1,1 & 0,1 & 1,07 & 1,03 & 0,913 & 0,932 & 0,902 & 0,110 & 0,988 & 1,006 & 0,629 & 0,542 & 0,456 & 0,247 & 0,280 & 144,746 & 406 \\
\hline 407 & 0,7 & 3,0 & 0,7 & 2,91 & 2,82 & 0,976 & 0,978 & 0,947 & 0,033 & 0,980 & 1,010 & 0,655 & 0,641 & 1,159 & 0,743 & 0,754 & 166,838 & 407 \\
\hline 408 & 55,9 & 36,2 & 66,6 & 31,60 & 30,59 & 0,839 & 0,872 & 0,844 & 0,205 & 0,535 & 1,368 & 0,472 & 0,585 & 12,271 & 7,175 & 5,525 & 33,025 & 408 \\
\hline 409 & 1,5 & 4,9 & 1,6 & 4,70 & 4,55 & 0,967 & 0,959 & 0,929 & 0,052 & 0,791 & 1,124 & 0,425 & 0,442 & 2,129 & 0,941 & 0,990 & 99,524 & 409 \\
\hline 410 & 0,2 & 1,7 & 0,2 & 1,52 & 1,47 & 0,950 & 0,920 & 0,890 & 0,095 & 0,858 & 1,080 & 0,479 & 0,392 & 0,704 & 0,276 & 0,280 & 38,659 & 410 \\
\hline 411 & 0,1 & 0,9 & 0,1 & 0,80 & 0,78 & 0,889 & 0,884 & 0,856 & 0,161 & 0,900 & 1,054 & 0,470 & 0,393 & 0,399 & 0,157 & 0,198 & 21,960 & 411 \\
\hline 412 & 0,2 & 1,8 & 0,2 & 1,66 & 1,60 & 0,868 & 0,940 & 0,910 & 0,144 & 0,655 & 1,236 & 0,327 & 0,283 & 0,794 & 0,225 & 0,221 & 4,656 & 412 \\
\hline 413 & 0,1 & 1,1 & 0,1 & 1,03 & 1,00 & 0,902 & 0,907 & 0,878 & 0,135 & 0,888 & 1,061 & 0,486 & 0,455 & 0,488 & 0,222 & 0,280 & 59,721 & 413 \\
\hline 414 & 3,4 & 7,9 & 3,8 & 7,44 & 7,20 & 0,909 & 0,943 & 0,913 & 0,107 & 0,694 & 1,200 & 0,486 & 0,493 & 3,000 & 1,479 & 1,390 & 61,756 & 414 \\
\hline 415 & 3,2 & 7,4 & 3,4 & 7,38 & 7,14 & 0,948 & 0,995 & 0,963 & 0,053 & 0,730 & 1,171 & 0,423 & 0,389 & 3,101 & 1,205 & 1,196 & 111,705 & 415 \\
\hline 416 & 6,6 & 10,2 & 7,0 & 9,74 & 9,43 & 0,941 & 0,951 & 0,920 & 0,077 & 0,786 & 1,128 & 0,670 & 0,690 & 3,532 & 2,437 & 2,171 & 121,553 & 416 \\
\hline 417 & 0,2 & 1,8 & 0,2 & 1,71 & 1,66 & 0,847 & 0,928 & 0,898 & 0,169 & 0,652 & 1,239 & 0,353 & 0,285 & 0,798 & 0,227 & 0,297 & 96,301 & 417 \\
\hline 418 & 0,3 & 2,5 & 0,3 & 2,27 & 2,20 & 0,938 & 0,899 & 0,871 & 0,118 & 0,585 & 1,308 & 0,281 & 0,297 & 1,159 & 0,345 & 0,396 & 15,402 & 418 \\
\hline 419 & 0,1 & 0,9 & 0,1 & 0,80 & 0,78 & 0,967 & 0,858 & 0,830 & 0,146 & 1,000 & 0,988 & 0,506 & 0,455 & 0,423 & 0,192 & 0,221 & 109,441 & 419 \\
\hline 420 & 4,9 & 8,9 & 5,2 & 8,71 & 8,43 & 0,937 & 0,978 & 0,946 & 0,067 & 0,776 & 1,135 & 0,535 & 0,589 & 3,413 & 2,012 & 2,005 & 158,260 & 420 \\
\hline 421 & 0,1 & 1,3 & 0,1 & 1,16 & 1,12 & 0,934 & 0,861 & 0,833 & 0,154 & 0,966 & 1,017 & 0,558 & 0,536 & 0,564 & 0,302 & 0,313 & 47,368 & 421 \\
\hline 422 & 0,4 & 3,1 & 0,5 & 3,08 & 2,98 & 0,833 & 0,979 & 0,948 & 0,168 & 0,544 & 1,356 & 0,281 & 0,276 & 1,395 & 0,385 & 0,357 & 109,352 & 422 \\
\hline 423 & 0,1 & 0,8 & 0,1 & 0,71 & 0,68 & 0,923 & 0,842 & 0,815 & 0,176 & 1,000 & 0,977 & 0,527 & 0,464 & 0,377 & 0,175 & 0,221 & 106,845 & 423 \\
\hline 424 & 0,4 & 2,4 & 0,4 & 2,25 & 2,18 & 0,954 & 0,934 & 0,904 & 0,081 & 0,761 & 1,146 & 0,406 & 0,406 & 1,052 & 0,427 & 0,420 & 32,331 & 424 \\
\hline 425 & 0,5 & 2,5 & 0,5 & 2,40 & 2,32 & 0,955 & 0,973 & 0,942 & 0,052 & 0,969 & 1,016 & 0,697 & 0,609 & 0,925 & 0,563 & 0,577 & 124,515 & 425 \\
\hline 426 & 5,4 & 9,4 & 5,8 & 9,19 & 8,90 & 0,926 & 0,976 & 0,945 & 0,078 & 0,759 & 1,148 & 0,544 & 0,572 & 3,540 & 2,024 & 1,980 & 1,571 & 426 \\
\hline 427 & 0,6 & 2,7 & 0,6 & 2,62 & 2,53 & 0,958 & 0,965 & 0,934 & 0,054 & 0,959 & 1,021 & 0,605 & 0,556 & 1,087 & 0,604 & 0,594 & 69,855 & 427 \\
\hline 428 & 0,1 & 1,0 & 0,1 & 0,84 & 0,81 & 0,917 & 0,874 & 0,846 & 0,151 & 1,000 & 0,953 & 1,025 & 0,781 & 0,317 & 0,248 & 0,280 & 107,170 & 428 \\
\hline 429 & 0,2 & 1,9 & 0,2 & 1,87 & 1,81 & 0,946 & 0,980 & 0,948 & 0,058 & 0,734 & 1,168 & 0,338 & 0,276 & 0,897 & 0,248 & 0,297 & 175,441 & 429 \\
\hline 430 & 0,9 & 3,5 & 0,9 & 3,41 & 3,30 & 0,976 & 0,989 & 0,957 & 0,027 & 0,940 & 1,031 & 0,590 & 0,551 & 1,387 & 0,764 & 0,798 & 144,908 & 430 \\
\hline 431 & 0,7 & 3,2 & 0,7 & 3,14 & 3,04 & 0,956 & 0,971 & 0,940 & 0,053 & 0,838 & 1,093 & 0,518 & 0,459 & 1,308 & 0,601 & 0,602 & 111,221 & 431 \\
\hline 432 & 1,0 & 3,7 & 1,0 & 3,66 & 3,54 & 0,959 & 0,982 & 0,951 & 0,045 & 0,884 & 1,064 & 0,563 & 0,582 & 1,485 & 0,864 & 0,846 & 130,504 & 432 \\
\hline 433 & 1,0 & 4,0 & 1,1 & 3,90 & 3,78 & 0,925 & 0,974 & 0,943 & 0,080 & 0,805 & 1,115 & 0,572 & 0,691 & 1,511 & 1,044 & 0,886 & 159,343 & 433 \\
\hline 434 & 1,5 & 4,4 & 1,5 & 4,35 & 4,21 & 0,960 & 0,991 & 0,959 & 0,041 & 0,954 & 1,024 & 0,716 & 0,705 & 1,612 & 1,136 & 1,155 & 140,226 & 434 \\
\hline 435 & 1,0 & 3,6 & 1,0 & 3,53 & 3,42 & 0,958 & 0,989 & 0,957 & 0,043 & 0,938 & 1,033 & 0,593 & 0,631 & 1,429 & 0,901 & 0,913 & 163,975 & 435 \\
\hline 436 & 0,1 & 1,1 & 0,1 & 0,90 & 0,87 & 0,950 & 0,827 & 0,800 & 0,180 & 0,999 & 1,001 & 0,569 & 0,434 & 0,456 & 0,198 & 0,280 & 172,304 & 436 \\
\hline 437 & 7,2 & 10,4 & 7,8 & 9,93 & 9,61 & 0,928 & 0,957 & 0,927 & 0,083 & 0,842 & 1,090 & 0,738 & 0,814 & 3,526 & 2,871 & 2,660 & 94,808 & 437 \\
\hline 438 & 0,2 & 1,5 & 0,2 & 1,45 & 1,41 & 0,929 & 0,956 & 0,926 & 0,084 & 1,000 & 0,980 & 0,680 & 0,634 & 0,598 & 0,379 & 0,408 & 146,306 & 438 \\
\hline 439 & 4,1 & 7,7 & 4,3 & 7,65 & 7,41 & 0,961 & 0,988 & 0,956 & 0,041 & 0,861 & 1,078 & 0,631 & 0,638 & 2,880 & 1,838 & 1,818 & 159,837 & 439 \\
\hline 440 & 2,2 & 6,1 & 2,3 & 6,04 & 5,85 & 0,960 & 0,994 & 0,962 & 0,041 & 0,735 & 1,166 & 0,447 & 0,413 & 2,481 & 1,026 & 1,052 & 142,176 & 440 \\
\hline 441 & 0,2 & 1,5 & 0,2 & 1,44 & 1,40 & 0,952 & 0,932 & 0,902 & 0,083 & 1,000 & 0,986 & 0,666 & 0,647 & 0,612 & 0,396 & 0,408 & 89,238 & 441 \\
\hline 442 & 0,2 & 1,7 & 0,2 & 1,61 & 1,56 & 0,940 & 0,945 & 0,915 & 0,081 & 0,993 & 1,003 & 0,661 & 0,566 & 0,666 & 0,377 & 0,408 & 31,991 & 442 \\
\hline 443 & 2,9 & 6,4 & 3,0 & 6,40 & 6,20 & 0,973 & 0,994 & 0,962 & 0,027 & 0,891 & 1,059 & 0,620 & 0,639 & 2,458 & 1,572 & 1,565 & 98,666 & 443 \\
\hline 444 & 0,3 & 2,4 & 0,3 & 2,23 & 2,16 & 0,885 & 0,944 & 0,914 & 0,128 & 0,681 & 1,212 & 0,372 & 0,433 & 1,016 & 0,440 & 0,408 & 35,046 & 444 \\
\hline 445 & 0,2 & 1,7 & 0,2 & 1,55 & 1,50 & 0,925 & 0,921 & 0,891 & 0,109 & 0,939 & 1,032 & 0,684 & 0,700 & 0,626 & 0,438 & 0,408 & 124,403 & 445 \\
\hline 446 & 17,7 & 16,1 & 18,1 & 15,95 & 15,44 & 0,980 & 0,993 & 0,961 & 0,021 & 0,862 & 1,077 & 0,614 & 0,658 & 6,062 & 3,991 & 4,010 & 29,861 & 446 \\
\hline 447 & 12,8 & 13,5 & 13,1 & 13,45 & 13,01 & 0,975 & 0,993 & 0,961 & 0,026 & 0,876 & 1,069 & 0,617 & 0,612 & 5,136 & 3,144 & 3,109 & 101,168 & 447 \\
\hline 448 & 0,1 & 0,8 & 0,1 & 0,75 & 0,72 & 0,960 & 0,964 & 0,934 & 0,054 & 1,000 & 0,900 & 0,745 & 0,552 & 0,317 & 0,175 & 0,221 & 135,000 & 448 \\
\hline 449 & 1,4 & 4,9 & 1,6 & 4,68 & 4,53 & 0,894 & 0,964 & 0,933 & 0,113 & 0,758 & 1,149 & 0,542 & 0,610 & 1,826 & 1,114 & 1,052 & 117,982 & 449 \\
\hline 450 & 1,5 & 5,4 & 1,9 & 4,91 & 4,75 & 0,814 & 0,915 & 0,886 & 0,204 & 0,671 & 1,221 & 0,564 & 0,687 & 1,860 & 1,277 & 0,990 & 117,902 & 450 \\
\hline 451 & 1,2 & 4,0 & 1,3 & 3,97 & 3,84 & 0,969 & 0,997 & 0,965 & 0,031 & 0,968 & 1,017 & 0,761 & 0,724 & 1,428 & 1,033 & 1,066 & 152,120 & 451 \\
\hline 452 & 0,2 & 1,5 & 0,2 & 1,39 & 1,34 & 0,984 & 0,954 & 0,924 & 0,048 & 0,904 & 1,052 & 0,576 & 0,574 & 0,579 & 0,332 & 0,357 & 153,330 & 452 \\
\hline 453 & 0,7 & 3,0 & 0,7 & 2,90 & 2,81 & 0,970 & 0,956 & 0,926 & 0,053 & 0,971 & 1,015 & 0,806 & 0,841 & 1,059 & 0,891 & 0,840 & 137,333 & 453 \\
\hline 454 & 0,2 & 1,7 & 0,2 & 1,60 & 1,55 & 0,937 & 0,938 & 0,908 & 0,088 & 0,941 & 1,031 & 0,535 & 0,549 & 0,721 & 0,396 & 0,396 & 100,020 & 454 \\
\hline
\end{tabular}




\begin{tabular}{|c|c|c|c|c|c|c|c|c|c|c|c|c|c|c|c|c|c|c|}
\hline 455 & 0,2 & 1,5 & 0,2 & 1,35 & 1,30 & 0,932 & 0,894 & 0,865 & 0,126 & 0,921 & 1,042 & 0,766 & 0,820 & 0,526 & 0,431 & 0,396 & 102,361 & 455 \\
\hline 456 & 0,1 & 1,2 & 0,1 & 1,09 & 1,06 & 0,904 & 0,921 & 0,891 & 0,124 & 1,000 & 0,987 & 0,665 & 0,566 & 0,470 & 0,266 & 0,280 & 24,305 & 456 \\
\hline 457 & 3,4 & 9,6 & 3,7 & 9,44 & 9,14 & 0,915 & 0,984 & 0,953 & 0,087 & 0,466 & 1,464 & 0,218 & 0,255 & 4,462 & 1,139 & 1,155 & 124,412 & 457 \\
\hline 458 & 0,7 & 3,1 & 0,8 & 3,07 & 2,97 & 0,947 & 0,988 & 0,956 & 0,054 & 0,971 & 1,015 & 0,743 & 0,694 & 1,130 & 0,784 & 0,798 & 43,012 & 458 \\
\hline 459 & 0,1 & 1,1 & 0,1 & 1,00 & 0,97 & 0,957 & 0,938 & 0,908 & 0,076 & 1,000 & 0,916 & 0,824 & 0,705 & 0,408 & 0,288 & 0,313 & 6,368 & 459 \\
\hline 460 & 0,3 & 2,0 & 0,3 & 1,90 & 1,83 & 0,952 & 0,961 & 0,930 & 0,062 & 0,934 & 1,035 & 0,578 & 0,620 & 0,798 & 0,495 & 0,495 & 103,823 & 460 \\
\hline 461 & 0,3 & 1,8 & 0,3 & 1,69 & 1,64 & 0,945 & 0,927 & 0,897 & 0,091 & 0,963 & 1,019 & 0,744 & 0,732 & 0,660 & 0,484 & 0,443 & 125,143 & 461 \\
\hline 462 & 10,3 & 13,1 & 11,1 & 11,98 & 11,59 & 0,935 & 0,912 & 0,882 & 0,110 & 0,753 & 1,152 & 0,635 & 0,690 & 4,556 & 3,144 & 3,011 & 134,266 & 462 \\
\hline 463 & 0,1 & 1,2 & 0,1 & 1,15 & 1,11 & 0,979 & 0,924 & 0,895 & 0,078 & 0,937 & 1,033 & 0,479 & 0,358 & 0,553 & 0,198 & 0,280 & 89,343 & 463 \\
\hline 464 & 22,3 & 21,5 & 24,1 & 20,87 & 20,20 & 0,927 & 0,971 & 0,940 & 0,078 & 0,608 & 1,282 & 0,339 & 0,370 & 9,168 & 3,390 & 3,164 & 98,459 & 464 \\
\hline 465 & 0,1 & 1,2 & 0,1 & 1,09 & 1,06 & 0,945 & 0,921 & 0,891 & 0,096 & 1,000 & 0,939 & 0,895 & 0,772 & 0,426 & 0,329 & 0,357 & 21,525 & 465 \\
\hline 466 & 0,1 & 1,4 & 0,1 & 1,34 & 1,29 & 0,965 & 0,953 & 0,922 & 0,059 & 0,861 & 1,078 & 0,412 & 0,307 & 0,645 & 0,198 & 0,297 & 178,685 & 466 \\
\hline 467 & 1,3 & 4,3 & 1,4 & 4,25 & 4,12 & 0,935 & 0,985 & 0,953 & 0,067 & 0,873 & 1,070 & 0,700 & 0,754 & 1,535 & 1,157 & 1,019 & 151,997 & 467 \\
\hline 468 & 0,2 & 1,4 & 0,2 & 1,29 & 1,25 & 0,971 & 0,932 & 0,902 & 0,073 & 1,000 & 0,958 & 0,746 & 0,722 & 0,533 & 0,385 & 0,396 & 26,152 & 468 \\
\hline 469 & 0,2 & 1,5 & 0,2 & 1,41 & 1,37 & 0,952 & 0,955 & 0,924 & 0,066 & 1,000 & 0,949 & 0,734 & 0,663 & 0,579 & 0,384 & 0,396 & 166,645 & 469 \\
\hline 470 & 17,5 & 18,4 & 20,4 & 17,84 & 17,26 & 0,855 & 0,971 & 0,940 & 0,147 & 0,651 & 1,239 & 0,456 & 0,591 & 6,986 & 4,130 & 3,390 & 132,352 & 470 \\
\hline 471 & 0,8 & 3,3 & 0,9 & 3,24 & 3,13 & 0,977 & 0,988 & 0,956 & 0,026 & 0,987 & 1,007 & 0,735 & 0,647 & 1,209 & 0,782 & 0,773 & 126,205 & 471 \\
\hline 472 & 0,1 & 1,0 & 0,1 & 0,71 & 0,68 & 0,929 & 0,736 & 0,712 & 0,274 & 0,867 & 1,074 & 0,453 & 0,391 & 0,423 & 0,165 & 0,198 & 75,911 & 472 \\
\hline 473 & 0,9 & 3,4 & 0,9 & 3,29 & 3,19 & 0,974 & 0,980 & 0,949 & 0,033 & 1,000 & 0,998 & 0,860 & 0,824 & 1,156 & 0,952 & 0,934 & 58,207 & 473 \\
\hline 474 & 0,4 & 2,1 & 0,4 & 1,96 & 1,90 & 0,980 & 0,954 & 0,924 & 0,050 & 1,000 & 0,966 & 0,777 & 0,709 & 0,769 & 0,545 & 0,577 & 14,394 & 474 \\
\hline 475 & 0,3 & 2,3 & 0,4 & 2,19 & 2,12 & 0,913 & 0,943 & 0,913 & 0,104 & 0,784 & 1,130 & 0,516 & 0,585 & 0,910 & 0,532 & 0,495 & 154,736 & 475 \\
\hline 476 & 0,1 & 1,2 & 0,1 & 1,13 & 1,10 & 0,944 & 0,954 & 0,923 & 0,072 & 1,000 & 0,948 & 0,730 & 0,619 & 0,467 & 0,289 & 0,313 & 136,344 & 476 \\
\hline 477 & 0,6 & 2,7 & 0,6 & 2,60 & 2,52 & 0,960 & 0,975 & 0,944 & 0,048 & 1,000 & 0,988 & 0,800 & 0,751 & 0,961 & 0,722 & 0,714 & 148,467 & 477 \\
\hline 478 & 0,1 & 1,1 & 0,1 & 0,96 & 0,93 & 0,941 & 0,837 & 0,810 & 0,174 & 1,000 & 0,945 & 0,719 & 0,679 & 0,456 & 0,310 & 0,357 & 131,909 & 478 \\
\hline 479 & 57,6 & 31,3 & 63,8 & 30,09 & 29,13 & 0,903 & 0,963 & 0,932 & 0,104 & 0,741 & 1,162 & 0,564 & 0,623 & 11,398 & 7,104 & 5,962 & 101,385 & 479 \\
\hline 480 & 0,1 & 1,3 & 0,1 & 1,12 & 1,09 & 0,881 & 0,875 & 0,846 & 0,173 & 0,694 & 1,201 & 0,346 & 0,294 & 0,577 & 0,170 & 0,198 & 66,718 & 480 \\
\hline 481 & 2,9 & 6,1 & 3,0 & 6,08 & 5,89 & 0,957 & 0,994 & 0,962 & 0,044 & 0,964 & 1,018 & 0,901 & 0,938 & 2,016 & 1,890 & 1,683 & 51,512 & 481 \\
\hline 482 & 0,1 & 0,7 & 0,1 & 0,64 & 0,62 & 0,913 & 0,872 & 0,844 & 0,155 & 1,000 & 0,914 & 0,723 & 0,493 & 0,301 & 0,149 & 0,198 & 0,000 & 482 \\
\hline 483 & 1,0 & 4,5 & 1,1 & 4,35 & 4,21 & 0,915 & 0,976 & 0,945 & 0,089 & 0,648 & 1,243 & 0,332 & 0,368 & 1,981 & 0,729 & 0,693 & 94,966 & 483 \\
\hline 484 & 0,1 & 1,1 & 0,1 & 0,80 & 0,78 & 0,925 & 0,759 & 0,735 & 0,252 & 1,000 & 0,988 & 0,693 & 0,606 & 0,408 & 0,248 & 0,280 & 12,744 & 484 \\
\hline 485 & 0,1 & 1,0 & 0,1 & 0,80 & 0,78 & 0,944 & 0,833 & 0,807 & 0,176 & 1,000 & 0,939 & 0,637 & 0,485 & 0,408 & 0,198 & 0,280 & 82,466 & 485 \\
\hline 486 & 2,8 & 6,6 & 3,0 & 6,32 & 6,11 & 0,939 & 0,961 & 0,930 & 0,073 & 0,806 & 1,114 & 0,615 & 0,570 & 2,395 & 1,365 & 1,287 & 137,961 & 486 \\
\hline 487 & 0,5 & 2,9 & 0,6 & 2,90 & 2,80 & 0,903 & 0,987 & 0,955 & 0,098 & 0,802 & 1,117 & 0,481 & 0,463 & 1,206 & 0,559 & 0,495 & 111,396 & 487 \\
\hline 488 & 4,7 & 8,3 & 4,8 & 8,19 & 7,93 & 0,966 & 0,989 & 0,957 & 0,035 & 0,853 & 1,083 & 0,617 & 0,570 & 3,100 & 1,768 & 1,763 & 148,523 & 488 \\
\hline 489 & 1,1 & 4,5 & 1,2 & 4,36 & 4,22 & 0,922 & 0,967 & 0,936 & 0,085 & 0,680 & 1,212 & 0,383 & 0,462 & 1,912 & 0,884 & 0,773 & 71,121 & 489 \\
\hline 490 & 0,1 & 0,8 & 0,1 & 0,74 & 0,71 & 0,967 & 0,875 & 0,847 & 0,130 & 1,000 & 0,889 & $\begin{array}{l}0,923 \\
0555\end{array}$ & 0,783 & 0,313 & 0,245 & 0,280 & $\begin{array}{l}159,699 \\
15770\end{array}$ & 490 \\
\hline 491 & 0,1 & 1,0 & 0,1 & 0,97 & 0,94 & 0,972 & 0,946 & 0,915 & 0,060 & 1,000 & 0,990 & 0,557 & 0,450 & 0,443 & 0,199 & 0,221 & 115,772 & 491 \\
\hline 492 & 0,2 & 1,7 & 0,2 & 1,52 & 1,48 & 0,949 & 0,920 & 0,890 & 0,095 & 0,841 & 1,090 & 0,450 & 0,412 & 0,721 & 0,297 & 0,357 & 77,783 & 492 \\
\hline 493 & 3,1 & 6,6 & 3,2 & 6,48 & 6,27 & 0,965 & 0,984 & 0,953 & 0,039 & 0,907 & 1,050 & 0,720 & 0,784 & 2,352 & 1,845 & 1,680 & 80,447 & 493 \\
\hline 494 & 0,3 & 2,1 & 0,4 & 2,07 & 2,00 & 0,914 & 0,969 & 0,938 & 0,091 & 0,941 & 1,031 & 0,708 & 0,595 & 0,783 & 0,466 & 0,443 & 120,321 & 494 \\
\hline 495 & 0,1 & 1,3 & 0,2 & 1,12 & 1,09 & 0,937 & 0,857 & 0,829 & 0,157 & 1,000 & 0,971 & 0,709 & 0,680 & 0,510 & 0,347 & 0,357 & 73,952 & 495 \\
\hline 496 & 0,2 & 1,6 & 0,2 & 1,55 & 1,50 & 0,968 & 0,976 & 0,944 & 0,040 & 1,000 & 0,942 & 0,937 & 0,822 & 0,553 & 0,455 & 0,495 & 142,207 & 496 \\
\hline 497 & 0,2 & 1,7 & 0,2 & 1,44 & 1,40 & 0,978 & 0,870 & 0,842 & 0,132 & 0,998 & 1,001 & 0,626 & 0,669 & 0,666 & 0,446 & 0,443 & 54,946 & 497 \\
\hline 498 & 0,5 & 3,4 & 0,6 & 3,29 & 3,18 & 0,880 & 0,972 & 0,941 & 0,123 & 0,592 & 1,299 & 0,315 & 0,299 & 1,477 & 0,441 & 0,495 & 130,628 & 498 \\
\hline 499 & 0,3 & 2,2 & 0,4 & 2,06 & 1,99 & 0,820 & 0,921 & 0,892 & 0,197 & 0,761 & 1,147 & 0,529 & 0,506 & 0,852 & 0,431 & 0,357 & 110,329 & 499 \\
\hline 500 & 2,8 & 6,7 & 3,1 & 6,62 & 6,41 & 0,902 & 0,986 & 0,955 & 0,099 & 0,771 & 1,139 & 0,594 & 0,640 & 2,435 & 1,560 & 1,428 & 54,503 & 500 \\
\hline 501 & 0,2 & 1,7 & 0,2 & 1,64 & 1,59 & 0,931 & 0,977 & 0,946 & 0,073 & 1,000 & 0,987 & 0,809 & 0,772 & 0,602 & 0,465 & 0,495 & 7,957 & 501 \\
\hline 502 & 0,1 & 0,7 & 0,1 & 0,55 & 0,53 & 0,958 & 0,744 & 0,720 & 0,259 & 1,000 & 0,873 & 0,714 & 0,552 & 0,317 & 0,175 & 0,198 & 29,035 & 502 \\
\hline 503 & 0,1 & 1,4 & 0,2 & 1,28 & 1,24 & 0,923 & 0,913 & 0,885 & 0,116 & 0,939 & 1,032 & 0,611 & 0,537 & 0,553 & 0,297 & 0,357 & 175,996 & 503 \\
\hline 504 & 0,5 & 2,8 & 0,6 & 2,72 & 2,64 & 0,884 & 0,972 & 0,941 & 0,119 & 0,871 & 1,071 & 0,758 & 0,823 & 0,956 & 0,787 & 0,664 & 108,348 & 504 \\
\hline 505 & 0,1 & 1,2 & 0,1 & 1,13 & 1,10 & 0,958 & 0,976 & 0,945 & 0,048 & 1,000 & 0,975 & 0,732 & 0,712 & 0,443 & 0,315 & 0,313 & 152,214 & 505 \\
\hline 506 & 0,1 & 1,4 & 0,1 & 1,24 & 1,20 & 0,918 & 0,904 & 0,875 & 0,127 & 0,912 & 1,047 & 0,482 & 0,411 & 0,602 & 0,248 & 0,280 & 84,701 & 506 \\
\hline 507 & 1,7 & 4,9 & 1,8 & 4,87 & 4,72 & 0,978 & 0,987 & 0,955 & 0,026 & 0,890 & 1,060 & 0,607 & 0,624 & 1,904 & 1,188 & 1,188 & 96,959 & 507 \\
\hline 508 & 0,1 & 1,0 & 0,1 & 0,77 & 0,75 & 0,889 & 0,782 & 0,757 & 0,244 & 1,000 & 0,996 & 0,668 & 0,605 & 0,387 & 0,234 & 0,221 & 133,693 & 508 \\
\hline 509 & 6,5 & 10,5 & 7,2 & 10,23 & 9,90 & 0,903 & 0,977 & 0,946 & 0,100 & 0,747 & 1,157 & 0,486 & 0,607 & 4,133 & 2,508 & 2,376 & 37,982 & 509 \\
\hline 510 & 0,2 & 1,9 & 0,3 & 1,75 & 1,69 & 0,917 & 0,929 & 0,899 & 0,109 & 0,873 & 1,070 & 0,546 & 0,550 & 0,756 & 0,416 & 0,420 & 129,759 & 510 \\
\hline 511 & 0,2 & 1,4 & 0,2 & 1,25 & 1,21 & 0,939 & 0,904 & 0,875 & 0,113 & 0,993 & 1,004 & 0,699 & 0,640 & 0,526 & 0,337 & 0,357 & 47,483 & 511 \\
\hline 512 & 0,2 & 2,7 & 0,4 & 2,13 & 2,06 & 0,658 & 0,777 & 0,752 & 0,409 & 0,400 & 1,581 & 0,499 & 0,773 & 0,783 & 0,605 & 0,198 & 162,006 & 512 \\
\hline
\end{tabular}




\begin{tabular}{|c|c|c|c|c|c|c|c|c|c|c|c|c|c|c|c|c|c|c|c|}
\hline 513 & 0,2 & 1,6 & 0,2 & 1,60 & 1,55 & 0,925 & 0,976 & 0,945 & 0,079 & 0,984 & 1,008 & 0,615 & 0,583 & 0,660 & 0,385 & 0,396 & 90,112 & 513 & $-2,7$ \\
\hline 514 & 0,2 & 1,5 & 0,2 & 1,44 & 1,40 & 0,986 & 0,981 & 0,950 & 0,023 & 1,000 & 0,994 & 0,723 & 0,626 & 0,553 & 0,347 & 0,396 & 164,193 & 514 & $-2,5$ \\
\hline 515 & 0,1 & 1,3 & 0,1 & 1,16 & 1,12 & 0,904 & 0,925 & 0,895 & 0,122 & 0,920 & 1,042 & 0,598 & 0,537 & 0,495 & 0,266 & 0,280 & 108,728 & 515 & $-2,3$ \\
\hline 516 & 0,7 & 3,0 & 0,7 & 2,98 & 2,89 & 0,952 & 0,987 & 0,956 & 0,049 & 0,944 & 1,029 & 0,659 & 0,619 & 1,151 & 0,712 & 0,754 & 42,336 & 516 & $-3,5$ \\
\hline 517 & 0,1 & 1,0 & 0,1 & 1,00 & 0,97 & 0,978 & 0,963 & 0,932 & 0,043 & 1,000 & 0,882 & 1,081 & 0,874 & 0,360 & 0,315 & 0,357 & 78,348 & 517 & $-1,8$ \\
\hline 518 & 0,2 & 1,7 & 0,2 & 1,64 & 1,59 & 0,919 & 0,946 & 0,916 & 0,097 & 0,931 & 1,036 & 0,640 & 0,532 & 0,666 & 0,354 & 0,396 & 115,411 & 518 & $-2,7$ \\
\hline 519 & 0,1 & 0,8 & 0,1 & 0,74 & 0,71 & 0,964 & 0,950 & 0,920 & 0,062 & 1,000 & 0,848 & 1,011 & 0,728 & 0,289 & 0,210 & 0,280 & 140,092 & 519 & $-1,5$ \\
\hline 520 & 0,1 & 1,3 & 0,2 & 1,11 & 1,07 & 0,968 & 0,838 & 0,811 & 0,166 & 1,000 & 0,975 & 0,659 & 0,464 & 0,533 & 0,248 & 0,297 & 1,987 & 520 & $-2,4$ \\
\hline 521 & 0,1 & 1,1 & 0,1 & 0,96 & 0,93 & 0,895 & 0,901 & 0,873 & 0,144 & 0,921 & 1,042 & 0,509 & 0,422 & 0,456 & 0,193 & 0,221 & 52,442 & 521 & $-2,2$ \\
\hline 522 & 0,2 & 1,8 & 0,2 & 1,69 & 1,64 & 0,973 & 0,941 & 0,911 & 0,064 & 0,697 & 1,198 & 0,323 & 0,250 & 0,840 & 0,210 & 0,280 & 133,183 & 522 & $-3,1$ \\
\hline 523 & 0,1 & 0,8 & 0,1 & 0,67 & 0,65 & 0,964 & 0,834 & 0,807 & 0,169 & 1,000 & 0,878 & 1,011 & 0,814 & 0,289 & 0,235 & 0,280 & 144,280 & 523 & $-1,5$ \\
\hline 524 & 0,6 & 3,3 & 0,7 & 3,17 & 3,06 & 0,916 & 0,960 & 0,929 & 0,093 & 0,745 & 1,159 & 0,449 & 0,476 & 1,351 & 0,644 & 0,664 & 91,579 & 524 & $-3,8$ \\
\hline 525 & 0,7 & 3,3 & 0,7 & 3,07 & 2,97 & 0,937 & 0,942 & 0,912 & 0,085 & 0,826 & 1,100 & 0,525 & 0,631 & 1,299 & 0,820 & 0,721 & 20,941 & 525 & $-3,7$ \\
\hline 526 & 1,7 & 5,2 & 1,8 & 5,08 & 4,92 & 0,933 & 0,980 & 0,948 & 0,070 & 0,778 & 1,134 & 0,464 & 0,465 & 2,138 & 0,993 & 0,975 & 146,534 & 526 & $-4,4$ \\
\hline 527 & 0,3 & 2,1 & 0,3 & 1,93 & 1,87 & 0,966 & 0,941 & 0,911 & 0,068 & 0,817 & 1,107 & 0,530 & 0,586 & 0,812 & 0,476 & 0,443 & 126,544 & 527 & $-3,0$ \\
\hline 528 & 0,1 & 0,9 & 0,1 & 0,80 & 0,78 & 0,914 & 0,858 & 0,830 & 0,166 & 1,000 & 0,941 & 0,668 & 0,533 & 0,387 & 0,206 & 0,221 & 138,430 & 528 & $-2,0$ \\
\hline 529 & 0,1 & 1,2 & 0,1 & 1,03 & 1,00 & 0,942 & 0,846 & 0,819 & 0,165 & 1,000 & 0,989 & 0,780 & 0,743 & 0,443 & 0,329 & 0,313 & 112,323 & 529 & $-2,1$ \\
\hline 530 & 0,0 & 0,7 & 0,1 & 0,51 & 0,49 & 0,952 & 0,719 & 0,696 & 0,285 & 1,000 & 0,901 & 0,688 & 0,493 & 0,301 & 0,149 & 0,198 & 4,837 & 530 & $-1,6$ \\
\hline 531 & 0,1 & 0,7 & 0,1 & 0,64 & 0,62 & 1,000 & 0,906 & 0,878 & 0,094 & 1,000 & 0,859 & 0,966 & 0,743 & 0,267 & 0,198 & 0,221 & 175,163 & 531 & $-1,4$ \\
\hline 532 & 0,1 & 1,0 & 0,1 & 0,83 & 0,80 & 0,937 & 0,838 & 0,811 & 0,174 & 0,945 & 1,029 & 0,562 & 0,485 & 0,408 & 0,198 & 0,221 & 79,898 & 532 & $-2,0$ \\
\hline 533 & 0,1 & 1,3 & 0,1 & 1,17 & 1,13 & 0,911 & 0,926 & 0,896 & 0,116 & 0,789 & 1,126 & 0,384 & 0,345 & 0,577 & 0,199 & 0,221 & 139,831 & 533 & $-2,5$ \\
\hline 534 & 0,2 & 1,7 & 0,2 & 1,61 & 1,56 & 0,951 & 0,924 & 0,894 & 0,091 & 0,778 & 1,134 & 0,444 & 0,392 & 0,736 & 0,289 & 0,313 & 33,471 & 534 & $-2,9$ \\
\hline 535 & 0,3 & 2,0 & 0,3 & 1,99 & 1,93 & 0,950 & 0,973 & 0,942 & 0,056 & 0,988 & 1,006 & 0,682 & 0,682 & 0,783 & 0,534 & 0,495 & 148,005 & 535 & $-3,0$ \\
\hline 536 & 0,7 & 3,1 & 0,8 & 3,06 & 2,96 & 0,935 & 0,979 & 0,948 & 0,069 & 0,904 & 1,052 & 0,700 & 0,697 & 1,129 & 0,787 & 0,721 & 145,696 & 536 & $-3,5$ \\
\hline 537 & 0,8 & 3,6 & 0,9 & 3,51 & 3,40 & 0,966 & 0,974 & 0,943 & 0,043 & 0,807 & 1,113 & 0,400 & 0,449 & 1,628 & 0,731 & 0,754 & 161,653 & 537 & $-4,0$ \\
\hline 538 & 0,3 & 1,9 & 0,3 & 1,66 & 1,60 & 0,969 & 0,860 & 0,832 & 0,144 & 1,000 & 0,982 & 0,730 & 0,610 & 0,731 & 0,446 & 0,495 & 90,291 & 538 & $-2,9$ \\
\hline 539 & 0,8 & 3,5 & 0,9 & 3,39 & 3,28 & 0,936 & 0,981 & 0,950 & 0,066 & 0,873 & 1,070 & 0,648 & 0,756 & 1,278 & 0,966 & 0,846 & 111,907 & 539 & $-3,7$ \\
\hline 540 & 6,1 & 10,1 & 6,5 & 9,35 & 9,05 & 0,932 & 0,929 & 0,899 & 0,098 & 0,755 & 1,151 & 0,630 & 0,681 & 3,506 & 2,387 & 2,137 & 104,014 & 540 & $-5,1$ \\
\hline 541 & 0,1 & 1,3 & 0,1 & 1,19 & 1,15 & 0,984 & 0,919 & 0,889 & 0,083 & 1,000 & 0,951 & 0,735 & 0,686 & 0,505 & 0,347 & 0,396 & 65,556 & 541 & $-2,3$ \\
\hline 542 & 0,1 & 1,3 & 0,1 & 1,22 & 1,18 & 0,872 & 0,909 & 0,880 & 0,157 & 0,707 & 1,190 & 0,360 & 0,312 & 0,596 & 0,186 & 0,198 & 0,114 & 542 & $-2,6$ \\
\hline 543 & 0,2 & 1,7 & 0,2 & 1,55 & 1,50 & 0,949 & 0,898 & 0,869 & 0,114 & 0,975 & 1,013 & 0,647 & 0,642 & 0,673 & 0,432 & 0,443 & 33,655 & 543 & $-2,8$ \\
\hline 544 & 0,1 & 1,2 & 0,1 & 1,03 & 1,00 & 0,929 & 0,853 & 0,826 & 0,163 & 1,000 & 0,951 & 0,828 & 0,671 & 0,443 & 0,297 & 0,297 & 176,644 & 544 & $-2,1$ \\
\hline 545 & 1,7 & 5,1 & 1,7 & 5,01 & 4,85 & 0,948 & 0,976 & 0,945 & 0,057 & 0,790 & 1,125 & 0,517 & 0,559 & 2,021 & 1,129 & 1,034 & 120,908 & 545 & $-4,3$ \\
\hline 546 & 0,7 & 3,3 & 0,8 & 3,16 & 3,06 & 0,932 & 0,968 & 0,937 & 0,075 & 0,829 & 1,098 & 0,556 & 0,605 & 1,269 & 0,767 & 0,714 & 155,528 & 546 & $-3,7$ \\
\hline 547 & 0,1 & 0,8 & 0,1 & 0,57 & 0,56 & 0,913 & 0,754 & 0,729 & 0,261 & 1,000 & 0,948 & 0,668 & 0,474 & 0,313 & 0,149 & 0,198 & 0,000 & 547 & $-1,6$ \\
\hline 548 & 0,1 & 1,1 & 0,1 & 1,00 & 0,97 & 0,956 & 0,914 & 0,885 & 0,097 & 1,000 & 0,951 & 0,740 & 0,658 & 0,426 & 0,280 & 0,297 & 138,281 & 548 & $-2,1$ \\
\hline 549 & 0,2 & 1,7 & 0,2 & 1,65 & 1,60 & 0,956 & 0,977 & 0,946 & 0,050 & 0,926 & 1,039 & 0,526 & 0,441 & 0,714 & 0,315 & 0,357 & 134,315 & 549 & $-2,8$ \\
\hline 550 & 0,1 & 1,0 & 0,1 & 0,84 & 0,81 & 0,939 & 0,840 & 0,813 & 0,171 & 0,955 & 1,023 & 0,464 & 0,383 & 0,456 & 0,175 & 0,221 & 43,051 & 550 & $-2,2$ \\
\hline 551 & 1,6 & 5,2 & 1,7 & 4,99 & 4,83 & 0,935 & 0,967 & 0,936 & 0,073 & 0,751 & 1,154 & 0,458 & 0,490 & 2,106 & 1,031 & 0,980 & 109,194 & 551 & $-4,4$ \\
\hline 552 & 1,3 & 4,3 & 1,4 & 4,20 & 4,07 & 0,960 & 0,978 & 0,947 & 0,046 & 0,917 & 1,044 & 0,700 & 0,747 & 1,565 & 1,170 & 1,107 & 132,582 & 552 & $-4,0$ \\
\hline 553 & 0,2 & 1,3 & 0,2 & 1,22 & 1,18 & 0,971 & 0,909 & 0,880 & 0,095 & 1,000 & 0,924 & 0,962 & 0,738 & 0,470 & 0,347 & 0,396 & 94,413 & 553 & $\begin{array}{l}-2,2 \\
\end{array}$ \\
\hline 554 & 0,1 & 0,8 & 0,1 & 0,70 & 0,67 & 0,917 & 0,900 & 0,872 & 0,130 & 1,000 & 0,940 & 0,757 & 0,638 & 0,301 & 0,192 & 0,221 & 5,316 & 554 & $-1,6$ \\
\hline 555 & 0,4 & 2,3 & 0,4 & 2,25 & 2,18 & 0,952 & 0,971 & 0,940 & 0,056 & 0,909 & 1,049 & 0,585 & 0,498 & 0,921 & 0,459 & 0,495 & 59,783 & 555 & $-3,2$ \\
\hline 556 & 0,1 & 1,1 & 0,1 & 1,07 & 1,03 & 1,000 & 0,941 & 0,911 & 0,059 & 1,000 & 0,973 & 0,700 & 0,601 & 0,443 & 0,266 & 0,280 & 144,848 & 556 & $-2,1$ \\
\hline 557 & 0,6 & 2,8 & 0,7 & 2,71 & 2,63 & 0,933 & 0,963 & 0,932 & 0,076 & 0,978 & 1,011 & 0,755 & 0,714 & 1,021 & 0,729 & 0,693 & 91,170 & 557 & $-3,4$ \\
\hline 558 & 0,2 & 1,5 & 0,2 & 1,32 & 1,28 & 0,963 & 0,882 & 0,854 & 0,124 & 1,000 & 0,966 & 0,795 & 0,626 & 0,553 & 0,347 & 0,396 & 0,000 & 558 & $-2,5$ \\
\hline 559 & 0,1 & 1,0 & 0,1 & 0,90 & 0,87 & 0,947 & 0,920 & 0,891 & 0,096 & 1,000 & 0,924 & 0,705 & 0,620 & $\begin{array}{l}0,353 \\
0,399\end{array}$ & 0,248 & $\begin{array}{l}0,580 \\
0,280\end{array}$ & 86,048 & $\begin{array}{l}500 \\
559\end{array}$ & $\begin{array}{l}-2,0 \\
-2,0\end{array}$ \\
\hline 560 & 0,3 & 2,0 & 0,3 & 1,96 & 1,90 & 0,936 & 0,981 & 0,949 & 0,067 & 1,000 & 0,996 & 0,814 & 0,838 & 0,709 & 0,594 & 0,560 & 11,590 & 560 & $-2,8$ \\
\hline 561 & 1,6 & 5,3 & 1,8 & 4,95 & $\begin{array}{l}4,79 \\
\end{array}$ & 0,862 & 0,938 & 0,908 & 0,151 & 0,710 & 1,187 & 0,640 & 0,767 & 1,769 & 1,356 & 1,066 & 176,205 & 561 & $-4,1$ \\
\hline 562 & 0,1 & 1,1 & 0,1 & 0,99 & 0,96 & 0,927 & 0,882 & 0,854 & 0,139 & 0,931 & 1,037 & 0,479 & 0,398 & 0,498 & 0,198 & 0,221 & 175,833 & 562 & $-2,3$ \\
\hline 563 & 0,4 & 2,5 & 0,5 & 2,47 & 2,39 & 0,932 & 0,989 & 0,957 & 0,069 & 0,874 & 1,069 & 0,530 & 0,566 & 1,021 & 0,578 & 0,577 & 168,975 & 563 & $-3,4$ \\
\hline 564 & 0,1 & 1,3 & 0,1 & 1,24 & 1,20 & 0,926 & 0,970 & 0,938 & 0,080 & 0,945 & 1,029 & 0,601 & 0,467 & 0,510 & 0,238 & 0,280 & 129,829 & 564 & $-2,4$ \\
\hline 565 & 0,1 & 1,2 & 0,1 & 1,03 & 1,00 & 0,939 & 0,886 & 0,858 & 0,130 & 1,000 & 0,975 & 0,861 & 0,805 & 0,408 & 0,329 & 0,297 & 2,329 & 565 & $-2,0$ \\
\hline 566 & 3,4 & 7,3 & 3,5 & 7,20 & 6,97 & 0,970 & 0,982 & 0,950 & 0,035 & 0,791 & 1,125 & 0,413 & 0,472 & 3,233 & 1,524 & 1,511 & 22,841 & 566 & $-5,0$ \\
\hline 567 & 0,1 & 0,7 & 0,1 & 0,64 & 0,62 & 0,957 & 0,859 & 0,832 & 0,148 & 1,000 & 0,906 & 0,966 & 0,748 & 0,267 & 0,199 & 0,198 & 140,062 & 567 & $-1,4$ \\
\hline 568 & 0,5 & 3,1 & 0,7 & 2,86 & 2,77 & 0,812 & 0,920 & 0,890 & 0,205 & 0,700 & 1,195 & 0,555 & 0,742 & 1,112 & 0,825 & 0,594 & 161,810 & 568 & $-3,5$ \\
\hline 569 & 0,1 & 1,0 & 0,1 & 0,80 & 0,78 & 0,897 & 0,810 & 0,784 & 0,216 & 1,000 & 0,952 & 0,731 & 0,608 & 0,387 & 0,235 & 0,280 & 64,268 & 569 & $-2,0$ \\
\hline 570 & 0,1 & 0,7 & 0,1 & 0,57 & 0,56 & 0,920 & 0,782 & 0,756 & 0,232 & 1,000 & 0,873 & 1,010 & 0,743 & 0,267 & 0,198 & $\begin{array}{l}0,221 \\
0,21\end{array}$ & 13,776 & 570 & $-1,4$ \\
\hline
\end{tabular}




\begin{tabular}{|c|c|c|c|c|c|c|c|c|c|c|c|c|c|c|c|c|c|c|}
\hline 571 & 1,4 & 5,0 & 1,4 & 4,94 & 4,78 & 0,949 & 0,987 & 0,955 & 0,053 & 0,682 & 1,211 & 0,340 & 0,339 & 2,258 & 0,764 & 0,773 & 46,232 & 571 \\
\hline 572 & 2,1 & 6,0 & 2,3 & 5,82 & 5,63 & 0,929 & 0,971 & 0,940 & 0,077 & 0,740 & 1,162 & 0,510 & 0,640 & 2,297 & 1,469 & 1,302 & 53,859 & 572 \\
\hline 573 & 0,5 & 2,7 & 0,6 & 2,52 & 2,44 & 0,931 & 0,931 & 0,901 & 0,098 & 0,903 & 1,052 & 0,668 & 0,707 & 1,002 & 0,709 & 0,664 & 63,694 & 573 \\
\hline 574 & 0,2 & 1,4 & 0,2 & 1,35 & 1,30 & 0,959 & 0,953 & 0,923 & 0,062 & 1,000 & 0,956 & 0,695 & 0,610 & 0,564 & 0,345 & 0,357 & 11,548 & 574 \\
\hline 575 & 2,8 & 6,7 & 2,9 & 6,61 & 6,40 & 0,966 & 0,986 & 0,954 & 0,037 & 0,791 & 1,125 & 0,476 & 0,503 & 2,751 & 1,383 & 1,347 & 28,438 & 575 \\
\hline 576 & 6,0 & 10,0 & 6,5 & 9,81 & 9,49 & 0,921 & 0,982 & 0,950 & 0,081 & 0,752 & 1,153 & 0,507 & 0,522 & 3,873 & 2,021 & 1,879 & 178,417 & 576 \\
\hline 577 & 0,4 & 2,4 & 0,4 & 2,15 & 2,08 & 0,906 & 0,905 & 0,876 & 0,134 & 0,895 & 1,057 & 0,642 & 0,707 & 0,892 & 0,631 & 0,533 & 23,659 & 577 \\
\hline 578 & 0,2 & 1,5 & 0,2 & 1,36 & 1,32 & 0,877 & 0,895 & 0,866 & 0,162 & 0,854 & 1,082 & 0,453 & 0,450 & 0,664 & 0,299 & 0,357 & 27,014 & 578 \\
\hline 579 & 0,1 & 1,0 & 0,1 & 0,93 & 0,90 & 0,943 & 0,899 & 0,870 & 0,116 & 0,942 & 1,030 & 0,494 & 0,383 & 0,456 & 0,175 & 0,221 & 144,066 & 579 \\
\hline 580 & 0,9 & 3,7 & 0,9 & 3,55 & 3,43 & 0,924 & 0,964 & 0,933 & 0,084 & 0,798 & 1,119 & 0,529 & 0,562 & 1,439 & 0,809 & 0,773 & 164,356 & 580 \\
\hline 581 & 6,2 & 11,1 & 7,7 & 10,62 & 10,28 & 0,805 & 0,956 & 0,926 & 0,200 & 0,632 & 1,258 & 0,560 & 0,805 & 3,757 & 3,025 & 1,973 & 30,536 & 581 \\
\hline 582 & 0,5 & 3,0 & 0,6 & 2,95 & 2,86 & 0,855 & 0,969 & 0,938 & 0,148 & 0,648 & 1,242 & 0,390 & 0,393 & 1,249 & 0,492 & 0,495 & 111,684 & 582 \\
\hline 583 & 0,1 & 1,6 & 0,2 & 1,32 & 1,28 & 0,884 & 0,845 & 0,818 & 0,194 & 0,769 & 1,140 & 0,460 & 0,538 & 0,644 & 0,347 & 0,313 & 69,339 & 583 \\
\hline 584 & 1,9 & 5,8 & 2,0 & 5,71 & 5,53 & 0,955 & 0,993 & 0,961 & 0,045 & 0,734 & 1,167 & 0,406 & 0,453 & 2,462 & 1,116 & 1,089 & 117,013 & 584 \\
\hline 585 & 0,4 & 2,5 & 0,4 & 2,28 & 2,21 & 0,930 & 0,924 & 0,895 & 0,103 & 0,742 & 1,161 & 0,417 & 0,443 & 1,049 & 0,465 & 0,495 & 18,809 & 585 \\
\hline 586 & 1,6 & 4,5 & 1,6 & 4,45 & 4,30 & 0,970 & 0,991 & 0,960 & 0,031 & 0,983 & 1,009 & 0,676 & 0,651 & 1,722 & 1,121 & 1,188 & 175,092 & 586 \\
\hline 587 & 0,2 & 1,7 & 0,3 & 1,68 & 1,63 & 0,951 & 0,962 & 0,931 & 0,062 & 0,990 & 1,005 & 0,816 & 0,833 & 0,612 & 0,510 & 0,443 & 169,243 & 587 \\
\hline 588 & 0,1 & 0,8 & 0,1 & 0,77 & 0,75 & 1,000 & 0,952 & 0,922 & 0,048 & 1,000 & 0,860 & 0,998 & 0,814 & 0,301 & 0,245 & 0,280 & 45,003 & 588 \\
\hline 589 & 0,1 & 1,4 & 0,1 & 1,27 & 1,22 & 0,932 & 0,931 & 0,901 & 0,097 & 0,917 & 1,044 & 0,480 & 0,468 & 0,598 & 0,280 & 0,313 & 34,037 & 589 \\
\hline 590 & 0,4 & 2,1 & 0,4 & 2,05 & 1,98 & 0,973 & 0,981 & 0,950 & 0,033 & 1,000 & 0,992 & 0,784 & 0,766 & 0,757 & 0,580 & 0,577 & 116,406 & 590 \\
\hline 591 & 0,1 & 1,2 & 0,1 & 1,06 & 1,02 & 0,900 & 0,888 & 0,860 & 0,150 & 0,982 & 1,009 & 0,541 & 0,478 & 0,510 & 0,244 & 0,280 & 112,731 & 591 \\
\hline 592 & 0,9 & 3,4 & 0,9 & 3,35 & 3,25 & 0,958 & 0,981 & 0,949 & 0,046 & 0,961 & 1,020 & 0,730 & 0,732 & 1,249 & 0,914 & 0,886 & 105,669 & 592 \\
\hline 593 & 0,5 & 2,8 & 0,6 & 2,56 & 2,48 & 0,907 & 0,901 & 0,872 & 0,135 & 0,784 & 1,129 & 0,519 & 0,535 & 1,112 & 0,595 & 0,602 & 170,147 & 593 \\
\hline 594 & 0,5 & 2,6 & 0,5 & 2,43 & 2,35 & 0,932 & 0,932 & 0,903 & 0,096 & 0,937 & 1,033 & 0,758 & 0,791 & 0,921 & 0,729 & 0,693 & 136,885 & 594 \\
\hline 595 & 1,8 & 6,8 & 2,2 & 5,59 & 5,41 & 0,800 & 0,827 & 0,800 & 0,265 & 0,486 & 1,435 & 0,430 & 0,492 & 2,286 & 1,125 & 0,913 & 24,708 & 595 \\
\hline 596 & 1,7 & 5,6 & 1,8 & 5,42 & 5,25 & 0,957 & 0,968 & 0,937 & 0,053 & 0,683 & 1,210 & 0,362 & 0,364 & 2,445 & 0,889 & 0,886 & 162,881 & 596 \\
\hline 597 & 0,1 & 1,1 & 0,1 & 1,03 & 1,00 & 1,000 & 0,949 & 0,919 & 0,051 & 1,000 & 0,890 & 0,940 & 0,744 & 0,399 & 0,297 & 0,357 & 108,298 & 597 \\
\hline 598 & 0,1 & 1,2 & 0,1 & 1,08 & 1,05 & 0,944 & 0,891 & 0,863 & 0,122 & 1,000 & 0,970 & 0,649 & 0,569 & 0,495 & 0,282 & 0,297 & 154,030 & 598 \\
\hline 599 & 0,2 & 1,5 & 0,2 & 1,39 & 1,34 & 0,886 & 0,896 & 0,868 & 0,154 & 0,798 & 1,119 & 0,481 & 0,520 & 0,634 & 0,329 & 0,297 & 143,453 & 599 \\
\hline 600 & 0,3 & 1,8 & 0,3 & 1,75 & 1,69 & 0,938 & 0,949 & 0,919 & 0,081 & 0,955 & 1,023 & 0,594 & 0,555 & 0,743 & 0,412 & 0,420 & 51,016 & 600 \\
\hline 601 & 24,7 & 19,1 & 25,9 & 18,80 & 18,20 & 0,953 & 0,986 & 0,955 & 0,049 & 0,855 & 1,081 & 0,702 & 0,686 & 6,693 & 4,594 & 4,397 & 120,954 & 601 \\
\hline 602 & 1,0 & 4,0 & 1,1 & 3,74 & 3,62 & 0,887 & 0,938 & 0,908 & 0,129 & 0,792 & 1,124 & 0,602 & 0,677 & 1,456 & 0,985 & 0,897 & 127,983 & 602 \\
\hline 603 & 0,1 & 1,1 & 0,1 & 1,02 & 0,98 & 1,000 & 0,885 & 0,856 & 0,115 & 1,000 & 0,917 & 0,812 & 0,671 & 0,443 & 0,297 & 0,313 & 106,575 & 603 \\
\hline 604 & 0,2 & 1,7 & 0,2 & 1,56 & 1,51 & 0,976 & 0,937 & 0,907 & 0,067 & 0,918 & 1,043 & 0,539 & 0,500 & 0,693 & 0,347 & 0,396 & 83,546 & 604 \\
\hline 605 & 0,2 & 1,7 & 0,2 & 1,60 & 1,55 & 0,872 & 0,960 & 0,930 & 0,134 & 0,752 & 1,153 & 0,408 & 0,388 & 0,721 & 0,279 & 0,297 & 76,996 & 605 \\
\hline 606 & 1,5 & 5,1 & 1,5 & 4,96 & 4,80 & 0,950 & 0,969 & 0,938 & 0,059 & 0,695 & 1,199 & 0,356 & 0,414 & 2,279 & 0,945 & 0,975 & 123,290 & 606 \\
\hline 607 & 0,1 & 0,9 & 0,1 & 0,84 & 0,81 & 0,943 & 0,927 & 0,897 & 0,093 & 1,000 & 0,899 & 0,840 & 0,707 & 0,350 & 0,248 & 0,280 & 2,650 & 607 \\
\hline 608 & 0,6 & 2,9 & 0,6 & 2,77 & 2,68 & 0,962 & 0,967 & 0,936 & 0,050 & 0,948 & 1,027 & 0,688 & 0,719 & 1,071 & 0,770 & 0,714 & 138,825 & 608 \\
\hline 609 & 0,1 & 1,0 & 0,1 & 0,90 & 0,87 & 0,905 & 0,871 & 0,843 & 0,160 & 1,000 & 0,950 & 0,913 & 0,824 & 0,360 & 0,297 & 0,297 & 109,802 & 609 \\
\hline 610 & 0,4 & 2,1 & 0,4 & 2,02 & 1,95 & 0,961 & 0,968 & 0,937 & 0,050 & 1,000 & 0,975 & 0,725 & 0,682 & 0,798 & 0,545 & 0,577 & 158,434 & 610 \\
\hline 611 & 0,1 & 1,3 & 0,1 & 1,16 & 1,12 & 0,900 & 0,897 & 0,868 & 0,143 & 0,995 & 1,003 & 0,661 & 0,686 & 0,505 & 0,347 & 0,357 & 169,669 & 611 \\
\hline 612 & 0,1 & 1,1 & 0,1 & 0,97 & 0,94 & 0,905 & 0,912 & 0,883 & 0,130 & 1,000 & 0,986 & 0,590 & 0,552 & 0,448 & 0,248 & 0,280 & 179,208 & 612 \\
\hline 613 & 25,0 & 22,4 & 28,4 & 20,22 & 19,57 & 0,879 & 0,902 & 0,873 & 0,156 & 0,624 & 1,266 & 0,507 & 0,544 & 7,922 & 4,309 & 3,488 & 1,259 & 613 \\
\hline 614 & 0,3 & 1,7 & 0,3 & 1,59 & 1,54 & 0,962 & 0,923 & 0,894 & 0,086 & 1,000 & 0,972 & 0,730 & 0,600 & 0,660 & 0,396 & 0,443 & 83,191 & 614 \\
\hline 615 & 0,6 & 3,1 & 0,6 & 3,00 & 2,90 & 0,937 & 0,966 & 0,935 & 0,071 & 0,766 & 1,143 & 0,499 & 0,515 & 1,223 & 0,630 & 0,602 & 53,294 & 615 \\
\hline 616 & 2,8 & 9,1 & 3,0 & 9,01 & 8,72 & 0,944 & 0,991 & 0,959 & 0,057 & 0,430 & 1,525 & 0,206 & 0,196 & 4,177 & 0,820 & 0,846 & 62,103 & 616 \\
\hline 617 & 0,2 & 1,4 & 0,2 & 1,28 & 1,24 & 0,940 & 0,924 & 0,895 & 0,097 & 1,000 & 0,996 & 0,710 & 0,631 & 0,526 & 0,332 & 0,357 & 118,809 & 617 \\
\hline 618 & 13,9 & 14,5 & 14,7 & 14,40 & 13,94 & 0,950 & 0,994 & 0,962 & 0,050 & 0,834 & 1,095 & 0,595 & 0,656 & 5,464 & 3,585 & 3,267 & 96,971 & 618 \\
\hline 619 & 0,4 & 2,5 & 0,4 & 2,28 & 2,21 & 0,931 & 0,924 & 0,895 & 0,102 & 0,823 & 1,102 & 0,550 & 0,549 & 0,961 & 0,527 & 0,495 & 72,154 & 619 \\
\hline 620 & 0,1 & 1,2 & 0,1 & 1,04 & 1,01 & 0,974 & 0,887 & 0,859 & 0,116 & 0,846 & 1,087 & 0,387 & 0,268 & 0,553 & 0,149 & 0,198 & 84,250 & 620 \\
\hline 621 & 0,4 & 2,5 & 0,5 & 2,40 & 2,33 & 0,947 & 0,973 & 0,942 & 0,059 & 0,904 & 1,052 & 0,523 & 0,527 & 1,034 & 0,545 & 0,560 & 2,435 & 621 \\
\hline 622 & 2,2 & 5,7 & 2,3 & 5,41 & 5,23 & 0,956 & 0,957 & 0,926 & 0,061 & 0,869 & 1,073 & 0,555 & 0,584 & 2,250 & 1,315 & 1,291 & 58,330 & 622 \\
\hline 623 & 0,9 & 3,6 & 1,0 & 3,51 & 3,40 & 0,913 & 0,971 & 0,940 & 0,091 & 0,870 & 1,072 & 0,596 & 0,603 & 1,390 & 0,838 & 0,792 & 174,229 & 623 \\
\hline 624 & 0,4 & 2,3 & 0,4 & 2,22 & 2,15 & 0,975 & 0,983 & 0,952 & 0,030 & 0,958 & 1,021 & 0,599 & 0,500 & 0,910 & 0,455 & 0,495 & 42,629 & 624 \\
\hline 625 & 0,1 & 1,1 & 0,1 & 0,91 & 0,88 & 0,932 & 0,859 & 0,831 & 0,157 & 1,000 & 0,939 & 0,803 & 0,666 & 0,399 & 0,266 & 0,297 & 69,473 & 625 \\
\hline 626 & 1,0 & 4,0 & 1,1 & 3,93 & 3,80 & 0,920 & 0,970 & 0,939 & 0,085 & 0,778 & 1,134 & 0,487 & 0,456 & 1,628 & 0,743 & 0,693 & 105,672 & 626 \\
\hline 627 & 0,4 & 2,4 & 0,4 & 2,25 & 2,18 & 0,945 & 0,934 & 0,904 & 0,086 & 0,904 & 1,052 & 0,531 & 0,553 & 1,002 & 0,554 & 0,577 & 146,954 & 627 \\
\hline 628 & 0,1 & 0,9 & 0,1 & 0,81 & 0,79 & 0,964 & 0,937 & 0,907 & 0,073 & 1,000 & 0,951 & 0,593 & 0,457 & $\begin{array}{l}0,377 \\
0,0\end{array}$ & 0,172 & 0,198 & 110,781 & 628 \\
\hline
\end{tabular}




\begin{tabular}{|c|c|c|c|c|c|c|c|c|c|c|c|c|c|c|c|c|c|c|c|}
\hline 629 & 0,5 & 2,5 & 0,5 & 2,38 & 2,30 & 0,943 & 0,941 & 0,911 & 0,082 & 0,957 & 1,022 & 0,748 & 0,753 & 0,909 & 0,684 & 0,626 & 9,873 & 629 & $-3,2$ \\
\hline 630 & 0,6 & 3,0 & 0,6 & 2,95 & 2,85 & 0,942 & 0,978 & 0,947 & 0,062 & 0,825 & 1,101 & 0,486 & 0,480 & 1,249 & 0,600 & 0,577 & 44,551 & 630 & $-3,6$ \\
\hline 631 & 0,4 & 2,4 & 0,4 & 2,33 & 2,26 & 0,971 & 0,984 & 0,952 & 0,033 & 0,915 & 1,045 & 0,519 & 0,454 & 1,002 & 0,455 & 0,495 & 141,164 & 631 & $-3,3$ \\
\hline 632 & 0,1 & 1,0 & 0,1 & 0,80 & 0,78 & 0,943 & 0,833 & 0,807 & 0,176 & 1,000 & 0,953 & 0,646 & 0,555 & 0,399 & 0,222 & 0,280 & 160,315 & 632 & $-2,0$ \\
\hline 633 & 0,2 & 1,9 & 0,2 & 1,72 & 1,67 & 0,847 & 0,929 & 0,899 & 0,169 & 0,644 & 1,246 & 0,367 & 0,441 & 0,783 & 0,345 & 0,313 & 171,252 & 633 & $-3,0$ \\
\hline 634 & 0,6 & 2,9 & 0,7 & 2,83 & 2,74 & 0,949 & 0,968 & 0,937 & 0,060 & 0,938 & 1,033 & 0,710 & 0,663 & 1,069 & 0,709 & 0,700 & 18,729 & 634 & $-3,4$ \\
\hline 635 & 0,3 & 2,1 & 0,3 & 1,99 & 1,93 & 0,930 & 0,968 & 0,937 & 0,077 & 0,868 & 1,073 & 0,483 & 0,481 & 0,877 & 0,422 & 0,443 & 6,703 & 635 & $-3,1$ \\
\hline 636 & 5,9 & 9,0 & 6,1 & 9,03 & 8,74 & 0,966 & 0,999 & 0,967 & 0,034 & 0,913 & 1,046 & 0,640 & 0,670 & 3,439 & 2,304 & 2,258 & 107,089 & 636 & $-5,1$ \\
\hline 637 & 0,7 & 3,1 & 0,7 & 3,08 & 2,98 & 0,955 & 0,979 & 0,948 & 0,050 & 0,854 & 1,082 & 0,550 & 0,474 & 1,249 & 0,592 & 0,626 & 152,946 & 637 & $-3,6$ \\
\hline 638 & 0,1 & 0,9 & 0,1 & 0,71 & 0,68 & 0,971 & 0,767 & 0,742 & 0,235 & 1,000 & 0,915 & 0,689 & 0,630 & 0,387 & 0,244 & 0,280 & 38,571 & 638 & $-2,0$ \\
\hline 639 & 0,7 & 3,1 & 0,7 & 3,04 & 2,94 & 0,964 & 0,967 & 0,936 & 0,049 & 0,835 & 1,094 & 0,471 & 0,447 & 1,332 & 0,595 & 0,626 & 138,486 & 639 & $-3,7$ \\
\hline 640 & 0,1 & 1,2 & 0,1 & 1,19 & 1,15 & 0,980 & 0,991 & 0,959 & 0,022 & 1,000 & 0,966 & 0,715 & 0,600 & 0,467 & 0,280 & 0,313 & 131,038 & 640 & $-2,2$ \\
\hline 641 & 0,2 & 1,5 & 0,2 & 1,43 & 1,38 & 0,937 & 0,956 & 0,925 & 0,077 & 1,000 & 0,988 & 0,637 & 0,658 & 0,602 & 0,396 & 0,408 & 2,854 & 641 & $-2,6$ \\
\hline 642 & 2,0 & 5,7 & 2,1 & 5,50 & 5,32 & 0,930 & 0,967 & 0,936 & 0,078 & 0,767 & 1,142 & 0,484 & 0,531 & 2,278 & 1,209 & 1,155 & 41,265 & 642 & $-4,5$ \\
\hline 643 & 0,4 & 2,2 & 0,4 & 2,17 & 2,10 & 0,950 & 0,982 & 0,951 & 0,053 & 0,964 & 1,018 & 0,658 & 0,563 & 0,852 & 0,480 & 0,495 & 127,719 & 643 & $-3,1$ \\
\hline 644 & 0,2 & 1,8 & 0,2 & 1,71 & 1,65 & 0,916 & 0,963 & 0,932 & 0,092 & 0,744 & 1,159 & 0,372 & 0,361 & 0,798 & 0,288 & 0,313 & 116,516 & 644 & $-3,0$ \\
\hline 645 & 0,1 & 0,7 & 0,1 & 0,64 & 0,62 & 1,000 & 0,921 & 0,892 & 0,079 & 1,000 & 0,865 & 0,922 & 0,557 & 0,267 & 0,149 & 0,198 & 169,357 & 645 & $-1,4$ \\
\hline 646 & 0,4 & 2,7 & 0,5 & 2,61 & 2,53 & 0,909 & 0,961 & 0,931 & 0,099 & 0,746 & 1,158 & 0,418 & 0,427 & 1,156 & 0,494 & 0,495 & 129,646 & 646 & $-3,5$ \\
\hline 647 & 0,1 & 1,1 & 0,1 & 0,92 & 0,89 & 0,952 & 0,852 & 0,824 & 0,155 & 1,000 & 0,975 & 0,599 & 0,434 & 0,456 & 0,198 & 0,280 & 85,782 & 647 & $-2,2$ \\
\hline 648 & 17,2 & 15,4 & 17,4 & 15,39 & 14,89 & 0,984 & 0,997 & 0,966 & 0,016 & 0,906 & 1,051 & 0,604 & 0,637 & 6,014 & 3,834 & 3,826 & 22,955 & 648 & $-5,9$ \\
\hline 649 & 0,5 & 2,7 & 0,6 & 2,59 & 2,50 & 0,904 & 0,942 & 0,912 & 0,112 & 0,845 & 1,088 & 0,622 & 0,652 & 1,019 & 0,665 & 0,560 & 140,756 & 649 & $-3,3$ \\
\hline 650 & 1,0 & 3,5 & 1,0 & 3,45 & 3,34 & 0,953 & 0,978 & 0,947 & 0,052 & 0,964 & 1,018 & 0,870 & 0,880 & 1,181 & 1,040 & 0,990 & 85,733 & 650 & $-3,6$ \\
\hline 651 & 0,1 & 1,2 & 0,1 & 1,09 & 1,06 & 0,982 & 0,892 & 0,863 & 0,110 & 1,000 & 0,934 & 0,977 & 0,910 & 0,423 & 0,385 & 0,396 & 20,636 & 651 & $-2,1$ \\
\hline 652 & 0,4 & 2,3 & 0,4 & 2,24 & 2,17 & 0,971 & 0,971 & 0,940 & 0,041 & 0,963 & 1,019 & 0,650 & 0,628 & 0,892 & 0,560 & 0,560 & 36,188 & 652 & $-3,2$ \\
\hline 653 & 0,1 & 1,1 & 0,1 & 0,93 & 0,90 & 0,886 & 0,833 & 0,807 & 0,202 & 0,955 & 1,023 & 0,764 & 0,744 & 0,399 & 0,297 & 0,280 & 33,097 & 653 & $-2,0$ \\
\hline 654 & 5,0 & 9,9 & 6,0 & 9,42 & 9,12 & 0,834 & 0,951 & 0,920 & 0,173 & 0,639 & 1,251 & 0,442 & 0,544 & 3,793 & 2,063 & 1,771 & 41,346 & 654 & $-5,2$ \\
\hline 655 & 0,2 & 2,0 & 0,3 & 1,86 & 1,80 & 0,907 & 0,946 & 0,916 & 0,108 & 0,777 & 1,135 & 0,377 & 0,387 & 0,897 & 0,347 & 0,357 & 91,763 & 655 & $-3,2$ \\
\hline 656 & 0,1 & 0,8 & 0,1 & 0,64 & 0,62 & 0,962 & 0,828 & 0,803 & 0,176 & 1,000 & 0,881 & 0,776 & 0,552 & 0,317 & 0,175 & 0,221 & 129,800 & 656 & $-1,7$ \\
\hline 657 & 0,4 & 2,5 & 0,4 & 2,36 & 2,29 & 0,872 & 0,937 & 0,907 & 0,143 & 0,759 & 1,148 & 0,434 & 0,413 & 1,063 & 0,439 & 0,408 & $\begin{array}{l}161,618 \\
\end{array}$ & 657 & $-3,4$ \\
\hline 658 & 1,4 & 4,7 & 1,5 & 4,60 & 4,45 & 0,891 & 0,980 & 0,949 & 0,111 & 0,785 & 1,129 & 0,510 & 0,542 & 1,854 & 1,004 & 0,913 & 23,271 & 658 & $-4,2$ \\
\hline 659 & 0,1 & 1,3 & 0,2 & 1,23 & 1,19 & 0,919 & 0,910 & 0,881 & 0,121 & 0,966 & 1,017 & 0,534 & 0,515 & 0,577 & 0,297 & 0,313 & 29,461 & 659 & $-2,5$ \\
\hline 660 & 0,1 & 0,8 & 0,1 & 0,71 & 0,68 & 1,000 & 0,853 & 0,826 & 0,147 & 1,000 & 0,909 & 0,764 & 0,596 & 0,332 & 0,198 & 0,221 & 73,831 & 660 & $-1,7$ \\
\hline 661 & 0,2 & 1,7 & 0,2 & 1,48 & 1,43 & 0,956 & 0,873 & 0,845 & 0,134 & 0,932 & 1,036 & 0,548 & 0,577 & 0,704 & 0,406 & 0,396 & 143,328 & 661 & $-2,8$ \\
\hline 662 & 0,1 & 1,1 & 0,1 & 0,99 & 0,96 & 0,972 & 0,937 & 0,907 & 0,069 & 0,968 & 1,016 & 0,441 & 0,299 & 0,498 & 0,149 & 0,198 & 5,100 & 662 & $-2,3$ \\
\hline 663 & 0,4 & 2,5 & 0,4 & 2,34 & 2,26 & 0,950 & 0,951 & 0,920 & 0,070 & 0,867 & 1,074 & 0,541 & 0,592 & 0,990 & 0,586 & 0,560 & 63,636 & 663 & $-3,3$ \\
\hline 664 & 0,1 & 0,9 & 0,1 & 0,77 & 0,75 & 0,969 & 0,839 & 0,812 & 0,164 & 1,000 & 0,944 & 0,963 & 0,781 & 0,317 & 0,248 & 0,280 & 61,098 & 664 & $-1,7$ \\
\hline 665 & 0,1 & 1,0 & 0,1 & 0,92 & 0,89 & 0,947 & 0,898 & 0,868 & 0,115 & 1,000 & 0,976 & 0,559 & 0,442 & 0,448 & 0,198 & 0,221 & 99,722 & 665 & $-2,2$ \\
\hline 666 & 0,2 & 1,5 & 0,2 & 1,32 & 1,28 & 0,987 & 0,899 & 0,870 & 0,102 & 1,000 & 0,967 & 0,654 & 0,579 & 0,598 & 0,347 & 0,396 & 78,164 & 666 & $-2,6$ \\
\hline 667 & 0,2 & 1,5 & 0,2 & 1,38 & 1,33 & 0,935 & 0,912 & 0,883 & 0,109 & 0,975 & 1,013 & 0,584 & 0,598 & 0,620 & 0,371 & 0,357 & 26,848 & 667 & $-2,6$ \\
\hline 668 & 0,1 & 1,5 & 0,2 & 1,32 & 1,28 & 0,924 & 0,892 & 0,863 & 0,132 & 0,857 & 1,080 & 0,457 & 0,404 & 0,645 & 0,261 & 0,280 & 121,719 & 668 & $-2,7$ \\
\hline 669 & 0,0 & 0,7 & 0,0 & 0,55 & 0,53 & 1,000 & 0,818 & 0,793 & 0,182 & 1,000 & 0,852 & 0,878 & 0,557 & 0,267 & 0,149 & 0,198 & 165,128 & 669 & $-1,4$ \\
\hline 670 & 0,1 & 1,3 & 0,1 & 1,16 & 1,12 & 0,898 & 0,886 & 0,858 & 0,153 & 0,952 & 1,025 & 0,649 & 0,588 & 0,505 & 0,297 & 0,297 & 13,812 & 670 & $-2,3$ \\
\hline 671 & 0,3 & 2,4 & 0,3 & 2,20 & 2,13 & 0,918 & 0,926 & 0,896 & 0,111 & 0,671 & 1,221 & 0,325 & 0,320 & 1,087 & 0,348 & 0,357 & 27,084 & 671 & $-3,4$ \\
\hline 672 & 0,8 & 4,1 & 0,9 & 3,93 & 3,80 & 0,925 & 0,948 & 0,918 & 0,091 & 0,599 & 1,292 & 0,291 & 0,314 & 1,892 & 0,594 & 0,594 & 0,531 & 672 & $-4,2$ \\
\hline 673 & 0,9 & 3,4 & 1,0 & 3,36 & 3,25 & 0,956 & 0,981 & 0,949 & 0,048 & 0,974 & 1,013 & 0,836 & 0,828 & 1,177 & 0,974 & 0,939 & 55,692 & 673 & $-3,6$ \\
\hline 674 & 0,1 & 0,9 & 0,1 & 0,76 & 0,74 & 0,867 & 0,852 & 0,824 & 0,199 & 1,000 & 1,000 & 0,625 & 0,549 & 0,360 & 0,198 & 0,221 & 93,698 & 674 & $-1,8$ \\
\hline 675 & 1,9 & 5,2 & 2,0 & 5,03 & 4,87 & 0,941 & 0,960 & 0,929 & 0,072 & 0,850 & 1,084 & 0,741 & 0,825 & 1,788 & 1,476 & 1,205 & 45,735 & 675 & $-4,2$ \\
\hline 676 & 47,9 & 27,9 & 51,7 & 26,75 & 25,90 & 0,927 & 0,960 & 0,929 & 0,083 & 0,776 & 1,135 & 0,635 & 0,733 & 9,804 & 7,191 & 6,199 & 62,821 & 676 & $-6,6$ \\
\hline 677 & 0,1 & 0,9 & 0,1 & 0,77 & 0,75 & 0,967 & 0,892 & 0,863 & 0,113 & 1,000 & 0,918 & 0,901 & 0,737 & 0,317 & 0,234 & 0,221 & 29,336 & 677 & $-1,7$ \\
\hline 678 & 0,6 & 2,7 & 0,6 & 2,61 & 2,53 & 0,962 & 0,985 & 0,954 & 0,041 & 1,000 & 0,999 & 0,686 & 0,641 & 1,021 & 0,654 & 0,664 & 30,372 & 678 & $-3,4$ \\
\hline 679 & 0,5 & 3,1 & 0,6 & 2,92 & 2,83 & 0,937 & 0,957 & 0,926 & 0,077 & 0,733 & 1,168 & 0,463 & 0,543 & 1,223 & 0,664 & 0,664 & 107,417 & 679 & $-3,6$ \\
\hline 680 & 14,4 & 15,9 & 15,7 & 15,67 & 15,17 & 0,919 & 0,985 & 0,954 & 0,083 & 0,715 & 1,183 & 0,535 & 0,501 & 5,855 & 2,931 & 2,631 & 16,184 & 680 & $-5,9$ \\
\hline 681 & 3,5 & 8,2 & 3,8 & 7,99 & 7,74 & 0,920 & 0,972 & 0,941 & 0,084 & 0,659 & 1,232 & 0,361 & 0,471 & 3,532 & 1,663 & 1,537 & 152,482 & 681 & $-5,1$ \\
\hline 682 & 0,1 & 0,9 & 0,1 & 0,70 & 0,67 & 0,958 & 0,788 & 0,762 & 0,217 & 0,907 & 1,050 & 0,431 & 0,243 & 0,408 & 0,099 & 0,198 & 85,307 & 682 & $-2,0$ \\
\hline 683 & 0,4 & 2,3 & 0,4 & 2,22 & 2,14 & 0,920 & 0,943 & 0,913 & 0,098 & 0,838 & 1,092 & 0,626 & 0,687 & 0,865 & 0,594 & 0,495 & 172,636 & 683 & $-3,1$ \\
\hline 684 & 1,0 & 3,9 & 1,0 & 3,85 & 3,72 & 0,951 & 0,983 & 0,952 & 0,052 & 0,813 & 1,109 & 0,442 & 0,439 & 1,690 & 0,743 & 0,754 & 81,392 & 684 & $-4,1$ \\
\hline 685 & 0,2 & 1,5 & 0,2 & 1,51 & 1,46 & 0,989 & 0,975 & 0,944 & 0,027 & 1,000 & 0,924 & 0,846 & 0,725 & 0,579 & 0,420 & 0,443 & 116,491 & 685 & $-2,5$ \\
\hline 686 & 0,6 & 3,0 & $\begin{array}{l}0,2 \\
0,7\end{array}$ & 2,75 & $\begin{array}{l}2,606 \\
2,66\end{array}$ & 0,929 & 0,927 & 0,898 & 0,101 & 0,878 & 1,067 & 0,625 & 0,674 & 1,117 & 0,753 & 0,721 & 97,340 & 686 & $-3,5$ \\
\hline
\end{tabular}




\begin{tabular}{|c|c|c|c|c|c|c|c|c|c|c|c|c|c|c|c|c|c|c|}
\hline 687 & 0,5 & 2,5 & 0,5 & 2,39 & 2,31 & 0,929 & 0,937 & 0,907 & 0,095 & 0,931 & 1,037 & 0,624 & 0,636 & 0,990 & 0,630 & 0,626 & 150,003 & 687 \\
\hline 688 & 0,6 & 2,8 & 0,6 & 2,66 & 2,57 & 0,962 & 0,956 & 0,926 & 0,058 & 1,000 & 0,999 & 0,752 & 0,767 & 1,021 & 0,783 & 0,754 & 53,833 & 688 \\
\hline 689 & 0,1 & 0,8 & 0,1 & 0,74 & 0,71 & 0,929 & 0,950 & 0,920 & 0,087 & 1,000 & 0,864 & 0,974 & 0,728 & 0,289 & 0,210 & 0,221 & 122,300 & 689 \\
\hline 690 & 1,2 & 5,2 & 1,4 & 4,97 & 4,81 & 0,841 & 0,961 & 0,931 & 0,164 & 0,549 & 1,349 & 0,304 & 0,343 & 2,211 & 0,759 & 0,693 & 131,812 & 690 \\
\hline 691 & 0,2 & 1,5 & 0,2 & 1,45 & 1,41 & 0,951 & 0,956 & 0,926 & 0,065 & 1,000 & 0,980 & 0,764 & 0,694 & 0,564 & 0,391 & 0,408 & 77,837 & 691 \\
\hline 692 & 2,1 & 6,2 & 2,3 & 6,02 & 5,82 & 0,933 & 0,976 & 0,945 & 0,072 & 0,695 & 1,199 & 0,369 & 0,468 & 2,693 & 1,260 & 1,209 & 126,589 & 692 \\
\hline 693 & 0,3 & 2,3 & 0,4 & 2,07 & 2,00 & 0,888 & 0,917 & 0,887 & 0,139 & 0,818 & 1,105 & 0,612 & 0,686 & 0,830 & 0,569 & 0,495 & 122,756 & 693 \\
\hline 694 & 18,0 & 16,8 & 19,2 & 15,85 & 15,34 & 0,936 & 0,943 & 0,913 & 0,085 & 0,799 & 1,119 & 0,774 & 0,802 & 5,439 & 4,365 & 3,804 & 46,821 & 694 \\
\hline 695 & 0,3 & 1,9 & 0,3 & 1,80 & 1,74 & 0,967 & 0,964 & 0,934 & 0,049 & 1,000 & 0,984 & 0,828 & 0,812 & 0,664 & 0,539 & 0,495 & 78,652 & 695 \\
\hline 696 & 2,5 & 6,9 & 2,7 & 6,81 & 6,59 & 0,913 & 0,990 & 0,959 & 0,088 & 0,661 & 1,230 & 0,373 & 0,468 & 2,916 & 1,365 & 1,321 & 51,988 & 696 \\
\hline 697 & 0,1 & 0,8 & 0,1 & 0,64 & 0,62 & 0,880 & 0,800 & 0,775 & 0,233 & 1,000 & 0,973 & 0,875 & 0,834 & 0,280 & 0,234 & 0,198 & 90,000 & 697 \\
\hline 698 & 0,5 & 2,8 & 0,6 & 2,71 & 2,62 & 0,965 & 0,976 & 0,945 & 0,042 & 0,881 & 1,066 & 0,622 & 0,707 & 1,050 & 0,743 & 0,693 & 177,137 & 698 \\
\hline 699 & 0,2 & 1,3 & 0,2 & 1,28 & 1,24 & 0,954 & 0,971 & 0,940 & 0,055 & 1,000 & 0,956 & 0,724 & 0,667 & 0,517 & 0,345 & 0,357 & 98,727 & 699 \\
\hline 700 & 0,0 & 0,7 & 0,0 & 0,48 & 0,47 & 1,000 & 0,691 & 0,669 & 0,309 & 1,000 & 0,887 & 0,749 & 0,514 & 0,289 & 0,149 & 0,198 & 100,643 & 700 \\
\hline 701 & 0,2 & 1,9 & 0,2 & 1,66 & 1,60 & 0,942 & 0,867 & 0,839 & 0,145 & 0,683 & 1,210 & 0,310 & 0,274 & 0,903 & 0,248 & 0,297 & 7,161 & 701 \\
\hline 702 & 0,2 & 1,5 & 0,2 & 1,40 & 1,35 & 0,939 & 0,913 & 0,884 & 0,106 & 1,000 & 0,994 & 0,662 & 0,634 & 0,602 & 0,382 & 0,408 & 69,806 & 702 \\
\hline 703 & 1,0 & 3,5 & 1,0 & 3,43 & 3,32 & 0,958 & 0,970 & 0,939 & 0,051 & 0,956 & 1,023 & 0,694 & 0,636 & 1,321 & 0,841 & 0,846 & 78,363 & 703 \\
\hline 704 & 0,1 & 1,3 & 0,1 & 1,08 & 1,05 & 0,854 & 0,863 & 0,836 & 0,200 & 0,803 & 1,116 & 0,580 & 0,527 & 0,470 & 0,248 & 0,221 & 90,274 & 704 \\
\hline 705 & 0,3 & 2,1 & 0,3 & 1,83 & 1,77 & 0,944 & 0,871 & 0,843 & 0,141 & 0,818 & 1,106 & 0,442 & 0,470 & 0,909 & 0,428 & 0,495 & 68,416 & 705 \\
\hline 706 & 0,2 & 1,7 & 0,2 & 1,59 & 1,54 & 0,926 & 0,960 & 0,929 & 0,084 & 0,993 & 1,004 & 0,767 & 0,658 & 0,598 & 0,393 & 0,408 & 121,609 & 706 \\
\hline 707 & 4,6 & 8,0 & 4,8 & 7,92 & 7,66 & 0,961 & 0,984 & 0,952 & 0,042 & 0,900 & 1,054 & 0,670 & 0,742 & 2,970 & 2,204 & 2,171 & 46,529 & 707 \\
\hline 708 & 0,1 & 1,2 & 0,1 & 1,06 & 1,02 & 0,947 & 0,868 & 0,841 & 0,142 & 1,000 & 0,942 & 0,773 & 0,706 & 0,467 & 0,330 & 0,357 & 60,490 & 708 \\
\hline 709 & 0,2 & 1,8 & 0,2 & 1,66 & 1,61 & 0,955 & 0,941 & 0,910 & 0,075 & 0,620 & 1,270 & 0,277 & 0,208 & 0,843 & 0,175 & 0,221 & 132,633 & 709 \\
\hline 710 & 1,1 & 4,3 & 1,2 & 4,22 & 4,08 & 0,904 & 0,978 & 0,947 & 0,098 & 0,738 & 1,164 & 0,425 & 0,450 & 1,807 & 0,813 & 0,798 & 106,830 & 710 \\
\hline 711 & 0,1 & 0,9 & 0,1 & 0,71 & 0,68 & 0,967 & 0,815 & 0,789 & 0,188 & 1,000 & 0,918 & 0,710 & 0,588 & 0,357 & 0,210 & 0,280 & 125,165 & 711 \\
\hline 712 & 0,2 & 1,5 & 0,2 & 1,41 & 1,37 & 0,927 & 0,914 & 0,885 & 0,113 & 0,978 & 1,011 & 0,744 & 0,744 & 0,564 & 0,420 & 0,408 & 37,870 & 712 \\
\hline 713 & 0,1 & 0,7 & 0,1 & 0,67 & 0,65 & 1,000 & 0,896 & 0,867 & 0,104 & 1,000 & 0,868 & 0,826 & 0,658 & 0,301 & 0,198 & 0,221 & 94,933 & 713 \\
\hline 714 & 0,2 & 1,9 & 0,2 & 1,67 & 1,62 & 0,950 & 0,894 & 0,865 & 0,117 & 0,668 & 1,224 & 0,299 & 0,222 & 0,891 & 0,198 & 0,297 & 90,336 & 714 \\
\hline 715 & 0,5 & 2,6 & 0,5 & 2,44 & 2,36 & 0,898 & 0,924 & 0,895 & 0,127 & 0,816 & 1,107 & 0,689 & 0,798 & 0,913 & 0,729 & 0,602 & 6,557 & 715 \\
\hline 716 & 1,0 & 3,6 & 1,0 & 3,52 & 3,41 & 0,959 & 0,967 & 0,936 & 0,053 & 0,913 & 1,047 & 0,542 & 0,596 & 1,506 & 0,897 & 0,886 & 49,913 & 716 \\
\hline 717 & 0,4 & 2,4 & 0,4 & 2,32 & 2,25 & 0,949 & 0,950 & 0,920 & 0,071 & 0,775 & 1,136 & 0,396 & 0,434 & 1,087 & 0,472 & 0,495 & 31,409 & 717 \\
\hline 718 & 1,2 & 3,8 & 1,2 & 3,72 & 3,60 & 0,977 & 0,968 & 0,937 & 0,039 & 0,990 & 1,005 & 0,726 & 0,693 & 1,429 & 0,990 & 1,010 & 7,086 & 718 \\
\hline 719 & 1,3 & 4,6 & 1,4 & 4,48 & 4,34 & 0,955 & 0,971 & 0,940 & 0,054 & 0,763 & 1,145 & 0,476 & 0,527 & 1,858 & 0,980 & 0,891 & 55,402 & 719 \\
\hline 720 & 1,2 & 4,4 & 1,3 & 4,01 & 3,88 & 0,927 & 0,914 & 0,885 & 0,113 & 0,789 & 1,126 & 0,653 & 0,698 & 1,535 & 1,072 & 0,995 & 15,593 & 720 \\
\hline 721 & 0,2 & 1,4 & 0,2 & 1,24 & 1,20 & 0,955 & 0,869 & 0,841 & 0,139 & 0,963 & 1,019 & 0,509 & 0,395 & 0,626 & 0,248 & 0,297 & 81,960 & 721 \\
\hline 722 & 0,1 & 1,3 & 0,1 & 1,09 & 1,06 & 0,950 & 0,854 & 0,826 & 0,155 & 1,000 & 0,967 & 0,748 & 0,698 & 0,488 & 0,341 & 0,313 & 75,600 & 722 \\
\hline 723 & 0,6 & 3,0 & 0,6 & 2,76 & 2,67 & 0,910 & 0,936 & 0,906 & 0,110 & 0,821 & 1,104 & 0,656 & 0,581 & 1,050 & 0,610 & 0,577 & 72,707 & 723 \\
\hline 724 & 5,9 & 8,9 & 6,1 & 8,85 & 8,57 & 0,969 & 0,990 & 0,958 & 0,033 & 0,920 & 1,042 & 0,624 & 0,688 & 3,459 & 2,380 & 2,279 & 69,665 & 724 \\
\hline 725 & 0,2 & 1,3 & 0,2 & 1,19 & 1,15 & 0,955 & 0,889 & 0,860 & 0,120 & 1,000 & 0,960 & 0,757 & 0,583 & 0,510 & 0,297 & 0,396 & 12,649 & 725 \\
\hline 726 & 2,3 & 5,9 & 2,4 & 5,88 & 5,69 & 0,958 & 0,989 & 0,957 & 0,044 & 0,812 & 1,110 & 0,521 & 0,564 & 2,362 & 1,331 & 1,332 & 32,611 & 726 \\
\hline 727 & 0,3 & 2,1 & 0,3 & 1,99 & 1,93 & 0,932 & 0,950 & 0,919 & 0,084 & 0,871 & 1,071 & 0,530 & 0,568 & 0,855 & 0,485 & 0,495 & 81,803 & 727 \\
\hline 728 & 0,2 & 1,6 & 0,2 & 1,48 & 1,43 & 0,989 & 0,902 & 0,874 & 0,098 & 0,995 & 1,002 & 0,599 & 0,560 & 0,673 & 0,377 & 0,396 & 138,186 & 728 \\
\hline 729 & 0,3 & 1,9 & 0,3 & 1,80 & 1,74 & 0,947 & 0,945 & 0,915 & 0,077 & 1,000 & 0,975 & 0,877 & 0,839 & 0,664 & 0,557 & 0,495 & 72,043 & 729 \\
\hline 730 & 2,8 & 6,8 & 3,1 & 6,54 & 6,33 & 0,914 & 0,956 & 0,926 & 0,096 & 0,756 & 1,150 & 0,513 & 0,579 & 2,641 & 1,528 & 1,540 & 60,050 & 730 \\
\hline 731 & 0,2 & 1,9 & 0,2 & 1,77 & 1,72 & 0,890 & 0,917 & 0,887 & 0,138 & 0,733 & 1,168 & 0,354 & 0,350 & 0,886 & 0,310 & 0,313 & 62,520 & 731 \\
\hline 732 & 0,1 & 0,8 & 0,1 & 0,67 & 0,65 & 0,913 & 0,877 & 0,849 & 0,151 & 1,000 & 0,948 & 0,594 & 0,447 & 0,332 & 0,149 & 0,198 & 107,993 & 732 \\
\hline 733 & 0,2 & 1,5 & 0,2 & 1,32 & 1,28 & 0,974 & 0,860 & 0,832 & 0,142 & 0,993 & 1,004 & 0,501 & 0,504 & 0,688 & 0,347 & 0,396 & 64,511 & 733 \\
\hline 734 & 12,1 & 13,4 & 12,8 & 13,00 & 12,58 & 0,944 & 0,972 & 0,941 & 0,062 & 0,853 & 1,083 & 0,777 & 0,809 & 4,460 & 3,607 & 3,266 & 24,806 & 734 \\
\hline 735 & 0,8 & 3,1 & 0,8 & 3,04 & 2,94 & 0,957 & 0,967 & 0,936 & 0,055 & 0,966 & 1,018 & 0,683 & 0,646 & 1,190 & 0,769 & 0,754 & 61,719 & 735 \\
\hline 736 & 0,2 & 1,5 & 0,2 & 1,32 & 1,28 & 0,905 & 0,876 & 0,847 & 0,156 & 0,907 & 1,050 & 0,627 & 0,629 & 0,577 & 0,363 & 0,313 & 45,882 & 736 \\
\hline 737 & 12,1 & 15,0 & 12,9 & 14,64 & 14,17 & 0,939 & 0,975 & 0,943 & 0,066 & 0,675 & 1,217 & 0,425 & 0,436 & 6,025 & 2,628 & 2,393 & 127,280 & 737 \\
\hline 738 & 0,2 & 1,6 & 0,2 & 1,50 & 1,45 & 0,895 & 0,919 & 0,889 & 0,133 & 0,789 & 1,126 & 0,433 & 0,372 & 0,700 & 0,260 & 0,280 & 171,423 & 738 \\
\hline 739 & 0,9 & 3,5 & 1,0 & 3,37 & 3,26 & 0,937 & 0,958 & 0,927 & 0,076 & 0,925 & 1,040 & 0,739 & 0,760 & 1,253 & 0,953 & 0,886 & 61,783 & 739 \\
\hline 740 & 1,5 & 4,4 & 1,5 & 4,34 & 4,20 & 0,976 & 0,991 & 0,960 & 0,026 & 0,975 & 1,013 & 0,683 & 0,659 & 1,664 & 1,096 & 1,107 & 23,938 & 740 \\
\hline 741 & 0,1 & 0,9 & 0,1 & 0,83 & 0,80 & 0,941 & 0,887 & 0,859 & 0,127 & 1,000 & 0,941 & 0,905 & 0,720 & 0,332 & 0,239 & 0,280 & 64,042 & 741 \\
\hline 742 & 0,9 & 3,9 & 1,0 & 3,81 & 3,69 & 0,936 & 0,976 & 0,945 & 0,068 & 0,741 & 1,162 & 0,395 & 0,448 & 1,703 & 0,763 & 0,773 & 144,877 & 742 \\
\hline 743 & 0,4 & 2,4 & 0,5 & 2,32 & 2,25 & 0,937 & 0,972 & 0,941 & 0,069 & 0,968 & 1,017 & 0,726 & 0,780 & 0,877 & 0,684 & 0,626 & 14,326 & 743 \\
\hline 744 & 0,6 & 2,8 & 0,6 & 2,62 & 2,53 & 0,961 & 0,920 & 0,891 & 0,089 & 0,929 & 1,038 & 0,566 & 0,592 & 1,160 & 0,687 & 0,714 & 136,112 & 744 \\
\hline
\end{tabular}




\begin{tabular}{|c|c|c|c|c|c|c|c|c|c|c|c|c|c|c|c|c|c|c|}
\hline 745 & 0,3 & 2,6 & 0,3 & 2,37 & 2,29 & 0,821 & 0,927 & 0,897 & 0,194 & 0,518 & 1,390 & 0,264 & 0,310 & 1,140 & 0,353 & 0,357 & 124,749 & 745 \\
\hline 746 & 0,7 & 3,4 & 0,8 & 3,27 & 3,16 & 0,907 & 0,953 & 0,923 & 0,104 & 0,795 & 1,121 & 0,667 & 0,752 & 1,190 & 0,894 & 0,754 & 35,849 & 746 \\
\hline 747 & 1,8 & 4,9 & 1,8 & 4,83 & 4,67 & 0,959 & 0,976 & 0,945 & 0,048 & 0,902 & 1,053 & 0,701 & 0,706 & 1,785 & 1,260 & 1,192 & 27,535 & 747 \\
\hline 748 & 0,7 & 3,5 & 0,8 & 3,44 & 3,33 & 0,939 & 0,981 & 0,950 & 0,064 & 0,733 & 1,168 & 0,397 & 0,391 & 1,515 & 0,593 & 0,626 & 41,852 & 748 \\
\hline 749 & 0,4 & 2,6 & 0,5 & 2,52 & 2,44 & 0,924 & 0,974 & 0,943 & 0,080 & 0,843 & 1,089 & 0,484 & 0,484 & 1,086 & 0,525 & 0,560 & 51,887 & 749 \\
\hline 750 & 0,2 & 1,7 & 0,3 & 1,47 & 1,42 & 0,971 & 0,872 & 0,844 & 0,131 & 1,000 & 0,960 & 0,703 & 0,669 & 0,666 & 0,446 & 0,495 & 59,383 & 750 \\
\hline 751 & 0,1 & 1,5 & 0,2 & 1,35 & 1,30 & 0,938 & 0,917 & 0,888 & 0,103 & 0,870 & 1,072 & 0,485 & 0,450 & 0,626 & 0,282 & 0,297 & 160,534 & 751 \\
\hline 752 & 0,2 & 1,7 & 0,2 & 1,55 & 1,50 & 0,946 & 0,936 & 0,906 & 0,083 & 0,993 & 1,004 & 0,700 & 0,615 & 0,626 & 0,385 & 0,408 & 131,397 & 752 \\
\hline 753 & 0,4 & 2,5 & 0,5 & 2,35 & 2,27 & 0,931 & 0,957 & 0,927 & 0,081 & 0,901 & 1,054 & 0,548 & 0,593 & 1,001 & 0,594 & 0,626 & 83,074 & 753 \\
\hline 754 & 0,2 & 1,6 & 0,2 & 1,41 & 1,37 & 0,899 & 0,883 & 0,854 & 0,155 & 0,960 & 1,021 & 0,815 & 0,869 & 0,553 & 0,481 & 0,408 & 3,593 & 754 \\
\hline 755 & 0,4 & 2,5 & 0,5 & 2,40 & 2,33 & 0,934 & 0,962 & 0,932 & 0,076 & 0,904 & 1,052 & 0,553 & 0,567 & 1,016 & 0,576 & 0,577 & 128,045 & 755 \\
\hline 756 & 0,3 & 1,9 & 0,3 & 1,80 & 1,74 & 0,985 & 0,959 & 0,928 & 0,044 & 1,000 & 0,939 & 0,930 & 0,848 & 0,660 & 0,560 & 0,577 & 66,873 & 756 \\
\hline 757 & 0,1 & 1,1 & 0,1 & 0,91 & 0,88 & 0,971 & 0,850 & 0,823 & 0,153 & 0,921 & 1,042 & 0,486 & 0,427 & 0,467 & 0,199 & 0,221 & 118,492 & 757 \\
\hline 758 & 1,6 & 4,7 & 1,6 & 4,62 & 4,47 & 0,975 & 0,992 & 0,960 & 0,026 & 0,902 & 1,053 & 0,520 & 0,567 & 1,953 & 1,107 & 1,107 & 117,567 & 758 \\
\hline 759 & 0,5 & 2,7 & 0,6 & 2,55 & 2,47 & 0,923 & 0,960 & 0,930 & 0,087 & 0,946 & 1,028 & 0,753 & 0,760 & 0,946 & 0,719 & 0,664 & 70,406 & 759 \\
\hline 760 & 0,1 & 1,1 & 0,1 & 1,00 & 0,97 & 0,957 & 0,938 & 0,908 & 0,076 & 1,000 & 0,916 & 0,862 & 0,744 & 0,399 & 0,297 & 0,313 & 173,567 & 760 \\
\hline 761 & 2,0 & 5,8 & 2,3 & 5,50 & 5,32 & 0,860 & 0,945 & 0,915 & 0,150 & 0,733 & 1,168 & 0,582 & 0,715 & 2,078 & 1,485 & 1,107 & 173,556 & 761 \\
\hline 762 & 0,2 & 1,7 & 0,3 & 1,54 & 1,49 & 0,943 & 0,891 & 0,863 & 0,123 & 1,000 & 0,987 & 0,741 & 0,662 & 0,645 & 0,428 & 0,443 & 77,434 & 762 \\
\hline 763 & 0,1 & 1,3 & 0,1 & 1,16 & 1,12 & 0,949 & 0,861 & 0,833 & 0,148 & 0,949 & 1,026 & 0,548 & 0,536 & 0,564 & 0,302 & 0,313 & 30,219 & 763 \\
\hline 764 & 0,1 & 1,1 & 0,1 & 0,87 & 0,84 & 0,947 & 0,822 & 0,796 & 0,186 & 0,996 & 1,002 & 0,628 & 0,501 & 0,423 & 0,212 & 0,221 & 163,077 & 764 \\
\hline 765 & 0,2 & 1,8 & 0,2 & 1,69 & 1,63 & 0,895 & 0,927 & 0,898 & 0,128 & 0,634 & 1,256 & 0,308 & 0,246 & 0,830 & 0,204 & 0,221 & 165,232 & 765 \\
\hline 766 & 0,2 & 1,7 & 0,2 & 1,67 & 1,61 & 0,947 & 0,956 & 0,925 & 0,069 & 0,909 & 1,049 & 0,507 & 0,491 & 0,744 & 0,366 & 0,357 & 171,797 & 766 \\
\hline 767 & 1,3 & 4,4 & 1,4 & 4,14 & 4,01 & 0,969 & 0,939 & 0,909 & 0,069 & 0,848 & 1,086 & 0,489 & 0,509 & 1,848 & 0,941 & 0,990 & 7,446 & 767 \\
\hline 768 & 4,8 & 8,0 & 5,0 & 7,94 & 7,68 & 0,970 & 0,992 & 0,960 & 0,031 & 0,946 & 1,028 & 0,737 & 0,789 & 2,887 & 2,277 & 2,234 & 20,886 & 768 \\
\hline 769 & 0,6 & 2,8 & 0,6 & 2,70 & 2,61 & 0,980 & 0,966 & 0,935 & 0,039 & 0,993 & 1,003 & 0,686 & 0,649 & 1,069 & 0,693 & 0,700 & 11,102 & 769 \\
\hline 770 & 2,1 & 6,1 & 2,4 & 5,80 & 5,62 & 0,866 & 0,948 & 0,918 & 0,143 & 0,710 & 1,187 & 0,471 & 0,553 & 2,390 & 1,322 & 1,034 & 170,378 & 770 \\
\hline 771 & 0,8 & 3,3 & 0,8 & 3,21 & 3,11 & 0,947 & 0,980 & 0,948 & 0,056 & 0,878 & 1,067 & 0,593 & 0,536 & 1,269 & 0,681 & 0,693 & 122,713 & 771 \\
\hline 772 & 0,4 & 2,2 & 0,4 & 2,12 & 2,05 & 0,931 & 0,946 & 0,915 & 0,088 & 0,906 & 1,051 & 0,616 & 0,594 & 0,866 & 0,515 & 0,505 & 156,740 & 772 \\
\hline 773 & 0,2 & 1,8 & 0,2 & 1,63 & 1,58 & 0,959 & 0,916 & 0,887 & 0,093 & 0,915 & 1,045 & 0,511 & 0,392 & 0,757 & 0,297 & 0,396 & 0,288 & 773 \\
\hline 774 & 0,1 & 1,1 & 0,1 & 0,95 & 0,91 & 0,885 & 0,877 & 0,849 & 0,169 & 0,610 & 1,280 & 0,276 & 0,261 & 0,510 & 0,133 & 0,198 & 60,329 & 774 \\
\hline 775 & 0,6 & 2,8 & 0,6 & 2,66 & 2,58 & 0,937 & 0,943 & 0,913 & 0,085 & 0,916 & 1,045 & 0,753 & 0,739 & 0,991 & 0,733 & 0,714 & 151,898 & 775 \\
\hline 776 & 0,2 & 1,5 & 0,2 & 1,28 & 1,24 & 0,928 & 0,879 & 0,851 & 0,141 & 0,927 & 1,039 & 0,627 & 0,526 & 0,564 & 0,297 & 0,297 & 174,621 & 776 \\
\hline 777 & 0,6 & 2,9 & 0,7 & 2,88 & 2,79 & 0,936 & 0,996 & 0,964 & 0,064 & 0,970 & 1,015 & 0,722 & 0,719 & 1,066 & 0,767 & 0,714 & 46,627 & 777 \\
\hline 778 & 0,2 & 1,7 & 0,2 & 1,57 & 1,52 & 0,920 & 0,944 & 0,913 & 0,097 & 0,896 & 1,056 & 0,579 & 0,509 & 0,660 & 0,336 & 0,357 & 103,822 & 778 \\
\hline 779 & 0,1 & 0,9 & 0,1 & 0,70 & 0,67 & 0,926 & 0,778 & 0,753 & 0,234 & 0,961 & 1,020 & 0,637 & 0,424 & 0,350 & 0,149 & 0,198 & 175,814 & 779 \\
\hline 780 & 1,1 & 3,9 & 1,1 & 3,81 & 3,69 & 0,931 & 0,983 & 0,951 & 0,071 & 0,878 & 1,067 & 0,606 & 0,622 & 1,486 & 0,925 & 0,913 & 11,696 & 780 \\
\hline 781 & 2,9 & 6,6 & 3,1 & 6,50 & 6,29 & 0,922 & 0,984 & 0,953 & 0,080 & 0,827 & 1,100 & 0,597 & 0,599 & 2,474 & 1,483 & 1,375 & 149,583 & 781 \\
\hline 782 & 0,4 & 3,0 & 0,5 & 2,84 & 2,75 & 0,803 & 0,947 & 0,916 & 0,204 & 0,544 & 1,356 & 0,324 & 0,441 & 1,238 & 0,545 & 0,495 & 66,511 & 782 \\
\hline 783 & 0,6 & 3,0 & 0,6 & 2,93 & 2,84 & 0,934 & 0,969 & 0,938 & 0,073 & 0,759 & 1,148 & 0,394 & 0,430 & 1,338 & 0,575 & 0,602 & 46,129 & 783 \\
\hline 784 & 0,1 & 1,1 & 0,1 & 0,90 & 0,87 & 1,000 & 0,827 & 0,800 & 0,173 & 1,000 & 0,930 & 0,659 & 0,602 & 0,456 & 0,275 & 0,280 & 53,407 & 784 \\
\hline 785 & 0,2 & 1,4 & 0,2 & 1,32 & 1,28 & 0,972 & 0,952 & 0,921 & 0,055 & 1,000 & 0,944 & 0,768 & 0,665 & 0,533 & 0,354 & 0,396 & 154,648 & 785 \\
\hline 786 & 0,6 & 2,8 & 0,6 & 2,77 & 2,68 & 0,948 & 0,977 & 0,945 & 0,057 & 0,901 & 1,053 & 0,550 & 0,511 & 1,155 & 0,590 & 0,594 & 105,726 & 786 \\
\hline 787 & 0,4 & 2,2 & 0,4 & 2,13 & 2,06 & 0,894 & 0,953 & 0,923 & 0,116 & 0,879 & 1,066 & 0,700 & 0,794 & 0,798 & 0,633 & 0,533 & 81,705 & 787 \\
\hline 788 & 0,6 & 2,8 & 0,6 & 2,72 & 2,64 & 0,946 & 0,972 & 0,941 & 0,061 & 0,895 & 1,057 & 0,556 & 0,628 & 1,131 & 0,710 & 0,693 & 117,886 & 788 \\
\hline 789 & 0,2 & 1,4 & 0,2 & 1,41 & 1,37 & 0,926 & 0,992 & 0,960 & 0,074 & 0,955 & 1,023 & 0,590 & 0,524 & 0,577 & 0,302 & 0,297 & 122,163 & 789 \\
\hline 790 & 2,2 & 7,5 & 2,8 & 6,15 & 5,96 & 0,781 & 0,817 & 0,791 & 0,285 & 0,487 & 1,433 & 0,474 & 0,618 & 2,428 & 1,501 & 0,773 & 110,312 & 790 \\
\hline 791 & 0,1 & 1,3 & 0,1 & 1,15 & 1,11 & 0,870 & 0,896 & 0,868 & 0,166 & 0,881 & 1,065 & 0,479 & 0,412 & 0,553 & 0,228 & 0,280 & 70,616 & 791 \\
\hline 792 & 9,5 & 12,3 & 10,2 & 12,25 & 11,86 & 0,928 & 0,999 & 0,967 & 0,072 & 0,793 & 1,123 & 0,489 & 0,555 & 4,971 & 2,758 & 2,693 & 93,273 & 792 \\
\hline 793 & 0,8 & 3,6 & 0,9 & 3,38 & 3,27 & 0,894 & 0,941 & 0,911 & 0,121 & 0,788 & 1,126 & 0,534 & 0,564 & 1,387 & 0,782 & 0,773 & 102,160 & 793 \\
\hline 794 & 0,1 & 1,0 & 0,1 & 0,97 & 0,94 & 0,919 & 0,972 & 0,941 & 0,086 & 1,000 & 0,977 & 0,666 & 0,571 & 0,399 & 0,228 & 0,221 & 140,108 & 794 \\
\hline 795 & 0,4 & 3,1 & 0,5 & 2,86 & 2,76 & 0,838 & 0,918 & 0,889 & 0,181 & 0,576 & 1,317 & 0,311 & 0,362 & 1,347 & 0,487 & 0,505 & 29,123 & 795 \\
\hline 796 & 2,0 & 5,3 & 2,2 & 5,26 & 5,09 & 0,940 & 0,988 & 0,956 & 0,061 & 0,904 & 1,052 & 0,826 & 0,894 & 1,773 & 1,584 & 1,321 & 178,900 & 796 \\
\hline 797 & 0,2 & 1,3 & 0,2 & 1,29 & 1,25 & 0,940 & 0,971 & 0,940 & 0,066 & 1,000 & 0,956 & 0,827 & 0,705 & 0,488 & 0,344 & 0,357 & 126,175 & 797 \\
\hline 798 & 0,7 & 3,4 & 0,8 & 3,29 & 3,19 & 0,904 & 0,969 & 0,938 & 0,100 & 0,808 & 1,112 & 0,510 & 0,631 & 1,362 & 0,859 & 0,816 & 105,443 & 798 \\
\hline 799 & 0,2 & 1,6 & 0,2 & 1,51 & 1,46 & 0,956 & 0,935 & 0,905 & 0,079 & 1,000 & 0,991 & 0,608 & 0,580 & 0,664 & 0,385 & 0,420 & 62,373 & 799 \\
\hline 800 & 0,9 & 3,6 & 0,9 & 3,47 & 3,36 & 0,965 & 0,963 & 0,932 & 0,051 & 0,852 & 1,083 & 0,494 & 0,497 & 1,506 & 0,749 & 0,754 & 104,929 & 800 \\
\hline 801 & 0,1 & 0,8 & 0,1 & 0,71 & 0,68 & 0,913 & 0,883 & 0,854 & 0,146 & 1,000 & 0,996 & 0,535 & 0,424 & 0,350 & 0,149 & 0,198 & 167,042 & 801 \\
\hline 802 & 0,8 & 3,7 & 0,9 & 3,52 & 3,41 & 0,922 & 0,949 & 0,919 & 0,093 & 0,742 & 1,161 & 0,427 & 0,471 & 1,554 & 0,733 & 0,664 & 135,490 & 802 \\
\hline
\end{tabular}




\begin{tabular}{|c|c|c|c|c|c|c|c|c|c|c|c|c|c|c|c|c|c|c|}
\hline 803 & 0,2 & 1,5 & 0,2 & 1,23 & 1,19 & 0,947 & 0,841 & 0,815 & 0,167 & 1,000 & 0,979 & 0,705 & 0,614 & 0,564 & 0,347 & 0,396 & 15,074 & 803 \\
\hline 804 & 0,1 & 1,3 & 0,1 & 1,17 & 1,13 & 0,878 & 0,906 & 0,877 & 0,154 & 0,792 & 1,123 & 0,378 & 0,323 & 0,596 & 0,193 & 0,221 & 126,536 & 804 \\
\hline 805 & 0,1 & 1,1 & 0,1 & 1,03 & 1,00 & 0,978 & 0,939 & 0,910 & 0,065 & 1,000 & 0,940 & 0,683 & 0,552 & 0,448 & 0,248 & 0,297 & 2,550 & 805 \\
\hline 806 & 0,2 & 1,5 & 0,2 & 1,41 & 1,37 & 0,960 & 0,948 & 0,917 & 0,066 & 0,996 & 1,002 & 0,632 & 0,632 & 0,596 & 0,377 & 0,357 & 80,439 & 806 \\
\hline 807 & 1,1 & 3,8 & 1,2 & 3,63 & 3,51 & 0,931 & 0,944 & 0,914 & 0,089 & 0,923 & 1,041 & 0,829 & 0,891 & 1,291 & 1,151 & 0,980 & 76,465 & 807 \\
\hline 808 & 0,1 & 0,9 & 0,1 & 0,74 & 0,71 & 0,935 & 0,821 & 0,795 & 0,190 & 1,000 & 0,947 & 0,710 & 0,606 & 0,357 & 0,216 & 0,221 & 158,414 & 808 \\
\hline 809 & 1,0 & 4,8 & 1,3 & 4,39 & 4,24 & 0,754 & 0,918 & 0,888 & 0,260 & 0,529 & 1,375 & 0,337 & 0,382 & 1,904 & 0,728 & 0,560 & 2,053 & 809 \\
\hline 810 & 0,2 & 1,7 & 0,2 & 1,68 & 1,63 & 0,938 & 0,962 & 0,931 & 0,072 & 0,768 & 1,141 & 0,353 & 0,336 & 0,819 & 0,275 & 0,280 & 62,116 & 810 \\
\hline 811 & 0,3 & 2,0 & 0,3 & 1,88 & 1,82 & 0,947 & 0,952 & 0,922 & 0,071 & 0,989 & 1,005 & 0,825 & 0,799 & 0,688 & 0,550 & 0,560 & 46,105 & 811 \\
\hline 812 & 0,4 & 2,5 & 0,5 & 2,36 & 2,29 & 0,923 & 0,947 & 0,916 & 0,093 & 0,894 & 1,058 & 0,576 & 0,621 & 0,990 & 0,615 & 0,602 & 133,915 & 812 \\
\hline 813 & 0,0 & 0,7 & 0,1 & 0,57 & 0,56 & 0,952 & 0,782 & 0,756 & 0,223 & 1,000 & 0,936 & 0,688 & 0,493 & 0,301 & 0,149 & 0,198 & 80,308 & 813 \\
\hline 814 & 1,6 & 5,7 & 1,9 & 4,85 & 4,69 & 0,854 & 0,849 & 0,822 & 0,210 & 0,635 & 1,255 & 0,637 & 0,722 & 1,816 & 1,310 & 0,891 & 179,764 & 814 \\
\hline 815 & 0,1 & 0,8 & 0,1 & 0,67 & 0,65 & 0,966 & 0,834 & 0,807 & 0,169 & 1,000 & 0,863 & 1,049 & 0,849 & 0,289 & 0,245 & 0,280 & 77,179 & 815 \\
\hline 816 & 25,9 & 20,5 & 27,3 & 20,01 & 19,37 & 0,951 & 0,976 & 0,945 & 0,054 & 0,776 & 1,135 & 0,500 & 0,566 & 8,128 & 4,601 & 4,252 & 89,651 & 816 \\
\hline 817 & 0,8 & 3,5 & 0,9 & 3,35 & 3,25 & 0,954 & 0,970 & 0,939 & 0,055 & 0,860 & 1,078 & 0,488 & 0,576 & 1,462 & 0,842 & 0,846 & 57,010 & 817 \\
\hline 818 & 0,5 & 2,8 & 0,6 & 2,69 & 2,60 & 0,961 & 0,962 & 0,932 & 0,054 & 0,875 & 1,069 & 0,516 & 0,481 & 1,156 & 0,556 & 0,577 & 104,136 & 818 \\
\hline 819 & 1,8 & 5,3 & 1,9 & 5,28 & 5,11 & 0,973 & 0,993 & 0,961 & 0,028 & 0,808 & 1,112 & 0,419 & 0,447 & 2,352 & 1,051 & 1,034 & 150,885 & 819 \\
\hline 820 & 0,1 & 0,7 & 0,1 & 0,55 & 0,53 & 1,000 & 0,744 & 0,720 & 0,256 & 1,000 & 0,893 & 0,683 & 0,552 & 0,317 & 0,175 & 0,221 & 37,820 & 820 \\
\hline 821 & 0,8 & 3,2 & 0,8 & 3,16 & 3,06 & 0,969 & 0,988 & 0,956 & 0,033 & 0,942 & 1,030 & 0,634 & 0,670 & 1,242 & 0,832 & 0,798 & 24,445 & 821 \\
\hline 822 & 7,8 & 11,5 & 8,6 & 11,03 & 10,68 & 0,907 & 0,958 & 0,927 & 0,102 & 0,741 & 1,162 & 0,616 & 0,729 & 4,021 & 2,931 & 2,536 & 28,846 & 822 \\
\hline 823 & 1,9 & 5,3 & 2,0 & 5,26 & 5,10 & 0,953 & 0,993 & 0,961 & 0,048 & 0,840 & 1,091 & 0,487 & 0,519 & 2,217 & 1,150 & 1,188 & 108,483 & 823 \\
\hline 824 & 0,1 & 1,0 & 0,1 & 0,80 & 0,78 & 0,971 & 0,810 & 0,784 & 0,192 & 1,000 & 0,981 & 0,618 & 0,485 & 0,408 & 0,198 & 0,280 & 98,171 & 824 \\
\hline 825 & 0,4 & 2,2 & 0,4 & 2,09 & 2,03 & 0,944 & 0,952 & 0,922 & 0,074 & 0,968 & 1,016 & 0,712 & 0,718 & 0,816 & 0,586 & 0,577 & 115,875 & 825 \\
\hline 826 & 0,4 & 2,1 & 0,4 & 2,03 & 1,96 & 0,961 & 0,956 & 0,926 & 0,059 & 1,000 & 0,990 & 0,675 & 0,656 & 0,830 & 0,545 & 0,577 & 79,273 & 826 \\
\hline 827 & 0,1 & 0,7 & 0,1 & 0,60 & 0,58 & 0,960 & 0,819 & 0,793 & 0,185 & 1,000 & 0,855 & 1,054 & 0,743 & 0,267 & 0,198 & 0,280 & 26,001 & 827 \\
\hline 828 & 0,1 & 0,9 & 0,1 & 0,76 & 0,74 & 0,929 & 0,852 & 0,824 & 0,165 & 1,000 & 1,000 & 0,487 & 0,364 & 0,408 & 0,149 & 0,198 & 77,004 & 828 \\
\hline 829 & 0,1 & 0,8 & 0,1 & 0,71 & 0,68 & 0,957 & 0,883 & 0,854 & 0,125 & 1,000 & 0,973 & 0,529 & 0,412 & 0,360 & 0,149 & 0,198 & 13,776 & 829 \\
\hline 830 & 5,5 & 9,1 & 5,6 & 9,04 & 8,75 & 0,980 & 0,993 & 0,961 & 0,022 & 0,838 & 1,092 & 0,580 & 0,682 & 3,485 & 2,376 & 2,178 & 3,007 & 830 \\
\hline 831 & 0,5 & 3,1 & 0,5 & 2,96 & 2,86 & 0,866 & 0,949 & 0,918 & 0,143 & 0,615 & 1,275 & 0,354 & 0,385 & 1,308 & 0,504 & 0,533 & 122,601 & 831 \\
\hline 832 & 5,7 & 9,6 & 6,1 & 9,48 & 9,17 & 0,942 & 0,992 & 0,960 & 0,059 & 0,787 & 1,127 & 0,556 & 0,652 & 3,617 & 2,357 & 2,060 & 39,006 & 832 \\
\hline 833 & 0,3 & 2,1 & 0,4 & 1,92 & 1,86 & 0,924 & 0,911 & 0,882 & 0,117 & 0,927 & 1,039 & 0,682 & 0,696 & 0,783 & 0,545 & 0,533 & 97,150 & 833 \\
\hline 834 & 0,2 & 1,7 & 0,3 & 1,54 & 1,49 & 0,980 & 0,906 & 0,877 & 0,096 & 1,000 & 0,966 & 0,656 & 0,574 & 0,690 & 0,396 & 0,495 & 99,870 & 834 \\
\hline 835 & 1,9 & 5,8 & 2,1 & 5,60 & 5,42 & 0,914 & 0,969 & 0,938 & 0,091 & 0,708 & 1,189 & 0,432 & 0,404 & 2,352 & 0,949 & 0,886 & 32,092 & 835 \\
\hline 836 & 0,1 & 1,3 & 0,1 & 1,09 & 1,06 & 0,964 & 0,854 & 0,826 & 0,151 & 1,000 & 0,994 & 0,608 & 0,599 & 0,526 & 0,315 & 0,297 & 50,036 & 836 \\
\hline 837 & 0,1 & 0,8 & 0,1 & 0,41 & 0,40 & 1,000 & 0,490 & 0,474 & 0,510 & 1,000 & 0,910 & 0,792 & 0,447 & 0,332 & 0,149 & 0,198 & 0,000 & 837 \\
\hline 838 & 0,1 & 1,3 & 0,1 & 1,18 & 1,14 & 0,964 & 0,899 & 0,870 & 0,108 & 0,952 & 1,025 & 0,540 & 0,447 & 0,553 & 0,248 & 0,297 & 88,231 & 838 \\
\hline 839 & 1,9 & 4,9 & 1,9 & 4,74 & 4,59 & 0,970 & 0,973 & 0,942 & 0,040 & 0,979 & 1,010 & 0,808 & 0,812 & 1,707 & 1,386 & 1,407 & 102,475 & 839 \\
\hline 840 & 1,0 & 3,7 & 1,1 & 3,60 & 3,49 & 0,955 & 0,972 & 0,941 & 0,053 & 0,946 & 1,028 & 0,776 & 0,798 & 1,302 & 1,040 & 0,990 & 100,573 & 840 \\
\hline 841 & 0,2 & 1,7 & 0,2 & 1,63 & 1,58 & 0,914 & 0,939 & 0,909 & 0,105 & 0,870 & 1,072 & 0,490 & 0,463 & 0,736 & 0,341 & 0,357 & 104,342 & 841 \\
\hline 842 & 0,2 & 1,5 & 0,2 & 1,38 & 1,33 & 0,886 & 0,905 & 0,876 & 0,148 & 0,934 & 1,035 & 0,686 & 0,682 & 0,564 & 0,385 & 0,396 & 30,094 & 842 \\
\hline 843 & 2,8 & 6,5 & 3,0 & 6,26 & 6,06 & 0,942 & 0,957 & 0,926 & 0,072 & 0,819 & 1,105 & 0,608 & 0,623 & 2,418 & 1,508 & 1,379 & 176,180 & 843 \\
\hline 844 & 0,1 & 0,8 & 0,1 & 0,76 & 0,74 & 0,964 & 0,952 & 0,921 & 0,060 & 1,000 & 0,878 & 0,859 & 0,632 & 0,313 & 0,198 & 0,221 & 76,968 & 844 \\
\hline 845 & 0,3 & 2,3 & 0,3 & 2,27 & 2,19 & 0,927 & 0,995 & 0,963 & 0,073 & 0,755 & 1,151 & 0,438 & 0,442 & 0,951 & 0,420 & 0,443 & 46,112 & 845 \\
\hline 846 & 0,6 & 2,9 & 0,6 & 2,67 & 2,58 & 0,980 & 0,925 & 0,896 & 0,077 & 0,926 & 1,039 & 0,612 & 0,570 & 1,129 & 0,644 & 0,693 & 89,167 & 846 \\
\hline 847 & 0,2 & 1,5 & 0,2 & 1,41 & 1,37 & 0,949 & 0,955 & 0,924 & 0,068 & 1,000 & 0,974 & 0,735 & 0,637 & 0,564 & 0,359 & 0,357 & 101,293 & 847 \\
\hline 848 & 0,2 & 1,7 & 0,2 & 1,51 & 1,46 & 0,865 & 0,883 & 0,855 & 0,178 & 0,814 & 1,109 & 0,490 & 0,544 & 0,700 & 0,381 & 0,357 & 48,035 & 848 \\
\hline 849 & 2,5 & 6,5 & 2,7 & 6,40 & 6,19 & 0,926 & 0,986 & 0,954 & 0,075 & 0,753 & 1,153 & 0,464 & 0,565 & 2,631 & 1,487 & 1,268 & 168,205 & 849 \\
\hline 850 & 0,2 & 1,7 & 0,2 & 1,57 & 1,52 & 0,944 & 0,944 & 0,913 & 0,079 & 0,941 & 1,031 & 0,541 & 0,495 & 0,700 & 0,347 & 0,396 & 0,249 & 850 \\
\hline 851 & 0,1 & 1,0 & 0,1 & 0,91 & 0,88 & 0,919 & 0,921 & 0,891 & 0,113 & 1,000 & 0,961 & 0,676 & 0,659 & 0,396 & 0,261 & 0,280 & 97,804 & 851 \\
\hline 852 & 5,6 & 10,2 & 6,0 & 9,95 & 9,63 & 0,935 & 0,976 & 0,945 & 0,070 & 0,673 & 1,219 & 0,379 & 0,402 & 4,323 & 1,736 & 1,633 & 127,656 & 852 \\
\hline 853 & 2,1 & 5,5 & 2,1 & 5,40 & 5,23 & 0,976 & 0,988 & 0,956 & 0,027 & 0,878 & 1,067 & 0,579 & 0,578 & 2,145 & 1,240 & 1,287 & 157,071 & 853 \\
\hline 854 & 0,3 & 2,1 & 0,3 & 1,90 & 1,83 & 0,894 & 0,922 & 0,893 & 0,132 & 0,860 & 1,078 & 0,515 & 0,509 & 0,846 & 0,430 & 0,420 & 65,765 & 854 \\
\hline 855 & 0,1 & 1,2 & 0,1 & 1,09 & 1,06 & 0,911 & 0,912 & 0,883 & 0,125 & 0,878 & 1,067 & 0,580 & 0,596 & 0,470 & 0,280 & 0,280 & 29,659 & 855 \\
\hline 856 & 0,6 & 3,3 & 0,7 & 2,97 & 2,87 & 0,878 & 0,888 & 0,859 & 0,166 & 0,712 & 1,185 & 0,461 & 0,482 & 1,321 & 0,636 & 0,577 & 90,666 & 856 \\
\hline 857 & 0,5 & 2,5 & 0,5 & 2,38 & 2,30 & 0,966 & 0,962 & 0,931 & 0,051 & 0,990 & 1,005 & 0,674 & 0,662 & 0,952 & 0,630 & 0,626 & 147,230 & 857 \\
\hline 858 & 2,2 & 6,0 & 2,3 & 5,85 & 5,66 & 0,944 & 0,980 & 0,948 & 0,059 & 0,773 & 1,138 & 0,422 & 0,456 & 2,573 & 1,173 & 1,192 & 121,380 & 858 \\
\hline 859 & 0,3 & 2,4 & 0,3 & 2,25 & 2,18 & 0,865 & 0,923 & 0,893 & 0,155 & 0,563 & 1,333 & 0,283 & 0,337 & 1,096 & 0,369 & 0,396 & 106,252 & 859 \\
\hline 860 & 0,4 & 2,4 & 0,5 & 2,40 & 2,32 & 0,951 & 0,984 & 0,953 & 0,051 & 0,908 & 1,050 & 0,561 & 0,496 & 0,986 & 0,489 & 0,495 & 28,653 & 860 \\
\hline
\end{tabular}




\begin{tabular}{|c|c|c|c|c|c|c|c|c|c|c|c|c|c|c|c|c|c|c|c|}
\hline 861 & 8,7 & 12,3 & 9,5 & 11,76 & 11,38 & 0,924 & 0,954 & 0,923 & 0,089 & 0,722 & 1,177 & 0,540 & 0,635 & 4,539 & 2,884 & 2,574 & 53,833 & 861 & $-5,5$ \\
\hline 862 & 0,4 & 2,3 & 0,4 & 2,18 & 2,11 & 0,933 & 0,943 & 0,912 & 0,088 & 0,889 & 1,060 & 0,643 & 0,687 & 0,865 & 0,594 & 0,560 & 1,236 & 862 & $-3,1$ \\
\hline 863 & 0,4 & 2,2 & 0,4 & 2,19 & 2,12 & 0,963 & 0,983 & 0,951 & 0,041 & 0,982 & 1,009 & 0,613 & 0,607 & 0,897 & 0,545 & 0,560 & 174,352 & 863 & $-3,2$ \\
\hline 864 & 0,1 & 1,3 & 0,2 & 1,20 & 1,16 & 0,937 & 0,908 & 0,879 & 0,112 & 1,000 & 0,980 & 0,665 & 0,590 & 0,526 & 0,310 & 0,357 & 35,960 & 864 & $-2,4$ \\
\hline 865 & 0,2 & 1,7 & 0,2 & 1,57 & 1,52 & 0,935 & 0,922 & 0,893 & 0,101 & 0,919 & 1,043 & 0,501 & 0,512 & 0,736 & 0,377 & 0,408 & 108,143 & 865 & $-2,9$ \\
\hline 866 & 0,5 & 2,8 & 0,6 & 2,57 & 2,49 & 0,900 & 0,932 & 0,902 & 0,121 & 0,871 & 1,072 & 0,668 & 0,658 & 1,002 & 0,660 & 0,626 & 136,396 & 866 & $-3,3$ \\
\hline 867 & 0,2 & 1,4 & 0,2 & 1,22 & 1,18 & 0,940 & 0,884 & 0,855 & 0,131 & 1,000 & 0,987 & 0,642 & 0,537 & 0,553 & 0,297 & 0,357 & 96,275 & 867 & $-2,5$ \\
\hline 868 & 1,0 & 4,9 & 1,2 & 4,31 & 4,17 & 0,816 & 0,880 & 0,852 & 0,220 & 0,513 & 1,396 & 0,389 & 0,529 & 1,789 & 0,947 & 0,721 & 76,983 & 868 & $-4,2$ \\
\hline 869 & 0,3 & 2,0 & 0,3 & 1,91 & 1,85 & 0,967 & 0,940 & 0,910 & 0,068 & 0,892 & 1,059 & 0,478 & 0,450 & 0,881 & 0,396 & 0,408 & 56,614 & 869 & $-3,1$ \\
\hline 870 & 0,2 & 1,6 & 0,2 & 1,41 & 1,37 & 0,940 & 0,905 & 0,876 & 0,112 & 0,794 & 1,122 & 0,391 & 0,354 & 0,709 & 0,251 & 0,280 & 159,471 & 870 & $-2,8$ \\
\hline 871 & 1,6 & 5,0 & 1,7 & 4,93 & 4,77 & 0,937 & 0,987 & 0,955 & 0,065 & 0,787 & 1,127 & 0,487 & 0,514 & 2,021 & 1,038 & 0,990 & 68,611 & 871 & $-4,3$ \\
\hline 872 & 0,1 & 0,9 & 0,1 & 0,74 & 0,71 & 0,931 & 0,821 & 0,795 & 0,192 & 1,000 & 0,981 & 0,593 & 0,465 & 0,377 & 0,175 & 0,198 & 165,983 & 872 & $-1,9$ \\
\hline 873 & 0,1 & 1,0 & 0,1 & 0,77 & 0,75 & 0,947 & 0,761 & 0,736 & 0,245 & 1,000 & 0,965 & 0,674 & 0,485 & 0,408 & 0,198 & 0,280 & 6,372 & 873 & $-2,0$ \\
\hline 874 & 2,5 & 5,9 & 2,6 & 5,72 & 5,54 & 0,949 & 0,966 & 0,935 & 0,061 & 0,890 & 1,060 & 0,729 & 0,807 & 2,082 & 1,680 & 1,462 & 147,180 & 874 & $-4,4$ \\
\hline 875 & 2,3 & 5,6 & 2,3 & 5,55 & 5,37 & 0,966 & 0,986 & 0,955 & 0,036 & 0,897 & 1,056 & 0,697 & 0,784 & 2,032 & 1,594 & 1,407 & 68,335 & 875 & $-4,3$ \\
\hline 876 & 0,7 & 3,1 & 0,8 & 3,04 & 2,94 & 0,959 & 0,979 & 0,947 & 0,047 & 0,961 & 1,020 & 0,737 & 0,648 & 1,129 & 0,731 & 0,754 & 116,728 & 876 & $-3,5$ \\
\hline 877 & 0,9 & 3,3 & 0,9 & 3,29 & 3,18 & 0,965 & 0,988 & 0,957 & 0,037 & 1,000 & 0,997 & 0,784 & 0,812 & 1,198 & 0,973 & 0,975 & 123,595 & 877 & $-3,6$ \\
\hline 878 & 0,3 & 2,0 & 0,3 & 1,84 & 1,78 & 0,947 & 0,938 & 0,908 & 0,082 & 0,993 & 1,004 & 0,678 & 0,649 & 0,756 & 0,490 & 0,533 & 48,909 & 878 & $-2,9$ \\
\hline 879 & 0,1 & 1,2 & 0,1 & 1,00 & 0,97 & 0,851 & 0,862 & 0,834 & 0,203 & 0,915 & 1,045 & 0,698 & 0,812 & 0,423 & 0,344 & 0,280 & 154,461 & 879 & $-2,1$ \\
\hline 880 & 0,2 & 1,5 & 0,2 & 1,38 & 1,33 & 0,880 & 0,912 & 0,883 & 0,149 & 0,894 & 1,058 & 0,568 & 0,546 & 0,602 & 0,329 & 0,357 & 86,537 & 880 & $-2,6$ \\
\hline 881 & 0,1 & 1,0 & 0,1 & 0,83 & 0,80 & 0,921 & 0,838 & 0,811 & 0,180 & 1,000 & 0,952 & 0,857 & 0,825 & 0,357 & 0,295 & 0,280 & 83,773 & 881 & $-1,8$ \\
\hline 882 & 0,7 & 3,3 & 0,7 & 2,98 & 2,89 & 0,917 & 0,907 & 0,878 & 0,124 & 0,790 & 1,125 & 0,522 & 0,500 & 1,287 & 0,644 & 0,626 & 82,616 & 882 & $-3,7$ \\
\hline 883 & 0,1 & 2,4 & 0,2 & 2,16 & 2,09 & 0,597 & 0,916 & 0,886 & 0,412 & 0,205 & 2,210 & 0,090 & 0,115 & 1,131 & 0,130 & 0,099 & 62,602 & 883 & $-3,5$ \\
\hline 884 & 0,2 & 1,4 & 0,2 & 1,23 & 1,19 & 0,912 & 0,867 & 0,840 & 0,159 & 0,955 & 1,023 & 0,607 & 0,614 & 0,564 & 0,347 & 0,357 & 20,436 & 884 & $-2,5$ \\
\hline 885 & 0,3 & 2,3 & 0,4 & 2,17 & 2,10 & 0,959 & 0,942 & 0,912 & 0,071 & 0,828 & 1,099 & 0,448 & 0,398 & 0,995 & 0,396 & 0,443 & 82,539 & 885 & $-3,3$ \\
\hline 886 & 0,3 & 2,1 & 0,4 & 2,00 & 1,94 & 0,932 & 0,955 & 0,925 & 0,081 & 0,962 & 1,019 & 0,641 & 0,633 & 0,816 & 0,517 & 0,533 & 176,449 & 886 & $-3,0$ \\
\hline 887 & 0,2 & 1,9 & 0,3 & 1,80 & 1,74 & 0,924 & 0,950 & 0,920 & 0,091 & 0,832 & 1,096 & 0,459 & 0,502 & 0,812 & 0,408 & 0,396 & 39,351 & 887 & $-3,0$ \\
\hline 888 & 3,2 & 7,0 & 3,4 & 6,92 & 6,70 & 0,925 & 0,987 & 0,955 & 0,077 & 0,812 & 1,110 & 0,655 & 0,706 & 2,486 & 1,755 & 1,472 & 143,167 & 888 & $-4,6$ \\
\hline 889 & 0,2 & 1,5 & 0,2 & 1,43 & 1,38 & 0,957 & 0,973 & 0,943 & 0,051 & 0,948 & 1,027 & 0,512 & 0,387 & 0,634 & 0,245 & 0,280 & 45,102 & 889 & $-2,7$ \\
\hline 890 & 0,1 & 0,9 & 0,1 & 0,80 & 0,78 & 1,000 & 0,884 & 0,856 & 0,116 & 1,000 & 0,927 & 0,745 & 0,583 & 0,360 & 0,210 & 0,280 & 148,344 & 890 & $-1,8$ \\
\hline 891 & 0,3 & 2,1 & 0,3 & 1,99 & 1,93 & 0,943 & 0,955 & 0,924 & 0,073 & 0,937 & 1,033 & 0,576 & 0,585 & 0,846 & 0,495 & 0,505 & 10,258 & 891 & $-3,1$ \\
\hline 892 & 0,3 & 1,8 & 0,3 & 1,77 & 1,72 & 0,958 & 0,973 & 0,941 & 0,050 & 1,000 & 0,978 & 0,778 & 0,688 & 0,673 & 0,463 & 0,495 & 132,823 & 892 & $-2,8$ \\
\hline 893 & 0,3 & 2,1 & 0,3 & 1,97 & 1,91 & 0,923 & 0,942 & 0,912 & 0,097 & 0,920 & 1,042 & 0,629 & 0,605 & 0,806 & 0,487 & 0,495 & 54,294 & 893 & $-3,0$ \\
\hline 894 & 0,3 & 2,3 & 0,3 & 2,11 & 2,04 & 0,908 & 0,936 & 0,906 & 0,112 & 0,721 & 1,177 & 0,378 & 0,400 & 0,991 & 0,396 & 0,420 & 88,058 & 894 & $-3,3$ \\
\hline 895 & 5,1 & 9,4 & 5,7 & 8,86 & 8,57 & 0,903 & 0,947 & 0,916 & 0,111 & 0,739 & 1,163 & 0,526 & 0,631 & 3,530 & 2,229 & 2,133 & 61,584 & 895 & $-5,1$ \\
\hline 896 & 0,1 & 1,2 & 0,1 & 1,16 & 1,12 & 0,981 & 0,968 & 0,937 & 0,037 & 1,000 & 0,938 & 0,696 & 0,574 & 0,488 & 0,280 & 0,357 & 34,425 & 896 & $-2,3$ \\
\hline 897 & 0,1 & 0,8 & 0,1 & 0,67 & 0,65 & 0,917 & 0,807 & 0,780 & 0,211 & 0,987 & 1,007 & 0,529 & 0,391 & 0,360 & 0,141 & 0,198 & 101,390 & 897 & $-1,8$ \\
\hline 898 & 0,1 & 1,1 & 0,1 & 0,97 & 0,94 & 0,977 & 0,926 & 0,896 & 0,077 & 1,000 & 0,923 & 0,786 & 0,600 & 0,408 & 0,245 & 0,280 & 59,853 & 898 & $-2,0$ \\
\hline 899 & 0,8 & 3,5 & 0,8 & 3,43 & 3,32 & 0,947 & 0,989 & 0,957 & 0,054 & 0,821 & 1,104 & 0,527 & 0,549 & 1,376 & 0,756 & 0,773 & 52,110 & 899 & $-3,8$ \\
\hline 900 & 0,1 & 1,0 & 0,1 & 0,83 & 0,80 & 0,931 & 0,838 & 0,811 & 0,176 & 0,851 & 1,084 & 0,419 & 0,331 & 0,448 & 0,149 & 0,198 & 87,586 & 900 & $-2,2$ \\
\hline 901 & 0,5 & 2,5 & 0,5 & 2,43 & 2,35 & 0,959 & 0,984 & 0,953 & 0,044 & 0,939 & 1,032 & 0,578 & 0,516 & 1,002 & 0,517 & 0,505 & 108,483 & 901 & $-3,3$ \\
\hline 902 & 0,1 & 1,0 & 0,1 & 0,86 & 0,83 & 0,976 & 0,866 & 0,838 & 0,136 & 1,000 & 0,891 & 0,979 & 0,807 & 0,357 & 0,288 & 0,313 & 125,450 & 902 & $-1,8$ \\
\hline 903 & 1,8 & 5,2 & 1,9 & 5,07 & 4,91 & 0,926 & 0,975 & 0,943 & 0,078 & 0,817 & 1,107 & 0,509 & 0,590 & 2,098 & 1,238 & 1,241 & 159,751 & 903 & $-4,4$ \\
\hline 904 & 2,0 & 5,0 & 2,0 & 4,95 & 4,79 & 0,969 & 0,992 & 0,960 & 0,032 & 0,996 & 1,002 & 0,840 & 0,859 & 1,729 & 1,485 & 1,400 & 109,693 & 904 & $-4,1$ \\
\hline 905 & 0,1 & 1,0 & 0,1 & 0,83 & 0,80 & 0,974 & 0,794 & 0,769 & 0,208 & 1,000 & 0,978 & 0,645 & 0,468 & 0,423 & 0,198 & 0,280 & 84,275 & 905 & $-2,1$ \\
\hline 906 & 0,6 & 2,7 & 0,6 & 2,60 & 2,52 & 0,963 & 0,965 & 0,934 & 0,051 & 1,000 & 0,998 & 0,659 & 0,623 & 1,059 & 0,660 & 0,693 & 61,550 & 906 & $-3,4$ \\
\hline 907 & 3,4 & 7,8 & 3,8 & 7,18 & 6,95 & 0,884 & 0,919 & 0,890 & 0,141 & 0,696 & 1,198 & 0,631 & 0,751 & 2,613 & 1,963 & 1,565 & 23,660 & 907 & $-4,7$ \\
\hline 908 & 0,9 & 3,6 & 1,0 & 3,61 & 3,49 & 0,949 & 0,989 & 0,958 & 0,052 & 0,859 & 1,079 & 0,539 & 0,534 & 1,466 & 0,783 & 0,798 & 27,815 & 908 & $-3,9$ \\
\hline 909 & 2,3 & 7,2 & 2,6 & 7,06 & 6,84 & 0,905 & 0,987 & 0,955 & 0,096 & 0,570 & 1,324 & 0,287 & 0,325 & 3,212 & 1,044 & 1,052 & 61,141 & 909 & $-5,0$ \\
\hline 910 & 2,4 & 7,1 & 2,7 & 6,89 & 6,67 & 0,901 & 0,973 & 0,942 & 0,103 & 0,613 & 1,277 & 0,324 & 0,340 & 3,100 & 1,055 & 0,995 & 80,355 & 910 & $-5,0$ \\
\hline 911 & 0,2 & 1,6 & 0,2 & 1,50 & 1,45 & 0,961 & 0,934 & 0,905 & 0,077 & 0,876 & 1,068 & 0,453 & 0,406 & 0,709 & 0,288 & 0,313 & 104,872 & 911 & $-2,8$ \\
\hline 912 & 0,2 & 2,3 & 0,2 & 2,16 & 2,09 & 0,837 & 0,947 & 0,916 & 0,171 & 0,426 & 1,532 & 0,191 & 0,203 & 1,086 & 0,221 & 0,280 & 112,405 & 912 & $-3,4$ \\
\hline 913 & 0,5 & 2,8 & 0,6 & 2,59 & 2,51 & 0,872 & 0,920 & 0,890 & 0,151 & 0,768 & 1,141 & 0,520 & 0,630 & 1,090 & 0,687 & 0,560 & 66,604 & 913 & $-3,4$ \\
\hline 914 & 0,4 & 2,6 & 0,5 & 2,45 & 2,37 & 0,887 & 0,935 & 0,905 & 0,130 & 0,809 & 1,112 & 0,563 & 0,571 & 1,001 & 0,572 & 0,560 & 65,107 & 914 & $-3,3$ \\
\hline 915 & 0,3 & 2,4 & 0,3 & 2,31 & 2,24 & 0,947 & 0,972 & 0,941 & 0,060 & 0,685 & 1,208 & 0,329 & 0,272 & 1,094 & 0,297 & 0,396 & 87,759 & 915 & $-3,5$ \\
\hline 916 & 0,3 & 2,0 & 0,3 & 1,79 & 1,73 & 0,866 & 0,918 & 0,889 & 0,158 & 0,834 & 1,095 & 0,593 & 0,605 & 0,736 & 0,446 & 0,408 & 75,148 & 916 & $-2,9$ \\
\hline 917 & 0,3 & 2,3 & 0,4 & 2,11 & 2,04 & 0,866 & 0,903 & 0,874 & 0,166 & 0,731 & 1,170 & 0,486 & 0,538 & 0,910 & 0,490 & 0,495 & 142,114 & 917 & $-3,2$ \\
\hline 918 & 0,1 & 1,3 & 0,1 & 1,23 & 1,19 & 0,947 & 0,929 & 0,900 & 0,088 & 0,954 & 1,024 & 0,502 & 0,459 & 0,579 & 0,266 & 0,280 & 112,470 & 918 & $-2,5$ \\
\hline
\end{tabular}




\begin{tabular}{|c|c|c|c|c|c|c|c|c|c|c|c|c|c|c|c|c|c|c|c|}
\hline 919 & 0,3 & 2,4 & 0,4 & 2,12 & 2,05 & 0,880 & 0,889 & 0,860 & 0,164 & 0,755 & 1,151 & 0,646 & 0,620 & 0,819 & 0,508 & 0,443 & 130,265 & 919 & $-3,0$ \\
\hline 920 & 1,1 & 4,3 & 1,2 & 4,14 & 4,01 & 0,930 & 0,965 & 0,934 & 0,078 & 0,760 & 1,147 & 0,437 & 0,425 & 1,802 & 0,766 & 0,754 & 35,804 & 920 & $-4,2$ \\
\hline 921 & 0,2 & 1,7 & 0,2 & 1,57 & 1,52 & 0,951 & 0,953 & 0,922 & 0,068 & 0,880 & 1,066 & 0,484 & 0,530 & 0,709 & 0,376 & 0,357 & 104,005 & 921 & $-2,8$ \\
\hline 922 & 0,2 & 1,5 & 0,2 & 1,32 & 1,28 & 0,875 & 0,866 & 0,838 & 0,183 & 0,835 & 1,094 & 0,511 & 0,436 & 0,620 & 0,270 & 0,297 & 103,270 & 922 & $-2,6$ \\
\hline 923 & 1,8 & 5,0 & 1,8 & 4,93 & 4,77 & 0,959 & 0,992 & 0,960 & 0,042 & 0,902 & 1,053 & 0,772 & 0,792 & 1,709 & 1,354 & 1,260 & 120,979 & 923 & $-4,1$ \\
\hline 924 & 0,3 & 2,2 & 0,3 & 1,99 & 1,93 & 0,920 & 0,914 & 0,885 & 0,118 & 0,748 & 1,156 & 0,388 & 0,407 & 0,961 & 0,391 & 0,396 & 11,432 & 924 & $-3,3$ \\
\hline 925 & 1,7 & 4,7 & 1,7 & 4,63 & 4,48 & 0,966 & 0,986 & 0,954 & 0,037 & 0,952 & 1,025 & 0,655 & 0,704 & 1,802 & 1,268 & 1,225 & 163,482 & 925 & $-4,2$ \\
\hline 926 & 0,3 & 1,8 & 0,3 & 1,70 & 1,64 & 0,943 & 0,919 & 0,890 & 0,099 & 1,000 & 0,980 & 0,791 & 0,735 & 0,673 & 0,495 & 0,577 & 66,852 & 926 & $-2,8$ \\
\hline 927 & 0,2 & 1,4 & 0,2 & 1,36 & 1,32 & 0,958 & 0,953 & 0,923 & 0,062 & 1,000 & 0,978 & 0,806 & 0,745 & 0,517 & 0,385 & 0,357 & 138,997 & 927 & $-2,4$ \\
\hline 928 & 0,2 & 1,7 & 0,2 & 1,63 & 1,57 & 0,921 & 0,945 & 0,916 & 0,096 & 0,970 & 1,015 & 0,800 & 0,705 & 0,602 & 0,425 & 0,408 & 143,027 & 928 & $-2,6$ \\
\hline 929 & 0,5 & 2,3 & 0,5 & 2,24 & 2,17 & 0,979 & 0,971 & 0,940 & 0,036 & 1,000 & 0,970 & 0,846 & 0,807 & 0,824 & 0,665 & 0,693 & 134,965 & 929 & $-3,0$ \\
\hline 930 & 0,2 & 2,0 & 0,2 & 2,01 & 1,95 & 0,959 & 1,014 & 0,981 & 0,043 & 0,736 & 1,166 & 0,352 & 0,307 & 0,913 & 0,280 & 0,313 & 129,635 & 930 & $-3,2$ \\
\hline 931 & 9,1 & 14,2 & 10,6 & 11,87 & 11,49 & 0,862 & 0,833 & 0,807 & 0,216 & 0,566 & 1,330 & 0,527 & 0,579 & 4,699 & 2,723 & 1,881 & 172,979 & 931 & $-5,6$ \\
\hline 932 & 0,4 & 2,4 & 0,5 & 2,28 & 2,21 & 0,946 & 0,945 & 0,915 & 0,077 & 0,925 & 1,040 & 0,561 & 0,584 & 0,986 & 0,576 & 0,594 & 105,696 & 932 & $-3,3$ \\
\hline 933 & 0,4 & 2,4 & 0,4 & 2,25 & 2,18 & 0,919 & 0,944 & 0,914 & 0,098 & 0,860 & 1,079 & 0,595 & 0,602 & 0,913 & 0,549 & 0,577 & 142,882 & 933 & $-3,2$ \\
\hline 934 & 0,1 & 1,4 & 0,1 & 1,19 & 1,15 & 0,947 & 0,871 & 0,843 & 0,140 & 0,894 & 1,058 & 0,471 & 0,414 & 0,598 & 0,248 & 0,297 & 10,276 & 934 & $-2,6$ \\
\hline 935 & 4,4 & 7,7 & 4,5 & 7,67 & 7,43 & 0,983 & 0,995 & 0,963 & 0,018 & 0,939 & 1,032 & 0,751 & 0,776 & 2,745 & 2,129 & 2,041 & 177,950 & 935 & $-4,8$ \\
\hline 936 & 1,6 & 4,8 & 1,8 & 4,75 & 4,60 & 0,927 & 0,992 & 0,960 & 0,073 & 0,891 & 1,059 & 0,644 & 0,690 & 1,793 & 1,238 & 1,155 & 2,577 & 936 & $-4,2$ \\
\hline 937 & 0,6 & 2,8 & 0,6 & 2,76 & 2,67 & 0,955 & 0,986 & 0,955 & 0,047 & 0,922 & 1,041 & 0,575 & 0,551 & 1,129 & 0,622 & 0,664 & 72,718 & 937 & $-3,5$ \\
\hline 938 & 0,2 & 2,4 & 0,3 & 2,23 & 2,16 & 0,835 & 0,918 & 0,889 & 0,184 & 0,474 & 1,452 & 0,235 & 0,273 & 1,099 & 0,300 & 0,313 & 73,876 & 938 & $-3,5$ \\
\hline 939 & 0,3 & 2,0 & 0,4 & 1,88 & 1,82 & 0,972 & 0,966 & 0,935 & 0,044 & 1,000 & 0,939 & 0,919 & 0,790 & 0,690 & 0,545 & 0,594 & 98,639 & 939 & $-2,8$ \\
\hline 940 & 0,1 & 1,0 & 0,1 & 0,74 & 0,71 & 0,937 & 0,773 & 0,749 & 0,235 & 1,000 & 0,988 & 0,562 & 0,364 & 0,408 & 0,149 & 0,198 & 0,720 & 940 & $-2,0$ \\
\hline 941 & 0,1 & 0,8 & 0,1 & 0,61 & 0,59 & 0,923 & 0,793 & 0,768 & 0,221 & 1,000 & 0,900 & 0,899 & 0,714 & 0,289 & 0,206 & 0,221 & 121,853 & 941 & $-1,5$ \\
\hline 942 & 0,2 & 1,6 & 0,2 & 1,55 & 1,50 & 0,916 & 0,983 & 0,951 & 0,086 & 0,944 & 1,029 & 0,616 & 0,598 & 0,620 & 0,371 & 0,357 & 151,491 & 942 & $-2,6$ \\
\hline 943 & 0,3 & 3,3 & 0,5 & 2,47 & 2,39 & 0,719 & 0,756 & 0,732 & 0,372 & 0,384 & 1,613 & 0,413 & 0,578 & 1,002 & 0,580 & 0,357 & 27,809 & 943 & $-3,3$ \\
\hline 944 & 0,3 & 1,9 & 0,3 & 1,76 & 1,71 & 0,976 & 0,949 & 0,919 & 0,056 & 1,000 & 0,961 & 0,752 & 0,629 & 0,709 & 0,446 & 0,495 & 179,805 & 944 & $-2,8$ \\
\hline 945 & 0,2 & 1,7 & 0,2 & 1,59 & 1,54 & 0,935 & 0,960 & 0,929 & 0,077 & 0,970 & 1,015 & 0,648 & 0,598 & 0,644 & 0,385 & 0,420 & 47,995 & 945 & $-2,7$ \\
\hline 946 & 0,1 & 0,8 & 0,1 & 0,75 & 0,72 & 0,964 & 0,888 & 0,860 & 0,117 & 1,000 & 0,921 & 0,764 & 0,632 & 0,332 & 0,210 & 0,221 & 142,543 & 946 & $-1,7$ \\
\hline 947 & 101,0 & 39,1 & 107,1 & 37,36 & 36,16 & 0,943 & 0,955 & 0,924 & 0,073 & 0,829 & 1,098 & 0,658 & 0,740 & 13,983 & 10,347 & 9,343 & 5,674 & 947 & $-7,1$ \\
\hline 948 & 3,1 & 6,8 & 3,2 & 6,69 & 6,48 & 0,956 & 0,985 & 0,953 & 0,047 & 0,842 & 1,090 & 0,565 & 0,575 & 2,641 & 1,517 & 1,472 & 2,564 & 948 & $-4,7$ \\
\hline 949 & 0,3 & 2,0 & 0,3 & 1,91 & 1,85 & 0,937 & 0,980 & 0,949 & 0,066 & 0,964 & 1,019 & 0,648 & 0,588 & 0,757 & 0,446 & 0,495 & 84,458 & 949 & $-2,9$ \\
\hline 950 & 4,6 & 8,6 & 4,9 & 8,52 & 8,25 & 0,931 & 0,989 & 0,957 & 0,070 & 0,774 & 1,137 & 0,484 & 0,482 & 3,466 & 1,671 & 1,565 & 108,871 & 950 & $-5,1$ \\
\hline 951 & 0,2 & 1,6 & 0,2 & 1,51 & 1,46 & 0,908 & 0,926 & 0,896 & 0,118 & 0,916 & 1,045 & 0,544 & 0,493 & 0,673 & 0,332 & 0,357 & 116,224 & 951 & $-2,8$ \\
\hline 952 & 1,7 & 6,8 & 2,2 & 5,27 & 5,10 & 0,801 & 0,777 & 0,752 & 0,299 & 0,476 & 1,449 & 0,544 & 0,760 & 2,019 & 1,535 & 1,034 & 58,044 & 952 & $-4,3$ \\
\hline 953 & 0,1 & 1,2 & 0,1 & 0,93 & 0,90 & 1,000 & 0,801 & 0,776 & 0,199 & 1,000 & 0,904 & 0,809 & 0,651 & 0,456 & 0,297 & 0,396 & 99,899 & 953 & $-2,2$ \\
\hline 954 & 0,1 & 1,1 & 0,1 & 0,99 & 0,96 & 0,872 & 0,861 & 0,833 & 0,189 & 0,957 & 1,022 & 0,614 & 0,535 & 0,456 & 0,244 & 0,280 & 91,766 & 954 & $-2,2$ \\
\hline 955 & 0,4 & 2,3 & 0,4 & 2,35 & 2,27 & 0,963 & 1,012 & 0,980 & 0,038 & 0,904 & 1,052 & 0,539 & 0,540 & 0,956 & 0,517 & 0,533 & 157,606 & 955 & $-3,3$ \\
\hline 956 & 0,6 & 3,5 & 0,7 & 3,42 & 3,31 & 0,931 & 0,981 & 0,949 & 0,072 & 0,646 & 1,244 & 0,310 & 0,333 & 1,603 & 0,535 & 0,577 & 6,773 & 956 & $-4,0$ \\
\hline 957 & 1,3 & 4,9 & 1,5 & 4,69 & 4,54 & 0,837 & 0,950 & 0,919 & 0,171 & 0,649 & 1,241 & 0,402 & 0,480 & 1,996 & 0,958 & 0,773 & 98,330 & 957 & $-4,3$ \\
\hline 958 & 1,8 & 6,3 & 2,2 & 5,57 & 5,40 & 0,824 & 0,890 & 0,861 & 0,208 & 0,571 & 1,323 & 0,411 & 0,555 & 2,352 & 1,305 & 1,089 & 112,970 & 958 & $-4,6$ \\
\hline 959 & 0,1 & 0,8 & 0,1 & 0,80 & 0,78 & 0,964 & 0,954 & 0,923 & 0,058 & 1,000 & 0,921 & 0,688 & 0,566 & 0,350 & 0,198 & 0,221 & 76,957 & 959 & $-1,8$ \\
\hline 960 & 0,3 & 2,4 & 0,4 & 2,27 & 2,20 & 0,927 & 0,956 & 0,925 & 0,085 & 0,764 & 1,144 & 0,384 & 0,417 & 1,066 & 0,444 & 0,495 & 163,660 & 960 & $-3,4$ \\
\hline 961 & 0,1 & 0,8 & 0,1 & 0,64 & 0,62 & 0,962 & 0,828 & 0,803 & 0,176 & 1,000 & 0,881 & 0,796 & 0,600 & 0,313 & 0,188 & 0,198 & 104,446 & 961 & $-1,6$ \\
\hline 962 & 0,1 & 0,8 & 0,1 & 0,70 & 0,67 & 0,926 & 0,840 & 0,813 & 0,176 & 1,000 & 0,944 & 0,601 & 0,412 & 0,360 & 0,149 & 0,198 & 95,375 & 962 & $-1,8$ \\
\hline 963 & 1,4 & 4,5 & 1,5 & 4,30 & 4,16 & 0,943 & 0,962 & 0,931 & 0,069 & 0,891 & 1,060 & 0,736 & 0,724 & 1,565 & 1,133 & 1,052 & 47,932 & 963 & $-4,0$ \\
\hline 964 & 0,4 & 2,5 & 0,5 & 2,27 & 2,20 & 0,873 & 0,913 & 0,884 & 0,154 & 0,823 & 1,103 & 0,557 & 0,579 & 0,961 & 0,556 & 0,594 & 106,980 & 964 & $-3,3$ \\
\hline 965 & 19,2 & 17,8 & 20,7 & 16,92 & 16,38 & 0,927 & 0,953 & 0,923 & 0,086 & 0,764 & 1,144 & 0,627 & 0,671 & 6,237 & $\begin{array}{l}\text {, J30 } \\
4,186\end{array}$ & $\begin{array}{l}3,856 \\
3,856\end{array}$ & $\begin{array}{l}119,320 \\
119\end{array}$ & $\begin{array}{l}0405 \\
965\end{array}$ & $\begin{array}{l}-3,0 \\
-6,0\end{array}$ \\
\hline 966 & 0,2 & 2,3 & 0,3 & 2,03 & 1,96 & 0,835 & 0,895 & 0,867 & 0,196 & 0,606 & 1,284 & 0,348 & 0,413 & 0,952 & 0,393 & 0,408 & 118,530 & 966 & $-3,3$ \\
\hline 967 & 0,1 & 1,2 & 0,1 & 1,13 & 1,10 & 0,981 & 0,923 & 0,894 & 0,079 & 1,000 & 0,960 & 0,668 & 0,597 & 0,498 & 0,297 & 0,357 & 0,546 & 967 & $-2,3$ \\
\hline 968 & 0,1 & 0,8 & 0,1 & 0,71 & 0,68 & 0,929 & 0,842 & 0,815 & 0,173 & 1,000 & 0,939 & 0,736 & 0,600 & 0,332 & 0,199 & 0,221 & 119,730 & 968 & $-1,7$ \\
\hline 969 & 0,3 & 1,7 & 0,3 & 1,57 & 1,52 & 0,991 & 0,914 & 0,884 & 0,087 & 1,000 & 0,941 & 0,816 & 0,767 & 0,645 & 0,495 & 0,505 & 14,836 & 969 & $-2,7$ \\
\hline 970 & 0,1 & 1,0 & 0,1 & 0,90 & 0,87 & 0,971 & 0,871 & 0,843 & 0,132 & 0,992 & 1,004 & 0,481 & 0,422 & 0,470 & 0,198 & 0,280 & 162,847 & 970 & $-2,2$ \\
\hline 971 & 1,4 & 5,0 & 1,6 & 4,85 & 4,69 & 0,899 & 0,977 & 0,945 & 0,104 & 0,734 & 1,167 & 0,460 & 0,523 & 1,996 & 1,044 & 0,980 & 169,079 & 971 & $-4,3$ \\
\hline 972 & 0,7 & 3,1 & 0,7 & 3,11 & 3,01 & 0,986 & 0,988 & 0,956 & 0,019 & 0,882 & 1,065 & 0,471 & 0,409 & 1,369 & 0,560 & 0,634 & 47,036 & 972 & $-3,8$ \\
\hline 973 & 0,2 & 1,3 & 0,2 & 1,19 & 1,15 & 0,984 & 0,947 & 0,917 & 0,055 & 1,000 & 0,908 & 0,887 & 0,750 & 0,467 & 0,350 & 0,396 & 43,763 & 973 & $-2,2$ \\
\hline 974 & 0,4 & 2,4 & 0,4 & 2,39 & 2,31 & 0,918 & 0,989 & 0,957 & 0,083 & 0,830 & 1,098 & 0,449 & 0,465 & 1,044 & 0,486 & 0,495 & 145,970 & 974 & $-3,4$ \\
\hline 975 & 0,1 & 0,8 & 0,1 & 0,75 & 0,72 & 1,000 & 0,951 & 0,920 & 0,049 & 1,000 & 0,877 & 0,895 & 0,697 & 0,301 & 0,210 & 0,280 & 51,526 & 975 & $-1,6$ \\
\hline 976 & 1,7 & 5,0 & 1,8 & 4,93 & 4,78 & 0,957 & 0,987 & 0,955 & 0,045 & 0,856 & 1,081 & 0,569 & 0,574 & 1,953 & 1,120 & 1,120 & 131,065 & 976 & $-4,3$ \\
\hline
\end{tabular}




\begin{tabular}{|c|c|c|c|c|c|c|c|c|c|c|c|c|c|c|c|c|c|c|c|}
\hline 977 & 1,8 & 4,9 & 1,8 & 4,81 & 4,66 & 0,957 & 0,984 & 0,953 & 0,046 & 0,920 & 1,043 & 0,712 & 0,668 & 1,769 & 1,182 & 1,192 & 62,698 & 977 & $-4,1$ \\
\hline 978 & 0,3 & 2,0 & 0,3 & 1,87 & 1,81 & 0,944 & 0,934 & 0,904 & 0,087 & 0,916 & 1,045 & 0,524 & 0,513 & 0,842 & 0,432 & 0,495 & 119,992 & 978 & $-3,1$ \\
\hline 979 & 0,0 & 0,7 & 0,1 & 0,57 & 0,56 & 0,952 & 0,782 & 0,756 & 0,223 & 1,000 & 0,936 & 0,621 & 0,531 & 0,317 & 0,168 & 0,198 & 152,367 & 979 & $-1,7$ \\
\hline 980 & 5,1 & 9,0 & 5,4 & 8,72 & 8,44 & 0,939 & 0,974 & 0,943 & 0,066 & 0,793 & 1,123 & 0,537 & 0,601 & 3,461 & 2,081 & 1,892 & 20,476 & 980 & $-5,1$ \\
\hline 981 & 1,8 & 5,1 & 1,9 & 5,04 & 4,88 & 0,952 & 0,992 & 0,961 & 0,049 & 0,877 & 1,068 & 0,589 & 0,621 & 1,972 & 1,225 & 1,209 & 51,950 & 981 & $-4,3$ \\
\hline 982 & 0,3 & 2,0 & 0,3 & 1,96 & 1,90 & 0,986 & 0,981 & 0,949 & 0,024 & 1,000 & 0,970 & 0,795 & 0,807 & 0,736 & 0,594 & 0,577 & 114,841 & 982 & $-2,9$ \\
\hline 983 & 0,3 & 2,3 & 0,3 & 2,19 & 2,12 & 0,902 & 0,932 & 0,902 & 0,119 & 0,620 & 1,270 & 0,271 & 0,316 & 1,131 & 0,358 & 0,357 & 61,772 & 983 & $-3,5$ \\
\hline 984 & 0,0 & 0,7 & 0,1 & 0,64 & 0,62 & 0,909 & 0,906 & 0,878 & 0,131 & 1,000 & 0,901 & 0,749 & 0,537 & 0,289 & 0,155 & 0,198 & 123,961 & 984 & $-1,5$ \\
\hline 985 & 0,3 & 1,8 & 0,3 & 1,57 & 1,52 & 0,937 & 0,899 & 0,870 & 0,119 & 1,000 & 0,978 & 0,817 & 0,707 & 0,630 & 0,446 & 0,495 & 83,878 & 985 & $-2,7$ \\
\hline 986 & 0,2 & 1,6 & 0,2 & 1,45 & 1,41 & 0,918 & 0,933 & 0,903 & 0,106 & 0,989 & 1,005 & 0,685 & 0,657 & 0,596 & 0,391 & 0,396 & 71,608 & 986 & $-2,6$ \\
\hline 987 & 0,4 & 2,2 & 0,4 & 2,12 & 2,05 & 0,945 & 0,958 & 0,927 & 0,069 & 0,973 & 1,014 & 0,819 & 0,792 & 0,769 & 0,608 & 0,626 & 26,378 & 987 & $-2,9$ \\
\hline 988 & 0,5 & 2,5 & 0,5 & 2,36 & 2,28 & 0,975 & 0,951 & 0,920 & 0,055 & 0,961 & 1,020 & 0,624 & 0,576 & 0,980 & 0,565 & 0,560 & 120,843 & 988 & $-3,3$ \\
\hline 989 & 0,1 & 0,7 & 0,1 & 0,64 & 0,62 & 1,000 & 0,872 & 0,844 & 0,128 & 1,000 & 0,914 & 0,594 & 0,447 & 0,332 & 0,149 & 0,198 & 107,137 & 989 & $-1,7$ \\
\hline 990 & 2,3 & 6,1 & 2,6 & 5,96 & 5,77 & 0,907 & 0,983 & 0,951 & 0,095 & 0,793 & 1,123 & 0,534 & 0,633 & 2,352 & 1,488 & 1,241 & 102,887 & 990 & $-4,6$ \\
\hline 991 & 1,2 & 4,7 & 1,4 & 4,61 & 4,46 & 0,867 & 0,983 & 0,952 & 0,134 & 0,696 & 1,199 & 0,445 & 0,527 & 1,864 & 0,983 & 0,798 & 86,509 & 991 & $-4,2$ \\
\hline 992 & 0,1 & 0,9 & 0,1 & 0,74 & 0,71 & 0,926 & 0,796 & 0,772 & 0,217 & 0,905 & 1,051 & 0,490 & 0,372 & 0,399 & 0,149 & 0,198 & 91,554 & 992 & $-2,0$ \\
\hline 993 & 0,1 & 0,8 & 0,1 & 0,74 & 0,71 & 0,958 & 0,917 & 0,888 & 0,093 & 1,000 & 0,952 & 0,732 & 0,474 & 0,313 & 0,149 & 0,198 & 178,903 & 993 & $-1,6$ \\
\hline 994 & 0,5 & 2,7 & 0,6 & 2,61 & 2,53 & 0,934 & 0,965 & 0,934 & 0,075 & 0,891 & 1,059 & 0,699 & 0,747 & 0,973 & 0,727 & 0,634 & 121,553 & 994 & $-3,3$ \\
\hline 995 & 0,1 & 1,5 & 0,2 & 1,27 & 1,23 & 0,797 & 0,849 & 0,821 & 0,253 & 0,701 & 1,194 & 0,439 & 0,378 & 0,602 & 0,228 & 0,221 & 95,831 & 995 & $-2,6$ \\
\hline 996 & 0,3 & 2,0 & 0,3 & 1,87 & 1,81 & 0,933 & 0,934 & 0,904 & 0,094 & 0,862 & 1,077 & 0,771 & 0,822 & 0,673 & 0,554 & 0,495 & 159,778 & 996 & $-2,8$ \\
\hline 997 & 0,1 & 0,9 & 0,1 & 0,75 & 0,72 & 0,967 & 0,860 & 0,833 & 0,144 & 1,000 & 0,918 & 0,738 & 0,600 & 0,350 & 0,210 & 0,221 & 126,953 & 997 & $-1,8$ \\
\hline 998 & 0,5 & 2,8 & 0,5 & 2,73 & 2,64 & 0,943 & 0,976 & 0,945 & 0,062 & 0,780 & 1,132 & 0,476 & 0,398 & 1,140 & 0,454 & 0,443 & 122,263 & 998 & $-3,5$ \\
\hline 999 & 0,6 & 3,0 & 0,7 & 3,00 & 2,90 & 0,970 & 0,987 & 0,956 & 0,032 & 0,880 & 1,066 & 0,533 & 0,451 & 1,241 & 0,560 & 0,626 & 130,679 & 999 & $-3,6$ \\
\hline 1000 & 0,1 & 1,4 & 0,1 & 1,25 & 1,21 & 0,880 & 0,887 & 0,858 & 0,165 & 0,677 & 1,215 & 0,331 & 0,286 & 0,644 & 0,184 & 0,198 & 110,403 & 1000 & $-2,7$ \\
\hline 1001 & 0,1 & 1,0 & 0,1 & 0,83 & 0,80 & 0,947 & 0,862 & 0,835 & 0,148 & 1,000 & 0,913 & 0,881 & 0,745 & 0,357 & 0,266 & 0,297 & 138,986 & 1001 & $-1,8$ \\
\hline 1002 & 0,4 & 2,3 & 0,4 & 2,11 & 2,04 & 0,958 & 0,918 & 0,889 & 0,092 & 0,931 & 1,036 & 0,599 & 0,618 & 0,910 & 0,563 & 0,560 & 121,906 & 1002 & $-3,2$ \\
\hline 1003 & 0,1 & 1,1 & 0,1 & 1,03 & 1,00 & 0,959 & 0,894 & 0,866 & 0,113 & 1,000 & 0,955 & 0,672 & 0,636 & 0,467 & 0,297 & 0,297 & 118,884 & 1003 & $-2,2$ \\
\hline 1004 & 0,3 & 2,0 & 0,3 & 1,88 & 1,82 & 0,972 & 0,953 & 0,922 & 0,055 & 1,000 & 0,959 & 0,870 & 0,801 & 0,704 & 0,564 & 0,577 & 63,608 & 1004 & $-2,8$ \\
\hline 1005 & 0,1 & 0,9 & 0,1 & 0,80 & 0,78 & 0,937 & 0,858 & 0,830 & 0,155 & 1,000 & 0,971 & 0,659 & 0,588 & 0,377 & 0,222 & 0,221 & 24,983 & 1005 & $-1,9$ \\
\hline 1006 & 0,1 & 1,1 & 0,1 & 1,03 & 1,00 & 0,932 & 0,916 & 0,888 & 0,108 & 1,000 & 0,998 & 0,715 & 0,696 & 0,423 & 0,295 & 0,280 & 76,840 & 1006 & $-2,1$ \\
\hline 1007 & 0,1 & 0,7 & 0,1 & 0,61 & 0,59 & 0,955 & 0,835 & 0,807 & 0,171 & 1,000 & 0,914 & 0,668 & 0,474 & 0,313 & 0,149 & 0,198 & 164,904 & 1007 & $-1,6$ \\
\hline 1008 & 0,0 & 0,6 & 0,0 & 0,61 & 0,59 & 1,000 & 0,957 & 0,926 & 0,043 & 1,000 & 0,816 & 1,019 & 0,707 & 0,248 & 0,175 & 0,221 & 135,000 & 1008 & $-1,3$ \\
\hline 1009 & 0,3 & 2,1 & 0,3 & 1,90 & 1,83 & 0,922 & 0,922 & 0,893 & 0,110 & 0,868 & 1,073 & 0,497 & 0,500 & 0,865 & 0,433 & 0,443 & 174,953 & 1009 & $-3,1$ \\
\hline 1010 & 0,1 & 1,3 & 0,1 & 1,11 & 1,07 & 0,918 & 0,855 & 0,828 & 0,166 & 0,823 & 1,102 & 0,441 & 0,351 & 0,564 & 0,198 & 0,198 & 80,975 & 1010 & $-2,5$ \\
\hline 1011 & 0,6 & 3,0 & 0,6 & 2,83 & 2,74 & 0,924 & 0,955 & 0,925 & 0,088 & 0,853 & 1,082 & 0,548 & 0,633 & 1,177 & 0,744 & 0,700 & 156,596 & 1011 & $-3,6$ \\
\hline 1012 & 2,1 & 5,2 & 2,2 & 5,18 & 5,01 & 0,976 & 0,993 & 0,961 & 0,025 & 0,982 & 1,009 & 0,892 & 0,903 & 1,741 & 1,572 & 1,418 & 58,026 & 1012 & $-4,1$ \\
\hline 1013 & 0,6 & 2,7 & 0,6 & 2,65 & 2,57 & 0,972 & 0,966 & 0,935 & 0,044 & 1,000 & 1,000 & 0,761 & 0,720 & 1,002 & 0,722 & 0,714 & 151,671 & 1013 & $-3,3$ \\
\hline 1014 & 0,8 & 3,2 & 0,8 & 3,18 & 3,08 & 0,942 & 0,980 & 0,948 & 0,061 & 0,904 & 1,052 & 0,683 & 0,719 & 1,190 & 0,856 & 0,754 & 137,751 & 1014 & $-3,6$ \\
\hline 1015 & 6,9 & 10,1 & 7,3 & 9,90 & 9,58 & 0,952 & 0,981 & 0,950 & 0,052 & 0,852 & 1,083 & 0,573 & 0,632 & 3,917 & 2,475 & 2,279 & 165,737 & 1015 & $-5,3$ \\
\hline 1016 & 0,1 & 1,2 & 0,1 & 1,02 & 0,98 & 0,961 & 0,864 & 0,836 & 0,142 & 1,000 & 0,958 & 0,693 & 0,527 & 0,470 & 0,248 & 0,297 & 88,964 & 1016 & $-2,2$ \\
\hline 1017 & 0,8 & 3,5 & 0,8 & 3,38 & 3,27 & 0,942 & 0,955 & 0,924 & 0,074 & 0,754 & 1,152 & 0,396 & 0,383 & 1,554 & 0,595 & 0,634 & 133,053 & 1017 & $-4,0$ \\
\hline 1018 & 0,4 & 2,4 & 0,4 & 2,36 & 2,28 & 0,932 & 0,968 & 0,937 & 0,075 & 0,851 & 1,084 & 0,502 & 0,517 & 1,010 & 0,522 & 0,560 & 66,780 & 1018 & $-3,3$ \\
\hline 1019 & 0,4 & 2,2 & 0,4 & 2,11 & 2,04 & 0,932 & 0,952 & 0,922 & 0,083 & 0,952 & 1,025 & 0,840 & 0,794 & 0,749 & 0,595 & 0,602 & 36,680 & 1019 & $-2,9$ \\
\hline 1020 & 0,1 & 1,5 & 0,2 & 1,28 & 1,24 & 0,806 & 0,866 & 0,838 & 0,236 & 0,702 & 1,193 & 0,374 & 0,452 & 0,645 & 0,292 & 0,280 & 85,175 & 1020 & $-2,7$ \\
\hline 1021 & 0,0 & 0,7 & 0,1 & 0,57 & 0,56 & 0,909 & 0,782 & 0,756 & 0,236 & 1,000 & 0,936 & 0,621 & 0,489 & 0,317 & 0,155 & 0,198 & 72,588 & 1021 & $-1,7$ \\
\hline 1022 & 0,4 & 2,2 & 0,4 & 2,17 & 2,10 & 0,988 & 0,982 & 0,951 & 0,021 & 1,000 & 0,963 & 0,866 & 0,759 & 0,783 & 0,594 & 0,626 & 88,500 & 1022 & $-3,0$ \\
\hline 1023 & 0,3 & 1,9 & 0,3 & 1,81 & 1,75 & 0,984 & 0,945 & 0,915 & 0,057 & 1,000 & 0,970 & 0,689 & 0,655 & 0,756 & 0,495 & 0,533 & 109,453 & 1023 & $-2,9$ \\
\hline 1024 & 2,6 & 6,9 & 2,8 & 6,65 & 6,43 & 0,928 & 0,968 & 0,937 & 0,079 & 0,693 & 1,201 & 0,397 & 0,480 & 2,887 & 1,386 & 1,321 & 90,681 & 1024 & $-4,9$ \\
\hline 1025 & 0,7 & 3,2 & 0,8 & 3,14 & 3,04 & 0,914 & 0,979 & 0,948 & 0,089 & 0,855 & 1,082 & 0,595 & 0,606 & 1,225 & 0,742 & 0,700 & 60,627 & 1025 & $-3,6$ \\
\hline 1026 & 1,2 & 3,8 & 1,2 & 3,71 & 3,59 & 0,977 & 0,982 & 0,951 & 0,029 & 1,000 & 0,989 & 0,753 & 0,720 & 1,401 & 1,009 & 0,995 & 17,015 & 1026 & $-3,8$ \\
\hline 1027 & 0,1 & 0,7 & 0,1 & 0,57 & 0,56 & 0,960 & 0,782 & 0,756 & 0,222 & 1,000 & 0,855 & 0,899 & 0,686 & 0,289 & 0,198 & 0,280 & 115,286 & 1027 & $-1,5$ \\
\hline 1028 & 4,9 & 8,4 & 5,2 & 8,27 & 8,01 & 0,943 & 0,979 & 0,948 & 0,061 & 0,857 & 1,081 & 0,668 & 0,684 & 3,046 & 2,084 & 2,060 & 114,818 & 1028 & $-4,9$ \\
\hline 1029 & 0,1 & 1,2 & 0,1 & 1,02 & 0,98 & 0,864 & 0,844 & 0,817 & 0,207 & 0,807 & 1,113 & 0,465 & 0,392 & 0,505 & 0,198 & 0,221 & 83,894 & 1029 & $-2,3$ \\
\hline 1030 & 0,9 & 3,7 & 1,0 & 3,55 & 3,44 & 0,898 & 0,964 & 0,933 & 0,109 & 0,814 & 1,108 & 0,559 & 0,642 & 1,416 & 0,908 & 0,891 & 47,119 & 1030 & $-3,8$ \\
\hline 1031 & 0,6 & 2,8 & 0,7 & 2,76 & 2,67 & 0,974 & 0,977 & 0,945 & 0,035 & 1,000 & 0,996 & 0,699 & 0,687 & 1,081 & 0,743 & 0,773 & 74,578 & 1031 & $-3,4$ \\
\hline 1032 & 0,1 & 0,9 & 0,1 & 0,71 & 0,68 & 1,000 & 0,815 & 0,789 & 0,185 & 1,000 & 0,902 & 0,849 & 0,738 & 0,332 & 0,245 & 0,280 & 57,572 & 1032 & $-1,7$ \\
\hline 1033 & 0,5 & 2,7 & 0,5 & 2,55 & 2,47 & 0,923 & 0,950 & 0,920 & 0,091 & 0,875 & 1,069 & 0,536 & 0,524 & 1,090 & 0,571 & 0,626 & 143,077 & 1033 & $-3,4$ \\
\hline 1034 & 0,1 & 0,8 & 0,1 & 0,48 & 0,47 & 0,923 & 0,631 & 0,611 & 0,377 & 1,000 & 0,887 & 0,899 & 0,686 & 0,289 & 0,198 & 0,221 & 28,110 & 1034 & $-1,5$ \\
\hline
\end{tabular}




\begin{tabular}{|c|c|c|c|c|c|c|c|c|c|c|c|c|c|c|c|c|c|c|c|}
\hline 1035 & 0,1 & 0,9 & 0,1 & 0,84 & 0,81 & 0,889 & 0,899 & 0,871 & 0,150 & 0,848 & 1,086 & 0,470 & 0,334 & 0,399 & 0,133 & 0,140 & 149,865 & 1035 & $-2,0$ \\
\hline 1036 & 0,1 & 1,0 & 0,1 & 0,96 & 0,93 & 1,000 & 0,935 & 0,905 & 0,065 & 1,000 & 0,914 & 0,768 & 0,606 & 0,408 & 0,248 & 0,297 & 96,118 & 1036 & $-2,0$ \\
\hline 1037 & 0,4 & 2,5 & 0,4 & 2,43 & 2,35 & 0,916 & 0,984 & 0,953 & 0,085 & 0,776 & 1,135 & 0,431 & 0,432 & 1,052 & 0,455 & 0,495 & 40,876 & 1037 & $-3,4$ \\
\hline 1038 & 0,3 & 2,1 & 0,4 & 2,03 & 1,96 & 0,946 & 0,968 & 0,937 & 0,063 & 0,976 & 1,012 & 0,646 & 0,648 & 0,819 & 0,531 & 0,495 & 147,704 & 1038 & $-3,0$ \\
\hline $\begin{array}{l}1039 \\
\end{array}$ & 0,1 & 0,8 & 0,1 & 0,64 & 0,62 & 0,962 & 0,800 & 0,775 & 0,204 & 1,000 & 0,913 & 0,707 & 0,596 & 0,332 & 0,198 & 0,221 & 168,293 & 1039 & $-1,7$ \\
\hline 1040 & 0,1 & 1,4 & 0,1 & 1,23 & 1,19 & 0,933 & 0,884 & 0,856 & 0,133 & 0,673 & 1,219 & 0,315 & 0,275 & 0,645 & 0,177 & 0,198 & 118,880 & 1040 & $-2,7$ \\
\hline 1041 & 0,2 & 1,6 & 0,2 & 1,47 & 1,42 & 0,914 & 0,902 & 0,872 & 0,131 & 0,858 & 1,079 & 0,486 & 0,477 & 0,690 & 0,329 & 0,357 & 105,350 & 1041 & $-2,8$ \\
\hline 1042 & 0,2 & 1,9 & 0,3 & 1,74 & 1,68 & 0,901 & 0,915 & 0,886 & 0,130 & 0,858 & 1,080 & 0,544 & 0,579 & 0,757 & 0,438 & 0,495 & 88,253 & 1042 & $-2,9$ \\
\hline 1043 & 0,4 & 2,5 & 0,5 & 2,43 & 2,35 & 0,926 & 0,973 & 0,942 & 0,078 & 0,873 & 1,070 & 0,665 & 0,731 & 0,909 & 0,664 & 0,594 & 58,006 & 1043 & $-3,2$ \\
\hline 1044 & 0,3 & 1,9 & 0,3 & 1,71 & 1,65 & 0,964 & 0,901 & 0,872 & 0,105 & 0,926 & 1,039 & 0,502 & 0,496 & 0,819 & 0,407 & 0,420 & 112,318 & 1044 & $-3,0$ \\
\hline 1045 & 0,2 & 1,5 & 0,2 & 1,38 & 1,33 & 0,951 & 0,912 & 0,883 & 0,101 & 1,000 & 0,979 & 0,672 & 0,592 & 0,598 & 0,354 & 0,396 & 65,876 & 1045 & $-2,6$ \\
\hline 1046 & 0,2 & 1,6 & 0,2 & 1,51 & 1,46 & 0,964 & 0,951 & 0,920 & 0,061 & 0,980 & 1,010 & 0,688 & 0,589 & 0,602 & 0,355 & 0,357 & 69,420 & 1046 & $-2,6$ \\
\hline 1047 & 0,1 & 0,8 & 0,1 & 0,74 & 0,71 & 1,000 & 0,950 & 0,920 & 0,050 & 1,000 & 0,900 & 0,679 & 0,527 & 0,332 & 0,175 & 0,198 & 117,065 & 1047 & $-1,7$ \\
\hline 1048 & 0,3 & 2,0 & 0,3 & 1,91 & 1,85 & 0,962 & 0,966 & 0,936 & 0,050 & 1,000 & 0,993 & 0,696 & 0,719 & 0,757 & 0,545 & 0,560 & 174,475 & 1048 & $-2,9$ \\
\hline 1049 & 0,2 & 1,5 & 0,2 & 1,32 & 1,28 & 0,970 & 0,909 & 0,879 & 0,096 & 0,934 & 1,035 & 0,652 & 0,632 & 0,553 & 0,350 & 0,357 & 136,868 & 1049 & $-2,5$ \\
\hline 1050 & 0,1 & 0,8 & 0,1 & 0,77 & 0,75 & 1,000 & 1,000 & 0,968 & 0,000 & 1,000 & 0,900 & 0,899 & 0,728 & 0,289 & 0,210 & 0,221 & 129,056 & 1050 & $-1,5$ \\
\hline 1051 & 0,5 & 2,5 & 0,5 & 2,32 & 2,25 & 0,964 & 0,946 & 0,916 & 0,065 & 0,972 & 1,014 & 0,663 & 0,679 & 0,946 & 0,642 & 0,664 & 95,526 & 1051 & $-3,2$ \\
\hline 1052 & 0,0 & 0,7 & 0,1 & 0,57 & 0,56 & 0,909 & 0,782 & 0,756 & 0,236 & 1,000 & 0,936 & 0,688 & 0,493 & 0,301 & 0,149 & 0,198 & 8,130 & 1052 & $-1,6$ \\
\hline 1053 & 0,2 & 1,7 & 0,2 & 1,51 & 1,46 & 0,921 & 0,904 & 0,875 & 0,124 & 0,907 & 1,050 & 0,541 & 0,506 & 0,688 & 0,348 & 0,357 & 18,239 & 1053 & $-2,8$ \\
\hline 1054 & 0,6 & 2,7 & 0,6 & 2,62 & 2,53 & 0,962 & 0,985 & 0,954 & 0,041 & 0,986 & 1,007 & 0,745 & 0,713 & 0,973 & 0,693 & 0,693 & 87,974 & 1054 & $-3,3$ \\
\hline 1055 & 0,4 & 2,6 & 0,5 & 2,55 & 2,47 & 0,883 & 0,975 & 0,944 & 0,119 & 0,785 & 1,129 & 0,492 & 0,464 & 1,050 & 0,487 & 0,495 & 110,414 & 1055 & $-3,4$ \\
\hline 1056 & 0,2 & 1,7 & 0,2 & 1,60 & 1,55 & 0,928 & 0,945 & 0,914 & 0,091 & 0,825 & 1,101 & 0,428 & 0,396 & 0,749 & 0,297 & 0,357 & 178,819 & 1056 & $-2,9$ \\
\hline 1057 & 0,1 & 0,7 & 0,1 & 0,51 & 0,49 & 0,957 & 0,730 & 0,707 & 0,273 & 1,000 & 0,845 & 1,120 & 0,800 & 0,248 & 0,198 & 0,221 & 76,224 & 1057 & $-1,3$ \\
\hline 1058 & 0,1 & 1,0 & 0,1 & 0.91 & 0,88 & 0,973 & 0,932 & 0,902 & 0,073 & 1,000 & 0,924 & 0,865 & 0,680 & 0,360 & 0,245 & 0,280 & 45,079 & 1058 & $-1,8$ \\
\hline
\end{tabular}

Bubbles' data

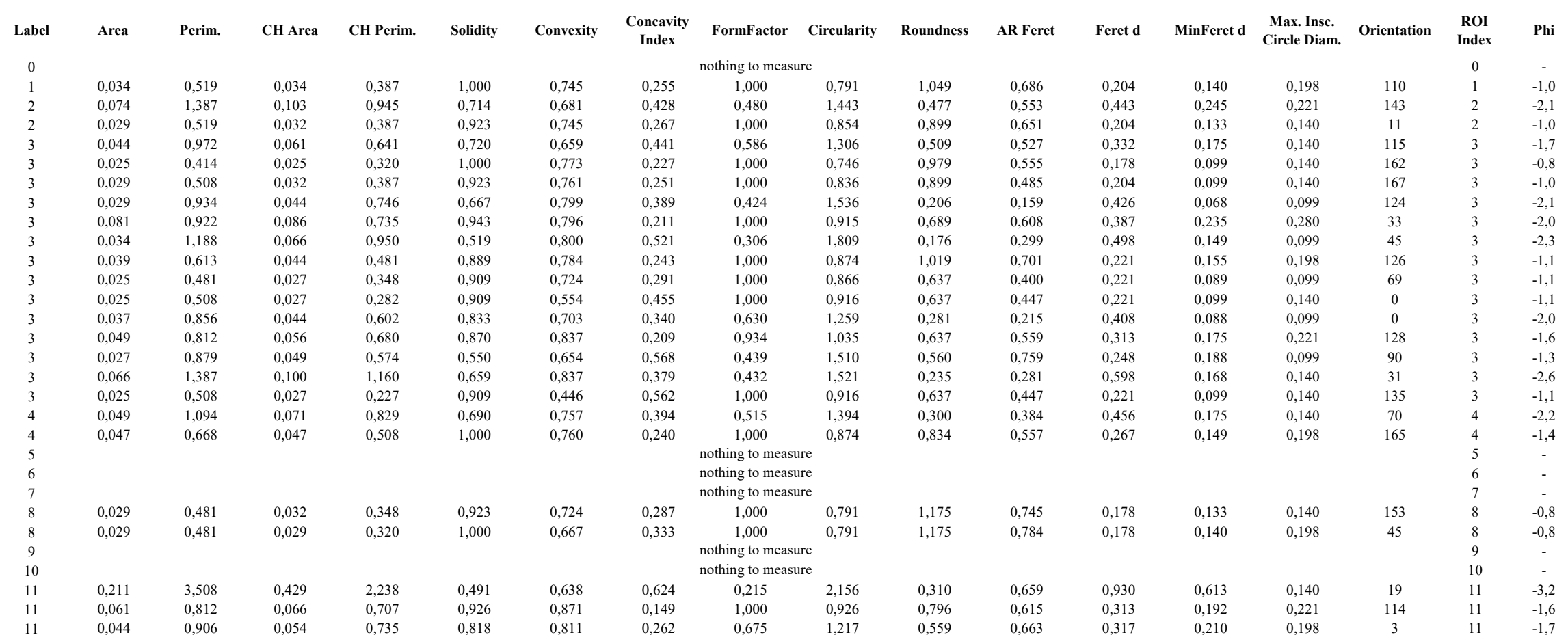




\begin{tabular}{|c|c|c|c|c|c|c|c|c|c|c|c|c|c|c|c|c|}
\hline 0,039 & 0,801 & 0,047 & 0,519 & 0,842 & 0,648 & 0,385 & 0,768 & 1,141 & 0,392 & 0,305 & 0,357 & 0,109 & 0,140 & 126 & 11 & $-1,8$ \\
\hline 0,071 & 1,508 & 0,110 & 1,066 & 0,644 & 0,707 & 0,461 & 0,393 & 1,596 & 0,355 & 0,588 & 0,505 & 0,297 & 0,198 & 2 & 11 & $-2,3$ \\
\hline 0,032 & 0,602 & 0,034 & 0,387 & 0,929 & 0,642 & 0,365 & 1,000 & 0,951 & 0,637 & 0,392 & 0,252 & 0,099 & 0,198 & 82 & 11 & $-1,3$ \\
\hline 0,074 & 1,293 & 0,108 & 1,028 & 0,682 & 0,795 & 0,379 & 0,553 & 1,345 & 0,429 & 0,560 & 0,467 & 0,262 & 0,198 & 146 & 11 & $-2,2$ \\
\hline 0,039 & 0,867 & 0,054 & 0,641 & 0,727 & 0,739 & 0,378 & 0,655 & 1,236 & 0,702 & 0,855 & 0,267 & 0,228 & 0,099 & 4 & 11 & $-1,4$ \\
\hline 0,027 & 0,718 & 0,044 & 0,547 & 0,611 & 0,761 & 0,456 & 0,656 & 1,234 & 0,483 & 0,499 & 0,267 & 0,133 & 0,099 & 62 & 11 & $-1,4$ \\
\hline 0,032 & 0,707 & 0,037 & 0,574 & 0,867 & 0,812 & 0,230 & 0,801 & 1,118 & 0,414 & 0,307 & 0,313 & 0,096 & 0,099 & 170 & 11 & $-1,6$ \\
\hline 0,049 & 1,265 & 0,086 & 0,801 & 0,571 & 0,633 & 0,564 & 0,385 & 1,612 & 0,509 & 0,667 & 0,350 & 0,234 & 0,099 & 109 & 11 & $-1,8$ \\
\hline 0,137 & 3,414 & 0,297 & 1,680 & 0,463 & 0,492 & 0,739 & 0,148 & 2,600 & 0,385 & 0,795 & 0,673 & 0,535 & 0,140 & 156 & 11 & $-2,8$ \\
\hline 0,037 & 1,171 & 0,061 & 0,668 & 0,600 & 0,570 & 0,587 & 0,337 & 1,724 & 0,516 & 0,658 & 0,301 & 0,198 & 0,099 & 53 & 11 & $-1,6$ \\
\hline 0,179 & 2,558 & 0,314 & 1,895 & 0,570 & 0,741 & 0,502 & 0,344 & 1,706 & 0,438 & 0,728 & 0,721 & 0,525 & 0,140 & 19 & 11 & $-2,8$ \\
\hline 0,029 & 0,613 & 0,034 & 0,481 & 0,857 & 0,784 & 0,259 & 0,983 & 1,009 & 0,588 & 0,392 & 0,252 & 0,099 & 0,099 & 6 & 11 & $-1,3$ \\
\hline \multirow[t]{9}{*}{0,047} & 0,801 & 0,054 & 0,613 & 0,864 & 0,766 & 0,271 & 0,912 & 1,047 & 0,590 & 0,531 & 0,317 & 0,168 & 0,140 & 127 & 11 & $-1,7$ \\
\hline & & & & & & & nothing to measure & & & & & & & & 12 & - \\
\hline & & & & & & & nothing to measure & & & & & & & & 13 & - \\
\hline & & & & & & & nothing to measure & & & & & & & & 14 & - \\
\hline & & & & & & & nothing to measure & & & & & & & & 15 & - \\
\hline & & & & & & & nothing to measure & & & & & & & & 16 & - \\
\hline & & & & & & & nothing to measure & & & & & & & & 17 & - \\
\hline & & & & & & & nothing to measure & & & & & & & & 18 & - \\
\hline & & & & & & & nothing to measure & & & & & & & & 19 & \\
\hline 0,025 & 0,625 & 0,025 & 0,492 & 1,000 & 0,788 & 0,212 & 0,790 & 1,125 & 0,311 & 0,110 & 0,317 & 0,035 & 0,099 & 45 & 20 & $-1,7$ \\
\hline \multirow[t]{5}{*}{0,029} & 0,547 & 0,037 & 0,320 & 0,800 & 0,586 & 0,460 & 1,000 & 0,900 & 0,899 & 0,691 & 0,204 & 0,141 & 0,140 & 167 & 21 & $-1,0$ \\
\hline & & & & & & & nothing to measure & & & & & & & & 22 & 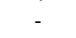 \\
\hline & & & & & & & nothing to measure & & & & & & & & 23 & - \\
\hline & & & & & & & nothing to measure & & & & & & & & 24 & - \\
\hline & & & & & & & nothing to measure & & & & & & & & 25 & - \\
\hline 0,027 & 0,547 & 0,032 & 0,414 & 0,846 & 0,757 & 0,287 & 1,000 & 0,940 & 0,560 & 0,424 & 0,248 & 0,105 & 0,140 & 148 & 26 & $-1,3$ \\
\hline \multirow[t]{4}{*}{0,047} & 1,028 & 0,066 & 0,746 & 0,704 & 0,726 & 0,404 & 0,554 & 1,343 & 0,397 & 0,410 & 0,387 & 0,159 & 0,140 & 142 & 26 & $-2,0$ \\
\hline & & & & & & & nothing to measure & & & & & & & & 27 & \\
\hline & & & & & & & nothing to measure & & & & & & & & 28 & - \\
\hline & & & & & & & nothing to measure & & & & & & & & 29 & - \\
\hline 0,034 & 0,961 & 0,056 & 0,508 & 0,609 & 0,529 & 0,613 & 0,467 & 1,464 & 0,557 & 0,707 & 0,280 & 0,198 & 0,099 & 45 & 30 & $-1,5$ \\
\hline 0,105 & 2,867 & 0,240 & 1,519 & 0,439 & 0,530 & 0,732 & 0,161 & 2,492 & 0,378 & 0,830 & 0,596 & 0,495 & 0,140 & 149 & 30 & $-2,6$ \\
\hline 0,025 & 0,481 & 0,027 & 0,414 & 0,909 & 0,862 & 0,165 & 1,000 & 0,866 & 0,637 & 0,447 & 0,221 & 0,099 & 0,140 & 108 & 30 & $-1,1$ \\
\hline \multirow[t]{2}{*}{0,034} & 0,906 & 0,044 & 0,481 & 0,778 & 0,530 & 0,520 & 0,525 & 1,380 & 0,686 & 0,588 & 0,252 & 0,149 & 0,099 & 115 & 31 & $-1,3$ \\
\hline & & & & & & & nothing to measure & & & & & & & & 32 & \\
\hline 0,108 & 1,790 & 0,145 & 1,430 & 0,746 & 0,799 & 0,324 & 0,423 & 1,537 & 0,273 & 0,266 & 0,709 & 0,189 & 0,198 & 96 & 33 & $-2,8$ \\
\hline 0,025 & 0,414 & 0,025 & 0,282 & 1,000 & 0,680 & 0,320 & 1,000 & 0,746 & 0,979 & 0,555 & 0,178 & 0,099 & 0,140 & 72 & 33 & $-0,8$ \\
\hline 0,032 & 0,613 & 0,039 & 0,387 & 0,812 & 0,631 & 0,414 & 1,000 & 0,969 & 0,828 & 0,671 & 0,221 & 0,149 & 0,140 & 77 & 34 & $-1,1$ \\
\hline 0,032 & 0,641 & 0,039 & 0,453 & 0,812 & 0,707 & 0,348 & 0,975 & 1,013 & 0,662 & 0,570 & 0,248 & 0,141 & 0,198 & 0 & 34 & $-1,3$ \\
\hline 0,029 & 0,574 & 0,034 & 0,348 & 0,857 & 0,606 & 0,419 & 1,000 & 0,945 & 0,764 & 0,447 & 0,221 & 0,099 & 0,099 & 159 & 34 & $-1,1$ \\
\hline 0,025 & 0,602 & 0,027 & 0,188 & 0,909 & 0,312 & 0,694 & 0,850 & 1,085 & 0,354 & 0,167 & 0,297 & 0,050 & 0,099 & 90 & 34 & $-1,6$ \\
\hline 0,032 & 0,680 & 0,037 & 0,586 & 0,867 & 0,862 & 0,192 & 0,867 & 1,074 & 0,487 & 0,364 & 0,289 & 0,105 & 0,099 & 144 & 34 & $-1,5$ \\
\hline 0,044 & 0,961 & 0,064 & 0,718 & 0,692 & 0,747 & 0,398 & 0,600 & 1,291 & 0,458 & 0,488 & 0,350 & 0,171 & 0,140 & 17 & 34 & $-1,8$ \\
\hline 0,127 & 1,430 & 0,149 & 1,133 & 0,852 & 0,792 & 0,255 & 0,783 & 1,130 & 0,676 & 0,707 & 0,490 & 0,347 & 0,297 & 96 & 34 & $-2,3$ \\
\hline \multirow[t]{9}{*}{0,025} & 0,481 & 0,027 & 0,320 & 0,909 & 0,667 & 0,346 & 1,000 & 0,866 & 0,707 & 0,500 & 0,210 & 0,105 & 0,140 & 140 & 35 & $-1,1$ \\
\hline & & & & & & & nothing to measure & & & & & & & & 36 & \\
\hline & & & & & & & nothing to measure & & & & & & & & 37 & \\
\hline & & & & & & & nothing to measure & & & & & & & & 38 & - \\
\hline & & & & & & & nothing to measure & & & & & & & & 39 & 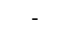 \\
\hline & & & & & & & nothing to measure & & & & & & & & 40 & - \\
\hline & & & & & & & nothing to measure & & & & & & & & 41 & - \\
\hline & & & & & & & nothing to measure & & & & & & & & 42 & 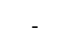 \\
\hline & & & & & & & nothing to measure & & & & & & & & 43 & 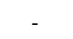 \\
\hline \multirow[t]{3}{*}{0,149} & 2,812 & 0,265 & 1,867 & 0,565 & 0,664 & 0,550 & 0,238 & 2,052 & 0,262 & 0,495 & 0,852 & 0,421 & 0,221 & 133 & 44 & -3, \\
\hline & & & & & & & nothing to measure & & & & & & & & 45 & \\
\hline & & & & & & & nothing to measure & & & & & & & & 46 & \\
\hline
\end{tabular}




\begin{tabular}{|c|c|c|c|c|c|c|c|c|c|c|c|c|c|c|c|c|}
\hline \multicolumn{17}{|c|}{ nothing to measure } \\
\hline 0,044 & 0,746 & 0,044 & 0,586 & 1,000 & 0,785 & 0,215 & 0,996 & 1,002 & 0,458 & 0,300 & 0,350 & 0,105 & 0,140 & 134 & 48 & $-1,8$ \\
\hline 0,037 & 0,840 & 0,056 & 0,613 & 0,652 & 0,730 & 0,440 & 0,655 & 1,236 & 0,562 & 0,584 & 0,289 & 0,168 & 0,099 & 130 & 48 & $-1,5$ \\
\hline 0,051 & 1,481 & 0,093 & 1,055 & 0,553 & 0,713 & 0,532 & 0,295 & 1,841 & 0,214 & 0,282 & 0,553 & 0,156 & 0,099 & 72 & 48 & $-2,5$ \\
\hline 0,025 & 0,547 & 0,029 & 0,453 & 0,833 & 0,828 & 0,239 & 1,000 & 0,986 & 0,509 & 0,280 & 0,248 & 0,069 & 0,099 & 126 & 48 & $-1,3$ \\
\hline 0,039 & 0,652 & 0,042 & 0,602 & 0,941 & 0,923 & 0,097 & 1,000 & 0,929 & 0,702 & 0,465 & 0,267 & 0,124 & 0,140 & 58 & 48 & $-1,4$ \\
\hline 0,027 & 0,481 & 0,027 & 0,414 & 1,000 & 0,862 & 0,138 & 1,000 & 0,826 & 1,077 & 0,745 & 0,178 & 0,133 & 0,198 & 30 & 48 & $-0,8$ \\
\hline 0,025 & 0,574 & 0,029 & 0,442 & 0,833 & 0,769 & 0,285 & 0,933 & 1,035 & 0,490 & 0,309 & 0,252 & 0,078 & 0,099 & 90 & 48 & $-1,3$ \\
\hline 0,039 & 0,680 & 0,042 & 0,547 & 0,941 & 0,805 & 0,204 & 1,000 & 0,968 & 0,497 & 0,331 & 0,317 & 0,105 & 0,140 & 46 & 48 & $-1,7$ \\
\hline 0,039 & 0,547 & 0,039 & 0,414 & 1,000 & 0,757 & 0,243 & 1,000 & 0,779 & 1,198 & 0,728 & 0,204 & 0,149 & 0,198 & 90 & 48 & $-1,0$ \\
\hline 0,071 & 1,586 & 0,118 & 1,176 & 0,604 & 0,742 & 0,473 & 0,355 & 1,678 & 0,318 & 0,458 & 0,533 & 0,244 & 0,198 & 101 & 48 & $-2,4$ \\
\hline 0,059 & 0,934 & 0,074 & 0,773 & 0,800 & 0,828 & 0,264 & 0,848 & 1,086 & 0,577 & 0,549 & 0,360 & 0,198 & 0,198 & 92 & 48 & $-1,8$ \\
\hline 0,025 & 0,453 & 0,025 & 0,320 & 1,000 & 0,707 & 0,293 & 1,000 & 0,816 & 0,796 & 0,500 & 0,198 & 0,099 & 0,140 & 72 & 48 & $-1,0$ \\
\hline 0,034 & 0,668 & 0,044 & 0,481 & 0,778 & 0,719 & 0,358 & 0,965 & 1,018 & 0,713 & 0,667 & 0,248 & 0,165 & 0,099 & 138 & 48 & $-1,3$ \\
\hline 0,042 & 1,094 & 0,069 & 0,707 & 0,607 & 0,646 & 0,529 & 0,438 & 1,512 & 0,416 & 0,497 & 0,357 & 0,177 & 0,099 & 110 & 48 & $-1,8$ \\
\hline 0,025 & 0,547 & 0,029 & 0,293 & 0,833 & 0,535 & 0,494 & $\begin{array}{c}1,000 \\
\text { nothing to measure }\end{array}$ & 0,986 & 0,707 & 0,590 & 0,210 & 0,124 & 0,140 & 108 & $\begin{array}{l}48 \\
49\end{array}$ & $\begin{array}{l}-1,1 \\
-\end{array}$ \\
\hline 0,059 & 1,414 & 0,086 & 1,238 & 0,686 & 0,875 & 0,338 & 0,370 & 1,645 & 0,180 & 0,172 & 0,645 & 0,111 & 0,140 & 126 & 50 & $-2,7$ \\
\hline 0,047 & 1,133 & 0,076 & 0,801 & 0,613 & 0,707 & 0,485 & 0,456 & 1,481 & 0,285 & 0,375 & 0,456 & 0,171 & 0,140 & 73 & 50 & $-2,2$ \\
\hline 0,027 & 0,641 & 0,034 & 0,387 & 0,786 & 0,604 & 0,451 & 0,825 & 1,101 & 0,560 & 0,566 & 0,248 & 0,140 & 0,140 & 108 & 50 & $-1,3$ \\
\hline 0,027 & 0,453 & 0,027 & 0,320 & 1,000 & 0,707 & 0,293 & 1,000 & 0,778 & 0,875 & 0,500 & 0,198 & 0,099 & 0,198 & 90 & 51 & $-1,0$ \\
\hline 0,037 & 0,801 & 0,044 & 0,519 & 0,833 & 0,648 & 0,389 & $\begin{array}{l}\quad 0,720 \\
\text { nothing to measure }\end{array}$ & 1,178 & 0,562 & 0,550 & 0,289 & 0,159 & 0,099 & 43 & $\begin{array}{l}51 \\
52\end{array}$ & $-1,5$ \\
\hline 0,025 & 0,707 & 0,034 & 0,453 & 0,714 & 0,641 & 0,459 & $\begin{array}{l}\quad 0,616 \\
\text { nothing to measure } \\
\text { nothing to measure } \\
\text { nothing to measure } \\
\text { nothing to measure } \\
\text { nothing to measure } \\
\text { nothing to measure } \\
\text { nothing to measure } \\
\text { nothing to measure }\end{array}$ & 1,274 & 0,398 & 0,375 & 0,280 & 0,105 & 0,099 & 54 & $\begin{array}{l}53 \\
54 \\
55 \\
56 \\
57 \\
58 \\
59 \\
60 \\
61\end{array}$ & $\begin{array}{c}-1,5 \\
- \\
- \\
- \\
- \\
- \\
- \\
- \\
-\end{array}$ \\
\hline 0,056 & 0,773 & 0,064 & 0,668 & 0,885 & 0,864 & 0,178 & 1,000 & 0,919 & 0,791 & 0,638 & 0,301 & 0,192 & 0,198 & 102 & 62 & $-1,6$ \\
\hline 0,027 & 0,508 & 0,029 & 0,320 & 0,917 & 0,631 & 0,379 & $\begin{array}{c}1,000 \\
\text { nothing to measure }\end{array}$ & 0,873 & 0,700 & 0,447 & 0,221 & 0,099 & 0,198 & 72 & $\begin{array}{l}62 \\
63\end{array}$ & $\begin{array}{l}-1,1 \\
-\end{array}$ \\
\hline 0,078 & 1,652 & 0,130 & 1,160 & 0,604 & 0,702 & 0,496 & 0,361 & 1,664 & 0,374 & 0,535 & 0,517 & 0,276 & 0,140 & 165 & 64 & $-2,4$ \\
\hline 0,039 & 0,989 & 0,061 & 0,773 & 0,640 & 0,782 & 0,421 & 0,504 & 1,408 & 0,279 & 0,301 & 0,423 & 0,127 & 0,099 & 122 & 64 & $-2,1$ \\
\hline 0,025 & 0,469 & 0,025 & 0,519 & 1,000 & 1,107 & 0,107 & 1,000 & 0,846 & 0,749 & 0,243 & 0,204 & 0,050 & 0,099 & 90 & 64 & $-1,0$ \\
\hline 0,032 & 0,586 & 0,034 & 0,934 & 0,929 & 1,594 & 0,598 & 1,000 & 0,926 & 0,662 & 0,424 & 0,248 & 0,105 & 0,140 & 31 & 64 & $-1,3$ \\
\hline & & & & & & & nothing to measure & & & & & & & & 65 & \\
\hline & & & & & & & nothing to measure & & & & & & & & 66 & - \\
\hline 0,034 & 0,879 & 0,047 & 0,558 & 0,737 & 0,635 & 0,450 & $\begin{array}{l}\text { nothing to measure } \\
0,559\end{array}$ & 1,338 & 0,435 & 0,442 & 0,317 & 0,140 & 0,099 & 140 & 68 & $-1,7$ \\
\hline 0,409 & 6,238 & 0,838 & 3,215 & 0,488 & 0,515 & 0,705 & 0,132 & 2,751 & 0,315 & 0,666 & 1,285 & 0,855 & 0,198 & 29 & 68 & $-3,7$ \\
\hline 0,037 & 0,641 & 0,042 & 0,414 & 0,882 & 0,646 & 0,373 & 1,000 & 0,943 & 0,764 & 0,600 & 0,248 & 0,149 & 0,198 & 45 & 68 & $-1,3$ \\
\hline & & & & & & & nothing to measure & & & & & & & & 69 & \\
\hline & & & & & & & nothing to measure & & & & & & & & 70 & 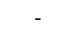 \\
\hline & & & & & & & nothing to measure & & & & & & & & 71 & \\
\hline & & & & & & & nothing to measure & & & & & & & & 72 & - \\
\hline & & & & & & & nothing to measure & & & & & & & & 73 & \\
\hline & & & & & & & nothing to measure & & & & & & & & 74 & - \\
\hline & & & & & & & nothing to measure & & & & & & & & 75 & \\
\hline & & & & & & & nothing to measure & & & & & & & & 76 & - \\
\hline & & & & & & & nothing to measure & & & & & & & & 77 & \\
\hline 0,051 & 0,707 & 0,056 & 0,586 & 0,913 & 0,828 & 0,192 & $\begin{array}{l}1,000 \\
\text { nothing to measure }\end{array}$ & 0,879 & 0,922 & 0,665 & 0,267 & 0,177 & 0,198 & 151 & $\begin{array}{l}78 \\
79\end{array}$ & $-1,4$ \\
\hline 0,025 & 0,625 & 0,025 & 0,586 & 1,000 & 0,938 & 0,062 & 0,790 & 1,125 & 0,311 & 0,110 & 0,317 & 0,035 & 0,099 & 135 & 80 & $-1,7$ \\
\hline 0,081 & 0,922 & 0,088 & 0,773 & 0,917 & 0,839 & 0,182 & 1,000 & 0,915 & 1,025 & 0,781 & 0,317 & 0,248 & 0,297 & 133 & 80 & $-1,7$ \\
\hline
\end{tabular}




\begin{tabular}{|c|c|c|c|c|c|c|c|c|c|c|c|c|c|c|c|c|}
\hline 0,025 & 0,519 & 0,027 & 0,442 & 0,909 & 0,851 & 0,175 & 1,000 & 0,936 & 0,749 & 0,485 & 0,204 & 0,099 & 0,140 & 165 & 80 & $-1,0$ \\
\hline 0,074 & 1,160 & 0,091 & 1,039 & 0,811 & 0,895 & 0,216 & 0,686 & 1,207 & 0,338 & 0,333 & 0,526 & 0,175 & 0,198 & 140 & 80 & $-2,4$ \\
\hline 0,039 & 0,895 & 0,056 & 0,707 & 0,696 & 0,790 & 0,370 & 0,615 & 1,275 & 0,407 & 0,472 & 0,350 & 0,165 & 0,140 & 106 & 80 & $-1,8$ \\
\hline 0,025 & 0,547 & 0,029 & 0,387 & 0,833 & 0,707 & 0,337 & 1,000 & 0,986 & 0,509 & 0,317 & 0,248 & 0,078 & 0,099 & 108 & 80 & $-1,3$ \\
\hline 0,034 & 0,680 & 0,042 & 0,519 & 0,824 & 0,764 & 0,294 & 0,934 & 1,035 & 0,524 & 0,434 & 0,289 & 0,125 & 0,140 & 162 & 80 & $-1,5$ \\
\hline 0,042 & 0,602 & 0,042 & 0,387 & 1,000 & 0,642 & 0,358 & 1,000 & 0,832 & 0,866 & 0,600 & 0,248 & 0,149 & 0,198 & 153 & 80 & $-1,3$ \\
\hline 0,029 & 0,547 & 0,034 & 0,414 & 0,857 & 0,757 & 0,282 & 1,000 & 0,900 & 0,764 & 0,559 & 0,221 & 0,124 & 0,140 & 69 & 81 & $-1,1$ \\
\hline 0,029 & 0,735 & 0,039 & 0,320 & 0,750 & 0,436 & 0,617 & 0,685 & 1,208 & 0,611 & 0,600 & 0,248 & 0,149 & 0,099 & 126 & 81 & $-1,3$ \\
\hline 0,029 & 0,668 & 0,034 & 0,375 & 0,857 & 0,562 & 0,461 & 0,827 & 1,099 & 0,588 & 0,392 & 0,252 & 0,099 & 0,099 & 175 & 81 & $-1,3$ \\
\hline 0,029 & 0,680 & 0,037 & 0,519 & 0,800 & 0,764 & 0,309 & 0,800 & 1,118 & 0,477 & 0,375 & 0,280 & 0,105 & 0,140 & 44 & 81 & $-1,5$ \\
\hline 0,113 & 2,000 & 0,169 & 1,453 & 0,667 & 0,727 & 0,431 & 0,354 & 1,680 & 0,293 & 0,443 & 0,700 & 0,310 & 0,198 & 48 & 81 & $-2,8$ \\
\hline 0,029 & 0,547 & 0,032 & 0,387 & 0,923 & 0,707 & 0,303 & 1,000 & 0,900 & 0,899 & 0,728 & 0,204 & 0,149 & 0,140 & 162 & 81 & $-1,0$ \\
\hline \multirow[t]{5}{*}{0,105} & 2,160 & 0,167 & 1,320 & 0,632 & 0,611 & 0,535 & 0,284 & 1,877 & 0,324 & 0,538 & 0,644 & 0,347 & 0,198 & 91 & 81 & $-2,7$ \\
\hline & & & & & & & nothing to measure & & & & & & & & 82 & - \\
\hline & & & & & & & nothing to measure & & & & & & & & 83 & - \\
\hline & & & & & & & nothing to measure & & & & & & & & 84 & - \\
\hline & & & & & & & nothing to measure & & & & & & & & 85 & \\
\hline 0,105 & 1,121 & 0,115 & 0,934 & 0,915 & 0,833 & 0,188 & 1,000 & 0,974 & 0,898 & 0,768 & 0,387 & 0,297 & 0,297 & 180 & 86 & $-2,0$ \\
\hline \multirow[t]{6}{*}{0,027} & 0,547 & 0,029 & 0,348 & 0,917 & 0,636 & 0,373 & 1,000 & 0,940 & 0,560 & 0,388 & 0,248 & 0,096 & 0,099 & 90 & 86 & $-1,3$ \\
\hline & & & & & & & nothing to measure & & & & & & & & 87 & - \\
\hline & & & & & & & nothing to measure & & & & & & & & 88 & - \\
\hline & & & & & & & nothing to measure & & & & & & & & 89 & - \\
\hline & & & & & & & nothing to measure & & & & & & & & 90 & - \\
\hline & & & & & & & nothing to measure & & & & & & & & 91 & \\
\hline 0,027 & 0,574 & 0,032 & 0,359 & 0,846 & 0,625 & 0,405 & 1,000 & 0,987 & 0,700 & 0,566 & 0,221 & 0,125 & 0,099 & 68 & 92 & $-1,1$ \\
\hline 0,069 & 1,426 & 0,108 & 1,094 & 0,636 & 0,767 & 0,432 & 0,424 & 1,535 & 0,327 & 0,394 & 0,517 & 0,204 & 0,198 & 122 & 92 & $-2,4$ \\
\hline 0,137 & 3,227 & 0,238 & 1,519 & 0,577 & 0,471 & 0,677 & 0,166 & 2,457 & 0,419 & 0,684 & 0,645 & 0,441 & 0,140 & 7 & 92 & $-2,7$ \\
\hline 0,074 & 1,066 & 0,086 & 0,801 & 0,857 & 0,751 & 0,287 & 0,813 & 1,109 & 0,466 & 0,442 & 0,448 & 0,198 & 0,280 & 97 & 92 & $-2,2$ \\
\hline 0,051 & 0,867 & 0,074 & 0,707 & 0,700 & 0,815 & 0,352 & 0,860 & 1,078 & 0,668 & 0,778 & 0,313 & 0,244 & 0,099 & 169 & 92 & $-1,6$ \\
\hline 0,027 & 0,481 & 0,029 & 0,320 & 0,917 & 0,667 & 0,344 & 1,000 & 0,826 & 1,077 & 0,745 & 0,178 & 0,133 & 0,198 & 37 & 92 & $-0,8$ \\
\hline 0,064 & 1,188 & 0,081 & 0,641 & 0,788 & 0,540 & 0,507 & 0,568 & 1,327 & 0,637 & 0,555 & 0,357 & 0,198 & 0,221 & 14 & 92 & $-1,8$ \\
\hline 0,025 & 0,442 & 0,027 & 1,055 & 0,909 & 2,388 & 1,391 & 1,000 & 0,796 & 0,979 & 0,555 & 0,178 & 0,099 & 0,198 & 0 & 92 & $-0,8$ \\
\hline \multirow[t]{2}{*}{0,064} & 1,574 & 0,115 & 0,680 & 0,553 & 0,432 & 0,723 & 0,323 & 1,760 & 0,285 & 0,464 & 0,533 & 0,248 & 0,198 & 94 & 92 & $-2,4$ \\
\hline & & & & & & & nothing to measure & & & & & & & & 93 & \\
\hline 0,083 & 0,895 & 0,086 & 0,801 & 0,971 & 0,895 & 0,109 & 1,000 & 0,874 & 0,962 & 0,745 & 0,332 & 0,248 & 0,297 & 99 & 94 & $-1,7$ \\
\hline \multirow[t]{6}{*}{0,029} & 0,773 & 0,044 & 0,586 & 0,667 & 0,757 & 0,412 & 0,618 & 1,272 & 0,449 & 0,471 & 0,289 & 0,136 & 0,099 & 135 & 95 & $-1,5$ \\
\hline & & & & & & & nothing to measure & & & & & & & & 96 & 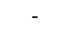 \\
\hline & & & & & & & nothing to measure & & & & & & & & 97 & - \\
\hline & & & & & & & nothing to measure & & & & & & & & 98 & - \\
\hline & & & & & & & nothing to measure & & & & & & & & 99 & \\
\hline & & & & & & & nothing to measure & & & & & & & & 100 & \\
\hline \multirow[t]{17}{*}{0,032} & 0,840 & 0,042 & 0,746 & 0,765 & 0,888 & 0,260 & 0,568 & 1,327 & 0,255 & 0,234 & 0,399 & 0,093 & 0,099 & 124 & 101 & $-2,0$ \\
\hline & & & & & & & nothing to measure & & & & & & & & 102 & - \\
\hline & & & & & & & nothing to measure & & & & & & & & 103 & - \\
\hline & & & & & & & nothing to measure & & & & & & & & 104 & 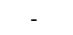 \\
\hline & & & & & & & nothing to measure & & & & & & & & 105 & 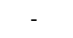 \\
\hline & & & & & & & nothing to measure & & & & & & & & 106 & - \\
\hline & & & & & & & nothing to measure & & & & & & & & 107 & - \\
\hline & & & & & & & nothing to measure & & & & & & & & 108 & \\
\hline & & & & & & & nothing to measure & & & & & & & & 109 & - \\
\hline & & & & & & & nothing to measure & & & & & & & & 110 & - \\
\hline & & & & & & & nothing to measure & & & & & & & & 111 & . \\
\hline & & & & & & & nothing to measure & & & & & & & & 112 & - \\
\hline & & & & & & & nothing to measure & & & & & & & & 113 & - \\
\hline & & & & & & & nothing to measure & & & & & & & & 114 & 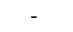 \\
\hline & & & & & & & nothing to measure & & & & & & & & 115 & \\
\hline & & & & & & & nothing to measure & & & & & & & & 116 & \\
\hline & & & & & & & nothing to measure & & & & & & & & 117 & \\
\hline
\end{tabular}




\begin{tabular}{|c|c|c|c|c|c|c|c|c|c|c|c|c|c|c|c|c|c|}
\hline $\begin{array}{l}118 \\
119\end{array}$ & 0,039 & 0,785 & 0,049 & 0,613 & 0,800 & 0,781 & 0,296 & $\begin{array}{c}0,800 \\
\text { nothing to measure }\end{array}$ & 1,118 & 0,551 & 0,468 & 0,301 & 0,141 & 0,140 & 165 & $\begin{array}{l}118 \\
119\end{array}$ & $-1,6$ \\
\hline 120 & & & & & & & & nothing to measure & & & & & & & & 120 & - \\
\hline 121 & & & & & & & & nothing to measure & & & & & & & & 121 & - \\
\hline 122 & & & & & & & & nothing to measure & & & & & & & & 122 & - \\
\hline 123 & 0,039 & 0,879 & 0,049 & 0,547 & 0,800 & 0,622 & 0,427 & 0,638 & 1,252 & 0,702 & 0,619 & 0,267 & 0,165 & 0,198 & 98 & 123 & $-1,4$ \\
\hline 124 & & & & & & & & nothing to measure & & & & & & & & 124 & - \\
\hline 125 & & & & & & & & nothing to measure & & & & & & & & 125 & - \\
\hline 126 & & & & & & & & nothing to measure & & & & & & & & 126 & - \\
\hline 127 & & & & & & & & nothing to measure & & & & & & & & 127 & - \\
\hline 128 & & & & & & & & nothing to measure & & & & & & & & 128 & - \\
\hline 129 & 0,027 & 0,707 & 0,034 & 0,387 & 0,786 & 0,547 & 0,501 & 0,677 & 1,215 & 0,412 & 0,364 & 0,289 & 0,105 & 0,140 & 90 & 129 & $-1,5$ \\
\hline 130 & & & & & & & & nothing to measure & & & & & & & & 130 & - \\
\hline 131 & & & & & & & & nothing to measure & & & & & & & & 131 & - \\
\hline 132 & & & & & & & & nothing to measure & & & & & & & & 132 & - \\
\hline 133 & & & & & & & & nothing to measure & & & & & & & & 133 & - \\
\hline 134 & & & & & & & & nothing to measure & & & & & & & & 134 & - \\
\hline 135 & & & & & & & & nothing to measure & & & & & & & & 135 & - \\
\hline 136 & & & & & & & & nothing to measure & & & & & & & & 136 & - \\
\hline 137 & & & & & & & & nothing to measure & & & & & & & & 137 & - \\
\hline 138 & 0,037 & 0,851 & 0,047 & 0,574 & 0,789 & 0,675 & 0,387 & 0,638 & 1,252 & 0,516 & 0,493 & 0,301 & 0,149 & 0,140 & 179 & 138 & $-1,6$ \\
\hline 139 & & & & & & & & nothing to measure & & & & & & & & 139 & - \\
\hline 140 & & & & & & & & nothing to measure & & & & & & & & 140 & - \\
\hline 141 & & & & & & & & nothing to measure & & & & & & & & 141 & - \\
\hline 142 & & & & & & & & nothing to measure & & & & & & & & 142 & - \\
\hline 143 & & & & & & & & nothing to measure & & & & & & & & 143 & - \\
\hline 144 & & & & & & & & nothing to measure & & & & & & & & 144 & - \\
\hline 145 & 0,029 & 0,574 & 0,032 & 0,442 & 0,923 & 0,769 & 0,243 & 1,000 & 0,945 & 0,764 & 0,447 & 0,221 & 0,099 & 0,140 & 5 & 145 & $-1,1$ \\
\hline 145 & 0,064 & 1,121 & 0,081 & 0,762 & 0,788 & 0,680 & 0,384 & 0,637 & 1,253 & 0,543 & 0,573 & 0,387 & 0,222 & 0,198 & 44 & 145 & $-2,0$ \\
\hline 145 & 0,064 & 1,160 & 0,088 & 0,906 & 0,722 & 0,781 & 0,354 & 0,595 & 1,297 & 0,414 & 0,473 & 0,443 & 0,209 & 0,198 & 38 & 145 & $-2,1$ \\
\hline 145 & 0,037 & 0,840 & 0,044 & 0,630 & 0,833 & 0,750 & 0,301 & 0,655 & 1,236 & 0,382 & 0,283 & 0,350 & 0,099 & 0,099 & 81 & 145 & $-1,8$ \\
\hline 145 & 0,047 & 1,171 & 0,071 & 0,801 & 0,655 & 0,684 & 0,468 & 0,426 & 1,531 & 0,336 & 0,417 & 0,420 & 0,175 & 0,099 & 24 & 145 & $-2,1$ \\
\hline 145 & 0,066 & 0,906 & 0,076 & 0,773 & 0,871 & 0,854 & 0,195 & 1,000 & 0,994 & 0,764 & 0,703 & 0,332 & 0,234 & 0,221 & 174 & 145 & $-1,7$ \\
\hline 145 & 0,027 & 0,680 & 0,034 & 0,574 & 0,786 & 0,845 & 0,264 & 0,733 & 1,168 & 0,379 & 0,329 & 0,301 & 0,099 & 0,099 & 90 & 145 & $-1,6$ \\
\hline 145 & 0,025 & 0,508 & 0,027 & 0,414 & 0,909 & 0,815 & 0,206 & 1,000 & 0,916 & 0,637 & 0,354 & 0,221 & 0,078 & 0,099 & 113 & 145 & $-1,1$ \\
\hline 146 & & & & & & & & nothing to measure & & & & & & & & 146 & - \\
\hline 147 & & & & & & & & nothing to measure & & & & & & & & 147 & - \\
\hline 148 & & & & & & & & nothing to measure & & & & & & & & 148 & - \\
\hline 149 & & & & & & & & nothing to measure & & & & & & & & 149 & - \\
\hline 150 & & & & & & & & nothing to measure & & & & & & & & 150 & - \\
\hline 151 & & & & & & & & nothing to measure & & & & & & & & 151 & - \\
\hline 152 & & & & & & & & nothing to measure & & & & & & & & 152 & - \\
\hline 153 & 0,027 & 0,602 & 0,029 & 0,414 & 0,917 & 0,688 & 0,323 & 0,935 & 1,034 & 0,379 & 0,164 & 0,301 & 0,050 & 0,099 & 90 & 153 & $-1,6$ \\
\hline 154 & & & & & & & & nothing to measure & & & & & & & & 154 & - \\
\hline 155 & & & & & & & & nothing to measure & & & & & & & & 155 & - \\
\hline 156 & 0,027 & 0,613 & 0,032 & 0,414 & 0,846 & 0,675 & 0,359 & 0,901 & 1,054 & 0,700 & 0,543 & 0,221 & 0,120 & 0,140 & 0 & 156 & $-1,1$ \\
\hline 157 & & & & & & & & nothing to measure & & & & & & & & 157 & - \\
\hline 158 & & & & & & & & nothing to measure & & & & & & & & 158 & - \\
\hline 159 & & & & & & & & nothing to measure & & & & & & & & 159 & - \\
\hline 160 & 0,162 & 2,984 & 0,343 & 2,039 & 0,471 & 0,683 & 0,616 & 0,228 & 2,093 & 0,349 & 0,713 & 0,769 & 0,548 & 0,221 & 116 & 160 & $-2,9$ \\
\hline 160 & 0,088 & 2,176 & 0,137 & 1,243 & 0,643 & 0,571 & 0,558 & 0,234 & 2,067 & 0,271 & 0,316 & 0,644 & 0,203 & 0,198 & 68 & 160 & $-2,7$ \\
\hline 160 & 0,025 & 0,442 & 0,025 & 0,254 & 1,000 & 0,575 & 0,425 & 1,000 & 0,796 & 0,979 & 0,555 & 0,178 & 0,099 & 0,198 & 108 & 160 & $-0,8$ \\
\hline 161 & & & & & & & & nothing to measure & & & & & & & & 161 & - \\
\hline 162 & & & & & & & & nothing to measure & & & & & & & & 162 & - \\
\hline 163 & & & & & & & & nothing to measure & & & & & & & & 163 & - \\
\hline 164 & & & & & & & & nothing to measure & & & & & & & & 164 & - \\
\hline 165 & & & & & & & & nothing to measure & & & & & & & & 165 & 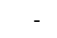 \\
\hline 166 & 0,025 & 0,453 & 0,027 & 0,320 & 0,909 & 0,707 & 0,307 & 1,000 & 0,816 & 0,979 & 0,588 & 0,178 & 0,105 & 0,140 & 135 & 166 & $-0,8$ \\
\hline
\end{tabular}




\begin{tabular}{|c|c|c|c|c|c|c|c|c|c|c|c|c|c|c|c|c|c|c|}
\hline 2 & 166 & 0,223 & 3,000 & 0,350 & 2,126 & 0,636 & 0,709 & 0,466 & 0,311 & 1,792 & 0,343 & 0,442 & 0,910 & 0,402 & 0,297 & 169 & 166 & $-3,2$ \\
\hline 3 & 166 & 0,025 & 0,707 & 0,037 & 0,453 & 0,667 & 0,641 & 0,490 & 0,616 & 1,274 & 0,374 & 0,352 & 0,289 & 0,102 & 0,099 & 140 & 166 & $-1,5$ \\
\hline 1 & 167 & 0,042 & 0,680 & 0,047 & 0,574 & 0,895 & 0,845 & 0,187 & 1,000 & 0,939 & 0,866 & 0,696 & 0,248 & 0,172 & 0,198 & 0 & 167 & $-1,3$ \\
\hline 2 & 167 & 0,047 & 1,453 & 0,083 & 0,977 & 0,559 & 0,673 & 0,549 & 0,277 & 1,900 & 0,295 & 0,331 & 0,448 & 0,149 & 0,099 & 102 & 167 & $-2,2$ \\
\hline 3 & 167 & 0,032 & 0,840 & 0,042 & 0,586 & 0,765 & 0,697 & 0,383 & 0,568 & 1,327 & 0,571 & 0,646 & 0,267 & 0,172 & 0,099 & 47 & 167 & $-1,4$ \\
\hline 4 & 167 & 0,051 & 1,188 & 0,076 & 0,829 & 0,677 & 0,698 & 0,442 & 0,458 & 1,477 & 0,411 & 0,481 & 0,399 & 0,192 & 0,140 & 18 & 167 & $-2,0$ \\
\hline 5 & 167 & 0,061 & 1,442 & 0,103 & 0,867 & 0,595 & 0,602 & 0,568 & 0,370 & 1,643 & 0,430 & 0,658 & 0,426 & 0,280 & 0,140 & 151 & 167 & $-2,1$ \\
\hline 6 & 167 & 0,025 & 0,492 & 0,027 & 0,387 & 0,909 & 0,786 & 0,232 & 1,000 & 0,886 & 0,979 & 0,588 & 0,178 & 0,105 & 0,140 & 139 & 167 & $-0,8$ \\
\hline 7 & 167 & 0,042 & 0,801 & 0,054 & 0,602 & 0,773 & 0,752 & 0,337 & 0,816 & 1,107 & 0,541 & 0,474 & 0,313 & 0,149 & 0,198 & 146 & 167 & $-1,6$ \\
\hline 8 & 167 & 0,034 & 0,547 & 0,034 & 0,414 & 1,000 & 0,757 & 0,243 & 1,000 & 0,833 & 0,891 & 0,600 & 0,221 & 0,133 & 0,198 & 63 & 167 & $-1,1$ \\
\hline 9 & 167 & 0,074 & 1,282 & 0,096 & 1,028 & 0,769 & 0,802 & 0,304 & 0,563 & 1,333 & 0,382 & 0,470 & 0,495 & 0,232 & 0,198 & 125 & 167 & $-2,3$ \\
\hline 10 & 167 & 0,029 & 0,536 & 0,032 & 0,922 & 0,923 & 1,722 & 0,726 & 1,000 & 0,881 & 0,764 & 0,447 & 0,221 & 0,099 & 0,140 & 11 & 167 & $-1,1$ \\
\hline 11 & 167 & 0,066 & 1,293 & 0,100 & 0,707 & 0,659 & 0,547 & 0,567 & 0,497 & 1,418 & 0,506 & 0,721 & 0,408 & 0,295 & 0,140 & 5 & 167 & $-2,0$ \\
\hline 12 & 167 & 0,047 & 0,851 & 0,054 & 0,547 & 0,864 & 0,643 & 0,383 & 0,808 & 1,113 & 0,465 & 0,388 & 0,357 & 0,139 & 0,140 & 124 & 167 & $-1,8$ \\
\hline 13 & 167 & 0,042 & 0,762 & 0,049 & 0,442 & 0,850 & 0,580 & 0,446 & 0,901 & 1,053 & 0,528 & 0,442 & 0,317 & 0,140 & 0,140 & 135 & 167 & $-1,7$ \\
\hline 14 & 167 & 0,032 & 0,547 & 0,034 & 0,558 & 0,929 & 1,021 & 0,074 & 1,000 & 0,864 & 0,828 & 0,566 & 0,221 & 0,125 & 0,140 & 59 & 167 & $-1,1$ \\
\hline 15 & 167 & 0,032 & 0,680 & 0,039 & 0,602 & 0,812 & 0,886 & 0,220 & 0,867 & 1,074 & 0,404 & 0,262 & 0,317 & 0,083 & 0,099 & 126 & 167 & $-1,7$ \\
\hline 16 & 167 & 0,034 & 0,934 & 0,051 & 0,414 & 0,667 & 0,444 & 0,649 & 0,495 & 1,422 & 0,396 & 0,447 & 0,332 & 0,149 & 0,099 & 164 & 167 & $-1,7$ \\
\hline 17 & 167 & 0,025 & 0,641 & 0,034 & 0,696 & 0,714 & 1,086 & 0,298 & 0,750 & 1,155 & 0,490 & 0,392 & 0,252 & 0,099 & 0,099 & 90 & 167 & $-1,3$ \\
\hline 18 & 167 & 0,034 & 0,961 & 0,064 & 0,519 & 0,538 & 0,540 & 0,651 & 0,467 & 1,464 & 0,336 & 0,389 & 0,360 & 0,140 & 0,099 & 8 & 167 & $-1,8$ \\
\hline 19 & 167 & 0,025 & 0,613 & 0,032 & 0,829 & 0,769 & 1,351 & 0,420 & 0,819 & 1,105 & 0,374 & 0,276 & 0,289 & 0,080 & 0,099 & 40 & 167 & $-1,5$ \\
\hline 20 & 167 & 0,081 & 1,121 & 0,088 & 0,453 & 0,917 & 0,404 & 0,602 & 0,808 & 1,112 & 0,584 & 0,566 & 0,420 & 0,238 & 0,280 & 160 & 167 & $-2,1$ \\
\hline 21 & 167 & 0,029 & 0,641 & 0,039 & 0,227 & 0,750 & 0,354 & 0,693 & 0,900 & 1,054 & 0,611 & 0,534 & 0,248 & 0,132 & 0,099 & 72 & 167 & $-1,3$ \\
\hline 22 & 167 & 0,025 & 0,442 & 0,025 & $\begin{array}{l}1,922 \\
\end{array}$ & 1,000 & 4,351 & 3,351 & 1,000 & 0,796 & 0,979 & 0,555 & 0,178 & 0,099 & 0,140 & 162 & 167 & $-0,8$ \\
\hline 23 & 167 & 0,149 & 2,867 & 0,297 & 0,414 & 0,504 & 0,144 & 0,989 & 0,228 & 2,092 & 0,265 & 0,506 & 0,847 & 0,429 & 0,198 & 1 & 167 & $-3,1$ \\
\hline 24 & 167 & 0,025 & 0,481 & 0,027 & 1,829 & 0,909 & 3,805 & 2,806 & 1,000 & 0,866 & 0,749 & 0,485 & 0,204 & 0,099 & 0,140 & 162 & 167 & $-1,0$ \\
\hline 25 & 167 & 0,076 & 2,016 & 0,162 & 0,586 & 0,470 & 0,291 & 0,886 & 0,235 & 2,064 & 0,112 & 0,193 & 0,930 & 0,180 & 0,099 & 163 & 167 & $-3,2$ \\
\hline 26 & 167 & 0,039 & 0,707 & 0,044 & 0,453 & 0,889 & 0,641 & 0,376 & 0,985 & 1,007 & 0,551 & 0,493 & 0,301 & 0,149 & 0,198 & 163 & 167 & $-1,6$ \\
\hline 27 & 167 & 0,034 & 0,586 & 0,037 & 0,801 & 0,933 & 1,367 & 0,373 & 1,000 & 0,892 & 0,891 & 0,632 & 0,221 & 0,140 & 0,198 & 110 & 167 & $-1,1$ \\
\hline 28 & 167 & 0,032 & 0,961 & 0,044 & 0,519 & 0,722 & 0,540 & 0,537 & 0,433 & 1,519 & 0,184 & 0,133 & 0,470 & 0,063 & 0,099 & 160 & 167 & $-2,2$ \\
\hline 29 & 167 & 0,034 & 0,801 & 0,042 & 0,453 & 0,824 & 0,566 & 0,469 & 0,672 & 1,220 & 0,615 & 0,557 & 0,267 & 0,149 & 0,099 & 162 & 167 & $-1,4$ \\
\hline 30 & 167 & 0,025 & 0,519 & 0,025 & 0,613 & 1,000 & 1,181 & 0,181 & 1,000 & 0,936 & 0,637 & 0,474 & 0,221 & 0,105 & 0,140 & 146 & 167 & $-1,1$ \\
\hline 31 & 167 & 0,051 & 0,895 & 0,064 & 0,668 & 0,808 & 0,747 & 0,318 & 0,808 & 1,113 & 0,786 & 0,767 & 0,289 & 0,222 & 0,198 & 9 & 167 & $-1,5$ \\
\hline 32 & 167 & 0,042 & 1,066 & 0,059 & 0,442 & 0,708 & 0,414 & 0,654 & 0,460 & 1,474 & 0,528 & 0,625 & 0,317 & 0,198 & 0,140 & 145 & 167 & $-1,7$ \\
\hline 33 & 167 & 0,027 & 0,668 & 0,037 & 0,254 & 0,733 & 0,380 & 0,675 & 0,758 & 1,148 & 0,483 & 0,365 & 0,267 & 0,097 & 0,099 & 0 & 167 & $-1,4$ \\
\hline 34 & 167 & 0,029 & 0,508 & 0,032 & 0,801 & 0,923 & 1,576 & 0,581 & 1,000 & 0,836 & 0,899 & 0,485 & 0,204 & 0,099 & 0,198 & 18 & 167 & $-1,0$ \\
\hline 35 & 167 & 0,044 & 0,867 & 0,056 & 0,867 & 0,783 & 1,000 & 0,217 & 0,737 & 1,165 & 0,353 & 0,245 & 0,399 & 0,098 & 0,099 & 8 & 167 & $-2,0$ \\
\hline 36 & 167 & 0,081 & 1,160 & 0,096 & 1,000 & 0,846 & 0,862 & 0,207 & 0,755 & 1,151 & 0,576 & 0,468 & 0,423 & 0,198 & 0,221 & 5 & 167 & $-2,1$ \\
\hline 37 & 167 & 0,103 & 1,105 & 0,108 & 0,801 & 0,955 & 0,725 & 0,279 & 1,000 & 0,972 & 0,652 & 0,642 & 0,448 & 0,288 & 0,313 & 72 & 167 & $-2,2$ \\
\hline 38 & 167 & 0,078 & 0,934 & 0,083 & 0,320 & 0,941 & 0,343 & 0,659 & 1,000 & 0,941 & 0,769 & 0,687 & 0,360 & 0,248 & 0,280 & 99 & 167 & $-1,8$ \\
\hline 39 & 167 & 0,029 & 0,630 & 0,034 & 0,574 & 0,857 & 0,913 & 0,167 & 0,933 & 1,036 & 0,527 & 0,371 & 0,267 & 0,099 & 0,198 & 162 & 167 & $-1,4$ \\
\hline 40 & 167 & 0,034 & 0,801 & 0,051 & 1,270 & 0,667 & 1,586 & 0,674 & 0,672 & 1,220 & 0,482 & 0,493 & 0,301 & 0,149 & 0,198 & 72 & 167 & $-1,6$ \\
\hline 41 & 167 & 0,086 & 1,657 & 0,149 & 1,227 & 0,574 & 0,740 & 0,499 & 0,393 & 1,596 & 0,250 & 0,300 & 0,660 & 0,198 & 0,198 & 7 & 167 & $-2,7$ \\
\hline 42 & 167 & 0,103 & 2,176 & 0,176 & 0,414 & 0,583 & 0,190 & 0,911 & 0,273 & 1,914 & 0,418 & 0,692 & 0,560 & 0,388 & 0,221 & 120 & 167 & $-2,5$ \\
\hline 43 & 167 & 0,025 & 0,481 & 0,027 & 0,348 & 0,909 & 0,724 & 0,291 & 1,000 & 0,866 & 0,637 & 0,447 & 0,221 & 0,099 & 0,140 & 148 & 167 & $-1,1$ \\
\hline 44 & 167 & 0,034 & 0,536 & 0,037 & 0,387 & 0,933 & 0,722 & 0,286 & 1,000 & 0,816 & 0,990 & 0,707 & 0,210 & 0,149 & 0,198 & 150 & 167 & $-1,1$ \\
\hline 45 & 167 & 0,037 & 0,668 & 0,042 & 0,359 & 0,882 & 0,537 & 0,477 & 1,000 & 0,983 & 0,764 & 0,707 & 0,248 & 0,175 & 0,140 & 114 & 167 & $-1,3$ \\
\hline 46 & 167 & 0,025 & 0,481 & 0,027 & 0,801 & 0,909 & 1,667 & 0,673 & 1,000 & 0,866 & 0,707 & 0,422 & 0,210 & 0,089 & 0,099 & 122 & 167 & $-1,1$ \\
\hline 47 & 167 & 0,059 & 1,000 & 0,076 & 0,602 & 0,774 & 0,602 & 0,458 & 0,739 & 1,163 & 0,679 & 0,626 & 0,332 & 0,208 & 0,221 & 28 & 167 & $-1,7$ \\
\hline 48 & 167 & 0,044 & 0,773 & 0,051 & 1,696 & 0,857 & 2,193 & 1,201 & 0,927 & 1,039 & 0,619 & 0,493 & 0,301 & 0,149 & 0,198 & 178 & 167 & $-1,6$ \\
\hline 49 & 167 & 0,157 & 2,851 & 0,260 & 1,133 & 0,604 & 0,397 & 0,721 & 0,242 & 2,031 & 0,453 & 0,671 & 0,664 & 0,446 & 0,221 & 89 & 167 & $-2,7$ \\
\hline 50 & 167 & 0,074 & 1,574 & 0,123 & 0,801 & 0,600 & 0,509 & 0,634 & 0,373 & 1,638 & 0,350 & 0,570 & 0,517 & 0,295 & 0,099 & 32 & 167 & $-2,4$ \\
\hline 51 & 167 & 0,054 & 1,000 & 0,069 & 2,531 & 0,786 & 2,531 & 1,546 & 0,678 & 1,215 & 0,412 & 0,419 & 0,408 & 0,171 & 0,198 & 170 & 167 & $-2,0$ \\
\hline 52 & 167 & 0,333 & 4,486 & 0,498 & 0,387 & 0,670 & 0,086 & 0,972 & 0,208 & 2,192 & 0,297 & 0,397 & 1,196 & 0,474 & 0,198 & 149 & 167 & $-3,6$ \\
\hline 53 & 167 & 0,029 & 0,707 & 0,034 & 0,867 & 0,857 & 1,227 & 0,268 & 0,739 & 1,163 & 0,611 & 0,537 & 0,248 & 0,133 & 0,140 & 149 & 167 & $-1,3$ \\
\hline 54 & 167 & 0,056 & 1,028 & 0,078 & 0,387 & 0,719 & 0,376 & 0,684 & 0,671 & 1,221 & 0,451 & 0,432 & 0,399 & 0,172 & 0,140 & 26 & 167 & $-2,0$ \\
\hline 55 & 167 & 0,027 & 0,668 & 0,034 & 0,320 & 0,786 & 0,479 & 0,563 & 0,758 & 1,148 & 0,412 & 0,352 & 0,289 & 0,102 & 0,099 & 0 & 167 & $-1,5$ \\
\hline 56 & 167 & 0,025 & 0,508 & 0,027 & 1,265 & 0,909 & 2,490 & 1,493 & 1,000 & 0,916 & 0,749 & 0,485 & 0,204 & 0,099 & 0,140 & 0 & 167 & $-1,0$ \\
\hline
\end{tabular}




\begin{tabular}{|c|c|c|c|c|c|c|c|c|c|c|c|c|c|c|c|c|c|}
\hline 167 & 0,105 & 1,867 & 0,174 & 0,613 & 0,606 & 0,328 & 0,779 & 0,380 & 1,623 & 0,502 & 0,679 & 0,517 & 0,351 & 0,221 & 108 & 167 & $-2,4$ \\
\hline 167 & 0,032 & 0,895 & 0,049 & 0,680 & 0,650 & 0,759 & 0,425 & 0,500 & 1,414 & 0,285 & 0,277 & 0,377 & 0,105 & 0,099 & 21 & 167 & $-1,9$ \\
\hline 167 & 0,034 & 0,961 & 0,054 & 0,574 & 0,636 & 0,598 & 0,542 & 0,467 & 1,464 & 0,274 & 0,311 & 0,399 & 0,124 & 0,099 & 134 & 167 & $-2,0$ \\
\hline 167 & 0,039 & 0,613 & 0,042 & 0,613 & 0,941 & 1,000 & 0,059 & 1,000 & 0,874 & 0,784 & 0,588 & 0,252 & 0,149 & 0,198 & 7 & 167 & $-1,3$ \\
\hline 167 & 0,044 & 0,801 & 0,054 & 0,801 & 0,818 & 1,000 & 0,182 & 0,864 & 1,076 & 0,432 & 0,367 & 0,360 & 0,132 & 0,140 & 165 & 167 & $-1,8$ \\
\hline 167 & 0,074 & 1,000 & 0,091 & 0,840 & 0,811 & 0,840 & 0,248 & 0,924 & 1,040 & 0,849 & 0,800 & 0,332 & 0,266 & 0,221 & 129 & 167 & $-1,7$ \\
\hline 167 & 0,069 & 1,188 & 0,081 & 0,348 & 0,848 & 0,293 & 0,723 & 0,611 & 1,279 & 0,524 & 0,606 & 0,408 & 0,248 & 0,198 & 68 & 167 & $-2,0$ \\
\hline 167 & 0,027 & 0,508 & 0,032 & 0,414 & 0,846 & 0,815 & 0,240 & 1,000 & 0,873 & 0,824 & 0,485 & 0,204 & 0,099 & 0,140 & 72 & 167 & $-1,0$ \\
\hline 167 & 0,025 & 0,613 & 0,034 & 0,414 & 0,714 & 0,675 & 0,432 & 0,819 & 1,105 & 0,490 & 0,392 & 0,252 & 0,099 & 0,099 & 90 & 167 & $-1,3$ \\
\hline 167 & 0,032 & 0,574 & 0,034 & 0,442 & 0,929 & 0,769 & 0,242 & 1,000 & 0,908 & 0,662 & 0,506 & 0,248 & 0,125 & 0,198 & 60 & 167 & $-1,3$ \\
\hline 167 & 0,032 & 0,574 & 0,037 & 0,574 & 0,867 & 1,000 & 0,133 & 1,000 & 0,908 & 0,828 & 0,597 & 0,221 & 0,132 & 0,198 & 26 & 167 & $-1,1$ \\
\hline 167 & 0,034 & 0,707 & 0,042 & 0,254 & 0,824 & 0,359 & 0,665 & 0,862 & 1,077 & 0,446 & 0,300 & 0,313 & 0,094 & 0,099 & 18 & 167 & $-1,6$ \\
\hline 167 & 0,027 & 0,481 & 0,029 & 0,856 & 0,917 & 1,781 & 0,786 & 1,000 & 0,826 & 1,077 & 0,702 & 0,178 & 0,125 & 0,140 & 162 & 167 & $-0,8$ \\
\hline 167 & 0,051 & 1,094 & 0,078 & 0,641 & 0,656 & 0,586 & 0,538 & 0,541 & 1,360 & 0,393 & 0,476 & 0,408 & 0,194 & 0,140 & 92 & 167 & $-2,0$ \\
\hline 167 & 0,059 & 1,028 & 0,071 & 0,387 & 0,828 & 0,376 & 0,647 & 0,700 & 1,195 & 0,745 & 0,625 & 0,317 & 0,198 & 0,198 & 112 & 167 & $-1,7$ \\
\hline 167 & 0,025 & 0,481 & 0,025 & 0,359 & 1,000 & 0,747 & 0,253 & 1,000 & 0,866 & 0,637 & 0,400 & 0,221 & 0,089 & 0,099 & 159 & 167 & $-1,1$ \\
\hline 168 & & & & & & & & nothing to measure & & & & & & & & 168 & - \\
\hline 169 & 0,034 & 1,066 & 0,059 & 0,773 & 0,583 & 0,725 & 0,499 & 0,379 & 1,624 & 0,343 & 0,452 & 0,357 & 0,161 & 0,099 & 110 & 169 & $-1,8$ \\
\hline 170 & & & & & & & & nothing to measure & & & & & & & & 170 & - \\
\hline 171 & & & & & & & & nothing to measure & & & & & & & & 171 & - \\
\hline 172 & & & & & & & & nothing to measure & & & & & & & & 172 & \\
\hline 173 & 0,034 & 0,558 & 0,037 & 0,481 & 0,933 & 0,861 & 0,154 & 1,000 & 0,850 & 1,049 & 0,686 & 0,204 & 0,140 & 0,198 & 114 & 173 & $-1,0$ \\
\hline 173 & 0,069 & 0,867 & 0,078 & 0,707 & 0,875 & 0,815 & 0,223 & 1,000 & 0,934 & 0,870 & 0,769 & 0,317 & 0,244 & 0,280 & 171 & 173 & $-1,7$ \\
\hline 173 & 0,049 & 0,895 & 0,061 & 0,574 & 0,800 & 0,642 & 0,410 & 0,769 & 1,140 & 0,749 & 0,686 & 0,289 & 0,198 & 0,198 & 176 & 173 & $-1,5$ \\
\hline 173 & 0,032 & 0,508 & 0,032 & 0,348 & 1,000 & 0,685 & 0,315 & 1,000 & 0,803 & 0,920 & 0,633 & 0,210 & 0,133 & 0,198 & 120 & 173 & $-1,1$ \\
\hline 173 & 0,027 & 0,442 & 0,027 & 0,188 & 1,000 & 0,425 & 0,575 & 1,000 & 0,759 & 1,077 & 0,555 & 0,178 & 0,099 & 0,198 & 0 & 173 & $-0,8$ \\
\hline 173 & 0,037 & 0,547 & 0,037 & 0,453 & 1,000 & 0,828 & 0,172 & 1,000 & 0,805 & 1,123 & 0,728 & 0,204 & 0,149 & 0,198 & 90 & 173 & $-1,0$ \\
\hline 173 & 0,049 & 0,972 & 0,064 & 0,773 & 0,769 & 0,795 & 0,308 & 0,651 & 1,239 & 0,392 & 0,334 & 0,399 & 0,133 & 0,140 & 149 & 173 & $-2,0$ \\
\hline 173 & 0,032 & 0,508 & 0,034 & 0,320 & 0,929 & 0,631 & 0,376 & 1,000 & 0,803 & 0,920 & 0,633 & 0,210 & 0,133 & 0,198 & 135 & 173 & $-1,1$ \\
\hline 173 & 0,056 & 1,348 & 0,088 & 1,078 & 0,639 & 0,799 & 0,413 & 0,390 & 1,602 & 0,202 & 0,234 & 0,596 & 0,139 & 0,099 & 141 & 173 & $-2,6$ \\
\hline 173 & 0,039 & 0,602 & 0,044 & 0,414 & 0,889 & 0,688 & 0,331 & 1,000 & 0,858 & 1,019 & 0,671 & 0,221 & 0,149 & 0,198 & 135 & 173 & $-1,1$ \\
\hline 173 & 0,029 & 0,707 & 0,037 & 0,680 & 0,800 & 0,961 & 0,204 & 0,739 & 1,163 & 0,340 & 0,201 & 0,332 & 0,067 & 0,099 & 155 & 173 & $-1,7$ \\
\hline 173 & 0,049 & 1,359 & 0,069 & 1,227 & 0,714 & 0,902 & 0,302 & 0,333 & 1,732 & 0,141 & 0,101 & 0,664 & 0,067 & 0,099 & 153 & 173 & $-2,7$ \\
\hline 173 & 0,037 & 0,641 & 0,044 & 0,453 & 0,833 & 0,707 & 0,337 & 1,000 & 0,943 & 0,735 & 0,527 & 0,252 & 0,133 & 0,140 & 145 & 173 & $-1,3$ \\
\hline 173 & 0,027 & 0,481 & 0,029 & 0,387 & 0,917 & 0,805 & 0,212 & 1,000 & 0,826 & 0,778 & 0,500 & 0,210 & 0,105 & 0,140 & 143 & 173 & $-1,1$ \\
\hline 173 & 0,044 & 0,668 & 0,047 & 0,547 & 0,947 & 0,818 & 0,189 & 1,000 & 0,898 & 0,674 & 0,514 & 0,289 & 0,149 & 0,198 & 156 & 173 & $-1,5$ \\
\hline 174 & & & & & & & & nothing to measure & & & & & & & & 174 & \\
\hline 175 & 0,034 & 0,972 & 0,049 & 0,746 & 0,700 & 0,767 & 0,380 & 0,456 & 1,481 & 0,292 & 0,284 & 0,387 & 0,110 & 0,099 & 118 & 175 & $-2,0$ \\
\hline 175 & 0,034 & 0,652 & 0,044 & 0,481 & 0,778 & 0,737 & 0,344 & 1,000 & 0,993 & 0,990 & 0,833 & 0,210 & 0,175 & 0,140 & 24 & 175 & $-1,1$ \\
\hline 175 & 0,025 & 0,414 & 0,025 & 0,227 & 1,000 & 0,547 & 0,453 & 1,000 & 0,746 & 1,273 & 0,632 & 0,157 & 0,099 & 0,140 & 90 & 175 & $-0,7$ \\
\hline 175 & 0,100 & 1,414 & 0,130 & 1,121 & 0,774 & 0,793 & 0,307 & 0,631 & 1,259 & 0,479 & 0,575 & 0,517 & 0,297 & 0,280 & 95 & 175 & $-2,4$ \\
\hline 175 & 0,137 & 1,718 & 0,179 & 1,359 & 0,767 & 0,791 & 0,313 & 0,584 & 1,309 & 0,686 & 0,790 & 0,505 & 0,399 & 0,297 & 170 & 175 & $-2,3$ \\
\hline 175 & 0,042 & 1,265 & 0,069 & 0,707 & 0,607 & 0,559 & 0,591 & 0,327 & 1,749 & 0,585 & 0,780 & 0,301 & 0,235 & 0,099 & 41 & 175 & $-1,6$ \\
\hline 175 & 0,047 & 1,055 & 0,047 & 0,641 & 1,000 & 0,607 & 0,393 & 0,526 & 1,379 & 0,456 & 0,412 & 0,360 & 0,149 & 0,198 & 0 & 175 & $-1,8$ \\
\hline 175 & 0,025 & 0,519 & 0,027 & 0,320 & 0,909 & 0,617 & 0,394 & 1,000 & 0,936 & 0,979 & 0,784 & 0,178 & 0,140 & 0,099 & 56 & 175 & $-0,8$ \\
\hline 175 & 0,098 & 1,547 & 0,135 & 1,105 & 0,727 & 0,714 & 0,395 & 0,515 & 1,394 & 0,467 & 0,606 & 0,517 & 0,313 & 0,198 & 26 & 175 & $-2,4$ \\
\hline 175 & 0,025 & 0,453 & 0,025 & 0,293 & 1,000 & 0,646 & 0,354 & 1,000 & 0,816 & 0,979 & 0,588 & 0,178 & 0,105 & 0,140 & 122 & 175 & $-0,8$ \\
\hline 175 & 0,044 & 0,906 & 0,066 & 0,707 & 0,667 & 0,780 & 0,399 & 0,675 & 1,217 & 0,619 & 0,758 & 0,301 & 0,228 & 0,198 & 98 & 175 & $-1,6$ \\
\hline 176 & 0,074 & 0,934 & 0,078 & 0,829 & 0,937 & 0,887 & 0,129 & 1,000 & 0,971 & 0,588 & 0,496 & 0,399 & 0,198 & 0,221 & 90 & 176 & $-2,0$ \\
\hline 176 & 0,027 & 0,652 & 0,034 & 0,414 & 0,786 & 0,635 & 0,423 & 0,797 & 1,120 & 0,539 & 0,392 & 0,252 & 0,099 & 0,140 & 78 & 176 & $-1,3$ \\
\hline 176 & 0,086 & 1,000 & 0,096 & 0,895 & 0,897 & 0,895 & 0,147 & 1,000 & 0,963 & 0,655 & 0,537 & 0,408 & 0,219 & 0,280 & 105 & 176 & $-2,0$ \\
\hline 177 & & & & & & & & nothing to measure & & & & & & & & 177 & 2,0 \\
\hline 178 & & & & & & & & nothing to measure & & & & & & & & 178 & - \\
\hline 179 & & & & & & & & nothing to measure & & & & & & & & 179 & - \\
\hline 180 & & & & & & & & nothing to measure & & & & & & & & 180 & - \\
\hline 181 & & & & & & & & nothing to measure & & & & & & & & 181 & - \\
\hline 182 & & & & & & & & nothing to measure & & & & & & & & 182 & - \\
\hline 183 & & & & & & & & nothing to measure & & & & & & & & 183 & - \\
\hline
\end{tabular}




\begin{tabular}{|c|c|c|c|c|c|c|c|c|c|c|c|c|c|c|c|c|c|}
\hline $\begin{array}{l}184 \\
185\end{array}$ & & & & & & & & $\begin{array}{l}\text { nothing to measure } \\
\text { nothing to measure }\end{array}$ & & & & & & & & $\begin{array}{l}184 \\
185\end{array}$ & - \\
\hline 186 & 0,029 & 0,519 & 0,029 & 0,387 & 1,000 & 0,745 & 0,255 & 1,000 & 0,854 & 0,764 & 0,474 & 0,221 & 0,105 & 0,140 & 108 & 186 & $-1,1$ \\
\hline 186 & 0,066 & 0,840 & 0,069 & 0,707 & 0,964 & 0,842 & 0,162 & 1,000 & 0,921 & 0,929 & 0,809 & 0,301 & 0,244 & 0,221 & 76 & 186 & $-1,6$ \\
\hline 187 & & & & & & & & nothing to measure & & & & & & & & 187 & - \\
\hline 188 & & & & & & & & nothing to measure & & & & & & & & 188 & - \\
\hline 189 & & & & & & & & nothing to measure & & & & & & & & 189 & - \\
\hline 190 & & & & & & & & nothing to measure & & & & & & & & 190 & - \\
\hline 191 & 0,032 & 0,586 & 0,034 & 0,453 & 0,929 & 0,773 & 0,238 & 1,000 & 0,926 & 0,662 & 0,424 & 0,248 & 0,105 & 0,140 & 135 & 191 & $-1,3$ \\
\hline 192 & & & & & & & & nothing to measure & & & & & & & & 192 & \\
\hline 193 & 0,039 & 0,574 & 0,042 & 0,442 & 0,941 & 0,769 & 0,238 & 1,000 & 0,818 & 1,132 & 0,707 & 0,210 & 0,149 & 0,198 & 90 & 193 & $-1,1$ \\
\hline 194 & & & & & & & & nothing to measure & & & & & & & & 194 & - \\
\hline 195 & & & & & & & & nothing to measure & & & & & & & & 195 & - \\
\hline 196 & & & & & & & & nothing to measure & & & & & & & & 196 & - \\
\hline 197 & & & & & & & & nothing to measure & & & & & & & & 197 & - \\
\hline 198 & & & & & & & & nothing to measure & & & & & & & & 198 & - \\
\hline 199 & & & & & & & & nothing to measure & & & & & & & & 199 & - \\
\hline 200 & & & & & & & & nothing to measure & & & & & & & & 200 & - \\
\hline 201 & & & & & & & & nothing to measure & & & & & & & & 201 & 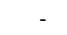 \\
\hline 202 & 0,029 & 0,508 & 0,029 & 0,320 & 1,000 & 0,631 & 0,369 & 1,000 & 0,836 & 0,764 & 0,447 & 0,221 & 0,099 & 0,140 & 103 & 202 & $-1,1$ \\
\hline 202 & 0,056 & 1,188 & 0,076 & 0,945 & 0,742 & 0,796 & 0,329 & 0,502 & 1,411 & 0,302 & 0,364 & 0,488 & 0,177 & 0,099 & 113 & 202 & $-2,3$ \\
\hline 202 & 0,081 & 1,094 & 0,091 & 0,867 & 0,892 & 0,793 & 0,234 & 0,849 & 1,085 & 0,840 & 0,849 & 0,350 & 0,297 & 0,221 & 52 & 202 & $-1,8$ \\
\hline 202 & 0,051 & 0,696 & 0,051 & 0,453 & 1,000 & 0,651 & 0,349 & 1,000 & 0,865 & 0,836 & 0,707 & 0,280 & 0,198 & 0,198 & 129 & 202 & $-1,5$ \\
\hline 202 & 0,032 & 0,696 & 0,037 & 0,254 & 0,867 & 0,365 & 0,649 & 0,827 & 1,100 & 0,571 & 0,371 & 0,267 & 0,099 & 0,099 & 98 & 202 & $-1,4$ \\
\hline 202 & 0,054 & 0,934 & 0,069 & 0,773 & 0,786 & 0,828 & 0,275 & 0,777 & 1,134 & 0,529 & 0,412 & 0,360 & 0,149 & 0,140 & 177 & 202 & $-1,8$ \\
\hline 202 & 0,034 & 0,735 & 0,044 & 0,508 & 0,778 & 0,692 & 0,380 & 0,799 & 1,119 & 0,396 & 0,362 & 0,332 & 0,120 & 0,198 & 72 & 202 & $-1,7$ \\
\hline 202 & 0,169 & 1,375 & 0,176 & 1,243 & 0,958 & 0,904 & 0,105 & 1,000 & 0,944 & 0,806 & 0,670 & 0,517 & 0,347 & 0,357 & 99 & 202 & $-2,4$ \\
\hline 202 & 0,049 & 0,773 & 0,056 & 0,602 & 0,870 & 0,778 & 0,257 & 1,000 & 0,986 & 0,878 & 0,743 & 0,267 & 0,198 & 0,198 & 94 & 202 & $-1,4$ \\
\hline 202 & 0,025 & 0,574 & 0,034 & 0,293 & 0,714 & 0,510 & 0,567 & 0,933 & 1,035 & 0,637 & 0,600 & 0,221 & 0,133 & 0,099 & 152 & 202 & $-1,1$ \\
\hline 202 & 0,025 & 0,414 & 0,025 & 0,227 & 1,000 & 0,547 & 0,453 & 1,000 & 0,746 & 1,273 & 0,632 & 0,157 & 0,099 & 0,198 & 72 & 202 & $-0,7$ \\
\hline 202 & 0,064 & 1,121 & 0,088 & 0,867 & 0,722 & 0,773 & 0,358 & 0,637 & 1,253 & 0,543 & 0,628 & 0,387 & 0,243 & 0,198 & 122 & 202 & $-2,0$ \\
\hline 202 & 0,059 & 1,094 & 0,083 & 0,801 & 0,706 & 0,732 & 0,398 & 0,618 & 1,272 & 0,588 & 0,693 & 0,357 & 0,248 & 0,198 & 134 & 202 & $-1,8$ \\
\hline 202 & 0,105 & 1,055 & 0,113 & 0,934 & 0,935 & 0,885 & 0,132 & 1,000 & 0,917 & 0,842 & 0,667 & 0,399 & 0,266 & 0,297 & 109 & 202 & $-2,0$ \\
\hline 202 & 0,137 & 1,359 & 0,152 & 1,282 & 0,903 & 0,943 & 0,112 & 0,934 & 1,035 & 0,584 & 0,606 & 0,547 & 0,331 & 0,357 & 78 & 202 & $-2,5$ \\
\hline 202 & 0,049 & 0,961 & 0,066 & 0,707 & 0,741 & 0,736 & 0,370 & 0,667 & 1,225 & 0,490 & 0,429 & 0,357 & 0,153 & 0,140 & 58 & 202 & $-1,8$ \\
\hline 202 & 0,027 & 0,547 & 0,029 & 0,254 & 0,917 & 0,465 & 0,542 & 1,000 & 0,940 & 0,700 & 0,447 & 0,221 & 0,099 & 0,198 & 0 & 202 & $-1,1$ \\
\hline 202 & 0,115 & 1,519 & 0,152 & 1,254 & 0,758 & 0,825 & 0,298 & 0,627 & 1,263 & 0,530 & 0,665 & 0,526 & 0,350 & 0,280 & 123 & 202 & $-2,4$ \\
\hline 202 & 0,074 & 0,934 & 0,086 & 0,829 & 0,857 & 0,887 & 0,182 & 1,000 & 0,971 & 0,735 & 0,583 & 0,357 & 0,208 & 0,221 & 49 & 202 & $-1,8$ \\
\hline 202 & 0,037 & 0,773 & 0,056 & 0,586 & 0,652 & 0,757 & 0,424 & 0,772 & 1,138 & 0,562 & 0,606 & 0,289 & 0,175 & 0,140 & 45 & 202 & $-1,5$ \\
\hline 202 & 0,029 & 0,453 & 0,029 & 0,387 & 1,000 & 0,854 & 0,146 & 1,000 & 0,745 & 1,528 & 0,894 & 0,157 & 0,140 & 0,140 & 90 & 202 & $-0,7$ \\
\hline 202 & 0,091 & 1,519 & 0,127 & 1,133 & 0,712 & 0,745 & 0,385 & 0,494 & 1,423 & 0,453 & 0,581 & 0,505 & 0,293 & 0,198 & 82 & 202 & $-2,3$ \\
\hline 202 & 0,069 & 1,171 & 0,096 & 0,972 & 0,718 & 0,830 & 0,329 & 0,628 & 1,262 & 0,419 & 0,460 & 0,456 & 0,210 & 0,198 & 41 & 202 & $-2,2$ \\
\hline 203 & 0,027 & 0,453 & 0,027 & 0,320 & 1,000 & 0,707 & 0,293 & 1,000 & 0,778 & 1,401 & 0,849 & 0,157 & 0,133 & 0,140 & 135 & 203 & $-0,7$ \\
\hline 204 & 0,027 & 0,879 & 0,027 & 0,680 & 1,000 & 0,773 & 0,227 & 0,439 & 1,510 & 0,269 & 0,272 & 0,357 & 0,097 & 0,099 & 37 & 204 & $-1,8$ \\
\hline 205 & & & & & & & & nothing to measure & & & & & & & & 205 & - \\
\hline 206 & & & & & & & & nothing to measure & & & & & & & & 206 & - \\
\hline 207 & & & & & & & & nothing to measure & & & & & & & & 207 & - \\
\hline 208 & 0,029 & 0,481 & 0,032 & 0,320 & 0,923 & 0,667 & 0,342 & 1,000 & 0,791 & 1,175 & 0,745 & 0,178 & 0,133 & 0,198 & 90 & 208 & $-0,8$ \\
\hline 208 & 0,029 & 0,481 & 0,032 & 0,320 & 0,923 & 0,667 & 0,342 & 1,000 & 0,791 & 1,175 & 0,745 & 0,178 & 0,133 & 0,140 & 135 & 208 & $-0,8$ \\
\hline 208 & 0,027 & 0,481 & 0,032 & 0,348 & 0,846 & 0,724 & 0,316 & 1,000 & 0,826 & 1,077 & 0,745 & 0,178 & 0,133 & 0,140 & 0 & 208 & $-0,8$ \\
\hline 208 & 0,027 & 0,536 & 0,034 & 0,254 & 0,786 & 0,474 & 0,568 & 1,000 & 0,920 & 0,700 & 0,447 & 0,221 & 0,099 & 0,099 & 98 & 208 & $-1,1$ \\
\hline 209 & & & & & & & & nothing to measure & & & & & & & & 209 & 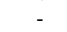 \\
\hline 210 & & & & & & & & nothing to measure & & & & & & & & 210 & - \\
\hline 211 & & & & & & & & nothing to measure & & & & & & & & 211 & - \\
\hline 212 & 0,025 & 0,414 & 0,025 & 0,227 & 1,000 & 0,547 & 0,453 & 1,000 & 0,746 & 1,273 & 0,632 & 0,157 & 0,099 & 0,198 & 72 & 212 & $-0,7$ \\
\hline 213 & & & & & & & & nothing to measure & & & & & & & & 213 & 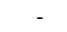 \\
\hline 214 & & & & & & & & nothing to measure & & & & & & & & 214 & - \\
\hline 215 & & & & & & & & nothing to measure & & & & & & & & 215 & 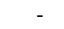 \\
\hline
\end{tabular}




\begin{tabular}{|c|c|c|c|c|c|c|c|c|c|c|c|c|c|c|c|c|c|c|}
\hline & 216 & & & & & & & & nothing to measure & & & & & & & & 216 & - \\
\hline & 217 & & & & & & & & nothing to measure & & & & & & & & 217 & - \\
\hline & 218 & & & & & & & & nothing to measure & & & & & & & & 218 & - \\
\hline & 219 & & & & & & & & nothing to measure & & & & & & & & 219 & \\
\hline \multirow[t]{2}{*}{1} & 220 & 0,037 & 0,613 & 0,044 & 0,508 & 0,833 & 0,829 & 0,239 & 1,000 & 0,902 & 0,955 & 0,791 & 0,221 & 0,175 & 0,099 & 45 & 220 & $-1,1$ \\
\hline & 221 & & & & & & & & nothing to measure & & & & & & & & 221 & \\
\hline \multirow{3}{*}{2} & 222 & 0,047 & 0,746 & 0,054 & 0,574 & 0,864 & 0,770 & 0,267 & 1,000 & 0,975 & 0,712 & 0,597 & 0,289 & 0,172 & 0,140 & 111 & 222 & $-1,5$ \\
\hline & 222 & 0,027 & 0,680 & 0,037 & 0,519 & 0,733 & 0,764 & 0,356 & 0,733 & 1,168 & 0,483 & 0,483 & 0,267 & 0,129 & 0,099 & 140 & 222 & $-1,4$ \\
\hline & 223 & & & & & & & & nothing to measure & & & & & & & & 223 & - \\
\hline 1 & 224 & 0,027 & 0,586 & 0,029 & 0,519 & 0,917 & 0,887 & 0,141 & 0,987 & 1,006 & 0,438 & 0,250 & 0,280 & 0,070 & 0,140 & 145 & 224 & $-1,5$ \\
\hline 2 & 224 & 0,025 & 0,773 & 0,032 & 0,613 & 0,769 & 0,793 & 0,310 & 0,515 & 1,394 & 0,220 & 0,176 & 0,377 & 0,067 & 0,099 & 135 & 224 & $-1,9$ \\
\hline \multirow[t]{5}{*}{3} & 224 & 0,029 & 0,762 & 0,042 & 0,481 & 0,706 & 0,631 & 0,472 & 0,636 & 1,254 & 0,306 & 0,223 & 0,350 & 0,078 & 0,099 & 15 & 224 & $-1,8$ \\
\hline & 225 & & & & & & & & nothing to measure & & & & & & & & 225 & \\
\hline & 226 & & & & & & & & nothing to measure & & & & & & & & 226 & - \\
\hline & 227 & & & & & & & & nothing to measure & & & & & & & & 227 & - \\
\hline & 228 & & & & & & & & nothing to measure & & & & & & & & 228 & \\
\hline 1 & 229 & 0,032 & 0,508 & 0,032 & 0,348 & 1,000 & 0,685 & 0,315 & 1,000 & 0,803 & 0,828 & 0,447 & 0,221 & 0,099 & 0,198 & 172 & 229 & $-1,1$ \\
\hline 1 & 230 & 0,032 & 0,746 & 0,044 & 0,508 & 0,722 & 0,681 & 0,423 & 0,720 & 1,179 & 0,637 & 0,588 & 0,252 & 0,149 & 0,140 & 91 & 230 & $-1,3$ \\
\hline 2 & 230 & 0,047 & 0,762 & 0,047 & 0,574 & 1,000 & 0,754 & 0,246 & 1,000 & 0,996 & 0,456 & 0,275 & 0,360 & 0,099 & 0,198 & 173 & 230 & $-1,8$ \\
\hline 3 & 230 & 0,029 & 0,934 & 0,049 & 0,547 & 0,600 & 0,586 & 0,576 & 0,424 & 1,536 & 0,449 & 0,647 & 0,289 & 0,187 & 0,099 & 45 & 230 & $-1,5$ \\
\hline \multirow[t]{5}{*}{4} & 230 & 0,025 & 0,519 & 0,027 & 0,293 & 0,909 & 0,564 & 0,446 & 1,000 & 0,936 & 1,273 & 0,849 & 0,157 & 0,133 & 0,099 & 148 & 230 & $-0,7$ \\
\hline & 231 & & & & & & & & nothing to measure & & & & & & & & 231 & \\
\hline & 232 & & & & & & & & nothing to measure & & & & & & & & 232 & - \\
\hline & 233 & & & & & & & & nothing to measure & & & & & & & & 233 & - \\
\hline & 234 & & & & & & & & nothing to measure & & & & & & & & 234 & \\
\hline 1 & 235 & 0,042 & 0,613 & 0,044 & 0,547 & 0,944 & 0,892 & 0,122 & 1,000 & 0,848 & 1,082 & 0,791 & 0,221 & 0,175 & 0,198 & 117 & 235 & $-1,1$ \\
\hline 2 & 235 & 0,025 & 0,602 & 0,032 & 0,414 & 0,769 & 0,688 & 0,388 & 0,850 & 1,085 & 0,439 & 0,256 & 0,267 & 0,068 & 0,099 & 75 & 235 & $-1,4$ \\
\hline 3 & 235 & 0,059 & 0,773 & 0,066 & 0,641 & 0,889 & 0,828 & 0,204 & 1,000 & 0,900 & 0,899 & 0,728 & 0,289 & 0,210 & 0,221 & 141 & 235 & $-1,5$ \\
\hline 4 & 235 & 0,032 & 0,652 & 0,034 & 0,586 & 0,929 & 0,898 & 0,124 & 0,942 & 1,031 & 0,404 & 0,221 & 0,317 & 0,070 & 0,140 & 141 & 235 & $-1,7$ \\
\hline 5 & 235 & 0,051 & 0,735 & 0,059 & 0,602 & 0,875 & 0,819 & 0,220 & 1,000 & 0,914 & 0,922 & 0,705 & 0,267 & 0,188 & 0,221 & 165 & 235 & $-1,4$ \\
\hline 6 & 235 & 0,039 & 1,094 & 0,064 & 0,746 & 0,615 & 0,682 & 0,499 & 0,412 & 1,558 & 0,334 & 0,453 & 0,387 & 0,175 & 0,099 & 56 & 235 & $-2,0$ \\
\hline \multirow[t]{2}{*}{7} & 235 & 0,044 & 0,735 & 0,054 & 0,547 & 0,818 & 0,744 & 0,314 & 1,000 & 0,987 & 0,790 & 0,705 & 0,267 & 0,188 & 0,198 & 117 & 235 & $-1,4$ \\
\hline & 236 & & & & & & & & nothing to measure & & & & & & & & 236 & \\
\hline 1 & 237 & 0,081 & 1,133 & 0,098 & 1,055 & 0,825 & 0,931 & 0,188 & 0,792 & 1,124 & 0,472 & 0,436 & 0,467 & 0,204 & 0,221 & 109 & 237 & $-2,2$ \\
\hline 2 & 237 & 0,032 & 0,641 & 0,037 & 0,320 & 0,867 & 0,500 & 0,517 & 0,975 & 1,013 & 0,571 & 0,371 & 0,267 & 0,099 & 0,140 & 100 & 237 & $-1,4$ \\
\hline \multirow[t]{4}{*}{3} & 237 & 0,025 & 0,680 & 0,032 & 0,414 & 0,769 & 0,610 & 0,454 & 0,667 & 1,225 & 0,637 & 0,495 & 0,221 & 0,110 & 0,140 & 45 & 237 & $-1,1$ \\
\hline & 238 & & & & & & & & nothing to measure & & & & & & & & 238 & 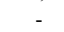 \\
\hline & 239 & & & & & & & & nothing to measure & & & & & & & & 239 & - \\
\hline & 240 & & & & & & & & nothing to measure & & & & & & & & 240 & \\
\hline \multirow[t]{2}{*}{1} & 241 & 0,029 & 0,481 & 0,032 & 0,348 & 0,923 & 0,724 & 0,287 & 1,000 & 0,791 & 1,175 & 0,745 & 0,178 & 0,133 & 0,140 & 153 & 241 & $-0,8$ \\
\hline & 242 & & & & & & & & nothing to measure & & & & & & & & 242 & \\
\hline 1 & 243 & 0,027 & 0,547 & 0,032 & 0,481 & 0,846 & 0,879 & 0,196 & 1,000 & 0,940 & 0,700 & 0,566 & 0,221 & 0,125 & 0,140 & 119 & 243 & $-1,1$ \\
\hline 2 & 243 & 0,032 & 1,094 & 0,064 & 0,773 & 0,500 & 0,707 & 0,579 & 0,335 & 1,729 & 0,207 & 0,351 & 0,443 & 0,155 & 0,099 & 0 & 243 & $-2,1$ \\
\hline 1 & 244 & 0,025 & 0,519 & 0,027 & 0,414 & 0,909 & 0,798 & 0,222 & 1,000 & 0,936 & 0,749 & 0,514 & 0,204 & 0,105 & 0,140 & 122 & 244 & $-1,0$ \\
\hline 2 & 244 & 0,069 & 0,934 & 0,076 & 0,801 & 0,903 & 0,858 & 0,172 & 0,989 & 1,005 & 0,548 & 0,496 & 0,399 & 0,198 & 0,221 & 103 & 244 & $-2,0$ \\
\hline 3 & 244 & 0,032 & 0,536 & 0,034 & 0,254 & 0,929 & 0,474 & 0,531 & 1,000 & 0,847 & 0,828 & 0,447 & 0,221 & 0,099 & 0,198 & 98 & 244 & $-1,1$ \\
\hline 4 & 244 & 0,025 & 0,547 & 0,029 & 0,320 & 0,833 & 0,586 & 0,446 & 1,000 & 0,986 & 0,749 & 0,485 & 0,204 & 0,099 & 0,099 & 90 & 244 & $-1,0$ \\
\hline 5 & 244 & 0,025 & 0,519 & 0,032 & 0,414 & 0,769 & 0,798 & 0,307 & 1,000 & 0,936 & 0,749 & 0,651 & 0,204 & 0,133 & 0,099 & 135 & 244 & $-1,0$ \\
\hline 6 & 244 & 0,047 & 1,055 & 0,071 & 0,519 & 0,655 & 0,492 & 0,614 & 0,526 & 1,379 & 0,465 & 0,547 & 0,357 & 0,195 & 0,099 & 156 & 244 & $-1,8$ \\
\hline 7 & 244 & 0,025 & 0,707 & 0,037 & 0,453 & 0,667 & 0,641 & 0,490 & 0,616 & 1,274 & 0,509 & 0,424 & 0,248 & 0,105 & 0,099 & 0 & 244 & $-1,3$ \\
\hline 8 & 244 & 0,025 & 0,481 & 0,027 & 0,320 & 0,909 & 0,667 & 0,346 & 1,000 & 0,866 & 0,707 & 0,500 & 0,210 & 0,105 & 0,140 & 140 & 244 & $-1,1$ \\
\hline 9 & 244 & 0,037 & 0,707 & 0,047 & 0,574 & 0,789 & 0,812 & 0,282 & 0,924 & 1,040 & 0,562 & 0,514 & 0,289 & 0,149 & 0,140 & 108 & 244 & $-1,5$ \\
\hline \multirow{4}{*}{10} & 244 & 0,025 & 0,574 & 0,027 & 0,188 & 0,909 & 0,327 & 0,679 & 0,933 & 1,035 & 0,490 & 0,196 & 0,252 & 0,050 & 0,099 & 90 & 244 & $-1,3$ \\
\hline & 245 & & & & & & & & nothing to measure & & & & & & & & 245 & - \\
\hline & 246 & & & & & & & & nothing to measure & & & & & & & & 246 & - \\
\hline & 247 & & & & & & & & nothing to measure & & & & & & & & 247 & - \\
\hline 1 & 248 & 0,088 & 1,586 & 0,135 & 1,171 & 0,655 & 0,739 & 0,433 & 0,441 & 1,506 & 0,468 & 0,571 & 0,490 & 0,280 & 0,198 & 122 & 248 & $-2,3$ \\
\hline 2 & 248 & 0,029 & 0,492 & 0,029 & 0,481 & 1,000 & 0,977 & 0,023 & 1,000 & 0,809 & 0,899 & 0,514 & 0,204 & 0,105 & 0,140 & 135 & 248 & $-1,0$ \\
\hline
\end{tabular}




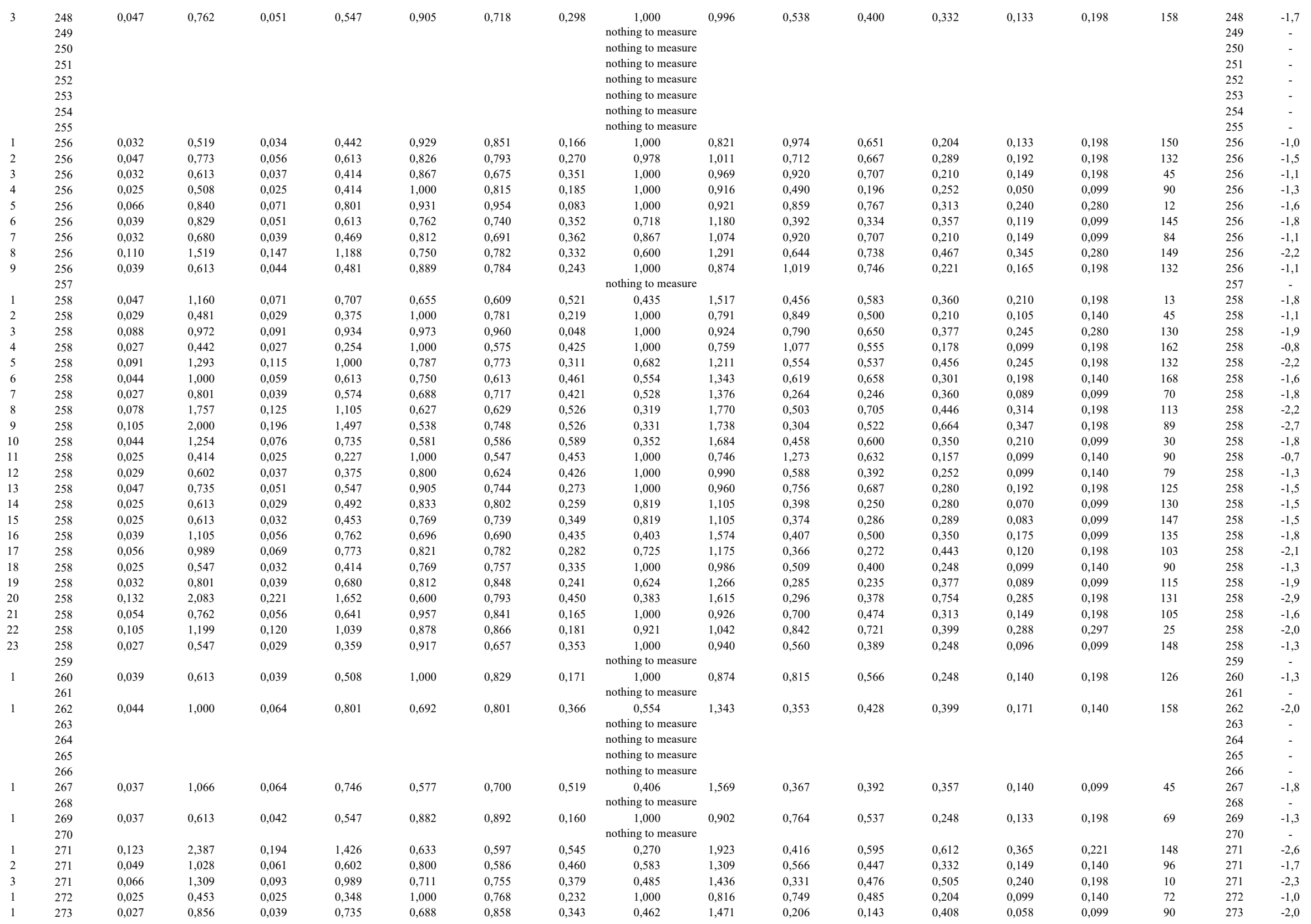




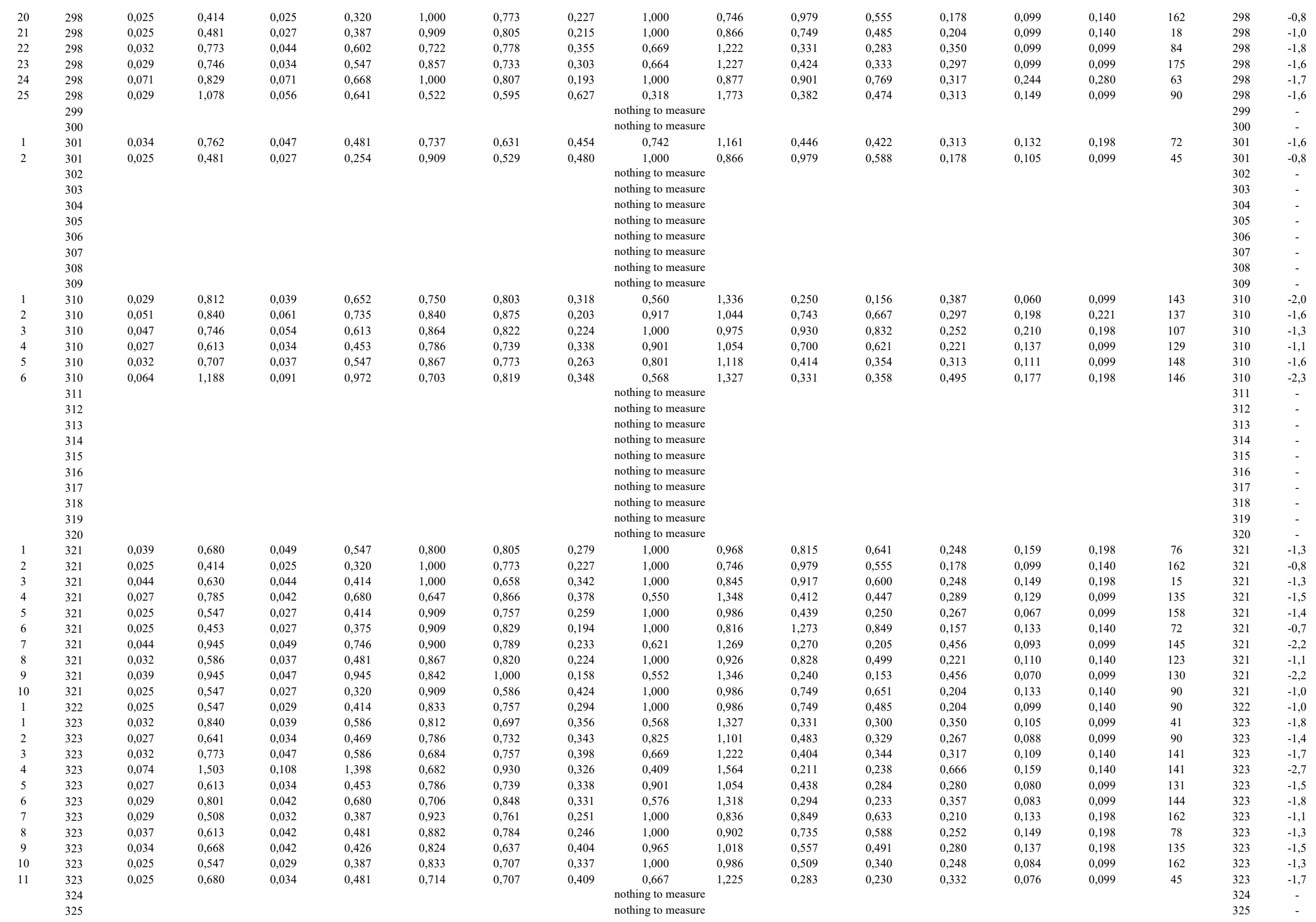


\begin{tabular}{lllllllcl} 
& & & & & \multicolumn{3}{c}{$\begin{array}{c}\text { nothing to measure } \\
\text { nothing to measure } \\
\text { nothing to measure }\end{array}$} \\
0,042 & 0,641 & 0,044 & 0,519 & 0,944 & 0,811 & 0,197 & 1,000 & 0,886 \\
0,037 & 0,668 & 0,044 & 0,508 & 0,833 & 0,760 & 0,292 & 1,000 & 0,983
\end{tabular}

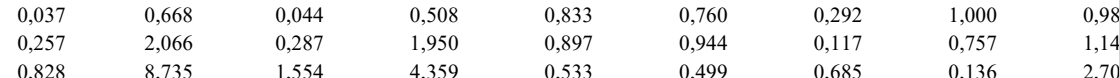

$\begin{array}{lllll}0,074 & 1,453 & 0,125 & 1,243 & 0,588\end{array}$

$\begin{array}{lllll}0,118 & 1,972 & 0,176 & 1,442 & 0,667\end{array}$

$\begin{array}{lll}0,118 & 1,972 & 0,176 \\ 0,025 & 0,414 & 0,025\end{array}$

$0,069-1,332-0,098$

$0,600 \quad 6,188$

$0,157 \quad 2,282$

0,025

$0,037 \quad 0,586$

$0,061 \quad 0,790$

$\begin{array}{ll}0,029 & 0,508 \\ 0,147 & 1,453\end{array}$

$0,032 \quad 0,613$

$\begin{array}{ll}0,707 \\ 0,064 & 1,481\end{array}$

$\begin{array}{ll}0,029 & 0,320 \\ 0,162 & 1,293\end{array}$

0,042

0,108

0,453

$0,855 \quad 0,436$

$\begin{array}{ll}0,731 & 0,428 \\ 0,867 & 0,133\end{array}$

0,820

$\begin{array}{ll}0,820 & 0,180 \\ 0,772 & 0,377\end{array}$

$\begin{array}{ll}1,000 & 0,820 \\ 0,510 & 0,772 \\ 0,503 & 0,703\end{array}$

$\begin{array}{lll}0,510 & 0,703 & 0,573 \\ 0,538 & 0,835 & 0,491\end{array}$

$\begin{array}{ll}0,835 & 0,491 \\ 0,831 & 0,311\end{array}$

$\begin{array}{lll}0,617 & 0,311 & 0,665\end{array}$

$\begin{array}{llll}0,937 & 0,934 & 0,091 & 1,000\end{array}$

$\begin{array}{llll}0,926 & 0,643 & 0,364 & 1,000 \\ 1,000 & 0,631 & 0,369 & 1,000\end{array}$

$\begin{array}{lll}1,000 & 0,631 & 0,369 \\ 0,909 & 0,890 & 0,143\end{array}$

0,765

0,739
0,773

$0,143 \quad 0,875$

0,246
0,466

1,000

0,365
nothing to measure

nothing to measure

nothing to measure

nothing to measure
nothing to measure

0,668

0,042

0,547

0,882

0,818

0,216

1,000

0,983

0,477

0,316

0,313

1,039
1,298

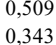

0,40

$\begin{array}{llllll}0,044 & 0,773 & 0,047 & 0,613 & 0,947 & 0,793 \\ 0,086 & 1,348 & 0,113 & 1,121 & 0,761 & 0,832 \\ 0,049 & 0,906 & 0,056 & 0,773 & 0,870 & 0,854\end{array}$

$\begin{array}{ll}0,054 & 0,707 \\ 0,027 & 0,442\end{array}$

$\begin{array}{lll}0,044 & 0,641 & 0,047\end{array}$

$0,025 \quad 0,481$

0,574

0,957

$0,854 \quad 0,19$

0,593
0,750

$\begin{array}{rrr}0,812 & 0,193 & \text { nothing to } \mathrm{m} \\ 0,575 & 0,425 & 1,000 \\ 0,94 & 0,1,1 & 1,000\end{array}$

to measure 1,155

$0,586 \quad 0,947$

0,425

$\begin{array}{ll}1,000 & 0,859 \\ 1,000 & 0,759\end{array}$

0,344

0,258

0,332
0,564

0,426

$\begin{array}{ll}1,000 & 0,861 \\ 0,799 & 1,119\end{array}$

0,824
1,077

0,606
0,555

0,289
0,178

0,178

0,692

0,346

1,000

1,146
0,524

0,555
0,894
0,584
0,588

0,221

0,289
0,178

nothing to measure
nothing to measure

$\begin{array}{lll}0,029 & 0,519 & 0,029\end{array}$

0,414

nothing to measure
nothing to measure

$\begin{array}{lll}0,051 & 0,680 & 0,051 \\ 0,044 & 0,879 & 0,0 \\ 0,027 & 0,508 & 0,032\end{array}$

$\begin{array}{lll}0,668 & 0,750\end{array}$

$\begin{array}{lll}0,108 & 1,691 & 0,108\end{array}$

$\begin{array}{lll}0,037 & 0,934 & 0,047\end{array}$

$\begin{array}{lll}0,042 & 0,613 & 0,044 \\ 0,027 & 0,453 & 0,027\end{array}$

$\begin{array}{llll}1,160 & 1,000 & 0,686 & 0,314 \\ 0,641 & 0,789 & 0,686 & 0,378 \\ 0,508 & 0,944 & 0,829 & 0,180\end{array}$

0,944
1,000

0,829
0,768

0,202

hing to measure

$0,761 \quad 0,346$

0,718
1,000

0,845
1,180

0,764

0,474

nothing to measure

nothing to measure

nothing to measure

$\begin{array}{lllllll}0,093 & 1,718 & 0,149 & 1,254 & 0,623 & 0,730 & 0,464\end{array}$

0,474
0,530

1,453

0,221
0,267
0,289

$0,099 \quad 0$,




\begin{tabular}{|c|c|c|c|c|c|c|c|c|c|c|c|c|c|c|c|c|}
\hline 0,029 & 0,906 & 0,051 & 0,625 & 0,571 & 0,689 & 0,529 & 0,450 & 1,491 & 0,413 & 0,536 & 0,301 & 0,161 & 0,099 & 28 & 355 & $-1,6$ \\
\hline 0,027 & 0,481 & 0,029 & 0,320 & 0,917 & 0,667 & 0,344 & 1,000 & 0,826 & 1,077 & 0,745 & 0,178 & 0,133 & 0,198 & 72 & 355 & $-0,8$ \\
\hline 0,025 & 0,481 & 0,027 & 0,359 & 0,909 & 0,747 & 0,268 & 1,000 & 0,866 & 0,749 & 0,485 & 0,204 & 0,099 & 0,140 & 90 & 355 & $-1,0$ \\
\hline 0,027 & 0,613 & 0,029 & 0,414 & 0,917 & 0,675 & 0,335 & 0,901 & 1,054 & 0,412 & 0,231 & 0,289 & 0,067 & 0,099 & 148 & 355 & $-1,5$ \\
\hline 0,066 & 1,227 & 0,093 & 1,028 & 0,711 & 0,838 & 0,332 & 0,553 & 1,345 & 0,382 & 0,485 & 0,470 & 0,228 & 0,198 & 171 & 355 & $-2,2$ \\
\hline 0,047 & 1,028 & 0,074 & 0,829 & 0,633 & 0,806 & 0,415 & 0,554 & 1,343 & 0,397 & 0,486 & 0,387 & 0,188 & 0,140 & 91 & 355 & $-2,0$ \\
\hline 0,169 & 1,934 & 0,221 & 1,574 & 0,767 & 0,814 & 0,298 & 0,568 & 1,326 & 0,453 & 0,523 & 0,690 & 0,360 & 0,313 & 165 & 355 & $-2,8$ \\
\hline 0,039 & 0,586 & 0,039 & 0,574 & 1,000 & 0,981 & 0,019 & 1,000 & 0,834 & 1,019 & 0,632 & 0,221 & 0,140 & 0,198 & 117 & 355 & $-1,1$ \\
\hline 0,027 & 0,613 & 0,029 & 0,426 & 0,917 & 0,694 & 0,317 & 0,901 & 1,054 & 0,560 & 0,424 & 0,248 & 0,105 & 0,099 & 135 & 355 & $-1,3$ \\
\hline 0,088 & 2,000 & 0,172 & 1,414 & 0,514 & 0,707 & 0,567 & 0,277 & 1,899 & 0,258 & 0,488 & 0,660 & 0,322 & 0,198 & 174 & 355 & $-2,7$ \\
\hline 0,061 & 0,895 & 0,071 & 0,735 & 0,862 & 0,821 & 0,226 & 0,961 & 1,020 & 0,707 & 0,596 & 0,332 & 0,198 & 0,198 & 178 & 355 & $-1,7$ \\
\hline 0,135 & 1,680 & 0,176 & 1,403 & 0,764 & 0,835 & 0,288 & 0,600 & 1,291 & 0,414 & 0,439 & 0,644 & 0,282 & 0,140 & 110 & 355 & $-2,7$ \\
\hline 0,047 & 0,879 & 0,061 & 0,680 & 0,760 & 0,773 & 0,330 & 0,758 & 1,149 & 0,605 & 0,528 & 0,313 & 0,165 & 0,140 & 161 & 355 & $-1,6$ \\
\hline 0,027 & 0,453 & 0,027 & 0,359 & 1,000 & 0,793 & 0,207 & 1,000 & 0,778 & 1,077 & 0,588 & 0,178 & 0,105 & 0,140 & 135 & 355 & $-0,8$ \\
\hline 0,027 & 0,668 & 0,032 & 0,442 & 0,846 & 0,661 & 0,372 & 0,758 & 1,148 & 0,350 & 0,269 & 0,313 & 0,084 & 0,099 & 0 & 355 & $-1,6$ \\
\hline 0,037 & 0,574 & 0,039 & 0,387 & 0,937 & 0,673 & 0,333 & 1,000 & 0,845 & 0,764 & 0,537 & 0,248 & 0,133 & 0,198 & 153 & 355 & $-1,3$ \\
\hline 0,029 & 0,801 & 0,044 & 0,558 & 0,667 & 0,697 & 0,451 & 0,576 & 1,318 & 0,449 & 0,516 & 0,289 & 0,149 & 0,099 & 140 & 355 & $-1,5$ \\
\hline 0,032 & 0,680 & 0,042 & 0,453 & 0,765 & 0,667 & 0,408 & 0,867 & 1,074 & 0,828 & 0,791 & 0,221 & 0,175 & 0,140 & 77 & 355 & $-1,1$ \\
\hline 0,056 & 1,613 & 0,096 & 0,972 & 0,590 & 0,603 & 0,571 & 0,272 & 1,917 & 0,299 & 0,407 & 0,490 & 0,199 & 0,099 & 132 & 355 & $-2,3$ \\
\hline 0,076 & 1,227 & 0,100 & 0,867 & 0,756 & 0,707 & 0,381 & 0,635 & 1,255 & 0,759 & 0,774 & 0,357 & 0,276 & 0,198 & 10 & 355 & $-1,8$ \\
\hline 0,025 & 0,414 & 0,025 & 0,227 & 1,000 & 0,547 & 0,453 & 1,000 & 0,746 & 0,979 & 0,555 & 0,178 & 0,099 & 0,140 & 108 & 355 & $-0,8$ \\
\hline 0,098 & 1,188 & 0,110 & 0,867 & 0,889 & 0,730 & 0,292 & 0,873 & 1,070 & 0,688 & 0,658 & 0,426 & 0,280 & 0,280 & 147 & 355 & $-2,1$ \\
\hline 0,064 & 1,171 & 0,086 & 0,922 & 0,743 & 0,787 & 0,334 & 0,583 & 1,309 & 0,571 & 0,623 & 0,377 & 0,235 & 0,198 & 142 & 355 & $-1,9$ \\
\hline 0,025 & 0,481 & 0,025 & 0,254 & 1,000 & 0,529 & 0,471 & 1,000 & 0,866 & 0,749 & 0,485 & 0,204 & 0,099 & 0,140 & 0 & 355 & $-1,0$ \\
\hline 0,071 & 1,016 & 0,081 & 0,829 & 0,879 & 0,815 & 0,221 & 0,865 & 1,075 & 0,506 & 0,351 & 0,423 & 0,149 & 0,198 & 1 & 355 & $-2,1$ \\
\hline 0,027 & 0,934 & 0,049 & 0,574 & 0,550 & 0,615 & 0,592 & 0,389 & 1,604 & 0,264 & 0,321 & 0,360 & 0,116 & 0,099 & 0 & 355 & $-1,8$ \\
\hline 0,027 & 0,641 & 0,034 & 0,414 & 0,786 & 0,646 & 0,413 & 0,825 & 1,101 & 0,700 & 0,671 & 0,221 & 0,149 & 0,140 & 0 & 355 & $-1,1$ \\
\hline 0,025 & 0,414 & 0,025 & 0,227 & 1,000 & 0,547 & 0,453 & 1,000 & 0,746 & 1,273 & 0,632 & 0,157 & 0,099 & 0,198 & 72 & 355 & $-0,7$ \\
\hline 0,029 & 0,773 & 0,039 & 0,426 & 0,750 & 0,550 & 0,515 & 0,618 & 1,272 & 0,611 & 0,555 & 0,248 & 0,137 & 0,099 & 135 & 355 & $-1,3$ \\
\hline 0,025 & 0,773 & 0,042 & 0,453 & 0,588 & 0,586 & 0,584 & 0,515 & 1,394 & 0,509 & 0,696 & 0,248 & 0,172 & 0,099 & 135 & 355 & $-1,3$ \\
\hline 0,025 & 0,414 & 0,025 & 0,227 & 1,000 & 0,547 & 0,453 & 1,000 & 0,746 & 1,273 & 0,632 & 0,157 & 0,099 & 0,140 & 90 & 355 & $-0,7$ \\
\hline 0,056 & 0,906 & 0,069 & 0,735 & 0,821 & 0,811 & 0,260 & 0,863 & 1,077 & 0,651 & 0,601 & 0,332 & 0,199 & 0,198 & 42 & 355 & $-1,7$ \\
\hline 0,034 & 0,812 & 0,044 & 0,586 & 0,778 & 0,721 & 0,357 & 0,653 & 1,237 & 0,615 & 0,516 & 0,267 & 0,137 & 0,140 & 148 & 355 & $-1,4$ \\
\hline 0,042 & 0,680 & 0,047 & 0,586 & 0,895 & 0,862 & 0,174 & 1,000 & 0,939 & 0,866 & 0,667 & 0,248 & 0,165 & 0,198 & 125 & 355 & $-1,3$ \\
\hline \multirow[t]{2}{*}{0,071} & 1,066 & 0,088 & 0,840 & 0,806 & 0,788 & 0,288 & $\begin{array}{c}0,785 \\
\text { nothing tomeasure }\end{array}$ & 1,128 & 0,697 & 0,737 & 0,360 & 0,266 & 0,221 & 27 & 355 & $-1,8$ \\
\hline & 0.547 & & & 0.917 & & & $\begin{array}{l}\text { nothing to measure } \\
1,000\end{array}$ & 0.940 & 0.560 & & 0248 & & 0099 & 122 & $\begin{array}{l}356 \\
357\end{array}$ & $\begin{array}{c}- \\
-1,3\end{array}$ \\
\hline \multirow{4}{*}{0,027} & 0,341 & 0,029 & 0,453 & 0,917 & 0,828 & 0,191 & $\begin{array}{c}1,000 \\
\text { nothing to measure }\end{array}$ & 0,940 & 0,560 & 0,358 & 0,248 & 0,089 & 0,099 & 122 & 358 & $\begin{array}{l}-1,3 \\
-\end{array}$ \\
\hline & & & & & & & nothing to measure & & & & & & & & 359 & - \\
\hline & & & & & & & nothing to measure & & & & & & & & 360 & - \\
\hline & & & & & & & nothing to measure & & & & & & & & 361 & - \\
\hline \multirow[t]{9}{*}{0,069} & 1,000 & 0,078 & 0,762 & 0,875 & 0,762 & 0,269 & 0,862 & 1,077 & 0,891 & 0,778 & 0,313 & 0,244 & 0,221 & 6 & 362 & $-1,6$ \\
\hline & & & & & & & nothing to measure & & & & & & & & 363 & - \\
\hline & & & & & & & nothing to measure & & & & & & & & 364 & - \\
\hline & & & & & & & nothing to measure & & & & & & & & 365 & - \\
\hline & & & & & & & nothing to measure & & & & & & & & 366 & - \\
\hline & & & & & & & nothing to measure & & & & & & & & 367 & - \\
\hline & & & & & & & nothing to measure & & & & & & & & 368 & - \\
\hline & & & & & & & nothing to measure & & & & & & & & 369 & - \\
\hline & & & & & & & nothing to measure & & & & & & & & 370 & - \\
\hline 0,027 & 0,586 & 0,034 & 0,414 & 0,786 & 0,707 & 0,363 & 0,987 & 1,006 & 0,700 & 0,601 & 0,221 & 0,133 & 0,140 & 108 & 371 & $-1,1$ \\
\hline \multirow[t]{7}{*}{0,029} & 0,613 & 0,034 & 0,348 & 0,857 & 0,567 & 0,456 & 0,983 & 1,009 & 0,849 & 0,707 & 0,210 & 0,149 & 0,140 & 135 & 371 & $-1,1$ \\
\hline & & & & & & & nothing to measure & & & & & & & & 372 & - \\
\hline & & & & & & & nothing to measure & & & & & & & & 373 & - \\
\hline & & & & & & & nothing to measure & & & & & & & & 374 & - \\
\hline & & & & & & & nothing to measure & & & & & & & & 375 & - \\
\hline & & & & & & & nothing to measure & & & & & & & & 376 & . \\
\hline & & & & & & & nothing to measure & & & & & & & & 377 & - \\
\hline
\end{tabular}


0,479

nothing to measure
nothing to measure

nothing to

nothing to measure

nothing to measure

nothing to measure

nothing to measure

nothing to measure

$\begin{array}{llllllll}0,039 & 0,801 & 0,044 & 0,519 & 0,889 & 0,648 & 0,369 & \text { nothing to me }\end{array}$

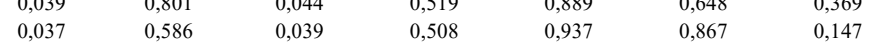

$\begin{array}{lll}0,081 & 2,011 & 0,152\end{array}$

$\begin{array}{lll}0,032 & 0,746 & 0,042\end{array}$

$\begin{array}{ll}0,029 & 0,757\end{array}$

1,105

0,029

$\begin{array}{ll}1,149 & 0,532\end{array}$

0,571

0,768
1,000

1,141

$\begin{array}{lll}0,356 & 0,251 & 1,995 \\ 0,020 & 1,179\end{array}$

0,718

0,03

1,133

0,047

0,790

1,000

0,949

0,051

0,766

0,373
0,352
0,601

$0,645 \quad 1,246$

$\begin{array}{ll}0,479 & 1,445 \\ 0,672 & 1,220\end{array}$

nothing to measure

nothing to measure

nothing to measure

$\begin{array}{lllllll}0,098 & 1,558 & 0,132 & 1,121 & 0,741 & 0,720 & 0,382\end{array}$

0,507

1,404

nothing to measure

nothing to measure

nothing to measure

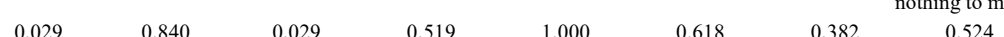

$\begin{array}{rrrr}0,618 & 0,382 & 0,524 & 1,381\end{array}$

$\begin{array}{lll}0,829 & 0,204 & 1,000\end{array}$

$\begin{array}{ll}1,000 & 0,874 \\ 0,919 & 1,043\end{array}$

$\begin{array}{lllll}0,039 & 0,613 & 0,044 & 0,508 & 0,889 \\ 0,064 & 0,934 & 0,078 & 0,829 & 0,812\end{array}$

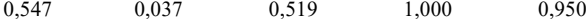

0,050

1,000

1,043

1,019

1,000

0,818

nothing to measure

$\begin{array}{lllllllcl}0,029 & 0,613 & 0,032 & 0,414 & 0,923 & 0,675 & 0,334 & 0,983 & 1,009 \\ 0,051 & 0,668 & 0,051 & 0,613 & 1,000 & 0,918 & 0,082 & 1,000 & 0,831\end{array}$

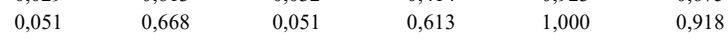

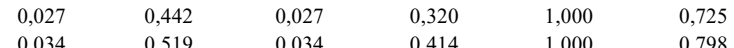

$0,042-0,586-0,0042$

$\begin{array}{rr}0,414 & 1,000 \\ 0,508 & 1,000\end{array}$

0,798

0,082

$\begin{array}{ll}1,000 & 0,759 \\ 1,000 & 0,791\end{array}$

1,009
0,831
0,759
0,791
0,810

0,611
0,922
1,077

$\begin{array}{ll}0,922 & 0,400 \\ 1,077 & 0,557\end{array}$

0,557

$1,273 \quad 0,857$

0,600

1,000
0,750

1,155

$\begin{array}{llll}0,025 & 0,641 & 0,034 & 0,453 \\ 0,027 & 0,519 & 0,029 & 0,426\end{array}$

$\begin{array}{lll}0,027 & 0,519 & 0,029 \\ 0,049 & 0,707 & 0,054\end{array}$

$\begin{array}{lll}0,027 & 0,652 & 0,053\end{array}$

$0,034 \quad 0,613$

$\begin{array}{lll}0,714 & 0,707 & 0,409\end{array}$

0,819
0,680

0,025

$0,481 \quad 0,039$

0,508

0,909

0,680
0,779

$\begin{array}{ll}0,025 & 0,574 \\ 0,360 & 3,685\end{array}$

0,032

0,320

0,875
0,833
0,769

0,720
0,667
0,558

1,000
1,000

1,000
0,797
1,000

0,892

0,398

0,297
0,514

1,000

1,120
0,934

$\begin{array}{ll}1,019 & 0,800 \\ 0,560 & 0,534\end{array}$

$\begin{array}{ll}1,019 & 0,800 \\ 0,686 & 0,559 \\ 0,979 & 0,702\end{array}$

$\begin{array}{ll}0,979 & 0,702 \\ 0,490 & 0,286\end{array}$

$\begin{array}{ll}0,149 & 0,145 \\ 0,702 & 0,657\end{array}$

$0,333 \quad 1,732$

$\begin{array}{ll}0,886 & 1,063 \\ 0,997 & 1,001\end{array}$

0,866

0,800

$\begin{array}{llllll}0,061 & 0,879 & 0,066 & 0,801 & 0,926 & 0,912 \\ 0,042 & 0,840 & 0,059 & 0,574 & 0,708 & 0,684\end{array}$

0,430

0,742

1,161 


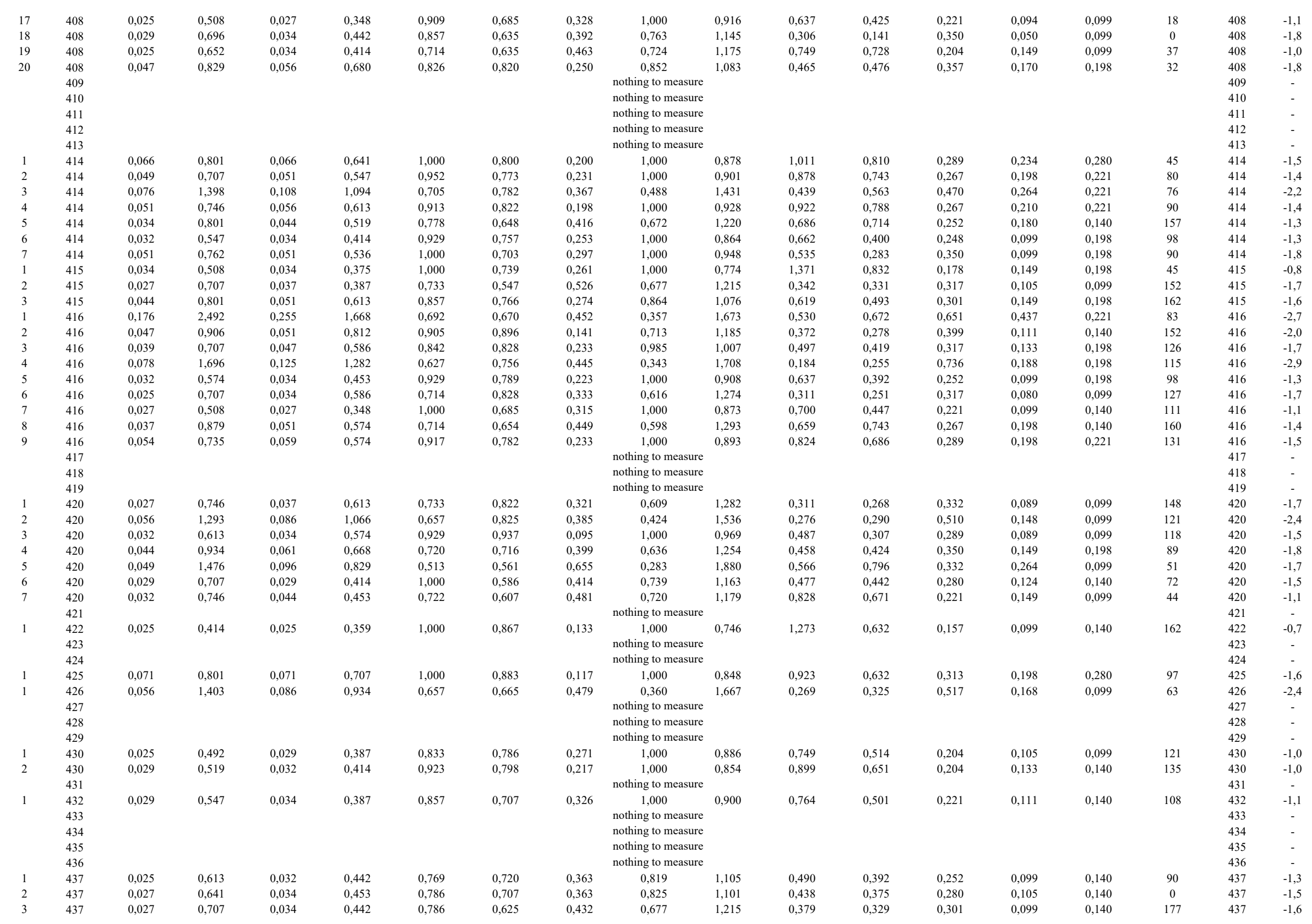




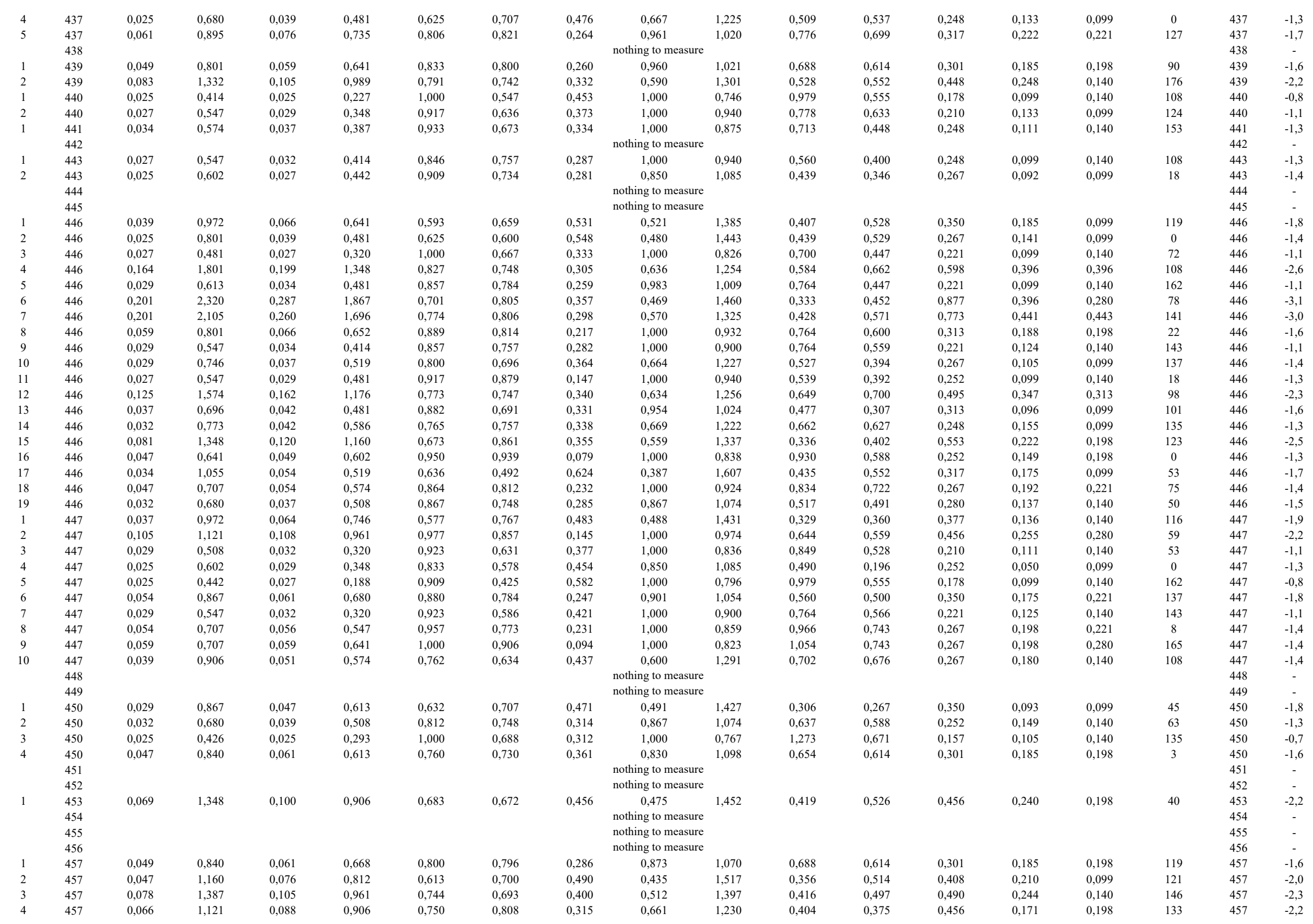




\begin{tabular}{|c|c|c|c|c|c|c|c|c|c|c|c|c|c|c|c|c|c|c|}
\hline 1 & $\begin{array}{l}458 \\
459 \\
460 \\
461\end{array}$ & 0,025 & 0,613 & 0,029 & 0,348 & 0,833 & 0,567 & 0,464 & $\begin{array}{c}0,819 \\
\text { nothing to measure } \\
\text { nothing to measure } \\
\text { nothing to measure }\end{array}$ & 1,105 & 0,979 & 0,784 & 0,178 & 0,140 & 0,099 & 62 & $\begin{array}{l}458 \\
459 \\
460 \\
461\end{array}$ & $\begin{array}{c}-0,8 \\
- \\
- \\
-\end{array}$ \\
\hline 1 & 462 & 0,034 & 0,680 & 0,037 & 0,519 & 0,933 & 0,764 & 0,245 & 0,934 & 1,035 & 0,524 & 0,460 & 0,289 & 0,133 & 0,198 & 147 & 462 & $-1,5$ \\
\hline 2 & 462 & 0,147 & 1,309 & 0,162 & 1,188 & 0,909 & 0,907 & 0,130 & 1,000 & 0,963 & 0,955 & 0,884 & 0,443 & 0,391 & 0,357 & 23 & 462 & $-2,1$ \\
\hline 3 & 462 & 0,029 & 0,547 & 0,032 & 0,387 & 0,923 & 0,707 & 0,303 & 1,000 & 0,900 & 0,611 & 0,424 & 0,248 & 0,105 & 0,140 & 140 & 462 & $-1,3$ \\
\hline 4 & 462 & 0,471 & 5,018 & 0,725 & 3,083 & 0,649 & 0,614 & 0,522 & 0,235 & 2,063 & 0,372 & 0,565 & 1,269 & 0,717 & 0,280 & 165 & 462 & $-3,7$ \\
\hline 5 & 462 & 0,025 & 0,426 & 0,025 & 0,320 & 1,000 & 0,753 & 0,247 & 1,000 & 0,767 & 1,273 & 0,671 & 0,157 & 0,105 & 0,140 & 108 & 462 & $-0,7$ \\
\hline 6 & 462 & 0,025 & 0,536 & 0,027 & 0,547 & 0,909 & 1,021 & 0,093 & 1,000 & 0,965 & 0,490 & 0,196 & 0,252 & 0,050 & 0,099 & 0 & 462 & $-1,3$ \\
\hline 7 & 462 & 0,037 & 0,668 & 0,042 & 1,105 & 0,882 & 1,654 & 0,664 & 1,000 & 0,983 & 0,562 & 0,404 & 0,289 & 0,117 & 0,198 & 110 & 462 & $-1,5$ \\
\hline 8 & 462 & 0,098 & 1,265 & 0,110 & 0,613 & 0,889 & 0,485 & 0,527 & 0,769 & 1,140 & 0,435 & 0,327 & 0,535 & 0,175 & 0,198 & 133 & 462 & $-2,4$ \\
\hline 9 & 462 & 0,037 & 0,707 & 0,042 & 1,270 & 0,882 & 1,796 & 0,805 & 0,924 & 1,040 & 0,466 & 0,331 & 0,317 & 0,105 & 0,140 & 139 & 462 & $-1,7$ \\
\hline 10 & 462 & 0,083 & 2,000 & 0,147 & 1,398 & 0,567 & 0,699 & 0,528 & 0,262 & 1,955 & 0,370 & 0,538 & 0,535 & 0,288 & 0,140 & 113 & 462 & $-2,4$ \\
\hline 11 & 462 & 0,108 & 1,652 & 0,140 & 0,453 & 0,772 & 0,274 & 0,761 & 0,496 & 1,419 & 0,269 & 0,294 & 0,714 & 0,210 & 0,280 & 141 & 462 & $-2,8$ \\
\hline 12 & 462 & 0,034 & 0,641 & 0,042 & 0,801 & 0,824 & 1,250 & 0,306 & 1,000 & 0,976 & 0,713 & 0,566 & 0,248 & 0,140 & 0,140 & 130 & 462 & $-1,3$ \\
\hline 13 & 462 & 0,042 & 1,066 & 0,061 & 0,453 & 0,680 & 0,425 & 0,658 & 0,460 & 1,474 & 0,333 & 0,388 & 0,399 & 0,155 & 0,140 & 140 & 462 & $-2,0$ \\
\hline 14 & $\begin{array}{l}462 \\
463\end{array}$ & 0,025 & 0,668 & 0,034 & 0,387 & 0,714 & 0,579 & 0,509 & $\begin{array}{l}\quad 0,690 \\
\text { nothing to measure }\end{array}$ & 1,204 & 0,509 & 0,566 & 0,248 & 0,140 & 0,099 & 108 & $\begin{array}{l}462 \\
463\end{array}$ & $-1,3$ \\
\hline 1 & 464 & 0,044 & 1,430 & 0,081 & 1,298 & 0,545 & 0,907 & 0,464 & 0,271 & 1,921 & 0,116 & 0,121 & 0,695 & 0,084 & 0,099 & 94 & 464 & $-2,8$ \\
\hline 2 & 464 & 0,032 & 0,840 & 0,044 & 0,492 & 0,722 & 0,586 & 0,499 & 0,568 & 1,327 & 0,571 & 0,657 & 0,267 & 0,175 & 0,140 & 122 & 464 & $-1,4$ \\
\hline 3 & 464 & 0,078 & 1,558 & 0,137 & 1,188 & 0,571 & 0,762 & 0,490 & 0,406 & 1,570 & 0,326 & 0,466 & 0,553 & 0,258 & 0,198 & 106 & 464 & $-2,5$ \\
\hline 4 & 464 & 0,027 & 0,801 & 0,037 & 0,641 & 0,733 & 0,800 & 0,333 & 0,528 & 1,376 & 0,241 & 0,125 & 0,377 & 0,047 & 0,099 & 72 & 464 & $-1,9$ \\
\hline 5 & 464 & 0,049 & 1,110 & 0,069 & 0,961 & 0,714 & 0,866 & 0,316 & 0,500 & 1,414 & 0,234 & 0,198 & 0,517 & 0,102 & 0,099 & 105 & 464 & $-2,4$ \\
\hline & 465 & & & & & & & & nothing to measure & & & & & & & & 465 & 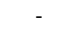 \\
\hline & 466 & & & & & & & & nothing to measure & & & & & & & & 466 & 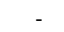 \\
\hline 1 & 467 & 0,034 & 1,000 & 0,061 & 0,574 & 0,560 & 0,574 & 0,612 & 0,431 & 1,523 & 0,446 & 0,632 & 0,313 & 0,198 & 0,099 & 40 & 467 & $-1,6$ \\
\hline 2 & 467 & 0,032 & 0,547 & 0,034 & 0,348 & 0,929 & 0,636 & 0,371 & 1,000 & 0,864 & 1,273 & 0,832 & 0,178 & 0,149 & 0,140 & 33 & 467 & $-0,8$ \\
\hline & 468 & & & & & & & & nothing to measure & & & & & & & & 468 & - \\
\hline & 469 & & & & & & & & nothing to measure & & & & & & & & 469 & - \\
\hline 1 & 470 & 0,029 & 0,707 & 0,039 & 0,586 & 0,750 & 0,828 & 0,303 & 0,739 & 1,163 & 0,373 & 0,260 & 0,317 & 0,083 & 0,099 & 122 & 470 & $-1,7$ \\
\hline 2 & 470 & 0,029 & 0,547 & 0,032 & 0,320 & 0,923 & 0,586 & 0,421 & 1,000 & 0,900 & 0,611 & 0,389 & 0,248 & 0,096 & 0,099 & 148 & 470 & $-1,3$ \\
\hline 3 & 470 & 0,049 & 0,840 & 0,059 & 0,613 & 0,833 & 0,730 & 0,317 & 0,873 & 1,070 & 0,749 & 0,667 & 0,289 & 0,192 & 0,221 & 101 & 470 & $-1,5$ \\
\hline 4 & 470 & 0,032 & 0,735 & 0,044 & 0,414 & 0,722 & 0,564 & 0,517 & 0,742 & 1,161 & 0,662 & 0,600 & 0,248 & 0,149 & 0,198 & 90 & 470 & $-1,3$ \\
\hline 5 & 470 & 0,039 & 0,641 & 0,047 & 0,481 & 0,842 & 0,750 & 0,296 & 1,000 & 0,913 & 1,019 & 0,791 & 0,221 & 0,175 & 0,140 & 134 & 470 & $-1,1$ \\
\hline 6 & 470 & 0,029 & 0,481 & 0,029 & 0,348 & 1,000 & 0,724 & 0,276 & 1,000 & 0,791 & 1,175 & 0,745 & 0,178 & 0,133 & 0,198 & 90 & 470 & $-0,8$ \\
\hline 7 & 470 & 0,042 & 0,840 & 0,056 & 0,641 & 0,739 & 0,763 & 0,352 & 0,742 & 1,161 & 0,481 & 0,512 & 0,332 & 0,170 & 0,140 & 99 & 470 & $-1,7$ \\
\hline 8 & 470 & 0,042 & 0,602 & 0,044 & 0,442 & 0,944 & 0,734 & 0,272 & 1,000 & 0,832 & 1,082 & 0,671 & 0,221 & 0,149 & 0,198 & 72 & 470 & $-1,1$ \\
\hline 9 & 470 & 0,034 & 0,613 & 0,037 & 0,442 & 0,933 & 0,720 & 0,287 & 1,000 & 0,934 & 0,990 & 0,707 & 0,210 & 0,149 & 0,140 & 5 & 470 & $-1,1$ \\
\hline 10 & 470 & 0,034 & 0,829 & 0,049 & 0,652 & 0,700 & 0,787 & 0,368 & 0,628 & 1,262 & 0,357 & 0,300 & 0,350 & 0,105 & 0,099 & 135 & 470 & $-1,8$ \\
\hline 11 & 470 & 0,118 & 1,387 & 0,132 & 1,227 & 0,889 & 0,884 & 0,160 & 0,769 & 1,141 & 0,446 & 0,460 & 0,579 & 0,267 & 0,280 & 109 & 470 & $-2,5$ \\
\hline 12 & 470 & 0,049 & 0,735 & 0,051 & 0,574 & 0,952 & 0,782 & 0,223 & 1,000 & 0,936 & 0,621 & 0,489 & 0,317 & 0,155 & 0,198 & 122 & 470 & $-1,7$ \\
\hline 13 & 470 & 0,029 & 0,707 & 0,037 & 0,680 & 0,800 & 0,961 & 0,204 & 0,739 & 1,163 & 0,340 & 0,250 & 0,332 & 0,083 & 0,099 & 156 & 470 & $-1,7$ \\
\hline 14 & 470 & 0,047 & 1,078 & 0,059 & 1,011 & 0,792 & 0,938 & 0,217 & 0,504 & 1,409 & 0,214 & 0,200 & 0,526 & 0,105 & 0,140 & 132 & 470 & $-2,4$ \\
\hline 15 & 470 & 0,047 & 0,773 & 0,051 & 0,707 & 0,905 & 0,914 & 0,128 & 0,978 & 1,011 & 0,654 & 0,589 & 0,301 & 0,177 & 0,198 & 113 & 470 & $-1,6$ \\
\hline 16 & 470 & 0,032 & 0,801 & 0,039 & 0,481 & 0,812 & 0,600 & 0,442 & 0,624 & 1,266 & 0,447 & 0,484 & 0,301 & 0,146 & 0,099 & 108 & 470 & $-1,6$ \\
\hline 17 & 470 & 0,044 & 1,149 & 0,076 & 0,812 & 0,581 & 0,707 & 0,512 & 0,420 & 1,543 & 0,310 & 0,411 & 0,426 & 0,175 & 0,099 & 47 & 470 & $-2,1$ \\
\hline 18 & 470 & 0,049 & 0,895 & 0,061 & 0,602 & 0,800 & 0,673 & 0,384 & 0,769 & 1,140 & 0,796 & 0,707 & 0,280 & 0,198 & 0,198 & 119 & 470 & $-1,5$ \\
\hline 19 & 470 & 0,054 & 0,840 & 0,059 & 0,707 & 0,917 & 0,842 & 0,179 & 0,961 & 1,020 & 0,700 & 0,550 & 0,313 & 0,172 & 0,198 & 146 & 470 & $-1,6$ \\
\hline & 471 & & & & & & & & nothing to measure & & & & & & & & 471 & . \\
\hline & 472 & & & & & & & & nothing to measure & & & & & & & & 472 & - \\
\hline 1 & 473 & 0,044 & 0,680 & 0,047 & 0,574 & 0,947 & 0,845 & 0,163 & 1,000 & 0,913 & 0,881 & 0,588 & 0,252 & 0,149 & 0,198 & 27 & 473 & $-1,3$ \\
\hline 2 & 473 & 0,061 & 0,773 & 0,066 & 0,707 & 0,926 & 0,914 & 0,113 & 1,000 & 0,881 & 1,098 & 0,831 & 0,267 & 0,222 & 0,221 & 137 & 473 & $-1,4$ \\
\hline & 474 & & & & & & & & nothing to measure & & & & & & & & 474 & . \\
\hline & 475 & & & & & & & & nothing to measure & & & & & & & & 475 & - \\
\hline & 476 & & & & & & & & nothing to measure & & & & & & & & 476 & - \\
\hline & 477 & & & & & & & & nothing to measure & & & & & & & & 477 & - \\
\hline & 478 & & & & & & & & nothing to measure & & & & & & & & 478 & - \\
\hline
\end{tabular}




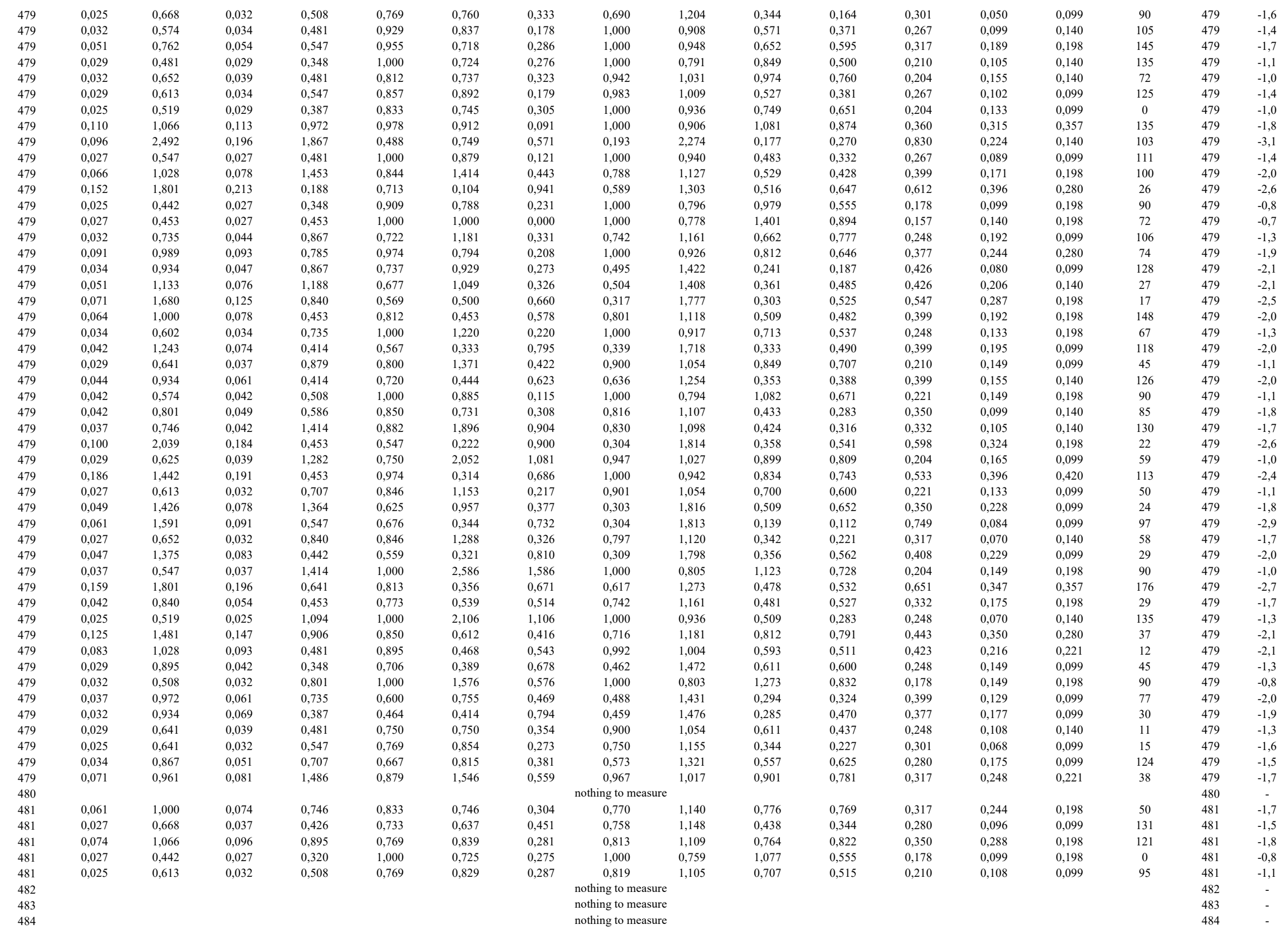




\begin{tabular}{|c|c|c|c|c|c|c|c|c|c|c|c|c|c|c|c|c|c|}
\hline $\begin{array}{l}485 \\
486\end{array}$ & & & & & & & & $\begin{array}{l}\text { nothing to measure } \\
\text { nothing to measure }\end{array}$ & & & & & & & & $\begin{array}{l}485 \\
486\end{array}$ & $\begin{array}{l}- \\
-\end{array}$ \\
\hline 487 & 0,025 & 0,773 & 0,034 & 0,387 & 0,714 & 0,500 & 0,576 & 0,515 & 1,394 & 0,637 & 0,632 & 0,221 & 0,140 & 0,099 & 45 & 487 & $-1,1$ \\
\hline 488 & 0,108 & 1,641 & 0,157 & 1,227 & 0,688 & 0,748 & 0,402 & 0,503 & 1,410 & 0,514 & 0,557 & 0,517 & 0,288 & 0,221 & 131 & 488 & $-2,4$ \\
\hline 488 & 0,054 & 1,320 & 0,086 & 0,812 & 0,629 & 0,615 & 0,535 & 0,389 & 1,604 & 0,483 & 0,650 & 0,377 & 0,245 & 0,140 & 157 & 488 & $-1,9$ \\
\hline 488 & 0,025 & 0,574 & 0,029 & 0,320 & 0,833 & 0,558 & 0,473 & 0,933 & 1,035 & 0,749 & 0,485 & 0,204 & 0,099 & 0,099 & 80 & 488 & $-1,0$ \\
\hline 488 & 0,029 & 1,039 & 0,047 & 0,613 & 0,632 & 0,590 & 0,551 & 0,342 & 1,709 & 0,250 & 0,301 & 0,387 & 0,116 & 0,099 & 0 & 488 & $-2,0$ \\
\hline 488 & 0,044 & 0,840 & 0,056 & 0,707 & 0,783 & 0,842 & 0,269 & 0,786 & 1,128 & 0,458 & 0,377 & 0,350 & 0,132 & 0,140 & 168 & 488 & $-1,8$ \\
\hline 488 & 0,025 & 0,481 & 0,027 & 0,414 & 0,909 & 0,862 & 0,165 & 1,000 & 0,866 & 0,637 & 0,400 & 0,221 & 0,089 & 0,099 & 162 & 488 & $-1,1$ \\
\hline 489 & & & & & & & & nothing to measure & & & & & & & & 489 & - \\
\hline 490 & & & & & & & & nothing to measure & & & & & & & & 490 & - \\
\hline 491 & & & & & & & & nothing to measure & & & & & & & & 491 & - \\
\hline 492 & & & & & & & & nothing to measure & & & & & & & & 492 & - \\
\hline 493 & & & & & & & & nothing to measure & & & & & & & & 493 & - \\
\hline 494 & & & & & & & & nothing to measure & & & & & & & & 494 & - \\
\hline 495 & & & & & & & & nothing to measure & & & & & & & & 495 & 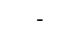 \\
\hline 496 & & & & & & & & nothing to measure & & & & & & & & 496 & - \\
\hline 497 & & & & & & & & nothing to measure & & & & & & & & 497 & - \\
\hline 498 & & & & & & & & nothing to measure & & & & & & & & 498 & - \\
\hline 499 & & & & & & & & nothing to measure & & & & & & & & 499 & - \\
\hline 500 & 0,025 & 0,602 & 0,029 & 0,188 & 0,833 & 0,312 & 0,708 & 0,850 & 1,085 & 0,490 & 0,196 & 0,252 & 0,050 & 0,099 & 90 & 500 & $-1,3$ \\
\hline 501 & & & & & & & & nothing to measure & & & & & & & & 501 & - \\
\hline 502 & & & & & & & & nothing to measure & & & & & & & & 502 & - \\
\hline 503 & & & & & & & & nothing to measure & & & & & & & & 503 & \\
\hline 504 & 0,027 & 0,680 & 0,037 & 0,387 & 0,733 & 0,569 & 0,507 & 0,733 & 1,168 & 0,560 & 0,424 & 0,248 & 0,105 & 0,140 & 90 & 504 & $-1,3$ \\
\hline 505 & & & & & & & & nothing to measure & & & & & & & & 505 & \\
\hline 506 & & & & & & & & nothing to measure & & & & & & & & 506 & - \\
\hline 507 & 0,091 & 1,066 & 0,108 & 0,895 & 0,841 & 0,839 & 0,226 & 1,000 & 0,999 & 0,772 & 0,724 & 0,387 & 0,280 & 0,280 & 93 & 507 & $-2,0$ \\
\hline 507 & 0,071 & 1,332 & 0,108 & 1,000 & 0,659 & 0,751 & 0,422 & 0,504 & 1,409 & 0,506 & 0,703 & 0,423 & 0,297 & 0,198 & 109 & 507 & $-2,1$ \\
\hline 507 & 0,034 & 0,602 & 0,037 & 0,453 & 0,933 & 0,753 & 0,256 & 1,000 & 0,917 & 0,891 & 0,651 & 0,221 & 0,144 & 0,198 & 82 & 507 & $-1,1$ \\
\hline 508 & & & & & & & & nothing to measure & & & & & & & & 508 & 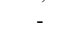 \\
\hline 509 & 0,145 & 1,652 & 0,181 & 1,332 & 0,797 & 0,806 & 0,281 & 0,666 & 1,226 & 0,709 & 0,744 & 0,510 & 0,379 & 0,313 & 49 & 509 & $-2,4$ \\
\hline 509 & 0,059 & 0,762 & 0,064 & 0,574 & 0,923 & 0,754 & 0,258 & 1,000 & 0,887 & 0,955 & 0,707 & 0,280 & 0,198 & 0,221 & 105 & 509 & $-1,5$ \\
\hline 509 & 0,130 & 1,751 & 0,167 & 1,497 & 0,779 & 0,855 & 0,264 & 0,532 & 1,370 & 0,329 & 0,349 & 0,709 & 0,248 & 0,297 & 1 & 509 & $-2,8$ \\
\hline 509 & 0,083 & 1,453 & 0,118 & 1,011 & 0,708 & 0,696 & 0,421 & 0,496 & 1,420 & 0,481 & 0,566 & 0,470 & 0,266 & 0,280 & 108 & 509 & $-2,2$ \\
\hline 509 & 0,061 & 0,735 & 0,061 & 0,574 & 1,000 & 0,782 & 0,218 & 1,000 & 0,837 & 0,936 & 0,686 & 0,289 & 0,198 & 0,280 & 107 & 509 & $-1,5$ \\
\hline 509 & 0,059 & 0,773 & 0,064 & 0,641 & 0,923 & 0,828 & 0,188 & 1,000 & 0,900 & 0,899 & 0,728 & 0,289 & 0,210 & 0,221 & 117 & 509 & $-1,5$ \\
\hline 509 & 0,091 & 1,801 & 0,157 & 1,160 & 0,578 & 0,644 & 0,552 & 0,351 & 1,687 & 0,417 & 0,639 & 0,526 & 0,336 & 0,221 & 153 & 509 & $-2,4$ \\
\hline 509 & 0,025 & 0,481 & 0,032 & 0,320 & 0,769 & 0,667 & 0,405 & 1,000 & 0,866 & 0,979 & 0,745 & 0,178 & 0,133 & 0,099 & 162 & 509 & $-0,8$ \\
\hline 510 & & & & & & & & nothing to measure & & & & & & & & 510 & - \\
\hline 511 & & & & & & & & nothing to measure & & & & & & & & 511 & - \\
\hline 512 & & & & & & & & nothing to measure & & & & & & & & 512 & - \\
\hline 513 & & & & & & & & nothing to measure & & & & & & & & 513 & - \\
\hline 514 & & & & & & & & nothing to measure & & & & & & & & 514 & - \\
\hline 515 & & & & & & & & nothing to measure & & & & & & & & 515 & - \\
\hline 516 & & & & & & & & nothing to measure & & & & & & & & 516 & - \\
\hline 517 & & & & & & & & nothing to measure & & & & & & & & 517 & - \\
\hline 518 & & & & & & & & nothing to measure & & & & & & & & 518 & - \\
\hline 519 & & & & & & & & nothing to measure & & & & & & & & 519 & - \\
\hline 520 & & & & & & & & nothing to measure & & & & & & & & 520 & - \\
\hline 521 & & & & & & & & nothing to measure & & & & & & & & 521 & - \\
\hline 522 & & & & & & & & nothing to measure & & & & & & & & 522 & . \\
\hline 523 & & & & & & & & nothing to measure & & & & & & & & 523 & \\
\hline 524 & 0,034 & 0,547 & 0,034 & 0,453 & 1,000 & 0,828 & 0,172 & 1,000 & 0,833 & 0,891 & 0,632 & 0,221 & 0,140 & 0,198 & 126 & 524 & $-1,1$ \\
\hline 524 & 0,059 & 1,028 & 0,078 & 0,735 & 0,750 & 0,715 & 0,379 & 0,700 & 1,195 & 0,611 & 0,707 & 0,350 & 0,248 & 0,198 & 179 & 524 & $-1,8$ \\
\hline 525 & 0,025 & 0,453 & 0,027 & 0,320 & 0,909 & 0,707 & 0,307 & 1,000 & 0,816 & 0,979 & 0,588 & 0,178 & 0,105 & 0,140 & 122 & 525 & $-0,8$ \\
\hline 526 & 0,032 & 0,707 & 0,039 & 0,481 & 0,812 & 0,680 & 0,371 & 0,801 & 1,118 & 0,414 & 0,251 & 0,313 & 0,079 & 0,099 & 159 & 526 & $-1,6$ \\
\hline 526 & 0,029 & 0,574 & 0,037 & 0,414 & 0,800 & 0,721 & 0,343 & 1,000 & 0,945 & 0,849 & 0,707 & 0,210 & 0,149 & 0,099 & 45 & 526 & $-1,1$ \\
\hline
\end{tabular}




\begin{tabular}{|c|c|c|c|c|c|c|c|c|c|c|c|c|c|c|c|c|c|}
\hline 527 & & & & & & & & nothing to measure & & & & & & & & 527 & - \\
\hline 528 & & & & & & & & nothing to measure & & & & & & & & 528 & \\
\hline 529 & 0,051 & 0,773 & 0,056 & 0,613 & 0,913 & 0,793 & 0,225 & 1,000 & 0,962 & 0,922 & 0,743 & 0,267 & 0,198 & 0,221 & 9 & 529 & $-1,4$ \\
\hline 530 & & & & & & & & nothing to measure & & & & & & & & 530 & 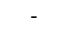 \\
\hline 531 & & & & & & & & nothing to measure & & & & & & & & 531 & - \\
\hline 532 & & & & & & & & nothing to measure & & & & & & & & 532 & - \\
\hline 533 & & & & & & & & nothing to measure & & & & & & & & 533 & - \\
\hline 534 & & & & & & & & nothing to measure & & & & & & & & 534 & - \\
\hline 535 & & & & & & & & nothing to measure & & & & & & & & 535 & - \\
\hline 536 & & & & & & & & nothing to measure & & & & & & & & 536 & - \\
\hline 537 & & & & & & & & nothing to measure & & & & & & & & 537 & 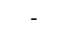 \\
\hline 538 & & & & & & & & nothing to measure & & & & & & & & 538 & - \\
\hline 539 & & & & & & & & nothing to measure & & & & & & & & 539 & - \\
\hline 540 & 0,027 & 0,453 & 0,027 & 0,348 & 1,000 & 0,768 & 0,232 & 1,000 & 0,778 & 1,401 & 0,894 & 0,157 & 0,140 & 0,198 & 108 & 540 & $-0,7$ \\
\hline 540 & 0,032 & 0,961 & 0,054 & 0,707 & 0,591 & 0,736 & 0,487 & 0,433 & 1,519 & 0,312 & 0,478 & 0,360 & 0,172 & 0,099 & 61 & 540 & $-1,8$ \\
\hline 540 & 0,027 & 0,691 & 0,027 & 0,519 & 1,000 & 0,752 & 0,248 & 0,710 & 1,187 & 0,280 & 0,100 & 0,350 & 0,035 & 0,099 & 135 & 540 & $-1,8$ \\
\hline 540 & 0,027 & 0,613 & 0,032 & 0,519 & 0,846 & 0,847 & 0,217 & 0,901 & 1,054 & 0,438 & 0,344 & 0,280 & 0,096 & 0,099 & 135 & 540 & $-1,5$ \\
\hline 540 & 0,110 & 1,094 & 0,118 & 0,961 & 0,937 & 0,879 & 0,136 & 1,000 & 0,929 & 0,988 & 0,823 & 0,377 & 0,310 & 0,313 & 45 & 540 & $-1,9$ \\
\hline 541 & & & & & & & & nothing to measure & & & & & & & & 541 & \\
\hline 542 & & & & & & & & nothing to measure & & & & & & & & 542 & - \\
\hline 543 & & & & & & & & nothing to measure & & & & & & & & 543 & - \\
\hline 544 & & & & & & & & nothing to measure & & & & & & & & 544 & - \\
\hline 545 & 0,047 & 0,773 & 0,059 & 0,613 & 0,792 & 0,793 & 0,294 & 0,978 & 1,011 & 0,834 & 0,811 & 0,267 & 0,216 & 0,198 & 25 & 545 & $-1,4$ \\
\hline 545 & 0,037 & 0,735 & 0,049 & 0,574 & 0,750 & 0,782 & 0,332 & 0,856 & 1,081 & 0,516 & 0,493 & 0,301 & 0,149 & 0,099 & 95 & 545 & $-1,6$ \\
\hline 546 & 0,076 & 0,867 & 0,081 & 0,707 & 0,939 & 0,815 & 0,194 & 1,000 & 0,888 & 0,987 & 0,791 & 0,313 & 0,248 & 0,297 & 40 & 546 & $-1,6$ \\
\hline 547 & & & & & & & & nothing to measure & & & & & & & & 547 & \\
\hline 548 & & & & & & & & nothing to measure & & & & & & & & 548 & - \\
\hline 549 & & & & & & & & nothing to measure & & & & & & & & 549 & - \\
\hline 550 & & & & & & & & nothing to measure & & & & & & & & 550 & - \\
\hline 551 & & & & & & & & nothing to measure & & & & & & & & 551 & - \\
\hline 552 & & & & & & & & nothing to measure & & & & & & & & 552 & - \\
\hline 553 & & & & & & & & nothing to measure & & & & & & & & 553 & - \\
\hline 554 & & & & & & & & nothing to measure & & & & & & & & 554 & - \\
\hline 555 & & & & & & & & nothing to measure & & & & & & & & 555 & - \\
\hline 556 & & & & & & & & nothing to measure & & & & & & & & 556 & - \\
\hline 557 & & & & & & & & nothing to measure & & & & & & & & 557 & - \\
\hline 558 & & & & & & & & nothing to measure & & & & & & & & 558 & - \\
\hline 559 & & & & & & & & nothing to measure & & & & & & & & 559 & - \\
\hline 560 & & & & & & & & nothing to measure & & & & & & & & 560 & - \\
\hline 561 & 0,037 & 0,613 & 0,042 & 0,481 & 0,882 & 0,784 & 0,246 & 1,000 & 0,902 & 0,735 & 0,588 & 0,252 & 0,149 & 0,198 & 0 & 561 & $-1,3$ \\
\hline 562 & & & & & & & & nothing to measure & & & & & & & & 562 & \\
\hline 563 & 0,025 & 0,602 & 0,032 & 0,359 & 0,769 & 0,597 & 0,465 & 0,850 & 1,085 & 0,509 & 0,424 & 0,248 & 0,105 & 0,099 & 140 & 563 & $-1,3$ \\
\hline 564 & & & & & & & & nothing to measure & & & & & & & & 564 & - \\
\hline 565 & & & & & & & & nothing to measure & & & & & & & & 565 & - \\
\hline 566 & 0,039 & 1,039 & 0,066 & 0,773 & 0,593 & 0,745 & 0,481 & 0,457 & 1,480 & 0,566 & 0,694 & 0,297 & 0,206 & 0,140 & 109 & 566 & $-1,6$ \\
\hline 566 & 0,044 & 0,680 & 0,047 & 0,602 & 0,947 & 0,886 & 0,126 & 1,000 & 0,913 & 0,619 & 0,493 & 0,301 & 0,149 & 0,198 & 84 & 566 & $-1,6$ \\
\hline 566 & 0,027 & 0,602 & 0,032 & 0,348 & 0,846 & 0,578 & 0,449 & 0,935 & 1,034 & 0,483 & 0,353 & 0,267 & 0,094 & 0,099 & 72 & 566 & $-1,4$ \\
\hline 567 & & & & & & & & nothing to measure & & & & & & & & 567 & \\
\hline 568 & & & & & & & & nothing to measure & & & & & & & & 568 & - \\
\hline 569 & & & & & & & & nothing to measure & & & & & & & & 569 & - \\
\hline 570 & & & & & & & & nothing to measure & & & & & & & & 570 & - \\
\hline 571 & & & & & & & & nothing to measure & & & & & & & & 571 & - \\
\hline 572 & & & & & & & & nothing to measure & & & & & & & & 572 & - \\
\hline 573 & & & & & & & & nothing to measure & & & & & & & & 573 & - \\
\hline 574 & & & & & & & & nothing to measure & & & & & & & & 574 & - \\
\hline 575 & 0,027 & 0,481 & 0,027 & 0,320 & 1,000 & 0,667 & 0,333 & 1,000 & 0,826 & 1,077 & 0,745 & 0,178 & 0,133 & 0,198 & 108 & 575 & $-0,8$ \\
\hline 576 & 0,032 & 0,668 & 0,039 & 0,508 & 0,812 & 0,760 & 0,304 & 0,896 & 1,056 & 0,447 & 0,329 & 0,301 & 0,099 & 0,140 & 169 & 576 & $-1,6$ \\
\hline
\end{tabular}




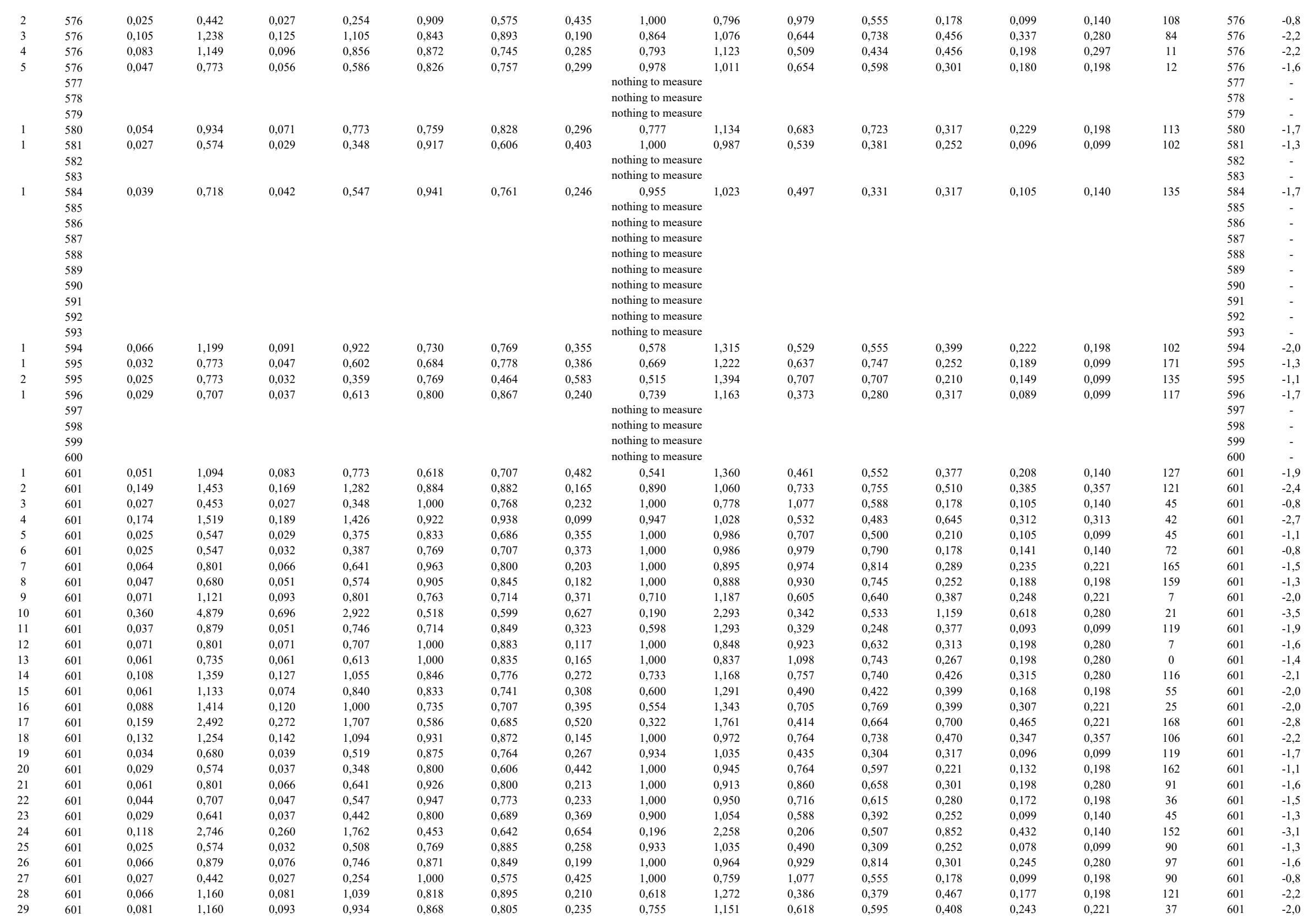




\begin{tabular}{|c|c|c|c|c|c|c|c|c|c|c|c|c|c|c|c|c|c|c|}
\hline 30 & 601 & 0,029 & 0,547 & 0,034 & 0,414 & 0,857 & 0,757 & 0,282 & 1,000 & 0,900 & 0,764 & 0,559 & 0,221 & 0,124 & 0,140 & 135 & 601 & $-1,1$ \\
\hline 31 & 601 & 0,025 & 0,519 & 0,027 & 0,387 & 0,909 & 0,745 & 0,271 & 1,000 & 0,936 & 0,637 & 0,373 & 0,221 & 0,083 & 0,140 & 58 & 601 & $-1,1$ \\
\hline 32 & 601 & 0,142 & 3,547 & 0,323 & 2,071 & 0,439 & 0,584 & 0,698 & 0,142 & 2,654 & 0,204 & 0,416 & 0,942 & 0,392 & 0,198 & 90 & 601 & $-3,2$ \\
\hline 33 & 601 & 0,054 & 0,867 & 0,066 & 0,707 & 0,815 & 0,815 & 0,262 & 0,901 & 1,054 & 0,778 & 0,707 & 0,297 & 0,210 & 0,198 & 135 & 601 & $-1,6$ \\
\hline 34 & 601 & 0,051 & 0,879 & 0,064 & 0,746 & 0,808 & 0,849 & 0,245 & 0,838 & 1,093 & 0,461 & 0,411 & 0,377 & 0,155 & 0,198 & 118 & 601 & $-1,9$ \\
\hline 35 & 601 & 0,147 & 2,426 & 0,265 & 1,696 & 0,556 & 0,699 & 0,537 & 0,314 & 1,784 & 0,340 & 0,573 & 0,743 & 0,426 & 0,198 & 154 & 601 & $-2,9$ \\
\hline 36 & 601 & 0,039 & 1,160 & 0,059 & 0,829 & 0,667 & 0,714 & 0,439 & 0,366 & 1,653 & 0,240 & 0,217 & 0,456 & 0,099 & 0,099 & 90 & 601 & $-2,2$ \\
\hline 37 & 601 & 0,027 & 0,547 & 0,029 & 0,453 & 0,917 & 0,828 & 0,191 & 1,000 & 0,940 & 0,539 & 0,392 & 0,252 & 0,099 & 0,140 & 5 & 601 & $-1,3$ \\
\hline 38 & 601 & 0,039 & 0,574 & 0,042 & 0,414 & 0,941 & 0,721 & 0,285 & 1,000 & 0,818 & 1,019 & 0,671 & 0,221 & 0,149 & 0,198 & 110 & 601 & $-1,1$ \\
\hline 39 & 601 & 0,100 & 1,028 & 0,103 & 0,906 & 0,976 & 0,882 & 0,121 & 1,000 & 0,914 & 0,803 & 0,702 & 0,399 & 0,280 & 0,297 & 48 & 601 & $-2,0$ \\
\hline 1 & 602 & 0,100 & 1,309 & 0,118 & 1,110 & 0,854 & 0,848 & 0,211 & 0,737 & 1,165 & 0,418 & 0,358 & 0,553 & 0,198 & 0,280 & 100 & 602 & $-2,5$ \\
\hline 2 & 602 & 0,029 & 0,801 & 0,047 & 0,641 & 0,632 & 0,800 & 0,419 & 0,576 & 1,318 & 0,340 & 0,289 & 0,332 & 0,096 & 0,099 & 105 & 602 & $-1,7$ \\
\hline \multirow[t]{4}{*}{3} & 602 & 0,025 & 0,680 & 0,037 & 0,481 & 0,667 & 0,707 & 0,444 & 0,667 & 1,225 & 0,374 & 0,352 & 0,289 & 0,102 & 0,099 & 135 & 602 & $-1,5$ \\
\hline & 603 & & & & & & & & nothing to measure & & & & & & & & 603 & \\
\hline & 604 & & & & & & & & nothing to measure & & & & & & & & 604 & - \\
\hline & 605 & & & & & & & & nothing to measure & & & & & & & & 605 & - \\
\hline 1 & 606 & 0,027 & 0,547 & 0,029 & 0,414 & 0,917 & 0,757 & 0,256 & 1,000 & 0,940 & 0,539 & 0,392 & 0,252 & 0,099 & 0,140 & 90 & 606 & $-1,3$ \\
\hline \multirow[t]{7}{*}{2} & 606 & 0,027 & 0,613 & 0,029 & 0,519 & 0,917 & 0,847 & 0,174 & 0,901 & 1,054 & 0,412 & 0,243 & 0,289 & 0,070 & 0,099 & 131 & 606 & $-1,5$ \\
\hline & 607 & & & & & & & & nothing to measure & & & & & & & & 607 & \\
\hline & 608 & & & & & & & & nothing to measure & & & & & & & & 608 & - \\
\hline & 609 & & & & & & & & nothing to measure & & & & & & & & 609 & - \\
\hline & 610 & & & & & & & & nothing to measure & & & & & & & & 610 & - \\
\hline & 611 & & & & & & & & nothing to measure & & & & & & & & 611 & \\
\hline & 612 & & & & & & & & nothing to measure & & & & & & & & 612 & - \\
\hline 1 & 613 & 0,027 & 0,680 & 0,034 & 0,508 & 0,786 & 0,748 & 0,331 & 0,733 & 1,168 & 0,389 & 0,283 & 0,297 & 0,084 & 0,099 & 90 & 613 & $-1,6$ \\
\hline 2 & 613 & 0,027 & 0,586 & 0,034 & 0,453 & 0,786 & 0,773 & 0,312 & 0,987 & 1,006 & 0,824 & 0,686 & 0,204 & 0,140 & 0,099 & 124 & 613 & $-1,0$ \\
\hline 3 & 613 & 0,042 & 0,680 & 0,047 & 0,652 & 0,895 & 0,959 & 0,113 & 1,000 & 0,939 & 0,637 & 0,429 & 0,289 & 0,124 & 0,140 & 147 & 613 & $-1,5$ \\
\hline 4 & 613 & 0,029 & 0,840 & 0,044 & 0,547 & 0,667 & 0,651 & 0,482 & 0,524 & 1,381 & 0,527 & 0,595 & 0,267 & 0,159 & 0,099 & 8 & 613 & $-1,4$ \\
\hline 5 & 613 & 0,037 & 0,879 & 0,054 & 0,680 & 0,682 & 0,773 & 0,391 & 0,598 & 1,293 & 0,367 & 0,392 & 0,357 & 0,140 & 0,140 & 68 & 613 & $-1,8$ \\
\hline 6 & 613 & 0,208 & 2,735 & 0,321 & 1,879 & 0,649 & 0,687 & 0,470 & 0,350 & 1,690 & 0,449 & 0,684 & 0,769 & 0,525 & 0,297 & 169 & 613 & $-2,9$ \\
\hline 7 & 613 & 0,049 & 0,906 & 0,059 & 0,773 & 0,833 & 0,854 & 0,222 & 0,750 & 1,155 & 0,509 & 0,566 & 0,350 & 0,198 & 0,198 & 22 & 613 & $-1,8$ \\
\hline 8 & 613 & 0,027 & 0,481 & 0,027 & 0,348 & 1,000 & 0,724 & 0,276 & 1,000 & 0,826 & 0,700 & 0,447 & 0,221 & 0,099 & 0,140 & 72 & 613 & $-1,1$ \\
\hline 9 & 613 & 0,027 & 0,442 & 0,027 & 0,320 & 1,000 & 0,725 & 0,275 & 1,000 & 0,759 & 1,077 & 0,555 & 0,178 & 0,099 & 0,198 & 90 & 613 & $-0,8$ \\
\hline 10 & 613 & 0,037 & 0,641 & 0,042 & 0,453 & 0,882 & 0,707 & 0,316 & 1,000 & 0,943 & 0,597 & 0,442 & 0,280 & 0,124 & 0,140 & 135 & 613 & $-1,5$ \\
\hline 11 & 613 & 0,032 & 0,508 & 0,032 & 0,320 & 1,000 & 0,631 & 0,369 & 1,000 & 0,803 & 0,974 & 0,485 & 0,204 & 0,099 & 0,198 & 90 & 613 & $-1,0$ \\
\hline 12 & 613 & 0,074 & 1,773 & 0,130 & 1,270 & 0,566 & 0,716 & 0,518 & 0,294 & 1,845 & 0,226 & 0,312 & 0,644 & 0,201 & 0,140 & 65 & 613 & $-2,7$ \\
\hline 13 & 613 & 0,267 & 4,354 & 0,573 & 2,746 & 0,466 & 0,631 & 0,649 & 0,177 & 2,377 & 0,273 & 0,541 & 1,117 & 0,605 & 0,198 & 21 & 613 & $-3,5$ \\
\hline 14 & 613 & 0,091 & 1,414 & 0,115 & 1,000 & 0,787 & 0,707 & 0,362 & 0,570 & 1,325 & 0,725 & 0,744 & 0,399 & 0,297 & 0,221 & 55 & 613 & $-2,0$ \\
\hline 15 & 613 & 0,059 & 0,867 & 0,071 & 0,641 & 0,828 & 0,739 & 0,313 & 0,983 & 1,009 & 0,745 & 0,692 & 0,317 & 0,219 & 0,221 & 120 & 613 & $-1,7$ \\
\hline 16 & 613 & 0,110 & 1,972 & 0,162 & 1,618 & 0,682 & 0,820 & 0,365 & 0,356 & 1,676 & 0,270 & 0,331 & 0,721 & 0,238 & 0,198 & 170 & 613 & $-2,8$ \\
\hline 17 & 613 & 0,088 & 1,254 & 0,110 & 0,895 & 0,800 & 0,714 & 0,349 & 0,705 & 1,191 & 0,881 & 0,832 & 0,357 & 0,297 & 0,198 & 144 & 613 & $-1,8$ \\
\hline 18 & 613 & 0,025 & 0,613 & 0,029 & 0,348 & 0,833 & 0,567 & 0,464 & 0,819 & 1,105 & 0,979 & 0,745 & 0,178 & 0,133 & 0,099 & 28 & 613 & $-0,8$ \\
\hline 19 & 613 & 0,071 & 1,293 & 0,103 & 1,028 & 0,690 & 0,795 & 0,371 & 0,534 & 1,368 & 0,434 & 0,478 & 0,456 & 0,218 & 0,198 & 61 & 613 & $-2,2$ \\
\hline 20 & 613 & 0,027 & 0,481 & 0,029 & 0,348 & 0,917 & 0,724 & 0,288 & 1,000 & 0,826 & 1,077 & 0,745 & 0,178 & 0,133 & 0,198 & 72 & 613 & $-0,8$ \\
\hline 21 & 613 & 0,027 & 0,453 & 0,027 & 0,320 & 1,000 & 0,707 & 0,293 & 1,000 & 0,778 & 1,401 & 0,849 & 0,157 & 0,133 & 0,140 & 135 & 613 & $-0,7$ \\
\hline 22 & 613 & 0,159 & 2,613 & 0,233 & 1,961 & 0,684 & 0,750 & 0,402 & 0,293 & 1,847 & 0,202 & 0,310 & 1,002 & 0,311 & 0,198 & 29 & 613 & $-3,3$ \\
\hline 23 & 613 & 0,152 & 3,061 & 0,287 & 2,542 & 0,530 & 0,830 & 0,500 & 0,204 & 2,215 & 0,115 & 0,204 & 1,296 & 0,264 & 0,140 & 49 & 613 & $-3,7$ \\
\hline 24 & 613 & 0,029 & 0,718 & 0,039 & 0,481 & 0,750 & 0,669 & 0,415 & 0,716 & 1,182 & 0,588 & 0,559 & 0,252 & 0,141 & 0,140 & 72 & 613 & $-1,3$ \\
\hline 25 & 613 & 0,120 & 1,691 & 0,152 & 1,243 & 0,790 & 0,735 & 0,338 & 0,528 & 1,376 & 0,511 & 0,543 & 0,547 & 0,297 & 0,198 & 86 & 613 & $-2,5$ \\
\hline 26 & 613 & 0,049 & 0,829 & 0,056 & 0,508 & 0,870 & 0,613 & 0,408 & 0,897 & 1,056 & 0,749 & 0,667 & 0,289 & 0,192 & 0,140 & 134 & 613 & $-1,5$ \\
\hline 27 & 613 & 0,103 & 1,718 & 0,135 & 1,171 & 0,764 & 0,682 & 0,396 & 0,438 & 1,511 & 0,594 & 0,596 & 0,470 & 0,280 & 0,198 & 154 & 613 & $-2,2$ \\
\hline 28 & 613 & 0,039 & 0,801 & 0,049 & 0,691 & 0,800 & 0,863 & 0,243 & 0,768 & 1,141 & 0,497 & 0,490 & 0,317 & 0,155 & 0,198 & 135 & 613 & $-1,7$ \\
\hline 29 & 613 & 0,260 & 3,149 & 0,392 & 2,105 & 0,662 & 0,669 & 0,473 & 0,329 & 1,743 & 0,461 & 0,677 & 0,847 & 0,574 & 0,357 & 60 & 613 & $-3,1$ \\
\hline 30 & 613 & 0,025 & 0,680 & 0,032 & 0,586 & 0,769 & 0,862 & 0,269 & 0,667 & 1,225 & 0,311 & 0,221 & 0,317 & 0,070 & 0,099 & 28 & 613 & $-1,7$ \\
\hline 31 & 613 & 0,025 & 0,746 & 0,025 & 0,652 & 1,000 & 0,874 & 0,126 & 0,553 & 1,344 & 0,255 & 0,170 & 0,350 & 0,060 & 0,099 & 63 & 613 & $-1,8$ \\
\hline 32 & 613 & 0,086 & 1,961 & 0,145 & 1,282 & 0,593 & 0,653 & 0,534 & 0,280 & 1,889 & 0,325 & 0,460 & 0,579 & 0,267 & 0,198 & 57 & 613 & $-2,5$ \\
\hline 33 & 613 & 0,037 & 0,895 & 0,054 & 0,536 & 0,682 & 0,599 & 0,512 & 0,577 & 1,317 & 0,360 & 0,275 & 0,360 & 0,099 & 0,140 & 167 & 613 & $-1,8$ \\
\hline 34 & 613 & 0,025 & 0,481 & 0,027 & 0,320 & 0,909 & 0,667 & 0,346 & 1,000 & 0,866 & 0,979 & 0,614 & 0,178 & 0,110 & 0,140 & 0 & 613 & $-0,8$ \\
\hline
\end{tabular}




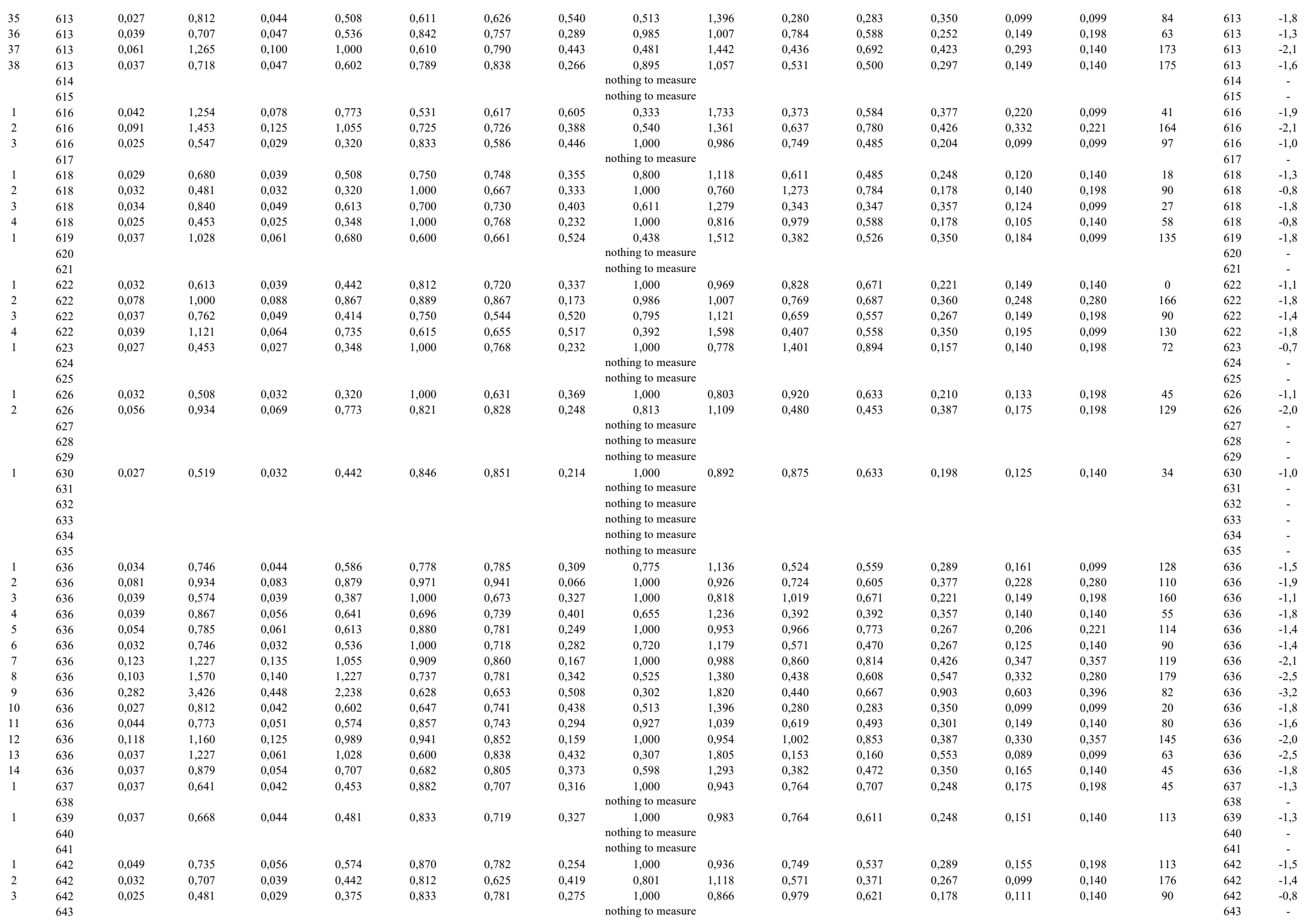


nothing to measure

$\begin{array}{lllll}0,029 & 0,586 & 0,037 & 0,508 & 0,800\end{array}$

0,488

1,432 $\begin{array}{llll}0,797 & 0,218 & 0,869 & 1,336 \\ 0,517 & 0,514 & 0,073\end{array}$ $\begin{array}{lll}0,517 & 0,514 & 0,672 \\ 0,847 & 0,187 & 1,00\end{array}$ nothing to measure

$\begin{array}{lllll}0,034 & 0,801 & 0,044 & 0,613 & 0,778 \\ 0,029 & 0,547 & 0,029 & 0,387 & 1,000\end{array}$

nothing to measure
nothing to measure

nothing to measure
nothing to measure

nothing to measure

nothing to measure

nothing to measure

nothing to measure

nothing to measure

nothing to measure

nothing to measure

0,718

$\begin{array}{ll}0,125 & 0,140 \\ & \\ 0,094 & 0,099 \\ 0,198 & 0,221 \\ 0,248 & 0,280 \\ 0,141 & 0,099 \\ 0,089 & 0,099 \\ 0,219 & 0,221 \\ 0,140 & 0,099 \\ 0,099 & 0,099 \\ 0,133 & 0,140 \\ 0,105 & 0,099 \\ 0,155 & 0,140 \\ 0,210 & 0,221\end{array}$




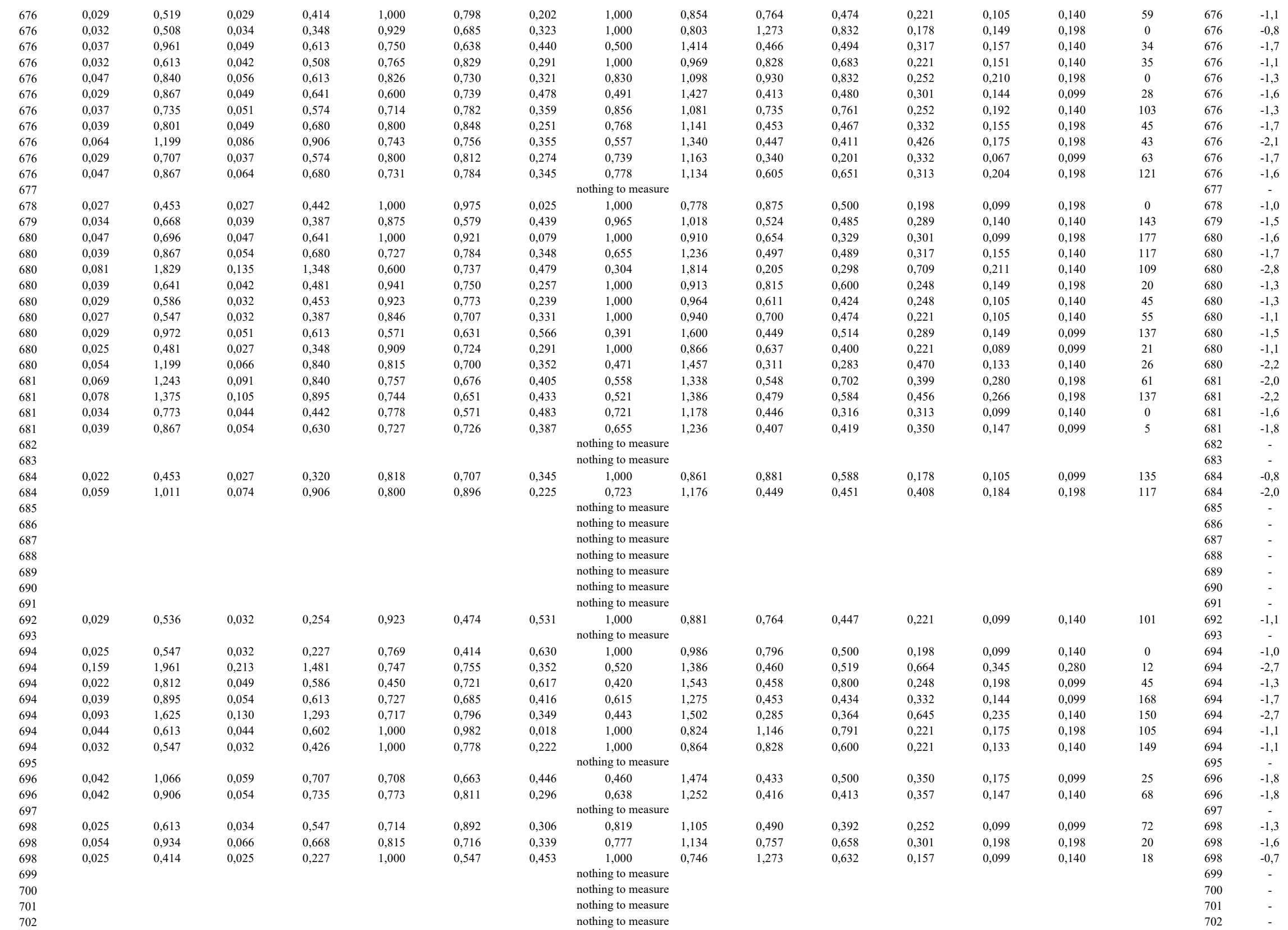




\begin{tabular}{|c|c|c|c|c|c|c|c|c|c|c|c|c|c|c|c|c|c|c|}
\hline 1 & $\begin{array}{l}703 \\
704 \\
705 \\
706\end{array}$ & 0,069 & 1,227 & 0,091 & 0,922 & 0,757 & 0,752 & 0,347 & $\begin{array}{l}0,573 \\
\text { nothing to measure } \\
\text { nothing to measure } \\
\text { nothing to measure }\end{array}$ & 1,321 & 0,419 & 0,434 & 0,456 & 0,198 & 0,221 & 84 & $\begin{array}{l}703 \\
704 \\
705 \\
706\end{array}$ & $\begin{array}{c}-2,2 \\
- \\
- \\
-\end{array}$ \\
\hline 1 & 707 & 0,034 & 0,586 & 0,039 & 0,519 & 0,875 & 0,887 & 0,169 & 1,000 & 0,892 & 0,891 & 0,632 & 0,221 & 0,140 & 0,140 & 137 & 707 & $-1,1$ \\
\hline 2 & 707 & 0,042 & 0,801 & 0,056 & 0,641 & 0,739 & 0,800 & 0,329 & 0,816 & 1,107 & 0,637 & 0,584 & 0,289 & 0,168 & 0,140 & 35 & 707 & $-1,5$ \\
\hline \multirow[t]{2}{*}{3} & 707 & 0,066 & 1,105 & 0,078 & 0,972 & 0,844 & 0,880 & 0,197 & 0,681 & 1,212 & 0,304 & 0,245 & 0,526 & 0,129 & 0,140 & 41 & 707 & $-2,4$ \\
\hline & $\begin{array}{l}708 \\
709\end{array}$ & & & & & & & & $\begin{array}{l}\text { nothing to measure } \\
\text { nothing to measure }\end{array}$ & & & & & & & & $\begin{array}{l}708 \\
709\end{array}$ & - \\
\hline \multirow[t]{9}{*}{1} & 710 & 0,061 & 1,094 & 0,081 & 0,840 & 0,758 & 0,768 & 0,336 & 0,643 & 1,247 & 0,398 & 0,369 & 0,443 & 0,163 & 0,198 & 112 & 710 & $-2,1$ \\
\hline & 711 & & & & & & & & nothing to measure & & & & & & & & 711 & \\
\hline & 712 & & & & & & & & nothing to measure & & & & & & & & 712 & - \\
\hline & 713 & & & & & & & & nothing to measure & & & & & & & & 713 & - \\
\hline & 714 & & & & & & & & nothing to measure & & & & & & & & 714 & - \\
\hline & 715 & & & & & & & & nothing to measure & & & & & & & & 715 & - \\
\hline & 716 & & & & & & & & nothing to measure & & & & & & & & 716 & - \\
\hline & 717 & & & & & & & & nothing to measure & & & & & & & & 717 & - \\
\hline & 718 & & & & & & & & nothing to measure & & & & & & & & 718 & - \\
\hline \multirow{5}{*}{1} & 719 & 0,025 & 0,547 & 0,027 & 0,414 & 0,909 & 0,757 & 0,259 & 1,000 & 0,986 & 0,439 & 0,294 & 0,267 & 0,078 & 0,099 & 72 & 719 & $-1,4$ \\
\hline & 720 & & & & & & & & nothing to measure & & & & & & & & 720 & - \\
\hline & 721 & & & & & & & & nothing to measure & & & & & & & & 721 & - \\
\hline & 722 & & & & & & & & nothing to measure & & & & & & & & 722 & - \\
\hline & 723 & & & & & & & & nothing to measure & & & & & & & & 723 & - \\
\hline 1 & 724 & 0,025 & 0,481 & 0,025 & 0,293 & 1,000 & 0,609 & 0,391 & 1,000 & 0,866 & 0,707 & 0,500 & 0,210 & 0,105 & 0,140 & 135 & 724 & $-1,1$ \\
\hline 2 & 724 & 0,027 & 0,613 & 0,029 & 0,426 & 0,917 & 0,694 & 0,317 & 0,901 & 1,054 & 0,438 & 0,317 & 0,280 & 0,089 & 0,099 & 148 & 724 & $-1,5$ \\
\hline 3 & 724 & 0,044 & 1,105 & 0,064 & 0,812 & 0,692 & 0,735 & 0,406 & 0,454 & 1,484 & 0,270 & 0,298 & 0,456 & 0,136 & 0,099 & 120 & 724 & $-2,2$ \\
\hline \multirow[t]{10}{*}{4} & 724 & 0,039 & 0,867 & 0,049 & 0,602 & 0,800 & 0,694 & 0,366 & 0,655 & 1,236 & 0,551 & 0,493 & 0,301 & 0,149 & 0,140 & 76 & 724 & $-1,6$ \\
\hline & 725 & & & & & & & & nothing to measure & & & & & & & & 725 & - \\
\hline & 726 & & & & & & & & nothing to measure & & & & & & & & 726 & - \\
\hline & 727 & & & & & & & & nothing to measure & & & & & & & & 727 & - \\
\hline & 728 & & & & & & & & nothing to measure & & & & & & & & 728 & - \\
\hline & 729 & & & & & & & & nothing to measure & & & & & & & & 729 & - \\
\hline & 730 & & & & & & & & nothing to measure & & & & & & & & 730 & - \\
\hline & 731 & & & & & & & & nothing to measure & & & & & & & & 731 & - \\
\hline & 732 & & & & & & & & nothing to measure & & & & & & & & 732 & - \\
\hline & 733 & & & & & & & & nothing to measure & & & & & & & & 733 & - \\
\hline 1 & 734 & 0,025 & 0,641 & 0,032 & 0,481 & 0,769 & 0,750 & 0,340 & 0,750 & 1,155 & 0,318 & 0,212 & 0,313 & 0,067 & 0,099 & 152 & 734 & $-1,6$ \\
\hline 2 & 734 & 0,034 & 0,934 & 0,049 & 0,641 & 0,700 & 0,686 & 0,434 & 0,495 & 1,422 & 0,343 & 0,385 & 0,357 & 0,137 & 0,099 & 39 & 734 & $-1,8$ \\
\hline 3 & 734 & 0,027 & 0,773 & 0,039 & 0,547 & 0,688 & 0,707 & 0,428 & 0,566 & 1,329 & 0,412 & 0,381 & 0,289 & 0,110 & 0,099 & 62 & 734 & $-1,5$ \\
\hline 4 & 734 & 0,034 & 0,680 & 0,037 & 0,613 & 0,933 & 0,902 & 0,118 & 0,934 & 1,035 & 0,435 & 0,331 & 0,317 & 0,105 & 0,140 & 45 & 734 & $-1,7$ \\
\hline 5 & 734 & 0,025 & 0,508 & 0,027 & 0,227 & 0,909 & 0,446 & 0,562 & 1,000 & 0,916 & 0,637 & 0,447 & 0,221 & 0,099 & 0,140 & 135 & 734 & $-1,1$ \\
\hline 6 & 734 & 0,027 & 0,547 & 0,032 & 0,320 & 0,846 & 0,586 & 0,442 & 1,000 & 0,940 & 0,700 & 0,474 & 0,221 & 0,105 & 0,140 & 108 & 734 & $-1,1$ \\
\hline 7 & 734 & 0,029 & 0,547 & 0,034 & 0,387 & 0,857 & 0,707 & 0,326 & 1,000 & 0,900 & 0,764 & 0,495 & 0,221 & 0,110 & 0,140 & 32 & 734 & $-1,1$ \\
\hline 8 & 734 & 0,027 & 0,668 & 0,034 & 0,481 & 0,786 & 0,719 & 0,353 & 0,758 & 1,148 & 0,412 & 0,334 & 0,289 & 0,096 & 0,099 & 122 & 734 & $-1,5$ \\
\hline 9 & 734 & 0,074 & 1,320 & 0,093 & 1,133 & 0,789 & 0,858 & 0,254 & 0,530 & 1,374 & 0,256 & 0,289 & 0,604 & 0,175 & 0,198 & 59 & 734 & $-2,6$ \\
\hline 10 & 734 & 0,088 & 1,469 & 0,132 & 1,133 & 0,667 & 0,771 & 0,404 & 0,514 & 1,395 & 0,473 & 0,619 & 0,488 & 0,302 & 0,198 & 126 & 734 & $-2,3$ \\
\hline 11 & 734 & 0,044 & 0,972 & 0,059 & 0,668 & 0,750 & 0,687 & 0,400 & 0,586 & 1,306 & 0,353 & 0,372 & 0,399 & 0,149 & 0,140 & 99 & 734 & $-2,0$ \\
\hline 12 & 734 & 0,049 & 1,066 & 0,066 & 0,735 & 0,741 & 0,689 & 0,405 & 0,542 & 1,359 & 0,688 & 0,676 & 0,301 & 0,204 & 0,198 & 109 & 734 & $-1,6$ \\
\hline 13 & 734 & 0,083 & 2,105 & 0,169 & 1,536 & 0,493 & 0,729 & 0,575 & 0,236 & 2,057 & 0,196 & 0,332 & 0,736 & 0,244 & 0,140 & 74 & 734 & $-2,9$ \\
\hline 14 & 734 & 0,039 & 0,906 & 0,054 & 0,696 & 0,727 & 0,768 & 0,358 & 0,600 & 1,291 & 0,407 & 0,424 & 0,350 & 0,149 & 0,140 & 175 & 734 & $-1,8$ \\
\hline \multirow{4}{*}{16} & 734 & 0,027 & 0,613 & 0,034 & 0,426 & 0,786 & 0,694 & 0,374 & 0,901 & 1,054 & 0,560 & 0,424 & 0,248 & 0,105 & 0,099 & 126 & 734 & $-1,3$ \\
\hline & 734 & 0,027 & 0,707 & 0,039 & 0,453 & 0,688 & 0,641 & 0,476 & 0,677 & 1,215 & 0,700 & 0,621 & 0,221 & 0,137 & 0,140 & 0 & 734 & $-1,1$ \\
\hline & 735 & & & & & & & & nothing to measure & & & & & & & & 735 & 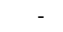 \\
\hline & 736 & & & & & & & & nothing to measure & & & & & & & & 736 & - \\
\hline 1 & 737 & 0,029 & 0,746 & 0,039 & 0,613 & 0,750 & 0,822 & 0,307 & 0,664 & 1,227 & 0,294 & 0,270 & 0,357 & 0,096 & 0,099 & 144 & 737 & $-1,8$ \\
\hline 2 & 737 & 0,039 & 0,762 & 0,054 & 0,574 & 0,727 & 0,754 & 0,367 & 0,848 & 1,086 & 0,599 & 0,614 & 0,289 & 0,177 & 0,099 & 72 & 737 & $-1,5$ \\
\hline 3 & 737 & 0,025 & 0,641 & 0,034 & 0,508 & 0,714 & 0,793 & 0,353 & 0,750 & 1,155 & 0,439 & 0,360 & 0,267 & 0,096 & 0,099 & 0 & 737 & $-1,4$ \\
\hline 4 & 737 & 0,025 & 0,481 & 0,027 & 0,387 & 0,909 & 0,805 & 0,215 & 1,000 & 0,866 & 0,979 & 0,555 & 0,178 & 0,099 & 0,140 & 108 & 737 & $-0,8$ \\
\hline
\end{tabular}




\begin{tabular}{|c|c|c|c|c|c|c|c|c|c|c|c|c|c|c|c|c|}
\hline 737 & 0,262 & 3,851 & 0,441 & 2,293 & 0,594 & 0,595 & 0,573 & 0,222 & 2,121 & 0,327 & 0,578 & 1,010 & 0,584 & 0,198 & 102 & 737 \\
\hline 737 & 0,027 & 0,613 & 0,034 & 0,414 & 0,786 & 0,675 & 0,389 & 0,901 & 1,054 & 0,824 & 0,691 & 0,204 & 0,141 & 0,099 & 27 & 737 \\
\hline 737 & 0,037 & 1,000 & 0,056 & 0,602 & 0,652 & 0,602 & 0,529 & 0,462 & 1,471 & 0,298 & 0,344 & 0,396 & 0,136 & 0,099 & 20 & 737 \\
\hline 737 & 0,047 & 1,171 & 0,064 & 0,812 & 0,731 & 0,693 & 0,408 & 0,426 & 1,531 & 0,327 & 0,329 & 0,426 & 0,140 & 0,140 & 129 & 737 \\
\hline 737 & 0,027 & 0,801 & 0,039 & 0,481 & 0,688 & 0,600 & 0,508 & 0,528 & 1,376 & 0,483 & 0,496 & 0,267 & 0,132 & 0,099 & 76 & 737 \\
\hline 737 & 0,037 & 0,840 & 0,049 & 0,602 & 0,750 & 0,717 & 0,378 & 0,655 & 1,236 & 0,659 & 0,657 & 0,267 & 0,175 & 0,140 & 118 & 737 \\
\hline 737 & 0,037 & 0,785 & 0,042 & 0,680 & 0,882 & 0,866 & 0,178 & 0,750 & 1,155 & 0,367 & 0,294 & 0,357 & 0,105 & 0,140 & 131 & 737 \\
\hline 737 & 0,032 & 0,680 & 0,042 & 0,442 & 0,765 & 0,650 & 0,422 & 0,867 & 1,074 & 0,662 & 0,537 & 0,248 & 0,133 & 0,140 & 45 & 737 \\
\hline 737 & 0,032 & 0,934 & 0,051 & 0,668 & 0,619 & 0,716 & 0,475 & 0,459 & 1,476 & 0,414 & 0,466 & 0,313 & 0,146 & 0,099 & 11 & 737 \\
\hline 737 & 0,039 & 0,906 & 0,056 & 0,718 & 0,696 & 0,793 & 0,368 & 0,600 & 1,291 & 0,334 & 0,272 & 0,387 & 0,105 & 0,099 & 131 & 737 \\
\hline 737 & 0,047 & 1,188 & 0,083 & 0,801 & 0,559 & 0,674 & 0,548 & 0,415 & 1,553 & 0,372 & 0,571 & 0,399 & 0,228 & 0,140 & 43 & 737 \\
\hline 737 & 0,054 & 1,387 & 0,096 & 0,934 & 0,564 & 0,673 & 0,545 & 0,352 & 1,685 & 0,311 & 0,422 & 0,470 & 0,198 & 0,140 & 11 & 737 \\
\hline 738 & & & & & & & & nothing to measure & & & & & & & & 738 \\
\hline 739 & 0,034 & 0,840 & 0,061 & 0,680 & 0,560 & 0,809 & 0,480 & 0,611 & 1,279 & 0,482 & 0,658 & 0,301 & 0,198 & 0,099 & 167 & 739 \\
\hline 740 & 0,034 & 0,641 & 0,039 & 0,481 & 0,875 & 0,750 & 0,280 & 1,000 & 0,976 & 0,615 & 0,557 & 0,267 & 0,149 & 0,198 & 73 & 740 \\
\hline 740 & 0,037 & 0,707 & 0,044 & 0,519 & 0,833 & 0,735 & 0,313 & 0,924 & 1,040 & 0,562 & 0,485 & 0,289 & 0,140 & 0,198 & 143 & 740 \\
\hline 740 & 0,025 & 0,481 & 0,027 & 0,348 & 0,909 & 0,724 & 0,291 & 1,000 & 0,866 & 0,979 & 0,588 & 0,178 & 0,105 & 0,099 & 108 & 740 \\
\hline 741 & & & & & & & & nothing to measure & & & & & & & & 741 \\
\hline 742 & & & & & & & & nothing to measure & & & & & & & & 742 \\
\hline 743 & & & & & & & & nothing to measure & & & & & & & & 743 \\
\hline 744 & & & & & & & & nothing to measure & & & & & & & & 744 \\
\hline 745 & & & & & & & & nothing to measure & & & & & & & & 745 \\
\hline 746 & 0,032 & 0,824 & 0,032 & 0,718 & 1,000 & 0,872 & 0,128 & 0,590 & 1,302 & 0,230 & 0,083 & 0,420 & 0,035 & 0,099 & 135 & 746 \\
\hline 747 & 0,047 & 1,133 & 0,081 & 0,707 & 0,576 & 0,624 & 0,567 & 0,456 & 1,481 & 0,590 & 0,781 & 0,317 & 0,248 & 0,099 & 158 & 747 \\
\hline 748 & & & & & & & & nothing to measure & & & & & & & & 748 \\
\hline 749 & & & & & & & & nothing to measure & & & & & & & & 749 \\
\hline 750 & & & & & & & & nothing to measure & & & & & & & & 750 \\
\hline 751 & & & & & & & & nothing to measure & & & & & & & & 751 \\
\hline 752 & & & & & & & & nothing to measure & & & & & & & & 752 \\
\hline 753 & & & & & & & & nothing to measure & & & & & & & & 753 \\
\hline 754 & & & & & & & & nothing to measure & & & & & & & & 754 \\
\hline 755 & & & & & & & & nothing to measure & & & & & & & & 755 \\
\hline 756 & & & & & & & & nothing to measure & & & & & & & & 756 \\
\hline 757 & & & & & & & & nothing to measure & & & & & & & & 757 \\
\hline 758 & & & & & & & & nothing to measure & & & & & & & & 758 \\
\hline 759 & & & & & & & & nothing to measure & & & & & & & & 759 \\
\hline 760 & & & & & & & & nothing to measure & & & & & & & & 760 \\
\hline 761 & 0,074 & 1,028 & 0,083 & 0,867 & 0,882 & 0,844 & 0,195 & 0,875 & 1,069 & 0,516 & 0,468 & 0,426 & 0,199 & 0,198 & 110 & 761 \\
\hline 762 & & & & & & & & nothing to measure & & & & & & & & 762 \\
\hline 763 & & & & & & & & nothing to measure & & & & & & & & 763 \\
\hline 764 & & & & & & & & nothing to measure & & & & & & & & 764 \\
\hline 765 & & & & & & & & nothing to measure & & & & & & & & 765 \\
\hline 766 & & & & & & & & nothing to measure & & & & & & & & 766 \\
\hline 767 & & & & & & & & nothing to measure & & & & & & & & 767 \\
\hline 768 & 0,029 & 0,547 & 0,032 & 0,414 & 0,923 & 0,757 & 0,254 & 1,000 & 0,900 & 0,611 & 0,400 & 0,248 & 0,099 & 0,140 & 108 & 768 \\
\hline 768 & 0,025 & 0,414 & 0,025 & 0,359 & 1,000 & 0,867 & 0,133 & 1,000 & 0,746 & 1,273 & 0,632 & 0,157 & 0,099 & 0,140 & 90 & 768 \\
\hline 768 & 0,032 & 0,574 & 0,034 & 0,414 & 0,929 & 0,721 & 0,288 & 1,000 & 0,908 & 0,828 & 0,597 & 0,221 & 0,132 & 0,140 & 79 & 768 \\
\hline 769 & & & & & & & & nothing to measure & & & & & & & & 769 \\
\hline 770 & 0,037 & 0,574 & 0,039 & 0,426 & 0,937 & 0,741 & 0,267 & 1,000 & 0,845 & 0,955 & 0,637 & 0,221 & 0,141 & 0,198 & 13 & 770 \\
\hline 770 & 0,049 & 0,785 & 0,056 & 0,735 & 0,870 & 0,936 & 0,145 & 1,000 & 1,000 & 0,749 & 0,606 & 0,289 & 0,175 & 0,198 & 48 & 770 \\
\hline 771 & & & & & & & & nothing to measure & & & & & & & & 771 \\
\hline 772 & & & & & & & & nothing to measure & & & & & & & & 772 \\
\hline 773 & & & & & & & & nothing to measure & & & & & & & & 773 \\
\hline 774 & & & & & & & & nothing to measure & & & & & & & & 774 \\
\hline 775 & & & & & & & & nothing to measure & & & & & & & & 775 \\
\hline 776 & & & & & & & & nothing to measure & & & & & & & & 776 \\
\hline 777 & & & & & & & & nothing to measure & & & & & & & & 777 \\
\hline 778 & & & & & & & & nothing to measure & & & & & & & & 778 \\
\hline
\end{tabular}




\begin{tabular}{|c|c|c|c|c|c|c|c|c|c|c|c|c|c|c|c|c|c|}
\hline 779 & & & & & & & & nothing to measure & & & & & & & & 779 & - \\
\hline 780 & & & & & & & & nothing to measure & & & & & & & & 780 & - \\
\hline 781 & & & & & & & & nothing to measure & & & & & & & & 781 & \\
\hline 782 & 0,034 & 0,906 & 0,047 & 0,547 & 0,737 & 0,604 & 0,476 & 0,525 & 1,380 & 0,524 & 0,606 & 0,289 & 0,175 & 0,099 & 39 & 782 & $-1,5$ \\
\hline 783 & & & & & & & & nothing to measure & & & & & & & & 783 & - \\
\hline 784 & & & & & & & & nothing to measure & & & & & & & & 784 & - \\
\hline 785 & & & & & & & & nothing to measure & & & & & & & & 785 & - \\
\hline 786 & & & & & & & & nothing to measure & & & & & & & & 786 & - \\
\hline 787 & & & & & & & & nothing to measure & & & & & & & & 787 & - \\
\hline 788 & & & & & & & & nothing to measure & & & & & & & & 788 & - \\
\hline 789 & & & & & & & & nothing to measure & & & & & & & & 789 & - \\
\hline 790 & 0,039 & 0,812 & 0,042 & 0,680 & 0,941 & 0,837 & 0,174 & 0,747 & 1,157 & 0,334 & 0,272 & 0,387 & 0,105 & 0,140 & 49 & 790 & $-2,0$ \\
\hline 790 & 0,032 & 0,961 & 0,051 & 0,547 & 0,619 & 0,569 & 0,575 & 0,433 & 1,519 & 0,487 & 0,614 & 0,289 & 0,177 & 0,099 & 90 & 790 & $-1,5$ \\
\hline 790 & 0,047 & 0,906 & 0,061 & 0,680 & 0,760 & 0,750 & 0,347 & 0,713 & 1,185 & 0,484 & 0,492 & 0,350 & 0,172 & 0,140 & 81 & 790 & $-1,8$ \\
\hline 790 & 0,025 & 0,547 & 0,029 & 0,348 & 0,833 & 0,636 & 0,400 & 1,000 & 0,986 & 0,637 & 0,425 & 0,221 & 0,094 & 0,140 & 45 & 790 & $-1,1$ \\
\hline 791 & & & & & & & & nothing to measure & & & & & & & & 791 & - \\
\hline 792 & 0,037 & 0,707 & 0,044 & 0,508 & 0,833 & 0,719 & 0,327 & 0,924 & 1,040 & 0,659 & 0,529 & 0,267 & 0,141 & 0,140 & 174 & 792 & $-1,4$ \\
\hline 792 & 0,025 & 0,453 & 0,025 & 0,348 & 1,000 & 0,768 & 0,232 & 1,000 & 0,816 & 0,749 & 0,485 & 0,204 & 0,099 & 0,140 & 90 & 792 & $-1,0$ \\
\hline 793 & 0,081 & 1,934 & 0,149 & 1,453 & 0,541 & 0,751 & 0,522 & 0,272 & 1,918 & 0,247 & 0,394 & 0,645 & 0,254 & 0,140 & 104 & 793 & $-2,7$ \\
\hline 793 & 0,042 & 0,735 & 0,044 & 0,574 & 0,944 & 0,782 & 0,225 & 0,970 & 1,015 & 0,481 & 0,400 & 0,332 & 0,133 & 0,198 & 63 & 793 & $-1,7$ \\
\hline 794 & & & & & & & & nothing to measure & & & & & & & & 794 & \\
\hline 795 & & & & & & & & nothing to measure & & & & & & & & 795 & - \\
\hline 796 & & & & & & & & nothing to measure & & & & & & & & 796 & - \\
\hline 797 & & & & & & & & nothing to measure & & & & & & & & 797 & - \\
\hline 798 & & & & & & & & nothing to measure & & & & & & & & 798 & - \\
\hline 799 & & & & & & & & nothing to measure & & & & & & & & 799 & - \\
\hline 800 & 0,025 & 0,586 & 0,032 & 0,481 & 0,769 & 0,820 & 0,292 & 0,898 & 1,055 & 0,749 & 0,651 & 0,204 & 0,133 & 0,099 & 90 & 800 & $-1,0$ \\
\hline 800 & 0,025 & 0,547 & 0,027 & 0,254 & 0,909 & 0,465 & 0,543 & 1,000 & 0,986 & 0,637 & 0,447 & 0,221 & 0,099 & 0,140 & 111 & 800 & $-1,1$ \\
\hline 801 & & & & & & & & nothing to measure & & & & & & & & 801 & - \\
\hline 802 & & & & & & & & nothing to measure & & & & & & & & 802 & - \\
\hline 803 & & & & & & & & nothing to measure & & & & & & & & 803 & - \\
\hline 804 & & & & & & & & nothing to measure & & & & & & & & 804 & - \\
\hline 805 & & & & & & & & nothing to measure & & & & & & & & 805 & - \\
\hline 806 & & & & & & & & nothing to measure & & & & & & & & 806 & - \\
\hline 807 & & & & & & & & nothing to measure & & & & & & & & 807 & - \\
\hline 808 & & & & & & & & nothing to measure & & & & & & & & 808 & - \\
\hline 809 & & & & & & & & nothing to measure & & & & & & & & 809 & - \\
\hline 810 & & & & & & & & nothing to measure & & & & & & & & 810 & - \\
\hline 811 & & & & & & & & nothing to measure & & & & & & & & 811 & - \\
\hline 812 & & & & & & & & nothing to measure & & & & & & & & 812 & - \\
\hline 813 & & & & & & & & nothing to measure & & & & & & & & 813 & - \\
\hline 814 & & & & & & & & nothing to measure & & & & & & & & 814 & - \\
\hline 815 & & & & & & & & nothing to measure & & & & & & & & 815 & - \\
\hline 816 & 0,061 & 0,812 & 0,066 & 0,735 & 0,926 & 0,904 & 0,121 & 1,000 & 0,926 & 0,776 & 0,552 & 0,317 & 0,175 & 0,221 & 48 & 816 & $-1,7$ \\
\hline 816 & 0,027 & 0,481 & 0,029 & 0,387 & 0,917 & 0,805 & 0,212 & 1,000 & 0,826 & 0,824 & 0,485 & 0,204 & 0,099 & 0,198 & 0 & 816 & $-1,0$ \\
\hline 816 & 0,064 & 1,254 & 0,083 & 0,829 & 0,765 & 0,661 & 0,413 & 0,509 & 1,401 & 0,637 & 0,693 & 0,357 & 0,248 & 0,140 & 170 & 816 & $-1,8$ \\
\hline 816 & 0,071 & 0,934 & 0,081 & 0,773 & 0,879 & 0,828 & 0,210 & 1,000 & 0,988 & 0,637 & 0,581 & 0,377 & 0,219 & 0,221 & 115 & 816 & $-1,9$ \\
\hline 816 & 0,034 & 0,735 & 0,042 & 0,348 & 0,824 & 0,474 & 0,555 & 0,799 & 1,119 & 0,713 & 0,600 & 0,248 & 0,149 & 0,198 & 153 & 816 & $-1,3$ \\
\hline 816 & 0,108 & 2,879 & 0,206 & 1,497 & 0,524 & 0,520 & 0,676 & 0,164 & 2,473 & 0,324 & 0,532 & 0,651 & 0,347 & 0,198 & 12 & 816 & $-2,7$ \\
\hline 816 & 0,042 & 0,922 & 0,054 & 0,547 & 0,773 & 0,593 & 0,466 & 0,615 & 1,275 & 0,528 & 0,559 & 0,317 & 0,177 & 0,099 & 150 & 816 & $-1,7$ \\
\hline 816 & 0,039 & 0,773 & 0,049 & 0,602 & 0,800 & 0,778 & 0,299 & 0,824 & 1,102 & 0,551 & 0,479 & 0,301 & 0,144 & 0,140 & 90 & 816 & $-1,6$ \\
\hline 816 & 0,034 & 0,895 & 0,047 & 0,547 & 0,737 & 0,611 & 0,469 & 0,538 & 1,363 & 0,713 & 0,696 & 0,248 & 0,172 & 0,099 & 144 & 816 & $-1,3$ \\
\hline 816 & 0,049 & 0,707 & 0,054 & 0,613 & 0,909 & 0,867 & 0,161 & 1,000 & 0,901 & 0,749 & 0,606 & 0,289 & 0,175 & 0,198 & 45 & 816 & $-1,5$ \\
\hline 816 & 0,074 & 0,961 & 0,091 & 0,829 & 0,811 & 0,862 & 0,234 & 1,000 & 1,000 & 0,764 & 0,728 & 0,350 & 0,255 & 0,221 & 22 & 816 & $-1,8$ \\
\hline 816 & 0,069 & 1,066 & 0,083 & 0,934 & 0,824 & 0,876 & 0,216 & 0,758 & 1,148 & 0,364 & 0,357 & 0,490 & 0,175 & 0,198 & 37 & 816 & $-2,3$ \\
\hline 816 & 0,027 & 0,519 & 0,029 & 0,414 & 0,917 & 0,798 & 0,219 & 1,000 & 0,892 & 0,824 & 0,651 & 0,204 & 0,133 & 0,140 & 57 & 816 & $-1,0$ \\
\hline 816 & 0,034 & 0,801 & 0,047 & 0,547 & 0,737 & 0,683 & 0,412 & 0,672 & 1,220 & 0,615 & 0,619 & 0,267 & 0,165 & 0,099 & 139 & 816 & $-1,4$ \\
\hline 816 & 0,152 & 2,210 & 0.211 & 1.602 & 0,721 & 0,725 & 0.392 & 0.391 & 1.600 & 0,385 & 0.466 & 0,709 & 0.331 & 0.221 & 70 & 816 & -2.8 \\
\hline
\end{tabular}




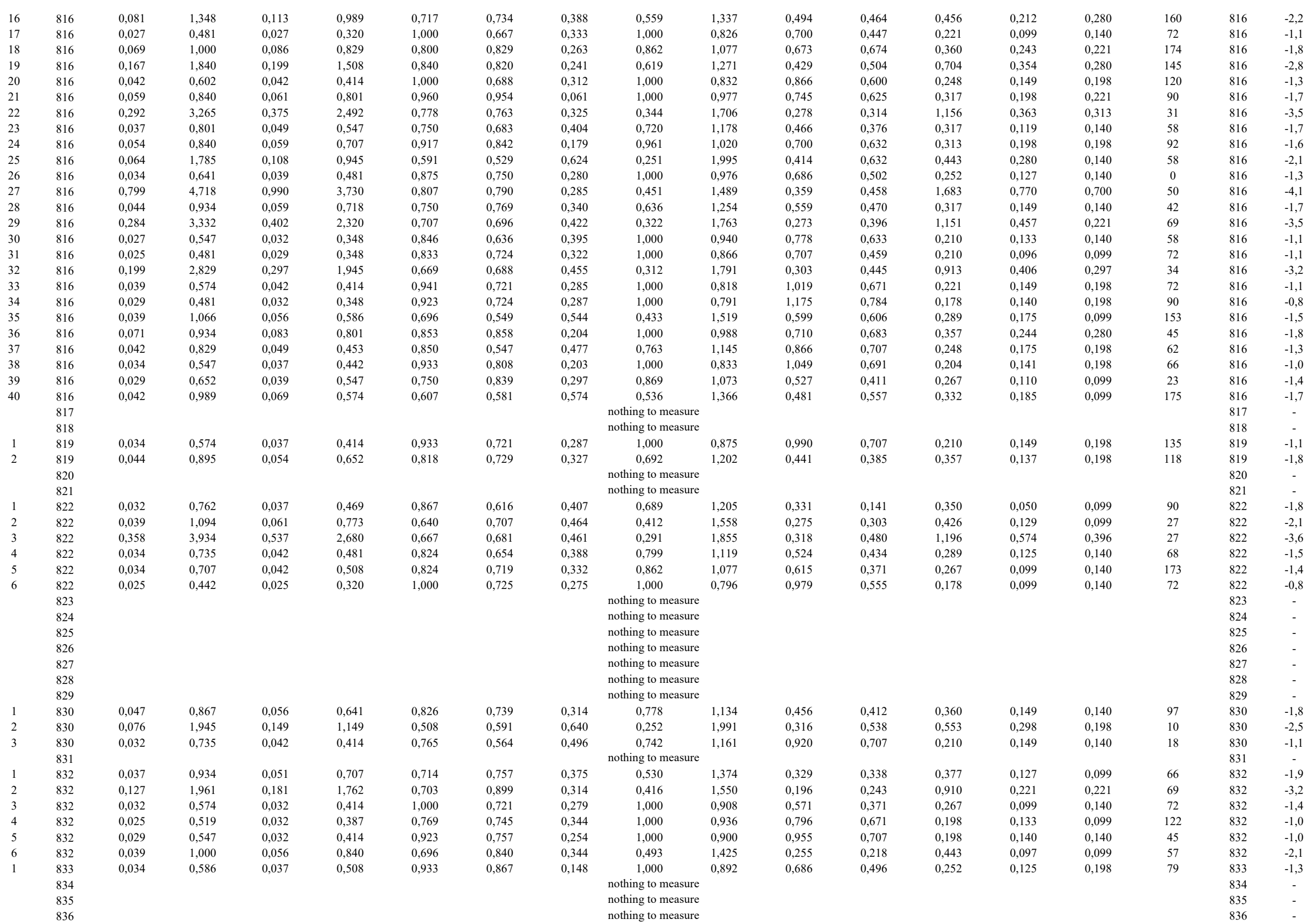




\begin{tabular}{|c|c|c|c|c|c|}
\hline 0,123 & 1,227 & 0,130 & 1,121 & 0,943 & 0,914 \\
\hline 0,027 & 0,707 & 0,042 & 0,508 & 0,647 & 0,719 \\
\hline 0,037 & 0,680 & 0,044 & 0,508 & 0,833 & 0,748 \\
\hline 0,029 & 0,519 & 0,029 & 0,453 & 1,000 & 0,872 \\
\hline 0,061 & 1,055 & 0,086 & 0,801 & 0,714 & 0,759 \\
\hline 0,086 & 1,028 & 0,096 & 0.867 & 0,897 & 0,844 \\
\hline 0,044 & 0,680 & 0,049 & 0,547 & 0,900 & 0,805 \\
\hline 0,032 & 0,707 & 0,039 & 0,453 & 0,812 & 0,641 \\
\hline 0,029 & 0,680 & 0,037 & 0,453 & 0,800 & 0,667 \\
\hline 0,025 & 0,641 & 0,032 & 0,442 & 0,769 & 0,689 \\
\hline 0,027 & 0,812 & 0,034 & 0,652 & 0,786 & 0,803 \\
\hline 0,027 & 0,547 & 0,032 & 0,414 & 0,846 & 0,757 \\
\hline 0,029 & 0,812 & 0,044 & 0,547 & 0,667 & 0,673 \\
\hline 0,027 & 0,707 & 0,037 & 0,547 & 0,733 & 0,773 \\
\hline 0,047 & 0,972 & 0,061 & 0,735 & 0,760 & 0,755 \\
\hline 0,103 & 1,746 & 0,140 & 1,387 & 0,737 & 0,794 \\
\hline 0,051 & 1,359 & 0,086 & 0,906 & 0,600 & 0,667 \\
\hline 0,034 & 0,680 & 0,039 & 0,547 & 0,875 & 0,805 \\
\hline 0,029 & 0,680 & 0,034 & 0,481 & 0,857 & 0,707 \\
\hline 0,029 & 0,547 & 0,034 & 0,414 & 0,857 & 0,757 \\
\hline 0,025 & 0,586 & 0,029 & 0,359 & 0,833 & 0,613 \\
\hline 0,029 & 0,680 & 0,044 & 0,481 & 0,667 & 0,707 \\
\hline 0,029 & 0,613 & 0,032 & 0,508 & 0,923 & 0,829 \\
\hline 0,025 & 0,707 & 0,034 & 0,442 & 0,714 & 0,625 \\
\hline 0,029 & 0,762 & 0,039 & 0,508 & 0,750 & 0,667 \\
\hline 0,029 & 0,574 & 0,034 & 0,387 & 0,857 & 0,673 \\
\hline 0,037 & 0,707 & 0,047 & 0,574 & 0,789 & 0,812 \\
\hline 0,331 & 4,989 & 0,645 & 2,790 & 0,513 & 0,559 \\
\hline 0,274 & 4,227 & 0,495 & 2,293 & 0,554 & 0,542 \\
\hline 0,042 & 1,133 & 0,061 & 0,707 & 0,680 & 0,624 \\
\hline
\end{tabular}

nothing to measure
nothing to measure

nothing to measure

1,000
0,677

nothing to measure
nothing to measure

$\begin{array}{lcc}0,302 & \text { nothing to measure } & \\ 0.000 & 1,000\end{array}$

$\begin{array}{lll}0,128 & 1,000 & 0,854 \\ 0,374 & 0,692 & 1,202\end{array}$

$\begin{array}{lll}0,187 & 1,000 & 0,990 \\ 0,219 & 1,000 & 0,913\end{array}$

$\begin{array}{lll}0,405 & 0,801 & 1,118\end{array}$

nothing to measure

nothing to measure
nothing to measure

nothing to measure

nothing to measure

nothing to measure

nothing to measure

0,389

0,800
thing to measure

nothing to measure

nothing to measure

0,750
nothing to measure

nothing to measure

$\begin{array}{lc}0,291 \quad 0,513 \\ 0,287 & 1,000\end{array}$

0,248

$\begin{array}{ll}0,513 & 1,396 \\ 1,000 & 0,940 \\ 0,560 & 1,336\end{array}$

$0,230 \quad 0,154$

$\begin{array}{lll}0,467 & 0,560 & 1,336 \\ 0,350 & 0,677 & 1,215\end{array}$

$\begin{array}{ll}0,700 & 0,495 \\ 0,588 & 0,599\end{array}$

$\begin{array}{ll}0,700 & 0,495 \\ 0,388 & 0,599 \\ 0,71 & 0,200\end{array}$

$0,619 \quad 1,271$

$\begin{array}{ll}0,424 & 1,535 \\ 0,350 & 1,69\end{array}$

$\begin{array}{ll}0,712 & 0,728 \\ 0,265 & 0,312\end{array}$

$\begin{array}{ll}0,265 & 0,312 \\ 0,334 & 0,474\end{array}$

$0,435 \quad 0,280$

$\begin{array}{lll}0,317 & 0,089 & 0,099\end{array}$

$0,232 \quad 0,934$

$\begin{array}{ll}0,326 & 0,800 \\ 0,282 & 1,000\end{array}$

$\begin{array}{rrr}0,421 & 0,898 & 0,900 \\ 0,444 & 0,8083 & 1,118\end{array}$

$0,188 \quad 0,800 \quad 1,118$

nothing to measure

nothing to measure

472

0,616

1,274

nothing to mease

nothing to measure

nothing to measure

$0,417 \quad 0,636$

1,2

nothing to measure

$\begin{array}{llllllll}0,357 & 1,000 & 0,945 & 0,764 & 0,559 & 0,221 & 0,124 & 0,099\end{array}$

$\begin{array}{llllllll}0,282 & 0,924 & 1,040 & 0,659 & 0,557 & 0,267 & 0,149 & 0,099 \\ 0,657 & 0,167 & 2,447 & 0,318 & 0,579 & 1,151 & 0,667 & 0,221\end{array}$

$\begin{array}{lll}0,639 & 0,193 & 2,276 \\ 0,494 & 0,408 & 1,56\end{array}$

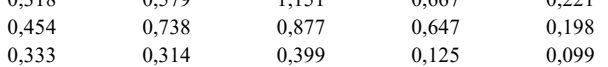

10,099
$\quad 12$

$\begin{array}{lc}837 & - \\ 838 & - \\ 839 & -2,3 \\ 840 & -1,3 \\ 841 & - \\ 842 & - \\ 843 & -1,4 \\ 843 & -1,1 \\ 843 & -1,8 \\ 843 & -2,0 \\ 843 & -1,3 \\ 843 & -1,3 \\ 844 & - \\ 845 & - \\ 846 & - \\ 847 & - \\ 848 & - \\ 849 & - \\ 850 & - \\ 851 & - \\ 852 & -1,3 \\ 853 & - \\ 854 & - \\ 855 & - \\ 856 & - \\ 857 & -1,4 \\ 858 & - \\ 859 & - \\ 860 & -2,0 \\ 860 & -1,1 \\ 861 & -1,3 \\ 861 & -1,7 \\ 861 & -1,5 \\ 861 & -2,8 \\ 861 & -2,1 \\ 861 & -1,7 \\ 861 & -1,7 \\ 861 & -1,3 \\ 861 & -1,0 \\ 861 & -1,3 \\ 861 & -1,5 \\ 862 & - \\ 863 & - \\ 864 & - \\ 865 & -1,3 \\ 866 & - \\ 867 & - \\ 868 & - \\ 869 & - \\ 870 & - \\ 871 & -1,8 \\ 872 & - \\ 873 & - \\ 874 & -1,1 \\ 874 & -1,4 \\ 875 & -3,5 \\ 876 & -3,1 \\ 876 & -2,0 \\ & \end{array}$ 


\begin{tabular}{|c|c|c|c|c|c|c|c|c|c|c|c|c|c|c|c|c|c|}
\hline 877 & & & & & & & & nothing to measure & & & & & & & & 877 & - \\
\hline 878 & & & & & & & & nothing to measure & & & & & & & & 878 & - \\
\hline 879 & & & & & & & & nothing to measure & & & & & & & & 879 & - \\
\hline 880 & & & & & & & & nothing to measure & & & & & & & & 880 & - \\
\hline 881 & & & & & & & & nothing to measure & & & & & & & & 881 & - \\
\hline 882 & & & & & & & & nothing to measure & & & & & & & & 882 & - \\
\hline 883 & & & & & & & & nothing to measure & & & & & & & & 883 & - \\
\hline 884 & & & & & & & & nothing to measure & & & & & & & & 884 & - \\
\hline 885 & & & & & & & & nothing to measure & & & & & & & & 885 & 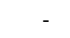 \\
\hline $\begin{array}{l}886 \\
887\end{array}$ & 0,037 & 0,801 & 0,051 & 0,519 & 0,714 & 0,648 & 0,453 & $\begin{array}{l}0,720 \\
\text { nothing to measure }\end{array}$ & 1,178 & 0,562 & 0,606 & 0,289 & 0,175 & 0,198 & 121 & $\begin{array}{l}886 \\
887\end{array}$ & $-1,5$ \\
\hline $\begin{array}{l}887 \\
888\end{array}$ & 0,064 & 0,906 & 0,071 & 0,812 & 0,897 & 0,896 & 0,146 & 0,975 & 1,013 & 0,509 & 0,380 & 0,399 & 0,152 & 0,198 & 124 & 888 & $-2,0$ \\
\hline 889 & & & & & & & & nothing to measure & & & & & & & & 889 & - \\
\hline 890 & & & & & & & & nothing to measure & & & & & & & & 890 & - \\
\hline 891 & & & & & & & & nothing to measure & & & & & & & & 891 & - \\
\hline 892 & & & & & & & & nothing to measure & & & & & & & & 892 & - \\
\hline 893 & & & & & & & & nothing to measure & & & & & & & & 893 & - \\
\hline 894 & & & & & & & & nothing to measure & & & & & & & & 894 & - \\
\hline 895 & 0,054 & 1,227 & 0,078 & 0,707 & 0,688 & 0,577 & 0,526 & 0,450 & 1,490 & 0,560 & 0,578 & 0,350 & 0,202 & 0,140 & 35 & 895 & $-1,8$ \\
\hline 895 & 0,025 & 0,630 & 0,034 & 0,414 & 0,714 & 0,658 & 0,446 & 0,777 & 1,134 & 0,439 & 0,371 & 0,267 & 0,099 & 0,099 & 135 & 895 & $-1,4$ \\
\hline 895 & 0,074 & 1,348 & 0,108 & 1,121 & 0,682 & 0,832 & 0,360 & 0,509 & 1,402 & 0,329 & 0,398 & 0,533 & 0,212 & 0,221 & 82 & 895 & $-2,4$ \\
\hline 895 & 0,039 & 0,989 & 0,054 & 0,696 & 0,727 & 0,704 & 0,403 & 0,504 & 1,408 & 0,300 & 0,310 & 0,408 & 0,127 & 0,099 & 80 & 895 & $-2,0$ \\
\hline 895 & 0,027 & 0,668 & 0,037 & 0,442 & 0,733 & 0,661 & 0,431 & 0,758 & 1,148 & 0,483 & 0,371 & 0,267 & 0,099 & 0,099 & 90 & 895 & $-1,4$ \\
\hline 895 & 0,025 & 0,481 & 0,027 & 0,348 & 0,909 & 0,724 & 0,291 & 1,000 & 0,866 & 0,979 & 0,555 & 0,178 & 0,099 & 0,140 & 162 & 895 & $-0,8$ \\
\hline 895 & 0,029 & 0,547 & 0,034 & 0,453 & 0,857 & 0,828 & 0,223 & 1,000 & 0,900 & 0,899 & 0,691 & 0,204 & 0,141 & 0,140 & 33 & 895 & $-1,0$ \\
\hline 895 & 0,054 & 0,934 & 0,061 & 0,735 & 0,880 & 0,787 & 0,245 & 0,777 & 1,134 & 0,560 & 0,424 & 0,350 & 0,149 & 0,198 & 85 & 895 & $-1,8$ \\
\hline 895 & 0,025 & 0,613 & 0,029 & 0,481 & 0,833 & 0,784 & 0,273 & 0,819 & 1,105 & 0,374 & 0,231 & 0,289 & 0,067 & 0,099 & 57 & 895 & $-1,5$ \\
\hline 895 & 0,034 & 1,066 & 0,066 & 0,801 & 0,519 & 0,751 & 0,542 & 0,379 & 1,624 & 0,262 & 0,383 & 0,408 & 0,156 & 0,099 & 102 & 895 & $-2,0$ \\
\hline 895 & 0,042 & 1,039 & 0,069 & 0,773 & 0,607 & 0,745 & 0,469 & 0,485 & 1,436 & 0,408 & 0,548 & 0,360 & 0,197 & 0,099 & 104 & 895 & $-1,8$ \\
\hline 896 & & & & & & & & nothing to measure & & & & & & & & 896 & - \\
\hline 897 & & & & & & & & nothing to measure & & & & & & & & 897 & - \\
\hline 898 & & & & & & & & nothing to measure & & & & & & & & 898 & - \\
\hline 899 & 0,074 & 1,332 & 0,098 & 0,906 & 0,750 & 0,680 & 0,406 & 0,521 & 1,385 & 0,721 & 0,799 & 0,360 & 0,288 & 0,221 & 67 & 899 & $-1,8$ \\
\hline 899 & 0,032 & 0,586 & 0,034 & 0,453 & 0,929 & 0,773 & 0,238 & 1,000 & 0,926 & 0,637 & 0,439 & 0,252 & 0,111 & 0,140 & 75 & 899 & $-1,3$ \\
\hline 900 & & & & & & & & nothing to measure & & & & & & & & 900 & - \\
\hline 901 & & & & & & & & nothing to measure & & & & & & & & 901 & - \\
\hline 902 & & & & & & & & nothing to measure & & & & & & & & 902 & 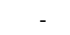 \\
\hline 903 & 0,042 & 1,000 & 0,059 & 0,680 & 0,708 & 0,680 & 0,433 & 0,524 & 1,382 & 0,355 & 0,381 & 0,387 & 0,147 & 0,198 & 58 & 903 & $-2,0$ \\
\hline 904 & 0,025 & 0,481 & 0,027 & 0,481 & 0,909 & 1,000 & 0,091 & 1,000 & 0,866 & 0,749 & 0,461 & 0,204 & 0,094 & 0,099 & 111 & 904 & $-1,0$ \\
\hline 904 & 0,032 & 0,773 & 0,042 & 0,481 & 0,765 & 0,621 & 0,446 & 0,669 & 1,222 & 0,662 & 0,566 & 0,248 & 0,140 & 0,140 & 140 & 904 & $-1,3$ \\
\hline 905 & & & & & & & & nothing to measure & & & & & & & & 905 & - \\
\hline 906 & 0,025 & 0,668 & 0,037 & 0,414 & 0,667 & 0,620 & 0,506 & 0,690 & 1,204 & 0,637 & 0,447 & 0,221 & 0,099 & 0,099 & 0 & 906 & $-1,1$ \\
\hline 906 & 0,037 & 0,801 & 0,044 & 0,414 & 0,833 & 0,517 & 0,511 & 0,720 & 1,178 & 0,764 & 0,600 & 0,248 & 0,149 & 0,140 & 52 & 906 & $-1,3$ \\
\hline 907 & & & & & & & & nothing to measure & & & & & & & & 907 & 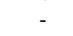 \\
\hline 908 & 0,037 & 0,707 & 0,047 & 0,519 & 0,789 & 0,735 & 0,339 & 0,924 & 1,040 & 0,735 & 0,588 & 0,252 & 0,149 & 0,140 & 117 & 908 & $-1,3$ \\
\hline 908 & 0,037 & 0,840 & 0,047 & 0,707 & 0,789 & 0,842 & 0,263 & 0,655 & 1,236 & 0,294 & 0,254 & 0,399 & 0,102 & 0,099 & 22 & 908 & $-2,0$ \\
\hline 908 & 0,039 & 1,011 & 0,051 & 0,586 & 0,762 & 0,579 & 0,483 & 0,482 & 1,441 & 0,599 & 0,485 & 0,289 & 0,140 & 0,099 & 123 & 908 & $-1,5$ \\
\hline 909 & & & & & & & & nothing to measure & & & & & & & & 909 & - \\
\hline 910 & 0,032 & 0,574 & 0,034 & 0,442 & 0,929 & 0,769 & 0,242 & 1,000 & 0,908 & 0,637 & 0,392 & 0,252 & 0,099 & 0,140 & 75 & 910 & $-1,3$ \\
\hline 911 & & & & & & & & nothing to measure & & & & & & & & 911 & 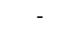 \\
\hline 912 & & & & & & & & nothing to measure & & & & & & & & 912 & - \\
\hline 913 & & & & & & & & nothing to measure & & & & & & & & 913 & - \\
\hline 914 & & & & & & & & nothing to measure & & & & & & & & 914 & - \\
\hline 915 & & & & & & & & nothing to measure & & & & & & & & 915 & - \\
\hline 916 & & & & & & & & nothing to measure & & & & & & & & 916 & - \\
\hline 917 & & & & & & & & nothing to measure & & & & & & & & 917 & - \\
\hline 918 & & & & & & & & nothing to measure & & & & & & & & 918 & - \\
\hline 919 & & & & & & & & nothing to measure & & & & & & & & 919 & 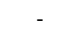 \\
\hline
\end{tabular}




$\begin{array}{llllllll}0,700 & 0,671 & 0,221 & 0,149 & 0,099 & 135 & 947 & -1,1 \\ 0,446 & 0,300 & 0,313 & 0,094 & 0,099 & 71 & 947 & -1,6 \\ 0,611 & 0,537 & 0,248 & 0,133 & 0,099 & 11 & 947 & -1,3 \\ 0,345 & 0,325 & 0,456 & 0,149 & 0,140 & 103 & 947 & -2,2 \\ 0,382 & 0,218 & 0,313 & 0,068 & 0,099 & 103 & 947 & -1,6 \\ 0,764 & 0,447 & 0,221 & 0,099 & 0,099 & 45 & 947 & -1,1 \\ 0,588 & 0,392 & 0,252 & 0,099 & 0,140 & 90 & 947 & -1,3 \\ 0,637 & 0,400 & 0,221 & 0,089 & 0,099 & 159 & 947 & -1,1 \\ 0,313 & 0,245 & 0,399 & 0,098 & 0,099 & 101 & 947 & -2,0 \\ 0,707 & 0,422 & 0,210 & 0,089 & 0,099 & 148 & 947 & -1,1 \\ 0,588 & 0,392 & 0,252 & 0,099 & 0,099 & 75 & 947 & -1,3 \\ 0,260 & 0,143 & 0,347 & 0,050 & 0,099 & 90 & 947 & -1,8 \\ 0,357 & 0,141 & 0,350 & 0,050 & 0,099 & 90 & 947 & -1,8 \\ 0,238 & 0,530 & 0,743 & 0,393 & 0,140 & 53 & 947 & -2,9 \\ 0,517 & 0,344 & 0,280 & 0,096 & 0,099 & 126 & 947 & -1,5 \\ 0,340 & 0,250 & 0,332 & 0,083 & 0,099 & 162 & 947 & -1,7 \\ 0,459 & 0,533 & 0,387 & 0,206 & 0,140 & 148 & 947 & -2,0 \\ 0,528 & 0,442 & 0,317 & 0,140 & 0,140 & 137 & 947 & -1,7 \\ 0,979 & 0,745 & 0,178 & 0,133 & 0,099 & 135 & 947 & -0,8 \\ 0,571 & 0,353 & 0,267 & 0,094 & 0,099 & 108 & 947 & -1,4 \\ 0,509 & 0,424 & 0,248 & 0,105 & 0,140 & 45 & 947 & -1,3 \\ 0,517 & 0,643 & 0,280 & 0,180 & 0,099 & 5 & 947 & -1,5 \\ 0,325 & 0,343 & 0,892 & 0,306 & 0,280 & 93 & 947 & -3,2 \\ 0,439 & 0,226 & 0,267 & 0,060 & 0,099 & 105 & 947 & -1,4\end{array}$




\begin{tabular}{|c|c|c|c|c|c|c|c|c|c|c|c|c|c|c|c|c|c|c|}
\hline 25 & 947 & 0,059 & 1,083 & 0,069 & 0,922 & 0,857 & 0,852 & 0,206 & 0,631 & 1,259 & 0,303 & 0,199 & 0,498 & 0,099 & 0,198 & 94 & 947 & $-2,3$ \\
\hline 26 & 947 & 0,069 & 1,320 & 0,088 & 1,044 & 0,778 & 0,790 & 0,305 & 0,495 & 1,422 & 0,327 & 0,282 & 0,517 & 0,146 & 0,140 & 81 & 947 & $-2,4$ \\
\hline 27 & 947 & 0,025 & 0,481 & 0,025 & 0,227 & 1,000 & 0,471 & 0,529 & 1,000 & 0,866 & 0,749 & 0,485 & 0,204 & 0,099 & 0,140 & 90 & 947 & $-1,0$ \\
\hline 28 & 947 & 0,027 & 0,574 & 0,032 & 0,481 & 0,846 & 0,837 & 0,224 & 1,000 & 0,987 & 0,483 & 0,294 & 0,267 & 0,078 & 0,099 & 163 & 947 & $-1,4$ \\
\hline 29 & 947 & 0,056 & 0,972 & 0,074 & 0,790 & 0,767 & 0,812 & 0,300 & 0,749 & 1,155 & 0,714 & 0,781 & 0,317 & 0,248 & 0,198 & 121 & 947 & $-1,7$ \\
\hline 30 & 947 & 0,025 & 0,442 & 0,027 & 0,254 & 0,909 & 0,575 & 0,435 & 1,000 & 0,796 & 0,979 & 0,555 & 0,178 & 0,099 & 0,198 & 162 & 947 & $-0,8$ \\
\hline 31 & 947 & 0,032 & 0,680 & 0,039 & 0,547 & 0,812 & 0,805 & 0,271 & 0,867 & 1,074 & 0,460 & 0,422 & 0,297 & 0,125 & 0,140 & 72 & 947 & $-1,6$ \\
\hline 32 & 947 & 0,025 & 0,508 & 0,027 & 0,320 & 0,909 & 0,631 & 0,380 & 1,000 & 0,916 & 0,637 & 0,425 & 0,221 & 0,094 & 0,099 & 90 & 947 & $-1,1$ \\
\hline 33 & 947 & 0,044 & 1,199 & 0,071 & 0,746 & 0,621 & 0,622 & 0,535 & 0,386 & 1,610 & 0,458 & 0,626 & 0,350 & 0,219 & 0,140 & 151 & 947 & $-1,8$ \\
\hline 34 & 947 & 0,029 & 0,481 & 0,029 & 0,320 & 1,000 & 0,667 & 0,333 & 1,000 & 0,791 & 0,899 & 0,485 & 0,204 & 0,099 & 0,198 & 90 & 947 & $-1,0$ \\
\hline 35 & 947 & 0,032 & 0,668 & 0,037 & 0,574 & 0,867 & 0,860 & 0,194 & 0,896 & 1,056 & 0,414 & 0,269 & 0,313 & 0,084 & 0,099 & 107 & 947 & $-1,6$ \\
\hline 36 & 947 & 0,039 & 0,746 & 0,047 & 0,547 & 0,842 & 0,733 & 0,310 & 0,886 & 1,063 & 0,509 & 0,425 & 0,313 & 0,133 & 0,198 & 151 & 947 & $-1,6$ \\
\hline 37 & 947 & 0,093 & 1,188 & 0,110 & 0,950 & 0,844 & 0,800 & 0,254 & 0,830 & $\begin{array}{l}1,098 \\
\text { S }\end{array}$ & 0,569 & 0,575 & 0,456 & 0,262 & 0,280 & 53 & 947 & $-2,2$ \\
\hline 38 & 947 & 0,029 & 0,801 & 0,039 & 0,613 & 0,750 & 0,766 & 0,343 & 0,576 & 1,318 & 0,294 & 0,249 & 0,357 & 0,089 & 0,099 & 113 & 947 & $-1,8$ \\
\hline 39 & 947 & 0,047 & 0,961 & 0,069 & 0,707 & 0,679 & 0,736 & 0,416 & 0,633 & $\begin{array}{l}1,257 \\
\text { S }\end{array}$ & 0,397 & 0,427 & 0,387 & 0,165 & 0,099 & 53 & 947 & $-2,0$ \\
\hline 40 & 947 & 0,051 & 1,121 & 0,081 & 0,785 & 0,636 & 0,700 & 0,472 & 0,514 & 1,394 & 0,438 & 0,527 & 0,387 & 0,204 & 0,140 & 133 & 947 & $-2,0$ \\
\hline 41 & 947 & 0,032 & 0,574 & 0,037 & 0,442 & 0,867 & 0,769 & 0,267 & 1,000 & 0,908 & 0,920 & 0,629 & 0,210 & 0,132 & 0,198 & 53 & 947 & $-1,1$ \\
\hline 42 & 947 & 0,037 & 0,762 & 0,051 & 0,453 & 0,714 & 0,594 & 0,496 & 0,795 & 1,121 & 0,562 & 0,572 & 0,289 & 0,165 & 0,198 & 117 & 947 & $-1,5$ \\
\hline 43 & 947 & 0,039 & 0,735 & 0,042 & 0,508 & 0,941 & 0,692 & 0,314 & 0,913 & 1,047 & 0,416 & 0,286 & 0,347 & 0,099 & 0,140 & 95 & 947 & $-1,8$ \\
\hline 44 & 947 & 0,029 & 0,481 & 0,029 & 0,320 & 1,000 & 0,667 & 0,333 & 1,000 & 0,791 & 1,175 & 0,784 & 0,178 & 0,140 & 0,198 & 45 & 947 & $-0,8$ \\
\hline 45 & 947 & 0,025 & 0,641 & 0,032 & 0,359 & 0,769 & 0,561 & 0,496 & 0,750 & 1,155 & 0,398 & 0,250 & 0,280 & 0,070 & 0,099 & 131 & 947 & $-1,5$ \\
\hline 46 & 947 & 0,025 & 0,453 & 0,027 & 0,320 & 0,909 & 0,707 & 0,307 & 1,000 & 0,816 & 1,273 & 0,849 & 0,157 & 0,133 & 0,140 & 34 & 947 & $-0,7$ \\
\hline 47 & 947 & 0,034 & 0,547 & 0,034 & 0,414 & 1,000 & 0,757 & 0,243 & 1,000 & 0,833 & 0,713 & 0,400 & 0,248 & 0,099 & 0,198 & 90 & 947 & $-1,3$ \\
\hline 48 & 947 & 0,037 & 0,574 & 0,037 & 0,453 & 1,000 & 0,789 & 0,211 & 1,000 & 0,845 & 0,955 & 0,671 & 0,221 & 0,149 & 0,198 & 72 & 947 & $-1,1$ \\
\hline 49 & 947 & 0,025 & 0,641 & 0,034 & 0,375 & 0,714 & 0,586 & 0,503 & 0,750 & 1,155 & 0,439 & 0,371 & 0,267 & 0,099 & 0,099 & 153 & 947 & $-1,4$ \\
\hline 50 & 947 & 0,039 & 0,707 & 0,044 & 0,613 & 0,889 & 0,867 & 0,173 & 0,985 & 1,007 & 0,551 & 0,493 & 0,301 & 0,149 & 0,198 & 111 & 947 & $-1,6$ \\
\hline 51 & 947 & 0,078 & 1,641 & 0,127 & 1,215 & 0,615 & 0,741 & 0,464 & 0,366 & 1,653 & 0,240 & 0,348 & 0,645 & 0,225 & 0,198 & 82 & 947 & $-2,7$ \\
\hline 52 & 947 & 0,027 & 0,519 & 0,029 & 0,414 & 0,917 & 0,798 & 0,219 & 1,000 & 0,892 & 0,824 & 0,543 & 0,204 & 0,111 & 0,140 & 108 & 947 & $-1,0$ \\
\hline 53 & 947 & 0,025 & 0,680 & 0,034 & 0,481 & 0,714 & 0,707 & 0,409 & 0,667 & 1,225 & 0,749 & 0,728 & 0,204 & 0,149 & 0,099 & 85 & 947 & $-1,0$ \\
\hline 54 & 947 & 0,029 & 0,574 & 0,032 & 0,508 & 0,923 & 0,885 & 0,139 & 1,000 & 0,945 & 0,588 & 0,392 & 0,252 & 0,099 & 0,140 & 94 & 947 & $-1,3$ \\
\hline 55 & 947 & 0,061 & 0,961 & 0,069 & 0,884 & 0,893 & 0,919 & 0,134 & 0,833 & 1,095 & 0,388 & 0,331 & 0,448 & 0,149 & 0,198 & 73 & 947 & $-2,2$ \\
\hline 56 & 947 & 0,059 & 1,243 & 0,081 & 1,016 & 0,727 & 0,818 & 0,328 & 0,479 & 1,446 & 0,206 & 0,173 & 0,602 & 0,104 & 0,140 & 93 & 947 & $-2,6$ \\
\hline 57 & 947 & 0,032 & 0,867 & 0,047 & 0,613 & 0,684 & 0,707 & 0,431 & 0,532 & 1,371 & 0,447 & 0,572 & 0,301 & 0,172 & 0,099 & 90 & 947 & $-1,6$ \\
\hline 58 & 947 & 0,025 & 0,453 & 0,027 & 0,320 & 0,909 & 0,707 & 0,307 & 1,000 & 0,816 & 1,273 & 0,708 & 0,157 & 0,111 & 0,140 & 146 & 947 & $-0,7$ \\
\hline 59 & 947 & 0,029 & 0,547 & 0,029 & 0,414 & 1,000 & 0,757 & 0,243 & 1,000 & 0,900 & 0,588 & 0,392 & 0,252 & 0,099 & 0,140 & 95 & 947 & $-1,3$ \\
\hline 60 & 947 & 0,025 & 0,547 & 0,027 & 0,481 & 0,909 & 0,879 & 0,152 & 1,000 & 0,986 & 0,509 & 0,400 & 0,248 & 0,099 & 0,140 & 90 & 947 & $-1,3$ \\
\hline 61 & 947 & 0,054 & 1,044 & 0,076 & 0,680 & 0,710 & 0,651 & 0,454 & 0,622 & 1,268 & 0,483 & 0,588 & 0,377 & 0,222 & 0,140 & 32 & 947 & $-1,9$ \\
\hline 62 & 947 & 0,027 & 0,574 & 0,034 & 0,414 & 0,786 & 0,721 & 0,352 & 1,000 & 0,987 & 0,560 & 0,358 & 0,248 & 0,089 & 0,099 & 121 & 947 & $-1,3$ \\
\hline 63 & 947 & 0,027 & 0,453 & 0,027 & 0,387 & 1,000 & 0,854 & 0,146 & 1,000 & 0,778 & 1,077 & 0,588 & 0,178 & 0,105 & 0,140 & 135 & 947 & $-0,8$ \\
\hline 64 & 947 & 0,034 & 0,547 & 0,037 & 0,414 & 0,933 & 0,757 & 0,252 & 1,000 & 0,833 & 1,049 & 0,728 & 0,204 & 0,149 & 0,198 & 90 & 947 & $-1,0$ \\
\hline 65 & 947 & 0,051 & 0,934 & 0,064 & 0,735 & 0,808 & 0,787 & 0,287 & 0,742 & 1,161 & 0,535 & 0,555 & 0,350 & 0,194 & 0,198 & 88 & 947 & $-1,8$ \\
\hline 66 & 947 & 0,025 & 0,641 & 0,034 & 0,469 & 0,714 & 0,732 & 0,391 & 0,750 & 1,155 & 0,490 & 0,381 & 0,252 & 0,096 & 0,099 & 83 & 947 & $-1,3$ \\
\hline 67 & 947 & 0,039 & 0,735 & 0,047 & 0,574 & 0,842 & 0,782 & 0,269 & 0,913 & 1,047 & 0,702 & 0,657 & 0,267 & 0,175 & 0,198 & 169 & 947 & $-1,4$ \\
\hline 68 & 947 & 0,032 & 0,707 & 0,039 & 0,519 & 0,812 & 0,735 & 0,325 & 0,801 & 1,118 & 0,487 & 0,447 & 0,289 & 0,129 & 0,140 & 56 & 947 & $-1,5$ \\
\hline 69 & 947 & 0,061 & 1,375 & 0,086 & 0,895 & 0,714 & 0,651 & 0,451 & 0,407 & 1,568 & 0,354 & 0,422 & 0,470 & 0,198 & 0,140 & 68 & 947 & $-2,2$ \\
\hline 70 & 947 & 0,027 & 0,574 & 0,032 & 0,320 & 0,846 & 0,558 & 0,468 & 1,000 & 0,987 & 0,539 & 0,392 & 0,252 & 0,099 & 0,140 & 90 & 947 & $-1,3$ \\
\hline 71 & 947 & 0,078 & 1,028 & 0,083 & 0,801 & 0,941 & 0,780 & 0,228 & 0,933 & 1,035 & 0,599 & 0,485 & 0,408 & 0,198 & 0,280 & 84 & 947 & $-2,0$ \\
\hline 72 & 947 & 0,025 & 0,680 & 0,037 & 0,387 & 0,667 & 0,569 & 0,545 & 0,667 & 1,225 & 0,398 & 0,344 & 0,280 & 0,096 & 0,099 & 17 & 947 & $-1,5$ \\
\hline 73 & 947 & 0,025 & 0,481 & 0,025 & 0,387 & 1,000 & 0,805 & 0,195 & 1,000 & 0,866 & 0,796 & 0,500 & 0,198 & 0,099 & 0,140 & 90 & 947 & $-1,0$ \\
\hline 74 & 947 & 0,034 & 0,829 & 0,044 & 0,574 & 0,778 & 0,693 & 0,379 & 0,628 & 1,262 & 0,307 & 0,260 & 0,377 & 0,098 & 0,099 & 61 & 947 & $-1,9$ \\
\hline 75 & 947 & 0,076 & 1,398 & 0,100 & 1,238 & 0,756 & 0,885 & 0,269 & 0,488 & 1,431 & 0,232 & 0,226 & 0,645 & 0,146 & 0,198 & 124 & 947 & $-2,7$ \\
\hline 76 & 947 & 0,069 & 1,403 & 0,123 & 0,961 & 0,560 & 0,685 & 0,541 & 0,438 & 1,511 & 0,419 & 0,607 & 0,456 & 0,277 & 0,099 & 146 & 947 & $-2,2$ \\
\hline 77 & 947 & 0,066 & 1,121 & 0,088 & 0,922 & 0,750 & 0,823 & 0,307 & 0,661 & 1,230 & 0,382 & 0,374 & 0,470 & 0,175 & 0,198 & 84 & 947 & $-2,2$ \\
\hline 78 & 947 & 0,027 & 0,519 & 0,027 & 0,414 & 1,000 & 0,798 & 0,202 & 1,000 & 0,892 & 0,700 & 0,474 & 0,221 & 0,105 & 0,140 & 50 & 947 & $-1,1$ \\
\hline 79 & 947 & 0,037 & 0,984 & 0,064 & 0,735 & 0,577 & 0,747 & 0,493 & 0,477 & 1,447 & 0,298 & 0,375 & 0,396 & 0,149 & 0,099 & 84 & 947 & $-2,0$ \\
\hline 80 & 947 & 0,027 & 0,641 & 0,032 & 0,320 & 0,846 & 0,500 & 0,523 & 0,825 & 1,101 & 0,539 & 0,392 & 0,252 & 0,099 & 0,099 & 108 & 947 & $-1,3$ \\
\hline 81 & 947 & 0,034 & 0,773 & 0,047 & 0,547 & 0,737 & 0,707 & 0,394 & 0,721 & 1,178 & 0,524 & 0,447 & 0,289 & 0,129 & 0,099 & 63 & 947 & $-1,5$ \\
\hline 82 & 947 & 0,032 & 0,641 & 0,037 & 0,442 & 0,867 & 0,689 & 0,338 & 0,975 & $\begin{array}{l}1,013 \\
\end{array}$ & 0,571 & 0,371 & 0,267 & 0,099 & 0,140 & 78 & 947 & $-1,4$ \\
\hline
\end{tabular}




\begin{tabular}{|c|c|c|c|c|c|c|c|c|c|c|c|c|c|c|c|c|c|}
\hline $\begin{array}{l}948 \\
949\end{array}$ & & & & & & & & $\begin{array}{l}\text { nothing to measure } \\
\text { nothing to measure }\end{array}$ & & & & & & & & $\begin{array}{l}948 \\
949\end{array}$ & - \\
\hline 950 & 0,025 & 0,453 & 0,029 & 0,348 & 0,833 & 0,768 & 0,286 & 1,000 & 0,816 & 1,273 & 0,849 & 0,157 & 0,133 & 0,140 & 146 & 950 & $-0,7$ \\
\hline 950 & 0,037 & 1,028 & 0,059 & 0,856 & 0,625 & 0,833 & 0,410 & 0,438 & 1,512 & 0,225 & 0,254 & 0,456 & 0,116 & 0,099 & 74 & 950 & $-2,2$ \\
\hline 951 & & & & & & & & nothing to measure & & & & & & & & 951 & \\
\hline $\begin{array}{l}952 \\
953\end{array}$ & 0,051 & 0,922 & 0,061 & 0,762 & 0,840 & 0,826 & 0,236 & $\begin{array}{c}0,760 \\
\text { nothing to measure }\end{array}$ & 1,147 & 0,411 & 0,426 & 0,399 & 0,170 & 0,140 & 68 & $\begin{array}{l}952 \\
953\end{array}$ & $\begin{array}{c}-2,0 \\
-\end{array}$ \\
\hline 954 & & & & & & & & nothing to measure & & & & & & & & 954 & - \\
\hline 955 & & & & & & & & nothing to measure & & & & & & & & 955 & - \\
\hline 956 & & & & & & & & nothing to measure & & & & & & & & 956 & - \\
\hline 957 & & & & & & & & nothing to measure & & & & & & & & 957 & - \\
\hline 958 & 0,130 & 1,547 & 0,157 & 1,277 & 0,828 & 0,825 & 0,245 & 0,682 & 1,211 & 0,465 & 0,428 & 0,596 & 0,255 & 0,280 & 128 & 958 & $-2,6$ \\
\hline 959 & & & & & & & & nothing to measure & & & & & & & & 959 & - \\
\hline 960 & & & & & & & & nothing to measure & & & & & & & & 960 & - \\
\hline 961 & & & & & & & & nothing to measure & & & & & & & & 961 & - \\
\hline 962 & & & & & & & & nothing to measure & & & & & & & & 962 & - \\
\hline 963 & & & & & & & & nothing to measure & & & & & & & & 963 & - \\
\hline 964 & & & & & & & & nothing to measure & & & & & & & & 964 & - \\
\hline 965 & 0,042 & 0,602 & 0,044 & 0,442 & 0,944 & 0,734 & 0,272 & 1,000 & 0,832 & 0,866 & 0,600 & 0,248 & 0,149 & 0,198 & 117 & 965 & $-1,3$ \\
\hline 965 & 0,037 & 0,696 & 0,042 & 0,481 & 0,882 & 0,691 & 0,331 & 0,954 & 1,024 & 0,516 & 0,329 & 0,301 & 0,099 & 0,140 & 90 & 965 & $-1,6$ \\
\hline 965 & 0,025 & 0,508 & 0,027 & 0,414 & 0,909 & 0,815 & 0,206 & 1,000 & 0,916 & 0,637 & 0,354 & 0,221 & 0,078 & 0,099 & 113 & 965 & $-1,1$ \\
\hline 965 & 0,054 & 1,348 & 0,086 & 0,707 & 0,629 & 0,525 & 0,603 & 0,373 & 1,638 & 0,529 & 0,674 & 0,360 & 0,243 & 0,140 & 178 & 965 & $-1,8$ \\
\hline 965 & 0,034 & 0,735 & 0,044 & 0,414 & 0,778 & 0,564 & 0,489 & 0,799 & 1,119 & 0,713 & 0,600 & 0,248 & 0,149 & 0,140 & 11 & 965 & $-1,3$ \\
\hline 965 & 0,076 & 0,945 & 0,081 & 0,867 & 0,939 & 0,918 & 0,102 & 1,000 & 0,967 & 0,745 & 0,664 & 0,360 & 0,239 & 0,221 & 151 & 965 & $-1,8$ \\
\hline 965 & 0,032 & 0,574 & 0,039 & 0,442 & 0,812 & 0,769 & 0,297 & 1,000 & 0,908 & 0,828 & 0,671 & 0,221 & 0,149 & 0,198 & 63 & 965 & $-1,1$ \\
\hline 965 & 0,037 & 0,773 & 0,049 & 0,547 & 0,750 & 0,707 & 0,385 & 0,772 & 1,138 & 0,466 & 0,465 & 0,317 & 0,147 & 0,140 & 103 & 965 & $-1,7$ \\
\hline 966 & & & & & & & & nothing to measure & & & & & & & & 966 & - \\
\hline 967 & & & & & & & & nothing to measure & & & & & & & & 967 & - \\
\hline 968 & & & & & & & & nothing to measure & & & & & & & & 968 & - \\
\hline 969 & & & & & & & & nothing to measure & & & & & & & & 969 & - \\
\hline 970 & & & & & & & & nothing to measure & & & & & & & & 970 & - \\
\hline 971 & 0,029 & 0,762 & 0,039 & 0,508 & 0,750 & 0,667 & 0,417 & 0,636 & 1,254 & 0,306 & 0,197 & 0,350 & 0,069 & 0,099 & 12 & 971 & $-1,8$ \\
\hline 972 & & & & & & & & nothing to measure & & & & & & & & 972 & - \\
\hline 973 & & & & & & & & nothing to measure & & & & & & & & 973 & - \\
\hline 974 & & & & & & & & nothing to measure & & & & & & & & 974 & - \\
\hline 975 & & & & & & & & nothing to measure & & & & & & & & 975 & - \\
\hline 976 & & & & & & & & nothing to measure & & & & & & & & 976 & - \\
\hline 977 & 0,042 & 0,895 & 0,056 & 0,574 & 0,739 & 0,642 & 0,443 & 0,654 & 1,237 & 0,297 & 0,284 & 0,423 & 0,120 & 0,198 & 70 & 977 & $-2,1$ \\
\hline 978 & & & & & & & & nothing to measure & & & & & & & & 978 & - \\
\hline 979 & & & & & & & & nothing to measure & & & & & & & & 979 & - \\
\hline 980 & 0,025 & 0,481 & 0,027 & 0,254 & 0,909 & 0,529 & 0,480 & 1,000 & 0,866 & 0,749 & 0,434 & 0,204 & 0,089 & 0,099 & 108 & 980 & $-1,0$ \\
\hline 980 & 0,047 & 1,055 & 0,074 & 0,773 & 0,633 & 0,733 & 0,454 & 0,526 & 1,379 & 0,336 & 0,405 & 0,420 & 0,170 & 0,099 & 27 & 980 & $-2,1$ \\
\hline 980 & 0,235 & 3,028 & 0,326 & 2,078 & 0,722 & 0,686 & 0,419 & 0,323 & 1,761 & 0,413 & 0,556 & 0,852 & 0,474 & 0,313 & 50 & 980 & $-3,1$ \\
\hline 980 & 0,049 & 0,961 & 0,061 & 0,801 & 0,800 & 0,833 & 0,260 & 0,667 & 1,225 & 0,344 & 0,303 & 0,426 & 0,129 & 0,198 & 65 & 980 & $-2,1$ \\
\hline 980 & 0,123 & 2,387 & 0,186 & 1,348 & 0,658 & 0,565 & 0,554 & 0,270 & 1,923 & 0,522 & 0,724 & 0,547 & 0,396 & 0,198 & 168 & 980 & $-2,5$ \\
\hline 981 & & & & & & & & nothing to measure & & & & & & & & 981 & 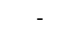 \\
\hline 982 & & & & & & & & nothing to measure & & & & & & & & 982 & - \\
\hline 983 & & & & & & & & nothing to measure & & & & & & & & 983 & - \\
\hline 984 & & & & & & & & nothing to measure & & & & & & & & 984 & - \\
\hline 985 & & & & & & & & nothing to measure & & & & & & & & 985 & - \\
\hline 986 & & & & & & & & nothing to measure & & & & & & & & 986 & - \\
\hline 987 & & & & & & & & nothing to measure & & & & & & & & 987 & - \\
\hline 988 & & & & & & & & nothing to measure & & & & & & & & 988 & - \\
\hline 989 & & & & & & & & nothing to measure & & & & & & & & 989 & 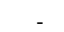 \\
\hline 990 & 0,032 & 0,707 & 0,037 & 0,547 & 0,867 & 0,773 & 0,263 & 0,801 & 1,118 & 0,404 & 0,313 & 0,317 & 0,099 & 0,099 & 51 & 990 & $-1,7$ \\
\hline 991 & & & & & & & & nothing to measure & & & & & & & & 991 & 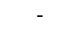 \\
\hline 992 & & & & & & & & nothing to measure & & & & & & & & 992 & - \\
\hline 993 & & & & & & & & nothing to measure & & & & & & & & 993 & - \\
\hline
\end{tabular}




\begin{tabular}{|c|c|c|c|c|c|c|c|c|c|c|c|c|c|c|c|c|c|}
\hline 994 & & & & & & & & nothing to measure & & & & & & & & 994 & - \\
\hline 995 & & & & & & & & nothing to measure & & & & & & & & 995 & - \\
\hline 996 & & & & & & & & nothing to measure & & & & & & & & 996 & - \\
\hline 997 & & & & & & & & nothing to measure & & & & & & & & 997 & - \\
\hline 998 & & & & & & & & nothing to measure & & & & & & & & 998 & - \\
\hline 999 & & & & & & & & nothing to measure & & & & & & & & 999 & - \\
\hline 1000 & & & & & & & & nothing to measure & & & & & & & & 1000 & - \\
\hline 1001 & & & & & & & & nothing to measure & & & & & & & & 1001 & - \\
\hline 1002 & & & & & & & & nothing to measure & & & & & & & & 1002 & - \\
\hline 1003 & & & & & & & & nothing to measure & & & & & & & & 1003 & - \\
\hline 1004 & & & & & & & & nothing to measure & & & & & & & & 1004 & - \\
\hline 1005 & & & & & & & & nothing to measure & & & & & & & & 1005 & - \\
\hline 1006 & & & & & & & & nothing to measure & & & & & & & & 1006 & - \\
\hline 1007 & & & & & & & & nothing to measure & & & & & & & & 1007 & - \\
\hline 1008 & & & & & & & & nothing to measure & & & & & & & & 1008 & - \\
\hline 1009 & & & & & & & & nothing to measure & & & & & & & & 1009 & - \\
\hline 1010 & & & & & & & & nothing to measure & & & & & & & & 1010 & - \\
\hline 1011 & & & & & & & & nothing to measure & & & & & & & & 1011 & - \\
\hline 1012 & & & & & & & & nothing to measure & & & & & & & & 1012 & - \\
\hline 1013 & & & & & & & & nothing to measure & & & & & & & & 1013 & - \\
\hline 1014 & & & & & & & & nothing to measure & & & & & & & & 1014 & . \\
\hline 1015 & 0,042 & 0,801 & 0,047 & 0,668 & 0,895 & 0,834 & 0,196 & 0,816 & 1,107 & 0,408 & 0,275 & 0,360 & 0,099 & 0,198 & 9 & 1015 & $-1,8$ \\
\hline 1015 & 0,027 & 0,707 & 0,037 & 0,481 & 0,733 & 0,680 & 0,417 & 0,677 & 1,215 & 0,539 & 0,588 & 0,252 & 0,149 & 0,099 & 18 & 1015 & $-1,3$ \\
\hline 1015 & 0,029 & 0,652 & 0,039 & 0,481 & 0,750 & 0,737 & 0,363 & 0,869 & 1,073 & 0,764 & 0,621 & 0,221 & 0,137 & 0,140 & 146 & 1015 & $-1,1$ \\
\hline 1015 & 0,049 & 1,000 & 0,064 & 0,840 & 0,769 & 0,840 & 0,281 & 0,616 & 1,274 & 0,344 & 0,312 & 0,426 & 0,133 & 0,198 & 116 & 1015 & $-2,1$ \\
\hline 1015 & 0,299 & 3,735 & 0,461 & 2,602 & 0,649 & 0,697 & 0,464 & 0,269 & 1,927 & 0,266 & 0,388 & 1,197 & 0,465 & 0,297 & 155 & 1015 & $-3,6$ \\
\hline 1015 & 0,037 & 0,746 & 0,049 & 0,519 & 0,750 & 0,696 & 0,393 & 0,830 & 1,098 & 0,659 & 0,631 & 0,267 & 0,168 & 0,140 & 102 & 1015 & $-1,4$ \\
\hline 1015 & 0,051 & 1,016 & 0,066 & 0,735 & 0,778 & 0,723 & 0,355 & 0,626 & 1,264 & 0,326 & 0,331 & 0,448 & 0,149 & 0,198 & 95 & 1015 & $-2,2$ \\
\hline 1015 & 0,039 & 0,840 & 0,047 & 0,519 & 0,842 & 0,618 & 0,413 & 0,699 & 1,196 & 0,599 & 0,485 & 0,289 & 0,140 & 0,140 & 127 & 1015 & $-1,5$ \\
\hline 1015 & 0,029 & 0,773 & 0,042 & 0,574 & 0,706 & 0,743 & 0,391 & 0,618 & 1,272 & 0,449 & 0,447 & 0,289 & 0,129 & 0,099 & 17 & 1015 & $-1,5$ \\
\hline 1015 & 0,032 & 0,922 & 0,054 & 0,453 & 0,591 & 0,491 & 0,653 & 0,471 & 1,458 & 0,404 & 0,589 & 0,317 & 0,187 & 0,099 & 160 & 1015 & $-1,7$ \\
\hline 1015 & 0,044 & 0,773 & 0,049 & 0,641 & 0,900 & 0,828 & 0,199 & 0,927 & 1,039 & 0,573 & 0,474 & 0,313 & 0,149 & 0,198 & 177 & 1015 & $-1,6$ \\
\hline 1015 & 0,025 & 0,547 & 0,027 & 0,414 & 0,909 & 0,757 & 0,259 & 1,000 & 0,986 & 0,490 & 0,310 & 0,252 & 0,078 & 0,099 & 162 & 1015 & $-1,3$ \\
\hline 1015 & 0,149 & 2,453 & 0,203 & 1,547 & 0,735 & 0,631 & 0,455 & 0,312 & 1,790 & 0,400 & 0,482 & 0,690 & 0,332 & 0,198 & 150 & 1015 & $-2,8$ \\
\hline 1015 & 0,096 & 1,613 & 0,147 & 1,359 & 0,650 & 0,843 & 0,384 & 0,461 & 1,472 & 0,336 & 0,533 & 0,602 & 0,321 & 0,221 & 10 & 1015 & $-2,6$ \\
\hline 1016 & & & & & & & & nothing to measure & & & & & & & & 1016 & \\
\hline 1017 & 0,025 & 0,508 & 0,029 & 0,348 & 0,833 & 0,685 & 0,357 & 1,000 & 0,916 & 0,637 & 0,425 & 0,221 & 0,094 & 0,099 & 108 & 1017 & $-1,1$ \\
\hline 1017 & 0,025 & 0,641 & 0,032 & 0,492 & 0,769 & 0,768 & 0,328 & 0,750 & 1,155 & 0,398 & 0,250 & 0,280 & 0,070 & 0,099 & 130 & 1017 & $-1,5$ \\
\hline 1018 & & & & & & & & nothing to measure & & & & & & & & 1018 & - \\
\hline 1019 & & & & & & & & nothing to measure & & & & & & & & 1019 & - \\
\hline 1020 & & & & & & & & nothing to measure & & & & & & & & 1020 & - \\
\hline 1021 & & & & & & & & nothing to measure & & & & & & & & 1021 & - \\
\hline 1022 & & & & & & & & nothing to measure & & & & & & & & 1022 & - \\
\hline 1023 & & & & & & & & nothing to measure & & & & & & & & 1023 & - \\
\hline 1024 & 0,027 & 0,519 & 0,029 & 0,387 & 0,917 & 0,745 & 0,269 & 1,000 & 0,892 & 0,700 & 0,474 & 0,221 & 0,105 & 0,140 & 122 & 1024 & $-1,1$ \\
\hline 1025 & & & & & & & & nothing to measure & & & & & & & & 1025 & \\
\hline 1026 & 0,051 & 0,879 & 0,061 & 0,668 & 0,840 & 0,761 & 0,288 & 0,838 & 1,093 & 0,668 & 0,632 & 0,313 & 0,198 & 0,140 & 10 & 1026 & $-1,6$ \\
\hline 1027 & & & & & & & & nothing to measure & & & & & & & & 1027 & - \\
\hline 1028 & & & & & & & & nothing to measure & & & & & & & & 1028 & - \\
\hline 1029 & & & & & & & & nothing to measure & & & & & & & & 1029 & - \\
\hline 1030 & & & & & & & & nothing to measure & & & & & & & & 1030 & - \\
\hline 1031 & & & & & & & & nothing to measure & & & & & & & & 1031 & - \\
\hline 1032 & & & & & & & & nothing to measure & & & & & & & & 1032 & - \\
\hline 1033 & & & & & & & & nothing to measure & & & & & & & & 1033 & . \\
\hline 1034 & & & & & & & & nothing to measure & & & & & & & & 1034 & - \\
\hline 1035 & & & & & & & & nothing to measure & & & & & & & & 1035 & - \\
\hline 1036 & & & & & & & & nothing to measure & & & & & & & & 1036 & - \\
\hline 1037 & & & & & & & & nothing to measure & & & & & & & & 1037 & - \\
\hline
\end{tabular}


nothing to measure

nothing to measure

nothing to measure

nothing to measure

nung to ncasure

nothing to measure

nothing to measure

nothing to measure

$0,027 \quad 0,64$

0,034

0,387

0,786

0,604

0,451

hing to mea
0,825

1,101

0,412

nothing to measure
nothing to measure

nothing to measure

nothing to measure

nothing to measure

nothing to measure
nothing to measure 
Clasts and bubbles' data for sample SM-82_Br04 used for shape parameters analysis

$\phi=-\log _{2}($ diam$)$, considering the Feret Diameter in millimeters

Clasts' data

\begin{tabular}{|c|c|c|c|c|c|c|c|c|c|c|c|c|c|c|c|c|c|c|c|}
\hline Label & Area $\left(\mathrm{cm}^{2}\right)$ & $\begin{array}{l}\text { Perimeter } \\
(\mathrm{cm})\end{array}$ & CH Area & CH Perim. & $\begin{array}{l}\text { CH Perim. } \\
\text { Correction }\end{array}$ & Solidity & Convexity & $\begin{array}{l}\text { Convexity } \\
\text { Correction }\end{array}$ & $\begin{array}{l}\text { Concavity } \\
\text { Index }\end{array}$ & Form Factor & Circularity & Roundness & $\begin{array}{l}\text { Axial Ratio } \\
\text { (Feret) }\end{array}$ & $\begin{array}{c}\text { Feret } \\
\text { diameter }(\mathbf{c m})\end{array}$ & $\begin{array}{l}\text { Min. Feret } \\
\text { diam. }\end{array}$ & $\begin{array}{l}\text { Max. } \\
\text { Insc. } \\
\text { Circle } \\
\text { Diam. }\end{array}$ & Orientation & $\begin{array}{l}\text { ROI } \\
\text { Index }\end{array}$ & Phi \\
\hline 0 & 16,4 & 14,6 & 16,9 & 14,6 & 14,09 & 0,973 & 0,996 & 0,964 & 0,027 & 0,968 & 1,017 & 0,851 & 0,853 & 4,959 & 4,231 & 4,029 & 81 & 0 & $-5,6$ \\
\hline 1 & 5,0 & 8,3 & 5,2 & 8,1 & 7,87 & 0,959 & 0,980 & 0,948 & 0,045 & 0,908 & 1,050 & 0,695 & 0,676 & 3,021 & 2,043 & 2,036 & 46 & 1 & $-4,9$ \\
\hline 2 & 8,6 & 11,5 & 9,1 & 11,2 & 10,84 & 0,938 & 0,974 & 0,943 & 0,067 & 0,814 & 1,109 & 0,541 & 0,599 & 4,487 & 2,688 & 2,515 & 80 & 2 & $-5,5$ \\
\hline 3 & 0,3 & 2,3 & 0,3 & 2,1 & 2,04 & 0,986 & 0,911 & 0,882 & 0,090 & 0,759 & 1,148 & 0,389 & 0,266 & 1,030 & 0,274 & 0,411 & 180 & 3 & $-3,4$ \\
\hline 4 & 1,8 & 5,4 & 1,9 & 5,4 & 5,19 & 0,938 & 0,990 & 0,959 & 0,062 & 0,767 & 1,142 & 0,439 & 0,481 & 2,277 & 1,096 & 1,070 & 89 & 4 & $-4,5$ \\
\hline 5 & 2,2 & 5,6 & 2,2 & 5,5 & 5,33 & 0,968 & 0,977 & 0,946 & 0,039 & 0,855 & 1,082 & 0,494 & 0,549 & 2,359 & 1,296 & 1,300 & 25 & 5 & $-4,6$ \\
\hline 6 & 13,2 & 15,3 & 14,4 & 14,6 & 14,12 & 0,920 & 0,956 & 0,925 & 0,091 & 0,712 & 1,185 & 0,443 & 0,562 & 6,160 & 3,462 & 2,906 & 134 & 6 & $-5,9$ \\
\hline 7 & 0,5 & 2,7 & 0,5 & 2,5 & 2,46 & 0,972 & 0,960 & 0,929 & 0,049 & 0,888 & 1,061 & 0,467 & 0,416 & 1,164 & 0,484 & 0,581 & 127 & 7 & $-3,5$ \\
\hline 8 & 0,6 & 2,9 & 0,7 & 2,8 & 2,75 & 0,951 & 0,969 & 0,938 & 0,058 & 0,924 & 1,040 & 0,564 & 0,550 & 1,196 & 0,658 & 0,685 & 61 & 8 & $-3,6$ \\
\hline 9 & 20,4 & 17,4 & 21,3 & 17,0 & 16,44 & 0,957 & 0,979 & 0,947 & 0,048 & 0,850 & 1,084 & 0,588 & 0,598 & 6,644 & 3,971 & 3,954 & 48 & 9 & $-6,1$ \\
\hline 10 & 0,2 & 1,6 & 0,3 & 1,5 & 1,43 & 0,926 & 0,919 & 0,890 & 0,110 & 1,000 & 0,935 & 0,749 & 0,614 & 0,632 & 0,387 & 0,494 & 52 & 10 & $\begin{array}{l}-2,7 \\
-2,7\end{array}$ \\
\hline 11 & 2,4 & 6,5 & 2,6 & 6,3 & 6,05 & 0,947 & 0,962 & 0,932 & 0,065 & 0,726 & 1,173 & 0,366 & 0,409 & 2,914 & 1,191 & 1,225 & 25 & 11 & $-4,9$ \\
\hline 12 & 1,4 & 5,2 & 1,7 & 4,5 & 4,36 & 0,829 & 0,873 & 0,845 & 0,213 & 0,654 & 1,237 & 0,592 & 0,729 & 1,729 & 1,261 & 0,877 & 63 & 12 & $-4,1$ \\
\hline 13 & 8,0 & 11,8 & 8,7 & 11,3 & 10,96 & 0,912 & 0,961 & 0,930 & 0,096 & 0,721 & 1,177 & 0,510 & 0,529 & 4,460 & 2,359 & 2,280 & 24 & 13 & $-5,5$ \\
\hline 14 & 0,3 & 2,0 & 0,3 & 1,8 & 1,77 & 0,913 & 0,892 & 0,864 & 0,139 & 0,885 & 1,063 & 0,642 & 0,721 & 0,766 & 0,552 & 0,433 & 50 & 14 & $-2,9$ \\
\hline 15 & 4,1 & 7,6 & 4,3 & 7,6 & 7,31 & 0,932 & 0,993 & 0,961 & 0,068 & 0,881 & 1,066 & 0,647 & 0,697 & 2,824 & 1,967 & 1,781 & 71 & 15 & $-4,8$ \\
\hline 16 & 0,3 & 2,3 & 0,3 & 2,2 & 2,12 & 0,925 & 0,938 & 0,908 & 0,097 & 0,668 & 1,224 & 0,298 & 0,261 & 1,115 & 0,291 & 0,387 & 140 & 16 & $-3,5$ \\
\hline 17 & 3,4 & 6,9 & 3,5 & 6,8 & 6,54 & 0,964 & 0,981 & 0,950 & 0,041 & 0,889 & 1,061 & 0,597 & 0,645 & 2,675 & 1,725 & 1,644 & 135 & 17 & $-4,7$ \\
\hline 18 & 0,8 & 4,0 & 0,9 & 3,9 & 3,75 & 0,895 & 0,977 & 0,946 & 0,108 & 0,671 & 1,221 & 0,351 & 0,390 & 1,746 & 0,682 & 0,685 & 77 & 18 & $-4,1$ \\
\hline 19 & 0,4 & 2,4 & 0,5 & 2,3 & 2,23 & 0,950 & 0,956 & 0,925 & 0,067 & 0,960 & 1,021 & 0,828 & 0,850 & 0,828 & 0,704 & 0,581 & 41 & 19 & $-3,0$ \\
\hline 20 & 0,3 & 2,3 & 0,3 & 2,2 & 2,09 & 0,940 & 0,937 & 0,907 & 0,087 & 0,701 & 1,194 & 0,329 & 0,272 & 1,070 & 0,291 & 0,387 & 134 & 20 & $-3,4$ \\
\hline 21 & 1,8 & 5,1 & 1,9 & 5,0 & 4,82 & 0,924 & 0,972 & 0,941 & 0,081 & 0,847 & $\begin{array}{l}1,087 \\
\end{array}$ & 0,556 & 0,577 & 2,016 & 1,164 & 1,104 & 102 & 21 & $-4,3$ \\
\hline 22 & 0,5 & 2,6 & 0,6 & 2,6 & 2,49 & 0,967 & 0,980 & 0,948 & 0,039 & 0,995 & 1,003 & 0,557 & 0,491 & 1,115 & 0,548 & 0,685 & 6 & 22 & $-3,5$ \\
\hline 23 & 0,3 & 1,8 & 0,3 & 1,7 & 1,64 & 0,931 & 0,921 & 0,892 & 0,105 & 0,938 & 1,033 & 0,588 & 0,523 & 0,741 & 0,387 & 0,494 & 124 & 23 & $-2,9$ \\
\hline 24 & 0,2 & 1,6 & 0,2 & 1,5 & 1,41 & 0,980 & 0,941 & 0,911 & 0,062 & 1,000 & 0,904 & 0,936 & 0,728 & 0,565 & 0,411 & 0,494 & 171 & 24 & $-2,5$ \\
\hline 25 & 0,2 & 1,6 & 0,2 & 1,5 & 1,46 & 0,942 & 0,921 & 0,891 & 0,098 & 1,000 & 0,967 & 0,843 & 0,800 & 0,589 & 0,471 & 0,411 & 144 & 25 & $-2,6$ \\
\hline 26 & 2,1 & 6,0 & 2,3 & 5,6 & 5,46 & 0,925 & 0,936 & 0,907 & 0,098 & 0,744 & 1,159 & 0,509 & 0,543 & 2,318 & $\begin{array}{l}1,259 \\
\text { S }\end{array}$ & 1,263 & 63 & 26 & $\begin{array}{l}-4,5 \\
\end{array}$ \\
\hline 27 & 0,3 & 1,8 & 0,3 & 1,6 & 1,55 & 0,932 & 0,917 & 0,887 & 0,107 & 1,000 & 0,972 & 0,693 & 0,697 & 0,688 & 0,479 & 0,548 & 91 & 27 & $-2,8$ \\
\hline 28 & 0,7 & 3,1 & 0,7 & 2,9 & 2,83 & 0,911 & 0,946 & 0,915 & 0,104 & 0,884 & 1,063 & 0,597 & 0,572 & 1,196 & 0,684 & 0,685 & 170 & 28 & $-3,6$ \\
\hline 29 & 0,9 & 3,4 & 1,0 & 3,3 & 3,16 & 0,941 & 0,957 & 0,927 & 0,073 & 0,969 & 1,016 & 0,833 & 0,758 & $\begin{array}{l}1,170 \\
\text {. }\end{array}$ & 0,887 & 0,866 & 34 & 29 & $-3,5$ \\
\hline 30 & 0,4 & 2,7 & 0,5 & 2,4 & 2,34 & 0,896 & 0,885 & 0,857 & 0,155 & 0,748 & 1,156 & 0,465 & 0,434 & 1,104 & 0,479 & 0,548 & 81 & 30 & $-3,5$ \\
\hline 31 & 0,1 & 1,2 & 0,1 & 1,0 & 1,00 & 0,962 & 0,860 & 0,832 & 0,145 & 1,000 & 0,988 & 0,522 & 0,453 & 0,535 & 0,242 & 0,306 & 48 & 31 & $-2,4$ \\
\hline 32 & 2,5 & 6,0 & 2,7 & 5,8 & 5,57 & 0,916 & 0,957 & 0,926 & 0,094 & 0,870 & 1,072 & 0,786 & 0,831 & 2,014 & 1,675 & 1,507 & 38 & 32 & $-4,3$ \\
\hline 33 & 5,6 & 8,4 & 5,8 & 8,4 & 8,09 & 0,980 & 0,998 & 0,966 & 0,020 & 1,000 & 0,994 & 0,829 & 0,860 & 2,946 & 2,534 & 2,424 & 2 & 33 & $-4,9$ \\
\hline 34 & 6,1 & 9,5 & 6,5 & 9,3 & 9,00 & 0,938 & 0,975 & 0,944 & 0,067 & 0,847 & $\begin{array}{l}1,086 \\
\end{array}$ & 0,660 & 0,728 & 3,440 & 2,505 & 2,166 & 148 & 34 & $-5,1$ \\
\hline 35 & 1,0 & 3,5 & 1,0 & 3,4 & 3,31 & 0,977 & 0,974 & 0,943 & 0,035 & 1,000 & 0,999 & 0,649 & 0,599 & 1,387 & 0,831 & 0,822 & 18 & 35 & $-3,8$ \\
\hline 36 & 0,2 & 1,4 & 0,2 & 1,0 & 0,98 & 0,946 & 0,723 & 0,699 & 0,282 & 1,000 & 0,979 & 0,891 & 0,707 & 0,484 & 0,342 & 0,387 & 63 & 36 & $-2,3$ \\
\hline 37 & 13,8 & 15,0 & 15,1 & 14,0 & 13,54 & 0,917 & 0,932 & 0,902 & 0,107 & 0,772 & 1,138 & 0,710 & 0,748 & 4,977 & 3,725 & 3,425 & 82 & 37 & $-5,6$ \\
\hline 38 & 0,8 & 3,3 & 0,8 & 3,2 & 3,14 & 0,972 & 0,984 & 0,952 & 0,032 & 0,946 & 1,028 & 0,526 & 0,515 & 1,410 & 0,727 & 0,775 & 120 & 38 & $-3,8$ \\
\hline 39 & 1,1 & 4,5 & 1,4 & 4,2 & 4,08 & 0,817 & 0,935 & 0,906 & 0,194 & 0,687 & 1,207 & 0,579 & 0,690 & 1,563 & 1,079 & 0,738 & 142 & 39 & $-4,0$ \\
\hline 40 & 0,6 & 3,1 & 0,7 & 2,9 & 2,82 & 0,896 & 0,929 & 0,900 & 0,126 & 0,774 & 1,137 & 0,551 & 0,656 & 1,182 & 0,776 & 0,685 & 21 & 40 & $-3,6$ \\
\hline 41 & 0,2 & 1,5 & 0,2 & 1,4 & 1,38 & 0,956 & 0,964 & 0,933 & 0,057 & 1,000 & 0,927 & 0,684 & 0,553 & 0,613 & 0,339 & 0,387 & 119 & 41 & $-2,6$ \\
\hline 42 & 1,8 & 5,5 & 1,9 & 5,2 & 5,04 & 0,947 & 0,952 & 0,922 & 0,071 & 0,776 & 1,135 & 0,453 & 0,447 & 2,277 & 1,018 & 1,043 & 71 & 42 & $-4,5$ \\
\hline 43 & 0,9 & 3,3 & 0,9 & 3,3 & 3,20 & 0,970 & 0,995 & 0,963 & 0,031 & 1,000 & 0,986 & 0,826 & 0,740 & 1,178 & 0,872 & 0,919 & 46 & 43 & $-3,6$ \\
\hline 44 & 0,7 & 2,9 & 0,7 & 2,8 & 2,74 & 0,986 & 0,981 & 0,950 & 0,023 & 1,000 & 0,992 & 0,611 & 0,521 & 1,182 & 0,616 & 0,685 & 82 & 44 & $-3,6$ \\
\hline 45 & 0,6 & 3,2 & 0,7 & 3,1 & 2,97 & 0,853 & 0,955 & 0,924 & 0,154 & 0,698 & 1,197 & 0,431 & 0,475 & 1,300 & 0,617 & 0,613 & 19 & 45 & $\begin{array}{l}-3,7 \\
\end{array}$ \\
\hline 46 & 0,3 & 2,1 & 0,4 & 2,0 & 1,98 & 0,961 & 0,957 & 0,927 & 0,058 & 0,946 & 1,028 & 0,550 & 0,435 & 0,890 & 0,387 & 0,494 & 137 & 46 & $-3,2$ \\
\hline 47 & 0,3 & 2,3 & 0,3 & 2,2 & 2,09 & 0,915 & 0,953 & 0,922 & 0,097 & 0,621 & 1,269 & 0,282 & 0,226 & 1,070 & 0,242 & 0,306 & 39 & 47 & $-3,4$ \\
\hline
\end{tabular}




\begin{tabular}{|c|c|c|c|c|c|c|c|c|c|c|c|c|c|c|c|c|c|c|c|}
\hline 48 & 0,2 & 1,6 & 0,3 & 1,5 & 1,43 & 0,893 & 0,898 & 0,869 & 0,148 & 1,000 & 0,957 & 0,860 & 0,772 & 0,589 & 0,455 & 0,433 & 22 & 48 & $-2,6$ \\
\hline 49 & 1,7 & 5,1 & 1,9 & 4,8 & 4,68 & 0,894 & 0,949 & 0,919 & 0,118 & 0,823 & 1,102 & 0,663 & 0,794 & 1,808 & 1,435 & 1,233 & 157 & 49 & $-4,2$ \\
\hline 50 & 0,6 & 2,9 & 0,6 & 2,7 & 2,60 & 0,912 & 0,924 & 0,894 & 0,117 & 0,862 & 1,077 & 0,493 & 0,510 & 1,225 & 0,624 & 0,581 & 148 & 50 & $-3,6$ \\
\hline 51 & 6,8 & 10,3 & 7,3 & 9,8 & 9,52 & 0,933 & 0,958 & 0,928 & 0,079 & 0,813 & 1,109 & 0,642 & 0,723 & 3,679 & 2,660 & 2,548 & 119 & 51 & $-5,2$ \\
\hline 52 & 0,4 & 2,9 & 0,5 & 2,8 & 2,72 & 0,931 & 0,968 & 0,937 & 0,076 & 0,667 & 1,224 & 0,327 & 0,279 & 1,317 & 0,368 & 0,387 & 64 & 52 & $-3,7$ \\
\hline 53 & 1,1 & 3,9 & 1,2 & 3,9 & 3,75 & 0,976 & 0,996 & 0,964 & 0,025 & 0,938 & 1,032 & 0,694 & 0,713 & 1,440 & 1,026 & 0,959 & 67 & 53 & $-3,8$ \\
\hline 54 & 0,7 & 3,4 & 0,7 & 3,4 & 3,25 & 0,953 & 0,984 & 0,953 & 0,049 & 0,725 & 1,174 & 0,344 & 0,338 & 1,577 & 0,533 & 0,581 & 37 & 54 & $-4,0$ \\
\hline 55 & 0,4 & 2,3 & 0,4 & 2,3 & 2,20 & 0,951 & 0,977 & 0,946 & 0,054 & 0,852 & 1,084 & 0,574 & 0,652 & 0,901 & 0,587 & 0,548 & 174 & 55 & $-3,2$ \\
\hline 56 & 4,5 & 7,6 & 4,6 & 7,6 & 7,31 & 0,985 & 0,998 & 0,966 & 0,015 & 0,994 & 1,003 & 0,723 & 0,686 & 2,824 & 1,937 & 1,947 & 35 & 56 & $-4,8$ \\
\hline 57 & 0,8 & 3,5 & 0,8 & 3,3 & 3,21 & 0,943 & 0,952 & 0,921 & 0,075 & 0,796 & 1,121 & 0,401 & 0,403 & 1,563 & 0,630 & 0,613 & 33 & 57 & $-4,0$ \\
\hline 58 & 3,7 & 7,3 & 3,9 & 7,1 & 6,91 & 0,928 & 0,975 & 0,944 & 0,076 & 0,855 & 1,081 & 0,633 & 0,732 & 2,710 & 1,984 & 1,672 & 156 & 58 & $-4,8$ \\
\hline 59 & 0,7 & 3,0 & 0,7 & 3,0 & 2,88 & 0,955 & 0,975 & 0,944 & 0,052 & 0,938 & 1,032 & 0,693 & 0,667 & 1,130 & 0,753 & 0,738 & 3 & 59 & $-3,5$ \\
\hline 60 & 0,3 & 2,0 & 0,4 & 1,9 & 1,82 & 0,935 & 0,918 & 0,889 & 0,105 & 1,000 & 0,994 & 0,841 & 0,813 & 0,715 & 0,581 & 0,548 & 176 & 60 & $-2,8$ \\
\hline 61 & 1,3 & 4,1 & 1,4 & 4,1 & 3,94 & 0,949 & 0,987 & 0,956 & 0,053 & 0,962 & 1,020 & 0,718 & 0,739 & 1,521 & 1,125 & 1,104 & 173 & 61 & $-3,9$ \\
\hline 62 & 8,1 & 11,7 & 8,8 & 11,3 & 10,91 & 0,924 & 0,962 & 0,931 & 0,085 & 0,744 & 1,160 & 0,488 & 0,574 & 4,607 & 2,643 & 2,548 & 3 & 62 & $-5,5$ \\
\hline 63 & 0,2 & 1,7 & 0,2 & 1,5 & 1,46 & 0,962 & 0,892 & 0,863 & 0,115 & 1,000 & 0,979 & 0,555 & 0,458 & 0,741 & 0,339 & 0,387 & 129 & 63 & $-2,9$ \\
\hline 64 & 0,4 & 2,3 & 0,4 & 2,2 & 2,09 & 0,966 & 0,950 & 0,919 & 0,061 & 0,984 & 1,008 & 0,592 & 0,528 & 0,932 & 0,492 & 0,494 & 143 & 64 & $-3,2$ \\
\hline 65 & 0,8 & 3,2 & 0,9 & 3,2 & 3,06 & 0,973 & 0,972 & 0,941 & 0,039 & 0,994 & 1,003 & 0,630 & 0,613 & 1,300 & 0,797 & 0,866 & 71 & 65 & $-3,7$ \\
\hline 66 & 49,5 & 26,6 & 52,1 & 26,5 & 25,69 & 0,950 & 0,997 & 0,965 & 0,050 & 0,877 & 1,068 & 0,726 & 0,800 & 9,320 & 7,457 & 6,712 & 179 & 66 & $-6,5$ \\
\hline 67 & 0,4 & 2,4 & 0,4 & 2,2 & 2,16 & 0,944 & 0,945 & 0,915 & 0,078 & 0,898 & 1,055 & 0,585 & 0,588 & 0,932 & 0,548 & 0,548 & 104 & 67 & $-3,2$ \\
\hline 68 & 3,1 & 6,7 & 3,3 & 6,4 & 6,24 & 0,947 & 0,964 & 0,934 & 0,064 & 0,872 & 1,071 & 0,663 & 0,727 & 2,439 & 1,774 & 1,650 & 127 & 68 & $-4,6$ \\
\hline 69 & 0,4 & 2,3 & 0,4 & 2,2 & 2,15 & 0,932 & 0,954 & 0,923 & 0,082 & 0,895 & 1,057 & 0,530 & 0,499 & 0,961 & 0,479 & 0,494 & 98 & 69 & $-3,3$ \\
\hline 70 & 17,0 & 22,4 & 21,1 & 21,4 & 20,74 & 0,806 & 0,958 & 0,928 & 0,198 & 0,427 & 1,531 & 0,221 & 0,318 & 9,892 & 3,148 & 3,042 & 47 & 70 & $-6,6$ \\
\hline 71 & 0,4 & 1,9 & 0,4 & 1,9 & 1,86 & 0,987 & 0,992 & 0,960 & 0,015 & 1,000 & 0,920 & 0,876 & 0,745 & 0,715 & 0,533 & 0,581 & 149 & 71 & $-2,8$ \\
\hline 72 & 3,2 & 6,4 & 3,3 & 6,4 & 6,15 & 0,979 & 0,992 & 0,960 & 0,023 & 0,988 & 1,006 & 0,696 & 0,695 & 2,432 & 1,690 & 1,667 & 159 & 72 & $-4,6$ \\
\hline 73 & 5,9 & 9,2 & 6,2 & 9,2 & 8,91 & 0,960 & 0,998 & 0,966 & 0,040 & 0,874 & 1,070 & 0,575 & 0,629 & 3,617 & 2,276 & 2,259 & 119 & 73 & $-5,2$ \\
\hline 74 & 0,8 & 4,9 & 0,9 & 4,8 & 4,63 & 0,893 & 0,978 & 0,947 & 0,109 & 0,431 & 1,523 & 0,191 & 0,185 & 2,341 & 0,434 & 0,433 & 162 & 74 & $-4,5$ \\
\hline 75 & 1,5 & 4,7 & 1,6 & 4,5 & 4,37 & 0,934 & 0,969 & 0,938 & 0,073 & 0,846 & 1,087 & 0,588 & 0,680 & 1,781 & 1,211 & 1,104 & 32 & 75 & $-4,2$ \\
\hline 76 & 0,6 & 3,4 & 0,7 & 3,2 & 3,13 & 0,938 & 0,957 & 0,926 & 0,076 & 0,702 & 1,193 & 0,343 & 0,338 & 1,539 & 0,521 & 0,565 & 110 & 76 & $-3,9$ \\
\hline 77 & 1,8 & 4,9 & 1,8 & 4,9 & 4,72 & 0,974 & 0,989 & 0,957 & 0,028 & 0,910 & 1,048 & 0,641 & 0,641 & 1,870 & 1,199 & 1,170 & 78 & 77 & $-4,2$ \\
\hline 78 & 8,5 & 11,2 & 9,2 & 11,1 & 10,73 & 0,928 & 0,986 & 0,954 & 0,073 & 0,848 & 1,086 & 0,573 & 0,649 & 4,354 & 2,827 & 2,635 & 16 & 78 & $-5,4$ \\
\hline 79 & 0,1 & 0,9 & 0,1 & 0,7 & 0,64 & 0,952 & 0,719 & 0,696 & 0,285 & 1,000 & 0,852 & 1,019 & 0,800 & 0,342 & 0,274 & 0,306 & 59 & 79 & $-1,8$ \\
\hline 80 & 0,5 & 2,5 & 0,5 & 2,4 & 2,32 & 0,966 & 0,963 & 0,932 & 0,050 & 1,000 & 0,965 & 0,730 & 0,641 & 0,961 & 0,616 & 0,685 & 89 & 80 & $-3,3$ \\
\hline 81 & 1,8 & 6,5 & 2,2 & 6,2 & 6,05 & 0,826 & 0,958 & 0,927 & 0,179 & 0,528 & 1,376 & 0,310 & 0,484 & 2,710 & 1,312 & 1,043 & 68 & 81 & $-4,8$ \\
\hline 82 & 0,5 & 2,8 & 0,6 & 2,7 & 2,59 & 0,917 & 0,948 & 0,918 & 0,098 & 0,815 & 1,108 & 0,458 & 0,476 & 1,198 & 0,570 & 0,565 & 35 & 82 & $-3,6$ \\
\hline 83 & 0,8 & 4,0 & 1,0 & 3,8 & 3,71 & 0,850 & 0,954 & 0,924 & 0,157 & 0,642 & 1,248 & 0,331 & 0,373 & 1,781 & 0,664 & 0,685 & 22 & 83 & $-4,2$ \\
\hline 84 & 1,4 & 4,2 & 1,4 & 4,1 & 3,98 & 0,974 & 0,987 & 0,956 & 0,029 & 1,000 & 0,996 & 0,699 & 0,664 & 1,593 & 1,057 & 1,043 & 29 & 84 & $-4,0$ \\
\hline 85 & 1,0 & 3,7 & 1,1 & 3,6 & 3,50 & 0,946 & 0,975 & 0,944 & 0,059 & 0,909 & 1,049 & 0,823 & 0,898 & 1,240 & 1,114 & 0,919 & 77 & 85 & $-3,6$ \\
\hline 86 & 9,1 & 11,4 & 9,6 & 11,2 & 10,83 & 0,953 & 0,985 & 0,954 & 0,050 & 0,890 & 1,060 & 0,766 & 0,758 & 3,894 & 2,951 & 2,617 & 130 & 86 & $-5,3$ \\
\hline 87 & 1,2 & 4,0 & 1,3 & 3,9 & 3,75 & 0,945 & 0,968 & 0,937 & 0,064 & 0,944 & 1,029 & 0,629 & 0,649 & 1,562 & 1,013 & 1,043 & 164 & 87 & $-4,0$ \\
\hline 88 & 0,6 & 3,0 & 0,7 & 2,9 & 2,79 & 0,936 & 0,969 & 0,938 & 0,071 & 0,880 & 1,066 & 0,512 & 0,497 & 1,240 & 0,616 & 0,685 & 179 & 88 & $-3,6$ \\
\hline 89 & 0,2 & 1,5 & 0,2 & 1,4 & 1,34 & 0,914 & 0,905 & 0,876 & 0,128 & 0,807 & 1,113 & 0,407 & 0,354 & 0,685 & 0,242 & 0,306 & 133 & 89 & $-2,8$ \\
\hline 90 & 2,4 & 5,8 & 2,6 & 5,6 & 5,47 & 0,931 & 0,969 & 0,937 & 0,076 & 0,891 & 1,059 & 0,723 & 0,733 & 2,060 & 1,511 & 1,430 & 11 & 90 & $-4,4$ \\
\hline 91 & 0,1 & 1,1 & 0,1 & 0,9 & 0,90 & 0,964 & 0,807 & 0,781 & 0,197 & 1,000 & 0,909 & 0,688 & 0,570 & 0,484 & 0,276 & 0,306 & 41 & 91 & $-2,3$ \\
\hline 92 & 86,7 & 37,3 & 92,5 & 35,4 & 34,30 & 0,937 & 0,951 & 0,920 & 0,080 & 0,784 & 1,129 & 0,648 & 0,663 & 13,055 & 8,657 & 8,329 & 119 & 92 & $-7,0$ \\
\hline 93 & 0,1 & 1,2 & 0,1 & 1,0 & 0,98 & 0,897 & 0,847 & 0,820 & 0,185 & 1,000 & 0,969 & 0,895 & 0,822 & 0,417 & 0,342 & 0,387 & 115 & 93 & $-2,1$ \\
\hline 94 & 1,9 & 5,3 & 2,0 & 5,1 & 4,91 & 0,944 & 0,958 & 0,927 & 0,070 & 0,853 & 1,083 & 0,652 & 0,732 & 1,929 & 1,412 & 1,263 & 170 & 94 & $-4,3$ \\
\hline 95 & 3,2 & 6,6 & 3,4 & 6,5 & 6,33 & 0,951 & 0,986 & 0,955 & 0,051 & 0,914 & 1,046 & 0,667 & 0,713 & 2,470 & 1,761 & 1,650 & 113 & 95 & $-4,6$ \\
\hline 96 & 0,2 & 2,1 & 0,3 & 1,9 & 1,87 & 0,796 & 0,937 & 0,907 & 0,213 & 0,595 & 1,296 & 0,296 & 0,265 & 0,932 & 0,247 & 0,274 & 144 & 96 & $-3,2$ \\
\hline 97 & 0,2 & 1,3 & 0,2 & 1,3 & 1,25 & 0,943 & 0,960 & 0,930 & 0,070 & 1,000 & 0,965 & 0,646 & 0,526 & 0,552 & 0,291 & 0,387 & 35 & 97 & $-2,5$ \\
\hline 98 & 0,4 & 2,8 & 0,5 & 2,6 & 2,54 & 0,827 & 0,935 & 0,905 & 0,185 & 0,644 & 1,246 & 0,359 & 0,387 & 1,196 & 0,462 & 0,387 & 69 & 98 & $-3,6$ \\
\hline 99 & 5,1 & 8,4 & 5,2 & 8,4 & 8,12 & 0,977 & 0,998 & 0,966 & 0,023 & 0,907 & 1,050 & 0,541 & 0,591 & 3,465 & 2,049 & 2,087 & 109 & 99 & $-5,1$ \\
\hline 100 & 13,5 & 15,9 & 15,5 & 14,7 & 14,21 & 0,871 & 0,922 & 0,893 & 0,151 & 0,668 & 1,223 & 0,476 & 0,568 & 6,004 & 3,409 & 2,635 & 68 & 100 & $-5,9$ \\
\hline 101 & 1,4 & 4,5 & 1,4 & 4,4 & 4,25 & 0,944 & 0,980 & 0,948 & 0,059 & 0,849 & 1,085 & 0,494 & 0,536 & 1,870 & 1,002 & 0,988 & 12 & 101 & $-4,2$ \\
\hline 102 & 1,6 & 5,0 & 1,7 & 4,9 & 4,77 & 0,944 & 0,986 & 0,955 & 0,058 & 0,797 & 1,120 & 0,441 & 0,456 & 2,140 & 0,976 & 0,919 & 140 & 102 & $-4,4$ \\
\hline 103 & 4,1 & 7,9 & 4,4 & 7,8 & 7,54 & 0,936 & 0,984 & 0,952 & 0,066 & 0,820 & 1,104 & 0,621 & 0,711 & 2,897 & 2,060 & 1,786 & 14 & 103 & $-4,9$ \\
\hline 104 & 0,4 & 2,5 & 0,4 & 2,4 & 2,34 & 0,895 & 0,949 & 0,919 & 0,116 & 0,701 & 1,195 & 0,376 & 0,349 & 1,107 & 0,386 & 0,387 & 27 & 104 & $-3,5$ \\
\hline 105 & 8,2 & 10,9 & 8,7 & 10,7 & 10,37 & 0,949 & 0,985 & 0,954 & 0,053 & 0,877 & 1,068 & 0,692 & 0,730 & 3,896 & 2,845 & 2,574 & 5 & 105 & $-5,3$ \\
\hline
\end{tabular}




\begin{tabular}{|c|c|c|c|c|c|c|c|c|c|c|c|c|c|c|c|c|c|c|c|}
\hline 106 & 2,4 & 6,1 & 2,5 & 6,0 & 5,84 & 0,968 & 0,985 & 0,953 & 0,036 & 0,797 & 1,120 & 0,457 & 0,444 & 2,574 & 1,142 & 1,104 & 63 & 106 & $-4,7$ \\
\hline 107 & 0,4 & 2,3 & 0,4 & 2,1 & 2,08 & 0,962 & 0,937 & 0,906 & 0,074 & 0,841 & 1,090 & 0,417 & 0,330 & 1,037 & 0,342 & 0,411 & 97 & 107 & $-3,4$ \\
\hline 108 & 1,6 & 4,8 & 1,6 & 4,6 & 4,49 & 0,946 & 0,970 & 0,939 & 0,062 & 0,852 & 1,084 & 0,548 & 0,510 & 1,899 & 0,969 & 0,988 & 137 & 108 & $-4,2$ \\
\hline 109 & 0,3 & 2,0 & 0,4 & 2,0 & 1,89 & 0,935 & 0,955 & 0,925 & 0,079 & 1,000 & 0,994 & 0,615 & 0,544 & 0,836 & 0,455 & 0,494 & 117 & 109 & $-3,1$ \\
\hline 110 & 0,9 & 3,5 & 1,0 & 3,4 & 3,28 & 0,971 & 0,974 & 0,942 & 0,039 & 0,974 & 1,013 & 0,623 & 0,629 & 1,385 & 0,872 & 0,919 & 23 & 110 & $-3,8$ \\
\hline 111 & 1,5 & 5,1 & 1,6 & 5,1 & 4,89 & 0,895 & 0,986 & 0,955 & 0,106 & 0,706 & 1,191 & 0,427 & 0,435 & 2,097 & 0,912 & 0,833 & 145 & 111 & $-4,4$ \\
\hline 112 & 19,0 & 16,3 & 19,5 & 16,2 & 15,64 & 0,973 & 0,992 & 0,960 & 0,028 & 0,898 & 1,055 & 0,675 & 0,665 & 5,981 & 3,977 & 3,982 & 39 & 112 & $-5,9$ \\
\hline 113 & 1,2 & 4,5 & 1,3 & 4,3 & 4,16 & 0,921 & 0,951 & 0,920 & 0,093 & 0,742 & 1,161 & 0,486 & 0,584 & 1,777 & 1,037 & 0,877 & 151 & 113 & $-4,2$ \\
\hline 114 & 3,5 & 7,4 & 3,8 & 7,2 & 6,96 & 0,932 & 0,967 & 0,936 & 0,075 & 0,805 & 1,115 & 0,577 & 0,614 & 2,795 & 1,716 & 1,532 & 34 & 114 & $-4,8$ \\
\hline 115 & 1,8 & 4,9 & 1,9 & 4,8 & 4,65 & 0,948 & 0,989 & 0,957 & 0,053 & 0,961 & 1,020 & 0,802 & 0,850 & 1,692 & 1,437 & 1,349 & 124 & 115 & $-4,1$ \\
\hline 116 & 1,2 & 4,1 & 1,3 & 3,9 & 3,79 & 0,901 & 0,955 & 0,925 & 0,108 & 0,868 & 1,074 & 0,675 & 0,724 & 1,479 & 1,070 & 0,919 & 171 & 116 & $-3,9$ \\
\hline 117 & 0,3 & 2,0 & 0,3 & 1,9 & 1,81 & 0,892 & 0,917 & 0,888 & 0,136 & 0,827 & 1,099 & 0,437 & 0,373 & 0,890 & 0,332 & 0,387 & 106 & 117 & $-3,2$ \\
\hline 118 & 0,5 & 2,6 & 0,5 & 2,4 & 2,36 & 0,947 & 0,930 & 0,900 & 0,088 & 0,918 & 1,044 & 0,497 & 0,503 & 1,134 & 0,570 & 0,613 & 108 & 118 & $-3,5$ \\
\hline 119 & 0,1 & 1,0 & 0,1 & 0,9 & 0,86 & 0,958 & 0,859 & 0,832 & 0,147 & 1,000 & 0,886 & 1,010 & 0,788 & 0,369 & 0,291 & 0,306 & 23 & 119 & $-1,9$ \\
\hline 120 & 3,1 & 6,9 & 3,3 & 6,6 & 6,41 & 0,934 & 0,965 & 0,935 & 0,075 & 0,816 & 1,107 & 0,566 & 0,620 & 2,622 & 1,626 & 1,475 & 32 & 120 & $-4,7$ \\
\hline 121 & 0,1 & 1,0 & 0,1 & 0,8 & 0,82 & 1,000 & 0,822 & 0,795 & 0,178 & 1,000 & 0,906 & 0,683 & 0,442 & 0,439 & 0,194 & 0,306 & 138 & 121 & $-2,1$ \\
\hline 122 & 0,8 & 3,1 & 0,8 & 3,0 & 2,91 & 0,960 & 0,970 & 0,939 & 0,049 & 1,000 & 0,978 & 0,898 & 0,865 & 1,063 & 0,920 & 0,877 & 50 & 122 & $-3,4$ \\
\hline 123 & 0,3 & 1,9 & 0,3 & 1,7 & 1,68 & 0,939 & 0,904 & 0,875 & 0,113 & 0,993 & 1,004 & 0,607 & 0,544 & 0,781 & 0,425 & 0,433 & 50 & 123 & $-3,0$ \\
\hline 124 & 0,1 & 0,9 & 0,1 & 0,6 & 0,61 & 1,000 & 0,678 & 0,656 & 0,322 & 1,000 & 0,852 & 0,878 & 0,557 & 0,369 & 0,205 & 0,274 & 17 & 124 & $-1,9$ \\
\hline 125 & 0,2 & 1,8 & 0,3 & 1,7 & 1,61 & 0,930 & 0,948 & 0,917 & 0,088 & 1,000 & 0,990 & 0,649 & 0,614 & 0,698 & 0,429 & 0,433 & 31 & 125 & $-2,8$ \\
\hline 126 & 0,5 & 3,2 & 0,6 & 3,1 & 3,00 & 0,880 & 0,955 & 0,925 & 0,128 & 0,654 & 1,237 & 0,375 & 0,350 & 1,365 & 0,477 & 0,548 & 16 & 126 & $-3,8$ \\
\hline 127 & 0,1 & 1,1 & 0,1 & 1,0 & 0,95 & 0,963 & 0,914 & 0,885 & 0,093 & 1,000 & 0,864 & 1,142 & 0,914 & 0,369 & 0,337 & 0,387 & 90 & 127 & $-1,9$ \\
\hline 128 & 0,3 & 1,8 & 0,3 & 1,7 & 1,61 & 0,982 & 0,927 & 0,898 & 0,075 & 1,000 & 0,984 & 0,706 & 0,692 & 0,688 & 0,477 & 0,494 & 21 & 128 & $-2,8$ \\
\hline 129 & 1,7 & 5,5 & 1,9 & 5,2 & 5,07 & 0,873 & 0,953 & 0,922 & 0,135 & 0,701 & 1,195 & 0,446 & 0,448 & 2,193 & 0,981 & 0,919 & 23 & 129 & $-4,5$ \\
\hline 130 & 13,6 & 14,7 & 14,3 & 14,6 & 14,10 & 0,950 & 0,989 & 0,957 & 0,052 & 0,785 & 1,129 & 0,483 & 0,540 & 5,978 & 3,228 & 2,912 & 12 & 130 & $-5,9$ \\
\hline 131 & 0,3 & 1,8 & 0,3 & 1,8 & 1,73 & 1,000 & 0,971 & 0,940 & 0,029 & 1,000 & 0,972 & 0,597 & 0,434 & 0,781 & 0,339 & 0,387 & 47 & 131 & $-3,0$ \\
\hline 132 & 8,0 & 11,2 & 8,7 & 10,6 & 10,31 & 0,921 & 0,948 & 0,918 & 0,095 & 0,797 & 1,120 & 0,681 & 0,732 & 3,865 & 2,831 & 2,345 & 105 & 132 & $-5,3$ \\
\hline 133 & 1,6 & 5,1 & 1,7 & 5,0 & 4,84 & 0,944 & 0,989 & 0,958 & 0,057 & 0,783 & 1,130 & 0,467 & 0,458 & 2,083 & 0,955 & 0,969 & 72 & 133 & $-4,4$ \\
\hline 134 & 0,6 & 3,2 & 0,6 & 3,1 & 2,97 & 0,912 & 0,971 & 0,940 & 0,092 & 0,740 & 1,163 & 0,389 & 0,351 & 1,385 & 0,487 & 0,548 & 14 & 134 & $-3,8$ \\
\hline 135 & 84,1 & 37,9 & 90,1 & 35,5 & 34,36 & 0,933 & 0,936 & 0,906 & 0,093 & 0,734 & 1,167 & 0,573 & 0,666 & 13,663 & 9,100 & 8,087 & 151 & 135 & $-7,1$ \\
\hline 136 & 0,3 & 2,6 & 0,4 & 2,4 & 2,29 & 0,863 & 0,928 & 0,899 & 0,155 & 0,624 & 1,265 & 0,300 & 0,260 & 1,172 & 0,304 & 0,306 & 80 & 136 & $-3,6$ \\
\hline 137 & 0,2 & 1,3 & 0,2 & 1,1 & 1,07 & 1,000 & 0,868 & 0,841 & 0,132 & 1,000 & 0,915 & 0,793 & 0,549 & 0,499 & 0,274 & 0,387 & 98 & 137 & $-2,3$ \\
\hline 138 & 0,4 & 2,5 & 0,4 & 2,3 & 2,23 & 0,957 & 0,926 & 0,897 & 0,086 & 0,836 & 1,094 & 0,399 & 0,418 & 1,148 & 0,479 & 0,494 & 162 & 138 & $-3,5$ \\
\hline 139 & 3,5 & 7,2 & 3,7 & 6,8 & 6,59 & 0,951 & 0,946 & 0,916 & 0,073 & 0,843 & 1,089 & 0,510 & 0,588 & 2,946 & 1,733 & 1,781 & 33 & 139 & $-4,9$ \\
\hline 140 & 10,6 & 12,9 & 11,0 & 12,6 & 12,17 & 0,961 & 0,973 & 0,942 & 0,047 & 0,797 & 1,120 & 0,530 & 0,518 & 5,045 & 2,615 & 2,585 & 51 & 140 & $-5,7$ \\
\hline 141 & 4,1 & 7,9 & 4,4 & 7,7 & 7,46 & 0,940 & 0,975 & 0,944 & 0,065 & 0,833 & 1,096 & 0,560 & 0,588 & 3,067 & 1,803 & 1,781 & 109 & 141 & $-4,9$ \\
\hline 142 & 9,2 & 12,1 & 9,9 & 11,9 & 11,56 & 0,926 & 0,983 & 0,952 & 0,076 & 0,785 & 1,129 & 0,596 & 0,566 & 4,436 & 2,510 & 2,392 & 119 & 142 & $-5,5$ \\
\hline 143 & 0,2 & 1,5 & 0,2 & 1,5 & 1,48 & 0,949 & 1,000 & 0,968 & 0,051 & 0,933 & 1,035 & 0,471 & 0,354 & 0,685 & 0,242 & 0,306 & 143 & 143 & $-2,8$ \\
\hline 144 & 0,3 & 1,8 & 0,3 & 1,6 & 1,59 & 0,967 & 0,927 & 0,897 & 0,080 & 1,000 & 0,959 & 0,710 & 0,588 & 0,698 & 0,411 & 0,494 & 7 & 144 & $-2,8$ \\
\hline 145 & 0,1 & 1,1 & 0,1 & 0,8 & 0,82 & 0,958 & 0,793 & 0,767 & 0,211 & 1,000 & 0,919 & 0,651 & 0,527 & 0,459 & 0,242 & 0,274 & 31 & 145 & $-2,2$ \\
\hline 146 & 0,2 & 1,5 & 0,2 & 1,4 & 1,38 & 1,000 & 0,939 & 0,910 & 0,061 & 1,000 & 0,929 & 1,081 & 0,953 & 0,499 & 0,475 & 0,433 & 53 & 146 & $-2,3$ \\
\hline 147 & 0,4 & 2,2 & 0,4 & 2,1 & 2,04 & 0,934 & 0,952 & 0,921 & 0,082 & 1,000 & 0,990 & 0,866 & 0,785 & 0,766 & 0,601 & 0,548 & 82 & 147 & $-2,9$ \\
\hline 148 & 0,3 & 2,0 & 0,3 & 1,8 & 1,77 & 0,958 & 0,916 & 0,886 & 0,094 & 1,000 & 0,996 & 0,597 & 0,587 & 0,825 & 0,484 & 0,548 & 45 & 148 & $-3,0$ \\
\hline 149 & 6,7 & 9,9 & 6,9 & 9,9 & 9,54 & 0,974 & 0,995 & 0,963 & 0,027 & 0,861 & 1,078 & 0,554 & 0,514 & 3,933 & 2,020 & 2,023 & 28 & 149 & $-5,3$ \\
\hline 150 & 1,0 & 3,5 & 1,0 & 3,3 & 3,23 & 0,962 & 0,963 & 0,932 & 0,053 & 1,000 & 0,998 & 0,705 & 0,652 & 1,317 & 0,859 & 0,919 & 16 & 150 & $-3,7$ \\
\hline 151 & 0,2 & 1,9 & 0,2 & 1,7 & 1,64 & 0,961 & 0,910 & 0,881 & 0,098 & 0,831 & 1,097 & 0,369 & 0,379 & 0,890 & 0,337 & 0,387 & 111 & 151 & $-3,2$ \\
\hline 152 & 3,3 & 7,6 & 3,6 & 7,4 & 7,14 & 0,907 & 0,967 & 0,936 & 0,098 & 0,714 & 1,183 & 0,450 & 0,503 & 3,058 & 1,540 & 1,532 & 162 & 152 & $-4,9$ \\
\hline 153 & 0,6 & 2,6 & 0,6 & 2,3 & 2,23 & 0,955 & 0,886 & 0,857 & 0,123 & 1,000 & 0,956 & 0,867 & 0,735 & 0,932 & 0,685 & 0,775 & 16 & 153 & $-3,2$ \\
\hline 154 & 0,7 & 3,0 & 0,7 & 2,9 & 2,79 & 0,955 & 0,969 & 0,938 & 0,054 & 0,994 & 1,003 & 0,814 & 0,826 & 1,045 & 0,864 & 0,799 & 167 & 154 & $-3,4$ \\
\hline 155 & 0,2 & 1,4 & 0,2 & 1,3 & 1,24 & 0,923 & 0,898 & 0,869 & 0,128 & 1,000 & 0,976 & 0,637 & 0,583 & 0,581 & 0,339 & 0,387 & 57 & 155 & $-2,5$ \\
\hline 156 & 0,2 & 1,7 & 0,2 & 1,6 & 1,55 & 0,920 & 0,946 & 0,916 & 0,097 & 0,942 & 1,030 & 0,537 & 0,520 & 0,715 & 0,372 & 0,387 & 19 & 156 & $-2,8$ \\
\hline 157 & 2,3 & 5,6 & 2,4 & 5,5 & 5,31 & 0,950 & 0,974 & 0,943 & 0,056 & 0,916 & 1,045 & 0,687 & 0,724 & 2,071 & 1,498 & 1,370 & 107 & 157 & $-4,4$ \\
\hline 158 & 0,1 & 1,3 & 0,2 & 1,2 & 1,18 & 0,969 & 0,970 & 0,939 & 0,044 & 1,000 & 0,927 & 0,759 & 0,588 & 0,494 & 0,291 & 0,387 & 132 & 158 & $-2,3$ \\
\hline 159 & 0,2 & 1,5 & 0,2 & 1,3 & 1,29 & 0,944 & 0,901 & 0,873 & 0,113 & 0,921 & 1,042 & 0,481 & 0,424 & 0,650 & 0,276 & 0,306 & 68 & 159 & $-2,7$ \\
\hline 160 & 2,0 & 5,9 & 2,1 & 5,8 & 5,60 & 0,945 & 0,984 & 0,953 & 0,057 & 0,710 & 1,187 & 0,359 & 0,353 & 2,631 & 0,930 & 0,969 & 131 & 160 & $-4,7$ \\
\hline 161 & 0,2 & 1,8 & 0,2 & 1,5 & 1,43 & 0,872 & 0,843 & 0,815 & 0,203 & 0,654 & 1,237 & 0,291 & 0,290 & 0,836 & 0,242 & 0,274 & 143 & 161 & $-3,1$ \\
\hline 162 & 1,3 & 4,1 & 1,3 & 4,1 & 3,98 & 0,979 & 0,996 & 0,964 & 0,022 & 0,951 & 1,025 & 0,590 & 0,610 & 1,668 & 1,017 & 1,043 & 154 & 162 & $-4,1$ \\
\hline 163 & 0,2 & 1,4 & 0,2 & 1,3 & 1,29 & 0,943 & 0,935 & 0,905 & 0,086 & 0,963 & 1,019 & 0,467 & 0,422 & 0,650 & 0,274 & 0,306 & 110 & 163 & $-2,7$ \\
\hline
\end{tabular}




\begin{tabular}{|c|c|c|c|c|c|c|c|c|c|c|c|c|c|c|c|c|c|c|c|}
\hline 164 & 6,7 & 9,7 & 6,9 & 9,6 & 9,30 & 0,967 & 0,994 & 0,963 & 0,034 & 0,901 & 1,053 & 0,579 & 0,589 & 3,839 & 2,260 & 2,247 & 151 & 164 & $-5,3$ \\
\hline 165 & 0,1 & 1,0 & 0,1 & 0,9 & 0,86 & 1,000 & 0,906 & 0,878 & 0,094 & 1,000 & 0,859 & 0,757 & 0,493 & 0,417 & 0,205 & 0,274 & 5 & 165 & $-2,1$ \\
\hline 166 & 0,6 & 2,8 & 0,7 & 2,6 & 2,55 & 0,951 & 0,953 & 0,923 & 0,068 & 1,000 & 0,974 & 0,851 & 0,808 & 0,981 & 0,792 & 0,738 & 34 & 166 & $-3,3$ \\
\hline 167 & 1,6 & 4,8 & 1,7 & 4,6 & 4,45 & 0,946 & 0,965 & 0,934 & 0,065 & 0,910 & 1,048 & 0,728 & 0,808 & 1,694 & 1,370 & 1,170 & 99 & 167 & $-4,1$ \\
\hline 168 & 1,5 & 5,0 & 1,6 & 4,8 & 4,68 & 0,915 & 0,966 & 0,935 & 0,091 & 0,736 & 1,166 & 0,412 & 0,496 & 2,128 & 1,055 & 0,969 & 174 & 168 & $-4,4$ \\
\hline 169 & 0,5 & 2,6 & 0,5 & 2,5 & 2,46 & 0,939 & 0,965 & 0,934 & 0,070 & 0,915 & 1,045 & 0,588 & 0,618 & 1,048 & 0,648 & 0,685 & 179 & 169 & $-3,4$ \\
\hline 170 & 0,2 & 1,5 & 0,2 & 1,3 & 1,29 & 0,956 & 0,879 & 0,851 & 0,129 & 1,000 & 0,951 & 0,615 & 0,525 & 0,646 & 0,339 & 0,387 & 148 & 170 & $-2,7$ \\
\hline 171 & 0,2 & 1,4 & 0,2 & 1,1 & 1,11 & 0,976 & 0,838 & 0,811 & 0,164 & 1,000 & 0,880 & 1,004 & 0,832 & 0,494 & 0,411 & 0,433 & 45 & 171 & $-2,3$ \\
\hline 172 & 4,6 & 8,6 & 4,7 & 8,6 & 8,32 & 0,977 & 0,994 & 0,962 & 0,024 & 0,768 & 1,141 & 0,459 & 0,478 & 3,562 & 1,704 & 1,716 & 151 & 172 & $-5,2$ \\
\hline 173 & 0,5 & 2,6 & 0,6 & 2,5 & 2,45 & 0,925 & 0,979 & 0,948 & 0,078 & 0,980 & 1,010 & 0,667 & 0,680 & 0,997 & 0,678 & 0,685 & 95 & 173 & $-3,3$ \\
\hline 174 & 11,2 & 12,3 & 11,6 & 12,2 & 11,78 & 0,967 & 0,986 & 0,955 & 0,036 & 0,923 & 1,041 & 0,707 & 0,710 & 4,488 & 3,185 & 3,163 & 24 & 174 & $-5,5$ \\
\hline 175 & 0,2 & 1,7 & 0,3 & 1,6 & 1,55 & 0,963 & 0,968 & 0,936 & 0,049 & 1,000 & 0,947 & 0,656 & 0,597 & 0,688 & 0,411 & 0,494 & 173 & 175 & $-2,8$ \\
\hline 176 & 27,8 & 20,2 & 28,6 & 20,2 & 19,56 & 0,970 & 0,999 & 0,967 & 0,030 & 0,853 & 1,083 & 0,586 & 0,581 & 7,770 & 4,517 & 4,360 & 6 & 176 & $-6,3$ \\
\hline 177 & 0,7 & 3,1 & 0,7 & 3,0 & 2,87 & 0,916 & 0,946 & 0,916 & 0,100 & 0,852 & 1,083 & 0,565 & 0,553 & 1,225 & 0,678 & 0,685 & 39 & 177 & $-3,6$ \\
\hline 178 & 0,1 & 1,0 & 0,1 & 0,8 & 0,77 & 0,957 & 0,812 & 0,787 & 0,193 & 1,000 & 0,859 & 0,824 & 0,606 & 0,399 & 0,242 & 0,306 & 109 & 178 & $-2,0$ \\
\hline 179 & 4,2 & 7,6 & 4,4 & 7,5 & 7,24 & 0,954 & 0,981 & 0,949 & 0,050 & 0,898 & 1,055 & 0,627 & 0,684 & 2,903 & 1,986 & 1,961 & 167 & 179 & $-4,9$ \\
\hline 180 & 0,6 & 3,4 & 0,7 & 3,2 & 3,09 & 0,877 & 0,950 & 0,920 & 0,133 & 0,707 & 1,190 & 0,424 & 0,439 & 1,378 & 0,606 & 0,581 & 54 & 180 & $-3,8$ \\
\hline 181 & 2,3 & 5,9 & 2,5 & 5,8 & 5,60 & 0,921 & 0,975 & 0,944 & 0,083 & 0,838 & 1,092 & 0,574 & 0,681 & 2,281 & 1,552 & 1,513 & 47 & 181 & $-4,5$ \\
\hline 182 & 0,1 & 1,1 & 0,1 & 1,0 & 0,98 & 0,962 & 0,950 & 0,919 & 0,063 & 1,000 & 0,881 & 1,098 & 0,881 & 0,369 & 0,325 & 0,306 & 105 & 182 & $-1,9$ \\
\hline 183 & 2,6 & 5,9 & 2,7 & 5,8 & 5,62 & 0,972 & 0,982 & 0,950 & 0,033 & 0,950 & 1,026 & 0,639 & 0,675 & 2,296 & 1,550 & 1,550 & 122 & 183 & $-4,5$ \\
\hline 184 & 0,8 & 3,4 & 0,9 & 3,3 & 3,18 & 0,968 & 0,973 & 0,942 & 0,042 & 0,925 & 1,040 & 0,605 & 0,618 & 1,330 & 0,822 & 0,866 & 7 & 184 & $-3,7$ \\
\hline 185 & 0,4 & 2,3 & 0,4 & 2,0 & 1,98 & 0,978 & 0,909 & 0,879 & 0,094 & 1,000 & 0,979 & 0,769 & 0,733 & 0,836 & 0,613 & 0,613 & 142 & 185 & $-3,1$ \\
\hline 186 & 0,3 & 1,7 & 0,3 & 1,5 & 1,48 & 0,982 & 0,922 & 0,892 & 0,080 & 1,000 & 0,930 & 1,011 & 0,857 & 0,565 & 0,484 & 0,494 & 104 & 186 & $-2,5$ \\
\hline 187 & 0,3 & 2,1 & 0,3 & 1,9 & 1,83 & 0,941 & 0,911 & 0,882 & 0,106 & 0,880 & 1,066 & 0,471 & 0,380 & 0,901 & 0,342 & 0,387 & 85 & 187 & $-3,2$ \\
\hline 188 & 0,3 & 2,3 & 0,3 & 2,2 & 2,11 & 0,973 & 0,937 & 0,907 & 0,068 & 0,786 & 1,128 & 0,376 & 0,344 & 1,070 & 0,368 & 0,387 & 56 & 188 & $-3,4$ \\
\hline 189 & 53,7 & 33,3 & 59,4 & 28,7 & 27,80 & 0,903 & 0,863 & 0,836 & 0,168 & 0,610 & 1,281 & 0,654 & 0,795 & 10,220 & 8,127 & 0,866 & 117 & 189 & $-6,7$ \\
\hline 190 & 0,3 & 2,0 & 0,3 & 2,0 & 1,93 & 0,942 & 1,000 & 0,968 & 0,058 & 0,963 & 1,019 & 0,637 & 0,682 & 0,781 & 0,533 & 0,494 & 131 & 190 & $-3,0$ \\
\hline 191 & 0,2 & 1,3 & 0,2 & 1,1 & 1,07 & 0,889 & 0,833 & 0,806 & 0,200 & 1,000 & 0,968 & 0,627 & 0,555 & 0,552 & 0,307 & 0,306 & 154 & 191 & $-2,5$ \\
\hline 192 & 0,8 & 3,8 & 0,9 & 3,5 & 3,43 & 0,885 & 0,926 & 0,896 & 0,137 & 0,714 & 1,183 & 0,389 & 0,405 & 1,650 & 0,668 & 0,685 & 34 & 192 & $-4,0$ \\
\hline 193 & 0,1 & 1,6 & 0,2 & 1,4 & 1,36 & 0,833 & 0,885 & 0,856 & 0,203 & 0,700 & 1,195 & 0,350 & 0,310 & 0,715 & 0,222 & 0,274 & 82 & 193 & $-2,8$ \\
\hline 194 & 1,1 & 4,0 & 1,1 & 3,9 & 3,82 & 0,959 & 0,996 & 0,964 & 0,042 & 0,872 & 1,071 & 0,634 & 0,680 & 1,479 & 1,005 & 0,877 & 56 & 194 & $-3,9$ \\
\hline 195 & 2,1 & 6,4 & 2,2 & 6,3 & 6,07 & 0,955 & 0,986 & 0,954 & 0,047 & 0,647 & 1,243 & 0,325 & 0,304 & 2,855 & 0,867 & 0,919 & 29 & 195 & $-4,8$ \\
\hline 196 & 0,3 & 1,9 & 0,3 & 1,7 & 1,68 & 0,958 & 0,923 & 0,893 & 0,088 & 1,000 & 0,939 & 0,833 & 0,763 & 0,698 & 0,533 & 0,581 & 169 & 196 & $-2,8$ \\
\hline 197 & 13,5 & 15,3 & 14,2 & 15,1 & 14,58 & 0,949 & 0,984 & 0,953 & 0,053 & 0,723 & 1,176 & 0,425 & 0,452 & 6,351 & 2,868 & 2,890 & 156 & 197 & $-6,0$ \\
\hline 198 & 0,5 & 2,6 & 0,5 & 2,4 & 2,32 & 0,955 & 0,929 & 0,899 & 0,084 & 0,936 & 1,034 & 0,493 & 0,510 & 1,134 & 0,578 & 0,685 & 160 & 198 & $-3,5$ \\
\hline 199 & 1,3 & 4,2 & 1,4 & 4,2 & 4,02 & 0,949 & 0,987 & 0,956 & 0,053 & 0,924 & 1,040 & 0,677 & 0,647 & 1,563 & 1,012 & 1,043 & 125 & 199 & $-4,0$ \\
\hline 200 & 0,1 & 1,2 & 0,1 & 1,0 & 0,98 & 1,000 & 0,847 & 0,820 & 0,153 & 1,000 & 0,951 & 0,688 & 0,566 & 0,484 & 0,274 & 0,306 & 82 & 200 & $-2,3$ \\
\hline 201 & 7,5 & 10,8 & 8,0 & 10,7 & 10,32 & 0,944 & 0,983 & 0,952 & 0,058 & 0,804 & 1,115 & 0,586 & 0,698 & 4,043 & 2,821 & 2,574 & 87 & 201 & $-5,3$ \\
\hline 202 & 3,6 & 7,2 & 3,8 & 6,9 & 6,69 & 0,949 & 0,957 & 0,926 & 0,067 & 0,862 & 1,077 & 0,703 & 0,780 & 2,545 & 1,986 & 1,828 & 173 & 202 & $-4,7$ \\
\hline 203 & 2,5 & 6,1 & 2,6 & 6,0 & 5,85 & 0,962 & 0,985 & 0,953 & 0,041 & 0,828 & 1,099 & 0,470 & 0,561 & 2,593 & 1,456 & 1,456 & 169 & 203 & $-4,7$ \\
\hline 204 & 4,2 & 7,5 & 4,3 & 7,3 & 7,10 & 0,970 & 0,981 & 0,949 & 0,036 & 0,944 & 1,029 & 0,685 & 0,709 & 2,795 & 1,983 & 2,036 & 158 & 204 & $-4,8$ \\
\hline 205 & 1,2 & 3,7 & 1,3 & 3,6 & 3,52 & 0,981 & 0,975 & 0,944 & 0,031 & 1,000 & 0,947 & 0,991 & 0,925 & 1,259 & 1,164 & 1,170 & 18 & 205 & $-3,7$ \\
\hline 206 & 5,2 & 8,4 & 5,4 & 8,3 & 8,03 & 0,954 & 0,983 & 0,951 & 0,049 & 0,915 & 1,045 & 0,738 & 0,783 & 2,992 & 2,344 & 2,209 & 122 & 206 & $-4,9$ \\
\hline 207 & 0,2 & 1,4 & 0,2 & 1,2 & 1,20 & 0,976 & 0,871 & 0,843 & 0,131 & 1,000 & 0,926 & 0,979 & 0,807 & 0,494 & 0,398 & 0,433 & 161 & 207 & $-2,3$ \\
\hline 208 & 0,5 & 2,4 & 0,5 & 2,3 & 2,20 & 0,962 & 0,961 & 0,930 & 0,054 & 1,000 & 0,968 & 0,841 & 0,808 & 0,847 & 0,685 & 0,685 & 15 & 208 & $-3,1$ \\
\hline 209 & 0,6 & 2,8 & 0,7 & 2,7 & 2,59 & 0,943 & 0,948 & 0,918 & 0,077 & 0,978 & 1,011 & 0,820 & 0,820 & 0,981 & 0,804 & 0,738 & 99 & 209 & $-3,3$ \\
\hline 210 & 0,1 & 1,1 & 0,1 & 0,9 & 0,91 & 0,920 & 0,879 & 0,850 & 0,145 & 1,000 & 0,919 & 1,010 & 0,831 & 0,369 & 0,307 & 0,274 & 173 & 210 & $-1,9$ \\
\hline 211 & 0,2 & 1,4 & 0,2 & 1,3 & 1,24 & 0,975 & 0,908 & 0,878 & 0,096 & 1,000 & 0,927 & 0,856 & 0,657 & 0,522 & 0,342 & 0,387 & 172 & 211 & $-2,4$ \\
\hline 212 & 2,2 & 5,8 & 2,4 & 5,5 & 5,34 & 0,914 & 0,955 & 0,925 & 0,097 & 0,828 & 1,099 & 0,660 & 0,720 & 2,060 & 1,484 & 1,263 & 19 & 212 & $-4,4$ \\
\hline 213 & 0,4 & 2,8 & 0,5 & 2,7 & 2,60 & 0,922 & 0,949 & 0,919 & 0,093 & 0,696 & 1,199 & 0,343 & 0,325 & 1,287 & 0,419 & 0,494 & 124 & 213 & $-3,7$ \\
\hline 214 & 0,7 & 3,4 & 0,8 & 3,1 & 2,98 & 0,868 & 0,918 & 0,889 & 0,156 & 0,790 & 1,125 & 0,645 & 0,773 & 1,182 & 0,914 & 0,685 & 69 & 214 & $-3,6$ \\
\hline 215 & 0,1 & 1,1 & 0,1 & 1,0 & 0,98 & 0,917 & 0,904 & 0,875 & 0,127 & 1,000 & 0,987 & 0,572 & 0,534 & 0,479 & 0,256 & 0,274 & 84 & 215 & $-2,3$ \\
\hline 216 & 2,4 & 5,9 & 2,6 & 5,6 & 5,44 & 0,915 & 0,947 & 0,917 & 0,100 & 0,853 & 1,083 & 0,637 & 0,733 & 2,185 & $\begin{array}{l}1,602 \\
\text {. }\end{array}$ & 1,377 & 138 & 216 & $-4,4$ \\
\hline 217 & 0,5 & 2,5 & 0,5 & 2,3 & 2,18 & 0,948 & 0,910 & 0,882 & 0,104 & 1,000 & 0,977 & 0,750 & 0,724 & 0,932 & 0,674 & 0,685 & 121 & 217 & $-3,2$ \\
\hline 218 & 0,2 & 1,5 & 0,2 & 1,1 & 1,11 & 0,842 & 0,785 & 0,760 & 0,267 & 0,885 & 1,063 & 0,558 & 0,567 & 0,585 & 0,332 & 0,306 & 74 & 218 & $-2,5$ \\
\hline 219 & 0,1 & 1,2 & 0,1 & 0,9 & 0,90 & 1,000 & 0,781 & 0,756 & 0,219 & 0,967 & 1,017 & 0,431 & 0,243 & 0,565 & 0,137 & 0,274 & 4 & 219 & $-2,5$ \\
\hline 220 & 0,1 & 1,1 & 0,1 & 0,9 & 0,90 & 0,962 & 0,864 & 0,837 & 0,141 & 1,000 & 0,881 & 0,796 & 0,632 & 0,433 & 0,274 & 0,306 & 78 & 220 & $-2,1$ \\
\hline 221 & 0,3 & 2,0 & 0,4 & 1,8 & 1,77 & 0,933 & 0,892 & 0,864 & 0,127 & 0,983 & 1,008 & 0,550 & 0,493 & 0,872 & 0,430 & 0,494 & 134 & 221 & $-3,1$ \\
\hline
\end{tabular}




\begin{tabular}{|c|c|c|c|c|c|c|c|c|c|c|c|c|c|c|c|c|c|c|c|}
\hline 222 & 0,3 & 1,9 & 0,3 & 1,8 & 1,77 & 0,953 & 0,971 & 0,940 & 0,055 & 1,000 & 0,992 & 0,597 & 0,549 & 0,781 & 0,429 & 0,494 & 158 & 222 & $-3,0$ \\
\hline 223 & 1,1 & 4,5 & 1,2 & 4,4 & 4,27 & 0,887 & 0,976 & 0,945 & 0,115 & 0,682 & 1,211 & 0,377 & 0,403 & 1,934 & 0,780 & 0,738 & 22 & 223 & $-4,3$ \\
\hline 224 & 0,6 & 3,0 & 0,7 & 2,9 & 2,80 & 0,864 & 0,957 & 0,926 & 0,143 & 0,856 & 1,081 & 0,649 & 0,775 & 1,107 & 0,858 & 0,698 & 156 & 224 & $-3,5$ \\
\hline 225 & 1,9 & 5,8 & 2,1 & 5,6 & 5,46 & 0,943 & 0,966 & 0,935 & 0,067 & 0,712 & 1,185 & 0,407 & 0,411 & 2,459 & 1,012 & 0,997 & 8 & 225 & $-4,6$ \\
\hline 226 & 4,8 & 8,3 & 5,0 & 8,3 & 7,99 & 0,964 & 0,989 & 0,957 & 0,037 & 0,871 & 1,072 & 0,617 & 0,672 & 3,157 & 2,123 & 1,976 & 175 & 226 & $-5,0$ \\
\hline 227 & 0,1 & 1,0 & 0,1 & 0,8 & 0,77 & 0,955 & 0,782 & 0,757 & 0,223 & 1,000 & 0,914 & 0,723 & 0,493 & 0,417 & 0,205 & 0,274 & 175 & 227 & $-2,1$ \\
\hline 228 & 0,2 & 1,7 & 0,2 & 1,5 & 1,43 & 0,870 & 0,869 & 0,841 & 0,185 & 0,819 & 1,105 & 0,467 & 0,485 & 0,715 & 0,347 & 0,387 & 103 & 228 & $-2,8$ \\
\hline 229 & 0,1 & 1,0 & 0,1 & 0,9 & 0,87 & 1,000 & 0,922 & 0,893 & 0,078 & 1,000 & 0,879 & 0,836 & 0,625 & 0,387 & 0,242 & 0,306 & 118 & 229 & $-2,0$ \\
\hline 230 & 0,1 & 1,3 & 0,1 & 1,1 & 1,11 & 0,933 & 0,872 & 0,844 & 0,144 & 0,956 & 1,023 & 0,548 & 0,372 & 0,552 & 0,205 & 0,274 & 173 & 230 & $-2,5$ \\
\hline 231 & 0,1 & 1,0 & 0,1 & 0,9 & 0,91 & 0,913 & 0,911 & 0,882 & 0,124 & 1,000 & 0,928 & 0,723 & 0,598 & 0,417 & 0,249 & 0,274 & 164 & 231 & $-2,1$ \\
\hline 232 & 0,3 & 2,0 & 0,3 & 1,7 & 1,68 & 0,955 & 0,887 & 0,858 & 0,122 & 0,970 & 1,015 & 0,617 & 0,549 & 0,781 & 0,429 & 0,433 & 64 & 232 & $-3,0$ \\
\hline 233 & 0,3 & 1,8 & 0,3 & 1,6 & 1,58 & 0,968 & 0,899 & 0,870 & 0,106 & 1,000 & 0,963 & 0,659 & 0,557 & 0,738 & 0,411 & 0,548 & 105 & 233 & $-2,9$ \\
\hline 234 & 0,1 & 1,5 & 0,2 & 1,1 & 1,11 & 0,806 & 0,765 & 0,741 & 0,305 & 0,762 & 1,146 & 0,434 & 0,395 & 0,632 & 0,249 & 0,274 & 171 & 234 & $-2,7$ \\
\hline 235 & 3,4 & 7,5 & 3,8 & 6,9 & 6,68 & 0,889 & 0,924 & 0,895 & 0,135 & 0,760 & 1,147 & 0,726 & 0,828 & 2,432 & 2,015 & 1,802 & 98 & 235 & $-4,6$ \\
\hline 236 & 1,9 & 5,5 & 2,1 & 5,4 & 5,23 & 0,927 & 0,990 & 0,959 & 0,074 & 0,807 & 1,113 & 0,546 & 0,581 & 2,110 & 1,225 & 1,104 & 152 & 236 & $-4,4$ \\
\hline 237 & 6,5 & 9,4 & 6,6 & 9,2 & 8,92 & 0,975 & 0,984 & 0,953 & 0,029 & 0,926 & 1,039 & 0,693 & 0,659 & 3,442 & 2,267 & 2,268 & 21 & 237 & $-5,1$ \\
\hline 238 & 0,2 & 1,7 & 0,3 & 1,6 & 1,52 & 0,945 & 0,945 & 0,914 & 0,078 & 1,000 & 0,947 & 0,807 & 0,773 & 0,620 & 0,479 & 0,494 & 179 & 238 & $-2,6$ \\
\hline 239 & 0,6 & 2,8 & 0,6 & 2,6 & 2,50 & 0,932 & 0,926 & 0,897 & 0,101 & 0,932 & 1,036 & 0,672 & 0,594 & 1,045 & 0,621 & 0,613 & 19 & 239 & $-3,4$ \\
\hline 240 & 3,0 & 7,7 & 3,7 & 7,2 & 6,99 & 0,820 & 0,933 & 0,903 & 0,193 & 0,636 & 1,254 & 0,450 & 0,633 & 2,926 & 1,852 & 1,562 & 28 & 240 & $-4,9$ \\
\hline 241 & 1,5 & 4,5 & 1,6 & 4,5 & 4,34 & 0,952 & 0,988 & 0,957 & 0,050 & 0,901 & 1,054 & 0,532 & 0,535 & 1,881 & 1,007 & 1,043 & 32 & 241 & $-4,2$ \\
\hline 242 & 0,8 & 3,5 & 0,9 & 3,4 & 3,34 & 0,946 & 0,985 & 0,953 & 0,056 & 0,842 & 1,090 & 0,476 & 0,432 & 1,482 & 0,641 & 0,685 & 135 & 242 & $-3,9$ \\
\hline 243 & 0,2 & 2,4 & 0,2 & 2,3 & 2,19 & 0,918 & 0,961 & 0,930 & 0,090 & 0,478 & 1,446 & 0,213 & 0,216 & 1,123 & 0,242 & 0,306 & 141 & 243 & $-3,5$ \\
\hline 244 & 0,4 & 2,4 & 0,5 & 2,2 & 2,12 & 0,939 & 0,900 & 0,871 & 0,117 & 0,922 & 1,041 & 0,526 & 0,566 & 1,027 & 0,581 & 0,613 & 35 & 244 & $-3,4$ \\
\hline 245 & 1,4 & 4,5 & 1,4 & 4,5 & 4,34 & 0,964 & 0,988 & 0,957 & 0,038 & 0,835 & 1,095 & 0,459 & 0,423 & 1,947 & 0,823 & 0,877 & 134 & 245 & $-4,3$ \\
\hline 246 & 1,5 & 4,6 & 1,6 & 4,3 & 4,14 & 0,921 & 0,935 & 0,905 & 0,102 & 0,883 & 1,064 & 0,615 & 0,686 & 1,746 & 1,198 & 1,104 & 41 & 246 & $-4,1$ \\
\hline 247 & 0,8 & 3,4 & 0,9 & 3,2 & 3,13 & 0,943 & 0,951 & 0,920 & 0,076 & 0,923 & 1,041 & 0,631 & 0,576 & 1,309 & 0,753 & 0,822 & 176 & 247 & $-3,7$ \\
\hline 248 & 3,9 & 7,5 & 4,2 & 7,3 & 7,07 & 0,945 & 0,975 & 0,944 & 0,060 & 0,884 & 1,064 & 0,630 & 0,632 & 2,824 & 1,784 & 1,828 & 40 & 248 & $-4,8$ \\
\hline 249 & 1,2 & 4,2 & 1,3 & 4,1 & 3,95 & 0,959 & 0,974 & 0,943 & 0,048 & 0,870 & 1,072 & 0,584 & 0,551 & 1,628 & 0,897 & 0,919 & 161 & 249 & $-4,0$ \\
\hline 250 & 24,0 & 20,2 & 25,8 & 19,5 & 18,91 & 0,931 & 0,969 & 0,938 & 0,075 & 0,743 & 1,160 & 0,570 & 0,705 & 7,321 & 5,162 & 4,353 & 61 & 250 & $-6,2$ \\
\hline 251 & 0,7 & 3,1 & 0,7 & 2,9 & 2,80 & 0,911 & 0,940 & 0,910 & 0,107 & 0,888 & 1,061 & 0,560 & 0,558 & 1,235 & 0,689 & 0,685 & 38 & 251 & $-3,6$ \\
\hline 252 & 0,4 & 2,6 & 0,5 & 2,3 & 2,25 & 0,913 & 0,881 & 0,853 & 0,147 & 0,805 & 1,114 & 0,456 & 0,434 & 1,115 & 0,484 & 0,565 & 36 & 252 & $-3,5$ \\
\hline 253 & 34,3 & 23,8 & 37,1 & 22,4 & 21,71 & 0,923 & 0,943 & 0,913 & 0,096 & 0,761 & 1,146 & 0,560 & 0,670 & 8,823 & 5,908 & 5,454 & 60 & 253 & $-6,5$ \\
\hline 254 & 1,0 & 4,0 & 1,1 & 3,8 & 3,66 & 0,933 & 0,945 & 0,915 & 0,087 & 0,772 & 1,138 & 0,428 & 0,384 & 1,712 & 0,657 & 0,685 & 121 & 254 & $-4,1$ \\
\hline 255 & 0,1 & 1,1 & 0,1 & 1,0 & 0,95 & 1,000 & 0,853 & 0,826 & 0,147 & 1,000 & 0,848 & 1,161 & 0,857 & 0,399 & 0,342 & 0,411 & 0 & 255 & $-2,0$ \\
\hline 256 & 1,1 & 4,8 & 1,4 & 4,1 & 3,98 & 0,829 & 0,862 & 0,834 & 0,220 & 0,627 & 1,263 & 0,520 & 0,620 & 1,668 & 1,034 & 0,866 & 58 & 256 & $-4,1$ \\
\hline 257 & 0,9 & 4,3 & 1,1 & 4,0 & 3,91 & 0,803 & 0,940 & 0,909 & 0,206 & 0,600 & 1,290 & 0,392 & 0,397 & 1,692 & 0,672 & 0,613 & 33 & 257 & $-4,1$ \\
\hline 258 & 1,1 & 3,9 & 1,2 & 3,7 & 3,57 & 0,960 & 0,953 & 0,922 & 0,062 & 0,942 & 1,030 & 0,669 & 0,655 & 1,464 & 0,959 & 0,959 & 180 & 258 & $-3,9$ \\
\hline 259 & 1,1 & 3,7 & 1,1 & 3,6 & 3,51 & 0,971 & 0,985 & 0,954 & 0,032 & 1,000 & 0,988 & 0,778 & 0,792 & 1,346 & 1,065 & 1,070 & 24 & 259 & $-3,8$ \\
\hline 260 & 0,2 & 1,3 & 0,2 & 1,1 & 1,11 & 0,974 & 0,862 & 0,834 & 0,141 & 1,000 & 0,900 & 0,725 & 0,610 & 0,552 & 0,337 & 0,387 & 157 & 260 & $-2,5$ \\
\hline 261 & 0,5 & 2,9 & 0,6 & 2,7 & 2,57 & 0,893 & 0,928 & 0,898 & 0,129 & 0,779 & 1,133 & 0,506 & 0,516 & 1,130 & 0,583 & 0,613 & 34 & 261 & $-3,5$ \\
\hline 262 & 7,4 & 10,4 & 7,8 & 10,3 & 9,93 & 0,949 & 0,990 & 0,958 & 0,052 & 0,865 & 1,075 & 0,612 & 0,631 & 3,923 & 2,474 & 2,396 & 68 & 262 & $-5,3$ \\
\hline 263 & 1,5 & 5,2 & 1,7 & 4,8 & 4,67 & 0,874 & 0,936 & 0,906 & 0,141 & 0,711 & 1,186 & 0,462 & 0,562 & 2,033 & 1,143 & 0,919 & 20 & 263 & $-4,3$ \\
\hline 264 & 0,9 & 3,5 & 0,9 & 3,4 & 3,32 & 0,929 & 0,985 & 0,953 & 0,073 & 0,888 & 1,061 & 0,587 & 0,566 & 1,365 & 0,772 & 0,738 & 27 & 264 & $-3,8$ \\
\hline 265 & 0,9 & 3,8 & 1,0 & 3,7 & 3,54 & 0,879 & 0,971 & 0,940 & 0,124 & 0,788 & 1,127 & 0,454 & 0,473 & 1,577 & 0,747 & 0,685 & 174 & 265 & $-4,0$ \\
\hline 266 & 0,6 & 3,0 & 0,7 & 2,7 & 2,63 & 0,889 & 0,908 & 0,879 & 0,144 & 0,845 & 1,088 & 0,702 & 0,764 & 1,043 & 0,797 & 0,685 & 57 & 266 & $-3,4$ \\
\hline 267 & 0,3 & 2,2 & 0,3 & 2,1 & 2,02 & 0,915 & 0,941 & 0,912 & 0,103 & 0,780 & 1,132 & 0,404 & 0,354 & 0,981 & 0,347 & 0,387 & 16 & 267 & $-3,3$ \\
\hline 268 & 1,9 & 5,0 & 2,0 & 4,9 & 4,76 & 0,967 & 0,982 & 0,950 & 0,038 & 0,953 & 1,025 & 0,665 & 0,682 & 1,908 & 1,301 & 1,263 & 18 & 268 & $-4,3$ \\
\hline 269 & 2,2 & 6,7 & 2,5 & 6,5 & 6,28 & 0,891 & 0,975 & 0,944 & 0,112 & 0,620 & 1,270 & 0,347 & 0,357 & 2,832 & 1,011 & 1,043 & 30 & 269 & $-4,8$ \\
\hline 270 & 0,1 & 1,2 & 0,1 & 1,1 & 1,09 & 0,966 & 0,924 & 0,895 & 0,083 & 1,000 & 0,946 & 0,713 & 0,600 & 0,484 & 0,291 & 0,387 & 160 & 270 & $-2,3$ \\
\hline 271 & 0,1 & 1,2 & 0,1 & 1,1 & 1,07 & 0,963 & 0,895 & 0,866 & 0,111 & 1,000 & 1,000 & 0,509 & 0,372 & 0,552 & 0,205 & 0,274 & 7 & 271 & $-2,5$ \\
\hline 272 & 1,0 & 3,5 & 1,1 & 3,5 & 3,37 & 0,961 & 0,985 & 0,954 & 0,042 & 1,000 & 0,978 & 0,810 & 0,750 & 1,280 & 0,959 & 0,969 & 149 & 272 & $-3,7$ \\
\hline 273 & 0,1 & 1,1 & 0,1 & 0,9 & 0,86 & 0,885 & 0,828 & 0,802 & 0,207 & 1,000 & 0,919 & 0,861 & 0,687 & 0,399 & 0,274 & 0,306 & 48 & 273 & $-2,0$ \\
\hline 274 & 0,4 & 2,1 & 0,4 & 2,0 & 1,95 & 0,951 & 0,939 & 0,909 & 0,078 & 1,000 & 0,998 & 0,633 & 0,621 & 0,858 & 0,533 & 0,581 & 31 & 274 & $-3,1$ \\
\hline 275 & 0,5 & 2,6 & 0,5 & 2,5 & 2,44 & 0,974 & 0,979 & 0,948 & 0,033 & 1,000 & 0,998 & 0,597 & 0,532 & 1,063 & 0,565 & 0,581 & 147 & 275 & $-3,4$ \\
\hline 276 & 0,1 & 1,0 & 0,1 & 0,8 & 0,73 & 0,955 & 0,744 & 0,721 & 0,260 & 1,000 & 0,914 & 0,668 & 0,474 & 0,433 & 0,205 & 0,274 & 100 & 276 & $-2,1$ \\
\hline 277 & 0,2 & 1,7 & 0,2 & 1,5 & 1,46 & 0,848 & 0,892 & 0,863 & 0,187 & 0,798 & 1,119 & 0,424 & 0,379 & 0,741 & 0,281 & 0,274 & 56 & 277 & $-2,9$ \\
\hline 278 & 0,5 & 2,8 & 0,5 & 2,6 & 2,54 & 0,919 & 0,940 & 0,910 & 0,101 & 0,773 & 1,138 & 0,384 & 0,374 & 1,259 & 0,471 & 0,548 & 19 & 278 & $-3,7$ \\
\hline 279 & 0,3 & 1,9 & 0,3 & 1,7 & 1,68 & 0,918 & 0,923 & 0,893 & 0,113 & 0,934 & 1,035 & 0,495 & 0,500 & 0,822 & 0,411 & 0,494 & 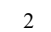 & 279 & $-3,0$ \\
\hline
\end{tabular}




\begin{tabular}{|c|c|c|c|c|c|c|c|c|c|c|c|c|c|c|c|c|c|c|c|}
\hline 280 & 10,8 & 14,3 & 11,9 & 13,8 & 13,34 & 0,908 & 0,966 & 0,935 & 0,098 & 0,665 & 1,226 & 0,402 & 0,390 & 5,841 & 2,275 & 2,166 & 39 & 280 & $-5,9$ \\
\hline 281 & 0,2 & 1,4 & 0,2 & 1,1 & 1,11 & 0,923 & 0,815 & 0,789 & 0,200 & 1,000 & 0,965 & 0,619 & 0,536 & 0,589 & 0,316 & 0,387 & 154 & 281 & $-2,6$ \\
\hline 282 & 14,0 & 14,3 & 14,4 & 14,2 & 13,73 & 0,969 & 0,988 & 0,957 & 0,033 & 0,854 & 1,082 & 0,755 & 0,825 & 4,859 & 4,010 & 3,492 & 144 & 282 & $-5,6$ \\
\hline 283 & 0,1 & 1,3 & 0,1 & 1,2 & 1,16 & 0,852 & 0,929 & 0,899 & 0,164 & 0,813 & 1,109 & 0,401 & 0,314 & 0,585 & 0,184 & 0,194 & 25 & 283 & $-2,5$ \\
\hline 284 & 0,2 & 1,4 & 0,2 & 1,3 & 1,30 & 0,974 & 0,936 & 0,906 & 0,069 & 1,000 & 0,973 & 0,645 & 0,553 & 0,585 & 0,323 & 0,387 & 30 & 284 & $-2,5$ \\
\hline 285 & 3,5 & 8,4 & 4,0 & 7,3 & 7,03 & 0,880 & 0,868 & 0,840 & 0,178 & 0,632 & 1,258 & 0,562 & 0,619 & 2,824 & 1,748 & 1,292 & 40 & 285 & $-4,8$ \\
\hline 286 & 0,1 & 1,4 & 0,1 & 1,3 & 1,27 & 0,867 & 0,934 & 0,904 & 0,149 & 0,783 & 1,130 & 0,389 & 0,272 & 0,632 & 0,172 & 0,194 & 52 & 286 & $-2,7$ \\
\hline 287 & 6,8 & 11,4 & 7,3 & 10,9 & 10,55 & 0,928 & 0,955 & 0,924 & 0,085 & 0,656 & 1,235 & 0,351 & 0,400 & 4,968 & 1,987 & 1,863 & 10 & 287 & $-5,6$ \\
\hline 288 & 0,2 & 1,6 & 0,2 & 1,6 & 1,50 & 0,961 & 0,953 & 0,923 & 0,061 & 1,000 & 0,958 & 0,643 & 0,508 & 0,675 & 0,342 & 0,387 & 103 & 288 & $-2,8$ \\
\hline 289 & 0,1 & 1,2 & 0,1 & 0,9 & 0,91 & 0,933 & 0,784 & 0,758 & 0,226 & 1,000 & 0,934 & 0,792 & 0,667 & 0,459 & 0,307 & 0,306 & 45 & 289 & $-2,2$ \\
\hline 290 & 7,5 & 11,1 & 7,9 & 11,1 & 10,70 & 0,955 & 0,992 & 0,960 & 0,045 & 0,763 & 1,145 & 0,438 & 0,507 & 4,682 & 2,375 & 2,140 & 7 & 290 & $-5,5$ \\
\hline 291 & 0,3 & 2,4 & 0,4 & 2,1 & 2,02 & 0,816 & 0,869 & 0,842 & 0,226 & 0,727 & 1,173 & 0,502 & 0,588 & 0,919 & 0,541 & 0,411 & 18 & 291 & $-3,2$ \\
\hline 292 & 5,4 & 10,2 & 5,8 & 10,1 & 9,75 & 0,942 & 0,991 & 0,959 & 0,059 & 0,661 & 1,230 & 0,335 & 0,320 & 4,546 & 1,454 & 1,456 & 38 & 292 & $-5,5$ \\
\hline 293 & 0,3 & 2,0 & 0,3 & 1,9 & 1,81 & 0,868 & 0,910 & 0,882 & 0,160 & 0,829 & 1,098 & 0,508 & 0,499 & 0,833 & 0,415 & 0,411 & 74 & 293 & $-3,1$ \\
\hline 294 & 0,2 & 1,6 & 0,2 & 1,4 & 1,39 & 0,973 & 0,887 & 0,858 & 0,116 & 0,808 & 1,113 & 0,392 & 0,327 & 0,741 & 0,242 & 0,306 & 53 & 294 & $-2,9$ \\
\hline 295 & 0,3 & 2,0 & 0,3 & 1,9 & 1,81 & 0,931 & 0,910 & 0,882 & 0,113 & 0,759 & 1,148 & 0,356 & 0,348 & 0,952 & 0,331 & 0,387 & 64 & 295 & $-3,3$ \\
\hline 296 & 2,1 & 6,0 & 2,3 & 5,9 & 5,70 & 0,922 & 0,982 & 0,951 & 0,080 & 0,737 & 1,165 & 0,423 & 0,447 & 2,518 & 1,125 & 1,096 & 26 & 296 & $-4,7$ \\
\hline 297 & 0,2 & 1,4 & 0,2 & 1,2 & 1,18 & 0,921 & 0,846 & 0,819 & 0,173 & 0,999 & 1,001 & 0,686 & 0,614 & 0,552 & 0,339 & 0,387 & 135 & 297 & $-2,5$ \\
\hline 298 & 0,1 & 1,0 & 0,1 & 0,7 & 0,68 & 1,000 & 0,692 & 0,670 & 0,308 & 1,000 & 0,914 & 0,594 & 0,447 & 0,459 & 0,205 & 0,274 & 163 & 298 & $-2,2$ \\
\hline 299 & 0,2 & 1,3 & 0,2 & 1,2 & 1,12 & 0,972 & 0,927 & 0,897 & 0,078 & 1,000 & 0,873 & 0,990 & 0,738 & 0,459 & 0,339 & 0,387 & 53 & 299 & $-2,2$ \\
\hline 300 & 1,0 & 3,7 & 1,0 & 3,6 & 3,52 & 0,963 & 0,975 & 0,944 & 0,045 & 0,873 & 1,070 & 0,525 & 0,520 & 1,532 & 0,797 & 0,799 & 101 & 300 & $-3,9$ \\
\hline 301 & 0,6 & 3,2 & 0,6 & 3,0 & 2,90 & 0,902 & 0,942 & 0,912 & 0,114 & 0,694 & 1,200 & 0,340 & 0,332 & 1,445 & 0,479 & 0,548 & 91 & 301 & $-3,9$ \\
\hline 302 & 0,3 & 2,2 & 0,4 & 2,0 & 1,91 & 0,933 & 0,915 & 0,885 & 0,108 & 0,888 & 1,061 & 0,492 & 0,416 & 0,922 & 0,384 & 0,387 & 39 & 302 & $-3,2$ \\
\hline 303 & 0,8 & 4,3 & 0,9 & 4,1 & 4,00 & 0,883 & 0,970 & 0,938 & 0,121 & 0,564 & 1,332 & 0,262 & 0,317 & 1,991 & 0,631 & 0,685 & 20 & 303 & $-4,3$ \\
\hline 304 & 0,3 & 2,0 & 0,3 & 1,8 & 1,72 & 0,910 & 0,889 & 0,860 & 0,143 & 0,904 & 1,052 & 0,532 & 0,481 & 0,828 & 0,398 & 0,433 & 63 & 304 & $-3,0$ \\
\hline 305 & 0,2 & 1,3 & 0,2 & 1,2 & 1,20 & 0,889 & 0,931 & 0,901 & 0,131 & 1,000 & 0,968 & 0,784 & 0,770 & 0,494 & 0,380 & 0,387 & 145 & 305 & $-2,3$ \\
\hline 306 & 0,2 & 1,5 & 0,2 & 1,3 & 1,29 & 0,977 & 0,911 & 0,882 & 0,092 & 1,000 & 0,928 & 0,877 & 0,687 & 0,535 & 0,368 & 0,387 & 28 & 306 & $-2,4$ \\
\hline 307 & 9,3 & 10,8 & 9,5 & 10,8 & 10,43 & 0,983 & 0,999 & 0,967 & 0,017 & 1,000 & 0,999 & 0,898 & 0,887 & 3,631 & 3,219 & 3,151 & 142 & 307 & $-5,2$ \\
\hline 308 & 0,1 & 1,1 & 0,1 & 0,9 & 0,91 & 0,923 & 0,848 & 0,821 & 0,170 & 1,000 & 0,932 & 0,679 & 0,566 & 0,459 & 0,260 & 0,274 & 27 & 308 & $-2,2$ \\
\hline 309 & 6,0 & 8,8 & 6,2 & 8,8 & 8,53 & 0,981 & 0,998 & 0,966 & 0,019 & 0,974 & 1,013 & 0,796 & 0,760 & 3,106 & 2,360 & 2,333 & 128 & 309 & $-5,0$ \\
\hline 310 & 4,0 & 7,7 & 4,1 & 7,5 & 7,29 & 0,964 & 0,983 & 0,952 & 0,040 & 0,856 & 1,081 & 0,550 & 0,518 & 3,040 & 1,575 & 1,644 & 179 & 310 & $-4,9$ \\
\hline 311 & 0,2 & 1,4 & 0,2 & 1,2 & 1,20 & 0,921 & 0,895 & 0,866 & 0,131 & 1,000 & 0,963 & 0,768 & 0,646 & 0,522 & 0,337 & 0,387 & 117 & 311 & $-2,4$ \\
\hline 312 & 8,9 & 11,3 & 9,3 & 11,2 & 10,84 & 0,958 & 0,992 & 0,960 & 0,043 & 0,879 & 1,067 & 0,709 & 0,741 & 4,001 & 2,965 & 2,824 & 124 & 312 & $-5,3$ \\
\hline 313 & 0,2 & 1,5 & 0,2 & 1,0 & 0,98 & 0,932 & 0,686 & 0,664 & 0,322 & 1,000 & 0,953 & 0,705 & 0,574 & 0,589 & 0,338 & 0,411 & 5 & 313 & $-2,6$ \\
\hline 314 & 6,5 & 11,4 & 7,0 & 11,1 & 10,73 & 0,935 & 0,973 & 0,942 & 0,070 & 0,630 & 1,260 & 0,313 & 0,320 & 5,144 & 1,644 & 1,781 & 178 & 314 & $-5,7$ \\
\hline 315 & 0,2 & 1,8 & 0,2 & 1,6 & 1,52 & 0,920 & 0,876 & 0,848 & 0,147 & 0,848 & 1,086 & 0,469 & 0,440 & 0,766 & 0,337 & 0,387 & 146 & 315 & $-2,9$ \\
\hline 316 & 0,6 & 2,7 & 0,6 & 2,6 & 2,52 & 0,969 & 0,980 & 0,948 & 0,037 & 1,000 & 0,970 & 0,809 & 0,778 & 0,969 & 0,754 & 0,775 & 18 & 316 & $-3,3$ \\
\hline 317 & 0,1 & 0,9 & 0,1 & 0,8 & 0,77 & 1,000 & 0,845 & 0,819 & 0,155 & 1,000 & 0,866 & 1,019 & 0,800 & 0,342 & 0,274 & 0,274 & 90 & 317 & $-1,8$ \\
\hline 318 & 0,1 & 1,1 & 0,1 & 0,9 & 0,86 & 0,917 & 0,800 & 0,775 & 0,217 & 1,000 & 0,973 & 0,560 & 0,500 & 0,484 & 0,242 & 0,274 & 141 & 318 & $-2,3$ \\
\hline 319 & 0,3 & 2,1 & 0,3 & 2,0 & 1,91 & 0,929 & 0,938 & 0,908 & 0,094 & 0,867 & 1,074 & 0,490 & 0,438 & 0,890 & 0,390 & 0,387 & 31 & 319 & $-3,2$ \\
\hline 320 & 5,2 & 8,8 & 5,3 & 8,8 & 8,53 & 0,974 & 0,998 & 0,966 & 0,026 & 0,833 & 1,096 & 0,634 & 0,754 & 3,220 & 2,427 & 2,073 & 81 & 320 & $-5,0$ \\
\hline 321 & 1,9 & 5,1 & 2,0 & 4,9 & 4,72 & 0,957 & 0,961 & 0,930 & 0,058 & 0,913 & 1,046 & 0,687 & 0,746 & 1,863 & 1,391 & 1,349 & 49 & 321 & $-4,2$ \\
\hline 322 & 0,4 & 2,2 & 0,4 & 2,0 & 1,95 & 0,926 & 0,923 & 0,893 & 0,107 & 0,932 & 1,036 & 0,735 & 0,784 & 0,781 & 0,612 & 0,548 & 7 & 322 & $-3,0$ \\
\hline 323 & 1,3 & 4,3 & 1,3 & 4,2 & 4,02 & 0,954 & 0,966 & 0,935 & 0,057 & 0,863 & 1,077 & 0,531 & 0,508 & 1,746 & 0,888 & 0,866 & 91 & 323 & $-4,1$ \\
\hline 324 & 0,1 & 1,1 & 0,1 & 0,8 & 0,81 & 0,920 & 0,778 & 0,754 & 0,236 & 1,000 & 0,919 & 1,010 & 0,743 & 0,369 & 0,274 & 0,306 & 122 & 324 & $-1,9$ \\
\hline 325 & 0,3 & 2,0 & 0,3 & 1,9 & 1,81 & 0,971 & 0,928 & 0,898 & 0,078 & 0,992 & 1,004 & 0,551 & 0,508 & 0,858 & 0,436 & 0,494 & 122 & 325 & $-3,1$ \\
\hline 326 & 2,7 & 7,2 & 3,3 & 6,7 & 6,46 & 0,824 & 0,932 & 0,902 & 0,189 & 0,659 & 1,232 & 0,456 & 0,609 & 2,741 & 1,669 & 0,775 & 101 & 326 & $-4,8$ \\
\hline 327 & 0,1 & 1,0 & 0,1 & 0,8 & 0,77 & 1,000 & 0,782 & 0,757 & 0,218 & 1,000 & 0,837 & 0,936 & 0,686 & 0,399 & 0,274 & 0,387 & 156 & 327 & $-2,0$ \\
\hline 328 & 0,1 & 1,2 & 0,1 & 1,1 & 1,11 & 0,968 & 0,926 & 0,896 & 0,081 & 1,000 & 0,931 & 0,588 & 0,496 & 0,552 & 0,274 & 0,387 & 111 & 328 & $-2,5$ \\
\hline 329 & 0,6 & 3,3 & 0,7 & 3,0 & 2,95 & 0,836 & 0,937 & 0,907 & 0,176 & 0,679 & 1,214 & 0,426 & 0,391 & $\begin{array}{l}1,309 \\
\text {. }\end{array}$ & 0,511 & 0,548 & 95 & 329 & $-3,7$ \\
\hline 330 & 12,9 & 15,5 & 14,1 & 14,9 & 14,44 & 0,918 & 0,962 & 0,931 & 0,091 & 0,674 & 1,218 & 0,376 & 0,423 & 6,607 & 2,796 & 2,774 & 30 & 330 & $-6,0$ \\
\hline 331 & 0,3 & 1,9 & 0,3 & 1,8 & 1,69 & 0,931 & 0,912 & 0,883 & 0,111 & 0,865 & 1,075 & 0,474 & 0,415 & 0,825 & 0,342 & 0,387 & 36 & 331 & $-3,0$ \\
\hline 332 & 0,3 & 1,8 & 0,3 & 1,6 & 1,55 & 0,966 & 0,917 & 0,887 & 0,090 & 1,000 & 0,964 & 0,735 & 0,574 & 0,675 & 0,387 & 0,433 & 40 & 332 & $-2,8$ \\
\hline 333 & 0,3 & 2,0 & 0,4 & 1,9 & 1,86 & 0,947 & 0,954 & 0,924 & 0,070 & 1,000 & 0,976 & 0,674 & 0,590 & 0,799 & 0,471 & 0,494 & 146 & 333 & $-3,0$ \\
\hline 334 & 0,8 & 3,2 & 0,8 & 3,2 & 3,07 & 0,960 & 0,983 & 0,952 & 0,043 & 0,963 & 1,019 & 0,620 & 0,565 & 1,280 & 0,722 & 0,738 & 115 & 334 & $-3,7$ \\
\hline 335 & 0,4 & 2,2 & 0,4 & 2,0 & 1,98 & 0,926 & 0,940 & 0,910 & 0,095 & 0,932 & 1,036 & 0,516 & 0,499 & 0,932 & 0,465 & 0,548 & 164 & 335 & $-3,2$ \\
\hline 336 & 0,3 & 1,9 & 0,3 & 1,8 & 1,77 & 0,958 & 0,971 & 0,940 & 0,051 & 1,000 & 0,939 & 0,746 & 0,657 & 0,738 & 0,484 & 0,565 & 146 & 336 & $-2,9$ \\
\hline 337 & 0,2 & 1,7 & 0,2 & 1,4 & 1,38 & 0,822 & 0,838 & 0,811 & 0,241 & 0,757 & 1,149 & 0,406 & 0,457 & 0,738 & 0,337 & 0,387 & 20 & 337 & $-2,9$ \\
\hline
\end{tabular}




\begin{tabular}{|c|c|c|c|c|c|c|c|c|c|c|c|c|c|c|c|c|c|c|c|}
\hline 338 & 1,5 & 4,3 & 1,5 & 4,2 & 4,08 & 0,951 & 0,979 & 0,947 & 0,053 & 0,997 & 1,002 & 0,727 & 0,721 & 1,603 & 1,156 & 1,104 & 174 & 338 & $-4,0$ \\
\hline 339 & 0,1 & 1,2 & 0,1 & 1,0 & 0,95 & 0,903 & 0,790 & 0,765 & 0,231 & 1,000 & 0,964 & 0,792 & 0,723 & 0,459 & 0,332 & 0,387 & 156 & 339 & $-2,2$ \\
\hline 340 & 3,1 & 7,3 & 3,4 & 7,1 & 6,90 & 0,919 & 0,982 & 0,951 & 0,083 & 0,745 & 1,159 & 0,436 & 0,505 & 3,017 & 1,523 & 1,410 & 141 & 340 & $-4,9$ \\
\hline 341 & 29,8 & 21,4 & 31,6 & 21,0 & 20,30 & 0,941 & 0,979 & 0,948 & 0,062 & 0,815 & 1,108 & 0,624 & 0,621 & 7,795 & 4,843 & 4,392 & 31 & 341 & $-6,3$ \\
\hline 342 & 0,2 & 1,6 & 0,2 & 1,5 & 1,48 & 0,900 & 0,943 & 0,913 & 0,115 & 1,000 & 0,995 & 0,699 & 0,584 & 0,620 & 0,362 & 0,387 & 25 & 342 & $-2,6$ \\
\hline 343 & 0,6 & 2,7 & 0,6 & 2,7 & 2,57 & 0,941 & 0,967 & 0,936 & 0,068 & 0,994 & 1,003 & 0,809 & 0,800 & 0,969 & 0,775 & 0,775 & 37 & 343 & $-3,3$ \\
\hline 344 & 0,3 & 1,9 & 0,3 & 1,7 & 1,65 & 1,000 & 0,903 & 0,874 & 0,097 & 1,000 & 0,972 & 0,627 & 0,439 & 0,781 & 0,342 & 0,411 & 92 & 344 & $-3,0$ \\
\hline 345 & 24,7 & 21,8 & 27,2 & 19,8 & 19,20 & 0,908 & 0,912 & 0,883 & 0,127 & 0,656 & 1,235 & 0,475 & 0,571 & 8,131 & 4,646 & 4,299 & 5 & 345 & $-6,3$ \\
\hline 346 & 0,1 & 1,2 & 0,1 & 1,1 & 1,04 & 0,962 & 0,921 & 0,891 & 0,088 & 1,000 & 0,957 & 0,549 & 0,464 & 0,522 & 0,242 & 0,274 & 149 & 346 & $-2,4$ \\
\hline 347 & 0,2 & 1,7 & 0,2 & 1,5 & 1,48 & 0,921 & 0,922 & 0,892 & 0,111 & 0,750 & 1,155 & 0,328 & 0,279 & 0,799 & 0,223 & 0,274 & 32 & 347 & $-3,0$ \\
\hline 348 & 28,7 & 20,8 & 30,5 & 20,2 & 19,55 & 0,943 & 0,969 & 0,938 & 0,065 & 0,831 & 1,097 & 0,710 & 0,783 & 7,179 & 5,621 & 5,024 & 2 & 348 & $-6,2$ \\
\hline 349 & 12,0 & 14,7 & 12,9 & 14,2 & 13,77 & 0,932 & 0,971 & 0,940 & 0,074 & 0,702 & 1,193 & 0,426 & 0,498 & 5,990 & 2,982 & 2,774 & 46 & 349 & $-5,9$ \\
\hline 350 & 41,4 & 26,1 & 44,0 & 24,3 & 23,50 & 0,941 & 0,929 & 0,900 & 0,092 & 0,763 & 1,145 & 0,668 & 0,678 & 8,883 & 6,019 & 5,833 & 178 & 350 & $-6,5$ \\
\hline 351 & 0,2 & 1,3 & 0,2 & 1,1 & 1,07 & 0,946 & 0,858 & 0,830 & 0,152 & 1,000 & 0,899 & 1,087 & 0,883 & 0,439 & 0,387 & 0,411 & 79 & 351 & $-2,1$ \\
\hline 352 & 0,2 & 1,6 & 0,2 & 1,4 & 1,34 & 0,958 & 0,892 & 0,863 & 0,116 & 1,000 & 0,942 & 0,658 & 0,559 & 0,646 & 0,361 & 0,387 & 61 & 352 & $-2,7$ \\
\hline 353 & 1,4 & 6,2 & 1,6 & 5,9 & 5,70 & 0,885 & 0,955 & 0,925 & 0,124 & 0,464 & 1,468 & 0,224 & 0,211 & 2,824 & 0,597 & 0,565 & 48 & 353 & $-4,8$ \\
\hline 354 & 1,3 & 4,6 & 1,4 & 4,6 & 4,44 & 0,943 & 0,988 & 0,957 & 0,059 & 0,764 & 1,144 & 0,418 & 0,381 & 1,997 & 0,760 & 0,775 & 35 & 354 & $-4,3$ \\
\hline 355 & 0,2 & 1,5 & 0,2 & 1,2 & 1,20 & 0,850 & 0,827 & 0,800 & 0,229 & 0,893 & 1,058 & 0,481 & 0,422 & 0,650 & 0,274 & 0,306 & 174 & 355 & $-2,7$ \\
\hline 356 & 0,2 & 1,6 & 0,2 & 1,5 & 1,41 & 0,980 & 0,918 & 0,889 & 0,084 & 1,000 & 0,935 & 0,734 & 0,542 & 0,632 & 0,342 & 0,411 & 4 & 356 & $-2,7$ \\
\hline 357 & 0,3 & 1,7 & 0,3 & 1,6 & 1,50 & 0,965 & 0,894 & 0,866 & 0,111 & 1,000 & 0,964 & 0,642 & 0,575 & 0,715 & 0,411 & 0,494 & 97 & 357 & $-2,8$ \\
\hline 358 & 0,9 & 3,6 & 1,0 & 3,4 & 3,30 & 0,884 & 0,939 & 0,909 & 0,131 & 0,854 & 1,082 & 0,676 & 0,703 & 1,300 & 0,914 & 0,822 & 63 & 358 & $-3,7$ \\
\hline 359 & 1,9 & 5,1 & 2,0 & 5,0 & 4,81 & 0,940 & 0,967 & 0,936 & 0,068 & 0,912 & 1,047 & 0,649 & 0,632 & 1,938 & 1,225 & 1,104 & 27 & 359 & $-4,3$ \\
\hline 360 & 2,9 & 6,9 & 3,1 & 6,5 & 6,32 & 0,914 & 0,939 & 0,909 & 0,106 & 0,748 & 1,156 & 0,598 & 0,728 & 2,475 & 1,802 & 1,456 & 176 & 360 & $-4,6$ \\
\hline 361 & 0,4 & 2,3 & 0,4 & 2,1 & 2,03 & 0,947 & 0,920 & 0,890 & 0,096 & 1,000 & 0,992 & 0,678 & 0,631 & 0,890 & 0,562 & 0,581 & 60 & 361 & $-3,2$ \\
\hline 362 & 5,9 & 9,1 & 6,1 & 9,0 & 8,70 & 0,959 & 0,988 & 0,957 & 0,042 & 0,890 & 1,060 & 0,690 & 0,652 & 3,288 & 2,145 & 2,166 & 17 & 362 & $-5,0$ \\
\hline 363 & 0,2 & 1,6 & 0,2 & 1,5 & 1,43 & 0,957 & 0,941 & 0,911 & 0,072 & 1,000 & 0,962 & 0,699 & 0,663 & 0,620 & 0,411 & 0,411 & 178 & 363 & $-2,6$ \\
\hline 364 & 1,7 & 5,3 & 1,9 & 5,3 & 5,09 & 0,916 & 0,986 & 0,954 & 0,086 & 0,764 & 1,144 & 0,434 & 0,479 & 2,253 & 1,080 & 1,096 & 18 & 364 & $-4,5$ \\
\hline 365 & 21,6 & 18,0 & 22,9 & 17,6 & 16,99 & 0,941 & 0,977 & 0,946 & 0,063 & 0,841 & 1,090 & 0,656 & 0,680 & 6,475 & 4,404 & 3,975 & 18 & 365 & $-6,0$ \\
\hline 366 & 0,3 & 1,6 & 0,3 & 1,4 & 1,36 & 0,982 & 0,864 & 0,836 & 0,137 & 1,000 & 0,904 & 0,946 & 0,814 & 0,589 & 0,479 & 0,548 & 61 & 366 & $-2,6$ \\
\hline 367 & 0,2 & 2,7 & 0,2 & 2,5 & 2,45 & 0,660 & 0,932 & 0,903 & 0,346 & 0,280 & 1,889 & 0,119 & 0,140 & 1,323 & 0,185 & 0,194 & 21 & 367 & $-3,7$ \\
\hline 368 & 0,1 & 1,3 & 0,1 & 1,1 & 1,02 & 1,000 & 0,826 & 0,800 & 0,174 & 1,000 & 0,960 & 0,523 & 0,351 & 0,585 & 0,205 & 0,274 & 172 & 368 & $-2,5$ \\
\hline 369 & 0,2 & 1,3 & 0,2 & 1,2 & 1,12 & 0,971 & 0,874 & 0,846 & 0,130 & 1,000 & 0,953 & 0,808 & 0,784 & 0,494 & 0,387 & 0,387 & 64 & 369 & $-2,3$ \\
\hline 370 & 1,3 & 4,3 & 1,4 & 4,2 & 4,02 & 0,969 & 0,970 & 0,939 & 0,043 & 0,914 & 1,046 & 0,663 & 0,716 & 1,599 & 1,145 & 1,070 & 8 & 370 & $-4,0$ \\
\hline 371 & 0,9 & 3,4 & 0,9 & 3,3 & 3,18 & 0,960 & 0,973 & 0,942 & 0,049 & 0,991 & 1,005 & 0,666 & 0,703 & 1,309 & 0,919 & 0,969 & 178 & 371 & $-3,7$ \\
\hline 372 & 0,6 & 3,0 & 0,6 & 3,0 & 2,86 & 0,949 & 0,970 & 0,939 & 0,059 & 0,831 & 1,097 & 0,432 & 0,402 & 1,346 & 0,541 & 0,548 & 167 & 372 & $-3,8$ \\
\hline 373 & 2,4 & 5,9 & 2,5 & 5,6 & 5,47 & 0,947 & 0,962 & 0,931 & 0,065 & 0,861 & 1,078 & 0,567 & 0,650 & 2,302 & 1,496 & 1,430 & 22 & 373 & $-4,5$ \\
\hline 374 & 0,6 & 2,9 & 0,6 & 2,7 & 2,64 & 0,938 & 0,949 & 0,919 & 0,080 & 0,856 & 1,081 & 0,499 & 0,485 & 1,198 & 0,581 & 0,613 & 48 & 374 & $-3,6$ \\
\hline 375 & 0,2 & 1,6 & 0,2 & 1,4 & 1,40 & 0,907 & 0,908 & 0,879 & 0,130 & 0,910 & 1,048 & 0,477 & 0,392 & 0,698 & 0,274 & 0,306 & 99 & 375 & $-2,8$ \\
\hline 376 & 3,6 & 7,1 & 3,8 & 6,8 & 6,63 & 0,950 & 0,969 & 0,938 & 0,059 & 0,903 & 1,052 & 0,775 & 0,839 & 2,429 & 2,039 & 1,828 & 77 & 376 & $-4,6$ \\
\hline 377 & 5,3 & 8,9 & 5,6 & 8,8 & 8,47 & 0,950 & 0,984 & 0,952 & 0,052 & 0,837 & 1,093 & 0,557 & 0,579 & 3,472 & 2,010 & 2,036 & 63 & 377 & $-5,1$ \\
\hline 378 & 0,1 & 1,1 & 0,1 & 0,7 & 0,70 & 1,000 & 0,681 & 0,660 & 0,319 & 1,000 & 0,887 & 0,745 & 0,625 & 0,439 & 0,274 & 0,306 & 154 & 378 & $-2,1$ \\
\hline 379 & 12,7 & 14,3 & 13,4 & 13,8 & 13,35 & 0,943 & 0,966 & 0,935 & 0,067 & 0,780 & 1,132 & 0,567 & 0,629 & 5,335 & 3,356 & 3,288 & 172 & 379 & $-5,7$ \\
\hline 380 & 2,9 & 6,2 & 3,0 & 6,1 & 5,89 & 0,967 & 0,989 & 0,957 & 0,035 & 0,954 & 1,024 & 0,818 & 0,778 & 2,116 & 1,647 & 1,655 & 139 & 380 & $-4,4$ \\
\hline 381 & 0,1 & 1,1 & 0,1 & 0,8 & 0,82 & 0,955 & 0,793 & 0,767 & 0,212 & 1,000 & 0,962 & 0,594 & 0,467 & 0,459 & 0,215 & 0,274 & 66 & 381 & $-2,2$ \\
\hline 382 & 0,2 & 1,6 & 0,2 & 1,5 & 1,43 & 0,952 & 0,919 & 0,890 & 0,094 & 0,915 & 1,045 & 0,451 & 0,399 & 0,728 & 0,291 & 0,387 & 142 & 382 & $-2,9$ \\
\hline 383 & 0,2 & 1,3 & 0,2 & 1,3 & 1,21 & 0,973 & 0,932 & 0,903 & 0,073 & 1,000 & 0,924 & 0,705 & 0,526 & 0,552 & 0,291 & 0,387 & 40 & 383 & $-2,5$ \\
\hline 384 & 6,2 & 10,2 & 6,9 & 9,7 & 9,37 & 0,891 & 0,946 & 0,915 & 0,122 & 0,739 & 1,163 & 0,480 & 0,611 & 4,043 & 2,471 & 2,427 & 64 & 384 & $-5,3$ \\
\hline 385 & 7,3 & 10,7 & 7,8 & 10,4 & 10,05 & 0,929 & 0,974 & 0,942 & 0,075 & 0,806 & 1,114 & 0,541 & 0,547 & 4,141 & 2,266 & 2,131 & 132 & 385 & $-5,4$ \\
\hline 386 & 0,1 & 1,3 & 0,1 & 1,2 & 1,12 & 0,897 & 0,899 & 0,871 & 0,144 & 0,919 & 1,043 & 0,447 & 0,411 & 0,589 & 0,242 & 0,306 & 44 & 386 & $-2,6$ \\
\hline 387 & 0,4 & 2,9 & 0,4 & 2,7 & 2,66 & 0,852 & 0,950 & 0,919 & 0,156 & 0,529 & 1,374 & 0,242 & 0,212 & 1,360 & 0,288 & 0,306 & 52 & 387 & $-3,8$ \\
\hline 388 & 1,0 & 3,4 & 1,0 & 3,4 & 3,25 & 0,981 & 0,984 & 0,953 & 0,025 & 1,000 & 0,981 & 0,772 & 0,692 & 1,259 & 0,872 & 0,969 & 41 & 388 & $-3,7$ \\
\hline 389 & 0,4 & 2,1 & 0,4 & 2,1 & 2,02 & 0,966 & 0,975 & 0,944 & 0,042 & 1,000 & 0,956 & 0,676 & 0,632 & 0,866 & 0,548 & 0,613 & 107 & 389 & $-3,1$ \\
\hline 390 & 0,1 & 1,2 & 0,1 & 1,0 & 0,98 & 0,935 & 0,847 & 0,820 & 0,166 & 1,000 & 0,918 & 0,754 & 0,571 & 0,479 & 0,274 & 0,387 & 81 & 390 & $-2,3$ \\
\hline 391 & 0,1 & 1,0 & 0,1 & 0,9 & 0,86 & 1,000 & 0,859 & 0,832 & 0,141 & 1,000 & 0,906 & 0,700 & 0,559 & 0,433 & 0,242 & 0,274 & 117 & 391 & $-2,1$ \\
\hline 392 & 10,3 & 13,4 & 11,0 & 12,9 & 12,50 & 0,936 & 0,962 & 0,932 & 0,074 & 0,716 & 1,182 & 0,449 & 0,495 & 5,395 & 2,669 & 2,515 & 24 & 392 & $-5,8$ \\
\hline 393 & 0,1 & 1,3 & 0,1 & 1,1 & 1,07 & 0,967 & 0,858 & 0,830 & 0,146 & 1,000 & 0,988 & 0,543 & 0,485 & 0,565 & 0,274 & 0,306 & 67 & 393 & $-2,5$ \\
\hline 394 & 0,2 & 1,6 & 0,2 & 1,3 & 1,25 & 0,939 & 0,813 & 0,787 & 0,197 & 1,000 & 0,965 & 0,689 & 0,679 & 0,632 & 0,429 & 0,433 & 130 & 394 & $-2,7$ \\
\hline 395 & 0,7 & 2,9 & 0,7 & 2,8 & 2,66 & 0,974 & 0,962 & 0,932 & 0,046 & 1,000 & 0,958 & 0,870 & 0,809 & 1,018 & 0,823 & 0,866 & 132 & 395 & $-3,3$ \\
\hline
\end{tabular}




\begin{tabular}{|c|c|c|c|c|c|c|c|c|c|c|c|c|c|c|c|c|c|c|c|}
\hline 396 & 0,3 & 1,8 & 0,3 & 1,6 & 1,57 & 0,947 & 0,926 & 0,896 & 0,091 & 1,000 & 0,981 & 0,809 & 0,693 & 0,632 & 0,438 & 0,433 & 24 & 396 & $-2,7$ \\
\hline 397 & 9,9 & 13,2 & 10,8 & 12,8 & 12,38 & 0,916 & 0,968 & 0,937 & 0,090 & 0,714 & 1,183 & 0,448 & 0,478 & 5,308 & 2,537 & 2,450 & 169 & 397 & $-5,7$ \\
\hline 398 & 0,2 & 1,4 & 0,2 & 1,4 & 1,32 & 0,895 & 0,962 & 0,931 & 0,112 & 0,992 & 1,004 & 0,666 & 0,620 & 0,552 & 0,342 & 0,387 & 95 & 398 & $-2,5$ \\
\hline 399 & 10,8 & 12,8 & 11,6 & 12,6 & 12,19 & 0,932 & 0,982 & 0,950 & 0,070 & 0,824 & 1,102 & 0,652 & 0,694 & 4,594 & 3,187 & 2,941 & 112 & 399 & $-5,5$ \\
\hline 400 & 0,5 & 2,7 & 0,6 & 2,6 & 2,52 & 0,958 & 0,966 & 0,935 & 0,054 & 0,936 & 1,033 & 0,561 & 0,525 & 1,107 & 0,581 & 0,613 & 31 & 400 & $-3,5$ \\
\hline 401 & 0,4 & 2,2 & 0,4 & 2,2 & 2,09 & 0,920 & 0,959 & 0,928 & 0,090 & 0,934 & 1,035 & 0,572 & 0,532 & 0,914 & 0,487 & 0,494 & 29 & 401 & $-3,2$ \\
\hline 402 & 4,2 & 7,3 & 4,3 & 7,2 & 6,98 & 0,963 & 0,983 & 0,952 & 0,041 & 0,972 & 1,014 & 0,834 & 0,816 & 2,518 & 2,055 & 1,961 & 82 & 402 & $-4,7$ \\
\hline 403 & 0,2 & 1,5 & 0,2 & 1,4 & 1,34 & 0,976 & 0,928 & 0,899 & 0,076 & 1,000 & 0,959 & 0,715 & 0,579 & 0,585 & 0,339 & 0,387 & 55 & 403 & $-2,5$ \\
\hline 404 & 0,3 & 2,1 & 0,3 & 2,1 & 2,00 & 0,918 & 0,992 & 0,961 & 0,083 & 0,913 & 1,046 & 0,584 & 0,477 & 0,828 & 0,395 & 0,387 & 125 & 404 & $-3,0$ \\
\hline 405 & 15,9 & 14,8 & 16,5 & 14,8 & 14,28 & 0,965 & 0,996 & 0,964 & 0,035 & 0,912 & 1,047 & 0,699 & 0,745 & 5,383 & 4,008 & 3,918 & 147 & 405 & $-5,8$ \\
\hline 406 & 0,1 & 1,0 & 0,1 & 0,8 & 0,82 & 0,909 & 0,867 & 0,839 & 0,161 & 1,000 & 0,901 & 0,749 & 0,537 & 0,399 & 0,215 & 0,274 & 27 & 406 & $-2,0$ \\
\hline 407 & 0,2 & 1,6 & 0,2 & 1,5 & 1,43 & 0,919 & 0,919 & 0,890 & 0,115 & 0,778 & 1,134 & 0,370 & 0,290 & 0,741 & 0,215 & 0,274 & 30 & 407 & $-2,9$ \\
\hline 408 & 0,6 & 2,9 & 0,7 & 2,9 & 2,77 & 0,945 & 0,982 & 0,950 & 0,058 & 0,952 & 1,025 & 0,595 & 0,619 & 1,172 & 0,726 & 0,685 & 84 & 408 & $-3,6$ \\
\hline 409 & 0,1 & 1,2 & 0,2 & 1,1 & 1,04 & 0,912 & 0,892 & 0,863 & 0,140 & 1,000 & 0,888 & 0,963 & 0,769 & 0,439 & 0,337 & 0,387 & 12 & 409 & $-2,1$ \\
\hline 410 & 9,1 & 11,6 & 9,3 & 11,5 & 11,13 & 0,974 & 0,995 & 0,963 & 0,027 & 0,855 & 1,082 & 0,518 & 0,534 & 4,723 & 2,524 & 2,518 & 34 & 410 & $-5,6$ \\
\hline 411 & 0,2 & 1,4 & 0,2 & 1,2 & 1,19 & 0,917 & 0,857 & 0,829 & 0,166 & 0,942 & 1,030 & 0,646 & 0,666 & 0,552 & 0,368 & 0,387 & 176 & 411 & $-2,5$ \\
\hline 412 & 1,6 & 4,6 & 1,7 & 4,5 & 4,38 & 0,941 & 0,980 & 0,949 & 0,062 & 0,924 & 1,040 & 0,610 & 0,636 & 1,808 & 1,150 & 1,104 & 37 & 412 & $-4,2$ \\
\hline 413 & 0,1 & 1,1 & 0,1 & 0,9 & 0,87 & 1,000 & 0,856 & 0,828 & 0,144 & 1,000 & 0,869 & 0,936 & 0,686 & 0,399 & 0,274 & 0,387 & 146 & 413 & $-2,0$ \\
\hline 414 & 0,9 & 3,8 & 1,0 & 3,5 & 3,40 & 0,879 & 0,931 & 0,901 & 0,139 & 0,810 & 1,111 & 0,689 & 0,743 & 1,303 & 0,969 & 0,866 & 91 & 414 & $-3,7$ \\
\hline 415 & 2,3 & 5,9 & 2,5 & 5,6 & 5,45 & 0,906 & 0,958 & 0,928 & 0,103 & 0,840 & 1,091 & 0,667 & 0,699 & 2,097 & 1,465 & 1,225 & 136 & 415 & $-4,4$ \\
\hline 416 & 2,0 & 5,6 & 2,1 & 5,5 & 5,33 & 0,937 & 0,977 & 0,946 & 0,067 & 0,777 & 1,135 & 0,414 & 0,486 & 2,455 & 1,194 & $\begin{array}{l}1,233 \\
\text { n }\end{array}$ & 26 & 416 & $-4,6$ \\
\hline 417 & 0,1 & 0,9 & 0,1 & 0,6 & 0,61 & 0,952 & 0,678 & 0,656 & 0,326 & 1,000 & 0,852 & 1,019 & 0,800 & 0,342 & 0,274 & 0,274 & 56 & 417 & $-1,8$ \\
\hline 418 & 2,9 & 6,5 & 3,2 & 6,3 & 6,09 & 0,925 & 0,964 & 0,933 & 0,083 & 0,869 & 1,073 & 0,724 & 0,787 & 2,277 & 1,792 & 1,532 & 51 & 418 & $-4,5$ \\
\hline 419 & 3,3 & 8,3 & 4,1 & 7,9 & 7,68 & 0,802 & 0,954 & 0,923 & 0,203 & 0,591 & 1,300 & 0,413 & 0,547 & 3,166 & 1,730 & 1,370 & 59 & 419 & $-5,0$ \\
\hline 420 & 1,8 & 6,2 & 2,0 & 5,8 & 5,65 & 0,905 & 0,949 & 0,919 & 0,108 & 0,593 & 1,298 & 0,303 & 0,310 & 2,741 & 0,850 & 0,919 & 4 & 420 & $-4,8$ \\
\hline 421 & 35,8 & 30,6 & 44,4 & 28,7 & 27,78 & 0,805 & 0,938 & 0,908 & 0,205 & 0,480 & 1,444 & 0,330 & 0,362 & 11,741 & 4,247 & 3,973 & 9 & 421 & $-6,9$ \\
\hline 422 & 0,2 & 2,0 & 0,3 & 1,8 & 1,71 & 0,875 & 0,889 & 0,860 & 0,167 & 0,731 & 1,170 & 0,380 & 0,331 & 0,877 & 0,291 & 0,306 & 138 & 422 & $-3,1$ \\
\hline 423 & 2,9 & 6,1 & 3,0 & 6,1 & 5,87 & 0,980 & 0,997 & 0,965 & 0,020 & 0,998 & 1,001 & 0,697 & 0,731 & 2,318 & 1,695 & 1,733 & 20 & 423 & $-4,5$ \\
\hline 424 & 0,4 & 2,3 & 0,4 & 2,1 & 2,06 & 0,908 & 0,920 & 0,891 & 0,122 & 0,874 & 1,070 & 0,521 & 0,511 & 0,952 & 0,486 & 0,494 & 121 & 424 & $-3,3$ \\
\hline 425 & 0,2 & 1,5 & 0,2 & 1,3 & 1,25 & 0,907 & 0,885 & 0,857 & 0,148 & 1,000 & 0,963 & 0,764 & 0,620 & 0,552 & 0,342 & 0,387 & 9 & 425 & $-2,5$ \\
\hline 426 & 0,2 & 1,4 & 0,2 & 1,3 & 1,25 & 1,000 & 0,934 & 0,904 & 0,066 & 1,000 & 0,963 & 0,731 & 0,634 & 0,535 & 0,339 & 0,387 & 134 & 426 & $-2,4$ \\
\hline 427 & 0,7 & 3,1 & 0,8 & 3,0 & 2,88 & 0,935 & 0,947 & 0,916 & 0,084 & 0,944 & 1,029 & 0,597 & 0,567 & 1,257 & 0,713 & 0,698 & 67 & 427 & $-3,7$ \\
\hline 428 & 0,2 & 1,4 & 0,2 & 1,2 & 1,16 & 0,927 & 0,867 & 0,839 & 0,152 & 1,000 & 0,924 & 0,834 & 0,705 & 0,522 & 0,368 & 0,433 & 68 & 428 & $-2,4$ \\
\hline 429 & 0,3 & 1,8 & 0,3 & 1,6 & 1,59 & 0,903 & 0,900 & 0,871 & 0,140 & 0,989 & 1,005 & 0,735 & 0,727 & 0,675 & 0,490 & 0,494 & 124 & 429 & $-2,8$ \\
\hline 430 & 0,2 & 1,6 & 0,2 & 1,3 & 1,21 & 0,857 & 0,808 & 0,782 & 0,239 & 0,882 & 1,065 & 0,515 & 0,525 & 0,646 & 0,339 & 0,387 & 134 & 430 & $-2,7$ \\
\hline 431 & 0,1 & 0,9 & 0,1 & 0,8 & 0,81 & 1,000 & 0,886 & 0,858 & 0,114 & 1,000 & 0,845 & 1,028 & 0,784 & 0,349 & 0,274 & 0,306 & 66 & 431 & $-1,8$ \\
\hline 432 & 0,1 & 1,4 & 0,1 & 1,3 & 1,21 & 0,967 & 0,896 & 0,868 & 0,109 & 0,873 & 1,070 & 0,415 & 0,300 & 0,646 & 0,194 & 0,306 & 36 & 432 & $-2,7$ \\
\hline 433 & 0,1 & 1,1 & 0,1 & 0,9 & 0,86 & 0,963 & 0,800 & 0,775 & 0,203 & 1,000 & 0,895 & 0,662 & 0,500 & 0,484 & 0,242 & 0,306 & 41 & 433 & $-2,3$ \\
\hline 434 & 1,5 & 4,8 & 1,6 & 4,7 & 4,51 & 0,937 & 0,973 & 0,942 & 0,068 & 0,842 & 1,090 & 0,577 & 0,553 & 1,843 & 1,018 & 0,919 & 48 & 434 & $-4,2$ \\
\hline 435 & 15,7 & 16,3 & 17,2 & 15,8 & 15,28 & 0,915 & 0,969 & 0,938 & 0,091 & 0,744 & 1,159 & 0,609 & 0,731 & 5,735 & 4,193 & 3,389 & 167 & 435 & $-5,8$ \\
\hline 436 & 0,8 & 3,3 & 0,8 & 3,3 & 3,18 & 0,983 & 0,984 & 0,953 & 0,023 & 0,941 & 1,031 & 0,561 & 0,547 & 1,377 & 0,753 & 0,822 & 3 & 436 & $-3,8$ \\
\hline 437 & 2,1 & 5,4 & 2,2 & 5,3 & 5,10 & 0,952 & 0,969 & 0,938 & 0,057 & 0,879 & 1,067 & 0,608 & 0,625 & 2,080 & 1,300 & 1,233 & 89 & 437 & $-4,4$ \\
\hline 438 & 3,4 & 7,3 & 3,7 & 7,1 & 6,85 & 0,901 & 0,976 & 0,944 & 0,102 & 0,802 & 1,116 & 0,522 & 0,538 & 2,861 & 1,538 & 1,300 & 94 & 438 & $-4,8$ \\
\hline 439 & 0,2 & 1,6 & 0,2 & 1,5 & 1,41 & 0,955 & 0,941 & 0,911 & 0,075 & 1,000 & 0,986 & 0,514 & 0,392 & 0,698 & 0,274 & 0,411 & 7 & 439 & $-2,8$ \\
\hline 440 & 8,3 & 11,7 & 8,9 & 11,4 & 11,05 & 0,931 & 0,971 & 0,940 & 0,074 & 0,755 & 1,151 & 0,487 & 0,496 & 4,655 & 2,307 & 2,144 & 144 & 440 & $-5,5$ \\
\hline 441 & 0,1 & 1,2 & 0,1 & 1,1 & 1,07 & 0,828 & 0,912 & 0,882 & 0,194 & 0,957 & 1,022 & 0,611 & 0,633 & 0,484 & 0,307 & 0,274 & 164 & 441 & $-2,3$ \\
\hline 442 & 2,9 & 6,3 & 3,0 & 6,1 & 5,90 & 0,956 & 0,974 & 0,943 & 0,051 & 0,917 & 1,044 & 0,619 & 0,622 & 2,422 & 1,507 & 1,513 & 172 & 442 & $-4,6$ \\
\hline 443 & 0,2 & 1,4 & 0,2 & 1,2 & 1,12 & 0,878 & 0,817 & 0,791 & 0,220 & 1,000 & 0,976 & 0,674 & 0,606 & 0,565 & 0,342 & 0,387 & 176 & 443 & $-2,5$ \\
\hline 444 & 0,1 & 1,0 & 0,1 & 0,8 & 0,77 & 0,957 & 0,782 & 0,757 & 0,222 & 1,000 & 0,893 & 0,683 & 0,552 & 0,439 & 0,242 & 0,274 & 152 & 444 & $-2,1$ \\
\hline 445 & $\begin{array}{l}3,1 \\
73,9\end{array}$ & 34,7 & 80,3 & 33,2 & 32,12 & 0,920 & 0,956 & 0,926 & 0,091 & 0,772 & 1,138 & 0,691 & 0,799 & 11,676 & 9,333 & 8,083 & 55 & 445 & $\begin{array}{l}-6,9 \\
-1,9\end{array}$ \\
\hline 446 & 11,8 & 13,5 & 13,0 & 13,0 & 12,57 & 0,913 & 0,964 & 0,933 & 0,094 & 0,820 & 1,104 & 0,602 & 0,686 & 5,004 & 3,431 & 2,821 & 21 & 446 & $-5,6$ \\
\hline 447 & 0,2 & 1,8 & 0,2 & 1,6 & 1,54 & 0,951 & 0,896 & 0,868 & 0,114 & 0,731 & 1,169 & 0,345 & 0,333 & 0,822 & 0,274 & 0,306 & 179 & 447 & $-3,0$ \\
\hline 448 & 0,6 & 2,7 & 0,6 & 2,6 & 2,55 & 0,947 & 0,966 & 0,935 & 0,063 & 0,989 & 1,005 & 0,601 & 0,614 & 1,115 & 0,685 & 0,685 & 172 & 448 & $-3,5$ \\
\hline 449 & 51,2 & 26,4 & 52,3 & 26,3 & 25,45 & 0,978 & 0,997 & 0,965 & 0,023 & 0,924 & 1,040 & 0,699 & 0,693 & 9,654 & 6,690 & 6,509 & 10 & 449 & $-6,6$ \\
\hline 450 & 0,3 & 2,1 & 0,3 & 1,9 & 1,83 & 0,972 & 0,895 & 0,866 & 0,109 & 0,928 & 1,038 & 0,501 & 0,375 & 0,914 & 0,342 & 0,411 & 84 & 450 & $-3,2$ \\
\hline 451 & 1,1 & 4,9 & 1,2 & 4,7 & 4,51 & 0,920 & 0,955 & 0,924 & 0,092 & 0,569 & 1,326 & 0,269 & 0,269 & 2,261 & 0,608 & 0,685 & 175 & 451 & $-4,5$ \\
\hline 452 & 0,2 & 1,4 & 0,2 & 1,3 & 1,25 & 0,905 & 0,909 & 0,880 & 0,132 & 1,000 & 0,950 & 0,712 & 0,606 & 0,565 & 0,342 & 0,411 & 11 & 452 & $-2,5$ \\
\hline 453 & 0,4 & 2,1 & 0,4 & 2,0 & 1,98 & 0,966 & 0,974 & 0,943 & 0,043 & 1,000 & 0,945 & 0,823 & 0,785 & 0,781 & 0,613 & 0,613 & 9 & 453 & $-3,0$ \\
\hline
\end{tabular}




\begin{tabular}{|c|c|c|c|c|c|c|c|c|c|c|c|c|c|c|c|c|c|c|c|}
\hline 454 & 0,3 & 2,0 & 0,3 & 1,8 & 1,72 & 0,959 & 0,896 & 0,867 & 0,112 & 1,000 & 0,974 & 0,651 & 0,513 & 0,802 & 0,411 & 0,548 & 81 & 454 & $-3,0$ \\
\hline 455 & 7,9 & 10,8 & 8,4 & 10,8 & 10,44 & 0,940 & 0,995 & 0,963 & 0,061 & 0,842 & 1,090 & 0,618 & 0,649 & 4,029 & 2,615 & 2,357 & 47 & 455 & $-5,3$ \\
\hline 456 & 0,2 & 1,6 & 0,2 & 1,5 & 1,41 & 0,960 & 0,888 & 0,860 & 0,119 & 1,000 & 0,977 & 0,577 & 0,522 & 0,705 & 0,368 & 0,387 & 113 & 456 & $-2,8$ \\
\hline 457 & 58,2 & 31,6 & 62,2 & 29,1 & 28,19 & 0,936 & 0,923 & 0,893 & 0,100 & 0,734 & 1,167 & 0,734 & 0,819 & 10,048 & 8,233 & 6,801 & 114 & 457 & $-6,7$ \\
\hline 458 & 0,5 & 2,6 & 0,5 & 2,4 & 2,34 & 0,884 & 0,935 & 0,905 & 0,133 & 0,874 & 1,069 & 0,541 & 0,523 & 1,045 & 0,547 & 0,548 & 31 & 458 & $-3,4$ \\
\hline 459 & 322,6 & 70,8 & 340,8 & 69,9 & 67,70 & 0,946 & 0,988 & 0,957 & 0,055 & 0,809 & 1,112 & 0,686 & 0,820 & 24,464 & 20,066 & 17,022 & 99 & 459 & $-7,9$ \\
\hline 460 & 1,6 & 5,1 & 1,7 & 5,1 & 4,89 & 0,942 & 0,989 & 0,958 & 0,059 & 0,775 & 1,136 & 0,447 & 0,475 & 2,140 & 1,017 & 1,070 & 46 & 460 & $-4,4$ \\
\hline 461 & 1,2 & 3,8 & 1,2 & 3,8 & 3,66 & 0,969 & 0,986 & 0,955 & 0,034 & 1,000 & 0,992 & 0,671 & 0,632 & 1,504 & 0,950 & 0,969 & 144 & 461 & $-3,9$ \\
\hline 462 & 0,1 & 1,3 & 0,1 & 1,2 & 1,12 & 1,000 & 0,889 & 0,861 & 0,111 & 0,897 & 1,056 & 0,447 & 0,329 & 0,589 & 0,194 & 0,274 & 52 & 462 & $-2,6$ \\
\hline 463 & 1,4 & 4,6 & 1,5 & 4,5 & 4,39 & 0,957 & 0,988 & 0,957 & 0,045 & 0,863 & 1,076 & 0,507 & 0,438 & 1,904 & 0,835 & 0,866 & 42 & 463 & $-4,3$ \\
\hline 464 & 0,1 & 1,1 & 0,1 & 0,9 & 0,91 & 0,880 & 0,848 & 0,821 & 0,193 & 1,000 & 0,973 & 0,560 & 0,424 & 0,484 & 0,205 & 0,274 & 167 & 464 & $-2,3$ \\
\hline 465 & 1,3 & 4,2 & 1,4 & 4,1 & 3,98 & 0,932 & 0,978 & 0,947 & 0,072 & 0,910 & 1,048 & 0,594 & 0,657 & 1,657 & 1,089 & 1,104 & 178 & 465 & $-4,1$ \\
\hline 466 & 1,3 & 4,1 & 1,4 & 4,0 & 3,84 & 0,952 & 0,965 & 0,934 & 0,060 & 0,965 & 1,018 & 0,665 & 0,695 & 1,577 & 1,096 & 1,162 & 86 & 466 & $-4,0$ \\
\hline 467 & 2,2 & 5,8 & 2,4 & 5,5 & 5,30 & 0,916 & 0,941 & 0,911 & 0,103 & 0,800 & 1,118 & 0,560 & 0,557 & 2,213 & 1,233 & 1,225 & 7 & 467 & $-4,5$ \\
\hline 468 & 0,1 & 1,0 & 0,1 & 0,8 & 0,78 & 0,955 & 0,828 & 0,802 & 0,178 & 1,000 & 0,879 & 0,786 & 0,606 & 0,399 & 0,242 & 0,274 & 157 & 468 & $-2,0$ \\
\hline 469 & 0,6 & 3,0 & 0,7 & 2,9 & 2,79 & 0,926 & 0,969 & 0,938 & 0,080 & 0,914 & 1,046 & 0,556 & 0,548 & 1,214 & 0,665 & 0,685 & 10 & 469 & $-3,6$ \\
\hline 470 & 0,4 & 2,2 & 0,4 & 2,1 & 2,05 & 0,938 & 0,942 & 0,912 & 0,085 & 0,887 & 1,062 & 0,479 & 0,390 & 0,973 & 0,380 & 0,387 & 133 & 470 & $-3,3$ \\
\hline 471 & 1,1 & 3,7 & 1,1 & 3,6 & 3,50 & 0,946 & 0,965 & 0,935 & 0,065 & 0,950 & 1,026 & 0,617 & 0,638 & 1,479 & 0,943 & 0,959 & 168 & 471 & $-3,9$ \\
\hline 472 & 1,1 & 4,0 & 1,2 & 3,8 & 3,68 & 0,922 & 0,945 & 0,915 & 0,096 & 0,861 & 1,078 & 0,675 & 0,737 & 1,445 & 1,064 & 0,969 & 34 & 472 & $-3,9$ \\
\hline 473 & 0,2 & 2,3 & 0,3 & 2,1 & 2,06 & 0,855 & 0,942 & 0,912 & 0,156 & 0,545 & 1,354 & 0,239 & 0,224 & 1,083 & 0,242 & 0,274 & 14 & 473 & $-3,4$ \\
\hline 474 & 0,6 & 2,9 & 0,7 & 2,8 & 2,72 & 0,925 & 0,956 & 0,925 & 0,087 & 0,931 & 1,037 & 0,644 & 0,592 & 1,123 & 0,665 & 0,685 & 59 & 474 & $-3,5$ \\
\hline 475 & 0,3 & 2,0 & 0,3 & 2,0 & 1,89 & 0,944 & 0,955 & 0,925 & 0,072 & 0,941 & 1,031 & 0,656 & 0,618 & 0,781 & 0,482 & 0,433 & 40 & 475 & $-3,0$ \\
\hline 476 & 2,2 & 5,3 & 2,2 & 5,3 & 5,14 & 0,987 & 0,997 & 0,965 & 0,013 & 0,970 & 1,015 & 0,645 & 0,621 & 2,080 & 1,292 & 1,292 & 152 & 476 & $-4,4$ \\
\hline 477 & 7,5 & 10,8 & 7,8 & 10,8 & 10,46 & 0,960 & 0,999 & 0,967 & 0,040 & 0,806 & 1,114 & 0,490 & 0,484 & 4,418 & 2,139 & 2,059 & 96 & 477 & $-5,5$ \\
\hline 478 & 3,2 & 6,7 & 3,4 & 6,6 & 6,39 & 0,926 & 0,992 & 0,960 & 0,075 & 0,894 & 1,058 & 0,679 & 0,740 & 2,432 & 1,799 & 1,532 & 165 & 478 & $-4,6$ \\
\hline 479 & 0,9 & 3,9 & 1,0 & 3,8 & 3,69 & 0,931 & 0,986 & 0,954 & 0,071 & 0,795 & 1,122 & 0,512 & 0,422 & 1,532 & 0,647 & 0,685 & 47 & 479 & $-3,9$ \\
\hline 480 & 0,2 & 1,4 & 0,2 & 1,3 & 1,25 & 1,000 & 0,909 & 0,880 & 0,091 & 1,000 & 0,963 & 0,645 & 0,576 & 0,585 & 0,337 & 0,387 & 12 & 480 & $-2,5$ \\
\hline 481 & 1,9 & 5,0 & 2,0 & 4,9 & 4,79 & 0,971 & 0,989 & 0,958 & 0,031 & 0,958 & 1,022 & 0,705 & 0,694 & 1,854 & 1,287 & 1,292 & 161 & 481 & $-4,2$ \\
\hline 482 & 0,7 & 3,1 & 0,7 & 2,9 & 2,80 & 0,954 & 0,945 & 0,915 & 0,071 & 0,916 & 1,045 & 0,550 & 0,567 & 1,259 & 0,715 & 0,738 & 20 & 482 & $-3,7$ \\
\hline 483 & 0,2 & 1,7 & 0,2 & 1,6 & 1,55 & 0,917 & 0,968 & 0,936 & 0,089 & 0,943 & 1,030 & 0,529 & 0,459 & 0,705 & 0,324 & 0,387 & 144 & 483 & $-2,8$ \\
\hline 484 & 0,2 & 1,5 & 0,2 & 1,5 & 1,43 & 1,000 & 0,965 & 0,934 & 0,035 & 1,000 & 0,939 & 0,644 & 0,450 & 0,646 & 0,291 & 0,387 & 49 & 484 & $-2,7$ \\
\hline 485 & 0,4 & 2,3 & 0,4 & 2,2 & 2,16 & 0,955 & 0,961 & 0,930 & 0,060 & 0,928 & 1,038 & 0,585 & 0,484 & 0,932 & 0,451 & 0,433 & 31 & 485 & $-3,2$ \\
\hline 486 & 1,3 & 4,1 & 1,3 & 4,0 & 3,88 & 0,964 & 0,978 & 0,946 & 0,042 & 0,948 & 1,027 & 0,612 & 0,648 & 1,624 & 1,052 & 0,969 & 144 & 486 & $-4,0$ \\
\hline 487 & 2,6 & 5,6 & 2,7 & 5,5 & 5,33 & 0,979 & 0,990 & 0,959 & 0,023 & 1,000 & 0,972 & 0,946 & 0,915 & 1,871 & 1,712 & 1,694 & 131 & 487 & $-4,2$ \\
\hline 488 & 0,3 & 1,9 & 0,3 & 1,8 & 1,72 & 0,912 & 0,924 & 0,894 & 0,116 & 0,993 & 1,004 & 0,877 & 0,796 & 0,650 & 0,517 & 0,494 & 119 & 488 & $-2,7$ \\
\hline 489 & 2,3 & 6,1 & 2,5 & 5,9 & 5,70 & 0,934 & 0,973 & 0,942 & 0,071 & 0,801 & 1,117 & 0,447 & 0,478 & 2,579 & 1,233 & 1,225 & 87 & 489 & $-4,7$ \\
\hline 490 & 1,0 & 3,5 & 1,0 & 3,5 & 3,38 & 0,968 & 0,985 & 0,954 & 0,035 & 0,994 & 1,003 & 0,649 & 0,588 & 1,397 & 0,822 & 0,833 & 96 & 490 & $-3,8$ \\
\hline 491 & 4,7 & 8,5 & 5,1 & 8,3 & 7,99 & 0,932 & 0,967 & 0,936 & 0,076 & 0,817 & 1,106 & 0,556 & 0,616 & 3,295 & 2,028 & 1,937 & 9 & 491 & $-5,0$ \\
\hline 492 & 8,7 & 11,0 & 9,3 & 10,9 & 10,53 & 0,944 & 0,985 & 0,953 & 0,058 & 0,901 & 1,054 & 0,746 & 0,732 & 3,863 & 2,830 & 2,518 & 110 & 492 & $-5,3$ \\
\hline 493 & 2,4 & 6,4 & 2,6 & 6,2 & 5,97 & 0,951 & 0,971 & 0,940 & 0,057 & 0,763 & 1,145 & 0,427 & 0,496 & 2,702 & 1,341 & 1,349 & 152 & 493 & $-4,8$ \\
\hline 494 & 3,2 & 6,9 & 3,4 & 6,7 & 6,48 & 0,939 & 0,976 & 0,944 & 0,066 & 0,845 & 1,088 & 0,650 & 0,679 & 2,490 & 1,690 & 1,532 & 29 & 494 & $-4,6$ \\
\hline 495 & 47,1 & 26,3 & 48,4 & 26,2 & 25,39 & 0,972 & 0,996 & 0,964 & 0,029 & 0,853 & 1,083 & 0,546 & 0,560 & 10,480 & 5,872 & 5,828 & 143 & 495 & $-6,7$ \\
\hline 496 & 0,2 & 1,4 & 0,2 & 1,2 & 1,20 & 0,951 & 0,905 & 0,876 & 0,107 & 1,000 & 0,902 & 0,937 & 0,687 & 0,499 & 0,342 & 0,411 & 79 & 496 & $-2,3$ \\
\hline 497 & 0,2 & 1,8 & 0,2 & 1,6 & 1,58 & 0,922 & 0,891 & 0,863 & 0,134 & 0,830 & 1,097 & 0,479 & 0,439 & 0,766 & 0,336 & 0,387 & 85 & 497 & $-2,9$ \\
\hline 498 & 7,1 & 9,9 & 7,3 & 9,8 & 9,44 & 0,969 & 0,987 & 0,955 & 0,034 & 0,912 & 1,047 & 0,841 & 0,835 & 3,278 & 2,738 & 2,660 & 150 & 498 & $-5,0$ \\
\hline 499 & 0,7 & 4,1 & 0,8 & 3,8 & 3,69 & 0,869 & 0,933 & 0,903 & 0,147 & 0,540 & 1,361 & 0,266 & 0,254 & 1,854 & 0,470 & 0,548 & 84 & 499 & $-4,2$ \\
\hline 500 & 0,8 & 3,2 & 0,8 & 3,2 & 3,05 & 0,977 & 0,983 & 0,952 & 0,029 & 0,959 & 1,021 & 0,631 & 0,539 & 1,257 & 0,678 & 0,775 & 134 & 500 & $-3,7$ \\
\hline 501 & 0,3 & 1,8 & 0,3 & 1,6 & 1,54 & 0,932 & 0,896 & 0,868 & 0,124 & 1,000 & 0,985 & 0,642 & 0,575 & 0,715 & 0,411 & 0,494 & 81 & 501 & $-2,8$ \\
\hline 502 & 0,2 & 1,3 & 0,2 & 1,2 & 1,16 & 0,941 & 0,957 & 0,926 & 0,073 & 1,000 & 0,913 & 1,019 & 0,834 & 0,433 & 0,361 & 0,387 & 123 & 502 & $-2,1$ \\
\hline 503 & 1,3 & 4,0 & 1,3 & 3,9 & 3,77 & 0,990 & 0,968 & 0,937 & 0,034 & 1,000 & 0,986 & 0,879 & 0,889 & 1,387 & 1,233 & 1,104 & 32 & 503 & $-3,8$ \\
\hline 504 & 17,2 & 18,8 & 19,7 & 15,9 & 15,38 & 0,877 & 0,847 & 0,820 & 0,197 & 0,615 & 1,275 & 0,642 & 0,763 & 5,848 & 4,461 & 3,572 & 115 & 504 & $-5,9$ \\
\hline 505 & 0,1 & 1,2 & 0,1 & 1,1 & 1,04 & 0,968 & 0,892 & 0,863 & 0,113 & 1,000 & 0,902 & 0,849 & 0,687 & 0,459 & 0,315 & 0,387 & 148 & 505 & $-2,2$ \\
\hline 506 & 2,1 & 5,7 & 2,3 & 5,5 & 5,36 & 0,936 & 0,968 & 0,937 & 0,071 & 0,824 & 1,102 & 0,530 & 0,586 & 2,269 & 1,330 & 1,225 & 141 & 506 & $-4,5$ \\
\hline 507 & 0,2 & 1,4 & 0,2 & 1,2 & 1,12 & 0,970 & 0,817 & 0,791 & 0,185 & 0,933 & 1,035 & 0,458 & 0,424 & 0,646 & 0,274 & 0,306 & 146 & 507 & $-2,7$ \\
\hline 508 & 0,3 & 2,0 & 0,3 & 1,8 & 1,77 & 0,937 & 0,892 & 0,864 & 0,125 & 0,829 & 1,098 & 0,478 & 0,500 & 0,858 & 0,429 & 0,433 & 115 & 508 & $-3,1$ \\
\hline 509 & 0,3 & 2,0 & 0,4 & 1,9 & 1,84 & 0,960 & 0,954 & 0,923 & 0,061 & 1,000 & 0,968 & 0,628 & 0,569 & 0,828 & 0,471 & 0,548 & 159 & 509 & $-3,0$ \\
\hline 510 & 6,3 & 10,9 & 7,2 & 10,4 & 10,10 & 0,874 & 0,960 & 0,929 & 0,132 & 0,669 & 1,222 & 0,447 & 0,470 & 4,234 & 1,990 & 1,370 & 84 & 510 & $-5,4$ \\
\hline 511 & 13,5 & 14,3 & 14,2 & 13,9 & 13,47 & 0,957 & 0,975 & 0,944 & 0,050 & 0,835 & 1,094 & 0,632 & 0,689 & 5,226 & 3,602 & 3,336 & 89 & 511 & $-5,7$ \\
\hline
\end{tabular}




\begin{tabular}{|c|c|c|c|c|c|c|c|c|c|c|c|c|c|c|c|c|c|c|c|}
\hline 512 & 23,8 & 18,6 & 24,9 & 18,4 & 17,81 & 0,957 & 0,987 & 0,955 & 0,045 & 0,861 & 1,078 & 0,614 & 0,716 & 7,024 & 5,029 & 4,506 & 22 & 512 & $-6,1$ \\
\hline 513 & 193,5 & 57,0 & 204,6 & 55,4 & 53,63 & 0,946 & 0,972 & 0,941 & 0,061 & 0,749 & 1,156 & 0,548 & 0,664 & 21,196 & 14,072 & 12,305 & 54 & 513 & $-7,7$ \\
\hline 514 & 0,2 & 1,7 & 0,2 & 1,5 & 1,46 & 0,841 & 0,872 & 0,844 & 0,204 & 0,725 & 1,175 & 0,377 & 0,390 & 0,766 & 0,299 & 0,306 & 120 & 514 & $-2,9$ \\
\hline 515 & 0,4 & 2,4 & 0,5 & 2,3 & 2,21 & 0,940 & 0,961 & 0,931 & 0,071 & 0,980 & 1,010 & 0,608 & 0,641 & 0,961 & 0,616 & 0,613 & 171 & 515 & $-3,3$ \\
\hline 516 & 0,5 & 2,7 & 0,6 & 2,6 & 2,54 & 0,928 & 0,966 & 0,936 & 0,080 & 0,929 & 1,038 & 0,591 & 0,558 & 1,083 & 0,604 & 0,565 & 65 & 516 & $-3,4$ \\
\hline 517 & 2,8 & 6,5 & 3,0 & 6,4 & 6,19 & 0,951 & 0,986 & 0,954 & 0,051 & 0,847 & 1,087 & 0,658 & 0,651 & 2,343 & 1,526 & 1,456 & 61 & 517 & $-4,6$ \\
\hline 518 & 0,6 & 2,9 & 0,6 & 2,8 & 2,70 & 0,978 & 0,968 & 0,937 & 0,039 & 0,937 & 1,033 & 0,744 & 0,732 & 1,030 & 0,753 & 0,738 & 5 & 518 & $-3,4$ \\
\hline 519 & 0,5 & 2,5 & 0,5 & 2,4 & 2,32 & 0,948 & 0,963 & 0,932 & 0,064 & 1,000 & 0,983 & 0,867 & 0,800 & 0,866 & 0,693 & 0,685 & 3 & 519 & $-3,1$ \\
\hline 520 & 12,7 & 13,9 & 13,5 & 13,6 & 13,14 & 0,938 & 0,974 & 0,943 & 0,067 & 0,819 & 1,105 & 0,572 & 0,555 & 5,310 & 2,949 & 2,712 & 137 & 520 & $-5,7$ \\
\hline 521 & 0,1 & 1,1 & 0,1 & 0,8 & 0,81 & 0,920 & 0,790 & 0,764 & 0,225 & 1,000 & 0,906 & 0,732 & 0,474 & 0,433 & 0,205 & 0,274 & 86 & 521 & $-2,1$ \\
\hline 522 & 0,3 & 1,8 & 0,3 & 1,7 & 1,68 & 0,953 & 0,950 & 0,919 & 0,069 & 1,000 & 0,963 & 0,670 & 0,581 & 0,738 & 0,429 & 0,494 & 67 & 522 & $-2,9$ \\
\hline 523 & 56,5 & 31,5 & 58,1 & 31,2 & 30,23 & 0,973 & 0,991 & 0,959 & 0,028 & 0,715 & 1,183 & 0,407 & 0,394 & 13,293 & 5,244 & 5,016 & 120 & 523 & $-7,1$ \\
\hline 524 & 0,2 & 1,4 & 0,2 & 1,2 & 1,15 & 1,000 & 0,842 & 0,815 & 0,158 & 1,000 & 0,927 & 0,680 & 0,468 & 0,585 & 0,274 & 0,411 & 175 & 524 & $-2,5$ \\
\hline 525 & 0,5 & 2,6 & 0,6 & 2,5 & 2,46 & 0,967 & 0,979 & 0,948 & 0,039 & 1,000 & 0,990 & 0,639 & 0,546 & 1,045 & 0,570 & 0,581 & 144 & 525 & $-3,4$ \\
\hline 526 & 0,2 & 1,7 & 0,3 & 1,6 & 1,54 & 0,930 & 0,945 & 0,916 & 0,089 & 1,000 & 0,951 & 0,823 & 0,663 & 0,620 & 0,411 & 0,494 & 167 & 526 & $-2,6$ \\
\hline 527 & 1,2 & 4,0 & 1,3 & 3,9 & 3,79 & 0,959 & 0,977 & 0,946 & 0,047 & 0,948 & 1,027 & 0,719 & 0,688 & 1,464 & 1,008 & 0,969 & 73 & 527 & $-3,9$ \\
\hline 528 & 0,5 & 2,5 & 0,6 & 2,5 & 2,41 & 0,958 & 0,979 & 0,948 & 0,047 & 1,000 & 0,982 & 0,719 & 0,647 & 0,973 & 0,630 & 0,685 & 133 & 528 & $-3,3$ \\
\hline 529 & 1,1 & 3,8 & 1,2 & 3,8 & 3,64 & 0,957 & 0,986 & 0,954 & 0,045 & 0,988 & 1,006 & 0,670 & 0,650 & 1,475 & 0,959 & 0,969 & 64 & 529 & $-3,9$ \\
\hline 530 & 1,7 & 4,6 & 1,8 & 4,6 & 4,47 & 0,965 & 0,997 & 0,965 & 0,035 & 0,989 & 1,005 & 0,816 & 0,831 & 1,624 & 1,349 & 1,233 & 150 & 530 & $-4,0$ \\
\hline 531 & 0,2 & 2,0 & 0,3 & 1,9 & 1,86 & 0,893 & 0,954 & 0,924 & 0,116 & 0,729 & 1,171 & 0,354 & 0,334 & 0,919 & 0,307 & 0,387 & 155 & 531 & $-3,2$ \\
\hline 532 & 1,0 & 3,8 & 1,0 & 3,6 & 3,51 & 0,972 & 0,965 & 0,934 & 0,045 & 0,858 & 1,079 & 0,560 & 0,498 & 1,479 & 0,737 & 0,799 & 74 & 532 & $-3,9$ \\
\hline 533 & 0,6 & 3,3 & 0,6 & 3,1 & 3,01 & 0,895 & 0,938 & 0,908 & 0,122 & 0,637 & 1,253 & 0,295 & 0,281 & 1,553 & 0,436 & 0,494 & 50 & 533 & $-4,0$ \\
\hline 534 & 0,1 & 1,1 & 0,1 & 1,0 & 0,96 & 0,893 & 0,884 & 0,856 & 0,158 & 1,000 & 0,926 & 0,796 & 0,637 & 0,433 & 0,276 & 0,274 & 144 & 534 & $-2,1$ \\
\hline 535 & 0,1 & 1,1 & 0,1 & 1,0 & 0,98 & 0,885 & 0,917 & 0,888 & 0,142 & 1,000 & 0,952 & 0,714 & 0,650 & 0,439 & 0,285 & 0,274 & 129 & 535 & $-2,1$ \\
\hline 536 & 77,9 & 32,8 & 80,3 & 32,4 & 31,34 & 0,970 & 0,986 & 0,954 & 0,033 & 0,907 & 1,050 & 0,679 & 0,704 & 12,081 & 8,509 & 8,167 & 14 & 536 & $-6,9$ \\
\hline 537 & 2,3 & 5,9 & 2,4 & 5,8 & 5,58 & 0,948 & 0,982 & 0,950 & 0,055 & 0,846 & $\begin{array}{l}1,087 \\
\text {. }\end{array}$ & 0,595 & 0,582 & 2,230 & 1,298 & 1,225 & 57 & 537 & $-4,5$ \\
\hline 538 & 0,5 & 2,7 & 0,6 & 2,5 & 2,43 & 0,924 & 0,932 & 0,902 & 0,102 & 0,883 & 1,064 & 0,532 & 0,486 & 1,107 & 0,538 & 0,548 & 98 & 538 & $-3,5$ \\
\hline 539 & 1,8 & 5,0 & 1,9 & 4,9 & 4,78 & 0,963 & 0,982 & 0,950 & 0,042 & 0,902 & 1,053 & 0,625 & 0,570 & 1,923 & 1,096 & 1,225 & 1 & 539 & $-4,3$ \\
\hline 540 & 4,1 & 7,3 & 4,3 & 7,3 & 7,02 & 0,958 & 0,988 & 0,956 & 0,044 & 0,966 & 1,018 & 0,876 & 0,923 & 2,455 & 2,265 & 1,947 & 125 & 540 & $-4,6$ \\
\hline 541 & 1,1 & 4,1 & 1,2 & 3,9 & 3,82 & 0,952 & 0,968 & 0,937 & 0,057 & 0,852 & 1,083 & 0,679 & 0,776 & 1,453 & 1,128 & 0,988 & 5 & 541 & $-3,9$ \\
\hline 542 & 3,7 & 6,9 & 3,9 & 6,8 & 6,63 & 0,962 & 0,987 & 0,955 & 0,040 & 0,973 & 1,014 & 0,711 & 0,731 & 2,585 & 1,889 & 1,802 & 56 & 542 & $-4,7$ \\
\hline 543 & 1,9 & 5,2 & 2,0 & 5,1 & 4,91 & 0,958 & 0,975 & 0,944 & 0,048 & 0,903 & 1,052 & 0,610 & 0,646 & 2,016 & 1,301 & 1,240 & 88 & 543 & $-4,3$ \\
\hline 544 & 3,5 & 7,0 & 3,6 & 7,0 & 6,76 & 0,973 & 0,992 & 0,961 & 0,028 & 0,895 & 1,057 & 0,610 & 0,553 & 2,717 & 1,503 & 1,532 & 38 & 544 & $-4,8$ \\
\hline 545 & 1,3 & 4,2 & 1,4 & 4,1 & 3,98 & 0,953 & 0,987 & 0,956 & 0,049 & 0,958 & 1,022 & 0,654 & 0,604 & 1,605 & 0,969 & 0,988 & 140 & 545 & $-4,0$ \\
\hline 546 & 1,5 & 5,7 & 1,5 & 5,5 & 5,36 & 0,948 & 0,977 & 0,946 & 0,057 & 0,573 & 1,321 & 0,256 & 0,303 & 2,698 & 0,818 & 0,866 & 152 & 546 & $-4,8$ \\
\hline 547 & 0,2 & 2,0 & 0,3 & 1,9 & 1,81 & 0,881 & 0,928 & 0,898 & 0,139 & 0,758 & 1,148 & 0,389 & 0,364 & 0,893 & 0,325 & 0,387 & 63 & 547 & $-3,2$ \\
\hline 548 & 1,1 & 3,6 & 1,1 & 3,5 & 3,36 & 0,966 & 0,974 & 0,943 & 0,042 & 1,000 & 0,969 & 0,819 & 0,779 & 1,292 & 1,007 & 0,988 & 153 & 548 & $-3,7$ \\
\hline 549 & 0,2 & 1,9 & 0,3 & 1,6 & 1,59 & 0,911 & 0,881 & 0,853 & 0,149 & 0,864 & 1,076 & 0,445 & 0,367 & 0,828 & 0,303 & 0,387 & 162 & 549 & $-3,0$ \\
\hline 550 & 0,2 & 1,9 & 0,3 & 1,8 & 1,69 & 0,898 & 0,931 & 0,901 & 0,123 & 0,884 & 1,064 & 0,453 & 0,372 & 0,836 & 0,311 & 0,387 & 40 & 550 & $-3,1$ \\
\hline 551 & 0,9 & 4,0 & 0,9 & 3,8 & 3,70 & 0,939 & 0,967 & 0,936 & 0,069 & 0,695 & 1,200 & 0,348 & 0,307 & 1,778 & 0,546 & 0,548 & 17 & 551 & $-4,2$ \\
\hline 552 & 0,2 & 1,5 & 0,2 & 1,2 & 1,20 & 0,973 & 0,827 & 0,800 & 0,175 & 0,946 & 1,028 & 0,473 & 0,406 & 0,675 & 0,274 & 0,387 & 107 & 552 & $-2,8$ \\
\hline 553 & 0,1 & 1,2 & 0,1 & 1,0 & 0,95 & 0,967 & 0,842 & 0,815 & 0,161 & 1,000 & 0,889 & 0,821 & 0,621 & 0,459 & 0,285 & 0,306 & 122 & 553 & $-2,2$ \\
\hline 554 & 1,9 & 5,4 & 1,9 & 5,1 & 4,95 & 0,959 & 0,952 & 0,921 & 0,063 & 0,811 & 1,111 & 0,470 & 0,542 & 2,247 & 1,218 & 1,225 & 50 & 554 & $-4,5$ \\
\hline 555 & 3,3 & 6,9 & 3,4 & 6,7 & 6,52 & 0,953 & 0,979 & 0,947 & 0,051 & 0,869 & 1,073 & 0,557 & 0,574 & 2,735 & 1,571 & 1,507 & 98 & 555 & $-4,8$ \\
\hline 556 & 0,4 & 2,3 & 0,4 & 2,2 & 2,18 & 0,930 & 0,961 & 0,930 & 0,080 & 0,862 & 1,077 & 0,497 & 0,494 & 0,981 & 0,484 & 0,494 & 39 & 556 & $-3,3$ \\
\hline 557 & 8,3 & 11,1 & 8,6 & 11,1 & 10,70 & 0,963 & 0,992 & 0,960 & 0,038 & 0,842 & 1,090 & 0,666 & 0,798 & 3,989 & 3,184 & 2,753 & 166 & 557 & $-5,3$ \\
\hline 558 & 0,4 & 2,2 & 0,4 & 2,2 & 2,09 & 0,947 & 0,959 & 0,928 & 0,067 & 1,000 & 0,976 & 0,881 & 0,824 & 0,781 & 0,644 & 0,685 & 137 & 558 & $-3,0$ \\
\hline 559 & 0,1 & 1,3 & 0,1 & 1,1 & 1,07 & 0,897 & 0,858 & 0,830 & 0,176 & 0,919 & 1,043 & 0,487 & 0,384 & 0,565 & 0,217 & 0,274 & 69 & 559 & $-2,5$ \\
\hline 560 & 0,6 & 3,0 & 0,7 & 2,8 & 2,66 & 0,912 & 0,902 & 0,874 & 0,132 & 0,850 & 1,085 & 0,493 & 0,481 & 1,274 & 0,613 & 0,685 & 153 & 560 & $-3,7$ \\
\hline 561 & 1,7 & 4,6 & 1,7 & 4,6 & 4,41 & 0,973 & 0,980 & 0,949 & 0,033 & 0,988 & 1,006 & 0,669 & 0,673 & 1,798 & 1,211 & 1,225 & 26 & 561 & $-4,2$ \\
\hline 562 & 19,1 & 22,9 & 22,6 & 22,7 & 21,96 & 0,845 & 0,990 & 0,958 & 0,155 & 0,456 & 1,480 & 0,239 & 0,272 & 10,074 & 2,740 & 2,470 & 153 & 562 & $-6,7$ \\
\hline 563 & 2,3 & 6,7 & 2,4 & 6,5 & 6,32 & 0,925 & 0,980 & 0,949 & 0,078 & 0,638 & 1,252 & 0,357 & 0,461 & 2,836 & 1,308 & 1,225 & 131 & 563 & $-4,8$ \\
\hline 564 & 23,1 & 18,3 & 23,8 & 18,3 & 17,67 & 0,971 & 0,997 & 0,965 & 0,029 & 0,868 & 1,073 & 0,577 & 0,684 & 7,147 & 4,889 & 4,539 & 39 & 564 & $-6,2$ \\
\hline 565 & 1,0 & 3,5 & 1,1 & 3,5 & 3,37 & 0,956 & 0,985 & 0,954 & 0,047 & 1,000 & 0,990 & 0,733 & 0,713 & 1,330 & 0,948 & 0,969 & 118 & 565 & $-3,7$ \\
\hline 566 & 2,2 & 6,6 & 2,5 & 6,3 & 6,10 & 0,897 & 0,957 & 0,927 & 0,111 & 0,642 & 1,248 & 0,347 & 0,394 & 2,851 & 1,123 & 1,070 & 33 & 566 & $-4,8$ \\
\hline 567 & 9,2 & 11,3 & 9,4 & 11,3 & 10,94 & 0,979 & 0,999 & 0,967 & 0,021 & 0,906 & 1,051 & 0,748 & 0,843 & 3,964 & 3,342 & 2,928 & 82 & 567 & $-5,3$ \\
\hline 568 & 1,4 & 4,4 & 1,5 & 4,1 & 3,99 & 0,916 & 0,946 & 0,915 & 0,100 & 0,910 & 1,048 & 0,757 & 0,791 & 1,521 & 1,203 & 1,130 & 125 & 568 & $-3,9$ \\
\hline 569 & 51,2 & 29,9 & 53,9 & 29,5 & 28,51 & 0,950 & 0,985 & 0,953 & 0,052 & 0,720 & 1,179 & 0,457 & 0,438 & 11,944 & 5,226 & 4,841 & 109 & 569 & $-6,9$ \\
\hline
\end{tabular}




\begin{tabular}{|c|c|c|c|c|c|c|c|c|c|c|c|c|c|c|c|c|c|c|c|}
\hline 570 & 15,3 & 14,8 & 16,3 & 14,6 & 14,15 & 0,936 & 0,987 & 0,955 & 0,066 & 0,876 & 1,068 & 0,811 & 0,814 & 4,901 & 3,991 & 3,492 & 64 & 570 & $-5,6$ \\
\hline 571 & 4,0 & 9,1 & 4,5 & 8,2 & 7,92 & 0,882 & 0,903 & 0,874 & 0,153 & 0,609 & 1,281 & 0,431 & 0,497 & 3,431 & 1,707 & 0,274 & 4 & 571 & $-5,1$ \\
\hline 572 & 0,5 & 2,6 & 0,6 & 2,5 & 2,38 & 0,959 & 0,950 & 0,919 & 0,065 & 1,000 & 0,988 & 0,738 & 0,680 & 0,969 & 0,659 & 0,685 & 78 & 572 & $-3,3$ \\
\hline 573 & 0,2 & 1,8 & 0,2 & 1,8 & 1,69 & 0,923 & 0,970 & 0,940 & 0,082 & 0,869 & 1,073 & 0,449 & 0,364 & 0,799 & 0,291 & 0,387 & 142 & 573 & $-3,0$ \\
\hline 574 & 1,4 & 6,6 & 1,8 & 6,1 & 5,94 & 0,783 & 0,933 & 0,903 & 0,227 & 0,409 & 1,563 & 0,208 & 0,255 & 2,932 & 0,747 & 0,685 & 128 & 574 & $-4,9$ \\
\hline 575 & 1,2 & 4,2 & 1,3 & 4,0 & 3,91 & 0,926 & 0,957 & 0,926 & 0,086 & 0,822 & 1,103 & 0,543 & 0,596 & 1,655 & 0,987 & 0,959 & 24 & 575 & $-4,0$ \\
\hline 576 & 1,9 & 5,6 & 2,1 & 5,5 & 5,28 & 0,903 & 0,967 & 0,936 & 0,103 & 0,756 & 1,150 & 0,451 & 0,576 & 2,326 & 1,339 & 1,263 & 105 & 576 & $-4,5$ \\
\hline 577 & 1,3 & 4,7 & 1,4 & 4,6 & 4,43 & 0,914 & 0,972 & 0,941 & 0,090 & 0,710 & 1,187 & 0,461 & 0,589 & 1,861 & 1,096 & 0,919 & 9 & 577 & $-4,2$ \\
\hline 578 & 1,5 & 4,7 & 1,6 & 4,4 & 4,29 & 0,942 & 0,952 & 0,922 & 0,075 & 0,890 & 1,060 & 0,594 & 0,567 & 1,813 & 1,027 & 1,043 & 93 & 578 & $-4,2$ \\
\hline 579 & 2,2 & 5,5 & 2,3 & 5,3 & 5,14 & 0,963 & 0,969 & 0,938 & 0,048 & 0,922 & 1,041 & 0,691 & 0,731 & 2,016 & 1,474 & 1,356 & 8 & 579 & $-4,3$ \\
\hline 580 & 0,6 & 3,0 & 0,7 & 2,7 & 2,57 & 0,901 & 0,879 & 0,851 & 0,157 & 0,817 & 1,106 & 0,558 & 0,528 & 1,166 & 0,616 & 0,613 & 89 & 580 & $-3,5$ \\
\hline 581 & 0,4 & 2,0 & 0,4 & 1,9 & 1,86 & 0,974 & 0,954 & 0,924 & 0,052 & 1,000 & 0,950 & 0,958 & 0,893 & 0,688 & 0,615 & 0,565 & 153 & 581 & $-2,8$ \\
\hline 582 & 0,2 & 1,4 & 0,2 & 1,2 & 1,18 & 1,000 & 0,889 & 0,860 & 0,111 & 1,000 & 0,966 & 0,817 & 0,687 & 0,499 & 0,342 & 0,411 & 176 & 582 & $-2,3$ \\
\hline 583 & 3,2 & 7,2 & 3,6 & 6,8 & 6,61 & 0,904 & 0,950 & 0,920 & 0,108 & 0,784 & 1,130 & 0,659 & 0,740 & 2,493 & 1,845 & 1,655 & 21 & 583 & $-4,6$ \\
\hline 584 & 4,8 & 9,4 & 5,4 & 9,1 & 8,83 & 0,897 & 0,971 & 0,940 & 0,107 & 0,685 & 1,208 & 0,395 & 0,468 & 3,933 & 1,840 & 1,475 & 176 & 584 & $-5,3$ \\
\hline 585 & 0,8 & 3,0 & 0,8 & 2,9 & 2,82 & 0,976 & 0,982 & 0,951 & 0,030 & 1,000 & 0,963 & 0,851 & 0,774 & 1,063 & 0,823 & 0,866 & 119 & 585 & $-3,4$ \\
\hline 586 & 0,7 & 3,2 & 0,7 & 3,1 & 2,97 & 0,933 & 0,971 & 0,940 & 0,073 & 0,822 & 1,103 & 0,474 & 0,416 & 1,323 & 0,550 & 0,548 & 160 & 586 & $-3,7$ \\
\hline 587 & 0,4 & 2,5 & 0,4 & 2,3 & 2,21 & 0,926 & 0,917 & 0,888 & 0,111 & 0,836 & 1,094 & 0,417 & 0,403 & 1,123 & 0,453 & 0,494 & 35 & 587 & $-3,5$ \\
\hline 588 & 1,6 & 5,4 & 1,8 & 5,2 & 5,08 & 0,931 & 0,976 & 0,945 & 0,073 & 0,713 & 1,185 & 0,380 & 0,368 & 2,341 & 0,862 & 0,866 & 16 & 588 & $-4,5$ \\
\hline 589 & 1,4 & 4,4 & 1,5 & 4,3 & 4,18 & 0,955 & 0,976 & 0,945 & 0,051 & 0,891 & 1,060 & 0,667 & 0,712 & 1,628 & 1,159 & 0,988 & 159 & 589 & $-4,0$ \\
\hline 590 & 0,4 & 2,3 & 0,5 & 2,2 & 2,11 & 0,969 & 0,960 & 0,929 & 0,051 & 1,000 & 0,964 & 0,820 & 0,778 & 0,828 & 0,644 & 0,685 & 140 & 590 & $-3,0$ \\
\hline 591 & 5,5 & 9,9 & 5,9 & 9,6 & 9,27 & 0,928 & 0,968 & 0,937 & 0,079 & 0,706 & 1,190 & 0,440 & 0,449 & 3,990 & 1,792 & 1,838 & 45 & 591 & $-5,3$ \\
\hline 592 & 0,8 & 3,2 & 0,8 & 3,2 & 3,07 & 0,978 & 0,979 & 0,947 & 0,031 & 0,976 & 1,012 & 0,599 & 0,515 & 1,317 & 0,678 & 0,775 & 133 & 592 & $-3,7$ \\
\hline 593 & 0,8 & 3,3 & 0,8 & 3,2 & 3,07 & 0,910 & 0,961 & 0,930 & 0,098 & 0,876 & 1,069 & 0,619 & 0,662 & 1,250 & 0,827 & 0,799 & 5 & 593 & $-3,6$ \\
\hline 594 & 0,8 & 3,9 & 0,8 & 3,7 & 3,55 & 0,922 & 0,952 & 0,922 & 0,092 & 0,655 & 1,235 & 0,315 & 0,338 & 1,768 & 0,597 & 0,613 & 142 & 594 & $-4,1$ \\
\hline 595 & 5,5 & 8,7 & 5,8 & 8,5 & 8,25 & 0,946 & 0,977 & 0,946 & 0,059 & 0,905 & $\begin{array}{l}1,051 \\
\text { S }\end{array}$ & 0,649 & 0,707 & 3,278 & 2,318 & 2,073 & 1 & 595 & $-5,0$ \\
\hline 596 & 3,8 & 7,5 & 4,1 & 7,2 & 7,02 & 0,933 & 0,966 & 0,935 & 0,075 & 0,849 & 1,085 & 0,601 & 0,665 & 2,838 & 1,887 & 1,838 & 11 & 596 & $-4,8$ \\
\hline 597 & 26,5 & 20,2 & 28,4 & 19,3 & 18,67 & 0,931 & 0,953 & 0,923 & 0,084 & 0,813 & 1,109 & 0,708 & 0,792 & 6,899 & 5,463 & 4,653 & 157 & 597 & $-6,1$ \\
\hline 598 & 0,3 & 1,9 & 0,3 & 1,7 & 1,63 & 0,933 & 0,902 & 0,872 & 0,119 & 0,949 & 1,026 & 0,615 & 0,617 & 0,738 & 0,455 & 0,494 & 114 & 598 & $-2,9$ \\
\hline 599 & 9,4 & 12,7 & 10,0 & 12,5 & 12,07 & 0,943 & 0,984 & 0,953 & 0,060 & 0,734 & 1,167 & 0,406 & 0,416 & 5,427 & 2,256 & 2,166 & 1 & 599 & $-5,8$ \\
\hline 600 & 0,5 & 2,4 & 0,5 & 2,2 & 2,16 & 0,935 & 0,924 & 0,894 & 0,100 & 1,000 & 0,995 & 0,860 & 0,773 & 0,833 & 0,644 & 0,613 & 56 & 600 & $-3,1$ \\
\hline 601 & 0,3 & 1,9 & 0,3 & 1,8 & 1,73 & 0,954 & 0,943 & 0,913 & 0,073 & 1,000 & 0,992 & 0,675 & 0,523 & 0,741 & 0,387 & 0,494 & 137 & 601 & $-2,9$ \\
\hline 602 & 0,3 & 2,3 & 0,3 & 2,1 & 2,06 & 0,806 & 0,920 & 0,891 & 0,210 & 0,642 & 1,248 & 0,348 & 0,363 & 0,997 & 0,362 & 0,306 & 167 & 602 & $-3,3$ \\
\hline 603 & 0,1 & 1,2 & 0,1 & 1,0 & 0,98 & 0,933 & 0,821 & 0,794 & 0,191 & 1,000 & 0,964 & 0,686 & 0,588 & 0,494 & 0,291 & 0,274 & 124 & 603 & $-2,3$ \\
\hline 604 & 0,1 & 1,1 & 0,1 & 1,0 & 0,95 & 1,000 & 0,914 & 0,885 & 0,086 & 1,000 & 0,864 & 0,828 & 0,632 & 0,433 & 0,274 & 0,387 & 24 & 604 & $-2,1$ \\
\hline 605 & 64,9 & 34,0 & 71,6 & 32,5 & 31,48 & 0,906 & 0,956 & 0,926 & 0,104 & 0,705 & 1,191 & 0,516 & 0,648 & 12,657 & 8,200 & 7,260 & 85 & 605 & $-7,0$ \\
\hline 606 & 0,5 & 2,6 & 0,6 & 2,4 & 2,34 & 0,944 & 0,943 & 0,913 & 0,080 & 1,000 & 0,978 & 0,876 & 0,844 & 0,893 & 0,753 & 0,698 & 78 & 606 & $-3,2$ \\
\hline 607 & 3,4 & 7,4 & 3,8 & 6,8 & 6,62 & 0,898 & 0,927 & 0,898 & 0,125 & 0,792 & 1,123 & 0,631 & 0,727 & 2,631 & 1,912 & 1,650 & 31 & 607 & $-4,7$ \\
\hline 608 & 0,2 & 2,0 & 0,2 & 1,7 & 1,68 & 0,880 & 0,887 & 0,858 & 0,165 & 0,677 & 1,215 & 0,303 & 0,296 & 0,932 & 0,276 & 0,306 & 72 & 608 & $-3,2$ \\
\hline 609 & 17,3 & 15,6 & 17,7 & 15,3 & 14,81 & 0,974 & 0,983 & 0,952 & 0,031 & 0,896 & 1,056 & 0,688 & 0,752 & 5,650 & 4,247 & 3,789 & 8 & 609 & $-5,8$ \\
\hline 610 & 0,5 & 2,6 & 0,5 & 2,5 & 2,43 & 0,955 & 0,951 & 0,920 & 0,067 & 0,890 & 1,060 & 0,483 & 0,451 & 1,140 & 0,514 & 0,494 & 37 & 610 & $-3,5$ \\
\hline 611 & 7,4 & 12,0 & 7,9 & 11,7 & 11,36 & 0,931 & 0,979 & 0,948 & 0,072 & 0,644 & 1,246 & 0,317 & 0,354 & 5,439 & 1,926 & 1,918 & 80 & 611 & $-5,8$ \\
\hline 612 & 0,2 & 1,6 & 0,2 & 1,5 & 1,46 & 0,940 & 0,921 & 0,891 & 0,099 & 1,000 & 0,987 & 0,598 & 0,528 & 0,685 & 0,362 & 0,387 & 43 & 612 & $-2,8$ \\
\hline 613 & 29,5 & 23,2 & 30,9 & 22,9 & 22,16 & 0,953 & 0,986 & 0,955 & 0,049 & 0,687 & 1,206 & 0,395 & 0,412 & 9,746 & 4,018 & 3,973 & 135 & 613 & $-6,6$ \\
\hline 614 & 1,2 & 4,0 & 1,2 & 3,8 & 3,71 & 0,939 & 0,954 & 0,924 & 0,076 & 0,897 & 1,056 & 0,562 & 0,553 & 1,617 & 0,894 & 0,919 & 119 & 614 & $-4,0$ \\
\hline 615 & 1,4 & 5,1 & 1,5 & 4,9 & 4,75 & 0,952 & 0,957 & 0,926 & 0,064 & 0,671 & 1,221 & 0,338 & 0,298 & 2,297 & 0,685 & 0,822 & 88 & 615 & $-4,5$ \\
\hline 616 & 0,2 & 1,6 & 0,2 & 1,4 & 1,34 & 0,917 & 0,862 & 0,835 & 0,161 & 1,000 & 0,997 & 0,560 & 0,461 & 0,685 & 0,315 & 0,387 & 40 & 616 & $-2,8$ \\
\hline 617 & 55,4 & 29,5 & 59,7 & 28,5 & 27,63 & 0,929 & 0,969 & 0,938 & 0,078 & 0,802 & 1,116 & 0,644 & 0,725 & 10,470 & 7,591 & 6,998 & 58 & 617 & $\begin{array}{l}-6,7 \\
-1\end{array}$ \\
\hline 618 & 0,3 & 2,3 & 0,4 & 2,0 & 1,98 & 0,909 & 0,902 & 0,873 & 0,133 & 0,801 & 1,118 & 0,396 & 0,388 & 1,027 & 0,398 & 0,433 & 34 & 618 & $-3,4$ \\
\hline 619 & 0,9 & 3,3 & 0,9 & 3,2 & 3,11 & 0,973 & 0,984 & 0,952 & 0,031 & 1,000 & 0,996 & 0,615 & 0,618 & 1,330 & 0,822 & 0,877 & 14 & 619 & $-3,7$ \\
\hline 620 & 0,4 & 2,3 & 0,5 & 2,2 & 2,16 & 0,938 & 0,954 & 0,924 & 0,078 & 0,969 & 1,016 & 0,637 & 0,579 & 0,919 & 0,532 & 0,565 & 54 & 620 & $-3,2$ \\
\hline 621 & 5,7 & 9,9 & 6,2 & 9,5 & 9,24 & 0,920 & 0,966 & 0,935 & 0,087 & 0,733 & 1,168 & 0,483 & 0,559 & 3,877 & 2,169 & 1,961 & 125 & 621 & $-5,3$ \\
\hline 622 & 0,4 & 2,3 & 0,4 & 2,2 & 2,11 & 0,979 & 0,960 & 0,929 & 0,046 & 1,000 & 0,975 & 0,604 & 0,546 & 0,954 & 0,521 & 0,565 & 21 & 622 & $-3,3$ \\
\hline 623 & 1,3 & 4,6 & 1,5 & 4,4 & 4,25 & 0,890 & 0,949 & 0,918 & 0,121 & 0,758 & 1,148 & 0,574 & 0,650 & 1,694 & 1,101 & 0,959 & 77 & 623 & $-4,1$ \\
\hline 624 & 0,4 & 2,5 & 0,4 & 2,5 & 2,38 & 0,957 & 0,979 & 0,948 & 0,048 & 0,825 & 1,101 & 0,409 & 0,399 & 1,134 & 0,453 & 0,494 & 149 & 624 & $-3,5$ \\
\hline 625 & 2,2 & 5,6 & 2,4 & 5,4 & 5,22 & 0,929 & 0,970 & 0,939 & 0,078 & 0,894 & 1,058 & 0,656 & 0,664 & 2,065 & 1,371 & 1,356 & 7 & 625 & $-4,4$ \\
\hline 626 & 0,5 & 2,7 & 0,5 & 2,6 & 2,48 & 0,937 & 0,952 & 0,921 & 0,079 & 0,847 & 1,087 & 0,433 & 0,404 & 1,198 & 0,484 & 0,565 & 37 & 626 & $-3,6$ \\
\hline 627 & 0,2 & 1,6 & 0,3 & 1,5 & 1,46 & 0,963 & 0,921 & 0,891 & 0,087 & 1,000 & 0,939 & 0,744 & 0,675 & 0,646 & 0,436 & 0,494 & 35 & 627 & $-2,7$ \\
\hline
\end{tabular}




\begin{tabular}{|c|c|c|c|c|c|c|c|c|c|c|c|c|c|c|c|c|c|c|c|}
\hline 628 & 5,2 & 8,7 & 5,4 & 8,6 & 8,30 & 0,962 & 0,989 & 0,958 & 0,040 & 0,871 & 1,072 & 0,572 & 0,555 & 3,403 & 1,889 & 1,903 & 49 & 628 & $-5,1$ \\
\hline 629 & 3,7 & 7,2 & 3,9 & 6,9 & 6,70 & 0,947 & 0,960 & 0,929 & 0,066 & 0,896 & 1,057 & 0,639 & 0,695 & 2,719 & 1,889 & 1,843 & 56 & 629 & $-4,8$ \\
\hline 630 & 10,6 & 12,2 & 11,0 & 12,0 & 11,60 & 0,963 & 0,982 & 0,950 & 0,041 & 0,898 & 1,056 & 0,760 & 0,784 & 4,223 & 3,311 & 3,210 & 66 & 630 & $-5,4$ \\
\hline 631 & 12,9 & 16,4 & 15,6 & 15,0 & 14,57 & 0,828 & 0,916 & 0,887 & 0,191 & 0,602 & 1,289 & 0,591 & 0,759 & 5,276 & 4,004 & 2,928 & 77 & 631 & $-5,7$ \\
\hline 632 & 1,2 & 4,3 & 1,3 & 4,1 & 3,94 & 0,926 & 0,957 & 0,926 & 0,085 & 0,858 & 1,079 & 0,538 & 0,650 & 1,712 & 1,113 & 1,043 & 84 & 632 & $-4,1$ \\
\hline 633 & 0,5 & 2,6 & 0,5 & 2,5 & 2,41 & 0,948 & 0,964 & 0,934 & 0,063 & 0,972 & 1,015 & 0,700 & 0,707 & 0,969 & 0,685 & 0,685 & 87 & 633 & $-3,3$ \\
\hline 634 & 7,1 & 11,5 & 7,5 & 11,2 & 10,80 & 0,951 & 0,974 & 0,943 & 0,056 & 0,678 & 1,214 & 0,361 & 0,408 & 5,000 & 2,041 & 1,961 & 54 & 634 & $-5,6$ \\
\hline 635 & 0,3 & 1,8 & 0,3 & 1,7 & 1,68 & 0,970 & 0,950 & 0,919 & 0,058 & 1,000 & 0,933 & 0,974 & 0,858 & 0,632 & 0,542 & 0,565 & 168 & 635 & $-2,7$ \\
\hline 636 & 0,5 & 2,4 & 0,5 & 2,2 & 2,15 & 0,945 & 0,909 & 0,880 & 0,106 & 1,000 & 0,985 & 0,732 & 0,736 & 0,922 & 0,678 & 0,685 & 131 & 636 & $-3,2$ \\
\hline 637 & 0,6 & 3,1 & 0,6 & 2,9 & 2,79 & 0,911 & 0,929 & 0,899 & 0,114 & 0,753 & 1,152 & 0,449 & 0,418 & 1,280 & 0,535 & 0,494 & 103 & 637 & $-3,7$ \\
\hline 638 & 0,8 & 4,5 & 1,2 & 4,1 & 3,99 & 0,704 & 0,917 & 0,887 & 0,307 & 0,508 & 1,403 & 0,306 & 0,467 & 1,844 & 0,862 & 0,548 & 62 & 638 & $-4,2$ \\
\hline 639 & 5,2 & 9,5 & 5,5 & 9,3 & 9,00 & 0,943 & 0,975 & 0,944 & 0,063 & 0,712 & 1,185 & 0,415 & 0,428 & 3,976 & 1,700 & 1,655 & 74 & 639 & $-5,3$ \\
\hline 640 & 13,5 & 16,3 & 15,0 & 16,0 & 15,50 & 0,904 & 0,984 & 0,953 & 0,097 & 0,642 & 1,248 & 0,376 & 0,488 & 6,768 & 3,302 & 3,186 & 61 & 640 & $-6,1$ \\
\hline 641 & 0,3 & 1,9 & 0,3 & 1,6 & 1,55 & 0,903 & 0,861 & 0,833 & 0,170 & 0,949 & 1,026 & 0,654 & 0,566 & 0,715 & 0,405 & 0,387 & 105 & 641 & $-2,8$ \\
\hline 642 & 1,0 & 3,8 & 1,1 & 3,6 & 3,48 & 0,938 & 0,951 & 0,921 & 0,079 & 0,873 & 1,070 & 0,589 & 0,649 & 1,466 & 0,951 & 0,919 & 114 & 642 & $-3,9$ \\
\hline 643 & 2,1 & 5,0 & 2,2 & 4,9 & 4,79 & 0,967 & 0,989 & 0,958 & 0,034 & 1,000 & 0,976 & 0,884 & 0,869 & 1,734 & 1,507 & 1,507 & 6 & 643 & $-4,1$ \\
\hline 644 & 3,8 & 7,5 & 3,9 & 7,5 & 7,24 & 0,975 & 0,998 & 0,966 & 0,026 & 0,842 & 1,090 & 0,490 & 0,500 & 3,130 & 1,563 & 1,598 & 150 & 644 & $-5,0$ \\
\hline 645 & 0,1 & 1,3 & 0,1 & 1,2 & 1,16 & 0,880 & 0,902 & 0,873 & 0,155 & 0,733 & 1,168 & 0,330 & 0,309 & 0,632 & 0,195 & 0,274 & 163 & 645 & $-2,7$ \\
\hline 646 & 2,2 & 5,8 & 2,3 & 5,7 & 5,53 & 0,948 & 0,984 & 0,953 & 0,055 & 0,827 & 1,100 & 0,472 & 0,459 & 2,445 & 1,123 & 1,130 & 4 & 646 & $-4,6$ \\
\hline 647 & 0,2 & 1,6 & 0,2 & 1,4 & 1,38 & 0,953 & 0,916 & 0,887 & 0,096 & 1,000 & 0,998 & 0,637 & 0,552 & 0,620 & 0,342 & 0,411 & 3 & 647 & $-2,6$ \\
\hline 648 & 1,4 & 4,1 & 1,4 & 4,0 & 3,91 & 0,967 & 0,987 & 0,955 & 0,036 & 1,000 & 0,989 & 0,838 & 0,800 & 1,440 & 1,152 & $\begin{array}{l}1,162 \\
\text {. }\end{array}$ & 31 & 648 & $-3,8$ \\
\hline 649 & 1,5 & 5,0 & 1,6 & 4,8 & 4,65 & 0,925 & 0,956 & 0,925 & 0,087 & 0,722 & 1,177 & 0,371 & 0,424 & 2,231 & 0,945 & 0,959 & 72 & 649 & $-4,5$ \\
\hline 650 & 0,2 & 1,7 & 0,2 & 1,6 & 1,55 & 0,868 & 0,946 & 0,916 & 0,143 & 0,942 & 1,030 & 0,563 & 0,536 & 0,698 & 0,375 & 0,411 & 12 & 650 & $-2,8$ \\
\hline 651 & 2,4 & 6,8 & 2,7 & 6,3 & 6,12 & 0,902 & 0,932 & 0,902 & 0,119 & 0,664 & 1,227 & 0,452 & 0,456 & 2,617 & 1,193 & 1,170 & 102 & 651 & $-4,7$ \\
\hline 652 & 1,3 & 4,4 & 1,4 & 4,2 & 4,07 & 0,943 & 0,967 & 0,936 & 0,066 & 0,872 & 1,071 & 0,747 & 0,791 & 1,496 & 1,183 & 0,988 & 129 & 652 & $-3,9$ \\
\hline 653 & 0,2 & 1,5 & 0,2 & 1,3 & 1,21 & 0,900 & 0,850 & 0,823 & 0,180 & 0,975 & 1,013 & 0,515 & 0,450 & 0,646 & 0,291 & 0,306 & 144 & 653 & $-2,7$ \\
\hline 654 & 0,3 & 2,1 & 0,3 & 2,0 & 1,96 & 0,891 & 0,957 & 0,926 & 0,118 & 0,749 & 1,155 & 0,427 & 0,434 & 0,893 & 0,387 & 0,387 & 41 & 654 & $-3,2$ \\
\hline 655 & 0,1 & 1,6 & 0,1 & 1,4 & 1,31 & 0,900 & 0,859 & 0,831 & 0,173 & 0,643 & 1,248 & 0,275 & 0,179 & 0,766 & 0,137 & 0,274 & 87 & 655 & $-2,9$ \\
\hline 656 & 0,6 & 2,6 & 0,6 & 2,5 & 2,38 & 0,922 & 0,950 & 0,919 & 0,092 & 1,000 & 0,975 & 0,947 & 0,867 & 0,866 & 0,751 & 0,738 & 85 & 656 & $-3,1$ \\
\hline 657 & 1,6 & 4,9 & 1,7 & 4,7 & 4,57 & 0,938 & 0,963 & 0,932 & 0,072 & 0,852 & 1,083 & 0,490 & 0,564 & 2,060 & 1,162 & 1,225 & 113 & 657 & $-4,4$ \\
\hline 658 & 0,5 & 2,6 & 0,6 & 2,5 & 2,46 & 0,951 & 0,965 & 0,934 & 0,060 & 0,983 & 1,008 & 0,720 & 0,713 & 0,981 & 0,699 & 0,685 & 41 & 658 & $-3,3$ \\
\hline 659 & 0,1 & 1,0 & 0,1 & 0,8 & 0,77 & 1,000 & 0,812 & 0,787 & 0,188 & 1,000 & 0,879 & 0,652 & 0,442 & 0,439 & 0,194 & 0,306 & 37 & 659 & $-2,1$ \\
\hline 660 & 5,1 & 9,5 & 5,3 & 9,4 & 9,11 & 0,957 & 0,994 & 0,963 & 0,043 & 0,716 & 1,182 & 0,421 & 0,399 & 3,930 & 1,568 & 1,598 & 37 & 660 & $-5,3$ \\
\hline 661 & 69,2 & 33,1 & 72,1 & 32,5 & 31,47 & 0,960 & 0,982 & 0,950 & 0,044 & 0,793 & 1,123 & 0,537 & 0,561 & 12,808 & 7,186 & 7,017 & 49 & 661 & $-7,0$ \\
\hline 662 & 42,8 & 27,6 & 45,7 & 26,9 & 25,99 & 0,937 & 0,974 & 0,942 & 0,068 & 0,708 & 1,189 & 0,429 & 0,498 & 11,271 & 5,608 & 5,330 & 70 & 662 & $-6,8$ \\
\hline 663 & 0,1 & 0,9 & 0,1 & 0,9 & 0,86 & 0,955 & 0,943 & 0,913 & 0,073 & 1,000 & 0,845 & 0,922 & 0,657 & 0,369 & 0,242 & 0,274 & 151 & 663 & $-1,9$ \\
\hline 664 & 0,1 & 1,0 & 0,1 & 0,8 & 0,73 & 0,958 & 0,744 & 0,721 & 0,259 & 1,000 & 0,873 & 0,861 & 0,614 & 0,399 & 0,245 & 0,274 & 61 & 664 & $-2,0$ \\
\hline 665 & 0,2 & 1,3 & 0,2 & 1,3 & 1,25 & 0,974 & 0,960 & 0,930 & 0,048 & 1,000 & 0,911 & 0,693 & 0,600 & 0,565 & 0,339 & 0,387 & 18 & 665 & $-2,5$ \\
\hline 666 & 0,9 & 3,2 & 0,9 & 3,1 & 3,00 & 0,995 & 0,983 & 0,951 & 0,018 & 1,000 & 0,936 & 0,850 & 0,790 & 1,164 & 0,920 & 0,919 & 57 & 666 & $-3,5$ \\
\hline 667 & 36,6 & 23,3 & 38,4 & 22,2 & 21,54 & 0,954 & 0,957 & 0,926 & 0,063 & 0,851 & 1,084 & 0,742 & 0,798 & 7,924 & 6,321 & 5,631 & 21 & 667 & $-6,3$ \\
\hline 668 & 5,2 & 9,1 & 5,8 & 8,6 & 8,30 & 0,897 & 0,937 & 0,907 & 0,121 & 0,781 & 1,131 & 0,650 & 0,670 & 3,193 & 2,138 & 1,918 & 80 & 668 & $-5,0$ \\
\hline 669 & 0,1 & 1,3 & 0,2 & 1,1 & 1,07 & 0,909 & 0,884 & 0,855 & 0,147 & 1,000 & 0,943 & 0,849 & 0,687 & 0,459 & 0,315 & 0,387 & 123 & 669 & $-2,2$ \\
\hline 670 & 3,4 & 7,1 & 3,6 & 7,0 & 6,79 & 0,942 & 0,987 & 0,956 & 0,060 & 0,847 & 1,087 & 0,610 & 0,538 & 2,665 & 1,433 & 1,356 & 130 & 670 & $-4,7$ \\
\hline 671 & 0,1 & 1,5 & 0,1 & 1,2 & 1,16 & 0,862 & 0,822 & 0,796 & 0,225 & 0,692 & 1,202 & 0,374 & 0,368 & 0,632 & 0,233 & 0,274 & 20 & 671 & $-2,7$ \\
\hline 672 & 0,1 & 1,6 & 0,2 & 1,5 & 1,46 & 0,838 & 0,943 & 0,913 & 0,172 & 0,709 & 1,187 & 0,362 & 0,314 & 0,715 & 0,225 & 0,274 & 24 & 672 & $-2,8$ \\
\hline 673 & 0,2 & 1,5 & 0,2 & 1,4 & 1,38 & 1,000 & 0,926 & 0,896 & 0,074 & 1,000 & 0,904 & 0,855 & 0,585 & 0,585 & 0,342 & 0,411 & 90 & 673 & $-2,5$ \\
\hline 674 & 1,4 & 4,5 & 1,4 & 4,4 & 4,23 & 0,958 & 0,979 & 0,948 & 0,047 & 0,873 & 1,070 & 0,556 & 0,499 & 1,781 & 0,889 & 0,919 & 63 & 674 & $-4,2$ \\
\hline 675 & 0,4 & 2,2 & 0,4 & 2,1 & 2,03 & 0,951 & 0,958 & 0,927 & 0,064 & 0,955 & 1,023 & 0,584 & 0,542 & 0,893 & 0,484 & 0,494 & 48 & 675 & $-3,2$ \\
\hline 676 & 0,3 & 1,9 & 0,3 & 1,7 & 1,68 & 0,938 & 0,912 & 0,883 & 0,108 & 0,993 & 1,004 & 0,621 & 0,510 & 0,766 & 0,390 & 0,411 & 11 & 676 & $-2,9$ \\
\hline 677 & 0,5 & 2,3 & 0,5 & 2,3 & 2,20 & 0,970 & 0,977 & 0,946 & 0,038 & 1,000 & 0,977 & 0,661 & 0,572 & 0,932 & 0,533 & 0,581 & 50 & 677 & $-3,2$ \\
\hline 678 & 0,2 & 1,8 & 0,2 & 1,6 & 1,58 & 0,878 & 0,906 & 0,877 & 0,154 & 0,658 & 1,233 & 0,300 & 0,233 & 0,847 & 0,197 & 0,274 & 5 & 678 & $-3,1$ \\
\hline 679 & 6,2 & 9,2 & 6,5 & 9,2 & 8,86 & 0,963 & 0,990 & 0,958 & 0,038 & 0,916 & 1,045 & 0,749 & 0,772 & 3,254 & 2,512 & 2,424 & 57 & 679 & $-5,0$ \\
\hline 680 & 0,5 & 3,2 & 0,6 & 3,0 & 2,86 & 0,891 & 0,935 & 0,905 & 0,127 & 0,624 & 1,266 & 0,300 & 0,268 & 1,453 & 0,390 & 0,411 & 3 & 680 & $-3,9$ \\
\hline 681 & 18,1 & 17,1 & 19,0 & 16,8 & 16,28 & 0,954 & 0,984 & 0,952 & 0,049 & 0,779 & 1,133 & 0,457 & 0,539 & 7,105 & 3,830 & 3,617 & 80 & 681 & $-6,2$ \\
\hline 682 & 0,7 & 3,0 & 0,8 & 2,9 & 2,83 & 0,940 & 0,957 & 0,927 & 0,073 & 1,000 & 0,999 & 0,726 & 0,656 & 1,140 & 0,748 & 0,775 & 107 & 682 & $-3,5$ \\
\hline 683 & 0,4 & 2,5 & 0,4 & 2,2 & 2,12 & 0,916 & 0,888 & 0,859 & 0,140 & 0,847 & 1,086 & 0,501 & 0,404 & 1,018 & 0,411 & 0,548 & 91 & 683 & $-3,3$ \\
\hline 684 & 0,6 & 3,3 & 0,7 & 3,1 & 3,00 & 0,853 & 0,933 & 0,903 & 0,161 & 0,651 & 1,240 & 0,359 & 0,387 & 1,425 & 0,552 & 0,565 & 66 & 684 & $-3,8$ \\
\hline 685 & 5,8 & 9,6 & 6,2 & 9,3 & 9,01 & 0,939 & 0,974 & 0,942 & 0,066 & 0,800 & 1,118 & 0,554 & 0,652 & 3,658 & 2,386 & 2,095 & 55 & 685 & $-5,2$ \\
\hline
\end{tabular}




\begin{tabular}{|c|c|c|c|c|c|c|c|c|c|c|c|c|c|c|c|c|c|c|c|}
\hline 686 & 0,6 & 2,6 & 0,6 & 2,6 & 2,48 & 0,961 & 0,979 & 0,948 & 0,044 & 1,000 & 0,971 & 0,739 & 0,680 & 0,997 & 0,678 & 0,738 & 62 & 686 & $-3,3$ \\
\hline 687 & 0,4 & 2,2 & 0,4 & 2,1 & 2,02 & 0,988 & 0,941 & 0,912 & 0,060 & 0,996 & 1,002 & 0,660 & 0,601 & 0,866 & 0,521 & 0,548 & 162 & 687 & $-3,1$ \\
\hline 688 & 0,4 & 2,7 & 0,5 & 2,5 & 2,39 & 0,816 & 0,905 & 0,876 & 0,207 & 0,633 & 1,257 & 0,344 & 0,372 & 1,178 & 0,438 & 0,387 & 142 & 688 & $-3,6$ \\
\hline 689 & 0,7 & 3,1 & 0,7 & 2,9 & 2,79 & 0,886 & 0,935 & 0,905 & 0,131 & 0,870 & 1,072 & 0,617 & 0,661 & 1,164 & 0,770 & 0,698 & 41 & 689 & $-3,5$ \\
\hline 690 & 4,1 & 7,8 & 4,3 & 7,7 & 7,43 & 0,953 & 0,983 & 0,952 & 0,050 & 0,837 & 1,093 & 0,539 & 0,610 & 3,095 & 1,889 & 1,786 & 33 & 690 & $-5,0$ \\
\hline 691 & 0,2 & 1,8 & 0,3 & 1,8 & 1,73 & 0,963 & 0,971 & 0,940 & 0,047 & 0,903 & 1,052 & 0,509 & 0,434 & 0,781 & 0,339 & 0,387 & 131 & 691 & $-3,0$ \\
\hline 692 & 0,5 & 2,6 & 0,6 & 2,5 & 2,46 & 0,950 & 0,979 & 0,948 & 0,054 & 0,986 & 1,007 & 0,597 & 0,604 & 1,063 & 0,642 & 0,685 & 71 & 692 & $-3,4$ \\
\hline 693 & 13,6 & 16,2 & 15,3 & 15,7 & 15,19 & 0,889 & 0,969 & 0,938 & 0,115 & 0,652 & 1,239 & 0,402 & 0,447 & 6,567 & 2,936 & 2,036 & 12 & 693 & $-6,0$ \\
\hline 694 & 3,3 & 7,5 & 3,7 & 7,0 & 6,81 & 0,880 & 0,935 & 0,905 & 0,136 & 0,725 & 1,175 & 0,616 & 0,717 & 2,599 & 1,864 & 1,475 & 103 & 694 & $-4,7$ \\
\hline 695 & 1,6 & 4,8 & 1,8 & 4,7 & 4,51 & 0,912 & 0,965 & 0,934 & 0,095 & 0,887 & 1,062 & 0,739 & 0,742 & 1,685 & 1,251 & 1,104 & 61 & 695 & $-4,1$ \\
\hline 696 & 0,4 & 2,4 & 0,4 & 2,1 & 2,03 & 0,854 & 0,890 & 0,861 & 0,183 & 0,803 & 1,116 & 0,523 & 0,502 & 0,932 & 0,468 & 0,433 & 135 & 696 & $-3,2$ \\
\hline 697 & 1,2 & 3,8 & 1,2 & 3,7 & 3,63 & 0,950 & 0,986 & 0,954 & 0,052 & 1,000 & 0,998 & 0,737 & 0,755 & 1,412 & 1,065 & 1,096 & 54 & 697 & $-3,8$ \\
\hline 698 & 0,2 & 1,5 & 0,2 & 1,3 & 1,25 & 1,000 & 0,876 & 0,848 & 0,124 & 0,975 & 1,013 & 0,458 & 0,354 & 0,685 & 0,242 & 0,306 & 142 & 698 & $-2,8$ \\
\hline 699 & 0,6 & 2,7 & 0,6 & 2,6 & 2,54 & 0,985 & 0,980 & 0,949 & 0,025 & 1,000 & 0,952 & 0,879 & 0,812 & 0,954 & 0,775 & 0,775 & 138 & 699 & $-3,3$ \\
\hline 700 & 0,2 & 2,1 & 0,3 & 1,7 & 1,61 & 0,845 & 0,804 & 0,779 & 0,250 & 0,674 & 1,218 & 0,361 & 0,304 & 0,901 & 0,274 & 0,306 & 93 & 700 & $-3,2$ \\
\hline 701 & 2,5 & 6,0 & 2,7 & 5,7 & 5,53 & 0,913 & 0,949 & 0,919 & 0,101 & 0,870 & 1,072 & 0,702 & 0,795 & 2,133 & 1,697 & 1,475 & 112 & 701 & $-4,4$ \\
\hline 702 & 0,3 & 1,9 & 0,3 & 1,7 & 1,64 & 0,950 & 0,902 & 0,873 & 0,110 & 0,950 & 1,026 & 0,626 & 0,540 & 0,738 & 0,398 & 0,433 & 118 & 702 & $-2,9$ \\
\hline 703 & 0,7 & 3,2 & 0,7 & 3,1 & 3,00 & 0,935 & 0,983 & 0,951 & 0,067 & 0,856 & 1,081 & 0,509 & 0,439 & 1,300 & 0,571 & 0,581 & 152 & 703 & $-3,7$ \\
\hline 704 & 1,0 & 4,7 & 1,1 & 4,5 & 4,38 & 0,925 & 0,972 & 0,941 & 0,080 & 0,607 & 1,284 & 0,291 & 0,316 & 2,141 & 0,676 & 0,685 & 10 & 704 & $-4,4$ \\
\hline 705 & 0,2 & 1,6 & 0,2 & 1,5 & 1,41 & 0,942 & 0,909 & 0,881 & 0,107 & 1,000 & 0,944 & 0,693 & 0,632 & 0,650 & 0,411 & 0,494 & 111 & 705 & $-2,7$ \\
\hline 706 & 4,8 & 10,3 & 5,6 & 9,4 & 9,06 & 0,850 & 0,912 & 0,883 & 0,174 & 0,568 & 1,326 & 0,426 & 0,450 & 3,775 & 1,700 & 1,430 & 166 & 706 & $-5,2$ \\
\hline 707 & 1,8 & 5,0 & 1,9 & 4,9 & 4,75 & 0,931 & 0,982 & 0,950 & 0,071 & 0,896 & 1,056 & 0,616 & 0,617 & 1,919 & 1,183 & 1,104 & 56 & 707 & $-4,3$ \\
\hline 708 & 3,2 & 6,8 & 3,4 & 6,5 & 6,29 & 0,932 & 0,951 & 0,920 & 0,084 & 0,848 & 1,086 & 0,710 & 0,672 & 2,378 & 1,598 & 1,410 & 44 & 708 & $-4,6$ \\
\hline 709 & 0,2 & 1,3 & 0,2 & 1,2 & 1,16 & 0,944 & 0,929 & 0,899 & 0,090 & 1,000 & 0,912 & 0,962 & 0,832 & 0,459 & 0,382 & 0,411 & 42 & 709 & $-2,2$ \\
\hline 710 & 20,5 & 18,9 & 22,1 & 17,2 & 16,61 & 0,928 & 0,910 & 0,881 & 0,116 & 0,724 & 1,176 & 0,733 & 0,812 & 5,966 & 4,845 & 4,253 & 51 & 710 & $-5,9$ \\
\hline 711 & 0,4 & 2,5 & 0,4 & 2,3 & 2,21 & 0,967 & 0,917 & 0,888 & 0,089 & 0,836 & 1,094 & 0,431 & 0,394 & 1,104 & 0,435 & 0,494 & 30 & 711 & $-3,5$ \\
\hline 712 & 0,3 & 2,0 & 0,3 & 1,9 & 1,81 & 0,903 & 0,953 & 0,922 & 0,108 & 0,862 & 1,077 & 0,466 & 0,409 & 0,847 & 0,347 & 0,387 & 11 & 712 & $-3,1$ \\
\hline 713 & 0,1 & 1,1 & 0,1 & 1,0 & 0,98 & 0,931 & 0,887 & 0,858 & 0,133 & 1,000 & 0,909 & 0,764 & 0,579 & 0,459 & 0,266 & 0,274 & 166 & 713 & $-2,2$ \\
\hline 714 & 29,9 & 21,6 & 32,0 & 20,8 & 20,11 & 0,933 & 0,963 & 0,932 & 0,077 & 0,807 & 1,113 & 0,564 & 0,655 & 8,211 & 5,376 & 5,207 & 62 & 714 & $-6,4$ \\
\hline 715 & 0,2 & 1,5 & 0,2 & 1,4 & 1,38 & 0,980 & 0,939 & 0,910 & 0,064 & 1,000 & 0,890 & 0,917 & 0,728 & 0,565 & 0,411 & 0,494 & 97 & 715 & $-2,5$ \\
\hline 716 & 0,3 & 2,0 & 0,3 & 1,8 & 1,72 & 0,914 & 0,906 & 0,877 & 0,127 & 0,985 & 1,007 & 0,627 & 0,620 & 0,781 & 0,484 & 0,494 & 56 & 716 & $-3,0$ \\
\hline 717 & 0,2 & 1,5 & 0,2 & 1,3 & 1,25 & 0,902 & 0,876 & 0,848 & 0,158 & 1,000 & 0,999 & 0,589 & 0,489 & 0,613 & 0,300 & 0,306 & 57 & 717 & $-2,6$ \\
\hline 718 & 0,3 & 2,0 & 0,3 & 1,9 & 1,86 & 0,969 & 0,954 & 0,924 & 0,055 & 0,919 & 1,043 & 0,446 & 0,422 & 0,919 & 0,387 & 0,433 & 27 & 718 & $-3,2$ \\
\hline 719 & 0,8 & 3,4 & 0,9 & 3,4 & 3,29 & 0,933 & 0,984 & 0,953 & 0,069 & 0,893 & 1,058 & 0,560 & 0,641 & 1,385 & 0,888 & 0,877 & 5 & 719 & $-3,8$ \\
\hline 720 & 0,1 & 0,9 & 0,1 & 0,7 & 0,70 & 1,000 & 0,777 & 0,752 & 0,223 & 1,000 & 0,831 & 0,836 & 0,625 & 0,387 & 0,242 & 0,306 & 45 & 720 & $-2,0$ \\
\hline 721 & 0,5 & 2,6 & 0,5 & 2,5 & 2,43 & 0,954 & 0,965 & 0,934 & 0,058 & 0,899 & 1,055 & 0,640 & 0,663 & 0,981 & 0,650 & 0,581 & 64 & 721 & $-3,3$ \\
\hline 722 & 3,5 & 7,6 & 3,7 & 7,5 & 7,25 & 0,938 & 0,988 & 0,956 & 0,064 & 0,754 & 1,151 & 0,469 & 0,400 & 3,061 & 1,225 & 1,263 & 50 & 722 & $-4,9$ \\
\hline 723 & 0,1 & 1,1 & 0,1 & 1,1 & 1,07 & 0,964 & 1,000 & 0,968 & 0,036 & 1,000 & 0,878 & 1,011 & 0,849 & 0,399 & 0,339 & 0,387 & 45 & 723 & $-2,0$ \\
\hline 724 & 0,2 & 1,8 & 0,2 & 1,5 & 1,49 & 0,854 & 0,831 & 0,804 & 0,224 & 0,707 & 1,190 & 0,353 & 0,329 & 0,833 & 0,274 & 0,306 & 83 & 724 & $-3,1$ \\
\hline 725 & 0,3 & 2,1 & 0,3 & 1,9 & 1,84 & 0,841 & 0,912 & 0,883 & 0,182 & 0,785 & 1,128 & 0,506 & 0,555 & 0,828 & 0,460 & 0,411 & 105 & 725 & $-3,0$ \\
\hline 726 & 1,1 & 4,1 & 1,1 & 4,0 & 3,87 & 0,951 & 0,969 & 0,938 & 0,059 & 0,799 & 1,119 & 0,468 & 0,479 & 1,718 & 0,823 & 0,866 & 31 & 726 & $-4,1$ \\
\hline 727 & 0,2 & 1,5 & 0,2 & 1,3 & 1,21 & 0,902 & 0,828 & 0,802 & 0,197 & 0,952 & 1,025 & 0,589 & 0,632 & 0,613 & 0,387 & 0,387 & 24 & 727 & $-2,6$ \\
\hline 728 & 2,5 & 5,7 & 2,6 & 5,6 & 5,45 & 0,980 & 0,991 & 0,959 & 0,022 & 0,980 & 1,010 & 0,789 & 0,789 & 2,014 & 1,590 & 1,562 & 139 & 728 & $-4,3$ \\
\hline 729 & 2,3 & 5,9 & 2,5 & 5,7 & 5,56 & 0,938 & 0,969 & 0,938 & 0,069 & 0,838 & 1,092 & 0,666 & 0,763 & 2,116 & 1,615 & 1,292 & 145 & 729 & $-4,4$ \\
\hline 730 & 3,3 & 6,5 & 3,4 & 6,4 & 6,21 & 0,968 & 0,992 & 0,960 & 0,033 & 0,977 & 1,012 & 0,742 & 0,779 & 2,362 & 1,841 & 1,802 & 124 & 730 & $-4,6$ \\
\hline 731 & 0,2 & 1,3 & 0,2 & 1,1 & 1,07 & 1,000 & 0,858 & 0,830 & 0,142 & 1,000 & 0,941 & 0,702 & 0,588 & 0,522 & 0,307 & 0,387 & 107 & 731 & $-2,4$ \\
\hline 732 & 2,5 & 6,6 & 2,7 & 6,4 & 6,23 & 0,916 & 0,980 & 0,949 & 0,086 & 0,729 & 1,171 & 0,426 & 0,524 & 2,735 & 1,433 & 1,300 & 68 & 732 & $-4,8$ \\
\hline 733 & 0,3 & 1,8 & 0,3 & 1,7 & 1,68 & 0,985 & 0,970 & 0,939 & 0,034 & 1,000 & 0,907 & 0,866 & 0,718 & 0,675 & 0,484 & 0,565 & 30 & 733 & $-2,8$ \\
\hline 734 & 8,8 & 12,5 & 9,2 & 12,1 & 11,72 & 0,953 & 0,968 & 0,937 & 0,057 & 0,705 & 1,191 & 0,612 & 0,808 & 4,274 & 3,453 & 2,470 & 123 & 734 & $-5,4$ \\
\hline 735 & 16,0 & 15,8 & 16,9 & 15,5 & 14,98 & 0,946 & 0,981 & 0,949 & 0,057 & 0,808 & 1,113 & 0,558 & 0,593 & 6,043 & 3,585 & 3,492 & 44 & 735 & $-5,9$ \\
\hline 736 & 0,4 & 2,3 & 0,4 & 2,0 & 1,93 & 0,944 & 0,885 & 0,856 & 0,128 & 0,974 & 1,013 & 0,629 & 0,583 & 0,893 & 0,521 & 0,548 & 74 & 736 & $-3,2$ \\
\hline 737 & 3,0 & 6,9 & 3,2 & 6,7 & 6,45 & 0,940 & 0,968 & 0,937 & 0,068 & 0,806 & 1,114 & 0,463 & 0,498 & 2,891 & 1,438 & 1,456 & 13 & 737 & $-4,9$ \\
\hline 738 & 0,2 & 1,7 & $\begin{array}{l}0,2 \\
0,3\end{array}$ & 1,5 & 1,41 & 0,870 & 0,868 & 0,841 & 0,185 & 0,980 & $\begin{array}{l}1,010 \\
1,01\end{array}$ & 0,598 & 0,620 & 0,685 & 0,425 & 0,387 & 128 & 738 & $-2,8$ \\
\hline & & & & & & & & & & & & & & & & & & & \\
\hline Label & Area & Perim. & CH Area & I Perim. & Solidity & Convexity & $\begin{array}{c}\text { Concavity } \\
\text { Index }\end{array}$ & FormFactor & Circularity & Roundness & AR Feret & Feret d & eret d & $\begin{array}{l}\text { Max. Insc. } \\
\text { Circle Diam. }\end{array}$ & ientation & $\begin{array}{l}\text { ROI } \\
\text { Index }\end{array}$ & Phi & & \\
\hline
\end{tabular}




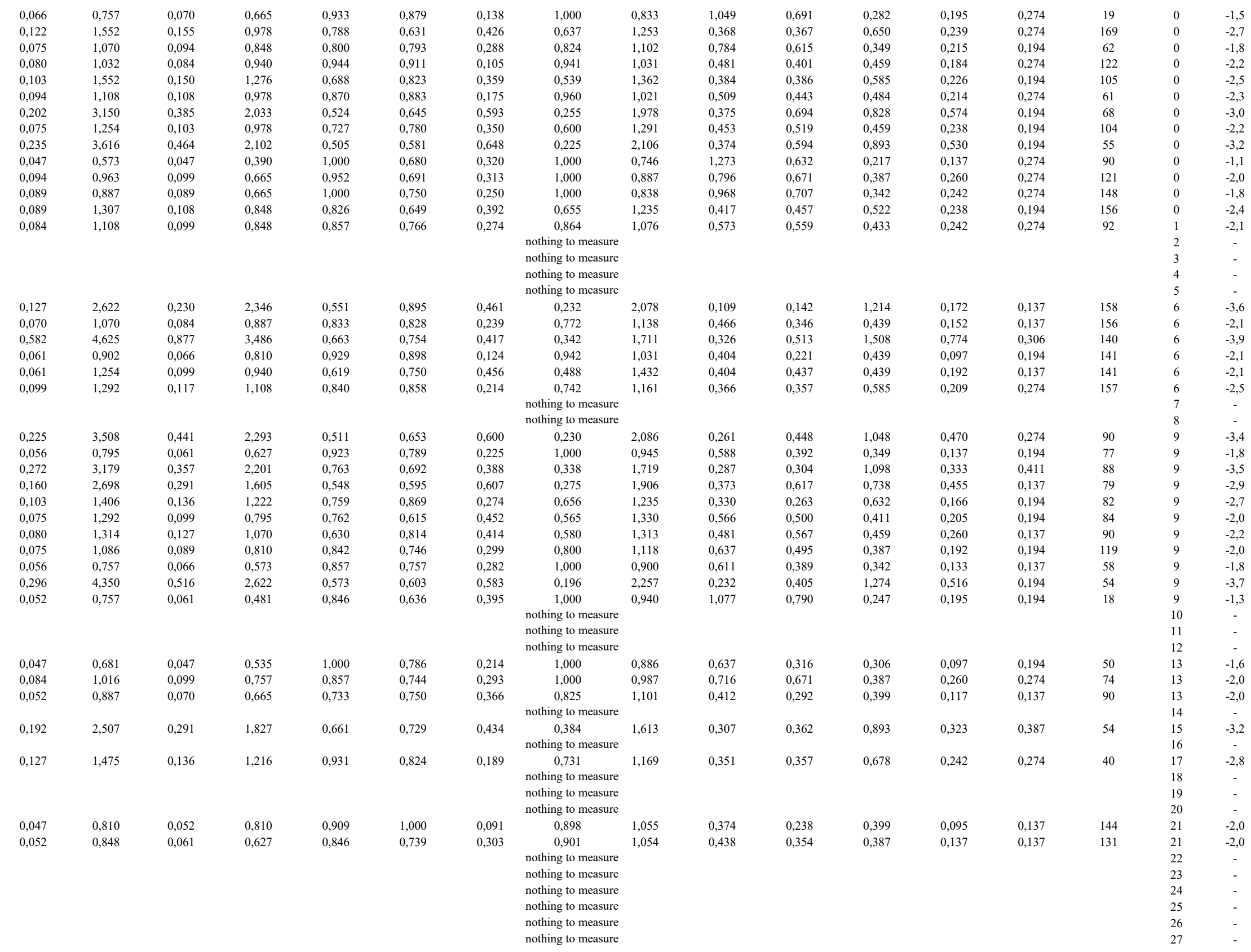




$\begin{array}{lllllll}0,136 & 1,865 & 0,206 & 1,513 & 0,659 & 0,812 & 0,390 \\ 0,141 & 2,362 & 0,225 & 1,384 & 0,625 & 0,586 & 0,559 \\ 0,075 & 1,108 & 0,099 & 0,795 & 0,762 & 0,717 & 0,370 \\ 0,052 & 0,795 & 0,070 & 0,535 & 0,733 & 0,673 & 0,422 \\ 0,141 & 2,584 & 0,258 & 1,605 & 0,545 & 0,621 & 0,592 \\ 0,066 & 0,887 & 0,080 & 0,703 & 0,824 & 0,793 & 0,272 \\ 0,070 & 1,162 & 0,103 & 0,925 & 0,682 & 0,796 & 0,378 \\ 0,113 & 1,422 & 0,150 & 1,162 & 0,750 & 0,817 & 0,310 \\ 0,075 & 1,583 & 0,113 & 0,887 & 0,667 & 0,560 & 0,552 \\ & & & & & & \\ 0,047 & 0,940 & 0,070 & 0,665 & 0,667 & 0,707 & 0,444 \\ 0,056 & 0,978 & 0,089 & 0,665 & 0,632 & 0,680 & 0,488 \\ 0,066 & 0,978 & 0,075 & 0,795 & 0,875 & 0,812 & 0,225 \\ 0,089 & 1,384 & 0,136 & 1,055 & 0,655 & 0,762 & 0,419 \\ 0,056 & 0,902 & 0,070 & 0,627 & 0,800 & 0,695 & 0,365 \\ 0,047 & 1,124 & 0,075 & 0,719 & 0,625 & 0,639 & 0,520 \\ 0,047 & 0,627 & 0,047 & 0,405 & 1,000 & 0,646 & 0,354 \\ 0,047 & 0,795 & 0,052 & 0,611 & 0,909 & 0,769 & 0,248 \\ 0,084 & 1,146 & 0,103 & 0,925 & 0,818 & 0,807 & 0,265\end{array}$

nothing to measure

nothing to measure

nothing to measure

nothing to measure

1,426

426

$\begin{array}{ll}0,768 & 1,141 \\ 1,000 & 0,987\end{array}$

$\begin{array}{lll}0,562 & 0,760 & 0,565\end{array}$

$\begin{array}{lll}0,781 & 0,292 & 0,274 \\ 0,565 & 0,429 & 0,274\end{array}$

$\begin{array}{ll}0,429 & 0,274 \\ 0,199 & 0,137\end{array}$

$\begin{array}{lll}0,565 & 0,429 & 0,274 \\ 0,342 & 0,152 & 0,137 \\ 0,794 & 0,407 & 0,194\end{array}$

$\begin{array}{lll}0,705 & 0,407 & 0,194 \\ 0,342 & 0,228 & 0,194\end{array}$

$0,360 \quad 0,577$

$\begin{array}{ll}0,407 & 0,194 \\ 0,228 & 0,194\end{array}$

1,000
1,976

$0,713 \quad 0,66$

0,439

$\begin{array}{ll}0,228 & 0,194 \\ 0,249 & 0,194\end{array}$

$0,700 \quad 1,195$

0,364

$\begin{array}{ll}0,230 & 0,274 \\ 0,229 & 0,137\end{array}$

ing to measure

$\begin{array}{lllllll}0,667 & 1,225 & 0,344 & 0,337 & 0,417 & 0,140 & 0,137\end{array}$

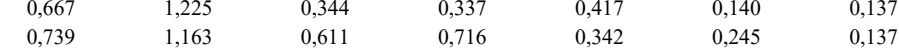

$\begin{array}{ll}0,739 & 1,163 \\ 0,862 & 1,077\end{array}$

0,611

$0,342 \quad 0,245 \quad 0,137$

$0,484 \quad 0,269 \quad 0,194$

$\begin{array}{ll}0,585 & 1,307 \\ 0,869 & 1,073\end{array}$

$0,484 \quad 0,55$

$0,611 \quad 0,566$

0,342
0,369

0,194

$\begin{array}{ll}0,467 & 1,464 \\ 1,000 & 0,816\end{array}$

0,439

0,291

0,808

0,196

0,459

$\begin{array}{ll}0,068 & 0,137 \\ 0,235 & 0,274\end{array}$

nothing to measure
nothing to measure

nothing to measure

mening to measuro

nothing to measure

nothing to measure

nothing to measure

nothing to measure

nothing to measure

nothing to measure

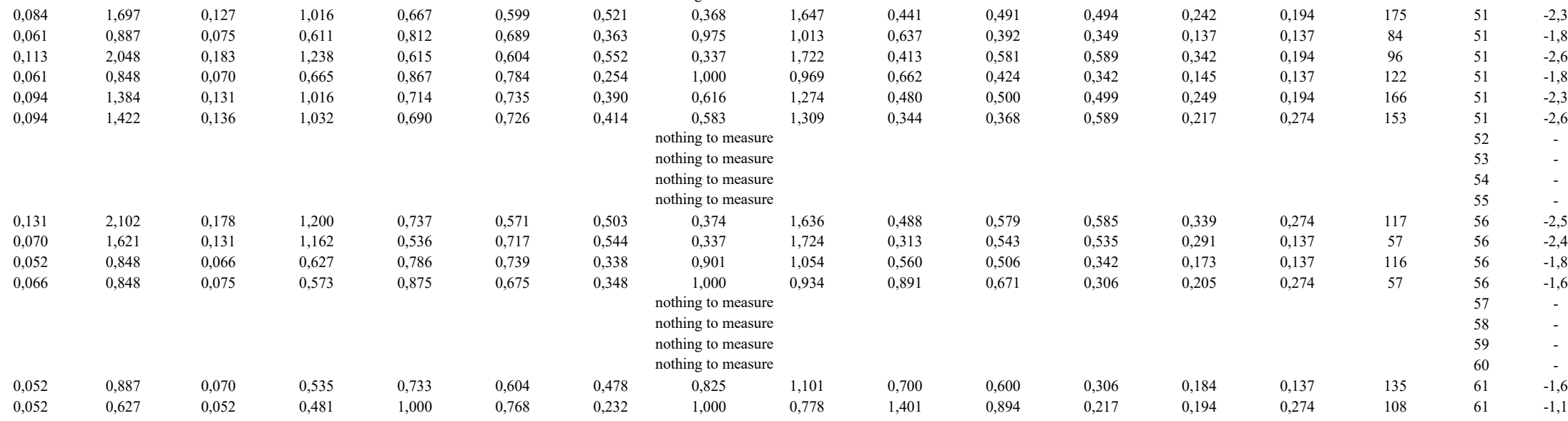




\begin{tabular}{|c|c|c|c|c|c|c|c|c|c|c|c|c|c|c|c|c|}
\hline 0,075 & 0,902 & 0,080 & 0,665 & 0,941 & 0,737 & 0,269 & $\begin{array}{l}1,000 \\
\text { nothing to measure } \\
\text { nothing to measure } \\
\text { nothing to measure }\end{array}$ & 0,929 & 0,784 & 0,588 & 0,349 & 0,205 & 0,274 & 90 & $\begin{array}{l}61 \\
62 \\
63 \\
64\end{array}$ & $\begin{array}{c}-1,8 \\
- \\
- \\
-\end{array}$ \\
\hline 0,047 & 0,573 & 0,047 & 0,313 & 1,000 & 0,547 & 0,453 & 1,000 & 0,746 & 1,273 & 0,632 & 0,217 & 0,137 & 0,274 & 72 & 65 & $-1,1$ \\
\hline 0,056 & 0,887 & 0,066 & 0,627 & 0,857 & 0,707 & 0,326 & 0,900 & 1,054 & 0,764 & 0,600 & 0,306 & 0,184 & 0,137 & 148 & 66 & $-1,6$ \\
\hline 0,052 & 0,757 & 0,061 & 0,497 & 0,846 & 0,657 & 0,376 & 1,000 & 0,940 & 0,560 & 0,334 & 0,342 & 0,114 & 0,137 & 121 & 66 & $-1,8$ \\
\hline 0,080 & 1,016 & 0,089 & 0,833 & 0,895 & 0,819 & 0,209 & 0,970 & 1,015 & 0,481 & 0,330 & 0,459 & 0,152 & 0,194 & 68 & 66 & $-2,2$ \\
\hline 0,305 & 3,638 & 0,521 & 2,850 & 0,586 & 0,783 & 0,468 & 0,290 & 1,859 & 0,206 & 0,261 & 1,372 & 0,358 & 0,274 & 167 & 66 & $-3,8$ \\
\hline 0,103 & 1,919 & 0,174 & 1,307 & 0,595 & 0,681 & 0,516 & 0,352 & 1,685 & 0,315 & 0,450 & 0,646 & 0,291 & 0,137 & 36 & 66 & $-2,7$ \\
\hline 0,056 & 1,254 & 0,094 & 0,887 & 0,600 & 0,707 & 0,496 & 0,450 & 1,491 & 0,340 & 0,377 & 0,459 & 0,173 & 0,137 & 148 & 66 & $-2,2$ \\
\hline 0,084 & 0,978 & 0,094 & 0,887 & 0,900 & 0,906 & 0,137 & 1,000 & 0,950 & 0,619 & 0,439 & 0,417 & 0,183 & 0,274 & 11 & 66 & $-2,1$ \\
\hline 0,047 & 0,627 & 0,047 & 0,535 & 1,000 & 0,854 & 0,146 & 1,000 & 0,816 & 0,749 & 0,485 & 0,282 & 0,137 & 0,194 & 18 & 66 & $-1,5$ \\
\hline 0,061 & 1,070 & 0,084 & 0,665 & 0,722 & 0,621 & 0,470 & $\begin{array}{c}0,669 \\
\text { nothing to measure }\end{array}$ & 1,222 & 0,487 & 0,458 & 0,399 & 0,183 & 0,137 & 18 & $\begin{array}{l}66 \\
67\end{array}$ & $-2,0$ \\
\hline 0,052 & 0,665 & 0,056 & 0,390 & 0,917 & 0,586 & 0,422 & 1,000 & 0,826 & 0,824 & 0,485 & 0,282 & 0,137 & 0,194 & 162 & 68 & $-1,5$ \\
\hline 0,075 & 0,925 & 0,080 & 0,611 & 0,941 & 0,661 & 0,344 & 1,000 & 0,952 & 0,509 & 0,316 & 0,433 & 0,137 & 0,274 & 171 & 68 & $-2,1$ \\
\hline 0,174 & 2,691 & 0,291 & 1,881 & 0,597 & 0,699 & 0,503 & 0,301 & 1,822 & 0,277 & 0,378 & 0,893 & 0,337 & 0,194 & 31 & 68 & $-3,2$ \\
\hline 0,056 & 0,940 & 0,070 & 0,665 & 0,800 & 0,707 & 0,355 & 0,800 & 1,118 & 0,611 & 0,424 & 0,342 & 0,145 & 0,194 & 45 & 68 & $-1,8$ \\
\hline 0,075 & 1,146 & 0,089 & 0,665 & 0,842 & 0,580 & 0,449 & $\begin{array}{c}0,718 \\
\text { nothing to measure }\end{array}$ & 1,180 & 0,702 & 0,557 & 0,369 & 0,205 & 0,194 & 15 & $\begin{array}{l}68 \\
69\end{array}$ & $\begin{array}{l}-1,9 \\
-\end{array}$ \\
\hline 0,047 & 1,070 & 0,080 & 0,665 & 0,588 & 0,621 & 0,559 & 0,515 & 1,394 & 0,490 & 0,588 & 0,349 & 0,205 & 0,137 & 90 & 70 & $-1,8$ \\
\hline 0,061 & 1,070 & 0,080 & 0,940 & 0,765 & 0,879 & 0,265 & 0,669 & 1,222 & 0,517 & 0,475 & 0,387 & 0,184 & 0,194 & 79 & 70 & $-2,0$ \\
\hline \multirow[t]{2}{*}{0,056} & 0,994 & 0,070 & 0,665 & 0,800 & 0,669 & 0,387 & $\begin{array}{c}0,716 \\
\text { nothing to measure }\end{array}$ & 1,182 & 0,373 & 0,331 & 0,439 & 0,145 & 0,137 & 141 & 70 & $-2,1$ \\
\hline & & & & & & & $\begin{array}{l}\text { nothing to measure } \\
\text { nothing to measure }\end{array}$ & & & & & & & & 71 & - \\
\hline 0,089 & 1,475 & 0,141 & 1,146 & 0,633 & 0,777 & 0,429 & 0,515 & 1,394 & 0,372 & 0,484 & 0,552 & 0,267 & 0,194 & 179 & 73 & $-2,5$ \\
\hline \multirow[t]{5}{*}{0,380} & 4,831 & 0,737 & 2,805 & 0,516 & 0,581 & 0,640 & 0,205 & 2,211 & 0,389 & 0,696 & 1,115 & 0,776 & 0,387 & 164 & 73 & $-3,5$ \\
\hline & & & & & & & nothing to measure & & & & & & & & 74 & - \\
\hline & & & & & & & nothing to measure & & & & & & & & 75 & - \\
\hline & & & & & & & nothing to measure & & & & & & & & 76 & - \\
\hline & & & & & & & nothing to measure & & & & & & & & 77 & - \\
\hline 0,047 & 0,719 & 0,056 & 0,481 & 0,833 & 0,670 & 0,370 & 1,000 & 0,936 & 0,749 & 0,485 & 0,282 & 0,137 & 0,137 & 90 & 78 & $-1,5$ \\
\hline 0,056 & 1,254 & 0,056 & 1,016 & 1,000 & 0,811 & 0,189 & 0,450 & 1,491 & 0,235 & 0,278 & 0,552 & 0,153 & 0,137 & 0 & 78 & $-2,5$ \\
\hline 0,056 & 0,795 & 0,061 & 0,627 & 0,923 & 0,789 & 0,225 & 1,000 & 0,945 & 0,611 & 0,424 & 0,342 & 0,145 & 0,137 & 133 & 78 & $-1,8$ \\
\hline 0,202 & 3,081 & 0,385 & 2,178 & 0,524 & 0,707 & 0,559 & 0,267 & 1,935 & 0,243 & 0,436 & 1,027 & 0,448 & 0,274 & 24 & 78 & $-3,4$ \\
\hline 0,061 & 1,070 & 0,084 & 0,665 & 0,722 & 0,621 & 0,470 & 0,669 & 1,222 & 0,571 & 0,496 & 0,369 & 0,183 & 0,137 & 12 & 78 & $-1,9$ \\
\hline 0,052 & 0,978 & 0,075 & 0,665 & 0,688 & 0,680 & 0,448 & 0,677 & 1,215 & 0,483 & 0,451 & 0,369 & 0,166 & 0,137 & 15 & 78 & $-1,9$ \\
\hline 0,324 & 3,011 & 0,441 & 2,232 & 0,734 & 0,741 & 0,371 & 0,449 & 1,493 & 0,301 & 0,369 & 1,170 & 0,432 & 0,274 & 23 & 78 & $-3,5$ \\
\hline 0,056 & 0,887 & 0,075 & 0,703 & 0,750 & 0,793 & 0,325 & 0,900 & 1,054 & 0,588 & 0,392 & 0,349 & 0,137 & 0,137 & 169 & 78 & $-1,8$ \\
\hline 0,075 & 0,940 & 0,084 & 0,703 & 0,889 & 0,748 & 0,276 & 1,000 & 0,968 & 1,019 & 0,791 & 0,306 & 0,242 & 0,194 & 73 & 78 & $-1,6$ \\
\hline \multirow[t]{8}{*}{0,047} & 0,833 & 0,061 & 0,573 & 0,769 & 0,688 & 0,388 & 0,850 & 1,085 & 0,439 & 0,315 & 0,369 & 0,116 & 0,137 & 90 & 78 & $-1,9$ \\
\hline & & & & & & & nothing to measure & & & & & & & & 79 & - \\
\hline & & & & & & & nothing to measure & & & & & & & & 80 & - \\
\hline & & & & & & & nothing to measure & & & & & & & & 81 & - \\
\hline & & & & & & & nothing to measure & & & & & & & & 82 & - \\
\hline & & & & & & & nothing to measure & & & & & & & & 83 & - \\
\hline & & & & & & & nothing to measure & & & & & & & & 84 & - \\
\hline & & & & & & & nothing to measure & & & & & & & & 85 & - \\
\hline 0,108 & 1,789 & 0,141 & 1,200 & 0,767 & 0,671 & 0,403 & 0,424 & 1,536 & 0,366 & 0,350 & 0,613 & 0,215 & 0,194 & 119 & 86 & $-2,6$ \\
\hline \multirow[t]{4}{*}{0,066} & 1,200 & 0,084 & 1,070 & 0,778 & 0,892 & 0,247 & 0,573 & 1,321 & 0,244 & 0,196 & 0,585 & 0,115 & 0,137 & 114 & 86 & $-2,5$ \\
\hline & & & & & & & nothing to measure & & & & & & & & 87 & - \\
\hline & & & & & & & nothing to measure & & & & & & & & 88 & - \\
\hline & & & & & & & nothing to measure & & & & & & & & 89 & - \\
\hline 0,056 & 0,887 & 0,080 & 0,627 & 0,706 & 0,707 & 0,415 & 0,900 & 1,054 & 0,477 & 0,392 & 0,387 & 0,152 & 0,137 & 126 & 90 & $-2,0$ \\
\hline \multirow[t]{2}{*}{0,066} & 1,422 & 0,113 & 0,833 & 0,583 & 0,586 & 0,587 & 0,408 & 1,565 & 0,336 & 0,412 & 0,499 & 0,205 & 0,137 & 0 & 90 & $-2,3$ \\
\hline & & & & & & & nothing to measure & & & & & & & & 91 & \\
\hline 0,253 & 4,258 & 0,497 & 2,584 & 0,509 & 0,607 & 0,629 & 0,176 & 2,387 & 0,203 & 0,412 & 1,259 & 0,519 & 0,274 & 126 & 92 & $-3,7$ \\
\hline
\end{tabular}




\begin{tabular}{|c|c|c|c|c|c|c|c|c|c|c|c|c|c|c|c|c|}
\hline 92 & 0,127 & 1,292 & 0,150 & 1,055 & 0,844 & 0,816 & 0,241 & 0,954 & 1,024 & 0,838 & 0,720 & 0,439 & 0,316 & 0,306 & 92 & 92 \\
\hline 92 & 0,061 & 0,887 & 0,070 & 0,535 & 0,867 & 0,604 & 0,418 & 0,975 & 1,013 & 0,487 & 0,404 & 0,399 & 0,162 & 0,274 & 172 & 92 \\
\hline 92 & 0,070 & 0,940 & 0,089 & 0,833 & 0,789 & 0,886 & 0,240 & 1,000 & 1,000 & 0,735 & 0,702 & 0,349 & 0,245 & 0,137 & 97 & 92 \\
\hline 92 & 0,281 & 1,903 & 0,319 & 1,719 & 0,882 & 0,904 & 0,152 & 0,977 & 1,012 & 0,764 & 0,671 & 0,685 & 0,460 & 0,494 & 164 & 92 \\
\hline 92 & 0,141 & 2,599 & 0,230 & 2,210 & 0,612 & 0,850 & 0,416 & 0,262 & 1,954 & 0,142 & 0,203 & 1,123 & 0,228 & 0,194 & 125 & 92 \\
\hline 92 & 0,108 & 1,124 & 0,117 & 0,887 & 0,920 & 0,789 & 0,226 & 1,000 & 0,965 & 0,791 & 0,658 & 0,417 & 0,274 & 0,306 & 84 & 92 \\
\hline 92 & 0,056 & 0,833 & 0,066 & 0,535 & 0,857 & 0,642 & 0,385 & 1,000 & 0,990 & 0,611 & 0,444 & 0,342 & 0,152 & 0,137 & 121 & 92 \\
\hline 92 & 0,145 & 1,475 & 0,164 & 1,254 & 0,886 & 0,850 & 0,189 & 0,840 & 1,091 & 0,548 & 0,500 & 0,581 & 0,291 & 0,306 & 133 & 92 \\
\hline 92 & 0,333 & 2,087 & 0,357 & 1,903 & 0,934 & 0,912 & 0,110 & 0,961 & 1,020 & 0,660 & 0,676 & 0,802 & 0,542 & 0,548 & 109 & 92 \\
\hline 92 & 1,243 & 6,237 & 1,553 & 5,664 & 0,801 & 0,908 & 0,220 & 0,402 & 1,578 & 0,213 & 0,240 & 2,727 & 0,654 & 0,387 & 114 & 92 \\
\hline 92 & 0,061 & 1,216 & 0,080 & 1,048 & 0,765 & 0,862 & 0,273 & 0,519 & 1,389 & 0,230 & 0,167 & 0,581 & 0,097 & 0,137 & 49 & 92 \\
\hline 92 & 0,267 & 3,248 & 0,483 & 2,362 & 0,553 & 0,727 & 0,523 & 0,318 & 1,772 & 0,290 & 0,470 & 1,083 & 0,509 & 0,274 & 108 & 92 \\
\hline 92 & 0,160 & 2,836 & 0,319 & 2,232 & 0,500 & 0,787 & 0,544 & 0,249 & 2,003 & 0,158 & 0,289 & 1,134 & 0,328 & 0,274 & 156 & 92 \\
\hline 92 & 0,075 & 1,216 & 0,094 & 0,887 & 0,800 & 0,729 & 0,337 & 0,638 & 1,252 & 0,551 & 0,581 & 0,417 & 0,242 & 0,194 & 27 & 92 \\
\hline 92 & 0,089 & 1,032 & 0,094 & 1,032 & 0,950 & 1,000 & 0,050 & 1,000 & 0,975 & 0,538 & 0,422 & 0,459 & 0,194 & 0,274 & 146 & 92 \\
\hline 92 & 0,169 & 2,102 & 0,249 & 1,643 & 0,679 & 0,782 & 0,388 & 0,480 & 1,443 & 0,376 & 0,483 & 0,757 & 0,366 & 0,274 & 109 & 92 \\
\hline 92 & 0,099 & 1,567 & 0,136 & 0,978 & 0,724 & 0,624 & 0,466 & 0,504 & 1,408 & 0,652 & 0,629 & 0,439 & 0,276 & 0,274 & 148 & 92 \\
\hline 92 & 0,056 & 0,810 & 0,066 & 0,573 & 0,857 & 0,707 & 0,326 & 1,000 & 0,964 & 0,764 & 0,501 & 0,306 & 0,153 & 0,194 & 55 & 92 \\
\hline 92 & 0,113 & 2,378 & 0,197 & 2,194 & 0,571 & 0,923 & 0,435 & 0,250 & 1,999 & 0,118 & 0,128 & 1,104 & 0,141 & 0,137 & 121 & 92 \\
\hline 92 & 0,220 & 2,102 & 0,310 & 1,666 & 0,712 & 0,792 & 0,355 & 0,627 & 1,263 & 0,617 & 0,711 & 0,675 & 0,479 & 0,306 & 71 & 92 \\
\hline 92 & 0,084 & 1,124 & 0,108 & 0,887 & 0,783 & 0,789 & 0,303 & 0,840 & 1,091 & 0,637 & 0,648 & 0,411 & 0,266 & 0,274 & 171 & 92 \\
\hline 92 & 0,052 & 1,124 & 0,075 & 0,848 & 0,688 & 0,755 & 0,397 & 0,513 & 1,396 & 0,350 & 0,345 & 0,433 & 0,150 & 0,137 & 110 & 92 \\
\hline 92 & 0,183 & 3,318 & 0,366 & 2,270 & 0,500 & 0,684 & 0,591 & 0,209 & 2,188 & 0,204 & 0,380 & 1,070 & 0,406 & 0,194 & 136 & 92 \\
\hline 92 & 0,174 & 1,865 & 0,220 & 1,460 & 0,787 & 0,783 & 0,304 & 0,627 & 1,263 & 0,466 & 0,494 & 0,688 & 0,340 & 0,274 & 90 & 92 \\
\hline 92 & 0,084 & 1,422 & 0,108 & 1,124 & 0,783 & 0,791 & 0,302 & 0,525 & 1,380 & 0,258 & 0,212 & 0,646 & 0,137 & 0,137 & 125 & 92 \\
\hline 92 & 0,084 & 1,254 & 0,117 & 0,848 & 0,720 & 0,677 & 0,428 & 0,675 & 1,217 & 0,509 & 0,566 & 0,459 & 0,260 & 0,194 & 106 & 92 \\
\hline 92 & 0,117 & 2,026 & 0,183 & 1,513 & 0,641 & 0,747 & 0,439 & 0,359 & 1,669 & 0,282 & 0,374 & 0,728 & 0,273 & 0,194 & 136 & 92 \\
\hline 92 & 0,061 & 0,848 & 0,075 & 0,665 & 0,812 & 0,784 & 0,286 & 1,000 & 0,969 & 0,662 & 0,537 & 0,342 & 0,184 & 0,194 & 117 & 92 \\
\hline 92 & 0,530 & 5,022 & 0,732 & 3,898 & 0,724 & 0,776 & 0,355 & 0,264 & 1,946 & 0,185 & 0,261 & 1,908 & 0,498 & 0,494 & 108 & 92 \\
\hline 92 & 0,084 & 0,887 & 0,084 & 0,665 & 1,000 & 0,750 & 0,250 & 1,000 & 0,861 & 0,716 & 0,500 & 0,387 & 0,194 & 0,274 & 135 & 92 \\
\hline 92 & 0,066 & 1,032 & 0,075 & 0,757 & 0,875 & 0,733 & 0,295 & 0,775 & 1,136 & 0,435 & 0,304 & 0,439 & 0,133 & 0,137 & 125 & 92 \\
\hline 92 & 0,089 & 1,368 & 0,122 & 0,810 & 0,731 & 0,592 & 0,488 & 0,599 & 1,293 & 0,456 & 0,486 & 0,499 & 0,242 & 0,274 & 77 & 92 \\
\hline 92 & 0,352 & 3,777 & 0,488 & 2,859 & 0,721 & 0,757 & 0,370 & 0,310 & 1,796 & 0,242 & 0,308 & 1,360 & 0,419 & 0,387 & 124 & 92 \\
\hline 92 & 0,047 & 0,978 & 0,047 & 0,573 & 1,000 & 0,586 & 0,414 & 0,616 & 1,274 & 0,490 & 0,392 & 0,349 & 0,137 & 0,137 & 135 & 92 \\
\hline 92 & 0,066 & 0,887 & 0,080 & 0,627 & 0,824 & 0,707 & 0,342 & 1,000 & 0,976 & 0,615 & 0,557 & 0,369 & 0,205 & 0,194 & 45 & 92 \\
\hline 92 & 0,244 & 2,668 & 0,328 & 1,896 & 0,743 & 0,711 & 0,387 & 0,431 & 1,524 & 0,483 & 0,544 & 0,802 & 0,436 & 0,306 & 140 & 92 \\
\hline 92 & 0,145 & 1,659 & 0,169 & 1,529 & 0,861 & 0,922 & 0,159 & 0,664 & 1,227 & 0,304 & 0,269 & 0,781 & 0,210 & 0,274 & 125 & 92 \\
\hline 92 & 0,084 & 1,437 & 0,131 & 1,162 & 0,643 & 0,808 & 0,405 & 0,514 & 1,395 & 0,318 & 0,417 & 0,581 & 0,242 & 0,194 & 138 & 92 \\
\hline 92 & 0,122 & 2,172 & 0,178 & 1,950 & 0,684 & 0,898 & 0,332 & 0,325 & 1,754 & 0,136 & 0,162 & 1,070 & 0,173 & 0,194 & 133 & 92 \\
\hline 92 & 0,178 & 2,270 & 0,281 & 1,659 & 0,633 & 0,731 & 0,455 & 0,435 & 1,517 & 0,479 & 0,623 & 0,688 & 0,429 & 0,274 & 92 & 92 \\
\hline 92 & 0,056 & 1,016 & 0,075 & 0,703 & 0,750 & 0,692 & 0,397 & 0,685 & 1,208 & 0,413 & 0,279 & 0,417 & 0,116 & 0,137 & 166 & 92 \\
\hline 92 & 0,061 & 1,292 & 0,094 & 0,887 & 0,650 & 0,686 & 0,470 & 0,459 & 1,476 & 0,227 & 0,247 & 0,585 & 0,145 & 0,137 & 0 & 92 \\
\hline 92 & 0,835 & 8,019 & 1,778 & 4,793 & 0,470 & 0,598 & 0,666 & 0,163 & 2,476 & 0,313 & 0,691 & 1,844 & 1,274 & 0,194 & 166 & 92 \\
\hline 92 & 0,089 & 1,697 & 0,089 & 1,384 & 1,000 & 0,815 & 0,185 & 0,389 & 1,603 & 0,249 & 0,319 & 0,675 & 0,215 & 0,194 & 36 & 92 \\
\hline 92 & 0,056 & 0,994 & 0,066 & 0,719 & 0,857 & 0,723 & 0,312 & 0,716 & 1,182 & 0,306 & 0,200 & 0,484 & 0,097 & 0,137 & 135 & 92 \\
\hline 92 & 0,497 & 5,565 & 0,863 & 3,669 & 0,576 & 0,659 & 0,544 & 0,202 & 2,226 & 0,233 & 0,426 & 1,650 & 0,703 & 0,274 & 121 & 92 \\
\hline 92 & 0,145 & 2,026 & 0,188 & 1,934 & 0,775 & 0,955 & 0,230 & 0,445 & 1,499 & 0,190 & 0,161 & 0,988 & 0,159 & 0,194 & 126 & 92 \\
\hline 92 & 0,052 & 1,292 & 0,089 & 0,627 & 0,579 & 0,485 & 0,665 & 0,389 & 1,604 & 0,438 & 0,504 & 0,387 & 0,195 & 0,137 & 0 & 92 \\
\hline 92 & 0,080 & 1,552 & 0,113 & 0,887 & 0,708 & 0,571 & 0,518 & 0,416 & 1,550 & 0,416 & 0,423 & 0,494 & 0,209 & 0,137 & 119 & 92 \\
\hline 92 & 0,056 & 1,032 & 0,084 & 0,665 & 0,667 & 0,644 & 0,487 & 0,664 & 1,227 & 0,588 & 0,527 & 0,349 & 0,184 & 0,137 & 126 & 92 \\
\hline 92 & 0,281 & 3,945 & 0,540 & 2,438 & 0,522 & 0,618 & 0,612 & 0,227 & 2,097 & 0,394 & 0,723 & 0,954 & 0,690 & 0,274 & 15 & 92 \\
\hline 92 & 0,080 & 1,842 & 0,164 & 1,276 & 0,486 & 0,693 & 0,599 & 0,295 & 1,840 & 0,214 & 0,296 & 0,688 & 0,203 & 0,137 & 5 & 92 \\
\hline 92 & 0,192 & 2,048 & 0,244 & 1,368 & 0,788 & 0,668 & 0,394 & 0,576 & 1,318 & 0,614 & 0,639 & 0,632 & 0,403 & 0,274 & 87 & 92 \\
\hline 92 & 0,141 & 1,567 & 0,188 & 1,216 & 0,750 & 0,776 & 0,336 & 0,720 & 1,178 & 0,721 & 0,825 & 0,499 & 0,411 & 0,274 & 145 & 92 \\
\hline 93 & & & & & & & & nothing to measure & & & & & & & & 93 \\
\hline 94 & & & & & & & & nothing to measure & & & & & & & & 94 \\
\hline 95 & & & & & & & & nothing to measure & & & & & & & & 95 \\
\hline 96 & & & & & & & & & & & & & & & & \\
\hline
\end{tabular}




\begin{tabular}{|c|c|c|c|c|c|c|c|c|c|c|c|c|c|c|c|c|c|}
\hline $\begin{array}{l}97 \\
98\end{array}$ & & & & & & & & $\begin{array}{l}\text { nothing to measure } \\
\text { nothing to measure }\end{array}$ & & & & & & & & $\begin{array}{l}97 \\
98\end{array}$ & - \\
\hline 99 & 0,070 & 1,292 & 0,103 & 1,108 & 0,682 & 0,858 & 0,348 & 0,530 & 1,374 & 0,262 & 0,234 & 0,585 & 0,137 & 0,137 & 72 & 99 & $-2,5$ \\
\hline 99 & 0,084 & 1,108 & 0,103 & 0,887 & 0,818 & 0,800 & 0,270 & 0,864 & 1,076 & 0,441 & 0,413 & 0,494 & 0,204 & 0,274 & 154 & 99 & $-2,3$ \\
\hline 99 & 0,183 & 2,973 & 0,357 & 2,133 & 0,513 & 0,718 & 0,563 & 0,260 & 1,961 & 0,203 & 0,306 & 1,072 & 0,328 & 0,194 & 137 & 99 & $-3,4$ \\
\hline 99 & 0,047 & 0,848 & 0,066 & 0,535 & 0,714 & 0,631 & 0,467 & 0,819 & 1,105 & 0,707 & 0,597 & 0,291 & 0,173 & 0,137 & 135 & 99 & $-1,5$ \\
\hline 100 & 0,084 & 1,070 & 0,099 & 0,833 & 0,857 & 0,778 & 0,264 & 0,927 & 1,039 & 0,619 & 0,493 & 0,417 & 0,205 & 0,274 & 82 & 100 & $-2,1$ \\
\hline 100 & 0,258 & 3,853 & 0,469 & 2,255 & 0,550 & 0,585 & 0,612 & 0,218 & 2,140 & 0,412 & 0,670 & 0,893 & 0,598 & 0,194 & 134 & 100 & $-3,2$ \\
\hline 100 & 0,225 & 3,410 & 0,389 & 1,903 & 0,578 & 0,558 & 0,611 & 0,243 & 2,027 & 0,470 & 0,789 & 0,781 & 0,616 & 0,306 & 142 & 100 & $-3,0$ \\
\hline 100 & 0,066 & 0,978 & 0,080 & 0,649 & 0,824 & 0,664 & 0,380 & 0,862 & 1,077 & 0,891 & 0,671 & 0,306 & 0,205 & 0,194 & 90 & 100 & $-1,6$ \\
\hline 100 & 0,089 & 1,422 & 0,127 & 1,093 & 0,704 & 0,769 & 0,376 & 0,554 & 1,343 & 0,356 & 0,332 & 0,565 & 0,187 & 0,194 & 97 & 100 & $-2,5$ \\
\hline 100 & 0,047 & 0,795 & 0,056 & 0,497 & 0,833 & 0,625 & 0,410 & 0,933 & 1,035 & 0,509 & 0,424 & 0,342 & 0,145 & 0,137 & 108 & 100 & $-1,8$ \\
\hline 101 & & & & & & & & nothing to measure & & & & & & & & 101 & \\
\hline 103 & 0,070 & 1,146 & 0,094 & 0,703 & 0,750 & 0,613 & 0,460 & 0,673 & 1,219 & 0,382 & 0,283 & 0,484 & 0,137 & 0,137 & 4 & 103 & $-2,3$ \\
\hline 104 & & & & & & & & nothing to measure & & & & & & & & 104 & 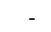 \\
\hline 105 & 0,089 & 1,827 & 0,150 & 1,475 & 0,594 & 0,808 & 0,450 & 0,336 & 1,726 & 0,186 & 0,275 & 0,781 & 0,214 & 0,137 & 169 & 105 & $-3,0$ \\
\hline 106 & & & & & & & & nothing to measure & & & & & & & & 106 & - \\
\hline 107 & & & & & & & & nothing to measure & & & & & & & & 107 & - \\
\hline 108 & & & & & & & & nothing to measure & & & & & & & & 108 & - \\
\hline 109 & & & & & & & & nothing to measure & & & & & & & & 109 & - \\
\hline 110 & & & & & & & & nothing to measure & & & & & & & & 110 & - \\
\hline 111 & 0,070 & 1,178 & 0,099 & 0,848 & 0,714 & 0,721 & 0,400 & 0,638 & 1,252 & 0,562 & 0,485 & 0,399 & 0,194 & 0,137 & 138 & 111 & $-2,0$ \\
\hline 112 & 0,136 & 2,216 & 0,202 & 2,087 & 0,674 & 0,941 & 0,331 & 0,348 & 1,695 & 0,159 & 0,174 & 1,043 & 0,182 & 0,137 & 161 & 112 & $-3,4$ \\
\hline 112 & 0,056 & 1,108 & 0,070 & 0,757 & 0,800 & 0,683 & 0,375 & 0,576 & 1,318 & 0,340 & 0,267 & 0,459 & 0,123 & 0,137 & 154 & 112 & $-2,2$ \\
\hline 112 & 0,061 & 0,978 & 0,070 & 0,757 & 0,867 & 0,773 & 0,263 & 0,801 & 1,118 & 0,368 & 0,250 & 0,459 & 0,115 & 0,137 & 158 & 112 & $-2,2$ \\
\hline 112 & 0,056 & 0,902 & 0,070 & 0,665 & 0,800 & 0,737 & 0,330 & 0,869 & 1,073 & 0,588 & 0,523 & 0,349 & 0,183 & 0,194 & 77 & 112 & $-1,8$ \\
\hline 112 & 0,629 & 6,329 & 1,285 & 4,287 & 0,489 & 0,677 & 0,604 & 0,197 & 2,252 & 0,208 & 0,416 & 1,961 & 0,815 & 0,411 & 168 & 112 & $-4,3$ \\
\hline 113 & 0,164 & 2,324 & 0,249 & 1,552 & 0,660 & 0,668 & 0,475 & 0,382 & 1,618 & 0,241 & 0,344 & 0,932 & 0,320 & 0,194 & 169 & 113 & $-3,2$ \\
\hline 113 & 0,075 & 0,871 & 0,080 & 0,443 & 0,941 & 0,509 & 0,495 & 1,000 & 0,897 & 0,815 & 0,600 & 0,342 & 0,205 & 0,274 & 150 & 113 & $-1,8$ \\
\hline 114 & 0,084 & 1,254 & 0,113 & 1,016 & 0,750 & 0,811 & 0,314 & 0,675 & 1,217 & 0,376 & 0,362 & 0,535 & 0,194 & 0,137 & 120 & 114 & $-2,4$ \\
\hline 115 & & & & & & & & nothing to measure & & & & & & & & 115 & - \\
\hline 116 & & & & & & & & nothing to measure & & & & & & & & 116 & - \\
\hline 117 & & & & & & & & nothing to measure & & & & & & & & 117 & - \\
\hline 118 & & & & & & & & nothing to measure & & & & & & & & 118 & - \\
\hline 119 & & & & & & & & nothing to measure & & & & & & & & 119 & - \\
\hline 120 & & & & & & & & nothing to measure & & & & & & & & 120 & - \\
\hline 121 & & & & & & & & nothing to measure & & & & & & & & 121 & - \\
\hline 122 & & & & & & & & nothing to measure & & & & & & & & 122 & - \\
\hline 123 & & & & & & & & nothing to measure & & & & & & & & 123 & - \\
\hline 124 & & & & & & & & nothing to measure & & & & & & & & 124 & - \\
\hline 125 & & & & & & & & nothing to measure & & & & & & & & 125 & - \\
\hline 126 & & & & & & & & nothing to measure & & & & & & & & 126 & - \\
\hline 127 & & & & & & & & nothing to measure & & & & & & & & 127 & - \\
\hline 128 & & & & & & & & nothing to measure & & & & & & & & 128 & - \\
\hline 129 & & & & & & & & nothing to measure & & & & & & & & 129 & - \\
\hline 130 & 0,347 & 3,600 & 0,488 & 2,416 & 0,712 & 0,671 & 0,438 & 0,337 & 1,724 & 0,300 & 0,375 & 1,214 & 0,455 & 0,274 & 17 & 130 & $-3,6$ \\
\hline 130 & 0,150 & 2,286 & 0,253 & 1,751 & 0,593 & 0,766 & 0,470 & 0,361 & 1,664 & 0,348 & 0,500 & 0,741 & 0,370 & 0,274 & 32 & 130 & $-2,9$ \\
\hline 130 & 0,052 & 0,703 & 0,056 & 0,222 & 0,917 & 0,315 & 0,690 & 1,000 & 0,873 & 0,700 & 0,447 & 0,306 & 0,137 & 0,194 & 18 & 130 & $-1,6$ \\
\hline 130 & 0,225 & 2,859 & 0,342 & 1,957 & 0,658 & 0,684 & 0,466 & 0,346 & 1,700 & 0,306 & 0,495 & 0,969 & 0,479 & 0,194 & 8 & 130 & $-3,3$ \\
\hline 130 & 0,080 & 1,384 & 0,131 & 0,940 & 0,607 & 0,680 & 0,507 & 0,524 & 1,382 & 0,585 & 0,757 & 0,417 & 0,315 & 0,194 & 0 & 130 & $-2,1$ \\
\hline 131 & & & & & & & & nothing to measure & & & & & & & & 131 & . \\
\hline 132 & 0,094 & 1,605 & 0,145 & 1,200 & 0,645 & 0,748 & 0,435 & 0,458 & 1,478 & 0,300 & 0,388 & 0,632 & 0,245 & 0,137 & 115 & 132 & $-2,7$ \\
\hline
\end{tabular}


nothing to measure

133

1,865

$\begin{array}{ll}0,183 & 1,330 \\ 0,052 & 0,519\end{array}$

$\begin{array}{ll}0,513 & 0,713 \\ 1,000 & 0,781\end{array}$

0,565

nothing to measure

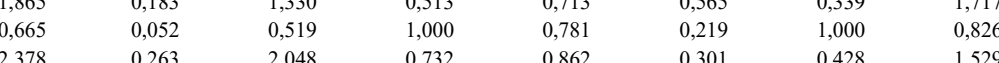

$\begin{array}{llll}0,080 & 1,345 & 0,099 & 1,269\end{array}$

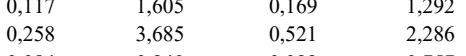

$0,084 \quad 0,940$

$0,099 \quad 1,108$

$\begin{array}{ll}0,066 & 1,070 \\ 0,940\end{array}$

$\begin{array}{ll}0,155 & 2,087 \\ 0,047 & 1,070\end{array}$

$0,066 \quad 1,200$

$\begin{array}{ll}0,122 & 1,934 \\ 0,052 & 0,665\end{array}$

$0,117 \quad 1,200$

$\begin{array}{ll}0,070 & 0,887 \\ 0,075 & 1,016\end{array}$

$\begin{array}{ll}0,075 & 1,016 \\ 0,084 & 1,513\end{array}$

$\begin{array}{ll}0,061 & 0,757 \\ 0,070 & 0,978\end{array}$

$\begin{array}{ll}0,099 & 1,406 \\ 0,052 & 0,757\end{array}$

$\begin{array}{ll}0,188 & 2,590 \\ 0,061 & 0,978\end{array}$

$0,113 \quad 1,475$

$\begin{array}{ll}0,089 & 1,368 \\ 0,047 & 0,627\end{array}$

$0,103 \quad 1,254$

\section{nothing to measure}

nothing to measure

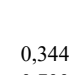

$0,344-0,626$ $\begin{array}{ll}0,740 & 0,447 \\ 0 & 0,239\end{array}$ $0,293 \quad 0,247$ $0,398 \quad 0,455$ $0,881 \quad 0,615$ $0,891 \quad 0,601$

$0,764 \quad 0,70$

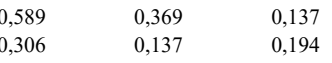

$\begin{array}{lll}0,017 & 0,245 & 0,274 \\ 0,589 & 0,145 & 0,137\end{array}$

$\begin{array}{lll}0,969 & 0,279 & 0,274\end{array}$

$\begin{array}{lll}0,349 & 0,215 & 0,274\end{array}$

0,242

$\begin{array}{lll}0,342 & 0,242 & 0,137\end{array}$

$\begin{array}{lll}0,781 & 0,259 & 0,274\end{array}$




\begin{tabular}{|c|c|c|c|c|c|c|c|c|c|c|c|c|c|c|c|c|}
\hline 0,056 & 0,864 & 0,080 & 0,795 & 0,706 & 0,920 & 0,305 & 0,947 & 1,027 & 0,611 & 0,627 & 0,342 & 0,215 & 0,137 & 162 & 140 & $-1,8$ \\
\hline 0,094 & 1,713 & 0,145 & 1,016 & 0,645 & 0,594 & 0,540 & 0,402 & 1,577 & 0,566 & 0,660 & 0,459 & 0,303 & 0,194 & 53 & 140 & $-2,2$ \\
\hline 0,047 & 0,665 & 0,052 & 0,497 & 0,909 & 0,747 & 0,268 & 1,000 & 0,866 & 0,979 & 0,555 & 0,247 & 0,137 & 0,194 & 90 & 140 & $-1,3$ \\
\hline 0,066 & 0,994 & 0,084 & 0,665 & 0,778 & 0,669 & 0,399 & 0,835 & 1,094 & 0,615 & 0,557 & 0,369 & 0,205 & 0,194 & 84 & 141 & $-1,9$ \\
\hline 0,244 & 1,972 & 0,305 & 1,697 & 0,800 & 0,860 & 0,244 & 0,788 & 1,126 & 0,637 & 0,702 & 0,698 & 0,490 & 0,387 & 112 & 142 & $-2,8$ \\
\hline 0,047 & 0,665 & 0,052 & 0,535 & 0,909 & 0,805 & 0,215 & 1,000 & 0,866 & 0,637 & 0,400 & 0,306 & 0,123 & 0,137 & 18 & 142 & $-1,6$ \\
\hline 0,066 & 0,810 & 0,066 & 0,627 & 1,000 & 0,773 & 0,227 & 1,000 & 0,892 & 0,990 & 0,667 & 0,291 & 0,194 & 0,194 & 63 & 142 & $-1,5$ \\
\hline \multirow[t]{7}{*}{0,047} & 0,864 & 0,061 & 0,627 & 0,769 & 0,725 & 0,359 & 0,790 & 1,125 & 0,490 & 0,476 & 0,349 & 0,166 & 0,137 & 118 & 142 & $-1,8$ \\
\hline & & & & & & & nothing to measure & & & & & & & & 143 & \\
\hline & & & & & & & nothing to measure & & & & & & & & 144 & - \\
\hline & & & & & & & nothing to measure & & & & & & & & 145 & - \\
\hline & & & & & & & nothing to measure & & & & & & & & 146 & - \\
\hline & & & & & & & nothing to measure & & & & & & & & 147 & - \\
\hline & & & & & & & nothing to measure & & & & & & & & 148 & - \\
\hline 0,047 & 1,070 & 0,075 & 0,573 & 0,625 & 0,536 & 0,597 & 0,515 & 1,394 & 0,490 & 0,571 & 0,349 & 0,199 & 0,137 & 90 & 149 & $-1,8$ \\
\hline 0,056 & 1,070 & 0,080 & 0,757 & 0,706 & 0,707 & 0,415 & 0,618 & 1,272 & 0,413 & 0,416 & 0,417 & 0,173 & 0,137 & 104 & 149 & $-2,1$ \\
\hline \multirow[t]{3}{*}{0,047} & 0,719 & 0,052 & 0,535 & 0,909 & 0,745 & 0,271 & 1,000 & 0,936 & 0,749 & 0,514 & 0,282 & 0,145 & 0,194 & 122 & 149 & $-1,5$ \\
\hline & & & & & & & nothing to measure & & & & & & & & 150 & - \\
\hline & & & & & & & nothing to measure & & & & & & & & 151 & \\
\hline \multirow[t]{16}{*}{0,047} & 0,848 & 0,061 & 0,535 & 0,769 & 0,631 & 0,436 & 0,819 & 1,105 & 0,749 & 0,686 & 0,282 & 0,194 & 0,137 & 135 & 152 & $-1,5$ \\
\hline & & & & & & & nothing to measure & & & & & & & & 153 & 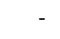 \\
\hline & & & & & & & nothing to measure & & & & & & & & 154 & - \\
\hline & & & & & & & nothing to measure & & & & & & & & 155 & - \\
\hline & & & & & & & nothing to measure & & & & & & & & 156 & - \\
\hline & & & & & & & nothing to measure & & & & & & & & 157 & - \\
\hline & & & & & & & nothing to measure & & & & & & & & 158 & - \\
\hline & & & & & & & nothing to measure & & & & & & & & 159 & - \\
\hline & & & & & & & nothing to measure & & & & & & & & 160 & - \\
\hline & & & & & & & nothing to measure & & & & & & & & 161 & - \\
\hline & & & & & & & nothing to measure & & & & & & & & 162 & - \\
\hline & & & & & & & nothing to measure & & & & & & & & 163 & - \\
\hline & & & & & & & nothing to measure & & & & & & & & 164 & - \\
\hline & & & & & & & nothing to measure & & & & & & & & 165 & - \\
\hline & & & & & & & nothing to measure & & & & & & & & 166 & - \\
\hline & & & & & & & nothing to measure & & & & & & & & 167 & . \\
\hline \multirow[t]{4}{*}{0,047} & 0,810 & 0,066 & 0,535 & 0,714 & 0,660 & 0,444 & 0,898 & 1,055 & 0,637 & 0,495 & 0,306 & 0,152 & 0,137 & 45 & 168 & $-1,6$ \\
\hline & & & & & & & nothing to measure & & & & & & & & 169 & - \\
\hline & & & & & & & nothing to measure & & & & & & & & 170 & - \\
\hline & & & & & & & nothing to measure & & & & & & & & 171 & \\
\hline 0,089 & 0,902 & 0,094 & 0,795 & 0,950 & 0,881 & 0,129 & 1,000 & 0,852 & 0,930 & 0,693 & 0,349 & 0,242 & 0,274 & 116 & 172 & $-1,8$ \\
\hline 0,070 & 1,345 & 0,094 & 1,124 & 0,750 & 0,835 & 0,299 & 0,488 & 1,431 & 0,225 & 0,209 & 0,632 & 0,132 & 0,137 & 39 & 172 & $-2,7$ \\
\hline 0,103 & 1,950 & 0,164 & 1,384 & 0,629 & 0,710 & 0,471 & 0,341 & 1,712 & 0,280 & 0,424 & 0,685 & 0,291 & 0,137 & 130 & 172 & $-2,8$ \\
\hline \multirow[t]{2}{*}{0,047} & 0,795 & 0,052 & 0,481 & 0,909 & 0,606 & 0,405 & 0,933 & 1,035 & 0,490 & 0,196 & 0,349 & 0,068 & 0,137 & 0 & 172 & $-1,8$ \\
\hline & & & & & & & nothing to measure & & & & & & & & 173 & - \\
\hline 0,052 & 1,238 & 0,084 & 0,757 & 0,611 & 0,611 & 0,550 & 0,423 & 1,537 & 0,483 & 0,581 & 0,369 & 0,214 & 0,137 & 56 & 174 & $-1,9$ \\
\hline 0,052 & 0,703 & 0,056 & 0,352 & 0,917 & 0,500 & 0,507 & 1,000 & 0,873 & 0,824 & 0,485 & 0,282 & 0,137 & 0,194 & 18 & 174 & $-1,5$ \\
\hline 0,094 & 1,345 & 0,122 & 0,848 & 0,769 & 0,631 & 0,436 & 0,651 & 1,239 & 0,749 & 0,714 & 0,399 & 0,285 & 0,274 & 129 & 174 & $-2,0$ \\
\hline \multirow[t]{2}{*}{0,052} & 0,940 & 0,070 & 0,611 & 0,733 & 0,650 & 0,440 & 0,733 & 1,168 & 0,539 & 0,392 & 0,349 & 0,137 & 0,137 & 90 & 174 & $-1,8$ \\
\hline & & & & & & & nothing to measure & & & & & & & & 175 & 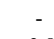 \\
\hline 1,098 & 4,204 & 1,224 & 3,707 & 0,897 & 0,882 & 0,157 & 0,780 & 1,132 & 0,750 & 0,842 & 1,365 & 1,148 & 0,997 & 142 & 176 & $-3,8$ \\
\hline 0,150 & 1,590 & 0,192 & 1,292 & 0,780 & 0,813 & 0,289 & 0,747 & 1,157 & 0,479 & 0,512 & 0,632 & 0,323 & 0,306 & 133 & 176 & $-2,7$ \\
\hline 0,084 & 1,070 & 0,099 & 0,925 & 0,857 & 0,864 & 0,197 & 0,927 & 1,039 & 0,468 & 0,416 & 0,479 & 0,199 & 0,274 & 8 & 176 & $-2,3$ \\
\hline 0,202 & 2,416 & 0,305 & 1,735 & 0,662 & 0,718 & 0,440 & 0,434 & 1,517 & 0,485 & 0,602 & 0,728 & 0,438 & 0,387 & 97 & 176 & $-2,9$ \\
\hline 0,267 & 2,821 & 0,385 & 2,156 & 0,695 & 0,764 & 0,385 & 0,422 & 1,539 & 0,354 & 0,500 & 0,981 & 0,490 & 0,274 & 116 & 176 & $-3,3$ \\
\hline 0,117 & 2,140 & 0,188 & 1,146 & 0,625 & 0,536 & 0,597 & 0,322 & 1,763 & 0,468 & 0,516 & 0,565 & 0,291 & 0,194 & 25 & 176 & $-2,5$ \\
\hline 0,047 & 0,573 & 0,047 & 0,390 & 1,000 & 0,680 & 0,320 & 1,000 & 0,746 & 0,979 & 0,555 & 0,247 & 0,137 & 0,194 & 72 & 176 & $-1,3$ \\
\hline 0,155 & 1,713 & 0,183 & 1,529 & 0,846 & 0,893 & 0,188 & 0,663 & 1,228 & 0,359 & 0,327 & 0,741 & 0,242 & 0,274 & 146 & 176 & $-2,9$ \\
\hline 0,418 & 6,414 & 1,074 & 4,364 & 0,389 & 0,680 & 0,690 & 0,128 & 2,800 & 0,125 & 0,299 & 2,059 & 0,616 & 0,194 & 5 & 176 & $-4,4$ \\
\hline
\end{tabular}




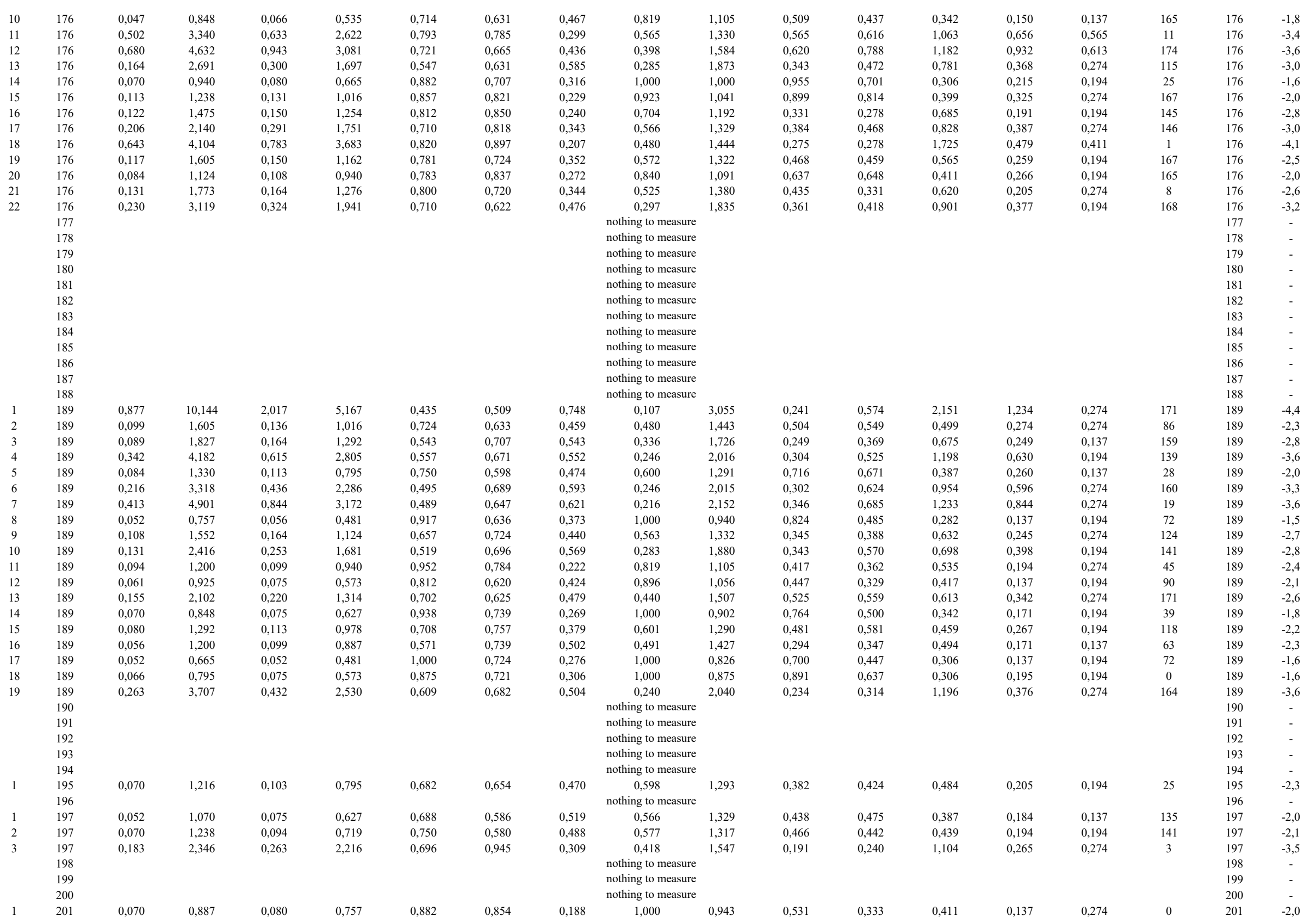




\begin{tabular}{|c|c|c|c|c|c|c|c|c|c|c|c|c|c|c|c|c|c|c|}
\hline 2 & $\begin{array}{l}201 \\
202 \\
203 \\
204\end{array}$ & 0,070 & 1,422 & 0,099 & 1,292 & 0,714 & 0,909 & 0,300 & $\begin{array}{l}\quad 0,438 \\
\text { nothing to measure } \\
\text { nothing to measure } \\
\text { nothing to measure }\end{array}$ & 1,512 & 0,197 & 0,174 & 0,675 & 0,118 & 0,137 & 122 & $\begin{array}{l}201 \\
202 \\
203 \\
204\end{array}$ & $\begin{array}{c}-2,8 \\
- \\
-\end{array}$ \\
\hline 1 & 205 & 0,066 & 1,032 & 0,084 & 0,757 & 0,778 & 0,733 & 0,347 & 0,775 & 1,136 & 0,713 & 0,696 & 0,342 & 0,238 & 0,194 & 101 & 205 & $-1,8$ \\
\hline 1 & 206 & 0,052 & 0,719 & 0,052 & 0,535 & 1,000 & 0,745 & 0,255 & 1,000 & 0,892 & 0,778 & 0,500 & 0,291 & 0,145 & 0,194 & 59 & 206 & $-1,5$ \\
\hline 2 & 206 & 0,061 & 0,848 & 0,075 & 0,665 & 0,812 & 0,784 & 0,286 & 1,000 & 0,969 & 0,662 & 0,555 & 0,342 & 0,190 & 0,194 & 34 & 206 & $-1,8$ \\
\hline 3 & $\begin{array}{l}206 \\
207\end{array}$ & 0,080 & 1,238 & 0,108 & 0,887 & 0,739 & 0,716 & 0,386 & $\begin{array}{c}0,654 \\
\text { nothing to measure }\end{array}$ & 1,237 & 0,373 & 0,287 & 0,522 & 0,150 & 0,194 & 106 & $\begin{array}{l}206 \\
207\end{array}$ & $-2,4$ \\
\hline & 208 & & & & & & & & nothing to measure & & & & & & & & 208 & - \\
\hline & 209 & & & & & & & & nothing to measure & & & & & & & & 209 & - \\
\hline & 210 & & & & & & & & nothing to measure & & & & & & & & 210 & - \\
\hline & 211 & & & & & & & & nothing to measure & & & & & & & & 211 & - \\
\hline & 212 & & & & & & & & nothing to measure & & & & & & & & 212 & - \\
\hline & 213 & & & & & & & & nothing to measure & & & & & & & & 213 & - \\
\hline & 214 & & & & & & & & nothing to measure & & & & & & & & 214 & - \\
\hline & 215 & & & & & & & & nothing to measure & & & & & & & & 215 & - \\
\hline & 216 & & & & & & & & nothing to measure & & & & & & & & 216 & - \\
\hline & 217 & & & & & & & & nothing to measure & & & & & & & & 217 & - \\
\hline & 218 & & & & & & & & nothing to measure & & & & & & & & 218 & - \\
\hline & 219 & & & & & & & & nothing to measure & & & & & & & & 219 & - \\
\hline & 220 & & & & & & & & nothing to measure & & & & & & & & 220 & - \\
\hline & 221 & & & & & & & & nothing to measure & & & & & & & & 221 & - \\
\hline & 222 & & & & & & & & nothing to measure & & & & & & & & 222 & - \\
\hline & 223 & & & & & & & & nothing to measure & & & & & & & & 223 & - \\
\hline & 224 & & & & & & & & nothing to measure & & & & & & & & 224 & - \\
\hline & 225 & & & & & & & & nothing to measure & & & & & & & & 225 & - \\
\hline & 226 & & & & & & & & nothing to measure & & & & & & & & 226 & - \\
\hline & 227 & & & & & & & & nothing to measure & & & & & & & & 227 & - \\
\hline & 228 & & & & & & & & nothing to measure & & & & & & & & 228 & - \\
\hline & 229 & & & & & & & & nothing to measure & & & & & & & & 229 & - \\
\hline & 230 & & & & & & & & nothing to measure & & & & & & & & 230 & - \\
\hline & 231 & & & & & & & & nothing to measure & & & & & & & & 231 & - \\
\hline & 232 & & & & & & & & nothing to measure & & & & & & & & 232 & - \\
\hline & 233 & & & & & & & & nothing to measure & & & & & & & & 233 & - \\
\hline & 234 & & & & & & & & nothing to measure & & & & & & & & 234 & - \\
\hline 1 & 235 & 0,061 & 1,491 & 0,113 & 0,940 & 0,542 & 0,631 & 0,589 & 0,345 & 1,703 & 0,243 & 0,384 & 0,565 & 0,217 & 0,137 & 135 & 235 & $-2,5$ \\
\hline 2 & 235 & 0,136 & 2,210 & 0,220 & 1,881 & 0,617 & 0,851 & 0,411 & 0,350 & 1,690 & 0,180 & 0,233 & 0,981 & 0,228 & 0,137 & 150 & 235 & $-3,3$ \\
\hline 3 & 235 & 0,056 & 1,032 & 0,075 & 0,902 & 0,750 & 0,874 & 0,280 & 0,664 & 1,227 & 0,306 & 0,255 & 0,484 & 0,124 & 0,137 & 126 & 235 & $-2,3$ \\
\hline 4 & 235 & 0,047 & 0,757 & 0,056 & 0,443 & 0,833 & 0,586 & 0,446 & 1,000 & 0,986 & 0,509 & 0,317 & 0,342 & 0,108 & 0,137 & 162 & 235 & $-1,8$ \\
\hline 1 & 236 & 0,047 & 0,665 & 0,052 & 0,443 & 0,909 & 0,667 & 0,346 & 1,000 & 0,866 & 0,749 & 0,485 & 0,282 & 0,137 & 0,194 & 175 & 236 & $-1,5$ \\
\hline & 237 & & & & & & & & nothing to measure & & & & & & & & 237 & - \\
\hline & 238 & & & & & & & & nothing to measure & & & & & & & & 238 & - \\
\hline & 239 & & & & & & & & nothing to measure & & & & & & & & 239 & - \\
\hline 1 & 240 & 0,080 & 1,070 & 0,099 & 0,649 & 0,810 & 0,607 & 0,437 & 0,875 & 1,069 & 0,833 & 0,588 & 0,349 & 0,205 & 0,274 & 17 & 240 & $-1,8$ \\
\hline & 241 & & & & & & & & nothing to measure & & & & & & & & 241 & \\
\hline & 242 & & & & & & & & nothing to measure & & & & & & & & 242 & - \\
\hline & 243 & & & & & & & & nothing to measure & & & & & & & & 243 & - \\
\hline & 244 & & & & & & & & nothing to measure & & & & & & & & 244 & - \\
\hline & 245 & & & & & & & & nothing to measure & & & & & & & & 245 & - \\
\hline & 246 & & & & & & & & nothing to measure & & & & & & & & 246 & - \\
\hline & 247 & & & & & & & & nothing to measure & & & & & & & & 247 & - \\
\hline 1 & 248 & 0,070 & 1,178 & 0,108 & 0,887 & 0,652 & 0,753 & 0,427 & 0,638 & 1,252 & 0,466 & 0,501 & 0,439 & 0,220 & 0,137 & 55 & 248 & $-2,1$ \\
\hline & 249 & & & & & & & & nothing to measure & & & & & & & & 249 & \\
\hline . & 250 & 0,047 & 0,757 & 0,052 & 0,535 & 0,909 & 0,707 & 0,307 & 1,000 & 0,986 & 0,637 & 0,435 & 0,306 & 0,133 & 0,137 & 54 & 250 & $-1,6$ \\
\hline 2 & 250 & 0,099 & 1,567 & 0,136 & 1,307 & 0,724 & 0,834 & 0,322 & 0,504 & 1,408 & 0,237 & 0,204 & 0,728 & 0,149 & 0,137 & 40 & 250 & $-2,9$ \\
\hline 3 & 250 & 0,094 & 1,513 & 0,127 & 1,238 & 0,741 & 0,818 & 0,317 & 0,515 & 1,394 & 0,283 & 0,249 & 0,650 & 0,162 & 0,194 & 12 & 250 & -27 \\
\hline 4 & 250 & 0,056 & 0,902 & 0,056 & 0,719 & 1,000 & 0,797 & 0,203 & 0,869 & 1,073 & 0,373 & 0,221 & 0,439 & 0,097 & 0,137 & 41 & 250 & $-2,1$ \\
\hline
\end{tabular}




\begin{tabular}{|c|c|c|c|c|c|c|c|c|c|c|c|c|c|c|c|c|c|}
\hline 250 & 0,314 & 3,463 & 0,535 & 2,966 & 0,588 & 0,856 & 0,437 & 0,329 & 1,743 & 0,201 & 0,241 & 1,412 & 0,340 & 0,306 & 122 & 250 & $-3,8$ \\
\hline 250 & 0,136 & 2,102 & 0,145 & 1,567 & 0,935 & 0,745 & 0,263 & 0,387 & 1,608 & 0,316 & 0,437 & 0,741 & 0,324 & 0,274 & 55 & 250 & $-2,9$ \\
\hline 250 & 0,047 & 0,757 & 0,052 & 0,535 & 0,909 & 0,707 & 0,307 & 1,000 & 0,986 & 0,509 & 0,334 & 0,342 & 0,114 & 0,194 & 32 & 250 & $-1,8$ \\
\hline 250 & 0,056 & 1,016 & 0,075 & 0,573 & 0,750 & 0,564 & 0,503 & 0,685 & 1,208 & 0,413 & 0,329 & 0,417 & 0,137 & 0,194 & 0 & 250 & $-2,1$ \\
\hline 250 & 0,113 & 1,681 & 0,160 & 1,368 & 0,706 & 0,814 & 0,348 & 0,500 & 1,414 & 0,280 & 0,321 & 0,715 & 0,230 & 0,194 & 103 & 250 & $-2,8$ \\
\hline 251 & & & & & & & & nothing to measure & & & & & & & & 251 & - \\
\hline 252 & & & & & & & & nothing to measure & & & & & & & & 252 & - \\
\hline 253 & 0,061 & 1,162 & 0,084 & 0,757 & 0,722 & 0,651 & 0,446 & 0,568 & 1,327 & 0,571 & 0,631 & 0,369 & 0,233 & 0,137 & 148 & 253 & $-1,9$ \\
\hline 253 & 0,089 & 1,055 & 0,099 & 0,665 & 0,905 & 0,631 & 0,382 & 1,000 & 0,996 & 0,538 & 0,392 & 0,459 & 0,180 & 0,274 & 93 & 253 & $-2,2$ \\
\hline 253 & 0,089 & 1,934 & 0,178 & 1,200 & 0,500 & 0,620 & 0,628 & 0,299 & 1,828 & 0,285 & 0,481 & 0,632 & 0,303 & 0,137 & 146 & 253 & $-2,7$ \\
\hline 253 & 0,084 & 1,384 & 0,113 & 1,162 & 0,750 & 0,840 & 0,297 & 0,554 & 1,343 & 0,270 & 0,211 & 0,632 & 0,134 & 0,137 & 147 & 253 & $-2,7$ \\
\hline 253 & 0,188 & 1,972 & 0,244 & $\begin{array}{l}1,659 \\
\text { S }\end{array}$ & 0,769 & 0,841 & 0,280 & 0,606 & 1,284 & 0,520 & 0,565 & 0,678 & 0,383 & 0,387 & 133 & 253 & $-2,8$ \\
\hline 253 & 0,127 & 1,307 & 0,155 & 1,200 & 0,818 & 0,918 & 0,200 & 0,931 & 1,036 & 0,593 & 0,547 & 0,522 & 0,285 & 0,306 & 127 & 253 & $\begin{array}{l}-2,4 \\
\end{array}$ \\
\hline 253 & 0,080 & 1,048 & 0,099 & 0,848 & 0,810 & 0,810 & 0,269 & 0,913 & 1,047 & 0,585 & 0,581 & 0,417 & 0,242 & 0,306 & 0 & 253 & $-2,1$ \\
\hline 253 & 0,094 & 1,789 & 0,155 & 1,238 & 0,606 & 0,692 & 0,500 & 0,368 & 1,647 & 0,344 & 0,439 & 0,589 & 0,258 & 0,137 & 166 & 253 & $-2,6$ \\
\hline 253 & 0,812 & 6,658 & 1,131 & 3,745 & 0,718 & 0,563 & 0,521 & 0,230 & 2,085 & 0,429 & 0,605 & 1,553 & 0,939 & 0,565 & 143 & 253 & $-4,0$ \\
\hline 253 & 0,141 & 2,668 & 0,281 & 1,643 & 0,500 & 0,616 & 0,631 & 0,248 & 2,007 & 0,263 & 0,528 & 0,825 & 0,436 & 0,274 & 37 & 253 & $-3,0$ \\
\hline 253 & 0,075 & 0,940 & 0,080 & 0,940 & 0,941 & 1,000 & 0,059 & 1,000 & 0,968 & 0,599 & 0,461 & 0,399 & 0,184 & 0,274 & 150 & 253 & $-2,0$ \\
\hline 253 & 0,056 & 0,848 & 0,061 & 0,627 & 0,923 & 0,739 & 0,272 & 0,983 & 1,009 & 0,449 & 0,364 & 0,399 & 0,145 & 0,194 & 145 & 253 & $-2,0$ \\
\hline 253 & 0,103 & 1,422 & 0,141 & 1,108 & 0,733 & 0,780 & 0,346 & 0,642 & 1,248 & 0,384 & 0,419 & 0,585 & 0,245 & 0,274 & 167 & 253 & $-2,5$ \\
\hline 253 & 0,108 & 1,475 & 0,141 & 0,994 & 0,767 & 0,674 & 0,401 & 0,623 & 1,267 & 0,791 & 0,814 & 0,417 & 0,339 & 0,274 & 81 & 253 & $-2,1$ \\
\hline 253 & 0,061 & 0,848 & 0,066 & 0,665 & 0,929 & 0,784 & 0,228 & 1,000 & 0,969 & 0,517 & 0,375 & 0,387 & 0,145 & 0,194 & 135 & 253 & $\begin{array}{l}-2,1 \\
-2,0\end{array}$ \\
\hline 253 & 0,070 & 1,200 & 0,084 & 1,032 & 0,833 & 0,860 & 0,218 & 0,614 & 1,276 & 0,294 & 0,242 & 0,552 & 0,133 & 0,137 & 145 & 253 & $-2,5$ \\
\hline 253 & 0,047 & 0,925 & 0,061 & 0,481 & 0,769 & 0,521 & 0,532 & 0,690 & 1,204 & 0,707 & 0,528 & 0,291 & 0,153 & 0,137 & 40 & 253 & $-1,5$ \\
\hline 253 & 0,080 & 1,162 & 0,094 & 0,978 & 0,850 & 0,842 & 0,218 & 0,742 & 1,161 & 0,408 & 0,369 & 0,499 & 0,184 & 0,194 & 110 & 253 & $-2,3$ \\
\hline 253 & 0,056 & 0,848 & 0,061 & 0,573 & 0,923 & 0,675 & 0,334 & 0,983 & 1,009 & 0,611 & 0,448 & 0,342 & 0,153 & 0,194 & 18 & 253 & $-1,8$ \\
\hline 253 & 0,230 & 2,416 & 0,347 & 2,064 & 0,662 & 0,854 & 0,368 & 0,495 & 1,421 & 0,367 & 0,512 & 0,893 & 0,457 & 0,274 & 135 & 253 & $\begin{array}{l}-3,0 \\
-3,2\end{array}$ \\
\hline 253 & 0,075 & 1,422 & 0,108 & 1,016 & 0,696 & 0,715 & 0,417 & 0,467 & 1,464 & 0,300 & 0,250 & 0,565 & 0,141 & 0,137 & 164 & 253 & $-2,5$ \\
\hline 253 & 0,103 & 1,804 & 0,131 & 1,491 & 0,786 & 0,826 & 0,276 & 0,398 & 1,584 & 0,215 & 0,186 & 0,781 & 0,145 & 0,194 & 43 & 253 & $-3,0$ \\
\hline 253 & 0,108 & 2,087 & 0,192 & $\begin{array}{l}1,108 \\
\text {. }\end{array}$ & 0,561 & 0,531 & 0,642 & 0,311 & $\begin{array}{l}1,792 \\
\end{array}$ & 0,431 & 0,606 & 0,565 & 0,342 & 0,274 & 128 & 253 & $-2,5$ \\
\hline 253 & 0,164 & 2,897 & 0,314 & 1,605 & 0,522 & 0,554 & 0,653 & 0,246 & 2,017 & 0,409 & 0,745 & 0,715 & 0,533 & 0,274 & 139 & 253 & $-2,8$ \\
\hline 253 & 0,047 & 0,757 & 0,052 & 0,535 & 0,909 & 0,707 & 0,307 & 1,000 & 0,986 & 0,509 & 0,283 & 0,342 & 0,097 & 0,137 & 126 & 253 & $-1,8$ \\
\hline 253 & 0,075 & 1,070 & 0,099 & 1,016 & 0,762 & 0,950 & 0,243 & 0,824 & 1,102 & 0,453 & 0,506 & 0,459 & 0,233 & 0,274 & 168 & 253 & $\begin{array}{l}-2,2 \\
-2\end{array}$ \\
\hline 253 & 0,136 & 1,475 & 0,169 & 1,200 & 0,806 & 0,813 & 0,270 & 0,785 & 1,128 & 0,506 & 0,520 & 0,585 & 0,304 & 0,306 & 163 & 253 & $-2,5$ \\
\hline 253 & 0,047 & 0,665 & 0,052 & 0,481 & 0,909 & 0,724 & 0,291 & 1,000 & 0,866 & 0,979 & 0,588 & 0,247 & 0,145 & 0,137 & 72 & 253 & $-1,3$ \\
\hline 253 & 0,047 & 0,940 & 0,066 & 0,627 & 0,714 & 0,667 & 0,439 & 0,667 & 1,225 & 0,439 & 0,295 & 0,369 & 0,109 & 0,137 & 163 & 253 & $-1,9$ \\
\hline 253 & 0,108 & 1,513 & 0,150 & 1,200 & 0,719 & 0,793 & 0,349 & 0,592 & 1,300 & 0,401 & 0,367 & 0,585 & 0,215 & 0,274 & 114 & 253 & $-2,5$ \\
\hline 254 & & & & & & & & nothing to measure & & & & & & & & 254 & - \\
\hline 255 & & & & & & & & nothing to measure & & & & & & & & 255 & - \\
\hline 256 & & & & & & & & nothing to measure & & & & & & & & 256 & - \\
\hline 257 & & & & & & & & nothing to measure & & & & & & & & 257 & - \\
\hline 258 & 0,061 & 0,902 & 0,070 & 0,703 & 0,867 & 0,779 & 0,258 & 0,942 & 1,031 & 0,571 & 0,451 & 0,369 & 0,166 & 0,194 & 98 & 258 & $-1,9$ \\
\hline 258 & 0,066 & 0,757 & 0,066 & 0,627 & 1,000 & 0,828 & 0,172 & 1,000 & 0,833 & 1,049 & 0,728 & 0,282 & 0,205 & 0,274 & 90 & 258 & $-1,5$ \\
\hline 259 & & & & & & & & nothing to measure & & & & & & & & 259 & - \\
\hline 260 & & & & & & & & nothing to measure & & & & & & & & 260 & - \\
\hline 261 & & & & & & & & nothing to measure & & & & & & & & 261 & - \\
\hline 262 & 0,047 & 0,757 & 0,056 & 0,665 & 0,833 & 0,879 & 0,206 & 1,000 & 0,986 & 0,490 & 0,333 & 0,349 & 0,116 & 0,137 & 162 & 262 & $-1,8$ \\
\hline 262 & 0,070 & 1,108 & 0,089 & 0,833 & 0,789 & 0,752 & 0,326 & 0,720 & 1,178 & 0,382 & 0,283 & 0,484 & 0,137 & 0,137 & 162 & 262 & $-2,3$ \\
\hline 262 & 0,052 & 1,184 & 0,070 & 0,519 & 0,733 & 0,439 & 0,622 & 0,462 & 1,471 & 0,215 & 0,124 & 0,552 & 0,068 & 0,137 & 0 & 262 & $-2,5$ \\
\hline 262 & 0,056 & 0,978 & 0,075 & 0,703 & 0,750 & 0,719 & 0,376 & 0,739 & 1,163 & 0,449 & 0,326 & 0,399 & 0,130 & 0,137 & 17 & 262 & $-2,0$ \\
\hline 262 & 0,113 & 1,789 & 0,174 & 1,475 & 0,649 & 0,825 & 0,393 & 0,442 & 1,504 & 0,235 & 0,286 & 0,781 & 0,223 & 0,274 & 61 & 262 & $-3,0$ \\
\hline 263 & & & & & & & & nothing to measure & & & & & & & & 263 & - \\
\hline 264 & & & & & & & & nothing to measure & & & & & & & & 264 & - \\
\hline 265 & & & & & & & & nothing to measure & & & & & & & & 265 & . \\
\hline 266 & & & & & & & & nothing to measure & & & & & & & & 266 & - \\
\hline 267 & & & & & & & & nothing to measure & & & & & & & & 267 & - \\
\hline 268 & & & & & & & & nothing to measure & & & & & & & & 268 & - \\
\hline 269 & & & & & & & & nothing to measure & & & & & & & & 269 & - \\
\hline
\end{tabular}




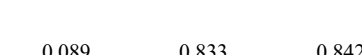

0,375

1,827

0,725

0,749

0,372

nothing to measure

nothing to measure

nothing to measure

nothing to measure

nothing to measure

nothing to measure

nothing to measure

nothing to meas
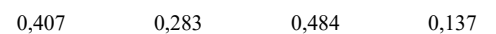

0,442
1,000

1,504
0,939

0,343

0,530

0,646

0,137

0,137

nothing to measure

nothing to measure
nothing to measure

nothing to measure

0,575
0

1,31

0,631

0,807

0,741

nothing to measure

nothing to measure

nothing to measure

nothing to measure

nothing to measure

nothing to measure

nothing to measure

nothing to measure

nothing to measure

nothing to measure

nothing to measure

nothing to measure

nothing to measure

nothing to measure

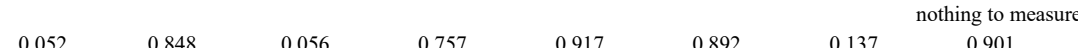

$\begin{array}{cccccccc}0,052 & 0,848 & 0,056 & 0,757 & 0,917 & 0,892 & 0,137 & 0,901\end{array}$

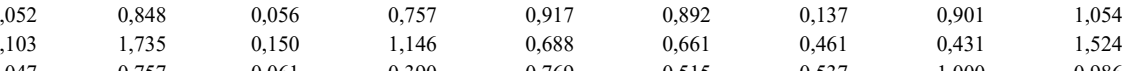

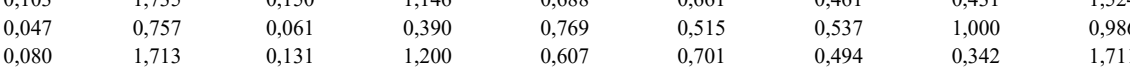

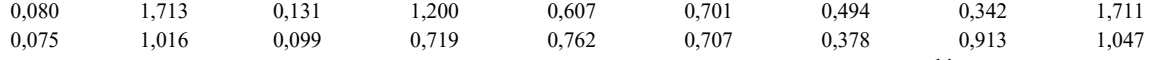

$\begin{array}{ll}0,061 & 0,757\end{array}$

$0,305 \quad 2,286$

$0,066-0.611-0.929$

$\begin{array}{ll}0,066 & 0,611 \\ 0,371 & 1,919\end{array}$

nothing to measure

$\begin{array}{ll}1,000 & 0,864 \\ 0,733 & 1,68\end{array}$

$\begin{array}{ll}0,720 & 1,179 \\ 0,353 & 1,682\end{array}$

nothing to measure

nothing to measure

$\begin{array}{lllllll}0,089 & 1,444 & 0,117 & 0,963 & 0,760 & 0,667 & 0,411\end{array}$

0,537

1,364

nothing to measure

nothing to measure

nothing to measure

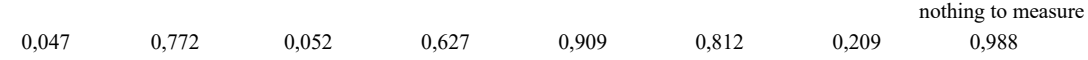

$1,006 \quad 0,749$

0,651

0,282

0,184

0,194 


\begin{tabular}{|c|c|c|c|c|c|c|c|c|c|c|c|c|c|c|c|c|c|c|}
\hline 2 & $\begin{array}{l}320 \\
321 \\
322\end{array}$ & 0,061 & 0,887 & 0,066 & 0,795 & 0,929 & 0,896 & 0,126 & $\begin{array}{l}\quad 0,975 \\
\text { nothing to measure } \\
\text { nothing to measure }\end{array}$ & 1,013 & 0,414 & 0,283 & 0,433 & 0,123 & 0,137 & 162 & $\begin{array}{l}320 \\
321 \\
322\end{array}$ & $\begin{array}{c}-2,1 \\
- \\
-\end{array}$ \\
\hline \multirow[t]{2}{*}{1} & $\begin{array}{l}323 \\
324\end{array}$ & 0,084 & 1,292 & 0,117 & 1,070 & 0,720 & 0,828 & 0,328 & $\begin{array}{c}0,636 \\
\text { nothing to measure }\end{array}$ & 1,254 & 0,337 & 0,326 & 0,565 & 0,184 & 0,137 & 69 & $\begin{array}{l}323 \\
324\end{array}$ & $\begin{array}{c}-2,5 \\
-\end{array}$ \\
\hline & 325 & & & & & & & & nothing to measure & & & & & & & & 325 & - \\
\hline \multirow{3}{*}{1} & 326 & 0,056 & 0,978 & 0,075 & 0,627 & 0,750 & 0,641 & 0,438 & 0,739 & 1,163 & 0,611 & 0,555 & 0,342 & 0,190 & 0,194 & 36 & 326 & $-1,8$ \\
\hline & $\begin{array}{l}327 \\
328\end{array}$ & & & & & & & & $\begin{array}{l}\text { nothing to measure } \\
\text { nothing to measure }\end{array}$ & & & & & & & & $\begin{array}{l}327 \\
328\end{array}$ & - \\
\hline & 329 & & & & & & & & nothing to measure & & & & & & & & 329 & - \\
\hline 1 & 330 & 0,056 & 0,887 & 0,066 & 0,703 & 0,857 & 0,793 & 0,252 & 0,900 & 1,054 & 0,382 & 0,269 & 0,433 & 0,116 & 0,137 & 12 & 330 & $-2,1$ \\
\hline 2 & 330 & 0,141 & 1,919 & 0,206 & 1,384 & 0,682 & 0,721 & 0,423 & 0,480 & 1,443 & 0,390 & 0,541 & 0,678 & 0,367 & 0,274 & 46 & 330 & $-2,8$ \\
\hline 3 & 330 & 0,174 & 2,691 & 0,291 & 1,751 & 0,597 & 0,651 & 0,534 & 0,301 & 1,822 & 0,466 & 0,774 & 0,688 & 0,533 & 0,137 & 93 & 330 & $-2,8$ \\
\hline 4 & 330 & 0,066 & 1,238 & 0,099 & 0,978 & 0,667 & 0,790 & 0,394 & 0,538 & 1,363 & 0,244 & 0,232 & 0,585 & 0,136 & 0,137 & 36 & 330 & $-2,5$ \\
\hline 5 & 330 & 0,174 & 2,660 & 0,347 & 1,957 & 0,500 & 0,736 & 0,566 & 0,308 & 1,801 & 0,377 & 0,684 & 0,766 & 0,524 & 0,274 & 101 & 330 & $-2,9$ \\
\hline \multirow[t]{5}{*}{6} & 330 & 0,070 & 1,345 & 0,103 & 0,887 & 0,682 & 0,659 & 0,466 & 0,488 & 1,431 & 0,382 & 0,424 & 0,484 & 0,205 & 0,194 & 12 & 330 & $-2,3$ \\
\hline & 331 & & & & & & & & nothing to measure & & & & & & & & 331 & - \\
\hline & 332 & & & & & & & & nothing to measure & & & & & & & & 332 & - \\
\hline & 333 & & & & & & & & nothing to measure & & & & & & & & 333 & - \\
\hline & 334 & & & & & & & & nothing to measure & & & & & & & & 334 & - \\
\hline & 335 & & & & & & & & nothing to measure & & & & & & & & 335 & - \\
\hline & 336 & & & & & & & & nothing to measure & & & & & & & & 336 & - \\
\hline & 337 & & & & & & & & nothing to measure & & & & & & & & 337 & - \\
\hline & 338 & & & & & & & & nothing to measure & & & & & & & & 338 & - \\
\hline & 339 & & & & & & & & nothing to measure & & & & & & & & 339 & - \\
\hline & 340 & & & & & & & & nothing to measure & & & & & & & & 340 & - \\
\hline 1 & 341 & 0,056 & 0,887 & 0,070 & 0,611 & 0,800 & 0,689 & 0,369 & 0,900 & 1,054 & 0,611 & 0,566 & 0,342 & 0,194 & 0,137 & 72 & 341 & $-1,8$ \\
\hline 2 & 341 & 0,056 & 0,994 & 0,075 & 0,611 & 0,750 & 0,615 & 0,459 & 0,716 & 1,182 & 0,611 & 0,570 & 0,342 & 0,195 & 0,194 & 72 & 341 & $-1,8$ \\
\hline 3 & 341 & 0,080 & 1,162 & 0,099 & 0,795 & 0,810 & 0,684 & 0,369 & 0,742 & 1,161 & 0,833 & 0,682 & 0,349 & 0,238 & 0,194 & 1 & 341 & $-1,8$ \\
\hline 4 & 341 & 0,103 & 1,827 & 0,164 & 1,292 & 0,629 & 0,707 & 0,473 & 0,389 & 1,604 & 0,280 & 0,348 & 0,685 & 0,239 & 0,274 & 27 & 341 & $-2,8$ \\
\hline 5 & 341 & 0,075 & 0,848 & 0,080 & 0,757 & 0,941 & 0,892 & 0,123 & 1,000 & 0,874 & 0,815 & 0,600 & 0,342 & 0,205 & 0,274 & 27 & 341 & $-1,8$ \\
\hline 6 & 341 & 0,103 & 1,200 & 0,113 & 0,978 & 0,917 & 0,815 & 0,203 & 0,901 & 1,054 & 0,539 & 0,392 & 0,494 & 0,194 & 0,274 & 39 & 341 & $-2,3$ \\
\hline 7 & 341 & 0,070 & 0,795 & 0,075 & 0,665 & 0,938 & 0,837 & 0,175 & 1,000 & 0,845 & 0,955 & 0,671 & 0,306 & 0,205 & 0,274 & 174 & 341 & $-1,6$ \\
\hline 8 & 341 & 0,066 & 0,757 & 0,066 & 0,573 & 1,000 & 0,757 & 0,243 & 1,000 & 0,833 & 0,891 & 0,632 & 0,306 & 0,194 & 0,274 & 17 & 341 & $-1,6$ \\
\hline 9 & 341 & 0,070 & 0,848 & 0,075 & 0,665 & 0,938 & 0,784 & 0,225 & 1,000 & 0,902 & 0,955 & 0,671 & 0,306 & 0,205 & 0,274 & 36 & 341 & $-1,6$ \\
\hline 10 & 341 & 0,052 & 0,665 & 0,052 & 0,519 & 1,000 & 0,781 & 0,219 & 1,000 & 0,826 & 0,700 & 0,447 & 0,306 & 0,137 & 0,194 & 18 & 341 & $-1,6$ \\
\hline 11 & 341 & 0,145 & 2,125 & 0,202 & 1,811 & 0,721 & 0,852 & 0,316 & 0,405 & 1,572 & 0,186 & 0,170 & 0,997 & 0,169 & 0,194 & 17 & 341 & $-3,3$ \\
\hline 12 & 341 & 0,066 & 0,978 & 0,075 & 0,810 & 0,875 & 0,828 & 0,212 & 0,862 & 1,077 & 0,396 & 0,267 & 0,459 & 0,123 & 0,137 & 30 & 341 & $-2,2$ \\
\hline 13 & 341 & 0,075 & 0,887 & 0,089 & 0,757 & 0,842 & 0,854 & 0,215 & 1,000 & 0,913 & 0,815 & 0,627 & 0,342 & 0,215 & 0,137 & 144 & 341 & $-1,8$ \\
\hline 14 & 341 & 0,056 & 1,200 & 0,080 & 0,703 & 0,706 & 0,586 & 0,508 & 0,491 & 1,427 & 0,588 & 0,540 & 0,349 & 0,189 & 0,137 & 90 & 341 & $-1,8$ \\
\hline 15 & 341 & 0,052 & 0,887 & 0,066 & 0,535 & 0,786 & 0,604 & 0,451 & 0,825 & 1,101 & 0,483 & 0,409 & 0,369 & 0,151 & 0,137 & 158 & 341 & $-1,9$ \\
\hline 16 & 341 & 0,056 & 0,978 & 0,066 & 0,573 & 0,857 & 0,586 & 0,438 & 0,739 & 1,163 & 0,611 & 0,566 & 0,342 & 0,194 & 0,194 & 0 & 341 & $-1,8$ \\
\hline 17 & 341 & 0,066 & 0,978 & 0,075 & 0,703 & 0,875 & 0,719 & 0,308 & 0,862 & 1,077 & 0,446 & 0,316 & 0,433 & 0,137 & 0,194 & 168 & 341 & $-2,1$ \\
\hline \multirow[t]{4}{*}{18} & 341 & 0,066 & 0,978 & 0,080 & 0,757 & 0,824 & 0,773 & 0,287 & 0,862 & 1,077 & 0,482 & 0,433 & 0,417 & 0,180 & 0,194 & 7 & 341 & $-2,1$ \\
\hline & 342 & & & & & & & & nothing to measure & & & & & & & & 342 & - \\
\hline & 343 & & & & & & & & nothing to measure & & & & & & & & 343 & - \\
\hline & 344 & & & & & & & & nothing to measure & & & & & & & & 344 & - \\
\hline 1 & 345 & 0,075 & 1,108 & 0,103 & 0,925 & 0,727 & 0,834 & 0,319 & 0,768 & 1,141 & 0,551 & 0,551 & 0,417 & 0,230 & 0,274 & 9 & 345 & $-2,1$ \\
\hline 2 & 345 & 0,084 & 1,368 & 0,108 & 0,871 & 0,783 & 0,637 & 0,423 & 0,567 & 1,328 & 0,353 & 0,248 & 0,552 & 0,137 & 0,194 & 177 & 345 & $-2,5$ \\
\hline 3 & 345 & 0,089 & 1,184 & 0,099 & 0,963 & 0,905 & 0,813 & 0,210 & 0,798 & 1,119 & 0,372 & 0,248 & 0,552 & 0,137 & 0,194 & 176 & 345 & $-2,5$ \\
\hline 4 & 345 & 0,084 & 1,330 & 0,117 & 0,963 & 0,720 & 0,724 & 0,393 & 0,600 & 1,291 & 0,458 & 0,400 & 0,484 & 0,194 & 0,137 & 80 & 345 & $-2,3$ \\
\hline 5 & 345 & 0,174 & 2,454 & 0,272 & 1,849 & 0,638 & 0,754 & 0,438 & 0,362 & 1,661 & 0,318 & 0,409 & 0,833 & 0,341 & 0,274 & 177 & 345 & $-3,1$ \\
\hline 6 & 345 & 0,131 & 1,384 & 0,145 & 1,200 & 0,903 & 0,867 & 0,164 & 0,862 & 1,077 & 0,488 & 0,419 & 0,585 & 0,245 & 0,306 & 155 & 345 & $-2,5$ \\
\hline 7 & 345 & 0,089 & 1,016 & 0,089 & 0,795 & 1,000 & 0,782 & 0,218 & 1,000 & 0,960 & 0,538 & 0,400 & 0,459 & 0,184 & 0,274 & 156 & 345 & $-2,2$ \\
\hline \multirow[t]{3}{*}{8} & 345 & 0,047 & 0,665 & 0,056 & 0,535 & 0,833 & 0,805 & 0,257 & 1,000 & 0,866 & 0,749 & 0,485 & 0,282 & 0,137 & 0,194 & 0 & 345 & $-1,5$ \\
\hline & 346 & & & & & & & & nothing to measure & & & & & & & & 346 & 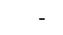 \\
\hline & 347 & & & & & & & & nothing to measure & & & & & & & & 347 & - \\
\hline 1 & 348 & 0,080 & 1,675 & 0,080 & 1,032 & 1,000 & 0,616 & 0,384 & 0,357 & 1,673 & 0,373 & 0,406 & 0,522 & 0,212 & 0,194 & 0 & 348 & $-2,4$ \\
\hline
\end{tabular}




\begin{tabular}{|c|c|c|c|c|c|c|c|c|c|c|c|c|c|c|c|c|}
\hline 0,160 & 1,934 & 0,235 & 1,460 & 0,680 & 0,755 & 0,403 & 0,536 & 1,366 & 0,637 & 0,815 & 0,565 & 0,460 & 0,306 & 103 & 348 & $-2,5$ \\
\hline 0,178 & 2,140 & 0,249 & 1,590 & 0,717 & 0,743 & 0,382 & 0,489 & 1,430 & 0,387 & 0,406 & 0,766 & 0,311 & 0,274 & 4 & 348 & $-2,9$ \\
\hline 0,070 & 1,070 & 0,089 & 0,611 & 0,789 & 0,571 & 0,478 & 0,772 & 1,138 & 0,659 & 0,529 & 0,369 & 0,195 & 0,194 & 4 & 348 & $-1,9$ \\
\hline 0,418 & 3,669 & 0,629 & 2,821 & 0,664 & 0,769 & 0,408 & 0,390 & $\begin{array}{l}1,602 \\
\end{array}$ & 0,340 & 0,547 & 1,250 & 0,684 & 0,433 & 9 & 348 & $-3,6$ \\
\hline 0,080 & 1,330 & 0,103 & 0,833 & 0,773 & 0,626 & 0,437 & 0,567 & 1,328 & 0,585 & 0,493 & 0,417 & 0,205 & 0,274 & 98 & 348 & $-2,1$ \\
\hline 0,056 & 1,254 & 0,094 & 0,940 & 0,600 & 0,750 & 0,472 & 0,450 & 1,491 & 0,235 & 0,293 & 0,552 & 0,162 & 0,137 & 135 & 348 & $-2,5$ \\
\hline 0,061 & 0,887 & 0,075 & 0,573 & 0,812 & 0,646 & 0,400 & 0,975 & 1,013 & 0,487 & 0,416 & 0,399 & 0,166 & 0,274 & 162 & 348 & $-2,0$ \\
\hline 0,047 & 0,627 & 0,047 & 0,535 & 1,000 & 0,854 & 0,146 & 1,000 & 0,816 & 0,796 & 0,500 & 0,274 & 0,137 & 0,194 & 18 & 349 & $-1,5$ \\
\hline 0,047 & 0,573 & 0,047 & 0,497 & 1,000 & 0,867 & 0,133 & 1,000 & 0,746 & 1,273 & 0,632 & 0,217 & 0,137 & 0,194 & 162 & 349 & $-1,1$ \\
\hline 0,070 & 1,108 & 0,089 & 0,795 & 0,789 & 0,717 & 0,353 & 0,720 & 1,178 & 0,562 & 0,514 & 0,399 & 0,205 & 0,274 & 35 & 350 & $-2,0$ \\
\hline 0,047 & 0,848 & 0,061 & 0,665 & 0,769 & 0,784 & 0,316 & 0,819 & 1,105 & 0,374 & 0,271 & 0,399 & 0,108 & 0,137 & 72 & 350 & $-2,0$ \\
\hline 0,047 & 1,162 & 0,084 & 0,757 & 0,556 & 0,651 & 0,565 & 0,437 & 1,513 & 0,318 & 0,416 & 0,433 & 0,180 & 0,137 & 0 & 350 & $-2,1$ \\
\hline 0,061 & 1,124 & 0,099 & 0,810 & 0,619 & 0,721 & 0,472 & 0,607 & 1,284 & 0,404 & 0,470 & 0,439 & 0,206 & 0,137 & 39 & 350 & $-2,1$ \\
\hline 0,136 & 2,232 & 0,239 & 1,919 & 0,569 & 0,860 & 0,454 & 0,343 & 1,707 & 0,231 & 0,401 & 0,866 & 0,347 & 0,274 & 17 & 350 & $-3,1$ \\
\hline 0,066 & 1,422 & 0,094 & 0,848 & 0,700 & 0,597 & 0,503 & 0,408 & 1,565 & 0,435 & 0,552 & 0,439 & 0,242 & 0,137 & 80 & 350 & $-2,1$ \\
\hline 0,052 & 0,994 & 0,089 & 0,795 & 0,579 & 0,800 & 0,466 & 0,656 & 1,234 & 0,379 & 0,439 & 0,417 & 0,183 & 0,137 & 84 & 350 & $-2,1$ \\
\hline 0,052 & 0,795 & 0,061 & 0,573 & 0,846 & 0,721 & 0,318 & 1,000 & 0,987 & 0,778 & 0,629 & 0,291 & 0,183 & 0,274 & 72 & 350 & $-1,5$ \\
\hline 0,342 & 4,005 & 0,530 & 2,637 & 0,646 & 0,658 & 0,492 & 0,268 & 1,931 & 0,219 & 0,337 & 1,412 & 0,475 & 0,387 & 31 & 350 & $-3,8$ \\
\hline 0,089 & 1,162 & 0,108 & 0,978 & 0,826 & 0,842 & 0,235 & 0,830 & 1,098 & 0,484 & 0,424 & 0,484 & 0,205 & 0,194 & 7 & 350 & $-2,3$ \\
\hline 0,080 & 1,330 & 0,099 & 1,108 & 0,810 & 0,833 & 0,253 & 0,567 & 1,328 & 0,271 & 0,200 & 0,613 & 0,123 & 0,137 & 28 & 350 & $-2,6$ \\
\hline 0,160 & 1,957 & 0,225 & 1,422 & 0,708 & 0,727 & 0,400 & 0,524 & 1,382 & 0,346 & 0,447 & 0,766 & 0,342 & 0,274 & 163 & 350 & $-2,9$ \\
\hline 0,174 & 1,773 & 0,220 & 1,475 & 0,787 & 0,832 & 0,271 & 0,694 & 1,201 & 0,444 & 0,478 & 0,705 & 0,337 & 0,387 & 43 & 350 & $-2,8$ \\
\hline \multirow[t]{15}{*}{0,066} & 1,605 & 0,127 & 1,032 & 0,519 & 0,643 & 0,599 & 0,320 & 1,767 & 0,292 & 0,516 & 0,535 & 0,276 & 0,137 & 90 & 350 & $-2,4$ \\
\hline & & & & & & & nothing to measure & & & & & & & & 351 & \\
\hline & & & & & & & nothing to measure & & & & & & & & 352 & - \\
\hline & & & & & & & nothing to measure & & & & & & & & 353 & - \\
\hline & & & & & & & nothing to measure & & & & & & & & 354 & - \\
\hline & & & & & & & nothing to measure & & & & & & & & 355 & - \\
\hline & & & & & & & nothing to measure & & & & & & & & 356 & - \\
\hline & & & & & & & nothing to measure & & & & & & & & 357 & - \\
\hline & & & & & & & nothing to measure & & & & & & & & 358 & - \\
\hline & & & & & & & nothing to measure & & & & & & & & 359 & - \\
\hline & & & & & & & nothing to measure & & & & & & & & 360 & - \\
\hline & & & & & & & nothing to measure & & & & & & & & 361 & - \\
\hline & & & & & & & nothing to measure & & & & & & & & 362 & - \\
\hline & & & & & & & nothing to measure & & & & & & & & 363 & - \\
\hline & & & & & & & nothing to measure & & & & & & & & 364 & - \\
\hline 0,113 & 1,789 & 0,164 & 1,292 & 0,686 & 0,722 & 0,419 & 0,442 & 1,504 & 0,340 & 0,424 & 0,650 & 0,276 & 0,274 & 26 & 365 & $-2,7$ \\
\hline 0,061 & 0,887 & 0,070 & 0,627 & 0,867 & 0,707 & 0,322 & 0,975 & 1,013 & 0,637 & 0,555 & 0,349 & 0,194 & 0,194 & 45 & 365 & $-1,8$ \\
\hline 0,094 & 1,697 & 0,141 & 1,032 & 0,667 & 0,608 & 0,514 & 0,409 & 1,563 & 0,374 & 0,462 & 0,565 & 0,261 & 0,274 & 138 & 365 & $\begin{array}{l}-2,5 \\
-2\end{array}$ \\
\hline 0,061 & 0,795 & 0,066 & 0,573 & 0,929 & 0,721 & 0,288 & 1,000 & 0,908 & 0,828 & 0,597 & 0,306 & 0,183 & 0,194 & 79 & 365 & $-1,6$ \\
\hline 0,089 & 2,010 & 0,150 & 1,330 & 0,594 & 0,661 & 0,529 & 0,277 & 1,900 & 0,269 & 0,333 & 0,650 & 0,216 & 0,137 & 17 & 365 & $-2,7$ \\
\hline 0,047 & 0,833 & 0,056 & 0,573 & 0,833 & 0,688 & 0,354 & 0,850 & 1,085 & 0,344 & 0,164 & 0,417 & 0,068 & 0,137 & 0 & 365 & $-2,1$ \\
\hline 0,099 & 1,529 & 0,141 & 1,032 & 0,700 & 0,675 & 0,442 & 0,530 & 1,374 & 0,546 & 0,674 & 0,479 & 0,323 & 0,194 & 71 & 365 & $-2,3$ \\
\hline 0,061 & 0,795 & 0,066 & 0,611 & 0,929 & 0,769 & 0,242 & 1,000 & 0,908 & 0,662 & 0,500 & 0,342 & 0,171 & 0,194 & 42 & 365 & $-1,8$ \\
\hline \multirow[t]{8}{*}{0,178} & 1,827 & 0,239 & 1,590 & 0,745 & 0,870 & 0,286 & 0,671 & 1,221 & 0,499 & 0,598 & 0,675 & 0,403 & 0,274 & 13 & 365 & $-2,8$ \\
\hline & & & & & & & nothing to measure & & & & & & & & 366 & 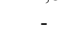 \\
\hline & & & & & & & nothing to measure & & & & & & & & 367 & - \\
\hline & & & & & & & nothing to measure & & & & & & & & 368 & - \\
\hline & & & & & & & nothing to measure & & & & & & & & 369 & - \\
\hline & & & & & & & nothing to measure & & & & & & & & 370 & - \\
\hline & & & & & & & nothing to measure & & & & & & & & 371 & - \\
\hline & & & & & & & nothing to measure & & & & & & & & 372 & \\
\hline \multirow[t]{3}{*}{0,150} & 1,865 & 0,211 & 1,552 & 0,711 & 0,832 & 0,334 & 0,542 & 1,358 & 0,279 & 0,333 & 0,828 & 0,276 & 0,274 & 162 & 373 & $-3,0$ \\
\hline & & & & & & & nothing to measure & & & & & & & & 374 & \\
\hline & & & & & & & nothing to measure & & & & & & & & 375 & \\
\hline 0,047 & 0,848 & 0,075 & 0,719 & 0,625 & 0,847 & 0,405 & 0,819 & 1,105 & 0,509 & 0,521 & 0,342 & 0,178 & 0,137 & 18 & 376 & $-1,8$ \\
\hline 047 & 0,589 & 0,047 & 0,443 & 1,000 & 0,753 & 0,247 & 1,000 & 0,767 & 1,273 & 0,671 & 0,217 & 0,145 & 0,194 & 72 & 377 & 1 \\
\hline
\end{tabular}




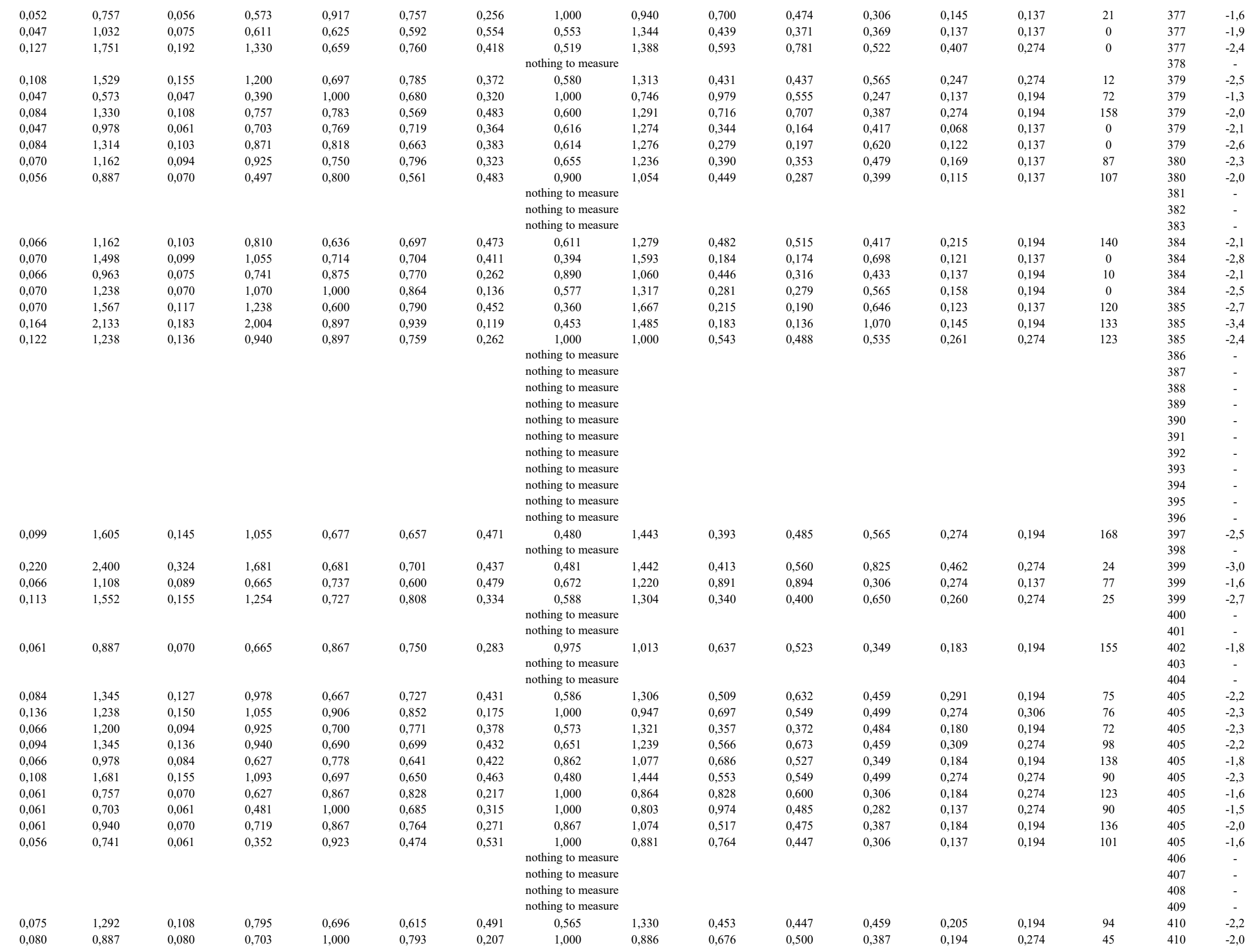




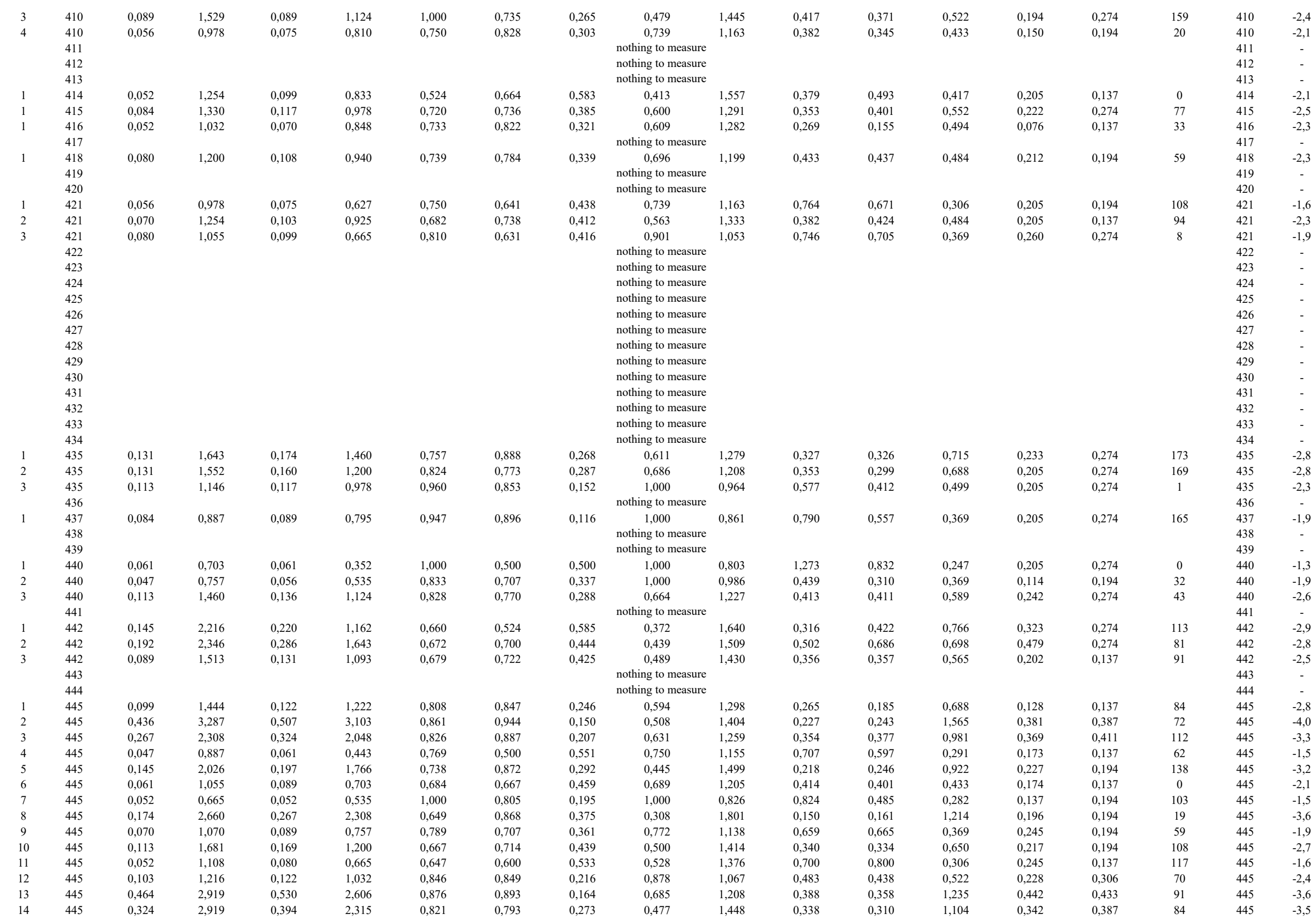




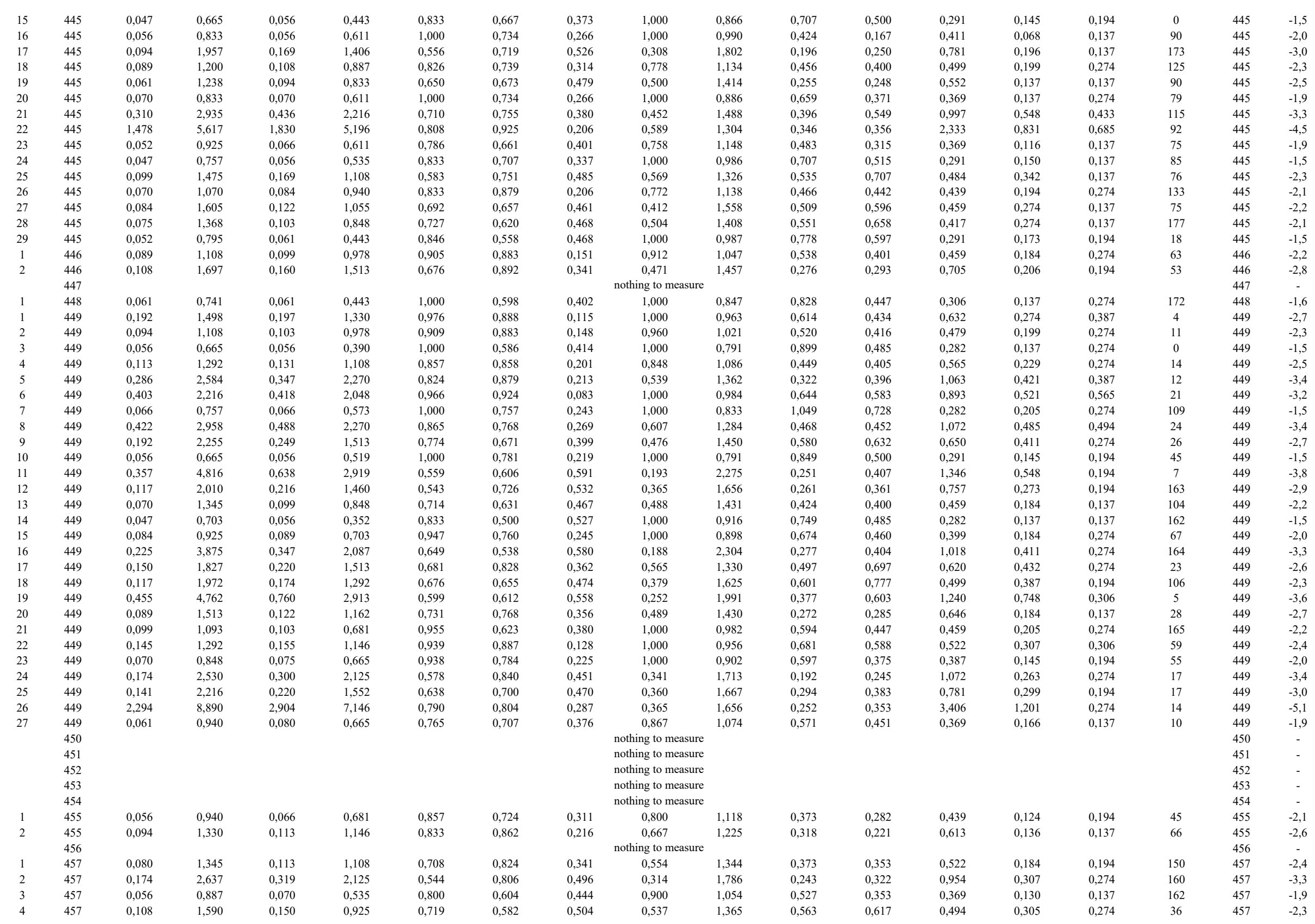




\begin{tabular}{|c|c|c|c|c|c|c|c|c|c|c|c|c|c|c|c|c|c|c|}
\hline 5 & 457 & 0,066 & 0,887 & 0,075 & 0,665 & 0,875 & 0,750 & 0,280 & 1,000 & 0,976 & 0,524 & 0,380 & 0,399 & 0,152 & 0,194 & 154 & 457 & $-2,0$ \\
\hline 6 & 457 & 0,061 & 1,124 & 0,080 & 0,887 & 0,765 & 0,789 & 0,316 & 0,607 & 1,284 & 0,368 & 0,268 & 0,459 & 0,123 & 0,137 & 123 & 457 & $-2,2$ \\
\hline 7 & 457 & 0,188 & 2,324 & 0,263 & 1,865 & 0,714 & 0,803 & 0,347 & 0,437 & 1,513 & 0,333 & 0,373 & 0,847 & 0,316 & 0,306 & 161 & 457 & $-3,1$ \\
\hline 8 & 457 & 0,052 & 1,070 & 0,084 & 0,887 & 0,611 & 0,828 & 0,425 & 0,566 & 1,329 & 0,342 & 0,407 & 0,439 & 0,178 & 0,137 & 18 & 457 & $-2,1$ \\
\hline 9 & 457 & 0,070 & 0,795 & 0,075 & 0,703 & 0,938 & 0,885 & 0,131 & 1,000 & 0,845 & 0,955 & 0,637 & 0,306 & 0,195 & 0,274 & 117 & 457 & $-1,6$ \\
\hline 10 & 457 & 0,197 & 1,681 & 0,220 & 1,406 & 0,894 & 0,836 & 0,195 & 0,876 & 1,069 & 0,529 & 0,398 & 0,688 & 0,274 & 0,411 & 96 & 457 & $-2,8$ \\
\hline 11 & 457 & 0,052 & 0,627 & 0,056 & 0,443 & 0,917 & 0,707 & 0,304 & 1,000 & 0,778 & 1,401 & 0,849 & 0,217 & 0,184 & 0,194 & 153 & 457 & $-1,1$ \\
\hline 12 & 457 & 0,103 & 1,200 & 0,122 & 0,963 & 0,846 & 0,802 & 0,251 & 0,901 & 1,054 & 0,700 & 0,632 & 0,433 & 0,274 & 0,274 & 1 & 457 & $-2,1$ \\
\hline 13 & 457 & 0,145 & 1,384 & 0,164 & 1,200 & 0,886 & 0,867 & 0,175 & 0,955 & 1,023 & 0,681 & 0,650 & 0,522 & 0,339 & 0,306 & 119 & 457 & $-2,4$ \\
\hline 14 & 457 & 0,164 & 1,406 & 0,183 & 1,146 & 0,897 & 0,815 & 0,211 & 1,000 & 0,979 & 0,731 & 0,648 & 0,535 & 0,347 & 0,387 & 160 & 457 & $-2,4$ \\
\hline 15 & 457 & 0,108 & 2,010 & 0,188 & 1,384 & 0,575 & 0,688 & 0,527 & 0,335 & 1,727 & 0,290 & 0,451 & 0,688 & 0,311 & 0,194 & 82 & 457 & $-2,8$ \\
\hline 16 & 457 & 0,047 & 0,795 & 0,066 & 0,535 & 0,714 & 0,673 & 0,434 & 0,933 & 1,035 & 0,637 & 0,501 & 0,306 & 0,153 & 0,137 & 118 & 457 & $-1,6$ \\
\hline 17 & 457 & 0,080 & 1,016 & 0,084 & 0,741 & 0,944 & 0,729 & 0,277 & 0,970 & 1,015 & 0,585 & 0,329 & 0,417 & 0,137 & 0,274 & 2 & 457 & $-2,1$ \\
\hline 18 & 457 & 0,061 & 0,848 & 0,066 & 0,795 & 0,929 & 0,937 & 0,095 & 1,000 & 0,969 & 0,571 & 0,416 & 0,369 & 0,153 & 0,194 & 30 & 457 & $-1,9$ \\
\hline 19 & 457 & 0,047 & 0,627 & 0,047 & 0,497 & 1,000 & 0,793 & 0,207 & 1,000 & 0,816 & 0,979 & 0,588 & 0,247 & 0,145 & 0,194 & 45 & 457 & $-1,3$ \\
\hline 20 & 457 & 0,117 & 1,108 & 0,122 & 0,848 & 0,962 & 0,766 & 0,237 & 1,000 & 0,913 & 0,637 & 0,500 & 0,484 & 0,242 & 0,306 & 135 & 457 & $-2,3$ \\
\hline 21 & 457 & 0,122 & 1,422 & 0,155 & 1,238 & 0,788 & 0,871 & 0,248 & 0,758 & 1,148 & 0,571 & 0,582 & 0,522 & 0,303 & 0,274 & 8 & 457 & $-2,4$ \\
\hline 22 & 457 & 0,052 & 1,254 & 0,089 & 0,719 & 0,579 & 0,573 & 0,600 & 0,413 & 1,557 & 0,483 & 0,631 & 0,369 & 0,233 & 0,137 & 117 & 457 & $-1,9$ \\
\hline 23 & 457 & 0,136 & 2,118 & 0,202 & 1,912 & 0,674 & 0,903 & 0,340 & 0,381 & 1,620 & 0,183 & 0,199 & 0,973 & 0,193 & 0,137 & 136 & 457 & $-3,3$ \\
\hline 24 & 457 & 0,089 & 0,940 & 0,099 & 0,795 & 0,905 & 0,845 & 0,182 & 1,000 & 0,888 & 0,968 & 0,800 & 0,342 & 0,274 & 0,274 & 37 & 457 & $-1,8$ \\
\hline 25 & 457 & 0,061 & 0,848 & 0,075 & 0,665 & 0,812 & 0,784 & 0,286 & 1,000 & 0,969 & 0,571 & 0,411 & 0,369 & 0,152 & 0,194 & 67 & 457 & $-1,9$ \\
\hline 26 & 457 & 0,113 & 1,016 & 0,113 & 0,795 & 1,000 & 0,782 & 0,218 & 1,000 & 0,855 & 0,899 & 0,686 & 0,399 & 0,274 & 0,306 & 32 & 457 & $-2,0$ \\
\hline 27 & 457 & 0,108 & 1,032 & 0,113 & 0,925 & 0,958 & 0,896 & 0,112 & 1,000 & 0,886 & 1,010 & 0,748 & 0,369 & 0,276 & 0,306 & 162 & 457 & $-1,9$ \\
\hline 28 & 457 & 0,075 & 0,887 & 0,084 & 0,665 & 0,889 & 0,750 & 0,274 & 1,000 & 0,913 & 0,815 & 0,626 & 0,342 & 0,214 & 0,274 & 31 & 457 & $-1,8$ \\
\hline 29 & 457 & 0,094 & 1,055 & 0,099 & 0,887 & 0,952 & 0,841 & 0,166 & 1,000 & 0,971 & 0,566 & 0,400 & 0,459 & 0,184 & 0,274 & 109 & 457 & $-2,2$ \\
\hline 30 & 457 & 0,263 & 2,324 & 0,347 & 2,087 & 0,757 & 0,898 & 0,264 & 0,611 & 1,279 & 0,412 & 0,415 & 0,901 & 0,374 & 0,387 & 17 & 457 & $-3,2$ \\
\hline 31 & 457 & 0,047 & 0,902 & 0,056 & 0,573 & 0,833 & 0,635 & 0,401 & $\begin{array}{c}0,724 \\
\end{array}$ & 1,175 & 0,439 & 0,371 & 0,369 & 0,137 & 0,137 & 165 & 457 & $-1,9$ \\
\hline & 458 & & & & & & & & nothing to measure & & & & & & & & 458 & - \\
\hline 1 & 459 & 0,052 & 0,848 & 0,066 & 0,535 & 0,786 & 0,631 & 0,427 & 0,901 & 1,054 & 0,560 & 0,570 & 0,342 & 0,195 & 0,194 & 90 & 459 & $-1,8$ \\
\hline 2 & 459 & 0,061 & 0,940 & 0,089 & 0,719 & 0,684 & 0,764 & 0,394 & 0,867 & 1,074 & 0,571 & 0,646 & 0,369 & 0,238 & 0,194 & 154 & 459 & $-1,9$ \\
\hline 3 & 459 & 0,075 & 0,887 & 0,080 & 0,795 & 0,941 & 0,896 & 0,119 & 1,000 & 0,913 & 0,599 & 0,485 & 0,399 & 0,194 & 0,274 & 36 & 459 & $-2,0$ \\
\hline 4 & 459 & 0,113 & 1,200 & 0,127 & 0,925 & 0,889 & 0,771 & 0,255 & 0,983 & 1,009 & 0,577 & 0,412 & 0,499 & 0,205 & 0,274 & 91 & 459 & $-2,3$ \\
\hline 5 & 459 & 0,174 & 1,643 & 0,197 & 1,422 & 0,881 & 0,865 & 0,180 & 0,808 & 1,113 & 0,575 & 0,552 & 0,620 & 0,342 & 0,306 & 161 & 459 & $-2,6$ \\
\hline 6 & 459 & 0,511 & 2,951 & 0,586 & 2,821 & 0,872 & 0,956 & 0,135 & 0,738 & 1,164 & 0,427 & 0,422 & 1,235 & 0,521 & 0,548 & 124 & 459 & $-3,6$ \\
\hline 7 & 459 & 0,066 & 0,810 & 0,070 & 0,810 & 0,933 & 1,000 & 0,067 & 1,000 & 0,892 & 0,713 & 0,424 & 0,342 & 0,145 & 0,194 & 140 & 459 & $-1,8$ \\
\hline 8 & 459 & 0,089 & 1,070 & 0,103 & 0,902 & 0,864 & 0,843 & 0,208 & 0,978 & 1,011 & 0,465 & 0,347 & 0,494 & 0,171 & 0,194 & 123 & 459 & $-2,3$ \\
\hline 9 & 459 & 0,366 & 2,729 & 0,436 & 2,454 & 0,839 & 0,899 & 0,190 & 0,617 & 1,273 & 0,344 & 0,372 & 1,164 & 0,434 & 0,433 & 115 & 459 & $-3,5$ \\
\hline 10 & 459 & 0,131 & 1,498 & 0,164 & 1,200 & 0,800 & 0,801 & 0,282 & 0,736 & 1,166 & 0,419 & 0,384 & 0,632 & 0,242 & 0,274 & 104 & 459 & $-2,7$ \\
\hline 11 & 459 & 0,047 & 0,833 & 0,061 & 0,443 & 0,769 & 0,532 & 0,522 & 0,850 & 1,085 & 0,439 & 0,256 & 0,369 & 0,094 & 0,137 & 165 & 459 & $-1,9$ \\
\hline 12 & 459 & 0,413 & 2,568 & 0,446 & 2,362 & 0,926 & 0,920 & 0,109 & 0,787 & 1,127 & 0,388 & 0,384 & 1,164 & 0,448 & 0,494 & 110 & 459 & $-3,5$ \\
\hline 13 & 459 & 0,061 & 1,178 & 0,075 & 1,124 & 0,812 & 0,954 & 0,193 & 0,553 & 1,345 & 0,224 & 0,146 & 0,589 & 0,086 & 0,137 & 129 & 459 & $-2,6$ \\
\hline 14 & 459 & 1,623 & 8,722 & 2,416 & 5,313 & 0,672 & 0,609 & 0,510 & 0,268 & 1,931 & 0,632 & 0,889 & 1,808 & 1,608 & 0,738 & 57 & 459 & $-4,2$ \\
\hline 15 & 459 & 0,056 & 0,757 & 0,061 & 0,573 & 0,923 & 0,757 & 0,254 & 1,000 & 0,900 & 0,588 & 0,392 & 0,349 & 0,137 & 0,194 & 108 & 459 & $-1,8$ \\
\hline 16 & 459 & 0,047 & 0,795 & 0,061 & 0,443 & 0,769 & 0,558 & 0,499 & 0,933 & 1,035 & 0,637 & 0,559 & 0,306 & 0,171 & 0,137 & 58 & 459 & $-1,6$ \\
\hline 17 & 459 & 0,052 & 0,757 & 0,061 & 0,573 & 0,846 & 0,757 & 0,287 & 1,000 & 0,940 & 0,560 & 0,358 & 0,342 & 0,123 & 0,137 & 125 & 459 & $-1,8$ \\
\hline 18 & 459 & 0,052 & 0,703 & 0,052 & 0,519 & 1,000 & 0,739 & 0,261 & 1,000 & 0,873 & 0,778 & 0,590 & 0,291 & 0,171 & 0,194 & 45 & 459 & $-1,5$ \\
\hline 19 & 459 & 0,099 & 1,016 & 0,103 & 0,810 & 0,955 & 0,797 & 0,208 & 1,000 & 0,914 & 0,668 & 0,474 & 0,433 & 0,205 & 0,274 & 102 & 459 & $-2,1$ \\
\hline 20 & 459 & 0,056 & 0,848 & 0,061 & 0,627 & 0,923 & 0,739 & 0,272 & 0,983 & 1,009 & 0,449 & 0,310 & 0,399 & 0,124 & 0,194 & 50 & 459 & $-2,0$ \\
\hline 21 & 459 & 0,047 & 0,978 & 0,070 & 0,741 & 0,667 & 0,757 & 0,412 & 0,616 & 1,274 & 0,354 & 0,295 & 0,411 & 0,121 & 0,137 & 90 & 459 & $-2,0$ \\
\hline 22 & 459 & 0,047 & 0,573 & 0,047 & 0,497 & 1,000 & 0,867 & 0,133 & 1,000 & 0,746 & 1,273 & 0,632 & 0,217 & 0,137 & 0,194 & 162 & 459 & $-1,1$ \\
\hline 23 & 459 & 0,155 & 1,498 & 0,160 & 1,314 & 0,971 & 0,877 & 0,126 & 0,867 & 1,074 & 0,416 & 0,299 & 0,688 & 0,205 & 0,274 & 84 & 459 & $-2,8$ \\
\hline 24 & 459 & 0,380 & 2,545 & 0,436 & 2,507 & 0,871 & 0,985 & 0,130 & 0,737 & 1,165 & 0,376 & 0,359 & 1,134 & 0,407 & 0,387 & 64 & 459 & $-3,5$ \\
\hline 25 & 459 & 0,216 & 1,773 & 0,244 & 1,475 & 0,885 & 0,832 & 0,204 & 0,863 & 1,077 & 0,604 & 0,609 & 0,675 & 0,411 & 0,411 & 80 & 459 & $-2,8$ \\
\hline 26 & 459 & 0,056 & 1,124 & 0,099 & 0,795 & 0,571 & 0,707 & 0,519 & 0,560 & 1,336 & 0,449 & 0,606 & 0,399 & 0,242 & 0,137 & 42 & 459 & $-2,0$ \\
\hline 27 & 459 & 0,202 & 1,865 & 0,244 & 1,681 & 0,827 & 0,902 & 0,199 & 0,729 & 1,171 & 0,375 & 0,377 & 0,828 & 0,312 & 0,387 & 72 & 459 & $-3,0$ \\
\hline 28 & 459 & 0,052 & 0,611 & 0,052 & 0,222 & 1,000 & 0,363 & 0,637 & 1,000 & 0,759 & 1,077 & 0,555 & 0,247 & 0,137 & 0,274 & 72 & 459 & $-1,3$ \\
\hline 29 & 459 & 0,066 & 0,994 & 0,084 & 0,757 & 0,778 & 0,761 & 0,326 & 0,835 & 1,094 & 0,686 & 0,654 & 0,349 & 0,228 & 0,194 & 33 & 459 & $-1,8$ \\
\hline 30 & 459 & 0,052 & 0,757 & 0,056 & 0,573 & 0,917 & 0,757 & 0,256 & 1,000 & 0,940 & 0,560 & 0,424 & 0,342 & 0,145 & 0,194 & 35 & 459 & $-1,8$ \\
\hline
\end{tabular}




\begin{tabular}{|c|c|c|c|c|c|c|c|c|c|c|c|c|c|c|c|c|c|c|}
\hline 31 & 459 & 0,160 & 2,362 & 0,258 & 1,957 & 0,618 & 0,828 & 0,419 & 0,359 & 1,668 & 0,243 & 0,266 & 0,914 & 0,243 & 0,274 & 157 & 459 & $-3,2$ \\
\hline 32 & 459 & 0,061 & 0,940 & 0,084 & 0,665 & 0,722 & 0,707 & 0,404 & 0,867 & 1,074 & 0,662 & 0,600 & 0,342 & 0,205 & 0,137 & 92 & 459 & $-1,8$ \\
\hline 33 & 459 & 0,047 & 0,795 & 0,056 & 0,443 & 0,833 & 0,558 & 0,473 & 0,933 & 1,035 & 0,439 & 0,250 & 0,369 & 0,092 & 0,137 & 28 & 459 & $-1,9$ \\
\hline 34 & 459 & 0,056 & 1,162 & 0,084 & 0,902 & 0,667 & 0,776 & 0,401 & 0,524 & 1,381 & 0,294 & 0,308 & 0,494 & 0,152 & 0,137 & 33 & 459 & $-2,3$ \\
\hline 35 & 459 & 0,080 & 1,422 & 0,127 & 1,238 & 0,630 & 0,871 & 0,392 & 0,496 & 1,420 & 0,223 & 0,214 & 0,675 & 0,145 & 0,137 & 15 & 459 & $-2,8$ \\
\hline 36 & 459 & 0,905 & 3,768 & 0,971 & 3,455 & 0,932 & 0,917 & 0,107 & 0,801 & 1,117 & 0,471 & 0,488 & 1,565 & 0,764 & 0,685 & 72 & 459 & $-4,0$ \\
\hline 37 & 459 & 0,150 & 1,384 & 0,164 & 1,238 & 0,914 & 0,895 & 0,136 & 0,986 & 1,007 & 0,479 & 0,369 & 0,632 & 0,233 & 0,306 & 53 & 459 & $-2,7$ \\
\hline 38 & 459 & 0,737 & 4,005 & 0,901 & 3,508 & 0,818 & 0,876 & 0,221 & 0,577 & 1,317 & 0,383 & 0,414 & 1,565 & 0,648 & 0,613 & 69 & 459 & $-4,0$ \\
\hline 39 & 459 & 0,052 & 1,216 & 0,094 & 0,795 & 0,550 & 0,654 & 0,568 & 0,439 & 1,510 & 0,264 & 0,321 & 0,499 & 0,160 & 0,137 & 0 & 459 & $-2,3$ \\
\hline 40 & 459 & 0,061 & 1,016 & 0,061 & 0,481 & 1,000 & 0,474 & 0,526 & 0,742 & 1,161 & 0,828 & 0,671 & 0,306 & 0,205 & 0,194 & 72 & 459 & $-1,6$ \\
\hline 41 & 459 & 0,056 & 1,070 & 0,070 & 0,940 & 0,800 & 0,879 & 0,234 & 0,618 & 1,272 & 0,263 & 0,176 & 0,522 & 0,092 & 0,137 & 23 & 459 & $-2,4$ \\
\hline 42 & 459 & 0,103 & 1,292 & 0,117 & 0,978 & 0,880 & 0,757 & 0,271 & 0,777 & 1,134 & 0,350 & 0,300 & 0,613 & 0,184 & 0,274 & 59 & 459 & $-2,6$ \\
\hline 43 & 459 & 0,164 & 2,064 & 0,211 & 1,896 & 0,778 & 0,919 & 0,237 & 0,484 & 1,437 & 0,214 & 0,190 & 0,988 & 0,187 & 0,194 & 36 & 459 & $-3,3$ \\
\hline 44 & 459 & 0,061 & 1,032 & 0,080 & 0,848 & 0,765 & 0,822 & 0,295 & 0,720 & 1,179 & 0,318 & 0,262 & 0,494 & 0,129 & 0,137 & 69 & 459 & $-2,3$ \\
\hline 45 & 459 & 0,052 & 0,665 & 0,052 & 0,443 & 1,000 & 0,667 & 0,333 & 1,000 & 0,826 & 0,700 & 0,447 & 0,306 & 0,137 & 0,194 & 72 & 459 & $-1,6$ \\
\hline 46 & 459 & 0,075 & 1,216 & 0,099 & 1,032 & 0,762 & 0,849 & 0,282 & 0,638 & 1,252 & 0,334 & 0,272 & 0,535 & 0,145 & 0,194 & 40 & 459 & $-2,4$ \\
\hline 47 & 459 & 0,052 & 0,887 & 0,061 & 0,665 & 0,846 & 0,750 & 0,294 & 0,825 & 1,101 & 0,412 & 0,286 & 0,399 & 0,114 & 0,137 & 63 & 459 & $-2,0$ \\
\hline 48 & 459 & 0,084 & 1,254 & 0,108 & 1,070 & 0,783 & 0,854 & 0,262 & 0,675 & 1,217 & 0,353 & 0,379 & 0,552 & 0,209 & 0,274 & 70 & 459 & $-2,5$ \\
\hline 49 & 459 & 0,450 & 2,324 & 0,455 & 2,270 & 0,990 & 0,977 & 0,025 & 1,000 & 0,977 & 0,633 & 0,559 & 0,952 & 0,532 & 0,548 & 35 & 459 & $-3,3$ \\
\hline 50 & 459 & 0,253 & 2,216 & 0,319 & 1,628 & 0,794 & 0,734 & 0,336 & 0,648 & 1,242 & 0,506 & 0,514 & 0,799 & 0,411 & 0,387 & 17 & 459 & $-3,0$ \\
\hline 51 & 459 & 0,131 & 1,108 & 0,141 & 0,887 & 0,933 & 0,800 & 0,211 & 1,000 & 0,863 & 1,049 & 0,844 & 0,399 & 0,337 & 0,387 & 17 & 459 & $-2,0$ \\
\hline 52 & 459 & 0,094 & 1,200 & 0,127 & 1,016 & 0,741 & 0,847 & 0,301 & 0,819 & 1,105 & 0,566 & 0,539 & 0,459 & 0,248 & 0,274 & 103 & 459 & $-2,2$ \\
\hline 53 & 459 & 0,075 & 1,330 & 0,122 & 1,070 & 0,615 & 0,805 & 0,431 & 0,533 & 1,369 & 0,334 & 0,393 & 0,535 & 0,210 & 0,194 & 36 & 459 & $-2,4$ \\
\hline 54 & 459 & 0,089 & 0,940 & 0,099 & 0,810 & 0,905 & 0,862 & 0,168 & 1,000 & 0,888 & 0,834 & 0,567 & 0,369 & 0,209 & 0,274 & 32 & 459 & $-1,9$ \\
\hline 55 & 459 & 0,150 & 2,118 & 0,211 & 1,950 & 0,711 & 0,921 & 0,300 & 0,421 & 1,542 & 0,184 & 0,179 & 1,018 & 0,183 & 0,194 & 41 & 459 & $-3,3$ \\
\hline 56 & 459 & 0,080 & 0,887 & 0,089 & 0,627 & 0,895 & 0,707 & 0,311 & 1,000 & 0,886 & 0,676 & 0,491 & 0,387 & 0,190 & 0,274 & 38 & 459 & $-2,0$ \\
\hline 57 & 459 & 0,249 & 1,865 & 0,286 & 1,643 & 0,869 & 0,881 & 0,177 & 0,898 & 1,055 & 0,750 & 0,724 & 0,650 & 0,471 & 0,411 & 116 & 459 & $-2,7$ \\
\hline 58 & 459 & 0,066 & 0,887 & 0,070 & 0,719 & 0,933 & 0,811 & 0,201 & 1,000 & 0,976 & 0,615 & 0,499 & 0,369 & 0,184 & 0,194 & 120 & 459 & $-1,9$ \\
\hline 59 & 459 & 0,047 & 1,093 & 0,061 & 0,573 & 0,769 & 0,525 & 0,528 & 0,494 & 1,423 & 0,199 & 0,125 & 0,548 & 0,068 & 0,137 & 0 & 459 & $-2,5$ \\
\hline 60 & 459 & 0,113 & 1,552 & 0,150 & 0,925 & 0,750 & 0,596 & 0,475 & 0,588 & 1,304 & 0,679 & 0,596 & 0,459 & 0,274 & 0,274 & 87 & 459 & $-2,2$ \\
\hline 61 & 459 & 0,291 & 3,264 & 0,394 & 2,469 & 0,738 & 0,757 & 0,358 & 0,343 & 1,707 & 0,272 & 0,357 & 1,166 & 0,417 & 0,306 & 52 & 459 & $-3,5$ \\
\hline 62 & 459 & 0,047 & 0,757 & 0,056 & 0,535 & 0,833 & 0,707 & 0,337 & 1,000 & 0,986 & 0,509 & 0,358 & 0,342 & 0,123 & 0,137 & 50 & 459 & $-1,8$ \\
\hline 63 & 459 & 0,521 & 2,752 & 0,558 & 2,584 & 0,933 & 0,939 & 0,091 & 0,864 & 1,076 & 0,487 & 0,498 & 1,166 & 0,581 & 0,411 & 51 & 459 & $-3,5$ \\
\hline 64 & 459 & 0,943 & 4,334 & 1,074 & 4,135 & 0,878 & 0,954 & 0,131 & 0,631 & 1,259 & 0,371 & 0,362 & 1,798 & 0,651 & 0,565 & 72 & 459 & $-4,2$ \\
\hline 65 & 459 & 0,084 & 1,307 & 0,113 & 0,940 & 0,750 & 0,719 & 0,376 & 0,621 & 1,269 & 0,432 & 0,381 & 0,499 & 0,190 & 0,194 & 63 & 459 & $-2,3$ \\
\hline 66 & 459 & 0,061 & 0,994 & 0,075 & 0,940 & 0,812 & 0,946 & 0,195 & 0,776 & 1,135 & 0,404 & 0,331 & 0,439 & 0,145 & 0,194 & 116 & 459 & $-2,1$ \\
\hline 67 & 459 & 0,216 & 1,773 & 0,253 & 1,513 & 0,852 & 0,854 & 0,208 & 0,863 & 1,077 & 0,518 & 0,497 & 0,728 & 0,362 & 0,387 & 36 & 459 & $-2,9$ \\
\hline 68 & 459 & 0,131 & 2,637 & 0,253 & 1,513 & 0,519 & 0,574 & 0,643 & 0,237 & 2,053 & 0,315 & 0,533 & 0,728 & 0,388 & 0,137 & 167 & 459 & $-2,9$ \\
\hline 69 & 459 & 0,047 & 0,978 & 0,066 & 0,719 & 0,714 & 0,735 & 0,390 & 0,616 & 1,274 & 0,311 & 0,260 & 0,439 & 0,114 & 0,137 & 90 & 459 & $-2,1$ \\
\hline 70 & 459 & 0,047 & 0,887 & 0,056 & 0,573 & 0,833 & 0,646 & 0,391 & 0,750 & 1,155 & 0,344 & 0,209 & 0,417 & 0,087 & 0,137 & 160 & 459 & $-2,1$ \\
\hline 71 & 459 & 0,483 & 3,302 & 0,544 & 2,821 & 0,888 & 0,854 & 0,184 & 0,557 & 1,340 & 0,303 & 0,288 & 1,425 & 0,410 & 0,411 & 56 & 459 & $-3,8$ \\
\hline 72 & 459 & 0,080 & 1,032 & 0,084 & 0,848 & 0,944 & 0,822 & 0,186 & 0,941 & 1,031 & 0,416 & 0,294 & 0,494 & 0,145 & 0,194 & 54 & 459 & $-2,3$ \\
\hline 73 & 459 & 1,131 & 4,471 & 1,290 & 4,028 & 0,876 & 0,901 & 0,158 & 0,711 & 1,186 & 0,440 & 0,518 & 1,808 & 0,936 & 0,919 & 80 & 459 & $-4,2$ \\
\hline 74 & 459 & 0,056 & 0,848 & 0,066 & 0,627 & 0,857 & 0,739 & 0,298 & 0,983 & 1,009 & 0,588 & 0,392 & 0,349 & 0,137 & 0,194 & 18 & 459 & $-1,8$ \\
\hline 75 & 459 & 0,056 & 1,399 & 0,108 & 0,810 & 0,522 & 0,579 & 0,637 & 0,361 & 1,664 & 0,312 & 0,511 & 0,479 & 0,245 & 0,137 & 0 & 459 & $-2,3$ \\
\hline 76 & 459 & 0,047 & 0,665 & 0,052 & 0,535 & 0,909 & 0,805 & 0,215 & 1,000 & 0,866 & 0,637 & 0,400 & 0,306 & 0,123 & 0,137 & 18 & 459 & $-1,6$ \\
\hline 77 & 459 & 0,366 & 2,469 & 0,432 & 2,118 & 0,848 & 0,858 & 0,208 & 0,754 & 1,152 & 0,633 & 0,656 & 0,858 & 0,563 & 0,565 & 137 & 459 & $-3,1$ \\
\hline 78 & 459 & 0,099 & 0,963 & 0,103 & 0,665 & 0,955 & 0,691 & 0,313 & 1,000 & 0,865 & 0,922 & 0,557 & 0,369 & 0,205 & 0,274 & 0 & 459 & $-1,9$ \\
\hline 79 & 459 & 0,127 & 1,070 & 0,127 & 0,978 & 1,000 & 0,914 & 0,086 & 1,000 & 0,848 & 0,929 & 0,658 & 0,417 & 0,274 & 0,387 & 177 & 459 & $-2,1$ \\
\hline 80 & 459 & 0,061 & 0,810 & 0,061 & 0,665 & 1,000 & 0,820 & 0,180 & 1,000 & 0,926 & 0,517 & 0,250 & 0,387 & 0,097 & 0,194 & 45 & 459 & $\begin{array}{l}-2,0 \\
\end{array}$ \\
\hline 81 & 459 & 0,136 & 1,621 & 0,150 & 1,437 & 0,906 & 0,887 & 0,147 & 0,651 & 1,240 & 0,327 & 0,266 & 0,728 & 0,194 & 0,306 & 41 & 459 & $-2,9$ \\
\hline 82 & 459 & 0,094 & 1,016 & 0,099 & 0,848 & 0,952 & 0,835 & 0,172 & 1,000 & 0,936 & 0,566 & 0,447 & 0,459 & 0,205 & 0,274 & 17 & 459 & $-2,2$ \\
\hline 83 & 459 & 0,056 & 0,757 & 0,066 & 0,627 & 0,857 & 0,828 & 0,223 & 1,000 & 0,900 & 0,611 & 0,389 & 0,342 & 0,133 & 0,137 & 32 & 459 & $-1,8$ \\
\hline 84 & 459 & 0,047 & 0,573 & 0,047 & 0,390 & 1,000 & 0,680 & 0,320 & 1,000 & 0,746 & 0,979 & 0,555 & 0,247 & 0,137 & 0,194 & 72 & 459 & $-1,3$ \\
\hline 85 & 459 & 0,061 & 1,086 & 0,089 & 0,810 & 0,684 & 0,746 & 0,405 & 0,650 & 1,240 & 0,404 & 0,347 & 0,439 & 0,152 & 0,137 & 125 & 459 & $-2,1$ \\
\hline 86 & 459 & 0,066 & 0,848 & 0,066 & 0,665 & 1,000 & 0,784 & 0,216 & 1,000 & 0,934 & 0,557 & 0,375 & 0,387 & 0,145 & 0,194 & 45 & 459 & $-2,0$ \\
\hline 87 & 459 & 0,075 & 0,940 & 0,089 & 0,757 & 0,842 & 0,805 & 0,251 & 1,000 & 0,968 & 0,637 & 0,491 & 0,387 & 0,190 & 0,194 & 55 & 459 & $-2,0$ \\
\hline 88 & 459 & 0,047 & 0,795 & 0,056 & 0,443 & 0,833 & 0,558 & 0,473 & 0,933 & 1,035 & 0,490 & 0,373 & 0,349 & 0,130 & 0,137 & 108 & 459 & $-1,8$ \\
\hline
\end{tabular}




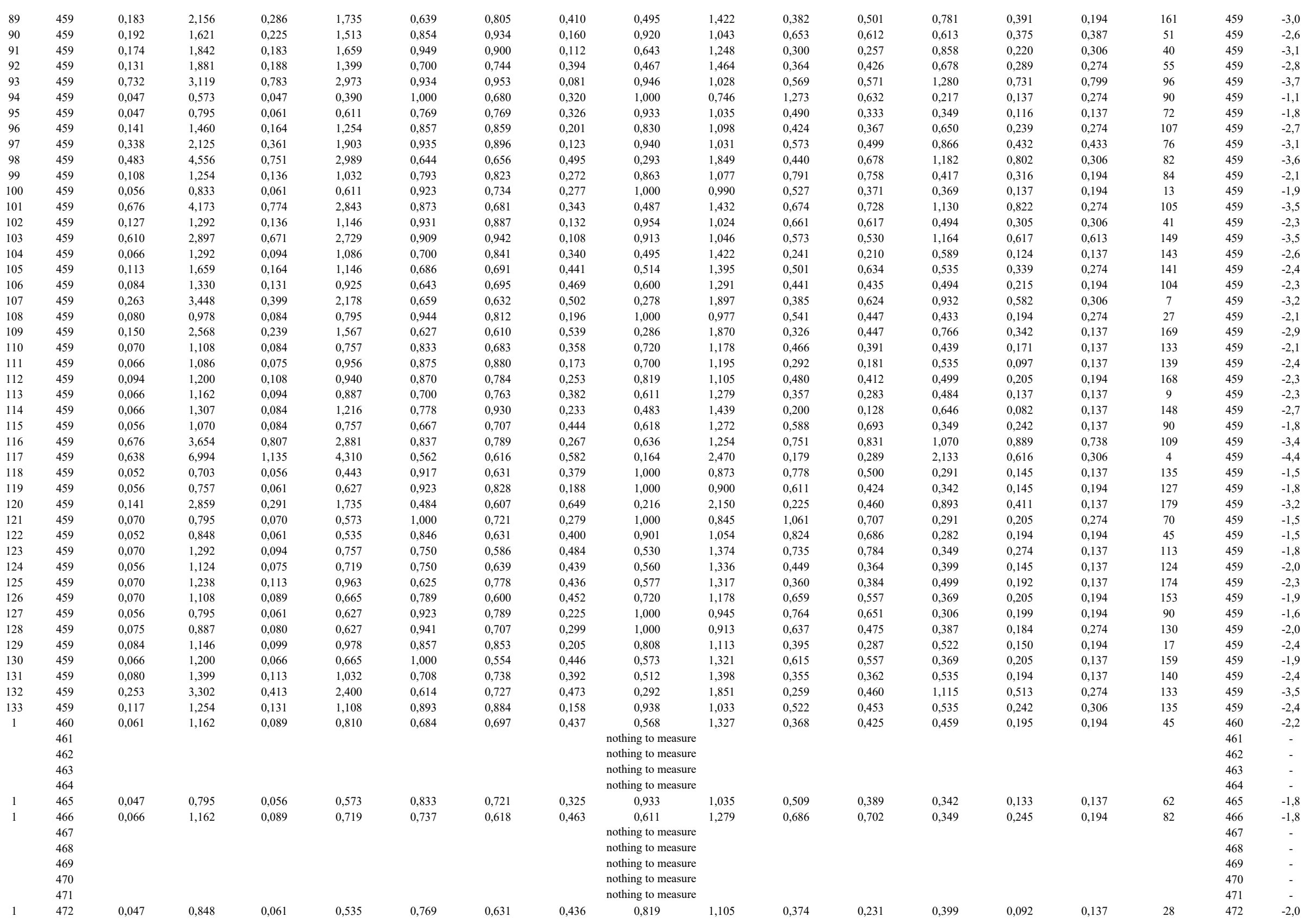


nothing to measure

nothing to measure

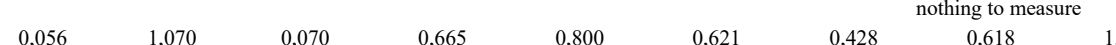

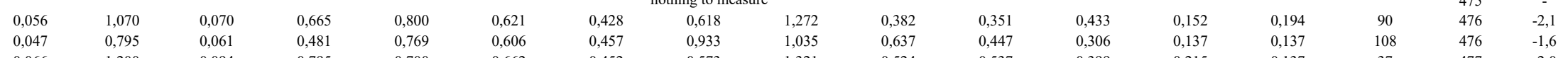

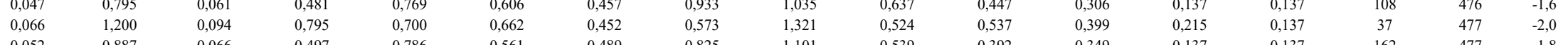

\section{nothing to measure}

nothing to measure

nothing to measure

nothing to measure

$\begin{array}{llllll}0,061 & 1,016 & 0,070 & 0,665 & 0,867 & 0,654\end{array}$

0,371

nothing to measu

nothing to measure

nothing to measure

$\begin{array}{llllll}0,150 & 1,811 & 0,220 & 1,460 & 0,681 & 0,806\end{array}$

$\begin{array}{lll}0,374 & 0,575 & 1,319\end{array}$

0,398

nothing to measure

$\begin{array}{llllll}0,108 & 1,016 & 0,108 & 0,757 & 1,000 & 0,744 \\ 0,047 & 0,665 & 0,052 & 0,352 & 0,909 & 0,529\end{array}$

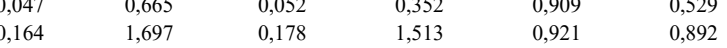

nothing to measure
nothing to measure 
nothing to measure

nothing to measure

$\begin{array}{llllllll}0,047 & 1,124 & 0,070 & 0,589 & 0,667 & 0,524 & 0,581 & 0,467\end{array}$

$0,909-0,650$

$\begin{array}{lll}0,362 & 1,000 & 1,464 \\ 0,965\end{array}$

1,464
0,965
1,006

$0,490 \quad 0,424$

$0,560-0,349$
0,360

$\begin{array}{lll}0,349 & 0,145 & 0,137 \\ 0,068 & 0,137\end{array}$

$\begin{array}{ll}0,987 & 1,006 \\ 0,433 & 1,519\end{array}$

$\begin{array}{lll}0,397 & 0,348 & 1,695 \\ 0,297 & 0,323 & 1,759\end{array}$

$\begin{array}{llll}0,474 & 4,135 & 0,713 & 3,255 \\ 0,150 & 2,416 & 0,211 & 2,248\end{array}$

$\begin{array}{llll}0,052 & 0,848 & 0,061 & 0,719\end{array}$

$0,664 \quad 0,78$

$\begin{array}{ll}0,711 & 0,930 \\ 0,846 & 0,847\end{array}$

0,297

0,323

1,054

$\begin{array}{lll}0,241 & 0,303 & 1,581\end{array}$

$0,412-0,131$
0,350

0,354
0,223
0,234

0,677

1,172
1,344
1,085

0,406

0,371

0,433

0,866

$\begin{array}{llll}0,188 & 2,064 & 0,230 & 1,766 \\ 0,047 & 0,833 & 0,052 & 0,260 \\ 0,127 & 1,491 & 0,136 & 1,475\end{array}$

$\begin{array}{llll}0,127 & 1,491 & 0,136 & 1,475 \\ 0,075 & 1,016 & 0,080 & 0,63\end{array}$

$\quad 0,856$

$0,931 \quad 0,990$

0,694

$\begin{array}{ll}0,342 & 0,348 \\ 0,354 & 0,167\end{array}$

$\begin{array}{ll}0,716 & 1,182 \\ 0,913 & 1,047\end{array}$

0,384

$\begin{array}{ll}0,167 & 0,411 \\ 0,275 & 0,705\end{array}$

nothing to measure

nothing to measure

$0,275 \quad 0,499$

$\begin{array}{llllll}0,047 & 0,703 & 0,052 & 0,352 & 0,909 & 0,500 \\ 0,052 & 1,093 & 0,066 & 0,573 & 0,86\end{array}$

0,508

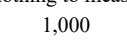




\begin{tabular}{|c|c|c|c|c|c|c|c|c|c|c|c|c|c|c|c|c|c|c|}
\hline 24 & 513 & 0,066 & 0,902 & 0,070 & 0,719 & 0,933 & 0,797 & 0,214 & 1,000 & 0,993 & 0,615 & 0,499 & 0,369 & 0,184 & 0,194 & 21 & 513 & $-1,9$ \\
\hline 25 & 513 & 0,056 & 0,795 & 0,061 & 0,665 & 0,923 & 0,837 & 0,181 & 1,000 & 0,945 & 0,527 & 0,332 & 0,369 & 0,123 & 0,137 & 21 & 513 & $-1,9$ \\
\hline 26 & 513 & 0,089 & 1,162 & 0,094 & 0,978 & 0,950 & 0,842 & 0,166 & 0,830 & 1,098 & 0,372 & 0,278 & 0,552 & 0,153 & 0,194 & 27 & 513 & $-2,5$ \\
\hline 27 & 513 & 0,089 & 1,162 & 0,103 & 0,978 & 0,864 & 0,842 & 0,209 & 0,830 & 1,098 & 0,397 & 0,272 & 0,535 & 0,145 & 0,194 & 38 & 513 & $-2,4$ \\
\hline 28 & 513 & 0,084 & 0,848 & 0,084 & 0,757 & 1,000 & 0,892 & 0,108 & 1,000 & 0,824 & 1,146 & 0,791 & 0,306 & 0,242 & 0,306 & 135 & 513 & $-1,6$ \\
\hline 29 & 513 & 0,047 & 0,665 & 0,052 & 0,573 & 0,909 & 0,862 & 0,165 & 1,000 & 0,866 & 0,637 & 0,447 & 0,306 & 0,137 & 0,194 & 108 & 513 & $-1,6$ \\
\hline 30 & 513 & 0,066 & 0,994 & 0,070 & 0,902 & 0,933 & 0,908 & 0,114 & 0,835 & 1,094 & 0,357 & 0,200 & 0,484 & 0,097 & 0,194 & 43 & 513 & $-2,3$ \\
\hline 31 & 513 & 0,164 & 1,903 & 0,206 & 1,529 & 0,795 & 0,803 & 0,284 & 0,570 & 1,325 & 0,284 & 0,322 & 0,858 & 0,276 & 0,274 & 35 & 513 & $-3,1$ \\
\hline 32 & 513 & 0,249 & 3,486 & 0,422 & 1,995 & 0,589 & 0,572 & 0,593 & 0,257 & 1,972 & 0,519 & 0,757 & 0,781 & 0,591 & 0,274 & 23 & 513 & $-3,0$ \\
\hline 33 & 513 & 0,089 & 1,184 & 0,108 & 0,925 & 0,826 & 0,781 & 0,280 & 0,798 & 1,119 & 0,456 & 0,384 & 0,499 & 0,192 & 0,274 & 96 & 513 & $-2,3$ \\
\hline 34 & 513 & 0,136 & 1,384 & 0,145 & 1,292 & 0,935 & 0,934 & 0,093 & 0,893 & 1,058 & 0,462 & 0,450 & 0,613 & 0,276 & 0,306 & 29 & 513 & $-2,6$ \\
\hline 35 & 513 & 0,328 & 2,416 & 0,375 & 2,210 & 0,875 & 0,915 & 0,151 & 0,707 & 1,189 & 0,336 & 0,304 & 1,115 & 0,339 & 0,387 & 45 & 513 & $-3,5$ \\
\hline 36 & 513 & 0,075 & 1,162 & 0,084 & 0,994 & 0,889 & 0,855 & 0,182 & 0,699 & 1,196 & 0,313 & 0,223 & 0,552 & 0,123 & 0,137 & 31 & 513 & $-2,5$ \\
\hline 37 & 513 & 0,047 & 0,810 & 0,047 & 0,665 & 1,000 & 0,820 & 0,180 & 0,898 & 1,055 & 0,398 & 0,250 & 0,387 & 0,097 & 0,137 & 45 & 513 & $-2,0$ \\
\hline \multirow[t]{7}{*}{38} & 513 & 0,056 & 0,810 & 0,061 & 0,665 & 0,923 & 0,820 & 0,195 & 1,000 & 0,964 & 0,477 & 0,250 & 0,387 & 0,097 & 0,194 & 50 & 513 & $-2,0$ \\
\hline & 514 & & & & & & & & nothing to measure & & & & & & & & 514 & - \\
\hline & 515 & & & & & & & & nothing to measure & & & & & & & & 515 & - \\
\hline & 516 & & & & & & & & nothing to measure & & & & & & & & 516 & - \\
\hline & 517 & & & & & & & & nothing to measure & & & & & & & & 517 & - \\
\hline & 518 & & & & & & & & nothing to measure & & & & & & & & 518 & - \\
\hline & 519 & & & & & & & & nothing to measure & & & & & & & & 519 & - \\
\hline 1 & 520 & 0,169 & 1,957 & 0,230 & 1,567 & 0,735 & 0,801 & 0,332 & 0,554 & 1,343 & 0,395 & 0,510 & 0,738 & 0,377 & 0,194 & 2 & 520 & $-2,9$ \\
\hline 2 & 520 & 0,070 & 0,978 & 0,094 & 0,795 & 0,750 & 0,812 & 0,313 & 0,924 & 1,040 & 0,659 & 0,557 & 0,369 & 0,205 & 0,194 & 5 & 520 & $-1,9$ \\
\hline 3 & 520 & 0,066 & 0,833 & 0,075 & 0,665 & 0,875 & 0,798 & 0,237 & 1,000 & 0,917 & 0,713 & 0,537 & 0,342 & 0,184 & 0,274 & 167 & 520 & $-1,8$ \\
\hline \multirow[t]{3}{*}{4} & 520 & 0,136 & 1,789 & 0,174 & 1,146 & 0,784 & 0,641 & 0,419 & 0,534 & 1,368 & 0,499 & 0,581 & 0,589 & 0,342 & 0,274 & 166 & 520 & $-2,6$ \\
\hline & 521 & & & & & & & & nothing to measure & & & & & & & & 521 & - \\
\hline & 522 & & & & & & & & nothing to measure & & & & & & & & 522 & - \\
\hline 1 & 523 & 0,352 & 2,675 & 0,441 & 2,308 & 0,798 & 0,863 & 0,244 & 0,618 & 1,272 & 0,412 & 0,479 & 1,043 & 0,500 & 0,433 & 164 & 523 & $-3,4$ \\
\hline 2 & 523 & 0,052 & 0,887 & 0,066 & 0,573 & 0,786 & 0,646 & 0,413 & 0,825 & 1,101 & 1,077 & 0,832 & 0,247 & 0,205 & 0,137 & 135 & 523 & $-1,3$ \\
\hline 3 & 523 & 0,183 & 1,590 & 0,216 & 1,330 & 0,848 & 0,837 & 0,223 & 0,910 & 1,048 & 0,690 & 0,746 & 0,581 & 0,433 & 0,411 & 49 & 523 & $-2,5$ \\
\hline 4 & 523 & 0,160 & 2,125 & 0,230 & 1,643 & 0,694 & 0,773 & 0,381 & 0,444 & 1,501 & 0,316 & 0,335 & 0,802 & 0,269 & 0,194 & 16 & 523 & $-3,0$ \\
\hline 5 & 523 & 0,047 & 0,757 & 0,052 & 0,535 & 0,909 & 0,707 & 0,307 & 1,000 & 0,986 & 0,509 & 0,358 & 0,342 & 0,123 & 0,137 & 144 & 523 & $-1,8$ \\
\hline 6 & 523 & 0,056 & 0,795 & 0,061 & 0,535 & 0,923 & 0,673 & 0,336 & 1,000 & 0,945 & 0,527 & 0,353 & 0,369 & 0,130 & 0,137 & 101 & 523 & $\begin{array}{r}-1,9 \\
\end{array}$ \\
\hline 7 & 523 & 0,652 & 6,396 & 1,023 & 5,631 & 0,638 & 0,880 & 0,382 & 0,200 & 2,234 & 0,096 & 0,116 & 2,945 & 0,342 & 0,387 & 2 & 523 & $-4,9$ \\
\hline 8 & 523 & 0,277 & 2,897 & 0,422 & 2,239 & 0,656 & 0,773 & 0,413 & 0,414 & 1,553 & 0,366 & 0,489 & 0,981 & 0,479 & 0,306 & 0 & 523 & $-3,3$ \\
\hline 9 & 523 & 0,066 & 1,222 & 0,080 & 0,833 & 0,824 & 0,681 & 0,364 & 0,552 & 1,346 & 0,217 & 0,110 & 0,620 & 0,068 & 0,137 & 0 & 523 & $-2,6$ \\
\hline 10 & 523 & 0,052 & 0,665 & 0,056 & 0,443 & $\begin{array}{l}0,917 \\
0,917\end{array}$ & 0,667 & 0,344 & 1,000 & 0,826 & 1,077 & 0,784 & 0,247 & 0,194 & 0,194 & 90 & 523 & $\begin{array}{l}-2,0 \\
-1,3\end{array}$ \\
\hline 11 & 523 & 0,164 & 2,324 & 0,220 & 1,957 & 0,745 & 0,842 & 0,300 & 0,382 & 1,618 & 0,217 & 0,219 & 0,981 & 0,215 & 0,274 & 23 & 523 & $-3,3$ \\
\hline 12 & 523 & 0,061 & 0,772 & 0,066 & 0,627 & 0,929 & 0,812 & 0,201 & 1,000 & 0,882 & 0,828 & 0,474 & 0,306 & 0,145 & 0,194 & 121 & 523 & $-1,6$ \\
\hline 13 & 523 & 0,155 & 1,330 & 0,174 & 1,162 & 0,892 & 0,874 & 0,166 & 1,000 & 0,953 & 0,793 & 0,666 & 0,499 & 0,332 & 0,387 & 175 & 523 & $-2,3$ \\
\hline 14 & 523 & 0,080 & 0,848 & 0,084 & 0,665 & 0,944 & 0,784 & 0,223 & 1,000 & 0,848 & 1,082 & 0,791 & 0,306 & 0,242 & 0,306 & 138 & 523 & $-1,6$ \\
\hline 15 & 523 & 0,122 & 2,026 & 0,160 & 1,858 & 0,765 & 0,917 & 0,249 & 0,373 & 1,636 & 0,164 & 0,165 & 0,973 & 0,161 & 0,194 & 126 & 523 & $-3,3$ \\
\hline 16 & 523 & 0,216 & 2,507 & 0,296 & 1,934 & 0,730 & 0,771 & 0,354 & 0,431 & $\begin{array}{l}1,523 \\
\text { S }\end{array}$ & 0,303 & 0,361 & 0,952 & 0,343 & 0,274 & 141 & 523 & $-3,3$ \\
\hline 17 & 523 & 0,056 & 0,902 & 0,070 & 0,611 & 0,800 & 0,677 & 0,379 & 0,869 & 1,073 & 0,527 & 0,465 & 0,369 & 0,171 & 0,194 & 156 & 523 & $-1,9$ \\
\hline \multirow[t]{7}{*}{18} & 523 & 0,070 & 1,108 & 0,089 & 0,940 & 0,789 & 0,848 & 0,259 & 0,720 & 1,178 & 0,329 & 0,220 & 0,522 & 0,115 & 0,137 & 112 & 523 & $-2,4$ \\
\hline & 524 & & & & & & & & nothing to measure & & & & & & & & 524 & - \\
\hline & 525 & & & & & & & & nothing to measure & & & & & & & & 525 & - \\
\hline & 526 & & & & & & & & nothing to measure & & & & & & & & 526 & - \\
\hline & 527 & & & & & & & & nothing to measure & & & & & & & & 527 & - \\
\hline & 528 & & & & & & & & nothing to measure & & & & & & & & 528 & - \\
\hline & 529 & & & & & & & & nothing to measure & & & & & & & & 529 & - \\
\hline \multirow[t]{2}{*}{1} & 530 & 0,047 & 1,124 & 0,075 & 0,573 & 0,625 & 0,510 & 0,617 & 0,467 & 1,464 & 0,374 & 0,319 & 0,399 & 0,127 & 0,137 & 105 & 530 & $-2,0$ \\
\hline & 531 & & & & & & & & nothing to measure & & & & & & & & 531 & - \\
\hline \multirow[t]{4}{*}{1} & 532 & 0,061 & 0,887 & 0,070 & 0,390 & 0,867 & 0,439 & 0,576 & 0,975 & 1,013 & 0,828 & 0,447 & 0,306 & 0,137 & 0,194 & 4 & 532 & $-1,6$ \\
\hline & 533 & & & & & & & & nothing to measure & & & & & & & & 533 & - \\
\hline & 534 & & & & & & & & nothing to measure & & & & & & & & 534 & - \\
\hline & 535 & & & & & & & & nothing to measure & & & & & & & & 535 & - \\
\hline 1 & 536 & 0,127 & 2,048 & 0,183 & 1,437 & 0,692 & 0,702 & 0,429 & 0,379 & 1,624 & 0,251 & 0,344 & 0,802 & 0,276 & 0,194 & 45 & 536 & $-3,0$ \\
\hline
\end{tabular}




\begin{tabular}{|c|c|c|c|c|c|c|c|c|c|c|c|c|c|c|c|c|}
\hline 0,070 & 1,254 & 0,094 & 1,032 & 0,750 & 0,823 & 0,306 & 0,563 & 1,333 & 0,294 & 0,298 & 0,552 & 0,165 & 0,194 & 30 & 536 & $-2,5$ \\
\hline 0,047 & 0,665 & 0,047 & 0,481 & 1,000 & 0,724 & 0,276 & 1,000 & 0,866 & 0,637 & 0,400 & 0,306 & 0,123 & 0,137 & 69 & 536 & $-1,6$ \\
\hline 0,066 & 1,751 & 0,122 & 1,307 & 0,538 & 0,747 & 0,526 & 0,269 & 1,927 & 0,158 & 0,219 & 0,728 & 0,159 & 0,137 & 61 & 536 & $-2,9$ \\
\hline 0,094 & 1,055 & 0,113 & 0,703 & 0,833 & 0,667 & 0,373 & 1,000 & 0,971 & 0,796 & 0,707 & 0,387 & 0,274 & 0,274 & 150 & 536 & $-2,0$ \\
\hline 0,220 & 3,172 & 0,422 & 1,919 & 0,522 & 0,605 & 0,620 & 0,275 & 1,906 & 0,402 & 0,672 & 0,836 & 0,562 & 0,137 & 18 & 536 & $-3,1$ \\
\hline 0,056 & 1,437 & 0,094 & 1,086 & 0,600 & 0,755 & 0,469 & 0,342 & 1,709 & 0,153 & 0,120 & 0,685 & 0,082 & 0,137 & 0 & 536 & $-2,8$ \\
\hline 0,056 & 0,925 & 0,070 & 0,573 & 0,800 & 0,620 & 0,430 & 0,827 & 1,099 & 0,382 & 0,250 & 0,433 & 0,108 & 0,137 & 75 & 536 & $-2,1$ \\
\hline 0,197 & 2,545 & 0,281 & 2,087 & 0,700 & 0,820 & 0,350 & 0,382 & 1,618 & 0,231 & 0,240 & 1,043 & 0,251 & 0,194 & 66 & 536 & $-3,4$ \\
\hline 0,253 & 4,144 & 0,493 & 3,318 & 0,514 & 0,801 & 0,525 & 0,185 & 2,322 & 0,111 & 0,162 & 1,701 & 0,275 & 0,194 & 58 & 536 & $-4,1$ \\
\hline 0,066 & 1,216 & 0,094 & 0,994 & 0,700 & 0,818 & 0,351 & 0,559 & 1,338 & 0,248 & 0,242 & 0,581 & 0,141 & 0,137 & 36 & 536 & $-2,5$ \\
\hline 0,253 & 4,885 & 0,643 & 3,463 & 0,394 & 0,709 & 0,672 & 0,133 & 2,738 & 0,109 & 0,258 & 1,716 & 0,443 & 0,274 & 59 & 536 & $-4,1$ \\
\hline 0,230 & 2,087 & 0,272 & 1,735 & 0,845 & 0,832 & 0,229 & 0,663 & 1,228 & 0,367 & 0,383 & 0,893 & 0,342 & 0,306 & 1 & 536 & $-3,2$ \\
\hline 0,066 & 0,925 & 0,075 & 0,703 & 0,875 & 0,760 & 0,270 & 0,965 & 1,018 & 0,482 & 0,319 & 0,417 & 0,133 & 0,137 & 169 & 536 & $-2,1$ \\
\hline 0,099 & 1,330 & 0,131 & 0,887 & 0,750 & 0,667 & 0,417 & 0,700 & 1,195 & 0,668 & 0,728 & 0,433 & 0,315 & 0,274 & 118 & 536 & $-2,1$ \\
\hline 0,052 & 0,994 & 0,061 & 0,902 & 0,846 & 0,908 & 0,179 & 0,656 & 1,234 & 0,269 & 0,187 & 0,494 & 0,092 & 0,137 & 32 & 536 & $-2,3$ \\
\hline 0,066 & 1,368 & 0,108 & 0,978 & 0,609 & 0,715 & 0,484 & 0,441 & 1,506 & 0,210 & 0,206 & 0,632 & 0,130 & 0,137 & 158 & 536 & $-2,7$ \\
\hline 0,080 & 1,475 & 0,117 & 1,032 & 0,680 & 0,700 & 0,439 & 0,460 & 1,474 & 0,355 & 0,452 & 0,535 & 0,242 & 0,137 & 131 & 536 & $-2,4$ \\
\hline 0,202 & 2,644 & 0,314 & 2,239 & 0,642 & 0,847 & 0,390 & 0,363 & 1,661 & 0,189 & 0,235 & 1,166 & 0,274 & 0,194 & 86 & 536 & $-3,5$ \\
\hline 0,052 & 0,795 & 0,061 & 0,611 & 0,846 & 0,769 & 0,278 & 1,000 & 0,987 & 0,483 & 0,332 & 0,369 & 0,123 & 0,137 & 58 & 536 & $-1,9$ \\
\hline 0,089 & 0,887 & 0,089 & 0,741 & 1,000 & 0,836 & 0,164 & 1,000 & 0,838 & 0,930 & 0,588 & 0,349 & 0,205 & 0,274 & 80 & 536 & $-1,8$ \\
\hline 0,164 & 1,552 & 0,183 & 1,292 & 0,897 & 0,833 & 0,196 & 0,857 & 1,080 & 0,459 & 0,353 & 0,675 & 0,238 & 0,274 & 69 & 536 & $-2,8$ \\
\hline 0,113 & 1,659 & 0,150 & 1,437 & 0,750 & 0,866 & 0,283 & 0,514 & 1,395 & 0,270 & 0,266 & 0,728 & 0,194 & 0,274 & 52 & 536 & $-2,9$ \\
\hline 0,061 & 0,887 & 0,070 & 0,703 & 0,867 & 0,793 & 0,246 & 0,975 & 1,013 & 0,487 & 0,307 & 0,399 & 0,123 & 0,137 & 62 & 536 & $-2,0$ \\
\hline 0,056 & 0,918 & 0,070 & 0,703 & 0,800 & 0,766 & 0,308 & 0,840 & 1,091 & 0,764 & 0,632 & 0,306 & 0,194 & 0,194 & 0 & 536 & $-1,6$ \\
\hline 0,089 & 1,513 & 0,131 & 1,146 & 0,679 & 0,757 & 0,403 & 0,489 & 1,430 & 0,331 & 0,299 & 0,585 & 0,175 & 0,274 & 103 & 536 & $-2,5$ \\
\hline 0,052 & 1,086 & 0,080 & 0,665 & 0,647 & 0,612 & 0,524 & 0,550 & 1,348 & 0,778 & 0,707 & 0,291 & 0,205 & 0,137 & 58 & 536 & $-1,5$ \\
\hline 0,244 & 3,707 & 0,436 & 2,324 & 0,559 & 0,627 & 0,578 & 0,223 & 2,117 & 0,422 & 0,783 & 0,858 & 0,672 & 0,194 & 24 & 536 & $-3,1$ \\
\hline 0,047 & 1,070 & 0,080 & 0,627 & 0,588 & 0,586 & 0,584 & 0,515 & 1,394 & 0,311 & 0,294 & 0,439 & 0,129 & 0,137 & 0 & 536 & $-2,1$ \\
\hline 0,084 & 1,422 & 0,127 & 0,940 & 0,667 & 0,661 & 0,475 & 0,525 & 1,380 & 0,559 & 0,773 & 0,439 & 0,339 & 0,194 & 96 & 536 & $-2,1$ \\
\hline \multirow[t]{4}{*}{0,127} & 1,200 & 0,145 & 0,940 & 0,871 & 0,784 & 0,252 & 1,000 & 0,951 & 0,838 & 0,769 & 0,439 & 0,337 & 0,387 & 46 & 536 & $-2,1$ \\
\hline & & & & & & & nothing to measure & & & & & & & & 537 & . \\
\hline & & & & & & & nothing to measure & & & & & & & & 538 & - \\
\hline & & & & & & & nothing to measure & & & & & & & & 539 & - \\
\hline \multirow{3}{*}{0,052} & 1,254 & 0,089 & 0,810 & 0,579 & 0,646 & 0,550 & 0,413 & 1,557 & 0,269 & 0,334 & 0,494 & 0,165 & 0,137 & 53 & 540 & $-2,3$ \\
\hline & & & & & & & nothing to measure & & & & & & & & 541 & - \\
\hline & & & & & & & nothing to measure & & & & & & & & 542 & - \\
\hline 0,056 & 0,848 & 0,066 & 0,611 & 0,857 & 0,720 & 0,314 & 0,983 & 1,009 & 0,764 & 0,637 & 0,306 & 0,195 & 0,194 & 103 & 543 & $-1,6$ \\
\hline \multirow[t]{2}{*}{0,150} & 1,842 & 0,225 & 1,590 & 0,667 & 0,863 & 0,360 & 0,556 & 1,341 & 0,392 & 0,439 & 0,698 & 0,307 & 0,274 & 169 & 544 & $-2,8$ \\
\hline & & & & & & & nothing to measure & & & & & & & & 545 & \\
\hline \multirow[t]{9}{*}{0,056} & 0,757 & 0,056 & 0,497 & 1,000 & 0,657 & 0,343 & 1,000 & 0,900 & 0,611 & 0,424 & 0,342 & 0,145 & 0,194 & 135 & 546 & $-1,8$ \\
\hline & & & & & & & nothing to measure & & & & & & & & 547 & \\
\hline & & & & & & & nothing to measure & & & & & & & & 548 & - \\
\hline & & & & & & & nothing to measure & & & & & & & & 549 & - \\
\hline & & & & & & & nothing to measure & & & & & & & & 550 & - \\
\hline & & & & & & & nothing to measure & & & & & & & & 551 & - \\
\hline & & & & & & & nothing to measure & & & & & & & & 552 & - \\
\hline & & & & & & & nothing to measure & & & & & & & & 553 & - \\
\hline & & & & & & & nothing to measure & & & & & & & & 554 & - \\
\hline 0,169 & 1,406 & 0,174 & 1,108 & 0,973 & 0,788 & 0,214 & 1,000 & 0,965 & 0,573 & 0,447 & 0,613 & 0,274 & 0,387 & 17 & 555 & $-2,6$ \\
\hline \multirow{2}{*}{0,286} & 2,637 & 0,352 & 2,378 & 0,813 & 0,902 & 0,211 & 0,517 & 1,391 & 0,262 & 0,307 & 1,178 & 0,361 & 0,306 & 148 & 555 & $-3,6$ \\
\hline & & & & & & & nothing to measure & & & & & & & & 556 & \\
\hline 0,066 & 0,940 & 0,080 & 0,703 & 0,824 & 0,748 & 0,308 & 0,934 & 1,035 & 0,524 & 0,380 & 0,399 & 0,152 & 0,194 & 24 & 557 & $-2,0$ \\
\hline 0,070 & 1,016 & 0,094 & 0,719 & 0,750 & 0,707 & 0,385 & 0,856 & 1,081 & 0,466 & 0,407 & 0,439 & 0,178 & 0,137 & 37 & 557 & $-2,1$ \\
\hline 0,052 & 0,940 & 0,075 & 0,703 & 0,688 & 0,748 & 0,402 & 0,733 & 1,168 & 0,560 & 0,537 & 0,342 & 0,184 & 0,194 & 58 & 557 & $-1,8$ \\
\hline 0,047 & 0,573 & 0,047 & 0,497 & 1,000 & 0,867 & 0,133 & 1,000 & 0,746 & 1,273 & 0,632 & 0,217 & 0,137 & 0,194 & 90 & 557 & $-1,1$ \\
\hline 0,052 & 0,719 & 0,056 & 0,535 & 0,917 & 0,745 & 0,269 & 1,000 & 0,892 & 0,700 & 0,474 & 0,306 & 0,145 & 0,194 & 122 & 557 & $-1,6$ \\
\hline 0,160 & 2,324 & 0,253 & 1,659 & 0,630 & 0,714 & 0,468 & 0,371 & 1,641 & 0,433 & 0,618 & 0,685 & 0,423 & 0,194 & 51 & 557 & $-2,8$ \\
\hline
\end{tabular}


nothing to measure nothing to measure

nothing to measure

\section{nothing to measure}

nothing to measure
nothing to measure

$\begin{array}{llllllll}0,075 & 1,422 & 0,108 & 1,238 & 0,696 & 0,871 & 0,331 & \text { nothing to meas }\end{array}$




\begin{tabular}{|c|c|c|c|c|c|c|c|c|c|c|c|c|c|c|c|c|c|}
\hline 605 & 0,122 & 1,475 & 0,155 & 1,032 & 0,788 & 0,700 & 0,368 & 0,704 & 1,192 & 0,543 & 0,543 & 0,535 & 0,291 & 0,274 & 136 & 605 & $-2,4$ \\
\hline 605 & 0,056 & 0,757 & 0,066 & 0,573 & 0,857 & 0,757 & 0,282 & 1,000 & 0,900 & 0,899 & 0,686 & 0,282 & 0,194 & 0,194 & 162 & 605 & $-1,5$ \\
\hline 605 & 0,066 & 0,978 & 0,089 & 0,719 & 0,737 & 0,735 & 0,374 & 0,862 & 1,077 & 0,435 & 0,420 & 0,439 & 0,184 & 0,274 & 146 & 605 & $-2,1$ \\
\hline 605 & 0,141 & 1,881 & 0,202 & 1,552 & 0,698 & 0,825 & 0,349 & 0,500 & 1,414 & 0,279 & 0,270 & 0,802 & 0,216 & 0,194 & 164 & 605 & $-3,0$ \\
\hline 605 & 0,108 & 1,238 & 0,127 & 0,887 & 0,852 & 0,716 & 0,320 & 0,885 & 1,063 & 0,505 & 0,447 & 0,522 & 0,233 & 0,274 & 2 & 605 & $-2,4$ \\
\hline 605 & 0,127 & 1,681 & 0,164 & 1,330 & 0,771 & 0,791 & 0,310 & 0,563 & 1,333 & 0,382 & 0,353 & 0,650 & 0,230 & 0,274 & 116 & 605 & $-2,7$ \\
\hline 605 & 0,108 & 1,659 & 0,164 & 1,216 & 0,657 & 0,733 & 0,435 & 0,493 & 1,425 & 0,345 & 0,452 & 0,632 & 0,285 & 0,194 & 141 & 605 & $-2,7$ \\
\hline 605 & 0,056 & 0,978 & 0,061 & 0,810 & 0,923 & 0,828 & 0,188 & 0,739 & 1,163 & 0,340 & 0,267 & 0,459 & 0,123 & 0,137 & 119 & 605 & $-2,2$ \\
\hline 605 & 0,141 & 2,485 & 0,235 & 2,118 & 0,600 & 0,852 & 0,426 & 0,286 & 1,869 & 0,139 & 0,135 & 1,134 & 0,153 & 0,194 & 124 & 605 & $-3,5$ \\
\hline 605 & 0,061 & 1,124 & 0,075 & 0,810 & 0,812 & 0,721 & 0,336 & 0,607 & 1,284 & 0,271 & 0,178 & 0,535 & 0,095 & 0,137 & 146 & 605 & $-2,4$ \\
\hline 605 & 0,052 & 0,795 & 0,056 & 0,665 & 0,917 & 0,837 & 0,183 & 1,000 & 0,987 & 0,483 & 0,294 & 0,369 & 0,108 & 0,137 & 107 & 605 & $-1,9$ \\
\hline 605 & 0,052 & 0,719 & 0,052 & 0,443 & 1,000 & 0,617 & 0,383 & 1,000 & 0,892 & 0,560 & 0,283 & 0,342 & 0,097 & 0,194 & 135 & 605 & $-1,8$ \\
\hline 605 & 0,066 & 1,032 & 0,075 & 0,902 & 0,875 & 0,874 & 0,177 & 0,775 & 1,136 & 0,357 & 0,200 & 0,484 & 0,097 & 0,137 & 137 & 605 & $-2,3$ \\
\hline 605 & 0,061 & 0,887 & 0,084 & 0,703 & 0,722 & 0,793 & 0,346 & 0,975 & 1,013 & 0,662 & 0,627 & 0,342 & 0,215 & 0,137 & 122 & 605 & $-1,8$ \\
\hline 605 & 0,070 & 1,384 & 0,108 & 1,070 & 0,652 & 0,773 & 0,415 & 0,462 & 1,471 & 0,233 & 0,257 & 0,620 & 0,159 & 0,137 & 6 & 605 & $-2,6$ \\
\hline 605 & 0,061 & 0,978 & 0,089 & 0,757 & 0,684 & 0,773 & 0,389 & 0,801 & 1,118 & 0,447 & 0,468 & 0,417 & 0,195 & 0,137 & 100 & 605 & $-2,1$ \\
\hline 605 & 0,056 & 0,719 & 0,056 & 0,535 & 1,000 & 0,745 & 0,255 & 1,000 & 0,854 & 0,764 & 0,474 & 0,306 & 0,145 & 0,194 & 108 & 605 & $-1,6$ \\
\hline 605 & 0,052 & 0,925 & 0,056 & 0,573 & 0,917 & 0,620 & 0,389 & 0,758 & 1,148 & 0,350 & 0,283 & 0,433 & 0,123 & 0,137 & 118 & 605 & $-2,1$ \\
\hline 606 & & & & & & & & nothing to measure & & & & & & & & 606 & 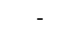 \\
\hline 607 & & & & & & & & nothing to measure & & & & & & & & 607 & - \\
\hline 608 & & & & & & & & nothing to measure & & & & & & & & 608 & - \\
\hline 609 & 0,873 & 8,913 & 1,586 & 4,585 & 0,550 & 0,514 & 0,662 & 0,138 & 2,692 & 0,271 & 0,474 & 2,024 & 0,959 & 0,387 & 156 & 609 & $-4,3$ \\
\hline 609 & 0,052 & 1,032 & 0,080 & 0,719 & 0,647 & 0,696 & 0,466 & 0,609 & 1,282 & 0,438 & 0,396 & 0,387 & 0,153 & 0,137 & 80 & 609 & $-2,0$ \\
\hline 609 & 0,099 & 0,978 & 0,099 & 0,795 & 1,000 & 0,812 & 0,188 & 1,000 & 0,879 & 0,668 & 0,474 & 0,433 & 0,205 & 0,274 & 165 & 609 & $-2,1$ \\
\hline 609 & 0,211 & 2,201 & 0,296 & 1,567 & 0,714 & 0,712 & 0,406 & 0,548 & 1,351 & 0,392 & 0,444 & 0,828 & 0,368 & 0,306 & 144 & 609 & $-3,0$ \\
\hline 609 & 0,117 & 2,010 & 0,188 & 1,345 & 0,625 & 0,669 & 0,500 & 0,365 & 1,656 & 0,274 & 0,332 & 0,738 & 0,245 & 0,274 & 33 & 609 & $-2,9$ \\
\hline 609 & 0,070 & 1,200 & 0,099 & 0,741 & 0,714 & 0,618 & 0,477 & 0,614 & 1,276 & 0,764 & 0,800 & 0,342 & 0,274 & 0,137 & 34 & 609 & $-1,8$ \\
\hline 609 & 0,089 & 1,055 & 0,094 & 0,795 & 0,950 & 0,754 & 0,251 & 1,000 & 0,996 & 0,538 & 0,447 & 0,459 & 0,205 & 0,274 & 153 & 609 & $-2,2$ \\
\hline 610 & & & & & & & & nothing to measure & & & & & & & & 610 & - \\
\hline 611 & 0,272 & 4,977 & 0,582 & 2,729 & 0,468 & 0,548 & 0,698 & 0,138 & 2,691 & 0,233 & 0,431 & 1,219 & 0,526 & 0,194 & 47 & 611 & $-3,6$ \\
\hline 611 & 0,066 & 1,032 & 0,084 & 0,848 & 0,778 & 0,822 & 0,285 & 0,775 & 1,136 & 0,495 & 0,522 & 0,411 & 0,215 & 0,194 & 108 & 611 & $-2,0$ \\
\hline 611 & 0,056 & 0,848 & 0,066 & 0,573 & 0,857 & 0,675 & 0,355 & 0,983 & 1,009 & 0,899 & 0,651 & 0,282 & 0,184 & 0,194 & 0 & 611 & $-1,5$ \\
\hline 611 & 0,066 & 1,254 & 0,094 & 0,833 & 0,700 & 0,664 & 0,450 & 0,525 & 1,380 & 0,343 & 0,385 & 0,494 & 0,190 & 0,194 & 37 & 611 & $-2,3$ \\
\hline 612 & & & & & & & & nothing to measure & & & & & & & & 612 & \\
\hline 613 & 0,047 & 0,757 & 0,056 & 0,443 & 0,833 & 0,586 & 0,446 & 1,000 & 0,986 & 0,637 & 0,425 & 0,306 & 0,130 & 0,137 & 161 & 613 & $-1,6$ \\
\hline 613 & 0,164 & 2,102 & 0,249 & 1,827 & 0,660 & 0,869 & 0,364 & 0,467 & 1,463 & 0,264 & 0,276 & 0,890 & 0,246 & 0,274 & 117 & 613 & $-3,2$ \\
\hline 613 & 0,056 & 0,940 & 0,075 & 0,703 & 0,750 & 0,748 & 0,355 & 0,800 & 1,118 & 0,611 & 0,570 & 0,342 & 0,195 & 0,137 & 26 & 613 & $-1,8$ \\
\hline 613 & 0,047 & 0,573 & 0,047 & 0,352 & 1,000 & 0,613 & 0,387 & 1,000 & 0,746 & 1,273 & 0,632 & 0,217 & 0,137 & 0,274 & 0 & 613 & $-1,1$ \\
\hline 613 & 0,070 & 1,254 & 0,108 & 0,833 & 0,652 & 0,664 & 0,483 & 0,563 & 1,333 & 0,516 & 0,479 & 0,417 & 0,199 & 0,194 & 12 & 613 & $-2,1$ \\
\hline 613 & 0,056 & 0,703 & 0,061 & 0,519 & 0,923 & 0,739 & 0,272 & 1,000 & 0,836 & 0,849 & 0,597 & 0,291 & 0,173 & 0,274 & 37 & 613 & $-1,5$ \\
\hline 613 & 0,052 & 0,848 & 0,066 & 0,573 & 0,786 & 0,675 & 0,389 & 0,901 & 1,054 & 0,560 & 0,437 & 0,342 & 0,150 & 0,194 & 79 & 613 & $-1,8$ \\
\hline 613 & 0,150 & 1,697 & 0,188 & 1,200 & 0,800 & 0,707 & 0,355 & 0,655 & 1,236 & 0,627 & 0,702 & 0,552 & 0,387 & 0,306 & 116 & 613 & $-2,5$ \\
\hline 613 & 0,080 & 1,108 & 0,103 & 0,940 & 0,773 & 0,848 & 0,273 & 0,816 & 1,107 & 0,433 & 0,421 & 0,484 & 0,204 & 0,274 & 63 & 613 & $-2,3$ \\
\hline 613 & 0,075 & 1,032 & 0,080 & 0,902 & 0,941 & 0,874 & 0,139 & 0,886 & 1,063 & 0,392 & 0,270 & 0,494 & 0,133 & 0,137 & 35 & 613 & $-2,3$ \\
\hline 613 & 0,113 & 1,384 & 0,141 & 1,070 & 0,800 & 0,773 & 0,302 & 0,739 & 1,163 & 0,470 & 0,496 & 0,552 & 0,274 & 0,306 & 108 & 613 & $-2,5$ \\
\hline 613 & 0,328 & 2,805 & 0,436 & 2,286 & 0,753 & 0,815 & 0,309 & 0,524 & 1,381 & 0,420 & 0,492 & 0,997 & 0,490 & 0,306 & 108 & 613 & $-3,3$ \\
\hline 613 & 0,052 & 0,719 & 0,056 & 0,573 & 0,917 & 0,798 & 0,219 & 1,000 & 0,892 & 0,700 & 0,474 & 0,306 & 0,145 & 0,194 & 48 & 613 & $-1,6$ \\
\hline 613 & 0,047 & 0,627 & 0,052 & 0,481 & 0,909 & 0,768 & 0,249 & 1,000 & 0,816 & 0,979 & 0,588 & 0,247 & 0,145 & 0,194 & 34 & 613 & $-1,3$ \\
\hline 613 & 0,094 & 1,032 & 0,103 & 0,848 & 0,909 & 0,822 & 0,200 & 1,000 & 0,950 & 0,621 & 0,434 & 0,439 & 0,190 & 0,274 & 37 & 613 & $-2,1$ \\
\hline 614 & & & & & & & & nothing to measure & & & & & & & & 614 & - \\
\hline 615 & & & & & & & & nothing to measure & & & & & & & & 615 & - \\
\hline 616 & & & & & & & & nothing to measure & & & & & & & & 616 & - \\
\hline 617 & & & & & & & & nothing to measure & & & & & & & & 617 & - \\
\hline 618 & & & & & & & & nothing to measure & & & & & & & & 618 & - \\
\hline 619 & & & & & & & & nothing to measure & & & & & & & & 619 & - \\
\hline 620 & & & & & & & & nothing to measure & & & & & & & & 620 & - \\
\hline 621 & & & & & & & & nothing to measure & & & & & & & & 621 & - \\
\hline 622 & & & & & & & & nothing to measure & & & & & & & & 622 & - \\
\hline
\end{tabular}




\section{nothing to measure} nothing to measure

0,996

\section{1,002}

nothing to measure

$\begin{array}{llllll}0,056 & 1,200 & 0,089 & 0,627 & 0,632 & 0,522 \\ 0,094 & 1,498 & 0,127 & 1,055 & 0,741 & 0,704\end{array}$

0,603

0, 127.055

$\begin{array}{ll}0,052 & 0,665 \\ 0,070 & 1,108 \\ 0,774 & 3,860 \\ 0,066 & 1,055 \\ 0,094 & 1,881 \\ 0,052 & 0,719 \\ 0,052 & 0,757 \\ 0,070 & 1,070 \\ 0,089 & 1,184 \\ 0,113 & 2,400 \\ 0,089 & 1,032 \\ 0,070 & 1,032 \\ 0,052 & 0,757 \\ 0,070 & 1,162 \\ 0,061 & 1,093\end{array}$

$\begin{array}{llll}0,052 & 0,481 & 1,000 & 0,724 \\ 0,080 & 0,795 & 0,882 & 0,717 \\ 0,887 & 3,363 & 0,873 & 0,871 \\ 0,075 & 0,795 & 0,875 & 0,754 \\ 0,192 & 1,345 & 0,488 & 0,715 \\ 0,061 & 0,573 & 0,846 & 0,798 \\ 0,061 & 0,573 & 0,846 & 0,757 \\ 0,089 & 0,757 & 0,789 & 0,707 \\ 0,103 & 0,887 & 0,864 & 0,749 \\ 0,244 & 1,919 & 0,462 & 0,799 \\ 0,089 & 0,887 & 1,000 & 0,859 \\ 0,080 & 0,902 & 0,882 & 0,874 \\ 0,061 & 0,665 & 0,846 & 0,879 \\ 0,099 & 0,887 & 0,714 & 0,763 \\ 0,084 & 0,573 & 0,722 & 0,525\end{array}$

$$
\begin{aligned}
& \text { no } \\
& \text { no } \\
& \text { no } \\
& \text { no } \\
& \text { no } \\
& \text { no } \\
& \text { no } \\
& \text { no } \\
& \text { no } \\
& \text { no } \\
& \text { no } \\
& \text { no } \\
& \text { no } \\
& \text { no } \\
& \text { no } \\
& \text { no } \\
& \hline \\
& \\
& \hline
\end{aligned}
$$

0,526
nothing to measure 1,427

0,917

nothing to measure

nothing to measure

nothing to measure

nothing to measure

nothing to measure

nothing to measure

nothing to measure

nothing to measure

nothing to measure
nothing to measure

nothing to measure
nothing to measure

\begin{tabular}{|c|c|c|c|c|c|}
\hline 0,527 & 0,557 & 0,369 & 0,205 & 0,137 & 153 \\
\hline 0,314 & 0,333 & 0,616 & 0,205 & 0,274 & 17 \\
\hline
\end{tabular}

$\begin{array}{ll}1,000 & 0,826\end{array}$

$\begin{array}{ll}0,720 & 1,178 \\ 0,653 & 1,238\end{array}$

$\begin{array}{ll}0,653 & 1,238 \\ 0,742 & 1,161 \\ 0,333 & 1,732\end{array}$

$\begin{array}{ll}0,333 & 1,732 \\ 1,000 & 0,892\end{array}$

$\begin{array}{ll}1,000 & 0,892 \\ 1,000 & 0,940 \\ 0,772 & 1,138\end{array}$

$\begin{array}{ll}0,772 & 1,138 \\ 0,798 & 1,119 \\ 0,246 & 2,018 \\ 1,000 & 0,975\end{array}$

$\begin{array}{ll}0,246 & 2,018 \\ 1,000 & 0,975\end{array}$

$\begin{array}{ll}1,000 & 1,098 \\ 1,000 & 0,940\end{array}$

$\begin{array}{ll}1,000 & 0,940 \\ 0,655 & 1,236\end{array}$

1,077
0,360
0,390
0,336
0,255
1,077
0,700
0,764
0,465
0,165
0,53
0,38
0,56
0,36
0,312

1,077
0,360
0,390
0,336
0,255
1,077
0,700
0,764
0,465
0,165
0,538
0,382
0,560
0,360
0,312

0,745
0,275
0,388
0,261
0,448
0,745
0,495
0,696
0,520
0,292
0,414
0,300
0,380
0,348
0,18

$\begin{array}{ll}0,745 & 0,247 \\ , 275 & 0,499 \\ , 388 & 1,589 \\ , 261 & 0,499 \\ , 048 & 0,685 \\ 0,745 & 0,247 \\ , 495 & 0,306 \\ , 696 & 0,342 \\ , 520 & 0,494 \\ , 292 & 0,932 \\ 0,414 & 0,459 \\ 0,300 & 0,484 \\ , 380 & 0,342 \\ , 348 & 0,499 \\ 0,181 & 0,499 \\ & \end{array}$

0,247
0,499
1,589
0,499
0,685
0,247
0,306
0,342
0,494
0,932
0,459
0,484
0,342
0,499
0,499

$\begin{array}{ll}0,184 & 0,194 \\ 0,137 & 0,194 \\ 0,616 & 0,685 \\ 0,130 & 0,137 \\ 0,307 & 0,137 \\ 0,184 & 0,194 \\ 0,152 & 0,194 \\ 0,238 & 0,137 \\ 0,257 & 0,274 \\ 0,272 & 0,194 \\ 0,190 & 0,274 \\ 0,145 & 0,194 \\ 0,130 & 0,137 \\ 0,174 & 0,194 \\ 0,090 & 0,137\end{array}$




\begin{tabular}{|c|c|c|c|c|c|c|c|c|c|c|c|c|c|c|c|c|}
\hline 0,056 & 0,810 & 0,061 & 0,665 & 0,923 & 0,820 & 0,195 & 1,000 & 0,964 & 0,611 & 0,424 & 0,342 & 0,145 & 0,194 & 50 & 662 & $-1,8$ \\
\hline 0,080 & 0,887 & 0,084 & 0,703 & 0,944 & 0,793 & 0,214 & 1,000 & 0,886 & 0,746 & 0,499 & 0,369 & 0,184 & 0,274 & 72 & 662 & $-1,9$ \\
\hline 0,047 & 0,665 & 0,052 & 0,535 & 0,909 & 0,805 & 0,215 & 1,000 & 0,866 & 0,749 & 0,485 & 0,282 & 0,137 & 0,194 & 162 & 662 & $-1,5$ \\
\hline 0,131 & 1,842 & 0,169 & 1,681 & 0,778 & 0,913 & 0,239 & 0,486 & 1,434 & 0,260 & 0,229 & 0,802 & 0,184 & 0,274 & 65 & 662 & $-3,0$ \\
\hline 0,056 & 1,070 & 0,080 & 0,681 & 0,706 & 0,636 & 0,468 & 0,618 & 1,272 & 0,449 & 0,447 & 0,399 & 0,178 & 0,137 & 90 & 662 & $-2,0$ \\
\hline 0,089 & 1,384 & 0,127 & 1,055 & 0,704 & 0,762 & 0,380 & 0,585 & 1,307 & 0,465 & 0,483 & 0,494 & 0,238 & 0,194 & 79 & 662 & $-2,3$ \\
\hline 0,052 & 0,741 & 0,061 & 0,481 & 0,846 & 0,650 & 0,383 & 1,000 & 0,920 & 0,700 & 0,434 & 0,306 & 0,133 & 0,137 & 90 & 662 & $-1,6$ \\
\hline 0,103 & 1,146 & 0,117 & 0,925 & 0,880 & 0,807 & 0,228 & 0,987 & 1,007 & 0,529 & 0,412 & 0,499 & 0,205 & 0,274 & 73 & 662 & $-2,3$ \\
\hline 0,047 & 0,757 & 0,056 & 0,481 & 0,833 & 0,636 & 0,400 & 1,000 & 0,986 & 0,979 & 0,784 & 0,247 & 0,194 & 0,194 & 43 & 662 & $-1,3$ \\
\hline \multirow[t]{5}{*}{0,084} & 0,887 & 0,084 & 0,665 & 1,000 & 0,750 & 0,250 & 1,000 & 0,861 & 0,881 & 0,588 & 0,349 & 0,205 & 0,274 & 90 & 662 & $-1,8$ \\
\hline & & & & & & & nothing to measure & & & & & & & & 663 & - \\
\hline & & & & & & & nothing to measure & & & & & & & & 664 & - \\
\hline & & & & & & & nothing to measure & & & & & & & & 665 & - \\
\hline & & & & & & & nothing to measure & & & & & & & & 666 & - \\
\hline 0,056 & 0,795 & 0,066 & 0,535 & 0,857 & 0,673 & 0,357 & 1,000 & 0,945 & 0,764 & 0,632 & 0,306 & 0,194 & 0,194 & 133 & 667 & $-1,6$ \\
\hline 0,061 & 0,703 & 0,061 & 0,443 & 1,000 & 0,631 & 0,369 & 1,000 & 0,803 & 0,828 & 0,447 & 0,306 & 0,137 & 0,274 & 98 & 667 & $-1,6$ \\
\hline 0,084 & 1,384 & 0,117 & 1,032 & 0,720 & 0,746 & 0,378 & 0,554 & 1,343 & 0,441 & 0,463 & 0,494 & 0,228 & 0,274 & 149 & 667 & $-2,3$ \\
\hline 0,164 & 2,064 & 0,211 & 1,858 & 0,778 & 0,900 & 0,244 & 0,484 & 1,437 & 0,223 & 0,200 & 0,969 & 0,194 & 0,274 & 139 & 667 & $-3,3$ \\
\hline 0,089 & 0,887 & 0,094 & 0,795 & 0,950 & 0,896 & 0,115 & 1,000 & 0,838 & 0,930 & 0,588 & 0,349 & 0,205 & 0,274 & 173 & 667 & $-1,8$ \\
\hline 0,216 & 2,637 & 0,342 & 2,232 & 0,630 & 0,846 & 0,401 & 0,390 & 1,601 & 0,224 & 0,285 & 1,107 & 0,315 & 0,194 & 157 & 668 & $-3,5$ \\
\hline 0,099 & 1,384 & 0,131 & 0,940 & 0,750 & 0,680 & 0,406 & 0,647 & 1,243 & 0,438 & 0,488 & 0,535 & 0,261 & 0,194 & 138 & 668 & $-2,4$ \\
\hline 0,136 & 1,957 & 0,197 & 1,422 & 0,690 & 0,727 & 0,413 & 0,447 & 1,496 & 0,381 & 0,415 & 0,675 & 0,280 & 0,194 & 150 & 668 & $-2,8$ \\
\hline 0,061 & 0,848 & 0,066 & 0,573 & 0,929 & 0,675 & 0,332 & 1,000 & 0,969 & 0,637 & 0,439 & 0,349 & 0,153 & 0,194 & 162 & 668 & $-1,8$ \\
\hline \multirow[t]{11}{*}{0,038} & 0,681 & 0,056 & 0,589 & 0,667 & 0,865 & 0,360 & 1,000 & 0,991 & 0,637 & 0,707 & 0,274 & 0,194 & 0,137 & 0 & 668 & $-1,5$ \\
\hline & & & & & & & nothing to measure & & & & & & & & 669 & - \\
\hline & & & & & & & nothing to measure & & & & & & & & 670 & - \\
\hline & & & & & & & nothing to measure & & & & & & & & 671 & - \\
\hline & & & & & & & nothing to measure & & & & & & & & 672 & - \\
\hline & & & & & & & nothing to measure & & & & & & & & 673 & . \\
\hline & & & & & & & nothing to measure & & & & & & & & 674 & - \\
\hline & & & & & & & nothing to measure & & & & & & & & 675 & - \\
\hline & & & & & & & nothing to measure & & & & & & & & 676 & . \\
\hline & & & & & & & nothing to measure & & & & & & & & 677 & - \\
\hline & & & & & & & nothing to measure & & & & & & & & 678 & - \\
\hline 0,174 & 1,896 & 0,188 & 1,896 & 0,925 & 1,000 & 0,075 & 0,607 & 1,284 & 0,277 & 0,228 & 0,893 & 0,203 & 0,274 & 56 & 679 & $-3,2$ \\
\hline 0,056 & 0,665 & 0,056 & 0,481 & 1,000 & 0,724 & 0,276 & 1,000 & 0,791 & 1,175 & 0,784 & 0,247 & 0,194 & 0,274 & 72 & 679 & $-1,3$ \\
\hline 0,056 & 0,757 & 0,056 & 0,627 & 1,000 & 0,828 & 0,172 & 1,000 & 0,900 & 0,611 & 0,424 & 0,342 & 0,145 & 0,194 & 45 & 679 & $-1,8$ \\
\hline \multirow[t]{2}{*}{0,075} & 1,529 & 0,117 & 1,070 & 0,640 & 0,700 & 0,469 & 0,403 & 1,574 & 0,407 & 0,444 & 0,484 & 0,215 & 0,137 & 62 & 679 & $-2,3$ \\
\hline & & & & & & & nothing to measure & & & & & & & & 680 & 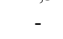 \\
\hline 0,117 & 1,384 & 0,131 & 1,254 & 0,893 & 0,906 & 0,142 & 0,770 & 1,140 & 0,358 & 0,316 & 0,646 & 0,204 & 0,274 & 28 & 681 & $-2,7$ \\
\hline 0,061 & 0,757 & 0,066 & 0,627 & 0,929 & 0,828 & 0,186 & 1,000 & 0,864 & 0,828 & 0,501 & 0,306 & 0,153 & 0,194 & 117 & 681 & $-1,6$ \\
\hline 0,164 & 1,934 & 0,211 & 1,605 & 0,778 & 0,830 & 0,280 & 0,552 & 1,347 & 0,305 & 0,260 & 0,828 & 0,215 & 0,274 & 64 & 681 & $-3,0$ \\
\hline 0,075 & 1,032 & 0,089 & 0,757 & 0,842 & 0,733 & 0,310 & 0,886 & 1,063 & 0,784 & 0,615 & 0,349 & 0,215 & 0,194 & 103 & 681 & $-1,8$ \\
\hline 0,061 & 0,887 & 0,070 & 0,535 & 0,867 & 0,604 & 0,418 & 0,975 & 1,013 & 0,662 & 0,600 & 0,342 & 0,205 & 0,194 & 23 & 681 & $-1,8$ \\
\hline \multirow{4}{*}{0,188} & 2,507 & 0,272 & 1,773 & 0,690 & 0,707 & 0,427 & 0,375 & 1,633 & 0,407 & 0,538 & 0,766 & 0,412 & 0,306 & 172 & 681 & $-2,9$ \\
\hline & & & & & & & nothing to measure & & & & & & & & 682 & - \\
\hline & & & & & & & nothing to measure & & & & & & & & 683 & - \\
\hline & & & & & & & nothing to measure & & & & & & & & 684 & . \\
\hline 0,047 & 0,573 & 0,047 & 0,390 & 1,000 & 0,680 & 0,320 & 1,000 & 0,746 & 1,273 & 0,632 & 0,217 & 0,137 & 0,274 & 90 & 685 & $-1,1$ \\
\hline \multirow[t]{5}{*}{0,089} & 0,940 & 0,089 & 0,940 & 1,000 & 1,000 & 0,000 & 1,000 & 0,888 & 0,712 & 0,485 & 0,399 & 0,194 & 0,274 & 139 & 685 & $-2,0$ \\
\hline & & & & & & & nothing to measure & & & & & & & & 686 & 2, \\
\hline & & & & & & & nothing to measure & & & & & & & & 687 & - \\
\hline & & & & & & & nothing to measure & & & & & & & & 688 & - \\
\hline & & & & & & & nothing to measure & & & & & & & & 689 & - \\
\hline 0,357 & 3,340 & 0,441 & 2,454 & 0,809 & 0,735 & 0,327 & 0,402 & 1,578 & 0,372 & 0,389 & 1,104 & 0,429 & 0,387 & 20 & 690 & $-3,5$ \\
\hline \multirow{3}{*}{0,094} & 1,162 & 0,108 & 0,940 & 0,870 & 0,809 & 0,231 & 0,873 & 1,070 & 0,566 & 0,527 & 0,459 & 0,242 & 0,274 & 141 & 690 & $-2,2$ \\
\hline & & & & & & & nothing to measure & & & & & & & & 691 & \\
\hline & & & & & & & nothing to measure & & & & & & & & 692 & \\
\hline
\end{tabular}




\begin{tabular}{|c|c|c|c|c|c|c|c|c|c|c|c|c|c|c|c|c|}
\hline 0,047 & 0,757 & 0,052 & 0,481 & 0,909 & 0,636 & 0,375 & 1,000 & 0,986 & 0,796 & 0,500 & 0,274 & 0,137 & 0,194 & 32 & 693 & $-1,5$ \\
\hline 0,061 & 0,719 & 0,066 & 0,665 & 0,929 & 0,925 & 0,103 & 1,000 & 0,821 & 0,974 & 0,686 & 0,282 & 0,194 & 0,194 & 90 & 693 & $-1,5$ \\
\hline 0,061 & 0,978 & 0,075 & 0,703 & 0,812 & 0,719 & 0,338 & 0,801 & 1,118 & 0,447 & 0,329 & 0,417 & 0,137 & 0,137 & 174 & 693 & $-2,1$ \\
\hline \multirow[t]{12}{*}{0,061} & 0,925 & 0,070 & 0,573 & 0,867 & 0,620 & 0,403 & $\begin{array}{c}0,896 \\
\text { nothing to measure }\end{array}$ & 1,056 & 0,414 & 0,283 & 0,433 & 0,123 & 0,137 & 21 & & $-2,1$ \\
\hline & & & & & & & $\begin{array}{l}\text { nothing to measure } \\
\text { nothing to measure }\end{array}$ & & & & & & & & $\begin{array}{l}694 \\
695\end{array}$ & - \\
\hline & & & & & & & nothing to measure & & & & & & & & 696 & - \\
\hline & & & & & & & nothing to measure & & & & & & & & 697 & - \\
\hline & & & & & & & nothing to measure & & & & & & & & 698 & - \\
\hline & & & & & & & nothing to measure & & & & & & & & 699 & - \\
\hline & & & & & & & nothing to measure & & & & & & & & 700 & - \\
\hline & & & & & & & nothing to measure & & & & & & & & 701 & - \\
\hline & & & & & & & nothing to measure & & & & & & & & 702 & - \\
\hline & & & & & & & nothing to measure & & & & & & & & 703 & - \\
\hline & & & & & & & nothing to measure & & & & & & & & 704 & - \\
\hline & & & & & & & nothing to measure & & & & & & & & 705 & \\
\hline \multirow[t]{4}{*}{0,056} & 0,757 & 0,061 & 0,665 & 0,923 & 0,879 & 0,144 & 1,000 & 0,900 & 0,611 & 0,400 & 0,342 & 0,137 & 0,194 & 162 & 706 & $-1,8$ \\
\hline & & & & & & & nothing to measure & & & & & & & & 707 & - \\
\hline & & & & & & & nothing to measure & & & & & & & & 708 & - \\
\hline & & & & & & & nothing to measure & & & & & & & & 709 & \\
\hline 0,178 & 2,232 & 0,249 & 1,957 & 0,717 & 0,877 & 0,309 & 0,450 & 1,491 & 0,222 & 0,236 & 1,011 & 0,239 & 0,274 & 161 & 710 & $-3,3$ \\
\hline \multirow[t]{4}{*}{0,047} & 0,719 & 0,056 & 0,443 & 0,833 & 0,617 & 0,418 & 1,000 & 0,936 & 0,979 & 0,702 & 0,247 & 0,173 & 0,137 & 15 & 710 & $-1,3$ \\
\hline & & & & & & & nothing to measure & & & & & & & & 711 & \\
\hline & & & & & & & nothing to measure & & & & & & & & 712 & - \\
\hline & & & & & & & nothing to measure & & & & & & & & 713 & - \\
\hline 0,150 & 2,545 & 0,267 & 1,659 & 0,561 & 0,652 & 0,560 & 0,291 & 1,853 & 0,348 & 0,580 & 0,741 & 0,430 & 0,274 & 147 & 714 & $-2,9$ \\
\hline 0,084 & 0,848 & 0,084 & 0,703 & 1,000 & 0,829 & 0,171 & 1,000 & 0,824 & 0,917 & 0,566 & 0,342 & 0,194 & 0,306 & 45 & 714 & $-1,8$ \\
\hline 0,164 & 1,399 & 0,183 & 1,254 & 0,897 & 0,896 & 0,146 & 1,000 & 0,974 & 0,891 & 0,800 & 0,484 & 0,387 & 0,387 & 97 & 714 & $-2,3$ \\
\hline 0,089 & 1,675 & 0,160 & 1,384 & 0,559 & 0,826 & 0,474 & 0,399 & 1,582 & 0,302 & 0,460 & 0,613 & 0,282 & 0,194 & 62 & 714 & $-2,6$ \\
\hline \multirow[t]{16}{*}{0,075} & 0,848 & 0,075 & 0,810 & 1,000 & 0,955 & 0,045 & 1,000 & 0,874 & 0,815 & 0,566 & 0,342 & 0,194 & 0,274 & 131 & 714 & $-1,8$ \\
\hline & & & & & & & nothing to measure & & & & & & & & 715 & \\
\hline & & & & & & & nothing to measure & & & & & & & & 716 & - \\
\hline & & & & & & & nothing to measure & & & & & & & & 717 & - \\
\hline & & & & & & & nothing to measure & & & & & & & & 718 & - \\
\hline & & & & & & & nothing to measure & & & & & & & & 719 & - \\
\hline & & & & & & & nothing to measure & & & & & & & & 720 & - \\
\hline & & & & & & & nothing to measure & & & & & & & & 721 & - \\
\hline & & & & & & & nothing to measure & & & & & & & & 722 & - \\
\hline & & & & & & & nothing to measure & & & & & & & & 723 & - \\
\hline & & & & & & & nothing to measure & & & & & & & & 724 & - \\
\hline & & & & & & & nothing to measure & & & & & & & & 725 & - \\
\hline & & & & & & & nothing to measure & & & & & & & & 726 & - \\
\hline & & & & & & & nothing to measure & & & & & & & & 727 & - \\
\hline & & & & & & & nothing to measure & & & & & & & & 728 & - \\
\hline & & & & & & & nothing to measure & & & & & & & & 729 & - \\
\hline 0,056 & 0,925 & 0,070 & 0,719 & 0,800 & 0,777 & 0,299 & 0,827 & 1,099 & 0,611 & 0,627 & 0,342 & 0,215 & 0,137 & 164 & 730 & $-1,8$ \\
\hline \multirow[t]{5}{*}{0,052} & 0,940 & 0,070 & 0,627 & 0,733 & 0,667 & 0,427 & 0,733 & 1,168 & 0,560 & 0,537 & 0,342 & 0,184 & 0,137 & 148 & 730 & $-1,8$ \\
\hline & & & & & & & nothing to measure & & & & & & & & 731 & - \\
\hline & & & & & & & nothing to measure & & & & & & & & 732 & - \\
\hline & & & & & & & nothing to measure & & & & & & & & 733 & - \\
\hline & & & & & & & nothing to measure & & & & & & & & 734 & \\
\hline 0,206 & 2,859 & 0,408 & 2,087 & 0,506 & 0,730 & 0,563 & 0,317 & 1,775 & 0,273 & 0,545 & 0,981 & 0,535 & 0,137 & 108 & 735 & $-3,3$ \\
\hline 0,080 & 1,238 & 0,113 & 0,871 & 0,708 & 0,703 & 0,416 & 0,654 & 1,237 & 0,408 & 0,412 & 0,499 & 0,205 & 0,194 & 90 & 735 & $-2,3$ \\
\hline 0,056 & 0,887 & 0,066 & 0,627 & 0,857 & 0,707 & 0,326 & 0,900 & 1,054 & 0,477 & 0,375 & 0,387 & 0,145 & 0,194 & 50 & 735 & $-2,0$ \\
\hline 0,047 & 0,757 & 0,056 & 0,443 & 0,833 & 0,586 & 0,446 & 1,000 & 0,986 & 0,439 & 0,310 & 0,369 & 0,114 & 0,194 & 58 & 735 & $-1,9$ \\
\hline 0,052 & 0,994 & 0,075 & 0,665 & 0,688 & 0,669 & 0,455 & 0,656 & 1,234 & 0,560 & 0,566 & 0,342 & 0,194 & 0,137 & 9 & 735 & $-1,8$ \\
\hline 0,066 & 0,978 & 0,075 & 0,719 & 0,875 & 0,735 & 0,293 & 0,862 & 1,077 & 0,396 & 0,267 & 0,459 & 0,123 & 0,137 & 62 & 735 & -2.2 \\
\hline 0,145 & 1,567 & 0,164 & 1,292 & 0,886 & 0,824 & 0,210 & 0,744 & 1,159 & 0,789 & 0,789 & 0,484 & 0,382 & 0,387 & 114 & 735 & $-2,3$ \\
\hline
\end{tabular}


Appendix V

Whole rock chemistry 


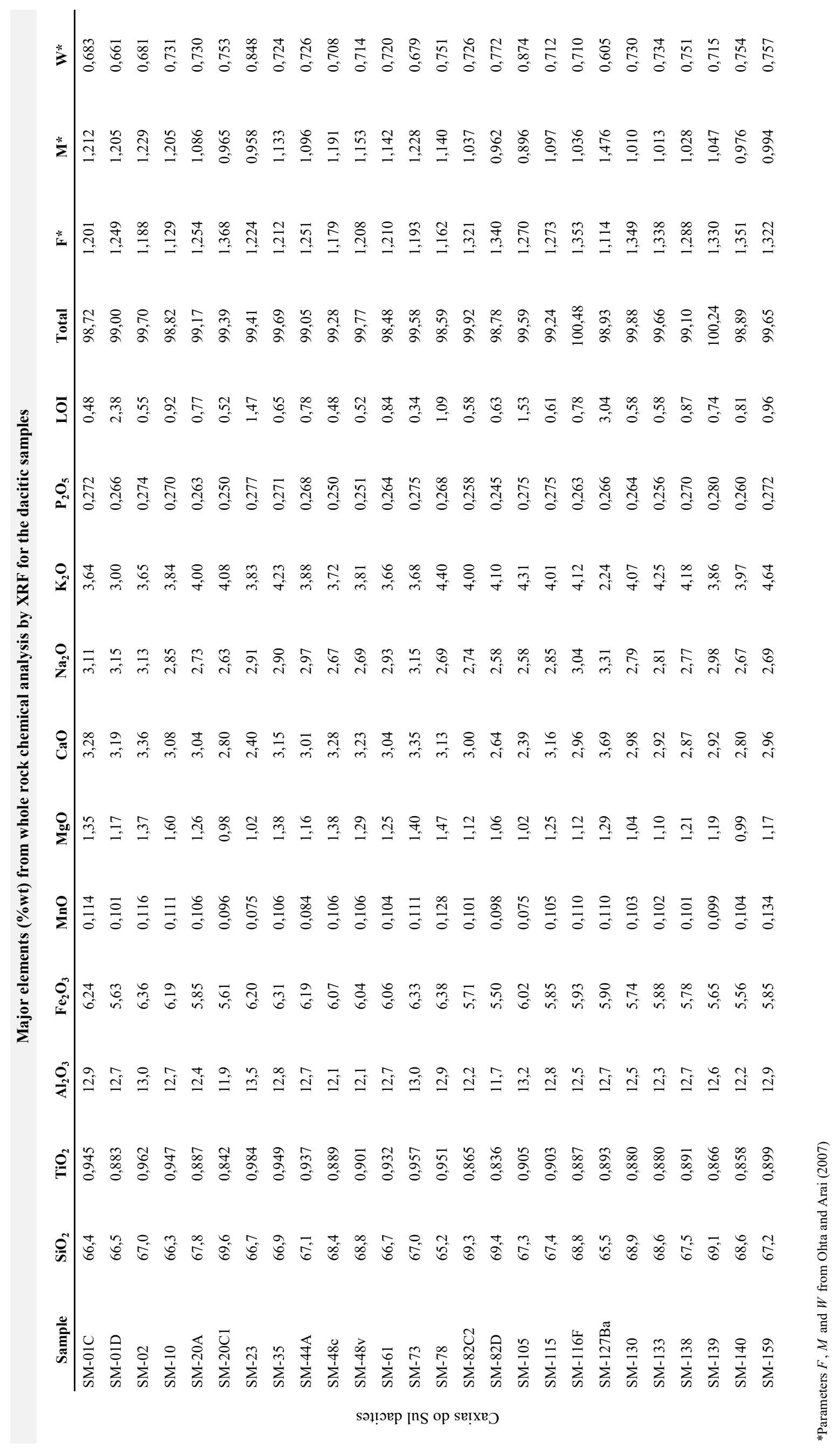




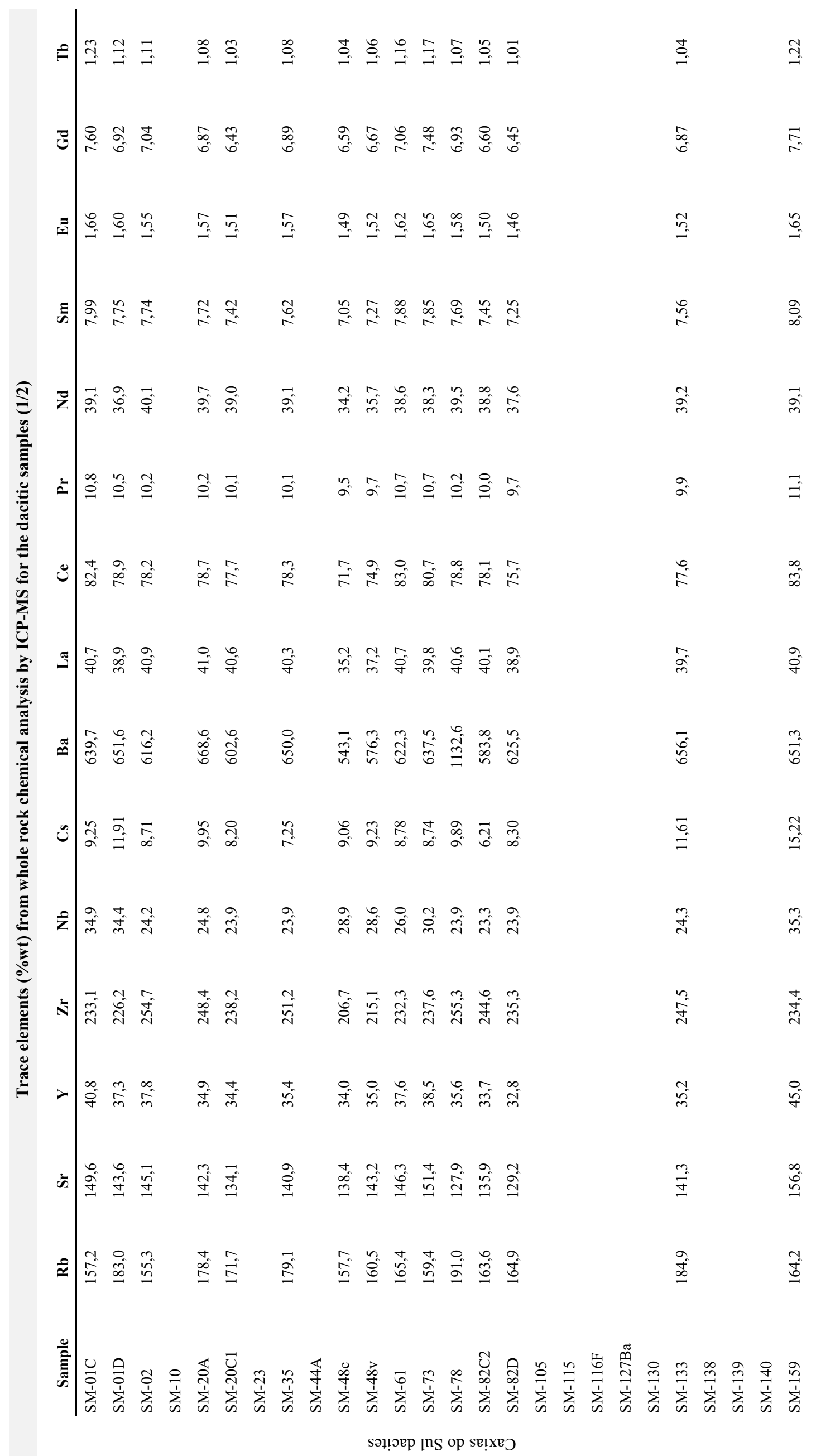




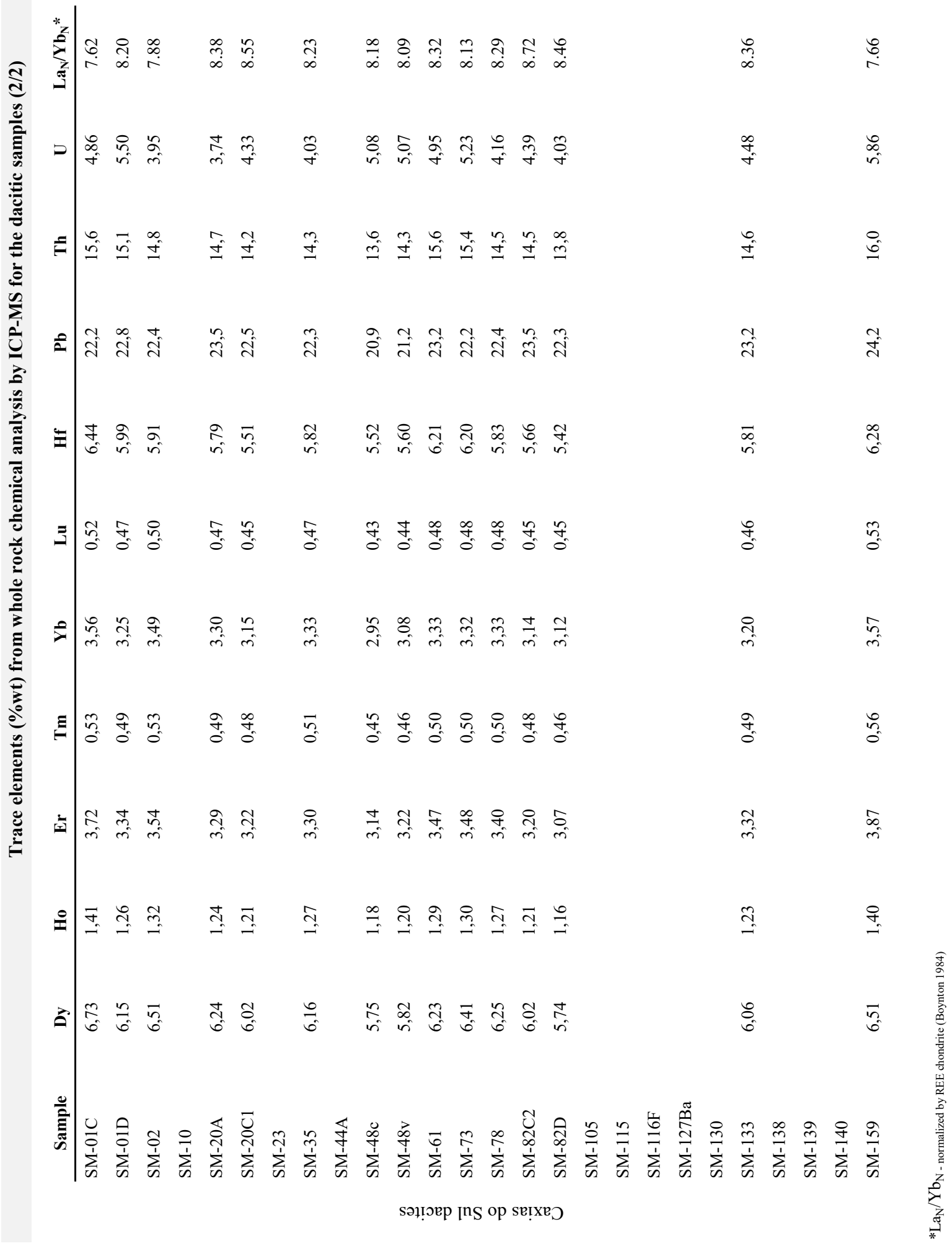




\begin{tabular}{|c|c|c|c|c|c|c|}
\hline$\approx$ & $\stackrel{9}{=}$ & $\stackrel{\circ}{\circ}$ & $\begin{array}{ccc}\infty & \infty \\
0 & \infty & \infty \\
0 & \infty & 0 \\
0 & 0\end{array}$ & 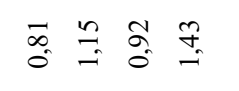 & $\stackrel{\infty}{\infty}$ & \\
\hline$\Xi$ & สู่ & $\stackrel{\infty}{\infty}$ & 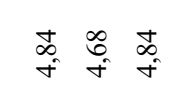 & 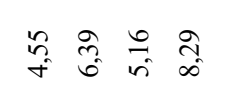 & $\stackrel{8}{+}$ & \\
\hline$=$ & $\stackrel{t}{=}$ & $\underset{g}{g}$ & $\stackrel{n}{=} \underset{f}{-}$ & 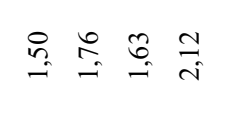 & $\stackrel{0}{9}$ & \\
\hline$\Xi$ & : & $\frac{n}{9} \frac{8}{7}$ & $\begin{array}{cc}3 \\
\text { in } \\
\text { in }\end{array}$ & 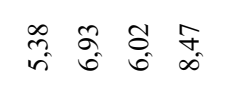 & $\stackrel{g}{q}$ & \\
\hline $\bar{z}$ & $i^{2}$ & $\stackrel{9}{\pi}$ & वें & 式 & $\stackrel{\circ}{i}$ & \\
\hline$=$ & $\bar{F}$ & $n^{2} 7$ & 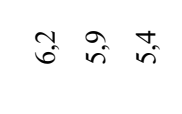 & 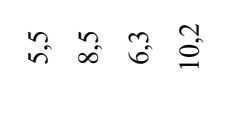 & $\therefore$ & \\
\hline 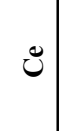 & 孛 & $\dot{m}$ & 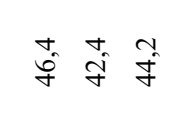 & $\vec{q} \vec{g} \vec{b} \bar{g}$ & $\infty$ & \\
\hline$s$ & ii & $\bar{g} \stackrel{0}{0}$ & 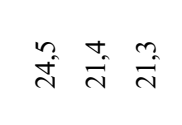 & 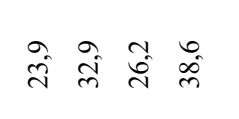 & $\stackrel{2}{g}$ & 8 \\
\hline$\cong$ & 等 & 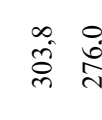 & 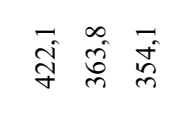 & 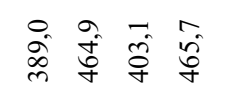 & के & \\
\hline 8 & ? & $\stackrel{\text { : }}{8}$ & 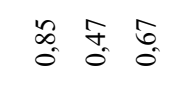 & 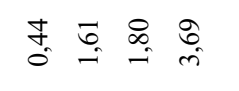 & $\stackrel{2}{=}$ & \\
\hline$\hat{z}$ & $\stackrel{+}{\Xi}$ & $\vec{\exists}$ & $\stackrel{0}{a} a_{a}^{2}$ & $\underset{\alpha}{+} \stackrel{f}{ \pm} \stackrel{\infty}{=} \overrightarrow{=}$ & $\infty$ & \\
\hline$s$ & 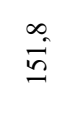 & 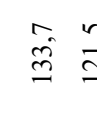 & 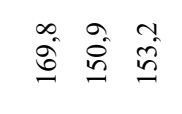 & 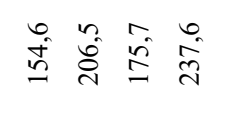 & 龺 & \\
\hline$>$ & in & 8 & $\frac{m}{m} \vec{i} \stackrel{\infty}{i}$ & 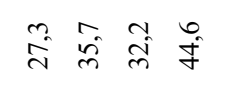 & $\underset{j}{\infty}$ & \\
\hline$i$ & 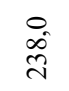 & 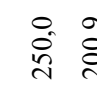 & 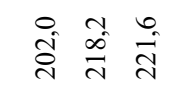 & 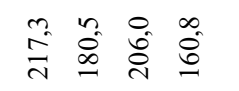 & $\stackrel{\circ}{\stackrel{n}{n}}$ & \\
\hline$\vec{\approx}$ & $\stackrel{\infty}{\infty}$ & 赵号 & 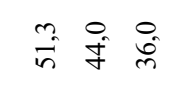 & $\begin{array}{ll}\hat{y} \\
\dot{y}\end{array}$ & $\stackrel{8}{q}$ & \\
\hline & & & & 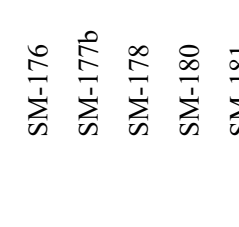 & 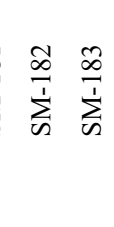 & \\
\hline
\end{tabular}




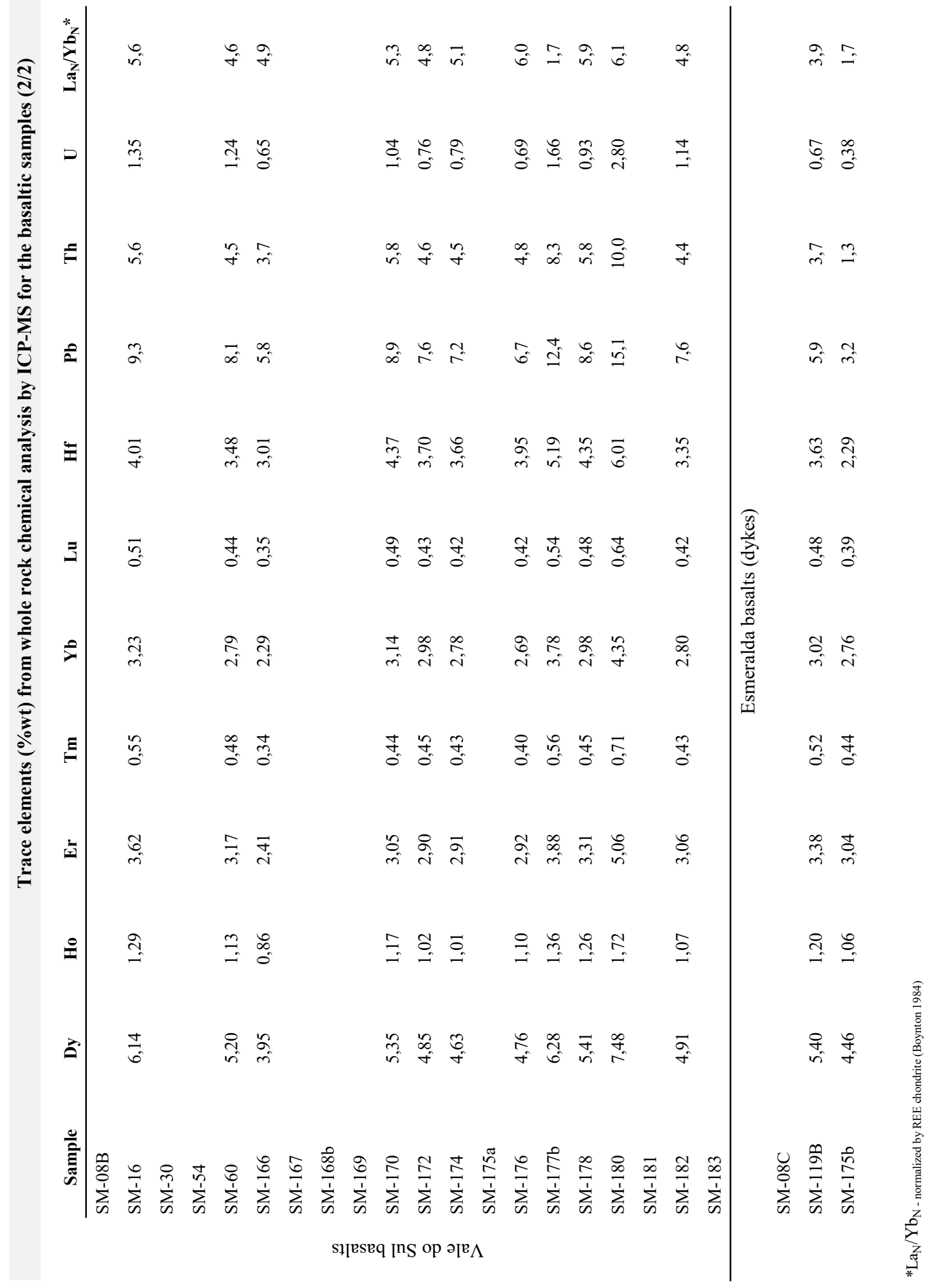

